\title{
Human Health and Ecological Risk Assessment for the Operation of the Explosives Waste Treatment Facility at Site 300 of the Lawrence Livermore National Laboratory
}

Volume 2: Input and Output Files for the Human Health Risk Assessment

Gretchen Gallegos

Jeffrey I. Daniels

U.S. Department of Energy Anthony Wegrecki

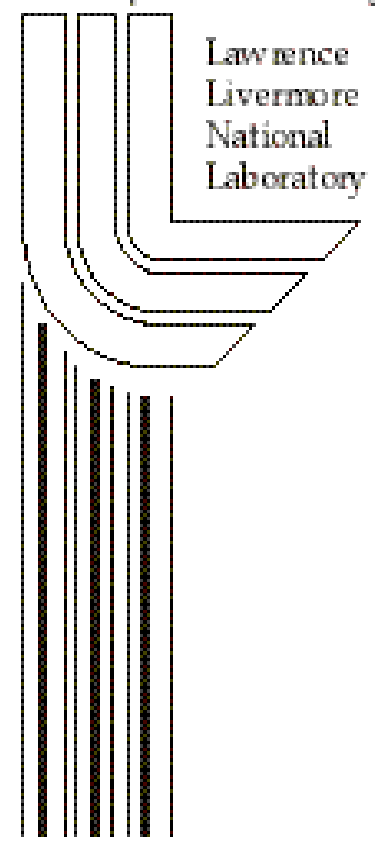

November 2005 
This work was performed under the auspices of the U. S. Department of Energy by the University of California, Lawrence Livermore National Laboratory under Contract No. W7405-Eng-48. 
List of documents in Volume 2

Model input folder

1. EWTF.src This file contains the UTM coordinates and elevations in feet of the Burn Pan, Burn Cage and Detonation Pad and the seven receptor locations, used in HARP.

2. EWTF.xoq This file contains the chi/Q data for the seven receptor locations; the annual average and 1-hour data are from OBODM. This file was used in HARP.

3. Sit3y5.vec This file contains 5 years of hourly meteorological data for Site 300 (wind towards) used in OBODM.

4. Site300.sit This file contains the site-specific ingestion pathway parameters used in HARP.

HARP output folder

Bystander worker folder Note: All HARP output files repeat the source term for the constituents of concern.

1. Calculation note.txt This file contains information about modification to the HARP output for the soil ingestion pathway for the worker.

2. Rep_Acu_Rec5_AllSrc_AllCh_BySrc_Site.txt This file contains the HARP output for the acute noncarcinogenic exposure for the bystander worker at B895.

3. Rep_Can_WRK_Avg_Rec5_AllSrc_AllCh_BySrc_ByChem_Site.txt This file contains the HĀRP output for the carcinogenic ris $\bar{k}$ for the bystander worker at B895, giving chemical specific results by source.

4. Rep_Can_WRK_Avg_Rec5_AllSrc_AllCh_BySrc_Site.txt This file contains the HARP output for the carcinogenic risk for the bystander worker at B895 by source, in summary form without chemical specific results.

5. Rep_Chr_Wrk_PtEst_Rec5_AllSrc_AllCh_BySrc_Site.txt This file contains the HARP output for the chronic noncarcinogenic exposure for the bystander worker at B895.

Carnegie resident folder Note: All HARP output files repeat the source term for the constituents of concern.

1. Rep_Acu_Rec2_AllSrc_AllCh_BySrc_Site.txt This file contains the HARP output for the acute noncarcinogenic exposure for the resident at the Carnegie State Vehicular Recreation Area. 2. Rep_Can_9yrC_Hi_Rec2_AllSrc_AllCh_BySrc_Site.txt This file contains the HAR̄P output for the carcinogeñic risk for the child receptor (9-year exposure) at the Carnegie State Vehicular Recreation Area.

3. Rep_Can_30yr_Hi_Rec2_AllSrc_AllCh_BySrc_ByChem_Site.txt This file contains the HARP output for the carcinogenic risk for the adult receptor (30-year exposure) at the Carnegie State Vehicular Recreation Area, giving chemical specific results by source. 4. Rep_Can_30yr_Hi_Rec2_AllSrc_AllCh_BySrc_Site.txt This file 
contains the HARP output for the carcinogenic risk for the adult receptor (30-year exposure) at the Carnegie state Vehicular Recreation Area, in summary form without chemical specific results. 5. Rep_Chr_Res_Hi_Rec2_AllSrc_AllCh_BySrc_Site.txt This file contains the HARP output for the chronic noncarcinogenic exposure for the receptors at the Carnegie State Vehicular Recreation Area.

Screening output folder Note: All HARP output files repeat the source term for the constituents of concern. 1. Rep_Acu_AllRec_AllSrc_AllCh_ByRec_Site.txt This file contains the HARP output for the acute noncarcinogenic exposure for all receptors. 2. Rep_Can_30yr_Hi_AllRec_AllSrc_AllCh_ByRec_Site.txt This file contains the HARP output for the carciñogenic risk for the adult receptor (30-year exposure) for all receptors. Sources cannot be distinguished and burn sources are over-represented at a rate of 300 burns per year.

3. Rep_Can_WRK_Avg_AllRec_AllSrc_AllCh_ByRec_Site.txt This file contains the HARP output for the carcinogenic risk for the worker exposure for all receptors. Sources cannot be distinguished and burn sources are over-represented at a rate of 300 burns per year. In addition, the ingestion exposure is over represented due to model parameters that could not be changed.

4. Rep_Chr_Res_Hi_AllRec_AllSrc_AllCh_ByRec_Site.txt This file contains the HARP output for the chronic noñcarcinogenic exposure for all receptors using residential exposure parameters.

5. Rep_Chr_Wrk_PtEst_AllRec_AllSrc_AllCh_ByRec_Site.txt This file contains the HARP output for the chronic noncarcinogenic exposure for all receptors using worker exposure parameters.

OBODM output folder Note: All OBODM output files repeat the input 1. FORM12.OUT This file is an OBODM output file for the barium emission factor of 0.0082 , representing forms 1 and 2 waste.

2. FORM34.OUT This file is an OBODM output file for the barium emission factor of 0.000086 , representing forms 3 and 4 waste. 
Model Input Folder EWTF.src

EWTF.xoq

Sit3y5.vec Site300.sit 
EWTF.src 


\section{SCREENINGMODE OFF C:IHARPExpress\Metfiles\Sit3y5.vec}

UTMZONE 10

SOURCE 1 OBPan 'LLNL Site 300' 137 SD SD $1 \quad 629304.5624168922 .7461083 .80010$

SOURCE 2 OBCage3 'LLNL Site 300' 137 SD SD 2629300.9624168914 .8371084 .74010

SOURCE 3 OBCage4 'LLNL Site 300' 137 SD SD 3629300.9624168914 .8371084 .74010

SOURCE 4 OD 'LLNL Site 300' 137 SD SD $4 \quad 629350.6744168674 .0751106 .28010$

RECEPTOR 1 PATHWAY PASTURE 'east pasture' 633000.004170500 .00898 .7210

RECEPTOR 2 SENSITIVE Carnegie 1 'Carnegie' 0 " " " 100628681.504165967 .60659 .4710

RECEPTOR 3 SENSITIVE Connolly ranch 2 'Connolly ranch' 0 " " 10632976.654166183 .04519 .6810

RECEPTOR 4 SENSITIVE B812 adult 3 'B812 adult' 0 " " " 100629950.004168674 .001015 .0510

RECEPTOR 5 SENSITIVE B895 ECP 4 'B895 ECP' 0 " " " $100630020.384168179 .23 \quad 1244.4110$

RECEPTOR 6 SENSITIVE East pasture 5 'East pasture' 0 " " $10 \quad 633000.004170500 .00898 .7210$

RECEPTOR 7 SENSITIVE Ecological 6 'Ecological' 0 " " " 10629500.004168500 .001259 .5210 
EWTF.xoq 


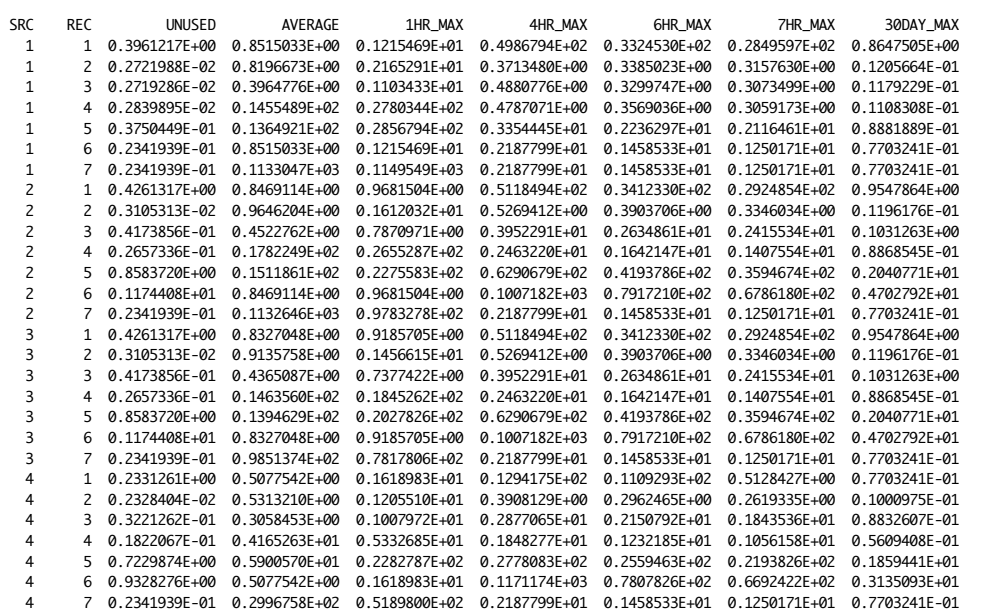

6HR_TIME 26985
14148 14148
14148
16860
34261

34261

43667
43667

43667
26985

26985
16860

16860
34261

34261
43667

43667
43670

43670
25595

43667

26985

16860

34261

43667

43670

25595

43667

32794

6860

9543
43667

43667
8536

8536
26314
43667
7HR_TIME 30DAY_TIME $\begin{array}{ll}26985 & 20500 \\ 14149 & 37025\end{array}$ $\begin{array}{ll}14149 & 37025 \\ 14149 & 37025 \\ 16861 & 3725\end{array}$

$\begin{array}{ll}14861 & 37025 \\ 10671 & 43500\end{array}$

43700

$\begin{array}{ll}16860 & 37025 \\ 10671 & 43500\end{array}$

$43671-43500$
43671

$\begin{array}{ll}43671 & 37650 \\ 25597 & 25825\end{array}$

43667

26985

1067

43671

43671
-25597

43667

32794

4149

9544
43671

8536

26314
43667 
Sit3y5.vec 


\begin{tabular}{|c|c|c|c|c|c|c|c|c|}
\hline \multicolumn{3}{|c|}{0300} & 2000 & \multicolumn{3}{|c|}{0300} & \multicolumn{2}{|c|}{2004} \\
\hline 00 & 1 & 11 & 69.7680 & 5.3500 & 280.0 & 4 & 600.0 & 600 \\
\hline 0 & 1 & 12 & 56.7581 & 500 & 279.9 & 5 & 0 & \\
\hline 0 & 1 & 13 & 51.9977 & 1.9750 & 280.0 & 4 & 00.0 & \\
\hline & 1 & 14 & 9.5375 & .4000 & 280.1 & & & \\
\hline 0 & 1 & 15 & 102.7525 & 0 & 280.3 & 5 & 0 & \\
\hline 0 & 1 & 16 & 90.4818 & 0 & 279.8 & 6 & 0.0 & \\
\hline & 1 & 17 & 126.9544 & 50 & 279.5 & 6 & 0.0 & \\
\hline 0 & 1 & 18 & 332.6797 & 50 & 279.6 & 6 & 0.0 & \\
\hline 0 & 1 & 19 & 336.9324 & & 279.3 & & 0.0 & \\
\hline & 1 & 110 & 343.5005 & 0 & 280.1 & 4 & 0.0 & \\
\hline & 1 & 111 & 359.4566 & & 281.8 & 3 & & \\
\hline 0 & 1 & 112 & 63.9353 & 0 & 282.3 & & 0.0 & \\
\hline 0 & 1 & 113 & 119.3991 & 50 & 282.2 & 1 & 00.0 & \\
\hline & 1 & 114 & 63.4290 & & 282.5 & 1 & & \\
\hline 0 & 1 & 115 & 055 & & 281.4 & 2 & 0 & \\
\hline & 1 & 116 & 57.7533 & 0 & 282.0 & 3 & 600.0 & \\
\hline & 1 & 117 & 5420 & & 281.0 & 4 & 0.0 & \\
\hline 0 & 1 & 118 & 84.7721 & & 280.6 & 4 & 0.0 & \\
\hline & 1 & 119 & 83.2479 & & 280.5 & 4 & 0.0 & \\
\hline & 1 & 120 & 75 & 0 & 280.3 & 4 & 0.0 & \\
\hline 0 & 1 & 121 & 5000 & & 280.5 & 4 & 0 & \\
\hline 0 & 1 & 122 & 83.4994 & 0 & 280.4 & 4 & 0.0 & \\
\hline & 1 & 123 & 021 & & 279.8 & 4 & 0.0 & \\
\hline 0 & 1 & 124 & 0 & & 279.6 & 4 & 0 & \\
\hline 0 & 1 & 21 & 000 & & 279.2 & 4 & 0.0 & \\
\hline & 1 & 22 & .5031 & & 278.8 & 4 & 0.0 & \\
\hline 0 & 1 & 23 & 69.75 & & 278.4 & 4 & 0.0 & \\
\hline 0 & 1 & 24 & 6.2727 & & 278.2 & 4 & 0.0 & \\
\hline & 1 & 25 & .50 & & 278.5 & 4 & 0.0 & \\
\hline 0 & 1 & 26 & .7483 & & 278.0 & 4 & 0.0 & \\
\hline 0 & 1 & 27 & 7504 & & 277.4 & 4 & 0.0 & \\
\hline & 1 & 28 & 84.7473 & & 277.2 & 4 & 0.0 & .0 \\
\hline 0 & 1 & 29 & 5000 & & 277.8 & 4 & 0.0 & \\
\hline & 1 & 210 & 634 & & 278.9 & 4 & 0.0 & \\
\hline & 1 & 211 & 85.4997 & & 279.4 & 4 & 0.0 & .0 \\
\hline & 1 & 212 & 000 & & 280.2 & 3 & 0.0 & \\
\hline 0 & 1 & 213 & 6.7548 & 0 & 281.3 & 3 & 0.0 & .0 \\
\hline 0 & 1 & 214 & 107.3010 & & 282.2 & 4 & 0.0 & .0 \\
\hline & 1 & 215 & . 491 & & 282.6 & 4 & 0.0 & \\
\hline$v$ & 1 & 216 & 111.5021 & & 282.7 & 4 & 0.0 & \\
\hline 00 & 1 & 217 & 122.2480 & 5 & 282.3 & 4 & 600.0 & .0 \\
\hline & 1 & 218 & 114.0005 & & 281.3 & 4 & 0.0 & \\
\hline . & $\perp$ & 219 & 85.9533 & 5 . & 280.8 & 4 & 600.0 & .0 \\
\hline 00 & 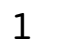 & 220 & 84.9945 & 6 & 280.4 & 4 & 600.0 & .0 \\
\hline & 1 & 221 & 7499 & & 279.9 & 4 & 600.0 & 600.0 \\
\hline 0 & 1 & 222 & 78.7510 & & 279.9 & 4 & 600.0 & \\
\hline & & 223 & 68.2486 & 5.7250 & 279.7 & 4 & 600.0 & 600 \\
\hline
\end{tabular}




\begin{tabular}{|c|c|c|c|c|c|c|c|c|}
\hline 0 & 1 & 24 & 9.0003 & 6.3500 & 279.7 & 4 & 00 & \\
\hline 0 & 1 & 31 & 0.0000 & 7.3750 & 279.3 & 4 & مn & \\
\hline & 1 & 32 & 73.4983 & 7.3500 & 278.9 & 4 & 00 & \\
\hline & 1 & 33 & 79.7468 & 7.4500 & 278.9 & & 00 & \\
\hline & 1 & 4 & 4.2505 & .5250 & 279.6 & & 0 & \\
\hline & 1 & 5 & 116.4982 & .1250 & 279.9 & & 00 & \\
\hline & 1 & 6 & 42.0000 & 7.4250 & 279.8 & & 00 & \\
\hline & 1 & 7 & 48.7500 & .6500 & 279.7 & & & \\
\hline & 1 & 8 & 154.5000 & 2.2750 & 278.8 & 5 & 0 & \\
\hline & 1 & 9 & 140.3224 & 0.5750 & 280.3 & & 00.0 & \\
\hline & 1 & 10 & 268.6220 & .9000 & 281.9 & & 00 & \\
\hline & 1 & & 234.74 & L. 9500 & & & & \\
\hline & 1 & 12 & 172.7670 & 2.7250 & 280.7 & & 00 & \\
\hline & 1 & 313 & 153.9192 & 2.6500 & 281.7 & 2 & 0.0 & 000 \\
\hline & 1 & 314 & 157.08 & & & 1 & & \\
\hline & 1 & 315 & 154.98 & 1.9250 & 284.0 & 1 & 0 & \\
\hline & 1 & 16 & 124.0242 & 9500 & 285.2 & & 0 & \\
\hline & 1 & 317 & 326.75 & 000 & 5 & $J$ & & \\
\hline & 1 & 318 & 134.45 & 750 & 282.5 & 4 & 0 & \\
\hline & 1 & 319 & 265.27 & 9000 & 281.5 & 5 & 0 & \\
\hline & 1 & 320 & 344.28 & 000 & 1.1 & 4 & & \\
\hline & 1 & 321 & 5.22 & 0 & 1.2 & 5 & & \\
\hline 0 & 1 & 322 & 17.4957 & 000 & 281.6 & 5 & 00 & 600 . \\
\hline & 1 & 323 & 13.49 & 1 . & 2.9 & 4 & 0 & \\
\hline & 1 & 324 & 72.26 & 0 & 6 & 5 & & \\
\hline & 1 & 1 & 120.5590 & 750 & 1.4 & 6 & & \\
\hline & 1 & 42 & 275.3583 & 500 & 280.6 & 6 & 0 & \\
\hline 0 & 1 & 43 & 336.35 & 0 & 281.2 & 5 & & \\
\hline 0 & 1 & 44 & 3.50 & 250 & 1.4 & 4 & 0 & 0 . \\
\hline & 1 & 45 & 107.17 & 500 & 1.5 & 5 & 0 & \\
\hline & 1 & 46 & 122.55 & 1. & 1.7 & 6 & & \\
\hline 0 & 1 & 47 & 104.57 & 50 & .8 & 6 & & . \\
\hline & 1 & 48 & 134.2680 & 1.6500 & 2.5 & 5 & 0 & 0 • \\
\hline 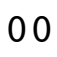 & 1 & 49 & 142.24 & 750 & 2.4 & 4 & 0 & \\
\hline & 1 & 410 & 38.23 & 0 & 2.2 & 3 & & \\
\hline & 1 & 411 & 96.5546 & 500 & 282.1 & 2 & 0 & 0 . \\
\hline & 1 & 412 & 16.89 & 500 & 282.3 & 1 & 0 & \\
\hline 0 & 1 & 413 & 154.3084 & 000 & 283.7 & 1 & 0 & • \\
\hline 0 & 1 & 414 & 163.23 & 1.8750 & 286.1 & 1 & 600.0 & 0 . \\
\hline & 1 & 415 & 157.50 & 500 & 5.2 & 2 & 600 & \\
\hline$v$ & 1 & 416 & 129.60 & 000 & 286.2 & 2 & & \\
\hline 00 & 1 & 417 & 82.2376 & 3.5750 & 285.2 & 3 & 600.0 & 600. \\
\hline & 1 & 418 & 89.00 & 250 & 283.8 & 4 & 600 & 600. \\
\hline 政 & 1 & 419 & 81.0034 & 5.8750 & 283.1 & 4 & 600 & 50 \\
\hline 00 & 1 & 420 & 73.0000 & 7.2250 & 282.8 & 4 & 600.0 & 00 \\
\hline & 1 & 421 & 82.7546 & 7.1500 & 282.5 & 4 & 600.0 & 600. \\
\hline & 1 & 422 & 90.7496 & 6.1250 & & 4 & 600 & \\
\hline & & 423 & 137.2548 & 5.1750 & 281.2 & 4 & 600.0 & 600 \\
\hline
\end{tabular}




\begin{tabular}{|c|c|c|c|c|c|c|c|c|}
\hline 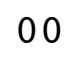 & & 4 & & & & & & \\
\hline 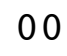 & 1 & 5 & 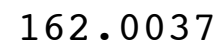 & 6250 & 0.2 & & ח & \\
\hline & 1 & 52 & 56.253 & 8250 & 79.5 & & 00 & \\
\hline & 1 & 5 & 145.2419 & 9250 & 279.3 & & 00 & \\
\hline & 1 & 4 & 0 & 250 & 79.4 & & & \\
\hline & 1 & 55 & & 00 & 79.4 & & 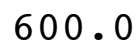 & \\
\hline & 1 & 6 & 501 & 500 & 279.4 & & 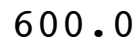 & \\
\hline & 1 & 7 & 00 & 8.6500 & 79.3 & & & \\
\hline & 1 & 8 & & 50 & 79.3 & & & \\
\hline & 1 & 9 & 999 & 7500 & 279.4 & & 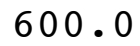 & \\
\hline & 1 & 510 & 006 & 13.5750 & 280.2 & & & \\
\hline & 1 & 11 & & & 80.9 & & & \\
\hline & 1 & 12 & 01 & 2.1500 & 281.9 & & 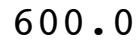 & \\
\hline & 1 & 13 & 74 & 11.35 & 282.8 & & & \\
\hline & 1 & 14 & & & 8 & & & \\
\hline & 1 & & & & 284.6 & & & \\
\hline & 1 & 16 & 13 & 00 & 284.8 & & 0 & \\
\hline & 1 & 17 & 63 & 6. & 84.4 & & 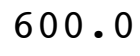 & \\
\hline & 1 & & & & 283.3 & & & \\
\hline & 1 & & & & 283.1 & 4 & & \\
\hline & 1 & 52 & 1 & 0 & 282.6 & - & & \\
\hline & 1 & 52 & & & 82.2 & & & \\
\hline & 1 & 22 & & 0 & 282.0 & 1 & & \\
\hline & 1 & 523 & 0 & 0 & 282.3 & - & & \\
\hline & 1 & 524 & & & 281.9 & 4 & & \\
\hline & 1 & 6 & & & 281.1 & 4 & & \\
\hline & 1 & 62 & 00 & & 280.9 & 4 & 0 & \\
\hline & 1 & 63 & 51 & & 280.5 & 4 & & \\
\hline & 1 & 64 & & 50 & 280.3 & 4 & & \\
\hline & 1 & 65 & 12 & 0 & 280.5 & 4 & & \\
\hline & 1 & 66 & & 0 & 280.6 & 4 & & \\
\hline & 1 & 67 & & 0 & 280.1 & 4 & & \\
\hline & 1 & 68 & 76 & & 280.0 & 4 & & \\
\hline & 1 & 69 & 69 & 00 & 280.9 & 2 & & \\
\hline & 1 & 610 & & & 282.1 & 2 & & \\
\hline & 1 & 61 & & & 1.4 & 2 & & \\
\hline & 1 & 612 & 06 & 1.52 & 283.2 & 1 & & \\
\hline & 1 & 61 & 75 & 1 . & 282.5 & 1 & & \\
\hline & 1 & 614 & & 2 . & 283.5 & 1 & 0 & \\
\hline & 1 & 61 & 28 & 2.0000 & 283.9 & 2 & & \\
\hline & 1 & 61 & 2 & 0 & 284.2 & 1 & & \\
\hline & 1 & 617 & & & 283.5 & 2 & .0 & \\
\hline & 1 & 61 & 3762 & 1.0000 & 282.2 & 3 & 0 & \\
\hline & 1 & 61 & 595 & 1.3750 & 281.9 & 4 & & \\
\hline 0 & 1 & 620 & 442 & & 281.0 & 5 & & \\
\hline & 1 & 62 & 3872 & 1.0000 & 281.3 & 6 & 600.0 & \\
\hline & 1 & 62 & & & & 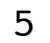 & & \\
\hline & & 2 & ( & 3.0000 & 279.8 & 4 & 600.0 & 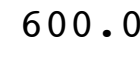 \\
\hline
\end{tabular}




\begin{tabular}{|c|c|c|c|c|c|c|c|c|}
\hline 0 & 1 & 24 & 5.5000 & 7000 & 279.0 & 5 & 0 & \\
\hline 0 & 1 & 1 & 4.7485 & .8250 & 279.3 & & 00.0 & \\
\hline 0 & 1 & 72 & 149.2501 & .7250 & 278.6 & & 00.0 & \\
\hline 0 & 1 & 73 & 33.7597 & 9250 & 279.3 & & 00.0 & \\
\hline 0 & 1 & 74 & 5.5184 & .9250 & 79.5 & & 0 & \\
\hline 0 & 1 & 75 & 4.7482 & 4750 & 279.0 & & 00 & \\
\hline 0 & 1 & 76 & 35.0071 & 6500 & 278.2 & & 00 & \\
\hline 0 & 1 & 77 & 07.9897 & .5500 & 278.0 & & .0 & \\
\hline 0 & 1 & 78 & 0000 & 2750 & 278.5 & & & \\
\hline 0 & 1 & 79 & 37.3537 & 1000 & 279.1 & & 0 & . \\
\hline 0 & 1 & 710 & 30.7461 & 0500 & 281.1 & & & \\
\hline 0 & 1 & 711 & 50.2486 & .2750 & 81.0 & & & \\
\hline 0 & 1 & 712 & 50.7356 & 3.6750 & 282.0 & & 00.0 & \\
\hline 0 & 1 & 713 & 58.0001 & 6500 & 282.9 & & 00.0 & \\
\hline & 1 & 714 & 53 & & 6 & & & \\
\hline 0 & 1 & 715 & 2 & & 28 & & & \\
\hline 0 & 1 & 716 & 42.5005 & 000 & 286.6 & & 0.0 & \\
\hline 0 & 1 & 717 & 9.2482 & 500 & .9 & & .0 & \\
\hline 0 & 1 & 718 & 54 & & 4.8 & & & \\
\hline 0 & 1 & 719 & 027 & 00 & 285.1 & 4 & .0 & \\
\hline 0 & 1 & 720 & 96.6824 & 2.5500 & 4.9 & & $\cdot 0$ & 600 \\
\hline 0 & 1 & 721 & 99. & & & & & \\
\hline 0 & 1 & 722 & 108.8476 & 50 & & & .0 & \\
\hline 0 & 1 & 723 & .0014 & 50 & .3 & 4 & .0 & \\
\hline & 1 & 724 & 06 & & & 4 & & \\
\hline 00 & 1 & 81 & .4446 & 0 & .6 & 5 & & \\
\hline 0 & 1 & 82 & 146.5389 & 000 & 1.7 & 6 & .0 & \\
\hline 0 & 1 & 83 & 9.2495 & 0 & .0 & 5 & .0 & \\
\hline 00 & 1 & 84 & 112.7076 & 0 & 2.2 & 6 & .0 & \\
\hline 0 & 1 & 85 & 10.0471 & 0 & 1.6 & 6 & .0 & 00 \\
\hline 00 & 1 & 86 & 162.5291 & 750 & 1.7 & 6 & .0 & \\
\hline 0 & 1 & 87 & 145.4972 & 0 & .9 & 5 & & 0 \\
\hline 00 & 1 & 88 & 087 & 0 & 0.8 & 6 & .0 & \\
\hline 00 & 1 & 89 & 135.9384 & 3000 & 2.5 & 5 & .0 & \\
\hline 0 & 1 & 810 & 100 & & 2 & 4 & & 0 \\
\hline 00 & 1 & 811 & 329.4018 & 000 & 4.2 & 3 & .0 & \\
\hline 00 & 1 & 812 & 305.9790 & .6000 & 5.7 & 2 & .0 & \\
\hline 0 & 1 & 813 & 186.2182 & 250 & 5.8 & 1 & .0 & 6 \\
\hline 00 & 1 & 814 & 169.7194 & 2.5500 & 5.7 & 1 & .0 & 60 \\
\hline 00 & 1 & 815 & 157.2505 & .9500 & 5.9 & 2 & .0 & \\
\hline 0 & 1 & 816 & 250.6587 & 500 & 286.1 & 1 & & \\
\hline 00 & 1 & 817 & 214.3536 & 1.5250 & 284.2 & 2 & .0 & \\
\hline 0 & 1 & 818 & 144.5034 & 2.2750 & 282.9 & 3 & .0 & 600 \\
\hline 00 & 1 & 819 & 113.0084 & 500 & 281.9 & 4 & .0 & \\
\hline 00 & 1 & 820 & 114.6441 & 1.3500 & 281.6 & 5 & .0 & \\
\hline 00 & 1 & 821 & 89.8854 & 2.2500 & 279.3 & 6 & 600.0 & 600 \\
\hline ac & 1 & 822 & 138 & & 280.2 & 6 & & \\
\hline & & 823 & 327.5379 & 0.6750 & 280.0 & & .0 & 600 \\
\hline
\end{tabular}




\begin{tabular}{|c|c|c|c|c|c|c|c|}
\hline 0 & 1824 & 6.2482 & 1.3750 & 280.1 & 5 & 00.0 & \\
\hline 0 & $\begin{array}{lll}1 & 9 & 1\end{array}$ & 22.9393 & 1.1500 & 80.1 & & 00.0 & \\
\hline & 192 & 105.0552 & 7000 & 280.7 & 5 & 00 & \\
\hline & 193 & 17.6113 & L. 2000 & 280.8 & & 00 & \\
\hline & $\begin{array}{lll}1 & 9 & 4\end{array}$ & 21.4521 & .72 & 81.0 & & 0 & \\
\hline & 195 & 111.8515 & .9000 & 81.1 & & 00 & \\
\hline & 196 & 23.0318 & 0.7750 & 281.1 & & 00 & \\
\hline & $\begin{array}{lll}1 & 9 & 7\end{array}$ & 101.0000 & .6000 & 80.7 & & & \\
\hline & 198 & 119.3205 & 2.1250 & 279.7 & & 0 & \\
\hline & 199 & 34.6531 & 1.8500 & 279.8 & & 0 & \\
\hline & 1910 & 53.4900 & 1.7000 & 282.9 & & 00 & \\
\hline & 1911 & 74.7663 & 160 & 84.0 & & & \\
\hline & 1912 & 106.6267 & 1.9750 & 284.8 & & 0 & \\
\hline & 1913 & 91.0452 & 4.2000 & 286.2 & & 0.0 & \\
\hline & 1914 & 85.8407 & & & & & \\
\hline & 1915 & 108.0754 & 3.9000 & 286.2 & & 0 & \\
\hline & 1916 & 78.0029 & 4.85 & 285.3 & & .0 & \\
\hline & 1917 & 79. & 0 & 284.3 & & & \\
\hline & 1918 & 75.7502 & 0 & 283.5 & 4 & 0 & \\
\hline & 1919 & 71.0002 & 0 & 4 & 4 & 0 & \\
\hline & 1920 & 75 . & 0 & 3 & 4 & & \\
\hline & 1921 & 78 . & & & 4 & & \\
\hline & 1922 & 80.5009 & 0 & 28 & 4 & 0 & 0. \\
\hline & 1923 & 78.4994 & 0 & 3 & 4 & 0 & \\
\hline & 1924 & 78 & & & 4 & & \\
\hline & 1101 & 81.5006 & 0 & 284 & 4 & & \\
\hline & 1102 & 81.2495 & 5.30 & 284 & 4 & 0 & \\
\hline 0 & 1103 & 74.2498 & 0 & 284.2 & 4 & & \\
\hline 0 & 1104 & 79.7499 & 0 & 284.2 & 4 & 0 & 0 . \\
\hline & 1105 & 78.7492 & 75 & 283.5 & 4 & 0 & \\
\hline & 1106 & 43.9066 & 1 . & 28 & 5 & & \\
\hline & 1107 & 95.8860 & 3 . & 28 & 4 & & \\
\hline & 1108 & 338.7058 & 1.05 & 281.9 & 5 & 0 & 0 • \\
\hline & 1109 & 357.4931 & 0 & & 4 & 0 & \\
\hline & 11010 & 279.2824 & 0 & & 3 & & \\
\hline & 11011 & 285.4034 & 1.3750 & 286.4 & 2 & & 0 . \\
\hline & 11012 & 163.7528 & 0 & 285.3 & 2 & 0 & \\
\hline 0 & 11013 & 225.8796 & 0 & 285.2 & 1 & 0 & 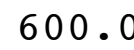 \\
\hline & 11014 & 163.0688 & 50 & 285.8 & 1 & 0 & 0 . \\
\hline & 11015 & 70.9954 & 0 & 284.9 & 2 & 0 & \\
\hline & 11016 & 69.9900 & 4 . & & 3 & & \\
\hline 00 & 11017 & 77.2503 & 4.8250 & 284.2 & 4 & 600.0 & 600 . \\
\hline & 11018 & 61.2166 & 3.3250 & 284.0 & 4 & 600 & 000 \\
\hline & 11019 & 34.4763 & 2.1000 & & 5 & 600 & $\cos$ \\
\hline 00 & 11020 & 30.2330 & 1.9750 & 283.5 & 6 & 600.0 & 00 . \\
\hline & 11021 & 34.2498 & 2.6500 & 283.4 & 5 & 600.0 & 600. \\
\hline & 11022 & 30.7465 & 2.72 & & 4 & 600 & \\
\hline & 11023 & 46.4959 & 4.1000 & 283.1 & כ & 600.0 & 600 \\
\hline
\end{tabular}




\begin{tabular}{|c|c|c|c|c|c|c|c|}
\hline 0 & 1024 & - & 2.6500 & 83.4 & 6 & ( & \\
\hline 0 & 1111 & 134.0059 & 1.1250 & 82.7 & & & \\
\hline & 112 & 49.6936 & 2.6500 & 283.0 & 5 & 0 & \\
\hline & 113 & 32.2516 & 4.9750 & 283.4 & & 00 & \\
\hline & 14 & 4.4799 & 2750 & 3.8 & & & \\
\hline & 115 & 342.2197 & 1.8250 & 83.9 & & 00 & \\
\hline & 116 & 328.1451 & 1.8750 & 283.7 & & & \\
\hline & 117 & 327.7500 & .5000 & & & & \\
\hline & 118 & 335.2522 & 2.5250 & 84.0 & 4 & 0 & \\
\hline & 119 & 356.5000 & 7750 & 284.5 & & 0 & \\
\hline & 110 & 353.5064 & .5250 & 285.6 & & & \\
\hline & 111 & 349.7504 & .9750 & & 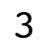 & & \\
\hline & 1112 & 19.2499 & 6.9250 & 287.5 & 4 & 0 & \\
\hline & 1113 & 25.2498 & 0.1000 & 287.8 & 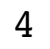 & 0 & \\
\hline & 114 & 35.7350 & & & 4 & & \\
\hline & 11 & 143.2733 & 000 & 283.7 & 4 & & \\
\hline & 1116 & 43.4481 & .8750 & 282.5 & 3 & & \\
\hline & 117 & 306.7621 & 2.5500 & 2.7 & 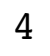 & & \\
\hline & 1118 & 104.1221 & 2.4250 & 284 & 5 & & \\
\hline & 119 & 89.0026 & 5500 & 282.3 & 4 & & \\
\hline & 20 & 82.0080 & 500 & 281.4 & 4 & & \\
\hline & 121 & 108.7021 & 50 & 31.5 & 5 & & \\
\hline & 122 & 90.9651 & 5750 & 281.7 & 4 & 0 & • \\
\hline & 123 & 4997 & 0 & 5 & 4 & & \\
\hline & 124 & 5290 & & & 4 & & \\
\hline & 121 & 63.2455 & 000 & 281.3 & 4 & & \\
\hline & 1122 & 88.7915 & 0 & .1 & 4 & & \\
\hline 0 & 123 & 99.4865 & 3. & 6 & 4 & & \\
\hline & 1124 & 80.5058 & 0 & 28 & 4 & & \\
\hline & 125 & 62.7495 & 500 & 280 & 5 & & \\
\hline & 1126 & 75.7386 & 4. & $2 \varepsilon$ & 5 & & \\
\hline & 1127 & 609 & 0 & 28 & 4 & & \\
\hline & 1128 & 89.2064 & 6500 & 280.2 & 5 & 0 & 0 . \\
\hline & 1129 & 6.9786 & 000 & 2 & 4 & & \\
\hline & 1210 & 341.2870 & & & 3 & & \\
\hline & 211 & 24.9531 & 500 & 281.4 & 2 & & 0 . \\
\hline & 1212 & 316.5474 & 50 & 281.5 & 1 & & \\
\hline 0 & 11213 & 220.5512 & 0 & 282.5 & 1 & & 。 \\
\hline & 11214 & 226.2649 & 000 & 282.1 & 1 & 0 & 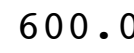 \\
\hline & 11215 & 292.7580 & 000 & 283.1 & 1 & & \\
\hline & 11216 & 271.2366 & 750 & & 2 & & \\
\hline 0 & 11217 & 285.2756 & 2.7500 & 281.2 & 3 & 600.0 & 0 \\
\hline & 218 & 326.7537 & 1.1250 & 280.8 & 4 & 0 & \\
\hline & 11219 & 21.0018 & 1.3000 & & 4 & & \\
\hline 00 & 11220 & 3.7501 & 1.5750 & 281.8 & 4 & 600 & 0 \\
\hline & 11221 & .9983 & 1.0250 & 281.9 & 4 & 600 & \\
\hline & 11222 & 339.7721 & & & 4 & 60 & \\
\hline & 1223 & 286.0490 & 1.6500 & 281.1 & & 600.0 & 600 \\
\hline
\end{tabular}




\begin{tabular}{|c|c|c|c|c|c|c|c|}
\hline 0 & 1224 & & 750 & 30.8 & & 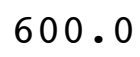 & \\
\hline & 1131 & 5 & 1.4250 & 81.1 & & 600.0 & \\
\hline & 1132 & 5.5029 & .3500 & 281.7 & 3 & 00 & \\
\hline & 133 & 4.0000 & 2.5750 & 282.7 & & 00.0 & \\
\hline & 134 & 59.2506 & 0000 & 82.8 & & 0 & \\
\hline & 135 & 38.7 & .2750 & 282.7 & & 00 & \\
\hline & 136 & 6.7856 & 2.2750 & 283.7 & & 500 & \\
\hline & 137 & 337.2499 & L. 7750 & 83.6 & & 0 & \\
\hline & 138 & 353.3364 & .6500 & 83.2 & & & \\
\hline & 139 & 8.6095 & 0.7000 & 284.8 & & 000 & 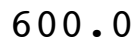 \\
\hline & 1310 & 219.9782 & 0.8000 & 287.1 & & 0 & \\
\hline & 1311 & 306.7146 & 1.3000 & 87.8 & & 0 & \\
\hline & 1312 & 174.9662 & 3.2000 & 287.9 & & 00.0 & \\
\hline & 1313 & 162.7494 & 3.8000 & 286.4 & & 0.0 & 00 \\
\hline & 1314 & 238.2765 & 2.72 & & & & \\
\hline & 1315 & 349.83 & 3.37 & 28 & & & \\
\hline & 1316 & 234.2619 & 2.1000 & 287.3 & L & .0 & 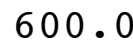 \\
\hline & 1317 & 255.1330 & 1.87 & .1 & & 0 & \\
\hline & 1318 & 316.5137 & 1.6000 & 28 & 3 & & \\
\hline & 1319 & 130.6305 & 0.8250 & 284.5 & 4 & .0 & 0 \\
\hline & 1320 & 26.8142 & 1.1500 & 284.7 & 5 & 0 & \\
\hline & 1 & 31.20 & 25 & & 6 & & \\
\hline & 1322 & 329.34 & 0.8250 & 28 & 6 & & \\
\hline & 1323 & 359.2948 & 0.6500 & 283.6 & 6 & 0 & 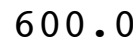 \\
\hline & 11324 & 327.7510 & 0 & & 5 & & \\
\hline & 114 & 159.7776 & 0.5750 & 28 & 6 & & \\
\hline & 1142 & 131.5094 & 5750 & 282.9 & 5 & 0 & U. \\
\hline & 1143 & 140.4332 & 6500 & 3.2 & 6 & & \\
\hline & 1144 & 213.8724 & 0 . & 28 & 6 & & $\bullet$ \\
\hline & 1145 & 126.2454 & 0.9500 & .4 & 6 & .0 & 0 • \\
\hline & 1146 & 153.3343 & 0.3750 & 283.2 & 6 & 0 & 0 . \\
\hline & 1147 & 36.0332 & 0.6750 & .3 & 5 & & \\
\hline & 1148 & 20.7726 & 1.0250 & 283.6 & 6 & & J \\
\hline & 1149 & 23.0134 & 1.3250 & 284.1 & 5 & 0 & 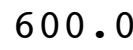 \\
\hline & 11410 & 53.1791 & 1.4750 & 28 & 4 & & \\
\hline & 11411 & 8.7293 & 1.4750 & 5.1 & 3 & & 0 . \\
\hline & 11412 & 276.3412 & 0.8500 & 287.8 & 2 & $\cdot 0$ & 0 . \\
\hline & 11413 & 241.7519 & 1.6500 & .8 & 1 & & 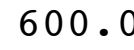 \\
\hline & 11414 & 231.7481 & 1.4000 & 287.3 & 2 & .0 & 0 . \\
\hline & 11415 & 226.5000 & 1.5500 & 287.7 & 1 & & o. \\
\hline & 11416 & 272.9261 & 1.2250 & 287.2 & 2 & & 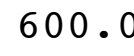 \\
\hline & 11417 & 277.5469 & 0.9500 & 286.0 & 3 & .0 & 0 . \\
\hline & 11418 & 159.8457 & 0.4250 & 285.2 & 4 & .0 & 00 . \\
\hline 8 & 11419 & 47.3401 & 0.2000 & 28 & 5 & & 0 \\
\hline 00 & 11420 & 76.5079 & 0.9750 & 285.4 & 6 & 60 & 600. \\
\hline & 11421 & 132.9064 & 1.4500 & 285.7 & 6 & 600.0 & 600 \\
\hline & & 187 & & & 6 & & \\
\hline & 1423 & 143.4428 & 0.9750 & 285.0 & r & 600.0 & 600 \\
\hline
\end{tabular}




\begin{tabular}{|c|c|c|c|c|c|c|c|}
\hline 0 & 1424 & 2900 & $\$ 750$ & 85.0 & 6 & - & \\
\hline 0 & 1151 & 353.7539 & 0.9000 & 285.2 & & 00.0 & \\
\hline 0 & 1152 & 349.9995 & 1.5000 & 285.1 & & 00 & \\
\hline & 153 & 264.5341 & 2.0750 & 285.1 & & 00.0 & \\
\hline & 154 & 279 & 2750 & & & & \\
\hline & 155 & 026 & 9750 & 3 & & S & \\
\hline & 156 & 348 . & 1.6750 & 82.5 & & 00 & \\
\hline & 157 & 319 & 1.8750 & & & & \\
\hline & 158 & 824 & .9750 & 9 & & & \\
\hline 0 & 159 & 349.7212 & 2.0500 & 85.9 & & 0 & \\
\hline & 1510 & 133.6982 & 1.3250 & 287 & & & \\
\hline & 511 & 199.89 & 17 & 3 & & & \\
\hline 0 & 1512 & 345 & 4.57 & 89.4 & & & \\
\hline 0 & 513 & 312 & 2.85 & 3 & & & \\
\hline & 514 & 300 & 2.82 & & & & \\
\hline 0 & - & 323. & & & & & \\
\hline 0 & 1516 & 291. & 3.07 & 89.3 & & .0 & \\
\hline & 517 & 275 . & 2.75 & & & & \\
\hline & 518 & 291. & & & - & & \\
\hline & 519 & 328 & & 288.3 & 4 & & \\
\hline 0 & 1520 & 345.7177 & 6.00 & 28 & 4 & .0 & \\
\hline 0 & 1521 & 357.2492 & & & 4 & & \\
\hline 0 & 1522 & 339. & 0 & 2 & 4 & & \\
\hline 0 & 1523 & 326 . & 4.82 & 2 & 4 & .0 & \\
\hline & 1524 & 294 . & & & 4 & & \\
\hline 0 & 1161 & 232 . & 2 . & & 5 & & \\
\hline & 1162 & 359 . & 0 & 8 & 4 & .0 & \\
\hline & 1163 & 359 . & 15 & & 4 & & \\
\hline 0 & 1164 & 63 & 7 . & 28 & 4 & & \\
\hline & 1165 & 59.2506 & 1.82 & 2.5 & 4 & & \\
\hline 0 & 1166 & 44.7837 & 2.12 & 282.1 & 4 & & \\
\hline 0 & 1167 & 33.7368 & 0 & 82.5 & 4 & & \\
\hline & 1168 & 891 & & 282.2 & 5 & & \\
\hline 0 & 1169 & 338.7854 & 0 & 282.2 & 4 & 0 & \\
\hline 0 & 11610 & 164 & 0 & & 3 & & \\
\hline 0 & & 301.6097 & & & 2 & & \\
\hline 0 & 11612 & 277.0616 & 0 & 282.8 & 2 & .0 & \\
\hline 0 & 11613 & 280 . & 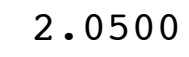 & & 2 & & \\
\hline 0 & 11614 & 278.9943 & 3.7250 & & 3 & .0 & \\
\hline 00 & 11615 & 285.7497 & & 283.7 & 3 & & \\
\hline 0 & 11616 & 277.5000 & 3. & & 3 & & \\
\hline 0 & 11617 & 302.9416 & 2.27 & & 4 & & \\
\hline 0 & 11618 & 289.7508 & 1.1250 & 282.4 & 5 & & 1 \\
\hline 20 & 11619 & 326.7204 & 0 & & 5 & & \\
\hline 00 & & 0.7787 & & & 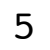 & & $\rho_{0}$ \\
\hline 00 & 21 & 37.2406 & 1.4750 & 282.1 & 5 & 600.0 & 500 \\
\hline & & 28 & & & 6 & & \\
\hline & 1623 & 343.3550 & 1.2250 & 281.5 & & .0 & 600 \\
\hline
\end{tabular}




\begin{tabular}{|c|c|c|c|c|c|c|c|}
\hline 0 & 624 & 334 & 9500 & 82.0 & & 00.0 & \\
\hline 0 & 1171 & 10 & 7250 & 281.7 & 6 & 600.0 & \\
\hline 0 & 1172 & 280.4259 & .7750 & 281.6 & 6 & 00 & \\
\hline & 1173 & 246.8055 & .7500 & 281.8 & & 00 & \\
\hline & 1174 & 186.2285 & 7250 & 81.3 & & 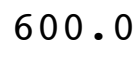 & \\
\hline & 1175 & 170 & .0250 & 80.7 & & 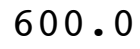 & \\
\hline & 1176 & 146.0000 & 9000 & 80.4 & & 0 & \\
\hline & 1177 & 738 & 8750 & & & & \\
\hline & 1178 & 43 & 3250 & 80.4 & & 0 & \\
\hline & 1179 & 855 & 1.6000 & 79.6 & & 0 & \\
\hline & 11710 & 143.7528 & 2.7750 & 1 & & 8 & \\
\hline & 11711 & 142 & 170 & 9.4 & & & \\
\hline & 11712 & 177 & 1.6500 & 80.1 & & 0 & \\
\hline & 11713 & 149.21 & 1.0 & .6 & & 0 & \\
\hline & 11714 & 13 & 0 & .1 & & & \\
\hline & 11715 & 15 & 2 . & 9.7 & & & \\
\hline & 11716 & 157 . & 2.72 & .5 & & 0 & \\
\hline & 11717 & 990 & 2.3 & .3 & & 0 & \\
\hline & 11718 & 15 & 1.2 & 9.4 & 3 & & \\
\hline & 11719 & 206 & 0.3 & & 6 & 0 & \\
\hline & 11720 & 26 & .4500 & 9.7 & & 0 & \\
\hline & 11721 & 31 & 0 . & 9.9 & & & \\
\hline & 11722 & 25 & 1 . & .9 & & & \\
\hline & 11723 & 279 . & 1.9 & 282.1 & & 0 & \\
\hline & 11724 & 162 & & 0.5 & & & \\
\hline & 1181 & 15 & 3 . & 0.0 & 4 & 0 & \\
\hline & 1182 & 161. & 3. & 280.1 & 5 & 0 & \\
\hline & 1183 & 166 & 2 . & . 1 & 4 & 0 & \\
\hline 0 & 1184 & 156 & 2 & .0 & 4 & & \\
\hline & 1185 & 146 . & 2.8000 & 280.1 & & 0 & \\
\hline & 1186 & 142 . & 1.6500 & 280.3 & 5 & 0 & \\
\hline & 1187 & 14 & 1.6 & 280.5 & 5 & & \\
\hline & 1188 & 152 & 0 & .5 & 4 & 0 & \\
\hline & 1189 & 140 & 2 . & 280.4 & 3 & 0 & \\
\hline & 11810 & 13 & 1 . & 280.8 & 2 & & \\
\hline & 11811 & 140 . & 3.0500 & . 7 & 3 & 600.0 & \\
\hline & 11812 & 121.1777 & 1.8500 & 281.7 & 2 & 0 & \\
\hline 0 & 11813 & 345 . & 1 . & $2 \varepsilon$ & 1 & 0 & \\
\hline 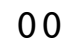 & 11814 & 169. & 1.6250 & 284.9 & 1 & 600.0 & \\
\hline & 11815 & 147 . & 2.1250 & 285.7 & 1 & 0 & \\
\hline & 11816 & 145 . & 2.4 & 285.7 & 2 & 600.0 & \\
\hline & 11817 & 157.9364 & 1.2750 & 286.9 & 3 & 600.0 & \\
\hline & 11818 & 141.0007 & 2.6250 & 284.9 & 4 & 600.0 & \\
\hline & 11819 & 162 . & 1.3750 & & 5 & 600 & \\
\hline 0 & 11820 & 248.3078 & 0.5000 & 284.6 & 6 & 600.0 & \\
\hline & 11821 & 140.3093 & 0.7750 & 284.6 & 6 & 600.0 & \\
\hline & $L$ & & 0 & & 0 & & \\
\hline & 1823 & 139.1061 & 2.2250 & 284.2 & 6 & 600.0 & 60 \\
\hline
\end{tabular}




\begin{tabular}{|c|c|c|c|c|c|c|c|}
\hline 0 & 18 & 0379 & 5250 & 284.4 & 6 & 00.0 & \\
\hline 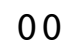 & 1191 & 101.7392 & 1000 & 284.2 & ( & 600 & \\
\hline & 1192 & 272.1116 & 4250 & 284.5 & ( & 00 & \\
\hline & 19 & 204.4284 & 7250 & 284.4 & & 00 & \\
\hline & 194 & 219.6736 & 5750 & 4.2 & & 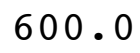 & \\
\hline & 195 & 342.3 & 500 & 84.0 & & 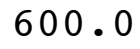 & \\
\hline & 196 & 353.7668 & 500 & 283.8 & & 0 & \\
\hline & 1197 & 92.5505 & 0000 & 3.6 & & & \\
\hline & 198 & 502 & L. 2250 & 83.5 & & 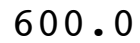 & \\
\hline & 199 & 344.9821 & 3500 & 283.2 & & 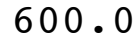 & \\
\hline & 1910 & 302.0686 & 1.1750 & 83.6 & & 0 & \\
\hline & 1911 & 348 & .77 & .1 & & & \\
\hline & 1912 & 275 . & 0.9250 & 286.3 & & 0 & \\
\hline & 1913 & 281.4884 & 1.3250 & 288.0 & & & \\
\hline & 1914 & 07 & 0 & 9.6 & & & \\
\hline & 1915 & 331 & 2 . & 38.7 & & & \\
\hline & 1916 & 127.2245 & 2.1750 & 287.9 & & 0 & \\
\hline & 1917 & 313.0267 & 1.80 & 9.1 & & 0 & \\
\hline & 1918 & 128 & 3.2 & 287.3 & & & \\
\hline & 1919 & 8.9103 & 2.5000 & 287.7 & \pm & 0 & \\
\hline & 1920 & 36.0081 & .5750 & 287.8 & 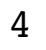 & 0 & \\
\hline & 1921 & 10 & 6 & 7.9 & 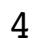 & & \\
\hline & 1922 & 7496 & 2.1250 & 287.8 & & 0 & \\
\hline & 923 & $3 \varepsilon$ & 7000 & 287.5 & 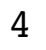 & 0 & \\
\hline & 11924 & 57 & 0 & 36.7 & 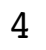 & & \\
\hline & 120 & 58 & 50 & 285.9 & 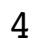 & & \\
\hline & 1202 & 355.2280 & 2.2000 & 285.2 & & 0 & \\
\hline & 1203 & 124.2912 & 1.80 & 284.2 & 6 & & \\
\hline & 1204 & 172.1919 & 1.2500 & 283.6 & 6 & & \\
\hline & 1205 & 275.5353 & 1.4750 & 283.3 & 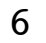 & 0 & \\
\hline & 1206 & 287.8158 & 1.0500 & 282.8 & 5 & 0 & \\
\hline & 1207 & 358 . & 0.9 & 282.8 & 6 & & \\
\hline & 1208 & 9.7198 & 1.0500 & 282.9 & 5 & 0 & \\
\hline & 1209 & 203.7154 & 0.6500 & 283.1 & 4 & 0 & \\
\hline & 120 & 481 & 000 & 283.6 & 3 & & \\
\hline & 12011 & 97.7476 & 4.5250 & 284.0 & 4 & & \\
\hline & 12012 & 76.2500 & .1500 & 283.9 & 4 & 0 & \\
\hline & 12013 & 90.2499 & 7.60 & 283.1 & 4 & & \\
\hline 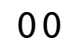 & 12014 & 82.4977 & 6.4000 & 283.9 & 4 & .0 & \\
\hline & 12015 & 103.2492 & .2500 & 284.4 & 3 & & \\
\hline & 12016 & 262 & 4.4250 & 284.9 & 3 & 0 & \\
\hline & 12017 & 106.0000 & 4.4750 & 284.0 & 4 & 600.0 & \\
\hline & 12018 & 88.5453 & .0000 & 283.1 & 4 & 600 & \\
\hline & 12019 & 1994 & .5250 & 282.5 & 4 & 600 & \\
\hline 8 & 12020 & 84.5040 & 5.7250 & 282.4 & 4 & 600.0 & \\
\hline & 1202 & 4969 & 5.0750 & 282.6 & 4 & 600.0 & \\
\hline & & & 00 & .8 & 4 & & \\
\hline & 12023 & 72.7498 & 5.9000 & 282.7 & 4 & 600.0 & 6 \\
\hline
\end{tabular}




\begin{tabular}{|c|c|c|c|c|c|c|c|}
\hline 00 & 12024 & 72.4945 & 5.5750 & 282.6 & 4 & 600.0 & 600.0 \\
\hline 00 & 1211 & 71.7500 & 6.3250 & 282.6 & 4 & 600.0 & 600.0 \\
\hline 00 & 1212 & 67.7439 & 5.9250 & 282.4 & 4 & 600.0 & 00.0 \\
\hline 00 & 1213 & 73.9963 & 5.5000 & 282.4 & 4 & 600.0 & 600.0 \\
\hline 00 & 1214 & 65.7476 & 6.3750 & 282.5 & 4 & 600.0 & 00.0 \\
\hline 00 & 1215 & 72.4945 & 4.5750 & 282.4 & 4 & 600.0 & 00.0 \\
\hline 00 & 1216 & 70.2547 & 5.1750 & 282.3 & 4 & 600.0 & 600.0 \\
\hline 00 & 1217 & 67.5046 & 5.3250 & 282.1 & 4 & 600.0 & 500.0 \\
\hline 00 & 1218 & 73.7500 & 5.4000 & 281.9 & 4 & 600.0 & 00.0 \\
\hline 00 & 1219 & 68.4995 & 5.0500 & 282.4 & 4 & 600.0 & 600.0 \\
\hline 00 & 12110 & 61.9221 & 4.1500 & 283.9 & 3 & 600.0 & 600.0 \\
\hline 00 & 12111 & 82.7323 & 3.4000 & 284.7 & 2 & 600.0 & \\
\hline 00 & 12112 & 119.0079 & 3.2000 & 285.6 & 2 & 600.0 & 600.0 \\
\hline 00 & 12113 & 253.4615 & 2.1750 & 285.9 & 1 & 600.0 & 600.0 \\
\hline 00 & 12114 & 279.5147 & 1.8000 & 286.1 & 1 & 600.0 & \\
\hline 00 & 12115 & 255.5712 & 2.3250 & 286.7 & 1 & 600.0 & 60 \\
\hline 00 & 12116 & 299.7499 & 2.2500 & 286.5 & 2 & 600.0 & 600.0 \\
\hline 00 & 12117 & 307.9941 & 1.6000 & 286.0 & 3 & 600.0 & \\
\hline 00 & 12118 & 202.8343 & 1.1500 & 285.3 & 4 & 600.0 & 60 \\
\hline 00 & 12119 & 68.2372 & 3.7750 & 284.8 & 4 & 600.0 & 600.0 \\
\hline 00 & 12120 & 78.2588 & 4.9000 & 284.3 & 4 & 600.0 & .0 \\
\hline 00 & 12121 & 67.2489 & 3.8000 & 284.0 & 4 & 600.0 & \\
\hline 00 & 12122 & 57.2903 & 2.4500 & 283.9 & 4 & 600.0 & 600.0 \\
\hline 00 & 12123 & 8.2497 & 2.0500 & 284.1 & 4 & 600.0 & .0 \\
\hline 00 & 12124 & 35.2701 & 2.8250 & 283.7 & 4 & 600.0 & \\
\hline 00 & 1221 & 51.7730 & 1.4750 & 282.5 & 5 & 600.0 & 600.0 \\
\hline 00 & 1222 & 22.0845 & 0.9250 & 282.1 & 6 & 600.0 & 600.0 \\
\hline 00 & 1223 & 145.4780 & 0.4500 & 282.4 & 6 & 600.0 & .0 \\
\hline 00 & 1224 & 166.2017 & 0.9500 & 281.9 & 6 & 600.0 & 60 \\
\hline 00 & 1225 & 331.9097 & 0.6750 & 281.2 & 6 & 600.0 & 600.0 \\
\hline 00 & 1226 & 6.9947 & 000 & 281.5 & 5 & 600.0 & .0 \\
\hline 00 & 1227 & 354.3759 & 1.7250 & 281.3 & 4 & 600.0 & 60 \\
\hline 00 & 1228 & 1.4998 & 1.2750 & 281.2 & 4 & 600.0 & 600.0 \\
\hline 00 & 1229 & 341.7342 & 1.3000 & 281.8 & 3 & 600.0 & .0 \\
\hline 00 & 12210 & 327.6387 & 000 & 282.8 & 3 & 600.0 & 60 \\
\hline 00 & 12211 & 282.8935 & 2.0000 & 284.2 & 2 & 600.0 & 600.0 \\
\hline 00 & 12212 & 263.2874 & 1.9750 & 284.7 & 1 & 600.0 & 600.0 \\
\hline 00 & 12213 & 228.6826 & 2.6750 & 284.7 & 2 & 600.0 & 600.0 \\
\hline 00 & 12214 & 224.4903 & 2.5000 & 284.9 & 1 & 600.0 & 600.0 \\
\hline 00 & 12215 & 222.5000 & 2.7500 & 284.7 & 2 & 600.0 & \\
\hline 00 & 12216 & 210.5684 & 2.7750 & 284.3 & 3 & 600.0 & 600.0 \\
\hline 00 & 12217 & 195.5185 & 1.9750 & 283.4 & 4 & 600.0 & 600.0 \\
\hline 00 & 12218 & 231.6115 & 1.2250 & 283.0 & 5 & 600.0 & 600.0 \\
\hline 00 & 12219 & 263.3149 & 1.2750 & 282.6 & 4 & 600.0 & 600.0 \\
\hline 00 & 12220 & 268.2856 & 0.9250 & 282.6 & 5 & 600.0 & 600.0 \\
\hline 00 & 12221 & 316.2489 & 1.2000 & 282.5 & 6 & 600.0 & 600.0 \\
\hline 00 & 12222 & 316.0081 & 1.475 & 282.4 & 5 & 600.0 & 600.0 \\
\hline 00 & 12223 & 301.2501 & 2.7500 & 282.2 & 4 & 600.0 & 600.0 \\
\hline
\end{tabular}




\begin{tabular}{|c|c|c|c|c|c|c|c|}
\hline 0 & 2224 & & 3000 & & & & \\
\hline 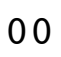 & 1231 & 1 & .0250 & 1.9 & & 0 & \\
\hline 0 & 1232 & 320.9980 & 2.6750 & 282.0 & & 00 & \\
\hline & 1233 & 19.0048 & 6.3750 & 283.3 & & 00.0 & \\
\hline 0 & 1234 & .5021 & .3000 & 83.2 & & & \\
\hline & 1235 & 9.4968 & .1500 & 83.4 & & 0 & \\
\hline 0 & 1236 & 322.2597 & 4.2250 & 83.1 & & 00 & \\
\hline & 1237 & 11.5006 & .8250 & 82.9 & & & \\
\hline & 1238 & 36.7489 & .4000 & 82.6 & & & \\
\hline 0 & 1239 & 30.0000 & 5.0750 & 83.2 & & 00.0 & \\
\hline 0 & 2310 & 25.2498 & 4.5500 & 283.9 & & & \\
\hline 0 & 12311 & 9.7666 & 3.2750 & 3.5 & & & \\
\hline 0 & 12312 & 323.4994 & 7.2750 & 83.3 & & .0 & \\
\hline 0 & 2313 & 6.2879 & 4.2750 & 282.8 & & 0.0 & \\
\hline & 12314 & 7.2353 & 500 & & & & \\
\hline U & 12315 & 293.2486 & & 282.4 & 3 & & \\
\hline 0 & 12316 & 305.9747 & 250 & 283.6 & & 0.0 & \\
\hline 0 & 12317 & 096 & 50 & 3 & 3 & .0 & \\
\hline 0 & 12318 & 555 & 2 . & 284.3 & 4 & & \\
\hline 0 & 12319 & 540 & 500 & 284 & 4 & & \\
\hline 0 & 12320 & 319.2382 & 1.9750 & 283.6 & 5 & . 0 & 000 \\
\hline 0 & 12321 & 355.1270 & & & 6 & & \\
\hline 0 & 12322 & 3702 & 750 & 28 & 6 & & \\
\hline 0 & 12323 & 167.0322 & 3000 & $2 \varepsilon$ & 5 & .0 & \\
\hline 0 & 12324 & 200 & & & 6 & & \\
\hline 00 & 1241 & 584 & 500 & 28 & 6 & & \\
\hline 0 & 1242 & 149.7498 & 750 & 284 & 5 & .0 & \\
\hline 0 & 1243 & 0.7493 & 0 & & 5 & .0 & \\
\hline 00 & 1244 & 176.5186 & 500 & 284 & 6 & .0 & \\
\hline 0 & 1245 & 9.7537 & 1 . & 28 & 5 & .0 & \\
\hline 0 & 1246 & 311.8840 & 1.1250 & 283.9 & 6 & .0 & \\
\hline 0 & 1247 & 279.1535 & 1.4500 & & 6 & & \\
\hline 0 & 1248 & 862 & 750 & 284 & 6 & .0 & \\
\hline 0 & 1249 & 306.2588 & 2.4000 & 28 & 5 & .0 & \\
\hline 0 & 12410 & 326.5038 & 0 & & 4 & & 0 \\
\hline 00 & 12411 & 887 & 3.4750 & .6 & 3 & .0 & \\
\hline 0 & 12412 & 300.2510 & 2.8750 & 285.9 & 3 & .0 & \\
\hline 0 & 12413 & 536 & 1 . & & 2 & .0 & \\
\hline 00 & 12414 & 199.6953 & 1.4000 & 285.8 & 1 & .0 & , \\
\hline 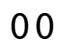 & 12415 & 166.1791 & 3.6000 & 285.1 & 2 & .0 & \\
\hline 0 & 12416 & 159.5000 & 250 & 28 & 3 & & \\
\hline 00 & 12417 & 160.7424 & 4.1750 & 284 & 4 & .0 & \\
\hline 0 & 12418 & 130.4748 & 3.1000 & 283.7 & 4 & .0 & 0 \\
\hline 00 & 12419 & 623 & 750 & & 5 & & \\
\hline 00 & 12420 & 263.1784 & 2.0750 & & 6 & 600.0 & 600 \\
\hline 00 & 12421 & 248.3555 & 1.8750 & 283.1 & 5 & 600.0 & 600 \\
\hline 00 & 12422 & 31 & & & 6 & & \\
\hline & 12423 & 341.4803 & 2.5000 & 28 & & .0 & 600 \\
\hline
\end{tabular}




\begin{tabular}{|c|c|c|c|c|c|c|c|}
\hline 0 & 2424 & & & & & & \\
\hline & 1251 & 07 & .4250 & 2.8 & & 600.0 & \\
\hline & 1252 & 138.7439 & 2.1500 & 282.7 & 4 & 00 & \\
\hline & 253 & 146.9853 & 2.1250 & 282.6 & & 00 & \\
\hline & 254 & 534 & 1500 & 82.6 & & 0 & \\
\hline & 255 & 169.2594 & 1.8500 & 82.5 & 4 & 00 & \\
\hline & 256 & 5027 & 1.4500 & 282.5 & & 00 & \\
\hline & 257 & 118.7788 & 0 & 82.6 & & & \\
\hline & 258 & 137.2515 & 1.5250 & 82.7 & & & \\
\hline & 259 & 103.3577 & 2.8500 & 282.9 & 4 & 00 & 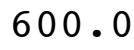 \\
\hline & 2510 & 92.2275 & 500 & 283.0 & 3 & & \\
\hline & 2511 & 90.7501 & 4.2500 & 83.6 & + & 0 & \\
\hline & 2512 & 92.2236 & 4.22 & 284.5 & 3 & 0 & \\
\hline & 2513 & 95.7468 & 4.70 & 284.8 & & 0.0 & $\bullet$ \\
\hline & 2514 & 4 & & & & & \\
\hline & 251 & 7 & & 9 & 3 & & \\
\hline & 2516 & 82.2580 & 0 & 285.3 & 3 & .0 & 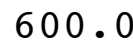 \\
\hline & 2517 & 72.5001 & 0 & .2 & 4 & & \\
\hline & 2518 & 82 & & & 4 & & \\
\hline & 2519 & 000 & 7 . & 282.9 & 4 & 0 & 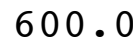 \\
\hline & 2520 & 69.7502 & 0 & 282.6 & 4 & 0 & \\
\hline & 125 & 64.7491 & & 282.4 & 4 & & \\
\hline & 2522 & 9998 & 0 & 282.3 & 4 & & \\
\hline & 12523 & 67.4969 & 0 & 282.1 & 4 & 0 & \\
\hline & 12524 & 66. & & .5 & 4 & & \\
\hline & 1261 & 59.2504 & 0 & 281 & 4 & & \\
\hline & 1262 & 60.7500 & 2000 & 28 & 4 & 0 & J. \\
\hline & 1263 & 63.0000 & 0 & .3 & 4 & & \\
\hline & 1264 & 66. & 0 & $2 \varepsilon$ & 4 & & \\
\hline & 1265 & 69.7500 & 250 & 0.0 & 4 & 0 & 0 • \\
\hline & 1266 & 73.7495 & 8.1250 & 280.1 & 4 & & 0 . \\
\hline & 1267 & 780 & 0 & & 4 & & \\
\hline & 1268 & 77.24 & & .1 & 4 & & 0 . \\
\hline & 1269 & 76.7500 & 50 & 280.2 & 4 & 0 & \\
\hline & 12610 & 71.7501 & & .4 & 4 & & \\
\hline & 12611 & 70.5003 & & 281.2 & 4 & & 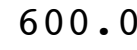 \\
\hline & 12612 & 106.1139 & 3.7250 & 283.1 & 3 & & . \\
\hline & 12613 & 146 . & 5 . & & 3 & & 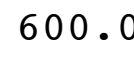 \\
\hline & 12614 & 135.9322 & 4.0000 & 283.4 & 3 & & 0 . \\
\hline & 12615 & 157.1719 & 5500 & 283.4 & 2 & & \\
\hline & 12616 & 170.2491 & 3.5000 & & 3 & & 0 \\
\hline & 12617 & 169.7461 & 3.3500 & 282.8 & 4 & & 0 . \\
\hline & 12618 & 116.7083 & 2.1500 & 282.6 & 5 & 0 & 0 . \\
\hline & 12619 & 72.5055 & 2500 & & 5 & & \\
\hline 00 & 12620 & 73.7545 & & 282.9 & 5 & & \\
\hline & 12621 & 75.2377 & 3.7500 & 283.1 & 5 & 600 & 00 . \\
\hline & & 2000 & & & 4 & & \\
\hline & 12623 & 0002 & 3.300 & 282.9 & 1 & 600.0 & 600 \\
\hline
\end{tabular}




\begin{tabular}{|c|c|c|c|c|c|c|c|}
\hline 0 & 2624 & 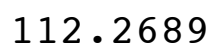 & 0 & 5 & & & \\
\hline & 1271 & 18 & .1250 & 2.1 & & ח ח & \\
\hline & 1272 & 34.7926 & 4250 & 282.1 & & 00 & \\
\hline & 1273 & 25.6759 & 4250 & 281.9 & & 00.0 & \\
\hline & 1274 & 246 & .5500 & 81.9 & & 0 & \\
\hline & 1275 & 2514 & 500 & 81.7 & & 00 & \\
\hline & 1276 & 106.1391 & 5750 & 281.8 & & 00 & \\
\hline & 1277 & 70.7352 & 250 & 81.5 & & & \\
\hline & 1278 & 113.8889 & 500 & 81.6 & & & \\
\hline & $127 \quad 9$ & 7603 & 000 & 82.3 & & 00 & 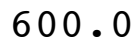 \\
\hline & 12710 & 154.9995 & 5.0000 & 281.5 & & & \\
\hline & 12711 & 153.7500 & 000 & 81.9 & & & \\
\hline & 12712 & 000 & 250 & 282.4 & & 00.0 & \\
\hline & 12713 & 142.7456 & 0 & 283.1 & & 0.0 & . \\
\hline & 12714 & 146.5046 & & 0 & & & \\
\hline & 12715 & 151.2498 & & 284 & & & \\
\hline & 12716 & 152.7492 & 4.8000 & 284.3 & & .0 & . \\
\hline & 12717 & 152.7466 & 4 . & 8 & & 0 & \\
\hline & 12718 & 140.1014 & 2 . & 282.7 & & & \\
\hline & 12719 & 87.9285 & 1.3500 & 282.6 & & 0 & 0 \\
\hline & 12720 & 116.9677 & 250 & 282.8 & & .0 & \\
\hline & 12721 & 112.9611 & & 283.2 & & & \\
\hline & 12722 & 007 & 500 & 283.3 & & & \\
\hline & 12723 & 99.7651 & 2 . & 283.4 & & . 0 & 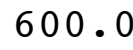 \\
\hline & 12724 & 121.3494 & & 0 & & & \\
\hline & 1281 & 147 . & 1 . & 281.7 & 5 & & \\
\hline & 1282 & 159.9296 & 750 & 280.3 & 6 & 0 & J. \\
\hline & 1283 & 57.8398 & 50 & 80.1 & & & \\
\hline & 1284 & 14.7429 & 750 & 279.9 & 5 & & \\
\hline & 1285 & 344.7308 & 000 & 280.0 & & 0 & 0 . \\
\hline & 1286 & 307.6428 & 250 & 279.6 & 5 & .0 & 0 . \\
\hline & 1287 & 142.9896 & 000 & 279.1 & 4 & & \\
\hline & 1288 & 144.2501 & 250 & 278.4 & 5 & & . \\
\hline & 1289 & 194.7092 & 3750 & 279.1 & 4 & 0 & . \\
\hline & 12810 & 337.4388 & 0 & & 3 & & \\
\hline & 12811 & 248.7219 & 1.4250 & 280.5 & 2 & & . \\
\hline & 12812 & 223.5097 & .5250 & 281.9 & 3 & & . \\
\hline & 12813 & 226.0347 & 500 & 282.4 & 2 & & 0 . \\
\hline & 12814 & 251.9452 & 2.0500 & 282.2 & 2 & .0 & 0 . \\
\hline & 12815 & 233.4765 & 5750 & 281.5 & 1 & & 0 . \\
\hline & 12816 & 279.2113 & 250 & 280 & 2 & & 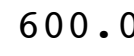 \\
\hline & 12817 & 305.2589 & 2.1750 & 280.8 & 3 & & 0 . \\
\hline & 12818 & 301.5138 & 1.2000 & 280.7 & 4 & 0 & 0 . \\
\hline & 12819 & 7.9447 & 0.7750 & 280.5 & 5 & & \\
\hline 00 & 12820 & 256.9540 & 0.3000 & 280.4 & 6 & & 0 . \\
\hline & 12821 & 145.0000 & 0.5750 & 280.3 & 5 & 600.0 & 600. \\
\hline & 12822 & 134 & & & 4 & & \\
\hline & 12823 & 60.0354 & 0.7000 & 280.1 & J & 600.0 & 600 \\
\hline
\end{tabular}




\begin{tabular}{|c|c|c|c|c|c|c|c|}
\hline 0 & 824 & 13.1649 & 4000 & 0.2 & & - & \\
\hline 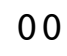 & 1291 & 284 . & 0.6250 & 80.2 & & 00.0 & \\
\hline & 1292 & 356.3796 & 1.0250 & 280.0 & & 00 & \\
\hline & 223 & 353.3313 & 1250 & 279.8 & & 00 & \\
\hline & 1294 & 527 & 250 & 79.8 & & 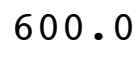 & \\
\hline & 1295 & 8.7502 & 5250 & 279.6 & & 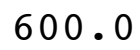 & \\
\hline & 1296 & 301.8388 & 3250 & 279.7 & & 0 & \\
\hline & 1297 & 468 & 1.0250 & 79.6 & & & \\
\hline & 1298 & 270.3275 & 0.7000 & 79.4 & & 0 & \\
\hline & 1299 & 187.0197 & 0.6500 & 279.9 & & 0 & \\
\hline & 12910 & 145.9936 & 2.1500 & 280.5 & & 0 & \\
\hline & 12911 & 959 & 2.4750 & 80.6 & & & \\
\hline & 12912 & 156 & 1.9750 & 282.4 & & 0 & \\
\hline & 12913 & 000 & 2.3250 & 282.5 & & 0 & \\
\hline & 12914 & 56 & 1.9500 & 2 & & & \\
\hline & 12915 & & 2.45 & 284.7 & & & \\
\hline & 12916 & 262 . & 2.2500 & 284.6 & & 0 & \\
\hline & 12917 & 06 & 1.4250 & .5 & & 0 & \\
\hline & 12918 & 32 & 1.5750 & 283.1 & & & \\
\hline & 12919 & 482 & 1.3250 & 283.0 & & & \\
\hline & 12920 & 320 & 0.5500 & 283.2 & & 0 & \\
\hline & 12921 & & 0.5750 & & & & \\
\hline & 12922 & 94 & 4.8250 & 285.4 & & & \\
\hline & 12923 & 296 . & 2.2000 & 284.2 & & 0 & \\
\hline & 12924 & & 2.3250 & & & & \\
\hline & 130 & 345 & 3.4000 & $2 \varepsilon$ & & & \\
\hline 0 & 1302 & 968 & 3.8750 & 284.3 & 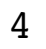 & 0 & \\
\hline & 1303 & 344 & 4.9000 & 2 & 1 & 0 & \\
\hline & 1304 & 33 & 4.8250 & $2 \xi$ & 4 & & \\
\hline & 1305 & 329 . & 4.4250 & 5.0 & 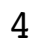 & 0 & \\
\hline & 1306 & 322 & 4.6250 & 285.1 & 4 & 0 & \\
\hline & 1307 & 31 & 7.3000 & & 4 & & \\
\hline & 1308 & 311 . & 7.2750 & 284.7 & 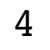 & 0 & \\
\hline & 1309 & 300 & 7.0500 & .5 & 4 & 0 & \\
\hline & 13010 & 27 & 5. & & 3 & & \\
\hline & 13011 & 33 & 4.2500 & 284.7 & 3 & 600.0 & \\
\hline & 13012 & 292.2053 & 2.8000 & 285.0 & 2 & 0 & \\
\hline 0 & 13013 & 212 & 4.3750 & 286.2 & 3 & 0 & \\
\hline 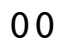 & 13014 & 040 & 7.5750 & 286.0 & 4 & 600.0 & \\
\hline & 13015 & 575 & .1500 & 285.0 & 3 & & \\
\hline & 1301 & 920 & 5.7500 & 284.4 & 3 & 60 & \\
\hline & 13017 & 470 & 6.5500 & 283.0 & 4 & 600.0 & \\
\hline & 13018 & 65.4994 & 6.9250 & 282.7 & 4 & 600 & \\
\hline & 13019 & 633 & 3.2000 & 282.4 & 5 & 600 & \\
\hline 0 & 13020 & 377 & 3.7250 & 282.3 & 4 & 600.0 & \\
\hline & 1302 & 2463 & 5.1500 & 282.1 & 4 & 600.0 & \\
\hline & & & 00 & & 4 & & \\
\hline & 13023 & 51.5907 & 2.9750 & 281.8 & 4 & 600.0 & 60 \\
\hline
\end{tabular}




\begin{tabular}{|c|c|c|c|c|c|c|c|}
\hline 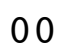 & 3024 & 7 & 250 & 81.6 & 4 & 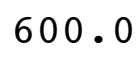 & \\
\hline 0 & 1311 & 3.9966 & 1500 & 281.5 & & 600.0 & \\
\hline & 1312 & 23.6328 & 1.4250 & 281.4 & & 00 & \\
\hline & 1313 & 27.5011 & 1.6750 & 281.7 & & 00 & \\
\hline & 1314 & 62.7493 & 7500 & 81.7 & & 0 & \\
\hline & 1315 & 26.0263 & .3250 & 81.2 & & 0 & \\
\hline & 1316 & 6.4062 & 9750 & 281.1 & & 00 & \\
\hline & 1317 & 0.2431 & 5500 & 81.3 & & 0 & \\
\hline & 1318 & 43.1773 & 9000 & 81.2 & & & \\
\hline & 319 & 10.0580 & 1.0000 & 281.2 & & 00 & U \\
\hline & 3110 & 268.4698 & 0.6250 & 282.3 & & 00.0 & \\
\hline & 3111 & 286.5000 & 102 & 82.5 & & & \\
\hline & 3112 & 268.4895 & 2.5250 & 283.3 & & 0 & \\
\hline & 3113 & 302.1039 & 2.12 & 283.7 & & 0.0 & . \\
\hline & 3114 & 351 . & 0 & & & & \\
\hline & 3115 & 278 . & 2 . & 282.0 & & & \\
\hline & 3116 & 261.4963 & 2.7500 & 282.3 & & .0 & \\
\hline & 3117 & 249 . & 1 . & 2.5 & & & \\
\hline & 3118 & 219.7501 & & 282.4 & 4 & & \\
\hline & 3119 & 257.6170 & 0 & 282.3 & & 0 & 0 \\
\hline & 13120 & 269.7848 & 3000 & 282.4 & 6 & 0 & \\
\hline & 13121 & 334.5000 & & 282.5 & 5 & & \\
\hline & 13122 & 301.2583 & 50 & 282.4 & 5 & & \\
\hline & 13123 & 250.5373 & 0.40 & 282.2 & 6 & 0 & 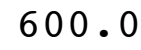 \\
\hline & 13124 & 140.7487 & & 281.7 & 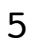 & & \\
\hline & $\begin{array}{lll}2 & 1 & 1\end{array}$ & 139.2509 & 3. & 280.8 & 5 & & \\
\hline & 212 & 137.2495 & 3 & 280.5 & 5 & 0 & J. \\
\hline & 213 & 147.7657 & 3. & 280.4 & 5 & & \\
\hline & $2 \quad 14$ & 151.2504 & 3 . & 280.3 & 5 & & 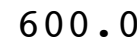 \\
\hline & 215 & 148.7502 & 3.7000 & 279.9 & 5 & & 0 . \\
\hline & 216 & 140.4989 & 1.5250 & 279.7 & 5 & & 0 . \\
\hline & $\begin{array}{lll}2 & 1 & 7\end{array}$ & 140.2500 & 2 . & 279.8 & 5 & & \\
\hline & 218 & 144.7520 & 9000 & 280.4 & 5 & 0 & . \\
\hline & $\begin{array}{lll}2 & 1 & 9\end{array}$ & 140.5023 & 2500 & 280.6 & 4 & 0 & 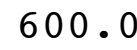 \\
\hline & 2110 & 220.1860 & 0 & & 3 & & \\
\hline & 2111 & 335.1272 & 1.5000 & 285.1 & 2 & & 0 . \\
\hline & 2112 & 250.7113 & 6750 & 285.8 & 1 & & 0 \\
\hline & 2113 & 225.0528 & 0 & .7 & 1 & & . \\
\hline 00 & 2114 & 220.9999 & 2.1500 & 286.2 & 1 & .0 & 0 . \\
\hline & 2115 & 222.4655 & 2.1250 & 286.7 & 1 & & 0 . \\
\hline & 2116 & 198.7571 & 500 & 286.2 & 2 & & 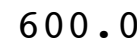 \\
\hline & 2117 & 180.5005 & 2.5750 & 285.8 & 3 & & 0 . \\
\hline & 2118 & 155.7488 & 1.9500 & 284.3 & 4 & 0 & 0 . \\
\hline 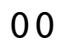 & 2119 & 156.2059 & 1.4750 & & 4 & & \\
\hline 0 & 2120 & 310.5394 & 0.9250 & & 5 & & 0 . \\
\hline & 2121 & 321.6701 & 1.0000 & 283.2 & 6 & 600 & 00 . \\
\hline & 2122 & 186.0124 & & & 5 & & \\
\hline & 2123 & 150.7521 & 2.2500 & 282.3 & 4 & 600.0 & 600 \\
\hline
\end{tabular}




\begin{tabular}{|c|c|c|c|c|c|c|c|c|}
\hline 0 & 2 & 24 & & 250 & 32.0 & & - & \\
\hline 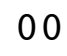 & 2 & 21 & 8.1371 & 1.0750 & 82.0 & & 00.0 & \\
\hline 0 & 2 & 22 & 199.4625 & 1.3250 & 281.8 & & 00 & \\
\hline & 2 & 23 & 291.3768 & 1.2250 & 281.7 & & 00 & \\
\hline & 2 & 24 & 249.8988 & 8000 & 81.6 & & 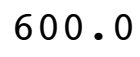 & \\
\hline & 2 & 25 & 246.8012 & .5000 & 81.4 & & 0 & \\
\hline & 2 & 26 & 285.7973 & 0250 & 281.1 & & 0( & \\
\hline & 2 & 2 & 288.2442 & .6000 & 80.9 & & 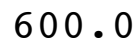 & \\
\hline & 2 & 28 & 325 . & .7500 & 80.8 & & 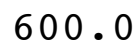 & \\
\hline & 2 & 29 & 133. & 1.2250 & 80.8 & & 0 & \\
\hline & 2 & 210 & 152.7510 & 1.9750 & 80.5 & & & \\
\hline & 2 & 211 & 159 & 1.4750 & 80.9 & & & \\
\hline & 2 & 212 & 241 & 0.8500 & 281.8 & & 0 & \\
\hline & 2 & 213 & 256 . & 1.5250 & 282.0 & & 0 & \\
\hline & 2 & 214 & 26 & 1 . & 82.2 & & & \\
\hline & 2 & 215 & 28 & 1 . & 282.4 & & & \\
\hline & 2 & 216 & 355 & 1.0250 & 282.9 & & 0 & \\
\hline & 2 & 217 & 53 & 1.25 & 3.2 & & 0 & \\
\hline & 2 & 218 & 41 & 3.22 & 282.8 & & & \\
\hline & 2 & 219 & 14 & 3750 & 283.1 & & & \\
\hline & 2 & 220 & 148 & 3.6500 & 283.6 & & 0 & \\
\hline & 2 & 221 & 145 & 1.2250 & 83.7 & & & \\
\hline & 2 & 222 & 61 & 2.0750 & 283.7 & & & \\
\hline & 2 & 223 & 31 & 1.2250 & 283.6 & & 0 & \\
\hline & 2 & 224 & & 000 & .3 & & & \\
\hline & 2 & 31 & 33 & 2.4250 & 283.2 & & & \\
\hline & 2 & 32 & 502 & 2.2000 & 283.4 & & 0 & \\
\hline & 2 & 33 & 40 & 2.7 & 284.3 & 4 & 0 & \\
\hline & 2 & 34 & 34 & 3.2 & 285.0 & 4 & & \\
\hline & 2 & 35 & 39 & 4.7500 & 285.5 & 4 & 0 & \\
\hline & 2 & 36 & 321 & 5.2000 & 287.0 & 4 & 0 & \\
\hline 0 & 2 & 37 & 31 & 5. & 288.0 & 4 & & \\
\hline & 2 & 38 & 86 & 5 . & 287.8 & 4 & & \\
\hline & 2 & 39 & 986 & 6.3000 & 288.0 & 1 & 0 & \\
\hline & 2 & 310 & 30 & 7 & 289.4 & 4 & & \\
\hline & 2 & 311 & 532 & 9. & 291.1 & 4 & .0 & \\
\hline & 2 & 312 & 777 & 5.4750 & 289.8 & 3 & 0 & \\
\hline & 2 & 313 & 32 & 6.5250 & 288.6 & 4 & 0 & \\
\hline 8 & 2 & 314 & 979 & 9.4750 & 288.3 & 4 & 600.0 & \\
\hline & 2 & 315 & 508 & 6.6750 & 285.7 & 4 & 0 & \\
\hline & 2 & 316 & 976 & 5.9750 & 286.1 & 3 & 600.0 & \\
\hline 0 & 2 & 317 & 245 & 6.4500 & 283.5 & 4 & 600.0 & \\
\hline & 2 & 318 & 4899 & 6.6250 & 283.8 & 4 & 600.0 & \\
\hline 0 & 2 & 319 & 997 & 4.1250 & 284.0 & 4 & 600 & \\
\hline 00 & 2 & 320 & 545 & 2.0750 & 283.7 & 5 & 600.0 & \\
\hline & 2 & 321 & 0001 & 2.9500 & 283.2 & 4 & 600.0 & 6 \\
\hline & 2 & 322 & 11 & 2 . & & 5 & & \\
\hline & 2 & 323 & 273.1990 & 1.6500 & 281.1 & 0 & 600.0 & 60 \\
\hline
\end{tabular}




\begin{tabular}{|c|c|c|c|c|c|c|c|c|}
\hline 0 & & 24 & 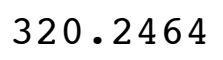 & 1000 & 280.8 & & (6) & \\
\hline 0 & 2 & 1 & 359.0209 & L. 1500 & 281.9 & & & \\
\hline 0 & 2 & 42 & 27.6872 & 2500 & 282.5 & 6 & 00 . & \\
\hline 0 & 2 & 3 & 52.2202 & .7000 & 283.0 & & 00.0 & \\
\hline 0 & 2 & 4 & 65.7895 & .3000 & 33.5 & & & \\
\hline & 2 & 5 & 150.4584 & L. 1000 & 283.4 & & 00 & \\
\hline 0 & 2 & 6 & 314.8851 & .6750 & 283.0 & & 00.0 & \\
\hline 0 & 2 & 7 & 10.1614 & .0000 & 82.9 & & & \\
\hline 0 & 2 & 8 & 341.3763 & .1750 & 282.3 & & 0 & \\
\hline 0 & 2 & 9 & 348.4677 & .7250 & 282.3 & & 0 & \\
\hline 0 & 2 & 410 & 325.0522 & .0250 & 284.3 & & 0 & \\
\hline 0 & 2 & 411 & 176.2808 & .5500 & 285.3 & & & \\
\hline 0 & 2 & 412 & 185.9444 & 1.3500 & 284.7 & & 0 & 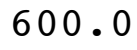 \\
\hline 0 & 2 & 413 & 213.0997 & .7250 & 284.2 & 1 & 0.0 & \\
\hline 0 & 2 & 414 & 169.7354 & .3250 & 283.7 & 2 & & \\
\hline 0 & 2 & 415 & 165.6679 & 1.3250 & 284 & 1 & & \\
\hline 0 & 2 & 416 & 288.0000 & .3250 & 285.3 & 1 & 0 & \\
\hline 0 & 2 & 417 & 316 . & .4500 & 6 & 1 & & \\
\hline 0 & 2 & 418 & 296.5000 & 4500 & 283 & 2 & & \\
\hline 0 & 2 & 419 & 316.3975 & .1000 & 282.9 & 3 & 0 & \\
\hline 0 & 2 & 420 & 8.2855 & .37 & 9 & 4 & & \\
\hline 0 & 2 & 421 & 53 & 0 & 28 & 5 & & \\
\hline 0 & 2 & 422 & 315.7986 & .1750 & 284 & 6 & & \\
\hline 0 & 2 & 423 & 349.5027 & .5500 & 284 & 5 & & \\
\hline 0 & 2 & 424 & 047 & 0 & 28 & 4 & & \\
\hline 0 & 2 & 51 & 426 & .7750 & 284 & 5 & & \\
\hline 0 & 2 & 52 & 291.9339 & .9750 & 283.4 & 6 & 0 & \\
\hline 0 & 2 & 53 & 170.5448 & 0 & 28 & 6 & & \\
\hline 00 & 2 & 54 & 31.9560 & 3.6500 & 284.9 & 5 & 0 & \\
\hline 0 & 2 & 55 & 4.5154 & 6500 & 284.6 & 6 & 0 & \\
\hline 0 & 2 & 56 & 47.7656 & 0 & 28 & 5 & & \\
\hline 0 & 2 & 57 & 344 . & 0 & 28 & 4 & & \\
\hline 0 & 2 & 58 & 334.4997 & .3000 & 284.8 & 4 & 0 & 00 \\
\hline 0 & 2 & 59 & 328.1558 & 5000 & 28 & 3 & & \\
\hline 0 & 2 & 510 & $34^{\circ}$ & 0 & 28 & 4 & & \\
\hline 00 & 2 & 511 & 344.4977 & 7.8000 & 287.6 & 4 & & \\
\hline 0 & 2 & 512 & 349.7529 & 6.8750 & 287.1 & 4 & & \\
\hline 0 & 2 & 513 & 348.9992 & 6.5000 & 287.9 & 4 & & 00 \\
\hline 00 & 2 & 514 & 8.5086 & 5.2750 & 288.3 & 3 & & 00 \\
\hline 0 & 2 & 515 & 18.5458 & 000 & 288.1 & 3 & & \\
\hline 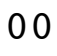 & 2 & 516 & 26.4982 & 500 & $28^{\circ}$ & 3 & & \\
\hline 00 & 2 & 517 & 59.2050 & 3.2000 & 285.6 & 3 & .0 & 00 \\
\hline 90 & 2 & 518 & 18.3704 & 1.5500 & 286.1 & 4 & .0 & 500 \\
\hline 00 & 2 & 519 & 126.3286 & 1.5250 & 285.8 & 5 & & 500 \\
\hline 00 & 2 & 520 & 10.0668 & 1.7750 & 285.6 & 6 & .0 & 500 \\
\hline 0 & 2 & 521 & 9.8899 & 1.2500 & 285.3 & 6 & 600.0 & 600 \\
\hline 00 & 2 & 522 & 2507 & 00 & & 5 & & \\
\hline & & 523 & 341.2475 & 1.7750 & 284.1 & 4 & 600.0 & 500 \\
\hline
\end{tabular}




\begin{tabular}{|c|c|c|c|c|c|c|c|c|}
\hline 0 & 2 & 24 & & 1.4750 & 3.7 & & & \\
\hline 0 & 2 & 1 & 279.9063 & 1.2750 & 282.8 & & & \\
\hline & 2 & 62 & 8.3281 & .1750 & 282.3 & & 00 & \\
\hline & 2 & 3 & 65.0089 & 5250 & 282.9 & & 00 & \\
\hline & 2 & 4 & 146.7297 & .92 & 83.1 & & 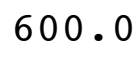 & \\
\hline & 2 & 5 & 283.9404 & .5250 & 282.4 & & 00 & \\
\hline & 2 & 6 & 314.6371 & 0.6750 & 282.3 & & 0 & \\
\hline & 2 & 7 & 352.0384 & .5250 & 82.4 & & & \\
\hline & 2 & 8 & 328.0015 & 1.0500 & 282.6 & & 0 & \\
\hline & 2 & 9 & 321.9931 & 0.9000 & 284.9 & & 00.0 & \\
\hline & 2 & 610 & 248.3605 & 1.2000 & 286.3 & & 00 & \\
\hline & 2 & 511 & 151.7474 & 525 & 82.1 & & & \\
\hline & 2 & 612 & 151.2523 & 4.8500 & 282.0 & & 0 & \\
\hline & 2 & 613 & 160.9999 & 3.5500 & 283.5 & & 0.0 & \\
\hline & 2 & 614 & 168.18 & & 85.1 & & & \\
\hline & 2 & $61 !$ & 205.0782 & 2.6750 & 285.9 & & 0 & \\
\hline & 2 & 616 & 202.0126 & .8500 & 285.9 & & 00.0 & \\
\hline & 2 & 617 & 215 . & 1.92 & 85.4 & & & \\
\hline 0 & 2 & 618 & 225 . & 0.45 & 284.9 & 4 & 0 & \\
\hline & 2 & 619 & 28.8327 & 0 & 284.1 & 5 & 0 & \\
\hline & 2 & 620 & 26.5701 & 0 & 284.1 & 5 & & \\
\hline & 2 & 621 & 153.7550 & 2.2 & 284.1 & 4 & & \\
\hline 0 & 2 & 622 & 142.4908 & 232 & 284 & 4 & 0 & U. \\
\hline 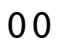 & 2 & 623 & 139.7484 & 2.42 & 28 & 5 & 0 & \\
\hline 0 & 2 & 624 & 154.7273 & 100 & 28 & 5 & & \\
\hline 0 & 2 & 71 & 292.1553 & 0.3500 & 283.4 & 6 & & \\
\hline 0 & 2 & 72 & 180.3462 & 0.37 & 283.8 & 6 & 0 & \\
\hline 0 & 2 & 73 & 223.4220 & 0 . & 5 & 6 & & \\
\hline 0 & 2 & 74 & 312.5937 & $0.78-2-3$ & 283.8 & 6 & 0 & 0 . \\
\hline & 2 & 75 & 301.1689 & 75 & 283.9 & 6 & 0 & \\
\hline 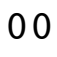 & 2 & 76 & 173.6226 & 0 & $\cdot 1$ & 6 & & \\
\hline 0 & 2 & 77 & 151.9968 & 0 & 282.7 & 5 & & • \\
\hline & 2 & 78 & 152.4914 & 0.9500 & 282.4 & 6 & 0 & U. \\
\hline 0 & 2 & 79 & 557 & 0 & 28 & 5 & 0 & \\
\hline & 2 & 710 & 321.4318 & 0 & 28 & 4 & & \\
\hline & 2 & 711 & 292.3633 & 1.3750 & 285.9 & 3 & 0 & 0 . \\
\hline 0 & 2 & 712 & 312.9846 & 8000 & 286.1 & 2 & 0 & \\
\hline 0 & 2 & 713 & 282.9243 & 2.70 & 287.3 & 1 & 0 & 60 \\
\hline 0 & 2 & 714 & 253.7465 & 2.6250 & 287.9 & 2 & 600.0 & 0 . \\
\hline 0 & 2 & 715 & 203.9147 & 000 & 287.9 & 2 & 0 & \\
\hline - & 2 & 716 & 138.3425 & 1 . & & 3 & & \\
\hline 00 & 2 & 717 & 143.5009 & 3.0000 & 286.0 & 4 & 600.0 & 600 . \\
\hline & 2 & 718 & 44.8110 & 0.6750 & 286.1 & 5 & 600 & 600. \\
\hline . & 2 & 719 & 359.2465 & 1.1000 & 286.3 & 5 & 600 & 10 \\
\hline 00 & 2 & 720 & 12.2820 & 0.8750 & 286.0 & 6 & 600.0 & 00 \\
\hline & 2 & 721 & 14.1739 & 2.1250 & 286.9 & 5 & 600.0 & 600. \\
\hline & 2 & 722 & 345.0674 & 1.2000 & 287.3 & 6 & 600 & 60 \\
\hline & & 723 & 183.6048 & 0.9500 & 287.2 & & 600.0 & 600 \\
\hline
\end{tabular}




\begin{tabular}{|c|c|c|c|c|c|c|c|c|}
\hline 0 & 2 & 24 & & 2.1750 & 86.0 & & 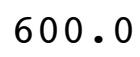 & \\
\hline & 2 & 1 & 168 & 2.3500 & 284.8 & & 600.0 & \\
\hline & 2 & 82 & 142.2539 & 2.0500 & 284.3 & & 00 & \\
\hline & 2 & 83 & 99.9249 & 9500 & 284.1 & & 00.0 & \\
\hline & 2 & 4 & 115.7960 & .0750 & 84.9 & & and & \\
\hline & 2 & 5 & 141.2500 & 3.5000 & 85.8 & & 0 & \\
\hline & 2 & 86 & 144.1988 & 1.8500 & 285.6 & & 500 & \\
\hline & 2 & 7 & 15.7015 & .5000 & 85.0 & & .0 & \\
\hline & 2 & 8 & 30.4937 & 9750 & 85.7 & & & \\
\hline & 2 & 9 & 71.3154 & 500 & 286.4 & & 00.0 & 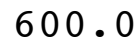 \\
\hline & 2 & 810 & 105.3150 & 7250 & 287 & & 0 & \\
\hline & 2 & 811 & 127.4211 & 2.7500 & 7 & & 0 & \\
\hline & 2 & 812 & 172.9248 & 4.5000 & 289.6 & & 0 & \\
\hline & 2 & 813 & 178.8288 & 4.0750 & 288.8 & & 0.0 & \\
\hline & 2 & 814 & 174.0849 & 250 & & & & \\
\hline & 2 & 815 & 200.7525 & & 291.3 & & & \\
\hline & 2 & 816 & 217.3796 & 1.6000 & 290.8 & & .0 & 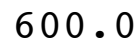 \\
\hline & 2 & 817 & 152.6800 & 000 & 1.2 & & 0 & \\
\hline & 2 & 818 & 157.2500 & 2 . & 288.5 & & & \\
\hline & 2 & 819 & 114.7931 & 500 & 28 & & & \\
\hline & 2 & 820 & 69.0027 & 5.0250 & 287.3 & 4 & 0 & • \\
\hline & 2 & 821 & 88.4893 & & & 4 & & \\
\hline & 2 & 822 & 90.8694 & 000 & 28 & 5 & & \\
\hline & 2 & 823 & 111.7464 & 500 & 287.1 & 4 & 0 & 00 \\
\hline & 2 & 824 & 121.7456 & & & 5 & & \\
\hline & 2 & 91 & 120.8507 & 1.3500 & 28 & 6 & & \\
\hline & 2 & 92 & 69.6541 & 2.8750 & 286.0 & 6 & 0 & U. \\
\hline & 2 & 93 & 63.4754 & 3250 & 28 & 5 & & \\
\hline & 2 & 94 & 11.4482 & 1.8500 & 28 & 4 & & 0 \\
\hline & 2 & 95 & 188.1405 & 1250 & 5.7 & 5 & .0 & 0 . \\
\hline & 2 & 96 & 146.8698 & 0.7000 & 285.6 & 6 & 0 & 0 . \\
\hline & 2 & 97 & 190.4032 & 1.1250 & 28 & 6 & & \\
\hline & 2 & 98 & 119.2406 & 1.1750 & 283.6 & 6 & & • \\
\hline & 2 & 99 & 98.7717 & 1.6500 & 282.7 & 5 & 0 & \\
\hline & 2 & 910 & 302.7301 & 000 & 285.3 & 4 & & 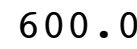 \\
\hline & 2 & 911 & 237.1983 & 1.8500 & 285.4 & 3 & & U \\
\hline & 2 & 912 & 241.2507 & 1.5750 & 285.8 & 2 & 0 & o. \\
\hline & 2 & 913 & 225.4954 & 500 & 28 & 1 & & . \\
\hline 00 & 2 & 914 & 180.8715 & 1.6750 & 285.2 & 2 & .0 & 0 . \\
\hline & 2 & 915 & 173.9685 & 1.3500 & 285.7 & 1 & & 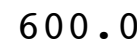 \\
\hline & 2 & 916 & 32.2021 & 250 & 286.0 & 1 & & 600. \\
\hline 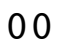 & 2 & 917 & 157.2293 & 2.2500 & 285.4 & 2 & .0 & 0 . \\
\hline & 2 & 918 & 130.5226 & 1.6250 & 285.1 & 3 & 0 & 00 . \\
\hline 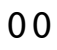 & 2 & 919 & 232.7977 & 250 & 284.7 & 4 & 600 & 0 \\
\hline 00 & 2 & 920 & 288.8965 & 1.0250 & 284.2 & 5 & & 600. \\
\hline & 2 & 921 & 3.8148 & 1.0000 & 284.1 & 6 & 600.0 & 600. \\
\hline & 2 & 922 & 141 . & & & 6 & & \\
\hline & & 923 & 274.8580 & 1.2750 & 283.8 & & 600.0 & 600 \\
\hline
\end{tabular}


$00 \quad 2 \quad 924 \quad 322.2888$

$\begin{array}{llll}00 & 210 & 1 & 329.4976\end{array}$

$00 \quad 210 \quad 2 \quad 322.5040$

$00 \quad 210 \quad 3 \quad 321.9952$

$\begin{array}{llll}00 & 210 & 4 & 322.5027\end{array}$

$\begin{array}{llll}00 & 210 & 5 & 319.2620\end{array}$

$\begin{array}{llll}00 & 210 & 6 & 310.2379\end{array}$

$\begin{array}{llll}00 & 210 & 7 & 314.9998\end{array}$

$00 \quad 210 \quad 8 \quad 326.0000$

$\begin{array}{llll}00 & 210 & 9 & 318.7391\end{array}$

$00 \quad 21010 \quad 317.7303$

$00 \quad 21011 \quad 355.5000$

$\begin{array}{lll}00 & 21012 & 68.2917\end{array}$

$\begin{array}{lll}00 & 21013 & 10.7013\end{array}$

$00 \quad 21014 \quad 12.7020$

$00 \quad 21015 \quad 88.4310$

$00 \quad 21016 \quad 293.1514$

$00 \quad 21017 \quad 256.0453$

$\begin{array}{llll}00 & 21018 & 227.2528\end{array}$

$\begin{array}{lll}00 & 21019 & 289.9823\end{array}$

$00 \quad 21020 \quad 339.6623$

$\begin{array}{lll}00 & 21021 & 332.4737\end{array}$

$\begin{array}{lll}00 & 21022 & 36.7749\end{array}$

$00 \quad 21023 \quad 24.8980$

$00 \quad 21024 \quad 14.5000$

$\begin{array}{llll}00 & 211 & 1 & 26.7379\end{array}$

$\begin{array}{llll}00 & 211 & 2 & 349.9339\end{array}$

$\begin{array}{llll}00 & 211 & 3 & 359.3450\end{array}$

$\begin{array}{llll}00 & 211 & 4 & 26.1123\end{array}$

$\begin{array}{llll}00 & 211 & 5 & 293.5701\end{array}$

$\begin{array}{llll}00 & 211 & 6 & 5.2549\end{array}$

$\begin{array}{llll}00 & 211 & 7 & 28.7432\end{array}$

$\begin{array}{llll}00 & 211 & 8 & 309.8881\end{array}$

$00 \quad 211 \quad 9 \quad 340.4894$

$\begin{array}{lll}00 & 21110 & 349.5000\end{array}$

$00 \quad 21111 \quad 289.1219$

$00 \quad 21112 \quad 313.5462$

$00 \quad 21113 \quad 312.0064$

$00 \quad 21114 \quad 314.7483$

$00 \quad 21115 \quad 321.5342$

$00 \quad 21116 \quad 328.5000$

$00 \quad 21117 \quad 329.0002$

$00 \quad 21118 \quad 317.3435$

$00 \quad 21119 \quad 354.5000$

$00 \quad 21120 \quad 351.0221$

$00 \quad 21121 \quad 349.7510$

$00 \quad 21122 \quad 71.7384$

$00 \quad 21123 \quad 44.1679$ $\begin{array}{lllll}3.0250 & 284.0 & 5 & 600.0 & 600.0\end{array}$

$\begin{array}{llllll}2.7750 & 283.9 & 4 & 600.0 & 600.0\end{array}$

$\begin{array}{lllll}3.5750 & 283.6 & 4 & 600.0 & 600.0\end{array}$

$\begin{array}{llllll}6.7750 & 283.6 & 4 & 600.0 & 600.0\end{array}$

$\begin{array}{lllll}7.2500 & 283.3 & 4 & 600.0 & 600.0\end{array}$

$\begin{array}{llllll}5.6250 & 283.3 \quad 4 & 600.0 & 600.0\end{array}$

$\begin{array}{lllll}5.2750 & 283.4 & 4 & 600.0 & 600.0\end{array}$

$6.3000 \quad 283.7 \quad 4 \quad 600.0 \quad 600.0$

$\begin{array}{lllll}4.2500 & 282.8 & 4 & 600.0 & 600.0\end{array}$

$\begin{array}{llllll}3.9250 & 282.7 & 4 & 600.0 & 600.0\end{array}$

$\begin{array}{lllll}3.2250 & 283.3 & 3 & 600.0 & 600.0\end{array}$

$2.2750 \quad 284.3 \quad 2 \quad 600.0 \quad 600.0$

$\begin{array}{lllll}1.8250 & 285.3 & 1 & 600.0 & 600.0\end{array}$

$\begin{array}{lllll}3.6750 & 283.6 & 2 & 600.0 & 600.0\end{array}$

$1.9000 \quad 283.4 \quad 1 \quad 600.0 \quad 600.0$

$2.8500 \quad 284.6 \quad 1 \quad 600.0 \quad 600.0$

$\begin{array}{lllll}4.0250 & 283.7 & 2 & 600.0 & 600.0\end{array}$

$\begin{array}{lllll}1.9750 & 283.2 & 1 & 600.0 & 600.0\end{array}$

$\begin{array}{lllll}3.2250 & 282.6 & 2 & 600.0 & 600.0\end{array}$

$2.4500 \quad 281.7 \quad 3 \quad 600.0 \quad 600.0$

$\begin{array}{lllll}1.4750 & 281.7 & 4 & 600.0 & 600.0\end{array}$

$\begin{array}{lllll}1.3000 & 281.4 & 4 & 600.0 & 600.0\end{array}$

$2.8500 \quad 281.5 \quad 5 \quad 600.0 \quad 600.0$

$2.7000 \quad 281.9 \quad 4 \quad 600.0 \quad 600.0$

$1.4000281 .3 \quad 5 \quad 600.0 \quad 600.0$

$\begin{array}{lllll}1.8500 & 281.7 & 6 & 600.0 & 600.0\end{array}$

$\begin{array}{lllll}2.8000 & 281.7 & 5 & 600.0 & 600.0\end{array}$

$2.7250 \quad 281.4 \quad 4 \quad 600.0 \quad 600.0$

$0.9000 \quad 281.8 \quad 5 \quad 600.0 \quad 600.0$

$\begin{array}{lllll}1.1750 & 281.4 & 6 & 600.0 & 600.0\end{array}$

$2.2250 \quad 280.9 \quad 6 \quad 600.0 \quad 600.0$

$\begin{array}{lllll}3.5750 & 282.2 & 5 & 600.0 & 600.0\end{array}$

$\begin{array}{lllll}1.2750 & 281.8 & 6 & 600.0 & 600.0\end{array}$

$\begin{array}{lllll}1.6750 & 281.8 & 5 & 600.0 & 600.0\end{array}$

$\begin{array}{lllll}1.5250 & 282.0 & 4 & 600.0 & 600.0\end{array}$

$\begin{array}{lllll}1.7500 & 282.3 & 3 & 600.0 & 600.0\end{array}$

$\begin{array}{lllll}2.2000 & 282.5 & 2 & 600.0 & 600.0\end{array}$

$\begin{array}{lllll}6.2500 & 283.7 & 3 & 600.0 & 600.0\end{array}$

$\begin{array}{lllll}7.9500 & 283.4 & 4 & 600.0 & 600.0\end{array}$

$8.4000 \quad 283.14600 .0 \quad 600.0$

$\begin{array}{llllll}7.8000 & 283.0 & 4 & 600.0 & 600.0\end{array}$

$\begin{array}{lllll}4.6750 & 283.2 & 3 & 600.0 & 600.0\end{array}$

$2.5500 \quad 283.0 \quad 4 \quad 600.0 \quad 600.0$

$\begin{array}{lllll}7.6500 & 282.8 & 4 & 600.0 & 600.0\end{array}$

$6.0500 \quad 282.1 \quad 4 \quad 600.0 \quad 600.0$

$\begin{array}{lllll}4.1500 & 281.5 & 4 & 600.0 & 600.0\end{array}$

$\begin{array}{lllll}5.6750 & 280.1 & 4 & 600.0 & 600.0\end{array}$

$6.6250 \quad 278.7 \quad 4 \quad 600.0 \quad 600.0$ 


\begin{tabular}{|c|c|c|c|c|c|c|c|}
\hline 0 & 1124 & 5.7893 & 3.6250 & 279.1 & 4 & 000.0 & \\
\hline 0 & 2121 & 1.7478 & 3.9250 & 280.5 & 4 & 600.0 & \\
\hline & 2122 & 53.9973 & 2000 & 280.9 & 4 & 00 & \\
\hline & 2123 & 41.5168 & 0500 & 281.5 & & 00 & \\
\hline & 2124 & 304.4439 & & 30.4 & & 0 & \\
\hline & 2125 & 809 & 6750 & 279.2 & & 00 & \\
\hline & 2126 & 194 & 1.2750 & 279.5 & & 0 & \\
\hline & 2127 & 9091 & .6000 & 79.8 & & & \\
\hline & 2128 & 344.5052 & .7000 & 279.7 & & 0 & \\
\hline & 2129 & 328.2490 & 3.5250 & 280.3 & & 0 & \\
\hline & 1210 & 309.9677 & 9500 & 281.5 & & 00 & \\
\hline & 1211 & 2.7512 & & 81.7 & & & \\
\hline & 21212 & 310.0007 & 3000 & 282.4 & 4 & 0 & \\
\hline & 21213 & 312.5017 & 7.0500 & 283.0 & 4 & 0.0 & \\
\hline & 21214 & 308.0092 & & & & & \\
\hline & 21215 & 298.2759 & 4. & 283.4 & 3 & 0 & \\
\hline & 21216 & 5.9639 & 0 & 284.4 & & 0 & \\
\hline & 21217 & 357.0007 & 2.72 & 4.6 & & & \\
\hline 0 & 21218 & 661 & 4 . & 284.2 & 2 & 0 & \\
\hline & 21219 & 12.5276 & 10 & 3.7 & 3 & & \\
\hline & 21220 & 51.5134 & 1 . & .1 & 4 & & \\
\hline & 21221 & 55.4881 & 0 & 2.6 & 5 & & \\
\hline 0 & 21222 & 221.5201 & 7500 & 282.4 & 6 & 0 & 0 • \\
\hline 0 & 21223 & 160.6458 & 500 & 282.1 & 6 & 0 & \\
\hline & 21224 & 294.4787 & & 282.7 & 6 & & \\
\hline & 2131 & 307.7236 & 0 & 282.5 & 6 & & \\
\hline & 2132 & 318.5000 & 4.10 & 281.9 & 5 & 0 & \\
\hline 0 & 2133 & 315.7590 & & 281.2 & 4 & & \\
\hline 0 & 2134 & 321.2496 & 0 & 1.3 & 4 & 0 & 0 . \\
\hline & 2135 & 313.4979 & 0 & 281.9 & 4 & & \\
\hline & 2136 & 299. & 0 & & 4 & & \\
\hline 0 & 2137 & 298 & 0 & .8 & 4 & & \\
\hline & 2138 & 302.0049 & 4000 & 282.4 & 4 & 0 & 0 • \\
\hline 0 & 2139 & 298 & 0 & & 4 & & \\
\hline & 21310 & 287 & 4 . & 3 & 3 & & \\
\hline & 21311 & 307.0000 & 750 & 283.8 & 4 & & 0 . \\
\hline & 21312 & 288.2642 & 500 & 284.2 & 3 & & \\
\hline 0 & 21313 & 272.2285 & 0 & & 3 & 0 & 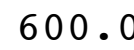 \\
\hline 0 & 21314 & 315.0076 & 4.3000 & 284.0 & 3 & 0 & 0 . \\
\hline & 21315 & 312.4862 & 250 & & 3 & 0 & \\
\hline C & 21316 & 322.7624 & 000 & & 4 & & \\
\hline 00 & 21317 & 314.9992 & 6.6750 & 283.9 & 4 & 600.0 & 600. \\
\hline & 21318 & 325.5000 & & 283.9 & 4 & 600 & 000 \\
\hline 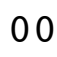 & 21319 & 327.4914 & 5.0250 & & 4 & & 50 \\
\hline 00 & 21320 & 332.5413 & 3.3250 & 284.3 & 5 & 600.0 & 00 \\
\hline & 21321 & 313.4933 & 1.2750 & 285.0 & 6 & 600 & 50 \\
\hline & 21322 & 242.5085 & 2000 & & 6 & 60 & \\
\hline & 21323 & 32.8403 & 3.5250 & 287.7 & 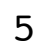 & 600.0 & 600 \\
\hline
\end{tabular}




\begin{tabular}{|c|c|c|c|c|c|c|c|}
\hline 0 & 324 & & 2250 & 7.2 & & & \\
\hline & 141 & 8. & .1750 & 287.8 & & 00.0 & \\
\hline & 2142 & 1.7495 & 1250 & 287.8 & & 00 & \\
\hline & 143 & 8.2501 & 7.4500 & 287.7 & & 00 & \\
\hline & 144 & 53.5000 & 3500 & 87.5 & & 0 & \\
\hline & 145 & 500 & .42 & 87.3 & & 0 & \\
\hline & 146 & 5000 & 8250 & 286.4 & & 00 & \\
\hline & 147 & 1.9675 & 500 & 86.0 & & & \\
\hline & 2148 & 28.0 & 8.85 & 2 & & & \\
\hline & 149 & 34.7497 & 0.5000 & 85.7 & & 0 & U \\
\hline & 1410 & 32.2500 & 5500 & 286.2 & & 00.0 & \\
\hline & 1411 & 27 & 0 & 8 & & & \\
\hline & 1412 & 172 & 12.3750 & 285.1 & & & \\
\hline & 1413 & 62.9353 & 9.5750 & 283 & & 0.0 & \\
\hline & 1414 & & 1 . & & & & \\
\hline & 1415 & & 1.4 & 6 & & & \\
\hline & 1416 & 57.5000 & 0 & 285 & & .0 & . \\
\hline & 417 & 00 & 6. & 3 & & & \\
\hline & 418 & 87 & 0 & 28 & & & \\
\hline & 419 & 80.2502 & 2.4250 & 284.1 & 4 & .0 & . \\
\hline & 21420 & 80.2384 & 3.35 & 283.6 & & 0 & \\
\hline & 21421 & 66.5092 & & & $\mathbf{I}$ & & \\
\hline & 1422 & 3885 & 0 & 282.8 & 1 & & \\
\hline & 21423 & 46.8255 & 5.70 & 282.8 & & 0 & \\
\hline & 21424 & -2502 & 6 . & 282.2 & 4 & & \\
\hline & 2151 & 70.6813 & 3.3 & 282.1 & 4 & & \\
\hline & 2152 & 23.8376 & 1.5250 & 281.3 & & 0 & J. \\
\hline & 2153 & 17.494 & 1. & 281.8 & 6 & & \\
\hline & 2154 & 63.8170 & & 281.5 & 6 & & \\
\hline & 2155 & 182.4639 & 750 & 281.1 & 6 & 0 & 0 . \\
\hline & 2156 & a 76 & 50 & 280.9 & 6 & & 0 . \\
\hline & 2157 & 160.10 & 0 & 28 & 6 & & \\
\hline & 2158 & 314.1535 & & 280.8 & - & & . \\
\hline & 2159 & 336.7070 & 250 & 282.3 & 5 & & \\
\hline & 21510 & 347.24 & 0 & 28 & 4 & & \\
\hline & 21511 & 274.5418 & 500 & 284.9 & 3 & & ( \\
\hline & 21512 & 274.2734 & 2250 & 285.0 & 2 & & \\
\hline & 21513 & 259.2727 & 500 & 28 & 1 & & \\
\hline & 21514 & 227.1697 & 5250 & 285.4 & 1 & & 0 . \\
\hline & 21515 & 222.2748 & 2.4500 & 285.3 & $Z$ & & \\
\hline & 21516 & 227.9899 & 000 & 284.9 & 3 & & 0 \\
\hline & 21517 & 216.5344 & 1.4750 & 284.8 & 2 & & 0 . \\
\hline & 21518 & 182.5064 & 2.2000 & 284.2 & 3 & 0 & 0 . \\
\hline & 21519 & 144.4810 & 1.7000 & 283.5 & 4 & & \\
\hline 00 & 21520 & 135.2116 & 0.9000 & 283.3 & 5 & & \\
\hline & 21521 & 245.0096 & 1.4250 & 283.1 & 5 & & 00 . \\
\hline & & כy & & & & & \\
\hline & 21523 & 325.7504 & 1.2250 & 282.9 & & 600.0 & 600 \\
\hline
\end{tabular}




\begin{tabular}{|c|c|c|c|c|c|c|c|}
\hline 0 & 1524 & & 5750 & 9 & & & \\
\hline 0 & 161 & 238 & 1750 & 2.7 & 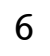 & 0 & \\
\hline 0 & 2162 & 134.2483 & 1.3500 & 282.7 & כ & 00 . & \\
\hline & 163 & 227.4033 & 1.2500 & 282.7 & & 00.0 & \\
\hline 0 & 164 & 342 & .3500 & 82.4 & & & \\
\hline & 165 & 55 & 1.7250 & 282.3 & & 00 & \\
\hline 0 & 166 & 825 & 1.3500 & 281.6 & & 00 & \\
\hline & 167 & 576 & .6500 & 81.1 & & & \\
\hline & 168 & 819 & 1.3500 & 81.0 & & & \\
\hline 0 & 169 & 49.9134 & 1.4500 & 281.2 & & 600.0 & 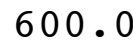 \\
\hline & 1610 & 5.7458 & 1.1250 & 281.8 & & & \\
\hline & 1611 & 44.5114 & 3.8000 & 31.2 & & & \\
\hline 0 & 1612 & 332.0530 & 2.2000 & 280.8 & 3 & 00.0 & \\
\hline 0 & 1613 & 267.2022 & 1.6750 & 281.4 & & 0.0 & \\
\hline & 21614 & 228.7568 & & 2.5 & & & \\
\hline 0 & 21615 & 203.4358 & 2 . & 281.7 & 2 & & \\
\hline 0 & 21616 & 173.2385 & 2.7750 & 280.9 & 1 & 0.0 & \\
\hline & 1617 & 329 & 3.3 & .1 & & .0 & \\
\hline 0 & 21618 & 123. & 3. & 280.1 & 3 & & \\
\hline 0 & 21619 & .5069 & 4.8000 & 280.3 & 4 & .0 & 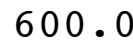 \\
\hline 0 & 21620 & 77.2299 & 6.2250 & 280.3 & 4 & $\cdot 0$ & 000 \\
\hline 0 & 216 & 80.5000 & 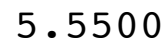 & 80.3 & 4 & & \\
\hline 0 & 21622 & 5000 & 5 . & 280.4 & 4 & .0 & \\
\hline 0 & 21623 & 74.9981 & 5.20 & .3 & 4 & .0 & \\
\hline 0 & 21624 & .2491 & 6 . & 1 & 4 & & \\
\hline 00 & 2171 & 67.2503 & 5.35 & 280.2 & 4 & & \\
\hline 0 & 2172 & 67.2500 & 5.5500 & 280.1 & 4 & .0 & 0 \\
\hline 0 & 2173 & 68.2499 & 5.2500 & .1 & 4 & .0 & \\
\hline 00 & 2174 & 7540 & 5.00 & 28 & 4 & .0 & \\
\hline 0 & 2175 & 69.2501 & 5.4000 & .9 & 4 & .0 & \\
\hline 0 & 2176 & 61.9999 & 5.6750 & 279.7 & 4 & .0 & 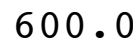 \\
\hline 0 & 2177 & 66.2491 & 55 & 279.9 & 4 & & \\
\hline 0 & 2178 & 7390 & 4 . & 280.1 & 4 & .0 & \\
\hline 0 & 2179 & 70.7499 & 3.9250 & 281.2 & 3 & .0 & \\
\hline 0 & 21710 & 9 & 1 & & 3 & & 0 \\
\hline 00 & 21711 & 002 & 2.4750 & 3.4 & 2 & .0 & \\
\hline 0 & 21712 & 229.2418 & 1.8000 & 283.0 & 1 & .0 & \\
\hline 0 & 21713 & 200.5000 & 2.42 & .2 & 1 & .0 & 6 \\
\hline 00 & 21714 & 168.2525 & 2.3000 & 283.6 & 1 & .0 & 60 \\
\hline 0 & 21715 & 180.2779 & 4.9500 & 283.8 & 2 & .0 & \\
\hline 0 & 21716 & 177.7565 & 4.4500 & & 3 & & \\
\hline 00 & 21717 & 172.7506 & 3.5750 & 283.0 & 3 & .0 & \\
\hline 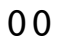 & 21718 & 177.0000 & 3.5250 & 282.5 & 4 & .0 & 00 \\
\hline 00 & 21719 & 128.2423 & 1.2500 & & 4 & .0 & \\
\hline 00 & 21720 & 72.2771 & 2.1750 & 282.2 & 4 & 600.0 & 600 \\
\hline 00 & 21721 & 113.8733 & 1.7500 & 282.1 & 4 & 600.0 & 600 \\
\hline 00 & 21722 & 15 & 1.3 & & 5 & & \\
\hline & 21723 & 147.0000 & 1.4250 & 281.2 & & .0 & 600 \\
\hline
\end{tabular}




\begin{tabular}{|c|c|c|c|c|c|c|c|}
\hline 0 & 1724 & & 2750 & & & 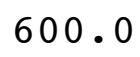 & \\
\hline 0 & 2181 & 120 & 1.1750 & 30.7 & \pm & 00.0 & \\
\hline & 2182 & 149.7496 & .6750 & 280.6 & 2 & 00 & \\
\hline & 2183 & 132.2543 & .7500 & 280.5 & & 00 & \\
\hline & 2184 & 31 & 3250 & 30.1 & & 0 & \\
\hline & 2185 & & 1250 & 80.1 & & O & \\
\hline & 2186 & 139.5000 & 2.4000 & 279.3 & & 0 & \\
\hline & 2187 & 75 & .9250 & 79.7 & & & \\
\hline & $218 \quad 8$ & 07 & .9500 & 80.2 & & 0 & \\
\hline & 2189 & 287 & 1.3250 & 81.9 & & v & \\
\hline & 21810 & 162.0050 & 1.7250 & 282.3 & & 0 & \\
\hline & 21811 & 61 & 750 & .0 & & & \\
\hline & 21812 & 87 & .9750 & 82.3 & & 0 & \\
\hline & 21813 & 180.7788 & 3.1750 & 283.1 & & & \\
\hline & 21814 & & 50 & 3 & & & \\
\hline & 21815 & 79 & 50 & 284.9 & & & \\
\hline & 21816 & 236 & 2.3000 & 85.4 & & 0 & \\
\hline & 21817 & 537 & .0250 & .2 & & 0 & \\
\hline & 21818 & 234 & .6000 & 4.6 & 2 & & \\
\hline & 21819 & 220.7375 & 0.6000 & 283.2 & 3 & 0 & \\
\hline & 21820 & 681 & .3750 & 282.6 & 4 & 0 & \\
\hline & 218 & 50 & 0.47 & 2.2 & 2 & & \\
\hline & 21822 & 765 & 0.3250 & 281.9 & 4 & 0 & \\
\hline & 21823 & 86 & 0.4750 & 281.5 & 5 & 0 & \\
\hline & 21824 & & 500 & 282.4 & 4 & & \\
\hline 0 & 219 & 120 & 1.1750 & 282.1 & 5 & & \\
\hline & 2192 & 374 & 0.3750 & 282.3 & 5 & 0 & \\
\hline & 2193 & 027 & 0.8250 & 3.0 & 6 & 0 & \\
\hline 0 & 2194 & 17 & 1.2500 & 283.1 & 5 & & \\
\hline & 2195 & 425 & 0.9750 & 282.7 & 6 & 0 & \\
\hline & 2196 & 500 & 2.2000 & 281.8 & 5 & 0 & \\
\hline & 2197 & & 3.0 & 280.7 & 5 & & \\
\hline & 2198 & 00 & 3.3500 & 280.6 & 5 & 0 & \\
\hline & 2199 & 002 & 2.9000 & 281.7 & 4 & 0 & \\
\hline & 21910 & & 3.4250 & & 4 & & \\
\hline & 21911 & 000 & 3.9000 & 284.4 & 4 & 600.0 & \\
\hline & 21912 & 968 & 2.8000 & 285.6 & 3 & 0 & \\
\hline 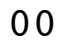 & 21913 & 72 & 2.4000 & 286.1 & 2 & 0 & \\
\hline$v$ & 21914 & & 2.3250 & 285.8 & 3 & 600.0 & \\
\hline & 21915 & 883 & 2.1250 & 285.8 & 3 & 0 & \\
\hline & 21916 & 00 & 2.9000 & 285.2 & 4 & 60 & \\
\hline 0 & 21917 & 500 & 3.7500 & 284.8 & 4 & 600.0 & \\
\hline & 21918 & 315.3276 & 2.7000 & 284.6 & 4 & 600.0 & \\
\hline 8 & 21919 & 034 & 2.0250 & 284 & 5 & 600 & \\
\hline 0 & 21920 & 480 & 1.3250 & 284.5 & 6 & 600.0 & \\
\hline & 21921 & 1080 & 0.9500 & 285.1 & 6 & 600.0 & \\
\hline & & & 50 & & 5 & & \\
\hline & 1923 & 2429 & 3.3250 & 286.8 & 4 & 600.0 & 60 \\
\hline
\end{tabular}




\begin{tabular}{|c|c|c|c|c|c|c|c|}
\hline & 24 & & & & & & \\
\hline 0 & 201 & & 1250 & 6.7 & & & \\
\hline & 2202 & 6.3239 & 2.1750 & 85.6 & & 00 & \\
\hline & 203 & 77.9779 & 1.3000 & 284.1 & & 00 & \\
\hline & 204 & 584 & 42 & & & & \\
\hline & 2205 & 7 & 42 & 84 & & 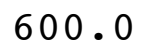 & \\
\hline & 2206 & L1.5999 & 9750 & 285.4 & & 00 & \\
\hline & 2207 & 337 & & & & & \\
\hline & 2208 & 5445 & 0 & 9 & & & \\
\hline & 2209 & 08.9897 & 4.3500 & 84.9 & & 0 & \\
\hline & 2010 & 0003 & .70 & $28 \varepsilon$ & & & \\
\hline & 22011 & 7.2522 & .55 & 6 & & & \\
\hline 0 & 22012 & 41.7497 & 0 & 90.1 & & & \\
\hline 0 & 22013 & 40.7499 & 11.20 & 5 & & & \\
\hline & 22014 & 995 & & & & & \\
\hline & 22015 & 85 & & 28 & & & \\
\hline & 22016 & 7492 & 9 . & 287.2 & & .0 & \\
\hline & 22017 & 041 & 0 & 5 & & & \\
\hline & 22018 & 506 & & 0 & & & \\
\hline & 22019 & 341 & 0 & & & & \\
\hline 0 & 22020 & 26.5678 & 5 . & 282.0 & & . 0 & \\
\hline 0 & 22021 & 356.5031 & 0 & & & & \\
\hline 0 & 22022 & 288 & 0 & 282 & & & \\
\hline 0 & 22023 & 157.4151 & 0 & 2 & & .0 & \\
\hline & 22024 & 275 & & & & & \\
\hline 0 & 2211 & 29 & & & & & \\
\hline & 2212 & .7496 & & 1 & 4 & .0 & \\
\hline & 2213 & 292.5024 & & 3 & 4 & & \\
\hline 0 & 2214 & 496 & 0 & 27 & 4 & & \\
\hline & 2215 & 320.9995 & & 279.7 & 4 & & \\
\hline 0 & 2216 & 328.5002 & 00 & 280.1 & 4 & & \\
\hline 0 & 2217 & 2 & & & 4 & & \\
\hline & 2218 & & & 4 & 4 & & \\
\hline 0 & 2219 & 338 . & & 282.8 & 4 & 0 & \\
\hline ח & 22110 & & & & 4 & & \\
\hline 0 & 22111 & & & & 4 & & \\
\hline & 22112 & 305.3958 & 500 & 286.4 & 3 & & \\
\hline 0 & 22113 & 760 & & & 4 & & \\
\hline 0 & 22114 & 301.4960 & & 286.8 & 4 & .0 & \\
\hline & 22115 & .9722 & 2000 & 287.7 & 4 & & \\
\hline 0 & 22116 & 080 & 500 & 287.3 & 3 & & \\
\hline 0 & 22117 & 306 . & & & 3 & & \\
\hline & 22118 & 326.0037 & 4.7000 & 286.7 & 4 & & 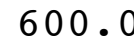 \\
\hline 00 & 22119 & 501 & 2 . & 28 & 4 & & \\
\hline 00 & 22120 & 341.7495 & & & $\Psi$ & .0 & 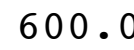 \\
\hline 00 & 22121 & 24.4699 & 3.5500 & 284.8 & 4 & .0 & 00 \\
\hline & 22122 & & & & 4 & & \\
\hline & 22123 & 75.7521 & 6.0000 & 284.0 & & .0 & 600 \\
\hline
\end{tabular}




\begin{tabular}{|c|c|c|c|c|c|c|c|}
\hline 0 & 2124 & 6.0000 & 6.3500 & 283.2 & 4 & 000.0 & \\
\hline & 2221 & 62.2498 & 5.9750 & 283.3 & 4 & 600.0 & \\
\hline & 2222 & 50.7183 & 4750 & 282.9 & 4 & 00 & \\
\hline & 2223 & 348.9874 & 1.8500 & 282.9 & & 00 & \\
\hline & 2224 & 8166 & .7500 & 33.1 & & 0 & \\
\hline & 2225 & 343.7489 & .5500 & 83.2 & & 00 & \\
\hline & 2226 & 352.2020 & 1000 & 283.3 & & 0 & \\
\hline & 2227 & 327.9176 & .9500 & 33.6 & & & \\
\hline & 2228 & 320.2493 & 2.6250 & 283.2 & & 0 & \\
\hline & 2229 & 16.7379 & 250 & 284.1 & & 0 & \\
\hline & 22210 & 342.0717 & 1.8000 & 284.5 & & 0 & \\
\hline & 22211 & 346.2931 & 2.8500 & 84.7 & & & \\
\hline & 22212 & 351.7556 & 4.8250 & 283.5 & 3 & 0 & \\
\hline & 22213 & 346.8093 & 5.8250 & 281.9 & 4 & 0.0 & \\
\hline & 22214 & 323.7505 & & 81.8 & 4 & & \\
\hline & 22215 & 323.7499 & 4.0250 & 281.5 & 4 & & \\
\hline & 22216 & 343.0021 & 6000 & 281.7 & 3 & 0 & \\
\hline & 22217 & 300.3026 & 000 & 1.9 & 3 & & \\
\hline 0 & 22218 & 327.7503 & 250 & 282.3 & 4 & & \\
\hline & 22219 & 327.0000 & 9000 & 281.8 & 4 & & \\
\hline & 22220 & 328.0006 & 000 & 1.0 & 4 & & \\
\hline & 22221 & 329.7500 & 50 & 1.1 & 4 & & \\
\hline 0 & 22222 & 24.9705 & 500 & 280.8 & 4 & 0 & 0 • \\
\hline & 22223 & 75.7483 & 2000 & 9.0 & 4 & 0 & \\
\hline & 22224 & 40.2403 & 50 & 278.9 & 4 & & \\
\hline & 2231 & 113.4331 & 500 & 278.8 & 5 & & \\
\hline & 2232 & 38.8309 & 1.9500 & 279.3 & 6 & & \\
\hline 0 & 2233 & 107.3078 & 0 & 279.1 & 5 & & \\
\hline 0 & 2234 & 84.1605 & 0 & 27 & 4 & & 0 . \\
\hline & 2235 & 80.7378 & 500 & 279.5 & 4 & & \\
\hline & 2236 & 90.000 & 0 & 279 & 5 & & \\
\hline & 2237 & 94 & 50 & 27 & 4 & & \\
\hline & 2238 & 90.7473 & 250 & 279.0 & 4 & 0 & 0 . \\
\hline & 2239 & 110.2427 & 0 & $2 \varepsilon$ & 3 & & \\
\hline & 22310 & 126.2463 & 50 & $2 \varepsilon$ & 3 & & \\
\hline & 22311 & 119.5000 & 750 & 281.0 & 3 & & \\
\hline & 22312 & 69.2714 & 000 & 281.8 & 2 & & \\
\hline 0 & 22313 & 109.4758 & 500 & 281.1 & 3 & & \\
\hline & 22314 & 74.3538 & 9000 & 278.6 & 2 & 0 & 0 . \\
\hline & 22315 & 97.4824 & 500 & 280.5 & 2 & & \\
\hline & 22316 & 104.7256 & 0 & & 3 & & \\
\hline 00 & 22317 & 83.8285 & 2.7500 & 280.2 & 2 & 600.0 & 00 . \\
\hline & 22318 & 88.9721 & 2750 & 280.2 & 3 & 600 & a \\
\hline & 22319 & 91.7494 & 5.1250 & 279.9 & 4 & & $\cos 20$ \\
\hline 0 & 22320 & 85.4981 & 5.6750 & 279.4 & 4 & 600.0 & 00 \\
\hline & 22321 & 70.2497 & 5.9000 & 278.9 & 4 & 600 & 50 \\
\hline & 22322 & 74.2489 & & 27 & 4 & 60 & \\
\hline & 22323 & 71.9866 & 6.1500 & 278.6 & 4 & 600.0 & 600 \\
\hline
\end{tabular}




\begin{tabular}{|c|c|c|c|c|c|c|c|}
\hline 0 & 22324 & 63.5000 & 6.8250 & 278.5 & 4 & 600.0 & 600.0 \\
\hline 00 & 2241 & 92.2477 & 3.4250 & 278.3 & 5 & 600.0 & 600.0 \\
\hline 00 & 2242 & 88.7853 & 2.9000 & 278.1 & 5 & 600.0 & 00.0 \\
\hline 0 & 2243 & 63.5000 & 5.9250 & 278.2 & 4 & 600.0 & 00.0 \\
\hline 00 & $224 \quad 4$ & 80.2460 & .2000 & 278.1 & 4 & 600.0 & 00.0 \\
\hline 00 & 2245 & 71.7473 & 4.5000 & 278.0 & 4 & 600.0 & 00.0 \\
\hline 00 & 2246 & 58.4991 & 5.3250 & 277.9 & 4 & 600.0 & 00.0 \\
\hline 00 & 2247 & 54.5000 & 5.2000 & 277.9 & 4 & 600.0 & 00.0 \\
\hline 00 & 2248 & 48.5000 & 4.8250 & 278.2 & 4 & 600.0 & 00.0 \\
\hline 00 & 2249 & 49.0000 & 4.2250 & 279.1 & 3 & 600.0 & 0 \\
\hline 00 & 22410 & 42.5133 & 2.6750 & 280.2 & - & 600.0 & 00.0 \\
\hline 00 & 22411 & 301.3208 & 2.2750 & 280.9 & 1 & 600.0 & \\
\hline 00 & 22412 & 259.7511 & 3.3500 & 280.4 & 2 & 600.0 & 00.0 \\
\hline 00 & 22413 & 233.2354 & 2.6000 & 280.5 & 2 & 600.0 & 00.0 \\
\hline 00 & 22414 & 251.7186 & 1.8750 & 280.9 & 1 & .0 & \\
\hline 00 & 22415 & 269.4297 & 2.1250 & 281.1 & 1 & 600.0 & 00.0 \\
\hline 00 & 22416 & 249.5504 & 2.3750 & 281.1 & 1 & 600.0 & 600.0 \\
\hline 00 & 22417 & 324.4336 & 2.5500 & 280.1 & 2 & .0 & \\
\hline 00 & 22418 & 350.7049 & 2.1250 & 279.3 & 3 & 600.0 & .0 \\
\hline 00 & 22419 & 341.4969 & 5.4500 & 278.6 & 4 & .0 & .0 \\
\hline 00 & 22420 & 315.2315 & 4.62 & 278.1 & 4 & 60 & \\
\hline 00 & 22421 & 304.4410 & 3.8250 & 278.0 & 4 & .0 & \\
\hline 00 & 22422 & 325.0023 & 3.7750 & 277.7 & 5 & 600.0 & .0 \\
\hline 00 & 22423 & 308.3530 & 2.37 & 278.0 & 5 & 60 & .0 \\
\hline 00 & 22424 & 298.7976 & 3.10 & 278.3 & 4 & .0 & \\
\hline 00 & 2251 & 318.0002 & 3.9000 & 278.0 & 4 & 600.0 & 600.0 \\
\hline 00 & 2252 & 319.9996 & 2.8750 & 278.0 & 4 & 600.0 & .0 \\
\hline 00 & 2253 & 309.9755 & 2.7250 & 277.9 & 4 & .0 & \\
\hline 00 & 2254 & 342.8118 & 2.0250 & 278.1 & 4 & .0 & 0.0 \\
\hline 00 & $225 \quad 5$ & 355.8020 & 0.9750 & 278.3 & 5 & .0 & .0 \\
\hline 00 & 2256 & 60.2151 & 1.1750 & 278.5 & 6 & .0 & \\
\hline 00 & 2257 & 174.8849 & 1.4500 & 278.1 & 6 & .0 & 600.0 \\
\hline 00 & 2258 & 195.6957 & 0.8750 & 277.7 & 6 & 600.0 & 600.0 \\
\hline 00 & 2259 & 110.0713 & 0.7750 & 279.7 & 5 & 600.0 & 0.0 \\
\hline 00 & 22510 & 231.3219 & 0.9250 & 282.7 & 4 & 600.0 & 600.0 \\
\hline 00 & 22511 & 223.9464 & 2.2250 & 282.9 & 3 & 600.0 & 600.0 \\
\hline 00 & 22512 & 252.8676 & 2.47 & 282.7 & 2 & 600.0 & 600.0 \\
\hline 00 & 22513 & 244.2618 & 2.1000 & 283.2 & 1 & 600.0 & 600.0 \\
\hline 00 & 22514 & 256.2388 & 2.2250 & 283.5 & 1 & 600.0 & 600.0 \\
\hline 00 & 22515 & 247.4717 & 2.3000 & 283.8 & 2 & 600.0 & 600.0 \\
\hline 00 & 22516 & 259.4227 & 2.3000 & 283.9 & 2 & 600.0 & 600.0 \\
\hline 00 & 22517 & 274.2696 & 2.1750 & 283.4 & 3 & 600.0 & 600.0 \\
\hline 00 & 22518 & 294.1320 & 2.1000 & 283.0 & 4 & 600.0 & 600.0 \\
\hline 00 & 22519 & 26.1240 & 0.9500 & 282.7 & 5 & 600.0 & 600.0 \\
\hline 00 & 22520 & 33.0004 & 2.2000 & 283.2 & 4 & 600.0 & 600.0 \\
\hline 00 & 22521 & 70.5284 & 3.5000 & 283.5 & 4 & 600.0 & 600.0 \\
\hline 00 & 22522 & 53.2556 & 3.825 & 283.3 & 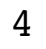 & 600.0 & 600.0 \\
\hline 00 & 22523 & 322.7919 & 0.750 & 283.4 & $J$ & 600.0 & 600.0 \\
\hline
\end{tabular}




\begin{tabular}{|c|c|c|c|c|c|c|c|}
\hline 0 & 2524 & & 2250 & 0 & & & \\
\hline & 2261 & 0 & 1.2500 & 3.3 & & $\Leftrightarrow \cap 00$ & \\
\hline & 2262 & 337.2558 & 9000 & 283.6 & 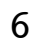 & 00 & \\
\hline & 2263 & 316.2295 & .4500 & 283.2 & & 00.0 & \\
\hline & 264 & 317.6938 & 9250 & 33.1 & & 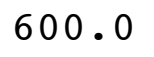 & \\
\hline & 2265 & 311.3087 & 1500 & 83.2 & & 0 & \\
\hline & 2266 & 140.2545 & 9000 & 83.1 & & 00 & \\
\hline & 2267 & 135.2403 & 5000 & 83.2 & & & \\
\hline & 2268 & 120.7630 & 0250 & 83.1 & & & \\
\hline & 2269 & 164.1449 & 1.1250 & 84.8 & & 00 & 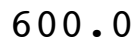 \\
\hline & 2610 & 244.2740 & 0.9500 & 286.7 & & & \\
\hline & 22611 & 253.7772 & 2.22 & 37.0 & & & \\
\hline & 22612 & 236.6037 & 2.2750 & 286.9 & & 00.0 & \\
\hline & 22613 & 210.9034 & 2.35 & 288.8 & & 0.0 & 00 \\
\hline & 22614 & 155.5018 & 0 & & & & \\
\hline & 22615 & 161.9251 & 4 . & 288.3 & & & \\
\hline & 22616 & 158.8476 & 3.00 & 288.0 & & .0 & . \\
\hline & 2617 & 324.3964 & 4 . & 5 & & 0 & \\
\hline & 22618 & 13.8644 & 2 . & 288.7 & & & \\
\hline & 22619 & 291.7911 & 1.4500 & 286.4 & 5 & 0 & U \\
\hline & 22620 & 28.8645 & 2.52 & 286.6 & 6 & .0 & 00 \\
\hline & 22621 & 335.1208 & 277 & & & & \\
\hline & 22622 & 345.5000 & 7.50 & 287.5 & 4 & & \\
\hline & 22623 & 350.7774 & 2.2500 & 287.1 & 5 & . 0 & . \\
\hline & 22624 & 10.4889 & 125 & & 4 & & \\
\hline & 2271 & 8.2515 & 7.72 & 284.3 & 4 & & \\
\hline & 2272 & 15.5008 & 0750 & 2 & 4 & 0 & U. \\
\hline & 2273 & 8.7501 & 0 & 7 & 4 & & \\
\hline & 2274 & 12.4966 & 925 & 28 & 4 & & • \\
\hline & 2275 & 7.1165 & 0 & 284.3 & 4 & 0 & 0 • \\
\hline & 2276 & 0.5000 & 3.3250 & 284.2 & 5 & .0 & 0 . \\
\hline & 2277 & 96.5891 & 167 & 283.5 & 6 & & \\
\hline & 2278 & 79.1148 & 2.4000 & 282.5 & 5 & & . \\
\hline & 2279 & 340.1960 & 1.0000 & 281.9 & 4 & 0 & 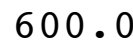 \\
\hline & 22710 & 15.1520 & 2 . & & 3 & & \\
\hline & 22711 & 63.3894 & 4.8750 & 2.6 & 3 & & . \\
\hline & 22712 & 40.4997 & .7000 & 283.9 & 4 & .0 & . \\
\hline 0 & 22713 & 51.1966 & 0 & 28 & 4 & & - \\
\hline ( & 22714 & 70.9339 & 7.1500 & 283.0 & 4 & .0 & 0 . \\
\hline & 22715 & 73.4149 & 3000 & 281.8 & 4 & & . \\
\hline & 22716 & 63.9992 & 750 & 282.3 & 4 & & 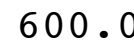 \\
\hline & 22717 & 84.5014 & 6.7750 & 282.4 & 4 & & 0 . \\
\hline & 22718 & 66.7502 & 8.9500 & 282.0 & 4 & 0 & 00 . \\
\hline & 22719 & 79.7694 & 5500 & 28 & 4 & & 0 \\
\hline 00 & 22720 & 71.2483 & 4.9500 & & 4 & & 0 . \\
\hline & 22721 & 70.7223 & 6.0000 & 280.8 & 4 & 600.0 & 600. \\
\hline & 22722 & 8000 & & & 4 & & \\
\hline & 22723 & 71.2429 & 5.1750 & 280.7 & $\mathbf{I}$ & 600.0 & 600 \\
\hline
\end{tabular}




\begin{tabular}{|c|c|c|c|c|c|c|c|}
\hline & 22724 & 8.5006 & 5.2750 & 280.8 & 4 & 600.0 & \\
\hline U & 2281 & 74.9991 & 7.0250 & 280.4 & 4 & 00.0 & \\
\hline & 2282 & 90.2455 & 500 & 280.6 & 4 & 0 & \\
\hline & 2283 & 1.2511 & .8000 & 280.7 & & 0 & \\
\hline & 2284 & 79.7501 & 0750 & 280.6 & & 00 & \\
\hline & 2285 & 77.9999 & 500 & 280.5 & & 0 & \\
\hline & 2286 & 91.7494 & & 280.3 & & & \\
\hline & 2287 & 89.7508 & & 80.1 & & & \\
\hline & 2288 & 88.0037 & 4.7250 & 280.2 & & 00.0 & 00 \\
\hline & 2289 & 92.0030 & 0 & 281.2 & & & \\
\hline & 22810 & 69.0011 & & 82.1 & & & \\
\hline & 22811 & 62.4997 & & 282.0 & & 0 & \\
\hline & 22812 & 66.7499 & & 283.1 & & & \\
\hline & 22813 & 67.2487 & & 83.4 & & & \\
\hline & 22814 & 28.4359 & & 283.8 & & & \\
\hline & 22815 & 279.7533 & 0 & 284.2 & & & \\
\hline & 22816 & 306.6723 & 2.27 & 283.5 & 1 & & \\
\hline & 22817 & 85.7506 & & 83.6 & 2 & & \\
\hline & 22818 & 75.2497 & 0 & 283.1 & 3 & 0 & 600. \\
\hline & 22819 & 77.9952 & 4.55 & 282.1 & 4 & & \\
\hline & 22820 & 76.2501 & & 281.8 & 4 & & \\
\hline & 22821 & 74.5000 & 0 & 281.8 & 4 & & \\
\hline & 22822 & 77.7500 & .83 & 281.7 & 4 & 0 & T \\
\hline & 22823 & 76.2500 & & 281.3 & 4 & & $\bullet$ \\
\hline & 22824 & 78.4908 & 0 & 281.2 & 4 & & \\
\hline & 2291 & 85.0000 & 4.85 & 281.0 & 4 & & ת \\
\hline & 2292 & 2.2546 & 0 & 0.1 & 4 & & \\
\hline & 2293 & 327.0000 & 4 . & 279.5 & 4 & & \\
\hline & 2294 & 296.7105 & 0 & 279.3 & 5 & & 0 . \\
\hline & 2295 & 331.2493 & & 278.9 & 4 & & \\
\hline & 2296 & 329.7501 & 0 & 279.6 & 4 & & \\
\hline & 2297 & 323.7505 & & 280.2 & 4 & & \\
\hline & 2298 & 320.9994 & 4.50 & 280.0 & 4 & 0 & \\
\hline 0 & 2299 & 307.4986 & 0 & 280.7 & 4 & & \\
\hline & 22910 & 309.4350 & & 281.0 & 3 & & \\
\hline & 22911 & 144.5615 & 3 . & 280.5 & 2 & & \\
\hline 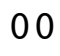 & 22912 & 81.2441 & 4 . & 281.5 & 3 & & \\
\hline 0 & 22913 & 102.9950 & 5. & 282.1 & 4 & 0 & 00 . \\
\hline & 22914 & 76.5000 & & 283.7 & 3 & 0 & \\
\hline & 22915 & 74 . & 0 & & 3 & & \\
\hline & 22916 & 82.5107 & 5.72 & 283.6 & 3 & & 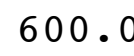 \\
\hline 0 & 22917 & 85.7506 & 5.3000 & 283.6 & 3 & 0 & م0 \\
\hline 0 & 22918 & 75.2497 & 4.31 & 283.1 & 4 & 0 & \\
\hline 0 & 22919 & 77.9952 & 4.5500 & 282.1 & 4 & 600.0 & 600 \\
\hline 0 & 22920 & 76.2501 & 5.3750 & 281.8 & 4 & 600.0 & 500 . \\
\hline & 22921 & 74 & & 281.8 & 4 & & \\
\hline & 22922 & 77.7500 & & 281.7 & 4 & & 600 \\
\hline & 2923 & 76.2500 & 6.4000 & 281.3 & 4 & 600.0 & 60 \\
\hline
\end{tabular}




\begin{tabular}{|c|c|c|c|c|c|c|c|}
\hline 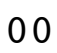 & 4 & 908 & 6.1000 & 81.2 & 4 & 600.0 & \\
\hline & $\begin{array}{lll}3 & 1 & 1\end{array}$ & 1.2482 & 5.0500 & 281.6 & 4 & 600.0 & \\
\hline & $\begin{array}{lll}3 & 1 & 2\end{array}$ & 121.0251 & 4.1500 & 282.0 & 4 & 00 & \\
\hline & $\begin{array}{lll}3 & 1 & 3\end{array}$ & 31.0000 & 0250 & 281.6 & & 00 & \\
\hline & $\begin{array}{lll}3 & 1 & 4\end{array}$ & 17.9729 & .6500 & 81.4 & & ac & \\
\hline & $\begin{array}{lll}3 & 1 & 5\end{array}$ & 124.7501 & 000 & 81.2 & & 0( & \\
\hline & $\begin{array}{lll}3 & 1 & 6\end{array}$ & 141.2494 & 1250 & 280.7 & & 00 & \\
\hline & $\begin{array}{lll}3 & 1 & 7\end{array}$ & 133.7441 & 2750 & 80.2 & & 0 & \\
\hline & $\begin{array}{lll}3 & 1 & 8\end{array}$ & 122.0107 & .4250 & 80.7 & & & \\
\hline & $\begin{array}{lll}3 & 1 & 9\end{array}$ & 153.7583 & 250 & 81.4 & 4 & 0 & U \\
\hline & 3110 & 155.0012 & 4.7750 & 281.6 & & 00.0 & \\
\hline & 3111 & 162.2518 & 3.6750 & 82.6 & & & \\
\hline & 112 & 162.2369 & 3 . & 283.2 & & 0 & \\
\hline & 3113 & 143.9353 & 3.4000 & 284.1 & & 0.0 & \\
\hline & 3114 & 147.7371 & & .2 & & & \\
\hline & 3115 & 158.7498 & & 285.5 & 2 & & \\
\hline & 3116 & 159.0002 & 3.7250 & 285.8 & 3 & .0 & \\
\hline & 3117 & 164.0145 & 000 & 5.7 & 3 & 0 & \\
\hline & 3118 & 180.2894 & 1 & 284.8 & 4 & & \\
\hline & 3119 & 303.4878 & 000 & 284.0 & 5 & 0 & 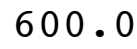 \\
\hline & 3120 & 37.8786 & 1.8000 & 283.9 & 5 & 0 & \\
\hline & 3121 & 64.7569 & 21 & 3.5 & 4 & & \\
\hline & 3122 & 78.5043 & 000 & 283.6 & 5 & & \\
\hline & 3123 & 54.4945 & 4.9000 & 283.3 & 4 & 0 & \\
\hline & 3124 & 66.9986 & & 282.4 & 4 & & \\
\hline & $\begin{array}{lll}3 & 2 & 1\end{array}$ & 63.0003 & 500 & 281.3 & 4 & & \\
\hline & 322 & 59.5002 & 0 & 281.9 & 4 & 0 & J. \\
\hline & 323 & 47.143 & 0 & 32.1 & 4 & & \\
\hline & 324 & 0.4968 & 0 & 281.8 & 4 & & 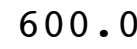 \\
\hline & 325 & 322.4284 & 2.7250 & 282.1 & 4 & 0 & 0 . \\
\hline & 326 & 5.5704 & 2.4750 & 281.5 & 5 & & 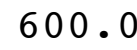 \\
\hline & 327 & 93.3993 & 0 & 280.6 & 4 & & \\
\hline & 328 & 87.2502 & 250 & 280.3 & 4 & & . \\
\hline & 329 & 79.0000 & 750 & 280.5 & 4 & 0 & 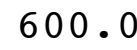 \\
\hline & 3210 & 97.7460 & 0 & 281.3 & 4 & & \\
\hline & 3211 & 125.7722 & 2.8500 & 281.9 & 3 & & . \\
\hline & 3212 & 201.6293 & 1.7750 & 280.9 & 2 & & . \\
\hline & 3213 & 213.1778 & 2.4250 & 280.6 & 2 & & ( \\
\hline - & 3214 & 154.5102 & 3.1250 & 280.5 & 3 & .0 & 0 . \\
\hline & 3215 & 116.2069 & 2.0750 & 281.1 & 2 & & \\
\hline & 3216 & 143.4515 & 500 & 281.1 & 3 & & 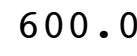 \\
\hline & 3217 & 115.5101 & 3.8250 & 280.8 & 2 & & 0 . \\
\hline & 3218 & 122.6890 & 3.4500 & 281.7 & 3 & 0 & 0 . \\
\hline 0 & 3219 & 149.5000 & 000 & 280.9 & 4 & & \\
\hline 00 & 3220 & 152.5002 & 5.1250 & 280.5 & 4 & & 0 . \\
\hline & 3221 & 152.2508 & 6.0750 & 280.1 & 4 & 600.0 & 600. \\
\hline & 3222 & 156. & & & 4 & & \\
\hline & 223 & 146.0002 & 4.6250 & 279.9 & 4 & 600.0 & 600 \\
\hline
\end{tabular}




\begin{tabular}{|c|c|c|c|c|c|c|c|}
\hline 0 & 24 & & 6000 & 279.6 & 4 & 000.0 & \\
\hline 0 & $\begin{array}{lll}3 & 3 & 1\end{array}$ & 150.2494 & 5.4000 & 279.4 & & 00.0 & \\
\hline 0 & 332 & 140.2503 & 5.5500 & 279.7 & & 00 & \\
\hline 0 & $\begin{array}{lll}3 & 3 & 3\end{array}$ & 78.3367 & 1500 & 280.0 & & 00.0 & \\
\hline 0 & 334 & 120.2631 & 3500 & 80.5 & & & \\
\hline 0 & 335 & 120.0751 & 3000 & 280.6 & & 00 & \\
\hline 0 & 336 & 080 & 0000 & 281.4 & & 00 & \\
\hline 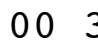 & 337 & 207 & 1500 & 81.8 & & 00.0 & \\
\hline 0 & 338 & 060 & 7250 & 82.2 & & & \\
\hline 0 & 339 & 29.9612 & 5.0500 & 283.1 & & 00.0 & 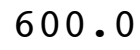 \\
\hline 0 & 3310 & 140.5097 & 9500 & 283.5 & & & \\
\hline 0 & 3 & 6.4962 & .62 & 83.9 & & & \\
\hline 0 & 3 & 5013 & 5.35 & 284.9 & 3 & .0 & \\
\hline 0 & 13 & 9989 & 5.5000 & 285.7 & & 0.0 & \\
\hline 0 & 3314 & 021 & & 28 & & & \\
\hline 0 & 3 & 523 & & 286 & 3 & & \\
\hline 0 & 16 & 7489 & 5.20 & 287.0 & a & .0 & \\
\hline 0 & 3317 & 81 & 4 . & 287.7 & & .0 & \\
\hline 0 & 18 & 8 & & 287.3 & 4 & & \\
\hline 0 & 19 & 10 & 50 & 286.0 & 4 & .0 & \\
\hline 0 & 3320 & 72.2499 & 6.8500 & 285.1 & 4 & .0 & 0 \\
\hline 0 & $3 \quad 321$ & 70.24 & & 284.6 & 4 & & \\
\hline 0 & 22 & 84 & 750 & 285.0 & 4 & .0 & \\
\hline 0 & $3 \quad 323$ & 81.0000 & 750 & 285.2 & 4 & .0 & \\
\hline 3 & $3 \quad 324$ & 1 & & 28 & 4 & & \\
\hline 003 & $\begin{array}{lll}3 & 4 & 1\end{array}$ & 51.31 & 2 . & 285 & 4 & & \\
\hline 0 & 342 & 81.2501 & 7000 & 284.2 & 4 & .0 & \\
\hline 3 & 343 & 500 & 4500 & 282.6 & 4 & .0 & \\
\hline 00 & $\begin{array}{lll}3 & 4 & 4\end{array}$ & 73 & 7 . & 282.8 & 4 & .0 & \\
\hline 0 & 345 & 4 & 4 . & 282.9 & 4 & .0 & \\
\hline 0 & 346 & 64.6214 & 3.3500 & 282.8 & 4 & .0 & \\
\hline 0 & 347 & 16.32 & 1.7250 & 282.2 & 5 & & \\
\hline 0 & 348 & 117 & 250 & 283.6 & 4 & .0 & \\
\hline 0 & $\begin{array}{lll}3 & 4 & 9\end{array}$ & 85.4783 & 6.4750 & 282.2 & 4 & .0 & \\
\hline 2 & 3410 & 1 & & $2 \varepsilon$ & 3 & & \\
\hline 0 & 3411 & 3 & 2 . & 286.1 & 2 & .0 & \\
\hline 3 & 3412 & 239.4664 & 2.2000 & 286.8 & 1 & .0 & \\
\hline 3 & 3413 & 19 & 500 & 286.3 & 1 & .0 & \\
\hline 00 & 3414 & 148 . & 2.7000 & 287.4 & 1 & .0 & 60 \\
\hline 3 & 3415 & 100.0970 & 2.9750 & 288.2 & 1 & .0 & \\
\hline 3 & 3416 & 433 & 5.2000 & 287.5 & 2 & & \\
\hline 00 & 3417 & 2438 & 5.0750 & 286.1 & 3 & .0 & 600 \\
\hline 0 & 3418 & 66.2550 & 5.3250 & 285.1 & 4 & .0 & 600 \\
\hline 00 & 3419 & 21.5579 & 3.4250 & 285.4 & 4 & & \\
\hline 00 & 3420 & 336.4826 & 2.7750 & 285.0 & 2 & .0 & \\
\hline 00 & 3421 & 330.5045 & 2.6250 & 284.8 & 4 & 600.0 & 600 \\
\hline 3 & 3422 & 32 & & .3 & 5 & & \\
\hline & 3423 & 305.9708 & 0.8750 & 283.4 & & .0 & 600 \\
\hline
\end{tabular}




\begin{tabular}{|c|c|c|c|c|c|c|c|c|}
\hline 0 & 3 & 24 & 1. & 1.0250 & 283.3 & 6 & . & \\
\hline 0 & 3 & 51 & 336.7556 & 1.2750 & 283.2 & & P & \\
\hline & 3 & 52 & 337.0851 & 1.7500 & 283.1 & 5 & 00 & \\
\hline & 2 & 3 & 69.0740 & 6.5500 & 281.4 & & 00 & \\
\hline & 3 & 4 & 498 & 00 & & & 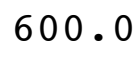 & \\
\hline & 3 & 5 & 53.0000 & 9.5000 & 80.4 & 4 & 00 & \\
\hline & 3 & 6 & 5000 & 1.0750 & 279.9 & & 0 & \\
\hline & 3 & 7 & 45.0092 & 9.4250 & 79.7 & & & \\
\hline & 3 & 8 & 40.7504 & 7.7750 & .79 .5 & 4 & 0 & \\
\hline & 3 & 9 & 9993 & 4.9250 & 279.8 & & 0 & \\
\hline & 3 & 10 & 39.9471 & 3.5250 & 279.8 & & 0 & \\
\hline & 3 & 1 & 27.7828 & 0 & 79.9 & & & \\
\hline & 3 & 12 & 66.9296 & 3.7500 & 279.9 & & 0 & \\
\hline & 3 & 13 & 44.9956 & 4.7250 & 280.4 & $3 .>3$ & 0.0 & \\
\hline & 3 & 14 & & $6 \quad 17$ & & & & \\
\hline & 3 & 15 & 44 . & 7.3250 & 280.4 & 4 & & \\
\hline & 3 & 16 & 45.2448 & 7.2000 & 280.8 & 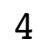 & & \\
\hline & 3 & 517 & 36.2500 & 4 . & 0 & 3 & & \\
\hline & 3 & 18 & 46.2350 & 750 & 280.6 & 4 & 0 & \\
\hline & 3 & 19 & 19.7476 & 0 & 280.1 & 5 & & \\
\hline & 3 & 20 & 19.1839 & 0 & 8 & 4 & & \\
\hline & 3 & 521 & 330 . & & 279.8 & 5 & & \\
\hline & 3 & 522 & 201.3960 & 000 & 279.0 & 4 & & • \\
\hline & 3 & 523 & 163.2852 & 3.40 & 278.6 & 4 & & \\
\hline & 3 & 524 & 139.4839 & & 278.4 & 5 & & \\
\hline & 3 & 61 & 26.7282 & 0 & 278.3 & 4 & & \\
\hline & 3 & 62 & 357.5502 & 1.7250 & 278.5 & 4 & & \\
\hline 0 & 3 & 63 & 323 . & 1. & 27 & 5 & & \\
\hline 0 & 3 & 64 & 333. & 1.4750 & 278.0 & 6 & & \\
\hline & 3 & 65 & 350.3706 & 500 & 277.8 & 6 & & \\
\hline & 3 & 66 & 223.7585 & 0 & 277 & 6 & & \\
\hline & 3 & 67 & 348 . & 0 & .7 & 6 & & \\
\hline & 3 & 68 & 19.5185 & 750 & 278.2 & 5 & 0 & 0 . \\
\hline & 3 & 69 & 251.1673 & 1. & 279.1 & 4 & & \\
\hline & 3 & 610 & 336 . & 0 & 28 & 3 & & \\
\hline & 3 & 611 & 182.2657 & 500 & 281.5 & 2 & & \\
\hline & 3 & 612 & 224 . & 50 & .5 & 2 & & \\
\hline 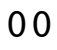 & 3 & 613 & 283.6876 & 2 . & 282.2 & 2 & & 0 . \\
\hline & 3 & 614 & 351.5059 & 250 & 282.9 & 1 & 0 & 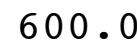 \\
\hline & 3 & 615 & 221 . & 000 & 282.2 & 2 & & \\
\hline & 3 & 616 & 255 . & 000 & & 1 & & \\
\hline 0 & 3 & 617 & 341.0000 & 2.2250 & 281.8 & 2 & 600.0 & 00 \\
\hline & 3 & 618 & 324.2542 & 3750 & 282.0 & 3 & 0 & \\
\hline & 3 & 619 & 334.8588 & 2.0000 & 281.1 & 4 & & \\
\hline 00 & 3 & 620 & 74.0884 & 2.9250 & 281.4 & 5 & 60 & 0 \\
\hline & 3 & 621 & 81.4923 & 5.7250 & 280.7 & 4 & 600.0 & 60 \\
\hline & 3 & 622 & 501 & 0 & & 4 & 600 & \\
\hline & & 623 & 67.7496 & 6.6000 & 280.0 & 4 & 600.0 & 600 \\
\hline
\end{tabular}




\begin{tabular}{|c|c|c|c|c|c|c|c|c|}
\hline 0 & 3 & 624 & 69.7502 & 6.9250 & 279.9 & 4 & 600.0 & 600.0 \\
\hline 00 & 3 & 71 & 77.2501 & 7.2500 & 279.7 & 4 & 600.0 & 00.0 \\
\hline 00 & 3 & 72 & 72.5000 & .3500 & 279.4 & 4 & 600.0 & 00.0 \\
\hline 00 & 3 & 73 & 68.7548 & 3.9500 & 279.4 & 4 & 600.0 & 00.0 \\
\hline 00 & 3 & 74 & 50.5569 & .8750 & 279.1 & 5 & 600.0 & 00.0 \\
\hline 00 & 3 & 75 & 312.2923 & 0.3750 & 278.8 & 6 & 600.0 & 00.0 \\
\hline 00 & 3 & 76 & 321.7787 & 1.0000 & 278.8 & 6 & 600.0 & 00.0 \\
\hline 00 & 3 & 77 & 331.9985 & 1.2750 & 278.3 & 5 & 600.0 & 00.0 \\
\hline 00 & 3 & 78 & 3.4982 & 1.0500 & 278.7 & 4 & 600.0 & 00.0 \\
\hline 00 & 3 & 79 & 19.0219 & 1.3000 & 279.9 & 4 & 600.0 & 0 \\
\hline 00 & 3 & 710 & 319.7808 & 0.5000 & 281.4 & 3 & 600.0 & 00.0 \\
\hline 00 & 3 & 711 & 288.9827 & 1.9750 & 281.6 & 2 & 600.0 & \\
\hline 00 & 3 & 712 & 307.2161 & 3.1750 & 282.0 & 2 & 600.0 & 00.0 \\
\hline 00 & 3 & 713 & 343.5000 & 5.5250 & 282.6 & 3 & 600.0 & 00.0 \\
\hline 00 & 3 & 714 & 324.9651 & 4.3000 & 282.3 & 3 & .0 & \\
\hline 00 & 3 & 715 & 332.8207 & 3.2500 & 282.5 & 2 & 600.0 & 00.0 \\
\hline 00 & 3 & 716 & 2.5028 & 3.6250 & 281.7 & 3 & 600.0 & 600.0 \\
\hline 00 & 3 & 717 & 348.2530 & 2.4500 & 280.9 & 4 & .0 & \\
\hline 00 & 3 & 718 & 346.7318 & 2.7250 & 280.2 & 4 & 600.0 & .0 \\
\hline 00 & 3 & 719 & 338.4997 & 2.5500 & 279.8 & 4 & 60 & .0 \\
\hline 00 & 3 & 720 & 333. & 2.4250 & 280.1 & 4 & 60 & \\
\hline 00 & 3 & 721 & 324.3052 & 3.1250 & 279.8 & 4 & 60 & \\
\hline 00 & 3 & 722 & 321.7502 & 5.0000 & 279.7 & 4 & 600.0 & 00.0 \\
\hline 00 & 3 & 723 & 308.7504 & 5.075 & 279.5 & 4 & 60 & .0 \\
\hline 00 & 3 & 724 & 318.5009 & 4.3750 & 279.2 & 4 & .0 & \\
\hline 00 & 3 & 81 & 358.4258 & 1.9750 & 279.1 & 5 & 600.0 & 600.0 \\
\hline 00 & 3 & 82 & 76.9984 & 1.4250 & 279.3 & 6 & 600.0 & .0 \\
\hline 00 & 3 & 83 & 47.6167 & 3.8500 & 279.5 & 5 & .0 & \\
\hline 00 & 3 & 84 & 114.9500 & 2.9750 & 278.5 & 5 & 600.0 & 600.0 \\
\hline 00 & 3 & 85 & 43.2472 & 4.0750 & 278.3 & 4 & 600.0 & 600.0 \\
\hline 00 & 3 & 86 & 19.3024 & 1.5000 & 277.9 & 5 & 0 & \\
\hline 00 & 3 & 87 & 34.8689 & 0.9250 & 277.8 & 6 & .0 & 600.0 \\
\hline 00 & 3 & 88 & 309.1930 & 1.4250 & 278.5 & 5 & 600.0 & 600.0 \\
\hline 00 & 3 & 89 & 322.7519 & 1.5750 & 280.3 & 4 & 600.0 & 0.0 \\
\hline 00 & 3 & 810 & 280.9197 & 2.7750 & 281.5 & 3 & 600.0 & 600.0 \\
\hline 00 & 3 & 811 & 264.7520 & 3.6000 & 281.3 & 3 & 600.0 & 600.0 \\
\hline 00 & 3 & 812 & 258.2504 & 2.7000 & 281.8 & 3 & 600.0 & 600.0 \\
\hline 00 & 3 & 813 & 316.4197 & 2.8250 & 282.3 & 2 & 600.0 & 600.0 \\
\hline 00 & 3 & 814 & 355.7492 & 5.6000 & 282.4 & 3 & 600.0 & 600.0 \\
\hline 00 & 3 & 815 & 355.0248 & 6.9000 & 280.6 & 4 & 600.0 & 600.0 \\
\hline 00 & 3 & 816 & 338.0057 & 4.8250 & 280.0 & 3 & 600.0 & 600.0 \\
\hline 00 & 3 & 817 & 337.0138 & 5.2250 & 280.7 & 4 & 600.0 & 600.0 \\
\hline 00 & 3 & 818 & 344.3440 & 3.6000 & 279.9 & 4 & 600.0 & 600.0 \\
\hline 00 & 3 & 819 & 328.0138 & 2.5500 & 279.3 & 4 & 600.0 & 600.0 \\
\hline 00 & 3 & 820 & 330.5005 & 3.4000 & 279.5 & 4 & 600.0 & 600.0 \\
\hline 00 & 3 & 821 & 332.7500 & 3.7250 & 278.9 & 4 & 600.0 & 600.0 \\
\hline 00 & 3 & 822 & 64.4024 & $2.325 c$ & 279.1 & 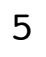 & 600.0 & 600.0 \\
\hline 00 & 3 & 823 & 330.8494 & 1.925 & 278.8 & 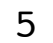 & 600.0 & 600.0 \\
\hline
\end{tabular}




\begin{tabular}{|c|c|c|c|c|c|c|c|}
\hline 0 & $3 \quad 824$ & 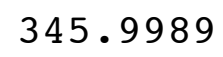 & 5000 & 79.0 & & 00.0 & \\
\hline 0 & 39 & 0.4982 & .8500 & 278.6 & & 500 & \\
\hline 0 & $\begin{array}{lll}3 & 9 & 2\end{array}$ & 349.5086 & .4500 & 278.7 & 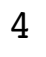 & 00 & \\
\hline & 39 & 333.5331 & 2.3750 & 278.6 & & 00 & \\
\hline & $\begin{array}{lll}3 & 9 & 4\end{array}$ & 931 & 1250 & 78.7 & & D & \\
\hline & $\begin{array}{lll}3 & 9 & 5\end{array}$ & 01 & 8000 & 278.7 & & 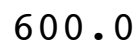 & \\
\hline & 39 & 355.2457 & 1.9750 & 279.3 & & 0( & \\
\hline & 39 & 296 & 1.5750 & 79.7 & & & \\
\hline & 39 & 00 & 2.2250 & 280.1 & & 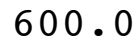 & \\
\hline & $\begin{array}{lll}3 & 9 & 9\end{array}$ & 320 & 1.6000 & 282.3 & & v & \\
\hline & 3910 & 343 & 2.3250 & 283.7 & & 0 & \\
\hline & 3911 & 15 & 2.9500 & 84.5 & & & \\
\hline & 3912 & 149 & 3.0250 & 284.7 & & 0 & \\
\hline & 3913 & 223 & 3.4500 & 284.1 & & & \\
\hline & $\begin{array}{ll}3 & 914\end{array}$ & 241 & 00 & 8 & & & \\
\hline & 3915 & 286 & 1 . & 283.0 & & & \\
\hline & 3916 & 310 . & 1.5000 & 284.0 & & 0 & \\
\hline & 3917 & 99 & 1.0250 & 283 & & 0 & \\
\hline & 3918 & 77 & 3 . & 283.3 & & & \\
\hline & 3919 & 62 & 4.6750 & 282.6 & 3 & & \\
\hline & 3920 & 00 & 4.5750 & 282.2 & 4 & 0 & \\
\hline & 3921 & & 0 & 281.8 & 4 & & \\
\hline & 3922 & 32 & 6.3750 & 281.4 & 4 & & \\
\hline & 3923 & 8 & 6.6500 & 281.3 & 4 & 0 & \\
\hline & 3924 & & 750 & 281.3 & 4 & & \\
\hline & 310 & 69 & 6.7250 & 281.7 & 4 & & \\
\hline & 3102 & 06 & .4750 & 281.8 & 4 & 0 & \\
\hline & 3103 & 56 & 750 & 281.0 & 5 & & \\
\hline & 3104 & 107 & 2.2250 & 281.3 & 6 & & \\
\hline & 3105 & 92 & 2.2250 & 281.5 & 6 & 0 & \\
\hline & 3106 & 817 & 1.4250 & 281.2 & 5 & 0 & \\
\hline & 3107 & & 1.3 & 280.0 & 4 & & \\
\hline & 3108 & 358 & 0.8000 & 282.1 & 3 & 0 & \\
\hline & 3109 & 329 & 0.5500 & 285.0 & 2 & 0 & \\
\hline & 31010 & 28 & 1.3 & 285.1 & 1 & & \\
\hline & 31011 & & 1.4000 & 285.3 & $\perp$ & .0 & \\
\hline & 31012 & 278 & 2.7500 & 285.3 & 2 & 0 & \\
\hline & 31013 & 26 & 3.6000 & 285.3 & 2 & & \\
\hline & 31014 & 22 & 1.6000 & 286.7 & 1 & 600.0 & \\
\hline & 31015 & 245 & 1.9500 & 287.5 & 1 & & \\
\hline & 31016 & 21 & 2.0750 & 287.7 & 1 & 600.0 & \\
\hline 0 & 31017 & 214 . & 2.5500 & 288.3 & 1 & 600.0 & \\
\hline & 31018 & 147.7624 & 4.1000 & 286.2 & 2 & 600.0 & \\
\hline & 31019 & & 3.4500 & 285 & 3 & 600 & \\
\hline 00 & 31020 & & 2.8250 & 284.9 & 4 & 600.0 & \\
\hline & 31021 & 9004 & 2.4250 & 284.5 & 4 & 600.0 & \\
\hline & & & & 28 & 4 & & \\
\hline & 31023 & 129.0080 & 1.0000 & 284.0 & 3 & 600.0 & 6 \\
\hline
\end{tabular}




\begin{tabular}{|c|c|c|c|c|c|c|c|}
\hline 0 & 1024 & 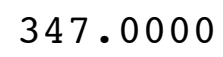 & 5250 & 33.4 & & 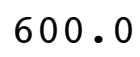 & \\
\hline 0 & 3111 & 47.5272 & .6000 & 83.8 & & 00 & \\
\hline & 3112 & 32.7068 & L. 0250 & 283.9 & & 00 & \\
\hline & 3113 & 56.0764 & 9500 & 283.4 & & 00 & \\
\hline & 3114 & 15.5015 & 750 & 83.8 & & 0 & \\
\hline & 3115 & & 500 & 83.8 & & 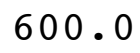 & \\
\hline & 3116 & 23.2657 & 000 & 83.7 & & 0 & \\
\hline & 3117 & 149.5432 & 4500 & & & & \\
\hline & 3118 & 143.2481 & 500 & 82.6 & & 0 & \\
\hline & 3119 & 127.2294 & 750 & 83.6 & & v & \\
\hline & 31110 & 217.3862 & 1.5500 & 85.7 & & 0 & \\
\hline & 31111 & 182.2519 & & & & & \\
\hline & 31112 & 164.9082 & 4 . & 285.2 & & 0 & \\
\hline & 31113 & 190.0092 & 0 & 84.5 & & 0 & \\
\hline & 31114 & 166 . & & & & & \\
\hline & 1115 & 171 . & & 4.5 & & & \\
\hline & 31116 & 166.5000 & 2.62 & 85.6 & & 0 & \\
\hline & 31117 & 152.7519 & 0 & .1 & & 0 & \\
\hline & 3111 & 167 . & 5 . & .3 & & & \\
\hline & 31119 & 175.5000 & 3.9000 & 284.8 & & 0 & \\
\hline & 31120 & 163.4885 & 250 & $2 \varepsilon$ & & 0 & \\
\hline & 31121 & 150.4977 & & & & & \\
\hline & 31122 & 122.2545 & 50 & $2 \varepsilon$ & & & \\
\hline & 1123 & 118.2542 & 4.1750 & 284.4 & & 0 & \\
\hline & 31124 & 125.5000 & 0 & & & & \\
\hline & 312 & 126.5001 & 5. & $2 \xi$ & & & \\
\hline & 3122 & 141.2924 & 3.6000 & 2 & & 0 & \\
\hline & 3123 & 138.7476 & 5250 & $2 \varepsilon$ & 1 & & \\
\hline & 3124 & 131.7496 & 00 & .7 & 4 & & \\
\hline & 3125 & 123.2492 & 0 & 3.8 & & 0 & \\
\hline & 3126 & 120.4843 & 3.5000 & 283.8 & 4 & 0 & \\
\hline & 3127 & 122 . & 0 & 28 & 4 & & \\
\hline & 3128 & 119.5092 & 50 & .9 & 4 & 0 & \\
\hline & 3129 & 99.4978 & 250 & 28 & 3 & 0 & \\
\hline & 31210 & 139.2499 & 0 & & 3 & & \\
\hline & 31211 & 145.7499 & & 5.9 & 4 & 600.0 & \\
\hline & 31212 & 151.2496 & 3250 & 286.6 & 3 & 0 & \\
\hline 0 & 31213 & 151. & 5 . & 28 & 3 & & \\
\hline & 31214 & 158.5000 & 3.9500 & 288.6 & 3 & 600.0 & \\
\hline & 31215 & 155.00 & 7500 & 289.4 & 2 & 0 & \\
\hline & 31216 & 150.25 & 500 & 289 & 3 & 600 & \\
\hline & 31217 & 138.5325 & 500 & 290.2 & 3 & 600.0 & \\
\hline & 31218 & 146.3033 & 3.2500 & 289.4 & 4 & 600 & \\
\hline & 31219 & 76.9839 & 500 & 287 & 4 & 600 & \\
\hline 0 & 31220 & 69.0000 & 6.6000 & 286.5 & 4 & 600.0 & \\
\hline & 31221 & 71.2503 & 7.1750 & 286.9 & 4 & 600.0 & \\
\hline & & 65 & & & 4 & & \\
\hline & $12<20$ & 70.2499 & 7.5500 & 286.8 & 4 & 600.0 & 60 \\
\hline
\end{tabular}




\begin{tabular}{|c|c|c|c|c|c|c|c|}
\hline 0 & 31224 & 74.0005 & 5.7250 & 287.6 & 4 & 600.0 & 600.0 \\
\hline 00 & 3131 & 77.2512 & 5.1750 & 287.8 & 4 & 600.0 & 600.0 \\
\hline 00 & 3132 & 78.4709 & 3.5000 & 288.2 & 5 & 600.0 & 00.0 \\
\hline 00 & 3133 & 92.5043 & 3.0250 & 287.2 & 4 & 600.0 & 00.0 \\
\hline 00 & 3134 & 77.5003 & 4.9500 & 286.5 & 5 & 600.0 & 00.0 \\
\hline 00 & 3135 & 75.2490 & 7.1000 & 285.8 & 4 & 600.0 & 00.0 \\
\hline 00 & 3136 & 74.7478 & 6.6750 & 286.2 & 4 & 600.0 & 00.0 \\
\hline 00 & 3137 & 105.8530 & 3.4000 & 286.3 & 4 & 600.0 & 00.0 \\
\hline 00 & 3138 & 164.8006 & 0.9750 & 286.7 & 3 & 600.0 & 00.0 \\
\hline 00 & 3139 & 183.3067 & 0.3500 & 289.2 & 2 & 600 & 0 \\
\hline 00 & 31310 & 131.0442 & 3.3000 & 288.8 & 2 & 600.0 & \\
\hline 00 & 31311 & 162.4832 & 1.8000 & 289.8 & 1 & 600.0 & \\
\hline 00 & 31312 & 191.1819 & 2.2500 & 290.9 & 1 & 600.0 & 00.0 \\
\hline 00 & 31313 & 170.5001 & 4.9000 & 290.2 & 2 & 600.0 & 00.0 \\
\hline 00 & 31314 & 138.0396 & 5.2500 & 290.7 & 3 & .0 & \\
\hline 00 & 31315 & 93.0014 & 4.5750 & 291.5 & 3 & 600.0 & 00.0 \\
\hline 00 & 31316 & 88.7518 & 5.7500 & 291.3 & 3 & 60 & .0 \\
\hline 00 & 31317 & 77.7473 & 7.6750 & 291.0 & 4 & .0 & \\
\hline 00 & 31318 & 70.2515 & 8.1750 & 290.2 & 4 & .0 & .0 \\
\hline 00 & 31319 & 78.7500 & 9.2250 & 288.2 & 4 & .0 & .0 \\
\hline 00 & 31320 & 73.2491 & 0.1000 & 287.5 & 4 & 6 & \\
\hline 00 & 31321 & 66.4995 & 9.3750 & 287.9 & 4 & .0 & \\
\hline 00 & 31322 & 60.7485 & 10.5250 & 287.1 & 4 & 600.0 & .0 \\
\hline 00 & 31323 & 70.2499 & 9.85 & 287.4 & 4 & 6 & \\
\hline 00 & 31324 & 68.7488 & 8.97 & 287.9 & 4 & .0 & \\
\hline 00 & 3141 & 73.5000 & 9.3000 & 286.9 & 4 & .0 & .0 \\
\hline 00 & 3142 & 96.1398 & 7.5000 & 288.0 & 4 & 600.0 & .0 \\
\hline 00 & 3143 & 85.2528 & 7.3750 & 288.6 & 4 & .0 & \\
\hline 00 & 3144 & 78.4992 & 7.2000 & 287.8 & 4 & .0 & 0 \\
\hline 00 & 3145 & 82.5003 & 7.9000 & 287.3 & 4 & .0 & .0 \\
\hline 00 & 3146 & 78.0015 & 7.57 & 286.8 & 4 & .0 & \\
\hline 00 & 3147 & 78.2501 & 8.3500 & 286.4 & 4 & .0 & 600.0 \\
\hline 00 & 3148 & 79.9998 & 8.2250 & 286.4 & 4 & 600.0 & 600.0 \\
\hline 00 & 3149 & 85.0001 & 7.5500 & 287.0 & 4 & .0 & 0.0 \\
\hline 00 & 31410 & 115.3461 & 5.8000 & 287.6 & 3 & 600.0 & 600.0 \\
\hline 00 & 31411 & 151.4912 & 5.6000 & 287.7 & 3 & 600.0 & 600.0 \\
\hline 00 & 31412 & 175.0005 & 4.47 & 288.4 & 3 & 600.0 & 600.0 \\
\hline 00 & 31413 & 158.5415 & 4.1000 & 289.3 & 3 & 600.0 & 600.0 \\
\hline 00 & 31414 & 160.0000 & 4.6000 & 290.0 & 3 & 600.0 & 600.0 \\
\hline 00 & 31415 & 153.7562 & 5.05 & 290.6 & 3 & 600.0 & 600.0 \\
\hline 00 & 31416 & 165.5055 & 4.3500 & 291.1 & 3 & 600.0 & 600.0 \\
\hline 00 & 31417 & 158.2528 & 4.2250 & 291.5 & 4 & 600.0 & 600.0 \\
\hline 00 & 31418 & 157.0000 & 6.0000 & 291.4 & 4 & 600.0 & 600.0 \\
\hline 00 & 31419 & 132.3324 & 4.5250 & 290.3 & 4 & 600.0 & 600.0 \\
\hline 00 & 31420 & 96.4369 & 5.7500 & 290.3 & 4 & 600.0 & 600.0 \\
\hline 00 & 31421 & 102.7713 & 5.2250 & 290.7 & 4 & 600.0 & 600.0 \\
\hline 00 & 31422 & 121.9830 & 5.9250 & 291.2 & 2 & 600.0 & 600.0 \\
\hline 00 & 31423 & 136.7503 & 6.850 & 291.1 & 4 & 600.0 & 600.0 \\
\hline
\end{tabular}




\begin{tabular}{|c|c|c|c|c|c|c|c|}
\hline 0 & 424 & & 4750 & & & & \\
\hline 0 & 3151 & 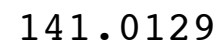 & 1000 & 290.3 & & ח0 & \\
\hline & 3152 & 27.1997 & 3250 & 290.7 & & 00 & \\
\hline & 315 & 151.5020 & 500 & 289.6 & & 00 & \\
\hline & 154 & & 50 & 39.2 & & & \\
\hline & 15 & 98 & 50 & 88.4 & & 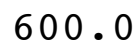 & \\
\hline & 156 & 539 & 8750 & 287.9 & & 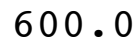 & \\
\hline & 157 & 41 & 7.3750 & 87.8 & & & \\
\hline & 3158 & 580 & 6.3500 & 87.8 & & & \\
\hline & 3159 & 500 & 5750 & 88.1 & & 0 & \\
\hline & 31510 & 160.2532 & 8.6750 & 288.4 & & 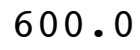 & \\
\hline & 1511 & 00 & 00 & 88.6 & & & \\
\hline & 2 & 00 & 8.0500 & 89.3 & & 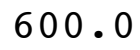 & \\
\hline & 1513 & 7474 & 5.7000 & 6 & & & \\
\hline & 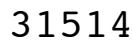 & & 4250 & 91.5 & & & \\
\hline & & & & 292.3 & & & \\
\hline & 5 & 8.7501 & 7.1750 & 293.0 & & 0 & \\
\hline & 517 & 44 & 4.3750 & 3.5 & & 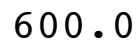 & \\
\hline & 5 & & 0 & 293.2 & & & \\
\hline & 1519 & 87 & 7.2000 & 292.2 & & & \\
\hline & 31520 & 84 & 750 & 291.4 & 4 & & \\
\hline & 0 & & 0 & 91.1 & & & \\
\hline & 2 & 95 & 7.3250 & 291.0 & & & \\
\hline & 523 & & .4250 & 290.8 & $I$ & & \\
\hline & 31524 & & & 290.3 & & & \\
\hline & 316 & & 8.7000 & .8 & 4 & & \\
\hline & 3162 & 89 & .3250 & 289.6 & 4 & & \\
\hline & 3163 & 5 & 0.6750 & 288.9 & 4 & & \\
\hline & 3164 & & 50 & 289.8 & 4 & & \\
\hline & 3165 & 63 & .1000 & 289.4 & 4 & & \\
\hline & 3166 & & .2250 & 289.1 & 4 & & \\
\hline & 3167 & & 8.6 & 289.0 & 4 & & \\
\hline & 3168 & & 000 & 287.3 & 4 & & \\
\hline & 3169 & 491 & 6.1750 & 287.2 & 4 & & \\
\hline & 316 & & 0 & & 4 & & \\
\hline & 31611 & & 8.6250 & 287.7 & 4 & & \\
\hline & 31612 & & 9.1250 & 288.4 & 4 & & \\
\hline & 31613 & 73 & 8.4250 & 288.5 & 4 & & \\
\hline & 31614 & & 8.1750 & 289.1 & 4 & & \\
\hline & & & 6.1750 & 290.1 & 4 & & \\
\hline & 31616 & & 7.2250 & 289.8 & 4 & & \\
\hline & 31617 & & 8.0250 & 287.8 & 4 & & \\
\hline & 31618 & 9999 & L. 1250 & 285.5 & 4 & 0 & \\
\hline & 31619 & & .3250 & 284 & 4 & 60 & \\
\hline 0 & & & 10.0250 & 284.9 & 4 & & \\
\hline & 31621 & 5001 & 10.3250 & 284.4 & 4 & 600.0 & \\
\hline & & & 50 & & 4 & & \\
\hline & 31623 & .9907 & 9.3250 & 285.3 & $\mathbf{I}$ & 600.0 & $r$ \\
\hline
\end{tabular}




\begin{tabular}{|c|c|c|c|c|c|c|c|}
\hline 0 & 24 & & & & & & \\
\hline 0 & 171 & 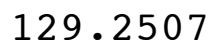 & .8250 & & & & \\
\hline & 3172 & 139.7499 & 10.9250 & 86.1 & & 00 & \\
\hline & 173 & 49.0000 & 1.7750 & 285.8 & & 00 & \\
\hline & 174 & 2.2498 & 0.2250 & & & & \\
\hline & 175 & 7.7500 & 1.15 & 84 & & 0 & \\
\hline & 176 & 47.0011 & 1.6250 & 283 & & 0 & \\
\hline & 177 & 44.7506 & 3250 & & & & \\
\hline & 178 & 7.0009 & .4750 & 9 & & & \\
\hline & 179 & 61.5107 & 9.8750 & 285.6 & & 0 & \\
\hline & 1710 & 57.4969 & 8.9750 & 285 & & & \\
\hline & 1711 & 54.2501 & 8.72 & & & & \\
\hline 0 & 1712 & 54.5000 & 9.3250 & 287.0 & & 0 & \\
\hline & 1713 & 54.7498 & 7.60 & 2 & & & \\
\hline & .714 & 8 & 6 . & & & & \\
\hline & 1715 & 8 & & 29 & & & \\
\hline & 1716 & 55.2455 & 5.60 & 9 & & .0 & \\
\hline & 1717 & 25 & 4 . & 2 & & & \\
\hline & 1718 & 1 & 4 . & & & & \\
\hline & 1719 & 70 & 2.42 & 29 & 4 & & \\
\hline 0 & 31720 & 141.2501 & 2.90 & $2 \varepsilon$ & 4 & . 0 & \\
\hline & 1721 & 89 & & & 4 & & \\
\hline & 31722 & 74 & 0 & $2 \varepsilon$ & 4 & & \\
\hline & 1723 & 25.5013 & 0 & 2 & 4 & . 0 & \\
\hline & 31724 & 21 & & & 4 & & \\
\hline 0 & 3181 & 112 & 4 . & & 4 & & \\
\hline & 3182 & 32.5001 & 500 & 29 & 4 & .0 & \\
\hline & 3183 & 41.7476 & 5.25 & & 4 & & \\
\hline & 3184 & 99 & 3 . & 28 & 4 & & \\
\hline & 3185 & 48.9998 & 7 . & 28 & 4 & & \\
\hline & 3186 & 157.7500 & 4.3500 & 288 & 5 & & \\
\hline 0 & 3187 & 4 & 332 & & 4 & & \\
\hline & 3188 & 220 & & 288 & 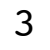 & & \\
\hline & 3189 & 171.1492 & 1.3500 & 291.2 & 2 & 0 & \\
\hline 唯 & 31810 & 164 . & & & 3 & & \\
\hline & 31811 & 15 & & & 3 & & \\
\hline & 31812 & 160.5248 & 4.5750 & 291.6 & 4 & .0 & \\
\hline & 31813 & 157.2410 & 5 . & 29 & 3 & & \\
\hline 00 & 31814 & 162.0000 & 5.4500 & 293.2 & 4 & .0 & \\
\hline & 31815 & 161.4990 & 4.7000 & 294.0 & 3 & & \\
\hline & 31816 & 154 . & 4.2500 & 29 & 3 & & \\
\hline 00 & 31817 & 141.5191 & 2.6 & & 3 & .0 & \\
\hline & 31818 & 98.9882 & 3.4500 & 294.2 & 4 & & 0 \\
\hline 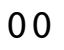 & 31819 & 81.2545 & 000 & & 4 & & \\
\hline 00 & & 75.4986 & & & 4 & .0 & \\
\hline מר & 31821 & 69.2508 & 7.8000 & 291.2 & 4 & .0 & 00 \\
\hline & & & & & 4 & & \\
\hline & 31823 & 74.5000 & 8.2250 & 291.5 & & .0 & 600 \\
\hline
\end{tabular}




\begin{tabular}{|c|c|c|c|c|c|c|c|}
\hline 0 & 1824 & 1.7500 & 9.2000 & 289.8 & 4 & 0 & \\
\hline & 3191 & 05003 & 8.5250 & & & & \\
\hline & 3192 & & 000 & & & & \\
\hline & 193 & 013 & 9750 & 89.4 & & 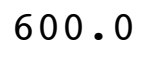 & \\
\hline & 194 & & & & & & \\
\hline & 195 & 528 & 500 & 87.9 & & r & \\
\hline & 196 & 5008 & 250 & 88.5 & & 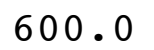 & \\
\hline & 197 & 01 & 0 & 88.0 & & & \\
\hline & 198 & 01 . & 4.1250 & 87.1 & & 0 & \\
\hline & 199 & 0.7518 & 0.7500 & 9 & & 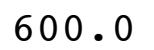 & \\
\hline & 1910 & 92 & & & & & \\
\hline & 1911 & 03. & & & & & \\
\hline & 912 & 74 . & 750 & 86.6 & & 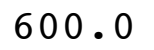 & \\
\hline & 913 & 64 & & & & & \\
\hline & 914 & & & & & & \\
\hline & 5 & 70 & 0 & 6 & & & \\
\hline & 916 & 76 & 0 & 7 & & & \\
\hline & 917 & & & & & & \\
\hline & 8 & & 7 . & 0 & & & \\
\hline & 919 & 77 & 0 & & & & \\
\hline & 920 & & & & & & \\
\hline & 921 & & & & & & \\
\hline & 922 & 80 & 0 & 7 & & & \\
\hline & 923 & 9 & & & & & \\
\hline & 1924 & & & & & & \\
\hline & 3201 & 00 & & & & & \\
\hline & 3202 & & 0 & 2.7 & 4 & & \\
\hline & 3203 & & 0 & & 4 & & \\
\hline & 3204 & & & & 4 & & \\
\hline & 3205 & & & & 4 & & \\
\hline & 3206 & & & & 4 & & \\
\hline & 3207 & & & & 4 & & \\
\hline & 3208 & 153. & 0 & 279.6 & 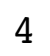 & & \\
\hline & 3209 & 1 & 0 & & 4 & & \\
\hline & 32010 & & & & 4 & & \\
\hline & 32011 & 175 . & & 3.0 & 4 & & \\
\hline & 32012 & & & & 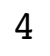 & & \\
\hline & 32013 & 17 & 0 & .0 & 4 & & \\
\hline & 32014 & 172 . & 7250 & 285.9 & 4 & & \\
\hline & 32015 & & & & 4 & & \\
\hline & 32016 & & & & 4 & & \\
\hline & 32017 & 172 . & 50 & 287.3 & 4 & & \\
\hline & 32018 & 158 . & 50 & 286 & 4 & & \\
\hline & 32019 & 153.0000 & & & $x^{2}$ & & \\
\hline & 32020 & 152.2500 & 14.0250 & 286.1 & 4 & 0 & $0_{0}>$ \\
\hline & 32 & & & & & & \\
\hline & 32022 & 152 . & & & & & \\
\hline & 20023 & 130.1499 & 12.3250 & 285.1 & & 600.0 & -0 \\
\hline
\end{tabular}




\begin{tabular}{|c|c|c|c|c|c|c|c|}
\hline 0 & 024 & & & & & & \\
\hline & 211 & 0 & 0.6500 & 83.4 & & & \\
\hline & 3212 & 160.2500 & 250 & 282.9 & & 00 & \\
\hline & 213 & 54.2498 & 10.3750 & 282.6 & & 00 & \\
\hline & 214 & & 0.0500 & 82.0 & & & \\
\hline & 215 & 0 & & 81.7 & & 0 & \\
\hline & 3216 & 7500 & 10.9750 & 82.0 & & 00 & \\
\hline & 3217 & 50.2500 & 1.9250 & 82.1 & & & \\
\hline & 3218 & 0 & 1.1250 & 82.4 & & & \\
\hline & 3219 & 2500 & 11.1500 & 83.3 & & 0 & \\
\hline & 32110 & 2501 & 8.6250 & 284 & & & \\
\hline & 32111 & 163.7499 & 8.0250 & 7 & & & \\
\hline & 321 & 172.24 & 8 . & 287.1 & & & \\
\hline & 2113 & 75.0005 & 9.52 & 5 & & & \\
\hline & 32114 & 6 & & & & & \\
\hline & 321 & 172 . & & 7 & 4 & & \\
\hline & 32116 & 162.7354 & 5.82 & 291.4 & 3 & 0 & \\
\hline & 32117 & 61 & 6 . & 0 & & & \\
\hline & 32118 & 151. & 6 . & .9 & 4 & & \\
\hline & 321 & 149.7474 & 000 & 291 & 4 & & \\
\hline & 32120 & .2497 & 0 & 291.1 & 4 & 0 & \\
\hline & 32 & 2.7496 & & & 4 & & \\
\hline & 321 & 14 & 3000 & 29 & 4 & & \\
\hline & 32123 & 149.5002 & 6.37 & 29 & 4 & 0 & \\
\hline & 32124 & 122.6188 & & & 4 & & \\
\hline & 3221 & 500 & 0 & 28 & 4 & & \\
\hline & 3222 & 85.2512 & 0 & 2 & 4 & & 0 \\
\hline & 3223 & 79.813 & 0 & 5 & 4 & & \\
\hline & 3224 & 662 & 0 & 28 & 5 & & \\
\hline & 3225 & 39.3069 & 250 & 287.9 & 6 & & J. \\
\hline & 3226 & 29.8032 & 500 & 288.0 & 6 & & . \\
\hline & 3227 & 138.7501 & 0 & & 5 & & \\
\hline & 3228 & 85.1674 & 000 & 289.8 & 4 & & 0 . \\
\hline & 3229 & 5580 & 50 & 292.7 & 3 & & 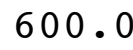 \\
\hline & 32210 & 26 & 0 & 292.1 & 2 & & \\
\hline & & 264.3031 & 750 & & 1 & & \\
\hline & 32212 & 234.9869 & 3000 & 291.9 & 1 & & \\
\hline & 32213 & 248.0424 & 500 & 29 & 1 & & \\
\hline & 32214 & 258.9641 & 2.0750 & 294.4 & 1 & & 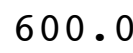 \\
\hline & 32215 & 219.43 & 250 & 295.2 & 1 & & \\
\hline & 32216 & 187.1416 & 250 & 294.4 & 1 & & \\
\hline & 32217 & 138.6372 & 000 & 293.8 & 2 & & 0 . \\
\hline & 32218 & 85.7293 & 4.0250 & 292.5 & 3 & & 0 . \\
\hline & 32219 & 5003 & 50 & & 4 & & \\
\hline 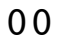 & 32220 & 80.5000 & 6.3250 & & 4 & & \\
\hline & 32221 & 72.2494 & 7.6750 & 288.7 & 4 & & 0 . \\
\hline & & 919 & & & 4 & & \\
\hline & 32223 & 63.2730 & 6.7500 & 288.6 & $\mathbf{4}$ & 600.0 & 600 \\
\hline
\end{tabular}




\begin{tabular}{|c|c|c|c|c|c|c|c|}
\hline v & 2224 & 0.0241 & 6.9500 & 287.5 & 4 & 政 & \\
\hline & 3231 & 0.1012 & 6.9500 & 287.6 & & & \\
\hline & 3232 & 5159 & 2250 & 289.0 & 4 & 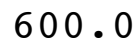 & \\
\hline & 3233 & 1.5117 & 1.3500 & 287.7 & & 00 & \\
\hline & 3234 & 7137 & & 32.8 & & & \\
\hline & 3235 & 5.0110 & 7500 & 83.3 & & 00 & \\
\hline & 3236 & 64.2518 & 6.8500 & 285.0 & & & \\
\hline & 3237 & 57.0000 & 1000 & 87.2 & & & \\
\hline & 3238 & 68.2443 & 6.6750 & 87.9 & & & \\
\hline & 3239 & 69.8002 & 2.0750 & 287.8 & & 0 & \\
\hline & 32310 & 261.9886 & 1.8000 & 288.4 & & & \\
\hline & 2311 & 243.5000 & 00 & 9 & & & \\
\hline & 2312 & 226.4954 & 1.8250 & 288.8 & & 0 & \\
\hline & 32313 & 69.4083 & 2.7250 & 289.5 & & 0 & \\
\hline & 32314 & 89. & 4.7250 & & & & \\
\hline & 323 & 94 . & 3.9500 & 289.6 & & & \\
\hline & 32316 & 74.4 & 5250 & 288.2 & & & \\
\hline & 32317 & 79. & 0 & .8 & & & \\
\hline & 32318 & 71 . & 50 & 286.1 & 4 & & \\
\hline & 32319 & 65.4997 & 6250 & 284.9 & 4 & & \\
\hline & 32320 & 71. & 0 & 284.2 & 4 & & \\
\hline & 32321 & 53. & & 1 & 4 & & \\
\hline & 32322 & 39.7528 & 5750 & 286.2 & 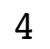 & & U. \\
\hline & 32323 & 88.2153 & 0 & .7 & 5 & & \\
\hline & 32324 & 53. & & 28 & 6 & & \\
\hline & 3241 & 38.5154 & 500 & 28 & 6 & & \\
\hline & 3242 & 32.9325 & .7000 & 284.1 & 6 & & \\
\hline & 3243 & 84.1511 & 1.4750 & 284 & 6 & & \\
\hline & 3244 & 793 & 0 & 28 & 6 & & \\
\hline & 3245 & 12.6181 & 750 & 282.6 & 6 & & \\
\hline & 3246 & 025 & 00 & 281.6 & 6 & & \\
\hline & 3247 & 95 . & 0 & 28 & 6 & & \\
\hline & 3248 & 94.9355 & 0.4750 & 282.1 & 5 & 0 & \\
\hline & 3249 & 102 & 50 & 285.3 & 4 & & \\
\hline & 32410 & 317 . & 50 & 28 & 3 & & \\
\hline & 32411 & 286.5176 & 750 & 287.3 & 2 & & \\
\hline & 32412 & 264.6870 & 250 & 287.5 & 1 & & \\
\hline 0 & 32413 & 206.1337 & 250 & 287.9 & 1 & & . \\
\hline & 32414 & 177.5038 & 2.3250 & 289.1 & 1 & & \\
\hline & 32415 & 492 & 00 & 289.8 & 2 & & \\
\hline & 32416 & 171.2537 & 00 & 289.8 & 2 & & \\
\hline 0 & 32417 & 132.5376 & 2.8250 & 290.3 & 1 & 0 & ( \\
\hline & 32418 & 96.5168 & 9750 & 288.3 & 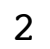 & 0 & \\
\hline & 32419 & 77.2500 & 7.0000 & 285.6 & 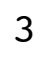 & & \\
\hline 0 & 32420 & 75.0000 & 8.9000 & 283.9 & 4 & 600 & 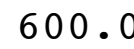 \\
\hline & 32421 & 000 & 9.7750 & 283.0 & 4 & 600 & \\
\hline & 324 & 5000 & & 282 & 4 & & \\
\hline & 32423 & 76.7500 & 10.3000 & 281.6 & 4 & 600.0 & 600 \\
\hline
\end{tabular}




\begin{tabular}{|c|c|c|c|c|c|c|c|}
\hline 0 & 32424 & 76.0000 & 9.4500 & 281.3 & 4 & 600.0 & 600.0 \\
\hline 00 & 3251 & 79.0000 & 8.2250 & 281.3 & 4 & 600.0 & 600.0 \\
\hline 00 & 3252 & 78.7500 & 9.0000 & 280.9 & 4 & 600.0 & 00.0 \\
\hline 0 & 3253 & 78.5002 & 9.9000 & 280.1 & 4 & 600.0 & 00.0 \\
\hline 00 & 3254 & 74.2501 & 9.8750 & 279.9 & 4 & 600.0 & 00.0 \\
\hline 00 & 3255 & 71.2500 & 10.1500 & 279.8 & 4 & 600.0 & 00.0 \\
\hline 00 & 3256 & 69.5000 & 11.2500 & 279.8 & 4 & 600.0 & 00.0 \\
\hline 00 & 3257 & 66.2499 & 10.4750 & 280.0 & 4 & 600.0 & 00.0 \\
\hline 00 & 3258 & 67.0000 & 9.2250 & 280.5 & 4 & 600.0 & 00.0 \\
\hline 00 & 3259 & 72.9986 & 7.2000 & 282.1 & 4 & 60 & 0 \\
\hline 00 & 32510 & 78.2450 & 4.5250 & 283.8 & 3 & 600.0 & \\
\hline 00 & 32511 & 297.8728 & 00 & 287.4 & 2 & 600.0 & \\
\hline 00 & 32512 & 207.2420 & 2.2000 & 287.1 & 1 & 600.0 & 00.0 \\
\hline 00 & 32513 & 211.6470 & 2.3500 & 288.1 & 1 & 600.0 & 00.0 \\
\hline 00 & 32514 & 261.0292 & 50 & 290.1 & 1 & .0 & \\
\hline 00 & 32515 & 350.7425 & 2.7750 & 290.3 & 1 & 600.0 & .0 \\
\hline 00 & 32516 & 74.4940 & 3.7500 & 289.6 & 2 & 60 & 0.0 \\
\hline 00 & 32517 & 91.4975 & 5. & 287.9 & 3 & .0 & \\
\hline 00 & 32518 & 75.2504 & 6.7000 & 285.7 & 4 & 60 & .0 \\
\hline 00 & 32519 & 75.0000 & 7.3000 & 284.4 & 4 & .0 & .0 \\
\hline 00 & 32520 & 75.7505 & 8 . & 283.5 & 4 & 6 & \\
\hline 00 & 32521 & 73.5000 & 000 & 283.4 & 4 & .0 & \\
\hline 00 & 32522 & 77.5017 & 7.9000 & 283.3 & 4 & 600.0 & .0 \\
\hline 00 & 32523 & 76.5000 & 8.3 & 283 & 4 & 6 & \\
\hline 00 & 32524 & 76.7499 & 7 . & 28 & 4 & .0 & \\
\hline 00 & 3261 & 75.2505 & 6.9250 & 283.7 & 4 & .0 & .0 \\
\hline 00 & 3262 & 78.6919 & 2.6750 & 285.1 & 5 & 60 & .0 \\
\hline 00 & 3263 & 90.2455 & 2 . & 284.9 & 6 & .0 & \\
\hline 00 & 3264 & 79.7499 & 3.3000 & 284.4 & 5 & .0 & .0 \\
\hline 00 & 3265 & 75.9998 & 3.7000 & 284.2 & 4 & 60 & .0 \\
\hline 00 & 3266 & 73.7449 & 2. & 284.2 & 5 & .0 & \\
\hline 00 & 3267 & 64.5823 & 1.92 & 284.1 & 6 & .0 & 600.0 \\
\hline 00 & 3268 & 145.5718 & 0.7750 & 286.4 & 5 & 600.0 & 600.0 \\
\hline 00 & 3269 & 23.7483 & 1.8250 & 286.2 & 4 & .0 & 0.0 \\
\hline 00 & 32610 & 302.6754 & 1.2250 & 28 & 3 & 600.0 & 600.0 \\
\hline 00 & 32611 & 231.2454 & 2.0750 & 289.1 & 2 & 600.0 & 600.0 \\
\hline 00 & 32612 & 187.4847 & 3.1500 & 288.7 & 2 & 600.0 & 0.0 \\
\hline 00 & 32613 & 179.0242 & 3.6750 & 289.2 & 2 & 600.0 & 600.0 \\
\hline 00 & 32614 & 161.2512 & 3.6750 & 289.8 & 3 & 600.0 & 600.0 \\
\hline 00 & 32615 & 156.0983 & 4.4500 & 289.8 & 3 & 600.0 & 600.0 \\
\hline 00 & 32616 & 85.2495 & 4.3750 & 289.4 & 4 & 600.0 & 600.0 \\
\hline 00 & 32617 & 82.7500 & 5.3500 & 288.4 & 4 & 600.0 & 600.0 \\
\hline 00 & 32618 & 78.7500 & 6.8250 & 287.0 & 4 & 600.0 & 600.0 \\
\hline 00 & 32619 & 76.2499 & 6.7000 & 286.0 & 4 & 600.0 & 600.0 \\
\hline 00 & 32620 & 78.7500 & 7.1500 & 285.2 & 4 & 600.0 & 600.0 \\
\hline 00 & 32621 & 75.2500 & 8.3250 & 284.8 & 4 & 600.0 & \\
\hline 00 & 32622 & 79.5005 & 10.0250 & 283.3 & 4 & 600.0 & 600.0 \\
\hline 00 & 32623 & 78.7500 & 11.275 & 282.1 & 4 & 600.0 & 600.0 \\
\hline
\end{tabular}




\begin{tabular}{|c|c|c|c|c|c|c|c|}
\hline 0 & 32624 & 77.2500 & 10.7000 & 282.0 & 4 & 600.0 & 600.0 \\
\hline 0 & 3271 & 77.7500 & 10.7000 & 281.5 & 4 & 600.0 & 00,0 \\
\hline 00 & 3272 & 77.7500 & 9.8000 & 281.4 & 4 & 600.0 & 00.0 \\
\hline 0 & 3273 & 80.7512 & 10.2000 & 281.0 & 4 & 600.0 & 0 \\
\hline 00 & 3274 & 72.5000 & 9.7500 & 280.8 & 4 & 00.0 & .0 \\
\hline 00 & 3275 & 69.7499 & 9.7750 & 280.5 & 4 & 600.0 & 00.0 \\
\hline 00 & 3276 & 65.5001 & 10.9000 & 280.6 & 4 & 600.0 & 0 \\
\hline 00 & 3277 & 62.2500 & 13.2750 & 280.8 & 4 & 600.0 & 00.0 \\
\hline 00 & 3278 & 61.0000 & 13.3750 & 281.0 & 4 & 600.0 & 00.0 \\
\hline 00 & 3279 & 61.5000 & 11.3750 & 281.6 & 4 & .0 & \\
\hline 00 & 32710 & 59.7499 & 11.2000 & 281.6 & 4 & .0 & \\
\hline 00 & 32711 & 60.2498 & 11.0250 & 282.0 & 4 & .0 & \\
\hline 00 & 32712 & 57.7491 & 11.1000 & 282.5 & 4 & 600.0 & \\
\hline 00 & 32713 & 60.5054 & 10.6250 & 282.8 & $I$ & 600.0 & \\
\hline 00 & 32714 & 494 & 11. & 283.1 & 4 & & \\
\hline 00 & 32715 & 62.0001 & 11.7250 & 282.8 & 4 & 60 & \\
\hline 00 & 32716 & 60.5000 & 12.2500 & 282.5 & 4 & .0 & .0 \\
\hline 00 & 32717 & 60.0000 & 11.30 & 282.1 & 4 & .0 & \\
\hline 00 & 32718 & 59.7499 & 11.3250 & 282.3 & 4 & 60 & \\
\hline 00 & 32719 & 60.4998 & 11.3750 & 282.1 & 4 & .0 & .0 \\
\hline 00 & 32720 & 62 . & 11.07 & .1 & 4 & 0 & \\
\hline 00 & 32721 & 02 & 9.4500 & 282.5 & 4 & .0 & \\
\hline 00 & 32722 & 69.0000 & 10.4000 & 282.3 & 4 & 60 & .0 \\
\hline 00 & 32723 & 002 & 10.95 & 282.0 & 4 & 0 & \\
\hline 00 & 32724 & 00 & 12 . & 281.0 & 4 & 0 & \\
\hline 00 & 3281 & 61.9998 & 13.5500 & 280.6 & 4 & 60 & .0 \\
\hline 00 & 3282 & 68.2500 & 10.1750 & 281.0 & 4 & .0 & .0 \\
\hline 00 & 3283 & 72.5000 & 7.85 & 282.1 & 4 & .0 & \\
\hline 00 & 3284 & 80.4994 & 10.6250 & 280.2 & 4 & .0 & \\
\hline 00 & 3285 & 61.7493 & 10.4000 & 279.6 & 4 & 6 & \\
\hline 00 & 3286 & 69.0025 & 980 & 279.5 & 4 & 0 & \\
\hline 00 & 3287 & 70.2498 & 8.87 & .5 & 4 & .0 & .0 \\
\hline 00 & 3288 & 75.7502 & 7.97 & 279.9 & 4 & .0 & 0.0 \\
\hline 00 & 3289 & 79.2500 & 6.32 & 280.9 & 4 & .0 & .0 \\
\hline 00 & 32810 & 91.4998 & 4.65 & 281.9 & 4 & 0 & 0 \\
\hline 00 & 32811 & 89.9924 & 2.8500 & 283.9 & 3 & 600.0 & 0.0 \\
\hline 00 & 32812 & 155.2508 & 5.75 & 284.6 & 3 & .0 & .0 \\
\hline 00 & 32813 & 152.4872 & 5.1000 & 284.7 & 3 & 600.0 & 600.0 \\
\hline 00 & 32814 & 166.7501 & 5.0000 & 285.1 & 3 & 600.0 & 600.0 \\
\hline 00 & 32815 & 153.4599 & 5.55 & 286.1 & 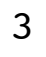 & .0 & \\
\hline 00 & 32816 & 118.7545 & 4.4250 & 287.2 & 3 & 600.0 & 600.0 \\
\hline 00 & 32817 & 110.6812 & 4.9000 & 286.9 & 3 & 600.0 & 600.0 \\
\hline 00 & 32818 & 102.7477 & 5.9750 & 285.2 & 4 & 600.0 & .0 \\
\hline 00 & 32819 & 78.9759 & 8.6750 & 283.2 & 4 & 600.0 & 600.0 \\
\hline 00 & 32820 & 79.2499 & 10.2750 & 281.6 & 4 & 600.0 & 600.0 \\
\hline 00 & 32821 & 66.0193 & 10.5250 & 280.7 & 4 & 600.0 & \\
\hline 00 & 32822 & 70.2542 & 10.4250 & 280.6 & 4 & 600.0 & 600.0 \\
\hline 00 & 32823 & 70.5000 & 10.650 & 280.2 & 4 & 600.0 & 600.0 \\
\hline
\end{tabular}




\begin{tabular}{|c|c|c|c|c|c|c|c|}
\hline & 24 & 3.0037 & 5250 & 80.8 & & ( & \\
\hline 0 & 3291 & 1.9996 & 5500 & 280.2 & & & \\
\hline & 3292 & 000 & 000 & 79.5 & & & \\
\hline & 3293 & 3.7499 & 3750 & .79 .3 & & & \\
\hline & 3294 & & 0 & 80.9 & & & \\
\hline & 3295 & 78 & 0 & 81.0 & & & \\
\hline & 3296 & 80 . & 500 & 80.6 & & & \\
\hline & 3297 & 78 & 500 & 81.1 & & & \\
\hline & 3298 & 92 & 500 & 83.0 & & & \\
\hline & 3299 & & 500 & 84.8 & & & \\
\hline & 32910 & 75 & 3750 & 87.2 & & & \\
\hline & 32911 & 6 & & 36.7 & & & \\
\hline & 329 & 169. & 2500 & 86.0 & & & \\
\hline & 32913 & 166. & 3.4000 & 287.3 & & & \\
\hline & 32914 & & & & & & \\
\hline & 32915 & 1 & 50 & 8.5 & & & \\
\hline & 32916 & 149.9823 & 3.7500 & 289.2 & & & \\
\hline & 3291 & 1 & 3 . & 7 & & & \\
\hline & 32918 & 153 & 0 & 9.4 & & & \\
\hline & 32919 & 920 & & 287.8 & & & \\
\hline & 32920 & & 0 & 6.1 & & & \\
\hline & 32921 & & & & & & \\
\hline & 325 & 73 & 0 & $2 \varepsilon$ & & & \\
\hline & 32923 & 69 & & 2 & & & \\
\hline & 32 & & & 287.4 & & & \\
\hline & 330 & & 50 & 287.8 & & & \\
\hline & 3302 & & & 2 & 4 & & \\
\hline & 3303 & & & 3 & 4 & & \\
\hline & 3304 & 7 & 0 & $2 \xi$ & 4 & & \\
\hline & 3305 & (17. & 0 & 285.5 & 4 & & \\
\hline & 3306 & & & 2 & 4 & & \\
\hline & 3307 & & & 2 & 4 & & \\
\hline & 3308 & 48. & 8.4500 & 284.3 & 1 & & \\
\hline & 3309 & 9 & 00 & 2 & 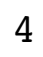 & & \\
\hline & 33 & & & $2 \varepsilon$ & 4 & & \\
\hline & 33 & 154 . & 250 & 287.6 & 4 & & \\
\hline & 33 & & & 2 & 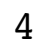 & & \\
\hline & 33 & & 0 & 290.1 & 4 & & \\
\hline & 33014 & 165. & 750 & 291.0 & 4 & & \\
\hline & 330 & & 00 & 25 & 4 & & \\
\hline & 33016 & 16 & 000 & 292.4 & 4 & & \\
\hline & 33017 & 163. & 11.2500 & 292.6 & 4 & & \\
\hline & & 130 & 00 & 292.1 & 4 & & \\
\hline & 33019 & & 0.1250 & 291 & 4 & & \\
\hline & 33020 & 147 . & 11.3250 & 291.7 & 4 & & \\
\hline & 33 & & 250 & 291 & 4 & & \\
\hline & & & & & 2 & & \\
\hline & נים & 151.2500 & 11.0000 & 289.7 & $\Psi$ & 600.0 & - \\
\hline
\end{tabular}




\begin{tabular}{|c|c|c|c|c|c|c|c|}
\hline 0 & 30 & & & & & & \\
\hline 0 & 331 & 1 & & 8.1 & & & \\
\hline & 3312 & 53. & 750 & 87.2 & & $0 c$ & \\
\hline & 3313 & 4.0000 & 750 & 86.8 & & 00 & \\
\hline & 3314 & & & 36.5 & & & \\
\hline & 331 & & & 36.6 & & & \\
\hline & 331 & 032 & 750 & 86.2 & & & \\
\hline & 331 & 2500 & 5500 & & & & \\
\hline & 331 & 7488 & 114 & 85.4 & & & \\
\hline & 3319 & 71.2488 & 11 & 86 & & & \\
\hline & 33110 & .9943 & 10.3000 & 87.7 & & & \\
\hline & 2. & & 50 & & & & \\
\hline & 31 & 97 & 13 & 0 & & & \\
\hline & 3113 & 7485 & 13 & & & & \\
\hline & 331 & & & & & & \\
\hline & 211 & & & & & & \\
\hline & 2. & .7500 & 13.1000 & .3 & & & \\
\hline & 3311 & 80 & 11 . & & & & \\
\hline & 3311 & & & & & & \\
\hline & 1 & 391 & 9.1750 & 4 & & & \\
\hline & 3312 & 000 & 9.4000 & 9 & & & \\
\hline & 3312 & & 9 . & & & & \\
\hline & 3122 & 93 & 10.1750 & 25 & & & \\
\hline & 3312 & 00 & 50 & 1.0 & & & \\
\hline & 33124 & & 8.22 & & & & \\
\hline & 41 & 5 & 6 . & & & & \\
\hline & $\begin{array}{lll}4 & 1 & 2\end{array}$ & 50 & 8 . & 2 & & & \\
\hline & $\begin{array}{ll}4 & 1\end{array}$ & & 9. & 2 & 4 & & \\
\hline & 41 & & 10. & 2 & 2 & & \\
\hline & $\begin{array}{lll}4 & 1 & 5\end{array}$ & & & 2 & & & \\
\hline & $\begin{array}{lll}4 & 1 & 6\end{array}$ & & 10 . & 28 & 4 & & \\
\hline & 41 & & & & 25 & & \\
\hline & $\begin{array}{lll}4 & 1 & 8\end{array}$ & & & 2 & 4 & & \\
\hline & $\begin{array}{lll}4 & 1 & 9\end{array}$ & & 50 & $2 \varepsilon$ & 4 & & \\
\hline & 4 & & & & 4 & & \\
\hline & 4111 & & & .2 & 4 & & \\
\hline & $4 \quad 112$ & & 0 & 293.0 & J & & \\
\hline & 4113 & & 0 & 29 & 3 & & \\
\hline & $\begin{array}{ll}4 & 114\end{array}$ & & & 29 & 4 & & \\
\hline & $4 \quad 11$ & & & 296 & 4 & & \\
\hline & 411 & & 50 & 29 & 4 & & \\
\hline & $\begin{array}{ll}4 & 117\end{array}$ & & & 297.5 & 4 & & \\
\hline & 411 & 0 & & 296.9 & 4 & & \\
\hline & $4 \quad 11$ & & & & 4 & & \\
\hline & $4 \quad 120$ & & & & 4 & & \\
\hline & $4 \quad 121$ & 5000 & $\$ 750$ & 296.7 & 4 & & \\
\hline & 4 & & & & 4 & & \\
\hline & $4 \quad 123$ & 7505 & 6.2500 & 296.9 & $I$ & 600.0 & \\
\hline
\end{tabular}




\begin{tabular}{|c|c|c|c|c|c|c|c|c|}
\hline 0 & 4 & 24 & & 500 & 5.7 & & & \\
\hline 0 & 4 & 21 & 00 & .1000 & 6.0 & & 0 & \\
\hline 0 & 4 & 22 & 138.2496 & .4500 & 296.4 & & 00 & \\
\hline 0 & 4 & 23 & 144.5000 & 7.6750 & 296.3 & & 00.0 & \\
\hline 0 & 4 & 4 & 5.2515 & .5250 & 94.6 & & & \\
\hline 0 & 4 & 25 & .00 & .1250 & 293.6 & & 00 & \\
\hline 0 & 4 & 26 & 44.2499 & 9.3000 & 293.5 & & 00 & \\
\hline & 4 & 7 & 47.9977 & .6000 & 292.6 & & .0 & \\
\hline & 4 & 8 & 9999 & .7500 & 292.9 & & & \\
\hline 0 & 4 & 9 & 49.0000 & 9.6750 & 293.3 & & 00 & 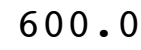 \\
\hline 0 & 4 & 210 & 49.5000 & 4.9250 & 294 & & & \\
\hline 0 & 4 & 211 & 10.78 & 170 & 295.6 & & & \\
\hline 0 & 4 & 212 & 279 & 5.1750 & 296 & & 00.0 & \\
\hline 0 & 4 & 213 & 9993 & 6.6750 & 29 & & 0.0 & \\
\hline & 4 & 214 & 9 & & 297 & & & \\
\hline 0 & 4 & 21 & & & 297.6 & 4 & & \\
\hline 0 & 4 & 216 & 152.2508 & 0 & 298.4 & 2 & 0.0 & \\
\hline 0 & 4 & 217 & 50.74 & 4 . & 298 & 4 & .0 & \\
\hline 0 & 4 & 218 & 6 & 3 . & 298 & 4 & & \\
\hline 0 & 4 & 219 & 24 & 0 & 296.8 & 5 & .0 & \\
\hline 00 & 4 & 220 & 88.9183 & 3.2750 & 296.8 & 4 & $\cdot 0$ & 0 \\
\hline 0 & 4 & 221 & 71. & & 296 & 4 & & \\
\hline 0 & 4 & 222 & 76 . & 0 & 29 & 4 & .0 & \\
\hline 0 & 4 & 223 & 73.7500 & 500 & 297 & 4 & .0 & \\
\hline 0 & 4 & 224 & 74 & & 29 & 4 & & \\
\hline 00 & 4 & 31 & 75 & 9 . & 29 & 4 & & \\
\hline 0 & 4 & 32 & 74.0001 & & 295 & 4 & .0 & \\
\hline 0 & 4 & 33 & 72 . & 0 & 29 & 4 & .0 & \\
\hline 00 & 4 & 34 & 71. & 0 & 295 & 4 & .0 & \\
\hline 0 & 4 & 35 & 62.99 & 6 & 29 & 4 & .0 & \\
\hline 00 & 4 & 36 & 57.3687 & 2.8250 & 294.9 & 5 & .0 & \\
\hline 0 & 4 & 37 & 13. & 0 & 294 & 4 & & 0 \\
\hline 00 & 4 & 38 & 353 . & & $29^{\circ}$ & 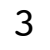 & .0 & \\
\hline 0 & 4 & 39 & 231.7352 & 0.9000 & 298.9 & 2 & .0 & \\
\hline 00 & 4 & 310 & 277 . & 1 . & 29 & 1 & & \\
\hline 00 & 4 & 311 & 241 & 750 & 29 & 2 & .0 & \\
\hline 00 & 4 & 312 & 233.9773 & 2.0000 & 297.6 & 1 & .0 & \\
\hline 0 & 4 & 313 & 227.5003 & 500 & 298 & 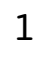 & .0 & \\
\hline 00 & 4 & 314 & 177.7483 & 3.7000 & 298.9 & 2 & .0 & 60 \\
\hline مח & 4 & 315 & 151.2912 & 3.9000 & 299.2 & 3 & .0 & \\
\hline . & 4 & 316 & 144.2293 & 000 & 299.2 & 3 & & \\
\hline 00 & 4 & 317 & 97.7473 & 4.2500 & 298.5 & 4 & .0 & \\
\hline 0 & 4 & 318 & 84.2455 & 4.5250 & 297.8 & 4 & .0 & 600 \\
\hline 00 & 4 & 319 & 73.0027 & 750 & 295.8 & 4 & .0 & \\
\hline 00 & 4 & 320 & 42.4927 & 4.7000 & 295.2 & $J$ & .0 & \\
\hline 00 & 4 & 321 & 23.7400 & 2.9750 & 295.1 & 4 & 600.0 & 600 \\
\hline$n g$ & 4 & 322 & 30 & & 294.6 & 4 & & \\
\hline & 4 & 323 & 328.8770 & 2.2000 & 293.0 & & .0 & 600 \\
\hline
\end{tabular}




\begin{tabular}{|c|c|c|c|c|c|c|c|c|}
\hline & 4 & 24 & 2.0780 & 250 & 89.0 & & 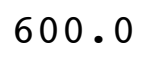 & \\
\hline 0 & 4 & 1 & 8.4500 & .0250 & 90.3 & & 00.0 & \\
\hline & 4 & 42 & 78.6041 & 0.6500 & 289.6 & & 00 & \\
\hline & 4 & 3 & 72.2174 & 2500 & 288.3 & & 00 & \\
\hline & 4 & 4 & 105.7248 & 5750 & 7.5 & & & \\
\hline & 4 & 5 & 127.4629 & 500 & 85.1 & & 0 & \\
\hline 0 & 4 & 6 & 87.9498 & 000 & 85.1 & & 00 & \\
\hline & 4 & 7 & 73.4204 & 1.1500 & 85.4 & & & \\
\hline & 4 & 8 & 309.2549 & 92 & 8 & & & \\
\hline 0 & 4 & 9 & 302.4788 & 1.2250 & 88.3 & & 0 & \\
\hline & 4 & 410 & 301.5000 & 1.3000 & 289.2 & & & \\
\hline & 4 & 411 & 36.6462 & 172 & 4 & & & \\
\hline 0 & 4 & 41 & 131.7923 & 2.15 & 291.5 & & & \\
\hline 0 & 4 & 413 & 204.2684 & 2.32 & 292 & & & \\
\hline & 4 & 414 & 36.2249 & & & & & \\
\hline 0 & 4 & 41 & 48.1502 & & 29 & & & \\
\hline 0 & 4 & 416 & 75.7499 & 7.40 & 292.5 & & .0 & \\
\hline & 4 & 417 & 77.4997 & 7 . & 3 & & .0 & \\
\hline 0 & 4 & 418 & 72 . & & 29 & 4 & & \\
\hline 0 & 4 & 419 & 74 . & 0 & 285 & 4 & & \\
\hline 0 & 4 & 420 & 71.7499 & 0.60 & 287.8 & 4 & .0 & \\
\hline 0 & 4 & 421 & 70.2185 & & & 2 & & \\
\hline 0 & 4 & 422 & 105.9943 & 0 & 287 & 5 & & \\
\hline 0 & 4 & 423 & 79.5001 & 0 & $2 \varepsilon$ & 4 & .0 & \\
\hline & 4 & 424 & 00 & & & & & \\
\hline 0 & 4 & 51 & 72.7253 & 1 . & 28 & 5 & & \\
\hline 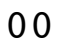 & 4 & 52 & 79.9906 & .1500 & 287 & 6 & .0 & \\
\hline & 4 & 53 & 93.2750 & 2. & & 5 & & \\
\hline 0 & 4 & 54 & 80.7295 & 6 & 28 & 4 & & \\
\hline & 4 & 55 & 507 & 0 & & 4 & .0 & \\
\hline 0 & 4 & 56 & 83.9930 & 6.1500 & 287.8 & 4 & & \\
\hline 0 & 4 & 57 & 82.9904 & 652 & 28 & 4 & & \\
\hline 0 & 4 & 58 & 88.7294 & 500 & $28^{\circ}$ & 3 & & \\
\hline 0 & 4 & 59 & 64.7302 & 3250 & 289.3 & 4 & 0 & \\
\hline 0 & 4 & 510 & 4 & 0 & & 3 & & \\
\hline 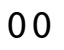 & 4 & 511 & 160.8534 & 0 & & 2 & & \\
\hline 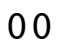 & 4 & 512 & 292.5406 & 1500 & 293.2 & 1 & .0 & \\
\hline 0 & 4 & 513 & 238 . & 0 & & 1 & & \\
\hline 0 & 4 & 514 & 58.7687 & 3.5000 & .3 & 2 & .0 & \\
\hline 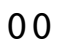 & 4 & 515 & 004 & 4000 & 292.3 & 3 & & \\
\hline 00 & 4 & 516 & 948 & 500 & 29 & 4 & & \\
\hline 00 & 4 & 517 & 86.9450 & 6.1000 & & 4 & .0 & 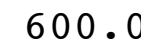 \\
\hline 00 & 4 & 518 & 73.4992 & 8.4250 & 290.2 & 4 & & 0 \\
\hline 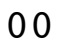 & 4 & 519 & 68 . & 5 & & 4 & & \\
\hline 00 & 4 & 520 & 61.5005 & 8.6250 & & 4 & .0 & \\
\hline 00 & 4 & 521 & 68.5011 & 7.2500 & 290.7 & 4 & .0 & 500 \\
\hline 00 & 4 & 522 & & & & 4 & & \\
\hline & 4 & 523 & 57.7499 & 6.4750 & 291.5 & $I$ & .0 & 600 \\
\hline
\end{tabular}




\begin{tabular}{|c|c|c|c|c|c|c|c|c|}
\hline 0 & 4 & 24 & & 250 & 0.9 & & & \\
\hline 0 & 4 & 61 & 104 & .2750 & 90.3 & & 0 & \\
\hline & 4 & 62 & 70.2506 & 8.0250 & 289.3 & & 00 & \\
\hline & 4 & 63 & 66.2491 & 0.5500 & 287.9 & & 00 & \\
\hline & 4 & 4 & 7.5000 & .2000 & 88.6 & & & \\
\hline & 4 & 65 & 9.0000 & .2750 & 288.2 & & 0 & \\
\hline 0 & 4 & 6 & 68.2500 & 8500 & 285.9 & & 00 & \\
\hline & 4 & 7 & 63.2500 & L. .0000 & 86.6 & & & \\
\hline & 4 & 8 & 9.5043 & .3000 & 85.7 & & & \\
\hline 0 & 4 & 9 & 74.2501 & 5500 & 286.8 & & 0 & \\
\hline & 4 & 610 & 69.7502 & 5.5250 & 285 & & & \\
\hline & 4 & & 217.6001 & 2 . & 7 & & & \\
\hline 0 & 4 & 12 & 241.7586 & 1.65 & 292.8 & & .0 & \\
\hline 0 & 4 & 3 & 107.4265 & 2.72 & 29 & & & \\
\hline & 4 & 4 & 2 & 0 & & & & \\
\hline 0 & 4 & & & & & 4 & & \\
\hline 0 & 4 & 61 & 000 & 7.20 & 292.6 & 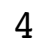 & .0 & \\
\hline & 4 & 17 & 73 . & 8 . & 0 & 4 & $\cdot 0$ & \\
\hline 0 & 4 & 61 & & 7 . & 29 & $\Psi$ & & \\
\hline 0 & 4 & 1 & 74 & 8. & 29 & 4 & & \\
\hline 0 & 4 & 620 & 77.7532 & 9.2250 & 28 & 4 & . 0 & \\
\hline 0 & 4 & 621 & & & & $\mathbf{4}$ & & \\
\hline 0 & 4 & 622 & 73 & 8. & 29 & 4 & & \\
\hline 0 & 4 & 623 & 68 . & 8 . & .2 & 4 & .0 & \\
\hline 0 & 4 & 624 & & & & $\mathbf{4}$ & & \\
\hline 00 & 4 & 71 & & 7 . & & 4 & & \\
\hline 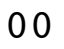 & 4 & 72 & 76.4931 & 0 & 289.2 & 4 & .0 & \\
\hline & 4 & 3 & 84 . & 0. & & 4 & & \\
\hline 0 & 4 & 74 & 74 . & 6 . & 28 & 4 & & \\
\hline & 4 & 75 & 84 . & & & 4 & & \\
\hline 0 & 4 & 76 & 96.00 & 5.02 & 288.8 & 4 & & \\
\hline 0 & 4 & 77 & 21 & 2 & & 4 & & \\
\hline & 4 & 78 & 150 & 0 & 29 & 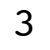 & & \\
\hline 0 & 4 & 79 & 180.6357 & 750 & 291.8 & 2 & 0 & \\
\hline 0 & 4 & 710 & 1 & & 29 & 2 & & \\
\hline 00 & 4 & 711 & 174 . & 0 & & 3 & & \\
\hline 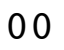 & 4 & 712 & 187.2835 & 3750 & 292.4 & 2 & .0 & \\
\hline 0 & 4 & 713 & 201 . & 3. & 29 & 2 & & \\
\hline 8 & 4 & 714 & 184.6035 & 2 . & 29 & 1 & .0 & \\
\hline 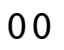 & 4 & 715 & 189.1827 & 2000 & 295.5 & 2 & & \\
\hline 00 & 4 & 716 & 195.4456 & 2 . & 29 & 1 & & \\
\hline 00 & 4 & 717 & 201.7776 & 1 . & & 1 & .0 & 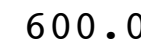 \\
\hline 0 & 4 & 718 & 228.7457 & 1.1750 & 296.5 & 2 & & 0 \\
\hline 00 & 4 & 719 & 187.5583 & & & 3 & & \\
\hline 00 & 4 & 720 & 81.7494 & & & 2 & & \\
\hline 00 & 4 & 721 & 75.7500 & 6.1750 & 292.7 & 4 & .0 & 500 \\
\hline 00 & 4 & 722 & & & & 4 & & \\
\hline & 4 & 723 & 70.2507 & 9.5000 & 289.3 & & .0 & 600 \\
\hline
\end{tabular}




\begin{tabular}{|c|c|c|c|c|c|c|c|c|}
\hline 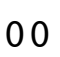 & 4 & 724 & 66.9985 & 8.2750 & 289.0 & 4 & 600.0 & 600.0 \\
\hline 00 & 4 & 81 & 72.2739 & 7.7750 & 288.1 & 4 & 600.0 & 00.0 \\
\hline 00 & 4 & 82 & 66.5009 & 7.3750 & 290.1 & 4 & 600.0 & 00.0 \\
\hline 0 & 4 & 83 & 52.5009 & 6.8500 & 290.2 & 4 & 600.0 & 00.0 \\
\hline 00 & 4 & 84 & 96.4187 & 1.8500 & 288.5 & 5 & 600.0 & 00.0 \\
\hline 00 & 4 & 85 & 82.9761 & 2.2500 & 286.7 & 6 & 600.0 & 00.0 \\
\hline 00 & 4 & 86 & 68.7820 & 4.7500 & 287.0 & 5 & 600.0 & 0 \\
\hline 00 & 4 & 87 & 79.9981 & 2.2750 & 287.8 & 6 & 600.0 & 00.0 \\
\hline 00 & 4 & 88 & 70.6395 & 3.9250 & 287.2 & 5 & 600.0 & 00.0 \\
\hline 00 & 4 & 89 & 79.2501 & 6.9250 & 284.5 & 4 & 60 & \\
\hline 00 & 4 & 810 & 83.7500 & 6.7500 & 285.4 & 4 & 600.0 & \\
\hline 00 & 4 & 811 & 88.5301 & 4.9000 & 287.3 & 3 & .0 & \\
\hline 00 & 4 & 812 & 83.2429 & 5.1000 & 289.4 & 3 & 600.0 & 00.0 \\
\hline 00 & 4 & 813 & 78.0011 & 8.3000 & 288.8 & 4 & 600.0 & \\
\hline 00 & 4 & 814 & 75.5023 & 10.3000 & 289.1 & 4 & .0 & \\
\hline 00 & 4 & 815 & 77.5009 & 11.7500 & 288.4 & 4 & 600.0 & .0 \\
\hline 00 & 4 & 816 & 131.5199 & 11.1750 & 288.0 & 4 & 60 & .0 \\
\hline 00 & 4 & 817 & 84.5163 & 9.9250 & 287.0 & 4 & .0 & \\
\hline 00 & 4 & 818 & 86.2450 & 8.1500 & 285.8 & 4 & 60 & \\
\hline 00 & 4 & 819 & 96.5012 & 7.5250 & 284.7 & 4 & .0 & .0 \\
\hline 00 & 4 & 820 & 106.8915 & 10.1250 & 282.8 & 4 & 0 & \\
\hline 00 & 4 & 821 & 128.7096 & 7.4500 & 282.7 & 4 & .0 & \\
\hline 00 & 4 & 822 & 168.5003 & 10.8250 & 282.2 & 4 & 600.0 & .0 \\
\hline 00 & 4 & 823 & 177.0024 & 12.27 & 281.6 & 4 & 0 & \\
\hline 00 & 4 & 824 & 176 . & 11 . & 281.5 & 4 & .0 & \\
\hline 00 & 4 & 91 & 179.5000 & 12.5500 & 281.4 & 4 & .0 & \\
\hline 00 & 4 & 92 & 179.7500 & 11.8500 & 282.0 & 4 & 60 & .0 \\
\hline 00 & 4 & 93 & 178.0002 & 11.3500 & 282.1 & 4 & .0 & \\
\hline 00 & 4 & 94 & 180.0000 & 12.1250 & 281.7 & 4 & .0 & .0 \\
\hline 00 & 4 & 95 & 180.5000 & 13.3750 & 281.4 & 4 & 6 & .0 \\
\hline 00 & 4 & 96 & 180.0001 & 12.1250 & 281.3 & 4 & .0 & \\
\hline 00 & 4 & 97 & 179.7500 & 5000 & 281.4 & 4 & .0 & 0.0 \\
\hline 00 & 4 & 98 & 179.7500 & 10.7750 & 282.0 & 4 & 600.0 & 0.0 \\
\hline 00 & 4 & 99 & 166.7616 & 7.4250 & 283.5 & 4 & 600.0 & 0.0 \\
\hline 00 & 4 & 910 & 150.2578 & 5 . & 285.1 & 3 & 600.0 & 600.0 \\
\hline 00 & 4 & 911 & 112.6756 & 4.3500 & 287.0 & 3 & 600.0 & 600.0 \\
\hline 00 & 4 & 912 & 86.5528 & 3.500 & 288.2 & 2 & 600.0 & 0.0 \\
\hline 00 & 4 & 913 & 85.2524 & 4.2500 & 289.4 & 3 & 600.0 & 600.0 \\
\hline 00 & 4 & 914 & 97.7505 & 5.3250 & 290.0 & 3 & 600.0 & 600.0 \\
\hline 00 & 4 & 915 & 97.5000 & 5 . & 290.0 & 3 & 600.0 & 600.0 \\
\hline 00 & 4 & 916 & 99.2626 & 6.8000 & 290.1 & 4 & 600.0 & 600.0 \\
\hline 00 & 4 & 917 & 89.2498 & 7.2750 & 289.5 & 4 & 600.0 & 600.0 \\
\hline 00 & 4 & 918 & 94.1884 & 7.6000 & 288.5 & 4 & 600.0 & 600.0 \\
\hline 00 & 4 & 919 & 153.7603 & 8.9500 & 286.6 & 4 & 600.0 & 600.0 \\
\hline 00 & 4 & 920 & 175.2443 & 9.6000 & 285.8 & 4 & 600.0 & 600.0 \\
\hline 00 & 4 & 921 & 173.2486 & 9.2250 & 285.8 & 4 & 600.0 & \\
\hline 00 & 4 & 922 & 178.7501 & 10.3000 & 285.4 & 4 & 600.0 & 600.0 \\
\hline 0 & 4 & 923 & 187.2501 & 11.275 & 284.5 & & 600.0 & 600.0 \\
\hline
\end{tabular}




\begin{tabular}{|c|c|c|c|c|c|c|c|}
\hline 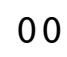 & 24 & & 50 & 84.9 & & & \\
\hline 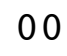 & 4101 & 180 & .6750 & 85.5 & & 00 & \\
\hline & 4102 & 177.0037 & 0750 & 85.0 & & 00 & \\
\hline & 4103 & 158.5000 & 8250 & 285.5 & & 00 & \\
\hline & $410 \quad 4$ & 00 & 6250 & 35.3 & & & \\
\hline & $410 \quad 5$ & 178 & & 85.5 & & 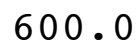 & \\
\hline & 4106 & 672 & 750 & 85.7 & & 0 & \\
\hline & 4107 & 389 & 250 & & & & \\
\hline & 4108 & 32 & & 87.0 & & & \\
\hline & 4109 & 129 & 50 & 88.1 & & & \\
\hline & 41010 & 143.5023 & .7250 & 89.2 & & 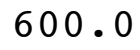 & \\
\hline & 41011 & 14 & 50 & 90.1 & & & \\
\hline & 41012 & 145 & 50 & 290.9 & & 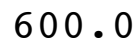 & \\
\hline & 41013 & 145 & 8750 & 291.9 & & & \\
\hline & 41014 & $14^{\circ}$ & & 0 & & & \\
\hline & 41015 & 11 & & 3.8 & & & \\
\hline & 41016 & 103.2534 & 0 & 294.2 & & 0 & \\
\hline & 41017 & 72 & 0 & 93.4 & & 0 & \\
\hline & 41018 & 66 & & 292.2 & & & \\
\hline & 41019 & 715 & 8000 & 291.2 & & & \\
\hline & 41020 & 153 & 50 & 291.1 & 4 & & \\
\hline & 41021 & 17 & & 0.6 & 2 & & \\
\hline & 41022 & 176 & 0 & 29 & 4 & & \\
\hline & 41023 & 16 & 50 & 290.7 & 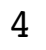 & & \\
\hline & 41024 & 12 & & 291.1 & 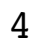 & & \\
\hline & 4111 & 110 & & 291.2 & 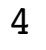 & & \\
\hline & 4112 & 67 . & 0 & 291.4 & 4 & 0 & \\
\hline & 4113 & 23 & 0 & 290.7 & 5 & & \\
\hline & 4114 & & & 288.6 & 4 & & \\
\hline & 4115 & & & 288.5 & & & \\
\hline & 4116 & 21. & 50 & 289.0 & 4 & & \\
\hline & 4117 & & & 290.4 & 5 & & \\
\hline & 4118 & 301 & & 292.8 & 4 & & \\
\hline & 4119 & 318 & 50 & 293.8 & 3 & & \\
\hline & 411 & 25 & & 29 & 2 & & \\
\hline & 41111 & 263. & & 294.0 & 2 & 0 & \\
\hline & 41112 & 230 & & 295.3 & 1 & & \\
\hline & 41113 & 256 & 3 . & 296.4 & 2 & & \\
\hline & 41114 & 220 & 3. & 297.1 & 2 & & \\
\hline & 41115 & 222 & 00 & 297.8 & 1 & & \\
\hline & 41116 & 207 & 2 . & 298.3 & 1 & & \\
\hline & 41117 & 195. & 3.4250 & 297.7 & 2 & 600.0 & \\
\hline & 41118 & 178 & 750 & 296.8 & 3 & 0 & \\
\hline & 41119 & 160 & 2.8500 & 295.5 & 4 & & \\
\hline 0 & 41120 & 145 . & 2.9500 & 294.1 & 5 & & \\
\hline & 41121 & 109.3123 & 2.5000 & 294.5 & 4 & 600.0 & \\
\hline & & & & 293 & 4 & & \\
\hline & $1+20$ & 74.5000 & 7.0750 & 293.1 & $\mathbf{4}$ & 600.0 & 6 \\
\hline
\end{tabular}




\begin{tabular}{|c|c|c|c|c|c|c|c|}
\hline 00 & 41124 & 78.0000 & 7.2750 & 292.5 & 4 & 600.0 & 00.0 \\
\hline 0 & 4121 & 69.7680 & 6.4750 & 292.2 & 4 & 600.0 & 600,0 \\
\hline 00 & 4122 & 72.2492 & 8.8750 & 291.2 & 4 & 600.0 & 00.0 \\
\hline 00 & 4123 & 72.2501 & 9.3500 & 290.8 & 4 & 600.0 & 00.0 \\
\hline 00 & 4124 & 63.9995 & 9.3250 & 290.7 & 4 & 600.0 & 00.0 \\
\hline 00 & 4125 & 68.4886 & 10.2250 & 290.0 & 4 & 600.0 & 00.0 \\
\hline 00 & 4126 & 60.0794 & 5.4000 & 291.8 & 4 & 600.0 & 600.0 \\
\hline 00 & 4127 & 230.0000 & 1.5250 & 291.9 & 5 & 600.0 & 00.0 \\
\hline 00 & 4128 & 326.0903 & 1.7500 & 291.7 & 4 & 600.0 & 00.0 \\
\hline 00 & 4129 & 266.1854 & 2.4250 & 293.7 & 3 & 600.0 & .0 \\
\hline 00 & 41210 & 238.5106 & 2.9750 & 294.4 & 2 & 600.0 & 500.0 \\
\hline 00 & 41211 & 271.0418 & 500 & 295.9 & 3 & 600.0 & \\
\hline 00 & 41212 & 279.5174 & 4.9000 & 297.1 & 3 & 600.0 & 600.0 \\
\hline 00 & 41213 & 226.9365 & 4.2250 & 297.1 & 3 & 600.0 & 0 \\
\hline 00 & 41214 & 178.5824 & 4.8250 & 297.6 & 3 & 600.0 & \\
\hline 00 & 41215 & 351.5997 & 5.4750 & 298.8 & 3 & 600.0 & 60 \\
\hline 00 & 41216 & 28.9973 & 6.7000 & 297.8 & 4 & 600.0 & 600.0 \\
\hline 00 & 41217 & 78.5015 & 6.9750 & 294.6 & 4 & 600.0 & \\
\hline 00 & 41218 & 77.0076 & 7.2250 & 293.3 & 4 & 600.0 & .0 \\
\hline 00 & 41219 & 66.3112 & 7.6250 & 292.7 & 4 & 600.0 & .0 \\
\hline 00 & 41220 & 79.7521 & 250 & 290.1 & 4 & 600.0 & .0 \\
\hline 00 & 41221 & 348.9753 & 250 & 289.2 & 4 & 600.0 & \\
\hline 00 & 41222 & 308.4991 & 3.0750 & 287.8 & 5 & 600.0 & 600.0 \\
\hline 00 & 41223 & 203.3603 & 1.1000 & 287.5 & 6 & 600.0 & .0 \\
\hline 00 & 41224 & 134.1209 & 000 & 286.5 & 6 & 600.0 & \\
\hline 00 & 4131 & 62.3344 & 000 & 286.0 & 5 & 600.0 & 60 \\
\hline 00 & 4132 & 330.2515 & 4.2750 & 285.1 & 4 & 600.0 & .0 \\
\hline 00 & 4133 & 317.7480 & 3.0500 & 285.3 & 4 & 00.0 & \\
\hline 00 & 4134 & 303.2189 & 3.3000 & 285.2 & 5 & 600.0 & 60 \\
\hline 00 & 4135 & 5.2548 & 1.1750 & 285.0 & 6 & 600.0 & 60 \\
\hline 00 & 4136 & 14.3427 & 1 . & 284.5 & 5 & 600.0 & \\
\hline 00 & 4137 & 60.0754 & 500 & 285.9 & 6 & 600.0 & .0 \\
\hline 00 & 4138 & 210.6517 & 0.9500 & 289.1 & 5 & 600.0 & 600.0 \\
\hline 00 & 4139 & 21.1769 & 1.550 & 289.9 & 4 & 600.0 & .0 \\
\hline 00 & 41310 & 28.4124 & 2.4500 & 290.6 & 3 & 600.0 & .0 \\
\hline 00 & 41311 & 69.1349 & 5.0250 & 289.6 & 3 & 600.0 & 600.0 \\
\hline 00 & 41312 & 25.8539 & 6.0750 & 289.9 & 4 & 600.0 & \\
\hline 00 & 41313 & 33.5029 & 6.5250 & 290.6 & 4 & 600.0 & 600.0 \\
\hline 00 & 41314 & 37.7502 & 8.5250 & 290.8 & 4 & 600.0 & 600.0 \\
\hline 00 & 41315 & 43.0000 & 10.8500 & 290.0 & 4 & 600.0 & \\
\hline 00 & 41316 & 56.9995 & 7.4000 & 289.4 & 4 & 600.0 & 600.0 \\
\hline 00 & 41317 & 79.2519 & 5.5250 & 289.0 & 3 & 600.0 & 600.0 \\
\hline 00 & 41318 & 61.2517 & 5.5750 & 288.0 & 3 & 600.0 & 600.0 \\
\hline 00 & 41319 & 106.0030 & 5.8500 & 286.5 & 4 & 600.0 & 600.0 \\
\hline 00 & 41320 & 98.5000 & 6.7250 & 285.2 & 4 & 600.0 & 600.0 \\
\hline 00 & 41321 & 103.2501 & 4.9500 & 284.3 & 4 & 600.0 & 600.0 \\
\hline 00 & 41322 & 89.2541 & 4.300 & 284 & 5 & 600.0 & 600.0 \\
\hline 00 & 41323 & 80.0000 & 4.0500 & 284.2 & 3 & 600.0 & 600.0 \\
\hline
\end{tabular}




\begin{tabular}{|c|c|c|c|c|c|c|c|}
\hline 0 & 41324 & 075 & 1250 & 84.4 & 4 & 600.0 & \\
\hline 0 & 4141 & 356.2249 & 1.8500 & 83.7 & & 600.0 & \\
\hline 0 & 4142 & 10.7549 & 2.6000 & 282.8 & & 00.0 & \\
\hline 0 & 4143 & 23.7330 & 1.3250 & 283.0 & & 00.0 & \\
\hline 0 & 144 & 307.8111 & .5750 & 82.7 & & 0 & \\
\hline & 145 & 319.80 & .9250 & 82.5 & & 00 & \\
\hline 0 & 146 & 338.2784 & 1.5500 & 282.4 & & 00 & \\
\hline & 147 & 335.4532 & .5000 & 82.6 & & & \\
\hline & 148 & 9.2575 & 1.7000 & 84.4 & & & \\
\hline 0 & 149 & 344.8433 & 1.7000 & 86.6 & & 00.0 & . \\
\hline 0 & 1410 & 151.4101 & 2.8250 & 287.4 & & & \\
\hline 0 & 1411 & 153.7503 & $31-$ & 86.8 & & & \\
\hline 0 & 1412 & 182.7861 & 3 & 287.6 & & 00.0 & \\
\hline 0 & 1413 & 126.4297 & 4.40 & 86.9 & & 0.0 & \\
\hline 0 & 414 & 92.2510 & 0 & & & & \\
\hline 0 & 5 & 41.80 & 2 . & 0 & & & \\
\hline 0 & 416 & 45.4585 & 2.92 & 288.6 & & 00.0 & \\
\hline 0 & 1417 & 7.8347 & 0 & .3 & & .0 & \\
\hline 0 & 418 & 66.65 & 4 . & 288.7 & & & \\
\hline 0 & 9 & 70.2505 & 0 & 286.4 & 3 & .0 & \\
\hline 0 & 41420 & 68.9564 & 9.30 & 284.3 & & .0 & 000 \\
\hline 0 & 41421 & 113.6667 & 070 & & I & & \\
\hline 0 & 41422 & 171.5037 & 8.65 & 28 & 4 & .0 & \\
\hline 0 & 41423 & 172.7654 & 7.97 & .3 & 4 & .0 & 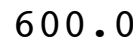 \\
\hline 0 & 41424 & 179. & & & 4 & & \\
\hline 00 & 4151 & 180.5000 & 8 . & & 4 & & \\
\hline 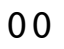 & 4152 & 180.2500 & 8.52 & 0 & 4 & .0 & \\
\hline 0 & 4153 & 175.74 & 6 . & .7 & 4 & .0 & \\
\hline 00 & 4154 & 177.9999 & 6 . & 28 & 4 & .0 & \\
\hline 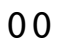 & 4155 & 45.2003 & 9500 & 282.3 & 4 & .0 & \\
\hline 0 & 4156 & 328.2524 & 0.8250 & 281.8 & 5 & .0 & \\
\hline 0 & 4157 & 354.0188 & 1 & & 5 & & \\
\hline 0 & 4158 & 336.0805 & 0 & .4 & 4 & .0 & \\
\hline 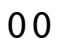 & 4159 & 201.0744 & 1.7250 & $2 \varepsilon$ & 3 & .0 & \\
\hline 0 & 41510 & 33. & 10 & & 2 & & 0 \\
\hline 00 & 41 & 91.0631 & 3.67 & .4 & 2 & .0 & \\
\hline 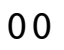 & 41512 & 99.5888 & 1.6750 & 285.4 & 1 & .0 & \\
\hline 0 & 41513 & 274.9433 & 0 & 3 & 2 & .0 & 0 \\
\hline 00 & 41514 & 253.5276 & 2.4500 & 287.2 & 1 & .0 & 60 \\
\hline 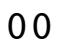 & 41515 & 217.3861 & 2.8500 & 288.0 & 1 & .0 & \\
\hline 00 & 41516 & 184.7476 & 000 & 287.3 & 1 & & \\
\hline 00 & 41517 & 84.5024 & 5.3000 & 286.4 & 2 & .0 & 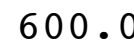 \\
\hline 00 & 41518 & 78.2472 & 5.2000 & 286.3 & 3 & .0 & 00 \\
\hline 00 & 41519 & 64.0584 & 5.3250 & 285.5 & 4 & & \\
\hline 00 & & 56.4946 & 5.7500 & 285.3 & 4 & 600.0 & 600 \\
\hline 00 & 41521 & 95.6218 & 4.8500 & 284.8 & 4 & 600.0 & 600 \\
\hline 00 & & & & & 4 & & \\
\hline & 41523 & 23.5008 & 2.2000 & 284.9 & J & 600.0 & 600 \\
\hline
\end{tabular}




\begin{tabular}{|c|c|c|c|c|c|c|c|}
\hline 0 & 524 & & 250 & & & & \\
\hline 0 & 161 & 9957 & .2500 & 34 & & & \\
\hline & 162 & 338 & 2.3750 & 83.2 & & 00 & \\
\hline & 163 & 22.1202 & 1.8500 & 83.1 & & 00 & \\
\hline & 64 & 22.1447 & 2500 & & & & \\
\hline & 65 & 51.45 & .3250 & 82.7 & & 0 & \\
\hline & 166 & 221 & 2.6500 & 83.0 & & 00 & \\
\hline & 167 & 2954 & 3.70 & & & & \\
\hline & 168 & 0000 & .15 & 1 & & & \\
\hline 0 & 169 & 01.7795 & 3.9250 & 86.1 & & 00 & \\
\hline & 1610 & 5.8176 & 4.0500 & 286 & & & \\
\hline & & 8.5001 & 7.22 & 7.0 & & & \\
\hline 0 & 16 & 7505 & 7.32 & 287.2 & & .0 & \\
\hline 0 & 613 & 2519 & 9.93 & 4 & & & \\
\hline & & 00 & & & & & \\
\hline 0 & & 0 & & 2 & & & \\
\hline 0 & 616 & 000 & 12.85 & 286.6 & & .0 & \\
\hline 0 & 1617 & 37 & 11.15 & 9 & & $\cdot 0$ & \\
\hline 0 & 0 & 12 & & & 4 & & \\
\hline 0 & 9 & 09 & 6 . & 7 & 4 & & \\
\hline 0 & 41620 & 312.2435 & 8.60 & 284.8 & 4 & .0 & \\
\hline 0 & 1 & .7500 & & & 4 & & \\
\hline 0 & 2 & 97 & 6 & 9 & 4 & & \\
\hline 0 & 41623 & .0000 & 6.72 & 2 & 4 & .0 & \\
\hline 0 & 41624 & 48 & & & 4 & & \\
\hline 0 & 417 & 2 & 0 & & 4 & & \\
\hline & 4172 & 989 & 0 & 4 & 4 & .0 & \\
\hline & 4173 & 781 & 2 . & & 5 & & \\
\hline 0 & 4174 & 42 & $1.3^{7}$ & & 6 & & \\
\hline & 4175 & 242 & 0 & & 6 & & \\
\hline 0 & 4176 & 7.1022 & 3.17 & & 5 & & \\
\hline 0 & $417 \quad 7$ & 87 & 2 & & 4 & & \\
\hline & 4178 & 379 & & 282 & 4 & & \\
\hline 0 & 4179 & 616 & 750 & & 2 & 0 & \\
\hline 0 & 41710 & 5 & & & 2 & & 0 \\
\hline 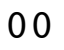 & 41711 & 28 & & & 1 & & \\
\hline 0 & 41712 & 308.2474 & 2.3500 & 282.8 & 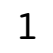 & .0 & \\
\hline 0 & 41713 & 393 & 2. & & 2 & & \\
\hline 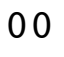 & 41714 & 241.5013 & 4.6250 & & 3 & .0 & \\
\hline & 41715 & 253.7444 & 9000 & 282.5 & 3 & & \\
\hline 00 & 41716 & 064 & 0 & & 4 & & \\
\hline 00 & 41717 & 263.4977 & 4.2750 & & 3 & & \\
\hline 0 & 41718 & 270.7504 & 5.2500 & 281.6 & 3 & & 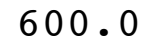 \\
\hline 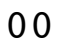 & 41719 & 501 & 5 . & & 4 & & \\
\hline 00 & 41720 & 307.8082 & 2.7000 & & 4 & .0 & \\
\hline 00 & 41721 & 332.8465 & 2.0250 & 280.4 & 4 & .0 & 500 \\
\hline & & & & & 5 & & \\
\hline & 41723 & 8918 & 3.0000 & 282.1 & & .0 & 600 \\
\hline
\end{tabular}




\begin{tabular}{|c|c|c|c|c|c|c|c|}
\hline 0 & 1724 & & 750 & 1.9 & & & \\
\hline & 4181 & 021 & .1000 & 30.9 & 4 & 00.0 & \\
\hline & 4182 & 84.2239 & 6000 & 280.8 & 5 & 00 & \\
\hline & 4183 & 126.5349 & 1.4250 & 279.7 & & 00 & \\
\hline & 184 & 75.2512 & 2750 & 79.7 & & 0 & \\
\hline & 4185 & & 7750 & 80.5 & & 0 & \\
\hline & 4186 & 466 & 1.7250 & 281.2 & & 00 & \\
\hline & 4187 & 54.4299 & .4750 & 82.6 & & 0 & \\
\hline & 4188 & 167.4601 & 9750 & 82.8 & & & \\
\hline & 4189 & 202.1223 & 1.8750 & 283.3 & 4 & 00 & 0 \\
\hline & 41810 & 245.9549 & .5750 & 285.6 & & 0.0 & \\
\hline & 811 & 3236 & 2.5750 & & & & \\
\hline & 1812 & 208.8946 & 500 & 286.6 & 1 & 0 & \\
\hline & 1813 & 216.2237 & .9750 & 287.2 & & 0 & 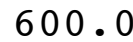 \\
\hline & 814 & 3970 & & & & & \\
\hline & 815 & 158.7514 & & 287.0 & 3 & & \\
\hline & 1816 & 168.0000 & 3.6000 & 287.1 & 2 & .0 & 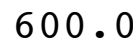 \\
\hline & 41817 & 145.7384 & 0 & .8 & 1 & & \\
\hline & 1818 & 137.9444 & & 288.0 & 2 & & \\
\hline & 1819 & 95.7424 & 3750 & 286.8 & 3 & 00.0 & $\checkmark$ \\
\hline & 41820 & 75.9832 & 250 & 285.0 & 4 & 0 & \\
\hline & 821 & 62.2494 & 0 & & 4 & & \\
\hline & 822 & 54.9998 & 000 & 28 & 4 & & \\
\hline & 41823 & 62.7509 & 8.8750 & 283.3 & 4 & 0 & \\
\hline & 41824 & 117.5860 & & .2 & 4 & & \\
\hline & 4191 & 167.0244 & 8 . & .1 & 4 & & \\
\hline & 4192 & 182.0000 & .5000 & 0 & 4 & 0 & . \\
\hline & 4193 & 176.8342 & 7.72 & .2 & 4 & & \\
\hline & 4194 & 182.7502 & 7 . & .1 & 4 & & \\
\hline & 4195 & 158.2334 & 7250 & 283.2 & 4 & 0 & 0 . \\
\hline & 4196 & 169.3344 & 5500 & 283.9 & 4 & & 0 . \\
\hline & 4197 & 145.5267 & 0 & 28 & 4 & & \\
\hline & 4198 & 105.5445 & 1.62 & 286.9 & 3 & & . \\
\hline & 4199 & 234.4997 & 2.3000 & 287.2 & 3 & 0 & . \\
\hline & 41910 & 227.5069 & 2 . & .3 & 2 & & \\
\hline & 11 & 208.7740 & 2.8000 & 287.8 & 1 & & 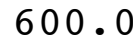 \\
\hline & 41912 & 172.3062 & 9000 & 288.2 & 2 & & . \\
\hline 0 & 41913 & 159.3461 & 2 . & 289 & 1 & & . \\
\hline 00 & 41914 & 204.1715 & 2.9250 & 289.6 & 1 & .0 & 0 . \\
\hline & 41915 & 205.4060 & 2.1750 & 289.9 & 1 & & \\
\hline & 41916 & 203.1607 & 2 . & 290.6 & 1 & & 0 \\
\hline & 41917 & 162.5162 & 3.0000 & 290.4 & 2 & & 0 . \\
\hline & 41918 & 176.6074 & 2.8250 & 290.4 & 2 & 0 & 0 . \\
\hline 00 & 41919 & 170.0107 & 750 & & 3 & & \\
\hline 0 & & 121.9176 & 4.5750 & 287.2 & 4 & & 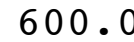 \\
\hline & 41921 & 79.0000 & 5.1500 & 286.1 & 4 & 600.0 & 00. \\
\hline & & 105 & & & 4 & & \\
\hline & 41923 & 170.0277 & 6.7250 & 285.7 & $I$ & 600.0 & 600 \\
\hline
\end{tabular}




\begin{tabular}{|c|c|c|c|c|c|c|c|}
\hline & 24 & & & & & & \\
\hline 0 & 201 & 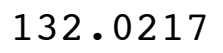 & 4.6500 & & & & \\
\hline & 4202 & 390 & 3500 & 86.1 & & 00 & \\
\hline & 4203 & L 6.6867 & 750 & 286 & & nan & \\
\hline & 204 & 094 & 750 & & & & \\
\hline & $420 \quad 5$ & 290 & 0 & 85 & & & \\
\hline & 4206 & 52.2591 & 500 & 284 & & 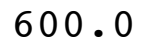 & \\
\hline & 4207 & 743 & 000 & & & & \\
\hline & $420 \quad 8$ & 8003 & 7500 & 6 & & & \\
\hline & 4209 & 54.2005 & 1000 & 87.4 & & 0 & \\
\hline & 2010 & 58.2587 & 3.8000 & $28 \varepsilon$ & & & \\
\hline & 42011 & 8.5000 & 4.7000 & & & & \\
\hline 0 & 42012 & 193 & 9750 & 9 & & & \\
\hline 0 & 2013 & 0011 & 4.3500 & & & & \\
\hline & 42014 & 491 & 0 & & & & \\
\hline 0 & 2015 & & & & & & \\
\hline 0 & 42016 & 1.2530 & 3750 & 292.2 & & .0 & \\
\hline & 42017 & 502 & 0 & 3 & & & \\
\hline 0 & 42018 & 7498 & 0 & 29 & 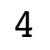 & & \\
\hline 0 & 42019 & 5299 & 250 & 29 & 4 & & \\
\hline 0 & 42020 & 76.9832 & .7250 & 28 & 4 & .0 & \\
\hline 0 & 42021 & 3.2311 & & & 4 & & \\
\hline 0 & 42022 & 291 & 750 & $2 \varepsilon$ & 4 & & \\
\hline 0 & 42023 & .2662 & 500 & & 4 & 0 & \\
\hline 0 & 42024 & 02 & & & 4 & & \\
\hline 0 & 4211 & 39 & 0 & 28 & 4 & & \\
\hline & 4212 & 869 & 750 & 287.2 & 4 & 0 & \\
\hline & 4213 & 000 & & & 4 & & \\
\hline 0 & 4214 & 485 & 0 & 28 & 4 & & \\
\hline & 4215 & 5.7491 & 750 & 4 & 4 & & \\
\hline 0 & 4216 & 180.2511 & 750 & & 4 & & \\
\hline 0 & 4217 & .1604 & 0 & & 4 & & \\
\hline & 4218 & 973 & & 287 & 4 & & \\
\hline 0 & 4219 & 997 & 50 & & 4 & & \\
\hline חס & 42110 & 41 & & & 4 & & \\
\hline & & 15 & 6.9250 & & 4 & & \\
\hline & 42112 & 00 & 4250 & 291.1 & 4 & & \\
\hline 0 & 42113 & 75 & 0 & & 4 & & \\
\hline 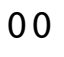 & 42114 & 902 & 7.4750 & & 4 & .0 & \\
\hline & 42115 & 505 & 3000 & 290.3 & 4 & & \\
\hline 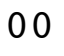 & 42116 & 19 & 250 & & 4 & & \\
\hline 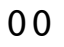 & 42117 & 125 . & 13.8750 & & 4 & & \\
\hline & 42118 & 152.7647 & 3.8750 & 285.4 & 4 & & 0 \\
\hline 00 & 42119 & 764 & 000 & & 4 & & \\
\hline 00 & 42120 & 141.5641 & 14.6000 & & 4 & .0 & \\
\hline 00 & 42121 & 124.9841 & 15.5500 & 284.1 & 4 & .0 & 500 \\
\hline & 42 & & & & 4 & & \\
\hline & 2123 & 7499 & 16.4500 & 282.8 & & .0 & 600 \\
\hline
\end{tabular}




\begin{tabular}{|c|c|c|c|c|c|c|c|}
\hline 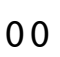 & 42124 & 61.5000 & 14.8500 & 283.4 & 4 & 600.0 & 600.0 \\
\hline 0 & 4221 & 64.0000 & 13.8000 & 282.9 & 4 & 600.0 & 00,0 \\
\hline 00 & 4222 & 57.9999 & 14.8250 & 282.5 & 4 & 600.0 & 00.0 \\
\hline 0 & 4223 & 59.0000 & 15.1500 & 282.8 & 4 & 600.0 & \\
\hline 00 & 4224 & 54.9998 & 13.2000 & 283.0 & 4 & 0.0 & 0 \\
\hline 00 & 4225 & 65.0118 & 11.3500 & 283.9 & 4 & 600.0 & 00.0 \\
\hline 00 & 4226 & 67.0000 & 11.4250 & 284.2 & 4 & 600 & 0 \\
\hline 00 & 4227 & 71.7485 & 10.5000 & 284.8 & 4 & 600.0 & 00.0 \\
\hline 00 & 4228 & 121.3904 & 9.4000 & 285.3 & 4 & 600.0 & 00.0 \\
\hline 00 & 4229 & 136.0404 & 3250 & 286.4 & 3 & & \\
\hline 00 & 42210 & 130.2424 & 5.1750 & 286.9 & 3 & .0 & \\
\hline 00 & 42211 & 173.0093 & 000 & 287.3 & 3 & .0 & \\
\hline 00 & 42212 & 158.4909 & 6.3250 & 288.1 & 4 & 600.0 & \\
\hline 00 & 42213 & 160.9406 & 7.1750 & 287.0 & 4 & 600.0 & \\
\hline 00 & 42214 & 115.0688 & 7 . & 286.7 & 4 & .0 & \\
\hline 00 & 42215 & 177.5031 & 10.3750 & 286.4 & 4 & 60 & \\
\hline 00 & 42216 & 181.7502 & 13.8500 & 285.6 & 4 & .0 & .0 \\
\hline 00 & 42217 & 177.5000 & 11. & 285.6 & 4 & .0 & \\
\hline 00 & 42218 & 179.5000 & 11.3000 & 285.4 & 4 & 60 & \\
\hline 00 & 42219 & 158.2518 & 12.9500 & 283.9 & 4 & .0 & .0 \\
\hline 00 & 42220 & 67.9999 & 14 . & 282.5 & 4 & 0 & \\
\hline 00 & 42221 & 66.7502 & 15.7250 & 281.6 & 4 & .0 & \\
\hline 00 & 42222 & 58.0000 & 13.6750 & 281.3 & 4 & .0 & .0 \\
\hline 00 & 42223 & 60.7500 & 13. & 281.3 & 4 & 0 & \\
\hline 00 & 42224 & 59.9998 & 13 & 281.3 & 4 & 0 & \\
\hline 00 & 4231 & 62.5000 & 11.7500 & 281.6 & 4 & .0 & .0 \\
\hline 00 & 4232 & 55.5092 & 11.5250 & 281.7 & 4 & 6 & \\
\hline 00 & 4233 & 52.7499 & 10.6750 & 281.6 & 4 & .0 & \\
\hline 00 & 4234 & 56.5000 & 5000 & 281.6 & 4 & .0 & \\
\hline 00 & 4235 & 60.5001 & 11.0250 & 281.7 & 4 & 6 & \\
\hline 00 & 4236 & 63.2495 & 11. & 281.4 & 4 & 0 & \\
\hline 00 & 4237 & 61.7498 & 11 . & 281.4 & 4 & .0 & .0 \\
\hline 00 & 4238 & 64.2501 & 11.1500 & 282.1 & 4 & 6 & .0 \\
\hline 00 & 4239 & 66.2479 & 9.6250 & 282.8 & 4 & .0 & .0 \\
\hline 00 & 42310 & 70.2480 & & & 4 & .0 & 0 \\
\hline 00 & 42311 & 117.4987 & 6.1000 & 285.4 & 4 & 600.0 & 0.0 \\
\hline 00 & 42312 & 137.2768 & 5 . & 286.6 & 3 & .0 & .0 \\
\hline 00 & 42313 & 144.7583 & 5.7250 & 287.5 & 3 & 600.0 & 600.0 \\
\hline 00 & 42314 & 125.2345 & 5.8750 & 287.9 & 3 & 600.0 & 600.0 \\
\hline 00 & 42315 & 137.7518 & 6 . & 288.2 & 4 & .0 & .0 \\
\hline 00 & 42316 & 142.9984 & & 288.4 & 4 & 600.0 & 600.0 \\
\hline 00 & 42317 & 145.4105 & 7.1500 & 287.9 & 4 & 600.0 & 600.0 \\
\hline 00 & 42318 & 163.6210 & 5.6750 & 287.5 & 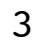 & 600.0 & 600.0 \\
\hline 00 & 42319 & 174.7506 & 6.6250 & 286.3 & 4 & 600.0 & 600.0 \\
\hline 00 & 42320 & 178.7502 & 7.5000 & 285.3 & 4 & 600.0 & 600.0 \\
\hline 00 & 42321 & 181.2500 & & 285 & 4 & 600.0 & \\
\hline 00 & 42322 & 180.5002 & 8.275 & 285.2 & 4 & 600.0 & 600.0 \\
\hline 0 & 42323 & 172.7919 & 8.075 & 285.1 & & 600.0 & 600.0 \\
\hline
\end{tabular}




\begin{tabular}{|c|c|c|c|c|c|c|c|}
\hline 0 & 2324 & & 0000 & 2 & & & \\
\hline & 4241 & & 4.3250 & 6.2 & & 0 & \\
\hline & 4242 & 120.000 & 000 & 287.3 & & 00 & \\
\hline & 4243 & 132.2503 & 8000 & 287.5 & & 00 & \\
\hline & $424 \quad 4$ & 6.5054 & 6250 & 86.4 & & & \\
\hline & 4245 & 129. & & 85.5 & & 0 & \\
\hline & $424 \quad 6$ & 128.9998 & 000 & 285.0 & & 00 & \\
\hline & 4247 & 28.7625 & 500 & 85.4 & & & \\
\hline & 4248 & 147.4999 & 0 & 85.7 & & & \\
\hline & 4249 & 165.5061 & 500 & 86.3 & & 0 & \\
\hline & 42410 & 169.7501 & 8.2750 & 287 & & 0 & \\
\hline & 42411 & 171.2494 & 500 & 8 & & & \\
\hline & 2412 & 168.0 & & 90.2 & & 0 & \\
\hline & 424 & 160.7505 & 9500 & 2 & & & \\
\hline & 424 & 160 & & & & & \\
\hline & 424 & 138.5 & & 293 & 3 & & \\
\hline & 42416 & 121.9 & 0 & 293.8 & & & \\
\hline & 42417 & 97. & 0 & 2 & & & \\
\hline & 42418 & 124 . & & 29 & 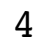 & & \\
\hline & 42419 & 155.1047 & 0 & 289.9 & 4 & & \\
\hline & 42420 & 19 & 500 & 289.3 & 4 & 0 & \\
\hline & 424 & 99.9 & & & 4 & & \\
\hline & 424 & 85 . & 0 & 28 & 4 & & \\
\hline & 42423 & 84.7 & 0 & 5 & 4 & 0 & \\
\hline & 42424 & 68. & & & 4 & & \\
\hline & 4251 & 64 . & 0 & & 4 & & \\
\hline & 4252 & 61.5000 & 000 & 288.2 & 4 & & o. \\
\hline & 4253 & .5009 & 0 & 287.4 & 4 & & \\
\hline & 4254 & 02 & 0 & 28 & 4 & & \\
\hline & 4255 & 500 & 0 & 2 & 4 & & J. \\
\hline & 4256 & 65.0002 & 10 & 28 & 4 & & 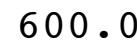 \\
\hline & 4257 & 69. & 0 & & 4 & & \\
\hline & 4258 & 66.9993 & 0 & 28 & 4 & & . \\
\hline & 4259 & 66.7501 & 8.5000 & & 4 & & \\
\hline & 42510 & 499 & 0 & & 4 & & \\
\hline & 42511 & 84.8101 & 1750 & 291.1 & 3 & & 0 . \\
\hline & 42512 & 96.3999 & 6500 & 291.3 & 3 & & \\
\hline & 42513 & 101.4968 & 0 & & 4 & & \\
\hline & 42514 & 104.7502 & 4750 & 292.5 & 3 & & 0 . \\
\hline & 425 & 106.5052 & 1000 & 292.9 & 4 & & \\
\hline & 42516 & 105.7500 & 5000 & 292 & 4 & & \\
\hline & 42517 & 108.8184 & 6.2750 & & 4 & & \\
\hline & 42518 & 144.6388 & 7.1750 & 291.0 & 4 & & 0 . \\
\hline & 42519 & 137.2652 & 8.4750 & & 4 & & \\
\hline 0 & 42520 & 174.7503 & & & 4 & & \\
\hline & 42521 & 175.2495 & 8.5750 & 288.5 & 4 & & 0 . \\
\hline & & 175 & & & 4 & & \\
\hline & 42523 & 180 & 9.5250 & $28 \varepsilon$ & & 60 & 8 \\
\hline
\end{tabular}




\begin{tabular}{|c|c|c|c|c|c|c|c|}
\hline 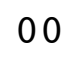 & 4 & & 500 & & & & \\
\hline 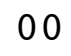 & 426 & -5 & 750 & 88.6 & & 0 & \\
\hline & 4262 & 52. & 9750 & 89.3 & & 00 & \\
\hline & 26 & 997 & 9750 & 89.6 & & 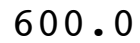 & \\
\hline & 4264 & 94 & 500 & & & & \\
\hline & 4265 & 102 & & 39.7 & & & \\
\hline & 4266 & 113 & 500 & 90.0 & & 0 & \\
\hline & 426 & 01 & 8250 & 0.6 & & & \\
\hline & 426 & 165 & & 1.0 & & & \\
\hline & 4269 & 155 & 000 & 91.4 & & & \\
\hline & 42610 & 161.2500 & 6.3250 & 91 & & & \\
\hline & 42611 & 163 & 0 & 8 & & & \\
\hline & 426 & 172 & 4.7750 & 93.9 & & & \\
\hline & 426 & 165 . & 4.3750 & 4 & & & \\
\hline & 426 & & & & & & \\
\hline & 4261 & & & & & & \\
\hline & 426 & 168 . & 4000 & $9 \varepsilon$ & & & \\
\hline & 42617 & 14 & 0 & 4 & & & \\
\hline & 426 & 11 & 5 . & & & & \\
\hline & 426 & 00 & 5.0750 & 29 & & & \\
\hline & 426 & 57 & 4.7000 & 7 & & & \\
\hline & 426 & & & & & & \\
\hline & 426 & 59 & 1500 & 25 & & & \\
\hline & 426 & 1 & 9750 & 292.0 & & & \\
\hline & 426 & & & & & & \\
\hline & 427 & & 8.8250 & 25 & & & \\
\hline & 4272 & & 8.1250 & 292.5 & 4 & & \\
\hline & 4273 & & 7.6250 & 291.8 & 4 & & \\
\hline & $427 \quad 4$ & & 7.7250 & 2 & 4 & & \\
\hline & 4275 & & 9.7000 & $2 \varepsilon$ & & & \\
\hline & 4276 & & 9750 & 28 & 4 & & \\
\hline & $427 \quad 7$ & & 0 & & 4 & & \\
\hline & 4278 & & 2250 & $2 \varepsilon$ & 4 & & \\
\hline & 4279 & & 5750 & 28 & 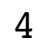 & & \\
\hline & 42710 & & 0 & & 4 & & \\
\hline & 42711 & & 6.5000 & .3 & 4 & & \\
\hline & 42712 & & 9.2750 & 290.3 & 4 & & \\
\hline & 42713 & & 8.5250 & 25 & 4 & & \\
\hline & 42714 & & 9.7000 & $2 \varepsilon$ & 4 & & \\
\hline & 42715 & 58 & .7500 & 289.3 & 4 & & \\
\hline & 42716 & & 6250 & 286.5 & 4 & & \\
\hline & 42717 & & 15.0750 & & 4 & & \\
\hline & 42718 & 50.2461 & 10.3500 & 284.5 & 4 & 0 & \\
\hline & 42719 & & 750 & 284 & 4 & & \\
\hline & 42720 & & 4.9000 & & 4 & & \\
\hline & 42721 & . 2716 & 7.7000 & 282.5 & 4 & 00.0 & \\
\hline & & & & & 4 & & \\
\hline & 2120 & 65.5000 & 13.6500 & 281.6 & & 600.0 & 6 \\
\hline
\end{tabular}




\begin{tabular}{|c|c|c|c|c|c|c|c|}
\hline & 42724 & 8.7470 & 8.3750 & 0.9 & 4 & 0.0 & \\
\hline U & 4281 & 85.7497 & 9.1750 & 280.6 & 4 & 00.0 & \\
\hline & 4282 & 91.8579 & 0500 & 280.4 & & ( & \\
\hline & 4283 & 1.7382 & .2500 & 80.1 & & 0 & \\
\hline & $428 \quad 4$ & 9.9319 & 0 & 279.9 & & 00 & \\
\hline & 4285 & 87.5001 & 0 & 279.7 & & 0 & \\
\hline & 4286 & 93.7510 & 00 & 279.6 & & 0 & \\
\hline & 4287 & 5.7499 & & 79.7 & & & \\
\hline & 4288 & 76.2500 & 7.7250 & 280.0 & & 00.0 & v \\
\hline & 4289 & 81.9917 & 6.47 & 281.1 & & & \\
\hline & 42810 & 102.1578 & & 83.1 & & & \\
\hline & 42811 & 154.5165 & 8 . & 284.6 & & 0 & \\
\hline & 42812 & 123.4945 & 0 & 285.4 & & & \\
\hline & 42813 & 130.7473 & 0 & 1 & & & 30 \\
\hline & 42814 & 119.0254 & & 286.8 & 4 & & \\
\hline & 42815 & 96.0586 & 0 & 287.2 & 4 & 0 & \\
\hline & 42816 & 65.2199 & 0 & 86.5 & 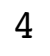 & & \\
\hline & 42817 & 90.6367 & & 287.0 & 4 & & \\
\hline & 42818 & 111.7319 & 0 & 286.3 & 4 & 0 & 0 \\
\hline & 42819 & 110.6460 & & .1 & 4 & 0 & \\
\hline & 42820 & 60.7499 & & 283.7 & 4 & & \\
\hline & 42821 & 59.4997 & & 283.2 & 4 & & \\
\hline & 42822 & 58.5000 & & 283.0 & 4 & 0 & 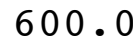 \\
\hline & 42823 & 92.4082 & & 282.9 & 4 & & \\
\hline & 42824 & 172.6041 & & 283.2 & 4 & & \\
\hline & 4291 & 173.2613 & & 282.9 & 4 & 0 & 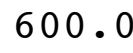 \\
\hline & 4292 & 130.1648 & & 284.3 & 4 & & \\
\hline & 4293 & 134.2390 & & 284.1 & 4 & & \\
\hline & 4294 & 140.9807 & 0 & 284.3 & 4 & 0 & 10 \\
\hline & 4295 & 137.7121 & & 28 & 4 & & \\
\hline & 4296 & 149 . & & 284.2 & 4 & & \\
\hline & 4297 & 138.7416 & & 284.3 & 4 & & \\
\hline & 4298 & 146.5009 & 0 & 285.2 & 4 & 0 & \\
\hline & 4299 & 158.4946 & 0 & 285.9 & 4 & & \\
\hline & 42910 & 151.8093 & & 287.0 & 4 & & \\
\hline & 42911 & 151.2502 & & 288.5 & 3 & & \\
\hline & 42912 & 163.2574 & & 289 & 3 & & \\
\hline & 42913 & 162.0032 & 4.97 & 290.6 & 3 & & - \\
\hline & 42914 & 163.7520 & 0 & 291.7 & 3 & 0 & \\
\hline & 42915 & 146.9748 & & 292.9 & 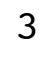 & & \\
\hline & 42916 & 150.5000 & 5.9 & 293.3 & 3 & & 00 . \\
\hline & 42917 & 138.0646 & 5.3500 & 293.5 & 3 & 0 & مח \\
\hline 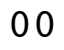 & 42918 & 137.0215 & 0 & 293 & 4 & & \\
\hline 0 & 42919 & 95.4514 & 3.95 & 291.9 & 4 & 600.0 & 600 \\
\hline 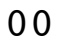 & 42920 & 94.3482 & 3.8000 & 290.2 & 4 & 600.0 & 00 \\
\hline & 42921 & 148.1169 & & 28 & a & & \\
\hline & 42922 & 133.6783 & & 289.2 & 4 & & 600 \\
\hline & $\lcm{2923}$ & 153.2672 & 8.5500 & 288.8 & 4 & 60 & 60 \\
\hline
\end{tabular}




\begin{tabular}{|c|c|c|c|c|c|c|c|}
\hline & 2924 & & & & & & \\
\hline 0 & 4301 & 592 & .2000 & 38.1 & & & \\
\hline & 4302 & 173.0007 & 8.6000 & 88.3 & & 00 & \\
\hline & 4303 & 167.7495 & 6.5750 & 289.0 & & 0 & \\
\hline & 304 & 50 . & & & & & \\
\hline & 305 & 8.99 & & 289 & & & \\
\hline & 306 & 3052 & 500 & 290 & & 00 & \\
\hline & 4307 & 890 & 4 . & & & & \\
\hline & $430 \quad 8$ & 61.8206 & $177^{1}$ & 92.7 & & & \\
\hline 0 & 309 & 919 & 2.42 & 292.6 & & & \\
\hline & 3010 & 2490 & 2.70 & 292 & & & \\
\hline & 3011 & 7521 & 0 & 3 & & & \\
\hline 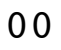 & 3012 & 272.3728 & 2 . & 95.4 & & & \\
\hline 0 & 3013 & 229.3251 & 2.5 & 3 & & & \\
\hline & 3014 & & & & & & \\
\hline 0 & 3015 & 224 & & & & & \\
\hline 0 & 3016 & 170 . & & 298 & & .0 & \\
\hline 0 & 43017 & 8 & & 4 & & & \\
\hline 0 & 3018 & 12 & & 29 & 2 & & \\
\hline 0 & 3019 & 53 & & 29 & 4 & & \\
\hline 0 & 43020 & 63.2470 & .10 & 291.8 & 4 & . 0 & \\
\hline 0 & 43021 & & 0 & & 2 & & \\
\hline 0 & 43022 & 51 & 0 & 29 & 4 & & \\
\hline 0 & 43023 & 68 & 9 . & 29 & 4 & . 0 & \\
\hline 0 & 43024 & & & & 4 & & \\
\hline 0 & 51 & & 8.4 & 29 & 4 & & \\
\hline 0 & $\begin{array}{lll}5 & 1 & 2\end{array}$ & 999 & 0 & 292.1 & 4 & 0 & \\
\hline & $\begin{array}{lll}5 & 1 & 3\end{array}$ & 71 . & 8 . & 291.7 & 4 & & \\
\hline 0 & $\begin{array}{lll}5 & 1 & 4\end{array}$ & 8 & 9 . & 29 & 4 & & \\
\hline & $\begin{array}{lll}5 & 1 & 5\end{array}$ & & 0 & 291.5 & 4 & . 0 & \\
\hline 0 & $\begin{array}{lll}5 & 1 & 6\end{array}$ & 6 & 7.90 & 291.8 & 4 & & \\
\hline 0 & $\begin{array}{lll}5 & 1 & 7\end{array}$ & 4 & 6 & 29 & 4 & & \\
\hline & $\begin{array}{lll}5 & 1 & 8\end{array}$ & 76 . & & 29 & 4 & & \\
\hline 0 & $\begin{array}{lll}5 & 1 & 9\end{array}$ & 506 & & 29 & 3 & & \\
\hline מ & 5110 & 15 & & & 2 & & \\
\hline 0 & $5 \quad 111$ & & & & 1 & & \\
\hline 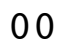 & $5 \quad 112$ & n & 4.6000 & 295.4 & 2 & & \\
\hline 0 & 5113 & 7 & & 29 & 3 & & \\
\hline 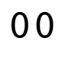 & $5 \quad 114$ & 81.0050 & & 295.8 & 4 & .0 & \\
\hline 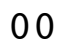 & 5115 & תחמ & & 295.8 & 4 & & \\
\hline 0 & 5116 & 62.2213 & 8 . & 295.5 & 4 & & \\
\hline 0 & $5 \quad 117$ & 62.4992 & 0 & & 4 & & \\
\hline 00 & 5118 & 67.7502 & 4.0250 & 289.7 & 4 & & 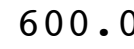 \\
\hline 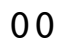 & 511 & 830 & 12 . & 288.9 & 4 & & \\
\hline 00 & 5120 & 76.9909 & 10.9500 & & 4 & . 0 & \\
\hline 00 & $5 \quad 121$ & 102.8357 & 7.9500 & 290.9 & 4 & .0 & 00 \\
\hline & $5 \quad 122$ & 11 & & & 4 & & \\
\hline & $5 \quad 123$ & 85.2202 & 9.5500 & 288.1 & & .0 & 600 \\
\hline
\end{tabular}




\begin{tabular}{|c|c|c|c|c|c|c|c|}
\hline 0 & 24 & & & & & & \\
\hline 0 & 21 & & 000 & 38.2 & & & \\
\hline 0 & 22 & 37.4957 & 9.9500 & 87.2 & & 00 & \\
\hline & 23 & 62.6379 & 3500 & 287.6 & & 00 & \\
\hline & 4 & & 000 & 37.3 & & & \\
\hline & 25 & 1 & & 87.8 & & & \\
\hline & 26 & 54. & 250 & 87.5 & & 0 & \\
\hline & 7 & & .4500 & 88.2 & & & \\
\hline & 28 & 10 & & 89.9 & & & \\
\hline & 29 & 45 . & 5000 & 91.4 & & 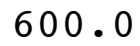 & \\
\hline & 210 & 25 & 6.1000 & 92.4 & & & \\
\hline & 211 & & & 0 & & & \\
\hline & 212 & 106 & 50 & 93.6 & & 0 & \\
\hline & 213 & 2 & 0 & 94.2 & & & \\
\hline & 214 & & & & & & \\
\hline & 215 & & & .7 & & & \\
\hline & 216 & I & 2500 & .7 & & & \\
\hline 5 & 217 & & 50 & .9 & & & \\
\hline & 218 & 15 & & .1 & & & \\
\hline & 219 & 1 & 4250 & .9 & & & \\
\hline ) & 220 & 179 . & 0 & $2 \varepsilon$ & & & \\
\hline & 221 & & & & & & \\
\hline & 222 & 1 & 50 & 2 & & & \\
\hline 5 & 223 & 1 & 50 & 2 & & & \\
\hline & 224 & & & 8 & & & \\
\hline & 31 & & & .8 & & & \\
\hline 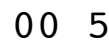 & 32 & .2216 & 50 & 287.6 & 2 & & \\
\hline 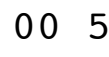 & 33 & & & 8.3 & 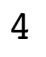 & & \\
\hline & 34 & & & .1 & & & \\
\hline 5 & 35 & & 50 & 287.2 & & & \\
\hline 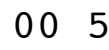 & 36 & & .6500 & 288.6 & 4 & & \\
\hline & 37 & & & 2 & 2 & & \\
\hline 5 & 38 & & & 29 & 1 & & \\
\hline 5 & 39 & & 250 & 291.7 & 4 & & \\
\hline 5 & 310 & & 0 & 292.1 & 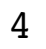 & & \\
\hline 5 & 311 & & & 3.0 & 4 & & \\
\hline 5 & 312 & & 1000 & 293.8 & 4 & & \\
\hline 5 & 313 & & 0 & 295.0 & 3 & & \\
\hline 5 & 314 & & & 294.6 & 4 & & \\
\hline 5 & 315 & & 50 & 294.6 & 4 & & \\
\hline 5 & 316 & & 50 & 295.6 & 4 & & \\
\hline 5 & 317 & & 750 & 295.3 & 4 & & \\
\hline 5 & 318 & 5008 & .4000 & 293.6 & 4 & 0 & \\
\hline 3 & 319 & & 5000 & 29 & 4 & & \\
\hline 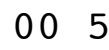 & 320 & & 14.4250 & 288.8 & 4 & & \\
\hline כ & 321 & 003 & 5.0750 & 288.4 & 4 & 600.0 & \\
\hline & 322 & & & & 4 & & \\
\hline & 323 & 7500 & 14.1000 & 288.3 & $\Psi$ & 600.0 & r \\
\hline
\end{tabular}




\begin{tabular}{|c|c|c|c|c|c|c|c|c|}
\hline & & 24 & & 3.7750 & 89.0 & & 准 & \\
\hline & 5 & 1 & 5994 & 2.0750 & 89.7 & & & \\
\hline & 5 & 2 & 000 & .750 & 39.1 & & 00 & \\
\hline & 5 & 3 & 2443 & 10.2750 & 288.2 & & 00 & \\
\hline & 5 & 4 & & & & & & \\
\hline & 5 & 5 & 1 & & 3 & & 0 & \\
\hline & 5 & 6 & 000 & 8.17 & & & & \\
\hline & 5 & 7 & 0 & & & & & \\
\hline & 5 & 8 & 484 & & 5 & & & \\
\hline & 5 & 9 & 73. & & 291.7 & & & \\
\hline & 5 & 10 & 2314 & 6 . & 6 & & & \\
\hline & 5 & 11 & & & & & & \\
\hline & 5 & 412 & 528 & & 93 & & & \\
\hline & 5 & 413 & 55.5095 & 8.75 & 0 & & & \\
\hline & 5 & 41 & & & & & & \\
\hline & 5 & 415 & 803 & & 291 & & & \\
\hline & 5 & 416 & 4.2 & 0 & & & & \\
\hline & 5 & 417 & 802 & & & & & \\
\hline & 5 & 418 & 501 & 0 & $28^{\circ}$ & & & \\
\hline & 5 & 419 & & 12 . & 28 & & & \\
\hline & 5 & 420 & 70 & & 0 & & & \\
\hline & 5 & 421 & 9 & & & 4 & & \\
\hline & 5 & 422 & 832 & & 28 & & & \\
\hline & 5 & 423 & 8 & & & & & \\
\hline & 5 & 424 & & & & & & \\
\hline & 5 & & & & & 4 & & \\
\hline & 5 & 52 & & 0 & 284.6 & 1 & & \\
\hline & 5 & 53 & 2 & 2. & 28 & & & \\
\hline & 5 & 54 & & 12 & $2 \varepsilon$ & 4 & & \\
\hline & 5 & 55 & & & $2 \varepsilon$ & $I$ & & \\
\hline & 5 & 56 & & & $2 \varepsilon$ & 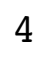 & & \\
\hline & 5 & 57 & & 2 & 28 & 4 & & \\
\hline & 5 & 58 & 497 & & 285.7 & $x$ & & \\
\hline & 5 & 59 & & & & 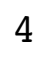 & & \\
\hline & 5 & 510 & & & & 4 & & \\
\hline & 5 & 511 & 56. & & 288.5 & 4 & & \\
\hline & 5 & 512 & טתנ & & & 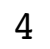 & & \\
\hline & 5 & 513 & 712515 & & 2800 & 1 & & \\
\hline & 5 & 514 & 545 & & 289.5 & 4 & & \\
\hline & 5 & 51 & 175 . & & 285 & 4 & & \\
\hline & 5 & 516 & 183. & 0 . & & 4 & & \\
\hline & 5 & 517 & 195.2527 & 10. & 289.1 & 4 & & \\
\hline & 5 & 518 & 231. & 0 & 288.2 & 4 & & \\
\hline & 5 & 519 & & 10 . & 286.4 & & & \\
\hline & 5 & 520 & 70.5000 & 11.5500 & & $\Psi$ & & \\
\hline & 5 & 52 & 999 & $11 . \varepsilon$ & 284.9 & 4 & & \\
\hline & 5 & 522 & & & & & & \\
\hline & & 523 & 78.4998 & 13.1000 & 283.4 & & 600.0 & 600 \\
\hline
\end{tabular}




\begin{tabular}{|c|c|c|c|c|c|c|c|c|}
\hline 0 & 5 & 524 & 75.9998 & 13.3750 & 282.2 & 4 & 600.0 & 600.0 \\
\hline 00 & 5 & 61 & 77.2500 & 12.4500 & 282.2 & 4 & 600.0 & 00.0 \\
\hline 00 & 5 & 62 & 72.7513 & 11.2250 & 282.1 & 4 & 600.0 & 00.0 \\
\hline 0 & 5 & 63 & 71.7499 & 11.5750 & 282.2 & 4 & 600.0 & 00.0 \\
\hline 00 & 5 & 64 & 71.2503 & 11.2000 & 282.1 & 4 & 600.0 & 00.0 \\
\hline 00 & 5 & 65 & 71.7498 & 10.9000 & 282.6 & 4 & 600.0 & 00.0 \\
\hline 00 & 5 & 66 & 73.2501 & 9.7250 & 283.8 & 4 & 600.0 & 00.0 \\
\hline 00 & 5 & 67 & 74.0000 & 10.3500 & 282.9 & 4 & 600.0 & 00.0 \\
\hline 00 & 5 & 68 & 74.0003 & 8.3500 & 284.0 & 4 & 600.0 & 00.0 \\
\hline 00 & 5 & 69 & 76.9999 & 8.4500 & 284.9 & 4 & 600.0 & 0 \\
\hline 00 & 5 & 610 & 73.7562 & 7.2500 & 286.3 & 4 & 600.0 & \\
\hline 00 & 5 & 611 & 62.7481 & 5.0750 & 287.5 & 3 & 600.0 & \\
\hline 00 & 5 & 612 & 65.7502 & 6.7000 & 287.4 & 4 & 600.0 & 00.0 \\
\hline 00 & 5 & 613 & 69.7504 & 8.3000 & 286.7 & 4 & 600.0 & 00.0 \\
\hline 00 & 5 & 614 & 82.8904 & 250 & 286.6 & 4 & .0 & \\
\hline 00 & 5 & 615 & 159.3603 & 8.5500 & 286.3 & 4 & 600.0 & .0 \\
\hline 00 & 5 & 616 & 82.6327 & 9.2750 & 284.9 & 4 & 60 & 0.0 \\
\hline 00 & 5 & 617 & 72.0021 & 9.9750 & 282.8 & 4 & .0 & \\
\hline 00 & 5 & 618 & 66.2502 & 9.6250 & 282.4 & 4 & 60 & .0 \\
\hline 00 & 5 & 619 & 87.0137 & 5.7250 & 281.8 & 4 & .0 & .0 \\
\hline 00 & 5 & 620 & 10.6114 & 2.8500 & 281.5 & 5 & 60 & \\
\hline 00 & 5 & 621 & 142.0105 & 3.2000 & 281.2 & 5 & .0 & \\
\hline 00 & 5 & 622 & 180.8953 & 2.0000 & 280.9 & 5 & 600.0 & .0 \\
\hline 00 & 5 & 623 & 276.3994 & 1.45 & 280.7 & J & 60 & \\
\hline 00 & 5 & 624 & 308.1999 & 1.5500 & 280.8 & 5 & .0 & \\
\hline 00 & 5 & 71 & 298.5029 & 2.4500 & 280.9 & 5 & 600.0 & 600.0 \\
\hline 00 & 5 & 72 & 346.9134 & 1.7000 & 281.0 & 5 & 60 & .0 \\
\hline 00 & 5 & 73 & 0.2657 & 1.3750 & 281.2 & 4 & .0 & \\
\hline 00 & 5 & 74 & 316.9989 & 1.4000 & 281.5 & 4 & .0 & 0.0 \\
\hline 00 & 5 & 75 & 320.9989 & 1.3500 & 281.4 & 4 & .0 & .0 \\
\hline 00 & 5 & 76 & 281.2980 & 0.1500 & 281.7 & 5 & .0 & \\
\hline 00 & 5 & 77 & 357.3900 & 0.7750 & 282.2 & 4 & 600.0 & 600.0 \\
\hline 00 & 5 & 78 & 335.8454 & 0.6250 & 282.7 & 3 & 600.0 & 600.0 \\
\hline 00 & 5 & 79 & 203.3689 & 1 . & 283.5 & 2 & 0 & 0.0 \\
\hline 00 & 5 & 710 & 333.4130 & 0.8750 & 283.9 & 3 & 600.0 & 600.0 \\
\hline 00 & 5 & 711 & 327.7569 & 0.8500 & 285.5 & 2 & 600.0 & 600.0 \\
\hline 00 & 5 & 712 & 254.3208 & 1.2250 & 286.1 & 1 & 600.0 & 600.0 \\
\hline 00 & 5 & 713 & 260.4118 & 1.0750 & 286.5 & 1 & 600.0 & 600.0 \\
\hline 00 & 5 & 714 & 250.6275 & 0.9500 & 286.7 & 2 & 600.0 & 600.0 \\
\hline 00 & 5 & 715 & 219.4986 & 1.5250 & 286.7 & 3 & 600.0 & 600.0 \\
\hline 00 & 5 & 716 & 227.4992 & 1.4000 & 286.7 & 3 & 600.0 & 600.0 \\
\hline 00 & 5 & 717 & 230.0000 & 1.0250 & 286.8 & 3 & 600.0 & 600.0 \\
\hline 00 & 5 & 718 & 270.7670 & 1.0000 & 286.8 & 3 & 600.0 & 600.0 \\
\hline 00 & 5 & 719 & 297.2482 & 1.4750 & 286.7 & 4 & 600.0 & 600.0 \\
\hline 00 & 5 & 720 & 299.4876 & 1.3000 & 286.7 & 4 & 600.0 & 600.0 \\
\hline 00 & 5 & 721 & 275.1999 & 1.4250 & 286.7 & 5 & 600.0 & 600.0 \\
\hline 00 & 5 & 722 & 287.9980 & 1.0250 & 286.6 & 5 & 600.0 & 600.0 \\
\hline 00 & J & 723 & 334.7841 & 0.900 & 286.6 & 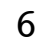 & 600.0 & 600.0 \\
\hline
\end{tabular}




\begin{tabular}{|c|c|c|c|c|c|c|c|}
\hline 0 & 24 & & & & & & \\
\hline 0 & 1 & & 2000 & 36.4 & & & \\
\hline 0 & 8 & 24.708 & 5500 & 86.3 & & 00 & \\
\hline & 8 & 546 & 250 & 286.2 & & 0 & \\
\hline & 4 & & & & & & \\
\hline & 85 & 5 & & 36.1 & & & \\
\hline & 8 & 338 . & 7000 & 86.3 & & 0 & \\
\hline & 7 & 4 & 500 & & & & \\
\hline & 8 & 300 & & 87.6 & & & \\
\hline & 89 & 276 . & 750 & 88.5 & & & \\
\hline & 810 & 236.7877 & 2.8000 & 88.4 & & & \\
\hline & 811 & 258 & 2.1250 & & & & \\
\hline & 812 & 275 & 1 . & 88.5 & & 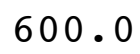 & \\
\hline & 813 & 246 . & 1.7000 & .9 & & & \\
\hline & 814 & 16 & & & & & \\
\hline & 815 & 15 & & 1.4 & & & \\
\hline & 816 & 141 . & 0 & 91.6 & & & \\
\hline & 817 & 122 & 0 & & & & \\
\hline & 818 & 10 & & & & & \\
\hline & 819 & 7 & 50 & & & & \\
\hline 0 & 820 & 80 & 9.3500 & 287.3 & & & \\
\hline & 821 & 14 & & & & & \\
\hline & 822 & 14 & 50 & .7 & & & \\
\hline 5 & 823 & 125 & 50 & .1 & & & \\
\hline 5 & 824 & & & & & & \\
\hline 0 & 9 & & 0 & & & & \\
\hline ) & 92 & 8 & 2000 & .5 & & & \\
\hline 5 & 93 & 7 & 0 & .4 & 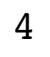 & & \\
\hline & 94 & $\pi$ & 0 & & & & \\
\hline 5 & 95 & 5 & 500 & .0 & & & \\
\hline 5 & 96 & & 1250 & .1 & 4 & & \\
\hline & 97 & 6 & 0 & & 4 & & \\
\hline 5 & 98 & & 50 & .7 & 4 & & \\
\hline 5 & 99 & & 50 & $2 \xi$ & 4 & & \\
\hline 5 & 910 & & & & 4 & & \\
\hline 5 & 911 & & & 7.7 & 3 & & \\
\hline 5 & 912 & 792 & 0 & 288.8 & 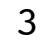 & & \\
\hline 5 & 913 & 15 & 5 . & 2 & 3 & & \\
\hline 5 & 914 & & 4 . & 291.3 & 3 & & \\
\hline 5 & 915 & & 500 & 291.0 & 4 & & \\
\hline 5 & 916 & & 0 & 29 & 4 & & \\
\hline 5 & 917 & & 1.5500 & 289.1 & 4 & & \\
\hline 5 & 918 & 180.9986 & .2000 & 287.6 & 4 & 0 & \\
\hline 5 & 919 & 153 & 500 & & 4 & & \\
\hline 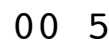 & 920 & & & 284.7 & 4 & & \\
\hline 3 & 921 & 5000 & 13.8000 & 284.1 & 4 & 00.0 & \\
\hline 3 & 92 & & & & 4 & & \\
\hline & 923 & 6.2515 & 12.6500 & 283.6 & $\mathbf{4}$ & 600.0 & 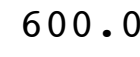 \\
\hline
\end{tabular}




\begin{tabular}{|c|c|c|c|c|c|c|c|}
\hline & 24 & 20 & 4500 & 33.1 & 4 & . & \\
\hline & 5101 & 8.2512 & 7.6250 & 282.8 & & 600.0 & \\
\hline & 102 & 118.2532 & 1250 & 282.4 & & 00 & \\
\hline & 103 & 34.7497 & 1000 & 282.0 & & 00 & \\
\hline & 104 & 35.7511 & 5500 & 81.7 & & & \\
\hline & 105 & 149.7473 & & 81.2 & & 0 & \\
\hline & 5106 & 151.2470 & 3500 & 81.3 & & 00 & \\
\hline & 5107 & 142.2522 & 750 & 81.6 & & & \\
\hline & 5108 & 132.2924 & 9250 & 82.3 & & & \\
\hline & 5109 & 132.5343 & 250 & 82.9 & & 0 & \\
\hline & 1010 & 0166 & 500 & 283.2 & & & \\
\hline & 51011 & 146.5095 & 7.0000 & 34.1 & & & \\
\hline & 51012 & 161.7795 & 6.8250 & 85.0 & & 0 & \\
\hline & 51013 & 162.0000 & 7.37 & 5 & & 0.0 & \\
\hline & 51014 & 157 & & & & & \\
\hline & 51015 & 154 . & & 5.7 & 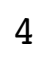 & & \\
\hline & 51016 & 153.5005 & 250 & 285.5 & 4 & 0 & \\
\hline & 51017 & 162.7628 & 0 & .1 & 4 & & \\
\hline & 51018 & 179.0016 & & .4 & 4 & & \\
\hline & 51019 & 162 . & 0 & 283.2 & 4 & & \\
\hline & 51020 & 173.7539 & 0 & 281.9 & 4 & 0 & \\
\hline & 51021 & 163.0196 & & 31.2 & 4 & & \\
\hline & 51022 & 151. & 0 & 81.1 & 4 & & \\
\hline & 51023 & 130.4954 & 8.40 & 0 & 4 & 0 & \\
\hline & 51024 & 97.4933 & & & 4 & & \\
\hline & 5111 & 84 . & 0 & & 4 & & \\
\hline & 5112 & 72.7501 & 0 & 0.0 & 4 & & 0 \\
\hline & 5113 & 67.5000 & 0 & 9.5 & 4 & & \\
\hline & 5114 & 69.2491 & 8 . & 279.3 & 4 & & \\
\hline & 5115 & 67.5000 & 0 & 279.1 & 4 & & J. \\
\hline & 5116 & 71.5002 & 8 . & 279.2 & 4 & & 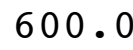 \\
\hline & 5117 & 79.5002 & & & 4 & & \\
\hline & 5118 & 78.5000 & 0 & 1.5 & 4 & & 0 . \\
\hline & 5119 & 86.9847 & 50 & .1 & 3 & 0 & 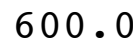 \\
\hline & 51110 & 126 . & 0 & & 3 & & \\
\hline & 51111 & 143.2499 & 0 & 5.2 & 3 & & \\
\hline & 51112 & 142.2541 & 750 & 286.0 & 4 & & \\
\hline & 51113 & 157.2496 & 0 & & 3 & & \\
\hline & 51114 & 135.8127 & 000 & & 3 & & 0 . \\
\hline & 51115 & 144.2616 & 250 & 288.9 & 3 & & \\
\hline & 51116 & 150.7528 & 000 & & 3 & & \\
\hline & 51117 & 148.9988 & 5.8750 & 289.6 & 3 & & 0 . \\
\hline & 51118 & 159.2496 & 6.3500 & 289.6 & 4 & & 0 . \\
\hline & 51119 & 92.0529 & 250 & & 4 & & \\
\hline 0 & 51120 & 67.9998 & 6.7250 & & 4 & & \\
\hline & 51121 & 69.0007 & 8.1750 & 284.7 & 4 & & 00 \\
\hline & 51122 & 70 & & & 4 & & \\
\hline & 51123 & 67.2500 & 8.8250 & 284.4 & $\Psi$ & 600.0 & 600 \\
\hline
\end{tabular}




\begin{tabular}{|c|c|c|c|c|c|c|c|}
\hline & 24 & 0 & 500 & 84.5 & 4 & . & \\
\hline & 5121 & 0.0000 & 3.8000 & 284.4 & 4 & 600.0 & \\
\hline & 5122 & 69.9998 & 9250 & 284.1 & 4 & 00 & \\
\hline & 5123 & 70.7499 & 8.5000 & 284.2 & & 00 & \\
\hline & 124 & 69.9999 & 500 & & & & \\
\hline & 125 & 993 & & 4 & & 0 & \\
\hline & 5126 & 2500 & 500 & 85.2 & & 00 & \\
\hline & 127 & 79.2501 & 4.6500 & 85.8 & & & \\
\hline & 128 & .9749 & 1.5750 & 87.3 & & & \\
\hline & 129 & 41.2504 & 1.9750 & 88.3 & & 0 & \\
\hline & 1210 & 225.2558 & 3.0750 & $28 \varepsilon$ & & & \\
\hline & 1211 & 211.0347 & 2.9500 & & & & \\
\hline & 1212 & 209 & 2.9500 & 90.6 & & & \\
\hline & 1213 & 204.0037 & 3.5750 & & & & \\
\hline & 1214 & 195. & 0 & & & & \\
\hline & 1215 & 182 . & & .5 & & & \\
\hline & 51216 & 176 . & 3.9500 & 292.2 & & & \\
\hline & 51217 & 173. & 0 & 6 & & & \\
\hline & 51218 & 177 . & 0 & & 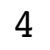 & & \\
\hline & 51219 & 164 & 250 & 290 & 4 & & \\
\hline & 51220 & 125.0370 & .4500 & 28 & 4 & 0 & \\
\hline & 51221 & 181.2 & & & 4 & & \\
\hline & 51222 & 179 . & 0 & 28 & 4 & & \\
\hline & 51223 & 180.7499 & 250 & 2 & 4 & 0 & \\
\hline & 51224 & 175.25 & & & 4 & & \\
\hline & 5131 & 144 & 2 . & & 5 & & \\
\hline & 5132 & 332 . & 750 & 0 & 6 & & 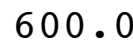 \\
\hline & 5133 & 359 . & 0 & & 5 & & \\
\hline & 5134 & 9 . & 0 & $2 \varepsilon$ & 6 & & \\
\hline & 5135 & 28.9960 & 0 & 7.0 & 6 & & 0 \\
\hline & 5136 & 19.2515 & 0 & $2 \varepsilon$ & 5 & & 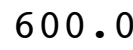 \\
\hline & 5137 & 7.5976 & 0 & & 4 & & \\
\hline & 5138 & 44.9316 & 0 & 28 & 3 & & 。 \\
\hline & 5139 & 96 . & 9000 & 28 & 0 & & \\
\hline & 51310 & 140 . & & & 3 & & \\
\hline & 51311 & 298.2974 & 1.7500 & .9 & 2 & & \\
\hline & 51312 & 233.1018 & 250 & 292.0 & 1 & & \\
\hline & 51313 & 200 . & 4 . & 29 & 2 & & \\
\hline & 51314 & 182.2443 & 4.9000 & 291.7 & 3 & & . \\
\hline & 51315 & 163.7132 & 500 & 292.4 & 2 & & \\
\hline & 51316 & 159.4825 & 3.7250 & & 2 & & \\
\hline & & 182.9998 & 4.1750 & & 3 & & \\
\hline & 51318 & 110.8335 & 5.2750 & 291.4 & 3 & & 0 . \\
\hline & 51319 & 136.2784 & 000 & & 4 & & \\
\hline 0 & 51320 & 162.7386 & 9.0750 & & 4 & & \\
\hline & 51321 & 137.5416 & 0.1500 & 284.5 & 4 & & - \\
\hline & & 79 & & & 4 & & \\
\hline & 51323 & 81.2382 & 10.1250 & 284.9 & $\Psi$ & 600.0 & 00 \\
\hline
\end{tabular}




\begin{tabular}{|c|c|c|c|c|c|c|c|}
\hline 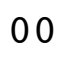 & 1324 & 5952 & .4750 & 83.9 & 4 & 000 & \\
\hline & 5141 & 106.1157 & 8.0000 & 284.0 & 4 & 600.0 & \\
\hline & 142 & 141.0667 & 4000 & 284.7 & 5 & 00 & \\
\hline & 143 & 169.7466 & 1.3250 & 283.2 & & 00 & \\
\hline & 144 & 5603 & 500 & 31.3 & & 0 & \\
\hline & 145 & 335 & & 80.8 & & 0 & \\
\hline & 146 & 331.0021 & 9250 & 81.4 & & 00 & \\
\hline & 147 & 344.0535 & 1.3750 & 83.1 & & & \\
\hline & 148 & 323.5022 & 1.1250 & .1 & & & \\
\hline & 149 & 285 & 2.5500 & 86.3 & & 00 & U \\
\hline & 1410 & 270.7499 & 3.5500 & 288.5 & & 00.0 & \\
\hline & 1411 & 267.0034 & 4.4250 & & & & \\
\hline & 2 & 169.3442 & 2.4250 & 290.0 & & 0 & \\
\hline & 1413 & 146.9637 & 0 & 6 & & 0.0 & 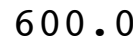 \\
\hline & 414 & 330 & & & & & \\
\hline & & 234 . & & 0 & & & \\
\hline & 1416 & 347.4873 & 3.15 & 90.8 & & .0 & \\
\hline & 1417 & 1.2498 & 7.32 & .0 & & & \\
\hline & 418 & 9.5627 & & & 3 & & \\
\hline & 1419 & 23.2476 & 7.3000 & 288.1 & 4 & .0 & . \\
\hline & 51420 & 11.2463 & 4.3750 & 287.4 & 4 & 0 & \\
\hline & 1421 & 352.9979 & & & 4 & & \\
\hline & 1422 & 347.7310 & 0 & 9 & 4 & & ? \\
\hline & 51423 & 38.5000 & 0 & 5.5 & 4 & 0 & 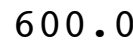 \\
\hline & 51424 & 177.3545 & & & 5 & & \\
\hline & 5151 & 198.2226 & 250 & $2 \varepsilon$ & 6 & & \\
\hline & 5152 & 260.5425 & 1.6500 & 7 & 6 & & J. \\
\hline & 5153 & 289 . & 1. & 2 & 6 & & \\
\hline & 5154 & 346 . & 50 & 28 & 6 & & \\
\hline & 5155 & 75.5793 & 50 & 4.2 & 6 & & 0 . \\
\hline & 5156 & 57.3902 & 0 & 284.3 & 6 & & 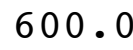 \\
\hline & 5157 & 192 . & 0 & & 5 & & \\
\hline & 5158 & 143 . & 0 & 3 & 4 & & . \\
\hline & 5159 & 057 & & 2 & 3 & & 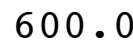 \\
\hline & 515 & 51. & & & 2 & & \\
\hline & 51511 & 67.5275 & 3.2250 & 5.5 & 2 & & . \\
\hline & 51512 & 298.4209 & 2000 & 287.1 & 1 & & 0 \\
\hline 0 & 51513 & 26 . & 4. & & 2 & & \\
\hline & 51514 & 34.1741 & 4.7750 & .4 & 3 & .0 & 0 . \\
\hline & 51515 & 80 . & 000 & 288.0 & 4 & & \\
\hline & 51516 & 60 . & 250 & 28 & 4 & & 0 \\
\hline & 51517 & 43.7490 & 6.4500 & 287.1 & 4 & & 0 . \\
\hline & 51518 & 94.5216 & 4.9500 & 286.9 & 3 & 0 & 0 . \\
\hline & 51519 & 128.2393 & 000 & & 4 & & \\
\hline 2 & 51520 & 102.4038 & & & 4 & & \\
\hline & 51521 & 57.4524 & 6.3000 & 283.0 & 4 & & 00 . \\
\hline & & 99 & & & 4 & & \\
\hline & 51523 & 145.4049 & 5.5750 & 282.2 & $\Psi$ & 600.0 & 600 \\
\hline
\end{tabular}




\begin{tabular}{|c|c|c|c|c|c|c|c|}
\hline 0 & 24 & & 0 & & & & \\
\hline 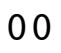 & 161 & 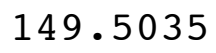 & 4.0000 & 32.3 & & & \\
\hline & 162 & 28.3716 & 6.6500 & 82.3 & & 00 & \\
\hline & 163 & 43.6898 & 6.5750 & 82.1 & & 00 & \\
\hline & 164 & 2.2 & 500 & 82.1 & & & \\
\hline & 65 & 1.24 & 1000 & 82.2 & & 0 & \\
\hline & 166 & 19.6721 & 8.2500 & 82.4 & & ( & \\
\hline & 167 & 034 & 9.1250 & 83.0 & & & \\
\hline & 168 & 5189 & 1750 & 83.7 & & & \\
\hline 0 & 169 & 34.2678 & 9.3000 & 84.3 & & 0 & \\
\hline & 1610 & 67.2585 & 8.8750 & 285.2 & & & \\
\hline & 611 & (9) 7545 & 7.2750 & & & & \\
\hline 0 & 2 & 48.0048 & 6.7750 & 86.7 & & .0 & \\
\hline 0 & 613 & 0.1856 & 6.8250 & 3 & & & \\
\hline & 4 & 36.2500 & 0 . & & & & \\
\hline 0 & & 33.7499 & & 9 & 4 & & \\
\hline 0 & 1616 & 82.7500 & 9.32 & 286.2 & 4 & .0 & \\
\hline 0 & 617 & 31.0003 & 8 . & & 4 & $\cdot 0$ & \\
\hline 0 & 8 & 500 & & & 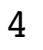 & & \\
\hline 0 & 9 & 8.7512 & 250 & & 4 & & \\
\hline 0 & 1620 & 8.7502 & 8.5500 & 284.3 & 4 & - 0 & \\
\hline 0 & 1 & 6.7010 & 9.42 & & 4 & & \\
\hline 0 & 2 & 507 & 0 & & 4 & & \\
\hline 0 & 1623 & 89.9605 & .3250 & & 4 & . 0 & \\
\hline 0 & 51624 & 100.2874 & 0 & & 4 & & \\
\hline 0 & 5171 & 999 & 9 . & & 4 & & \\
\hline 0 & 5172 & 105.7629 & 8.20 & 4 & 4 & .0 & \\
\hline 0 & 5173 & 136.8668 & 7. & & 4 & & \\
\hline 0 & 5174 & 2496 & 8 . & & 4 & & \\
\hline & 5175 & 5000 & 0 & 2.8 & 4 & & \\
\hline 0 & 5176 & 107.2527 & 7.9750 & 0 & 4 & & \\
\hline 0 & 5177 & 2363 & 0 & & 4 & & \\
\hline & 5178 & 2527 & 000 & & 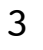 & & \\
\hline 0 & 5179 & 158.7468 & 4.7250 & & 3 & 0 & \\
\hline 0 & 51710 & 7120 & 0 & & 3 & & 0 \\
\hline 0 & 51711 & 16 & & & 3 & & \\
\hline 0 & 51712 & 152.0395 & 4.8750 & 9.6 & 3 & .0 & \\
\hline 0 & 51713 & 491 & 000 & & 3 & & \\
\hline 0 & 51714 & 161.7756 & 5.6750 & 291.2 & 3 & .0 & \\
\hline 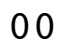 & 51715 & 146.7521 & 000 & 292.4 & 3 & & \\
\hline 0 & 51716 & 459 & 250 & & 4 & & \\
\hline 0 & 51717 & 154.3506 & 7.2500 & & 4 & & \\
\hline 0 & 51718 & 116.0850 & 5.2750 & 293.0 & 3 & & 0 \\
\hline 5 & 51719 & 124.1561 & 250 & & 4 & & \\
\hline 00 & 51720 & 201.5046 & 8.1500 & & 4 & .0 & \\
\hline 00 & 51721 & 192.9998 & 9.0000 & 289.1 & 4 & .0 & 500 \\
\hline & & & & & 4 & & \\
\hline & 51723 & 179.7670 & 8.9750 & 289.8 & & .0 & 600 \\
\hline
\end{tabular}




\begin{tabular}{|c|c|c|c|c|c|c|c|}
\hline 0 & 24 & & 3750 & 90.3 & & & \\
\hline 0 & 181 & 309 & 7.4750 & 91.1 & & 0 & \\
\hline & 182 & 126.5021 & 6.3500 & 293.0 & & 00 & \\
\hline & 183 & 32.7608 & 7.0750 & 292.1 & & 00 & \\
\hline & 184 & 12.7539 & .5250 & 90.9 & & & \\
\hline & 185 & 39.0019 & & 290.4 & & 0 & \\
\hline & 186 & 53.7513 & 0.3750 & 290.2 & & 00 & \\
\hline & 187 & 55.7501 & 0 & 90.6 & & & \\
\hline & 188 & 3.4982 & 0 & 91.4 & & & \\
\hline 0 & 189 & 66.5120 & 6.8250 & 292.4 & & 0 & \\
\hline 0 & 1810 & 56.0053 & 6.7000 & 292.5 & & & \\
\hline 0 & 1811 & 54.5000 & 6.0000 & 7 & & & \\
\hline 0 & 1812 & 0011 & 5.72 & 295 & & & \\
\hline 0 & 1813 & 0018 & 5.1000 & 29 & & & \\
\hline & 814 & 70.7482 & & & & & \\
\hline 0 & 15 & 670 & & 29 & 3 & & \\
\hline 0 & 1816 & 121.1050 & 4.9500 & 299 & 3 & .0 & \\
\hline 0 & 817 & 09.7533 & 5.32 & 6 & & $\cdot 0$ & \\
\hline 0 & 818 & 94.0023 & 5.95 & $29^{\circ}$ & 3 & & \\
\hline 0 & 819 & 156.7984 & 4500 & 29 & 4 & & \\
\hline 0 & 51820 & 185.2500 & 7.5500 & 294.4 & 4 & .0 & \\
\hline 0 & 1 & 177.7670 & 0 & & 4 & & \\
\hline 0 & 1822 & 175.0000 & 0 & 29 & 4 & & \\
\hline 0 & 51823 & 183.2496 & 7 . & 29 & 4 & .0 & \\
\hline 0 & 51824 & 181.7500 & & & 4 & & \\
\hline 0 & 5191 & 7500 & 0 & 29 & 4 & & \\
\hline 0 & 5192 & 174.0048 & 7.95 & 29 & 4 & .0 & \\
\hline 0 & 5193 & 168.5031 & 7. & 29 & 4 & & \\
\hline 0 & 5194 & 193 & 6 . & 29 & 4 & & \\
\hline & 5195 & 143.7082 & 2 . & 294 & r & & \\
\hline 0 & 5196 & 113.1886 & 1.80 & 292 & 6 & & \\
\hline 0 & 5197 & 3860 & 170 & 29 & 5 & & \\
\hline & 5198 & 7500 & & & 4 & & \\
\hline 0 & 5199 & 7507 & 8 . & & 4 & 0 & \\
\hline 0 & 51910 & 1 & 0 & & 4 & & \\
\hline 0 & 51911 & & 7.22 & & 4 & .0 & \\
\hline م & 51912 & 149.9989 & 7.65 & 297.8 & 4 & .0 & \\
\hline 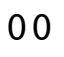 & 51913 & 2486 & 7 . & 29 & 4 & & \\
\hline 0 & 51914 & 158.5017 & 6.7500 & 300.3 & 4 & .0 & \\
\hline 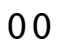 & 51915 & 158.4992 & 6 . & 301.0 & 4 & & \\
\hline 00 & 51916 & 154.4952 & 5 . & 30 & 3 & & \\
\hline 00 & 51917 & 150.5027 & 4.52 & & 3 & .0 & \\
\hline 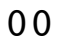 & 51918 & 156.7495 & 5.2750 & 301.9 & 3 & & 0 \\
\hline 0 & 51919 & 3192 & 5 . & 30 & 4 & & \\
\hline 00 & 51920 & 100.3750 & 6.8750 & & 4 & .0 & \\
\hline 00 & 51921 & 172.7580 & 8.0000 & 297.2 & 4 & .0 & 00 \\
\hline & & 18 & & & 4 & & \\
\hline & 51923 & 132.1320 & 7.5250 & 298.0 & & .0 & 600 \\
\hline
\end{tabular}




\begin{tabular}{|c|c|c|c|c|c|c|c|}
\hline 0 & 924 & & 750 & 98.2 & & & \\
\hline 0 & 201 & 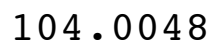 & 5.8500 & 98.1 & & 0 & \\
\hline 0 & 202 & 128.0130 & 4000 & 298.1 & & 00 & \\
\hline & 5203 & 107.2518 & 1250 & 298.9 & & 00.0 & \\
\hline & 204 & 24.42 & 2500 & 298.6 & & & \\
\hline & 5205 & 45.999 & 4250 & 296.9 & & 0 & \\
\hline 0 & 5206 & 43.4973 & 7.4000 & 296.8 & & 00 & \\
\hline & 5207 & 51.7501 & 47 & 297.2 & & & \\
\hline & 5208 & 8.7505 & 75 & 297.7 & & & \\
\hline 0 & 5209 & 48.7503 & 7.5000 & 298.1 & & 0 & \\
\hline J & 52010 & 48.7390 & 7.7750 & 298.2 & & & \\
\hline 0 & 52011 & 59.5000 & .72 & 298 & & & \\
\hline 0 & 52012 & 54.2658 & 4.85 & 300.4 & & .0 & \\
\hline 0 & 52013 & 57.7529 & $\cdot 23$ & 301.6 & & 0.0 & \\
\hline 0 & 52014 & 000 & 0 & 302 & & & \\
\hline 0 & 52015 & 046 & & 303 & 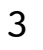 & & \\
\hline 0 & 52016 & 181.5000 & 0 & 303.8 & 4 & 0.0 & \\
\hline 0 & 52017 & 77.4989 & 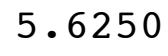 & 30 & & .0 & \\
\hline 0 & 52018 & 74.7507 & 0 & 30 & 3 & & \\
\hline 0 & 52019 & 171.5815 & 3.42 & 30 & 4 & & \\
\hline 0 & 52020 & 90.9831 & 4.35 & 30 & 4 & .0 & 000 \\
\hline 0 & 52021 & 88.6739 & & 300 & 4 & & \\
\hline 0 & 52022 & 137.4249 & 0 & 30 & 4 & & \\
\hline 0 & 52023 & 126.4101 & 0 & 30 & 4 & .0 & \\
\hline 0 & 52024 & 90 & & 30 & 4 & & \\
\hline 00 & 5211 & 98.5897 & & 30 & 4 & & \\
\hline 0 & 5212 & 122.3312 & 1500 & 301.0 & 4 & .0 & \\
\hline 0 & 5213 & 112.252 & 20 & 301.5 & 4 & .0 & \\
\hline 00 & 5214 & 9983 & 3 . & 30 & 5 & .0 & \\
\hline 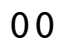 & 5215 & 134.0410 & 9500 & 300 & 4 & .0 & \\
\hline 0 & 5216 & 145.5043 & 500 & 299.7 & 5 & .0 & \\
\hline 0 & 5217 & 132.2374 & 0 & 300.2 & 4 & & \\
\hline 0 & 5218 & 153. & & 30 & 3 & .0 & \\
\hline 0 & 5219 & 165.7485 & 0 & 30 & 3 & .0 & \\
\hline 0 & 52110 & 164.4989 & 0 & 30 & 4 & & \\
\hline 00 & 52111 & 162.2500 & 7500 & 302.8 & 3 & .0 & \\
\hline 0 & 52112 & 165.9991 & 7.1250 & 303.6 & 4 & .0 & \\
\hline 0 & 52113 & 163.7488 & 7 . & 304 & 4 & .0 & \\
\hline 00 & 52114 & 168.0002 & 7.4750 & 305.5 & 4 & .0 & 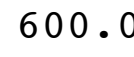 \\
\hline 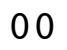 & 52115 & 164.7501 & 6.7000 & 306.3 & 4 & .0 & \\
\hline 8 & 52116 & 169.7666 & 000 & 306.8 & 4 & & \\
\hline 00 & 52117 & 180.7497 & 7.0500 & 306.7 & 4 & .0 & \\
\hline 0 & 52118 & 185.7501 & 6.0500 & 306.4 & 4 & .0 & 0 \\
\hline 0 & 52119 & 176.0208 & 7500 & 305.5 & 4 & & \\
\hline 00 & 52120 & 87.3431 & 4.5250 & 303.7 & 4 & 600.0 & 600 \\
\hline 00 & 52121 & 73.7525 & 6.6750 & 302.5 & 4 & .0 & 600 \\
\hline 00 & 52122 & 63 & & 302.2 & 4 & & \\
\hline & 52123 & 56.7505 & 8.0500 & 302.0 & 1 & .0 & 600 \\
\hline
\end{tabular}




\begin{tabular}{|c|c|c|c|c|c|c|c|}
\hline 00 & 2124 & 58.5024 & 8.0750 & 302.3 & 4 & 600.0 & 00.0 \\
\hline 0 & 5221 & 62.4991 & 7.5750 & 302.8 & 4 & 600.0 & 600,0 \\
\hline 00 & 5222 & 66.4999 & 6.6000 & 302.7 & 4 & 600.0 & 00.0 \\
\hline 00 & 5223 & 66.9999 & 5.9750 & 302.8 & 4 & 600.0 & 00.0 \\
\hline 00 & 5224 & 70.2492 & 5.6500 & 302.8 & 4 & 600.0 & 00.0 \\
\hline 00 & 5225 & 80.0249 & 4.8250 & 303.0 & 4 & 600.0 & 00.0 \\
\hline 00 & 5226 & 85.2090 & 4.5250 & 302.8 & 4 & 600.0 & 0.0 \\
\hline 00 & 5227 & 153.7121 & 1.2500 & 302.2 & 3 & 600.0 & 00.0 \\
\hline 00 & 5228 & 338.3249 & 0.6750 & 303.5 & 2 & 600.0 & 00.0 \\
\hline 00 & 5229 & 184.7356 & 4.6750 & 303.1 & 3 & 600.0 & \\
\hline 00 & 52210 & 167.0000 & 3.6000 & 302.6 & 3 & 600.0 & 500.0 \\
\hline 00 & 52211 & 165.5319 & 3. & 304.2 & 2 & 600.0 & \\
\hline 00 & 52212 & 181.8815 & 5.0000 & 305.5 & 3 & 600.0 & 00.0 \\
\hline 00 & 52213 & 171.5083 & 6.0000 & 305.0 & 4 & 600.0 & 0 \\
\hline 00 & 52214 & 172.2554 & & 305.6 & 3 & 600.0 & \\
\hline 00 & 52215 & 169.9807 & 4 . & 306.2 & 3 & 600.0 & 60 \\
\hline 00 & 52216 & 162.5005 & 4.35 & 306.8 & 3 & 600.0 & 600.0 \\
\hline 00 & 52217 & 123.4690 & 4.3 & 307.1 & 3 & 600.0 & \\
\hline 00 & 52218 & 85.9796 & 4.9 & 305.9 & 3 & 600.0 & .0 \\
\hline 00 & 52219 & 72.0000 & 5.25 & 304.5 & 4 & 600.0 & .0 \\
\hline 00 & 52220 & 67.9982 & 6 . & 304.1 & 4 & 600.0 & .0 \\
\hline 00 & 52221 & 64.5000 & 7 . & 303.8 & 4 & 0.0 & \\
\hline 00 & 52222 & 65.7500 & 7.3250 & 303.5 & 4 & 600.0 & 600.0 \\
\hline 00 & 52223 & 67.7473 & 7.5 & 303.4 & 4 & 600.0 & .0 \\
\hline 00 & 52224 & 003 & 6 . & .5 & 4 & 0 & \\
\hline 00 & 5231 & 82.5018 & 5.6750 & 302.8 & 4 & 600.0 & 60 \\
\hline 00 & 5232 & 132.6558 & 5.6500 & 302.1 & 4 & 600.0 & .0 \\
\hline 00 & 5233 & 131.2515 & 3.8 & 301.0 & 4 & 600.0 & \\
\hline 00 & 5234 & 136.5000 & 4.47 & 299.9 & 4 & 600.0 & 60 \\
\hline 00 & 5235 & 144.0000 & 7.60 & 299.1 & 4 & 600.0 & .0 \\
\hline 00 & 5236 & 156.2532 & 9. & 298.6 & 4 & 600.0 & \\
\hline 00 & 5237 & 159.2502 & 10.15 & 298.5 & 4 & 600.0 & .0 \\
\hline 00 & 5238 & 159.4985 & 9.3000 & 298.8 & 4 & 600.0 & 600.0 \\
\hline 00 & 5239 & 162.5015 & 10.1 & 299.4 & 4 & 600.0 & .0 \\
\hline 00 & 52310 & 160.2504 & 6 . & 300.5 & 4 & 600.0 & .0 \\
\hline 00 & 52311 & 159.5155 & 4.92 & 301.4 & 3 & 600.0 & 600.0 \\
\hline 00 & 52312 & 154.4862 & 5.87 & 303.2 & 3 & 600.0 & \\
\hline 00 & 52313 & 166.0049 & 5.0 & 302.8 & 3 & 600.0 & .0 \\
\hline 00 & 52314 & 149.2209 & 3.6750 & 303.2 & 3 & 600.0 & 600.0 \\
\hline 00 & 52315 & 118.0984 & 3.37 & 303.4 & 2 & 600.0 & \\
\hline 00 & 52316 & 60.7781 & 4.17 & 303.1 & 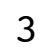 & 600.0 & 600.0 \\
\hline 00 & 52317 & 67.5153 & 4.3250 & 302.6 & 3 & 600.0 & 600.0 \\
\hline 00 & 52318 & 66.2757 & 6.2500 & 301.9 & 4 & 600.0 & 600.0 \\
\hline 00 & 52319 & 73.2584 & 6.5250 & 301.6 & 4 & 600.0 & 600.0 \\
\hline 00 & 52320 & 69.7545 & 9.7750 & 299.6 & 4 & 600.0 & 600.0 \\
\hline 00 & 52321 & 63.8432 & 8.6500 & 299.9 & 4 & 600.0 & 600.0 \\
\hline 00 & 52322 & 114.8408 & 7 . & 300.7 & 4 & .0 & 600.0 \\
\hline 00 & 52323 & 96.5023 & 7.9500 & 301.4 & 4 & 600.0 & 600.0 \\
\hline
\end{tabular}




\begin{tabular}{|c|c|c|c|c|c|c|c|}
\hline 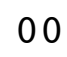 & 24 & & & & & & \\
\hline 0 & 241 & 795 & 000 & & & & \\
\hline & 242 & 467 & 4500 & 99.6 & & 00 & \\
\hline & 243 & 153.0011 & 750 & 297.2 & & 00 & \\
\hline & $24 \quad 4$ & e & & & & & \\
\hline & 245 & 10 & & 4.5 & & & \\
\hline & 246 & 3209 & 250 & 94.6 & & & \\
\hline & 5247 & 96 & & & & & \\
\hline & 5248 & 938 & & & & & \\
\hline & 249 & 173 & 2750 & 95.4 & & & \\
\hline & 52410 & 152.7128 & 0 & 5 & & & \\
\hline & 52411 & 143 & & & & & \\
\hline & 2412 & 13 & 6 . & .5 & & & \\
\hline & 52413 & 897 & ובנ. & 98.3 & & & \\
\hline & 2414 & & & & & & \\
\hline & 241 & & & .6 & & & \\
\hline & 2416 & 20.20 & 1.7 & 98.3 & & & \\
\hline & 2417 & 1 & 0 & 3.2 & & & \\
\hline & 241 & & & 296.7 & & & \\
\hline & 24 & 1 & 50 & 29 & & & \\
\hline & 524 & 4 & .1250 & 2 & & & \\
\hline & 242 & & & 292.9 & & & \\
\hline & 24 & 0 & 0 & 25 & & & \\
\hline & 52423 & 5 & 0 & 292.4 & & & \\
\hline & 52424 & & & 291.5 & & & \\
\hline & 525 & 0 & 0 & & & & \\
\hline & 5252 & 000 & 0 & 289.8 & & & \\
\hline & 5253 & 5 & 50 & 288.8 & & & \\
\hline & 5254 & & 0 & $2 \varepsilon$ & & & \\
\hline & 5255 & & .0250 & 287.5 & & & \\
\hline & 5256 & $\epsilon$ & .47 & 286.5 & 1 & & \\
\hline & 5257 & & 0 & 286.3 & 4 & & \\
\hline & 5258 & & 0 & 286.6 & 1 & & \\
\hline & 5259 & & 0 & 287.9 & 然 & & \\
\hline & 525 & & & $2 \varepsilon$ & 4 & & \\
\hline & 52511 & & & 289.8 & 4 & & \\
\hline & 52512 & & 8.5250 & 291.1 & 4 & & \\
\hline & 52513 & & 0 & 291.4 & 4 & & \\
\hline & 52514 & & 10.2250 & 292.5 & 4 & & \\
\hline & 52515 & & & 293.3 & 4 & & \\
\hline & 52516 & & 9.2500 & 293.8 & 4 & & \\
\hline & 52517 & & 9.9500 & 293.2 & 4 & & \\
\hline & 52518 & & .2750 & 292.8 & 4 & & \\
\hline & 52519 & & & 292.4 & 4 & & \\
\hline & 52520 & & 10.9500 & 291.9 & 4 & & \\
\hline & 52521 & & 1.4250 & 291.8 & 4 & & \\
\hline & & & & & 4 & & \\
\hline & 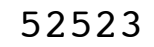 & 10. & 10.9500 & 291.1 & 1 & 600.0 & 6 \\
\hline
\end{tabular}




\begin{tabular}{|c|c|c|c|c|c|c|c|}
\hline 0 & 52524 & 75.7509 & 10.9750 & 291.2 & 4 & 600.0 & 00.0 \\
\hline 00 & 5261 & 72.7505 & 11.0000 & 290.9 & 4 & 600.0 & 00 \\
\hline 00 & 5262 & 71.5000 & 10.9250 & 290.1 & 4 & 600.0 & 00.0 \\
\hline 00 & 5263 & 70.7505 & 11.9500 & 289.1 & 4 & 600.0 & 00.0 \\
\hline 00 & 5264 & 70.0000 & 11.8250 & 288.4 & 4 & 600.0 & 00.0 \\
\hline 00 & 5265 & 69.7505 & 11.3000 & 288.5 & 4 & 600.0 & 00.0 \\
\hline 00 & 5266 & 64.7500 & 11.2750 & 288.7 & 4 & 600.0 & .0 \\
\hline 00 & 5267 & 61.5000 & 11.5250 & 288.3 & 4 & 600.0 & 00.0 \\
\hline 00 & 5268 & 71.2486 & 11.0250 & 290.1 & 4 & 600.0 & .0 \\
\hline 00 & 5269 & 81.0000 & 11.2500 & 290.3 & 4 & 600.0 & .0 \\
\hline 00 & 52610 & 74.5025 & 9.1500 & 291.3 & 4 & 600.0 & 00.0 \\
\hline 00 & 52611 & 69.4987 & 00 & 293.3 & 4 & 600.0 & \\
\hline 00 & 52612 & 67.4969 & 7.3250 & 294.2 & 4 & 600.0 & .0 \\
\hline 00 & 52613 & 71.4895 & 8.2250 & 294.8 & 4 & 600.0 & .0 \\
\hline 00 & 52614 & 76.2441 & 7.8250 & 295.4 & 4 & 600.0 & \\
\hline 00 & 52615 & 61.5024 & 9.0250 & 295.5 & 4 & 600.0 & 60 \\
\hline 00 & 52616 & 89.0131 & 10.1250 & 295.1 & 4 & 600.0 & .0 \\
\hline 00 & 52617 & 77.0025 & 11.5250 & 294.0 & 4 & 600.0 & \\
\hline 00 & 52618 & 70.7503 & 12.6250 & 292.9 & 4 & 600.0 & \\
\hline 00 & 52619 & 66.2500 & 13.2250 & 291.2 & 4 & 600.0 & .0 \\
\hline 00 & 52620 & 66. & 14.6750 & 290.3 & 4 & 600.0 & .0 \\
\hline 00 & 52621 & 63.2500 & 12 . & 291.1 & 4 & 0 & \\
\hline 00 & 52622 & 64.0000 & 12.7250 & 291.3 & 4 & 600.0 & 60 \\
\hline 00 & 52623 & 004 & 11.97 & 291.2 & 4 & 600.0 & .0 \\
\hline 00 & 52624 & 68.2500 & 10 . & 291.4 & 4 & 0.0 & \\
\hline 00 & 5271 & 65.7496 & 10.4250 & 291.2 & 4 & 600.0 & 60 \\
\hline 00 & 5272 & 66.5000 & 10.2750 & 291.4 & 4 & 600.0 & .0 \\
\hline 00 & 5273 & 69.7495 & 10 . & 291.6 & 4 & 0.0 & \\
\hline 00 & 5274 & 75.0000 & 11.0750 & 291.4 & 4 & 600.0 & 60 \\
\hline 00 & 5275 & 77.5000 & 11.7250 & 290.1 & 4 & 600.0 & .0 \\
\hline 00 & 5276 & 72.7499 & 9.07 & 290.2 & 4 & 600.0 & \\
\hline 00 & 5277 & 76.2494 & 6.7750 & 292.4 & 4 & 600.0 & .0 \\
\hline 00 & 5278 & 79.2498 & 6.1500 & 294.0 & 4 & 600.0 & 600.0 \\
\hline 00 & 5279 & 78.2310 & 5.20 & 295.2 & 3 & 600.0 & \\
\hline 00 & 52710 & 140.1085 & 2.92 & 297.7 & 2 & 600.0 & .0 \\
\hline 00 & 52711 & 101.0388 & 5.5750 & 297.6 & 3 & 600.0 & 600.0 \\
\hline 00 & 52712 & 115.0077 & 6.0500 & 298.1 & 4 & 600.0 & \\
\hline 00 & 52713 & 166.5485 & 8.1750 & 298.1 & 4 & 600.0 & .0 \\
\hline 00 & 52714 & 156.2491 & 8.1000 & 298.4 & 4 & 600.0 & 600.0 \\
\hline 00 & 52715 & 119.2328 & 8.9000 & 298.6 & 4 & 600.0 & \\
\hline 00 & 52716 & 110.5741 & 9.6750 & 298.5 & 4 & 600.0 & 60 \\
\hline 00 & 52717 & 56.2517 & 11.6000 & 298.1 & 4 & 600.0 & 600.0 \\
\hline 00 & 52718 & 59.7502 & 13.8000 & 296.1 & 4 & 600.0 & 600.0 \\
\hline 00 & 52719 & 63.2500 & 13.2000 & 295 & 4 & 600.0 & 600.0 \\
\hline 00 & 52720 & 65.0000 & 11.4250 & 294.9 & 4 & 600.0 & 600.0 \\
\hline 00 & 52721 & 63.7499 & 10.9000 & 295.0 & 4 & 600.0 & 600.0 \\
\hline 00 & 52722 & 67.7489 & 11.125 & & 4 & 600.0 & 600.0 \\
\hline 00 & 52723 & 72.2400 & 11.0500 & 294.2 & 4 & 600.0 & 600.0 \\
\hline
\end{tabular}




\begin{tabular}{|c|c|c|c|c|c|c|c|}
\hline & 2724 & 0.7500 & 9.1250 & 294.2 & 4 & . & \\
\hline & 281 & 67.7498 & 8.5750 & 294.3 & & 00.0 & \\
\hline & 282 & 65.0001 & 9500 & 294.4 & & 0 & \\
\hline & 283 & 3.5014 & .5000 & 293.8 & & & \\
\hline & 284 & 7500 & 9750 & 93.5 & & 00 . & \\
\hline & 285 & & 250 & 294 & & & \\
\hline & 286 & 5005 & & 294 & & & \\
\hline & 287 & & & 5 & & & \\
\hline & 5288 & 71.2508 & 8.6250 & 295.8 & & 0 & \\
\hline & 289 & 91 & & 29 & & & \\
\hline & 2810 & & & 4 & & & \\
\hline & 2811 & 89. & 4 . & 298.8 & & & \\
\hline & 2812 & 78.7550 & 0 & 299.8 & & & \\
\hline & 52813 & 98.7481 & & 95 & & & \\
\hline & 52814 & 0 & & & & & \\
\hline & 52815 & 76 . & & 300.0 & & & \\
\hline & 52816 & 66.7494 & 0 & 298 & & & \\
\hline & 52817 & 71.9986 & & 298 & & & \\
\hline & 52818 & 81.2501 & & 298 & & 0 & \\
\hline 0 & 52819 & 04.26 & & 29 & & & \\
\hline & 52820 & 74.32 & & & & & \\
\hline 0 & 52821 & 59. & & 29 & & & \\
\hline ) & 52822 & 65.0000 & 0 & 294.3 & & 0 & \\
\hline & 52823 & 63.2500 & 0 & 293.5 & & & \\
\hline 0 & 52824 & 67 & 0 & 29 & 4 & & \\
\hline & 5291 & 68. & 0 & 291.8 & & & \\
\hline & 5292 & 64 . & & 29 & 4 & & \\
\hline 0 & 5293 & 68 . & 0 & 29 & 4 & & \\
\hline & 5294 & 77 . & 0 & & & 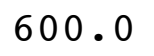 & \\
\hline & 5295 & & & 29 & 4 & & \\
\hline & 5296 & 6 & 0 & 29 & 4 & & \\
\hline & 5297 & & & & 4 & & \\
\hline & 5298 & 000 & 5500 & 291.6 & 4 & 0 & \\
\hline 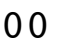 & 5299 & 3 & 0 & 293 & 4 & & \\
\hline & 52910 & & & & 4 & & \\
\hline & 52911 & & 000 & 294.4 & 4 & . 0 & \\
\hline & 52912 & & & 294 & 4 & & \\
\hline & 52913 & 87 . & 7 . & 296.0 & 4 & & \\
\hline & 52914 & & & 296.3 & 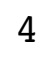 & & \\
\hline & 52915 & 102 & & 295 & 4 & & \\
\hline 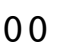 & 52916 & & 0.5250 & 294.9 & + & & \\
\hline 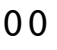 & 52917 & 70 . & .6500 & 293.5 & a & & \\
\hline 0 & 52918 & 70 . & 750 & 293.0 & 4 & & \\
\hline 00 & 52919 & 76.0046 & & 292.2 & 2 & .0 & \\
\hline 0 & 52920 & 62.2511 & 10.4250 & 292.2 & 4 & .0 & مח \\
\hline & 52921 & & & 292.2 & & & \\
\hline & 52922 & 63.2500 & 0.2750 & 291.9 & & & \\
\hline & 52923 & 66.7501 & 7.9500 & 291.5 & & .0 & -0 \\
\hline
\end{tabular}




\begin{tabular}{|c|c|c|c|c|c|c|c|}
\hline 00 & 52924 & 73.2501 & 9.1000 & 290.6 & 4 & 600.0 & 00.0 \\
\hline 0 & 5301 & 68.7499 & 10.0750 & 289.1 & 4 & 600.0 & 600,0 \\
\hline 00 & 5302 & 67.0005 & 10.3000 & 288.6 & 4 & 600.0 & 00.0 \\
\hline 00 & 5303 & 67.7494 & 10.8000 & 288.3 & 4 & 600.0 & 00.0 \\
\hline 0 & $530 \quad 4$ & 74.2501 & 13.0250 & 288.0 & 4 & 600.0 & 0.0 \\
\hline 00 & 5305 & 73.2502 & 13.3500 & 287.2 & 4 & 600.0 & 00.0 \\
\hline 00 & 5306 & 73.5000 & 13.1500 & 286.3 & 4 & 600.0 & 0.0 \\
\hline 0 & 5307 & 75.2500 & 11.1250 & 286.8 & 4 & 600.0 & 00.0 \\
\hline 00 & 5308 & 74.0000 & 9.7000 & 287.3 & 4 & 600.0 & 00.0 \\
\hline 00 & 5309 & 79.5000 & 9.1250 & 288.1 & 4 & 600.0 & 600.0 \\
\hline 00 & 53010 & 85.7504 & 6.3750 & 289.7 & 4 & 600.0 & 600.0 \\
\hline 00 & 53011 & 91.0000 & 000 & 291.1 & 3 & 600.0 & \\
\hline 00 & 53012 & 103.4945 & 5.3500 & 292.3 & 3 & 600.0 & 00.0 \\
\hline 00 & 53013 & 101.0215 & 6.3250 & 293.1 & 4 & 600.0 & 600.0 \\
\hline 0 & 53014 & 97.5015 & 00 & 293.5 & 4 & .0 & \\
\hline 00 & 53015 & 121.2503 & 250 & 294.2 & 4 & 60 & 00.0 \\
\hline 00 & 53016 & 123.3071 & 7.2750 & 294.2 & 4 & 60 & 0.0 \\
\hline 00 & 53017 & 119.0223 & 7.7250 & 293.5 & 4 & 60 & \\
\hline 00 & 53018 & 92.2513 & 7.7750 & 292.6 & 4 & .0 & .0 \\
\hline 00 & 53019 & 71.2571 & 7.1250 & 291.4 & 4 & 60 & 0.0 \\
\hline 0 & 53020 & 68.9995 & 750 & 290.2 & 4 & .0 & \\
\hline 00 & 53021 & 72.7500 & 8 . & 289.7 & 4 & 0 & \\
\hline 00 & 53022 & 69.5017 & 9.1750 & 289.2 & 4 & 60 & \\
\hline 00 & 53023 & 74.4999 & 8.4000 & 289.7 & 4 & 6 & $\cdot 0$ \\
\hline 00 & 53024 & 73.5000 & 8 . & 289.7 & 4 & 0 & \\
\hline 00 & 5311 & 71.9999 & 8 . & 289.3 & 4 & 60 & 0.0 \\
\hline 00 & 5312 & 101.8519 & 6.8500 & 290.3 & 4 & 60 & 0.0 \\
\hline 00 & 5313 & 150.2455 & 9.2 & 290.5 & 4 & .0 & \\
\hline 00 & 5314 & 154.7483 & 12.2500 & 288.1 & 4 & 60 & 0.0 \\
\hline 00 & 5315 & 157.5000 & 11.9000 & 287.6 & 4 & 6 & 0.0 \\
\hline 00 & 5316 & 158.5000 & 12 . & 287.2 & 4 & 6 & \\
\hline 00 & 5317 & 158.4986 & 14.7500 & 286.9 & 4 & .0 & .0 \\
\hline 00 & 5318 & 167.0000 & 14.0250 & 287.4 & 4 & 600.0 & 0.0 \\
\hline 00 & 5319 & 170.0001 & 12.7000 & 288.4 & 4 & 6 & \\
\hline 00 & 53110 & 170.9997 & 12 . & 289.7 & 4 & .0 & .0 \\
\hline 00 & 53111 & 170.0000 & 12.9000 & 291.1 & 4 & 600.0 & 600.0 \\
\hline 00 & 53112 & 174.2343 & 9.4750 & 292.6 & 4 & .0 & 0.0 \\
\hline 00 & 53113 & 174.4986 & 7.6250 & 294.0 & 4 & 600.0 & 600.0 \\
\hline 00 & 53114 & 150.2494 & 7.1750 & 295.1 & 4 & 600.0 & 600.0 \\
\hline 00 & 53115 & 159.4991 & 7 . & 295.8 & 4 & 60 & 0.0 \\
\hline 00 & 53116 & 164.5003 & 7.3750 & 296.3 & 4 & 600.0 & 0.0 \\
\hline 00 & 53117 & 168.7478 & 6.5250 & 297.0 & 4 & 600.0 & 600.0 \\
\hline 00 & 53118 & 168.2421 & 6.4000 & 297.1 & 4 & 600.0 & 600.0 \\
\hline 00 & 53119 & 178.5000 & 5.3750 & 296.5 & 4 & 600.0 & 600.0 \\
\hline 00 & 53120 & 152.5024 & 2.5500 & 295.1 & 5 & 600.0 & 600.0 \\
\hline 00 & 53121 & 99.9222 & 2.4000 & 294.5 & 4 & 600.0 & 600.0 \\
\hline 00 & 53122 & 79.7458 & 4 . & 293 & 4 & 600.0 & 60 \\
\hline 00 & 53123 & 70.7485 & 7.3500 & 292.5 & 4 & 600.0 & 600.0 \\
\hline
\end{tabular}




\begin{tabular}{|c|c|c|c|c|c|c|c|c|}
\hline & & 124 & 9.2496 & 6.7750 & 292.7 & 4 & 0.0 & \\
\hline 0 & 6 & 11 & 78.0008 & 7.3500 & 293.1 & 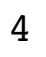 & 00.0 & \\
\hline & 6 & 12 & 76.0000 & 4750 & 293.4 & & 70 & \\
\hline & 6 & 13 & 6.2501 & 7.5250 & 293.3 & & 0 & \\
\hline & 6 & 14 & 76.2500 & & 293.2 & & 0 & \\
\hline & 6 & 15 & 000 & & 293.3 & & 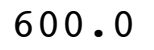 & \\
\hline & 6 & 16 & 72.5000 & & & & & \\
\hline & 6 & 17 & 73 & & 94.8 & & & \\
\hline & 0 & 18 & 65.4779 & 2.8250 & 296.9 & & 00.0 & \\
\hline & 6 & 19 & 244 & & 297.3 & & & \\
\hline & 6 & 110 & 367 & & 297.5 & & & \\
\hline & 6 & 111 & 230.2483 & 7500 & 298.6 & & & \\
\hline 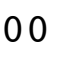 & 6 & 112 & 232.3776 & 0000 & 299.8 & & & \\
\hline & 6 & 113 & 242.3482 & 0 & 300.3 & & & \\
\hline & 6 & 114 & 185.6251 & & 301.3 & & & \\
\hline & 6 & 115 & 177.5717 & 000 & 301.8 & & 0 & \\
\hline & 6 & 116 & 99.61 & 0 & 302.4 & & $\cdot 0$ & \\
\hline & 6 & 117 & 101.27 & & 301.8 & & & \\
\hline 0 & 6 & 118 & 034 & 0 & 299.8 & & .0 & \\
\hline 0 & 6 & 11 & 83. & & 297.8 & 4 & - 0 & \\
\hline & 6 & 120 & 71 . & & 295 & - & & \\
\hline 0 & 6 & 121 & 71 . & & 295.2 & 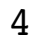 & & \\
\hline 0 & 6 & 122 & 73.7500 & 0 & 295.1 & 4 & .0 & \\
\hline 0 & 6 & 123 & 68.75 & & 294.4 & 4 & 0 & \\
\hline 0 & 6 & 124 & 70 & 0 & 29 & 4 & & \\
\hline 0 & 6 & 21 & 69.9999 & & 293 & 4 & .0 & \\
\hline 0 & 6 & 22 & 71. & & 293 & 4 & & \\
\hline 0 & 6 & 23 & 73 & 0 & 293 & 4 & & \\
\hline & 6 & 24 & 76 . & & 294.2 & 4 & 0.0 & \\
\hline 0 & 6 & 25 & & & 29 & 4 & & \\
\hline 0 & 6 & 26 & & & 293 & 4 & & \\
\hline & 6 & 27 & & & 295.0 & 4 & .0 & \\
\hline 0 & 6 & 28 & 62.5445 & 0 & 298.0 & 3 & .0 & \\
\hline 0 & 6 & 29 & .7385 & 0 & 297 & 2 & & \\
\hline & 6 & 210 & 257 . & & 298.2 & 1 & .0 & \\
\hline & 6 & 211 & 267 . & 0 & 299.9 & 1 & .0 & \\
\hline & 6 & 212 & 340 . & 0 & 30 & 1 & & \\
\hline 0 & 6 & 213 & 88 . & 3. & & 2 & .0 & \\
\hline 0 & 6 & 214 & 92 . & & 299.3 & 3 & 0.0 & \\
\hline & 6 & 215 & 91 & 0 & 29 & 3 & & \\
\hline 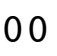 & 6 & 216 & 89.7502 & 5.9250 & 298.9 & 3 & .0 & \\
\hline 00 & 6 & 217 & 79.5055 & 6.8500 & 297.3 & 4 & 0.0 & \\
\hline 0 & 6 & 218 & 82.5138 & 9.3250 & 295.2 & 4 & & \\
\hline 00 & 6 & 219 & 70.5000 & 0.8750 & 293.1 & 4 & 600.0 & \\
\hline 00 & 6 & 220 & 72.0001 & 0.7250 & 291.7 & 4 & 600.0 & م0 \\
\hline 00 & 6 & 221 & & & 291.7 & 4 & & \\
\hline & 6 & 222 & 77.2501 & 1.1000 & 292.1 & 4 & 00.0 & \\
\hline & 0 & 223 & 72.2494 & 11.6750 & 291.7 & & 600.0 & 500 \\
\hline
\end{tabular}




\begin{tabular}{|c|c|c|c|c|c|c|c|c|}
\hline 0 & 6 & 224 & 73.5000 & 11.0750 & 292.4 & 4 & 600.0 & 00.0 \\
\hline 00 & 6 & 31 & 75.0000 & 10.6500 & 292.5 & 4 & 600.0 & 00.0 \\
\hline 00 & 6 & 32 & 79.2427 & 6.9000 & 294.2 & 4 & 600.0 & 00.0 \\
\hline 00 & 6 & 33 & 88.7390 & 4.8000 & 294.9 & 4 & 600.0 & 00.0 \\
\hline 00 & 6 & 34 & 91.2269 & 3.8750 & 293.8 & $I$ & 600.0 & 00.0 \\
\hline 00 & 6 & 35 & 77.0000 & 5.2750 & 294.0 & 4 & 600.0 & 00.0 \\
\hline 00 & 6 & 36 & 82.2497 & 5.3750 & 293.7 & 4 & 600.0 & 600.0 \\
\hline 00 & 6 & 37 & 134.9768 & 2.6000 & 294.6 & 3 & 600.0 & 00.0 \\
\hline 00 & 6 & 38 & 219.5151 & 1.0500 & 296.9 & 2 & 600.0 & 00.0 \\
\hline 00 & 6 & 39 & 174.7496 & 3.6000 & 295.9 & 2 & 600.0 & .0 \\
\hline 00 & 6 & 310 & 170.0000 & 4.9750 & 295.8 & 3 & 600.0 & 00.0 \\
\hline 00 & 6 & 311 & 163.2501 & 5.9250 & 295.7 & 3 & 600.0 & \\
\hline 00 & 6 & 312 & 173.5067 & 6.0750 & 297.4 & 4 & 600.0 & 600.0 \\
\hline 00 & 6 & 313 & 171.4959 & 6.5250 & 299.1 & 4 & 600.0 & 0 \\
\hline 00 & 6 & 314 & 176.7501 & & 299.8 & 4 & 600.0 & \\
\hline 00 & 6 & 315 & 123.4172 & 4.6750 & 301.1 & 3 & 600.0 & 60 \\
\hline 00 & 6 & 316 & 73.0456 & 4.6000 & 301.1 & 3 & 600.0 & 600.0 \\
\hline 00 & 6 & 317 & 79.7687 & 5. & 300.2 & 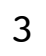 & 600.0 & \\
\hline 00 & 6 & 318 & 62.7449 & 6.8750 & 299.0 & 4 & 600.0 & .0 \\
\hline 00 & 6 & 319 & 57.5000 & 8.3500 & 297.4 & 4 & 600.0 & .0 \\
\hline 00 & 6 & 320 & 66.2483 & 9.15 & 296.0 & 4 & 600.0 & .0 \\
\hline 00 & 6 & 321 & 66.7492 & 9.5 & 295.2 & 4 & 600.0 & \\
\hline 00 & 6 & 322 & 70.5000 & 9.4500 & 295.7 & 4 & 600.0 & 600.0 \\
\hline 00 & 6 & 323 & 81.6700 & 6.62 & 295.9 & 4 & 600.0 & .0 \\
\hline 00 & 6 & 324 & 97.6506 & 3. & 295.9 & 5 & 600.0 & \\
\hline 00 & 6 & 41 & 75.5801 & 0.9500 & 296.0 & 6 & 600.0 & 60 \\
\hline 00 & 6 & 42 & 124.2506 & 0.52 & 294.9 & 5 & 600.0 & .0 \\
\hline 00 & 6 & 43 & 40.6397 & $0 . \varepsilon$ & 295.1 & 6 & 600.0 & \\
\hline 00 & 6 & 44 & 41.9714 & 1.1750 & 295.6 & 6 & 600.0 & 60 \\
\hline 00 & 6 & 45 & 99.2590 & 2.2250 & 295.2 & 6 & 600.0 & 60 \\
\hline 00 & 6 & 46 & 216.1904 & 0.3 & 293.8 & 6 & 600.0 & \\
\hline 00 & 6 & 47 & 311.6561 & 0.2500 & 296.2 & 5 & 600.0 & .0 \\
\hline 00 & 6 & 48 & 270.9410 & 1.2250 & 296.2 & 4 & 600.0 & 600.0 \\
\hline 00 & 6 & 49 & 234.7425 & 2.67 & 295.5 & 3 & 600.0 & .0 \\
\hline 00 & 6 & 410 & 241.7539 & 2.6 & 295.2 & 2 & 600.0 & .0 \\
\hline 00 & 6 & 411 & 272.7980 & 2.7000 & 296.6 & 1 & 600.0 & 600.0 \\
\hline 00 & 6 & 412 & 233.6515 & 2.8500 & 297.5 & 1 & 600.0 & \\
\hline 00 & 6 & 413 & 190.3886 & 3.4500 & 298.5 & 2 & 600.0 & 600.0 \\
\hline 00 & 6 & 414 & 170.7504 & 6.3750 & 298.3 & 3 & 600.0 & 600.0 \\
\hline 00 & 6 & 415 & 133.9737 & 4.875 & 299.4 & 3 & 600.0 & \\
\hline 00 & 6 & 416 & 78.2486 & 6.8750 & 297.7 & 4 & 600.0 & 600.0 \\
\hline 00 & 6 & 417 & 100.6142 & 8.5500 & 295.6 & 4 & 600.0 & 600.0 \\
\hline 00 & 6 & 418 & 89.7437 & 11.3000 & 291.8 & 4 & 600.0 & 600.0 \\
\hline 00 & 6 & 419 & 71.2503 & 12.2500 & 289.4 & 4 & 600.0 & 600.0 \\
\hline 00 & 6 & 420 & 69.7521 & 11.6000 & 288.9 & 4 & 600.0 & 600.0 \\
\hline 00 & 6 & 421 & 72.7500 & 12.1750 & 286.7 & 4 & 600.0 & 600.0 \\
\hline 00 & 6 & 422 & 68.2498 & 12.650 & 284.2 & 4 & 600.0 & 600.0 \\
\hline 00 & 6 & 423 & 66.9999 & 8.9500 & 283.9 & 4 & 600.0 & 600.0 \\
\hline
\end{tabular}




\begin{tabular}{|c|c|c|c|c|c|c|c|c|}
\hline 0 & 6 & 424 & 59.2501 & 12.1500 & 282.8 & 4 & 600.0 & 00.0 \\
\hline 00 & 6 & 51 & 63.4983 & 17.3250 & 282.6 & 4 & 600.0 & 600.0 \\
\hline 00 & 6 & 52 & 68.7500 & 17.8500 & 282.7 & 4 & 600.0 & 00.0 \\
\hline 00 & 6 & 53 & 61.7503 & 13.0750 & 283.5 & 4 & 600.0 & 00.0 \\
\hline 00 & 6 & 54 & 66.9995 & 13.6500 & 282.8 & $I$ & 600.0 & 00.0 \\
\hline 00 & 6 & 55 & 62.5000 & 14.5250 & 283.0 & 4 & 600.0 & 00.0 \\
\hline 00 & 6 & 56 & 64.5000 & 16.3500 & 283.1 & 4 & 600.0 & 600.0 \\
\hline 00 & 6 & 57 & 63.0000 & 13.2750 & 284.1 & 4 & 600.0 & 500.0 \\
\hline 00 & 6 & 58 & 64.7494 & 11.6000 & 285.8 & 4 & 600.0 & 00.0 \\
\hline 00 & 6 & 59 & 68.0000 & 11.8500 & 287.2 & 4 & 600.0 & .0 \\
\hline 00 & 6 & 510 & 69.2505 & 11.0000 & 288.6 & 4 & 600.0 & 00.0 \\
\hline 00 & 6 & 511 & 72.5000 & 9.92 & 289.7 & 4 & 600.0 & \\
\hline 00 & 6 & 512 & 72.4991 & 8.0000 & 291.1 & 4 & 600.0 & 600.0 \\
\hline 00 & 6 & 513 & 76.2503 & 6.7000 & 292.9 & 4 & 600.0 & 600.0 \\
\hline 00 & 6 & 514 & 82.7493 & 7.6750 & 293.7 & 4 & 600.0 & \\
\hline 00 & 6 & 515 & 79.5000 & 7.5000 & 294.2 & 4 & 600.0 & 60 \\
\hline 00 & 6 & 516 & 76.5014 & 7.2250 & 294.3 & 4 & 600.0 & 600.0 \\
\hline 00 & 6 & 517 & 116.1954 & 8.9000 & 293.5 & 4 & 600.0 & .0 \\
\hline 00 & 6 & 518 & 114.1376 & 10.7250 & 292.9 & 4 & 600.0 & .0 \\
\hline 00 & 6 & 519 & 68.2504 & 11.0750 & 291.7 & 4 & 600.0 & .0 \\
\hline 00 & 6 & 520 & 68.0000 & 12.9000 & 289.7 & 4 & 600.0 & .0 \\
\hline 00 & 6 & 521 & 72.0000 & 12.2750 & 289.2 & 4 & 600.0 & \\
\hline 00 & 6 & 522 & 71.9998 & 11.4750 & 289.7 & 4 & 600.0 & 60 \\
\hline 00 & 6 & 523 & 80.9815 & 11.2750 & 288.8 & 4 & 600.0 & .0 \\
\hline 00 & 6 & 524 & 67.7500 & 11.5750 & 289.4 & 4 & 600.0 & \\
\hline 00 & 6 & 61 & 67.5000 & 10.3000 & 289.7 & 4 & 600.0 & 60 \\
\hline 00 & 6 & 62 & 73.2500 & 9.3500 & 290.6 & 4 & 600.0 & .0 \\
\hline 00 & 6 & 63 & 74.0001 & 7.8750 & 290.6 & 4 & 600.0 & \\
\hline 00 & 6 & 64 & 70.2501 & 7.9500 & 290.4 & 4 & 600.0 & 60 \\
\hline 00 & 6 & 65 & 71.2501 & 7.3250 & 290.7 & 4 & 600.0 & 600.0 \\
\hline 00 & 6 & 66 & 73.0000 & 7.32 & 291.1 & 4 & 600.0 & \\
\hline 00 & 6 & 67 & 81.0025 & 6.4000 & 291.9 & 4 & 600.0 & .0 \\
\hline 00 & 6 & 68 & 87.0034 & 6.2250 & 292.8 & 4 & 600.0 & 600.0 \\
\hline 00 & 6 & 69 & 73.7495 & 7.825 & 293.0 & 4 & 600.0 & .0 \\
\hline 00 & 6 & 610 & 75.0000 & 7.4750 & 293.7 & 4 & 600.0 & .0 \\
\hline 00 & 6 & 611 & 80.2496 & 6.9000 & 294.7 & 4 & 600.0 & 600.0 \\
\hline 00 & 6 & 612 & 75.0000 & 8.1250 & 295.3 & 4 & 600.0 & \\
\hline 00 & 6 & 613 & 75.0064 & 7.4750 & 295.9 & 4 & 600.0 & 600.0 \\
\hline 00 & 6 & 614 & 82.9916 & 8.3500 & 295.9 & 4 & 600.0 & 600.0 \\
\hline 00 & 6 & 615 & 79.7338 & 8.7000 & 295.5 & 4 & 600.0 & \\
\hline 00 & 6 & 616 & 73.5000 & 7.60 & 296.0 & 7 & 600.0 & 600.0 \\
\hline 00 & 6 & 617 & 72.0000 & 8.2000 & 295.5 & 4 & 600.0 & 600.0 \\
\hline 00 & 6 & 618 & 66.0002 & 8.5750 & 294.7 & 4 & 600.0 & 600.0 \\
\hline 00 & 6 & 619 & 64.2498 & 9.8250 & 293.6 & 4 & 600.0 & 600.0 \\
\hline 00 & 6 & 620 & 63.5009 & 11.0500 & 292.4 & 4 & 600.0 & 600.0 \\
\hline 00 & 6 & 621 & 66.7499 & 9.2750 & 291.1 & 4 & 600.0 & 600.0 \\
\hline 00 & 6 & 622 & 68.2500 & 9.775 & & 4 & 600.0 & 600.0 \\
\hline 00 & 6 & 623 & 54.7605 & 5.9500 & 289.9 & 4 & 600.0 & 600.0 \\
\hline
\end{tabular}




\begin{tabular}{|c|c|c|c|c|c|c|c|c|}
\hline 00 & 6 & 624 & 66.4872 & 8.2000 & 289.3 & 4 & 600.0 & 600.0 \\
\hline 00 & 6 & 71 & 58.7511 & 8.4250 & 288.8 & 4 & 600.0 & 600.0 \\
\hline 00 & 6 & 72 & 62.7481 & 10.4500 & 289.1 & 4 & 600.0 & 00.0 \\
\hline 00 & 6 & 73 & 67.2506 & 10.3250 & 288.0 & 4 & 600.0 & 600.0 \\
\hline 00 & 6 & 74 & 73.7500 & 11.4750 & 285.6 & 4 & 600.0 & 00.0 \\
\hline 00 & 6 & 75 & 72.9992 & 10.9250 & 286.1 & 4 & 600.0 & 00.0 \\
\hline 00 & 6 & 76 & 78.0000 & 10.2000 & 286.0 & 4 & 600.0 & 600.0 \\
\hline 00 & 6 & 77 & 74.7500 & 13.1000 & 285.2 & 4 & 600.0 & 500.0 \\
\hline 00 & 6 & 78 & 69.5029 & 10.4250 & 286.0 & 4 & 600.0 & 00.0 \\
\hline 00 & 6 & 79 & 60.9969 & 11.6000 & 286.9 & 4 & 600.0 & .0 \\
\hline 00 & 6 & 710 & 68.2502 & 8.9750 & 288.4 & 4 & 600.0 & 600.0 \\
\hline 00 & 6 & 711 & 79.0000 & 000 & 290.2 & 4 & 600.0 & \\
\hline 00 & 6 & 712 & 63.7494 & 6.8750 & 292.0 & 4 & 600.0 & 600.0 \\
\hline 00 & 6 & 713 & 80.0070 & 7.7250 & 292.5 & 4 & 600.0 & 600.0 \\
\hline 00 & 6 & 714 & 88.5000 & 9. & 292.1 & 4 & 600.0 & \\
\hline 00 & 6 & 715 & 93.2506 & 8.3500 & 292.0 & 4 & 600.0 & 60 \\
\hline 00 & 6 & 716 & 96.2506 & 7.6750 & 291.9 & 4 & 600.0 & 600.0 \\
\hline 00 & 6 & 717 & 86.5107 & 9. & 290.8 & 4 & 600.0 & .0 \\
\hline 00 & 6 & 718 & 66.2500 & 9.4750 & 290.3 & 4 & 600.0 & 60 \\
\hline 00 & 6 & 719 & 64.5001 & 000 & 289.1 & 4 & 600.0 & 600.0 \\
\hline 00 & 6 & 720 & 69.7491 & 8 . & 288.1 & 4 & 600.0 & .0 \\
\hline 00 & 6 & 721 & 92.3606 & 4. & 287.4 & 4 & 600.0 & \\
\hline 00 & 6 & 722 & 84.0733 & 5.9750 & 287.0 & 4 & 600.0 & 600.0 \\
\hline 00 & 6 & 723 & 59.5000 & 8.9000 & 287.2 & 4 & 600.0 & .0 \\
\hline 00 & 6 & 724 & 53.5011 & 6 & 287.7 & 4 & 600.0 & \\
\hline 00 & 6 & 81 & 60.2595 & 7.9250 & 286.5 & 4 & 600.0 & 600.0 \\
\hline 00 & 6 & 82 & 70.2425 & 5.4250 & 286.4 & 4 & 600.0 & 600.0 \\
\hline 00 & 6 & 83 & 60.2526 & 7. & 284.5 & 4 & 600.0 & .0 \\
\hline 00 & 6 & 84 & 41.7284 & 5.4000 & 284.1 & 4 & 600.0 & 60 \\
\hline 00 & 6 & 85 & 63.9998 & 7.2500 & 283.4 & 4 & 600.0 & 600.0 \\
\hline 00 & 6 & 86 & 64.2481 & 7 . & 283.1 & 4 & 600.0 & .0 \\
\hline 00 & 6 & 87 & 57.5003 & 750 & 283.7 & 4 & 600.0 & 60 \\
\hline 00 & 6 & 88 & 74.2251 & 7.3500 & 284.5 & 4 & 600.0 & 600.0 \\
\hline 00 & 6 & 89 & 83.9723 & 6.6500 & 285.2 & 4 & 600.0 & 60 \\
\hline 00 & 6 & 810 & 58.0001 & 9 . & 285.1 & 4 & 600.0 & 60 \\
\hline 00 & 6 & 811 & 61.7506 & 9.2750 & 285.5 & 4 & 600.0 & 600.0 \\
\hline 00 & 6 & 812 & 60.4995 & 8.1500 & 286.5 & 4 & 600.0 & 600.0 \\
\hline 00 & 6 & 813 & 54.2518 & 8.7750 & 286.4 & 4 & 600.0 & 600.0 \\
\hline 00 & 6 & 814 & 63.5000 & 9.6750 & 286.9 & 4 & 600.0 & 600.0 \\
\hline 00 & 6 & 815 & 61.2501 & 25 & 288.3 & 4 & 600.0 & \\
\hline 00 & 6 & 816 & 60.2498 & 8.6500 & 288.4 & 4 & 600.0 & 600.0 \\
\hline 00 & 6 & 817 & 63.7455 & 7.9000 & 288.6 & 4 & 600.0 & 600.0 \\
\hline 00 & 6 & 818 & 67.5000 & 7.4000 & 288.2 & 4 & 600.0 & 600.0 \\
\hline 00 & 6 & 819 & 66.0000 & 8.1500 & 286.9 & 4 & 600.0 & 600.0 \\
\hline 00 & 6 & 820 & 63.2500 & 8.3500 & 286.0 & 4 & 600.0 & 600.0 \\
\hline 00 & 6 & 821 & 63.5000 & 8.2500 & 286.1 & 4 & 600.0 & 600.0 \\
\hline 00 & 6 & 822 & 70.2498 & 8.400 & 285.6 & 4 & 600.0 & 600.0 \\
\hline 00 & 6 & 823 & 77.0000 & 10.1250 & 284.5 & 4 & 600.0 & 600.0 \\
\hline
\end{tabular}




\begin{tabular}{|c|c|c|c|c|c|c|c|}
\hline 0 & 6824 & 9.7483 & .7250 & 284.7 & 4 & 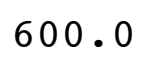 & \\
\hline & $\begin{array}{lll}6 & 9 & 1\end{array}$ & 24998 & 9.7750 & 284.5 & & & \\
\hline & $\begin{array}{lll}6 & 9 & 2\end{array}$ & 68 & 3500 & & & & \\
\hline & $\begin{array}{lll}6 & 9 & 3\end{array}$ & 8.7497 & 1.8000 & 83.2 & & 0 & \\
\hline & $\begin{array}{lll}6 & 9 & 4\end{array}$ & & & & & & \\
\hline & 695 & 63 & 50 & 82.9 & & & \\
\hline & 696 & 7.2499 & 500 & 83.2 & & 0 & \\
\hline & $\begin{array}{lll}6 & 9 & 7\end{array}$ & 7 & & & & & \\
\hline & $\begin{array}{lll}6 & 9 & 8\end{array}$ & 6 & & 85.3 & & 0 & \\
\hline & $\begin{array}{lll}6 & 9 & 9\end{array}$ & & & 85.8 & & 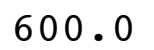 & \\
\hline & 6910 & & & & & & \\
\hline & 6911 & & & & & & \\
\hline & 6912 & 6 & & & & 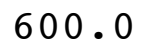 & \\
\hline & 6913 & & & & & & \\
\hline & 6914 & & & & & & \\
\hline & 6915 & 9 & & 8 & & & \\
\hline & 6916 & 5 & 8.50 & & & & \\
\hline & 6917 & & & & & & \\
\hline & 6918 & & 0 & 6 & & & \\
\hline & 6919 & 9 & 0 & 0 & & & \\
\hline & 6920 & & & & & & \\
\hline & 6921 & & & & & & \\
\hline & 6922 & 0 & 0 & 3 & & & \\
\hline & 6923 & & & & & & \\
\hline & 6924 & & & & 4 & & \\
\hline & 6101 & & & & & & \\
\hline & 6102 & 0 & 13. & & $I$ & & \\
\hline & 6103 & 0 & 12. & & 4 & & \\
\hline & 6104 & 8 & & & 4 & & \\
\hline & 6105 & 1 & & & 4 & & \\
\hline & 6106 & 2 & & & 4 & & \\
\hline & 6107 & & & & 4 & & \\
\hline & 6108 & & & 4 & 4 & & \\
\hline & 6109 & & & & 4 & & \\
\hline & 61010 & & & & 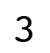 & & \\
\hline & 61011 & 07.2636 & & 289.2 & & & \\
\hline & 61012 & & & & 3 & & \\
\hline & 61013 & 7 & & & 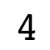 & & \\
\hline & 61014 & & & 291.9 & 4 & & \\
\hline & 61015 & & & & 4 & & \\
\hline & 61016 & & & & 4 & & \\
\hline & 61017 & 2 & 50 & 291.1 & 4 & & \\
\hline & 61018 & 1 & 10 & & 4 & & \\
\hline & 61019 & & 10 . & & 2 & & \\
\hline & 61020 & 70.9995 & 9.8500 & 288.3 & 4 & 0 & P \\
\hline & & & & & 4 & & \\
\hline & 61022 & & & & & & \\
\hline & $010<3$ & 66.4992 & 10.1000 & 288.2 & & 600.0 & $=0$ \\
\hline
\end{tabular}




\begin{tabular}{|c|c|c|c|c|c|c|c|}
\hline 00 & 61024 & 66.5000 & 9.0000 & 289.1 & 4 & 600.0 & 600.0 \\
\hline 00 & 6111 & 71.7496 & 9.0750 & 289.1 & 4 & 600.0 & 600,0 \\
\hline 00 & 6112 & 64.2504 & 10.1500 & 287.4 & 4 & 600.0 & 00.0 \\
\hline 00 & 6113 & 70.2500 & 9.4750 & 287.5 & 4 & 600.0 & 00.0 \\
\hline 00 & 6114 & 71.9995 & 9.1750 & 287.4 & 4 & 600.0 & 00.0 \\
\hline 00 & 6115 & 74.2498 & 8.1500 & 287.9 & 4 & 600.0 & 00.0 \\
\hline 00 & 6116 & 75.2500 & 9.2250 & 287.1 & 4 & 600.0 & 600.0 \\
\hline 00 & 6117 & 75.9996 & 7.9500 & 287.3 & 4 & 600.0 & 00.0 \\
\hline 00 & 6118 & 108.3079 & 3.7750 & 291.2 & 3 & 600.0 & 00.0 \\
\hline 00 & 6119 & 172.7498 & 4.8750 & 292.4 & 4 & 600.0 & .0 \\
\hline 00 & 61110 & 173.7505 & 6.0750 & 292.5 & 4 & 600.0 & 500.0 \\
\hline 00 & 61111 & 168.7499 & 6.2250 & 293.0 & 4 & 600.0 & \\
\hline 00 & 61112 & 165.4959 & 5.2750 & 294.8 & 3 & 600.0 & 00.0 \\
\hline 00 & 61113 & 158.7502 & 6.1500 & 296.1 & 4 & 600.0 & 600.0 \\
\hline 00 & 61114 & 156.9999 & 6.4500 & 297.2 & 4 & 600.0 & \\
\hline 00 & 61115 & 144.2819 & 6.3500 & 297.9 & 4 & 600.0 & 60 \\
\hline 00 & 61116 & 91.4986 & 6.0750 & 297.5 & 4 & 600.0 & 600.0 \\
\hline 00 & 61117 & 85.4997 & 7.1000 & 296.2 & 4 & 600.0 & \\
\hline 00 & 61118 & 77.2494 & 7.8500 & 294.2 & 4 & 600.0 & .0 \\
\hline 00 & 61119 & 72.7485 & 8.0000 & 293.1 & 4 & 600.0 & 60 \\
\hline 00 & 61120 & 66.9986 & 8.80 & 292.3 & 4 & 600.0 & .0 \\
\hline 00 & 61121 & 74.2499 & 9.6000 & 292.3 & 4 & 600.0 & \\
\hline 00 & 61122 & 70.7501 & 9.1000 & 292.3 & 4 & 600.0 & 600.0 \\
\hline 00 & 61123 & 68.5010 & 9.05 & 292.3 & 4 & 600.0 & .0 \\
\hline 00 & 61124 & 66.0000 & 9.4000 & 292.1 & 4 & 600.0 & \\
\hline 00 & 6121 & 62.7502 & 10.2250 & 291.9 & 4 & 600.0 & 60 \\
\hline 00 & 6122 & 67.5000 & 9.9250 & 291.5 & 4 & 600.0 & .0 \\
\hline 00 & 6123 & 65.2506 & 9.67 & 291.6 & 4 & 600.0 & \\
\hline 00 & 6124 & 76.7499 & 10.2000 & 291.6 & 4 & 600.0 & 60 \\
\hline 00 & 6125 & 72.7499 & 9.9250 & 291.5 & 4 & 600.0 & 60 \\
\hline 00 & 6126 & 73.2502 & 9.75 & 291.1 & 4 & 600.0 & \\
\hline 00 & 6127 & 70.7496 & 8.7000 & 291.3 & 4 & 600.0 & .0 \\
\hline 00 & 6128 & 83.7521 & 7.6500 & 292.2 & 4 & 600.0 & 600.0 \\
\hline 00 & 6129 & 89.7483 & 6.37 & 293.3 & 4 & 600.0 & .0 \\
\hline 00 & 61210 & 139.2979 & 6.2000 & 295.8 & 4 & 600.0 & 60 \\
\hline 00 & 61211 & 148.9992 & 6.6250 & 297.3 & 4 & 600.0 & 600.0 \\
\hline 00 & 61212 & 140.7050 & 6.1000 & 298.7 & 4 & 600.0 & \\
\hline 00 & 61213 & 142.0359 & 7.2500 & 299.7 & 4 & 600.0 & 600.0 \\
\hline 00 & 61214 & 138.4933 & 6.4000 & 300.5 & 4 & 600.0 & 600.0 \\
\hline 00 & 61215 & 132.8473 & 5.200 & 301.5 & 3 & 600.0 & \\
\hline 00 & 61216 & 108.4925 & 5.5750 & 301.6 & 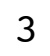 & 600.0 & 600.0 \\
\hline 00 & 61217 & 78.6569 & 6.4750 & 300.8 & 4 & 600.0 & 600.0 \\
\hline 00 & 61218 & 64.7498 & 7.5500 & 299.5 & 4 & 600.0 & 600.0 \\
\hline 00 & 61219 & 59.7600 & 8.0000 & 298.2 & 4 & 600.0 & 600.0 \\
\hline 00 & 61220 & 64.5000 & 9.5500 & 297.0 & 4 & 600.0 & 600.0 \\
\hline 00 & 61221 & 68.0000 & 8.7500 & 295.9 & 4 & 600.0 & 600.0 \\
\hline 00 & 61222 & 67.7504 & 8.600 & 295.4 & 4 & 600.0 & 600.0 \\
\hline 00 & 61223 & 77.9924 & 8.1250 & 294.9 & 4 & 600.0 & 600.0 \\
\hline
\end{tabular}




\begin{tabular}{|c|c|c|c|c|c|c|c|}
\hline & 224 & & 0000 & 96.6 & & & \\
\hline 0 & 131 & 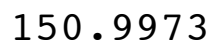 & .3750 & & & & \\
\hline & 132 & 2509 & .3750 & 96.5 & & 00 & \\
\hline & 133 & 74.2499 & 0.3000 & 295.5 & & nan & \\
\hline & 134 & 9.7499 & .5250 & & & & \\
\hline & 135 & 00 & & 2 & & & \\
\hline & 136 & 9.4999 & 8.6500 & 295.1 & & 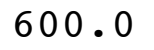 & \\
\hline & 137 & 2502 & 500 & 95 & & & \\
\hline & 138 & 7498 & 0 & 5 & & & \\
\hline 0 & 139 & 59.0002 & 0.2750 & 297.5 & & & \\
\hline & 1310 & 2500 & 8.9750 & 298 & & & \\
\hline & 1311 & & 7.6750 & 1 & & & \\
\hline 0 & 13 & 75 & 8 . & 301.4 & & & \\
\hline ) & 313 & 9 & 7.2000 & 302 & & & \\
\hline & & & 0 & 30 & & & \\
\hline 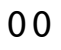 & & & & 30 & & & \\
\hline 0 & 13 & 00 & 7.6500 & 305 & & .0 & \\
\hline & 17 & 01 & 250 & 30 & & & \\
\hline 0 & & & 7 . & 30 & $x$ & & \\
\hline 0 & 9 & 08 & 500 & 30 & $\Delta$ & & \\
\hline 0 & 0 & 153. & 3.2000 & 30 & & • 0 & \\
\hline 0 & & & & & 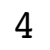 & & \\
\hline 0 & 2 & 85 & 0 & 30 & 4 & & \\
\hline 0 & 1323 & 46 & 500 & 304.1 & & 0 & \\
\hline 0 & 61324 & & & 30 & & & \\
\hline 0 & 6141 & & & 30 & 4 & & \\
\hline & 6142 & 140 . & 750 & 30 & 4 & 0 & \\
\hline & 6143 & 63 & 500 & 30 & 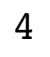 & & \\
\hline 0 & 6144 & & 9 . & 29 & 4 & & \\
\hline & 6145 & 09 & 250 & 299.8 & 4 & & \\
\hline & 6146 & 21 & 8.1250 & 299.5 & 4 & & \\
\hline 0 & 6147 & & 0 & 300.5 & 4 & & \\
\hline & 6148 & 57 & & 1.5 & 4 & & \\
\hline 0 & 6149 & 171.2503 & 0 & 302.1 & 4 & & \\
\hline 0 & & 4 & 0 & 303.1 & 4 & & \\
\hline & & 00 & 0.9000 & 304.5 & 4 & & \\
\hline & 61412 & 002 & 2500 & 305.8 & 4 & & \\
\hline 0 & & 1 & 7. & 307 & 4 & & \\
\hline 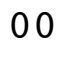 & & 167 . & 7.3750 & 308.0 & 4 & .0 & \\
\hline & & 170.7302 & 000 & 308.8 & 4 & & \\
\hline 0 & & 621 & 6 . & 309.4 & 4 & & \\
\hline 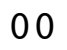 & & 173.2527 & & & 3 & & \\
\hline & 61418 & 169.4785 & 4.2000 & 309.9 & 3 & & 0 \\
\hline 00 & & 506 & 750 & 309.8 & & & \\
\hline 00 & & 137.4511 & & 307.9 & 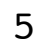 & .0 & \\
\hline 0 & & 514 & 1.9750 & 307.5 & 5 & & مص \\
\hline & & 13 & & & o & & \\
\hline & 61423 & 7379 & 6.3000 & 305.9 & & .0 & 600 \\
\hline
\end{tabular}




\begin{tabular}{|c|c|c|c|c|c|c|c|}
\hline 0 & 61424 & 52.9966 & 7.1250 & 305.7 & 4 & 600.0 & 600.0 \\
\hline 0 & 6151 & 5.2495 & 6.7250 & 306.1 & 4 & 600.0 & 00,0 \\
\hline 00 & 6152 & 75.8754 & 6.3750 & 306.1 & 4 & 600.0 & 00.0 \\
\hline 0 & 6153 & 167.7493 & 10.3750 & 306.6 & 4 & 600.0 & 00.0 \\
\hline 00 & 6154 & 160.7431 & 8.1500 & 305.6 & 4 & 600.0 & 00.0 \\
\hline 00 & 6155 & 103.5467 & 3.9750 & 304.9 & 4 & 600.0 & 00.0 \\
\hline 00 & 6156 & 169.7539 & 7.0000 & 305.1 & 4 & 600.0 & 00.0 \\
\hline 00 & 6157 & 166.8796 & 8.4000 & 305.7 & 4 & 600.0 & 00.0 \\
\hline 00 & 6158 & 173.2534 & 9.1250 & 306.2 & 4 & 600.0 & 00.0 \\
\hline 00 & 6159 & 167.4750 & 6.2750 & 306.6 & 4 & 600 & 0 \\
\hline 00 & 61510 & 173.7488 & 4.7000 & 306.6 & 3 & 600.0 & \\
\hline 00 & 61511 & 187.7537 & 5.9000 & 306.8 & 3 & 600.0 & \\
\hline 00 & 61512 & 183.7590 & 4.7250 & 307.2 & 3 & 600.0 & 00.0 \\
\hline 00 & 61513 & 176.7503 & 4.9250 & 308.2 & 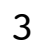 & 600.0 & 00.0 \\
\hline 00 & 61514 & 173.7495 & 7.0000 & 8.3 & 4 & .0 & \\
\hline 00 & 61515 & 170.7667 & 6.8000 & 308.7 & 4 & 600.0 & .0 \\
\hline 00 & 61516 & 173.2490 & 6.2000 & 308.8 & 4 & 60 & .0 \\
\hline 00 & 61517 & 171.2559 & 5.8500 & 309.3 & 3 & .0 & \\
\hline 00 & 61518 & 127.2315 & 4.5250 & 309.9 & 3 & 60 & \\
\hline 00 & 61519 & 114.2713 & 4.2500 & 309.0 & 4 & .0 & .0 \\
\hline 00 & 61520 & 86.2019 & 6.2500 & .5 & 4 & 6 & \\
\hline 00 & 61521 & 74.7503 & 7.5750 & .7 & 4 & .0 & \\
\hline 00 & 61522 & 61.2486 & 8.8500 & 302.9 & 4 & 600.0 & .0 \\
\hline 00 & 61523 & 70.7485 & 9.07 & 2.6 & 4 & 6 & \\
\hline 00 & 61524 & 96.0037 & 8. & 302.4 & 4 & .0 & \\
\hline 00 & 6161 & 123.2968 & 7.1250 & 304.5 & 4 & 600.0 & .0 \\
\hline 00 & 6162 & 174.2724 & 9.7750 & 304.5 & 4 & 600.0 & .0 \\
\hline 00 & 6163 & 179.9963 & 9.2500 & 303.6 & 4 & .0 & \\
\hline 00 & 6164 & 184.5015 & 10.3500 & 302.4 & 4 & .0 & .0 \\
\hline 00 & 6165 & 187.2527 & 10.9500 & 300.8 & 4 & 60 & .0 \\
\hline 00 & 6166 & 175.2539 & 10.5750 & 300.0 & 4 & .0 & \\
\hline 00 & 6167 & 174.0000 & 9.6000 & 300.0 & 4 & .0 & 0.0 \\
\hline 00 & 6168 & 176.5000 & 12.7250 & 300.2 & 4 & 600.0 & 0.0 \\
\hline 00 & 6169 & 181.2491 & 14.0000 & 300.3 & 4 & 0.0 & 0.0 \\
\hline 00 & 61610 & 176.2405 & 10.4250 & 300.9 & 4 & 600.0 & 600.0 \\
\hline 00 & 61611 & 175.0007 & 8.8500 & 301.7 & 4 & 600.0 & 600.0 \\
\hline 00 & 61612 & 169.4997 & 7.250 & 302.9 & 4 & 600.0 & 600.0 \\
\hline 00 & 61613 & 166.0000 & 7.5000 & 303.7 & 4 & 600.0 & 600.0 \\
\hline 00 & 61614 & 164.4939 & 7.6750 & 304.4 & 4 & 600.0 & 600.0 \\
\hline 00 & 61615 & 168.5013 & 7.5750 & 305.0 & 4 & 600.0 & 600.0 \\
\hline 00 & 61616 & 168.5000 & 7.3000 & 305.2 & 4 & 600.0 & 600.0 \\
\hline 00 & 61617 & 169.7494 & 7.3500 & 305.4 & 4 & 600.0 & 600.0 \\
\hline 00 & 61618 & 143.0009 & 6.1500 & 305.3 & 4 & 600.0 & 600.0 \\
\hline 00 & 61619 & 80.7539 & 5.2750 & 303.4 & 4 & 600.0 & 600.0 \\
\hline 00 & 61620 & 71.7502 & 7.7500 & 300.7 & 4 & 600.0 & 600.0 \\
\hline 00 & 61621 & 87.6138 & 9 . & 299.3 & 4 & 600.0 & \\
\hline 00 & 61622 & 112.1946 & 9.4250 & 298.5 & 4 & 600.0 & 600.0 \\
\hline 00 & 61623 & 146.9044 & 8.725 & 298.6 & & 600.0 & 600.0 \\
\hline
\end{tabular}




\begin{tabular}{|c|c|c|c|c|c|c|c|}
\hline & 24 & & & & & & \\
\hline & 6171 & & 0 & 8.7 & & & \\
\hline & 172 & 885 & 250 & 297.8 & & 00 & \\
\hline & 173 & 47.6772 & 1.0250 & 296.7 & & 0 & \\
\hline & 174 & & .4500 & 5.7 & & & \\
\hline & 175 & 0 & & 5.7 & & & \\
\hline & 176 & 16 & 8.4750 & 95.4 & & 0 & \\
\hline & 177 & 34. & 6. & 95.7 & & & \\
\hline & 178 & $20-7$ & & .9 & & & \\
\hline & 179 & 5569 & 0 & 97.7 & & 0 & \\
\hline & 1710 & 3049 & 5.85 & 298.3 & & & \\
\hline & 1711 & 607688 & & 99.2 & & & \\
\hline & 1712 & 5975 & 5 . & 300.1 & & & \\
\hline & 1713 & 86.9998 & 0 & 299.7 & & & \\
\hline & 1714 & 91 & & 9 & & & \\
\hline & 1715 & 89 . & & 299.7 & & & \\
\hline & 1716 & 105.8451 & 9.32 & 298.6 & 4 & & \\
\hline & 1717 & 70 & 1 . & 97.7 & & & \\
\hline & 1718 & 133. & 0 & 296 & 4 & & \\
\hline & 1719 & 130.2503 & 50 & 294.3 & 4 & & \\
\hline & 1720 & 737 & 0 & 292.7 & 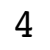 & 0 & \\
\hline & 1721 & 73. & & 291.7 & 4 & & \\
\hline & 1722 & 02 & 0 & 29 & 4 & & \\
\hline & 61723 & 487 & 3.77 & 290.4 & 4 & & \\
\hline & 61724 & & 0 & 29 & $\mathbf{4}$ & & \\
\hline & $618 \quad 1$ & 00 & 12 . & 28 & 4 & & \\
\hline & 6182 & 81.7499 & 12 . & 288.2 & 4 & & . \\
\hline & 6183 & 80 & 11 . & 28 & 4 & & \\
\hline & 6184 & & 0 & 28 & 4 & & \\
\hline & 6185 & 498 & 0 & 288.2 & 4 & & \\
\hline & 6186 & 002 & 9.3000 & 288.0 & 4 & & \\
\hline & 6187 & 0 & & 28 & 4 & & \\
\hline & 6188 & 000 & 0 & 289.1 & 4 & & . \\
\hline & 6189 & 859 & 500 & 291.1 & 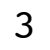 & & \\
\hline & 61810 & & & & 2 & & \\
\hline & 61811 & & & 296.1 & & & \\
\hline & 61812 & 377 & 6250 & 297.0 & & & \\
\hline & 61813 & 502 & 3 . & 297.4 & 2 & & \\
\hline & 61814 & 59.5937 & 3.1750 & 299.3 & 2 & & 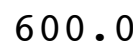 \\
\hline & 61815 & 77.7706 & 500 & 298.7 & 3 & & \\
\hline & 61816 & 113.1396 & 00 & 296 & 4 & & \\
\hline & 61817 & 145.5526 & 6.4750 & & 4 & & \\
\hline & 61818 & 169.9742 & 0250 & 294.7 & 4 & & 0 \\
\hline & 61819 & 159.5572 & 50 & 293 & 4 & & \\
\hline & 61820 & 141.4693 & & & 4 & & \\
\hline & 61821 & 161.7519 & 9.0250 & 289.8 & 4 & & 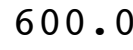 \\
\hline & & 137 & & & & & \\
\hline & 61823 & 59.3855 & 2.7750 & 291.5 & & 60 & 600 \\
\hline
\end{tabular}




\begin{tabular}{|c|c|c|c|c|c|c|c|}
\hline 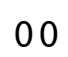 & 1824 & 2.7566 & 1.9750 & 92.1 & 6 & 00.0 & \\
\hline & 6191 & 0.7500 & 3.4250 & 292.9 & & & \\
\hline & 6192 & 50 & 3750 & 1.9 & & & \\
\hline & 193 & 24.2651 & 750 & 89.8 & & 00 & \\
\hline & 194 & 3 & & 7.5 & & & \\
\hline & 195 & 140 & 3500 & 88.9 & & 0 & \\
\hline & 196 & 212.2766 & 500 & 87.3 & & 0 & \\
\hline & 197 & 160.7828 & & 90.0 & & & \\
\hline & 198 & 176.7511 & & 291.3 & & 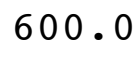 & \\
\hline & 199 & 174.4994 & & 291.6 & & 0 & \\
\hline & 910 & 166 & & 92 & & & \\
\hline & 911 & 167 . & & 95 & & & \\
\hline & 1912 & 171 . & 0 & 296.6 & & 0 & \\
\hline & 1913 & 169 & 0 & 6 & & & \\
\hline & 1914 & & & & & & \\
\hline & 1915 & 160 & & 300.5 & 3 & & \\
\hline & 61916 & 173.25 & 0 & 301.4 & & & \\
\hline & 61917 & 173. & & 301.9 & 4 & & \\
\hline & 1918 & 15 & & 301.9 & 3 & & \\
\hline & 61919 & 134 & 0 & 300.2 & & & \\
\hline & 61920 & & & & & & \\
\hline & 61921 & 173 & & 9 & 4 & & \\
\hline & 1922 & 107.1557 & 0 & 29 & 4 & & \\
\hline & 61923 & & & & & & \\
\hline & 61924 & & & & 4 & & \\
\hline & 6201 & 88 & & 297 & 4 & & \\
\hline & 6202 & & & & 4 & & \\
\hline & 6203 & 100 . & 0 & 297 & 4 & & \\
\hline & 6204 & 94 & & 297.8 & 1 & & \\
\hline & 6205 & 128 . & & 298 & 4 & & \\
\hline & 6206 & 121. & & 29 & 4 & & \\
\hline & 6207 & 12 & & 29 & 4 & & \\
\hline & 6208 & 143. & & 298.3 & 4 & & \\
\hline & 6209 & 156 & & 298 & 4 & & \\
\hline & 62010 & & & & 4 & & \\
\hline & 62011 & 151.2446 & & 300.5 & 3 & & \\
\hline & 62012 & 16 & & 301.7 & 4 & & \\
\hline & 62013 & 163. & & 303.1 & 4 & & \\
\hline & 62014 & 172.4998 & & 304.2 & 4 & & \\
\hline & 62015 & 17 & & & 4 & & \\
\hline & 62016 & 177 . & & 305.2 & 4 & & \\
\hline & 62017 & 174.2500 & & 305.8 & 4 & & \\
\hline & 62018 & 124.8202 & & 305.3 & 3 & & \\
\hline & 62019 & 147.5265 & & & 4 & & \\
\hline & 62020 & 171.0005 & 7.7750 & 301.4 & 4 & .0 & م0 \\
\hline & 62 & 16 & & & 4 & & \\
\hline & 62022 & 185.0016 & & & & & \\
\hline & 52023 & 5752 & 1.0000 & 299. & & 600.0 & 500 \\
\hline
\end{tabular}




\begin{tabular}{|c|c|c|c|c|c|c|c|}
\hline 00 & 62024 & 69.5000 & 11.0000 & 299.4 & 4 & 600.0 & 00.0 \\
\hline 0 & 6211 & 62.5000 & 10.1750 & 299.5 & 4 & 600.0 & 600,0 \\
\hline 00 & 6212 & 69.5000 & 9.4750 & 300.0 & 4 & 600.0 & 00.0 \\
\hline 00 & 6213 & 72.5000 & 7.8750 & 300.2 & 4 & 600.0 & 00.0 \\
\hline 00 & 6214 & 72.5001 & 7.5250 & 300.3 & 4 & 600.0 & 00.0 \\
\hline 00 & 6215 & 74.0000 & 7.7000 & 300.1 & 4 & 600.0 & 00.0 \\
\hline 00 & 6216 & 77.7501 & 7.7500 & 300.0 & 4 & 600.0 & 00.0 \\
\hline 00 & 6217 & 72.4994 & 6.0000 & 300.6 & 4 & 600.0 & 00.0 \\
\hline 00 & 6218 & 84.7481 & 3.6500 & 302.6 & 3 & 600.0 & 00.0 \\
\hline 00 & 6219 & 160.3311 & 3.4000 & 303.8 & 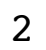 & 600.0 & .0 \\
\hline 00 & 62110 & 197.7328 & 3.7250 & 303.2 & 2 & 600.0 & 00.0 \\
\hline 00 & 62111 & 173.9977 & 3.9750 & 303.6 & 2 & 600.0 & \\
\hline 00 & 62112 & 168.1982 & 5.9250 & 304.7 & 3 & 600.0 & .0 \\
\hline 00 & 62113 & 77.3786 & 4.1500 & 307.0 & 3 & 600.0 & 0 \\
\hline 00 & 62114 & 103.2580 & 5.4000 & 306.5 & 3 & 600.0 & \\
\hline 00 & 62115 & 101.0000 & 5.7750 & 306.6 & 3 & 600.0 & 60 \\
\hline 00 & 62116 & 91.2447 & 6.5750 & 306.4 & 4 & 600.0 & 600.0 \\
\hline 00 & 62117 & 73.7445 & 7.4000 & 305.6 & 4 & 600.0 & \\
\hline 00 & 62118 & 69.2511 & 8.4500 & 304.6 & 4 & 600.0 & .0 \\
\hline 00 & 62119 & 66.7509 & 7.6000 & 303.4 & 4 & 600.0 & .0 \\
\hline 00 & 62120 & 61.2499 & 10.97 & 301.9 & 4 & 600.0 & .0 \\
\hline 00 & 62121 & 63.4999 & 11. & 300.7 & 4 & 0.0 & \\
\hline 00 & 62122 & 62.9999 & 11.9000 & 299.7 & 4 & 600.0 & 600.0 \\
\hline 00 & 62123 & 63.2500 & 11.0500 & 300.3 & 4 & 600.0 & .0 \\
\hline 00 & 62124 & 66.5001 & 10.2000 & 301.0 & 4 & 00.0 & \\
\hline 00 & 6221 & 66.5001 & 9.9500 & 301.0 & 4 & 600.0 & 60 \\
\hline 00 & 6222 & 65.0000 & 9.9000 & 300.9 & 4 & 600.0 & .0 \\
\hline 00 & 6223 & 67.7499 & 8.62 & 301.2 & 4 & 00.0 & \\
\hline 00 & 6224 & 70.2500 & 7.7250 & 301.5 & 4 & 600.0 & 60 \\
\hline 00 & 6225 & 64.2517 & 7.1500 & 301.4 & 4 & 600.0 & .0 \\
\hline 00 & 6226 & 66.7499 & 5.57 & 301.3 & 4 & 600.0 & \\
\hline 00 & 6227 & 6.2066 & 1.55 & 299.8 & 3 & 600.0 & .0 \\
\hline 00 & 6228 & 269.0753 & 1.0500 & 300.5 & 2 & 600.0 & 600.0 \\
\hline 00 & 6229 & 250.7536 & 1.95 & 301.2 & 1 & 600.0 & .0 \\
\hline 00 & 62210 & 261.6792 & 2.0000 & 301.8 & 1 & 600.0 & .0 \\
\hline 00 & 62211 & 232.6618 & 2.9250 & 303.1 & 1 & 600.0 & 600.0 \\
\hline 00 & 62212 & 229.1979 & 3.0250 & 303.8 & 2 & 600.0 & \\
\hline 00 & 62213 & 103.1052 & 5.4250 & 304.7 & 3 & 600.0 & 600.0 \\
\hline 00 & 62214 & 76.9482 & 5.6750 & 304.8 & 3 & 600.0 & 600.0 \\
\hline 00 & 62215 & 84.7364 & 6.825 & 303.9 & 4 & 600.0 & \\
\hline 00 & 62216 & 74.5000 & 7.1000 & 303.5 & 4 & 600.0 & 60 \\
\hline 00 & 62217 & 68.7401 & 9.9500 & 301.7 & 4 & 600.0 & 600.0 \\
\hline 00 & 62218 & 62.7485 & 12.0500 & 299.6 & 4 & 600.0 & 600.0 \\
\hline 00 & 62219 & 68.7482 & 11.4000 & 298.0 & 4 & 600.0 & 600.0 \\
\hline 00 & 62220 & 66.2443 & 10.9000 & 297.4 & 4 & 600.0 & 600.0 \\
\hline 00 & 62221 & 65.2500 & 11.2250 & 297.0 & 4 & 600.0 & 600.0 \\
\hline 00 & 62222 & 68.4998 & 9.875 & 297.6 & 4 & 600.0 & 600.0 \\
\hline 00 & 62223 & 69.0000 & 9.7000 & 297.1 & 4 & 600.0 & 600.0 \\
\hline
\end{tabular}




\begin{tabular}{|c|c|c|c|c|c|c|c|}
\hline 0 & 52224 & 0.2504 & 9.3500 & 296.6 & 4 & 600.0 & \\
\hline & 6231 & 72.2503 & 9.3750 & 296.4 & 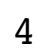 & $c 00$ & \\
\hline & 6232 & 7513 & 3750 & 6.3 & 1 & 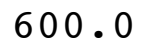 & \\
\hline & 6233 & 5.0002 & 750 & 95.3 & & 00 & \\
\hline & 6234 & 1.2501 & & 5.6 & & & \\
\hline & 6235 & 0001 & 500 & 95.2 & & $x^{2}+2$ & \\
\hline & 6236 & 67.0112 & 7.8250 & 95.1 & & S & \\
\hline & 6237 & 690 & 00 & 95.3 & & 0 & \\
\hline & 6238 & 93.0621 & 1.3750 & 296.2 & & 0 & \\
\hline & 6239 & 41.8344 & & 295 & & 0 & \\
\hline & 2310 & 258.5065 & & & & & \\
\hline & 62311 & 263. & & 3 & & & \\
\hline & 62312 & 231.9228 & 5000 & 299.2 & & 0 & \\
\hline & 62313 & 233. & & 99 & & & \\
\hline & 62314 & 199. & & & 3 & & \\
\hline & 62315 & 174 . & & 300.1 & 4 & & \\
\hline & 62316 & 160.0107 & 0 & 30 & 2 & U & \\
\hline & 62317 & 167.4983 & & & 3 & & \\
\hline & 62318 & 101. & 0 & 30 & 2 & & \\
\hline & 62319 & 67.3150 & 0 & & 3 & & \\
\hline & 62320 & 22 . & & & & & \\
\hline & 62321 & 58 . & & 29 & 5 & & \\
\hline & 62322 & 38.9600 & 0 & 296.1 & 6 & & \\
\hline & 62323 & 33.6436 & & 2 & 6 & & \\
\hline & 62324 & 328 . & & 29 & 6 & & \\
\hline & 6241 & 104.1712 & & 291 & 6 & & \\
\hline & 6242 & 29.5674 & & 295.1 & 6 & & \\
\hline & 6243 & 103.4742 & & 29 & 6 & & \\
\hline & $624 \quad 4$ & 167.6669 & & 292 & 6 & & \\
\hline & 6245 & 167.5328 & & 292.1 & 5 & & \\
\hline & 6246 & 155.9062 & & 293 & 4 & & \\
\hline & 6247 & 83. & & & 3 & & \\
\hline & 6248 & 323.6091 & & 297.9 & 2 & & \\
\hline & 6249 & 247 . & & 29 & 1 & & \\
\hline & 62410 & 23 & & & 2 & & \\
\hline & 62411 & 229.9996 & & 297.8 & & & \\
\hline & 62412 & 222 . & & 300.4 & 2 & & \\
\hline & 62413 & 158.3065 & & 300.7 & 3 & & \\
\hline & 62414 & 89.4776 & & 301.5 & 3 & & \\
\hline & 62415 & 99. & & 301.5 & 3 & & \\
\hline & 62416 & 100.2491 & & 301.0 & 3 & & \\
\hline & 62417 & 93.0015 & 500 & 300.0 & 4 & & 0 \\
\hline & 62418 & 76.5014 & & 298.4 & 4 & & \\
\hline 0 & 62419 & 71.5000 & 9.1750 & & 4 & & 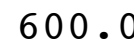 \\
\hline & 62420 & 73.0003 & 1.3750 & 293.9 & 4 & .0 & ה \\
\hline & & 63 & & .2 & 4 & & \\
\hline & 62422 & 72.2527 & & 295.8 & 4 & & \\
\hline & $0<4<3$ & 74.7498 & 6.5250 & 297.6 & & 600.0 & 60 \\
\hline
\end{tabular}




\begin{tabular}{|c|c|c|c|c|c|c|c|}
\hline & 2424 & .2004 & 8.9000 & 297.1 & 4 & . & \\
\hline & 6251 & 7.2500 & 10.1000 & 295.8 & & & \\
\hline & 6252 & 7502 & 8750 & 296.3 & & 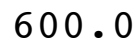 & \\
\hline & 5253 & 8.9994 & 5500 & 296.2 & & 00 & \\
\hline & 254 & 500 & & & & & \\
\hline & 255 & 2518 & & 3 & & 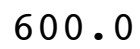 & \\
\hline & 256 & 74.7485 & 000 & 96.5 & & & \\
\hline & 257 & 3899 & 3.40 & & & & \\
\hline & 6258 & 23.4949 & 1.77 & 9 & & & \\
\hline & 259 & 51.2507 & 1.4250 & 29 & & & \\
\hline & 2510 & 227.2440 & 2.9750 & 6 & & & \\
\hline & 2511 & 201.1171 & & & & & \\
\hline & 2512 & 169.4925 & 6 . & 5 & & 0 & \\
\hline & 62513 & 173.2494 & 6.3500 & 5 & & 0 & \\
\hline & 62514 & 168.7535 & & & & & \\
\hline & 62515 & 120 . & & 8 & 3 & & \\
\hline & 62516 & 93.7518 & 5.95 & & & & \\
\hline & 62517 & 81 . & & & & & \\
\hline & 62518 & 61. & & & 4 & & \\
\hline & 62519 & 480 & & & 4 & & \\
\hline & 62520 & 64 & & & 4 & & \\
\hline & 62521 & & & & 4 & & \\
\hline & 62522 & 64.2503 & & 29 & 4 & & \\
\hline & 62523 & 67.5006 & & & 4 & & \\
\hline & 62524 & 0 & & & 4 & & \\
\hline & 6261 & 2495 & & & 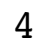 & & \\
\hline & 6262 & 72.2500 & & 29 & $x^{2}$ & & \\
\hline & 6263 & 71.2499 & & 0 & 4 & & \\
\hline & 6264 & 77 . & & 25 & 4 & & \\
\hline & 6265 & 77.7494 & & 29 & 4 & & \\
\hline & 6266 & 74.7506 & & & 4 & & \\
\hline & 6267 & 1 & & & 4 & & \\
\hline & 6268 & 01.0059 & 0 & 30 & & & \\
\hline & 6269 & 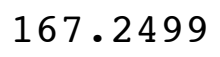 & & & 2 & & \\
\hline & 62610 & 2 & & & 3 & & \\
\hline & 62611 & 173.9931 & & & 3 & & \\
\hline & 62612 & 498 & & & 3 & & \\
\hline & 62613 & 171. & & & 4 & & \\
\hline & 62614 & 164.7498 & & .4 & 4 & & \\
\hline & 62615 & 034 & & & 4 & & \\
\hline & 62616 & 103. & & & 3 & & \\
\hline & 62617 & 79.2500 & 6.9000 & 305.1 & 4 & & 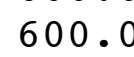 \\
\hline & 62618 & 67 . & 7.1500 & 304.4 & 4 & 0 & \\
\hline & 62619 & 71.0000 & & 303.4 & 2 & & \\
\hline 0 & 62620 & 66.7500 & 8.4000 & 302.3 & 4 & 600 & \\
\hline & 62621 & 7500 & 0.6000 & 301.3 & 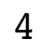 & & \\
\hline & 626 & & & 30 & & & \\
\hline & 62623 & 74.7497 & 10.5000 & 300.0 & & 600.0 & 600 \\
\hline
\end{tabular}




\begin{tabular}{|c|c|c|c|c|c|c|c|}
\hline 0 & 62624 & 72.2502 & 10.8750 & 00.1 & 4 & 600.0 & 600.0 \\
\hline 00 & 6271 & 73.7499 & 10.4250 & 300.6 & 4 & 600.0 & 00.0 \\
\hline 00 & 6272 & 75.5000 & 1750 & 300.8 & 4 & 600.0 & 00.0 \\
\hline 0 & 6273 & 70.7504 & 8.7250 & 301.2 & 4 & 600.0 & 00.0 \\
\hline 00 & 6274 & 69.5000 & 8.2750 & 301.5 & 4 & 600.0 & .0 \\
\hline 00 & 6275 & 72.7501 & 9.0000 & 301.4 & 4 & 600.0 & 00.0 \\
\hline 00 & $627 \quad 6$ & 74.0012 & 8.7250 & 301.7 & 4 & 600.0 & 0 \\
\hline 00 & 6277 & 63.4810 & 5.0250 & 302.4 & 3 & 600.0 & 00.0 \\
\hline 00 & 6278 & 68.7496 & 5.0250 & 303.3 & 3 & 600.0 & 00.0 \\
\hline 00 & 6279 & 105.7687 & 4.7500 & 303.6 & 3 & 60 & 0 \\
\hline 00 & 62710 & 142.7590 & 3.8750 & 304.5 & 2 & 600.0 & \\
\hline 00 & 62711 & 83.2224 & 3.5750 & 306.0 & 2 & .0 & \\
\hline 00 & 62712 & 62.7578 & 3.7750 & 307.0 & 2 & 600.0 & 00.0 \\
\hline 00 & 62713 & 90.0000 & 4.6500 & 306.9 & 3 & 600.0 & \\
\hline 00 & 62714 & 83.7483 & 5.2250 & 306.7 & 3 & .0 & \\
\hline 00 & 62715 & 87.7477 & 6.4250 & 306.4 & 4 & 600.0 & .0 \\
\hline 00 & 62716 & 80.2500 & 7.1250 & 306.2 & 4 & .0 & .0 \\
\hline 00 & 62717 & 68.7485 & 8.2000 & 305.6 & 4 & .0 & \\
\hline 00 & 62718 & 58.2499 & 9.1500 & 304.7 & 4 & .0 & \\
\hline 00 & 62719 & 59.7500 & 10.3500 & 303.9 & 4 & .0 & .0 \\
\hline 00 & 62720 & 66.7498 & 9.47 & .8 & 4 & 6 & \\
\hline 00 & 62721 & 66.7494 & 9.7750 & 302.4 & 4 & .0 & \\
\hline 00 & 62722 & 71.0000 & 9.7250 & 302.6 & 4 & 60 & .0 \\
\hline 00 & 62723 & 70.7500 & 10.30 & .7 & 4 & 6 & \\
\hline 00 & 62724 & 72.7500 & 10.6250 & .5 & 4 & 0 & \\
\hline 00 & 6281 & 74.7500 & 9.4250 & 302.7 & 4 & 600.0 & .0 \\
\hline 00 & 6282 & 76.0000 & 8.675 & 302.4 & 4 & 0 & .0 \\
\hline 00 & 6283 & 73.9999 & 8.5750 & 302.3 & 4 & .0 & \\
\hline 00 & 6284 & 73.7498 & 7.6000 & 302.5 & 4 & .0 & .0 \\
\hline 00 & 6285 & 77.0550 & 3.7750 & 301.4 & 4 & 6 & .0 \\
\hline 00 & 6286 & 82.2508 & 4.5750 & 301.1 & 4 & .0 & \\
\hline 00 & 6287 & 78.2499 & 6.2000 & 301.9 & 4 & .0 & 0.0 \\
\hline 00 & 6288 & 96.2312 & 4.8750 & 303.2 & 3 & 0 & 0.0 \\
\hline 00 & 6289 & 130.2620 & 3.0750 & 304.7 & 2 & 0 & 0.0 \\
\hline 00 & 62810 & 157.0610 & 3.5000 & 304.9 & 2 & 600.0 & 600.0 \\
\hline 00 & 62811 & 153.0869 & 3.3250 & 305.7 & 2 & 600.0 & 600.0 \\
\hline 00 & 62812 & 25.8429 & 2.82 & 307.2 & 1 & 600.0 & 0.0 \\
\hline 00 & 62813 & 81.3735 & 4.5750 & 306.9 & 2 & 600.0 & 600.0 \\
\hline 00 & 62814 & 93.2500 & 6.9000 & 306.0 & 3 & 600.0 & 600.0 \\
\hline 00 & 62815 & 69.2434 & 6.3500 & 306.6 & 4 & 600.0 & 600.0 \\
\hline 00 & 62816 & 71.9874 & 6.9500 & 305.9 & 4 & 600.0 & 600.0 \\
\hline 00 & 62817 & 63.4999 & 8.4750 & 304.9 & 4 & 600.0 & 600.0 \\
\hline 00 & 62818 & 68.0001 & 10.1750 & 303.5 & 4 & 600.0 & 600.0 \\
\hline 00 & 62819 & 70.2500 & 11.5000 & 302.0 & 4 & 600.0 & 600.0 \\
\hline 00 & 62820 & 69.5006 & 12.4000 & 301.3 & 4 & 600.0 & 600.0 \\
\hline 00 & 62821 & 69.0000 & 11.8000 & 300.8 & 4 & 600.0 & \\
\hline 00 & 62822 & 70.0000 & 11.4000 & 301.9 & 4 & 600.0 & 600.0 \\
\hline 00 & 62823 & 71.5000 & 10.500 & 302.4 & 4 & 600.0 & 600.0 \\
\hline
\end{tabular}




\begin{tabular}{|c|c|c|c|c|c|c|c|}
\hline 0 & 62824 & 71.9999 & 11.1250 & 302.3 & 4 & 600.0 & 00.0 \\
\hline 00 & 6291 & 70.7499 & 9.2000 & 302.4 & 4 & 600.0 & 600.0 \\
\hline 00 & 6292 & 71.9997 & 8.6750 & 302.2 & 4 & 600.0 & 00.0 \\
\hline 00 & 6293 & 66.2500 & 8.8750 & 301.8 & 4 & 600.0 & 00.0 \\
\hline 00 & 6294 & 42.3134 & 4.4750 & 300.2 & 4 & 600.0 & 00.0 \\
\hline 00 & 6295 & 43.1974 & 3.9250 & 297.4 & 4 & 600.0 & 00.0 \\
\hline 00 & 6296 & 75.7495 & 7.5250 & 300.7 & 4 & 600.0 & .0 \\
\hline 00 & 6297 & 356.0517 & 1.9500 & 297.8 & 3 & 600.0 & 00.0 \\
\hline 00 & 6298 & 275.6986 & 0.7250 & 298.7 & 2 & 600.0 & .0 \\
\hline 00 & 6299 & 298.2515 & 1.2750 & 300.7 & 1 & 600.0 & \\
\hline 00 & 62910 & 261.4842 & 2.0250 & 301.8 & 1 & 600.0 & 00.0 \\
\hline 00 & 62911 & 231.0107 & 2.8750 & 301.7 & 2 & 600.0 & \\
\hline 00 & 62912 & 236.9586 & 2.7250 & 303.2 & 1 & 600.0 & .0 \\
\hline 00 & 62913 & 111.0593 & 3.8750 & 304.4 & 2 & 600.0 & .0 \\
\hline 00 & 62914 & 69.9966 & 7.7250 & 303.4 & 3 & 600.0 & \\
\hline 00 & 62915 & 76.2450 & 6.8500 & 303.7 & 4 & 600.0 & 60 \\
\hline 00 & 62916 & 60.7492 & 8.4000 & 303.0 & 4 & 600.0 & 600.0 \\
\hline 00 & 62917 & 56.9991 & 9.0250 & 302.5 & 4 & 600.0 & \\
\hline 00 & 62918 & 61.7518 & 9.1000 & 301.6 & 4 & 600.0 & .0 \\
\hline 00 & 62919 & 68.2501 & 9.5750 & 300.0 & 4 & 600.0 & .0 \\
\hline 00 & 62920 & 67.9994 & 9.90 & 299.0 & 4 & 600.0 & .0 \\
\hline 00 & 62921 & 69.7500 & 9.9250 & 298.5 & 4 & 0.0 & \\
\hline 00 & 62922 & 71.2501 & 9.8000 & 298.0 & 4 & 600.0 & 60 \\
\hline 00 & 62923 & 64.5000 & 11.45 & 299.0 & 4 & 600.0 & .0 \\
\hline 00 & 62924 & 67.0000 & 11. & 298.0 & 4 & 0.0 & \\
\hline 00 & 6301 & 74.7503 & 8.7250 & 296.2 & 4 & 600.0 & 60 \\
\hline 00 & 6302 & 73.2435 & 8.6000 & 293.9 & 4 & 600.0 & .0 \\
\hline 00 & 6303 & 74.2517 & 8.7000 & 294.2 & 4 & 0.0 & \\
\hline 00 & 6304 & 71.7490 & 9.0500 & 293.3 & 4 & 600.0 & 60 \\
\hline 00 & 6305 & 63.0000 & 12.4500 & 293.7 & 4 & 600.0 & .0 \\
\hline 00 & 6306 & 62.0000 & 11.92 & 293.4 & 4 & 600.0 & \\
\hline 00 & 6307 & 74.5014 & 9.2500 & 293.5 & 4 & 600.0 & .0 \\
\hline 00 & 6308 & 86.2301 & 5.6750 & 295.8 & 3 & 600.0 & 600.0 \\
\hline 00 & 6309 & 79.3827 & 5.30 & 297.6 & 3 & 600.0 & \\
\hline 00 & 63010 & 78.2542 & 6.47 & 298.1 & 4 & 600.0 & .0 \\
\hline 00 & 63011 & 97.2467 & 6.7500 & 298.0 & 4 & 600.0 & 600.0 \\
\hline 00 & 63012 & 87.7265 & 7.2500 & 298.2 & 4 & 600.0 & .0 \\
\hline 00 & 63013 & 95.3922 & 6.7000 & 299.5 & 4 & 600.0 & .0 \\
\hline 00 & 63014 & 72.7502 & 11.2250 & 299.8 & 4 & 600.0 & 600.0 \\
\hline 00 & 63015 & 77.7499 & 11.4250 & 300.2 & 4 & 600.0 & \\
\hline 00 & 63016 & 70.7508 & 11.1750 & 299.7 & 4 & 600.0 & 60 \\
\hline 00 & 63017 & 71.2506 & 10.1500 & 299.1 & 4 & 600.0 & 600.0 \\
\hline 00 & 63018 & 69.2489 & 9.2000 & 298.2 & 4 & 600.0 & 600.0 \\
\hline 00 & 63019 & 65.2501 & 13.2250 & 295.9 & 7 & 600.0 & 600.0 \\
\hline 00 & 63020 & 66.5000 & 13.4750 & 293.8 & 4 & 600.0 & 600.0 \\
\hline 00 & 63021 & 61.5005 & 12.8500 & 294.0 & 4 & 600.0 & 600.0 \\
\hline 00 & 63022 & 63.0002 & 11.925 & 294.0 & 4 & 600.0 & 600.0 \\
\hline 00 & 63023 & 68.2503 & 12.1250 & 291.9 & 4 & 600.0 & 600.0 \\
\hline
\end{tabular}




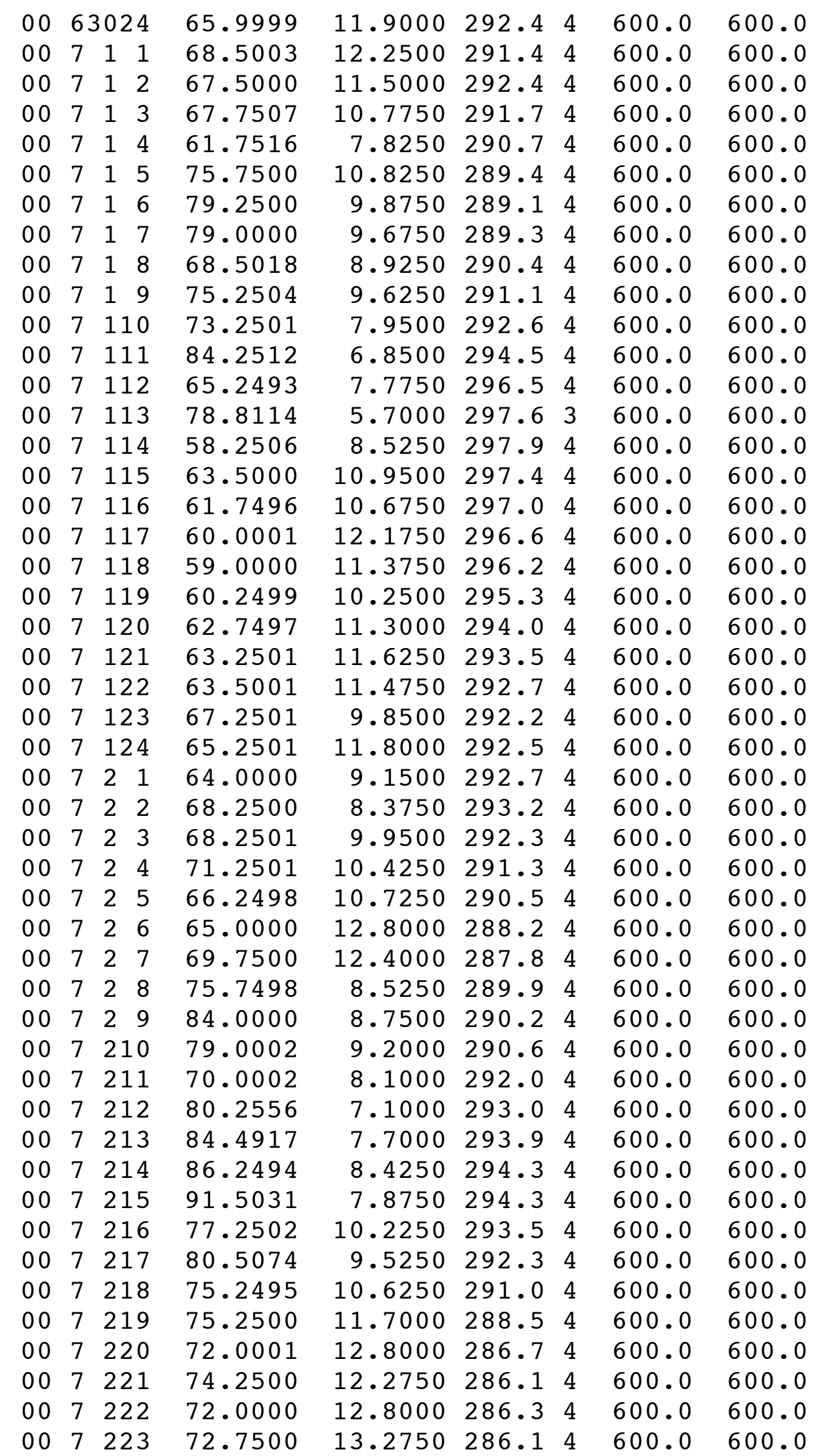




\begin{tabular}{|c|c|c|c|c|c|c|c|c|}
\hline 0 & 7 & 224 & 75.0000 & 12.7250 & 285.3 & 4 & 600.0 & 600.0 \\
\hline 00 & 7 & 31 & 73.2495 & 11.9000 & 286.1 & 4 & 600.0 & 00.0 \\
\hline 00 & 7 & 32 & 78.2503 & 10.2000 & 287.0 & 4 & 600.0 & 00.0 \\
\hline 0 & 7 & 33 & 79.4998 & 10.3750 & 287.3 & 4 & 600.0 & 00.0 \\
\hline 00 & 7 & 34 & 80.2501 & 10.9250 & 286.2 & 4 & 600.0 & 00.0 \\
\hline 00 & 7 & 35 & 75.2501 & 11.3000 & 285.2 & 4 & 600.0 & 00.0 \\
\hline 00 & 7 & 36 & 75.5000 & 11.3500 & 285.1 & 4 & 600.0 & 0 \\
\hline 00 & 7 & 37 & 74.0000 & 8.9500 & 285.8 & 4 & 600.0 & 00.0 \\
\hline 00 & 7 & 38 & 87.7485 & 5.4250 & 287.0 & 3 & 600.0 & 00.0 \\
\hline 00 & 7 & 39 & 75.2668 & 2.1500 & 290.0 & 2 & 600.0 & 0 \\
\hline 00 & 7 & 310 & 255.1398 & 2.0750 & 292.0 & 1 & 600.0 & \\
\hline 00 & 7 & 311 & 240.9932 & 2.2500 & 293.5 & 1 & 600.0 & \\
\hline 00 & 7 & 312 & 237.1561 & 2.6750 & 294.1 & 1 & 600.0 & .0 \\
\hline 00 & 7 & 313 & 329.5639 & 3.2750 & 295.3 & - & 600.0 & 00.0 \\
\hline 00 & 7 & 314 & 67.0105 & 5.4500 & 294.8 & 3 & .0 & \\
\hline 00 & 7 & 315 & 77.9924 & 7.3500 & 293.8 & 4 & 600.0 & .0 \\
\hline 00 & 7 & 316 & 76.7485 & 7.7000 & 293.5 & 4 & .0 & .0 \\
\hline 00 & 7 & 317 & 78.7500 & 10.1250 & 291.7 & 4 & .0 & \\
\hline 00 & 7 & 318 & 75.7502 & 11.7250 & 289.9 & 4 & .0 & \\
\hline 00 & 7 & 319 & 68.0006 & 11.3500 & 289.7 & 4 & .0 & .0 \\
\hline 00 & 7 & 320 & 67.9998 & 12.80 & 289.0 & 4 & 6 & \\
\hline 00 & 7 & 321 & 63.5000 & 12.4000 & 289.1 & 4 & .0 & \\
\hline 00 & 7 & 322 & 69.5003 & 9.9500 & 288.9 & 4 & 600.0 & .0 \\
\hline 00 & 7 & 323 & 70.0000 & 10.37 & 288.6 & 4 & 6 & \\
\hline 00 & 7 & 324 & 70.5003 & 10.1500 & 288.5 & 4 & .0 & \\
\hline 00 & 7 & 41 & 70.0005 & 9.9000 & 288.8 & 4 & 600.0 & .0 \\
\hline 00 & 7 & 42 & 73.4997 & 9.8250 & 288.9 & 4 & .0 & .0 \\
\hline 00 & 7 & 43 & 77.7500 & 9.3250 & 288.9 & 4 & .0 & \\
\hline 00 & 7 & 44 & 78.2501 & 8.8750 & 288.1 & 4 & .0 & 0.0 \\
\hline 00 & 7 & 45 & 82.5000 & 7.4250 & 288.8 & 4 & 6 & .0 \\
\hline 00 & 7 & 46 & 82.9930 & 5.90 & 289.3 & 4 & .0 & \\
\hline 00 & 7 & 47 & 97.7493 & 3.7750 & 290.4 & 4 & .0 & 0.0 \\
\hline 00 & 7 & 48 & 186.2591 & 2.4250 & 292.1 & 3 & 600.0 & 0.0 \\
\hline 00 & 7 & 49 & 237.9030 & 2.1000 & 292.6 & 2 & 600.0 & 0.0 \\
\hline 00 & 7 & 410 & 247.4328 & 2.4750 & 293.8 & 1 & 600.0 & 600.0 \\
\hline 00 & 7 & 411 & 194.5096 & 3.7250 & 294.9 & 2 & 600.0 & 600.0 \\
\hline 00 & 7 & 412 & 104.8277 & 4.60 & 295.8 & 3 & 600.0 & 600.0 \\
\hline 00 & 7 & 413 & 99.6836 & 4.0500 & 297.0 & 3 & 600.0 & 600.0 \\
\hline 00 & 7 & 414 & 105.2448 & 4.9250 & 297.5 & 3 & 600.0 & 600.0 \\
\hline 00 & 7 & 415 & 90.9973 & 5.1500 & 298.1 & 3 & 600.0 & 600.0 \\
\hline 00 & 7 & 416 & 100.2431 & 6.8750 & 297.7 & 4 & 600.0 & 600.0 \\
\hline 00 & 7 & 417 & 86.2502 & 7.8750 & 296.7 & 4 & 600.0 & 600.0 \\
\hline 00 & 7 & 418 & 78.5005 & 9.5000 & 294.0 & 4 & 600.0 & 600.0 \\
\hline 00 & 7 & 419 & 69.4999 & 10.3750 & 291.9 & 4 & 600.0 & 600.0 \\
\hline 00 & 7 & 420 & 66.7492 & 10.7000 & 291.2 & 4 & 600.0 & 600.0 \\
\hline 00 & 7 & 421 & 65.0000 & 11.7500 & 290.8 & 4 & 600.0 & 600.0 \\
\hline 00 & 7 & 422 & 71.7501 & 9.4500 & 290.2 & 4 & 600.0 & 600.0 \\
\hline 00 & 7 & 423 & 72.0002 & 10.000 & 289.3 & $\Psi$ & 600.0 & 600.0 \\
\hline
\end{tabular}




\begin{tabular}{|c|c|c|c|c|c|c|c|c|}
\hline 00 & 7 & 424 & 66.2498 & 11.1000 & 288.5 & 4 & 600.0 & 00.0 \\
\hline 0 & 7 & 51 & 4.5000 & 12.1000 & 288.8 & 4 & 600.0 & 600.0 \\
\hline 00 & 7 & 52 & 64.7500 & 11.1500 & 288.4 & 4 & 600.0 & 00.0 \\
\hline 00 & 7 & 53 & 62.5000 & 11.2250 & 287.8 & 4 & 600.0 & 600.0 \\
\hline 00 & 7 & 54 & 66.4995 & 10.7750 & 287.1 & 4 & 600.0 & 00.0 \\
\hline 00 & 7 & 55 & 69.2504 & 11.1250 & 286.8 & 4 & 600.0 & 00.0 \\
\hline 00 & 7 & 56 & 68.0000 & 11.2500 & 285.9 & 4 & 600.0 & 600.0 \\
\hline 00 & 7 & 57 & 67.5002 & 12.2750 & 286.1 & 4 & 600.0 & 500.0 \\
\hline 00 & 7 & 58 & 65.7500 & 11.7500 & 287.0 & 4 & 600.0 & 00.0 \\
\hline 00 & 7 & 59 & 61.7500 & 11.0500 & 288.0 & 4 & 600.0 & .0 \\
\hline 00 & 7 & 510 & 69.7548 & 10.0000 & 289.0 & 4 & 600.0 & 600.0 \\
\hline 00 & 7 & 511 & 68.4997 & 10.3750 & 289.5 & 4 & 600.0 & \\
\hline 00 & 7 & 512 & 69.9976 & 10.2500 & 290.1 & 4 & 600.0 & 600.0 \\
\hline 00 & 7 & 513 & 68.5000 & 10.7750 & 290.1 & 4 & 600.0 & 600.0 \\
\hline 00 & 7 & 514 & 70.7478 & 10.1500 & 290.8 & 4 & 600.0 & \\
\hline 00 & 7 & 515 & 74.7505 & 10.6000 & 291.3 & 4 & 600.0 & 60 \\
\hline 00 & 7 & 516 & 62.9996 & 11.2000 & 291.2 & 4 & 600.0 & 600.0 \\
\hline 00 & 7 & 517 & 64.4997 & 12.1000 & 290.4 & 4 & 600.0 & .0 \\
\hline 00 & 7 & 518 & 66.9998 & 11.2250 & 289.5 & 4 & 600.0 & 60 \\
\hline 00 & 7 & 519 & 70.7554 & 11.3500 & 288.3 & 4 & 600.0 & 60 \\
\hline 00 & 7 & 520 & 60.7485 & 12.8000 & 287.2 & 4 & 600.0 & .0 \\
\hline 00 & 7 & 521 & 68.5006 & 12.7500 & 287.0 & 4 & 600.0 & \\
\hline 00 & 7 & 522 & 66.2500 & 13.3500 & 286.4 & 4 & 600.0 & 600.0 \\
\hline 00 & 7 & 523 & 66.9999 & 13.1250 & 286.7 & 4 & 600.0 & .0 \\
\hline 00 & 7 & 524 & 65. & 11.7500 & 286.7 & 4 & 600.0 & \\
\hline 00 & 7 & 61 & 70.7505 & 10.5500 & 286.8 & 4 & 600.0 & 60 \\
\hline 00 & 7 & 62 & 74.0000 & 10.6250 & 286.5 & 4 & 600.0 & .0 \\
\hline 00 & 7 & 63 & 73.2500 & 10.0000 & 286.8 & 4 & 600.0 & .0 \\
\hline 00 & 7 & 64 & 74.0000 & 9.7250 & 287.0 & 4 & 600.0 & 60 \\
\hline 00 & 7 & 65 & 76.2500 & 9.4250 & 286.4 & 4 & 600.0 & 600.0 \\
\hline 00 & 7 & 66 & 77.7500 & 10.8500 & 285.2 & 4 & 600.0 & .0 \\
\hline 00 & 7 & 67 & 78.7500 & 10.3000 & 285.4 & 4 & 600.0 & 60 \\
\hline 00 & 7 & 68 & 78.7500 & 8.3750 & 287.1 & 4 & 600.0 & 600.0 \\
\hline 00 & 7 & 69 & 87.5000 & 6.9250 & 288.5 & 4 & 600.0 & .0 \\
\hline 00 & 7 & 610 & 506 & 6.1250 & 289.7 & 4 & 600.0 & \\
\hline 00 & 7 & 611 & 83.0000 & 5.7000 & 290.6 & 3 & 600.0 & 600.0 \\
\hline 00 & 7 & 612 & 95.7495 & 5.4000 & 291.6 & 3 & 600.0 & \\
\hline 00 & 7 & 613 & 87.2983 & 5.8500 & 293.0 & 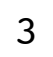 & 600.0 & 600.0 \\
\hline 00 & 7 & 614 & 73.2492 & 6.7500 & 293.4 & 4 & 600.0 & 600.0 \\
\hline 00 & 7 & 615 & 81.2620 & 7.8000 & 293.1 & 4 & 600.0 & \\
\hline 00 & 7 & 616 & 75.0006 & 8.4000 & 293.1 & 4 & 600.0 & 600.0 \\
\hline 00 & 7 & 617 & 70.9991 & 10.6250 & 292.0 & 4 & 600.0 & 600.0 \\
\hline 00 & 7 & 618 & 70.0007 & 11.6750 & 290.0 & 4 & 600.0 & 600.0 \\
\hline 00 & 7 & 619 & 66.7499 & 12.3000 & 288.4 & 4 & 600.0 & 600.0 \\
\hline 00 & 7 & 620 & 68.2496 & 13.8500 & 287.3 & 4 & 600.0 & 600.0 \\
\hline 00 & 7 & 621 & 69.0000 & 14.5250 & 286.7 & 4 & 600.0 & 600.0 \\
\hline 00 & 7 & 622 & 72.0000 & 14.075 & 286.2 & 4 & 600.0 & 600.0 \\
\hline 00 & 7 & 623 & 71.7500 & 13.8250 & 285.8 & 4 & 600.0 & 600.0 \\
\hline
\end{tabular}




\begin{tabular}{|c|c|c|c|c|c|c|c|c|}
\hline 0 & 7 & 624 & 72.7500 & 13.3250 & 285.5 & 4 & 600.0 & 600.0 \\
\hline 00 & 7 & 71 & 68.7492 & 12.5750 & 285.0 & 4 & 600.0 & 00.0 \\
\hline 00 & 7 & 72 & 65.2501 & 12.4750 & 284.7 & 4 & 600.0 & 00.0 \\
\hline 0 & 7 & 73 & 67.7500 & 12.1250 & 284.4 & 4 & 600.0 & 00.0 \\
\hline 00 & 7 & 74 & 69.9999 & 11.9000 & 284.6 & 4 & 600.0 & 00.0 \\
\hline 00 & 7 & 75 & 72.7500 & 11.7000 & 284.6 & 4 & 600.0 & 00.0 \\
\hline 00 & 7 & 76 & 68.0006 & 11.8250 & 284.1 & 4 & 600.0 & 0 \\
\hline 00 & 7 & 77 & 67.4995 & 11.1750 & 284.7 & 4 & 600.0 & 00.0 \\
\hline 00 & 7 & 78 & 72.7495 & 9.6000 & 286.1 & 4 & 600.0 & 00.0 \\
\hline 00 & 7 & 79 & 76.7499 & 9.6250 & 287.1 & 4 & 600.0 & 0 \\
\hline 00 & 7 & 710 & 73.0000 & 8.4500 & 288.2 & 4 & 600.0 & \\
\hline 00 & 7 & 711 & 72.2500 & 8.1750 & 289.5 & 4 & 600.0 & \\
\hline 00 & 7 & 712 & 74.0000 & 7.6250 & 290.5 & 4 & 600.0 & 00.0 \\
\hline 00 & 7 & 713 & 68.5000 & 9.8500 & 290.9 & 4 & 600.0 & 00.0 \\
\hline 00 & 7 & 714 & 493 & & 291.9 & 4 & .0 & \\
\hline 00 & 7 & 715 & 73.4963 & 000 & 292.5 & 4 & 600.0 & .0 \\
\hline 00 & 7 & 716 & 70.4994 & 10.4000 & 292.5 & 4 & .0 & .0 \\
\hline 00 & 7 & 717 & 68.9996 & 9.8 & 292.3 & 4 & .0 & \\
\hline 00 & 7 & 718 & 76.2500 & 9.8000 & 291.3 & 4 & 60 & .0 \\
\hline 00 & 7 & 719 & 71.0027 & 12.1000 & 289.7 & 4 & .0 & .0 \\
\hline 00 & 7 & 720 & 000 & 13. & 287.8 & 4 & 6 & \\
\hline 00 & 7 & 721 & 58.7500 & 12 . & 286.9 & 4 & .0 & \\
\hline 00 & 7 & 722 & 61.9828 & 9.6750 & 286.6 & 4 & 600.0 & .0 \\
\hline 00 & 7 & 723 & 85.4994 & 8.85 & 286.6 & 4 & 6 & \\
\hline 00 & 7 & 724 & 998 & 10 & 286.5 & 4 & .0 & \\
\hline 00 & 7 & 81 & 61.7496 & 11.3500 & 285.8 & 4 & .0 & 600.0 \\
\hline 00 & 7 & 82 & 63.7472 & 11.8500 & 285.9 & 4 & 60 & .0 \\
\hline 00 & 7 & 83 & 76.0004 & 10 & 286.5 & 4 & .0 & \\
\hline 00 & 7 & 84 & 72.0000 & 10.4750 & 286.3 & 4 & .0 & 0.0 \\
\hline 00 & 7 & 85 & 79.0011 & 10.2250 & 285.6 & 4 & 60 & .0 \\
\hline 00 & 7 & 86 & 82.5000 & 9.4750 & 285.4 & 4 & .0 & \\
\hline 00 & 7 & 87 & 77.9998 & 8.22 & 286.0 & 4 & .0 & 0.0 \\
\hline 00 & 7 & 88 & 79.9994 & 6.5000 & 287.4 & 4 & 600.0 & 0.0 \\
\hline 00 & 7 & 89 & 150.3783 & 2. & 290.9 & 3 & 600.0 & 0.0 \\
\hline 00 & 7 & 810 & 247.7438 & 2.55 & 293.0 & 2 & 600.0 & 600.0 \\
\hline 00 & 7 & 811 & 233.3218 & 2.3000 & 294.4 & 1 & 600.0 & 600.0 \\
\hline 00 & 7 & 812 & 175.5000 & 5.60 & 295.0 & 2 & 600.0 & 0.0 \\
\hline 00 & 7 & 813 & 174.5317 & 7.0000 & 295.4 & 3 & 600.0 & 600.0 \\
\hline 00 & 7 & 814 & 99.5103 & 3.7000 & 297.3 & 2 & 600.0 & 600.0 \\
\hline 00 & 7 & 815 & 115.0000 & 3 . & 298.0 & 2 & 600.0 & 600.0 \\
\hline 00 & 7 & 816 & 97.2298 & 5.5500 & 298.1 & 3 & 600.0 & 600.0 \\
\hline 00 & 7 & 817 & 113.2406 & 6.4000 & 297.7 & 4 & 600.0 & 600.0 \\
\hline 00 & 7 & 818 & 117.2497 & 6.7500 & 297.0 & 4 & 600.0 & 600.0 \\
\hline 00 & 7 & 819 & 74.7442 & 6.8250 & 295.7 & 4 & 600.0 & 600.0 \\
\hline 00 & 7 & 820 & 71.2503 & 7.8250 & 293.9 & 4 & 600.0 & 600.0 \\
\hline 00 & 7 & 821 & 71.7500 & & 293.2 & 4 & 600.0 & 600.0 \\
\hline 00 & 7 & 822 & 71.4998 & 9.0250 & 293.0 & 4 & 600.0 & 600.0 \\
\hline 00 & 7 & 823 & 69.9998 & 9.125 & 293.0 & $\Psi$ & 600.0 & 600.0 \\
\hline
\end{tabular}




\begin{tabular}{|c|c|c|c|c|c|c|c|}
\hline 0 & 24 & 3.7501 & 8.5500 & 292.8 & 4 & 00 & \\
\hline 0 & $\begin{array}{lll}7 & 9 & 1\end{array}$ & 4.5000 & 8.3250 & 293.1 & 4 & 00.0 & \\
\hline & $\begin{array}{lll}7 & 9 & 2\end{array}$ & 74.4998 & .9000 & 293.5 & 4 & 00 & \\
\hline & $\begin{array}{lll}7 & 9 & 3\end{array}$ & 76.7499 & 5000 & 294.2 & & 00 & \\
\hline & $\begin{array}{lll}7 & 9 & 4\end{array}$ & 4.3333 & L. 1500 & 93.7 & & 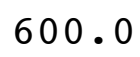 & \\
\hline & 795 & 129.3268 & .9750 & 92.4 & & 00 & \\
\hline & 796 & 52.5910 & 0.6500 & 292.1 & & 0 & \\
\hline & $\begin{array}{lll}7 & 9 & 7\end{array}$ & 98.4189 & .9000 & 94.8 & & & \\
\hline & $\begin{array}{lll}7 & 9 & 8\end{array}$ & 260.6596 & 1.4250 & 296.6 & 4 & 0 & \\
\hline & $\begin{array}{lll}7 & 9 & 9\end{array}$ & 230.0000 & 2.9750 & 295.7 & & 00 & \\
\hline & 7910 & 186.7515 & .1750 & 295.4 & & 00 & \\
\hline & 7911 & 178.7521 & & 96.6 & & & \\
\hline & 7912 & 177.7498 & 6.2000 & 297.8 & 4 & 0 & \\
\hline & 7913 & 175.9592 & 5.0750 & 298.9 & 3 & 0.0 & \\
\hline & 7914 & 169.19 & & 300 & & & \\
\hline & 7915 & 138.3002 & 750 & 300.9 & 3 & 0 & \\
\hline & 7916 & 107.7693 & 0 & 300.9 & 3 & 0 & \\
\hline & 7917 & 91 . & 0 & 300.3 & 4 & & \\
\hline & 7918 & 106.7425 & 7. & 299.5 & 4 & 0 & \\
\hline & 7919 & 94.6809 & 0 & 298 & 4 & 0 & \\
\hline & 7920 & 69 . & 000 & 29 & 4 & & \\
\hline & 7921 & 69. & & 29 & 4 & & \\
\hline & 7922 & 71.5000 & 250 & 295.3 & 4 & 0 & 0. \\
\hline & 7923 & 63. & 0 & 295.2 & 4 & 0 & \\
\hline & 7924 & & & 295.1 & 4 & & \\
\hline & 7101 & 60.4990 & 000 & 295 & 4 & & \\
\hline & 7102 & 64.4997 & 8.6000 & 295.7 & 4 & 0 & \\
\hline 0 & 7103 & 61.4073 & 0 & 295.3 & 4 & & \\
\hline 0 & 7104 & 165 & 0 & .1 & 4 & 0 & 0 . \\
\hline & 7105 & 25.5626 & 250 & 291.7 & 5 & & \\
\hline & 7106 & 84.4944 & 0 & 294.1 & 4 & & \\
\hline & 7107 & 388 & 5 . & 295.2 & 3 & & \\
\hline & 7108 & 42.8074 & 1.8250 & 294.9 & 2 & 0 & J. \\
\hline & 7109 & 82.4858 & 000 & 297.1 & 1 & 0 & \\
\hline & 71010 & 243 . & 2 . & 29 & 1 & & \\
\hline & 71011 & 234.7412 & 500 & 299.3 & 1 & & 0 . \\
\hline & 71012 & 82 . & 500 & 299.8 & 2 & 0 & \\
\hline 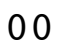 & 71013 & 87.7540 & 500 & 299.9 & 3 & 0 & 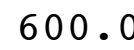 \\
\hline & 71014 & 85.2429 & 750 & 300.4 & 4 & 0 & 0 . \\
\hline & 71015 & 100.4671 & 50 & 300.8 & 4 & 0 & \\
\hline 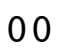 & 71016 & 89.4946 & 7.5250 & 300.4 & 4 & & \\
\hline 00 & 71017 & 92.2517 & 7.6750 & 299.9 & 4 & 600.0 & 600 . \\
\hline & 71018 & 86.2495 & 7.4500 & 299.1 & 4 & 600 & 000 \\
\hline & 71019 & 74.5010 & 8.2000 & 298.1 & 4 & & s \\
\hline 00 & 71020 & 71.7501 & 8.9750 & 295.9 & 4 & 600.0 & 00 \\
\hline & 71021 & 67.2502 & 8.9750 & 295.4 & 4 & 600.0 & 0 \\
\hline & 71022 & 65.2500 & & 29 & 4 & 60 & \\
\hline & 71023 & 61.7499 & 7.7250 & 296.6 & 4 & 600.0 & 600 \\
\hline
\end{tabular}




\begin{tabular}{|c|c|c|c|c|c|c|c|}
\hline & 1024 & 0.5005 & 7.4250 & 297.0 & 4 & . & \\
\hline 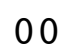 & 7111 & 5.2499 & 4.4250 & 296.7 & 4 & 00.0 & \\
\hline & 7112 & 954 & 1.2500 & 294.3 & & 0 & \\
\hline & 113 & 08.4998 & .5750 & 292.8 & & 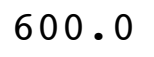 & \\
\hline & 114 & 62.6435 & & 293.4 & & 0 & \\
\hline & 115 & 47 & 750 & 295.2 & & 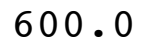 & \\
\hline & 116 & 42 . & & 294.8 & & 0 & \\
\hline & 117 & 147.2 & & 96.2 & & & \\
\hline & 118 & 278.5000 & 9750 & 298.0 & & 0 & 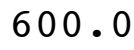 \\
\hline & 119 & 270.1789 & 0 & 298.9 & & & \\
\hline & 1110 & 183.6007 & & 3 & & & \\
\hline & 1111 & 164.5470 & 3.8250 & 299.9 & & & \\
\hline & 1112 & 173.0827 & .6250 & 301.2 & & & \\
\hline & 1113 & 95.2499 & 0 & 301 & & & \\
\hline & 1114 & 108.5681 & & 302.1 & & & \\
\hline & 1115 & 113.8067 & 8000 & 302.1 & & & \\
\hline & 1116 & 102.7650 & 0 & 301.4 & & & \\
\hline & 1117 & 83.5000 & & & - & & \\
\hline & 1118 & 78.0000 & 8750 & 299.7 & 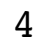 & 0 & U \\
\hline & 71119 & 69 . & 0 & 298.2 & $I$ & & \\
\hline & 71120 & 72 . & & 6 & 4 & & \\
\hline & 71121 & 72 . & 0 & 29 & 4 & & \\
\hline & 71122 & 72.4999 & 0 & 296.6 & 4 & U & \\
\hline & 71123 & 72.2500 & & 29 & 4 & & \\
\hline & 711 & 69 . & 0 & 29 & 4 & & \\
\hline & 7121 & 72.7495 & .7500 & 296.6 & 4 & & \\
\hline & 7122 & 76.750 & 0 & 29 & 4 & & \\
\hline & 7123 & 001 & 0 & 295.8 & 4 & & \\
\hline & 7124 & 75.2500 & 0 & 294.3 & 4 & & \\
\hline & 7125 & 250 & 0 & 294.4 & 4 & & \\
\hline & 7126 & 71 & 0 & 294.1 & 4 & & \\
\hline & 7127 & 500 & & & 4 & & \\
\hline & 7128 & 78.9985 & 4.6250 & 295.5 & 3 & & \\
\hline & 7129 & 4.9998 & 0 & 294.6 & 3 & & \\
\hline & 71210 & 118.5283 & 0 & 295.5 & 2 & & \\
\hline & 71211 & .2491 & 1750 & 296.0 & 3 & & \\
\hline & 71212 & 509 & & $29^{\circ}$ & 3 & & \\
\hline & 71213 & 000 & 0 & .0 & 4 & & \\
\hline & 71214 & .2499 & 1250 & 298.2 & 4 & & \\
\hline & 71215 & 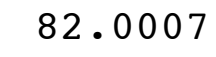 & 0 & 29 & 4 & & \\
\hline & 71216 & 81.7501 & 7.5750 & 298.7 & 4 & & \\
\hline & 71217 & 81.7499 & 8.5000 & 297.8 & 4 & 0 & م0 \\
\hline & 71218 & 75.7500 & 8.8500 & 296.5 & 4 & & \\
\hline 0 & 71219 & 74.2498 & 0.3500 & 294.6 & 4 & & \\
\hline & 71220 & 76.0000 & 1.2500 & 292.6 & 4 & 600 & 00 \\
\hline & 71221 & 76 & & 291.0 & 4 & & \\
\hline & 71222 & 73.5000 & 1.6750 & 290.2 & $\Psi$ & & 600 \\
\hline & 71223 & 75.0000 & 9.5500 & 290.3 & 4 & 60 & 60 \\
\hline
\end{tabular}




\begin{tabular}{|c|c|c|c|c|c|c|c|}
\hline 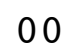 & 12 & 7 & 250 & 90.4 & 2 & 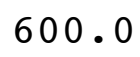 & \\
\hline 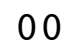 & 7131 & 8.2500 & .6000 & 288.5 & 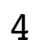 & 500 & \\
\hline & 7132 & 7.7500 & 12.7750 & 287.1 & & 00 & \\
\hline & 7133 & 6.7500 & 11.7250 & 286.8 & & 00 & \\
\hline & 134 & 6.2501 & 6750 & 87.4 & & & \\
\hline & 7135 & 98 & & 87.7 & & & \\
\hline & 7136 & 000 & 500 & 87.4 & & 00 & \\
\hline & 7137 & 95 & 3250 & 89.3 & & & \\
\hline & 7138 & 8 & & 89.9 & & & \\
\hline & 7139 & 15 & 2500 & 91.1 & & & \\
\hline & 1310 & 6 & 500 & 92.0 & & & \\
\hline & 11 & & & 4 & & & \\
\hline & 1312 & 7 & 4 . & 94.5 & & & \\
\hline & 13 & $y$ & 5.4250 & 295.8 & & & \\
\hline & 14 & & & & & & \\
\hline & 121 & & & & & & \\
\hline & 13 & 88 . & 00 & 97.7 & & 0 & \\
\hline & 1317 & 97 & 4 . & 8 & & 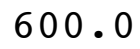 & \\
\hline & 713 & & & & & & \\
\hline & 713 & & 00 & 295.4 & & & \\
\hline & 71320 & 00 & & 92.9 & & & \\
\hline & 71321 & & & 2.3 & & & \\
\hline & 71322 & 62 & & 294.2 & & & \\
\hline & 71323 & 00 & 0 & .1 & & & \\
\hline & 71324 & & & & & & \\
\hline & 714 & 96 & 1 . & .3 & & & \\
\hline & $714 \quad 2$ & 21 & 0 & 293.8 & & & \\
\hline & 7143 & 41 & 0 . & 293.3 & 6 & & \\
\hline & $714 \quad 4$ & & 0 . & 2 & 6 & & \\
\hline & 7145 & 106 & 5000 & 293.0 & & & \\
\hline & 7146 & 58 & 0.47 & 293.9 & 6 & & \\
\hline & 7147 & & & 29 & 5 & & \\
\hline & 7148 & 03 & & 299.7 & 4 & & \\
\hline & 7149 & 08 & 1. & 298.1 & 2 & & \\
\hline & 71 & & 0 & 29 & 2 & & \\
\hline & & & 4 . & 297.6 & 3 & & \\
\hline & & 501 & 0 & 298.5 & 3 & & \\
\hline & 71413 & 16 & 6 . & 300.3 & 4 & & \\
\hline & 71 & 171 . & 6 . & 301.4 & 4 & & \\
\hline & & 08 & & 302.1 & 4 & & \\
\hline & & 097 & 5 . & 302.9 & 3 & & \\
\hline & 71417 & 105 . & 5.6750 & 302.7 & 3 & & \\
\hline & 714 & 373 & 6.0000 & 301.5 & 4 & 0 & \\
\hline & & 000 & & 29 & 4 & & \\
\hline & & 499 & 9.4500 & 297.3 & 4 & & \\
\hline & 71 & 000 & 0.5250 & 296.9 & 4 & 600.0 & \\
\hline & & & & & 4 & & \\
\hline & 11420 & 68.7488 & 11.0000 & 296.4 & $\Psi$ & 600.0 & $c_{1}$ \\
\hline
\end{tabular}




\begin{tabular}{|c|c|c|c|c|c|c|c|}
\hline 00 & 71424 & 72.4998 & 10.9000 & 296.4 & 4 & 600.0 & 00.0 \\
\hline 00 & 7151 & 68.2501 & 10.5250 & 296.6 & 4 & 600.0 & 600,0 \\
\hline 00 & 7152 & 68.7500 & 10.1750 & 296.3 & 4 & 600.0 & 00.0 \\
\hline 00 & 7153 & 70.2501 & 9.2000 & 296.4 & 4 & 600.0 & 00.0 \\
\hline 00 & 7154 & 74.7489 & 9.6500 & 296.2 & 4 & 600.0 & 00.0 \\
\hline 00 & 7155 & 68.5000 & 9.8750 & 296.0 & 4 & 600.0 & 00.0 \\
\hline 00 & 7156 & 76.2501 & 9.4750 & 295.8 & 4 & 600.0 & 600.0 \\
\hline 00 & $\begin{array}{ll}715 & 7\end{array}$ & 76.2500 & 9.1250 & 296.1 & 4 & 600.0 & 00.0 \\
\hline 00 & 7158 & 78.7501 & 8.2250 & 296.7 & 4 & 600.0 & 00.0 \\
\hline 00 & 7159 & 81.7458 & 6.1000 & 298.5 & 4 & 600.0 & .0 \\
\hline 00 & 71510 & 119.5457 & 4.1500 & 299.7 & 3 & 600.0 & 00.0 \\
\hline 00 & 71511 & 91.9551 & 4.9500 & 299.4 & 3 & 600.0 & \\
\hline 00 & 71512 & 75.9848 & 5.3000 & 299.2 & 3 & 600.0 & 600.0 \\
\hline 00 & 71513 & 81.9989 & 6.4250 & 299.6 & 4 & 600.0 & 600.0 \\
\hline 00 & 71514 & 72.0004 & 7. & 299.9 & 4 & 600.0 & \\
\hline 00 & 71515 & 70.5008 & 8.1250 & 299.9 & 4 & 600.0 & 60 \\
\hline 00 & 71516 & 76.0007 & 8.42 & 299.4 & 4 & 600.0 & 600.0 \\
\hline 00 & 71517 & 76.9988 & 8.9750 & 298.3 & 4 & 600.0 & \\
\hline 00 & 71518 & 70.0002 & 10.7500 & 297.4 & 4 & 600.0 & .0 \\
\hline 00 & 71519 & 79.7446 & 8.1000 & 296.1 & 4 & 600.0 & 60 \\
\hline 00 & 71520 & 72.2482 & 10. & 294.6 & 4 & 600.0 & .0 \\
\hline 00 & 71521 & 66.9986 & 11. & 293.6 & 4 & 600.0 & \\
\hline 00 & 71522 & 66.0000 & 11.1000 & 293.9 & 4 & 600.0 & 600.0 \\
\hline 00 & 71523 & 65.0000 & 11.7000 & 293.7 & 4 & 600.0 & .0 \\
\hline 00 & 71524 & 64.0000 & 11. & 293.3 & 4 & 600.0 & \\
\hline 00 & 7161 & 67.2507 & 000 & 293.9 & 4 & 600.0 & 60 \\
\hline 00 & 7162 & 65.0000 & 10.1500 & 293.6 & 4 & 600.0 & .0 \\
\hline 00 & 7163 & 71.7499 & 8.0 & 293.1 & 4 & 600.0 & \\
\hline 00 & 7164 & 71.5000 & 10.4250 & 291.7 & 4 & 600.0 & 60 \\
\hline 00 & 7165 & 73.0000 & 11.7000 & 290.1 & 4 & 600.0 & 60 \\
\hline 00 & 7166 & 77.7498 & 12.90 & 288.4 & 4 & 600.0 & \\
\hline 00 & 7167 & 77.5000 & 11 . & 289.2 & 4 & 600.0 & 60 \\
\hline 00 & 7168 & 72.0073 & 9.1750 & 288.8 & 4 & 600.0 & 600.0 \\
\hline 00 & 7169 & 77.2504 & 6 . & 289.8 & 4 & 600.0 & .0 \\
\hline 00 & 71610 & 81.2530 & 4 . & 292.1 & 3 & 600.0 & .0 \\
\hline 00 & 71611 & 67.7516 & 4.8250 & 294.2 & 3 & 600.0 & 600.0 \\
\hline 00 & 71612 & 71.0003 & 7.9500 & 292.3 & 4 & 600.0 & \\
\hline 00 & 71613 & 75.2505 & 9.9500 & 291.8 & 4 & 600.0 & 600.0 \\
\hline 00 & 71614 & 81.5000 & 9.1500 & 293.2 & 4 & 600.0 & 600.0 \\
\hline 00 & 71615 & 72.7509 & 9.8000 & 293.7 & 4 & 600.0 & \\
\hline 00 & 71616 & 74.7519 & 8.5750 & 293.8 & 4 & 600.0 & 600.0 \\
\hline 00 & 71617 & 69.9993 & 8.8750 & 293.1 & 4 & 600.0 & 600.0 \\
\hline 00 & 71618 & 76.7501 & 11.8000 & 290.2 & 4 & 600.0 & 600.0 \\
\hline 00 & 71619 & 74.7519 & 12.5250 & 288.9 & 4 & 600.0 & 600.0 \\
\hline 00 & 71620 & 71.4991 & 13.5750 & 287.1 & 4 & 600.0 & 600.0 \\
\hline 00 & 71621 & 79.2501 & 13.5750 & 286.5 & 4 & 600.0 & 600.0 \\
\hline 00 & 71622 & 78.7499 & 13.7500 & 286.0 & 4 & 600.0 & 600.0 \\
\hline 00 & 71623 & 72.5011 & 14.3000 & 285.9 & 4 & 600.0 & 600.0 \\
\hline
\end{tabular}




\begin{tabular}{|c|c|c|c|c|c|c|c|}
\hline 00 & 71624 & 68.5001 & 13.8750 & 285.6 & 4 & 600.0 & 0.0 \\
\hline 00 & 7171 & 70.2500 & 14.4250 & 285.8 & 4 & 600.0 & 600.0 \\
\hline 00 & 7172 & 69.7499 & 14.3750 & 285.9 & 4 & 600.0 & 00.0 \\
\hline 0 & 7173 & 68.7500 & 13.8750 & 285.6 & 2 & 600.0 & 00.0 \\
\hline 00 & $717 \quad 4$ & 65.2499 & 12.6000 & 285.5 & 4 & 600.0 & 00.0 \\
\hline 00 & 7175 & 999 & 10.8750 & 285.3 & 4 & 600.0 & 00.0 \\
\hline 00 & 7176 & 65.9989 & 11.2500 & 285.3 & 4 & 600.0 & 00.0 \\
\hline 00 & $\begin{array}{ll}717 & 7\end{array}$ & 62.7499 & 10.4000 & 285.8 & 4 & 600.0 & \\
\hline 00 & 7178 & 67.0000 & 9.0000 & 286.7 & 4 & 600.0 & 00.0 \\
\hline 00 & 7179 & 80.2495 & 8.0000 & 287.6 & 4 & 600.0 & 00.0 \\
\hline 00 & 71710 & 87.4995 & 6.9750 & 288.9 & 4 & 600.0 & \\
\hline 00 & 71711 & 87.5001 & 6.0250 & 290.3 & 4 & 600.0 & 60 \\
\hline 00 & 71712 & 78.2492 & 5.9500 & 291.7 & 3 & 600.0 & \\
\hline 00 & 71713 & 82.5027 & 6.9750 & 292.3 & 4 & 0 & \\
\hline 00 & 71714 & 75.2462 & 6.4250 & 293.5 & 4 & .0 & 60 \\
\hline 00 & 71715 & 85.2502 & 7.1000 & 293.9 & 4 & 600.0 & 600.0 \\
\hline 00 & 71716 & 85.0000 & 7.7500 & 293.8 & 4 & 600.0 & .0 \\
\hline 00 & 71717 & 81.5000 & 8.97 & 293.1 & 4 & .0 & \\
\hline 00 & 71718 & 80.7501 & 8.9500 & 292.2 & 4 & 600.0 & 600.0 \\
\hline 00 & 71719 & 76.7500 & 9.27 & 291.1 & 4 & .0 & .0 \\
\hline 00 & 71720 & 76.7502 & 11.2250 & 288.9 & 4 & .0 & \\
\hline 00 & 71721 & 72.2505 & 12.1750 & 288.0 & 4 & .0 & 60 \\
\hline 00 & 71722 & 76.0000 & 11.12 & . 1 & 4 & 60 & .0 \\
\hline 00 & 71723 & 69.7499 & 8.57 & 292.6 & 4 & .0 & \\
\hline 00 & 71724 & 69.2498 & 9.7250 & 291.2 & 4 & 60 & \\
\hline 00 & 7181 & 77.2501 & 9.0250 & 291.4 & 4 & .0 & 600.0 \\
\hline 00 & 7182 & 73.2500 & 8.02 & 291.1 & 4 & .0 & .0 \\
\hline 00 & 7183 & 74.9986 & 8.0000 & 291.4 & 4 & 600.0 & .0 \\
\hline 00 & 7184 & 75.7500 & 8.70 & 291.1 & 4 & 600.0 & 600.0 \\
\hline 00 & 7185 & 87.0098 & 6.47 & 291.6 & 4 & .0 & .0 \\
\hline 00 & 7186 & 94.9996 & 3.97 & 291.5 & 4 & .0 & \\
\hline 00 & 7187 & 98.2497 & 4.0750 & 292.9 & 3 & .0 & .0 \\
\hline 00 & $718 \quad 8$ & 130.5532 & 2.82 & 294.5 & 2 & .0 & .0 \\
\hline 00 & 7189 & 208.1013 & 2.70 & 296.0 & 1 & 600.0 & 60 \\
\hline 00 & 71810 & 170.7497 & 5.27 & 295.7 & 2 & .0 & .0 \\
\hline 00 & 71811 & 170.9984 & 6.95 & 295.7 & 3 & .0 & .0 \\
\hline 00 & 71812 & 172.2495 & 6.8250 & 297.4 & 4 & .0 & .0 \\
\hline 00 & 71813 & 166.7500 & 7.2000 & 299.2 & 4 & 600.0 & 600.0 \\
\hline 00 & 71814 & 167.0057 & 6.4000 & 300.3 & 4 & 600.0 & 600.0 \\
\hline 00 & 71815 & 173.2515 & 6.40 & 301.0 & 4 & 600.0 & 60 \\
\hline 00 & 71816 & 167.7509 & 5.7750 & 301.6 & 3 & 600.0 & 600.0 \\
\hline 00 & 71817 & 158.7024 & 4.8250 & 302.3 & 3 & 600.0 & 600.0 \\
\hline 00 & 71818 & 88.4686 & 5.4000 & 301.5 & 3 & 600.0 & 600.0 \\
\hline 00 & 71819 & 75.0000 & 6.6000 & 299.6 & 4 & 600.0 & 600.0 \\
\hline 00 & 71820 & 66.0002 & 7.7000 & 298.1 & 4 & 600.0 & 600.0 \\
\hline 00 & 71821 & 66.9994 & 9.4500 & & 4 & 600.0 & \\
\hline 00 & 71822 & 63.4991 & 9.8750 & 295.9 & 4 & 600.0 & 600.0 \\
\hline 00 & 71823 & 67.9999 & 9.750 & 295.9 & 4 & 600.0 & 600.0 \\
\hline
\end{tabular}




\begin{tabular}{|c|c|c|c|c|c|c|c|}
\hline 00 & 71824 & 69.0001 & 9.7750 & 296.3 & 4 & 600.0 & 00.0 \\
\hline 00 & 7191 & 74.7491 & 9.9000 & 296.7 & 4 & 600.0 & 600,0 \\
\hline 00 & 7192 & 73.7505 & 10.0750 & 297.1 & 4 & 600.0 & 00.0 \\
\hline 00 & 7193 & 68.4998 & 9.3250 & 297.2 & 4 & 600.0 & 00.0 \\
\hline 00 & 7194 & 71.7501 & 8.0250 & 297.3 & 4 & 600.0 & 00.0 \\
\hline 00 & 7195 & 75.2500 & 8.0250 & 296.9 & 4 & 600.0 & 00.0 \\
\hline 00 & 7196 & 77.0000 & 8.6750 & 297.0 & 4 & 600.0 & 600.0 \\
\hline 00 & 7197 & 72.2495 & 8.2250 & 297.0 & 4 & 600.0 & 00.0 \\
\hline 00 & 7198 & 84.5055 & 6.6750 & 298.0 & 4 & 600.0 & 00.0 \\
\hline 00 & 7199 & 85.2497 & 5.9500 & 298.6 & 4 & 600.0 & .0 \\
\hline 00 & 71910 & 169.5000 & 3.7250 & 300.5 & 3 & 600.0 & 600.0 \\
\hline 00 & 71911 & 93.4879 & 4.4250 & 300.9 & 3 & 600.0 & \\
\hline 00 & 71912 & 99.2599 & 4.3000 & 302.0 & 3 & 600.0 & 600.0 \\
\hline 00 & 71913 & 81.2502 & 4.4000 & 302.9 & 3 & 600.0 & 0 \\
\hline 00 & 71914 & 148.7732 & 6.1250 & 303.5 & 4 & 600.0 & \\
\hline 00 & 71915 & 119.7548 & 5.2750 & 303.9 & 3 & 600.0 & 60 \\
\hline 00 & 71916 & 97.9947 & 5.6250 & 303.6 & 3 & 600.0 & 600.0 \\
\hline 00 & 71917 & 88.7052 & 6.5250 & 303.2 & 4 & 600.0 & \\
\hline 00 & 71918 & 74.2570 & 7.6000 & 301.8 & 4 & 600.0 & .0 \\
\hline 00 & 71919 & 81.0000 & 8.9000 & 299.5 & 4 & 600.0 & 60 \\
\hline 00 & 71920 & 73.5000 & 10.50 & 297.8 & 4 & 600.0 & .0 \\
\hline 00 & 71921 & 70.0000 & 10 . & 296.6 & 4 & 0.0 & \\
\hline 00 & 71922 & 63.5000 & 10.9000 & 296.4 & 4 & 600.0 & 600.0 \\
\hline 00 & 71923 & 67.2501 & 10.07 & 297.8 & 4 & 600.0 & .0 \\
\hline 00 & 71924 & 998 & 9 & 298.0 & 4 & 0 & \\
\hline 00 & 7201 & 69.7500 & 8.9000 & 298.3 & 4 & 600.0 & 60 \\
\hline 00 & 7202 & 71.5000 & 8.5000 & 298.1 & 4 & 600.0 & .0 \\
\hline 00 & 7203 & 78.9997 & 8.20 & 298.0 & 4 & 600.0 & \\
\hline 00 & 7204 & 80.5000 & 8.2500 & 297.5 & 4 & 600.0 & 60 \\
\hline 00 & 7205 & 79.9998 & 7.5750 & 297.7 & 4 & 600.0 & .0 \\
\hline 00 & 7206 & 141.4893 & 2.37 & 296.8 & 5 & 600.0 & \\
\hline 00 & 7207 & 180.2799 & 0.8500 & 297.9 & 4 & 600.0 & .0 \\
\hline 00 & 7208 & 190.2088 & 0.5750 & 300.2 & 3 & 600.0 & 600.0 \\
\hline 00 & 7209 & 269.7279 & 1.30 & 301.3 & 2 & 600.0 & .0 \\
\hline 00 & 72010 & 223.0511 & 2 . & 300.7 & 1 & 600.0 & .0 \\
\hline 00 & 72011 & 199.2975 & 3.3750 & 302.2 & 2 & 600.0 & 600.0 \\
\hline 00 & 72012 & 184.7495 & 4.3000 & 302.9 & 3 & 600.0 & \\
\hline 00 & 72013 & 173.0030 & 6.3750 & 302.9 & 4 & 600.0 & 600.0 \\
\hline 00 & 72014 & 169.2506 & 6.6000 & 303.5 & 4 & 600.0 & 600.0 \\
\hline 00 & 72015 & 157.9551 & 5.45 & 304.6 & 3 & 600.0 & \\
\hline 00 & 72016 & 122.2429 & 5.4000 & 304.9 & 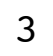 & 600.0 & 600.0 \\
\hline 00 & 72017 & 88.2507 & 6.4500 & 303.2 & 4 & 600.0 & 600.0 \\
\hline 00 & 72018 & 70.0000 & 8.9000 & 300.8 & 4 & 600.0 & 600.0 \\
\hline 00 & 72019 & 69.5000 & 8.8750 & 300.4 & 4 & 600.0 & 600.0 \\
\hline 00 & 72020 & 66.0000 & 9.2000 & 298.8 & 4 & 600.0 & 600.0 \\
\hline 00 & 72021 & 69.5000 & 8.7250 & 299.3 & 4 & 600.0 & 600.0 \\
\hline 00 & 72022 & 68.2500 & 8.500 & 299.8 & 4 & 600.0 & 600.0 \\
\hline 00 & 72023 & 67.2501 & 8.4500 & 300.0 & 4 & 600.0 & 600.0 \\
\hline
\end{tabular}




\begin{tabular}{|c|c|c|c|c|c|c|c|}
\hline 00 & 72024 & 66.2500 & 8.9000 & 300.4 & 4 & 600.0 & 600.0 \\
\hline 0 & 7211 & 4.5000 & 7.9250 & 300.7 & 4 & 600.0 & 600,0 \\
\hline 00 & 7212 & 65.2500 & 7.4000 & 300.1 & 4 & 600.0 & 00.0 \\
\hline 00 & 7213 & 64.7501 & 8.2000 & 300.5 & 4 & 600.0 & 600.0 \\
\hline 00 & 7214 & 68.0000 & 8.1250 & 299.6 & 4 & 600.0 & 00.0 \\
\hline 00 & 7215 & 75.2499 & 8.5250 & 298.5 & 4 & 600.0 & 00.0 \\
\hline 00 & 7216 & 75.2500 & 10.4000 & 297.7 & 4 & 600.0 & 00.0 \\
\hline 00 & 7217 & 75.0000 & 10.0000 & 297.1 & 4 & 600.0 & 00.0 \\
\hline 00 & 7218 & 71.4998 & 8.1500 & 297.4 & 4 & 600.0 & 00.0 \\
\hline 00 & 7219 & 78.5399 & 5.1500 & 297.6 & 3 & 600.0 & .0 \\
\hline 00 & 72110 & 74.0143 & 3.1750 & 299.3 & 2 & 600.0 & 500.0 \\
\hline 00 & 72111 & 105.5129 & 5.9750 & 299.3 & 3 & 600.0 & \\
\hline 00 & 72112 & 98.7487 & 6.0000 & 300.0 & 4 & 600.0 & 600.0 \\
\hline 00 & 72113 & 89.7415 & 6.7000 & 299.6 & 4 & 600.0 & 0 \\
\hline 00 & 72114 & 67.0012 & 10.0000 & 299.6 & 4 & 600.0 & \\
\hline 00 & 72115 & 60.7500 & 11.5000 & 299.5 & 4 & 600.0 & 60 \\
\hline 00 & 72116 & 64.2500 & 10.6250 & 299.9 & 4 & 600.0 & 600.0 \\
\hline 00 & 72117 & 65.0000 & 10.9250 & 300.0 & 4 & 600.0 & \\
\hline 00 & 72118 & 64.9998 & 10.6500 & 298.4 & 4 & 600.0 & .0 \\
\hline 00 & 72119 & 62.7497 & 13.6750 & 296.2 & 4 & 600.0 & 60 \\
\hline 00 & 72120 & 61.5000 & 14.2750 & 295.5 & 4 & 600.0 & .0 \\
\hline 00 & 72121 & 61.7500 & 14.1000 & 295.0 & 4 & 0.0 & \\
\hline 00 & 72122 & 63.0000 & 13.6750 & 295.4 & 4 & 600.0 & 600.0 \\
\hline 00 & 72123 & 65.5000 & 12.2250 & 295.8 & 4 & 600.0 & .0 \\
\hline 00 & 72124 & 71.7501 & 10.60 & 295.4 & 4 & 600.0 & \\
\hline 00 & 7221 & 76.5000 & 10.1000 & 294.6 & 4 & 600.0 & 60 \\
\hline 00 & 7222 & 77.0069 & 9.4750 & 294.2 & 4 & 600.0 & .0 \\
\hline 00 & 7223 & 72.5009 & 9.85 & 294.5 & 4 & 00.0 & \\
\hline 00 & 7224 & 68.2500 & 11.2000 & 293.8 & 4 & 600.0 & 60 \\
\hline 00 & 7225 & 66.7500 & 11.0500 & 293.2 & 4 & 600.0 & .0 \\
\hline 00 & 7226 & 66.2504 & 11.00 & 293.3 & 4 & 600.0 & \\
\hline 00 & 7227 & 66.7502 & 11.5250 & 294.1 & 4 & 600.0 & 60 \\
\hline 00 & 7228 & 67.7502 & 9.6000 & 295.1 & 4 & 600.0 & 600.0 \\
\hline 00 & 7229 & 83.2620 & 8.100 & 295.7 & 4 & 600.0 & .0 \\
\hline 00 & 72210 & 103.9954 & 5.8250 & 297.2 & 3 & 600.0 & .0 \\
\hline 00 & 72211 & 95.9920 & 5.7750 & 298.7 & 3 & 600.0 & 600.0 \\
\hline 00 & 72212 & 72.0084 & 6.5000 & 299.0 & 4 & 600.0 & \\
\hline 00 & 72213 & 71.9878 & 6.5500 & 300.2 & 4 & 600.0 & 600.0 \\
\hline 00 & 72214 & 76.5038 & 6.9000 & 300.2 & 4 & 600.0 & 600.0 \\
\hline 00 & 72215 & 85.0050 & 7.850 & 300.3 & 4 & 600.0 & \\
\hline 00 & 72216 & 88.2501 & 8.0500 & 300.4 & 4 & 600.0 & 600.0 \\
\hline 00 & 72217 & 83.0002 & 7.5250 & 300.5 & 4 & 600.0 & 600.0 \\
\hline 00 & 72218 & 72.7514 & 7.4250 & 300.1 & 4 & 600.0 & 600.0 \\
\hline 00 & 72219 & 86.7505 & 5.8250 & 299.9 & 4 & 600.0 & 600.0 \\
\hline 00 & 72220 & 75.4880 & 6.8500 & 299.0 & 4 & 600.0 & 600.0 \\
\hline 00 & 72221 & 68.9998 & 9.2000 & 298.9 & 4 & 600.0 & 600.0 \\
\hline 00 & 72222 & 68.4999 & 8.675 & 299.0 & 4 & 600.0 & 600.0 \\
\hline 00 & 72223 & 68.9995 & 8.3000 & 298.8 & 4 & 600.0 & 600.0 \\
\hline
\end{tabular}




\begin{tabular}{|c|c|c|c|c|c|c|c|}
\hline & 2224 & 9.0 & & 8.6 & 4 & 0 & \\
\hline U & 7231 & 68.7500 & 7.4500 & 298.5 & 4 & 00.0 & \\
\hline & 7232 & 66.7496 & 500 & 298.2 & & 0 & \\
\hline & 7233 & 5.9993 & 3.5000 & 97.6 & & 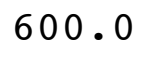 & \\
\hline & $723 \quad 4$ & & & 297.4 & & 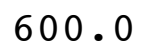 & \\
\hline & 7235 & 000 & & 298.5 & & 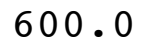 & \\
\hline & 7236 & 2506 & & 299.3 & & 0 & \\
\hline & 7237 & 2.7225 & & 00.2 & & & \\
\hline & 7238 & 68.8478 & 1.5500 & 301.3 & & 0 & 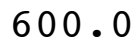 \\
\hline & 7239 & 195.5885 & & 299.3 & & & \\
\hline & 72310 & 169.49 & & 299.4 & & & \\
\hline & 72311 & 166.49 & & 300.0 & & 0 & \\
\hline & 2312 & 165 & 0 & 301.7 & & & \\
\hline & 72313 & 173.9977 & & 302.7 & & & \\
\hline & 72314 & 170 . & & 303.3 & 3 & & \\
\hline & 72315 & 169.4997 & & 304.0 & & & \\
\hline & 72316 & 171 . & & 30 & & & \\
\hline & 72317 & 168 . & & 30 & 3 & & \\
\hline & 72318 & 113.5300 & 0 & 305.3 & 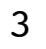 & 0 & 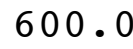 \\
\hline & 72319 & 0 & & 9 & 4 & & \\
\hline & 72320 & 68 . & & & 4 & & \\
\hline & 72321 & 67 . & & & 4 & & \\
\hline & 72322 & 64.50 & 0 & 301.1 & 4 & 0 & \\
\hline & 72323 & 68.24 & & & 4 & & \\
\hline & 72324 & 69 & & 9 & 4 & & \\
\hline & $724 \quad 1$ & 74.0000 & & 301.6 & 4 & & \\
\hline & 7242 & & & .1 & 4 & & \\
\hline & 7243 & 90 & & .4 & 5 & & \\
\hline & $724 \quad 4$ & 42 . & & .6 & 4 & & • \\
\hline & 7245 & 51 & & 6 & 5 & & \\
\hline & 7246 & 84 . & & 30 & 6 & & \\
\hline & 7247 & 138 . & & 1.3 & 5 & & \\
\hline & 7248 & 229.94 & & 302.7 & 4 & & \\
\hline & 7249 & 229. & & 302.3 & 3 & & \\
\hline & 72410 & 182 . & & 302.2 & 2 & & \\
\hline & 72411 & 169. & & 302.3 & 3 & & \\
\hline & 72412 & 176 . & & .1 & 4 & & \\
\hline & 72413 & 179.25 & & 304.2 & 4 & & \\
\hline & 72414 & 171.99 & & 304.8 & 3 & & \\
\hline & 72415 & 172 . & & .3 & 4 & & \\
\hline & 72416 & 129.4599 & & 306.2 & 3 & & \\
\hline & 72417 & 103.2473 & 6.3500 & 306.1 & 4 & 0 & \\
\hline & 72418 & 87 & 6 . & 304.7 & 4 & & \\
\hline 0 & 72419 & 66.7478 & 6.8500 & 303.0 & 4 & 0 & \\
\hline & 72420 & 66.7506 & 0.4500 & 301.2 & 4 & 600 & 00 \\
\hline & 72421 & & & 30 & 4 & & \\
\hline & 72422 & 69.50 & & 300.5 & 4 & & 600 \\
\hline & 72423 & 67.2518 & 8.2500 & 300.9 & & 60 & 60 \\
\hline
\end{tabular}




\begin{tabular}{|c|c|c|c|c|c|c|c|}
\hline & 2424 & 1.4702 & 8.8000 & 1.1 & 4 & 000 & \\
\hline & $725 \quad 1$ & 17499 & 9.1000 & 300.4 & & & \\
\hline & 252 & 7502 & 250 & 299.8 & & 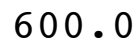 & \\
\hline & 253 & 8.7501 & 9.1000 & 299.0 & & 00 & \\
\hline & 254 & 505 & 9.3500 & 8.4 & & & \\
\hline & 255 & 7500 & .7250 & 98.2 & & 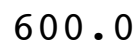 & \\
\hline & 256 & 76.7500 & 9.3000 & 98.1 & & & \\
\hline & 257 & 0000 & 500 & & & & \\
\hline & 258 & 84.5000 & 500 & 99.6 & & & \\
\hline & 259 & 002 & 000 & 300.4 & & & \\
\hline & 2510 & 21.5469 & 250 & 302.0 & & & \\
\hline & 2511 & 02 & & & & & \\
\hline & 2512 & 100. & 750 & 302.9 & & 0 & \\
\hline & 2513 & 98.4998 & 5.32 & 6 & & & \\
\hline & 2514 & & & & & & \\
\hline & 2515 & 023 & 6 . & 303.7 & 4 & & \\
\hline & 2516 & 2526 & 7 . & & & & \\
\hline & 2517 & 502 & & & & & \\
\hline & 2518 & 497 & & & & & \\
\hline & 2519 & 5000 & & 8 & & & \\
\hline & 2520 & 9 & 0 & & & & \\
\hline & 72521 & & & & $I$ & & \\
\hline & 2522 & 998 & 0 & 29 & 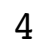 & & \\
\hline & 72523 & 000 & & 25 & 4 & & \\
\hline & 72524 & & & & 4 & & \\
\hline & 7261 & 502 & & & 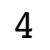 & & \\
\hline & 7262 & 000 & 0 & 297.2 & & & \\
\hline & 7263 & 411 & & 294 & 4 & & \\
\hline & 7264 & 0 & 0 & .1 & 4 & & \\
\hline & 7265 & 000 & & 294.2 & $I$ & & \\
\hline & 7266 & U & & & 4 & & \\
\hline & 7267 & 9 & & & 4 & & \\
\hline & 7268 & 66.7507 & 0 & 295.7 & $x$ & & \\
\hline & 7269 & 00 & & & 4 & & \\
\hline & 72610 & & 0 & & 4 & & \\
\hline & 72611 & 509 & & & 4 & & \\
\hline & 72612 & .2501 & 0 & 298.1 & 4 & & \\
\hline & 72613 & 551 & 0 & & 4 & & \\
\hline & 72614 & 494 & 750 & 3.1 & 4 & & \\
\hline & 726 & 500 & 0 & 29 & 4 & & \\
\hline & 72616 & 999 & & & 4 & & \\
\hline & 72617 & 66.2499 & 2.4500 & 294.7 & 4 & & \\
\hline & 726 & 000 & 750 & 292.5 & 4 & & \\
\hline & 72619 & 67.7500 & & 291.6 & 4 & & \\
\hline & 72620 & 69.7500 & 14.2500 & 290.2 & 4 & 60 & \\
\hline & 72621 & 500 & 4.5000 & 291.1 & 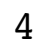 & & \\
\hline & 72622 & & & & & & \\
\hline & 72623 & 72.7499 & 12.1500 & 292.4 & & 600.0 & 500 \\
\hline
\end{tabular}




\begin{tabular}{|c|c|c|c|c|c|c|c|}
\hline 0 & 2624 & 3.0000 & 12.1500 & 290.8 & 4 & 000 & \\
\hline & $727 \quad 1$ & 2.2495 & 11.8500 & 291.0 & & & \\
\hline & $727 \quad 2$ & 0003 & 12.9500 & 291.0 & 4 & 0 & \\
\hline & 7273 & 1.5000 & 2.0750 & 291.2 & & 00 & \\
\hline & $727 \quad 4$ & 2467 & 500 & 90.5 & & & \\
\hline & 7275 & 82.0034 & 2750 & 89.7 & & J & \\
\hline & 7276 & 0009 & 5000 & 289.5 & & & \\
\hline & $\begin{array}{ll}727 & 7\end{array}$ & 82.7501 & 3000 & & & & \\
\hline & 7278 & 95.8354 & .6500 & 92.4 & & & \\
\hline & 7279 & 14.9931 & 750 & .5 & & & \\
\hline & 72710 & 215.3195 & 750 & 2 & & & \\
\hline & 72711 & 4.4973 & & 6 & & & \\
\hline & 72712 & 170.7497 & 6.2250 & 296 & & 0 & \\
\hline & 72713 & 176.0000 & 7.6500 & 0 & & 0 & \\
\hline & 72714 & 172.2493 & & & 4 & & \\
\hline & 72715 & 141.9389 & & 301.0 & 4 & & \\
\hline & 72716 & 122.9044 & 0 & 302.0 & 3 & & \\
\hline & 72717 & 122.9683 & 0 & & & & \\
\hline & 72718 & 87.7500 & 0 & 29 & 4 & & \\
\hline & 72719 & 84.4963 & 0 & 298 & 4 & & \\
\hline & 72720 & 75.7500 & 0 & 6 & 4 & & \\
\hline & 72721 & 78.25 & & & 4 & & \\
\hline & 72722 & 70.0000 & 0 & 297 & 4 & & U • \\
\hline & 72723 & 66.5002 & 0 & 2 & 4 & & \\
\hline & 72724 & 64.7499 & & & 4 & & \\
\hline & 7281 & 71.7459 & & & 4 & & \\
\hline & 7282 & 77.9992 & 0 & 2 & 4 & & \\
\hline & 7283 & 79.7500 & & 29 & 4 & & \\
\hline & 7284 & 81.7499 & 0 & 29 & 4 & & \\
\hline & 7285 & 76.4994 & 0 & 296.7 & 4 & & \\
\hline & 7286 & 73.7498 & & & 4 & & \\
\hline & 7287 & 502 & 0 & 29 & 4 & & \\
\hline & 7288 & 16.2116 & 0 & 298.7 & 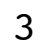 & 0 & . \\
\hline & 7289 & 070 & 1. & 25 & 2 & & \\
\hline & 72810 & 37 & & & 2 & & \\
\hline & 72811 & 171.2496 & & 300.2 & 3 & & \\
\hline & 72812 & 171.0000 & 0 & 301.0 & 4 & & \\
\hline 0 & 72813 & 174.4979 & 0 & 302.1 & 3 & & • \\
\hline & 72814 & 174.2498 & 50 & 302.8 & 3 & & 0 . \\
\hline & 72815 & 170.5017 & 0 & 303 & 4 & & \\
\hline & 72816 & 148.2444 & 0 & & 4 & & \\
\hline 0 & 72817 & 138.7508 & 4.7500 & 304.8 & 3 & 0 & 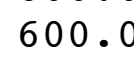 \\
\hline & 72818 & 91.7634 & & 304.3 & 3 & 0 & \\
\hline & 72819 & 74.2489 & & 302.5 & 4 & & \\
\hline 0 & 72820 & 74.5005 & 7.0250 & 301.0 & 4 & 600 & 00 . \\
\hline & 72821 & 71.5000 & 8.1000 & 300.2 & 4 & 600 & \\
\hline & 72822 & 0002 & & 30 & 4 & & \\
\hline & 72823 & 74.7500 & 9.0250 & 299.5 & 4 & 600.0 & 600 \\
\hline
\end{tabular}




\begin{tabular}{|c|c|c|c|c|c|c|c|}
\hline 0 & 2824 & 4.0001 & 9.1500 & 299.6 & 4 & 600.0 & \\
\hline 0 & 7291 & 4.5000 & 8.4500 & 300.2 & 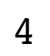 & 600.0 & \\
\hline & 7292 & 74.7500 & 9250 & 300.2 & 4 & 00 & \\
\hline & 7293 & 5.9999 & 6.9250 & 300.3 & & 00 & \\
\hline & 7294 & 2500 & .9500 & 0.7 & & 0 & \\
\hline & 7295 & 74.2499 & 000 & 300 & 4 & 00 & \\
\hline & 7296 & 74.2500 & 7000 & 300.2 & & 0 & \\
\hline & 7297 & 39.1928 & .1500 & 300.5 & & & \\
\hline & 7298 & 6.0146 & 2.0750 & 302.0 & 2 & 0 & \\
\hline & 7299 & 8.0007 & 3750 & 301.6 & & 0 & \\
\hline & 72910 & 170.2504 & .7500 & 300.9 & & 00 & \\
\hline & 72911 & 1.2500 & .8500 & & 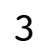 & & \\
\hline & 72912 & 170.0000 & 5.6750 & 302.4 & 3 & 0 & \\
\hline & 72913 & 165.4940 & 4.3750 & 304.0 & 3 & 0.0 & \\
\hline & 72914 & 168.9976 & & & & & \\
\hline & 72915 & 166.7606 & 4.3250 & 306.3 & 3 & 0 & \\
\hline & 72916 & 160.0188 & 3.7500 & 307.0 & & 0 & \\
\hline & 72917 & 131.15 & 3. & .2 & & & \\
\hline 0 & 72918 & 98.98 & 5 . & .1 & 3 & 0 & \\
\hline & 72919 & 84.00 & 6500 & 305.1 & 4 & & \\
\hline & 72920 & 70 . & 0 & 2.7 & 4 & & \\
\hline & 72921 & 72 . & 50 & 1.6 & 4 & & \\
\hline 0 & 72922 & 69.7500 & 3000 &. .4 & 4 & 0 & 0 • \\
\hline 0 & 72923 & 77.25 & 000 & .1 & 4 & 0 & \\
\hline & 72924 & & & & 4 & & \\
\hline & 7301 & 79.2500 & 500 & .6 & 4 & & \\
\hline & 7302 & 76.25 & .6000 & .6 & 4 & & \\
\hline 0 & 7303 & 77.75 & 0 & 301.0 & 4 & & \\
\hline 0 & 7304 & 72 . & 0 & L. 1 & 4 & 0 & 0 . \\
\hline & $730 \quad 5$ & 70.74 & 7000 & 301.7 & 4 & & \\
\hline & 7306 & 73.74 & 0 & 1.7 & 4 & & \\
\hline & 7307 & 81 . & 0 & .8 & 4 & & \\
\hline & 7308 & 100.5771 & 2000 & 304.2 & 3 & 0 & J. \\
\hline 0 & 7309 & 221 . & 0 & .6 & 2 & 0 & \\
\hline & 73010 & 164 . & 0 & .2 & 3 & & \\
\hline & 73011 & 162.2497 & 500 & 303.4 & 3 & & . \\
\hline & 73012 & 169.24 & 5.7000 & 304.2 & 3 & & \\
\hline 0 & 73013 & 166.50 & 500 & 305.3 & 4 & 0 & \\
\hline & 73014 & 161.7618 & 7.2750 & 306.4 & 4 & 0 & 0 . \\
\hline & 73015 & 108.25 & 250 & 30 & 3 & 0 & \\
\hline & 73016 & 158.6478 & 5.1250 & & 3 & & \\
\hline 00 & 73017 & 141.4237 & 4.9250 & 307.2 & 3 & 600.0 & 600 . \\
\hline & 73018 & 87.4992 & 4.1000 & 306.9 & 3 & 600 & 000 \\
\hline & 73019 & 91.7539 & 5.4250 & 304.6 & 4 & & s \\
\hline 00 & 73020 & 75.5067 & 6.1000 & 303.1 & 4 & 600.0 & 00 . \\
\hline & 73021 & 85.5092 & 4.9500 & 302.7 & 4 & 600.0 & 50 \\
\hline & 73022 & & 00 & 302.5 & 4 & 60 & \\
\hline & 73023 & 67.7518 & 7.3500 & 302.1 & 4 & 600.0 & 600 \\
\hline
\end{tabular}




\begin{tabular}{|c|c|c|c|c|c|c|c|c|}
\hline 00 & \multicolumn{2}{|c|}{73024} & 64.0000 & 7.9500 & 302.5 & 4 & 600.0 & 00.0 \\
\hline 00 & 73 & & 67.2501 & 7.9750 & 302.6 & 4 & 600.0 & 00.0 \\
\hline 00 & 73 & 12 & 69.9988 & 7.5250 & 302.4 & 4 & 600.0 & 00.0 \\
\hline 00 & 73 & 13 & 76.9998 & 7.6500 & 302.2 & 4 & 600.0 & 00.0 \\
\hline 00 & 73 & 14 & 75.9991 & 7.8750 & 301.0 & 4 & 600.0 & 00.0 \\
\hline 00 & 73 & 15 & 73.7501 & 7.4250 & 300.9 & 4 & 600.0 & 00.0 \\
\hline 00 & 73 & 16 & 74.7500 & 7.0750 & 300.9 & 4 & 600.0 & 00.0 \\
\hline 00 & 73 & 17 & 74.2500 & 5.6750 & 302.0 & 3 & 600.0 & 00.0 \\
\hline 00 & 73 & 8 & 179.0682 & 2.4500 & 303.7 & 2 & 600.0 & 00.0 \\
\hline 00 & 73 & 19 & 169.5000 & 5.1500 & 303.2 & 3 & 600.0 & 600.0 \\
\hline 0 & 73 & 110 & 164.0000 & 5.0750 & 304.1 & 3 & 600.0 & 00.0 \\
\hline 0 & 73 & 111 & 166.2498 & 50 & 305.0 & 3 & 600.0 & \\
\hline 00 & 73 & 112 & 176.7485 & 6.0250 & 305.6 & 4 & 600.0 & 00.0 \\
\hline 00 & 73 & 113 & 181.0000 & 6.2000 & 306.0 & 4 & 600.0 & 600.0 \\
\hline 00 & 73 & 114 & 177.7390 & 6.2000 & 306.7 & 4 & .0 & \\
\hline 00 & 73 & 115 & 178.0002 & 6.3500 & 307.2 & 4 & .0 & 00.0 \\
\hline 00 & 73 & 116 & 176.7605 & 6.1250 & 307.6 & 4 & 600.0 & 00.0 \\
\hline 00 & 73 & 117 & 177.4994 & 6.12 & 308.2 & 4 & .0 & \\
\hline 00 & 73 & 118 & 177.0011 & 5.3000 & 308.3 & 3 & .0 & 00.0 \\
\hline 00 & 73 & 119 & 112.2197 & 4.2000 & 307.4 & 4 & 60 & 0.0 \\
\hline 00 & 73 & 120 & 72.2504 & 6.1 & 30 & 4 & .0 & .0 \\
\hline 00 & 73 & 121 & 64.7500 & 7.1750 & 304.5 & 4 & 0 & \\
\hline 00 & 73 & 122 & 66.5000 & 8.8750 & 304.7 & 4 & 600.0 & 600.0 \\
\hline 00 & 73 & 123 & 70.0002 & 9.1500 & 305.3 & 4 & 6 & .0 \\
\hline 00 & 73 & 124 & 67.4999 & 0 & 305.6 & 4 & 6 & \\
\hline 00 & 8 & 11 & 67.9999 & 8.1500 & 305.8 & 4 & .0 & 600.0 \\
\hline 00 & 8 & 12 & 70.0002 & 7.5250 & 305.9 & 4 & .0 & 0.0 \\
\hline 00 & 8 & 13 & 75.6617 & 38 & 304.9 & 4 & 0 & 0.0 \\
\hline 00 & 8 & 14 & 23.2223 & 1.6500 & 303.0 & 5 & .0 & 600.0 \\
\hline 00 & 8 & 15 & 252.5215 & 0.9750 & 301.6 & 6 & 60 & 0.0 \\
\hline 00 & 8 & 16 & 75.4196 & 1.2000 & 302.2 & 6 & .0 & \\
\hline 00 & 8 & 17 & 47.6938 & 4.5250 & 305.1 & 5 & 600.0 & 0.0 \\
\hline 00 & 8 & 18 & 339.8184 & 2.7250 & 299.1 & 4 & 600.0 & 600.0 \\
\hline 00 & 8 & 19 & 301.7622 & 1.9000 & 304.1 & 3 & .0 & 0.0 \\
\hline 00 & 8 & 110 & 181.2277 & 500 & 303.7 & 2 & .0 & 0 \\
\hline 00 & 8 & 111 & 144.3249 & 3.1750 & 307.2 & 2 & 600.0 & 600.0 \\
\hline 00 & 8 & 112 & 174.7540 & 6.4500 & 305.8 & 3 & 600.0 & 0.0 \\
\hline 00 & 8 & 113 & 162.7533 & 5.1750 & 306.9 & 3 & 600.0 & 600.0 \\
\hline 00 & 8 & 114 & 164.2506 & 6.2250 & 307.8 & 4 & 600.0 & 600.0 \\
\hline 00 & 8 & 115 & 166.2495 & 7.9500 & 308.1 & 4 & 600.0 & 0.0 \\
\hline 00 & 8 & 116 & 173.4928 & 6.4250 & 308.2 & 4 & 600.0 & 0.0 \\
\hline 00 & 8 & 117 & 177.0000 & 6.0750 & 308.1 & 4 & 600.0 & 600.0 \\
\hline 00 & 8 & 118 & 83.7170 & 6.2000 & 307.9 & 4 & 600.0 & 600.0 \\
\hline 00 & 8 & 119 & 74.5003 & 7.7750 & 306.0 & 4 & 600.0 & 600.0 \\
\hline 00 & 8 & 120 & 70.2500 & 8.9000 & 304.4 & 4 & 600.0 & 600.0 \\
\hline 00 & 8 & 121 & 71.2481 & 9.3750 & 304.0 & 4 & 600.0 & 600.0 \\
\hline 00 & 8 & 122 & 73.7505 & .450 & 303.9 & 4 & 600.0 & 600.0 \\
\hline 00 & 8 & 123 & 72.7500 & 10.4000 & 304.2 & 4 & 600.0 & 600.0 \\
\hline
\end{tabular}




\begin{tabular}{|c|c|c|c|c|c|c|c|c|}
\hline 0 & & 24 & 2.7500 & 9.7750 & 304.2 & 4 & 0 & \\
\hline 0 & 8 & 1 & 2.0000 & 9.1000 & 304.1 & & חم & \\
\hline & 8 & 22 & 70.0001 & 9750 & 303.9 & & 0 & \\
\hline & 8 & 3 & 71.5000 & 2750 & 304.0 & & 00 & \\
\hline & 8 & 4 & 4.7499 & .6250 & & & 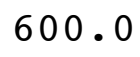 & \\
\hline & 8 & 5 & 72.2500 & 50 & .4 & & 00 & \\
\hline & 8 & 6 & 73.0642 & 3500 & 304.0 & & 0 & \\
\hline & 8 & 7 & 11.8619 & 1.2000 & 301.5 & & & \\
\hline & 8 & 8 & 67.2276 & 1.3500 & 03.8 & & 0 & \\
\hline & 8 & 9 & 47.9981 & 1.9750 & 304.7 & & 00 & \\
\hline & 8 & 210 & 241.8228 & 1.6750 & 305.9 & & . & \\
\hline & 8 & 11 & 225.7478 & & & & & \\
\hline & 8 & 12 & 230.7581 & 50 & 307.1 & & 0 & \\
\hline & 8 & 213 & 235.9544 & 2.8500 & 307.4 & & 0 & \\
\hline & 8 & 214 & 175 . & & & & & \\
\hline & 8 & 215 & 157.2455 & 6.3750 & 308 & 3 & & \\
\hline & 8 & 216 & 143.5000 & 6.32 & 308 & & 0 & \\
\hline & 8 & 217 & 104 . & 0 & 30 & & & \\
\hline & 8 & 218 & 80 . & 6 . & 30 & 4 & 0 & \\
\hline & 8 & 219 & 70.0004 & 0 & & 4 & & \\
\hline & 8 & 20 & 67 . & 0 & 3 & 4 & & \\
\hline & 8 & 221 & 62 . & & & 4 & & \\
\hline 0 & 8 & 222 & 59.2503 & 0 & 30 & 4 & 0 & • \\
\hline & 8 & 223 & 51. & 0 & & 4 & 0 & \\
\hline & 8 & 224 & 83 & & & 5 & & \\
\hline & 8 & 31 & 132.7225 & 0 & & 6 & & \\
\hline & 8 & 32 & 58.4733 & 0 & & 0 & & \\
\hline 0 & 8 & 33 & 123. & 0 & 29 & 6 & & \\
\hline 0 & 8 & 34 & 145 . & 0 & 29 & 6 & 0 & 0 . \\
\hline & 8 & 35 & 21 . & 0 & & 6 & & \\
\hline & 8 & 36 & 355 . & 0 & & 6 & & \\
\hline & 8 & 37 & 7 . & 0 & & 5 & & \\
\hline & 8 & 38 & 699 & 9500 & 30 & 4 & 0 & 0 . \\
\hline 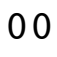 & 8 & 39 & 266 . & 0 & & 3 & & \\
\hline & 8 & 310 & 277 . & & & 2 & & \\
\hline & 8 & 311 & 232.7501 & 500 & & 2 & & \\
\hline & 8 & 312 & 209 . & & & 1 & & \\
\hline 0 & 8 & 313 & 167 . & 0 & 4 & 2 & & 0 . \\
\hline & 8 & 314 & 168.7499 & 0 & .1 & 3 & 0 & 0 \\
\hline & 8 & 315 & 177 . & & & 4 & & \\
\hline 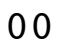 & 8 & 316 & 193. & 0 & & 4 & & \\
\hline 00 & 8 & 317 & 187.8535 & 6.0750 & 305.1 & 4 & 600.0 & 0. \\
\hline & 8 & 318 & 217.0180 & & 30 & 3 & 0 & 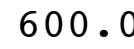 \\
\hline 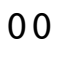 & 8 & 319 & 244.4981 & 2.7250 & & 4 & & \\
\hline 0 & 8 & 320 & 2.5293 & 1.7500 & & 5 & 600 & 0 \\
\hline & 8 & 321 & 35.9814 & 1.9500 & 298.6 & - & 600 & 60 \\
\hline & 8 & 322 & 171 & & & & 60 & \\
\hline & & 323 & 350.0052 & 0.4250 & 296.7 & & 600.0 & 600 \\
\hline
\end{tabular}




\begin{tabular}{|c|c|c|c|c|c|c|c|c|}
\hline 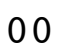 & 8 & 24 & 8 & 0750 & 296.6 & 6 & 00 & \\
\hline & 8 & 1 & 0.0761 & .5750 & 295.5 & & 00.0 & \\
\hline & 8 & 2 & 77.0383 & 3750 & 295.0 & & 00 & \\
\hline & 8 & 3 & 53.0968 & 4000 & 295.9 & & 00 & \\
\hline & 8 & 4 & 39 & .3250 & 95.4 & & & \\
\hline & 8 & 5 & 356.2 & & 92.0 & & 0 & \\
\hline & 8 & 46 & 165.8923 & 000 & 291.3 & & 00 & \\
\hline & 8 & 7 & 50.9937 & 50 & 93.9 & & & \\
\hline & 8 & 8 & 4.5670 & & 3 & & & \\
\hline & 8 & 9 & 241.2403 & 500 & 296.2 & & 0 & \\
\hline & 8 & 410 & 240.7667 & 750 & 297.2 & & & \\
\hline & 8 & 411 & 234.9988 & & 9 & & & \\
\hline & 8 & 412 & 214.5000 & 2 . & 301.2 & & 0 & \\
\hline & 8 & 413 & 160.0002 & 2000 & 30 & & & \\
\hline & 8 & 414 & 165. & & & & & \\
\hline & 8 & 415 & $170.7 \leq$ & & & 4 & & \\
\hline & 8 & 416 & 143.7784 & 50 & 305 & 3 & & \\
\hline & 8 & 417 & 101.5000 & 4 . & & & & \\
\hline & 8 & 418 & 108 & & & 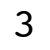 & & \\
\hline & 8 & 419 & 74.2059 & 750 & & 4 & .0 & \\
\hline & 8 & 420 & 72.9993 & 000 & & 4 & 0 & \\
\hline & 8 & 421 & 77. & & & 4 & & \\
\hline & 8 & 422 & 59. & 0 & 30 & 4 & & \\
\hline & 8 & 423 & 64.94 & 0 & .3 & 4 & 0 & \\
\hline & 8 & 424 & 68.4 & & & 4 & & \\
\hline & 8 & 51 & 72 . & & & 4 & & \\
\hline & 8 & 52 & 70.74 & 0 & 299.7 & 4 & & 0 \\
\hline & 8 & 53 & 73.74 & 0 & .2 & 4 & & \\
\hline & 8 & 54 & 78 . & 50 & & 4 & & \\
\hline & 8 & 55 & 75.50 & & 29 & 4 & & J. \\
\hline & 8 & 56 & 77.2487 & & .5 & 4 & & . \\
\hline & 8 & 57 & 78.2 & & & 4 & & \\
\hline & 8 & 58 & 74.25 & & .9 & 4 & & 0 . \\
\hline & 8 & 59 & 81.2509 & & .4 & 4 & & \\
\hline & 8 & 510 & 84.7 & & & 4 & & \\
\hline & 8 & 511 & 93.7504 & & & 3 & & 0 . \\
\hline & 8 & 512 & 91.9979 & & 304.5 & 3 & & \\
\hline & 8 & 513 & 91. & & 30 & 4 & & \\
\hline & 8 & 514 & 85 . & 6 . & 305.2 & 4 & & 0 . \\
\hline & 8 & 51 & 88 . & & 305.3 & 4 & & \\
\hline & 8 & 516 & 91.5000 & 8 . & 304.6 & 4 & & \\
\hline & 8 & 517 & 82.4969 & 7 . & & 4 & & 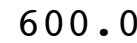 \\
\hline & 8 & 518 & 101.7539 & 5.8500 & 303.6 & 3 & 0 & 0 . \\
\hline & 8 & 519 & 69.1966 & & 302.9 & 4 & & \\
\hline חמח & 8 & 520 & 64.2500 & & & 4 & & \\
\hline & 8 & 521 & 65.5000 & 1.6750 & 300.8 & 4 & & 0 \\
\hline & 8 & 522 & 64 & & & 4 & & \\
\hline & 0 & 523 & 67.2503 & 10.0750 & 301.3 & $\Psi$ & 600.0 & 60 \\
\hline
\end{tabular}




\begin{tabular}{|c|c|c|c|c|c|c|c|c|}
\hline 0 & 8 & 524 & 68.0000 & 10.0250 & 301.2 & 4 & 600.0 & 600.0 \\
\hline 00 & 8 & 61 & 76.5000 & 8.7250 & 301.0 & 4 & 600.0 & 600.0 \\
\hline 00 & 8 & 62 & 73.5005 & 10.4750 & 300.8 & 4 & 600.0 & 00.0 \\
\hline 0 & 8 & 63 & 5.0000 & 11.3250 & 300.6 & 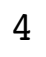 & 600.0 & 00.0 \\
\hline 00 & 8 & 64 & 64.5000 & 10.5500 & 300.6 & 4 & 600.0 & 00.0 \\
\hline 00 & 8 & 65 & 60.5000 & 9.2000 & 300.6 & 4 & 600.0 & 00.0 \\
\hline 00 & 8 & 66 & 63.5008 & 9.6000 & 300.7 & 4 & 600.0 & 0 \\
\hline 00 & 8 & 67 & 75.0000 & 10.0500 & 301.1 & 4 & 600.0 & 00.0 \\
\hline 00 & 8 & 68 & 73.4995 & 10.0500 & 301.3 & 4 & 600.0 & 00.0 \\
\hline 00 & 8 & 69 & 78.2508 & 10.5750 & 300.8 & 4 & 600.0 & 0 \\
\hline 00 & 8 & 610 & 77.2499 & 8.3000 & 301.2 & 4 & 600.0 & \\
\hline 00 & 8 & 611 & 87.2503 & 7.5750 & 301.8 & 4 & .0 & \\
\hline 00 & 8 & 612 & 81.0007 & 7.4000 & 301.9 & 4 & 600.0 & 00.0 \\
\hline 00 & 8 & 613 & 83.5013 & 7.7500 & 302.8 & 4 & 600.0 & 00.0 \\
\hline 00 & 8 & 614 & 91.7663 & 000 & 303.4 & 4 & .0 & \\
\hline 00 & 8 & 615 & 90.7466 & 7.8750 & 303.4 & 4 & 600.0 & .0 \\
\hline 00 & 8 & 616 & 75.2680 & 8.1500 & 303.1 & 4 & 60 & .0 \\
\hline 00 & 8 & 617 & 64.5000 & 11.6250 & 302.2 & 4 & .0 & \\
\hline 00 & 8 & 618 & 66.5000 & 11.6000 & 301.1 & 4 & 60 & .0 \\
\hline 00 & 8 & 619 & 64.2498 & 11.0250 & 300.1 & 4 & 60 & .0 \\
\hline 00 & 8 & 620 & 61.4999 & 11.57 & 29 & 4 & 6 & \\
\hline 00 & 8 & 621 & 62.0000 & 11.9250 & 29 & 4 & .0 & \\
\hline 00 & 8 & 622 & 61.5000 & 11.9500 & 298.8 & 4 & 600.0 & .0 \\
\hline 00 & 8 & 623 & 62.2500 & 12.17 & 298.5 & 4 & 6 & \\
\hline 00 & 8 & 624 & 68.5000 & 12.1750 & 297.5 & 4 & .0 & \\
\hline 00 & 8 & 71 & 80.0001 & 10.5750 & 296.2 & 4 & .0 & .0 \\
\hline 00 & 8 & 72 & 80.5002 & 10.7500 & 295.1 & 4 & 60 & .0 \\
\hline 00 & 8 & 73 & 77.5000 & 10.9000 & 295.0 & 4 & .0 & \\
\hline 00 & 8 & 74 & 73.2496 & 12.1750 & 294.2 & 4 & .0 & 0 \\
\hline 00 & 8 & 75 & 73.7500 & 12.6250 & 293.3 & 4 & 6 & .0 \\
\hline 00 & 8 & 76 & 69.7500 & 13.8750 & 292.5 & 4 & 0 & \\
\hline 00 & 8 & 77 & 70.0000 & 13.9500 & 291.6 & 4 & .0 & 0.0 \\
\hline 00 & 8 & 78 & 71.2503 & 14.2750 & 291.4 & 4 & 600.0 & 0.0 \\
\hline 00 & 8 & 79 & 69.7500 & 12.6500 & 292.1 & 4 & .0 & 0.0 \\
\hline 00 & 8 & 710 & 76.7502 & 10.5750 & 293.3 & 4 & 600.0 & 600.0 \\
\hline 00 & 8 & 711 & 78.2492 & 10.3000 & 293.9 & 4 & 600.0 & 600.0 \\
\hline 00 & 8 & 712 & 83.2484 & 8.8750 & 294.9 & 4 & 600.0 & 600.0 \\
\hline 00 & 8 & 713 & 64.5000 & 10.6000 & 295.7 & 4 & 600.0 & 600.0 \\
\hline 00 & 8 & 714 & 65.2495 & 10.2750 & 296.6 & 4 & 600.0 & 600.0 \\
\hline 00 & 8 & 715 & 67.5003 & 10.4000 & 297.1 & 4 & 600.0 & 600.0 \\
\hline 00 & 8 & 716 & 69.4995 & 9.6000 & 297.3 & 4 & 600.0 & 600.0 \\
\hline 00 & 8 & 717 & 68.2501 & 11.2250 & 296.5 & 4 & 600.0 & 600.0 \\
\hline 00 & 8 & 718 & 67.7497 & 11.6500 & 296.0 & 4 & 600.0 & 600.0 \\
\hline 00 & 8 & 719 & 71.2500 & 11.2250 & 294.3 & 4 & 600.0 & 600.0 \\
\hline 00 & 8 & 720 & 66.0019 & 11.7250 & 293.6 & 4 & 600.0 & 600.0 \\
\hline 00 & 8 & 721 & 58.5023 & 7.450 & 293.8 & 4 & 600.0 & \\
\hline 00 & 8 & 722 & 67.7504 & 8.6250 & 294.6 & 4 & 600.0 & 600.0 \\
\hline 00 & 0 & 723 & 79.5017 & 8.975 & 293.9 & & 600.0 & 600.0 \\
\hline
\end{tabular}




\begin{tabular}{|c|c|c|c|c|c|c|c|c|}
\hline & 3 & 724 & 0.000 & 9.3500 & 4.6 & 4 & 10 & \\
\hline & 8 & 81 & 73.5014 & 10.0000 & 294.8 & & 00.0 & \\
\hline & 8 & 82 & 66.2501 & 7250 & 294.7 & & 0 & \\
\hline & 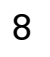 & 3 & 6.2500 & 1.1000 & 293.9 & & & \\
\hline & & 4 & 9.9999 & 1750 & 94.3 & & 00 . & \\
\hline & & 5 & 253 & 3750 & 293.9 & & & \\
\hline & 8 & 6 & .78 .4358 & 8750 & 290.2 & & & \\
\hline & 8 & 7 & 76 & & 91.4 & & & \\
\hline & 0 & 8 & 51.4814 & 0.9750 & 293.1 & & 0 & \\
\hline & 8 & 9 & 22 & 1.5250 & 295.2 & & & \\
\hline & 8 & 10 & 275.2935 & & 3 & & & \\
\hline & 3 & 11 & 251.9554 & 2.4000 & 297.6 & & & \\
\hline & 8 & 12 & 259 . & 3.0250 & 298.0 & & & \\
\hline & 8 & 13 & 220.4992 & 3 . & 3 & & & \\
\hline & 3 & 14 & 204 . & & 299 & & & \\
\hline & 8 & 15 & 163 & 000 & 300.9 & & 0 & \\
\hline & 8 & 816 & 84 . & 4.1750 & 301.3 & & & \\
\hline & 8 & 17 & 86. & & 299.4 & & & \\
\hline & 8 & 18 & 76 . & 7.4000 & $29^{\circ}$ & 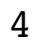 & 0 & \\
\hline & 8 & 81 & 72 . & 8.12 & 29 & 4 & & \\
\hline & 8 & 20 & 73 . & & & - & & \\
\hline 0 & 8 & 21 & 75 & 9.3250 & 29 & 4 & & \\
\hline 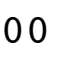 & 8 & 822 & 74.5001 & 8.4500 & 294.2 & 4 & 0 & \\
\hline & 8 & 823 & 68.5161 & & 294.0 & 4 & & \\
\hline 0 & 8 & 824 & & 0 & 29 & 4 & & \\
\hline 0 & 8 & 91 & 48 . & 250 & 29 & 4 & 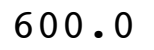 & \\
\hline & 8 & 2 & $06 \quad 92$ & 500 & 29 & 5 & & \\
\hline 0 & 8 & 93 & 6 & 50 & 29 & 6 & & \\
\hline & 8 & 94 & 120 & 250 & & 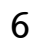 & 0.0 & \\
\hline & 8 & 95 & 56 . & 0 & 29 & 6 & & \\
\hline & 8 & 96 & 98. & & 29 & 6 & & \\
\hline & 8 & 97 & 885 & & 292 & 5 & & \\
\hline 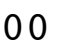 & 8 & 98 & 248.6257 & 500 & 294.8 & 4 & 0 & \\
\hline 0 & 8 & 99 & 1200 & 0 & 294.1 & 3 & & \\
\hline & 8 & 910 & 249.7292 & & & 2 & & \\
\hline & 8 & 911 & 230.7445 & 000 & 296.5 & 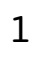 & .0 & \\
\hline & 8 & 912 & 230 . & & 297.6 & 2 & & \\
\hline - & 8 & 913 & 209 . & 5 . & 29 & 3 & & \\
\hline & 8 & 914 & 173.6275 & & 299.4 & 3 & & \\
\hline & 8 & 915 & 157. & & 299 & 3 & & \\
\hline 0 & 8 & 916 & 120 . & & 300.0 & 3 & & \\
\hline 0 & 8 & 917 & 86.9999 & 5.8000 & 298.7 & 3 & .0 & \\
\hline 0 & 8 & 918 & 80 . & 750 & 296.4 & 4 & & \\
\hline 00 & 8 & 919 & 78.2500 & 9.9000 & 293.9 & 4 & 600.0 & \\
\hline 0 & 8 & 920 & 77.2496 & 11.0000 & 292.1 & 4 & 600.0 & 600 \\
\hline & 8 & 921 & & & 290.4 & 4 & & \\
\hline & 8 & 922 & 73.4997 & 1.3000 & 289.4 & 4 & & \\
\hline & & 23 & 74.7493 & 9.4750 & 289.0 & & 600.0 & 50 \\
\hline
\end{tabular}




\begin{tabular}{|c|c|c|c|c|c|c|c|}
\hline & 924 & 997 & 0250 & 88.7 & 4 & 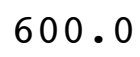 & \\
\hline & 8101 & 000 & 11.2500 & 89.3 & & 00.0 & \\
\hline & 8102 & 74.2500 & .9500 & 288.2 & & 00 & \\
\hline & 8103 & 74.5000 & 1.7750 & 288.0 & & 00 & \\
\hline & 104 & 501 & & & & & \\
\hline & 8105 & 89 & & 86.7 & & 0 & \\
\hline & 8106 & 76.7501 & 1.5500 & 86.4 & & 00 & \\
\hline & 8107 & 7.5000 & 0 & 9 & & & \\
\hline & 8108 & 7.2504 & .6250 & 3 & & & \\
\hline & 8109 & 91.5229 & 4.9500 & 90.2 & & 0 & \\
\hline & 81010 & 6.2500 & 3.27 & 291.8 & & & \\
\hline & 81011 & 9.6031 & 17 & 5 & & & \\
\hline & 81012 & 256.7244 & 1.80 & 96 & & 0 & \\
\hline & 81013 & 269.3522 & 2.00 & 7 & & & \\
\hline & 81014 & 51 & & & & & \\
\hline & 81015 & 86 . & & 295 & & & \\
\hline & 81016 & 81.2373 & 5.52 & 299 & & & \\
\hline & 81017 & 85 & 6 . & 3 & & & \\
\hline & 81018 & 74 . & 6 . & 7 & & & \\
\hline & 81019 & 82.2496 & 8.12 & 295 & 4 & & \\
\hline & 81020 & .5040 & 20 & 294 & 4 & 0 & \\
\hline & 81021 & .7494 & & & 4 & & \\
\hline & 81022 & 500 & 0 & 29 & 4 & & \\
\hline & 81023 & 76.0006 & 8.20 & 4 & 4 & & \\
\hline & 81024 & & 0 & & 4 & & \\
\hline & 8111 & 00 & 7 . & 29 & 4 & & \\
\hline & 8112 & 81.9989 & 0 & 29 & 4 & & 0 \\
\hline & 8113 & 84.2500 & 5.70 & 25 & 4 & & \\
\hline & 8114 & 84.7498 & 5.47 & 29 & 4 & & \\
\hline & 8115 & 92.2503 & 0 & 294.8 & 4 & & . \\
\hline & 8116 & 28.5119 & 2.6000 & 294.6 & 4 & & . \\
\hline & 8117 & 150 & 282 & & 4 & & \\
\hline & 8118 & 154.9947 & 5000 & 6 & 3 & & 。 \\
\hline & 8119 & 161.9982 & 4.7500 & 25 & 3 & & \\
\hline & 81110 & 168.2522 & 0 & & 3 & & \\
\hline & 81111 & 162.4977 & & & 3 & & \\
\hline & 81112 & 167.5003 & 4.7500 & 299.8 & 3 & & \\
\hline & 81113 & 169. & 500 & & 3 & & \\
\hline & 81114 & 172.7498 & 4.9750 & 302.1 & 3 & & 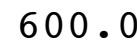 \\
\hline & 81115 & 166.7384 & 000 & 303.2 & 3 & & \\
\hline & 81116 & 171.2492 & 500 & & 3 & & \\
\hline & 81117 & 178.0161 & 5.6250 & & 3 & & \\
\hline & 81118 & 183.7516 & 5.2500 & 303.6 & 3 & & 0 \\
\hline & 81119 & 148.1093 & 750 & & 4 & & \\
\hline 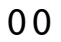 & 81120 & 73.4190 & 5.1000 & & 4 & & \\
\hline & 81121 & 72.7499 & 7.0750 & 299.8 & 4 & & 0 . \\
\hline & 81122 & & & & 4 & & \\
\hline & 81123 & 63.2501 & 8.3500 & 299.0 & $\Psi$ & 600.0 & 6 \\
\hline
\end{tabular}




\begin{tabular}{|c|c|c|c|c|c|c|c|}
\hline 0 & 124 & 2 & 3750 & 99.4 & 4 & 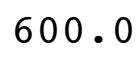 & \\
\hline & 8121 & 8.5000 & 8.2250 & 299.6 & 4 & 600.0 & \\
\hline & 8122 & 7499 & 8.0500 & 300.2 & 4 & 00 & \\
\hline & 123 & 78.2500 & 6.7000 & 300.7 & & 00 & \\
\hline & 124 & 5002 & 5.8750 & 00.3 & & 0 & \\
\hline & 125 & 2500 & & 99.7 & & 0 & \\
\hline & 8126 & 007 & 3250 & 299.6 & & 00 & \\
\hline & 127 & 72.7491 & 6000 & 00.3 & & & \\
\hline & 128 & 22.0 & 2.4500 & 1.9 & & & \\
\hline & 129 & 002 & 2.3000 & 302.2 & & 0 & o \\
\hline & 1210 & 232.4986 & 2.7500 & 301.8 & & & \\
\hline & 1211 & 231 . & 2.8000 & 03.2 & & & \\
\hline & 81212 & 170 . & 500 & 304.1 & & 0 & \\
\hline & 1213 & 177.5 & 4.4250 & 304.6 & & & \\
\hline & 1214 & 93. & & & & & \\
\hline & 1215 & 114 . & & 6 & 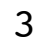 & & \\
\hline & 1216 & 100.7 & 0 & 306.2 & 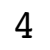 & .0 & \\
\hline & 1217 & 86 . & 0 & .2 & 4 & & \\
\hline & 1218 & 81 . & & & 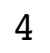 & & \\
\hline & 1219 & 64.7 & 0 & & 4 & & \\
\hline & 81220 & 63.4997 & 0.7500 & 9 & 4 & 0 & \\
\hline & 81221 & 64. & 0 & & 4 & & \\
\hline & 81222 & 61 & 1.3500 & & 4 & & \\
\hline & 81223 & 62.5001 & .5000 & 8 & 4 & 0 & \\
\hline & 81224 & 63. & 9250 & & 4 & & \\
\hline & 8131 & 62 . & 000 & 29 & 4 & & \\
\hline & 8132 & 61.2500 & 0000 & 30 & 4 & & . \\
\hline & 8133 & 70 . & 2500 & .4 & 4 & & \\
\hline & 8134 & 70 . & 0 & & 4 & & \\
\hline & 8135 & 000 & 3250 & 298.8 & 4 & & J. \\
\hline & 8136 & 67.5005 & 5250 & 297.9 & 4 & & 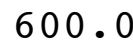 \\
\hline & 8137 & 0 & 0 & & 4 & & \\
\hline & 8138 & & 000 & 1.0 & 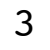 & & 0 . \\
\hline & 8139 & $y$ & 9250 & 302.6 & 2 & & \\
\hline & 81310 & 23 & 0 & & 2 & & \\
\hline & 81311 & 236.7485 & 4750 & 1.7 & 3 & & \\
\hline & 81312 & 766 & 9000 & 304.5 & 2 & & \\
\hline & 81313 & 073 & $\$ 750$ & 304.4 & 3 & & \\
\hline & 81314 & 138.4984 & 6.5250 & 305.1 & 4 & & 0 . \\
\hline & 81315 & 133.8893 & 250 & 305.2 & 4 & & \\
\hline & 81316 & 111.1996 & 250 & 305.2 & 3 & & \\
\hline & 81317 & 88.9984 & 7.2250 & 304.3 & 4 & & 0 . \\
\hline & 81318 & 94.5138 & 7.4000 & 303.2 & 4 & & 0 . \\
\hline & 81319 & 5024 & 750 & & 4 & & \\
\hline 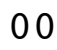 & 81320 & 65.7496 & 8.5000 & & 4 & & \\
\hline & 81321 & 68.4998 & 7.4500 & 300.6 & 4 & & 00 \\
\hline & & 6 & & & 4 & & \\
\hline & 81323 & 67.7505 & 8.9000 & 299.4 & $\Psi$ & 600.0 & 600 \\
\hline
\end{tabular}




\begin{tabular}{|c|c|c|c|c|c|c|c|}
\hline 00 & 81324 & 63.2501 & 10.1750 & 298.6 & 4 & 600.0 & 600.0 \\
\hline 0 & 8141 & 66.7498 & 10.5250 & 298.2 & 4 & 600.0 & 600.0 \\
\hline 00 & 8142 & 75.7500 & 10.3000 & 297.7 & 4 & 600.0 & 00.0 \\
\hline 00 & 8143 & 76.2502 & 9.6750 & 297.5 & 4 & 600.0 & 00.0 \\
\hline 00 & 8144 & 69.7500 & 9.2500 & 297.3 & 4 & 600.0 & 00.0 \\
\hline 00 & 8145 & 69.5000 & 8.5750 & 297.4 & 4 & 600.0 & 00.0 \\
\hline 00 & 8146 & 72.5000 & 8.2000 & 297.6 & 4 & 600.0 & 600.0 \\
\hline 00 & 8147 & 80.2434 & 6.9750 & 298.7 & 4 & 600.0 & 00.0 \\
\hline 00 & 8148 & 156.0228 & 2.9250 & 300.1 & 3 & 600.0 & \\
\hline 00 & 8149 & 166.7504 & 4.9500 & 300.5 & 3 & 600.0 & 600.0 \\
\hline 00 & 81410 & 170.9952 & 5.4500 & 300.5 & 3 & 600.0 & .0 \\
\hline 00 & 81411 & 172.9999 & 6.2500 & 301.3 & 4 & 600.0 & 600.0 \\
\hline 00 & 81412 & 174.4994 & 6.7500 & 302.1 & 4 & 600.0 & 60 \\
\hline 00 & 81413 & 168.4995 & 6.2000 & 303.3 & 4 & 600.0 & \\
\hline 00 & 81414 & 169.2530 & 5.9500 & 304.6 & 3 & 600.0 & .0 \\
\hline 00 & 81415 & 159.6076 & 6.3000 & 305.1 & 4 & 600.0 & \\
\hline 00 & 81416 & 108.2495 & 5.3250 & 305.4 & 3 & 600.0 & 600.0 \\
\hline 00 & 81417 & 110.2490 & 5.8000 & 304.9 & 3 & 600.0 & .0 \\
\hline 00 & 81418 & 72.7420 & 8.0750 & 303.3 & 4 & 600.0 & \\
\hline 00 & 81419 & 68.5000 & 9.0000 & 302.1 & 4 & 600.0 & 600.0 \\
\hline 00 & 81420 & 69.7504 & 7.7000 & 300.6 & 4 & 600.0 & .0 \\
\hline 00 & 81421 & 64.7500 & 9.1750 & 300.4 & 4 & 600.0 & \\
\hline 00 & 81422 & 62.4973 & 8.3250 & 300.5 & 4 & 600.0 & 60 \\
\hline 00 & 81423 & 69.2489 & 7.4500 & 300.5 & 4 & 600.0 & .0 \\
\hline 00 & 81424 & 69.4991 & 7.2250 & 300.5 & 4 & 600.0 & \\
\hline 00 & 8151 & 68.0002 & 7.8250 & 300.6 & 4 & 600.0 & 60 \\
\hline 00 & 8152 & 71.7500 & 8.7250 & 299.8 & 4 & 600.0 & .0 \\
\hline 00 & 8153 & 69.7500 & 9.0500 & 298.8 & 4 & 600.0 & .0 \\
\hline 00 & 8154 & 65.2501 & 8.0500 & 298.7 & 4 & 600.0 & .0 \\
\hline 00 & 8155 & 63.7497 & 6.1000 & 299.4 & 4 & 600.0 & 600.0 \\
\hline 00 & 8156 & 75.5000 & 7.0000 & 299.3 & 4 & 600.0 & 60 \\
\hline 00 & 8157 & 83.7394 & 4.4000 & 299.3 & 4 & 600.0 & 60 \\
\hline 00 & 8158 & 177.4112 & 1.3500 & 300.4 & 3 & 600.0 & 60 \\
\hline 00 & 8159 & 244.9376 & 1.1750 & 298.9 & 2 & 600.0 & .0 \\
\hline 00 & 81510 & 231.2532 & 1 . & 299.9 & 2 & 600.0 & \\
\hline 00 & 81511 & 168.4987 & 4.6750 & 301.4 & 3 & 600.0 & 600.0 \\
\hline 00 & 81512 & 167.9947 & 6.4250 & 301.8 & 4 & 600.0 & 600.0 \\
\hline 00 & 81513 & 176.2501 & 5.1250 & 302.6 & 3 & 600.0 & 60 \\
\hline 00 & 81514 & 168.5008 & 4.7000 & 303.2 & 3 & 600.0 & 600.0 \\
\hline 00 & 81515 & 172.5029 & 5.7750 & 303.8 & 3 & 600.0 & 600.0 \\
\hline 00 & 81516 & 158.9996 & 5.9250 & 305.0 & 3 & 600.0 & 600.0 \\
\hline 00 & 81517 & 130.1480 & 5.6250 & 305.5 & 3 & 600.0 & 600.0 \\
\hline 00 & 81518 & 95.2505 & 6.3750 & 304.7 & 4 & 600.0 & 600.0 \\
\hline 00 & 81519 & 81.2508 & 5.3250 & 303.7 & 4 & 600.0 & 600.0 \\
\hline 00 & 81520 & 64.5013 & 7.1750 & 302.2 & 4 & 600.0 & 600.0 \\
\hline 00 & 81521 & 71.4991 & 8.2000 & 301.9 & 4 & 600.0 & 600.0 \\
\hline 00 & 81522 & 74.7497 & 8 . & 301.4 & $\Psi$ & 600.0 & .0 \\
\hline 00 & 81523 & 69.5000 & 8.9000 & 301.4 & $\mathbf{4}$ & 600.0 & 600.0 \\
\hline
\end{tabular}




\begin{tabular}{|c|c|c|c|c|c|c|c|}
\hline 0 & 524 & 500 & 750 & 01.4 & 4 & 000 & \\
\hline & 8161 & 7500 & .6750 & 301.1 & 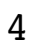 & 00.0 & \\
\hline & 162 & 4991 & 250 & 300.9 & 4 & 00 & \\
\hline & 163 & 78.2500 & 8250 & 300.5 & & 00.0 & \\
\hline & 164 & 5000 & 0000 & 00.3 & & 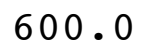 & \\
\hline & 165 & 89.2486 & & 00.0 & & 0 & \\
\hline & 8166 & 91.2479 & 7500 & 299.7 & & 00 & \\
\hline & 167 & 64.6265 & 5000 & 300.0 & & & \\
\hline & 168 & 2 & & 00.3 & & & \\
\hline & 169 & 62 . & 750 & 299.6 & & 00 & \\
\hline & 1610 & 164.4998 & & 300.1 & & 0.0 & \\
\hline & 1611 & 163.2 & & 300.9 & & & \\
\hline & 81612 & 164. & & 301.7 & 4 & 0 & \\
\hline & 1613 & 168 & 0 & 303.1 & 3 & & \\
\hline & 1614 & 175 & & & & & \\
\hline & 1615 & 171. & & 305 & 3 & & \\
\hline & 1616 & 171. & 0 & 306.1 & 3 & .0 & . \\
\hline & 617 & 147 . & & 4 & 3 & & \\
\hline & 1618 & 88 . & & 305.6 & 1 & & \\
\hline & 1619 & & & 303.6 & 4 & .0 & . \\
\hline & 81620 & 68.7 & & 301.4 & 4 & .0 & \\
\hline & 816 & 75.2 & & & 4 & & \\
\hline & 81622 & 75 & & 301.8 & 4 & & \\
\hline & 81623 & 74.7 & 0 & 301.9 & 4 & 0 & \\
\hline & 81624 & 77.7 & & 302.1 & 4 & & \\
\hline & 8171 & 75 & & 301.6 & 4 & & \\
\hline & 8172 & 73.7 & & .1 & 4 & 0 & J. \\
\hline & 8173 & 72.4 & & 30 & 4 & & \\
\hline & 8174 & 78 . & & 29 & 4 & & \\
\hline & 8175 & 69. & & 297.7 & 4 & & J. \\
\hline & 8176 & 70 & 9 . & 297.8 & 4 & & 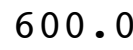 \\
\hline & 8177 & 80 . & & 299.3 & 4 & & \\
\hline & 8178 & 45 . & & 301.3 & 2 & & 0 . \\
\hline & 8179 & in & 0 & 301.6 & 2 & & 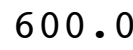 \\
\hline & 81710 & 1 & & & 3 & & \\
\hline & 81711 & 167 . & & 301.9 & 3 & & 0 . \\
\hline & 81712 & 171.750 & & 303.0 & 3 & & \\
\hline & 81713 & 100 & & 30 & 2 & & \\
\hline & 81714 & 84.29 & 50 & 305.4 & 3 & & 0 . \\
\hline & 81715 & 81.250 & & 304.8 & 4 & & \\
\hline & 81716 & 89.74 & 0 & 304.3 & 4 & & \\
\hline & 81717 & 91.9887 & 8.5500 & 303.4 & 4 & & 0 . \\
\hline & 81718 & 65.9755 & 8.4250 & 302.8 & 4 & 0 & 0 . \\
\hline & 81719 & 66.4885 & 0 & & 4 & & \\
\hline - & 81720 & 73.0000 & 8.2750 & & 4 & & \\
\hline & 81721 & 60.2468 & 8.9250 & 300.2 & 4 & & 00 . \\
\hline & & & & & 4 & & \\
\hline & 81723 & 59.7496 & 11.8750 & 299.1 & $x$ & 600.0 & $600+8$ \\
\hline
\end{tabular}




\begin{tabular}{|c|c|c|c|c|c|c|c|}
\hline 00 & 81724 & 58.4997 & 11.5500 & 298.6 & 4 & 600.0 & 600.0 \\
\hline 00 & 8181 & 59.5017 & 12.1000 & 297.9 & 4 & 600.0 & 600,0 \\
\hline 00 & 8182 & 58.0000 & 11.6750 & 297.4 & 4 & 600.0 & 00.0 \\
\hline 00 & 8183 & 63.7485 & 9.1250 & 297.1 & 4 & 600.0 & 00.0 \\
\hline 00 & 8184 & 81.9285 & 6.9250 & 296.9 & 4 & 600.0 & 00.0 \\
\hline 00 & 8185 & 94.1287 & 4.3250 & 295.7 & 4 & 600.0 & 00.0 \\
\hline 00 & 8186 & 78.9276 & 2.9500 & 295.9 & 5 & 600.0 & 600.0 \\
\hline 00 & 8187 & 72.4985 & 7.0250 & 295.1 & 4 & 600.0 & 00.0 \\
\hline 00 & 8188 & 80.2633 & 7.8250 & 295.0 & 4 & 600.0 & 00.0 \\
\hline 00 & 8189 & 78.4505 & 6.0500 & 296.4 & 4 & 600.0 & .0 \\
\hline 00 & 81810 & 74.0007 & 7.4750 & 296.7 & 4 & 600.0 & 600.0 \\
\hline 00 & 81811 & 67.4968 & 6.9500 & 298.5 & 4 & 600.0 & \\
\hline 00 & 81812 & 77.5046 & 6.9000 & 299.2 & 4 & 600.0 & 600.0 \\
\hline 00 & 81813 & 70.7476 & 7.9750 & 299.6 & 4 & 600.0 & 0 \\
\hline 00 & 81814 & 77.2500 & 7.8250 & 300.0 & 4 & 600.0 & \\
\hline 00 & 81815 & 64.2499 & 8.4500 & 300.1 & 4 & 600.0 & 60 \\
\hline 00 & 81816 & 71.5000 & 8.9750 & 300.1 & 4 & 600.0 & 600.0 \\
\hline 00 & 81817 & 65.7499 & 9.9500 & 298.9 & 4 & 600.0 & \\
\hline 00 & 81818 & 64.2500 & 8.9250 & 298.4 & 4 & 600.0 & .0 \\
\hline 00 & 81819 & 65.5000 & 10.9250 & 296.8 & 4 & 600.0 & .0 \\
\hline 00 & 81820 & 65.2500 & 11.5500 & 296.3 & 4 & 600.0 & .0 \\
\hline 00 & 81821 & 66.0007 & 12.3000 & 296.2 & 4 & 0.0 & \\
\hline 00 & 81822 & 68.5014 & 8.9500 & 296.0 & 4 & 600.0 & 600.0 \\
\hline 00 & 81823 & 66.2501 & 7.900 & 296.4 & 4 & 600.0 & .0 \\
\hline 00 & 81824 & 70.5005 & 8.5250 & 295.7 & 4 & 600.0 & \\
\hline 00 & 8191 & 69.7478 & 8.4250 & 294.7 & 4 & 600.0 & 60 \\
\hline 00 & 8192 & 69.0000 & 8.5000 & 294.1 & 4 & 600.0 & .0 \\
\hline 00 & 8193 & 72.2501 & 8.4750 & 293.1 & 4 & 600.0 & \\
\hline 00 & 8194 & 62.7495 & 8.7750 & 293.2 & 4 & 600.0 & 60 \\
\hline 00 & 8195 & 66.2500 & 9.3000 & 292.6 & 4 & 600.0 & 60 \\
\hline 00 & 8196 & 70.5000 & 9.17 & 292.3 & 4 & 600.0 & \\
\hline 00 & 8197 & 68.5000 & 10.1000 & 292.8 & 4 & 600.0 & 60 \\
\hline 00 & 8198 & 75.7496 & 7.1500 & 293.6 & 4 & 600.0 & 600.0 \\
\hline 00 & 8199 & 81.2500 & 7.425 & 293.5 & 4 & 600.0 & .0 \\
\hline 00 & 81910 & 76.7500 & 6.8000 & 293.9 & 4 & 600.0 & .0 \\
\hline 00 & 81911 & 77.2508 & 5.5750 & 295.6 & 3 & 600.0 & 600.0 \\
\hline 00 & 81912 & 67.7504 & 7.1500 & 296.8 & 4 & 600.0 & \\
\hline 00 & 81913 & 93.0037 & 8.2250 & 297.1 & 4 & 600.0 & 600.0 \\
\hline 00 & 81914 & 69.5185 & 8.8000 & 297.7 & 4 & 600.0 & 600.0 \\
\hline 00 & 81915 & 58.7500 & 12.4500 & 297.5 & 4 & 600.0 & \\
\hline 00 & 81916 & 61.2501 & 12.4250 & 296.7 & 4 & 600.0 & 600.0 \\
\hline 00 & 81917 & 64.0000 & 13.0500 & 294.7 & 4 & 600.0 & 600.0 \\
\hline 00 & 81918 & 74.2497 & 11.5000 & 293.1 & 4 & 600.0 & 600.0 \\
\hline 00 & 81919 & 71.0023 & 11.4750 & 292.2 & 4 & 600.0 & 600.0 \\
\hline 00 & 81920 & 65.7501 & 12.6250 & 292.1 & 4 & 600.0 & 600.0 \\
\hline 00 & 81921 & 58.2502 & 12.3500 & 292.8 & 4 & 600.0 & 600.0 \\
\hline 00 & 81922 & 63.2500 & 11.7750 & 293.2 & 4 & 600.0 & 600.0 \\
\hline 00 & 81923 & 66.2500 & 10.0000 & 293.3 & 4 & 600.0 & 600.0 \\
\hline
\end{tabular}




\begin{tabular}{|c|c|c|c|c|c|c|c|}
\hline 0 & 81924 & 70.9994 & 9.3250 & 292.9 & 4 & 600.0 & 600.0 \\
\hline 00 & 8201 & 70.5000 & 8.7500 & 292.8 & 4 & 600.0 & 600.0 \\
\hline 00 & 8202 & 71.7493 & 500 & 291.7 & 4 & 600.0 & 00.0 \\
\hline 0 & 8203 & 74.4945 & 8.0250 & 291.1 & 4 & 600.0 & 00.0 \\
\hline 00 & 8204 & 79.2532 & .6000 & 291.1 & 4 & 600.0 & 00.0 \\
\hline 00 & 8205 & 76.4963 & 7.7250 & 291.2 & 4 & 600.0 & 00.0 \\
\hline 00 & 8206 & 83.5000 & 7.1000 & 291.2 & 4 & 600.0 & 0 \\
\hline 00 & 8207 & 85.0000 & 5.6750 & 291.7 & 4 & 600.0 & 00.0 \\
\hline 00 & 8208 & 108.5629 & 3.6500 & 293.1 & 3 & 600.0 & 00.0 \\
\hline 00 & 8209 & 258.2835 & 1.8250 & 293.9 & 2 & 600.0 & 0 \\
\hline 00 & 82010 & 182.1945 & 3.8250 & 294.2 & 2 & 600.0 & 00.0 \\
\hline 00 & 82011 & 171.2606 & 4.5500 & 295.3 & 3 & 600.0 & \\
\hline 00 & 82012 & 172.2493 & 5.7000 & 296.2 & 3 & 600.0 & 00.0 \\
\hline 00 & 82013 & 170.0000 & .9000 & 297.5 & 4 & 600.0 & 00.0 \\
\hline 00 & 82014 & 167.9952 & 00 & 298.6 & 4 & .0 & \\
\hline 00 & 82015 & 176.7725 & 5.4250 & 299.3 & 3 & 600.0 & .0 \\
\hline 00 & 82016 & 170.4997 & 5.3750 & 300.0 & 3 & 60 & 600.0 \\
\hline 00 & 82017 & 113.7751 & 4. & 300.5 & 3 & .0 & \\
\hline 00 & 82018 & 88.2438 & 5.8000 & 299.2 & 4 & 600.0 & .0 \\
\hline 00 & 82019 & 80.7498 & 7.0250 & 297.0 & 4 & 60 & .0 \\
\hline 00 & 82020 & 77.5013 & 7.7500 & 295.7 & 4 & 60 & \\
\hline 00 & 82021 & 69.7500 & 7.9250 & 295.4 & 4 & .0 & \\
\hline 00 & 82022 & 73.5009 & 9.1750 & 295.0 & 4 & 600.0 & .0 \\
\hline 00 & 82023 & 71.7502 & .7500 & 295.3 & 4 & 6 & \\
\hline 00 & 82024 & 74.7500 & 7. & 295.6 & 4 & .0 & \\
\hline 00 & 8211 & 73.7492 & 6.4500 & 296.0 & 4 & 600.0 & 600.0 \\
\hline 00 & 8212 & 78.7500 & 3.5250 & 296.5 & 4 & 600.0 & .0 \\
\hline 00 & 8213 & 76.7499 & 4.2000 & 296.6 & 5 & .0 & \\
\hline 00 & 8214 & 90.4948 & 2.3000 & 296.2 & 6 & .0 & 0.0 \\
\hline 00 & 8215 & 219.9463 & 0.5500 & 294.9 & 6 & .0 & .0 \\
\hline 00 & 8216 & 192.9654 & 0.0000 & 293.6 & 6 & .0 & \\
\hline 00 & 8217 & 80.4047 & 0.4750 & 293.8 & 5 & .0 & 600.0 \\
\hline 00 & 8218 & 218.5470 & 0.1250 & 295.9 & 4 & 600.0 & 600.0 \\
\hline 00 & 8219 & 240.4969 & 0.9000 & 297.0 & 3 & 600.0 & 0.0 \\
\hline 00 & 82110 & 236.7076 & 1.9000 & 298.2 & 2 & 600.0 & 600.0 \\
\hline 00 & 82111 & 232.2580 & 2.6000 & 298.5 & 2 & 600.0 & 600.0 \\
\hline 00 & 82112 & 193.9649 & 2.7750 & 299.7 & 1 & 600.0 & 600.0 \\
\hline 00 & 82113 & 199.7401 & 4.1250 & 300.3 & 2 & 600.0 & 600.0 \\
\hline 00 & 82114 & 182.7386 & 3.3750 & 301.3 & 2 & 600.0 & 600.0 \\
\hline 00 & 82115 & 191.0563 & 250 & 302.3 & 2 & 600.0 & 600.0 \\
\hline 00 & 82116 & 210.9845 & 3.6250 & 302.6 & 2 & 600.0 & 600.0 \\
\hline 00 & 82117 & 146.4677 & 3.5500 & 302.7 & 2 & 600.0 & 600.0 \\
\hline 00 & 82118 & 97.2319 & 4.7750 & 301.7 & 3 & 600.0 & 600.0 \\
\hline 00 & 82119 & 77.0012 & 5.5250 & 299.4 & 4 & 600.0 & 600.0 \\
\hline 00 & 82120 & 75.7500 & 6.9500 & 297.5 & 4 & 600.0 & 600.0 \\
\hline 00 & 82121 & 74.2500 & 500 & 297.1 & 4 & 600.0 & 600.0 \\
\hline 00 & 82122 & 72.2500 & 7.7750 & 296.6 & 2 & 600.0 & 600.0 \\
\hline 00 & 82123 & 71.0001 & 7.775 & 296.9 & 4 & 600.0 & 600.0 \\
\hline
\end{tabular}




\begin{tabular}{|c|c|c|c|c|c|c|c|}
\hline 0 & 2124 & 65.0014 & .9250 & 297.8 & 4 & 600.0 & \\
\hline & 8221 & 3.8383 & 0.3500 & 297.6 & & 600 & \\
\hline & 8222 & 58.1753 & .0250 & 296.9 & 6 & 00 & \\
\hline & 8223 & 110.5811 & .2750 & 295.6 & & 00.0 & \\
\hline & 224 & 00 & .4750 & 93.7 & & 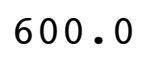 & \\
\hline & 8225 & 124.4780 & 500 & 293.5 & & 00 & \\
\hline & 8226 & 102.8058 & 0000 & 293.7 & & 0 & \\
\hline & 8227 & 85.9267 & .5250 & 95.3 & & & \\
\hline & 8228 & 287.0882 & .0500 & 298.6 & & 0 & \\
\hline & 8229 & 253.7380 & 1.7750 & 298.0 & & 0 & \\
\hline & 82210 & 240.4987 & 3.2750 & 296.4 & & 0 & \\
\hline & 82211 & 239.0005 & 2.7250 & .5 & & & \\
\hline & 82212 & 195.7010 & 3.8000 & 298.8 & & 0 & \\
\hline & 82213 & 184.2553 & 5.2500 & 300.1 & 3 & 0.0 & \\
\hline & 82214 & 155.29 & & & & & \\
\hline & 82215 & 155.2570 & 500 & 301.1 & 3 & 0 & \\
\hline & 82216 & 133.4260 & 4.45 & 30 & 3 & 0 & \\
\hline & 82217 & 105 . & .67 & .9 & & & \\
\hline 0 & 82218 & 96.5006 & 5.35 & 29 & 4 & 0 & \\
\hline & 82219 & 75.7499 & 0 & 297.3 & 4 & & \\
\hline & 82220 & 74 . & 0 & 29 & 4 & & \\
\hline & 82221 & 74.75 & 0 & 29 & 4 & & \\
\hline 0 & 82222 & 72.9993 & 2.1750 & 296.4 & 5 & & U. \\
\hline & 82223 & 54 & 0 & .4 & 4 & & \\
\hline & 82224 & 45. & 3. & 29 & 4 & & \\
\hline & 8231 & 7460 & 1.6000 & 296.2 & 5 & & \\
\hline & 8232 & 38. & .6000 & 295.1 & 6 & & \\
\hline 0 & 8233 & 76 . & .57 & 29 & 6 & & \\
\hline & 8234 & 81 . & 50 & 292.8 & 6 & & 0 . \\
\hline & 8235 & 150.4610 & 3250 & 292.4 & 6 & & \\
\hline & 8236 & 75 . & 0 & 292.0 & 6 & & \\
\hline 0 & 8237 & 42 . & 750 & 291.8 & 5 & & \\
\hline & 8238 & 243.1887 & 0.7750 & 293.3 & 4 & 0 & 0 . \\
\hline & 8239 & 283. & 1.17 & 29 & 3 & & \\
\hline & 82310 & 239. & 0 & 29 & 2 & & \\
\hline & 82311 & 230.2498 & 2.5750 & 295.8 & 1 & & \\
\hline & 82312 & 231.4983 & 2.4500 & 297.0 & 1 & & \\
\hline 0 & 82313 & 76.2532 & 000 & 298.3 & 2 & & 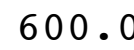 \\
\hline & 82314 & 74.7504 & 5.5000 & 298.1 & 3 & 0 & 0 . \\
\hline & 82315 & 75 . & 000 & 297.2 & 4 & & \\
\hline 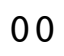 & 82316 & 78.50 & 500 & 297.2 & 4 & & \\
\hline 0 & 82317 & 67.9979 & 9.1500 & 295.8 & 4 & 600.0 & 00 . \\
\hline & 82318 & 74.7500 & 250 & 294.6 & 4 & 600 & 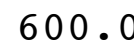 \\
\hline 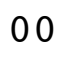 & 82319 & 79.2500 & 9.5750 & 293.2 & 4 & & ros \\
\hline 00 & 82320 & 75.5000 & 9.7500 & 291.8 & 4 & 600.0 & 00 \\
\hline & 82321 & 79.7505 & 6.9500 & 292.9 & 4 & 600 & 0 \\
\hline & 82322 & 75 & 000 & 294.3 & 4 & & \\
\hline & 82323 & 78.7441 & 4.5000 & 294.5 & 4 & 600.0 & 600 \\
\hline
\end{tabular}




\begin{tabular}{|c|c|c|c|c|c|c|c|}
\hline 0 & 2324 & 8.5000 & 2.7250 & 294.6 & 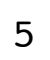 & 00 & \\
\hline 0 & 8241 & 62481 & 1.2750 & 292.7 & & & \\
\hline & 8242 & 000 & 5750 & 292.8 & & 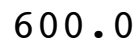 & \\
\hline & 8243 & 108.4395 & 3000 & 292.6 & & 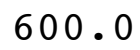 & \\
\hline & $824 \quad 4$ & & 500 & 93.6 & & & \\
\hline & 8245 & 67 & 3000 & 92.8 & & & \\
\hline & 8246 & 209 & 4250 & 291.9 & & & \\
\hline & 8247 & 163 & 750 & 93.5 & & & \\
\hline & 8248 & 196. & 0.8000 & 295.9 & & & \\
\hline & 8249 & 210 . & 750 & 295.7 & & & \\
\hline & 82410 & 162 . & 250 & 295.0 & & & \\
\hline & 82411 & 167 & & 295.9 & & & \\
\hline & 82412 & 163. & 4.8250 & 297.5 & & & \\
\hline & 82413 & 169. & 1500 & 299.2 & & & \\
\hline & 82414 & & & & & & \\
\hline & 82415 & 162 & 500 & 301.7 & & & \\
\hline & 82416 & 173. & .8250 & 302.7 & & & \\
\hline & 82417 & 164. & 50 & 02.7 & & & \\
\hline & 82418 & 170 . & 0 & 302.2 & & & \\
\hline & 82419 & 163 & 0 & 301.1 & & & \\
\hline & 82420 & 365 & 0 & .8 & & & \\
\hline & 82421 & 71 & & 9.4 & 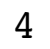 & & \\
\hline & 82422 & 500 & 250 & 299.1 & & & \\
\hline & 82423 & 000 & 0 & 9.2 & & & \\
\hline & 82424 & & & & : & & \\
\hline & 825 & 75 & 750 & 299.5 & & & \\
\hline & 8252 & 86 & 50 & 300.0 & & & \\
\hline & 8253 & 67 & 0 & 299.4 & 6 & & \\
\hline & 8254 & 58 & 50 & .3 & & & \\
\hline & 8255 & & .750 & 299.4 & & & \\
\hline & 8256 & 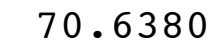 & 750 & 25 & 4 & & \\
\hline & 8257 & 12 & 50 & .3 & 3 & & \\
\hline & 8258 & 242 . & 3500 & 302.3 & & & \\
\hline & 8259 & 20 & 750 & 302.1 & 1 & & \\
\hline & 82510 & 16 & 50 & 299.9 & 2 & & \\
\hline & 82511 & 164 . & 5.7250 & 299.9 & 3 & & \\
\hline & 82 & 16 & 3000 & 301.1 & 4 & & \\
\hline 0 & 82513 & 162. & 500 & 302.0 & 4 & & \\
\hline & 82514 & 161. & 5.4000 & 303.6 & 3 & & \\
\hline & 82515 & 160 & 250 & 304 & 3 & & \\
\hline & 82516 & 112 & 500 & 304 & 3 & & \\
\hline & 82517 & 92 . & 6.1250 & 304.0 & 4 & & \\
\hline & 82518 & 8 & 6.7250 & 302.3 & 4 & & \\
\hline & 82519 & 72 . & 7.4000 & 301.0 & 4 & & \\
\hline 0 & 82520 & 000 & 9.7000 & 300.4 & 4 & & \\
\hline & 82 & 67. & 9.6750 & 299.6 & 4 & & \\
\hline & 82 & 70 & .2750 & & 4 & & \\
\hline & 82523 & 65.0001 & 10.8750 & 298.1 & 4 & 600.0 & 00 \\
\hline
\end{tabular}




\begin{tabular}{|c|c|c|c|c|c|c|c|}
\hline 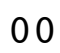 & 2524 & 2500 & 8500 & 297.7 & 4 & 000 & \\
\hline & 8261 & 2.5000 & 9.8750 & 298.2 & \pm & 600.0 & \\
\hline & 8262 & 60.7501 & 250 & 298.5 & 4 & 00 & \\
\hline & 8263 & 61.0011 & 4000 & 298.4 & & 00 & \\
\hline & 264 & 7.1491 & 1000 & 98.0 & & & \\
\hline & 8265 & 101.9970 & 750 & 97.8 & & 0 & \\
\hline & 8266 & 4786 & 9750 & 299.5 & & 00 & \\
\hline & 8267 & 80.2492 & 2750 & 99.5 & & & \\
\hline & 8268 & 228.6575 & 3500 & 01.4 & & & \\
\hline & 269 & 271.9777 & 1.1750 & 301.7 & & 0 & \\
\hline & 2610 & 241.2132 & 2.8000 & 29 & & 0.0 & \\
\hline & 82611 & 233.75 & 2.4500 & .3 & & & \\
\hline & 82612 & 234.2507 & 2500 & 301.4 & 3 & 0 & \\
\hline & 2613 & 196.9924 & 3.8250 & 302.8 & & 0.0 & \\
\hline & 2614 & 79 . & 0 & & & & \\
\hline & 2615 & 97 . & & 9 & 3 & & \\
\hline & 2616 & 82.7497 & 8500 & 303.3 & 3 & .0 & \\
\hline & 2617 & 80 & 250 & 0 & 4 & & \\
\hline & 82618 & 89.2 & 750 & 4 & 4 & & \\
\hline & 82619 & 58.0000 & 7.0750 & 298.6 & 4 & 0 & . \\
\hline & 82620 & 69.5037 & 3250 & 297.2 & 4 & 0 & \\
\hline & 82621 & 66.24 & 0 & 297.1 & 4 & & \\
\hline & 82622 & 60.50 & 3250 & 29 & 4 & & \\
\hline & 82623 & 67.4994 & 9.3750 & 296.7 & 4 & 0 & \\
\hline & 82624 & 69.7 & & & 4 & & \\
\hline & 8271 & 73. & 50 & 29 & 4 & & \\
\hline & 8272 & 62.7511 & 7500 & 298.6 & 4 & & . \\
\hline & 8273 & 92.239 & 250 & 297 & 5 & & \\
\hline & 8274 & 114 & 500 & 29 & 5 & & \\
\hline & 8275 & 69.6529 & 000 & 297.3 & 6 & & J. \\
\hline & 8276 & 91.0138 & 5000 & 297.1 & 6 & & 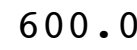 \\
\hline & 8277 & 317.13 & 0 & $29 \varepsilon$ & 5 & & \\
\hline & 8278 & 233.8079 & 0 & 30 & 4 & & 0 . \\
\hline & 8279 & 195.9286 & 3500 & 300.7 & 3 & 0 & 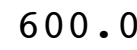 \\
\hline & 82710 & 189. & 0 & & 2 & & \\
\hline & 82711 & 166.2403 & 750 & 299.3 & 3 & & \\
\hline & 82712 & 165.4724 & 1000 & 300.6 & 4 & & \\
\hline & 82713 & 170.2823 & 6 . & 302.4 & 4 & & \\
\hline & 82714 & 120.5302 & 4.6750 & 304.0 & 3 & & 0 . \\
\hline & 82715 & 144.2542 & 250 & 303.6 & 4 & & \\
\hline & 82716 & 118.7727 & 000 & 304.5 & 3 & & \\
\hline & 82717 & 98.0009 & 5.8500 & 303.5 & 3 & & 0 . \\
\hline & 82718 & 78.4997 & 6.7250 & 301.4 & 4 & 0 & 0 . \\
\hline & 82719 & 78.4998 & 500 & 299.2 & 4 & & \\
\hline r & 82720 & 76.5001 & 7.5250 & 298.3 & 4 & & \\
\hline & 82721 & 72.2500 & 7.2500 & 298.4 & 4 & & 00 \\
\hline & & 69 . & & 298.3 & 4 & & \\
\hline & 82723 & 69.7500 & 8.5500 & 299.1 & $\Psi$ & 600.0 & $60 \Omega$ \\
\hline
\end{tabular}




\begin{tabular}{|c|c|c|c|c|c|c|c|}
\hline 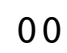 & 2724 & 676 & 750 & 9.8 & $\Lambda$ & 证 & \\
\hline 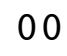 & 3281 & 0.7484 & 6250 & 299.7 & 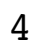 & 600 & \\
\hline & 8282 & 78.7650 & 1000 & 298.6 & & 00 & \\
\hline & 8283 & 149.5298 & 5250 & 296.2 & & 00 & \\
\hline & 8284 & 86 & 750 & & & & \\
\hline & 8285 & 74 & & 94.6 & & 0 & \\
\hline & 8286 & 77.7298 & 2250 & 294.5 & & 0 & \\
\hline & 8287 & 170.5109 & 1.7250 & 95.9 & & & \\
\hline & $828 \quad 8$ & 323 & 0.3500 & 98.4 & & & \\
\hline & 8289 & 323. & 1.5500 & 299.4 & & 0 & \\
\hline & 82810 & 273 & 1.7750 & 99.8 & & & \\
\hline & 82811 & 270 & 2.0500 & 00.1 & & & \\
\hline & 32812 & 735 & 3.3250 & 300.6 & & 0 & \\
\hline & 82813 & 89.7488 & 4.4000 & 9 & & & \\
\hline & 82814 & & & 2 & & & \\
\hline & 8281 & & & 01.9 & & & \\
\hline & 828 & 75.2472 & 6.4750 & 01.6 & & P & \\
\hline & 82817 & 00 & 50 & 0.4 & & 0 & \\
\hline & 8281 & & & 9.4 & & & \\
\hline & 82819 & 999 & 1250 & 298 & & & \\
\hline & 82820 & 000 & 3000 & 5 & 2 & & \\
\hline & 82821 & & 0 & & 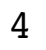 & & \\
\hline & 82822 & 73 & 9.1250 & $29^{\circ}$ & & & \\
\hline & 82823 & 73 & 9.8000 & 297.3 & 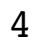 & & \\
\hline & 82824 & & 9.9000 & 296.8 & 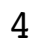 & & \\
\hline & 829 & 74 & 50 & 29 & 4 & & \\
\hline & 8292 & 75 & 6750 & 296.4 & 4 & & \\
\hline & 8293 & 75 & 9250 & 294.8 & 4 & & \\
\hline & 8294 & 69 & 00 & 29 & 4 & & \\
\hline & 8295 & 029 & 0.1750 & 292.2 & 4 & & \\
\hline & 8296 & 497 & 6.6750 & 292.3 & 4 & & \\
\hline & 8297 & & 50 & 292 & 3 & & \\
\hline & 8298 & 37 & 1.4250 & 25 & 2 & & \\
\hline & 8299 & 73 & 3750 & 29 & 1 & & \\
\hline & 82910 & & 250 & & 1 & & \\
\hline & 82911 & & 2.1500 & & 2 & & \\
\hline & 82912 & & .9500 & 289.7 & 2 & & \\
\hline & 82913 & & 7.5000 & 292.4 & 3 & & \\
\hline & 82914 & 21 & 2.5250 & 292.3 & 2 & & \\
\hline & 82915 & 45 & 2.7000 & 289.6 & 2 & & \\
\hline & 82916 & 27 & 2.8750 & 289.3 & 2 & & \\
\hline & 82917 & 205 & 1.9250 & 288.5 & 1 & & \\
\hline & 82918 & 241 & 5.0000 & 288.4 & 2 & 0 & \\
\hline & 82919 & 00 & 5.4000 & 290.3 & 3 & & \\
\hline & 82920 & 430 & 3.0500 & 292.0 & 4 & & \\
\hline & 8292 & 100 & 3.8500 & 293.2 & 4 & 600.0 & \\
\hline & 8 & & & & 4 & & \\
\hline & 202 & 0000 & 8.1500 & 293.5 & 4 & 600.0 & r \\
\hline
\end{tabular}




\begin{tabular}{|c|c|c|c|c|c|c|c|}
\hline & 2924 & 499 & 3250 & 294.8 & 4 & 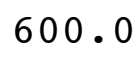 & \\
\hline & 8301 & 5.7449 & 5.6000 & 295.3 & & 600.0 & \\
\hline & 3302 & 1.1178 & 3000 & 293.4 & & 00 & \\
\hline & 303 & 30.7077 & 3250 & 289.1 & & 00 & \\
\hline & 304 & 7.9382 & 000 & 88.8 & & & \\
\hline & 8305 & 9752 & .4250 & 87.8 & & 0 & \\
\hline & 8306 & 7641 & 000 & 287.5 & & 00 & \\
\hline & 307 & 5.4979 & 0 & 87.4 & & & \\
\hline & 308 & 950 & & 87.7 & & & \\
\hline & 309 & 993 & 3000 & 88.3 & & 0 & \\
\hline & 3010 & 9.5000 & 8.1000 & $28 \varepsilon$ & & & \\
\hline & 11 & 1.7502 & 0 & & & & \\
\hline & 2 & 77.2505 & 750 & 290 & & & \\
\hline & 3013 & 88.2517 & 4.30 & 292 & & & \\
\hline & 14 & 97 & & 7 & & & \\
\hline & 5 & 97 . & & 293.3 & & & \\
\hline & 16 & 78.2498 & 0 & 292.8 & 4 & & \\
\hline & 17 & 89 & 0 & .1 & & & \\
\hline & 3018 & 72 . & 0 & 0 & & & \\
\hline & 9 & 71.7500 & 0 & 285 & 4 & & \\
\hline & 83020 & 67.2500 & 9750 & 288.8 & 4 & & \\
\hline & 830 & 71.7495 & 0 & & $\mathbf{4}$ & & \\
\hline & 83022 & 71. & 3000 & 28 & 4 & & \\
\hline & 83023 & 508 & 9.82 & .5 & 4 & & \\
\hline & 83024 & 999 & & & 4 & & \\
\hline & 8311 & 76 . & 0 & 28 & 4 & & \\
\hline & 8312 & 71.5003 & 5250 & 287.9 & 4 & & 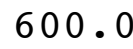 \\
\hline & 8313 & 88.2924 & & 7.3 & 4 & & \\
\hline & 8314 & 99 & 0 & 28 & 4 & & \\
\hline & 8315 & 71. & 0 & 2 & 4 & & 0 \\
\hline & 8316 & 69.7500 & 7.90 & .8 & 4 & & . \\
\hline & 8317 & 83 & & & 4 & & \\
\hline & 8318 & 506 & & 28 & 4 & & 。 \\
\hline & 8319 & 506 & 0 & 28 & 4 & & \\
\hline & & 32 & & & 3 & & \\
\hline & & 114 . & & 291.8 & $?$ & & \\
\hline & 83112 & 317 & 7250 & 293.2 & 2 & & \\
\hline & 83113 & 6 & & 293 & 3 & & \\
\hline & 83114 & 89. & 4.7250 & 294.3 & 3 & & . \\
\hline & & 42 & 00 & 293.8 & 4 & & \\
\hline & & 380 & 500 & 292.8 & 4 & & \\
\hline & & 69.2534 & 8.0000 & 292.0 & 4 & & \\
\hline & 83118 & 74.7494 & 9.0250 & 290.8 & 4 & & 0 . \\
\hline & & 982 & & 285 & 4 & & \\
\hline & & 63.5000 & 1.2750 & 288.8 & 4 & & \\
\hline & 83121 & 499 & 0.3500 & 288.1 & 4 & & 0 . \\
\hline & & & & & 4 & & \\
\hline & 83123 & 59.2792 & 9.6250 & 287.6 & $I$ & 600.0 & 600 \\
\hline
\end{tabular}




\begin{tabular}{|c|c|c|c|c|c|c|c|c|}
\hline 00 & & 3124 & 41.0000 & 8.0000 & 287.4 & 4 & 600.0 & 600.0 \\
\hline 00 & 9 & 11 & 60.0023 & 9.6500 & 286.9 & 4 & 600.0 & 600.0 \\
\hline 00 & 9 & 12 & 66.2500 & 8.1500 & 286.8 & 4 & 600.0 & 00.0 \\
\hline 00 & 9 & 13 & 78.2562 & 6.6750 & 286.6 & 4 & 600.0 & 600.0 \\
\hline 00 & 9 & 14 & 76.9932 & 4.9000 & 286.4 & 4 & 600.0 & 00.0 \\
\hline 00 & 9 & 15 & 39.0025 & 2.5000 & 286.7 & 4 & 600.0 & 500.0 \\
\hline 00 & 9 & 16 & 96.5765 & 1.1750 & 286.8 & 5 & 600.0 & 600.0 \\
\hline 00 & 9 & 17 & 220.1738 & 0.7000 & 287.0 & 0 & 600.0 & 500.0 \\
\hline 00 & 9 & 18 & 227.6229 & 0.8750 & 289.7 & 5 & 600.0 & 00.0 \\
\hline 00 & 9 & 19 & 232.4510 & 2.8000 & 289.6 & 4 & 600.0 & 600.0 \\
\hline 00 & 9 & 110 & 270.9671 & 4.0750 & 289.8 & 3 & 600.0 & 600.0 \\
\hline 00 & 9 & 111 & 277.9857 & 2.3500 & 290.3 & 2 & 600.0 & \\
\hline 00 & 9 & 112 & 95.3301 & 2.0750 & 290.4 & 1 & 600.0 & 600.0 \\
\hline 00 & 9 & 113 & 58.1633 & 2.0500 & 288.5 & 2 & 600.0 & 600.0 \\
\hline 00 & 9 & 114 & 52.2315 & 2.3750 & 288.7 & 2 & 600.0 & \\
\hline 00 & 9 & 115 & 336.8139 & 0.7000 & 288.9 & 1 & 600.0 & 60 \\
\hline 00 & 9 & 116 & 81.0258 & 4.2750 & 288.1 & 2 & 600.0 & 600.0 \\
\hline 00 & 9 & 117 & 92.0000 & 5.2500 & 287.3 & 3 & 600.0 & .0 \\
\hline 00 & 9 & 118 & 91.7519 & 5.6250 & 287.1 & 4 & 600.0 & 60 \\
\hline 00 & 9 & 119 & 92.2494 & 4.3750 & 286.7 & 4 & 600.0 & 600.0 \\
\hline 00 & 9 & 120 & 86.2719 & 3.8000 & 287.1 & 4 & 600.0 & .0 \\
\hline 00 & 9 & 121 & 86.5018 & 6.1000 & 286.0 & 4 & 600.0 & \\
\hline 00 & 9 & 122 & 67.5000 & 7.9250 & 285.5 & 4 & 600.0 & 600.0 \\
\hline 00 & 9 & 123 & 53.7602 & 5.7500 & 285.9 & 4 & 600.0 & .0 \\
\hline 00 & 9 & 124 & 42.5005 & 3.8500 & 286.6 & 4 & 600.0 & \\
\hline 00 & 9 & 21 & 306.4284 & 1.0750 & 286.7 & 5 & 600.0 & 600.0 \\
\hline 00 & 9 & 22 & 189.2217 & 0.9000 & 286.3 & 6 & 600.0 & 600.0 \\
\hline 00 & 9 & 23 & 164.9915 & 1.8250 & 285.7 & 5 & 600.0 & .0 \\
\hline 00 & 9 & 24 & 348.0589 & 0.5500 & 285.8 & 5 & 600.0 & 600.0 \\
\hline 00 & 9 & 25 & 333.5000 & 1.1250 & 285.5 & 4 & 600.0 & 600.0 \\
\hline 00 & 9 & 26 & 22.9828 & 1.1500 & 285.4 & 5 & 600.0 & .0 \\
\hline 00 & 9 & 27 & 338.7880 & 0.2750 & 285.5 & 5 & 600.0 & 60 \\
\hline 00 & 9 & 28 & 275.2235 & 0.2500 & 286.1 & 4 & 600.0 & 600.0 \\
\hline 00 & 9 & 29 & 333.9050 & 0.9750 & 288.1 & 3 & 600.0 & .0 \\
\hline 00 & 9 & 210 & 304.7507 & 2.0000 & 290.2 & 2 & 600.0 & 60 \\
\hline 00 & 9 & 211 & 189.0414 & 3.5500 & 290.0 & 2 & 600.0 & 600.0 \\
\hline 00 & 9 & 212 & 259.5379 & 2.9750 & 290.2 & 1 & 600.0 & 600.0 \\
\hline 00 & 9 & 213 & 281.0000 & 3.3500 & 291.4 & 2 & 600.0 & 600.0 \\
\hline 00 & 9 & 214 & 290.0332 & 2.5500 & 291.2 & 2 & 600.0 & 600.0 \\
\hline 00 & 9 & 215 & 269.0484 & 1.7500 & 292.2 & 1 & 600.0 & \\
\hline 00 & 9 & 216 & 230.3737 & 1.7250 & 293.0 & 1 & 600.0 & 600.0 \\
\hline 00 & 9 & 217 & 256.8177 & 1.9250 & 292.3 & 1 & 600.0 & 600.0 \\
\hline 00 & 9 & 218 & 158.7168 & 4.2250 & 291.3 & 2 & 600.0 & 600.0 \\
\hline 00 & 9 & 219 & 97.0005 & 4.8000 & 290.5 & 3 & 600.0 & 600.0 \\
\hline 00 & 9 & 220 & 76.9993 & 6.1500 & 289.2 & 4 & 600.0 & 600.0 \\
\hline 00 & 9 & 221 & 71.0000 & 6.4250 & 288.3 & 4 & 600.0 & 600.0 \\
\hline 00 & 9 & 222 & 67.5000 & 7.7500 & 287.8 & 4 & 600.0 & 600.0 \\
\hline 00 & 9 & 223 & 67.9998 & 8.125 & 287.3 & 4 & 600.0 & 600.0 \\
\hline
\end{tabular}




\begin{tabular}{|c|c|c|c|c|c|c|c|c|}
\hline 0 & 9 & 224 & 64.7505 & 7.9000 & 287.2 & 4 & 600.0 & 600.0 \\
\hline 00 & 9 & 31 & 68.7504 & 8.4250 & 287.4 & 4 & 600.0 & 600.0 \\
\hline 00 & 9 & 32 & 68.7502 & 9.0500 & 287.2 & 4 & 600.0 & 00.0 \\
\hline 00 & 9 & 33 & 70.0000 & 8.7250 & 287.1 & 4 & 600.0 & 00.0 \\
\hline 00 & 9 & 34 & 66.2500 & 8.2250 & 286.9 & 4 & 600.0 & 00.0 \\
\hline 00 & 9 & 35 & 68.0000 & 8.9500 & 286.9 & 4 & 600.0 & 00.0 \\
\hline 00 & 9 & 36 & 69.5000 & 9.8500 & 286.3 & 4 & 600.0 & 00.0 \\
\hline 00 & 9 & 37 & 67.2504 & 9.5000 & 286.3 & 4 & 600.0 & .0 \\
\hline 00 & 9 & 38 & 68.5005 & 8.2500 & 286.8 & 4 & 600.0 & 600.0 \\
\hline 00 & 9 & 39 & 68.2503 & 5.5750 & 288.8 & 3 & 600.0 & 600.0 \\
\hline 00 & 9 & 310 & 72.5018 & 5.6000 & 289.6 & 3 & 600.0 & \\
\hline 00 & 9 & 311 & 87.2511 & 5.5750 & 290.4 & 3 & 600.0 & 600.0 \\
\hline 00 & 9 & 312 & 95.7441 & 5.9000 & 290.8 & 3 & 600.0 & 0 \\
\hline 00 & 9 & 313 & 97.2239 & 6.0500 & 291.7 & 4 & 600.0 & 00.0 \\
\hline 00 & 9 & 314 & 90.4982 & 6.3250 & 292.0 & 4 & 600.0 & 60 \\
\hline 00 & 9 & 315 & 95.7479 & 6.3000 & 292.3 & 4 & 600.0 & 600.0 \\
\hline 00 & 9 & 316 & 74.5006 & 6.7000 & 292.0 & 4 & 600.0 & .0 \\
\hline 00 & 9 & 317 & 72.2480 & 7.0250 & 291.4 & 4 & .0 & \\
\hline 00 & 9 & 318 & 60.2499 & 9.1750 & 289.9 & 4 & 600.0 & 600.0 \\
\hline 00 & 9 & 319 & 61.0001 & 0.3750 & 288.1 & 4 & 600.0 & .0 \\
\hline 00 & 9 & 320 & 61.9998 & 9.4500 & 287.3 & 4 & .0 & \\
\hline 00 & 9 & 321 & 69.0021 & 8.9500 & 286.8 & 4 & 600.0 & 60 \\
\hline 00 & 9 & 322 & 65.2500 & 8.1250 & 286.5 & 4 & 600.0 & .0 \\
\hline 00 & 9 & 323 & 77.3131 & 6.3250 & 286.3 & 4 & .0 & \\
\hline 00 & 9 & 324 & 77.5032 & 7.1500 & 286.5 & 4 & .0 & 60 \\
\hline 00 & 9 & 41 & 68.0000 & 8.2000 & 286.3 & 4 & 600.0 & 600.0 \\
\hline 00 & 9 & 42 & 69.0002 & 7.3000 & 286.1 & 4 & 600.0 & .0 \\
\hline 00 & 9 & 43 & 66.7485 & 6.9250 & 286.0 & 4 & 600.0 & 60 \\
\hline 00 & 9 & 44 & 65.2502 & 7.2500 & 286.1 & 4 & 600.0 & 600.0 \\
\hline 00 & 9 & 45 & 68.2500 & 6.4750 & 286.1 & 4 & 600.0 & 60 \\
\hline 00 & 9 & 46 & 69.9897 & 5.8250 & 286.0 & 4 & 600.0 & 60 \\
\hline 00 & 9 & 47 & 99.7546 & 4.5750 & 286.1 & 4 & 600.0 & 600.0 \\
\hline 00 & 9 & 48 & 69.4992 & 7.7000 & 287.0 & 4 & 600.0 & 600.0 \\
\hline 00 & 9 & & 89.4293 & 7.4750 & 287.8 & 4 & 600.0 & 600.0 \\
\hline 00 & 9 & 410 & 61.5067 & 7.6500 & 288.2 & 4 & 600.0 & 600.0 \\
\hline 00 & 9 & 411 & 61.0002 & 7.3000 & 289.4 & 4 & 600.0 & 600.0 \\
\hline 00 & 9 & 412 & 70.1920 & 6.5750 & 290.7 & 4 & 600.0 & 600.0 \\
\hline 00 & 9 & 413 & 82.9936 & 6.8500 & 291.2 & 4 & 600.0 & 600.0 \\
\hline 00 & 9 & 414 & 92.5008 & 5.9000 & 292.0 & 3 & 600.0 & 600.0 \\
\hline 00 & 9 & 415 & 89.7483 & 5.0500 & 292.8 & 3 & 600.0 & 600.0 \\
\hline 00 & 9 & 416 & 92.9997 & 5.7250 & 292.7 & 3 & 600.0 & 600.0 \\
\hline 00 & 9 & 417 & 76.4992 & 6.9000 & 291.8 & 4 & 600.0 & 600.0 \\
\hline 00 & 9 & 418 & 74.2506 & 8.7250 & 289.8 & 4 & 600.0 & 600.0 \\
\hline 00 & 9 & 419 & 76.2502 & 8.5000 & 289.0 & 4 & 600.0 & 600.0 \\
\hline 00 & 9 & 420 & 72.9979 & 7.9750 & 288.9 & 4 & 600.0 & 600.0 \\
\hline 00 & 9 & 421 & 70.4999 & 8.1250 & 288.3 & 4 & 600.0 & 600.0 \\
\hline 00 & 9 & 422 & 70.7493 & 8.3000 & 288.3 & 4 & 600.0 & 600.0 \\
\hline 00 & y & 423 & 72.2501 & 7.875 & 288.4 & 4 & 600.0 & 600.0 \\
\hline
\end{tabular}




\begin{tabular}{|c|c|c|c|c|c|c|c|c|}
\hline 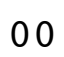 & 9 & 24 & 78.5000 & 7.2750 & 288.7 & \pm & 0 & \\
\hline 0 & 9 & 51 & 1.2332 & $; .1000$ & 289.3 & & ח & \\
\hline 0 & 9 & 52 & 1033 & 4500 & 289.9 & & 0 & \\
\hline & 9 & 53 & 55.0006 & 4500 & 288.8 & & 00 & \\
\hline & 9 & 54 & 50 & 50 & 88.0 & & & \\
\hline & 9 & 5 & 03 & 00 & 287.3 & & 0 & \\
\hline & 9 & 56 & 160 . & 7.6250 & 286.9 & & & \\
\hline & 9 & 57 & 161. & 6000 & 86.9 & & 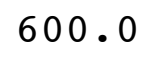 & \\
\hline & 9 & 58 & 159. & .7250 & 87.7 & & 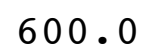 & \\
\hline & 9 & 59 & 161.7499 & 3.4000 & 289.0 & & & \\
\hline & 9 & 510 & 160.9977 & .1000 & 89.9 & & 0 & \\
\hline & 9 & 511 & 166 & & 90.9 & & & \\
\hline & 9 & 512 & 171 . & 7.5250 & 292.3 & & 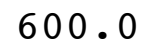 & \\
\hline & 9 & 513 & 174.4971 & 6.5750 & 293.8 & & 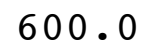 & \\
\hline & 9 & 514 & 181 & & & & & \\
\hline & 9 & 515 & 181. & 5250 & 295.3 & 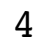 & & \\
\hline & 9 & 516 & 179 . & 7.7250 & 295.6 & 4 & & \\
\hline & 9 & 517 & 178 & 0 & 29 & 4 & & \\
\hline & 9 & 518 & 179 & 7 . & 295.9 & 4 & & \\
\hline & 9 & 519 & 176. & 4.6250 & 294.7 & 4 & & \\
\hline & 9 & 520 & 03 & 0 & 293.6 & 4 & & \\
\hline & 9 & 521 & 20 & 50 & 293.2 & 4 & & \\
\hline & 9 & 522 & 167 . & 6.0750 & 293.3 & 4 & & \\
\hline & 9 & 523 & 88 & 0 & 293.7 & 4 & & \\
\hline & 9 & 524 & 17 & & 293.7 & 4 & & \\
\hline & 9 & 61 & 500 & 50 & 293 & 4 & & \\
\hline & 9 & 62 & 000 & 8.8500 & 293.1 & 4 & & \\
\hline 0 & 9 & 63 & 18 & 50 & 292.1 & 4 & & \\
\hline & 9 & 64 & 179 & 50 & 291.2 & 4 & & \\
\hline & 9 & 65 & 180 & 4000 & 290.9 & 4 & & \\
\hline & 9 & 66 & 180 & 0 & 290.6 & 4 & & \\
\hline & 9 & 67 & 18 & 50 & 290.7 & 4 & & \\
\hline & 9 & 68 & 180 . & 9.2500 & 291.1 & 4 & 0 & \\
\hline & 9 & 69 & 175 & 00 & 292.0 & 4 & & \\
\hline & 9 & 610 & 175 & 0 & 29 & 4 & & \\
\hline & 9 & 611 & 175 . & 000 & 294.0 & 4 & & \\
\hline & 9 & 612 & 982 & 6.4500 & 295.0 & 4 & & \\
\hline 0 & 9 & 613 & 166 & 50 & 296.2 & 4 & 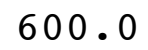 & \\
\hline & 9 & 614 & 166. & 6.3500 & 297.5 & 4 & & \\
\hline & 9 & 615 & 173 & 2500 & 297.9 & 4 & & \\
\hline & 9 & 616 & 182 & 3000 & 298.3 & 4 & & \\
\hline 0 & 9 & 617 & 186 & 5.0250 & 298.4 & 3 & 0 & \\
\hline & 9 & 618 & 184.9990 & 3.2500 & 298.4 & 4 & & \\
\hline & 9 & 619 & 164 & 1.7750 & 297 & 5 & & \\
\hline 0 & 9 & 620 & 128.4914 & 1.2000 & 296.4 & 4 & 60 & \\
\hline & 9 & 621 & 122.9959 & 1.2250 & 296.8 & 4 & & \\
\hline & 9 & 622 & 474 & 1.7500 & 29 & 5 & & \\
\hline & 9 & 623 & 85.0000 & 3.1250 & 296.8 & 4 & 600.0 & $c$ \\
\hline
\end{tabular}




\begin{tabular}{|c|c|c|c|c|c|c|c|c|}
\hline & 9 & 24 & & & 297.0 & 5 & & \\
\hline 0 & 9 & 71 & 74.9166 & .6500 & 296.7 & 0 & 00 & \\
\hline & 9 & 72 & 50.9587 & 1.2250 & 296.4 & & 0 & \\
\hline & 9 & 3 & 0.7677 & L. 3250 & 296.7 & & 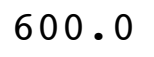 & \\
\hline & 9 & 74 & 129.2893 & 5000 & 294.8 & & 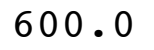 & \\
\hline & 9 & 75 & 53.7653 & 000 & 294.6 & & 0 & \\
\hline & 9 & 6 & 55.5093 & 2.7250 & 293.5 & & & \\
\hline & 9 & 77 & 7 & .6750 & 94.2 & & & \\
\hline & 9 & 78 & 150.5415 & 9000 & 295.9 & & 0 & \\
\hline & 9 & 9 & 0000 & 3750 & 296.5 & & & \\
\hline & 9 & 710 & 161.49 & & & & & \\
\hline & 9 & 711 & 162 . & 6.7750 & 297.8 & & 0 & \\
\hline ) & 9 & 712 & 165.5000 & .7250 & 299 & & 8.0 & \\
\hline & 9 & 713 & 173.7409 & 0 & 300.3 & & .0 & \\
\hline 0 & 9 & 714 & 185 & 0 & & & & \\
\hline & 9 & 715 & 175.5000 & .7250 & 301.9 & & 0.0 & \\
\hline & 9 & 716 & 181.2682 & 4 . & 4 & & & \\
\hline & 9 & 717 & 181.5 & & & & & \\
\hline ) & 9 & 718 & 182.4959 & 2000 & 30 & & 0.0 & 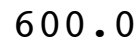 \\
\hline & 9 & 719 & 161. & 0 & 4 & & 0 & \\
\hline & 9 & 720 & 114 . & & & & & \\
\hline 0 & 9 & 721 & 73.23 & 0 & 29 & & & \\
\hline 0 & 9 & 722 & 69.75 & 0 & 298.2 & & .0 & \\
\hline & 9 & 723 & 66.74 & & & & & \\
\hline 0 & 9 & 724 & 66 & 50 & 2 & 4 & & \\
\hline 0 & 9 & 81 & 67.74 & 0 & 298.3 & 4 & $\cdot 0$ & \\
\hline & 9 & 82 & 70 . & 0 & 4 & 4 & & \\
\hline 0 & 9 & 83 & 70 . & 50 & .5 & 4 & & \\
\hline 0 & 9 & 84 & 68 . & 0 & 298.3 & 4 & 0.0 & \\
\hline & 9 & 85 & 68 . & 0 & .4 & 4 & & \\
\hline 0 & 9 & 86 & 64 & & 0 & 4 & & \\
\hline & 9 & 87 & 999 & & .2 & 4 & • 0 & \\
\hline 0 & 9 & 88 & 35.7616 & 1.8500 & 299.9 & 2 & 0 & \\
\hline 0 & 9 & 89 & 279 . & 0 & 4 & 2 & & \\
\hline & 9 & 810 & 238.5000 & 000 & 300.3 & - & .0 & \\
\hline 0 & 9 & 811 & 227.2495 & 3750 & 301.0 & 1 & .0 & \\
\hline 0 & 9 & 812 & 240 . & 0 & .1 & 2 & & \\
\hline 0 & 9 & 813 & 97 . & 4.5500 & 302.8 & 3 & .0 & \\
\hline 0 & 9 & 814 & 77 . & 9250 & .1 & 2 & .0 & \\
\hline & 9 & 815 & 83 . & 0 & .8 & 3 & & \\
\hline 0 & 9 & 816 & 88 . & 8.0250 & 302.6 & 4 & & \\
\hline 0 & 9 & 817 & 85.9982 & 7.6250 & 301.1 & 4 & 0.0 & \\
\hline 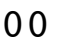 & 9 & 818 & 78.2508 & 500 & 298 & 4 & & \\
\hline 00 & 9 & 819 & 74.0000 & 9.1750 & 298.1 & 4 & 600.0 & \\
\hline 0 & 9 & 820 & 70.0000 & 0.1500 & 296.1 & 4 & 600.0 & \\
\hline & 9 & 821 & & & & & & \\
\hline 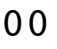 & 9 & 822 & 60. & & 297.3 & 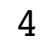 & 600 & \\
\hline & & 823 & 64.2500 & 9.6750 & 296.8 & 4 & 600.0 & 0 \\
\hline
\end{tabular}




\begin{tabular}{|c|c|c|c|c|c|c|c|}
\hline 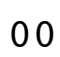 & 24 & 3.0000 & 9.1000 & 296.3 & 4 & . & \\
\hline & $\begin{array}{lll}9 & 9 & 1\end{array}$ & 0.7498 & .1750 & 96.0 & & & \\
\hline & $\begin{array}{lll}9 & 9 & 2\end{array}$ & 68.7501 & 10.9750 & 295.6 & 1 & 0 & \\
\hline & $\begin{array}{lll}9 & 9 & 3\end{array}$ & 67.2500 & 8500 & 295.3 & & 00 & \\
\hline & $\begin{array}{lll}9 & 9 & 4\end{array}$ & 0 & & & & & \\
\hline & 995 & 62.9962 & 500 & 95.6 & & 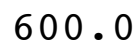 & \\
\hline & 996 & 67.2500 & 3250 & 295.7 & & & \\
\hline & $\begin{array}{lll}9 & 9 & 7\end{array}$ & 0.4933 & 7500 & 95.2 & & & \\
\hline & $\begin{array}{lll}9 & 9 & 8\end{array}$ & 269.8283 & 0.3000 & 296.9 & & 0 & \\
\hline & $\begin{array}{lll}9 & 9 & 9\end{array}$ & 299.6827 & 3250 & 297.7 & & 0 & \\
\hline & 9910 & 230.7496 & 250 & 95.7 & & & \\
\hline & 9911 & 228.0005 & & 7 & & & \\
\hline & 9912 & 184.6791 & 500 & 298.1 & & 0 & \\
\hline & 9913 & 170.4683 & 4.3250 & 298.7 & & 0 & \\
\hline & 9914 & 8.4967 & & & & & \\
\hline & 9915 & 187.2417 & 250 & 300 & 3 & & \\
\hline & 9916 & 134.1120 & 0 & 301.5 & & & \\
\hline & 9917 & 110 . & 0 & 30 & & & \\
\hline & 9918 & 79. & 4 . & 29 & 3 & & \\
\hline & 9919 & 75 & 0 & 296.2 & 4 & & \\
\hline & 9920 & 73. & 0 & 29 & 4 & & \\
\hline & 9921 & 60 . & & 29 & 4 & & \\
\hline & 9922 & 38.0005 & 750 & 29 & 4 & 0 & U. \\
\hline & 9923 & 45 . & 0 & 29 & 5 & & \\
\hline & 9924 & & & 29 & 4 & & \\
\hline & 9101 & 47.4724 & 750 & 29 & 5 & & \\
\hline & 9102 & 56.1549 & 2000 & 294.5 & 6 & & \\
\hline 0 & 9103 & 113.9753 & 0 & 293.1 & 6 & & \\
\hline 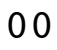 & 9104 & 142 . & 0 & 291.7 & 5 & & \\
\hline & 9105 & 176.2501 & 000 & 290.5 & 5 & & \\
\hline & 9106 & 146.6826 & 0 & 291.0 & 4 & & \\
\hline & 9107 & 147 . & 0 & 292 & 4 & & \\
\hline & 9108 & 156.7445 & 000 & 293.2 & 3 & 0 & 0 . \\
\hline & 9109 & 289.5816 & 0 & 295.6 & 2 & & \\
\hline & 91010 & 239. & 0 & 29 & 3 & & \\
\hline & 91011 & 214.6277 & 500 & 297.5 & 2 & & \\
\hline & 91012 & 171.7585 & 000 & 297.7 & 3 & & \\
\hline 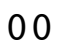 & 91013 & 168.4992 & 0 & 298.4 & 3 & & 0 • \\
\hline & 91014 & 169.0000 & 000 & 299.7 & 3 & 0 & 0 . \\
\hline & 91015 & 155.2573 & & 300.9 & 4 & & \\
\hline & 91016 & 135.4673 & 0 & 300 & 3 & & \\
\hline 0 & 91017 & 124.9887 & 3.8750 & 300.9 & 2 & 0 & . \\
\hline & 91018 & 115.4920 & 500 & 300.2 & 3 & 0 & \\
\hline & 91019 & 79.4983 & 4.3000 & 298.4 & 4 & & \\
\hline 0 & 91020 & 70.7502 & 6.4250 & 297.3 & 4 & 600 & 0 \\
\hline & 91021 & 69.7497 & 500 & 297.7 & 4 & 600 & \\
\hline & 91022 & 77 & & 298.1 & 4 & & \\
\hline & 91023 & 72.0011 & 4.8250 & 298.0 & 4 & 600.0 & 600 \\
\hline
\end{tabular}




\begin{tabular}{|c|c|c|c|c|c|c|c|}
\hline 0 & 1024 & 7229 & 2.2000 & 297.8 & 5 & 600.0 & \\
\hline & 9111 & 112.0001 & 0.4500 & 296.3 & & 00.0 & \\
\hline & 9112 & 130.4156 & 4250 & 295.6 & & 00 & \\
\hline & 9113 & 47.2359 & 3000 & 295.7 & & 00.0 & \\
\hline & 114 & 30.7485 & .3000 & 96.5 & & 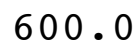 & \\
\hline & 115 & 44.4066 & .8750 & 4 & & 00 & \\
\hline & 116 & 107.2581 & 0.4500 & 293.9 & & 00 & \\
\hline & 117 & 13.9438 & 750 & 94.2 & & & \\
\hline & 118 & 356.4275 & 1250 & 98.4 & & & \\
\hline & 119 & 281.1699 & 000 & 299.7 & & 00 & 0 \\
\hline & 1110 & 210.9769 & 1000 & 298.4 & & 0.0 & \\
\hline & 1111 & 175.5015 & .1500 & 98.7 & & 0 & \\
\hline & 1112 & 191.5960 & 2.8000 & 300.0 & & 0 & \\
\hline & 1113 & 169.4506 & 3.6000 & 301.3 & & 0.0 & 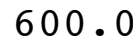 \\
\hline & 91114 & 187. & & & & & \\
\hline & 1115 & 194. & & .6 & & & \\
\hline & 91116 & 199.7503 & 0250 & 303.0 & & .0 & 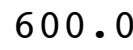 \\
\hline & 1117 & 190 . & 0 & .9 & & & \\
\hline & 91118 & 195 . & 50 & .3 & & & \\
\hline & 91119 & 158 . & 2.5250 & 300.9 & 4 & 0 & $\checkmark$ \\
\hline & 91120 & 144.0000 & .9250 & .8 & & 0 & \\
\hline & 91121 & 90.7113 & & & 4 & & \\
\hline & 91122 & 86.74 & 00 & 29 & 4 & & \\
\hline & 91123 & 71.4997 & 2.9000 & 299.6 & 4 & 0 & \\
\hline & 91124 & 91 . & & 299.3 & 5 & & \\
\hline & 9121 & 73. & 2.3000 & .1 & 5 & & \\
\hline & 9122 & 65.2488 & 1.2750 & 299.1 & 5 & 0 & . \\
\hline & 9123 & 50.3410 & 2750 & 29 & 5 & & \\
\hline & 9124 & 11 . & 0.9250 & .7 & 5 & & \\
\hline & 9125 & 10.5000 & 1.1000 & 297.3 & 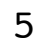 & 0 & U. \\
\hline & 9126 & 21.2448 & 0.3250 & 297.0 & 4 & & 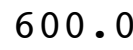 \\
\hline & 9127 & 10.5083 & .1500 & 29 & 5 & & \\
\hline & 9128 & 318.5652 & 1750 & 300.8 & 4 & 0 & . \\
\hline & 9129 & 236.2522 & 5000 & 300.8 & 3 & 0 & . \\
\hline & 91210 & 243.0103 & 000 & & 2 & & \\
\hline & 91211 & 234.0000 & 5000 & 300.4 & 3 & & 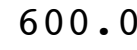 \\
\hline & 91212 & 155.6823 & 3.5750 & 298.3 & 2 & & 0 . \\
\hline 0 & 91213 & 159. & 1.9250 & 30 & 1 & & . \\
\hline 00 & 91214 & 160.7491 & 3.9250 & 300.5 & 2 & .0 & 0 . \\
\hline & 91215 & 165.2542 & 2.3250 & 300.4 & 1 & & 0 . \\
\hline & 91216 & 185.8749 & 1.5500 & 300.4 & $\perp$ & & 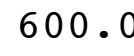 \\
\hline & & 163.2793 & 2.9250 & 299.5 & 1 & & 0 . \\
\hline & 91218 & 189.6463 & 2.6250 & 299.7 & 2 & 0 & 0 \\
\hline & 91219 & 131.4839 & 3250 & 299.1 & 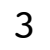 & & \\
\hline 0 & 91220 & 95.4763 & 3.4750 & 299.9 & 4 & & 0 . \\
\hline & 91221 & 129.7559 & 3.2750 & 299.9 & 5 & 600 & 00 . \\
\hline & & 151.5025 & & 300.5 & 5 & & \\
\hline & 91223 & 152.2475 & 2.6000 & 300.4 & & 600.0 & 60 \\
\hline
\end{tabular}




\begin{tabular}{|c|c|c|c|c|c|c|c|}
\hline 0 & 24 & & & & & & \\
\hline 0 & 131 & 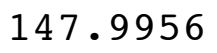 & 9250 & 0.8 & & & \\
\hline & 9132 & 155.7451 & 1000 & 299.8 & & 00 & \\
\hline & 133 & 35.9915 & 0.0000 & 299.4 & & 00 & \\
\hline & 134 & 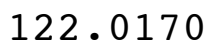 & .750 & & & & \\
\hline & 135 & 29.51 & 0 & 299.3 & & 0 & \\
\hline & 136 & 33.4651 & 500 & 299.1 & & (n) & \\
\hline & 137 & 51. & 2.22 & 98.5 & & & \\
\hline & 138 & 4 & 22 & 97.5 & & & \\
\hline 0 & 139 & 53. & 3 . & 298.2 & & 0 & \\
\hline O & 1310 & 2.24 & 4.32 & 299 & & & \\
\hline 0 & 1311 & 772 & 0 & 300.3 & & & \\
\hline 0 & 13 & 59 & 0 & 301 & & & \\
\hline 0 & 1313 & 57.25 & & 302 & & & \\
\hline & 1314 & 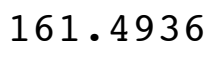 & & 303 & & & \\
\hline 0 & 13 & & & 303 & & & \\
\hline 0 & 1316 & 72 & & 304.1 & & .0 & \\
\hline 0 & 1317 & 1 & & 304.7 & & & \\
\hline 0 & 1318 & & & 303.1 & & & \\
\hline 0 & 13 & & & 300.8 & 4 & & 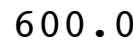 \\
\hline 0 & 913 & 994 & 0 & 300.3 & 4 & . 0 & \\
\hline 0 & 913 & & & 300.2 & 4 & & \\
\hline 0 & 913 & & & 30 & 4 & & \\
\hline 0 & 91323 & 4 & 6 . & 299.5 & 4 & 0 & \\
\hline 0 & 91324 & & & 297.9 & & & \\
\hline 0 & 914 & 221 & & 296 & 6 & & \\
\hline 0 & 9142 & 709 & & 295.3 & 6 & .0 & \\
\hline 0 & 9143 & 67. & & 294.8 & 5 & & \\
\hline 0 & 9144 & 46 & & 294.3 & 4 & & \\
\hline & 9145 & 69 & & 293.4 & 4 & & \\
\hline 0 & 9146 & 772 & 4 . & 293.6 & 4 & & \\
\hline 0 & 9147 & 66. & 0 & 292.9 & 4 & & \\
\hline & 9148 & 89 & & 293.4 & 4 & & \\
\hline 0 & 9149 & 005 & 0 & 293.9 & 4 & 0 & \\
\hline ח & 91410 & 84 & & 295.4 & 3 & & \\
\hline 0 & & 7 & & 294.2 & 4 & & \\
\hline 0 & 91412 & 498 & & 294.4 & 4 & & \\
\hline 0 & 91413 & 7 & & 294.9 & 4 & & \\
\hline 0 & & 98 & & 295.6 & 4 & .0 & \\
\hline & 91415 & 7 & 7.7250 & 296.3 & 4 & & \\
\hline 0 & & 81 . & 8 . & 295.8 & 4 & & \\
\hline 0 & & & & 295.2 & 4 & & \\
\hline 0 & 91418 & 70.9989 & 9.3000 & 294.3 & 4 & & 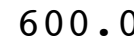 \\
\hline 00 & & 500 & .4250 & 293.0 & 4 & & \\
\hline 00 & & & 10.2500 & 292.2 & 4 & .0 & \\
\hline 00 & 91421 & 68.9998 & 10.8500 & 292.1 & 4 & .0 & 500 \\
\hline & & & 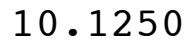 & 291.8 & 4 & & \\
\hline & 91423 & 69.4998 & 10.3500 & 291.1 & & .0 & 600 \\
\hline
\end{tabular}




\begin{tabular}{|c|c|c|c|c|c|c|c|}
\hline 0 & 91424 & 68.7499 & 10.5500 & 291.1 & 4 & 600.0 & 600.0 \\
\hline 00 & 9151 & 68.7496 & 10.6250 & 290.6 & 4 & 600.0 & 00.0 \\
\hline 00 & 9152 & 70.7500 & 10.8750 & 290.6 & 4 & 600.0 & 00.0 \\
\hline 0 & 9153 & 69.7500 & 10.6250 & 290.6 & 4 & 600.0 & 00.0 \\
\hline 00 & 9154 & 74.5005 & 500 & 290.2 & 4 & 600.0 & 00.0 \\
\hline 00 & 9155 & 72.7502 & 12.7750 & 289.3 & 4 & 600.0 & 00.0 \\
\hline 00 & 9156 & 63.0001 & 11.2750 & 289.3 & 4 & 600.0 & 00.0 \\
\hline 00 & 9157 & 70.7500 & 10.3000 & 289.9 & 4 & 600.0 & 00.0 \\
\hline 00 & 9158 & 72.7499 & 10.2000 & 290.8 & 4 & 600.0 & 00.0 \\
\hline 00 & 9159 & 80.2515 & 750 & 291.4 & 4 & 600 & 0 \\
\hline 00 & 91510 & 81.2508 & 250 & 292.9 & 4 & 600.0 & \\
\hline 00 & 91511 & 96.5013 & 500 & 293.9 & 4 & .0 & \\
\hline 00 & 91512 & 95.7500 & 500 & 295.1 & 4 & 600.0 & 00.0 \\
\hline 00 & 91513 & 101.9986 & 6.8500 & 296.4 & 4 & 600.0 & 00.0 \\
\hline 00 & 91514 & 102 . & 250 & 296.7 & 4 & .0 & \\
\hline 00 & 91515 & 101.0000 & 7.2250 & 296.5 & 4 & 600.0 & .0 \\
\hline 00 & 91516 & 82.5107 & 7.2000 & 296.6 & 4 & 60 & .0 \\
\hline 00 & 91517 & 73.7488 & 7. & 296.2 & 4 & .0 & \\
\hline 00 & 91518 & 69.2438 & 9.1250 & 294.8 & 4 & .0 & \\
\hline 00 & 91519 & 65.0002 & 10.4500 & 293.5 & 4 & .0 & .0 \\
\hline 00 & 91520 & 64.5000 & 10 . & 293 & 4 & 6 & \\
\hline 00 & 91521 & 66.7500 & 750 & 293.3 & 4 & .0 & \\
\hline 00 & 91522 & 68.5000 & 9.7750 & 292.8 & 4 & 600.0 & .0 \\
\hline 00 & 91523 & 68.7485 & 9 . & 292.6 & 4 & 0 & \\
\hline 00 & 91524 & 63.5000 & 10 . & 291.9 & 4 & .0 & \\
\hline 00 & 9161 & 67.2500 & 000 & 291.3 & 4 & 600.0 & .0 \\
\hline 00 & 9162 & 66.4990 & 9.3000 & 291.5 & 4 & 600.0 & .0 \\
\hline 00 & 9163 & 73.7501 & 8 . & 292.8 & 4 & .0 & \\
\hline 00 & 9164 & 74.7500 & 500 & 291.5 & 4 & .0 & .0 \\
\hline 00 & 9165 & 71.2498 & 8.0500 & 291.3 & 4 & 6 & .0 \\
\hline 00 & 9166 & 79.0115 & 750 & 291.8 & 4 & .0 & \\
\hline 00 & 9167 & 82.7500 & 5 . & 292.6 & 4 & .0 & 0.0 \\
\hline 00 & 9168 & 38.0375 & 2.1000 & 293.8 & 3 & 600.0 & 0.0 \\
\hline 00 & 9169 & 267.9415 & 1.3250 & 294.8 & 2 & .0 & 0.0 \\
\hline 00 & 91610 & 199.9425 & 3. & 294.7 & 2 & 600.0 & 600.0 \\
\hline 00 & 91611 & 165.2499 & 6.4000 & 294.8 & 3 & 600.0 & 600.0 \\
\hline 00 & 91612 & 163.7501 & 5.800 & 295.5 & 3 & 600.0 & 600.0 \\
\hline 00 & 91613 & 170.2483 & 5.8250 & 297.3 & 3 & 600.0 & 600.0 \\
\hline 00 & 91614 & 174.9931 & 5.9000 & 297.9 & 3 & 600.0 & 600.0 \\
\hline 00 & 91615 & 174.5010 & 5 . & 298.8 & 3 & 600.0 & 600.0 \\
\hline 00 & 91616 & 178.5017 & 5.7000 & 299.1 & 3 & 600.0 & 600.0 \\
\hline 00 & 91617 & 167.4994 & 5.8000 & 299.1 & 3 & 600.0 & 600.0 \\
\hline 00 & 91618 & 175.5193 & 5.9500 & 298.7 & 4 & 600.0 & 600.0 \\
\hline 00 & 91619 & 140.0000 & 3.0250 & 297.6 & 5 & 600.0 & 600.0 \\
\hline 00 & 91620 & 79.7229 & 2.7250 & 297.5 & 4 & 600.0 & 600.0 \\
\hline 00 & 91621 & 85.7519 & 2.7250 & 297.7 & 5 & 600.0 & 600.0 \\
\hline 00 & 91622 & 79.4945 & 2.9500 & 297.7 & 5 & 600.0 & 600.0 \\
\hline 00 & 91623 & 72.4992 & 3.525 & 297.8 & 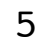 & 600.0 & 600.0 \\
\hline
\end{tabular}




\begin{tabular}{|c|c|c|c|c|c|c|c|}
\hline 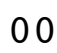 & 1624 & 290 & 3000 & 297.7 & 5 & 600.0 & \\
\hline & 9171 & 129.2498 & 2.5000 & 296.8 & & 00.0 & \\
\hline & 9172 & 126.2504 & 1000 & 297.1 & & 00 & \\
\hline & 9173 & 128.4997 & 9000 & 296.7 & & 00.0 & \\
\hline & 174 & 118 . & 3500 & 96.5 & & $v$ & \\
\hline & 175 & 129.5023 & 50 & 295.8 & & 0 & \\
\hline & 9176 & 122.7324 & 8750 & 295.8 & & 00 & \\
\hline & 177 & 157.7908 & 3000 & 95.6 & & & \\
\hline & 178 & 150.2718 & .5000 & .0 & & & \\
\hline & 9179 & 170.0046 & 8.5250 & 296.0 & & 0 & U \\
\hline & 1710 & 160.9979 & 6.6500 & 297.2 & & 00.0 & \\
\hline & 1711 & 163.9995 & 507 & 98.3 & & & \\
\hline & 91712 & 5202 & 6.3000 & 299.6 & & .0 & \\
\hline & 1713 & 165.2505 & 6.7500 & 300.9 & & 0.0 & \\
\hline & 91714 & 163 & & 302.3 & & & \\
\hline & 1715 & 157 . & & 30 & & & \\
\hline & 91716 & 161.2557 & 4.40 & 303.6 & & .0 & . \\
\hline & 1717 & 158.2473 & 0 & .8 & & 0 & \\
\hline & 91718 & 155.99 & & 303.3 & & & \\
\hline & 91719 & 153.5901 & 2.4750 & 301.8 & & .0 & 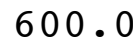 \\
\hline & 91720 & 126.9945 & 1.2000 & 301.0 & & .0 & \\
\hline & 91721 & 97 . & & .2 & & & \\
\hline & 91722 & 84 . & 500 & .6 & & & \\
\hline & 91723 & 93.2983 & 2.02 & 301.6 & & 0 & \\
\hline & 91724 & 74.4928 & & 301 & & & \\
\hline & $918 \quad 1$ & 40.2 & 2 . & 302.2 & 5 & & \\
\hline & 9182 & 26.4999 & 5250 & 302.8 & & 0 & J. \\
\hline & 9183 & 113.6095 & 0 & 1.1 & & & \\
\hline & 9184 & 158.2049 & 3 . & 298.3 & 4 & & e. \\
\hline & 9185 & 108.2192 & 2.9000 & 298.8 & & 0 & 0. \\
\hline & 9186 & 153.7294 & 3.4000 & 298.3 & & & s. \\
\hline & 9187 & 152 . & 3 & 298.2 & 5 & & \\
\hline & 9188 & 153. & 2.25 & 299.9 & 4 & 0 & . \\
\hline & 9189 & 204.1565 & 50 & 302.4 & 3 & 0 & 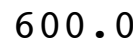 \\
\hline & 91810 & 243.2506 & 2 . & 302.5 & 3 & & \\
\hline & 91811 & 250.9927 & 2 . & 303.5 & 2 & & . \\
\hline & 91812 & 209.7181 & 2750 & 304.1 & 2 & & 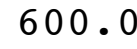 \\
\hline 2 & 91813 & 176 . & 4 . & 304.5 & 3 & & 0 . \\
\hline 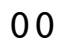 & 91814 & 167.7209 & 4.3000 & 305.6 & 3 & .0 & 0 . \\
\hline & 91815 & 168.2488 & 1250 & 306.2 & 3 & & 0 . \\
\hline & 91816 & 175.9995 & 5.7000 & 306.2 & 3 & & 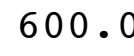 \\
\hline & 91817 & 171.0000 & 5.4000 & 306.2 & 3 & & 0 . \\
\hline & 91818 & 160.7781 & 3.3500 & 305.8 & 4 & 0 & 0 \\
\hline & 91819 & 119.8131 & 1.8500 & 304.0 & 5 & & \\
\hline 00 & 91820 & 121.7778 & 2.3000 & 303.8 & 6 & & 0 . \\
\hline & 91821 & 137.0000 & 3.0000 & 303.9 & 5 & 0 & 00 . \\
\hline & 91822 & 85 & & 303.7 & 4 & & \\
\hline & 91823 & 73.0000 & 6.6000 & 302.9 & $\Psi$ & 600.0 & 60 \\
\hline
\end{tabular}




\begin{tabular}{|c|c|c|c|c|c|c|c|}
\hline 0 & 1824 & 2.7502 & 8.1500 & 302.8 & 4 & 600.0 & \\
\hline 0 & 9191 & 0.4998 & 8.5750 & 303.3 & . & 600.0 & \\
\hline & 9192 & 72.2496 & 3000 & 303.9 & 4 & 0 & \\
\hline & 193 & 75.7500 & .4000 & 303.7 & & 00 & \\
\hline & 194 & 799 & .3500 & 3.9 & & 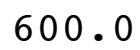 & \\
\hline & 9195 & 7425 & 750 & 302.9 & & 0 & \\
\hline & 196 & 23.8281 & 250 & 302.7 & & 00 & \\
\hline & 9197 & 101.7483 & .3000 & 301.7 & & 0 & \\
\hline & 9198 & 122.5097 & 6.7250 & 302.0 & 4 & 00 & \\
\hline & 9199 & 155.5107 & 7.2500 & 302.2 & & 0 & \\
\hline & 1910 & 160.7497 & 00 & 302.9 & & & \\
\hline & 1911 & 165.2501 & & 303.7 & 3 & & \\
\hline 0 & 91912 & 158.2505 & 750 & 305.2 & 3 & 0 & \\
\hline & 1913 & 152 . & 0 & & 3 & & \\
\hline & 1914 & 151. & & & 3 & & \\
\hline 0 & 91915 & 152 . & & 30 & 3 & 0 & \\
\hline 0 & 91916 & 166.0023 & 4.87 & 308.5 & 3 & 0 & \\
\hline & 91917 & 170 . & & & 3 & & \\
\hline 0 & 91918 & 136 . & & 30 & 4 & & \\
\hline 0 & 91919 & 76 . & 0 & 30 & 4 & 0 & \\
\hline & 91920 & 72 & & & 4 & & \\
\hline 0 & 91921 & 64 . & 7 . & & 4 & & \\
\hline 0 & 91922 & 70.7545 & 0 & 30 & 4 & 0 & \\
\hline & 91923 & 72 . & 0 & & 4 & & \\
\hline 0 & 91924 & & & & 4 & & \\
\hline & 9201 & 74 . & & & 4 & & \\
\hline 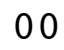 & 9202 & 943 & 7 . & 4.2 & 4 & & \\
\hline & 9203 & 125 . & 2 . & & 5 & & \\
\hline & 9204 & 534 & & 301.0 & 6 & & \\
\hline & 9205 & 157 . & 0 & .9 & 5 & & \\
\hline & 9206 & 156. & 0 & & 4 & & \\
\hline & 9207 & 149. & 1 . & .3 & 5 & & \\
\hline & $920 \quad 8$ & 88.9197 & 0 & 2.3 & 4 & & \\
\hline 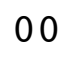 & 9209 & 112 . & 0 & 30 & 3 & & \\
\hline & 92010 & 145 & & & 3 & & \\
\hline & 92011 & 163.2515 & & 4.0 & 4 & & \\
\hline & 92012 & 174 . & 6 . & 4.7 & 4 & & \\
\hline & 92013 & 185.2499 & 7. & 306.0 & 4 & & 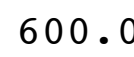 \\
\hline & 92014 & 179.0000 & 5.6750 & 307.3 & 3 & & 6 \\
\hline & 92015 & 125 . & & & 3 & & \\
\hline & 92016 & 78 & & 7.8 & 3 & & \\
\hline & 92017 & 77.2450 & 5.4750 & 307.0 & 3 & 0 & 0 \\
\hline & 92018 & 69.0011 & 250 & 304.2 & 4 & & \\
\hline 0 & 92019 & 62.0000 & 9.2500 & 301.9 & 4 & & 600 \\
\hline 0 & 92020 & 59.7500 & 9.0500 & 302.6 & 4 & 600.0 & 00 \\
\hline & 92021 & & & & 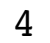 & & \\
\hline & 92022 & 64.4997 & 9.6750 & 299.8 & 4 & & 600 \\
\hline & 92023 & 67.2539 & 9.6750 & 296.3 & & 600.0 & 60 \\
\hline
\end{tabular}




\begin{tabular}{|c|c|c|c|c|c|c|c|}
\hline 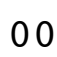 & 2024 & 9.9099 & 5.9750 & 295.9 & 4 & 0 & \\
\hline & 9211 & 32.7512 & 8.7750 & 292.1 & & & \\
\hline & 9212 & 80.4928 & .9250 & 290.2 & 4 & v & \\
\hline & 9213 & 3.7624 & 1.1500 & 291.4 & & 00 & \\
\hline & 214 & 8.2720 & 0 & 90.5 & & & \\
\hline & 9215 & 177.8047 & 50 & 90.7 & & 0 & \\
\hline & 9216 & 137.0768 & & 87.1 & & & \\
\hline & 9217 & 9.7505 & 6.8250 & 87.7 & & & \\
\hline & 9218 & 79.7304 & 7.10 & 88.6 & & & \\
\hline & 9219 & 57.8657 & & 290.7 & & & \\
\hline & 92110 & 356.6033 & 1.87 & 93.6 & & & \\
\hline & 92111 & 226.3506 & & 93.0 & & & \\
\hline & 92112 & 208.3567 & 0 & 294.4 & & 0 & \\
\hline & 92113 & 195.6907 & 0 & 296 & & 0 & \\
\hline & 92114 & 344.5304 & & 2 & & & \\
\hline & 92115 & 345.2701 & & 297.4 & & & \\
\hline & 92116 & 46.1704 & 3.77 & 297.0 & & & \\
\hline & 92117 & 79.72 & 4 . & 296 & & & \\
\hline & 92118 & 85.7554 & & 294.1 & 4 & & \\
\hline & 92119 & 76.0021 & 0 & 292.2 & 4 & & \\
\hline & 92120 & 39.75 & 0 & 291.3 & 4 & & \\
\hline & 92121 & 63. & & 29 & 4 & & \\
\hline & 92122 & 63.0430 & 0 & 289 & 4 & & U. \\
\hline & 92123 & 79 & & 2 & 4 & & \\
\hline & 92124 & 3 & & 28 & 5 & & \\
\hline & 9221 & 5035 & 0 & 287.6 & 4 & & \\
\hline & 9222 & 339.50 & 0 & 287 & 5 & & \\
\hline 0 & 9223 & 310 . & & 28 & 5 & & \\
\hline & 9224 & 297 . & & 28 & 4 & & \\
\hline & 9225 & 280.0221 & & 287.5 & 5 & & \\
\hline & 9226 & 288.78 & & & 6 & & \\
\hline & 9227 & 260 & & 28 & 6 & & \\
\hline & 9228 & 23.8156 & & 288.9 & 5 & 0 & . \\
\hline & 9229 & 318 . & 0 & 25 & 4 & & \\
\hline & 92210 & 31 & & & 3 & & \\
\hline & 92211 & 229.8846 & & 290.7 & 2 & & \\
\hline & 92212 & 220.1806 & 0 & 291.8 & 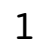 & & \\
\hline 0 & 92213 & 111.9355 & 0 & 291.7 & 2 & & \\
\hline & 92214 & 94.5518 & & 293.2 & 2 & 0 & 0 . \\
\hline & 92215 & 086 & & 292.5 & 3 & & \\
\hline & 92216 & 123.1281 & & 292.2 & 3 & & \\
\hline 0 & 92217 & 91.0021 & 5.9500 & 291.2 & 3 & 60 & 0. \\
\hline & 92218 & 70.74 & & 289.9 & 4 & 0 & \\
\hline & 92219 & 69.7499 & 7.2250 & 288.6 & 4 & & \\
\hline 0 & 92220 & 76.7504 & 6.6000 & 288.4 & 4 & 600 & 0 \\
\hline & 92221 & 70.5009 & 4.6750 & 288 & 4 & 600 & \\
\hline & 92222 & .7477 & & & 4 & & \\
\hline & 92223 & 78.5008 & 3.3750 & 289.5 & 4 & 600.0 & 600 \\
\hline
\end{tabular}




\begin{tabular}{|c|c|c|c|c|c|c|c|}
\hline 0 & 2224 & 102 & 500 & & & & \\
\hline 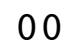 & 9231 & 86.7390 & .9750 & 289.0 & 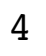 & 600.0 & \\
\hline & 9232 & 129.2937 & 5500 & 289.5 & 5 & 00 & \\
\hline & 9233 & 156.2515 & 4500 & 289.2 & & 00 & \\
\hline & 9234 & 144.0277 & 250 & 88.9 & & & \\
\hline & 9235 & 163 & 250 & 88.7 & & 0 & \\
\hline & 9236 & 104.1814 & 500 & 288.4 & & 00 & \\
\hline & 9237 & 150.2987 & 500 & 88.5 & & & \\
\hline & 9238 & 157.7495 & 3500 & 89.7 & & 0 & \\
\hline & 9239 & 171.0000 & 9250 & 90.5 & & $v$ & \\
\hline & 92310 & 167.2501 & 5.6000 & 91.1 & & 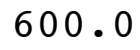 & \\
\hline & 92311 & 165 & 0 & 91.7 & & & \\
\hline & 92312 & 168 & 50 & 292.9 & & 0 & \\
\hline & 92313 & 170 . & 5.8250 & 294.0 & & 0 & \\
\hline & 92314 & 17 & & 9 & & & \\
\hline & 9231 & 17 & & 295.7 & & & \\
\hline & 92316 & 181 . & 0 & 295.9 & 3 & 0 & \\
\hline & 92317 & 17 & 50 & 9 & 3 & 0 & \\
\hline & 92318 & 18 & & 295.4 & 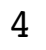 & & \\
\hline & 92319 & 180.7533 & 4.1250 & 294.2 & 4 & 0 & \\
\hline & 92320 & 160 & 1.8500 & 294.0 & J & 0 & \\
\hline & 9232 & 79 & & & 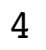 & & \\
\hline & 92322 & 499 & 0 & 292.9 & 4 & & \\
\hline & 92323 & 77 & 5.9000 & 292.2 & 4 & 0 & \\
\hline & 92324 & 0 & & 291.4 & 4 & & \\
\hline & 924 & 00 & 7 . & 291.3 & 4 & & \\
\hline & 9242 & 000 & 7.1250 & 291.1 & 4 & 0 & \\
\hline & 9243 & 499 & 0 & 290.9 & 4 & & \\
\hline & $924 \quad 4$ & 01 & 5 . & 291.7 & 4 & & \\
\hline & 9245 & 501 & 00 & 291.6 & 4 & 0 & \\
\hline & 9246 & 946 & 3.5000 & 291.5 & 5 & 0 & \\
\hline & 9247 & 3 & 1 . & 291.9 & 6 & & \\
\hline & 9248 & 367 & 0 . & 294.3 & 5 & 0 & \\
\hline & 9249 & 004 & 7750 & 295.1 & 4 & 0 & \\
\hline & 92410 & 16 & & & 3 & & \\
\hline & 92411 & 551 & 4.6000 & 5.2 & 3 & 600 & \\
\hline & 92412 & 177 & 4.8750 & 295.6 & 3 & 0 & \\
\hline & 92413 & 510 & 4.1500 & 296.7 & 3 & 0 & \\
\hline 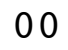 & 92414 & 702 & 4.1250 & 297.6 & 3 & 600.0 & \\
\hline & 92415 & 515 & 3000 & 297.8 & 3 & & \\
\hline & 92416 & 969 & 750 & 298.2 & 2 & 60 & \\
\hline 0 & 92417 & 595 & 4.1500 & 297.9 & 3 & 600.0 & \\
\hline & 92418 & 198.5110 & 3.7000 & 297.2 & 4 & 600 & \\
\hline & 92419 & 109 & 3.1250 & 296 & 5 & 600 & \\
\hline 0 & 92420 & 152.2479 & 3.1000 & 295.2 & 5 & 600.0 & \\
\hline & 92421 & 127.3204 & 1.5500 & 295.3 & 5 & 600.0 & \\
\hline & 92 & & 00 & & 0 & & \\
\hline & 32420 & 6586 & 0.7500 & 295.2 & 0 & 600.0 & 60 \\
\hline
\end{tabular}




\begin{tabular}{|c|c|c|c|c|c|c|c|}
\hline 0 & 2424 & 7404 & 2750 & 295.4 & 5 & 600.0 & \\
\hline & 9251 & 114.4199 & 0.1000 & 294.3 & & 600.0 & \\
\hline & 9252 & 28.7312 & 9250 & 294.8 & & 00 & \\
\hline & 9253 & 50.6900 & 9000 & 295.1 & & 00.0 & \\
\hline & 9254 & 50.7951 & .2250 & 94.5 & & 0 & \\
\hline & 9255 & 138.2515 & .1000 & 93.8 & & 00 & \\
\hline & 9256 & 084 & 2.1750 & 293.6 & & 00 & \\
\hline & 9257 & 161.5009 & 3.7000 & 92.3 & & 0 & \\
\hline & 9258 & 170.5013 & .7250 & 92.0 & & & \\
\hline & 9259 & 172.7502 & 6500 & 292.7 & & 0 & U \\
\hline & 92510 & 177.2527 & 9500 & 294.1 & & 00.0 & \\
\hline & 92511 & 177.5000 & 500 & 1 & & & \\
\hline & 92512 & 168.7499 & 4.9250 & 296.8 & & .0 & \\
\hline & 92513 & 177.7520 & 4.3750 & 298.0 & & 0 & 8 \\
\hline & 92514 & 199. & 50 & & & & \\
\hline & 92515 & 209 . & & 300.9 & & & \\
\hline & 92516 & 213.9606 & 2.3000 & 301.5 & & .0 & 8 \\
\hline & 92517 & 212 . & 2.1000 & .2 & & 0 & \\
\hline & 92518 & 163. & 1.4000 & 00.5 & 3 & & \\
\hline & 92519 & 144.9996 & 2.1000 & 298.8 & 4 & .0 & 0 \\
\hline & 92520 & 96.4673 & 3.0000 & 298.4 & 4 & 000 & \\
\hline & 92521 & 80 . & 500 & & 4 & & \\
\hline & 92522 & 76 & 7750 & 297.3 & 4 & & \\
\hline & 92523 & 79.7508 & 750 & 296.8 & 4 & 0 & 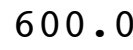 \\
\hline & 92524 & 77 & & & 4 & & \\
\hline & 9261 & 79 & 250 & 29 & 4 & & \\
\hline & 9262 & 545 & 3.0250 & 297.3 & 5 & 0 & U. \\
\hline & 9263 & 80 . & 2.2000 & 297.3 & 6 & & \\
\hline & 9264 & 81 . & 1.3000 & 29 & 6 & & \\
\hline & 9265 & 135 . & 750 & 295.8 & & 0 & 0 . \\
\hline & 9266 & 180.2501 & .0000 & 294.9 & 6 & & 0 . \\
\hline & 9267 & 151. & 50 & 295.1 & 6 & & \\
\hline & 9268 & 116 . & 5000 & 298.2 & 5 & 0 & . \\
\hline & 9269 & 172 & 5250 & 297.5 & 4 & 0 & 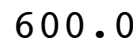 \\
\hline & 92610 & 168. & 000 & 297.2 & 3 & & \\
\hline & 92611 & 175 . & 5.6500 & 297.2 & 3 & & 0 . \\
\hline & 92612 & 170 . & 0000 & 298.1 & 4 & & \\
\hline & 92613 & 172 . & 5 . & 299.2 & 3 & & . \\
\hline 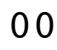 & 92614 & 173. & 4.8250 & 300.4 & 3 & .0 & 0 . \\
\hline & 92615 & 180 . & 0250 & 301.1 & 3 & & 0 . \\
\hline & 92616 & 183. & 250 & 301.2 & 3 & & 0 . \\
\hline & 92617 & 176.2448 & 4.5500 & 301.0 & 3 & & 0 . \\
\hline & 92618 & 120.1020 & 3.7750 & 299.7 & 4 & 0 & 0 . \\
\hline 00 & 92619 & 498 & 750 & 29 & 4 & & \\
\hline 0 & 92620 & 80.7507 & 6.6250 & 296.7 & 4 & & 0 . \\
\hline & 92621 & 83.0004 & 4.8000 & 297.2 & 4 & 600.0 & 00. \\
\hline & & & & & 4 & & \\
\hline & 92623 & 72.7500 & 4.2250 & 297.3 & $\Psi$ & 600.0 & 600 \\
\hline
\end{tabular}




\begin{tabular}{|c|c|c|c|c|c|c|c|}
\hline 0 & 92624 & 78.4936 & 3.6000 & 297.4 & 4 & 600.0 & 600.0 \\
\hline 00 & 9271 & 77.2392 & 3.8250 & 297.1 & 4 & 600.0 & 600.0 \\
\hline 00 & 9272 & 85.0000 & 1.4500 & 297.5 & 5 & 600.0 & 00.0 \\
\hline 0 & 9273 & 6.2158 & 1.4750 & 297.0 & 6 & 600.0 & 00.0 \\
\hline 00 & 9274 & 80.5000 & 6.9750 & 294.5 & o & 600.0 & 00.0 \\
\hline 00 & 9275 & 77.9968 & 9.8250 & 292.4 & 4 & 600.0 & 00.0 \\
\hline 00 & 9276 & 96.7524 & 9.6250 & 292.1 & 4 & 600.0 & 00.0 \\
\hline 00 & 9277 & 79.7497 & 7.0250 & 293.5 & 4 & 600.0 & 00.0 \\
\hline 00 & 9278 & 77.7476 & 5.8750 & 293.6 & 3 & 600.0 & 00.0 \\
\hline 00 & 9279 & 83.2498 & 4.7250 & 294.4 & 3 & 60 & 0 \\
\hline 00 & 92710 & 121.0515 & 3.8250 & 295.6 & - & 600.0 & \\
\hline 00 & 92711 & 91.0000 & 5.9250 & 293.5 & 3 & .0 & \\
\hline 00 & 92712 & 85.2501 & 6.7250 & 293.8 & 4 & 600.0 & 00.0 \\
\hline 00 & 92713 & 77.4991 & 6.6000 & 294.9 & 4 & 600.0 & 00.0 \\
\hline 00 & 92714 & 68.2498 & 6.8000 & 295.4 & 4 & .0 & \\
\hline 00 & 92715 & 70.2502 & 7.7500 & 295.4 & 4 & 600.0 & .0 \\
\hline 00 & 92716 & 78.7504 & 9.1250 & 294.4 & 4 & .0 & 0.0 \\
\hline 00 & 92717 & 75.0004 & 9.7250 & 292.9 & 4 & .0 & \\
\hline 00 & 92718 & 73.7500 & 11.0750 & 291.1 & 4 & .0 & \\
\hline 00 & 92719 & 69.4986 & 9.9750 & 289.2 & 4 & .0 & .0 \\
\hline 00 & 92720 & 64.4990 & 11.1250 & 288.1 & 4 & 6 & \\
\hline 00 & 92721 & 72.5000 & 12.5250 & 287.6 & 4 & .0 & \\
\hline 00 & 92722 & 71.2500 & 13.2500 & 287.1 & 4 & 60 & .0 \\
\hline 00 & 92723 & 66.2498 & 13.25 & 286.4 & 4 & 6 & \\
\hline 00 & 92724 & 64.7499 & 12.5500 & 286.3 & 4 & 6 & \\
\hline 00 & 9281 & 66.5000 & 13.2250 & 286.2 & 4 & 600.0 & .0 \\
\hline 00 & 9282 & 64.5000 & 13.0750 & 286.3 & 4 & .0 & .0 \\
\hline 00 & 9283 & 67.7500 & 14.0250 & 286.0 & 4 & .0 & \\
\hline 00 & 9284 & 65.2501 & 14.9750 & 285.9 & 4 & .0 & .0 \\
\hline 00 & 9285 & 65.4998 & 14.8250 & 285.5 & 4 & 6 & .0 \\
\hline 00 & 9286 & 68.2500 & 13.0500 & 285.4 & 4 & .0 & \\
\hline 00 & 9287 & 69.0000 & 11.1250 & 285.6 & 4 & .0 & 0.0 \\
\hline 00 & 9288 & 64.7501 & 9.8000 & 286.4 & 4 & 600.0 & 0.0 \\
\hline 00 & 9289 & 71.7500 & 8.3000 & 287.6 & 4 & 0 & 0.0 \\
\hline 00 & 92810 & 77.4998 & 7.6750 & 288.5 & 4 & 600.0 & 600.0 \\
\hline 00 & 92811 & 84.5018 & 6.9500 & 289.3 & 4 & 600.0 & 600.0 \\
\hline 00 & 92812 & 72.7495 & 8.625 & 289.7 & 4 & 600.0 & 0.0 \\
\hline 00 & 92813 & 81.4979 & 6.3500 & 290.7 & 4 & 600.0 & 600.0 \\
\hline 00 & 92814 & 93.9994 & 7.3750 & 290.7 & 4 & 600.0 & 600.0 \\
\hline 00 & 92815 & 80.2507 & 7.4250 & 290.5 & 4 & 600.0 & 600.0 \\
\hline 00 & 92816 & 83.7501 & 7.6500 & 290.2 & 4 & 600.0 & 600.0 \\
\hline 00 & 92817 & 78.7499 & 6.2000 & 290.1 & 4 & 600.0 & 600.0 \\
\hline 00 & 92818 & 71.4983 & 8.4750 & 288.5 & 4 & 600.0 & 600.0 \\
\hline 00 & 92819 & 73.7494 & 8.2750 & 287.4 & 4 & 600.0 & 600.0 \\
\hline 00 & 92820 & 78.7494 & 9.3500 & 287.0 & 4 & 600.0 & 600.0 \\
\hline 00 & 92821 & 78.7509 & 9.6250 & 286.9 & 4 & 600.0 & \\
\hline 00 & 92822 & 74.0000 & 9.00 & 289.0 & 4 & 600.0 & 600.0 \\
\hline 00 & 92823 & 86.2501 & 6.575 & 290.2 & & 600.0 & 600.0 \\
\hline
\end{tabular}




\begin{tabular}{|c|c|c|c|c|c|c|c|}
\hline 0 & 2824 & 8.9989 & 6.2250 & 290.8 & 4 & 000 & \\
\hline 0 & 9291 & 5.4968 & 4.6250 & 291.3 & 4 & 600.0 & \\
\hline & 9292 & 7634 & .6250 & 291.9 & 4 & 00 & \\
\hline & 9293 & 98.8811 & 4.2500 & 292.3 & & 00 & \\
\hline & 9294 & 26 . & .2750 & 92.2 & & 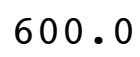 & \\
\hline & 9295 & 6.5699 & .4250 & 91.9 & & 00 & \\
\hline & 9296 & 040 & 500 & 292.7 & & & \\
\hline & 9297 & 135.2483 & .8500 & 92.5 & & & \\
\hline & 9298 & 135.5000 & .4000 & 293.6 & & 0 & \\
\hline & 9299 & 136.4895 & 3500 & 294.3 & & 0 & \\
\hline & 92910 & 152.0000 & .3250 & 294.8 & & 0 & \\
\hline & 92911 & 155.2455 & .6000 & 95.4 & 4 & & \\
\hline & 92912 & 159.0005 & 500 & 296.3 & 4 & 0 & \\
\hline & 92913 & 156.0055 & 7.1250 & 297.5 & 4 & 0.0 & \\
\hline & 92914 & 162.5000 & & 298.7 & 4 & & \\
\hline & 92915 & 161.5012 & 3000 & 300.0 & 3 & 0 & \\
\hline & 92916 & 171.9966 & .2000 & 301.1 & 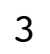 & 0 & \\
\hline & 92917 & 147.5523 & 000 & 0 & & & \\
\hline 0 & 92918 & 69.7445 & 750 & 299.9 & 4 & 0 & \\
\hline & 92919 & 70.5000 & 7000 & 298.9 & 4 & & \\
\hline & 92920 & 72.7508 & 0 & .2 & 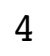 & & \\
\hline & 92921 & 74 . & 50 & 299.4 & 4 & & \\
\hline 0 & 92922 & 78.9979 & 250 & 299.7 & 4 & 0 & U • \\
\hline 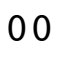 & 92923 & 22.3418 & 0 & 301.0 & 4 & 0 & \\
\hline 0 & 92924 & 7.2508 & 50 & & 4 & & \\
\hline & 9301 & 8365 & 3500 & 298.8 & 5 & & \\
\hline & 9302 & 150.8438 & .4000 & 298.7 & 4 & & \\
\hline 0 & 9303 & 128.4721 & 500 & 29 & 4 & & \\
\hline 0 & 9304 & 000 & 250 & 297.2 & 4 & & 0 . \\
\hline & 9305 & 134.8871 & 4.5500 & 296.8 & 4 & & \\
\hline & 9306 & 174.7508 & 2000 & .3 & 4 & & \\
\hline 0 & 9307 & 160.8789 & 00 & .5 & 4 & & \\
\hline & 9308 & 139.4172 & 4250 & 297.9 & 3 & 0 & 0 . \\
\hline & 9309 & 168.2506 & 50 & .5 & 3 & & \\
\hline & 93010 & 178 . & 50 & .9 & 4 & & \\
\hline & 93011 & 177.7503 & 500 & 298.2 & 4 & & \\
\hline & 93012 & 169.7504 & 250 & 299.9 & 3 & & \\
\hline 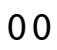 & 93013 & 162.2512 & 250 & 301.4 & 3 & & \\
\hline & 93014 & 155.7489 & 2000 & 302.4 & 3 & 0 & 0 . \\
\hline & 93015 & 171. & 750 & 302.6 & 2 & & \\
\hline & 93016 & 167 . & 500 & & 2 & & \\
\hline 0 & 93017 & 144.6633 & 3.1000 & 303.6 & 2 & 600.0 & 00 . \\
\hline & 93018 & 124.2382 & 5750 & 303.8 & 3 & 600 & 000 \\
\hline , & 93019 & 172.2479 & 2.2250 & 301.3 & 4 & & \\
\hline 00 & 93020 & 146.2114 & 1.3000 & 300.4 & 5 & 600.0 & 00 . \\
\hline & 93021 & 118.3616 & 2.2500 & 299.9 & 6 & 600.0 & 50 \\
\hline & 93022 & 79.2507 & 5500 & 300.3 & 5 & 60 & \\
\hline & 93023 & 75.4908 & 6.6000 & 300.7 & 4 & 600.0 & 600 \\
\hline
\end{tabular}




\begin{tabular}{|c|c|c|c|c|c|c|c|}
\hline 0 & & ב & .7750 & 301.2 & 4 & 80.0 & \\
\hline & 1 & 104.2216 & 2.9250 & 300.3 & & 600.0 & \\
\hline 1 & 12 & 69.0996 & 1.2500 & 298.8 & & 00 & \\
\hline 1 & 1 & 26.5258 & 2.4500 & 298.6 & & 00 & \\
\hline & 14 & 88.3835 & 500 & 98.7 & & 0 & \\
\hline & 1 & 155 . & & 96.8 & & 0 & \\
\hline 1 & 1 & 159.6813 & 1.6000 & 296.3 & & 00 & \\
\hline & 17 & 140 . & .5000 & 96.8 & & & \\
\hline & 18 & 158.9279 & 4750 & 97.5 & & & \\
\hline & 19 & 165.7150 & 1.8250 & 297.9 & & 0 & - \\
\hline & 110 & 161.1786 & 3.8000 & 299.7 & & & \\
\hline & 111 & 172.4963 & 0 & 99.6 & & & \\
\hline 0 & 112 & 177.24 & 6 . & 300.1 & & 0 & \\
\hline & 113 & 175.7497 & 5.5000 & 301.1 & & 0.0 & \\
\hline & 114 & 177.7501 & & & & & \\
\hline & 11 & 178.9 & & 30 & & & \\
\hline & 116 & 165.33 & .30 & 303.7 & & .0 & \\
\hline & 117 & 87 . & 0 & 9 & & & \\
\hline & 118 & 79 . & & & & & \\
\hline & 119 & 78 . & 000 & 299.2 & 4 & & 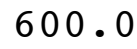 \\
\hline & 120 & 65.9997 & 9.7250 & 298.6 & & 0 & \\
\hline & 121 & 64 . & 0 & & 4 & & \\
\hline & 122 & 60 . & 50 & 29 & 4 & & \\
\hline 0 & 123 & 65.5009 & 7.4250 & 29 & 4 & 0 & \\
\hline & 124 & 66. & & 29 & $\mathbf{4}$ & & \\
\hline 0 & 21 & 46.5024 & 750 & 299.3 & 4 & & \\
\hline & 22 & 102.2087 & 500 & 297.2 & 5 & & J. \\
\hline & 23 & 66.2479 & 0 & 297.2 & 4 & & \\
\hline & 24 & 77.2 & 0 & 29 & 4 & & \\
\hline & 25 & 68.7500 & 500 & 297.0 & 4 & 0 & 0. \\
\hline & 26 & 69.5000 & 750 & 296.5 & 4 & & 0 . \\
\hline & 27 & 62.7513 & 0 & & 4 & & \\
\hline & 28 & 83.9705 & & 29 & 2 & & 0 . \\
\hline & 29 & 74.2534 & 000 & 29 & 2 & & \\
\hline & 210 & 236.8107 & 0 & 29 & 2 & & \\
\hline & 211 & 254.3849 & 750 & 297.6 & 1 & & . \\
\hline & 212 & 118.8871 & 1500 & 297.8 & & & 0 . \\
\hline & 213 & 68.9318 & 3 . & 29 & 2 & & \\
\hline & 214 & 65.7063 & 4.5250 & 299.3 & 3 & & 0 . \\
\hline & 215 & 79.7490 & 750 & 298.5 & 4 & & \\
\hline & 216 & 75.0011 & 250 & 297.3 & 4 & & \\
\hline & 217 & 75.0000 & 9.1750 & 296.0 & 4 & & 0 . \\
\hline & 218 & 70.2524 & 9.3750 & 294.7 & 4 & 0 & 0 . \\
\hline & 219 & 65.2495 & 2000 & 29 & 2 & & \\
\hline 001 & 220 & 62.4998 & 0.8500 & & 4 & & \\
\hline & 221 & 65.0002 & 9.3000 & 294.2 & 4 & 600 & 600. \\
\hline & 222 & 67 & & & 4 & & \\
\hline & 223 & 71.5003 & 8.0250 & 294.2 & & 600.0 & 60 \\
\hline
\end{tabular}




\begin{tabular}{|c|c|c|c|c|c|c|c|}
\hline r & 24 & 67.9991 & 9.2750 & 292.8 & 4 & 600.0 & \\
\hline 01 & 31 & 1.2497 & 8.1500 & 293.1 & 4 & 600.0 & \\
\hline J1 & 32 & 84.4994 & .2000 & 293.5 & 4 & 00.0 & \\
\hline 01 & 3 & 134.8771 & 2.2750 & 292.6 & & 00 & \\
\hline & 4 & 6.9312 & .3750 & 90.9 & & 00 & \\
\hline & 5 & 133.7187 & .7000 & 290.2 & & 00 & \\
\hline 0 & 6 & 136.1825 & 0.7500 & 289.3 & & 00.0 & \\
\hline & 7 & 151.0781 & .6750 & 89.4 & & & \\
\hline 01 & 8 & 194.7062 & .20 & 291.9 & 5 & 0 & \\
\hline 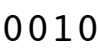 & 9 & 212.7051 & 1.2750 & 293.5 & & 00.0 & \\
\hline & 10 & 175.5014 & 3.77 & 293.0 & 3 & 0 & \\
\hline & & 170.5015 & 12 & 93.7 & 3 & & \\
\hline 0 & 12 & 169.4908 & 4.50 & 294.9 & 3 & 00 & \\
\hline 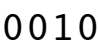 & 13 & 168.2493 & 4.67 & 295.6 & 3 & 0.0 & \\
\hline & 4 & 167.7952 & & 296.9 & 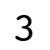 & & \\
\hline 01 & 15 & 163.7979 & 4.80 & 297.7 & 3 & 0 & \\
\hline & 16 & 129.4245 & 4.50 & 298.1 & 3 & 0 & \\
\hline 0 & 17 & 89.2483 & 4 . & 297.6 & 4 & & \\
\hline 0 & 18 & 79.2491 & 0 & 295.6 & 4 & 0 & \\
\hline & 19 & 72.7511 & 0 & 294.1 & 4 & 0 & \\
\hline & 20 & 77.2499 & 0 & 293.6 & 4 & & \\
\hline 0 & 21 & 74.7500 & & 292.9 & 4 & & \\
\hline 0 & 22 & 73.2500 & 0 & 292.6 & 4 & 00 & 600. \\
\hline 0 & 323 & 72.7496 & & 292.6 & 4 & 0 & \\
\hline 0 & 324 & 76.2498 & & 292.6 & 4 & & \\
\hline 0. & 41 & 74.7500 & & 292.9 & 4 & & \\
\hline 0 & 2 & 72.9936 & 0 & 292.8 & 4 & 0 & \\
\hline 0 & 43 & 74.7514 & & 292.1 & 4 & & \\
\hline 0 & 44 & 58.7579 & 0 & 291.7 & 5 & 0 & 0 . \\
\hline 0 & 45 & 91.0203 & 0 & 290.2 & 6 & 0 & \\
\hline 0 & 46 & 96.7682 & & 289.2 & 6 & & \\
\hline 0 & 47 & 146.7496 & 0 & 288.3 & 6 & & $\bullet$ \\
\hline & 48 & 36.6889 & 0.7500 & 290.2 & 5 & 0 & 0 • \\
\hline 0 & 49 & 310.9465 & 1 . & 291.3 & 4 & 0 & \\
\hline 0 & 410 & 282.1624 & 1. & 292.6 & 3 & & \\
\hline 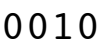 & 411 & 267.8293 & 1.8500 & 292.9 & 2 & & . \\
\hline & 412 & 249.0763 & 0 & 294.8 & 1 & 0 & \\
\hline 00 & 413 & 267.7025 & 2 . & 296.6 & 1 & & 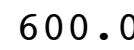 \\
\hline 0 & 414 & 193.5438 & 3.7750 & 296.7 & 2 & .0 & 0 . \\
\hline & 415 & 131.8104 & & 297.2 & 3 & 0 & \\
\hline 0 & 416 & 88.7597 & 3 & $29^{\circ}$ & 2 & & \\
\hline 00 & 417 & 87.0006 & 5.1250 & 296.4 & 3 & 600.0 & 600. \\
\hline & 418 & 86.0000 & & 295.1 & 4 & 600 & 600 \\
\hline 00 & 419 & 76.2483 & 6.6000 & 293.2 & 4 & 600 & con \\
\hline 001 & 420 & 76.4990 & 6.2750 & 293.5 & 4 & 600.0 & 00 \\
\hline & 421 & 77.5000 & 6.1750 & 293.6 & 4 & 600.0 & 600. \\
\hline & 422 & 0000 & & 29 & 4 & 600 & \\
\hline & 423 & 82.0000 & 5.1750 & 295.2 & 4 & 600.0 & 600 \\
\hline
\end{tabular}




\begin{tabular}{|c|c|c|c|c|c|c|c|}
\hline & 24 & & 0 & 4.9 & & & \\
\hline & 1 & 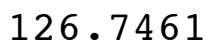 & 1.7500 & 93.8 & & 0 & \\
\hline & 52 & 83.8850 & 5750 & 293.5 & & 00 & \\
\hline & 53 & 77.5000 & 8500 & 293.6 & & 00.0 & \\
\hline & 4 & 000 & 6000 & 93.4 & & & \\
\hline & 5 & 81 & 250 & 93.6 & & 0 & \\
\hline & 56 & 3285 & 2.6250 & 292.4 & & 00 & \\
\hline & 7 & 188 & 2000 & 91.6 & & & \\
\hline & 8 & 304.5269 & 0 & 94.1 & & & \\
\hline 0 & 9 & 290.7877 & 0.5250 & 296.8 & & 00 & 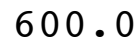 \\
\hline & 10 & 244.7494 & 1.7500 & 296.3 & & & \\
\hline & 11 & 165.7304 & 377 & 95.4 & & & \\
\hline 0 & 12 & 162.74 & 500 & 295.9 & & .0 & \\
\hline & 13 & 165.0000 & 4.9250 & 297.2 & & & \\
\hline & 14 & 169. & & & & & \\
\hline & 1 & 167. & & 7 & & & \\
\hline & 16 & 169.0000 & 0 & 298.9 & & .0 & \\
\hline & 17 & 173. & 4 . & 4 & & & \\
\hline & 18 & 120 . & & 4 & 4 & & \\
\hline & 19 & 86 . & & 296 & 4 & & \\
\hline & 20 & 71.7505 & .72 & 296 & 4 & 0 & \\
\hline & 21 & 70 . & & & 4 & & \\
\hline & 22 & 74 . & & 29 & 4 & & \\
\hline & 523 & 79.5000 & 0 & 29 & 4 & 0 & \\
\hline & 524 & 79.7500 & & & 4 & & \\
\hline & 61 & 87 . & & 29 & 4 & & \\
\hline & 62 & 92.0236 & 5000 & 297.2 & 5 & 0 & 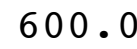 \\
\hline & 63 & 112.1329 & 0 & 29 & 6 & & \\
\hline & 64 & 90. & 5 . & 29 & 5 & & \\
\hline & 65 & 92.7498 & 750 & 295.8 & 4 & & . \\
\hline & 66 & 99.5000 & 4.72 & 295.7 & 4 & & 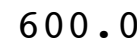 \\
\hline & 67 & 118.7592 & 247 & 29 & 5 & & \\
\hline & 68 & 130.3337 & & & 4 & & 0 . \\
\hline & 69 & 164.7518 & 3 . & 296 & 3 & 0 & \\
\hline & 610 & 172.7505 & & & 3 & & \\
\hline & 611 & 172.2498 & & 295.8 & 3 & & \\
\hline & 612 & 170.0011 & 4.4000 & 297.0 & 3 & & \\
\hline & 613 & 169.4997 & 5. & 29 & 3 & & \\
\hline & 614 & 164.2501 & 5 . & .7 & 3 & & 0 . \\
\hline & 615 & 119.9308 & 7750 & 300.3 & 3 & & \\
\hline & 616 & 82.5069 & 5 . & 29 & 3 & & \\
\hline & 617 & 72.5000 & 7.90 & 297.5 & 4 & & 0 . \\
\hline & 618 & 75.5002 & 9.7250 & 295.2 & 4 & 0 & 0 . \\
\hline & 619 & 72.2501 & 750 & 294 & 4 & & \\
\hline 00 & 620 & 67.2498 & 1.4750 & 294.3 & 4 & & \\
\hline & 621 & 68.5002 & 11.6000 & 295.2 & 4 & 600 & 600. \\
\hline & 622 & 71.2499 & 12.1 & 29 & 4 & & \\
\hline & 623 & 74 & 11.4750 & 294.5 & & 600.0 & 60 \\
\hline
\end{tabular}




\begin{tabular}{|c|c|c|c|c|c|c|c|}
\hline & 24 & 0.3000 & 3.2000 & 292.4 & 4 & 0.0 & \\
\hline 1 & 71 & 4.7494 & 3.7750 & 289.3 & & 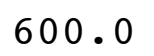 & \\
\hline & 72 & 79.7501 & .3500 & 287.6 & & 0 & \\
\hline 01 & 73 & 80.5014 & 2.5750 & 287.1 & & 00 & \\
\hline & 74 & 8.50 & 2.0250 & 36.9 & & & \\
\hline & 75 & 5000 & 1.6500 & 87.2 & & 00 & \\
\hline & 76 & 82.2500 & .8250 & 287.2 & & 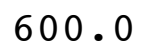 & \\
\hline & 77 & 31.5003 & 500 & 86.8 & & & \\
\hline & 78 & 68.9989 & 250 & 87.3 & & & \\
\hline 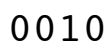 & 79 & 68.7356 & .750 & 288.1 & & 00 & \\
\hline & 710 & 87.7548 & 9000 & 289.1 & & 0 & \\
\hline & 711 & & & 90.0 & & & \\
\hline 01 & 712 & 2441 & 0 & 292.4 & & 0 & \\
\hline 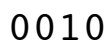 & 713 & 81.7498 & 0000 & 292.9 & & 0.0 & \\
\hline & 714 & & & 92.8 & & & \\
\hline 0 & 715 & 85.5027 & 0 & 293.3 & 4 & & \\
\hline & 716 & 83.0000 & 0 & 293.5 & 4 & 0 & \\
\hline & 717 & 79 . & & 292.4 & & & \\
\hline 0 & 718 & 77.2500 & & 291.8 & 4 & & \\
\hline & 719 & 74.7498 & 0 & 292.2 & 4 & & \\
\hline & 720 & 74 & 0 & 291.6 & $I$ & & \\
\hline & 721 & & & 291.5 & 4 & & \\
\hline 0 & 722 & 2499 & 0 & 291.3 & 4 & & \\
\hline 0 & 723 & 78.4995 & 0 & 29 & 4 & & \\
\hline & 724 & & & 29 & 4 & & \\
\hline & 81 & 426 & & 29 & 4 & & \\
\hline & 82 & 83.9408 & 0 & 289.4 & 4 & & \\
\hline 0 & 83 & 757 & 2. & 29 & 4 & & \\
\hline & 84 & 936 & 1 . & 289 & 5 & & \\
\hline & 85 & 7.2504 & 000 & 289.6 & 4 & & \\
\hline & 86 & 4.9925 & 0 & 288.3 & 5 & & \\
\hline 0 & 87 & 109. & 0 & 28 & 6 & & \\
\hline & 88 & 29.4111 & 500 & 290.9 & J & 0 & 0 . \\
\hline & 89 & 261.8751 & 1. & 292.1 & 4 & & \\
\hline & 810 & 209 . & 0 & 294.5 & 3 & & \\
\hline & 811 & 170.0000 & 500 & 294.6 & 2 & & \\
\hline & 812 & 158.7525 & 50 & 295.1 & 2 & & \\
\hline & 813 & 160.9993 & 0 & 296.2 & 2 & & \\
\hline 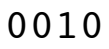 & 814 & 165.9952 & 500 & 297.7 & 3 & 0 & 0 . \\
\hline & 815 & 156.7384 & & 298.7 & 3 & & \\
\hline & 816 & 114.3992 & 4 . & 299 & 3 & & \\
\hline 00 & 817 & 65.7430 & 5.7500 & 298.3 & 4 & 0 & 0. \\
\hline & 818 & 62.9981 & & 297.5 & 4 & 0 & \\
\hline & 819 & 45.0103 & 5.2000 & 296.2 & 4 & & \\
\hline 01 & 820 & 67.2551 & 5.8500 & 294.6 & 4 & 60 & 0 \\
\hline & 821 & 60.9899 & 3500 & 294.4 & 4 & 600 & \\
\hline & 822 & 2471 & 0 & 292 & 4 & & \\
\hline & 823 & 63.9989 & 9.6750 & 292.5 & 4 & 600.0 & 600 \\
\hline
\end{tabular}




\begin{tabular}{|c|c|c|c|c|c|c|c|}
\hline 10 & 24 & 8.9999 & 11.2250 & 291.4 & 4 & 00 & \\
\hline 0 & 91 & 7.2501 & 11.1750 & 291.2 & & 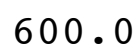 & \\
\hline & 92 & 986 & 6500 & 8.4 & & & \\
\hline & 3 & 3.2499 & 2.5750 & 86.5 & & 00 & \\
\hline & 94 & & 50 & & & & \\
\hline & 95 & 75 & .2750 & 86.8 & & 0 & \\
\hline & 96 & 67.2509 & 1.5500 & 86.8 & & 0 & \\
\hline & 97 & 62.7501 & 1.30 & 87.3 & & & \\
\hline & 98 & 59.48 & 8.2250 & 288.1 & & 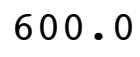 & \\
\hline & 99 & 58.99 & 9.5750 & 288.8 & & 0 & \\
\hline & 910 & 61. & 7.2250 & 7 & & & \\
\hline & 911 & & 5.4750 & 92.5 & & & \\
\hline 0 & 912 & 68.1850 & 6.6250 & 291.3 & & 0 & \\
\hline & 913 & 85 . & .6750 & 89.7 & & & \\
\hline & 914 & 0 & & 6 & & & \\
\hline 01 & 915 & 65. & 750 & 287.0 & & & \\
\hline & 916 & 63.75 & 10.4750 & 288.5 & & & \\
\hline & 917 & 55.74 & 12 . & 288.4 & 4 & & \\
\hline 01 & 918 & 63. & 000 & 287.6 & 4 & & \\
\hline & 919 & 76 & .7750 & 286 & & & \\
\hline & 920 & 76 & 0 & & & & \\
\hline 01 & 921 & 42 & & 28 & 4 & & \\
\hline & 922 & 98.86 & 1000 & 286.0 & & & \\
\hline & 923 & 8 & 0 & & & & \\
\hline 0 & 924 & & & & 5 & & \\
\hline & & 332 . & 4000 & 284.6 & & & \\
\hline & 102 & 85.9288 & 1.2000 & 284.6 & 6 & & \\
\hline & & 69. & 1.2750 & 284.4 & 6 & & \\
\hline & 104 & 62.24 & 3250 & 284.4 & 5 & & \\
\hline & 105 & 46.71 & 250 & 284.4 & 4 & & \\
\hline & & 4 & & & 4 & & \\
\hline & 107 & 3 . & & & 5 & & \\
\hline . & 108 & 37.4038 & 3.4000 & 283.1 & 4 & & \\
\hline 01 & 109 & 4 & 2.2250 & 282 & 3 & & \\
\hline & 1010 & 358 . & & & 3 & & \\
\hline & & 44.4801 & & 284.1 & & & \\
\hline & 012 & 48 . & 4 . & 284.7 & 3 & & \\
\hline & 13 & 68. & 250 & 284.8 & 2 & & \\
\hline & 1014 & 60.9981 & 4000 & 284.9 & 3 & & \\
\hline & & 64. & 000 & & 4 & & \\
\hline & 16 & & & & 3 & & \\
\hline 001 & 1017 & 75.3832 & 3.3750 & 285.8 & 4 & & \\
\hline & 1018 & 107 . & 250 & 285.0 & 5 & & \\
\hline 00101 & 1019 & 116.7077 & 2.5250 & 284.6 & 5 & & \\
\hline 01 & 1020 & 65.2120 & 3.3750 & 284.5 & 4 & .0 & ה \\
\hline & & 60 . & & & 3 & & \\
\hline & 1022 & 0.00 & 1.3250 & 284.2 & & & 每 \\
\hline . & $0<3$ & $\partial 1.0000$ & $1 \cdot 1300$ & 284 & & 600.0 & 50 \\
\hline
\end{tabular}




\begin{tabular}{|c|c|c|c|c|c|c|}
\hline 1024 & 6.6874 & 0.3750 & 284.2 & 6 & 600 & \\
\hline 111 & 144.4982 & 1.8250 & 284.3 & כ & תח & \\
\hline 010112 & 169.1634 & 6500 & 284.2 & 5 & 00 & \\
\hline 010113 & 187.7523 & .0750 & 284.1 & & 00 & \\
\hline 0114 & 135.7787 & 50 & 33.5 & & 0 & \\
\hline 0115 & 5.6127 & 1.2000 & 83.4 & & 00 & \\
\hline 010116 & 329.7536 & 0.4500 & 283.3 & & 0 & \\
\hline 0117 & 40.3533 & .3250 & & & & \\
\hline 010118 & 129.2392 & .7750 & 283.3 & & 0 & \\
\hline 010119 & 170.9958 & 1.5750 & 285.3 & & 0 & \\
\hline 01110 & 169.2522 & 0 & 85.1 & & 0 & \\
\hline & 224.7544 & & & & & \\
\hline 01 & 323.0104 & 2.3250 & 284.5 & & 0 & \\
\hline 113 & 355.2253 & .07 & 284.7 & & 0 & \\
\hline & 313.1703 & & & & & \\
\hline 5 & 315.9931 & 1.42 & 284 & 1 & & \\
\hline 116 & 337.3460 & 1.47 & 283.8 & & 0 & \\
\hline 17 & 353.4624 & 19 & 83.4 & 2 & & \\
\hline 0 & 182.2406 & 0 & 28 & 3 & & \\
\hline 0101119 & 205.2060 & 0 & $2 \varepsilon$ & 4 & & \\
\hline 20 & 300.4780 & 0 & 1 & 5 & & \\
\hline & 10.4609 & & 9 & 4 & & \\
\hline 122 & 1.0929 & 0 & 282.9 & 4 & 0 & 0 . \\
\hline 123 & 317.5005 & 0 & 282.9 & 5 & 0 & \\
\hline 24 & 312.4036 & & 282.7 & 6 & & \\
\hline 010121 & 38.2475 & 0 & 282 & 6 & & \\
\hline 010122 & 150.2570 & 0 & 282.5 & 5 & & \\
\hline 0010123 & 179.9474 & 0 & 5 & 6 & & \\
\hline 010124 & 28.7391 & 4750 & 282 & 6 & & 0 . \\
\hline 010125 & 234.1402 & 30 & 282.6 & 6 & & \\
\hline 010126 & 130.6762 & 0 & 282.5 & 5 & & \\
\hline 0010127 & 132.7503 & 0 & 282.4 & 5 & & \\
\hline 0010128 & 150.2480 & 0 & 282.6 & 4 & 0 & J. \\
\hline 010129 & 160.5017 & 0 & 28 & 4 & & \\
\hline 10 & 224 . & 0 & .8 & 3 & & \\
\hline 211 & 197.2515 & 3. & 284.8 & 2 & 0 & 0 . \\
\hline & 185 . & & & 3 & & \\
\hline 00 & 217 . & 0 & & 2 & & \\
\hline 00101214 & 234.2662 & 1.6250 & 287.4 & 1 & 0 & 。 \\
\hline 215 & 215.0073 & 0 & $28^{\circ}$ & 2 & & \\
\hline 00101216 & 206.5021 & 0 & & 2 & & \\
\hline 00101217 & 179.3813 & 2.1750 & 287.7 & 3 & 600.0 & 600. \\
\hline 1218 & 147.7500 & 2.3500 & 286.8 & 4 & 600 & 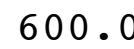 \\
\hline 00101219 & 145.2502 & 2.2500 & & 5 & & \\
\hline 00101220 & 152.0000 & 2.2750 & 285.5 & 5 & 600.0 & 00 \\
\hline 01221 & 124.7403 & 1.5250 & 285.6 & 4 & 600.0 & 50 \\
\hline 1222 & 76.7332 & & & 4 & 60 & \\
\hline 0101223 & 92.7350 & 2.5750 & 285.8 & 4 & 600.0 & 600 \\
\hline
\end{tabular}




\begin{tabular}{|c|c|c|c|c|c|c|}
\hline 224 & 80.7460 & 5.1250 & 85.4 & 4 & 600.0 & \\
\hline 131 & 7.2504 & 5.9500 & 284.9 & 4 & 600.0 & \\
\hline 10132 & 73.9988 & 4500 & 285.1 & 4 & 00.0 & \\
\hline 10133 & 74.7502 & 6000 & 285.1 & & 00 & \\
\hline 0134 & 3.2501 & .1500 & 85.2 & & 0 & \\
\hline 0135 & 75.4992 & 4500 & 85.7 & & 00 & \\
\hline 010136 & 77.5001 & 9750 & 85.9 & & 00 & \\
\hline 010137 & 82.2153 & 7000 & & & 0 & \\
\hline 010138 & 0.5857 & 5250 & 0 & & & \\
\hline 010139 & 160.2363 & 1.5000 & 88.9 & & 00 & 0 \\
\hline 0101310 & 182.3965 & 1.2000 & 290.6 & & & \\
\hline & 168.2492 & 100 & 89.3 & & & \\
\hline 12 & 166.2494 & 3 . & 290.0 & 2 & 0 & \\
\hline 313 & 167.4853 & 4.45 & .9 & 3 & 0.0 & \\
\hline & 174.5000 & & .3 & & & \\
\hline & 161.4985 & & 291.7 & 3 & & \\
\hline 316 & 170.7524 & 5000 & 291.8 & 3 & .0 & . \\
\hline 17 & 170.2499 & 4 . & 1.4 & 4 & & \\
\hline & 150.2684 & 4. & 0.4 & 4 & & \\
\hline 19 & 77.7511 & 0 & & 4 & & \\
\hline 20 & 82.9988 & 5.8000 & 289.1 & 4 & 0 & 00 . \\
\hline & 82.7500 & & 88.9 & 4 & & \\
\hline 22 & 77.9998 & 0 & 289 & 4 & & \\
\hline 323 & 70.2501 & .7500 & 3.7 & 4 & 0 & \\
\hline 24 & 79.5031 & & & 4 & & \\
\hline 001 & 80.2502 & 0 & 289.2 & 4 & & \\
\hline 010142 & 81.7501 & .2500 & 9.5 & 4 & 0 & . \\
\hline 010143 & 85.7498 & 500 & 4 & 4 & & \\
\hline 44 & 145 . & 1 . & .5 & 5 & & \\
\hline 010145 & 142.4935 & 500 & 8.0 & 6 & 0 & 0. \\
\hline 010146 & 89.0806 & 3500 & 287.7 & 6 & 0 & 0 . \\
\hline 47 & 145.5165 & 1 . & 287.7 & 5 & & \\
\hline 010148 & 149.5239 & 500 & 288.7 & 4 & & 0 . \\
\hline 010149 & 170.7496 & 750 & 289.7 & 3 & 0 & 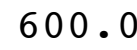 \\
\hline 10 & 172.5668 & 0 & & 2 & & \\
\hline & 169.5000 & 4.3750 & 291.1 & 3 & & 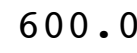 \\
\hline 01412 & 164.4989 & 750 & 291.1 & 3 & & 0 \\
\hline 413 & 151.9979 & 3. & 29 & 2 & & 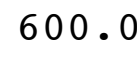 \\
\hline 001 & 164.7509 & 4.7750 & 293.2 & 3 & .0 & 0 . \\
\hline 0101415 & 167.9959 & 4.2750 & 293.6 & 3 & & \\
\hline & 151.2460 & 000 & 29 & 2 & & 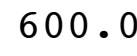 \\
\hline 001 & 172.2855 & 3.4750 & 293.4 & 3 & .0 & 0 . \\
\hline 0101418 & 149.5763 & 2.7500 & 292.1 & 4 & 0 & 0 . \\
\hline 0010 & 79.7496 & 250 & & 4 & & \\
\hline 00101420 & 76.7489 & 6.1750 & & 4 & & 0 . \\
\hline 0101421 & 73.2500 & 7.0250 & 290.0 & 4 & 600 & 600 \\
\hline & 5003 & & & 4 & & \\
\hline 0101423 & 70.5003 & 7.9250 & 290.0 & & 600.0 & $r_{0}$ \\
\hline
\end{tabular}




\begin{tabular}{|c|c|c|c|c|c|c|}
\hline 1424 & 3.2500 & 7.9000 & 289.9 & 4 & 600.0 & \\
\hline 0151 & 8.5000 & 7.3250 & 90.2 & . & 600.0 & \\
\hline 010152 & 002 & 9750 & 290.5 & 4 & 00 & \\
\hline 010153 & 04.9295 & 5750 & 289.1 & & 00 & \\
\hline 0154 & 5.6287 & .8750 & 38.5 & & 0 & \\
\hline 0155 & 0076 & 3250 & 88.7 & & 00 & \\
\hline 0156 & 49.4700 & 1000 & 288.5 & & 0 & \\
\hline 0157 & 373 & .8250 & 87.8 & & & \\
\hline 010158 & 9759 & .6000 & 289.2 & & 0 & \\
\hline 010159 & 309.2554 & 0.7500 & 290.8 & & 00 & \\
\hline 510 & 266.7618 & 1.7000 & 291.4 & & 0 & \\
\hline & 243.2491 & 2.3250 & 90.5 & & & \\
\hline 12 & 247.5046 & 0000 & 291.9 & & 0 & \\
\hline 513 & 208.9987 & 2.9500 & 292.5 & & 0 & \\
\hline & 208.4830 & & 292.8 & $z$ & & \\
\hline 15 & 230.4986 & 5250 & 293.4 & 2 & 0 & \\
\hline 516 & 222.1643 & .4500 & 293.8 & 3 & 0 & \\
\hline 517 & 218.7499 & .7250 & 293.7 & 4 & & \\
\hline 18 & 171.5693 & 2.0000 & 292.0 & 5 & 0 & \\
\hline 519 & 152.9874 & 1.4500 & 291.3 & 5 & & \\
\hline 20 & 136. & 1.3500 & 291.2 & 4 & & \\
\hline 21 & 58. & 50 & 291.4 & 4 & & \\
\hline 522 & 2566 & 5000 & 291 & 4 & 0 & 0. \\
\hline 523 & 73. & 4 . & 291.6 & 4 & & \\
\hline 24 & 89. & & 292.0 & 4 & & \\
\hline 010161 & 115 . & 3000 & 290.8 & 4 & & \\
\hline 010162 & 63.4921 & .2500 & 291.1 & 5 & & \\
\hline 0010163 & 118.2952 & 0 & 291.2 & 5 & & \\
\hline 0010164 & 155 & 250 & 29 & 6 & & 0 . \\
\hline 010165 & 152.000 & 2000 & 289.8 & $J$ & & \\
\hline 010166 & 90.251 & 0 & 289.1 & 6 & & \\
\hline 0010167 & 43. & 0 & 288.6 & 6 & & \\
\hline 0010168 & 156.5005 & 7000 & 289.4 & 5 & 0 & J. \\
\hline 010169 & 172 . & 00 & 291.5 & 4 & 0 & \\
\hline 610 & 265 . & 0 & 293.9 & 3 & & \\
\hline 1611 & 241.2216 & 1.9750 & 293.6 & 2 & & 0 . \\
\hline 1612 & 229.7519 & 4250 & 293.6 & 2 & & \\
\hline 001 & 228.0123 & 0 & 293.9 & 2 & & 0 . \\
\hline 00101614 & 217.7430 & 2.9750 & 294.4 & 1 & 0 & 0. \\
\hline 1615 & 222.4061 & 000 & 295.1 & 1 & 0 & \\
\hline 1616 & 212 . & 000 & 295.2 & 2 & & \\
\hline 00101617 & 213.2594 & 1.7000 & 295.0 & 3 & 600.0 & 600. \\
\hline 1618 & 174.4505 & 0.8000 & 293.7 & 4 & 600 & 000 \\
\hline 00101619 & 158.7505 & 1.2000 & 292.9 & 4 & & s \\
\hline 00101620 & 134.7533 & 0.6000 & 292.6 & 5 & 600.0 & 00 \\
\hline 01621 & 128. & 0.0000 & 291.7 & 6 & 600 & 50 \\
\hline & 37 & 0.37 & 291.7 & 6 & 60 & \\
\hline 0101623 & 27.7494 & 0.4000 & 291.8 & & 600.0 & 600 \\
\hline
\end{tabular}




\begin{tabular}{|c|c|c|c|c|c|c|}
\hline 4 & $1 . / 482$ & 0.7750 & 92.5 & 4 & - & \\
\hline 171 & 6.7897 & 0.2500 & 292.3 & 4 & 600.0 & \\
\hline 0172 & 82.7372 & 250 & 291.9 & & 00 & \\
\hline 10173 & 89.6828 & 0000 & 291.6 & & 00 & \\
\hline 0174 & 69.2497 & 5500 & 90.9 & & & \\
\hline 0175 & 0.1875 & & 89.7 & & 0 & \\
\hline 0176 & 9872 & 500 & 289.5 & & 00 & \\
\hline 0177 & 3009 & 0 & 90.1 & & & \\
\hline 10178 & 2592 & 0 & 91.6 & & & \\
\hline 010179 & 298.6368 & 1.2750 & 93.6 & & 0 & \\
\hline 0101710 & 278.3176 & 1.6750 & 294 & & & \\
\hline 711 & 240.2275 & 0 & 94.1 & & & \\
\hline .712 & 167.7103 & 750 & 294.3 & & & \\
\hline 1713 & 160.2468 & 0 & 29 & & & \\
\hline 714 & 169. & & & & & \\
\hline 715 & 173. & & 9 & 2 & & \\
\hline .716 & 170.7525 & 1750 & 296.2 & 3 & & \\
\hline 717 & 165 . & 500 & 4 & & & \\
\hline & 155 . & 0 & 29 & 5 & & \\
\hline 719 & 145 & 250 & 293 & 6 & & \\
\hline 720 & 129.7489 & 2.3500 & 294.1 & 5 & 0 & \\
\hline 721 & 87.9573 & & & 4 & & \\
\hline 22 & 77 . & 0 & 29 & 4 & & \\
\hline 723 & 71.7499 & 250 & 292.3 & 4 & 0 & \\
\hline 724 & 69.0000 & & 29 & 4 & & \\
\hline 81 & 70 . & 0 & 292 & 4 & & \\
\hline 010182 & 72.0000 & 500 & 292 & 4 & & . \\
\hline 010183 & 74.5000 & 500 & & 4 & & \\
\hline 184 & 109.3123 & 0 & 29 & 4 & & \\
\hline 010185 & 147.7515 & 750 & 292.2 & 4 & & . \\
\hline 010186 & 109.2682 & 000 & 292.7 & 5 & & . \\
\hline 187 & 107.4699 & 0 & & 4 & & \\
\hline 010188 & 93.9904 & 0 & 290.6 & 4 & & 0 . \\
\hline 010189 & 133.8074 & 500 & 292.1 & 4 & & \\
\hline 810 & 147.7496 & & & 3 & & \\
\hline 811 & 162.9985 & 6.3000 & 293.8 & 4 & & \\
\hline 1812 & 168.2502 & 3750 & 294.2 & 3 & & \\
\hline 813 & 161.4174 & 0 & 295.4 & 3 & & 0 \\
\hline 814 & 86.5000 & 4.2500 & 297.0 & 3 & & 0 . \\
\hline 1815 & 114.4931 & & 297.2 & 3 & & \\
\hline 816 & 97.5028 & 4 . & $29^{\circ}$ & 3 & & \\
\hline 317 & 85.0008 & 5.1250 & 296.3 & 4 & & 0 . \\
\hline 818 & 76.2501 & 6.5250 & 294.3 & 4 & & 0 . \\
\hline 819 & 69.0000 & 000 & & 4 & & \\
\hline 00101820 & 68.5010 & 7.9250 & & 4 & & \\
\hline 0101821 & 74.0000 & 9.7250 & 293.1 & 4 & & 00 . \\
\hline & 71 & & & 4 & & \\
\hline 0101823 & 74 & 9.6750 & 293.0 & & 60 & 600 \\
\hline
\end{tabular}




\begin{tabular}{|c|c|c|c|c|c|c|}
\hline & 3.9994 & & 2.8 & 4 & 10.0 & \\
\hline 010191 & 69.7506 & 9.3750 & 292.3 & 4 & 00.0 & \\
\hline 10192 & 71.0002 & 9500 & 292.1 & 4 & 0 & \\
\hline 0193 & 0.9998 & .7750 & 91.0 & & 0 & \\
\hline 0194 & 69.5000 & 0 & 90.4 & & $c$ & \\
\hline 0195 & 497 & 3750 & 290.5 & & 0 & \\
\hline 0196 & 8.2489 & & 290.0 & & 0 & \\
\hline 0197 & 5.5024 & & 90.2 & & & \\
\hline 010198 & 16.9676 & 3.02 & 292.1 & & 00.0 & 0 \\
\hline 010199 & 154.9428 & & 292.2 & & & \\
\hline 910 & 161.7507 & & 92.5 & & & \\
\hline 911 & 164.2472 & & 293.0 & & 0 & \\
\hline 912 & 164.7501 & 0 & 293.6 & & & \\
\hline 913 & 167.2714 & & 94.3 & & & \\
\hline 4 & 161.5000 & & 295.1 & 3 & & \\
\hline 5 & 162.4960 & 3 . & 295.6 & & 0 & \\
\hline 16 & 160.2917 & & 96.2 & & & \\
\hline & 164.2547 & & 295.8 & & & \\
\hline 918 & 176.0003 & & 294.0 & & 0 & 000 \\
\hline 19 & 8 & & .5 & 4 & & \\
\hline & 65 . & & .3 & 4 & & \\
\hline 21 & 51 & & 293.1 & 4 & & \\
\hline 922 & 73.0009 & & 293.7 & 4 & 0 & \\
\hline 23 & 76.9998 & & 292.9 & 4 & & \\
\hline 4 & 73 & & 29 & 4 & & \\
\hline 201 & 78.7500 & & 293.2 & 4 & 0 & \\
\hline 0202 & 79.00 & & .7 & 4 & & \\
\hline 0203 & 46 . & 1 . & 29 & 5 & & \\
\hline 010204 & 45.5707 & & 292.4 & 6 & 0 & • \\
\hline 010205 & 351.2480 & & 291.8 & 5 & & \\
\hline 010206 & 7 & & .1 & 6 & & \\
\hline 010207 & 155.9586 & & .1 & 6 & & \\
\hline 010208 & 99.6280 & 4 . & 291.5 & 5 & & \\
\hline 010209 & 91.7282 & & 291.5 & 4 & & \\
\hline 010 & & & .7 & 4 & & \\
\hline 0102011 & 80.5351 & & 290.8 & 4 & & \\
\hline 02012 & 76 . & & 292.5 & 3 & & \\
\hline 00102013 & 67.2400 & & 292.7 & 4 & & 0 . \\
\hline 0102014 & 63.2495 & 0 & 291.3 & 4 & 0 & \\
\hline 015 & 87.1070 & & 291.3 & 4 & & \\
\hline 0010 & 75.4960 & & .9 & 4 & & \\
\hline 0102017 & 81.2511 & 7.10 & 289.0 & 4 & 0 & ח \\
\hline 0102018 & 823 & 4 . & 28 & 4 & & \\
\hline 00102019 & 85.0160 & 8. & 288.4 & 4 & 0 & 600. \\
\hline 0102020 & 74.5000 & 7.4000 & 287.4 & 4 & 600 & 00 \\
\hline & & & & 4 & & \\
\hline 00102022 & 74.2507 & 9 . & & 4 & 600 & 600 \\
\hline 02 & 68.2509 & 8.5000 & 286.5 & & 60 & 60 \\
\hline
\end{tabular}




\begin{tabular}{|c|c|c|c|c|c|c|}
\hline & שעקטים & 3250 & 86.3 & & & \\
\hline 0211 & 2498 & 9000 & 35.8 & & & \\
\hline 10212 & 2 & & & & & \\
\hline 3 & 79.7502 & 8000 & 85.5 & & & \\
\hline 0214 & 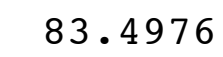 & & & & & \\
\hline 215 & 8 & & & & & \\
\hline 010216 & & & 85.5 & & & \\
\hline 010217 & 92 & & & & & \\
\hline 8 & 75 & 250 & 5.7 & & & \\
\hline 010219 & 161. & 250 & & & & \\
\hline 02110 & 149 & 1750 & & & & \\
\hline & 170 & & & & & \\
\hline 0102112 & 175 & 00 & 88.5 & & & \\
\hline 02113 & 158 & 1500 & 4 & & & \\
\hline & & & & & & \\
\hline $0-1-2=0$ & 164 & 50 & & & & \\
\hline 02116 & 162 & & 290.9 & & & \\
\hline 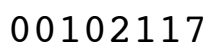 & 156 & & & & & \\
\hline 0 & 15 & 0 & 5 & & & \\
\hline 2119 & 15 & & & & & \\
\hline 02120 & 1 & & & & & \\
\hline & 16 & & 2 & & & \\
\hline 1 & 156 & & 28 & & & \\
\hline 123 & & 0 & & & & \\
\hline & & & & & & \\
\hline 01022 & & & & 4 & & \\
\hline 010222 & 1 & 0 & & 4 & & \\
\hline 010223 & 15 & & & 4 & & \\
\hline 010224 & & & & 4 & & \\
\hline 010225 & & 0 & 2 & 4 & & \\
\hline 010226 & & & & 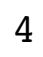 & & \\
\hline 01022 & & & & 4 & & \\
\hline 010228 & & 0 & 3.3 & 1 & & \\
\hline 010229 & & 0 & & 4 & & \\
\hline 210 & & & & 4 & & \\
\hline 2211 & & & 287.5 & 4 & & \\
\hline & & & & 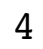 & & \\
\hline & & & & 4 & & \\
\hline 214 & 171 . & 750 & 290.7 & 4 & & \\
\hline 2 & 2 & & 291.3 & 4 & & \\
\hline 2216 & & 250 & 291 & 4 & & \\
\hline 0102217 & 167 . & 500 & 291.2 & 4 & & \\
\hline 21 & 3 & 750 & 290.2 & 4 & & \\
\hline 0102219 & 150 & & & 4 & & \\
\hline 0102220 & 150 . & .0250 & 289.2 & 4 & & \\
\hline 221 & 15 & & 288 & 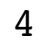 & & \\
\hline & & & & & & \\
\hline 0102223 & 839 & 7.6000 & 288.2 & & 600.0 & \\
\hline
\end{tabular}




\begin{tabular}{|c|c|c|c|c|c|c|}
\hline 2224 & 5.8235 & 4.3000 & 287.5 & 4 & 600.0 & \\
\hline 010231 & 5.2727 & 5.6750 & 287.2 & 4 & 600.0 & \\
\hline 10232 & 2508 & 3250 & 287.4 & 4 & 0 & \\
\hline 10233 & 310.6846 & 2.1500 & 286.7 & & 00 & \\
\hline 0234 & 261.8348 & 1.0500 & 84.5 & & 0 & \\
\hline 010235 & 2499 & 3250 & 83.1 & & 0 & \\
\hline 010236 & 141.5000 & 1250 & 283.5 & & 00 & \\
\hline 010237 & 91.9556 & .2000 & 83.8 & & 0 & \\
\hline 010238 & 129.6415 & 2.6500 & 284.6 & & 0 & \\
\hline 010239 & 152.9989 & 4.7250 & 284.6 & & 0 & \\
\hline 0102310 & 155.7459 & 750 & 1 & & & \\
\hline 02311 & 159.7498 & & 87.8 & & & \\
\hline 0102312 & 160.5037 & 2250 & 289.3 & & 0 & \\
\hline 313 & 165 . & 000 & 0 & & & \\
\hline & 185 . & & & & & \\
\hline 02315 & 180 . & 000 & 29 & & 0 & \\
\hline 0102316 & 352.7562 & 1.5500 & 295.1 & & 0 & \\
\hline & 33.6445 & 117 & 294.8 & $\boldsymbol{J}$ & & \\
\hline 318 & 135. & 1.1750 & 292 & 4 & & \\
\hline 0102319 & 292.0152 & 9250 & 291 & 5 & 0 & \\
\hline 20 & 356 . & 0 & 29 & 4 & & \\
\hline 21 & 16. & 1.7250 & 291.4 & 4 & & \\
\hline 0102322 & 25.5067 & .0000 & 292.3 & 5 & 0 & \\
\hline 02323 & 40.9700 & 0 & 29 & 5 & & \\
\hline 24 & 72 . & & 29 & 5 & & \\
\hline 010241 & 502 & 3250 & 292.6 & 4 & & \\
\hline 0010242 & 94.5071 & 3.42 & 292.1 & 5 & & \\
\hline 010243 & 21.6692 & 0 & 290.8 & 5 & & \\
\hline 010244 & 375 & & 290.2 & 4 & & \\
\hline 0010245 & 452 & 000 & 290.9 & 5 & & \\
\hline 0010246 & 58.1489 & & 290.7 & 6 & & \\
\hline 0010247 & 141 . & & 290.3 & 6 & & \\
\hline 0010248 & 153.2610 & .8500 & 291.8 & 5 & & \\
\hline 0010249 & 191.4181 & 0 & 294.2 & 4 & & \\
\hline 0010 & 294 & & 294.8 & 3 & & \\
\hline & 234.2503 & 2.4500 & 294.1 & 3 & & \\
\hline & 227 . & & 294.0 & 2 & & \\
\hline 0010 & 224.9995 & 2 . & 295.1 & 1 & & 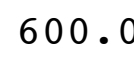 \\
\hline 0102414 & 228.6541 & 2.6000 & 295.5 & 1 & & 6 \\
\hline 15 & 234.2644 & 1. & 296.1 & 1 & & \\
\hline 00102 & 239.4877 & 1 . & 295.6 & 1 & & \\
\hline 00102417 & 217.1396 & 1.8000 & 295.2 & 2 & 0 & 0 \\
\hline 00102 & 70.9947 & 250 & 293.0 & 3 & & \\
\hline 00102419 & 79.8811 & 4.9750 & 291.9 & 4 & .0 & 600 \\
\hline 00102420 & 69.5009 & 6.9750 & 290.6 & 4 & 600.0 & 600 \\
\hline & 58 & & 290.1 & a & & \\
\hline 0102422 & 56.3987 & & 290.7 & 4 & & 600 \\
\hline $010<4<2$ & 64.9057 & 2.1250 & 290. & & 600.0 & 60 \\
\hline
\end{tabular}




\begin{tabular}{|c|c|c|c|c|c|c|}
\hline 424 & 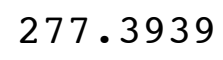 & .0500 & 87.3 & & - & \\
\hline 251 & 279 . & .2500 & 84.9 & 5 & 600.0 & \\
\hline 0252 & 104.0673 & 1.0000 & 283.9 & 6 & 00 & \\
\hline 10253 & 351.5128 & 8000 & 283.6 & 6 & 00 & \\
\hline 0254 & 2.9610 & 9500 & 83.4 & & 0 & \\
\hline $025 \quad 5$ & 8.2446 & 4000 & 84.1 & & 00 & \\
\hline 010256 & 344.7496 & 0250 & 284.1 & 6 & 00 & \\
\hline 010257 & 311.1531 & .5750 & 84.3 & & 0 & \\
\hline 010258 & 225.9941 & .3500 & 84.5 & 5 & & \\
\hline 010259 & 201.0391 & 0.7750 & 85.7 & 4 & 600.0 & 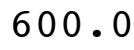 \\
\hline 0102510 & 327.0184 & 3.3250 & 287.7 & 3 & 0 & \\
\hline & 7.9024 & 6.9750 & 87.8 & 4 & 00.0 & \\
\hline 2 & 6.4187 & 4.5500 & 286.9 & 3 & $\cdot 0$ & ( \\
\hline 13 & 341.6563 & 3.3250 & 287.4 & 2 & 00.0 & \\
\hline & 000 & 0 & & 3 & & \\
\hline & 5055 & 3250 & 28 & 4 & & \\
\hline 6 & 0.4990 & 9250 & 288.8 & 3 & .0 & . \\
\hline 17 & 358.5003 & .3750 & 8.4 & 4 & 0 & \\
\hline & 355.7515 & 3.5000 & 287.0 & 4 & & \\
\hline 19 & 2.9507 & 2.9750 & 286.3 & 4 & 0 & \\
\hline 20 & 340.7401 & 2.2750 & 285.8 & 5 & 0 & - \\
\hline & 353.1002 & 2.4000 & & 5 & & \\
\hline 22 & 329.9989 & 2.4750 & 285.7 & 6 & & \\
\hline 23 & 346.2463 & 2.2750 & 285.1 & 5 & 0 & 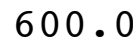 \\
\hline 24 & 330.4969 & 1.6250 & 28 & 5 & & \\
\hline 001 & 330.5169 & 3.4250 & 28 & 4 & & \\
\hline 010262 & 315.2517 & 4.4250 & 285.4 & 4 & 0 & U. \\
\hline 010263 & 319.7468 & 4250 & 0 & 4 & & \\
\hline 264 & 303.7344 & 3.6250 & 28 & 4 & & • \\
\hline 010265 & 309.7194 & 4.4750 & 283.2 & 4 & 0 & 0 . \\
\hline 010266 & 315.2511 & 6250 & 283.2 & 4 & 0 & 0 . \\
\hline 267 & 311.5013 & 4.7500 & 282 & 4 & & \\
\hline 268 & 303.9977 & 4.2000 & 28 & 4 & & U. \\
\hline 010269 & 293.9947 & 5.5500 & 283.4 & 4 & 0 & 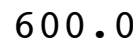 \\
\hline 0 & 307.7649 & 0 & & 4 & & \\
\hline & 289.7502 & 7.5500 & 283.8 & 4 & & ( \\
\hline 512 & 327.7513 & 7.5250 & 284.2 & 4 & $\cdot 0$ & 0 . \\
\hline 00 & 318.5170 & 7.4250 & 28 & 4 & & - \\
\hline 001 & 278.2497 & 6.0000 & 283.9 & 4 & .0 & 0 . \\
\hline 515 & 270.7499 & 4.5250 & 283.8 & 3 & & \\
\hline & 291.7363 & 3.7750 & 283.8 & 2 & & 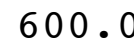 \\
\hline 0010 & 282.7314 & 3.6250 & 283.7 & 3 & .0 & 0 . \\
\hline 0102618 & 309.5294 & 1.6750 & 283.6 & 4 & .0 & 00 . \\
\hline 00102619 & 235.3126 & 1.3500 & & 5 & & \\
\hline 00102620 & 220.2472 & 1.5750 & & 4 & & 600. \\
\hline 0102621 & 193.7597 & 2.0250 & 283.0 & 4 & 600.0 & 600 \\
\hline & 196 & & & 4 & & \\
\hline 0102623 & 182.9998 & 7.6000 & 282.7 & & 600.0 & 60 \\
\hline
\end{tabular}




\begin{tabular}{|c|c|c|c|c|c|c|}
\hline 524 & & .0000 & 2.7 & & & \\
\hline 271 & 176 & 5.8000 & 32.5 & 4 & 600.0 & \\
\hline 0272 & 7500 & 750 & 282.4 & 4 & 00 & \\
\hline 10273 & 173.2523 & 7.1250 & 282.3 & & 00 & \\
\hline $027 \quad 4$ & 175.7502 & 8000 & 82.3 & & 0 & \\
\hline 0275 & 173.7583 & 3000 & 286.2 & & 00 & \\
\hline 010276 & 159.7502 & 4750 & 290.5 & & 00 & \\
\hline 010277 & 159.7456 & 250 & 90.7 & & 0 & \\
\hline 010278 & 163.7499 & 6750 & 90.5 & 4 & & \\
\hline 010279 & 164.2483 & 6.6750 & 290.2 & 4 & 00 & 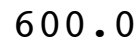 \\
\hline 0102710 & 153.1172 & 3.4250 & 290.0 & & 0 & \\
\hline 2711 & 152.5530 & 1.2500 & 91.7 & & 0 & \\
\hline 2712 & 84.6287 & 0.5250 & 292.9 & 1 & 0 & \\
\hline 02713 & 173.7332 & 0.7250 & 294.4 & & 0.0 & \\
\hline 2714 & 152 & 500 & 294.6 & & & \\
\hline 715 & 181 & & 292.5 & 1 & & \\
\hline 2716 & 190 & 2.7500 & 290.3 & & .0 & 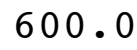 \\
\hline 2717 & 278.5274 & 0.72 & 8.8 & 3 & & \\
\hline 2718 & 69.5416 & 750 & 287.6 & 4 & & \\
\hline 2719 & 72 . & $\$ 750$ & 286.5 & 4 & & \\
\hline 2720 & 67.7501 & 5.8000 & 285.9 & 4 & 0 & • \\
\hline 721 & 67.9999 & & & 4 & & \\
\hline 722 & 66 & 0 & 285.7 & 4 & & \\
\hline 2723 & 71.0024 & 8.0500 & 285.1 & 4 & 0 & 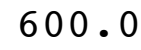 \\
\hline 724 & 63. & & 28 & 4 & & \\
\hline 281 & 55 & 4.6000 & 28 & 4 & & \\
\hline 010282 & 67.0000 & .3000 & 284.1 & 4 & 0 & U. \\
\hline 010283 & 74 . & 0 & 284.3 & 5 & & \\
\hline 0010284 & 289 & 50 & 28 & 6 & & • \\
\hline 010285 & 164 . & 0. & 283.0 & 6 & 0 & U. \\
\hline 0010286 & 43.9234 & 1.6750 & 283.2 & 6 & 0 & 0 . \\
\hline 0010287 & 9.7477 & 0 & 282.9 & 5 & & \\
\hline 010288 & 353 . & 1. & 284 & 4 & & 0 \\
\hline 010289 & 350.9826 & 000 & 286.0 & 3 & 0 & \\
\hline 2810 & 348 . & 3 . & & 2 & & \\
\hline 811 & 339.0185 & 4.5500 & 287.7 & 3 & & 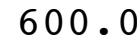 \\
\hline 0102812 & 338.1457 & 5.0250 & 288.1 & 3 & 0 & 0. \\
\hline 0102813 & 350.3816 & 3 . & 28 & 2 & & . \\
\hline 00102814 & 344.2863 & 4.2750 & 288.1 & 3 & .0 & 0 . \\
\hline 0102815 & 348.7427 & 4.9500 & 288.3 & 3 & & \\
\hline 00102816 & 359.2500 & 500 & 288.3 & 4 & & 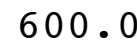 \\
\hline 00102817 & 49.1774 & 4.3250 & 286.8 & 4 & .0 & 0 . \\
\hline 0102818 & 131.5775 & 4.7000 & 284.8 & 4 & 0 & 00 . \\
\hline 00102819 & 5009 & 750 & & 4 & & \\
\hline 00102820 & 91.5150 & 3.9000 & & 4 & & 600. \\
\hline 0102821 & 2498 & 5.2500 & 284.1 & 4 & 600 & 600 \\
\hline & & 4. & & 4 & & \\
\hline 0102823 & 764 & 4.2000 & 284.2 & 7 & 600.0 & 60 \\
\hline
\end{tabular}




\begin{tabular}{|c|c|c|c|c|c|c|}
\hline 2824 & 49.9105 & 2.3750 & 284.5 & 5 & 600.0 & \\
\hline 291 & 4.4995 & 4.1000 & 284.4 & 4 & 600.0 & \\
\hline 010292 & 5347 & 3500 & 284.3 & 4 & 00 & \\
\hline 010293 & 299.1412 & 1.2000 & 283.3 & & 00 & \\
\hline 0294 & 126.4594 & L.8500 & 3.4 & & 0 & \\
\hline 0295 & 66.3643 & .1250 & 283.0 & 6 & 00 & \\
\hline 010296 & 57.2210 & 2.9250 & 282.9 & & 0 & \\
\hline 010297 & 64.7310 & .3500 & 82.9 & & & \\
\hline 010298 & 68.5067 & 3.5250 & 283.0 & 4 & 0 & \\
\hline 010299 & 74.4739 & 2.4750 & 283.5 & & 00.0 & \\
\hline 910 & 95.7441 & 2.1750 & 284.3 & & 0 & \\
\hline 911 & 140.5717 & 265 & 84.3 & 1 & & \\
\hline 0102912 & 108 & 000 & 285.3 & & 0 & \\
\hline 2913 & 61.3308 & 4.0500 & 286.9 & 3 & 0.0 & \\
\hline 914 & 101.5000 & & & 3 & & \\
\hline 2915 & 106 & 5750 & 286.0 & 3 & 0 & \\
\hline 0102916 & 90.0640 & 3.33 & 286.1 & 3 & 0 & \\
\hline 17 & 80 . & 4 . & .3 & 4 & & \\
\hline 2918 & 637 & 3. & 284.2 & 4 & 0 & \\
\hline 0102919 & 5000 & .70 & 283.6 & 4 & 0 & \\
\hline 920 & 130 & 0 & 9 & 5 & & \\
\hline 921 & 228 & & 282.5 & 6 & & \\
\hline 0102922 & 51.2077 & 0 & 282.7 & 5 & 0 & \\
\hline 0102923 & 21.9989 & 2 . & 282.2 & 5 & 0 & \\
\hline 924 & 73. & & 282.9 & 4 & & \\
\hline 010301 & 2506 & & 282.1 & 4 & & \\
\hline 010302 & 49.5000 & 3.25 & 282.5 & 5 & 0 & \\
\hline 0010303 & 031 & & 282.5 & 4 & & \\
\hline 010304 & 998 & 4 & 28 & 4 & & 0 . \\
\hline 010305 & 57.2475 & 3 . & 283.0 & 4 & 0 & \\
\hline 010306 & 23.8500 & & 282 & 5 & & \\
\hline 0010307 & 633 & 1 . & 282.2 & 5 & & \\
\hline 0010308 & 310.8651 & 0 & 281.9 & 4 & 0 & J. \\
\hline 010309 & 290 & & 282.7 & 3 & 0 & \\
\hline 010 & 263 & 0 & 282.6 & 2 & & \\
\hline 00103011 & 274 . & & 284.1 & 2 & & 0 . \\
\hline 03012 & 264 . & 0 & 283.4 & 2 & & \\
\hline 001 & 272 . & 0 & 28 & 2 & & 0 . \\
\hline 00103014 & 256.8074 & 3 . & 283.9 & 2 & .0 & ) \\
\hline 3015 & 240.5000 & 2 . & 283.6 & 2 & & \\
\hline 00103016 & 310.7815 & 1 . & & 3 & & \\
\hline 00103017 & 11.2481 & 1.4000 & 283.9 & 4 & 600.0 & 600 . \\
\hline 03018 & 73.0813 & 750 & 284.0 & 4 & 600 & 000 \\
\hline 00103019 & 142.7491 & 2.2500 & & 5 & & $c_{0}$ \\
\hline 00103020 & 172.8259 & 6.0000 & 283.2 & 4 & 600.0 & 00 \\
\hline 0103021 & 176.5002 & 7.2000 & 282.3 & 4 & 600.0 & 60 \\
\hline 0103 & 162.2510 & 0 & 28 & 4 & 60 & \\
\hline 0103023 & 149.5003 & 5.8000 & 281.6 & 4 & 600.0 & 600 \\
\hline
\end{tabular}




\begin{tabular}{|c|c|c|c|c|c|c|}
\hline 024 & & 0 & & & & \\
\hline 311 & 9 & 2750 & 2.4 & 4 & 0 & \\
\hline 10312 & 93.5017 & 4500 & 282.5 & 4 & 00 & \\
\hline 10313 & 106.2575 & 8000 & 283.2 & & 00 & \\
\hline 0314 & 130.7519 & 1000 & 33.6 & & & \\
\hline 0315 & 122.02 & 5000 & 83.4 & & 0 & \\
\hline 10316 & 143.2708 & 3750 & 83.7 & & 00 & \\
\hline 0317 & 145.2473 & 8250 & 3.7 & & & \\
\hline 031 & 144.0008 & 5000 & 83.8 & & & \\
\hline 319 & 152.2504 & 6250 & 84.7 & & 0 & 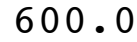 \\
\hline 0103110 & 156.9982 & 8.4500 & 285.2 & & & \\
\hline 17 & 164.7500 & .2250 & & 4 & & \\
\hline 12 & 003 & 7.9250 & 86.1 & 4 & 0 & \\
\hline 113 & 168.7509 & 7.77 & 287.4 & & .0 & \\
\hline 114 & 167 & & & 4 & & \\
\hline & 164 . & & 2 & 4 & & \\
\hline 16 & 170.2495 & 9750 & 288.6 & 4 & 0 & 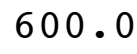 \\
\hline 117 & 166.5000 & 500 & 3 & 4 & & \\
\hline 18 & 166.7568 & & 4 & 4 & & \\
\hline 19 & 7545 & 250 & 287.2 & 4 & 0 & 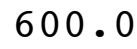 \\
\hline 120 & 174.7504 & 3. & 287.1 & 4 & 0 & \\
\hline 21 & 175.4998 & & & 5 & & \\
\hline 22 & 801 & 50 & 28 & 4 & & \\
\hline 123 & 126.9165 & 9250 & 287 & 4 & 0 & \\
\hline 124 & 100. & 0 & 28 & 4 & & \\
\hline 001111 & 175 . & 5250 & 287 & 5 & & \\
\hline 01112 & 169.2732 & 1500 & 2 & 6 & & J. \\
\hline 01113 & 174.0019 & 0 & 3 & 5 & & \\
\hline 001114 & 166. & 0 & 28 & 4 & & \\
\hline 01115 & 168.0616 & 3.2000 & 6 & 5 & & U. \\
\hline 01116 & 129.3298 & 2.9500 & 28 & 5 & & 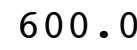 \\
\hline 01117 & 163.6252 & 3 & & 4 & & \\
\hline 01118 & 180.9963 & 0 & 4 & 5 & & . \\
\hline 01119 & 298.8306 & 3000 & $2 \varepsilon$ & 4 & & 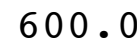 \\
\hline 011 & 193 & 0 & & 3 & & \\
\hline 0011111 & 219.4815 & 0.3500 & & 2 & & 0 . \\
\hline 011112 & 175.7573 & 1.3250 & 288.2 & 1 & & \\
\hline 011113 & 168.4997 & 3 . & 28 & 2 & & \\
\hline 0011114 & 175.5003 & 2.2500 & 288.9 & 1 & .0 & 0 . \\
\hline $0011 \quad 115$ & 173.2524 & 1.5750 & 288.3 & 1 & & \\
\hline 011116 & 179.7468 & 000 & 287 & 1 & & \\
\hline 0011117 & 179.9992 & 2.4250 & 286.9 & 2 & & 0 . \\
\hline 011118 & 158.7405 & 1.8250 & 286.1 & 3 & 0 & 0 . \\
\hline 0011119 & 152.7256 & 000 & & 4 & & \\
\hline 0011120 & 124.8415 & 2.4750 & & 5 & & 0 . \\
\hline 0011121 & 109.4637 & 2.9500 & 286.0 & 5 & & 00 \\
\hline 011122 & 171. & & & 5 & & \\
\hline 011123 & 169.0018 & 4.4000 & 285.1 & & 600.0 & 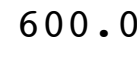 \\
\hline
\end{tabular}




\begin{tabular}{|c|c|c|c|c|c|c|c|}
\hline 011 & 24 & & & & & & \\
\hline & 1 & 2 & .5750 & 4.4 & & 0 & \\
\hline 1 & 2 & 165.7502 & 2.5750 & 284.1 & & 00 . & \\
\hline 1 & 3 & 138.5882 & 4.1500 & 284.4 & & 00.0 & \\
\hline & 4 & 65.8233 & .9750 & & & 0 & \\
\hline & 5 & 2.9678 & 0000 & 83.8 & & 00 & \\
\hline 1 & 6 & 151.8842 & 2.7250 & 284.4 & & 00 & 0 \\
\hline & 7 & 147.3130 & 2.3000 & 7 & & .0 & \\
\hline & 8 & 80.5031 & 3.2250 & 86.0 & & & \\
\hline 01 & 9 & 131.7900 & 1.4750 & 87.5 & & 600.0 & 00 \\
\hline 01 & 210 & 174.5000 & 3.2000 & 288.2 & & .0 & \\
\hline & 11 & 170.4890 & 3.5250 & 9 & & .0 & \\
\hline 01 & 12 & 167.2555 & 2 . & 288.4 & & .0 & \\
\hline 1 & 13 & 158.7855 & 3.15 & 289.4 & & 0.0 & \\
\hline & 14 & 168.4999 & & & & & \\
\hline 01 & 15 & 168.7578 & & 90.2 & & & \\
\hline 01 & 16 & 149.0688 & 2.2000 & 290.4 & & .0 & $\bullet$ \\
\hline & 17 & 148.4415 & 1 . & 0.3 & & .0 & \\
\hline 0 & 18 & 105.7641 & & 9.3 & 3 & & \\
\hline 1 & 219 & 103.1967 & 250 & 289.2 & 4 & 0 & 0 \\
\hline 01 & 220 & 97.7618 & 1.9000 & 289.0 & & .0 & 00 \\
\hline 0 & 221 & 75.2292 & & 289.2 & & & \\
\hline 1 & 222 & 105.5000 & 50 & 289.3 & 6 & & \\
\hline 01 & 223 & 139.2705 & 750 & 288.7 & 6 & 0 & 00 \\
\hline 01 & 224 & 154.9927 & & & 5 & & \\
\hline 00 & 31 & 174.2503 & 4 . & 287.6 & 4 & & \\
\hline 01 & 32 & 179.7514 & 9000 & 287.0 & 4 & .0 & U. \\
\hline 0 & 33 & 169.8215 & 0 & $2 \varepsilon$ & 5 & & \\
\hline 00 & 34 & 175.5002 & 3 . & 28 & 4 & & $\bullet$ \\
\hline 10 & 35 & 178.5000 & 7000 & 284.8 & 4 & 0 & 0 . \\
\hline 01 & 36 & 167.3349 & 4.3750 & 284.4 & 4 & .0 & 0 . \\
\hline 01 & 37 & 180.5000 & 0 & 284.3 & 4 & & \\
\hline . & 38 & 170.3960 & & 285.0 & 4 & & U. \\
\hline 01 & 39 & 177.5010 & 500 & 28 & 4 & 0 & 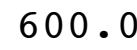 \\
\hline 0 & 310 & 180.5000 & 0 & & 4 & & \\
\hline 001 & 311 & 179 . & 6.4500 & 286.7 & 4 & & 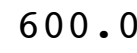 \\
\hline 01 & 312 & 179.0001 & 9500 & 287.6 & 3 & .0 & 0 . \\
\hline 0 & 313 & 178 . & 6 . & 288 & 4 & & . \\
\hline 001 & 314 & 176.7500 & 5.1000 & 289.4 & 3 & .0 & 600 . \\
\hline 001 & 315 & 175.7552 & 3.7750 & 290.2 & 2 & & 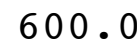 \\
\hline & 316 & 175.7499 & 250 & 290.1 & 3 & & 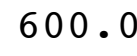 \\
\hline 001 & 317 & 177.5000 & 4.2250 & 289.7 & 4 & .0 & 0 . \\
\hline 101 & 318 & 154.5668 & 2.7750 & 289.5 & 5 & 0 & 00 . \\
\hline 00 & 319 & 117.3036 & 2.7000 & 289 & 6 & & \\
\hline 0011 & 320 & 145.6649 & 3.9750 & 290.4 & 5 & & 600. \\
\hline 0 & 321 & 162.3238 & 5.1500 & 290.5 & 4 & 600.0 & 600 \\
\hline & 322 & 181 & & & 4 & & \\
\hline & 323 & 7845 & 5.4000 & 290.6 & $\mathbf{4}$ & 600.0 & 600 \\
\hline
\end{tabular}




\begin{tabular}{|c|c|c|c|c|c|c|c|}
\hline- & 24 & & & & & & \\
\hline & 1 & 8 & 3.6500 & 90.0 & 4 & 0 & \\
\hline 1 & 42 & 89.0000 & 0000 & 289.9 & 4 & 00 & \\
\hline 01 & 4 & 73.2397 & 5.2000 & 289.5 & & 00.0 & \\
\hline & 4 & 84.7450 & .5250 & 89.3 & & 0 & \\
\hline & 45 & 164.0024 & .5500 & 88.0 & & $0 C$ & \\
\hline 1 & 46 & 218.5064 & 7000 & 286.5 & & 00 & 0 \\
\hline & 7 & 3.2573 & 1.6750 & 86.5 & & 00.0 & \\
\hline & 48 & 18.5097 & .9500 & 5 & & & \\
\hline 01 & 49 & 113.0269 & 0.6500 & 290.5 & 4 & 000 & 0 \\
\hline 01 & 410 & 211.8256 & 1.1000 & 291.1 & & 00.0 & \\
\hline 0 & 411 & 177.5288 & 2.4500 & 7 & & 00.0 & \\
\hline 01 & 412 & 235.9984 & 2.42 & 289.7 & 3 & .0 & \\
\hline 1 & 413 & 282.2540 & 1.4750 & 291.6 & 2 & 0.0 & 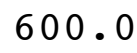 \\
\hline & 414 & 295.5037 & 2 . & 92.2 & & & \\
\hline 01 & 415 & 92.8294 & 1 . & 294.0 & 2 & & \\
\hline 01 & 416 & 68.2868 & 4.3000 & 293.3 & 3 & .0 & 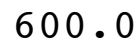 \\
\hline & 417 & 63.7425 & 3.8500 & 92.6 & -1 & .0 & \\
\hline ? & 418 & 63.7496 & 5 . & 291.3 & 4 & & \\
\hline 0 & 419 & 71.7344 & 0 & 290.1 & 4 & & \\
\hline 01 & 420 & 54.7488 & 3.1250 & 288.8 & 4 & . 0 & 00 . \\
\hline 0 & 421 & 41.2513 & & & 5 & & \\
\hline 0. & 422 & 67.4920 & 7.3750 & 288.0 & 4 & & \\
\hline 01 & 423 & 77.2495 & 9.7750 & 285.2 & 4 & 0 & ) \\
\hline 01 & 424 & 000 & 0 & & 4 & & \\
\hline 001 & 51 & 72.4997 & 7 . & 285.6 & 4 & & \\
\hline 01 & 52 & 79.2501 & 3500 & 284.8 & 4 & 0 & U. \\
\hline 0 & 53 & 75.997 & 0 & 283.4 & 4 & & \\
\hline 0 & 54 & 72.7500 & 000 & 283.3 & 4 & & $\bullet$ \\
\hline 19 & 55 & 77.9996 & 5750 & 283.6 & 4 & 0 & 0 . \\
\hline 01 & 56 & 82.0042 & 6.8500 & 283.4 & 4 & .0 & 0 . \\
\hline 01 & 57 & 83.7510 & 0 & 283.2 & 4 & & \\
\hline 政 & 58 & 91.2484 & 6.2000 & 283.6 & 4 & & o. \\
\hline 01 & 59 & 132.3742 & 6.7750 & 284.7 & 4 & 0 & 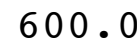 \\
\hline $0-$ & 510 & 112.2598 & 125 & & 3 & & \\
\hline 001 & 511 & 165.2461 & 5.0500 & 286.6 & 3 & & . \\
\hline 01 & 512 & 174.7583 & 4.9000 & 287.8 & 3 & .0 & 0 . \\
\hline 0 & 513 & 164.5133 & 4.8750 & 288.8 & 3 & & ( \\
\hline 001 & 514 & 165.6165 & 4.7250 & 289.5 & 3 & .0 & 0 . \\
\hline 1201 & 515 & 98.2236 & 3.9750 & 290.2 & 2 & .0 & 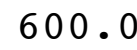 \\
\hline & 516 & 78.2512 & 6.5500 & 289.4 & 3 & & 60 \\
\hline 001 & 517 & 68.0008 & 7.0250 & 288.8 & 4 & & 0 . \\
\hline 01 & 518 & 71.2467 & 7.9250 & 288.1 & 4 & 0 & 00 . \\
\hline 00 & 519 & 79.9977 & 7.7750 & 288.1 & 4 & & \\
\hline 001 & 520 & 74.7496 & 8.0750 & 288.1 & 4 & & 600. \\
\hline 01 & 521 & 81.4992 & 8.1000 & 287.8 & 4 & 600.0 & 600 \\
\hline & 522 & 70.4997 & & 287.2 & 4 & & \\
\hline & 523 & 71.0008 & 8.8000 & 286.9 & $\mathbf{4}$ & 600.0 & 600 \\
\hline
\end{tabular}




\begin{tabular}{|c|c|c|c|c|c|c|c|}
\hline & 24 & m. & 9.2000 & 286.5 & 4 & 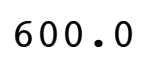 & \\
\hline & 1 & -00 & 9.3500 & 286.1 & & & \\
\hline & 62 & 000 & 500 & 35.9 & & . & \\
\hline & 3 & 3.2496 & 9750 & 85.7 & & 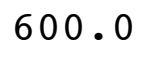 & \\
\hline & 4 & & & & & & \\
\hline & 65 & 0001 & 9250 & 84.6 & & 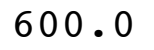 & \\
\hline & 6 & 84.9966 & 5000 & 83.7 & & - & \\
\hline & 7 & 527 & .6250 & & & & \\
\hline & 8 & 81.2498 & 0.3000 & 85.8 & & 0 & \\
\hline & 9 & 171.5000 & 8.3500 & 85.7 & & & \\
\hline & 10 & 166 & & & & & \\
\hline & 11 & 177 . & & & & & \\
\hline & 12 & 175.7510 & 000 & 87.6 & & 0 & \\
\hline & 13 & 160 & 0 & & & & \\
\hline & 1 & & & & & & \\
\hline & 15 & 170 & 0 & 89.1 & & & \\
\hline & 616 & 169.2507 & 3.9250 & 9 & & & \\
\hline & 617 & 180. & & & & & \\
\hline & 18 & 168 & 0 & 0 & 4 & & \\
\hline & 619 & 15 & 0 & $2 \varepsilon$ & & & \\
\hline & 620 & & & & & & \\
\hline & 621 & & & & 4 & & \\
\hline & 622 & 151. & 2 . & 3 & 4 & & \\
\hline & 623 & & & & & & \\
\hline & 624 & & 0 & & 4 & & \\
\hline & 71 & 153 & & & 4 & & \\
\hline & 72 & & 0 & & 4 & & \\
\hline & 73 & 15 & 0 & & 4 & & \\
\hline & 74 & 151 . & & & 4 & & \\
\hline & 75 & & 0 & & 4 & & \\
\hline & 76 & & & & 4 & & \\
\hline & 77 & & 0 & & 4 & & \\
\hline & 78 & 155 . & 3500 & 1 & 4 & & \\
\hline & 79 & & 0 & & 4 & & \\
\hline & 710 & 16 & & & 4 & & \\
\hline & 711 & 160.2499 & & 28 & 4 & & \\
\hline & 712 & 16 & & & 4 & & \\
\hline & 713 & 165 . & 750 & 2 & 4 & & \\
\hline & 714 & 163.0000 & & 288.5 & 3 & & \\
\hline & 715 & 15 & & & 3 & & \\
\hline & 716 & 164 . & 50 & & 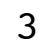 & & \\
\hline & 717 & 164.2473 & 2.2000 & 289.2 & 4 & & \\
\hline & 718 & & 250 & & J & & \\
\hline & 719 & & 1.6500 & & 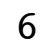 & & \\
\hline & 720 & 35.0000 & 1.7000 & 288.1 & 5 & 0 & P \\
\hline & 721 & & & & & & \\
\hline & 722 & 147.9876 & & & & & \\
\hline & 723 & 158.4830 & 0.1750 & 287.2 & & .0 & - \\
\hline
\end{tabular}




\begin{tabular}{|c|c|c|c|c|c|c|c|}
\hline & 24 & & & 7.7 & & & \\
\hline 1 & 1 & 1 & 0.9750 & 287.3 & & & \\
\hline $1+2$ & 82 & 122.0850 & 6250 & 287.2 & & . & \\
\hline 01 & 3 & 38.4740 & 1.6000 & 287.1 & & 00.0 & \\
\hline & 4 & 20.7453 & 1.3500 & 7.2 & & & \\
\hline & 5 & 34.1428 & 1.0750 & 87.3 & & s & \\
\hline & 6 & 125.1877 & 0.6250 & 287.0 & & 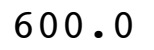 & \\
\hline & 7 & 133.0249 & 1.1750 & 87.0 & & & \\
\hline & 8 & 51.3959 & 0.6500 & 87.2 & & 0 & \\
\hline n & 9 & 5577 & 0.5000 & 289.1 & & 00 & \\
\hline & 10 & 22.7156 & 1.07 & 290 & & & \\
\hline & 11 & 75.8864 & & 2 & & & \\
\hline 0 & 12 & 71.0023 & 7.1000 & 288.7 & & 0 & \\
\hline 0 & 813 & 87.0436 & 5.20 & 288.8 & & 0.0 & \\
\hline & 814 & & & & & & \\
\hline 0 & 15 & 66.5002 & 6.27 & 288.3 & 4 & & \\
\hline & 816 & 60.2494 & 2.87 & 288.0 & 4 & 0 & \\
\hline & 17 & 55.7500 & 0 & 28 & - & & \\
\hline 0 & 18 & 56.4997 & 0 & 28 & 4 & & \\
\hline & 819 & 64.2501 & 11.72 & 283.0 & 4 & & \\
\hline & 820 & 61.2492 & 11. & 282.5 & 4 & & \\
\hline & 821 & 66.0027 & & 282.6 & 4 & & \\
\hline 0 & 822 & 59.2498 & 0 & 28 & 4 & & \\
\hline 0 & 823 & 62.7499 & 0 & 282.1 & 4 & & \\
\hline & 824 & 67.7497 & & 281 & 4 & & \\
\hline & 91 & 2498 & $\begin{array}{ll}9 & 7\end{array}$ & 281.1 & 4 & & \\
\hline & 92 & 5000 & 9.52 & 280.8 & 4 & & \\
\hline 0 & 93 & 62 & 0 & 280 & 4 & & \\
\hline & 94 & 000 & 0 & 280.1 & 4 & & \\
\hline & 95 & 998 & 0 & 279.8 & 4 & & \\
\hline & 96 & 002 & 0 & 27 & 4 & & \\
\hline 00 & 97 & 5 & 0 & 27 & 4 & & \\
\hline & 98 & 64.7500 & 7.8750 & 279.8 & 4 & 0 & J. \\
\hline 0 & 99 & 569 & & 280.3 & 4 & & \\
\hline 0 & 910 & 34 & 0 & 28 & 4 & & \\
\hline & 911 & 79.0018 & 7.4000 & 282.0 & 4 & & \\
\hline & 912 & 74.2486 & 0 & 282.8 & 4 & & \\
\hline & 913 & 82.7538 & 0 & 283.6 & 3 & & 0 . \\
\hline 0 & 914 & 66.8935 & 500 & 284.0 & 3 & 0 & 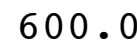 \\
\hline & 915 & 494 & & 28 & 3 & & \\
\hline & 916 & 839 & 6 . & 283.0 & 4 & & \\
\hline 00 & 917 & 59.9989 & 6.5250 & 282.2 & 4 & 600.0 & 0. \\
\hline & 918 & 66.7509 & 6.4250 & 281.1 & 4 & 600 & \\
\hline & 919 & 61.7509 & 9.1500 & 280.6 & 4 & & \\
\hline 00 & 920 & 61.2499 & 8.9000 & 280.4 & 4 & 600.0 & 00 \\
\hline & 921 & 61.7500 & 9.1500 & 280.4 & 4 & 600 & \\
\hline & 922 & .2501 & & 280.2 & 4 & & \\
\hline & 923 & 58.5003 & 9.5750 & 280.0 & $I$ & 600.0 & 600 \\
\hline
\end{tabular}




\begin{tabular}{|c|c|c|c|c|c|c|}
\hline 011 & 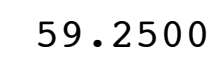 & 9.5500 & 279.9 & 4 & 000.0 & \\
\hline 011101 & 3.0008 & 8.6250 & 279.4 & 4 & 0 & \\
\hline 11102 & 995 & $\$ 750$ & 279.3 & 1 & 0 & \\
\hline 11103 & 5.7500 & 7.5500 & 279.1 & & 00 & \\
\hline 11104 & 27643 & 1.8500 & 78.8 & & & \\
\hline 011105 & 428 & 500 & 279.0 & & 0 & \\
\hline 011106 & 9.2903 & 1.0500 & 278.7 & & 00 & \\
\hline 011107 & 41.0241 & 3.6250 & 78.9 & & 0 & \\
\hline 011108 & 4.9989 & 3.9000 & 279.4 & & 0 & \\
\hline 011109 & 0.4963 & 1.4750 & 281.4 & & 0 & \\
\hline 11010 & 62.5244 & 500 & 1.8 & & & \\
\hline 11011 & 2.7426 & 00 & & & & \\
\hline 11012 & 225.5493 & 2.3750 & 282.0 & & 0 & \\
\hline 1013 & 285.2821 & 1.3000 & 9.5 & 1 & & \\
\hline 014 & 236.4871 & & 2.7 & 1 & & \\
\hline 1015 & 199. & 500 & 282.3 & 1 & 0 & \\
\hline 11016 & 126.2119 & 3.3750 & 280.4 & 2 & 0 & \\
\hline 017 & 25.5275 & & 9.1 & 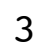 & & \\
\hline 018 & 871 & 50 & .2 & 4 & & \\
\hline 11019 & 72.4816 & 2000 & 0.2 & 4 & 0 & \\
\hline 020 & 66. & & & 4 & & \\
\hline 021 & 72 . & 50 & .5 & 4 & & \\
\hline 11022 & 80.2502 & 2000 & 9.5 & 4 & 0 & \\
\hline 023 & 85.500 & 4000 & & 4 & & \\
\hline 024 & 78 . & & & 4 & & \\
\hline 1111 & 000 & 750 & 3.9 & 4 & & \\
\hline 0011112 & 96.5045 & 3.7000 & 9.1 & 4 & & \\
\hline 011113 & 142.2495 & 50 & 8.4 & 5 & & \\
\hline 011114 & 105 & 5000 & 3.5 & 4 & & \\
\hline 0011115 & 131.6886 & 500 & 8.4 & 5 & & \\
\hline 0011116 & 143.5000 & 0 & & 5 & & \\
\hline 0011117 & 140 & & .9 & 6 & & \\
\hline 0011118 & 119.5000 & 1.9750 & 278.6 & 6 & & \\
\hline 011119 & 138.1033 & 2 . & 279.1 & 5 & & \\
\hline 00111110 & 164.2517 & & 9.7 & 4 & & \\
\hline 00111111 & 167.9998 & 4.7500 & 280.0 & 3 & & \\
\hline 1112 & 172 . & 4 . & .7 & 3 & & \\
\hline 00111113 & 176.2542 & 5.3500 & 281.0 & 3 & & s \\
\hline 00111114 & 177.0018 & 5250 & 281.5 & 3 & & 6 \\
\hline 1115 & 171.2616 & & 281.8 & 3 & & \\
\hline 1116 & 171.5000 & 5 . & 282.0 & 3 & & \\
\hline 00111117 & 169.7503 & 4.6000 & 281.6 & 4 & 0 & 0 \\
\hline 1118 & 170.2490 & & 280.7 & 4 & & \\
\hline 00111119 & 152.7359 & & 280.6 & 4 & 600.0 & 600 \\
\hline 00111120 & 135.2495 & 5.7500 & 280.9 & 4 & 600.0 & 600 \\
\hline 1121 & 144 & & & 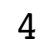 & & \\
\hline 1122 & 143.7500 & 00 & 280.9 & 4 & & 600 \\
\hline 1123 & 121.2531 & 6.3750 & 281 . & & 600.0 & 600 \\
\hline
\end{tabular}




\begin{tabular}{|c|c|c|c|c|c|c|}
\hline 1124 & & .2750 & & & & \\
\hline 11121 & 138.7500 & 7.0500 & 1.2 & 4 & 0 & \\
\hline 11122 & 5.2504 & 6.2250 & 280.9 & 4 & 00.0 & \\
\hline 11123 & 142.2499 & 7.0750 & 280.4 & & 00 & \\
\hline 11124 & 37501 & .6250 & 79.9 & & 0 & \\
\hline 11125 & .628 & 2000 & 279.6 & 4 & 00 & \\
\hline 011126 & 29.0000 & 5000 & 279.3 & 4 & 00 & \\
\hline 011127 & 28.2461 & 9250 & 279.5 & & & \\
\hline 011128 & 80064 & 3250 & 79.6 & 4 & & \\
\hline 011129 & 47.0046 & 9500 & 280.4 & 4 & 00 & 8 \\
\hline 0111210 & 166.7494 & 2750 & 280.8 & & & \\
\hline 1211 & 1719950 & 1.4750 & 82.1 & & 0 & \\
\hline 0111212 & 70 & 1.9000 & 283.3 & 1 & .0 & \\
\hline 0111213 & 61.7024 & 2.8250 & 284 & & 00.0 & \\
\hline 11214 & 39.2 & 0 & 84.2 & & & \\
\hline 215 & 96 . & & 284.4 & 2 & & \\
\hline 0111216 & 208.2563 & 2.6250 & 284.3 & 2 & .0 & \\
\hline 0111217 & 227.2415 & 0 & 3.7 & 3 & & \\
\hline 0111218 & 199.9053 & 50 & 282.1 & 4 & & \\
\hline 0111219 & 1.0702 & 500 & 281.5 & 5 & & \\
\hline 0111220 & 40.6375 & 1.7750 & 281.4 & 4 & 0 & 00 \\
\hline 0111221 & 73.2513 & & 281.1 & 4 & & \\
\hline 222 & 72.50 & 0 & 281.1 & 4 & & \\
\hline 0111223 & 70.7499 & 250 & 28 & 4 & 0 & \\
\hline 00111224 & 74.5000 & & & 4 & & \\
\hline 0011131 & 72.0000 & 750 & 28 & 4 & & \\
\hline 0011132 & 71.2556 & 4.8000 & 280.1 & 4 & 0 & J. \\
\hline 011133 & 60.5271 & 4.3250 & 279 & 4 & & \\
\hline 0011134 & 12.7533 & 000 & 27 & 4 & & \\
\hline 0011135 & 0.7498 & 2000 & 278.6 & 4 & & \\
\hline 0011136 & 346.9938 & 2.1750 & 278.0 & 4 & & 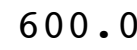 \\
\hline 0011137 & 85.1558 & 1.9250 & 277.5 & 5 & & \\
\hline 0011138 & 103.4938 & 500 & 277.9 & 4 & & 0 . \\
\hline 0011139 & 77.2600 & 500 & 278.3 & 4 & 0 & \\
\hline 0111310 & 70.5459 & 0 & & 3 & & \\
\hline 0011 & 323.6396 & 1.6000 & 281.8 & 2 & & 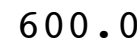 \\
\hline 0111312 & 238.7543 & 2.6250 & 281.4 & 2 & 0 & 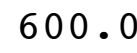 \\
\hline 0111313 & 285.5009 & 500 & 282 & 2 & & . \\
\hline 00111314 & 295.2500 & 3.5500 & 282.0 & 3 & .0 & 0 . \\
\hline 0111315 & 293.4892 & 1.3500 & 282.0 & 2 & & \\
\hline 00111316 & 289.2677 & 750 & 281.5 & 1 & & 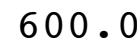 \\
\hline 00111317 & 337.3139 & 1.0750 & 280.2 & 2 & .0 & 0 . \\
\hline 0111318 & 31.2207 & 1.7500 & 279.7 & 3 & 0 & 00 . \\
\hline 00111319 & 86.8776 & 250 & 27 & 4 & & \\
\hline 00111320 & 81.2511 & 5.1250 & 279.8 & 4 & & 600. \\
\hline 0111321 & 76.2487 & 5.7250 & 279.4 & 4 & 600.0 & 600 \\
\hline 122 & 70.7560 & & & 4 & & \\
\hline 0111323 & 67.7485 & 5.2250 & 279.0 & & 600.0 & 600 \\
\hline
\end{tabular}




\begin{tabular}{|c|c|c|c|c|c|c|}
\hline $112<4$ & 62.5001 & 5.5000 & 279.1 & 4 & 600.0 & \\
\hline 1141 & 2.2502 & 4.6750 & 279.0 & 4 & 600.0 & \\
\hline 011142 & 82.7253 & 1.8750 & 278.7 & 5 & 00 & \\
\hline 011143 & 56.7493 & 0500 & 278.8 & & 00 & \\
\hline $01114 \quad 4$ & 1.9979 & & 279.0 & & 0 & \\
\hline 1145 & 64.7502 & 500 & 278.8 & 5 & 00 & \\
\hline 011146 & 74.2502 & 1750 & 278.6 & & 00 & \\
\hline 011147 & 79.2495 & .5000 & 278.5 & & & \\
\hline 011148 & 71.2259 & 3750 & 278.7 & & 0 & \\
\hline 011149 & 65.7499 & 1000 & 279.5 & & 0 & \\
\hline 410 & 82.0001 & 250 & 280.5 & 3 & 0 & \\
\hline & 92.2543 & & & 3 & & \\
\hline 01 & 110.4982 & 5000 & 281.5 & 3 & 0 & \\
\hline 13 & 163.3377 & 4.7750 & $2 \varepsilon$ & 3 & 0 & \\
\hline & 159.2503 & & & 3 & & \\
\hline 5 & 170.7548 & 250 & & 3 & 0 & \\
\hline 416 & 160.5263 & 3.6000 & 280.3 & & 0 & \\
\hline 17 & 151.2345 & 000 & 279.8 & & & \\
\hline 0 & 123.5282 & 2 . & 279.2 & 4 & 0 & \\
\hline 19 & 87.4908 & 0 & 280.2 & 4 & 0 & \\
\hline 20 & 72.7500 & 0 & 9.7 & 4 & & \\
\hline & 77.2500 & & & 4 & & \\
\hline 422 & 98.8045 & 750 & 8.5 & 4 & 0 & 0. \\
\hline 23 & 69.2513 & 0 & 9.2 & 4 & 0 & \\
\hline 24 & 73.4747 & & 3.9 & 4 & & \\
\hline 011151 & 61.5002 & 000 & & 4 & & \\
\hline 011152 & 66.5000 & 5.37 & 9.0 & 4 & 0 & \\
\hline 0011153 & 69.5000 & 0 & 9.0 & 4 & & \\
\hline 0011154 & 654 & 4 & 27 & 4 & & 0 . \\
\hline 011155 & 64.3012 & 1.8000 & 7.4 & 5 & 0 & \\
\hline 011156 & 85 & 0 & .8 & 4 & & \\
\hline 0011157 & 75 . & 0 & .3 & 5 & & \\
\hline 0011158 & 103.7761 & 5500 & 279.4 & 6 & 0 & J. \\
\hline 011159 & 132.5000 & 00 & 9.9 & 5 & 0 & \\
\hline 510 & 22 & 0 & .3 & 4 & & \\
\hline 511 & 313.6208 & 750 & 279.8 & 3 & & 0 . \\
\hline 0111512 & 239.0512 & 50 & 1.6 & 2 & 0 & \\
\hline 001 & 172.2591 & 0 & 0.5 & 1 & & \\
\hline 514 & 176.5041 & 000 & 281.2 & 1 & 0 & 0 . \\
\hline 1515 & 170.0228 & 500 & 281.4 & 1 & 0 & \\
\hline 516 & 189.2519 & 0 & 281.5 & 2 & & \\
\hline 00111517 & 195.7640 & 1.8000 & 280.6 & 3 & 600.0 & 600 . \\
\hline 0111518 & 170.1519 & 0.7500 & 279.9 & 4 & 600 & 000 \\
\hline 00111519 & 129.7224 & & 279.9 & 5 & & s \\
\hline 00111520 & 39.5756 & 0.7250 & 279.9 & 6 & 600.0 & 00 \\
\hline 0111521 & 311.9528 & 0.5250 & 279.3 & 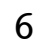 & 600 & 0 \\
\hline & 358.2180 & & 279.0 & 6 & 60 & \\
\hline 0111523 & 160.2825 & 3.2250 & 278.5 & & 600.0 & 600 \\
\hline
\end{tabular}




\begin{tabular}{|c|c|c|c|c|c|c|}
\hline 1524 & & 2750 & $8 \cdot 3$ & & & \\
\hline 1161 & 7332 & 2.4250 & 278.2 & 0 & & \\
\hline 011162 & . & .8250 & 278.2 & 6 & 0 & \\
\hline 011163 & 155.4903 & 2.8500 & 278.0 & & 00 & \\
\hline 11164 & 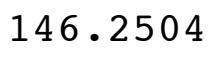 & 00 & 277.9 & & 0 & \\
\hline 165 & 347 & .6750 & 277.9 & 6 & 00 & \\
\hline 011166 & 2713 & .3250 & 277.8 & & 0 & \\
\hline 1167 & 0057 & .1500 & 277.8 & & & \\
\hline 011168 & 5076 & .9500 & 277.8 & 4 & 0 & \\
\hline 011169 & 17.5376 & 1.5750 & 279.2 & & 00 & \\
\hline 610 & 67.2602 & 3.4500 & 280.0 & & 0 & \\
\hline & 3.2555 & 4.6750 & 280.2 & & & \\
\hline 512 & 71.2606 & 4.8000 & 280.8 & & 0 & \\
\hline 613 & 57.4960 & 4.3250 & 281.8 & 3 & 0.0 & \\
\hline & & & & & & \\
\hline 15 & 170.7481 & 3.4000 & 282.6 & 2 & 0 & \\
\hline 616 & 79.5023 & 3.17 & 282.6 & 2 & 0 & \\
\hline 17 & 32.4999 & 3.1500 & 281.8 & & & \\
\hline 0 & 178 . & 2.7500 & .5 & 4 & 0 & \\
\hline 619 & 142.6607 & .2000 & 280.4 & 5 & 0 & \\
\hline 20 & 4.749 & 2.27 & 280.5 & 4 & & \\
\hline 21 & 5 & 3. & 280.7 & 4 & & \\
\hline 22 & 116.9981 & 2.0000 & 281.3 & 5 & 600 & 000. \\
\hline 523 & 3.2484 & 5500 & 281.9 & 6 & 0 & \\
\hline 24 & 110.9826 & 0 & 281.9 & 6 & & \\
\hline 71 & 7499 & 2.1500 & 281.8 & 6 & & \\
\hline 011172 & 143.5913 & 4.3500 & 280.9 & 5 & 0 & \\
\hline 0011173 & 151.4917 & 0 & 279.7 & 4 & & \\
\hline 174 & 158.2513 & 50 & 279.2 & 4 & & 0 . \\
\hline 011175 & 162.7604 & 7500 & 279.0 & 4 & 0 & \\
\hline 011176 & 161.2555 & 0 & 279.8 & 4 & & \\
\hline 177 & 173.0023 & 0 & 278.8 & 4 & & . \\
\hline 0011178 & 156.8897 & 4.5250 & 279.6 & 4 & 0 & U. \\
\hline 011179 & 160.9873 & 0 & 281.3 & 3 & 0 & \\
\hline 10 & 170 . & 8 . & .9 & 4 & & \\
\hline 711 & 171.0000 & 500 & 281.2 & 4 & 0 & • \\
\hline 001 & 169.0000 & 9500 & 282.1 & 3 & 0 & \\
\hline 001 & 175.2522 & 000 & 283.1 & 3 & & \\
\hline 001 & 175.0000 & 5.1750 & 284.0 & 3 & 600.0 & 0 . \\
\hline 715 & 177.5000 & 3250 & 284.7 & 3 & 600 & \\
\hline 716 & 177.5003 & 750 & 284.9 & 3 & & \\
\hline 00111717 & 179.0000 & 3.8750 & 284.3 & 4 & 600.0 & 600 . \\
\hline 1718 & 158.5443 & 3.2000 & 283.2 & 5 & 600 & 600. \\
\hline 0011 & 151.2582 & 3.2000 & 283.4 & 5 & 600 & 600 \\
\hline 00111720 & 135.6666 & 3.8000 & 283.7 & 4 & 600.0 & 00 \\
\hline 1721 & 109.2447 & 4.2250 & 284.0 & 4 & 600.0 & 600 . \\
\hline & 106.5037 & 3.2000 & 283.8 & 4 & 60 & \\
\hline 723 & 118.0209 & 1.9750 & 283.3 & & 600.0 & 600 \\
\hline
\end{tabular}




\begin{tabular}{|c|c|c|c|c|c|c|}
\hline 11724 & 3.9705 & 1.7000 & 283.6 & 5 & 000.0 & \\
\hline 011181 & 6.7381 & 0.8250 & 82.9 & 6 & - & \\
\hline 011182 & 101.8481 & 4500 & 282.2 & 6 & 0 & \\
\hline 011183 & 59.4964 & .8000 & 283.1 & & 00 & \\
\hline 011184 & 6.2728 & .7500 & 32.4 & & 0 & \\
\hline 1185 & 29.9179 & 7500 & 82.1 & & 00 & \\
\hline 011186 & 12.2498 & 1.3750 & 282.1 & & 0 & \\
\hline 1187 & 358.9966 & 7000 & 81.8 & & & \\
\hline 011188 & 355.4917 & 6750 & 82.4 & & 0 & \\
\hline 011189 & 337.9995 & 3000 & 285.1 & & 0 & \\
\hline 1810 & 256.7537 & 250 & 286.4 & & & \\
\hline 311 & 235.4579 & & & & & \\
\hline 1812 & 169.1562 & 500 & 285.4 & & 0 & \\
\hline 813 & 170.4991 & 4.8500 & 284.6 & & 0 & \\
\hline & 193.1271 & & & & & \\
\hline 15 & 284.6188 & 1.5250 & 286.3 & L & & \\
\hline 816 & 279.5330 & 0 & 286 & & 0 & \\
\hline 17 & 297 . & 0 & .3 & & & \\
\hline 18 & 235.8339 & 750 & 283.6 & 3 & & \\
\hline 819 & 173.7519 & 50 & 283.3 & 4 & & \\
\hline 320 & 164.75 & 0 & 82.6 & 5 & & \\
\hline 21 & 171.5 & & 281.8 & 6 & & \\
\hline 822 & 164.1002 & 500 & 281.8 & 6 & 0 & U • \\
\hline 323 & 146.1675 & 500 & 1.4 & 6 & 0 & \\
\hline 24 & 147.2137 & 0 & 1.0 & 6 & & \\
\hline 011191 & 78.2282 & 500 & 281.0 & 5 & & \\
\hline 011192 & 148.7967 & 0 & 281.3 & 6 & & \\
\hline 0011193 & 145.33 & & 280.8 & 6 & & \\
\hline 011194 & 148.4994 & 50 & 27 & 5 & & \\
\hline 011195 & 165.7411 & 0 & 279.7 & 6 & & \\
\hline 011196 & 175.7497 & & & 6 & & \\
\hline 0011197 & 148.68 & 0 & .5 & 6 & & \\
\hline 0011198 & 155.7550 & 1000 & 280.3 & 6 & 0 & J. \\
\hline 011199 & 8.1292 & 0 & 282.8 & 5 & & \\
\hline 10 & 349.2500 & 0 & .3 & 4 & & \\
\hline 11 & 243.0526 & 000 & 285.0 & 3 & & 0 . \\
\hline 912 & 219.0533 & 500 & 285.4 & 2 & & \\
\hline 001 & 218.0 & 2 . & & 1 & & \\
\hline 14 & 223.2496 & 1.9500 & 286.0 & 2 & 0 & . \\
\hline 915 & 220.8152 & 500 & 286.3 & 2 & & \\
\hline 916 & 191.2497 & 000 & & 3 & & \\
\hline 0011 & 177.7439 & 2.6000 & 284.8 & 4 & 600.0 & 600 . \\
\hline 1918 & 176.5003 & 1750 & 283.5 & 5 & 600 & 000 \\
\hline 919 & 177.2500 & 500 & & 6 & & 50 \\
\hline 00111920 & 171.0747 & 0.4250 & 282.7 & 6 & 600.0 & 00 \\
\hline 921 & 2641 & 0.5750 & 282.5 & - & 600 & 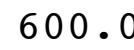 \\
\hline & 30.2501 & 1.3500 & & 5 & 60 & \\
\hline 011 & 47.1696 & 1.1000 & 282.8 & 4 & 600.0 & 600 \\
\hline
\end{tabular}




\begin{tabular}{|c|c|c|c|c|c|c|}
\hline 924 & & .1750 & & & & \\
\hline 1201 & 9 & .0750 & 1.8 & & 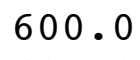 & \\
\hline 11202 & 140.1560 & 4000 & 280.8 & & 00 & \\
\hline 11203 & 58.0000 & 1.2500 & 281.0 & & 00 & \\
\hline $1120 \quad 4$ & 355.4997 & .9250 & 80.9 & & 0 & \\
\hline 11205 & 548 & 5500 & 81.1 & & 0 & \\
\hline 011206 & 9993 & 3000 & 281.2 & & 00 & \\
\hline 011207 & 4850 & L. 4000 & 81.1 & & 0 & \\
\hline 011208 & 1360 & 4250 & 81.6 & & & \\
\hline 011209 & 218.5000 & 0.4750 & 282.9 & & 00.0 & 8 \\
\hline 0112010 & 220.9124 & 1.3500 & 282.7 & & 0 & \\
\hline 011 & 156.4982 & 3.0500 & 81.8 & & & \\
\hline 0112012 & 185.6415 & 2.1500 & 282.4 & & .0 & \\
\hline 0112013 & 268.4138 & 1.2250 & 284.5 & & 0.0 & \\
\hline 014 & 5 & 1.5250 & & & & \\
\hline 15 & 8 & 2.22 & 286.3 & & & \\
\hline 0112016 & 288.25 & 1.7000 & 286.2 & & .0 & 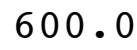 \\
\hline 12017 & 264 . & 1.1500 & 5.1 & & 0 & \\
\hline 12018 & 165 . & 750 & 283.7 & & & \\
\hline 019 & 126 & 0.1750 & 283.2 & 5 & & \\
\hline 0112020 & 102.7794 & 0.4750 & 283.2 & 6 & 0 & 00. \\
\hline 0112021 & 73.3080 & 0.1500 & 283.1 & 6 & & \\
\hline 022 & 05 & 7000 & 282.7 & 6 & & \\
\hline 0112023 & 104.2533 & 2.7750 & 283.3 & 5 & 0 & \\
\hline 0112024 & 76.7499 & 4.72 & .0 & 4 & & \\
\hline 0011211 & 7505 & 5750 & 283.2 & 4 & & \\
\hline 011212 & 79.7500 & 9250 & 282.4 & 4 & 0 & . \\
\hline 011213 & 499 & 0 & 282.4 & 4 & & \\
\hline 0011214 & 500 & 6.7500 & 282.3 & 4 & & \\
\hline 0011215 & 82.5000 & 6.2250 & 282.7 & 4 & 0 & 0 • \\
\hline 0011216 & 91.4724 & 4.6250 & 282.8 & 4 & 0 & 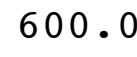 \\
\hline 0011217 & 81.7501 & 3.7250 & 283.0 & 5 & & \\
\hline 0011218 & 048 & 7000 & 282.9 & 4 & & 0 • \\
\hline 0011219 & 3093 & 4.0000 & 283.3 & 4 & 0 & 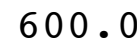 \\
\hline 0112110 & 5590 & 0 & & 3 & & \\
\hline 00112111 & 159.3875 & 1.3500 & 284.2 & 2 & & 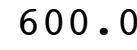 \\
\hline 0112112 & 127.9702 & 8500 & 282.6 & 1 & $\cdot 0$ & . \\
\hline 00112113 & 28.2780 & 1 . & 281.9 & 1 & & . \\
\hline 00112114 & 159.0034 & 4.6000 & 279.9 & 2 & .0 & 0 . \\
\hline 00112115 & 88.7416 & 2.0500 & 280.3 & 1 & & \\
\hline 00112116 & 344.4652 & 0.7500 & 282.0 & 2 & & 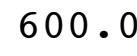 \\
\hline 00112117 & 49.2334 & 1.1250 & 281.5 & 3 & .0 & 0 . \\
\hline 0112118 & 83.2898 & 2.9750 & 281.5 & 4 & .0 & 00 . \\
\hline 00112119 & 982 & 4250 & 282.0 & 4 & & \\
\hline 00112120 & 93.0339 & 3.9000 & 281.8 & 4 & 0 & 0 . \\
\hline 0112121 & .0015 & 4.0750 & 281.7 & 4 & 600 & 600 \\
\hline & \pm 218 & & & 5 & & \\
\hline 0112123 & 48.4413 & 2.5500 & 281.5 & 7 & 600.0 & 600 \\
\hline
\end{tabular}




\begin{tabular}{|c|c|c|c|c|c|c|}
\hline 2124 & 3.7181 & 2.5500 & 281.6 & 5 & 600.0 & \\
\hline 1221 & 5.9964 & 0.1500 & 280.7 & 6 & 600.0 & \\
\hline 011222 & 106.2860 & 250 & 280.4 & 6 & 00 & \\
\hline 011223 & 349.2534 & 1000 & 280.3 & & 00 & \\
\hline 011224 & 2.6077 & .6500 & 30.4 & & 0 & \\
\hline 1225 & 159.8324 & 9250 & 279.9 & & 00 & \\
\hline 011226 & 166.9999 & 6500 & 278.5 & & 0 & \\
\hline 011227 & 150 . & 4000 & 78.7 & & & \\
\hline 011228 & 164.2511 & 0750 & 278.8 & 4 & 0 & \\
\hline 011229 & 161.7499 & 7000 & 278.8 & & 0 & \\
\hline 2210 & 152.9969 & 3500 & 279.0 & & 0 & \\
\hline 211 & 131.30 & 2.8000 & 79.3 & 1 & & \\
\hline 2212 & 132. & 9000 & 279.5 & & 0 & \\
\hline 0112213 & 138.2472 & 4.5750 & 280.4 & 2 & 0.0 & \\
\hline 214 & 124.3728 & & 281.8 & & & \\
\hline 15 & 138.5000 & 500 & 282.9 & 3 & 0 & \\
\hline 216 & 139.7522 & 9500 & 283.3 & & 0 & \\
\hline 17 & 131.1173 & 250 & .5 & & & \\
\hline 01 & 91.2460 & 500 & 282.3 & 4 & 0 & \\
\hline 219 & 77.2501 & 750 & 282.1 & 4 & & \\
\hline 20 & 5000 & 500 & 281.7 & 4 & & \\
\hline 21 & 69. & 50 & 282.2 & 4 & & \\
\hline 01 & 999 & 750 & 282 & 5 & 0 & \\
\hline 23 & 69.0245 & 250 & 283.0 & 6 & & \\
\hline 24 & 66. & 0 & 281.8 & 6 & & \\
\hline 011231 & 100 . & 500 & 281.8 & 5 & & \\
\hline 011232 & 96.1186 & 250 & 280.7 & 4 & & \\
\hline 0011233 & 8.6713 & 0 & 280.2 & 5 & & \\
\hline 011234 & 346 . & 00 & 28 & 5 & & 0 . \\
\hline 011235 & 4.9246 & 500 & 279.4 & 5 & & \\
\hline 011236 & 10 . & 50 & 279.8 & 4 & & \\
\hline 0011237 & 348 . & 00 & 280.2 & 5 & & \\
\hline 0011238 & 313.2517 & 750 & 281.0 & 5 & 0 & J. \\
\hline 011239 & 172.3073 & 00 & 282.3 & 4 & 0 & \\
\hline 10 & 245 . & 50 & 282.9 & 3 & & \\
\hline 0011 & 278.0745 & 500 & 282.2 & 2 & & 0 . \\
\hline 011 & 200.1995 & 5000 & 281.8 & 1 & & \\
\hline 001 & 143.7221 & 500 & 281.7 & 1 & & 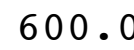 \\
\hline 0011 & 153.2030 & 250 & 282.1 & 1 & 60 & 0 . \\
\hline 0112315 & 230.3920 & 000 & 282.2 & 1 & 0 & \\
\hline 316 & 164.9693 & 500 & 282.4 & 1 & & \\
\hline 00112 & 141.0000 & 1.8500 & 281.6 & 2 & 600.0 & 600 . \\
\hline 0112318 & 274.5799 & 0.7750 & 281.0 & 3 & 600 & . \\
\hline 00112319 & 271.3327 & 0.1500 & 280.9 & 4 & & 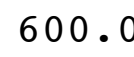 \\
\hline 00112320 & 285.9173 & 0.9500 & 280.8 & 5 & 600.0 & 00 \\
\hline 0112321 & 9942 & 0.4500 & 280.9 & 4 & 600 & 促 \\
\hline & 890 & 500 & 280.5 & 5 & 60 & \\
\hline 0112323 & 136.5027 & 2.3750 & 279.9 & & 600.0 & 600 \\
\hline
\end{tabular}




\begin{tabular}{|c|c|c|c|c|c|c|}
\hline 324 & & 2250 & 9.8 & & & \\
\hline 24 & 00 & 7500 & 279.7 & & 0 & \\
\hline 1242 & 158.7491 & 3250 & 279.4 & & 00 & \\
\hline 11243 & 153.3337 & 8750 & 279.1 & & 00.0 & \\
\hline $1124 \quad 4$ & 684 & 1250 & 279.2 & & 0 & \\
\hline $1124 \quad 5$ & 159.5481 & 3250 & 279.4 & & 00 & \\
\hline 011246 & 156.2423 & 1500 & 279.1 & & 00 & \\
\hline 011247 & 157.2492 & 6000 & 279.2 & & .0 & \\
\hline 011248 & 4.2369 & 9500 & 279.0 & & & \\
\hline 011249 & 161.2438 & 3.0750 & 279.2 & & 00.0 & 0 \\
\hline 0112410 & 162.4946 & 2.8000 & 279.3 & & 00.0 & \\
\hline 11 & 167.7549 & 220 & 279.5 & & & \\
\hline 01 & 171.7499 & 750 & 280.1 & & 0 & \\
\hline 0112 & 164.5003 & 3.7250 & 280.2 & & 0.0 & \\
\hline 14 & 151.5000 & & & & & \\
\hline 5 & 140.7790 & & 283.1 & & & \\
\hline 01 & 123.9556 & 1.27 & 284.2 & & .0 & U \\
\hline 17 & 110.4604 & 1 . & .6 & & .0 & \\
\hline 18 & 66.5484 & & 3.1 & & & \\
\hline 01 & 108.2069 & 2. & 3.8 & & 0 & \\
\hline 0112 & 74.4862 & 1.8750 & 284.2 & & .0 & 00 . \\
\hline 21 & 120.3767 & & & 6 & & \\
\hline 01 & 101 & 0 & .3 & 6 & & \\
\hline 0112423 & 136.4053 & 1.7750 & 3.1 & 6 & . 0 & 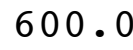 \\
\hline 011 & 22.5781 & & .4 & 6 & & \\
\hline 51 & 73.6820 & 50 & & 6 & & \\
\hline 011252 & 124.4954 & 500 & 1.6 & 5 & 0 & . \\
\hline 011253 & 150.2301 & 0 & .0 & 6 & & \\
\hline 0011254 & 110 & 0 . & .3 & 6 & & • \\
\hline 0011255 & 139.5000 & 2 . & 2.2 & 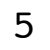 & & 0 . \\
\hline 0011256 & 119.5204 & 1.8500 & 281.8 & 6 & & S \\
\hline 0011257 & 96.2488 & 0 & 1.5 & 6 & & \\
\hline 011258 & 149.7449 & 0 & 1.6 & 6 & & . \\
\hline 0011259 & 132.8313 & 1.0250 & 2.9 & 5 & 0 & \\
\hline 011 & 02113 & 50 & & 4 & & \\
\hline 0011 & 274.7176 & 1.0750 & 5.1 & 3 & & . \\
\hline 011 & 149.7513 & 6.3000 & 9.2 & 4 & .0 & 0. \\
\hline 00112 & 21.8206 & 500 & .9 & 3 & & - \\
\hline 00112 & 120.6512 & 2.8250 & 282.8 & 2 & .0 & 600 . \\
\hline 0112515 & 168.2495 & 3.4500 & 279.8 & 2 & .0 & \\
\hline 0011 & 32.0337 & 1.8250 & 281.1 & 1 & & 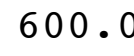 \\
\hline 0011 & 89.7139 & 2.3000 & 279.9 & 2 & .0 & 0 . \\
\hline 0112518 & 42.4943 & 0.6250 & 281.4 & 3 & 0 & 00 . \\
\hline 00112519 & 125.8143 & 1.2500 & 281.4 & 4 & & \\
\hline 00112520 & 61.7374 & 3.1250 & 279.0 & 5 & .0 & 600. \\
\hline 00112521 & 103.8235 & 3.6500 & 279.6 & 4 & 600.0 & 600 \\
\hline & 294 & & & 5 & & \\
\hline 0112523 & 146.8687 & 1.4250 & 275.8 & & .0 & 60 \\
\hline
\end{tabular}




\begin{tabular}{|c|c|c|c|c|c|c|}
\hline 2524 & 94.0131 & 2.0750 & 276.5 & 6 & 600.0 & \\
\hline 1261 & 147.0393 & 2.3500 & 275.8 & 0 & 600.0 & \\
\hline 011262 & 317.1532 & 4500 & 275.8 & 6 & 00 & \\
\hline 011263 & 147.5813 & 2.6750 & 275.6 & 6 & 00 & \\
\hline 011264 & 115.6 & 500 & 275.9 & & 0 & \\
\hline 1265 & 163.0 & 00 & 276.1 & 6 & 00 & \\
\hline 011266 & 31.3900 & 1.2500 & 276.6 & 6 & 0 & \\
\hline 011267 & 29.9752 & .9000 & 78.0 & & & \\
\hline 011268 & 103.7926 & 0 & 279.8 & 6 & 0 & \\
\hline 011269 & 96.1658 & 3 & 276.3 & & 0 & \\
\hline 610 & 89.3017 & 0 & 277.6 & 4 & 0 & \\
\hline & 213.3737 & & 78.3 & 3 & & \\
\hline 612 & 219.1398 & 1.1 & 279.1 & 2 & 0 & \\
\hline 613 & 185.3834 & 1.90 & 280.1 & 1 & 0 & \\
\hline & 1.4712 & & & 1 & & \\
\hline 01 & 235.8964 & 2 . & 285.0 & 1 & 0 & \\
\hline 616 & 228.7474 & 1.8 & 281.6 & 2 & 0 & \\
\hline 17 & 183.1480 & .4 & 8 & 3 & & \\
\hline 0 & 22.94 & 1.6 & 279.9 & 4 & 0 & \\
\hline 19 & 94.7176 & 0 & 279.2 & 5 & & \\
\hline 20 & 182 . & 0 & .4 & 6 & & \\
\hline 21 & 348 . & & .9 & 6 & & \\
\hline 22 & 5.4883 & .70 & 7.4 & 5 & 00 & 0 \\
\hline 23 & 148 . & 0 & 7 & 6 & 0 & \\
\hline 24 & & & & 5 & & \\
\hline 271 & 2482 & 0 & .2 & 6 & & \\
\hline 011272 & 68.3981 & 1.05 & 2.9 & 6 & 0 & \\
\hline 0011273 & 49.91 & & 2 & 6 & & \\
\hline 0011274 & 68 . & 0 & 1.7 & 6 & & 0 . \\
\hline 011275 & 97.5327 & 1.3 & 281.8 & 6 & & \\
\hline 011276 & 56.6055 & 0 & & 6 & & \\
\hline $001127 \quad 7$ & 272 & 0 & .4 & 6 & & \\
\hline 0011278 & 94.0000 & 1.35 & 1.0 & 6 & 0 & J. \\
\hline 011279 & 313.1095 & 0 & .5 & 5 & & \\
\hline 710 & 248 & 0 & .6 & 4 & & \\
\hline 711 & 228.2495 & 2.30 & 9.7 & 4 & & • \\
\hline 0112712 & 224.7737 & 0 & 9.8 & 3 & 0 & \\
\hline 0011 & 217.5649 & 2 . & 0.1 & 3 & & \\
\hline 0011 & 196.9147 & 1.4500 & 279.9 & 2 & $\cdot 0$ & 0 . \\
\hline 00112715 & 222.2644 & 0 & $2 \varepsilon$ & 1 & 0 & \\
\hline 716 & 204.5527 & 1 . & 279.8 & 2 & & \\
\hline 00112717 & 172.4890 & 2.8000 & 279.2 & 3 & 600.0 & 600 . \\
\hline 0112718 & 94.5869 & 0.9 & 279.0 & 4 & 600 & 00 \\
\hline 00112719 & 54.7017 & & 279.2 & 5 & & 10 \\
\hline 00112720 & 140.1462 & 1.1000 & 278.7 & 6 & 600.0 & 00 \\
\hline 12721 & 106.6259 & 1.4250 & 278.4 & 6 & 600 & 0 \\
\hline 00112722 & 115.1620 & & 278.2 & 6 & & \\
\hline 0112723 & 99.0643 & 0.5250 & 278.1 & & 600.0 & 600 \\
\hline
\end{tabular}




\begin{tabular}{|c|c|c|c|c|c|c|}
\hline 2724 & & .4750 & 78.0 & & & \\
\hline 11281 & 7 & 1.0500 & 277.9 & & 600.0 & \\
\hline 1282 & 254.6900 & 5000 & 277.8 & 6 & 00 & \\
\hline 11283 & 349.1553 & 1.3250 & 277.8 & 6 & 00 & \\
\hline 1284 & 353 & 3750 & 277.8 & & & \\
\hline 11285 & 333 & 5000 & 277.8 & & 0 & \\
\hline 011286 & 3824 & 5000 & 277.8 & & 00 & \\
\hline 011287 & 354.7364 & 500 & 277.8 & & 0 & \\
\hline 011288 & 343.9989 & 50 & .77 .9 & 5 & & \\
\hline 011289 & 325.9986 & 1.6750 & 278.0 & 4 & 0 & 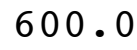 \\
\hline 0112810 & 332.3092 & 1.9000 & 278.3 & & & \\
\hline 11 & 318.5121 & 2.67 & 78.7 & & & \\
\hline 812 & 273 & 2.3500 & 278.9 & & 0 & \\
\hline 813 & 261.6509 & 1.2750 & 279.3 & & & \\
\hline 14 & 281 & & 279.6 & & & \\
\hline 15 & 261 & & 279.8 & & & \\
\hline 816 & 287.0006 & 2.9750 & 279.6 & 3 & 0 & \\
\hline 17 & 243.1137 & 0 & 279.5 & & & \\
\hline 18 & 267.1449 & 0 & 279.4 & 5 & & \\
\hline 01 & 248.0644 & 5750 & 279.3 & 6 & & \\
\hline 20 & 268.4994 & 1.3500 & 279.2 & 6 & 0 & \\
\hline 21 & 346.5008 & & 279.0 & 5 & & \\
\hline 22 & 21.7240 & 1.4250 & 278.9 & 6 & & \\
\hline 0112 & 150.7390 & 3.8750 & 278.7 & 5 & 0 & \\
\hline 24 & 48.5769 & & 278.4 & 6 & & \\
\hline 001 & 144.5220 & 1.1000 & 278.0 & 6 & & \\
\hline 011292 & 29.2158 & 9000 & 279.1 & 6 & 0 & J. \\
\hline 011293 & 238.9216 & 0 & 280.3 & 6 & & \\
\hline 0011294 & 177.1337 & 00 & 280.9 & 6 & & \\
\hline 011295 & 75.8457 & 1250 & 283.2 & 5 & 0 & U. \\
\hline 011296 & 5.2569 & 250 & 283.6 & 4 & & 0 . \\
\hline 011297 & 0.2619 & 0 & 285.6 & 4 & & \\
\hline 011298 & 358.9963 & & 285.2 & 4 & & 0 . \\
\hline 011299 & 26.0458 & .8250 & 285.4 & 4 & 0 & \\
\hline 01 & 33.7502 & 0 & 285.6 & 4 & & \\
\hline 0011 & 42.4729 & 8500 & 286.0 & 3 & & 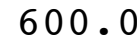 \\
\hline 12 & 56.5069 & 6.4500 & 285.5 & 4 & & . \\
\hline 001 & 56.7626 & 00 & 286.6 & 3 & & 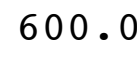 \\
\hline 0011 & 67.2986 & 4.6250 & 286.7 & 3 & .0 & 0 . \\
\hline 0112915 & 78.6425 & 8750 & 286.1 & 2 & & \\
\hline 916 & 58.2302 & 500 & 286.4 & 2 & & 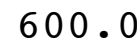 \\
\hline 0011 & 69.4886 & 2.0000 & 285.8 & 3 & & 0 . \\
\hline 0112918 & 175.3291 & 1.8500 & 283.7 & 4 & 0 & 0 . \\
\hline 0011 & 86.6060 & 500 & 283.7 & 4 & & \\
\hline 00112920 & 66.7500 & 5.7000 & 283.6 & 4 & & 0 . \\
\hline 011292 & 40.4802 & 4.0250 & 282.6 & 4 & & 600 \\
\hline & 96 & & 283.3 & 5 & & \\
\hline 0112 & 66.5008 & 5.2250 & 283.2 & & 600.0 & 5 \\
\hline
\end{tabular}




\begin{tabular}{|c|c|c|c|c|c|c|c|}
\hline \multicolumn{2}{|c|}{00112924} & 1.002 & .3250 & 283.2 & 4 & 800 & \\
\hline \multicolumn{2}{|c|}{0011301} & 108.5000 & 4.3500 & 282.7 & 4 & & \\
\hline \multicolumn{2}{|c|}{001130} & 128.9767 & 8750 & 282.1 & & 0 & \\
\hline \multicolumn{2}{|c|}{001130} & 113.2374 & 4000 & 281.8 & & 00 & \\
\hline \multicolumn{2}{|c|}{001130} & 125 & 2000 & 2.1 & & & \\
\hline \multicolumn{2}{|c|}{0011305} & 950 & 4500 & 82.1 & & 0 & \\
\hline \multicolumn{2}{|c|}{001130} & 123.5258 & 8750 & 281.7 & & & \\
\hline \multicolumn{2}{|c|}{001130} & 133. & .2500 & 2.2 & & & \\
\hline \multicolumn{2}{|c|}{001130} & 143 & .4750 & 82.5 & & & \\
\hline \multicolumn{2}{|c|}{0011309} & 136 & & 283.2 & & & \\
\hline \multicolumn{2}{|c|}{00113010} & 159.7495 & 0 & 83.6 & & & \\
\hline \multicolumn{2}{|c|}{00113011} & 158 . & & & & & \\
\hline \multicolumn{2}{|c|}{00113012} & 154 & 0 & 82.1 & & & \\
\hline \multicolumn{2}{|c|}{00113013} & 166.4994 & 0 & 283.1 & & 0 & \\
\hline \multicolumn{2}{|c|}{00113014} & 168 . & & & & & \\
\hline \multicolumn{2}{|c|}{00113015} & 172.2502 & 0 & & & & \\
\hline \multicolumn{2}{|c|}{00113016} & 170.75 & 0 & 3.6 & & & \\
\hline \multicolumn{2}{|c|}{00113017} & 160 & & & & & \\
\hline \multicolumn{2}{|c|}{00113018} & 150 & & 4 & 5 & & \\
\hline \multicolumn{2}{|c|}{00113019} & 125.09 & 0 & 2.5 & & & \\
\hline \multicolumn{2}{|c|}{00113020} & 141.24 & 0 & 2.8 & & & \\
\hline 00113 & 021 & 135 . & & 2.7 & 6 & & \\
\hline 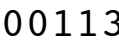 & 22 & 143 . & 0 & .7 & 6 & & . \\
\hline 0. & & 86 . & & 282.8 & 5 & & \\
\hline & 024 & 107 & & & 6 & & \\
\hline 01 & 1 & 110.7306 & 0 & .1 & 6 & & \\
\hline 012 & 2 & 160.25 & 0 & 2.1 & 6 & & \\
\hline 012 & 13 & 155.57 & & & 6 & & \\
\hline 012 & 14 & 151.22 & & 8 & 6 & & \\
\hline 012 & 5 & 158.50 & 0 & 1.6 & 5 & & \\
\hline 012 & 16 & 158 . & & 7 & 6 & & \\
\hline 0012 & 17 & 161 & & & 6 & & \\
\hline 0012 & 18 & 172.74 & 0 & .7 & 5 & 0 & \\
\hline 012 & 19 & 161. & 0 & & 4 & & \\
\hline 012 & 110 & 164 & & & 3 & & \\
\hline 012 & 111 & 178.00 & 0 & 1.6 & 2 & & \\
\hline 012 & 112 & 178 & & .7 & 2 & & \\
\hline 0012 & 113 & 179 & 0 & & 2 & & \\
\hline 012 & 114 & 177.7500 & $\$ 750$ & 283.7 & 2 & 0 & 00 . \\
\hline 012 & 115 & 178 & 0 & & 0 & & \\
\hline 012 & 116 & 177 . & 0 & & 2 & & \\
\hline 0012 & 117 & 148.9941 & 1750 & 283.0 & 3 & 0 & 0 . \\
\hline 012 & 118 & 164.76 & & 282.4 & 4 & 0 & \\
\hline 0012 & 119 & 149.3577 & 250 & & 5 & & \\
\hline 0012 & 120 & 164.5000 & 1.6750 & 282.4 & 6 & 600.0 & 00 \\
\hline 012 & 121 & 5879 & & 282.8 & 6 & 600 & 0 \\
\hline 012 & 122 & 62.2591 & 500 & & 5 & 60 & \\
\hline 012 & 123 & 41.0000 & 1.0750 & 283.2 & & 600.0 & 50 \\
\hline
\end{tabular}




\begin{tabular}{|c|c|c|c|c|c|c|c|}
\hline & 24 & - & 1.0000 & 83.5 & & - & \\
\hline 012 & 21 & 171.7488 & 2.3500 & 282.4 & & & \\
\hline 1 & 22 & 173.5009 & 4500 & 282.0 & 5 & 00 & \\
\hline ] & 23 & 167.0039 & .2250 & 282.1 & & 00 & \\
\hline & 24 & 179.75 & .9250 & 81.7 & & 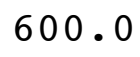 & \\
\hline & 5 & 118.3184 & .2500 & 81.2 & 6 & 00 & \\
\hline 01 & 6 & 44.0435 & 0.2250 & 281.3 & & 0 & \\
\hline & 7 & 171.4839 & .3750 & 81.1 & & & \\
\hline 政 & 8 & 173.5110 & 1.5000 & 281.7 & 6 & 0 & \\
\hline 01 & 9 & 160.4739 & 2.3250 & 281.6 & & 00 & \\
\hline 10 & 210 & 341.1562 & 1.0000 & & $\mathbf{4}$ & 0 & \\
\hline & 211 & 214.82 & 1.9250 & & 3 & & \\
\hline 01 & 12 & 243 & 0.4000 & 283.8 & 2 & 0 & \\
\hline 1 & 213 & 278.1515 & 0.5750 & 286.0 & $\perp$ & 0.0 & \\
\hline & 214 & 243.49 & & & 1 & & \\
\hline 01 & 15 & 260. & 1.2500 & 286.5 & 2 & 0 & \\
\hline 2 & 216 & 242.2040 & 1.3500 & 285.8 & 1 & 0 & \\
\hline 01 & 217 & 212 . & .5500 & 4.2 & 2 & & \\
\hline 01 & 218 & 231.16 & 0.1750 & 282.6 & 3 & 0 & \\
\hline 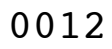 & 219 & 343.5242 & 1.4500 & 282.2 & 4 & & \\
\hline U. & 220 & 12.74 & 1 . & 282.1 & 4 & & \\
\hline 0 & 221 & 19. & 0 & 282.5 & 4 & & \\
\hline 0 & 222 & 6.7402 & 4750 & 283.3 & 4 & 0 & \\
\hline 01 & 223 & 342.4977 & 0.85 & 282.9 & 4 & 0 & \\
\hline 0 & 224 & & 50 & 283.6 & 5 & & \\
\hline 0. & 31 & 283 . & 7750 & 283.7 & 5 & & \\
\hline 01 & 32 & 307.2568 & 8000 & 282.6 & 6 & 0 & \\
\hline 01 & 33 & 188.40 & 0 & 282.2 & 6 & & \\
\hline 0 & 34 & 152.1167 & 00 & 281.5 & 6 & 0 & 0 . \\
\hline 01 & 35 & 180.24 & 1.5000 & 281.0 & 6 & 0 & \\
\hline 0 & 36 & 141.74 & 1.2500 & 277.7 & 6 & & \\
\hline 0 & 37 & 149 . & 00 & 274.5 & 6 & & 政 \\
\hline 0 & 38 & 129.9300 & 1.7750 & 274.2 & 6 & 0 & U. \\
\hline 01 & 39 & 159 . & 4750 & 275.3 & 5 & & \\
\hline 0 & 310 & 144 . & 50 & 27 & 4 & & \\
\hline 001 & 311 & 238.7694 & 0.7750 & 279.3 & 3 & & 0 . \\
\hline & 312 & 321.4316 & 1.2250 & 279.8 & 2 & 0 & \\
\hline 001 & 313 & 281.56 & 0.7500 & 281.1 & 1 & 0 & . \\
\hline 001 & 314 & 274.3233 & 0.8250 & 283.8 & 1 & 0 & 0 . \\
\hline 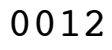 & 315 & 193.3221 & 250 & 283.3 & 1 & 0 & \\
\hline 001 & 316 & 358.0490 & 000 & & 2 & & \\
\hline 001 & 317 & 14.5120 & 0.0000 & 283.3 & 3 & 600.0 & 600 . \\
\hline & 318 & 69.7938 & 0.4500 & 282.4 & 4 & 600 & 600. \\
\hline 0012 & 319 & 117.9319 & 0.4000 & & 5 & & 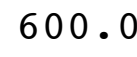 \\
\hline 0012 & 320 & 19.7492 & 1.3750 & 283.3 & 4 & 600.0 & 500 . \\
\hline & 321 & 13.7466 & 0.5500 & 283.7 & 5 & 600.0 & 600. \\
\hline & 322 & 84.5199 & & & 6 & & 60 \\
\hline 01 & 323 & 140.5046 & 2.4500 & 281.6 & & 600.0 & 600 \\
\hline
\end{tabular}




\begin{tabular}{|c|c|c|c|c|c|c|c|}
\hline & 24 & & 2.9000 & & & & \\
\hline 01 & 4 & 730 & 2.1250 & 282.2 & & & \\
\hline 1 & 42 & 117.4792 & 1250 & 282.5 & 5 & 00 & \\
\hline ] & 43 & 128.0024 & .0000 & 282.9 & & 00 & \\
\hline & 4 & 4.8178 & L. 0250 & 82.8 & & 0 & \\
\hline & 4 & 152.2743 & 1.6250 & 282.8 & & 00 & \\
\hline 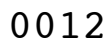 & 6 & 136.5046 & 0.9250 & 283.3 & & 0 & \\
\hline & 7 & 62.2695 & 2000 & 83.2 & & & \\
\hline 01 & 8 & 162.2487 & 2.4000 & 82.6 & & 0 & \\
\hline 01 & 9 & 167.7537 & 3000 & 282.7 & & 00 & \\
\hline 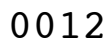 & 410 & 174.0003 & .9500 & 283.6 & & 0 & \\
\hline & 411 & 172.2500 & 500 & 84.6 & & & \\
\hline 01 & 412 & 172.7499 & 750 & 285.8 & & 0 & \\
\hline 07 & 413 & 172.4997 & 3.8000 & 286.4 & & 0.0 & \\
\hline & 414 & 175.7500 & & 5 & & & \\
\hline 01 & 415 & 179.3351 & 500 & 287.8 & & 0 & \\
\hline 2 & 416 & 205.6216 & 750 & 286.8 & & 0 & \\
\hline 01 & 417 & 58 . & 00 & 7 & & & \\
\hline 01 & 418 & 43.9127 & 750 & 284.6 & 3 & 0 & \\
\hline 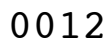 & 419 & 87.7568 & 750 & 286.0 & 4 & 0 & \\
\hline 0 & 420 & 138.4917 & 00 & 286.1 & 5 & & \\
\hline 0 & 421 & 153.7857 & 50 & 284.4 & 6 & & \\
\hline 0 & 422 & 166.0053 & 500 & 284.8 & 6 & 0 & \\
\hline 01 & 423 & 68.4540 & 500 & 285 & 6 & 0 & \\
\hline 0 & 424 & 71.2713 & 00 & & 6 & & \\
\hline 01 & 51 & 2504 & 1.2250 & 286.8 & 5 & & \\
\hline 01 & 52 & 30.4560 & 3750 & 287.2 & 5 & 0 & \\
\hline 0 & 53 & 24 . & 0 & 287 & 5 & & \\
\hline 0 & 54 & 154 . & 500 & .9 & 6 & 0 & 0 . \\
\hline 01 & 55 & 160.2543 & 2250 & 283.2 & 6 & 0 & \\
\hline 0 & 56 & 163.75 & 5000 & 283.3 & 6 & & \\
\hline 00 & 57 & 273 & 00 & .1 & 6 & & \\
\hline 00 & 58 & 45.1548 & 1250 & 282.7 & 6 & 0 & U. \\
\hline 01 & 59 & 139.4132 & 500 & 285.6 & 5 & 0 & \\
\hline 0 & 510 & 161 & 50 & 28 & 4 & & \\
\hline 001 & 511 & 76.9476 & 1.5000 & 286.0 & 3 & & 0 . \\
\hline 01 & 512 & 161.2482 & 2.2250 & 284.4 & 2 & 0 & \\
\hline 001 & 513 & 160.2701 & 3.6250 & 285.0 & 2 & 0 & 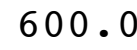 \\
\hline 001 & 514 & 162.7506 & 2.7500 & 285.9 & 1 & 600.0 & 0 . \\
\hline 0 & 515 & 148.2926 & 1.5500 & 285.5 & 1 & 60 & \\
\hline 001 & 516 & 163.5193 & 000 & 284 & 2 & & \\
\hline 001 & 517 & 132.2910 & 0.5750 & 282.7 & 3 & 600.0 & 600 . \\
\hline & 518 & 45.2345 & 0.4500 & 282.6 & 4 & 600 & 600. \\
\hline 0012 & 519 & 359.3813 & 0.2500 & 284.1 & 4 & 600 & 60 \\
\hline 0012 & 520 & 14.5625 & 1.9500 & 285.2 & 4 & 600.0 & 500 \\
\hline & 521 & 110.8486 & 1.0000 & 285.6 & 5 & 600.0 & 600 \\
\hline & 522 & 281.5000 & 0.3250 & 28 & 6 & 600 & 60 \\
\hline 01 & 523 & 45.6740 & 1.1000 & 283.2 & & 600.0 & 600 \\
\hline
\end{tabular}




\begin{tabular}{|c|c|c|c|c|c|c|c|}
\hline 12 & 24 & 38.25 & 750 & 9 & 6 & 10.0 & \\
\hline 01 & 61 & 7.4697 & 0.4250 & 284.5 & 0 & 0 & \\
\hline 1 & 62 & 294 & 6500 & 34.1 & 6 & 00 & \\
\hline 01 & 3 & 199.3882 & .1500 & 282.5 & & 00 & \\
\hline & 4 & 273.34 & .3500 & 83.1 & & 0 & \\
\hline 01 & 65 & 289.1676 & 500 & 84.5 & & 0 & \\
\hline 01 & 6 & 4.2626 & 0.3000 & 284.4 & & 00 & \\
\hline 01 & 7 & 277.9181 & 0.2500 & 82.8 & & 0 & \\
\hline U & 8 & 139.7313 & 1.6250 & 282.8 & 6 & 00 & \\
\hline 01 & 9 & 151.2515 & 2.5250 & 283.0 & & 0 & \\
\hline & 610 & 164.2086 & 0 & 3 & & & \\
\hline 01 & 11 & 117.3771 & & 83.6 & 3 & & \\
\hline 01 & 12 & 7.6773 & 0 & 285.8 & 2 & 0 & \\
\hline 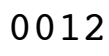 & 613 & 313.9632 & .27 & 3 & 1 & 0 & \\
\hline & 614 & 327 . & & 9 & 1 & & \\
\hline 1 & 615 & 350.7445 & 0 . & 286.0 & 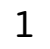 & 0 & \\
\hline 1 & 616 & 254.8824 & 0.3000 & 285.9 & 1 & 0 & \\
\hline 0 & 617 & 123.3988 & & 83.7 & 2 & & \\
\hline 01 & 618 & 143.6004 & 0 & 282.4 & 3 & 0 & \\
\hline 01 & 619 & 32.6630 & 0.95 & 281.7 & 4 & 0 & \\
\hline J & 620 & 3.4747 & & & 5 & & \\
\hline 0 & 621 & 108 . & 1. & 28 & 6 & & \\
\hline 01 & 622 & 142.2671 & 1.07 & 283.1 & 6 & 0 & \\
\hline 0 & 623 & 109. & 1 . & 2 & 6 & & \\
\hline 01 & 624 & 56. & 1 & 28 & 6 & & \\
\hline & 71 & 137.4899 & 20 & 284 & 6 & & \\
\hline 01 & 72 & 136.2497 & 2 & 283.5 & 6 & & \\
\hline $0-$ & 73 & 135.8841 & 0 & 3 & 6 & & \\
\hline 01 & 74 & 140.2489 & 50 & 285.3 & 6 & & \\
\hline 01 & 75 & 135.7995 & 2 . & 285.1 & 6 & & \\
\hline 0 & 76 & 121. & 0 & & 6 & & \\
\hline 0 & 77 & 83 & 0 & 28 & 6 & & \\
\hline 001 & 78 & 81.9713 & 1.1750 & 284.4 & 6 & & \\
\hline 01 & 79 & 265 . & 0 . & 28 & 5 & & \\
\hline 01 & 710 & 143 . & 1 . & & 4 & & \\
\hline & 711 & 49.1566 & 0.4750 & 284.5 & 3 & & \\
\hline 01 & 712 & 223.8798 & 0 & 28 & 2 & & \\
\hline 00 & 713 & 240.2353 & 0.8750 & 288.5 & 1 & & 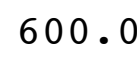 \\
\hline 001 & 714 & 252.4898 & 1.9500 & 286.4 & 2 & & 6 \\
\hline 0 & 715 & 325 . & 0 . & 28 & 2 & & \\
\hline 001 & 716 & 298.9971 & 0 . & 287.6 & 2 & & \\
\hline 001 & 717 & 191.0559 & 0.0750 & 286.7 & 3 & 0 & 0 \\
\hline 001 & 718 & 310.7891 & & 284.9 & 4 & & \\
\hline 0012 & 719 & 80.7091 & 0.8750 & 285.2 & 5 & 600.0 & 600 \\
\hline 001 & 720 & 181.9793 & 0.5750 & 285.1 & 6 & 600.0 & 600 \\
\hline & 721 & 110 . & & & 6 & & \\
\hline 01 & 722 & 115.2113 & 0.4000 & & 6 & & 600 \\
\hline . & 723 & 107.5672 & 0.5000 & 286.8 & J & 600.0 & 600 \\
\hline
\end{tabular}




\begin{tabular}{|c|c|c|c|c|c|c|c|}
\hline & 24 & & 0.8000 & 287.0 & & & \\
\hline 1 & 81 & 151. & 0.9500 & 286.4 & 4 & & \\
\hline 1 & 82 & 103.8493 & 1.6750 & 284.5 & 5 & 00 & \\
\hline 1 & 3 & 133.5321 & 1.0000 & 285.2 & & 00 & \\
\hline & 4 & & 1.7750 & & & 0 & \\
\hline & 5 & 5155 & 1.6750 & 283.7 & & 00 & \\
\hline 01 & 6 & 133.2351 & 1.2750 & 283.0 & & 0 & \\
\hline & 7 & . 9446 & 2. 3000 & 82.7 & & & \\
\hline 01 & 8 & 126.6758 & 1.7500 & 281.9 & 6 & 0 & \\
\hline 01 & 9 & 63.5280 & 1.2750 & 283.0 & & 00.0 & \\
\hline 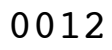 & 810 & 319.1183 & 1.3250 & 285 & & 0 & \\
\hline & 11 & 11.2453 & 0.4750 & & 3 & & \\
\hline 01 & 12 & 227.3636 & 1.1750 & 288.3 & 2 & 00 & \\
\hline 01 & 813 & 173.4158 & 4.0750 & 287.8 & 3 & 0.0 & \\
\hline & 814 & 182.3308 & & & 3 & & \\
\hline 01 & 15 & 3653 & 2.8000 & 288.9 & 2 & 0 & \\
\hline 2 & 816 & 87.1500 & 1.5250 & 289.8 & 1 & 0 & \\
\hline 01 & 817 & 877 & 2.9250 & 28 & 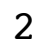 & & \\
\hline 0 & 18 & 74.0011 & 4.5750 & 287.3 & 3 & 0 & \\
\hline 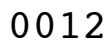 & 819 & 71.7500 & 5750 & 286 & 4 & 0 & \\
\hline 0. & 820 & 969 & 500 & 28 & 4 & & \\
\hline 0 & 821 & 000 & .2000 & 28 & 4 & & \\
\hline 0 & 822 & 2513 & 3.2250 & 286.2 & 4 & 0 & U. \\
\hline 01 & 823 & 71.006 & 0.9750 & 286.2 & 5 & 0 & \\
\hline 0 & 824 & 74.2506 & 0 & 28 & 4 & & \\
\hline 0 & 91 & 2500 & 3.4500 & 28 & 4 & & \\
\hline 01 & 92 & 85.0333 & .5000 & 28 & 4 & 0 & \\
\hline 0 & 93 & 52.3197 & 50 & 28 & 5 & & \\
\hline 01 & 94 & 125 . & 0.3750 & 28 & 6 & 0 & 0 . \\
\hline 01 & 95 & 125.4243 & 7500 & 283.0 & 6 & 0 & \\
\hline 0 & 96 & 115.7511 & 250 & 28 & 5 & & \\
\hline 00 & 97 & 202 & 500 & .6 & 6 & & \\
\hline 00 & 98 & 90.5135 & 0.5750 & 283.6 & 6 & 0 & U • \\
\hline 01 & 99 & 305.6357 & 750 & 283.1 & 5 & 0 & \\
\hline 0 & 910 & 354 . & 50 & 28 & 4 & & \\
\hline 001 & 911 & 335.7760 & 0.6750 & 284.4 & 3 & & 0 . \\
\hline 00 & 912 & 346.6554 & 0.4250 & 285.2 & 2 & 0 & \\
\hline 001 & 913 & 8.3156 & 0.4750 & 286.0 & 1 & 0 & 0 \\
\hline 001 & 914 & 205.5000 & 2.0250 & 285.6 & 1 & 600.0 & 0 . \\
\hline 00 & 915 & 145.8930 & 250 & 285.3 & 2 & 0 & \\
\hline 001 & 916 & 66.9989 & 000 & 284.7 & 3 & & \\
\hline 001 & 917 & 67.4969 & 4.6250 & 284.3 & 4 & 600.0 & 600 . \\
\hline & 918 & 72.0323 & 5000 & 284.0 & 4 & 600 & 600. \\
\hline 0012 & 919 & 68.9620 & 4.9500 & 284.0 & 4 & 600 & 600 \\
\hline 0012 & 920 & 74.2455 & 4.0750 & 284.2 & 4 & 600.0 & 600 \\
\hline & 921 & 64.5000 & 4.6750 & 284.4 & 4 & 600.0 & 600. \\
\hline & 922 & 6613 & 1.47 & 284.3 & 5 & 600 & 60 \\
\hline 01 & 923 & 54.0001 & 3.5750 & 284.3 & 4 & 600.0 & 600 \\
\hline
\end{tabular}




\begin{tabular}{|c|c|c|c|c|c|c|}
\hline 012 & & 4.0750 & 284.0 & & & \\
\hline 012101 & 8.2501 & 6.3000 & 283.7 & 4 & & \\
\hline 012102 & 59.5012 & .8250 & 283.5 & 4 & 0 & \\
\hline 012103 & 60.2610 & .3750 & 283.6 & & 00 & \\
\hline $01210 \quad 4$ & 7.75 & .3500 & 33.6 & & & \\
\hline 012105 & 5000 & 7250 & 83.5 & & 0 & \\
\hline 012106 & 62.0002 & 5.3250 & 283.2 & & 0 & \\
\hline 012107 & 66.7499 & .8500 & 33.3 & & & \\
\hline 012108 & 72.2519 & .6000 & 283.4 & 4 & 0 & \\
\hline 012109 & 81.9986 & 50 & 283.4 & & 00 & \\
\hline 1010 & 97.2450 & 4.72 & 283.8 & 4 & & \\
\hline 011 & 88.7248 & $12=$ & 83.0 & 3 & & \\
\hline 0121012 & 68.0037 & 5.3250 & 284.0 & 3 & 0 & \\
\hline 21013 & 76.6436 & 4.70 & 284.3 & 3 & 0 & \\
\hline 014 & 0726 & & & 4 & & \\
\hline 0121015 & 107.7504 & 6 . & 283.8 & 4 & & \\
\hline 0121016 & 122.7513 & 0 & 283.8 & 3 & 0 & \\
\hline 1017 & 112.74 & 5. & 1 & 4 & & \\
\hline 1018 & 110.70 & $4.8^{\circ}$ & 282.4 & 4 & 0 & \\
\hline 0121019 & 147.47 & 4 . & 281.8 & 4 & & \\
\hline 020 & 143.88 & 4 . & 281.7 & 4 & & \\
\hline 1021 & & & 281.7 & 5 & & \\
\hline 0121022 & 56.75 & 0 & 281 & 4 & 0 & \\
\hline 21023 & 42.36 & 1.70 & 281.2 & 5 & 0 & \\
\hline 1024 & & & 281.3 & 6 & & \\
\hline 012111 & 61 & 0 & 28 & 5 & & \\
\hline 012112 & 48.0370 & 3. & 280.8 & 4 & 0 & \\
\hline 0012113 & 54.53 & 0 & 280 & 4 & & \\
\hline 0012114 & 79 & 0 & 28 & 4 & & \\
\hline 012115 & 76.3072 & 95 & 280.6 & 5 & 0 & \\
\hline 012116 & 21 . & 0 & $2 \varepsilon$ & 4 & & \\
\hline 0012117 & 345 . & 0 & 28 & 4 & & \\
\hline 0012118 & 18.9398 & 205 & 281.1 & 5 & 0 & 0 . \\
\hline 012119 & 1.95 & 0 & $2 \varepsilon$ & 4 & 0 & \\
\hline 1110 & 229 . & 0 & 28 & 3 & & \\
\hline 00121111 & 245.7146 & 1.95 & 282.8 & 2 & & 0 . \\
\hline 0121112 & 255.3170 & 750 & 283.2 & 1 & 0 & \\
\hline 00121113 & 227.2438 & 2 . & 282.8 & 2 & & 0 . \\
\hline 00121114 & 267.9264 & 1.2750 & 282.7 & 1 & 0 & 0 . \\
\hline 00121115 & 213.7181 & 1 . & 282.5 & 1 & 0 & \\
\hline 00121116 & 315.3581 & 1.75 & & 1 & & \\
\hline 00121117 & 306.5000 & 2.3000 & 282.2 & 2 & 600.0 & 600. \\
\hline 0121118 & 145.2465 & 1.7750 & 281.8 & 3 & 600 & 000 \\
\hline 00121119 & 32.6871 & 1.3000 & & 4 & & s \\
\hline 00121120 & 309.5422 & 1.0250 & 280.6 & 5 & 600.0 & 00 \\
\hline 0121121 & 355.0023 & 1.3500 & 280.4 & 5 & 600 & 60 \\
\hline 00121122 & 320.3010 & 0 & & 6 & 60 & \\
\hline 0121123 & 88.3130 & 4.3500 & 281.7 & & 600.0 & 600 \\
\hline
\end{tabular}




\begin{tabular}{|c|c|c|c|c|c|c|c|}
\hline-1 & & 6.1791 & 3750 & 82.1 & $\Lambda$ & 00.0 & \\
\hline 01212 & 1 & 3.4994 & 5.8500 & 281.3 & 4 & 600.0 & 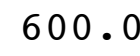 \\
\hline 1212 & 2 & 5.2441 & 8750 & 81.1 & 4 & 00 & \\
\hline 1212 & 3 & 93.7518 & 4250 & 280.6 & 4 & 00 & \\
\hline 01212 & 4 & 977 & 750 & 30.2 & & 0( & \\
\hline 1212 & 5 & 74.2232 & 750 & 80.3 & & 0 & \\
\hline 01212 & 6 & 80.2450 & 3250 & 80.2 & & 00 & \\
\hline 01212 & 7 & 4.5000 & 5000 & & & 0 & \\
\hline 01212 & 8 & 478 & 500 & 80.2 & & & \\
\hline 01212 & 9 & 7491 & 9750 & 80.7 & & 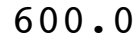 & \\
\hline 012121 & & 85.5000 & 6.8000 & 81.2 & & 0 & \\
\hline 212 & & 528 & .3250 & 31.7 & & & \\
\hline 01212 & & 158 & 00 & 82.4 & & 0 & \\
\hline 012121 & & 752 & 4.2250 & 32.8 & & 0 & \\
\hline 212 & & 39 & & & & & \\
\hline 1 & & & & 4.1 & & & \\
\hline 01212 & & 161.9828 & 4.7500 & .4 & & 0 & \\
\hline 212 & & 655 & 3 . & 1.9 & & 0 & \\
\hline 212 & & 12 & & 0.7 & & & \\
\hline 01212 & & 000 & 1.6750 & 30.8 & 4 & 0 & \\
\hline 212 & & 081 & 2.4250 & 281.0 & & 0 & \\
\hline 12 & & 571 & 50 & 1.2 & & & \\
\hline 1 & & 154 & 9750 & 281.3 & 4 & & \\
\hline 212 & & 883 & 2.6250 & 281.0 & 4 & 0 & \\
\hline 122 & & 77 & 50 & 31.6 & 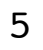 & & \\
\hline 13 & 1 & 89 & 00 & & 5 & & \\
\hline 01213 & 2 & 297 & 6000 & 280.3 & 0 & 0 & \\
\hline 01213 & 3 & 029 & 3250 & 279.9 & 5 & 0 & \\
\hline 01213 & 4 & 562 & 00 & .9 & 6 & & \\
\hline 01213 & 5 & 549 & 1.7250 & 9.3 & 6 & 0 & \\
\hline 01213 & 6 & 065 & 2.1250 & 280.0 & 5 & 0 & \\
\hline 213 & 7 & 45 & 00 & & 6 & & \\
\hline 01213 & 8 & 890 & 00 & .5 & 6 & 0 & \\
\hline 01213 & 9 & 250 & 500 & 280.7 & 5 & 0 & \\
\hline 213 & & 32 & 0 & & 4 & & \\
\hline & & 305.9969 & 2.4250 & 2.3 & 4 & 600 & \\
\hline 213 & & 287.1142 & 2.2250 & 282.7 & 3 & 0 & \\
\hline 21 & & 282.7028 & 2.2500 & 284.2 & 2 & 0 & \\
\hline 01213 & & 359.2479 & 2.9250 & 5.8 & 1 & 600.0 & \\
\hline 213 & & 000 & 9750 & 284.3 & 1 & & \\
\hline 213 & & 320 . & 3.3250 & 28 & 2 & 600 & \\
\hline & & 311. & 4.4500 & 283.9 & 3 & 600.0 & \\
\hline 1213 & & 331.5000 & 3.1250 & 283.7 & 4 & 600 & \\
\hline 01213 & & 176 & 2.2500 & & 5 & 600 & \\
\hline 0012132 & & 144.7519 & 3.2250 & 282.2 & 4 & 600.0 & \\
\hline 1213 & & 5037 & 3.0500 & 281.9 & 4 & 600.0 & \\
\hline & & 17 & 50 & & 4 & & \\
\hline 012132 & & 18.9205 & 3.2500 & 282.7 & 4 & 600.0 & 6 \\
\hline
\end{tabular}




\begin{tabular}{|c|c|c|c|c|c|c|}
\hline 1324 & 8.4069 & 3.0250 & 284.0 & 5 & 600.0 & \\
\hline 2141 & 3.3168 & 3.1000 & 283.5 & D & 600.0 & \\
\hline 12142 & 327.2066 & 2500 & 282.5 & 6 & 00 & \\
\hline 012143 & 291.6453 & .5000 & 282.7 & & 00 & \\
\hline $01214 \quad 4$ & 74.1527 & .8750 & 83.4 & & 0 & \\
\hline 12145 & 275.8465 & 7250 & 82.7 & & 00 & \\
\hline 012146 & 101.5000 & .1250 & 283.1 & & 0 & \\
\hline 012147 & 67.5865 & .9250 & 82.8 & & & \\
\hline 012148 & 71.1337 & .5000 & 282.5 & & 0 & \\
\hline 012149 & 343.2524 & 2.2250 & 282.6 & & 00 & \\
\hline 410 & 98.5820 & 1.9000 & 284.0 & & 0 & \\
\hline & 89.8184 & 3.6250 & 84.2 & & & \\
\hline 01 & 81.7199 & $\$ 500$ & 284.6 & & 0 & \\
\hline 0121413 & 120.5038 & 2.5250 & 284.5 & & 0 & \\
\hline & 241.2410 & & 83.2 & & & \\
\hline 01 & 349.4914 & 5500 & 282.9 & & 0 & \\
\hline 416 & 315.3945 & 0.82 & 283.2 & & 0 & \\
\hline 17 & 301.3339 & L. 3000 & .1 & & & \\
\hline 01 & 331.9893 & 2.6500 & 282.9 & 3 & 0 & \\
\hline 419 & 318.5000 & 1.9000 & 283.3 & 4 & 0 & \\
\hline 420 & 337.2435 & 0 & .6 & 5 & & \\
\hline 21 & 82.48 & 0 & 28 & 6 & & \\
\hline 422 & 62.9979 & 4.4750 & 284.8 & 5 & 0 & 00 . \\
\hline 423 & 77.9864 & 4 & 284.7 & 4 & & \\
\hline 24 & 61.7423 & 0 & 28 & 4 & & \\
\hline 012151 & 59.9962 & 6.4500 & 285.1 & 4 & & \\
\hline 012152 & 81.5000 & 4.7000 & 285.0 & 4 & 0 & \\
\hline 0012153 & 83.00 & 0 & 284.5 & 4 & & \\
\hline 0012154 & 58.7439 & 4250 & 284.4 & 4 & & 0 . \\
\hline 012155 & 71.7218 & 7250 & 284.4 & 4 & 0 & \\
\hline 012156 & 95.7489 & 0 & 28 & 5 & & \\
\hline 0012157 & 114.4914 & 3 . & 284.4 & 5 & & \\
\hline 0012158 & 115.5625 & 1000 & 284.2 & 4 & 0 & J. \\
\hline 012159 & 93.9982 & 500 & 284.2 & 3 & 0 & \\
\hline 510 & 78 . & & 28 & 4 & & \\
\hline 00121511 & 72.0004 & 4000 & 285.3 & 3 & & 0 . \\
\hline 00121512 & 94.6769 & 000 & 286.7 & 2 & 0 & \\
\hline 00121513 & 122.7727 & 500 & 287.5 & 1 & & \\
\hline 00121514 & 133.5031 & 4.3000 & 286.3 & 2 & 0 & 0 . \\
\hline 0121515 & 169.5033 & 750 & 286.3 & 2 & 0 & \\
\hline 00121516 & 161.5003 & 5 . & & 3 & & \\
\hline 00121517 & 167.9998 & 7.2750 & 284.4 & 4 & 600.0 & 600. \\
\hline 00121518 & 150.5314 & 7.7500 & 283.8 & 4 & 600 & 000 \\
\hline 00121519 & 144.0624 & 7.1250 & 284.9 & 4 & & s \\
\hline 00121520 & 172.0038 & 7.5750 & 284.1 & 4 & 600.0 & 00 \\
\hline 0121521 & 176.5000 & 5.4500 & 281.9 & 4 & 600.0 & 50 \\
\hline 00121522 & 179.0000 & 000 & & 4 & 60 & \\
\hline 0121523 & 175.0193 & 4.8500 & 282.9 & 4 & 600.0 & 600 \\
\hline
\end{tabular}




\begin{tabular}{|c|c|c|c|c|c|c|}
\hline 24 & & & & & & \\
\hline 2161 & 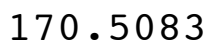 & & 3.1 & 4 & & \\
\hline 2162 & 177.0002 & 2000 & 82.7 & 4 & 00 & \\
\hline 12163 & 175.5000 & 9250 & 281.9 & & 00 & \\
\hline 2164 & 6 & 000 & 1.3 & & & \\
\hline 2165 & 180 & 500 & 81.7 & & 0 & \\
\hline 12166 & 181.5001 & 500 & 81.8 & & 00 & \\
\hline 12167 & 2500 & 3500 & 81.4 & & & \\
\hline 12168 & 181.2500 & & 81.3 & & & \\
\hline 012169 & 176.5415 & 3500 & 81.8 & & 0 & \\
\hline 0121610 & 126.8481 & 9250 & 282.8 & & & \\
\hline 11 & 168.4933 & & 83.8 & & & \\
\hline 2 & 181 & 00 & 284.0 & 4 & 0 & \\
\hline 21613 & 179.0000 & 4.0000 & 8 & & & \\
\hline & 259 . & & & & & \\
\hline & 231 . & & 9 & 1 & & \\
\hline 616 & 303.7597 & 0.7500 & 286.7 & & 0 & \\
\hline 617 & 185 . & 1.2000 & .4 & & & \\
\hline & 158. & & 2 & 3 & & \\
\hline 619 & 170 . & & 284 & 4 & & \\
\hline 620 & 134.8092 & 2 . & 285.5 & 5 & 0 & \\
\hline & 174.75 & & & 5 & & \\
\hline 2 & 90 & 00 & 283.8 & 6 & & \\
\hline 623 & 108.0000 & 1.32 & 3 & 6 & 0 & \\
\hline 24 & 114 . & & & & & \\
\hline 1 & 66. & 1 . & 28 & 6 & & \\
\hline 012172 & 129.9375 & 1.3750 & 8.5 & 6 & & 0 \\
\hline 012173 & 147.9084 & 0 & & 6 & & \\
\hline 174 & 137 . & 00 & 28 & 5 & & \\
\hline 012175 & 76.995 & & 4 & 4 & & J. \\
\hline 012176 & 163.7900 & 500 & 284.2 & 4 & & 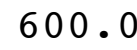 \\
\hline $01217 \quad 7$ & 174.2515 & 0 & 7 & 4 & & \\
\hline 012178 & 164.7602 & 0 & 27 & 4 & & 0 . \\
\hline 012179 & 174.7888 & 500 & 279.7 & 4 & & 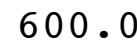 \\
\hline .710 & 169.9205 & & & 3 & & \\
\hline & 181.7497 & 000 & 0.7 & 4 & & \\
\hline 0121712 & 183.0000 & 3.9000 & 282.3 & 4 & & \\
\hline 00121713 & 181.0000 & 0 & 283.4 & 4 & & 0 . \\
\hline 00121714 & 177.2499 & 9.0500 & 4.5 & 4 & .0 & 0 . \\
\hline 0121715 & 177.7500 & 6.1000 & 285.3 & 4 & & \\
\hline 00121716 & 173.7495 & 250 & & 4 & & \\
\hline 00121717 & 171.8150 & 5.1500 & & 4 & & 0 . \\
\hline 0121718 & 171.2500 & 6.6250 & 283.9 & 4 & 0 & 0 . \\
\hline 00121719 & 158.7505 & 500 & & 4 & & \\
\hline 00121720 & 162.5086 & & & 4 & & \\
\hline 0121721 & 141.7449 & 8.5500 & 282.9 & 4 & & 00 . \\
\hline & & & & 4 & & \\
\hline 0121723 & 156.5000 & 8.9750 & 282.7 & & .0 & 600 \\
\hline
\end{tabular}




\begin{tabular}{|c|c|c|c|c|c|c|}
\hline 724 & & 5000 & & & & \\
\hline 2181 & 152.8117 & 3250 & 1.8 & 4 & 0 & \\
\hline 12182 & 188 & 5000 & 281.0 & 4 & 00 & \\
\hline 12183 & 92.4948 & 9250 & 282.0 & & 00 & \\
\hline 2184 & 115 . & 8500 & 82.1 & & 0 & \\
\hline 12185 & 146 & 000 & 279.9 & & 0 & \\
\hline 012186 & 7494 & 8750 & 279.5 & & 00 & \\
\hline 012187 & 148.2500 & 2500 & 278.6 & & & \\
\hline 012188 & 141.4 & 1750 & 78.1 & & & \\
\hline 012189 & 50.7773 & 1.3000 & 278.9 & 4 & 00 & 0 \\
\hline 0121810 & 320.7607 & 2.3000 & 280.5 & & 0 & \\
\hline 811 & 288.2 & .9000 & 80.6 & & & \\
\hline 0121812 & 270 & 2.3000 & 280.7 & 2 & 0 & \\
\hline 0121813 & 290 & 2.9250 & 281.3 & & 00.0 & \\
\hline 814 & 298 & 00 & 82.5 & & & \\
\hline 815 & 275 & & 282.6 & 3 & & \\
\hline 1816 & 227.4917 & 1.7750 & 282.2 & 3 & 0 & \\
\hline 1817 & 253 & 50 & 81.3 & 4 & & \\
\hline 1818 & 254 . & 00 & 280.5 & 5 & & \\
\hline 819 & 325.4 & 500 & 280.1 & 4 & & \\
\hline 1820 & 31.7 & 0.5250 & 280.2 & 4 & 0 & 00 \\
\hline 21 & 2.8 & & & 5 & & \\
\hline 822 & 357 . & 1.6500 & 280.9 & 4 & & \\
\hline 0121823 & 352.8 & 3500 & 281.1 & 4 & 0 & \\
\hline 824 & 275.1 & & & 5 & & \\
\hline 91 & 315 . & 2 . & 280.8 & 5 & & \\
\hline 012192 & 73.5 & 3500 & 280.2 & 6 & 0 & U. \\
\hline 012193 & 162.51 & 000 & 79.5 & 6 & & \\
\hline 0012194 & 157 & 50 & 279.3 & 5 & & \\
\hline 0012195 & 94 . & 50 & 280.0 & 6 & & 0 • \\
\hline 0012196 & 143.4183 & 0.8750 & 280.4 & 6 & & 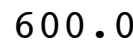 \\
\hline 012197 & 101 & 00 & 280.4 & 6 & & \\
\hline 012198 & 104 . & 50 & 280.3 & 5 & & 0 . \\
\hline 0012199 & 52.7479 & 2.9250 & 281.6 & 4 & 0 & \\
\hline 0121910 & 290.7829 & 50 & & 3 & & \\
\hline 001 & 312.9607 & 1.4500 & 283.9 & 2 & & 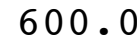 \\
\hline 0121912 & 324.7310 & 1.9000 & 284.9 & 3 & 0 & 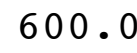 \\
\hline 00121913 & 234.3866 & 000 & 285.4 & 2 & & . \\
\hline 00121914 & 237.4898 & 1.9250 & 285.4 & 3 & .0 & 0 . \\
\hline 0121915 & 182.1554 & 3.5750 & 284.9 & 2 & & \\
\hline 00121916 & 173.7500 & 3.8250 & 284.0 & 2 & & 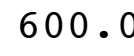 \\
\hline 00121917 & 133.3476 & 2.1000 & 283.3 & 3 & .0 & 0 . \\
\hline 0121918 & 78.9053 & 1.5250 & 282.7 & 4 & .0 & 00 . \\
\hline 00121919 & 126.9718 & 500 & & 5 & & \\
\hline 00121920 & 139.9494 & 1.6750 & & 6 & & 0 . \\
\hline 00121921 & 155.0000 & 1.8250 & 283.4 & 6 & 600 & 600 \\
\hline 21 & & & & 6 & & \\
\hline 0121923 & 127.9961 & 1.3750 & 283.5 & & 60 & 60 \\
\hline
\end{tabular}




\begin{tabular}{|c|c|c|c|c|c|c|}
\hline 924 & & $9 / 50$ & & & & \\
\hline 201 & 24 & .7000 & 3.1 & 5 & 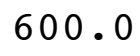 & \\
\hline 12202 & 155.5835 & 2750 & 81.9 & 5 & 00 & \\
\hline 12203 & 164.7585 & 2.8750 & 281.6 & & 00 & \\
\hline 12204 & 164.2606 & 6500 & 1.1 & & 0 & \\
\hline 12205 & 108.2498 & 0 & 81.6 & & 0 & \\
\hline 012206 & 156.7173 & 9750 & 81.7 & & 00 & \\
\hline 012207 & 69.9084 & 0 & 81.6 & & & \\
\hline 012208 & 149.3076 & 0250 & 81.8 & & & \\
\hline 012209 & 169.9994 & 1.5250 & 82.6 & & 00 & 0 \\
\hline 0122010 & 169.2501 & 2.2750 & 283.7 & & 0 & \\
\hline 2011 & 189.7733 & 0.9500 & 84.5 & & & \\
\hline 0122012 & 274.0730 & 0 . & 286.1 & & 0 & \\
\hline 0122013 & 233.2499 & 2.20 & 285.8 & & 0.0 & \\
\hline 2014 & 244 . & & & & & \\
\hline 2015 & 227 . & & 286.1 & 3 & & \\
\hline 22016 & 288.9581 & 1.1750 & 285.8 & 3 & .0 & . \\
\hline 2017 & 318.6211 & 0 & 2 & 4 & & \\
\hline 2018 & 350.3211 & 1 . & 284.2 & 4 & & \\
\hline 2019 & 1792 & 1.5250 & 283 & 4 & & \\
\hline 2020 & 7.7535 & 1.3250 & 284.8 & 4 & 0 & . \\
\hline 2021 & 11.9535 & & 285.2 & 4 & & \\
\hline 022 & 301.6105 & 0 & 285.1 & 5 & & \\
\hline 0122023 & 243.0171 & 0.2750 & 284.1 & 6 & 0 & 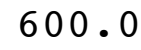 \\
\hline 22024 & 155.2376 & & 284.2 & 6 & & \\
\hline 211 & 107.2667 & 0 . & .3 & 6 & & \\
\hline 012212 & 127.4393 & 2000 & 2 & 6 & 0 & . \\
\hline 012213 & 45.4083 & 0 & 9 & 6 & & \\
\hline 0012214 & 173.0339 & 0 . & $2 \varepsilon$ & 6 & & \\
\hline 012215 & 40.6212 & 00 & 5.8 & 6 & 0 & 0 • \\
\hline 0012216 & 134.1316 & 2.8000 & 284.1 & 6 & 0 & 0 . \\
\hline 0012217 & 64.4000 & 2 . & 283.3 & 6 & & \\
\hline 0012218 & 39.8963 & 500 & 1.1 & 6 & & 0 . \\
\hline 0012219 & 90.3654 & 5.7250 & 282.6 & 5 & 0 & \\
\hline 00122110 & 23.5515 & 2 . & & 4 & & \\
\hline 0012 & 80.8156 & 1.6750 & 285.5 & 3 & & . \\
\hline 0122112 & 83.5452 & 9500 & 288.6 & 3 & $\cdot 0$ & . \\
\hline 00122113 & 88.0535 & 4 . & 286.8 & 3 & & . \\
\hline 00122114 & 82.7213 & 3.8250 & 5.9 & 2 & .0 & 0 . \\
\hline 0122115 & 80.2279 & 4.3750 & 286.9 & 3 & 0 & 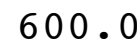 \\
\hline 00122116 & 95.7323 & 3 . & $28^{\circ}$ & 2 & & 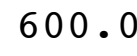 \\
\hline 00122117 & 108.9995 & 5.5250 & 285.1 & 3 & .0 & 0 . \\
\hline 0122118 & 106.2506 & 5.6000 & 284.4 & 4 & 0 & 00 . \\
\hline 00122119 & 73.7497 & 500 & & 4 & & \\
\hline 00122120 & 61.4243 & 2.6000 & & 5 & 0 & 0 . \\
\hline 00122121 & 345.9902 & 2.3500 & 283.3 & 5 & 600 & 600 \\
\hline 221 & & & & 4 & & \\
\hline 0122123 & 34.7519 & 4.0250 & 283.5 & & 600.0 & 60 \\
\hline
\end{tabular}




\begin{tabular}{|c|c|c|c|c|c|c|}
\hline 2124 & 9.5002 & 4.8750 & 284.1 & 4 & 600.0 & \\
\hline 2221 & 4.2501 & 6.4250 & 284.2 & 1 & 600.0 & \\
\hline 012222 & 7497 & 5250 & 284.0 & 4 & 00 & \\
\hline 012223 & 61.0009 & 5.8250 & 284.1 & & 00 & \\
\hline 012224 & 64.7502 & & 84.0 & & 0 & \\
\hline 012225 & 1966 & .3750 & 282.8 & & 00 & \\
\hline 012226 & 175.9465 & 0.8250 & 281.5 & & 0 & \\
\hline 012227 & 355.8090 & .2500 & 81.0 & & & \\
\hline 012228 & 85.9700 & .7000 & 281.6 & 6 & 0 & \\
\hline 012229 & 86.0571 & 2.6250 & 282.7 & & 00 & \\
\hline 0122210 & 71.2334 & 3.1000 & 284.0 & & & \\
\hline 2211 & 131.7058 & 3.0250 & 85.1 & 3 & & \\
\hline 0122212 & 126.2642 & 4.4250 & 285.2 & 3 & 0 & \\
\hline 0122213 & 140.2949 & 5.0500 & 285.2 & 3 & 0 & \\
\hline 22214 & 167.7755 & & & $z$ & & \\
\hline 0122215 & 171.2496 & 500 & 284.5 & 2 & 0 & \\
\hline 0122216 & 164.9995 & 0 & 284.5 & 2 & 0 & \\
\hline 22217 & 159.4983 & 0 & 4.6 & 3 & & \\
\hline 0122218 & 131.99 & 3.7250 & 283.7 & 4 & 0 & \\
\hline 0122219 & 96.24 & 0 & 283.1 & 4 & 0 & \\
\hline 2220 & 80.99 & 0 & 282.6 & 4 & & \\
\hline 221 & 74 . & 0 & 282.3 & 4 & & \\
\hline 222 & 71.0002 & 3250 & 282.1 & 4 & 0 & \\
\hline 2223 & 67.50 & 5.92 & 282.1 & 4 & & \\
\hline 224 & 75. & 0 & 282.1 & 4 & & \\
\hline 012231 & 82.9984 & 7500 & 282.3 & 5 & & \\
\hline 012232 & 102.5408 & 6750 & 282.3 & 6 & 0 & \\
\hline 0012233 & 126.56 & 0 & 281.8 & 6 & & 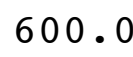 \\
\hline 012234 & 117 . & 0 & 281.7 & 6 & & 0 . \\
\hline 012235 & 114.7573 & 1.5000 & 281.4 & 6 & 0 & \\
\hline 012236 & 156.6906 & 500 & 28 & 6 & & \\
\hline 0012237 & 38. & 0 & .4 & 6 & & \\
\hline 0012238 & 87.7368 & 0.3000 & 280.4 & 6 & 0 & J. \\
\hline 012239 & 149.52 & 500 & 281.8 & 5 & & \\
\hline 2310 & 165 . & 0 & 281.2 & 4 & & \\
\hline 00122311 & 163.0118 & 2.9750 & 282.2 & 3 & & 0 . \\
\hline 0122312 & 168.4997 & 500 & 283.1 & 2 & 0 & \\
\hline 00122313 & 164.5369 & 0 & 283.3 & 1 & & 0 . \\
\hline 00122314 & 182.1621 & 2.8750 & 284.3 & 1 & 0 & . \\
\hline 0122315 & 134.5865 & 000 & 283.4 & 2 & 0 & \\
\hline 00122316 & 136.0411 & 3 . & & 2 & & \\
\hline 00122317 & 137.3605 & 1.0500 & 283.9 & 3 & 600.0 & 600 . \\
\hline 0122318 & 109.3667 & 1.7750 & 283.2 & 4 & 600 & 000 \\
\hline 00122319 & 123.2610 & 4.7000 & & 4 & & s \\
\hline 00122320 & 87.7491 & 7.3250 & 282.9 & 4 & 600.0 & 00 \\
\hline 0122321 & 85.0295 & 5.9250 & 283.2 & 4 & 600 & 60 \\
\hline 0122322 & 78.5018 & & 282.9 & 4 & 60 & \\
\hline 0122323 & 79.7502 & 5.1250 & 283.2 & 4 & 600.0 & 600 \\
\hline
\end{tabular}




\begin{tabular}{|c|c|c|c|c|c|c|}
\hline 2024 & 0.0049 & 3.8500 & 283.0 & 4 & 800 & \\
\hline 2241 & 0.2489 & 3.9500 & 83.0 & $t$ & 600.0 & \\
\hline 12242 & 7518 & 1250 & 282.9 & 4 & 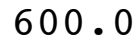 & \\
\hline 012243 & 31.4985 & 8500 & 282.3 & & 00 & \\
\hline $224 \quad 4$ & & & & & & \\
\hline 2245 & 161 & 500 & 80.4 & & 0 & \\
\hline 012246 & 172.5000 & 3250 & 280.1 & & & \\
\hline 012247 & 175 . & .2750 & & & & \\
\hline 012248 & 174.2499 & 3.7250 & 78.7 & & & \\
\hline 012249 & 150.2336 & 0500 & 278.8 & & & \\
\hline 2410 & 162.0016 & 750 & 79.5 & & & \\
\hline & 171.04 & & 79.7 & & & \\
\hline 2 & 174. & 4250 & 80.7 & & 0 & \\
\hline 2413 & 177.7507 & 7250 & 281.9 & & 0 & \\
\hline & 180 . & & 82.9 & & & \\
\hline 5 & 180 & 7.4250 & 283.8 & 4 & & \\
\hline 2416 & 181.0000 & 000 & 284.3 & 4 & & \\
\hline 17 & 173.5034 & & 0 & & & \\
\hline 8 & 180 & 0 & 283 & 4 & & \\
\hline 2419 & 171.9998 & 50 & 8 & 4 & & \\
\hline 20 & 166 . & 0 & & 4 & & \\
\hline & 162 . & & & 4 & & \\
\hline 22 & 162.5001 & 000 & 282 & 4 & & \\
\hline 23 & 166 . & 250 & 282 & 4 & & \\
\hline 24 & 171. & & & 4 & & \\
\hline 012251 & 174 & 000 & 282 & 4 & & \\
\hline 012252 & 176.7499 & 3000 & 8 & 4 & & \\
\hline 012253 & 174.00 & 0 & & 4 & & \\
\hline 012254 & 165 & 50 & 8 & 4 & & \\
\hline 012255 & 161.4954 & 5500 & 6 & 4 & & \\
\hline 012256 & 160 . & 0 & & 4 & & \\
\hline 012257 & 15 & 0 & 7 & 4 & & \\
\hline 012258 & 149.5000 & 000 & 279.4 & 4 & 0 & \\
\hline 012259 & 138 . & 0 & & 4 & & \\
\hline 2510 & 12 & 0 & & 4 & & \\
\hline 0122511 & 134.2907 & 250 & 281.2 & 4 & & \\
\hline 22512 & 150.0000 & 250 & 282.2 & 4 & & \\
\hline 0122513 & 153.2492 & 0 & 281.8 & 4 & & \\
\hline 0122514 & 151.5000 & 500 & 282.0 & 4 & & \\
\hline 22515 & 155.747 & 000 & 282.2 & 3 & & \\
\hline 0122516 & 152.2495 & 750 & & 3 & & \\
\hline 00122517 & 137.3233 & 2.0750 & 282.5 & 4 & $6 c$ & . \\
\hline 2518 & .3274 & 7500 & 282.2 & 5 & 0 & \\
\hline 00122519 & 91.7494 & 500 & & 4 & & \\
\hline 00122520 & 129.2446 & 0.6500 & 283.1 & 5 & 60 & 00 \\
\hline 0122521 & 161.0186 & 2.2750 & & $c$ & 0 & \\
\hline r & 71 & & & & & \\
\hline 012252 & 60.0275 & 1.1750 & 281.3 & & 600.0 & 600 \\
\hline
\end{tabular}




\begin{tabular}{|c|c|c|c|c|c|c|}
\hline $3<4$ & & .1000 & & & & \\
\hline 261 & 24 & L. 7500 & 0.5 & 6 & n & \\
\hline 12262 & 151.4089 & 6000 & 279.6 & 6 & 00 & \\
\hline 12263 & 44.2883 & 5000 & 279.0 & & 00 & \\
\hline 12264 & .5009 & .7000 & 79.4 & & 0 & \\
\hline 12265 & 41 & .6000 & 80.4 & & 0 & \\
\hline 012266 & 338.2863 & 1.7250 & 280.5 & 4 & 00 & \\
\hline 012267 & 334.2506 & 1.8250 & 80.6 & & 0 & \\
\hline 012268 & 336.2654 & 2.2750 & 80.5 & 4 & & \\
\hline 012269 & 311.4976 & 1.6000 & 81.4 & 3 & 00.0 & 8 \\
\hline 0122610 & 279.9608 & 1.7250 & 282.3 & & 0 & \\
\hline 11 & 275.4930 & 1.4250 & 82.5 & 1 & & \\
\hline 612 & 268.7539 & 1.7750 & 283.3 & 2 & 0 & \\
\hline 613 & 302.7575 & 2.2750 & 283.5 & 3 & 0.0 & \\
\hline 14 & 293. & & & 2 & & \\
\hline 15 & 286 . & & 284.8 & 2 & & \\
\hline 01 & 300.5024 & 1.2750 & 285.4 & 3 & .0 & 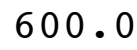 \\
\hline 17 & 303 . & 0 & .3 & 4 & & \\
\hline 18 & 329. & 0 & 282.4 & 5 & & \\
\hline 01 & 354 & 000 & 281.8 & 5 & & \\
\hline 20 & 6.9997 & 1.1250 & 281.8 & 5 & 0 & . \\
\hline 21 & 6.7485 & & & 5 & & \\
\hline 01 & 24 . & 0 & 281.4 & 4 & & \\
\hline 523 & 133.0718 & 0.2000 & $2 \varepsilon$ & 5 & 0 & 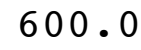 \\
\hline 24 & 145.7833 & & & 6 & & \\
\hline 71 & 15 & 00 & 281.1 & 6 & & \\
\hline 012272 & 0.2494 & 500 & 281 & 6 & 0 & . \\
\hline 012273 & 175.7940 & 0 & 9 & 6 & & \\
\hline 0012274 & 277.2500 & 0 . & 28 & 6 & & $\bullet$ \\
\hline 012275 & 345.4417 & 000 & 280.8 & 6 & 0 & U. \\
\hline 012276 & 1.2651 & 1.3250 & 281.2 & 5 & 0 & 0 . \\
\hline 012277 & 4.2302 & 0 & 281.1 & 4 & & \\
\hline 012278 & 358.8907 & 000 & 281.5 & 4 & & 0 . \\
\hline 012279 & 318.2402 & 250 & 281.9 & 4 & 0 & \\
\hline 0122710 & 308.8369 & 0 & & 3 & & \\
\hline 0012 & 149.8356 & 0.3000 & 282.8 & 2 & & 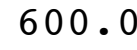 \\
\hline 0122712 & 270.5442 & 6750 & 284.7 & 1 & $\cdot 0$ & 0 . \\
\hline 00122713 & 167.2630 & 000 & 285.0 & 1 & & . \\
\hline 0012 & 158.1603 & 1.4500 & 286.6 & 1 & .0 & 0 . \\
\hline 0122715 & 290.1325 & 1.2250 & 287.8 & 1 & & \\
\hline 00122716 & 235.6139 & 000 & 287.3 & 2 & & 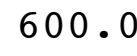 \\
\hline 00122717 & 152.0454 & 2.0000 & 284.9 & 3 & .0 & 0 . \\
\hline 0122718 & 157.7763 & 1.7000 & 282.7 & 4 & .0 & 00 . \\
\hline 00122719 & 141.4982 & 500 & 281.9 & 5 & & \\
\hline 00122720 & 151.0407 & 1.6000 & 282.2 & 6 & & 0 . \\
\hline 0122721 & 166.2495 & 2.6000 & 282.1 & 6 & & 600 \\
\hline 2 & 10 & & & 6 & & \\
\hline 0122723 & 111.8288 & 0.4500 & 283.7 & & 60 & $=0$ \\
\hline
\end{tabular}




\begin{tabular}{|c|c|c|c|c|c|c|}
\hline 2724 & & 2250 & 4.0 & & & \\
\hline 2281 & 0 & 3500 & 33.3 & 5 & 1000 & \\
\hline 12282 & 83.2955 & 0000 & 83.3 & 6 & 00.0 & \\
\hline 012283 & 208.1621 & 0000 & 283.9 & & 00 & \\
\hline 012284 & 336.9234 & 0750 & & & & \\
\hline 012285 & 145.4992 & 4000 & 9 & 6 & 00 & \\
\hline 012286 & 39.5116 & 5500 & 283.0 & & 00 & \\
\hline 012287 & 129 & 750 & & & 0 & \\
\hline 0012288 & 125.9951 & 3750 & 3 & 4 & & \\
\hline 0012289 & 152.5031 & 2.7000 & 283.9 & & 00.0 & \\
\hline 00122810 & 159.5029 & 1.6750 & 285.5 & & & \\
\hline 001 & 163.4962 & 0 & & $\perp$ & & \\
\hline 00122812 & 150.51 & 1.4 & 286.8 & 1 & .0 & \\
\hline 00122813 & 198.0249 & 0 & 287.9 & 1 & .0 & \\
\hline 314 & 156 . & & & 1 & & \\
\hline 001 & 225 . & & 287.9 & 1 & & \\
\hline 816 & 257.8431 & 0 & 288.1 & 2 & .0 & 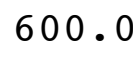 \\
\hline 817 & 265.3188 & 0 & 1 & 3 & .0 & \\
\hline 818 & 350.27 & & 284 & 4 & & \\
\hline 001 & 139.4625 & & 284.8 & 5 & .0 & \\
\hline 820 & 136.7429 & .52 & 285 & 6 & .0 & 000 \\
\hline 00 & 138.2600 & & & 6 & & \\
\hline 001 & 136 & 0 & 28 & 6 & & \\
\hline 00122823 & 147.7531 & 0 & 28 & 5 & .0 & 000 \\
\hline 24 & 145.2450 & & & 5 & & \\
\hline 91 & 33.7537 & 0 & 28 & 6 & & \\
\hline 0012292 & 14.0000 & 0 & 282.9 & 6 & .0 & 000 \\
\hline 012293 & 18.0000 & 0 & 4 & 6 & .0 & \\
\hline 0012294 & 18.0000 & 0 & 28 & 6 & .0 & \\
\hline 0012295 & 66.3842 & 0 & 28 & 6 & .0 & 00 \\
\hline 0012296 & 121.4989 & 0.3500 & 283.5 & 5 & .0 & \\
\hline 0012297 & 136.9982 & 2 . & & 6 & & \\
\hline 0012298 & 159.2584 & & 28 & 6 & .0 & \\
\hline 0012299 & 160.2500 & 0.3000 & 282.6 & 5 & .0 & \\
\hline 00122910 & 256.5934 & 020 & & 4 & & \\
\hline 0012 & 287.3475 & 0.9750 & & 3 & .0 & \\
\hline 912 & 167.1202 & 3.0500 & 282.8 & 2 & .0 & \\
\hline 0012 & 161.6938 & 1 . & & 1 & .0 & 6 \\
\hline 00122 & 158.0000 & 2.8750 & 284.0 & 1 & .0 & 60 \\
\hline 00122915 & 154.2555 & 1.8750 & 284.4 & 1 & .0 & \\
\hline 00122916 & 165.0011 & 2.9000 & 28 & 1 & & 600 \\
\hline 00122917 & 106.0094 & 2.4000 & 28 & 2 & .0 & 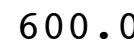 \\
\hline 00122918 & 93.2713 & 1.9750 & 284.7 & 3 & .0 & 00 \\
\hline 00122919 & 137.5515 & 1.1000 & & 4 & & \\
\hline 00122920 & 142.4453 & 2.7500 & & 5 & 600.0 & 600 \\
\hline 00122921 & 127.5098 & 1.8500 & 283.3 & 6 & 600.0 & 600 \\
\hline 22 & 13 & & & 6 & & \\
\hline 0122923 & 141.5000 & 0.5750 & 282.9 & & .0 & 600 \\
\hline
\end{tabular}




\begin{tabular}{|c|c|c|c|c|c|c|}
\hline 924 & & & & & & \\
\hline 301 & 532 & .3750 & 2.1 & 6 & 0 & \\
\hline 12302 & 159.7506 & 1000 & 281.4 & 6 & 00 & \\
\hline 12303 & 98.0116 & 2000 & 280.9 & & 00 & \\
\hline 12304 & 000 & .7500 & 81.9 & & & \\
\hline 12305 & 2567 & .2750 & 83.1 & & 0 & \\
\hline 012306 & 109. & 1250 & 282.2 & & 00 & \\
\hline 012307 & 0170 & 1500 & 282.0 & & 0 & \\
\hline 012308 & 353 & 1250 & 82.0 & 6 & & \\
\hline 012309 & 248.4460 & 0.0000 & 84.1 & & 00 & 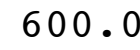 \\
\hline 0123010 & 240.9253 & 1500 & 285.1 & 4 & & \\
\hline 11 & 254.7379 & 1.3250 & 84.0 & 3 & & \\
\hline 12 & 251.2 & 1.7500 & 283.8 & 4 & 0 & \\
\hline 13 & 189 & 2.2500 & 284.3 & 3 & & \\
\hline 14 & 152 . & 0 & & 2 & & \\
\hline 15 & 154 . & & 284.9 & 2 & & \\
\hline 16 & 142.7476 & 2.6500 & 285.4 & 1 & 0 & 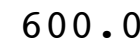 \\
\hline 17 & 122 . & 1.9000 & .1 & 2 & 0 & \\
\hline 18 & 122 . & 1.7250 & 28 & 3 & & \\
\hline 19 & 118 & 9750 & 28 & 4 & & \\
\hline 20 & 122.2366 & 3.0750 & 284.8 & 5 & 0 & 00. \\
\hline 21 & 105.7550 & & 285.2 & 6 & & \\
\hline 22 & 114.4436 & 2.1250 & 285.3 & 6 & & \\
\hline 023 & 140.2524 & 2.4250 & 28 & 6 & 0 & \\
\hline 24 & 140.0295 & 1.57 & & 6 & & \\
\hline 11 & 121. & 0.6750 & 28 & 6 & & \\
\hline 012312 & 104.5548 & 0.4000 & .7 & 6 & 0 & J. \\
\hline 012313 & 33.3394 & 0.8000 & .1 & 5 & & \\
\hline 0012314 & 141.2505 & 1.5250 & .3 & 6 & & \\
\hline 0012315 & 24.7658 & 0.2750 & 281.9 & 6 & & U. \\
\hline 0012316 & 122.2638 & 0.0250 & .4 & 6 & & 0 . \\
\hline 012317 & 183.0 & 0 & & 6 & & \\
\hline 012318 & 192.4870 & 500 & 280.5 & 6 & & 0 . \\
\hline 0012319 & 14.0329 & 0.7500 & 282.4 & 5 & 0 & \\
\hline 01 & 352 . & 1.3 & & 4 & & \\
\hline 001 & 305.5059 & 1.2750 & .5 & 3 & & 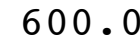 \\
\hline 12 & 255.8257 & 1.8500 & 284.5 & 3 & & . \\
\hline 0012 & 163.3446 & 3.4750 & & 2 & & . \\
\hline 0012 & 159.0000 & 3.9500 & 283.3 & 2 & .0 & 0 . \\
\hline 0123115 & 132.2496 & 2.0750 & 284.7 & 1 & & \\
\hline 00123116 & 26.4249 & 2.0250 & & 2 & & 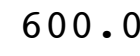 \\
\hline 00123 & 110.2388 & 1.9375 & 285.3 & 3 & .0 & 0 . \\
\hline 0123118 & 122.7457 & 1.7250 & 284.0 & 4 & 0 & 0 . \\
\hline 00123119 & 118.0001 & 3.9750 & & 4 & & \\
\hline 00123120 & 122.2366 & 3.0750 & & 5 & & 0 . \\
\hline 0123121 & 105.7550 & 2.8000 & 285.2 & 6 & 600 & 600. \\
\hline & 114 . & 2.1250 & & 6 & & \\
\hline 0123123 & 140.2524 & 2.4250 & 284.9 & & .0 & 60 \\
\hline
\end{tabular}




\begin{tabular}{|c|c|c|c|c|c|c|c|c|}
\hline & & 4 & & & & & & \\
\hline 1 & 1 & 11 & 32 & 5750 & 4.2 & 6 & 00.0 & \\
\hline & 1 & 12 & 271.8284 & .8250 & 283.2 & 6 & 00.0 & \\
\hline & 1 & 13 & 219.0028 & 5500 & 281.6 & & 00.0 & \\
\hline & 1 & 14 & $\llcorner 82$ & .4500 & 81.3 & & & \\
\hline & 1 & 15 & 49.7862 & 0 & 81.7 & & 0( & \\
\hline & 1 & 16 & 65.4678 & 2.3000 & 281.6 & & 00 & \\
\hline & 1 & 7 & 3981 & L. 2750 & 81.1 & & & \\
\hline & 1 & 18 & 66.7906 & .2000 & 81.1 & & & \\
\hline & 1 & 19 & 73.7893 & 0.4500 & 81.5 & & 00 & 0 \\
\hline & 1 & 110 & 36.0106 & 0.1750 & 283.8 & & & \\
\hline & 1 & 111 & 81.9513 & 1.2250 & 85.0 & & & \\
\hline & 1 & 112 & 252.4759 & 2.2250 & 284.4 & 2 & .0 & 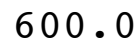 \\
\hline & 1 & 113 & 49.3034 & 2.6000 & 283.1 & 3 & 0.0 & \\
\hline & 1 & 114 & 50.2315 & 2.47 & & & & \\
\hline & 1 & 115 & 63.9271 & 1.42 & 283.4 & 2 & & \\
\hline & 1 & 116 & 215.5153 & .3500 & 285.9 & 1 & .0 & 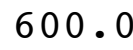 \\
\hline & 1 & 117 & 24.5791 & .8000 & .1 & & 0 & \\
\hline & 1 & 118 & 45.2455 & 0.2500 & 283.6 & 3 & & \\
\hline & 1 & 119 & 82.1708 & 1.0500 & 283.9 & 4 & & . \\
\hline$\perp$ & 1 & 120 & 137.7500 & 0.4250 & 283.7 & 5 & 0 & \\
\hline & 1 & 121 & 59.0679 & & & 6 & & \\
\hline 1 & 1 & 122 & 5443 & 50 & 28 & 5 & & \\
\hline 1 & 1 & 123 & 4.0034 & 500 & .5 & 5 & 0 & \\
\hline & 1 & 124 & 318.6551 & & & 5 & & \\
\hline 1 & 1 & 21 & 4110 & 000 & .8 & 6 & & \\
\hline & 1 & 22 & 308.0416 & 9000 & .4 & 6 & 0 & J \\
\hline & 1 & 23 & 249 . & 0 & .1 & 6 & & \\
\hline 1 & 1 & 24 & 143.9878 & 50 & 282.4 & 6 & & 0 \\
\hline & 1 & 25 & 175.5343 & 0.4000 & 281.9 & 6 & 0 & 0 . \\
\hline & 1 & 26 & 305.7583 & 3500 & 282.0 & 6 & .0 & 0 . \\
\hline 1 & 1 & 27 & 3.7491 & 0 & 282.0 & 5 & & \\
\hline & 1 & 28 & 000 & & 282.1 & 6 & & . \\
\hline 1 & 1 & 29 & 4.5083 & 1.0750 & 284.6 & 5 & 0 & \\
\hline & 1 & 210 & 325.0775 & 0 & & 4 & & \\
\hline 1 & 1 & 211 & 629 & 1.2750 & 0 & 3 & & 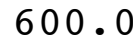 \\
\hline & 1 & 212 & 240.0004 & 2.0750 & 285.3 & 3 & & . \\
\hline 1 & 1 & 213 & 164 . & 4 . & 283.7 & 3 & & 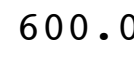 \\
\hline$U_{1}$ & 1 & 214 & 236.1098 & 1.0000 & 285.2 & 2 & & 0 . \\
\hline 1 & 1 & 215 & 248.7018 & 0.6750 & 286.9 & 1 & & \\
\hline & 1 & 216 & 295.6206 & 1.1000 & 286.2 & 2 & & 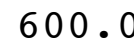 \\
\hline 1 & 1 & 217 & 354.7501 & 1.2000 & 285.9 & 3 & & 0 . \\
\hline 1 & 1 & 218 & 2.2983 & 1.0000 & 283.5 & 4 & 0 & 00 . \\
\hline 01 & 1 & 219 & 22.2144 & 1.7000 & & 4 & & 0 \\
\hline 01 & 1 & 220 & 3.5000 & 0.9500 & 285.4 & 4 & & 500. \\
\hline & 1 & 221 & 335.5041 & 0.3750 & 284.7 & 4 & 600.0 & 600. \\
\hline & 1 & 222 & 14.7617 & & & 5 & & \\
\hline & & 223 & 339.4848 & 0.0750 & 285.7 & $J$ & 600.0 & 600 \\
\hline
\end{tabular}




\begin{tabular}{|c|c|c|c|c|c|c|c|}
\hline & 24 & & & & & & \\
\hline & 13 & 2 & .6750 & 3.6 & & 00.0 & \\
\hline & 132 & 291.2267 & .3500 & 282.1 & 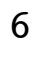 & 00.0 & \\
\hline & 133 & 344.0782 & L. 5000 & 282.1 & & 00.0 & \\
\hline & 134 & 626 & .1250 & 83.1 & & 0 & \\
\hline & 135 & 36.2457 & 2250 & 83.4 & & 00 & \\
\hline & 13 & 243.1613 & 9000 & 82.8 & & 50 & U \\
\hline & 1 & 47.4998 & L. 0500 & 81.5 & & 0.0 & \\
\hline & 1 & 33.2671 & 8000 & 80.2 & & & \\
\hline & 1 & 16.5029 & 1.6000 & 81.8 & & 000 & 0 \\
\hline & 10 & 24.7570 & 0.8000 & 285.1 & & & \\
\hline & 1311 & 278.1143 & 0.9750 & 85.9 & & 00.0 & \\
\hline & 12 & 298.9162 & 1.3750 & 287.0 & & 0 & 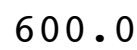 \\
\hline & 13 & 167.3930 & 3.2250 & 285.7 & & 0.0 & 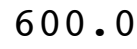 \\
\hline & 1314 & 145.7509 & 2.47 & & & & \\
\hline & 13 & 153.7498 & 3.65 & 286.6 & 2 & & \\
\hline & 16 & 151.5938 & 4.2000 & 286.0 & 3 & .0 & 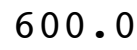 \\
\hline & 1317 & 95.1802 & .5250 & 4 & & 0 & \\
\hline & 1318 & 117.4128 & 0.9250 & 28 & 5 & & \\
\hline & 19 & 96.8855 & 1.5250 & 286.9 & 6 & 00.0 & . \\
\hline & 1320 & 41.0096 & 1.6500 & 287.8 & 5 & 0 & . \\
\hline & 1321 & 14.2494 & 0 & 287.6 & 5 & & \\
\hline & 22 & 32.5003 & 2.9750 & 288.8 & 5 & & \\
\hline & 1323 & 350.2512 & 1.3000 & 288.8 & 6 & 0 & 0 • \\
\hline & 1324 & 57.9070 & 2.12 & & 6 & & \\
\hline & $\begin{array}{lll}1 & 4 & 1\end{array}$ & 143.9989 & 3.5000 & 28 & 5 & & \\
\hline & 142 & 126.6813 & 9000 & 284.6 & 6 & 0 & U. \\
\hline & 143 & 91.1872 & 0 & 9 & 5 & & \\
\hline & $\begin{array}{lll}14 & 4\end{array}$ & 93.6361 & 0.2500 & 28 & 6 & & $\bullet$ \\
\hline & 145 & 139.7491 & 1.9500 & 284.1 & 6 & .0 & 0 . \\
\hline & 146 & 124.6686 & 1.6250 & 284.0 & 6 & 0 & 0 . \\
\hline & $\begin{array}{lll}1 & 4 & 7\end{array}$ & 38.9246 & 015 & 283.1 & 5 & & \\
\hline & 148 & 22.5019 & 1 . & 284.2 & 5 & & 0 • \\
\hline & $\begin{array}{lll}1 & 4 & 9\end{array}$ & 195.1750 & 0.8000 & 285.5 & 4 & 0 & \\
\hline & 1410 & 265.9038 & 1.47 & & 3 & & 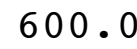 \\
\hline & 1411 & 303.5986 & 1.3500 & 285.3 & 2 & & 0 . \\
\hline & 1412 & 264.9093 & 1.6250 & 286.3 & 2 & 0 & 0 . \\
\hline & 1413 & 254.2678 & 250 & 28 & 2 & & . \\
\hline & 1414 & 268.9271 & 0.8250 & 288.6 & 1 & .0 & 0 . \\
\hline & 1415 & 218.2050 & 1.8750 & 287.6 & 2 & & 0 . \\
\hline & 1416 & 140.0021 & 1.5750 & 286 & 3 & & 600. \\
\hline & 1417 & 135.2500 & 0.7250 & 285.9 & 4 & .0 & 0 . \\
\hline & 1418 & 118.8706 & 0.5500 & 283.8 & 5 & .0 & 00 . \\
\hline & 1419 & 12.0052 & 1.3750 & & 5 & & 0 \\
\hline 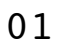 & 1420 & 68.8045 & 0.0250 & 283.2 & 6 & 60 & 600. \\
\hline & 421 & 138.9988 & 0.3500 & 284.7 & 6 & 600.0 & 600 \\
\hline & 422 & 158 & 0 . & & 5 & & \\
\hline & 423 & 141.4342 & 0.0000 & 285.0 & & 600.0 & 600 \\
\hline
\end{tabular}




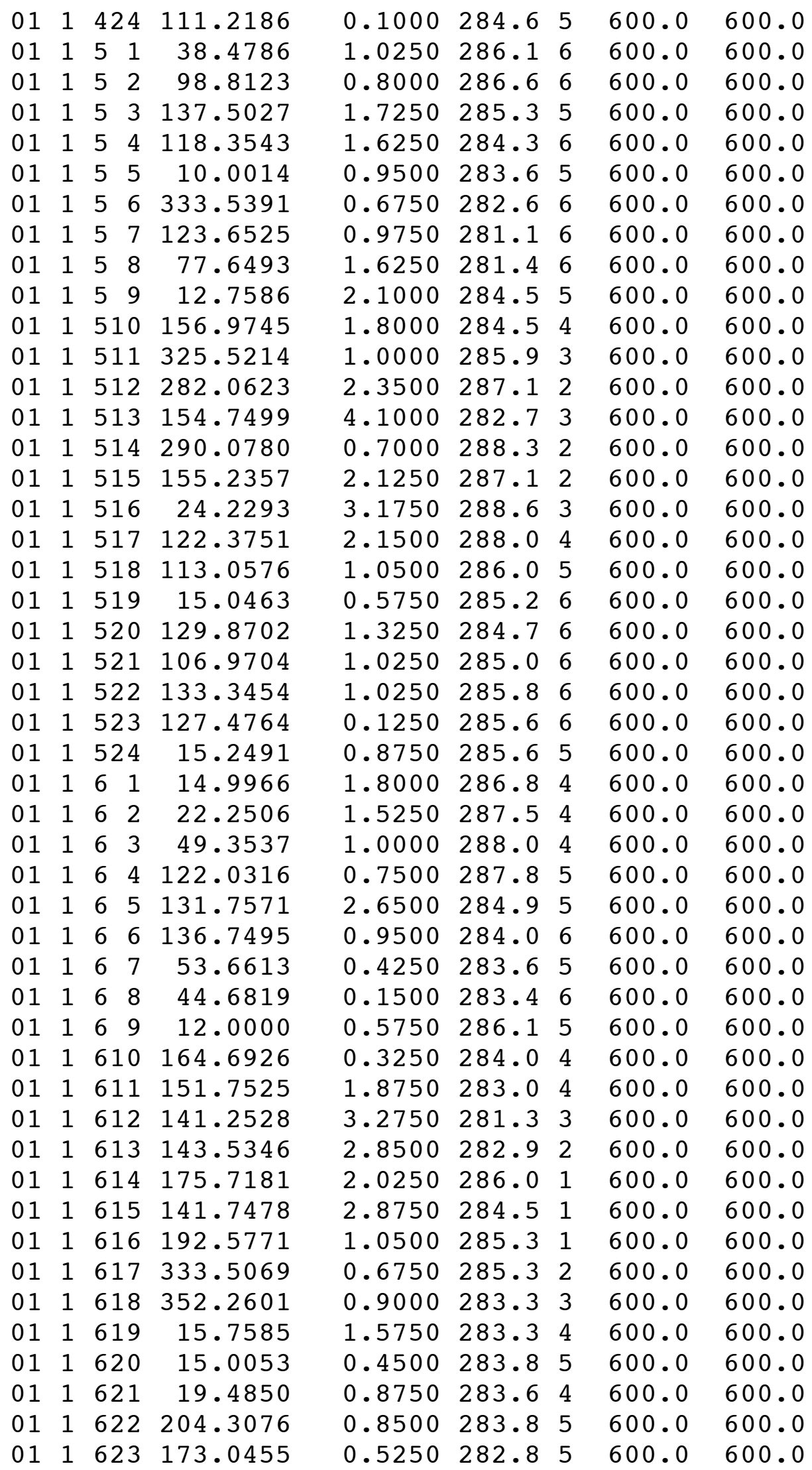




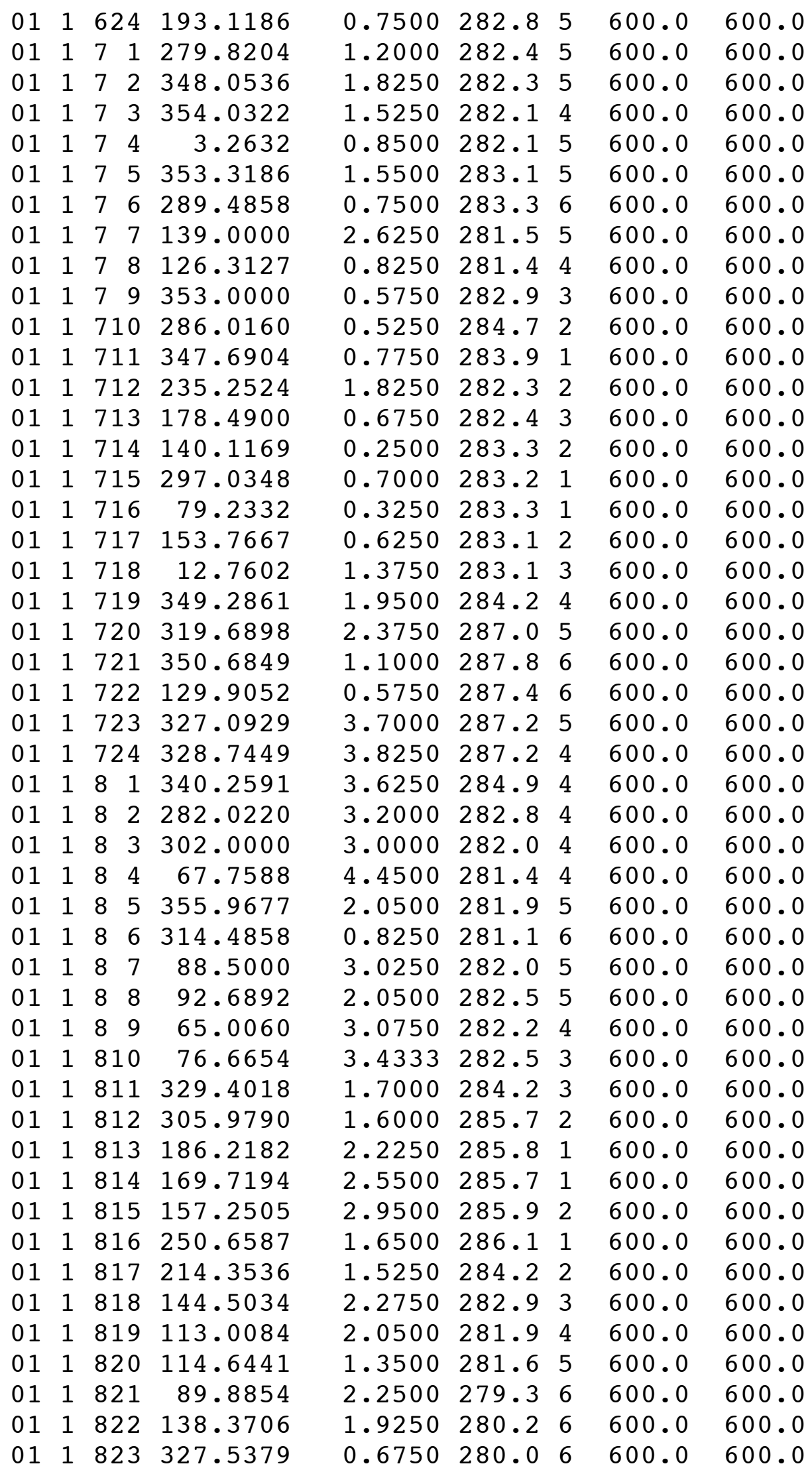




\begin{tabular}{|c|c|c|c|c|c|c|c|}
\hline & 1824 & 6.2482 & & 0.1 & 5 & 0.0 & \\
\hline & $\begin{array}{lll}1 & 9 & 1\end{array}$ & 22.9393 & 1.1500 & 280.1 & & 00 & \\
\hline & 192 & 105.0552 & 7000 & 280.7 & & , & \\
\hline & $\begin{array}{lll}1 & 9 & 3\end{array}$ & 7.6113 & .2000 & 80.8 & & & \\
\hline & $\begin{array}{lll}1 & 9 & 4\end{array}$ & 21.4521 & & 81.0 & & 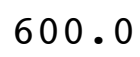 & \\
\hline & 195 & 111.8515 & 0 & 281.1 & & 0 & \\
\hline & 196 & 23.0318 & & 81.1 & & 0 & \\
\hline & 197 & 101 . & & & & & \\
\hline & 198 & 119.3205 & 2.1250 & 279.7 & & 0 & 0 \\
\hline & 199 & 34.6531 & 1.85 & 279.8 & & & \\
\hline & 1910 & 53.4900 & 0 & 82.9 & & & \\
\hline & 11 & 286.3022 & 1.23 & 281.7 & & 0 & \\
\hline & 1912 & 333.5000 & 0 & 282.6 & & & \\
\hline & 1913 & 163.4582 & & 82.2 & & & \\
\hline & 1914 & 259.5423 & & 81.9 & & & \\
\hline & 1915 & 334.1712 & 0 & 282.3 & & & \\
\hline & 1916 & 68.0823 & & 2.1 & & & \\
\hline & 1917 & 325 . & & 1.5 & & & \\
\hline & 1918 & 308.1439 & 2.72 & 281.0 & & 0 & 600 \\
\hline & 1919 & 320.7568 & & 5 & & & \\
\hline & 1920 & 339.1793 & & & & & \\
\hline & 1921 & 353 . & & 28 & & & \\
\hline & 1922 & 2.0000 & 0 & 281.7 & & & \\
\hline & 1923 & 8.2467 & & 81.8 & & & \\
\hline & 1924 & 352.9882 & & 282.1 & & & \\
\hline & 1101 & 7.5870 & 0 & 282.1 & & & \\
\hline & 1102 & 342.9661 & & 281.7 & & & \\
\hline & 1103 & 347.7433 & & 28 & 6 & & \\
\hline & 1104 & 286.4214 & 0 & 280.3 & 6 & 0 & $c$ \\
\hline & 1105 & 318.5000 & & 280.2 & & & \\
\hline & 1106 & 303 . & & $2 \varepsilon$ & 4 & & \\
\hline & 1107 & 315.7857 & & 282.0 & 4 & & \\
\hline & 1108 & 223.6643 & 0 & 282.3 & 5 & & \\
\hline & 1109 & 258.2295 & 3. & 281.1 & 4 & & \\
\hline & 11010 & 282.1982 & & 282.6 & 3 & & \\
\hline & 11011 & 253.1984 & 0 & 280.0 & 3 & & \\
\hline & 11012 & 306.3812 & 0 & 281.2 & 4 & & \\
\hline & 11013 & 302.6828 & 1.2750 & 281.5 & 4 & & \\
\hline & 11014 & 303.0120 & & 283.2 & 4 & & \\
\hline & 11015 & 328.7508 & & & 4 & & \\
\hline & 11016 & 274.1734 & & 281.7 & 3 & & \\
\hline & 11017 & 309.0872 & 3.7750 & 282.1 & 4 & 0 & 0 \\
\hline & 11018 & 236.5424 & 2 . & 281.2 & 5 & & \\
\hline & 11019 & 266.4906 & 2.1500 & 281.1 & 6 & 0 & . \\
\hline & 11020 & 0.4945 & 4.6500 & 281.4 & 5 & 600 & 00 \\
\hline & 11021 & 327.5206 & & & & & \\
\hline & 11022 & 178.4551 & 2.0750 & & & & 600 \\
\hline & 1023 & 343.0831 & 2.2500 & 280.6 & & 60 & 60 \\
\hline
\end{tabular}




\begin{tabular}{|c|c|c|c|c|c|c|c|}
\hline & 11024 & 9.5766 & 2.1750 & 280.9 & 6 & 0.0 & \\
\hline & 1111 & 27.1051 & 3.2250 & 279.9 & 5 & 00 & \\
\hline & 112 & 20.0471 & 1250 & 279.4 & & 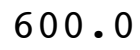 & \\
\hline & 113 & 55.5680 & .7250 & 279.3 & & & \\
\hline & 114 & 15.7828 & 2.2 & 279.4 & & $0 c$ & \\
\hline & 115 & 5415 & 1.6250 & 279.2 & & 0 & \\
\hline & 116 & 309.4721 & & 279.0 & & & \\
\hline & 117 & 331.9 & & 78.8 & & & \\
\hline & 118 & 334.0150 & 3.8750 & 278.6 & 4 & 00 & 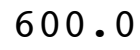 \\
\hline & 119 & 326.3543 & 0 & 278.9 & & & \\
\hline & 110 & 287 . & & 79.0 & & & \\
\hline & 1111 & 295 . & 6.3250 & 280.3 & . & 0 & \\
\hline & 1112 & 299. & 7.8500 & 280.5 & 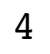 & & \\
\hline & 113 & 322.2499 & 947 & 81.0 & & & \\
\hline & 1114 & 326 . & & & 4 & & \\
\hline & 1115 & 326 . & 3000 & 280.5 & 4 & & \\
\hline & 1116 & 326 . & 0 & 30.4 & 4 & & \\
\hline & 1117 & 326 . & 0 & 9.7 & 4 & & \\
\hline & 1118 & 328.5002 & 1.4750 & 278.6 & 4 & 0 & 00 \\
\hline & 1119 & 327.2561 & & 279.0 & 4 & & \\
\hline & 1120 & 320.24 & & 279.3 & 4 & & \\
\hline & 121 & 319. & & 279 & 4 & & \\
\hline & 122 & 326.0041 & 0 & 279.3 & 4 & 0 & \\
\hline & 123 & 317.5040 & & 279.2 & 4 & & \\
\hline & 124 & 318 & & 27 & 4 & & \\
\hline & 121 & 305.7582 & 0 & 278.7 & 4 & & \\
\hline & 122 & 271.44 & & 278.6 & 4 & & \\
\hline & 123 & 250 . & & .5 & 4 & & \\
\hline & 124 & 228.2092 & 0 & 278.4 & 4 & 0 & . \\
\hline & 1125 & 219.75 & & 278.3 & 4 & & \\
\hline & 126 & 201 . & & 278.2 & 4 & & \\
\hline & 127 & 189. & & 277.9 & 4 & & \\
\hline & 1128 & 209.0000 & 0 & 278.0 & 4 & & \\
\hline & 1129 & 189. & 0 & 277.9 & 4 & & \\
\hline & 210 & 170 . & & 277.9 & 4 & & \\
\hline & 211 & 138.5161 & 0 & 278.2 & 3 & & \\
\hline & 1212 & 130 . & 1 . & 279.2 & 2 & & \\
\hline & 213 & 140.2446 & 3 . & 280.4 & 3 & & \\
\hline & 1214 & 157.2498 & 000 & 281.7 & 3 & 0 & \\
\hline & 11215 & 154 . & & 281.9 & 4 & & \\
\hline & 11216 & 156.9989 & & 282.2 & 3 & & \\
\hline & 11217 & 154.5292 & 1.9250 & 282.8 & 4 & 0 & (n) \\
\hline & 11218 & 135.1629 & 1.20 & 281.6 & 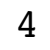 & & \\
\hline & 11219 & 69.9946 & 3.3250 & 281.3 & 5 & 600.0 & 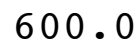 \\
\hline & 11220 & 70.7502 & 5.4000 & 281.0 & 4 & 600.0 & 00 \\
\hline & 11221 & 73. & & & 4 & & \\
\hline & 1222 & 68.2499 & & & 4 & & 600 \\
\hline & 1223 & 0 & 8.4500 & 280.4 & 4 & 60 & 60 \\
\hline
\end{tabular}




\begin{tabular}{|c|c|c|c|c|c|c|c|}
\hline 01 & 11224 & 71.4999 & 8.6500 & 280.3 & 4 & 600.0 & 600.0 \\
\hline 01 & 1131 & 65.7500 & 8.9250 & 280.5 & 4 & 600.0 & 600.0 \\
\hline 01 & 1132 & 53.2518 & 6.5000 & 280.6 & 4 & 600.0 & 00.0 \\
\hline 01 & 1133 & 42.7500 & 6.2250 & 280.9 & 4 & 600.0 & 00.0 \\
\hline 01 & 1134 & 48.2503 & 5.8500 & 280.8 & 4 & 600.0 & 00.0 \\
\hline 01 & 1135 & 14.2512 & 3.3000 & 280.6 & 4 & 600.0 & 00.0 \\
\hline 01 & 1136 & 11.7000 & 2.1750 & 280.6 & 5 & 600.0 & 00.0 \\
\hline 01 & 1137 & 56.2504 & 5.0750 & 280.3 & 4 & 600.0 & 00.0 \\
\hline 01 & 1138 & 61.0021 & 6.4500 & 280.2 & 4 & 600.0 & 00.0 \\
\hline 01 & 1139 & 60.4995 & 7.5250 & 280.2 & 4 & 600.0 & 00.0 \\
\hline 01 & 11310 & 72.7497 & 5.8000 & 281.1 & 3 & 600.0 & 00.0 \\
\hline 01 & 11311 & 64.9828 & 6.6000 & 281.3 & 4 & 600.0 & \\
\hline 01 & 11312 & 56.2494 & 6.3250 & 282.0 & 4 & 600.0 & 00.0 \\
\hline 01 & 11313 & 64.9920 & 5.6500 & 282.6 & 3 & 600.0 & 00.0 \\
\hline 01 & 11314 & 79.2541 & 5.7750 & 283.0 & 3 & .0 & \\
\hline 01 & 11315 & 83.2513 & 5.3000 & 283.3 & 3 & 600.0 & 00.0 \\
\hline 01 & 11316 & 82.2636 & 5.9250 & 282.9 & 3 & 60 & .0 \\
\hline 01 & 11317 & 68.0000 & 5.7250 & 282.3 & 4 & .0 & \\
\hline 01 & 11318 & 64.9985 & 6.3500 & 281.3 & 4 & 60 & .0 \\
\hline 01 & 11319 & 68.0005 & 6.6750 & 280.6 & 4 & 60 & .0 \\
\hline 01 & 11320 & 092 & 6 . & 280.5 & 4 & 60 & \\
\hline 01 & 11321 & 75.7505 & 7.4000 & 280.5 & 4 & .0 & \\
\hline 01 & 11322 & 79.0000 & 7.0500 & 280.5 & 4 & 600.0 & 0.0 \\
\hline 01 & 11323 & 80.4994 & 5.97 & 280.8 & 4 & 60 & .0 \\
\hline 01 & 11324 & 81.5031 & 5 . & 280.8 & 4 & .0 & \\
\hline 01 & 1141 & 79.2463 & 4.8250 & 280.7 & 4 & 600.0 & 600.0 \\
\hline 01 & 1142 & 78.7569 & 4.0000 & 280.7 & 4 & 600.0 & .0 \\
\hline 01 & 1143 & 101.6210 & 4.0750 & 280.9 & 4 & .0 & \\
\hline 01 & 1144 & 146.2501 & 3.3000 & 280.5 & 4 & 600.0 & 600.0 \\
\hline 01 & 1145 & 141.9807 & 3.3250 & 280.3 & 5 & 600.0 & 600.0 \\
\hline 01 & 1146 & 159.9966 & 4.9500 & 279.5 & 4 & .0 & \\
\hline 01 & 1147 & 159.7504 & 5 . & 279.4 & 4 & .0 & 600.0 \\
\hline 01 & 1148 & 161.7312 & 4.7500 & 279.4 & 4 & 600.0 & 600.0 \\
\hline 01 & 1149 & 173.0003 & 4.4500 & 278.7 & 3 & 600.0 & 0.0 \\
\hline 01 & 11410 & 179.2500 & 6.8250 & 278.4 & 4 & 600.0 & 600.0 \\
\hline 01 & 11411 & 180.7500 & 7.4500 & 278.5 & 4 & 600.0 & 600.0 \\
\hline 01 & 11412 & 180.7500 & 7.9250 & 279.0 & 4 & 600.0 & 600.0 \\
\hline 01 & 11413 & 174.7521 & 5.8500 & 280.0 & 3 & 600.0 & 600.0 \\
\hline 01 & 11414 & 176.7502 & 4.6250 & 280.7 & 3 & 600.0 & 600.0 \\
\hline 01 & 11415 & 180.2500 & 5.2250 & 281.2 & 3 & 600.0 & 600.0 \\
\hline 01 & 11416 & 180.0007 & 5.0250 & 281.2 & 3 & 600.0 & 600.0 \\
\hline 01 & 11417 & 181.2500 & 4.5250 & 280.2 & 4 & 600.0 & 600.0 \\
\hline 01 & 11418 & 181.5000 & 4.1000 & 279.6 & 4 & 600.0 & 600.0 \\
\hline 01 & 11419 & 153.5402 & 4.8250 & 280.0 & 4 & 600.0 & 600.0 \\
\hline 01 & 11420 & 160.8416 & 5.4000 & 280.2 & 4 & 600.0 & 600.0 \\
\hline 01 & 11421 & 164.2524 & 7.1750 & & 4 & 600.0 & 600.0 \\
\hline 01 & 11422 & 167.2541 & 0.1500 & 280.6 & 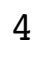 & 600.0 & 600.0 \\
\hline 01 & 1423 & 141.7475 & 8.975 & 280.1 & 4 & 600.0 & 600.0 \\
\hline
\end{tabular}




\begin{tabular}{|c|c|c|c|c|c|c|c|}
\hline & & & & & & & \\
\hline & 151 & 000 & 0 & 9.3 & & & \\
\hline & 152 & 000 & 250 & 279.4 & & 0 & \\
\hline & 153 & 000 & 500 & 279.5 & & 0 & \\
\hline & 54 & & & 79.2 & & & \\
\hline & 55 & & 0 & 78.8 & & & \\
\hline & 156 & 500 & 11.2250 & 278.3 & & $\Omega_{2}$ & \\
\hline & 15 & 0 & 9.5250 & 78.0 & & & \\
\hline & $\begin{array}{ll}5 & 8\end{array}$ & & 5750 & 78.1 & & & \\
\hline & 159 & 390 & 0.0250 & 278.4 & & 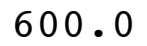 & \\
\hline & 510 & 2657 & 8.7500 & 278.8 & & & \\
\hline & 11 & 0 & 3.4250 & 9.5 & & & \\
\hline & 5 & 56 & 00 & 280.2 & & & \\
\hline & 5 & 7 & 4.7750 & 280.8 & & & \\
\hline & 1 & & 500 & & & & \\
\hline & & & 14. & & & & \\
\hline & 516 & - & 12.9750 & 1.0 & & & \\
\hline & 517 & & 000 & & & & \\
\hline & 518 & & 0 & & & & \\
\hline & 9 & & 1.9750 & & 4 & & \\
\hline & 520 & 999 & 500 & & & 0 & \\
\hline & 1 & & 12. & & 4 & & \\
\hline & 2 & & 0 & & 4 & & \\
\hline & 523 & 97 & .8500 & & & & \\
\hline & 524 & & 0 & & 2 & & \\
\hline & 16 & & 0 & .6 & 4 & & \\
\hline & 162 & 98 & 1.5000 & 7.2 & 4 & & \\
\hline & 163 & י & 11.3000 & & 4 & & \\
\hline & 164 & 2 & 50 & & 4 & & \\
\hline & 165 & 998 & 500 & .1 & 2 & & 0 . \\
\hline & 166 & 1 & 11.6250 & & 4 & & \\
\hline & 167 & & 50 & & 4 & & \\
\hline & 168 & & 750 & & 4 & & . \\
\hline & 169 & 00 & 500 & 4 & 4 & 0 & \\
\hline & 0 & 1 & 0 & & 4 & & \\
\hline & & & & 7.7 & 4 & & \\
\hline & 612 & 167 . & 500 & 8.7 & 4 & & \\
\hline & 1613 & 17 & 9. & 27 & 4 & & \\
\hline & 1614 & 164.7501 & & & 4 & & 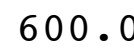 \\
\hline & 6 & $\perp$ & 50 & 5 & 4 & & \\
\hline & 11616 & 560 & 00 & & 4 & & \\
\hline & & & 6.6750 & 280.4 & 4 & & \\
\hline & 618 & 140.7548 & 7.4250 & 279.8 & 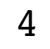 & & 0 \\
\hline & 11619 & 925 & 750 & & 4 & & \\
\hline & & 146 & 11.0000 & 279.2 & 4 & & \\
\hline & & .5000 & 9.1000 & 278.2 & 4 & & 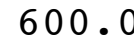 \\
\hline & & & & & & & \\
\hline & $\begin{array}{l}1020 \\
\end{array}$ & 19080 & 5.9000 & 277.5 & & .0 & s \\
\hline
\end{tabular}




\begin{tabular}{|c|c|c|c|c|c|c|c|}
\hline & 4 & & & 7.2 & & & \\
\hline & 171 & 9 & .5000 & 277.2 & & 00.0 & \\
\hline & 172 & 139.7500 & 8.5750 & 276.9 & 4 & 00 & \\
\hline & 173 & 129.0193 & 6.3750 & 276.9 & & 00 & \\
\hline & 174 & 107 & .8500 & 276.9 & & 0 & \\
\hline & 175 & 93 & & 276.6 & & 0 & \\
\hline & 176 & 138.2500 & 8.0250 & 276.6 & & 00 & \\
\hline & 177 & 51.2503 & 1.1500 & 276.1 & & 0 & \\
\hline & 178 & 24.7862 & 6.6250 & 76.2 & & & \\
\hline & 179 & 09.7557 & 5.5250 & 276.2 & & 00 & 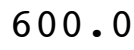 \\
\hline & 1710 & 123.2398 & 5.2500 & 277.7 & & & \\
\hline & 1711 & 143.4986 & 7.9250 & 278.0 & & & \\
\hline & 1712 & 0000 & 7.3750 & 278.8 & 4 & 0 & \\
\hline & 1713 & 151.5003 & 4.2000 & 280.3 & & 0.0 & \\
\hline & $\lcm{714}$ & 48.2501 & 0 & 281.1 & & & \\
\hline & 1715 & 1100 & 4 . & 282.2 & 4 & & \\
\hline & 1716 & 154.7560 & 4.7500 & 282.4 & 4 & .0 & \\
\hline & 1717 & 172.5000 & 2.9000 & 281.5 & 4 & & \\
\hline & 1718 & 159.7504 & 0.4250 & 279.5 & 4 & & \\
\hline & 1719 & 135.3437 & 0.8000 & 279.0 & 5 & 0 & . \\
\hline & 1720 & 031 & 2.5250 & 280.3 & 4 & 0 & \\
\hline & 1721 & 109.9744 & 2.0750 & 280.7 & 5 & & \\
\hline & 1722 & 238 & 3.8250 & 280.9 & 4 & & \\
\hline & 1723 & 97.1779 & 3.6250 & 280.8 & 4 & 0 & \\
\hline & 11724 & 109.5172 & 000 & 280.7 & 5 & & \\
\hline & 1181 & 163.5927 & 1.3000 & 278.7 & 6 & & \\
\hline & 182 & 63.4108 & 0.7000 & 278.1 & 6 & 0 & J. \\
\hline & 1183 & 358.7504 & 1.0000 & 277.4 & 5 & & \\
\hline & 1184 & 16.9925 & 500 & 278.1 & 4 & & \\
\hline & 1185 & 144.3012 & 0.7250 & 278.1 & 5 & 0 & 0 . \\
\hline & 1186 & 146.2501 & 1.9250 & 277.9 & 6 & & 0 . \\
\hline & 1187 & 144.5345 & 1.4250 & 277.9 & 6 & & \\
\hline & 1188 & 130.4332 & 0.8000 & 278.1 & 5 & & 0 . \\
\hline & 1189 & 154 . & 1.3000 & 279.2 & 4 & 0 & \\
\hline & 11810 & 156 & 210 & 279.4 & 4 & & \\
\hline & 11811 & 206.1458 & 1.7250 & 280.4 & 3 & & . \\
\hline & 1812 & 252.2587 & 2.3250 & 281.2 & 2 & & 0 . \\
\hline & 11813 & 174.6057 & 250 & 280.4 & 2 & & 0 . \\
\hline & 11814 & 163.2269 & 2.7500 & 281.0 & 2 & .0 & 0 . \\
\hline & 11815 & 215.6509 & 1.6000 & 282.0 & 2 & & \\
\hline & 11816 & 156.7618 & 2.9250 & 280.8 & 3 & & 0 \\
\hline & 11817 & 145.2488 & 2.2750 & 280.5 & 4 & & 0 . \\
\hline & 11818 & 131.7497 & 1.0000 & 279.6 & 5 & 0 & 0 \\
\hline & 11819 & 3603 & 250 & 279.6 & 6 & & \\
\hline & 11820 & 62.6903 & 0.6750 & 279.6 & 6 & & \\
\hline & 1182 & 24.7304 & 0.6750 & 279.6 & 5 & 600 & 600. \\
\hline & & & & 279.3 & 6 & & \\
\hline & 1823 & 152.5021 & 2.0250 & 279.1 & & 600.0 & 60 \\
\hline
\end{tabular}




\begin{tabular}{|c|c|c|c|c|c|c|c|}
\hline & 824 & & 4000 & 79.4 & & 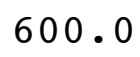 & \\
\hline & 191 & 10 & 0.2250 & 279.2 & & 600.0 & \\
\hline & 1192 & 13.0000 & .7000 & 279.2 & & 00 & \\
\hline & 193 & 356.5587 & 0.6250 & 279.6 & & 00 & \\
\hline & 194 & 597 & .3000 & 279.8 & & 0 & \\
\hline & 195 & 149 . & .4750 & 280.0 & & 00 & \\
\hline & 196 & 148.7517 & 1.7000 & 278.3 & & 500 & \\
\hline & 197 & 149.9920 & .6750 & 277.6 & & 0 & \\
\hline & 198 & 143.4998 & .5750 & .77 .6 & & & \\
\hline & 199 & 3958 & 0.3250 & 278.1 & & 00 & 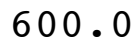 \\
\hline & 1910 & 23.4334 & 1.4750 & 278.8 & & 00.0 & \\
\hline & 911 & 179.1816 & 1.6750 & 280.0 & & 0 & \\
\hline & 1912 & 290.2669 & 0.9500 & 283.0 & & 00.0 & \\
\hline & 913 & 157.9586 & 2.0250 & 282.3 & & 0.0 & \\
\hline & $\mathbf{L} 14$ & 246 . & 5250 & 284.1 & & & \\
\hline & 915 & 285 . & 2.50 & 283.9 & & & \\
\hline & 1916 & 267.0436 & 3.0750 & 283.6 & & .0 & 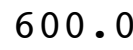 \\
\hline & 1917 & 297 . & 1.6750 & 283.0 & & 0 & \\
\hline & 1918 & 238.3645 & 1.2750 & 281.7 & & & \\
\hline & 1919 & 187.8541 & 2.4000 & 280.6 & 5 & 0 & . \\
\hline & 920 & 267.2108 & .4750 & 280.3 & & .0 & \\
\hline & 1921 & 343.5000 & 2.4000 & 280.0 & 4 & & \\
\hline & 1922 & 1.5131 & 0750 & 280.3 & 4 & & \\
\hline & 1923 & 1.0005 & 3.3000 & 282.5 & 4 & 0 & 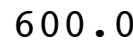 \\
\hline & 11924 & 54.3553 & 1.2250 & 283.6 & 5 & & \\
\hline & 1201 & 146.7529 & 1.7750 & 282.5 & 6 & & \\
\hline & 1202 & 158.1679 & 5750 & 281.4 & 6 & 0 & U. \\
\hline & 1203 & 48.3103 & 5750 & 281.6 & 6 & & \\
\hline & 1204 & 349.7550 & 1.6500 & 282.2 & 5 & & 0 \\
\hline & 1205 & 18.3931 & 0.4000 & 281.2 & 6 & 0 & 0 • \\
\hline & 1206 & 350.5572 & 1.0000 & 281.9 & 5 & 0 & 0 . \\
\hline & 1207 & 303.6037 & .3750 & 281.2 & 6 & & \\
\hline & 1208 & 312 . & 1.0250 & 280.7 & 6 & & 0 . \\
\hline & 1209 & 128.5772 & 2.9000 & 280.2 & 5 & 0 & o. \\
\hline & 12010 & 130.7925 & 2 . & 278.1 & 4 & & \\
\hline & 12011 & 28.4081 & 1.3750 & 279.2 & 3 & & 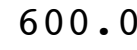 \\
\hline & 12012 & 343.9689 & 1.5750 & 280.9 & 2 & $\cdot 0$ & 0 . \\
\hline & 12013 & 309.9634 & 1.5500 & 281.0 & 3 & & 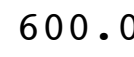 \\
\hline & 12014 & 189.6785 & 1.4000 & 281.0 & 2 & .0 & 0 . \\
\hline & 12015 & 172.5222 & 2.2500 & 280.4 & 1 & & o. \\
\hline & 12016 & 150.3304 & 1.5250 & 280.3 & 1 & & 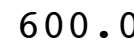 \\
\hline & 12017 & 125.0048 & 0.4250 & 281.6 & 2 & .0 & 0 . \\
\hline & 12018 & 331.8706 & 0.5500 & 279.6 & 3 & 0 & 00 . \\
\hline & 12019 & 20.4758 & 0.5500 & 281.1 & 4 & & 0 \\
\hline & 12020 & 113.0426 & 0.6250 & 282.2 & 5 & & 600. \\
\hline & 12021 & 346.4531 & 0.3750 & 280.7 & 6 & 600.0 & 600 \\
\hline & . & 3215 & & 281.1 & 6 & & \\
\hline & 2023 & 172.0080 & 1.7500 & 279.3 & & 600.0 & 60 \\
\hline
\end{tabular}




\begin{tabular}{|c|c|c|c|c|c|c|c|}
\hline 1 & 2024 & & 0 & 8.9 & & $x^{2}+2$ & \\
\hline & 1211 & 209 & .6250 & 278.9 & 6 & 600.0 & \\
\hline & 1212 & 99.5000 & 7750 & 279.0 & 6 & 00 & \\
\hline & 213 & 165.3583 & L. 1750 & 279.2 & & 00 & \\
\hline & 214 & 372 & .8500 & 278.7 & & 0 & \\
\hline & 215 & 146.7499 & 1.1000 & 278.8 & 6 & 00 & \\
\hline & 216 & 141.2479 & 0750 & 277.6 & & 00 & \\
\hline & 217 & 149.0002 & 500 & 277.6 & & 0 & \\
\hline & 218 & 152.0002 & 3.6000 & 278.3 & 4 & & \\
\hline & 219 & 159.7581 & 3.6000 & 278.9 & 3 & 00 & 0 \\
\hline & 2110 & 167.5001 & 4.2750 & 279.0 & & 00.0 & \\
\hline & 2111 & 172.7500 & 3.4250 & 79.3 & & 0 & \\
\hline & 12112 & 172.2501 & 2250 & 280.4 & 2 & 0 & \\
\hline & 2113 & 175.0001 & 3.9750 & 280.4 & 2 & 0.0 & r \\
\hline & 2114 & 173.2652 & & 281.6 & 2 & & \\
\hline & 12115 & 177.2500 & & 3.0 & 2 & & \\
\hline & 2116 & 178.2500 & 9000 & 282.4 & 2 & .0 & U \\
\hline & 2117 & 179.7500 & 0 & 1.6 & 3 & 0 & \\
\hline & 2118 & 154.2982 & & 1.6 & 4 & & \\
\hline & 2119 & 63.6898 & 8000 & 281.5 & 5 & 00.0 & U \\
\hline & 2120 & 30.7161 & 000 & 1.8 & 4 & 0 & \\
\hline & 2121 & 111.4048 & & & 5 & & \\
\hline & 2122 & 7636 & 750 & $2 \varepsilon$ & 6 & & \\
\hline & 12123 & 133.4919 & 0.8000 & 282.0 & 6 & 0 & 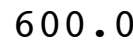 \\
\hline & 12124 & 46.5986 & & 281.4 & 6 & & \\
\hline & 1221 & 39.2717 & 00 & 1.5 & 6 & & \\
\hline & 1222 & 28.5775 & 750 & 282.3 & 6 & 0 & U. \\
\hline & 1223 & 68.3783 & 500 & 281.8 & 6 & & \\
\hline & 1224 & 162.0226 & 000 & 0 & 6 & & \\
\hline & 1225 & 171.9973 & 3250 & 0.8 & 6 & 0 & 0 . \\
\hline & 1226 & 147.2089 & 1.1000 & 280.9 & 6 & 0 & 0 . \\
\hline & 1227 & 138.6824 & 000 & 1.0 & 6 & & \\
\hline & 1228 & 162.0033 & 1.9500 & 281.2 & 6 & 0 & . \\
\hline & 1229 & 176.5002 & 2.5250 & 283.0 & 5 & 0 & 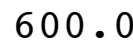 \\
\hline & 12210 & 178.5000 & 750 & 3.9 & 4 & & \\
\hline & 12211 & 170.7540 & 1.4250 & 5.7 & 3 & & ( \\
\hline & 12212 & 176.4985 & 5000 & 286.1 & 2 & $\cdot 0$ & 0 . \\
\hline & 12213 & 172.0003 & 750 & 5.1 & 1 & & ( \\
\hline & 12214 & 178.2504 & 4.1500 & 286.6 & 2 & .0 & 0 . \\
\hline & 12215 & 173.2505 & 2.3500 & 287.8 & 1 & .0 & 0 \\
\hline & 12216 & 235.5459 & 2.3250 & 287.1 & 2 & & 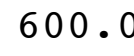 \\
\hline & 12217 & 229.6403 & 1.4250 & 286.2 & 3 & .0 & 0 . \\
\hline & 12218 & 243.1289 & 0.4000 & 285.0 & 4 & 0 & 00 . \\
\hline & 12219 & 11.5403 & 0.7250 & 284.4 & 4 & & \\
\hline & 12220 & 49.1423 & 1.2000 & 284.6 & 5 & & 600. \\
\hline & 12221 & 132.7512 & 1.4750 & 284.3 & 4 & 600.0 & 600 \\
\hline & & 39 & 1.2250 & & 5 & & \\
\hline & 12220 & 64.2584 & 2.7250 & 284.8 & 1 & 600.0 & 60 \\
\hline
\end{tabular}




\begin{tabular}{|c|c|c|c|c|c|c|c|}
\hline 1 & 2224 & & 0.1750 & 83.6 & & & \\
\hline & 1231 & 346 & 1.7500 & 283.0 & & & \\
\hline & 1232 & 319.5773 & 7500 & 282.1 & & 00 & \\
\hline & 1233 & 336.7120 & .0250 & 281.3 & & 00 & \\
\hline & 1234 & 0 & .0500 & 82.7 & & 0 & \\
\hline & 1235 & 8.0000 & 0.9000 & 283.7 & & 00 & \\
\hline & 1236 & 123.0911 & 0.8750 & 283.6 & & 0 & \\
\hline & 1237 & 351.6653 & 1.4500 & 84.2 & & & \\
\hline & 1238 & 42.7068 & 1.1000 & 283.5 & & 0 & \\
\hline & 1239 & 345.5372 & 2.2000 & 284.0 & & 00.0 & \\
\hline & 2310 & 355.7501 & 2.2250 & 284.5 & & 00 & \\
\hline & 2311 & 338.1010 & & 85.1 & & & \\
\hline & 2312 & 306.1845 & 2.2250 & 285.2 & & 00 & \\
\hline & 2313 & 323.1671 & 1.9500 & 285.6 & & 00.0 & . \\
\hline & 12314 & 182.67 & & 83.0 & & & \\
\hline & 12315 & 300.7397 & 1 . & 284.2 & & 0 & \\
\hline & 2316 & 342.5000 & 2.3000 & 284.0 & & 0 & \\
\hline & 12317 & 4.7509 & 2 . & .8 & & & \\
\hline & 123 & 49.3428 & 3 . & 282.9 & 4 & 0 & \\
\hline & 2319 & 11.5221 & 2.97 & 282.0 & 5 & 0 & \\
\hline & 12320 & 11 & 1 . & 281.3 & 6 & & \\
\hline & 12321 & 336 . & 0 & 282.0 & 6 & & \\
\hline & 12322 & 58.8006 & 750 & 282.3 & 6 & 0 & . \\
\hline & 12323 & 25.2560 & 1.70 & 282.1 & 6 & 0 & \\
\hline & 12324 & 48.76 & & 281.8 & 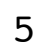 & & \\
\hline & 1241 & 9.1349 & & 281.4 & 6 & & \\
\hline & 1242 & 70.5173 & 1.62 & 280.5 & 6 & 0 & \\
\hline & 1243 & 91.2238 & & 279.5 & 5 & & \\
\hline & 1244 & 68 . & 4 & 278.6 & 4 & 0 & 0 . \\
\hline & 1245 & 63.2979 & & 278.8 & 4 & 0 & \\
\hline & 1246 & 67.5235 & 4 & 278.7 & 4 & & \\
\hline & 1247 & 7605 & 5. & 278.6 & 4 & & 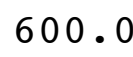 \\
\hline & 1248 & 69.7496 & 750 & 278.7 & 4 & 600 & U • \\
\hline 1 & 1249 & 02 & 6 . & 278.8 & 4 & 0 & \\
\hline & 12410 & 67. & 0 & 279.3 & 4 & & \\
\hline & 12411 & 76.4994 & & 279.9 & 3 & & 0 . \\
\hline & 12412 & 82.4983 & & 280.5 & 4 & 0 & \\
\hline 1 & 12413 & 91.2503 & 0 & 281.1 & 4 & 0 & • \\
\hline & 12414 & 81.9013 & 000 & 281.2 & 4 & 60 & 0 . \\
\hline & 12415 & 431 & & 281.8 & 4 & 6 & \\
\hline & 12416 & 66. & & 282.4 & 4 & & \\
\hline I & 12417 & 71.5003 & 5.5500 & 281.5 & 4 & 600.0 & 600 . \\
\hline & 12418 & 69.0016 & 500 & 280.5 & 4 & 600 & 600. \\
\hline & 12419 & 81.5000 & 5.2750 & 279.9 & 4 & 600 & 600 \\
\hline 01 & 12420 & 76.0554 & 3.4750 & 279.5 & 5 & 600.0 & 00 \\
\hline & 12421 & 188.9091 & 1.2750 & 278.9 & 6 & 600.0 & 600. \\
\hline & 12422 & .3076 & 000 & 279.2 & 3 & 600 & 60 \\
\hline & 12423 & 31.8805 & 1.7250 & 278.8 & & 600.0 & 600 \\
\hline
\end{tabular}




\begin{tabular}{|c|c|c|c|c|c|c|c|}
\hline & 24 & 6 & 0250 & 78.7 & 6 & 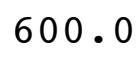 & \\
\hline & 1251 & 3.2768 & 2.2250 & 278.7 & & 600.0 & \\
\hline & 1252 & 7.5681 & 0.8500 & 278.2 & & 00 & \\
\hline & 253 & 45.7240 & 1.5000 & 278.4 & & 00 & \\
\hline & 254 & 3031 & 000 & 78.2 & & 0 & \\
\hline & 255 & 19943 & 4500 & 278.7 & & 0 & \\
\hline & 256 & 357.5559 & 2.3000 & 279.0 & & 00 & \\
\hline & 257 & 42.0053 & 2.2750 & 278.8 & & & \\
\hline & 258 & 354.2614 & .5500 & 78.7 & & & \\
\hline & 259 & 334.0000 & 000 & 278.8 & & 0 & 0 \\
\hline & 2510 & 325.2127 & 2.6250 & 280.0 & & & \\
\hline & 2511 & 322.3679 & 4.27 & 1.8 & & & \\
\hline & 2512 & 326.8189 & 8.3000 & 282.3 & & 0 & \\
\hline & 2513 & 331.9541 & 8.7250 & 282.9 & & 0.0 & \\
\hline & 2514 & 344 . & 0 & 3.4 & & & \\
\hline & 2515 & 353 . & 0 & 2.2 & 4 & & \\
\hline & 2516 & 351.5005 & 10.7500 & 280.6 & 4 & .0 & \\
\hline & 2517 & 182 & 10.30 & & 4 & & \\
\hline & 2518 & 32.8726 & 3.20 & 7.6 & 4 & & \\
\hline & 2519 & 344.4937 & 3.27 & 277.1 & 5 & 0 & . \\
\hline & 2520 & 8.6511 & 2.52 & 7.7 & 6 & 0 & \\
\hline & 2521 & 344.6365 & 1.67 & 7.8 & 6 & & \\
\hline & 2522 & 3227 & 1.92 & 3.0 & 6 & & \\
\hline & 2523 & 61.0042 & 5.30 & 278.8 & 5 & 0 & \\
\hline & 12524 & 64. & 0 & 9.1 & 4 & & \\
\hline & 1261 & 2454 & 0 & 279.4 & 4 & & \\
\hline & 1262 & 54.7496 & 1250 & 279.3 & 4 & 0 & J. \\
\hline & 1263 & 25.7702 & 0 & & 5 & & \\
\hline & 1264 & 17 . & 1.6750 & 27 & 4 & & \\
\hline & 1265 & 36.0002 & 3.0750 & 278.8 & 5 & & 0. \\
\hline & 1266 & 43.0002 & 2.3000 & 278.9 & 5 & & 0 . \\
\hline & 1267 & 32.5000 & 105 & 279.2 & 4 & & \\
\hline & 1268 & 45.5905 & 0.42 & 27 & 5 & & . \\
\hline & 1269 & 109.1891 & 3250 & 279.0 & 4 & 0 & 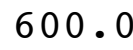 \\
\hline & 12610 & 140.7495 & 0.7250 & 280.2 & 3 & & \\
\hline & 12611 & 221.6696 & 1.1250 & 1.8 & 2 & & \\
\hline & 12612 & 195.9620 & 3.8750 & 0.5 & 3 & & \\
\hline & 12613 & 194.1589 & 337 & 27 & 3 & & \\
\hline & 12614 & 208.0055 & 1.0250 & 280.2 & 2 & & 0 . \\
\hline & 12615 & 202.1506 & 1.7750 & 281.0 & 1 & & \\
\hline & 12616 & 168.4415 & 2.5500 & 280.6 & 1 & & \\
\hline & & 148.0023 & 2.3500 & 280.8 & 2 & & 0 . \\
\hline & 12618 & 169.2517 & 4.1500 & 279.1 & 3 & & 0 . \\
\hline & 12619 & 172.7468 & 6.9750 & 278.3 & 4 & & \\
\hline & 12620 & 160.7444 & 8.6500 & 277.0 & 4 & & \\
\hline & 12621 & 154.2505 & 8.0500 & 276.4 & 4 & & 00 . \\
\hline & & 142 . & & & 4 & & \\
\hline & 12623 & 150.2470 & 8.5750 & 276.7 & & 60 & 60 \\
\hline
\end{tabular}




\begin{tabular}{|c|c|c|c|c|c|c|c|}
\hline & 24 & & & 7.6 & & & \\
\hline & 271 & 4 & .2000 & 278.6 & & & \\
\hline & 272 & 141.0000 & 0.7500 & 278.9 & & 00 & \\
\hline & 273 & 35.4997 & 1000 & 278.8 & & 0 & \\
\hline & 274 & & & 78.6 & & & \\
\hline & 275 & & & 78.6 & & 0 & \\
\hline & 276 & 7499 & 9000 & 278.3 & & 0 & \\
\hline & 277 & 1.7499 & 9.4250 & 278.0 & & & \\
\hline & 1278 & 2500 & 0 & 78.3 & & & \\
\hline & 279 & 2450 & 8.7750 & 278.8 & & & \\
\hline & 2710 & 1.7495 & 9.7750 & 279.2 & & & \\
\hline & 2711 & 2370 & 0 & 9.1 & & & \\
\hline & 12712 & 56.5006 & 0 & 279.7 & & & \\
\hline & 2713 & 2500 & 12.35 & 280 & & & \\
\hline & 2714 & 21 & & & & & \\
\hline & 12715 & 32 & & & 4 & & \\
\hline & 2716 & 2509 & 9.25 & 3.2 & 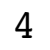 & & \\
\hline & 2717 & 5222 & 0 & & 4 & & \\
\hline & 2718 & 2507 & & & 4 & & \\
\hline & 2719 & 2501 & 7.9500 & 282.5 & 4 & & \\
\hline & 2720 & 0006 & 250 & 2.3 & 4 & & \\
\hline & 12721 & & 0 & & 4 & & \\
\hline & 12722 & 04 & 0 & .2 & 4 & & \\
\hline & 12723 & 146.4999 & .52 & .0 & 4 & 0 & \\
\hline & 12724 & 0 & 10 . & & 4 & & \\
\hline & 1281 & 0 & & & 4 & & \\
\hline & 1282 & 27.9897 & 0 & .5 & 4 & & . \\
\hline & 1283 & 0 & & 6 & 4 & & \\
\hline & 1284 & 0 & 50 & 3 & 4 & & \\
\hline & 1285 & 33.2656 & 2.7000 & 7.8 & 4 & & . \\
\hline & 1286 & 146.0177 & 2.9000 & 277.5 & 5 & & . \\
\hline & 1287 & 29 & 0 & & 6 & & \\
\hline & 1288 & 61 . & & 7.3 & 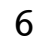 & & . \\
\hline & 1289 & 23.2482 & 750 & 279.3 & 5 & & \\
\hline & 12810 & 49.6337 & & 1.9 & 4 & & \\
\hline & 12811 & & & 1.2 & 3 & & \\
\hline & 12812 & 283.3831 & 00 & 0.9 & 3 & & \\
\hline & 12813 & 272 . & & 1.1 & 2 & & \\
\hline & 12814 & 284.9625 & & 2.7 & 1 & & . \\
\hline & 12815 & 280 . & 2 . & 282.8 & 1 & & \\
\hline & 12816 & 290.5069 & 1 . & 283.1 & 1 & & \\
\hline & 12817 & 292.4998 & 2.6750 & 282.0 & 2 & & \\
\hline & 12818 & 320.7145 & 1.9750 & 281.0 & 3 & & 0 \\
\hline & 12819 & 22.2182 & 500 & 280.9 & 4 & & \\
\hline & 12820 & 106.1629 & 1.5750 & 281.3 & 5 & & \\
\hline & 12821 & 2507 & 3.8000 & 281.8 & 4 & & 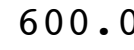 \\
\hline & 12822 & & & & כ & & \\
\hline & 12823 & 3880 & 1.1250 & 280.4 & & .0 & 60 \\
\hline
\end{tabular}




\begin{tabular}{|c|c|c|c|c|c|c|c|}
\hline & 2824 & 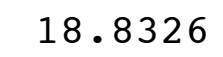 & 7500 & 80.4 & 6 & (6. & \\
\hline & 1291 & 2.5321 & 0.7500 & 280.3 & 6 & 600.0 & \\
\hline & 1292 & 118.8176 & 1500 & 280.1 & 5 & 00 & \\
\hline & 293 & 83.9537 & 3.4250 & 280.5 & & 00 & \\
\hline & 294 & 321 & .8250 & 80.6 & & 0 & \\
\hline & 1295 & 94.4543 & .8250 & 280.7 & & 00 & \\
\hline & 296 & 5941 & 1.6250 & 279.4 & & 500 & \\
\hline & 297 & 826 & 2.8000 & 79.9 & & & \\
\hline & 298 & 85.2397 & 4.9000 & 79.9 & & & \\
\hline & 299 & 72.7503 & 500 & 280.1 & 3 & 500 & 0 \\
\hline & 2910 & 82.7683 & 1500 & 280.3 & & 00.0 & \\
\hline & 2911 & 100.9429 & 4.5250 & 81.2 & & 0 & \\
\hline & 12912 & 132 . & 5000 & 281.4 & 3 & 00.0 & \\
\hline & 2913 & 146 & 4.5250 & 282.0 & 3 & 00.0 & 00 \\
\hline & 2914 & 164 . & & 82.8 & & & \\
\hline & 12915 & 175 . & & 282.6 & 4 & & \\
\hline & 12916 & 181 & 1000 & 282.1 & 4 & .0 & . \\
\hline & 12917 & 185 . & 0 & 81.9 & 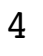 & & \\
\hline & 12918 & 172 . & & 280.6 & 5 & & \\
\hline & 12919 & 142.9481 & 2750 & 280.3 & 4 & 0 & U. \\
\hline & 12920 & 114.4449 & .7000 & 280.4 & 4 & 0 & \\
\hline & 12921 & 141.7533 & 750 & 280.2 & 4 & & \\
\hline & 12922 & 133. & 250 & 280.0 & 4 & & \\
\hline & 12923 & 135.0000 & 250 & 280.3 & 4 & 0 & . \\
\hline & 12924 & 141.2498 & & & 4 & & \\
\hline & 1301 & 150 . & 250 & 28 & 4 & & \\
\hline & 1302 & 149.7496 & 750 & 279.3 & 4 & 0 & U. \\
\hline & 1303 & 153. & 500 & 278.4 & 4 & & \\
\hline & 1304 & 153. & 000 & 278.1 & 4 & & \\
\hline & 1305 & 148.2501 & 000 & 278.1 & 4 & 0 & 0 • \\
\hline & 1306 & 147.9998 & 000 & 277.7 & 4 & 0 & 0 . \\
\hline & 1307 & 148 . & 50 & 277.4 & 4 & & \\
\hline & 1308 & 147 . & 000 & 277.4 & 4 & & o. \\
\hline & 1309 & 144.0014 & $\$ 750$ & 277.7 & 4 & 0 & 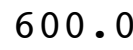 \\
\hline & 13010 & 144.2494 & 0 & 278.1 & 4 & & \\
\hline & 13011 & 144.4997 & 7 . & 278.6 & 4 & & . \\
\hline & 13012 & 144.5000 & 2250 & 279.6 & 4 & & . \\
\hline & 13013 & 152.2398 & 500 & 280.3 & 4 & & 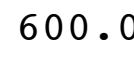 \\
\hline & 13014 & 152.0061 & 5.6250 & 280.7 & 4 & .0 & 0 . \\
\hline & 13015 & 144.2512 & 4.9250 & 280.4 & 4 & & . \\
\hline & 13016 & 152.0110 & 250 & 280.3 & 3 & & 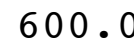 \\
\hline & 13017 & 154.2438 & 2.8500 & 280.4 & 4 & .0 & 0 . \\
\hline & 13018 & 123.4903 & 1.2250 & 280.1 & 5 & 0 & 0 . \\
\hline & 13019 & 138.9980 & 250 & 280.0 & 5 & & \\
\hline & 13020 & 132.9919 & 2.9000 & 279.3 & 5 & & 0 . \\
\hline & 13021 & 84.0172 & 3.6000 & 279.2 & 4 & 600.0 & 600 \\
\hline & & 118.7218 & & & 4 & & \\
\hline & 13023 & 90.6968 & 3.2750 & 279.1 & & 600.0 & 600 \\
\hline
\end{tabular}




\begin{tabular}{|c|c|c|c|c|c|c|c|}
\hline & 3024 & & 0 & 9.0 & & & \\
\hline & 1311 & 00 & 2.7000 & 278.9 & & 0 & \\
\hline & 1312 & 143.2500 & 3.1000 & 278.3 & & 00 & \\
\hline & 1313 & 163.9966 & 2.3250 & 278.1 & & 00.0 & \\
\hline & 314 & 150.2278 & .3750 & 278.5 & & 0 & \\
\hline & 315 & 149.4998 & 500 & 276.7 & & 0 & \\
\hline & 316 & 5001 & 5.0750 & 276.1 & & 00 & \\
\hline & 31 & 43.2482 & 500 & 276.8 & & .0 & \\
\hline & 318 & 107.5538 & 8500 & 77.4 & & & \\
\hline & 31 & 49.4771 & 4.5000 & 278.1 & & 00 & 0 \\
\hline & 3110 & 47.5003 & 8750 & 278.4 & & 00.0 & \\
\hline & 111 & חמחמת & .1000 & 279.5 & & & \\
\hline & 3112 & 152 & 3.8500 & 281.1 & & 0 & \\
\hline & 3113 & 158.2495 & 6.0500 & 281.9 & & 0.0 & . \\
\hline & 3114 & 155 & 0 & & & & \\
\hline & 3115 & 153. & & 3.5 & & & \\
\hline & 3116 & 158.0032 & 3.9500 & 284.0 & & .0 & 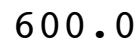 \\
\hline & 3117 & 160 . & 000 & & & 0 & \\
\hline & 3118 & 148 & & .8 & & & \\
\hline & 3119 & 89.2480 & 2.7000 & 3.2 & & .0 & 0 \\
\hline & 13120 & 111.7204 & 2.7750 & 3.6 & & .0 & \\
\hline & 13121 & 155.2419 & & & & & \\
\hline & 13122 & 158 & 750 & .7 & 6 & & \\
\hline & 13123 & 94.3686 & 1.3500 & 3.8 & 6 & 0 & . \\
\hline & 13124 & 38.7519 & 1.2500 & & & & \\
\hline & $\begin{array}{lll}2 & 1 & 1\end{array}$ & 9814 & 0.5000 & .8 & 5 & & \\
\hline & 212 & 144.2541 & 2.8000 & 1.7 & 6 & 0 & J. \\
\hline & 213 & 152.998 & 2.8500 & 1.2 & 6 & & \\
\hline & $\begin{array}{lll}2 & 1 & 4\end{array}$ & 7551 & 1.1750 & .2 & 6 & & $\bullet$ \\
\hline & 215 & 94.2012 & 0.1750 & L. 0 & 6 & 0 & 0 • \\
\hline & 216 & 35.2128 & 2.1000 & .5 & 5 & .0 & 0 . \\
\hline & $\begin{array}{lll}2 & 1 & 7\end{array}$ & 20.4999 & 1.6750 & .8 & 4 & & \\
\hline & 218 & 3165 & 1.6500 & .0 & 5 & 0 & . \\
\hline & $\begin{array}{lll}2 & 1 & 9\end{array}$ & 80.2587 & 7500 & .3 & 4 & .0 & . \\
\hline & 2110 & 196.5392 & 67 & & 3 & & \\
\hline & 2111 & 203.6346 & 2.1000 & 3.9 & 2 & & 0 . \\
\hline & 2112 & 254.7176 & .6750 & 4.3 & 1 & .0 & . \\
\hline & 2113 & 202.62 & 750 & $2 \varepsilon$ & 1 & & . \\
\hline- & 2114 & 239.0000 & 1.9750 & 4.4 & 2 & .0 & 0 . \\
\hline & 2115 & 186.6287 & 2.2000 & 4.7 & 1 & .0 & 0 . \\
\hline & 2116 & 178.0000 & 2.1250 & 283.5 & $\perp$ & & 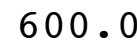 \\
\hline & 2117 & 176.2580 & 0.7500 & 283.8 & 1 & & 0 . \\
\hline & 2118 & 165.3595 & 2.1250 & 282.4 & 2 & 0 & 00 . \\
\hline & 2119 & 167.0146 & 0.1500 & 281.8 & 3 & & \\
\hline - & 2120 & 56.7604 & 0.0500 & 281.3 & 4 & & 00 . \\
\hline & 2121 & 38.7499 & 0.0000 & 280.7 & 4 & 600.0 & 00. \\
\hline & 2122 & 1092 & & 281.1 & 5 & & \\
\hline & 2123 & 6.7502 & 1.2250 & 281.8 & 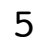 & 600.0 & 600 \\
\hline
\end{tabular}




\begin{tabular}{|c|c|c|c|c|c|c|c|c|}
\hline 1 & 2 & 24 & & 0 & & & & \\
\hline & 2 & 1 & 1 & .3250 & 1.0 & & 00.0 & \\
\hline & 2 & 22 & 142.2643 & 2.3500 & 280.1 & 6 & 00.0 & \\
\hline & 2 & 23 & 151.7374 & 2.0000 & 279.6 & & 00.0 & \\
\hline & 2 & 4 & 62.2556 & .2250 & 278.8 & & 0 & \\
\hline & 2 & 25 & 161.5202 & .9500 & 279.0 & & 00 & \\
\hline & 2 & 26 & 162.0115 & 1.8000 & 279.2 & & 500 & 0 \\
\hline & 2 & 7 & 58.2382 & L. 8500 & 279.9 & & 00.0 & \\
\hline & 2 & 8 & 48.5258 & L. 2500 & 80.3 & $v$ & & \\
\hline & 2 & 9 & 146.0745 & 2.1250 & 280.8 & & 500.0 & 00 \\
\hline & 2 & 210 & 140.3046 & 2.1250 & 280.6 & & 0 & \\
\hline & 2 & 211 & 155.7935 & 177 & 81.9 & 3 & 00.0 & \\
\hline & 2 & 212 & 196.49 & 0.9750 & 285.0 & 2 & 0 & . \\
\hline & 2 & 213 & 66.0795 & 2.8250 & 284.5 & 1 & 00.0 & 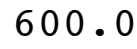 \\
\hline & 2 & 214 & 174 & & 83.4 & 2 & & \\
\hline & 2 & 215 & 171.5 & & 285.3 & 1 & & \\
\hline & 2 & 216 & 165.6692 & 2.72 & 285.1 & 1 & .0 & 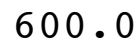 \\
\hline & 2 & 217 & 126.5904 & .50 & 285.5 & 1 & 0 & \\
\hline & 2 & 218 & 97.2464 & 40 & 284.5 & 2 & & \\
\hline & 2 & 219 & 57 . & $\begin{array}{lll}0 & 87\end{array}$ & 285.2 & 3 & 0 & 0 \\
\hline & 2 & 220 & 164.5000 & 2.72 & 284.9 & 4 & .0 & 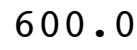 \\
\hline & 2 & 221 & 121.6942 & 2.27 & 284.7 & 5 & & \\
\hline & 2 & 222 & 172.7509 & 3.67 & 283.5 & 4 & & \\
\hline & 2 & 223 & 135.8861 & 2.4250 & 283.4 & 5 & 0 & . \\
\hline & 2 & 224 & 152 . & & 28 & 6 & & \\
\hline & 2 & 31 & 175.50 & 4 . & 282.0 & 5 & & \\
\hline & 2 & 32 & 104.9498 & 2.0000 & 281.7 & 6 & 0 & U. \\
\hline & 2 & 33 & 174.501 & 1.72 & 281.9 & 6 & & 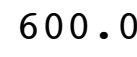 \\
\hline & 2 & 34 & 167.812 & 2.32 & 282.0 & 6 & & $\bullet$ \\
\hline & 2 & 35 & 38.2858 & 5000 & 282.8 & 6 & .0 & 0 • \\
\hline & 2 & 36 & 144.3412 & 1.0750 & 284.7 & 6 & 0 & 0 . \\
\hline & 2 & 37 & 142.2395 & 1.5250 & 284.2 & 6 & & \\
\hline & 2 & 38 & 141.0982 & 2.1250 & 282.8 & 6 & & 0 • \\
\hline & 2 & 39 & 177.7500 & 4.2250 & 283.1 & 5 & 0 & \\
\hline & 2 & 310 & 176.2509 & & 283.5 & 4 & & 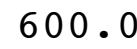 \\
\hline & 2 & 311 & 179.5000 & 4.0750 & 284.3 & 3 & & U \\
\hline & 2 & 312 & 178.7502 & 3.4500 & 285.8 & 2 & 0 & 0 . \\
\hline & 2 & 313 & 177.2500 & 3 . & 287 & 2 & & . \\
\hline- & 2 & 314 & 177.5031 & 4.4000 & 288.4 & 3 & .0 & 0 . \\
\hline & 2 & 315 & 179.2500 & 4.8000 & 288.3 & 3 & 0 & 0 . \\
\hline & 2 & 316 & 178.7506 & 3500 & 288.6 & 3 & & 600. \\
\hline & 2 & 317 & 171.0007 & 5.2750 & 288.1 & 3 & .0 & 00 . \\
\hline & 2 & 318 & 150.9749 & 6.3000 & 288.7 & 4 & .0 & 00 . \\
\hline & 2 & 319 & 135.3116 & 3.8500 & 289.1 & 4 & 600 & 0 \\
\hline 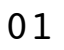 & 2 & 320 & 171.2455 & 0.9250 & 288.2 & 5 & & 600. \\
\hline & 2 & 321 & 176.2599 & 0.7500 & 287.2 & 6 & 600.0 & 600. \\
\hline & 2 & 322 & 179.8564 & 2250 & 28 & 6 & & \\
\hline & & 323 & 106.4126 & 1.1000 & 287.8 & & 600.0 & 600 \\
\hline
\end{tabular}




\begin{tabular}{|c|c|c|c|c|c|c|c|c|}
\hline 1 & 2 & 24 & 9.2504 & 1.6750 & 288.6 & 6 & 00.0 & \\
\hline & 2 & 41 & 146.9852 & 1.2000 & 288.6 & & 00.0 & \\
\hline & 2 & 42 & 71.3551 & 7500 & 288.4 & 6 & 0 & \\
\hline & 2 & 3 & 62.9347 & 2.0000 & 288.7 & & 00.0 & \\
\hline & 2 & 44 & 110.5323 & 1.3500 & 38.8 & & 0.0 & \\
\hline & 2 & 5 & 82.0000 & 1.4250 & 288.8 & & 00 & \\
\hline & 2 & 6 & 79.4941 & 1.3000 & 288.9 & & 00 & \\
\hline & 2 & 47 & 120 . & 1.2750 & 87.4 & & 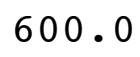 & \\
\hline & 2 & 8 & 170.7562 & 3.3250 & 285.9 & & 00 & \\
\hline & 2 & 9 & 151.8712 & 1.8250 & 28 & & 0 & \\
\hline & 2 & 410 & 198 . & 1.5000 & 89.4 & & & \\
\hline & 2 & 411 & 176 . & 3.15 & 88.2 & & & \\
\hline & 2 & 412 & 178 . & 3.2250 & 288.4 & & 0 & \\
\hline & 2 & 413 & 179. & .0500 & 89.6 & & 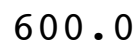 & \\
\hline & 2 & 414 & 179. & & 90.8 & & & \\
\hline & 2 & 415 & 178 . & 3.2750 & 291.2 & 2 & 0 & \\
\hline & 2 & 416 & 174.5086 & 2.7750 & 291.5 & 1 & 0 & \\
\hline & 2 & 417 & 179.2500 & 21 & 290.8 & 2 & & \\
\hline & 2 & 418 & 170 . & 0 & 289.1 & 3 & & \\
\hline & 2 & 419 & 173.7842 & .40 & 288.3 & 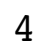 & 0 & \\
\hline & 2 & 420 & 127 . & 0 & 289.4 & 5 & & \\
\hline & 2 & 421 & 158 . & 0 & 289.4 & 6 & & \\
\hline & 2 & 422 & 160.5008 & 97 & 289.8 & 5 & 0 & \\
\hline & 2 & 423 & 172 . & 0 & 289.6 & & & \\
\hline & 2 & 424 & 157. & & 288.4 & 5 & & \\
\hline & 2 & 51 & 123.6810 & 2.0250 & 289.1 & 6 & & \\
\hline & 2 & 52 & 166.5000 & 1.60 & 288.3 & 6 & & \\
\hline & 2 & 53 & 150 . & 170 & 288.0 & 6 & & \\
\hline & 2 & 54 & 160.4485 & 2.6000 & 288.2 & 6 & & \\
\hline & 2 & 55 & 127 . & 1.07 & 287.3 & 6 & & \\
\hline & 2 & 56 & 139. & & 287.8 & 6 & & \\
\hline & 2 & 57 & 114 . & 1 . & 287.4 & 6 & & \\
\hline & 2 & 58 & 135.2889 & 1.6000 & 287.6 & 6 & & \\
\hline & 2 & 59 & 119 . & 2 . & 287.0 & 5 & & \\
\hline & 2 & 510 & 167.7891 & 1.6750 & 287.7 & 4 & & \\
\hline & 2 & 511 & 174.2486 & 2.2250 & 287.6 & 3 & & \\
\hline & 2 & 512 & 177 . & 0 & 285.8 & 3 & & \\
\hline & 2 & 513 & 178.5005 & 4.5250 & 286.1 & 3 & & \\
\hline & 2 & 514 & 180.0000 & 4.0750 & 288.2 & 3 & & 6 \\
\hline & 2 & 515 & 179. & 4 . & 288.7 & 3 & & \\
\hline & 2 & 516 & 181. & 4.1500 & 289.2 & 3 & & 0 . \\
\hline+ & 2 & 517 & 180.0000 & 3.6250 & 288.8 & 2 & 0 & 0 \\
\hline & 2 & 518 & 150.4955 & 1.4750 & 287.6 & 3 & & \\
\hline 01 & 2 & 519 & 59.6821 & 1.7000 & 287.2 & 4 & & 600 \\
\hline+ & 2 & 520 & 81.2500 & 5.7250 & 288.0 & 4 & 600.0 & 00 \\
\hline & 2 & 521 & 00 & & 288.4 & 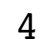 & & \\
\hline & 2 & 522 & 67.7491 & 7.2000 & 288.3 & 4 & & 600 \\
\hline & & 523 & 70.0242 & 4.8500 & 288.0 & & 600.0 & 600 \\
\hline
\end{tabular}




\begin{tabular}{|c|c|c|c|c|c|c|c|c|}
\hline 1 & 2 & 524 & 60.4613 & 3.4250 & 287.4 & 5 & 600.0 & 600.0 \\
\hline 01 & 2 & 61 & 71.5153 & 5.0500 & 286.9 & 4 & 600.0 & 00.0 \\
\hline 01 & 2 & 62 & 54.5009 & 10.3500 & 286.1 & 4 & 600.0 & 00.0 \\
\hline 01 & 2 & 63 & 60.7499 & 13.5750 & 284.8 & 4 & 600.0 & 00.0 \\
\hline 01 & 2 & 64 & 58.2501 & 13. & 284.2 & 4 & 600.0 & 00.0 \\
\hline 01 & 2 & 65 & 65.9839 & 9.6750 & 282.7 & 4 & 600.0 & 00.0 \\
\hline 01 & 2 & 66 & 69.2512 & 10. & 281.5 & 4 & 600.0 & 00.0 \\
\hline 01 & 2 & 67 & 74.8133 & 10.0250 & 279.5 & 4 & 600.0 & 00.0 \\
\hline 01 & 2 & 68 & 65.2510 & 11.3250 & 278.8 & 4 & 600.0 & 00.0 \\
\hline 01 & 2 & 69 & 45.2513 & 12 . & 279.0 & 4 & 600.0 & .0 \\
\hline 01 & 2 & 610 & 58.7603 & 11. & 279.5 & 4 & 600.0 & \\
\hline 01 & 2 & 611 & 67.0000 & 10 . & 280.0 & 4 & 600.0 & \\
\hline 01 & 2 & 612 & 56.2658 & 10. & 280.7 & 4 & 600.0 & 00.0 \\
\hline 01 & 2 & 613 & 50.0000 & 8.2250 & 281.3 & 4 & 600.0 & 00.0 \\
\hline 01 & 2 & 614 & 56.2515 & 10 . & 281.8 & 4 & .0 & \\
\hline 01 & 2 & 615 & 56.2503 & 11 . & 281.5 & 4 & 600.0 & .0 \\
\hline 01 & 2 & 616 & 71.9296 & 250 & 281.3 & 4 & 60 & .0 \\
\hline 01 & 2 & 617 & 89.9997 & 9. & 280.7 & 4 & .0 & \\
\hline 01 & 2 & 618 & 87.5018 & 000 & 280.0 & 4 & .0 & \\
\hline 01 & 2 & 619 & 101.6533 & 7.7750 & 279.3 & 4 & .0 & .0 \\
\hline 01 & 2 & 620 & 149.3418 & 5 . & 278.0 & 4 & 6 & \\
\hline 01 & 2 & 621 & 98.7505 & 750 & 278.5 & 4 & .0 & \\
\hline 01 & 2 & 622 & 97.0000 & 6.7 & 278.5 & 4 & 600.0 & .0 \\
\hline 01 & 2 & 623 & 121.0088 & 8 . & 279.1 & 4 & 6 & \\
\hline 01 & 2 & 624 & 81.2517 & 7 . & 277.6 & 4 & .0 & \\
\hline 01 & 2 & 71 & 86.0002 & 8.2000 & 277.2 & 4 & .0 & \\
\hline 01 & 2 & 72 & 79.2500 & 8.1000 & 276.6 & 4 & 60 & .0 \\
\hline 01 & 2 & 73 & 85.2501 & 8. & 276.5 & 4 & .0 & \\
\hline 01 & 2 & 74 & 101.4971 & 8.7750 & 276.6 & 4 & .0 & 0.0 \\
\hline 01 & 2 & 75 & 125.7503 & 10.5000 & 277.1 & 4 & 60 & .0 \\
\hline 01 & 2 & 76 & 137.0000 & 11 . & 276.8 & 4 & .0 & \\
\hline 01 & 2 & 77 & 146.0016 & 12.7000 & 276.6 & 4 & .0 & 600.0 \\
\hline 01 & 2 & 78 & 151.7500 & 13.4250 & 276.2 & 4 & 600.0 & 600.0 \\
\hline 01 & 2 & 79 & 152.2500 & 12.7250 & 276.6 & 4 & 600.0 & 0.0 \\
\hline 01 & 2 & 710 & 153.7500 & 14.6000 & 277.5 & 4 & 600.0 & 600.0 \\
\hline 01 & 2 & 711 & 158.2502 & 15.9000 & 278.2 & 4 & 600.0 & 600.0 \\
\hline 01 & 2 & 712 & 163.5041 & 16 . & 279.0 & 4 & 600.0 & .0 \\
\hline 01 & 2 & 713 & 164.2498 & 15.5000 & 279.7 & 4 & 600.0 & 600.0 \\
\hline 01 & 2 & 714 & 161.2486 & 16.0500 & 280.2 & 4 & 600.0 & 00.0 \\
\hline 01 & 2 & 715 & 171.7497 & 15 . & 280.9 & 4 & 60 & 0.0 \\
\hline 01 & 2 & 716 & 174.7482 & 14.8750 & 281.4 & 4 & 600.0 & 600.0 \\
\hline 01 & 2 & 717 & 174.2505 & 14.7750 & 281.5 & 4 & 600.0 & 600.0 \\
\hline 01 & 2 & 718 & 158.4988 & 11.4500 & 281.0 & 4 & 600.0 & 600.0 \\
\hline 01 & 2 & 719 & 148.7500 & 10.8750 & 280.2 & 4 & 600.0 & 600.0 \\
\hline 01 & 2 & 720 & 147.2500 & 10.9500 & 280.1 & 4 & 600.0 & 600.0 \\
\hline 01 & 2 & 721 & 145.2503 & 10.7500 & 280.1 & . & 600.0 & 600.0 \\
\hline 01 & 2 & 722 & 147.7502 & 10.8500 & 279.7 & 4 & 600.0 & 600.0 \\
\hline . & 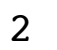 & 723 & 147.2500 & 11.550 & 279.2 & & 600.0 & 600.0 \\
\hline
\end{tabular}




\begin{tabular}{|c|c|c|c|c|c|c|c|c|}
\hline & & 24 & & & 8.9 & & & \\
\hline & 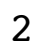 & 1 & 500 & 3.2250 & 278.8 & & & \\
\hline & 2 & 2 & 47.0000 & 2000 & 278.6 & & 00 & \\
\hline & 2 & 3 & 151.7505 & 1.7750 & 278.3 & & 00 & \\
\hline & & 4 & 149.2522 & 9.8750 & 78.1 & & & \\
\hline & 2 & 5 & 147.2503 & 8.8250 & 277.7 & & 0 & \\
\hline & & 6 & 43.7498 & 7500 & 277.4 & & & \\
\hline & 2 & 7 & 40.7502 & 9.2250 & 77.2 & & & \\
\hline & 2 & 8 & 7533 & 7.3750 & 277.0 & & 0 & \\
\hline & & 9 & 45.5019 & 9.1000 & 277.8 & & 0 & \\
\hline & 2 & 10 & 48.2499 & 8.2250 & 278.8 & & & \\
\hline & $?$ & 11 & 9.2505 & 7.4500 & 79.8 & & & \\
\hline & & 12 & 52.5021 & 7.3750 & 280.6 & 4 & 0 & \\
\hline & 2 & 813 & 49.4892 & 5.4000 & 281.5 & & 00.0 & \\
\hline & 2 & 14 & & & & & & \\
\hline & 2 & 315 & 59.4729 & 2 . & 283.6 & & & \\
\hline & 2 & 816 & 32.0000 & 0 & 285.2 & & 0 & \\
\hline & 2 & 17 & 502 & 1 . & 0 & & & \\
\hline & 2 & 818 & 025 & 000 & 282.4 & 3 & 0 & \\
\hline & 2 & 819 & 324.5039 & & 281.2 & 4 & 0 & \\
\hline & 2 & 820 & 520 & 750 & $2 \varepsilon$ & $J$ & & \\
\hline & 2 & 821 & 80.7482 & 00 & 4 & 4 & & \\
\hline & 2 & 822 & 72.9952 & 50 & 279.5 & 4 & 0 & \\
\hline & 2 & 823 & 70.2394 & 5.7250 & 9.5 & 4 & & \\
\hline & 2 & 824 & 21.3230 & 50 & 9.3 & 4 & & \\
\hline & 2 & 91 & 696 & 500 & 279.4 & 5 & & \\
\hline & 2 & 92 & 60.9685 & 1.9750 & 279.5 & 5 & & \\
\hline & 2 & 93 & 47.9712 & 250 & 279.4 & 6 & & \\
\hline & 2 & 94 & 74 & 00 & 279.5 & 6 & & \\
\hline & 2 & 95 & 93.8477 & 3000 & 278.9 & 6 & & \\
\hline & 2 & 96 & 661 & 250 & 27 & 6 & & \\
\hline & 2 & 97 & 255 & 00 & .2 & 5 & & \\
\hline & 2 & 98 & 304.9244 & 2.8750 & 279.6 & 6 & 0 & 0 . \\
\hline 1 & 2 & 99 & 311.9827 & 500 & .7 & 5 & & \\
\hline & 2 & 910 & 319. & 50 & 281.0 & 4 & & \\
\hline & 2 & 911 & 315.2620 & & 280.1 & 4 & & \\
\hline & 2 & 912 & 328.2496 & 000 & 279.0 & 4 & & \\
\hline 1 & 2 & 913 & 337.7405 & 750 & 278.9 & 3 & & 0 . \\
\hline & 2 & 914 & 6.9981 & 750 & 280.6 & 4 & 60 & 0 \\
\hline & 2 & 915 & .7501 & 750 & 282.0 & 3 & & \\
\hline & 2 & 916 & 356.2301 & 250 & 281.1 & 2 & & \\
\hline 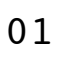 & 2 & 917 & 26.0053 & 4.4250 & 278.8 & 3 & 600.0 & 00 . \\
\hline & 2 & 918 & 16.2515 & 4.6500 & 279.0 & 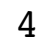 & 0 & \\
\hline & 2 & 919 & 319.1154 & 2.1250 & 278.9 & 5 & & \\
\hline 01 & 2 & 920 & 334.6605 & 3.4000 & 278.7 & 5 & 600 & 0 \\
\hline & 2 & 921 & 346.7568 & 3.4750 & 278.3 & 5 & 600.0 & 00 \\
\hline & & 922 & 354.5037 & 000 & 278.2 & 4 & & \\
\hline & & 923 & 302.0185 & 2.1000 & 278.5 & & 600.0 & 600 \\
\hline
\end{tabular}


$\begin{array}{llll}01 & 2 & 924 & 314.6772\end{array}$

$\begin{array}{llll}01 & 210 & 1 & 329.3681\end{array}$

$\begin{array}{llll}01 & 210 & 2 & 351.2915\end{array}$

$\begin{array}{llll}01 & 210 & 3 & 340.6090\end{array}$

$\begin{array}{llll}01 & 210 & 4 & 0.3011\end{array}$

$\begin{array}{llll}01 & 210 & 5 & 356.5002\end{array}$

$\begin{array}{llll}01 & 210 & 6 & 357.9722\end{array}$

$\begin{array}{llll}01 & 210 & 7 & 13.4956\end{array}$

$\begin{array}{llll}01 & 210 & 8 & 356.7441\end{array}$

$\begin{array}{llll}01 & 210 & 9 & 357.0547\end{array}$

$\begin{array}{lll}01 & 21010 & 331.8879\end{array}$

$\begin{array}{lll}01 & 21011 & 14.9032\end{array}$

$\begin{array}{lll}01 & 21012 & 33.2501\end{array}$

$\begin{array}{llll}01 & 21013 & 343.4983\end{array}$

$01 \quad 21014 \quad 359.5146$

$\begin{array}{lll}01 & 21015 & 33.5744\end{array}$

$\begin{array}{lll}01 & 21016 & 64.0021\end{array}$

$\begin{array}{lll}01 & 21017 & 63.2501\end{array}$

$\begin{array}{lll}01 & 21018 & 49.2151\end{array}$

$\begin{array}{lll}01 & 21019 & 32.6226\end{array}$

$\begin{array}{lll}01 & 21020 & 337.7503\end{array}$

$01 \quad 21021351.7902$

$\begin{array}{lll}01 & 21022 & 7.5105\end{array}$

$\begin{array}{lll}01 & 21023 & 335.2718\end{array}$

$\begin{array}{llll}01 & 21024 & 320.8719\end{array}$

$\begin{array}{llll}01 & 211 & 1 & 328.7343\end{array}$

$\begin{array}{llll}01 & 211 & 2 & 312.2541\end{array}$

$\begin{array}{llll}01 & 211 & 3 & 315.5000\end{array}$

$\begin{array}{llll}01 & 211 & 4 & 317.0007\end{array}$

$\begin{array}{llll}01 & 211 & 5 & 298.5308\end{array}$

$\begin{array}{llll}01 & 211 & 6 & 329.2137\end{array}$

$\begin{array}{llll}01 & 211 & 7 & 357.4583\end{array}$

$\begin{array}{llll}01 & 211 & 8 & 267.7949\end{array}$

$\begin{array}{llll}01 & 211 & 9 & 324.2146\end{array}$

$\begin{array}{llll}01 & 21110 & 323.7347\end{array}$

$\begin{array}{lll}01 & 21111 & 67.8242\end{array}$

$0121112 \quad 40.6156$

$\begin{array}{lll}01 & 21113 & 97.9993\end{array}$

$\begin{array}{lll}01 & 21114 & 43.6083\end{array}$

$\begin{array}{lll}01 & 21115 & 14.7530\end{array}$

$\begin{array}{lll}01 & 21116 & 47.5926\end{array}$

$\begin{array}{lll}01 & 21117 & 62.2869\end{array}$

$\begin{array}{lll}01 & 21118 & 27.7501\end{array}$

$\begin{array}{lll}01 & 21119 & 10.2332\end{array}$

$\begin{array}{lll}01 & 21120 & 347.2504\end{array}$

$\begin{array}{llll}01 & 21121 & 350.7482\end{array}$

$01 \quad 21122 \quad 354.2145$

$0121123 \quad 331.0519$
$2.8500 \quad 278.6 \quad 6 \quad 600.0 \quad 600.0$

$2.7000 \quad 278.4 \quad 6 \quad 600.0 \quad 600.0$

$4.5000 \quad 277.8 \quad 5 \quad 600.0 \quad 600.0$

$1.9500 \quad 277.3 \quad 6 \quad 600.0 \quad 600.0$

$3.9500278 .0 \quad 5 \quad 600.0 \quad 600.0$

$4.2250 \quad 278.144600 .0 \quad 600.0$

$2.4750 \quad 277.155600 .0 \quad 600.0$

$1.9500 \quad 276.6 \quad 6 \quad 600.0 \quad 600.0$

$1.7000 \quad 276.5 \quad 6 \quad 600.0 \quad 600.0$

$2.8500 \quad 277.0 \quad 5 \quad 600.0 \quad 600.0$

$3.1500 \quad 277.9 \quad 4 \quad 600.0 \quad 600.0$

$3.6250 \quad 279.4 \quad 3 \quad 600.0 \quad 600.0$

$\begin{array}{llllll}4.3000 & 279.7 & 3 & 600.0 & 600.0\end{array}$

$3.5250 \quad 278.5 \quad 2 \quad 600.0 \quad 600.0$

$\begin{array}{llllll}4.1250 & 280.4 & 3 & 600.0 & 600.0\end{array}$

$5.2250 \quad 281.0 \quad 3 \quad 600.0 \quad 600.0$

$\begin{array}{llllll}4.7250 & 280.8 & 3 & 600.0 & 600.0\end{array}$

$\begin{array}{llllll}5.6750 & 280.9 & 3 & 600.0 & 600.0\end{array}$

$4.3750 \quad 279.6 \quad 4 \quad 600.0 \quad 600.0$

$2.4750 \quad 278.7 \quad 4 \quad 600.0 \quad 600.0$

$2.3000 \quad 278.5 \quad 5 \quad 600.0 \quad 600.0$

$2.1250 \quad 278.7 \quad 6 \quad 600.0 \quad 600.0$

$3.0250 \quad 279.0 \quad 5 \quad 600.0 \quad 600.0$

$2.0750 \quad 278.6 \quad 6 \quad 600.0 \quad 600.0$

$\begin{array}{lllll}1.8500 & 278.7 & 6 & 600.0 & 600.0\end{array}$

$3.7750 \quad 278.3 \quad 5 \quad 600.0 \quad 600.0$

$3.7500 \quad 277.4 \quad 4 \quad 600.0 \quad 600.0$

$4.2750 \quad 277.2 \quad 4 \quad 600.0 \quad 600.0$

$3.6500 \quad 277.144600 .0 \quad 600.0$

$\begin{array}{llllll}4.7250 & 277.4 & 4 & 600.0 & 600.0\end{array}$

$3.2750 \quad 277.5 \quad 5 \quad 600.0 \quad 600.0$

$1.4000 \quad 277.0 \quad 6 \quad 600.0 \quad 600.0$

$1.0500 \quad 276.8 \quad 6 \quad 600.0 \quad 600.0$

$1.7500 \quad 277.155600 .0 \quad 600.0$

$\begin{array}{lllll}1.5000 & 278.7 & 4 & 600.0 & 600.0\end{array}$

$\begin{array}{llllll}2.0750 & 279.5 & 3 & 600.0 & 600.0\end{array}$

$4.1500 \quad 280.0 \quad 3 \quad 600.0 \quad 600.0$

$\begin{array}{llllll}3.3500 & 278.8 & 2 & 600.0 & 600.0\end{array}$

$5.0500 \quad 279.4 \quad 3 \quad 600.0 \quad 600.0$

$6.0500 \quad 280.6 \quad 4 \quad 600.0 \quad 600.0$

$6.1500280 .6 \quad 4 \quad 600.0 \quad 600.0$

$6.1750 \quad 279.8 \quad 4 \quad 600.0 \quad 600.0$

$3.4500 \quad 279.2 \quad 4 \quad 600.0 \quad 600.0$

$2.7250 \quad 278.6 \quad 4 \quad 600.0 \quad 600.0$

$2.7500 \quad 278.4 \quad 5 \quad 600.0 \quad 600.0$

$2.8000 \quad 278.2 \quad 6 \quad 600.0 \quad 600.0$

$2.6750 \quad 277.6 \quad 6 \quad 600.0 \quad 600.0$

$3.0750277 .4 \quad 5 \quad 600.0 \quad 600.0$ 
$01 \quad 21124 \quad 341.2494$

$\begin{array}{llll}01 & 212 & 1 & 333.2500\end{array}$

$01 \quad 212 \quad 2 \quad 340.7511$

012123342.0000

$\begin{array}{llll}01 & 212 & 4 & 348.2461\end{array}$

$\begin{array}{llll}01 & 212 & 5 & 350.7444\end{array}$

$\begin{array}{llll}01 & 212 & 6 & 2.3759\end{array}$

$\begin{array}{llll}01 & 212 & 7 & 314.7130\end{array}$

$\begin{array}{llll}01 & 212 & 8 & 329.4418\end{array}$

$01 \quad 21299325.0046$

$0121210 \quad 296.0846$

$\begin{array}{llll}01 & 21211 & 285.2643\end{array}$

$01 \quad 21212 \quad 247.0437$

0121213162.0000

$01 \quad 21214 \quad 159.4692$

$01 \quad 21215135.0341$

$0121216 \quad 173.0221$

$01 \quad 21217 \quad 159.9996$

$\begin{array}{lll}01 & 21218 & 170.5000\end{array}$

$\begin{array}{lll}01 & 21219 & 171.7489\end{array}$

$\begin{array}{lll}01 & 21220 & 156.4995\end{array}$

$\begin{array}{lll}01 & 21221 & 151.4999\end{array}$

$0121222 \quad 157.2500$

$\begin{array}{lll}01 & 21223 & 150.7497\end{array}$

$0121224 \quad 143.0002$

$\begin{array}{llll}01 & 213 & 1 & 135.7525\end{array}$

$\begin{array}{llll}01 & 213 & 2 & 146.9998\end{array}$

$\begin{array}{llll}01 & 213 & 3 & 144.5000\end{array}$

$\begin{array}{llll}01 & 213 & 4 & 150.5000\end{array}$

$\begin{array}{llll}01 & 213 & 5 & 153.0000\end{array}$

$\begin{array}{llll}01 & 213 & 6 & 154.2500\end{array}$

$\begin{array}{llll}01 & 213 & 7 & 155.5000\end{array}$

$\begin{array}{llll}01 & 213 & 8 & 152.4991\end{array}$

$\begin{array}{llll}01 & 213 & 9 & 154.0001\end{array}$

$\begin{array}{lll}01 & 21310 & 154.4999\end{array}$

$0121311 \quad 153.2500$

0121312150.5000

$\begin{array}{lll}01 & 21313 & 153.2500\end{array}$

$\begin{array}{lll}01 & 21314 & 157.7499\end{array}$

0121315154.4997

$0121316 \quad 164.5005$

$\begin{array}{lll}01 & 21317 & 154.7494\end{array}$

$\begin{array}{lll}01 & 21318 & 74.2507\end{array}$

$\begin{array}{lll}01 & 21319 & 5.8547\end{array}$

$\begin{array}{lll}01 & 21320 & 28.2411\end{array}$

$0121321 \quad 38.9121$

$0121322 \quad 53.2188$

$0121323 \quad 33.1314$ $\begin{array}{lllll}3.2000 & 276.4 \quad 4 & 600.0 & 600.0\end{array}$

$4.0000 \quad 276.4 \quad 4 \quad 600.0 \quad 600.0$

$\begin{array}{lllll}5.1000 & 276.8 \quad 4 & 600.0 & 600.0\end{array}$

$\begin{array}{lllll}4.1250 & 277.4 & 4 & 600.0 & 600.0\end{array}$

$\begin{array}{lllll}4.8000 & 277.8 & 4 & 600.0 & 600.0\end{array}$

$\begin{array}{lllll}2.8500 & 277.9 & 5 & 600.0 & 600.0\end{array}$

$2.9750 \quad 277.7 \quad 5 \quad 600.0 \quad 600.0$

$\begin{array}{lllll}1.3000 & 277.1 & 6 & 600.0 & 600.0\end{array}$

$4.2750 \quad 276.8 \quad 5 \quad 600.0 \quad 600.0$

$\begin{array}{lllll}7.0250 & 276.9 & 4 & 600.0 & 600.0\end{array}$

$\begin{array}{lllll}3.7750 & 276.3 & 3 & 600.0 & 600.0\end{array}$

$6.6750 \quad 276.9 \quad 4 \quad 600.0 \quad 600.0$

$\begin{array}{lllll}4.4000 & 277.6 & 3 & 600.0 & 600.0\end{array}$

$2.6750 \quad 279.8 \quad 2 \quad 600.0 \quad 600.0$

$\begin{array}{lllll}5.6000 & 278.9 & 3 & 600.0 & 600.0\end{array}$

$\begin{array}{lllll}3.7750 & 279.3 & 2 & 600.0 & 600.0\end{array}$

$\begin{array}{lllll}6.0750 & 278.8 & 3 & 600.0 & 600.0\end{array}$

$6.6500 \quad 278.14600 .0 \quad 600.0$

$6.9000 \quad 277.4 \quad 4 \quad 600.0 \quad 600.0$

$6.8000 \quad 277.0 \quad 4 \quad 600.0 \quad 600.0$

$6.6000 \quad 276.8 \quad 4 \quad 600.0 \quad 600.0$

$\begin{array}{lllll}7.1250 & 276.7 & 4 & 600.0 & 600.0\end{array}$

$\begin{array}{llllll}6.1500 & 276.3 & 4 & 600.0 & 600.0\end{array}$

$\begin{array}{lllll}4.6750 & 276.1 & 4 & 600.0 & 600.0\end{array}$

$5.1000276 .2 \quad 4 \quad 600.0 \quad 600.0$

$\begin{array}{lllll}6.5750 & 276.7 & 4 & 600.0 & 600.0\end{array}$

$9.1500 \quad 276.6 \quad 4 \quad 600.0 \quad 600.0$

$10.4000 \quad 276.7 \quad 4 \quad 600.0 \quad 600.0$

$10.8750 \quad 277.144600 .0 \quad 600.0$

$13.0750 \quad 276.9 \quad 4 \quad 600.0 \quad 600.0$

$14.5500 \quad 276.7 \quad 4 \quad 600.0 \quad 600.0$

$13.2000 \quad 276.7 \quad 4 \quad 600.0 \quad 600.0$

$12.7750 \quad 276.7 \quad 4 \quad 600.0 \quad 600.0$

$13.9250 \quad 277.144600 .0 \quad 600.0$

$14.5000 \quad 277.5 \quad 4 \quad 600.0 \quad 600.0$

$13.1500 \quad 278.3 \quad 4 \quad 600.0 \quad 600.0$

$10.7250 \quad 279.4 \quad 4 \quad 600.0 \quad 600.0$

$10.7750 \quad 280.8 \quad 4 \quad 600.0 \quad 600.0$

$\begin{array}{llllll}9.2750 & 282.1 & 4 & 600.0 & 600.0\end{array}$

$8.0000 \quad 282.8 \quad 4 \quad 600.0 \quad 600.0$

$\begin{array}{llllll}6.2250 & 283.7 & 4 & 600.0 & 600.0\end{array}$

$\begin{array}{lllll}5.4250 & 283.9 & 4 & 600.0 & 600.0\end{array}$

$3.5750 \quad 283.4 \quad 5 \quad 600.0 \quad 600.0$

$\begin{array}{lllll}2.0250 & 283.5 & 6 & 600.0 & 600.0\end{array}$

$\begin{array}{lllll}1.3750 & 283.6 & 6 & 600.0 & 600.0\end{array}$

$\begin{array}{lllll}1.0500 & 283.4 & 6 & 600.0 & 600.0\end{array}$

$\begin{array}{lllll}1.2750 & 283.2 & 5 & 600.0 & 600.0\end{array}$

$0.6000 \quad 282.4 \quad 6 \quad 600.0 \quad 600.0$ 


\begin{tabular}{|c|c|c|c|c|c|c|c|}
\hline & 1324 & & 750 & 7 & & & \\
\hline & 14 & 314 & .2000 & 31.6 & & 00.0 & \\
\hline & 2142 & 138.1868 & 1.0250 & 280.9 & 4 & 00 & \\
\hline & 143 & 86.4474 & 2500 & 280.4 & & 00 & \\
\hline & 144 & 62 & 7250 & 80.0 & & 0 & \\
\hline & 14 & 136 & 2.5000 & 277.9 & & 0 & \\
\hline & 146 & 7539 & 2.7750 & 277.7 & & 00 & \\
\hline & 147 & 141 . & 3.5250 & 278.0 & & 0 & \\
\hline & 148 & 146.5000 & 5.4000 & 78.1 & & & \\
\hline & 14 & 7506 & 4.9000 & 278.1 & 4 & 00 & 0 \\
\hline & 1410 & 164.2501 & 2.4750 & 279.7 & & 000 & \\
\hline & 1411 & 189.2062 & 1.2250 & 81.5 & & 0 & \\
\hline & 14 & 219.2474 & 1.6250 & 281.9 & 1 & 0 & \\
\hline & 1413 & 186.8093 & 2.6000 & 282.3 & & 0.0 & . \\
\hline & 1414 & 168 & 2.7250 & 5 & & & \\
\hline & 14 & 174 . & 1.3000 & 283.4 & 1 & & \\
\hline & 1416 & 184.5443 & 2.0250 & 283.2 & 1 & .0 & 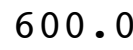 \\
\hline & 417 & 181 & 2.0000 & 282.8 & 1 & 0 & \\
\hline & 1418 & 179 & 1.0250 & 282.4 & 2 & & \\
\hline & 1419 & 149.9832 & 1.1250 & 281.8 & 3 & .0 & 0 \\
\hline & 1420 & 71.7518 & 3.6250 & 281.5 & 4 & 0 & \\
\hline & 1421 & 74.5000 & 5.2250 & & 4 & & \\
\hline & 422 & 72 . & 5.4250 & 280.7 & 4 & & 0 . \\
\hline & 21423 & 77.0009 & 5.2250 & 280.7 & 4 & 0 & J. \\
\hline & 21424 & 75 . & 4.9500 & & 5 & & \\
\hline & 2151 & 065 & 1.9750 & 28 & 6 & & \\
\hline & 2152 & 50.7425 & 0.9250 & 280.2 & 5 & 0 & U. \\
\hline & 2153 & 73.5023 & 1.2500 & 280.5 & 4 & & \\
\hline & 2154 & 63. & 0.0250 & 279.3 & 5 & & $\bullet$ \\
\hline & 2155 & 56.3450 & 0.0000 & 279.0 & 5 & 0 & 0 • \\
\hline & 2156 & 359.0000 & 0.0000 & 279.0 & 6 & 0 & 0 . \\
\hline & 2157 & 350.7351 & 0.0500 & 279.0 & 5 & & \\
\hline & 2158 & 320.2287 & 2500 & 279.4 & 6 & 0 & 0 • \\
\hline & 2159 & 340.0000 & 8250 & 281.2 & 5 & 0 & 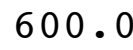 \\
\hline & 21510 & 309.9551 & 000 & 281.8 & 4 & & \\
\hline & 21511 & 273.0017 & 2.2000 & 282.0 & 3 & & . \\
\hline & 21512 & 270.2574 & 3.5000 & 281.4 & 2 & $\cdot 0$ & 0 . \\
\hline & 21513 & 256.7413 & 2.7750 & 281.9 & 1 & & 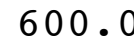 \\
\hline & 21514 & 244.1230 & 1.4500 & 282.7 & 1 & .0 & 0 . \\
\hline & 21515 & 275.9605 & 1.9000 & 283.5 & 2 & & 0 \\
\hline & 21516 & 281.0000 & 1.6500 & 283.6 & 2 & & 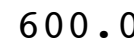 \\
\hline & 21517 & 284.7727 & 1.7750 & 283.5 & 2 & .0 & 0 . \\
\hline & 21518 & 298.9632 & 1.5250 & 283.2 & 3 & .0 & 00 . \\
\hline & 21519 & 347.0765 & 0.9750 & 282.6 & 4 & 0 & \\
\hline 01 & 21520 & 65.7578 & 3.0750 & 282.5 & 4 & 600.0 & 00 . \\
\hline & 2152 & 74.0006 & 4.8500 & 282.1 & 5 & 600.0 & 00 \\
\hline & & טבנס & 3. & & 4 & & \\
\hline & 1523 & 103.4727 & 0.5500 & 281.5 & 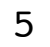 & 600.0 & 60 \\
\hline
\end{tabular}




\begin{tabular}{|c|c|c|c|c|c|c|c|}
\hline 1 & 1524 & 207 & 9500 & 81.9 & 5 & 600.0 & \\
\hline & 2161 & 0.2336 & 1.3750 & 281.6 & & 600.0 & \\
\hline & 162 & 132.6158 & 2500 & 280.8 & & 00 & \\
\hline & 163 & 328.2963 & .9250 & 280.0 & & 00.0 & \\
\hline & 164 & 14.5009 & .7500 & 279.9 & & 00 & \\
\hline & 165 & 3.7790 & .0250 & 279.4 & & 00 & \\
\hline & 166 & 42.7581 & 0.3250 & 279.4 & & 500 & \\
\hline & 167 & 21.1510 & .2250 & 279.2 & & .0 & \\
\hline & 168 & 17.7331 & .3250 & 79.8 & & & \\
\hline & 169 & 25.7130 & 0.1500 & 282.6 & & 00.0 & 0 \\
\hline & 1610 & 267.1387 & .6000 & 284.4 & & 00.0 & \\
\hline & 1611 & 280.5367 & 1.7500 & 83.5 & & & \\
\hline & 16 & 277.2476 & 2.6750 & 283.6 & & 0 & \\
\hline & 1613 & 274.2676 & 3.5750 & 283.7 & & 0.0 & 0 \\
\hline & 1614 & 263. & 500 & & & & \\
\hline & 16 & 267.2335 & & 286.2 & & & \\
\hline & 1616 & 330.9168 & 4.0750 & 287.5 & & 0.0 & 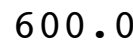 \\
\hline & 1617 & 74 . & 000 & 287.2 & & 0 & \\
\hline & 1618 & 148.7675 & 2.5000 & 285.4 & & & \\
\hline & 1619 & 83.4871 & 4.6250 & 284.2 & & .0 & 0 \\
\hline & 21620 & 75.7645 & 7750 & 283.8 & & 0 & U. \\
\hline & 1621 & 92.3 & .4500 & & & & \\
\hline & 622 & 347 . & 1.4500 & 283.5 & & & 0 . \\
\hline & 21623 & 318.44 & 1.1250 & 283.9 & & 0 & . \\
\hline & 21624 & 306.73 & & & & & \\
\hline & 2171 & 317 . & 3.5750 & 28 & 5 & & \\
\hline & 2172 & 330.7539 & .0000 & 284 & 4 & 0 & U. \\
\hline & 2173 & 326.2 & .3250 & 284 & 4 & & \\
\hline & 2174 & 338.9 & 500 & 284 & 4 & & $\bullet$ \\
\hline & 2175 & 338.0000 & 5500 & 284.5 & 4 & 0 & 0 • \\
\hline & 2176 & 331.2455 & 3.1750 & 284.5 & 4 & 0 & 0 . \\
\hline & 2177 & 321.24 & 250 & 28 & 4 & & \\
\hline & 2178 & 318 . & 000 & 284.2 & 4 & 0 & 0 • \\
\hline & 2179 & 326.746 & 750 & 284.1 & 4 & 0 & 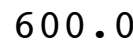 \\
\hline & 21710 & 325.25 & 0 & & 4 & & \\
\hline & 21711 & 315.7509 & 3.2500 & 285.4 & 3 & & 0 . \\
\hline & 21712 & 274.7230 & 1.6250 & 285.7 & 2 & $\cdot 0$ & 0 . \\
\hline & 21713 & 316.2219 & 4.6000 & 287.5 & 3 & & . \\
\hline & 21714 & 349.7504 & 7.0500 & 287.8 & 4 & .0 & 0 . \\
\hline & 21715 & 346.9993 & 6.4000 & 287.9 & 4 & & 0 \\
\hline & 21716 & 228.6720 & 1.5250 & 286.2 & 3 & & 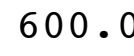 \\
\hline & 21717 & 169.5214 & 2.9250 & 285.8 & 2 & .0 & 0 . \\
\hline & 21718 & 160.8292 & 3.9250 & 285.0 & 3 & 0 & 00 . \\
\hline & 21719 & 169.5014 & 5.6250 & 282.1 & 4 & & 0 \\
\hline 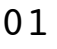 & 21720 & 149.6759 & 2.6250 & 281.8 & 5 & & 00 . \\
\hline & 21721 & 124.0348 & 1.1750 & 282.4 & 6 & 600.0 & 00. \\
\hline & 21722 & 154 . & & & 0 & & \\
\hline & 21723 & 49.6060 & 1.8000 & 281.9 & & 60 & 600 \\
\hline
\end{tabular}




\begin{tabular}{|c|c|c|c|c|c|c|c|}
\hline & 1724 & 000 & 4000 & 83.8 & 5 & 00.0 & \\
\hline & 2181 & 34.3374 & 2.2500 & 284.5 & & 600.0 & \\
\hline & 2182 & 335.0000 & 2.7750 & 284.3 & & 00 & \\
\hline & 183 & 323.0333 & L. 7500 & 283.1 & & 00 & \\
\hline & 184 & 213.5254 & .1750 & 82.3 & & 0 & \\
\hline & 185 & 140.1904 & 1.5500 & 281.3 & & 00 & \\
\hline & 186 & 152.6874 & 1.5000 & 281.1 & & 500 & U \\
\hline & 187 & 48.8222 & L. 3000 & 81.6 & & .0 & \\
\hline & 188 & 193.6850 & .6750 & 81.4 & & & \\
\hline & 189 & 191.7076 & 1.4250 & 281.0 & & 00 & 0 \\
\hline & 1810 & 302.4556 & 0.9750 & 281.1 & & 00.0 & \\
\hline & 1811 & 312.0000 & 1.3250 & 82.0 & & & \\
\hline & 1812 & 230 & 2.0000 & 282.1 & & 0 & \\
\hline & 1813 & 356.1027 & 1.3500 & 283.7 & & 0.0 & 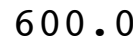 \\
\hline & 1814 & 264 & 2.22 & .1 & & & \\
\hline & 1815 & 228 & 3.32 & 285.4 & & & \\
\hline & 1816 & 153.4438 & 1.5250 & 285.7 & & .0 & 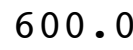 \\
\hline & 817 & 170 . & 3.4250 & .5 & & 0 & \\
\hline & 1818 & 154.0732 & 1.8750 & 283.7 & & & \\
\hline & 1819 & 70.5000 & 1.1500 & 283.6 & 4 & 0 & $\checkmark$ \\
\hline & 820 & 347.6624 & 1.1250 & 283.4 & & 0 & \\
\hline & 821 & 314.9749 & 1.2500 & 283.3 & 6 & & \\
\hline & 1822 & 330.6103 & 1.8750 & 282.6 & 6 & & \\
\hline & 21823 & 332.5000 & 1.3750 & 282.6 & 6 & 0 & . \\
\hline & 21824 & 314.7424 & 500 & 282.7 & 5 & & \\
\hline & 2191 & 328.5008 & 500 & 283.3 & 4 & & \\
\hline & 2192 & 317.7490 & 4750 & 283.3 & 4 & 0 & U. \\
\hline & 2193 & 295.7416 & 4.7000 & 0 & 4 & & \\
\hline & 2194 & 326.7502 & 9.10 & 28 & 4 & & • \\
\hline & 2195 & 330.4973 & 7.4000 & 283.3 & 4 & 0 & 0 • \\
\hline & 2196 & 349.0019 & 9750 & 281.8 & 4 & & 0 . \\
\hline & 2197 & 344.7403 & 0 & 279.8 & 4 & & \\
\hline & 2198 & 345.4280 & 1.6750 & 280.6 & 5 & 0 & 0 • \\
\hline & 2199 & 12.9474 & 6000 & 281.4 & 4 & 0 & 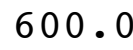 \\
\hline & 21910 & 331.8182 & 2.65 & & 3 & & \\
\hline & 21911 & 311.9409 & 2.9250 & 283.5 & 2 & & 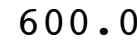 \\
\hline & 21912 & 318.4012 & 3.2000 & 283.2 & 2 & & 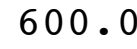 \\
\hline & 21913 & 347.3672 & 4.1750 & 284.6 & 3 & & . \\
\hline & 21914 & 325.3018 & 6.5750 & 285.5 & 4 & .0 & 0 . \\
\hline & 21915 & 340.5000 & 4500 & 285.8 & 3 & & 0 . \\
\hline & 21916 & 329.6494 & 5.8000 & 285.1 & 3 & & 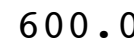 \\
\hline & 21917 & 330.7894 & 5.6250 & 283.7 & 3 & .0 & 0 . \\
\hline & 21918 & 318.1818 & 3.1000 & 282.4 & 4 & 0 & 00 . \\
\hline & 21919 & 345.6683 & 3.9500 & & 4 & & \\
\hline & 21920 & 341.5657 & 1.4000 & 281.5 & 5 & & 600. \\
\hline & 21921 & 259.5448 & 2.0500 & 281.4 & 6 & 600.0 & 00. \\
\hline & & 332 & & & o & & \\
\hline & 1923 & 202.7434 & 3.9000 & 281.3 & & 60 & 60 \\
\hline
\end{tabular}


$\begin{array}{lll}01 & 21924 & 221.3699\end{array}$

$\begin{array}{llll}01 & 220 & 1 & 231.2701\end{array}$

$\begin{array}{llll}01 & 220 & 2 & 224.0914\end{array}$

$\begin{array}{llll}01 & 220 & 3 & 234.4419\end{array}$

$\begin{array}{llll}01 & 220 & 4 & 211.3431\end{array}$

$\begin{array}{llll}01 & 220 & 5 & 237.5796\end{array}$

$\begin{array}{llll}01 & 220 & 6 & 245.6920\end{array}$

$\begin{array}{llll}01 & 220 & 7 & 237.3457\end{array}$

$\begin{array}{llll}01 & 220 & 8 & 202.4940\end{array}$

$\begin{array}{llll}01 & 220 & 9 & 201.7930\end{array}$

$\begin{array}{lll}01 & 22010 & 219.9126\end{array}$

$0122011 \quad 292.4695$

$\begin{array}{lll}01 & 22012 & 0.6284\end{array}$

$\begin{array}{lll}01 & 22013 & 27.5147\end{array}$

$\begin{array}{lll}01 & 22014 & 16.7655\end{array}$

$\begin{array}{lll}01 & 22015 & 350.8343\end{array}$

$\begin{array}{llll}01 & 22016 & 282.5631\end{array}$

$\begin{array}{lll}01 & 22017 & 233.6611\end{array}$

$\begin{array}{lll}01 & 22018 & 15.2562\end{array}$

$\begin{array}{lll}01 & 22019 & 357.0852\end{array}$

$\begin{array}{lll}01 & 22020 & 209.4227\end{array}$

0122021300.2066

$\begin{array}{lll}01 & 22022 & 10.8015\end{array}$

$\begin{array}{lll}01 & 22023 & 251.9296\end{array}$

$0122024 \quad 258.6610$

$\begin{array}{llll}01 & 221 & 1 & 279.7198\end{array}$

$\begin{array}{llll}01 & 221 & 2 & 251.3934\end{array}$

$\begin{array}{llll}01 & 221 & 3 & 257.6504\end{array}$

$\begin{array}{llll}01 & 221 & 4 & 275.0000\end{array}$

$\begin{array}{llll}01 & 221 & 5 & 273.4889\end{array}$

$\begin{array}{llll}01 & 221 & 6 & 187.5171\end{array}$

$\begin{array}{llll}01 & 221 & 7 & 271.5032\end{array}$

$\begin{array}{llll}01 & 221 & 8 & 265.0008\end{array}$

$\begin{array}{llll}01 & 221 & 9 & 284.9890\end{array}$

$01 \quad 22110 \quad 304.1550$

$\begin{array}{llll}01 & 22111 & 243.2488\end{array}$

$\begin{array}{lll}01 & 22112 & 241.7255\end{array}$

$\begin{array}{lll}01 & 22113 & 264.9483\end{array}$

$\begin{array}{lll}01 & 22114 & 352.2636\end{array}$

$\begin{array}{lll}01 & 22115 & 329.3128\end{array}$

$0122116 \quad 319.4760$

$\begin{array}{lll}01 & 22117 & 28.2499\end{array}$

$\begin{array}{lll}01 & 22118 & 200.1370\end{array}$

$\begin{array}{lll}01 & 22119 & 112.9290\end{array}$

$\begin{array}{lll}01 & 22120 & 149.4182\end{array}$

$\begin{array}{lll}01 & 22121 & 17.4989\end{array}$

$\begin{array}{lll}01 & 22122 & 21.0007\end{array}$

$\begin{array}{lll}01 & 22123 & 246.5308\end{array}$
$3.3500 \quad 281.0 \quad 5$

$3.1000280 .7 \quad 5$

$2.9750280 .7 \quad 5$

2.9750281 .05

2.9000281 .35

1.9250281 .26

$3.0250281 .7 \quad 5$

1.8500281 .36

2.8250281 .26

3.0250281 .65

$2.4000 \quad 283.0 \quad 4$

$2.3000284 .2 \quad 3$

$5.7000 \quad 284.4 \quad 3$

$5.3500 \quad 284.4 \quad 3$

$4.8500283 .6 \quad 3$

$2.8500 \quad 283.22$

$2.1500 \quad 284.01$

$\begin{array}{ll}1.6250 & 284.31\end{array}$

$3.6750 \quad 283.7 \quad 2$

$3.0000 \quad 282.8 \quad 3$

$3.1500 \quad 282.4 \quad 4$

$3.5500 \quad 282.4 \quad 5$

$3.3000 \quad 282.7 \quad 5$

$1.8500 \quad 282.86$

$3.8750 \quad 283.1 \quad 5$

2.8500282 .66

2.4250282 .36

$4.6250 \quad 282.4 \quad 5$

$4.0500282 .8 \quad 4$

$2.4750 \quad 282.8 \quad 5$

$1.9250 \quad 282.76$

$2.9750 \quad 282.5 \quad 5$

$4.9500 \quad 282.04$

$\begin{array}{llll}7.4500 & 282.7 \quad 4\end{array}$

$6.5500 \quad 282.8 \quad 4$

$5.1500 \quad 283.2 \quad 3$

$4.7750 \quad 283.9 \quad 3$

6.0000284 .84

$6.6250286 .8 \quad 4$

$5.3750 \quad 286.8 \quad 3$

$5.2500286 .6 \quad 3$

$4.7750 \quad 286.13$

$\begin{array}{llll}1.4250 & 285.1 \quad 4\end{array}$

1.3500283 .95

1.4250283 .26

$1.3500 \quad 283.56$

$4.7500 \quad 283.3 \quad 5$

4.2500283 .34
600.0600 .0

600.0600 .0

600.0600 .0

600.0600 .0

600.0600 .0

600.0600 .0

600.0600 .0

600.0600 .0

600.0600 .0

$600.0 \quad 600.0$

600.0600 .0

600.0600 .0

$600.0 \quad 600.0$

$600.0 \quad 600.0$

$600.0 \quad 600.0$

$600.0 \quad 600.0$

$600.0 \quad 600.0$

600.0600 .0

600.0600 .0

$600.0 \quad 600.0$

$600.0 \quad 600.0$

600.0600 .0

$600.0 \quad 600.0$

600.0600 .0

600.0600 .0

600.0600 .0

600.0600 .0

600.0600 .0

$600.0 \quad 600.0$

600.0600 .0

600.0600 .0

600.0600 .0

$600.0 \quad 600.0$

600.0600 .0

$600.0 \quad 600.0$

$600.0 \quad 600.0$

$600.0 \quad 600.0$

$600.0 \quad 600.0$

$600.0 \quad 600.0$

$600.0 \quad 600.0$

$600.0 \quad 600.0$

600.0600 .0

$600.0 \quad 600.0$

$600.0 \quad 600.0$

600.0600 .0

600.0600 .0

600.0600 .0

600.0600 .0 
$\begin{array}{lll}01 & 22124 & 257.9619\end{array}$

$\begin{array}{llll}01 & 222 & 1 & 280.5000\end{array}$

$\begin{array}{llll}01 & 222 & 2 & 344.5722\end{array}$

$\begin{array}{llll}01 & 222 & 3 & 333.7962\end{array}$

$\begin{array}{llll}01 & 222 & 4 & 274.7552\end{array}$

$\begin{array}{llll}01 & 222 & 5 & 231.2799\end{array}$

$\begin{array}{llll}01 & 222 & 6 & 246.4127\end{array}$

$\begin{array}{llll}01 & 222 & 7 & 194.3629\end{array}$

$\begin{array}{llll}01 & 222 & 8 & 37.2467\end{array}$

$\begin{array}{llll}01 & 222 & 9 & 59.2496\end{array}$

$\begin{array}{llll}01 & 22210 & 64.4747\end{array}$

$\begin{array}{lll}01 & 22211 & 66.3484\end{array}$

$\begin{array}{lll}01 & 22212 & 46.2535\end{array}$

$\begin{array}{lll}01 & 22213 & 58.2378\end{array}$

$\begin{array}{lll}01 & 22214 & 51.7506\end{array}$

$\begin{array}{lll}01 & 22215 & 54.7500\end{array}$

$\begin{array}{lll}01 & 22216 & 70.5000\end{array}$

$\begin{array}{lll}01 & 22217 & 57.0027\end{array}$

$\begin{array}{lll}01 & 22218 & 52.0017\end{array}$

$\begin{array}{lll}01 & 22219 & 8.4971\end{array}$

$\begin{array}{lll}01 & 22220 & 357.5000\end{array}$

$\begin{array}{llll}01 & 22221 & 340.9814\end{array}$

$\begin{array}{lll}01 & 22222 & 332.2494\end{array}$

$\begin{array}{lll}01 & 22223 & 339.9221\end{array}$

$\begin{array}{llll}01 & 22224 & 289.1455\end{array}$

$\begin{array}{llll}01 & 223 & 1 & 282.8007\end{array}$

$\begin{array}{llll}01 & 223 & 2 & 296.9320\end{array}$

$\begin{array}{llll}01 & 223 & 3 & 329.5000\end{array}$

$\begin{array}{llll}01 & 223 & 4 & 295.7020\end{array}$

$\begin{array}{llll}01 & 223 & 5 & 327.9478\end{array}$

$\begin{array}{llll}01 & 223 & 6 & 81.3856\end{array}$

$\begin{array}{llll}01 & 223 & 7 & 167.2398\end{array}$

$\begin{array}{llll}01 & 223 & 8 & 197.7197\end{array}$

$\begin{array}{llll}01 & 223 & 9 & 194.9969\end{array}$

$\begin{array}{lll}01 & 22310 & 175.8093\end{array}$

$0122311 \quad 154.4845$

$01 \quad 22312 \quad 155.7503$

$\begin{array}{lll}01 & 22313 & 140.5037\end{array}$

$0122314 \quad 155.0000$

$\begin{array}{lll}01 & 22315 & 167.0041\end{array}$

$0122316 \quad 164.7499$

$\begin{array}{lll}01 & 22317 & 105.2455\end{array}$

$\begin{array}{lll}01 & 22318 & 82.5166\end{array}$

$\begin{array}{lll}01 & 22319 & 61.7497\end{array}$

$01 \quad 22320 \quad 68.7500$

$\begin{array}{lll}01 & 22321 & 68.0000\end{array}$

$\begin{array}{lll}01 & 22322 & 57.7653\end{array}$

$0122323 \quad 41.2504$ $\begin{array}{lllll}3.0500 & 283.0 & 5 & 600.0 & 600.0\end{array}$

$3.1500 \quad 282.7 \quad 5 \quad 600.0 \quad 600.0$

$4.4500 \quad 282.3 \quad 4 \quad 600.0 \quad 600.0$

$5.2500281 .9 \quad 4 \quad 600.0 \quad 600.0$

$\begin{array}{llllll}4.8750 & 281.5 & 4 & 600.0 & 600.0\end{array}$

$5.7500 \quad 280.8 \quad 4 \quad 600.0 \quad 600.0$

$3.9250 \quad 281.2 \quad 4 \quad 600.0 \quad 600.0$

$2.3750 \quad 281.0 \quad 5 \quad 600.0 \quad 600.0$

$4.1500281 .4 \quad 4 \quad 600.0 \quad 600.0$

$6.7750 \quad 278.9 \quad 4 \quad 600.0 \quad 600.0$

$\begin{array}{lllll}5.0250 & 278.4 & 3 & 600.0 & 600.0\end{array}$

$3.7000 \quad 280.4 \quad 2 \quad 600.0 \quad 600.0$

$6.5000 \quad 280.7 \quad 3 \quad 600.0 \quad 600.0$

$6.7000 \quad 281.0 \quad 4 \quad 600.0 \quad 600.0$

$7.6250 \quad 281.6 \quad 4 \quad 600.0 \quad 600.0$

$6.3750 \quad 281.944600 .0 \quad 600.0$

$\begin{array}{llllll}7.0750 & 281.3 & 4 & 600.0 & 600.0\end{array}$

$6.5000280 .6 \quad 4 \quad 600.0 \quad 600.0$

$3.1000 \quad 281.144600 .0 \quad 600.0$

$3.4250 \quad 280.6 \quad 4 \quad 600.0 \quad 600.0$

$\begin{array}{llllll}4.6500 & 280.4 & 4 & 600.0 & 600.0\end{array}$

$5.9500 \quad 279.2 \quad 4 \quad 600.0 \quad 600.0$

$5.1000 \quad 278.0 \quad 4 \quad 600.0 \quad 600.0$

$3.9750 \quad 277.0 \quad 4 \quad 600.0 \quad 600.0$

$3.5500 \quad 276.7 \quad 5 \quad 600.0 \quad 600.0$

$\begin{array}{llllll}4.1750 & 277.0 & 4 & 600.0 & 600.0\end{array}$

$4.9750 \quad 277.4 \quad 4 \quad 600.0 \quad 600.0$

$\begin{array}{llllll}4.8500 & 276.9 & 4 & 600.0 & 600.0\end{array}$

$2.3250 \quad 276.6 \quad 5 \quad 600.0 \quad 600.0$

$1.9750 \quad 276.5 \quad 6 \quad 600.0 \quad 600.0$

$2.6750 \quad 276.6 \quad 6 \quad 600.0 \quad 600.0$

$2.7750 \quad 276.6 \quad 5 \quad 600.0 \quad 600.0$

$3.0250 \quad 276.6 \quad 4 \quad 600.0 \quad 600.0$

$\begin{array}{llllll}5.6250 & 276.4 & 4 & 600.0 & 600.0\end{array}$

$5.5000 \quad 276.3 \quad 3 \quad 600.0 \quad 600.0$

$\begin{array}{llllll}4.4250 & 276.5 & 3 & 600.0 & 600.0\end{array}$

$\begin{array}{lllll}4.3250 & 277.4 & 3 & 600.0 & 600.0\end{array}$

$\begin{array}{lllll}4.2250 & 279.1 & 3 & 600.0 & 600.0\end{array}$

$\begin{array}{llllll}4.9250 & 279.8 & 3 & 600.0 & 600.0\end{array}$

$\begin{array}{llllll}4.1250 & 280.7 & 3 & 600.0 & 600.0\end{array}$

$4.4500 \quad 281.3 \quad 3 \quad 600.0 \quad 600.0$

$\begin{array}{llllll}3.9250 & 281.7 & 2 & 600.0 & 600.0\end{array}$

$3.2250 \quad 281.0 \quad 3 \quad 600.0 \quad 600.0$

$5.1000 \quad 280.144600 .0 \quad 600.0$

$6.5750 \quad 279.9 \quad 4 \quad 600.0 \quad 600.0$

$\begin{array}{lllll}7.8250 & 279.5 & 4 & 600.0 & 600.0\end{array}$

$6.1000 \quad 279.8 \quad 4 \quad 600.0 \quad 600.0$

$4.5000 \quad 280.144600 .0 \quad 600.0$ 


\begin{tabular}{|c|c|c|c|c|c|c|c|}
\hline & 24 & 32.4765 & 5750 & 79.8 & & 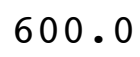 & \\
\hline & 241 & 8.2383 & 0.4500 & 279.6 & & 00.0 & \\
\hline & 2242 & 1.8335 & 9000 & 279.5 & & 00 & \\
\hline & 243 & 2481 & 3.7750 & 279.6 & & 00 & \\
\hline & $24 \quad 4$ & 335.6249 & .2500 & 278.7 & & & \\
\hline & 2245 & 328 . & & 277.6 & & 0 & \\
\hline & 246 & 323.7677 & 750 & 277.1 & & 0 & \\
\hline & 2247 & 314.5021 & 6.3000 & 276.5 & & & \\
\hline & 2248 & 318.2494 & 0 & 8 & & & \\
\hline & 249 & 311.2487 & 500 & 277.0 & & 0 & \\
\hline & 2410 & 301.0018 & 5000 & 277.0 & & & \\
\hline & 2411 & 9902 & 0 & 7.6 & & & \\
\hline & 2412 & 300.7485 & 8 . & 279.0 & & & \\
\hline & 2413 & 311.7483 & 9750 & 1 & & 0 & \\
\hline & 22414 & 663 & & & & & \\
\hline & 224 & 252 . & & & & & \\
\hline & 22416 & 217.7186 & 2.0500 & 281.1 & & 0 & \\
\hline & 22417 & 283.4443 & 1 . & 1.3 & & & \\
\hline & 2418 & 103.7263 & & & & & \\
\hline & 22419 & 358.0216 & 1.4000 & .1 & & & 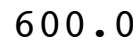 \\
\hline & 22420 & 356.5054 & 2 . & .8 & & 0 & \\
\hline & 22421 & 332.5411 & & & & & \\
\hline & 22422 & 316.7458 & 0 & & 4 & & \\
\hline & 22423 & 340.5064 & 00 & .8 & 4 & 0 & \\
\hline & 22424 & 352.2494 & & & $\mathbf{4}$ & & \\
\hline & 2251 & 15.0824 & 0 & & 4 & & \\
\hline & 2252 & 332.3912 & 0 & 9.6 & & & . \\
\hline & 2253 & 311.4306 & 0 & .6 & 5 & & \\
\hline & 2254 & 356 . & 0 & & 6 & & \\
\hline & 2255 & 3.4624 & 000 & 0 & & & J. \\
\hline & 2256 & 301.8315 & 9750 & .1 & 6 & & 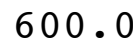 \\
\hline & 2257 & 227.5891 & 0 & & 6 & & \\
\hline & 2258 & 312.7597 & & .9 & 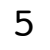 & & 0 . \\
\hline & 2259 & 229.1336 & 000 & 1.1 & 4 & & 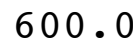 \\
\hline & 22510 & 240.1548 & 0 & & 3 & & \\
\hline & 22511 & 251.7546 & 1.6000 & .3 & 2 & & . \\
\hline & 22512 & 236.0572 & 750 & 4.6 & 1 & & \\
\hline & 22513 & 215 . & 0 & 3 & 1 & & \\
\hline & 22514 & 199.9622 & 000 & 4.4 & 2 & & 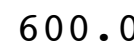 \\
\hline & 22515 & 214.3131 & 500 & 5.0 & $\perp$ & & \\
\hline & 22516 & 116.4880 & 500 & 4.0 & 2 & & \\
\hline & 22517 & 157.2497 & 4.4750 & 283.8 & 3 & & 0 . \\
\hline & 22518 & 164.2500 & 3.5250 & 283.7 & 4 & 0 & 0 . \\
\hline & 22519 & 167.2498 & 50 & 283.2 & 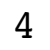 & & \\
\hline & 22520 & 171.4998 & $\$ 750$ & 283.0 & 4 & & \\
\hline & 22521 & 170.0000 & 7.7750 & 281.9 & 4 & & 00 \\
\hline & 22 & 176 & & 1.3 & 4 & & \\
\hline & 22523 & 178.2501 & 9.6500 & 280.9 & & .0 & 60 \\
\hline
\end{tabular}


$0122524 \quad 178.0002$

$\begin{array}{llll}01 & 226 & 1 & 174.5023\end{array}$

$01226 \quad 2 \quad 158.0007$

$\begin{array}{llll}01 & 226 & 3 & 156.9995\end{array}$

$\begin{array}{llll}01 & 226 & 4 & 142.7425\end{array}$

$\begin{array}{llll}01 & 226 & 5 & 146.9862\end{array}$

$\begin{array}{llll}01 & 226 & 6 & 156.2529\end{array}$

$\begin{array}{llll}01 & 226 & 7 & 146.2517\end{array}$

$01226 \quad 8 \quad 144.1764$

$01 \quad 226 \quad 9 \quad 164.5065$

$0122610 \quad 179.0000$

$0122611 \quad 178.2500$

$0122612 \quad 172.7498$

$0122613 \quad 173.5017$

$01 \quad 22614 \quad 174.4997$

0122615168.0339

$0122616 \quad 177.2501$

$\begin{array}{llll}01 & 22617 & 177.7501\end{array}$

$0122618 \quad 158.9281$

$0122619 \quad 92.2573$

$0122620 \quad 80.9986$

$0122621 \quad 98.0000$

$0122622 \quad 145.9848$

$0122623 \quad 126.6428$

$\begin{array}{lll}01 & 22624 & 76.4997\end{array}$

$\begin{array}{llll}01 & 227 & 1 & 75.2493\end{array}$

$\begin{array}{llll}01 & 227 & 2 & 101.9704\end{array}$

$\begin{array}{llll}01 & 227 & 3 & 118.4724\end{array}$

$\begin{array}{llll}01 & 227 & 4 & 121.3512\end{array}$

$\begin{array}{llll}01 & 227 & 5 & 106.9410\end{array}$

$\begin{array}{llll}01 & 227 & 6 & 109.9945\end{array}$

$\begin{array}{llll}01 & 227 & 7 & 143.5081\end{array}$

$\begin{array}{llll}01 & 227 & 8 & 163.0000\end{array}$

$01227 \quad 9 \quad 141.2500$

$0122710 \quad 146.5000$

$0122711 \quad 152.7498$

$\begin{array}{lll}01 & 22712 & 152.4999\end{array}$

$0122713 \quad 147.9981$

$0122714 \quad 158.0032$

$0122715 \quad 163.0002$

$0122716 \quad 164.0000$

$\begin{array}{lll}01 & 22717 & 153.7476\end{array}$

$01 \quad 22718 \quad 144.7502$

$0122719 \quad 141.2513$

$\begin{array}{lll}01 & 22720 & 148.7496\end{array}$

$\begin{array}{lll}01 & 22721 & 155.9999\end{array}$

$0122722 \quad 163.5009$

$\begin{array}{lll}01 & 22723 & 149.9998\end{array}$
$10.4750 \quad 281.144600 .0 \quad 600.0$

$9.8250 \quad 281.6 \quad 4 \quad 600.0 \quad 600.0$

$9.9000 \quad 282.0 \quad 4 \quad 600.0 \quad 600.0$

$9.6000 \quad 282.1 \quad 4 \quad 600.0 \quad 600.0$

$\begin{array}{lllll}7.0500 & 282.1 & 4 & 600.0 & 600.0\end{array}$

$\begin{array}{llllll}6.2000 & 282.3 & 4 & 600.0 & 600.0\end{array}$

$\begin{array}{lllll}5.8500 & 282.5 & 4 & 600.0 & 600.0\end{array}$

$\begin{array}{llllll}5.5250 & 282.5 \quad 4 & 600.0 & 600.0\end{array}$

$5.1000 \quad 282.6 \quad 4 \quad 600.0 \quad 600.0$

$\begin{array}{lllll}4.8500 & 283.4 & 3 & 600.0 & 600.0\end{array}$

$\begin{array}{lllll}5.9500 & 283.6 & 3 & 600.0 & 600.0\end{array}$

$\begin{array}{lllll}5.2000 & 283.6 & 3 & 600.0 & 600.0\end{array}$

$5.8500 \quad 284.4 \quad 3 \quad 600.0 \quad 600.0$

$\begin{array}{lllll}7.0750 & 285.3 & 4 & 600.0 & 600.0\end{array}$

$\begin{array}{llllll}6.1000 & 285.7 & 4 & 600.0 & 600.0\end{array}$

$\begin{array}{lllll}5.5250 & 286.5 & 3 & 600.0 & 600.0\end{array}$

$\begin{array}{lllll}5.5250 & 286.0 & 3 & 600.0 & 600.0\end{array}$

$\begin{array}{lllll}5.2250 & 286.6 & 3 & 600.0 & 600.0\end{array}$

$\begin{array}{lllll}4.3000 & 285.8 \quad 4 & 600.0 & 600.0\end{array}$

$3.4250 \quad 285.3 \quad 4 \quad 600.0 \quad 600.0$

$\begin{array}{lllll}4.2500 & 285.5 & 4 & 600.0 & 600.0\end{array}$

$\begin{array}{lllll}3.6250 & 285.8 & 4 & 600.0 & 600.0\end{array}$

$3.2000 \quad 285.6 \quad 5 \quad 600.0 \quad 600.0$

$\begin{array}{lllll}3.9500 & 285.3 & 4 & 600.0 & 600.0\end{array}$

$\begin{array}{lllll}6.9500 & 284.1 & 4 & 600.0 & 600.0\end{array}$

$6.6750 \quad 284.2 \quad 4 \quad 600.0 \quad 600.0$

$\begin{array}{lllll}5.0750 & 283.9 & 4 & 600.0 & 600.0\end{array}$

$\begin{array}{llllll}5.1250 & 283.5 & 4 & 600.0 & 600.0\end{array}$

$\begin{array}{lllll}5.2500 & 282.9 & 4 & 600.0 & 600.0\end{array}$

$\begin{array}{lllll}5.3250 & 282.5 & 4 & 600.0 & 600.0\end{array}$

$5.2000 \quad 282.0 \quad 4 \quad 600.0 \quad 600.0$

$\begin{array}{lllll}6.9750 & 280.6 & 4 & 600.0 & 600.0\end{array}$

$10.0000 \quad 280.144600 .0 \quad 600.0$

$\begin{array}{lllll}9.5000 & 280.5 \quad 4 & 600.0 & 600.0\end{array}$

$\begin{array}{llllll}9.2750 & 281.5 & 4 & 600.0 & 600.0\end{array}$

$10.3750 \quad 283.0 \quad 4 \quad 600.0 \quad 600.0$

$10.1000 \quad 284.4 \quad 4 \quad 600.0 \quad 600.0$

$8.3250 \quad 285.6 \quad 4 \quad 600.0 \quad 600.0$

$9.6000 \quad 286.9 \quad 4 \quad 600.0 \quad 600.0$

$8.8000 \quad 288.0 \quad 4 \quad 600.0 \quad 600.0$

$10.0250 \quad 288.7 \quad 4 \quad 600.0 \quad 600.0$

$8.3750 \quad 289.0 \quad 4 \quad 600.0 \quad 600.0$

$\begin{array}{lllll}8.4250 & 288.4 \quad 4 & 600.0 & 600.0\end{array}$

$9.4500 \quad 287.4 \quad 4 \quad 600.0 \quad 600.0$

$10.7500 \quad 286.7 \quad 4 \quad 600.0 \quad 600.0$

$9.0750 \quad 285.4 \quad 4 \quad 600.0 \quad 600.0$

$\begin{array}{lllll}7.1750 & 284.3 & 4 & 600.0 & 600.0\end{array}$

$5.7750 \quad 283.4 \quad 4 \quad 600.0 \quad 600.0$ 


\begin{tabular}{|c|c|c|c|c|c|c|c|c|}
\hline \multicolumn{3}{|c|}{22724} & & & & & & \\
\hline 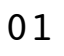 & 22 & 1 & 88 & 7.6750 & 2.5 & & 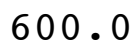 & \\
\hline & $22 \xi$ & 82 & 54.2502 & 7000 & 81.7 & & 00 & \\
\hline & 22 & 83 & 50.5000 & 7.2250 & 81.3 & & 00 & \\
\hline & $22 \varepsilon$ & 84 & 500 & 5000 & 81.2 & & & \\
\hline & $22\}$ & 85 & & 4500 & 30.8 & & & \\
\hline & $22 \xi$ & 86 & 147 . & 000 & 79.7 & & 0 & \\
\hline & 22 & 87 & 003 & 9500 & & & & \\
\hline & $22 \varepsilon$ & 8 & 499 & 2750 & 9.8 & & & \\
\hline & 22 & 8 & 505 & 8.4250 & 80.5 & & 0 & \\
\hline & 2 & 810 & 562 & 8.6500 & 81.2 & & & \\
\hline & $22 \xi$ & 8 & 92 & 500 & 1.7 & & & \\
\hline & 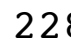 & 8 & 997 & 0 & 82.6 & & & \\
\hline & $22 \varepsilon$ & 813 & 966 & 10.7000 & 83.2 & & & \\
\hline & $22 \xi$ & 8 & & & & & & \\
\hline & ? & 815 & & & 4.5 & & & \\
\hline & & 8 & 503 & 7.5750 & 84.8 & & 0 & \\
\hline & 2 & 817 & 51 & 4 . & & & ) & \\
\hline & 8 & 818 & 23 & & 2.1 & & & \\
\hline & & 819 & 124.7083 & 1.6500 & 81.6 & & 0 & \\
\hline & 22 & 820 & 590 & .7000 & .6 & & & \\
\hline & 22 & 8 & 59 & & 0.4 & & & \\
\hline & & 822 & 30 & 500 & .1 & & & \\
\hline & 22 & 823 & 000 & 750 & 280.1 & & 0 & \\
\hline & 22 & 824 & 357 & & 80.8 & & & \\
\hline & 3 & 1 & 31 & 50 & 281.6 & 5 & & \\
\hline & 3 & 12 & 295 & 3750 & 282.0 & 6 & 0 & \\
\hline & 3 & 13 & 20 & 2.5500 & 282.0 & 6 & & \\
\hline & 3 & 14 & 35 & 0 & 282.5 & 6 & & \\
\hline & 3 & 15 & 169. & 2.8000 & 281.3 & 6 & & \\
\hline & 3 & 16 & 110.3610 & 3.3000 & 278.2 & 5 & & \\
\hline & 3 & 17 & 12 & 0 & .1 & 6 & & \\
\hline & 3 & 18 & 39 & 750 & 280.2 & 5 & 0 & \\
\hline & 3 & 19 & 11 & 4000 & 282.6 & 4 & & \\
\hline & 3 & 110 & & .4250 & & 3 & & \\
\hline & 3 & 111 & & 750 & 3.8 & 2 & & \\
\hline & 3 & 112 & 167 & 1750 & 284.8 & 2 & & \\
\hline & 3 & 113 & 17 & 3 . & 285.0 & 2 & & \\
\hline & 3 & 114 & 197 . & 3.0500 & 285.8 & 2 & 0 & \\
\hline & 3 & 115 & & 1.7000 & 286.6 & 1 & & \\
\hline & 3 & 116 & 75 & 1.1250 & 286.6 & 1 & 0 & \\
\hline & 3 & 117 & 654 & 3.1000 & 285.4 & 2 & 600.0 & \\
\hline & 3 & 118 & 334 & 4.2250 & 284.3 & 3 & 0 & \\
\hline & 3 & 119 & & 4.9750 & & 4 & 600.0 & \\
\hline & 3 & 120 & & 6.5250 & 282.0 & 4 & 600.0 & \\
\hline & 3 & 121 & 989 & 7.6750 & 281.4 & 4 & 600.0 & \\
\hline & & 12 & & & & 4 & & \\
\hline & & 123 & 5000 & 6.2500 & 281.3 & 4 & 600.0 & 60 \\
\hline
\end{tabular}




\begin{tabular}{|c|c|c|c|c|c|c|c|c|}
\hline 1 & 3 & 124 & 57.2460 & 4.5250 & 281.8 & 4 & 600.0 & 600.0 \\
\hline 01 & 3 & 21 & 3.7735 & 2.9500 & 280.7 & 5 & 600.0 & 600.0 \\
\hline 01 & 3 & 22 & 339.7606 & 2.8000 & 280.0 & 6 & 600.0 & 00.0 \\
\hline 01 & 3 & 23 & 100.0188 & 2.1500 & 279.4 & 6 & 600.0 & 00.0 \\
\hline 01 & 3 & 24 & 302.4410 & 2.0000 & 278.6 & 6 & 600.0 & 00.0 \\
\hline 01 & 3 & 25 & 302.7450 & 1.7750 & 278.3 & 5 & 600.0 & 00.0 \\
\hline 01 & 3 & 26 & 298.2492 & 2.1750 & 278.3 & 4 & 600.0 & 00.0 \\
\hline 01 & 3 & 27 & 327.5346 & 1.1250 & 278.4 & 5 & 600.0 & 00.0 \\
\hline 01 & 3 & 28 & 182.9568 & 1.6000 & 278.6 & 4 & 600.0 & 500.0 \\
\hline 01 & 3 & 29 & 154.7554 & 3.3500 & 278.6 & 3 & 600.0 & 00.0 \\
\hline 01 & 3 & 210 & 191.0623 & 1.6750 & 279.1 & 2 & 600.0 & 00.0 \\
\hline 01 & 3 & 211 & 264.8189 & 1.2250 & 280.3 & 1 & 600.0 & 00.0 \\
\hline 01 & 3 & 212 & 273.1414 & 1.4750 & 280.9 & 1 & 600.0 & 00.0 \\
\hline 01 & 3 & 213 & 305.2513 & 2.1750 & 282.4 & 1 & 600.0 & .0 \\
\hline 01 & 3 & 214 & 189.1121 & 2 & 283.5 & 1 & .0 & \\
\hline 01 & 3 & 215 & 83.2965 & 5.1250 & 283.7 & 2 & 600.0 & 00.0 \\
\hline 01 & 3 & 216 & 69.2424 & 5.7250 & 284.0 & 3 & 600.0 & 600.0 \\
\hline 01 & 3 & 217 & 94.4495 & 3.4250 & 284.2 & 2 & .0 & \\
\hline 01 & 3 & 218 & 161.3007 & 3.6750 & 282.3 & 3 & 600.0 & 00.0 \\
\hline 01 & 3 & 219 & 168.9992 & 2.0000 & 281.0 & 4 & 600.0 & .0 \\
\hline 01 & 3 & 220 & 99.5302 & 1 . & 281.0 & 5 & .0 & .0 \\
\hline 01 & 3 & 221 & 94.9995 & 3. & 281.2 & 5 & 600.0 & .0 \\
\hline 01 & 3 & 222 & 85.2429 & 4.6000 & 280.7 & 4 & 600.0 & 600.0 \\
\hline 01 & 3 & 223 & 124.6595 & 3. & 280.3 & 5 & .0 & .0 \\
\hline 01 & 3 & 224 & 132.0033 & 3. & 279.8 & 5 & .0 & \\
\hline 01 & 3 & 31 & 146.3196 & 2.5000 & 279.2 & 6 & 600.0 & 600.0 \\
\hline 01 & 3 & 32 & 167.0011 & 3.2500 & 279.1 & 5 & 600.0 & 600.0 \\
\hline 01 & 3 & 33 & 172.2049 & 2.2 & 279.1 & 6 & 600.0 & \\
\hline 01 & 3 & 34 & 112.7363 & 0.5750 & 278.9 & 6 & 600.0 & 600.0 \\
\hline 01 & 3 & 35 & 7.5937 & 0.1750 & 278.9 & 5 & 600.0 & 600.0 \\
\hline 01 & 3 & 36 & 338.0150 & 1. & 278.7 & 6 & .0 & .0 \\
\hline 01 & 3 & 37 & 326.4893 & 2.0750 & 279.0 & 5 & 600.0 & 600.0 \\
\hline 01 & 3 & 38 & 326.5000 & 2.4750 & 279.2 & 4 & 600.0 & 600.0 \\
\hline 01 & 3 & 39 & 294.7491 & 2.2750 & 279.1 & 4 & 600.0 & 0.0 \\
\hline 01 & 3 & 310 & 264.0704 & 2.0750 & 280.4 & 3 & 600.0 & 600.0 \\
\hline 01 & 3 & 311 & 242.4601 & 2.5000 & 280.7 & 2 & 600.0 & 600.0 \\
\hline 01 & 3 & 312 & 244.5110 & 2.8500 & 280.7 & 3 & 600.0 & 600.0 \\
\hline 01 & 3 & 313 & 230.4966 & 3.7000 & 281.2 & 3 & 600.0 & 600.0 \\
\hline 01 & 3 & 314 & 241.0729 & 4.6750 & 281.4 & 3 & 600.0 & 600.0 \\
\hline 01 & 3 & 315 & 243.2494 & 3.9250 & 282.1 & 3 & 600.0 & 600.0 \\
\hline 01 & 3 & 316 & 237.4948 & 3.6750 & 281.9 & 3 & 600.0 & 600.0 \\
\hline 01 & 3 & 317 & 174.7877 & 1.5250 & 282.0 & 2 & 600.0 & 600.0 \\
\hline 01 & 3 & 318 & 154.4941 & 1.4500 & 281.9 & 3 & 600.0 & 600.0 \\
\hline 01 & 3 & 319 & 268.3813 & 0.7750 & 281.7 & 4 & 600.0 & 600.0 \\
\hline 01 & 3 & 320 & 327.2454 & 4.6000 & 282.2 & 4 & 600.0 & 600.0 \\
\hline 01 & 3 & 321 & 314.4916 & 5.2500 & 282.5 & 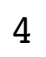 & 600.0 & 600.0 \\
\hline 01 & 3 & 322 & 324.0004 & 8.5000 & 282.4 & 4 & 600.0 & 600.0 \\
\hline & 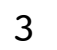 & 323 & 329.7500 & 9.875 & 282.1 & 4 & 600.0 & 600.0 \\
\hline
\end{tabular}




\begin{tabular}{|c|c|c|c|c|c|c|c|}
\hline I & 21 & & & & & & \\
\hline 1 & 4 & 500 & 000 & 31.7 & & 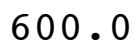 & \\
\hline 1 & 4 & 327.2501 & 7.9250 & 81.5 & & 00 & \\
\hline & 4 & 27.5000 & 7.9750 & 81.3 & & 00 & \\
\hline & 4 & 323.0080 & 7500 & 81.5 & & & \\
\hline & 4 & 315 . & 8500 & 81.9 & & 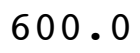 & \\
\hline & 4 & 282.7930 & 5250 & 82.1 & & 00 & \\
\hline & 7 & 37.2392 & 0500 & 82.4 & & & \\
\hline & 4 & 27 & 9.3500 & 82.0 & & & \\
\hline & 49 & 241.2684 & 250 & 82.5 & & 0 & 8 \\
\hline & 410 & 279.4940 & 10.0250 & 84.0 & & & \\
\hline & 411 & 321.2495 & 1.4250 & 84.9 & & & \\
\hline & 2 & 324 & 12.8000 & 85.4 & & & \\
\hline & 3 & 326.7500 & 13.2750 & 84.0 & & & \\
\hline & 414 & 326 & 12 . & & & & \\
\hline & 41 & 332 & 14 . & 84.0 & & & \\
\hline & 416 & 334.9996 & 15 . & 84.6 & & 0 & \\
\hline & 417 & 329 & 14 . & .7 & 2 & & \\
\hline & 41 & 33 & 10 . & 84.2 & 4 & & \\
\hline & 419 & 332 & 500 & 284.6 & 4 & & \\
\hline 3 & 420 & 295 . & 2.30 & 283.8 & & & \\
\hline & 421 & & & & & & \\
\hline & 422 & 281 & 1 . & 282.4 & & & \\
\hline & 423 & 233 & 2 . & 282.0 & & & \\
\hline & 424 & 16 & & 1.5 & & & \\
\hline & 5 & 15 & 1 . & 281.2 & 6 & & \\
\hline & 5 & 200.2302 & 0 . & 281.2 & & & \\
\hline & 53 & 278 & 2 . & 281.3 & 6 & & \\
\hline & 5 & 29 & 0 & 281.4 & 5 & & \\
\hline & 5 & 288 & 0 & 281.1 & & & \\
\hline 3 & 56 & 309 . & 5.9750 & 281.8 & 4 & & \\
\hline & 57 & 32 & 0 & 281.9 & 4 & & \\
\hline 3 & 58 & 325 . & .6750 & 282.0 & 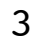 & & \\
\hline 3 & 59 & 284.2121 & 250 & 282.8 & 3 & & \\
\hline & 510 & 29 & & 28 & 4 & & \\
\hline & 511 & 31 & & 284.6 & 4 & & \\
\hline 3 & 512 & 316.7516 & 6.5000 & 285.6 & 4 & & \\
\hline 3 & 51 & 251 . & 0 & 285.2 & 4 & & \\
\hline & 514 & 279 & 4.6750 & 285.7 & 3 & & \\
\hline & 51 & 239.8470 & 6.4750 & 285.6 & 4 & & \\
\hline 3 & 51 & 211 . & 4 . & 284.6 & 3 & & \\
\hline & 517 & 202.4810 & 4.4500 & 275.9 & 3 & & \\
\hline 3 & 51 & 218.2432 & 3.3500 & 284.2 & 4 & 00.0 & \\
\hline 3 & 519 & 873 & .2250 & 283.9 & 5 & & \\
\hline & 520 & 204.2730 & 1.4500 & 283.8 & 6 & & \\
\hline & 521 & 217.7680 & 1.4250 & 283.7 & 6 & 600.0 & 00 \\
\hline & 32 & & & & J & & \\
\hline & 2 & .5073 & 3.6250 & 282.8 & $\Psi$ & 600.0 & \\
\hline
\end{tabular}




\begin{tabular}{|c|c|c|c|c|c|c|c|c|}
\hline & 3 & 7 & & & & & & \\
\hline & 3 & 1 & 34 & 3.5000 & 2.6 & & 0 & \\
\hline & 3 & 2 & 150.7472 & 0250 & 282.7 & & 00 & \\
\hline & 3 & 3 & 140.1246 & 8250 & 282.2 & & 00.0 & \\
\hline & 3 & 4 & .8502 & 4.3250 & 82.8 & & & \\
\hline & 3 & 5 & 4.2506 & 0 & 83.0 & & 0 & \\
\hline & 3 & 6 & 41.5018 & 8.3000 & 283.5 & & 00 & \\
\hline & 3 & 7 & 1.4969 & 6.2250 & 84.0 & & 0.0 & \\
\hline & 3 & 8 & 8.7505 & 1500 & 84.7 & & & \\
\hline & - & 9 & 35.3741 & 5.6000 & 85.4 & & 00 & 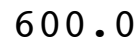 \\
\hline & 3 & 610 & 37.2325 & 6.0750 & 286 & & & \\
\hline & 3 & 611 & 6.7993 & 3.9000 & 7 & & .0 & \\
\hline & 3 & 12 & 11.2605 & 4.8750 & 287.4 & & .0 & \\
\hline & 3 & 13 & 75.5011 & 5.9500 & 287.3 & & 0.0 & \\
\hline & 3 & 14 & 496 & 0 & & & & \\
\hline & 3 & & & & 5 & & & \\
\hline & 3 & 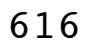 & 73.7488 & 5250 & 288.4 & & .0 & 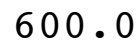 \\
\hline & 3 & 17 & 91.2185 & 6.8500 & 0 & & & \\
\hline & 3 & & 5789 & 6.8250 & $28^{\circ}$ & & & \\
\hline & 3 & & 65.2479 & 4.8250 & 287.3 & 4 & & 0 \\
\hline & 3 & 62 & 5700 & 4.1750 & 286.9 & 4 & .0 & \\
\hline & 3 & 621 & 99.2498 & 4.10 & & 4 & & \\
\hline & 3 & 622 & 87.7470 & 392 & 285.7 & 4 & & \\
\hline & 3 & 623 & .7647 & 8.25 & 2 & 4 & 0 & \\
\hline & 3 & 624 & 995 & 0 & & 4 & & \\
\hline & 3 & 71 & 494 & 7250 & 28 & 4 & & \\
\hline & 3 & 72 & 4945 & 2250 & 285.8 & 4 & 0 & J. \\
\hline & 3 & 73 & 989 & 1500 & 7 & 4 & & \\
\hline & 3 & 74 & 000 & 755 & 28 & 4 & & \\
\hline & 3 & 75 & 84.0000 & 0000 & 284.4 & 4 & 0 & 0 • \\
\hline & 3 & 76 & 80.7501 & 8.7500 & 283.8 & 4 & 0 & 0 . \\
\hline & 3 & 77 & 80.7493 & 652 & 28 & 4 & & \\
\hline & 3 & 78 & 110.3335 & 750 & 285.2 & 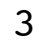 & & . \\
\hline & 3 & 79 & 305.8317 & 0.5500 & 287.4 & 0 & 0 & \\
\hline & 3 & 710 & 179.7521 & 20 & & 1 & & \\
\hline & 3 & 711 & 237.7192 & 2.3250 & .5 & 1 & & 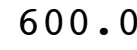 \\
\hline & 3 & 712 & 260.5181 & 1.8250 & 288.0 & 1 & & 0 . \\
\hline & 3 & 713 & 236 . & 1.8000 & 28 & 1 & & 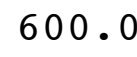 \\
\hline & 3 & 714 & 223.2456 & 1.9500 & 288.9 & 1 & & 0 . \\
\hline & 3 & 715 & 255.6267 & 1.5500 & 289.6 & 1 & & 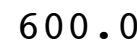 \\
\hline & 3 & 716 & 213.5021 & 1.3000 & 291.2 & $\perp$ & & 0 \\
\hline & 3 & 717 & 92.2489 & 2.4000 & 290.9 & 1 & & 0 . \\
\hline & 3 & 718 & 86.2532 & 3.5500 & 289.7 & 2 & 0 & 0 . \\
\hline & 3 & 719 & 927 & 250 & & 3 & & \\
\hline 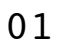 & 3 & 720 & 82.2748 & 3.4500 & & 4 & & 500. \\
\hline & 3 & 721 & 70.7532 & 3.5750 & 286.1 & 5 & 600.0 & 600. \\
\hline & & 722 & 00 & 0 & & 4 & & \\
\hline & & 125 & 71.4982 & 9.7750 & 283.1 & $\mathbf{4}$ & 600.0 & 600 \\
\hline
\end{tabular}




\begin{tabular}{|c|c|c|c|c|c|c|c|c|}
\hline 1 & 3 & 724 & 79.5000 & 9.4500 & 282.9 & 4 & 600.0 & 600.0 \\
\hline 01 & 3 & 81 & 73.5023 & 8.9250 & 282.8 & 4 & 600.0 & 600.0 \\
\hline 01 & 3 & 82 & 72.2494 & 7.4250 & 282.9 & 4 & 600.0 & 00.0 \\
\hline 01 & 3 & 83 & 80.2502 & 9.4750 & 281.7 & 4 & 600.0 & 00.0 \\
\hline 01 & 3 & 84 & 73.7494 & 11.5250 & 281.2 & 4 & 600.0 & 00.0 \\
\hline 01 & 3 & 85 & 68.2503 & 11.0250 & 280.9 & 4 & 600.0 & 00.0 \\
\hline 01 & 3 & 86 & 75.5000 & 250 & 281.0 & 4 & 600.0 & 00.0 \\
\hline 01 & 3 & 87 & 75.5001 & 8.0000 & 281.1 & 4 & 600.0 & 00.0 \\
\hline 01 & 3 & 88 & 74.0002 & 7.4750 & 281.2 & 4 & 600.0 & 00.0 \\
\hline 01 & 3 & 89 & 77.2497 & 500 & 282.0 & 4 & 600.0 & 00.0 \\
\hline 01 & 3 & 810 & 78.0037 & 00 & 284.4 & 3 & 600.0 & \\
\hline 01 & 3 & 811 & 59.3455 & 250 & 285.7 & 2 & 600.0 & \\
\hline 01 & 3 & 812 & 45.7301 & 3.0750 & 286.4 & 2 & 600.0 & 00.0 \\
\hline 01 & 3 & 813 & 73.0895 & 4.7500 & 286.4 & 3 & .0 & 00.0 \\
\hline 01 & 3 & 814 & 63.2054 & 00 & 286.2 & 4 & .0 & \\
\hline 01 & 3 & 815 & 59.7501 & 250 & 287.0 & 4 & 600.0 & 00.0 \\
\hline 01 & 3 & 816 & 69.9998 & 8.7250 & 286.4 & 4 & 600.0 & 600.0 \\
\hline 01 & 3 & 817 & 61.5000 & 8.3 & 285.9 & 4 & .0 & \\
\hline 01 & 3 & 818 & 68.0000 & 250 & 284.6 & 4 & 600.0 & .0 \\
\hline 01 & 3 & 819 & 64.7500 & 10 . & 283.0 & 4 & 60 & .0 \\
\hline 01 & 3 & 820 & 70.7500 & 10 . & 282.2 & 4 & 60 & \\
\hline 01 & 3 & 821 & 70.2481 & 50 & 281.6 & 4 & .0 & \\
\hline 01 & 3 & 822 & 69.2500 & 12.7500 & 280.7 & 4 & 600.0 & 00.0 \\
\hline 01 & 3 & 823 & 67.2498 & 12 . & 280.2 & 4 & 60 & .0 \\
\hline 01 & 3 & 824 & 66.2500 & 14 & 280.0 & 4 & .0 & \\
\hline 01 & 3 & 91 & 69.7506 & 500 & 279.9 & 4 & 600.0 & 600.0 \\
\hline 01 & 3 & 92 & 67.5000 & 10.2250 & 279.6 & 4 & 600.0 & 600.0 \\
\hline 01 & 3 & 93 & 62.7502 & 10 . & 279.6 & 4 & .0 & \\
\hline 01 & 3 & 94 & 66.0000 & 9. & 279.6 & 4 & 600.0 & 600.0 \\
\hline 01 & 3 & 95 & 75.7512 & 8.5750 & 279.8 & 4 & .0 & 600.0 \\
\hline 01 & 3 & 96 & 70.7499 & 7 . & 279.7 & 4 & .0 & \\
\hline 01 & 3 & 97 & 82.4968 & 6. & 280.1 & 4 & .0 & 600.0 \\
\hline 01 & 3 & 98 & 86.7476 & 6.6000 & 280.1 & 4 & 600.0 & 600.0 \\
\hline 01 & 3 & 99 & 92.2499 & 7. & 280.4 & 4 & 600.0 & 0.0 \\
\hline 01 & 3 & 910 & 94.7501 & 7 . & 280.3 & 4 & 600.0 & 600.0 \\
\hline 01 & 3 & 911 & 128.3880 & 6.8750 & 281.7 & 4 & 600.0 & 600.0 \\
\hline 01 & 3 & 912 & 157.7919 & 750 & 283.1 & 4 & 600.0 & 0.0 \\
\hline 01 & 3 & 913 & 160.0834 & 7.8500 & 283.8 & 4 & 600.0 & 600.0 \\
\hline 01 & 3 & 914 & 167.2502 & 7.2000 & 284.4 & 4 & 600.0 & 600.0 \\
\hline 01 & 3 & 915 & 163.0661 & 6 . & 284.9 & 4 & 600.0 & 0.0 \\
\hline 01 & 3 & 916 & 151.2661 & 750 & 285.3 & 4 & 600.0 & 600.0 \\
\hline 01 & 3 & 917 & 122.1821 & 4.5500 & 284.2 & 3 & 600.0 & 600.0 \\
\hline 01 & 3 & 918 & 96.5000 & 5.1000 & 283.8 & 4 & 600.0 & 600.0 \\
\hline 01 & 3 & 919 & 73.2500 & 5.8750 & 282.3 & 4 & 600.0 & 600.0 \\
\hline 01 & 3 & 920 & 74.5000 & 7.7500 & 282.0 & 4 & 600.0 & 600.0 \\
\hline 01 & 3 & 921 & 71.0002 & 8.2250 & 281.9 & . & 600.0 & 600.0 \\
\hline 01 & 3 & 922 & 72.4986 & 8.6750 & 281.7 & 4 & 600.0 & 600.0 \\
\hline . & 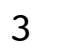 & 923 & 92.9957 & 9.075 & 281.3 & 4 & 600.0 & 600.0 \\
\hline
\end{tabular}


$\begin{array}{llll}01 & 3 & 924 & 118.7514\end{array}$

$01310 \quad 1 \quad 148.5000$

$01310 \quad 2 \quad 149.2500$

$013103 \quad 152.5000$

$\begin{array}{llll}01 & 310 & 4 & 145.2498\end{array}$

$01310 \quad 5 \quad 137.7511$

$01310 \quad 6 \quad 145.0000$

$01310 \quad 7 \quad 144.2501$

$01310 \quad 8 \quad 151.0005$

$01310 \quad 9 \quad 152.5000$

$0131010 \quad 156.7501$

0131011161.2503

0131012159.2660

$0131013 \quad 156.7533$

$0131014 \quad 155.2500$

0131015155.2502

$0131016 \quad 166.4745$

$0131017 \quad 156.7473$

$0131018 \quad 110.7909$

$\begin{array}{lll}01 & 31019 & 69.7478\end{array}$

$\begin{array}{lll}01 & 31020 & 76.4982\end{array}$

$\begin{array}{lll}01 & 31021 & 76.9998\end{array}$

$0131022 \quad 76.0000$

$\begin{array}{lll}01 & 31023 & 79.7499\end{array}$

$\begin{array}{lll}01 & 31024 & 77.2500\end{array}$

$\begin{array}{llll}01 & 311 & 1 & 92.4582\end{array}$

$\begin{array}{llll}01 & 311 & 2 & 206.8682\end{array}$

$\begin{array}{llll}01 & 311 & 3 & 334.0258\end{array}$

$\begin{array}{llll}01 & 311 & 4 & 15.4171\end{array}$

$\begin{array}{llll}01 & 311 & 5 & 124.8585\end{array}$

$\begin{array}{llll}01 & 311 & 6 & 135.0310\end{array}$

$\begin{array}{llll}01 & 311 & 7 & 128.2427\end{array}$

$\begin{array}{llll}01 & 311 & 8 & 115.7535\end{array}$

$\begin{array}{llll}01 & 311 & 9 & 132.3367\end{array}$

$\begin{array}{lll}01 & 31110 & 147.2500\end{array}$

$\begin{array}{llll}01 & 31111 & 149.2496\end{array}$

$\begin{array}{lll}01 & 31112 & 159.7510\end{array}$

$0131113 \quad 151.2472$

$\begin{array}{llll}01 & 31114 & 147.7520\end{array}$

0131115151.0000

$0131116 \quad 148.4991$

$0131117 \quad 145.7444$

$0131118 \quad 151.4767$

$\begin{array}{lll}01 & 31119 & 87.8527\end{array}$

$\begin{array}{lll}01 & 31120 & 97.5166\end{array}$

$\begin{array}{lll}01 & 31121 & 133.0740\end{array}$

$01 \quad 31122 \quad 140.7489$

$0131123 \quad 150.4992$

\begin{tabular}{|c|c|c|c|c|}
\hline 8.9000 & 281.7 & 4 & 600.0 & \\
\hline 9.9000 & 281.5 & 4 & 600.0 & 00 \\
\hline 11.0250 & 281.1 & 4 & 600.0 & 600.0 \\
\hline 1.6750 & 280.8 & 4 & 600.0 & 600 . \\
\hline 2.1500 & 281.0 & 4 & 600.0 & 600.0 \\
\hline 9.9500 & 281.3 & 4 & 600.0 & 600 . \\
\hline 11.3750 & 281.2 & 4 & 600.0 & 600.0 \\
\hline 1.075 & 281.3 & 4 & 600.0 & 600.0 \\
\hline 10.4750 & 281.7 & 4 & 600.0 & 600.0 \\
\hline 10.0750 & 282.5 & 4 & 600.0 & 600.0 \\
\hline 0.450 & 283.1 & 4 & 600.0 & 600.0 \\
\hline 8.950 & 283.9 & 4 & 600.0 & 600.0 \\
\hline 7.7750 & 284.8 & 4 & 600.0 & 600.0 \\
\hline 7.7750 & 285.6 & 4 & 600.0 & 600.0 \\
\hline 7.100 & 286 & 4 & 600.0 & 600.0 \\
\hline 6.400 & 287.0 & 4 & 600.0 & 600.0 \\
\hline 5.375 & 287.5 & 3 & 600.0 & 600.0 \\
\hline 4.225 & 287.5 & 3 & 600.0 & 600.0 \\
\hline 3.950 & 287.0 & 4 & 600.0 & 600.0 \\
\hline 4.825 & 285.5 & 5 & 600.0 & 600.0 \\
\hline 5.650 & 285.2 & 4 & 600.0 & 600.0 \\
\hline 6.10 & 284 . & 4 & 0.0 & 600.0 \\
\hline 5.6250 & 284.9 & 4 & 600.0 & 600.0 \\
\hline 5.625 & 284.9 & 4 & 600.0 & 600.0 \\
\hline 7.07 & 284 & 4 & 0.0 & 600.0 \\
\hline 5.9750 & 285 & 4 & 600.0 & 600.0 \\
\hline 1.775 & 283.4 & 5 & 600.0 & 600.0 \\
\hline 0.625 & 282.7 & 6 & 600.0 & 600.0 \\
\hline 0.3000 & 283. & 6 & 600.0 & 600.0 \\
\hline 0.625 & 283.0 & 5 & 600.0 & 600.0 \\
\hline 3.100 & 283. & 4 & 600.0 & 600.0 \\
\hline 3.875 & 283.3 & 4 & 600.0 & 600.0 \\
\hline 4.350 & 283.7 & 3 & 600.0 & 600.0 \\
\hline 4.800 & 283 . & 4 & 600.0 & 600.0 \\
\hline 6.525 & 28 & 4 & 600.0 & 600.0 \\
\hline 7.225 & 283.7 & 4 & 600.0 & 600.0 \\
\hline 6.750 & 284. & 4 & 600.0 & 600.0 \\
\hline 6.075 & 284 . & 4 & 600.0 & 600.0 \\
\hline 6.425 & 285.8 & 4 & 600.0 & 600.0 \\
\hline 7.500 & 286 . & 4 & 600.0 & 600.0 \\
\hline 6.625 & 28 & 4 & 600.0 & 600.0 \\
\hline 4.9750 & 287.4 & 3 & 600.0 & 600.0 \\
\hline 2.750 & 287.2 & 4 & 600.0 & 600.0 \\
\hline 2.350 & 286 & 5 & 600.0 & 600.0 \\
\hline 3.000 & 286.5 & 4 & 600.0 & 600.0 \\
\hline 5.00 & 287 & 4 & 600.0 & 600.0 \\
\hline 6.10 & 28 & 4 & 600.0 & 600.0 \\
\hline 5.42 & 28 & 4 & 600.0 & 60 \\
\hline
\end{tabular}




\begin{tabular}{|c|c|c|c|c|c|c|c|}
\hline 1 & 24 & & 0250 & 6.3 & & & \\
\hline & 3121 & 501 & 5.9250 & 85.7 & 4 & 00.0 & \\
\hline & 3122 & 136.2431 & 4.2750 & 285.5 & 4 & 00 & \\
\hline & 3123 & 151.2501 & 6.0250 & 284.3 & & 00.0 & \\
\hline & 124 & 45.5003 & 7750 & 84.5 & & 0 & \\
\hline & 125 & 5850 & 3250 & 284.4 & 4 & 00 & \\
\hline & 126 & 138.8030 & 3.2250 & 283.0 & & 500 & 0 \\
\hline & 3127 & 034 & .3000 & 81.6 & & 00.0 & \\
\hline & 3128 & 2.2504 & 4.5250 & 82.9 & 4 & & \\
\hline & 129 & 51.7504 & 500 & 285.1 & 3 & 500.0 & 0 \\
\hline & 1210 & 53.5031 & 4.0000 & 278.8 & & 00.0 & \\
\hline & 1211 & 78.7424 & 3.7750 & 87.8 & & 0 & \\
\hline & 31212 & 76.7670 & 4.7750 & 288.0 & 3 & 00.0 & \\
\hline & 1213 & 64.2528 & .2500 & 288.6 & 3 & 00.0 & 00 \\
\hline & 1214 & 7892 & 0 & 0 & 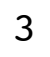 & & \\
\hline & 1215 & 276 & & 290.0 & 3 & & \\
\hline & 1216 & 177.5009 & 3750 & 290.2 & 3 & 00.0 & 00 \\
\hline & 31217 & 0.0129 & 4.5000 & 0.0 & 3 & 0 & \\
\hline & 1218 & 06 & 000 & 289.4 & 4 & & \\
\hline & 31219 & 104 & 4.4250 & 288.6 & 4 & .0 & 0 \\
\hline & 31220 & 75.4945 & 5.4750 & 287.7 & 4 & 0 & 00. \\
\hline & 1221 & 75.7527 & 0 & 287.1 & 4 & & \\
\hline & 31222 & 73 & 0 & 286.8 & 4 & & \\
\hline & 31223 & 68.9999 & 3.3750 & 286.6 & 4 & .0 & . \\
\hline & 31224 & 73.2499 & & & 4 & & \\
\hline & 3131 & 68.5000 & 250 & 28 & 4 & & \\
\hline & 3132 & 64.2500 & 6500 & 286.0 & 4 & 0 & U. \\
\hline & 3133 & 67.2502 & 5500 & 1 & 4 & & . \\
\hline & 3134 & 68.0000 & 9.2000 & 28 & 4 & & $\bullet$ \\
\hline & 3135 & 72.2498 & 8.2000 & 285.9 & 4 & .0 & 0 • \\
\hline & 3136 & 79.7500 & 7.8500 & 286.0 & 4 & 0 & 0 . \\
\hline & 3137 & 82.7476 & 6.6750 & 28 & 4 & & \\
\hline & 3138 & 87.7496 & 000 & 286.5 & 4 & & 0 • \\
\hline & 3139 & 171.7173 & 1.2000 & 288.7 & 3 & 0 & J \\
\hline & 31310 & 238.9062 & 750 & 288.6 & 2 & & \\
\hline & 31311 & 189.7071 & 5750 & 288.3 & 1 & & 0 . \\
\hline & 31312 & 175.7505 & 3.8500 & 288.4 & 2 & .0 & 0 . \\
\hline & 31313 & 257.0000 & 2.2250 & 290.8 & 1 & & 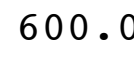 \\
\hline & 31314 & 174.2498 & 4.2750 & 291.2 & 2 & .0 & 600 . \\
\hline & 31315 & 170.7501 & 4.5750 & 291.8 & 3 & 0 & 0 . \\
\hline & 31316 & 167.7634 & 3.7500 & 292.6 & 2 & & 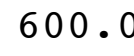 \\
\hline & 31317 & 133.5787 & 3.4750 & 292.6 & 2 & .0 & 00 . \\
\hline & 31318 & 102.7527 & 3.6500 & 291.8 & 3 & .0 & 00 . \\
\hline & 31319 & 77.2514 & 250 & 290.1 & 4 & 600 & 0 \\
\hline 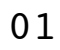 & 31320 & 75.4914 & 6.3500 & 289.8 & 4 & 60 & 600 \\
\hline & 31321 & 65.5000 & 5.7500 & 289.7 & 4 & 600.0 & 600 \\
\hline & & 9165 & & & 5 & & \\
\hline & 31323 & 19.7489 & 2.0500 & 287.9 & J & 600.0 & 600 \\
\hline
\end{tabular}




\begin{tabular}{|c|c|c|c|c|c|c|c|}
\hline & 24 & & 0 & 1.4 & & & \\
\hline & 3141 & -7 & 2.1750 & 286.9 & & 600.0 & \\
\hline & 3142 & 114.0258 & 0250 & 287.1 & 5 & 00 & \\
\hline & 143 & 150.9751 & 1.3500 & 286.1 & & 00 & \\
\hline & 144 & 93.7261 & 3.2000 & 86.6 & & & \\
\hline & 145 & 139 & 9750 & 85.9 & & 0 & \\
\hline & 146 & 110.4919 & 1.9000 & 284.0 & & 00 & \\
\hline & 147 & 52.4764 & 1.5000 & 83.8 & & & \\
\hline & 148 & 137.6 & 6750 & 5 & & & \\
\hline & 149 & 269.94 & 0.2000 & 89.2 & & 0 & 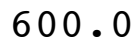 \\
\hline & 1410 & 177.9092 & 1.7250 & 289.5 & & 0 & \\
\hline & 11 & 178.2501 & 4.4250 & & & & \\
\hline & 2 & 171.9998 & 6.9000 & 288.9 & 4 & 0 & \\
\hline & 1413 & 170.9988 & 6.4500 & 289.1 & 4 & 0.0 & \\
\hline & 14 & 167 . & & & & & \\
\hline & & 165 . & & .7 & 3 & & \\
\hline & 416 & 166.7595 & 4.6250 & 290.5 & 3 & .0 & 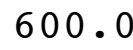 \\
\hline & 417 & 177.2500 & 0 & 90.6 & & & \\
\hline & 418 & 173.4995 & 0 & 290.0 & & & \\
\hline & 1419 & 125.2241 & 3.8500 & 288.9 & 4 & 0 & 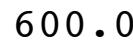 \\
\hline & 31420 & 79.5000 & 5250 & 288.3 & 4 & 0 & \\
\hline & 1 & 86.7554 & 0 & 37.9 & 4 & & \\
\hline & 2 & 2517 & 50 & $28 \varepsilon$ & 4 & & \\
\hline & 31423 & 80.7496 & .2250 & 288.6 & 4 & 0 & \\
\hline & 31424 & 77.5000 & & 9.0 & 4 & & \\
\hline & 3151 & 72 & 0 & .1 & 4 & & \\
\hline & 3152 & 73.9999 & 250 & 287.4 & 4 & 0 & J. \\
\hline & 3153 & 82.4998 & 7 . & 287.2 & 4 & & \\
\hline & 3154 & 82 . & 50 & 286.7 & 4 & & \\
\hline & 3155 & 80.5005 & $\$ 750$ & 287.5 & 4 & & 0 • \\
\hline & 3156 & 83.49 & 6.2250 & 286.7 & 4 & & 0 . \\
\hline & 3157 & 83. & 50 & 28 & 4 & & \\
\hline & 3158 & 80 . & & 285.8 & 4 & & . \\
\hline & 3159 & 521 & 3500 & 286.5 & 4 & 0 & 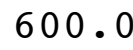 \\
\hline & 31510 & 80 . & 0 & & 4 & & \\
\hline & 31511 & & & 286.9 & 3 & & . \\
\hline & 31512 & 73.4895 & 7.7750 & 286.4 & 4 & & 0 . \\
\hline & 31513 & 901 & 7.6250 & 286 & 4 & & \\
\hline & 31514 & 73.749 & 8.5500 & 285.4 & 4 & & 0 . \\
\hline & 31515 & 64 . & 3250 & 285.1 & 4 & & \\
\hline & 31516 & 72.6425 & 500 & 287.3 & 3 & & \\
\hline & 31517 & & 6.6500 & 287.0 & 4 & & 0 . \\
\hline & 31518 & 69.0000 & 7.7750 & 285.4 & 4 & 0 & 0 . \\
\hline & 31519 & 67 . & 9.4000 & 282.9 & 4 & & \\
\hline & 31520 & 65.9999 & 0.2250 & 281.7 & 4 & & \\
\hline & 31521 & 67.2500 & 0.2500 & 282.1 & 4 & & 00 . \\
\hline & & & & 282.3 & 4 & & \\
\hline & 31523 & 71.2500 & 10.2500 & 282.5 & $I$ & 600.0 & 600 \\
\hline
\end{tabular}




\begin{tabular}{|c|c|c|c|c|c|c|c|}
\hline 01 & 31524 & 68.7494 & 10.6250 & 282.3 & 4 & 600.0 & 600.0 \\
\hline 01 & 3161 & 67.7501 & 10.4250 & 282.0 & 4 & 600.0 & 600.0 \\
\hline 01 & 3162 & 67.5000 & 9.5500 & 281.9 & 4 & 600.0 & 00.0 \\
\hline 01 & 3163 & 67.0002 & 8.9250 & 281.8 & 4 & 600.0 & 00.0 \\
\hline 01 & 3164 & 70.4995 & 9.1500 & 281.7 & 4 & 600.0 & 00.0 \\
\hline 01 & 3165 & 73.0000 & 9.2000 & 281.5 & 4 & 600.0 & 00.0 \\
\hline 01 & 3166 & 78.5002 & 9.1250 & 281.5 & 4 & 600.0 & 00.0 \\
\hline 01 & 3167 & 72.7500 & 8.3500 & 281.2 & 4 & 600.0 & 00.0 \\
\hline 01 & 3168 & 64.9988 & 7.6000 & 281.3 & 4 & 600.0 & 00.0 \\
\hline 01 & 3169 & 59.7487 & 7.3000 & 282.4 & 4 & 600.0 & 00.0 \\
\hline 01 & 31610 & 95.9432 & 5.1250 & 283.7 & 3 & 600.0 & 00.0 \\
\hline 01 & 31611 & 147.7502 & 7.1000 & 284.3 & 4 & 600.0 & \\
\hline 01 & 31612 & 159.5069 & 7.2250 & 284.8 & 4 & 600.0 & 00.0 \\
\hline 01 & 31613 & 148.3228 & 5.4000 & 286.1 & 3 & 600.0 & 00.0 \\
\hline 01 & 31614 & 157.7367 & 6.4000 & 287.0 & 4 & .0 & \\
\hline 01 & 31615 & 167.7497 & 7.2250 & 287.7 & 4 & 600.0 & 00.0 \\
\hline 01 & 31616 & 164.2563 & 6.6500 & 288.0 & 4 & 600.0 & 600.0 \\
\hline 01 & 31617 & 166.5061 & 6.3500 & 288.1 & 4 & .0 & \\
\hline 01 & 31618 & 100.6023 & 5.6750 & 287.0 & 4 & 600.0 & .0 \\
\hline 01 & 31619 & 72.7500 & 7.5250 & 285.5 & 4 & 60 & .0 \\
\hline 01 & 31620 & 71.5006 & 9.0500 & 285.2 & 4 & 60 & \\
\hline 01 & 31621 & 67.0000 & 8.9500 & 285.1 & 4 & .0 & \\
\hline 01 & 31622 & 69.0000 & 8.7500 & 285.4 & 4 & 600.0 & 00.0 \\
\hline 01 & 31623 & 67.4999 & 7.82 & 285.9 & 4 & 60 & \\
\hline 01 & 31624 & 69.2492 & 7.2250 & 286.2 & 4 & .0 & \\
\hline 01 & 3171 & 75.0000 & 8.4000 & 285.8 & 4 & 600.0 & 600.0 \\
\hline 01 & 3172 & 73.9991 & 8.7750 & 285.1 & 4 & 600.0 & .0 \\
\hline 01 & 3173 & 72.5015 & 8.7000 & 284.5 & 4 & .0 & \\
\hline 01 & 3174 & 68.9996 & 8.4000 & 284.1 & 4 & 600.0 & 600.0 \\
\hline 01 & 3175 & 76.5000 & 7.1250 & 283.9 & 4 & .0 & .0 \\
\hline 01 & 3176 & 77.2499 & 6.1750 & 284.3 & 4 & .0 & \\
\hline 01 & 3177 & 101.0000 & 4.0000 & 285.4 & 4 & .0 & 600.0 \\
\hline 01 & 3178 & 79.2496 & 6.1500 & 285.0 & 4 & 600.0 & 600.0 \\
\hline 01 & 3179 & 113.0006 & 3.2250 & 287.8 & 3 & 600.0 & 0.0 \\
\hline 01 & 31710 & 137.6285 & 3.5250 & 289.5 & 2 & 600.0 & 600.0 \\
\hline 01 & 31711 & 169.9954 & 3.3750 & 290.2 & 2 & 600.0 & 600.0 \\
\hline 01 & 31712 & 112.8438 & 4.2000 & 290.9 & 3 & 600.0 & 0.0 \\
\hline 01 & 31713 & 67.2551 & 5.7250 & 291.1 & 3 & 600.0 & 600.0 \\
\hline 01 & 31714 & 99.4839 & 4.5000 & 292.3 & 3 & 600.0 & 600.0 \\
\hline 01 & 31715 & 100.2037 & 4.6250 & 292.6 & 3 & 600.0 & 600.0 \\
\hline 01 & 31716 & 86.7505 & 5.3500 & 292.6 & 3 & 600.0 & 600.0 \\
\hline 01 & 31717 & 67.2500 & 5.8250 & 292.4 & 3 & 600.0 & 600.0 \\
\hline 01 & 31718 & 70.9862 & 5.4750 & 291.8 & 4 & 600.0 & 600.0 \\
\hline 01 & 31719 & 70.7491 & 5.8250 & 290.9 & 4 & 600.0 & 600.0 \\
\hline 01 & 31720 & 69.7501 & 6.4500 & 290.3 & 4 & 600.0 & 600.0 \\
\hline 01 & 31721 & 71.7507 & 6.4250 & 290.0 & 4 & 600.0 & 600.0 \\
\hline 01 & 31722 & 102.2963 & 3.5750 & 290.7 & 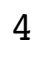 & 600.0 & 600.0 \\
\hline 01 & 31723 & 85.7498 & 5.150 & 290.1 & 4 & 600.0 & 600.0 \\
\hline
\end{tabular}




\begin{tabular}{|c|c|c|c|c|c|c|c|}
\hline & 724 & 004 & 6000 & 89.7 & 4 & 600.0 & \\
\hline & 3181 & 102.7476 & 4.3250 & 289.6 & 4 & 600.0 & \\
\hline & 3182 & 93.2549 & 1250 & 289.3 & 4 & 00 & \\
\hline & 3183 & 107.9684 & 8000 & 289.5 & & 00 & \\
\hline & 184 & 136.7452 & 6500 & 89.2 & & 0 & \\
\hline & 185 & 159.5038 & 2750 & 88.4 & & 00 & \\
\hline & 186 & 144.2323 & 500 & 288.0 & & 500 & \\
\hline & 3187 & 121.7501 & 250 & 88.1 & & & \\
\hline & 188 & 156.0000 & 5000 & 89.1 & & & \\
\hline & 189 & 168.4994 & 500 & 90.0 & & 00 & 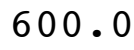 \\
\hline & 1810 & 177.5010 & 3.8250 & 290.7 & & & \\
\hline & 1811 & 176.4999 & 4.1000 & 90.7 & & & \\
\hline & 31812 & 169.4994 & 500 & 291.1 & 3 & 0 & \\
\hline & 1813 & 167.5055 & 4.4750 & 291.6 & & 0.0 & \\
\hline & 814 & 173.5000 & & 8 & & & \\
\hline & 815 & 177.4999 & & 293 & 3 & & \\
\hline & 1816 & 168.7020 & 8750 & 293.9 & 2 & .0 & 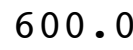 \\
\hline & 817 & 173.5009 & 00 & 94.4 & 1 & & \\
\hline & 818 & 150.7421 & & 293.9 & 2 & & \\
\hline & 31819 & 109.3334 & 0 & 293.4 & 3 & & 0 \\
\hline & 820 & 162.0123 & .0500 & 292.0 & 4 & 0 & \\
\hline & 821 & 135.4580 & & 291.9 & 5 & & \\
\hline & 822 & 150.5102 & 50 & 291.5 & 6 & & \\
\hline & 31823 & 78.4994 & 750 & 291.8 & 5 & 0 & \\
\hline & 31824 & 73.2498 & & 292.3 & 4 & & \\
\hline & 3191 & 79.7499 & 0 & 293 & 4 & & \\
\hline & 3192 & 108.6865 & 9250 & 292.8 & 5 & 0 & J. \\
\hline & 3193 & 185.9638 & 0 & 291.3 & 6 & & \\
\hline & 3194 & 7.7648 & 1.4750 & 290.2 & 5 & & \\
\hline & 3195 & 351.2966 & 4500 & 290.2 & 5 & 0 & 0 . \\
\hline & 3196 & 11.0000 & 0 & 290.6 & 5 & 0 & 0 . \\
\hline & 3197 & 348.8447 & 0 & 290.5 & 5 & & \\
\hline & 3198 & 338.5092 & & 292.2 & 4 & & . \\
\hline & 3199 & 305.9990 & 1.2000 & 293.3 & 3 & 0 & 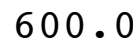 \\
\hline & 31910 & 256.9643 & 2 . & 29 & 3 & & \\
\hline & 31911 & 248.0139 & 2 . & 293.2 & 2 & & 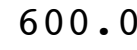 \\
\hline & 31912 & 196.7270 & 3.2500 & 292.4 & 2 & & 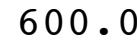 \\
\hline & 31913 & 219.6463 & 1 . & 294.3 & 1 & & 0 . \\
\hline & 31914 & 224.9896 & 3.0250 & 295.0 & 2 & .0 & 0 . \\
\hline & 31915 & 219.3096 & 3.0250 & 295.6 & 2 & & 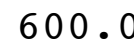 \\
\hline & 31916 & 219.5542 & 2 . & 295.8 & 1 & & 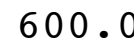 \\
\hline & 31917 & 204.5480 & 2.4500 & 295.7 & 2 & & 0 . \\
\hline & 31918 & 167.6686 & 3.6000 & 294.3 & 3 & 0 & 0 . \\
\hline & 31919 & 163.7508 & 3.2000 & 292.6 & 4 & & \\
\hline 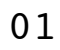 & 31920 & 171.2508 & 2.2500 & 292.3 & 5 & & 0 . \\
\hline & 31921 & 178.7552 & 0.4750 & 292.0 & 6 & 600 & 600 \\
\hline & & 572 & & 29 & 6 & & \\
\hline & 31923 & 23.7099 & 0.8250 & 292.0 & J & 600.0 & 600 \\
\hline
\end{tabular}




\begin{tabular}{|c|c|c|c|c|c|c|c|}
\hline & 31924 & 7.5000 & 8500 & 291.7 & 4 & 0.0 & \\
\hline & 3201 & 12.2833 & 0.3750 & 291.8 & 5 & 00.0 & \\
\hline & 3202 & 247 & 250 & 291.4 & & 0 & \\
\hline & 3203 & 09.0211 & .9500 & 291.4 & & 0 & \\
\hline & 3204 & 312.0000 & 250 & 290.9 & & 00 & \\
\hline & 3205 & 206.2017 & 250 & 290.1 & & 0 & \\
\hline & 3206 & 173.7557 & 000 & 290.1 & & & \\
\hline & 207 & 159.0127 & & 89.9 & & & \\
\hline & 3208 & 171.2463 & 0.7750 & 290.4 & & 00.0 & 00 \\
\hline & 3209 & 181.7180 & 1000 & 292.4 & & & \\
\hline & 32010 & 230.0478 & & 94.6 & & & \\
\hline & 32011 & 183.5547 & 3 . & 293.5 & & .0 & \\
\hline & 32012 & 187.9860 & .5250 & 293.4 & & & \\
\hline & 32013 & 164.7856 & 2.1000 & 93.8 & & & \\
\hline & 32014 & 179.2616 & 3.4250 & 294.8 & & & \\
\hline & 32015 & 199.6095 & 3.7000 & 295.3 & & 0 & \\
\hline & 32016 & 235.7628 & 1.57 & 296.6 & & & \\
\hline & 2017 & 242.6638 & 250 & 296.9 & & & \\
\hline & 32018 & 228.8588 & 1.7750 & 296.0 & & 0 & 600. \\
\hline & 32019 & 148.5217 & 2.2000 & 294.6 & 3 & & \\
\hline & 32020 & 85.5009 & & 294.2 & $\mathbf{4}$ & & \\
\hline & 32021 & 91.2971 & 750 & 293.5 & 4 & & \\
\hline & 32022 & 86.4966 & .8750 & 292.8 & 4 & 0 & 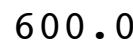 \\
\hline & 32023 & 76.0000 & .4500 & 292.5 & 4 & & $\bullet$ \\
\hline & 32024 & 80.2501 & 750 & 292.6 & 5 & & \\
\hline & 3211 & 80.5000 & 750 & 292.2 & 5 & 0 & 0 \\
\hline & 3212 & 84.4994 & 0 & 292.1 & 4 & & \\
\hline & 3213 & 86.7519 & 50 & 291.8 & 4 & & \\
\hline & 3214 & 125.7465 & 5000 & 291.1 & 5 & 0 & 10 \\
\hline & 3215 & 137 . & 0 & 290.8 & 5 & & \\
\hline & 3216 & 108 & 50 & 29 & 6 & & \\
\hline & 3217 & 139.7659 & 000 & 290.2 & 6 & & \\
\hline & 3218 & 148.5075 & 0.3250 & 291.5 & 5 & 0 & \\
\hline & 3219 & 167.2595 & 2 . & 292.0 & 4 & & \\
\hline & 32110 & 174.5008 & 000 & 291.2 & 3 & & \\
\hline & 32111 & 166.2451 & $\$ 750$ & 291.0 & 3 & & \\
\hline & 32112 & 173. & 0 & 292 & 3 & & \\
\hline & 32113 & 176.2658 & 4.7250 & 292.9 & 3 & & 0 \\
\hline & 32114 & 174.5105 & 3500 & 293.8 & 3 & 0 & \\
\hline & 32115 & 173.5012 & 4 . & 293 & 3 & & \\
\hline & 32116 & 128.7391 & 4.0250 & 293.7 & 3 & & 00 . \\
\hline & 32117 & 86.0000 & 4.0250 & 293.4 & 4 & 0 & 00 \\
\hline & 32118 & 78.7464 & 000 & 292.4 & 4 & 0 & \\
\hline & 32119 & 70.5000 & 6.7000 & 290.9 & 4 & 600.0 & 600 \\
\hline & 32120 & 71.7499 & 8.0500 & 290.0 & 4 & 600.0 & 00 \\
\hline & 32121 & & & $28 \varepsilon$ & a & & \\
\hline & 32122 & 73.5000 & 9.1000 & 289.4 & 4 & 600 & 600 \\
\hline & 2123 & 75.7500 & 8.4750 & 290.0 & 4 & 600.0 & 60 \\
\hline
\end{tabular}




\begin{tabular}{|c|c|c|c|c|c|c|c|}
\hline 1 & 32124 & 73.0000 & 8.5750 & 289.7 & 4 & 600.0 & 600.0 \\
\hline 01 & 3221 & 75.5001 & 11.2750 & 287.1 & 4 & 600.0 & 600.0 \\
\hline 01 & 3222 & 76.2498 & 12.7750 & 284.6 & 4 & 600.0 & 00.0 \\
\hline 01 & 3223 & 76.0000 & 12.8750 & 284.0 & 4 & 600.0 & 00.0 \\
\hline 01 & 3224 & 76.2500 & 12.3750 & 283.5 & 4 & 600.0 & 00.0 \\
\hline 01 & 3225 & 78.5003 & 10.6750 & 283.2 & 4 & 600.0 & 00.0 \\
\hline 01 & 3226 & 76.7500 & 11.1750 & 282.9 & 4 & 600.0 & 00.0 \\
\hline 01 & 3227 & 77.5000 & 10.5500 & 283.1 & 4 & 600.0 & 00.0 \\
\hline 01 & 3228 & 76.5000 & 10.4000 & 283.4 & 4 & 600.0 & 00.0 \\
\hline 01 & 3229 & 75.5000 & 9.9250 & 284.1 & 4 & 600.0 & 0 \\
\hline 01 & 32210 & 78.7500 & 8.2250 & 285.2 & 4 & 600.0 & \\
\hline 01 & 32211 & 81.2463 & 6.9000 & 286.2 & 4 & 600.0 & \\
\hline 01 & 32212 & 84.9992 & 7.0000 & 286.6 & 4 & 600.0 & 00.0 \\
\hline 01 & 32213 & 78.2438 & 6.5250 & 287.5 & 4 & 600.0 & 00.0 \\
\hline 01 & 32214 & 79.7493 & & 288.4 & 4 & .0 & \\
\hline 01 & 32215 & 78.9993 & 6.2500 & 289.3 & 4 & 600.0 & 00.0 \\
\hline 01 & 32216 & 68.5000 & 8.0500 & 289.2 & 4 & 60 & 0.0 \\
\hline 01 & 32217 & 67.0000 & 7.5 & 289.3 & 4 & .0 & \\
\hline 01 & 32218 & 68.5000 & 7.7000 & 288.6 & 4 & 60 & \\
\hline 01 & 32219 & 70.2501 & 7.6000 & 287.7 & 4 & .0 & .0 \\
\hline 01 & 32220 & 67.7500 & 9. & 286.2 & 4 & 6 & \\
\hline 01 & 32221 & 68.5006 & 10.02 & 285.1 & 4 & .0 & \\
\hline 01 & 32222 & 69.2526 & 8.7000 & 285.0 & 4 & 600.0 & .0 \\
\hline 01 & 32223 & 74.5052 & $6.9^{\circ}$ & 28 & 4 & 6 & \\
\hline 01 & 32224 & 80.7500 & 8. & 284.2 & 4 & .0 & \\
\hline 01 & 3231 & 77.5000 & 7.7500 & 283.6 & 4 & .0 & \\
\hline 01 & 3232 & 76.2506 & 8.3500 & 283.3 & 4 & 600.0 & .0 \\
\hline 01 & 3233 & 70.5000 & 8.3 & 283.1 & 4 & .0 & \\
\hline 01 & 3234 & 65.9989 & 8.37 & 283.0 & 4 & .0 & 0.0 \\
\hline 01 & 3235 & 71.0000 & 7.8750 & 282.9 & 4 & .0 & .0 \\
\hline 01 & 3236 & 70.0000 & 7. & 282.8 & 4 & .0 & \\
\hline 01 & 3237 & 78.9999 & 6.32 & 282.8 & 4 & .0 & 600.0 \\
\hline 01 & 3238 & 72.0103 & 5.2500 & 283.5 & 4 & 600.0 & 0.0 \\
\hline 01 & 3239 & 91.6594 & 3.27 & 284.9 & 3 & 0.0 & 0.0 \\
\hline 01 & 32310 & 231.0381 & 1.6750 & 287.3 & 2 & 600.0 & 600.0 \\
\hline 01 & 32311 & 290.8050 & 1.3500 & 288.6 & 1 & 600.0 & 600.0 \\
\hline 01 & 32312 & 288.2663 & 1.70 & 289.4 & 1 & 600.0 & 0.0 \\
\hline 01 & 32313 & 233.5958 & 2.7250 & 289.7 & 1 & 600.0 & 600.0 \\
\hline 01 & 32314 & 283.8956 & 2.0500 & 291.5 & 1 & 600.0 & 600.0 \\
\hline 01 & 32315 & 291.1225 & 1.9500 & 292.7 & 1 & 600.0 & 600.0 \\
\hline 01 & 32316 & 179.0076 & 2.6250 & 291.8 & 1 & 600.0 & 600.0 \\
\hline 01 & 32317 & 331.1215 & 1.9250 & 292.0 & 1 & 600.0 & 600.0 \\
\hline 01 & 32318 & 78.7502 & 4.4750 & 289.6 & - & 600.0 & 600.0 \\
\hline 01 & 32319 & 71.9995 & 4.9750 & 288.4 & 3 & 600.0 & 600.0 \\
\hline 01 & 32320 & 60.7685 & 1.9750 & 288.1 & 4 & 600.0 & 600.0 \\
\hline 01 & 32321 & 34.6715 & 1.5750 & 287.6 & 5 & 600.0 & 600.0 \\
\hline 01 & 32322 & 14.5165 & 1.4250 & 287.4 & 4 & 600.0 & 600.0 \\
\hline J & 32323 & 29.4158 & 1.1500 & 287.2 & 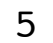 & 600.0 & 600.0 \\
\hline
\end{tabular}




\begin{tabular}{|c|c|c|c|c|c|c|c|}
\hline 1 & 32324 & 88.2401 & 3.4250 & 287.4 & 5 & 600.0 & 600.0 \\
\hline 01 & 3241 & 59.0299 & 3.8000 & 286.7 & 4 & 600.0 & 600.0 \\
\hline 01 & 3242 & 56.5724 & 1.6000 & 286.6 & 5 & 600.0 & 00.0 \\
\hline 1 & 3243 & 74.2713 & 5.7750 & 285.5 & 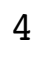 & 600.0 & 00.0 \\
\hline 01 & $324 \quad 4$ & 72.7505 & 8.4750 & 283.5 & 4 & 600.0 & 00.0 \\
\hline 01 & 3245 & 84.4270 & 3.1500 & 284.5 & 4 & 600.0 & 00.0 \\
\hline 01 & 3246 & 62.1991 & 1.1750 & 283.7 & 5 & 600.0 & 600.0 \\
\hline 01 & 3247 & 69.6224 & 1.3750 & 284.5 & 6 & 600.0 & .0 \\
\hline 01 & 3248 & 22.1178 & 1.4500 & 285.7 & 5 & 600.0 & 600.0 \\
\hline 01 & 3249 & 329.9544 & 0.8500 & 289.4 & 4 & 600.0 & 600.0 \\
\hline 01 & 32410 & 289.9121 & 2.1000 & 290.0 & 3 & 600.0 & \\
\hline 01 & 32411 & 245.0250 & 2.5500 & 290.1 & 2 & 600.0 & 60 \\
\hline 01 & 32412 & 265.4112 & 2.5500 & 291.0 & 1 & 600.0 & 0 \\
\hline 01 & 32413 & 263.1488 & 2.2750 & 292.5 & 1 & 600.0 & 00.0 \\
\hline 01 & 32414 & 269.9665 & 2.5750 & 293.1 & 1 & 600.0 & 60 \\
\hline 01 & 32415 & 272.3629 & 2.6250 & 292.8 & 1 & 600.0 & 600.0 \\
\hline 01 & 32416 & 338.0672 & 2.3750 & 293.5 & 1 & 600.0 & .0 \\
\hline 01 & 32417 & 16.3086 & 3.3000 & 293.4 & 2 & .0 & \\
\hline 01 & 32418 & 35.5799 & 3.2000 & 291.9 & 3 & 600.0 & 600.0 \\
\hline 01 & 32419 & 16.0000 & 5.5250 & 290.7 & 4 & 600.0 & .0 \\
\hline 01 & 32420 & 31.0018 & 6.3 & 290.0 & 4 & .0 & \\
\hline 01 & 32421 & 41.7519 & 6.8750 & 289.3 & 4 & .0 & 60 \\
\hline 01 & 32422 & 43.3188 & 4.1750 & 288.8 & 4 & 600.0 & 600.0 \\
\hline 01 & 32423 & 88.7565 & 4.2750 & 287.6 & 4 & .0 & \\
\hline 01 & 32424 & 63.5023 & 6.0000 & 286.8 & 4 & .0 & 60 \\
\hline 01 & 3251 & 60.1041 & 4.0250 & 286.6 & 4 & 600.0 & 600.0 \\
\hline 01 & 3252 & 31.5000 & 4.1000 & 286.6 & 4 & 600.0 & .0 \\
\hline 01 & 3253 & 43.2650 & 4.1250 & 285.9 & 4 & 600.0 & 60 \\
\hline 01 & 3254 & 219.9172 & 1.1750 & 285.4 & 5 & 600.0 & 600.0 \\
\hline 01 & 3255 & 32.9069 & 2.9250 & 285.1 & 5 & 600.0 & .0 \\
\hline 01 & 3256 & 44.1766 & 3.8000 & 285.6 & 4 & 600.0 & 60 \\
\hline 01 & 3257 & 155.0046 & 3.4000 & 283.6 & 4 & 600.0 & 600.0 \\
\hline 01 & 3258 & 144.2580 & 3.2000 & 283.4 & 4 & 600.0 & 600.0 \\
\hline 01 & 3259 & 110.5000 & 3.6500 & 284.6 & 3 & 600.0 & 600.0 \\
\hline 01 & 32510 & 113.4986 & 4.7000 & 286.1 & 3 & 600.0 & 600.0 \\
\hline 01 & 32511 & 116.5023 & 5.6250 & 286.3 & 3 & 600.0 & 600.0 \\
\hline 01 & 32512 & 147.2402 & 6.6750 & 286.6 & 4 & 600.0 & 600.0 \\
\hline 01 & 32513 & 149.4548 & 5.5000 & 287.2 & 3 & 600.0 & 600.0 \\
\hline 01 & 32514 & 160.9989 & 5.8000 & 287.6 & 3 & 600.0 & 600.0 \\
\hline 01 & 32515 & 160.7439 & 5.7000 & 287.6 & 3 & 600.0 & 600.0 \\
\hline 01 & 32516 & 151.9908 & 6.2250 & 288.1 & 4 & 600.0 & 600.0 \\
\hline 01 & 32517 & 105.0353 & 6.2250 & 287.2 & 4 & 600.0 & 600.0 \\
\hline 01 & 32518 & 85.2405 & 5.7500 & 286.2 & 4 & 600.0 & 600.0 \\
\hline 01 & 32519 & 75.2788 & 7.0250 & 285.0 & 4 & 600.0 & 600.0 \\
\hline 01 & 32520 & 59.5000 & 7.5000 & 284.4 & 4 & 600.0 & 600.0 \\
\hline 01 & 32521 & 62.5000 & 8.1250 & 283 & 4 & 600.0 & \\
\hline 01 & 32522 & 69.0034 & 8.7750 & 283.0 & 4 & 600.0 & 600.0 \\
\hline 01 & 32523 & 69.2504 & 8.800 & 282.7 & 4 & 600.0 & 600.0 \\
\hline
\end{tabular}




\begin{tabular}{|c|c|c|c|c|c|c|c|}
\hline 1 & 32524 & 66.7500 & 10.1750 & 282.5 & 4 & 600.0 & 600.0 \\
\hline 01 & 3261 & 74.7519 & 9.5250 & 282.8 & 4 & 600.0 & 600.0 \\
\hline 01 & 3262 & 74.4997 & 9.1750 & 282.9 & 4 & 600.0 & 00.0 \\
\hline 01 & 3263 & 70.5000 & 8.5500 & 282.7 & 4 & 600.0 & 00.0 \\
\hline 01 & 3264 & 72.0000 & 8.6000 & 282.3 & 4 & 600.0 & 00.0 \\
\hline 01 & 3265 & 80.7496 & 8.2750 & 282.1 & 4 & 600.0 & 00.0 \\
\hline 01 & 3266 & 82.0000 & 8.8000 & 282.0 & 4 & 600.0 & 00.0 \\
\hline 01 & 3267 & 88.7504 & 8.0250 & 282.0 & 4 & 600.0 & 00.0 \\
\hline 01 & 3268 & 100.4997 & 6.1250 & 283.3 & 4 & 600.0 & 00.0 \\
\hline 01 & 3269 & 128.2480 & 500 & 285.4 & 4 & 600.0 & .0 \\
\hline 01 & 32610 & 156.5530 & 000 & 285.8 & 4 & 600.0 & \\
\hline 01 & 32611 & 169.2499 & 250 & 286.3 & 4 & 600.0 & \\
\hline 01 & 32612 & 168.7483 & 5.3000 & 287.4 & 3 & 600.0 & 00.0 \\
\hline 01 & 32613 & 159.5274 & 750 & 288.7 & 3 & .0 & .0 \\
\hline 01 & 32614 & 156.5107 & 50 & 289.8 & 4 & .0 & \\
\hline 01 & 32615 & 132.1619 & 250 & 290.5 & 4 & 600.0 & 00.0 \\
\hline 01 & 32616 & 107.5034 & 6.0250 & 290.4 & 4 & 600.0 & 600.0 \\
\hline 01 & 32617 & 88.2535 & 6 . & 289.7 & 4 & .0 & \\
\hline 01 & 32618 & 69.5014 & 500 & 288.9 & 4 & 600.0 & .0 \\
\hline 01 & 32619 & 75.0069 & 7.7750 & 288.0 & 4 & .0 & .0 \\
\hline 01 & 32620 & 79.2532 & 8 . & 287.5 & 4 & .0 & \\
\hline 01 & 32621 & 72.7516 & 250 & 286.4 & 4 & .0 & \\
\hline 01 & 32622 & 70.0023 & 8.3250 & 286.8 & 4 & 600.0 & 0 \\
\hline 01 & 32623 & 79.0011 & 7 . & 287.6 & 4 & 60 & .0 \\
\hline 01 & 32624 & 000 & 7 . & 287.5 & 4 & .0 & \\
\hline 01 & 3271 & 81.2500 & 8.2750 & 286.9 & 4 & 600.0 & 600.0 \\
\hline 01 & 3272 & 78.2492 & 7.9000 & 287.0 & 4 & 600.0 & 600.0 \\
\hline 01 & 3273 & 93.6908 & 7. & 287.3 & 4 & .0 & \\
\hline 01 & 3274 & 124.7161 & 6 . & 287.8 & 4 & .0 & 0.0 \\
\hline 01 & 3275 & 135.5000 & 6.6250 & 287.4 & 4 & 600.0 & 0.0 \\
\hline 01 & 3276 & 138.5000 & 6 . & 286.8 & 4 & .0 & \\
\hline 01 & 3277 & 136.4994 & 8.3500 & 286.6 & 4 & .0 & 600.0 \\
\hline 01 & 3278 & 136.7460 & 7.6250 & 286.9 & 4 & 600.0 & 600.0 \\
\hline 01 & 3279 & 148.2482 & 7.6500 & 287.3 & 4 & 600.0 & 0.0 \\
\hline 01 & 32710 & 150.4994 & 6.8750 & 288.5 & 4 & 600.0 & 600.0 \\
\hline 01 & 32711 & 149.0518 & 5.6000 & 289.6 & 3 & 600.0 & 600.0 \\
\hline 01 & 32712 & 138.5000 & 4 . & 290.7 & 3 & 600.0 & 0.0 \\
\hline 01 & 32713 & 147.5011 & 5.0750 & 291.5 & 3 & 600.0 & 600.0 \\
\hline 01 & 32714 & 148.2616 & 4.8000 & 293.0 & 3 & 600.0 & 600.0 \\
\hline 01 & 32715 & 130.0000 & 4.9500 & 293.6 & 3 & 600.0 & 600.0 \\
\hline 01 & 32716 & 130.2618 & 5.4750 & 294.1 & 3 & 600.0 & 600.0 \\
\hline 01 & 32717 & 92.5560 & 5.6500 & 293.6 & 3 & 600.0 & 600.0 \\
\hline 01 & 32718 & 72.4998 & 7.1250 & 292.0 & 4 & 600.0 & 600.0 \\
\hline 01 & 32719 & 75.4994 & 9.2750 & 291.6 & 4 & 600.0 & 600.0 \\
\hline 01 & 32720 & 74.7497 & 8.7500 & 291.3 & 4 & 600.0 & 600.0 \\
\hline 01 & 32721 & 70.7499 & 8.8750 & 291.5 & 4 & 600.0 & 600.0 \\
\hline 01 & 32722 & 69.7499 & 8.6250 & 290.7 & 4 & 600.0 & 600.0 \\
\hline & 32723 & 67.9977 & 9.750 & 289.2 & 4 & 600.0 & 600.0 \\
\hline
\end{tabular}




\begin{tabular}{|c|c|c|c|c|c|c|c|}
\hline 1 & 32724 & 74.7499 & 10.4000 & 288.5 & 4 & 600.0 & 600.0 \\
\hline 01 & 3281 & 73.0001 & 10.0250 & 288.4 & 4 & 600.0 & 00,0 \\
\hline 01 & 3282 & 71.2497 & 9.7000 & 288.0 & 4 & 600.0 & 00.0 \\
\hline 1 & 3283 & 65.0007 & 9.6500 & 288.2 & 4 & 600.0 & 00.0 \\
\hline 01 & 3284 & 81.3393 & 500 & 288.9 & 4 & 600.0 & 0.0 \\
\hline 01 & 3285 & 73.9816 & 8.2500 & 288.7 & 4 & 600.0 & 00.0 \\
\hline 01 & 3286 & 83.9989 & 9.3750 & 289.5 & 4 & 600.0 & 0 \\
\hline 01 & 3287 & 87.7573 & 9.9750 & 288.5 & 4 & 600.0 & 00.0 \\
\hline 01 & 3288 & 84.7543 & 9.6750 & 288.3 & 4 & 600.0 & 00.0 \\
\hline 01 & 3289 & 79.2515 & 8.3750 & 288.4 & 4 & 60 & 0 \\
\hline 01 & 32810 & 73.0193 & 6.0750 & 289.9 & 4 & 600.0 & \\
\hline 01 & 32811 & 68.4906 & 750 & 290.8 & 4 & .0 & \\
\hline 01 & 32812 & 86.6830 & 6.1750 & 291.4 & 4 & 600.0 & .0 \\
\hline 01 & 32813 & 118.5161 & 5.2750 & 293.0 & 3 & 600.0 & \\
\hline 01 & 32814 & 89.0169 & 4 . & 293.6 & 3 & .0 & \\
\hline 01 & 32815 & 87.7491 & 6.4750 & 293.4 & 4 & 600.0 & .0 \\
\hline 01 & 32816 & 86.2522 & 6.9250 & 293.4 & 4 & 60 & .0 \\
\hline 01 & 32817 & 69.2498 & 7.6250 & 293.3 & 4 & .0 & \\
\hline 01 & 32818 & 68.4914 & 8.9000 & 291.7 & 4 & .0 & \\
\hline 01 & 32819 & 64.2492 & 9.3500 & 291.0 & 4 & .0 & .0 \\
\hline 01 & 32820 & 70.7498 & 10.07 & 291.0 & 4 & 0 & \\
\hline 01 & 32821 & 71.4982 & 10.0250 & 290.4 & 4 & .0 & \\
\hline 01 & 32822 & 80.7439 & 11.4500 & 290.7 & 4 & 600.0 & .0 \\
\hline 01 & 32823 & 74.2528 & 10.70 & 289.4 & 4 & 0 & \\
\hline 01 & 32824 & 77.7514 & 12 . & 28 & 4 & 0 & \\
\hline 01 & 3291 & 74.5000 & 11.6750 & 288.5 & 4 & .0 & \\
\hline 01 & 3292 & 74.9999 & 11.5750 & 288.2 & 4 & 60 & .0 \\
\hline 01 & 3293 & 73.7502 & 11.5500 & 287.4 & 4 & .0 & \\
\hline 01 & 3294 & 67.5011 & 10.4500 & 286.8 & 4 & .0 & .0 \\
\hline 01 & 3295 & 65.5000 & 9.4750 & 286.3 & 4 & .0 & \\
\hline 01 & 3296 & 90.3410 & 8.2750 & 287.2 & 4 & .0 & \\
\hline 01 & 3297 & 101.2507 & 7.2500 & 288.3 & 4 & .0 & 0.0 \\
\hline 01 & 3298 & 111.2228 & 6.9250 & 288.7 & 4 & 600.0 & 0.0 \\
\hline 01 & 3299 & 140.9975 & 8.3250 & 289.3 & 4 & 0.0 & 0.0 \\
\hline 01 & 32910 & 144.2500 & 11.0500 & 289.6 & 4 & 600.0 & 0.0 \\
\hline 01 & 32911 & 154.5000 & 11.6500 & 289.9 & 4 & 600.0 & 0.0 \\
\hline 01 & 32912 & 158.7530 & 11.975 & 290.7 & 4 & 600.0 & .0 \\
\hline 01 & 32913 & 155.0000 & 9.8750 & 291.7 & 4 & 600.0 & 600.0 \\
\hline 01 & 32914 & 149.0023 & 9.2750 & 292.7 & 4 & 600.0 & 600.0 \\
\hline 01 & 32915 & 140.5064 & 9.1750 & 293.6 & 4 & 600.0 & .0 \\
\hline 01 & 32916 & 115.2542 & 6.7000 & 293.8 & 4 & 600.0 & 600.0 \\
\hline 01 & 32917 & 107.2556 & 6.4250 & 293.7 & 4 & 600.0 & 600.0 \\
\hline 01 & 32918 & 86.2542 & 6.4000 & 293.0 & 4 & 600.0 & 600.0 \\
\hline 01 & 32919 & 71.7451 & 8.8250 & 291.0 & 4 & 600.0 & 600.0 \\
\hline 01 & 32920 & 70.7509 & 8.1750 & 290.8 & 4 & 600.0 & 600.0 \\
\hline 01 & 32921 & 80.9996 & 7.8000 & 291.4 & 4 & 600.0 & 600.0 \\
\hline 01 & 32922 & 100.9247 & 6.2000 & 292.3 & 4 & 600.0 & 600.0 \\
\hline 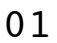 & 32923 & 99.9826 & 5.200 & 292.7 & 4 & 600.0 & 600.0 \\
\hline
\end{tabular}




\begin{tabular}{|c|c|c|c|c|c|c|c|}
\hline & 24 & & & & & & \\
\hline & 301 & 9 & 0.1500 & 3.3 & & & \\
\hline & 302 & 50.7500 & .4250 & 292.2 & & 00 & \\
\hline & 303 & 51.2501 & 1.4000 & 291.6 & & 0 & \\
\hline & 304 & 01 & 1.3000 & 91.1 & & & \\
\hline & 305 & & 9.8000 & 90.3 & & 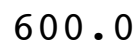 & \\
\hline & 306 & 5000 & 12.0750 & 290.0 & & 00 & \\
\hline & 307 & 7.7488 & 500 & 89.5 & & & \\
\hline & 308 & 4.7500 & 8.4750 & 89.9 & & & \\
\hline & 309 & 66.2515 & 8.2500 & 90.7 & & 0 & \\
\hline & 3010 & 65.0014 & 9.3750 & 91.1 & & & \\
\hline & & 55.5002 & 970 & 91.5 & & & \\
\hline & 2 & 62.2480 & 8.15 & 292.8 & & 0 & \\
\hline & 013 & 56.7461 & 7.42 & 8 & & 0.0 & \\
\hline & 14 & 5046 & & & & & \\
\hline & 5 & 1.7521 & & 295 & 4 & & \\
\hline & 16 & 67.2523 & 8.17 & 296.5 & 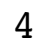 & 0 & \\
\hline & 17 & 062 & 500 & 6 & 4 & & \\
\hline & 018 & 563 & & 4 & $\Psi$ & & \\
\hline & 019 & 011 & 3.70 & 295.5 & 4 & & \\
\hline & 20 & 89.1791 & 750 & 294.3 & 4 & 0 & \\
\hline & 21 & 92.2519 & & & 4 & & \\
\hline & 3022 & 2489 & 0 & 29 & 4 & & \\
\hline & 33023 & 106.4383 & 5 . & 294.9 & 4 & 0 & \\
\hline & 33024 & 127 . & & & 4 & & \\
\hline & 3311 & 928 & & 29 & 4 & & \\
\hline & 3312 & 142.9989 & 0 & 29 & 4 & & 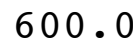 \\
\hline & 3313 & 149.2501 & & & 4 & & \\
\hline & 3314 & 148.5000 & 0 & & 4 & & \\
\hline & 3315 & 49.4944 & 6 . & 292.4 & 4 & & . \\
\hline & 3316 & 147.9963 & 4.57 & 291.3 & 4 & & 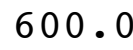 \\
\hline & 3317 & 144 . & 0 & 291.7 & 4 & & \\
\hline & 3318 & 145.5064 & 0 & .7 & 4 & & . \\
\hline & 3319 & 151.7499 & 3500 & 292.1 & 4 & & \\
\hline & & 154.7497 & 13. & & 4 & & \\
\hline & & 157.5054 & 9 . & 3.7 & 4 & & \\
\hline & 33112 & 156.7451 & 0 & 295.1 & 4 & & \\
\hline & 33113 & 143.4829 & 5. & & 3 & & \\
\hline & 33114 & 139.7473 & 4.3000 & 297.2 & 3 & & . \\
\hline & 33115 & 140.9977 & 0 & 297.3 & 3 & & \\
\hline & & 136.3756 & 000 & 297 & 3 & & \\
\hline & & 112.8915 & 4.1000 & 297.8 & 3 & & \\
\hline & 33118 & 77.9989 & 6.3000 & 296.0 & 4 & & 0 . \\
\hline & & 000 & 6 . & 294 & 4 & & \\
\hline & & 70.0000 & 8.2750 & & 4 & & \\
\hline & 33121 & 63.2500 & 8.9250 & 293.3 & 4 & & 2 \\
\hline & & 66 & & & 4 & & \\
\hline & 3123 & 69.9785 & 7.9000 & 291.5 & 1 & 600.0 & 60 \\
\hline
\end{tabular}




\begin{tabular}{|c|c|c|c|c|c|c|c|}
\hline & 64 & 0.1000 & 7.1500 & 291.2 & & & \\
\hline & $\begin{array}{lll}4 & 1 & 1\end{array}$ & 4.2495 & 8.7000 & 290.9 & & & \\
\hline & $\begin{array}{lll}4 & 1 & 2\end{array}$ & 2463 & 9750 & 290.3 & & 5 & \\
\hline & $\begin{array}{lll}4 & 1 & 3\end{array}$ & 6.7499 & 0.8250 & 289.3 & & 00 & \\
\hline & 14 & 8.7501 & & 38.8 & & & \\
\hline & 15 & 018 & 5500 & 88.6 & & 00 . & \\
\hline & 416 & 998 & 3500 & 287.3 & & & \\
\hline & $\begin{array}{lll}4 & 1 & 7\end{array}$ & 7496 & 9.5500 & & & & \\
\hline & $\begin{array}{lll}4 & 1 & 8\end{array}$ & 2500 & 250 & 83 & & & \\
\hline & 9 & 995 & 10.9000 & 282.8 & & & \\
\hline & 110 & 7537 & 7750 & 4 & & & \\
\hline & 111 & & & & & & \\
\hline & 12 & 010 & 750 & 89.4 & & 0 & \\
\hline & 4113 & 81.5000 & 7.0500 & 3 & & 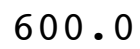 & \\
\hline & $4 \quad 114$ & & & & & & \\
\hline & 15 & 66 & 50 & 289.6 & & & \\
\hline & 16 & $/ 494$ & 8.4500 & 289.6 & & & \\
\hline & $4 \quad 117$ & 7 & 0 & & & & \\
\hline & $4 \quad 118$ & 998 & 0 & 28 & & & \\
\hline & 19 & 6 & & & & & \\
\hline & 4120 & 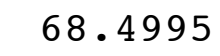 & & 2 & & & \\
\hline & 4121 & 1 & & 2 & 4 & & \\
\hline & 22 & 996 & & 280 & & & • \\
\hline & 4123 & 000 & & 2 & 4 & & \\
\hline & $4 \quad 124$ & & & & $\boldsymbol{I}$ & & \\
\hline & $\begin{array}{lll}4 & 2 & 1\end{array}$ & 54 & 50 & 27 & 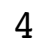 & & \\
\hline & 422 & 501 & 500 & 2 & $I$ & & \\
\hline & 423 & 0 & & 279 & 4 & & \\
\hline & 424 & 9 & 0 & 27 & 4 & & \\
\hline & 425 & 031 & 00 & 278.9 & $I$ & & \\
\hline & 426 & 7 & & & 4 & & \\
\hline & $\begin{array}{lll}4 & 2 & 7\end{array}$ & & 0 & & 4 & & \\
\hline & 428 & 69. & 000 & 278.7 & $x$ & & \\
\hline & 429 & 70 & & & 4 & & \\
\hline & 4210 & & 0 & & 3 & & \\
\hline & 4211 & & & & 3 & & \\
\hline & 4212 & 2.7540 & 0 & & 3 & & \\
\hline & 4213 & 17548 & 0 & & $3+3>$ & & \\
\hline & 4214 & 121.2705 & & $2 \varepsilon$ & 3 & & \\
\hline & 4215 & 74 & & & 3 & & \\
\hline & 4216 & 105.5583 & & & & & \\
\hline & 4217 & 119.5259 & 6.3750 & 284.6 & 4 & & \\
\hline & 4218 & 79.4487 & & 283.0 & 4 & & \\
\hline & 4219 & 54.7476 & & & 4 & & \\
\hline s & 4220 & 59.0000 & 9.1000 & 279.9 & 4 & 60 & \\
\hline & 4221 & 000 & 9.2000 & 279.5 & 4 & 600 & \\
\hline & 222 & 1500 & & & 4 & & \\
\hline & 223 & 57.7500 & 9.5250 & 278.9 & & 600.0 & 600 \\
\hline
\end{tabular}




\begin{tabular}{|c|c|c|c|c|c|c|c|c|}
\hline 1 & 4 & 224 & 63.0000 & 9.5500 & 278.5 & 4 & 600.0 & 600.0 \\
\hline 01 & 4 & 31 & 66.2512 & 8.8500 & 278.0 & 4 & 600.0 & 600.0 \\
\hline 01 & 4 & 32 & 65.9997 & 8.3500 & 277.6 & 4 & 600.0 & 00.0 \\
\hline 01 & 4 & 33 & 71.0000 & 5.8750 & 277.2 & 4 & 600.0 & 00.0 \\
\hline 01 & 4 & 34 & 79.0038 & 6.6750 & 276.7 & 4 & 600.0 & 00.0 \\
\hline 01 & 4 & 35 & 68.2501 & 7.6000 & 276.4 & 4 & 600.0 & 00.0 \\
\hline 01 & 4 & 36 & 74.2539 & 7.9250 & 276.2 & 4 & 600.0 & 00.0 \\
\hline 01 & 4 & 37 & 82.7505 & 7.5750 & 276.3 & 4 & 600.0 & 00.0 \\
\hline 01 & 4 & 38 & 74.4990 & 5.9250 & 277.3 & 4 & 600.0 & 00.0 \\
\hline 01 & 4 & 39 & 90.7827 & 2.9000 & 279.6 & 3 & 600.0 & 00.0 \\
\hline 01 & 4 & 310 & 167.5464 & 2.5750 & 281.6 & 2 & 600.0 & 00.0 \\
\hline 01 & 4 & 311 & 142.7229 & 2.3750 & 282.6 & 1 & 600.0 & 00.0 \\
\hline 01 & 4 & 312 & 156.4616 & 3.8750 & 282.4 & 2 & 600.0 & 00.0 \\
\hline 01 & 4 & 313 & 141.1167 & 4.0500 & 283.5 & 3 & .0 & 00.0 \\
\hline 01 & 4 & 314 & 136.4413 & 750 & 284.4 & 3 & .0 & \\
\hline 01 & 4 & 315 & 143.2869 & 4.9500 & 284.5 & 3 & 600.0 & 00.0 \\
\hline 01 & 4 & 316 & 127.1766 & 4.9250 & 284.7 & 3 & 600.0 & 600.0 \\
\hline 01 & 4 & 317 & 104.6966 & 5.0250 & 284.4 & 3 & .0 & \\
\hline 01 & 4 & 318 & 74.9628 & 6.7250 & 283.1 & 4 & 600.0 & .0 \\
\hline 01 & 4 & 319 & 61.7499 & 8.1500 & 281.0 & 4 & 60 & .0 \\
\hline 01 & 4 & 320 & 60.7500 & 9.57 & 279.6 & 4 & 60 & \\
\hline 01 & 4 & 321 & 63.2500 & 9.6250 & 278.9 & 4 & .0 & \\
\hline 01 & 4 & 322 & 67.7491 & 8.9000 & 278.7 & 4 & 600.0 & 500.0 \\
\hline 01 & 4 & 323 & 71.7500 & 8.30 & 278.6 & 4 & 60 & .0 \\
\hline 01 & 4 & 324 & 74.0001 & 8.6750 & 278.7 & 4 & .0 & \\
\hline 01 & 4 & 41 & 72.5000 & 8.2500 & 278.8 & 4 & 600.0 & 600.0 \\
\hline 01 & 4 & 42 & 76.2501 & 7.3000 & 278.7 & 4 & 600.0 & .0 \\
\hline 01 & 4 & 43 & 78.7500 & 5.8000 & 278.9 & 4 & 0 & \\
\hline 01 & 4 & 44 & 87.9966 & 2.7250 & 279.0 & 4 & 600.0 & 600.0 \\
\hline 01 & 4 & 45 & 116.5046 & 1.5500 & 278.8 & 5 & 600.0 & 600.0 \\
\hline 01 & 4 & 46 & 97.4896 & 4.0750 & 278.1 & 4 & .0 & \\
\hline 01 & 4 & 47 & 113.2505 & 3.3000 & 278.6 & 4 & .0 & 600.0 \\
\hline 01 & 4 & 48 & 122.4951 & 3.2750 & 278.9 & 4 & 600.0 & 600.0 \\
\hline 01 & 4 & 49 & 154.7133 & 4.3250 & 280.3 & 3 & 600.0 & 0.0 \\
\hline 01 & 4 & 410 & 158.2591 & 5.2750 & 281.2 & 3 & 600.0 & 600.0 \\
\hline 01 & 4 & 411 & 164.2535 & 5.4250 & 282.3 & 3 & 600.0 & 600.0 \\
\hline 01 & 4 & 412 & 176.2539 & 4.5500 & 283.6 & 3 & 600.0 & 600.0 \\
\hline 01 & 4 & 413 & 181.4042 & 4.3500 & 284.2 & 3 & 600.0 & 600.0 \\
\hline 01 & 4 & 414 & 206.1782 & 3.3250 & 285.1 & 2 & 600.0 & 600.0 \\
\hline 01 & 4 & 415 & 142.5459 & 3.5500 & 286.1 & 2 & 600.0 & 600.0 \\
\hline 01 & 4 & 416 & 184.7621 & 3.9250 & 286.5 & 2 & 600.0 & 600.0 \\
\hline 01 & 4 & 417 & 179.5278 & 4.6250 & 285.5 & 3 & 600.0 & 600.0 \\
\hline 01 & 4 & 418 & 163.8201 & 3.7750 & 285.0 & 2 & 600.0 & 600.0 \\
\hline 01 & 4 & 419 & 91.7032 & 4.4000 & 284.0 & 3 & 600.0 & 600.0 \\
\hline 01 & 4 & 420 & 73.7478 & 6.1000 & 282.7 & 4 & 600.0 & 600.0 \\
\hline 01 & 4 & 421 & 71.2512 & 6.6500 & 282.1 & 4 & 600.0 & 600.0 \\
\hline 01 & 4 & 422 & 66.2500 & 7.4750 & 281.8 & 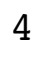 & 600.0 & 600.0 \\
\hline . & 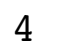 & 423 & 68.7498 & 7.750 & 282.1 & 4 & 600.0 & 600.0 \\
\hline
\end{tabular}




\begin{tabular}{|c|c|c|c|c|c|c|c|c|}
\hline 1 & 4 & 424 & 65.5001 & 8.7750 & 282.4 & 4 & 600.0 & 600.0 \\
\hline 0 & 4 & 51 & 67.7502 & 8.1000 & 282.5 & 4 & 600.0 & 600.0 \\
\hline 01 & 4 & 52 & 69.9996 & 7.6000 & 282.7 & 4 & 600.0 & 00.0 \\
\hline 01 & 4 & 53 & 70.7494 & 7.7250 & 282.7 & 4 & 600.0 & 600.0 \\
\hline 01 & 4 & 54 & 73.9993 & 7.4250 & 282.6 & 4 & 600.0 & 00.0 \\
\hline 01 & 4 & 55 & 69.9993 & 7.5000 & 282.5 & 4 & 600.0 & 500.0 \\
\hline 01 & 4 & 56 & 76.4983 & 7.9000 & 282.5 & 4 & 600.0 & 600.0 \\
\hline 01 & 4 & 57 & 72.7514 & 7.2250 & 282.4 & 4 & 600.0 & 500.0 \\
\hline 01 & 4 & 58 & 77.2479 & 6.1000 & 282.4 & 4 & 600.0 & 00.0 \\
\hline 01 & 4 & 59 & 86.5000 & 5.5750 & 283.7 & 3 & 600.0 & 600.0 \\
\hline 01 & 4 & 510 & 86.7476 & 4.4750 & 284.8 & 3 & 600.0 & 600.0 \\
\hline 01 & 4 & 511 & 122.3651 & 4.7750 & 286.2 & 3 & 600.0 & \\
\hline 01 & 4 & 512 & 125.4960 & 4.5500 & 287.1 & 3 & 600.0 & 600.0 \\
\hline 01 & 4 & 513 & 123.9987 & 5.0750 & 287.9 & 3 & 600.0 & 600.0 \\
\hline 01 & 4 & 514 & 90.7379 & 5.9500 & 288.4 & 3 & 600.0 & \\
\hline 01 & 4 & 515 & 88.0028 & 6.6250 & 288.6 & 4 & 600.0 & 60 \\
\hline 01 & 4 & 516 & 81.7519 & 7.6750 & 288.1 & 4 & 600.0 & 600.0 \\
\hline 01 & 4 & 517 & 64.9166 & 7.9250 & 287.0 & 4 & 600.0 & \\
\hline 01 & 4 & 518 & 66.7509 & 9.1500 & 285.3 & 4 & 600.0 & 60 \\
\hline 01 & 4 & 519 & 69.4999 & 10.9500 & 283.5 & 4 & 600.0 & 600.0 \\
\hline 01 & 4 & 520 & 65.9998 & 12.8500 & 281.1 & 4 & 600.0 & .0 \\
\hline 01 & 4 & 521 & 60.4983 & 14.4000 & 280.2 & 4 & 600.0 & \\
\hline 01 & 4 & 522 & 65.7503 & 11.5250 & 280.3 & 4 & 600.0 & 600.0 \\
\hline 01 & 4 & 523 & 64.7497 & 12.1000 & 279.8 & 4 & 600.0 & .0 \\
\hline 01 & 4 & 524 & 60.5000 & 13.5250 & 279.7 & 4 & 600.0 & \\
\hline 01 & 4 & 61 & 60.0000 & 12.8000 & 279.9 & 4 & 600.0 & 60 \\
\hline 01 & 4 & 62 & 61.2500 & 12.9000 & 279.7 & 4 & 600.0 & 600.0 \\
\hline 01 & 4 & 63 & 59.7502 & 12.2250 & 279.8 & 4 & 600.0 & .0 \\
\hline 01 & 4 & 64 & 60.7501 & 11.4750 & 279.8 & 4 & 600.0 & 60 \\
\hline 01 & 4 & 65 & 62.7496 & 10.3250 & 280.0 & 4 & 600.0 & 600.0 \\
\hline 01 & 4 & 66 & 54.2517 & 7.62 & 280.3 & 4 & 600.0 & .0 \\
\hline 01 & 4 & 67 & 61.5000 & 7.3000 & 279.4 & 4 & 600.0 & 60 \\
\hline 01 & 4 & 68 & 4.7939 & 3.4250 & 278.8 & 4 & 600.0 & 600.0 \\
\hline 01 & 4 & 69 & 328.5242 & 3.6500 & 279.5 & 4 & 600.0 & .0 \\
\hline 01 & 4 & 610 & 305.2489 & 3.82 & 280.5 & 4 & 600.0 & 6 \\
\hline 01 & 4 & 611 & 307.2500 & 3.0750 & 281.5 & 3 & 600.0 & 600.0 \\
\hline 01 & 4 & 612 & 319.2354 & 2.3000 & 282.6 & 2 & 600.0 & 600.0 \\
\hline 01 & 4 & 613 & 281.3044 & 1.1000 & 284.1 & 1 & 600.0 & 600.0 \\
\hline 01 & 4 & 614 & 14.5628 & 3.0750 & 283.9 & 2 & 600.0 & 600.0 \\
\hline 01 & 4 & 615 & 3.7489 & 3.6500 & 283.7 & 2 & 600.0 & \\
\hline 01 & 4 & 616 & 0.4926 & 3.6750 & 283.8 & 2 & 600.0 & 600.0 \\
\hline 01 & 4 & 617 & 0.2502 & 4.2500 & 283.9 & 3 & 600.0 & 600.0 \\
\hline 01 & 4 & 618 & 12.2502 & 4.2250 & 283.7 & 3 & 600.0 & 600.0 \\
\hline 01 & 4 & 619 & 71.4527 & 3.9000 & 282.2 & 4 & 600.0 & 600.0 \\
\hline 01 & 4 & 620 & 79.7491 & 5.2250 & 280.4 & 4 & 600.0 & 600.0 \\
\hline 01 & 4 & 621 & 74.2493 & 6.6500 & 279.8 & 4 & 600.0 & 600.0 \\
\hline 01 & 4 & 622 & 55.5000 & 6.275 & 279.8 & 4 & 600.0 & 600.0 \\
\hline 01 & 4 & 623 & 60.7030 & 5.2250 & 279.5 & 4 & 600.0 & 600.0 \\
\hline
\end{tabular}




\begin{tabular}{|c|c|c|c|c|c|c|c|c|}
\hline 01 & 4 & 624 & 89.4807 & 4.4250 & 279.6 & 4 & 600.0 & 600.0 \\
\hline 01 & 4 & 71 & 80.2539 & 3.7250 & 279.3 & 4 & 600.0 & 600.0 \\
\hline 01 & 4 & 72 & 66.2497 & 4.8750 & 279.1 & 4 & 600.0 & 00.0 \\
\hline 01 & 4 & 73 & 75.0000 & 4.2750 & 278.0 & 4 & 600.0 & 00.0 \\
\hline 01 & 4 & 74 & 69.0034 & .0250 & 277.7 & 4 & 600.0 & 00.0 \\
\hline 01 & 4 & 75 & 73.3076 & 4.1500 & 278.0 & 4 & 600.0 & 00.0 \\
\hline 01 & 4 & 76 & 67.0034 & 5.8250 & 278.3 & 4 & 600.0 & 00.0 \\
\hline 01 & 4 & 77 & 77.7621 & 5.2750 & 277.7 & 4 & 600.0 & 00.0 \\
\hline 01 & 4 & 78 & 66.2429 & 5.6000 & 278.3 & 3 & 600.0 & 00.0 \\
\hline 01 & 4 & 79 & 69.4686 & 6.1750 & 279.0 & 4 & 600.0 & 00.0 \\
\hline 01 & 4 & 710 & 79.5145 & 4.0750 & 280.0 & 3 & 600.0 & 00.0 \\
\hline 01 & 4 & 711 & 66.7453 & 6.7000 & 280.7 & 4 & 600.0 & 00.0 \\
\hline 01 & 4 & 712 & 72.2547 & 6.3000 & 281.2 & 4 & 600.0 & 00.0 \\
\hline 01 & 4 & 713 & 70.7191 & 7.0500 & 281.7 & 4 & 600.0 & 00.0 \\
\hline 01 & 4 & 714 & 71.4681 & 7.2750 & 282.6 & 4 & .0 & \\
\hline 01 & 4 & 715 & 100.1788 & 7.7500 & 282.4 & 4 & 600.0 & 00.0 \\
\hline 01 & 4 & 716 & 145.4488 & 6.9250 & 278.9 & 4 & 600.0 & 600.0 \\
\hline 01 & 4 & 717 & 56.2775 & 2.8750 & 277.1 & 3 & .0 & \\
\hline 01 & 4 & 718 & 187.9256 & 2.6500 & 278.0 & 2 & 600.0 & 00.0 \\
\hline 01 & 4 & 719 & 42.2187 & 4.2250 & 278.7 & 3 & 60 & .0 \\
\hline 01 & 4 & 720 & 77.2497 & 6.6250 & 279.4 & 4 & .0 & \\
\hline 01 & 4 & 721 & 70.2488 & 6.8250 & 278.7 & 4 & 600.0 & .0 \\
\hline 01 & 4 & 722 & 65.7496 & 7.3250 & 278.4 & 4 & 600.0 & 600.0 \\
\hline 01 & 4 & 723 & 63.2500 & .1500 & 278.2 & 4 & .0 & .0 \\
\hline 01 & 4 & 724 & 61.5000 & 8.7750 & 278.0 & 4 & .0 & \\
\hline 01 & 4 & 81 & 59.5001 & 9.3750 & 277.9 & 4 & 600.0 & 600.0 \\
\hline 01 & 4 & 82 & 58.5000 & 9.9250 & 277.7 & 4 & 600.0 & .0 \\
\hline 01 & 4 & 83 & 61.2515 & 8.7000 & 277.6 & 4 & 600.0 & \\
\hline 01 & 4 & 84 & 66.4962 & 6.8500 & 277.5 & 4 & 600.0 & 600.0 \\
\hline 01 & 4 & 85 & 70.2499 & 7.1250 & 277.6 & 4 & 600.0 & 600.0 \\
\hline 01 & 4 & 86 & 62.2498 & 7.3500 & 277.6 & 4 & .0 & .0 \\
\hline 01 & 4 & 87 & 59.2504 & 5.6750 & 277.9 & 4 & 600.0 & 600.0 \\
\hline 01 & 4 & 88 & 43.5172 & 3.8750 & 279.0 & 3 & 600.0 & 600.0 \\
\hline 01 & 4 & 89 & 55.4995 & 4.7250 & 279.9 & 3 & 600.0 & 0.0 \\
\hline 01 & 4 & 810 & 67.2008 & 3.4500 & 281.1 & 2 & 600.0 & 600.0 \\
\hline 01 & 4 & 811 & 106.1249 & 3.3000 & 281.5 & 2 & 600.0 & 600.0 \\
\hline 01 & 4 & 812 & 107.5276 & 3.6250 & 282.1 & 2 & 600.0 & 600.0 \\
\hline 01 & 4 & 813 & 335.4299 & 3.7750 & 281.7 & 2 & 600.0 & 600.0 \\
\hline 01 & 4 & 814 & 71.0540 & 2.6500 & 283.4 & 1 & 600.0 & 600.0 \\
\hline 01 & 4 & 815 & 304.7257 & $5.1 / 50$ & 281.7 & 2 & 600.0 & 600.0 \\
\hline 01 & 4 & 816 & 329.4566 & 3.8500 & 280.8 & 3 & 600.0 & 600.0 \\
\hline 01 & 4 & 817 & 321.5000 & 2.9750 & 281.1 & 3 & 600.0 & 600.0 \\
\hline 01 & 4 & 818 & 320.4960 & 3.1750 & 281.0 & 2 & 600.0 & 600.0 \\
\hline 01 & 4 & 819 & 36.5139 & 3.4500 & 280.4 & 3 & 600.0 & 600.0 \\
\hline 01 & 4 & 820 & 94.8137 & 3.1500 & 279.4 & 4 & 600.0 & 600.0 \\
\hline 01 & 4 & 821 & 144.2256 & 5.1250 & 279.0 & 4 & 600.0 & 600.0 \\
\hline 01 & 4 & 822 & 145.6976 & 3.2750 & 277.7 & 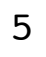 & 600.0 & 600.0 \\
\hline - & 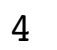 & 823 & 93.8614 & 1.375 & 277.0 & 6 & 600.0 & 600.0 \\
\hline
\end{tabular}




\begin{tabular}{|c|c|c|c|c|c|c|c|}
\hline & 24 & - & 2.0750 & 277.2 & & & \\
\hline & $\begin{array}{lll}4 & 9 & 1\end{array}$ & 07333 & .2250 & 277.4 & & & \\
\hline & $\begin{array}{lll}4 \quad 92\end{array}$ & 72.233 & 500 & 277.5 & & 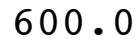 & \\
\hline & 493 & 82.9920 & 750 & 277.7 & & 00 & \\
\hline & $\begin{array}{lll}4 & 9 & 4\end{array}$ & 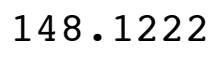 & & & & & \\
\hline & 495 & 7494 & 0 & 277.6 & & 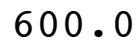 & \\
\hline & 496 & 163.4968 & 3.72 & 277.2 & & & \\
\hline & $\begin{array}{lll}4 & 9 & 7\end{array}$ & 160 & .4000 & & & & \\
\hline & 49 & 157. & 8.45 & & & & \\
\hline & $\begin{array}{lll}4 & 9 & 9\end{array}$ & 164 . & & & & & \\
\hline & 4910 & 057 & 0 & .4 & & & \\
\hline & 4911 & & & & & & \\
\hline & 4912 & 159.4991 & 0 & & & 0 & \\
\hline & 4913 & 155.0002 & 0 & & & 0 & \\
\hline & 4914 & & & & & & \\
\hline & 4915 & 155 . & & & 4 & & \\
\hline & 4916 & 158.7519 & 0 & & & & \\
\hline & 4917 & 155 . & & & & & \\
\hline & 4918 & 137 . & & & & & \\
\hline & 4919 & 102.75 & & & & & \\
\hline & 4920 & 129 . & 0 & & & & \\
\hline & $4 \quad 921$ & 14 & & & & & \\
\hline & 4922 & 140 . & & 2 & & & \\
\hline & 4923 & 1 & & & & & \\
\hline & 4924 & & & & 4 & & \\
\hline & 4101 & 142 & & & 4 & & \\
\hline & 4102 & 142.7512 & 0 & & - & & \\
\hline & 4103 & 142.250 & & & 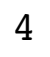 & & \\
\hline & 4104 & 146 . & 0 & & 4 & & \\
\hline & $410 \quad 5$ & 150.2501 & 0 & & 4 & & \\
\hline & 4106 & 149.500 & & & A & & \\
\hline & 4107 & 150 . & & & 4 & & \\
\hline & 4108 & 155.9996 & & & 4 & & \\
\hline & 4109 & 156 . & & & 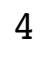 & & \\
\hline & 41010 & & & & 4 & & \\
\hline & 41011 & 161.4945 & & & 4 & & \\
\hline & & 285 & & & 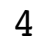 & & \\
\hline & 41013 & 121. & & & 3 & & \\
\hline & 41014 & 500 & & .7 & 3 & & \\
\hline & & 66 . & & & $\mathbf{Y}$ & & \\
\hline & 41016 & 64 . & & & 4 & & \\
\hline & 41017 & 77 . & 7 . & 5.3 & 4 & & \\
\hline & & 998 & & & 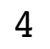 & & \\
\hline & 41019 & 80.5000 & & & & & \\
\hline & 41020 & 80.0012 & 10.0750 & 282.5 & 4 & & \\
\hline & 21 & 506 & & & 4 & & \\
\hline & 410 & 1505 & 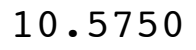 & & & & \\
\hline & $\$ 1023$ & 64.2500 & 9.2750 & 282.0 & & 600.0 & 600 \\
\hline
\end{tabular}




\begin{tabular}{|c|c|c|c|c|c|c|c|}
\hline & 41024 & .0004 & 9.7750 & 281.6 & 4 & (6) & \\
\hline & 4111 & 1.0002 & 11.6000 & 280.5 & 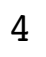 & 00,0 & \\
\hline & 4112 & 7499 & 500 & 280.3 & & & \\
\hline & 4113 & 3.2682 & 0.4250 & 80.4 & & & \\
\hline & 114 & & & 80.0 & & 00.0 & \\
\hline & 115 & 002 & & 80.1 & & 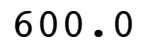 & \\
\hline & 116 & 123 & & 280.3 & & & \\
\hline & 117 & & & & & & \\
\hline & 118 & 66.4963 & 6.9000 & 80.8 & & 00 & 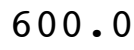 \\
\hline & 119 & 75.2504 & & 281.5 & & & \\
\hline & 1110 & 67.4969 & & & & & \\
\hline & 1111 & 64 . & & 82.9 & & & \\
\hline & 1112 & 63.9963 & 9.2500 & 283.2 & & & \\
\hline & 1113 & 63.2434 & & & & & \\
\hline & & 57 . & & & & & \\
\hline & 5 & 57.0084 & 0 & & & & \\
\hline & 1116 & 963 & & & & & \\
\hline & 1117 & 53. & 0 & & & & \\
\hline & 1118 & 55 . & 50 & 282.3 & 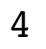 & & 00 \\
\hline & 41119 & 60.7500 & 0 & 281.0 & 4 & & \\
\hline & 1120 & 62.25 & 0 & & 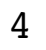 & & \\
\hline & 1 & 73 & 0 & 0 & 1 & & \\
\hline & 1122 & 71.0001 & 11.1500 & 280.3 & 4 & & \\
\hline & 1123 & 71.4991 & & & 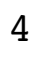 & & \\
\hline & 4 & 78 & 0 & & 4 & & \\
\hline & 4121 & 79.0000 & 500 & 281.4 & 4 & & \\
\hline & 4122 & 98.2585 & & & 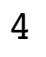 & & \\
\hline & 4123 & 28.7706 & 0 & 9 & 4 & & \\
\hline & 4124 & 38.5002 & & 282.3 & 4 & & \\
\hline & 4125 & 675 & & 1.8 & 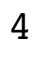 & & \\
\hline & 4126 & 1 & & & 4 & & \\
\hline & 4127 & & & 281.3 & 4 & & \\
\hline & 4128 & 151.2500 & 750 & 281.8 & 4 & & \\
\hline & 4129 & 7 & 0 & 282.5 & 4 & & \\
\hline & 41210 & 161.4997 & & .4 & 4 & & \\
\hline & 41211 & 160.2487 & & 284.3 & 4 & & \\
\hline & 41212 & 161. & 5. & & 3 & & \\
\hline & 41213 & 16 & 000 & 5.8 & 3 & & \\
\hline & 41214 & 173.00 & & 288.1 & 2 & & \\
\hline & 41215 & 166. & & & & & \\
\hline & 41216 & 139.9855 & 750 & 3.7 & 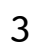 & & \\
\hline & 41217 & 101.5918 & 750 & 288.7 & 3 & 0 & \\
\hline & 41218 & 84 . & & 287.4 & 0 & & \\
\hline & 41219 & 71.0000 & 750 & 285.1 & 4 & 0 & \\
\hline & 41220 & 73.7466 & 7.5500 & 284.1 & 4 & 600.0 & 00 \\
\hline & 41221 & & & & 4 & & \\
\hline & & 59.2383 & & & 4 & & \\
\hline & $12<2$ & 75.7506 & 8.2250 & 282.0 & 4 & .0 & 60 \\
\hline
\end{tabular}




\begin{tabular}{|c|c|c|c|c|c|c|c|}
\hline 1 & 1224 & 2.2501 & 8.7750 & 282.0 & 4 & 000.0 & \\
\hline & 4131 & 7.5000 & 9.7250 & 281.5 & a & 600.0 & \\
\hline & 4132 & 000 & 3000 & 81.3 & 4 & 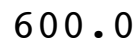 & \\
\hline & 4133 & 71.2501 & 9.3500 & 280.9 & & 00 & \\
\hline & 4134 & 6.5000 & 00 & 30.8 & & & \\
\hline & 135 & 7500 & 1.3500 & 79.8 & & 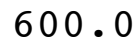 & \\
\hline & 4136 & 71.4973 & 9.7000 & 279.2 & & 00 & \\
\hline & 137 & 69.5000 & 2.37 & 79.1 & & 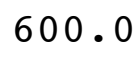 & \\
\hline & 138 & 500 & 12.6500 & 279.2 & & 0 & \\
\hline & 139 & 057 & 12.0750 & 279.9 & & 0 & \\
\hline & 1310 & 65 & & 81.1 & & & \\
\hline & 1311 & 00 & & 81.8 & & & \\
\hline & 1312 & 021 & & 282.7 & & 0 & \\
\hline & 313 & 982 & 0 & & & & \\
\hline & & 68 & 0 & 2 & & & \\
\hline & 5 & 997 & & 284.3 & 4 & & \\
\hline & 1316 & 76.7506 & 9.97 & 283.3 & & 0 & \\
\hline & 7 & 70.2500 & 0 & 282.8 & $\Psi$ & & \\
\hline & 8 & 14 & 0 & 281.6 & 4 & & \\
\hline & 1319 & 70.2498 & 11. & 280.3 & 1 & & \\
\hline & 20 & 69 . & 11. & & & & \\
\hline & 1 & 73. & & 279.8 & 4 & & \\
\hline & 322 & 78.5001 & 8 . & 279.6 & 4 & & \\
\hline & 323 & 78.2498 & & & a & & \\
\hline & 24 & 80 . & & & 4 & & \\
\hline & 141 & 75.4885 & & 281.8 & 4 & & \\
\hline & 4142 & 77.7500 & 0 & 281.5 & 4 & & \\
\hline & 143 & 81.7500 & & 79.9 & 4 & & \\
\hline & 144 & 79.9988 & & 280.2 & A & & \\
\hline & 4145 & 00.4238 & & 281.4 & 5 & & \\
\hline & 4146 & 1.2535 & & 4 & 5 & & \\
\hline & $\begin{array}{ll}414 & 7\end{array}$ & 127 . & & 281.2 & 6 & & \\
\hline & 4148 & 75.8314 & 0 & 282.3 & 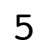 & & \\
\hline & 4149 & 9.1852 & & 284 & 4 & & \\
\hline & 41410 & 1 & & & 3 & & \\
\hline & 411 & 109.2177 & & 286.4 & & & \\
\hline & 41412 & 175.5002 & 5 . & 286 & 3 & & \\
\hline & 41413 & 177.5202 & & 5.8 & 3 & & \\
\hline & 41414 & 138.7123 & 2.8000 & 288.4 & 2 & & \\
\hline & & 165 . & & & 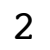 & & \\
\hline & & 138.0164 & & 288.3 & 3 & & \\
\hline & 41417 & 102.0034 & 4.4500 & 288.2 & 3 & & \\
\hline & 41418 & 85.7531 & 50 & 287.1 & 4 & & \\
\hline & 41419 & 73.7476 & & & 4 & & $\cos _{1}+2$ \\
\hline & 41420 & 80.4997 & 5.7750 & 285.3 & 4 & 0 & 00 . \\
\hline & & & & & 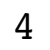 & & \\
\hline & & 73.5003 & 8.4000 & & 4 & & \\
\hline & $4<3$ & 76.5000 & 6.9000 & 283.5 & & 600.0 & 600 \\
\hline
\end{tabular}




\begin{tabular}{|c|c|c|c|c|c|c|c|}
\hline & 24 & 7499 & 250 & 82.4 & 4 & 600.0 & \\
\hline & 4151 & 6.9423 & 2.6750 & 283.3 & & 600.0 & \\
\hline & 4152 & 52.2931 & 0.8000 & 283.2 & & 00 & \\
\hline & 153 & 105.8072 & L. 2000 & 282.7 & & 00 & \\
\hline & 154 & 0.5370 & .8250 & 81.0 & & 00 & \\
\hline & 155 & 105.9614 & .3750 & 81.3 & & 00 & \\
\hline & 156 & 114.5365 & 0.8000 & 280.2 & & 500 & 0 \\
\hline & 157 & 141.4236 & 1.0500 & 79.7 & & 00.0 & \\
\hline & 158 & 285.1970 & .5750 & 82.6 & & & \\
\hline & 159 & 239.4734 & 1.3500 & 284.2 & & 500 & 0 \\
\hline & 1510 & 256.0030 & 2.0500 & 285.0 & & 00.0 & \\
\hline & 1511 & 272.9345 & 1.8000 & 86.4 & & 0 & \\
\hline & 1512 & 227.4298 & 1.8500 & 288.6 & & 0 & . \\
\hline & 1513 & 216.9465 & 2.9500 & 289.1 & & 0.0 & 0 \\
\hline & 1514 & 182 . & 0 & 9.8 & & & \\
\hline & 515 & 154 . & & 290.8 & 1 & & \\
\hline & 1516 & 132.3070 & 3.5250 & 290.8 & & .0 & 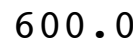 \\
\hline & 1517 & 145 . & 0 & 0.4 & & 0 & \\
\hline & 518 & 91. & 4 . & 288.7 & 3 & & \\
\hline & 519 & 75.7557 & 4.2500 & 287.3 & 4 & 000.0 & U. \\
\hline & 1520 & 69.7514 & 2.9750 & 286.9 & 4 & 0 & 0. \\
\hline & 521 & 83.6089 & & 286.1 & 5 & & \\
\hline & 1522 & 53. & 0 & 285.8 & 6 & & \\
\hline & 41523 & 28.9919 & 1.40 & 286.1 & 6 & 0 & . \\
\hline & 41524 & 42.4968 & & 285.8 & 6 & & \\
\hline & 4161 & 354 . & 7750 & 285.3 & 6 & & \\
\hline & 4162 & 59.3853 & 2000 & 284.8 & 6 & 0 & U. \\
\hline & 4163 & 134.3170 & 4750 & 283.7 & 6 & & \\
\hline & 4164 & 80.2693 & 0.4750 & 283.0 & 6 & & $\bullet$ \\
\hline & 4165 & 88.2572 & 5000 & 283.4 & 6 & 0 & 0 • \\
\hline & 4166 & 110.7866 & .1250 & 282.6 & 6 & 0 & 0 . \\
\hline & 4167 & 108.3877 & 50 & 283.3 & 6 & & \\
\hline & 4168 & 44.1735 & 0.4250 & 285.0 & 5 & 0 & 0 • \\
\hline & 4169 & 163.0944 & 500 & 285.8 & 4 & 0 & 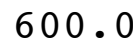 \\
\hline & 41610 & 99.2484 & 2 . & & 3 & & \\
\hline & 41611 & 106.4954 & 2.1000 & 287.5 & 2 & & 0 . \\
\hline & 41612 & 179.1508 & 2.0250 & 289.0 & 1 & .0 & 0 . \\
\hline & 41613 & 70.3604 & 0 & 290.1 & 2 & & 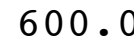 \\
\hline & 41614 & 101.6040 & 5.7500 & 289.7 & 3 & .0 & 0 . \\
\hline & 41615 & 105.0009 & 7.1750 & 289.6 & 4 & & . \\
\hline & 41616 & 100.6232 & 6.7750 & 289.7 & 4 & & 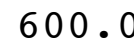 \\
\hline & 41617 & 88.7657 & 7.0500 & 289.4 & 4 & .0 & 600 . \\
\hline & 41618 & 88.9994 & 7.8000 & 288.5 & 4 & .0 & 00 . \\
\hline & 41619 & 79.7504 & 8.5750 & & 4 & 600 & 0 \\
\hline & & 68.4971 & 8.2750 & 286.6 & 4 & 600.0 & 00 . \\
\hline & 41621 & 61.9999 & 7.9500 & 286.6 & 4 & 600.0 & 500. \\
\hline & & 123 & & & 5 & & \\
\hline & 1623 & 66.2402 & 3.6500 & 285.4 & $I$ & 600.0 & 600 \\
\hline
\end{tabular}




\begin{tabular}{|c|c|c|c|c|c|c|c|}
\hline & 1624 & 0.3092 & 4.6250 & 285.0 & 4 & . & \\
\hline & $417 \quad 1$ & 32506 & .0750 & 284.8 & & & \\
\hline & 4172 & 0885 & 2.0750 & 284.2 & & 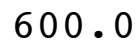 & \\
\hline & 173 & 85.2707 & 2.8750 & 284.3 & & 00 & \\
\hline & 174 & 154.5777 & 1.5250 & & & & \\
\hline & 175 & 133.7518 & 0 & 82.8 & & 00 & \\
\hline & 176 & 96.7499 & 3.4250 & 283.0 & & & \\
\hline & 177 & 113.4851 & 50 & & & & \\
\hline & 178 & 311.3871 & 0.8250 & 9 & & 0 & \\
\hline & 179 & 227.8296 & 1.7750 & 288.1 & & 0 & \\
\hline & 1710 & 230.0000 & 2.2000 & 288 & & & \\
\hline & 1711 & 182.4066 & & & & & \\
\hline & 1712 & 193.6673 & 3.6250 & 291.2 & & 0 & \\
\hline & 1713 & 174.8561 & 4.82 & 291.4 & 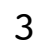 & 0 & \\
\hline & 1714 & 134 . & & 92.3 & & & \\
\hline & 1715 & 146 . & 0 & 292.9 & 3 & & \\
\hline & 1716 & 138.5111 & 0 & 293.3 & 3 & & \\
\hline & .717 & 140 . & 0 & 29 & & & \\
\hline & 1718 & 184 . & 5. & 291.8 & 4 & & \\
\hline & 1719 & 144.4245 & 0 & 291.1 & 4 & & \\
\hline & 1720 & 89 . & 0 & 29 & 4 & & \\
\hline & 1721 & 130. & & 28 & 4 & & \\
\hline & 1722 & 69.2512 & 0 & 288.2 & 4 & & U. \\
\hline & 1723 & 64 . & .15 & 287.8 & 4 & & \\
\hline & 1724 & 59. & & 287.9 & 4 & & \\
\hline & 181 & 117.2792 & 0 & 287 & 5 & & \\
\hline & 4182 & 102.00 & 0 & 287.4 & 4 & & \\
\hline & 4183 & 83. & & 287 & 4 & & \\
\hline & 4184 & 81 . & 0 & 28 & 4 & & \\
\hline & 4185 & 77. & 0 & 286.5 & 4 & & \\
\hline & 4186 & 76 . & & 28 & 4 & & \\
\hline & 4187 & 78 & 0 & 28 & 4 & & \\
\hline & 4188 & 84.2502 & 500 & 288.0 & 4 & 0 & 0 . \\
\hline & 4189 & 90 . & & 28 & 3 & & \\
\hline & 41810 & 264 . & 0 & 29 & 2 & & \\
\hline & 41811 & 291.1314 & & 290.2 & 1 & & \\
\hline & 41812 & 278 . & 0 & 291.9 & 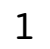 & & \\
\hline & 41813 & 143.7758 & 0 & 291.7 & 2 & & 0 • \\
\hline & 41814 & 77.7485 & 8.2250 & 289.6 & 3 & & 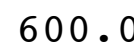 \\
\hline & 41815 & 74 . & 0 & 287.6 & 4 & & \\
\hline & 41816 & 72 . & 0 & 288.2 & 4 & & \\
\hline 1 & 41817 & 90.0059 & 8.7500 & 287.3 & 4 & 0 & 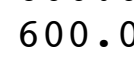 \\
\hline & 41818 & 88 . & & 285.7 & 4 & 0 & \\
\hline & 41819 & 86.7482 & & 283.7 & 4 & & \\
\hline 01 & 41820 & 72.7384 & 10.1750 & 282.3 & 4 & 60 & 00 . \\
\hline & 41821 & 46.9097 & 9.7250 & 281.9 & 4 & 600 & \\
\hline & 41822 & 66 & & 282.0 & 4 & & \\
\hline & 1823 & 45.9686 & 3.9250 & 282.0 & 4 & 600.0 & 600 \\
\hline
\end{tabular}




\begin{tabular}{|c|c|c|c|c|c|c|c|}
\hline 01 & 41824 & 90.7514 & 5.7000 & 281.1 & 4 & 600.0 & 600.0 \\
\hline 01 & 4191 & 86.5031 & 7.2500 & 281.0 & 4 & 600.0 & 600.0 \\
\hline 01 & 4192 & 78.5014 & 7.8750 & 281.0 & 4 & 600.0 & 00.0 \\
\hline 01 & 4193 & 67.7501 & 7.7000 & 280.9 & 4 & 600.0 & 00.0 \\
\hline 01 & 4194 & 63.5015 & 7.0500 & 280.9 & 4 & 600.0 & 00.0 \\
\hline 01 & 4195 & 63.7489 & 8.2000 & 280.9 & 4 & 600.0 & 00.0 \\
\hline 01 & 4196 & 68.2503 & 8.1000 & 281.0 & 4 & 600.0 & 00.0 \\
\hline 01 & 4197 & 58.9989 & 7.2250 & 281.0 & 4 & 600.0 & 00.0 \\
\hline 01 & 4198 & 71.5006 & 5.0000 & 281.5 & 4 & 600.0 & 00.0 \\
\hline 01 & 4199 & 70.7485 & 4.6750 & 281.8 & 3 & 600.0 & 00.0 \\
\hline 01 & 41910 & 84.7630 & 4.7250 & 282.3 & 3 & 600.0 & 00.0 \\
\hline 01 & 41911 & 71.2211 & 4.9250 & 282.6 & 3 & 600.0 & \\
\hline 01 & 41912 & 55.2532 & 4.5750 & 283.2 & 3 & 600.0 & 00.0 \\
\hline 01 & 41913 & 80.9326 & 4.8500 & 284.2 & 3 & 600.0 & 00.0 \\
\hline 01 & 41914 & 69.5092 & 5.6500 & 285.1 & 3 & .0 & \\
\hline 01 & 41915 & 92.7498 & 7.8750 & 284.9 & 4 & 600.0 & 00.0 \\
\hline 01 & 41916 & 101.5042 & 7.3750 & 284.3 & 4 & 600.0 & 600.0 \\
\hline 01 & 41917 & 105.0000 & 7.4750 & 283.4 & 4 & .0 & \\
\hline 01 & 41918 & 90.0000 & 7.3000 & 283.1 & 4 & 600.0 & .0 \\
\hline 01 & 41919 & 85.5006 & 6.8250 & 282.2 & 4 & .0 & .0 \\
\hline 01 & 41920 & 74.7502 & 8.92 & 281.4 & 4 & 60 & \\
\hline 01 & 41921 & 78.4914 & 6.6250 & 280.9 & 4 & .0 & \\
\hline 01 & 41922 & 85.2432 & 5.5750 & 280.5 & 4 & 600.0 & 00.0 \\
\hline 01 & 41923 & 91.2222 & 6.20 & 280.3 & 4 & 60 & .0 \\
\hline 01 & 41924 & 69.9920 & 6.1000 & 280.4 & 4 & .0 & \\
\hline 01 & 4201 & 81.9501 & 3.2250 & 280.2 & 4 & 600.0 & 600.0 \\
\hline 01 & $420 \quad 2$ & 41.8679 & 1.5500 & 280.0 & 5 & 600.0 & 600.0 \\
\hline 01 & 4203 & 353.0569 & 1.7000 & 279.5 & 6 & .0 & \\
\hline 01 & $420 \quad 4$ & 39.1381 & 1.1500 & 280.1 & 6 & .0 & 600.0 \\
\hline 01 & $420 \quad 5$ & 344.2208 & 1.7250 & 279.7 & 5 & 600.0 & 600.0 \\
\hline 01 & 4206 & 346.9908 & 2.2500 & 279.9 & 4 & .0 & \\
\hline 01 & 4207 & 335.2698 & 2.0500 & 279.9 & 5 & .0 & 600.0 \\
\hline 01 & 4208 & 307.7646 & 2.6750 & 280.8 & 4 & 600.0 & 600.0 \\
\hline 01 & $420 \quad 9$ & 314.4987 & 3.4000 & 280.6 & 4 & 600.0 & 0.0 \\
\hline 01 & 42010 & 303.0004 & 5.3250 & 280.7 & 4 & 600.0 & 600.0 \\
\hline 01 & 42011 & 311.5088 & 5.5000 & 280.9 & 4 & 600.0 & 600.0 \\
\hline 01 & 42012 & 322.2707 & 6.1250 & 280.7 & 4 & 600.0 & 600.0 \\
\hline 01 & 42013 & 296.5092 & 3.2750 & 281.1 & 4 & 600.0 & 600.0 \\
\hline 01 & 42014 & 290.5258 & 3.4000 & 281.7 & 3 & 600.0 & 600.0 \\
\hline 01 & 42015 & 302.7022 & 2.0750 & 282.8 & 2 & 600.0 & 600.0 \\
\hline 01 & 42016 & 104.9426 & 5.5500 & 281.9 & 3 & 600.0 & 600.0 \\
\hline 01 & 42017 & 79.3567 & 10.1000 & 278.9 & 4 & 600.0 & 600.0 \\
\hline 01 & 42018 & 68.5011 & 7.3500 & 277.9 & 4 & 600.0 & 600.0 \\
\hline 01 & 42019 & 65.4995 & 8.9750 & 278.9 & 4 & 600.0 & 600.0 \\
\hline 01 & 42020 & 60.0000 & 8.8500 & 278.4 & 4 & 600.0 & 600.0 \\
\hline 01 & 42021 & 79.9350 & 6.1750 & 278.4 & 4 & 600.0 & 600.0 \\
\hline 01 & 42022 & 83.5012 & 4.8750 & 278.1 & 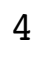 & 600.0 & 600.0 \\
\hline J & 42023 & 78.7357 & 4.3500 & 278.0 & 4 & 600.0 & 600.0 \\
\hline
\end{tabular}




\begin{tabular}{|c|c|c|c|c|c|c|c|}
\hline+ & 42024 & 3.2500 & 5.7500 & 277.9 & 4 & 600.0 & \\
\hline & 4211 & 3.4991 & 6.1250 & 277.8 & 4 & O & \\
\hline & 4212 & 011 & 6000 & 277.7 & 4 & 0 & \\
\hline & 4213 & 73.2467 & .3250 & 277.6 & & 00.0 & \\
\hline & 4214 & 30.7550 & .3250 & 77.6 & & (2) & \\
\hline & 4215 & 5009 & .8000 & 277.6 & 4 & 00 & \\
\hline & 4216 & 79.4944 & 1000 & 277.3 & & 00 & \\
\hline & 4217 & 21.7232 & .5750 & 278.2 & & 0.0 & \\
\hline & 4218 & 42.8304 & 4.8750 & 279.5 & & 00.0 & \\
\hline & 4219 & 111.7380 & 1250 & 280.8 & & 0 & \\
\hline & 42110 & 144.0000 & 000 & 282.6 & & & \\
\hline & 42111 & 137.4991 & 750 & & 4 & & \\
\hline & 2112 & 150.7240 & 7000 & 284.4 & 4 & 0 & \\
\hline & 2113 & 162.2499 & 250 & .3 & 4 & & \\
\hline & 2114 & 159. & 250 & & 4 & & \\
\hline & 2115 & 158 . & 750 & 287.2 & 4 & 0 & \\
\hline & 42116 & 148.7533 & 7.9750 & 288.4 & 4 & 0 & \\
\hline & 42117 & 98.3460 & 500 & & 3 & & \\
\hline & 2118 & 80.4798 & 250 & .5 & 4 & & \\
\hline & 42119 & 71.7461 & 250 & 5.8 & 4 & 0 & \\
\hline & 2120 & 73 . & 500 & & 4 & & \\
\hline & 2121 & 71. & 250 & .7 & 4 & & \\
\hline & 42122 & 74.7497 & 750 & .7 & 4 & 0 & \\
\hline & 42123 & 71.9999 & 750 & & 4 & & \\
\hline & 42124 & 00 & 50 & & 4 & & \\
\hline & 4221 & 79.7502 & 250 & 5.5 & 4 & & \\
\hline & 4222 & 145.7489 & 2.9000 & 3.7 & 4 & & \\
\hline & 4223 & 502 & 000 & 1.1 & 4 & & \\
\hline & 4224 & 166.0517 & 500 & 2.2 & 5 & & \\
\hline & 4225 & 102.1014 & 750 & 2.3 & 6 & & \\
\hline & 4226 & 109.4048 & 750 & .5 & 6 & & \\
\hline & 4227 & 13 & 750 & 3.1 & 5 & & \\
\hline & 4228 & 335.4806 & 500 & 286.1 & 4 & & \\
\hline & 4229 & 277.2646 & 750 & $2 \varepsilon$ & 3 & & 0 . \\
\hline & 42210 & 23 & 50 & .8 & 2 & & \\
\hline & 42211 & 224.4769 & 2.6000 & 287.4 & 1 & & \\
\hline & 42212 & 240 . & 000 & 9.0 & 1 & & \\
\hline & 42213 & 183. & 500 & 290.4 & 1 & & 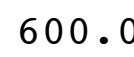 \\
\hline & 42214 & 66.2271 & 3.6250 & 291.3 & 2 & .0 & 6 \\
\hline & 42215 & 77.3752 & 500 & 291.5 & 2 & & \\
\hline & 42216 & 100.0084 & 250 & 291.2 & 3 & & \\
\hline & 42217 & 88.1907 & 5.2500 & 290.4 & 3 & .0 & 0 \\
\hline & 42218 & 74.9991 & 5.9000 & 289.1 & 4 & & \\
\hline 01 & 42219 & 75.7489 & 6.6750 & 287.6 & 4 & .0 & 600 \\
\hline 1 & 42220 & 77.9997 & 6.9500 & 286.8 & 4 & 600.0 & 00 \\
\hline & 42221 & 73 & & 285.8 & 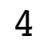 & & \\
\hline & 42222 & 78.2494 & 000 & 285.7 & 4 & & 600 \\
\hline & $2 \angle 20$ & 75.2500 & 9.0500 & 286.4 & & 600.0 & 600 \\
\hline
\end{tabular}




\begin{tabular}{|c|c|c|c|c|c|c|c|}
\hline+ & 42224 & 0.4999 & 8.9250 & 286.6 & 4 & 600.0 & \\
\hline & 4231 & 5.5000 & 8.6000 & 286.6 & 4 & 600 & \\
\hline & 4232 & 000 & 5000 & 286.6 & 4 & 0 & \\
\hline & 4233 & 80.7506 & 5.6000 & 286.8 & & 00 & \\
\hline & 4234 & 2.7496 & .7250 & 87.1 & & 0 & \\
\hline & 4235 & 89.0000 & 6.2000 & 286.7 & & 0 & \\
\hline & 4236 & 85.0000 & 5.2500 & 286.2 & & 00 & \\
\hline & 4237 & 116 & 3.1250 & 87.0 & & 0 & \\
\hline & 4238 & 295.4037 & 1.1750 & 287.8 & & 00 & \\
\hline & 4239 & 252.0853 & 1.2500 & 289.0 & & 0 & \\
\hline & 42310 & 211.7731 & 2.5000 & 89.5 & & & \\
\hline & 42311 & 171.49 & & 289.7 & & & \\
\hline & 42312 & 169.5031 & 4.5750 & 290.5 & 3 & 00 & \\
\hline & 42313 & 170.24 & 4 . & 91.6 & & 0 & \\
\hline & 42314 & 169.97 & & 292.7 & 2 & & \\
\hline & 42315 & 172.2531 & 250 & 293.6 & 2 & 0 & \\
\hline & 42316 & 172.2495 & 4.20 & 293.9 & 3 & 0 & \\
\hline & 42317 & 184.08 & & 293.7 & 3 & & \\
\hline & 42318 & 170 . & 0 & 293.5 & 3 & & \\
\hline & 42319 & 153.99 & 2.80 & 292.6 & 4 & 0 & \\
\hline & 42320 & 141. & 1 . & 291.4 & 5 & & \\
\hline & 42321 & 101 . & 1 . & 291.5 & 4 & & \\
\hline & 42322 & 101.8060 & 3.3000 & 291.9 & 5 & 0 & \\
\hline & 42323 & 78 . & & 290.8 & 4 & & \\
\hline & 42324 & 77 . & & 291.1 & 4 & & \\
\hline & 4241 & 76.50 & 0 & 291.3 & 4 & & \\
\hline & 4242 & 78.75 & 0 & 291.7 & 4 & & \\
\hline & 4243 & 81.0 & 0 & 291.5 & 5 & & \\
\hline & $424 \quad 4$ & 101.44 & $2 \quad 1$ & 291.4 & 4 & & \\
\hline & 4245 & 114.47 & 0 . & 290.7 & 5 & & \\
\hline & 4246 & 54 & 0 . & 290.4 & 5 & & \\
\hline & 4247 & 132 . & & 292.8 & 6 & & \\
\hline & 4248 & 333. & 0.55 & 295.9 & 5 & & \\
\hline & 4249 & 247.62 & 127 & 295.9 & 4 & & • \\
\hline & 42410 & 234 . & & 294.7 & 3 & & \\
\hline & 42411 & 218.5777 & 3.20 & 295.2 & 2 & & \\
\hline & 42412 & 210 . & 3 . & 295.6 & 2 & & \\
\hline & 42413 & 206 . & 3.37 & 296.3 & 2 & & 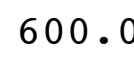 \\
\hline & 42414 & 202.7266 & 3.9000 & 297.0 & 2 & & 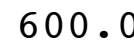 \\
\hline & 42415 & 187.50 & 4 . & 297.5 & 3 & & \\
\hline & 42416 & 175.25 & & 298.0 & 3 & & \\
\hline & 42417 & 186.3067 & 3.7500 & 298.1 & 3 & 0 & 0 \\
\hline & 42418 & 185 . & 3.93 & 297.8 & 3 & & \\
\hline 01 & 42419 & 184.9091 & 2.9750 & 296.9 & 4 & & 600 \\
\hline 01 & 42420 & 148.2577 & 2.0500 & 295.6 & 5 & 600.0 & 00 \\
\hline & 42421 & 98 . & & 295.5 & 4 & & \\
\hline & 42422 & 92.9596 & 1.1500 & 295.7 & 5 & & 600 \\
\hline & $244<2$ & 46.2619 & 0.2750 & 294.8 & & 600.0 & 600 \\
\hline
\end{tabular}




\begin{tabular}{|c|c|c|c|c|c|c|c|}
\hline 1 & 2424 & 6.7522 & 2.7250 & 295.2 & 5 & 00.0 & \\
\hline & 4251 & 45011 & 6.7500 & 295.0 & & & \\
\hline & 4252 & 82.9897 & 7250 & 95.3 & & ח & \\
\hline & 4253 & 138.1391 & 1.2500 & 94.7 & & 00 & \\
\hline & $425 \quad 4$ & 3 & .5500 & & & & \\
\hline & 4255 & 102.0735 & 3000 & 93.8 & & 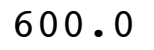 & \\
\hline & 4256 & 78.2289 & 1.9250 & 94.1 & & 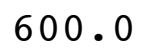 & \\
\hline & 4257 & 24.7938 & .9750 & 95.4 & & 0 & \\
\hline & 4258 & 327.7563 & 0.7750 & 297.4 & & 0.0 & \\
\hline & 4259 & 294.9252 & 1.5500 & 297.8 & & 0 & \\
\hline & 2510 & 266.5027 & & 6 & & & \\
\hline & 2511 & 234 . & & 0 & & & \\
\hline & 2512 & 213.0588 & 250 & 299.0 & & 0 & \\
\hline & 2513 & 198. & & 5 & & & \\
\hline & 2514 & 177. & & 5 & & & \\
\hline & 2515 & 154 . & 50 & 301.1 & & & \\
\hline & 2516 & 144.2595 & .2000 & 300.8 & & 0 & \\
\hline & 2517 & 129.2490 & & & & & \\
\hline & 8 & 105 . & 0 & 3 & & & \\
\hline & 2519 & 79.4920 & 0 & 297 & & U & \\
\hline & 20 & 71 . & & & & & \\
\hline & 21 & 74 . & & 29 & 4 & & \\
\hline & 2522 & 81 . & 0 & 29 & 4 & & \\
\hline & 2523 & 70 . & & & & & \\
\hline & 2524 & 71 . & & & 4 & & \\
\hline & 261 & 72 . & 750 & 29 & 4 & & \\
\hline & 4262 & 70 . & 9.50 & 294 & 4 & & \\
\hline & 263 & 72 . & 0 & 294 & 4 & & \\
\hline & 264 & 499 & & 294 & 4 & & \\
\hline & 4265 & 73. & 500 & 294 & 4 & & \\
\hline & 4266 & 81 . & & 294 & 4 & & \\
\hline & 4267 & 76 . & & & 4 & & \\
\hline & 4268 & 77 . & 0 & 294.7 & 4 & & \\
\hline & 4269 & 80 . & & 295 & 4 & & \\
\hline & 2610 & & & & 4 & & \\
\hline & 42611 & 92.4986 & & 295.7 & 4 & & \\
\hline & 42612 & 81 . & & 296 & 3 & & \\
\hline & 426 & 67. & & 29 & 4 & & \\
\hline & 42614 & 79.2483 & & 296.2 & 4 & & \\
\hline & 42615 & 107 . & & 296 & 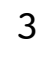 & & \\
\hline & 42616 & 101. & & & 4 & & \\
\hline & 42617 & 93.2892 & 8.2500 & 295.5 & 4 & & \\
\hline & 42618 & 64. & & 293 & 4 & & \\
\hline & 42619 & 69.7502 & 0.4500 & 293.7 & 4 & & \\
\hline & 42620 & 72.2502 & 10.7250 & 292.9 & 4 & 0 & ה חم \\
\hline & & & & & 4 & & \\
\hline & 42622 & 66.9999 & 0.5750 & & & & \\
\hline & $20<5$ & 65.7500 & 11.4500 & 291.3 & & 600.0 & 60 \\
\hline
\end{tabular}




\begin{tabular}{|c|c|c|c|c|c|c|c|}
\hline 1 & 42624 & 65.5000 & 11.5750 & 291.2 & 4 & 600.0 & 600.0 \\
\hline 01 & $427 \quad 1$ & 66.0002 & 11.5000 & 291.2 & 4 & 600.0 & 00.0 \\
\hline 01 & 4272 & 67.0001 & 11.2000 & 290.9 & 4 & 600.0 & 00.0 \\
\hline 01 & 4273 & 71.2475 & 9.8500 & 290.9 & 4 & 600.0 & 00.0 \\
\hline 01 & $427 \quad 4$ & 70.9995 & 10.0750 & 290.5 & 4 & 600.0 & 00.0 \\
\hline 01 & $427 \quad 5$ & 72.7497 & 9.2500 & 290.4 & 4 & 600.0 & 00.0 \\
\hline 01 & 4276 & 73.2506 & 11.0500 & 289.1 & 4 & 600.0 & 00.0 \\
\hline 01 & $427 \quad 7$ & 66.9989 & 11.4250 & 287.2 & 4 & 600.0 & 00.0 \\
\hline 01 & 4278 & 70.4997 & 11.4500 & 286.2 & 4 & 600.0 & 00.0 \\
\hline 01 & 4279 & 72.4991 & 10. & 287.1 & 4 & 600.0 & .0 \\
\hline 01 & 42710 & 82.9998 & 500 & 288.6 & 4 & 600.0 & \\
\hline 01 & 42711 & 80.2501 & 250 & 290.0 & 4 & 600.0 & \\
\hline 01 & 42712 & 76.2498 & 500 & 291.2 & 4 & 600.0 & 00.0 \\
\hline 01 & 42713 & 73.9989 & 750 & 290.7 & 4 & 600.0 & 00.0 \\
\hline 01 & 42714 & 73.4985 & 9 . & 291.4 & 4 & .0 & \\
\hline 01 & 42715 & 68.5009 & 10.2250 & 291.6 & 4 & 600.0 & .0 \\
\hline 01 & 42716 & 65.7505 & 10.5500 & 291.4 & 4 & 60 & 0.0 \\
\hline 01 & 42717 & 67.7505 & 10. & 290.7 & 4 & .0 & \\
\hline 01 & 42718 & 69.5008 & 10. & 289.5 & 4 & 60 & \\
\hline 01 & 42719 & 115.6463 & 5.6500 & 287.6 & 4 & .0 & .0 \\
\hline 01 & 42720 & 161. & 2 . & 285.8 & 5 & 6 & \\
\hline 01 & 42721 & 125.0215 & 5 . & 285.1 & 4 & .0 & \\
\hline 01 & 42722 & 122.5206 & 5. & 284.0 & 4 & 600.0 & .0 \\
\hline 01 & 42723 & 111.5147 & 5 . & 283 & 4 & 0 & \\
\hline 01 & 42724 & 88.0401 & & 28 & 5 & .0 & \\
\hline 01 & 4281 & 98.2379 & & 283.5 & 4 & .0 & .0 \\
\hline 01 & 4282 & 96.5040 & 5.8000 & 283.5 & 4 & .0 & .0 \\
\hline 01 & 4283 & 82.7459 & 5 & 283.3 & 4 & .0 & \\
\hline 01 & 4284 & 79.7499 & & 282.9 & 4 & .0 & 0.0 \\
\hline 01 & $428 \quad 5$ & 64.9973 & 6.8 & 282.5 & 4 & 60 & .0 \\
\hline 01 & 4286 & 73.5000 & 6 . & 282.1 & 4 & .0 & \\
\hline 01 & 4287 & 95.5092 & 5 . & 282.1 & 4 & .0 & 600.0 \\
\hline 01 & 4288 & 107.5005 & 5.2750 & 282.8 & 3 & 600.0 & 600.0 \\
\hline 01 & 4289 & 86.4816 & 6. & 283.8 & 4 & .0 & 0.0 \\
\hline 01 & 42810 & 79.7478 & 5.72 & 285.2 & 3 & 600.0 & 600.0 \\
\hline 01 & 42811 & 82.9866 & 6.2000 & 286.0 & 4 & 600.0 & 600.0 \\
\hline 01 & 42812 & 75.9966 & 5.7 & 287.2 & 3 & 600.0 & 0.0 \\
\hline 01 & 42813 & 98.7519 & 6.2750 & 287.7 & 4 & 600.0 & 600.0 \\
\hline 01 & 42814 & 90.0031 & 6.3250 & 287.9 & 4 & 600.0 & 600.0 \\
\hline 01 & 42815 & 60.2494 & 7.2000 & 287.4 & 4 & 600.0 & 600.0 \\
\hline 01 & 42816 & 66.7529 & 7.4750 & 286.9 & 4 & 600.0 & 600.0 \\
\hline 01 & 42817 & 89.0018 & 7.9500 & 286.8 & 4 & 600.0 & 600.0 \\
\hline 01 & 42818 & 72.4982 & 8.1750 & 286.5 & 4 & 600.0 & 600.0 \\
\hline 01 & 42819 & 73.9888 & 7.0250 & 285.3 & 4 & 600.0 & 600.0 \\
\hline 01 & 42820 & 72.2502 & 8.1250 & 284.0 & 4 & 600.0 & 600.0 \\
\hline 01 & 42821 & 73.7500 & 8.1500 & 283.7 & 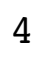 & 600.0 & 600.0 \\
\hline 01 & 42822 & 68.9999 & 9.5750 & 283.5 & 4 & 600.0 & 600.0 \\
\hline 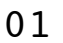 & 42823 & 69.5005 & 9.825 & 283.6 & 4 & 600.0 & 600.0 \\
\hline
\end{tabular}




\begin{tabular}{|c|c|c|c|c|c|c|c|}
\hline & 2824 & 2.7487 & 9.5000 & 283.4 & 4 & - & \\
\hline & 4291 & 4.5000 & 8.3500 & 283.2 & & & \\
\hline & 4292 & 2499 & 9250 & 283.2 & 4 & r & \\
\hline & 4293 & 80.0000 & 8.1750 & 283.4 & & 00 & \\
\hline & 4294 & 1.0000 & .8750 & 3.0 & & & \\
\hline & 295 & 5000 & 6750 & 82.7 & & 00 & \\
\hline & 4296 & 998 & 9.2500 & 282.7 & & 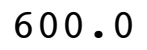 & \\
\hline & 4297 & 2490 & .9750 & 33.7 & & & \\
\hline & 298 & 8.2937 & 4.7500 & 86.1 & & 0 & \\
\hline & 4299 & 000 & 7.1500 & 287.1 & & 0 & \\
\hline & 2910 & 155.7485 & 6.2750 & 287.8 & & 0 & \\
\hline & 2911 & 277 & & 89.4 & 3 & & \\
\hline & 2912 & 168.7500 & 4.6000 & 290.9 & & 0 & \\
\hline & 2913 & 155.5000 & 4.7500 & 292.2 & & 00.0 & \\
\hline & 2914 & 151.49 & & 93.2 & & & \\
\hline & 2915 & 129.9926 & 6.3000 & 293.7 & 1 & & \\
\hline & 2916 & 96.9941 & 0 & 293.9 & 3 & 0 & \\
\hline & 2917 & 89 . & 0 & .6 & 4 & & \\
\hline & 2918 & 85 . & 6 . & 292.6 & 4 & & \\
\hline & 2919 & 67.75 & 0 & 29 & 4 & & \\
\hline & 2920 & 70 . & 0 & 4 & 4 & & \\
\hline & 2921 & & & 4 & 4 & & \\
\hline & 2922 & 75 & & 290 & 4 & & \\
\hline & 42923 & 76 . & & 290 & 4 & & \\
\hline & 42924 & & & 29 & 4 & & \\
\hline & 4301 & 2511 & & 290 & 4 & & \\
\hline & $430 \quad 2$ & 85.24 & 0 & 290.7 & 4 & & \\
\hline & 4303 & 97 . & & 291.7 & 5 & & \\
\hline & $430 \quad 4$ & 109 & 0 & 29 & 6 & & \\
\hline & 4305 & 0 & 0 & 289.5 & 6 & & \\
\hline & 4306 & 10 & & 29 & 6 & & \\
\hline & 4307 & 97 & 0 & 29 & 6 & & \\
\hline & 4308 & 220.8671 & 000 & 294.8 & 2 & 0 & 0 . \\
\hline & 4309 & 238 . & & 29 & 4 & & \\
\hline & 43010 & 20 & 0 & 29 & 3 & & \\
\hline & 43011 & 263.5871 & & 295.9 & 2 & & \\
\hline & 43012 & 70 . & 0 & 297.2 & 2 & & \\
\hline & 43013 & 110 . & 0 & 297.2 & 3 & & U. \\
\hline & 43014 & 98.9995 & 000 & 297.3 & 3 & & 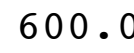 \\
\hline & 430 & 9 & & 29 & 4 & & \\
\hline & 43016 & 85 . & & & 4 & & \\
\hline & 43017 & 80.2435 & 5.9750 & 297.1 & 3 & 0 & 0 \\
\hline & 430 & 67.99 & & 296.0 & 4 & 0 & \\
\hline & 43019 & 75.5005 & 9.2250 & 294.2 & 4 & & \\
\hline . & 43020 & 69.9995 & 0.0000 & 293.2 & 4 & 60 & 00 . \\
\hline & & 66.4983 & 9.3750 & 292.8 & 4 & 600 & \\
\hline & & & 0.7 & 291.9 & 4 & & \\
\hline & $\$ 3023$ & 71.7495 & 9.9750 & 291.6 & & 600.0 & 600 \\
\hline
\end{tabular}




\begin{tabular}{|c|c|c|c|c|c|c|c|}
\hline & & 0.0000 & .3750 & 91.6 & & & \\
\hline & $\begin{array}{lll}5 & 1 & 1\end{array}$ & 19984 & .7500 & 2908 & & & \\
\hline & 12 & 499 & 500 & 290.0 & & 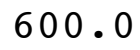 & \\
\hline & 13 & 2.5000 & 11.1750 & 289.0 & & 00 & \\
\hline & 14 & & & 88.2 & & & \\
\hline & 5 & 0 & & 87.6 & & & \\
\hline & 6 & 75.7502 & & 286.7 & & & \\
\hline & 7 & 5.7460 & & & & & \\
\hline & 8 & 2.7055 & & 88.8 & & & \\
\hline & 9 & 1.4990 & 0 & 89.5 & & & \\
\hline & 110 & .5000 & 0 . & 290.2 & & & \\
\hline & 111 & & & & & & \\
\hline & 12 & 52.5001 & & 92.5 & & & \\
\hline & 113 & 4.2542 & 10 . & 5 & & & \\
\hline & 114 & & & & & & \\
\hline & 15 & 52 & 0 & 6 & & & \\
\hline & 16 & 159.25 & & 29 & & & \\
\hline & 117 & 4 & & 6 & & & \\
\hline & 118 & & & 29 & & & \\
\hline & 119 & 0 & & & & & \\
\hline & 120 & & & 8 & & & \\
\hline & 121 & & & & & & \\
\hline & 22 & 7 & & 291.8 & & & \\
\hline & 123 & & & & & & \\
\hline & 124 & & & & & & \\
\hline & $\begin{array}{lll}5 & 2 & 1\end{array}$ & & & 28 & & & \\
\hline & $\begin{array}{lll}5 & 2 & 2\end{array}$ & 0 & 0 & 2 & & & \\
\hline & $\begin{array}{lll}5 & 2 & 3\end{array}$ & 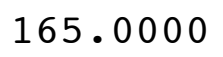 & 0 & 8 & & & \\
\hline & 524 & 170 . & 0 & .2 & 4 & & \\
\hline & 525 & 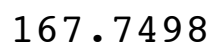 & & .2 & & & \\
\hline & 526 & & & & 4 & & \\
\hline & $\begin{array}{lll}5 & 2 & 7\end{array}$ & & 0 & & 4 & & \\
\hline & 528 & 162 . & & 284.8 & & & \\
\hline & $\begin{array}{lll}5 & 2 & 9\end{array}$ & & & & 4 & & \\
\hline & 10 & & 0 & & 4 & & \\
\hline & 11 & & & .7 & 4 & & \\
\hline & 5212 & 8 & 18 . & 288.7 & 4 & & \\
\hline & 213 & 1 & 17 & 290.1 & 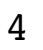 & & \\
\hline & $5 \quad 214$ & 171 . & 17 . & .1 & 4 & & \\
\hline & 5215 & 1 & 0 & 29 & 4 & & \\
\hline & 5216 & 178 . & & & 4 & & \\
\hline & 5217 & 176.2512 & 18 . & 292.9 & 4 & & \\
\hline & 5218 & 1 & 17 . & 292.8 & 4 & & \\
\hline & 5219 & 175.4997 & & 292.2 & & & \\
\hline & 5220 & 169.7504 & 10.75 & 291.1 & $I$ & & \\
\hline & 221 & 171.2508 & & 289.8 & 4 & 0 & \\
\hline & 222 & 16 & & & & & \\
\hline & 223 & 173.5649 & 7.6250 & 288.5 & & 600.0 & 600 \\
\hline
\end{tabular}




\begin{tabular}{|c|c|c|c|c|c|c|c|}
\hline 1 & 24 & & & & & & \\
\hline 1 & 31 & 000 & 500 & & & 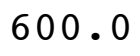 & \\
\hline & 32 & 182.5000 & 11.3750 & 87.0 & & 00 & \\
\hline & 33 & 69.5194 & 3000 & 86.3 & & 00 & \\
\hline & 4 & & 9000 & & & & \\
\hline & 35 & 01 & 3500 & 84.1 & & 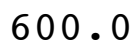 & \\
\hline & 36 & 63.2493 & 500 & 83.7 & & 00 & \\
\hline & 7 & 61.2502 & 9.8750 & & & & \\
\hline & 3 & 58.2504 & 10.2250 & 85.6 & & & \\
\hline & 39 & 67.0000 & 11.7250 & 87.1 & & 0 & \\
\hline & 310 & 178.5027 & 10.3500 & 289 & & & \\
\hline & 311 & 185 & 10.3500 & .9 & & & \\
\hline & 312 & 178 . & 8.7000 & 91.9 & & 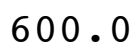 & \\
\hline & 313 & 175.7508 & 7.4500 & 93.2 & & & \\
\hline & 314 & 169 & & 1 & & & \\
\hline & 315 & & & 9 & & & \\
\hline & 316 & 158.9995 & 7.5000 & 95.7 & & 0 & \\
\hline & 317 & 16 & 7 . & 1 & & & \\
\hline & 318 & 167 & & 0 & & & \\
\hline & 319 & 161.23 & 250 & 29 & & & \\
\hline & 320 & 146.00 & 6.9000 & 294.6 & 4 & 0 & 0 \\
\hline & 321 & 146 & 7 . & & & & \\
\hline & 322 & 14 & 7 . & 25 & & & \\
\hline & 323 & 136. & 7.8000 & 29 & 4 & & \\
\hline & 324 & & & & 4 & & \\
\hline & 41 & 12 & 6 . & & 4 & & \\
\hline & 42 & 109.7499 & 000 & 293.1 & 4 & & \\
\hline & 43 & 109.2136 & 5 . & 292 & 4 & & \\
\hline & 44 & 120 & 3 . & 292.1 & 5 & & \\
\hline & 45 & 104 & 4 . & 292.0 & 4 & & \\
\hline & 46 & 125.54 & 2.4250 & 291.4 & 5 & & \\
\hline & 47 & 15 & 3.2750 & 291.9 & 4 & & \\
\hline & 48 & 158 & 500 & 292 & 3 & & \\
\hline & 49 & 162.9998 & 3250 & 293.3 & 3 & & \\
\hline & 410 & & 0 & 29 & 4 & & \\
\hline & 411 & & & 295.0 & 4 & & \\
\hline & 412 & 169.5013 & 5.7000 & 296.0 & 3 & & \\
\hline & 413 & 16 & 6 . & 297.2 & 4 & & \\
\hline & 414 & 16 & 5.2250 & 298.6 & 3 & & \\
\hline & 415 & 174.2503 & 6.8500 & 299.2 & 4 & & \\
\hline & 416 & & 500 & 299.3 & 4 & & \\
\hline & 417 & & 5.0250 & 299.8 & 3 & .0 & \\
\hline & 418 & 4947 & 7.4500 & 298.3 & 4 & 00.0 & 00 . \\
\hline & 419 & 429 & 000 & 296.5 & 4 & 00 & \\
\hline & 420 & .4784 & 6.9000 & 294.6 & 4 & 600.0 & 500 \\
\hline & 421 & 2500 & 9.4000 & 294.3 & 4 & 600.0 & 0 \\
\hline & 422 & & 50 & 29 & 4 & & \\
\hline & 423 & 0000 & 9.0000 & 294.7 & $\Psi$ & 600.0 & \\
\hline
\end{tabular}




\begin{tabular}{|c|c|c|c|c|c|c|c|c|}
\hline 1 & 5 & 424 & 76.5000 & 8.4250 & 294.4 & 4 & 600.0 & 600.0 \\
\hline 01 & 5 & 51 & 69.5021 & 8.3000 & 294.2 & 4 & 600.0 & 600.0 \\
\hline 01 & 5 & 52 & 69.0002 & 8.9250 & 293.6 & 4 & 600.0 & 00.0 \\
\hline 01 & 5 & 53 & 67.7500 & 8.7500 & 292.7 & 4 & 600.0 & 00.0 \\
\hline 01 & 5 & 54 & 76.0005 & 9.1000 & 292.2 & 4 & 600.0 & 00.0 \\
\hline 01 & 5 & 55 & 81.2496 & 9.3500 & 291.7 & 4 & 600.0 & 00.0 \\
\hline 01 & 5 & 56 & 88.7485 & 9.2250 & 291.4 & 4 & 600.0 & 00.0 \\
\hline 01 & 5 & 57 & 72.9864 & 6.2000 & 291.6 & 4 & 600.0 & 00.0 \\
\hline 01 & 5 & 58 & 133.7130 & 3.5750 & 293.8 & 3 & 600.0 & 00.0 \\
\hline 01 & 5 & 59 & 120.8398 & 500 & 294.3 & 3 & 600.0 & 00.0 \\
\hline 01 & 5 & 510 & 143.3939 & 5.9000 & 294.4 & 3 & 600.0 & 00.0 \\
\hline 01 & 5 & 511 & 163.0092 & 9 & 294.3 & 4 & 600.0 & 00.0 \\
\hline 01 & 5 & 512 & 163.2526 & 6.7500 & 295.1 & 4 & 600.0 & 00.0 \\
\hline 01 & 5 & 513 & 158.2410 & 4.7750 & 296.4 & 3 & 600.0 & 00.0 \\
\hline 01 & 5 & 514 & 163.7527 & 5 . & 297.8 & 3 & .0 & \\
\hline 01 & 5 & 515 & 166.0023 & 5 & 298.7 & 3 & 600.0 & 00.0 \\
\hline 01 & 5 & 516 & 158.2522 & 5.3500 & 299.6 & 3 & 600.0 & 600.0 \\
\hline 01 & 5 & 517 & 160.9973 & 5. & 300.0 & 3 & .0 & \\
\hline 01 & 5 & 518 & 112.3744 & 5.9500 & 299.3 & 3 & 600.0 & 00.0 \\
\hline 01 & 5 & 519 & 77.4994 & 6.0000 & 297.3 & 4 & .0 & .0 \\
\hline 01 & 5 & 520 & 78.5000 & 7. & 295.5 & 4 & .0 & \\
\hline 01 & 5 & 521 & 77.4992 & 8. & 295.0 & 4 & 600.0 & \\
\hline 01 & 5 & 522 & 72.9981 & 8.7750 & 295.2 & 4 & 600.0 & 500.0 \\
\hline 01 & 5 & 523 & 73.0000 & 8.2 & 295.7 & 4 & 60 & .0 \\
\hline 01 & 5 & 524 & 74.0001 & 7 . & 296.0 & 4 & .0 & \\
\hline 01 & 5 & 61 & 64.7505 & 8.2750 & 295.7 & 4 & 600.0 & 600.0 \\
\hline 01 & 5 & 62 & 68.5000 & 8.5000 & 294.9 & 4 & 600.0 & 600.0 \\
\hline 01 & 5 & 63 & 70.5000 & 8.7 & 294.0 & 4 & 600.0 & \\
\hline 01 & 5 & 64 & 78.0006 & 8.7500 & 293.6 & 4 & 600.0 & 600.0 \\
\hline 01 & 5 & 65 & 79.7455 & 7.4250 & 293.6 & 4 & 600.0 & 600.0 \\
\hline 01 & 5 & 66 & 84.5000 & 6 & 293.0 & 4 & .0 & \\
\hline 01 & 5 & 67 & 106.4729 & 4.2500 & 293.8 & 3 & 600.0 & 600.0 \\
\hline 01 & 5 & 68 & 141.8985 & 4.8250 & 295.1 & 3 & 600.0 & 600.0 \\
\hline 01 & 5 & 69 & 166.5069 & 6.9750 & 295.1 & 4 & 600.0 & 0.0 \\
\hline 01 & 5 & 610 & 168.9988 & 5.1750 & 296.1 & 4 & 600.0 & 600.0 \\
\hline 01 & 5 & 611 & 166.9835 & 4.5250 & 297.2 & 3 & 600.0 & 600.0 \\
\hline 01 & 5 & 612 & 168.7505 & 5.2 & 298.2 & 3 & 600.0 & 600.0 \\
\hline 01 & 5 & 613 & 172.2513 & 5.8750 & 299.2 & 3 & 600.0 & 600.0 \\
\hline 01 & 5 & 614 & 169.4995 & 5.5000 & 300.2 & 3 & 600.0 & 600.0 \\
\hline 01 & 5 & 615 & 173.2508 & 5.2500 & 301.1 & 3 & 600.0 & 600.0 \\
\hline 01 & 5 & 616 & 177.2515 & 5.4250 & 301.4 & 3 & 600.0 & 600.0 \\
\hline 01 & 5 & 617 & 180.9997 & 5.6000 & 301.3 & 3 & 600.0 & 600.0 \\
\hline 01 & 5 & 618 & 173.2512 & 5.0750 & 301.1 & 3 & 600.0 & 600.0 \\
\hline 01 & 5 & 619 & 127.5898 & 4.5250 & 300.3 & 4 & 600.0 & 600.0 \\
\hline 01 & 5 & 620 & 86.0057 & 5.6500 & 298.7 & 4 & 600.0 & 600.0 \\
\hline 01 & 5 & 621 & 80.9998 & 6.7000 & 297.9 & 4 & 600.0 & 600.0 \\
\hline 01 & 5 & 622 & 82.2498 & 6.1500 & 298.1 & 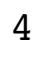 & 600.0 & 600.0 \\
\hline . & & 623 & 104.7325 & 3.275 & 298.6 & 4 & 600.0 & 600.0 \\
\hline
\end{tabular}




\begin{tabular}{|c|c|c|c|c|c|c|c|}
\hline+ & 24 & 3.9993 & 4.5250 & 298.2 & 5 & 00.0 & \\
\hline 1 & $\begin{array}{lll}5 & 7 & 1\end{array}$ & 3.4986 & 4.8750 & 298.4 & & 0 & \\
\hline & $\begin{array}{lll}5 & 7 & 2\end{array}$ & 000 & 5250 & 98.5 & & 0 & \\
\hline & $\begin{array}{lll}5 & 7\end{array}$ & 97.8784 & .4750 & 98.4 & & 00.0 & \\
\hline & $\begin{array}{lll}5 & 7 & 4\end{array}$ & 2 & & 7.3 & & 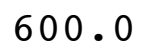 & \\
\hline & 575 & 46.7500 & 4.0750 & 96.3 & & 00 & \\
\hline & 576 & 149.2500 & 000 & 295.8 & & 00 & \\
\hline & $\begin{array}{lll}5 & 7 & 7\end{array}$ & 156.2500 & .3500 & 96.0 & & 00.0 & \\
\hline & $\begin{array}{lll}5 & 7 & 8\end{array}$ & 155.7499 & 3.7250 & 296.3 & & 00.0 & \\
\hline & $\begin{array}{lll}5 & 7 & 9\end{array}$ & 160.2483 & 3500 & 296.6 & & 0 & \\
\hline & 5710 & 160.5000 & 50 & 298.4 & & & \\
\hline & $5 \quad 711$ & 164.2494 & & 299.6 & & & \\
\hline & 5712 & 161.5021 & 250 & 300.8 & & 0 & \\
\hline & 5713 & 164.5000 & & 302.1 & & U & \\
\hline & $5 \quad 714$ & 176 . & & 303.2 & & & \\
\hline & 5715 & 188 . & 7 . & 303.4 & & 0 & \\
\hline & 5716 & 184.0000 & 6.8500 & 303.7 & & 600.0 & \\
\hline & $5 \quad 717$ & 178.49 & & 303.8 & & & \\
\hline & 5718 & 185 . & 50 & 303.6 & & & \\
\hline & 5719 & 172.73 & 50 & 303.0 & & 0 & \\
\hline & 5720 & 84 . & & 3 & & & \\
\hline & $5 \quad 721$ & 72.75 & & 30 & & & \\
\hline & 5722 & 74.2503 & 50 & 29 & & 0 & \\
\hline & 5723 & 71.25 & & 9 & & & \\
\hline & $5 \quad 724$ & 71. & & 30 & & & \\
\hline & $\begin{array}{lll}5 & 8 & 1\end{array}$ & 70.7497 & 50 & 300.0 & 4 & & \\
\hline & 582 & 73.5000 & 0 & 300.1 & 4 & & \\
\hline & 583 & 68.75 & & 300.4 & 4 & & \\
\hline & $\begin{array}{lll}5 & 8 & 4\end{array}$ & 67.0000 & & 300.5 & 1 & & \\
\hline & 585 & 71.4890 & & 300.5 & & & \\
\hline & 586 & 116.41 & & 300.1 & & & \\
\hline & $\begin{array}{lll}5 & 8 & 7\end{array}$ & 235 . & 0 & 301.9 & 4 & & \\
\hline & 588 & 176.57 & 0 & 301.4 & 2 & & \\
\hline & $\begin{array}{lll}5 & 8 & 9\end{array}$ & 169. & 0 & 30 & 2 & & \\
\hline & 5810 & 167 . & & 30 & 3 & & \\
\hline & 5811 & 214.4350 & & 302.4 & & & \\
\hline & 5812 & 213 . & & 302.8 & 2 & & \\
\hline & 5813 & 189.5080 & 3 . & 303.5 & 2 & & 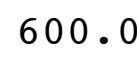 \\
\hline 1 & 5814 & 191.0061 & & 304.3 & 3 & & \\
\hline & 5815 & 193. & & 304.8 & 3 & & \\
\hline 01 & 5816 & 196.7320 & 4 . & 305.3 & 3 & & 0 . \\
\hline 01 & 5817 & 178.2701 & 5.6500 & 305.3 & 3 & .0 & 0 \\
\hline & 5818 & 161.3817 & & 304.9 & 3 & & \\
\hline 01 & 5819 & 100.4810 & 5.1750 & 303.8 & 4 & 600.0 & 600 \\
\hline+ & 5820 & 95.7505 & 5.4500 & 301.5 & 4 & 600.0 & 00 \\
\hline & 5821 & & & 300.8 & 4 & & \\
\hline & 822 & 84.0000 & 7.1000 & 300.5 & 4 & & 600 \\
\hline & 823 & 67.9993 & 6.6000 & 300.5 & & 600.0 & 600 \\
\hline
\end{tabular}




\begin{tabular}{|c|c|c|c|c|c|c|c|}
\hline & $5 \quad 824$ & 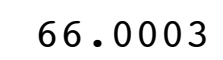 & 7.2750 & 0.8 & 4 & 0.0 & \\
\hline & $\begin{array}{lll}5 & 9 & 1\end{array}$ & 6.5001 & 7.0750 & 301.2 & 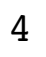 & 00 & \\
\hline & $\begin{array}{lll}5 & 9 & 2\end{array}$ & 005 & 3750 & 1.2 & & 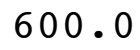 & \\
\hline & $\begin{array}{lll}5 & 9 & 3\end{array}$ & 6.0000 & .4750 & 300.1 & & & \\
\hline & $\begin{array}{lll}5 & 9 & 4\end{array}$ & 000 & 015 & 99.1 & & 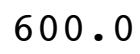 & \\
\hline & 595 & 77.7500 & & 298.6 & & 0 & \\
\hline & 596 & 89.7589 & 500 & 297.5 & & 0 & \\
\hline & $\begin{array}{lll}5 & 9 & 7\end{array}$ & 8 & & 98.5 & & & \\
\hline & $\begin{array}{lll}5 & 9 & 8\end{array}$ & 177.7534 & 3.3500 & 299.7 & & 00.0 & 00 \\
\hline & $\begin{array}{lll}5 & 9 & 9\end{array}$ & 165.7501 & 6.60 & 298.8 & & & \\
\hline & 5910 & 165.00 & & 99.6 & & & \\
\hline & 11 & 166. & & 300.9 & & 0 & \\
\hline & 5912 & 158.4924 & 6.97 & 301.6 & & & \\
\hline & 5913 & 143.2494 & & 302.4 & & & \\
\hline & $5 \quad 914$ & 124.50 & & 303.4 & & & \\
\hline & 5915 & 96.7578 & 0 & 304.2 & & & \\
\hline & 5916 & 87.74 & 0 & 302.5 & 4 & & \\
\hline & 5917 & 76 . & & 301.8 & - & & \\
\hline & 5918 & 76.24 & 7 . & 301.5 & 4 & 0 & . \\
\hline & 5919 & 69. & & 300.6 & 4 & & \\
\hline & 5920 & 65 . & & 298.8 & 4 & & \\
\hline & 5921 & 68 . & 0 & 298 & 4 & & \\
\hline & $5 \quad 922$ & 68.00 & & 298.8 & 4 & 0 & 5 \\
\hline & 5923 & 66.49 & & 299.0 & 4 & & \\
\hline & $5 \quad 924$ & 69 & & 29 & 4 & & \\
\hline & 5101 & 139.1052 & 0 & 298.5 & 5 & & \\
\hline & 5102 & 149 . & & 297.5 & 5 & & \\
\hline & 5103 & 95 . & & 297.6 & 6 & & \\
\hline & 5104 & 115.9594 & 0 & 297.4 & 5 & 0 & 0 \\
\hline & 5105 & 150.24 & & 295.2 & 4 & & \\
\hline & 5106 & 143 . & & 294.1 & 4 & & \\
\hline & 5107 & 144 & & .1 & 4 & & \\
\hline & 5108 & 140.9977 & 0 & 295.2 & 4 & & \\
\hline & 5109 & 146.25 & 0 & 296.3 & 4 & & \\
\hline & 51010 & & & .9 & 4 & & \\
\hline & 51011 & 159.9989 & & 298.4 & 4 & & \\
\hline & 51012 & 170 . & & 300.2 & 3 & & \\
\hline & 51013 & 169. & 0 & 301.7 & 3 & & . \\
\hline & 51014 & 170.4982 & & 302.8 & 3 & 0 & \\
\hline & 51015 & 177 . & & & 0 & & \\
\hline & 51016 & 165.7524 & & 304.6 & 3 & & \\
\hline & 51017 & 184.7485 & 4.8000 & 304.6 & 3 & 0 & ח \\
\hline & 51018 & 158.3978 & 0 & 304.2 & 3 & & \\
\hline & 51019 & 91.7468 & 4.15 & 303.0 & 4 & 600.0 & 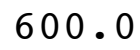 \\
\hline & 51020 & 78.4876 & 5.1000 & 301.4 & 4 & 600.0 & 00 \\
\hline & & & & & 4 & & \\
\hline & 51022 & 75.5001 & 8. & & 4 & & 600 \\
\hline & 1023 & 78.2500 & 8.8000 & 98.3 & 4 & 60 & 60 \\
\hline
\end{tabular}




\begin{tabular}{|c|c|c|c|c|c|c|c|}
\hline & 51024 & 3.9999 & 8.0250 & 8.7 & 4 & 0.0 & \\
\hline & 5111 & 77.4998 & 7.8500 & 299.2 & 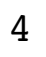 & 00.0 & \\
\hline & 5112 & 77.5000 & 000 & 299.3 & & 0 & \\
\hline & 113 & 8.0001 & .4500 & 99.9 & & & \\
\hline & 114 & 141.0458 & 0 & 297.9 & & J & \\
\hline & 115 & 3937 & & 294.9 & & 0 & \\
\hline & 116 & 24.4903 & & 297.2 & & 0 & \\
\hline & 117 & 306.9888 & & 00.9 & & & \\
\hline & 118 & 245.4113 & 0.8000 & 300.5 & & 00.0 & 0 \\
\hline & 119 & 238.9855 & 2.27 & 299.1 & & & \\
\hline & 1110 & 224.25 & & 99.4 & & & \\
\hline & 1111 & 250.6675 & 1.9750 & 300 & & & \\
\hline & 1112 & 194.47 & 3.8500 & 301 & & & \\
\hline & 1113 & 163. & 4 . & 301 & & & \\
\hline & 1114 & 158 . & & & 3 & & \\
\hline & 1115 & 159.4917 & 0 & 302.5 & 1 & 0 & \\
\hline & 51116 & 141 . & & 5 & & & \\
\hline & 1117 & 120 . & & & 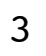 & & \\
\hline & 1118 & 76.2498 & 0 & 29 & 4 & 0 & (6) \\
\hline & 1119 & 79 . & & 297 & 4 & & \\
\hline & 1120 & 75 . & & 297.2 & 4 & & \\
\hline & 21 & 79 . & & 297.8 & 4 & & \\
\hline & 51122 & 79.00 & 0 & 297.4 & 4 & 0 & \\
\hline & 51123 & 77.24 & & 296.6 & 4 & & \\
\hline & 511 & 78 . & & 29 & 4 & & \\
\hline & 5121 & 79.0000 & 0 & 296.3 & 4 & & \\
\hline & 5122 & 76 . & & 29 & 4 & & \\
\hline & 5123 & 69. & & 29 & 4 & & \\
\hline & 5124 & 71.7499 & 0 & 292.9 & 4 & 0 & . \\
\hline & 5125 & 0326 & & 29 & 5 & & \\
\hline & 5126 & 26 . & & $28^{\circ}$ & 6 & & \\
\hline & 5127 & 240.23 & & 287.4 & 5 & & \\
\hline & 5128 & 156.1879 & 2 . & 288.1 & 4 & & \\
\hline & 5129 & 137. & 2 . & 291.0 & 3 & & \\
\hline & 51210 & 83.74 & & 291.6 & 3 & & \\
\hline & 51211 & 84.24 & 0 & 292.0 & 3 & & \\
\hline & 51212 & 90 . & 4. & 293.6 & 3 & & \\
\hline & 51213 & 68 . & 6 . & 294.7 & 4 & & \\
\hline & 51214 & 64.249 & & 294.4 & 4 & 0 & \\
\hline & 51215 & 66. & & 295 & 4 & & \\
\hline & 51216 & 70.7502 & 6 . & 294.2 & 4 & & \\
\hline & 51217 & 77.4995 & 6.9500 & 292.7 & 4 & 0 & (n) \\
\hline & 51218 & 76.25 & 50 & 289.9 & 4 & & \\
\hline & 51219 & 73.7501 & 9.9250 & 288.0 & 4 & 600.0 & 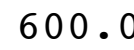 \\
\hline & 51220 & 73.5000 & 1.1000 & 286.6 & 4 & 600.0 & 00 \\
\hline & 51221 & 77 . & 0 & 286 & 4 & & \\
\hline & 51222 & 62.8349 & & 287.3 & 4 & & 600 \\
\hline & $12 \angle 2$ & 75.2498 & 2.7000 & 288.7 & & 60 & 60 \\
\hline
\end{tabular}




\begin{tabular}{|c|c|c|c|c|c|c|c|}
\hline 01 & 51224 & 85.5000 & 6.5750 & 286.6 & 4 & 600.0 & 600.0 \\
\hline 01 & 5131 & 82.0000 & 8.8000 & 284.5 & 4 & 600.0 & 600.0 \\
\hline 01 & 5132 & 75.5009 & 8.2000 & 284.5 & 4 & 600.0 & 00.0 \\
\hline 01 & 5133 & 75.7502 & 8.7250 & 284.1 & 4 & 600.0 & 00.0 \\
\hline 01 & 5134 & 80.2501 & 9.8000 & 284.1 & 4 & 600.0 & 00.0 \\
\hline 01 & 5135 & 76.0012 & 10.0500 & 283.4 & 4 & 600.0 & 00.0 \\
\hline 01 & 5136 & 70.0000 & 9.7750 & 283.2 & 4 & 600.0 & 00.0 \\
\hline 01 & 5137 & 71.4982 & 8.2250 & 283.8 & 4 & 600.0 & 00.0 \\
\hline 01 & 5138 & 76.5041 & 6.4500 & 285.2 & 4 & 600.0 & 00.0 \\
\hline 01 & 5139 & 86.2497 & 4.5500 & 286.7 & 3 & 600.0 & 00.0 \\
\hline 01 & 51310 & 89.7756 & 4.6250 & 287.6 & 3 & 600.0 & 00.0 \\
\hline 01 & 51311 & 90.5185 & 4.4500 & 289.3 & 3 & 600.0 & \\
\hline 01 & 51312 & 84.9899 & 4.9250 & 290.5 & 3 & 600.0 & 00.0 \\
\hline 01 & 51313 & 89.0027 & 5.6750 & 291.5 & 3 & 600.0 & 00.0 \\
\hline 01 & 51314 & 89.2488 & 7 . & 291.9 & 4 & .0 & \\
\hline 01 & 51315 & 81.4997 & 9.6750 & 291.2 & 4 & 600.0 & .0 \\
\hline 01 & 51316 & 74.5000 & 9.2250 & 291.2 & 4 & 60 & .0 \\
\hline 01 & 51317 & 74.0005 & 10.8750 & 291.0 & 4 & .0 & \\
\hline 01 & 51318 & 71.2499 & 10.8750 & 290.3 & 4 & 60 & .0 \\
\hline 01 & 51319 & 67.4998 & 11.4250 & 288.7 & 4 & .0 & .0 \\
\hline 01 & 51320 & 71.7505 & 9.9 & 286.9 & 4 & 6 & \\
\hline 01 & 51321 & 69.7500 & 11.6000 & 287.4 & 4 & .0 & \\
\hline 01 & 51322 & 72.7501 & 12.1000 & 287.9 & 4 & 600.0 & .0 \\
\hline 01 & 51323 & 74.7499 & 11.37 & 287.8 & 4 & 60 & \\
\hline 01 & 51324 & 72.5014 & 10. & 287.5 & 4 & .0 & \\
\hline 01 & 5141 & 72.9995 & 10.1500 & 287.6 & 4 & .0 & 600.0 \\
\hline 01 & 5142 & 75.7500 & 10.2750 & 288.0 & 4 & 600.0 & .0 \\
\hline 01 & 5143 & 67.0014 & 9.7500 & 287.7 & 4 & .0 & \\
\hline 01 & 5144 & 69.7505 & 10.4000 & 286.5 & 4 & .0 & 0.0 \\
\hline 01 & $514 \quad 5$ & 72.5043 & 10.7750 & 286.6 & 4 & 60 & .0 \\
\hline 01 & 5146 & 74.5001 & 9.77 & 286.8 & 4 & .0 & \\
\hline 01 & 5147 & 79.0000 & 8. & 288.3 & 4 & .0 & 600.0 \\
\hline 01 & 5148 & 79.7497 & 9.2500 & 288.0 & 4 & 600.0 & 600.0 \\
\hline 01 & 5149 & 76.7495 & 7.9250 & 289.4 & 4 & .0 & 0.0 \\
\hline 01 & 51410 & 89.7497 & 5.70 & 292.5 & 3 & 600.0 & 600.0 \\
\hline 01 & 51411 & 89.5484 & 5.6250 & 292.8 & 3 & 600.0 & 600.0 \\
\hline 01 & 51412 & 80.2489 & 5.0750 & 294.4 & 3 & 600.0 & 0.0 \\
\hline 01 & 51413 & 88.7462 & 6.4750 & 295.0 & 4 & 600.0 & 600.0 \\
\hline 01 & 51414 & 95.4030 & 5.1750 & 295.7 & 3 & 600.0 & 600.0 \\
\hline 01 & 51415 & 75.2506 & 7.1250 & 296.0 & 4 & 600.0 & 600.0 \\
\hline 01 & 51416 & 90.5000 & 6.9750 & 294.5 & 4 & 600.0 & 600.0 \\
\hline 01 & 51417 & 68.4959 & 9.6250 & 293.2 & 4 & 600.0 & 600.0 \\
\hline 01 & 51418 & 80.4709 & 9.0250 & 292.8 & 4 & 600.0 & 600.0 \\
\hline 01 & 51419 & 76.5000 & 8.3750 & 291.7 & 4 & 600.0 & 600.0 \\
\hline 01 & 51420 & 85.0086 & 8.0750 & 291.2 & 4 & 600.0 & 600.0 \\
\hline 01 & 51421 & 70.2500 & 11.4000 & 289.7 & 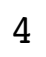 & 600.0 & 600.0 \\
\hline 01 & 51422 & 64.7500 & 9.8250 & 289.6 & 4 & 600.0 & 600.0 \\
\hline . & 51423 & 70.9993 & 9.650 & 289.6 & 4 & 600.0 & 600.0 \\
\hline
\end{tabular}




\begin{tabular}{|c|c|c|c|c|c|c|c|}
\hline 01 & 51424 & 72.2503 & 10.6250 & 289.6 & 4 & 600.0 & 600.0 \\
\hline 01 & $\begin{array}{ll}515 & 1\end{array}$ & 66.0000 & 8.9000 & 291.1 & 4 & 600.0 & 600.0 \\
\hline 01 & 5152 & 68.7502 & 8.6000 & 290.9 & 4 & 600.0 & 00.0 \\
\hline 01 & 5153 & 66.7507 & 8.9750 & 289.8 & 4 & 600.0 & 00.0 \\
\hline 01 & 5154 & 70.7519 & 8.9250 & 289.3 & 4 & 600.0 & 00.0 \\
\hline 01 & 5155 & 78.7499 & 8.7750 & 289.7 & 4 & 600.0 & 00.0 \\
\hline 01 & 5156 & 81.5003 & 7.8000 & 290.4 & 4 & 600.0 & 00.0 \\
\hline 01 & $\begin{array}{ll}515 & 7\end{array}$ & 74.2504 & 8.0500 & 290.8 & 4 & 600.0 & 00.0 \\
\hline 01 & 5158 & 80.2539 & 6.3750 & 292.0 & 4 & 600.0 & 00.0 \\
\hline 01 & 5159 & 72.7429 & 4.8500 & 294.0 & 3 & 600.0 & 00.0 \\
\hline 01 & 1510 & 83.2524 & 5.3000 & 294.4 & 3 & 600.0 & 00.0 \\
\hline 01 & 1511 & 91.9979 & 500 & 295.5 & 3 & 600.0 & \\
\hline 01 & 1512 & 88.5046 & 6.4250 & 296.1 & 4 & 600.0 & 00.0 \\
\hline 01 & 1513 & 92.9958 & 6.2500 & 296.9 & 4 & .0 & .0 \\
\hline 01 & 1514 & 94.2495 & 7 . & 296.9 & 4 & .0 & \\
\hline 01 & 1515 & 97.8039 & 5.0000 & 297.1 & 3 & 600.0 & 00.0 \\
\hline 01 & 1516 & 105.2620 & 6.1000 & 296.2 & 4 & 600.0 & 600.0 \\
\hline 01 & 1517 & 90.0025 & 7.7 & 295.9 & 4 & .0 & \\
\hline 01 & 1518 & 85.7820 & 9.5000 & 294.3 & 4 & 600.0 & .0 \\
\hline 01 & 1519 & 74.7504 & 9.7500 & 291.9 & 4 & 60 & .0 \\
\hline 01 & 1520 & 73.2501 & 8.2 & 290.7 & 4 & 60 & \\
\hline 01 & 1521 & 68.7511 & 10.5 & 290.4 & 4 & .0 & \\
\hline 01 & 1522 & 63.2494 & 11.1500 & 290.4 & 4 & 600.0 & 600.0 \\
\hline 01 & 51523 & 64.0000 & 11.3 & 290.0 & 4 & .0 & \\
\hline 01 & 51524 & 67.7501 & 10 . & 289.7 & 4 & .0 & \\
\hline 01 & 5161 & 70.2498 & 10.8250 & 289.6 & 4 & 600.0 & 600.0 \\
\hline 01 & 5162 & 69.9998 & 9.1750 & 289.4 & 4 & 600.0 & 600.0 \\
\hline 01 & 5163 & 73.0001 & 10 & 289.0 & 4 & .0 & \\
\hline 01 & 5164 & 75.2500 & 10. & 288.6 & 4 & 600.0 & 600.0 \\
\hline 01 & 5165 & 75.9996 & 10.0500 & 288.2 & 4 & .0 & 600.0 \\
\hline 01 & 5166 & 83.0005 & 9 . & 287.6 & 4 & .0 & \\
\hline 01 & 5167 & 85.2501 & 8 . & 287.9 & 4 & .0 & 600.0 \\
\hline 01 & 5168 & 96.3725 & 5.4500 & 289.5 & 3 & 600.0 & 600.0 \\
\hline 01 & 5169 & 146.0007 & 7. & 291.0 & 4 & 0.0 & 0.0 \\
\hline 01 & 51610 & 156.0236 & 6.7000 & 292.0 & 4 & 600.0 & 600.0 \\
\hline 01 & 51611 & 158.0092 & 5.7750 & 293.1 & 3 & 600.0 & 600.0 \\
\hline 01 & 51612 & 161.5005 & 5.1 & 294.5 & 3 & 600.0 & 0.0 \\
\hline 01 & 51613 & 162.2689 & 5.0750 & 295.9 & 3 & 600.0 & 600.0 \\
\hline 01 & 51614 & 155.2498 & 5.0500 & 296.9 & 3 & 600.0 & 600.0 \\
\hline 01 & 51615 & 144.2562 & 5.6 & 297.1 & 3 & 600.0 & 600.0 \\
\hline 01 & 51616 & 94.7685 & 5.8750 & 296.9 & 3 & 600.0 & 600.0 \\
\hline 01 & 51617 & 88.0027 & 6.7500 & 295.9 & 4 & 600.0 & 600.0 \\
\hline 01 & 51618 & 75.2496 & 6.5250 & 294.4 & 4 & 600.0 & 600.0 \\
\hline 01 & 51619 & 76.7485 & 7.6250 & 292.9 & 4 & 600.0 & 600.0 \\
\hline 01 & 51620 & 81.2616 & 8.4000 & 291.5 & 4 & 600.0 & 600.0 \\
\hline 01 & 51621 & 76.7489 & 9.9500 & 291.6 & 4 & 600.0 & 600.0 \\
\hline 01 & 51622 & 78.0048 & 9.5500 & 291.9 & 4 & 600.0 & 600.0 \\
\hline . & 51623 & 71.7500 & 8.125 & 292.5 & 4 & 600.0 & 600.0 \\
\hline
\end{tabular}




\begin{tabular}{|c|c|c|c|c|c|c|c|}
\hline & 51624 & 6.7500 & 8.5250 & 2.5 & 4 & . & \\
\hline & 5171 & 72.7492 & 2000 & 292.0 & 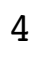 & 00.0 & \\
\hline & 172 & 989 & 5750 & 291.7 & & 0 & \\
\hline & 173 & 7.0000 & .7750 & 92.2 & & & \\
\hline & 174 & 6.2698 & 4750 & 93.4 & & J & \\
\hline & 175 & 7 . & 000 & 293.3 & & 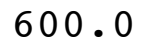 & \\
\hline & 176 & 05.7683 & & 292.5 & & & \\
\hline & 177 & 3 & & 93.5 & & & \\
\hline & 178 & 148.7478 & 6.9750 & 293.9 & & 00 & 0 \\
\hline & 179 & 153.24 & 5750 & 294 & & & \\
\hline & 1710 & 163. & & 95 & & & \\
\hline & 1711 & 171 . & 50 & 296 & & & \\
\hline & 1712 & 178 . & 4.8750 & 297.6 & & & \\
\hline & 1713 & 186. & & 299 & & & \\
\hline & 1714 & 151. & & 300 & 3 & & \\
\hline & 1715 & 134 . & 50 & 301.1 & & & \\
\hline & 1716 & 97 . & 0 & 300 & & & \\
\hline & 1717 & 97 . & & 300 & 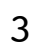 & & \\
\hline & 1718 & 88.73 & 0 & 298.8 & 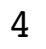 & 0 & \\
\hline & 1719 & 72.25 & 0 & 296 & 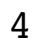 & & \\
\hline & 1720 & 77.26 & & 296 & 4 & & \\
\hline & 1721 & 70 & 50 & 296 & 4 & & \\
\hline & 51722 & 71.50 & 50 & 295.5 & 4 & 0 & \\
\hline & 1723 & 72.75 & & 295.2 & 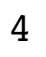 & & \\
\hline & 517 & 72 . & & 29 & 4 & & \\
\hline & 5181 & 65.4997 & 000 & 293.6 & 4 & & \\
\hline & 5182 & 68 . & & 29 & 4 & & \\
\hline & 5183 & 76 . & 0 & 29 & 4 & & \\
\hline & 5184 & 90.2522 & 0 & 292.4 & 4 & 0 & 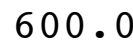 \\
\hline & 5185 & & & 292.2 & 4 & & \\
\hline & 5186 & 1 & & 292.4 & 4 & & \\
\hline & 5187 & 107 . & & 292.0 & 4 & & \\
\hline & 5188 & 136.7800 & 50 & 292.5 & 4 & & \\
\hline & 5189 & 155.9 & 0 & 293.1 & 4 & & \\
\hline & 51810 & 166.2501 & & 294.5 & 4 & & \\
\hline & 51811 & 157.2447 & & 296.1 & 3 & & \\
\hline & 51812 & 161. & & 297 & 4 & & \\
\hline & 51813 & 153.2467 & & 299.2 & 4 & & \\
\hline & 51814 & 157.500 & & 300.4 & 4 & & \\
\hline & 51815 & 157 . & & 301.3 & 4 & & \\
\hline & 51816 & 112.4296 & & 301.6 & 4 & & \\
\hline & 51817 & 89.0021 & 6.8000 & 300.9 & 4 & 0 & n? \\
\hline & 51818 & 81.0083 & 000 & 300.0 & 4 & & \\
\hline & 51819 & 68.7461 & 7.2000 & 298.7 & 4 & 0 & \\
\hline & 51820 & 66.0000 & 8.3500 & 297.4 & 4 & 600.0 & 00 \\
\hline & 51821 & & & 297 & 4 & & \\
\hline & 51822 & 76.2524 & & 297.8 & & & 600 \\
\hline & 1823 & 78.5008 & 7.9750 & 297.8 & & .0 & 60 \\
\hline
\end{tabular}




\begin{tabular}{|c|c|c|c|c|c|c|c|}
\hline & 1824 & 0.7497 & 8.6000 & 298.0 & 4 & . & \\
\hline & 5191 & 0.7501 & 8.8000 & 298.0 & & & \\
\hline & 5192 & 87.2070 & 9000 & 97.7 & 4 & 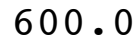 & \\
\hline & 193 & 22.7468 & 6.2500 & 299.1 & & 00 & \\
\hline & 94 & 5.7637 & .17 & 8.3 & & & \\
\hline & 195 & 116.2788 & .3500 & 97.2 & & 0 & \\
\hline & 196 & 116.2481 & 6.0750 & 296.8 & & & \\
\hline & 197 & 136.2501 & .8250 & & & & \\
\hline & 198 & 143.7514 & 7.4500 & 296 & & 0 & \\
\hline & 199 & 154.2493 & 5250 & 297.7 & & 0 & \\
\hline & 1910 & 157.9979 & 7.8500 & 298.8 & & & \\
\hline & 911 & 169.50 & & & & & \\
\hline & 1912 & 171.0000 & 750 & 301.2 & 4 & 0 & \\
\hline & 1913 & 165.0038 & 7.22 & 302.3 & & 0 & \\
\hline & & 166. & & & & & \\
\hline & 1915 & 160.7497 & 0 & 30 & 4 & & \\
\hline & 1916 & 168.0024 & 7.92 & 30 & 4 & & \\
\hline & 917 & 170 . & 7 . & 3 & & & \\
\hline & 19 & 119 . & 0 & 30 & & & \\
\hline & 1919 & 9890 & 0 & 30 & & & \\
\hline & 920 & 76 . & 0 & 3 & 4 & & \\
\hline & 921 & 74 . & & 29 & 4 & & \\
\hline & 1922 & 74.9999 & 0 & 29 & 4 & & • \\
\hline & 923 & 89 & & & 4 & & \\
\hline & 1924 & 10 & & & 4 & & \\
\hline & 5201 & 127.4977 & 0 & 30 & 4 & & \\
\hline & 5202 & 138.75 & 0 & 299.7 & - & & \\
\hline & 5203 & 145.5 & 0 & 298.5 & 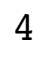 & & \\
\hline & $520 \quad 4$ & 142 . & 50 & 29 & 4 & & \\
\hline & 5205 & 143.2470 & 0 & 297.2 & 4 & & \\
\hline & 5206 & 151.74 & & 29 & 4 & & \\
\hline & 5207 & 160 . & 50 & 29 & 4 & & \\
\hline & 5208 & 169.2498 & 0 & 297.6 & 4 & 0 & 0 . \\
\hline & 5209 & 165 . & 0 & .1 & 4 & & \\
\hline & 52010 & 164. & & & 4 & & \\
\hline & 52011 & 166.5000 & & 300.0 & 4 & & \\
\hline & 52012 & 167.5000 & 0 & 301.5 & 4 & & \\
\hline & 52013 & 160.5064 & 0 & 302.6 & 4 & & \\
\hline & 52014 & 162.5037 & 0 & 304.1 & 4 & & \\
\hline & 52015 & 170 . & & 304.7 & 4 & & \\
\hline & 52016 & 162 . & & 305.4 & 4 & & \\
\hline & 52017 & 164.7508 & 6.8000 & 306.0 & 4 & 0 & . \\
\hline & 52018 & 168.2504 & 4250 & 306.0 & 4 & 0 & \\
\hline & 52019 & 151.4723 & & 305.1 & 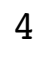 & & \\
\hline . & 52020 & 82.4816 & 6.3500 & 302.6 & 4 & 600 & 00 . \\
\hline & 52 & 500 & 7.6000 & 301.2 & 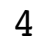 & 600 & \\
\hline & 52022 & 74 & & 300 & & & \\
\hline & 2023 & 73.5000 & 8.7250 & 300.7 & & 600.0 & 600 \\
\hline
\end{tabular}




\begin{tabular}{|c|c|c|c|c|c|c|c|}
\hline 01 & 52024 & 81.2503 & 8.3750 & 301.2 & 4 & 600.0 & 600.0 \\
\hline 01 & 5211 & 112.2690 & 7.9750 & 302.3 & 4 & 600.0 & 00,0 \\
\hline 01 & 5212 & 119.2523 & 6.9750 & 301.8 & 4 & 600.0 & 00.0 \\
\hline 01 & 5213 & 115.8018 & 6.3500 & 300.3 & 4 & 600.0 & 00.0 \\
\hline 01 & 5214 & 116.9812 & 6.9750 & 299.4 & 4 & 600.0 & 00.0 \\
\hline 01 & 5215 & 140.7501 & 7.4750 & 299.0 & 4 & 600.0 & 00.0 \\
\hline 01 & 5216 & 148.2524 & 7.2750 & 298.4 & 4 & 600.0 & 00.0 \\
\hline 01 & 5217 & 157.7496 & 8.4500 & 298.6 & 4 & 600.0 & 00.0 \\
\hline 01 & 5218 & 160.0000 & 8.3500 & 298.9 & 4 & 600.0 & 00.0 \\
\hline 01 & 5219 & 164.2502 & 9.9500 & 299.4 & 4 & 600.0 & 0 \\
\hline 01 & 52110 & 165.2488 & 9.0500 & 300.3 & 4 & 600.0 & \\
\hline 01 & 52111 & 165.7583 & 8.1250 & 301.8 & 4 & 600.0 & \\
\hline 01 & 52112 & 156.7460 & 6.9750 & 303.0 & 4 & 600.0 & 00.0 \\
\hline 01 & 52113 & 164.4990 & 6.0750 & 304.0 & 4 & 600.0 & 00.0 \\
\hline 01 & 52114 & 169.2504 & 6.5250 & 304.7 & 4 & .0 & \\
\hline 01 & 52115 & 164.2435 & 7.0250 & 305.6 & 4 & 600.0 & .0 \\
\hline 01 & 52116 & 158.0034 & 7.5750 & 306.4 & 4 & 60 & .0 \\
\hline 01 & 52117 & 166.0057 & 7.4000 & 306.5 & 4 & .0 & \\
\hline 01 & 52118 & 92.7504 & 5.8750 & 305.5 & 4 & 60 & .0 \\
\hline 01 & 52119 & 77.2461 & 6.2500 & 303.4 & 4 & .0 & .0 \\
\hline 01 & 52120 & 74.5017 & 8.07 & 301.2 & 4 & 60 & \\
\hline 01 & 52121 & 73.0005 & 9.1250 & 299.9 & 4 & .0 & \\
\hline 01 & 52122 & 78.0004 & 10.5000 & 298.7 & 4 & 600.0 & .0 \\
\hline 01 & 52123 & 71.5000 & 10.10 & 299.3 & 4 & 60 & \\
\hline 01 & 52124 & 76.5003 & 9.35 & 299.5 & 4 & .0 & \\
\hline 01 & 5221 & 73.2500 & 9.7000 & 299.5 & 4 & .0 & \\
\hline 01 & 5222 & 72.2500 & 9.2000 & 299.5 & 4 & 600.0 & .0 \\
\hline 01 & 5223 & 75.0000 & 8.7750 & 299.4 & 4 & .0 & \\
\hline 01 & 5224 & 78.0001 & 8.8250 & 299.6 & 4 & .0 & .0 \\
\hline 01 & 5225 & 79.5002 & 8.7250 & 299.7 & 4 & 60 & .0 \\
\hline 01 & 5226 & 81.7498 & 7.6500 & 299.3 & 4 & .0 & \\
\hline 01 & 5227 & 79.0000 & 7.00 & 299.8 & 4 & .0 & 0.0 \\
\hline 01 & 5228 & 103.3994 & 3.1000 & 301.3 & 3 & 600.0 & 0.0 \\
\hline 01 & 5229 & 219.3681 & 2.3750 & 300.7 & 2 & .0 & 0.0 \\
\hline 01 & 52210 & 183.0446 & 2.7250 & 301.6 & 1 & 600.0 & 600.0 \\
\hline 01 & 52211 & 94.0038 & 4.4000 & 302.6 & 2 & 600.0 & 600.0 \\
\hline 01 & 52212 & 85.0055 & 4.3250 & 303.3 & 3 & 600.0 & 600.0 \\
\hline 01 & 52213 & 92.7519 & 5.3000 & 303.5 & 3 & 600.0 & 600.0 \\
\hline 01 & 52214 & 91.0000 & 6.3250 & 303.7 & 4 & 600.0 & 600.0 \\
\hline 01 & 52215 & 88.4969 & 6.8500 & 303.8 & 4 & 600.0 & 600.0 \\
\hline 01 & 52216 & 74.7502 & 8.7750 & 303.5 & 4 & 600.0 & 600.0 \\
\hline 01 & 52217 & 69.2500 & 9.7000 & 302.8 & 4 & 600.0 & 600.0 \\
\hline 01 & 52218 & 68.2498 & 10.4000 & 301.6 & 4 & 600.0 & 600.0 \\
\hline 01 & 52219 & 91.5000 & 6.0500 & 300.9 & 4 & 600.0 & 600.0 \\
\hline 01 & 52220 & 73.2498 & 10.0750 & 299.2 & 4 & 600.0 & 600.0 \\
\hline 01 & 52221 & 73.2500 & 10.9000 & 298.4 & 4 & 600.0 & 600.0 \\
\hline 01 & 52222 & 72.7500 & 10.0250 & 298.2 & 1 & 600.0 & 600.0 \\
\hline . & 52223 & 72.0000 & 10.1250 & 298.1 & 4 & 600.0 & 600.0 \\
\hline
\end{tabular}




\begin{tabular}{|c|c|c|c|c|c|c|c|}
\hline 01 & 52224 & 73.2500 & 10.5250 & 298.1 & 4 & 600.0 & 600.0 \\
\hline 01 & 5231 & 73.0014 & 10.9750 & 297.3 & 4 & 600.0 & 600.0 \\
\hline 01 & 5232 & 66.9997 & 11.0500 & 297.4 & 4 & 600.0 & 00.0 \\
\hline 01 & 5233 & 71.5002 & 10.4500 & 297.5 & 4 & 600.0 & 00.0 \\
\hline 01 & 5234 & 72.7500 & 10.6000 & 297.2 & 4 & 600.0 & 00.0 \\
\hline 01 & 5235 & 71.7496 & 11.0000 & 297.0 & 4 & 600.0 & 00.0 \\
\hline 01 & 5236 & 75.0000 & 10.4250 & 297.4 & 4 & 600.0 & 00.0 \\
\hline 01 & 5237 & 73.5000 & 000 & 298.3 & 4 & 600.0 & 00.0 \\
\hline 01 & 5238 & 71.0000 & 7.2750 & 299.4 & 4 & 600.0 & 00.0 \\
\hline 01 & 5239 & 69.0001 & 750 & 300.5 & 3 & 600.0 & 0 \\
\hline 01 & 52310 & 74.2366 & 00 & 301.2 & 3 & 600.0 & \\
\hline 01 & 52311 & 80.7524 & 50 & 302.1 & 3 & 600.0 & \\
\hline 01 & 52312 & 84.4979 & 250 & 302.7 & 3 & 600.0 & 00.0 \\
\hline 01 & 52313 & 89.7592 & 250 & 303.3 & 3 & 600.0 & 00.0 \\
\hline 01 & 52314 & 97.0000 & 7. & 303.9 & 4 & .0 & \\
\hline 01 & 52315 & 103.7476 & 250 & 304.0 & 4 & 600.0 & 00.0 \\
\hline 01 & 52316 & 108.5083 & 7.3500 & 304.3 & 4 & 60 & 00.0 \\
\hline 01 & 52317 & 101.9995 & 7 . & 303.7 & 4 & .0 & \\
\hline 01 & 52318 & 105.4987 & 500 & 302.8 & 4 & .0 & \\
\hline 01 & 52319 & 97.2542 & 50 & 300.8 & 4 & .0 & .0 \\
\hline 01 & 52320 & 75.2489 & 7 . & 298.9 & 4 & 6 & \\
\hline 01 & 52321 & 70.0000 & 8 . & 298.1 & 4 & .0 & \\
\hline 01 & 52322 & 75.0004 & 8.9 & 298.0 & 4 & 600.0 & .0 \\
\hline 01 & 52323 & 72.0000 & 9 . & 298.0 & 4 & 6 & \\
\hline 01 & 52324 & 74.2501 & & 298.0 & 4 & .0 & \\
\hline 01 & 5241 & 70.7500 & 9 . & 297.8 & 4 & .0 & 0 \\
\hline 01 & 5242 & 71.5000 & 8.7000 & 297.4 & 4 & 600.0 & .0 \\
\hline 01 & 5243 & 67.9998 & 8 & 297.3 & 4 & .0 & \\
\hline 01 & 5244 & 68.7501 & 7 . & 297.3 & 4 & .0 & 0.0 \\
\hline 01 & $524 \quad 5$ & 72.5023 & 8.1750 & 297.4 & 4 & .0 & .0 \\
\hline 01 & 5246 & 77.2501 & 8.2 & 297.7 & 4 & .0 & \\
\hline 01 & 5247 & 87.0021 & 7. & 299.1 & 4 & .0 & 600.0 \\
\hline 01 & 5248 & 88.7500 & 5.7250 & 300.5 & 3 & 600.0 & 600.0 \\
\hline 01 & 5249 & 94.0000 & 5.2 & 301.7 & 3 & 0 & 0.0 \\
\hline 01 & 52410 & 121.2624 & 5.4 & 302.8 & 3 & 600.0 & 600.0 \\
\hline 01 & 52411 & 110.2481 & 4.6750 & 303.2 & 3 & 600.0 & 600.0 \\
\hline 01 & 52412 & 92.9981 & 5 . & 303.1 & 3 & 600.0 & 0.0 \\
\hline 01 & 52413 & 96.5006 & 6.7500 & 303.3 & 4 & 600.0 & 600.0 \\
\hline 01 & 52414 & 97.7499 & 7.4250 & 303.2 & 4 & 600.0 & 600.0 \\
\hline 01 & 52415 & 89.2523 & 7. & 303.1 & 4 & 600.0 & 600.0 \\
\hline 01 & 52416 & 98.0034 & 7.8250 & 302.8 & 4 & 600.0 & 600.0 \\
\hline 01 & 52417 & 100.5000 & 7.6000 & 302.3 & 4 & 600.0 & 600.0 \\
\hline 01 & 52418 & 90.5006 & 6.4250 & 301.9 & 4 & 600.0 & 600.0 \\
\hline 01 & 52419 & 78.5000 & 6.9750 & 300.8 & 4 & 600.0 & 600.0 \\
\hline 01 & 52420 & 71.2500 & 7.7500 & 299.1 & 4 & 600.0 & 600.0 \\
\hline 01 & 52421 & 73.0001 & 8.3750 & 298.1 & 4 & 600.0 & 600.0 \\
\hline 01 & 52422 & 75.0002 & 9.3250 & 297.9 & 4 & 600.0 & 600.0 \\
\hline J & 52423 & 74.9999 & 9.4000 & 298.0 & $\Psi$ & 600.0 & 600.0 \\
\hline
\end{tabular}




\begin{tabular}{|c|c|c|c|c|c|c|c|}
\hline 01 & 52424 & 75.5000 & 8.8500 & 298.1 & 4 & 600.0 & 600.0 \\
\hline 01 & 5251 & 74.0000 & 8.1250 & 297.9 & 4 & 600.0 & 600.0 \\
\hline 01 & 5252 & 71.2500 & 9.1000 & 297.7 & 4 & 600.0 & 00.0 \\
\hline 01 & 5253 & 70.2495 & 8.7500 & 297.6 & 4 & 600.0 & 00.0 \\
\hline 01 & 5254 & 73.2502 & 9.2000 & 297.5 & 4 & 600.0 & 00.0 \\
\hline 01 & 5255 & 66.7502 & 9.1750 & 297.3 & 4 & 600.0 & 00.0 \\
\hline 01 & 5256 & 66.5005 & 8.7250 & 297.7 & 4 & 600.0 & 00.0 \\
\hline 01 & $\begin{array}{ll}525 & 7\end{array}$ & 72.7500 & 7.9500 & 298.6 & 4 & 600.0 & 00.0 \\
\hline 01 & 5258 & 82.7483 & 7.3500 & 300.2 & 4 & 600.0 & 00.0 \\
\hline 01 & 5259 & 85.2496 & 8.6000 & 299.8 & 4 & 600.0 & 00.0 \\
\hline 01 & 52510 & 85.9998 & 8.2000 & 301.0 & 4 & 600.0 & \\
\hline 01 & 52511 & 87.7506 & 7.6000 & 302.1 & 4 & 600.0 & \\
\hline 01 & 52512 & 94.7494 & 5.5250 & 302.6 & 3 & 600.0 & 00.0 \\
\hline 01 & 52513 & 95.0150 & 5.0250 & 303.5 & 3 & .0 & 00.0 \\
\hline 01 & 52514 & 80.2394 & 6.3500 & 303.3 & 4 & .0 & \\
\hline 01 & 52515 & 69.7494 & 7.9750 & 302.5 & 4 & 600.0 & 00.0 \\
\hline 01 & 52516 & 71.5000 & 8.2250 & 302.1 & 4 & 600.0 & 600.0 \\
\hline 01 & 52517 & 71.0000 & 7.9500 & 301.6 & 4 & .0 & \\
\hline 01 & 52518 & 74.0000 & 8.8250 & 300.4 & 4 & .0 & .0 \\
\hline 01 & 52519 & 70.9998 & 9.5750 & 298.7 & 4 & .0 & .0 \\
\hline 01 & 52520 & 67 . & 8 . & 297.9 & 4 & 60 & \\
\hline 01 & 52521 & 61.0000 & 8.0500 & 298.4 & 4 & .0 & \\
\hline 01 & 52522 & 63.5000 & 7.9750 & 298.6 & 4 & 600.0 & .0 \\
\hline 01 & 52523 & 64.5000 & 7.45 & 298.2 & 4 & 60 & .0 \\
\hline 01 & 52524 & 78.5284 & 250 & 296.8 & 4 & .0 & \\
\hline 01 & 5261 & 76.7530 & 6.5500 & 295.5 & 4 & 600.0 & 600.0 \\
\hline 01 & 5262 & 70.4957 & 3.7000 & 294.6 & 4 & 600.0 & 600.0 \\
\hline 01 & 5263 & 62.9999 & 7.5750 & 295.7 & 4 & 0 & \\
\hline 01 & 5264 & 66.5003 & 7.8250 & 296.1 & 4 & .0 & 0.0 \\
\hline 01 & 5265 & 78.0000 & 6.3000 & 296.7 & 4 & 60 & .0 \\
\hline 01 & 5266 & 82.4991 & 5.9250 & 297.1 & 4 & .0 & \\
\hline 01 & 5267 & 79.0000 & 6.4500 & 297.7 & 4 & .0 & 600.0 \\
\hline 01 & 5268 & 71.9982 & 7.2250 & 298.0 & 4 & 600.0 & 600.0 \\
\hline 01 & 5269 & 87.2319 & 6.2250 & 298.2 & 4 & 0 & 0.0 \\
\hline 01 & 52610 & 48.9553 & 3.6500 & 298.6 & 3 & 600.0 & 600.0 \\
\hline 01 & 52611 & 108.1008 & 2.5750 & 299.9 & 2 & 600.0 & 600.0 \\
\hline 01 & 52612 & 72.4731 & 3.6500 & 300.3 & 2 & 600.0 & 0.0 \\
\hline 01 & 52613 & 71.7827 & 5.9750 & 299.3 & 3 & 600.0 & 600.0 \\
\hline 01 & 52614 & 88.7765 & 5.8000 & 299.3 & 3 & 600.0 & 600.0 \\
\hline 01 & 52615 & 97.7497 & 8.5750 & 298.6 & 4 & 600.0 & 600.0 \\
\hline 01 & 52616 & 103.5000 & 8.6250 & 298.5 & 4 & 600.0 & 600.0 \\
\hline 01 & 52617 & 96.0000 & 7.0000 & 297.9 & 4 & 600.0 & 600.0 \\
\hline 01 & 52618 & 76.4997 & 7.3500 & 296.5 & 4 & 600.0 & 600.0 \\
\hline 01 & 52619 & 83.5000 & 7.6750 & 295.7 & 4 & 600.0 & 600.0 \\
\hline 01 & 52620 & 78.5078 & 10.4000 & 294.4 & 4 & 600.0 & 600.0 \\
\hline 01 & 52621 & 64.2499 & & 294.2 & 4 & 600.0 & 600.0 \\
\hline 01 & 52622 & 66.9998 & 10.2500 & 293.9 & 4 & 600.0 & 600.0 \\
\hline . & 52623 & 63.0000 & 9.125 & 294.4 & 4 & 600.0 & 600.0 \\
\hline
\end{tabular}




\begin{tabular}{|c|c|c|c|c|c|c|c|}
\hline 1 & 52624 & 64.7508 & 7.2500 & 295.2 & 4 & 600.0 & 600.0 \\
\hline 01 & 5271 & 76.0011 & 8.2000 & 294.1 & 4 & 600.0 & 00.0 \\
\hline 01 & 5272 & 74.9994 & 8.8750 & 293.0 & 4 & 600.0 & 00.0 \\
\hline 1 & 5273 & 75.2480 & 9.6500 & 290.6 & 4 & 600.0 & 00.0 \\
\hline 01 & 5274 & 79.0000 & 11.9750 & 287.5 & 4 & 600.0 & 0.0 \\
\hline 01 & 5275 & 74.5000 & 13.8750 & 287.3 & 4 & 600.0 & 00.0 \\
\hline 01 & 5276 & 79.2501 & 13.7750 & 287.1 & 4 & 600.0 & 0 \\
\hline 01 & 5277 & 75.2512 & 12.5750 & 287.6 & 4 & 600.0 & 00.0 \\
\hline 01 & 5278 & 71.0001 & 13.5500 & 286.1 & 4 & 600.0 & 00.0 \\
\hline 01 & 5279 & 69.7500 & 14.2000 & 286.6 & 4 & 60 & 0 \\
\hline 1 & 52710 & 72.7498 & 11.9250 & 288.3 & 4 & 600.0 & \\
\hline 01 & 52711 & 83.7514 & 9.6250 & 290.2 & 4 & .0 & \\
\hline 01 & 52712 & 93.2507 & 8.9000 & 292.3 & 4 & 600.0 & .0 \\
\hline 01 & 52713 & 78.2518 & 7.9750 & 295.0 & 4 & 600.0 & \\
\hline 01 & 52714 & 77.2526 & 11. & 294.0 & 4 & .0 & \\
\hline 01 & 52715 & 79.4979 & 11.4500 & 292.9 & 4 & 60 & \\
\hline 01 & 52716 & 76.7545 & 10.9000 & 292.1 & 4 & 60 & .0 \\
\hline 01 & 52717 & 66.4994 & 12.7250 & 291.4 & 4 & .0 & \\
\hline 01 & 52718 & 71.5001 & 14.5000 & 290.3 & 4 & .0 & \\
\hline 01 & 52719 & 68.2503 & 15.5000 & 289.2 & 4 & .0 & .0 \\
\hline 01 & 52720 & 67.5000 & 14 & 288.4 & 4 & 6 & \\
\hline 01 & 52721 & 65.4998 & 14 . & 288.2 & 4 & .0 & \\
\hline 01 & 52722 & 65.7500 & 14.7250 & 288.1 & 4 & 600.0 & .0 \\
\hline 01 & 52723 & 64.2501 & 14 . & 288.1 & 4 & 0 & \\
\hline 01 & 52724 & 65.2500 & 15 . & 285.3 & 4 & 0 & \\
\hline 01 & 5281 & 67.7499 & 15.3250 & 284.7 & 4 & .0 & \\
\hline 01 & 5282 & 69.5003 & 12.8750 & 287.0 & 4 & 60 & .0 \\
\hline 01 & 5283 & 64.7499 & 12.6750 & 287.4 & 4 & .0 & \\
\hline 01 & 5284 & 68.7500 & 11.7750 & 285.8 & 4 & .0 & .0 \\
\hline 01 & $528 \quad 5$ & 63.7500 & 12.1500 & 286.0 & 4 & .0 & \\
\hline 01 & 5286 & 66.7466 & 13.3250 & 286.8 & 4 & .0 & \\
\hline 01 & $528 \quad 7$ & 72.5000 & 12.6500 & 286.3 & 4 & .0 & .0 \\
\hline 01 & 5288 & 74.0000 & 9.4250 & 286.3 & 4 & 0 & 0.0 \\
\hline 01 & 5289 & 77.2501 & 10.7250 & 286.9 & 4 & .0 & .0 \\
\hline 01 & 52810 & 74.5000 & 9.5750 & 288.5 & 4 & 0 & 0.0 \\
\hline 01 & 52811 & 87.7592 & 7.6750 & 289.7 & 4 & 600.0 & 0.0 \\
\hline 01 & 52812 & 87.2486 & 7.775 & 291.7 & 4 & 600.0 & .0 \\
\hline 01 & 52813 & 80.2265 & 8.0750 & 292.4 & 4 & 600.0 & 600.0 \\
\hline 01 & 52814 & 75.0002 & 8.8500 & 292.4 & 4 & 600.0 & 600.0 \\
\hline 01 & 52815 & 75.5003 & 10.3250 & 292.1 & 4 & 600.0 & .0 \\
\hline 01 & 52816 & 72.4969 & 9.8000 & 292.2 & 4 & 600.0 & 600.0 \\
\hline 01 & 52817 & 75.4990 & 10.3750 & 291.3 & 4 & 600.0 & 600.0 \\
\hline 01 & 52818 & 71.7501 & 10.6500 & 291.0 & 4 & 600.0 & 600.0 \\
\hline 01 & 52819 & 71.5009 & 10.5000 & 290.7 & 4 & 600.0 & 600.0 \\
\hline 01 & 52820 & 75.5000 & 10.9250 & 289.9 & 4 & 600.0 & 600.0 \\
\hline 01 & 52821 & 78.0000 & 9.3500 & 290.9 & 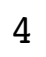 & 600.0 & 600.0 \\
\hline 01 & 52822 & 78.0000 & 8.425 & 291.7 & 4 & 600.0 & 600.0 \\
\hline J & 52823 & 80.0002 & 8.950 & 291.4 & & 600.0 & 600.0 \\
\hline
\end{tabular}




\begin{tabular}{|c|c|c|c|c|c|c|c|}
\hline & 24 & . & 5000 & 90.9 & 4 & 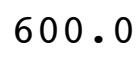 & \\
\hline & 5291 & 7.2500 & 9.5750 & 290.6 & 4 & 600.0 & \\
\hline & 292 & 74.0000 & 2500 & 290.5 & 4 & 00 & \\
\hline & 293 & 0000 & 0500 & 290.4 & & 00 & \\
\hline & 5294 & 500 & 5250 & 90.3 & & & \\
\hline & 5295 & 593 & & 90.7 & & 0 & \\
\hline & 296 & 251 & 250 & 91.1 & & 00 & \\
\hline & 297 & 32 & 500 & 92.8 & & & \\
\hline & 298 & 59 . & & 3 & & & \\
\hline & 299 & 157.4985 & 7500 & 93.5 & & 0 & \\
\hline & 2910 & 163.99 & 6.0750 & 29 & & & \\
\hline & 2911 & 71 . & & 2 & & & \\
\hline & 2912 & 172 . & & 297.2 & & & \\
\hline & 2913 & 170.4 & 3750 & 29 & & & \\
\hline & 2914 & 161. & & & & & \\
\hline & 2915 & 168 . & & 2 & 3 & & \\
\hline & 2916 & 177.7 & 4.95 & 301.2 & & & \\
\hline & 2917 & 177.4 & 4. & .7 & & & \\
\hline & 2918 & 117 . & & & 3 & & \\
\hline & 2919 & 82.5000 & 4.42 & .5 & 4 & & \\
\hline & 2920 & 83.2514 & 0 & 298.7 & $I$ & & \\
\hline & 2921 & 77.74 & & & 4 & & \\
\hline & 922 & 77 . & & 29 & 4 & & \\
\hline & 2923 & 82.23 & 6.55 & .1 & 4 & 0 & \\
\hline & 52924 & 100.2 & & & 4 & & \\
\hline & 530 & 114.6 & 0 & & 4 & & \\
\hline & 5302 & 150.4998 & 0 & 298.3 & 4 & & 0 \\
\hline & 5303 & 152 & 0 & 297.2 & 4 & & \\
\hline & 5304 & 147 & 0 & & 4 & & \\
\hline & 5305 & 149.2498 & 3000 & 29 & 4 & & . \\
\hline & 5306 & 152.5000 & 2500 & 5 & 4 & & 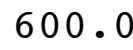 \\
\hline & 5307 & 158 & 10 . & & 4 & & \\
\hline & 5308 & 165 . & & .4 & 4 & & 。 \\
\hline & 5309 & 154 & 0 & 25 & 4 & & \\
\hline & 530 & 153. & & & 4 & & \\
\hline & & 164 . & & .8 & 4 & & 0 • \\
\hline & 53012 & 167 . & & 301.0 & 4 & & \\
\hline & 53013 & 167 . & & 30 & 4 & & \\
\hline & 53014 & 161. & & .3 & 3 & & 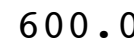 \\
\hline & 530 & 167.0000 & & 304.4 & 3 & & \\
\hline & 53016 & 168.7492 & 0 & & 3 & & \\
\hline & & 165.0077 & 000 & & 3 & & \\
\hline & 53018 & 181.4994 & 5.3500 & 305.5 & 3 & & 0 . \\
\hline & 53019 & 180 . & 000 & & 4 & & \\
\hline & 53020 & 153.9585 & 2.1250 & & 4 & & \\
\hline & 53021 & 115.0162 & 2.1500 & 303.6 & 4 & & ר. \\
\hline & & & & & 4 & & \\
\hline & 3023 & 111.4912 & 3.1500 & 304.0 & & .0 & 60 \\
\hline
\end{tabular}




\begin{tabular}{|c|c|c|c|c|c|c|c|}
\hline 01 & 53024 & 91.3321 & 2.3250 & 304.2 & 4 & 600.0 & 600.0 \\
\hline 01 & 5311 & 77.4954 & 3.4500 & 304.5 & 4 & 600.0 & 00.0 \\
\hline 01 & 5312 & 103.2455 & 3.6000 & 304.6 & 4 & 600.0 & 00.0 \\
\hline 01 & 5313 & 114.2501 & 4.3500 & 304.4 & 4 & 600.0 & 00.0 \\
\hline 01 & 5314 & 109.0000 & 4.0000 & 303.4 & 4 & 600.0 & 00.0 \\
\hline 01 & 5315 & 108.9781 & 3.2000 & 302.9 & 4 & 600.0 & 00.0 \\
\hline 01 & 5316 & 136.5021 & 1.4500 & 302.8 & 4 & 600.0 & 00.0 \\
\hline 01 & 5317 & 165.2489 & 1.6500 & 304.3 & 3 & 600.0 & 00.0 \\
\hline 01 & 5318 & 155.9968 & 6.5250 & 303.4 & 4 & 600.0 & 00.0 \\
\hline 01 & 5319 & 161.9908 & 7.3000 & 303.4 & 4 & 600.0 & .0 \\
\hline 01 & 53110 & 162.2485 & 6.8750 & 303.7 & 4 & 600.0 & \\
\hline 01 & 3111 & 167.7499 & 6.2250 & 304.7 & 4 & 600.0 & \\
\hline 01 & 3112 & 173.4991 & 5.4000 & 306.2 & 3 & 600.0 & 00.0 \\
\hline 01 & 3113 & 184.5031 & 5.0750 & 307.4 & 3 & 600.0 & 00.0 \\
\hline 01 & 3114 & 191.0049 & 00 & 308.5 & 3 & .0 & \\
\hline 01 & 53115 & 190.4968 & 4.4750 & 309.6 & 3 & 600.0 & .0 \\
\hline 01 & 53116 & 182.5225 & 4.7000 & 309.6 & 3 & 600.0 & 600.0 \\
\hline 01 & 53117 & 112.2327 & 6.5000 & 308.9 & 4 & .0 & \\
\hline 01 & 3118 & 112.2482 & 5.6500 & 307.4 & 3 & .0 & .0 \\
\hline 01 & 53119 & 101.1970 & 4.7750 & 305.9 & 4 & 60 & .0 \\
\hline 01 & 53120 & 73.2495 & 9 . & 303.7 & 4 & 60 & \\
\hline 01 & 53121 & 65.4986 & 9.0500 & 302.5 & 4 & .0 & \\
\hline 01 & 3122 & 65.2504 & 7.9250 & 301.7 & 4 & 600.0 & .0 \\
\hline 01 & 53123 & 70.2500 & 9.8500 & 301.9 & 4 & 60 & \\
\hline 01 & 53124 & 74.7507 & 000 & 301.1 & 4 & .0 & \\
\hline 01 & $\begin{array}{lll}6 & 1 & 1\end{array}$ & 78.4991 & 7.1250 & 300.6 & 4 & .0 & 600.0 \\
\hline 01 & $\begin{array}{lll}6 & 1 & 2\end{array}$ & 77.9998 & 6.6500 & 300.2 & 4 & 600.0 & .0 \\
\hline 01 & $\begin{array}{lll}6 & 1 & 3\end{array}$ & 82.2501 & 6.5750 & 299.3 & 4 & .0 & \\
\hline 01 & $\begin{array}{lll}6 & 1 & 4\end{array}$ & 80.7500 & 6.5500 & 299.2 & 4 & .0 & 0.0 \\
\hline 01 & 615 & 83.2500 & 7.2000 & 299.3 & 4 & 60 & .0 \\
\hline 01 & 616 & 56.8460 & 3.1500 & 300.1 & 5 & .0 & \\
\hline 01 & $\begin{array}{lll}6 & 1 & 7\end{array}$ & 153.0722 & 0.7750 & 302.3 & 4 & .0 & 600.0 \\
\hline 01 & $\begin{array}{lll}6 & 1 & 8\end{array}$ & 301.6290 & 1.3500 & 301.9 & 3 & 600.0 & 600.0 \\
\hline 01 & $\begin{array}{lll}6 & 1 & 9\end{array}$ & 243.7708 & 2.5250 & 301.3 & 2 & 600.0 & 0.0 \\
\hline 01 & 6110 & 220.5108 & 2.4750 & 301.5 & 1 & 600.0 & 600.0 \\
\hline 01 & 6111 & 74.5077 & 4.2000 & 301.9 & 2 & 600.0 & 600.0 \\
\hline 01 & 6112 & 74.4596 & 6.7000 & 302.0 & 3 & 600.0 & .0 \\
\hline 01 & 6113 & 90.7711 & 6.0750 & 300.9 & 4 & 600.0 & 600.0 \\
\hline 01 & $6 \quad 114$ & 88.7721 & 7.5750 & 298.1 & 4 & 600.0 & 600.0 \\
\hline 01 & 6115 & 85.0023 & 7.2500 & 298.6 & 4 & 600.0 & 0 \\
\hline 01 & 6116 & 98.4957 & 7.6250 & 298.1 & 4 & 600.0 & 600.0 \\
\hline 01 & 6117 & 80.0562 & 5.9000 & 297.9 & 3 & 600.0 & 600.0 \\
\hline 01 & 6118 & 65.7498 & 8.1750 & 296.8 & 4 & 600.0 & 600.0 \\
\hline 01 & 6119 & 73.7545 & 7.6750 & 295.0 & 4 & 600.0 & 600.0 \\
\hline 01 & 6120 & 50.2462 & 9.4250 & 293.9 & 4 & 600.0 & 600.0 \\
\hline 01 & $6 \quad 121$ & 61.7502 & 13.6000 & 293.0 & 4 & 600.0 & 600.0 \\
\hline 01 & 6122 & 63.5000 & 12.2250 & 292.3 & 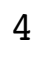 & 600.0 & 600.0 \\
\hline J & 6123 & 62.7496 & 13.6250 & 291.4 & 4 & 600.0 & 600.0 \\
\hline
\end{tabular}




\begin{tabular}{|c|c|c|c|c|c|c|c|c|}
\hline 1 & 6 & 124 & 70.4985 & 8.0500 & 291.8 & 4 & 600.0 & 00.0 \\
\hline 01 & 6 & 21 & 92.5727 & 5.4750 & 291.3 & 4 & 600.0 & 00.0 \\
\hline 01 & 6 & 22 & 71.9460 & 6.4750 & 290.2 & 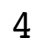 & 600.0 & 00.0 \\
\hline 01 & 6 & 23 & 114.7508 & 4.5250 & 289.3 & 4 & 600.0 & 600.0 \\
\hline 01 & 6 & 24 & 82.3888 & 5.6750 & 289.2 & 4 & 600.0 & 00.0 \\
\hline 01 & 6 & 25 & 71.9365 & 9.2250 & 289.0 & 4 & 600.0 & 00.0 \\
\hline 01 & 6 & 26 & 80.4432 & 7.3250 & 289.2 & 4 & 600.0 & 600.0 \\
\hline 01 & 6 & 27 & 67.7356 & 8.9000 & 288.4 & 4 & 600.0 & 500.0 \\
\hline 01 & 6 & 28 & 71.4999 & 9.1500 & 288.6 & 4 & 600.0 & 00.0 \\
\hline 01 & 6 & 29 & 74.4990 & 8.6250 & 290.0 & 4 & 600.0 & .0 \\
\hline 01 & 6 & 210 & 84.9507 & 6.4750 & 292.2 & 4 & 600.0 & 600.0 \\
\hline 01 & 6 & 211 & 83.4861 & 7.4500 & 292.5 & 4 & 600.0 & \\
\hline 01 & 6 & 212 & 89.0049 & 7.5250 & 293.6 & 4 & 600.0 & 600.0 \\
\hline 01 & 6 & 213 & 72.0000 & 9.1000 & 294.5 & 4 & 600.0 & 600.0 \\
\hline 01 & 6 & 214 & 64.7500 & 11.0500 & 294.3 & 4 & 600.0 & \\
\hline 01 & 6 & 215 & 66.5000 & 10.5750 & 294.1 & 4 & 600.0 & 60 \\
\hline 01 & 6 & 216 & 66.0002 & 11.9750 & 292.6 & 4 & 600.0 & 600.0 \\
\hline 01 & 6 & 217 & 62.0001 & 13.0250 & 292.0 & 4 & 600.0 & \\
\hline 01 & 6 & 218 & 58.4991 & 11.3500 & 292.4 & 4 & 600.0 & 60 \\
\hline 01 & 6 & 219 & 66.0000 & 11.1750 & 291.3 & 4 & 600.0 & \\
\hline 01 & 6 & 220 & 69.9904 & 11.0250 & 289.5 & 4 & 600.0 & .0 \\
\hline 01 & 6 & 221 & 73.7502 & 9.1000 & 288.7 & 4 & 600.0 & \\
\hline 01 & 6 & 222 & 70.0000 & 10.0750 & 288.9 & 4 & 600.0 & 60 \\
\hline 01 & 6 & 223 & 67.2501 & 10.6500 & 289.0 & 4 & 600.0 & .0 \\
\hline 01 & 6 & 224 & 69.9998 & 11.0500 & 288.5 & 4 & 600.0 & \\
\hline 01 & 6 & 31 & 72.7500 & 10.4500 & 287.9 & 4 & 600.0 & 60 \\
\hline 01 & 6 & 32 & 72.9999 & 10.0000 & 286.8 & 4 & 600.0 & 600.0 \\
\hline 01 & 6 & 33 & 76.0007 & 9.2750 & 286.5 & 4 & 600.0 & .0 \\
\hline 01 & 6 & 34 & 80.7521 & 9.9500 & 285.9 & 4 & 600.0 & 60 \\
\hline 01 & 6 & 35 & 87.4908 & 9.3750 & 285.4 & 4 & 600.0 & 600.0 \\
\hline 01 & 6 & 36 & 112.2530 & 9.1000 & 286.6 & 4 & 600.0 & .0 \\
\hline 01 & 6 & 37 & 135.9999 & 10.1750 & 287.4 & 4 & 600.0 & 60 \\
\hline 01 & 6 & 38 & 144.9968 & 10.6250 & 287.7 & 4 & 600.0 & 600.0 \\
\hline 01 & 6 & 39 & 158.5046 & 10.7750 & 288.4 & 4 & 600.0 & .0 \\
\hline 01 & 6 & 310 & 158.7502 & 8.0250 & 289.6 & 4 & 600.0 & 60 \\
\hline 01 & 6 & 311 & 161.0000 & 7.4250 & 290.9 & 4 & 600.0 & 600.0 \\
\hline 01 & 6 & 312 & 162.7476 & 7.1500 & 292.3 & 4 & 600.0 & \\
\hline 01 & 6 & 313 & 165.5054 & 6.3750 & 293.7 & 4 & 600.0 & 600.0 \\
\hline 01 & 6 & 314 & 169.0000 & 7.0750 & 294.7 & 4 & 600.0 & 600.0 \\
\hline 01 & 6 & 315 & 176.2701 & 5.7250 & 295.8 & 3 & 600.0 & \\
\hline 01 & 6 & 316 & 174.5207 & 6.4250 & 296.3 & 7 & 600.0 & 600.0 \\
\hline 01 & 6 & 317 & 118.4274 & 6.0750 & 296.2 & 4 & 600.0 & 600.0 \\
\hline 01 & 6 & 318 & 80.0011 & 6.3250 & 294.7 & 4 & 600.0 & 600.0 \\
\hline 01 & 6 & 319 & 72.4928 & 7.0250 & 293.1 & 4 & 600.0 & 600.0 \\
\hline 01 & 6 & 320 & 64.4998 & 7.1500 & 291.8 & 4 & 600.0 & 600.0 \\
\hline 01 & 6 & 321 & 68.0014 & 7.8000 & 291.4 & 4 & 600.0 & 600.0 \\
\hline 01 & 6 & 322 & 75.5000 & 8.025 & .7 & 4 & 600.0 & 600.0 \\
\hline 01 & 6 & 323 & 89.1741 & 7.8500 & 292.1 & 4 & 600.0 & 600.0 \\
\hline
\end{tabular}




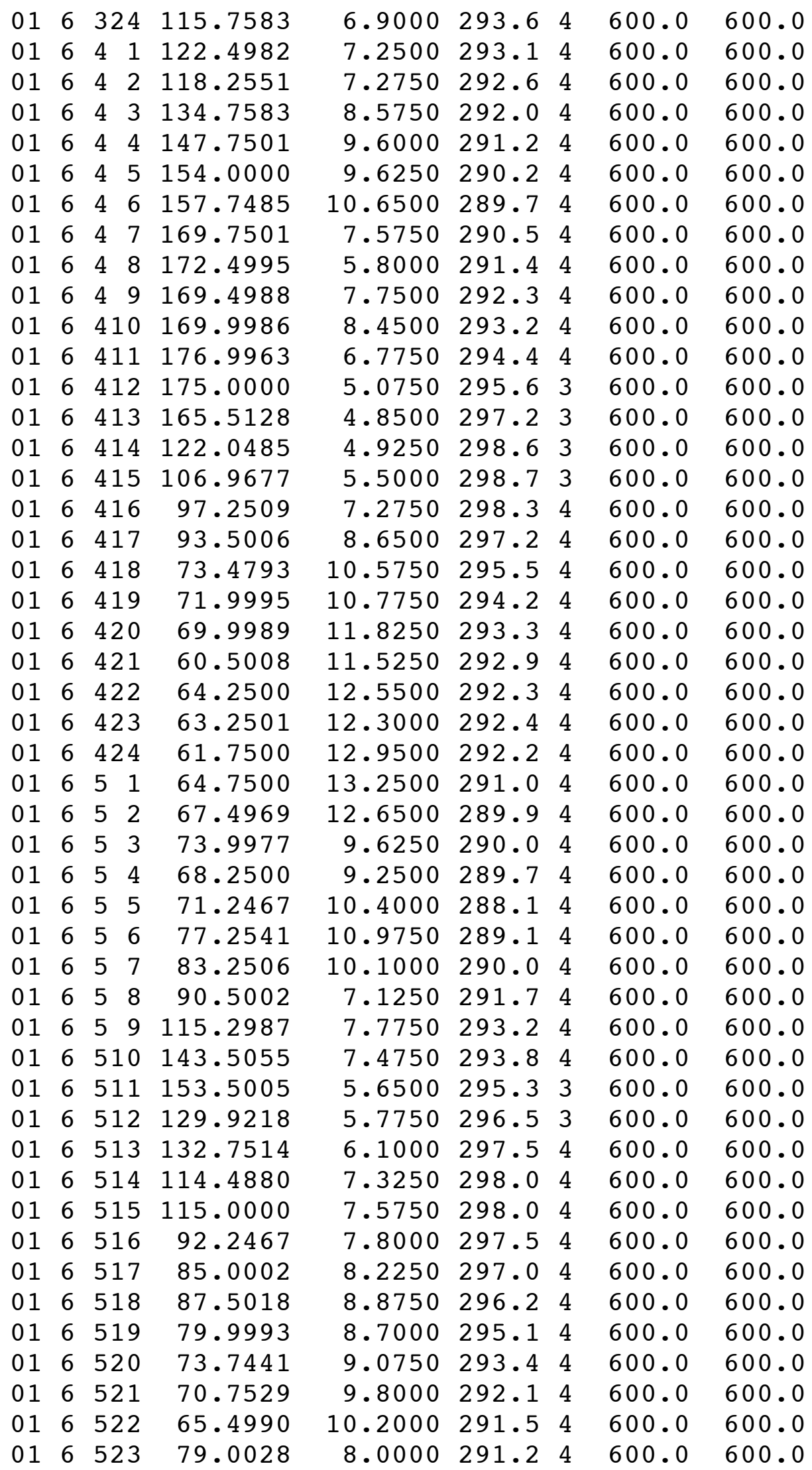




\begin{tabular}{|c|c|c|c|c|c|c|c|c|}
\hline 1 & 6 & 524 & 83.7494 & 9.2750 & 291.7 & 4 & 600.0 & 600.0 \\
\hline 01 & 6 & 61 & 76.7482 & 10.3500 & 290.9 & 4 & 600.0 & 600.0 \\
\hline 01 & 6 & 62 & 73.2501 & 10.6000 & 291.0 & 4 & 600.0 & 00.0 \\
\hline 01 & 6 & 63 & 72.4991 & 10.2000 & 290.7 & 4 & 600.0 & 00.0 \\
\hline 01 & 6 & 64 & 75.7499 & 9.5250 & 291.0 & 4 & 600.0 & 00.0 \\
\hline 01 & 6 & 65 & 69.0037 & 7.8750 & 290.8 & 4 & 600.0 & 00.0 \\
\hline 01 & 6 & 66 & 75.0034 & 7.4000 & 291.0 & 4 & 600.0 & 00.0 \\
\hline 01 & 6 & 67 & 90.4752 & 5.2750 & 292.3 & 4 & 600.0 & 00.0 \\
\hline 01 & 6 & 68 & 148.7063 & 3.7750 & 294.7 & 3 & 600.0 & 00.0 \\
\hline 01 & 6 & 69 & 159.2680 & 4.5000 & 296.0 & 3 & 600.0 & 00.0 \\
\hline 01 & 6 & 610 & 165.7485 & 6.3250 & 296.1 & 4 & 600.0 & 00.0 \\
\hline 01 & 6 & 611 & 159.0034 & 5.8500 & 296.9 & 3 & 600.0 & \\
\hline 01 & 6 & 612 & 155.0080 & 5.1250 & 298.7 & 3 & 600.0 & 00.0 \\
\hline 01 & 6 & 613 & 165.4992 & 6.0250 & 299.8 & 4 & 600.0 & 00.0 \\
\hline 01 & 6 & 614 & 173.4989 & 6.9250 & 300.9 & 4 & .0 & \\
\hline 01 & 6 & 615 & 176.9927 & 7.7000 & 301.7 & 4 & 600.0 & 00.0 \\
\hline 01 & 6 & 616 & 129.4644 & 6.2000 & 302.0 & 4 & 600.0 & 600.0 \\
\hline 01 & 6 & 617 & 100.7335 & 6.0250 & 301.7 & 4 & .0 & \\
\hline 01 & 6 & 618 & 88.5017 & 7.1000 & 300.7 & 4 & 600.0 & .0 \\
\hline 01 & 6 & 619 & 87.7437 & 6.8500 & 299.6 & 4 & .0 & .0 \\
\hline 01 & 6 & 620 & 80.4991 & 7.7500 & 298.1 & 4 & 60 & \\
\hline 01 & 6 & 621 & 77.0000 & 8.1750 & 297.5 & 4 & .0 & \\
\hline 01 & 6 & 622 & 73.2500 & 8.4500 & 297.2 & 4 & 600.0 & 0 \\
\hline 01 & 6 & 623 & 75.7501 & 9.02 & 296.8 & 4 & 60 & .0 \\
\hline 01 & 6 & 624 & 79.2508 & 9.3250 & 296.5 & 4 & .0 & \\
\hline 01 & 6 & 71 & 75.5000 & 8.9000 & 296.2 & 4 & 600.0 & 600.0 \\
\hline 01 & 6 & 72 & 73.7496 & 9.5250 & 295.7 & 4 & 600.0 & .0 \\
\hline 01 & 6 & 73 & 76.7502 & 8.9250 & 295.8 & 4 & .0 & \\
\hline 01 & 6 & 74 & 78.7499 & 8.5250 & 295.4 & 4 & 600.0 & 600.0 \\
\hline 01 & 6 & 75 & 83.0000 & 8.4500 & 295.3 & 4 & .0 & 600.0 \\
\hline 01 & 6 & 76 & 82.7597 & 6.8500 & 295.7 & 4 & .0 & \\
\hline 01 & 6 & 77 & 91.9961 & 4.4750 & 296.9 & 4 & .0 & 600.0 \\
\hline 01 & 6 & 78 & 147.7134 & 5.2000 & 298.4 & 3 & 600.0 & 600.0 \\
\hline 01 & 6 & 79 & 157.2527 & 6.8750 & 298.0 & 4 & 600.0 & 0.0 \\
\hline 01 & 6 & 710 & 165.2467 & 6.4250 & 298.8 & 4 & 600.0 & 600.0 \\
\hline 01 & 6 & 711 & 169.2502 & 6.2500 & 300.1 & 4 & 600.0 & 600.0 \\
\hline 01 & 6 & 712 & 172.9990 & 6.0000 & 301.5 & 4 & 600.0 & 600.0 \\
\hline 01 & 6 & 713 & 181.2522 & 6.5000 & 302.8 & 4 & 600.0 & 600.0 \\
\hline 01 & 6 & 714 & 178.7502 & 6.1000 & 303.7 & 4 & 600.0 & 600.0 \\
\hline 01 & 6 & 715 & 187.2501 & 750 & 304.2 & 4 & 600.0 & 600.0 \\
\hline 01 & 6 & 716 & 168.7376 & 6.8000 & 304.9 & 4 & 600.0 & 600.0 \\
\hline 01 & 6 & 717 & 129.5340 & 6.2250 & 304.9 & 4 & 600.0 & 600.0 \\
\hline 01 & 6 & 718 & 79.7451 & 6.6000 & 303.9 & 4 & 600.0 & 600.0 \\
\hline 01 & 6 & 719 & 74.2498 & 6.7000 & 301.9 & 4 & 600.0 & 600.0 \\
\hline 01 & 6 & 720 & 72.7485 & 6.9000 & 300.5 & 4 & 600.0 & 600.0 \\
\hline 01 & 6 & 721 & 69.7485 & 7.7000 & 299.9 & 4 & 600.0 & 600.0 \\
\hline 01 & 6 & 722 & 70.0000 & 8.5250 & 299.1 & 4 & 600.0 & 600.0 \\
\hline . & - & 723 & 69.5000 & 8.400 & 298.5 & 4 & 600.0 & 600.0 \\
\hline
\end{tabular}




\begin{tabular}{|c|c|c|c|c|c|c|c|c|}
\hline 1 & 6 & 24 & 5.0005 & 8.6250 & 98.1 & 4 & 00.0 & \\
\hline & 6 & 81 & 8.4995 & .3500 & 298.2 & & & \\
\hline & 6 & 82 & 64 & 750 & 8.0 & & 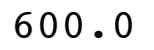 & \\
\hline & 6 & 3 & 000 & 000 & 97.5 & & 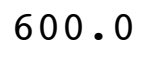 & \\
\hline & 6 & 4 & & & & & & \\
\hline & 6 & 85 & 0 & 500 & 96.8 & & 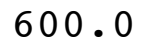 & \\
\hline & 6 & 6 & & & 97.5 & & 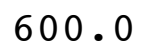 & \\
\hline & 6 & 7 & & 0 & 97.5 & & 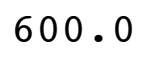 & \\
\hline & 6 & 8 & 75 . & 7 . & 297.9 & & 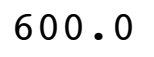 & \\
\hline & 6 & 9 & & 500 & & & 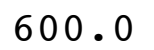 & \\
\hline & 6 & 10 & & & & & & \\
\hline & 6 & 11 & & & & & & \\
\hline & 6 & 12 & 105 . & 7. & 300.8 & & 0 & \\
\hline & 6 & 813 & & & & & & \\
\hline & 6 & 814 & & & & & & \\
\hline & 6 & 815 & & & .6 & & & \\
\hline & 6 & 816 & & 0.15 & .2 & & v & \\
\hline & 6 & 817 & & & & & & \\
\hline & 6 & 818 & 62 & 0 & & & & \\
\hline & 6 & 819 & 6 & 0 & 29 & & & \\
\hline & 6 & 820 & & & & & & \\
\hline & 6 & 821 & & & & & & \\
\hline & 6 & 822 & 86 & 0 & 29 & & & \\
\hline & 6 & 823 & & & & & & \\
\hline & 6 & 824 & & & & & & \\
\hline & 6 & 91 & 012 & & & 4 & & \\
\hline & 6 & 92 & 7 & & & 4 & & \\
\hline & 6 & 93 & 62 & 0 & & 4 & & \\
\hline & 6 & 94 & & 0 & & 4 & & \\
\hline & 6 & 95 & 64. & 0 & 29 & 4 & & \\
\hline & 6 & 96 & & 0 & 4 & 4 & & \\
\hline & 6 & 97 & 77 . & & & 4 & & \\
\hline & 6 & 98 & 86. & & 293.8 & $\Psi$ & & \\
\hline & 6 & 99 & 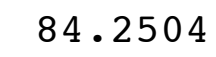 & & 29 & 4 & & \\
\hline & 6 & 910 & & & & 4 & & \\
\hline & 6 & 911 & & & 295.8 & & & \\
\hline & 6 & 912 & & & 29 & 3 & & \\
\hline & 6 & 913 & & & 297.8 & 4 & & \\
\hline & 6 & 914 & 90. & & 297.9 & 4 & & \\
\hline & 6 & 915 & 7 & & & 4 & & \\
\hline & 6 & 916 & & 0 & 297.3 & $\Psi$ & & \\
\hline & 6 & 917 & 68.2500 & 10.7000 & 297.0 & 4 & & \\
\hline & 6 & 918 & 69.75 & 0 & & 4 & & \\
\hline & 6 & 919 & & 11.1500 & & 7 & & \\
\hline & 6 & 920 & 65.7497 & 10.8250 & 293.6 & 4 & 0 & م0 \\
\hline & 6 & 921 & b. & & & 4 & & \\
\hline & 6 & 922 & 64.4982 & 12.1500 & $293 . \varepsilon$ & & & \\
\hline & & 923 & 70.0000 & 11.5750 & 293. & & 600.0 & 500 \\
\hline
\end{tabular}




\begin{tabular}{|c|c|c|c|c|c|c|c|}
\hline & 24 & 6.7505 & .3500 & 93.4 & 4 & 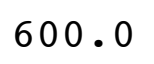 & \\
\hline & 6101 & 07384 & & 92.8 & & & \\
\hline & 6102 & 994 & & 1.3 & & & \\
\hline & 6103 & 6.2501 & 750 & 0.4 & & 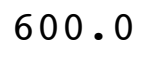 & \\
\hline & 6104 & & & & & & \\
\hline & 6105 & 984 & 750 & 88.5 & & 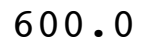 & \\
\hline & 6106 & 994 & 000 & 88.8 & & 0 & \\
\hline & 6107 & 97 & 00 & & & & \\
\hline & 6108 & 91 & 250 & 90.0 & & 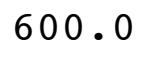 & \\
\hline & 6109 & & & 91.2 & & & \\
\hline & 1010 & & & & & & \\
\hline & 61011 & & & 0 & & & \\
\hline & 1012 & & 750 & 94.9 & & 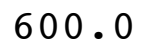 & \\
\hline & 61013 & & & & & & \\
\hline & 61014 & & & & & & \\
\hline & 51015 & & & 95.9 & & & \\
\hline & 61016 & $y$ & 9.8250 & 295.2 & & & \\
\hline & 61017 & & & & & & \\
\hline & 61018 & & 50 & & & & \\
\hline & 61019 & & 50 & 292.7 & & & \\
\hline & 61020 & & & & & & \\
\hline & 61021 & & 0 & 29 & & & \\
\hline & 61022 & & 750 & 29 & & & \\
\hline & 61023 & & & & & & \\
\hline & 61024 & & & 29 & & & \\
\hline & 6111 & & 500 & & & & \\
\hline & 6112 & 0 & 50 & & & & \\
\hline & 6113 & & 0 & 2 & & & \\
\hline & 6114 & & & $28 \varepsilon$ & & & \\
\hline & 6115 & $y$ & 000 & 288.2 & 4 & & \\
\hline & 6116 & 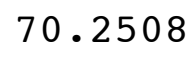 & & & 4 & & \\
\hline & $\begin{array}{ll}611 & 7\end{array}$ & & 0 & & 4 & & \\
\hline & 6118 & & 11 & 287.8 & & & \\
\hline & 6119 & & 11 & & 4 & & \\
\hline & 61110 & & & & 4 & & \\
\hline & 61111 & 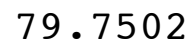 & & 292.5 & 4 & & \\
\hline & 61112 & & & & 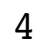 & & \\
\hline & 61113 & & & & 4 & & \\
\hline & 61114 & 00 & & 294.9 & 4 & & \\
\hline & 61115 & & & 29 & 4 & & \\
\hline & 61116 & & & & 4 & & \\
\hline & 61117 & 73. & 000 & 294.2 & 4 & & \\
\hline & 61118 & 73 & 0 & 293 & 4 & & \\
\hline & 61119 & & & & 4 & & \\
\hline & 61120 & 70.2551 & 10.5000 & 290.6 & 4 & 0 & (2) \\
\hline & 61121 & & 0 & & 4 & & \\
\hline & 61122 & & 12.4250 & & & & \\
\hline & $11<3$ & 2000 & 12.7500 & 288.9 & & 600.0 & $=0$ \\
\hline
\end{tabular}




\begin{tabular}{|c|c|c|c|c|c|c|c|}
\hline & & 2 & 1250 & 39.3 & & & \\
\hline & 6121 & 0.7493 & 1.1000 & 288.5 & & 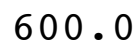 & \\
\hline & 6122 & 6.2501 & .8500 & 287.3 & & 00 & \\
\hline & 123 & 7.4966 & 1.8000 & 287.7 & & 0 & \\
\hline & 124 & 999 & 2000 & & & & \\
\hline & 125 & 500 & .7250 & 87 & & & \\
\hline & 126 & 77.2498 & 3500 & 87.0 & & 0 & \\
\hline & 127 & 82.7501 & & 89.4 & & & \\
\hline & 128 & 5.2495 & & 4 & & & \\
\hline & 129 & 01.6019 & 6.2250 & 92 & & & \\
\hline & 1210 & 1.7505 & 0 & 93.9 & & & \\
\hline & 1211 & 30120 & & 7 & & & \\
\hline & 1212 & 010 & 5 . & 295.2 & & & \\
\hline & 1213 & 3.8986 & 5.90 & 296 & & & \\
\hline & 214 & 7511 & & & & & \\
\hline & 215 & & & 29 & & & \\
\hline & 1216 & 76.4793 & 6.50 & 297.1 & & & \\
\hline & 217 & 74.5000 & 0 & 4 & & & \\
\hline & 218 & 72.749 & & & & & \\
\hline & 219 & 74.5000 & 6.27 & 29 & 4 & & \\
\hline & 1220 & 68.7500 & 0 & 294.6 & 1 & 0 & \\
\hline & 221 & 67.0002 & & & 2 & & \\
\hline & 2 & 73 & 8 . & 29 & 4 & & \\
\hline & 1223 & 72.7500 & 8.70 & 292.7 & & & \\
\hline & 1224 & 72.2489 & & 0 & 4 & & \\
\hline & 6131 & 76.9998 & 0 & 291.6 & 4 & & \\
\hline & 6132 & 81.9629 & 5000 & 291.2 & 4 & & . \\
\hline & 6133 & 32.2519 & 0 & 29 & 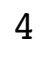 & & \\
\hline & 6134 & 2.5000 & 0 & 292.7 & 4 & & \\
\hline & 6135 & 150.7502 & .4750 & 291.8 & $I$ & & 0 . \\
\hline & 6136 & 156.0000 & 12.8500 & 291.3 & 4 & & \\
\hline & 6137 & .5000 & 0 & 291.3 & 4 & & \\
\hline & 6138 & 158.4995 & 750 & 25 & 4 & & . \\
\hline & 6139 & 68.249 & 0 & 292.7 & 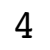 & & \\
\hline & $611^{3}$ & 501 & 0 & 293.4 & 4 & & \\
\hline & & 162.9936 & 9.7750 & 294.9 & 4 & & \\
\hline & 61312 & 170.5041 & 10.8500 & 296.4 & 4 & & \\
\hline & 61313 & 172.2498 & 13. & 297.2 & 4 & & \\
\hline & 61314 & 170.0000 & 12.1500 & 297.7 & 4 & & ( \\
\hline & 1315 & 169.4931 & 10.7750 & 298.5 & 4 & & \\
\hline & & 165.5000 & 10.9250 & 299.1 & 4 & & \\
\hline & & 167.7496 & 10.2000 & 299.7 & 4 & & \\
\hline & 61318 & 180.2515 & 8.5500 & 299.9 & 4 & & 0 \\
\hline & & 184.2500 & & 299.2 & 4 & & \\
\hline & & 113.7577 & & 298.1 & 4 & & \\
\hline & 61321 & 74.5111 & 5.7250 & 297.0 & 1 & & \\
\hline & & 1 & & & & & \\
\hline & 1323 & 82.2500 & 5.3500 & 296.9 & & 60 & 0 \\
\hline
\end{tabular}




\begin{tabular}{|c|c|c|c|c|c|c|c|}
\hline & 1324 & & 3750 & 97.9 & & & \\
\hline & $614 \quad 1$ & 9 & 5.0000 & 297.9 & 4 & 600.0 & \\
\hline & 6142 & 82.4998 & 6000 & 297.7 & 4 & 00 & \\
\hline & 6143 & 88.4982 & 1250 & 297.9 & & 00 & \\
\hline & 144 & 99 & .9500 & 97.8 & & 0 & \\
\hline & 145 & 115 & 250 & 297.8 & & 00 & \\
\hline & 146 & 115.3595 & 2750 & 297.4 & 4 & 00 & \\
\hline & 147 & 771 & .2250 & 297.9 & & 0 & \\
\hline & 148 & 155.7488 & .1250 & 8 & & & \\
\hline & 149 & 158.5023 & 250 & 297.5 & 4 & 00 & 0 \\
\hline & 1410 & 158.2487 & 0000 & 297.4 & 4 & 000 & \\
\hline & 411 & 170.2501 & 0 & .8 & 3 & & \\
\hline & 412 & 170.50 & 0 & 30 & 3 & 0 & \\
\hline & 1413 & 179.7872 & 4500 & 302.4 & 3 & 0 & 8 \\
\hline & 1414 & 182.24 & & 1 & & & \\
\hline & 5 & 189.97 & & & 3 & & \\
\hline & 1416 & 190.5399 & 4.2750 & 304.6 & 3 & 00.0 & . \\
\hline & 417 & 188.1968 & 4 . & .8 & 3 & 0 & \\
\hline & 418 & 187.36 & & .5 & 3 & & \\
\hline & 1419 & 99.7203 & $\$ 750$ & 302.9 & 4 & .0 & 00 \\
\hline & 1420 & 82.0034 & 500 & .7 & 4 & 0 & \\
\hline & 421 & 69.00 & & & 4 & & \\
\hline & 422 & 68.4998 & 250 & 299.4 & 4 & 0 & 0 . \\
\hline & 1423 & 72.2524 & 750 & .1 & 4 & .0 & 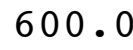 \\
\hline & 61424 & 61.2501 & 0 & & 4 & & \\
\hline & 6151 & 45.2576 & 750 & .5 & 4 & & \\
\hline & 6152 & 41.7506 & 7500 & 299.7 & 4 & 0 & J. \\
\hline & 6153 & 29.74 & 250 & .2 & 4 & & \\
\hline & 6154 & 25.24 & 00 & 297.8 & 5 & & \\
\hline & 6155 & 184.8690 & 0.9750 & 294.9 & 6 & 0 & 0 . \\
\hline & 6156 & 291.48 & 1.3500 & 295.6 & 6 & & 0 . \\
\hline & 6157 & 335.53 & 50 & 297.8 & 5 & & \\
\hline & 6158 & 286.55 & 500 & .2 & 4 & 0 & 0 . \\
\hline & 6159 & 225.65 & 500 & 300.2 & 3 & 0 & 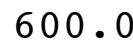 \\
\hline & 615 & 178.78 & 0 & 299.4 & 2 & & \\
\hline & 61511 & 194.74 & 4.1500 & 300.7 & 3 & & 0 . \\
\hline & 61512 & 196.9989 & 250 & 301.8 & 2 & & \\
\hline & 61513 & 176.7601 & 3. & 303.1 & 2 & & . \\
\hline & 61514 & 172.9200 & 5 . & 304.0 & 3 & .0 & 0 . \\
\hline & 61515 & 109.76 & 7000 & 305.2 & 3 & & 0 . \\
\hline & 61516 & 108.1873 & 250 & 305.2 & 3 & & 0 . \\
\hline & 61517 & 97.2265 & 5.3500 & 304.6 & 3 & .0 & 0 . \\
\hline & 61518 & 76.4526 & 5.1750 & 303.8 & 3 & .0 & 00 . \\
\hline & 61519 & 70.9999 & 500 & 301.9 & 4 & & \\
\hline 01 & 61520 & 73.2539 & 8.0250 & 299.5 & 4 & & 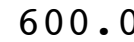 \\
\hline & 61521 & 76.2507 & 8.5000 & 298.8 & 4 & 600.0 & 00. \\
\hline & & 70.75 & & 298.2 & 4 & & \\
\hline & 61523 & 65.9986 & 6.9500 & 299.2 & $\Psi$ & 600.0 & 60 \\
\hline
\end{tabular}




\begin{tabular}{|c|c|c|c|c|c|c|c|}
\hline & 1524 & 4.5064 & 6.3000 & 300.0 & 4 & . & \\
\hline & 6161 & 3.7557 & 7.2750 & 300.3 & & & \\
\hline & 6162 & 5046 & 4500 & 300.1 & 4 & 0 & \\
\hline & 6163 & 91.4714 & 0500 & 300.4 & & 00 & \\
\hline & 164 & 2.5786 & & & & & \\
\hline & 165 & 920 & 50 & 98.9 & & 00 & \\
\hline & 166 & 11.1710 & 3250 & 299.5 & & 0 & \\
\hline & 167 & 9602 & .4500 & 01.3 & & & \\
\hline & 168 & 3730 & 1.4000 & 02.0 & & 0 & \\
\hline & 169 & 176.4273 & 500 & 300.7 & & 00 & \\
\hline & 1610 & 189.7592 & 4.2000 & 300.6 & & 0 & \\
\hline & 611 & 174.2449 & & & & & \\
\hline & 1612 & 182.0000 & 500 & 302.4 & & 0 & \\
\hline & 1613 & 169.8815 & 3. & 303.8 & & 0 & \\
\hline & 614 & 171.2502 & & & & & \\
\hline & 1615 & 172.0041 & 00 & 305.4 & 3 & & \\
\hline & 1616 & 142.1954 & 0 & 306 & 3 & 0 & \\
\hline & 1617 & 104.7492 & & 30 & 4 & & \\
\hline & 1618 & 90.4624 & & 304.7 & 4 & 0 & \\
\hline & 619 & 76.9940 & 0 & 302.7 & 4 & & \\
\hline & 620 & 77.7489 & 0 & 6 & 4 & & \\
\hline & 1621 & 69. & 0 & & 4 & & \\
\hline & 1622 & 57.9982 & 250 & 295 & 4 & 0 & U. \\
\hline & 61623 & 52.7500 & 0 & 0.2 & 4 & & \\
\hline & 61624 & 59. & & & 4 & & \\
\hline & 6171 & 63.0000 & & & 4 & & \\
\hline & 6172 & 68.2500 & 0 & 3 & 4 & & \\
\hline & 6173 & 73 & & & 4 & & \\
\hline & 6174 & 78 . & 0 & & 4 & & \\
\hline & $617 \quad 5$ & 75.7500 & 0 & .0 & 4 & & \\
\hline & 6176 & 74.2470 & 0 & & 4 & & \\
\hline & 6177 & 1 & 0 & & 3 & & \\
\hline & 6178 & 268.9607 & 4500 & 301.6 & 2 & 0 & 0 . \\
\hline & 6179 & 95.1203 & 0 & 2.3 & 2 & & \\
\hline & 61710 & 148 . & 0 & .3 & 3 & & \\
\hline & 61711 & 175.5055 & 750 & 301.8 & 4 & & \\
\hline & 61712 & 180.7510 & 50 & 2.6 & 4 & & \\
\hline & 61713 & 161.6919 & 0 & & 3 & & \\
\hline & 61714 & 138.9962 & 000 & 5.7 & 3 & & 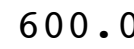 \\
\hline & 61715 & 9976 & & & 4 & & \\
\hline & 61716 & 127.0417 & & & 4 & & \\
\hline 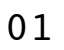 & 61717 & 97.9989 & 6.6750 & 305.0 & 4 & 600.0 & 0. \\
\hline & 61718 & 82.7495 & & 304.0 & 4 & 0 & \\
\hline & 61719 & 85.7605 & 5.5000 & & 4 & & \\
\hline I & 61720 & 78.9988 & 6.3500 & 301.9 & 4 & 600 & 0 \\
\hline & 61721 & 72.7503 & 7.4250 & & 4 & 600 & \\
\hline & & 9989 & 0 & & 4 & & \\
\hline & 61723 & 73.5000 & 9.7000 & 299.7 & & 600.0 & 600 \\
\hline
\end{tabular}




\begin{tabular}{|c|c|c|c|c|c|c|c|}
\hline & 1724 & 2.9994 & .8500 & 299.2 & 4 & 0 & \\
\hline & 6181 & 1.7500 & .5250 & 298.8 & & & \\
\hline & 6182 & 77.5000 & 1000 & 98.3 & & 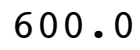 & \\
\hline & 6183 & 87.2325 & 8.9750 & 298.0 & & 00 & \\
\hline & 184 & 07.2508 & .3000 & 97.6 & & & \\
\hline & 185 & 37.7608 & .7750 & 96.7 & & 00 & \\
\hline & 186 & 145.7499 & 0.8500 & 296.2 & & & \\
\hline & 187 & 151.7500 & 0.1500 & 96.0 & & & \\
\hline & 188 & 149.2499 & 9.4000 & 296.2 & & 0 & \\
\hline & 6189 & 159.2505 & 0750 & 297.0 & & 0 & \\
\hline & 1810 & 168.7482 & 7.0500 & 297.8 & & & \\
\hline & 811 & 160.7240 & & 99 & & & \\
\hline & 1812 & 162.7504 & 5.7000 & 301.0 & & 0 & \\
\hline & 1813 & 171.7483 & 6.3000 & 302.2 & & 0 & \\
\hline & 814 & 177.7506 & & & 4 & & \\
\hline & 1815 & 179.7581 & 5.6750 & 304 & 3 & & \\
\hline & 1816 & 174.7413 & 0 & 305 & & 0 & \\
\hline & 817 & 167.7520 & 5.42 & 30 & & & \\
\hline & 1818 & 126.8196 & 0 & 30 & 3 & & \\
\hline & 819 & 101.0184 & 0 & 30 & 4 & & \\
\hline & 820 & 83.4991 & 0 & 6 & 4 & & \\
\hline & 821 & 67.4989 & & & 4 & & \\
\hline & 1822 & 66.4997 & 250 & 29 & 4 & 0 & U. \\
\hline & 1823 & 71.2501 & 0 & 30 & 4 & & \\
\hline & 61824 & 71.4998 & & & 4 & & \\
\hline & 6191 & 0000 & 500 & 30 & 4 & & \\
\hline & 6192 & 78.7500 & 0 & 300.2 & 4 & & \\
\hline & 6193 & 82.2506 & 0 & 299.4 & 4 & & \\
\hline & 6194 & 07 & 0 & 29 & 4 & & \\
\hline & 6195 & 2 & 0 & 300.3 & 4 & & \\
\hline & 6196 & 957 & & 299.1 & 5 & & \\
\hline & 6197 & 8 & 0 & 29 & 4 & & \\
\hline & 6198 & 151.2502 & 250 & 299.7 & 4 & 0 & 0 . \\
\hline & 6199 & 160.49 & 0 & 299.8 & 4 & & \\
\hline & 61910 & 6 & & 301.2 & 4 & & \\
\hline & 61911 & 167.5009 & & 302.3 & 4 & & \\
\hline & 61912 & 171.7500 & 0 & 303.2 & 4 & & \\
\hline & 61913 & 174.7498 & 0 & 304.0 & 4 & & \\
\hline & 61914 & 187.9998 & 50 & 304.7 & 4 & & 0 . \\
\hline & 61915 & 170.9882 & 0 & 305.8 & 3 & & \\
\hline & 61916 & 116.2527 & 0 & 306.2 & 4 & & \\
\hline & 61917 & 99.2509 & 6.7250 & 305.3 & 4 & 600 & 0. \\
\hline & 61918 & 91.0041 & & 304.2 & 4 & 0 & \\
\hline & 61919 & 77.2491 & 7.2750 & 303.2 & 4 & & \\
\hline I & 61920 & 77.5002 & 7.1250 & 302.3 & 4 & 600 & 0 \\
\hline & 921 & 2518 & 5000 & 302.3 & 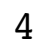 & 600 & \\
\hline & & 60.2508 & 50 & 302.7 & 4 & & \\
\hline & 61923 & 63.2522 & 5.4250 & 302.6 & & 600.0 & 600 \\
\hline
\end{tabular}




\begin{tabular}{|c|c|c|c|c|c|c|c|}
\hline & 61924 & 4.2479 & 2.7500 & 302.3 & 4 & 000 & \\
\hline & 6201 & 7.2506 & 0.9250 & 301.8 & & ل & \\
\hline & 6202 & 63.2210 & .3500 & 300.7 & & 00 & \\
\hline & 6203 & 116.4617 & 3000 & 300.4 & & 00 & \\
\hline & $620 \quad 4$ & 37.3385 & .1500 & 00.3 & & 0 & \\
\hline & 6205 & 143.3122 & .2750 & 299.4 & & 00 & \\
\hline & 6206 & 129.7475 & 5.2000 & 299.4 & & 0 & \\
\hline & 6207 & 137.7500 & .9250 & 99.9 & & & \\
\hline & 6208 & 149.0050 & 8.3500 & 300.3 & & 0 & \\
\hline & 6209 & 156.9986 & 8.5500 & 301.5 & & 600.0 & \\
\hline & 62010 & 163.2506 & 7.0500 & 302.2 & & 0 & \\
\hline & 2011 & 161.2480 & 250 & & & & \\
\hline & 2012 & 166.5000 & 4.6250 & 305.2 & & 0 & \\
\hline & 62013 & 177.2524 & 5.1500 & 306.5 & & 00.0 & 皮 \\
\hline & 2014 & 184.5052 & & & & & \\
\hline & 62015 & 183.0401 & 500 & 307.8 & 3 & 0 & \\
\hline & 62016 & 171.7843 & 4250 & 308.2 & & 0 & \\
\hline & 62017 & 110.2460 & 0 & 3.6 & & & \\
\hline & 62018 & 86.7302 & 6.3250 & 307.2 & 4 & 0 & \\
\hline & 62019 & 70.7473 & 7.1750 & 305.3 & 4 & 0 & \\
\hline & 62020 & 66.7500 & 0 & 7 & 4 & & \\
\hline & 62021 & 66.4998 & 00 & 1 & 4 & & \\
\hline & 62022 & 66.2499 & 7500 & & 4 & 0 & 00. \\
\hline & 62023 & 68.2509 & 250 & .1 & 4 & 0 & \\
\hline & 62024 & 69.2500 & & & 4 & & \\
\hline & 6211 & 72.5000 & 50 & & 4 & & \\
\hline & 6212 & 70.2507 & 7 . & 3.8 & 4 & 0 & \\
\hline & 6213 & 140.8700 & 0 & 2.7 & 5 & & \\
\hline & 6214 & 298.4751 & 00 & 25 & 6 & & 0 . \\
\hline & 6215 & 354.0903 & 500 & 3.4 & J & 0 & \\
\hline & 6216 & 30.1874 & 00 & $2 \varsigma$ & 6 & & \\
\hline & 6217 & 127 . & 250 & .9 & 5 & & \\
\hline & 6218 & 231.9197 & 1.9750 & 3.0 & 4 & 0 & U • \\
\hline & 6219 & 189.6640 & 50 & .2 & 3 & 0 & \\
\hline & 62110 & 172 . & 0 & & 3 & & \\
\hline & 62111 & 177.5043 & 250 & 5.3 & 2 & & 0 . \\
\hline & 62112 & 190.0311 & 500 & 306.2 & 2 & 0 & \\
\hline 1 & 62113 & 170.9975 & 4 . & 5.9 & 3 & & . \\
\hline & 62114 & 172.2493 & 5.2250 & 307.6 & 3 & 0 & 0 . \\
\hline & 62115 & 177.2699 & 750 & 30 & 4 & 0 & \\
\hline & 62116 & 130.7168 & 250 & & 3 & & \\
\hline r & 62117 & 93.5194 & 6.5500 & 307.9 & 4 & 600.0 & 600 . \\
\hline & 62118 & 80.9993 & 7.1250 & 306.4 & 4 & 600 & 600. \\
\hline & 62119 & 73.7498 & 8.2750 & 305.1 & 4 & & 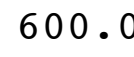 \\
\hline r & 62120 & 76.2500 & 8.8000 & 303.8 & 4 & 600.0 & 00 . \\
\hline & 62121 & 73.7501 & 9.1250 & 302.7 & 4 & 600.0 & 600 \\
\hline & 62122 & 71.0002 & & 302.5 & 4 & 600 & \\
\hline & 62123 & 65.7500 & 8.4250 & 303.2 & 4 & 600.0 & 600 \\
\hline
\end{tabular}




\begin{tabular}{|c|c|c|c|c|c|c|c|}
\hline 01 & 62124 & 59.7502 & 7.8500 & 303.4 & 4 & 600.0 & 600.0 \\
\hline 01 & 6221 & 6.2479 & 6.0750 & 303.7 & 4 & 600.0 & 600.0 \\
\hline 01 & 6222 & 172.8345 & 0.5250 & 302.0 & 5 & 600.0 & 00.0 \\
\hline 01 & 6223 & 85.4659 & 1.2250 & 302.1 & 6 & 600.0 & 00.0 \\
\hline 01 & 6224 & 119.4914 & 1.2500 & 301.1 & 6 & 600.0 & 00.0 \\
\hline 01 & 6225 & 98.3124 & 0.3250 & 300.6 & 6 & 600.0 & 00.0 \\
\hline 01 & 6226 & 125.7287 & 0.2000 & 301.3 & 6 & 600.0 & 0 \\
\hline 01 & 6227 & 194.3689 & 1.0000 & 302.9 & 5 & 600.0 & 00.0 \\
\hline 01 & 6228 & 211.3491 & 2.2750 & 303.0 & 4 & 600.0 & 00.0 \\
\hline 01 & 6229 & 234.5230 & 2.2500 & 303.6 & 3 & 60 & \\
\hline 01 & 62210 & 216.7346 & 2.3500 & 304.5 & 2 & 600.0 & \\
\hline 01 & 62211 & 207.2398 & 3.4500 & 305.4 & 2 & 600.0 & \\
\hline 01 & 62212 & 216.3481 & 3.7000 & 305.7 & 2 & 600.0 & .0 \\
\hline 01 & 62213 & 196.5010 & 3.5500 & 307.0 & 2 & .0 & 00.0 \\
\hline 01 & 62214 & 144.2300 & 5.4750 & 307.4 & 3 & .0 & \\
\hline 01 & 62215 & 120.2434 & 6.1500 & 308.2 & 4 & 600.0 & .0 \\
\hline 01 & 62216 & 98.0005 & 7.5000 & 306.7 & 4 & 60 & .0 \\
\hline 01 & 62217 & 91.2496 & 8.9250 & 304.1 & 4 & .0 & \\
\hline 01 & 62218 & 80.2498 & 10.8250 & 302.7 & 4 & .0 & \\
\hline 01 & 62219 & 76.7573 & 10.4500 & 300.9 & 4 & .0 & .0 \\
\hline 01 & 62220 & 68.7502 & 14 & 8.5 & 4 & 6 & \\
\hline 01 & 62221 & 65.2500 & 500 & 298.5 & 4 & .0 & \\
\hline 01 & 62222 & 63.2500 & 13.5000 & 298.2 & 4 & 600.0 & .0 \\
\hline 01 & 62223 & 002 & 13.52 & 298.0 & 4 & 0 & \\
\hline 01 & 62224 & 000 & 11. & .5 & 4 & 0 & \\
\hline 01 & 6231 & 68.7500 & 11.7500 & 298.2 & 4 & .0 & \\
\hline 01 & 6232 & 68.7501 & 10.4750 & 298.3 & 4 & 60 & .0 \\
\hline 01 & 6233 & 68.7496 & 8.8000 & 298.8 & 4 & .0 & \\
\hline 01 & 6234 & 67.7494 & 8.3500 & 299.1 & 4 & .0 & .0 \\
\hline 01 & 6235 & 70.7502 & 7.7750 & 299.0 & 4 & .0 & \\
\hline 01 & 6236 & 75.2501 & 8.3 & 298.5 & 4 & .0 & \\
\hline 01 & 6237 & 73.7501 & 250 & .3 & 4 & .0 & 0 \\
\hline 01 & 6238 & 75.6655 & 4.2500 & 299.5 & 3 & 0.0 & 0.0 \\
\hline 01 & 6239 & 75.8636 & 2.8000 & 300.9 & 2 & 0 & .0 \\
\hline 01 & 62310 & 101.0061 & 5.3000 & 298.5 & 3 & 600.0 & 600.0 \\
\hline 01 & 62311 & 98.2549 & 3.7500 & 300.1 & 2 & 600.0 & 600.0 \\
\hline 01 & 62312 & 114.6762 & 4.7250 & 301.5 & 3 & 600.0 & .0 \\
\hline 01 & 62313 & 80.7505 & 7.2000 & 301.4 & 4 & 600.0 & 600.0 \\
\hline 01 & 62314 & 83.2633 & 7.6500 & 301.6 & 4 & 600.0 & 600.0 \\
\hline 01 & 62315 & 71.7494 & 9.4500 & 301.3 & 4 & 600.0 & .0 \\
\hline 01 & 62316 & 83.7502 & 11.6500 & 300.2 & 4 & 600.0 & 600.0 \\
\hline 01 & 62317 & 79.5006 & 13.0750 & 298.8 & 4 & 600.0 & 600.0 \\
\hline 01 & 62318 & 71.5001 & 12.2000 & 298.9 & 4 & 600.0 & 600.0 \\
\hline 01 & 62319 & 67.2498 & 12.4250 & 296.7 & 4 & 600.0 & 600.0 \\
\hline 01 & 62320 & 62.5001 & 13.0000 & 295.6 & 4 & 600.0 & 600.0 \\
\hline 01 & 62321 & 68.2534 & 13.7000 & 294.5 & . & 600.0 & 600.0 \\
\hline 01 & 62322 & 70.7500 & 14.2500 & 293.6 & 4 & 600.0 & 600.0 \\
\hline 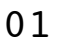 & 62323 & 74.4992 & 12.5000 & 293.0 & 4 & 600.0 & 600.0 \\
\hline
\end{tabular}




\begin{tabular}{|c|c|c|c|c|c|c|c|}
\hline 1 & 62324 & 2.7550 & 10.0500 & 92.5 & 4 & 0 & \\
\hline & 6241 & 72500 & 12.9500 & 293.0 & & & \\
\hline & 6242 & 0 & 750 & 2.7 & & & \\
\hline & 6243 & 000 & 3.3500 & 92.1 & & 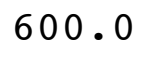 & \\
\hline & $624 \quad 4$ & & & 2.4 & & & \\
\hline & 6245 & 66 & 000 & 92.1 & & 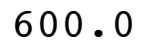 & \\
\hline & 6246 & 998 & 3.3500 & 91.8 & & - & \\
\hline & 6247 & 4 & 2. & 91.9 & & 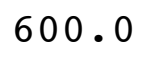 & \\
\hline & 6248 & 62.25 & 0.1750 & 93.0 & & 0 & \\
\hline & 249 & 62.74 & & 94.9 & & 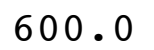 & \\
\hline & 2410 & 5 . & & & & & \\
\hline & 2411 & 9. & & 0 & & & \\
\hline & 2412 & 87. & 8.3750 & 296.3 & & 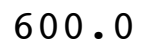 & \\
\hline & 2413 & $77.4 \varepsilon$ & 10 . & & & & \\
\hline & 2414 & & & & & & \\
\hline & 5 & 71. & & 297.2 & & & \\
\hline & 62416 & 68.25 & 10.0750 & 29 & & U & \\
\hline & 2417 & 64 & 10 & & & & \\
\hline & 24 & 74 . & 13 & 3 & 4 & & \\
\hline & 62419 & 72 & 12 . & & & & \\
\hline & 62420 & & & & & & \\
\hline & 62421 & & 13 & & & & \\
\hline & 2422 & 76 . & 13 & 29 & & & \\
\hline & 62423 & $\pi$ & & & & & \\
\hline & 62424 & & & & 4 & & \\
\hline & 6251 & 67 . & & & 4 & & \\
\hline & 6252 & & & 290 & 4 & & \\
\hline & 6253 & 70 & & 291 & 4 & & \\
\hline & 6254 & 62 . & & 290 & 4 & & \\
\hline & 6255 & 67. & 0 & 290 & a & & \\
\hline & 6256 & 0 & 0 & & 4 & & \\
\hline & 6257 & 69. & & & 4 & & \\
\hline & 6258 & 69. & 0 & 290.5 & 4 & & \\
\hline & 6259 & 72 & & 291 & 4 & & \\
\hline & 62510 & & & & 4 & & \\
\hline & 62511 & 85 . & & 293.4 & 4 & & \\
\hline & 62512 & 70 . & & & 4 & & \\
\hline & 62513 & 80 . & 50 & 294.2 & 4 & & \\
\hline & 62514 & 90.49 & & 294.8 & 4 & & \\
\hline & 62515 & 101. & & & 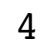 & & \\
\hline & 62516 & & & & 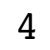 & & \\
\hline & 62517 & 101.500 & 7.8500 & 292.1 & 4 & & \\
\hline & 62518 & 91. & & & 4 & & \\
\hline & 62519 & & & & 2 & & \\
\hline & 62520 & 97.7608 & 7.0750 & 286.8 & 4 & 0 & م. \\
\hline & & & & & 4 & & \\
\hline & & 14.992 & & & & & \\
\hline & 2525 & 69.2501 & 6.6250 & 287.6 & & 600.0 & 50 \\
\hline
\end{tabular}




\begin{tabular}{|c|c|c|c|c|c|c|c|}
\hline 1 & 62524 & 7.2502 & 6.3750 & 287.6 & 4 & 600.0 & \\
\hline & 6261 & 0.4997 & 7.8000 & 286.8 & 4 & מn & \\
\hline & 6262 & 001 & 5000 & 287.1 & 4 & 0 & \\
\hline & 6263 & 71.7500 & .2250 & 286.4 & & 00 & \\
\hline & 6264 & 5.0002 & .5000 & & & & \\
\hline & 6265 & 77.7499 & 6250 & 287.0 & & 0 & \\
\hline & 6266 & 76.2500 & 7.6000 & 287.2 & & 00 & \\
\hline & 6267 & 80.2527 & 4.9250 & 88.6 & & 0 & \\
\hline & 6268 & 39.4632 & 2.4250 & 290.7 & & 00 & \\
\hline & 6269 & 279.9823 & 9750 & 291.4 & & 0 & \\
\hline & 62610 & 260 . & .2250 & 92.3 & & & \\
\hline & 62611 & 290 . & & 293.7 & & & \\
\hline & 2612 & 279.1607 & 0250 & 294.8 & & 0 & \\
\hline & 62613 & 194.6794 & 0 & 95 & & 0 & \\
\hline & 62614 & 202 . & & .6 & & & \\
\hline & 2615 & 189. & 0 & 297.0 & 2 & 0 & \\
\hline & 62616 & 215.4976 & 5.12 & 297.5 & 3 & 0 & \\
\hline & 62617 & 205.9504 & $12=$ & 297.9 & 3 & & \\
\hline & 62618 & 148 . & 0 & 296.6 & 2 & & \\
\hline & 62619 & 70.9390 & 32 & 295.5 & 3 & 0 & \\
\hline & 62620 & 70 . & 0 & 294.5 & 4 & & \\
\hline & 62621 & 341 . & 2 . & 294.2 & 5 & & \\
\hline & 62622 & 312.9355 & 50 & 291.7 & 5 & 0 & \\
\hline & 62623 & 290 . & & 290.5 & 6 & & \\
\hline & 62624 & 303. & & 289.1 & 5 & & \\
\hline & 6271 & 300 & 0 & 288.5 & 4 & & \\
\hline & 6272 & 295.7473 & 3.55 & 288.2 & 4 & & \\
\hline & 6273 & 284 . & 0 & 288.0 & 5 & & \\
\hline & 6274 & 296.2365 & 010 & 287.5 & 4 & & \\
\hline & 6275 & 326 . & 1.72 & 287.4 & 4 & & \\
\hline & 6276 & 325 . & 0 & 287.4 & 4 & & \\
\hline & 6277 & 319 . & 0 & 287.6 & 4 & & \\
\hline & 6278 & 263.1289 & 1.30 & 289.1 & 3 & & \\
\hline & 6279 & 192.7261 & 2.57 & 289.5 & 2 & & - \\
\hline & 62710 & 177 . & & 289.6 & 3 & & \\
\hline & 62711 & 180.7710 & & 291.6 & 2 & & \\
\hline & 62712 & 201.8788 & 3 . & 293.7 & 2 & & \\
\hline & 62713 & 188. & 4.87 & 293.9 & 3 & & 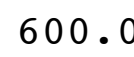 \\
\hline & 62714 & 162.2756 & 4.7250 & 293.2 & 3 & & 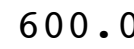 \\
\hline & 62715 & 172.7520 & & 293.7 & 2 & & \\
\hline & 62716 & 175.4977 & & 294.3 & 3 & & \\
\hline & 62717 & 152.7501 & 7.3250 & 293.1 & 4 & 0 & 0 \\
\hline & 62718 & 163.7872 & 0 & 292.3 & 3 & & \\
\hline 01 & 62719 & 151.2504 & 2.4250 & 292.4 & 4 & 600.0 & 600 \\
\hline 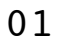 & 62720 & 155.4982 & 5.8000 & 290.9 & 4 & 600.0 & 00 \\
\hline & 62721 & 162.5046 & & 289.7 & 4 & & \\
\hline & 62722 & 133.6307 & & 289.5 & 4 & & 600 \\
\hline & 21203 & 81.2490 & 3.4250 & 289.6 & & 600.0 & 600 \\
\hline
\end{tabular}




\begin{tabular}{|c|c|c|c|c|c|c|c|}
\hline 1 & 62724 & 2.2492 & 3.2000 & 289.5 & 5 & 600.0 & \\
\hline & 6281 & 9.7499 & 5.3000 & 288.4 & & 1000 & \\
\hline & 6282 & 7505 & 500 & 8.1 & & מ & \\
\hline & 6283 & 82.2486 & 7.0250 & 88.1 & & 00 & \\
\hline & 6284 & 2.7504 & 0 & 8.2 & & & \\
\hline & 6285 & 74.9991 & 9000 & 87.7 & & 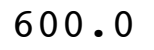 & \\
\hline & 6286 & 73.7499 & 250 & 87.6 & & 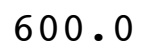 & \\
\hline & 6287 & 79.2495 & .4250 & 88.2 & & 0 & \\
\hline & 6288 & 89.4990 & 3.5500 & 90.4 & & 00 & \\
\hline & 6289 & 172.1385 & 3750 & 293.5 & & 0 & \\
\hline & 62810 & 178.3874 & 50 & 1 & & & \\
\hline & 62811 & 166.8638 & & 294 & & & \\
\hline & 2812 & 158.7506 & 3500 & 294.7 & & 0 & \\
\hline & 2813 & 151.2719 & 500 & 3 & & & \\
\hline & 2814 & 139.1159 & & 1 & & & \\
\hline & 2815 & 132.4997 & 0 & 297 & 4 & & \\
\hline & 62816 & 117.8256 & 5.7500 & 297.6 & & 0 & \\
\hline & 62817 & 102.4948 & & 297.2 & & & \\
\hline & 2818 & 79.7493 & 50 & 295.7 & & & \\
\hline & 62819 & 85.2518 & 50 & 293.7 & & & \\
\hline & 820 & 960 & & 5 & & & \\
\hline & 62821 & 71.2479 & & 29 & & & \\
\hline & 2822 & 79.4288 & .2500 & 291.4 & 4 & & \\
\hline & 62823 & 500 & 0 & 291 & & & \\
\hline & 62824 & 02 & & 29 & 4 & & \\
\hline & 6291 & 000 & .750 & 291.2 & 4 & & \\
\hline & 6292 & 79.0002 & .7000 & 292.4 & 4 & & \\
\hline & 6293 & 80.2500 & & 292 & 4 & & \\
\hline & 6294 & 82.2498 & 7500 & 292 & 4 & & \\
\hline & 6295 & 82.7501 & 0 & 291.8 & 4 & & \\
\hline & 6296 & 80.7500 & & 291.9 & 4 & & \\
\hline & 6297 & & & 29 & 4 & & \\
\hline & 6298 & 02.3481 & 3500 & 295.6 & 2 & & \\
\hline & 6299 & 184.4752 & 0 & 296 & 4 & & \\
\hline & 62910 & & & & 3 & & \\
\hline & 62911 & 168.7505 & 3250 & 297.8 & 3 & & \\
\hline & 62912 & 174.9966 & 0 & 298 & 4 & & \\
\hline & 62913 & 172.2555 & 50 & & 4 & & \\
\hline & 62914 & 177.2499 & 5000 & 300.9 & 4 & & \\
\hline & 62915 & 180.7497 & & 301.6 & 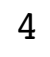 & & \\
\hline & 62916 & 180.2500 & & 302.2 & 4 & & \\
\hline & 62917 & 148.5193 & 5500 & 302.9 & 3 & & \\
\hline & 62918 & 101.4140 & 00 & 302.0 & 3 & & \\
\hline & 62919 & 78.2484 & 5.2250 & & 4 & & \\
\hline & 62920 & 70.0002 & 7.3500 & 297.5 & 4 & 0 & ה \\
\hline & & & & & 4 & & \\
\hline & & 68.7501 & 8.9000 & & & & ( \\
\hline & 2923 & 70.5003 & 8.6250 & 297.7 & & 600.0 & 60 \\
\hline
\end{tabular}




\begin{tabular}{|c|c|c|c|c|c|c|c|}
\hline 1 & 62924 & 71.7496 & 8.3500 & 297.7 & 4 & 600.0 & 600.0 \\
\hline 01 & 6301 & 69.5000 & 8.4500 & 297.5 & 4 & 600.0 & 600.0 \\
\hline 01 & 6302 & 71.5000 & 7.7000 & 297.7 & 4 & 600.0 & 00.0 \\
\hline 01 & 6303 & 78.2509 & 6.0250 & 298.3 & 4 & 600.0 & 00.0 \\
\hline 01 & 6304 & 78.5000 & .2250 & 298.6 & 4 & 600.0 & 00.0 \\
\hline 01 & 6305 & 78.2527 & 3.0750 & 298.6 & 4 & 600.0 & 00.0 \\
\hline 01 & 6306 & 80.7468 & 2.0000 & 298.8 & 5 & 600.0 & 00.0 \\
\hline 01 & 6307 & 206.4023 & 0.4250 & 300.4 & 4 & 600.0 & 00.0 \\
\hline 01 & 6308 & 323.5520 & 0.9750 & 301.8 & 3 & 600.0 & 00.0 \\
\hline 01 & 6309 & 209.4248 & 2.5750 & 300.9 & 2 & 600.0 & 00.0 \\
\hline 01 & 63010 & 229.2498 & 3.5500 & 300.6 & 3 & 600.0 & 00.0 \\
\hline 01 & 63011 & 190.0000 & 3.9500 & 301.5 & 2 & 600.0 & \\
\hline 01 & 63012 & 180.6087 & 4.8250 & 302.6 & 3 & 600.0 & 00.0 \\
\hline 01 & 63013 & 94.7493 & .0250 & 303.5 & 3 & 600.0 & 00.0 \\
\hline 01 & 63014 & 102.9917 & 750 & 303.5 & 4 & .0 & \\
\hline 01 & 63015 & 98.5153 & 6.2000 & 304.0 & 4 & 600.0 & 00.0 \\
\hline 01 & 63016 & 100.0092 & 6.1750 & 304.0 & 4 & 600.0 & 600.0 \\
\hline 01 & 63017 & 107.9946 & 6.5250 & 303.5 & 4 & .0 & \\
\hline 01 & 63018 & 102.8792 & 6.6000 & 302.6 & 4 & 600.0 & .0 \\
\hline 01 & 63019 & 79.2246 & 8.7250 & 301.1 & 4 & .0 & .0 \\
\hline 01 & 63020 & 72.4778 & .6500 & 300.3 & 4 & 60 & \\
\hline 01 & 63021 & 83.0069 & 8.8250 & 299.0 & 4 & .0 & \\
\hline 01 & 63022 & 69.7494 & 8.3750 & 298.6 & 4 & 600.0 & 00.0 \\
\hline 01 & 63023 & 63.7500 & .5250 & 299.0 & 4 & 60 & .0 \\
\hline 01 & 63024 & 68.5000 & 8.2750 & 299.4 & 4 & .0 & \\
\hline 01 & $\begin{array}{lll}7 & 1 & 1\end{array}$ & 76.5001 & 8.1250 & 299.3 & 4 & 600.0 & 600.0 \\
\hline 01 & $\begin{array}{lll}7 & 1 & 2\end{array}$ & 78.5002 & 9.0000 & 298.6 & 4 & 600.0 & .0 \\
\hline 01 & $\begin{array}{lll}7 & 1 & 3\end{array}$ & 73.4994 & 8.2250 & 298.3 & 4 & .0 & \\
\hline 01 & $\begin{array}{lll}7 & 1 & 4\end{array}$ & 78.7505 & 8.3250 & 298.2 & 4 & 600.0 & 600.0 \\
\hline 01 & $\begin{array}{lll}7 & 1 & 5\end{array}$ & 77.5000 & 8.3250 & 297.6 & 4 & 600.0 & .0 \\
\hline 01 & $\begin{array}{lll}7 & 1 & 6\end{array}$ & 79.0001 & 9.0500 & 297.3 & 4 & .0 & \\
\hline 01 & $\begin{array}{lll}7 & 1 & 7\end{array}$ & 81.0000 & 7.0750 & 298.5 & 4 & .0 & 600.0 \\
\hline 01 & $\begin{array}{lll}7 & 1 & 8\end{array}$ & 85.2495 & 5.7250 & 299.4 & 4 & 600.0 & 600.0 \\
\hline 01 & $\begin{array}{lll}7 & 1 & 9\end{array}$ & 240.0009 & 2.4750 & 300.7 & 3 & 600.0 & 0.0 \\
\hline 01 & 7110 & 205.8037 & 2.9250 & 300.6 & 2 & 600.0 & 600.0 \\
\hline 01 & $7 \quad 111$ & 173.2509 & 6.4750 & 302.5 & 3 & 600.0 & 600.0 \\
\hline 01 & 7112 & 155.4986 & 7.4250 & 302.5 & 4 & 600.0 & 600.0 \\
\hline 01 & 7113 & 163.5064 & 6.3750 & 303.9 & 4 & 600.0 & 600.0 \\
\hline 01 & $7 \quad 114$ & 166.0118 & 6.4750 & 304.7 & 4 & 600.0 & 600.0 \\
\hline 01 & 7115 & 138.4798 & 6.0000 & 306.0 & 4 & 600.0 & 600.0 \\
\hline 01 & 7116 & 100.7499 & 5.5000 & 306.4 & 3 & 600.0 & 600.0 \\
\hline 01 & 7117 & 108.5032 & 6.1750 & 305.9 & 4 & 600.0 & 600.0 \\
\hline 01 & 7118 & 87.2500 & 6.3750 & 304.9 & 4 & 600.0 & 600.0 \\
\hline 01 & $7 \quad 119$ & 84.0000 & 6.8250 & 303.6 & 4 & 600.0 & 600.0 \\
\hline 01 & 7120 & 78.4991 & 8.2750 & 302.3 & 4 & 600.0 & 600.0 \\
\hline 01 & $7 \quad 121$ & 71.4992 & 7.9500 & 301.7 & 4 & 600.0 & 600.0 \\
\hline 01 & 7122 & 76.0012 & 8.3250 & 301.6 & 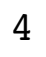 & 600.0 & 600.0 \\
\hline 01 & $7 \quad 123$ & 71.7500 & 9.5500 & 301.2 & 4 & 600.0 & 600.0 \\
\hline
\end{tabular}




\begin{tabular}{|c|c|c|c|c|c|c|c|c|}
\hline 1 & 7 & 24 & 5.2499 & 8.6250 & 301.5 & 4 & 600.0 & \\
\hline & 7 & 21 & 2.7500 & 8.3500 & 301.7 & & 6000 & \\
\hline & 7 & 22 & 76.2501 & 500 & 301.6 & 4 & 0 & \\
\hline & 7 & 23 & 79.7499 & 5.6750 & 301.4 & & 00.0 & \\
\hline & 7 & 24 & 30.00 & .0250 & 01.4 & & 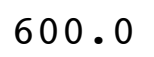 & \\
\hline & 7 & 25 & 92.6891 & 4.9250 & 01.6 & & 00 & \\
\hline & 7 & 26 & 08.9722 & .2000 & 301.8 & & 00 & \\
\hline & 7 & 27 & 0.8940 & .9000 & 302.8 & & 00.0 & \\
\hline & 7 & 28 & 64.2501 & 5.5250 & 302.3 & & 00.0 & \\
\hline & 7 & 29 & 71.5003 & 750 & 302.3 & & 0 & \\
\hline & 7 & 210 & 170.7500 & .7750 & 302.4 & & & \\
\hline & 7 & 211 & 172.2501 & 5.7000 & 303.6 & 4 & & \\
\hline & 7 & 212 & 174.4924 & 5.7250 & 304.8 & 3 & 0 & \\
\hline & 7 & 213 & 169.99 & 5.6000 & 5.8 & 3 & & \\
\hline & 7 & 214 & 171.75 & & & 3 & & \\
\hline & 7 & 215 & 176.2482 & 750 & 308.0 & 0 & 0 & \\
\hline & 7 & 216 & 181.5107 & 6.1500 & 307.5 & 4 & 600.0 & \\
\hline & 7 & 217 & 190.2512 & & 7.2 & 4 & & \\
\hline & 7 & 218 & 172.6597 & 3000 & 307.3 & 4 & & \\
\hline & 7 & 219 & 154.4991 & 3.3750 & 307.3 & 4 & 0 & \\
\hline & 7 & 220 & 92 . & 4 . & & 4 & & \\
\hline & 7 & 221 & 77 . & 5 . & .0 & 4 & & \\
\hline & 7 & 222 & 77.7500 & 5000 & 306.0 & 4 & 0 & \\
\hline & 7 & 223 & 77.7499 & 0 & .8 & 4 & & \\
\hline & 7 & 224 & 78 . & & .7 & 4 & & \\
\hline & 7 & 31 & 77.7500 & 500 & 306.0 & 4 & & \\
\hline & 7 & 32 & 76.0000 & 6.4250 & 5.5 & 4 & & \\
\hline & 7 & 33 & 80.9632 & 750 & 7.2 & 5 & & \\
\hline & 7 & 34 & 144.3230 & 250 & 305.6 & 6 & & \\
\hline & 7 & 35 & 178.5497 & 750 & 5.1 & 6 & & \\
\hline & 7 & 36 & 34.5967 & 0 & 30 & 6 & & \\
\hline & 7 & 37 & 157.6366 & 1 . & 7.8 & 5 & & \\
\hline & 7 & 38 & 184.1788 & 9500 & 307.8 & 4 & & \\
\hline & 7 & 39 & 170 . & 0 & 30 & 4 & & \\
\hline & 7 & 310 & 166. & 4 . & 5.2 & 4 & & \\
\hline & 7 & 311 & 165.7500 & 4.7250 & 305.8 & 4 & & \\
\hline & 7 & 312 & 166. & 5 . & 307.4 & 4 & & \\
\hline & 7 & 313 & 173.2379 & 4 . & 309.0 & 3 & & 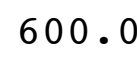 \\
\hline & 7 & 314 & 173.4983 & 4.7750 & 310.4 & 3 & & 6 \\
\hline & 7 & 315 & 176.2480 & & 31 & 3 & & \\
\hline & 7 & 316 & 160.4675 & 250 & 310.7 & 3 & & \\
\hline 1 & 7 & 317 & 171.9728 & 5.4250 & 311.2 & 3 & 0 & 0 \\
\hline & 7 & 318 & 95.6127 & 500 & 311.2 & 3 & & \\
\hline & 7 & 319 & 78.0015 & 5.4000 & 310.1 & 4 & .0 & \\
\hline+ & 7 & 320 & 70.2501 & 7.7750 & 308.6 & 4 & 600.0 & 00 \\
\hline & 7 & 321 & 70 . & & & 4 & & \\
\hline & 7 & 322 & 72.0000 & 500 & 307.6 & 4 & & 600 \\
\hline & & 323 & 72.7499 & 8.3000 & 307.5 & & 600.0 & 600 \\
\hline
\end{tabular}




\begin{tabular}{|c|c|c|c|c|c|c|c|c|}
\hline 01 & 7 & 324 & 71.5000 & 8.1500 & 307.6 & 4 & 600.0 & 600.0 \\
\hline 01 & 7 & 41 & 63.2500 & 6.5750 & 307.5 & 4 & 600.0 & 600.0 \\
\hline 01 & 7 & 42 & 61.9998 & 5.6000 & 307.5 & 4 & 600.0 & 00.0 \\
\hline 01 & 7 & 43 & 62.2499 & 5.6000 & 307.3 & 4 & 600.0 & 00.0 \\
\hline 01 & 7 & 44 & 62.2498 & 6.3750 & 307.1 & 4 & 600.0 & 00.0 \\
\hline 01 & 7 & 45 & 64.0000 & 6.7250 & 307.3 & 4 & 600.0 & 00.0 \\
\hline 01 & 7 & 46 & 74.4986 & 7.1750 & 306.2 & 4 & 600.0 & 00.0 \\
\hline 01 & 7 & 47 & 51.7190 & 4.2750 & 305.8 & 3 & 600.0 & 00.0 \\
\hline 01 & 7 & 48 & 148.7511 & 1.9750 & 304.7 & 2 & 600.0 & 00.0 \\
\hline 01 & 7 & 49 & 16.6453 & 1.8500 & 305.5 & 1 & 600.0 & 00.0 \\
\hline 01 & 7 & 410 & 250.3514 & 2.5500 & 306.9 & 1 & 600.0 & 00.0 \\
\hline 01 & 7 & 411 & 239.3986 & 3.2250 & 307.7 & 2 & 600.0 & \\
\hline 01 & 7 & 412 & 253.8077 & 2.7250 & 307.4 & 1 & 600.0 & 00.0 \\
\hline 01 & 7 & 413 & 227.1100 & 1.9000 & 307.4 & 1 & 60 & .0 \\
\hline 01 & 7 & 414 & 215.2539 & 50 & 307.2 & 2 & .0 & \\
\hline 01 & 7 & 415 & 161.2498 & 2.3500 & 306.8 & 1 & 600.0 & 00.0 \\
\hline 01 & 7 & 416 & 223.1070 & 2.5250 & 305.0 & 1 & 600.0 & 600.0 \\
\hline 01 & 7 & 417 & 104.6867 & 2.5250 & 306.0 & 1 & .0 & \\
\hline 01 & 7 & 418 & 142.4510 & 3.4750 & 304.5 & 2 & 600.0 & .0 \\
\hline 01 & 7 & 419 & 32.7956 & 2.0750 & 302.4 & 3 & 60 & .0 \\
\hline 01 & 7 & 420 & 5.6713 & 1.6 & 302.1 & 4 & 60 & \\
\hline 01 & 7 & 421 & 336.4867 & 1.3750 & 302.4 & 5 & .0 & \\
\hline 01 & 7 & 422 & 7.9693 & 1.9500 & 301.8 & 6 & 600.0 & 00.0 \\
\hline 01 & 7 & 423 & 22.4665 & $1.6^{\circ}$ & 302.5 & 6 & .0 & .0 \\
\hline 01 & 7 & 424 & 297.4492 & 1.3 & 302.4 & 6 & .0 & \\
\hline 01 & 7 & 51 & 237.9766 & 0.2500 & 301.7 & 6 & 600.0 & 600.0 \\
\hline 01 & 7 & 52 & 197.3544 & 0.6000 & 302.0 & 6 & 600.0 & 600.0 \\
\hline 01 & 7 & 53 & 45.1719 & 1.0 & 301.6 & 6 & 0 & \\
\hline 01 & 7 & 54 & 1.6535 & 0.7500 & 301.5 & 6 & 600.0 & 600.0 \\
\hline 01 & 7 & 55 & 39.4572 & 0.9250 & 298.6 & 6 & 600.0 & 600.0 \\
\hline 01 & 7 & 56 & 211.6820 & 0. & 298.9 & 6 & .0 & \\
\hline 01 & 7 & 57 & 46.3078 & 0. & 302.1 & 5 & 600.0 & 600.0 \\
\hline 01 & 7 & 58 & 309.2000 & 1.0000 & 304.6 & 4 & 600.0 & 600.0 \\
\hline 01 & 7 & 59 & 257.3900 & 2.1 & 305.1 & 3 & 600.0 & 0.0 \\
\hline 01 & 7 & 510 & 228.7501 & 3.00 & 303.7 & 2 & 600.0 & 600.0 \\
\hline 01 & 7 & 511 & 213.8575 & 3.5250 & 303.9 & 2 & 600.0 & 600.0 \\
\hline 01 & 7 & 512 & 165.2504 & & 304.7 & 3 & 600.0 & 0.0 \\
\hline 01 & 7 & 513 & 171.4991 & 5.1000 & 306.1 & 3 & 600.0 & 600.0 \\
\hline 01 & 7 & 514 & 156.7140 & 4.5250 & 307.4 & 3 & 600.0 & 600.0 \\
\hline 01 & 7 & 515 & 85.2417 & 4.6500 & 308.2 & 3 & 600.0 & 600.0 \\
\hline 01 & 7 & 516 & 68.9943 & 5.4250 & 308.1 & 3 & 600.0 & 600.0 \\
\hline 01 & 7 & 517 & 74.2450 & 7.3750 & 306.2 & 4 & 600.0 & 600.0 \\
\hline 01 & 7 & 518 & 74.5041 & 8.7250 & 303.9 & 4 & 600.0 & 600.0 \\
\hline 01 & 7 & 519 & 73.2524 & 11.1750 & 301.0 & 4 & 600.0 & 600.0 \\
\hline 01 & 7 & 520 & 63.5157 & 9.5500 & 299.8 & 4 & 600.0 & 600.0 \\
\hline 01 & 7 & 521 & 32.0782 & 3.7500 & 300.7 & 4 & 600.0 & 600.0 \\
\hline 01 & 7 & 522 & 46.5042 & 4.2000 & 300.8 & 4 & 600.0 & 600.0 \\
\hline 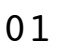 & 7 & 523 & 45.4151 & 3.175 & 301.4 & 3 & 600.0 & 600.0 \\
\hline
\end{tabular}




\begin{tabular}{|c|c|c|c|c|c|c|c|c|}
\hline 1 & 7 & 24 & 0.7497 & 4.3250 & 301.4 & 4 & 600.0 & \\
\hline & 7 & 1 & 4.2503 & 8.2000 & 301.1 & 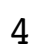 & 60 & \\
\hline & 7 & 62 & 75.7499 & 1250 & 300.4 & 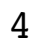 & 0 & \\
\hline & 7 & 3 & 79.7489 & .9500 & 300.4 & & 00.0 & \\
\hline & 7 & 4 & 7.2498 & & 9.8 & & (2) & \\
\hline & 7 & 65 & 76.5001 & 7.6250 & 300.8 & & 00 & \\
\hline & 7 & 6 & 29.2582 & 5000 & 300.4 & & 00 & \\
\hline & 7 & 7 & 348.7951 & .0500 & 98.5 & & 0.0 & \\
\hline & 7 & 8 & 8.2035 & 7750 & 300.7 & & 00.0 & \\
\hline & 7 & 9 & 241.7835 & 0000 & 297.4 & & 0 & \\
\hline & 7 & 610 & 249.4911 & 5750 & 298.1 & & & \\
\hline & 7 & 611 & 282.0065 & 750 & & & & \\
\hline & 7 & 612 & 280.5653 & 2.3250 & 302.0 & & 0 & \\
\hline & 7 & 613 & 236 . & 500 & 3.1 & & 0 & \\
\hline & 7 & 614 & 253. & & & & & \\
\hline & 7 & 615 & 219. & 000 & 304.6 & & 0 & \\
\hline & 7 & 616 & 127.6623 & 4.5250 & 304.2 & 2 & 10.0 & \\
\hline & 7 & 617 & 83. & $-\quad 1$ & 301.7 & 3 & & \\
\hline & 7 & 618 & 494 & 750 & .1 & 4 & & \\
\hline & 7 & 619 & 82.4973 & 6000 & 9.7 & 4 & 0 & \\
\hline & 7 & 620 & 74 . & 0 & .2 & 4 & & \\
\hline & 7 & 621 & 61. & 50 & .2 & 4 & & \\
\hline & 7 & 622 & 50.7571 & 6500 & 299.2 & 4 & 0 & \\
\hline & 7 & 623 & 40.3886 & & .4 & 5 & & \\
\hline & 7 & 624 & 328 . & & & 5 & & \\
\hline & 7 & 71 & 326.7435 & 4000 & 293.7 & 5 & & \\
\hline & 7 & 72 & 352.3653 & 1.3250 & 293.5 & 6 & & \\
\hline & 7 & 73 & 346.1504 & .7500 & 292.6 & 5 & & \\
\hline & 7 & 74 & 80.4939 & 0.4750 & 293.7 & 6 & & \\
\hline & 7 & 75 & 168 . & 0 & 294.2 & 5 & & \\
\hline & 7 & 76 & 205 . & & 293.8 & 6 & & \\
\hline & 7 & 77 & 306 . & 0 & .2 & 5 & & \\
\hline & 7 & 78 & 295.0001 & .1500 & 296.2 & 4 & & \\
\hline & 7 & 79 & 239. & 0 & 25 & 3 & & \\
\hline & 7 & 710 & 209 . & & & 2 & & \\
\hline & 7 & 711 & 171.2511 & 500 & 298.8 & 3 & & \\
\hline & 7 & 712 & 178.5031 & 0 & 9.4 & 3 & & \\
\hline & 7 & 713 & 181.7493 & 500 & .0 & 3 & & \\
\hline & 7 & 714 & 171.7497 & 5250 & 300.7 & 3 & & 6 \\
\hline & 7 & 715 & 172 . & 0 & 1.5 & 4 & & \\
\hline & 7 & 716 & 175.2498 & 6 . & 301.9 & 4 & & 0 . \\
\hline+ & 7 & 717 & 177.0000 & 5.9500 & 302.2 & 3 & 0 & 0 \\
\hline & 7 & 718 & 177.4992 & 50 & 301.4 & 4 & & \\
\hline 01 & 7 & 719 & 175.7460 & 000 & & 4 & & 600 \\
\hline+ & 7 & 720 & 93.6337 & 3.7250 & 299.8 & 4 & 600.0 & 00 \\
\hline & 7 & 721 & 499 & & & 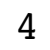 & & \\
\hline & 7 & 722 & 60.1744 & 4.3250 & & 4 & & 600 \\
\hline & 1 & 723 & 31.0023 & 1.6750 & 297.8 & & 600.0 & 600 \\
\hline
\end{tabular}




\begin{tabular}{|c|c|c|c|c|c|c|c|c|}
\hline & 7 & 24 & ש & & 8.3 & 6 & 10 & \\
\hline & 7 & 81 & 7.3806 & 4.3000 & 299.1 & & 00.0 & \\
\hline & 7 & 82 & 657 & 250 & 298.6 & & 0 & \\
\hline & 7 & 3 & 0.9973 & .7000 & 99.4 & & & \\
\hline & 7 & 4 & 83. & 750 & 99.3 & & 00 . & \\
\hline & 7 & 5 & 505 & 250 & 299.3 & & 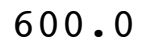 & \\
\hline & 7 & 6 & 101.7911 & 2.3000 & 298.9 & & & \\
\hline & 7 & 7 & 6.4266 & & 5 & & & \\
\hline & 7 & 8 & 240.6294 & 1.0500 & 301.4 & & 0 & 政 \\
\hline & 7 & 9 & 179.3254 & 9750 & 298 & & & \\
\hline & 7 & 10 & 166.7500 & & 98 & & & \\
\hline & 7 & 811 & 169. & 750 & 300 & & & \\
\hline & 7 & 812 & 159.2522 & 0 & 302.5 & & & \\
\hline & 7 & 813 & 84.9927 & & 5 & & & \\
\hline & 7 & 814 & 101.7507 & & 304 & & & \\
\hline & 7 & 815 & 90.2515 & 50 & 304 & & & \\
\hline & 7 & 81 & 78 . & 0 & 8 & 4 & & \\
\hline & 7 & 817 & 80 . & & & - & & \\
\hline & 7 & 818 & 000 & 500 & 30 & 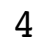 & & 00 \\
\hline & 7 & 819 & 69.7502 & 0 & 3 & 4 & & \\
\hline & 7 & 820 & 72.2500 & & 297.3 & 4 & & \\
\hline & 7 & 821 & 74 & 0 & $29^{\circ}$ & 4 & & \\
\hline & 7 & 822 & 71.7500 & 0 & 297.8 & 4 & & \\
\hline & 7 & 823 & 67.7498 & & 298.0 & 4 & & \\
\hline & 7 & 824 & 68 . & & 29 & 4 & & \\
\hline & 7 & 91 & 67.0000 & 2500 & 298.2 & 4 & & \\
\hline & 7 & 92 & 67.2499 & & 29 & a & & \\
\hline & 7 & 93 & 72 & & $29^{\circ}$ & 4 & & \\
\hline & 7 & 94 & 000 & 0 & 297.7 & 4 & & \\
\hline & 7 & 95 & 001 & & 29 & 4 & & \\
\hline & 7 & 96 & 8 & & 29 & 4 & & \\
\hline & 7 & 97 & & & 29 & 4 & & \\
\hline & 7 & 98 & 45.6241 & 0 & 298.1 & 2 & & \\
\hline & 7 & 99 & 3 & 0 & 298.1 & 2 & & \\
\hline & 7 & 910 & & & & & & \\
\hline & 7 & 911 & 101.4215 & 500 & 300.4 & & & \\
\hline & 7 & 912 & & 0 & 295 & 3 & & \\
\hline & 7 & 913 & 80 . & & 299.8 & 4 & & \\
\hline & 7 & 914 & 75.7485 & & 300.6 & 4 & & \\
\hline & 7 & 915 & 74 . & 0 & 300.5 & 4 & & \\
\hline & 7 & 916 & 70 . & 0 . & 300.1 & 4 & & \\
\hline & 7 & 917 & 70.0000 & .1750 & 299.4 & 4 & & (2) \\
\hline & 7 & 918 & 73.2500 & 0 & 298.0 & 4 & & \\
\hline & 7 & 919 & 74.5000 & 11.9750 & 297.0 & 4 & & \\
\hline & 7 & 920 & 72.7500 & 12.7000 & 297.0 & 4 & 0 & 00 \\
\hline & 7 & 921 & & 0 & 297.2 & 4 & & \\
\hline & 7 & 922 & & 11 . & 295.8 & & & \\
\hline & & 923 & 73.2500 & 10.9500 & 295.4 & & 60 & 50 \\
\hline
\end{tabular}




\begin{tabular}{|c|c|c|c|c|c|c|c|}
\hline & 24 & & 9.9750 & 294.9 & 4 & 政 & \\
\hline & 101 & 47500 & 0.0500 & 96.2 & & & \\
\hline & 102 & 556 & & 5.0 & & & \\
\hline & 103 & 2.9711 & 8.0250 & 91.1 & & 00 & \\
\hline & 104 & . & & & & & \\
\hline & 105 & 2500 & 3250 & 90.2 & & & \\
\hline & 106 & 3174 & & 91.6 & & 0 & \\
\hline & 107 & 608 & & 90.7 & & & \\
\hline & 108 & 751 & & 91.1 & & 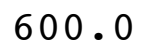 & \\
\hline & 109 & 492 & & & & & \\
\hline & 1010 & 494 & & & & & \\
\hline & 1011 & & & 9 & & & \\
\hline & 1012 & 818 & & 98.2 & & 0 & \\
\hline & 1013 & 68. & & & & & \\
\hline & 014 & & & & & & \\
\hline & 1015 & 500 & & 99.2 & 4 & & \\
\hline & 1016 & 5000 & 8.20 & 298 & & & \\
\hline & 1017 & 997 & & 5 & & & \\
\hline & 1018 & 500 & 0 & 4 & 4 & & \\
\hline & 1019 & 500 & 0 & & & & \\
\hline & 1020 & 01 & & & & & \\
\hline & 1021 & & & 29 & 4 & & \\
\hline & 1022 & 2504 & & 291 & 4 & & \\
\hline & 1023 & 500 & & & & & \\
\hline & 1024 & & & & 4 & & \\
\hline & 111 & 500 & & 288 & 4 & & \\
\hline & 7112 & 000 & 0 & 287 & 4 & & \\
\hline & 7113 & 00 & 12 & 6 & 4 & & \\
\hline & 7114 & 501 & & 286.2 & A & & \\
\hline & 7115 & 005 & & & 4 & & \\
\hline & 7116 & 500 & & & 4 & & \\
\hline & $\begin{array}{ll}711 & 7\end{array}$ & 79.2500 & & 5.2 & 4 & & \\
\hline & 7118 & 74.9994 & & 287.1 & 4 & & \\
\hline & 7119 & & & $2 \varepsilon$ & 4 & & \\
\hline & 71110 & & & & 3 & & \\
\hline & 71111 & 74.9984 & & 293.2 & & & \\
\hline & 71112 & 39 & & 29 & 3 & & \\
\hline & 71113 & 63 & & 29 & 3 & & \\
\hline & 71114 & 88. & & 296.0 & 3 & & \\
\hline & 71115 & 011 & & 29 & 4 & & \\
\hline & 71116 & & & & 4 & & \\
\hline & 71117 & 71.0000 & 500 & 294.2 & 4 & & \\
\hline & 71118 & 72.4999 & 10 & 292 & 4 & & \\
\hline & 71119 & 75.2500 & 11.9250 & & 7 & & \\
\hline & 71120 & 73.5018 & 12.3250 & 289.0 & 4 & 0 & P0 \\
\hline & 71121 & & & & 4 & & \\
\hline & 71122 & 77.7497 & 13. & & & & \\
\hline & 1123 & 76.7500 & 12.8750 & 286 . & & 600.0 & 500 \\
\hline
\end{tabular}




\begin{tabular}{|c|c|c|c|c|c|c|c|}
\hline 01 & 71124 & 74.0000 & 12.9500 & 286.3 & 4 & 600.0 & 600.0 \\
\hline 01 & 7121 & 73.2501 & 13.5000 & 286.2 & 4 & 600.0 & 600.0 \\
\hline 01 & 7122 & 77.5000 & 12.4250 & 286.2 & 4 & 600.0 & 00.0 \\
\hline 01 & 7123 & 76.5002 & 11.4250 & 286.0 & 4 & 600.0 & 00.0 \\
\hline 01 & 7124 & 78.2500 & 11.6250 & 285.7 & 4 & 600.0 & 00.0 \\
\hline 01 & 7125 & 78.0000 & 11.0250 & 285.7 & 4 & 600.0 & 00.0 \\
\hline 01 & 7126 & 82.9998 & 10.0250 & 285.6 & 4 & 600.0 & 00.0 \\
\hline 01 & 7127 & 80.2502 & 7.8000 & 286.3 & 4 & 600.0 & 00.0 \\
\hline 01 & 7128 & 84.7498 & 6.1250 & 287.5 & 4 & 600.0 & 00.0 \\
\hline 01 & 7129 & 84.2435 & 500 & 289.2 & 3 & 600.0 & .0 \\
\hline 01 & 71210 & 84.7507 & 3.7250 & 291.0 & 3 & 600.0 & 00.0 \\
\hline 01 & 71211 & 66.4960 & 000 & 293.0 & 2 & 600.0 & \\
\hline 01 & 71212 & 140.9802 & 3.8000 & 295.8 & 2 & 600.0 & 00.0 \\
\hline 01 & 71213 & 172.2554 & 6.8250 & 295.2 & 3 & .0 & 00.0 \\
\hline 01 & 71214 & 180.2463 & 00 & 297.0 & 4 & .0 & \\
\hline 01 & 71215 & 173.2500 & 00 & 298.7 & 4 & 600.0 & 00.0 \\
\hline 01 & 71216 & 87.0236 & 4.7250 & 299.5 & 3 & 600.0 & 600.0 \\
\hline 01 & 71217 & 86.7521 & 6.6 & 298.4 & 4 & .0 & \\
\hline 01 & 71218 & 78.5005 & 8.5000 & 297.1 & 4 & 600.0 & .0 \\
\hline 01 & 71219 & 72.5000 & 9.6000 & 295.9 & 4 & 60 & .0 \\
\hline 01 & 71220 & 72.2460 & 9.7 & 294.3 & 4 & 60 & \\
\hline 01 & 71221 & 74.7500 & 9.2750 & 293.4 & 4 & .0 & \\
\hline 01 & 71222 & 78.5000 & 8.4500 & 295.3 & 4 & 600.0 & .0 \\
\hline 01 & 71223 & 78.7511 & 8. & 295.7 & 4 & 60 & \\
\hline 01 & 71224 & 76.5000 & 8 . & 295.1 & 4 & .0 & \\
\hline 01 & 7131 & 76.2498 & 000 & 294.7 & 4 & .0 & 600.0 \\
\hline 01 & 7132 & 74.5000 & 8.2000 & 294.8 & 4 & 600.0 & .0 \\
\hline 01 & 7133 & 74.0000 & 8.2 & 294.7 & 4 & .0 & \\
\hline 01 & 7134 & 79.2526 & 8.1500 & 294.8 & 4 & .0 & 600.0 \\
\hline 01 & 7135 & 83.0002 & 7.6000 & 294.9 & 4 & 600.0 & 0.0 \\
\hline 01 & 7136 & 78.9994 & 7 . & 295.1 & 4 & .0 & \\
\hline 01 & 7137 & 78.4997 & 6 . & 295.9 & 4 & .0 & 600.0 \\
\hline 01 & 7138 & 89.8779 & 3.3500 & 297.7 & 3 & 600.0 & 600.0 \\
\hline 01 & 7139 & 248.1219 & 1.3 & 299.4 & 2 & 600.0 & 0.0 \\
\hline 01 & 71310 & 179.9183 & 4.9000 & 297.9 & 3 & 600.0 & 600.0 \\
\hline 01 & 71311 & 167.5001 & 5.6750 & 298.6 & 3 & 600.0 & 600.0 \\
\hline 01 & 71312 & 173.9995 & 7.3000 & 299.1 & 4 & 600.0 & 0.0 \\
\hline 01 & 71313 & 170.9979 & 7.6500 & 300.2 & 4 & 600.0 & 600.0 \\
\hline 01 & 71314 & 158.7545 & 6.2750 & 301.6 & 4 & 600.0 & 600.0 \\
\hline 01 & 71315 & 131.5401 & 5.4750 & 302.3 & 3 & 600.0 & 600.0 \\
\hline 01 & 71316 & 94.2481 & 5.5500 & 302.5 & 3 & 600.0 & 600.0 \\
\hline 01 & 71317 & 82.7502 & 6.8750 & 301.8 & 4 & 600.0 & 600.0 \\
\hline 01 & 71318 & 77.2502 & 7.7500 & 300.7 & 4 & 600.0 & 600.0 \\
\hline 01 & 71319 & 84.2496 & 7.2750 & 299.4 & 4 & 600.0 & 600.0 \\
\hline 01 & 71320 & 73.5000 & 6.8250 & 297.9 & 4 & 600.0 & 600.0 \\
\hline 01 & 71321 & 78.7476 & 7.2500 & 297.1 & 4 & 600.0 & 600.0 \\
\hline 01 & 71322 & 72.4998 & 9.0500 & 296.8 & 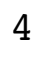 & 600.0 & 600.0 \\
\hline . & 71323 & 69.0000 & 8.500 & 296.6 & 4 & 600.0 & 600.0 \\
\hline
\end{tabular}




\begin{tabular}{|c|c|c|c|c|c|c|c|}
\hline 01 & 71324 & 68.7499 & 9.2500 & 296.4 & 4 & 600.0 & 600.0 \\
\hline 01 & $714 \quad 1$ & 75.0000 & 8.9750 & 296.1 & 4 & 600.0 & 600.0 \\
\hline 01 & 7142 & 70.7496 & 8.4250 & 296.0 & 4 & 600.0 & 00.0 \\
\hline 01 & 7143 & 68.5011 & 8.6000 & 295.3 & 4 & 600.0 & 00.0 \\
\hline 01 & $714 \quad 4$ & 70.5000 & 9.4750 & 295.5 & 4 & 600.0 & 00.0 \\
\hline 01 & 7145 & 70.7499 & 10.7000 & 293.9 & 4 & 600.0 & 00.0 \\
\hline 01 & 7146 & 75.2503 & 11.2500 & 292.5 & 4 & 600.0 & 00.0 \\
\hline 01 & $\begin{array}{ll}714 & 7\end{array}$ & 78.4997 & 10.9500 & 292.7 & 4 & 600.0 & 00.0 \\
\hline 01 & 7148 & 82.9985 & 8.6000 & 293.9 & 4 & 600.0 & 00.0 \\
\hline 01 & 7149 & 76.4992 & 7.4000 & 294.3 & 4 & 600.0 & .0 \\
\hline 01 & 71410 & 88.5002 & 7.1750 & 294.3 & 4 & 600.0 & \\
\hline 01 & 71411 & 77.2502 & 8.9000 & 294.8 & 4 & 600.0 & \\
\hline 01 & 71412 & 83.2497 & 7.9500 & 295.8 & 4 & 600.0 & 00.0 \\
\hline 01 & 71413 & 78.2499 & 8.5750 & 296.2 & 4 & 600.0 & 00.0 \\
\hline 01 & 71414 & 76.2503 & 50 & 296.6 & 4 & .0 & \\
\hline 01 & 71415 & 80.5000 & 8.1250 & 296.8 & 4 & 600.0 & .0 \\
\hline 01 & 71416 & 78.7500 & 9.1750 & 296.4 & 4 & 60 & .0 \\
\hline 01 & 71417 & 78.7500 & 10.8250 & 295.0 & 4 & .0 & \\
\hline 01 & 71418 & 79.7506 & 10.5750 & 293.9 & 4 & .0 & \\
\hline 01 & 71419 & 76.5000 & 11.3500 & 292.6 & 4 & .0 & .0 \\
\hline 01 & 71420 & 78.7500 & 12 . & 289.6 & 4 & 6 & \\
\hline 01 & 71421 & 77.5000 & 13.3500 & 288.4 & 4 & .0 & \\
\hline 01 & 71422 & 72.2500 & 12.2500 & 290.1 & 4 & 600.0 & .0 \\
\hline 01 & 71423 & 73.5000 & 11.02 & 292.1 & 4 & 60 & \\
\hline 01 & 71424 & 73.7500 & 10 & 290.8 & 4 & .0 & \\
\hline 01 & 7151 & 75.5000 & 10.2750 & 289.8 & 4 & .0 & \\
\hline 01 & 7152 & 74.2495 & 8.7000 & 290.4 & 4 & 600.0 & .0 \\
\hline 01 & 7153 & 74.4994 & 8.0000 & 289.1 & 4 & .0 & \\
\hline 01 & 7154 & 71.7503 & 8.6750 & 290.0 & 4 & .0 & 0.0 \\
\hline 01 & $715 \quad 5$ & 77.7501 & 7.9500 & 289.6 & 4 & 60 & .0 \\
\hline 01 & 7156 & 78.5000 & 6.0500 & 289.7 & 4 & .0 & \\
\hline 01 & $\begin{array}{ll}715 & 7\end{array}$ & 89.8745 & 500 & 290.1 & 3 & .0 & 600.0 \\
\hline 01 & 7158 & 71.3429 & 3.2000 & 290.0 & 2 & 600.0 & 600.0 \\
\hline 01 & 7159 & 53.5386 & 2.2000 & 292.2 & 1 & 0 & 0.0 \\
\hline 01 & 71510 & 19.5097 & 2.7750 & 293.8 & 1 & 600.0 & 600.0 \\
\hline 01 & 71511 & 33.8551 & 2.7500 & 295.2 & 1 & 600.0 & 600.0 \\
\hline 01 & 71512 & 51.0000 & 3.000 & 296.3 & 2 & 600.0 & 600.0 \\
\hline 01 & 71513 & 20.3344 & 2.7750 & 297.9 & 1 & 600.0 & 600.0 \\
\hline 01 & 71514 & 51.0000 & 5.4500 & 297.3 & 2 & 600.0 & 600.0 \\
\hline 01 & 71515 & 64.5000 & 5.1500 & 297.8 & 3 & 600.0 & 600.0 \\
\hline 01 & 71516 & 74.0059 & 5.8250 & 297.7 & 3 & 600.0 & 600.0 \\
\hline 01 & 71517 & 81.7498 & 8.1500 & 295.9 & 4 & 600.0 & 600.0 \\
\hline 01 & 71518 & 75.7500 & 10.7250 & 293.2 & 4 & 600.0 & 600.0 \\
\hline 01 & 71519 & 74.5000 & 11.8250 & 290.4 & 4 & 600.0 & 600.0 \\
\hline 01 & 71520 & 70.2512 & 11.6250 & 288.2 & 4 & 600.0 & 600.0 \\
\hline 01 & 71521 & 75.0027 & 12.6250 & 287.8 & 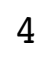 & 600.0 & 600.0 \\
\hline 01 & 71522 & 75.2503 & 11.5500 & 288.5 & 4 & 600.0 & 600.0 \\
\hline J & 71523 & 74.7501 & 10.9750 & 289.5 & 4 & 600.0 & 600.0 \\
\hline
\end{tabular}




\begin{tabular}{|c|c|c|c|c|c|c|c|}
\hline 01 & 71524 & 73.9993 & 10.8000 & 288.9 & 4 & 600.0 & 00.0 \\
\hline 01 & 7161 & 73.5000 & 11.6250 & 287.7 & 4 & 600.0 & 00,0 \\
\hline 01 & 7162 & 71.7496 & 10.9250 & 287.4 & 4 & 600.0 & 00.0 \\
\hline 01 & 7163 & 68.0005 & 12.4500 & 287.3 & 4 & 600.0 & 00.0 \\
\hline 01 & 7164 & 66.7500 & 12.8000 & 286.9 & 4 & 600.0 & 00.0 \\
\hline 01 & 7165 & 69.7500 & 14.4500 & 286.0 & 4 & 600.0 & 00.0 \\
\hline 01 & 7166 & 71.0000 & 13.2500 & 285.9 & 4 & 600.0 & 600.0 \\
\hline 1 & 7167 & 67.5003 & 11.3750 & 286.6 & 4 & 600.0 & 00.0 \\
\hline 01 & 7168 & 71.0005 & 10.8250 & 287.3 & 4 & 600.0 & 00.0 \\
\hline 01 & 7169 & 71.5029 & 10.6000 & 287.5 & 4 & 600.0 & .0 \\
\hline 01 & 71610 & 72.5005 & 9.2250 & 288.3 & 4 & 600.0 & 500.0 \\
\hline 01 & 71611 & 75.2501 & 8.6750 & 289.9 & 4 & 600.0 & \\
\hline 01 & 71612 & 81.0007 & 9.0250 & 290.8 & 4 & 600.0 & 600.0 \\
\hline 01 & 71613 & 72.7497 & 9.0000 & 291.4 & 4 & 600.0 & 600 \\
\hline 01 & 71614 & 74.2501 & 9. & 291.7 & 4 & 600.0 & \\
\hline 01 & 71615 & 67.5000 & 9.9750 & 291.5 & 4 & 600.0 & 60 \\
\hline 01 & 71616 & 69.0002 & 10.5250 & 291.0 & 4 & 600.0 & 600.0 \\
\hline 01 & 71617 & 69.4998 & 11.3250 & 289.7 & 4 & 600.0 & \\
\hline 01 & 71618 & 72.5000 & 12.1000 & 288.5 & 4 & 600.0 & .0 \\
\hline 01 & 71619 & 73.4995 & 11.3500 & 287.9 & 4 & 600.0 & 60 \\
\hline 01 & 71620 & 70.9968 & 12.37 & 286.7 & 4 & 600.0 & .0 \\
\hline 01 & 71621 & 74.9986 & 11. & 286.6 & 4 & 600.0 & \\
\hline 01 & 71622 & 75.0002 & 10.3000 & 286.0 & 4 & 600.0 & 600 \\
\hline 01 & 71623 & 74.2500 & 10.9750 & 285.4 & 4 & 600.0 & .0 \\
\hline 01 & 71624 & 73.2503 & 10. & 285.4 & 4 & 600.0 & \\
\hline 01 & 7171 & 75.0003 & 10.1000 & 286.1 & 4 & 600.0 & 60 \\
\hline 01 & 7172 & 78.2498 & 9.1000 & 286.0 & 4 & 600.0 & 600.0 \\
\hline 01 & 7173 & 61.7914 & 4.10 & 287.4 & 4 & 600.0 & \\
\hline 01 & 7174 & 90.8819 & 2.3500 & 286.8 & 5 & 600.0 & 60 \\
\hline 01 & 7175 & 71.2532 & 2.2500 & 286.5 & 5 & 600.0 & 600.0 \\
\hline 01 & 7176 & 62.1744 & 0.52 & 287.0 & 6 & 600.0 & \\
\hline 01 & $717 \quad 7$ & 134.8067 & 1.0250 & 288.6 & 5 & 600.0 & 60 \\
\hline 01 & 7178 & 231.2912 & 1.6500 & 290.0 & 4 & 600.0 & 600.0 \\
\hline 01 & 7179 & 234.5083 & 2.3750 & 290.2 & 3 & 600.0 & .0 \\
\hline 01 & 71710 & 241.4986 & 2 . & 291.5 & 2 & 600.0 & \\
\hline 01 & 71711 & 244.0080 & 2.3500 & 293.6 & 1 & 600.0 & 600.0 \\
\hline 01 & 71712 & 212.6902 & 3.9500 & 294.8 & 2 & 600.0 & \\
\hline 01 & 71713 & 177.7539 & 4.8000 & 295.9 & 3 & 600.0 & 600.0 \\
\hline 01 & 71714 & 94.2148 & 2.7500 & 298.3 & 2 & 600.0 & 600.0 \\
\hline 01 & 71715 & 107.7279 & 3.9500 & 298.8 & 2 & 600.0 & \\
\hline 01 & 71716 & 116.2410 & 5.57 & 298.8 & 3 & 600.0 & 600.0 \\
\hline 01 & 71717 & 106.2500 & 6.6000 & 298.1 & 4 & 600.0 & 600.0 \\
\hline 01 & 71718 & 84.7468 & 6.7500 & 297.0 & 4 & 600.0 & 600.0 \\
\hline 01 & 71719 & 72.9585 & 7.0750 & 295.2 & 4 & 600.0 & 600.0 \\
\hline 01 & 71720 & 69.5005 & 9.8250 & 293.3 & 4 & 600.0 & 600.0 \\
\hline 01 & 71721 & 72.2501 & 9.5500 & 292.7 & 4 & 600.0 & 600.0 \\
\hline 01 & 71722 & 67.2501 & 8.87 & 29 & 4 & 600.0 & 600.0 \\
\hline 01 & 71723 & 68.4997 & 7.7250 & 293.7 & 4 & 600.0 & 600.0 \\
\hline
\end{tabular}




\begin{tabular}{|c|c|c|c|c|c|c|c|}
\hline 01 & 71724 & 67.7485 & 7.5750 & 293.8 & 4 & 600.0 & 600.0 \\
\hline 01 & 7181 & 73.0000 & 7.5000 & 294.2 & 4 & 600.0 & 00.0 \\
\hline 01 & 7182 & 79.4995 & 6.1000 & 294.8 & 4 & 600.0 & 00.0 \\
\hline 01 & 7183 & 124.2507 & 2.1500 & 294.1 & 5 & 600.0 & 00.0 \\
\hline 01 & 7184 & 102.3009 & 2.4500 & 293.2 & J & 600.0 & 00.0 \\
\hline 01 & 7185 & 76.5179 & 4.0750 & 293.0 & 4 & 600.0 & 00.0 \\
\hline 01 & 7186 & 72.2491 & 000 & 293.2 & 4 & 600.0 & 00.0 \\
\hline 01 & 7187 & 62.3377 & 2.5500 & 294.1 & 3 & 600.0 & 00.0 \\
\hline 01 & 7188 & 296.9634 & 1.8000 & 293.9 & 2 & 600.0 & 00.0 \\
\hline 01 & 7189 & 282.4891 & 2.1750 & 294.9 & 1 & 600.0 & .0 \\
\hline 01 & 71810 & 254.6756 & 500 & 296.2 & 1 & 600.0 & \\
\hline 01 & 71811 & 226.4784 & 500 & 297.3 & 1 & 600.0 & \\
\hline 01 & 71812 & 194.9354 & 500 & 298.3 & 2 & 600.0 & 00.0 \\
\hline 01 & 71813 & 147.3272 & 500 & 300.2 & 3 & .0 & 00.0 \\
\hline 01 & 71814 & 111.7425 & 50 & 301.2 & 3 & .0 & \\
\hline 01 & 71815 & 101.7025 & 750 & 301.3 & 3 & 600.0 & .0 \\
\hline 01 & 71816 & 109.5000 & 6.3750 & 301.2 & 4 & 60 & .0 \\
\hline 01 & 71817 & 94.0007 & 7 . & 300.2 & 4 & .0 & \\
\hline 01 & 71818 & 87.0002 & 8. & 298.3 & 4 & 60 & \\
\hline 01 & 71819 & 73.7485 & 9. & 296.9 & 4 & .0 & .0 \\
\hline 01 & 71820 & 69 . & 9 . & 295.8 & 4 & 6 & \\
\hline 01 & 71821 & 72.4998 & 9. & 295.5 & 4 & .0 & \\
\hline 01 & 71822 & 74.4997 & 8.8 & 295.3 & 4 & 600.0 & .0 \\
\hline 01 & 71823 & 69.5001 & 9 . & 295.1 & 4 & 6 & \\
\hline 01 & 71824 & 72.2500 & 9 . & 293.9 & 4 & 0 & \\
\hline 01 & 7191 & 72.7499 & 9.7 & 293.8 & 4 & .0 & \\
\hline 01 & 7192 & 70.7500 & 9.3000 & 293.4 & 4 & .0 & .0 \\
\hline 01 & 7193 & 71.7505 & 8. & 293.2 & 4 & .0 & \\
\hline 01 & 7194 & 75.4999 & 9.7000 & 291.6 & 4 & .0 & 0.0 \\
\hline 01 & 7195 & 66.7492 & 10.0250 & 291.6 & 4 & 60 & .0 \\
\hline 01 & 7196 & 70.7501 & 9 . & 291.0 & 4 & .0 & \\
\hline 01 & 7197 & 73.7500 & 9.77 & 290.9 & 4 & .0 & 0.0 \\
\hline 01 & 7198 & 69.9995 & 10.7750 & 291.0 & 4 & 600.0 & 0.0 \\
\hline 01 & 7199 & 67.2501 & 9.57 & 292.3 & 4 & 600.0 & .0 \\
\hline 01 & 71910 & 74.5000 & 8.7500 & 293.4 & 4 & 600.0 & 600.0 \\
\hline 01 & 71911 & 76.2500 & 9.0500 & 294.2 & 4 & 600.0 & 600.0 \\
\hline 01 & 71912 & 77.2366 & 8. & 295.7 & 4 & 600.0 & 0.0 \\
\hline 01 & 71913 & 76.2555 & 8.1750 & 296.4 & 4 & 600.0 & 600.0 \\
\hline 01 & 71914 & 71.9986 & 8.0500 & 296.9 & 4 & 600.0 & 600.0 \\
\hline 01 & 71915 & 72.5003 & 9.5250 & 296.6 & 4 & 600.0 & 600.0 \\
\hline 01 & 71916 & 77.4998 & 9.3250 & 296.8 & 4 & 600.0 & 600.0 \\
\hline 01 & 71917 & 78.2500 & 9.5250 & 295.6 & 4 & 600.0 & 600.0 \\
\hline 01 & 71918 & 75.7499 & 11.7500 & 293.8 & 4 & 600.0 & 600.0 \\
\hline 01 & 71919 & 73.2495 & 12.8500 & 292.8 & 4 & 600.0 & 600.0 \\
\hline 01 & 71920 & 68.0000 & 12.1750 & 291.7 & 4 & 600.0 & 600.0 \\
\hline 01 & 71921 & 61.2498 & 12.1500 & 290.1 & 4 & 600.0 & 600.0 \\
\hline 01 & 71922 & 60.2500 & 13.5500 & 289.8 & 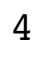 & 600.0 & 600.0 \\
\hline . & 71923 & 65.4973 & 12.325 & 290.5 & 4 & 600.0 & 600.0 \\
\hline
\end{tabular}




\begin{tabular}{|c|c|c|c|c|c|c|c|}
\hline 01 & 71924 & 68.4994 & 10.0250 & 290.4 & 4 & 600.0 & 600.0 \\
\hline 01 & 7201 & 65.7502 & 11.6000 & 289.8 & 4 & 600.0 & 00,0 \\
\hline 01 & 7202 & 71.0000 & 11.2250 & 288.8 & 4 & 600.0 & 0.0 \\
\hline 1 & 7203 & 74.0000 & 11.2000 & 287.9 & 4 & 600.0 & 00.0 \\
\hline 01 & 7204 & 71.7501 & 11.3500 & 288.1 & 4 & 600.0 & 0.0 \\
\hline 01 & 7205 & 75.2502 & 10.5250 & 289.0 & 4 & 600.0 & 00.0 \\
\hline 01 & 7206 & 75.2495 & 9.0000 & 288.6 & 4 & 600.0 & 0 \\
\hline 01 & 7207 & 72.5000 & 10.2250 & 287.7 & 4 & 600.0 & 00.0 \\
\hline 01 & 7208 & 74.0000 & 10.2500 & 288.8 & 4 & 600.0 & 00.0 \\
\hline 01 & 7209 & 77.2500 & 9.5000 & 289.4 & 4 & 60 & 0 \\
\hline 1 & 72010 & 79.7506 & 7.8000 & 290.9 & 4 & 600.0 & \\
\hline 01 & 72011 & 92.2499 & 6.2750 & 293.2 & 4 & .0 & \\
\hline 01 & 72012 & 91.2501 & 5.3500 & 295.2 & 3 & 600.0 & .0 \\
\hline 01 & 72013 & 84.5018 & 6.6750 & 295.3 & 4 & 600.0 & \\
\hline 01 & 72014 & 71.0002 & 7 . & 295.2 & 4 & .0 & \\
\hline 01 & 72015 & 75.2508 & 7.1500 & 295.8 & 4 & 60 & \\
\hline 01 & 72016 & 71.7500 & 10.2500 & 294.8 & 4 & .0 & .0 \\
\hline 01 & 72017 & 73.7497 & 11.5250 & 293.2 & 4 & .0 & \\
\hline 01 & 72018 & 78.7500 & 11.5500 & 291.3 & 4 & .0 & \\
\hline 01 & 72019 & 79.7511 & 12.3500 & 289.0 & 4 & .0 & .0 \\
\hline 01 & 72020 & 74.2500 & 13.87 & .3 & 4 & 6 & \\
\hline 01 & 72021 & 70.2502 & 13.3250 & 288.2 & 4 & .0 & \\
\hline 01 & 72022 & 72.9999 & 11.5750 & 289.3 & 4 & 600.0 & .0 \\
\hline 01 & 72023 & 72.5000 & 11.47 & .6 & 4 & 0 & \\
\hline 01 & 72024 & 76.2500 & 10 & 28 & 4 & 0 & \\
\hline 01 & 7211 & 75.4997 & 10.7000 & 288.4 & 4 & .0 & \\
\hline 01 & 7212 & 71.5005 & 11.0000 & 288.7 & 4 & 60 & .0 \\
\hline 01 & 7213 & 72.2498 & 11.5000 & 288.2 & 4 & .0 & \\
\hline 01 & 7214 & 73.9998 & 11.1250 & 288.4 & 4 & .0 & .0 \\
\hline 01 & 7215 & 73.9989 & 11.3500 & 287.7 & 4 & .0 & \\
\hline 01 & 7216 & 78.0024 & 11.1000 & 287.5 & 4 & .0 & \\
\hline 01 & $\begin{array}{ll}721 & 7\end{array}$ & 78.9998 & 10 & 287.4 & 4 & .0 & 0 \\
\hline 01 & 7218 & 76.5009 & 7.8750 & 289.5 & 4 & 0.0 & 0.0 \\
\hline 01 & 7219 & 86.7533 & 8. & 289.7 & 4 & .0 & .0 \\
\hline 01 & 72110 & 92.2415 & 7.82 & 290.1 & 4 & 600.0 & 0.0 \\
\hline 01 & 72111 & 87.5003 & 5.0000 & 292.9 & 3 & 600.0 & 600.0 \\
\hline 01 & 72112 & 84.7481 & 6.70 & 293.4 & 4 & 600.0 & .0 \\
\hline 01 & 72113 & 93.2512 & 6.0750 & 294.4 & 4 & 600.0 & 600.0 \\
\hline 01 & 72114 & 83.0038 & 6.8000 & 295.3 & 4 & 600.0 & 600.0 \\
\hline 01 & 72115 & 78 . & 8.3250 & 295.3 & 4 & 600.0 & 600.0 \\
\hline 01 & 72116 & 70.9999 & 9.2000 & 295.2 & 4 & 600.0 & 600.0 \\
\hline 01 & 72117 & 74.5005 & 9.8250 & 294.6 & 4 & 600.0 & 600.0 \\
\hline 01 & 72118 & 76.2500 & 9.7000 & 294.0 & 4 & 600.0 & 600.0 \\
\hline 01 & 72119 & 74.2498 & 10.9500 & 293.2 & 4 & 600.0 & 600.0 \\
\hline 01 & 72120 & 72.5000 & 11.3000 & 292.4 & 4 & 600.0 & 600.0 \\
\hline 01 & 72121 & 73.2501 & 11.0250 & 292.0 & 4 & 600.0 & 600.0 \\
\hline 01 & 72122 & 67.9999 & 10.6000 & 292.5 & 4 & 600.0 & 600.0 \\
\hline 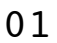 & 72123 & 71.5000 & 9.800 & 293.1 & & 600.0 & 600.0 \\
\hline
\end{tabular}




\begin{tabular}{|c|c|c|c|c|c|c|c|}
\hline 1 & 2124 & 2.7500 & 9.2500 & 93.1 & 4 & 600.0 & \\
\hline & 7221 & 69.7505 & 7.8500 & 293.3 & 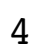 & 1000 & \\
\hline & 7222 & 499 & 250 & & 4 & 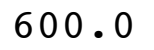 & \\
\hline & 7223 & 6.2500 & 750 & 94.0 & & 00 & \\
\hline & 7224 & 2.2503 & & 93.8 & & & \\
\hline & 7225 & 81.2498 & 250 & 93.8 & & 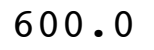 & \\
\hline & 7226 & 12.7686 & 1250 & 93.7 & & S & \\
\hline & 7227 & 66.7349 & .8000 & 93.9 & & 0 & \\
\hline & 7228 & 230.9487 & 1.5250 & 95.0 & & 00 & \\
\hline & 229 & 241.4963 & 2.2000 & & & 0 & \\
\hline & 2210 & 169. & 000 & & & & \\
\hline & 2211 & 170 . & 250 & 7 & & & \\
\hline & 2212 & 164 . & 750 & 298.1 & & 0 & \\
\hline & 72213 & 177 . & 750 & & & & \\
\hline & 72214 & & & & & & \\
\hline & 72215 & 177 . & 500 & 301.2 & & & \\
\hline & 72216 & 176.2481 & 6.1750 & .5 & & 0 & \\
\hline & 72217 & 126.4066 & 5.1250 & 302.3 & 3 & & \\
\hline & 72218 & 84 & 750 & 4 & 3 & & \\
\hline & 72219 & 78 & 5500 & & 4 & & \\
\hline & 72220 & 74 . & 0 & & 4 & & \\
\hline & 72221 & 65 . & 750 & 29 & 4 & & \\
\hline & 72222 & 63. & 0000 & 29 & 4 & & \\
\hline & 72223 & 68 & 0 & & 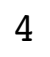 & & \\
\hline & 72224 & & 50 & & 4 & & \\
\hline & 7231 & 02 & 250 & 297.8 & 4 & & \\
\hline & 7232 & 70 . & 7.2250 & 298.1 & 4 & & \\
\hline & 7233 & 59. & 3.3750 & 297 & 5 & & \\
\hline & 7234 & 98 & 5000 & & 6 & & \\
\hline & 7235 & 74 & 000 & & 5 & & \\
\hline & 7236 & 16 & 500 & & 6 & & \\
\hline & 7237 & 106 . & & & 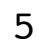 & & \\
\hline & 7238 & 45.5112 & 1.2250 & 298.5 & 4 & & \\
\hline & 7239 & 201.7233 & 0 & 297 & 3 & & \\
\hline & 72310 & 182 & 750 & & 3 & & \\
\hline & 72311 & 182.2444 & 6.2750 & 298.5 & 4 & & \\
\hline & 72312 & 173. & 750 & 300.3 & 4 & & \\
\hline & 72313 & 171. & 500 & 1.7 & 4 & & \\
\hline & 72314 & 168.0011 & 2500 & 302.5 & 4 & & \\
\hline & 72315 & 163. & 000 & & 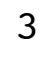 & & \\
\hline & 72316 & 156. & 250 & & 4 & & \\
\hline & 72317 & 110.4673 & 4.2750 & 304.2 & 3 & & \\
\hline & 72318 & 98.9752 & 4.4250 & 303.8 & 3 & & \\
\hline & 72319 & 76.7502 & 6.2250 & & 4 & & \\
\hline & 72320 & 79.0002 & 9.4500 & 299.3 & 4 & 600.0 & 00 . \\
\hline & 72321 & & & & 4 & & \\
\hline & 72322 & 75.0000 & 1.9250 & & 4 & & 600 \\
\hline & 2323 & 66.2505 & 11.3500 & 298.3 & & 600.0 & 60 \\
\hline
\end{tabular}




\begin{tabular}{|c|c|c|c|c|c|c|c|}
\hline 01 & 72324 & 67.0021 & 9.7750 & 299.1 & 4 & 600.0 & 600.0 \\
\hline 01 & 7241 & 71.9989 & 8.0250 & 299.7 & 4 & 600.0 & 600.0 \\
\hline 01 & 7242 & 83.7498 & 7.0000 & 300.0 & 4 & 600.0 & 00.0 \\
\hline 01 & 7243 & 85.2501 & 8.0750 & 299.1 & 4 & 600.0 & 00.0 \\
\hline 01 & $724 \quad 4$ & 81.4994 & 5.4500 & 298.1 & 4 & 600.0 & 00.0 \\
\hline 01 & 7245 & 109.3601 & 3.8250 & 296.4 & 4 & 600.0 & 00.0 \\
\hline 01 & 7246 & 100.5599 & 2.4750 & 295.6 & 5 & 600.0 & 00.0 \\
\hline 01 & 7247 & 83.7497 & 5.6000 & 297.2 & 4 & 600.0 & 00.0 \\
\hline 01 & 7248 & 294.5332 & 1.9750 & 298.5 & 3 & 600.0 & 00.0 \\
\hline 01 & 7249 & 223.8794 & 2.2250 & 299.6 & 2 & 600.0 & 00.0 \\
\hline 01 & 72410 & 209.4431 & 3.0500 & 299.5 & 2 & 600.0 & 00.0 \\
\hline 01 & 72411 & 188.8597 & 3.3000 & 301.5 & 2 & 600.0 & .0 \\
\hline 01 & 72412 & 39.7052 & 3.7000 & 303.4 & 2 & 600.0 & 00.0 \\
\hline 01 & 72413 & 15.0000 & 3.9000 & 304.0 & 2 & .0 & 00.0 \\
\hline 01 & 72414 & 85.6451 & 250 & 304.5 & 3 & .0 & \\
\hline 01 & 72415 & 92.2502 & 5 & 304.3 & 3 & 600.0 & 00.0 \\
\hline 01 & 72416 & 89.2503 & 6.4500 & 303.7 & 4 & 600.0 & 600.0 \\
\hline 01 & 72417 & 89.4997 & 6 & 303.5 & 4 & .0 & \\
\hline 01 & 72418 & 81.9973 & 6.7250 & 302.8 & 4 & 600.0 & .0 \\
\hline 01 & 72419 & 79.2500 & 6.1000 & 302.4 & 4 & 60 & .0 \\
\hline 01 & 72420 & 73.2500 & 7.22 & 300.6 & 4 & .0 & \\
\hline 01 & 72421 & 73.7500 & 7.9000 & 301.2 & 4 & .0 & \\
\hline 01 & 72422 & 82.4841 & 5.8250 & 301.3 & 4 & 600.0 & 0.0 \\
\hline 01 & 72423 & 88.8629 & 3.57 & 300.4 & 5 & 60 & .0 \\
\hline 01 & 72424 & 60.8926 & 2.2750 & 299.7 & 6 & .0 & \\
\hline 01 & 7251 & 9.2063 & 0.9250 & 296.8 & 6 & 600.0 & 600.0 \\
\hline 01 & 7252 & 70.9646 & 2.7250 & 299.2 & 5 & 600.0 & .0 \\
\hline 01 & 7253 & 56.4959 & 1.5500 & 297.2 & 6 & 0 & \\
\hline 01 & 7254 & 67.9767 & 1.0750 & 298.1 & 6 & 600.0 & 600.0 \\
\hline 01 & $725 \quad 5$ & 259.3240 & 0.5000 & 294.7 & 6 & 600.0 & .0 \\
\hline 01 & 7256 & 45.1010 & 1.0250 & 292.1 & 6 & .0 & \\
\hline 01 & $\begin{array}{ll}725 & 7\end{array}$ & 119.7039 & 0.7750 & 295.9 & 5 & .0 & 600.0 \\
\hline 01 & 7258 & 161.1969 & 0.9000 & 299.5 & 4 & 600.0 & 600.0 \\
\hline 01 & 7259 & 261.3122 & 2.0000 & 299.1 & 3 & 600.0 & 0.0 \\
\hline 01 & 72510 & 224.5566 & 2.9500 & 298.3 & 2 & 600.0 & 600.0 \\
\hline 01 & 72511 & 202.2495 & 3.0500 & 299.7 & 2 & 600.0 & 600.0 \\
\hline 01 & 72512 & 240.0402 & 3.2750 & 301.6 & 2 & 600.0 & 600.0 \\
\hline 01 & 72513 & 80.0120 & 4.4500 & 302.8 & 3 & 600.0 & 600.0 \\
\hline 01 & 72514 & 83.5086 & 6.2250 & 302.9 & 4 & 600.0 & 600.0 \\
\hline 01 & 72515 & 83.2495 & 6.1000 & 304.2 & 4 & 600.0 & 600.0 \\
\hline 01 & 72516 & 82.4985 & 7.6500 & 304.4 & 4 & 600.0 & 600.0 \\
\hline 01 & 72517 & 82.4466 & 7.4500 & 303.6 & 4 & 600.0 & 600.0 \\
\hline 01 & 72518 & 78.4815 & 8.0250 & 302.4 & 4 & 600.0 & 600.0 \\
\hline 01 & 72519 & 53.0353 & 5.6250 & 301.9 & 4 & 600.0 & 600.0 \\
\hline 01 & 72520 & 38.0000 & 3.6750 & 299.1 & 4 & 600.0 & 600.0 \\
\hline 01 & 72521 & 70.0000 & 4.7000 & 298.3 & 4 & 600.0 & 600.0 \\
\hline 01 & 72522 & 60.7545 & 4.9000 & 297.4 & 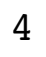 & 600.0 & 600.0 \\
\hline . & 72523 & 72.5009 & 5.675 & 300.6 & 4 & 600.0 & 600.0 \\
\hline
\end{tabular}




\begin{tabular}{|c|c|c|c|c|c|c|c|}
\hline 01 & 72524 & 73.2504 & 6.3250 & 300.3 & 4 & 600.0 & 600.0 \\
\hline 01 & 7261 & 79.2486 & 5.2500 & 299.9 & 4 & 600.0 & 600.0 \\
\hline 01 & 7262 & 67.7512 & 3.9750 & 299.8 & 4 & 600.0 & 00.0 \\
\hline 01 & 7263 & 102.0954 & 3.3000 & 298.5 & 5 & 600.0 & 00.0 \\
\hline 01 & 7264 & 21.2669 & 0.5750 & 295.9 & 6 & 600.0 & 00.0 \\
\hline 01 & 7265 & 9.2257 & 0.1500 & 295.9 & 5 & 600.0 & 00.0 \\
\hline 01 & 7266 & 85.8074 & 1.3750 & 294.7 & 5 & 600.0 & 00.0 \\
\hline 01 & 7267 & 157.0268 & 0.7750 & 294.8 & 4 & 600.0 & 00.0 \\
\hline 01 & 7268 & 296.0366 & 0.6750 & 297.4 & 3 & 600.0 & 00.0 \\
\hline 01 & 7269 & 265.0056 & 2.1750 & 297.0 & 2 & 600.0 & 00.0 \\
\hline 01 & 72610 & 238.7506 & 2.9250 & 297.1 & 3 & 600.0 & 00.0 \\
\hline 01 & 72611 & 204.1818 & 3.5250 & 298.7 & 2 & 600.0 & \\
\hline 01 & 72612 & 162.7533 & 3.7500 & 299.9 & 3 & 600.0 & 00.0 \\
\hline 01 & 72613 & 167.9984 & 4.4500 & 301.2 & 3 & 600.0 & 00.0 \\
\hline 01 & 72614 & 168.0023 & 250 & 302.6 & 3 & .0 & \\
\hline 01 & 72615 & 181.5131 & 6.4750 & 304.0 & 4 & 600.0 & 00.0 \\
\hline 01 & 72616 & 129.4112 & 4.6500 & 305.8 & 3 & 600.0 & 600.0 \\
\hline 01 & 72617 & 100.3716 & 4.0750 & 305.8 & 3 & .0 & \\
\hline 01 & 72618 & 11.0042 & 2.2750 & 305.1 & 2 & 600.0 & .0 \\
\hline 01 & 72619 & 54.8023 & 1.9500 & 303.0 & 3 & 60 & .0 \\
\hline 01 & 72620 & 55.5480 & 1.3500 & 299.6 & 4 & 60 & \\
\hline 01 & 72621 & 338.6737 & 1.3250 & 298.3 & 5 & .0 & \\
\hline 01 & 72622 & 83.3705 & 1.9500 & 299.4 & 6 & 600.0 & 500.0 \\
\hline 01 & 72623 & 71.8043 & 0.92 & 298.8 & 6 & 60 & .0 \\
\hline 01 & 72624 & 90.0424 & 000 & 298.9 & 6 & .0 & \\
\hline 01 & 7271 & 78.4185 & 3.2500 & 299.0 & 5 & 600.0 & 600.0 \\
\hline 01 & 7272 & 66.2502 & 2.4000 & 298.6 & 4 & 600.0 & .0 \\
\hline 01 & 7273 & 103.9712 & 2.6750 & 298.5 & 5 & 0 & \\
\hline 01 & 7274 & 105.3666 & 1.7500 & 297.5 & 6 & 600.0 & 600.0 \\
\hline 01 & $727 \quad 5$ & 310.1970 & 0.3000 & 295.8 & 5 & 600.0 & 600.0 \\
\hline 01 & 7276 & 55.9996 & 0.3250 & 296.8 & 6 & .0 & \\
\hline 01 & $\begin{array}{ll}727 & 7\end{array}$ & 150.3514 & 0.8000 & 298.1 & 5 & .0 & 600.0 \\
\hline 01 & 7278 & 257.2781 & 1.1500 & 299.1 & 4 & 600.0 & 600.0 \\
\hline 01 & 7279 & 294.4990 & 1.7750 & 298.3 & 3 & 600.0 & 0.0 \\
\hline 01 & 72710 & 248.2349 & 2.1500 & 299.7 & 2 & 600.0 & 600.0 \\
\hline 01 & 72711 & 234.4995 & 3.0250 & 300.5 & 2 & 600.0 & 600.0 \\
\hline 01 & 72712 & 177.9797 & 3.5250 & 301.8 & 2 & 600.0 & 600.0 \\
\hline 01 & 72713 & 167.7508 & 4.1500 & 303.0 & 3 & 600.0 & 600.0 \\
\hline 01 & 72714 & 170.0000 & 5.5500 & 303.7 & 3 & 600.0 & 600.0 \\
\hline 01 & 72715 & 173.9941 & 5.1250 & 305.0 & 3 & 600.0 & 600.0 \\
\hline 01 & 72716 & 105.1864 & 4.0500 & 306.4 & 3 & 600.0 & 600.0 \\
\hline 01 & 72717 & 86.5003 & 5.4000 & 305.7 & 3 & 600.0 & 600.0 \\
\hline 01 & 72718 & 68.2574 & 6.0750 & 304.6 & 4 & 600.0 & 600.0 \\
\hline 01 & 72719 & 66.2489 & 7.4250 & 303.3 & 4 & 600.0 & 600.0 \\
\hline 01 & 72720 & 67.5000 & 8.2000 & 301.8 & 4 & 600.0 & 600.0 \\
\hline 01 & 72721 & 75.0012 & 7.1750 & 301.6 & 4 & 600.0 & 600.0 \\
\hline 01 & 72722 & 70.5000 & 6.9250 & 301.4 & 4 & 600.0 & 600.0 \\
\hline . & 72723 & 71.2500 & 5.8250 & 300.6 & 4 & 600.0 & 600.0 \\
\hline
\end{tabular}




\begin{tabular}{|c|c|c|c|c|c|c|c|}
\hline 1 & 2724 & 0.2496 & 5.8750 & 1.1 & 4 & 000.0 & \\
\hline & 7281 & 67.2488 & 5.7000 & 300.8 & & & \\
\hline & 7282 & 318 & 3250 & 1.2 & & & \\
\hline & 7283 & 64.4669 & 750 & 1.5 & & 00 & \\
\hline & $728 \quad 4$ & 9.75 & & & & & \\
\hline & 7285 & 96.1634 & 250 & 99.2 & & 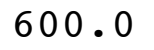 & \\
\hline & 7286 & 80.3210 & 2250 & 297.8 & & 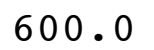 & \\
\hline & 7287 & 193.9109 & 50 & 97.6 & & & \\
\hline & 7288 & 254.8630 & 0.8250 & 299.5 & & 0 & \\
\hline & 289 & 292.4341 & 1.7500 & & & 0 & \\
\hline & 2810 & 241 . & 0 & & & & \\
\hline & 2811 & 221 . & & & & & \\
\hline & 2812 & 180.6963 & 750 & 301.8 & & 0 & \\
\hline & 72813 & 67 & & & & & \\
\hline & 2814 & & & & & & \\
\hline & 2815 & 108 . & 0 & & & & \\
\hline & 72816 & 96.2539 & 0.42 & 304.2 & & U & \\
\hline & 72817 & 100.5023 & & 3.4 & + & & \\
\hline & 2818 & 76 & & & 4 & & \\
\hline & 72819 & 69 & 0 & & & & \\
\hline & 72820 & 68 . & 0 & & & & \\
\hline & 72821 & 67. & & & 4 & & \\
\hline & 2822 & 56.0000 & 5000 & 7 & 4 & & \\
\hline & 72823 & 63. & & & & & \\
\hline & 72824 & 63. & & & 4 & & \\
\hline & 7291 & 501 & 0 & & 4 & & \\
\hline & 7292 & 70.99 & 0 & 9 & 4 & & \\
\hline & 7293 & 66. & 0 & & 4 & & \\
\hline & 7294 & 66. & 0 & & 1 & & \\
\hline & 7295 & 64.5000 & 0 & 8.2 & 4 & & \\
\hline & 7296 & 71.5086 & & & 4 & & \\
\hline & 7297 & & & & 4 & & \\
\hline & 7298 & 51.7729 & 0 & 7.9 & 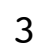 & & \\
\hline & 7299 & 259.7272 & 0 & 1 & 2 & & \\
\hline & 72910 & 236 . & & & 2 & & \\
\hline & 72911 & 252.2578 & 2.5750 & 0.3 & & & \\
\hline & 72912 & 247 . & & & 2 & & \\
\hline & 72913 & 209 & 3. & 1.6 & 2 & & \\
\hline & 72914 & 75.8660 & & 301.5 & 3 & & \\
\hline & 72915 & 8 & 0 & 1.2 & 4 & & \\
\hline & 72916 & & & & 4 & & \\
\hline & 72917 & 76.7508 & 9500 & 296.8 & 4 & & \\
\hline & 72918 & 72.7499 & 0 & 294.8 & 4 & & \\
\hline & 72919 & 69.2501 & 12.4000 & & 4 & & \\
\hline & 72920 & 67.7500 & 13.4000 & 292.1 & 4 & 0 & م. \\
\hline & 72921 & & & & 4 & & \\
\hline & 72922 & 67.2495 & 11.4500 & & & & \\
\hline & 2923 & 75.2549 & 10.9250 & 292.3 & & 600.0 & 60 \\
\hline
\end{tabular}




\begin{tabular}{|c|c|c|c|c|c|c|c|}
\hline 01 & 72924 & 65.2496 & 13.3000 & 291.8 & 4 & 600.0 & 600.0 \\
\hline 01 & 7301 & 68.7500 & 13.8500 & 291.6 & 4 & 600.0 & 00.0 \\
\hline 01 & 7302 & 68.2486 & 13.1750 & 290.9 & 4 & 600.0 & 00.0 \\
\hline 01 & 7303 & 66.7500 & 13.3000 & 290.8 & 4 & 600.0 & 00.0 \\
\hline 01 & 7304 & 73.5003 & 9.9000 & 290.6 & 4 & 600.0 & 00.0 \\
\hline 01 & 7305 & 69.7497 & 11.5000 & 289.7 & 4 & 600.0 & 00.0 \\
\hline 01 & 7306 & 72.0023 & 12.2000 & 289.2 & 4 & 600.0 & 0 \\
\hline 01 & $\begin{array}{ll}730 & 7\end{array}$ & 63.5000 & 13.9250 & 289.2 & 4 & 600.0 & 00.0 \\
\hline 01 & 7308 & 62.5000 & 14.3500 & 289.1 & 4 & 600.0 & 00.0 \\
\hline 01 & 7309 & 65.7518 & 13.7500 & 289.2 & 4 & 600.0 & .0 \\
\hline 01 & 73010 & 59.7500 & 12.5250 & 290.1 & 4 & 600.0 & \\
\hline 01 & 73011 & 67.9997 & 10.8250 & 291.2 & 4 & 600.0 & \\
\hline 01 & 73012 & 77.9992 & 9.3000 & 292.1 & 4 & 600.0 & 00.0 \\
\hline 01 & 73013 & 68.9775 & 9.6750 & 292.5 & 4 & .0 & 00.0 \\
\hline 01 & 73014 & 82.9992 & 00 & 293.5 & 4 & .0 & \\
\hline 01 & 73015 & 81.0000 & 10.3500 & 293.3 & 4 & 600.0 & .0 \\
\hline 01 & 73016 & 74.0000 & 9.7000 & 293.1 & 4 & 60 & .0 \\
\hline 01 & 73017 & 75.2499 & 9.2250 & 292.5 & 4 & .0 & \\
\hline 01 & 73018 & 73.7498 & 11.3500 & 291.0 & 4 & .0 & \\
\hline 01 & 73019 & 71.7500 & 11.3500 & 289.7 & 4 & .0 & .0 \\
\hline 01 & 73020 & 75.0000 & 12 . & .1 & 4 & 6 & \\
\hline 01 & 73021 & 77.0000 & 12 . & 287.6 & 4 & .0 & \\
\hline 01 & 73022 & 80.0000 & 11.3250 & 288.2 & 4 & 600.0 & .0 \\
\hline 01 & 73023 & 81.4998 & 9 . & 288.4 & 4 & 0 & \\
\hline 01 & 73024 & 79.2485 & 10 . & 289.1 & 4 & 0 & \\
\hline 01 & 7311 & 75.0002 & 8.9500 & 289.7 & 4 & .0 & \\
\hline 01 & 7312 & 77.2501 & 6.8250 & 291.0 & 4 & 60 & .0 \\
\hline 01 & 7313 & 76.9999 & 5. & 291.1 & 4 & .0 & \\
\hline 01 & 7314 & 80.9986 & 4.2000 & 291.9 & 5 & .0 & 0.0 \\
\hline 01 & 7315 & 115.0313 & 2.6000 & 291.4 & - & 60 & .0 \\
\hline 01 & 7316 & 109.0042 & 2.62 & 292.2 & 5 & 0 & \\
\hline 01 & $\begin{array}{ll}731 & 7\end{array}$ & 104.1256 & & .1 & 4 & .0 & 0.0 \\
\hline 01 & 7318 & 300.1077 & 0.4000 & 298.5 & 3 & 600.0 & 0.0 \\
\hline 01 & 7319 & 254.4917 & 1.7250 & 298.2 & 2 & 0.0 & .0 \\
\hline 01 & 73110 & 203.2990 & 3.77 & 297.2 & 2 & 600.0 & 600.0 \\
\hline 01 & 73111 & 170.7521 & 4.8000 & 296.6 & 3 & 600.0 & 600.0 \\
\hline 01 & 73112 & 175.4783 & 4 . & 298.1 & 3 & 600.0 & .0 \\
\hline 01 & 73113 & 186.0021 & 4.7500 & 299.2 & 3 & 600.0 & 600.0 \\
\hline 01 & 73114 & 174.5432 & 4.4500 & 300.4 & 3 & 600.0 & 600.0 \\
\hline 01 & 73115 & 178.7386 & 4 . & 301.1 & 3 & 600.0 & 600.0 \\
\hline 01 & 73116 & 148.5331 & 5. & 301.9 & 3 & 600.0 & 600.0 \\
\hline 01 & 73117 & 103.2486 & 5.7250 & 301.7 & 3 & 600.0 & 600.0 \\
\hline 01 & 73118 & 98.0005 & 6.4750 & 300.2 & 4 & 600.0 & 600.0 \\
\hline 01 & 73119 & 93.0015 & 5.9000 & 298.5 & 4 & 600.0 & 600.0 \\
\hline 01 & 73120 & 69.2475 & 8.8250 & 297.2 & 4 & 600.0 & 600.0 \\
\hline 01 & 73121 & 70.7498 & & 296.5 & 4 & 600.0 & 600.0 \\
\hline 01 & 73122 & 70.7500 & 8.8250 & 296.1 & 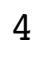 & 600.0 & 600.0 \\
\hline 01 & 73123 & 68.5000 & 8.300 & 296.3 & 4 & 600.0 & 600.0 \\
\hline
\end{tabular}




\begin{tabular}{|c|c|c|c|c|c|c|c|c|}
\hline & & & 0.2501 & 8.7000 & 296.9 & 4 & 000 & \\
\hline & 8 & 11 & חمח 4 & 8.0250 & 297.0 & 4 & & \\
\hline & 8 & 12 & 68.2487 & 5.6500 & 297.2 & 4 & 00 & \\
\hline & 8 & 13 & 354.8005 & 4000 & 296.6 & & 00 & \\
\hline & 8 & 14 & & 0.1250 & & & & \\
\hline & 8 & 15 & 0 & 0.1750 & & & 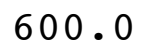 & \\
\hline & 8 & 16 & 6.3169 & 0.5750 & & & & \\
\hline & 8 & 17 & 2514 & 1.2000 & & & & \\
\hline & 8 & 18 & 9456 & 0.5500 & 299.0 & & & \\
\hline & 8 & 19 & 232.2509 & 2.6000 & 297.8 & & 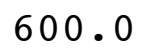 & \\
\hline & 8 & 110 & 220.7690 & 2.7250 & 298.2 & & & \\
\hline & 8 & 111 & 227.0274 & & & & & \\
\hline & 8 & 112 & 209 . & 250 & 300.0 & & 0 & \\
\hline & 8 & 113 & 179.0500 & 2.85 & 301.7 & & $\cdot 0$ & \\
\hline & 8 & 114 & & & & & & \\
\hline & 8 & 115 & 87 & 6 . & 301.7 & & & \\
\hline & 8 & 116 & 84.5000 & 0 & 1.1 & 4 & & \\
\hline & 8 & 117 & 42 & & & & & \\
\hline & 8 & 118 & 01 & 8 . & .3 & 4 & & \\
\hline & 8 & 119 & 2501 & & 29 & 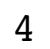 & & \\
\hline & 8 & 120 & 14 & & 7 & 4 & & \\
\hline & 8 & 121 & & & & 4 & & \\
\hline & 8 & 122 & 494 & & & 4 & & • \\
\hline & 8 & 123 & 006 & & 25 & 4 & & \\
\hline & 8 & 124 & & & & 4 & & \\
\hline & 8 & 21 & 36 & & 29 & 4 & & \\
\hline & 8 & 22 & 505 & 0 & 297.6 & 4 & & \\
\hline & 8 & 23 & 515 & & 29 & 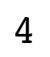 & & \\
\hline & 8 & 24 & 7 & 0 & 25 & 4 & & \\
\hline & 8 & 25 & & 0 & 25 & 4 & & \\
\hline & 8 & 26 & & & & 4 & & \\
\hline & 8 & 27 & 7 & 4 . & 25 & 4 & & \\
\hline & 8 & 28 & 32 . & 750 & 298.9 & 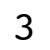 & 0 & \\
\hline & 8 & 29 & 88 & & 2 & 2 & & \\
\hline & 8 & 210 & 2 & & 25 & 2 & & \\
\hline & 8 & 211 & & & 299.7 & & & \\
\hline & 8 & 212 & 197.7107 & & 301.9 & 1 & & \\
\hline & 8 & 213 & 002575 & 0 & 303.0 & 2 & & \\
\hline & 8 & 214 & 006 & 500 & 302.9 & 3 & & \\
\hline & 8 & $21 !$ & & & .1 & 4 & & \\
\hline & 8 & 216 & 525 & 8 . & 302.4 & 4 & & \\
\hline & 8 & 217 & 75.2490 & 0.7250 & 300.9 & 4 & & 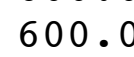 \\
\hline & 8 & 218 & & 50 & 0.0 & 4 & 0 & \\
\hline & 8 & 219 & 67.7500 & 2.6000 & 298.9 & 4 & & \\
\hline+ & 8 & 220 & 70.4990 & 1.9500 & 297.1 & 4 & 600 & \\
\hline & 8 & 221 & 5000 & 3.3500 & 295.3 & 4 & 600 & \\
\hline & 8 & 222 & 7500 & & 295.1 & 4 & & \\
\hline & & 223 & 72.2501 & 14.0500 & 294.5 & & 600.0 & 600 \\
\hline
\end{tabular}




\begin{tabular}{|c|c|c|c|c|c|c|c|c|}
\hline & 8 & 224 & 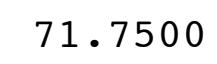 & & 4.6 & 4 & & \\
\hline & 8 & 31 & 7.7497 & 12.1750 & 295.0 & & 0,0 & \\
\hline & 8 & 32 & 992 & 000 & 294.9 & & & \\
\hline & 8 & 3 & 3.2498 & 9.6500 & 94.8 & & & \\
\hline & 8 & 4 & 1 & & 1 & & 0 & \\
\hline & 8 & 5 & 500 & 10. & 5 & & r & \\
\hline & 8 & 6 & 8.7500 & 2 . & & & & \\
\hline & 8 & 7 & & 0 & & & & \\
\hline & 8 & 8 & 76.5000 & 8.9500 & 94.0 & & ( & 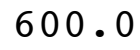 \\
\hline & 8 & 9 & & 500 & & & & \\
\hline & 8 & 10 & & & & & & \\
\hline & 8 & 11 & 73. & & 1 & & & \\
\hline & 8 & & 70 & 10.97 & & & & \\
\hline & 8 & 13 & 0 & 9.62 & & & & \\
\hline & 8 & 1 & 70 & 10 . & & & & \\
\hline & 8 & & 72.24 & 10.17 & 6 & & & \\
\hline & 8 & 16 & 01 & 1 . & & & & \\
\hline & 8 & 7 & & 1 . & & & & \\
\hline & 8 & 18 & 69.7500 & 13.30 & 29 & & & 0 \\
\hline & 8 & $J$ & 97 & 14 & & & & \\
\hline & 8 & 20 & & & & & & \\
\hline & 8 & 21 & & & & & & \\
\hline & 8 & 322 & 13 & 0 & 29 & & & \\
\hline & 8 & 323 & 71 & 8.5 & & & & \\
\hline & 8 & 324 & & & & 4 & & \\
\hline & 8 & 41 & 66. & 12 . & 28 & 4 & & \\
\hline & 8 & 42 & 5 & 11 & & & & \\
\hline & 8 & 43 & 0 & 12 & & 4 & & \\
\hline & 8 & 44 & 0 & 11 . & & 4 & & \\
\hline & 8 & 45 & & & & 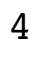 & & \\
\hline & 8 & 46 & & 0 & & 4 & & \\
\hline & 8 & 47 & & & & 4 & & \\
\hline & 8 & 48 & 00 & 9 . & 28 & 4 & & \\
\hline & 8 & 49 & 4 & 9 & & 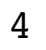 & & \\
\hline & 8 & 410 & & & & 4 & & \\
\hline & 8 & 411 & 86. & & & 4 & & \\
\hline & 8 & 412 & & 0 & & 4 & & \\
\hline & 8 & 413 & & 6 . & & 4 & & \\
\hline & 8 & 414 & & & 29 & 4 & & \\
\hline & 8 & 415 & & & & 4 & & \\
\hline & 8 & 416 & & & & 7 & & \\
\hline & 8 & 417 & 71.5003 & & $29^{\circ}$ & 4 & & \\
\hline & 8 & 418 & 00 & & 29 & 4 & & \\
\hline & 8 & 419 & 74.7495 & & & 4 & & \\
\hline & 8 & 420 & 75.4999 & 9.6250 & 294.1 & 4 & 0 & 00 \\
\hline & 8 & 42 & & & & & & \\
\hline & 8 & 422 & ים הים & 11.7 & 291.9 & & & \\
\hline & 8 & 423 & 74.0000 & 13.7000 & 291.4 & & .0 & 0 \\
\hline
\end{tabular}




\begin{tabular}{|c|c|c|c|c|c|c|c|c|}
\hline 1 & 8 & 424 & 71.9992 & 11.7250 & 291.9 & 4 & 600.0 & 600.0 \\
\hline 01 & 8 & 51 & 74.2496 & 10.6000 & 292.3 & 4 & 600.0 & 00.0 \\
\hline 01 & 8 & 52 & 80.2500 & 10.2000 & 292.8 & 4 & 600.0 & 00.0 \\
\hline 1 & 8 & 53 & 79.2495 & 9.3250 & 292.1 & 4 & 600.0 & 00.0 \\
\hline 01 & 8 & 54 & 73.9963 & 9.4500 & 291.6 & 4 & 600.0 & 00.0 \\
\hline 01 & 8 & 55 & 74.2500 & 9.5500 & 291.4 & 4 & 600.0 & 00.0 \\
\hline 01 & 8 & 56 & 74.5000 & 9.7000 & 290.8 & 4 & 600.0 & 00.0 \\
\hline 01 & 8 & 57 & 71.5000 & 8.7750 & 291.9 & 4 & 600.0 & 00.0 \\
\hline 01 & 8 & 58 & 72.7500 & 8.5000 & 293.2 & 4 & 600.0 & 00.0 \\
\hline 01 & 8 & 59 & 80.7501 & 6.6250 & 294.8 & 4 & 600.0 & .0 \\
\hline 01 & 8 & 510 & 94.0012 & 4.8500 & 296.3 & 3 & 600.0 & \\
\hline 01 & 8 & 511 & 92.7528 & 3.5250 & 297.7 & 2 & 600.0 & \\
\hline 01 & 8 & 512 & 102.0392 & 3.0250 & 299.3 & 2 & 600.0 & 00.0 \\
\hline 01 & 8 & 513 & 98.2587 & 4.2500 & 300.1 & 3 & 600.0 & 00.0 \\
\hline 01 & 8 & 514 & 96.4979 & 4.9500 & 300.6 & 3 & .0 & \\
\hline 01 & 8 & 515 & 94.2503 & 5.5750 & 300.9 & 3 & 600.0 & .0 \\
\hline 01 & 8 & 516 & 71.0032 & 6.7750 & 300.8 & 4 & 60 & 600.0 \\
\hline 01 & 8 & 517 & 71.0000 & 7.5250 & 300.0 & 4 & .0 & \\
\hline 01 & 8 & 518 & 69.2500 & 9.2250 & 298.3 & 4 & 600.0 & .0 \\
\hline 01 & 8 & 519 & 73.2500 & 10.3250 & 296.5 & 4 & .0 & .0 \\
\hline 01 & 8 & 520 & 74 . & 10.9250 & 295.8 & 4 & 60 & \\
\hline 01 & 8 & 521 & 72.2503 & 10.1500 & 296.0 & 4 & .0 & \\
\hline 01 & 8 & 522 & 75.0000 & 9.4250 & 296.4 & 4 & 600.0 & .0 \\
\hline 01 & 8 & 523 & 76.2500 & 9.7750 & 296.4 & 4 & 6 & \\
\hline 01 & 8 & 524 & 77.2501 & 10.0750 & 296.4 & 4 & .0 & \\
\hline 01 & 8 & 61 & 78.5000 & 9.9250 & 296.4 & 4 & 600.0 & 600.0 \\
\hline 01 & 8 & 62 & 80.7500 & 8.0500 & 296.9 & 4 & 600.0 & .0 \\
\hline 01 & 8 & 63 & 82.9999 & 7.7250 & 297.2 & 4 & .0 & \\
\hline 01 & 8 & 64 & 80.0000 & 8.0000 & 297.2 & 4 & .0 & 0.0 \\
\hline 01 & 8 & 65 & 79.7500 & 7.7250 & 297.1 & 4 & 60 & .0 \\
\hline 01 & 8 & 66 & 79.2500 & 8.4250 & 296.6 & 4 & .0 & \\
\hline 01 & 8 & 67 & 81.5000 & 7.0250 & 297.3 & 4 & .0 & 600.0 \\
\hline 01 & 8 & 68 & 37.5904 & 3.6000 & 299.1 & 3 & 600.0 & 600.0 \\
\hline 01 & 8 & 69 & 178.2351 & 3.8500 & 300.2 & 2 & 600.0 & 0.0 \\
\hline 01 & 8 & 610 & 176.7500 & 5.5000 & 300.4 & 3 & 600.0 & 600.0 \\
\hline 01 & 8 & 611 & 173.0000 & 6.6000 & 301.1 & 4 & 600.0 & 600.0 \\
\hline 01 & 8 & 612 & 175.2505 & 7.7250 & 302.1 & 4 & 600.0 & 600.0 \\
\hline 01 & 8 & 613 & 163.2457 & 7.6000 & 302.5 & 4 & 600.0 & 600.0 \\
\hline 01 & 8 & 614 & 152.9861 & 6.3250 & 303.7 & 4 & 600.0 & 600.0 \\
\hline 01 & 8 & 615 & 144.5014 & 5.2750 & 304.6 & 3 & 600.0 & 600.0 \\
\hline 01 & 8 & 616 & 113.2505 & 5.3000 & 305.2 & 3 & 600.0 & 600.0 \\
\hline 01 & 8 & 617 & 96.0193 & 5.7000 & 304.8 & 3 & 600.0 & 600.0 \\
\hline 01 & 8 & 618 & 92.7517 & 6.4750 & 303.5 & 4 & 600.0 & 600.0 \\
\hline 01 & 8 & 619 & 77.7503 & 8.4750 & 301.3 & 4 & 600.0 & 600.0 \\
\hline 01 & 8 & 620 & 71.7499 & 10.7250 & 299.6 & 4 & 600.0 & 600.0 \\
\hline 01 & 8 & 621 & 76.0000 & 10.2250 & 299.3 & 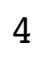 & 600.0 & 600.0 \\
\hline 01 & 8 & 622 & 76.5000 & 9.4500 & 299.8 & 4 & 600.0 & 600.0 \\
\hline J & 8 & 623 & 76.5000 & 9.275 & 300.1 & 4 & 600.0 & 600.0 \\
\hline
\end{tabular}




\begin{tabular}{|c|c|c|c|c|c|c|c|c|}
\hline 1 & 8 & 24 & 5.5000 & 0.2250 & 300.4 & 4 & 600.0 & \\
\hline & 8 & 71 & 6.2500 & 9.5250 & 300.9 & & 600 & \\
\hline & 8 & 72 & 2503 & 0750 & 301.3 & & 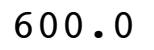 & \\
\hline & 8 & 73 & 3.2501 & 7.7000 & 301.2 & & 00.0 & \\
\hline & 8 & 74 & 8.2501 & 7.5000 & & & & \\
\hline & 8 & 75 & 78.5000 & 7.3250 & 01.6 & & 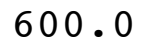 & \\
\hline & 8 & 76 & 89.2478 & 5000 & 301.5 & & 00 & \\
\hline & 8 & 77 & 32.0000 & 2.3750 & & & 0 & \\
\hline & 8 & 78 & 97.9516 & 0.2500 & 304.6 & & 0 & \\
\hline & 8 & 79 & 37.3592 & 5250 & & & 0 & \\
\hline & 8 & 710 & 72.2495 & 50 & & & & \\
\hline & 8 & 711 & 165.7499 & & & & & \\
\hline & 8 & 712 & 171.4994 & 5.8750 & 303.5 & & 0 & \\
\hline & 8 & 713 & 167.2500 & 0 & 4.2 & 4 & 0 & \\
\hline & 8 & 714 & & & & 4 & & \\
\hline & 8 & 715 & 173. & 50 & & 3 & & \\
\hline & 8 & 716 & 177.7497 & 5.6250 & 7.0 & & 10.0 & \\
\hline & 8 & 717 & 177.2561 & & & & & \\
\hline & 8 & 718 & 173.4533 & 50 & .8 & 3 & & \\
\hline & 8 & 719 & 117.2650 & 250 & 306.2 & & U & \\
\hline & 8 & 720 & 503 & & & 4 & & \\
\hline & 8 & 721 & 69. & & & 4 & & \\
\hline & 8 & 722 & 73.5000 & 750 & 4.1 & 4 & 0 & \\
\hline & 8 & 723 & 74.2500 & 0 & 1 & 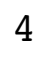 & & \\
\hline & 8 & 724 & 500 & & & 4 & & \\
\hline & 8 & 81 & 71.5003 & 3000 & & 4 & & \\
\hline & 8 & 82 & 76.7500 & 7.4750 & 4.2 & 4 & & \\
\hline & 8 & 83 & 77.5000 & 50 & & 4 & & \\
\hline & 8 & 84 & 78.5000 & & & 4 & & \\
\hline & 8 & 85 & 73.2492 & & .5 & 4 & & \\
\hline & 8 & 86 & 05.7724 & & & 5 & & \\
\hline & 8 & 87 & 0 & & .1 & 4 & & \\
\hline & 8 & 88 & 319.8977 & 0 & 6.8 & 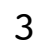 & & \\
\hline & 8 & 89 & 562 & 0 & & 2 & & \\
\hline & 8 & 810 & 234 . & 2 . & .3 & 2 & & \\
\hline & 8 & 811 & 216.0000 & & 306.5 & & & \\
\hline & 8 & 812 & 180 . & 3 . & 7.2 & 2 & & \\
\hline & 8 & 813 & 173. & 5 . & 7.6 & 3 & & \\
\hline & 8 & 814 & 147.5449 & 5.5250 & 309.0 & 3 & & \\
\hline & 8 & 815 & 584 & 0 & 3 & 3 & & \\
\hline & 8 & 816 & 89.0000 & 6 . & 3.1 & 4 & & \\
\hline+ & 8 & 817 & 82.9993 & 6.7250 & 307.2 & 4 & & n \\
\hline & 8 & 818 & 73.5003 & 7 . & 305.7 & 4 & & \\
\hline 01 & 8 & 819 & 72.4997 & 8.5000 & & 4 & & 600 \\
\hline I & 8 & 820 & 75.7501 & 9.1750 & 302.8 & 4 & 600.0 & 00 . \\
\hline & 8 & 821 & 73 & & & 4 & & \\
\hline & 8 & 822 & 74.2500 & 8.6500 & & 4 & & 600 \\
\hline & & 823 & 75.2508 & 8.5250 & 304.2 & & 600.0 & 600 \\
\hline
\end{tabular}




\begin{tabular}{|c|c|c|c|c|c|c|c|}
\hline & $8 \quad 824$ & 4977 & 6.4250 & 303.0 & 4 & 600.0 & \\
\hline & $\begin{array}{lll}8 & 9 & 1\end{array}$ & 141.3307 & 1.1500 & 299.7 & & 600.0 & \\
\hline & 892 & 138.0903 & 1.2500 & 298.2 & & 00 & \\
\hline & $\begin{array}{lll}8 & 9 & 3\end{array}$ & 85.4891 & 1.0750 & 296.5 & & 00.0 & \\
\hline & $\begin{array}{lll}8 & 9 & 4\end{array}$ & 280 . & .1500 & 96.8 & & 0 & \\
\hline & 895 & 23. & .5250 & 296.5 & & 00 & \\
\hline & 896 & 481 & 1.6250 & 297.1 & & 00 & \\
\hline & $\begin{array}{lll}8 & 9 & 7\end{array}$ & 33.6010 & .7250 & 298.9 & & 0 & \\
\hline & 898 & 320.8707 & .9000 & 298 & & & \\
\hline & $\begin{array}{lll}8 & 9 & 9\end{array}$ & 261.4982 & 1.3500 & 300.5 & & $0 c$ & U \\
\hline & 8910 & 258.5683 & 1.9500 & 300.6 & & 00.0 & \\
\hline & 8911 & 229.7500 & 77 & 300.8 & & & \\
\hline & 8912 & 236.9498 & 3.2500 & 301.7 & & 0 & \\
\hline & 8913 & 50.5464 & 0.02 & 303.0 & & 0.0 & 00 \\
\hline & 8914 & 67.2383 & & 303 & & & \\
\hline & 8915 & 81.7499 & & 302.8 & & & \\
\hline & 8916 & 88.0001 & 7 . & 301.9 & & .0 & . \\
\hline & 8917 & 000 & 0 & 301.3 & & 0 & \\
\hline & 8918 & 78 . & & 300.3 & & & \\
\hline & 8919 & 73.4998 & 0 & 298.5 & 4 & 00.0 & 0 \\
\hline & 8920 & 76.2467 & .6250 & 296 & 4 & 0 & \\
\hline & 8921 & 82 . & & 296 & 4 & & \\
\hline & 8922 & 79. & 0 & 296.2 & 4 & & \\
\hline & 8923 & 74.2500 & 8.7750 & 295.6 & 4 & 0 & 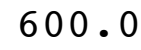 \\
\hline & 8924 & 44.3508 & & 296.3 & 4 & & \\
\hline & 8101 & 78.0081 & 4 . & 295.3 & 4 & & \\
\hline & 8102 & 79.4928 & 4.1000 & 295.2 & 4 & 0 & U. \\
\hline & 8103 & 73.6873 & 1.82 & 294.6 & 5 & & \\
\hline & 8104 & 82.2590 & 0 & 291.8 & 4 & & $\bullet$ \\
\hline & 8105 & 80.7501 & 500 & 291.4 & 4 & 0 & 0 . \\
\hline & 8106 & 80.0000 & 3.3750 & 291.0 & 4 & & 0 . \\
\hline & 8107 & 79.7502 & 50 & 290.9 & 4 & & \\
\hline & 8108 & 81.7499 & 50 & 291.2 & 4 & 0 & . \\
\hline & 8109 & 82.7501 & 750 & 293.2 & 4 & 0 & 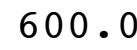 \\
\hline & 81010 & 93.9369 & 2 . & 295.6 & 3 & & \\
\hline & 81011 & 136.0129 & 1.8500 & 298.3 & 2 & & . \\
\hline & 81012 & 161.9483 & 3.3500 & 299.1 & 2 & $\cdot 0$ & 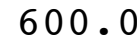 \\
\hline & 81013 & 62.3679 & 3 . & 300.3 & 2 & & 0 . \\
\hline & 81014 & 81.7500 & 5.1000 & 300.7 & 3 & .0 & 0 . \\
\hline & 81015 & 85.4997 & 7000 & 300.7 & 4 & & 0 . \\
\hline & 81016 & 84.2497 & 5.7500 & 301.0 & 4 & & 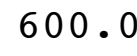 \\
\hline & 81017 & 79.7494 & 6.4500 & 300.1 & 4 & & 0 . \\
\hline & 81018 & 74.5000 & 5.9750 & 299.0 & 4 & 0 & 0 . \\
\hline & 81019 & 78.5000 & 8.3000 & 29 & 4 & & \\
\hline- & 81020 & 85.7491 & 7.6250 & & 4 & & 0 . \\
\hline & 81021 & 87.5010 & 3.5750 & 296.9 & 4 & 600.0 & 00 . \\
\hline & 81022 & 145 & 00 & & 5 & & \\
\hline & 81023 & 102.9878 & 3.1750 & 297.9 & & 600.0 & 60 \\
\hline
\end{tabular}




\begin{tabular}{|c|c|c|c|c|c|c|c|}
\hline & 81024 & 0.2489 & 7.7500 & 8.4 & 4 & 0 & \\
\hline & 8111 & 77.0001 & 8.3750 & 298.1 & 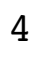 & 00.0 & \\
\hline & 3112 & 97.8849 & 000 & 297.6 & & 0 & \\
\hline & 113 & 32.8084 & .6000 & 295.8 & & & \\
\hline & 114 & 81.2489 & 3500 & & & 5 & \\
\hline & 115 & 2500 & 000 & 297.0 & & 0 & \\
\hline & 116 & 81.0000 & & 297.6 & & & \\
\hline & 117 & 6.7477 & & & & & \\
\hline & 118 & 258.5149 & 1.7250 & 298.4 & & 00.0 & 0 \\
\hline & 119 & 349.3429 & 4000 & $29^{\circ}$ & & & \\
\hline & 1110 & 201.2519 & & 98 & & & \\
\hline & 1111 & 168.7504 & 4.8250 & 298.4 & & & \\
\hline & 1112 & 164.7501 & 9000 & 30 & & & \\
\hline & 1113 & 168.4936 & 0 & 301 & & & \\
\hline & 1114 & 173. & & 30 & 4 & & \\
\hline & 1115 & 160.3173 & 8000 & 303.8 & 1 & & \\
\hline & 1116 & 80.2462 & 0 & 30 & & & \\
\hline & 1117 & 80 & & 30 & & & \\
\hline & 1118 & 001 & 0 & .3 & 4 & & 000 \\
\hline & 31119 & 76 . & 0 & 30 & 4 & & \\
\hline & 1120 & 77 . & & & 4 & & \\
\hline & 21 & 75 & & 29 & 4 & & \\
\hline & 81122 & 002 & 0 & 300.0 & 4 & 0 & \\
\hline & 81123 & 002 & & 299.8 & 4 & & \\
\hline & 811 & 00 & & 29 & 4 & & \\
\hline & 8121 & 75.7500 & & 298.6 & 4 & & \\
\hline & 8122 & 75 . & & 29 & 4 & & \\
\hline & 8123 & 73. & & 29 & 4 & & \\
\hline & 8124 & 69.7470 & & 298.0 & 4 & 0 & . \\
\hline & 8125 & 66.5225 & & 29 & 5 & & \\
\hline & 8126 & 58 . & & 29 & 5 & & \\
\hline & 8127 & 585 & & & 4 & & \\
\hline & 8128 & 184.2374 & 0 & 297.8 & 2 & & \\
\hline & 8129 & 288.8416 & 0 & 298.5 & 2 & & \\
\hline & 81210 & 746 & & 298.3 & 1 & & \\
\hline & 81211 & 35.1139 & 0 & 298.2 & 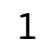 & & \\
\hline & 81212 & 40 . & & 29 & 2 & & \\
\hline & 81213 & 48 . & & 299.3 & 3 & & \\
\hline & 81214 & 69.2539 & & 298.8 & 4 & & \\
\hline & 81215 & 81 . & 7 . & 298 & 4 & & \\
\hline & 81216 & 80.5002 & 8.9000 & 297.8 & 4 & & \\
\hline & 81217 & 79.2501 & 9.1250 & 297.0 & 4 & 0 & 0 \\
\hline & 81218 & 81.4997 & 0 & 295.2 & 4 & & \\
\hline & 81219 & 79.7501 & 1.0750 & 293.3 & 4 & 0 & 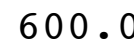 \\
\hline & 81220 & 78.0002 & 12.4500 & 290.8 & 4 & 600.0 & 00 \\
\hline & 81221 & 79 . & & 289 & 4 & & \\
\hline & 81222 & 81.7501 & 10.5750 & 290.0 & & & 600 \\
\hline & 1223 & 82.5000 & 6.4250 & 292.0 & & .0 & 60 \\
\hline
\end{tabular}




\begin{tabular}{|c|c|c|c|c|c|c|c|}
\hline 1 & 1224 & 6.5000 & 7.2500 & 292.4 & 4 & 00 & \\
\hline & 8131 & 35002 & 10.2500 & 293.1 & & & \\
\hline & 132 & 500 & 500 & & & & \\
\hline & 133 & 3.7504 & 0.4750 & 92.9 & & 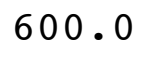 & \\
\hline & 134 & & 8.0500 & 2.7 & & & \\
\hline & 135 & 743 & 6.3250 & 91.3 & & 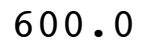 & \\
\hline & 136 & 005 & 7750 & 91.7 & & 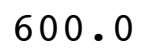 & \\
\hline & 137 & 503 & 8.7250 & 92.1 & & 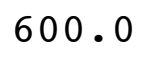 & \\
\hline & 138 & 80.5002 & 0.0250 & 91.1 & & 0 & \\
\hline & 139 & 2472 & 1250 & & & & \\
\hline & 1310 & 7.3919 & & & & & \\
\hline & 1311 & 5.8020 & & & & & \\
\hline & 1312 & 103.1532 & 000 & 297.7 & & 0 & \\
\hline & 1313 & 457 & & & & & \\
\hline & 1314 & & & & & & \\
\hline & 1315 & 84 & 50 & 5 & & & \\
\hline & 1316 & 83.4968 & 0 & 299.7 & & U & \\
\hline & 1217 & 76.4997 & & 298.6 & 4 & & \\
\hline & 8 & 76 & 50 & 0 & 4 & & \\
\hline & 1319 & 5000 & 0 & 25 & & & \\
\hline & 320 & 66. & & & & & \\
\hline & 1321 & 69 . & & 29 & 4 & & \\
\hline & 1322 & 2500 & 500 & 297.6 & 4 & & \\
\hline & 1323 & 78. & & & & & \\
\hline & 81324 & & & & 4 & & \\
\hline & 141 & 500 & & 297 & 4 & & \\
\hline & 8142 & 78 . & 0 & 298.1 & 4 & & \\
\hline & 143 & 0 & & 297 & 4 & & \\
\hline & 8144 & 998 & & 297.2 & 4 & & \\
\hline & 8145 & 82.74 & & 296 & 4 & & \\
\hline & 8146 & & & 29 & 4 & & \\
\hline & 8147 & 130 . & & & & & \\
\hline & 8148 & 7.8501 & 50 & 297.1 & & & \\
\hline & 8149 & 2 & & & & & \\
\hline & 81410 & 250 & & & & & \\
\hline & 1411 & 187 . & & 299.4 & & & \\
\hline & 1412 & 194 . & 00 & & 3 & & \\
\hline & 81413 & 183. & & .5 & 3 & & \\
\hline & 1414 & 180.0168 & 500 & 302.7 & 4 & & \\
\hline & 1415 & 142 . & & & 3 & & \\
\hline & 81416 & & & & 3 & & \\
\hline & 81417 & 88.0132 & 5.7500 & 304.2 & 3 & & \\
\hline & 81418 & 92.6305 & 250 & 303.1 & 3 & & \\
\hline & 81419 & & & & 4 & & \\
\hline & 81420 & 68.2500 & 10.4000 & 298.8 & 4 & 0 & م0 \\
\hline & & & & & 4 & & \\
\hline & 81422 & 76.9999 & 8.9250 & & & & \\
\hline & 1425 & 71.5000 & 8.5250 & 298.0 & & 600.0 & 500 \\
\hline
\end{tabular}




\begin{tabular}{|c|c|c|c|c|c|c|c|}
\hline 1 & 1424 & 5.2512 & 8.0250 & 298.1 & 4 & 600.0 & \\
\hline 1 & 8151 & 6.9989 & 8.1250 & 298.3 & 4 & 600.0 & \\
\hline & 8152 & 000 & 000 & 298.8 & 4 & 0 & \\
\hline & 153 & 74.5000 & .1250 & 299.4 & & 00.0 & \\
\hline & 154 & 6.2771 & .6250 & 98.8 & & 0.0 & \\
\hline & 155 & 66.0028 & 3.1500 & 299.1 & & 00 & \\
\hline & 156 & 07.8172 & 2.7750 & 297.6 & & 00 & \\
\hline & 157 & 4.2013 & .1250 & 97.0 & & 00 & \\
\hline & 8158 & 89.8769 & 0.6500 & 297.1 & & 00 & \\
\hline & 159 & 261.4462 & 1.2000 & 300.0 & & 0 & \\
\hline & 1510 & 234 . & .0000 & 299.8 & & & \\
\hline & 1511 & 225 . & 500 & 299.8 & & & \\
\hline & 1512 & 194.8205 & 4.4250 & 301.0 & & 00 & \\
\hline & 1513 & 180.2539 & 000 & 302.3 & 3 & 0 & \\
\hline & 1514 & 155.1767 & & & 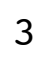 & & \\
\hline & 1515 & 103.9991 & 4.6250 & 304.5 & 3 & 0 & \\
\hline & 81516 & 87.5032 & 4.4500 & 304.7 & 3 & 0 & \\
\hline & 81517 & 88.7903 & 6.1500 & 303.6 & 4 & & \\
\hline & 1518 & 71.2492 & 7.9000 & 30 & 4 & & \\
\hline & 81519 & 79.5002 & 5750 & 301.0 & 4 & 0 & \\
\hline & 1520 & 72 . & 0 & 0 & 4 & & \\
\hline & 81521 & 71 . & 8 . & 298.1 & 4 & & \\
\hline & 1522 & 65.9986 & 8750 & 299.0 & 4 & 0 & \\
\hline & 81523 & 500 & 000 & 3 & 4 & & \\
\hline & 81524 & 67. & 50 & 29 & 4 & & \\
\hline & 8161 & 71.2509 & 500 & 299.8 & 4 & & \\
\hline & 8162 & 77.2500 & 6.3250 & 300.2 & 4 & & \\
\hline & 8163 & 004 & 500 & 300.5 & 4 & & \\
\hline & 8164 & 64.7498 & 7250 & 300.4 & 5 & & \\
\hline & 8165 & 80.7511 & 4.8000 & 300.4 & 5 & & \\
\hline & 8166 & 13.4304 & 750 & 299.2 & 5 & & \\
\hline & 8167 & 160 . & 750 & 299.0 & 4 & & \\
\hline & 8168 & 80.7738 & 8250 & 299.0 & 3 & & \\
\hline 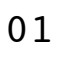 & 8169 & 281 . & 0 & 301.4 & 2 & & \\
\hline & 81610 & 165 & 4.7000 & & 3 & & \\
\hline & 81611 & 165.0000 & 4.7500 & 300.3 & 4 & & \\
\hline & 81612 & 166. & 500 & 301.4 & 4 & & \\
\hline & 81613 & 166. & 750 & 302.6 & 3 & & ( \\
\hline & 81614 & 167.0004 & 4.4000 & 303.9 & 3 & & 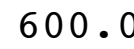 \\
\hline & 81615 & 165. & & 305.3 & 2 & & \\
\hline & 81616 & 181.0729 & 4 . & 306.3 & 3 & & \\
\hline$\perp$ & 81617 & 195.0034 & 4.8000 & 306.3 & 3 & 0 & 0 \\
\hline & 81618 & 179.5203 & 4.4000 & 305.8 & 3 & & \\
\hline 01 & 81619 & 134.7791 & 4.1500 & 304.5 & 4 & 600.0 & 600 \\
\hline 0 & 81620 & 73.2506 & 5.7000 & 302.4 & 4 & 600.0 & 600 \\
\hline & 81621 & 75 & & 1.8 & 4 & & \\
\hline & 81622 & 77.2500 & 8.4750 & 301.7 & 4 & & 600 \\
\hline & $10<3$ & 76.0000 & 8.6250 & 301.4 & & 600.0 & 600 \\
\hline
\end{tabular}




\begin{tabular}{|c|c|c|c|c|c|c|c|}
\hline & 24 & 498 & 500 & 300.9 & 4 & (6. & \\
\hline & 8171 & 5.0000 & 3.5000 & 301.6 & 4 & 600.0 & \\
\hline & 172 & 0001 & 9750 & 302.3 & 4 & 00 & \\
\hline & 173 & 73.2500 & 6.7500 & 302.9 & & 00 & \\
\hline & 174 & 2500 & .4250 & 02.4 & & 0 & \\
\hline & 175 & 0 & & 02.6 & & 0 & \\
\hline & 176 & 913 & 2.2250 & 300.9 & & 00 & \\
\hline & 177 & 207 & 1.6250 & 00.6 & & & \\
\hline & 178 & 516 & 500 & 04.1 & & & \\
\hline & 179 & 272.9878 & 1.6000 & 304.6 & & 0 & o \\
\hline & 1710 & 212.4000 & 3.6000 & 302.9 & & & \\
\hline & 1711 & 165.2500 & 3.0500 & 01.5 & & & \\
\hline & 1712 & 52.6245 & 1.9250 & 305.3 & & 0 & \\
\hline & 1713 & 173.9995 & 4.95 & 305.6 & & & \\
\hline & 1714 & 161 . & & .8 & & & \\
\hline & 1715 & 92 . & & 307.7 & 3 & & \\
\hline & 1716 & 85.7459 & 0 & 307.1 & 3 & .0 & \\
\hline & 1717 & 88 . & 0 & 305.7 & 1 & & \\
\hline & 1718 & 71 . & & 304.1 & 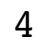 & & \\
\hline & 1719 & 501 & 8000 & 302.8 & 4 & 0 & \\
\hline & 81720 & 69.7501 & 7.7750 & 301.8 & 4 & 0 & \\
\hline & 1721 & 63. & & 301.8 & 4 & & \\
\hline & 1722 & 68 & 0 & 301.6 & 4 & & \\
\hline & 81723 & 70.2500 & 0 & 301.8 & 4 & 0 & \\
\hline & 81724 & 00 & & 301.9 & 4 & & \\
\hline & 8181 & 71 . & 8 . & 1.6 & 4 & & \\
\hline & 8182 & 73.2503 & 7.77 & 1.6 & 4 & & J. \\
\hline & 8183 & 76.500 & 6.27 & 301.2 & 4 & & \\
\hline & 8184 & 76 . & 0 & .6 & 4 & & \\
\hline & 8185 & 503 & & 300.6 & 4 & & 0. \\
\hline & 8186 & 72.2504 & 6.8000 & 301.4 & 4 & & 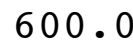 \\
\hline & 8187 & 76.7499 & & 302.0 & 4 & & \\
\hline & 8188 & 716 & & .1 & 3 & & . \\
\hline & 8189 & 278.2948 & 1.3500 & 304.1 & 2 & & \\
\hline & 81810 & 238.4742 & & & 2 & & \\
\hline & 81811 & 232.3321 & & 3.0 & 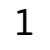 & & \\
\hline & 81812 & 183.6489 & 3.5000 & 304.6 & 2 & & \\
\hline & 81813 & 80 & 5.17 & 305.1 & 3 & & \\
\hline & 81814 & 83.0005 & 5.7000 & 305.7 & 3 & & 0 . \\
\hline & 81815 & 84.5005 & 7.3500 & 306.0 & 4 & & \\
\hline & 81816 & 465 & 8.5250 & 305.9 & 4 & & \\
\hline & 81817 & 70.7494 & 9.2750 & 305.3 & 4 & & 0 . \\
\hline & 81818 & 67.7500 & 0.0000 & 304.5 & 4 & & 0 . \\
\hline & 81819 & 000 & 10.7500 & & $I$ & & \\
\hline & 81820 & 69.2500 & 9.7000 & & 4 & & \\
\hline & 81821 & 71.5003 & 8.4000 & 303.1 & 4 & & 00 . \\
\hline & 822 & & & & 4 & & \\
\hline & 31823 & 64.4997 & 7.3250 & 303.1 & & 600.0 & 60 \\
\hline
\end{tabular}




\begin{tabular}{|c|c|c|c|c|c|c|c|}
\hline & 1824 & לע & 5.6500 & 302.8 & 4 & 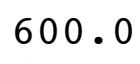 & \\
\hline & 8191 & 103.6402 & 1.9250 & 302.0 & & & \\
\hline & 192 & 58 & 250 & 300.3 & & 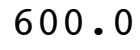 & \\
\hline & 193 & 31.0000 & 4250 & 300.7 & & 00 & \\
\hline & 194 & 22.3905 & & & & & \\
\hline & 195 & 4043 & 750 & 00.3 & & 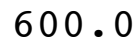 & \\
\hline & 196 & 143.7468 & 500 & 98.0 & & & \\
\hline & 197 & 5420 & .7250 & 8.2 & & & \\
\hline & 198 & 170.7808 & 1.2000 & 299.8 & & & \\
\hline & 199 & 232.8360 & 2.2500 & 301.0 & & & \\
\hline & 1910 & 237.4931 & 2.9750 & 300.8 & & & \\
\hline & 911 & 232.7498 & 2.9750 & & & & \\
\hline & 1912 & 219.7800 & 3.6750 & 302.0 & & 0 & \\
\hline & 1913 & 214.7928 & 3.5250 & & & 0 & \\
\hline & 914 & 103. & & & & & \\
\hline & 1915 & 85 & 500 & & & & \\
\hline & 1916 & 80.7460 & 6.2500 & & & & \\
\hline & 17 & 82 . & 250 & & & & \\
\hline & 1918 & 000 & 750 & .8 & & & \\
\hline & 1919 & 2500 & 2000 & & & & \\
\hline & 920 & 959 & 0 & & & & \\
\hline & 1921 & & 0 & & & & \\
\hline & 1922 & 2500 & 9.1250 & 29 & 4 & & \\
\hline & 1923 & 60.2498 & .2000 & & & & \\
\hline & 81924 & & & & & & \\
\hline & 8201 & 2555 & 4.4250 & & 4 & & \\
\hline & 8202 & 006 & 250 & .5 & & & \\
\hline & 8203 & 001 & 750 & .2 & 4 & & \\
\hline & $820 \quad 4$ & 574 & 0 & & 4 & & \\
\hline & $820 \quad 5$ & 508 & 50 & & $t^{2}$ & & \\
\hline & 8206 & 989 & 0 & & a & & \\
\hline & 8207 & 7 & 0 & & 4 & & \\
\hline & 8208 & 61.8747 & 4250 & 292.8 & & & \\
\hline & 8209 & $y$ & 00 & 2.7 & 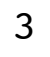 & & \\
\hline & 82010 & 6 & & & 2 & & \\
\hline & 82011 & 871 & 4.4250 & .9 & 3 & & \\
\hline & 82012 & 505 & 250 & .0 & 3 & & \\
\hline & 82013 & 010 & 500 & & 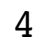 & & \\
\hline & 82014 & 491 & 750 & 5.7 & 4 & & \\
\hline & 82015 & 300 & 000 & & 4 & & \\
\hline & 82016 & 7 & 000 & & 4 & & \\
\hline & 82017 & 69.0000 & L. 8000 & 294.7 & 4 & & 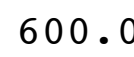 \\
\hline & 820 & 001 & 000 & 292.4 & 4 & & \\
\hline & 82019 & 69.2508 & 15.3750 & & 4 & & \\
\hline & 82020 & 69.0000 & 15.5000 & & $\Psi$ & 60 & \\
\hline & & 7500 & 3.8250 & 291.4 & 1 & & \\
\hline & & 00 & 000 & & & & \\
\hline & 2023 & 71.5000 & 10.9500 & 292.0 & $I$ & 600.0 & 600 \\
\hline
\end{tabular}




\begin{tabular}{|c|c|c|c|c|c|c|c|}
\hline & 2024 & 3.9983 & 8.0250 & 92.0 & 4 & 000 & \\
\hline & 8211 & 7.4946 & 6.3000 & 291.2 & & & \\
\hline & 3212 & & & 0.5 & & & \\
\hline & 8213 & $0=0$ & 2.5500 & 90.4 & & 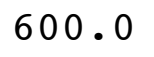 & \\
\hline & 3214 & & & 91.1 & & & \\
\hline & 8215 & 9 & 750 & 90.0 & & 0 & \\
\hline & 8216 & 0 & & 89.1 & & 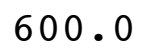 & \\
\hline & 217 & & 50 & 89.0 & & & \\
\hline & 8218 & 6 . & 8.2500 & 88.7 & & 0 & \\
\hline & 219 & & 9750 & & & 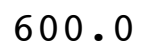 & \\
\hline & 2110 & & & & & & \\
\hline & 2111 & & & 2 & & & \\
\hline & 2112 & 0 & 750 & 95.3 & & 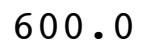 & \\
\hline & 2113 & 0 & & & & & \\
\hline & 2114 & & & & & & \\
\hline & 21 & & & 96.8 & & & \\
\hline & 2116 & 0 & 9.77 & 295.9 & & & \\
\hline & 2117 & & 0 & 93.1 & & & \\
\hline & 8 & & 50 & 91.8 & & & \\
\hline & 2119 & & 0 & & & & \\
\hline & 2120 & & & & & & \\
\hline & 82121 & & & 29 & 4 & & \\
\hline & 2122 & & 3750 & 7 & 4 & & \\
\hline & 82123 & & & & & & \\
\hline & 82124 & & & & 4 & & \\
\hline & 8221 & & 3750 & & 4 & & \\
\hline & 8222 & & 2.35 & & 4 & & \\
\hline & 8223 & & 0 & 1 & 4 & & \\
\hline & 8224 & & & & 4 & & \\
\hline & 8225 & & 0 & & a & & \\
\hline & 8226 & & 0 & & 4 & & \\
\hline & 8227 & & 0 & & 4 & & \\
\hline & 8228 & $\pi+\sigma_{0}$ & 0 & 8.3 & 4 & & \\
\hline & 8229 & & 0 & 8 & 4 & & \\
\hline & 82210 & & & & 4 & & \\
\hline & 82211 & & & 290.9 & 4 & & \\
\hline & 82212 & & 0 . & 29 & 4 & & \\
\hline & 82213 & & & & 4 & & \\
\hline & 82214 & 50 & .7250 & 292.7 & 4 & & \\
\hline & 82215 & & & 292 & 4 & & \\
\hline & 82216 & & & & 4 & & \\
\hline & 82217 & & 10.0000 & 293.9 & 4 & & \\
\hline & 82218 & & 0 & 292 & 4 & & \\
\hline & 82219 & & & & $x^{2}$ & & \\
\hline & 82220 & 66. & 13.1250 & 289.6 & 4 & 0 & (2) \\
\hline & 82221 & & & & 4 & & \\
\hline & 82222 & & & & & & \\
\hline & $2<20$ & 08.3000 & 12.8500 & 287.8 & & 600.0 & -0 \\
\hline
\end{tabular}




\begin{tabular}{|c|c|c|c|c|c|c|c|}
\hline & 2224 & 0.2500 & 11.9500 & 287.8 & 4 & . & \\
\hline & 8231 & 0.2501 & 11.2500 & 287.6 & & & \\
\hline & 3232 & 7497 & 12.1500 & 287.4 & & 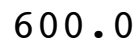 & \\
\hline & 8233 & 5.4998 & 1.6750 & 287.4 & & 00 & \\
\hline & 8234 & & .6750 & & & & \\
\hline & 8235 & 82 & 1000 & 87.8 & & 0 & \\
\hline & 8236 & 2500 & 5500 & 288.2 & & & \\
\hline & 237 & 999 & 8.9500 & 88.6 & & & \\
\hline & 8238 & 511 & 7.4750 & 89.7 & & & \\
\hline & 8239 & 999 & 6.2500 & 291.3 & & & \\
\hline & 2310 & 91.5000 & 5.5250 & 92.7 & & & \\
\hline & 2311 & 22.7516 & & & & & \\
\hline & 2312 & 9.9963 & 4.6750 & .9 & & 0 & \\
\hline & 2313 & 93.2403 & 6.3500 & 296.7 & & 0 & \\
\hline & 2314 & & & & & & \\
\hline & 82315 & 76 & 250 & 29 & 4 & & \\
\hline & 2316 & 103 & 7.9500 & 297 & & & \\
\hline & 82317 & 02 & 0 & 8 & & & \\
\hline & 82318 & 79 . & & 29 & 4 & & \\
\hline & 2319 & 77 & 0 & 293.0 & & & \\
\hline & 82320 & 3 & 0 & 292.4 & 4 & & \\
\hline & 2321 & & & 292.3 & 4 & & \\
\hline & 82322 & 68.5000 & 0 & 29 & 4 & & • \\
\hline & 82323 & 67.7492 & 0 & 292.2 & 4 & & \\
\hline & 82324 & 69. & & 292.4 & 4 & & \\
\hline & 8241 & 71.2498 & & 29 & 4 & & \\
\hline & $824 \quad 2$ & .5000 & 0 & 292.0 & 4 & & \\
\hline & 8243 & 72 . & & 291.7 & 4 & & \\
\hline & $824 \quad 4$ & 69. & 0 & 29 & 4 & & \\
\hline & 8245 & 74.2500 & 0 & 292.0 & 4 & & \\
\hline & 8246 & & 0 & 29 & 4 & & \\
\hline & 8247 & 0 & & 29 & 4 & & \\
\hline & 8248 & 79.5000 & 500 & 29 & 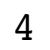 & & \\
\hline & 8249 & 42 & 0 & 29 & 3 & & \\
\hline & 82410 & 3 & & & 2 & & \\
\hline & 82411 & 74.2597 & & 298.3 & 2 & & \\
\hline & 82412 & 26 & 0 & 298.2 & 3 & & \\
\hline & 82413 & 141 . & 0 & 298.8 & J & & \\
\hline & 82414 & 144.2637 & 000 & 299.3 & 4 & & \\
\hline & 824 & 1 & 0 & 300.1 & 4 & & \\
\hline & 82416 & 104 . & 6 . & 300.2 & 4 & & \\
\hline & 82417 & 90.9973 & 6.8500 & 300.1 & 4 & & 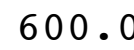 \\
\hline & 824 & 80 . & & 299.5 & 4 & & \\
\hline & 82419 & 78.7500 & 7.8750 & 298.2 & 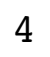 & & \\
\hline & 82420 & 71.4995 & .0250 & 296.9 & 4 & 60 & \\
\hline & & 7499 & 0.0750 & 297.0 & 4 & & \\
\hline & & 0 & & & & & \\
\hline & 32423 & 74.5000 & 8.9500 & 297.4 & & 600.0 & 600 \\
\hline
\end{tabular}




\begin{tabular}{|c|c|c|c|c|c|c|c|}
\hline 1 & 82424 & 1.5000 & 8.2000 & 297.1 & 4 & 600.0 & \\
\hline 1 & 8251 & 5.7496 & 7.5500 & 297.4 & 4 & 600.0 & \\
\hline & 8252 & 81.7499 & 1750 & 298.0 & 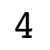 & 0 & \\
\hline & 8253 & 80.0000 & 4.9250 & 297.8 & & 00.0 & \\
\hline & 8254 & 4.4933 & 3.7250 & 7.9 & & 0.0 & \\
\hline & 8255 & 5008 & 2.8500 & 297.9 & & 00 & \\
\hline & 8256 & 5.2343 & 4.4750 & 297.4 & & 00 & \\
\hline & 8257 & 100.3972 & 1.9250 & 98.7 & & 0 & \\
\hline & 8258 & 84.0600 & 1.1250 & 300.4 & & 00 & \\
\hline & 3259 & 243.9235 & 1.6750 & 300.5 & & 0 & \\
\hline & 2510 & 237.2587 & 500 & 300.2 & & & \\
\hline & 2511 & 217.0211 & 250 & & & & \\
\hline & 2512 & 164.7560 & 2.7000 & 302.5 & & 0 & \\
\hline & 2513 & 173.2515 & 3.7500 & & 2 & 0 & \\
\hline & 2514 & 164.50 & & & 3 & & \\
\hline & 2515 & 172.7496 & 500 & 304.4 & 3 & 0 & \\
\hline & 82516 & 151.4997 & 5.3000 & 305.0 & 3 & 600.0 & \\
\hline & 82517 & 97.2866 & 5.2500 & & 3 & & \\
\hline & 2518 & 78.5002 & 6.5250 & .8 & 4 & & \\
\hline & 82519 & 74.7459 & 2500 & 30 & 4 & 0 & \\
\hline & 2520 & 70.99 & 0 & & 4 & & \\
\hline & 82521 & 66.7502 & 7.9000 & .6 & 4 & & \\
\hline & 82522 & 67.2498 & 7.5750 & 1.0 & 4 & 0 & \\
\hline & 82523 & 74.50 & 7.5500 & .5 & 4 & & \\
\hline & 82524 & 79.2501 & 50 & .4 & 4 & & \\
\hline & 8261 & 77.2500 & 7.7000 & & 4 & & \\
\hline & 8262 & 75.5000 & 7.7000 & 301.2 & 4 & & \\
\hline & 8263 & 75.5010 & 7.0750 & .2 & 4 & & \\
\hline & 8264 & 72.4995 & 6.3000 & & 4 & & \\
\hline & 8265 & 77.50 & 5.8000 & .7 & 4 & & \\
\hline & 8266 & 79 . & 7.7250 & 8 & 4 & & \\
\hline & 8267 & 82 . & 250 & .4 & 4 & & \\
\hline & 8268 & 244.00 & 5500 & 3.2 & 3 & & \\
\hline 1 & 8269 & 229 . & 000 & 2.1 & 2 & & - \\
\hline & 82610 & 173.5138 & & 302.1 & 2 & & \\
\hline & 82611 & 168.2503 & 3.4500 & 302.8 & 2 & & \\
\hline & 82612 & 174.5000 & 250 & .7 & 3 & & \\
\hline & 82613 & 179.4986 & 6.3000 & 304.8 & 4 & & 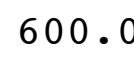 \\
\hline & 82614 & 169.2507 & 6.6750 & 305.6 & 4 & & 6 \\
\hline & 82615 & 164.5000 & 500 & 5.3 & 4 & & \\
\hline & 82616 & 156.2542 & 250 & & 3 & & \\
\hline$\perp$ & 82617 & 107.8178 & 4.7250 & 306.8 & 3 & 0 & 0 \\
\hline & 82618 & 86.7552 & 5.4000 & 305.2 & 3 & & \\
\hline 1 & 82619 & 81.0000 & 6.9000 & 303.3 & 4 & & 600 \\
\hline 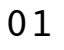 & 82620 & 73.5000 & 8.2750 & 302.4 & 4 & 600.0 & 00 \\
\hline & 82 & & & .0 & 4 & & \\
\hline & 82622 & 67.0000 & 8.7500 & 302.1 & 4 & & 600 \\
\hline & $200<3$ & 76.7506 & 8.7500 & 302. & & 600.0 & 600 \\
\hline
\end{tabular}




\begin{tabular}{|c|c|c|c|c|c|c|c|}
\hline 1 & 82624 & 65.5000 & 7.0000 & 302.4 & 4 & 600.0 & 600.0 \\
\hline 01 & 8271 & 69.2500 & 6.6250 & 302.6 & 4 & 600.0 & 600.0 \\
\hline 01 & 8272 & 85.2522 & 6.7500 & 302.4 & 4 & 600.0 & 00.0 \\
\hline 01 & 8273 & 91.2498 & 7.1250 & 301.5 & 4 & 600.0 & 00.0 \\
\hline 01 & 8274 & 84.0005 & 6.2500 & 301.4 & 4 & 600.0 & 00.0 \\
\hline 01 & 8275 & 80.9989 & 4.0750 & 302.2 & 4 & 600.0 & 00.0 \\
\hline 01 & 8276 & 113.8210 & 2.6500 & 302.0 & 4 & 600.0 & 00.0 \\
\hline 01 & 8277 & 142.4055 & 2.9750 & 301.1 & 4 & 600.0 & 00.0 \\
\hline 01 & 8278 & 155.7527 & 5.0500 & 300.9 & 4 & 600.0 & 00.0 \\
\hline 01 & 8279 & 150.7539 & 5.5500 & 301.5 & 4 & 600 & 0 \\
\hline 01 & 82710 & 159.7504 & 5.8500 & 302.5 & 4 & 600.0 & \\
\hline 01 & 82711 & 162.7492 & 6.6500 & 303.2 & 4 & 600.0 & \\
\hline 01 & 82712 & 158.9984 & 6.5500 & 304.3 & 4 & 600.0 & 00.0 \\
\hline 01 & 82713 & 168.5000 & 7.1000 & 305.9 & 4 & 600.0 & 00.0 \\
\hline 01 & 82714 & 162.2542 & 6.6750 & 307.1 & 4 & .0 & \\
\hline 01 & 82715 & 167.9997 & 6.1000 & 307.9 & 4 & 600.0 & .0 \\
\hline 01 & 82716 & 115.4077 & 5.7750 & 308.4 & 3 & 60 & 0.0 \\
\hline 01 & 82717 & 78.5207 & 6.8250 & 307.8 & 4 & .0 & \\
\hline 01 & 82718 & 68.9989 & 7.7750 & 306.6 & 4 & 60 & \\
\hline 01 & 82719 & 70.0002 & 7.8000 & 305.3 & 4 & .0 & .0 \\
\hline 01 & 82720 & 67.5000 & 9.02 & 304.9 & 4 & 6 & \\
\hline 01 & 82721 & 70.7501 & 8.7500 & 304.7 & 4 & .0 & \\
\hline 01 & 82722 & 74.0001 & 8.5000 & 304.3 & 4 & 600.0 & .0 \\
\hline 01 & 82723 & 70.5000 & 0.12 & 303.1 & 4 & 6 & \\
\hline 01 & 82724 & 73.0000 & 9.9750 & 303.0 & 4 & .0 & \\
\hline 01 & 8281 & 75.7499 & 10.1750 & 303.4 & 4 & .0 & \\
\hline 01 & 8282 & 80.4998 & 8.6000 & 303.5 & 4 & 600.0 & .0 \\
\hline 01 & 8283 & 125.6766 & 2.8750 & 301.5 & 5 & .0 & \\
\hline 01 & 8284 & 94.9656 & 6.7750 & 301.2 & 4 & .0 & .0 \\
\hline 01 & 8285 & 112.0166 & 5.4250 & 301.5 & 4 & .0 & .0 \\
\hline 01 & 8286 & 133.9705 & 4.4750 & 301.6 & 4 & .0 & \\
\hline 01 & 8287 & 127.5065 & 4.2000 & 302.4 & 3 & .0 & 600.0 \\
\hline 01 & 8288 & 144.2484 & 6.1250 & 302.9 & 4 & 600.0 & 0.0 \\
\hline 01 & 8289 & 158.2494 & 7.0000 & 302.8 & 4 & .0 & 0.0 \\
\hline 01 & 82810 & 158.4963 & 6.7000 & 303.5 & 4 & 600.0 & 600.0 \\
\hline 01 & 82811 & 155.0049 & 6.2750 & 304.3 & 4 & 600.0 & 600.0 \\
\hline 01 & 82812 & 160.7478 & 5.10 & 305.4 & 3 & 600.0 & .0 \\
\hline 01 & 82813 & 162.5609 & 5.5750 & 307.1 & 3 & 600.0 & 600.0 \\
\hline 01 & 82814 & 106.7538 & 5.5750 & 308.2 & 3 & 600.0 & 600.0 \\
\hline 01 & 82815 & 84.9651 & 6.7500 & 308.0 & 4 & 600.0 & 600.0 \\
\hline 01 & 82816 & 88.2721 & 6.0500 & 307.6 & 4 & 600.0 & 600.0 \\
\hline 01 & 82817 & 77.6228 & 6.8500 & 307.0 & 4 & 600.0 & 600.0 \\
\hline 01 & 82818 & 63.0001 & 9.4500 & 305.4 & 4 & 600.0 & 600.0 \\
\hline 01 & 82819 & 63.2500 & 9.2750 & 304.3 & 4 & 600.0 & 600.0 \\
\hline 01 & 82820 & 69.4968 & 8.0250 & 303.6 & 4 & 600.0 & 600.0 \\
\hline 01 & 82821 & 70.5013 & 6.7500 & 303.1 & 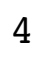 & 600.0 & 600.0 \\
\hline 01 & 82822 & 69.5000 & 7.1750 & 302.5 & 4 & 600.0 & 600.0 \\
\hline J & 82823 & 70.2523 & 10.4500 & 301.1 & & 600.0 & 600.0 \\
\hline
\end{tabular}




\begin{tabular}{|c|c|c|c|c|c|c|c|}
\hline & 2824 & 8.7473 & .5750 & 300.4 & 4 & 800 & \\
\hline & 8291 & 3.5000 & 9.2750 & & & & \\
\hline & 3292 & 76.0034 & 3500 & 299.4 & 4 & r & \\
\hline & 8293 & 77.7509 & 5250 & 298.6 & & 00 & \\
\hline & 294 & 2.2511 & & & & & \\
\hline & 8295 & 27.7513 & 1.52 & 0 & & 00 & \\
\hline & 8296 & 62.2778 & 0.9000 & 294.9 & & & \\
\hline & 297 & 75.5312 & 1.3000 & 95.9 & & & \\
\hline & 8298 & 57.2699 & 1.07 & 298.2 & & 0 & \\
\hline & 8299 & 281.8474 & 1.4750 & 298.8 & & 0 & \\
\hline & 2910 & 262.8170 & 2.1000 & 299.5 & & 0 & \\
\hline & 2911 & 250.6035 & & 00.3 & & & \\
\hline & 2912 & 233.5227 & 2.42 & 301.1 & & 0 & \\
\hline & 2913 & 49.7539 & 4.32 & 301.5 & & 0 & \\
\hline & 2914 & 86.00 & & & & & \\
\hline & 2915 & 85.7508 & & 301.4 & 4 & & \\
\hline & 2916 & 84.5009 & 7 . & 0 & 4 & & \\
\hline & 2917 & 72 . & & & & & \\
\hline & 2918 & 75 . & & 297 & 4 & & \\
\hline & 2919 & 5000 & 1.20 & 29 & 4 & & \\
\hline & 2920 & 67 . & 0 & 5 & 4 & & \\
\hline & 2921 & 78 . & 0 & 9 & 4 & & \\
\hline & 82922 & 76.9999 & 0 & & 4 & & \\
\hline & 82923 & 9 & 0 & 2 & 4 & & \\
\hline & 82924 & & 0 & & 4 & & \\
\hline & 8301 & 79 . & & & 4 & & \\
\hline & 8302 & & & 289.7 & + & & \\
\hline & 8303 & 79. & & 288 & 4 & & \\
\hline & 8304 & 82 . & 0 & 28 & 4 & & \\
\hline & $830 \quad 5$ & 80.2499 & & 286.7 & 4 & & \\
\hline & 8306 & 79.24 & & 28 & 4 & & \\
\hline & 8307 & 80 & & 28 & 4 & & \\
\hline & 8308 & 20.1645 & & 290.3 & 3 & 0 & \\
\hline & 8309 & 1 . & & 29 & 2 & & \\
\hline & 83010 & 235 . & & 29 & 1 & & \\
\hline & 83011 & 228.2502 & & 292.9 & & & \\
\hline & 83012 & 230 . & & 294 & 2 & & \\
\hline & 83013 & 102 . & 0 & 1 & 2 & & \\
\hline & 83014 & 149 & & 296.7 & 3 & & \\
\hline & 830 & 99. & & 296 & 3 & & \\
\hline & 83016 & 93. & & & 4 & & \\
\hline & 83017 & 86.7512 & 6.6250 & 295.2 & 4 & 0 & . \\
\hline & 830 & 80 . & & 293.6 & 4 & 0 & \\
\hline & 83019 & 78.7502 & & 291 & 4 & & \\
\hline & 83020 & 76.0000 & & 290 & 4 & 60 & 00 . \\
\hline & & & & 289.9 & 4 & 0 & \\
\hline & & & & & & & \\
\hline & 83023 & .73 .0334 & 0.6000 & 291.9 & & 600.0 & 600 \\
\hline
\end{tabular}




\begin{tabular}{|c|c|c|c|c|c|c|c|}
\hline & 3024 & 22.2103 & 500 & 292.0 & 5 & 0 & \\
\hline 1 & 8311 & 2.8556 & 0.5250 & 291.2 & & 0 & \\
\hline & 8312 & 60.9874 & 2750 & 290.6 & & 0 & \\
\hline & 8313 & 40.9415 & .3750 & 290.4 & & 00 & \\
\hline & 8314 & 01.6321 & .2750 & 88.6 & & 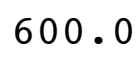 & \\
\hline & 8315 & 85.6615 & .8000 & 289.4 & & 00 & \\
\hline & 8316 & 671 & 1.8000 & 290.6 & & 0 & \\
\hline & 8317 & 68.3290 & .8750 & 91.8 & & & \\
\hline & 8318 & 176.9407 & 1.0500 & 294.0 & & 0 & \\
\hline & 8319 & 241.6560 & 1.5250 & 294.5 & & 0 & \\
\hline & 3110 & 242.5000 & 2.4250 & 294.8 & & 0 & \\
\hline & 717 & 234.2422 & .0000 & 96.0 & & & \\
\hline & 3112 & 250.8375 & 2.2000 & 297.9 & & 0 & \\
\hline & 3113 & 242.4044 & 2.4250 & 299.4 & & 0.0 & \\
\hline & 3114 & 223.7021 & & 300.3 & & & \\
\hline & 83115 & 176.2907 & 250 & 300.8 & & 0 & \\
\hline & 3116 & 111.7451 & 4.7750 & 300.8 & & 0 & \\
\hline & 3117 & 91.2523 & .7250 & 300.4 & & & \\
\hline & 831 & 87.5000 & .4750 & 299.4 & 4 & 0 & \\
\hline & 3119 & 81.0000 & 5000 & 297.2 & 4 & & \\
\hline & 83120 & 76 . & 72 & .5 & 4 & & \\
\hline & 3121 & 81 . & 250 & 294.7 & 4 & & \\
\hline & 83122 & 73.7509 & 9750 & 294 & 4 & 0 & U. \\
\hline & 83123 & 001 & 7.32 & 295.3 & 4 & 0 & \\
\hline & 83124 & 73. & 0 & 297.0 & 4 & & \\
\hline & $\begin{array}{lll}9 & 1 & 1\end{array}$ & 83.2109 & 2.2250 & 297.1 & 5 & & \\
\hline & 912 & 140.5069 & 1.1500 & 296.5 & 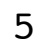 & & \\
\hline & 913 & 153.2504 & $10 ?$ & 295.1 & 4 & & \\
\hline$\perp$ & $\begin{array}{lll}9 & 1 & 4\end{array}$ & 356 . & 0 & 294.8 & 5 & & 0 . \\
\hline & 915 & 80.2609 & 500 & 294.6 & 6 & & \\
\hline & 916 & 87.2481 & 0 & 294.6 & 6 & & \\
\hline & $9 \begin{array}{ll}9 & 1\end{array}$ & 161 . & 0 & 294.4 & 6 & & \\
\hline & $\begin{array}{lll}9 & 1 & 8\end{array}$ & 331.3833 & 0.7000 & 296.4 & 5 & 0 & 0 . \\
\hline & $9 \begin{array}{ll}9 & 1\end{array}$ & 217 . & 500 & 297.2 & 4 & & \\
\hline & 9110 & 166. & 3 . & 297.2 & 3 & & \\
\hline & $9 \quad 111$ & 169.9984 & 500 & 297.2 & 3 & & \\
\hline & 9112 & 170.2496 & 4.2500 & 298.2 & 3 & & \\
\hline 1 & 9113 & 165 . & 1250 & 299.6 & 3 & & 0 . \\
\hline & $9 \quad 114$ & 179.7488 & 4.1500 & 301.0 & 3 & 0 & 0 \\
\hline & 9115 & 185 . & 250 & 302.1 & 2 & & \\
\hline & 9116 & 166. & 750 & 302.9 & 2 & & \\
\hline 01 & $9 \quad 117$ & 120.2580 & 4.8000 & 303.3 & 3 & 600.0 & 00 . \\
\hline & 9118 & 105.1982 & 7750 & 302.2 & 4 & 600 & . \\
\hline & 9119 & 86.7613 & 4.8000 & 299.9 & 4 & & \\
\hline 01 & 9120 & 73.7500 & 7.5000 & 298.3 & 4 & 600 & 00 \\
\hline & $\begin{array}{ll}9 & 121\end{array}$ & 65.9983 & 7.3250 & 297.5 & 4 & 600.0 & 500 . \\
\hline & $9 \quad 122$ & 51.5003 & 000 & 298.1 & 4 & 60 & \\
\hline & 123 & 58.7260 & 4.2500 & 298.0 & & 600.0 & 600 \\
\hline
\end{tabular}




\begin{tabular}{|c|c|c|c|c|c|c|c|c|}
\hline & & 24 & 2.5140 & 2.3500 & 98.5 & & - & \\
\hline & y & 1 & 100.9319 & .2500 & 298.9 & & 500.0 & \\
\hline & 9 & 22 & 69.7736 & 2000 & 298.7 & & 00 & \\
\hline & 9 & 3 & 124.1199 & 4750 & 298.2 & & 00 & \\
\hline & $y$ & 4 & 137.2499 & 000 & 97.8 & & & \\
\hline & 9 & 5 & 148.4928 & 5750 & 97.3 & & 0 & \\
\hline & 9 & 6 & 319 & 3750 & 296.5 & & 00 & \\
\hline & 9 & 7 & 147.2558 & 1750 & 97.0 & & & \\
\hline & 9 & 8 & 193.6897 & 8250 & 00.1 & & & \\
\hline & 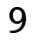 & 9 & 9348 & 1.3250 & 301.3 & & 0 & \\
\hline & 9 & 210 & 198.3926 & 3.1000 & 301.9 & & & \\
\hline & 9 & 11 & 181.0000 & 157 & 01.8 & & & \\
\hline & 9 & 12 & 170 & 5.2000 & 302.5 & & 0 & \\
\hline & 9 & 213 & 165.2032 & 5.9000 & 304.1 & & & \\
\hline & 9 & 214 & 912 & 0 & 305 & & & \\
\hline & 9 & 15 & 113.7121 & & 304 & & & \\
\hline & 9 & 216 & 96.2522 & 6.7750 & 304.1 & & .0 & \\
\hline & 9 & 217 & 83.1935 & 7.7500 & 303.2 & & & \\
\hline & 9 & 18 & 64.7506 & & 301.8 & & & \\
\hline & 9 & 219 & 70.5000 & 9.55 & 300 & 4 & & 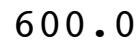 \\
\hline & 9 & 220 & 74.2500 & .1000 & 299.3 & & 0 & \\
\hline & 9 & 221 & 76.5000 & & 299.5 & & & \\
\hline & 9 & 222 & 000 & 250 & 298.8 & 4 & & \\
\hline & 9 & 223 & 56.2727 & 8.2250 & 298.1 & & 0 & \\
\hline & 9 & 224 & 79.7499 & & $29^{\circ}$ & & & \\
\hline & 9 & 31 & 83. & & 298 & 4 & & \\
\hline & 9 & 32 & 85.4946 & 8500 & 298.7 & 4 & & J. \\
\hline & 9 & 33 & 76 . & 4500 & 298.8 & 4 & & \\
\hline & 9 & 34 & 74 . & 50 & 298.7 & 4 & & \\
\hline & 9 & 35 & 74.7499 & 4000 & 298.8 & 4 & & J. \\
\hline & 9 & 36 & 78.2500 & 7.7250 & 298.8 & A & & 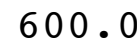 \\
\hline & 9 & 37 & 80.2504 & 750 & 299.4 & 4 & & \\
\hline & 9 & 38 & 80.2502 & 750 & 300.8 & 4 & & 0 . \\
\hline & 9 & 39 & 92.5000 & 4.3500 & 302.4 & 4 & & \\
\hline & 9 & 310 & 179.0941 & 2 . & 303.5 & 3 & & \\
\hline & 9 & 311 & 174.2346 & 4750 & 303.6 & 2 & & \\
\hline & 9 & 312 & 153.3165 & 750 & 304.1 & 3 & & \\
\hline & 9 & 313 & 57.5170 & 0 & 305.2 & 3 & & \\
\hline & 9 & 314 & 81.2621 & 5.6750 & 305.0 & 3 & & 0 . \\
\hline & 9 & 315 & 94.7502 & 3000 & 304.4 & 4 & & \\
\hline & 9 & 316 & 99.7506 & 6.7500 & 304.2 & 4 & & \\
\hline & 9 & 317 & 98.2555 & 6.3750 & 303.8 & 4 & & \\
\hline & 9 & 318 & 88.4976 & 7.6250 & 302.7 & 4 & & 0 . \\
\hline & 9 & 319 & 83.2496 & 9250 & 301.0 & 4 & & \\
\hline & 9 & 320 & 76.7502 & 1.0250 & 300.2 & 4 & & \\
\hline & 9 & 321 & 74.7499 & 1.7500 & 299.9 & 4 & & 00 . \\
\hline & 9 & 322 & 74 & & 299.9 & & & \\
\hline & & 323 & 79.0005 & 9.0750 & 299.8 & & 600.0 & $c^{\circ}$ \\
\hline
\end{tabular}




\begin{tabular}{|c|c|c|c|c|c|c|c|c|}
\hline & 9 & 324 & 76.5005 & 9.1000 & 299.8 & 4 & 600.0 & 600.0 \\
\hline 01 & 9 & 41 & 75.7499 & 7.2000 & 299.8 & 4 & 600.0 & 00.0 \\
\hline 01 & 9 & 42 & 70.9976 & 6.9250 & 299.7 & 4 & 600.0 & 00.0 \\
\hline 01 & 9 & 43 & 67.5000 & 7.9250 & 300.1 & 4 & 600.0 & 00.0 \\
\hline 01 & 9 & 44 & 39.5856 & 2.7500 & 299.9 & 4 & 600.0 & 00.0 \\
\hline 01 & 9 & 45 & 102.8884 & 0.9500 & 299.2 & 5 & 600.0 & 00.0 \\
\hline 01 & 9 & 46 & 16.1383 & 1.2000 & 298.2 & 6 & 600.0 & 00.0 \\
\hline 01 & 9 & 47 & 51.0934 & 1.2000 & 299.4 & 6 & 600.0 & 00.0 \\
\hline 01 & 9 & 48 & 298.3282 & 0.5250 & 301.8 & 5 & 600.0 & 00.0 \\
\hline 01 & 9 & 49 & 283.6639 & 1.0750 & 304.0 & 4 & 600.0 & .0 \\
\hline 01 & 9 & 410 & 238.2496 & 2.6750 & 303.0 & 3 & 600.0 & \\
\hline 01 & 9 & 411 & 234.2501 & 3.0750 & 303.0 & 2 & 600.0 & \\
\hline 01 & 9 & 412 & 249.7041 & 2.6000 & 304.2 & 1 & 600.0 & 00.0 \\
\hline 01 & 9 & 413 & 72.0000 & 5.5500 & 304.6 & 2 & 600.0 & 00.0 \\
\hline 01 & 9 & 414 & 87.0034 & 7 . & 303.9 & 3 & .0 & \\
\hline 01 & 9 & 415 & 88.7511 & 8.7500 & 303.7 & 4 & 600.0 & .0 \\
\hline 01 & 9 & 416 & 81.2504 & 9.4000 & 303.0 & 4 & 60 & 0.0 \\
\hline 01 & 9 & 417 & 74.2500 & 11.7250 & 302.0 & 4 & .0 & \\
\hline 01 & 9 & 418 & 65.0000 & 13.0750 & 300.7 & 4 & .0 & \\
\hline 01 & 9 & 419 & 71.0004 & 11.4250 & 299.2 & 4 & .0 & .0 \\
\hline 01 & 9 & 420 & 67.2499 & 11.92 & 297.9 & 4 & 6 & \\
\hline 01 & 9 & 421 & 61.2499 & 10.2500 & 297.7 & 4 & .0 & \\
\hline 01 & 9 & 422 & 63.9996 & 10.3750 & 297.3 & 4 & 600.0 & .0 \\
\hline 01 & 9 & 423 & 81.0954 & 6. & 297.3 & 4 & 6 & \\
\hline 01 & 9 & 424 & 128.7423 & 2 . & 296.5 & 4 & 60 & \\
\hline 01 & 9 & 51 & 78.4841 & 4. & 297.3 & 4 & .0 & .0 \\
\hline 01 & 9 & 52 & 61.0000 & 10.3250 & 297.7 & 4 & 60 & .0 \\
\hline 01 & 9 & 53 & 66.2470 & 10 & 297.2 & 4 & .0 & \\
\hline 01 & 9 & 54 & 73.9998 & 8.6500 & 296.4 & 4 & .0 & 0.0 \\
\hline 01 & 9 & 55 & 65.4991 & 9.8500 & 296.9 & 4 & 60 & .0 \\
\hline 01 & 9 & 56 & 76.5000 & 8.3 & 296.7 & 4 & .0 & \\
\hline 01 & 9 & 57 & 75.7504 & 9.5000 & 296.8 & 4 & .0 & 600.0 \\
\hline 01 & 9 & 58 & 82.5018 & 8.2500 & 297.0 & 4 & 600.0 & 600.0 \\
\hline 01 & 9 & 59 & 83.5009 & 6.2750 & 297.7 & 4 & .0 & 0.0 \\
\hline 01 & 9 & 510 & 74.5005 & 7.5750 & 297.9 & 4 & 600.0 & 600.0 \\
\hline 01 & 9 & 511 & 65.0011 & 10.0750 & 298.0 & 4 & 600.0 & 600.0 \\
\hline 01 & 9 & 512 & 60.4983 & 9.25 & 299.1 & 4 & 600.0 & 600.0 \\
\hline 01 & 9 & 513 & 68.2540 & 8.1500 & 299.9 & 4 & 600.0 & 600.0 \\
\hline 01 & 9 & 514 & 72.4992 & 9.7750 & 300.1 & 4 & 600.0 & 600.0 \\
\hline 01 & 9 & 515 & 74.5000 & 10.1500 & 299.9 & 4 & 600.0 & 600.0 \\
\hline 01 & 9 & 516 & 64.2496 & 9.5250 & 299.8 & 4 & 600.0 & 600.0 \\
\hline 01 & 9 & 517 & 62.7502 & 9.8000 & 300.3 & 4 & 600.0 & 600.0 \\
\hline 01 & 9 & 518 & 65.5000 & 10.0000 & 299.2 & 4 & 600.0 & 600.0 \\
\hline 01 & 9 & 519 & 82.9895 & 7.1000 & 297.1 & 4 & 600.0 & 600.0 \\
\hline 01 & 9 & 520 & 68.9989 & 10.0750 & 295.6 & 4 & 600.0 & 600.0 \\
\hline 01 & 9 & 521 & 67.0000 & 11.5500 & 295.7 & 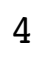 & 600.0 & 600.0 \\
\hline 01 & 9 & 522 & 69.7461 & 10.1000 & 296.2 & 4 & 600.0 & 600.0 \\
\hline J & 3 & 523 & 76.7533 & 9.5000 & 294.8 & & 600.0 & 600.0 \\
\hline
\end{tabular}




\begin{tabular}{|c|c|c|c|c|c|c|c|c|}
\hline 1 & 9 & 524 & 67.9998 & 8.8250 & 293.9 & 4 & 600.0 & 600.0 \\
\hline 01 & 9 & 61 & 75.7501 & 8.3250 & 294.1 & 4 & 600.0 & 00.0 \\
\hline 01 & 9 & 62 & 92.8104 & 6.2250 & 294.3 & 4 & 600.0 & 00.0 \\
\hline 01 & 9 & 63 & 108.1999 & 7.0750 & 293.6 & 4 & 600.0 & 00.0 \\
\hline 01 & 9 & 64 & 142.7502 & 13.6500 & 292.7 & 4 & 600.0 & 00.0 \\
\hline 01 & 9 & 65 & 150.7497 & 13.0000 & 291.9 & 4 & 600.0 & 00.0 \\
\hline 01 & 9 & 66 & 151.7500 & 12.1500 & 291.3 & 4 & 600.0 & 00.0 \\
\hline 01 & 9 & 67 & 154.5014 & 11.9750 & 291.2 & 4 & 600.0 & 00.0 \\
\hline 01 & 9 & 68 & 151.2501 & 8.2500 & 291.7 & 4 & 600.0 & 00.0 \\
\hline 01 & 9 & 69 & 158.2517 & 8.2000 & 292.6 & 4 & 600.0 & .0 \\
\hline 01 & 9 & 610 & 148.2499 & 5.6750 & 293.9 & 3 & 600.0 & \\
\hline 01 & 9 & 611 & 150.5000 & 6.1750 & 295.5 & 4 & 600.0 & \\
\hline 01 & 9 & 612 & 162.5018 & 7.6000 & 296.7 & 4 & 600.0 & 00.0 \\
\hline 01 & 9 & 613 & 161.2475 & 6.5250 & 298.1 & 4 & 600.0 & 00.0 \\
\hline 01 & 9 & 614 & 165.0006 & 6.0250 & 299.9 & 4 & .0 & \\
\hline 01 & 9 & 615 & 160.7514 & 5.0500 & 301.1 & 3 & 600.0 & .0 \\
\hline 01 & 9 & 616 & 166.7126 & 5.9500 & 302.2 & 3 & 60 & 0.0 \\
\hline 01 & 9 & 617 & 181.4995 & 6.9500 & 302.1 & 4 & .0 & \\
\hline 01 & 9 & 618 & 167.0128 & 4.6000 & 301.8 & 4 & 60 & \\
\hline 01 & 9 & 619 & 93.2265 & 4.4250 & 300.4 & 4 & .0 & .0 \\
\hline 01 & 9 & 620 & 78.5006 & 6.20 & 298.9 & 4 & 6 & \\
\hline 01 & 9 & 621 & 73.9995 & 6.4750 & 298.3 & 4 & .0 & \\
\hline 01 & 9 & 622 & 73.5000 & 6.4750 & 298.4 & 4 & 600.0 & .0 \\
\hline 01 & 9 & 623 & 72.2499 & 7.37 & 297.8 & 4 & 6 & \\
\hline 01 & 9 & 624 & 73.0000 & 7.7250 & 297.5 & 4 & 0 & \\
\hline 01 & 9 & 71 & 73.7496 & 7.8250 & 297.6 & 4 & .0 & 600.0 \\
\hline 01 & 9 & 72 & 76.4991 & 7.0250 & 298.0 & 4 & 60 & .0 \\
\hline 01 & 9 & 73 & 77.2500 & 5.9500 & 298.4 & 4 & .0 & \\
\hline 01 & 9 & 74 & 78.7491 & 4.7750 & 298.7 & 5 & .0 & 0.0 \\
\hline 01 & 9 & 75 & 101.4697 & 4.4000 & 298.7 & 4 & 60 & .0 \\
\hline 01 & 9 & 76 & 112.1827 & 3.1250 & 298.3 & 4 & .0 & \\
\hline 01 & 9 & 77 & 110.0607 & 1.6500 & 298.5 & 5 & .0 & 600.0 \\
\hline 01 & 9 & 78 & 156.1937 & 1.1500 & 299.8 & 4 & 600.0 & 0.0 \\
\hline 01 & 9 & 79 & 245.9369 & 1.5500 & 301.3 & 3 & 600.0 & 0.0 \\
\hline 01 & 9 & 710 & 241.7499 & 3.1750 & 299.8 & 3 & 600.0 & 600.0 \\
\hline 01 & 9 & 711 & 224.0534 & 2.0500 & 301.4 & 2 & 600.0 & 600.0 \\
\hline 01 & 9 & 712 & 173.2483 & 4.1750 & 301.5 & 3 & 600.0 & 0.0 \\
\hline 01 & 9 & 713 & 169.7489 & 4.9000 & 302.0 & 3 & 600.0 & 600.0 \\
\hline 01 & 9 & 714 & 160.0000 & 4.8500 & 303.4 & 3 & 600.0 & 600.0 \\
\hline 01 & 9 & 715 & 162.0148 & 5.2250 & 304.2 & 3 & 600.0 & 600.0 \\
\hline 01 & 9 & 716 & 122.0324 & 4.9250 & 304.5 & 3 & 600.0 & 600.0 \\
\hline 01 & 9 & 717 & 79.4696 & 6.5250 & 302.9 & 4 & 600.0 & 600.0 \\
\hline 01 & 9 & 718 & 81.5000 & 6.9000 & 301.1 & 4 & 600.0 & 600.0 \\
\hline 01 & 9 & 719 & 78.5003 & 9.3500 & 299.1 & 4 & 600.0 & 600.0 \\
\hline 01 & 9 & 720 & 76.0000 & 8.9750 & 298.6 & 4 & 600.0 & 600.0 \\
\hline 01 & 9 & 721 & 76.5018 & 9.0250 & 298.7 & 4 & 600.0 & 600.0 \\
\hline 01 & 9 & 722 & 77.2500 & 10.0500 & 298.6 & 4 & 600.0 & 600.0 \\
\hline J & 9 & 723 & 80.0001 & 10.150 & 298.4 & 4 & 600.0 & 600.0 \\
\hline
\end{tabular}




\begin{tabular}{|c|c|c|c|c|c|c|c|c|}
\hline 1 & 9 & 724 & 71.5000 & 9.0750 & 298.9 & 4 & 600.0 & 600.0 \\
\hline 01 & 9 & 81 & 70.0000 & 8.2250 & 299.3 & 4 & 600.0 & 600.0 \\
\hline 01 & 9 & 82 & 102.3060 & 3.7000 & 298.3 & 4 & 600.0 & 00.0 \\
\hline 01 & 9 & 83 & 18.2801 & 1.7500 & 295.7 & 5 & 600.0 & 00.0 \\
\hline 01 & 9 & 84 & 105.0525 & 3.4750 & 297.0 & 5 & 600.0 & 00.0 \\
\hline 01 & 9 & 85 & 94.9851 & 4.9000 & 297.7 & 4 & 600.0 & 00.0 \\
\hline 01 & 9 & 86 & 137.5676 & 1.1500 & 295.1 & 5 & 600.0 & 00.0 \\
\hline 01 & 9 & 87 & 123.6050 & 0.7500 & 295.2 & 6 & 600.0 & 00.0 \\
\hline 01 & 9 & 88 & 88.2493 & 1.6000 & 295.6 & 5 & 600.0 & 00.0 \\
\hline 01 & 9 & 89 & 327.3922 & 1.0250 & 298.0 & 4 & 600.0 & 00.0 \\
\hline 01 & 9 & 810 & 202.1540 & 4.0500 & 298.0 & 3 & 600.0 & 00.0 \\
\hline 01 & 9 & 811 & 168.5001 & 5.9500 & 298.0 & 4 & 600.0 & .0 \\
\hline 01 & 9 & 812 & 166.2491 & 5.0500 & 299.4 & 3 & 600.0 & 00.0 \\
\hline 01 & 9 & 813 & 159.5001 & 6.0000 & 300.0 & 4 & 600.0 & 00.0 \\
\hline 01 & 9 & 814 & 110.3887 & 4.2500 & 301.2 & 3 & .0 & \\
\hline 01 & 9 & 815 & 99.0030 & 4.3250 & 301.5 & 3 & 600.0 & 00.0 \\
\hline 01 & 9 & 816 & 92.0002 & 4.8000 & 301.2 & 3 & 600.0 & 600.0 \\
\hline 01 & 9 & 817 & 78.7430 & 4.9750 & 300.2 & 3 & .0 & \\
\hline 01 & 9 & 818 & 77.7499 & 7.0500 & 298.1 & 4 & 600.0 & .0 \\
\hline 01 & 9 & 819 & 77.0000 & 9.0250 & 295.2 & 4 & .0 & .0 \\
\hline 01 & 9 & 820 & 75.0005 & 8. & 294.7 & 4 & 60 & \\
\hline 01 & 9 & 821 & 77.5000 & 8.8250 & 294.8 & 4 & .0 & \\
\hline 01 & 9 & 822 & 76.7502 & 7.0000 & 296.5 & 4 & 600.0 & 00.0 \\
\hline 01 & 9 & 823 & 83.4073 & 5.50 & 297.1 & 4 & 60 & .0 \\
\hline 01 & 9 & 824 & 100.2375 & 2.2750 & 294.0 & 5 & .0 & \\
\hline 01 & 9 & 91 & 59.7192 & 1.9000 & 292.6 & 6 & 600.0 & 600.0 \\
\hline 01 & 9 & 92 & 89.2081 & 3.0750 & 291.9 & 5 & 600.0 & .0 \\
\hline 01 & 9 & 93 & 80.9705 & 2.7750 & 291.1 & 6 & 600.0 & \\
\hline 01 & 9 & 94 & 78.5000 & 5.7250 & 290.1 & 5 & 600.0 & 600.0 \\
\hline 01 & 9 & 95 & 79.2501 & 4.7500 & 290.1 & 4 & 600.0 & 600.0 \\
\hline 01 & 9 & 96 & 80.2500 & 6.7250 & 289.0 & 4 & .0 & \\
\hline 01 & 9 & 97 & 80.0000 & 5.2500 & 289.0 & 4 & .0 & 600.0 \\
\hline 01 & 9 & 98 & 80.5005 & 3.5250 & 290.3 & 3 & 600.0 & 600.0 \\
\hline 01 & 9 & 99 & 88.9935 & 2.7750 & 291.5 & 2 & 600.0 & 0.0 \\
\hline 01 & 9 & 910 & 90.6651 & 3.0250 & 292.0 & 2 & 600.0 & 600.0 \\
\hline 01 & 9 & 911 & 239.0589 & 2.2000 & 295.2 & 1 & 600.0 & 600.0 \\
\hline 01 & 9 & 912 & 79.9303 & 3.8750 & 294.6 & 2 & 600.0 & 600.0 \\
\hline 01 & 9 & 913 & 72.9846 & 4.4750 & 294.6 & 3 & 600.0 & 600.0 \\
\hline 01 & 9 & 914 & 84.8355 & 3.8250 & 296.4 & 2 & 600.0 & 600.0 \\
\hline 01 & 9 & 915 & 79.9952 & 4.1750 & 297.2 & 3 & 600.0 & 600.0 \\
\hline 01 & 9 & 916 & 76.0000 & 6.1250 & 296.4 & 4 & 600.0 & 600.0 \\
\hline 01 & 9 & 917 & 75.9998 & 7.0750 & 295.0 & 4 & 600.0 & 600.0 \\
\hline 01 & 9 & 918 & 79.2502 & 8.1500 & 292.9 & 4 & 600.0 & 600.0 \\
\hline 01 & 9 & 919 & 78.7500 & 9.2500 & 291.0 & 4 & 600.0 & 600.0 \\
\hline 01 & 9 & 920 & 75.7501 & 9.5500 & 289.7 & 4 & 600.0 & 600.0 \\
\hline 01 & 9 & 921 & 75.7500 & 9.8250 & 288.9 & 4 & 600.0 & 600.0 \\
\hline 01 & 9 & 922 & 71.2498 & 9.8750 & 288.4 & 4 & 600.0 & 600.0 \\
\hline J & ? & 923 & 71.5000 & 9.7000 & 288.3 & 4 & 600.0 & 600.0 \\
\hline
\end{tabular}




\begin{tabular}{|c|c|c|c|c|c|c|c|}
\hline 1 & 9924 & 0.3000 & 9.3000 & 288.2 & 4 & 100 & \\
\hline & 9101 & 67500 & 9.3250 & 287.6 & & & \\
\hline & 9102 & 7502 & 2750 & 287.3 & & מ & \\
\hline & 9103 & 7.5000 & 3500 & 287.0 & & 00 & \\
\hline & 104 & & & & & & \\
\hline & 9105 & 77.2499 & 750 & 86.8 & & (20 0 & \\
\hline & 9106 & 75.5000 & 5000 & 286.7 & & 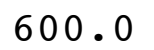 & \\
\hline & 9107 & 7.5000 & 8500 & 87.1 & & 0 & \\
\hline & 9108 & 81.0000 & 000 & 288.3 & & 0 & \\
\hline & 9109 & & & 289 & & 0 & \\
\hline & 91010 & 4.8228 & & 5 & & & \\
\hline & 91011 & 8.9422 & & 4 & & & \\
\hline & 91012 & 42.0380 & 3250 & 295.1 & & 0 & \\
\hline & 91013 & . & & 1 & & & \\
\hline & 91014 & & & & & & \\
\hline & 91015 & 71 & 0 & 297 & & & \\
\hline & 91016 & 157.2498 & 0 & 297 & & 0 & \\
\hline & 91017 & 1.28 & & & & & \\
\hline & 91018 & 117 & & 29 & 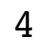 & & \\
\hline & 91019 & 83 & 0 & 292 & 4 & & \\
\hline & 91020 & & & 3 & & & \\
\hline & 91021 & 69 & & 28 & 4 & & \\
\hline & 91022 & 70.7497 & .80 & 289.3 & 4 & & \\
\hline & 91023 & 710 & 0 & 4 & 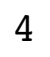 & & \\
\hline & 91024 & & & & 4 & & \\
\hline & 9111 & 75 & 500 & 28 & 4 & & \\
\hline & 9112 & 80.00 & 0 & 289.7 & 4 & & \\
\hline & 9113 & 78 . & & & 4 & & \\
\hline & 9114 & 76.7499 & & 28 & 4 & & \\
\hline & 9115 & 72 . & 0 & 29 & 4 & & \\
\hline & 9116 & 76 & & 7 & 4 & & \\
\hline & 9117 & 71 . & & 29 & 4 & & \\
\hline & 9118 & 53.26 & 0 & 291.2 & 2 & & \\
\hline & 9119 & 92 . & & 290 & 3 & & \\
\hline & 91110 & 328 . & & & 2 & & \\
\hline & 91111 & 97.7548 & 1.9750 & 294.9 & & & \\
\hline & 91112 & 5 . & 0 & 295.8 & 1 & & \\
\hline & 91113 & 118 . & 0 & 296.6 & 2 & & \\
\hline & 91114 & 96.6228 & & 296.7 & 3 & & \\
\hline & 91115 & 109 . & & & 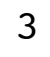 & & \\
\hline & 91116 & 91.9893 & & 297.3 & 3 & & \\
\hline & 91117 & 78.4925 & 5.7750 & 296.6 & 3 & & \\
\hline & 91118 & 73.2502 & & 294.5 & 4 & & \\
\hline & 91119 & 71.7503 & & 292.3 & 4 & & \\
\hline & 91120 & 77.2500 & 9.6750 & 291.6 & 4 & 600.0 & ה \\
\hline & 91121 & & & 291.4 & 4 & & \\
\hline & 91122 & 78.7499 & & 291.1 & & & \\
\hline & 1123 & 77.7500 & 10.1500 & 290.5 & & 600.0 & 50 \\
\hline
\end{tabular}




\begin{tabular}{|c|c|c|c|c|c|c|c|}
\hline 1 & 124 & 5.2500 & 11.4000 & 289.9 & 4 & 00.0 & \\
\hline & 9121 & 4.7500 & 12.1750 & 289.1 & & & \\
\hline & 9122 & 000 & 9000 & 8.8 & & & \\
\hline & 123 & 2.5000 & 2.3250 & 88.7 & & 00 & \\
\hline & 124 & 3.5008 & & & & & \\
\hline & 125 & 000 & 1.0250 & 89.5 & & 0 & \\
\hline & 126 & 68.2500 & 9.8250 & 90.4 & & 0 & \\
\hline & 127 & 0.2500 & 8.7250 & 91.3 & & 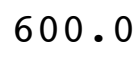 & \\
\hline & 128 & 77.0019 & 8.0000 & 291.6 & & $c$ & \\
\hline & 129 & 88.2502 & 7.4750 & 292.7 & & 0 & \\
\hline & 210 & 4.4987 & 7 . & 92.7 & & & \\
\hline & 211 & 1.2498 & & 92 & & & \\
\hline & 1212 & 0.4709 & 6.3750 & 294 & & 0 & \\
\hline & 213 & 5 . & 0 & 95.2 & & & \\
\hline & 214 & & & 1 & & & \\
\hline & 215 & 72 . & 8 . & 295.9 & 4 & & \\
\hline & 1216 & 65.0006 & 8.9500 & 295.0 & & 0 & \\
\hline & 217 & 69. & 9.27 & 293.9 & - & & \\
\hline & 8 & 68. & 0 & 1 & 4 & & \\
\hline & 1219 & 68.5000 & .50 & 292.0 & & & \\
\hline & 220 & 89 & & 4 & & & \\
\hline & 1221 & & 50 & 29 & 4 & & \\
\hline & 1222 & 71.4995 & 12.15 & 289.8 & 4 & & \\
\hline & 223 & 533 & & 9 & & & \\
\hline & 1224 & & 0 & 28 & 4 & & \\
\hline & 9131 & 84.7501 & 11.2250 & 287.4 & 4 & & \\
\hline & 9132 & 85.0000 & 0 & 287.2 & 4 & & \\
\hline & 9133 & 84.4998 & 0 & 288.4 & 4 & & \\
\hline & 9134 & 84.4992 & 0 & 289.7 & 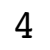 & & \\
\hline & 9135 & 756 & 0 & 289.1 & & & \\
\hline & 9136 & o 0711 & & 3 & 6 & & \\
\hline & 9137 & & & $2 \varepsilon$ & 6 & & \\
\hline & 9138 & 777 & 1.1000 & 290.3 & J & & \\
\hline & 9139 & 954 & & 293 & 4 & & \\
\hline & 91310 & 285 . & & & 3 & & \\
\hline & 91311 & 254.4095 & 2.5500 & 295.2 & & & \\
\hline & 91312 & 226 . & 0 & 295.6 & 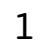 & & \\
\hline & 91 & 184 . & & 297.7 & $\perp$ & & \\
\hline & 91314 & 211.2719 & .750 & 299.4 & & & \\
\hline & & 16 & 0 & 29 & 3 & & \\
\hline & & 162.0011 & & 299.8 & 4 & & \\
\hline & 91317 & 161.9938 & 5.0500 & 299.8 & 3 & & \\
\hline & 91318 & 161.5000 & 50 & 298.6 & 4 & & \\
\hline & 91319 & 154.2499 & & & 4 & & \\
\hline & 91320 & 78.9081 & 2.5750 & 295.8 & 5 & 0 & م. \\
\hline & & & & & 5 & & \\
\hline & & 83.7493 & 4.1000 & & & & \\
\hline & 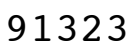 & 80.4997 & 4.8750 & 296.8 & & 600.0 & 60 \\
\hline
\end{tabular}




\begin{tabular}{|c|c|c|c|c|c|c|c|}
\hline & 91324 & 3.9931 & 2.5000 & 297.6 & 4 & 0.0 & \\
\hline & $914 \quad 1$ & 87.7470 & 1.0750 & 297.7 & 5 & 00.0 & \\
\hline & 9142 & 97.9539 & 500 & 296.5 & & 0 & \\
\hline & 143 & 60.2636 & .4500 & 296.2 & & 0 & \\
\hline & 144 & 122.0779 & 50 & 295.2 & & $0 c$ & \\
\hline & 9145 & 5279 & 000 & 294.5 & & 00 & \\
\hline & 9146 & 351.8811 & 3750 & 295.1 & & 0.0 & \\
\hline & 147 & 189.2180 & & 95.8 & & & \\
\hline & 9148 & 235.2512 & 0.3250 & 298.6 & & 00.0 & 00 \\
\hline & 9149 & 243.4969 & 1.37 & 299.4 & & & \\
\hline & 1410 & 239.0002 & & 99.1 & & & \\
\hline & 1411 & 235.7494 & 2 & 299.5 & & 0 & 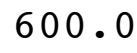 \\
\hline & 1412 & 217.2482 & 3.72 & 299.7 & & & \\
\hline & 91413 & 194.7445 & 3.77 & 300.5 & & & \\
\hline & 91414 & 205.9908 & & 301.6 & 2 & & \\
\hline & 1415 & 202.7279 & 2.92 & 302.6 & 1 & 0 & \\
\hline & 91416 & 194.0015 & 4 . & 302.7 & 2 & 0 & \\
\hline & 1417 & 179.5202 & & 302.6 & 2 & & \\
\hline & 91418 & 145.8272 & 0 & 302.0 & 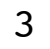 & 0 & 50 \\
\hline & 91419 & 85.7413 & 4 . & 300.2 & 4 & & \\
\hline & 91420 & 67.7506 & & 298.8 & 4 & & \\
\hline & 91421 & 71.0005 & & 298.2 & 4 & & \\
\hline & 91422 & 71.5000 & .20 & 297.9 & 4 & 0 & 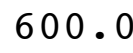 \\
\hline & 91423 & 75.0000 & & 297.4 & 4 & & $\bullet$ \\
\hline & 91424 & 984 & & 29 & 4 & & \\
\hline & 9151 & 71.7508 & 7 . & 297.5 & 4 & 0 & 0 \\
\hline & 9152 & 70.9995 & & 29 & 4 & & \\
\hline & 9153 & 69.7573 & & 29 & 4 & & \\
\hline & 9154 & 112.6877 & 2.22 & 297.7 & 5 & 0 & 0 \\
\hline & 9155 & 65.6665 & & 295.3 & 6 & & \\
\hline & 9156 & 806 & & 29 & 6 & & \\
\hline & 9157 & 207.0937 & & 295.8 & 5 & & \\
\hline & 9158 & 325.8055 & 0.75 & 296.2 & 4 & 0 & \\
\hline & 9159 & 207.9492 & 0 & 299.0 & 3 & & \\
\hline & 91510 & 213.7718 & & 300.2 & 2 & & \\
\hline & 91511 & 217.1077 & 4.10 & 299.4 & 3 & & \\
\hline & 91512 & 229.5018 & 3 . & 299.6 & 2 & & \\
\hline & 91513 & 252.2624 & 3 . & 301.5 & 2 & 0 & 00 . \\
\hline & 91514 & 63.0844 & 0 & 302.5 & 3 & .0 & \\
\hline & 91515 & 85.2448 & 0 & 301.5 & 3 & & \\
\hline & 91516 & 78.2526 & & 300.3 & 4 & & . \\
\hline & 91517 & 75.4998 & 7.5250 & 299.7 & 4 & 0 & 00 \\
\hline & 91518 & 75.2499 & 7 . & 298.7 & 4 & 0 & \\
\hline 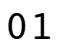 & 91519 & 75.5000 & 9.0000 & 297.8 & 4 & 600.0 & 600 \\
\hline & 91520 & 74.7501 & 9.8750 & 296.5 & 4 & 600.0 & 00 \\
\hline & 91521 & 74.2527 & & 296.2 & - & & \\
\hline & 91522 & 71.7502 & 9.4250 & 296.5 & 4 & .0 & 600 \\
\hline & 1523 & 72.2498 & 9.1500 & 296.3 & 4 & 600.0 & 60 \\
\hline
\end{tabular}




\begin{tabular}{|c|c|c|c|c|c|c|c|}
\hline 1 & 1524 & 3.7499 & 8.2500 & 96.1 & 4 & 0.0 & \\
\hline & 9161 & 2.7500 & 8.4750 & 96.1 & & & \\
\hline & 9162 & 9999 & 5000 & 6.1 & & 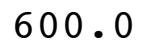 & \\
\hline & 163 & 8.7498 & 500 & 94.8 & & 00 & \\
\hline & 164 & & & 3.3 & & & \\
\hline & 165 & 5000 & 5750 & 92.8 & & 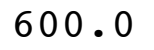 & \\
\hline & 166 & 1.7502 & 3750 & 91.2 & & 00 & \\
\hline & 167 & 6.0001 & 1.2250 & 90.5 & & 0 & \\
\hline & 168 & 72.7525 & 1.2250 & 290.7 & & 0 & \\
\hline & 169 & 75.7502 & 9.8000 & 292.2 & & 0 & \\
\hline & 610 & 032 & 500 & 93.0 & & & \\
\hline & 611 & 0 & & 94.5 & & & \\
\hline & 1612 & 77.7511 & 250 & 296.5 & & 0 & \\
\hline & 613 & 0 & .1250 & 96.0 & & & \\
\hline & 614 & & & 97.0 & & & \\
\hline & 615 & 71 . & 8.4250 & 296.9 & & & \\
\hline & 1616 & 76 & 9.7000 & 295.7 & & 0 & \\
\hline & 617 & 83. & 9.8500 & 294.2 & & & \\
\hline & 8 & 76 . & 1.0250 & 293.7 & 4 & & \\
\hline & 1619 & 77.75 & 250 & 292.8 & & & \\
\hline & 620 & & & 4 & & & \\
\hline & 1621 & & 50 & 29 & 4 & & \\
\hline & 1622 & 68.74 & 0.7500 & 293 & 4 & & \\
\hline & 623 & 76 . & 000 & 29 & & & \\
\hline & 1624 & & & 29 & 4 & & \\
\hline & 9171 & 86 . & 3000 & 294 & 4 & & \\
\hline & 9172 & 86.50 & 000 & 293.7 & 5 & & \\
\hline & 9173 & 74. & 750 & 292 & 6 & & \\
\hline & 9174 & 74 . & 4750 & 291.7 & 6 & & \\
\hline & 9175 & 22. & 250 & 289.8 & 6 & & \\
\hline & 9176 & 20 & 250 & & 6 & & \\
\hline & 9177 & & 50 & 29 & 6 & & \\
\hline & 9178 & 93. & 750 & 292.5 & 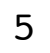 & & \\
\hline & 9179 & 1 . & 500 & 294.8 & 4 & & \\
\hline & 91710 & 166. & & & 4 & & \\
\hline & 91711 & 161.50 & 500 & 296.2 & 3 & & \\
\hline & 91712 & 185. & 750 & 297 & 3 & & \\
\hline & 91713 & 169. & 250 & 298.1 & 3 & & \\
\hline & 91714 & 175.2501 & 4.6000 & 299.4 & 3 & & \\
\hline & 91715 & 169 . & 500 & 300 & 3 & & \\
\hline & 91716 & 164 . & & & 3 & & \\
\hline & 91717 & 144.4216 & 5.0000 & 300.8 & 3 & & \\
\hline & 91718 & 81 . & 000 & 299 & 4 & & \\
\hline & 91719 & & & & 4 & & \\
\hline & 91720 & 76.2495 & 7.2750 & 297.2 & 4 & 0.0 & م0 \\
\hline & $91 / 21$ & & & & 4 & & \\
\hline & 91722 & 69.7500 & 8.7000 & 297.2 & 4 & & \\
\hline & $1 / 23$ & 75.7502 & 8.9000 & 297.6 & & 600.0 & 600 \\
\hline
\end{tabular}




\begin{tabular}{|c|c|c|c|c|c|c|c|}
\hline & 1724 & 7.2499 & 8.7750 & 297.6 & 4 & 600.0 & \\
\hline & 9181 & 7.7500 & 8.7750 & 297.6 & 4 & 600.0 & \\
\hline & 9182 & 5000 & .2750 & 297.3 & 4 & 00 & \\
\hline & 9183 & 78.0000 & 5.9750 & 297.1 & & 00 & \\
\hline & 184 & 21.2655 & 0.8750 & 94.3 & & 0 & \\
\hline & 185 & 0.7349 & .6000 & 291.9 & & 00 & \\
\hline & 9186 & 92.0919 & 0.6750 & 292.3 & & 00 & \\
\hline & 187 & 78.5138 & .5000 & 95.3 & & & \\
\hline & 9188 & 155.2924 & 0.9000 & 297.2 & & 0 & \\
\hline & 9189 & 215.7798 & 1.5750 & 297.2 & & 00.0 & \\
\hline & 1810 & 238.7499 & 2.3500 & 296.5 & & 0 & \\
\hline & 811 & 205.7042 & & & & & \\
\hline & 1812 & 191.2500 & 4 . & 297.7 & 3 & 0 & \\
\hline & 1813 & 192.2500 & 5.27 & 299.2 & 2 & 0.0 & \\
\hline & 814 & 64.3305 & & & & & \\
\hline & 1815 & 89.2498 & & 303.0 & 2 & 0 & \\
\hline & 1816 & 82.5000 & 0 & 302.2 & 3 & 0 & \\
\hline & 91817 & 73.7494 & 0 & 30 & 4 & & \\
\hline & 91818 & 67.2500 & 7 . & 30 & 4 & 0 & \\
\hline & 91819 & 71.2498 & 7. & 298.7 & 4 & 0 & \\
\hline & 91820 & 73.5040 & 7 . & 298.3 & 4 & & \\
\hline & 91821 & 75.9989 & & .4 & 4 & & \\
\hline & 91822 & 72.2500 & & 29 & 4 & 0 & U. \\
\hline & 91823 & 75.2500 & 7 . & 29 & 4 & 0 & \\
\hline & 91824 & 73.5000 & & 29 & 4 & & \\
\hline & 9191 & 80.7509 & & 29 & 4 & & \\
\hline & 9192 & 78.5037 & 6 . & 298.7 & 4 & 0 & \\
\hline & 9193 & 81.4991 & & 29 & 4 & & \\
\hline & 9194 & 120.7623 & & .7 & 5 & 0 & 0 . \\
\hline & 9195 & 92.5505 & & 29 & 6 & 0 & \\
\hline & 9196 & 126.7713 & & .5 & 5 & & \\
\hline & 9197 & 129. & 1 . & .6 & 4 & & $\bullet$ \\
\hline & 9198 & 163.6850 & & 296.8 & 3 & 0 & 0 • \\
\hline & 9199 & 246.8266 & 1 . & 29 & 2 & 0 & \\
\hline & 91910 & 179.7080 & & 0 & 2 & & \\
\hline & 91911 & 163.2506 & & 296.7 & 3 & & 0 . \\
\hline & 91912 & 166.7505 & & 297.8 & 3 & 0 & \\
\hline 1 & 91913 & 173.7495 & 4 . & 299.3 & 3 & 0 & \\
\hline & 91914 & 166.7502 & 5 . & 300.7 & 3 & 60 & 0 . \\
\hline & 91915 & 159.3822 & & 302.5 & 3 & 0 & \\
\hline & 91916 & 123.6243 & & 303.3 & 3 & & \\
\hline$V_{1}$ & 91917 & 111.5048 & 4.4250 & 302.8 & 3 & 600.0 & 600 . \\
\hline & 91918 & 77.9562 & & 301.4 & 4 & 600 & 600 \\
\hline & 91919 & 74.2495 & 7.5250 & 299.8 & 4 & & 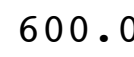 \\
\hline 01 & 91920 & 75.5000 & 7.6750 & 299.5 & 4 & 600.0 & 00 \\
\hline & 91921 & 76.5000 & & 299.4 & 4 & 600.0 & 600. \\
\hline & 22 & 79.2500 & & 299.3 & 4 & 60 & \\
\hline & 1923 & 80.2500 & 8.1250 & 299.0 & 4 & 600.0 & 600 \\
\hline
\end{tabular}




\begin{tabular}{|c|c|c|c|c|c|c|c|}
\hline 01 & 91924 & 79.5000 & 8.4000 & 298.3 & 4 & 600.0 & 600.0 \\
\hline 01 & 9201 & 80.7501 & 7.7000 & 298.4 & 4 & 600.0 & 600.0 \\
\hline 01 & 9202 & 78.2500 & 7.1500 & 298.8 & 4 & 600.0 & 00.0 \\
\hline 01 & 9203 & 77.9998 & 7.2750 & 299.0 & 4 & 600.0 & 00.0 \\
\hline 01 & 9204 & 77.5000 & 7.4750 & 298.8 & 4 & 600.0 & 00.0 \\
\hline 01 & 9205 & 76.7497 & 4.5000 & 298.1 & 4 & 600.0 & 00.0 \\
\hline 01 & 9206 & 45.7185 & 2.6250 & 297.9 & 4 & 600.0 & 00.0 \\
\hline 01 & 9207 & 83.5849 & 0.8750 & 298.0 & 5 & 600.0 & 00.0 \\
\hline 01 & $920 \quad 8$ & 122.5351 & 0.4000 & 299.9 & 4 & 600.0 & 00.0 \\
\hline 01 & 9209 & 252.8588 & 1.0750 & 300.6 & 3 & 600.0 & 00.0 \\
\hline 01 & 92010 & 236.0070 & 2.5000 & 298.6 & 2 & 600.0 & 00.0 \\
\hline 01 & 92011 & 164.7512 & 3.4750 & 298.4 & 3 & 600.0 & \\
\hline 01 & 92012 & 161.0004 & 3.9000 & 298.9 & 4 & 600.0 & 00.0 \\
\hline 01 & 92013 & 166.0001 & 3.8500 & 300.5 & 3 & 600.0 & 00.0 \\
\hline 01 & 92014 & 170.7540 & 50 & 301.5 & 3 & .0 & \\
\hline 01 & 92015 & 130.9439 & 000 & 302.8 & 3 & 600.0 & 00.0 \\
\hline 01 & 92016 & 113.7458 & 3.7750 & 302.7 & 2 & 600.0 & 600.0 \\
\hline 01 & 92017 & 90.7244 & 4.3 & 301.9 & 3 & .0 & \\
\hline 01 & 92018 & 93.9966 & 6.9500 & 299.7 & 4 & 600.0 & .0 \\
\hline 01 & 92019 & 80.0002 & 8.4000 & 297.5 & 4 & .0 & .0 \\
\hline 01 & 92020 & 77.0000 & 9.0 & 297.4 & 4 & 60 & \\
\hline 01 & 92021 & 78.7502 & 9.2500 & 297.6 & 4 & .0 & .0 \\
\hline 01 & 92022 & 80.4998 & 9.5750 & 297.6 & 4 & 600.0 & 0 \\
\hline 01 & 92023 & 73.5002 & 8.72 & 297.7 & 4 & 6 & .0 \\
\hline 01 & 92024 & 78.9450 & 4.4 & 297.5 & 4 & .0 & \\
\hline 01 & 9211 & 62.2435 & 7.3000 & 297.7 & 4 & 600.0 & 600.0 \\
\hline 01 & 9212 & 70.5000 & 8.3750 & 297.7 & 4 & 600.0 & 0.0 \\
\hline 01 & 9213 & 74.7501 & 8.3 & 297.0 & 4 & 0 & \\
\hline 01 & 9214 & 76.0000 & 8.8750 & 296.9 & 4 & .0 & 0.0 \\
\hline 01 & 9215 & 79.2492 & 8.3250 & 296.7 & 4 & .0 & 0.0 \\
\hline 01 & 9216 & 86.2500 & 8.8750 & 296.7 & 4 & .0 & \\
\hline 01 & 9217 & 85.5018 & 8.1250 & 297.1 & 4 & .0 & 600.0 \\
\hline 01 & 9218 & 81.2494 & 6.3750 & 298.0 & 4 & 600.0 & 600.0 \\
\hline 01 & 9219 & 92.6107 & 4.6250 & 299.4 & 3 & 600.0 & 0.0 \\
\hline 01 & 92110 & 166.7501 & 6.1500 & 298.7 & 4 & 600.0 & 600.0 \\
\hline 01 & 92111 & 166.2501 & 5.9500 & 298.1 & 4 & 600.0 & 600.0 \\
\hline 01 & 92112 & 167.7499 & 6.4500 & 299.5 & 4 & 600.0 & 600.0 \\
\hline 01 & 92113 & 166.7494 & 6.2500 & 300.1 & 4 & 600.0 & 600.0 \\
\hline 01 & 92114 & 167.2614 & 6.9750 & 301.7 & 4 & 600.0 & 600.0 \\
\hline 01 & 92115 & 93.0052 & 4.3250 & 303.1 & 3 & 600.0 & 600.0 \\
\hline 01 & 92116 & 97.7502 & 6.1000 & 302.2 & 4 & 600.0 & 600.0 \\
\hline 01 & 92117 & 109.2634 & 5.8750 & 301.7 & 4 & 600.0 & 600.0 \\
\hline 01 & 92118 & 75.7439 & 8.0250 & 300.7 & 4 & 600.0 & 600.0 \\
\hline 01 & 92119 & 72.2500 & 7.2250 & 300.2 & 4 & 600.0 & 600.0 \\
\hline 01 & 92120 & 62.9931 & 7.0750 & 299.2 & 4 & 600.0 & 600.0 \\
\hline 01 & 92121 & 72.7502 & 7.9000 & 299.2 & 4 & 600.0 & 600.0 \\
\hline 01 & 92122 & 74.2498 & 7.3500 & 299.5 & 4 & 600.0 & 600.0 \\
\hline . & 92123 & 79.7500 & 7.7500 & 299.1 & 4 & 600.0 & 600.0 \\
\hline
\end{tabular}




\begin{tabular}{|c|c|c|c|c|c|c|c|}
\hline 01 & 92124 & 77.2500 & 8.0000 & 299.4 & 4 & 600.0 & 600.0 \\
\hline 01 & 9221 & 75.2502 & 7.2250 & 299.5 & 4 & 600.0 & 600.0 \\
\hline 01 & 9222 & 76.7500 & 5.8500 & 299.6 & 4 & 600.0 & 00.0 \\
\hline 01 & 9223 & 62.8498 & 3.3750 & 298.8 & 5 & 600.0 & 00.0 \\
\hline 01 & 9224 & 10.2524 & 1.4250 & 297.9 & 4 & 600.0 & 00.0 \\
\hline 01 & 9225 & 29.2980 & 0.9500 & 297.8 & 5 & 600.0 & 00.0 \\
\hline 01 & 9226 & 127.4898 & 0.8250 & 297.1 & 6 & 600.0 & 00.0 \\
\hline 01 & 9227 & 358.0000 & 0.5750 & 297.0 & 6 & 600.0 & 00.0 \\
\hline 01 & 9228 & 313.8450 & 0.5500 & 299.0 & 5 & 600.0 & 00.0 \\
\hline 01 & 9229 & 244.5344 & 0.7500 & 299.4 & 4 & 600.0 & 0 \\
\hline 01 & 92210 & 243.4999 & 1.9250 & 300.1 & 3 & 600.0 & \\
\hline 01 & 92211 & 247.2530 & 2.2500 & 299.8 & 3 & 600.0 & \\
\hline 01 & 92212 & 198.5000 & 2.8000 & 300.3 & 2 & 600.0 & 00.0 \\
\hline 01 & 92213 & 157.2472 & 3.0500 & 299.9 & 3 & 600.0 & 00.0 \\
\hline 01 & 92214 & 231.5508 & 1.8000 & 303.2 & 2 & .0 & \\
\hline 01 & 92215 & 190.0037 & 3.1750 & 303.5 & 2 & 600.0 & 00.0 \\
\hline 01 & 92216 & 164.9764 & 3.2250 & 302.9 & 2 & 600.0 & 600.0 \\
\hline 01 & 92217 & 156.2544 & 3.3250 & 301.7 & 3 & .0 & \\
\hline 01 & 92218 & 116.7470 & 3.5750 & 301.2 & 4 & 600.0 & .0 \\
\hline 01 & 92219 & 101.3775 & 4.4000 & 299.3 & 4 & .0 & .0 \\
\hline 01 & 92220 & 63.0107 & 5.8250 & 298.7 & 4 & 60 & \\
\hline 01 & 92221 & 60.7493 & 9.0000 & 298.6 & 4 & .0 & \\
\hline 01 & 92222 & 68.0000 & 8.6250 & 299.1 & 4 & 600.0 & .0 \\
\hline 01 & 92223 & 68.5009 & .90 & 299.3 & 4 & 6 & \\
\hline 01 & 92224 & 47.8149 & 3.3750 & 299.0 & 5 & .0 & \\
\hline 01 & 9231 & 15.0330 & 1.1000 & 296.9 & 6 & 600.0 & .0 \\
\hline 01 & 9232 & 44.0344 & 0.6500 & 297.1 & 6 & 600.0 & .0 \\
\hline 01 & 9233 & 148.7504 & 1.2500 & 297.3 & 5 & .0 & \\
\hline 01 & 9234 & 147.2524 & 2.0500 & 296.5 & 4 & .0 & 600.0 \\
\hline 01 & 9235 & 61.8442 & 1.1250 & 296.0 & 5 & .0 & 0.0 \\
\hline 01 & 9236 & 16.7408 & 0.2500 & 296.3 & 6 & .0 & \\
\hline 01 & 9237 & 181.8386 & 0.2000 & 295.4 & 6 & .0 & 600.0 \\
\hline 01 & 9238 & 159.9224 & 1.0500 & 295.6 & 5 & 600.0 & 600.0 \\
\hline 01 & 9239 & 220.7846 & 0.5000 & 297.6 & 4 & 600.0 & 0.0 \\
\hline 01 & 92310 & 159.2563 & 2.4750 & 297.7 & 3 & 600.0 & 600.0 \\
\hline 01 & 92311 & 199.4137 & 3.0000 & 298.7 & 2 & 600.0 & 600.0 \\
\hline 01 & 92312 & 200.6868 & 2.5750 & 299.2 & 1 & 600.0 & 600.0 \\
\hline 01 & 92313 & 164.7515 & 3.9500 & 298.7 & 2 & 600.0 & 600.0 \\
\hline 01 & 92314 & 166.5000 & 4.2000 & 298.8 & 3 & 600.0 & 600.0 \\
\hline 01 & 92315 & 167.4982 & 5.1750 & 299.7 & 3 & 600.0 & 600.0 \\
\hline 01 & 92316 & 171.7505 & 5.4500 & 299.6 & 3 & 600.0 & 600.0 \\
\hline 01 & 92317 & 162.7506 & 5.1250 & 297.6 & 4 & 600.0 & 600.0 \\
\hline 01 & 92318 & 148.5005 & 3.1000 & 297.2 & 5 & 600.0 & 600.0 \\
\hline 01 & 92319 & 133.5182 & 1.8250 & 295.3 & 5 & 600.0 & 600.0 \\
\hline 01 & 92320 & 34.9957 & 1.2000 & 294.8 & 4 & 600.0 & 600.0 \\
\hline 01 & 92321 & 52.5008 & 2.1250 & 295.3 & 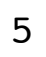 & 600.0 & 600.0 \\
\hline 01 & 92322 & 85.6514 & 1.6750 & 295.8 & 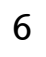 & 600.0 & 600.0 \\
\hline . & 92323 & 38.1104 & 0.5000 & 295.1 & 0 & 600.0 & 600.0 \\
\hline
\end{tabular}




\begin{tabular}{|c|c|c|c|c|c|c|c|}
\hline & 2324 & & 0.3750 & 94.4 & & & \\
\hline & 9241 & 289 & 0.3500 & 293.5 & & & \\
\hline & 9242 & 0799 & 500 & 294.3 & & . & \\
\hline & 9243 & 344.0000 & 0.8000 & 293.8 & & 00 & \\
\hline & $924 \quad 4$ & 018 & 1.2500 & 93.5 & & & \\
\hline & 9245 & 2473 & 1.5750 & 93.4 & & 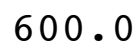 & \\
\hline & 9246 & 356 . & 1.4000 & 293.3 & & & \\
\hline & 9247 & 335 . & 1.57 & 93.8 & & & \\
\hline & 9248 & 333.6621 & 1 . & 295.4 & & 0 & \\
\hline & 9249 & 335.7447 & 1.1750 & 297.2 & & 0 & \\
\hline & 92410 & 302.3207 & 2.02 & 296.4 & & & \\
\hline & 2411 & 228.7326 & & 7 & & & \\
\hline & 92412 & 246.2515 & 1.8750 & 297.5 & & 0 & \\
\hline & 92413 & 114.8702 & 2.8000 & 299.2 & & 0 & \\
\hline & 92414 & 78.25 & & & & & \\
\hline & 92415 & 77.7478 & 9.3750 & 294 & & & \\
\hline & 92416 & 75.5000 & 8.5500 & 29 & & & \\
\hline & 92417 & 78.25 & 9 . & 1 & & & \\
\hline & 924 & 77 . & 0 & 291.7 & 4 & & \\
\hline & 92419 & 74.2495 & .5500 & 29 & 4 & & \\
\hline & 92420 & 72 . & 0 & 29 & 4 & & \\
\hline & 92421 & 73. & & & 4 & & \\
\hline & 92422 & 112.7780 & 250 & 289.2 & 5 & & U. \\
\hline & 92423 & 127 . & 0 & 289 & 3 & & \\
\hline & 92424 & 120 . & & 28 & 4 & & \\
\hline & 9251 & 82.7494 & 0 & 287.8 & 5 & & \\
\hline & 9252 & 75.2484 & 0 & 287.5 & 5 & & \\
\hline & 9253 & 89 . & 0 & $2 \varepsilon$ & 4 & & \\
\hline & 9254 & 36 . & 0 & 28 & 5 & & \\
\hline & $925 \quad 5$ & 346.9072 & 3000 & 286.8 & 6 & & \\
\hline & 9256 & 341.7771 & & 28 & 6 & & \\
\hline & 9257 & 134 . & 0 & 28 & 6 & & \\
\hline & 9258 & 356.2595 & 3250 & 289.9 & 5 & 0 & 0 . \\
\hline & 9259 & 297 . & 0 & 292.4 & 4 & & \\
\hline & 92510 & 27 & & & 3 & & \\
\hline & 92511 & 232.5009 & & 290.7 & 3 & & \\
\hline & 92512 & 225 . & 0 & 292.1 & 2 & & \\
\hline & 92513 & 187.2561 & 0 & 292.9 & 2 & & \\
\hline & 92514 & 168.2003 & 3.2250 & 293.9 & 2 & 0 & 0 . \\
\hline & 92515 & 164 . & & 294.5 & 3 & & \\
\hline & 92516 & 179.7498 & 0 & 294 & 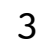 & & \\
\hline 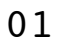 & 92517 & 181.7505 & 6.6250 & 293.6 & 4 & 600.0 & 8 \\
\hline & 92518 & 177.5020 & & 293.0 & 4 & 0 & \\
\hline & 92519 & 160.5000 & 4.2750 & 291.6 & 5 & & \\
\hline 01 & 92520 & 136.3184 & 4.1500 & 291.8 & 5 & 600 & 0 \\
\hline & 92521 & 4973 & 5.2250 & 291.1 & 4 & 600 & \\
\hline & 92522 & & & 290.8 & 4 & & \\
\hline & 2523 & 79.7500 & 6.5000 & 290.8 & $I$ & 600.0 & 600 \\
\hline
\end{tabular}




\begin{tabular}{|c|c|c|c|c|c|c|c|}
\hline 01 & 92524 & 86.7550 & 5.3750 & 291.2 & 4 & 600.0 & 600.0 \\
\hline 01 & 9261 & 3.2281 & 4.8250 & 290.9 & 4 & 600.0 & 00.0 \\
\hline 01 & 9262 & 108.7501 & 4.5500 & 291.4 & 4 & 600.0 & 00.0 \\
\hline 01 & 9263 & 102.9976 & 3.8500 & 291.2 & 4 & 600.0 & 00.0 \\
\hline 01 & 9264 & 109.0032 & 3.0750 & 291.1 & 4 & 600.0 & 00.0 \\
\hline 01 & 9265 & 113.2713 & 4.0000 & 290.9 & 4 & 600.0 & 00.0 \\
\hline 01 & 9266 & 102.8976 & 4.2250 & 290.4 & 4 & 600.0 & 00.0 \\
\hline 01 & 9267 & 117.8081 & 2.5750 & 291.0 & 4 & 600.0 & 00.0 \\
\hline 01 & 9268 & 147.3440 & 1.5500 & 293.0 & 3 & 600.0 & 00.0 \\
\hline 01 & 9269 & 172.7492 & 2.7750 & 294.0 & 3 & 600.0 & .0 \\
\hline 01 & 92610 & 168.0000 & 4.1500 & 294.5 & 3 & 600.0 & \\
\hline 01 & 92611 & 157.2561 & 5.5500 & 295.0 & 3 & 600.0 & \\
\hline 01 & 92612 & 158.7476 & 6.3250 & 296.2 & 4 & 600.0 & 00.0 \\
\hline 01 & 92613 & 151.2536 & 6.0000 & 297.1 & 4 & 600.0 & 00.0 \\
\hline 01 & 92614 & 164.0034 & 5 . & 298.5 & 3 & .0 & \\
\hline 01 & 92615 & 172 . & 5.7500 & 299.1 & 3 & 600.0 & .0 \\
\hline 01 & 92616 & 166.0651 & 5.4750 & 299.4 & 3 & 60 & .0 \\
\hline 01 & 92617 & 115.9553 & 4.6250 & 299.3 & 3 & .0 & \\
\hline 01 & 92618 & 966 & 4.8000 & 298.1 & 4 & 60 & .0 \\
\hline 01 & 92619 & 74.2503 & 5.9750 & 296.6 & 4 & .0 & .0 \\
\hline 01 & 92620 & 000 & 6 . & 295.4 & 4 & 60 & \\
\hline 01 & 92621 & 76.7497 & 8.3250 & 294.7 & 4 & .0 & \\
\hline 01 & 92622 & 71.2499 & 9.2500 & 294.1 & 4 & 600.0 & .0 \\
\hline 01 & 92623 & 75.2500 & 10.15 & 293.5 & 4 & 6 & \\
\hline 01 & 92624 & 76.4998 & 10 . & 293.0 & 4 & .0 & \\
\hline 01 & 9271 & 81.0002 & 8.4750 & 293.9 & 4 & .0 & 600.0 \\
\hline 01 & 9272 & 79.5000 & 8.7250 & 293.2 & 4 & 60 & .0 \\
\hline 01 & 9273 & 78.4999 & 9.2000 & 292.4 & 4 & .0 & \\
\hline 01 & 9274 & 76.5000 & 9.7500 & 292.2 & 4 & .0 & 0 \\
\hline 01 & $927 \quad 5$ & 75.5000 & 9.5500 & 292.4 & 4 & .0 & .0 \\
\hline 01 & 9276 & 70.2499 & 750 & 293.0 & 4 & .0 & \\
\hline 01 & $927 \quad 7$ & 64.2503 & 7.92 & 293.6 & 4 & .0 & 0.0 \\
\hline 01 & 9278 & 58.7664 & 5.1250 & 293.4 & 4 & 600.0 & 600.0 \\
\hline 01 & 9279 & 84.0793 & 2.5000 & 294.7 & 3 & .0 & 0.0 \\
\hline 01 & 92710 & 77.4998 & 6.5000 & 292.6 & 4 & 600.0 & 600.0 \\
\hline 01 & 92711 & 74.5002 & 9.2500 & 292.3 & 4 & 600.0 & 600.0 \\
\hline 01 & 92712 & 73.4979 & 8.87 & 293.9 & 4 & 600.0 & 600.0 \\
\hline 01 & 92713 & 82.2509 & 7.1000 & 294.9 & 4 & 600.0 & 600.0 \\
\hline 01 & 92714 & 80.2555 & 9.0250 & 294.5 & 4 & 600.0 & 600.0 \\
\hline 01 & 92715 & 79.7500 & 9 . & 294.8 & 4 & 600.0 & 600.0 \\
\hline 01 & 92716 & 90.5128 & 8.4000 & 294.1 & 4 & 600.0 & 600.0 \\
\hline 01 & 92717 & 83.9977 & 11.5750 & 291.7 & 4 & 600.0 & 600.0 \\
\hline 01 & 92718 & 77.5000 & 13.1500 & 290.8 & 4 & 600.0 & 600.0 \\
\hline 01 & 92719 & 70.7504 & 11.2000 & 290.4 & 4 & 600.0 & 600.0 \\
\hline 01 & 92720 & 63.5005 & 11.3500 & 289.6 & 4 & 600.0 & 600.0 \\
\hline 01 & 92721 & 69.7499 & 11.6000 & 290.3 & 4 & 600.0 & 600.0 \\
\hline 01 & 92722 & 68.7500 & 11.1750 & 290.2 & 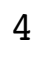 & 600.0 & 600.0 \\
\hline . & 92723 & 65.2500 & 12.3500 & 289.9 & 4 & 600.0 & 600.0 \\
\hline
\end{tabular}




\begin{tabular}{|c|c|c|c|c|c|c|c|}
\hline 1 & 92724 & 64.7500 & 12.7250 & 288.9 & 4 & 600.0 & 600.0 \\
\hline 01 & 9281 & 63.7500 & 12.8750 & 288.4 & 4 & 600.0 & 600.0 \\
\hline 01 & 9282 & 64.7501 & 9000 & 288.9 & 4 & 600.0 & 00.0 \\
\hline 01 & 9283 & 65.7487 & 9.8500 & 289.0 & 4 & 600.0 & 00.0 \\
\hline 01 & 9284 & 63.5000 & 9.8500 & 289.3 & 4 & 600.0 & 00.0 \\
\hline 01 & 9285 & 65.2501 & 10.3500 & 289.1 & 4 & 600.0 & 00.0 \\
\hline 01 & 9286 & 65.5000 & 10.3500 & 289.1 & 4 & 600.0 & 00.0 \\
\hline 01 & 9287 & 74.4995 & 9.2250 & 289.3 & 4 & 600.0 & 00.0 \\
\hline 01 & $928 \quad 8$ & 83.4994 & 9.8500 & 289.3 & 4 & 600.0 & 00.0 \\
\hline 01 & 9289 & 84.7497 & 7.6500 & 289.7 & 4 & 600 & 0 \\
\hline 01 & 92810 & 76.5008 & 4.0000 & 291.7 & 3 & 600.0 & \\
\hline 01 & 92811 & 138.4427 & 3.6500 & 293.4 & 2 & 600.0 & \\
\hline 01 & 92812 & 100.4929 & 3.9000 & 294.4 & 3 & 600.0 & 00.0 \\
\hline 01 & 92813 & 111.5075 & 5.1750 & 295.7 & 3 & 600.0 & 00.0 \\
\hline 01 & 92814 & 142.0000 & 500 & 295.6 & 4 & .0 & \\
\hline 01 & 92815 & 142.4914 & 5.6250 & 296.3 & 3 & 600.0 & .0 \\
\hline 01 & 92816 & 131.8382 & 4.7500 & 296.8 & 3 & 60 & 0.0 \\
\hline 01 & 92817 & 101.2498 & 4.7000 & 296.4 & 3 & .0 & \\
\hline 01 & 92818 & 85.7501 & 6.8500 & 294.5 & 4 & 60 & \\
\hline 01 & 92819 & 83.2554 & 8.8000 & 292.7 & 4 & .0 & .0 \\
\hline 01 & 92820 & 001 & 9.07 & 292.0 & 4 & 6 & \\
\hline 01 & 92821 & 71.5002 & 9.2000 & 292.1 & 4 & .0 & \\
\hline 01 & 92822 & 77.2503 & 8.9000 & 292.3 & 4 & 600.0 & .0 \\
\hline 01 & 92823 & 78.2501 & 8.62 & 292.4 & 4 & 6 & \\
\hline 01 & 92824 & 81.4994 & 8.3500 & 292.1 & 4 & .0 & \\
\hline 01 & 9291 & 83.7511 & 7.7000 & 292.2 & 4 & .0 & .0 \\
\hline 01 & 9292 & 80.0002 & 7.8000 & 292.0 & 4 & .0 & .0 \\
\hline 01 & 9293 & 89.5000 & 7.8250 & 292.4 & 4 & .0 & \\
\hline 01 & 9294 & 98.0000 & 7.0750 & 292.6 & 4 & .0 & .0 \\
\hline 01 & 9295 & 132.0808 & 5.6000 & 294.2 & 4 & .0 & .0 \\
\hline 01 & 9296 & 135.2638 & 4.4250 & 293.7 & 4 & .0 & \\
\hline 01 & 9297 & 132.0000 & 3.6500 & 293.4 & 4 & .0 & 0.0 \\
\hline 01 & 9298 & 129.6503 & 4.4750 & 294.5 & 3 & 600.0 & 0.0 \\
\hline 01 & 9299 & 148.0000 & 6.8000 & 295.2 & 4 & .0 & .0 \\
\hline 01 & 92910 & 160.2499 & 7.57 & 29 & 4 & 600.0 & 0.0 \\
\hline 01 & 92911 & 160.7482 & 7.2250 & 296.8 & 4 & 600.0 & 600.0 \\
\hline 01 & 92912 & 155.5031 & 6.60 & 297.3 & 4 & 600.0 & .0 \\
\hline 01 & 92913 & 160.2153 & 5.6000 & 298.2 & 3 & 600.0 & 600.0 \\
\hline 01 & 92914 & 157.4994 & 5.1500 & 299.2 & 3 & 600.0 & 600.0 \\
\hline 01 & 92915 & 162.0028 & 5.2500 & 300.4 & 3 & 600.0 & 600.0 \\
\hline 01 & 92916 & 181.7467 & 4.6750 & 301.0 & 3 & 600.0 & 600.0 \\
\hline 01 & 92917 & 194.2437 & 3.7750 & 300.8 & 3 & 600.0 & 600.0 \\
\hline 01 & 92918 & 202.3815 & 1.8250 & 300.0 & 4 & 600.0 & 600.0 \\
\hline 01 & 92919 & 27.9766 & 0.8750 & 298.5 & 5 & 600.0 & 600.0 \\
\hline 01 & 92920 & 35.7495 & 1.2500 & 298.5 & 4 & 600.0 & 600.0 \\
\hline 01 & 92921 & 41.2494 & 1.6250 & 298.7 & 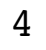 & 600.0 & 600.0 \\
\hline 01 & 92922 & 53.0434 & 1.3750 & 299.1 & 5 & 600.0 & 600.0 \\
\hline J & 92923 & 31.5000 & 1.7250 & 298.9 & 4 & 600.0 & 600.0 \\
\hline
\end{tabular}




\begin{tabular}{|c|c|c|c|c|c|c|c|}
\hline 1 & & 32.7618 & 500 & 99.2 & 4 & 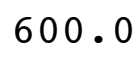 & \\
\hline 1 & 1 & 4.6338 & 3250 & 298.3 & & 600.0 & \\
\hline & 2 & 298.2492 & 500 & 297.4 & & 00 & \\
\hline & 3 & 325.5000 & 1.2750 & 297.0 & & 00 & \\
\hline & 4 & 314.2446 & 750 & 96.5 & & & \\
\hline & 5 & 288.1343 & 50 & 96 & & 0 & \\
\hline & 6 & 282.0000 & 0000 & 295.6 & & 00 & \\
\hline & 7 & 331.0000 & 6500 & 95.8 & & & \\
\hline & 8 & 296 & 50 & 97.9 & & & \\
\hline & 9 & 277.6056 & 1.4250 & 299.0 & & 0 & \\
\hline & 10 & 266.2950 & 5750 & 298.9 & & & \\
\hline & & 280.1371 & .7750 & 0 & & & \\
\hline & 2 & 229. & 3.1250 & .1 & & & \\
\hline & 3 & 230.9481 & 2.7750 & 301.4 & & 0 & \\
\hline & & 972 & & & & & \\
\hline & 5 & 224 . & & 3 & & & \\
\hline & 16 & 219.2501 & 2.1500 & 303 & & 0 & \\
\hline & & 171.7156 & 0 & 9 & & & \\
\hline & 18 & 148.4998 & 3 . & & & & \\
\hline & 19 & 142.5000 & 3.0500 & 301.1 & & 0 & 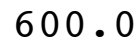 \\
\hline & & 140.7505 & 2.8000 & 0 & & & \\
\hline & 21 & 134.0177 & & & & & \\
\hline & 22 & 102 . & 50 & .1 & & & \\
\hline & 23 & 88.9992 & 3.8500 & 303.2 & & 0 & \\
\hline & 24 & 121.3114 & 270 & & & & \\
\hline & 1 & 146 . & 2.9 & .8 & 5 & & \\
\hline & 12 & 138.5002 & 3.5000 & 302.0 & & & . \\
\hline & 13 & 140.003 & 4.20 & 302.2 & 5 & & \\
\hline & 14 & 134 . & 5 . & 30 & 4 & & \\
\hline & 15 & 000 & 4 . & 1.7 & 4 & & J. \\
\hline & 16 & 135 . & 500 & 301.0 & 4 & & 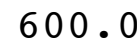 \\
\hline & 17 & 13 & 0 & 30 & 4 & & \\
\hline & 18 & 117 . & 50 & 300.6 & 3 & & 。 \\
\hline & 19 & 163 & 0 & 302.1 & & & \\
\hline & 110 & 15 & 0 & & 2 & & \\
\hline & 111 & 156.5002 & 6.2750 & 3.7 & 3 & & \\
\hline & 112 & 159 . & 8500 & 304.3 & 4 & & \\
\hline & 113 & 157.4996 & 7 . & 30 & 4 & & \\
\hline & 114 & 157.5000 & 6.9750 & 306.2 & 4 & & ?. \\
\hline & 115 & 160 . & 5.9000 & 307.3 & 3 & & \\
\hline & 116 & 126.7661 & 500 & 307.9 & 3 & & \\
\hline & 117 & 91.9992 & 5.8250 & 307.0 & 4 & & 0 . \\
\hline & 118 & 000 & 7.5250 & 305.0 & 4 & & 0 . \\
\hline & 119 & 000 & 8.7500 & 304.0 & 4 & & \\
\hline & 120 & 0002 & 0.0250 & 303.3 & 4 & & \\
\hline & 121 & 500 & 0.5000 & 303.1 & 4 & & 00 \\
\hline & 12 & & 0 & & 4 & & \\
\hline & 120 & 72.2501 & 9.5500 & 303.3 & & 60 & 20 \\
\hline
\end{tabular}




\begin{tabular}{|c|c|c|c|c|c|c|c|}
\hline & 124 & 5.2500 & 9750 & 3.1 & 4 & 0.0 & \\
\hline 11 & 21 & 78.5000 & 6000 & 302.7 & 4 & 00.0 & \\
\hline & 2 & 2500 & 7250 & 302.5 & & 0 & \\
\hline & 3 & 87.7500 & .3000 & 302.7 & & 0 & \\
\hline & 4 & 2.2455 & 8000 & 02.5 & & $c$ & \\
\hline & 5 & 9910 & & 303.4 & & 00 & \\
\hline & 6 & 5023 & & & & & \\
\hline & 7 & 2 & & 2.6 & & & \\
\hline & 8 & 133.7511 & 3.7750 & 303.1 & & 00.0 & 00 \\
\hline & 9 & 2503 & 0 & 303.2 & & & \\
\hline & 10 & 154.5006 & 0 & & & & \\
\hline & 11 & 162 . & 5.40 & 304.1 & & $\cdot 0$ & \\
\hline & 12 & 4941 & 5.67 & 304.8 & & & \\
\hline & 13 & 165 . & 0 & & & & \\
\hline & 214 & & & & 4 & & \\
\hline & 215 & 164.4997 & 0 & 307.1 & 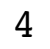 & 0 & \\
\hline & 216 & 158 . & 4 . & 30 & 4 & & \\
\hline & 217 & 150 . & & & $I$ & & \\
\hline & 18 & 76 & 0 & 306.0 & 1 & 0 & 600. \\
\hline & 219 & 63. & & & 4 & & \\
\hline & 220 & & & & 4 & & \\
\hline & 221 & 64 & & & 4 & & \\
\hline & 222 & 68.2500 & 0 & 303.5 & 4 & 0 & 1 \\
\hline & 223 & 60.2501 & & & 4 & & \\
\hline & 224 & 501 & & & 4 & & \\
\hline & 31 & 65.7498 & 0 & 30 & 4 & 0 & • \\
\hline & 32 & 70.9998 & & & 4 & & \\
\hline & 33 & 86 & & & 4 & & \\
\hline & 34 & 84.7492 & & 3.8 & 4 & & 10 \\
\hline & 35 & 4.5092 & & & 4 & & \\
\hline & 36 & 83 & & 30 & 5 & & \\
\hline & 37 & 923 & & 302.8 & 6 & & \\
\hline & 38 & 131.9878 & 1 . & 301.0 & 5 & 0 & \\
\hline & 39 & 280.3486 & 0 & 302.3 & 4 & & \\
\hline & 310 & 246.8251 & & 305.7 & 3 & & \\
\hline & 311 & 219.3068 & & 3.9 & 2 & & \\
\hline & 312 & 164 . & 4 . & & 3 & & \\
\hline & 313 & 170 . & 4 . & 304.6 & 3 & & 0 . \\
\hline & 314 & 165.5005 & & 305.0 & 4 & .0 & \\
\hline & 315 & 163. & & & 3 & & \\
\hline & 316 & 160.0005 & & 305.8 & 4 & & 00 . \\
\hline & 317 & 118.5815 & 4.4000 & 305.6 & 4 & 0 & (n) \\
\hline & 318 & 80.4995 & 6.9250 & 303.3 & 4 & 0 & \\
\hline & 319 & 82.5001 & 9.0500 & 301.4 & 4 & 600.0 & $\operatorname{con}$ \\
\hline & 320 & 77.7499 & 9.8500 & 301.0 & 4 & 600.0 & 00 \\
\hline & 321 & $7^{\circ}$ & & & 4 & & \\
\hline & 32 & 76.7500 & 50 & & $\Psi$ & & 600 \\
\hline & 23 & 76.7500 & 10.4500 & 299.7 & 4 & 60 & 60 \\
\hline
\end{tabular}




\begin{tabular}{|c|c|c|c|c|c|c|c|}
\hline & 24 & (..200 & 0.3250 & 99.5 & & & \\
\hline & 1 & 9.5000 & 10.1500 & 299.4 & & & \\
\hline & 2 & 0 & 500 & 98.7 & & 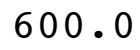 & \\
\hline & 3 & 0.2500 & 10.3750 & 298.4 & & 00 & \\
\hline & 4 & & 9.8250 & & & & \\
\hline & 5 & 1 & 8.55 & 97.2 & & 00 . & \\
\hline & 6 & 006 & 10.1750 & 95.7 & & & \\
\hline & 7 & 2504 & 1.20 & & & & \\
\hline & 8 & 48.7839 & 5.70 & 9 & & & \\
\hline & 9 & 5550 & 2.1000 & 296.0 & & 0 & \\
\hline & 410 & 011 & 4.70 & 295.2 & & & \\
\hline & 411 & & & & & & \\
\hline & 12 & 86. & 4 . & 297.2 & & 0 & \\
\hline & 413 & 88.00 & 8.00 & 295.8 & & 0 & \\
\hline & 414 & & & & & & \\
\hline & 415 & 79 . & & 295 & 4 & & \\
\hline & 416 & 75 & 0 & 29 & & & \\
\hline & 417 & 3. & 12. & & & & \\
\hline & 418 & 73. & 13. & 29 & 4 & & \\
\hline & 419 & & 13. & 29 & & & \\
\hline & 420 & & & $28 \varepsilon$ & & & \\
\hline & 421 & & & 7 & $I$ & & \\
\hline & 422 & 76 & & 28 & 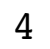 & & \\
\hline & 423 & & 0 & 28 & 4 & & \\
\hline & 424 & & & & 4 & & \\
\hline & 51 & 74 & & 28 & 4 & & \\
\hline & 52 & 74 & 2500 & 285.2 & $I$ & & \\
\hline & 53 & 72 & & 285 & 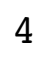 & & \\
\hline & 54 & 65 & & 28 & 4 & & \\
\hline & 55 & & & 28 & 4 & & \\
\hline & 56 & & & 28 & 4 & & \\
\hline & 57 & & 10 & 28 & 4 & & \\
\hline & 58 & 68.50 & & 286.2 & 4 & 0 & \\
\hline & 59 & 0 & & 28 & 4 & & \\
\hline & 510 & & 0 & 2 & 4 & & \\
\hline & 511 & & & 288.3 & 4 & & \\
\hline & 512 & 66. & & 289.6 & 4 & & \\
\hline & 513 & 75710 & 0 & 291.0 & 3 & & \\
\hline & 514 & 71.7503 & & 291.3 & 4 & & \\
\hline & 51 & 81 . & & 290.9 & 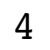 & & \\
\hline & 516 & 65 & & 291.1 & 4 & & \\
\hline & 517 & 72.2606 & 7.6750 & 290.3 & 4 & & 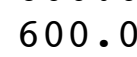 \\
\hline & & 76 & 12 . & 287.5 & 4 & 0 & \\
\hline & 519 & & 13. & 286.0 & 4 & & \\
\hline & 520 & 72.9999 & 13.4500 & 285.7 & 4 & 60 & \\
\hline & 521 & 998 & 11.8250 & 285.4 & 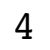 & 600 & \\
\hline & 522 & & & 28 & & & \\
\hline & 523 & 68.0001 & 12.1000 & 285.1 & & 600.0 & $c_{0}$ \\
\hline
\end{tabular}




\begin{tabular}{|c|c|c|c|c|c|c|c|}
\hline & 524 & 8.7500 & 11.6250 & 285.2 & 4 & 600.0 & \\
\hline 11 & 61 & 9.2500 & 10.0250 & 285.2 & 1 & 00.0 & \\
\hline & 62 & 68.7500 & 000 & 285.1 & & 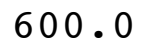 & \\
\hline & 3 & 0.5000 & 8.2000 & 85.0 & & 0 & \\
\hline & 4 & 000 & 8.2500 & 84.8 & & 00 & \\
\hline & 5 & 01 & 7.8250 & 284.7 & & 00 & \\
\hline & 6 & 70.9998 & 7.1750 & 84.5 & & 0.0 & \\
\hline & 7 & 6 & 0 & 84.5 & & & \\
\hline & 8 & 74.7501 & 4.9500 & 285.2 & & 00.0 & 00 \\
\hline & 9 & 89.2492 & 2.7000 & 287.0 & & & \\
\hline & 610 & 245.3257 & 1.3500 & & & & \\
\hline & 611 & 236 . & 2.52 & 289.6 & & .0 & ( \\
\hline & 612 & 224 . & 2.27 & 290.6 & & & \\
\hline & 613 & 230.5255 & 0 & 91.5 & & & \\
\hline & 614 & 200 . & & 291.8 & & & \\
\hline & 615 & 158.7429 & 0 & 292.0 & & 0.0 & \\
\hline & 616 & 147.7384 & 4.20 & 291.9 & & .0 & \\
\hline & 617 & 152.2644 & & 291.7 & 4 & & \\
\hline 1 & 618 & 123.3391 & 2.2500 & 291.4 & 5 & 0 & 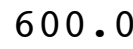 \\
\hline & 619 & $0 \cdot 24$ & 0 & 290.2 & 5 & & \\
\hline & 620 & & & & 4 & & \\
\hline & 621 & 53. & 0 & 289.4 & 4 & & \\
\hline & 622 & 59.0080 & 0 & 289.3 & 4 & 0 & 5 \\
\hline & 623 & 55.2377 & 2.47 & & 4 & & \\
\hline & 624 & 0 & 0 & 28 & 4 & & \\
\hline & 71 & 009 & .75 & 288.3 & 4 & 0 & 1 \\
\hline & 72 & 57 . & 0 & 288 & 4 & & \\
\hline & 73 & 60 . & 0 & 28 & 5 & & \\
\hline & 74 & 134.9367 & 0 & 288.1 & 5 & 0 & 10 \\
\hline & 75 & & & 287.5 & 6 & & \\
\hline & 76 & 12 & 0 & 28 & 6 & & \\
\hline & 77 & & 0 & 286.8 & 5 & & \\
\hline & 78 & 47.1860 & 1.15 & 288.6 & 4 & 0 & \\
\hline & 79 & 323 . & 11 & 291.0 & 3 & 0 & \\
\hline & 710 & 259 . & 1.3750 & 292.2 & 2 & & \\
\hline & 711 & 273.6851 & 2.3000 & 292.2 & 1 & & \\
\hline & 712 & 223. & 0 & 292.9 & 1 & & \\
\hline & 713 & 249.2448 & 2. & 294.4 & 1 & 0 & 0 . \\
\hline & 714 & 222.9162 & 3.2250 & 295.3 & 2 & .0 & \\
\hline & 715 & 249 . & 4 . & 295.4 & 3 & & \\
\hline & 716 & 33. & 2.6500 & 296.1 & 2 & & 00 . \\
\hline & 717 & 63.6354 & 4.9000 & 294.8 & 3 & 0 & 00 \\
\hline & 718 & 80.2492 & 7.3500 & 291.7 & 4 & 0 & \\
\hline & 719 & 72.7504 & 8.4500 & 290.6 & 4 & 600.0 & 600 \\
\hline 1 & 720 & 78.0002 & 7.6750 & 290.8 & 4 & 600.0 & 00 \\
\hline & 721 & 72.7497 & & 291.1 & 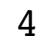 & & \\
\hline & 722 & 73.2447 & 6.1500 & 291.5 & 4 & 600 & 600 \\
\hline 1 & 723 & 63.9827 & 4.9250 & 291. & 4 & 600.0 & 60 \\
\hline
\end{tabular}




\begin{tabular}{|c|c|c|c|c|c|c|c|}
\hline 0 & 24 & 3.2545 & 4.7000 & 290.6 & 4 & 00.0 & \\
\hline 11 & 81 & 9.2511 & 10.7750 & 285.8 & & 00 & \\
\hline & 82 & 501 & 7250 & 289.4 & & 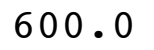 & \\
\hline & 3 & 3.5014 & 6.9500 & 90.8 & & 00 & \\
\hline & 4 & 0.997 & & 89.8 & & & \\
\hline & 85 & 750 & 6.6500 & 89.4 & & s & \\
\hline & 6 & 77.249 & 7500 & 86.4 & & 00 & \\
\hline & 7 & 3.50 & 8.5750 & 87.1 & & 0 & \\
\hline & 8 & 70 & 4250 & 288.4 & & 0 & \\
\hline & 9 & 5092 & & 90.3 & & 0 & \\
\hline & 810 & & 2.2000 & 90.8 & & & \\
\hline & 11 & & & 0 & & & \\
\hline & 12 & 512 & 2.0250 & 293.2 & & 0 & \\
\hline & 813 & . & 00 & 4 & & & \\
\hline & 814 & & & & & & \\
\hline & 815 & 129. & 500 & 294 & & & \\
\hline & 816 & 112.48 & 5.3500 & 294.7 & & 0.0 & \\
\hline & 817 & 104 . & & 294.2 & & & \\
\hline & 818 & 88 & 0 & 292.8 & 1 & & \\
\hline & 819 & 81. & 0 & 291.9 & & 0 & \\
\hline & 820 & & & & & & \\
\hline & 821 & 77 & & 29 & 4 & & \\
\hline & 822 & 77 . & 9500 & 292.8 & 4 & & \\
\hline & 823 & 73 . & 7. & 2.9 & 4 & & \\
\hline & 824 & & & 29 & 4 & & \\
\hline & 91 & 84 & 000 & 291.9 & 4 & & \\
\hline & 92 & 85.253 & 500 & 291.0 & 4 & & \\
\hline & 93 & 1 & 0 & 290.8 & 4 & & \\
\hline & 94 & 54 . & 500 & 288.8 & 4 & & \\
\hline & 95 & 148.49 & 750 & 288.1 & 4 & & \\
\hline & 96 & 143. & & 6 & 4 & & \\
\hline & 97 & 1 & & 287.3 & 4 & & \\
\hline & 98 & 168 . & 000 & 287.2 & 4 & & \\
\hline & 99 & 158 . & 0 & 287.9 & 4 & & \\
\hline & 910 & & & & 4 & & \\
\hline & 911 & 151.25 & & 290.4 & 3 & & \\
\hline & 912 & 153. & & 29 & 3 & & \\
\hline & 913 & 158 . & 5. & 292.9 & 3 & & \\
\hline & 914 & 153.50 & & 293.9 & 3 & & \\
\hline & 915 & 153. & & 29 & 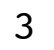 & & \\
\hline & 916 & 145 . & 5 . & 295.7 & 3 & & \\
\hline & 917 & 141.499 & 4.6250 & 296.0 & 4 & & \\
\hline & 918 & 138.252 & & 295.3 & - & & \\
\hline & 919 & 97.1765 & & 293.9 & 4 & & 600. \\
\hline & 920 & 74.499 & 6.4750 & 292.7 & 4 & 600.0 & ה חم \\
\hline & 921 & & & 292.2 & 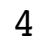 & & \\
\hline & 922 & 77.5000 & & 292.3 & 4 & & \\
\hline & 923 & 82.4995 & 7.4000 & 292.7 & & 600.0 & 600 \\
\hline
\end{tabular}




\begin{tabular}{|c|c|c|c|c|c|c|}
\hline 10 & & & & & & \\
\hline 11010 & 0 & 5500 & 5.0 & 4 & 0 & \\
\hline 0102 & 147.5010 & 250 & 294.3 & 4 & 00 & \\
\hline 10103 & 154.2492 & 4750 & 292.8 & & 00 & \\
\hline 0104 & 02 & 000 & 92.0 & & 0 & \\
\hline 0105 & 133.7 & 3500 & 91.6 & & 00 & \\
\hline 0106 & 118.1910 & 3000 & 291.3 & & 00 & \\
\hline 0107 & 16.3076 & 250 & 291.2 & & 0 & \\
\hline 0108 & 132.7502 & 4000 & 91.5 & & & \\
\hline 10109 & 143.2501 & 9750 & 292.3 & 4 & 00 & 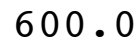 \\
\hline 1010 & 157.7500 & 9000 & 293.2 & & 0 & \\
\hline 011 & 155.7504 & 6.6750 & 93.6 & 4 & 00.0 & \\
\hline 012 & 162.5014 & $\$ 750$ & 294.7 & 4 & 0 & ( \\
\hline 013 & 166.0002 & 6.3250 & 295.8 & 4 & 0.0 & \\
\hline 014 & 163.2544 & & & 4 & & \\
\hline 015 & 155.9864 & & 297.9 & 3 & & \\
\hline 016 & 149.1905 & 1.2500 & 299.5 & 2 & .0 & 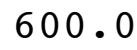 \\
\hline 017 & 244.4816 & 1.2000 & 9.0 & 3 & 0 & \\
\hline 018 & 171.5573 & 1.65 & 297.3 & 4 & & \\
\hline 019 & 96.5037 & .1250 & 296.0 & 4 & .0 & . \\
\hline 020 & 69.0011 & 6.4250 & 294.9 & 4 & .0 & . \\
\hline 021 & 71.7498 & 110 & & 4 & & \\
\hline 022 & 0000 & 4.7750 & 29 & 4 & & \\
\hline 023 & 60.7527 & 4.8250 & 295.6 & 5 & 0 & 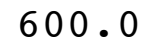 \\
\hline 024 & 0237 & & 29 & 4 & & \\
\hline 111 & 1300 & 4.6750 & 29 & 4 & & \\
\hline 110112 & 75.5072 & 8250 & 293.5 & 4 & 0 & U. \\
\hline 110113 & 78.0000 & 6.8000 & 293.4 & 4 & & . \\
\hline 10114 & 68.2524 & 6.3750 & 29 & 4 & & \\
\hline 110115 & 83.7529 & 5000 & 293.4 & 4 & .0 & 0 • \\
\hline 110116 & 80.7502 & 7.9250 & 292.3 & 4 & 0 & 0 . \\
\hline 110117 & 83.0000 & 707 & 292.4 & 4 & & \\
\hline 0110118 & 82.5000 & 000 & 293.2 & 4 & & 0 • \\
\hline 110119 & 107.4766 & 3.6000 & 294.3 & 3 & 0 & 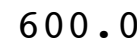 \\
\hline & 99.6015 & 0 & & 3 & & \\
\hline 111 & 87.7500 & 5.4750 & 295.4 & 3 & & 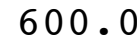 \\
\hline 1112 & 131.7300 & 5.9250 & 295.8 & 3 & .0 & 0 . \\
\hline 113 & 139.2698 & 6 . & 295.6 & 4 & & . \\
\hline 114 & 152.5037 & 6.5250 & 296.1 & 4 & .0 & 600. \\
\hline 1115 & 138.4539 & 4.4250 & 296.5 & 3 & .0 & 0 \\
\hline 116 & 123.5063 & 750 & 296.6 & 3 & & 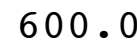 \\
\hline 117 & 85.2450 & 4.5500 & 296.1 & 4 & .0 & 600 . \\
\hline 1118 & 82.0000 & 5.6000 & 294.8 & 4 & .0 & 00 . \\
\hline 1119 & 76.0001 & 6.9500 & 293.1 & 4 & & \\
\hline 01101120 & 80.5000 & 6.3000 & 292.8 & 4 & 0 & 600. \\
\hline 1121 & 114.8854 & 3.8750 & 293.4 & 4 & 600.0 & 600 \\
\hline 22 & 147 & & 29 & 4 & & \\
\hline 1123 & 147.0000 & 6.4250 & 293.8 & & 600.0 & $=0$ \\
\hline
\end{tabular}




\begin{tabular}{|c|c|c|c|c|c|c|}
\hline 24 & & & & & & \\
\hline 121 & 2503 & 0 & 2.0 & & & \\
\hline 0122 & 100 & 500 & 91.3 & & 00 & \\
\hline 0123 & 5002 & 2.5000 & 290.6 & & 0 & \\
\hline 0124 & & & 89.9 & & & \\
\hline 0125 & 502 & 0 & 89.6 & & & \\
\hline 0126 & 7501 & 3250 & 89.2 & & 00 & \\
\hline 10127 & 00 & 50 & & & & \\
\hline 10128 & 00 & 0 & & & & \\
\hline 10129 & 51.4997 & 000 & 89.9 & & 0 & \\
\hline 1210 & 164.2502 & 0750 & 91.2 & & & \\
\hline 211 & 57.9962 & & 92.8 & & & \\
\hline 212 & 98 & 8. & 293.9 & & & \\
\hline 213 & 2475 & 7 . & 3 & & & \\
\hline 214 & & & & & & \\
\hline & & & 29 & 4 & & \\
\hline 216 & 5000 & 0 & 298.6 & 2 & & \\
\hline 217 & 04 & & 9 & & & \\
\hline & & & 3 & 4 & & \\
\hline 9 & 271 & & 298.2 & 4 & & \\
\hline 220 & 145.7299 & 4 . & 298.1 & 4 & & \\
\hline & & & & 4 & & \\
\hline 2 & 17 & 4. & $29^{\circ}$ & 5 & & \\
\hline 223 & 141.0000 & 5.7250 & 8 & 4 & & \\
\hline 224 & 119. & & & 4 & & \\
\hline 31 & 126 . & & 29 & 4 & & \\
\hline 110132 & 125.0115 & 750 & 294 & 4 & & \\
\hline 110133 & 129 . & 0 & & 4 & & \\
\hline 134 & 13 & 8 . & & 4 & & \\
\hline 110135 & 121.7545 & & 29 & 4 & & . \\
\hline 110136 & 130.7485 & 7.8000 & 293.4 & 4 & & \\
\hline 110137 & 142 . & 0 & & 4 & & \\
\hline 110138 & & & & 4 & & \\
\hline 110139 & 148.7499 & 750 & 293.0 & 4 & & \\
\hline & 144.9994 & & & 4 & & \\
\hline & 152.2499 & & & 4 & & \\
\hline 312 & 147.7478 & 9250 & 296.6 & 4 & & \\
\hline & 143 . & 5 . & & 3 & & \\
\hline 314 & 153.2492 & 750 & .6 & 3 & & . \\
\hline 01315 & 147.5006 & & 300.2 & 3 & & \\
\hline & 158.0050 & 000 & & 0 & & \\
\hline & 140.7550 & 2.1750 & 299.9 & 4 & & \\
\hline 01318 & 124.7988 & 1.5500 & 299.0 & 5 & & 0 \\
\hline & 553 & 500 & & 4 & & \\
\hline & 98.9266 & & & 5 & & \\
\hline 01201 & 7595 & 1.9750 & 298.9 & 6 & & 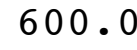 \\
\hline & & & & 3 & & \\
\hline 01323 & 10 & 5.1000 & 299.0 & & & \\
\hline
\end{tabular}




\begin{tabular}{|c|c|c|c|c|c|c|}
\hline 324 & & 6.4750 & 8.7 & & & \\
\hline 0141 & 04 & 7.6000 & 297.4 & 4 & 00.0 & \\
\hline 0142 & 148.7499 & 8.6250 & 295.7 & 4 & 00 & \\
\hline 10143 & 139.0096 & 7250 & 295.3 & & 00 & \\
\hline $014 \quad 4$ & 99.5199 & .8250 & 95.7 & & 0 & \\
\hline 0145 & 97.9943 & & 95.1 & & 0 & \\
\hline 0146 & 111.5000 & 2000 & 295.0 & 4 & 00 & \\
\hline 10147 & 132.7666 & 3.6500 & 294.4 & & 00.0 & \\
\hline 10148 & 148.9917 & 0 & 94.4 & 4 & & \\
\hline $014 \quad 9$ & 152.5170 & 2.1000 & 295.6 & 4 & 00 & 0 \\
\hline 1410 & 156.0138 & 2.5000 & 297.1 & 3 & & \\
\hline & 229.0877 & 2.7750 & 98.9 & & & \\
\hline 2 & 4994 & 4.5750 & 297.9 & 3 & 0 & \\
\hline 413 & 163.4982 & 4.1750 & 298.8 & 3 & 0.0 & \\
\hline & 159 . & & 30 & & & \\
\hline & 169. & & 30 & 3 & & \\
\hline 16 & 162.0000 & 4.1750 & 300.2 & 3 & .0 & 8 \\
\hline 17 & 137. & 0 & 99.7 & 4 & & \\
\hline & 101. & & 299.6 & 4 & & \\
\hline 19 & 000 & 500 & 299.3 & 5 & & . \\
\hline 420 & 105.7545 & 1.7500 & 298.8 & 5 & 0 & \\
\hline & 104.9605 & & 299.2 & 4 & & \\
\hline 22 & 5017 & 50 & 298.7 & 4 & & \\
\hline 423 & 127.5049 & 2.9750 & 298.4 & 4 & 0 & \\
\hline 424 & 132.2343 & & 298 & 4 & & \\
\hline 51 & 143.7505 & 2 . & 297.6 & 5 & & \\
\hline 110152 & 144.9995 & 500 & 296.3 & 5 & 0 & U. \\
\hline 110153 & 118.9015 & 0 & 296.2 & 6 & & \\
\hline 154 & 278.8130 & 50 & 295 & 6 & & \\
\hline 110155 & 349.7445 & 00 & 295.0 & 5 & 0 & U. \\
\hline 110156 & 298.8244 & 000 & 294.6 & 6 & 0 & 0 . \\
\hline 110157 & 128.9673 & 50 & 294.9 & 5 & & \\
\hline 110158 & 189.2954 & 0 & 296.6 & 4 & & . \\
\hline 110159 & 224.9656 & 5000 & 298.4 & 3 & 0 & 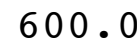 \\
\hline & 279.7423 & 250 & 298.9 & 2 & & \\
\hline & 256.4837 & 1.9000 & 298.9 & 1 & & 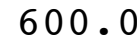 \\
\hline 512 & 224.9768 & 1.8750 & 299.6 & 1 & & 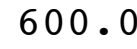 \\
\hline 513 & 281.7642 & 1. & 300.7 & 1 & & 0 . \\
\hline 514 & 272.6527 & 1.7250 & 302.4 & 1 & .0 & 0 . \\
\hline 01515 & 227.0290 & 5000 & 302.3 & 1 & & \\
\hline 516 & 207.5347 & 250 & 301.8 & 2 & & 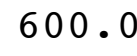 \\
\hline 17 & 176.7564 & 3.4000 & 300.2 & 3 & .0 & 0 . \\
\hline 1101518 & 145.0000 & 3.3750 & 298.8 & 4 & 0 & 00 . \\
\hline 01101519 & 114.7787 & 500 & 298.2 & 4 & & \\
\hline 01101520 & 83.7499 & 4.8250 & 297.7 & 5 & 0 & 600. \\
\hline 1101521 & 114.2600 & 2.1500 & 298.0 & 5 & 600.0 & 00. \\
\hline & & & 297 & 5 & & \\
\hline 1101523 & 86.4966 & 1.1250 & 297.0 & 0 & 600.0 & 60 \\
\hline
\end{tabular}




\begin{tabular}{|c|c|c|c|c|c|c|}
\hline 524 & ע & 1.5500 & 296.9 & 5 & 600.0 & \\
\hline 10161 & 8.5226 & 1.4500 & 297.4 & & 600.0 & \\
\hline 0162 & 329.8969 & 5750 & 296.4 & 0 & 00 & \\
\hline 10163 & 334.1999 & 5250 & 296.5 & & 00 & \\
\hline 0164 & 120.9056 & 1500 & 295.4 & & 0 & \\
\hline 0165 & 133.8487 & 4000 & 294.5 & & 00 & \\
\hline 0166 & 155.2550 & 5500 & 294.2 & & 00 & \\
\hline 0167 & 52.8371 & 500 & 293.9 & & 0 & \\
\hline 0168 & 125.2566 & 2000 & 294.7 & & & \\
\hline 0169 & 317.3371 & 9500 & 296.8 & & 00 & 0 \\
\hline 1610 & 269.9527 & 1.4500 & 297.3 & & 0 & \\
\hline & 262.2608 & 1.6750 & 297.1 & & 00.0 & \\
\hline 12 & 218 & 750 & 297.7 & & 0 & \\
\hline 613 & 164.7505 & 4.0000 & 297.2 & & 0.0 & \\
\hline 14 & 185 . & & 297 & & & \\
\hline & 203 . & & 298.1 & & & \\
\hline 16 & 178.5000 & 4.9500 & 296.3 & & 0.0 & . \\
\hline 17 & 86.4207 & 50 & 296.0 & & 0 & \\
\hline & 21 . & & 293.7 & & & \\
\hline 519 & 924 & 2.3 & 293.4 & & 0 & 0 \\
\hline 520 & 108.1307 & 1.5750 & 294.1 & & .0 & 0. \\
\hline & 96.4288 & & 292.5 & 6 & & \\
\hline 22 & 38 & 50 & 291.2 & 5 & & \\
\hline 523 & 19.8599 & 2.6750 & 292.3 & 6 & 0 & \\
\hline 24 & 16 . & & 291.9 & 6 & & \\
\hline 71 & 20 & 500 & 292.3 & 5 & & \\
\hline 110172 & 58.4328 & 2.5750 & 291.5 & 6 & 0 & U. \\
\hline 110173 & 78.3636 & 2.7500 & 290.5 & 6 & & \\
\hline 174 & 91 & 5 . & 289.2 & 5 & & \\
\hline 110175 & 79.9963 & 250 & 288.5 & 4 & 0 & 0 . \\
\hline 110176 & 76.7520 & 9250 & 287.9 & 4 & 0 & 0 . \\
\hline 177 & 80.4998 & 50 & 286.6 & 4 & & \\
\hline 110178 & 000 & 3500 & 286.6 & 4 & 0 & 0 • \\
\hline 110179 & 84.9996 & 250 & 288.1 & 3 & 0 & 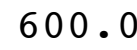 \\
\hline & 166. & 2 . & 290.2 & 2 & & \\
\hline 711 & 244.8318 & 2.0750 & 291.3 & 1 & & ( \\
\hline 1712 & 194.3312 & 3.3250 & 291.8 & 2 & $\cdot 0$ & 0 . \\
\hline 713 & 168.7541 & 2.8000 & 292.9 & 2 & & 00 . \\
\hline 714 & 162.0000 & 3.9500 & 293.5 & 3 & .0 & 0 . \\
\hline 01715 & 174.7498 & 3250 & 293.8 & 3 & & \\
\hline & 163.0005 & 750 & 294.0 & 4 & & 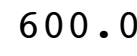 \\
\hline 717 & 155.7506 & 3.7250 & 293.7 & 5 & .0 & 0 . \\
\hline 1101718 & 167.2544 & 3.1750 & 292.9 & 4 & .0 & 00 . \\
\hline 01719 & 141.0892 & 1.2250 & 291.2 & 4 & & \\
\hline 01101720 & 105.9984 & 1.2250 & 290.8 & 5 & & 00 \\
\hline 1101721 & 75.5012 & 4.0500 & 290.9 & 4 & 600.0 & 00. \\
\hline & & 4.1500 & 290.8 & 4 & & \\
\hline 01723 & 95.8169 & 2.2000 & 292.0 & & 600.0 & $60 \Omega$ \\
\hline
\end{tabular}




\begin{tabular}{|c|c|c|c|c|c|c|}
\hline 724 & 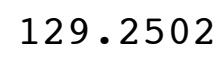 & 1.5750 & 1.5 & & & \\
\hline 110181 & 511 & 2.1750 & 291.0 & & & \\
\hline 10182 & 133 & 9000 & 290.8 & 4 & 00 & \\
\hline 110183 & 136.4613 & 6000 & 291.0 & & 00 & \\
\hline 0184 & 141 . & 0 & 290.6 & & 0 & \\
\hline 0185 & 0000 & .9750 & 290.7 & & 00 & \\
\hline 110186 & 143.2489 & 3.1750 & 290.0 & & 00 & \\
\hline 0187 & 142.2500 & 0 & 89.5 & & & \\
\hline 10188 & 129.5780 & 1.6750 & 291.1 & & 0 & \\
\hline 110189 & 156.7775 & 2.8500 & 293.0 & & 00.0 & \\
\hline 01810 & 157.2490 & 4.17 & 293.3 & & 0 & \\
\hline 811 & 163.2506 & & 293.9 & & & \\
\hline 812 & 164.5000 & 6.0750 & 294.4 & 4 & 0 & \\
\hline 813 & 160.0000 & 4.87 & 295.6 & 3 & 0.0 & \\
\hline & 157.7483 & & 296.5 & 3 & & \\
\hline 315 & 165.7538 & 4 . & 297.3 & 3 & 0 & \\
\hline 816 & 145.7498 & 0 & 298.2 & 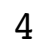 & 0 & \\
\hline 317 & 146.7496 & 0 & 298.2 & 5 & & \\
\hline 318 & 125.2835 & 2 . & 297.1 & 5 & 0 & \\
\hline 1819 & 143.7518 & 0 & 296.1 & 5 & 0 & \\
\hline 820 & 91.8976 & 4 . & 296.7 & 4 & 0 & \\
\hline 21 & 91.9943 & & 296.6 & 4 & & \\
\hline 822 & 89.7430 & 0 & 296.9 & 4 & 0 & \\
\hline 823 & 81.1933 & 2 & 296.9 & 4 & 0 & \\
\hline 324 & 019 & & 296.4 & 5 & & \\
\hline 110191 & 918 & 0 & 295.6 & 6 & & \\
\hline 110192 & 224.9897 & 1 . & 294.9 & 6 & 0 & \\
\hline 0110193 & 358.2574 & & 293.8 & 5 & & $\bullet^{\circ}$ \\
\hline 0110194 & 79 & 0 & .9 & 4 & 0 & 0 . \\
\hline 110195 & 342.8150 & 0.27 & 293.7 & 5 & 0 & \\
\hline 110196 & 16.3119 & & 293.1 & 5 & & \\
\hline 0197 & 196 & 0 & 293.3 & 6 & & $\sigma_{0}$ \\
\hline 0110198 & 292.7122 & $\begin{array}{lll}0 & 12\end{array}$ & 296.3 & 5 & 0 & U. \\
\hline 110199 & 296.9769 & 0 . & 297.8 & 4 & 0 & \\
\hline 10 & 278 & 1 . & 297.3 & 3 & & \\
\hline 11 & 279.4768 & 1.7750 & 297.0 & 2 & & 0 . \\
\hline 01912 & 227.1839 & 1.6000 & 297.1 & 1 & 0 & \\
\hline 913 & 177.6945 & 1 . & 296.9 & 2 & 0 & \\
\hline 914 & 232.7374 & 1.4750 & 298.2 & 1 & 60 & 0 . \\
\hline 1915 & 233. & 0 & 298.7 & 1 & 600 & \\
\hline 916 & 207.6129 & 2 . & 299.0 & 2 & & \\
\hline 01101917 & 153.0012 & 2.6000 & 297.5 & 3 & 600.0 & 600 . \\
\hline 01918 & 122.1357 & 2.5750 & 296.6 & 4 & 600 & 600. \\
\hline 01101919 & 106.6450 & 2.4750 & 296.8 & 5 & 600 & 60 \\
\hline 01101920 & 121.0743 & 1.9500 & 295.9 & 6 & 600.0 & 00 . \\
\hline 0192 & 9979 & 4.4500 & 296.5 & 5 & 600.0 & 600 \\
\hline & 005 & & 296.1 & 4 & 600 & 60 \\
\hline 01923 & 104.8496 & 1.9250 & 295.1 & $J$ & 600.0 & 600 \\
\hline
\end{tabular}




\begin{tabular}{|c|c|c|c|c|c|c|}
\hline 924 & 3.7626 & 3.0250 & 295.4 & 4 & 600.0 & \\
\hline 110201 & $2 \quad 2512$ & 5.2750 & 295.3 & 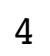 & 6000 & \\
\hline 10202 & 018 & 500 & 95.2 & 4 & 0 & \\
\hline 0203 & 1.0000 & 000 & 5.5 & & 00 & \\
\hline $020 \quad 4$ & 0.4819 & & & & & \\
\hline 10205 & 865 & & & & 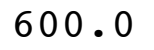 & \\
\hline 110206 & 722 & & 91.3 & & 0 & \\
\hline 110207 & 7888 & 50 & 1.1 & & 0 & \\
\hline 110208 & 537 & 7500 & 92.9 & & 0 & \\
\hline 110209 & 303.1144 & & 95.6 & & 0 & \\
\hline 2010 & 276 . & & & & & \\
\hline 011 & 270 . & & 4 & & & \\
\hline 012 & 274 . & 9500 & 297.2 & & 0 & \\
\hline 013 & 264 & 0 & & & & \\
\hline 014 & 245 & & & & & \\
\hline 015 & 218 & & 298.9 & & & \\
\hline 02016 & 166.7083 & 3.55 & 298 & & 0 & \\
\hline 017 & 169.1961 & & 8 & & & \\
\hline 018 & 100 & & 0 & & & \\
\hline 019 & 700 & 0 & 294.2 & & & \\
\hline 20 & 35 & & 2 & & & \\
\hline 021 & 69 & & 29 & 4 & & \\
\hline 022 & 67.2862 & 0 & 293 & & & \\
\hline 023 & $70 \quad 25$ & & & & & \\
\hline 24 & & & 29 & 4 & & \\
\hline 211 & 7288 & & 292 & 2 & & \\
\hline 110212 & 77.7342 & & 292.7 & 4 & & \\
\hline 110213 & 64.5018 & & 292.1 & 4 & & \\
\hline 110214 & & & & 4 & & \\
\hline 110215 & 68 . & 8 . & & 4 & & \\
\hline 110216 & 70 & & & 4 & & \\
\hline 110217 & & & & 4 & & \\
\hline 110218 & 68.7500 & 0 & 286.5 & 4 & & \\
\hline 0110219 & 74.2500 & 0 & 286 & 4 & & \\
\hline 110 & & & & 4 & & \\
\hline 1102111 & 290 & & 289.9 & 3 & & \\
\hline 12 & & & & 4 & & \\
\hline 1102113 & 75.7461 & & 289.4 & 4 & & \\
\hline 1102114 & 81.5000 & & 289.7 & 4 & & \\
\hline 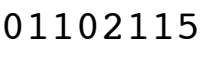 & 80 . & & & 4 & & \\
\hline & 505 & 0 & 289.0 & 4 & & \\
\hline 1102117 & 69.4985 & 10. & 287.9 & 4 & & \\
\hline 110 & 69 . & & 288.0 & 4 & & \\
\hline 01102119 & 76.0002 & 10.8250 & 285.9 & 4 & & $\cos _{1}+2$ \\
\hline 1102120 & 67.7498 & 9.0000 & 288.7 & 4 & .0 & 00 . \\
\hline & & & & 4 & & \\
\hline 2122 & 76.9963 & & 288.4 & & & 600 \\
\hline 02123 & 84.2544 & 6.7750 & 290 . & & 600.0 & 50 \\
\hline
\end{tabular}




\begin{tabular}{|c|c|c|c|c|c|c|}
\hline 124 & 8.5005 & 9.0250 & 288.4 & 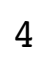 & 600.0 & \\
\hline 110221 & 2.7493 & 7.5750 & & 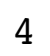 & 1000 & \\
\hline 0222 & 82.9973 & 750 & 39.6 & 4 & 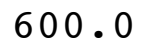 & \\
\hline 10223 & 88.2534 & 50 & 89.5 & & 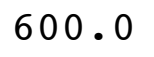 & \\
\hline 0224 & 9.7487 & & & & & \\
\hline 10225 & 105.2715 & & 89.8 & & O & \\
\hline 110226 & 3.0000 & & 88.1 & & - & \\
\hline 110227 & 5.5000 & & 7.9 & & & \\
\hline 110228 & 22.6463 & 4 . & 89.4 & & 0 & \\
\hline 110229 & 161.2575 & & 290.4 & & 0 & \\
\hline 2210 & 165.0009 & & 9 & & & \\
\hline 2211 & 162.5000 & & 5 & & & \\
\hline 2212 & 162.4995 & & 293.0 & & 0 & \\
\hline 213 & 164 . & 0 & 4 & & & \\
\hline & 152 . & & 94 & & & \\
\hline 215 & 154 . & & 295.6 & & & \\
\hline 2216 & 150.7508 & 0 & 295.6 & & 0 & \\
\hline 217 & 107.2655 & & 295.0 & & & \\
\hline 218 & 73. & & 293 & a & & \\
\hline 219 & 76.9999 & & 292.9 & & & \\
\hline & 74 . & & 292.3 & & & \\
\hline & 76 . & & 292.1 & 4 & & \\
\hline & 71.7500 & & 291.4 & 4 & & \\
\hline 223 & 68.25 & & 291 & 4 & & \\
\hline 24 & & & 290 & 4 & & \\
\hline 231 & 69. & & 289.6 & 4 & & \\
\hline 110232 & 71.9993 & 0 & 288.9 & 4 & & \\
\hline 110233 & 77 . & & 288.4 & 4 & & \\
\hline 110234 & 73. & & 287.8 & 4 & & \\
\hline 110235 & 68.75 & & 287.5 & 4 & & \\
\hline 110236 & 67 . & & 287.7 & 4 & & \\
\hline 110237 & 67 . & & 28 & 4 & & \\
\hline 110238 & 70.2506 & & 288.1 & 4 & & \\
\hline 110239 & 73.50 & 0 & 288.6 & 4 & & \\
\hline & & & & 4 & & \\
\hline & 90.5000 & & 290.4 & 4 & & \\
\hline & 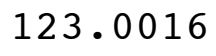 & & 291.1 & 3 & & \\
\hline 313 & 145.7437 & & 291.5 & 4 & & \\
\hline 1102314 & 160.9998 & & 292.0 & 4 & & \\
\hline & 164 . & & 292.6 & 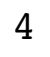 & & \\
\hline & 173. & & 293.0 & 4 & & \\
\hline 02317 & 174.4945 & 8.3 & 293.0 & 4 & & \\
\hline & 161. & & 291.9 & 4 & & \\
\hline 01102319 & 152.7500 & & 291.1 & 4 & & \\
\hline 1102320 & 149.0002 & 7.9000 & 291.0 & 4 & 0 & م. \\
\hline & & & 4 & 4 & & \\
\hline & 156.2500 & 00 & 290.9 & & & ( \\
\hline 23 & 139.2300 & 10.9500 & 290.4 & & 600.0 & 50 \\
\hline
\end{tabular}




\begin{tabular}{|c|c|c|c|c|c|c|}
\hline 324 & & & & & & \\
\hline 241 & 1 & 6.2500 & 9.5 & 4 & ) & \\
\hline 242 & 165.7501 & 4750 & 289.0 & 4 & 00 & \\
\hline 0243 & 159.9995 & 5500 & 288.0 & & 00 & \\
\hline $024 \quad 4$ & & 10.3500 & & & & \\
\hline 0245 & & 7.97 & 86.8 & & 0 & \\
\hline 0246 & 147 & 6.1750 & 287.0 & & 00 & \\
\hline 10247 & 10 & 7.2250 & 86.8 & & 0 & \\
\hline 10248 & 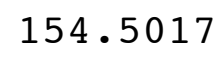 & 7000 & .9 & & & \\
\hline 1024 & 146.0000 & 4.8500 & 287.6 & & 00 & 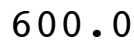 \\
\hline 410 & 145.4556 & 5.7750 & 288.5 & & & \\
\hline & 141 & 5.1000 & & 4 & & \\
\hline 2 & 143 & 4.3750 & 291.9 & 3 & 0 & \\
\hline & 146.7502 & 5.6250 & 292.7 & 3 & 0.0 & \\
\hline & -2 & 0 & 7 & 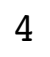 & & \\
\hline & & & 294.5 & 4 & & \\
\hline 6 & 5000 & 3.7500 & 295.0 & 4 & .0 & \\
\hline & 7540 & 1.7750 & .0 & 5 & & \\
\hline & 62 . & 1 . & 293.9 & 6 & & \\
\hline & 3163 & 1.3250 & 293.2 & 5 & & \\
\hline 20 & 24.5015 & 1.6750 & 293.5 & 4 & 0 & \\
\hline & 56.7103 & & & 5 & & \\
\hline 2 & 67 & 0 & 294.4 & 5 & & \\
\hline 23 & 141.7124 & 1.8750 & 293.9 & 5 & 0 & \\
\hline 24 & 151.5207 & & 293.2 & 4 & & \\
\hline 51 & 74.1166 & 0 & 292.6 & 5 & & \\
\hline 110252 & 112.4251 & 0.9500 & 292.0 & 6 & 0 & . \\
\hline 110253 & 135.0021 & 1.5000 & 291.6 & 5 & & \\
\hline 25 & 138.5075 & 3 . & .1 & 4 & & \\
\hline 110255 & 145.0000 & 750 & 289.9 & 4 & 0 & U. \\
\hline 110256 & 146.0000 & 500 & 290.0 & 4 & & 0 \\
\hline 257 & 138.7253 & 8 . & 289.0 & 4 & & \\
\hline 110258 & 130.79 & 5000 & 289.4 & 4 & & 0 . \\
\hline 110259 & 152.2498 & 000 & 290.8 & 4 & 0 & \\
\hline & 161. & 0 & 292.1 & 4 & & \\
\hline & 163.2500 & 000 & 292.5 & 4 & & . \\
\hline & 162.2505 & 8000 & 293.1 & 4 & & \\
\hline 13 & 170 . & 250 & 294.2 & 4 & & \\
\hline & 170.0032 & 4.0750 & 294.8 & 3 & .0 & 0 \\
\hline 15 & 173.7480 & 250 & 295.4 & 2 & & \\
\hline & 212.2573 & 1.7500 & 295.9 & 2 & & \\
\hline & 225.0000 & 1.2750 & 295.5 & 3 & & 0 . \\
\hline 02518 & 275.2939 & 1.0750 & 293.9 & 4 & 0 & 0 . \\
\hline 02519 & 355.7851 & 500 & & 5 & & \\
\hline & 4.7478 & 1.7500 & 293.3 & 4 & & \\
\hline & 21.7733 & 1.8250 & 293.7 & 4 & & 00 . \\
\hline & & & & 5 & & \\
\hline & 3182 & 0.2750 & 293.4 & & .0 & 60 \\
\hline
\end{tabular}




\begin{tabular}{|c|c|c|c|c|c|c|}
\hline $3<4$ & & . 6750 & & & & \\
\hline 10261 & 1 & 1.9000 & 3.9 & & 00 & \\
\hline 0262 & 147.2534 & 1.6750 & 292.5 & 4 & 00 & \\
\hline 10263 & 88.0548 & 0.7250 & 292.5 & & 00 & \\
\hline 0264 & 137.0281 & .3000 & 92.7 & & 0 & \\
\hline 0265 & 89.9160 & 1750 & 290.8 & & 00 & \\
\hline 10266 & 129.4402 & 1.6000 & 290.4 & & 00 & U \\
\hline 0267 & 159.6513 & 1.4500 & 290.3 & & 0 & \\
\hline 10268 & 136.2495 & 8000 & 89.5 & & & \\
\hline 10269 & 153.0008 & 3.2000 & 291.2 & & 600.0 & 0 \\
\hline 102610 & 158.0138 & 3.0750 & 292.1 & & 0 & \\
\hline 611 & 151.2398 & 3.1750 & 292.7 & & 00.0 & \\
\hline 612 & 162.0008 & 4.3750 & 293.2 & 4 & 0 & 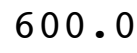 \\
\hline 2613 & 158.5009 & 3.6250 & 294.8 & & 0.0 & \\
\hline 614 & 158.2506 & 0 & 7 & & & \\
\hline & 161.0 & 3 . & 296.1 & 4 & & \\
\hline 616 & 151.4986 & 2.7750 & 296.4 & 4 & 0.0 & . \\
\hline 617 & 145.5003 & 2.6000 & 295.8 & & 0 & \\
\hline & 130.7613 & 500 & 294.4 & 5 & & \\
\hline 619 & 90.6360 & 1.0000 & 293.9 & 6 & 0 & \\
\hline 2620 & 138.8674 & 1.0250 & 293.6 & 6 & 0 & 00. \\
\hline & 64.3394 & 0.35 & & 6 & & \\
\hline 2 & 2.3244 & 1.2250 & 293.0 & 5 & & \\
\hline 623 & 12.9983 & 1.6500 & 293.3 & 4 & 0 & 0 • \\
\hline 524 & 1.9998 & & 29 & 4 & & \\
\hline 271 & 354.5551 & 1.5500 & 291.9 & 5 & & \\
\hline 110272 & 312.6431 & 0.2000 & 290.3 & 6 & 0 & . \\
\hline 110273 & 91.999 & 1.4250 & 28 & 6 & & \\
\hline $27 \quad 4$ & 102.2346 & 000 & 28 & 6 & & \\
\hline 110275 & 96.2465 & $\$ 750$ & 287.7 & 5 & .0 & 0 • \\
\hline 110276 & 92.7478 & 250 & 286.6 & 4 & 0 & 0 . \\
\hline $11027 \quad 7$ & 85.9999 & 0 & 286.2 & 4 & & \\
\hline 110278 & 78.4998 & 0 & 286.2 & 4 & & 0 • \\
\hline 110279 & 85.0007 & 3.7500 & 287.7 & 3 & 0 & 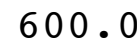 \\
\hline 2710 & 25 . & 2 . & & 2 & & \\
\hline & 246.7186 & 1.8500 & 290.0 & 1 & & 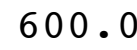 \\
\hline 1102712 & 232.5142 & 2.0500 & 290.7 & 1 & $\cdot 0$ & 0 . \\
\hline 713 & 181.1897 & 250 & 291.4 & 2 & & . \\
\hline 714 & 121.5287 & 5 . & 291.4 & 3 & .0 & 0 . \\
\hline 1102715 & 118.1102 & 5000 & 291.2 & 3 & 0 & . \\
\hline 2716 & 101.7683 & 500 & 290 & 3 & & 0 . \\
\hline 17 & 82.2524 & 5.9500 & 288.6 & 4 & .0 & 00 . \\
\hline 1102718 & 76.0002 & 6.0500 & 287.6 & 4 & .0 & 00 . \\
\hline 02719 & 78.2528 & 500 & 286.8 & 4 & & 0 \\
\hline & 64.2512 & 5.9000 & 286.4 & 4 & 0 & 600. \\
\hline 0272 & 66.5074 & 4.0000 & 286.3 & 4 & 600.0 & 600 \\
\hline & 94.0182 & & & 5 & & \\
\hline 120 & 84.2517 & 4.6000 & 286.1 & & 600.0 & 60 \\
\hline
\end{tabular}




\begin{tabular}{|c|c|c|c|c|c|c|}
\hline 724 & 4.2556 & 4.8750 & 85.8 & 5 & 600.0 & \\
\hline 110281 & 2.4675 & 2.9500 & 285.5 & 4 & 600.0 & \\
\hline 10282 & 27.5023 & 1.0250 & 285.2 & 5 & 00 & \\
\hline 10283 & 21.8137 & 1.3250 & 285.3 & & 00 & \\
\hline 0284 & 66.5255 & 7250 & & & 0 & \\
\hline 0285 & 110.2333 & .2750 & 85.9 & & 00 & \\
\hline 10286 & 56.5288 & 1.5250 & 284.9 & & 00 & \\
\hline 0287 & 57.7479 & 500 & & & & \\
\hline 10288 & 352.9768 & 7000 & 0 & & & \\
\hline 110289 & 262.3833 & 1.0000 & 88.1 & & 00 & 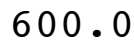 \\
\hline 1102810 & 231.2287 & 1.9000 & 288.6 & & & \\
\hline 811 & 281.3151 & 2.0500 & 39.1 & & & \\
\hline 812 & 295.6315 & 2.0250 & 290.2 & & 0 & \\
\hline 813 & 251.2259 & 2.3250 & 290.2 & & 0.0 & \\
\hline 814 & 141.8378 & 0 & .2 & & & \\
\hline 315 & 158.8863 & 750 & 290.6 & & & \\
\hline 816 & 162.4721 & 2.7250 & 289.9 & & .0 & 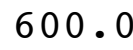 \\
\hline 817 & 106.6185 & 250 & 8 & & & \\
\hline 318 & 154 & 7250 & 288.7 & & & \\
\hline 819 & 152.9992 & 9250 & 287.9 & & & \\
\hline 820 & 153.0018 & 2.8000 & 287.1 & & 0 & 00 \\
\hline 21 & 109.7409 & 1.4500 & 287.4 & & & \\
\hline 322 & 76.74 & 3.3250 & 287 & & & \\
\hline 323 & 77.9998 & 2.5500 & 287.5 & & 0 & \\
\hline 24 & 90.0201 & 2.1500 & & & & \\
\hline 291 & 71.2756 & 1.8750 & 28 & 6 & & \\
\hline 110292 & 80.7502 & 3.5750 & 287.5 & & 0 & U. \\
\hline 110293 & 73.0100 & .4500 & 287 & 4 & & \\
\hline 294 & 8.5041 & 0.7500 & 28 & 5 & & \\
\hline 110295 & 326.9165 & 0.6250 & 286.4 & 6 & 0 & 0 • \\
\hline 110296 & 220.7242 & 0.2750 & 286.2 & 6 & & 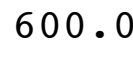 \\
\hline 297 & 358.5444 & 0 & 28 & 5 & & \\
\hline 110298 & 345.4890 & 5500 & 286.5 & 4 & 0 & 0 • \\
\hline 110299 & 317.2923 & 1.5750 & 287.9 & 3 & 0 & 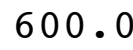 \\
\hline 910 & 269.0194 & 500 & 28 & 2 & & \\
\hline & 257.7733 & 2.7250 & 289.3 & 2 & & 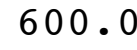 \\
\hline 912 & 265.0021 & .9000 & 289.7 & 2 & & 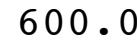 \\
\hline 13 & 259.3001 & .6500 & 290.1 & 1 & & 00 . \\
\hline 14 & 263.6152 & 1.8250 & 290.5 & 1 & .0 & 0 . \\
\hline 02915 & 288.0000 & 2.0250 & 290.0 & 2 & & \\
\hline 916 & 284.4510 & 750 & 289 & 3 & & 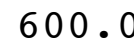 \\
\hline 17 & 279.2437 & 2.3250 & 289.7 & 4 & & 0 . \\
\hline 1102918 & 291.4778 & 2.4750 & 289.3 & 4 & 0 & 0 . \\
\hline 1102919 & 297.5043 & 1.6250 & & 5 & & \\
\hline 01102920 & 327.4992 & 1.9750 & 288.7 & 4 & & 0 . \\
\hline 1102921 & 351.6696 & 1.7500 & 288.8 & 5 & 600.0 & 00. \\
\hline & 288.9659 & & & b & & \\
\hline 02923 & 245.9429 & 0.7250 & 288.7 & & .0 & 60 \\
\hline
\end{tabular}




\begin{tabular}{|c|c|c|c|c|c|c|}
\hline 924 & & $3 / 50$ & & & & \\
\hline 0301 & 276.9493 & .7500 & 8.4 & 5 & 600.0 & \\
\hline 0302 & 254.0000 & 3000 & 288.0 & 6 & 00 & \\
\hline 10303 & 118.2672 & 8000 & 288.1 & & 00 & \\
\hline 030 & 242.1942 & .8750 & & & 0 & \\
\hline 0305 & 319.3108 & 250 & 86.9 & & 00 & \\
\hline 0306 & 321.5000 & 9750 & 286.8 & 4 & 00 & \\
\hline 0307 & 336.2488 & 000 & 287.2 & & 00 & \\
\hline 0308 & 30.7498 & 000 & 87.1 & 4 & & \\
\hline 10309 & 329.4986 & 2.6500 & 286.7 & 3 & 600.0 & 0 \\
\hline 3010 & 289.5706 & 1.8750 & 287.8 & & 0 & \\
\hline 11 & 290.4883 & 2.7750 & 88.8 & $\perp$ & 00.0 & \\
\hline 2 & 10.5316 & 3.2250 & 290.1 & 2 & 0 & 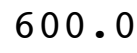 \\
\hline 13 & 29.2481 & 3.4250 & 292.1 & 2 & 0.0 & \\
\hline & 88.2877 & & 290 & 3 & & \\
\hline 5 & 80.497 & & 290 & 4 & & \\
\hline 16 & 76.9945 & 3.6500 & 290.1 & 3 & .0 & 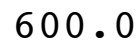 \\
\hline 17 & 96.0046 & 500 & 289.0 & 4 & & \\
\hline & 70.499 & & 287.3 & 4 & & \\
\hline 19 & 72.2503 & 0 & 286.9 & 4 & & \\
\hline 020 & 63.5005 & 7.2500 & 286.8 & 4 & 0 & • \\
\hline & 351.2658 & & & 5 & & \\
\hline 022 & 100.4650 & 1.3000 & 28 & 6 & & \\
\hline 023 & 47.6613 & 3.0250 & 287.0 & 5 & 0 & \\
\hline 24 & 118.9366 & & 3 & 6 & & \\
\hline 311 & 377 & 0 . & 28 & 6 & & \\
\hline 110312 & 193.8791 & 1.1500 & 285.5 & 6 & 0 & U. \\
\hline 110313 & 100.9221 & 000 & 284.8 & 6 & & \\
\hline 110314 & 140.2633 & 2 . & 284 & 6 & & \\
\hline 110315 & 67.6705 & 250 & 284.1 & 6 & 0 & 0 • \\
\hline 110316 & 102.4606 & 1.4000 & 284.5 & 6 & 0 & 0 . \\
\hline 317 & 81.1453 & 250 & 284.4 & 6 & & \\
\hline 0110318 & 91.9006 & 750 & 285.5 & 5 & & 0 \\
\hline 110319 & 180.2539 & 0.4000 & 288.7 & 4 & 0 & $\bullet$ \\
\hline & 167.0339 & 250 & & 3 & & \\
\hline & 167.9989 & 4.1750 & 287.3 & 4 & & 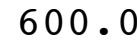 \\
\hline 112 & 169.9848 & 1000 & 288.0 & 3 & & 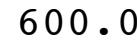 \\
\hline 113 & 175.2272 & 000 & 289.1 & 2 & & . \\
\hline 114 & 164.5000 & 2.7750 & 289.9 & 1 & .0 & 0 . \\
\hline 115 & 158.9992 & 4.2250 & 290.0 & 2 & & \\
\hline & 165.2486 & 000 & 290.0 & 3 & & 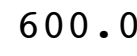 \\
\hline 17 & 164.7367 & 3.9750 & 289.6 & 4 & .0 & 00 . \\
\hline 1103118 & 158.0000 & 2.6750 & 288.2 & 4 & .0 & 00 . \\
\hline 01103119 & 108.3382 & 750 & 287.5 & 5 & & \\
\hline & 98.5009 & 0.8500 & 287.5 & 5 & 0 & 600. \\
\hline 0312 & 85.7050 & 1.5750 & 287.6 & 4 & 600.0 & 00 \\
\hline & 87 & 00 & 7 & 4 & & \\
\hline 03123 & 95.1884 & 2.2000 & 287.5 & 7 & 600.0 & 600 \\
\hline
\end{tabular}




\begin{tabular}{|c|c|c|c|c|c|c|c|}
\hline 17 & 24 & 77.2492 & 3.9500 & 287.7 & 5 & 600.0 & \\
\hline 11 & 11 & 88.2391 & 3.1500 & 287.8 & & 600.0 & \\
\hline 1 & 1 & 149.7644 & 5000 & 286.8 & 4 & 600.0 & \\
\hline 11 & 13 & 155.5000 & 0000 & 286.0 & & 00.0 & \\
\hline & 14 & 152.7499 & .0500 & & & 00 & \\
\hline & 15 & 164.6445 & 250 & 285.4 & & 00 & \\
\hline & 16 & 28.9990 & 1750 & 285.3 & & 00 & \\
\hline & 17 & 151.5000 & 7000 & 85.0 & & & \\
\hline & 18 & 142.6886 & 0.1750 & 287.3 & & 0 & \\
\hline 1. & 19 & 209.0607 & 0.3500 & 290.1 & & 00.0 & \\
\hline & 110 & 161.7910 & 0500 & 289.2 & & 0 & \\
\hline & 111 & 161.0023 & & 88.0 & & & \\
\hline 1 & 112 & 172.2455 & 7000 & 288.1 & & 0 & \\
\hline 1. & 113 & 187.3334 & 3.8000 & 289.0 & & 0.0 & \\
\hline & 114 & 358.6667 & & & & & \\
\hline 1 & 11 & 170 . & 2.5000 & 290.2 & & 0 & \\
\hline & 116 & 167.7519 & 1.9250 & 290.2 & & 0 & \\
\hline & 117 & 174.9930 & 0 & 289.6 & & & \\
\hline 1 & 118 & 172.2265 & 0 . & 288.3 & 4 & 0 & \\
\hline 1 & 119 & 152.9940 & 0000 & 287.8 & 5 & 0 & \\
\hline L & 120 & 151.2504 & 0 & 287.7 & 4 & & \\
\hline & 121 & 132.4908 & & 287.7 & 4 & & \\
\hline & 122 & 130.0007 & 0 & 288.0 & 4 & 0 & \\
\hline 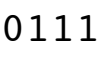 & 123 & 134.2869 & 0 & 287.9 & 5 & 0 & \\
\hline & 124 & 138.2503 & & 28 & 5 & & \\
\hline & 21 & 104.5764 & 0 & 287.2 & 4 & & \\
\hline 1 & 22 & 100.1879 & 0.32 & 287.7 & 4 & 0 & \\
\hline & 23 & 118.5688 & & 287 & 5 & 0 & $\mathrm{C}$ \\
\hline & 24 & 57.4898 & 0 & 286.7 & 6 & 0 & 0 . \\
\hline & 25 & 165.5299 & 0 & 286.0 & 5 & 0 & \\
\hline & 26 & 171.5122 & & 285.6 & 4 & & \\
\hline & 27 & 80.2332 & 0 & 285.0 & 5 & & \\
\hline & 28 & 101.4728 & 000 & 285.9 & 6 & 600 & 0 • \\
\hline 1 & 29 & 155.9823 & 0 & 287.2 & 5 & 0 & \\
\hline & 210 & 165 . & 0 & 289.2 & 4 & & \\
\hline & 211 & 185.5000 & 1.9750 & 289.0 & 3 & 0 & 0 . \\
\hline & 212 & 187 . & 2.7000 & 289.8 & 4 & 0 & \\
\hline & 213 & 188.0001 & 3.12 & 290.0 & 4 & 0 & 0 \\
\hline & 214 & 174.0000 & 3.3000 & 290.3 & 3 & 600.0 & 0 . \\
\hline & 215 & 166.7395 & 750 & 291.0 & 3 & 600 & \\
\hline & 216 & 183.5121 & 000 & 290.9 & 3 & & \\
\hline & 217 & 170.4908 & 3.5500 & 290.2 & 4 & 600.0 & 600 . \\
\hline & 218 & 158.5000 & 2.2000 & 289.2 & 4 & 600 & 600 \\
\hline & 219 & 133.5000 & 1.3250 & 288.8 & 4 & 600 & 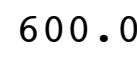 \\
\hline & 220 & 76.8773 & 1.7250 & 289.2 & 4 & 600.0 & 00 . \\
\hline & 221 & 102.3416 & 1.7750 & 289.2 & 5 & 600.0 & 600. \\
\hline & 222 & 9804 & 00 & 289.3 & 4 & 600 & \\
\hline & 223 & 78.2508 & 3.3750 & 289.2 & & 600.0 & 600 \\
\hline
\end{tabular}




\begin{tabular}{|c|c|c|c|c|c|c|c|}
\hline 1 & 24 & ועל & 3.9750 & 9.5 & & 000 & \\
\hline 111 & 31 & 100.9218 & 2.5000 & 290.0 & 4 & 600.0 & \\
\hline & 32 & 4990 & 0000 & 289.2 & 1 & 00 & \\
\hline & 33 & 133.5000 & 1.5500 & 288.7 & & 00.0 & \\
\hline & 34 & 132.7 & 1.3500 & 88.5 & & 0 & \\
\hline & 35 & 131.7521 & 1.8000 & 288.7 & & 00 & \\
\hline & 6 & 130.7514 & 3.0500 & 288.3 & & 00 & \\
\hline & 7 & 136.5000 & .4500 & 88.2 & & 00 & \\
\hline & 8 & 135.4957 & 2.0000 & 288.6 & 4 & 00.0 & \\
\hline & 9 & 139.4778 & 1.4500 & 289.3 & & 0 & \\
\hline & 10 & 168.9999 & & 89.8 & & & \\
\hline & 11 & 168 . & & 289.1 & 4 & & \\
\hline 1. & 12 & 165.0000 & 5.1250 & 290.0 & 3 & 00.0 & \\
\hline & 313 & 169.9995 & 4 . & 291.6 & 4 & 0 & \\
\hline & 14 & 165 . & & 292.8 & 4 & & \\
\hline & 15 & 159.2232 & 50 & 293.7 & 3 & 0 & \\
\hline$\perp$ & 316 & 138.0012 & 2.27 & 294.1 & 3 & 0 & \\
\hline & 317 & 137.00 & & 293.4 & 4 & & \\
\hline 1 & 18 & 126 . & 0 & 292.7 & 4 & 0 & \\
\hline & 319 & 124.0245 & 2.20 & 292.5 & 5 & 0 & \\
\hline & 320 & 108 . & & 293.2 & & & \\
\hline & 21 & 118.26 & 2 . & 293.5 & 5 & & \\
\hline 1 & 322 & 89.3150 & 1.37 & 293.6 & 5 & 0 & \\
\hline & 323 & 86.5034 & 0 & 293.7 & 5 & & \\
\hline & 324 & 102 & & 293 & 5 & & \\
\hline & 41 & 116.2429 & 007 & 293.7 & 6 & & \\
\hline 1 & 42 & 126.5046 & 0.90 & 293.4 & 6 & & \\
\hline & 43 & 132.2515 & 0 & 292.7 & 6 & & \\
\hline & 44 & 142.7501 & 150 & 291.7 & 5 & & \\
\hline & 45 & 124.5511 & 1.22 & 291.1 & 6 & & \\
\hline & 46 & 119.74 & & 291.5 & 5 & & \\
\hline & 47 & 125 . & 0 & 291.5 & 4 & & \\
\hline 1 & 48 & 108.6461 & 1.9 & 292.9 & 5 & & \\
\hline & 49 & 121.2432 & 0 & 294 & 4 & & \\
\hline & 410 & 165. & & 293.4 & 4 & & \\
\hline & 411 & 160.2661 & & 292.7 & 4 & & \\
\hline & 412 & 162.00 & 4 . & 293.5 & 4 & & \\
\hline & 413 & 166.5000 & 4.8250 & 294.9 & 4 & & 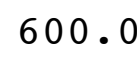 \\
\hline & 414 & 166.5000 & 9750 & 295.3 & 4 & & 6 \\
\hline & 415 & 160.0005 & 0 & 296.0 & 4 & & \\
\hline & 416 & 162.0002 & 5 . & 296.4 & 4 & & 6 \\
\hline & 417 & 158.7504 & 4.3750 & 295.6 & 4 & .0 & 0 \\
\hline & 418 & 110.4444 & & 294.6 & 4 & & \\
\hline & 419 & 80.5003 & 5.4500 & 294.8 & 4 & 600.0 & 600 \\
\hline & 420 & 67.2490 & 4.7250 & 294.6 & 4 & 600.0 & 600 \\
\hline & 421 & 71 & & 294.6 & 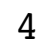 & & \\
\hline & 422 & 73.5000 & 5.7000 & 294.5 & 4 & & 600 \\
\hline & 423 & 66.7497 & 4.6750 & 294.0 & & 600.0 & 600 \\
\hline
\end{tabular}




\begin{tabular}{|c|c|c|c|c|c|c|c|}
\hline & 24 & & 4.3750 & 94.1 & & & \\
\hline 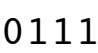 & 51 & 02363 & 4.8500 & 293.7 & & & \\
\hline & 52 & 75.9998 & 7.0250 & 293.4 & $\Lambda$ & 00 & \\
\hline & 3 & 72.5009 & 7.2750 & 292.6 & & 00.0 & \\
\hline & 4 & 7.0007 & .0250 & 292.9 & & & \\
\hline & 5 & 88.7588 & 50 & 92.3 & & 00 & \\
\hline & 6 & 89.7987 & 000 & 291.9 & & 00 & \\
\hline & 7 & 12.1057 & 0.82 & 91.3 & & & \\
\hline & 8 & 190.2000 & 1.1000 & 288.8 & & 0 & \\
\hline & 9 & 121.5366 & 1.2750 & 288.6 & & 00.0 & \\
\hline & 10 & 281.9434 & 0.4000 & 292.0 & & & \\
\hline & 11 & 263.2434 & 50 & 92.5 & & & \\
\hline & 12 & 168.7453 & 750 & 292.3 & & 0 & \\
\hline 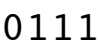 & 13 & 163.7528 & 5.0000 & 291.9 & & 00.0 & \\
\hline & 14 & 165.7504 & & 292.9 & 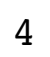 & & \\
\hline & 1 ? & 166.0000 & 5.12 & 293.2 & 4 & 0 & \\
\hline & 16 & 160.7529 & 0 & 293.1 & 4 & 0 & \\
\hline & 17 & 149.7498 & 0 & 292.3 & & & \\
\hline & 18 & 74.2802 & 0 . & 291.0 & 6 & 0 & \\
\hline & 19 & 76.3944 & 0 & 290.6 & 6 & & \\
\hline & 20 & 93.9250 & 0 & 291.7 & 5 & & \\
\hline & 21 & 82 . & & 293.0 & 4 & & \\
\hline & 22 & 5461 & 50 & 292.5 & 5 & 0 & \\
\hline 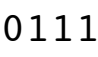 & 523 & 80.2509 & 3 . & 291.6 & 4 & 0 & \\
\hline & 524 & 33.1973 & & 290.8 & 4 & & \\
\hline & 61 & 007 & 0 & 290.2 & 4 & & \\
\hline & 62 & 25.5000 & 1.65 & 290.2 & 5 & 0 & \\
\hline & 63 & 55. & 03. & 289.4 & 6 & & \\
\hline & 64 & 83.4238 & 1 . & 289.5 & 6 & 0 & • \\
\hline & 65 & 92.7473 & 0 & 289.2 & 6 & 0 & \\
\hline & 66 & 82.5000 & & 286.3 & 5 & & \\
\hline & 67 & 97 & 4 . & 28 & 4 & & \\
\hline & 68 & 80.7514 & 000 & 286.2 & 4 & 0.0 & 0 • \\
\hline & 69 & 84.4994 & 0 & 286.6 & 3 & 0 & \\
\hline & 610 & 500 & 0 & 28 & 4 & & \\
\hline & 611 & 86.0000 & 7.7750 & 286.5 & 4 & & 0 . \\
\hline & 612 & 86.9998 & 0 & 286.8 & 4 & 0 & \\
\hline & 613 & 80.2467 & 0 & 288.9 & 4 & 0 & 0 . \\
\hline & 614 & 77.9996 & 500 & 289.4 & 4 & 0 & 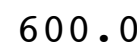 \\
\hline & 615 & 82.2696 & 0 & 289.7 & 4 & 0 & \\
\hline & 616 & 86.4936 & 7 . & 289.6 & 4 & & \\
\hline & 617 & 75.0011 & 8.8000 & 287.0 & 4 & 600.0 & 600. \\
\hline & 618 & 92.0000 & 4.8500 & 287.4 & 4 & 600 & 05 \\
\hline & 619 & 78.7498 & 8.5000 & 285.0 & 4 & & $(\cos$ \\
\hline & 620 & 80.2501 & 1.1500 & 283.7 & 4 & 600.0 & 00. \\
\hline & 621 & 78.7500 & 10.2250 & 283.6 & 4 & 600.0 & 60 \\
\hline & 622 & 78.9994 & 8.2 & 28 & 4 & .0 & \\
\hline & 623 & 79.4998 & 7.0750 & 285.0 & 4 & 600.0 & 600 \\
\hline
\end{tabular}




\begin{tabular}{|c|c|c|c|c|c|c|c|}
\hline & 24 & & 5500 & 86.2 & & & \\
\hline & 71 & 67 & .9250 & 286.8 & 4 & 600.0 & \\
\hline & 72 & 144.5006 & 2.8500 & 286.0 & 5 & 00.0 & \\
\hline & 73 & 124.5157 & 6250 & 286.3 & & 00 & \\
\hline & 74 & 131.2516 & .8250 & 85.8 & & 0 & \\
\hline & 75 & 142.2482 & 3000 & 85.3 & & 0 & \\
\hline & 76 & 145.9999 & 7000 & 284.8 & & 00 & \\
\hline & 77 & 144.2508 & 3.0750 & 84.5 & & 0 & \\
\hline & 78 & 145.7500 & .2500 & 84.4 & & & \\
\hline & 79 & 151.7499 & 8.6000 & 284.7 & & 00 & 0 \\
\hline & 710 & 148.9993 & 8.3000 & 285.3 & & & \\
\hline & 711 & 154.2502 & 8.8500 & & & 0 & \\
\hline & 712 & 153.7500 & 5000 & 286.6 & 4 & 00.0 & 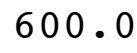 \\
\hline & 713 & 157.2509 & 6.7000 & 288.2 & 4 & 0.0 & 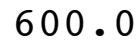 \\
\hline & 714 & 159.2480 & 500 & & 4 & & \\
\hline & 715 & 158.7498 & 0 & 1 & 4 & & \\
\hline & 716 & 163.9998 & .7500 & 290.3 & 4 & 0.0 & 6 \\
\hline & 717 & 146.5110 & 3.8000 & 9 & 5 & 0 & \\
\hline & 718 & 112.2673 & 2.5000 & 3 & 4 & & \\
\hline & 719 & 84.9889 & 2.6000 & 289.3 & 4 & .0 & . \\
\hline 11 & 720 & 94.7529 & .9500 & 289.3 & 4 & 0 & \\
\hline & 721 & 136.0124 & & & 4 & & \\
\hline & 722 & 146 . & 2.4500 & 28 & 5 & & \\
\hline 11 & 723 & 122.3487 & 1.7500 & .3 & 6 & 0 & 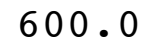 \\
\hline & 724 & 27.4284 & .8250 & & 5 & & \\
\hline 1 & 81 & 15.7517 & 250 & 28 & 6 & & \\
\hline 1. & 82 & 356.4732 & 1000 & 287.5 & 6 & 0 & U. \\
\hline & 83 & 247.5101 & 0 & 287.2 & 6 & & \\
\hline & 84 & 304.4245 & 750 & 28 & 5 & & \\
\hline 11 & 85 & 281.7181 & 250 & 286.9 & 6 & 0 & 0 • \\
\hline & 86 & 267.8549 & .7250 & 28 & 6 & 0 & 0 . \\
\hline & 87 & 261.1163 & .6750 & & 6 & & \\
\hline & 88 & 261.7776 & 0.6250 & 287.3 & 6 & & 0 • \\
\hline 1 & 89 & 309.0496 & 1.0500 & 289.4 & 5 & 0 & . \\
\hline & 810 & 276.3716 & 0 & & 4 & & \\
\hline & 811 & 283.8261 & 2.4250 & 290.2 & 3 & & 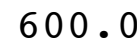 \\
\hline & 812 & 244.1671 & .2750 & 290.7 & 2 & $\cdot 0$ & . \\
\hline & 813 & 242.9981 & 3000 & 290 & 2 & & . \\
\hline & 814 & 233.3363 & 2.5750 & 291.0 & 2 & .0 & 0 . \\
\hline & 815 & 230.7474 & 2.2250 & 291.5 & 1 & & 0 . \\
\hline & 816 & 234.0073 & 1.3500 & 292.0 & 2 & & 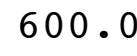 \\
\hline & 817 & 159.4912 & 1.8250 & 291.1 & 3 & .0 & 0 . \\
\hline & 818 & 149.5006 & 1.7000 & 289.7 & 4 & 0 & 00 . \\
\hline & 819 & 156.2281 & 1.4250 & 28 & 4 & & \\
\hline & 820 & 155.4714 & 0.8750 & 289.7 & 4 & & 600. \\
\hline & 821 & 85.0892 & 0.3500 & 289.6 & 5 & 600.0 & 600 \\
\hline & 822 & 21.7508 & & & 6 & & \\
\hline & 823 & 38.4915 & 1.2250 & 290.4 & 0 & 600.0 & 60 \\
\hline
\end{tabular}




\begin{tabular}{|c|c|c|c|c|c|c|}
\hline 11824 & $2 \cdot 1204$ & 0.7000 & 90.2 & 6 & 000.0 & \\
\hline $\begin{array}{lll}11 & 9 & 1\end{array}$ & 4.4985 & 1.6500 & 291.2 & & 600.0 & \\
\hline 192 & 13.2735 & 1750 & 90.6 & & 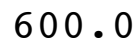 & \\
\hline 193 & 296.0249 & .2750 & 89.4 & & 00 & \\
\hline 194 & 338. & .6000 & 89.9 & & & \\
\hline 195 & 280.4572 & 0.0750 & 89.3 & & 0 & \\
\hline 196 & 309.9865 & .0500 & 89.1 & & 00 & \\
\hline $\begin{array}{lll}1 & 9 & 7\end{array}$ & 312.6867 & .1000 & 89.1 & & 0 & \\
\hline 1198 & 257.8325 & 0.0750 & 289.8 & & 0 & \\
\hline $\begin{array}{lll}19 & 9\end{array}$ & 238.8936 & 250 & 292.5 & & 0 & \\
\hline 1910 & 263 & 00 & 92.0 & & & \\
\hline 1911 & 257 . & & 291.3 & 3 & & \\
\hline 11912 & 271.2230 & 1.5250 & 291.5 & & 0 & \\
\hline 11913 & 306 . & 500 & 2 & 1 & & \\
\hline 11914 & 264 . & & 94 & & & \\
\hline 11915 & 312 . & 750 & 295.0 & & 0 & \\
\hline 11916 & 253.5214 & 1.9000 & 294.6 & 1 & 0 & \\
\hline 1917 & 151.24 & & 292.5 & 2 & & \\
\hline 11918 & 137.4948 & 0 & 291.4 & 3 & & \\
\hline 11919 & 147.7468 & 750 & 291.0 & 4 & 0 & \\
\hline 1920 & 147 . & & 290 & & & \\
\hline 921 & 146 . & 2 . & 290 & 5 & & \\
\hline 1922 & 143.7501 & 2.1000 & 289.8 & 4 & 0 & \\
\hline 923 & 189. & 0 & 285 & D & & \\
\hline 924 & & & 28 & 4 & & \\
\hline 1101 & 111.9363 & 50 & 289.5 & 5 & & \\
\hline 11102 & 143.0000 & 1.5750 & 289.6 & 4 & & \\
\hline 1103 & 144.9968 & 50 & 289.1 & 4 & & \\
\hline $11110 \quad 4$ & 157.0317 & 5000 & 288.5 & 5 & & \\
\hline 111105 & 7.4158 & 250 & 288.2 & 5 & & \\
\hline 111106 & 126 & & 288.3 & 5 & & \\
\hline 111107 & 0 & & 288.2 & 6 & & \\
\hline 111108 & 359.5006 & 500 & 288.9 & 6 & & \\
\hline 111109 & 318.0253 & 0 & 285 & 5 & & \\
\hline 010 & 300 . & & 290.3 & 4 & & \\
\hline 1011 & 267.1356 & 750 & 291.2 & 3 & & \\
\hline 012 & 287 . & 00 & 290.7 & 2 & & \\
\hline 013 & 119 . & 2 . & 291.5 & 1 & & 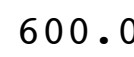 \\
\hline 01111014 & 170.2338 & 2000 & 290.9 & 2 & & 6 \\
\hline 015 & 177.2777 & & 29 & 3 & & \\
\hline 1016 & 168.2533 & 00 & 289.3 & 4 & & \\
\hline 1017 & 131.8532 & 3.3750 & 288.5 & 4 & 0 & 0 \\
\hline 018 & 106.4149 & 50 & 288.1 & 5 & & \\
\hline 01111019 & 290.6345 & 0.9750 & 287.3 & 6 & .0 & 600 \\
\hline 11020 & 275.1227 & 2.0500 & 286.4 & 6 & 600.0 & 00 \\
\hline 021 & 300 . & & & 6 & & \\
\hline 022 & 320.4155 & 1 . & 285.9 & & & 600 \\
\hline 1023 & 325.7543 & 3.0000 & 286.8 & & 600.0 & 600 \\
\hline
\end{tabular}




\begin{tabular}{|c|c|c|c|c|c|c|}
\hline .024 & & 3250 & 7.5 & & & \\
\hline 11111 & 02 & .5500 & 37.7 & 4 & 00 & \\
\hline 11112 & 304.0025 & 0000 & 287.6 & 4 & 00 & \\
\hline 11113 & 320.0000 & 4250 & 287.5 & 4 & 00 & \\
\hline 1114 & 335.8597 & 2000 & 86.4 & & 0 & \\
\hline 1115 & & 6500 & 286.3 & & 00 & \\
\hline 11116 & 350.5031 & 3250 & 286.4 & & 000 & 0 \\
\hline 11117 & 568 & .3250 & 86.6 & & 00.0 & \\
\hline 11118 & 3576 & 2250 & 86.4 & & & \\
\hline 11119 & 323.4403 & 2.5500 & 287.8 & & 600.0 & 00 \\
\hline 111110 & 291.9919 & 3.3250 & 289.5 & & 0 & \\
\hline & 318.6157 & & 90.1 & & 00.0 & \\
\hline 2 & 340 & 7 . & 291.4 & 4 & 00.0 & 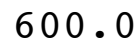 \\
\hline 113 & 321.0779 & 0 & 291.4 & 4 & 0.0 & \\
\hline & 306 . & & 291.3 & 4 & & \\
\hline & 294 . & & 291.0 & 4 & & \\
\hline 1 & 302.2654 & 0 & 291.0 & 4 & .0 & 0 \\
\hline 17 & 00 & & 291.0 & 4 & 0 & \\
\hline & 316.4945 & & 290.5 & 4 & & \\
\hline 19 & 327.7500 & 0 & 290.3 & 4 & 0 & \\
\hline 111 & 327.7573 & 6 . & 289.9 & 4 & 0 & . \\
\hline & 322.9051 & & 289.7 & 4 & & \\
\hline 22 & 303.9223 & 3. & 28 & 4 & & \\
\hline 123 & 267.1776 & 4 . & 288.6 & 4 & 0 & 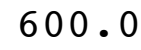 \\
\hline 124 & 218 . & & 28 & 5 & & \\
\hline 21 & 212.8541 & 1. & 287.7 & 6 & & \\
\hline 111122 & 216.3865 & 1.22 & 287.5 & 6 & 0 & . \\
\hline 111123 & 342.5002 & 3.72 & 288.6 & 5 & & \\
\hline 111124 & 348.2500 & 3 . & 28 & 4 & & 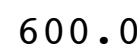 \\
\hline 111125 & 339.0000 & 3 . & 288.3 & 4 & & 0 . \\
\hline 111126 & 333.0003 & 5.0250 & 288.2 & 4 & & ( \\
\hline 111127 & 333.4997 & 0 & 288.3 & 4 & & \\
\hline 111128 & 326.5031 & 6. & 288.8 & 4 & & U. \\
\hline 111129 & 322.9962 & 8.8250 & 289.5 & 4 & 0 & \\
\hline 210 & 330. & 0 & & 4 & & \\
\hline 11 & 33.0059 & 6 . & 286.9 & 4 & & 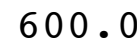 \\
\hline 1111212 & 67.2501 & 8.5750 & 285.8 & 4 & $\cdot 0$ & 0 . \\
\hline 213 & 65.2276 & 000 & 285.6 & 4 & & . \\
\hline 01111214 & 37.1631 & 7.1250 & 286.1 & 4 & .0 & 00 . \\
\hline 1111215 & 55.1545 & 3.9250 & 286.2 & 3 & 0 & \\
\hline 216 & 335.7222 & 3.3000 & 285.8 & 2 & & 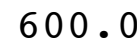 \\
\hline 01 & 290.2271 & 2.0250 & 284.7 & 3 & .0 & 00 . \\
\hline 1111218 & 61.5853 & 1.5750 & 284.7 & 4 & 600 & 00 . \\
\hline 219 & .2443 & 3.7500 & 284.7 & 4 & & 0 \\
\hline 01111220 & 53.7839 & 2.8000 & 284.3 & 5 & 60 & 600. \\
\hline 11221 & 355.4508 & 1.1500 & 283.6 & 6 & 600.0 & 600 \\
\hline & 17 & & & 6 & & \\
\hline & 351.0541 & 0.6000 & 283.7 & & 600.0 & 60 \\
\hline
\end{tabular}




\begin{tabular}{|c|c|c|c|c|c|c|}
\hline 11224 & 5.9912 & 1.7500 & 284.4 & 6 & 600.0 & \\
\hline 111131 & 6.1698 & 2.9500 & 284.7 & J & 600.0 & \\
\hline 11132 & 7853 & 2250 & 284.7 & 4 & 0 & \\
\hline 11133 & 61.3597 & 4.4500 & 284.4 & & 00.0 & \\
\hline 11134 & 187. & 1.3000 & 83.9 & & 0.0 & \\
\hline 11135 & 29.0902 & 1.2250 & 283.5 & & 0 & \\
\hline 111136 & 30.2402 & 2.3250 & 283.9 & & 00 & \\
\hline 111137 & 70.3421 & .5250 & 84.3 & & 0 & \\
\hline 111138 & 273.1474 & 0.7750 & 284.0 & 6 & 00 & \\
\hline 111139 & 330.3860 & 0.1750 & 285.8 & & 0 & \\
\hline 111310 & 264.1799 & 000 & 7 & & & \\
\hline 111311 & 287.8365 & 250 & 286 & 3 & & \\
\hline 1111312 & 284.0123 & 2.0500 & 286.5 & 3 & 0 & \\
\hline 313 & 282.0000 & .2000 & 9 & 2 & & \\
\hline & 272.2491 & & 9 & 3 & & \\
\hline 315 & 279.4993 & 500 & 286.6 & 3 & 0 & \\
\hline 1111316 & 279.7522 & 2.3250 & 286.6 & 3 & 0 & \\
\hline & 306.9553 & & 286.2 & 4 & & \\
\hline 18 & 7.8640 & 00 & 286.0 & 5 & & \\
\hline 1319 & 10.6822 & 1250 & 285.8 & 6 & 0 & \\
\hline 20 & 162 . & & 4 & 6 & & \\
\hline & 124.7131 & & 28 & 6 & & \\
\hline 1111322 & 328.3355 & 500 & 285.4 & 6 & 0 & \\
\hline 323 & 347.4110 & 0 & 4 & 6 & & \\
\hline 24 & 14 & & 28 & 5 & & \\
\hline 141 & 69.8090 & 50 & 285.6 & 6 & & \\
\hline 111142 & 59.0048 & 000 & 286.0 & 5 & & \\
\hline 111143 & 188.0148 & 0 & 6 & 6 & & \\
\hline $11114 \quad 4$ & 12.2475 & 00 & 284.8 & 5 & & \\
\hline $11114 \quad 5$ & 33.4945 & 250 & 284.9 & 4 & & \\
\hline 111146 & 66 & & 7 & 5 & & \\
\hline 1147 & 95 . & & 9 & 6 & & \\
\hline $11114 \quad 8$ & 97.9984 & 7500 & 287.0 & 5 & & \\
\hline 111149 & 98.7379 & 0 & 287 & 4 & & - \\
\hline 1111410 & 234.9827 & & & 3 & & \\
\hline 1111411 & 271.9728 & 1.0500 & 286.3 & 2 & & \\
\hline 412 & 189.8688 & 00 & 286.8 & 1 & & \\
\hline 413 & 236.4472 & 750 & 287.1 & 1 & & 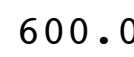 \\
\hline 01111414 & 262.9102 & 0.6000 & 289.6 & 1 & & 6 \\
\hline & 234.2843 & 1 . & $28 \varepsilon$ & 1 & & \\
\hline & 205.3818 & 2 . & 288.8 & 2 & & \\
\hline 01111417 & 240.2370 & 1.9750 & 287.6 & 3 & 0 & 0 \\
\hline 418 & 329.3441 & 00 & 286.7 & 4 & & \\
\hline 01111419 & 349.9973 & 1.6750 & 286.4 & 4 & 600.0 & 600 \\
\hline 01111420 & 5.0090 & 1.2750 & 286.3 & 5 & 600.0 & 600 \\
\hline & & & 286.3 & 4 & & \\
\hline & 9.7605 & 1 . & 286.4 & 4 & & 600 \\
\hline 11423 & 54.8173 & 0.7500 & 286.6 & & 600.0 & 600 \\
\hline
\end{tabular}




\begin{tabular}{|c|c|c|c|c|c|c|}
\hline-1 & & 0 & & & & \\
\hline 11151 & 3 & 0.6500 & 5.6 & 6 & 00.0 & \\
\hline 11152 & 128.1235 & .1500 & 285.5 & 6 & 00 & \\
\hline 11153 & 152.9018 & 3000 & 285.3 & & 00 & \\
\hline 1154 & 73.5000 & .2000 & & & 0 & \\
\hline 1155 & 354.2334 & 5500 & 84.5 & & 00 & \\
\hline 11156 & 10.5031 & 1.4250 & 284.7 & 4 & 500 & U \\
\hline 11157 & 3.9897 & 1.4250 & 84.7 & & 0 & \\
\hline 11158 & 1.5032 & 1250 & 85.3 & 4 & & \\
\hline 11159 & 356.2543 & 1.9250 & 86.7 & 4 & 600.0 & 8 \\
\hline 1510 & 339.9897 & 2.4250 & 287.3 & 3 & 0 & \\
\hline & 303.2502 & 2.80 & 88.1 & & 00.0 & \\
\hline 512 & 274.7504 & 3.3250 & 288.2 & 2 & 0 & \\
\hline 513 & 278.3709 & 2.2000 & 289.1 & & 0.0 & \\
\hline 14 & 290.2625 & & & 2 & & \\
\hline & 245.3699 & 1 . & 289.3 & 2 & & \\
\hline 516 & 250.8651 & 1.9500 & 288.7 & 2 & .0 & 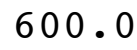 \\
\hline 517 & 273.0736 & 1.17 & 8.2 & 3 & 0 & \\
\hline 518 & 321.5000 & 1 . & 287.3 & 4 & & \\
\hline 519 & 0020 & 1.7250 & 286.6 & 4 & & \\
\hline 1111520 & 13.6519 & 0.5250 & 286.6 & 5 & 0 & . \\
\hline 21 & 340.4656 & 0.12 & & 6 & & \\
\hline 22 & 5.7140 & 7250 & 28 & 6 & & \\
\hline 523 & 14.5129 & 0.4750 & 286.6 & 5 & 0 & 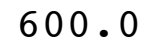 \\
\hline 524 & 0.0070 & 0 & & 4 & & \\
\hline 61 & 16.7474 & 1.3000 & 28 & 4 & & \\
\hline 111162 & 91.4715 & 6750 & 28 & 5 & 0 & . \\
\hline 111163 & 179.6264 & 5000 & 2 & 6 & & \\
\hline 111164 & 147.4998 & 2 . & 28 & 5 & & \\
\hline 111165 & 137.8980 & 1.1750 & 285.1 & 6 & 0 & U. \\
\hline 111166 & 147.5000 & 1.7000 & 285.2 & 5 & 0 & 0 \\
\hline 111167 & 23.2285 & 0 & & 6 & & \\
\hline 111168 & 132.6867 & & 285.4 & 6 & & 0 • \\
\hline 111169 & 25.3652 & 0.4500 & 287.7 & 5 & 0 & 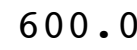 \\
\hline & 310.9002 & 0 & & 4 & & \\
\hline & 254.6151 & 1.1250 & 9.1 & 3 & & 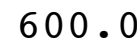 \\
\hline 1111612 & 245.5184 & 1.9000 & 289.0 & 2 & & 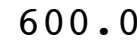 \\
\hline 613 & 227.0681 & 2.1750 & 28 & 1 & & 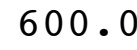 \\
\hline 14 & 265.8630 & 2.2250 & 289.0 & 1 & .0 & 0 . \\
\hline 11615 & 268.9571 & 1.4750 & 289.2 & 1 & & \\
\hline & 275.6004 & 000 & 289 & 1 & & 0 \\
\hline 17 & 162.0344 & 1.6000 & 288.6 & 2 & .0 & 0 . \\
\hline 111618 & 230.2431 & 1.1000 & 287.5 & 3 & .0 & 00 . \\
\hline 619 & 22.1969 & 0.5500 & 287 & 4 & 60 & 0 \\
\hline 01111620 & 112.9949 & 2.3750 & 287.6 & 5 & 60 & 600. \\
\hline 1162 & 55.7861 & 3.9500 & 287.7 & 4 & 600.0 & 00 \\
\hline & 79.5445 & & & 4 & & \\
\hline & 79.7562 & 5.8000 & 286.8 & 1 & 600.0 & $=0$ \\
\hline
\end{tabular}




\begin{tabular}{|c|c|c|c|c|c|c|}
\hline $110<4$ & 3.7521 & 5.1500 & 286.5 & 4 & 600.0 & \\
\hline 111171 & 0.0235 & 2.2750 & 86.5 & 4 & תח & \\
\hline 11172 & 4299 & 6500 & 286.2 & 5 & 00 & \\
\hline 11173 & 72.7967 & .3250 & 285.7 & & 00 & \\
\hline 1174 & 89.2237 & 3.5250 & 6.3 & & 0 & \\
\hline 1175 & 77.2532 & 000 & 86.6 & 4 & 00 & \\
\hline 11176 & 75.0174 & 2.4000 & 286.4 & & 00 & \\
\hline 1177 & 79.2912 & .1750 & 85.6 & & & \\
\hline 1178 & 70.8244 & .4750 & 284.8 & & 0 & \\
\hline 111179 & 221.7143 & 1.1250 & 285.3 & & 0 & \\
\hline 1710 & 280.7997 & 0.9250 & 285.2 & & 0 & \\
\hline 1711 & 265.9525 & 1.7750 & & 3 & & \\
\hline 1712 & 289.2844 & 0.9000 & 284.9 & 2 & 0 & \\
\hline 11713 & 200.5023 & 0.9250 & 285.0 & 1 & 0 & \\
\hline 714 & 220.4681 & 1.0250 & & & & \\
\hline .715 & 286.2161 & 0.8000 & 286.9 & 1 & 0 & \\
\hline 1716 & 232.7553 & 1.9250 & 286.7 & 2 & 0 & \\
\hline 1717 & 245.7411 & 000 & 86.1 & & & \\
\hline 718 & 10.7386 & .1750 & 285.2 & 4 & 0 & \\
\hline 1719 & 29.1397 & 5250 & 284.6 & 5 & 0 & \\
\hline 720 & 81.9147 & 000 & 284.8 & 6 & & \\
\hline 721 & 123.4833 & 750 & 284.8 & 6 & & \\
\hline 1722 & 43.3334 & 000 & 284.6 & 5 & 00 & 0. \\
\hline 723 & 57.4717 & 0 & 284.3 & 6 & 0 & \\
\hline 724 & 164.4940 & 50 & 284.4 & 5 & & \\
\hline 81 & 179.9278 & 750 & 284 & 5 & & \\
\hline 111182 & 147.7413 & .6250 & 283.8 & 5 & 0 & \\
\hline 111183 & 151.0000 & 0 & 283.6 & 4 & & \\
\hline 184 & 137.8918 & 50 & 28 & 4 & & 0 . \\
\hline 1185 & 119.2451 & 4500 & 283.2 & 5 & 0 & \\
\hline 186 & 126.0209 & 500 & 283.8 & 4 & & \\
\hline 187 & 145 & 0 & 283.1 & 4 & & \\
\hline 111188 & 146.2748 & 5500 & 283.0 & 5 & 0 & J. \\
\hline 189 & 156.7468 & 00 & 283.0 & 4 & & \\
\hline 310 & 138. & 3. & 282.6 & 3 & & \\
\hline 811 & 313.8157 & 1.4500 & 284.6 & 2 & & 0 . \\
\hline 812 & 298.2351 & 1.9000 & 286.2 & 2 & 0 & \\
\hline 813 & 292.6073 & 1.3750 & 288.2 & 1 & & 0 . \\
\hline 814 & 295.9966 & 250 & 289.0 & 1 & 0 & 0 . \\
\hline 1815 & 297.5139 & 750 & 289.7 & 1 & 0 & \\
\hline 816 & 250.3241 & 750 & 289.4 & 1 & & \\
\hline 817 & 254.9122 & 1.5500 & 287.9 & 2 & 600.0 & 600 . \\
\hline 1818 & 283.7609 & 1.7750 & 286.6 & 3 & 600 & 000 \\
\hline 819 & 201.3238 & 0.4250 & 285.6 & 4 & 600 & s \\
\hline 820 & 123.6245 & 1.4500 & 285.0 & 5 & 600.0 & 00 \\
\hline 821 & 356.8867 & 0.9250 & 284.8 & 6 & 600.0 & 500. \\
\hline & 312.6978 & & 284.9 & 5 & 60 & \\
\hline 823 & 314.7791 & 1.5250 & 284.6 & כ & 600.0 & 600 \\
\hline
\end{tabular}




\begin{tabular}{|c|c|c|c|c|c|c|}
\hline 824 & & .7500 & 54.4 & & & \\
\hline 11191 & 00 & .5000 & 34.6 & 4 & 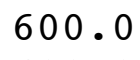 & \\
\hline 1192 & 257.5000 & 1.0750 & 284.9 & 5 & 00 & \\
\hline 11193 & 150.7499 & 2.7750 & 284.7 & & 00 & \\
\hline 1194 & 132.3006 & .7250 & 84.9 & & 0 & \\
\hline 1195 & 58.7906 & 5500 & 84.7 & & 00 & \\
\hline 11196 & 306.8596 & 1250 & 284.2 & & 00 & \\
\hline 11197 & 308.3644 & .8000 & 83.5 & & 00 & \\
\hline 11198 & 355.4651 & 9000 & 83.0 & & & \\
\hline 11199 & 3.9029 & 0.6000 & 284.2 & 4 & 600.0 & 0 \\
\hline 111910 & 89.0946 & 0.8500 & 285.7 & & & \\
\hline 911 & 249.8494 & 0.7000 & 87.2 & & 00.0 & \\
\hline 912 & 352 & 500 & 289.0 & 1 & 0 & \\
\hline 1111913 & 243.6950 & 1.0500 & 290.9 & & 0.0 & \\
\hline 914 & 299 & & & & & \\
\hline 915 & 354 & & 289.5 & 1 & & \\
\hline 1916 & 80.7496 & 250 & 290.2 & 2 & .0 & 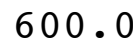 \\
\hline 1917 & 63.7540 & 50 & 5 & 3 & & \\
\hline 4918 & 124.4696 & 1 . & 290.0 & 4 & & \\
\hline 919 & 2260 & 500 & 288.9 & 5 & & \\
\hline 1111920 & 322.4456 & 0.9500 & 288.1 & 6 & 0 & . \\
\hline 921 & 16.7234 & & & 6 & & \\
\hline 922 & 107.9240 & 00 & 28 & 6 & & \\
\hline 1111923 & 5.8590 & 1.0500 & 286.4 & 6 & 0 & \\
\hline 1924 & 10.9963 & & & 5 & & \\
\hline 201 & 31.2498 & 2 . & 28 & 4 & & \\
\hline 111202 & 29.7490 & 2.0750 & 287.8 & 5 & 0 & J. \\
\hline 111203 & 64.7618 & 2000 & .1 & 5 & & \\
\hline 111204 & 37.9622 & 2.4750 & 28 & 6 & & \\
\hline 111205 & 289.5200 & 750 & 8.1 & 6 & & 0 . \\
\hline 111206 & 9.8974 & 0.2750 & 286.9 & 6 & & 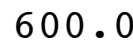 \\
\hline 111207 & 168.0994 & 0 & $2 \varepsilon$ & 6 & & \\
\hline 111208 & 341.9924 & 1.4250 & 286.7 & 6 & & 0 . \\
\hline 111209 & 337.3313 & 2.5500 & 286.2 & 5 & 0 & \\
\hline 1112010 & 335.4875 & 2 . & & 4 & & \\
\hline 2011 & 354.6639 & 000 & 288.4 & 3 & & 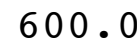 \\
\hline 1112012 & 119.4995 & 3.2000 & 291.4 & 2 & & o. \\
\hline 2013 & 199.1955 & 500 & 291.1 & 2 & & 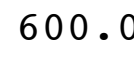 \\
\hline 1112014 & 213.0293 & 1.6250 & 291.5 & 1 & .0 & 0 . \\
\hline 1112015 & 184.9286 & 4.2000 & 291.3 & 2 & & \\
\hline 1112016 & 236.4043 & 2.7500 & 289.6 & 3 & & 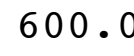 \\
\hline 01112017 & 237.7192 & 1.7250 & 289.0 & 4 & .0 & 0 . \\
\hline 1112018 & 306.4047 & 1.3500 & 287.6 & 5 & 0 & 00 . \\
\hline 1112019 & 4.2366 & 1.7250 & 287.3 & 4 & & \\
\hline 01112020 & 96.0521 & 1.2000 & 287.9 & 5 & & 600. \\
\hline 1202 & 79.2408 & 2.8000 & 288.0 & 4 & 600.0 & 600. \\
\hline 20 & 20 & & 9 & 4 & & \\
\hline 12023 & 196.7087 & 1.8000 & 288.0 & & 60 & 60 \\
\hline
\end{tabular}




\begin{tabular}{|c|c|c|c|c|c|c|}
\hline 2024 & & 0500 & 8.0 & & & \\
\hline 11211 & 12 & .2000 & 7.3 & & 600.0 & \\
\hline 1212 & 313.5064 & 1.3250 & 286.6 & 6 & 00 & \\
\hline 11213 & 342.5046 & 2.2250 & 286.1 & & 00 & \\
\hline 1214 & 121 & .1500 & 86.4 & & 0 & \\
\hline 1215 & 85.6789 & .2500 & 287.2 & & 00 & \\
\hline 11216 & 9336 & 2.4250 & 287.8 & & 00 & U \\
\hline 11217 & 62.65 & .8000 & 86.5 & & 00.0 & \\
\hline 11218 & 32 & 1.3250 & 86.4 & 0 & & \\
\hline 11219 & 110.7972 & 1.6000 & 287.6 & & 600.0 & 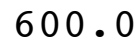 \\
\hline 1112110 & 313.9625 & 1.4000 & 287.8 & & & \\
\hline & 299.75 & 1.6000 & 87.9 & & 00.0 & \\
\hline 2 & 286 & 2.9250 & 288.2 & 2 & 0 & 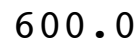 \\
\hline 112113 & 307.29 & 2.8750 & 287.5 & 3 & 0.0 & \\
\hline & 331 & 250 & 7 & & & \\
\hline & & 1.5250 & 289.0 & 3 & & \\
\hline 1 & 214.21 & 1.8250 & 289.0 & 2 & .0 & 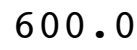 \\
\hline 17 & 131. & 2.7500 & 288.4 & 3 & 0 & \\
\hline & 298 . & 1.1500 & 289.2 & 4 & & \\
\hline 19 & 140 & 2.3000 & 288.6 & 5 & & \\
\hline 111 & 33.2248 & 2.6250 & 289.0 & 6 & 0 & 00 . \\
\hline 21 & 349.5724 & 1.5000 & & 6 & & \\
\hline 22 & 331 & 1.9250 & 289.2 & 6 & & \\
\hline 23 & 56.6238 & 1.6750 & 289.3 & 6 & 0 & 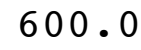 \\
\hline 24 & & 2 & .6 & 6 & & \\
\hline 21 & 28 & 3.2750 & 289.0 & 5 & & \\
\hline 111222 & 49.5524 & 6.8500 & 288.8 & 4 & 0 & . \\
\hline 111223 & 018 & 7.8750 & 288.1 & 4 & & \\
\hline 111224 & 7466 & 7.2250 & 288.2 & 4 & & 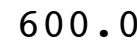 \\
\hline 111225 & 7426 & 5250 & 287.7 & 4 & 0 & 0 • \\
\hline 111226 & .2452 & 7.6750 & 287.6 & 4 & 0 & 0 . \\
\hline 111227 & 64.2512 & 770 & 287.2 & 4 & & \\
\hline 111228 & 2507 & 500 & 286.4 & 4 & & U • \\
\hline 111229 & 86.7525 & 6.2000 & 285.9 & 4 & 0 & 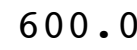 \\
\hline 210 & $10 \varepsilon$ & 4 . & & 3 & & \\
\hline 11 & 118.0000 & 4.7000 & 286.9 & 3 & & 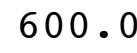 \\
\hline 12 & 131.8764 & 5.8250 & 287.1 & 3 & $\cdot 0$ & 0 . \\
\hline 13 & 105.7463 & 5.3250 & 287.6 & 3 & & 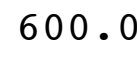 \\
\hline 14 & 102.5842 & 4.3250 & 288.2 & 3 & .0 & 00 . \\
\hline 215 & 88.5198 & 4.8250 & 287.8 & 3 & 0 & o. \\
\hline & 68.9972 & 6.8750 & 287.2 & 4 & & . \\
\hline 17 & 63.7497 & 8.2500 & 286.2 & 4 & .0 & 00 . \\
\hline 112218 & 61.9958 & 6.3000 & 285.7 & 4 & .0 & 00 . \\
\hline 219 & 67.2438 & 6.7000 & 285.2 & 4 & 60 & 0 \\
\hline 01112220 & 73.2522 & 6.8500 & 284.8 & 4 & 0 & 600. \\
\hline 1222 & .2527 & 4.5750 & 284.7 & 4 & 600.0 & 600 \\
\hline & 4474 & 0 & 284.6 & 4 & & \\
\hline & 88.2504 & 7.7000 & 284.2 & 1 & 600.0 & 600 \\
\hline
\end{tabular}




\begin{tabular}{|c|c|c|c|c|c|c|}
\hline 2224 & 1.0004 & 6.9500 & 83.4 & 4 & 0 & \\
\hline 111231 & 70007 & 6.8000 & 83.1 & & & \\
\hline 11232 & 4971 & 500 & & 4 & 0 & \\
\hline 11233 & 97.5005 & 8250 & 283.2 & & 00 & \\
\hline 1234 & 06 & & 3.2 & & & \\
\hline 1235 & 0 & 500 & 82.8 & & $0 c$ & \\
\hline 11236 & 92.2499 & 000 & 282.6 & & & \\
\hline 11237 & 124.5072 & 9750 & 32.6 & & & \\
\hline 11238 & 129.1598 & 5.4250 & 82.4 & & & \\
\hline 111239 & 144.9899 & 000 & 282.2 & & 0 & \\
\hline 10 & 140.2952 & 5 . & 82.5 & & & \\
\hline & 131.12 & & 83.8 & & & \\
\hline 2 & 163.74 & 4000 & 284.3 & & 0 & \\
\hline 13 & 172.4954 & 5.47 & 284.0 & & 0 & \\
\hline & 169.75 & & & & & \\
\hline & 190.2512 & 1.7750 & 284.7 & 3 & & \\
\hline & 234.2215 & 0.6750 & 285.2 & & & \\
\hline & 212.9163 & 1.22 & & & & \\
\hline 8 & 199.36 & 0.85 & 283.7 & 4 & & \\
\hline 19 & 279.2797 & 1.17 & 283.2 & 5 & & \\
\hline 20 & 289.29 & 1 . & 2.9 & & & \\
\hline & 258.47 & & 2.6 & 6 & & \\
\hline 22 & 313.9031 & 0.8500 & 282.6 & 6 & & • \\
\hline 23 & 341.75 & 2 . & 282.8 & 6 & & \\
\hline 24 & 316.23 & & & 5 & & \\
\hline 1 & 337.00 & 2.97 & & 5 & & \\
\hline 111242 & 31 & 2.45 & 284.3 & 6 & & \\
\hline 111243 & 316.24 & $71-12$ & & & & \\
\hline 244 & 30 & 0 & 8 & 4 & & \\
\hline 111245 & 325.64 & 8.2000 & 6 & 4 & & \\
\hline 111246 & 312 . & 1.70 & & 4 & & \\
\hline 24 & 33 & 6 . & & 4 & & \\
\hline 111248 & 344.70 & 7.2250 & 284.0 & 4 & 0 & \\
\hline 111249 & 5 & 0 & & 4 & & \\
\hline & 8 & 0 & & 4 & & \\
\hline & 70.4998 & .2250 & & 4 & & \\
\hline & 70.24 & .7500 & 9 & 4 & & \\
\hline & 01 & 9.5500 & 282.9 & 4 & & \\
\hline & 87.7586 & 9.1500 & 282.6 & 4 & & \\
\hline & 90 . & 000 & 283.0 & 4 & & \\
\hline & 71.6968 & 9.80 & & 4 & & \\
\hline 17 & 71.4939 & 10.2500 & 281.2 & 4 & 0 & 0. \\
\hline & 94. & & 281.2 & 4 & 0 & \\
\hline & 98.0168 & 7.2250 & & 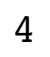 & & \\
\hline & 86.7634 & 5.7750 & 280.3 & 4 & 600 & 00 . \\
\hline & $\$ 750$ & 6.6250 & 279.0 & 4 & 600 & \\
\hline & 87 & & & 4 & & \\
\hline 423 & 92.2374 & 5.6500 & 280.0 & & 600.0 & 600 \\
\hline
\end{tabular}




\begin{tabular}{|c|c|c|c|c|c|c|}
\hline 2424 & 5.4569 & 6.6250 & 280.0 & 4 & 000.0 & \\
\hline 111251 & 0084 & 8.4000 & 279.9 & 4 & 600.0 & \\
\hline 11252 & 487 & 7250 & 280.0 & 4 & 00 & \\
\hline 11253 & 72.0014 & 8.7750 & 280.1 & & 00 & \\
\hline 254 & 014 & .1250 & 80.0 & & 0 & \\
\hline 1255 & 71 & 7.1500 & 79.9 & & 00 & \\
\hline 11256 & 71.4957 & 6.0500 & 280.1 & & 0 & \\
\hline 11257 & 69 . & .6750 & 80.2 & & & \\
\hline 11258 & 4997 & .8250 & 80.4 & 4 & 0 & \\
\hline 111259 & 55.7477 & 500 & 280.7 & & 00 & \\
\hline 10 & 67.0186 & 4.3250 & 281.5 & & 0 & \\
\hline & 20 & 50 & 82.2 & & & \\
\hline 2 & 94.7533 & 2.9250 & 282.5 & & 0 & \\
\hline 13 & 80.1968 & 3.10 & 282.4 & & 0 & \\
\hline & 62.9794 & & 82.9 & & & \\
\hline 15 & 105.2454 & 1.8000 & 282.8 & 1 & & \\
\hline & 86.9206 & 1.95 & 282.5 & & 0 & \\
\hline & 68.2542 & 2 . & 2.0 & & & \\
\hline 8 & 65.2563 & 2 . & 281.7 & 4 & 0 & \\
\hline 19 & 192.0540 & 0 & 280.8 & 5 & & \\
\hline 20 & 83 & & 280 & 6 & & \\
\hline & 59. & & 4 & 6 & & \\
\hline 2 & 57.7468 & 0 & 280.8 & 5 & 0 & \\
\hline 23 & 65.5003 & & 281 & 5 & & \\
\hline & 69. & & & 5 & & \\
\hline 61 & 67 . & & 280 & 5 & & \\
\hline 111262 & 77.2508 & 3.22 & 280.9 & 5 & & \\
\hline 111263 & 94.5115 & 0 & 280.7 & 5 & & \\
\hline 6 & 02 & 0 & 4 & 5 & & \\
\hline 111265 & 133.0161 & 2.70 & 280.1 & 4 & & \\
\hline 111266 & 102.2494 & & 280.2 & 5 & & \\
\hline 67 & 724 & 4. & 28 & 5 & & \\
\hline 111268 & 138.8994 & 0 & 279.8 & 4 & 0 & 0 . \\
\hline 111269 & 126.4337 & 2 . & 280.0 & 4 & & \\
\hline & 157. & 3. & 28 & 3 & & \\
\hline & 167.7495 & & 280.3 & 4 & & 0 . \\
\hline & 166.7495 & 50 & 280.1 & 4 & & \\
\hline & 157.2493 & 0 & 280.7 & 4 & & \\
\hline & 159.5115 & 5 . & 281.4 & 3 & 0 & 0 . \\
\hline & 151.4995 & & 281.8 & 4 & & \\
\hline & 148 . & 4 . & 281.7 & 4 & & \\
\hline 17 & 151.0000 & 5.0750 & 281.1 & 4 & 600.0 & 00 . \\
\hline 618 & 157.7502 & & 280.4 & 5 & 600 & \\
\hline 619 & 165.5124 & 4.2000 & & 5 & & \\
\hline 620 & 168.2502 & 4.4000 & 280.2 & 4 & 600.0 & 00 \\
\hline 621 & 153.0002 & 6.9750 & 280.4 & 4 & 600 & \\
\hline & 148 . & 0 & & 4 & & \\
\hline 23 & 150.0000 & 0.0000 & 280.7 & & 600.0 & 600 \\
\hline
\end{tabular}




\begin{tabular}{|c|c|c|c|c|c|c|}
\hline$<4$ & & & & & & \\
\hline $1127 \quad 1$ & 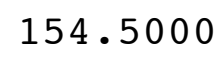 & 0 & 9.5 & & & \\
\hline 1272 & 149.5000 & 1000 & 279.2 & & 00 & \\
\hline 11273 & 5000 & 5500 & 278.7 & & 0 & \\
\hline $127 \quad 4$ & & 3750 & 78.2 & & & \\
\hline 1275 & & & .77 .9 & & 0 & \\
\hline 11276 & 997 & .750 & 277.8 & & 00 & \\
\hline $1127 \quad 7$ & 501 & 0 & 277.7 & & & \\
\hline 11278 & 2534 & 0 & 77.7 & & & \\
\hline 11279 & 041 & 3000 & 278.3 & & 0 & 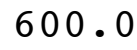 \\
\hline 112710 & 154.7512 & 7.8000 & 278.6 & & & \\
\hline 711 & $=1901$ & 7.3500 & 79.0 & & & \\
\hline 712 & 63 & 50 & 280 & 4 & 0 & \\
\hline 2713 & 154.5052 & 50 & 280.9 & & & \\
\hline 714 & & & 6 & & & \\
\hline 1 & & & 28 & 3 & & \\
\hline 716 & 170.2 & 2.8000 & 282.1 & & & \\
\hline 717 & & 0 & 281.2 & & & \\
\hline $718=18$ & 151. & & 28 & 4 & & \\
\hline 19 & 586 & 000 & 279.8 & 5 & & \\
\hline 720 & 39.2524 & 1.5750 & 280.1 & 4 & 0 & \\
\hline 721 & 62130 & & 279.6 & 4 & & \\
\hline 22 & 12 . & 0 & 27 & 4 & & \\
\hline 723 & 352.0263 & 1.5250 & 279.6 & 4 & 0 & \\
\hline 724 & 17.9249 & & 279 & 5 & & \\
\hline 81 & 5.4 & 0 & 280 & 6 & & \\
\hline 111282 & 49.4861 & 3000 & 280.0 & 6 & & . \\
\hline 11283 & 340.246 & 500 & 279 & 6 & & \\
\hline 284 & $344 . \varepsilon$ & 50 & 28 & 5 & & \\
\hline 111285 & 336.6238 & 250 & 279.8 & 5 & & . \\
\hline 111286 & 1.7291 & 1.8250 & 280.3 & 4 & & \\
\hline 111287 & 326.7760 & 50 & 281.1 & 5 & & \\
\hline 111288 & 7.6881 & & 28 & 6 & & \\
\hline 111289 & 317.8886 & 250 & 281.7 & 5 & & \\
\hline 810 & 312.2459 & 0 & 282.1 & 4 & & \\
\hline & 339.49 & & & 3 & & \\
\hline 12 & 326.5890 & 750 & 282.6 & 2 & & \\
\hline 313 & 335.1566 & 2. & 282 & 1 & & \\
\hline & 292.9895 & & 2.7 & 1 & & 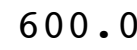 \\
\hline 315 & 309.763 & & 282.3 & 1 & & \\
\hline 816 & 269.76 & 000 & 281.5 & 1 & & \\
\hline & 339.7509 & 4.2750 & 283.2 & 2 & & 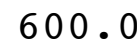 \\
\hline 1281 & 269.5000 & 3.3000 & 281.3 & 3 & & 0 \\
\hline 819 & 297.5964 & 000 & & 4 & & \\
\hline & 323.2492 & 5.7000 & & 4 & & \\
\hline 282 & 323.7583 & 9.9000 & 280.6 & 4 & & (2) \\
\hline & & & & 4 & & \\
\hline $0<5$ & 296.5008 & 12.3000 & 279.6 & & & \\
\hline
\end{tabular}




\begin{tabular}{|c|c|c|c|c|c|c|}
\hline $0<4$ & & & & & & \\
\hline 11291 & 8 & 0 & 9.4 & & & \\
\hline 1292 & 88.6717 & 6.4500 & 278.8 & 4 & 00 & \\
\hline 11293 & 314.2019 & 7.1250 & 278.3 & & 00 & \\
\hline 1294 & 01 & 6.6250 & 78.4 & & & \\
\hline 1295 & 098 & 0 & 278.3 & & 0 & \\
\hline 11296 & 323.9563 & 2.1750 & 278.4 & & 00 & \\
\hline 11297 & 2577 & 1.4750 & 279.5 & & & \\
\hline 11298 & 29.9680 & 1500 & 80.9 & & & \\
\hline 11299 & 43.0170 & 3.4250 & 281.6 & & 00 & 00 \\
\hline 910 & 69.2419 & 4.4250 & 282.6 & & & \\
\hline 11 & 65.9984 & 5.0250 & 83.2 & & & \\
\hline 912 & 86.7542 & 750 & 283.1 & & 0 & \\
\hline 913 & 80.1148 & 3.4750 & 283.7 & & 0.0 & \\
\hline 1 & 81 . & 0 & & & & \\
\hline 5 & 97 . & & & 2 & & \\
\hline 16 & 168.0221 & 4.0250 & 283.3 & 3 & .0 & \\
\hline 17 & 162.5418 & 0 & & 4 & & \\
\hline & 144 . & 250 & & 5 & & \\
\hline 19 & 2976 & 750 & 2.7 & 4 & & \\
\hline 20 & 67.9989 & 4.2250 & 2.5 & 4 & 0 & \\
\hline & 77.5000 & & & 4 & & \\
\hline 22 & 7485 & 750 & 281.8 & 4 & & \\
\hline 23 & 81.0012 & 6.4000 & 1.4 & 4 & 0 & \\
\hline 24 & 106.2135 & & & 4 & & \\
\hline 01 & 102.2483 & 2. & & 4 & & \\
\hline 111302 & 79.9699 & 2.6500 & 0.2 & 4 & 0 & 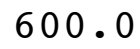 \\
\hline 111303 & 73.2505 & 250 & 6 & 4 & & \\
\hline 111304 & 73. & 6 . & 2 & 4 & & \\
\hline 111305 & 61.5006 & 750 & 0.1 & 4 & 0 & 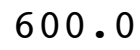 \\
\hline 111306 & 88.6849 & 3.7000 & 0.5 & 4 & & 0 . \\
\hline 111307 & 78 . & 250 & & 4 & & \\
\hline 111308 & 57.2535 & 500 & 9.9 & 4 & & \\
\hline 111309 & 60.0000 & 250 & 0.7 & 3 & 0 & \\
\hline & 55 . & 2 . & & 2 & & \\
\hline & 61.2055 & 1.2750 & 3.1 & 1 & & \\
\hline 12 & 157.0097 & 2.1750 & 3.0 & 1 & & \\
\hline 13 & 33. & 500 & & 1 & & \\
\hline 14 & 266.3064 & 2.5750 & 3.8 & 1 & & 0 . \\
\hline 1113015 & 225.3314 & 2.1250 & 3.5 & 1 & & \\
\hline 16 & 306.2370 & 250 & & 2 & & \\
\hline 17 & 302.6318 & 2.0000 & 282.8 & 3 & & . \\
\hline 13018 & 335.2508 & 2.7500 & 281.7 & 4 & 0 & 0 . \\
\hline 019 & 320.5530 & 000 & & 4 & & \\
\hline 01113020 & 340.9426 & 0.6250 & 281.5 & 5 & & \\
\hline 00 & 30.0762 & 1.4000 & 281.7 & 4 & 600.0 & 00 . \\
\hline & & & & כ & & \\
\hline & 234.8178 & 0.0750 & 281.7 & & .0 & 60 \\
\hline
\end{tabular}




\begin{tabular}{|c|c|c|c|c|c|c|c|}
\hline & & & & & & & \\
\hline & 1 & 343.3152 & 2.0750 & 1.2 & & 600.0 & \\
\hline & 1 & 329. & 1250 & 280.3 & 4 & 00 & \\
\hline & 1 & 327.7506 & 4.2250 & 280.8 & & 00 & \\
\hline & 1 & 345.2839 & .2750 & 81.2 & & & \\
\hline & 1 & & & 81.2 & & 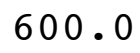 & \\
\hline & 16 & 5005 & 7500 & 81.3 & & 00 & \\
\hline & 1 & 2516 & 7.2750 & 81.3 & & 0 & \\
\hline & 1 & 2500 & .7750 & 81.8 & & & \\
\hline & 19 & 33.7500 & 9.3250 & 82.1 & & 0 & 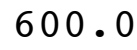 \\
\hline & 110 & 0000 & 8.8500 & 282.2 & & & \\
\hline & 111 & 336.2511 & 9.2000 & 82.8 & & & \\
\hline & 112 & 785 & 8.4250 & 83.1 & & & \\
\hline & 113 & 1.5028 & 7.8500 & 283.3 & & & \\
\hline & 11 & 81 & 0 & & & & \\
\hline & 11 & & & 282.3 & $I$ & & \\
\hline & 11 & 302.2499 & 7.7500 & 282.6 & 4 & & \\
\hline & 117 & .2494 & 0 & 2.6 & 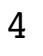 & & \\
\hline & 11 & .7528 & 0 & 82.4 & 4 & & \\
\hline & 11 & 998 & 500 & 282.2 & 4 & & \\
\hline 11 & 120 & 305.7557 & 1.2750 & 281.8 & $I$ & & \\
\hline & 121 & 301.2500 & & 82.1 & 4 & & \\
\hline & 122 & 040 & 0 & 282.2 & 4 & & \\
\hline 1 & 123 & 301.7502 & 0.3500 & 282.2 & 4 & & \\
\hline & 124 & 292.7497 & & & 4 & & \\
\hline 11 & 21 & 29 & 0 & 282 & 4 & & \\
\hline 1$]$ & 22 & 328.5328 & 50 & 283.2 & 4 & & 0 • \\
\hline & 23 & 350.9994 & 500 & & 4 & & \\
\hline & 24 & 337 . & 50 & & 4 & & \\
\hline & 25 & 352.2541 & 750 & 5.8 & 4 & & J. \\
\hline 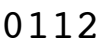 & 26 & 343.7494 & 5500 & 285.2 & 4 & & 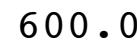 \\
\hline & 27 & 7.4997 & 0 & & 4 & & \\
\hline & 28 & 356.2443 & & .7 & 4 & & \\
\hline 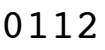 & 29 & .0562 & 250 & 284.3 & 4 & & \\
\hline & 210 & 345 & 250 & & 4 & & \\
\hline & 211 & .2491 & 1.5000 & & 4 & & \\
\hline 1 & 212 & 15.7489 & 4000 & 283.8 & 4 & & \\
\hline & 213 & 50 & 500 & & 4 & & \\
\hline- & 214 & & 8.8250 & .3 & 4 & & 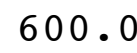 \\
\hline & 215 & .2504 & 3500 & 284.3 & 4 & & \\
\hline & 216 & 7073 & 000 & & 4 & & \\
\hline & 217 & 0007 & 9.2250 & 282.7 & 4 & & 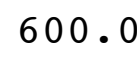 \\
\hline & 218 & .2461 & 7.1500 & 282.3 & 4 & & 0 \\
\hline & 219 & 810 & 000 & & 4 & & \\
\hline & 220 & .7501 & & & 4 & & \\
\hline & 221 & 47.2508 & 7.2500 & 282.0 & 4 & 600 & 00. \\
\hline & 222 & I & & & 4 & & \\
\hline & $2 \pi$ & 38.4963 & 6.1750 & 281.3 & 4 & 600.0 & 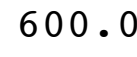 \\
\hline
\end{tabular}




\begin{tabular}{|c|c|c|c|c|c|c|c|}
\hline 112 & 24 & & 2.9750 & 79.9 & & & \\
\hline 1 & 1 & 78.7794 & 3.0000 & 279.2 & & & \\
\hline & 32 & 6.0000 & 1.6500 & 279.2 & & 00 & \\
\hline 1. & 3 & 318.6109 & 1.3250 & 279.4 & & 600.0 & \\
\hline & 4 & 28.1518 & 1.62 & 279.6 & & 0 & \\
\hline & 5 & 351.7056 & 1.5500 & 279.2 & & 00 & \\
\hline & 6 & 37.3851 & 2.7500 & 280.0 & & 00.0 & \\
\hline & 7 & 54.2534 & .7000 & 279.9 & & & \\
\hline & 8 & 25.4518 & 1.7500 & 279.6 & & .0 & \\
\hline 11 & 9 & 56.2672 & 0.7250 & 281.4 & & 00.0 & \\
\hline & 10 & 326.9248 & 1.0000 & 282.8 & & 00.0 & \\
\hline & & 33.9929 & 20 & 82.6 & & & \\
\hline 1 & 12 & 57.1339 & 3.7750 & 282.9 & & 00 & \\
\hline 11 & 13 & 82.7891 & 3.1250 & 282.8 & & 600.0 & . \\
\hline & & 79.9900 & & 282.0 & & & \\
\hline & 1 & 56.4977 & 4.0750 & 282.4 & & 0 & \\
\hline & 16 & 120.3926 & 3.45 & 283.1 & & 0 & \\
\hline & 17 & 145.0451 & .50 & 282.1 & & & \\
\hline 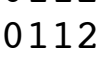 & 18 & 143.7491 & 67 & 281.3 & & 0 & \\
\hline & 19 & 146.2501 & .97 & 281.1 & 4 & 0 & \\
\hline L. & 20 & 151.7500 & 0 & 0.8 & 4 & 0 & \\
\hline & 21 & 152.0000 & 0 & .2 & 4 & & \\
\hline & 22 & 147.2499 & 9.7250 & 280.0 & 4 & 0 & 0 . \\
\hline 1 & 323 & 150.7499 & 7.97 & 0.0 & 4 & 0 & \\
\hline & 324 & 141.7382 & & & 4 & & \\
\hline & 1 & 145.9995 & 9.3500 & 280.0 & 4 & & 0 . \\
\hline 1 & 2 & 142.2482 & 7.65 & 279.9 & 4 & 0 & \\
\hline & 43 & 128.2481 & 0 & 279.9 & 4 & & • \\
\hline 1 & 44 & 101.7456 & 6 . & 9.3 & 4 & 0 & 0 . \\
\hline 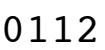 & 5 & 97.5067 & 0 & 279.2 & 4 & 0 & \\
\hline & 46 & 97.5000 & 0 & 9.3 & 4 & & \\
\hline & 47 & 88. & 6 . & 3.7 & 4 & & $\bullet$ \\
\hline 1. & 48 & 71.0027 & 7.3500 & 278.0 & 4 & 600.0 & 0 . \\
\hline 1. & 49 & 77.2497 & 7 . & 8.3 & 4 & 0 & \\
\hline & 410 & 555 & 5. & .6 & 4 & & \\
\hline & 411 & 98.9854 & 4.5750 & 280.7 & 4 & 0 & 600 . \\
\hline & 412 & 82.8034 & 750 & 281.0 & 4 & 0 & \\
\hline & 413 & 81.2435 & 7. & 282.1 & 4 & 0 & \\
\hline 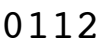 & 414 & 93.0083 & 6 & 283.1 & 4 & 600.0 & 0 . \\
\hline & 415 & 79.9982 & 50 & $2 \varepsilon$ & 3 & 600 & \\
\hline & 416 & 65.2500 & 5 . & & 3 & & \\
\hline 011 & 417 & 85.1328 & 2.7500 & 282.5 & 4 & 600.0 & 600 . \\
\hline & 418 & 43.6331 & 1.9000 & 281.9 & 5 & 600 & 600 \\
\hline & 419 & 8.1212 & 2.7250 & 282.0 & 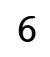 & 600 & 600 \\
\hline 01 & 420 & 337.2337 & 1.3750 & 281.7 & 6 & 600.0 & 500 . \\
\hline & 421 & 117.6054 & 2.3000 & 281.8 & 6 & 600.0 & 600. \\
\hline & 422 & 55.2910 & 4.4500 & 281 & 5 & 600.0 & 600 \\
\hline & 423 & 37.4925 & 3.5000 & 281.3 & 4 & 600.0 & 600 \\
\hline
\end{tabular}




\begin{tabular}{|c|c|c|c|c|c|c|c|}
\hline 12 & 24 & $=2424$ & 2.8500 & 1.3 & 4 & 600.0 & \\
\hline 112 & 51 & 7.5308 & 3.7500 & 280.9 & כ & 0 & \\
\hline 112 & 52 & 48.2429 & 500 & 280.9 & & 00 & \\
\hline & 3 & 50.2504 & 3.8750 & 280.6 & & 00.0 & \\
\hline & 4 & 3.2637 & 3.9000 & 80.8 & & 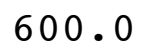 & \\
\hline & 55 & 37.7534 & 2.4250 & 281.2 & & 00 & \\
\hline & 6 & 24.7533 & 1.6750 & 281.0 & & 00 & \\
\hline & 7 & 343.8233 & 1.4500 & 81.1 & & 0.0 & \\
\hline 2 & 8 & 348.2917 & 1.3250 & 81.1 & & 00.0 & \\
\hline & 9 & 21.7618 & 2.5000 & 281.7 & & 0 & \\
\hline & 10 & 326.2977 & 1.4500 & 82.7 & & & \\
\hline & 11 & 309.3214 & 1.4000 & 83.8 & 3 & & \\
\hline 117 & 12 & 22.6916 & 2.0000 & 284.5 & & 00.0 & \\
\hline 112 & 13 & 8.0000 & 2.8250 & 284.2 & & 0 & \\
\hline & 14 & 13.2521 & 750 & 0 & & & \\
\hline 2 & 15 & 016 & 500 & 284.0 & & 0 & \\
\hline 112 & 516 & 60.0440 & 3.2500 & 284.7 & C & 0.0 & \\
\hline & 517 & 258.9359 & 1.5500 & 282.1 & 3 & & \\
\hline 2 & 18 & 214 . & 000 & 282.3 & 4 & 0 & \\
\hline 2 & 519 & 348.2127 & 5750 & 281.5 & 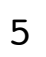 & 0 & \\
\hline & 20 & 125 & 250 & & 6 & & \\
\hline & 521 & 153 & 000 & 282.8 & 6 & & \\
\hline 11 & 522 & 84.8099 & 0.3750 & 283.3 & 6 & & \\
\hline & 523 & 70.5952 & 5000 & 9 & 6 & & \\
\hline & 524 & 245 . & 00 & & 6 & & \\
\hline & 61 & 252.9913 & 5000 & 283.9 & 6 & & \\
\hline 112 & 62 & 258.4690 & 0.5250 & 283.9 & 6 & & \\
\hline & 63 & 268.0461 & 250 & 283.7 & 6 & & \\
\hline & 64 & 299. & 0.4500 & 284.0 & 6 & & \\
\hline 112 & 65 & 357 . & 0.2000 & 284.1 & 6 & & \\
\hline & 66 & 22 . & 250 & & 6 & & \\
\hline & 67 & 331.3551 & 1 . & 283.6 & 6 & & \\
\hline 112 & 68 & 320.2685 & 0.7750 & 283.9 & 5 & & \\
\hline 112 & 69 & 983 & 0 & 28 & 4 & & \\
\hline & 610 & 346 . & 1.1750 & 28 & 3 & & \\
\hline & 611 & 86.9186 & 1.2000 & 287.0 & 2 & & \\
\hline & 612 & 155.2503 & 00 & 286.7 & 3 & & \\
\hline & 613 & 168.5015 & 3.7000 & 286.8 & 3 & & 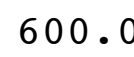 \\
\hline 110 & 614 & 174.2891 & 1.6500 & 287.7 & 2 & & 6 \\
\hline & 615 & 183. & & 287 & 3 & & \\
\hline & 616 & 141 . & 500 & 287.6 & 3 & 0 & 6 \\
\hline & 617 & 124.1850 & 3.9000 & 286.6 & 4 & .0 & 0 \\
\hline & 618 & 152.0034 & 750 & 285.2 & 4 & & \\
\hline 0112 & 619 & 115.7556 & 5.4500 & 285.4 & 4 & 600.0 & 600 \\
\hline 01 & 620 & 143.2545 & 6.3750 & 285.7 & 4 & 600.0 & 500. \\
\hline & 621 & 139. & & & 4 & & \\
\hline & 622 & 143.2465 & 5.4250 & & 4 & & 600 \\
\hline & 623 & 158.4959 & 5.9500 & 283.2 & & 600.0 & 600 \\
\hline
\end{tabular}




\begin{tabular}{|c|c|c|c|c|c|c|c|}
\hline & 24 & & 4.9500 & $2 \cdot 8$ & & & \\
\hline 17 & 71 & 992 & 4.8750 & 282.3 & 4 & & \\
\hline & 72 & 139.9710 & .9750 & 281.8 & 4 & 00 & \\
\hline & 73 & 142.7737 & 5.1750 & 281.3 & & 00 & \\
\hline & 74 & 159.5032 & .0750 & 81.0 & & 0 & \\
\hline & 75 & 151.7505 & 1000 & 280.8 & & 00 & \\
\hline & 76 & 154.2504 & 6.6500 & 280.4 & & 0 & \\
\hline & 77 & 37.5366 & .9250 & 80.3 & & & \\
\hline & 78 & 144.2449 & 2.8000 & 280.4 & 5 & 0 & \\
\hline 11 & 79 & 147.2944 & 9000 & 281.2 & & 00 & \\
\hline & 710 & 156.2629 & .5250 & 282.0 & & & \\
\hline & 711 & 157.9632 & 4.8500 & 82.3 & + & & \\
\hline 1 & 712 & 150.0000 & 5250 & 283.1 & 4 & 0 & \\
\hline 1. & 713 & 141.2496 & 5.5250 & 284.2 & 4 & 0.0 & \\
\hline & 714 & 143.7512 & & 285.1 & 4 & & \\
\hline & 715 & 127.5223 & 3.2500 & 285.9 & 4 & 0 & \\
\hline & 716 & 135.2819 & 1.8250 & 286.4 & 3 & 0 & \\
\hline 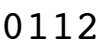 & 717 & 117.9578 & .5750 & 0 & 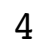 & & \\
\hline & 718 & 216.7056 & .1750 & 284.1 & 5 & 0 & \\
\hline & 719 & 260.1161 & .5000 & 282.8 & 6 & & \\
\hline L. & 720 & 354.8248 & 1.1000 & 282.8 & 5 & & \\
\hline & 721 & 343.8235 & 1.8750 & 282.4 & 5 & & \\
\hline & 722 & 318.3723 & 9500 & 282.9 & 6 & 0 & \\
\hline 1 & 723 & 300.0551 & 1.1250 & 283.3 & 6 & 0 & \\
\hline & 724 & 174.7535 & 0 & 282.6 & 6 & & \\
\hline & 81 & 213.4806 & 1.4250 & 281.2 & 5 & & \\
\hline 1. & 82 & 249.0000 & .0250 & 280.2 & 6 & 0 & \\
\hline & 83 & 221.2429 & 0 & 280.6 & 6 & 0 & C \\
\hline & 84 & 181.6174 & .6500 & 280.0 & 6 & 0 & 0 . \\
\hline 1. & 85 & 136.7710 & .3250 & 279.5 & 5 & 0 & \\
\hline & 86 & 126.2783 & 0 & $27 s$ & 6 & & \\
\hline & 87 & 34.6628 & 0 & 27 & 6 & & . \\
\hline & 88 & 351.7362 & 0.1250 & 279.4 & 6 & 0 & U. \\
\hline 1. & 89 & 61.2528 & 0 & 280.8 & 5 & 0 & \\
\hline & 810 & 219.5436 & 0 & 281.2 & 4 & & \\
\hline & 811 & 156.5017 & 2.1500 & 279.6 & 4 & & 0 . \\
\hline & 812 & 158.7702 & 2.8250 & 279.3 & 3 & 0 & \\
\hline & 813 & 133.9651 & 1.7250 & 280.4 & 2 & 0 & • \\
\hline- & 814 & 335.2179 & 0.7000 & 282.4 & 1 & 60 & 0 . \\
\hline & 815 & 2.7569 & 500 & 283.8 & 1 & 60 & \\
\hline & 816 & 78.9630 & 1.9000 & & 1 & & \\
\hline 011 & 817 & 133.9862 & 2.4000 & 283.8 & 2 & 600.0 & 600 . \\
\hline & 818 & 88.5845 & 2.4000 & 283.0 & 3 & 600 & 600. \\
\hline & 819 & 117.1468 & 1.6000 & 282.8 & 4 & 600 & 600 \\
\hline 011 & 820 & 99.8978 & 6.0250 & 284.2 & 4 & 600.0 & 500. \\
\hline & 821 & 83.4977 & 7.6500 & 284.8 & 4 & 600.0 & 600. \\
\hline & 822 & 9996 & 8.7000 & 28 & 4 & 60 & 60 \\
\hline & 823 & 78.7390 & 8.0250 & 283.0 & 4 & 600.0 & 600 \\
\hline
\end{tabular}




\begin{tabular}{|c|c|c|c|c|c|c|}
\hline 28 & 7. 2431 & & 2.2 & 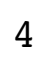 & & \\
\hline 129 & 5.4127 & 2000 & 281.8 & & 0 & \\
\hline 29 & 145 & 0 & 5 & & & \\
\hline 29 & 188 & 1250 & 79.5 & & & \\
\hline 9 & 6 & & 79.4 & & & \\
\hline 29 & 941 & & 279.3 & & & \\
\hline 29 & 966 & & 279.4 & & & \\
\hline 29 & & & 78.7 & & & \\
\hline 129 & 908 & 6.7500 & 278.6 & & 0 & \\
\hline 29 & 936 & 0 & 278.1 & & & \\
\hline 2910 & 30 & & & & & \\
\hline 11 & 54 & & 277.5 & & & \\
\hline 12 & 97.2307 & 250 & 277.8 & & & \\
\hline 2913 & 53. & & & & & \\
\hline 12914 & 153 & & 278 & & & \\
\hline 2915 & 157 & 0 & 279.8 & & & \\
\hline 2916 & 123 & 0 & & & & \\
\hline 2917 & 105 & & 81.2 & & & \\
\hline 2918 & 141 & 0 & 281 & & & \\
\hline 2919 & 1 & & & & & \\
\hline 2920 & & & & & & \\
\hline 2921 & 15 & & 281.2 & & & \\
\hline 2922 & 161. & 0 & 280.8 & & & \\
\hline 2923 & 159 . & & & & & \\
\hline 924 & 160 & & 28 & 4 & & \\
\hline 10 & 160.0000 & 750 & 279 & & & \\
\hline 102 & 156.2801 & & & & & \\
\hline 103 & 124.7282 & & 27 & 4 & & \\
\hline 2104 & 144.7617 & & 27 & & & \\
\hline 2105 & & & & 4 & & \\
\hline 2106 & 110 & & & 4 & & \\
\hline 12107 & 86.7430 & & & 4 & & \\
\hline 112108 & 88.7685 & 000 & 279.2 & 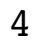 & & \\
\hline 112109 & 124.3460 & 0 & 2 & 4 & & \\
\hline 1010 & 136.5290 & & & 4 & & \\
\hline 121011 & 150.2524 & 750 & 28 & 4 & & \\
\hline 1012 & 128 . & & & 4 & & \\
\hline 013 & 137.4979 & & $2 \varepsilon$ & 4 & & \\
\hline 1121014 & 152.5000 & 250 & 3.7 & 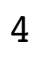 & & \\
\hline 015 & 150 . & & & 4 & & \\
\hline 016 & 144 . & 10.2500 & & 7 & & \\
\hline 1121017 & 148.5002 & 1.3250 & 284.0 & 4 & & \\
\hline 21018 & 134.2963 & 000 & & 4 & & \\
\hline 1121019 & 113.9992 & & 283.4 & 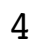 & & \\
\hline 1121020 & 96.7527 & 8.8750 & 282.4 & 4 & 0 & \\
\hline & & & & & & \\
\hline & 123. & 0.4250 & & & & \\
\hline 1121023 & 113.4950 & 8.9000 & $\angle 0$ & & .0 & 600 \\
\hline
\end{tabular}




\begin{tabular}{|c|c|c|c|c|c|c|}
\hline $0<4$ & & & & & & \\
\hline 2111 & 0 & .4000 & 31.7 & & & \\
\hline 2112 & - & 1000 & 81.3 & & 0 & \\
\hline 12113 & 46.4998 & 3.9750 & 281.3 & & 00 & \\
\hline 2114 & & & & & & \\
\hline 115 & 75 & & 80.9 & & 0 & \\
\hline 12116 & 48.7500 & 500 & 280.7 & & & \\
\hline 12117 & 1.7500 & 0 & & & & \\
\hline 12118 & 51.7496 & 2.35 & 80.2 & & 0 & \\
\hline 112119 & 158.4995 & 50 & 280.3 & & 0 & \\
\hline 110 & 161.5000 & 10.92 & 280.6 & & & \\
\hline & $5=0$ & & 81.1 & & & \\
\hline 11 & 50 . & 8.22 & 281.9 & 4 & 0 & \\
\hline 113 & 59.5000 & 7. & 282.7 & & 0 & \\
\hline & & & 33.0 & 4 & & \\
\hline 1. & 50 & 5.35 & 283.3 & 4 & & \\
\hline 6 & I & 3 . & 283.3 & 3 & 0 & \\
\hline & 04.90 & 3 . & 2.8 & & & \\
\hline & 72 . & 4 . & 282.0 & 4 & & \\
\hline & 71.50 & & 281.9 & 4 & & \\
\hline 20 & 75.25 & & 2.0 & 4 & & \\
\hline & 74.22 & & 281.7 & 4 & & \\
\hline 22 & 71 & & 281.6 & 4 & 0 & \\
\hline 123 & 77.25 & & 281.8 & 4 & & \\
\hline 24 & 82.25 & & 81.6 & 4 & & \\
\hline 21 & 83 & & 281.7 & 4 & & \\
\hline 112122 & 99.25 & & 281.8 & 4 & & \\
\hline 112123 & 02.49 & & 1.4 & 4 & & \\
\hline 112124 & 147 & & .9 & 5 & & \\
\hline 112125 & 111.65 & & 280.8 & 4 & & \\
\hline 112126 & 94.46 & & 280.3 & 4 & & \\
\hline 2127 & 149 & 0 & .0 & 4 & & \\
\hline 112128 & 143.7459 & & 277.4 & 4 & 0 & 0 . \\
\hline 112129 & 134.74 & & 277.9 & 2 & & \\
\hline & 121. & & .6 & 2 & & \\
\hline 211 & 112.9789 & & 280.0 & 1 & & \\
\hline 21212 & 265.1976 & & 282.6 & 1 & & \\
\hline 213 & 260.37 & 0 & 283.5 & 1 & & J • \\
\hline 112 & 241.2793 & & 284.9 & 1 & 0 & 0 . \\
\hline 21215 & 272.57 & 1. & 284.3 & 1 & 0 & \\
\hline 216 & 243.7485 & 1 . & & 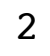 & & \\
\hline 01121217 & 256.4327 & 1.3 & 282.3 & 3 & 600.0 & 00 . \\
\hline 2121 & 269.48 & & 281.4 & 4 & 0 & \\
\hline 01121219 & 348.4601 & & & 4 & & \\
\hline 01121220 & 35.9260 & 1.1750 & 281.1 & 5 & 600.0 & 0 \\
\hline 21221 & 137.6408 & 0.1000 & & 6 & 600 & \\
\hline 010 & 142.24 & & & ? & & \\
\hline 21223 & 80.0652 & 0.2500 & 281.8 & & 600.0 & 600 \\
\hline
\end{tabular}




\begin{tabular}{|c|c|c|c|c|c|c|}
\hline 224 & & & & & & \\
\hline 2131 & 5 & .7000 & 0.9 & & & \\
\hline 2132 & 5 & 1.3250 & 280.9 & 6 & 00 & \\
\hline 12133 & 3891 & 1.2750 & 281.0 & & 00 & \\
\hline 2134 & 84 & .9000 & 0.6 & & & \\
\hline 2135 & 2 & 0750 & 80.1 & & 0 & \\
\hline 12136 & 06 & $\$ 750$ & 80.1 & & 00 & \\
\hline 12137 & 34 . & 500 & 80.3 & & & \\
\hline 12138 & 142.274 & 50 & 80.2 & & & \\
\hline 12139 & 55.425 & 0.5250 & 82.2 & & 00 & 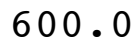 \\
\hline 310 & 2694 & 0.8000 & 282.8 & & & \\
\hline & 316.7533 & 1.3000 & 83.7 & & & \\
\hline 2 & 300 & 2.7250 & 282.8 & & 0 & \\
\hline 13 & 282.20 & 2.5250 & 282.7 & & & \\
\hline & 265 & & & & & \\
\hline & 216 & 1 . & & 1 & & \\
\hline 16 & 155.4 & 1.75 & 283.1 & & & \\
\hline 17 & 125 . & 3.20 & 2.5 & & & \\
\hline & 87 . & 5 & 281.5 & 3 & & \\
\hline 9 & 0 & & 281.3 & 4 & & \\
\hline 20 & 162.5155 & 3.25 & 280.8 & 5 & 0 & \\
\hline & & & & 4 & & \\
\hline 2 & 68 & 27 & 7 & 5 & & \\
\hline 23 & 329.9526 & 2.2500 & 0.4 & 6 & 0 & \\
\hline & 304 . & & & & & \\
\hline 1 & 30 & 4 . & 6 & 4 & & \\
\hline 42 & 341 . & 3.9 & 2.0 & 4 & & 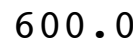 \\
\hline 112143 & 23. & 3 . & 2.6 & 5 & & \\
\hline $4 \quad 4$ & 15 & 6 & 0 & 4 & & \\
\hline 112 & 100 . & 9.0 & & 4 & & . \\
\hline $11214 \quad 6$ & 7622 & 9.0 & 280.1 & 4 & & \\
\hline 112 & 88 & 0 & 79.5 & 4 & & \\
\hline 112 & & 0 & .3 & 4 & & \\
\hline 112149 & 488 & 00 & 279.2 & 4 & & \\
\hline 10 & 81 . & 0 & & 4 & & \\
\hline & & 8.3750 & .9 & 4 & & \\
\hline 12 & 108.0023 & 7.8750 & 280.4 & 4 & & \\
\hline 13 & 118 . & 7.1 & 9 & 4 & & \\
\hline 14 & 138. & 8.7500 & 281.4 & 4 & & 0 . \\
\hline & 147.1970 & 0.6000 & 281.4 & 4 & & \\
\hline & 172 . & 50 & & 4 & & \\
\hline & 164 . & 0.5750 & 280.8 & 4 & & . \\
\hline 121418 & 160.0000 & 4.7000 & 280.3 & 4 & & 0 . \\
\hline 19 & 158 . & 750 & & I & & \\
\hline 01121420 & 155.7500 & 15.5500 & & 4 & & \\
\hline 21 & 157.2504 & 14.9500 & 279.6 & 4 & 0 & 00 \\
\hline & & 5.3 & & 4 & & \\
\hline 1121423 & 158.750 & 13.9250 & • & & & 8 \\
\hline
\end{tabular}




\begin{tabular}{|c|c|c|c|c|c|c|}
\hline & & & & & & \\
\hline 2151 & 8 & & 8.3 & & & \\
\hline 2152 & 156.0002 & 6750 & 278.5 & 4 & 00 & \\
\hline 12153 & 154.2506 & 1.9750 & 278.6 & & 00 & \\
\hline 2154 & 152.5009 & & 8.6 & & & \\
\hline 2155 & 110 & & 278.7 & & 0 & \\
\hline 12156 & 152.7500 & 0.7250 & 278.5 & & 00 & \\
\hline 12157 & 999 & .2250 & 278.3 & & & \\
\hline 12158 & 7500 & 6750 & 78.3 & & & \\
\hline 12159 & 156.0011 & 8.2750 & 278.7 & & 0 & 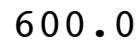 \\
\hline 1510 & 161.4880 & 0500 & 279.3 & & & \\
\hline & 170.7502 & 2500 & 79.8 & & & \\
\hline 512 & 158 & 7750 & 280.2 & 4 & 0 & \\
\hline 513 & 163.0000 & 0000 & 281.0 & & & \\
\hline & 164 . & & & & & \\
\hline & 181. & & 2.1 & 3 & & \\
\hline 516 & 167.5209 & 8750 & 282.2 & 3 & 0 & \\
\hline 517 & 168 & 0 & .5 & & & \\
\hline & 313. & & & 5 & & \\
\hline 9 & 351 & & 9.9 & 4 & & \\
\hline 520 & 18.7380 & 1.6000 & 280.2 & 4 & 0 & \\
\hline & 14.7347 & & & 4 & & \\
\hline 2 & 13 & 0 & & 4 & & \\
\hline 523 & 10.5000 & 0 & .6 & 4 & 0 & \\
\hline 524 & 12.7501 & & & 4 & & \\
\hline 61 & 6. & 0 & & 5 & & \\
\hline 112162 & 13.2447 & 0 & 2 & 4 & & 0 • \\
\hline 112163 & 290.8135 & 0 & 2 & 5 & & \\
\hline 112164 & 157.2556 & & 2 & 6 & & \\
\hline 112165 & 27.7154 & 0 & 9.8 & 6 & & 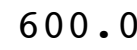 \\
\hline 112166 & 9.5105 & 1.4750 & 9.9 & 5 & & 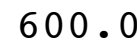 \\
\hline 112167 & 050 & 0 & 2 & 4 & & \\
\hline 112168 & 27.1690 & & .9 & 5 & & \\
\hline 112169 & 141.2576 & 250 & 1.4 & 4 & & \\
\hline & 161.2448 & & & 4 & & \\
\hline & 156.2492 & 1.9750 & 2.8 & 4 & & \\
\hline 612 & 358.4346 & 1.2250 & 3.5 & 3 & & \\
\hline 613 & 327.4213 & 1. & & 2 & & \\
\hline 1121614 & 303.6073 & 1.7500 & 2.4 & 3 & & . \\
\hline 1121615 & 301.0055 & 1.1500 & 2.4 & 3 & & \\
\hline & 334.5505 & 1 . & 282.3 & 3 & & \\
\hline 1121617 & 358.0129 & 0 . & 282.3 & 4 & & 0 . \\
\hline 121618 & 18.0624 & 1.8000 & 282.6 & 4 & & 0 . \\
\hline 21619 & 17.7537 & 750 & & 4 & & \\
\hline 01121620 & 26.5221 & 1.6500 & & 4 & & \\
\hline 2162 & 5.1564 & 1.9250 & 285.0 & 5 & & 00 . \\
\hline & 359 & & & O & & \\
\hline 1121623 & 67.2728 & 1.5500 & 285.4 & & 600.0 & $r^{2}$ \\
\hline
\end{tabular}




\begin{tabular}{|c|c|c|c|c|c|c|}
\hline 624 & & .4750 & & & & \\
\hline 2171 & 0 & .9000 & 3 & & (20 & \\
\hline 2172 & 31.8025 & 7000 & 285.8 & 4 & 00 & \\
\hline 12173 & 357.8177 & .8000 & 285.4 & 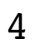 & 00 & \\
\hline 2174 & 331.2658 & .7750 & & & 0 & \\
\hline 2175 & 250.50 & 9000 & 84.0 & & 00 & \\
\hline 12176 & 256.4894 & 0.6250 & 283.7 & & 00 & \\
\hline $1217 \quad 7$ & 331.7056 & L. 6500 & 83.6 & & & \\
\hline 12178 & 352.2444 & 8000 & 83.9 & & & \\
\hline 112179 & 137.3012 & 3.0250 & 83.2 & 4 & 00 & 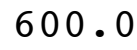 \\
\hline 1121710 & 162.8501 & 1.4750 & 282.0 & 3 & & \\
\hline 1711 & 336.5268 & 2.7750 & 82.5 & & & \\
\hline 121712 & 181.0841 & 2.8500 & 282.9 & 2 & 0 & \\
\hline 1121713 & 179.6358 & 3.1750 & 282.1 & 2 & 0.0 & \\
\hline 1714 & 260.8979 & & 32.2 & & & \\
\hline 1715 & 233.7119 & 0 . & 281.7 & 1 & & \\
\hline 1121716 & 160.2478 & 2.1000 & 282.2 & 1 & .0 & 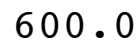 \\
\hline 1717 & 82.1464 & 2.30 & .5 & 2 & & \\
\hline 1718 & 7.50 & 1 . & 281.5 & 3 & & \\
\hline 1719 & 10.9945 & 1.9250 & 282.0 & 4 & & $\bullet$ \\
\hline 1121720 & 25.9995 & 2.1250 & 282.8 & 4 & 0 & 0 \\
\hline 1121721 & 68.75 & & 282.8 & 5 & & \\
\hline .722 & 85.0221 & 3 . & 282.9 & 4 & & \\
\hline 1121723 & 77.7538 & 6.8000 & 281.5 & 4 & 0 & \\
\hline 1121724 & 59.42 & 0 & & 4 & & \\
\hline 0112181 & 86.99 & 750 & 28 & 4 & & \\
\hline 112182 & 78.9998 & 7500 & 280.7 & 4 & 0 & . \\
\hline 112183 & 71.2562 & 4.7500 & 7 & 4 & & \\
\hline 112184 & 60.2056 & 50 & 27 & 5 & & \\
\hline 112185 & 43.6964 & 2.0500 & 279.5 & 6 & 0 & U. \\
\hline 112186 & 353.2965 & 1.1750 & 280.0 & 6 & 0 & 0 . \\
\hline 112187 & 9376 & 0 & 28 & 6 & & \\
\hline 0112188 & 105.7575 & & 279.6 & 5 & & 0 . \\
\hline 112189 & 85.7500 & 6.5500 & 280.1 & 4 & 0 & \\
\hline 1121810 & 83.7499 & 0 & & 4 & & \\
\hline 811 & 76.1283 & 2 . & 281.9 & 3 & & 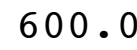 \\
\hline 1121812 & 269.9108 & 1.9750 & 282.0 & 2 & & 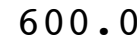 \\
\hline 01121813 & 200.640 & 1 . & 282.3 & 1 & & ( \\
\hline 01121814 & 309.8662 & 1.9500 & 282.6 & 1 & .0 & 0 . \\
\hline 1121815 & 343.5210 & 2.6000 & 282.2 & 2 & & \\
\hline 1816 & 327.8175 & 2.2000 & 282.4 & 1 & & 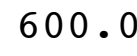 \\
\hline 01121817 & 116.9393 & 1.5250 & 282.1 & 2 & .0 & 0 . \\
\hline 1121818 & 144.3367 & 2.9250 & 281.1 & 3 & 0 & 00 . \\
\hline 1121819 & 100.2646 & 500 & 28 & 4 & & \\
\hline 01121820 & 205.7353 & 1.3000 & 281.2 & 5 & & 500. \\
\hline 1121821 & 5.8589 & 1.3750 & 280.4 & 5 & 600.0 & 600 \\
\hline & 355 & 1 . & & 5 & & \\
\hline 1121823 & 20.5000 & 2.1000 & 280.4 & 4 & 600.0 & 600 \\
\hline
\end{tabular}




\begin{tabular}{|c|c|c|c|c|c|c|}
\hline 1121824 & 3.2486 & 1.7000 & 280.3 & 5 & 600.0 & \\
\hline 112191 & 18.5008 & 1.0250 & 280.5 & 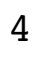 & 00.0 & \\
\hline 12192 & 27.4536 & 1250 & 280.4 & 4 & 0 & \\
\hline 2193 & 6.2374 & .0750 & 80.3 & & 0 & \\
\hline 2194 & 22.6943 & 1.6250 & 280.7 & & 00 & \\
\hline 12195 & 2523 & 1.7500 & 281.2 & & 00 & \\
\hline 12196 & 10.2133 & 3750 & 280.7 & & & \\
\hline 12197 & 356.5542 & 1.5500 & 80.3 & & & \\
\hline 112198 & 270.3455 & 0.7000 & 280.3 & & 00.0 & 00 \\
\hline 112199 & 158.7637 & 6250 & 280.0 & & & \\
\hline 21910 & 149.2547 & 0 & 79.6 & & & \\
\hline 21911 & 177.0628 & 0.4500 & 282.2 & & 0 & \\
\hline 21912 & 117.8014 & 1.6750 & 281.3 & & & \\
\hline 121913 & 156.2415 & 2.60 & 30.0 & & & \\
\hline 121914 & 206.6070 & 1.37 & 280.2 & & & \\
\hline 1121915 & 277.4982 & 9750 & 279.2 & & & \\
\hline 21916 & 317.3109 & 0 & 279.6 & & & \\
\hline 21917 & 7.4470 & & 79.3 & & & \\
\hline 1121918 & 106.4404 & 1.5250 & 280.7 & & 0 & 600. \\
\hline 1121919 & 101.4903 & & 280.9 & & & \\
\hline 1920 & 335.3032 & & 281.2 & 5 & & \\
\hline 21921 & 325 . & 0 & 281.6 & 4 & & \\
\hline 1121922 & 293.0649 & .82 & 282.1 & 5 & 0 & 1 \\
\hline 1121923 & 182.9609 & & 82.2 & 4 & & $\bullet$ \\
\hline 21924 & 96. & & 280 & 4 & & \\
\hline 112201 & 71.5765 & 0 & 280.3 & 4 & 0 & 0 \\
\hline 112202 & 1.1192 & & 7 & 4 & & \\
\hline 112203 & 26.2494 & 6 & 28 & 4 & & \\
\hline 112204 & 21.7752 & 0 & 281.7 & 4 & & • \\
\hline 112205 & 337.4453 & & 0.5 & 5 & & \\
\hline 112206 & 226.4173 & & 27 & 6 & & \\
\hline 112207 & 356.7586 & & 279.4 & 5 & & \\
\hline 112208 & 314.4127 & 2.42 & 280.1 & 6 & 0 & \\
\hline 112209 & 304.3322 & 0 & 280.7 & 5 & & \\
\hline 1122010 & 308.7583 & & 281.5 & 4 & & \\
\hline 1122011 & 292.0006 & 6 . & 281.0 & 4 & & \\
\hline 1122012 & 332 . & 7 . & 281.2 & 4 & & \\
\hline 01122013 & 336 . & 4 . & 0.8 & 3 & & 0 \\
\hline 1122014 & 357.0404 & 600 & 280.4 & 4 & 0 & \\
\hline 1122015 & 357.5010 & & & 3 & & \\
\hline 01122016 & 10.7550 & 3.57 & 281.1 & 2 & & . \\
\hline 01122017 & 15.2580 & 4.6000 & 280.7 & 3 & 0 & م0 \\
\hline 01122018 & 0.7509 & 4.4500 & 280.6 & 4 & 0 & \\
\hline 01122019 & 358.0062 & 4.0500 & 280.5 & 4 & 600.0 & 500. \\
\hline 01122020 & 343.5046 & 3.7500 & 280.2 & 4 & 600.0 & 500 . \\
\hline 22021 & 314 . & & & 4 & & \\
\hline 1122022 & 321.4915 & & & 4 & 600 & 600 \\
\hline 1122023 & 299.6406 & 5.2000 & 278 ." & & .0 & 60 \\
\hline
\end{tabular}




\begin{tabular}{|c|c|c|c|c|c|c|}
\hline 024 & & 8000 & 8.8 & & & \\
\hline 2211 & 4 & 8000 & 279.2 & 4 & (2) & \\
\hline 2212 & 0000 & 6250 & 278.0 & 4 & 00 & \\
\hline 12213 & 5014 & 7250 & 277.9 & & 00 & \\
\hline 2214 & 87 & .8750 & 278.2 & & 0 & \\
\hline 2215 & 160.4493 & 1250 & 277.8 & & 00 & \\
\hline 12216 & 147.4991 & 1.7000 & 277.4 & & 00 & \\
\hline 12217 & 58.2499 & .9250 & 277.7 & & & \\
\hline 12218 & 50 & 6750 & 278.0 & 4 & & \\
\hline 12219 & 150.7506 & 3.3750 & 278.3 & 4 & 00 & 0 \\
\hline 1122110 & 160.0000 & 2.2500 & 278.7 & 4 & 0 & \\
\hline & 155.9800 & 1.0250 & 279.1 & 3 & & \\
\hline 12 & 171.9931 & 1.3000 & 279.9 & 2 & 0 & \\
\hline 122113 & 178.0545 & 2.4500 & 280.6 & 3 & 0.0 & \\
\hline & 79.12 & 500 & & & & \\
\hline & 224.25 & 500 & 280.7 & 3 & & \\
\hline 12 & 236.4821 & 1.1000 & 280.5 & 3 & .0 & U \\
\hline 17 & 74.6166 & 2000 & .5 & 4 & 0 & \\
\hline 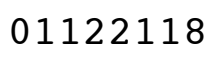 & 38 . & 500 & .4 & 5 & & \\
\hline 1 & 8.1762 & 3500 & 280.3 & 4 & & • \\
\hline 112 & 348.2414 & 1.7250 & 280.3 & 4 & 0 & 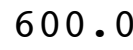 \\
\hline 21 & 334.5684 & 1.3500 & 0.6 & 5 & & \\
\hline 22 & 311 & 250 & .4 & 6 & & \\
\hline 112 & 317.2143 & 1.7500 & 0.3 & 5 & 0 & \\
\hline 24 & 334.54 & 1.1250 & 9.7 & 5 & & \\
\hline 21 & 353.9 & 1000 & 9.5 & 5 & & \\
\hline 112222 & 17.7554 & 1.7500 & 280.6 & 4 & 0 & . \\
\hline 112223 & 9.6621 & 1.4250 & 1.0 & 4 & & \\
\hline 112224 & 358.7314 & 250 & .7 & 5 & & \\
\hline 112225 & 12.4148 & 9500 & 1.4 & 6 & 0 & 0 • \\
\hline 112226 & 343.9438 & 2.5750 & 282.1 & 5 & 0 & 0 \\
\hline 112227 & 3590 & 000 & 282.4 & 6 & & \\
\hline 112228 & 340.25 & 0 & 282.7 & 5 & & . \\
\hline 112229 & 339.4783 & 8.0250 & 283.0 & 4 & 0 & \\
\hline 1122210 & 306.0160 & 50 & 3.1 & 4 & & \\
\hline 11 & 307.1668 & 5 . & 3.2 & 3 & & ( \\
\hline 212 & 357.7506 & 6.6000 & 284.4 & 4 & 0 & 0 . \\
\hline 213 & 30.9091 & 000 & 0 & 3 & & 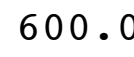 \\
\hline 214 & 149.7525 & 4.3500 & 280.7 & 4 & .0 & 0 \\
\hline 1122215 & 287.0200 & 2.3000 & 280.6 & 3 & & \\
\hline 1122216 & 312.4393 & 0.6500 & 280.9 & 2 & & 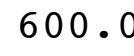 \\
\hline 01122217 & 107.1959 & 2.5500 & 281.4 & 3 & .0 & 0 . \\
\hline 1122218 & 290.9227 & 1.1500 & 281.2 & 4 & .0 & 00 . \\
\hline 1122219 & 322.9438 & 1.5000 & 281.0 & 5 & & \\
\hline 01122220 & 7.8207 & 1.5250 & 281.1 & 4 & 60 & 600. \\
\hline 122221 & 8.6532 & 1.5500 & 281.6 & 5 & 600.0 & 600 \\
\hline 1900 & & & 282.2 & 6 & & \\
\hline 122223 & 212.6402 & 0.6750 & 281.4 & & .0 & 60 \\
\hline
\end{tabular}




\begin{tabular}{|c|c|c|c|c|c|c|}
\hline 224 & & 6000 & & & & \\
\hline 2231 & 6 & 2.0000 & 1.5 & & 00 & \\
\hline 12232 & 67.0536 & 1.6000 & 281.9 & 5 & 00 & \\
\hline 12233 & 80.3206 & 0.4000 & 281.7 & & 00 & \\
\hline 2234 & 123.9848 & .5000 & 81.4 & & 0 & \\
\hline 2235 & 100 & 4750 & 81.5 & & 0 & \\
\hline 12236 & 102.2810 & 250 & 281.3 & & 00 & \\
\hline 12237 & 77.0022 & 3250 & 81.0 & & & \\
\hline 12238 & 138.4699 & 2500 & 80.5 & & & \\
\hline 12239 & 88.8896 & 3.8000 & 281.1 & 4 & 600.0 & 0 \\
\hline 1122310 & 168.3076 & 3.4250 & 281.0 & & 0 & \\
\hline 11 & 153.2500 & 3.1250 & 80.8 & & & \\
\hline 12 & 162.9962 & 3 . & 281.7 & 4 & 0 & \\
\hline 122313 & 165.7545 & 4.50 & 281.2 & & 0.0 & \\
\hline 14 & 159.2506 & & & & & \\
\hline & 165.95 & & 280.8 & 3 & & \\
\hline 16 & 147.7456 & 7500 & 280.7 & 4 & .0 & . \\
\hline 17 & 134.9770 & 0 & .7 & 4 & & \\
\hline & 132.2489 & & 280.8 & 4 & & \\
\hline 19 & 135.4945 & 0 & 281.1 & 4 & 0 & v. \\
\hline 20 & 151.0001 & 500 & 281.4 & 4 & .0 & \\
\hline 21 & 152 . & & 81.6 & 4 & & \\
\hline 22 & 156 & 0 & 281.7 & 4 & & \\
\hline 1122323 & 148.9989 & 6.6000 & 281.3 & 4 & 0 & 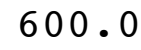 \\
\hline 324 & 153. & & 81.0 & 4 & & \\
\hline 41 & 146.7546 & & 28 & 5 & & \\
\hline 112242 & 123.2523 & 3 & 279.5 & 4 & 0 & . \\
\hline 112243 & 139.2524 & 3 & 9 & 4 & & \\
\hline $11224 \quad 4$ & 130.4557 & 1 . & 278.3 & 5 & & \\
\hline 112245 & 158.7663 & 0 & 278.3 & 6 & 0 & 0 • \\
\hline 112246 & 270.101 & 0 & 277.5 & 6 & & 0 . \\
\hline 112247 & 10.72 & 0 & 277.3 & 6 & & \\
\hline 112248 & 337.8089 & 0 & 277.5 & 5 & 0 & . \\
\hline 112249 & 316.8497 & 1. & 278.1 & 4 & 0 & 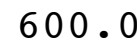 \\
\hline 410 & 306 . & 0 & & 3 & & \\
\hline 11 & 289. & 1 . & 278.9 & 2 & & ( \\
\hline 412 & 298.0499 & 1.5500 & 279.7 & 1 & & 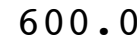 \\
\hline 413 & 316 . & 1 . & 278.9 & 2 & & ( \\
\hline 0112 & 288.3724 & 1.6250 & 279.5 & 1 & .0 & 0 . \\
\hline 1122415 & 279.7113 & 1.7250 & 279.4 & 2 & & \\
\hline 22416 & 304.7073 & 1.4750 & 279.2 & 3 & & 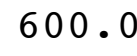 \\
\hline 01122417 & 331.4729 & 2.1250 & 279.3 & 4 & .0 & 0 . \\
\hline 1122418 & 345.6961 & 1.6000 & 279.2 & 5 & 0 & 0 . \\
\hline 1122419 & 87.3750 & 500 & $27 \xi$ & 6 & & \\
\hline 01122420 & 190.2019 & 0.5750 & 278.9 & 6 & & 0 . \\
\hline 122421 & 141.4982 & 1.6500 & 278.2 & 6 & 600.0 & 00. \\
\hline 172012 & 127 . & & & 6 & & \\
\hline 1122423 & 149.9846 & 1.7500 & 277.7 & & 60 & $60 \Omega$ \\
\hline
\end{tabular}




\begin{tabular}{|c|c|c|c|c|c|c|}
\hline $24<4$ & & & 17.3 & & & \\
\hline 2251 & 173.2575 & .8000 & 277.6 & 6 & n & \\
\hline 2252 & 317.8028 & 2500 & 277.3 & 6 & 00 & \\
\hline 12253 & 167.3530 & 3750 & 278.2 & & 00 & \\
\hline 2254 & 4.3890 & .3250 & 278.3 & & 0 & \\
\hline $225 \quad 5$ & 258.0000 & & 279.1 & 6 & 0 & \\
\hline 12256 & 158.2595 & 9750 & 278.4 & 6 & 00 & \\
\hline 12257 & 829 & .2750 & 278.3 & & 0 & \\
\hline 12258 & 155.4998 & .9000 & 277.8 & & & \\
\hline 12259 & 152.7511 & 2.1250 & 277.9 & 4 & 000 & 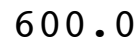 \\
\hline 122510 & 158.4971 & 1.6250 & 278.4 & 4 & & \\
\hline & 162.4966 & 1.5000 & 278.7 & 3 & 00.0 & \\
\hline 2 & 257 & 0.6250 & 279.7 & 2 & 0 & \\
\hline 12 & 169.9 & 2.9750 & 279.5 & 3 & & \\
\hline 14 & 169. & 0 & 280.1 & & & \\
\hline & 160 . & 1.25 & 280.1 & 3 & & \\
\hline 1 & 155.8466 & 1.3250 & 280.2 & 2 & .0 & \\
\hline 17 & 274.5895 & 0.77 & 280.2 & 3 & & \\
\hline & 154.7457 & 2 . & 279.8 & 4 & & \\
\hline 1 & 120 . & 1.3250 & 279.7 & 5 & & \\
\hline 20 & 159.4990 & 4.0000 & 279.6 & 5 & 0 & \\
\hline 21 & 153. & $10-7$ & 279.4 & 4 & & \\
\hline 22 & 145 & 1.0500 & 279.4 & 4 & & \\
\hline 23 & 161.2656 & 0.9250 & 279.6 & 4 & 0 & \\
\hline 24 & 151.5107 & 1 & 279.9 & 4 & & \\
\hline 11 & 159. & 1.2750 & 280.0 & 4 & & \\
\hline 112262 & 154.874 & 3500 & 280.1 & 5 & 0 & . \\
\hline 112263 & 149.50 & 1.5000 & 280.2 & 4 & & \\
\hline 112264 & 145.7486 & 1 . & 279.7 & 4 & & \\
\hline 112265 & 147.0355 & 000 & 279.3 & 4 & 0 & 0. \\
\hline 112266 & 156.4990 & 2.3250 & 279.5 & 5 & 0 & s. \\
\hline 112267 & 151.2498 & 130 & 279.2 & 5 & & \\
\hline 11226 & 152.2500 & 0 & 279.0 & 5 & & 0 . \\
\hline 112269 & 157.9976 & 2.5500 & 279.0 & 4 & 0 & \\
\hline 10 & 157.5046 & 0 & 279.9 & 3 & & \\
\hline 11 & 166.7726 & 3.1500 & 280.4 & 4 & & . \\
\hline 12 & 162.7513 & 2.6750 & 280.6 & 3 & & 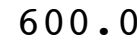 \\
\hline 13 & 201.3202 & 2 . & 281.6 & 2 & & 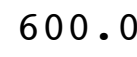 \\
\hline 14 & 175.5027 & 3.9500 & 280.1 & 3 & .0 & 0 \\
\hline 12261 & 158.4912 & 4.4500 & 280.1 & 4 & & \\
\hline 2616 & 151.5623 & 2.2000 & 280.1 & 3 & & 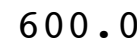 \\
\hline 0112 & 150.5000 & 1.6250 & 280.1 & 4 & .0 & 00 . \\
\hline 1122618 & 168.5606 & 2.4750 & 280.0 & 5 & 0 & 00 . \\
\hline 1122619 & 345.2801 & 000 & 280.2 & 6 & & \\
\hline 01122620 & 351.0145 & 2.1000 & 280.2 & 5 & & 00 \\
\hline 2262 & 15.4458 & 1.9000 & 280.2 & 4 & 600.0 & 600 \\
\hline & 24 & & 280.6 & 5 & & \\
\hline 112262 & 334.9836 & 1.4250 & 282.4 & & .0 & -1 \\
\hline
\end{tabular}




\begin{tabular}{|c|c|c|c|c|c|c|}
\hline $6 \angle 4$ & & & & & & \\
\hline 2271 & 168 & & .2 & & & \\
\hline 2272 & 38 & 5250 & 79.5 & 0 & 00 & \\
\hline 12273 & 348.0244 & 1.7750 & 279.1 & & 00 & \\
\hline $227 \quad 4$ & 424 & & 79.0 & & & \\
\hline 2275 & 60 & & 78.7 & & 0 & \\
\hline 12276 & 091 & 4500 & 278.6 & & 00 & \\
\hline $1227 \quad 7$ & 680 & & 78.3 & & & \\
\hline 12278 & 18 & 0 & 78.1 & & & \\
\hline 12279 & 142.2260 & 1.0250 & 80.0 & & 0 & 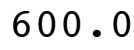 \\
\hline 122710 & 217.5664 & 0.7500 & 280.0 & & & \\
\hline 711 & 63.5094 & 0.9000 & 81.0 & & & \\
\hline 712 & 340 & 1.0750 & 280.7 & & 0 & \\
\hline 2713 & 252.3843 & 0.7000 & 283.3 & & & \\
\hline 714 & 346 & 0 & & & & \\
\hline 715 & 309 & & 287.1 & & & \\
\hline 716 & 322.6080 & 2.00 & 287.4 & & .0 & \\
\hline 717 & 347 . & 0 & 8 & & & \\
\hline 718 & 348 . & & 288.7 & $x$ & & \\
\hline 719 & 931 & 8000 & 288.2 & 4 & 0 & \\
\hline 720 & 952 & .1000 & 284.8 & 5 & .0 & \\
\hline 721 & 332 . & & 281.8 & 4 & & \\
\hline 22 & 334 & 0 & 282 & 4 & & \\
\hline 723 & 321.0080 & 4.90 & 284.9 & 4 & 0 & \\
\hline 724 & 317.2040 & & & 4 & & \\
\hline 81 & 333 & & 28 & 4 & & \\
\hline 112282 & 270.8322 & 9250 & 286.2 & 5 & & 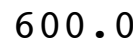 \\
\hline 112283 & 157.6426 & 0 & 8 & 6 & & \\
\hline 284 & 129 & 0 & 28 & 5 & & \\
\hline 112285 & 19.7602 & 1.9750 & 0.7 & 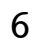 & 0 & 0 \\
\hline 112286 & 344.1007 & 2.1250 & 280.9 & 6 & & 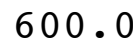 \\
\hline 112287 & 333. & 0 & 281.7 & 6 & & \\
\hline 112288 & & 1.6 & 281.9 & 6 & & \\
\hline 112289 & 200.2229 & 1.1250 & 281.4 & 5 & & \\
\hline 810 & 238 & & & 4 & & \\
\hline & & & 1.1 & 3 & & \\
\hline 812 & 300.3685 & 2.7750 & 281.4 & 3 & & \\
\hline 813 & 321 . & 5.5 & 282 & 4 & & \\
\hline 314 & 321.5000 & 6.1000 & 283.1 & 4 & & 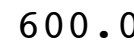 \\
\hline 815 & 298.7451 & & 282.9 & 4 & & \\
\hline 816 & 282.0032 & 4 . & & 3 & & \\
\hline 817 & 291.0012 & 5.8250 & 281.6 & 4 & & 0 . \\
\hline 12281 & 306.5000 & 7.6750 & 281.9 & 4 & & 0 . \\
\hline 22819 & 309 . & 8 . & & 4 & & \\
\hline 01122820 & 325.9963 & 8.1750 & 282.7 & 4 & & \\
\hline 2282 & 327.7509 & 0.7250 & 282.4 & 4 & & 00 . \\
\hline & 32 & & & 4 & & \\
\hline 122823 & 323.7571 & 0.7500 & 282.0 & & .0 & s \\
\hline
\end{tabular}




\begin{tabular}{|c|c|c|c|c|c|c|}
\hline 824 & & 9000 & & & & \\
\hline 2291 & 305.4983 & .7500 & 1.3 & 4 & b & \\
\hline 2292 & 02.5249 & 4750 & 281.3 & 4 & 00 & \\
\hline 12293 & 6.2500 & 5750 & 281.6 & & 00 & \\
\hline 2294 & 5.5000 & .0250 & 81.5 & & 0 & \\
\hline 2295 & 592 & 0250 & 81.0 & & 0 & \\
\hline 12296 & 09.7533 & 0000 & 280.9 & & 00 & \\
\hline 12297 & 7.7507 & 6000 & 81.0 & & & \\
\hline 12298 & 4.0000 & 2000 & 81.5 & 4 & & \\
\hline 12299 & 309.5138 & 1250 & 282.2 & 4 & 00 & 8 \\
\hline 122910 & 300.9887 & 9500 & 282.5 & & 0 & \\
\hline 911 & 299.4865 & 9500 & 82.4 & 4 & & \\
\hline 912 & 298.2417 & 000 & 283.0 & 4 & 0 & \\
\hline 122913 & 322.7592 & 5.9250 & 284.3 & 4 & 0.0 & \\
\hline 914 & 330.2501 & & 1 & 4 & & \\
\hline 5 & 8.7429 & & 284.2 & 4 & & \\
\hline 916 & 312.2526 & 3.9000 & 283.9 & 4 & .0 & 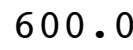 \\
\hline 917 & 8.0110 & 000 & 9 & 4 & & \\
\hline 918 & 331.5428 & 00 & 283.6 & 4 & & \\
\hline 19 & 1.1340 & 1.2000 & 283 & 5 & & \\
\hline 920 & 23.6425 & 0.8500 & 283.6 & 6 & 0 & . \\
\hline 921 & 134.1361 & 1.8750 & & 6 & & \\
\hline 22 & 148.0000 & 50 & 28 & 5 & & \\
\hline 923 & 139.2499 & 3.2250 & 283.6 & 5 & 0 & \\
\hline 924 & 144.0001 & 750 & & 5 & & \\
\hline 01 & 149.5061 & 500 & 28 & 5 & & \\
\hline 112302 & 153.5080 & 2.0750 & .4 & 4 & 0 & . \\
\hline 112303 & 134.5252 & 5500 & 4 & 5 & & \\
\hline $11230 \quad 4$ & 844 & 750 & 28 & 5 & & \\
\hline 112305 & 316.0000 & 0.4000 & 3.4 & 6 & 0 & U. \\
\hline 112306 & 24.3926 & 750 & 283.5 & 5 & 0 & 0 \\
\hline 112307 & 130.5978 & 50 & & 6 & & \\
\hline 112308 & 241.4001 & 500 & 283.4 & 6 & & 0 . \\
\hline 112309 & 296.5738 & 0.0250 & 283.6 & 5 & 0 & \\
\hline 1123010 & .3788 & 50 & & 4 & & \\
\hline 11 & 298.0785 & 0.6500 & .9 & 3 & & 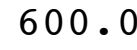 \\
\hline 1123012 & 293.7237 & 0.9500 & 284.0 & 2 & & 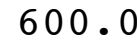 \\
\hline 01123013 & 313.3243 & 1.1250 & & 2 & & 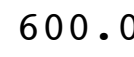 \\
\hline 01123014 & 301.5000 & 3.5500 & 283.8 & 3 & .0 & 0 . \\
\hline 1123015 & 358.3731 & 2.0500 & 284.5 & 3 & & \\
\hline 1123016 & 268.5834 & 1.7500 & 284.8 & 2 & & 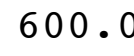 \\
\hline 01123017 & 310.8429 & 0.5250 & 284.8 & 3 & .0 & 0 . \\
\hline 1123018 & 324.5075 & 2.2750 & 284.6 & 4 & 0 & 00 . \\
\hline 01123019 & 337.5028 & 3.1000 & & 4 & & \\
\hline 01123020 & 311.2728 & 1.2250 & & 5 & & 0 . \\
\hline 12302 & 171.0172 & 1.7000 & 284.5 & 6 & 600.0 & 600 \\
\hline & 499 & .150 & & 5 & & \\
\hline 112302 & 6252 & 1.0000 & 284.1 & & .0 & 60 \\
\hline
\end{tabular}




\begin{tabular}{|c|c|c|c|c|c|c|c|c|}
\hline \multicolumn{3}{|c|}{01123024} & & 0 & & & & \\
\hline \multicolumn{3}{|c|}{0112311} & 92 & 7250 & & & & \\
\hline \multicolumn{3}{|c|}{11231} & 173.6940 & 2000 & 284.9 & 6 & 00 & \\
\hline \multicolumn{3}{|c|}{11231} & 960 & 3500 & 284.5 & & 0 & \\
\hline \multicolumn{2}{|c|}{11231} & 14 & 239 & 8750 & & & & \\
\hline \multicolumn{3}{|c|}{011231} & 650 & 500 & 5 & & & \\
\hline \multicolumn{3}{|c|}{011231} & 853 & 500 & 84.8 & & 0 & \\
\hline \multicolumn{2}{|c|}{11231} & 17 & 320 & & & & & \\
\hline \multicolumn{3}{|c|}{011231} & 351.5707 & 500 & 3 & & & \\
\hline \multicolumn{3}{|c|}{0112319} & 408 & 000 & 85.8 & & 0 & \\
\hline \multicolumn{3}{|c|}{1123110} & 9634 & 1.2250 & 0 & & & \\
\hline \multicolumn{3}{|c|}{01123111} & 336.2396 & 1.7500 & & & & \\
\hline \multicolumn{3}{|c|}{01123112} & 272 & 0 & 2 & & & \\
\hline \multicolumn{3}{|c|}{01123113} & 226.2520 & .7750 & 5 & & .0 & \\
\hline \multicolumn{3}{|c|}{01123114} & 180 & & & & & \\
\hline \multicolumn{3}{|c|}{01123115} & 177 . & & & & & \\
\hline \multicolumn{3}{|c|}{01123116} & 174.7545 & 0 & 5 & & 0 & \\
\hline \multicolumn{3}{|c|}{01123117} & 171.8636 & 0 & & & & \\
\hline \multicolumn{3}{|c|}{01123118} & 180 & & & & & \\
\hline \multicolumn{3}{|c|}{01123119} & 267 & & 0 & & 0 & \\
\hline \multicolumn{3}{|c|}{01123120} & 339.5543 & 0 & 1 & 6 & 0 & \\
\hline & & & 339. & & & 6 & & \\
\hline & & & 313 & 0 & & 5 & & \\
\hline & 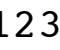 & 123 & 334.3225 & 1.25 & 2 & 5 & 0 & \\
\hline 1 & & 124 & 321. & & & 5 & & \\
\hline 02 & 1 & 1 & 768 & 1 . & & 5 & & \\
\hline 2 & 1 & 12 & 346.5276 & 0 & 4 & 5 & 0 & . \\
\hline 2 & 1 & 13 & 336 & 0 & & 6 & & \\
\hline 02 & 1 & 14 & 0 . & 50 & & 6 & & \\
\hline 2 & 1 & 15 & 14.7160 & 0 & 9 & 6 & & 0 . \\
\hline 2 & 1 & 16 & 353.257 & 3000 & & 5 & & \\
\hline 02 & 1 & 17 & 338 & 0 & & 6 & & \\
\hline 2 & 1 & 18 & 14 . & 0 & 8 & 6 & & ? \\
\hline 2 & 1 & 9 & 298 . & 50 & & 5 & & \\
\hline 2 & 1 & 110 & 26 & & & 4 & & \\
\hline 02 & 1 & 111 & 162 . & 000 & & 3 & & . \\
\hline 2 & 1 & 112 & 324.2624 & 2000 & 6 & 2 & & \\
\hline 02 & 1 & 113 & 283 & & & 1 & & \\
\hline 02 & 1 & 114 & 164.9907 & 3500 & $2 \varepsilon$ & 1 & & 0 . \\
\hline 02 & 1 & 115 & 166.4103 & 8000 & 286.0 & $Z$ & & \\
\hline 02 & 1 & 116 & 213 . & 000 & 28 & 2 & & \\
\hline 02 & 1 & 117 & 200.6680 & 0.5000 & 285.8 & 3 & & 0 . \\
\hline 02 & 1 & 118 & 156.6522 & 0.3250 & 285.6 & 4 & 0 & 0 \\
\hline 02 & 1 & 119 & 317 & 5000 & & 5 & & \\
\hline & 1 & 120 & 316.9183 & 3.1500 & & 4 & & 0 . \\
\hline 02 & 1 & 121 & 347.2369 & 1.8000 & 286.0 & 5 & & 0 . \\
\hline 0 & 1 & 122 & 94 & & & 0 & & \\
\hline 2 & 1 & 123 & 346.0000 & 5.1000 & 287.7 & $J$ & 600.0 & $=0$ \\
\hline
\end{tabular}




\begin{tabular}{|c|c|c|c|c|c|c|c|c|}
\hline- & 1 & 24 & & 1750 & 87.6 & & 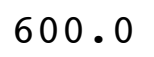 & \\
\hline 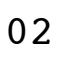 & 1 & 21 & 138 & .7750 & 286.6 & & 600.0 & \\
\hline 2 & 1 & 22 & 180.0395 & .3500 & 286.3 & 6 & 00 & \\
\hline 2 & 1 & 23 & 57.4714 & 1000 & 285.9 & & 00.0 & \\
\hline 2 & 1 & 24 & 71.4995 & .4500 & 84.6 & & 0 & \\
\hline & 1 & 25 & 26.0661 & 5250 & 284.3 & & 00 & \\
\hline 2 & 1 & 26 & 93.0496 & 2.9500 & 284.3 & & 00.0 & \\
\hline & 1 & 7 & 662 & .4000 & 84.5 & & .0 & \\
\hline & 1 & 8 & 1185 & 0750 & 285.0 & & & \\
\hline 2 & 1 & 29 & 07.0061 & 4.1750 & 284.9 & & 600.0 & (5) \\
\hline 2 & 1 & 210 & 91.9931 & .9750 & 284 & & & \\
\hline & 1 & 211 & 8.2512 & .5000 & 85.4 & & .0 & \\
\hline 2 & 1 & 212 & 10.9992 & .6500 & 286.0 & & 00.0 & \\
\hline 2 & 1 & 213 & 21.9635 & .5500 & 285.6 & & .0 & \\
\hline & 1 & 214 & 2.6111 & .2000 & 1 & & & \\
\hline 2 & 1 & 215 & 4.2174 & .9250 & 285.4 & & & \\
\hline 02 & 1 & 216 & 273.9690 & 0.5750 & 285.7 & & 0.0 & \\
\hline 2 & 1 & 217 & 4.7675 & 500 & 285.8 & & .0 & \\
\hline 02 & 1 & 218 & 0.3942 & 500 & 285.2 & & & \\
\hline 02 & 1 & 219 & 1.2501 & 000 & 285.2 & 4 & .0 & \\
\hline 02 & 1 & 220 & 126.4983 & 3.6500 & 285.0 & 4 & $\cdot 0$ & 600 \\
\hline 02 & 1 & 221 & 116.4946 & 2250 & & 4 & & \\
\hline 02 & 1 & 222 & 7762 & 750 & 28 & 4 & .0 & \\
\hline 02 & 1 & 223 & 90.7494 & 500 & .1 & 4 & .0 & \\
\hline 2 & 1 & 224 & 48 & & & 4 & & \\
\hline 02 & 1 & 31 & 2499 & 7.7500 & 28 & 4 & & \\
\hline 02 & 1 & 32 & 76.2360 & 7750 & 28 & 4 & .0 & \\
\hline 2 & 1 & 33 & 2503 & 0 & .3 & 4 & .0 & \\
\hline 02 & 1 & 34 & 78.7511 & 6 . & 282.9 & 4 & .0 & \\
\hline 02 & 1 & 35 & 80.0107 & 500 & 282.8 & 4 & .0 & 60 \\
\hline 02 & 1 & 36 & 84.00 & 5.9000 & 282.7 & 4 & .0 & \\
\hline 02 & 1 & 37 & 88.5002 & 6 . & 282.8 & 4 & & 0 \\
\hline 02 & 1 & 38 & 79.2427 & 0 & 282.3 & 4 & .0 & \\
\hline 02 & 1 & 39 & 72.2455 & $\$ 500$ & 282.3 & 4 & .0 & \\
\hline 22 & 1 & 310 & 4 & & 282.9 & 4 & & \\
\hline 02 & 1 & 311 & 104.4472 & 3.87 & 284.1 & 3 & .0 & \\
\hline 02 & 1 & 312 & 124.7430 & 4.9750 & 284.4 & 3 & .0 & \\
\hline 2 & 1 & 313 & 110.1005 & 4 . & 284.6 & 3 & .0 & 6 \\
\hline 02 & 1 & 314 & 148.2515 & 5.1750 & 284.4 & 4 & .0 & 600 \\
\hline 02 & 1 & 315 & 148.8786 & 9000 & 284.4 & 4 & .0 & \\
\hline 02 & 1 & 316 & 173.2500 & 000 & 283.4 & 4 & & \\
\hline 02 & 1 & 317 & 174.7519 & 4.0500 & 282.8 & 4 & .0 & 600 \\
\hline 02 & 1 & 318 & 153.9941 & 3.0250 & 282.0 & 4 & .0 & 600 \\
\hline 02 & 1 & 319 & 127.4987 & 1.5250 & 281.5 & - & & \\
\hline 02 & 1 & 320 & 147.5097 & 2.1500 & 281.7 & 2 & .0 & \\
\hline 02 & 1 & 321 & 127.0000 & 2.8500 & 282.8 & 4 & .0 & 600 \\
\hline 02 & 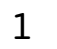 & 322 & 7427 & & 283.1 & 4 & & \\
\hline & & 323 & 143.7496 & 2.6250 & 282.3 & $\Psi$ & 600.0 & 600 \\
\hline
\end{tabular}




\begin{tabular}{|c|c|c|c|c|c|c|c|c|}
\hline 2 & 1 & 24 & 4.4344 & 2250 & 81.9 & & 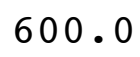 & \\
\hline 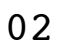 & 1 & 1 & 98.4861 & 2.1000 & 282.3 & & 500.0 & \\
\hline & 1 & 2 & 126.3395 & 4250 & 282.4 & & 00 & \\
\hline & 1 & 43 & 147.2576 & 1500 & 281.4 & & 00.0 & \\
\hline & 1 & 4 & 137.0000 & .0750 & 81.5 & & 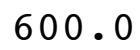 & \\
\hline & 1 & 5 & 121.7668 & .8000 & 81.2 & & 00 & \\
\hline & 1 & 46 & 131.7387 & 9000 & 280.7 & & 00 & \\
\hline & 1 & 7 & 135.7511 & 3500 & 80.0 & & 0 & \\
\hline & 1 & 8 & 134.2698 & 8000 & 79.6 & & & \\
\hline & 1 & 9 & 139.4420 & 1500 & 80.6 & & $0 c$ & U \\
\hline & 1 & 410 & 146.7244 & 2.3750 & 281.2 & & 00.0 & \\
\hline & 1 & 411 & 164.4957 & .3500 & 82.3 & 4 & 0 & \\
\hline & 1 & 412 & 194.4390 & 1.8000 & 283.2 & 3 & 0 & \\
\hline & 1 & 413 & 232.5376 & .4250 & 283.1 & & 0.0 & 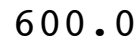 \\
\hline & 1 & 414 & 264 . & 0 & 33.6 & & & \\
\hline & 1 & 415 & 288 . & & 283.2 & 3 & & \\
\hline & 1 & 416 & 285.0001 & 0 & 282.1 & 4 & .0 & 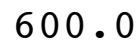 \\
\hline & 1 & 417 & 280 . & 0 & 31.3 & 4 & & \\
\hline & 1 & 418 & 27 . & 50 & 281.1 & 5 & & \\
\hline & 1 & 419 & 142.6400 & 7750 & 281.6 & 6 & 0 & $\checkmark$ \\
\hline 2 & 1 & 420 & 140.7491 & .0250 & 282.1 & 5 & 0 & \\
\hline & 1 & 421 & 66.5000 & & 281.1 & 6 & & \\
\hline & 1 & 422 & 77.5527 & 9250 & 280.8 & 6 & & \\
\hline & 1 & 423 & 322.8890 & .8250 & 280.5 & 5 & 0 & \\
\hline & 1 & 424 & 348 & 0 & .3 & 4 & & \\
\hline 2 & 1 & 51 & 313 . & 0750 & 280.1 & 5 & & \\
\hline 2 & 1 & 52 & 309.8437 & 3250 & 279 & 6 & 0 & J \\
\hline & 1 & 53 & 12.7351 & 500 & 9 & 6 & & \\
\hline 2 & 1 & 54 & 156.2348 & .4250 & 283.8 & 6 & & $\bullet$ \\
\hline & 1 & 55 & 120.6732 & 9250 & 282.9 & 5 & 0 & 0 . \\
\hline 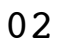 & 1 & 56 & 150.7514 & 7750 & 280.9 & 4 & 0 & 0 . \\
\hline 2 & 1 & 57 & 150.2360 & 00 & 279.0 & 4 & & \\
\hline & 1 & 58 & 140.1554 & 000 & 278.9 & 5 & & . \\
\hline 2 & 1 & 59 & 13.0647 & 5500 & 278.8 & 4 & 0 & 0 \\
\hline 2 & 1 & 510 & 357.2542 & 2.1250 & 279.3 & 3 & & \\
\hline 2 & 1 & 511 & 312.1233 & 1.1250 & 280.5 & 2 & & 0 . \\
\hline 2 & 1 & 512 & 206.8596 & 5500 & 281.0 & 1 & & 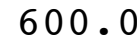 \\
\hline 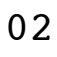 & 1 & 513 & 162.2587 & 000 & 281.4 & 2 & & 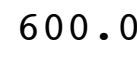 \\
\hline 2 & 1 & 514 & 167.9986 & 2.7500 & 281.0 & 3 & .0 & 0 . \\
\hline 2 & 1 & 515 & 171.5060 & 1.9000 & 280.9 & 3 & & 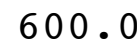 \\
\hline 2 & 1 & 516 & 225.2534 & 000 & 280.9 & 2 & & 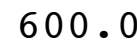 \\
\hline 02 & 1 & 517 & 219.0850 & 1.5500 & 280.8 & 3 & & 0 . \\
\hline 12 & 1 & 518 & 243.7288 & 0.5750 & 280.7 & 4 & 0 & 0 . \\
\hline 02 & 1 & 519 & 296.6582 & 0.3000 & 280.7 & 5 & & \\
\hline 02 & 1 & 520 & 350.3080 & 1.5000 & 280.8 & 4 & & 0 . \\
\hline 2 & 1 & 521 & 85.1037 & 1.3250 & 281.1 & 5 & 600 & 600. \\
\hline & 1 & 522 & 73 & & & 6 & & \\
\hline & & 523 & 42.2569 & 1.3500 & 285.7 & 0 & 600.0 & 600 \\
\hline
\end{tabular}




\begin{tabular}{|c|c|c|c|c|c|c|c|c|}
\hline 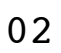 & 1 & 24 & & 3000 & 86.4 & & - & \\
\hline 2 & 1 & 61 & 19.0090 & 0.6250 & 86.2 & & 600.0 & \\
\hline 2 & 1 & 62 & 43.1852 & 1.3750 & 286.3 & 6 & 00 & \\
\hline 2 & 1 & 63 & 155.9032 & 0.9750 & 286.4 & & 00.0 & \\
\hline & 1 & 64 & 126.8671 & 1.6750 & 86.5 & & 0 & \\
\hline & 1 & 65 & 123.5860 & .9250 & 286.6 & & 00 & \\
\hline 2 & 1 & 66 & 258.1491 & 0.6250 & 286.5 & & 500 & \\
\hline 2 & 1 & 7 & 18.3693 & 1.6250 & 286.2 & & .0 & \\
\hline & 1 & 8 & 30.7402 & 1.2250 & 86.5 & & & \\
\hline & 1 & 9 & 199.2177 & 1.8500 & 284.9 & & 600.0 & 0 \\
\hline 2 & 1 & 610 & 4.9222 & 0.9250 & 284 & & & \\
\hline & 1 & & 19.6112 & 1.6750 & 85.1 & & .0 & \\
\hline 2 & 1 & 12 & 15.2514 & 1.3250 & 286.5 & & 00.0 & \\
\hline 2 & 1 & 613 & 340.0356 & 0.8250 & 287.2 & & 0.0 & \\
\hline & 1 & 4 & 09 & 0.30 & 287.9 & & & \\
\hline 02 & 1 & & 151. & 1.9000 & 288.1 & & & \\
\hline 02 & 1 & 16 & 129.2523 & 2.8000 & 286.1 & & 0.0 & 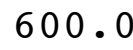 \\
\hline 2 & 1 & 7 & 151.7477 & 000 & 7 & 2 & .0 & \\
\hline 2 & 1 & 8 & 118.5127 & 1.2750 & 283.1 & 4 & & \\
\hline 02 & 1 & 619 & 357 & 750 & 282.9 & 5 & .0 & \\
\hline 02 & 1 & 620 & 76.10 & 1.1500 & 282.6 & 6 & .0 & 000 \\
\hline 2 & 1 & 621 & 142.7554 & & 284.2 & 5 & & \\
\hline 02 & 1 & 622 & 140 & 1.7750 & 285.5 & 5 & .0 & \\
\hline 2 & 1 & 623 & 137.0100 & .9250 & 28 & 4 & .0 & \\
\hline 2 & 1 & 624 & 205 & 0 & & 5 & & \\
\hline 02 & 1 & 71 & 184.1760 & 1. & 283.4 & 4 & & \\
\hline 02 & 1 & 72 & 329.2468 & 0.7250 & 282.7 & 5 & .0 & 0 \\
\hline 2 & 1 & 73 & 359.9977 & 1.8250 & 282.4 & 4 & .0 & \\
\hline 02 & 1 & 74 & 1.7411 & 1.4000 & 28 & 4 & .0 & \\
\hline 02 & 1 & 75 & 1.0196 & 750 & 283.0 & 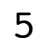 & .0 & \\
\hline 02 & 1 & 76 & 224.8965 & 0.6250 & 282.4 & 6 & .0 & \\
\hline 02 & 1 & 77 & 158 & 1.1250 & 281.9 & 6 & & 0 \\
\hline 02 & 1 & 78 & 340.2906 & 0.7750 & 281.2 & 6 & .0 & \\
\hline 02 & 1 & 79 & 100.4165 & 2000 & 281.0 & 5 & .0 & \\
\hline 02 & 1 & 710 & 103 & 0 & & 4 & & \\
\hline 02 & 1 & 711 & 160.3124 & 750 & 283.3 & 3 & .0 & \\
\hline 02 & 1 & 712 & 333.7015 & 0.7500 & 284.4 & 2 & .0 & \\
\hline 2 & 1 & 713 & 175.9639 & 500 & 28 & 1 & .0 & 6( \\
\hline 02 & 1 & 714 & 155.7835 & 1.8500 & 283.5 & 1 & .0 & 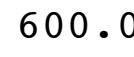 \\
\hline 02 & 1 & 715 & 242.8975 & 9000 & 283.1 & 1 & .0 & \\
\hline 02 & 1 & 716 & 329 . & 500 & 283.7 & 1 & & \\
\hline 02 & 1 & 717 & 22.2515 & 1.0750 & 284.0 & 2 & .0 & 0 \\
\hline 02 & 1 & 718 & 51.2455 & 2.4250 & 285.0 & 3 & .0 & 00 \\
\hline 02 & 1 & 719 & 124.6084 & 500 & 285.7 & 4 & & \\
\hline 02 & 1 & 720 & 140.5716 & 2.3500 & 284.4 & 5 & .0 & \\
\hline 02 & 1 & 721 & 109.8927 & 2.6250 & 285.3 & 4 & 600.0 & 500 \\
\hline 02 & 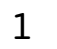 & 722 & 11 & & 284.7 & 5 & & \\
\hline & & 120 & 355.4332 & 1.2500 & 283.7 & & .0 & 600 \\
\hline
\end{tabular}




\begin{tabular}{|c|c|c|c|c|c|c|c|c|}
\hline 2 & 1 & 724 & 10.5154 & 1.5000 & 284.0 & 5 & 600.0 & 600.0 \\
\hline 02 & 1 & 81 & 14.7485 & 1.9500 & 284.5 & 4 & 600.0 & 600.0 \\
\hline 02 & 1 & 82 & 18.7451 & 1.8000 & 284.2 & 5 & 600.0 & 00.0 \\
\hline 02 & 1 & 83 & 22.5393 & 1.0500 & 284.2 & 6 & 600.0 & 00.0 \\
\hline 02 & 1 & 84 & 70.0132 & 1.8750 & 285.0 & 6 & 600.0 & 00.0 \\
\hline 02 & 1 & 85 & 106.6713 & 2.8000 & 285.2 & 5 & 600.0 & 00.0 \\
\hline 02 & 1 & 86 & 121.5754 & 3.3000 & 284.5 & 4 & 600.0 & 00.0 \\
\hline 02 & 1 & 87 & 95.4980 & 3.3000 & 285.2 & 4 & 600.0 & 00.0 \\
\hline 02 & 1 & 88 & 122.0000 & 1.5500 & 284.9 & 5 & 600.0 & 00.0 \\
\hline 02 & 1 & 89 & 96.3508 & 2.2000 & 285.3 & 4 & 600.0 & 00.0 \\
\hline 02 & 1 & 810 & 41.4317 & 2.7250 & 286.5 & 4 & 600.0 & 00.0 \\
\hline 02 & 1 & 811 & 353.5000 & 1.1000 & 287.4 & 3 & 600.0 & 00.0 \\
\hline 02 & 1 & 812 & 342.0415 & 0.7250 & 288.8 & 2 & 600.0 & 00.0 \\
\hline 02 & 1 & 813 & 277.2083 & 1.0750 & 289.4 & 1 & 600.0 & 00.0 \\
\hline 02 & 1 & 814 & 163.5008 & 50 & 286.0 & 2 & .0 & \\
\hline 02 & 1 & 815 & 164.2555 & 000 & 285.1 & 3 & 600.0 & 00.0 \\
\hline 02 & 1 & 816 & 164.0012 & 3.3000 & 283.8 & 4 & 600.0 & 600.0 \\
\hline 02 & 1 & 817 & 164.2479 & 2.7 & 283.4 & 4 & .0 & \\
\hline 02 & 1 & 818 & 154.1949 & 2.6000 & 283.3 & 5 & 600.0 & 00.0 \\
\hline 02 & 1 & 819 & 47.2718 & 0.8500 & 283.4 & 6 & 60 & .0 \\
\hline 02 & 1 & 820 & 153.0000 & 3. & 282.8 & 5 & 60 & \\
\hline 02 & 1 & 821 & 156.0000 & 4 & 282.4 & 5 & .0 & \\
\hline 02 & 1 & 822 & 165.4982 & 4.5500 & 282.5 & 4 & 600.0 & 600.0 \\
\hline 02 & 1 & 823 & 156.5027 & 4.2 & 282.5 & 5 & .0 & .0 \\
\hline 02 & 1 & 824 & 130.3878 & 2.2 & 282.8 & 5 & .0 & \\
\hline 02 & 1 & 91 & 115.2508 & 3.4000 & 283.4 & 4 & 600.0 & 600.0 \\
\hline 02 & 1 & 92 & 128.7805 & 3.1250 & 283.4 & 4 & 600.0 & .0 \\
\hline 02 & 1 & 93 & 157.0011 & 3.5 & 283.2 & 5 & 0 & \\
\hline 02 & 1 & 94 & 152.7496 & 2.6250 & 283.0 & 4 & 600.0 & 600.0 \\
\hline 02 & 1 & 95 & 147.8516 & 2.8250 & 282.7 & 4 & 600.0 & 600.0 \\
\hline 02 & 1 & 96 & 134.4957 & 5 & 283.5 & 4 & .0 & \\
\hline 02 & 1 & 97 & 120.4489 & 5.1 & 283.8 & 4 & 600.0 & 600.0 \\
\hline 02 & 1 & 98 & 99.5029 & 3.3750 & 283.5 & 4 & 600.0 & 600.0 \\
\hline 02 & 1 & 99 & 112.5221 & 1.7 & 284.1 & 3 & 600.0 & 0.0 \\
\hline 02 & 1 & 910 & 178.7163 & 1.00 & 285.4 & 2 & 600.0 & 600.0 \\
\hline 02 & 1 & 911 & 161.7240 & 1.4250 & 285.6 & 2 & 600.0 & 600.0 \\
\hline 02 & 1 & 912 & 132.7763 & 3.8 & 285.5 & 2 & 600.0 & 600.0 \\
\hline 02 & 1 & 913 & 151.2494 & 5.8750 & 285.7 & 3 & 600.0 & 600.0 \\
\hline 02 & 1 & 914 & 160.4896 & 5.8000 & 286.0 & 4 & 600.0 & 600.0 \\
\hline 02 & 1 & 915 & 157.9874 & 4.6000 & 285.9 & 4 & 600.0 & 600.0 \\
\hline 02 & 1 & 916 & 158.5015 & 5.8000 & 286.6 & 4 & 600.0 & 600.0 \\
\hline 02 & 1 & 917 & 148.9982 & 4.1250 & 286.0 & 4 & 600.0 & 600.0 \\
\hline 02 & 1 & 918 & 137.7465 & 3.2000 & 285.0 & 4 & 600.0 & 600.0 \\
\hline 02 & 1 & 919 & 105.7495 & 6.1500 & 285.3 & 4 & 600.0 & 600.0 \\
\hline 02 & 1 & 920 & 116.2472 & 7.4000 & 285.8 & 4 & 600.0 & 600.0 \\
\hline 02 & 1 & 921 & 121.8735 & 8.7750 & 285.8 & 4 & 600.0 & 600.0 \\
\hline 02 & 1 & 922 & 89.0028 & 7.3500 & 284.5 & 4 & 600.0 & 600.0 \\
\hline 04 & & 923 & 115.5023 & 5.575 & 285.6 & 4 & 600.0 & 600.0 \\
\hline
\end{tabular}




\begin{tabular}{|c|c|c|c|c|c|c|c|}
\hline 2 & 924 & & 0 & 3 & & & \\
\hline & 1101 & 15 & 6500 & 34.2 & 4 & 00.0 & \\
\hline & 1102 & 143.5259 & 3750 & 282.8 & 4 & 00 & \\
\hline & 1103 & 94.1806 & 4750 & 282.2 & & 00 & \\
\hline & 104 & 149.2539 & 6000 & 81.2 & & 0 & \\
\hline & 1105 & 31.1858 & 1500 & 81.2 & & 00 & \\
\hline & 1106 & 131.2032 & 5500 & 281.2 & & 00 & \\
\hline & 1107 & 142.9448 & 000 & 81.2 & & & \\
\hline & 1108 & 48.5208 & 0 & 81.2 & & & \\
\hline & 1109 & 351.6736 & 1.1750 & 82.5 & & 00 & 0 \\
\hline & 1010 & 197.1487 & .5000 & 283.5 & & & \\
\hline & 11011 & 278.9954 & .8000 & 83.8 & & & \\
\hline & 11012 & 298.5174 & 0.9250 & 285.4 & & 0 & \\
\hline & 11013 & 242.7502 & 2.1000 & 284.8 & & 0.0 & \\
\hline & 11014 & 276.2587 & & & & & \\
\hline & 11015 & 299.1597 & & 28 & 1 & & \\
\hline & 11016 & 201.4007 & 1.1500 & 285.4 & 1 & .0 & \\
\hline & 11017 & 144.9866 & 0 & .5 & & & \\
\hline & 11018 & 80.5799 & 50 & 282.7 & 3 & & \\
\hline & 11019 & 0588 & 4250 & 283.2 & 4 & & 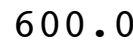 \\
\hline 2 & 11020 & 17.4935 & 0.7250 & 282.6 & 5 & 0 & \\
\hline & 11021 & 124.1021 & .9250 & 283.7 & 6 & & \\
\hline & 11022 & 19.3092 & 000 & 283.1 & 5 & & \\
\hline 2 & 11023 & 18.2472 & 2.4250 & 283.8 & 4 & 0 & \\
\hline & 11024 & 121.3127 & & 2.7 & 5 & & \\
\hline 2 & 1111 & 134.7999 & 2000 & 281.7 & 6 & & \\
\hline & 1112 & 257.7797 & 9500 & 281.1 & 6 & 0 & . \\
\hline & 1113 & 275.3857 & 0 & 1 & 6 & & \\
\hline 2 & 1114 & 352.1889 & 50 & 279.7 & 6 & & \\
\hline & 1115 & 10.0000 & 000 & 280.2 & 6 & 0 & 0 . \\
\hline & 1116 & 214.2293 & 5500 & 280.0 & 6 & 0 & 0 . \\
\hline 2 & 1117 & 153.5043 & 0 & 277.6 & 6 & & \\
\hline & 1118 & 267.3585 & & 276.5 & 6 & & . \\
\hline 2 & 1119 & 243.7712 & 000 & 276.5 & 5 & 0 & 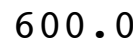 \\
\hline 2 & 11110 & 332 & 0 & 27 & 4 & & \\
\hline 02 & 11111 & 345.0635 & 1.8500 & 278.1 & 3 & & 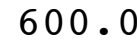 \\
\hline 2 & 11112 & 305.4611 & 5500 & 278.8 & 2 & & 0 . \\
\hline 2 & 11113 & 318 . & 0 & 279.1 & 2 & & \\
\hline 2 & 11114 & 314.5002 & 0.7000 & 279.7 & 1 & .0 & 0 . \\
\hline 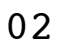 & 11115 & 302.2485 & 1.6750 & 279.7 & 2 & & \\
\hline 2 & 11116 & 274.1974 & 50 & 279 & 1 & & 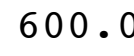 \\
\hline 2 & 11117 & 237.2819 & 0.6250 & 279.7 & 2 & & 0 . \\
\hline 2 & 11118 & 282.7157 & 0.4500 & 279.5 & 3 & 0 & 0 . \\
\hline 02 & 11119 & 351.5000 & 750 & & 4 & & \\
\hline 02 & 11120 & 39.3366 & 2.0000 & 279.1 & 5 & & 0 . \\
\hline 0 & 11121 & 13.6028 & 1.4250 & 278.9 & 6 & 600 & 600 \\
\hline & 11122 & 9722 & & & 6 & & \\
\hline & 1123 & 146.4630 & 1.5500 & 278.8 & 0 & 600.0 & 60 \\
\hline
\end{tabular}




\begin{tabular}{|c|c|c|c|c|c|c|c|}
\hline 02 & 1124 & 6.6831 & 1.0750 & 279.3 & 6 & 600.0 & 0.0 \\
\hline 02 & 1121 & 107.1108 & 1.5750 & 279.1 & 6 & 600.0 & 600.0 \\
\hline 02 & 1122 & 157.0572 & 2.0250 & 277.8 & 6 & 600.0 & 600.0 \\
\hline 02 & 1123 & 353.7269 & 0.8750 & 276.8 & 6 & 600.0 & 600.0 \\
\hline 02 & 1124 & 138.5208 & 2.4500 & 276.7 & 5 & 600.0 & 00.0 \\
\hline 02 & 125 & 9.7449 & 1.7750 & 277.3 & 6 & 600.0 & 00.0 \\
\hline 02 & 1126 & 131.4046 & 1.1500 & 279.2 & 6 & 600.0 & 600.0 \\
\hline 02 & 1127 & 114.7273 & 1.9000 & 278.1 & 6 & 600.0 & 00.0 \\
\hline 02 & 1128 & 93.8375 & 4.1000 & 278.2 & 5 & 600.0 & 00.0 \\
\hline 02 & 1129 & 109.4027 & 3.1250 & 280.0 & 4 & 600.0 & 600.0 \\
\hline 02 & 11210 & 99.0159 & 2.6250 & 282.1 & 3 & 600.0 & 600 \\
\hline 02 & 11211 & 106.2619 & 2.2250 & 280.0 & 2 & 600.0 & \\
\hline 02 & 11212 & 141.2312 & 2.5250 & 278.9 & 1 & 600.0 & 600.0 \\
\hline 02 & 11213 & 125.1675 & 3.1500 & 280.1 & 2 & 600.0 & 600.0 \\
\hline 02 & 11214 & 56.2958 & 1.4500 & 282.2 & 1 & 600.0 & \\
\hline 02 & 11215 & 141.1753 & 2.7750 & 282.3 & 1 & 600.0 & 60 \\
\hline 02 & 11216 & 119.7576 & 2.4500 & 280.7 & 1 & 600.0 & 600.0 \\
\hline 02 & 11217 & 140.5339 & 2.65 & 281.0 & 2 & 600.0 & \\
\hline 02 & 11218 & 129.4588 & 2.7000 & 279.5 & 3 & 600.0 & 60 \\
\hline 02 & 11219 & 106.1818 & 2.1750 & 280.2 & 4 & 600.0 & 60 \\
\hline 02 & 11220 & 115.7552 & 2.35 & 282.0 & 5 & 600.0 & .0 \\
\hline 02 & 11221 & 137.4730 & 2.2250 & 281.2 & 5 & 600.0 & \\
\hline 02 & 11222 & 112.9309 & 1.8250 & 281.7 & 6 & 600.0 & 600.0 \\
\hline 02 & 11223 & 116.7552 & 2.1250 & 278.9 & 6 & 600.0 & .0 \\
\hline 02 & 11224 & 128.0134 & 1.85 & 281.0 & 6 & 600.0 & \\
\hline 02 & 1131 & 133.9051 & 1.8750 & 281.8 & 6 & 600.0 & 600.0 \\
\hline 02 & 1132 & 116.0682 & 2.1500 & 281.7 & 6 & 600.0 & 600.0 \\
\hline 02 & 1133 & 135.5000 & 3.3 & 281.5 & 5 & 600.0 & .0 \\
\hline 02 & 1134 & 122.2468 & 2.6750 & 280.7 & 5 & 600.0 & 600.0 \\
\hline 02 & 1135 & 94.9990 & 3.4000 & 280.7 & 5 & 600.0 & 600.0 \\
\hline 02 & 1136 & 102.5000 & 3.80 & 280.9 & 4 & 600.0 & .0 \\
\hline 02 & 1137 & 95.6721 & 3.6000 & 280.4 & 4 & 600.0 & 600.0 \\
\hline 02 & 1138 & 95.9939 & 3.1000 & 279.9 & 4 & 600.0 & 600.0 \\
\hline 02 & 1139 & 349.2730 & 1.5250 & 279.2 & 3 & 600.0 & .0 \\
\hline 02 & 11310 & 298.6225 & 0.7250 & 280.1 & 2 & 600.0 & 600.0 \\
\hline 02 & 11311 & 325.2494 & 1.3750 & 279.7 & 2 & 600.0 & 600.0 \\
\hline 02 & 11312 & 231.4437 & 0.8000 & 282.4 & 1 & 600.0 & 600.0 \\
\hline 02 & 11313 & 178.9466 & 1.9000 & 282.8 & 1 & 600.0 & 600.0 \\
\hline 02 & 11314 & 172.4962 & 2.4750 & 283.3 & 2 & 600.0 & 600.0 \\
\hline 02 & 11315 & 181.5000 & 1.6250 & 283.9 & 1 & 600.0 & \\
\hline 02 & 11316 & 272.6844 & 1.1000 & 284.5 & 1 & 600.0 & 600.0 \\
\hline 02 & 11317 & 293.5013 & 1.3750 & 283.0 & 2 & 600.0 & 600.0 \\
\hline 02 & 11318 & 304.2655 & 0.8750 & 280.8 & 3 & 600.0 & 600.0 \\
\hline 02 & 11319 & 63.4897 & 0.8500 & 280.3 & 4 & 600.0 & 600.0 \\
\hline 02 & 11320 & 142.5101 & 1.4500 & 280.9 & 4 & 600.0 & 600.0 \\
\hline 02 & 11321 & 134.7597 & 1.2000 & 281.1 & 5 & 600.0 & 600.0 \\
\hline 02 & 11322 & 106.6607 & 2.575 & 280.6 & 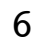 & 600.0 & 600.0 \\
\hline 02 & 11323 & 137.3315 & 1.5750 & 279.2 & 0 & 600.0 & 600.0 \\
\hline
\end{tabular}




\begin{tabular}{|c|c|c|c|c|c|c|c|}
\hline 02 & 1324 & 864 & 0250 & 278.5 & 6 & 600.0 & 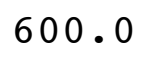 \\
\hline 2 & 1141 & 28.8484 & 1.4250 & 278.6 & 6 & 600.0 & 600.0 \\
\hline 02 & 1142 & 51.4401 & 1.8750 & 279.6 & 6 & 600.0 & 00.0 \\
\hline 02 & 1143 & 142.9939 & 1.8750 & 278.8 & 5 & 600.0 & 00.0 \\
\hline 02 & 1144 & 150.5031 & 3.3000 & 278.2 & & 600.0 & 00.0 \\
\hline 02 & 1145 & 145.2503 & 4.1500 & 277.0 & 3 & 600.0 & 00.0 \\
\hline 02 & 1146 & 147.0002 & 4.8750 & 275.8 & 5 & 600.0 & 600.0 \\
\hline 02 & 1147 & 159.9984 & 4.3500 & 275.7 & 4 & 600.0 & .0 \\
\hline 02 & 1148 & 158.7501 & 2.8000 & 275.5 & 4 & 600.0 & \\
\hline 02 & 1149 & 103.8976 & 2.3500 & 275.4 & 3 & 600.0 & 600.0 \\
\hline 02 & 11410 & 57.2475 & 1.6000 & 275.7 & 2 & 600.0 & 0 \\
\hline 02 & 11411 & 151.4937 & 0.8500 & 276.4 & 1 & 600.0 & \\
\hline 02 & 11412 & 156.5092 & 2.0500 & 276.5 & 2 & 600.0 & .0 \\
\hline 02 & 11413 & 132.4387 & 1.9000 & 276.9 & 2 & 600.0 & \\
\hline 02 & 11414 & 142.4933 & 5.2000 & 277.2 & 3 & 600.0 & .0 \\
\hline 02 & 11415 & 109.7559 & 2.6500 & 278.3 & 2 & 600.0 & \\
\hline 02 & 11416 & 186.6353 & 2.2000 & 279.3 & 1 & 600.0 & 00.0 \\
\hline 02 & 11417 & 226.0025 & 2.1000 & 278.2 & 2 & 600.0 & .0 \\
\hline 02 & 11418 & 338.0667 & 1.3500 & 277.5 & 3 & 600.0 & \\
\hline 02 & 11419 & 88.1228 & 1.7250 & 277.3 & 4 & 600.0 & 600.0 \\
\hline 02 & 11420 & 113.8966 & 2.4000 & 279.0 & 5 & 600.0 & 600.0 \\
\hline 02 & 11421 & 108.9965 & 3.0000 & 279.3 & 4 & 600.0 & \\
\hline 02 & 11422 & 148.5138 & 2.7750 & 279.0 & 4 & 600.0 & 60 \\
\hline 02 & 11423 & 109.2475 & 4.9750 & 279.8 & 4 & 600.0 & .0 \\
\hline 02 & 11424 & 134.0777 & 5.3250 & 280.1 & 4 & 600.0 & \\
\hline 02 & 1151 & 144.4969 & 8.8750 & 279.8 & 4 & 600.0 & 60 \\
\hline 02 & 1152 & 149.2482 & 9.9750 & 278.3 & 4 & 600.0 & .0 \\
\hline 02 & 1153 & 152.5001 & 1.6750 & 277.3 & 4 & 600.0 & .0 \\
\hline 02 & 1154 & 156.7502 & 10.4250 & 277.0 & 4 & 600.0 & .0 \\
\hline 02 & 1155 & 154.7499 & 10.5250 & 276.3 & 4 & 600.0 & .0 \\
\hline 02 & 1156 & 147.0443 & 7.8000 & 275.6 & 4 & 600.0 & 60 \\
\hline 02 & 1157 & 144.7517 & 8.4750 & 275.9 & 4 & 600.0 & 60 \\
\hline 02 & 1158 & 146.2534 & 9.7000 & 276.0 & 4 & 600.0 & 60 \\
\hline 02 & 1159 & 159.0000 & 9.6250 & 276.2 & 4 & 600.0 & .0 \\
\hline 02 & 11510 & 159.5000 & 000 & 276.7 & 4 & 600.0 & 60 \\
\hline 02 & 11511 & 163.0006 & 8.9500 & 277.1 & 4 & 600.0 & 600.0 \\
\hline 02 & 11512 & 159.2500 & 7.2250 & 278.0 & 4 & 600.0 & 600.0 \\
\hline 02 & 11513 & 164.7535 & 9.2750 & 279.1 & 4 & 600.0 & 600.0 \\
\hline 02 & 11514 & 172.4982 & 8.3000 & 280.1 & 4 & 600.0 & 600.0 \\
\hline 02 & 11515 & 168.4986 & 7.2250 & 280.6 & 4 & 600.0 & 600.0 \\
\hline 02 & 11516 & 165.9988 & 7.8250 & 281.0 & 4 & 600.0 & 600.0 \\
\hline 02 & 11517 & 162.5040 & 5.1250 & 280.9 & 4 & 600.0 & 600.0 \\
\hline 02 & 11518 & 139.9984 & 3.6000 & 280.2 & 4 & 600.0 & 600.0 \\
\hline 02 & 11519 & 146.0000 & 4.1250 & 280.2 & 4 & 600.0 & 600.0 \\
\hline 02 & 11520 & 146.7498 & 4.6500 & 280.4 & 4 & 600.0 & 600.0 \\
\hline 02 & 11521 & 146.2500 & 6.2250 & 280.6 & 4 & 600.0 & 600.0 \\
\hline 02 & 11522 & 5009 & 7 . & 280.2 & 4 & 600.0 & .0 \\
\hline 02 & 11523 & 141.2498 & 7.7000 & 279.7 & $I$ & 600.0 & 600.0 \\
\hline
\end{tabular}




\begin{tabular}{|c|c|c|c|c|c|c|c|}
\hline 2 & 24 & & & 8.7 & & & \\
\hline & 161 & 000 & .7250 & 277.9 & & 0 & \\
\hline & 162 & 132.7470 & 7000 & 277.7 & 4 & 00 & \\
\hline & 163 & 152.5037 & 7250 & 276.7 & & 00 & \\
\hline & 164 & & 3500 & 276.4 & & & \\
\hline & 165 & 1 & & 276.9 & & 0 & \\
\hline & 166 & 48.9989 & 500 & 277.5 & & 00 & \\
\hline & 167 & 51.9916 & 0 & 276.8 & & & \\
\hline & 168 & 81057 & & 76.7 & & & \\
\hline & 169 & 5002 & 4750 & 276.6 & & 0 & \\
\hline & 1610 & 54.5000 & 0 & 276.4 & & & \\
\hline & 611 & 000 & & 277.4 & 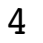 & & \\
\hline & 1612 & 59.7499 & 0 & 277.8 & 4 & & \\
\hline & 1613 & 55.7520 & 0 & $27 \xi$ & 4 & & \\
\hline & 614 & 7119 & & 3 & & & \\
\hline & 615 & 0 & & 280.5 & 2 & & \\
\hline & 1616 & 32.9849 & 0 & 280.8 & 3 & & \\
\hline & 1617 & 3572 & 0 & 280.5 & 4 & & \\
\hline & 1618 & 85.5035 & & 280.2 & 5 & & \\
\hline & 1619 & 2019 & 0 & 280.5 & 4 & .0 & \\
\hline & 620 & .249 & 2.30 & 280.8 & 5 & 0 & \\
\hline & 621 & 24 & & 281.0 & 4 & & \\
\hline & 622 & 34.2558 & & 281.1 & 5 & & \\
\hline & 1623 & 56.6328 & 0 & 281.2 & 6 & 0 & \\
\hline & 11624 & 94.7532 & & 281.8 & 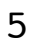 & & \\
\hline & 1171 & 36 & 0 & 281.7 & 4 & & \\
\hline & 1172 & 79.5090 & & 281.3 & 4 & & . \\
\hline & 1173 & 51.058 & & 281.3 & 4 & & \\
\hline$?$ & 1174 & 121.3687 & 0 & 280.1 & 5 & & \\
\hline & 1175 & .917 & & 279.2 & 6 & & J. \\
\hline & 1176 & .24 & 0 & 279.7 & 5 & & 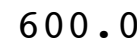 \\
\hline & 1177 & $50-2$ & & 280.1 & 4 & & \\
\hline & 1178 & 67.0076 & & 280.0 & 4 & & . \\
\hline & 1179 & .4963 & 50 & 279.9 & 4 & 0 & 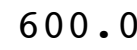 \\
\hline & 11710 & 13 & 0 & 278.8 & 4 & & \\
\hline & 11711 & 166.2606 & 4000 & 276.7 & 4 & & \\
\hline & 11712 & 166.5000 & 4000 & 276.0 & 4 & & \\
\hline 2 & 11713 & 155.00 & & 275.4 & 4 & & \\
\hline 02 & 11714 & 149.7501 & 0000 & 276.3 & 4 & & 0 . \\
\hline & 11715 & 151.4994 & 500 & 277.4 & 4 & & \\
\hline 2 & 11716 & 144.5000 & 50 & 278.2 & 4 & & \\
\hline 0 & 11717 & 92.0523 & 3.2000 & 278.7 & 4 & & 0 \\
\hline 2 & 11718 & 104.2545 & 3.6750 & 279.2 & 4 & 0 & 0 . \\
\hline 2 & 11719 & 135.0185 & 000 & 280.4 & 4 & & \\
\hline 02 & 11720 & 152.7468 & 5 & 280.5 & 4 & & $0^{2}$ \\
\hline 02 & 11721 & 116.7789 & 4.8500 & 281.0 & 4 & & 600. \\
\hline & 11722 & & & 281.1 & 4 & & \\
\hline & 1723 & 135.5554 & 6.4000 & 282.1 & $x$ & 600.0 & $\cos 8$ \\
\hline
\end{tabular}




\begin{tabular}{|c|c|c|c|c|c|c|c|}
\hline$?$ & 24 & & & & & & \\
\hline & 181 & 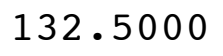 & 0000 & 2.4 & & & \\
\hline & 182 & 40.7557 & 2750 & 282.3 & & 00 & \\
\hline & 183 & 5003 & 3.6250 & 281.6 & & 00 & \\
\hline & 184 & & 9250 & & & & \\
\hline & 185 & 50 & 9750 & 79.9 & & 0 & \\
\hline & 186 & 52.2498 & 8250 & 279.1 & & 0 & \\
\hline & 187 & 2500 & 2750 & 78.8 & & & \\
\hline & 188 & 7499 & .4750 & 78.5 & & & \\
\hline & 189 & 4991 & 6.3250 & 278.9 & & 0 & \\
\hline & 1810 & 59.7500 & 4250 & 278.9 & & & \\
\hline & 1811 & 8.2501 & .0250 & 78.8 & & & \\
\hline & 1812 & 7514 & 7.2000 & 279.6 & & & \\
\hline & 1813 & 000 & 5.9500 & 280.9 & & & \\
\hline & 814 & & 0 & 81.3 & & & \\
\hline & 815 & & & 282.3 & & & \\
\hline & 1816 & .7708 & 2.2750 & 283.6 & & & \\
\hline & 817 & 07 & 500 & 3.5 & & & \\
\hline & 1818 & 82 & & 281.9 & 2 & & \\
\hline & 819 & 51 & 000 & & 4 & & \\
\hline & 1820 & 5.7031 & .3500 & 281.2 & & 0 & \\
\hline & 1821 & 7.8102 & & & 0 & & \\
\hline & 822 & 28 & 195 & 281.6 & 5 & & \\
\hline & 1823 & 477 & 250 & 281.0 & 6 & 0 & \\
\hline & 11824 & 18 . & & .4 & 3 & & \\
\hline & 1191 & 22 & 0 & 281.2 & 5 & & \\
\hline & 1192 & 13.2878 & 250 & 281.0 & 5 & & \\
\hline & 1193 & 22.2503 & 0 & 31.3 & 5 & & \\
\hline & 1194 & 10.4272 & 0 & 28 & 4 & & \\
\hline & 1195 & 47.4190 & 2000 & 280.0 & 5 & & 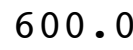 \\
\hline & 1196 & 5.5749 & 2500 & 280.3 & 6 & & \\
\hline & 1197 & .2448 & 0 & & 5 & & \\
\hline & 1198 & 109. & 750 & 280.5 & 4 & & \\
\hline & 1199 & 90.2866 & 250 & 280.1 & 4 & & \\
\hline & 11910 & 429 & & & 4 & & \\
\hline & 11911 & & & 281.8 & 4 & & \\
\hline & 912 & 102.2506 & 750 & 281.4 & 4 & & \\
\hline 2 & 11913 & 118.9429 & 6 . & 281.2 & 4 & & \\
\hline 02 & 11914 & 165.9993 & & 279.6 & 4 & & 0 . \\
\hline & 11915 & 152.7545 & $\$ 750$ & 277.8 & 4 & & \\
\hline & 11916 & 151.0000 & 000 & & 4 & & \\
\hline & 11917 & 150.9936 & 4.4250 & 279.8 & 4 & & \\
\hline & 11918 & 152.5000 & 8.3000 & 279.4 & 4 & & 0 \\
\hline 4 & 11919 & 155.2496 & 500 & & 4 & & \\
\hline 02 & 11920 & 152.7499 & 9.3500 & & 4 & & \\
\hline & 11921 & 150.5000 & 1.0750 & 280.6 & 4 & 0 & 00 \\
\hline & & & 0 & & 4 & & \\
\hline & 1923 & 148.2500 & 11.9500 & 281.3 & & .0 & 60 \\
\hline
\end{tabular}




\begin{tabular}{|c|c|c|c|c|c|c|c|}
\hline 2 & 924 & & & & & & \\
\hline & 1201 & & 1.8250 & 0.9 & & & \\
\hline & 1202 & 144.4995 & 000 & 280.7 & $x$ & 00 & \\
\hline & 1203 & 139.0005 & 8.6000 & 280.7 & & 00 & \\
\hline & 204 & .2502 & 3750 & 80.8 & & & \\
\hline & 1205 & 127.7500 & 500 & 80.9 & & 0 & \\
\hline & 1206 & 5021 & 250 & 80.6 & & 00 & \\
\hline & 207 & 30.4859 & 500 & 80.0 & & & \\
\hline & 1208 & 1.0161 & 8750 & 80.5 & & & \\
\hline & 1209 & 480 & 5000 & 81.3 & & 0 & v \\
\hline & 2010 & 1175 & 1.2250 & 282.4 & & & \\
\hline & 12011 & 5.0006 & 1.27 & 82.6 & & & \\
\hline & 12012 & 3246 & 1.9500 & 283.3 & & 0 & \\
\hline & 2013 & 78.0410 & 1.97 & 282.6 & & 0.0 & \\
\hline & 2014 & 60 & 1.77 & & & & \\
\hline & 12015 & 300 . & 1.47 & 28 & & & \\
\hline & 12016 & 275.7029 & 1.4000 & 284 & & 0 & \\
\hline & 12017 & 302.4007 & 0 . & & & & \\
\hline & 12018 & 235.5456 & 0 . & 7 & 3 & & \\
\hline & 12019 & 5507 & 1.17 & 280 & 4 & & 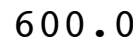 \\
\hline 2 & 12020 & 39.6897 & 1.37 & 280.4 & 5 & 0 & \\
\hline & 12021 & 12.2688 & 0 & 280.2 & 4 & & \\
\hline & 12022 & 44 & 117 & 280 & 5 & & \\
\hline & 12023 & 46.1971 & 1.77 & 280.4 & 6 & 0 & \\
\hline & 12024 & 75.6339 & & & 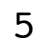 & & \\
\hline & 1211 & 83.7533 & 0 & 28 & 4 & & \\
\hline & 1212 & 7941 & 0 & 9 & 4 & 0 & . \\
\hline & 1213 & 32.6966 & 0 & & 4 & & \\
\hline & 1214 & 227 & 0 & $2 \varepsilon$ & 4 & & \\
\hline & 1215 & 82.753 & 0 & 9.3 & 4 & & • \\
\hline & 1216 & 76.501 & 0 & 279.8 & 4 & & 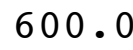 \\
\hline & 1217 & 60.2571 & 0 & 8 & 4 & & \\
\hline & 1218 & 7608 & 0 & 28 & 4 & & 0 . \\
\hline 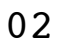 & 1219 & 095 & 5000 & 28 & 4 & & 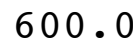 \\
\hline & 12110 & 489 & & & 4 & & \\
\hline & 12111 & 86.8081 & & & 3 & & \\
\hline 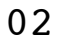 & 12112 & 82.7933 & 4000 & 283.9 & 3 & & \\
\hline 2 & 12113 & 71.2633 & 5. & & 3 & & \\
\hline 02 & 12114 & 54.0043 & 6.8500 & 283.7 & 4 & & 0 . \\
\hline & 12115 & 74.6696 & 4.3000 & 284.6 & 3 & & \\
\hline 2 & 12116 & 24.0734 & 500 & 283 & 2 & & \\
\hline 2 & 12117 & 64.4822 & 2.6250 & 282.3 & 3 & & 0 . \\
\hline & 12118 & 94.7175 & 4.9500 & 281.3 & 4 & & 0 . \\
\hline 2 & 12119 & 118.4946 & 6.9750 & & 4 & & \\
\hline 02 & 12120 & 91.4973 & 7.3500 & & 4 & & \\
\hline & 12121 & 92.2471 & 8.0000 & 279.1 & 4 & 600 & 600. \\
\hline & 12122 & 84.2517 & 7 . & & 4 & & \\
\hline & 12123 & 74.7506 & 8.3250 & 277.9 & $\mathbf{4}$ & 600.0 & 600 \\
\hline
\end{tabular}




\begin{tabular}{|c|c|c|c|c|c|c|c|}
\hline 02 & 12124 & 84.5000 & 8.7500 & 277.4 & 4 & 600.0 & 600.0 \\
\hline 02 & 1221 & 86.5000 & 8.5750 & 276.8 & 4 & 600.0 & 600.0 \\
\hline 02 & 1222 & 86.4998 & 7.8250 & 276.7 & 4 & 600.0 & 00.0 \\
\hline 02 & 1223 & 84.4954 & 6.7250 & 276.5 & 4 & 600.0 & 00.0 \\
\hline 02 & 1224 & 84.0000 & .7750 & 276.4 & 4 & 600.0 & 00.0 \\
\hline 02 & 1225 & 85.0000 & 7.3000 & 276.1 & 4 & 600.0 & 00.0 \\
\hline 02 & 1226 & 81.7507 & 8.1500 & 275.8 & 4 & 600.0 & 00.0 \\
\hline 02 & 1227 & 86.5000 & 8.2000 & 275.9 & 4 & 600.0 & 00.0 \\
\hline 02 & 1228 & 92.4979 & 8.4000 & 276.0 & 4 & 600.0 & 00.0 \\
\hline 02 & 1229 & 88.2501 & 7.3000 & 276.2 & 4 & 600.0 & 0 \\
\hline 02 & 12210 & 91.2489 & 5.6500 & 276.8 & 4 & 600.0 & \\
\hline 02 & 12211 & 98.7530 & 4.0500 & 277.7 & 3 & 600.0 & \\
\hline 02 & 12212 & 123.1981 & 4.2750 & 278.4 & 3 & 600.0 & 00.0 \\
\hline 02 & 12213 & 120.7721 & 4.5750 & 278.7 & 3 & 600.0 & 00.0 \\
\hline 02 & 12214 & 142.1266 & 250 & 279.5 & 3 & .0 & \\
\hline 02 & 12215 & 155.7500 & 4.9000 & 279.1 & 4 & 600.0 & 00.0 \\
\hline 02 & 12216 & 163.7518 & 5.3250 & 279.4 & 4 & 60 & .0 \\
\hline 02 & 12217 & 156.7478 & 5.1250 & 279.0 & 4 & .0 & \\
\hline 02 & 12218 & 136.5097 & 5.6000 & 278.6 & 4 & 600.0 & .0 \\
\hline 02 & 12219 & 110.2470 & 5.9250 & 278.3 & 4 & .0 & .0 \\
\hline 02 & 12220 & 94.7506 & 5.9250 & 278.1 & 4 & 60 & \\
\hline 02 & 12221 & 88.7478 & 6.6000 & 277.9 & 4 & .0 & \\
\hline 02 & 12222 & 88.4959 & 6.4250 & 277.3 & 4 & 600.0 & .0 \\
\hline 02 & 12223 & 117.0000 & 5.6250 & 277.3 & 4 & 6 & .0 \\
\hline 02 & 12224 & 133.2501 & 5.0000 & 277.2 & 4 & .0 & \\
\hline 02 & 1231 & 122.2180 & 4.9500 & 277.5 & 4 & 600.0 & 600.0 \\
\hline 02 & 1232 & 132.2589 & 5.3000 & 277.3 & 4 & 600.0 & .0 \\
\hline 02 & 1233 & 120.7204 & 6.0250 & 277.5 & 4 & .0 & \\
\hline 02 & 1234 & 138.9966 & 7.9250 & 277.9 & 4 & .0 & 600.0 \\
\hline 02 & 1235 & 150.0007 & 7.7750 & 277.4 & 4 & 60 & 0.0 \\
\hline 02 & 1236 & 149.5000 & 7.8500 & 277.2 & 4 & .0 & \\
\hline 02 & 1237 & 151.5001 & 8.5750 & 277.2 & 4 & .0 & 600.0 \\
\hline 02 & 1238 & 152.2498 & 7.9000 & 277.0 & 4 & 600.0 & 600.0 \\
\hline 02 & 1239 & 149.2517 & 6.7250 & 277.3 & 4 & 600.0 & 0.0 \\
\hline 02 & 12310 & 150.7498 & 6.2500 & 278.1 & 4 & 600.0 & 600.0 \\
\hline 02 & 12311 & 157.9993 & 6.3750 & 278.9 & 4 & 600.0 & 600.0 \\
\hline 02 & 12312 & 166.7498 & 7.4500 & 279.4 & 4 & 600.0 & 600.0 \\
\hline 02 & 12313 & 156.5000 & 7.9500 & 280.0 & 4 & 600.0 & 600.0 \\
\hline 02 & 12314 & 162.2501 & 7.1500 & 280.8 & 4 & 600.0 & 600.0 \\
\hline 02 & 12315 & 155.5000 & 5.8250 & 281.7 & 4 & 600.0 & 600.0 \\
\hline 02 & 12316 & 160.0006 & 5.9250 & 282.5 & 3 & 600.0 & 600.0 \\
\hline 02 & 12317 & 161.2494 & 5.1250 & 282.3 & 4 & 600.0 & 600.0 \\
\hline 02 & 12318 & 151.2481 & 2.7250 & 281.0 & 5 & 600.0 & 600.0 \\
\hline 02 & 12319 & 152.2506 & 3.5250 & 280.7 & 5 & 600.0 & 600.0 \\
\hline 02 & 12320 & 149.7502 & 3.3750 & 280.7 & 5 & 600.0 & 600.0 \\
\hline 02 & 12321 & 138.5009 & 2.2000 & 280.2 & 5 & 600.0 & 600.0 \\
\hline 02 & 12322 & 151.5056 & 3.2500 & 280.6 & 5 & 600.0 & 600.0 \\
\hline $0<$ & 12323 & 156.0000 & 4.275 & 280.6 & J & 600.0 & 600.0 \\
\hline
\end{tabular}




\begin{tabular}{|c|c|c|c|c|c|c|c|}
\hline 2 & 2324 & & 0 & 9 & & & \\
\hline & 1241 & 993 & 4.2500 & 30.7 & & 0 & \\
\hline & 1242 & 151.5000 & 3.8250 & 280.2 & & 00 & \\
\hline & 1243 & 137.7477 & 4.3500 & 280.0 & & 00 & \\
\hline & 1244 & 125.7485 & .2500 & 79.8 & & & \\
\hline & 1245 & 133.7715 & .7250 & 279.4 & & 0 & \\
\hline & $\lfloor 246$ & 142.2503 & 0500 & 278.9 & & 00 & \\
\hline & 1247 & 20.0595 & 1000 & 78.3 & & & \\
\hline & 1248 & 11.2766 & .7250 & 79.1 & & & \\
\hline & 1249 & 2575 & 2.2250 & 280.0 & & 00 & 0 \\
\hline & 2410 & 34.4744 & 1.3000 & 281.8 & & & \\
\hline & 12411 & 275.9271 & 1.6250 & 81.5 & & & \\
\hline & 12412 & 235.7508 & 2.0250 & 281.7 & & 0 & \\
\hline & 2413 & 227.7565 & .4750 & 281.6 & & 0.0 & 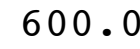 \\
\hline & 2414 & 166.7461 & 000 & 82.3 & & & \\
\hline & 12415 & 164.2501 & 2.32 & 282.8 & & & \\
\hline & 12416 & 161.5029 & 2.3500 & 282.2 & & .0 & $\bullet$ \\
\hline & 12417 & 71.7841 & 1.1000 & 282.9 & & & \\
\hline & 12418 & 18.5006 & 3750 & 281.4 & & & \\
\hline & 12419 & 60.3015 & 9000 & 281.4 & & 0 & 0 \\
\hline 2 & 12420 & 152.5240 & 3750 & 281.1 & & 0 & \\
\hline & 12421 & 355.2716 & 2750 & 280.0 & & & \\
\hline & 12422 & 351.7667 & 2500 & 279.9 & 5 & & \\
\hline$?$ & 12423 & 320.0947 & .1250 & 280.1 & & 0 & \\
\hline & 12424 & 335.7501 & 1.5500 & & 4 & & \\
\hline 2 & 1251 & 330.5547 & 7750 & 27 & 5 & & \\
\hline & 1252 & 244.0185 & 3500 & 279.6 & 6 & 0 & J. \\
\hline & 1253 & 305.4342 & 2750 & 279.0 & 6 & & \\
\hline 2 & 1254 & 153.0348 & 500 & 278.4 & 6 & & 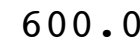 \\
\hline & 1255 & 86.9331 & 7750 & 278.6 & 6 & 0 & 0 • \\
\hline 2 & 1256 & 147.0011 & 2.7750 & 277.6 & 5 & 0 & 0 . \\
\hline 2 & 1257 & 140.7521 & 2.8250 & 277.3 & 4 & & \\
\hline & 1258 & 66.2131 & 2250 & 278.5 & 5 & & D. \\
\hline 2 & 1259 & 70.2625 & L. 1750 & 279.5 & 4 & 0 & \\
\hline 2 & 12510 & 33045 & 7500 & 281.9 & 3 & & \\
\hline 2 & 12511 & 330.8243 & 1.7500 & 282.9 & 2 & & 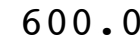 \\
\hline 2 & 12512 & 267.1287 & .0000 & 283.3 & 1 & & 0 . \\
\hline 2 & 12513 & 180.8513 & 4000 & 281.9 & 2 & & 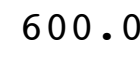 \\
\hline 2 & 12514 & 296.5936 & 1.7000 & 282.6 & 2 & .0 & 0 . \\
\hline 02 & 12515 & 322.4689 & 1.3750 & 283.5 & 1 & & 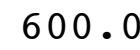 \\
\hline 2 & 12516 & 320.5572 & 1.1500 & 283.3 & 2 & & 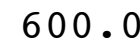 \\
\hline 2 & 12517 & 21.3959 & 0.4000 & 282.7 & 3 & & 0 . \\
\hline 2 & 12518 & 106.5879 & 3.5000 & 282.1 & 4 & 0 & 0 . \\
\hline 02 & 12519 & 90.5000 & 5250 & 282.4 & 5 & & \\
\hline 02 & 12520 & 126.5000 & 2.5750 & 281.6 & 4 & & 0 . \\
\hline 0 & 12521 & 47.7687 & 3.1250 & 281.4 & 4 & 600.0 & 600. \\
\hline & & 2503 & & & 4 & & \\
\hline & 12523 & 35.3701 & 2.6500 & 281.9 & 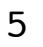 & 600.0 & 600 \\
\hline
\end{tabular}




\begin{tabular}{|c|c|c|c|c|c|c|c|}
\hline 02 & 12524 & 4.4206 & 0.5750 & 281.4 & 6 & 600.0 & 00.0 \\
\hline 02 & 1261 & 109.1015 & 0.9250 & 282.1 & 6 & 600.0 & 600.0 \\
\hline 02 & 1262 & 185.5280 & 1.4750 & 281.8 & 5 & 600.0 & 00.0 \\
\hline 02 & 1263 & 167.8944 & 1.2000 & 281.3 & 6 & 600.0 & 600.0 \\
\hline 02 & 1264 & 166.9970 & 0.8250 & 281.8 & 6 & 600.0 & 00.0 \\
\hline 02 & 1265 & 329.1722 & 0.5000 & 282.0 & 6 & 600.0 & 000.0 \\
\hline 02 & 1266 & 33.4669 & 1.1750 & 282.2 & 6 & 600.0 & 600.0 \\
\hline 02 & 1267 & 108.0989 & 0.7000 & 282.4 & 6 & 600.0 & 00.0 \\
\hline 02 & 1268 & 109.7221 & 1.2500 & 282.2 & 6 & 600.0 & 00.0 \\
\hline 02 & 1269 & 15.7556 & 1.4250 & 282.3 & 5 & 600.0 & 600.0 \\
\hline 02 & 12610 & 335.9817 & 0.2250 & 282.9 & 4 & 600.0 & 600.0 \\
\hline 02 & 12611 & 343.0107 & 0.7750 & 283.7 & 3 & 600.0 & \\
\hline 02 & 12612 & 341.0037 & 1.0750 & 284.0 & 2 & 600.0 & 600.0 \\
\hline 02 & 12613 & 330.2494 & 1.5500 & 284.6 & 3 & 600.0 & 600.0 \\
\hline 02 & 12614 & 182.8439 & 2.9750 & 284.8 & 2 & 600.0 & \\
\hline 02 & 12615 & 93.0000 & 5.6250 & 282.5 & 3 & 600.0 & 60 \\
\hline 02 & 12616 & 84.0041 & 5.6250 & 281.8 & 3 & 600.0 & 600.0 \\
\hline 02 & 12617 & 76.7525 & 6.2750 & 280.5 & 4 & 600.0 & \\
\hline 02 & 12618 & 83.7488 & 5.9750 & 279.7 & 4 & 600.0 & 60 \\
\hline 02 & 12619 & 98.5232 & 6.2750 & 279.2 & 4 & 600.0 & 600.0 \\
\hline 02 & 12620 & 71.5000 & 6.2500 & 279.3 & 4 & 600.0 & .0 \\
\hline 02 & 12621 & 82.9982 & 4.8500 & 279.2 & 4 & 600.0 & \\
\hline 02 & 12622 & 71.9862 & 6.2250 & 278.9 & 4 & 600.0 & 600.0 \\
\hline 02 & 12623 & 72.9998 & 5.9500 & 278.7 & 4 & 600.0 & .0 \\
\hline 02 & 12624 & 75.7501 & 6.4250 & 278.6 & 4 & 600.0 & \\
\hline 02 & 1271 & 84.5005 & 5.5750 & 278.6 & 4 & 600.0 & 600.0 \\
\hline 02 & 1272 & 86.5003 & 5.1250 & 278.7 & 4 & 600.0 & 600.0 \\
\hline 02 & 1273 & 88.9924 & 6.1750 & 278.3 & 4 & 600.0 & \\
\hline 02 & 1274 & 72.7499 & 7.1500 & 277.6 & 4 & 600.0 & 600.0 \\
\hline 02 & 1275 & 74.9996 & 7.0500 & 277.6 & 4 & 600.0 & 600.0 \\
\hline 02 & 1276 & 118.1578 & 5.0000 & 277.2 & 4 & 600.0 & .0 \\
\hline 02 & 1277 & 118.7578 & 5.0250 & 276.9 & 4 & 600.0 & 60 \\
\hline 02 & 1278 & 125.2506 & 4.4250 & 276.9 & 4 & 600.0 & 600.0 \\
\hline 02 & 1279 & 131.5009 & 3.7750 & 277.4 & 4 & 600.0 & .0 \\
\hline 02 & 12710 & 151.0000 & 4 . & 278.4 & 4 & 600.0 & 60 \\
\hline 02 & 12711 & 156.4981 & 5.1500 & 278.2 & 3 & 600.0 & 600.0 \\
\hline 02 & 12712 & 157.7543 & 4.3750 & 278.4 & 3 & 600.0 & 600 \\
\hline 02 & 12713 & 157.8017 & 4.4500 & 278.8 & 3 & 600.0 & 600.0 \\
\hline 02 & 12714 & 141.7546 & 4.8250 & 279.6 & 3 & 600.0 & 600.0 \\
\hline 02 & 12715 & 170.2299 & 4.2500 & 279.7 & 3 & 600.0 & \\
\hline 02 & 12716 & 178.7426 & 4.3250 & 279.5 & 3 & 600.0 & 600.0 \\
\hline 02 & 12717 & 171.2353 & 3.7750 & 279.1 & 4 & 600.0 & 600.0 \\
\hline 02 & 12718 & 159.2585 & 2.2500 & 278.0 & 4 & 600.0 & 600.0 \\
\hline 02 & 12719 & 75.0785 & 2.5250 & 277.6 & 4 & 600.0 & 600.0 \\
\hline 02 & 12720 & 63.5002 & 5.0000 & 277.2 & 4 & 600.0 & 600.0 \\
\hline 02 & 12721 & 56.9998 & 4.8750 & 276.9 & 5 & 600.0 & 600.0 \\
\hline 02 & 12722 & 64.4853 & 4.975 & 276.9 & 5 & 600.0 & 600.0 \\
\hline 02 & 12723 & 63.5303 & 4.0000 & 276.8 & $\mathrm{~J}$ & 600.0 & 600.0 \\
\hline
\end{tabular}




\begin{tabular}{|c|c|c|c|c|c|c|c|}
\hline 02 & 12724 & 21.2522 & 1.7000 & 276.6 & 5 & 600.0 & 00.0 \\
\hline 02 & 1281 & 78.0294 & 2.4750 & 277.2 & 4 & 600.0 & 600.0 \\
\hline 02 & 1282 & 32.2658 & 2.6500 & 277.2 & 4 & 600.0 & 00.0 \\
\hline 02 & 1283 & 18.9969 & 3.8000 & 276.7 & 4 & 600.0 & 600.0 \\
\hline 02 & 1284 & 10.0000 & 2.7250 & 276.5 & 4 & 600.0 & 00.0 \\
\hline 02 & 1285 & 31.9974 & 2.0750 & 275.7 & 5 & 600.0 & 00.0 \\
\hline 02 & 1286 & 27.7573 & 3.2000 & 274.8 & 4 & 600.0 & 600.0 \\
\hline 02 & 1287 & 48.8182 & 4.0250 & 275.9 & 4 & 600.0 & 600.0 \\
\hline 02 & 1288 & 354.6631 & 1.1000 & 275.4 & 5 & 600.0 & 00.0 \\
\hline 02 & 1289 & 53.1249 & 1.7500 & 276.0 & 4 & 600.0 & 600.0 \\
\hline 02 & 12810 & 56.2663 & 4.8500 & 276.9 & 3 & 600.0 & 600.0 \\
\hline 02 & 12811 & 30.7571 & 4.5 & 277.7 & 3 & 600.0 & \\
\hline 02 & 12812 & 57.4847 & 4.8000 & 277.9 & 3 & 600.0 & 600.0 \\
\hline 02 & 12813 & 51.4506 & 4.5250 & 278.8 & 3 & 600.0 & 600.0 \\
\hline 02 & 12814 & 97.8407 & 3 & 279.0 & 2 & 600.0 & \\
\hline 02 & 12815 & 60.2300 & 4.1750 & 279.7 & 3 & 600.0 & 60 \\
\hline 02 & 12816 & 117.7138 & 4.8500 & 278.9 & 3 & 600.0 & 600.0 \\
\hline 02 & 12817 & 157.2509 & 5.5500 & 277.2 & 4 & 600.0 & \\
\hline 02 & 12818 & 160.2506 & 5.4500 & 275.6 & 4 & 600.0 & 60 \\
\hline 02 & 12819 & 145.4079 & 750 & 274.9 & 5 & 600.0 & 600 \\
\hline 02 & 12820 & 42.6057 & 3.2000 & 275.3 & 4 & 600.0 & .0 \\
\hline 02 & 12821 & 82.2504 & 250 & 274.5 & 4 & 600.0 & \\
\hline 02 & 12822 & 83.9924 & 000 & 273.9 & 4 & 600.0 & 600.0 \\
\hline 02 & 12823 & 130.7897 & 1.5250 & 273.4 & 5 & 600.0 & .0 \\
\hline 02 & 12824 & 153.0004 & 50 & 273.3 & 5 & 600.0 & \\
\hline 02 & 1291 & 146.5018 & 3.4750 & 273.7 & 4 & 600.0 & 600.0 \\
\hline 02 & 1292 & 149.2499 & 3.3500 & 273.8 & 5 & 600.0 & 60 \\
\hline 02 & 1293 & 171.0000 & 2 . & 273.7 & 4 & 600.0 & .0 \\
\hline 02 & 1294 & 144.9313 & 2.0750 & 273.7 & 5 & 600.0 & 60 \\
\hline 02 & 1295 & 165.9988 & 5.1500 & 273.5 & 4 & 600.0 & 600.0 \\
\hline 02 & 1296 & 159.2498 & 5 . & 273.2 & 4 & 600.0 & .0 \\
\hline 02 & 1297 & 161.7499 & 250 & 273.0 & 4 & 600.0 & 60 \\
\hline 02 & 1298 & 160.4990 & 4.6250 & 272.7 & 4 & 600.0 & 600.0 \\
\hline 02 & 1299 & 161.4995 & 900 & 272.7 & 4 & 600.0 & .0 \\
\hline 02 & 12910 & 154.7520 & 6.1250 & 272.2 & 4 & 600.0 & 60 \\
\hline 02 & 12911 & 144.5031 & 5.6250 & 272.6 & 4 & 600.0 & 600.0 \\
\hline 02 & 12912 & 144.0009 & 5.1750 & 273.4 & 3 & 600.0 & 600.0 \\
\hline 02 & 12913 & 142.5000 & 5 . & 274.8 & 4 & 600.0 & 600.0 \\
\hline 02 & 12914 & 147.2502 & 5.4500 & 276.4 & 4 & 600.0 & 600.0 \\
\hline 02 & 12915 & 156.0043 & 7.6250 & 277.3 & 4 & 600.0 & \\
\hline 02 & 12916 & 165.0000 & 9.2500 & 278.1 & 4 & 600.0 & 600.0 \\
\hline 02 & 12917 & 160.5006 & 8.1500 & 278.1 & 4 & 600.0 & 600.0 \\
\hline 02 & 12918 & 145.0070 & 7.3250 & 277.8 & 4 & 600.0 & 600.0 \\
\hline 02 & 12919 & 136.4982 & 7.2750 & 278.0 & 4 & 600.0 & 600.0 \\
\hline 02 & 12920 & 140.0000 & 7.0750 & 278.0 & 4 & 600.0 & 600.0 \\
\hline 02 & 12921 & 140.7502 & 7.5750 & 278.2 & 4 & 600.0 & 600.0 \\
\hline 02 & 12922 & 137.2485 & 8.3500 & 278.3 & 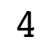 & 600.0 & 600.0 \\
\hline 02 & 12923 & 137.5000 & 8.1000 & 278.2 & 4 & 600.0 & 600.0 \\
\hline
\end{tabular}




\begin{tabular}{|c|c|c|c|c|c|c|c|}
\hline 2 & 2924 & & 7.9500 & 78.2 & & & \\
\hline & 1301 & 537 & 6.8250 & 277.9 & 4 & & \\
\hline & 1302 & 5000 & 3250 & 277.6 & 4 & 00 & \\
\hline & 1303 & 135.7546 & 7.9500 & 277.2 & & 00 & \\
\hline & 1304 & 144.2512 & .5000 & 276.6 & & 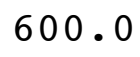 & \\
\hline & 1305 & 5001 & 9500 & 275.8 & & 00 & \\
\hline & 1306 & 0000 & 1000 & 275.3 & & 0 & \\
\hline & 307 & 996 & .3000 & 74.9 & & & \\
\hline & 1308 & 001 & 7.3250 & 274.6 & 4 & 0 & \\
\hline & 1309 & 157.2495 & 7.2500 & 275.5 & & 0 & \\
\hline & 3010 & 149.2503 & 7.8000 & 276.1 & $I$ & 0 & \\
\hline & 3011 & 0.0008 & 9.7250 & 76.2 & 4 & & \\
\hline & 3012 & 156.2499 & 9.8000 & 276.6 & 4 & 0 & \\
\hline 2 & 3013 & 161.5005 & 8.2500 & 277.4 & $\mathbf{4}$ & 0.0 & \\
\hline & 3014 & 155.7504 & 5.4750 & 278.5 & 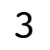 & & \\
\hline 2 & 13015 & 160.7498 & 4.7500 & 279.1 & 3 & 0 & \\
\hline & 3016 & 154.2360 & 5.2750 & 279.3 & 4 & 0 & \\
\hline 2 & 3017 & 973 & 5.5500 & 279.2 & 4 & & \\
\hline 2 & 13018 & 138.9910 & 3.1750 & 278.1 & 4 & 0 & \\
\hline 2 & 3019 & 144.0081 & .4000 & 277.2 & 5 & & \\
\hline , & 3020 & 005 & 250 & 277.4 & 4 & & \\
\hline 2 & 3021 & .4982 & 500 & 277.9 & 5 & & \\
\hline 2 & 13022 & 117.5112 & 1500 & 278.0 & 6 & 0 & U. \\
\hline 2 & 13023 & 5.4202 & 1.7250 & 278.1 & 5 & & \\
\hline 2 & 13024 & 1.7260 & 2.6750 & $27 \xi$ & 4 & & \\
\hline 2 & 1311 & 242 & 1.2000 & 277.9 & 5 & & \\
\hline 2 & 1312 & 90.7050 & .3250 & 277.4 & 6 & & \\
\hline 2 & 1313 & 113.8428 & .1500 & 27 & 6 & & \\
\hline 2 & 1314 & 32.2442 & .6750 & 277.4 & 5 & 0 & 0 . \\
\hline 2 & 1315 & 25.0002 & 8000 & 277.3 & 4 & & \\
\hline 2 & 1316 & 22.2500 & 750 & 27 & 4 & & \\
\hline 2 & 1317 & 42.0329 & 250 & 27 & 4 & & \\
\hline 2 & 1318 & 51.1226 & 500 & 276.7 & 5 & 0 & 0 . \\
\hline 2 & 1319 & 249.5650 & 750 & 28 & 4 & & \\
\hline 2 & 13110 & 302 . & 750 & 28 & 3 & & \\
\hline & 13111 & 269.3060 & 1.3500 & 280.0 & 3 & & \\
\hline 2 & 13112 & 244.4999 & 1.9750 & 279.2 & 3 & & \\
\hline 2 & 13113 & 246.3900 & 750 & 279.6 & 2 & 0 & 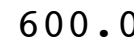 \\
\hline 2 & 13114 & 282.2470 & 2.1000 & 280.8 & 2 & 0 & 0 . \\
\hline 2 & 13115 & 293.0000 & 500 & 281.7 & 1 & & \\
\hline- & 13116 & 242.4476 & 1.6000 & 281.4 & 1 & & \\
\hline 02 & 13117 & 247.1901 & 0.7500 & 281.4 & 2 & 600.0 & 00 . \\
\hline & 13118 & 278.3671 & 0.7000 & 280.0 & 3 & 600 & s \\
\hline . & 13119 & 317.9252 & 0.3000 & 278.8 & 4 & & -0 \\
\hline 02 & 13120 & 6.7505 & 1.2500 & 278.7 & 5 & 600.0 & 00 \\
\hline 20 & 13121 & 15.7505 & 0.5500 & 278.3 & 4 & 600.0 & 60 \\
\hline & 13122 & 6.2467 & 1.3500 & 278.2 & 4 & 600 & \\
\hline & 13123 & 356.0017 & 1.0250 & 278.1 & 4 & 600.0 & 600 \\
\hline
\end{tabular}




\begin{tabular}{|c|c|c|c|c|c|c|c|c|}
\hline 02 & & 3124 & 1.7562 & 0.1500 & 277.8 & 4 & 600.0 & 600.0 \\
\hline 02 & 2 & 11 & 264.5168 & 0.2500 & 277.5 & 5 & 600.0 & 600.0 \\
\hline 02 & 2 & 12 & 352.0586 & 0.3000 & 277.4 & 4 & 600.0 & 00.0 \\
\hline 02 & 2 & 13 & 170.7682 & 0.7750 & 277.3 & 5 & 600.0 & 600.0 \\
\hline 02 & 2 & 14 & 71.5692 & 0.5250 & 276.9 & 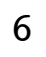 & 600.0 & 00.0 \\
\hline 02 & 2 & 15 & 136.7689 & 1.0250 & 276.9 & 6 & 600.0 & 000 \\
\hline 02 & 2 & 16 & 119.9963 & 0.8500 & 276.7 & 6 & 600.0 & 600.0 \\
\hline 02 & 2 & 17 & 336.1735 & 0.7250 & 276.8 & 0 & 600.0 & 00.0 \\
\hline 02 & 2 & 18 & 0.0573 & 0.5500 & 276.7 & 6 & 600.0 & 00.0 \\
\hline 02 & 2 & 19 & 84.5604 & 0.4000 & 278.2 & 5 & 600.0 & 600.0 \\
\hline 02 & 2 & 110 & 87.8500 & 1.2750 & 278.5 & 4 & 600.0 & 600.0 \\
\hline 02 & 2 & 111 & 163.6472 & 1.6250 & 278.3 & 3 & 600.0 & .0 \\
\hline 02 & 2 & 112 & 180.2472 & 3.0500 & 280.8 & 2 & 600.0 & 600.0 \\
\hline 02 & 2 & 113 & 167.7500 & 4.5500 & 279.4 & 3 & 600.0 & 600.0 \\
\hline 02 & 2 & 114 & 166.7505 & 3.8000 & 280.6 & 4 & 600.0 & \\
\hline 02 & 2 & 115 & 160.9977 & 4.1750 & 281.0 & 4 & 600.0 & 60 \\
\hline 02 & 2 & 116 & 153.4994 & 4.2250 & 281.3 & 4 & 600.0 & 600.0 \\
\hline 02 & 2 & 117 & 152.2496 & 4.2250 & 281.1 & 4 & 600.0 & \\
\hline 02 & 2 & 118 & 155.0006 & 4.3250 & 280.1 & 5 & 600.0 & 60 \\
\hline 02 & 2 & 119 & 151.7504 & 3.6000 & 279.4 & 5 & 600.0 & 60 \\
\hline 02 & 2 & 120 & 153.5001 & 3.6000 & 278.9 & 5 & 600.0 & .0 \\
\hline 02 & 2 & 121 & 155.5002 & 3.6250 & 278.5 & 5 & 600.0 & \\
\hline 02 & 2 & 122 & 138.0536 & 3.2250 & 278.4 & 4 & 600.0 & 600.0 \\
\hline 02 & 2 & 123 & 121.0000 & 3.4000 & 278.7 & 4 & 600.0 & .0 \\
\hline 02 & 2 & 124 & 124.4991 & 3.2500 & 278.7 & 4 & 600.0 & \\
\hline 02 & 2 & 21 & 126.5003 & 3.0500 & 279.1 & 4 & 600.0 & 600.0 \\
\hline 02 & 2 & 22 & 144.0016 & 2.7250 & 278.3 & 5 & 600.0 & 600.0 \\
\hline 02 & 2 & 23 & 120.3149 & 2.4000 & 278.5 & 4 & 600.0 & \\
\hline 02 & 2 & 24 & 147.5202 & 1.9000 & 278.4 & 4 & 600.0 & 600.0 \\
\hline 02 & 2 & 25 & 135.5015 & 1.9250 & 278.4 & 5 & 600.0 & 600.0 \\
\hline 02 & 2 & 26 & 93.1910 & 2.0750 & 278.8 & 5 & 600.0 & .0 \\
\hline 02 & 2 & 27 & 118.2048 & 1.2000 & 278.7 & 6 & 600.0 & 60 \\
\hline 02 & 2 & 28 & 91.0907 & 0.3750 & 278.7 & 5 & 600.0 & 600.0 \\
\hline 02 & 2 & 29 & 7.8971 & 0.2250 & 281.8 & 4 & 600.0 & 60 \\
\hline 02 & 2 & 210 & 335.2576 & 0.6500 & 283.1 & 3 & 600.0 & 60 \\
\hline 02 & 2 & 211 & 294.7670 & 1.7250 & 281.9 & 2 & 600.0 & 600.0 \\
\hline 02 & 2 & 212 & 225.1913 & 1.2500 & 282.9 & 1 & 600.0 & 600.0 \\
\hline 02 & 2 & 213 & 210.9946 & 2.9000 & 282.5 & 2 & 600.0 & 600.0 \\
\hline 02 & 2 & 214 & 229.5092 & 2.1000 & 283.5 & 1 & 600.0 & 600.0 \\
\hline 02 & 2 & 215 & 207.3372 & 2.4500 & 283.7 & 1 & 600.0 & \\
\hline 02 & 2 & 216 & 173.4950 & 2.6250 & 283.7 & 2 & 600.0 & 600.0 \\
\hline 02 & 2 & 217 & 145.4986 & 2.5000 & 283.5 & 3 & 600.0 & 600.0 \\
\hline 02 & 2 & 218 & 146.5064 & 1.9750 & 282.1 & 4 & 600.0 & 600.0 \\
\hline 02 & 2 & 219 & 136.4213 & 1.2750 & 281.4 & 4 & 600.0 & 600.0 \\
\hline 02 & 2 & 220 & 106.3954 & 1.4250 & 281.5 & 5 & 600.0 & 600.0 \\
\hline 02 & 2 & 221 & 68.8005 & 0.8000 & 281.5 & 6 & 600.0 & 600.0 \\
\hline 02 & 2 & 222 & 112.5404 & 1.0750 & 281.4 & 5 & 600.0 & 600.0 \\
\hline 02 & 2 & 223 & 92.0593 & 0.6750 & 280.8 & 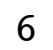 & 600.0 & 600.0 \\
\hline
\end{tabular}




\begin{tabular}{|c|c|c|c|c|c|c|c|c|}
\hline 2 & 2 & 24 & 2.2122 & 0.5500 & 280.7 & 6 & 80 & \\
\hline 2 & 2 & 31 & 152.7537 & 1.1000 & 280.4 & & 00.0 & \\
\hline & 2 & 32 & 116.6401 & 4500 & 280.6 & 4 & 00 & \\
\hline & 2 & 33 & 81.3572 & 2.8500 & 281.0 & & 00 & \\
\hline & 2 & 4 & 42.8451 & .1000 & 30.5 & & 00 & \\
\hline & 2 & 5 & 73.5539 & .1250 & 280.6 & & 00 & \\
\hline & 2 & 6 & 149.7502 & 4.5000 & 278.8 & & 0 & \\
\hline & 2 & 7 & 130.2632 & .1750 & 78.3 & & & \\
\hline & 2 & 8 & 81.0141 & .7750 & 279.0 & & 0 & \\
\hline & 2 & 9 & 142.7868 & 3.0250 & 278.3 & & 00 & \\
\hline & 2 & 10 & 167.5000 & 1.3000 & 281.2 & & 0 & \\
\hline & 2 & & 165.5000 & 3.5250 & 81.2 & 4 & & \\
\hline 2 & 2 & 12 & 158.2465 & 4.8500 & 279.9 & 4 & 0 & \\
\hline 2 & 2 & 13 & 165.0012 & 4.0250 & 282.2 & 4 & 0.0 & \\
\hline & 2 & 4 & 152.2515 & 3.3500 & 83.2 & 4 & & \\
\hline 2 & 2 & $1:$ & 136.4386 & 1.8000 & 285.4 & 3 & 0 & \\
\hline & 2 & 16 & 133.0000 & 6000 & 286.6 & & 0 & \\
\hline 2 & 2 & 17 & 146 . & 0 & 284.0 & 3 & & \\
\hline 2 & 2 & 18 & 141.7500 & 00 & 282.4 & 4 & 0 & \\
\hline 2 & 2 & 19 & 144.2500 & 7000 & 282.0 & 5 & 0 & \\
\hline 2 & 2 & 20 & 107.1903 & 0 & 281.5 & 6 & & \\
\hline 2 & 2 & 21 & 106.2504 & & 281.6 & 5 & & \\
\hline 2 & 2 & 322 & 59.9521 & 2250 & 281.9 & 5 & 0 & 00. \\
\hline 2 & 2 & 323 & 93.2402 & .52 & 282.1 & 6 & & \\
\hline 2 & 2 & 324 & 131.0050 & 0 & 282.6 & 5 & & \\
\hline 2 & 2 & 41 & 111.6265 & 3000 & 282.1 & 5 & & \\
\hline 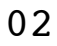 & 2 & 42 & 139.9985 & 5500 & 282.2 & 4 & 0 & \\
\hline 2 & 2 & 43 & 150.5000 & 0 & 281.8 & 4 & & \\
\hline 2 & 2 & 44 & 112.1990 & 1.3750 & 281.6 & 5 & 0 & e \\
\hline 2 & 2 & 45 & 147.9989 & 4750 & 280.9 & 4 & & \\
\hline 0 & 2 & 46 & 39.5284 & 0 & 281.3 & 5 & & \\
\hline 2 & 2 & 47 & 130 . & 0 & 280.9 & 6 & & \\
\hline 2 & 2 & 48 & 31.0000 & 0.9750 & 281.7 & 5 & 0 & • \\
\hline 2 & 2 & 49 & 16.4954 & .2250 & 283.8 & 4 & & \\
\hline 2 & 2 & 410 & 336 . & 50 & 285.7 & 3 & & \\
\hline & 2 & 411 & 250.1554 & 3750 & 284.3 & 3 & & 0 . \\
\hline 2 & 2 & 412 & 255.2604 & 1.7000 & 284.7 & 2 & & \\
\hline 2 & 2 & 413 & 205.2552 & 2.0250 & 285.1 & 1 & 0 & 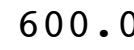 \\
\hline 02 & 2 & 414 & 205.5072 & 2.6750 & 285.8 & 2 & 0 & 0 . \\
\hline 2 & 2 & 415 & 239.2213 & 7500 & 286.6 & 1 & 0 & \\
\hline- & 2 & 416 & 223.6455 & 750 & 287.8 & 1 & & \\
\hline 02 & 2 & 417 & 266.3286 & 1.0250 & 287.0 & 2 & 600.0 & 600 . \\
\hline 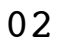 & 2 & 418 & 204.2272 & 5250 & 284.6 & 3 & 600 & 600. \\
\hline . & 2 & 419 & 349.5968 & 1.2500 & 283.6 & 4 & & 10 \\
\hline 02 & 2 & 420 & 25.8848 & 1.5000 & 283.4 & 5 & 600.0 & 00 . \\
\hline 02 & 2 & 421 & 134.8390 & 1.2250 & 283.7 & 5 & 600.0 & 600. \\
\hline & 2 & 422 & 115.2363 & & & 4 & & \\
\hline & & 423 & 133.5608 & 1.9250 & 284.2 & 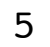 & 600.0 & 600 \\
\hline
\end{tabular}




\begin{tabular}{|c|c|c|c|c|c|c|c|c|}
\hline 2 & 2 & 424 & 43.9090 & 0.8500 & 283.0 & 6 & 600.0 & 600.0 \\
\hline 02 & 2 & 51 & 33.7539 & 1.4750 & 284.2 & 6 & 600.0 & 600.0 \\
\hline 02 & 2 & 52 & 26.2432 & .8500 & 284.4 & 6 & 600.0 & 00.0 \\
\hline 02 & 2 & 53 & 65.3505 & 0.4750 & 283.3 & 6 & 600.0 & 00.0 \\
\hline 02 & 2 & 54 & 23.9994 & 1.3000 & 284.3 & 5 & 600.0 & 00.0 \\
\hline 02 & 2 & 55 & 119.5645 & 1.3750 & 283.6 & 6 & 600.0 & 00.0 \\
\hline 02 & 2 & 56 & 142.7509 & 2.8250 & 281.9 & 5 & 600.0 & 00.0 \\
\hline 02 & 2 & 57 & 139.7426 & 2.2250 & 281.4 & 4 & 600.0 & 00.0 \\
\hline 02 & 2 & 58 & 102.4857 & 0.9000 & 281.7 & 5 & 600.0 & 00.0 \\
\hline 02 & 2 & 59 & 146.5008 & 2.2500 & 282.6 & 4 & 600.0 & 00.0 \\
\hline 02 & 2 & 510 & 117.1843 & 1.4000 & 284.0 & 3 & 600.0 & 00.0 \\
\hline 02 & 2 & 511 & 333.7671 & 0.9750 & 285.2 & 2 & 600.0 & 00.0 \\
\hline 02 & 2 & 512 & 218.3993 & 1.4500 & 284.7 & 1 & 600.0 & 00.0 \\
\hline 02 & 2 & 513 & 179.8092 & 3.1000 & 284.1 & 2 & 600.0 & 00.0 \\
\hline 02 & 2 & 514 & 156.4999 & 4.3000 & 283.1 & 3 & .0 & \\
\hline 02 & 2 & 515 & 154.0000 & 4.0250 & 282.9 & 4 & 600.0 & 00.0 \\
\hline 02 & 2 & 516 & 152.4994 & 3.8500 & 283.3 & 4 & 60 & .0 \\
\hline 02 & 2 & 517 & 158.3529 & 1.2250 & 284.3 & 3 & .0 & \\
\hline 02 & 2 & 518 & 95.1376 & 0.6000 & 283.8 & 4 & 600.0 & 00.0 \\
\hline 02 & 2 & 519 & 142.9442 & 1.4500 & 282.2 & 4 & 60 & .0 \\
\hline 02 & 2 & 520 & 141.2501 & 2.5500 & 282.2 & 4 & 60 & \\
\hline 02 & 2 & 521 & 121.3815 & 2.0500 & 282.2 & 5 & .0 & \\
\hline 02 & 2 & 522 & 132.4724 & 1.0500 & 282.0 & 6 & 600.0 & 600.0 \\
\hline 02 & 2 & 523 & 117.0115 & 1.12 & 282.3 & 6 & .0 & .0 \\
\hline 02 & 2 & 524 & 91.7200 & 0.4250 & 282.3 & 6 & .0 & \\
\hline 02 & 2 & 61 & 148.5000 & 1.6000 & 282.3 & 5 & 600.0 & 600.0 \\
\hline 02 & 2 & 62 & 151.2523 & 2.1500 & 281.5 & 5 & 600.0 & 600.0 \\
\hline 02 & 2 & 63 & 185.2039 & 1.3000 & 280.8 & 6 & 600.0 & \\
\hline 02 & 2 & 64 & 348.2515 & 2.1000 & 279.7 & 5 & 600.0 & 600.0 \\
\hline 02 & 2 & 65 & 4.5466 & 2.9500 & 279.3 & 5 & 600.0 & 600.0 \\
\hline 02 & 2 & 66 & 216.5569 & 0.9750 & 279.7 & 6 & .0 & \\
\hline 02 & 2 & 67 & 138.0585 & 1.6500 & 279.4 & 6 & 600.0 & 600.0 \\
\hline 02 & 2 & 68 & 143.0161 & 1.9500 & 280.6 & 5 & 600.0 & 600.0 \\
\hline 02 & 2 & 69 & 157.2499 & 3.0000 & 280.6 & 4 & 600.0 & 0.0 \\
\hline 02 & 2 & 610 & 168.2948 & 2.4500 & 282.7 & 3 & 600.0 & 600.0 \\
\hline 02 & 2 & 611 & 185.5141 & 1.6000 & 283.6 & 2 & 600.0 & 600.0 \\
\hline 02 & 2 & 612 & 185.7656 & 2.8750 & 283.9 & 1 & 600.0 & 600.0 \\
\hline 02 & 2 & 613 & 168.4917 & 2.9250 & 284.0 & 2 & 600.0 & 600.0 \\
\hline 02 & 2 & 614 & 230.4606 & 2.4000 & 285.3 & 1 & 600.0 & 600.0 \\
\hline 02 & 2 & 615 & 275.1636 & 1.5750 & 285.8 & 1 & 600.0 & 600.0 \\
\hline 02 & 2 & 616 & 240.5299 & 0.9000 & 287.1 & 1 & 600.0 & 600.0 \\
\hline 02 & 2 & 617 & 323.0831 & 1.6000 & 285.6 & 2 & 600.0 & 600.0 \\
\hline 02 & 2 & 618 & 117.1845 & 2.7000 & 284.4 & 3 & 600.0 & 600.0 \\
\hline 02 & 2 & 619 & 103.9696 & 3.8500 & 283.4 & 4 & 600.0 & 600.0 \\
\hline 02 & 2 & 620 & 39.1945 & 4.0500 & 282.9 & 4 & 600.0 & 600.0 \\
\hline 02 & 2 & 621 & 332.7515 & 3.4000 & 282.5 & 4 & 600.0 & 600.0 \\
\hline 02 & 2 & 622 & 337.5268 & 1.6250 & 282.7 & - & 600.0 & 600.0 \\
\hline 04 & 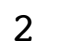 & 623 & 352.2507 & 2.125 & 282.6 & 3 & 600.0 & 600.0 \\
\hline
\end{tabular}




\begin{tabular}{|c|c|c|c|c|c|c|c|c|}
\hline 2 & 2 & 24 & 1.7495 & 9250 & 33.1 & & 0 & \\
\hline 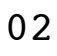 & 2 & 71 & 6.5170 & .2750 & 283.3 & & 600.0 & \\
\hline & 2 & 72 & 211.9164 & 1750 & 283.2 & & 00 & \\
\hline & 2 & 73 & 33.2631 & 9250 & 283.6 & & 00.0 & \\
\hline & 2 & 4 & 32.0002 & .7750 & 85.0 & & 00 & \\
\hline & 2 & 75 & 7.5021 & .6500 & 84.9 & & 00 & \\
\hline & 2 & 76 & 29.2633 & 1.1750 & 284.8 & & 00 & \\
\hline & 2 & 7 & 20.6333 & .4750 & 84.7 & & 0 & \\
\hline & 2 & 78 & 17.9224 & .7750 & 85.0 & & & \\
\hline & 2 & 79 & 30.1291 & 1.5000 & 86.5 & & 00 & 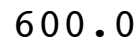 \\
\hline & 2 & 710 & 349.9480 & 3.3250 & 286.6 & & & \\
\hline & 2 & 711 & 4.5713 & 3.1500 & 88.4 & & 0 & \\
\hline & 2 & 712 & 172.3713 & 2.1750 & 287.1 & & $\cdot 0$ & \\
\hline & 2 & 713 & 236.0025 & 1.2000 & 287.1 & & 0.0 & 00 \\
\hline & 2 & 714 & 9.1050 & 3.2500 & & & & \\
\hline & 2 & 715 & 17.9947 & 3.7750 & 289.2 & & & \\
\hline & 2 & 716 & 355.7494 & 3.3500 & 287.9 & & .0 & 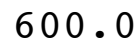 \\
\hline & 2 & 717 & 336.5985 & 3.2000 & 0 & & 0 & \\
\hline & 2 & 718 & 303 . & 2.2750 & 28 & & & \\
\hline & 2 & 719 & 51.5000 & 3500 & 286.4 & 4 & .0 & 0. \\
\hline 2 & 2 & 720 & 10.7414 & .9250 & 285.9 & & 0 & U. \\
\hline & 2 & 721 & 350.4841 & 0 & & & & \\
\hline & 2 & 722 & 76.5378 & 0 & 284.9 & & & \\
\hline$?$ & 2 & 723 & 84.5927 & 6.4000 & 282.2 & & 0 & 0 • \\
\hline & 2 & 724 & 108.7473 & 0 & 5 & & & \\
\hline 2 & 2 & 81 & 87.7573 & 7750 & 28 & 4 & & \\
\hline , & 2 & 82 & 56.4994 & 5000 & 280.3 & 4 & 0 & U. \\
\hline & 2 & 83 & 68.4968 & 4000 & 279.8 & 4 & & \\
\hline 2 & 2 & 84 & 81.0000 & 6.5750 & 279.8 & 4 & & 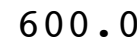 \\
\hline , & 2 & 85 & 79.7514 & $\$ 750$ & 279.3 & 4 & 0 & 0 . \\
\hline 2 & 2 & 86 & 77.5013 & 1500 & 278.7 & 4 & & 0 . \\
\hline 2 & 2 & 87 & 104.9531 & 3750 & 279.1 & 4 & & \\
\hline 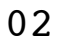 & 2 & 88 & 121.3399 & 250 & 279.4 & 4 & & 0 • \\
\hline 2 & 2 & 89 & 152.5000 & 9500 & 279.9 & 4 & 0 & 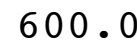 \\
\hline 2 & 2 & 810 & 146.0016 & 250 & 280.1 & 4 & & \\
\hline 2 & 2 & 811 & 150.5000 & 750 & 0.0 & 4 & & 0 . \\
\hline 2 & 2 & 812 & 158.7441 & 7.7000 & 280.4 & 4 & & . \\
\hline 2 & 2 & 813 & 159.2472 & 7.1000 & 28 & 4 & & . \\
\hline 02 & 2 & 814 & 155.7505 & 7.1500 & 282.3 & 4 & .0 & 0 . \\
\hline 2 & 2 & 815 & 151.0005 & 6250 & 283.4 & 4 & & 0 . \\
\hline 02 & 2 & 816 & 161.9991 & 9.3500 & 284 & 4 & & 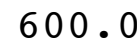 \\
\hline 02 & 2 & 817 & 158.0006 & 8.8250 & 284.0 & 4 & .0 & 0 . \\
\hline 2 & 2 & 818 & 152.2460 & 8.4750 & 283.4 & 4 & 0 & 0 . \\
\hline 02 & 2 & 819 & 157.7476 & 500 & 282 & 4 & & \\
\hline 02 & 2 & 820 & 147.7492 & 5.7500 & 282.5 & 4 & & 00 . \\
\hline 2 & 2 & 821 & 144.2511 & 5.2500 & 282.9 & 4 & 600.0 & 600. \\
\hline & 2 & 822 & 147.7499 & & & 4 & & \\
\hline & & 823 & 146.9999 & 8.7000 & 283.0 & 7 & 600.0 & 600 \\
\hline
\end{tabular}




\begin{tabular}{|c|c|c|c|c|c|c|c|}
\hline & 24 & & .4000 & 282.8 & & & \\
\hline 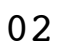 & $\begin{array}{lll}2 & 9 & 1\end{array}$ & 154.0000 & .0250 & 282.3 & & & \\
\hline & 292 & 7348 & 3000 & 281.4 & & 00 & \\
\hline & 293 & 152.4994 & 9.6750 & 281.2 & & 00 & \\
\hline & 94 & & .6500 & & & & \\
\hline & 295 & 5000 & 250 & 80.5 & & 0 & \\
\hline & 296 & 997 & 7250 & 279.8 & & & \\
\hline & $\begin{array}{lll}2 & 9 & 7\end{array}$ & 999 & 0750 & 79.5 & & & \\
\hline & 298 & 2495 & 8.2750 & 79.0 & & 0 & \\
\hline & 299 & 150.2496 & 0.1750 & 278.8 & & 0 & \\
\hline & 2910 & .4798 & 0 & 279.2 & & & \\
\hline & 2911 & & & 80.6 & & & \\
\hline & 2912 & 559 & 5.8750 & 281.7 & 4 & 0 & \\
\hline & 2913 & 153.0000 & 5.57 & 282.2 & & 0 & \\
\hline & 2914 & & & & & & \\
\hline 2 & 2915 & 973 & 0 & 284.9 & & & \\
\hline & 2916 & 201.7448 & 3.25 & 284.6 & & & \\
\hline & 2917 & 27 & 2 & .8 & & & \\
\hline 2 & 2918 & 303 & 1. & 284.1 & 4 & 0 & \\
\hline & 2919 & 300.7995 & 0 & 283.1 & 4 & & \\
\hline & 2920 & 318 . & 0 & 2.8 & & & \\
\hline & 2921 & 30 & & 3.2 & 6 & & \\
\hline 2 & 2922 & 327 . & 0 & .3 & 5 & & U. \\
\hline 2 & 2923 & 356 . & 1 . & .1 & 4 & & \\
\hline & 2924 & 330 . & & & 3 & & \\
\hline & 2101 & 301 & 187 & .1 & 4 & & \\
\hline & $210 \quad 2$ & 267.3845 & 0 & 282.5 & 5 & & \\
\hline 2 & 2103 & 299.2459 & 057 & 282.4 & 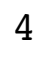 & & \\
\hline 2 & 2104 & 224 . & 0 & 281.8 & 5 & & \\
\hline & 2105 & 220.7117 & 0 & 1.6 & 6 & & \\
\hline 2 & 2106 & 323.8554 & & & 6 & & \\
\hline 2 & 2107 & 145 & 0 & .1 & 6 & & \\
\hline & 2108 & 146.4999 & 7250 & 280.0 & 5 & 0 & 0 . \\
\hline 2 & 2109 & 151.4994 & & 1.1 & 4 & & \\
\hline & 21010 & 162 . & 3. & 282.4 & 4 & & \\
\hline & 21011 & 158 . & & 282.9 & 4 & & \\
\hline & 21012 & 163.0007 & 0 & 284.0 & 4 & & \\
\hline 2 & 21013 & 165.4997 & 0 & 284.6 & 4 & & 0 . \\
\hline & 21014 & 157.2610 & 3.3250 & 286.1 & 4 & 0 & 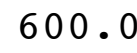 \\
\hline & 21015 & 32 . & 0 & 28 & 3 & & \\
\hline- & 21016 & 168.3047 & 2 . & & 2 & & \\
\hline 02 & 21017 & 77.7405 & 1.8250 & 288.0 & 3 & 600.0 & 00 . \\
\hline & 21018 & 85.8959 & 1.4000 & 287.1 & 4 & 0 & \\
\hline- & 21019 & 126.2364 & 1.1250 & & 5 & & \\
\hline 02 & 21020 & 62.0744 & 1.1500 & 286.6 & 6 & 600 & 00 \\
\hline & 21021 & 140.3907 & 0.7000 & 285.9 & 6 & 600 & 60 \\
\hline & 21022 & 5552 & 50 & & 6 & & \\
\hline & 21023 & 63.5541 & 0.9500 & 286.8 & & 600.0 & 600 \\
\hline
\end{tabular}




\begin{tabular}{|c|c|c|c|c|c|c|c|}
\hline 2 & 1024 & & 1750 & 37.0 & & & \\
\hline & 2111 & & .9000 & 85.5 & & $r \cap 0$ & \\
\hline & 2112 & 91.2583 & 1.4000 & 284.8 & & 00 & \\
\hline & 113 & 194.3292 & 0.3750 & 284.8 & & 00.0 & \\
\hline & 114 & 156.5000 & .1750 & & & 0 & \\
\hline & 2115 & 117.3901 & 1.1000 & 83.5 & & 00 & \\
\hline & 2116 & 50.2448 & 0.0250 & 283.3 & & 00 & \\
\hline & 2117 & 23.9963 & .3750 & 83.2 & & & \\
\hline & 2118 & 66.3068 & .1750 & 83.7 & & & \\
\hline & 119 & 31.2503 & 0.8500 & 84.7 & & 00 & U \\
\hline & 1110 & 334.1549 & 0.5500 & 287.2 & & 00.0 & \\
\hline & 1111 & 179.6841 & 1.7250 & 8 & & & \\
\hline & 1112 & 164.2013 & 1.8750 & 287.8 & & .0 & \\
\hline & 1113 & 159.7445 & 2.8250 & 287.6 & & 0.0 & \\
\hline & 1114 & 161.2498 & 0 & & & & \\
\hline & 21115 & 164.75 & 4 . & 287.7 & & & \\
\hline & 21116 & 141.4962 & 2.2000 & 288.1 & & .0 & . \\
\hline & 1117 & 133.7505 & 1.8000 & 1 & & 0 & \\
\hline & 21118 & 151.2496 & 1.85 & 0 & & & \\
\hline & 21119 & 160.7752 & 1.9000 & 286.0 & & 0 & . \\
\hline 2 & 21120 & 153.7952 & 2.3250 & 5 & & .0 & \\
\hline & 21121 & 107.0663 & $151-7$ & & & & \\
\hline & 1122 & 126.7637 & 7000 & 28 & & & \\
\hline 2 & 21123 & 82.2494 & 3.9250 & 287.2 & & 0 & 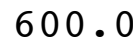 \\
\hline & 21124 & 122.6142 & 0 & & & & \\
\hline 2 & 2121 & 153.7493 & 1.9750 & 28 & 5 & & \\
\hline & 2122 & 136.4740 & 2.2500 & 286 & & 0 & J. \\
\hline & 2123 & 130.7766 & 1.1500 & 8 & & & \\
\hline 2 & 2124 & 129.9632 & 1.0750 & 28 & 5 & & \\
\hline & 2125 & 106.3275 & 2.4000 & 286.7 & 4 & 0 & 0 . \\
\hline 2 & 2126 & 107.7631 & 2.1500 & 287.1 & 5 & & 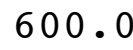 \\
\hline 2 & 2127 & 136.4979 & 3.10 & 28 & 5 & & \\
\hline & 2128 & 139.5000 & 3750 & 286.3 & 4 & 0 & . \\
\hline 2 & 2129 & 145.5120 & 500 & 28 & 4 & 0 & 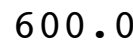 \\
\hline 2 & 21210 & 152.5046 & 0 & 28 & 4 & & \\
\hline 2 & 21211 & 155.7492 & 8.7500 & 287.0 & 4 & & . \\
\hline 2 & 21212 & 152.2532 & . 9750 & 287.1 & 4 & & 0 . \\
\hline 2 & 21213 & 153.0001 & 9 . & 287.2 & 4 & & 0 . \\
\hline 2 & 21214 & 156.2500 & 8.7000 & 287.9 & 4 & .0 & 0 . \\
\hline 2 & 21215 & 157.7504 & 7.6000 & 288.4 & 4 & & 0 . \\
\hline 2 & 21216 & 147.2522 & 5.3250 & 288.9 & 4 & & 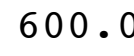 \\
\hline 2 & 21217 & 128.8374 & 3.4750 & 288.8 & 3 & & 0 . \\
\hline 2 & 21218 & 114.9113 & 3.4250 & 288.8 & 4 & 0 & 0 . \\
\hline 2 & 21219 & 87.0090 & 500 & 288.7 & 4 & & \\
\hline 02 & 21220 & 109.4378 & 3.1000 & 288.5 & 4 & & 0 . \\
\hline & 21221 & 90.4927 & 4.9500 & 288.4 & 4 & 600 & 600 \\
\hline & & 80 & & & 4 & & \\
\hline & & 76.7499 & 5.8750 & 288.0 & $\mathbf{I}$ & 600.0 & 600 \\
\hline
\end{tabular}




\begin{tabular}{|c|c|c|c|c|c|c|c|}
\hline 2 & 1224 & 270 & 3000 & 287.0 & 5 & 600.0 & \\
\hline 2 & 2131 & 11.4990 & 1.8750 & 285.8 & & 600.0 & \\
\hline & 2132 & 14.2523 & 2250 & 286.4 & & 00 & \\
\hline & 2133 & 70.5021 & 2.8250 & 286.9 & & 00 & \\
\hline & 134 & 57.6135 & .8500 & 86.8 & & 0 & \\
\hline & 2135 & & 000 & 85.9 & & 00 & \\
\hline & 2136 & 5398 & 0.6750 & 285.5 & & 00 & \\
\hline & 137 & 175.6016 & 4000 & 85.3 & & 0 & \\
\hline & 2138 & 153.7122 & .9750 & 85.5 & & & \\
\hline & 139 & 139.5995 & 1.6250 & 285.3 & & 00 & 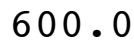 \\
\hline & 1310 & 258.5000 & 0.8250 & 284.8 & & 0 & \\
\hline & 1311 & 336.4481 & 1.1250 & & & & \\
\hline & 1312 & 223. & 2.1750 & 284 & & 0 & \\
\hline & 1313 & 128.2541 & 2.9000 & 28 & & 0.0 & 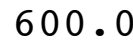 \\
\hline & 1314 & 220.7952 & 0 & & & & \\
\hline & 21315 & & 2.2750 & .8 & & & \\
\hline & 1316 & 203.1021 & 2.7750 & 283 & & .0 & 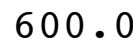 \\
\hline & 317 & 158.7476 & 3.0750 & & & & \\
\hline & 21318 & 158.2498 & 500 & 28 & & & \\
\hline & 1319 & 144.4991 & 4.1250 & 282.0 & & 0 & $\checkmark$ \\
\hline 2 & 21320 & 148.4986 & 3.4250 & 282.7 & & 0 & \\
\hline & 1 & 152.7 & 500 & & & & \\
\hline & 1322 & 157 . & 1750 & 28 & & & \\
\hline 2 & 21323 & 149.2455 & 3.2500 & .1 & & 0 & 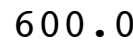 \\
\hline & 21324 & 140.25 & 4.2250 & & & & \\
\hline 2 & 2141 & 147.5008 & 2500 & & 4 & & \\
\hline & 2142 & 106.3417 & .1250 & 2 & 4 & 0 & U. \\
\hline & 2143 & 99.25 & 5750 & & 4 & & \\
\hline 2 & 2144 & 114.4979 & 750 & $2 \varepsilon$ & 4 & & 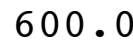 \\
\hline & 2145 & 110.0267 & 3.9000 & 2 & 4 & 0 & 0 • \\
\hline & 2146 & 86.5092 & 3.0250 & 28 & 4 & & 0 . \\
\hline 2 & 2147 & 142.4990 & 500 & & 4 & & \\
\hline & 2148 & 150.7499 & 7000 & .3 & 4 & 0 & . \\
\hline 2 & 2149 & 145.5001 & 4.8750 & 28 & 4 & 0 & . \\
\hline 2 & 21410 & 160.25 & 0 & & 3 & & \\
\hline 2 & & 165.0000 & 4.3000 & .7 & 3 & & U \\
\hline 2 & 21412 & 173.2527 & 4250 & 286.1 & 3 & & 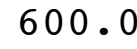 \\
\hline 2 & 21413 & 174.2527 & 250 & 28 & 3 & & . \\
\hline 02 & 21414 & 171.7473 & 3.9250 & 287.8 & 4 & .0 & 0 . \\
\hline 20 & 21415 & 163.2498 & 3.1500 & 288.5 & 3 & & 0 . \\
\hline 2 & 21416 & 164.2502 & 3.3250 & 288.8 & 3 & & 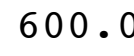 \\
\hline 02 & 21417 & 155.7514 & 3.0750 & 288.6 & 4 & .0 & 0 . \\
\hline 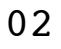 & 21418 & 171.0111 & 1.5500 & 287.4 & 4 & 0 & 0 . \\
\hline 2 & 21419 & 29.9844 & 1.4500 & 28 & 5 & & \\
\hline 02 & 21420 & 38.4595 & 0.8250 & & 6 & & 0 . \\
\hline 0 & 21421 & 30.4899 & 0.8500 & 286.1 & 5 & 600.0 & 600 \\
\hline & & 16 . & & & 4 & & \\
\hline & 21423 & 36.2638 & 1.4500 & 285.5 & $J$ & 600.0 & 600 \\
\hline
\end{tabular}




\begin{tabular}{|c|c|c|c|c|c|c|c|}
\hline 02 & 21424 & 33.4749 & 1.5750 & 284.6 & 6 & 600.0 & 600.0 \\
\hline 02 & 2151 & 69.4308 & 1.9250 & 284.0 & 6 & 600.0 & 600.0 \\
\hline 02 & 2152 & 38.3387 & 1.2750 & 283.3 & 6 & 600.0 & 00.0 \\
\hline 02 & 2153 & 30.9989 & 2.4750 & 283.1 & 5 & 600.0 & 600.0 \\
\hline 02 & 2154 & 30.9986 & 2.2000 & 282.8 & 4 & 600.0 & 00.0 \\
\hline 02 & 2155 & 46.0030 & 2.6500 & 282.3 & 4 & 600.0 & 00.0 \\
\hline 02 & 2156 & 33.2532 & 2.4250 & 282.2 & 4 & 600.0 & 600.0 \\
\hline 02 & 2157 & 35.7132 & 1.8000 & 282.1 & 5 & 600.0 & 500.0 \\
\hline 02 & 2158 & 21.1853 & 0.9000 & 281.7 & 6 & 600.0 & 00.0 \\
\hline 02 & 2159 & 316.3498 & 0.8000 & 281.9 & 5 & 600.0 & 600.0 \\
\hline 02 & 21510 & 326.6212 & 0.7250 & 282.4 & 4 & 600.0 & 600.0 \\
\hline 02 & 21511 & 338.9560 & 0.6750 & 283.5 & 3 & 600.0 & \\
\hline 02 & 21512 & 225.8973 & 1.6750 & 283.1 & 2 & 600.0 & 600.0 \\
\hline 02 & 21513 & 217.4404 & 1.4250 & 284.0 & 1 & 600.0 & 600.0 \\
\hline 02 & 21514 & 143.3321 & 3.2500 & 284.4 & 2 & 600.0 & \\
\hline 02 & 21515 & 107.2545 & 3.8000 & 285.3 & 3 & 600.0 & 60 \\
\hline 02 & 21516 & 96.6504 & 2.9500 & 285.4 & 2 & 600.0 & 600.0 \\
\hline 02 & 21517 & 134.2939 & 4.4500 & 284.0 & 3 & 600.0 & \\
\hline 02 & 21518 & 100.2110 & 3.4750 & 283.4 & 4 & 600.0 & 60 \\
\hline 02 & 21519 & 82.9989 & 4.6750 & 282.7 & 5 & 600.0 & 600.0 \\
\hline 02 & 21520 & 85.0000 & 5.37 & 282.4 & 4 & 600.0 & .0 \\
\hline 02 & 21521 & 79.0000 & 5.5750 & 282.1 & 4 & 600.0 & \\
\hline 02 & 21522 & 74.4999 & 5.2500 & 281.9 & 4 & 600.0 & 600.0 \\
\hline 02 & 21523 & 42.7660 & 2.5500 & 281.9 & 5 & 600.0 & .0 \\
\hline 02 & 21524 & 334.1907 & 1.9250 & 281.4 & 4 & 600.0 & \\
\hline 02 & 2161 & 337.8372 & 1.1250 & 280.7 & 4 & 600.0 & 600.0 \\
\hline 02 & 2162 & 105.5306 & 0.8000 & 280.6 & 5 & 600.0 & 600.0 \\
\hline 02 & 2163 & 20.3352 & 0.4000 & 280.9 & 6 & 600.0 & .0 \\
\hline 02 & 2164 & 73.2455 & 2.4500 & 281.3 & 5 & 600.0 & 600.0 \\
\hline 02 & 2165 & 86.2010 & 0.8750 & 281.1 & 6 & 600.0 & 600.0 \\
\hline 02 & 2166 & 14.2136 & 0.4500 & 280.7 & 6 & 600.0 & .0 \\
\hline 02 & 2167 & 353.0261 & 1.4000 & 280.5 & 5 & 600.0 & 600.0 \\
\hline 02 & 2168 & 350.5055 & 1.2500 & 280.2 & 6 & 600.0 & 600.0 \\
\hline 02 & 2169 & 346.6898 & 1.1250 & 280.9 & 5 & 600.0 & .0 \\
\hline 02 & 21610 & 279.8270 & 1.2500 & 282.8 & 4 & 600.0 & 600.0 \\
\hline 02 & 21611 & 278.0541 & 1.8250 & 283.7 & 3 & 600.0 & 600.0 \\
\hline 02 & 21612 & 286.7431 & 2.2000 & 284.2 & 2 & 600.0 & 600.0 \\
\hline 02 & 21613 & 269.4760 & 1.3750 & 284.8 & 1 & 600.0 & 600.0 \\
\hline 02 & 21614 & 247.6950 & 0.9750 & 285.9 & 1 & 600.0 & 600.0 \\
\hline 02 & 21615 & 249.0512 & 1.2000 & 284.7 & 1 & 600.0 & \\
\hline 02 & 21616 & 317.3258 & 1.3250 & 285.0 & 1 & 600.0 & 600.0 \\
\hline 02 & 21617 & 320.9736 & 2.5000 & 284.6 & 2 & 600.0 & 600.0 \\
\hline 02 & 21618 & 336.7456 & 3.8250 & 284.7 & 3 & 600.0 & 600.0 \\
\hline 02 & 21619 & 352.9233 & 3.4750 & 283.7 & 4 & 600.0 & 600.0 \\
\hline 02 & 21620 & 347.2230 & 2.5500 & 281.9 & 5 & 600.0 & 600.0 \\
\hline 02 & 21621 & 105.4922 & 2.3000 & 281.8 & 6 & 600.0 & 600.0 \\
\hline 02 & 21622 & 28.8019 & 1.6500 & 281.9 & 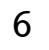 & 600.0 & 600.0 \\
\hline 02 & 21623 & 356.1799 & 1.9500 & 281.5 & 0 & 600.0 & 600.0 \\
\hline
\end{tabular}




\begin{tabular}{|c|c|c|c|c|c|c|c|}
\hline 02 & 21624 & 6.4957 & 2.4500 & 281.0 & 5 & 600.0 & 600.0 \\
\hline 02 & 2171 & 353.5138 & 1.6750 & 281.1 & 6 & 600.0 & 600.0 \\
\hline 02 & 2172 & 329.2163 & 4250 & 280.8 & 6 & 600.0 & 00.0 \\
\hline 02 & 2173 & 299.2801 & 0.9000 & 280.5 & 6 & 600.0 & 00.0 \\
\hline 02 & 2174 & 234.2385 & 1.2000 & 279.2 & 6 & 600.0 & 00.0 \\
\hline 02 & 2175 & 108.9654 & 1.5250 & 278.9 & 6 & 600.0 & 00.0 \\
\hline 02 & 2176 & 165.9047 & 2.3000 & 278.5 & 6 & 600.0 & 00.0 \\
\hline 02 & 2177 & 171.4910 & 1.9250 & 278.0 & 6 & 600.0 & 00.0 \\
\hline 02 & $217 \quad 8$ & 185.0129 & 3.6750 & 278.1 & 5 & 600.0 & 00.0 \\
\hline 02 & 2179 & 150.9966 & 5.3250 & 277.4 & 4 & 600.0 & 00.0 \\
\hline 02 & 21710 & 159.7769 & 3.8000 & 277.7 & 4 & 600.0 & 00.0 \\
\hline 02 & 21711 & 167.2555 & 3.7 & 278.2 & 4 & 600.0 & \\
\hline 02 & 21712 & 166.4959 & 4.0250 & 278.8 & 4 & 600.0 & 00.0 \\
\hline 02 & 21713 & 121.6337 & 5.4000 & 279.8 & 3 & 600.0 & 00.0 \\
\hline 02 & 21714 & 145.7585 & 5 . & 280.3 & 3 & .0 & \\
\hline 02 & 21715 & 105.9489 & 5 & 281.2 & 3 & 600.0 & 00.0 \\
\hline 02 & 21716 & 110.2463 & 4.8750 & 281.5 & 3 & 600.0 & 600.0 \\
\hline 02 & 21717 & 75.9931 & 6. & 281.3 & 4 & .0 & \\
\hline 02 & 21718 & 69.2323 & 5.7500 & 280.6 & 4 & 600.0 & .0 \\
\hline 02 & 21719 & 66.7485 & 6.9500 & 280.5 & 4 & 60 & .0 \\
\hline 02 & 21720 & 64.0000 & 6 . & 280.2 & 4 & 60 & \\
\hline 02 & 21721 & 64.5002 & 7.3 & 279.9 & 4 & .0 & \\
\hline 02 & 21722 & 66.7500 & 7.9250 & 279.9 & 4 & 600.0 & 500.0 \\
\hline 02 & 21723 & 67.2499 & 7.8 & 279.9 & 4 & 60 & .0 \\
\hline 02 & 21724 & 66.0000 & 6. & 280.0 & 4 & .0 & \\
\hline 02 & 2181 & 60.9988 & 5.9 & 280.1 & 4 & 600.0 & 600.0 \\
\hline 02 & 2182 & 64.5005 & 4.5750 & 280.2 & 4 & 600.0 & .0 \\
\hline 02 & 2183 & 75.7779 & 2.6 & 280.1 & 5 & 600.0 & \\
\hline 02 & 2184 & 63.5000 & 5.1 & 279.8 & 4 & 600.0 & 600.0 \\
\hline 02 & 2185 & 50.5086 & 3.6 & 279.9 & 4 & 600.0 & 600.0 \\
\hline 02 & 2186 & 172.5434 & 1. & 279.5 & 5 & 0 & \\
\hline 02 & 2187 & 141.4895 & 1.07 & 279.4 & 6 & 600.0 & 600.0 \\
\hline 02 & 2188 & 62.9979 & 2.9250 & 279.8 & 5 & 600.0 & 600.0 \\
\hline 02 & 2189 & 83.4944 & 3.2250 & 280.8 & 4 & 600.0 & 0.0 \\
\hline 02 & 21810 & 80.7513 & 3.5750 & 281.8 & 3 & 600.0 & 600.0 \\
\hline 02 & 21811 & 102.0116 & 3.0500 & 282.5 & 3 & 600.0 & 600.0 \\
\hline 02 & 21812 & 125.9356 & 2.4 & 283.3 & 2 & 600.0 & 600.0 \\
\hline 02 & 21813 & 294.4096 & 1.8750 & 283.7 & 1 & 600.0 & 600.0 \\
\hline 02 & 21814 & 339.1361 & 2.0750 & 283.9 & 1 & 600.0 & 600.0 \\
\hline 02 & 21815 & 302.0780 & 2.4750 & 284.0 & 1 & 600.0 & 600.0 \\
\hline 02 & 21816 & 278.1619 & 1.9000 & 283.9 & 1 & 600.0 & 600.0 \\
\hline 02 & 21817 & 283.7729 & 1.2000 & 283.4 & 2 & 600.0 & 600.0 \\
\hline 02 & 21818 & 0.7923 & 0.6250 & 283.2 & 3 & 600.0 & 600.0 \\
\hline 02 & 21819 & 69.4319 & 1.2250 & 283.1 & 4 & 600.0 & 600.0 \\
\hline 02 & 21820 & 189.0764 & 0.1000 & 282.9 & 5 & 600.0 & 600.0 \\
\hline 02 & 21821 & 84.1194 & 0.7000 & 282.7 & 6 & 600.0 & 600.0 \\
\hline 02 & 21822 & 34.6724 & 0.7000 & 282.8 & 6 & 600.0 & 600.0 \\
\hline 0 & 21823 & 159.2358 & 0.975 & 282.3 & 3 & 600.0 & 600.0 \\
\hline
\end{tabular}




\begin{tabular}{|c|c|c|c|c|c|c|c|}
\hline 2 & 1824 & & 500 & 2.2 & & & \\
\hline 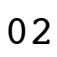 & 2191 & 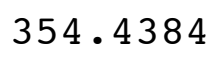 & 1.2000 & 81.8 & & 0 & \\
\hline & 2192 & 3.2766 & 1.0000 & 282.0 & & 00.0 & \\
\hline 2 & 193 & 84.6706 & .6000 & 281.4 & & 00.0 & \\
\hline & 194 & 28.7870 & .7500 & 80.9 & & & \\
\hline & 195 & 326.9205 & 1.5750 & 280.8 & & 00 & \\
\hline & 2196 & 343.7464 & 1.4250 & 281.2 & & 50 & \\
\hline & 2197 & 45.2070 & 1.2500 & 281.2 & & 00.0 & \\
\hline & 198 & 327.1134 & 1.1000 & 81.4 & & & \\
\hline & 2199 & 49.7670 & 1.4000 & 281.8 & & 600.0 & 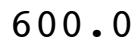 \\
\hline 2 & 1910 & 331.9007 & 1.5500 & 283.1 & & & \\
\hline & 1911 & 218.2519 & 0.8250 & 84.2 & & & \\
\hline 2 & 21912 & 183.04 & 0.9500 & 284.2 & & 00.0 & \\
\hline 2 & 1913 & 321.1806 & 1.2250 & 284 & & 0.0 & \\
\hline & 1914 & 257 & & & & & \\
\hline 2 & 1915 & 228 & & 283 & 1 & & \\
\hline 2 & 1916 & 298.14 & 9000 & 283.8 & & 0.0 & \\
\hline 2 & 917 & 95.54 & 0 & 5 & 1 & .0 & \\
\hline 2 & 21918 & 60 . & 0 & 28 & 2 & & \\
\hline 2 & 1919 & 90 . & 0 & 285.7 & 3 & .0 & \\
\hline 02 & 21920 & 97.4765 & 2.4500 & 285.6 & 4 & .0 & 000 \\
\hline 2 & 21921 & 81.81 & & & 5 & & \\
\hline 02 & 21922 & 0. & 0 & 28 & 5 & .0 & \\
\hline 02 & 21923 & 241.33 & 000 & 9 & 6 & .0 & \\
\hline 2 & 21924 & 319.17 & & & 6 & & \\
\hline 02 & 2201 & 357.2 & 1 . & 28 & 5 & & \\
\hline 02 & 2202 & 13.9982 & 1.9750 & 284.8 & 5 & .0 & 10 \\
\hline 2 & 2203 & 110.048 & 0 & 8 & 6 & .0 & \\
\hline 02 & 2204 & 69. & 3 & 28 & 5 & .0 & \\
\hline 02 & 2205 & 128.1361 & 50 & & 6 & .0 & \\
\hline 02 & 2206 & 274.2623 & 0.7750 & 284.0 & 6 & .0 & \\
\hline 02 & 2207 & 5.1296 & 2 . & 283.1 & 5 & & \\
\hline 02 & 2208 & 64.5712 & & 284 & 6 & .0 & \\
\hline 02 & 2209 & 25.4788 & 0.4000 & & 5 & .0 & \\
\hline 02 & 22010 & 309 . & 0 & & 4 & & \\
\hline 02 & 22011 & 324.5000 & 1.3500 & .7 & 3 & .0 & \\
\hline 02 & 22012 & 137.9408 & 2.6000 & 288.6 & 2 & .0 & \\
\hline 2 & 22013 & 112.9604 & 3 . & & 2 & .0 & \\
\hline 02 & 22014 & 126.2700 & 5.1500 & .2 & 3 & .0 & 600 \\
\hline 02 & 22015 & 117.9665 & 4.7750 & 288.7 & 3 & .0 & \\
\hline 02 & 22016 & 116.9584 & 3.3000 & 289 & 2 & .0 & \\
\hline 02 & 22017 & 87.0347 & 2.9250 & 289.4 & 2 & .0 & $6 c^{2}$ \\
\hline 02 & 22018 & 125.5859 & 3.7750 & 288.6 & 3 & .0 & 00 \\
\hline 02 & 22019 & 96.4979 & 5.3500 & 287 & 4 & & \\
\hline 02 & 22020 & 88.2485 & 6.6250 & 287 & 4 & .0 & \\
\hline 02 & 22021 & 90.0007 & 6.7000 & 287.3 & 4 & .0 & 500 \\
\hline 02 & 22022 & 92 & & & 4 & & \\
\hline & 22023 & 139.7876 & 3.2250 & 287.3 & & .0 & 600 \\
\hline
\end{tabular}




\begin{tabular}{|c|c|c|c|c|c|c|c|}
\hline- & 2024 & & 0000 & $6 \cdot 8$ & & & \\
\hline & 2211 & 171 . & 4.9250 & 85.5 & 4 & 600.0 & \\
\hline & 2212 & 164.0005 & 0500 & 284.9 & 4 & 00 & \\
\hline & 2213 & 128.1283 & 0000 & 284.9 & & 00 & \\
\hline & 214 & 125.6009 & .4500 & & & 0 & \\
\hline & 2215 & 152.2517 & 250 & 85.0 & & 00 & \\
\hline & 2216 & 136.7864 & 500 & 284.6 & & 00 & \\
\hline & 2217 & 98.1135 & 250 & 83.8 & & & \\
\hline & 2218 & 11.7204 & 3250 & 85.5 & & & \\
\hline & 2219 & 337.5426 & 1.2250 & 287.0 & 4 & 0 & 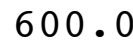 \\
\hline & 22110 & 286.0388 & 1.9500 & 288.1 & & & \\
\hline & 22111 & 238.2489 & 2.4750 & 5 & & & \\
\hline & 22112 & 187.3545 & 3.5750 & 287.2 & & 0 & \\
\hline & 22113 & 233.2491 & 2.9750 & 287.5 & & 0.0 & \\
\hline & 22114 & 246.9610 & 0 & & & & \\
\hline & 22115 & 246.5000 & 1. & 28 & 1 & & \\
\hline & 22116 & 273.5913 & .7250 & 288.6 & 1 & .0 & \\
\hline & 22117 & 273.0956 & 000 & 3 & 1 & & \\
\hline & 22118 & 316.4871 & 0 & 7 & 2 & & \\
\hline & 22119 & 357.6784 & .4750 & 285.8 & 3 & 0 & 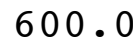 \\
\hline 2 & 22120 & 19.0554 & 750 & 285.6 & 4 & 0 & \\
\hline & 22121 & 358.5030 & & & 5 & & \\
\hline & 221 & 3372 & 0 & 28 & 6 & & \\
\hline 2 & 22123 & 248.1713 & 0.9750 & 2 & 6 & 0 & \\
\hline & 22124 & 329.5028 & & & 5 & & \\
\hline 2 & 2221 & 356.7323 & 500 & 28 & 4 & & \\
\hline & 2222 & 321.0203 & .7500 & 28 & 5 & 0 & . \\
\hline & 2223 & 185.2758 & 2250 & 4 & 6 & & \\
\hline 2 & 2224 & 207.8134 & 750 & 28 & 5 & & \\
\hline & 2225 & 224.3323 & 2000 & 2 & 5 & 0 & U. \\
\hline & 2226 & 254.4023 & 4000 & 28 & 6 & & 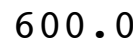 \\
\hline 2 & 2227 & 241.6486 & 250 & & 6 & & \\
\hline & 2228 & 301.8693 & 500 & 28 & 6 & & . \\
\hline 2 & 2229 & 357.9979 & 750 & 28 & 5 & 0 & 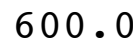 \\
\hline 2 & 22210 & 314.2417 & 0 & & 4 & & \\
\hline 02 & 22211 & 317.5350 & 0.9250 & & 3 & & • \\
\hline 2 & 22212 & 216.0607 & .4000 & 286.9 & 2 & & 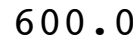 \\
\hline 2 & 22213 & 227 . & 2500 & 28 & 1 & & 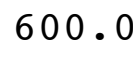 \\
\hline 2 & 22214 & 150.9650 & 2.1250 & 289.2 & 1 & .0 & 0 . \\
\hline 2 & 22215 & 243.2525 & 7500 & 290.1 & 1 & & 0 . \\
\hline 2 & 22216 & 259.5857 & 2.3250 & 290.8 & 1 & & . \\
\hline 2 & 22217 & 18.8583 & 1.2250 & 291.0 & 1 & & 0 . \\
\hline ? & 22218 & 35.0698 & .0500 & 292.7 & 2 & 0 & 0 . \\
\hline 2 & 22219 & 31.6123 & 750 & & 3 & & \\
\hline 02 & 22220 & 93.7496 & 9.0500 & & 4 & & 0 . \\
\hline 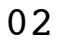 & 22221 & 107.4994 & 8.2000 & 283.6 & 4 & 600 & 600 \\
\hline & 22222 & 2602 & & & 4 & & \\
\hline & 22223 & 116.4673 & 4.0000 & 283.9 & & 600.0 & 60 \\
\hline
\end{tabular}




\begin{tabular}{|c|c|c|c|c|c|c|c|}
\hline 2 & 2224 & & 0 & & & & \\
\hline 2 & 2231 & 205 & 3.2250 & 2.7 & 4 & 0 & \\
\hline & 2232 & 88.2472 & 9000 & 282.9 & 4 & 00 & \\
\hline & 2233 & 99.4954 & 4.4000 & 282.7 & & 00 & \\
\hline & 2234 & 04 & 1250 & 82.6 & & & \\
\hline & 2235 & 88.2589 & 750 & 282.3 & 4 & 0 & \\
\hline & 2236 & 81.7602 & 500 & 281.6 & 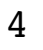 & 00 & \\
\hline & 2237 & 91.9966 & 500 & 81.6 & & & \\
\hline & 2238 & 87.4973 & 3500 & 81.4 & 4 & & \\
\hline & 2239 & 99.2475 & 500 & 82.2 & & .0 & 0 \\
\hline & 22310 & 100.4907 & 9750 & 282.7 & & & \\
\hline & 22311 & 88.3891 & 0 & 84.2 & & & \\
\hline & 22312 & 105.2206 & 000 & 283.5 & 4 & 0 & \\
\hline & 22313 & 79.6953 & .9250 & 284.7 & 3 & 0.0 & \\
\hline & 22314 & 89.5583 & & & & & \\
\hline & 22315 & 75.0023 & & 28 & 4 & & \\
\hline & 22316 & 87.5823 & 5250 & 284.8 & 4 & .0 & \\
\hline & 22317 & 72.7521 & 500 & .2 & 4 & & \\
\hline & 22318 & 85.4985 & 6 . & 283.4 & 4 & & \\
\hline & 22319 & 74.9724 & 5750 & 282.4 & 4 & & 0 \\
\hline 2 & 22320 & 74.4976 & 0250 & 282.1 & 4 & 0 & \\
\hline & 22321 & 75.5000 & & 81.7 & 4 & & \\
\hline & 22322 & 77.5000 & 0 & 281.4 & 4 & & \\
\hline$?$ & 22323 & 76.2496 & 7.8500 & 281.2 & 4 & 0 & 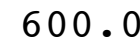 \\
\hline & 22324 & 83.0011 & $7 \quad 17$ & .2 & 4 & & \\
\hline 2 & 2241 & 77.2502 & 3250 & 281.4 & 4 & & \\
\hline & 2242 & 79.5003 & 1000 & 281.2 & 4 & 0 & J. \\
\hline & 2243 & 84.2500 & 3000 & 81.2 & 4 & & \\
\hline 2 & $224 \quad 4$ & 98.4968 & 6.1750 & 281.7 & 4 & & \\
\hline & 2245 & 102.9989 & 2250 & 282.3 & 4 & 0 & 0 . \\
\hline 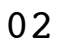 & 2246 & 122.5000 & 0500 & 282.7 & 4 & & 0 . \\
\hline 2 & 2247 & 115.9958 & 000 & 282.2 & 4 & & \\
\hline & 2248 & 108.9237 & 250 & 282.4 & 4 & & 0 . \\
\hline 2 & 2249 & 138.4999 & 000 & 283.4 & 4 & 0 & \\
\hline 2 & 22410 & 141.9963 & 0 & & 4 & & \\
\hline 2 & 22411 & 162.0057 & 3500 & .6 & 4 & & 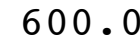 \\
\hline 2 & 22412 & 163.9959 & 7750 & 285.3 & 3 & & 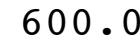 \\
\hline 2 & 22413 & 156 . & 250 & & 3 & & \\
\hline 2 & 22414 & 145.7367 & 4.1000 & 286.7 & 3 & .0 & 0 . \\
\hline 2 & 22415 & 146.2801 & 3.2000 & 287.6 & 3 & & \\
\hline 2 & 22416 & 150.4730 & 2.4250 & 28 & 2 & & 0 \\
\hline 2 & 22417 & 158.7499 & 2.3500 & .0 & 3 & & 0 . \\
\hline 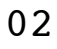 & 22418 & 145.0193 & 2.5500 & 287.7 & 4 & 0 & 0 . \\
\hline 2 & 22419 & 48.9686 & 1.6250 & & 5 & & \\
\hline 02 & 22420 & 86.1112 & 1.0750 & & 6 & & 0 . \\
\hline 0 & 22421 & 27.9931 & 0.8000 & 286.6 & 6 & 600 & 600. \\
\hline & 22422 & 29.9927 & & & 5 & & \\
\hline & 22423 & 51.7495 & 0.9000 & 287.3 & 4 & 600.0 & 600 \\
\hline
\end{tabular}




\begin{tabular}{|c|c|c|c|c|c|c|c|}
\hline 2 & 2424 & 2852 & 1750 & 87.6 & ? & 00.0 & \\
\hline 2 & 2251 & 105.1245 & 1.4750 & 288.2 & & 600.0 & \\
\hline & 2252 & 94.5699 & L. 4750 & 288.0 & & 00 & \\
\hline & 253 & 116.9645 & 0.7750 & 287.8 & & 00.0 & \\
\hline & 254 & 110.1206 & .2000 & 87.1 & & 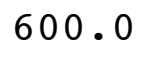 & \\
\hline & 2255 & 141.0000 & .3000 & 1 & & 0 & \\
\hline & 2256 & 4960 & 6500 & 286.2 & & 00 & \\
\hline & 2257 & 142.5000 & .1250 & 83.9 & & & \\
\hline & 2258 & 146.2501 & 150 & 83.7 & & & \\
\hline & 2259 & 152.5014 & 5500 & 284.3 & & 00 & 0 \\
\hline & 2510 & 159.2491 & 4.8750 & 285.1 & & & \\
\hline & 22511 & 162.5000 & 5.5500 & 86.4 & & & \\
\hline & 22512 & 168. & 50 & 287.6 & & .0 & \\
\hline & 22513 & 160.5000 & 5.4000 & 288.4 & & 0.0 & \\
\hline & 22514 & 163. & & & & & \\
\hline & 22515 & 146 . & & 290.3 & & & \\
\hline & 22516 & 134.8281 & .1750 & 290.7 & & .0 & \\
\hline & 2517 & 111 . & 0 & .0 & & & \\
\hline & 22518 & 108 . & 2 . & 6 & & & \\
\hline & 22519 & 111.2511 & 2.2750 & 289.6 & & 0 & 0 \\
\hline 2 & 22520 & 81.5013 & 2.5750 & 289.9 & & .0 & \\
\hline & 22521 & 108.1059 & 0 & & & & \\
\hline & 22522 & 77 . & 50 & 290.2 & 4 & & \\
\hline & 22523 & 85.0090 & 2.4500 & 290.8 & & 0 & \\
\hline & 22524 & 192.1676 & & 289.3 & & & \\
\hline & 2261 & 349 . & 250 & 28 & 5 & & \\
\hline & 2262 & 70.6209 & 4500 & 288.0 & 6 & 0 & . \\
\hline & 2263 & 172.945 & 0 & 7.7 & 5 & & \\
\hline 2 & 2264 & 268.3421 & 750 & 28 & 6 & & \\
\hline & 2265 & 345.6995 & 1.1250 & .9 & 5 & 0 & 0. \\
\hline & 2266 & 6.4997 & 0.7750 & .7 & 4 & 0 & 0 . \\
\hline 2 & 2267 & 298 . & 50 & & 5 & & \\
\hline & 2268 & 167 . & 3750 & .3 & 5 & & . \\
\hline r & 2269 & 174 . & 000 & .6 & 4 & 0 & 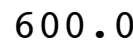 \\
\hline 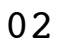 & 22610 & 249 . & 0 & & 3 & & \\
\hline 2 & 22611 & 263.5707 & 1.8750 & & 2 & & . \\
\hline 2 & 22612 & 248.2402 & 1750 & 288.9 & 1 & & 0 . \\
\hline 2 & 22613 & 215 . & 0 & 8 & 1 & & \\
\hline 2 & 22614 & 176.9710 & 2.9500 & 289.5 & 1 & .0 & 0 . \\
\hline 2 & 22615 & 174.2434 & 500 & 289.5 & 2 & & 0 . \\
\hline 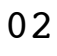 & 22616 & 162.2509 & 3 . & 28 & 3 & & 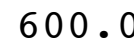 \\
\hline 2 & 22617 & 152.2733 & 2.3250 & 289.1 & 4 & & 0 . \\
\hline 2 & 22618 & 144.4973 & 1.0750 & 288.4 & 5 & 0 & 0 . \\
\hline 2 & 22619 & 84.8674 & 50 & 287.8 & 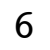 & & \\
\hline 02 & 22620 & 128.2496 & 1.5750 & 287.9 & 5 & & 0 . \\
\hline & 22621 & 145.7476 & 1.7500 & 287.8 & 4 & & 600. \\
\hline & & 18 & & & 5 & & \\
\hline & 22623 & 59.5656 & 1.6000 & 288.7 & & 600.0 & 60 \\
\hline
\end{tabular}




\begin{tabular}{|c|c|c|c|c|c|c|c|}
\hline 2 & 2624 & & & & & & \\
\hline & 2271 & 7 & & & & & \\
\hline & 2272 & 131.3257 & 5750 & 289.0 & & 00 & \\
\hline & 2273 & 97.3382 & 0.8750 & 288.3 & & 00.0 & \\
\hline & 2274 & 952 & & 38.0 & & & \\
\hline & 2275 & 51 & .4750 & 88.6 & & 0 & \\
\hline & 2276 & 375 & 000 & 87.7 & & 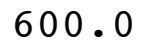 & \\
\hline & 2277 & 02.6313 & 2250 & 86.8 & & & \\
\hline & 2278 & 104.3403 & 0 & 5 & & & \\
\hline & 2279 & 2515 & 3.0000 & 86.8 & & 0 & \\
\hline & 22710 & 154.0000 & 9750 & 286 & & & \\
\hline & 22711 & 147.5000 & 007 & 6 & & & \\
\hline & 22712 & 995 & 6 . & 288.5 & 4 & 0 & \\
\hline & 22713 & 145.0000 & 5.8750 & 3 & & & \\
\hline & 22714 & 151.2500 & & & & & \\
\hline & 22715 & 149 . & & 290.5 & 4 & & \\
\hline & 22716 & 135.2591 & 4.10 & 291.1 & 4 & & \\
\hline & 22717 & 119.2507 & 4 . & 4 & 4 & & \\
\hline & 22718 & 122.2515 & & & 4 & & \\
\hline & 22719 & 7670 & 750 & 29 & 4 & & \\
\hline & 22720 & 133.5000 & 2.7250 & 290 & & 0 & \\
\hline & 22721 & 115.9904 & 21 & & & & \\
\hline & 22722 & 7020 & 170 & 291.1 & 6 & & \\
\hline & 22723 & 83.4997 & 2.70 & 291.6 & & 0 & \\
\hline & 22724 & 87.2489 & & & & & \\
\hline & 2281 & 120 & 0 & 29 & 4 & & \\
\hline & 2282 & 148.5006 & .4000 & 291.3 & 4 & & 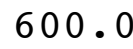 \\
\hline & 2283 & 130.9993 & 0 & 8 & 4 & & \\
\hline 2 & 2284 & 116 . & 0 & 29 & 4 & & \\
\hline & 2285 & 119.0101 & 0 & 290.2 & 4 & & 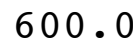 \\
\hline & 2286 & 123.2340 & 0 & 289.7 & 4 & & 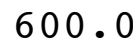 \\
\hline & 2287 & 124.5907 & 0 & $2 \varepsilon$ & 4 & & \\
\hline & 2288 & 147 . & & 28 & 4 & & 0 . \\
\hline 2 & 2289 & 140.7493 & 250 & 28 & 4 & & \\
\hline & 22810 & 120 & & & 4 & & \\
\hline & 22811 & 152 . & & .5 & 4 & & \\
\hline & 22812 & 151.5000 & 4250 & 288.6 & 4 & & \\
\hline 2 & 22813 & 146.2575 & 7 . & 289 & 4 & & \\
\hline 02 & 22814 & 159.2494 & 9.8500 & 290.0 & 4 & & 0 . \\
\hline & 22815 & 161.7460 & 8.2500 & 290.9 & 4 & & \\
\hline 2 & 22816 & 159 . & 7 . & 291.2 & 4 & & \\
\hline 2 & 22817 & 160.9973 & 6.1250 & 291.2 & 4 & & 0 . \\
\hline & 22818 & 136.8161 & 4.4000 & 290.9 & 5 & & 0 \\
\hline 12 & 22819 & 128.3266 & 500 & 290 & 4 & & \\
\hline 02 & 22820 & 146.2495 & & 290.3 & 4 & & \\
\hline & 22821 & 148.4995 & 6.6500 & 290.0 & 4 & 60 & 00 . \\
\hline & 22822 & 15 & & & 4 & & \\
\hline & 22823 & 169.2489 & 9.3500 & 287.6 & & 600.0 & 60 \\
\hline
\end{tabular}




\begin{tabular}{|c|c|c|c|c|c|c|c|c|}
\hline & & & & & & & & \\
\hline & 3 & 1 & 0 & 0 & 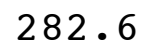 & & & \\
\hline & 3 & 12 & 61.5000 & 5.4250 & 81.2 & & 00 & \\
\hline & 3 & 13 & 52.4973 & 2.2750 & 280.7 & & ro & \\
\hline & 3 & 14 & & & & & & \\
\hline & 3 & 15 & & 0 & 79.1 & & & \\
\hline & 2 & 16 & 51.9998 & 1.3250 & 278.6 & & 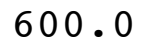 & \\
\hline & $\mathrm{c}$ & 17 & 997 & & 77.9 & & & \\
\hline & 3 & 18 & 0 & 0 & 77.9 & & & \\
\hline & 3 & 19 & 55.2495 & 0250 & 78.9 & & 0 & \\
\hline & 3 & 110 & 2501 & 0 & 280.2 & & & \\
\hline & 3 & 111 & 157 & 0 & 81.7 & & & \\
\hline 2 & 3 & 112 & 74.24 & 0 & 82.7 & & 0 & \\
\hline 2 & 3 & 11. & 7505 & 0 & 283.7 & & & \\
\hline & 3 & 114 & & & & & & \\
\hline 2 & 3 & 11 & & & 285 & & & \\
\hline 2 & 3 & 116 & 2523 & 0 & 286.2 & & .0 & \\
\hline & 3 & 117 & 5 & & 7 & & & \\
\hline & 3 & 118 & & & 4 & 4 & & \\
\hline & 3 & 119 & 0 & & & 1 & & \\
\hline 2 & 3 & 120 & 7.7508 & 0 & & 1 & . 0 & \\
\hline & 3 & 121 & & & & 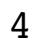 & & \\
\hline 2 & 3 & 122 & 8 & 0 & 0 & 4 & & \\
\hline 2 & 3 & 123 & 6 & 0 & & 4 & 0 & \\
\hline & 3 & 124 & & & & & & \\
\hline 02 & 3 & 21 & 0 & & & 4 & & \\
\hline 0 & 3 & 22 & 1.9999 & 500 & 9 & 4 & .0 & \\
\hline & 3 & 23 & 1 & 0 & & 4 & & \\
\hline 02 & 3 & 24 & 3 & 0 & 3 & 4 & & \\
\hline 02 & 3 & 25 & 0 & & & 4 & & \\
\hline 02 & 3 & 26 & 21.49 & & & 4 & & \\
\hline 02 & 3 & 27 & 9 & & & 4 & & \\
\hline 02 & 3 & 28 & 1 & & & - & & \\
\hline 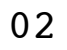 & 3 & 29 & 5 & 0 & & 1 & & \\
\hline 22 & 3 & 210 & & & & 4 & & \\
\hline . & 3 & 211 & & & & 3 & & \\
\hline 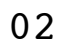 & 3 & 212 & 168.00 & 0500 & 283.4 & 3 & & \\
\hline 2 & 3 & 213 & 162 . & 0 & & 3 & & \\
\hline 02 & 3 & 214 & 177 . & 4.5750 & & 3 & .0 & \\
\hline 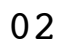 & 3 & 215 & 175.5129 & 1500 & 286.8 & 3 & & \\
\hline 02 & 3 & 216 & 161 & 0 & & 2 & & \\
\hline 02 & 3 & 217 & 164.7701 & 750 & & 3 & & \\
\hline 02 & 3 & 218 & 151.7494 & 3.1250 & 286.9 & 4 & & 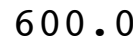 \\
\hline 02 & 3 & 219 & & & & 4 & & \\
\hline 02 & 3 & 220 & 120.5918 & & & & & \\
\hline 02 & 3 & 221 & 47.2801 & 0.2250 & 285.5 & 6 & .0 & 00 \\
\hline & 3 & 222 & & & & 6 & & \\
\hline & & 223 & 37.2169 & 1.0000 & 285.4 & & .0 & 600 \\
\hline
\end{tabular}




\begin{tabular}{|c|c|c|c|c|c|c|c|c|}
\hline 02 & 3 & 224 & 17.0024 & 0.9000 & 285.5 & 6 & 600.0 & 600.0 \\
\hline 02 & 3 & 31 & 1.8508 & 0.3250 & 284.9 & 6 & 600.0 & 600.0 \\
\hline 02 & 3 & 32 & 270.6367 & 0.1750 & 284.5 & 6 & 600.0 & 00.0 \\
\hline 02 & 3 & 33 & 27.7445 & 1.0750 & 284.9 & 6 & 600.0 & 500.0 \\
\hline 02 & 3 & 34 & 75.4779 & 0.3250 & 284.3 & 6 & 600.0 & 00.0 \\
\hline 02 & 3 & 35 & 138.6674 & 0.5000 & 284.1 & 5 & 600.0 & 00.0 \\
\hline 02 & 3 & 36 & 72.4391 & 0.4000 & 283.5 & 6 & 600.0 & 600.0 \\
\hline 02 & 3 & 37 & 56.0095 & 0.2750 & 284.1 & 6 & 600.0 & 00.0 \\
\hline 02 & 3 & 38 & 140.2499 & 1.1500 & 284.3 & 5 & 600.0 & 600.0 \\
\hline 02 & 3 & 39 & 155.9770 & 0.8500 & 285.6 & 4 & 600.0 & 600.0 \\
\hline 02 & 3 & 310 & 250.5167 & 0.8250 & 287.8 & 3 & 600.0 & \\
\hline 02 & 3 & 311 & 250.0134 & 1.7000 & 287.1 & 2 & 600.0 & 600.0 \\
\hline 02 & 3 & 312 & 256.2450 & 1.8750 & 287.7 & 1 & 600.0 & 60 \\
\hline 02 & 3 & 313 & 253.0019 & 1.9750 & 288.0 & 1 & 600.0 & 600.0 \\
\hline 02 & 3 & 314 & 228.4696 & 2.2000 & 289.3 & 1 & 600.0 & 60 \\
\hline 02 & 3 & 315 & 228.4954 & 2.7000 & 289.2 & 2 & 600.0 & 60 \\
\hline 02 & 3 & 316 & 232.7287 & 2.4250 & 289.6 & 1 & 600.0 & .0 \\
\hline 02 & 3 & 317 & 230.4937 & 2.0000 & 289.6 & 2 & 600.0 & .0 \\
\hline 02 & 3 & 318 & 296.5255 & 1.4000 & 289.2 & 3 & 600.0 & 60 \\
\hline 02 & 3 & 319 & 1.5172 & 1.3250 & 287.7 & 4 & 600.0 & 600.0 \\
\hline 02 & 3 & 320 & 23.7605 & 2.10 & 287.6 & 5 & .0 & \\
\hline 02 & 3 & 321 & 20.2383 & 1.6500 & 287.6 & 6 & 600.0 & 60 \\
\hline 02 & 3 & 322 & 16.4973 & 0.1000 & 286.4 & 5 & 600.0 & 600.0 \\
\hline 02 & 3 & 323 & 148.3363 & 1.1000 & 286.4 & 6 & 600.0 & 600.0 \\
\hline 02 & 3 & 324 & 150.7497 & 0.7500 & 286.1 & 5 & .0 & 60 \\
\hline 02 & 3 & 41 & 119.3223 & 0.0000 & 284.9 & 4 & 600.0 & 600.0 \\
\hline 02 & 3 & 42 & 102.2494 & .0000 & 284.6 & 4 & 600.0 & .0 \\
\hline 02 & 3 & 43 & 158.7623 & 0.7500 & 285.0 & 4 & 600.0 & 60 \\
\hline 02 & 3 & 44 & 110.8385 & 0.5250 & 284.6 & 5 & 600.0 & 600.0 \\
\hline 02 & 3 & 45 & 100.0409 & 8000 & 284.3 & 6 & 600.0 & .0 \\
\hline 02 & 3 & 46 & 151.5000 & 2 . & 284.2 & 5 & .0 & 60 \\
\hline 02 & 3 & 47 & 165.1494 & 1.3250 & 283.9 & 4 & 600.0 & 600.0 \\
\hline 02 & 3 & 48 & 42.0244 & 0.5500 & 284.3 & 3 & 600.0 & 600.0 \\
\hline 02 & 3 & 49 & 347.7546 & 0 . & 287.9 & 3 & 600.0 & 600.0 \\
\hline 02 & 3 & 410 & 331.8400 & 0.8250 & 289.1 & 2 & 600.0 & 600.0 \\
\hline 02 & 3 & 411 & 247.7425 & 2.1750 & 288.5 & 2 & 600.0 & 600.0 \\
\hline 02 & 3 & 412 & 187.7489 & 750 & 287.7 & 2 & 600.0 & 60 \\
\hline 02 & 3 & 413 & 213.2491 & 2.7500 & 288.4 & 1 & 600.0 & 600.0 \\
\hline 02 & 3 & 414 & 217.7508 & 2.8000 & 289.2 & 1 & 600.0 & 600.0 \\
\hline 02 & 3 & 415 & 229.5150 & 000 & 289.5 & 1 & 600.0 & 600.0 \\
\hline 02 & 3 & 416 & 245.2657 & 1.8750 & 290.2 & 1 & 600.0 & 600.0 \\
\hline 02 & 3 & 417 & 228.7426 & 1.8500 & 289.8 & 2 & 600.0 & 600.0 \\
\hline 02 & 3 & 418 & 296.2406 & 1.0250 & 289.5 & 3 & 600.0 & 600.0 \\
\hline 02 & 3 & 419 & 352.7107 & 0.7000 & 288.0 & 4 & 600.0 & 600.0 \\
\hline 02 & 3 & 420 & 17.2654 & 1.3500 & 287.7 & 4 & 600.0 & 600.0 \\
\hline 02 & 3 & 421 & 60.4469 & 3.5000 & 287.4 & 4 & 600.0 & 600.0 \\
\hline 02 & 3 & 422 & 77.7500 & 6.6000 & 286.1 & 4 & 600.0 & 600.0 \\
\hline 02 & 3 & 423 & 79.7499 & 7.7500 & 285.2 & 4 & 600.0 & 600.0 \\
\hline
\end{tabular}




\begin{tabular}{|c|c|c|c|c|c|c|c|c|}
\hline 02 & 3 & 424 & 77.7500 & 8.2750 & 284.8 & 4 & 600.0 & 600.0 \\
\hline 02 & 3 & 51 & 78.0006 & 10.2500 & 282.5 & 4 & 600.0 & 600.0 \\
\hline 02 & 3 & 52 & 77.5043 & 9.8000 & 282.5 & 4 & 600.0 & 00.0 \\
\hline 02 & 3 & 53 & 77.5000 & 9.6500 & 282.3 & 4 & 600.0 & 00.0 \\
\hline 02 & 3 & 54 & 74.7500 & 9.9250 & 281.9 & 4 & 600.0 & 00.0 \\
\hline 02 & 3 & 55 & 71.2801 & 6.6000 & 282.4 & 4 & 600.0 & 00.0 \\
\hline 02 & 3 & 56 & 82.2501 & 7.8750 & 282.0 & 4 & 600.0 & 00.0 \\
\hline 02 & 3 & 57 & 78.2492 & 9.0250 & 281.0 & 4 & 600.0 & 00.0 \\
\hline 02 & 3 & 58 & 78.2504 & 7.4000 & 281.8 & 4 & 600.0 & 00.0 \\
\hline 02 & 3 & 59 & 82.0048 & 5.4750 & 283.0 & 4 & 600.0 & 00.0 \\
\hline 02 & 3 & 510 & 20.7758 & 2.4250 & 285.1 & 3 & 600.0 & \\
\hline 02 & 3 & 511 & 271.0022 & 2.3750 & 285.7 & 2 & 600.0 & \\
\hline 02 & 3 & 512 & 191.5985 & 1.7000 & 286.4 & 1 & 600.0 & 00.0 \\
\hline 02 & 3 & 513 & 256.5000 & 1.9750 & 287.5 & 1 & .0 & 00.0 \\
\hline 02 & 3 & 514 & 230.4785 & 1.4750 & 289.1 & 1 & .0 & \\
\hline 02 & 3 & 515 & 265.0177 & 1.4750 & 289.4 & 1 & 600.0 & 00.0 \\
\hline 02 & 3 & 516 & 82.5059 & 2.3500 & 289.1 & 1 & 60 & .0 \\
\hline 02 & 3 & 517 & 38.3172 & 2.0250 & 288.7 & 1 & .0 & \\
\hline 02 & 3 & 518 & 315.2517 & 2.1500 & 287.8 & 2 & 600.0 & .0 \\
\hline 02 & 3 & 519 & 248.6269 & 1.5000 & 287.3 & 3 & 60 & .0 \\
\hline 02 & 3 & 520 & 144.3820 & 1.8500 & 285.5 & 4 & 60 & \\
\hline 02 & 3 & 521 & 356.2542 & 1.3000 & 285.4 & 5 & .0 & \\
\hline 02 & 3 & 522 & 350.2377 & 1.6250 & 285.5 & 5 & 600.0 & 00.0 \\
\hline 02 & 3 & 523 & 212.2473 & 0.8750 & 285.1 & 6 & 60 & \\
\hline 02 & 3 & 524 & 142.9738 & 0.5500 & 284.7 & 6 & .0 & \\
\hline 02 & 3 & 61 & 177.4121 & 0.4500 & 284.7 & 6 & 600.0 & 600.0 \\
\hline 02 & 3 & 62 & 303.8060 & 0.9750 & 284.6 & 6 & 600.0 & .0 \\
\hline 02 & 3 & 63 & 321.1945 & 1.1250 & 285.3 & 6 & 0 & \\
\hline 02 & 3 & 64 & 238.6307 & 1.1250 & 285.2 & 6 & 600.0 & 600.0 \\
\hline 02 & 3 & 65 & 258.4432 & 1.2000 & 285.6 & 6 & 600.0 & 600.0 \\
\hline 02 & 3 & 66 & 331.0005 & 3.0000 & 286.6 & 5 & .0 & \\
\hline 02 & 3 & 67 & 353.7178 & 3.2250 & 286.4 & 4 & .0 & 600.0 \\
\hline 02 & 3 & 68 & 341.6872 & 2.2750 & 285.6 & 3 & 600.0 & 600.0 \\
\hline 02 & 3 & 69 & 331.7438 & 2.2750 & 285.7 & 2 & 600.0 & 0.0 \\
\hline 02 & 3 & 610 & 267.9238 & 2.02 & 285.2 & 1 & 600.0 & 600.0 \\
\hline 02 & 3 & 611 & 78.3683 & 5.5000 & 285.4 & 2 & 600.0 & 600.0 \\
\hline 02 & 3 & 612 & 34.2486 & 5.1500 & 287.0 & 3 & 600.0 & 600.0 \\
\hline 02 & 3 & 613 & 46.2310 & 2.5750 & 287.4 & 2 & 600.0 & 600.0 \\
\hline 02 & 3 & 614 & 324.2851 & 4.1000 & 287.5 & 3 & 600.0 & 600.0 \\
\hline 02 & 3 & 615 & 60.2760 & 5.7250 & 286.8 & 3 & 600.0 & 0.0 \\
\hline 02 & 3 & 616 & 47.5495 & 4.7000 & 286.6 & 3 & 600.0 & 600.0 \\
\hline 02 & 3 & 617 & 47.2150 & 3.4000 & 286.6 & 3 & 600.0 & 600.0 \\
\hline 02 & 3 & 618 & 25.3560 & 2.7000 & 285.5 & 4 & 600.0 & 600.0 \\
\hline 02 & 3 & 619 & 16.4692 & 1.9500 & 285.0 & 5 & 600.0 & 600.0 \\
\hline 02 & 3 & 620 & 49.6057 & 2.7250 & 284.7 & 6 & 600.0 & 600.0 \\
\hline 02 & 3 & 621 & 37.5000 & 3.3250 & 284.3 & 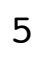 & 600.0 & 600.0 \\
\hline 02 & 3 & 622 & 88.0023 & 3.5500 & 283.2 & 5 & 600.0 & 600.0 \\
\hline 04 & 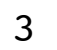 & 623 & 69.7512 & 4.850 & 283.3 & 4 & 600.0 & 600.0 \\
\hline
\end{tabular}




\begin{tabular}{|c|c|c|c|c|c|c|c|c|}
\hline 2 & 3 & 624 & 72.2501 & 4.4750 & 283.0 & 4 & 600.0 & 00.0 \\
\hline 02 & 3 & 71 & 1.7390 & 2.1750 & 282.5 & 5 & 600.0 & 600.0 \\
\hline 02 & 3 & 72 & 311.5268 & 1.4000 & 282.3 & 6 & 600.0 & 00.0 \\
\hline 02 & 3 & 73 & 224.2428 & 1.1000 & 281.9 & 6 & 600.0 & 600.0 \\
\hline 02 & 3 & 74 & 301.7512 & 1.4250 & 281.8 & 0 & 600.0 & 00.0 \\
\hline 02 & 3 & 75 & 321.8053 & 0.8000 & 281.6 & 6 & 600.0 & 00.0 \\
\hline 02 & 3 & 76 & 353.3859 & 1.1000 & 281.4 & 5 & 600.0 & 600.0 \\
\hline 02 & 3 & 77 & 340.3106 & 0.8250 & 281.5 & 6 & 600.0 & 00.0 \\
\hline 02 & 3 & 78 & 338.4076 & 1.5250 & 282.2 & 5 & 600.0 & 00.0 \\
\hline 02 & 3 & 79 & 332.2686 & 2.0750 & 282.8 & 4 & 600.0 & 600.0 \\
\hline 02 & 3 & 710 & 328.3493 & 1.6000 & 283.7 & 3 & 600.0 & .0 \\
\hline 02 & 3 & 711 & 14.4985 & 3.9500 & 284.9 & 2 & 600.0 & \\
\hline 02 & 3 & 712 & 6.7497 & 5.9500 & 285.8 & 3 & 600.0 & 600.0 \\
\hline 02 & 3 & 713 & 48.0282 & 6.1250 & 285.1 & 4 & 600.0 & 600.0 \\
\hline 02 & 3 & 714 & 65.5006 & 10 . & 282.9 & 4 & 600.0 & \\
\hline 02 & 3 & 715 & 56.7505 & 10 . & 280.7 & 4 & 600.0 & 60 \\
\hline 02 & 3 & 716 & 59.5120 & 15.2000 & 280.4 & 4 & 600.0 & 600.0 \\
\hline 02 & 3 & 717 & 67.9999 & 13. & 280.4 & 4 & 600.0 & .0 \\
\hline 02 & 3 & 718 & 67.4995 & 13.7250 & 280.0 & 4 & 600.0 & .0 \\
\hline 02 & 3 & 719 & 91.6478 & 13.5750 & 279.1 & 4 & 600.0 & 60 \\
\hline 02 & 3 & 720 & 91.0000 & 9 . & 278.7 & 4 & 600.0 & .0 \\
\hline 02 & 3 & 721 & 86.7501 & 10 . & 278.6 & 4 & 600.0 & \\
\hline 02 & 3 & 722 & 79.7499 & 7.6250 & 278.2 & 4 & 600.0 & 60 \\
\hline 02 & 3 & 723 & 80.7476 & 7.2000 & 277.8 & 4 & 600.0 & .0 \\
\hline 02 & 3 & 724 & 78.7499 & 7. & 277.1 & 4 & 600.0 & \\
\hline 02 & 3 & 81 & 75.2498 & 7.7500 & 276.5 & 4 & 600.0 & 60 \\
\hline 02 & 3 & 82 & 72.0002 & 7.6750 & 276.3 & 4 & 600.0 & .0 \\
\hline 02 & 3 & 83 & 77.4997 & 7. & 276.0 & 4 & 600.0 & \\
\hline 02 & 3 & 84 & 86.7504 & 750 & 276.1 & 4 & 600.0 & 60 \\
\hline 02 & 3 & 85 & 112.6437 & 4.0000 & 276.7 & 4 & 600.0 & 600.0 \\
\hline 02 & 3 & 86 & 145.2699 & 2 . & 276.6 & 4 & 600.0 & .0 \\
\hline 02 & 3 & 87 & 70.5733 & 750 & 276.4 & 5 & 600.0 & .0 \\
\hline 02 & 3 & 88 & 175.5000 & 0.9500 & 278.3 & 4 & 600.0 & 600.0 \\
\hline 02 & 3 & 89 & 169.5055 & 2 . & 279.3 & 3 & 600.0 & .0 \\
\hline 02 & 3 & 810 & 172.7459 & 4 . & 27 & 3 & 600.0 & 60 \\
\hline 02 & 3 & 811 & 162.7458 & 6.7000 & 279.1 & 4 & 600.0 & 600.0 \\
\hline 02 & 3 & 812 & 163.7513 & 6.0750 & 279.7 & 4 & 600.0 & \\
\hline 02 & 3 & 813 & 165.4997 & 5 . & 280.3 & 3 & 600.0 & 600.0 \\
\hline 02 & 3 & 814 & 167.4944 & 4.6000 & 281.1 & 3 & 600.0 & 600.0 \\
\hline 02 & 3 & 815 & 162.2549 & 0000 & 281.8 & 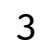 & 600.0 & \\
\hline 02 & 3 & 816 & 160.2366 & 3.1500 & 282.4 & - & 600.0 & 600.0 \\
\hline 02 & 3 & 817 & 165.2510 & 2.9250 & 282.5 & 3 & 600.0 & 600.0 \\
\hline 02 & 3 & 818 & 174.4995 & 2.6250 & 281.9 & 4 & 600.0 & 600.0 \\
\hline 02 & 3 & 819 & 161.5000 & 1.4000 & 280.6 & 5 & 600.0 & 600.0 \\
\hline 02 & 3 & 820 & 105.2789 & 0.4250 & 280.1 & 4 & 600.0 & 600.0 \\
\hline 02 & 3 & 821 & 8.0546 & 0.3500 & 279.6 & 5 & 600.0 & 600.0 \\
\hline 02 & 3 & 822 & 10.5001 & 1. & & 5 & 600.0 & 600.0 \\
\hline 02 & 3 & 823 & 355.6961 & 1.2500 & 279.7 & 4 & 600.0 & 600.0 \\
\hline
\end{tabular}




\begin{tabular}{|c|c|c|c|c|c|c|c|}
\hline 2 & 24 & & 1250 & 79.5 & 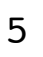 & (6. & \\
\hline 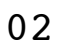 & $\begin{array}{lll}3 & 9 & 1\end{array}$ & 326.5217 & 2.3750 & 279.1 & & 600.0 & \\
\hline & $\begin{array}{lll}3 & 9 & 2\end{array}$ & 327.7530 & 0500 & 278.7 & & 00 & \\
\hline & $\begin{array}{lll}3 & 9 & 3\end{array}$ & 35.7555 & 1.6000 & 279.0 & & 00.0 & \\
\hline & $\begin{array}{lll}3 & 9 & 4\end{array}$ & 245.9419 & .7250 & 79.2 & & 0 & \\
\hline & 395 & 154.5000 & 500 & 278.8 & & 00 & \\
\hline & 396 & 332.9494 & 7250 & 278.7 & & 00 & \\
\hline & $\begin{array}{lll}3 & 9 & 7\end{array}$ & 16.9168 & .6500 & 279.5 & & 0 & \\
\hline & 398 & 8.7585 & .2750 & 80.3 & & & \\
\hline & $\begin{array}{lll}3 & 9 & 9\end{array}$ & 129.8081 & 2.2750 & 280.4 & & 00 & v \\
\hline & 3910 & 278.6325 & 0.7750 & 281.9 & & & \\
\hline & 3911 & 250.3616 & 1.2750 & 84.3 & & 0 & \\
\hline & 3912 & 279.1322 & 2.2000 & 284.9 & & 0 & \\
\hline & 3913 & 181.6406 & 3.4250 & 285.1 & & 0.0 & \\
\hline & 3914 & 200 & 0 & & & & \\
\hline & 3915 & 182 . & 2 . & 285.8 & & & \\
\hline & 3916 & 154.2498 & .65 & 285.1 & & .0 & \\
\hline & 3917 & 126 . & 0 & 3 & & & \\
\hline & 3918 & 44 . & 2 & 6 & & & \\
\hline & 3919 & 258 . & 8250 & 3.8 & & & 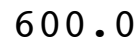 \\
\hline 2 & 3920 & 294.2363 & 0.4250 & 283.3 & & 0 & 0 \\
\hline & 3921 & 8.2383 & 11 & & & & \\
\hline & 3922 & 34.5219 & 750 & 282.8 & 6 & & \\
\hline$?$ & 3923 & 32.8679 & 3.6500 & $2 \varepsilon$ & 5 & 0 & ) \\
\hline & 3924 & 51.4758 & & & & & \\
\hline 2 & 3101 & 219 . & 1 & $2 \varepsilon$ & 5 & & \\
\hline & 3102 & 335.1739 & .9500 & 281.9 & 5 & 0 & J. \\
\hline & 3103 & 9.0307 & 0 & & 5 & & \\
\hline 2 & 3104 & 339.0007 & 0 & $2 \varepsilon$ & 4 & & • \\
\hline & 3105 & 317.5514 & 1.7250 & 0.9 & 5 & 0 & 0 . \\
\hline 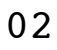 & 3106 & 72.3320 & 1500 & 2.7 & 6 & 0 & 0 . \\
\hline 2 & 3107 & 61.7790 & 0 & 3 & 6 & & \\
\hline & 3108 & 1104 & 500 & 282.2 & 5 & & J • \\
\hline 2 & 3109 & 90.5989 & 500 & 282.6 & 4 & 0 & \\
\hline 2 & 31010 & 79.2682 & 0 & & 3 & & \\
\hline 2 & 31011 & 95.6649 & 5000 & 4.3 & 2 & & . \\
\hline 2 & 31012 & 114.1142 & 4000 & 4.7 & 3 & & . \\
\hline 2 & 31013 & 155 . & 3 . & & 2 & & 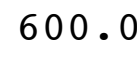 \\
\hline 2 & 31014 & 167.0000 & 4.1750 & 285.9 & 3 & .0 & 0 . \\
\hline 2 & 31015 & 175.1832 & 4500 & 285.8 & 2 & & \\
\hline 2 & 31016 & 197.0081 & 000 & $2 \varepsilon$ & 3 & & 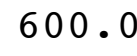 \\
\hline 2 & 31017 & 186.2498 & 000 & 285.8 & 3 & .0 & 0 . \\
\hline 2 & 31018 & 173.4760 & 4.0500 & 285.3 & 4 & 0 & 0 . \\
\hline 2 & 31019 & 160.5000 & 750 & & 5 & & \\
\hline 02 & 31020 & 133.5135 & 4.0250 & 284.6 & 4 & & 0 . \\
\hline 0 & 31021 & 85.2518 & 5.1250 & 283.7 & 4 & 600.0 & 600 \\
\hline & 310 & 8 & & & 4 & & \\
\hline & 31023 & 73.4997 & 7.4250 & 282.9 & 4 & 600.0 & 600 \\
\hline
\end{tabular}




\begin{tabular}{|c|c|c|c|c|c|c|c|}
\hline 02 & 31024 & 80.5004 & 5.5250 & 283.3 & 4 & 600.0 & 600.0 \\
\hline 02 & 3111 & 78.9966 & 5.1750 & 283.5 & 4 & 600.0 & 600.0 \\
\hline 02 & 3112 & 77.7497 & 6.4500 & 283.2 & 4 & 600.0 & 00.0 \\
\hline 02 & 3113 & 96.9671 & 3.8250 & 283.2 & 4 & 600.0 & 600.0 \\
\hline 02 & 3114 & 111.0039 & 3.0250 & 284.1 & 4 & 600.0 & 00.0 \\
\hline 02 & 3115 & 134.5097 & 2.6000 & 284.0 & 4 & 600.0 & 00.0 \\
\hline 02 & 3116 & 129.2013 & 2.6250 & 283.5 & 4 & 600.0 & 600.0 \\
\hline 02 & 3117 & 90.7497 & 3.5500 & 283.9 & 5 & 600.0 & 500.0 \\
\hline 02 & 3118 & 86.7332 & 2.7250 & 284.7 & 4 & 600.0 & 00.0 \\
\hline 02 & 3119 & 88.2099 & 1.9000 & 286.0 & 3 & 600.0 & 600.0 \\
\hline 02 & 31110 & 172.2638 & 1.7250 & 288.2 & 2 & 600.0 & .0 \\
\hline 02 & 31111 & 168.7499 & 4.1750 & 287.6 & 3 & 600.0 & \\
\hline 02 & 31112 & 171.5000 & 4.1250 & 288.0 & 4 & 600.0 & 600.0 \\
\hline 02 & 31113 & 167.4997 & 4.4000 & 288.9 & 4 & 600.0 & 600 \\
\hline 02 & 31114 & 170.5000 & & 289.1 & 4 & 600.0 & \\
\hline 02 & 31115 & 160.2386 & 500 & 289.3 & 4 & 600.0 & 60 \\
\hline 02 & 31116 & 147.7980 & 5.1500 & 288.6 & 3 & 600.0 & 600.0 \\
\hline 02 & 31117 & 111.0027 & 5.1000 & 287.9 & 4 & 600.0 & \\
\hline 02 & 31118 & 100.7552 & 4.1000 & 287.7 & 4 & 600.0 & 60 \\
\hline 02 & 31119 & 88.9945 & 5.4250 & 286.8 & 4 & 600.0 & 60 \\
\hline 02 & 31120 & 81.2473 & 7.55 & 286.7 & 4 & 600.0 & .0 \\
\hline 02 & 31121 & 71.0001 & 250 & 285.7 & 4 & 600.0 & \\
\hline 02 & 31122 & 67.4998 & 7.7500 & 285.3 & 4 & 600.0 & 600.0 \\
\hline 02 & 31123 & 67.4969 & 7.6000 & 285.7 & 4 & 600.0 & .0 \\
\hline 02 & 31124 & 81.7382 & 7.2000 & 286.5 & 4 & 600.0 & \\
\hline 02 & 3121 & 76.7439 & 7.5500 & 286.3 & 4 & 600.0 & 60 \\
\hline 02 & 3122 & 77.0002 & 7.1750 & 287.1 & 4 & 600.0 & 60 \\
\hline 02 & 3123 & 71.5010 & 8.27 & 285.7 & 4 & 600.0 & \\
\hline 02 & 3124 & 75.5000 & 8.3000 & 285.2 & 4 & 600.0 & 60 \\
\hline 02 & 3125 & 70.7501 & 8.7250 & 284.4 & 4 & 600.0 & 600.0 \\
\hline 02 & 3126 & 77.5000 & 8.10 & 284.4 & 4 & 600.0 & .0 \\
\hline 02 & 3127 & 76.2499 & 6.9250 & 284.9 & 4 & 600.0 & 60 \\
\hline 02 & 3128 & 77.7483 & 6.4000 & 285.6 & 4 & 600.0 & 600.0 \\
\hline 02 & 3129 & 80.7505 & 6.400 & 286.0 & 4 & 600.0 & .0 \\
\hline 02 & 31210 & 78.2521 & 6.5500 & 285.8 & 4 & 600.0 & 60 \\
\hline 02 & 31211 & 75.2392 & 7.8750 & 285.3 & 4 & 600.0 & 600.0 \\
\hline 02 & 31212 & 68.7514 & 7.4750 & 286.1 & 4 & 600.0 & \\
\hline 02 & 31213 & 66.0027 & 7.3500 & 286.6 & 4 & 600.0 & 600.0 \\
\hline 02 & 31214 & 83.9381 & 4.9000 & 286.8 & 3 & 600.0 & 600.0 \\
\hline 02 & 31215 & 78.9927 & 11.0250 & 285.9 & 4 & 600.0 & \\
\hline 02 & 31216 & 69.2492 & 11.3500 & 285.7 & 4 & 600.0 & 600.0 \\
\hline 02 & 31217 & 66.7497 & 11.2750 & 285.7 & 4 & 600.0 & 600.0 \\
\hline 02 & 31218 & 65.7500 & 14.1500 & 284.5 & 4 & 600.0 & 600.0 \\
\hline 02 & 31219 & 67.5001 & 14.9500 & 283.1 & 4 & 600.0 & 600.0 \\
\hline 02 & 31220 & 66.2500 & 15.4000 & 282.4 & 4 & 600.0 & 600.0 \\
\hline 02 & 31221 & 63.7497 & 14.1250 & 281.8 & 4 & 600.0 & 600.0 \\
\hline 02 & 31222 & 70.2222 & 11.8500 & 28 & 4 & 600.0 & 600.0 \\
\hline 02 & 31223 & 77.7502 & 7.7500 & 280.9 & 4 & 600.0 & 600.0 \\
\hline
\end{tabular}




\begin{tabular}{|c|c|c|c|c|c|c|c|}
\hline 02 & 31224 & 71.5023 & 8.3750 & 280.4 & 4 & 600.0 & 00.0 \\
\hline 02 & 3131 & 83.2493 & 8.1750 & 279.9 & 4 & 600.0 & 600.0 \\
\hline 02 & 3132 & 84.4881 & 6.4000 & 279.4 & 4 & 600.0 & 00.0 \\
\hline 02 & 3133 & 117.0000 & 5.1000 & 278.9 & 4 & 600.0 & 600.0 \\
\hline 02 & 3134 & 118.9767 & 4.8750 & 278.6 & 4 & 600.0 & 00.0 \\
\hline 02 & 3135 & 107.2656 & 7.3500 & 278.6 & 4 & 600.0 & 00.0 \\
\hline 02 & 3136 & 109.4601 & 6.6750 & 278.3 & 4 & 600.0 & 600.0 \\
\hline 02 & 3137 & 96.7505 & 6.8000 & 278.1 & 4 & 600.0 & 00.0 \\
\hline 02 & 3138 & 93.5003 & 6.9500 & 278.4 & 4 & 600.0 & 00.0 \\
\hline 02 & 3139 & 89.7260 & 8.6000 & 278.9 & 4 & 600.0 & 600.0 \\
\hline 02 & 31310 & 96.2508 & 7.5250 & 279.9 & 4 & 600.0 & .0 \\
\hline 02 & 31311 & 102.2501 & 8.2000 & 280.6 & 4 & 600.0 & \\
\hline 02 & 31312 & 108.5014 & 8.2750 & 281.4 & 4 & 600.0 & 600.0 \\
\hline 02 & 31313 & 113.0021 & 8.2000 & 282.0 & 4 & 600.0 & 600.0 \\
\hline 02 & 31314 & 112.9904 & 8.4000 & 282.8 & 4 & 600.0 & \\
\hline 02 & 31315 & 105.7413 & 8.1250 & 282.7 & 4 & 600.0 & 60 \\
\hline 02 & 31316 & 83.5000 & 7.7750 & 282.2 & 4 & 600.0 & 600.0 \\
\hline 02 & 31317 & 74.4731 & 8.8250 & 281.9 & 4 & 600.0 & \\
\hline 02 & 31318 & 70.5005 & 7.9750 & 281.1 & 4 & 600.0 & .0 \\
\hline 02 & 31319 & 87.2539 & 7.5000 & 280.5 & 4 & 600.0 & 600 \\
\hline 02 & 31320 & 87.0002 & 7.95 & 279.6 & 4 & 600.0 & .0 \\
\hline 02 & 31321 & 86.2499 & 8 . & 279.0 & 4 & 600.0 & \\
\hline 02 & 31322 & 83.0019 & 8.3750 & 278.7 & 4 & 600.0 & 600 \\
\hline 02 & 31323 & 74.2499 & 6. & 278.3 & 4 & 600.0 & .0 \\
\hline 02 & 31324 & 76.4994 & & 277.9 & 4 & 600.0 & \\
\hline 02 & 3141 & 79.0000 & 7.1000 & 277.8 & 4 & 600.0 & 60 \\
\hline 02 & 3142 & 85.2503 & 7.8500 & 277.9 & 4 & 600.0 & 60 \\
\hline 02 & 3143 & 79.7509 & $6.8=2.8$ & 277.6 & 4 & 600.0 & \\
\hline 02 & 3144 & 128.2102 & 6.4000 & 278.0 & 4 & 600.0 & 60 \\
\hline 02 & 3145 & 113.4478 & 5.90 & 277.8 & 4 & 600.0 & 600 \\
\hline 02 & 3146 & 124.9773 & 6 . & 277.8 & 4 & 600.0 & \\
\hline 02 & 3147 & 140.0000 & 7 . & 278.2 & 4 & 600.0 & 60 \\
\hline 02 & 3148 & 144.2515 & 9.8500 & 278.6 & 4 & 600.0 & 600.0 \\
\hline 02 & 3149 & 161.2503 & 11.2250 & 279.1 & 4 & 600.0 & .0 \\
\hline 02 & 31410 & 165.9993 & 12 . & 27 & 4 & 600.0 & .0 \\
\hline 02 & 31411 & 165.0015 & 11.9500 & 280.5 & 4 & 600.0 & 600.0 \\
\hline 02 & 31412 & 163.7511 & 11.4250 & 281.5 & 4 & 600.0 & \\
\hline 02 & 31413 & 160.5000 & 11.30 & 282.3 & 4 & 600.0 & 600.0 \\
\hline 02 & 31414 & 161.2485 & 10.6000 & 283.2 & 4 & 600.0 & 600.0 \\
\hline 02 & 31415 & 151.0005 & 9.2000 & 283.8 & 4 & 600.0 & \\
\hline 02 & 31416 & 149.2482 & 7.8250 & 284.4 & 4 & 600.0 & 600.0 \\
\hline 02 & 31417 & 144.5008 & 7.3250 & 284.3 & 4 & 600.0 & 600.0 \\
\hline 02 & 31418 & 125.4407 & 6.0000 & 284.0 & 4 & 600.0 & 600.0 \\
\hline 02 & 31419 & 114.6216 & 6.4000 & 282.9 & 4 & 600.0 & 600.0 \\
\hline 02 & 31420 & 89.0000 & 7.5500 & 281.7 & 4 & 600.0 & 600.0 \\
\hline 02 & 31421 & 85.5014 & 7.9500 & 281.6 & 4 & 600.0 & 600.0 \\
\hline 02 & 31422 & 77.9988 & 7 . & 280.8 & 4 & 600.0 & 600.0 \\
\hline 02 & 31423 & 81.9996 & 7.6750 & 280.7 & 4 & 600.0 & 600.0 \\
\hline
\end{tabular}




\begin{tabular}{|c|c|c|c|c|c|c|c|}
\hline 02 & 31424 & 73.0001 & 7.3750 & 280.4 & 4 & 600.0 & 00.0 \\
\hline 02 & 3151 & 71.7498 & 7.8000 & 279.9 & 4 & 600.0 & 600.0 \\
\hline 02 & 3152 & 77.2502 & 8.9250 & 279.6 & 4 & 600.0 & 00.0 \\
\hline 02 & 3153 & 81.2500 & 9.4500 & 279.4 & 4 & 600.0 & 600.0 \\
\hline 02 & 3154 & 83.4994 & 9.8500 & 279.0 & 4 & 600.0 & 00.0 \\
\hline 02 & 3155 & 74.7493 & 10.3500 & 278.3 & 4 & 600.0 & 00.0 \\
\hline 02 & 3156 & 68.7548 & 8.2750 & 278.2 & 4 & 600.0 & 600.0 \\
\hline 02 & 3157 & 69.7479 & 4.5000 & 277.7 & 4 & 600.0 & 00.0 \\
\hline 02 & 3158 & 61.7152 & 2.8000 & 278.4 & 3 & 600.0 & 00.0 \\
\hline 02 & 3159 & 282.0507 & 1.3250 & 280.0 & 2 & 600.0 & 600.0 \\
\hline 02 & 31510 & 222.4398 & 2.4000 & 279.8 & 1 & 600.0 & .0 \\
\hline 02 & 31511 & 37.9521 & 4.3250 & 281.7 & 2 & 600.0 & \\
\hline 02 & 31512 & 57.4872 & 6.2750 & 281.6 & 3 & 600.0 & 600.0 \\
\hline 02 & 31513 & 50.5170 & 6.5000 & 282.1 & 4 & 600.0 & 600 \\
\hline 02 & 31514 & 61.4994 & & 282.7 & 4 & 600.0 & \\
\hline 02 & 31515 & 59.2512 & 8.2250 & 282.2 & 4 & 600.0 & 60 \\
\hline 02 & 31516 & 60.0000 & 7.4250 & 281.6 & 4 & 600.0 & 600.0 \\
\hline 02 & 31517 & 64.2415 & 8. & 281.6 & 4 & 600.0 & \\
\hline 02 & 31518 & 65.7500 & 000 & 280.4 & 4 & 600.0 & .0 \\
\hline 02 & 31519 & 64.7505 & 9.7000 & 279.4 & 4 & 600.0 & 600 \\
\hline 02 & 31520 & 61.7499 & 9. & 278.9 & 4 & 600.0 & .0 \\
\hline 02 & 31521 & 68.5015 & 8 . & 278.8 & 4 & 600.0 & \\
\hline 02 & 31522 & 71.7511 & 8.3000 & 278.7 & 4 & 600.0 & 600 \\
\hline 02 & 31523 & 72.2509 & 6.72 & 278.6 & 4 & 600.0 & .0 \\
\hline 02 & 31524 & 71.0000 & & 278.3 & 4 & 600.0 & \\
\hline 02 & 3161 & 66.4994 & 9.1750 & 278.1 & 4 & 600.0 & 600.0 \\
\hline 02 & 3162 & 73.2491 & 7.2250 & 278.1 & 4 & 600.0 & 60 \\
\hline 02 & 3163 & 93.7492 & 5.12 & 277.9 & 4 & 600.0 & \\
\hline 02 & 3164 & 106.2499 & 5.3000 & 277.8 & 4 & 600.0 & 60 \\
\hline 02 & 3165 & 96.3301 & 6.35 & 277.4 & 4 & 600.0 & 600.0 \\
\hline 02 & 3166 & 71.7488 & 8 . & 277.0 & 4 & 600.0 & \\
\hline 02 & 3167 & 79.2551 & 7 . & 276.8 & 4 & 600.0 & 60 \\
\hline 02 & 3168 & 71.9862 & 8.3500 & 277.0 & 4 & 600.0 & 600.0 \\
\hline 02 & 3169 & 83.0000 & 7 . & 277.9 & 4 & 600.0 & .0 \\
\hline 02 & 31610 & 92.2499 & 7 . & 278.7 & 4 & 600.0 & 60 \\
\hline 02 & 31611 & 110.7391 & 8.1000 & 279.9 & 4 & 600.0 & 600.0 \\
\hline 02 & 31612 & 114.9997 & 8.6000 & 280.6 & 4 & 600.0 & \\
\hline 02 & 31613 & 129.7514 & 9.4250 & 281.2 & 4 & 600.0 & 600.0 \\
\hline 02 & 31614 & 132.0064 & 8.7000 & 282.0 & 4 & 600.0 & 600.0 \\
\hline 02 & 31615 & 104.9685 & 6.3250 & 282.0 & 4 & 600.0 & \\
\hline 02 & 31616 & 84.9353 & 6.4250 & 281.8 & 4 & 600.0 & 600.0 \\
\hline 02 & 31617 & 61.9984 & 8.1000 & 281.4 & 4 & 600.0 & 600.0 \\
\hline 02 & 31618 & 64.4985 & 8.7500 & 280.5 & 4 & 600.0 & 600.0 \\
\hline 02 & 31619 & 65.5000 & 10.9750 & 279.4 & 4 & 600.0 & 600.0 \\
\hline 02 & 31620 & 66.9998 & 10.8750 & 279.1 & 4 & 600.0 & 600.0 \\
\hline 02 & 31621 & 66.5002 & 10.3000 & 278.7 & 4 & 600.0 & 600.0 \\
\hline 02 & 31622 & 63.4999 & 9.525 & 278.6 & 4 & 600.0 & 600.0 \\
\hline 02 & 31623 & 61.5005 & 8.8250 & 278.5 & 4 & 600.0 & 600.0 \\
\hline
\end{tabular}




\begin{tabular}{|c|c|c|c|c|c|c|c|}
\hline 2 & 31624 & 62.5000 & 8.5000 & 278.5 & 4 & 600.0 & 00.0 \\
\hline 02 & 3171 & 0.6005 & 6.0000 & 278.5 & 4 & 600.0 & 600,0 \\
\hline 02 & 3172 & 70.4331 & 2.5500 & 278.2 & 5 & 600.0 & 00.0 \\
\hline 02 & 3173 & 59.9896 & 5.6250 & 277.8 & 4 & 600.0 & 00.0 \\
\hline 02 & 3174 & 91.4441 & 3.4000 & 278.3 & 4 & 600.0 & 00.0 \\
\hline 02 & 3175 & 98.7545 & 3.9500 & 278.4 & 4 & 600.0 & 00.0 \\
\hline 02 & 3176 & 80.9982 & 6.2000 & 278.4 & 4 & 600.0 & 00.0 \\
\hline 02 & 3177 & 48.5654 & 2.7750 & 278.1 & 5 & 600.0 & 00.0 \\
\hline 02 & 3178 & 345.9776 & 1.0500 & 277.3 & 4 & 600.0 & 00.0 \\
\hline 02 & 3179 & 318.5211 & 3.3750 & 276.4 & 3 & 600.0 & 600.0 \\
\hline 02 & 31710 & 264.3169 & 2.9250 & 276.3 & 3 & 600.0 & 0 \\
\hline 02 & 31711 & 243.9261 & 4.3 & 275.4 & 3 & 600.0 & \\
\hline 02 & 31712 & 326.7710 & 1.9500 & 274.9 & 3 & 600.0 & 00.0 \\
\hline 02 & 31713 & 332.2519 & 3.4750 & 273.4 & 4 & 600.0 & 0 \\
\hline 02 & 31714 & 341.9947 & & 274.8 & 4 & 600.0 & \\
\hline 02 & 31715 & 346.4945 & 5.2500 & 278.0 & 3 & 600.0 & 60 \\
\hline 02 & 31716 & 25.2763 & 2.4250 & 280.3 & 2 & 600.0 & 600.0 \\
\hline 02 & 31717 & 108.5605 & 4 & 279.8 & 3 & 600.0 & \\
\hline 02 & 31718 & 81.0084 & 00 & 278.6 & 4 & 600.0 & .0 \\
\hline 02 & 31719 & 209.1667 & 50 & 277.6 & 5 & 600.0 & .0 \\
\hline 02 & 31720 & 113.3761 & 5 . & 275.2 & 4 & 600.0 & .0 \\
\hline 02 & 31721 & 121.1550 & 6 . & 276.3 & 4 & 0.0 & \\
\hline 02 & 31722 & 124.0000 & 500 & 276.9 & 4 & 600.0 & 600.0 \\
\hline 02 & 31723 & 129.9983 & 7 . & 277.2 & 4 & 600.0 & \\
\hline 02 & 31724 & 123.7481 & & 277.9 & 4 & 00.0 & \\
\hline 02 & 3181 & 143.7497 & 12 . & 278.1 & 4 & 600.0 & 60 \\
\hline 02 & 3182 & 151.2500 & 11. & 277.3 & 4 & 600.0 & .0 \\
\hline 02 & 3183 & 150.5000 & 11 . & 276.7 & 4 & 0.0 & \\
\hline 02 & 3184 & 148.5000 & 12 . & 276.7 & 4 & 600.0 & 60 \\
\hline 02 & 3185 & 152.5000 & 14. & 276.5 & 4 & 600.0 & .0 \\
\hline 02 & 3186 & 153.0000 & 14 . & 276.6 & 4 & 600.0 & \\
\hline 02 & 3187 & 152.5000 & 14 . & 276.4 & 4 & 600.0 & .0 \\
\hline 02 & 3188 & 156.7499 & 14.0250 & 276.6 & 4 & 600.0 & 600.0 \\
\hline 02 & 3189 & 158.7511 & 12 . & 277.2 & 4 & 600.0 & .0 \\
\hline 02 & 31810 & 171.2498 & 11. & 278.3 & 4 & 600.0 & .0 \\
\hline 02 & 31811 & 172.9960 & 10.8500 & 279.2 & 4 & 600.0 & 600.0 \\
\hline 02 & 31812 & 173.4928 & 10.5500 & 280.4 & 4 & 600.0 & \\
\hline 02 & 31813 & 175.5014 & 8.3500 & 281.5 & 4 & 600.0 & 600.0 \\
\hline 02 & 31814 & 167.2505 & 7.6500 & 282.7 & 4 & 600.0 & 600.0 \\
\hline 02 & 31815 & 160.0019 & 7.250 & 283.4 & 4 & 600.0 & \\
\hline 02 & 31816 & 163.7585 & 5.7250 & 284.3 & 3 & 600.0 & 600.0 \\
\hline 02 & 31817 & 175.2515 & 5.9000 & 284.6 & 3 & 600.0 & 600.0 \\
\hline 02 & 31818 & 165.7537 & 2.7250 & 284.7 & 4 & 600.0 & 600.0 \\
\hline 02 & 31819 & 106.4321 & 0.6500 & 283.7 & 4 & 600.0 & 600.0 \\
\hline 02 & 31820 & 88.4553 & 1.5000 & 283.6 & 5 & 600.0 & 600.0 \\
\hline 02 & 31821 & 120.9988 & 1.2000 & 283.5 & 5 & 600.0 & 600.0 \\
\hline 02 & 31822 & 95.9979 & & 284.0 & 5 & 600.0 & 600.0 \\
\hline 02 & 31823 & 109.9376 & 2.875 & 283.7 & 4 & 600.0 & 600.0 \\
\hline
\end{tabular}




\begin{tabular}{|c|c|c|c|c|c|c|c|}
\hline 2 & 824 & 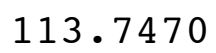 & 0 & & & & \\
\hline 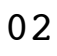 & 3191 & 28.2511 & .3000 & 3.6 & & 00.0 & \\
\hline & 3192 & 60.2617 & 1.2750 & 283.3 & & 00 & \\
\hline & 3193 & 123.2257 & 0.0500 & 282.5 & & 00 & \\
\hline & 3194 & 67.1093 & .1000 & 82.1 & & 0 & \\
\hline & 3195 & 65.1655 & 2500 & 82.1 & & 0 & \\
\hline & 3196 & 56.4504 & 000 & 281.7 & & 00 & \\
\hline & 3197 & 328.7638 & 0000 & 81.6 & & & \\
\hline & 3198 & 336.0359 & 1500 & 84.7 & & & \\
\hline & 3199 & 329.3211 & 500 & 85.8 & & 0 & U \\
\hline & 1910 & 287.7598 & 1.6250 & 286.2 & & 00.0 & \\
\hline & 1911 & 252.1578 & 1.92 & .9 & & & \\
\hline & 1912 & 228.5029 & 2.4750 & 86.1 & & 0 & \\
\hline & 1913 & 254.7794 & 2.3500 & 287.3 & & 0 & \\
\hline & 1914 & 305 & & .8 & & & \\
\hline & 31915 & 281 . & & 289.2 & & & \\
\hline & 31916 & 289.8241 & 2.9000 & 289.0 & & 0 & . \\
\hline & 31917 & 249 . & 000 & .7 & & & \\
\hline & 31918 & 285 . & & 287.3 & & & \\
\hline & 31919 & 324.6081 & 1.7750 & 285.5 & & .0 & 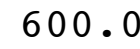 \\
\hline & 31920 & 341.2561 & 250 & .8 & & 0 & \\
\hline & 31921 & 5.7657 & & & & & \\
\hline & 31922 & 19 . & 0 & 28 & & & \\
\hline$?$ & 31923 & 46.5734 & 1.92 & .6 & & 0 & \\
\hline & 31924 & 120.7371 & & & & & \\
\hline 2 & 3201 & 161.42 & 0 & .1 & 4 & & \\
\hline & 3202 & 317.6975 & 27 & .6 & & 0 & J. \\
\hline & 3203 & 11.4488 & 5500 & .1 & & & \\
\hline 2 & 3204 & 20.7513 & 1.32 & .0 & 6 & & \\
\hline & 3205 & 13.0307 & 02 & .0 & & 0 & 0 . \\
\hline & 3206 & 294.9804 & .3250 & 5.1 & 6 & & 0 . \\
\hline 2 & 3207 & 238.3725 & 47 & & 6 & & \\
\hline & 3208 & 318.1066 & 0 & .1 & 5 & 0 & . \\
\hline 2 & 3209 & 150.2577 & 97 & .4 & 4 & 0 & 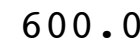 \\
\hline 2 & 32010 & 162.5010 & 0 & & 4 & & \\
\hline 2 & 32011 & 163.4997 & 4.6750 & .0 & 4 & & . \\
\hline 2 & 32012 & 160.0110 & 2250 & 284.8 & 3 & & 0 \\
\hline 2 & 32013 & 179.4960 & 2 . & .5 & 2 & & 0 . \\
\hline 02 & 32014 & 220.8685 & 1.3500 & 289.2 & 1 & .0 & 0 . \\
\hline 02 & 32015 & 227.9705 & 1.5500 & 289.6 & 1 & & 0 . \\
\hline 2 & 32016 & 258.0000 & 1.2250 & 29 & 2 & & 0 \\
\hline 2 & 32017 & 235.5041 & 1.1750 & 290.1 & 2 & & 0 . \\
\hline 2 & 32018 & 248.7354 & 0.4500 & 289.2 & 3 & 0 & 0 . \\
\hline 02 & 32019 & 334.5154 & 0.3500 & & 4 & & \\
\hline 02 & 32020 & 10.5046 & 1.5500 & & 4 & & 0 \\
\hline 02 & 32021 & 14.2512 & 1.3000 & 288.1 & 5 & & 00. \\
\hline & & 91 & & & 5 & & \\
\hline & 32023 & 67.3234 & 0.4000 & 287.7 & 0 & 600.0 & 600 \\
\hline
\end{tabular}




\begin{tabular}{|c|c|c|c|c|c|c|c|}
\hline 2 & 2024 & & 000 & 7.4 & & & \\
\hline & 3211 & 9876 & .5500 & 36.9 & & 0 & \\
\hline & 3212 & 118.2088 & 4750 & 286.4 & & 00 & \\
\hline & 3213 & 348.9976 & .6000 & 286.1 & & 00.0 & \\
\hline & 214 & 358.2205 & .2000 & 86.0 & & & \\
\hline & 3215 & 343.7690 & .8750 & 85.6 & & 0 & \\
\hline & 3216 & 355.5005 & 1250 & 285.3 & & 00 & \\
\hline & 3217 & 341.7453 & 1.4000 & 85.3 & & & \\
\hline & 3218 & 350.4088 & .2500 & .0 & & & \\
\hline & 3219 & 213.1355 & 0.2750 & 87.5 & & 0 & U \\
\hline & 32110 & 238.2678 & 0.8000 & 288.5 & & 00.0 & \\
\hline & 32111 & 232.2960 & 2.1750 & 88.2 & & & \\
\hline & 32112 & 233.2844 & 2.4250 & 288.3 & & 0 & \\
\hline & 32113 & 217.7223 & 2.0750 & 289 & & .0 & \\
\hline & 32114 & 155.7790 & 2 & 6 & & & \\
\hline & 32115 & 168.7511 & & 291.0 & & & \\
\hline & 32116 & 172.7473 & 4.3750 & 291.1 & & .0 & \\
\hline & 32117 & 126.7193 & 4 . & 90.8 & & & \\
\hline & 32118 & 90.7403 & & 289.1 & & & \\
\hline & 32119 & 78.0007 & 9000 & 287.1 & 4 & .0 & 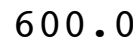 \\
\hline & 32120 & 81.5000 & 500 & 28 & & .0 & \\
\hline & 321 & 74.8221 & & & & & \\
\hline & 32122 & 77.0810 & 1.0500 & 287.0 & 5 & & \\
\hline 2 & 32123 & 80.5027 & 6.7250 & 284.9 & & 0 & \\
\hline & 32124 & 79.2501 & 0 & 8 & 4 & & \\
\hline & 3221 & 65.2728 & 2750 & 28 & 4 & & \\
\hline & 3222 & 71.7486 & 2000 & 284.7 & 4 & 0 & J. \\
\hline & 3223 & 81.0000 & 7.97 & 282.7 & 4 & & \\
\hline 2 & 3224 & 47.7359 & 250 & 282.6 & 4 & & \\
\hline & 3225 & 3.9874 & 2.32 & 280.8 & & 0 & U. \\
\hline & 3226 & 351.9976 & 2.9000 & 280.9 & 5 & & 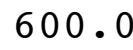 \\
\hline 2 & 3227 & 359.1847 & 0 & 281.0 & 6 & & \\
\hline & 3228 & 334.6272 & 1.5000 & 280.6 & 5 & & . \\
\hline 2 & 3229 & 342.4692 & 2.7250 & 281.2 & 4 & 0 & 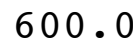 \\
\hline 2 & 32210 & 307.6565 & 2.17 & 282.2 & 3 & & \\
\hline 2 & & 275.5221 & 2.6500 & 283.7 & 2 & & 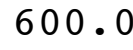 \\
\hline 2 & 32212 & 273.7552 & .3250 & 285.5 & 1 & & 0 . \\
\hline 2 & 32213 & 241.2197 & 2.12 & 286.2 & 1 & & \\
\hline 2 & 32214 & 213.2941 & 1.5750 & 284.8 & 1 & .0 & 0 . \\
\hline 2 & 32215 & 145.9422 & 0.4750 & 284.1 & 1 & & 0 . \\
\hline 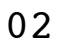 & 32216 & 199.2968 & 1.2000 & 285.0 & 1 & & \\
\hline 2 & 32217 & 240.0369 & 1.1750 & 284.5 & 1 & & 0 . \\
\hline 2 & 32218 & 176.3549 & 0.8000 & 284.5 & 2 & 0 & 0 . \\
\hline 2 & 32219 & 125.0122 & 1.4750 & 284.2 & 3 & & \\
\hline 02 & 32220 & 49.8936 & 4.1750 & 283.8 & 4 & & 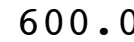 \\
\hline & 32221 & 46.7889 & 2.4000 & 283.6 & 5 & 600 & 600. \\
\hline & & 40.2576 & & & 4 & & \\
\hline & 32223 & 39.2454 & 3.6750 & 283.7 & 7 & 600.0 & 600 \\
\hline
\end{tabular}




\begin{tabular}{|c|c|c|c|c|c|c|c|}
\hline 02 & 32224 & 29.3636 & 1.3250 & 283.6 & 5 & 600.0 & 600.0 \\
\hline 02 & 3231 & 57.5066 & 4.2000 & 283.7 & 4 & 600.0 & 600.0 \\
\hline 02 & 3232 & 85.8551 & 6.6750 & 280.8 & 4 & 600.0 & 00.0 \\
\hline 02 & 3233 & 30.9930 & 2.3250 & 280.9 & 5 & 600.0 & 00.0 \\
\hline 02 & 3234 & 341.9710 & 2.7250 & 281.5 & 6 & 600.0 & 00.0 \\
\hline 02 & 3235 & 159.2805 & 1.1750 & 281.4 & 6 & 600.0 & 00.0 \\
\hline 02 & 3236 & 94.7136 & 0.9000 & 281.7 & 6 & 600.0 & 00.0 \\
\hline 02 & 3237 & 133.0020 & 1.4500 & 281.2 & 6 & 600.0 & 00.0 \\
\hline 02 & 3238 & 353.2902 & 1.4750 & 280.9 & 5 & 600.0 & 00.0 \\
\hline 02 & 3239 & 312.5771 & 1.0750 & 282.3 & 4 & 600.0 & 0 \\
\hline 02 & 32310 & 308.4627 & 1.1500 & 282.8 & 3 & 600.0 & \\
\hline 02 & 32311 & 305.1129 & 1.6750 & 284.3 & 2 & 600.0 & \\
\hline 02 & 32312 & 68.0245 & 4.1000 & 284.9 & 3 & 600.0 & 00.0 \\
\hline 02 & 32313 & 82.2299 & 4.1250 & 285.9 & 3 & 600.0 & 00.0 \\
\hline 02 & 32314 & 70.9613 & 5. & 285.6 & 3 & .0 & \\
\hline 02 & 32315 & 88.0453 & 500 & 285.3 & 4 & 600.0 & 00.0 \\
\hline 02 & 32316 & 67.0186 & 5.4250 & 285.0 & 3 & 60 & .0 \\
\hline 02 & 32317 & 80.7517 & 5. & 284.8 & 3 & .0 & \\
\hline 02 & 32318 & 83.9951 & 6.9250 & 283.8 & 4 & 60 & .0 \\
\hline 02 & 32319 & 85.2644 & 7.2750 & 282.8 & 4 & 60 & .0 \\
\hline 02 & 32320 & 91.2604 & 5.20 & 282.1 & 4 & 60 & \\
\hline 02 & 32321 & 86.0118 & 6.5750 & 281.7 & 4 & .0 & \\
\hline 02 & 32322 & 68.9987 & 6.5750 & 281.5 & 4 & 600.0 & 00.0 \\
\hline 02 & 32323 & 65.9998 & 8.87 & 281.4 & 4 & 60 & \\
\hline 02 & 32324 & 62.5000 & 10. & 281.0 & 4 & .0 & \\
\hline 02 & 3241 & 65.7521 & 9.3000 & 280.6 & 4 & 600.0 & 600.0 \\
\hline 02 & 3242 & 52.7690 & 8.8500 & 280.4 & 4 & 600.0 & .0 \\
\hline 02 & 3243 & 52.5094 & 9.7 & 280.1 & 4 & .0 & \\
\hline 02 & 3244 & 58.0000 & 9.0250 & 280.1 & 4 & .0 & 0.0 \\
\hline 02 & 3245 & 68.9627 & 8.3500 & 280.0 & 4 & 60 & 0.0 \\
\hline 02 & 3246 & 83.7507 & 7. & 279.9 & 4 & .0 & \\
\hline 02 & 3247 & 79.5014 & 8. & 279.7 & 4 & .0 & 600.0 \\
\hline 02 & 3248 & 78.4358 & 7.8250 & 280.3 & 4 & 600.0 & 600.0 \\
\hline 02 & 3249 & 84.7473 & 7.7250 & 280.9 & 4 & 0.0 & 0.0 \\
\hline 02 & 32410 & 76.7496 & 6.40 & 281.9 & 4 & 600.0 & 600.0 \\
\hline 02 & 32411 & 92.7535 & 6.0750 & 282.8 & 4 & 600.0 & 600.0 \\
\hline 02 & 32412 & 101.5038 & 5.82 & 283.5 & 3 & 600.0 & 600.0 \\
\hline 02 & 32413 & 109.0023 & 5.3750 & 284.1 & 3 & 600.0 & 600.0 \\
\hline 02 & 32414 & 130.5045 & 5.5250 & 284.7 & 3 & 600.0 & 600.0 \\
\hline 02 & 32415 & 116.2192 & 5.6500 & 285.3 & 3 & 600.0 & 600.0 \\
\hline 02 & 32416 & 123.9677 & 5.4250 & 285.3 & 3 & 600.0 & 600.0 \\
\hline 02 & 32417 & 97.2398 & 5.4000 & 284.5 & 3 & 600.0 & 600.0 \\
\hline 02 & 32418 & 79.2492 & 5.6250 & 283.6 & 4 & 600.0 & 600.0 \\
\hline 02 & 32419 & 71.7494 & 6.8000 & 282.1 & 4 & 600.0 & 600.0 \\
\hline 02 & 32420 & 74.2501 & 8.7000 & 281.2 & 4 & 600.0 & 600.0 \\
\hline 02 & 32421 & 77.0000 & 7.4000 & 281.4 & 4 & 600.0 & 600.0 \\
\hline 02 & 32422 & 78.5005 & 8.1750 & 281.2 & 4 & 600.0 & 600.0 \\
\hline 42 & 32423 & 82.2501 & 8.5750 & 280.6 & 4 & 600.0 & 600.0 \\
\hline
\end{tabular}




\begin{tabular}{|c|c|c|c|c|c|c|c|}
\hline 2 & 32424 & 77.7500 & 9.5250 & 280.0 & 4 & 600.0 & 600.0 \\
\hline 02 & 3251 & 78.5000 & 9.9500 & 279.6 & 4 & 600.0 & 600.0 \\
\hline 02 & 3252 & 75.9994 & 9.9500 & 279.4 & 4 & 600.0 & 00.0 \\
\hline 02 & 3253 & 81.5000 & 7.7250 & 280.0 & 4 & 600.0 & 00.0 \\
\hline 02 & 3254 & 82.0000 & 9.1750 & 279.3 & 4 & 600.0 & 00.0 \\
\hline 02 & 3255 & 80.2501 & 8.3250 & 279.4 & 4 & 600.0 & 00.0 \\
\hline 02 & 3256 & 83.2500 & 7.1000 & 279.5 & 4 & 600.0 & 00.0 \\
\hline 02 & 3257 & 78.7497 & 3.8250 & 280.2 & 4 & 600.0 & 00.0 \\
\hline 02 & 3258 & 84.9917 & 3.2500 & 280.9 & 3 & 600.0 & 00.0 \\
\hline 02 & 3259 & 257.2498 & 250 & 282.0 & 2 & 600.0 & 0 \\
\hline 02 & 32510 & 260.5000 & 500 & 282.8 & 1 & 600.0 & 00.0 \\
\hline 02 & 32511 & 263.2237 & 500 & 283.1 & 1 & 600.0 & \\
\hline 02 & 32512 & 245.7565 & 2.2000 & 284.1 & 1 & 600.0 & 00.0 \\
\hline 02 & 32513 & 209.3340 & 2.4 & 285.1 & 1 & 600.0 & 00.0 \\
\hline 02 & 32514 & 223.9937 & 00 & 285.0 & 1 & .0 & \\
\hline 02 & 32515 & 203.0453 & 250 & 285.3 & 1 & 600.0 & 00.0 \\
\hline 02 & 32516 & 201.7641 & 2.3500 & 285.0 & 2 & 600.0 & 600.0 \\
\hline 02 & 32517 & 192.7502 & 2 . & 284.9 & 3 & .0 & \\
\hline 02 & 32518 & 194.7501 & 2.1500 & 284.7 & 4 & 600.0 & .0 \\
\hline 02 & 32519 & 158.8202 & 1.3750 & 284.3 & 4 & 60 & .0 \\
\hline 02 & 32520 & 136.7585 & 0 . & 283.7 & 4 & 60 & \\
\hline 02 & 32521 & 140.9157 & 0 . & 283.2 & 4 & .0 & \\
\hline 02 & 32522 & 149.7518 & 2.6000 & 283.1 & 5 & 600.0 & 0.0 \\
\hline 02 & 32523 & 150.0019 & 1. & 2.8 & 5 & 60 & .0 \\
\hline 02 & 32524 & 131.4219 & 0 . & 282.6 & 6 & .0 & \\
\hline 02 & 3261 & 134.4806 & 0.3250 & 282.5 & 6 & 600.0 & 600.0 \\
\hline 02 & 3262 & 218.8390 & 0.3000 & 282.1 & 6 & 600.0 & .0 \\
\hline 02 & 3263 & 26.2391 & 1.4 & 282.8 & 5 & 0 & \\
\hline 02 & 3264 & 59.0346 & 1.0250 & 283.1 & 5 & .0 & 600.0 \\
\hline 02 & 3265 & 137.7521 & 1. & 282.9 & 5 & .0 & 0.0 \\
\hline 02 & 3266 & 143.2501 & 2 . & 282.0 & 5 & .0 & \\
\hline 02 & 3267 & 143.5000 & & 281.6 & 5 & .0 & 600.0 \\
\hline 02 & 3268 & 139.2528 & 1.3000 & 283.7 & 4 & 600.0 & 600.0 \\
\hline 02 & 3269 & 170.0000 & 3.2250 & 284.9 & 3 & 600.0 & 0.0 \\
\hline 02 & 32610 & 175.5021 & 4.2500 & 284.7 & 3 & 600.0 & 600.0 \\
\hline 02 & 32611 & 171.7495 & 3.7250 & 285.9 & 3 & 600.0 & 600.0 \\
\hline 02 & 32612 & 170.7519 & 3.6500 & 286.5 & 3 & 600.0 & 600.0 \\
\hline 02 & 32613 & 182.7423 & 3.5750 & 287.6 & 2 & 600.0 & 600.0 \\
\hline 02 & 32614 & 176.2487 & 3.9500 & 288.6 & 2 & 600.0 & 600.0 \\
\hline 02 & 32615 & 163.0000 & 500 & 289.0 & 3 & 600.0 & 600.0 \\
\hline 02 & 32616 & 168.4954 & 3.6500 & 289.5 & 2 & 600.0 & 600.0 \\
\hline 02 & 32617 & 168.8850 & 2.8750 & 289.8 & 3 & 600.0 & 600.0 \\
\hline 02 & 32618 & 169.7506 & 2.6750 & 289.3 & 4 & 600.0 & 600.0 \\
\hline 02 & 32619 & 147.2748 & 1.3750 & 287.9 & 5 & 600.0 & 600.0 \\
\hline 02 & 32620 & 126.3616 & 1.4000 & 287.4 & 4 & 600.0 & 600.0 \\
\hline 02 & 32621 & 98.9718 & & 288.0 & 4 & 600.0 & 600.0 \\
\hline 02 & 32622 & 112.0000 & 4.1250 & 287.9 & 5 & 600.0 & 600.0 \\
\hline $0<$ & 32623 & 82.7494 & 5.475 & 287.6 & 4 & 600.0 & 600.0 \\
\hline
\end{tabular}




\begin{tabular}{|c|c|c|c|c|c|c|c|}
\hline 02 & 32624 & 82.2511 & 4.3500 & 288.1 & 5 & 600.0 & 600.0 \\
\hline 02 & 3271 & 83.2498 & 3.6250 & 288.7 & כ & 600.0 & 00,0 \\
\hline 02 & 3272 & 78.7499 & 4.9250 & 288.7 & & 600.0 & 00.0 \\
\hline 02 & 3273 & 78.5000 & 3.5750 & 288.9 & 4 & 600.0 & 00.0 \\
\hline 02 & 3274 & 103.6754 & 1.2750 & 288.3 & 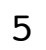 & 600.0 & 0.0 \\
\hline 02 & 3275 & 169.0242 & 1.4500 & 287.5 & 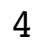 & 600.0 & 00.0 \\
\hline 02 & 3276 & 151.4998 & 3.4500 & 285.9 & 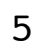 & 600.0 & 0 \\
\hline 02 & 3277 & 142.7482 & 3.1000 & 285.4 & 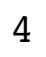 & 600.0 & 00.0 \\
\hline 02 & 3278 & 138.2931 & 3.6250 & 286.8 & 4 & 600.0 & 00.0 \\
\hline 02 & 3279 & 165.5110 & 3.4750 & 287.6 & 4 & .0 & \\
\hline 02 & 32710 & 166.9993 & 4.5250 & 287.5 & 4 & 600.0 & \\
\hline 02 & 32711 & 163.2498 & 4.4000 & 288.2 & 3 & .0 & \\
\hline 02 & 32712 & 164.0000 & 4.3500 & 288.8 & 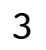 & 600.0 & \\
\hline 02 & 32713 & 161.2505 & 5.3750 & 289.3 & 3 & 600.0 & \\
\hline 02 & 32714 & 152.7499 & 5 . & 290.2 & 3 & .0 & \\
\hline 02 & 32715 & 152.7820 & 5.5500 & 291.1 & 3 & 600.0 & \\
\hline 02 & 32716 & 152.5001 & 5.8000 & 291.7 & 4 & 60 & .0 \\
\hline 02 & 32717 & 139.7586 & 4.5500 & 292.3 & 4 & .0 & \\
\hline 02 & 32718 & 143.5055 & 4.1000 & 292.2 & 5 & .0 & \\
\hline 02 & 32719 & 100.4008 & 4.7750 & 291.2 & 4 & .0 & .0 \\
\hline 02 & 32720 & 000 & 6. & 290.4 & 4 & 0 & \\
\hline 02 & 32721 & 76.4985 & 6.7500 & 289.9 & 4 & .0 & \\
\hline 02 & 32722 & 82.2545 & 7.4250 & 289.7 & 4 & 600.0 & .0 \\
\hline 02 & 32723 & 81.2506 & 7.05 & 289.4 & 4 & 0 & \\
\hline 02 & 32724 & 83.7506 & 7.3250 & 290.0 & 4 & 0 & \\
\hline 02 & 3281 & 117.6409 & 8.1250 & 291.2 & 4 & .0 & \\
\hline 02 & 3282 & 131.7476 & 8.2000 & 291.2 & 4 & 6 & \\
\hline 02 & 3283 & 145.7525 & 10.6750 & 289.9 & 4 & .0 & \\
\hline 02 & 3284 & 145.2500 & 11.0750 & 289.6 & 4 & .0 & \\
\hline 02 & 3285 & 144.0004 & 9.7000 & 289.5 & 4 & .0 & \\
\hline 02 & 3286 & 154.5000 & 11.0750 & 289.1 & 4 & 0 & \\
\hline 02 & 3287 & 156.7500 & 12.2250 & 288.4 & 4 & .0 & 0 \\
\hline 02 & 3288 & 159.2500 & 9.5000 & 288.5 & 4 & 600.0 & 0.0 \\
\hline 02 & 3289 & 165.5040 & 12.1750 & 289.4 & 4 & .0 & .0 \\
\hline 02 & 32810 & 161.9989 & 12.80 & 289.9 & 4 & 0 & 0.0 \\
\hline 02 & 32811 & 172.0000 & 12.3250 & 290.7 & 4 & 600.0 & 600.0 \\
\hline 02 & 32812 & 160.7395 & 10.57 & 291.6 & 4 & 600.0 & .0 \\
\hline 02 & 32813 & 161.7442 & 8.6500 & 292.8 & 4 & 600.0 & 600.0 \\
\hline 02 & 32814 & 162.7520 & 9.4750 & 294.0 & 4 & 600.0 & 600.0 \\
\hline 02 & 32815 & 166.9969 & 9.47 & 29 & 4 & 600.0 & \\
\hline 02 & 32816 & 170.5012 & 9.2500 & 296.1 & 4 & 600.0 & .0 \\
\hline 02 & 32817 & 156.9778 & 8.4750 & 296.2 & 4 & 600.0 & 600.0 \\
\hline 02 & 32818 & 134.7529 & 7.2000 & 295.9 & 4 & 600.0 & 600.0 \\
\hline 02 & 32819 & 127.2500 & 8.5750 & 295.5 & 4 & 600.0 & 600.0 \\
\hline 02 & 32820 & 131.2495 & 9.8000 & 295.4 & 4 & 600.0 & 600.0 \\
\hline 02 & 32821 & 136.2501 & 11.1250 & 295.2 & . & 600.0 & 600.0 \\
\hline 02 & 32822 & 148.7527 & 12.8500 & 295.1 & 4 & 600.0 & 600.0 \\
\hline 48 & 32823 & 157.0000 & 13.475 & 294.2 & 4 & 600.0 & 600.0 \\
\hline
\end{tabular}




\begin{tabular}{|c|c|c|c|c|c|c|c|}
\hline [ & 24 & & & & & & \\
\hline 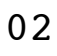 & 3291 & 9 & 2.3250 & 2.9 & & & \\
\hline & 3292 & 152.0002 & 2.6000 & 292.3 & & 00 & \\
\hline & 293 & 52.2503 & 2.4500 & 291.9 & & 0 & \\
\hline & 294 & 0.9989 & 00 & 91.2 & & & \\
\hline & 3295 & 7.7491 & & 90.6 & & 0 & \\
\hline & 3296 & 42.0016 & 3500 & 289.9 & & 0 & \\
\hline & 3297 & 38.5027 & 0 & 89.3 & & & \\
\hline & 3298 & 3.7500 & 0 & 89.2 & & & \\
\hline & 3299 & 47.7517 & 1.5750 & 89.4 & & 0 & \\
\hline & 2910 & 152.5000 & 0.8000 & 90.0 & & & \\
\hline & 2911 & 0.2556 & 8.6500 & 91.2 & & & \\
\hline & 32912 & 51.0005 & 7 . & 292.2 & 4 & 0 & \\
\hline & 2913 & 8.2461 & 6.52 & 93.2 & & $\bullet$ & \\
\hline & 2914 & 8 & & 5 & & & \\
\hline & 32915 & 8 & & 295.4 & 2 & & \\
\hline & 2916 & 139.2463 & 5.05 & 296.2 & 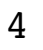 & .0 & \\
\hline & 2917 & 3.2494 & 4 . & .8 & & & \\
\hline & 2918 & 91.4734 & & 295.8 & & & \\
\hline & 2919 & 79 & 8 . & 294.0 & 4 & & \\
\hline & 32920 & 72.7499 & 9.00 & 293.3 & 4 & 0 & \\
\hline & 32921 & 72.5 & & & 4 & & \\
\hline & 32922 & 78 & 0 & 29 & 4 & & \\
\hline & 32923 & 84.7566 & 7 . & 294.6 & 4 & 0 & \\
\hline & 32924 & 86.7504 & & & 4 & & \\
\hline & 3301 & 96.5021 & & 29 & 4 & & \\
\hline & 3302 & 107.5000 & 0 & 296.1 & 4 & & . \\
\hline & 3303 & 2.7592 & 3. & & 4 & & \\
\hline & 3304 & 153. & 4. & 29 & 4 & & \\
\hline & 3305 & 157.2505 & & 292.4 & 4 & & 0 \\
\hline & 3306 & 136.5411 & 000 & 290.9 & 4 & & \\
\hline & 3307 & 123.2526 & 0 & & 4 & & \\
\hline & 3308 & 129.9963 & & .1 & 4 & & \\
\hline & 3309 & 130.5090 & 0 & 290.7 & 3 & & \\
\hline & 330 & 149.253 & & & 4 & & \\
\hline & & 154.5001 & & 291.8 & 4 & & \\
\hline & 33012 & 156.2503 & 500 & 292.8 & 4 & & \\
\hline 2 & 33013 & 157 . & 7. & & 4 & & \\
\hline 2 & 33014 & 155.5000 & & 294.9 & 4 & & . \\
\hline & 33015 & 141.0908 & 0 & 296.1 & 4 & & \\
\hline & 33016 & 141.7499 & 5 . & 29 & 4 & & \\
\hline & & 130.9975 & 4 . & & 3 & & 0 . \\
\hline & 33018 & 124.5207 & 5.3000 & 296.9 & 4 & & 0 \\
\hline 4 & 33019 & 89.2492 & 000 & & 4 & & \\
\hline 0 & 33020 & 73.7502 & 7.4250 & & 4 & & \\
\hline & 33021 & 73.9997 & 7.6500 & 294.2 & 4 & & 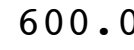 \\
\hline & & 74 & & & 4 & & \\
\hline & 33023 & 78.0002 & 7.2750 & 295.2 & & 600.0 & 60 \\
\hline
\end{tabular}




\begin{tabular}{|c|c|c|c|c|c|c|c|}
\hline 02 & 33024 & 87.7482 & 6.3000 & 295.7 & 4 & 600.0 & 00.0 \\
\hline 02 & 3311 & 81.2506 & 5.3750 & 296.3 & 4 & 600.0 & 600.0 \\
\hline 02 & 3312 & 118.6128 & 3.4250 & 296.1 & 4 & 600.0 & 00.0 \\
\hline 02 & 3313 & 91.2273 & 4.2250 & 296.5 & 4 & 600.0 & 600.0 \\
\hline 02 & 3314 & 135.5000 & 2.9250 & 292.8 & 4 & 600.0 & 00.0 \\
\hline 02 & 3315 & 155.2497 & 4.2000 & 293.4 & 4 & 600.0 & 000.0 \\
\hline 02 & 3316 & 133.6734 & 2.7000 & 293.3 & 4 & 600.0 & 600.0 \\
\hline 02 & 3317 & 144.2688 & 4.3750 & 292.7 & 4 & 600.0 & 00.0 \\
\hline 02 & 3318 & 120.5477 & 1.9500 & 293.1 & 3 & 600.0 & 00.0 \\
\hline 02 & 3319 & 146.0104 & 1.6500 & 293.1 & 2 & 600.0 & .0 \\
\hline 02 & 33110 & 153.2103 & 4.5500 & 294.0 & 3 & 600.0 & 600.0 \\
\hline 02 & 33111 & 157.4998 & 5.6 & 293.7 & 4 & 600.0 & \\
\hline 02 & 33112 & 158.5000 & 6.4000 & 293.8 & 4 & 600.0 & 600.0 \\
\hline 02 & 33113 & 156.0006 & 6.1000 & 295.1 & 4 & 600.0 & 600.0 \\
\hline 02 & 33114 & 155.5023 & 5.5250 & 295.9 & 3 & 600.0 & \\
\hline 02 & 33115 & 167.7530 & 5.8750 & 296.5 & 4 & 600.0 & 60 \\
\hline 02 & 33116 & 164.9984 & 6.0500 & 296.8 & 4 & 600.0 & 600.0 \\
\hline 02 & 33117 & 150.4999 & 6.1500 & 296.7 & 4 & 600.0 & \\
\hline 02 & 33118 & 144.5107 & 4.4250 & 296.3 & 5 & 600.0 & 60 \\
\hline 02 & 33119 & 133.3321 & 3.1750 & 295.4 & 4 & 600.0 & 600 \\
\hline 02 & 33120 & 90.2486 & 5. & 294.8 & 4 & 600.0 & .0 \\
\hline 02 & 33121 & 87.2516 & 6 . & 294.6 & 4 & 600.0 & \\
\hline 02 & 33122 & 79.2498 & 6.8500 & 294.1 & 4 & 600.0 & 600.0 \\
\hline 02 & 33123 & 80.7501 & 7.1500 & 294.1 & 4 & 600.0 & .0 \\
\hline 02 & 33124 & 79.0000 & 7.3750 & 293.8 & 4 & 600.0 & \\
\hline 02 & $\begin{array}{lll}4 & 1 & 1\end{array}$ & 80.2501 & 6.4750 & 294.4 & 4 & 600.0 & 600.0 \\
\hline 02 & $\begin{array}{lll}4 & 1 & 2\end{array}$ & 84.2500 & 5.2250 & 295.3 & 4 & 600.0 & 600.0 \\
\hline 02 & 413 & 104.6668 & 3. & 295.4 & 4 & 600.0 & .0 \\
\hline 02 & $\begin{array}{lll}4 & 1 & 4\end{array}$ & 140.2302 & 1.0500 & 294.5 & 4 & 600.0 & 600.0 \\
\hline 02 & 415 & 235.6919 & 0.4500 & 291.8 & 5 & 600.0 & 600.0 \\
\hline 02 & 416 & 0.3566 & 0.0000 & 291.7 & 6 & 600.0 & .0 \\
\hline 02 & $\begin{array}{lll}4 & 1 & 7\end{array}$ & 174.6805 & 1.2750 & 292.9 & 5 & 600.0 & 600.0 \\
\hline 02 & $\begin{array}{lll}4 & 1 & 8\end{array}$ & 162.7502 & 2.9750 & 292.6 & 4 & 600.0 & 600.0 \\
\hline 02 & 419 & 161.5131 & 3.7000 & 291.9 & 4 & 600.0 & .0 \\
\hline 02 & 4110 & 164.7499 & 4.9250 & 292.5 & 4 & 600.0 & 60 \\
\hline 02 & 4111 & 164.2486 & 5.5000 & 293.1 & 4 & 600.0 & 600.0 \\
\hline 02 & 4112 & 168.0002 & 5.1500 & 294.2 & 4 & 600.0 & 600 \\
\hline 02 & 4113 & 169.7501 & 5.1750 & 295.3 & 4 & 600.0 & 600.0 \\
\hline 02 & 4114 & 166.2500 & 5.2750 & 296.2 & 3 & 600.0 & 600.0 \\
\hline 02 & 4115 & 164.7533 & 4.8500 & 296.9 & 4 & 600.0 & \\
\hline 02 & 4116 & 161.4997 & 4.8500 & 297.6 & 4 & 600.0 & 600.0 \\
\hline 02 & $4 \quad 117$ & 159.4990 & 4.6000 & 297.9 & 4 & 600.0 & 600.0 \\
\hline 02 & 4118 & 147.3416 & 4.5000 & 296.8 & 3 & 600.0 & 600.0 \\
\hline 02 & 4119 & 92.2482 & 5.0750 & 296.2 & 4 & 600.0 & 600.0 \\
\hline 02 & 4120 & 78.0000 & 7.9750 & 294.9 & 4 & 600.0 & 600.0 \\
\hline 02 & 4121 & 80.0001 & 8.7500 & 294.4 & 4 & 600.0 & 600.0 \\
\hline 02 & 4122 & 76.0000 & 7.9500 & 294.1 & 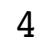 & 600.0 & 600.0 \\
\hline 02 & 4123 & 79.2499 & 9.1500 & 293.4 & 4 & 600.0 & 600.0 \\
\hline
\end{tabular}




\begin{tabular}{|c|c|c|c|c|c|c|c|c|}
\hline 2 & 4 & 124 & 76.7500 & 9.6000 & 293.2 & 4 & 600.0 & 600.0 \\
\hline 02 & 4 & 21 & 77.9998 & 9.4250 & 293.7 & 4 & 600.0 & 00.0 \\
\hline 02 & 4 & 22 & 79.4995 & 9.1000 & 293.9 & 4 & 600.0 & 00.0 \\
\hline 02 & 4 & 23 & 78.7498 & 9.4000 & 293.6 & 4 & 600.0 & 00.0 \\
\hline 02 & 4 & 24 & 73.0000 & 9.3250 & 292.8 & 4 & 600.0 & 00.0 \\
\hline 02 & 4 & 25 & 78.5000 & 8.5500 & 292.5 & 4 & 600.0 & 00.0 \\
\hline 02 & 4 & 26 & 95.4983 & 7.9750 & 292.5 & 4 & 600.0 & 00.0 \\
\hline 02 & 4 & 27 & 137.2450 & 6.7000 & 293.3 & 4 & 600.0 & 00.0 \\
\hline 02 & 4 & 28 & 158.2690 & 1.6750 & 292.9 & 3 & 600.0 & 00.0 \\
\hline 02 & 4 & 29 & 172.2500 & 500 & 292.8 & 4 & 600.0 & \\
\hline 02 & 4 & 210 & 158.2443 & 5.6250 & 293.1 & 4 & 600.0 & \\
\hline 02 & 4 & 211 & 159.4997 & 5.0500 & 293.6 & 4 & 600.0 & \\
\hline 02 & 4 & 212 & 153.2495 & 5.9750 & 294.0 & 4 & 600.0 & 00.0 \\
\hline 02 & 4 & 213 & 161.9995 & 5.4500 & 295.2 & 4 & .0 & .0 \\
\hline 02 & 4 & 214 & 121.7408 & 500 & 295.0 & 4 & .0 & \\
\hline 02 & 4 & 215 & 88.7502 & 7.5750 & 293.7 & 4 & 600.0 & 00.0 \\
\hline 02 & 4 & 216 & 84.7511 & 7.4500 & 293.7 & 4 & 600.0 & 0.0 \\
\hline 02 & 4 & 217 & 77.7496 & 8.5750 & 293.1 & 4 & .0 & \\
\hline 02 & 4 & 218 & 72.5000 & 9.6750 & 292.6 & 4 & .0 & 0 \\
\hline 02 & 4 & 219 & 76.2502 & 11.4000 & 290.6 & 4 & .0 & 0.0 \\
\hline 02 & 4 & 220 & 80.5000 & 11.5250 & 289.5 & 4 & .0 & \\
\hline 02 & 4 & 221 & 76.2501 & 11.0000 & 289.7 & 4 & .0 & .0 \\
\hline 02 & 4 & 222 & 76.7500 & 10.4250 & 290.8 & 4 & 600.0 & 0.0 \\
\hline 02 & 4 & 223 & 78.0000 & 9.75 & 290.9 & 4 & .0 & \\
\hline 02 & 4 & 224 & 75.0002 & 10.1000 & 289.9 & 4 & .0 & \\
\hline 02 & 4 & 31 & 76.5001 & 10.9500 & 288.7 & 4 & .0 & 0.0 \\
\hline 02 & 4 & 32 & 80.2500 & 11.6750 & 286.7 & 4 & .0 & 0.0 \\
\hline 02 & 4 & 33 & 81.5000 & 11.7250 & 285.8 & 4 & .0 & \\
\hline 02 & 4 & 34 & 82.0000 & 12.4000 & 284.9 & 4 & .0 & 0.0 \\
\hline 02 & 4 & 35 & 82.0000 & 12.2750 & 284.0 & 4 & 60 & \\
\hline 02 & 4 & 36 & 83.5000 & 9.5000 & 283.8 & 4 & .0 & \\
\hline 02 & 4 & 37 & 82.5000 & 8.5250 & 284.2 & 4 & .0 & 0.0 \\
\hline 02 & 4 & 38 & 84.7502 & 9.5750 & 283.3 & 4 & 600.0 & 0.0 \\
\hline 02 & 4 & 39 & 81.7500 & 9.2750 & 283.4 & 4 & 600.0 & 0.0 \\
\hline 02 & 4 & 310 & 84.0001 & 8.90 & 284.1 & 4 & .0 & 0.0 \\
\hline 02 & 4 & 311 & 89.0000 & 7.5000 & 285.2 & 4 & 600.0 & 600.0 \\
\hline 02 & 4 & 312 & 89.0000 & 6.9500 & 286.2 & 4 & .0 & \\
\hline 02 & 4 & 313 & 86.5000 & 6.8250 & 287.3 & 4 & 600.0 & 600.0 \\
\hline 02 & 4 & 314 & 88.2494 & 7.1250 & 288.2 & 4 & 600.0 & 600.0 \\
\hline 02 & 4 & 315 & 89.2499 & 6.5500 & 288.8 & 4 & 600.0 & \\
\hline 02 & 4 & 316 & 78.2495 & 7.6500 & 288.4 & 4 & 600.0 & 600.0 \\
\hline 02 & 4 & 317 & 78.0000 & 8.2250 & 288.0 & 4 & 600.0 & 600.0 \\
\hline 02 & 4 & 318 & 76.0000 & 9.0750 & 286.8 & 4 & 600.0 & 600.0 \\
\hline 02 & 4 & 319 & 75.9999 & 9.4000 & 285.2 & 4 & 600.0 & 600.0 \\
\hline 02 & 4 & 320 & 79.0000 & 10.0250 & 284.1 & 4 & 600.0 & 600.0 \\
\hline 02 & 4 & 321 & 80.7500 & 10.3250 & 283.6 & 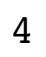 & 600.0 & \\
\hline 02 & 4 & 322 & 77.0000 & 10.8750 & 283.1 & 4 & 600.0 & 600.0 \\
\hline 42 & 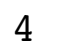 & 323 & 70.7498 & 10.700 & 282.8 & 4 & 600.0 & 600.0 \\
\hline
\end{tabular}




\begin{tabular}{|c|c|c|c|c|c|c|c|c|}
\hline 2 & 4 & 324 & 74.7500 & 10.0250 & 282.7 & 4 & 600.0 & 600.0 \\
\hline 02 & 4 & 41 & 76.0000 & 9.5250 & 282.7 & 4 & 600.0 & 600.0 \\
\hline 02 & 4 & 42 & 77.2501 & 8.9000 & 282.5 & 4 & 600.0 & 00.0 \\
\hline 02 & 4 & 43 & 77.5000 & 8.5000 & 282.3 & 4 & 600.0 & 00.0 \\
\hline 02 & 4 & 44 & 75.7500 & 8.9000 & 282.2 & 4 & 600.0 & 00.0 \\
\hline 02 & 4 & 45 & 77.5000 & 7.7750 & 282.1 & 4 & 600.0 & 00.0 \\
\hline 02 & 4 & 46 & 74.2500 & 7.1750 & 282.1 & 4 & 600.0 & 00.0 \\
\hline 02 & 4 & 47 & 75.0002 & 7.7250 & 282.1 & 4 & 600.0 & 00.0 \\
\hline 02 & 4 & 48 & 79.2500 & 7.8750 & 282.4 & 4 & 600.0 & 00.0 \\
\hline 02 & 4 & 49 & 80.7505 & 6.4000 & 283.3 & 4 & 600.0 & 0 \\
\hline 02 & 4 & 410 & 88.9984 & 5.7500 & 283.9 & 3 & 600.0 & \\
\hline 02 & 4 & 411 & 95.7710 & 4. & 284.4 & 3 & 600.0 & \\
\hline 02 & 4 & 412 & 70.8062 & 3.6250 & 286.2 & 2 & 600.0 & 00.0 \\
\hline 02 & 4 & 413 & 66.8815 & 3.7750 & 286.5 & 2 & 600.0 & 00.0 \\
\hline 02 & 4 & 414 & 69.2483 & 4. & 286.8 & 3 & .0 & \\
\hline 02 & 4 & 415 & 83.2527 & 5.5000 & 286.4 & 3 & 600.0 & 00.0 \\
\hline 02 & 4 & 416 & 88.4963 & 6.1750 & 286.2 & 4 & 60 & 00.0 \\
\hline 02 & 4 & 417 & 88.0011 & 6. & 285.4 & 4 & .0 & \\
\hline 02 & 4 & 418 & 82.7478 & 6.2750 & 284.9 & 4 & 600.0 & .0 \\
\hline 02 & 4 & 419 & 77.7511 & 5.5750 & 284.3 & 4 & .0 & .0 \\
\hline 02 & 4 & 420 & 73.5000 & 6. & 283.9 & 4 & 60 & \\
\hline 02 & 4 & 421 & 75.2498 & 5.7500 & 284.1 & 4 & .0 & \\
\hline 02 & 4 & 422 & 84.2506 & 5.1000 & 284.1 & 4 & 600.0 & 00.0 \\
\hline 02 & 4 & 423 & 18.7971 & 2.6 & 283.8 & 4 & 60 & .0 \\
\hline 02 & 4 & 424 & 284.5401 & 0.1750 & 283.4 & 5 & .0 & \\
\hline 02 & 4 & 51 & 12.7458 & 1.8500 & 283.0 & 4 & 600.0 & 600.0 \\
\hline 02 & 4 & 52 & 336.9586 & 0.9250 & 282.2 & 5 & 600.0 & .0 \\
\hline 02 & 4 & 53 & 354.5045 & 1.8500 & 281.5 & 4 & .0 & \\
\hline 02 & 4 & 54 & 4.6995 & 1.7250 & 281.5 & 4 & 600.0 & 600.0 \\
\hline 02 & 4 & 55 & 59.4650 & 1.7250 & 281.9 & 5 & 600.0 & 600.0 \\
\hline 02 & 4 & 56 & 79.5577 & 1.4250 & 282.3 & 5 & .0 & \\
\hline 02 & 4 & 57 & 36.3331 & 0.20 & 282.5 & 6 & .0 & 600.0 \\
\hline 02 & 4 & 58 & 306.7078 & 0.1500 & 283.0 & 5 & 600.0 & 600.0 \\
\hline 02 & 4 & 59 & 229.7379 & 0.4750 & 283.6 & 4 & 600.0 & 0.0 \\
\hline 02 & 4 & 510 & 255.2000 & 1.22 & 284.9 & 3 & 600.0 & 600.0 \\
\hline 02 & 4 & 511 & 241.1995 & 2.4000 & 285.7 & 2 & 600.0 & 600.0 \\
\hline 02 & 4 & 512 & 219.6368 & 2.4500 & 286.6 & 1 & 600.0 & 600.0 \\
\hline 02 & 4 & 513 & 180.2027 & 2.5250 & 286.3 & 2 & 600.0 & 600.0 \\
\hline 02 & 4 & 514 & 158.0206 & 0.8250 & 286.6 & 1 & 600.0 & 600.0 \\
\hline 02 & 4 & 515 & 69.7322 & 1.4000 & 287.2 & 1 & 600.0 & 600.0 \\
\hline 02 & 4 & 516 & 178.4798 & 3.1000 & 288.5 & 2 & 600.0 & 600.0 \\
\hline 02 & 4 & 517 & 140.9864 & 5.3000 & 286.7 & 3 & 600.0 & 600.0 \\
\hline 02 & 4 & 518 & 150.4994 & 6.1500 & 285.7 & 4 & 600.0 & 600.0 \\
\hline 02 & 4 & 519 & 134.0415 & 3.9250 & 285.0 & 4 & 600.0 & 600.0 \\
\hline 02 & 4 & 520 & 129.7394 & 3.1000 & 284.6 & 4 & 600.0 & 600.0 \\
\hline 02 & 4 & 521 & 86.4982 & 4.8750 & 284.7 & 4 & 600.0 & 600.0 \\
\hline 02 & 4 & 522 & 78.5005 & 6.4750 & 284.4 & 4 & 600.0 & 600.0 \\
\hline 42 & 4 & 523 & 71.2500 & 7.9000 & 283.9 & 4 & 600.0 & 600.0 \\
\hline
\end{tabular}




\begin{tabular}{|c|c|c|c|c|c|c|c|c|}
\hline 2 & 4 & 524 & 71.7501 & 7.5250 & 283.9 & 4 & 600.0 & 00.0 \\
\hline 2 & 4 & 61 & 3.7499 & 7.8500 & 283.7 & 4 & 600.0 & 600.0 \\
\hline 02 & 4 & 62 & 72.4997 & 8.6500 & 283.2 & 4 & 600.0 & 00.0 \\
\hline 02 & 4 & 63 & 70.2501 & 8.0500 & 283.3 & 4 & 600.0 & 00.0 \\
\hline 12 & 4 & 64 & 79.1869 & 6.5250 & 283.1 & 4 & 600.0 & 00.0 \\
\hline 02 & 4 & 65 & 82.4954 & 8.3750 & 282.6 & 4 & 600.0 & 00.0 \\
\hline 02 & 4 & 66 & 74.9996 & 7.7250 & 282.8 & 4 & 600.0 & 600.0 \\
\hline 02 & 4 & 67 & 75.2501 & 7.7750 & 282.8 & 4 & 600.0 & .0 \\
\hline 02 & 4 & 68 & 77.7499 & 7.1250 & 283.4 & 4 & 600.0 & \\
\hline 02 & 4 & 69 & 76.0000 & 7.2000 & 284.1 & 4 & 600.0 & 00.0 \\
\hline 02 & 4 & 610 & 86.2658 & 5.8750 & 285.5 & 3 & 600.0 & 0 \\
\hline 02 & 4 & 611 & 81.7508 & 6.2250 & 286.6 & 4 & 600.0 & \\
\hline 02 & 4 & 612 & 82.9981 & 6.2500 & 287.4 & 4 & 600.0 & .0 \\
\hline 02 & 4 & 613 & 100.2486 & 5.1250 & 288.1 & 3 & 600.0 & \\
\hline 02 & 4 & 614 & 13.2876 & 5.3000 & 288.9 & 3 & 600.0 & \\
\hline 02 & 4 & 615 & 119.9353 & 5.4250 & 289.5 & 3 & 600.0 & \\
\hline 02 & 4 & 616 & 90.5014 & 6.4000 & 289.2 & 4 & 600.0 & 600.0 \\
\hline 02 & 4 & 617 & 78.5010 & 7.02 & 288.6 & 4 & 600.0 & .0 \\
\hline 02 & 4 & 618 & 77.5000 & 7.7000 & 287.5 & 4 & 0.0 & \\
\hline 02 & 4 & 619 & 78.2518 & 8.9000 & 286.0 & 4 & 600.0 & \\
\hline 02 & 4 & 620 & 74.2500 & 10.6500 & 285.1 & 4 & 600.0 & .0 \\
\hline 02 & 4 & 621 & 79.2498 & 11.7500 & 283.7 & 4 & 600.0 & \\
\hline 02 & 4 & 622 & 79.5000 & 10.8750 & 284.2 & 4 & .0 & .0 \\
\hline 02 & 4 & 623 & 78.2500 & 10.4500 & 284.9 & 4 & 600.0 & .0 \\
\hline 02 & 4 & 624 & 78.7502 & 12.0500 & 284.0 & 4 & 600.0 & \\
\hline 02 & 4 & 71 & 79.7500 & 8.5750 & 285.9 & 4 & 600.0 & 60 \\
\hline 02 & 4 & 72 & 81.5000 & 9.1250 & 284.9 & 4 & 600.0 & .0 \\
\hline 02 & 4 & 73 & 81.5000 & 10.70 & 282.8 & 4 & 0.0 & .0 \\
\hline 02 & 4 & 74 & 80.5000 & 9.9500 & 282.7 & 4 & 600.0 & .0 \\
\hline 02 & 4 & 75 & 78.2501 & 8.1500 & 283.0 & 4 & 600.0 & 600.0 \\
\hline 02 & 4 & 76 & 102.5335 & 2.7000 & 283.7 & 5 & 600.0 & .0 \\
\hline 02 & 4 & 77 & 77.0002 & 3.6000 & 283.4 & 4 & 600.0 & .0 \\
\hline 02 & 4 & 78 & 238.3755 & 0.6500 & 286.1 & 3 & 600.0 & 600.0 \\
\hline 02 & 4 & 79 & 356.7000 & 1.5750 & 287.3 & 2 & 600.0 & .0 \\
\hline 02 & 4 & 710 & 279.1061 & 1.7500 & 288.9 & 1 & 0 & \\
\hline 02 & 4 & 711 & 234.0306 & 2.4000 & 289.4 & 1 & 600.0 & 600.0 \\
\hline 02 & 4 & 712 & 174.1856 & 4.5000 & 288.9 & 2 & 600.0 & 600.0 \\
\hline 02 & 4 & 713 & 160.9998 & 5.1500 & 289.8 & 3 & 600.0 & 600.0 \\
\hline 02 & 4 & 714 & 157.9989 & 5.4500 & 291.2 & 4 & 600.0 & 600.0 \\
\hline 02 & 4 & 715 & 162.5009 & 5.1250 & 292.4 & 3 & 600.0 & 600.0 \\
\hline 02 & 4 & 716 & 161.7446 & 4.7500 & 293.3 & 3 & 600.0 & 600.0 \\
\hline 02 & 4 & 717 & 145.2432 & 4.9750 & 293.3 & 4 & 600.0 & 600.0 \\
\hline 02 & 4 & 718 & 103.2490 & 3.9250 & 292.4 & 3 & 600.0 & 600.0 \\
\hline 02 & 4 & 719 & 88.7959 & 5.2500 & 291.0 & 4 & 600.0 & 600.0 \\
\hline 02 & 4 & 720 & 70.2467 & 7.3250 & 290.0 & 4 & 600.0 & 600.0 \\
\hline 02 & 4 & 721 & 74.5006 & 7.1750 & 289.5 & 4 & 600.0 & 600.0 \\
\hline 02 & 4 & 722 & 71.2498 & 7.37 & 28 & 4 & 600.0 & .0 \\
\hline 02 & 4 & 723 & 70.7499 & 7.3750 & 289.9 & 4 & 600.0 & 600.0 \\
\hline
\end{tabular}




\begin{tabular}{|c|c|c|c|c|c|c|c|c|}
\hline 02 & 4 & 724 & 71.7502 & 7.2500 & 289.9 & 4 & 600.0 & 00.0 \\
\hline 2 & 4 & 81 & 74.5000 & 7.5500 & 289.9 & $\Psi$ & 600.0 & 600.0 \\
\hline 02 & 4 & 82 & 69.5009 & 6.6000 & 290.6 & 4 & 600.0 & 00.0 \\
\hline 02 & 4 & 83 & 77.7485 & 5.8500 & 290.2 & 4 & 600.0 & 00.0 \\
\hline 02 & 4 & 84 & 84.6364 & .7500 & 288.5 & 5 & 600.0 & 00.0 \\
\hline 02 & 4 & 85 & 342.0123 & 0.8500 & 287.1 & 6 & 600.0 & 00.0 \\
\hline 02 & 4 & 86 & 9.0112 & 0.7000 & 286.8 & 5 & 600.0 & 600.0 \\
\hline 02 & 4 & 87 & 66.2260 & 0.9500 & 287.9 & 6 & 600.0 & 00.0 \\
\hline 02 & 4 & 88 & 207.4904 & 1.4000 & 288.1 & 5 & 600.0 & \\
\hline 02 & 4 & 89 & 265.4396 & 1.1000 & 290.3 & 4 & 600.0 & 600.0 \\
\hline 02 & 4 & 810 & 224.7647 & 2.3250 & 290.7 & 3 & 600.0 & 0 \\
\hline 02 & 4 & 811 & 157.1723 & 2.8250 & 291.3 & 2 & 600.0 & 00.0 \\
\hline 02 & 4 & 812 & 47.7367 & 2.6250 & 291.8 & 1 & 600.0 & 600.0 \\
\hline 02 & 4 & 813 & 66.2855 & 3.0250 & 293.2 & 2 & 600.0 & 0 \\
\hline 02 & 4 & 814 & 93.5015 & $5 \cdot$ & 292.1 & 3 & 600.0 & \\
\hline 02 & 4 & 815 & 79.5009 & 6 & 292.2 & 4 & 600.0 & \\
\hline 02 & 4 & 816 & 78.5000 & 8.2500 & 291.6 & 4 & 600.0 & 600.0 \\
\hline 02 & 4 & 817 & 79.0034 & 7. & 291.0 & 4 & 600.0 & .0 \\
\hline 02 & 4 & 818 & 82.9984 & 7.9000 & 289.3 & 4 & 600.0 & \\
\hline 02 & 4 & 819 & 77.4986 & 8.0750 & 289.1 & 4 & 600.0 & 60 \\
\hline 02 & 4 & 820 & 85.5021 & 6.1500 & 288.3 & 4 & 600.0 & 600.0 \\
\hline 02 & 4 & 821 & 80.5000 & 6.40 & 287.6 & 4 & 600.0 & \\
\hline 02 & 4 & 822 & 64.7384 & 7.7250 & 287.0 & 4 & 600.0 & 600.0 \\
\hline 02 & 4 & 823 & 75.3630 & 5.0500 & 286.6 & 4 & 600.0 & .0 \\
\hline 02 & 4 & 824 & 67.6569 & 3.0250 & 285.7 & 4 & 600.0 & \\
\hline 02 & 4 & 91 & 41.1696 & 1.1750 & 286.0 & 5 & 600.0 & .0 \\
\hline 02 & 4 & 92 & 96.2440 & 1.6500 & 286.0 & 6 & 600.0 & 600.0 \\
\hline 02 & 4 & 93 & 99.3886 & 1.70 & 283.7 & 6 & 600.0 & .0 \\
\hline 02 & 4 & 94 & 135.9183 & 1.2000 & 285.1 & 6 & 600.0 & 600.0 \\
\hline 02 & 4 & 95 & 130.1687 & 2.7500 & 284.1 & 6 & 600.0 & 600.0 \\
\hline 02 & 4 & 96 & 99.5121 & 2.1750 & 283.7 & 6 & 600.0 & 60 \\
\hline 02 & 4 & 97 & 64.5303 & 1.3750 & 284.9 & 6 & 600.0 & 600.0 \\
\hline 02 & 4 & 98 & 270.2900 & 0.7500 & 285.3 & 5 & 600.0 & 600.0 \\
\hline 02 & 4 & 99 & 276.7576 & 1.6500 & 284.3 & 4 & 600.0 & 600.0 \\
\hline 02 & 4 & 910 & 214.8121 & 1.7250 & 286.3 & 3 & 600.0 & 600.0 \\
\hline 02 & 4 & 911 & 209.3289 & 1.6750 & 286.7 & 2 & 600.0 & 600.0 \\
\hline 02 & 4 & 912 & 296.0069 & 1.7500 & 288.2 & 1 & 600.0 & 600.0 \\
\hline 02 & 4 & 913 & 242.3876 & 2.2750 & 288.9 & 1 & 600.0 & 600.0 \\
\hline 02 & 4 & 914 & 275.1206 & 2.4250 & 289.5 & 1 & 600.0 & 600.0 \\
\hline 02 & 4 & 915 & 225.2506 & 3.2750 & 288.4 & 2 & 600.0 & 600.0 \\
\hline 02 & 4 & 916 & 175.2092 & 4.4000 & 287.2 & 3 & 600.0 & 600.0 \\
\hline 02 & 4 & 917 & 166.0120 & 3.0250 & 287.2 & 4 & 600.0 & 600.0 \\
\hline 02 & 4 & 918 & 189.7648 & 2.9750 & 286.8 & 3 & 600.0 & 600.0 \\
\hline 02 & 4 & 919 & 164.7520 & 2.4000 & 286.5 & 4 & 600.0 & 600.0 \\
\hline 02 & 4 & 920 & 155.5092 & 2.6750 & 286.3 & 5 & 600.0 & 600.0 \\
\hline 02 & 4 & 921 & 129.3541 & 3.1000 & 286.1 & 4 & 600.0 & 600.0 \\
\hline 02 & 4 & 922 & 92.2620 & 3. & 28 & 4 & 600.0 & .0 \\
\hline 02 & 4 & 923 & 87.7470 & 4.6250 & 285.9 & 4 & 600.0 & 600.0 \\
\hline
\end{tabular}




\begin{tabular}{|c|c|c|c|c|c|c|c|}
\hline 2 & 4924 & 97.8276 & 5.0250 & 285.7 & 4 & 600.0 & 00.0 \\
\hline 02 & 4101 & 9.2475 & 4.9500 & 285.4 & 4 & 600.0 & 600.0 \\
\hline 02 & $410 \quad 2$ & 106.8860 & 3.5750 & 285.7 & 5 & 600.0 & 00.0 \\
\hline 02 & 4103 & 75.0000 & 4.9750 & 285.5 & 4 & 600.0 & 00.0 \\
\hline 02 & $410 \quad 4$ & 80.5004 & 5.0000 & 285.6 & 4 & 600.0 & 00.0 \\
\hline 02 & 4105 & 73.7499 & 6.6250 & 285.1 & 4 & 600.0 & 00.0 \\
\hline 02 & 4106 & 74.7476 & 8.5000 & 284.7 & 4 & 600.0 & 600.0 \\
\hline 02 & 4107 & 75.2502 & 7.6000 & 284.7 & 4 & 600.0 & 00.0 \\
\hline 02 & 4108 & 75.9997 & 6.9750 & 285.4 & 4 & 600.0 & 00.0 \\
\hline 02 & 4109 & 74.0023 & 6.3250 & 286.6 & 4 & 600.0 & .0 \\
\hline 02 & 41010 & 79.5046 & 4.7250 & 28 & 3 & 600.0 & 00.0 \\
\hline 02 & 41011 & 103.5314 & 50 & 289.1 & 3 & 600.0 & \\
\hline 02 & 41012 & 127.5005 & 4.8000 & 289.8 & 3 & 600.0 & 600.0 \\
\hline 02 & 41013 & 132.8958 & 5.3750 & 291.2 & 3 & 600.0 & 0 \\
\hline 02 & 41014 & 152.7502 & & 291.3 & 4 & 600.0 & \\
\hline 02 & 41015 & 146.0341 & 00 & 291.8 & 4 & 600.0 & 60 \\
\hline 02 & 41016 & 129.0221 & 5.2750 & 292.1 & 3 & 600.0 & 600.0 \\
\hline 02 & 41017 & 142.8007 & 5 . & 2.3 & 3 & 600.0 & .0 \\
\hline 02 & 41018 & 114.7472 & 4.9750 & 291.7 & 3 & 600.0 & .0 \\
\hline 02 & 41019 & 83.7429 & 5.9250 & 289.8 & 4 & 600.0 & 60 \\
\hline 02 & 41020 & 73.2498 & 6. & .2 & 4 & 600.0 & .0 \\
\hline 02 & 41021 & 77.5000 & 9. & 287.6 & 4 & 600.0 & \\
\hline 02 & 41022 & 79.0005 & 9.2250 & 286.5 & 4 & 600.0 & 600.0 \\
\hline 02 & 41023 & 74.0000 & 10 . & 286.5 & 4 & 600.0 & .0 \\
\hline 02 & 41024 & 70.7489 & & 286.9 & 4 & 600.0 & \\
\hline 02 & 4111 & 69.5000 & 9 . & 286.6 & 4 & 600.0 & 60 \\
\hline 02 & 4112 & 74.7500 & 10 . & 286.4 & 4 & 600.0 & .0 \\
\hline 02 & 4113 & 70.0000 & 10 . & 286.2 & 4 & 00.0 & \\
\hline 02 & 4114 & 67.7500 & 50 & 286 & 4 & 600.0 & 60 \\
\hline 02 & 4115 & 72.9995 & 8 . & 286.7 & 4 & 600.0 & .0 \\
\hline 02 & 4116 & 75.0000 & 8 . & 287.2 & 4 & 600.0 & \\
\hline 02 & 4117 & 76.9999 & 9.27 & 287.2 & 4 & 600.0 & 60 \\
\hline 02 & 4118 & 68.4828 & 6.6750 & 288.5 & 4 & 600.0 & 600.0 \\
\hline 02 & 4119 & 87.0000 & 7 . & 28 & 4 & 600.0 & .0 \\
\hline 02 & 41110 & 77.7371 & 5 . & 290.2 & 3 & 600.0 & 60 \\
\hline 02 & 41111 & 90.4828 & 4.2000 & 291.9 & 3 & 600.0 & 600.0 \\
\hline 02 & 41112 & 101.9007 & 5 . & 292.0 & 3 & 600.0 & \\
\hline 02 & 41113 & 86.2501 & 6. & 291.6 & 4 & 600.0 & 600.0 \\
\hline 02 & 41114 & 91.9966 & 7.3500 & 292.0 & 4 & 600.0 & 600.0 \\
\hline 02 & 41115 & 100.2486 & 8 . & 291.8 & 4 & 600.0 & \\
\hline 02 & 41116 & 76.1904 & 9. & 29 & 4 & 600.0 & 600.0 \\
\hline 02 & 41117 & 70.7515 & 11.0750 & 290.9 & 4 & 600.0 & 600.0 \\
\hline 02 & 41118 & 66.2515 & 10.8500 & 289.8 & 4 & 600.0 & 600.0 \\
\hline 02 & 41119 & 75.5002 & 8.5750 & 289.5 & 4 & 600.0 & 600.0 \\
\hline 02 & 41120 & 80.4413 & 8.3250 & 288.8 & 4 & 600.0 & 600.0 \\
\hline 02 & 41121 & 75.2520 & 7.1750 & 288.9 & 4 & 600.0 & 600.0 \\
\hline 02 & 41122 & 76.4998 & & & 4 & .0 & 600.0 \\
\hline 02 & 41123 & 81.5000 & 9.3250 & 288.7 & 4 & 600.0 & 600.0 \\
\hline
\end{tabular}




\begin{tabular}{|c|c|c|c|c|c|c|c|}
\hline 2 & 41124 & 82.2511 & 8.9000 & 288.4 & 4 & 600.0 & 00.0 \\
\hline 02 & 4121 & 80.7500 & 8.7500 & 288.2 & 4 & 600.0 & 600.0 \\
\hline 02 & 4122 & 80.5000 & 8.4000 & 288.2 & 4 & 600.0 & 00.0 \\
\hline 02 & 4123 & 87.5000 & 8.2500 & 288.3 & 4 & 600.0 & 00.0 \\
\hline 02 & 4124 & 84.2504 & 7.8750 & 287.9 & 4 & 600.0 & 00.0 \\
\hline 02 & 4125 & 108.5275 & 6.1250 & 288.5 & 4 & 600.0 & 00.0 \\
\hline 02 & 4126 & 137.4925 & 5.7500 & 288.9 & 4 & 600.0 & 600.0 \\
\hline 02 & 4127 & 147.5092 & 7.5000 & 288.6 & 4 & 600.0 & 00.0 \\
\hline 02 & 4128 & 155.2517 & 7.9500 & 288.7 & 4 & 600.0 & 00.0 \\
\hline 02 & 4129 & 155.2499 & 8.3000 & 288.9 & 4 & 600.0 & .0 \\
\hline 02 & 41210 & 159.7571 & 6.6250 & 290.0 & 4 & 600.0 & 00.0 \\
\hline 02 & 41211 & 164.5000 & 6.5500 & 291.5 & 4 & 600.0 & \\
\hline 02 & 41212 & 127.5005 & 4.8000 & 289.8 & 3 & 600.0 & 600.0 \\
\hline 02 & 41213 & 132.8958 & 5.3750 & 291.2 & 3 & 600.0 & 0 \\
\hline 02 & 41214 & 152.7502 & 6.5750 & 291.3 & 4 & 600.0 & \\
\hline 02 & 41215 & 146.0341 & 6.0500 & 291.8 & 4 & 600.0 & 60 \\
\hline 02 & 41216 & 129.0221 & 5.2750 & 292.1 & 3 & 600.0 & 600.0 \\
\hline 02 & 41217 & 142.8007 & 5.3000 & 292.3 & 3 & 600.0 & \\
\hline 02 & 41218 & 114.7472 & 4.9750 & 291.7 & 3 & 600.0 & .0 \\
\hline 02 & 41219 & 83.7429 & 5.9250 & 289.8 & 4 & 600.0 & 600 \\
\hline 02 & 41220 & 73.2498 & 6.8500 & 288.2 & 4 & 600.0 & .0 \\
\hline 02 & 41221 & 77.5000 & 9.5750 & 287.6 & 4 & 600.0 & \\
\hline 02 & 41222 & 79.0005 & 9.2250 & 286.5 & 4 & 600.0 & 60 \\
\hline 02 & 41223 & 74.0000 & 10.3750 & 286.5 & 4 & 600.0 & .0 \\
\hline 02 & 41224 & 70.7489 & 9.5750 & 286.9 & 4 & 600.0 & \\
\hline 02 & 4131 & 69.5000 & 9.8750 & 286.6 & 4 & 600.0 & 60 \\
\hline 02 & 4132 & 74.7500 & 10.4750 & 286.4 & 4 & 600.0 & .0 \\
\hline 02 & 4133 & 70.0000 & 10 . & 286.2 & 4 & 600.0 & \\
\hline 02 & 4134 & 67.7500 & 9.4750 & 286.4 & 4 & 600.0 & 60 \\
\hline 02 & 4135 & 72.9995 & 8.9000 & 286.7 & 4 & 600.0 & .0 \\
\hline 02 & 4136 & 75.0000 & 8.15 & 287.2 & 4 & 600.0 & \\
\hline 02 & 4137 & 76.9999 & 9.2750 & 287.2 & 4 & 600.0 & 60 \\
\hline 02 & 4138 & 68.4828 & 6.6750 & 288.5 & 4 & 600.0 & 600.0 \\
\hline 02 & 4139 & 87.0000 & 7.10 & 289.1 & 4 & 600.0 & .0 \\
\hline 02 & 41310 & 77.7371 & $5.0^{\circ}$ & 290.2 & 3 & 600.0 & 60 \\
\hline 02 & 41311 & 90.4828 & 4.2000 & 291.9 & 3 & 600.0 & 600.0 \\
\hline 02 & 41312 & 101.9007 & 5.9500 & 292.0 & 3 & 600.0 & \\
\hline 02 & 41313 & 86.2501 & 6.4250 & 291.6 & 4 & 600.0 & 600.0 \\
\hline 02 & 41314 & 91.9966 & 7.3500 & 292.0 & 4 & 600.0 & 600.0 \\
\hline 02 & 41315 & 100.2486 & 8.4000 & 291.8 & 4 & 600.0 & \\
\hline 02 & 41316 & 76.1904 & 9.0500 & 291.4 & 4 & 600.0 & 600.0 \\
\hline 02 & 41317 & 146.0000 & 3.9000 & 299.6 & 3 & 600.0 & 600.0 \\
\hline 02 & 41318 & 149.2497 & 3.9500 & 299.2 & 4 & 600.0 & 600.0 \\
\hline 02 & 41319 & 139.0440 & 4.2500 & 298.1 & 4 & 600.0 & 600.0 \\
\hline 02 & 41320 & 78.2500 & 7.3000 & 296.6 & 4 & 600.0 & 600.0 \\
\hline 02 & 41321 & 76.7501 & 8.3250 & 296.0 & 4 & 600.0 & 600.0 \\
\hline 02 & 41322 & 77.9992 & 8.000 & 296.3 & 4 & 600.0 & 600.0 \\
\hline 02 & 41323 & 82.7506 & 7.4250 & 296.7 & 4 & 600.0 & 600.0 \\
\hline
\end{tabular}




\begin{tabular}{|c|c|c|c|c|c|c|c|}
\hline 02 & 41324 & 70.7505 & 7.7750 & 296.8 & 4 & 600.0 & 600.0 \\
\hline 02 & $414 \quad 1$ & 72.4994 & 8.9750 & 295.6 & 4 & 600.0 & 600.0 \\
\hline 02 & $414 \quad 2$ & 70.2501 & 9.8000 & 294.4 & 4 & 600.0 & 00.0 \\
\hline 02 & 4143 & 81.7500 & 10.6000 & 292.9 & 4 & 600.0 & 00.0 \\
\hline 02 & $414 \quad 4$ & 79.2501 & 10.5250 & 292.8 & 4 & 600.0 & 00.0 \\
\hline 02 & $414 \quad 5$ & 78.2498 & 9.0500 & 293.6 & 4 & 600.0 & 00.0 \\
\hline 02 & 4146 & 74.9993 & 8.5250 & 294.1 & 4 & 600.0 & 00.0 \\
\hline 02 & 4147 & 67.2517 & 9.3500 & 293.1 & 4 & 600.0 & 00.0 \\
\hline 02 & 4148 & 73.7499 & 8.0500 & 293.5 & 4 & 600.0 & 00.0 \\
\hline 02 & 4149 & 78.5067 & 7.5250 & 294.6 & 4 & 600 & 0 \\
\hline 02 & 41410 & 73.2511 & 8.0250 & 295.2 & 4 & 600.0 & \\
\hline 02 & 41411 & 80.2499 & 5.7750 & 295.8 & 3 & .0 & \\
\hline 02 & 41412 & 68.7483 & 10.8000 & 294.9 & 4 & 600.0 & 00.0 \\
\hline 02 & 41413 & 74.7509 & 10.6500 & 294.8 & 4 & 600.0 & 00.0 \\
\hline 02 & 41414 & 96.5122 & 8.2500 & 294.6 & 4 & .0 & \\
\hline 02 & 41415 & 64.2366 & 9.4000 & 294.2 & 4 & 600.0 & .0 \\
\hline 02 & 41416 & 67.2504 & 14.5250 & 291.2 & 4 & 60 & .0 \\
\hline 02 & 41417 & 69.7500 & 17.6500 & 289.2 & 4 & .0 & \\
\hline 02 & 41418 & 67.5110 & 13.4500 & 286.6 & 4 & 60 & \\
\hline 02 & 41419 & 81.0000 & 17.4000 & 282.5 & 4 & .0 & .0 \\
\hline 02 & 41420 & 77.2498 & 16.67 & 280.9 & 4 & 6 & \\
\hline 02 & 41421 & 65.7500 & 21.8250 & 280.3 & 4 & .0 & \\
\hline 02 & 41422 & 59.2505 & 23.3250 & 279.9 & 4 & 600.0 & .0 \\
\hline 02 & 41423 & 65.7501 & 21.5500 & 279.9 & 4 & 6 & \\
\hline 02 & 41424 & 64.7500 & 20.3250 & 279.6 & 4 & .0 & \\
\hline 02 & 4151 & 54.5000 & 16.3500 & 279.3 & 4 & .0 & \\
\hline 02 & 4152 & 79.5663 & 6.7500 & 279.3 & 4 & 60 & .0 \\
\hline 02 & 4153 & 91.7495 & 8.5750 & 279.2 & 4 & .0 & \\
\hline 02 & 4154 & 82.9970 & 10.4250 & 278.9 & 4 & .0 & 0 \\
\hline 02 & 4155 & 78.2480 & 10.9000 & 278.6 & 4 & 6 & .0 \\
\hline 02 & 4156 & 90.2376 & 10.0250 & 278.6 & 4 & .0 & \\
\hline 02 & 4157 & 98.5002 & 9.7250 & 278.7 & 4 & .0 & 600.0 \\
\hline 02 & 4158 & 91.7501 & 8.9250 & 278.8 & 4 & 600.0 & 600.0 \\
\hline 02 & 4159 & 89.5138 & 6.0250 & 279.7 & 4 & .0 & 0.0 \\
\hline 02 & 41510 & 89.2310 & 5.4000 & 281.5 & 3 & 600.0 & 600.0 \\
\hline 02 & 41511 & 125.8228 & 5.4250 & 282.3 & 3 & 600.0 & 600.0 \\
\hline 02 & 41512 & 154.0982 & 5.2750 & 283.7 & 3 & 600.0 & .0 \\
\hline 02 & 41513 & 158.7413 & 5.1500 & 284.7 & 3 & 600.0 & 600.0 \\
\hline 02 & 41514 & 150.3529 & 4.2000 & 285.9 & 3 & 600.0 & 600.0 \\
\hline 02 & 41515 & 152.6958 & 3.3750 & 286.1 & 2 & 600.0 & 600.0 \\
\hline 02 & 41516 & 101.2446 & 4.1250 & 286.8 & 3 & 600.0 & 600.0 \\
\hline 02 & 41517 & 89.2671 & 5.6500 & 286.4 & 3 & 600.0 & 600.0 \\
\hline 02 & 41518 & 79.7527 & 6.9250 & 284.9 & 4 & 600.0 & 600.0 \\
\hline 02 & 41519 & 78.7502 & 8.5500 & 282.8 & 4 & 600.0 & 600.0 \\
\hline 02 & 41520 & 77.5000 & 9.5500 & 281.6 & 4 & 600.0 & 600.0 \\
\hline 02 & 41521 & 75.7494 & 9.3750 & 281.1 & 4 & 600.0 & 600.0 \\
\hline 02 & 41522 & 78.5005 & 9.1250 & 280.9 & 4 & 600.0 & 600.0 \\
\hline 02 & 41523 & 74.0000 & 9.1750 & 280.5 & 4 & 600.0 & 600.0 \\
\hline
\end{tabular}




\begin{tabular}{|c|c|c|c|c|c|c|c|}
\hline 02 & 41524 & 69.0002 & 8.4000 & 280.9 & 4 & 600.0 & 600.0 \\
\hline 02 & 4161 & 69.2500 & 7.8250 & 281.0 & 4 & 600.0 & 600.0 \\
\hline 02 & 4162 & 67.0000 & 5.3500 & 281.5 & 4 & 600.0 & 00.0 \\
\hline 02 & 4163 & 81.2504 & 5.1750 & 281.1 & 4 & 600.0 & 600.0 \\
\hline 02 & 4164 & 85.2502 & 5.4500 & 280.5 & 4 & 600.0 & 00.0 \\
\hline 02 & 4165 & 83.7499 & 7.7750 & 279.5 & 4 & 600.0 & 00.0 \\
\hline 02 & 4166 & 79.5000 & 7.5250 & 279.6 & 4 & 600.0 & 600.0 \\
\hline 02 & 4167 & 67.9885 & 10.5000 & 279.0 & 4 & 600.0 & 500.0 \\
\hline 02 & 4168 & 52.2505 & 8.2500 & 280.0 & 4 & 600.0 & 00.0 \\
\hline 02 & 4169 & 71.5119 & 5.8500 & 281.7 & 3 & 600.0 & 600.0 \\
\hline 02 & 41610 & 83.0123 & 6.7500 & 282.4 & 4 & 600.0 & 600.0 \\
\hline 02 & 41611 & 81.9908 & 7.5000 & 283.2 & 4 & 600.0 & \\
\hline 02 & 41612 & 81.7446 & 8.4250 & 283.4 & 4 & 600.0 & 600.0 \\
\hline 02 & 41613 & 74.4990 & 9.6000 & 283.6 & 4 & 600.0 & 600.0 \\
\hline 02 & 41614 & 70.9960 & 8.6500 & 283.2 & 4 & 600.0 & \\
\hline 02 & 41615 & 74.5080 & 7.7250 & 283.1 & 4 & 600.0 & 60 \\
\hline 02 & 41616 & 94.5000 & 7.2250 & 283.2 & 4 & 600.0 & 600.0 \\
\hline 02 & 41617 & 88.2472 & 7.2500 & 283.2 & 4 & 600.0 & \\
\hline 02 & 41618 & 76.5006 & 6.0500 & 282.5 & 4 & 600.0 & 60 \\
\hline 02 & 41619 & 51.9500 & 5.3250 & 281.4 & 4 & 600.0 & 60 \\
\hline 02 & 41620 & 35.5027 & 5. & 281.2 & 4 & 600.0 & .0 \\
\hline 02 & 41621 & 25.2771 & 4. & 281.3 & 4 & 600.0 & \\
\hline 02 & 41622 & 36.5000 & 5.7000 & 281.0 & 4 & 600.0 & 600.0 \\
\hline 02 & 41623 & 35.0000 & 3.0250 & 280.4 & 5 & 600.0 & .0 \\
\hline 02 & 41624 & 63.0033 & 5. & 279.9 & 4 & 600.0 & \\
\hline 02 & 4171 & 70.7503 & 4.6250 & 278.7 & 4 & 600.0 & 600.0 \\
\hline 02 & 4172 & 98.2561 & 5.6250 & 279.5 & 4 & 600.0 & 600.0 \\
\hline 02 & 4173 & 79.0146 & 6.9250 & 279.2 & 4 & 600.0 & \\
\hline 02 & $417 \quad 4$ & 66.4954 & 8.4000 & 278.9 & 4 & 600.0 & 60 \\
\hline 02 & 4175 & 75.0000 & 8.3500 & 278.8 & 4 & 600.0 & 600.0 \\
\hline 02 & $417 \quad 6$ & 85.2372 & 6 . & 278.6 & 4 & 600.0 & .0 \\
\hline 02 & $417 \quad 7$ & 90.2319 & 5.3500 & 279.1 & 4 & 600.0 & 60 \\
\hline 02 & 4178 & 89.5740 & 5.2250 & 279.8 & 3 & 600.0 & 600.0 \\
\hline 02 & 4179 & 86.7400 & 5.125 & 280.8 & 3 & 600.0 & 60 \\
\hline 02 & 41710 & 81.0000 & 6. & 281.4 & 4 & 600.0 & 60 \\
\hline 02 & 41711 & 69.6696 & 6.2250 & 282.5 & 4 & 600.0 & 600.0 \\
\hline 02 & 41712 & 64.0057 & 6.1500 & 283.3 & 4 & 600.0 & .0 \\
\hline 02 & 41713 & 56.9927 & 7 . & 283.7 & 4 & 600.0 & 600.0 \\
\hline 02 & 41714 & 57.5554 & 7.5000 & 284.1 & 4 & 600.0 & 600.0 \\
\hline 02 & 41715 & 58.9902 & 8.8250 & 284.0 & 4 & 600.0 & \\
\hline 02 & 41716 & 63.9985 & 9.7500 & 283.8 & 4 & 600.0 & 600.0 \\
\hline 02 & 41717 & 63.2558 & 9.9500 & 283.1 & 4 & 600.0 & 600.0 \\
\hline 02 & 41718 & 66.5000 & 11.4750 & 282.0 & 4 & 600.0 & 600.0 \\
\hline 02 & 41719 & 62.5000 & 11.9250 & 281.0 & 4 & 600.0 & 600.0 \\
\hline 02 & 41720 & 61.2500 & 11.2750 & 280.3 & 4 & 600.0 & 600.0 \\
\hline 02 & 41721 & 58.0002 & 9.2000 & 280.0 & 4 & 600.0 & 600.0 \\
\hline 02 & 41722 & 59.9993 & 8.7000 & & 4 & 600.0 & 600.0 \\
\hline 02 & 41723 & 66.2495 & 7.1500 & 279.5 & 4 & 600.0 & 600.0 \\
\hline
\end{tabular}




\begin{tabular}{|c|c|c|c|c|c|c|c|}
\hline 2 & 41724 & 3.2498 & 8.2250 & 279.3 & 4 & 600.0 & 00.0 \\
\hline 02 & 4181 & 8.0011 & 9.0000 & 279.3 & 4 & 600.0 & 600.0 \\
\hline 02 & 4182 & 79.7493 & 8.0000 & 279.0 & 4 & 600.0 & 00.0 \\
\hline 02 & 4183 & 88.2705 & 7.2000 & 278.7 & 4 & 600.0 & 00.0 \\
\hline 02 & 4184 & 93.5055 & 6.5750 & 278.3 & 4 & 600.0 & 00.0 \\
\hline 02 & 4185 & 80.2501 & 6.6250 & 278.2 & 4 & 600.0 & 00.0 \\
\hline 02 & 4186 & 79.2465 & 7.2750 & 278.1 & 4 & 600.0 & 600.0 \\
\hline 02 & 4187 & 86.4995 & 7.7500 & 278.4 & 4 & 600.0 & 00.0 \\
\hline 02 & $418 \quad 8$ & 121.6362 & 6.5250 & 279.8 & 4 & 600.0 & 00.0 \\
\hline 02 & 4189 & 156.5018 & 9.0000 & 280.4 & 4 & 600.0 & 600.0 \\
\hline 02 & 41810 & 162.7505 & 8.4500 & 281.5 & 4 & 600.0 & 0 \\
\hline 02 & 41811 & 174.7439 & 7.1500 & 282.2 & 4 & 600.0 & \\
\hline 02 & 41812 & 161.2616 & 7.1000 & 282.6 & 4 & 600.0 & 00.0 \\
\hline 02 & 41813 & 166.5006 & 7.6250 & 283.8 & 4 & 600.0 & 0 \\
\hline 02 & 41814 & 159.2302 & 7.7 & 284.6 & 4 & 600.0 & \\
\hline 02 & 41815 & 154.5001 & 7.7500 & 285.4 & 4 & 600.0 & 60 \\
\hline 02 & 41816 & 151.7473 & 8.3750 & 286.1 & 4 & 600.0 & .0 \\
\hline 02 & 41817 & 162.2482 & 8.6250 & 286.0 & 4 & 600.0 & \\
\hline 02 & 41818 & 130.3942 & 6.8500 & 285.4 & 4 & 600.0 & .0 \\
\hline 02 & 41819 & 139.0589 & 250 & 283.9 & 4 & 600.0 & .0 \\
\hline 02 & 41820 & 85.0003 & 6 . & 282.8 & 4 & 600.0 & .0 \\
\hline 02 & 41821 & 89.5000 & 8 . & 282.7 & 4 & 0.0 & \\
\hline 02 & 41822 & 89.2485 & 7.7000 & 282.5 & 4 & 600.0 & 600.0 \\
\hline 02 & 41823 & 97.7507 & 7.7500 & 283.0 & 4 & 600.0 & .0 \\
\hline 02 & 41824 & 100.4948 & 8.2250 & 282.9 & 4 & 600.0 & \\
\hline 02 & 4191 & 130.0104 & 8.4500 & 283.8 & 4 & 600.0 & 60 \\
\hline 02 & 4192 & 144.4991 & 11.1250 & 283.4 & 4 & 600.0 & .0 \\
\hline 02 & 4193 & 152.2500 & 11.50 & 283.1 & 4 & 0.0 & \\
\hline 02 & 4194 & 149.4997 & 750 & 282.8 & 4 & 600.0 & 60 \\
\hline 02 & 4195 & 153.7504 & 11.5000 & 282.8 & 4 & 600.0 & .0 \\
\hline 02 & 4196 & 155.2498 & 10 & 283.1 & 4 & 600.0 & \\
\hline 02 & 4197 & 160.5000 & 750 & 283.1 & 4 & 600.0 & .0 \\
\hline 02 & 4198 & 158.0000 & 11.7750 & 283.3 & 4 & 600.0 & 600.0 \\
\hline 02 & 4199 & 167.2501 & 11 . & 284.6 & 4 & 600.0 & .0 \\
\hline 02 & 41910 & 170.9982 & 11. & 285.9 & 4 & 600.0 & .0 \\
\hline 02 & 41911 & 175.0009 & 11.5000 & 287.2 & 4 & 600.0 & 600.0 \\
\hline 02 & 41912 & 179.5055 & 11.2000 & 288.0 & 4 & 600.0 & \\
\hline 02 & 41913 & 175.7565 & 10.6000 & 288.9 & 4 & 600.0 & .0 \\
\hline 02 & 41914 & 177.7509 & 10.8500 & 289.4 & 4 & 600.0 & 600.0 \\
\hline 02 & 41915 & 177.4839 & 10.9250 & 290.1 & 4 & 600.0 & \\
\hline 02 & 41916 & 171.0000 & 10.8250 & 290.7 & 4 & 600.0 & 60 \\
\hline 02 & 41917 & 170.0001 & 10.2000 & 290.9 & 4 & 600.0 & 600.0 \\
\hline 02 & 41918 & 173.4983 & 9.9000 & 290.9 & 4 & 600.0 & 600.0 \\
\hline 02 & 41919 & 161.0274 & 7.5750 & 290.1 & 4 & 600.0 & 600.0 \\
\hline 02 & 41920 & 139.5000 & 7.5000 & 289.5 & 4 & 600.0 & 600.0 \\
\hline 02 & 41921 & 126.7787 & 7.8000 & 289.4 & 4 & 600.0 & 600.0 \\
\hline 02 & 41922 & 98.5027 & 7.325 & 288.5 & 4 & 600.0 & 600.0 \\
\hline 02 & 41923 & 109.7502 & 7.9500 & 288.5 & 4 & 600.0 & 600.0 \\
\hline
\end{tabular}




\begin{tabular}{|c|c|c|c|c|c|c|c|}
\hline 2 & 924 & & & & & & \\
\hline & 4201 & & .3250 & 8.1 & 4 & 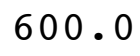 & \\
\hline & 4202 & 91.5000 & 1250 & 287.8 & 4 & 00 & \\
\hline & 1203 & 109.5122 & 6.8250 & 287.8 & & 00 & \\
\hline & 204 & 909 & 750 & 7.7 & & & \\
\hline & 4205 & 135 & 500 & 87.2 & & 0 & \\
\hline & 4206 & 9812 & 7250 & 86.0 & & 00 & \\
\hline & 4207 & 114 & 3250 & 85.9 & & & \\
\hline & $420 \quad 8$ & 108.4482 & 8500 & 87.3 & & & \\
\hline & 4209 & 169.7502 & 4750 & 88.5 & & 0 & 0 \\
\hline & 2010 & 165.0000 & 9250 & 289 & & & \\
\hline & 42011 & 178 & & 89.9 & & & \\
\hline & 42012 & 116 & 5000 & 290.8 & 3 & 0 & \\
\hline & 2013 & 100.0000 & 0 & 291.5 & & 0.0 & \\
\hline & 42014 & 99 & & 91.7 & & & \\
\hline & 2015 & 100 . & & 291.5 & 4 & & \\
\hline & 2016 & 93.2505 & 0 & 291.0 & 4 & 0 & \\
\hline & 42017 & 02 & & 289.6 & 4 & & \\
\hline & 2018 & 83. & & 287.3 & 4 & & \\
\hline & 42019 & 81.7500 & & 284.5 & 4 & 0 & \\
\hline & 42020 & 79.7500 & 0 & 283.6 & 4 & 0 & \\
\hline & 42021 & 77.7500 & & & 4 & & \\
\hline & 42022 & 5000 & & 28 & 4 & & \\
\hline & 42023 & 62.2506 & 0 & 285.0 & 4 & 0 & \\
\hline & 42024 & 99.4967 & & & 4 & & \\
\hline & $421 \quad 1$ & 152. & & 28 & 5 & & \\
\hline & 4212 & 148.1839 & & 281.5 & 6 & & o. \\
\hline & 4213 & 141.247 & & 281.5 & 6 & & \\
\hline 2 & 4214 & 149.9 & & 281.1 & 6 & & \\
\hline & 4215 & 15.0008 & & 281.5 & 6 & & J. \\
\hline & 4216 & 23.2506 & 0 & 283.8 & 5 & & 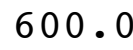 \\
\hline & 4217 & 13.7492 & & 285.7 & 4 & & \\
\hline & 4218 & 310.9976 & & 289.0 & 3 & & . \\
\hline 2 & 4219 & 250.2492 & & 288.1 & 3 & & \\
\hline 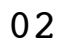 & 421 & 241.4997 & & 288.5 & 3 & & \\
\hline & & 235.2563 & & 289.4 & 2 & & \\
\hline & 42112 & 226.2810 & & 290.7 & 2 & & \\
\hline 2 & 42113 & 227.7509 & & 291.2 & 2 & & \\
\hline 2 & 42114 & 209.2013 & 2250 & 292.0 & 3 & & 0 . \\
\hline & 42115 & 216.9417 & 9000 & 293.0 & 2 & & \\
\hline 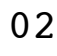 & 42116 & 192.0075 & 4. & 293.3 & 3 & & \\
\hline 2 & 42117 & 176.7370 & 4.0000 & 293.5 & 3 & & 0 . \\
\hline & 42118 & 189.0000 & 6500 & 293.3 & 3 & & 0 . \\
\hline 2 & 42119 & 168.2475 & 000 & 292.3 & 4 & & \\
\hline 02 & 42120 & 161.2501 & & 290.9 & 5 & & \\
\hline & 42121 & 152.0359 & 3.8000 & 290.6 & 5 & & 00 . \\
\hline & 42 & 14 & & 290.6 & 4 & & \\
\hline & 42123 & 113.1026 & 2.9500 & 291.0 & & 600.0 & 8 \\
\hline
\end{tabular}




\begin{tabular}{|c|c|c|c|c|c|c|c|}
\hline 2 & 42124 & 82.2499 & 6.3250 & 290.0 & 4 & 600.0 & 00.0 \\
\hline 02 & 4221 & 78.0000 & 7.3250 & 289.2 & 4 & 600.0 & 600.0 \\
\hline 02 & 4222 & 79.7500 & 6.6250 & 289.5 & 4 & 600.0 & 00.0 \\
\hline 02 & 4223 & 84.7295 & 5.1250 & 290.0 & 4 & 600.0 & 600.0 \\
\hline 02 & 4224 & 100.4973 & 2.9000 & 290.5 & 4 & 600.0 & 00.0 \\
\hline 02 & 4225 & 116.7525 & 1.2000 & 290.3 & 4 & 600.0 & 00.0 \\
\hline 02 & 4226 & 147.7425 & 0.8750 & 289.6 & 5 & 600.0 & 600.0 \\
\hline 02 & 4227 & 79.3936 & 0.1250 & 290.8 & 0 & 600.0 & 00.0 \\
\hline 02 & 4228 & 321.4064 & 0.3500 & 293.5 & 5 & 600.0 & 00.0 \\
\hline 02 & 4229 & 239.9989 & 1.3000 & 293.9 & 4 & 600.0 & .0 \\
\hline 02 & 42210 & 196.4239 & 3.2000 & 293.8 & 3 & 600.0 & 00.0 \\
\hline 02 & 42211 & 209.2020 & 00 & 294.1 & 2 & 600.0 & \\
\hline 02 & 42212 & 212.2444 & 3.5000 & 294.9 & 2 & 600.0 & 600.0 \\
\hline 02 & 42213 & 202.2041 & 4.7250 & 295.1 & 3 & 600.0 & .0 \\
\hline 02 & 42214 & 180.7495 & 4.4750 & 295.8 & 3 & 600.0 & \\
\hline 02 & 42215 & 164.2423 & 3.9000 & 297.0 & 2 & 600.0 & 60 \\
\hline 02 & 42216 & 175.2465 & 4.9500 & 297.2 & 3 & 600.0 & 600.0 \\
\hline 02 & 42217 & 175.7515 & 4.6500 & 297.3 & 3 & 600.0 & \\
\hline 02 & 42218 & 180.0000 & 5.9250 & 296.7 & 4 & 600.0 & .0 \\
\hline 02 & 42219 & 168.4998 & 4.8250 & 295.1 & 5 & 600.0 & 600 \\
\hline 02 & 42220 & 136.1988 & 3.4000 & 293.7 & 4 & 600.0 & .0 \\
\hline 02 & 42221 & 88.8001 & 500 & 293.2 & 4 & 600.0 & \\
\hline 02 & 42222 & 77.5000 & 6.5750 & 292.2 & 4 & 600.0 & 600.0 \\
\hline 02 & 42223 & 74.7494 & 6 . & 292.2 & 4 & 600.0 & .0 \\
\hline 02 & 42224 & 79.2499 & 7 . & 292.5 & 4 & 600.0 & \\
\hline 02 & 4231 & 78.7500 & 7.2750 & 292.5 & 4 & 600.0 & 60 \\
\hline 02 & 4232 & 81.5000 & 6.4500 & 293.1 & 4 & 600.0 & .0 \\
\hline 02 & 4233 & 72.8814 & 3.7500 & 293.5 & 4 & 600.0 & \\
\hline 02 & 4234 & 56.0046 & 0.5750 & 293.0 & 5 & 600.0 & 60 \\
\hline 02 & 4235 & 180.4945 & 0.7000 & 291.9 & 6 & 600.0 & .0 \\
\hline 02 & 4236 & 87.4277 & 0 & 291.0 & 6 & 600.0 & \\
\hline 02 & 4237 & 111.8962 & 000 & 293.0 & 6 & 600.0 & 60 \\
\hline 02 & 4238 & 327.4637 & 0.0500 & 295.7 & 5 & 600.0 & 600.0 \\
\hline 02 & 4239 & 198.6460 & 1.7500 & 296.1 & 4 & 600.0 & .0 \\
\hline 02 & 42310 & 166.5000 & 250 & 295.0 & 3 & 600.0 & 60 \\
\hline 02 & 42311 & 180.2298 & 5.8000 & 295.1 & 3 & 600.0 & 600.0 \\
\hline 02 & 42312 & 187.4982 & 5.0500 & 295.6 & 3 & 600.0 & \\
\hline 02 & 42313 & 195.0046 & 4.6250 & 296.7 & 3 & 600.0 & 600.0 \\
\hline 02 & 42314 & 195.9927 & 4.1750 & 297.6 & 3 & 600.0 & 600.0 \\
\hline 02 & 42315 & 196.2614 & 5000 & 298.3 & 3 & 600.0 & \\
\hline 02 & 42316 & 187.4977 & 4.3750 & 299.0 & 3 & 600.0 & 600.0 \\
\hline 02 & 42317 & 193.9981 & 4.3250 & 299.3 & 3 & 600.0 & 600.0 \\
\hline 02 & 42318 & 176.7249 & 4.7500 & 299.2 & 3 & 600.0 & 600.0 \\
\hline 02 & 42319 & 163.2500 & 4.6250 & 297.7 & 4 & 600.0 & 600.0 \\
\hline 02 & 42320 & 153.8034 & 3.7000 & 296.0 & 4 & 600.0 & 600.0 \\
\hline 02 & 42321 & 81.2480 & 10.0500 & 292.2 & 4 & 600.0 & 600.0 \\
\hline 02 & 42322 & 77.4998 & 11.225 & 291.2 & 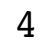 & 600.0 & 600.0 \\
\hline 02 & 42323 & 77.0001 & 12.2500 & 289.7 & 4 & 600.0 & 600.0 \\
\hline
\end{tabular}




\begin{tabular}{|c|c|c|c|c|c|c|c|}
\hline 2 & 42324 & 77.0001 & 12.9250 & 288.7 & 4 & 600.0 & 600.0 \\
\hline 02 & $424 \quad 1$ & 75.0000 & 13.6750 & 287.3 & 4 & 600.0 & 600.0 \\
\hline 02 & $424 \quad 2$ & 74.0001 & 500 & 286.8 & 4 & 600.0 & 00.0 \\
\hline 02 & 4243 & 77.5000 & 11.4250 & 286.4 & 4 & 600.0 & 00.0 \\
\hline 02 & $424 \quad 4$ & 105.4768 & 8.8500 & 284.6 & 4 & 600.0 & 00.0 \\
\hline 02 & 4245 & 78.4988 & 11.5000 & 283.8 & 4 & 600.0 & 00.0 \\
\hline 02 & $424 \quad 6$ & 81.2507 & 10.5000 & 284.0 & 4 & 600.0 & 0 \\
\hline 02 & 4247 & 79.4997 & 8.5750 & 283.4 & 4 & 600.0 & 00.0 \\
\hline 02 & 4248 & 80.7500 & 7.1500 & 284.2 & 4 & 600.0 & 00.0 \\
\hline 02 & $424 \quad 9$ & 99.2019 & 4.5500 & 285.8 & 3 & 60 & 0 \\
\hline 02 & 42410 & 150.4433 & 1.6750 & 288.8 & 2 & 600.0 & \\
\hline 02 & 42411 & 250.7149 & 2.7500 & 290.1 & 1 & .0 & \\
\hline 02 & 42412 & 253.9032 & 2.4500 & 291.4 & 1 & 600.0 & .0 \\
\hline 02 & 42413 & 181.6076 & 4.4000 & 291.9 & 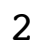 & 600.0 & \\
\hline 02 & 42414 & 166.6040 & & 292.5 & 2 & .0 & \\
\hline 02 & 42415 & 101.7917 & 3.1000 & 293.6 & 2 & 600.0 & \\
\hline 02 & 42416 & 136.2095 & 5.1500 & 292.8 & 3 & .0 & .0 \\
\hline 02 & 42417 & 101.2727 & 5.97 & 291.3 & 3 & .0 & \\
\hline 02 & 42418 & 96.2509 & 5.8500 & 290.7 & 3 & .0 & \\
\hline 02 & 42419 & 88.0023 & 7.8000 & 288.2 & 4 & .0 & .0 \\
\hline 02 & 42420 & 77.2491 & 9. & 286 & 4 & 0 & \\
\hline 02 & 42421 & 73.2495 & 10.4750 & 285.8 & 4 & .0 & \\
\hline 02 & 42422 & 73.2500 & 10.8250 & 285.5 & 4 & 600.0 & .0 \\
\hline 02 & 42423 & 73.7500 & 10.52 & 285 & 4 & 0 & \\
\hline 02 & 42424 & 75.0000 & 10 . & 28 & 4 & 0 & \\
\hline 02 & 4251 & 74.5000 & 10.2750 & 285.3 & 4 & .0 & .0 \\
\hline 02 & 4252 & 75.7500 & 9.7000 & 285.5 & 4 & 60 & .0 \\
\hline 02 & 4253 & 73.5000 & 9.5000 & 285.4 & 4 & .0 & \\
\hline 02 & 4254 & 71.2500 & 9.1250 & 285.1 & 4 & .0 & .0 \\
\hline 02 & 4255 & 80.5000 & 9.8500 & 284.4 & 4 & 6 & \\
\hline 02 & 4256 & 79.0000 & 10.0750 & 283.8 & 4 & 0 & \\
\hline 02 & 4257 & 71.2496 & 9.1500 & 284.5 & 4 & .0 & 0.0 \\
\hline 02 & 4258 & 72.0000 & 7.0500 & 286.5 & 4 & 600.0 & 0.0 \\
\hline 02 & 4259 & 78.5008 & 6.1500 & 288.4 & 4 & .0 & 0.0 \\
\hline 02 & 42510 & 83.5025 & 6.1000 & 289.2 & 4 & 600.0 & 0.0 \\
\hline 02 & 42511 & 87.7508 & 5.8000 & 290.6 & 3 & 600.0 & 600.0 \\
\hline 02 & 42512 & 92.5003 & 6.1250 & 291.5 & 4 & 600.0 & .0 \\
\hline 02 & 42513 & 79.0000 & 7.4000 & 291.5 & 4 & 600.0 & 600.0 \\
\hline 02 & 42514 & 86.2527 & 7.7750 & 291.4 & 4 & 600.0 & 600.0 \\
\hline 02 & 42515 & 77.4982 & 8.8750 & 290.8 & 4 & 600.0 & 600.0 \\
\hline 02 & 42516 & 72.7495 & 10.1750 & 290.0 & 4 & 600.0 & 600.0 \\
\hline 02 & 42517 & 64.0000 & 10.4750 & 289.4 & 4 & 600.0 & 600.0 \\
\hline 02 & 42518 & 63.0000 & 10.0750 & 288.2 & 4 & 600.0 & 600.0 \\
\hline 02 & 42519 & 68.2500 & 11.5250 & 287.0 & 4 & 600.0 & 600.0 \\
\hline 02 & 42520 & 67.7500 & 12.1250 & 285.6 & 4 & 600.0 & 600.0 \\
\hline 02 & 42521 & 68.0000 & 12.5000 & 285.8 & . & 600.0 & 600.0 \\
\hline 02 & 42522 & 70.0000 & 11.5250 & 285.9 & 4 & 600.0 & 600.0 \\
\hline 02 & 42523 & 72.5000 & 12.200 & 284.2 & 4 & 600.0 & 600.0 \\
\hline
\end{tabular}




\begin{tabular}{|c|c|c|c|c|c|c|c|}
\hline 02 & 42524 & 74.9998 & 12.2750 & 283.0 & 4 & 600.0 & 00.0 \\
\hline 02 & 4261 & 71.9988 & 13.1000 & 282.5 & 4 & 600.0 & 600.0 \\
\hline 02 & 4262 & 70.0000 & 13.7500 & 281.7 & 4 & 600.0 & 00.0 \\
\hline 02 & 4263 & 73.7499 & 10.0000 & 281.4 & 4 & 600.0 & 600.0 \\
\hline 02 & 4264 & 72.5005 & 10.2750 & 281.3 & 4 & 600.0 & 00.0 \\
\hline 02 & 4265 & 74.2501 & 10.2500 & 281.4 & 4 & 600.0 & 00.0 \\
\hline 02 & 4266 & 73.7499 & 8.3750 & 281.7 & 4 & 600.0 & 600.0 \\
\hline 02 & 4267 & 75.0001 & 7.1500 & 281.9 & 4 & 600.0 & 00.0 \\
\hline 02 & 4268 & 58.2265 & 5.2750 & 282.6 & 3 & 600.0 & 00.0 \\
\hline 02 & 4269 & 44.9117 & 3.0500 & 284.0 & 2 & 600.0 & 600.0 \\
\hline 02 & 42610 & 77.5406 & 5.0000 & 285.2 & 3 & 600.0 & 600.0 \\
\hline 02 & 42611 & 90.9022 & 4.7750 & 285.5 & 3 & 600.0 & \\
\hline 02 & 42612 & 74.7404 & 6.2750 & 285.4 & 4 & 600.0 & 600.0 \\
\hline 02 & 42613 & 75.2808 & 5.0500 & 285.6 & 3 & 600.0 & 600 \\
\hline 02 & 42614 & 67.0000 & 7.1250 & 285.8 & 4 & 600.0 & \\
\hline 02 & 42615 & 77.2351 & 7.9250 & 285.1 & 4 & 600.0 & 60 \\
\hline 02 & 42616 & 75.5008 & 8.5500 & 284.3 & 4 & 600.0 & 600.0 \\
\hline 02 & 42617 & 69.7512 & 8.1000 & 284.0 & 4 & 600.0 & \\
\hline 02 & 42618 & 73.7492 & 9.6250 & 283.4 & 4 & 600.0 & 60 \\
\hline 02 & 42619 & 74.5000 & 9.7250 & 282.8 & 4 & 600.0 & 600 \\
\hline 02 & 42620 & 74.5000 & 9.1250 & 282.4 & 4 & 600.0 & .0 \\
\hline 02 & 42621 & 75.0007 & 8.2000 & 282.5 & 4 & 600.0 & \\
\hline 02 & 42622 & 71.0018 & 7.7250 & 282.4 & 4 & 600.0 & 600.0 \\
\hline 02 & 42623 & 69.9996 & 6.8000 & 282.2 & 4 & 600.0 & .0 \\
\hline 02 & 42624 & 37.0827 & 000 & 282.2 & 5 & 600.0 & \\
\hline 02 & 4271 & 38.4895 & 500 & 281.6 & 4 & 600.0 & 60 \\
\hline 02 & 4272 & 60.7512 & 15.0000 & 281.6 & 4 & 600.0 & .0 \\
\hline 02 & 4273 & 62.2499 & 14.7250 & 281.3 & 4 & 600.0 & \\
\hline 02 & $427 \quad 4$ & 63.9996 & 14.4250 & 281.4 & 4 & 600.0 & 60 \\
\hline 02 & $427 \quad 5$ & 60.5000 & 12.5250 & 281.0 & 4 & 600.0 & 600.0 \\
\hline 02 & 4276 & 47.0096 & 9 & 280.8 & 4 & 600.0 & .0 \\
\hline 02 & $427 \quad 7$ & 32.5008 & 500 & 281.1 & 4 & 600.0 & 60 \\
\hline 02 & 4278 & 57.2224 & 7.0500 & 282.0 & 4 & 600.0 & 600.0 \\
\hline 02 & 4279 & 51.2511 & 5.6500 & 282.4 & 3 & 600.0 & .0 \\
\hline 02 & 42710 & 32.2534 & 250 & 283.1 & 4 & 600.0 & 60 \\
\hline 02 & 42711 & 56.6252 & 5.4250 & 283.7 & 3 & 600.0 & 600.0 \\
\hline 02 & 42712 & 81.2629 & 5.0750 & 284.9 & 3 & 600.0 & .0 \\
\hline 02 & 42713 & 72.7534 & 500 & 285.6 & 4 & 600.0 & 600.0 \\
\hline 02 & 42714 & 67.7500 & 6.9500 & 285.5 & 4 & 600.0 & 600.0 \\
\hline 02 & 42715 & 68.7501 & 7.6250 & 285.6 & 4 & 600.0 & \\
\hline 02 & 42716 & 70.5000 & 7.6250 & 285.2 & 4 & 600.0 & 600.0 \\
\hline 02 & 42717 & 68.2528 & 7.5500 & 284.7 & 4 & 600.0 & 600.0 \\
\hline 02 & 42718 & 70.2497 & 7.6000 & 284.2 & 4 & 600.0 & 600.0 \\
\hline 02 & 42719 & 74.0007 & 7.9000 & 282.9 & 4 & 600.0 & 600.0 \\
\hline 02 & 42720 & 72.0006 & 9.6000 & 281.5 & 4 & 600.0 & 600.0 \\
\hline 02 & 42721 & 60.0003 & 8.9750 & 280.6 & 4 & 600.0 & 600.0 \\
\hline 02 & 42722 & 64.2503 & 7.250 & 28 & 4 & 600.0 & 600.0 \\
\hline 02 & 42723 & 64.7501 & 5.2000 & 280.0 & 4 & 600.0 & 600.0 \\
\hline
\end{tabular}




\begin{tabular}{|c|c|c|c|c|c|c|c|}
\hline 02 & 42724 & 74.7521 & 4.4250 & 279.9 & 4 & 600.0 & 00.0 \\
\hline 02 & 4281 & 74.2518 & 4.5000 & 279.8 & 4 & 600.0 & 600.0 \\
\hline 02 & 4282 & 65.7481 & 3.2750 & 279.6 & 4 & 600.0 & 00.0 \\
\hline 02 & 4283 & 84.7396 & 2.1000 & 279.4 & 5 & 600.0 & 600.0 \\
\hline 02 & 4284 & 78.7502 & 2.6250 & 279.3 & J & 600.0 & 00.0 \\
\hline 02 & 4285 & 65.9555 & 3.4750 & 279.2 & 4 & 600.0 & 00.0 \\
\hline 02 & 4286 & 67.0103 & 4.9250 & 279.4 & 4 & 600.0 & 600.0 \\
\hline 02 & 4287 & 69.5000 & 4.9000 & 279.6 & 4 & 600.0 & 00.0 \\
\hline 02 & $428 \quad 8$ & 62.0138 & 3.8250 & 280.5 & 4 & 600.0 & 00.0 \\
\hline 02 & 4289 & 94.2502 & 1.3750 & 282.7 & 3 & 600.0 & 600.0 \\
\hline 02 & 42810 & 260.5511 & 2.2500 & 284.0 & 2 & 600.0 & 600.0 \\
\hline 02 & 42811 & 282.0706 & 2.3250 & 285.4 & 1 & 600.0 & \\
\hline 02 & 42812 & 266.3265 & 2.5750 & 285.7 & 1 & 600.0 & 600.0 \\
\hline 02 & 42813 & 258.4374 & 2.4750 & 286.0 & 1 & 600.0 & 600 \\
\hline 02 & 42814 & 201.9931 & 3.4750 & 286.0 & 2 & 600.0 & \\
\hline 02 & 42815 & 236.7386 & 3.1000 & 287.0 & 2 & 600.0 & 60 \\
\hline 02 & 42816 & 167.0170 & 4.0250 & 286.4 & 3 & 600.0 & 600.0 \\
\hline 02 & 42817 & 173.2522 & 3.3750 & 287.1 & 2 & 600.0 & \\
\hline 02 & 42818 & 160.4976 & 5.1000 & 286.6 & 3 & 600.0 & 60 \\
\hline 02 & 42819 & 156.7511 & 5.5500 & 285.3 & 4 & 600.0 & 600 \\
\hline 02 & 42820 & 105.1011 & 5.5750 & 284.1 & 4 & 600.0 & .0 \\
\hline 02 & 42821 & 75.0000 & 7.4000 & 282.6 & 4 & 600.0 & \\
\hline 02 & 42822 & 79.0018 & 6.9250 & 282.3 & 4 & 600.0 & 600.0 \\
\hline 02 & 42823 & 76.7501 & 7.1000 & 281.8 & 4 & 600.0 & .0 \\
\hline 02 & 42824 & 75.5000 & 7.3750 & 281.7 & 4 & 600.0 & \\
\hline 02 & 4291 & 78.2500 & 7.9000 & 281.7 & 4 & 600.0 & 60 \\
\hline 02 & 4292 & 77.5000 & 5.3000 & 281.9 & 4 & 600.0 & 600.0 \\
\hline 02 & 4293 & 76.7500 & 7.2000 & 282.0 & 4 & 600.0 & \\
\hline 02 & 4294 & 77.2500 & 8.1000 & 281.5 & 4 & 600.0 & 60 \\
\hline 02 & 4295 & 74.2501 & 8.7500 & 281.1 & 4 & 600.0 & 600.0 \\
\hline 02 & 4296 & 74.5002 & .625 & 280.7 & 4 & 600.0 & .0 \\
\hline 02 & 4297 & 76.2500 & 8.4250 & 280.2 & 4 & 600.0 & 60 \\
\hline 02 & 4298 & 62.7495 & 6.5750 & 280.9 & 4 & 600.0 & 600.0 \\
\hline 02 & 4299 & 31.5000 & 3.8500 & 282.5 & 4 & 600.0 & .0 \\
\hline 02 & 42910 & 278.8407 & 2.6750 & 282.6 & 3 & 600.0 & 60 \\
\hline 02 & 42911 & 255.2609 & 2.6250 & 282.4 & 2 & 600.0 & 600.0 \\
\hline 02 & 42912 & 287.4229 & 1.0000 & 282.8 & 1 & 600.0 & .0 \\
\hline 02 & 42913 & 312.1200 & 1.0250 & 282.4 & 1 & 600.0 & 600.0 \\
\hline 02 & 42914 & 348.2575 & 2.1500 & 282.5 & 2 & 600.0 & 600.0 \\
\hline 02 & 42915 & 32.0034 & 2.6000 & 284.8 & 1 & 600.0 & \\
\hline 02 & 42916 & 110.4128 & 5.4000 & 285.9 & 2 & 600.0 & 600.0 \\
\hline 02 & 42917 & 117.2530 & 5.7250 & 285.8 & 3 & 600.0 & 600.0 \\
\hline 02 & 42918 & 123.5351 & 5.6250 & 285.2 & 3 & 600.0 & 600.0 \\
\hline 02 & 42919 & 88.7487 & 5.1000 & 284.3 & 4 & 600.0 & 600.0 \\
\hline 02 & 42920 & 70.4997 & 5.3500 & 283.3 & 4 & 600.0 & 600.0 \\
\hline 02 & 42921 & 78.7493 & 6.7500 & 281.9 & 4 & 600.0 & 600.0 \\
\hline 02 & 42922 & 72.5002 & 8.175 & 281.1 & 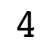 & 600.0 & 600.0 \\
\hline 02 & 42923 & 71.7521 & 8.7250 & 280.8 & 4 & 600.0 & 600.0 \\
\hline
\end{tabular}




\begin{tabular}{|c|c|c|c|c|c|c|c|}
\hline 2 & 42924 & 65.2499 & 9.1500 & 280.3 & 4 & 600.0 & 600.0 \\
\hline 02 & 4301 & 76.0011 & 8.0000 & 280.2 & 4 & 600.0 & 600.0 \\
\hline 02 & 4302 & 68.7500 & 8.8250 & 280.2 & 4 & 600.0 & 00.0 \\
\hline 02 & 4303 & 64.7501 & 8.8750 & 280.3 & 4 & 600.0 & 00.0 \\
\hline 02 & $430 \quad 4$ & 61.2501 & 7.5250 & 280.1 & 4 & 600.0 & 00.0 \\
\hline 02 & 4305 & 72.4707 & 5.5000 & 279.9 & 4 & 600.0 & 00.0 \\
\hline 02 & 4306 & 90.9197 & 4.0750 & 279.9 & 4 & 600.0 & 600.0 \\
\hline 02 & 4307 & 69.2156 & 4.8500 & 280.7 & 4 & 600.0 & .0 \\
\hline 02 & 4308 & 70.0073 & 6.0250 & 282.1 & 4 & 600.0 & 600.0 \\
\hline 02 & 4309 & 65.7535 & 5.7500 & 283.3 & 3 & 600.0 & 60 \\
\hline 02 & 43010 & 72.1064 & 750 & 285.0 & 3 & 600.0 & \\
\hline 02 & 43011 & 90.2745 & 250 & 285.2 & 3 & 600.0 & 60 \\
\hline 02 & 43012 & 84.9752 & 5.8250 & 284.6 & 3 & 600.0 & \\
\hline 02 & 43013 & 61.7052 & 5.7750 & 284.8 & 3 & 600.0 & 500.0 \\
\hline 02 & 43014 & 72.4779 & 00 & 285.4 & 3 & 600.0 & 60 \\
\hline 02 & 43015 & 65.7537 & 5.2 & 285.4 & 3 & 600.0 & 600.0 \\
\hline 02 & 43016 & 66.7506 & 00 & 284.3 & 4 & 600.0 & .0 \\
\hline 02 & 43017 & 76.7530 & 6 . & 283.7 & 4 & .0 & \\
\hline 02 & 43018 & 67.7468 & 6.6000 & 283.0 & 4 & 600.0 & \\
\hline 02 & 43019 & 62.7496 & 6 . & 282.5 & 4 & .0 & .0 \\
\hline 02 & 43020 & 56.7502 & 8 . & 281.5 & 4 & .0 & \\
\hline 02 & 43021 & 59.2504 & 500 & 281.2 & 4 & .0 & 60 \\
\hline 02 & 43022 & 65.2500 & 10.6250 & 280.9 & 4 & 60 & 600.0 \\
\hline 02 & 43023 & 64.7499 & 10 & 280.7 & 4 & .0 & \\
\hline 02 & 43024 & 60.2495 & 500 & 280.6 & 4 & .0 & 60 \\
\hline 02 & $\begin{array}{lll}5 & 1 & 1\end{array}$ & 60.5018 & 6.7000 & 280.7 & 4 & 600.0 & 600.0 \\
\hline 02 & $\begin{array}{lll}5 & 1 & 2\end{array}$ & 70.2496 & 6 . & 280.7 & 4 & .0 & .0 \\
\hline 02 & $\begin{array}{lll}5 & 1 & 3\end{array}$ & 71.0001 & 6 & 280.8 & 4 & 600.0 & 60 \\
\hline 02 & $\begin{array}{lll}5 & 1 & 4\end{array}$ & 68.2496 & 6.7750 & 280.9 & 4 & 600.0 & 600.0 \\
\hline 02 & 515 & 70.2496 & 6 . & 280.8 & 4 & .0 & .0 \\
\hline 02 & 516 & 68.7468 & 6 . & 280.6 & 4 & .0 & \\
\hline 02 & $\begin{array}{lll}5 & 1 & 7\end{array}$ & 61.7514 & 500 & 280.9 & 4 & .0 & 600.0 \\
\hline 02 & $\begin{array}{lll}5 & 1 & 8\end{array}$ & 71.7489 & 6.0500 & 281.8 & 4 & 600.0 & 600.0 \\
\hline 02 & $\begin{array}{lll}5 & 1 & 9\end{array}$ & 52.4545 & 4 . & 283.6 & 3 & 600.0 & 600.0 \\
\hline 02 & 5110 & 100.7559 & 2.0500 & 285.7 & 2 & 600.0 & 600.0 \\
\hline 02 & $5 \quad 111$ & 162.7942 & 2.7250 & 286.3 & 1 & 600.0 & 600.0 \\
\hline 02 & 5112 & 157.2431 & 5 . & 286.7 & 2 & 600.0 & 60 \\
\hline 02 & 5113 & 161.2319 & 5.3250 & 287.2 & 3 & 600.0 & 600.0 \\
\hline 02 & $5 \quad 114$ & 156.4990 & 5.0500 & 287.8 & 3 & 600.0 & 600.0 \\
\hline 02 & 5115 & 137.7640 & 000 & 288.2 & 3 & 600.0 & 600.0 \\
\hline 02 & 5116 & 129.6250 & 5.5500 & 288.4 & 3 & 600.0 & 600.0 \\
\hline 02 & 5117 & 131.7795 & 6.0000 & 288.6 & 4 & 600.0 & 600.0 \\
\hline 02 & 5118 & 94.9839 & 5.3750 & 287.9 & 3 & 600.0 & 600.0 \\
\hline 02 & 5119 & 72.2543 & 6.7500 & 286.1 & 4 & 600.0 & 600.0 \\
\hline 02 & 5120 & 72.0024 & 7.8250 & 284.5 & 4 & 600.0 & 600.0 \\
\hline 02 & 5121 & 75.2500 & 9.7500 & .9 & 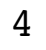 & 600.0 & 600.0 \\
\hline 02 & 5122 & 74.0000 & 10.2000 & 283.6 & 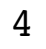 & 600.0 & 600.0 \\
\hline 02 & 5123 & 74.0003 & 10.4750 & 283.5 & 4 & 600.0 & 600.0 \\
\hline
\end{tabular}




\begin{tabular}{|c|c|c|c|c|c|c|c|c|}
\hline 02 & 5 & 124 & 72.7499 & 10.2750 & 283.6 & 4 & 600.0 & 600.0 \\
\hline 02 & 5 & 21 & 71.9998 & 10.9500 & 283.6 & 4 & 600.0 & 600.0 \\
\hline 02 & 5 & 22 & 71.2500 & 11.7000 & 283.4 & 4 & 600.0 & 00.0 \\
\hline 02 & 5 & 23 & 5.5000 & 11.5000 & 282.9 & . & 600.0 & 00.0 \\
\hline 02 & 5 & 24 & 72.0000 & 11.8500 & 282.5 & 4 & 600.0 & 00.0 \\
\hline 02 & 5 & 25 & 72.7500 & 12.3500 & 282.1 & 4 & 600.0 & 00.0 \\
\hline 02 & 5 & 26 & 0.7461 & 10.3250 & 282.4 & 4 & 600.0 & 600.0 \\
\hline 02 & 5 & 27 & 68.7499 & 9.9250 & 282.9 & 4 & 600.0 & .0 \\
\hline 02 & 5 & 28 & 86.0101 & 7.6000 & 284.0 & 4 & 600.0 & 600.0 \\
\hline 02 & 5 & 29 & 94.2507 & 7.5500 & 284.4 & 4 & 600.0 & 60 \\
\hline 02 & 5 & 210 & 97.2500 & 6.9750 & 285.8 & 4 & 600.0 & \\
\hline 02 & 5 & 211 & 99.5023 & 6.5000 & 287.0 & 4 & 600.0 & 60 \\
\hline 02 & 5 & 212 & 88.0003 & 6.9750 & 288.3 & 4 & 600.0 & \\
\hline 02 & 5 & 213 & 89.5000 & 6.6750 & 289.6 & 4 & 600.0 & 600.0 \\
\hline 02 & 5 & 214 & 80.7509 & 7 . & 290.3 & 4 & 600.0 & 60 \\
\hline 02 & 5 & 215 & 71.5000 & 8.8500 & 289.9 & 4 & 600.0 & 600 \\
\hline 02 & 5 & 216 & 75.4998 & 10.22 & 289.6 & 4 & 600.0 & .0 \\
\hline 02 & 5 & 217 & 76.2502 & 10.4750 & 288.8 & 4 & .0 & \\
\hline 02 & 5 & 218 & 76.0011 & 11.4750 & 286.7 & 4 & 600.0 & 60 \\
\hline 02 & 5 & 219 & 81.0000 & 12.52 & 284.7 & 4 & .0 & .0 \\
\hline 02 & 5 & 220 & 78.5006 & 13.2 & 283.4 & 4 & .0 & \\
\hline 02 & 5 & 221 & 76.7500 & 13.1500 & 283.2 & 4 & .0 & 60 \\
\hline 02 & 5 & 222 & 74.0000 & 13.8500 & 282.7 & 4 & .0 & 600.0 \\
\hline 02 & 5 & 223 & 79.0007 & 12.8000 & 283.0 & 4 & .0 & \\
\hline 02 & 5 & 224 & 78.2500 & 12.6500 & 282.8 & 4 & .0 & 60 \\
\hline 02 & 5 & 31 & 77.7500 & 13.2500 & 282.3 & 4 & 600.0 & .0 \\
\hline 02 & 5 & 32 & 79.5000 & 11.8500 & 282.1 & 4 & .0 & .0 \\
\hline 02 & 5 & 33 & 81.7500 & 11.4250 & 281.7 & 4 & 600.0 & 60 \\
\hline 02 & 5 & 34 & 82.0000 & 10.2000 & 281.2 & 4 & 600.0 & 600.0 \\
\hline 02 & 5 & 35 & 82.7499 & 11.5250 & 281.1 & 4 & 600.0 & .0 \\
\hline 02 & 5 & 36 & 500 & 10 . & 281.6 & 4 & .0 & \\
\hline 02 & 5 & 37 & 84.0001 & 9.2500 & 282.3 & 4 & 600.0 & 600.0 \\
\hline 02 & 5 & 38 & 85.5000 & 8.6500 & 283.5 & 4 & 600.0 & 600.0 \\
\hline 02 & 5 & & 89.9866 & 000 & 284.2 & 4 & 600.0 & 600.0 \\
\hline 02 & 5 & 310 & 88.0000 & 6.4500 & 285.4 & 4 & .0 & 600.0 \\
\hline 02 & 5 & 311 & 84.2523 & 5.1500 & 287.1 & 3 & 600.0 & 600.0 \\
\hline 02 & 5 & 312 & 88.7518 & 250 & 288.6 & 3 & 600.0 & 60 \\
\hline 02 & 5 & 313 & 88.0003 & 6.5500 & 289.3 & 4 & 600.0 & 600.0 \\
\hline 02 & 5 & 314 & 91.1724 & 6.2000 & 290.4 & 4 & 600.0 & 600.0 \\
\hline 02 & 5 & 315 & 81.9910 & 250 & 291.0 & 4 & 600.0 & 600.0 \\
\hline 02 & 5 & 316 & 76.4991 & 7.3750 & 290.8 & 4 & 600.0 & 600.0 \\
\hline 02 & 5 & 317 & 77.0009 & 6.7250 & 291.3 & 4 & 600.0 & 600.0 \\
\hline 02 & 5 & 318 & 80.2501 & 7.6750 & 290.1 & 4 & 600.0 & 600.0 \\
\hline 02 & 5 & 319 & 78.4999 & 8.6750 & 288.9 & 4 & 600.0 & 600.0 \\
\hline 02 & 5 & 320 & 76.2501 & 8.4000 & 288.5 & 4 & 600.0 & 600.0 \\
\hline 02 & 5 & 321 & 73.7499 & 10.2000 & 287.0 & 4 & 600.0 & \\
\hline 02 & 5 & 322 & 78.0002 & 10.5000 & 286.4 & 4 & 600.0 & 600.0 \\
\hline 02 & 5 & 323 & 80.5003 & 10.575 & 286.3 & 4 & 600.0 & 600.0 \\
\hline
\end{tabular}




\begin{tabular}{|c|c|c|c|c|c|c|c|c|}
\hline 2 & 5 & 324 & 78.7500 & 9.9500 & 286.9 & 4 & 600.0 & 600.0 \\
\hline 02 & 5 & 41 & 84.0002 & 8.7750 & 286.8 & 4 & 600.0 & 600.0 \\
\hline 02 & 5 & 42 & 81.0000 & 6.6000 & 287.5 & 4 & 600.0 & 00.0 \\
\hline 02 & 5 & 43 & 90.4991 & 4.2000 & 288.1 & 4 & 600.0 & 00.0 \\
\hline 02 & 5 & 44 & 112.6708 & 2.9250 & 287.6 & 4 & 600.0 & 00.0 \\
\hline 02 & 5 & 45 & 40.9645 & 0.7500 & 285.8 & 5 & 600.0 & 00.0 \\
\hline 02 & 5 & 46 & 127.4997 & 1.4500 & 285.6 & 5 & 600.0 & 00.0 \\
\hline 02 & 5 & 47 & 148.4972 & 2.1250 & 287.1 & 4 & 600.0 & 00.0 \\
\hline 02 & 5 & 48 & 189.0715 & 1.2000 & 289.9 & 3 & 600.0 & 00.0 \\
\hline 02 & 5 & 49 & 179.4195 & 2.7000 & 291.1 & 2 & 600.0 & .0 \\
\hline 02 & 5 & 410 & 177.7506 & 3.8000 & 291.2 & 3 & 600.0 & \\
\hline 02 & 5 & 411 & 180.9986 & 000 & 291.9 & 3 & 600.0 & \\
\hline 02 & 5 & 412 & 171.7499 & 4.6000 & 292.9 & 3 & 600.0 & 00.0 \\
\hline 02 & 5 & 413 & 185.7499 & 4.9500 & 293.9 & 3 & 600.0 & 00.0 \\
\hline 02 & 5 & 414 & 171.0005 & 50 & 294.8 & 3 & .0 & \\
\hline 02 & 5 & 415 & 172.2629 & 5.9250 & 296.0 & 3 & 600.0 & 00.0 \\
\hline 02 & 5 & 416 & 138.0023 & 4.7000 & 297.1 & 3 & 60 & .0 \\
\hline 02 & 5 & 417 & 113.8156 & 5. & 296.2 & 3 & .0 & \\
\hline 02 & 5 & 418 & 92.7516 & 6.0250 & 294.9 & 4 & 600.0 & .0 \\
\hline 02 & 5 & 419 & 76.7503 & 7.0750 & 292.9 & 4 & .0 & .0 \\
\hline 02 & 5 & 420 & 77.5000 & 7 . & 291.7 & 4 & 6 & \\
\hline 02 & 5 & 421 & 73.2499 & 000 & 290.8 & 4 & .0 & \\
\hline 02 & 5 & 422 & 76.0000 & 9.6250 & 290.8 & 4 & 600.0 & .0 \\
\hline 02 & 5 & 423 & 75.7501 & 9. & 291.0 & 4 & 6 & \\
\hline 02 & 5 & 424 & 74.5000 & 000 & 291.5 & 4 & .0 & \\
\hline 02 & 5 & 51 & 71.7488 & 7.5750 & 292.7 & 4 & 600.0 & 600.0 \\
\hline 02 & 5 & 52 & 79.2487 & 7.4750 & 293.1 & 4 & 600.0 & .0 \\
\hline 02 & 5 & 53 & 73.7685 & 5. & 291.8 & 4 & .0 & \\
\hline 02 & 5 & 54 & 137.8862 & 2.5000 & 289.8 & 5 & .0 & 600.0 \\
\hline 02 & 5 & 55 & 52.8189 & 0.9750 & 287.3 & 6 & 60 & 0.0 \\
\hline 02 & 5 & 56 & 124.4653 & 2.2750 & 288.3 & 6 & .0 & \\
\hline 02 & 5 & 57 & 161.8664 & 2.1500 & 288.9 & 5 & .0 & 600.0 \\
\hline 02 & 5 & 58 & 172.0000 & 4.4500 & 290.6 & 4 & 600.0 & 600.0 \\
\hline 02 & 5 & 59 & 167.0177 & 5.8000 & 291.4 & 4 & 600.0 & 0.0 \\
\hline 02 & 5 & 510 & 166.2498 & 7.27 & 291.9 & 4 & 600.0 & 600.0 \\
\hline 02 & 5 & 511 & 167.7505 & 6.7750 & 293.2 & 4 & 600.0 & 600.0 \\
\hline 02 & 5 & 512 & 162.2501 & 7.1250 & 294.3 & 4 & 600.0 & 0.0 \\
\hline 02 & 5 & 513 & 164.7481 & 7.3000 & 295.8 & 4 & 600.0 & 600.0 \\
\hline 02 & 5 & 514 & 158.0096 & 6.3750 & 297.2 & 4 & 600.0 & 600.0 \\
\hline 02 & 5 & 515 & 168.5094 & 6.2250 & 298.2 & 4 & 600.0 & .0 \\
\hline 02 & 5 & 516 & 142.5855 & 6.1250 & 298.5 & 4 & 600.0 & 600.0 \\
\hline 02 & 5 & 517 & 88.7514 & 7.0250 & 297.2 & 4 & 600.0 & 600.0 \\
\hline 02 & 5 & 518 & 89.4986 & 7.1250 & 296.1 & 4 & 600.0 & 600.0 \\
\hline 02 & 5 & 519 & 78.2213 & 6.9250 & 294.8 & 4 & 600.0 & 600.0 \\
\hline 02 & 5 & 520 & 73.7519 & 8.0000 & 293.5 & 4 & 600.0 & 600.0 \\
\hline 02 & 5 & 521 & 71.7501 & 9.1500 & 292.4 & 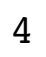 & 600.0 & 600.0 \\
\hline 02 & 5 & 522 & 74.7499 & 9.7000 & 291.9 & 4 & 600.0 & 600.0 \\
\hline 0 & & 523 & 72.5000 & 10.700 & 291.0 & 4 & 600.0 & 600.0 \\
\hline
\end{tabular}




\begin{tabular}{|c|c|c|c|c|c|c|c|c|}
\hline 2 & 5 & 524 & 72.9993 & 9.9500 & 290.8 & 4 & 600.0 & 600.0 \\
\hline 02 & 5 & 61 & 75.4999 & 9.6250 & 290.7 & 4 & 600.0 & 00.0 \\
\hline 02 & 5 & 62 & 74.5032 & 10.1000 & 289.9 & 4 & 600.0 & 00.0 \\
\hline 02 & 5 & 63 & 76.2502 & 10.2250 & 289.4 & 4 & 600.0 & 00.0 \\
\hline 02 & 5 & 64 & 73.2498 & 10.0500 & 288.8 & 4 & 600.0 & 00.0 \\
\hline 02 & 5 & 65 & 76.7502 & 9.8750 & 288.3 & 4 & 600.0 & 00.0 \\
\hline 02 & 5 & 66 & 73.7499 & 750 & 287.8 & 4 & 600.0 & 00.0 \\
\hline 02 & 5 & 67 & 76.0011 & 10.1500 & 287.8 & 4 & 600.0 & 00.0 \\
\hline 02 & 5 & 68 & 71.5003 & 9.4500 & 288.5 & 4 & 600.0 & 00.0 \\
\hline 02 & 5 & 69 & 74.2500 & 9.2500 & 288.5 & 4 & 600.0 & .0 \\
\hline 02 & 5 & 610 & 76.2500 & 7.5000 & 289.8 & 4 & 600.0 & \\
\hline 02 & 5 & 611 & 87.7505 & 5.7000 & 291.8 & 3 & 600.0 & \\
\hline 02 & 5 & 612 & 81.9973 & 7.4250 & 292.0 & 4 & 600.0 & 00.0 \\
\hline 02 & 5 & 613 & 70.5014 & 8.4750 & 292.5 & 4 & 600.0 & 00.0 \\
\hline 02 & 5 & 614 & 82.7507 & 00 & 292.6 & 4 & .0 & \\
\hline 02 & 5 & 615 & 76.4979 & 000 & 292.3 & 4 & 600.0 & .0 \\
\hline 02 & 5 & 616 & 67.7444 & 9.5750 & 292.3 & 4 & 60 & .0 \\
\hline 02 & 5 & 617 & 71.2501 & 10 & 291.0 & 4 & .0 & \\
\hline 02 & 5 & 618 & 69.2495 & 11.4500 & 289.3 & 4 & 60 & .0 \\
\hline 02 & 5 & 619 & 75.7500 & 11.8500 & 287.3 & 4 & .0 & .0 \\
\hline 02 & 5 & 620 & 77.2500 & 13. & 285.6 & 4 & 6 & \\
\hline 02 & 5 & 621 & 76.5000 & 13. & 285.3 & 4 & .0 & \\
\hline 02 & 5 & 622 & 75.7502 & 13.6750 & 284.6 & 4 & 600.0 & .0 \\
\hline 02 & 5 & 623 & 73.2500 & 14 . & 284.4 & 4 & 6 & \\
\hline 02 & 5 & 624 & 72.2482 & 14 & 284.2 & 4 & 60 & \\
\hline 02 & 5 & 71 & 75.4997 & 13.0500 & 283.8 & 4 & .0 & \\
\hline 02 & 5 & 72 & 77.2500 & 13.7750 & 284.3 & 4 & 60 & .0 \\
\hline 02 & 5 & 73 & 69.4997 & 13 & 285.0 & 4 & .0 & \\
\hline 02 & 5 & 74 & 70.0000 & 13.7750 & 284.6 & 4 & .0 & 0.0 \\
\hline 02 & 5 & 75 & 72.2498 & 14.0000 & 284.3 & 4 & 60 & \\
\hline 02 & 5 & 76 & 70.5003 & 14 . & 283.6 & 4 & .0 & \\
\hline 02 & 5 & 77 & 72.5000 & 12.90 & 284.0 & 4 & .0 & 0.0 \\
\hline 02 & 5 & 78 & 71.5003 & 11.4250 & 284.7 & 4 & 600.0 & 600.0 \\
\hline 02 & 5 & 79 & 72.7502 & 11.0000 & 285.3 & 4 & 600.0 & 0.0 \\
\hline 02 & 5 & 710 & 79.2317 & 9.1500 & 286.2 & 4 & 600.0 & 600.0 \\
\hline 02 & 5 & 711 & 95.0023 & 7.6000 & 287.0 & 4 & 600.0 & 600.0 \\
\hline 02 & 5 & 712 & 97.0001 & 7.6250 & 287.8 & 4 & 600.0 & .0 \\
\hline 02 & 5 & 713 & 118.9544 & 6.5250 & 289.2 & 4 & 600.0 & 600.0 \\
\hline 02 & 5 & 714 & 146.9976 & 7.3750 & 289.9 & 4 & 600.0 & 00.0 \\
\hline 02 & 5 & 715 & 158.2382 & 6.8000 & 290.9 & 4 & 60 & .0 \\
\hline 02 & 5 & 716 & 154.2235 & 6.6750 & 291.9 & 4 & 600.0 & 600.0 \\
\hline 02 & 5 & 717 & 179.2610 & 8.7500 & 292.2 & 4 & 600.0 & 600.0 \\
\hline 02 & 5 & 718 & 181.0016 & 9.4000 & 291.9 & 4 & 600.0 & 600.0 \\
\hline 02 & 5 & 719 & 166.7416 & 7.3750 & 291.2 & 4 & 600.0 & 600.0 \\
\hline 02 & 5 & 720 & 141.7412 & 5.4500 & 289.8 & 4 & 600.0 & 600.0 \\
\hline 02 & 5 & 721 & 133.7535 & 6.0750 & 289.7 & . & 600.0 & 600.0 \\
\hline 02 & 5 & 722 & 148.2503 & 10.1000 & 289.6 & 4 & 600.0 & 600.0 \\
\hline 4 & & 723 & 143.0004 & 9.925 & 289.3 & & 600.0 & 600.0 \\
\hline
\end{tabular}




\begin{tabular}{|c|c|c|c|c|c|c|c|c|}
\hline & 5 & 4 & & & & & & \\
\hline & 5 & 1 & 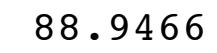 & .8250 & & & & \\
\hline & 5 & 2 & 117.2019 & 8.5250 & 287.8 & & 00 & \\
\hline & 5 & 83 & 39.5005 & 9.1250 & 287.8 & & 00 & \\
\hline & & 4 & 40.4998 & & & & & \\
\hline & 5 & 5 & 48.7495 & & 87.4 & & 0 & \\
\hline & 5 & 6 & 157.7499 & 000 & 287.3 & & 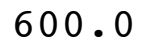 & \\
\hline & 5 & $r$ & .71 .0096 & & 87.7 & & & \\
\hline & 5 & 8 & 177.2500 & 9 & 3 & & & \\
\hline & & 9 & 174.2502 & 750 & 288.9 & & & \\
\hline & 5 & 810 & 169.2580 & & 285 & & & \\
\hline & 5 & 1 & a $\cap \cap 11$ & & 90.6 & & & \\
\hline & J & 12 & 950 & 4 . & 292 & & & \\
\hline & - & 13 & 171.2337 & 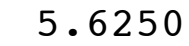 & 29 & & & \\
\hline & 5 & 14 & 171 & & 5 & & & \\
\hline & 5 & 15 & 171 & & 29 & & & \\
\hline & $\Gamma$ & 816 & 157. & & 29 & & & \\
\hline & 5 & 17 & 119 . & - & 7 & & & \\
\hline & 5 & - & 94. & & 29 & & & \\
\hline & 5 & 19 & 77 & & 291 & 4 & & \\
\hline 2 & 5 & 820 & 68.7500 & U & 290.3 & 1 & - 0 & \\
\hline & 5 & 821 & 61.9977 & & & & & \\
\hline 2 & 5 & 822 & 497 & & 29 & 1 & & \\
\hline & 5 & 823 & 643 & U & 28 & & 0 & \\
\hline & 5 & 824 & & & & & & \\
\hline 02 & 5 & 91 & 67 & & 28 & 4 & & \\
\hline 0 & 5 & 92 & 008 & 0 & 288.4 & 2 & 0 & \\
\hline & 5 & 93 & & & 28 & 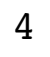 & & \\
\hline 02 & 5 & 94 & 74 & & 28 & 4 & & \\
\hline & 5 & 95 & & & & 4 & & \\
\hline 02 & 5 & 96 & 7 & 0 & 28 & 4 & & \\
\hline 02 & 5 & 97 & & & 2 & 4 & & \\
\hline . & 5 & 98 & & & 28 & 4 & & \\
\hline 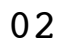 & 5 & 99 & & 0 & 28 & 4 & & \\
\hline 02 & 5 & 910 & & & & 4 & & \\
\hline 02 & 5 & 911 & & & 291.0 & 4 & & \\
\hline 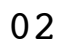 & 5 & 912 & & 7 . & 291.6 & 4 & & \\
\hline 2 & 5 & 913 & 6 & 7 . & 292.3 & 4 & & \\
\hline 02 & 5 & 914 & & & 292.4 & 4 & .0 & \\
\hline 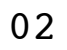 & 5 & 91 & & & 292.5 & a & & \\
\hline 02 & 5 & 91 & & 0 & 291.8 & 4 & & \\
\hline 02 & 5 & 917 & & 0 & 291.3 & 4 & & \\
\hline 02 & 5 & 918 & 019 & 750 & 289.6 & 4 & & 0 \\
\hline 02 & 5 & 919 & 74 . & 11 . & 287.7 & & & \\
\hline 02 & 5 & 920 & & 11.17 & 285.9 & & & \\
\hline 02 & 5 & 921 & 68.4862 & 10.0250 & 286.5 & 4 & & 0 \\
\hline & 5 & 922 & & & & 4 & & \\
\hline & & 923 & 62.7500 & 11.3500 & 286.2 & & .0 & 600 \\
\hline
\end{tabular}




\begin{tabular}{|c|c|c|c|c|c|c|c|}
\hline 2 & $5 \quad 924$ & 62.5034 & 6.3250 & 285.7 & 4 & 600.0 & 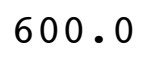 \\
\hline 2 & 5101 & 69.7497 & 10.7500 & 285.6 & 4 & 600.0 & 600.0 \\
\hline 02 & 5102 & 71.0000 & 11.4000 & 284.8 & 4 & 600.0 & 00.0 \\
\hline 02 & 5103 & 72.2499 & 13.0500 & 283.6 & 4 & 600.0 & 00.0 \\
\hline 2 & 5104 & 68.9998 & 12.9250 & 282.5 & 4 & 600.0 & 00.0 \\
\hline 02 & 5105 & 68.2482 & 11.5500 & 281.9 & 4 & 600.0 & .0 \\
\hline 02 & 5106 & 67.5000 & 9.9000 & 281.8 & 4 & 600.0 & 00.0 \\
\hline 02 & 5107 & 68.7492 & 10.5500 & 281.8 & 4 & 600.0 & .0 \\
\hline 02 & 5108 & 72.9995 & 10.5750 & 282.2 & 4 & 600.0 & \\
\hline 02 & 5109 & 83.5009 & 8.4250 & 283.6 & 4 & 600.0 & 600.0 \\
\hline 02 & 51010 & 103.5452 & 6.5000 & 285.4 & 4 & 600.0 & 0 \\
\hline 02 & 51011 & 102.5038 & 5.3250 & 286.7 & 3 & 600.0 & \\
\hline 02 & 51012 & 106.5037 & 5.9000 & 287.5 & 3 & 600.0 & .0 \\
\hline 02 & 51013 & 106.2504 & 7.07 & 288.1 & 4 & 600.0 & \\
\hline 02 & 51014 & 111.5000 & 8.10 & 287.9 & 4 & 600.0 & \\
\hline 02 & 51015 & 105.4931 & 9.25 & 287.6 & 4 & 600.0 & \\
\hline 02 & 51016 & 80.0069 & 8.7500 & 287.3 & 4 & 600.0 & 00.0 \\
\hline 02 & 51017 & 70.7310 & 7.6750 & 287.5 & 4 & 600.0 & .0 \\
\hline 02 & 51018 & 80.2499 & 7 . & 287.4 & 4 & 600.0 & \\
\hline 02 & 51019 & 71.2512 & 7.5000 & 286.0 & 4 & 600.0 & 600.0 \\
\hline 02 & 51020 & 83.2507 & 7.5750 & 285.8 & 4 & 600.0 & .0 \\
\hline 02 & 51021 & 87.5000 & 8.22 & 285.9 & 4 & 600.0 & \\
\hline 02 & 51022 & 94.7504 & 8.0250 & 286.0 & 4 & 600.0 & 60 \\
\hline 02 & 51023 & 104.7508 & 7.8250 & 286.4 & 4 & 600.0 & .0 \\
\hline 02 & 51024 & 100.5010 & 7.45 & 286.4 & 4 & 600.0 & \\
\hline 02 & 5111 & 93.2393 & 8.3250 & 286.3 & 4 & 600.0 & .0 \\
\hline 02 & 5112 & 93.0004 & 8.5750 & 285.7 & 4 & 600.0 & .0 \\
\hline 02 & 5113 & 109.2153 & 7.22 & 286.5 & 4 & 600.0 & .0 \\
\hline 02 & 5114 & 133.7595 & 7.4000 & 286.7 & 4 & 600.0 & .0 \\
\hline 02 & 5115 & 140.9993 & 8.8250 & 286.5 & 4 & 600.0 & .0 \\
\hline 02 & 5116 & 146.7500 & 8.3750 & 286.6 & 4 & 600.0 & .0 \\
\hline 02 & 5117 & 148.2498 & 9.1250 & 287.6 & 4 & 600.0 & \\
\hline 02 & 5118 & 166.0023 & 10.4750 & 288.5 & 4 & 600.0 & .0 \\
\hline 02 & 5119 & 165.4990 & 10.3500 & 289.2 & 4 & 600.0 & .0 \\
\hline 02 & 51110 & 171.4997 & 10.5250 & 290.1 & 4 & 600.0 & \\
\hline 02 & 51111 & 174.0000 & 9.4000 & 291.5 & 4 & 600.0 & 600.0 \\
\hline 02 & 51112 & 177.2431 & 7.6250 & 292.9 & 4 & 600.0 & 600.0 \\
\hline 02 & 51113 & 171.2556 & 6 . & 294.0 & 4 & 600.0 & 60 \\
\hline 02 & 51114 & 170.0000 & 7.0250 & 294.9 & 4 & 600.0 & 600.0 \\
\hline 02 & 51115 & 169.2269 & 6.9250 & 295.4 & 4 & 600.0 & 600 \\
\hline 02 & 51116 & 162.3015 & 6.3250 & 296.1 & 4 & 600.0 & 600.0 \\
\hline 02 & 51117 & 158.7511 & 6.4500 & 296.7 & 4 & 600.0 & 600.0 \\
\hline 02 & 51118 & 98.7785 & 5.4250 & 296.0 & 3 & 600.0 & 600.0 \\
\hline 02 & 51119 & 72.5000 & 5.9000 & 294.3 & 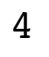 & 600.0 & 600.0 \\
\hline 02 & 51120 & 69.5000 & 6.9500 & 292.7 & 4 & 600.0 & 600.0 \\
\hline 02 & 51121 & 72.0004 & 7.8250 & 292.1 & 4 & 600.0 & 600.0 \\
\hline 02 & 51122 & 80.5005 & & .0 & 4 & 600.0 & .0 \\
\hline 02 & 51123 & 75.7500 & 9.075 & 291.4 & $I$ & 600.0 & 600.0 \\
\hline
\end{tabular}




\begin{tabular}{|c|c|c|c|c|c|c|c|}
\hline 02 & 51124 & 74.0001 & 9.3250 & 291.3 & 4 & 600.0 & 00.0 \\
\hline 2 & 5121 & 71.5000 & 9.4250 & 291.4 & 4 & 600.0 & 600.0 \\
\hline 02 & 5122 & 72.5002 & 9.1250 & 291.5 & 4 & 600.0 & 00.0 \\
\hline 02 & 5123 & 74.0001 & 8.4750 & 291.6 & 4 & 600.0 & 00.0 \\
\hline 02 & 124 & 69.2494 & 6.2000 & 292.2 & 4 & 600.0 & 00.0 \\
\hline 02 & 125 & 108.7289 & 1.2500 & 291.9 & 5 & 600.0 & 00.0 \\
\hline 02 & 126 & 126.7298 & 0.7500 & 290.5 & 6 & 600.0 & 500.0 \\
\hline 02 & 5127 & 160.7349 & 0.1750 & 294.0 & 5 & 600.0 & .0 \\
\hline 02 & 5128 & 213.2958 & 1.7750 & 295.1 & 4 & 600.0 & \\
\hline 02 & 5129 & 242.7425 & 2.4250 & 295.0 & 3 & 600.0 & 600.0 \\
\hline 02 & 51210 & 246.2621 & 2.7750 & 295.7 & 2 & 600.0 & 0 \\
\hline 02 & 1211 & 236.9903 & 3.5250 & 296.1 & 2 & 600.0 & \\
\hline 02 & 51212 & 236.7218 & 3.4250 & 297.2 & 2 & 600.0 & .0 \\
\hline 02 & 51213 & 214.0929 & 4.8750 & 297.5 & 3 & 600.0 & \\
\hline 02 & 51214 & 223.0166 & 3 & 298.1 & 2 & 600.0 & .0 \\
\hline 02 & 1215 & 191.7461 & 4 . & 298.4 & 3 & 600.0 & \\
\hline 02 & 51216 & 191.3666 & 4.8750 & 298.5 & 3 & 600.0 & 600.0 \\
\hline 02 & 51217 & 191.5089 & 2 . & 299.2 & 2 & 600.0 & .0 \\
\hline 02 & 1218 & 198.3080 & 2.4750 & 298.8 & 1 & 600.0 & \\
\hline 02 & 1219 & 156.7868 & 4.3750 & 296.8 & 2 & 600.0 & \\
\hline 02 & 51220 & 93.4188 & 5.9750 & 295.3 & 3 & 600.0 & .0 \\
\hline 02 & 51221 & 75.9995 & 7.62 & 293.6 & 4 & 600.0 & \\
\hline 02 & 51222 & 72.7492 & 10.1750 & 292.5 & 4 & 600.0 & 60 \\
\hline 02 & 51223 & 74.5000 & 10. & 291.5 & 4 & 600.0 & .0 \\
\hline 02 & 51224 & 76.0002 & 10 & 290.4 & 4 & 600.0 & \\
\hline 02 & 5131 & 76.5000 & 11 . & 289.9 & 4 & 600.0 & 60 \\
\hline 02 & 5132 & 76.5002 & 11.4500 & 289.8 & 4 & 600.0 & .0 \\
\hline 02 & 5133 & 76.5000 & 10.30 & 290.6 & 4 & 600.0 & .0 \\
\hline 02 & 5134 & 80.0002 & 10 . & 290.5 & 4 & 600.0 & .0 \\
\hline 02 & 5135 & 77.7496 & 10.9750 & 289.2 & 4 & 600.0 & .0 \\
\hline 02 & 5136 & 73.4998 & 10.3750 & 289.6 & 4 & 600.0 & 60 \\
\hline 02 & 5137 & 76.5002 & 9.9250 & 289.8 & 4 & 600.0 & \\
\hline 02 & 5138 & 82.5000 & 8.8750 & 290.2 & 4 & 600.0 & .0 \\
\hline 02 & 5139 & 83.4998 & 8.0500 & 290.9 & 4 & 600.0 & .0 \\
\hline 02 & 51310 & 83.7504 & 7 . & 290.7 & 4 & 600.0 & \\
\hline 02 & 51311 & 82.0000 & 9.6000 & 290.4 & 4 & 600.0 & 600.0 \\
\hline 02 & 51312 & 78.0023 & 8.8750 & 291.9 & 4 & 600.0 & 600.0 \\
\hline 02 & 51313 & 77.5002 & 9.4500 & 293.0 & 4 & 600.0 & 60 \\
\hline 02 & 51314 & 79.2502 & 11.0750 & 292.6 & 4 & 600.0 & 600.0 \\
\hline 02 & 51315 & 71.7494 & 11.8500 & 293.0 & 4 & 600.0 & 600.0 \\
\hline 02 & 51316 & 71.5120 & 12.7250 & 293.0 & 4 & 600.0 & 600.0 \\
\hline 02 & 51317 & 62.2511 & 12.5750 & 292.2 & 4 & 600.0 & 600.0 \\
\hline 02 & 51318 & 68.7501 & 12.5500 & 291.3 & 4 & 600.0 & 600.0 \\
\hline 02 & 51319 & 66.0000 & 13.5250 & 291.0 & 4 & 600.0 & 600.0 \\
\hline 02 & 51320 & 78.9924 & 7.2500 & 289.5 & 4 & 600.0 & 600.0 \\
\hline 02 & 51321 & 68.7500 & 11.4750 & 289.8 & 4 & 600.0 & 600.0 \\
\hline 02 & 51322 & 69.2498 & 10 . & 290.4 & 4 & 600.0 & 600.0 \\
\hline 02 & 51323 & 70.9991 & 11.025 & 289.1 & $\mathbf{4}$ & 600.0 & 600.0 \\
\hline
\end{tabular}




\begin{tabular}{|c|c|c|c|c|c|c|c|}
\hline 02 & 51324 & 73.2500 & 11.4000 & 287.9 & 4 & 600.0 & 600.0 \\
\hline 02 & 5141 & 75.5003 & 12.2000 & 286.6 & 4 & 600.0 & 600.0 \\
\hline 02 & 5142 & 73.7498 & 11.6250 & 286.4 & 4 & 600.0 & 00.0 \\
\hline 02 & 5143 & 72.2500 & 12.6250 & 285.9 & 4 & 600.0 & 00.0 \\
\hline 02 & 5144 & 75.5000 & 11.9000 & 285.6 & 4 & 600.0 & 00.0 \\
\hline 02 & 5145 & 81.2500 & 10.3750 & 284.9 & 4 & 600.0 & 00.0 \\
\hline 02 & 5146 & 78.2499 & 9.6500 & 284.7 & 4 & 600.0 & 00.0 \\
\hline 02 & 5147 & 91.2455 & 8.4250 & 285.8 & 4 & 600.0 & 00.0 \\
\hline 02 & 5148 & 127.0000 & 7.0250 & 288.1 & 4 & 600.0 & 00.0 \\
\hline 02 & 5149 & 152.4988 & 6.8250 & 289.1 & 4 & 600.0 & 0 \\
\hline 02 & 51410 & 154.7501 & 7.5500 & 290.6 & 4 & 600.0 & \\
\hline 02 & 1411 & 161.4872 & 000 & 291.3 & 4 & 600.0 & \\
\hline 02 & 51412 & 159.7851 & 4.6500 & 293.2 & 3 & 600.0 & 00.0 \\
\hline 02 & 51413 & 163.7494 & 4.3750 & 295.1 & 3 & 600.0 & \\
\hline 02 & 1414 & 163.2228 & 4 . & 296.4 & 3 & .0 & \\
\hline 02 & 51415 & 153.4990 & 5.3000 & 297.2 & 3 & 600.0 & .0 \\
\hline 02 & 51416 & 121.6883 & 6.2000 & 297.3 & 4 & 60 & .0 \\
\hline 02 & 51417 & 106.2461 & 7.20 & 296.5 & 4 & .0 & \\
\hline 02 & 51418 & 88.7514 & 7.1250 & 294.8 & 4 & 60 & .0 \\
\hline 02 & 51419 & 69.9904 & 8.6000 & 293.1 & 4 & .0 & .0 \\
\hline 02 & 51420 & 76.2504 & 10.95 & 292.1 & 4 & 6 & \\
\hline 02 & 51421 & 71.5000 & 9.7000 & 292.5 & 4 & .0 & \\
\hline 02 & 51422 & 73.2500 & 8.9000 & 293.2 & 4 & 600.0 & .0 \\
\hline 02 & 51423 & 78.2498 & 8.47 & 293.5 & 4 & 0 & \\
\hline 02 & 51424 & 79.0000 & 9.02 & 293.2 & 4 & .0 & \\
\hline 02 & 5151 & 76.7502 & 8.8250 & 292.8 & 4 & .0 & \\
\hline 02 & 5152 & 80.2500 & 8.9250 & 292.6 & 4 & 60 & .0 \\
\hline 02 & 5153 & 81.7499 & 8.47 & 292.7 & 4 & .0 & \\
\hline 02 & 5154 & 78.9995 & 9.7500 & 291.4 & 4 & .0 & 0.0 \\
\hline 02 & 5155 & 79.9998 & 8.92 & 291.0 & 4 & 60 & \\
\hline 02 & 5156 & 82.0000 & 8.47 & 290.5 & 4 & .0 & \\
\hline 02 & $\begin{array}{ll}5157 & 7\end{array}$ & 87.7501 & 6.02 & 291.1 & 4 & .0 & 600.0 \\
\hline 02 & 5158 & 119.5913 & 3.1750 & 293.4 & 3 & 600.0 & 600.0 \\
\hline 02 & 5159 & 168.1836 & $3 . \varepsilon$ & 294.3 & 2 & .0 & 0.0 \\
\hline 02 & 51510 & 171.0007 & 5.52 & 294.3 & 3 & 600.0 & 600.0 \\
\hline 02 & 51511 & 163.4992 & 6.6500 & 294.4 & 4 & 600.0 & 600.0 \\
\hline 02 & 51512 & 156.5003 & 6.8250 & 294.9 & 4 & 600.0 & .0 \\
\hline 02 & 51513 & 160.7472 & 6.1750 & 296.3 & 4 & 600.0 & 600.0 \\
\hline 02 & 51514 & 149.1025 & 6.2000 & 297.5 & 4 & 600.0 & 600.0 \\
\hline 02 & 51515 & 103.2377 & 6.7500 & 297.3 & 4 & 600.0 & 600.0 \\
\hline 02 & 51516 & 94.5009 & 7.7750 & 296.8 & 4 & 600.0 & 600.0 \\
\hline 02 & 51517 & 77.5000 & 7.5750 & 295.8 & 4 & 600.0 & 600.0 \\
\hline 02 & 51518 & 78.2512 & 6.6500 & 295.6 & 4 & 600.0 & 600.0 \\
\hline 02 & 51519 & 86.2492 & 6.8500 & 294.4 & 4 & 600.0 & 600.0 \\
\hline 02 & 51520 & 77.7500 & 7.9500 & 293.1 & 4 & 600.0 & 600.0 \\
\hline 02 & 51521 & 76.2500 & 9.5750 & 292.5 & . & 600.0 & 600.0 \\
\hline 02 & 51522 & 74.0000 & 10.2000 & 292.4 & 4 & 600.0 & 600.0 \\
\hline 48 & 51523 & 71.0001 & 10.9500 & 292.0 & 4 & 600.0 & 600.0 \\
\hline
\end{tabular}




\begin{tabular}{|c|c|c|c|c|c|c|c|}
\hline 02 & 51524 & 67.0000 & 11.2000 & 291.5 & 4 & 600.0 & 00.0 \\
\hline 02 & 5161 & 67.5000 & 11.4000 & 291.5 & 4 & 600.0 & 600,0 \\
\hline 02 & 5162 & 74.4807 & 12.1500 & 291.1 & 4 & 600.0 & 00.0 \\
\hline 02 & 5163 & 89.7647 & 9.1000 & 291.0 & 4 & 600.0 & 00.0 \\
\hline 02 & 164 & 101.7473 & 7.0250 & 292.1 & 4 & 600.0 & 00.0 \\
\hline 02 & 165 & 103.9876 & 6.8500 & 291.6 & 4 & 600.0 & 00.0 \\
\hline 02 & 166 & 112.5006 & 6.8000 & 292.0 & 4 & 600.0 & 600.0 \\
\hline 02 & 167 & 127.7496 & 7.0250 & 293.0 & 4 & 600.0 & 00.0 \\
\hline 02 & 168 & 133.2446 & 7.3000 & 293.5 & 4 & 600.0 & 00.0 \\
\hline 02 & 169 & 136.5017 & 7.1000 & 293.9 & 4 & 600.0 & 600.0 \\
\hline 02 & 1610 & 137.2505 & 7.3500 & 294.4 & 4 & 600.0 & .0 \\
\hline 02 & 611 & 139.7528 & 6. & 295.3 & 4 & 600.0 & \\
\hline 02 & 1612 & 146.7507 & 5.2250 & 296.7 & 3 & 600.0 & 600.0 \\
\hline 02 & 1613 & 98.6850 & 5.5750 & 297.6 & 3 & 600.0 & 600.0 \\
\hline 02 & 14 & 78.4931 & 7.3250 & 297.5 & 4 & 600.0 & \\
\hline 02 & 51615 & 95.7482 & 7.2000 & 297.8 & 4 & 600.0 & 60 \\
\hline 02 & 51616 & 91.5008 & 7.2500 & 298.0 & 4 & 600.0 & 600.0 \\
\hline 02 & 51617 & 97.0004 & 7.8000 & 296.5 & 4 & 600.0 & \\
\hline 02 & 51618 & 72.7466 & 9.1750 & 294.6 & 4 & 600.0 & 60 \\
\hline 02 & 51619 & 68.2500 & 10.7500 & 293.6 & 4 & 600.0 & 60 \\
\hline 02 & 51620 & 76.7491 & 9 . & 292.9 & 4 & 600.0 & .0 \\
\hline 02 & 51621 & 70.9998 & 10 . & 292.8 & 4 & 600.0 & \\
\hline 02 & 1622 & 70.7501 & 750 & 292.4 & 4 & 600.0 & 600 \\
\hline 02 & 51623 & 70.7502 & 11. & 292.2 & 4 & 600.0 & .0 \\
\hline 02 & 51624 & 75.4998 & 11. & 292.3 & 4 & 600.0 & \\
\hline 02 & 5171 & 78.7500 & 11. & 291.7 & 4 & 600.0 & 60 \\
\hline 02 & 5172 & 81.5001 & 10.4250 & 291.6 & 4 & 600.0 & 60 \\
\hline 02 & 5173 & 83.7484 & 9 . & 291.4 & 4 & 600.0 & \\
\hline 02 & 5174 & 82.4973 & 750 & 291.1 & 4 & 600.0 & 60 \\
\hline 02 & 5175 & 82.9997 & 8.7500 & 290.6 & 4 & 600.0 & 600.0 \\
\hline 02 & 5176 & 81.7504 & 8 . & 290.4 & 4 & 600.0 & \\
\hline 02 & 5177 & 86.2505 & 7.3250 & 290.7 & 4 & 600.0 & 60 \\
\hline 02 & 5178 & 81.2504 & 7.3250 & 292.2 & 4 & 600.0 & 600.0 \\
\hline 02 & 5179 & 95.6692 & 6 . & 293.5 & 4 & 600.0 & .0 \\
\hline 02 & 51710 & 110.3289 & 5 . & 294.3 & 3 & 600.0 & .0 \\
\hline 02 & 51711 & 102.0010 & 5.2750 & 294.8 & 3 & 600.0 & 600.0 \\
\hline 02 & 51712 & 103.5005 & 6.5250 & 295.9 & 4 & 600.0 & \\
\hline 02 & 51713 & 84.4985 & 7.4250 & 295.8 & 4 & 600.0 & 600.0 \\
\hline 02 & 51714 & 80.2501 & 8.4000 & 296.2 & 4 & 600.0 & 600.0 \\
\hline 02 & 51715 & 85.7439 & 10.1750 & 295.4 & 4 & 600.0 & \\
\hline 02 & 51716 & 87.1965 & 8.5000 & 295.7 & 4 & 600.0 & 600.0 \\
\hline 02 & 51717 & 67.9986 & 9.1250 & 295.6 & 4 & 600.0 & 600.0 \\
\hline 02 & 51718 & 67.5000 & 11.3000 & 294.3 & 4 & 600.0 & 600.0 \\
\hline 02 & 51719 & 66.0001 & 12.1750 & 292.9 & 4 & 600.0 & 600.0 \\
\hline 02 & 51720 & 66.5000 & 12.8500 & 291.8 & 4 & 600.0 & 600.0 \\
\hline 02 & 51721 & 67.2500 & 14.1750 & 290.6 & 4 & 600.0 & 600.0 \\
\hline 02 & 51722 & 68.2504 & 14.52 & 290.4 & 4 & 600.0 & 600.0 \\
\hline 02 & 51723 & 73.7501 & 13.4500 & 290.1 & 4 & 600.0 & 600.0 \\
\hline
\end{tabular}




\begin{tabular}{|c|c|c|c|c|c|c|c|}
\hline 02 & 51724 & 78.5000 & 12.2250 & 290.3 & 4 & 600.0 & 600.0 \\
\hline 02 & 5181 & 75.0000 & 12.3250 & 289.6 & 4 & 600.0 & 600.0 \\
\hline 02 & 5182 & 73.2500 & 11.6500 & 288.9 & 4 & 600.0 & 00.0 \\
\hline 2 & 5183 & 75.0000 & 11.5000 & 288.5 & 4 & 600.0 & 00.0 \\
\hline 02 & 5184 & 74.2500 & 12.9000 & 287.6 & 4 & 600.0 & 00.0 \\
\hline 02 & 5185 & 74.0001 & 12.7250 & 286.6 & 4 & 600.0 & 00.0 \\
\hline 02 & 5186 & 74.0000 & 12.0000 & 286.6 & 4 & 600.0 & 0 \\
\hline 02 & 5187 & 72.2501 & 11.4250 & 287.0 & 4 & 600.0 & \\
\hline 02 & 5188 & 71.7501 & 11.4500 & 286.8 & 4 & 600.0 & 000.0 \\
\hline 02 & 5189 & 70.2500 & 11.1250 & 287.3 & 4 & 600.0 & \\
\hline 02 & 51810 & 83.2258 & 9.9 & 288.1 & 4 & 600.0 & \\
\hline 02 & 51811 & 81.5000 & 8.7250 & 289.0 & 4 & 600.0 & 60 \\
\hline 02 & 51812 & 77.2496 & 10.4750 & 289.8 & 4 & 600.0 & \\
\hline 02 & 51813 & 81.2501 & 8.27 & 291.4 & 4 & 600.0 & 600.0 \\
\hline 02 & 51814 & 79.4862 & 8 . & 291.6 & 4 & 600.0 & .0 \\
\hline 02 & 51815 & 82.0021 & 7.9750 & 291.9 & 4 & 600.0 & \\
\hline 02 & 51816 & 76.2507 & 11.9250 & 290.8 & 4 & .0 & .0 \\
\hline 02 & 51817 & 64.7492 & 10.5 & 289.6 & 4 & .0 & \\
\hline 02 & 51818 & 63.7506 & 11.4250 & 289.3 & 4 & 600.0 & \\
\hline 02 & 51819 & 61.9814 & 10 & 288.4 & 4 & .0 & .0 \\
\hline 02 & 51820 & 112.9670 & & 286.5 & 4 & .0 & \\
\hline 02 & 51821 & 164.0098 & 00 & 285.1 & 5 & .0 & \\
\hline 02 & 51822 & 120.2636 & 3.3000 & 284.6 & 4 & .0 & 600.0 \\
\hline 02 & 51823 & 89.4939 & 5.3 & 284.6 & 4 & .0 & \\
\hline 02 & 51824 & 092 & 250 & 284.2 & 4 & .0 & \\
\hline 02 & 5191 & 78.0011 & 6.7500 & 285.0 & 4 & 600.0 & .0 \\
\hline 02 & 5192 & 83.7510 & 6 . & 285.0 & 4 & .0 & .0 \\
\hline 02 & 5193 & 75.7416 & 750 & 285.3 & 4 & .0 & .0 \\
\hline 02 & 5194 & 20.8412 & 500 & 284.0 & 5 & 60 & .0 \\
\hline 02 & 5195 & 316.2205 & 1.27 & 283.2 & 6 & 0 & .0 \\
\hline 02 & 5196 & 193.6348 & 1 . & 283.3 & 6 & .0 & \\
\hline 02 & 5197 & 228.8992 & 000 & 283.1 & 5 & .0 & .0 \\
\hline 02 & 5198 & 268.5005 & 2.5250 & 285.5 & 4 & .0 & 600.0 \\
\hline 02 & 5199 & 265.9998 & 3 . & 286.4 & 3 & .0 & .0 \\
\hline 02 & 51910 & 261.2479 & 500 & 288.0 & 2 & .0 & .0 \\
\hline 02 & 51911 & 273.9761 & 1.3750 & 289.2 & 1 & 600.0 & 600.0 \\
\hline 02 & 51912 & 12.1922 & 1 . & 290.0 & 1 & .0 & .0 \\
\hline 02 & 51913 & 22.9170 & 6.8750 & 288.8 & 2 & 600.0 & 600.0 \\
\hline 02 & 51914 & 24.0000 & 10.1250 & 288.9 & 3 & 600.0 & 600.0 \\
\hline 02 & 51915 & 50.2771 & 9 . & 287.4 & 4 & 600.0 & 600.0 \\
\hline 02 & 51916 & 66.5005 & 10.3500 & 286.7 & 4 & 600.0 & 600.0 \\
\hline 02 & 51917 & 71.2501 & 7.0000 & 287.3 & 4 & 600.0 & 600.0 \\
\hline 02 & 51918 & 60.2049 & 3.8750 & 288.1 & 3 & 600.0 & 600.0 \\
\hline 02 & 51919 & 27.4977 & 5.9500 & 287.2 & 4 & 600.0 & 600.0 \\
\hline 02 & 51920 & 7.7379 & 4.1250 & 286.0 & 4 & 600.0 & 600.0 \\
\hline 02 & 51921 & 350.5187 & 3.8000 & 285.8 & 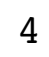 & 600.0 & \\
\hline 02 & 51922 & 354.2486 & 2.3250 & 285.2 & 5 & 600.0 & 600.0 \\
\hline 02 & 51923 & 357.0096 & 2.050 & 284.8 & o & 600.0 & 600.0 \\
\hline
\end{tabular}




\begin{tabular}{|c|c|c|c|c|c|c|c|}
\hline 02 & 51924 & 193.4 & 9250 & 284.5 & 6 & 600 & \\
\hline 02 & 5201 & 351.2098 & 1.8000 & 284.6 & 6 & 600.0 & 600.0 \\
\hline 02 & 5202 & 359.0018 & 3.3500 & 284.5 & 5 & 600.0 & 00.0 \\
\hline 02 & 5203 & 348.2493 & 3.6500 & 284.1 & 4 & 600.0 & 00.0 \\
\hline 02 & $520 \quad 4$ & 350.7731 & 1.9750 & 283.0 & 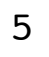 & 600.0 & 00.0 \\
\hline 02 & 5205 & 329.2350 & 2.7750 & 282.6 & 5 & 600.0 & 00.0 \\
\hline 02 & 5206 & 14.8353 & 2.1500 & 281.7 & 6 & 600.0 & 600.0 \\
\hline 02 & 5207 & 355.1336 & 1.8000 & 281.7 & 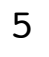 & 600.0 & 00.0 \\
\hline 02 & $520 \quad 8$ & 28.6820 & 2.5750 & 281.8 & 4 & 600.0 & \\
\hline 02 & 5209 & 347.5023 & 2.8000 & 283.3 & 3 & 600.0 & 600.0 \\
\hline 02 & 52010 & 11.7822 & 3.7500 & 285.2 & 2 & 600.0 & 60 \\
\hline 02 & 52011 & 25.5119 & 5.0750 & 284.2 & 3 & 600.0 & 600.0 \\
\hline 02 & 52012 & 3.7466 & 5.2750 & 285.4 & 3 & 600.0 & 60 \\
\hline 02 & 52013 & 13.5054 & 6.4500 & 285.6 & 4 & 600.0 & 0 \\
\hline 02 & 52014 & 54.0264 & 6.1250 & 282.9 & 4 & 600.0 & .0 \\
\hline 02 & 52015 & 18.7440 & 4.4250 & 284.3 & 3 & 600.0 & .0 \\
\hline 02 & 52016 & 31.5554 & 8.7750 & 285.5 & 4 & 600.0 & 600.0 \\
\hline 02 & 52017 & 24.5388 & 8.3250 & 285.9 & 4 & 600.0 & .0 \\
\hline 02 & 52018 & 5.9995 & 4.1250 & 284.8 & 3 & 600.0 & \\
\hline 02 & 52019 & 349.9910 & 3.9250 & 283.6 & 4 & 600.0 & 60 \\
\hline 02 & 52020 & 10.5000 & 1.7500 & 283.2 & 5 & 600.0 & .0 \\
\hline 02 & 52021 & 358.5477 & 0.9250 & 282.2 & 6 & 600.0 & \\
\hline 02 & 52022 & 73.5512 & 2.9250 & 282.4 & 5 & 600.0 & 60 \\
\hline 02 & 52023 & 351.6695 & 0.9000 & 281.6 & 6 & 600.0 & .0 \\
\hline 02 & 52024 & 353.1462 & 0.9750 & 281.8 & 6 & 600.0 & \\
\hline 02 & 5211 & 336.9498 & 1.4250 & 281.7 & 5 & 600.0 & 60 \\
\hline 02 & 5212 & 6.6998 & 0.4500 & 282.0 & 6 & 600.0 & .0 \\
\hline 02 & 5213 & 248.9881 & 0.57 & $281 \cdot 6$ & 6 & 600.0 & .0 \\
\hline 02 & 5214 & 108.4022 & 1.0250 & 281.8 & 6 & 600.0 & 60 \\
\hline 02 & 5215 & 329.9689 & 0.1750 & 281.7 & 6 & 600.0 & .0 \\
\hline 02 & 5216 & 49.7172 & 0.7000 & $281 \cdot 6$ & 6 & 600.0 & 60 \\
\hline 02 & $\begin{array}{ll}5217\end{array}$ & 64.4717 & 1.2000 & 281.9 & 5 & 600.0 & 60 \\
\hline 02 & 5218 & 22.8382 & 2.0250 & 281.8 & 4 & 600.0 & 600.0 \\
\hline 02 & 5219 & 359.6485 & 1.4750 & 283.7 & 3 & 600.0 & 600.0 \\
\hline 02 & 52110 & 50.4038 & 2.5000 & 285.5 & 2 & 600.0 & \\
\hline 02 & 52111 & 136.3510 & 2.4500 & 286.6 & 1 & 600.0 & 600.0 \\
\hline 02 & 52112 & 123.2350 & 3.6500 & 286.3 & 2 & 600.0 & 600.0 \\
\hline 02 & 52113 & 44.7653 & 3.8250 & 287.3 & 2 & 600.0 & 600.0 \\
\hline 02 & 52114 & 81.5862 & 4.4000 & 288.3 & 3 & 600.0 & 600.0 \\
\hline 02 & 52115 & 103.7521 & 5.1250 & 288.1 & 3 & 600.0 & 600.0 \\
\hline 02 & 52116 & 107.7794 & 4.9500 & 288.2 & 3 & 600.0 & 600.0 \\
\hline 02 & 52117 & 96.9508 & 5.0250 & 288.8 & 3 & 600.0 & 600.0 \\
\hline 02 & 52118 & 107.2410 & 6.4500 & 287.7 & 4 & 600.0 & 600.0 \\
\hline 02 & 52119 & 85.7481 & 5.9000 & 286.3 & 4 & 600.0 & 600.0 \\
\hline 02 & 52120 & 75.5000 & 7.4250 & 284.2 & 4 & 600.0 & 600.0 \\
\hline 02 & 52121 & 64.7498 & 9.1250 & 283.0 & 4 & 600.0 & 600.0 \\
\hline 02 & 52122 & 64.2500 & 10.175 & .7 & 4 & 600.0 & 600.0 \\
\hline 02 & 52123 & 66.5000 & 9.9500 & 282.2 & $I$ & 600.0 & 600.0 \\
\hline
\end{tabular}




\begin{tabular}{|c|c|c|c|c|c|c|c|}
\hline 2 & 52124 & 65.7500 & 9.6750 & 282.2 & 4 & 600.0 & 600.0 \\
\hline 02 & 5221 & 70.2500 & 9.1000 & 282.2 & 4 & 600.0 & 600.0 \\
\hline 02 & 5222 & 9994 & 8.3750 & 282.0 & 4 & 600.0 & 00.0 \\
\hline 02 & 5223 & 74.0000 & 8.8500 & 281.8 & 4 & 600.0 & 00.0 \\
\hline 02 & 5224 & 70.5000 & 8.0750 & 281.9 & 4 & 600.0 & 00.0 \\
\hline 02 & 5225 & 74.5009 & 8.2000 & 282.1 & 4 & 600.0 & 00.0 \\
\hline 02 & 5226 & 73.7504 & 8.1250 & 281.9 & 4 & 600.0 & 0 \\
\hline 02 & 5227 & 77.0002 & 7.5250 & 282.5 & 4 & 600.0 & 00.0 \\
\hline 02 & 5228 & 92.7815 & 4.5250 & 284.6 & 3 & 600.0 & 00.0 \\
\hline 02 & 5229 & 110.7473 & 3.1000 & 286.9 & 2 & 60 & 0 \\
\hline 02 & 52210 & 166.6960 & 5.2250 & 287.4 & 3 & 600.0 & \\
\hline 02 & 52211 & 170.5003 & 5.9750 & 287.8 & 3 & 600.0 & \\
\hline 02 & 52212 & 174.7561 & 5.1250 & 288.8 & 3 & 600.0 & 00.0 \\
\hline 02 & 52213 & 167.2469 & 4.2500 & 290.1 & 3 & 600.0 & \\
\hline 02 & 52214 & 174.5772 & 5.4000 & 290.6 & 3 & .0 & \\
\hline 02 & 52215 & 176.5048 & 5.5500 & 291.4 & 3 & 600.0 & .0 \\
\hline 02 & 52216 & 162.3335 & 6.5250 & 291.4 & 4 & 60 & .0 \\
\hline 02 & 52217 & 145.2496 & 5.9 & 291.5 & 3 & .0 & \\
\hline 02 & 52218 & 112.4062 & 6.1500 & 290.7 & 4 & 60 & \\
\hline 02 & 52219 & 86.4995 & 6.9250 & 289.1 & 4 & .0 & .0 \\
\hline 02 & 52220 & 77.2499 & 7 . & 287.0 & 4 & 6 & \\
\hline 02 & 52221 & 72.0000 & 8.1000 & 285.4 & 4 & .0 & \\
\hline 02 & 52222 & 72.7501 & 10.0750 & 285.3 & 4 & 600.0 & .0 \\
\hline 02 & 52223 & 73.2497 & 9.20 & 285.6 & 4 & 0 & \\
\hline 02 & 52224 & 79.7520 & 7.77 & 286.3 & 4 & .0 & \\
\hline 02 & 5231 & 88.7229 & 6.2250 & 286.8 & 4 & .0 & \\
\hline 02 & 5232 & 102.7381 & 5.1500 & 287.4 & 4 & 60 & .0 \\
\hline 02 & 5233 & 131.2696 & 5.5000 & 288.0 & 4 & .0 & \\
\hline 02 & 5234 & 143.5006 & 7.9000 & 287.5 & 4 & .0 & .0 \\
\hline 02 & 5235 & 142.2489 & 8.92 & 285.9 & 4 & .0 & \\
\hline 02 & 5236 & 141.7478 & 8.77 & 285.8 & 4 & .0 & \\
\hline 02 & 5237 & 160.7513 & 10.80 & 286.7 & 4 & .0 & 0.0 \\
\hline 02 & 5238 & 174.5009 & 12.4250 & 287.5 & 4 & 600.0 & 0.0 \\
\hline 02 & 5239 & 170.7502 & 10.6500 & 288.4 & 4 & .0 & 0.0 \\
\hline 02 & 52310 & 168.2494 & 9.0500 & 289.6 & 4 & 600.0 & 0.0 \\
\hline 02 & 52311 & 163.7344 & 7.4500 & 290.7 & 4 & 600.0 & 600.0 \\
\hline 02 & 52312 & 158.4980 & 6.70 & 292.0 & 4 & 600.0 & .0 \\
\hline 02 & 52313 & 177.4742 & 7.9750 & 293.2 & 4 & 600.0 & 600.0 \\
\hline 02 & 52314 & 178.9645 & 7.9750 & 294.2 & 4 & 600.0 & 600.0 \\
\hline 02 & 52315 & 173.4310 & 7.75 & 295.1 & 4 & 600.0 & 600.0 \\
\hline 02 & 52316 & 177.9835 & 7.1250 & 295.8 & 4 & 600.0 & 600.0 \\
\hline 02 & 52317 & 179.0000 & 6.3000 & 296.2 & 4 & 600.0 & 600.0 \\
\hline 02 & 52318 & 150.2274 & 5.1250 & 296.3 & 3 & 600.0 & 600.0 \\
\hline 02 & 52319 & 84.0048 & 6.0750 & 294.4 & 4 & 600.0 & 600.0 \\
\hline 02 & 52320 & 75.2461 & 6.5000 & 292.7 & 4 & 600.0 & 600.0 \\
\hline 02 & 52321 & 75.0001 & 7.5750 & 292.0 & 4 & 600.0 & 600.0 \\
\hline 02 & 52322 & 77.5000 & 8.0500 & 291.8 & 4 & 600.0 & 600.0 \\
\hline $0<$ & 52323 & 73.2500 & 7.950 & 291.8 & 4 & 600.0 & 600.0 \\
\hline
\end{tabular}




\begin{tabular}{|c|c|c|c|c|c|c|c|}
\hline 02 & 52324 & 74.5002 & 7.7500 & 292.3 & 4 & 600.0 & 600.0 \\
\hline 02 & 5241 & 73.2500 & 7.1500 & 292.7 & 4 & 600.0 & 600.0 \\
\hline 02 & 5242 & 73.7500 & 7.5000 & 292.8 & 4 & 600.0 & 00.0 \\
\hline 02 & 5243 & 74.0000 & 7.6500 & 292.9 & 4 & 600.0 & 00.0 \\
\hline 02 & 5244 & 78.0000 & 7.3000 & 293.2 & 4 & 600.0 & 00.0 \\
\hline 02 & 5245 & 78.7500 & 7.2250 & 293.2 & 4 & 600.0 & 00.0 \\
\hline 02 & 5246 & 105.8011 & 3.3250 & 292.9 & 5 & 600.0 & 0 \\
\hline 02 & 5247 & 163.7428 & 0.7250 & 293.3 & 4 & 600.0 & 00.0 \\
\hline 02 & 5248 & 251.4891 & 1.3750 & 293.6 & 3 & 600.0 & 00.0 \\
\hline 02 & 5249 & 247.0000 & 2.1500 & 294.7 & 2 & 600.0 & 0 \\
\hline 02 & 52410 & 268.7500 & 2.2000 & 296.2 & 1 & 600.0 & \\
\hline 02 & 52411 & 244.2489 & 3.0250 & 297.6 & 2 & 600.0 & \\
\hline 02 & 52412 & 228.7164 & 3.6500 & 297.8 & 2 & 600.0 & 00.0 \\
\hline 02 & 52413 & 207.7654 & 3.8000 & 298.6 & 2 & 600.0 & 00.0 \\
\hline 02 & 52414 & 206.7169 & 3.5500 & 299.7 & 2 & .0 & \\
\hline 02 & 52415 & 190.9754 & 4.7000 & 300.1 & 3 & 600.0 & .0 \\
\hline 02 & 52416 & 195.0000 & 5.3750 & 300.2 & 3 & 60 & .0 \\
\hline 02 & 52417 & 176.7710 & 5.75 & 300.1 & 3 & .0 & \\
\hline 02 & 52418 & 127.0000 & 5.9500 & 299.3 & 3 & 60 & .0 \\
\hline 02 & 52419 & 97.5000 & 5.6750 & 298.0 & 4 & .0 & .0 \\
\hline 02 & 52420 & 81 . & 6 . & 296.3 & 4 & 6 & \\
\hline 02 & 52421 & 000 & 7.0000 & 295.4 & 4 & .0 & \\
\hline 02 & 52422 & 67.7494 & 7.3500 & 295.0 & 4 & 600.0 & .0 \\
\hline 02 & 52423 & 969 & 7.50 & 295.0 & 4 & 0 & \\
\hline 02 & 52424 & 513 & 7.95 & 294.7 & 4 & .0 & \\
\hline 02 & 5251 & 77.0000 & 8.3500 & 294.5 & 4 & .0 & \\
\hline 02 & 5252 & 73.7499 & 8.2750 & 294.3 & 4 & 60 & .0 \\
\hline 02 & 5253 & 74.2500 & 7.7250 & 294.3 & 4 & .0 & \\
\hline 02 & 5254 & 78.5000 & 7.0500 & 294.4 & 4 & .0 & .0 \\
\hline 02 & $525 \quad 5$ & 76.2501 & 6.3000 & 294.8 & 4 & 60 & .0 \\
\hline 02 & 5256 & 78.7512 & 58500 & 295.5 & 4 & .0 & \\
\hline 02 & 5257 & 75.2500 & 5.1250 & 296.1 & 4 & .0 & 0.0 \\
\hline 02 & 5258 & 82.9705 & 3.2750 & 297.7 & 3 & 600.0 & 600.0 \\
\hline 02 & 5259 & 96.2472 & 3.2000 & 299.1 & 2 & .0 & 0.0 \\
\hline 02 & 52510 & 169.4068 & 5.6500 & 299.0 & 3 & 600.0 & 600.0 \\
\hline 02 & 52511 & 127.1217 & 4.4000 & 299.3 & 3 & 600.0 & 600.0 \\
\hline 02 & 52512 & 110.3972 & 4.2500 & 299.7 & 3 & 600.0 & 600.0 \\
\hline 02 & 52513 & 131.2501 & 6.0750 & 298.9 & 4 & 600.0 & 600.0 \\
\hline 02 & 52514 & 107.9772 & 5.7500 & 298.9 & 3 & 600.0 & 600.0 \\
\hline 02 & 52515 & 105.2394 & 6.6250 & 299.1 & 4 & 600.0 & 600.0 \\
\hline 02 & 52516 & 82.7488 & 7.4250 & 298.7 & 4 & 600.0 & 600.0 \\
\hline 02 & 52517 & 77.5000 & 8.1250 & 297.9 & 4 & 600.0 & 600.0 \\
\hline 02 & 52518 & 72.2502 & 8.0750 & 296.8 & 4 & 600.0 & 600.0 \\
\hline 02 & 52519 & 65.9994 & 9.6000 & 295.8 & 4 & 600.0 & 600.0 \\
\hline 02 & 52520 & 70.0000 & 10.2250 & 294.6 & 4 & 600.0 & 600.0 \\
\hline 02 & 52521 & 73.4998 & 11.5500 & 293.1 & 4 & 600.0 & 600.0 \\
\hline 02 & 52522 & 84.4948 & 9.2750 & 291.5 & 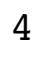 & 600.0 & 600.0 \\
\hline $0<$ & 52523 & 70.0000 & 11.1500 & 290.9 & 4 & 600.0 & 600.0 \\
\hline
\end{tabular}




\begin{tabular}{|c|c|c|c|c|c|c|c|}
\hline 02 & 52524 & 73.7502 & 10.6500 & 292.1 & 4 & 600.0 & 600.0 \\
\hline 02 & 5261 & 72.0000 & 11.3500 & 291.7 & 4 & 600.0 & 600.0 \\
\hline 02 & 5262 & 65.7497 & 10.8250 & 291.4 & 4 & 600.0 & 00.0 \\
\hline 02 & 5263 & 69.9979 & 10.2000 & 290.7 & 4 & 600.0 & 00.0 \\
\hline 02 & 5264 & 77.7500 & 11.2000 & 291.1 & 4 & 600.0 & 00.0 \\
\hline 02 & 5265 & 73.2495 & 10.8500 & 291.0 & 4 & 600.0 & 00.0 \\
\hline 02 & 5266 & 73.2499 & 11.1250 & 291.2 & 4 & 600.0 & 00.0 \\
\hline 02 & 5267 & 79.2502 & 9.7000 & 291.1 & 4 & 600.0 & 00.0 \\
\hline 02 & 5268 & 82.4999 & 10.1500 & 291.9 & 4 & 600.0 & 00.0 \\
\hline 02 & 5269 & 84.0023 & 8.9750 & 292.1 & 4 & 600.0 & 0 \\
\hline 02 & 52610 & 78.7506 & 9.9750 & 292.7 & 4 & 600.0 & \\
\hline 02 & 52611 & 71.4998 & 9.0750 & 294.3 & 4 & 600.0 & \\
\hline 02 & 52612 & 76.0000 & 8.4000 & 294.7 & 4 & 600.0 & .0 \\
\hline 02 & 52613 & 71.7494 & 8.5250 & 295.9 & 4 & 600.0 & 00.0 \\
\hline 02 & 52614 & 64.9945 & 9.6250 & 295.7 & 4 & .0 & \\
\hline 02 & 52615 & 74.5000 & 10.8000 & 295.3 & 4 & 600.0 & .0 \\
\hline 02 & 52616 & 66.7500 & 10.9250 & 294.8 & 4 & 60 & .0 \\
\hline 02 & 52617 & 66.0000 & 12.4750 & 294.4 & 4 & .0 & \\
\hline 02 & 52618 & 65.0000 & 12.5250 & 293.4 & 4 & 60 & .0 \\
\hline 02 & 52619 & 67.0000 & 11.2750 & 292.2 & 4 & .0 & .0 \\
\hline 02 & 52620 & 69.4998 & 10.5250 & 291.1 & 4 & 6 & \\
\hline 02 & 52621 & 000 & 11.3750 & 290.3 & 4 & .0 & \\
\hline 02 & 52622 & 80.7153 & 9.0250 & 290.2 & 4 & 600.0 & .0 \\
\hline 02 & 52623 & 73.7483 & 8.70 & 290.2 & 4 & 60 & \\
\hline 02 & 52624 & 5003 & 8.0250 & 290.0 & 4 & .0 & \\
\hline 02 & 5271 & 66.5000 & 10.9750 & 289.9 & 4 & .0 & \\
\hline 02 & 5272 & 67.5000 & 11.5500 & 289.6 & 4 & 60 & .0 \\
\hline 02 & 5273 & 66.0001 & 10.4750 & 289.7 & 4 & .0 & \\
\hline 02 & 5274 & 69.0000 & 10.0000 & 289.9 & 4 & .0 & .0 \\
\hline 02 & 5275 & 65.0000 & 8.0250 & 289.6 & 4 & .0 & .0 \\
\hline 02 & 5276 & 67.0003 & 7.70 & 289.7 & 4 & .0 & \\
\hline 02 & 5277 & 70.5000 & 5.8250 & 290.5 & 3 & .0 & 600.0 \\
\hline 02 & 5278 & 99.7516 & 4.4500 & 291.2 & 3 & 600.0 & 600.0 \\
\hline 02 & 5279 & 87.9996 & 5.5750 & 292.3 & 3 & .0 & 0.0 \\
\hline 02 & 52710 & 87.7500 & 4.7000 & 293.6 & 3 & 600.0 & 600.0 \\
\hline 02 & 52711 & 75.5010 & 5.4500 & 294.3 & 3 & 600.0 & 600.0 \\
\hline 02 & 52712 & 78.5184 & 6.6500 & 295.2 & 4 & 600.0 & 600.0 \\
\hline 02 & 52713 & 98.1214 & 6.7250 & 295.1 & 4 & 600.0 & 600.0 \\
\hline 02 & 52714 & 83.5000 & 7.2750 & 295.4 & 4 & 600.0 & 600.0 \\
\hline 02 & 52715 & 72.4945 & 8.4250 & 295.3 & 4 & 600.0 & 600.0 \\
\hline 02 & 52716 & 79.0001 & 9.4000 & 294.7 & 4 & 600.0 & 600.0 \\
\hline 02 & 52717 & 83.7405 & 8.3250 & 293.6 & 4 & 600.0 & 600.0 \\
\hline 02 & 52718 & 76.5010 & 9.3500 & 292.7 & 4 & 600.0 & 600.0 \\
\hline 02 & 52719 & 87.5702 & 8.6750 & 290.9 & 4 & 600.0 & 600.0 \\
\hline 02 & 52720 & 93.2616 & 6.2750 & 289.3 & 4 & 600.0 & 600.0 \\
\hline 02 & 52721 & 76.0000 & 9.0250 & 288.8 & 4 & 600.0 & 600.0 \\
\hline 02 & 52722 & 68.2500 & 12.5250 & 287.7 & 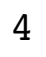 & 600.0 & 600.0 \\
\hline 02 & 52723 & 72.0000 & 12.425 & 287.5 & 4 & 600.0 & 600.0 \\
\hline
\end{tabular}




\begin{tabular}{|c|c|c|c|c|c|c|c|}
\hline 02 & 52724 & 75.7489 & 9.8750 & 288.4 & 4 & 600.0 & 600.0 \\
\hline 02 & 5281 & 72.7501 & 9.9000 & 287.8 & 4 & 600.0 & 600.0 \\
\hline 02 & 5282 & 81.9992 & 9.8750 & 288.0 & 4 & 600.0 & 00.0 \\
\hline 02 & 5283 & 75.7482 & 11.2500 & 286.6 & 4 & 600.0 & 00.0 \\
\hline 02 & 5284 & 72.2496 & 8.7000 & 288.6 & 4 & 600.0 & 00.0 \\
\hline 02 & 5285 & 80.5005 & 5.9750 & 290.0 & 4 & 600.0 & 00.0 \\
\hline 02 & 5286 & 80.2502 & 5.7500 & 290.0 & 4 & 600.0 & 0 \\
\hline 02 & 5287 & 82.7501 & 4.8000 & 290.2 & 4 & 600.0 & 00.0 \\
\hline 02 & 5288 & 128.4826 & 2.7750 & 292.1 & 3 & 600.0 & 00.0 \\
\hline 02 & 5289 & 140.5344 & 2.3000 & 294.0 & 2 & 60 & 0 \\
\hline 02 & 52810 & 139.1885 & 4.8750 & 294.0 & 3 & 600.0 & \\
\hline 02 & 52811 & 96.9862 & 4.9250 & 294.2 & 3 & .0 & \\
\hline 02 & 52812 & 106.6904 & 5.5000 & 295.2 & 3 & 600.0 & .0 \\
\hline 02 & 52813 & 140.6073 & 7.2500 & 296.0 & 4 & 600.0 & \\
\hline 02 & 52814 & 129.9278 & 7.0750 & 296.8 & 4 & .0 & \\
\hline 02 & 52815 & 107.4986 & 6.6750 & 296.8 & 4 & 600.0 & .0 \\
\hline 02 & 52816 & 100.2493 & 7.4750 & 296.4 & 4 & 60 & .0 \\
\hline 02 & 52817 & 95.0173 & 6.1250 & 295.7 & 4 & .0 & \\
\hline 02 & 52818 & 76.5015 & 6.7500 & 295.3 & 4 & 60 & \\
\hline 02 & 52819 & 75.4989 & 7.8250 & 294.1 & 4 & .0 & .0 \\
\hline 02 & 52820 & 999 & 9.1 & 292.5 & 4 & 0 & \\
\hline 02 & 52821 & 021 & 8.7000 & 292.1 & 4 & .0 & \\
\hline 02 & 52822 & 76.2500 & 10.0500 & 291.9 & 4 & 600.0 & .0 \\
\hline 02 & 52823 & 74.7500 & 9.72 & 291.7 & 4 & 0 & \\
\hline 02 & 52824 & 71.0000 & 9. & 291.5 & 4 & 0 & \\
\hline 02 & 5291 & 69.5000 & 9.7000 & 291.6 & 4 & .0 & \\
\hline 02 & 5292 & 73.2501 & 8.4750 & 292.1 & 4 & 60 & .0 \\
\hline 02 & 5293 & 73.2495 & 7.6750 & 292.2 & 4 & .0 & \\
\hline 02 & 5294 & 79.4998 & 6.8250 & 292.4 & 4 & .0 & .0 \\
\hline 02 & 5295 & 76.7474 & 6.9750 & 292.5 & 4 & .0 & \\
\hline 02 & 5296 & 81.0000 & 6 & 292.4 & 4 & 0 & \\
\hline 02 & 5297 & 81.4999 & 5.72 & 293.2 & 4 & .0 & 0 \\
\hline 02 & 5298 & 100.7568 & 3.5500 & 295.6 & 3 & 600.0 & 0.0 \\
\hline 02 & 5299 & 221.2403 & 3.1750 & 298.3 & 2 & .0 & .0 \\
\hline 02 & 52910 & 190.2505 & 5.02 & 297.7 & 3 & 600.0 & 0.0 \\
\hline 02 & 52911 & 176.2495 & 5.6500 & 297.8 & 3 & 600.0 & 600.0 \\
\hline 02 & 52912 & 177.9977 & 5.10 & 299.2 & 3 & 600.0 & .0 \\
\hline 02 & 52913 & 170.7535 & 4.6250 & 300.2 & 3 & 600.0 & 600.0 \\
\hline 02 & 52914 & 173.7666 & 4.7500 & 301.2 & 3 & 600.0 & 600.0 \\
\hline 02 & 52915 & 170.9950 & 5.20 & 301.7 & 3 & 600.0 & 600.0 \\
\hline 02 & 52916 & 177.0023 & 6.1000 & 302.3 & 4 & 600.0 & 600.0 \\
\hline 02 & 52917 & 180.0177 & 6.3250 & 302.7 & 4 & 600.0 & 600.0 \\
\hline 02 & 52918 & 127.4719 & 5.1250 & 302.7 & 3 & 600.0 & 600.0 \\
\hline 02 & 52919 & 86.5207 & 5.6500 & 301.1 & 4 & 600.0 & 600.0 \\
\hline 02 & 52920 & 72.2499 & 6.7750 & 299.5 & 4 & 600.0 & 600.0 \\
\hline 02 & 52921 & 81.7500 & 8.5250 & 298.9 & 4 & 600.0 & 600.0 \\
\hline 02 & 52922 & 70.7498 & 7.7750 & 298.8 & 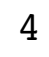 & 600.0 & 600.0 \\
\hline 02 & 52923 & 72.0000 & 7.5500 & 298.9 & $\Psi$ & 600.0 & 600.0 \\
\hline
\end{tabular}




\begin{tabular}{|c|c|c|c|c|c|c|c|}
\hline 02 & 52924 & 73.7499 & 8.1500 & 298.9 & 4 & 600.0 & 600.0 \\
\hline 02 & 5301 & 77.2502 & 8.5250 & 298.5 & 4 & 600.0 & 600.0 \\
\hline 02 & 5302 & 78.2500 & 8.5750 & 297.7 & 4 & 600.0 & 00.0 \\
\hline 02 & 5303 & 82.5000 & 6.6500 & 298.0 & 4 & 600.0 & 00.0 \\
\hline 02 & 5304 & 88.5023 & 4.4750 & 298.3 & 5 & 600.0 & 00.0 \\
\hline 02 & 5305 & 107.6676 & 2.6000 & 298.3 & 5 & 600.0 & 00.0 \\
\hline 02 & 5306 & 110.4599 & 3.0750 & 297.6 & 4 & 600.0 & 00.0 \\
\hline 02 & 5307 & 140.6820 & 1.8250 & 299.5 & 3 & 600.0 & 00.0 \\
\hline 02 & 5308 & 229.2417 & 1.4000 & 301.8 & 2 & 600.0 & 00.0 \\
\hline 02 & 5309 & 228.0247 & 2.0500 & 302.7 & 1 & 600.0 & 0 \\
\hline 02 & 3010 & 228.4861 & 3.9250 & 302.9 & 2 & 600.0 & \\
\hline 02 & 3011 & 220.2491 & 5.2250 & 303.1 & 3 & 600.0 & \\
\hline 02 & 3012 & 197.0096 & 6.3750 & 303.6 & 4 & 600.0 & 00.0 \\
\hline 02 & 3013 & 180.2524 & 6.1000 & 304.2 & 4 & 600.0 & 00.0 \\
\hline 02 & 3014 & 169.9904 & 6 . & 304.7 & 4 & .0 & \\
\hline 02 & 3015 & 151.7546 & 6.0000 & 305.4 & 4 & 600.0 & .0 \\
\hline 02 & 53016 & 107.9691 & 6.5750 & 304.9 & 4 & 60 & .0 \\
\hline 02 & 3017 & 98.4972 & 6. & 303.7 & 4 & .0 & \\
\hline 02 & 53018 & 82.2688 & 5.9500 & 303.2 & 3 & 60 & .0 \\
\hline 02 & 53019 & 73.4963 & 8.1000 & 302.0 & 4 & .0 & .0 \\
\hline 02 & 53020 & 75.2501 & 8.22 & 301.4 & 4 & 6 & \\
\hline 02 & 53021 & 75.2503 & 9.4500 & 300.1 & 4 & .0 & \\
\hline 02 & 53022 & 76.0002 & 9.9000 & 299.8 & 4 & 600.0 & .0 \\
\hline 02 & 53023 & 76.5003 & 0.125 & 300.2 & 4 & 6 & \\
\hline 02 & 53024 & 72.2500 & 9.5500 & 300.5 & 4 & .0 & \\
\hline 02 & 5311 & 71.2500 & 8.4250 & 301.1 & 4 & .0 & \\
\hline 02 & 5312 & 69.2480 & 6.1500 & 301.5 & 4 & .0 & .0 \\
\hline 02 & 5313 & 52.9756 & 4.7500 & 301.8 & 5 & .0 & \\
\hline 02 & 5314 & 38.0000 & 4.4750 & 301.3 & 5 & .0 & .0 \\
\hline 02 & 5315 & 352.6030 & 1.9500 & 299.6 & 5 & 60 & .0 \\
\hline 02 & 5316 & 326.4419 & 06500 & 296.8 & 6 & .0 & \\
\hline 02 & 5317 & 165.5887 & 0.1000 & 300.5 & 5 & .0 & 600.0 \\
\hline 02 & 5318 & 243.0174 & 1.6750 & 299.9 & 4 & 600.0 & 600.0 \\
\hline 02 & 5319 & 237.2573 & 2.6000 & 299.9 & 3 & .0 & 0.0 \\
\hline 02 & 53110 & 207.4291 & 3.8750 & 300.1 & 2 & 600.0 & 600.0 \\
\hline 02 & 53111 & 180.4971 & 4.5750 & 300.9 & 3 & 600.0 & 600.0 \\
\hline 02 & 53112 & 183.0057 & 4.7250 & 302.0 & 3 & 600.0 & 0.0 \\
\hline 02 & 53113 & 186.9966 & 5.4000 & 303.0 & 3 & 600.0 & 600.0 \\
\hline 02 & 53114 & 126.0528 & 5.3500 & 304.3 & 3 & 600.0 & 600.0 \\
\hline 02 & 53115 & 104.5000 & 6.2250 & 304.5 & 4 & 600.0 & 600.0 \\
\hline 02 & 53116 & 85.9759 & 8.8000 & 303.6 & 4 & 600.0 & 600.0 \\
\hline 02 & 53117 & 83.4983 & 8.9500 & 303.0 & 4 & 600.0 & 600.0 \\
\hline 02 & 53118 & 81.0007 & 8.1250 & 301.1 & 4 & 600.0 & 600.0 \\
\hline 02 & 53119 & 76.0000 & 7.8000 & 298.8 & 4 & 600.0 & 600.0 \\
\hline 02 & 53120 & 46.7506 & 6.0250 & 299.7 & 4 & 600.0 & 600.0 \\
\hline 02 & 53121 & 32.2498 & 3.9000 & 298.4 & 4 & 600.0 & 600.0 \\
\hline 02 & 53122 & 29.4972 & 2.4750 & 298.7 & 5 & 600.0 & 600.0 \\
\hline 48 & 53123 & 63.5331 & 1.2500 & 297.8 & 0 & 600.0 & 600.0 \\
\hline
\end{tabular}




\begin{tabular}{|c|c|c|c|c|c|c|c|c|}
\hline 2 & & 124 & 76.1640 & 4.6500 & 298.2 & 5 & 600.0 & 00.0 \\
\hline 2 & 6 & 11 & 78.2505 & 5.5000 & 295.3 & 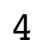 & 600.0 & 600.0 \\
\hline 02 & 6 & 12 & 29.3072 & 4.7250 & 292.3 & 4 & 600.0 & 00.0 \\
\hline 02 & 6 & 13 & 78.5573 & 2.3000 & 292.2 & 5 & 600.0 & 00.0 \\
\hline 02 & 6 & 14 & 64.3763 & 2.6500 & 289.7 & & 600.0 & 00.0 \\
\hline 02 & 6 & 15 & 86.5122 & 10.9000 & 287.2 & J & 600.0 & 00.0 \\
\hline 02 & 6 & 16 & 62.2462 & 8.1000 & 290.3 & 4 & 600.0 & 500.0 \\
\hline 02 & 6 & 17 & 75.5000 & 11.2750 & 289.6 & 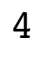 & 600.0 & .0 \\
\hline 02 & 6 & 18 & 72.7474 & 10.6750 & 290.8 & 4 & 600.0 & \\
\hline 02 & 6 & 19 & 83.5032 & 9.7750 & 291.1 & 4 & 600.0 & 600.0 \\
\hline 02 & 6 & 110 & 83.2500 & 9.7500 & 290.3 & 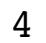 & 600.0 & 0 \\
\hline 02 & 6 & 111 & 88.5000 & 9.6250 & 291.6 & 4 & 600.0 & \\
\hline 02 & 6 & 112 & 84.5000 & 9.6250 & 293.5 & 4 & 600.0 & .0 \\
\hline 02 & 6 & 113 & 73.7494 & 10.6500 & 294.0 & 4 & 600.0 & 0 \\
\hline 02 & 6 & 114 & 69.2485 & 12.12 & 293.4 & 4 & 600.0 & \\
\hline 02 & 6 & 115 & 68.5008 & 11.2 & 293.4 & 4 & 600.0 & \\
\hline 02 & 6 & 116 & 74.7503 & 9.8750 & 293.4 & 4 & 600.0 & .0 \\
\hline 02 & 6 & 117 & 67.5000 & 10.5500 & 292.0 & 4 & 600.0 & .0 \\
\hline 02 & 6 & 118 & 79.9770 & 10.6750 & 288.4 & 4 & 600.0 & \\
\hline 02 & 6 & 119 & 70.7498 & 14.9250 & 286.7 & 4 & 600.0 & 600.0 \\
\hline 02 & 6 & 120 & 68.7499 & 15.7750 & 287.3 & 4 & 600.0 & .0 \\
\hline 02 & 6 & 121 & 70.2500 & 15.17 & 288.5 & 4 & 600.0 & \\
\hline 02 & 6 & 122 & 71.5000 & 14.4250 & 287.0 & 4 & 600.0 & 60 \\
\hline 02 & 6 & 123 & 73.2500 & 12.2000 & 285.8 & 4 & 600.0 & .0 \\
\hline 02 & 6 & 124 & 74.2498 & 12.9000 & 286.0 & 4 & 600.0 & \\
\hline 02 & 6 & 21 & 71.2495 & 3000 & 285.6 & 4 & 600.0 & 60 \\
\hline 02 & 6 & 22 & 74.4985 & 14.2250 & 285.5 & 4 & 600.0 & .0 \\
\hline 02 & 6 & 23 & 86.0000 & 10.7250 & 284.6 & 4 & 600.0 & .0 \\
\hline 02 & 6 & 24 & 84.5003 & 10.1250 & 283.9 & 4 & 600.0 & 60 \\
\hline 02 & 6 & 25 & 83.2527 & 11.1500 & 283.1 & 4 & 600.0 & .0 \\
\hline 02 & 6 & 26 & 81.9998 & 10.7500 & 283.0 & 4 & 600.0 & .0 \\
\hline 02 & 6 & 27 & 81.4995 & 9.5000 & 284.2 & 4 & 600.0 & \\
\hline 02 & 6 & 28 & 87.7500 & 5.8500 & 286.6 & 4 & 600.0 & 600.0 \\
\hline 02 & 6 & 29 & 96.4968 & 4.3500 & 288.5 & 3 & 600.0 & .0 \\
\hline 02 & 6 & 210 & 94.7476 & 250 & 290.1 & 2 & 600.0 & \\
\hline 02 & 6 & 211 & 95.0000 & 3.8500 & 291.2 & 2 & 600.0 & 600.0 \\
\hline 02 & 6 & 212 & 121.4945 & 4.7250 & 292.3 & 3 & 600.0 & 600.0 \\
\hline 02 & 6 & 213 & 118.9979 & 5.3500 & 293.9 & 3 & 600.0 & 60 \\
\hline 02 & 6 & 214 & 103.0011 & 7.1750 & 294.4 & 4 & 600.0 & 600.0 \\
\hline 02 & 6 & 215 & 98.5072 & 7.3750 & 294.3 & 4 & 600.0 & 600.0 \\
\hline 02 & 6 & 216 & 90.9780 & 7.1250 & 294.2 & 4 & 600.0 & 600.0 \\
\hline 02 & 6 & 217 & 93.5031 & 9.2000 & 293.3 & 4 & 600.0 & 600.0 \\
\hline 02 & 6 & 218 & 81.2515 & 8.4250 & 292.5 & 4 & 600.0 & 600.0 \\
\hline 02 & 6 & 219 & 73.0000 & 10.8500 & 290.7 & 4 & 600.0 & 600.0 \\
\hline 02 & 6 & 220 & 72.5000 & 10.7250 & 290.9 & 4 & 600.0 & 600.0 \\
\hline 02 & 6 & 221 & 73.4999 & 9.8500 & 291.2 & 4 & 600.0 & 600.0 \\
\hline 02 & 6 & 222 & 77.2513 & 11. & & 4 & 600.0 & .0 \\
\hline 02 & 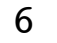 & 223 & 70.5000 & 12.5250 & 290.1 & 4 & 600.0 & 600.0 \\
\hline
\end{tabular}




\begin{tabular}{|c|c|c|c|c|c|c|c|c|}
\hline 2 & 6 & 224 & 71.0000 & 10.2750 & 290.2 & 4 & 600.0 & 600.0 \\
\hline 02 & 6 & 31 & 74.7500 & 10.0000 & 290.7 & 4 & 600.0 & 00.0 \\
\hline 02 & 6 & 32 & 72.7500 & 10.4750 & 290.3 & 4 & 600.0 & 00.0 \\
\hline 02 & 6 & 33 & 78.5000 & 11.2250 & 289.6 & 4 & 600.0 & 00.0 \\
\hline 02 & 6 & 34 & 75.7499 & 10.3500 & 289.5 & 4 & 600.0 & 00.0 \\
\hline 02 & 6 & 35 & 77.0005 & 9.7500 & 288.5 & 4 & 600.0 & 00.0 \\
\hline 02 & 6 & 36 & 79.4998 & 9.0500 & 288.3 & 4 & 600.0 & 00.0 \\
\hline 02 & 6 & 37 & 86.9993 & 6.1000 & 289.8 & 4 & 600.0 & 00.0 \\
\hline 02 & 6 & 38 & 95.6699 & 2.5250 & 293.2 & 3 & 600.0 & 00.0 \\
\hline 02 & 6 & 39 & 225.3284 & 2.1750 & 295.7 & 2 & 600.0 & 0 \\
\hline 02 & 6 & 310 & 158.7795 & 3.5250 & 296.0 & - & 600.0 & \\
\hline 02 & 6 & 311 & 158.5015 & 6.5250 & 295.6 & 3 & 600.0 & \\
\hline 02 & 6 & 312 & 151.5000 & 5.4500 & 296.7 & 3 & 600.0 & 00.0 \\
\hline 02 & 6 & 313 & 145.4995 & 6.2250 & 298.0 & 4 & 600.0 & 00.0 \\
\hline 02 & 6 & 314 & 138.5115 & 6.1250 & 299.2 & 4 & .0 & \\
\hline 02 & 6 & 315 & 89.2382 & 6.6250 & 299.6 & 4 & 600.0 & .0 \\
\hline 02 & 6 & 316 & 72.7496 & 8.5250 & 298.2 & 4 & 60 & .0 \\
\hline 02 & 6 & 317 & 79.0000 & 8. & 298.7 & 4 & .0 & \\
\hline 02 & 6 & 318 & 77.2498 & 8.5750 & 298.4 & 4 & 60 & .0 \\
\hline 02 & 6 & 319 & 72.5000 & 8.3750 & 297.6 & 4 & .0 & .0 \\
\hline 02 & 6 & 320 & 69.9999 & 9.67 & 296.0 & 4 & 6 & \\
\hline 02 & 6 & 321 & 73.7501 & 10.3500 & 295.7 & 4 & .0 & \\
\hline 02 & 6 & 322 & 70.5000 & 10.0250 & 295.1 & 4 & 600.0 & .0 \\
\hline 02 & 6 & 323 & 69.2500 & 10.57 & 294.7 & 4 & 60 & \\
\hline 02 & 6 & 324 & 73.7500 & 11.1750 & 294.0 & 4 & .0 & \\
\hline 02 & 6 & 41 & 76.7498 & 10.3250 & 293.4 & 4 & .0 & .0 \\
\hline 02 & 6 & 42 & 84.7502 & 8.8000 & 293.7 & 4 & 60 & .0 \\
\hline 02 & 6 & 43 & 95.2417 & 7.1750 & 294.1 & 4 & .0 & \\
\hline 02 & 6 & 44 & 111.7427 & 6.2250 & 295.0 & 4 & .0 & 0.0 \\
\hline 02 & 6 & 45 & 123.2460 & 6.2000 & 295.3 & 4 & 60 & .0 \\
\hline 02 & 6 & 46 & 124.4824 & 4.3750 & 295.6 & 4 & .0 & \\
\hline 02 & 6 & 47 & 111.9722 & 3.4750 & 297.0 & 3 & .0 & 600.0 \\
\hline 02 & 6 & 48 & 150.9792 & 7.1250 & 297.5 & 4 & 600.0 & 600.0 \\
\hline 02 & 6 & 49 & 167.5105 & 6.2000 & 297.7 & 4 & .0 & 0.0 \\
\hline 02 & 6 & 410 & 175.0000 & 6.87 & 298.4 & 4 & 600.0 & 600.0 \\
\hline 02 & 6 & 411 & 173.7533 & 5.9500 & 299.7 & 3 & 600.0 & 600.0 \\
\hline 02 & 6 & 412 & 168.5010 & 6.5500 & 300.4 & 4 & 600.0 & 600.0 \\
\hline 02 & 6 & 413 & 179.0037 & 6.3000 & 301.9 & 4 & 600.0 & 600.0 \\
\hline 02 & 6 & 414 & 180.5046 & 6.1250 & 303.0 & 4 & 600.0 & 600.0 \\
\hline 02 & 6 & 415 & 178.7506 & 000 & 303.5 & 4 & 600.0 & 600.0 \\
\hline 02 & 6 & 416 & 182.7406 & 4.9750 & 304.2 & 3 & 600.0 & 600.0 \\
\hline 02 & 6 & 417 & 175.4991 & 6.3750 & 304.7 & 4 & 600.0 & 600.0 \\
\hline 02 & 6 & 418 & 112.8351 & 5.8000 & 304.4 & 3 & 600.0 & 600.0 \\
\hline 02 & 6 & 419 & 80.0014 & 7.2250 & 302.1 & 4 & 600.0 & 600.0 \\
\hline 02 & 6 & 420 & 75.7496 & 8.0000 & 300.6 & 4 & 600.0 & 600.0 \\
\hline 02 & 6 & 421 & 71.7468 & 7.8750 & 300.3 & 4 & 600.0 & 600.0 \\
\hline 02 & 6 & 422 & 70.2498 & 8.1000 & 300.5 & 4 & 600.0 & 600.0 \\
\hline $0<$ & 6 & 423 & 77.7500 & 8.625 & 300.4 & 4 & 600.0 & 600.0 \\
\hline
\end{tabular}




\begin{tabular}{|c|c|c|c|c|c|c|c|c|}
\hline 2 & 6 & 424 & 75.0000 & 8.2750 & 299.7 & 4 & 600.0 & 00.0 \\
\hline 02 & 6 & 51 & 79.2496 & 7.7250 & 299.8 & 4 & 600.0 & 600.0 \\
\hline 02 & 6 & 52 & 85.2488 & 7.1500 & 299.7 & 4 & 600.0 & 00.0 \\
\hline 02 & 6 & 53 & 119.7290 & 5.8500 & 301.4 & 4 & 600.0 & 600.0 \\
\hline 02 & 6 & 54 & 142.2325 & 7.6500 & 301.1 & 4 & 600.0 & 00.0 \\
\hline 02 & 6 & 55 & 135.4765 & 5.5500 & 300.2 & 4 & 600.0 & 00.0 \\
\hline 02 & 6 & 56 & 150.9988 & 7.8250 & 300.5 & 4 & 600.0 & 600.0 \\
\hline 02 & 6 & 57 & 157.7499 & 9.6750 & 300.2 & 4 & 600.0 & 00.0 \\
\hline 02 & 6 & 58 & 166.5037 & 10.1000 & 300.4 & 4 & 600.0 & 00.0 \\
\hline 02 & 6 & 59 & 168.5009 & 9.2500 & 300.7 & 4 & 600.0 & 600.0 \\
\hline 02 & 6 & 510 & 156.7481 & 7.2000 & 301.6 & 4 & 600.0 & .0 \\
\hline 02 & 6 & 511 & 160.9997 & 00 & 302.7 & 4 & 600.0 & \\
\hline 02 & 6 & 512 & 157.7496 & 7.0250 & 303.8 & 4 & 600.0 & 600.0 \\
\hline 02 & 6 & 513 & 164.4990 & 7.5500 & 305.1 & 4 & 600.0 & 600.0 \\
\hline 02 & 6 & 514 & 168.0007 & 6 . & 306.3 & 4 & 600.0 & \\
\hline 02 & 6 & 515 & 174.2561 & 250 & 307.4 & 4 & 600.0 & 60 \\
\hline 02 & 6 & 516 & 177.5678 & 6.2750 & 308.1 & 4 & 600.0 & 600.0 \\
\hline 02 & 6 & 517 & 97.5043 & 5.8250 & 307.8 & 3 & 600.0 & \\
\hline 02 & 6 & 518 & 80.4985 & 5.7000 & 306.5 & 3 & 600.0 & 60 \\
\hline 02 & 6 & 519 & 74.2584 & 6.1000 & 305.4 & 4 & 600.0 & 600 \\
\hline 02 & 6 & 520 & 80.4986 & 6.2000 & $30<$ & 4 & 600.0 & .0 \\
\hline 02 & 6 & 521 & 80.7500 & 7.3750 & 302.3 & 4 & 600.0 & \\
\hline 02 & 6 & 522 & 71.2489 & 9.1750 & 302.0 & 4 & 600.0 & 600 \\
\hline 02 & 6 & 523 & 70.7500 & 8 . & 301.2 & 4 & 600.0 & .0 \\
\hline 02 & 6 & 524 & 72.7500 & 9 . & 300.6 & 4 & 600.0 & \\
\hline 02 & 6 & 61 & 76.2500 & 9.4750 & 299.8 & 4 & 600.0 & 60 \\
\hline 02 & 6 & 62 & 124.5840 & 6.9000 & 301.2 & 4 & 600.0 & .0 \\
\hline 02 & 6 & 63 & 111.4893 & 3 . & 300.3 & 4 & 00.0 & \\
\hline 02 & 6 & 64 & 135.7471 & 4.7500 & 300.3 & 4 & 600.0 & 60 \\
\hline 02 & 6 & 65 & 147.7508 & 5.9500 & 299.2 & 4 & 600.0 & 600.0 \\
\hline 02 & 6 & 66 & 140.7612 & 5.70 & 299.1 & 4 & 600.0 & .0 \\
\hline 02 & 6 & 67 & 155.2503 & 6.9750 & 299.2 & 4 & 600.0 & 60 \\
\hline 02 & 6 & 68 & 170.7504 & 8.4250 & 299.1 & 4 & 600.0 & 600.0 \\
\hline 02 & 6 & 69 & 171.4959 & 7.2500 & 299.8 & 4 & 600.0 & .0 \\
\hline 02 & 6 & 610 & 169.6647 & 5 . & 30 & 3 & 600.0 & .0 \\
\hline 02 & 6 & 611 & 169.6647 & 5.5000 & 300.8 & 3 & 600.0 & 600.0 \\
\hline 02 & 6 & 612 & 178.0000 & 5.4000 & 304.1 & 3 & 600.0 & \\
\hline 02 & 6 & 613 & 146.2128 & 5.9250 & 305.4 & 3 & 600.0 & 600.0 \\
\hline 02 & 6 & 614 & 130.9304 & 7.0000 & 305.4 & 4 & 600.0 & 600.0 \\
\hline 02 & 6 & 615 & 103.7530 & 6.4000 & 305.3 & 4 & 600.0 & \\
\hline 02 & 6 & 616 & 103.2492 & 6.4000 & 30 & 4 & 600.0 & 600.0 \\
\hline 02 & 6 & 617 & 76.1684 & 8.1000 & 303.8 & 4 & 600.0 & 600.0 \\
\hline 02 & 6 & 618 & 68.0000 & 10.7250 & 302.0 & 4 & 600.0 & 600.0 \\
\hline 02 & 6 & 619 & 65.2502 & 12.1750 & 301.2 & 4 & 600.0 & 600.0 \\
\hline 02 & 6 & 620 & 70.9804 & 11.4250 & 300.8 & 4 & 600.0 & 600.0 \\
\hline 02 & 6 & 621 & 94.2381 & 7.5500 & 299.7 & 4 & 600.0 & 600.0 \\
\hline 02 & 6 & 622 & 69.4999 & 8 . & & 4 & 600.0 & 600.0 \\
\hline 02 & 6 & 623 & 71.7500 & 11.2750 & 297.6 & 4 & 600.0 & 600.0 \\
\hline
\end{tabular}




\begin{tabular}{|c|c|c|c|c|c|c|c|c|}
\hline 2 & 6 & 624 & 79.5000 & 10.5000 & 296.2 & 4 & 600.0 & 00.0 \\
\hline 02 & 6 & 71 & 73.2500 & 11.7000 & 295.6 & 4 & 600.0 & 00.0 \\
\hline 02 & 6 & 72 & 74.4995 & 11.2250 & 294.8 & 4 & 600.0 & 00.0 \\
\hline 12 & 6 & 73 & 72.7492 & 10.4000 & 294.0 & 4 & 600.0 & 0 \\
\hline 02 & 6 & 74 & 82.7505 & 10.1000 & 293.1 & 4 & 600.0 & 00.0 \\
\hline 02 & 6 & 75 & 87.5001 & 8.8000 & 293.3 & 4 & 600.0 & 00.0 \\
\hline 02 & 6 & 76 & 101.2457 & 250 & 293.8 & 4 & 600.0 & 0 \\
\hline 02 & 6 & 77 & 02.7371 & 7.5000 & 293.6 & 4 & 600.0 & 00.0 \\
\hline 02 & 6 & 78 & 136.0249 & 8.3250 & 294.7 & 4 & 600.0 & 00.0 \\
\hline 02 & 6 & 79 & 138.2509 & 7.3500 & 295.4 & 4 & .0 & \\
\hline 02 & 6 & 710 & 149.4939 & 7.3500 & 296.2 & 4 & 600.0 & \\
\hline 02 & 6 & 711 & 153.2571 & 250 & 298.0 & 3 & .0 & \\
\hline 02 & 6 & 712 & 160.5184 & 5.6000 & 299.3 & 3 & 600.0 & 00.0 \\
\hline 02 & 6 & 713 & 156.9989 & 5.7000 & 300.5 & 3 & 600.0 & \\
\hline 02 & 6 & 714 & 109.9995 & 5.2500 & 301.1 & 3 & .0 & \\
\hline 02 & 6 & 715 & 94.2521 & 7.7250 & 300.2 & 4 & 600.0 & \\
\hline 02 & 6 & 716 & 88.2507 & 7.7250 & 299.5 & 4 & .0 & .0 \\
\hline 02 & 6 & 717 & 77.5046 & 9.45 & 298.1 & 4 & .0 & \\
\hline 02 & 6 & 718 & 73.2500 & 10.2000 & 297.6 & 4 & .0 & \\
\hline 02 & 6 & 719 & 70.0000 & 10.2000 & 298.2 & 4 & 0 & .0 \\
\hline 02 & 6 & 720 & 69.5000 & 9.95 & 296.4 & 4 & 0 & \\
\hline 02 & 6 & 721 & 72.7492 & 9.7500 & 295.7 & 4 & .0 & \\
\hline 02 & 6 & 722 & 71.0001 & 9.4500 & 295.3 & 4 & 600.0 & .0 \\
\hline 02 & 6 & 723 & 71.9993 & 10.62 & 294.7 & 4 & 0 & \\
\hline 02 & 6 & 724 & 79.2481 & 11.3750 & 293.7 & 4 & 0 & \\
\hline 02 & 6 & 81 & 99.4164 & 9.1250 & 293.6 & 4 & .0 & \\
\hline 02 & 6 & 82 & 142.7655 & 11.3750 & 293.9 & 4 & 60 & .0 \\
\hline 02 & 6 & 83 & 152.2495 & 9.9500 & 292.8 & 4 & .0 & \\
\hline 02 & 6 & 84 & 161.2532 & 8.7250 & 291.6 & 4 & .0 & .0 \\
\hline 02 & 6 & 85 & 161.7503 & 9.1750 & 290.4 & 4 & 6 & \\
\hline 02 & 6 & 86 & 163.7500 & 8.37 & 289.6 & 4 & .0 & \\
\hline 02 & 6 & 87 & 163.5000 & 9.0750 & 290.1 & 4 & .0 & .0 \\
\hline 02 & 6 & 88 & 172.2488 & 12.0500 & 290.4 & 4 & 600.0 & 0.0 \\
\hline 02 & 6 & 89 & 168.5002 & 13.4250 & 290.8 & 4 & .0 & .0 \\
\hline 02 & 6 & 810 & 169.2503 & 11.12 & 291.7 & 4 & 0 & 0.0 \\
\hline 02 & 6 & 811 & 171.0002 & 9.3000 & 292.9 & 4 & 600.0 & 600.0 \\
\hline 02 & 6 & 812 & 178.2510 & 8.80 & 293.9 & 4 & 600.0 & .0 \\
\hline 02 & 6 & 813 & 173.2498 & 11.3750 & 295.0 & 4 & 600.0 & 600.0 \\
\hline 02 & 6 & 814 & 179.4987 & 11.0750 & 295.6 & 4 & 600.0 & 600.0 \\
\hline 02 & 6 & 815 & 186.4994 & 8.07 & 296.1 & 4 & 600.0 & .0 \\
\hline 02 & 6 & 816 & 182.2566 & 5.2250 & 296.9 & 3 & 600.0 & 600.0 \\
\hline 02 & 6 & 817 & 105.1337 & 6.8750 & 296.7 & 4 & 600.0 & 600.0 \\
\hline 02 & 6 & 818 & 68.4992 & 8.3500 & 294.8 & 4 & 600.0 & 600.0 \\
\hline 02 & 6 & 819 & 71.0012 & 9.0500 & 293.1 & 4 & 600.0 & 600.0 \\
\hline 02 & 6 & 820 & 69.4991 & 8.6750 & 290.7 & 4 & 600.0 & 600.0 \\
\hline 02 & 6 & 821 & 75.2500 & 9.8750 & 288.7 & . & 600.0 & 600.0 \\
\hline 02 & 6 & 822 & 87.4847 & 10.8750 & 288.0 & 4 & 600.0 & 600.0 \\
\hline 48 & 0 & 823 & 136.1584 & 12.5000 & 288.7 & & 600.0 & 600.0 \\
\hline
\end{tabular}




\begin{tabular}{|c|c|c|c|c|c|c|c|}
\hline & 24 & & & 8.4 & & & \\
\hline & $\begin{array}{lll}6 & 9 & 1\end{array}$ & 2.2500 & 1.9250 & & & 20 & \\
\hline & $6 \quad 92$ & 7553 & 7750 & 287.7 & & 0 & \\
\hline & $\begin{array}{lll}6 & 9 & 3\end{array}$ & 40.5096 & 1.7500 & 287.7 & & 00 & \\
\hline & $\begin{array}{lll}6 & 9 & 4\end{array}$ & & .4750 & & & & \\
\hline & 695 & & & & & & \\
\hline & 696 & 7500 & 4250 & .9 & & 0 & \\
\hline & $\begin{array}{lll}6 & 9 & 7\end{array}$ & 55.7502 & 250 & & & & \\
\hline & $\begin{array}{lll}6 & 9 & 8\end{array}$ & 00 & 500 & & & & \\
\hline & $\begin{array}{lll}6 & 9 & 9\end{array}$ & 2509 & 3250 & 0 & & & \\
\hline & 6910 & 011 & 4.8500 & 90.1 & & & \\
\hline & 6911 & 78 & 0 & & & & \\
\hline & $\begin{array}{ll}6 & 912\end{array}$ & 06 & 0 & 92.1 & & & \\
\hline & 6913 & 4.2556 & 0.12 & 1 & & & \\
\hline & 6914 & & 0 & 2 & & & \\
\hline & 6915 & & 0 & 3 & & & \\
\hline & 6916 & 2499 & 0 & 295.7 & & & \\
\hline & 6917 & 17 & 0 & 8 & & & \\
\hline & 6918 & & 0 & & & & \\
\hline & 6919 & 5000 & & 295.6 & 4 & & \\
\hline & 6920 & 5000 & 5.75 & 94.8 & & & \\
\hline & 6921 & & & & $I$ & & \\
\hline & 6922 & 0 & 0 & 29 & 4 & & \\
\hline & 6923 & 1.5002 & 0 & 293.9 & & & \\
\hline & 6924 & & & 6 & 4 & & \\
\hline & 6101 & 00 & & .3 & 4 & & \\
\hline & 6102 & 000 & 0 & 292.9 & 4 & & \\
\hline & 6103 & 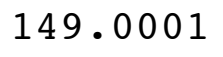 & & .3 & 4 & & \\
\hline & 6104 & 08 & 0 & .7 & 4 & & \\
\hline & 6105 & 00 & & .9 & 4 & & \\
\hline & 6106 & 002 & 0 & 290.5 & 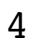 & & \\
\hline & 6107 & & & & 4 & & \\
\hline & 6108 & 0 & & .2 & 1 & & . \\
\hline & 6109 & 09 & 0 & 292.1 & 4 & & \\
\hline & 61010 & & & & 4 & & \\
\hline & 61011 & & & 294.8 & 4 & & \\
\hline & 61012 & 055 & & 296.0 & 4 & & \\
\hline 2 & 61013 & & & 29 & 4 & & \\
\hline & 61014 & 175.9834 & & 298.3 & 4 & & 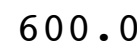 \\
\hline & 61015 & 2561 & 0 & 299.2 & 4 & & \\
\hline & 61016 & $1 \varepsilon$ & 0 & 299.7 & 4 & & \\
\hline & 61017 & 178.5000 & & 299.8 & 4 & & \\
\hline & 61018 & 4.7543 & 000 & 299.2 & 4 & & \\
\hline & 61019 & 1 & & 29 & & & \\
\hline 2 & 61020 & 3259 & & 298.2 & - & & \\
\hline & 61021 & 655 & 1.3750 & 297.6 & 4 & & \\
\hline & & & & & & & \\
\hline & 61023 & נו & 4.3250 & 280.0 & & .0 & 00 \\
\hline
\end{tabular}




\begin{tabular}{|c|c|c|c|c|c|c|c|}
\hline & 24 & & $\$ 250$ & 8.7 & & & \\
\hline & 111 & 21 . & 6.8500 & 98.2 & & & \\
\hline & 112 & 89.7500 & 000 & 96.8 & & 00 & \\
\hline & 113 & 91.2495 & 1000 & 296.9 & & 00 & \\
\hline & 114 & 114.1949 & 000 & 6.7 & & & \\
\hline & 115 & 23.75 & 500 & 96.1 & & & \\
\hline & 116 & 120.9814 & 000 & 295.4 & & 0 & \\
\hline & 117 & 137 . & 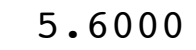 & 95.8 & & & \\
\hline & 118 & 38.25 & 0 & 96.1 & & & \\
\hline & 119 & 153.2597 & 500 & 297.5 & & 0 & \\
\hline & 1110 & 162.5000 & 6.75 & 298.3 & & & \\
\hline & 1111 & 172.5000 & 625 & 3 & & & \\
\hline 2 & 1 & 175 . & 6. & 300.2 & & & \\
\hline 2 & 113 & 170.7466 & 0.7 & 30 & & & \\
\hline & & 31 . & & 30 & & & \\
\hline 2 & & 190 & & 302 & & & \\
\hline 2 & 1116 & 180 . & 6 & 303 & & .0 & \\
\hline & 17 & 92 . & 5. & 4 & & & \\
\hline & & 84 & & 9 & & & \\
\hline & 9 & 77 & 7.4 & 298 & 4 & & \\
\hline 2 & 1120 & 72 & 8.72 & 29 & 4 & .0 & \\
\hline & & & & & $I$ & & \\
\hline 2 & 2 & & & 29 & 4 & & \\
\hline 2 & 1123 & 70 . & 0 & & 4 & 0 & \\
\hline 2 & 124 & & & & 4 & & \\
\hline 02 & 6121 & 71 . & 12 & 29 & 4 & & \\
\hline 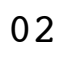 & 6122 & 008 & 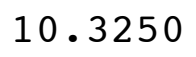 & 29 & 4 & 0 & \\
\hline & 6123 & 000 & 1 & 29 & 4 & & \\
\hline 2 & 6124 & 76 & 0 & 29 & 4 & & \\
\hline & 6125 & 000 & 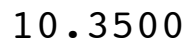 & 29 & 4 & & \\
\hline 2 & 6126 & 0 & 0 & 29 & 4 & & \\
\hline 2 & 6127 & 82 & & 29 & 4 & & \\
\hline & 6128 & & & & 4 & & \\
\hline 2 & 6129 & 9 & 5 . & & 0 & & \\
\hline 02 & 61210 & 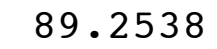 & & & 3 & & \\
\hline . & & & & & 3 & & \\
\hline & 61212 & 90 . & 5500 & 298.2 & 3 & & \\
\hline 2 & 61213 & 89 . & & 29 & 3 & & \\
\hline 02 & 61214 & 89.4976 & 6.8500 & & 4 & .0 & \\
\hline 02 & 61215 & 86. & 7.1000 & 299.4 & 4 & & \\
\hline 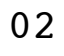 & 61216 & 91. & 7 . & 29 & 4 & & \\
\hline 02 & 61217 & 80.4962 & & & 4 & & \\
\hline & 61218 & 76.2505 & 9.3500 & 296.8 & 4 & & 0 \\
\hline 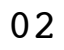 & 61219 & & 000 & & 4 & & \\
\hline 02 & 61220 & 76.5000 & 11.4500 & & 4 & .0 & \\
\hline 02 & 61221 & 74.2501 & 11.5500 & 293.8 & 4 & & החم \\
\hline & & & & & 4 & & \\
\hline & & 73.7499 & 11.0250 & 294.5 & & .0 & 600 \\
\hline
\end{tabular}




\begin{tabular}{|c|c|c|c|c|c|c|c|}
\hline 02 & 61224 & 76.7500 & 10.8000 & 294.0 & 4 & 600.0 & 600.0 \\
\hline 02 & 6131 & 77.5000 & 10.6250 & 293.6 & 4 & 600.0 & 00,0 \\
\hline 02 & 6132 & 76.0000 & 9.6750 & 293.1 & 4 & 600.0 & 00.0 \\
\hline 02 & 6133 & 81.7508 & 10.3250 & 293.0 & 4 & 600.0 & 00.0 \\
\hline 02 & 6134 & 76.5000 & 9.4250 & 292.3 & 4 & 600.0 & 00.0 \\
\hline 02 & 6135 & 77.7500 & 9.4750 & 291.8 & 4 & 600.0 & 00.0 \\
\hline 02 & 6136 & 79.7501 & 9.5500 & 292.0 & 4 & 600.0 & 00.0 \\
\hline 02 & 6137 & 78.0000 & 8.3000 & 292.1 & 4 & 600.0 & 00.0 \\
\hline 02 & 6138 & 80.2500 & 8.6500 & 292.6 & 4 & 600.0 & 00.0 \\
\hline 02 & 6139 & 89.7241 & 7.0750 & 293.3 & 4 & 600 & 0 \\
\hline 02 & 61310 & 6.2500 & 5.6500 & 293.8 & 4 & 600.0 & \\
\hline 02 & 61311 & 96.5000 & 5.6500 & 294.8 & 3 & .0 & \\
\hline 02 & 61312 & 86.4936 & 6.0500 & 295.6 & 4 & 600.0 & 00.0 \\
\hline 02 & 61313 & 91.2489 & 6.7750 & 295.8 & 4 & 600.0 & 00.0 \\
\hline 02 & 61314 & 93.2844 & 6.4250 & 296.8 & 4 & .0 & \\
\hline 02 & 61315 & 77.0006 & 8.0000 & 296.6 & 4 & 600.0 & .0 \\
\hline 02 & 61316 & 71.7500 & 11.0750 & 295.0 & 4 & 60 & .0 \\
\hline 02 & 61317 & 73.9998 & 12.7250 & 294.1 & 4 & .0 & \\
\hline 02 & 61318 & 75.7496 & 12.6000 & 292.6 & 4 & 60 & .0 \\
\hline 02 & 61319 & 74.5000 & 14.4250 & 290.1 & 4 & .0 & .0 \\
\hline 02 & 61320 & 75.2503 & 15.4750 & 288.3 & 4 & 6 & \\
\hline 02 & 61321 & 72.0000 & 14.2000 & 288.0 & 4 & .0 & \\
\hline 02 & 61322 & 71.5001 & 14.0500 & 288.7 & 4 & 600.0 & .0 \\
\hline 02 & 61323 & 79.7499 & 12.07 & 290.0 & 4 & 0 & \\
\hline 02 & 61324 & 72.2501 & 12.7000 & 289.3 & 4 & .0 & \\
\hline 02 & 6141 & 73.5000 & 12.0250 & 289.3 & 4 & .0 & \\
\hline 02 & 6142 & 77.5000 & 11.4750 & 288.7 & 4 & 60 & .0 \\
\hline 02 & 6143 & 79.4995 & 11.8750 & 287.6 & 4 & .0 & \\
\hline 02 & 6144 & 73.7497 & 13.4500 & 287.4 & 4 & .0 & .0 \\
\hline 02 & 6145 & 83.0000 & 11.8750 & 286.9 & 4 & 6 & .0 \\
\hline 02 & 6146 & 84.7488 & 10.6000 & 286.7 & 4 & .0 & \\
\hline 02 & 6147 & 81.5000 & 11.4750 & 286.7 & 4 & .0 & 0.0 \\
\hline 02 & 6148 & 84.4847 & 8.9250 & 288.9 & 4 & 600.0 & 600.0 \\
\hline 02 & 6149 & 96.0016 & 7.0000 & 289.5 & 4 & .0 & 0.0 \\
\hline 02 & 61410 & 88. & 7.9500 & 290.1 & 4 & 600.0 & 600.0 \\
\hline 02 & 61411 & 94.5031 & 7.6000 & 291.2 & 4 & 600.0 & 600.0 \\
\hline 02 & 61412 & 87.4992 & 8.3250 & 292.3 & 4 & 600.0 & 600.0 \\
\hline 02 & 61413 & 85.7530 & 7.9750 & 294.2 & 4 & 600.0 & 600.0 \\
\hline 02 & 61414 & 81.4977 & 7.6250 & 294.9 & 4 & 600.0 & 600.0 \\
\hline 02 & 61415 & 72.5000 & 9.4250 & 294.7 & 4 & 600.0 & 600.0 \\
\hline 02 & 61416 & 76.2501 & 10.3750 & 294.8 & 4 & 600.0 & 600.0 \\
\hline 02 & 61417 & 78.5000 & 8.9500 & 294.7 & 4 & 600.0 & 600.0 \\
\hline 02 & 61418 & 68.7488 & 8.8000 & 294.0 & 4 & 600.0 & 600.0 \\
\hline 02 & 61419 & 68.7497 & 10.0500 & 293.0 & 4 & 600.0 & 600.0 \\
\hline 02 & 61420 & 68.5000 & 10.8500 & 292.2 & 4 & 600.0 & 600.0 \\
\hline 02 & 61421 & 69.7500 & 12.0750 & 292.1 & 4 & 600.0 & 600.0 \\
\hline 02 & 61422 & 71.0001 & 11.5500 & 292.5 & 4 & 600.0 & 600.0 \\
\hline 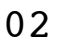 & $0 \perp 4<3$ & 72.7502 & 11.050 & 293.0 & 4 & 600.0 & 600.0 \\
\hline
\end{tabular}




\begin{tabular}{|c|c|c|c|c|c|c|c|}
\hline 02 & 61424 & 71.9998 & 10.7250 & 292.9 & 4 & 600.0 & 00.0 \\
\hline 02 & 6151 & 75.2500 & 10.1750 & 293.0 & 4 & 600.0 & 600.0 \\
\hline 02 & 6152 & 75.0000 & 9.8250 & 293.1 & 4 & 600.0 & 00.0 \\
\hline 02 & 6153 & 75.7500 & 9.7250 & 292.7 & 4 & 600.0 & 00.0 \\
\hline 02 & 6154 & 78.5000 & 9.6000 & 292.2 & 4 & 600.0 & 00.0 \\
\hline 02 & 6155 & 79.5000 & 9.2000 & 292.4 & 4 & 600.0 & 00.0 \\
\hline 02 & 6156 & 80.0000 & 7.4500 & 293.2 & 4 & 600.0 & 600.0 \\
\hline 02 & 6157 & 84.7500 & 5.6250 & 294.6 & 4 & 600.0 & 00.0 \\
\hline 02 & 6158 & 65.3195 & 2.9000 & 296.3 & 3 & 600.0 & 00.0 \\
\hline 02 & 6159 & 261.1070 & 1.6750 & 296.9 & 2 & 600.0 & 600.0 \\
\hline 02 & 61510 & 249.2526 & 1.9250 & 298.4 & 1 & 600.0 & .0 \\
\hline 02 & 61511 & 273.9420 & 500 & 300.0 & 1 & 600.0 & \\
\hline 02 & 61512 & 79.2511 & 3.2750 & 300.4 & 2 & 600.0 & 600.0 \\
\hline 02 & 61513 & 77.5000 & 4.9250 & 300.2 & 3 & 600.0 & .0 \\
\hline 02 & 61514 & 88.9963 & & 299.3 & 4 & 600.0 & \\
\hline 02 & 61515 & 88.4973 & 7.4250 & 298.6 & 4 & 600.0 & 60 \\
\hline 02 & 61516 & 93.9992 & 8.1250 & 298.7 & 4 & 600.0 & 600.0 \\
\hline 02 & 61517 & 84.5076 & 9.8250 & 297.8 & 4 & 600.0 & \\
\hline 02 & 61518 & 70.7499 & 12.0500 & 296.5 & 4 & 600.0 & .0 \\
\hline 02 & 61519 & 71.2503 & 12.9000 & 295.5 & 4 & 600.0 & 600 \\
\hline 02 & 61520 & 78.7524 & 11 . & 295.0 & 4 & 600.0 & .0 \\
\hline 02 & 61521 & 76.2360 & 11 . & 294.0 & 4 & 600.0 & \\
\hline 02 & 61522 & 72.9988 & 10. & 294.6 & 4 & 600.0 & 600.0 \\
\hline 02 & 61523 & 70.9988 & 9 . & 295.3 & 4 & 600.0 & .0 \\
\hline 02 & 61524 & 70.5000 & 9 . & 295.7 & 4 & 600.0 & \\
\hline 02 & 6161 & 72.0001 & 250 & 295.5 & 4 & 600.0 & 60 \\
\hline 02 & 6162 & 67.5000 & 9.6250 & 295.7 & 4 & 600.0 & 60 \\
\hline 02 & 6163 & 69.7500 & 9. & 295.8 & 4 & 0.0 & \\
\hline 02 & 6164 & 70.5000 & 9.1250 & 296.1 & 4 & 600.0 & 60 \\
\hline 02 & 6165 & 68.2502 & 7.8750 & 295.7 & 4 & 600.0 & 600 \\
\hline 02 & 6166 & 109.5774 & 2 . & 294.4 & 5 & 600.0 & \\
\hline 02 & 6167 & 91.0000 & 5.3250 & 295.3 & 4 & 600.0 & 60 \\
\hline 02 & 6168 & 101.7516 & 5.5250 & 296.3 & 3 & 600.0 & 600.0 \\
\hline 02 & 6169 & 92.5266 & 8 . & 295.2 & 4 & 600.0 & .0 \\
\hline 02 & 61610 & 77.0006 & 9. & 295.2 & 4 & 600.0 & .0 \\
\hline 02 & 61611 & 76.5014 & 8.5750 & 295.9 & 4 & 600.0 & 600.0 \\
\hline 02 & 61612 & 72.5002 & 9 . & 296.5 & 4 & 600.0 & \\
\hline 02 & 61613 & 76.4997 & 10 & 296.8 & 4 & 600.0 & .0 \\
\hline 02 & 61614 & 73.7499 & 11.8500 & 296.9 & 4 & 600.0 & 600.0 \\
\hline 02 & 61615 & 76.5015 & 10.5500 & 297.4 & 4 & 600.0 & \\
\hline 02 & 61616 & 77.2501 & 12.3500 & 296.2 & 4 & 600.0 & 600.0 \\
\hline 02 & 61617 & 76.5005 & 12.7250 & 295.1 & 4 & 600.0 & 600.0 \\
\hline 02 & 61618 & 78.0002 & 12.8750 & 294.3 & 4 & 600.0 & 600.0 \\
\hline 02 & 61619 & 72.0000 & 12.7750 & 294.4 & 4 & 600.0 & 600.0 \\
\hline 02 & 61620 & 65.7499 & 13.1750 & 293.3 & 4 & 600.0 & 600.0 \\
\hline 02 & 61621 & 67.0000 & 13.8000 & 294.2 & 4 & 600.0 & 600.0 \\
\hline 02 & 61622 & 69.0000 & 12.7750 & & 4 & 600.0 & 600.0 \\
\hline 02 & 61623 & 68.9998 & 12.4000 & 294.9 & 4 & 600.0 & 600.0 \\
\hline
\end{tabular}




\begin{tabular}{|c|c|c|c|c|c|c|c|}
\hline 2 & 61624 & 67.2499 & 11.2250 & 295.1 & 4 & 600.0 & 00.0 \\
\hline 02 & 6171 & 5.0001 & 9.9000 & 295.3 & 4 & 600.0 & 600.0 \\
\hline 02 & 6172 & 66.9995 & 9.5750 & 294.8 & 4 & 600.0 & 00.0 \\
\hline 02 & 6173 & 71.7499 & 9.9500 & 294.4 & 4 & 600.0 & 00.0 \\
\hline 02 & 6174 & 72.7500 & 9.5000 & 293.5 & 4 & 600.0 & 00.0 \\
\hline 02 & 6175 & 71.2500 & 9.8000 & 292.5 & 4 & 600.0 & 00.0 \\
\hline 02 & 6176 & 80.2275 & 9.4000 & 292.5 & 4 & 600.0 & 600.0 \\
\hline 02 & 6177 & 72.0002 & 11.4750 & 292.4 & 4 & 600.0 & 00.0 \\
\hline 02 & 6178 & 80.5009 & 10.9250 & 293.0 & 4 & 600.0 & 00.0 \\
\hline 02 & 6179 & 83.5000 & 7.7750 & 294.1 & 4 & 600.0 & .0 \\
\hline 02 & 61710 & 87.5002 & 7.3500 & 294.6 & 4 & 600.0 & 00.0 \\
\hline 02 & 61711 & 88.5000 & 00 & 295.6 & 4 & 600.0 & \\
\hline 02 & 61712 & 100.9977 & 6.3000 & 297.6 & 4 & 600.0 & 00.0 \\
\hline 02 & 61713 & 95.9966 & 6.8500 & 298.6 & 4 & 600.0 & .0 \\
\hline 02 & 61714 & 92.7502 & & 299.2 & 4 & 600.0 & \\
\hline 02 & 61715 & 87.9991 & 7.2500 & 300.0 & 4 & 600.0 & 60 \\
\hline 02 & 61716 & 85.2511 & 8.3750 & 299.4 & 4 & 600.0 & 600.0 \\
\hline 02 & 61717 & 75.0149 & 9 . & 298.4 & 4 & 600.0 & \\
\hline 02 & 61718 & 74.7595 & 8.4750 & 298.2 & 4 & 600.0 & .0 \\
\hline 02 & 61719 & 66.7487 & 250 & 297.2 & 4 & 600.0 & 600 \\
\hline 02 & 61720 & 71.4979 & 10 . & 295.3 & 4 & 600.0 & .0 \\
\hline 02 & 61721 & 65.4952 & 10. & 294.9 & 4 & 0.0 & \\
\hline 02 & 61722 & 73.0001 & 10. & 294.8 & 4 & 600.0 & 600.0 \\
\hline 02 & 61723 & 68.2501 & 10.7000 & 294.3 & 4 & 600.0 & .0 \\
\hline 02 & 61724 & 74.9998 & 11. & 294.2 & 4 & 00.0 & \\
\hline 02 & 6181 & 75.2501 & 11.7750 & 293.8 & 4 & 600.0 & 60 \\
\hline 02 & 6182 & 79.7499 & 11.9500 & 293.5 & 4 & 600.0 & .0 \\
\hline 02 & 6183 & 83.0000 & 12.70 & 292.8 & 4 & 0.0 & \\
\hline 02 & 6184 & 79.2500 & 11.2500 & 292.0 & 4 & 600.0 & 60 \\
\hline 02 & 6185 & 78.7519 & 11.3000 & 291.7 & 4 & 600.0 & .0 \\
\hline 02 & 6186 & 78.7500 & 10 . & 292.0 & 4 & 600.0 & \\
\hline 02 & 6187 & 78.0023 & 250 & 292.3 & 4 & 600.0 & 60 \\
\hline 02 & 6188 & 82.5027 & 10.3250 & 292.9 & 4 & 600.0 & 600.0 \\
\hline 02 & 6189 & 80.2496 & 9.22 & 293.8 & 4 & 600.0 & .0 \\
\hline 02 & 61810 & 74.0000 & 750 & 294.9 & 4 & 600.0 & .0 \\
\hline 02 & 61811 & 76.7520 & 7.9500 & 295.8 & 4 & 600.0 & 600.0 \\
\hline 02 & 61812 & 82.7505 & 9.5000 & 296.1 & 4 & 600.0 & \\
\hline 02 & 61813 & 86.5009 & 9.3500 & 296.4 & 4 & 600.0 & 600.0 \\
\hline 02 & 61814 & 83.7519 & 7.8000 & 297.0 & 4 & 600.0 & 600.0 \\
\hline 02 & 61815 & 70.2508 & 9.4250 & 297.0 & 4 & 600.0 & \\
\hline 02 & 61816 & 66.2500 & 10.5500 & 296.4 & 4 & 600.0 & 600.0 \\
\hline 02 & 61817 & 65.9995 & 9.9250 & 295.9 & 4 & 600.0 & 600.0 \\
\hline 02 & 61818 & 79.7521 & 7.9250 & 296.3 & 4 & 600.0 & 600.0 \\
\hline 02 & 61819 & 76.2498 & 8.6500 & 295.7 & 4 & 600.0 & 600.0 \\
\hline 02 & 61820 & 71.0001 & 8.9750 & 294.9 & 4 & 600.0 & 600.0 \\
\hline 02 & 61821 & 70.9999 & 9.2750 & 294.7 & 4 & 600.0 & 600.0 \\
\hline 02 & 61822 & 74.7500 & 9.125 & 295.0 & 4 & 600.0 & 600.0 \\
\hline 02 & 61823 & 73.9999 & 8.6000 & 294.8 & 4 & 600.0 & 600.0 \\
\hline
\end{tabular}




\begin{tabular}{|c|c|c|c|c|c|c|c|}
\hline 02 & 61824 & 78.0000 & 10.0000 & 294.1 & 4 & 600.0 & 600.0 \\
\hline 02 & 6191 & 82.9998 & 10.6250 & 293.5 & 4 & 600.0 & 600.0 \\
\hline 02 & 6192 & 82.4999 & 10.7250 & 293.5 & 4 & 600.0 & 00.0 \\
\hline 02 & 6193 & 81.5000 & 9.6250 & 293.7 & 4 & 600.0 & 00.0 \\
\hline 02 & 6194 & 104.1736 & 7.0250 & 294.7 & 4 & 600.0 & 00.0 \\
\hline 02 & 6195 & 134.7509 & 7.3750 & 295.6 & 4 & 600.0 & 00.0 \\
\hline 02 & 6196 & 146.9992 & 9.9500 & 295.0 & 4 & 600.0 & 0 \\
\hline 02 & 6197 & 156.0001 & 11.4000 & 295.0 & 4 & 600.0 & 00.0 \\
\hline 02 & 6198 & 168.2498 & 12.2750 & 295.5 & 4 & 600.0 & 00.0 \\
\hline 02 & 6199 & 169.2515 & 12.4250 & 296.1 & 4 & 60 & \\
\hline 02 & 61910 & 175.5003 & 11.8500 & 297.1 & 4 & 600.0 & \\
\hline 02 & 61911 & 178.0075 & 8.5500 & 298.7 & 4 & .0 & \\
\hline 02 & 61912 & 165.5135 & 5.3000 & 300.4 & 3 & 600.0 & 00.0 \\
\hline 02 & 61913 & 176.2274 & 5.4000 & 301.4 & 3 & 600.0 & \\
\hline 02 & 61914 & 126.7123 & 5.3750 & 302.0 & 3 & .0 & \\
\hline 02 & 61915 & 84.2535 & 5.9750 & 302.4 & 3 & 600.0 & .0 \\
\hline 02 & 61916 & 83.7354 & 7.1000 & 302.0 & 4 & 60 & .0 \\
\hline 02 & 61917 & 79.2654 & 8.2250 & 301.2 & 4 & .0 & \\
\hline 02 & 61918 & 75.2496 & 7.0750 & 301.0 & 4 & .0 & \\
\hline 02 & 61919 & 70.9988 & 6.6500 & 300.4 & 4 & .0 & .0 \\
\hline 02 & 61920 & 75.2502 & 8 . & 299.0 & 4 & 6 & \\
\hline 02 & 61921 & 80.7501 & 8.0500 & 298.7 & 4 & .0 & \\
\hline 02 & 61922 & 77.5000 & 7.8750 & 298.6 & 4 & 600.0 & .0 \\
\hline 02 & 61923 & 79.0000 & 8.02 & 298.2 & 4 & 0 & \\
\hline 02 & 61924 & 78.7497 & 750 & 297.5 & 4 & 0 & \\
\hline 02 & 6201 & 82.0007 & 9.1250 & 296.4 & 4 & .0 & \\
\hline 02 & 6202 & 79.0000 & 9.8000 & 296.3 & 4 & 60 & .0 \\
\hline 02 & 6203 & 75.4988 & 9.0500 & 296.0 & 4 & .0 & \\
\hline 02 & 6204 & 73.7508 & 8.5250 & 296.2 & 4 & .0 & .0 \\
\hline 02 & 6205 & 79.2500 & 9.1750 & 295.1 & 4 & 6 & \\
\hline 02 & 6206 & 80.9999 & 9.7750 & 293.8 & 4 & .0 & \\
\hline 02 & 6207 & 77.7500 & 7.7750 & 295.2 & 4 & .0 & 0 \\
\hline 02 & 6208 & 164.1861 & 3.1250 & 297.0 & 3 & 600.0 & 0.0 \\
\hline 02 & 6209 & 229.9022 & 2.2000 & 297.5 & 2 & .0 & 0.0 \\
\hline 02 & 62010 & 88.7530 & 5.6750 & 295.7 & 3 & 600.0 & 600.0 \\
\hline 02 & 62011 & 89.7488 & 6.5500 & 296.2 & 4 & 600.0 & 600.0 \\
\hline 02 & 62012 & 83.0000 & 6.9250 & 296.8 & 4 & 600.0 & 0.0 \\
\hline 02 & 62013 & 81.5009 & 6.7750 & 297.8 & 4 & 600.0 & 600.0 \\
\hline 02 & 62014 & 77.5010 & 8.4500 & 296.8 & 4 & 600.0 & 600.0 \\
\hline 02 & 62015 & 76.5002 & 10.5500 & 296.6 & 4 & 600.0 & 600.0 \\
\hline 02 & 62016 & 72.7489 & 11.7750 & 296.0 & 4 & 600.0 & 600.0 \\
\hline 02 & 62017 & 67.7499 & 11.8750 & 294.5 & 4 & 600.0 & 600.0 \\
\hline 02 & 62018 & 67.0000 & 13.0500 & 292.8 & 4 & 600.0 & 600.0 \\
\hline 02 & 62019 & 70.7504 & 14.0000 & 291.5 & 4 & 600.0 & 600.0 \\
\hline 02 & 62020 & 72.7500 & 13.9250 & 290.1 & 4 & 600.0 & 600.0 \\
\hline 02 & 62021 & 72.0000 & 14.2250 & 289.9 & . & 600.0 & 600.0 \\
\hline 02 & 62022 & 71.7504 & 13.7250 & 289.6 & 4 & 600.0 & 600.0 \\
\hline 02 & 62023 & 70.0001 & 14.050 & 289.1 & $\Psi$ & 600.0 & 600.0 \\
\hline
\end{tabular}




\begin{tabular}{|c|c|c|c|c|c|c|c|}
\hline 02 & 62024 & 72.2501 & 14.3500 & 287.4 & 4 & 600.0 & 00.0 \\
\hline 02 & 6211 & 73.9999 & 15.7750 & 286.3 & 4 & 600.0 & 600.0 \\
\hline 02 & 6212 & 78.5009 & 15.8500 & 285.3 & 4 & 600.0 & 00.0 \\
\hline 02 & 6213 & 75.7478 & 14.5750 & 284.5 & 4 & 600.0 & 00.0 \\
\hline 02 & 6214 & 73.2501 & 13.0000 & 284.2 & 4 & 600.0 & 00.0 \\
\hline 02 & 6215 & 78.0016 & 12.7500 & 284.3 & 4 & 600.0 & 00.0 \\
\hline 02 & 6216 & 82.7500 & 13.2750 & 284.2 & 4 & 600.0 & 600.0 \\
\hline 02 & 6217 & 74.5000 & 12.6250 & 284.0 & 4 & 600.0 & 00.0 \\
\hline 02 & 6218 & 73.0000 & 10.7500 & 284.7 & 4 & 600.0 & 00.0 \\
\hline 02 & 6219 & 75.2500 & 9.0250 & 286.4 & 4 & 600.0 & .0 \\
\hline 02 & 62110 & 71.5023 & 5.1000 & 289.0 & 3 & 600.0 & 00.0 \\
\hline 02 & 62111 & 75.7917 & 00 & 291.1 & 3 & 600.0 & \\
\hline 02 & 62112 & 64.1940 & 4.5750 & 292.4 & 3 & 600.0 & 00.0 \\
\hline 02 & 62113 & 62.5000 & 5.5250 & 293.2 & 3 & 600.0 & .0 \\
\hline 02 & 62114 & 68.2490 & & 293.6 & 4 & 600.0 & \\
\hline 02 & 62115 & 72.7497 & 10.2000 & 291.5 & 4 & 600.0 & 60 \\
\hline 02 & 62116 & 69.9998 & 12.6250 & 289.9 & 4 & 600.0 & 600.0 \\
\hline 02 & 62117 & 73.2506 & 13.2750 & 288.9 & 4 & 600.0 & \\
\hline 02 & 62118 & 77.0000 & 12.7750 & 288.1 & 4 & 600.0 & .0 \\
\hline 02 & 62119 & 75.0000 & 13.4500 & 286.7 & 4 & 600.0 & 600 \\
\hline 02 & 62120 & 0.5002 & 13. & 286.0 & 4 & 600.0 & .0 \\
\hline 02 & 62121 & 77.2499 & 12 . & 285.5 & 4 & 600.0 & \\
\hline 02 & 62122 & 75.2507 & 13.6750 & 285.3 & 4 & 600.0 & 600.0 \\
\hline 02 & 62123 & 77.4998 & 15 . & 284.5 & 4 & 600.0 & .0 \\
\hline 02 & 62124 & 77.2500 & 14. & 284.1 & 4 & 600.0 & \\
\hline 02 & 6221 & 78.9998 & 13.9250 & 284.4 & 4 & 600.0 & 60 \\
\hline 02 & 6222 & 80.7499 & 12.7750 & 284.2 & 4 & 600.0 & .0 \\
\hline 02 & 6223 & 79.0000 & 11.37 & 284.2 & 4 & 600.0 & \\
\hline 02 & 6224 & 80.0000 & 11.1750 & 284.0 & 4 & 600.0 & 60 \\
\hline 02 & 6225 & 79.7500 & 9.9000 & 284.0 & 4 & 600.0 & .0 \\
\hline 02 & 6226 & 81.0000 & 9 . & 284.3 & 4 & 600.0 & \\
\hline 02 & 6227 & 82.5000 & 9 . & 284.8 & 4 & 600.0 & 60 \\
\hline 02 & 6228 & 81.9999 & 7.5500 & 286.3 & 4 & 600.0 & 600.0 \\
\hline 02 & 6229 & 81.5000 & 5 . & 287.9 & 4 & 600.0 & .0 \\
\hline 02 & 62210 & 94.3944 & 2 . & 291.0 & 3 & 600.0 & 60 \\
\hline 02 & 62211 & 44.9417 & 2.6500 & 293.3 & 2 & 600.0 & 600.0 \\
\hline 02 & 62212 & 18.8506 & 2.4750 & 295.0 & 1 & 600.0 & \\
\hline 02 & 62213 & 48.0227 & 4.0250 & 295.9 & 2 & 600.0 & 600.0 \\
\hline 02 & 62214 & 90.4999 & 4.5500 & 296.6 & 3 & 600.0 & 600.0 \\
\hline 02 & 62215 & 98.7518 & 5.1500 & 297.6 & 3 & 600.0 & \\
\hline 02 & 62216 & 92.2470 & 6.9750 & 297.7 & 4 & 600.0 & 600.0 \\
\hline 02 & 62217 & 89.7477 & 6.5250 & 297.0 & 4 & 600.0 & 600.0 \\
\hline 02 & 62218 & 80.5002 & 8.2000 & 295.5 & 4 & 600.0 & 600.0 \\
\hline 02 & 62219 & 73.9916 & 10.0750 & 294.6 & 4 & 600.0 & 600.0 \\
\hline 02 & 62220 & 72.9998 & 10.4250 & 293.7 & 4 & 600.0 & 600.0 \\
\hline 02 & 62221 & 75.0003 & 10.9250 & 292.6 & 4 & 600.0 & 600.0 \\
\hline 02 & 62222 & 72.0002 & 10.175 & 29 & 4 & 600.0 & 600.0 \\
\hline 02 & 62223 & 70.0000 & 9.2250 & 294.6 & 4 & 600.0 & 600.0 \\
\hline
\end{tabular}




\begin{tabular}{|c|c|c|c|c|c|c|c|}
\hline 2 & 62224 & 71.7501 & 9.0000 & 294.6 & 4 & 600.0 & 00.0 \\
\hline 02 & 6231 & 73.0001 & 10.4250 & 293.6 & 4 & 600.0 & 600.0 \\
\hline 02 & 6232 & 68.2500 & 9.4750 & 293.2 & 4 & 600.0 & 00.0 \\
\hline 02 & 6233 & 69.2492 & 9.1250 & 292.6 & 4 & 600.0 & 00.0 \\
\hline 02 & 6234 & 71.2502 & 9.2750 & 292.6 & 4 & 600.0 & 00.0 \\
\hline 02 & 6235 & 75.2503 & 9.5000 & 292.2 & 4 & 600.0 & 00.0 \\
\hline 02 & 6236 & 70.0001 & 9.6750 & 292.3 & 4 & 600.0 & 600.0 \\
\hline 02 & 6237 & 78.0007 & 8.1000 & 292.7 & 4 & 600.0 & 00.0 \\
\hline 02 & 6238 & 91.7519 & 5.9000 & 293.6 & 3 & 600.0 & 00.0 \\
\hline 02 & 6239 & 94.9916 & 7.3500 & 293.7 & 4 & 600.0 & 600.0 \\
\hline 02 & 62310 & 91.4926 & 7.5500 & 294.5 & 4 & 600.0 & .0 \\
\hline 02 & 62311 & 75.2503 & 7.6000 & 295.2 & 4 & 600.0 & \\
\hline 02 & 62312 & 77.7489 & 7.7250 & 296.3 & 4 & 600.0 & 00.0 \\
\hline 02 & 62313 & 79.0000 & 7.7500 & 297.6 & 4 & 600.0 & .0 \\
\hline 02 & 62314 & 70.7518 & 9.5750 & 297.6 & 4 & 600.0 & \\
\hline 02 & 62315 & 76.0021 & 10.1250 & 297.2 & 4 & 600.0 & 60 \\
\hline 02 & 62316 & 67.7497 & 10.7750 & 296.9 & 4 & 600.0 & .0 \\
\hline 02 & 62317 & 68.7502 & 10.1000 & 296.3 & 4 & 600.0 & \\
\hline 02 & 62318 & 61.7506 & 10.5000 & 295.8 & 4 & 600.0 & .0 \\
\hline 02 & 62319 & 70.5002 & 13.0500 & 294.2 & 4 & 600.0 & 600 \\
\hline 02 & 62320 & 74.4997 & 12.57 & 292.8 & 4 & 600.0 & .0 \\
\hline 02 & 62321 & 73.5000 & 12.0250 & 293.1 & 4 & 0.0 & \\
\hline 02 & 62322 & 74.0001 & 11.4500 & 293.8 & 4 & 600.0 & 60 \\
\hline 02 & 62323 & 74.2500 & 11.1250 & 293.8 & 4 & 600.0 & .0 \\
\hline 02 & 62324 & 75.5001 & 11. & 293.2 & 4 & 600.0 & \\
\hline 02 & 6241 & 71.7500 & 10.0250 & 293.5 & 4 & 600.0 & 60 \\
\hline 02 & 6242 & 75.2500 & 8.7500 & 293.5 & 4 & 600.0 & .0 \\
\hline 02 & 6243 & 74.5000 & 8.30 & 294.7 & 4 & 600.0 & \\
\hline 02 & 6244 & 75.4966 & 6.5750 & 295.1 & 4 & 600.0 & 60 \\
\hline 02 & 6245 & 90.7496 & 4.5250 & 294.7 & 5 & 600.0 & .0 \\
\hline 02 & 6246 & 50.3329 & 1.37 & 293.9 & 6 & 600.0 & \\
\hline 02 & 6247 & 106.7467 & 3 . & 295.1 & 5 & 600.0 & .0 \\
\hline 02 & 6248 & 90.2363 & 3.8500 & 296.2 & 4 & 600.0 & 600.0 \\
\hline 02 & 6249 & 258.2300 & 2.350 & 296.9 & 3 & 600.0 & .0 \\
\hline 02 & 62410 & 210.8912 & 3.8250 & 296.4 & 3 & 600.0 & .0 \\
\hline 02 & 62411 & 177.4991 & 6.5500 & 297.0 & 4 & 600.0 & 600.0 \\
\hline 02 & 62412 & 173.4995 & 5.3250 & 298.2 & 3 & 600.0 & \\
\hline 02 & 62413 & 173.0008 & 4.9250 & 299.4 & 3 & 600.0 & .0 \\
\hline 02 & 62414 & 181.9993 & 5.1500 & 301.0 & 3 & 600.0 & 600.0 \\
\hline 02 & 62415 & 178.5083 & 5.800 & 301.5 & 3 & 600.0 & \\
\hline 02 & 62416 & 169.2489 & 6.3750 & 301.8 & 4 & 600.0 & 600.0 \\
\hline 02 & 62417 & 157.7085 & 5.7500 & 302.3 & 3 & 600.0 & 600.0 \\
\hline 02 & 62418 & 94.0037 & 5.0500 & 302.4 & 3 & 600.0 & 600.0 \\
\hline 02 & 62419 & 82.7494 & 5.8250 & 301.0 & 4 & 600.0 & 600.0 \\
\hline 02 & 62420 & 79.5001 & 7.4250 & 298.5 & 4 & 600.0 & 600.0 \\
\hline 02 & 62421 & 77.2501 & 9.1750 & 297.3 & 4 & 600.0 & 600.0 \\
\hline 02 & 62422 & 77.0000 & 8.950 & 297.4 & 4 & 600.0 & 600.0 \\
\hline 02 & 62423 & 79.9998 & 8.6250 & 297.6 & 4 & 600.0 & 600.0 \\
\hline
\end{tabular}




\begin{tabular}{|c|c|c|c|c|c|c|c|}
\hline 2 & 62424 & 78.5000 & 9.1000 & 297.2 & 4 & 600.0 & 600.0 \\
\hline 02 & 6251 & 77.7501 & 9.1750 & 296.9 & 4 & 600.0 & 600.0 \\
\hline 02 & 6252 & 75.7500 & 8.5000 & 296.9 & 4 & 600.0 & 00.0 \\
\hline 02 & 6253 & 75.2500 & 7.7000 & 297.1 & 4 & 600.0 & 00.0 \\
\hline 02 & 6254 & 75.2501 & 6.8250 & 297.4 & 4 & 600.0 & 00.0 \\
\hline 02 & 6255 & 76.0000 & 6.8750 & 297.6 & 4 & 600.0 & 00.0 \\
\hline 02 & 6256 & 77.4995 & 5.7000 & 298.1 & 4 & 600.0 & 00.0 \\
\hline 02 & 6257 & 91.7492 & 3.7750 & 299.2 & 4 & 600.0 & 00.0 \\
\hline 02 & 6258 & 140.6246 & 1.9750 & 301.0 & 3 & 600.0 & 00.0 \\
\hline 02 & 6259 & 232.2499 & 2.1000 & 301.4 & 2 & 600.0 & 0 \\
\hline 02 & 62510 & 189.1637 & 4.3000 & 300.4 & 3 & 600.0 & \\
\hline 02 & 62511 & 181.0023 & 6.3500 & 300.7 & 4 & 600.0 & \\
\hline 02 & 62512 & 178.4992 & 6.5500 & 302.0 & 4 & 600.0 & 00.0 \\
\hline 02 & 62513 & 168.7725 & 6.6750 & 303.1 & 4 & 600.0 & 00.0 \\
\hline 02 & 62514 & 159.0000 & 50 & 304.5 & 3 & .0 & \\
\hline 02 & 62515 & 98.2302 & 4.9250 & 305.6 & 3 & 600.0 & 00.0 \\
\hline 02 & 62516 & 91.7411 & 6.3750 & 305.3 & 4 & 60 & .0 \\
\hline 02 & 62517 & 83.2527 & 6. & 304.5 & 4 & .0 & \\
\hline 02 & 62518 & 83.7539 & 6.9250 & 303.4 & 4 & 600.0 & .0 \\
\hline 02 & 62519 & 76.0004 & 7.7750 & 301.7 & 4 & .0 & .0 \\
\hline 02 & 62520 & 77.0000 & 9.42 & 300.0 & 4 & 60 & \\
\hline 02 & 62521 & 71.7501 & 9.8250 & 299.4 & 4 & .0 & \\
\hline 02 & 62522 & 67.5002 & 9.4750 & 299.2 & 4 & 600.0 & .0 \\
\hline 02 & 62523 & 65.2500 & 9.57 & 299.3 & 4 & 6 & \\
\hline 02 & 62524 & 66.2500 & 9.0750 & 299.4 & 4 & .0 & \\
\hline 02 & 6261 & 63.2489 & 8.9250 & 299.4 & 4 & 600.0 & 600.0 \\
\hline 02 & 6262 & 60.7511 & 8.4250 & 299.0 & 4 & 60 & .0 \\
\hline 02 & 6263 & 52.2500 & 4.85 & 298.2 & 5 & .0 & \\
\hline 02 & 6264 & 62.7504 & 4.8750 & 298.3 & 5 & .0 & 0.0 \\
\hline 02 & 6265 & 65.4991 & 4.7750 & 298.5 & 5 & 60 & .0 \\
\hline 02 & 6266 & 28.5554 & 2.1250 & 298.2 & 4 & .0 & \\
\hline 02 & 6267 & 343.8320 & 0.1000 & 301.2 & 3 & .0 & 600.0 \\
\hline 02 & 6268 & 338.2602 & 0.6000 & 301.0 & 2 & 600.0 & 600.0 \\
\hline 02 & 6269 & 285.5462 & 1.3250 & 300.6 & 1 & 0 & 0.0 \\
\hline 02 & 62610 & 244.7511 & 2.1750 & 300.3 & 1 & 600.0 & 600.0 \\
\hline 02 & 62611 & 239.9989 & 2.6250 & 300.2 & 1 & 600.0 & 600.0 \\
\hline 02 & 62612 & 260.2557 & 3.0000 & 301.0 & 2 & 600.0 & 600.0 \\
\hline 02 & 62613 & 321.3019 & 3.3250 & 302.3 & 2 & 600.0 & 600.0 \\
\hline 02 & 62614 & 62.7525 & 4.3750 & 302.8 & 3 & 600.0 & 600.0 \\
\hline 02 & 62615 & 89.7499 & 6.7000 & 302.2 & 4 & 600.0 & 600.0 \\
\hline 02 & 62616 & 88.4954 & 8.5000 & 301.9 & 4 & 600.0 & 600.0 \\
\hline 02 & 62617 & 85.9871 & 8.9250 & 300.5 & 4 & 600.0 & 600.0 \\
\hline 02 & 62618 & 81.2495 & 9.7750 & 297.4 & 4 & 600.0 & 600.0 \\
\hline 02 & 62619 & 82.5001 & 12.1000 & 294.6 & 4 & 600.0 & 600.0 \\
\hline 02 & 62620 & 77.0000 & 13.3000 & 292.3 & 4 & 600.0 & 600.0 \\
\hline 02 & 62621 & 78.7500 & 14.2000 & 290.6 & 4 & 600.0 & 600.0 \\
\hline 02 & 62622 & 80.0000 & 14.8250 & 289.7 & 4 & 600.0 & 600.0 \\
\hline 02 & 62623 & 78.9995 & 13.0250 & 290.2 & 4 & 600.0 & 600.0 \\
\hline
\end{tabular}




\begin{tabular}{|c|c|c|c|c|c|c|c|}
\hline 2 & 62624 & 4.7502 & 11.9750 & 292.4 & 4 & 600.0 & 00.0 \\
\hline 02 & 6271 & 76.2500 & 10.6750 & 293.3 & 4 & 600.0 & 00.0 \\
\hline 02 & 6272 & 71.7500 & 10.9000 & 292.3 & 4 & 600.0 & 00.0 \\
\hline 02 & 6273 & 76.5000 & 12.9500 & 290.5 & 4 & 600.0 & 00.0 \\
\hline 02 & 6274 & 78.2501 & 13.2500 & 288.5 & 4 & 600.0 & 00.0 \\
\hline 02 & 6275 & 77.5000 & 13.3000 & 288.6 & 4 & 600.0 & 00.0 \\
\hline 02 & 6276 & 78.5000 & 11.0750 & 291.2 & 4 & 600.0 & 00.0 \\
\hline 02 & 6277 & 32.7494 & 9.2250 & 292.5 & 4 & 600.0 & 00.0 \\
\hline 02 & 6278 & 83.2500 & 9.4750 & 291.6 & 4 & 600.0 & 00.0 \\
\hline 02 & 6279 & 87.7485 & 8.3500 & 293.5 & 4 & 600.0 & .0 \\
\hline 02 & 62710 & 35.9977 & 7.8750 & 294.6 & 4 & 600.0 & 00.0 \\
\hline 02 & 62711 & 87.4998 & 6.2500 & 296.8 & 4 & 600.0 & \\
\hline 02 & 62712 & 79.4954 & 5.5750 & 298.8 & 3 & 600.0 & .0 \\
\hline 02 & 62713 & 84.7494 & 6.5250 & 299.5 & 4 & 600.0 & 00.0 \\
\hline 02 & 62714 & 81.9998 & & 298.9 & 4 & 600.0 & \\
\hline 02 & 62715 & 73.0000 & 10.9250 & 299.2 & 4 & 600.0 & 60 \\
\hline 02 & 62716 & 76.7501 & 9.45 & 299.6 & 4 & 600.0 & 600.0 \\
\hline 02 & 62717 & 74.4998 & 9.97 & 298.8 & 4 & 600.0 & \\
\hline 02 & 62718 & 70.0001 & 11.0750 & 297.6 & 4 & 600.0 & .0 \\
\hline 02 & 62719 & 66.2501 & 12.7500 & 296.4 & 4 & 600.0 & .0 \\
\hline 02 & 62720 & 67.2500 & 13.10 & 295.1 & 4 & 600.0 & .0 \\
\hline 02 & 62721 & 68.5000 & 13.2 & 295.0 & 4 & 0.0 & \\
\hline 02 & 62722 & 68.5000 & 12.8000 & 294.5 & 4 & 600.0 & 60 \\
\hline 02 & 62723 & 66.2500 & 12.25 & 294.9 & 4 & 600.0 & .0 \\
\hline 02 & 62724 & 69.5000 & 12.3 & 294.4 & 4 & 600.0 & \\
\hline 02 & 6281 & 66.9998 & 12.9250 & 293.7 & 4 & 600.0 & 60 \\
\hline 02 & $628 \quad 2$ & 65.0000 & 13.7500 & 292.4 & 4 & 600.0 & . 0 \\
\hline 02 & 6283 & 69.2500 & 13.77 & 292.2 & 4 & 0.0 & \\
\hline 02 & 6284 & 68.0000 & 12.1250 & 293.2 & 4 & 600.0 & 60 \\
\hline 02 & 6285 & 69.7500 & 12.37 & 292.7 & 4 & 600.0 & .0 \\
\hline 02 & 6286 & 72.7499 & 11.57 & 291.9 & 4 & 600.0 & \\
\hline 02 & 6287 & 72.5002 & 9.7750 & 292.8 & 4 & 600.0 & 60 \\
\hline 02 & 6288 & 89.0000 & 9.6250 & 291.9 & 4 & 600.0 & 600.0 \\
\hline 02 & 6289 & 80.2498 & 9.10 & 292.1 & 4 & 600.0 & .0 \\
\hline 02 & 62810 & 79.2503 & 9.3 & 293.2 & 4 & 600.0 & 60 \\
\hline 02 & 62811 & 74.9999 & 9.1500 & 293.9 & 4 & 600.0 & 600.0 \\
\hline 02 & 62812 & 79.0000 & 9.67 & 294.7 & 4 & 600.0 & \\
\hline 02 & 62813 & 76.7499 & 9.15 & 295.9 & 4 & 600.0 & .0 \\
\hline 02 & 62814 & 82.2495 & 10.4000 & 295.9 & 4 & 600.0 & 600.0 \\
\hline 02 & 62815 & 80.2633 & 9.6000 & 297.3 & 4 & 600.0 & \\
\hline 02 & 62816 & 77.0000 & 9.5500 & 297.8 & 4 & 600.0 & 60 \\
\hline 02 & 62817 & 72.7509 & 11.3750 & 297.6 & 4 & 600.0 & 600.0 \\
\hline 02 & 62818 & 68.0002 & 10.9000 & 296.1 & 4 & 600.0 & 600.0 \\
\hline 02 & 62819 & 70.0000 & 10.9750 & 295.4 & 4 & 600.0 & 600.0 \\
\hline 02 & 62820 & 74.5001 & 12.1500 & 293.3 & 4 & 600.0 & 600.0 \\
\hline 02 & 62821 & 68.7492 & 12.5500 & 293.1 & 4 & 600.0 & 600.0 \\
\hline 02 & 62822 & 70.2501 & 11.82 & 292.9 & 4 & 600.0 & 600.0 \\
\hline 02 & 62823 & 72.2500 & 13.0750 & 292.6 & 4 & 600.0 & 600.0 \\
\hline
\end{tabular}




\begin{tabular}{|c|c|c|c|c|c|c|c|}
\hline 2 & 62824 & 70.7500 & 12.8000 & 292.9 & 4 & 600.0 & 600.0 \\
\hline 02 & 6291 & 70.9995 & 12.1500 & 293.0 & 4 & 600.0 & 600.0 \\
\hline 02 & 6292 & 72.0006 & 000 & 292.7 & 4 & 600.0 & 00.0 \\
\hline 02 & 6293 & 77.0000 & 11.8250 & 292.3 & 4 & 600.0 & 00.0 \\
\hline 02 & 6294 & 71.2503 & 750 & 292.4 & 4 & 600.0 & 00.0 \\
\hline 02 & 6295 & 72.5000 & 10.2250 & 292.7 & 4 & 600.0 & 00.0 \\
\hline 02 & 6296 & 73.7500 & 10.4250 & 292.9 & 4 & 600.0 & 0 \\
\hline 02 & 6297 & 76.7502 & 10.8250 & 293.2 & 4 & 600.0 & 00.0 \\
\hline 02 & 6298 & 77.7499 & 9.5000 & 294.2 & 4 & 600.0 & 00.0 \\
\hline 02 & 6299 & 78.7493 & 8.1750 & 295.8 & 4 & 60 & 0 \\
\hline 02 & 62910 & 84.0000 & 7.7500 & 298.0 & 4 & 600.0 & \\
\hline 02 & 62911 & 87.2508 & 7.3500 & 299.0 & 4 & .0 & \\
\hline 02 & 62912 & 93.7485 & 5.5250 & 300.5 & 3 & 600.0 & .0 \\
\hline 02 & 62913 & 97.7537 & 5.6250 & 301.9 & 3 & 600.0 & 00.0 \\
\hline 02 & 62914 & 87.2498 & 7.2750 & 301.8 & 4 & .0 & \\
\hline 02 & 62915 & 78.7571 & 8.0000 & 301.2 & 4 & 600.0 & .0 \\
\hline 02 & 62916 & 62.2503 & 9.5750 & 300.3 & 4 & .0 & .0 \\
\hline 02 & 62917 & 66.9996 & 10.5250 & 299.2 & 4 & .0 & \\
\hline 02 & 62918 & 70.4986 & 9.3000 & 298.9 & 4 & 60 & .0 \\
\hline 02 & 62919 & 68.7502 & 8.8750 & 298.5 & 4 & .0 & .0 \\
\hline 02 & 62920 & 72.9999 & 11.3250 & 297.3 & 4 & 6 & \\
\hline 02 & 62921 & 77.2377 & 10.6750 & 296.6 & 4 & .0 & \\
\hline 02 & 62922 & 76.7478 & 8.7500 & 297.0 & 4 & 600.0 & .0 \\
\hline 02 & 62923 & 72.5001 & 10.92 & 296.8 & 4 & 0 & \\
\hline 02 & 62924 & 70.7504 & 10.3750 & 296.5 & 4 & .0 & \\
\hline 02 & 6301 & 73.7500 & 10.2500 & 296.3 & 4 & .0 & \\
\hline 02 & 6302 & 74.5000 & 10.7500 & 295.6 & 4 & 60 & .0 \\
\hline 02 & 6303 & 76.5003 & 8.7500 & 296.1 & 4 & .0 & \\
\hline 02 & 6304 & 80.0000 & 7.9250 & 296.0 & 4 & .0 & .0 \\
\hline 02 & 6305 & 81.0000 & 7.5250 & 295.2 & 4 & 6 & \\
\hline 02 & 6306 & 100.2724 & 5.0500 & 296.2 & 4 & .0 & \\
\hline 02 & 6307 & 109.2509 & 4.3500 & 298.0 & 3 & .0 & 0.0 \\
\hline 02 & 6308 & 153.2513 & 5.2000 & 299.2 & 3 & 600.0 & 0.0 \\
\hline 02 & 6309 & 167.4997 & 5.6500 & 299.7 & 3 & .0 & .0 \\
\hline 02 & 63010 & 167.0021 & 5.90 & 300.1 & 3 & 600.0 & 600.0 \\
\hline 02 & 63011 & 170.7367 & 4.7750 & 301.1 & 3 & 600.0 & 600.0 \\
\hline 02 & 63012 & 173.0021 & 6.02 & 302.3 & 4 & 600.0 & .0 \\
\hline 02 & 63013 & 166.7583 & 5.2250 & 303.6 & 3 & 600.0 & 600.0 \\
\hline 02 & 63014 & 174.7503 & 6.0000 & 304.3 & 4 & 600.0 & 600.0 \\
\hline 02 & 63015 & 172.0021 & 6.0000 & 305.3 & 4 & 600.0 & 600.0 \\
\hline 02 & 63016 & 178.4912 & 7.2500 & 305.8 & 4 & 600.0 & 600.0 \\
\hline 02 & 63017 & 125.6201 & 4.9500 & 306.4 & 3 & 600.0 & 600.0 \\
\hline 02 & 63018 & 101.7515 & 5.1500 & 305.9 & 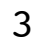 & 600.0 & 600.0 \\
\hline 02 & 63019 & 79.2452 & 6.1000 & 303.8 & 4 & 600.0 & 600.0 \\
\hline 02 & 63020 & 73.2500 & 6.8250 & 301.8 & 4 & 600.0 & 600.0 \\
\hline 02 & 63021 & 69.2508 & 7.8000 & 301.1 & 4 & 600.0 & 600.0 \\
\hline 02 & 63022 & 74.2499 & 7.8750 & 301.4 & 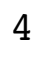 & 600.0 & 600.0 \\
\hline 02 & 63023 & 78.2500 & 7.9000 & 301.4 & $\Psi$ & 600.0 & 600.0 \\
\hline
\end{tabular}




\begin{tabular}{|c|c|c|c|c|c|c|c|c|}
\hline 02 & & 3024 & 79.5000 & 7.2250 & 301.4 & 4 & 600.0 & 600.0 \\
\hline 02 & 7 & 11 & 92.9970 & 3.4750 & 302.1 & 4 & 600.0 & 600.0 \\
\hline 02 & 7 & 12 & 93.2483 & 2.3250 & 301.8 & 4 & 600.0 & 00.0 \\
\hline 02 & 7 & 13 & 102.5000 & 1.0750 & 301.0 & 5 & 600.0 & 00.0 \\
\hline 02 & 7 & 14 & 102.3177 & 1.0750 & 300.7 & 6 & 600.0 & 00.0 \\
\hline 02 & 7 & 15 & 28.3839 & 0.5500 & 299.8 & 6 & 600.0 & 00.0 \\
\hline 02 & 7 & 16 & 155.2480 & 1.1250 & 300.6 & 5 & 600.0 & 00.0 \\
\hline 02 & 7 & 17 & 173.7497 & 1.7750 & 301.2 & 4 & 600.0 & 00.0 \\
\hline 02 & 7 & 18 & 174.4986 & 3.7000 & 301.0 & 4 & 600.0 & 00.0 \\
\hline 02 & 7 & 19 & 169.4997 & 6.2750 & 300.6 & 4 & 600.0 & .0 \\
\hline 02 & 7 & 110 & 169.5000 & 6.9250 & 301.3 & 4 & 600.0 & 00.0 \\
\hline 02 & 7 & 111 & 170.7500 & 6.3000 & 302.5 & 4 & 600.0 & .0 \\
\hline 02 & 7 & 112 & 176.0002 & 6.3000 & 303.9 & 4 & 600.0 & 00.0 \\
\hline 02 & 7 & 113 & 168.7350 & 5.6500 & 305.0 & 3 & 600.0 & 00.0 \\
\hline 02 & 7 & 114 & 170.0288 & 5.9500 & 305.8 & 3 & .0 & \\
\hline 02 & 7 & 115 & 173.2549 & 5.7750 & 306.5 & 3 & 600.0 & 00.0 \\
\hline 02 & 7 & 116 & 182.9983 & 6.1000 & 306.8 & 4 & 600.0 & 600.0 \\
\hline 02 & 7 & 117 & 144.7806 & 5.8 & 307.2 & 3 & .0 & \\
\hline 02 & 7 & 118 & 93.4885 & 5.9000 & 306.6 & 3 & 600.0 & .0 \\
\hline 02 & 7 & 119 & 87.5002 & 7.1500 & 304.8 & 4 & 60 & .0 \\
\hline 02 & 7 & 120 & 93.0014 & 5.5 & 302.5 & 4 & 60 & \\
\hline 02 & 7 & 121 & 72.2500 & 7.7250 & 300.9 & 4 & .0 & \\
\hline 02 & 7 & 122 & 105.7485 & 2.9000 & 300.2 & 5 & 600.0 & 500.0 \\
\hline 02 & 7 & 123 & 94.2501 & 2.02 & 300.2 & 5 & 60 & .0 \\
\hline 02 & 7 & 124 & 45.3797 & 1.9250 & 301.4 & 6 & .0 & \\
\hline 02 & 7 & 21 & 55.1645 & 1.9750 & 301.5 & 6 & 600.0 & 600.0 \\
\hline 02 & 7 & 22 & 39.0000 & 3.1250 & 302.1 & 5 & 600.0 & .0 \\
\hline 02 & 7 & 23 & 70.2676 & 4.5250 & 301.2 & 5 & 600.0 & \\
\hline 02 & 7 & 24 & 73.2574 & 5.9250 & 300.4 & 4 & 600.0 & 600.0 \\
\hline 02 & 7 & 25 & 62.4976 & 8.0250 & 299.7 & 4 & 600.0 & 600.0 \\
\hline 02 & 7 & 26 & 123.1618 & 1.3750 & 298.7 & 5 & .0 & \\
\hline 02 & 7 & 27 & 278.1621 & 1.2750 & 295.8 & 4 & 600.0 & 600.0 \\
\hline 02 & 7 & 28 & 260.4947 & 1.1250 & 297.0 & 3 & 600.0 & 600.0 \\
\hline 02 & 7 & 29 & 182.7172 & 2.7500 & 298.2 & 2 & 600.0 & 0.0 \\
\hline 02 & 7 & 210 & 178.2450 & 3.8000 & 298.8 & 3 & 600.0 & 600.0 \\
\hline 02 & 7 & 211 & 177.2541 & 3.2500 & 301.1 & 2 & 600.0 & 600.0 \\
\hline 02 & 7 & 212 & 173.2410 & 4.6000 & 302.5 & 3 & 600.0 & 600.0 \\
\hline 02 & 7 & 213 & 147.9421 & 5.5750 & 304.5 & 3 & 600.0 & 600.0 \\
\hline 02 & 7 & 214 & 105.2541 & 4.9500 & 305.5 & 3 & 600.0 & 600.0 \\
\hline 02 & 7 & 215 & 92.7719 & 6.1750 & 305.3 & 4 & 600.0 & 600.0 \\
\hline 02 & 7 & 216 & 91.7500 & 6.5250 & 305.3 & 4 & 600.0 & 600.0 \\
\hline 02 & 7 & 217 & 87.4962 & 7.2250 & 303.8 & 4 & 600.0 & 600.0 \\
\hline 02 & 7 & 218 & 78.2495 & 7.1500 & 302.3 & 4 & 600.0 & 600.0 \\
\hline 02 & 7 & 219 & 80.4997 & 7.7000 & 301.1 & 4 & 600.0 & 600.0 \\
\hline 02 & 7 & 220 & 69.5032 & 5.5250 & 300.6 & 4 & 600.0 & 600.0 \\
\hline 02 & 7 & 221 & 28.2492 & 3.1250 & 298.8 & 4 & 600.0 & 600.0 \\
\hline 02 & 7 & 222 & 70.9120 & 2.4750 & 298.0 & 5 & 600.0 & 600.0 \\
\hline 0 & 7 & 223 & 67.0103 & 5.250 & 298.6 & 4 & 600.0 & 600.0 \\
\hline
\end{tabular}




\begin{tabular}{|c|c|c|c|c|c|c|c|c|}
\hline 2 & 7 & 224 & 72.5000 & 6.9500 & 298.8 & 4 & 600.0 & 600.0 \\
\hline 02 & 7 & 31 & 75.0000 & 9.6500 & 298.3 & 4 & 600.0 & 600.0 \\
\hline 02 & 7 & 32 & 71.7494 & 9.5500 & 299.1 & 4 & 600.0 & 00.0 \\
\hline 02 & 7 & 33 & 72.7413 & 7.8000 & 298.7 & 4 & 600.0 & 00.0 \\
\hline 02 & 7 & 34 & 71.2550 & 7.9250 & 297.7 & 4 & 600.0 & 00.0 \\
\hline 02 & 7 & 35 & 68.2501 & 9.4250 & 296.9 & 4 & 600.0 & 00.0 \\
\hline 02 & 7 & 36 & 69.7506 & 9.7750 & 296.5 & 4 & 600.0 & 00.0 \\
\hline 02 & 7 & 37 & 66.2504 & 9.8500 & 296.8 & 4 & 600.0 & 00.0 \\
\hline 02 & 7 & 38 & 58.2405 & 8.0500 & 297.2 & 4 & 600.0 & 00.0 \\
\hline 02 & 7 & 39 & 86.8949 & 250 & 297.0 & 3 & 600.0 & .0 \\
\hline 02 & 7 & 310 & 133.4573 & 4.7250 & 297.1 & 3 & 600.0 & \\
\hline 02 & 7 & 311 & 96.2355 & 5.5750 & 298.8 & 3 & 600.0 & \\
\hline 02 & 7 & 312 & 98.5420 & 5.0500 & 300.1 & 3 & 600.0 & 00.0 \\
\hline 02 & 7 & 313 & 87.9973 & 6.5500 & 301.4 & 4 & 600.0 & 00.0 \\
\hline 02 & 7 & 314 & 86.5046 & 00 & 301.6 & 4 & .0 & \\
\hline 02 & 7 & 315 & 78.2492 & 8.3000 & 301.9 & 4 & 600.0 & .0 \\
\hline 02 & 7 & 316 & 81.0001 & 9.3250 & 301.5 & 4 & 60 & .0 \\
\hline 02 & 7 & 317 & 80.2468 & 8.07 & 301.0 & 4 & .0 & \\
\hline 02 & 7 & 318 & 68.2509 & 11.1250 & 300.1 & 4 & 60 & .0 \\
\hline 02 & 7 & 319 & 64.2504 & 12.4500 & 298.7 & 4 & .0 & .0 \\
\hline 02 & 7 & 320 & 60.2500 & 12.10 & 298.0 & 4 & 60 & \\
\hline 02 & 7 & 321 & 64.7391 & 9.4750 & 297.4 & 4 & .0 & \\
\hline 02 & 7 & 322 & 69.9977 & 9.7250 & 296.8 & 4 & 600.0 & 0 \\
\hline 02 & 7 & 323 & 72.5000 & 9.82 & 295.4 & 4 & 60 & \\
\hline 02 & 7 & 324 & 69.2501 & 10.2000 & 295.9 & 4 & .0 & \\
\hline 02 & 7 & 41 & 72.4998 & 9.0250 & 295.6 & 4 & 600.0 & 600.0 \\
\hline 02 & 7 & 42 & 71.2495 & 10.4500 & 295.4 & 4 & 60 & .0 \\
\hline 02 & 7 & 43 & 66.5000 & 10.3500 & 295.3 & 4 & .0 & \\
\hline 02 & 7 & 44 & 64.5000 & 8.2500 & 295.2 & 4 & .0 & 0.0 \\
\hline 02 & 7 & 45 & 70.4880 & 7.3750 & 295.4 & 4 & 60 & 0.0 \\
\hline 02 & 7 & 46 & 90.4903 & 7.6250 & 295.0 & 4 & .0 & \\
\hline 02 & 7 & 47 & 90.2501 & 7.3000 & 294.7 & 4 & .0 & 600.0 \\
\hline 02 & 7 & 48 & 83.2501 & 8.4250 & 295.4 & 4 & 600.0 & 600.0 \\
\hline 02 & 7 & 49 & 95.5159 & 5.0750 & 297.7 & 3 & .0 & 0.0 \\
\hline 02 & 7 & 410 & 105.5040 & 4.6500 & 298.2 & 3 & 600.0 & 600.0 \\
\hline 02 & 7 & 411 & 93.4954 & 5.2750 & 298.1 & 3 & 600.0 & 600.0 \\
\hline 02 & 7 & 412 & 101.7273 & 6.7500 & 298.9 & 4 & 600.0 & 0.0 \\
\hline 02 & 7 & 413 & 109.2392 & 7.4000 & 299.9 & 4 & 600.0 & 600.0 \\
\hline 02 & 7 & 414 & 109.7545 & 6.1750 & 301.1 & 4 & 600.0 & 600.0 \\
\hline 02 & 7 & 415 & 96.2542 & 7.7500 & 301.3 & 4 & 600.0 & 600.0 \\
\hline 02 & 7 & 416 & 90.2500 & 8.7000 & 301.1 & 4 & 600.0 & 600.0 \\
\hline 02 & 7 & 417 & 86.7497 & 8.2250 & 300.8 & 4 & 600.0 & 600.0 \\
\hline 02 & 7 & 418 & 79.2513 & 9.5250 & 299.9 & 4 & 600.0 & 600.0 \\
\hline 02 & 7 & 419 & 71.9995 & 9.8750 & 298.3 & 4 & 600.0 & 600.0 \\
\hline 02 & 7 & 420 & 66.5003 & 10.9000 & 297.7 & 4 & 600.0 & 600.0 \\
\hline 02 & 7 & 421 & 63.5000 & 11.4750 & 297.2 & 4 & 600.0 & 600.0 \\
\hline 02 & 7 & 422 & 69.2494 & 9.7750 & 297.2 & 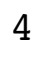 & 600.0 & 600.0 \\
\hline 02 & 7 & 423 & 77.0000 & 8.9500 & 296.7 & 4 & 600.0 & 600.0 \\
\hline
\end{tabular}




\begin{tabular}{|c|c|c|c|c|c|c|c|c|}
\hline 2 & 7 & 424 & 77.0000 & 9.9000 & 295.7 & 4 & 600.0 & 600.0 \\
\hline 02 & 7 & 51 & 76.7500 & 9.5500 & 295.5 & 4 & 600.0 & 600.0 \\
\hline 02 & 7 & 52 & 74.0000 & 9.0500 & 295.4 & 4 & 600.0 & 00.0 \\
\hline 02 & 7 & 53 & 78.9995 & 8.0750 & 295.4 & 4 & 600.0 & 00.0 \\
\hline 02 & 7 & 54 & 79.0003 & 7.8750 & 295.3 & 4 & 600.0 & 00.0 \\
\hline 02 & 7 & 55 & 80.2504 & 7.7250 & 294.7 & 4 & 600.0 & 00.0 \\
\hline 02 & 7 & 56 & 79.7498 & 6.8750 & 294.6 & 4 & 600.0 & 00.0 \\
\hline 02 & 7 & 57 & 89.2495 & 6.0000 & 295.9 & 4 & 600.0 & 00.0 \\
\hline 02 & 7 & 58 & 87.9993 & 6.0000 & 296.4 & 4 & 600.0 & 00.0 \\
\hline 02 & 7 & 59 & 200.8277 & 2.8250 & 297.3 & 3 & 600.0 & 00.0 \\
\hline 02 & 7 & 510 & 160.8699 & 3.2500 & 298.3 & 2 & 600.0 & \\
\hline 02 & 7 & 511 & 94.2523 & 4.7500 & 298.8 & 3 & 600.0 & \\
\hline 02 & 7 & 512 & 91.2506 & 5.6250 & 299.6 & 3 & 600.0 & 00.0 \\
\hline 02 & 7 & 513 & 101.4997 & 6.5500 & 299.3 & 4 & 600.0 & 00.0 \\
\hline 02 & 7 & 514 & 96.9994 & 7 . & 299.5 & 4 & .0 & \\
\hline 02 & 7 & 515 & 97.2501 & 7.4750 & 300.1 & 4 & 600.0 & 00.0 \\
\hline 02 & 7 & 516 & 86.9968 & 8.2250 & 300.0 & 4 & 60 & 600.0 \\
\hline 02 & 7 & 517 & 81.2503 & 9.3 & 299.4 & 4 & .0 & \\
\hline 02 & 7 & 518 & 84.4991 & 8.6750 & 298.6 & 4 & 600.0 & .0 \\
\hline 02 & 7 & 519 & 72.9998 & 10.0500 & 297.9 & 4 & .0 & .0 \\
\hline 02 & 7 & 520 & 70.2492 & 10.2 & 297.6 & 4 & 60 & \\
\hline 02 & 7 & 521 & 71.4999 & 9.5500 & 297.7 & 4 & .0 & \\
\hline 02 & 7 & 522 & 81.5000 & 9.0000 & 297.4 & 4 & 600.0 & 00.0 \\
\hline 02 & 7 & 523 & 76.2488 & 8.5 & 297.1 & 4 & 60 & \\
\hline 02 & 7 & 524 & 76.2498 & 8.97 & 296.6 & 4 & .0 & \\
\hline 02 & 7 & 61 & 82.7500 & 9.2500 & 295.8 & 4 & 600.0 & 600.0 \\
\hline 02 & 7 & 62 & 73.7498 & 9.7750 & 295.8 & 4 & 600.0 & .0 \\
\hline 02 & 7 & 63 & 76.7501 & 10.10 & 295.4 & 4 & .0 & \\
\hline 02 & 7 & 64 & 80.2504 & 11.1500 & 295.4 & 4 & 600.0 & 600.0 \\
\hline 02 & 7 & 65 & 81.0000 & 10.9500 & 295.5 & 4 & .0 & 600.0 \\
\hline 02 & 7 & 66 & 80.9999 & 8.50 & 295.7 & 4 & .0 & \\
\hline 02 & 7 & 67 & 90.7488 & 7.5500 & 296.6 & 4 & .0 & 600.0 \\
\hline 02 & 7 & 68 & 91.2489 & 8.0500 & 297.4 & 4 & 600.0 & 600.0 \\
\hline 02 & 7 & 69 & 96.5000 & 7.10 & 298.0 & 4 & 600.0 & 0.0 \\
\hline 02 & 7 & 610 & 101.2539 & 6.55 & 299.3 & 4 & 600.0 & 600.0 \\
\hline 02 & 7 & 611 & 107.5008 & 4.6500 & 301.0 & 3 & 600.0 & 600.0 \\
\hline 02 & 7 & 612 & 91.2501 & 4.5500 & 302.1 & 3 & 600.0 & 600.0 \\
\hline 02 & 7 & 613 & 86.2517 & 5.0500 & 303.2 & 3 & 600.0 & 600.0 \\
\hline 02 & 7 & 614 & 98.2527 & 6.8250 & 302.7 & 4 & 600.0 & 600.0 \\
\hline 02 & 7 & 615 & 101.7508 & 7.6000 & 302.5 & 4 & 600.0 & 0.0 \\
\hline 02 & 7 & 616 & 74.6866 & 9.4000 & 301.5 & 4 & 600.0 & 600.0 \\
\hline 02 & 7 & 617 & 65.2502 & 12.0250 & 300.1 & 4 & 600.0 & 600.0 \\
\hline 02 & 7 & 618 & 64.7500 & 12.5750 & 299.1 & 4 & 600.0 & 600.0 \\
\hline 02 & 7 & 619 & 64.5001 & 11.6750 & 298.3 & 4 & 600.0 & 600.0 \\
\hline 02 & 7 & 620 & 67.7496 & 11.8000 & 297.4 & 4 & 600.0 & 600.0 \\
\hline 02 & 7 & 621 & 67.0000 & 11.2500 & 297.4 & 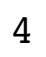 & 600.0 & 600.0 \\
\hline 02 & 7 & 622 & 69.7500 & 11.8000 & 296.8 & 4 & 600.0 & 600.0 \\
\hline 04 & 7 & 623 & 67.5000 & 12.575 & 295.8 & 4 & 600.0 & 600.0 \\
\hline
\end{tabular}




\begin{tabular}{|c|c|c|c|c|c|c|c|c|}
\hline 02 & 7 & 624 & 67.7499 & 14.1750 & 295.3 & 4 & 600.0 & 600.0 \\
\hline 02 & 7 & 71 & 65.2500 & 13.6000 & 294.5 & 4 & 600.0 & 00.0 \\
\hline 02 & 7 & 72 & 64.7500 & 13.3500 & 294.0 & 4 & 600.0 & 00.0 \\
\hline 02 & 7 & 73 & 66.7500 & 12.6250 & 294.4 & 4 & 600.0 & 00.0 \\
\hline 02 & 7 & 74 & 66.7500 & 12.4000 & 294.2 & 4 & 600.0 & 00.0 \\
\hline 02 & 7 & 75 & 67.2501 & 12.6500 & 294.0 & 4 & 600.0 & 00.0 \\
\hline 02 & 7 & 76 & 66.2500 & 13.4750 & 293.6 & 4 & 600.0 & 00.0 \\
\hline 02 & 7 & 77 & 66.5000 & 12.2500 & 293.9 & 4 & 600.0 & 00.0 \\
\hline 02 & 7 & 78 & 69.5000 & 10.5250 & 294.2 & 4 & 600.0 & 00.0 \\
\hline 02 & 7 & 79 & 69.7500 & 10.3250 & 294.9 & 4 & 600.0 & 0 \\
\hline 02 & 7 & 710 & 68.2500 & 10.7 & 295.9 & 4 & 600.0 & \\
\hline 02 & 7 & 711 & 73.4973 & 8 & 297.3 & 4 & 600.0 & \\
\hline 02 & 7 & 712 & 80.2497 & 7.4500 & 298.0 & 4 & 600.0 & 00.0 \\
\hline 02 & 7 & 713 & 78.7497 & 7.6000 & 298.7 & 4 & 600.0 & 00.0 \\
\hline 02 & 7 & 714 & 93.5119 & 8 . & 298.2 & 4 & .0 & \\
\hline 02 & 7 & 715 & 85.7223 & 8.2250 & 298.5 & 4 & 600.0 & .0 \\
\hline 02 & 7 & 716 & 92.9998 & 10.7750 & 298.0 & 4 & 60 & .0 \\
\hline 02 & 7 & 717 & 100.2500 & 11.12 & 297.4 & 4 & .0 & \\
\hline 02 & 7 & 718 & 97.2504 & 9.0250 & 296.7 & 4 & .0 & .0 \\
\hline 02 & 7 & 719 & 94.4959 & 7.0500 & 295.7 & 4 & .0 & .0 \\
\hline 02 & 7 & 720 & 973 & 8.1 & 294.0 & 4 & 6 & \\
\hline 02 & 7 & 721 & 67.4756 & 8.7750 & 293.3 & 4 & .0 & \\
\hline 02 & 7 & 722 & 68.0000 & 10.9750 & 293.3 & 4 & 600.0 & .0 \\
\hline 02 & 7 & 723 & 67.5000 & 10.92 & 293.3 & 4 & 0 & \\
\hline 02 & 7 & 724 & 70.7500 & 10 . & 293.7 & 4 & .0 & \\
\hline 02 & 7 & 81 & 71.5006 & 9.5750 & 294.1 & 4 & 0 & 600.0 \\
\hline 02 & 7 & 82 & 72.2503 & 8.2750 & 294.0 & 4 & 60 & .0 \\
\hline 02 & 7 & 83 & 75.7657 & 9.7750 & 293.8 & 4 & .0 & \\
\hline 02 & 7 & 84 & 79.2491 & 8.5500 & 294.1 & 4 & .0 & 0.0 \\
\hline 02 & 7 & 85 & 84.7525 & 8.0500 & 294.4 & 4 & 60 & .0 \\
\hline 02 & 7 & 86 & 83.7491 & 7. & 293.8 & 4 & .0 & \\
\hline 02 & 7 & 87 & 93.1935 & 4.7 & 295.5 & 3 & .0 & 600.0 \\
\hline 02 & 7 & 88 & 164.5031 & 5.1500 & 296.8 & 3 & 600.0 & 600.0 \\
\hline 02 & 7 & 89 & 162.7508 & 8.25 & 295.7 & 4 & .0 & 0.0 \\
\hline 02 & 7 & 810 & 162.9982 & 7.57 & 296.2 & 4 & 600.0 & 600.0 \\
\hline 02 & 7 & 811 & 167.0014 & 7.1500 & 297.2 & 4 & 600.0 & 600.0 \\
\hline 02 & 7 & 812 & 163.2503 & 6.9500 & 298.0 & 4 & 600.0 & 0.0 \\
\hline 02 & 7 & 813 & 167.2509 & 5.8000 & 299.6 & 3 & 600.0 & 600.0 \\
\hline 02 & 7 & 814 & 164.2917 & 6.1250 & 301.1 & 4 & 600.0 & 600.0 \\
\hline 02 & 7 & 815 & 160.5000 & 5.725 & 302.3 & 3 & 600.0 & 600.0 \\
\hline 02 & 7 & 816 & 160.4954 & 5.2500 & 303.3 & 3 & 600.0 & 600.0 \\
\hline 02 & 7 & 817 & 170.5009 & 5.5500 & 304.0 & 3 & 600.0 & 600.0 \\
\hline 02 & 7 & 818 & 185.2499 & 4.0000 & 303.9 & 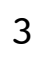 & 600.0 & 600.0 \\
\hline 02 & 7 & 819 & 165.4500 & 4.1250 & 303.5 & 4 & 600.0 & 600.0 \\
\hline 02 & 7 & 820 & 127.0521 & 3.2500 & 302.3 & 4 & 600.0 & 600.0 \\
\hline 02 & 7 & 821 & 77.5002 & 5.3250 & 301.6 & . & 600.0 & 600.0 \\
\hline 02 & 7 & 822 & 78.2505 & 6.9250 & 301.2 & 4 & 600.0 & 600.0 \\
\hline 0 & 7 & 823 & 84.0006 & 6.925 & 301.2 & & 600.0 & 600.0 \\
\hline
\end{tabular}




\begin{tabular}{|c|c|c|c|c|c|c|c|}
\hline [ & 24 & 1.7489 & 3000 & 01.5 & 4 & 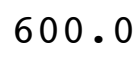 & \\
\hline & $\begin{array}{lll}7 & 9 & 1\end{array}$ & 80.7501 & .9250 & 301.1 & & 00.0 & \\
\hline & $\begin{array}{lll}7 & 9 & 2\end{array}$ & 79.0000 & 3750 & 301.7 & & 00 & \\
\hline & $\begin{array}{lll}7 & 9 & 3\end{array}$ & 81.7503 & 9250 & 301.6 & & 00 & \\
\hline & $\begin{array}{lll}7 & 9 & 4\end{array}$ & 5.5000 & .4250 & & & & \\
\hline & $\begin{array}{lll}7 & 9 & 5\end{array}$ & 2491 & 0 & 02.2 & & 0 & \\
\hline & $\begin{array}{lll}7 & 9 & 6\end{array}$ & 983 & 9750 & 302.8 & & 0 & \\
\hline & $\begin{array}{lll}7 & 9 & 7\end{array}$ & 16.5114 & .7750 & 04.0 & & & \\
\hline & $\begin{array}{lll}7 & 9 & 8\end{array}$ & 1 & .4250 & & & & \\
\hline & $\begin{array}{lll}7 & 9 & 9\end{array}$ & 906 & 250 & 306.5 & & 0 & \\
\hline & 7910 & 231.4994 & 3.3000 & 306.2 & & & \\
\hline & 7911 & 225.7 & 3.3750 & 06.6 & & & \\
\hline & 7912 & 214 . & 000 & 307.1 & & 0 & \\
\hline & 7913 & 215.2551 & 4. 2750 & 307.4 & & & \\
\hline & 7914 & 219. & & & & & \\
\hline & 7915 & 184 . & & & 3 & & \\
\hline & 7916 & 194. & 50 & 309.0 & 3 & & \\
\hline & 7917 & 186 . & 0 & & & & \\
\hline & 7918 & 183. & & & 3 & & \\
\hline & 7919 & 162.7519 & 250 & 308.2 & 4 & & \\
\hline & 7920 & 101.8907 & 500 & 30 & 4 & 0 & \\
\hline & 7921 & 78 . & & & 4 & & \\
\hline & 7922 & 69 . & 50 & & 4 & & \\
\hline & 7923 & 68.74 & 0 & 30 & 4 & & \\
\hline & 7924 & 73. & & & 4 & & \\
\hline & 7101 & 79. & & 30 & 4 & & \\
\hline & 7102 & 79.2500 & 0 & 4 & 4 & & . \\
\hline & 7103 & 79.2501 & 0 & & 4 & & \\
\hline & 7104 & 81.2487 & 0 & & 4 & & \\
\hline & 7105 & 105.8561 & 250 & .2 & 5 & & 0 \\
\hline & 7106 & 135.5086 & 750 & 30 & 5 & & \\
\hline & 7107 & 154.2470 & 0 & & 4 & & \\
\hline & 7108 & 168.9973 & & 5.2 & 3 & & 0 . \\
\hline$z$ & 7109 & 169.2595 & 3.2750 & .3 & 2 & & \\
\hline & 71010 & 167.4998 & 0 & & 3 & & \\
\hline & 71011 & 171.5000 & 750 & 7.3 & 3 & & \\
\hline & 71012 & 173.9989 & 8500 & 308.2 & 3 & & \\
\hline 2 & 71013 & 177.4954 & 0 & 30 & 3 & & \\
\hline 2 & 71014 & 171.2527 & 5.7250 & 310.9 & 3 & & 0 . \\
\hline & 71015 & 178.2515 & 000 & 311.4 & 4 & & \\
\hline 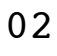 & 71016 & 175.7453 & 000 & & 4 & & \\
\hline 2 & 71017 & 160.4979 & 6.0500 & 312.3 & 4 & & 0 . \\
\hline & 71018 & 90.2501 & 5.8750 & 312.2 & 4 & & 0 \\
\hline 2 & 71019 & 74.2489 & 000 & & $I$ & & \\
\hline 2 & 71020 & 72.7491 & 6.8250 & & 4 & & \\
\hline & 71021 & 72.2504 & 9.7000 & 307.3 & 4 & & 6. \\
\hline & 710 & 98 & & & 4 & & \\
\hline & 71023 & 64.5000 & 0.8000 & 306.4 & & 600.0 & 5 \\
\hline
\end{tabular}




\begin{tabular}{|c|c|c|c|c|c|c|c|}
\hline 02 & 71024 & 68.5005 & 10.0500 & 306.6 & 4 & 600.0 & 600.0 \\
\hline 02 & 7111 & 71.2500 & 9.7500 & 306.8 & 4 & 600.0 & 600.0 \\
\hline 02 & 7112 & 70.5000 & 9.4750 & 307.1 & 4 & 600.0 & 00.0 \\
\hline 02 & 7113 & 72.9995 & 8.5500 & 307.2 & 4 & 600.0 & 00.0 \\
\hline 02 & 7114 & 84.9986 & 4.9750 & 307.0 & 4 & 600.0 & 00.0 \\
\hline 02 & 7115 & 105.7700 & 1.6500 & 304.6 & 5 & 600.0 & 00.0 \\
\hline 02 & 7116 & 344.1770 & 1.0500 & 303.1 & 6 & 600.0 & 00.0 \\
\hline 02 & 7117 & 0.5876 & 0.8000 & 305.8 & 5 & 600.0 & .0 \\
\hline 02 & 7118 & 246.9752 & 1.7500 & 305.9 & 4 & 600.0 & 000 \\
\hline 02 & 7119 & 225.5887 & 2.2500 & 303.1 & 3 & 600.0 & 0 \\
\hline 02 & 71110 & 166.9989 & 3.1250 & 305.1 & 3 & 600.0 & \\
\hline 02 & 71111 & 179.2410 & 00 & 305.3 & 3 & 600.0 & .0 \\
\hline 02 & 71112 & 173.7288 & 4.9750 & 305.1 & 3 & 600.0 & \\
\hline 02 & 71113 & 193.7498 & 5.8250 & 306.1 & 3 & 600.0 & 00.0 \\
\hline 02 & 71114 & 185.2987 & 00 & 307.0 & 3 & 600.0 & 60 \\
\hline 02 & 71115 & 171.7382 & 5.6750 & 308.1 & 3 & 600.0 & .0 \\
\hline 02 & 71116 & 189.9804 & 5.0250 & 309.5 & 3 & 600.0 & .0 \\
\hline 02 & 71117 & 131.2388 & 4 . & 309.1 & 3 & .0 & \\
\hline 02 & 71118 & 82.2515 & 5.7500 & 307.9 & 4 & 600.0 & 600.0 \\
\hline 02 & 71119 & 75.4908 & 6.0500 & 306.5 & 4 & .0 & .0 \\
\hline 02 & 71120 & 5.4091 & 3. & 304.9 & 5 & .0 & \\
\hline 02 & 71121 & 323.5000 & 1.5250 & 301.4 & 6 & .0 & 60 \\
\hline 02 & 71122 & 40.7425 & 4.3500 & 303.8 & 5 & .0 & 600.0 \\
\hline 02 & 71123 & 67.8439 & 6.0 & 304.9 & 4 & .0 & \\
\hline 02 & 71124 & 352.3745 & 3. & 301.3 & 4 & .0 & 60 \\
\hline 02 & 7121 & 315.0787 & 2.0250 & 297.4 & 5 & 600.0 & .0 \\
\hline 02 & 7122 & 71.1400 & 2 . & 298.5 & 6 & .0 & .0 \\
\hline 02 & 7123 & 97.5658 & 1.3750 & 299.6 & 6 & 600.0 & .0 \\
\hline 02 & 7124 & 150.9254 & 1.0250 & 300.0 & 6 & 600.0 & 600.0 \\
\hline 02 & 7125 & 10.3789 & 2 . & 300.2 & 6 & .0 & .0 \\
\hline 02 & 7126 & 7.1550 & 50 & 301.5 & 6 & .0 & \\
\hline 02 & 7127 & 36.5054 & 0.7750 & 300.9 & 5 & .0 & 600.0 \\
\hline 02 & 7128 & 221.9748 & 1.3000 & 301.2 & 4 & 600.0 & 600.0 \\
\hline 02 & 7129 & 290.0000 & 1.2750 & 299.8 & 3 & 600.0 & 600.0 \\
\hline 02 & 71210 & 296.2587 & 1.9000 & 302.8 & 2 & .0 & 600.0 \\
\hline 02 & 71211 & 285.5291 & 2.1500 & 304.7 & 1 & 600.0 & 600.0 \\
\hline 02 & 71212 & 252.9639 & 3.2250 & 306.2 & 2 & .0 & 60 \\
\hline 02 & 71213 & 233.2893 & 2.8750 & 306.6 & 1 & 600.0 & 600.0 \\
\hline 02 & 71214 & 208.9828 & 3.7250 & 307.7 & 2 & 600.0 & 600.0 \\
\hline 02 & 71215 & 191.4826 & 5.3000 & 307.4 & 3 & 600.0 & 600.0 \\
\hline 02 & 71216 & 185.7477 & 5.8750 & 306.8 & 3 & 600.0 & 600.0 \\
\hline 02 & 71217 & 176.7545 & 5.6000 & 306.2 & 3 & 600.0 & 600.0 \\
\hline 02 & 71218 & 181.2497 & 4.7750 & 305.4 & 3 & 600.0 & 600.0 \\
\hline 02 & 71219 & 156.4904 & 3.5750 & 304.7 & 4 & 600.0 & 600.0 \\
\hline 02 & 71220 & 99.0702 & 3.6500 & 304.7 & 4 & 600.0 & 600.0 \\
\hline 02 & 71221 & 57.5115 & 3.6750 & 304.9 & 4 & 600.0 & 600.0 \\
\hline 02 & 71222 & 40.6439 & 2.9500 & 304.6 & 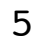 & 600.0 & 600.0 \\
\hline 02 & 71223 & 15.2437 & 2.000 & 303.5 & 0 & 600.0 & 600.0 \\
\hline
\end{tabular}




\begin{tabular}{|c|c|c|c|c|c|c|c|}
\hline 02 & 71224 & 4.9747 & 1.9000 & 301.1 & 6 & 600.0 & 00.0 \\
\hline 02 & 7131 & 59.0991 & 1.9000 & 302.4 & 6 & 600.0 & 600.0 \\
\hline 02 & 7132 & 36.7210 & 1.5750 & 302.4 & 6 & 600.0 & 00.0 \\
\hline 02 & 7133 & 71.9938 & 2.8750 & 303.4 & 6 & 600.0 & 00.0 \\
\hline 02 & 7134 & 92.3570 & 1.0000 & 300.3 & 0 & 600.0 & 00.0 \\
\hline 02 & 7135 & 10.9892 & 1.7000 & 299.1 & 6 & 600.0 & .0 \\
\hline 02 & 7136 & 104.5564 & 3.8250 & 302.7 & 5 & 600.0 & 500.0 \\
\hline 02 & 7137 & 342.9322 & 1.0750 & 301.7 & 4 & 600.0 & .0 \\
\hline 02 & 7138 & 313.1714 & 0.6250 & 302.1 & 3 & 600.0 & \\
\hline 02 & 7139 & 256.9985 & 1.9250 & 302.9 & 2 & 600.0 & 600.0 \\
\hline 02 & 71310 & 241.0007 & 2.5500 & 303.4 & 2 & 600.0 & 0 \\
\hline 02 & 71311 & 243.9976 & 2.5750 & 305.0 & 1 & 600.0 & \\
\hline 02 & 71312 & 256.0198 & 2.6250 & 306.8 & 1 & 600.0 & .0 \\
\hline 02 & 71313 & 104.7513 & 4.3000 & 307.2 & 2 & 600.0 & \\
\hline 02 & 71314 & 91.9998 & 5.3000 & 307.0 & 3 & 600.0 & .0 \\
\hline 02 & 71315 & 85.4997 & 8.8750 & 306.5 & 4 & 600.0 & \\
\hline 02 & 71316 & 88.0003 & 8.7500 & 305.5 & 4 & 600.0 & 600.0 \\
\hline 02 & 71317 & 85.0012 & 9.0500 & 304.8 & 4 & 600.0 & .0 \\
\hline 02 & 71318 & 77.2500 & 10.5000 & 302.5 & 4 & 600.0 & \\
\hline 02 & 71319 & 76.5000 & 12.0250 & 299.6 & 4 & 600.0 & 600.0 \\
\hline 02 & 71320 & 77.7501 & 11.7500 & 298.2 & 4 & 600.0 & .0 \\
\hline 02 & 71321 & 72.9993 & 11.2500 & 298.2 & 4 & 600.0 & \\
\hline 02 & 71322 & 74.5013 & 10.9000 & 299.1 & 4 & 600.0 & 60 \\
\hline 02 & 71323 & 79.2496 & 9.9750 & 299.7 & 4 & 600.0 & .0 \\
\hline 02 & 71324 & 75.0000 & 8.75 & 300.4 & 4 & 600.0 & \\
\hline 02 & 7141 & 79.5000 & 9.4000 & 300.0 & 4 & 600.0 & 60 \\
\hline 02 & 7142 & 65.2854 & 8.0250 & 301.0 & 4 & 600.0 & .0 \\
\hline 02 & 7143 & 64.5000 & 7.3500 & 299.8 & 4 & 600.0 & .0 \\
\hline 02 & 7144 & 73.7499 & 9.2000 & 299.2 & 4 & 600.0 & .0 \\
\hline 02 & 7145 & 73.7107 & 5.8750 & 299.5 & 4 & 600.0 & .0 \\
\hline 02 & 7146 & 43.0254 & 1.675 & 297.6 & 5 & 600.0 & .0 \\
\hline 02 & 7147 & 72.6176 & 4.50 & 298.4 & 4 & 600.0 & \\
\hline 02 & 7148 & 74.2495 & 4.3750 & 299.3 & 3 & 600.0 & .0 \\
\hline 02 & 7149 & 90.7076 & 3.4750 & 302.1 & 2 & 600.0 & .0 \\
\hline 02 & 71410 & 101.2492 & 3.9250 & 303.0 & 3 & 600.0 & \\
\hline 02 & 71411 & 97.4751 & 3.2500 & 304.4 & 2 & 600.0 & 600.0 \\
\hline 02 & 71412 & 80.4977 & 5.7750 & 305.1 & 3 & 600.0 & 600.0 \\
\hline 02 & 71413 & 80.5000 & 6.3500 & 304.9 & 4 & 600.0 & 60 \\
\hline 02 & 71414 & 71.5000 & 9.0750 & 304.6 & 4 & 600.0 & 600.0 \\
\hline 02 & 71415 & 65.5000 & 9.1250 & 304.4 & 4 & 600.0 & 600 \\
\hline 02 & 71416 & 65.2499 & 9.2500 & 304.2 & 4 & 600.0 & 600.0 \\
\hline 02 & 71417 & 64.2500 & 12.0500 & 303.6 & 4 & 600.0 & 600.0 \\
\hline 02 & 71418 & 65.0000 & 12.6000 & 301.9 & 4 & 600.0 & 600.0 \\
\hline 02 & 71419 & 67.5000 & 14.5750 & 300.9 & 4 & 600.0 & 600.0 \\
\hline 02 & 71420 & 65.2500 & 12.4250 & 300.1 & 4 & 600.0 & 600.0 \\
\hline 02 & 71421 & 64.2498 & 11.3750 & 299.6 & 4 & 600.0 & 600.0 \\
\hline 02 & 71422 & 67.5008 & 11.2000 & 29 & 4 & 600.0 & .0 \\
\hline 02 & 71423 & 59.2500 & 9.9250 & 298.8 & 4 & 600.0 & 600.0 \\
\hline
\end{tabular}




\begin{tabular}{|c|c|c|c|c|c|c|c|}
\hline 02 & 71424 & 61.7501 & 9.5750 & 299.4 & 4 & 600.0 & 600.0 \\
\hline 02 & $\begin{array}{ll}715 & 1\end{array}$ & 61.5000 & 8.9000 & 299.9 & 4 & 600.0 & 600.0 \\
\hline 02 & 7152 & 66.5000 & 8.6000 & 300.1 & 4 & 600.0 & 00.0 \\
\hline 02 & 7153 & 65.5002 & 8.3500 & 300.5 & 4 & 600.0 & 600.0 \\
\hline 02 & 7154 & 71.9908 & 4.5750 & 298.0 & 4 & 600.0 & 00.0 \\
\hline 02 & 7155 & 68.4928 & 2.3500 & 296.9 & 5 & 600.0 & 00.0 \\
\hline 02 & 7156 & 76.2796 & 3.6250 & 297.2 & 4 & 600.0 & 600.0 \\
\hline 02 & 7157 & 68.2662 & 2.8000 & 297.6 & 3 & 600.0 & 500.0 \\
\hline 02 & 7158 & 59.3267 & 2.1000 & 298.4 & 2 & 600.0 & 00.0 \\
\hline 02 & 7159 & 36.9183 & 1.4750 & 297.4 & 1 & 600.0 & 600.0 \\
\hline 02 & 71510 & 350.9439 & 1.8500 & 298.5 & 1 & 600.0 & 600.0 \\
\hline 02 & 71511 & 177.9645 & 2.1000 & 298.4 & 1 & 600.0 & .0 \\
\hline 02 & 71512 & 62.1017 & 3.2500 & 300.9 & 2 & 600.0 & 600.0 \\
\hline 02 & 71513 & 91.2502 & 4.6500 & 300.3 & 3 & 600.0 & 600.0 \\
\hline 02 & 71514 & 79.7261 & 3.9500 & 302.4 & 2 & 600.0 & \\
\hline 02 & 71515 & 75.9976 & 6.1750 & 301.5 & 3 & 600.0 & 60 \\
\hline 02 & 71516 & 78.7497 & 6.3500 & 301.3 & 4 & 600.0 & 600.0 \\
\hline 02 & 71517 & 81.2500 & 7.9500 & 299.9 & 4 & 600.0 & \\
\hline 02 & 71518 & 79.0000 & 8.9250 & 298.3 & 4 & 600.0 & 60 \\
\hline 02 & 71519 & 78.0000 & 8.4750 & 297.0 & 4 & 600.0 & 600.0 \\
\hline 02 & 71520 & 80.5000 & 8.70 & 294.9 & 4 & 600.0 & .0 \\
\hline 02 & 71521 & 78.0000 & 5.8250 & 295.4 & 4 & 600.0 & \\
\hline 02 & 71522 & 75.7499 & 6.2500 & 296.3 & 4 & 600.0 & 600.0 \\
\hline 02 & 71523 & 82.5000 & 3.1000 & 298.0 & 4 & 600.0 & .0 \\
\hline 02 & 71524 & 79.2500 & 6.6 & 296.3 & 4 & 600.0 & \\
\hline 02 & 7161 & 78.5018 & 8.4250 & 295.5 & 4 & 600.0 & 600.0 \\
\hline 02 & 7162 & 61.2472 & 6.5000 & 295.8 & 4 & 600.0 & 600.0 \\
\hline 02 & 7163 & 67.8544 & 2.8 & 293.8 & 5 & 600.0 & .0 \\
\hline 02 & 7164 & 50.9802 & 0.7750 & 292.1 & 6 & 600.0 & 600.0 \\
\hline 02 & 7165 & 124.2639 & 0.8500 & 290.8 & 6 & 600.0 & 600.0 \\
\hline 02 & 7166 & 106.5197 & 0.75 & 290.4 & 6 & 600.0 & 60 \\
\hline 02 & 7167 & 192.1644 & 0.6500 & 292.1 & 5 & 600.0 & 600.0 \\
\hline 02 & 7168 & 333.5743 & 1.3250 & 293.3 & 4 & 600.0 & 600.0 \\
\hline 02 & 7169 & 259.5098 & 1.92 & 294.2 & 3 & 600.0 & .0 \\
\hline 02 & 71610 & 231.2494 & 2.5750 & 294.5 & 2 & 600.0 & 600.0 \\
\hline 02 & 71611 & 241.2587 & 2.6000 & 296.2 & 1 & 600.0 & 600.0 \\
\hline 02 & 71612 & 228.7482 & 3.0250 & 297.3 & 2 & 600.0 & 600.0 \\
\hline 02 & 71613 & 229.0176 & 3.6750 & 298.9 & 2 & 600.0 & 600.0 \\
\hline 02 & 71614 & 163.4386 & 5.4500 & 301.0 & 3 & 600.0 & 600.0 \\
\hline 02 & 71615 & 134.0557 & 5.1000 & 302.5 & 3 & 600.0 & \\
\hline 02 & 71616 & 137.4458 & 5.8750 & 302.2 & 3 & 600.0 & 600.0 \\
\hline 02 & 71617 & 84.9977 & 4.1250 & 302.7 & 3 & 600.0 & 600.0 \\
\hline 02 & 71618 & 79.7461 & 4.8500 & 301.3 & 3 & 600.0 & 600.0 \\
\hline 02 & 71619 & 83.4997 & 5.4250 & 299.4 & 4 & 600.0 & 600.0 \\
\hline 02 & 71620 & 73.7476 & 5.6500 & 296.9 & 4 & 600.0 & 600.0 \\
\hline 02 & 71621 & 73.2496 & 9.0000 & 294.3 & 4 & 600.0 & 600.0 \\
\hline 02 & 71622 & 76.9988 & 7.5750 & 294.3 & 4 & 600.0 & 600.0 \\
\hline 02 & 71623 & 75.7562 & 6.5000 & 295.3 & 4 & 600.0 & 600.0 \\
\hline
\end{tabular}




\begin{tabular}{|c|c|c|c|c|c|c|c|}
\hline 02 & 71624 & 83.4502 & 4.7250 & 295.5 & 4 & 600.0 & 00.0 \\
\hline 02 & $\begin{array}{ll}717 & 1\end{array}$ & 83.4986 & 2.7500 & 295.9 & 5 & 600.0 & 600.0 \\
\hline 02 & 7172 & 75.7693 & 0.4000 & 294.8 & 6 & 600.0 & 00.0 \\
\hline 02 & 7173 & 93.9744 & 0.5250 & 294.6 & 6 & 600.0 & 00.0 \\
\hline 02 & 7174 & 50.6581 & 0.5250 & 294.2 & 6 & 600.0 & 00.0 \\
\hline 02 & 7175 & 97.6457 & 1.1750 & 293.6 & 6 & 600.0 & 00.0 \\
\hline 02 & 7176 & 98.3462 & 1.1250 & 295.1 & 6 & 600.0 & 600.0 \\
\hline 02 & $\begin{array}{ll}717 & 7\end{array}$ & 168.7626 & 0.7500 & 293.7 & 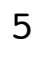 & 600.0 & 00.0 \\
\hline 02 & 7178 & 281.4225 & 0.9750 & 294.7 & 4 & 600.0 & 00.0 \\
\hline 02 & 7179 & 265.8404 & 1.7000 & 296.2 & 3 & 600.0 & 600.0 \\
\hline 02 & 71710 & 240.2317 & 2.7750 & 296.4 & 2 & 600.0 & 0 \\
\hline 02 & 71711 & 232.2518 & 3.0000 & 297.3 & 2 & 600.0 & \\
\hline 02 & 71712 & 203.0493 & 4.2500 & 299.3 & 3 & 600.0 & 600.0 \\
\hline 02 & 71713 & 82.1098 & 3.6250 & 301.2 & 2 & 600.0 & 600 \\
\hline 02 & 71714 & 90.9986 & 5.5750 & 301.1 & 3 & 600.0 & \\
\hline 02 & 71715 & 93.2472 & 6.9000 & 301.0 & 4 & 600.0 & 60 \\
\hline 02 & 71716 & 118.4936 & 6.9250 & 301.4 & 4 & 600.0 & 600.0 \\
\hline 02 & 71717 & 83.2606 & 9.0250 & 299.6 & 4 & 600.0 & \\
\hline 02 & 71718 & 76.7500 & 10.2250 & 297.5 & 4 & 600.0 & .0 \\
\hline 02 & 71719 & 74.7484 & 10.6250 & 296.4 & 4 & 600.0 & 600 \\
\hline 02 & 71720 & 75.2498 & 11.6500 & 295.9 & 4 & 600.0 & .0 \\
\hline 02 & 71721 & 74.2503 & 8.7500 & 296.6 & 4 & 600.0 & \\
\hline 02 & 71722 & 72.7506 & 9.2000 & 296.0 & 4 & 600.0 & 600.0 \\
\hline 02 & 71723 & 70.2506 & 9.4000 & 296.0 & 4 & 600.0 & .0 \\
\hline 02 & 71724 & 74.2498 & 9.8000 & 295.0 & 4 & 600.0 & \\
\hline 02 & 7181 & 71.2499 & 9.6250 & 294.8 & 4 & 600.0 & 60 \\
\hline 02 & 7182 & 73.2502 & 9.9250 & 294.7 & 4 & 600.0 & 60 \\
\hline 02 & 7183 & 74.5018 & 9.5250 & 293.7 & 4 & 600.0 & \\
\hline 02 & 7184 & 73.2502 & 8.4000 & 294.2 & 4 & 600.0 & 60 \\
\hline 02 & 7185 & 80.3459 & 6.2500 & 294.3 & 4 & 600.0 & 600 \\
\hline 02 & 7186 & 470 & 4.05 & 293.4 & 4 & 600.0 & \\
\hline 02 & 7187 & 84.5120 & 4.1250 & 292.9 & 3 & 600.0 & 60 \\
\hline 02 & 7188 & 100.4908 & 3.8750 & 293.4 & 2 & 600.0 & 600.0 \\
\hline 02 & 7189 & 74.8548 & 4.025 & 295.8 & 3 & 600.0 & .0 \\
\hline 02 & 71810 & 129.9168 & 2.2500 & 298.2 & 2 & 600.0 & 60 \\
\hline 02 & 71811 & 237.5451 & 2.5500 & 299.2 & 1 & 600.0 & 600.0 \\
\hline 02 & 71812 & 100.0016 & 4.0500 & 299.1 & 2 & 600.0 & \\
\hline 02 & 71813 & 126.4048 & 6.9000 & 298.7 & 3 & 600.0 & 600.0 \\
\hline 02 & 71814 & 93.9985 & 7.6250 & 299.7 & 4 & 600.0 & 600.0 \\
\hline 02 & 71815 & 98.2688 & 7.7750 & 300.1 & 4 & 600.0 & \\
\hline 02 & 71816 & 93.9854 & 7.9250 & 300.3 & 4 & 600.0 & 600.0 \\
\hline 02 & 71817 & 91.0012 & 7.5000 & 300.0 & 4 & 600.0 & 600.0 \\
\hline 02 & 71818 & 76.7021 & 7.4000 & 300.2 & 4 & 600.0 & 600.0 \\
\hline 02 & 71819 & 71.9916 & 8.4250 & 298.6 & 4 & 600.0 & 600.0 \\
\hline 02 & 71820 & 72.2492 & 9.3250 & 296.5 & 4 & 600.0 & 600.0 \\
\hline 02 & 71821 & 70.2500 & 8.6750 & 297.7 & 4 & 600.0 & 600.0 \\
\hline 02 & 71822 & 71.2498 & 9.225 & 297.7 & 4 & 600.0 & 600.0 \\
\hline 02 & 71823 & 78.2485 & 8.6500 & 297.4 & 4 & 600.0 & 600.0 \\
\hline
\end{tabular}




\begin{tabular}{|c|c|c|c|c|c|c|c|}
\hline 02 & 71824 & 79.0000 & 7.7000 & 297.7 & 4 & 600.0 & 600.0 \\
\hline 02 & 7191 & 80.2460 & 5.8750 & 298.4 & 4 & 600.0 & 600.0 \\
\hline 02 & 7192 & 87.0000 & 5.8250 & 298.2 & 4 & 600.0 & 00.0 \\
\hline 02 & 7193 & 81.9998 & 5.6750 & 297.8 & 4 & 600.0 & 00.0 \\
\hline 02 & 7194 & 81.7505 & .6000 & 297.6 & 4 & 600.0 & 00.0 \\
\hline 02 & 7195 & 89.2447 & 4.6000 & 297.6 & 4 & 600.0 & 00.0 \\
\hline 02 & 7196 & 125.2447 & 0.9000 & 295.4 & 5 & 600.0 & 00.0 \\
\hline 02 & 7197 & 126.1814 & 1.9250 & 297.4 & 4 & 600.0 & 00.0 \\
\hline 02 & 7198 & 230.6754 & 1.2750 & 298.8 & 3 & 600.0 & 00.0 \\
\hline 02 & 7199 & 218.6643 & 2.3250 & 299.0 & 2 & 600.0 & 00.0 \\
\hline 02 & 71910 & 227.8490 & 3.4250 & 299.6 & 2 & 600.0 & 00.0 \\
\hline 02 & 71911 & 188.9993 & 4.2500 & 300.4 & 3 & 600.0 & .0 \\
\hline 02 & 71912 & 183.4991 & 5.3250 & 301.6 & 3 & 600.0 & 00.0 \\
\hline 02 & 71913 & 180.0024 & 5.7000 & 302.4 & 3 & 600.0 & 00.0 \\
\hline 02 & 71914 & 175.7540 & 6.6500 & 303.7 & 4 & .0 & \\
\hline 02 & 71915 & 153.7460 & 6.1750 & 304.4 & 4 & 600.0 & 00.0 \\
\hline 02 & 71916 & 148.0007 & 5.7250 & 304.5 & 3 & 600.0 & 600.0 \\
\hline 02 & 71917 & 109.2724 & 5.2000 & 304.8 & 3 & .0 & \\
\hline 02 & 71918 & 90.2460 & 5.8750 & 304.0 & 4 & 600.0 & .0 \\
\hline 02 & 71919 & 88.7497 & 6.2250 & 302.5 & 4 & .0 & .0 \\
\hline 02 & 71920 & 80.2516 & .55 & 300.6 & 4 & 60 & \\
\hline 02 & 71921 & 80.9882 & 7.2500 & 299.7 & 4 & .0 & \\
\hline 02 & 71922 & 72.7500 & 6.9750 & 300.0 & 4 & 600.0 & 00.0 \\
\hline 02 & 71923 & 73.0002 & 6.57 & 300.5 & 4 & 60 & .0 \\
\hline 02 & 71924 & 73.9966 & 6.6250 & 300.6 & 4 & .0 & \\
\hline 02 & 7201 & 76.7502 & 6.9250 & 300.7 & 4 & 600.0 & 600.0 \\
\hline 02 & 7202 & 78.5000 & 6.3000 & 300.7 & 4 & 600.0 & .0 \\
\hline 02 & 7203 & 85.9995 & 3.9500 & 300.9 & 4 & 0 & \\
\hline 02 & 7204 & 86.5489 & 2.1250 & 300.1 & 5 & 600.0 & 600.0 \\
\hline 02 & 7205 & 17.5117 & 0.9500 & 298.5 & 6 & 600.0 & .0 \\
\hline 02 & 7206 & 26.7446 & 1.0500 & 299.5 & 5 & .0 & \\
\hline 02 & 7207 & 23.9871 & 0.4500 & 302.2 & 4 & .0 & 600.0 \\
\hline 02 & 7208 & 265.3826 & 1.4000 & 302.5 & 3 & 600.0 & 600.0 \\
\hline 02 & 7209 & 234.2809 & 2.4250 & 301.0 & 2 & 600.0 & 0.0 \\
\hline 02 & 72010 & 198.2501 & 2.4000 & 303.0 & 1 & 600.0 & 600.0 \\
\hline 02 & 72011 & 186.5023 & 4.8500 & 302.6 & 2 & 600.0 & 600.0 \\
\hline 02 & 72012 & 179.4963 & 5.7250 & 303.2 & 3 & 600.0 & 600.0 \\
\hline 02 & 72013 & 171.7528 & 5.2000 & 304.1 & 3 & 600.0 & 600.0 \\
\hline 02 & 72014 & 170.5069 & 5.8250 & 305.3 & 3 & 600.0 & 600.0 \\
\hline 02 & 72015 & 104.7229 & 4.5250 & 306.4 & 3 & 600.0 & 600.0 \\
\hline 02 & 72016 & 88.4985 & 6.3750 & 305.5 & 4 & 600.0 & 600.0 \\
\hline 02 & 72017 & 80.9998 & 6.8000 & 304.3 & 4 & 600.0 & 600.0 \\
\hline 02 & 72018 & 77.7483 & 7.4500 & 302.7 & 4 & 600.0 & 600.0 \\
\hline 02 & 72019 & 75.4998 & 8.6750 & 300.6 & 4 & 600.0 & 600.0 \\
\hline 02 & 72020 & 76.7499 & 9.3250 & 299.6 & 4 & 600.0 & 600.0 \\
\hline 02 & 72021 & 75.2501 & 8.8500 & 300.6 & 4 & 600.0 & 600.0 \\
\hline 02 & 72022 & 76.0000 & 9.4250 & 301.3 & 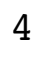 & 600.0 & 600.0 \\
\hline $0<$ & 72023 & 77.0000 & 9.4250 & 301.2 & 4 & 600.0 & 600.0 \\
\hline
\end{tabular}




\begin{tabular}{|c|c|c|c|c|c|c|c|}
\hline 02 & 72024 & 78.0000 & 8.8250 & 00.5 & 4 & 600.0 & 600.0 \\
\hline 02 & 7211 & 77.7487 & 7.2250 & 300.1 & 4 & 600.0 & 00,0 \\
\hline 02 & 7212 & 82.4976 & 5.3750 & 298.7 & 4 & 600.0 & 00.0 \\
\hline 02 & 7213 & 83.4998 & 6.9500 & 297.1 & 4 & 600.0 & 0 \\
\hline 02 & 7214 & 86.2494 & 7.9250 & 298.2 & 4 & 600.0 & 00.0 \\
\hline 02 & 7215 & 123.9325 & 3.5750 & 297.2 & 5 & 600.0 & 00.0 \\
\hline 02 & 7216 & 95.1142 & 5.7250 & 296.9 & 4 & 600.0 & 0 \\
\hline 02 & 7217 & 81.5005 & 7.8000 & 297.5 & 4 & 600.0 & 00.0 \\
\hline 02 & 7218 & 116.1084 & 5.1250 & 296.6 & 3 & 600.0 & 00.0 \\
\hline 02 & 7219 & 232.2795 & 1.6750 & 295.7 & 2 & 60 & \\
\hline 02 & 72110 & 312.7021 & 2.1750 & 297.8 & 1 & 600.0 & \\
\hline 02 & 72111 & 97.5045 & 4.6750 & 297.9 & 2 & .0 & \\
\hline 02 & 72112 & 95.4989 & 4.2000 & 299.1 & 3 & 600.0 & .0 \\
\hline 02 & 72113 & 88.4997 & 4.8500 & 300.2 & J & 600.0 & \\
\hline 02 & 72114 & 84.7735 & 6.3000 & 300.5 & 4 & .0 & \\
\hline 02 & 72115 & 82.4991 & 7.5500 & 299.6 & 4 & 600.0 & \\
\hline 02 & 72116 & 74.2505 & 8.0750 & 298.8 & 4 & .0 & .0 \\
\hline 02 & 72117 & 73.0000 & 10.0750 & 297.4 & 4 & .0 & \\
\hline 02 & 72118 & 74.5000 & 11.2000 & 296.0 & 4 & .0 & \\
\hline 02 & 72119 & 77.7504 & 9.8750 & 294.6 & 4 & .0 & .0 \\
\hline 02 & 72120 & 77.2498 & 10.27 & 293.1 & 4 & 0 & \\
\hline 02 & 72121 & 73.9984 & 11.5000 & 291.6 & 4 & .0 & \\
\hline 02 & 72122 & 77.5003 & 12.0750 & 290.7 & 4 & 600.0 & .0 \\
\hline 02 & 72123 & 82.4997 & 11.20 & 290.8 & 4 & 0 & \\
\hline 02 & 72124 & 87.5000 & 10.7750 & .3 & 4 & 0 & \\
\hline 02 & 7221 & 87.7500 & 11.2250 & 289.2 & 4 & .0 & \\
\hline 02 & 7222 & 84.2501 & 10.3000 & 289.0 & 4 & 60 & \\
\hline 02 & 7223 & 83.7500 & 9.4000 & 288.9 & 4 & .0 & \\
\hline 02 & 7224 & 82.5000 & 9.0000 & 288.7 & 4 & .0 & \\
\hline 02 & $722 \quad 5$ & 81.7500 & 10.1250 & 288.0 & 4 & .0 & \\
\hline 02 & 7226 & 83.2500 & 9.5250 & 287.7 & 4 & .0 & \\
\hline 02 & 7227 & 82.0000 & 7.4750 & .5 & 4 & .0 & .0 \\
\hline 02 & 7228 & 96.0188 & 3.4000 & 291.3 & 3 & 0.0 & 0.0 \\
\hline 02 & 7229 & 241.0000 & 2.0250 & 292.9 & 2 & .0 & .0 \\
\hline 02 & 72210 & 230.7596 & 2.5000 & 294.2 & 1 & 600.0 & 0.0 \\
\hline 02 & 72211 & 228.5009 & 3.1000 & 295.9 & 2 & 600.0 & 0.0 \\
\hline 02 & 72212 & 217.9965 & 3.22 & 297.7 & 2 & 600.0 & .0 \\
\hline 02 & 72213 & 28.2885 & 3.4000 & 299.2 & 2 & 600.0 & 600.0 \\
\hline 02 & 72214 & 103.4968 & 4.7750 & 298.8 & 3 & 600.0 & 600.0 \\
\hline 02 & 72215 & 101.7506 & 5 . & 298.3 & 3 & 600.0 & .0 \\
\hline 02 & 72216 & 91.2487 & 6.3000 & 298.2 & 4 & 600.0 & 600.0 \\
\hline 02 & 72217 & 88.2504 & 7.1750 & 297.6 & 4 & 600.0 & 600.0 \\
\hline 02 & 72218 & 81.7500 & 7.5750 & 296.3 & 4 & 600.0 & 600.0 \\
\hline 02 & 72219 & 78.2498 & 8.9250 & 294.3 & 4 & 600.0 & 600.0 \\
\hline 02 & 72220 & 80.0000 & 10.3500 & 292.3 & 4 & 600.0 & 600.0 \\
\hline 02 & 72221 & 81.9999 & 11.1500 & 291.2 & 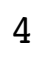 & 600.0 & 600.0 \\
\hline 02 & 72222 & 80.5000 & 11.0250 & 290.9 & 4 & 600.0 & 600.0 \\
\hline 02 & 72223 & 79.0001 & 11.3000 & 290.6 & 4 & 600.0 & 600.0 \\
\hline
\end{tabular}




\begin{tabular}{|c|c|c|c|c|c|c|c|}
\hline 2 & 72224 & 79.7500 & 10.9000 & 290.9 & 4 & 600.0 & 600.0 \\
\hline 02 & 7231 & 81.0000 & 10.5500 & 290.9 & 4 & 600.0 & 600.0 \\
\hline 02 & 7232 & 80.7500 & 9.6750 & 291.0 & 4 & 600.0 & 00.0 \\
\hline 02 & 7233 & 76.0000 & 7.3500 & 291.8 & 4 & 600.0 & 00.0 \\
\hline 02 & 7234 & 76.0005 & 6.4000 & 291.7 & 4 & 600.0 & 00.0 \\
\hline 02 & 7235 & 80.2500 & 6.8500 & 290.4 & 4 & 600.0 & 00.0 \\
\hline 02 & 7236 & 81.0002 & 5.2750 & 290.2 & 4 & 600.0 & 0 \\
\hline 02 & 7237 & 80.7501 & 7.0750 & 289.4 & 4 & 600.0 & 00.0 \\
\hline 02 & 7238 & 83.7497 & 5.1500 & 290.0 & 4 & 600.0 & 00.0 \\
\hline 02 & 7239 & 85.0000 & 3.7750 & 291.7 & 4 & 60 & \\
\hline 02 & 72310 & 88.5642 & 2.5500 & 293.9 & 3 & 600.0 & \\
\hline 02 & 72311 & 257.4871 & 2.3250 & 295.9 & 2 & .0 & \\
\hline 02 & 72312 & 247.8042 & 3.0750 & 297.0 & 2 & 600.0 & .0 \\
\hline 02 & 72313 & 216.5096 & 3. & 298.5 & 2 & 600.0 & \\
\hline 02 & 72314 & 189.6287 & & 298.8 & 3 & .0 & \\
\hline 02 & 72315 & 168.2506 & 7.0750 & 298.6 & 4 & 600.0 & \\
\hline 02 & 72316 & 147.9769 & 5.4500 & 299.1 & 3 & 60 & .0 \\
\hline 02 & 72317 & 97.2499 & 5.77 & 298.6 & 3 & .0 & \\
\hline 02 & 72318 & 118.0141 & 7.5750 & 296.9 & 4 & .0 & \\
\hline 02 & 72319 & 178.2707 & 9.4 & 294.9 & 4 & .0 & .0 \\
\hline 02 & 72320 & 182.2500 & 10 & 292.5 & 4 & 0 & \\
\hline 02 & 72321 & 181.7500 & 10. & 291.0 & 4 & .0 & \\
\hline 02 & 72322 & 97.5781 & 11.2750 & 289.8 & 4 & 600.0 & .0 \\
\hline 02 & 72323 & 76.7500 & 11.77 & 289.5 & 4 & 0 & \\
\hline 02 & 72324 & 73.4998 & 11.7 & 28 & 4 & 0 & \\
\hline 02 & 7241 & 76.7500 & 11.2500 & 289.4 & 4 & .0 & \\
\hline 02 & 7242 & 78.0000 & 10.1500 & 289.7 & 4 & 60 & \\
\hline 02 & 7243 & 79.7500 & 9.95 & 289.5 & 4 & .0 & \\
\hline 02 & $724 \quad 4$ & 82.4998 & 9.0250 & 289.8 & 4 & .0 & .0 \\
\hline 02 & $724 \quad 5$ & 85.7499 & 8.92 & 289.5 & 4 & .0 & \\
\hline 02 & 7246 & 93.9053 & 7. & 290.4 & 4 & 0 & \\
\hline 02 & 7247 & 153.7755 & 5.95 & 291.3 & 3 & .0 & .0 \\
\hline 02 & 7248 & 141.5314 & 3.6500 & 292.9 & 2 & 600.0 & 0.0 \\
\hline 02 & 7249 & 257.3309 & 1.60 & 296.3 & 1 & .0 & .0 \\
\hline 02 & 72410 & 237.6334 & 2.47 & 297.6 & 1 & 600.0 & 0.0 \\
\hline 02 & 72411 & 227.7400 & 2.6500 & 297.8 & 1 & 600.0 & 0.0 \\
\hline 02 & 72412 & 212.4914 & 4.20 & 299.1 & 2 & 600.0 & .0 \\
\hline 02 & 72413 & 191.4933 & 5.3500 & 300.2 & 3 & 600.0 & 600.0 \\
\hline 02 & 72414 & 179.9992 & 5.4750 & 301.3 & 3 & 600.0 & 600.0 \\
\hline 02 & 72415 & 170.2454 & 6.95 & 302.1 & 4 & 600.0 & .0 \\
\hline 02 & 72416 & 106.3718 & 4.6500 & 303.0 & 3 & 600.0 & 600.0 \\
\hline 02 & 72417 & 85.0019 & 6.3000 & 301.4 & 4 & 600.0 & 600.0 \\
\hline 02 & 72418 & 78.5001 & 8.1000 & 299.6 & 4 & 600.0 & 600.0 \\
\hline 02 & 72419 & 77.2499 & 10.5750 & 297.0 & 4 & 600.0 & 600.0 \\
\hline 02 & 72420 & 80.7503 & 11.9750 & 294.9 & 4 & 600.0 & 600.0 \\
\hline 02 & 72421 & 105.1990 & 8.5250 & & . & 600.0 & 600.0 \\
\hline 02 & 72422 & 166.0112 & 6.000 & 294.8 & 4 & 600.0 & 600.0 \\
\hline 48 & 72423 & 125.1704 & 2.475 & 296.9 & 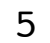 & 600.0 & 600.0 \\
\hline
\end{tabular}




\begin{tabular}{|c|c|c|c|c|c|c|c|}
\hline 02 & 72424 & 75.7502 & 7.5250 & 297.8 & 4 & 600.0 & 600.0 \\
\hline 02 & 7251 & 81.0000 & 9.0500 & 297.1 & 4 & 600.0 & 600.0 \\
\hline 02 & 7252 & 79.2500 & 9.9000 & 296.3 & 4 & 600.0 & 00.0 \\
\hline 02 & 7253 & 80.0000 & 10.2750 & 294.9 & 4 & 600.0 & 00.0 \\
\hline 02 & 7254 & 77.5000 & 9.7000 & 295.2 & 4 & 600.0 & 00.0 \\
\hline 02 & 7255 & 79.2501 & 9.5500 & 295.6 & 4 & 600.0 & 00.0 \\
\hline 02 & 7256 & 81.0005 & 7.8500 & 296.9 & 4 & 600.0 & 0 \\
\hline 02 & $\begin{array}{ll}725 & 7\end{array}$ & 85.4999 & 5.9500 & 297.6 & 4 & 600.0 & 00.0 \\
\hline 02 & 7258 & 24.4363 & 2.0250 & 299.4 & 3 & 600.0 & 00.0 \\
\hline 02 & 7259 & 270.4354 & 1.8750 & 297.5 & 2 & 60 & 0 \\
\hline 02 & 72510 & 282.3045 & 500 & 296.9 & 1 & 600.0 & \\
\hline 02 & 72511 & 239.1900 & 250 & 299.3 & 1 & 600.0 & \\
\hline 02 & 72512 & 201.6634 & 500 & 300.0 & 2 & 600.0 & .0 \\
\hline 02 & 72513 & 165.2653 & 5.8250 & 301.2 & 3 & 600.0 & \\
\hline 02 & 72514 & 114.9691 & 4 . & 302.8 & 3 & .0 & \\
\hline 02 & 72515 & 97.5000 & 000 & 302.4 & 4 & 600.0 & \\
\hline 02 & 72516 & 81.2475 & 7.3000 & 302.3 & 4 & 60 & .0 \\
\hline 02 & 72517 & 71.2503 & 8.5 & 301.3 & 4 & .0 & \\
\hline 02 & 72518 & 75.7501 & 750 & 299.4 & 4 & .0 & \\
\hline 02 & 72519 & 77.0002 & 10.9500 & 297.2 & 4 & .0 & .0 \\
\hline 02 & 72520 & 73.7500 & 11 . & 296.8 & 4 & 6 & \\
\hline 02 & 72521 & 73.2498 & 11.2 & 296.4 & 4 & .0 & \\
\hline 02 & 72522 & 76.0000 & 10.9000 & 296.8 & 4 & 600.0 & .0 \\
\hline 02 & 72523 & 73.2498 & 10.3 & 296.4 & 4 & 6 & \\
\hline 02 & 72524 & 74.0000 & 9 . & 297.0 & 4 & 0 & \\
\hline 02 & 7261 & 79.0003 & 9.3750 & 297.5 & 4 & .0 & \\
\hline 02 & 7262 & 78.2500 & 9.1250 & 297.2 & 4 & 60 & .0 \\
\hline 02 & 7263 & 77.5000 & 8.3 & 297.6 & 4 & .0 & \\
\hline 02 & 7264 & 80.5000 & 8 & 297.6 & 4 & .0 & .0 \\
\hline 02 & 7265 & 81.2501 & 9.1250 & 296.2 & 4 & 6 & \\
\hline 02 & 7266 & 81.9999 & 8 . & 295.3 & 4 & .0 & \\
\hline 02 & 7267 & 103.6528 & 3 . & 296.8 & 3 & .0 & 0 \\
\hline 02 & 7268 & 356.1311 & 0.8750 & 297.3 & 2 & 600.0 & 600.0 \\
\hline 02 & 7269 & 183.0872 & 3.3250 & 297.9 & 2 & .0 & 0.0 \\
\hline 02 & 72610 & 168.7497 & 5.07 & 298.3 & 3 & 600.0 & 0.0 \\
\hline 02 & 72611 & 168.7500 & 4.6750 & 299.4 & 3 & 600.0 & 600.0 \\
\hline 02 & 72612 & 172.0000 & 4 . & 302.1 & 3 & 600.0 & .0 \\
\hline 02 & 72613 & 176.2505 & 4.5500 & 303.5 & 3 & 600.0 & 600.0 \\
\hline 02 & 72614 & 176.0005 & 6.4250 & 304.0 & 4 & 600.0 & 600.0 \\
\hline 02 & 72615 & 155.0048 & 6.7250 & 304.4 & 4 & 600.0 & 600.0 \\
\hline 02 & 72616 & 117.1053 & 5.8250 & 305.1 & 3 & 600.0 & 600.0 \\
\hline 02 & 72617 & 87.5169 & 5.6500 & 305.1 & 3 & 600.0 & 600.0 \\
\hline 02 & 72618 & 85.2500 & 6.8250 & 304.2 & 4 & 600.0 & 600.0 \\
\hline 02 & 72619 & 84.7501 & 8.2000 & 302.2 & 4 & 600.0 & 600.0 \\
\hline 02 & 72620 & 77.7499 & 9.5000 & 300.9 & 4 & 600.0 & 600.0 \\
\hline 02 & 72621 & 77.7502 & 11.0250 & 300.2 & 4 & 600.0 & 600.0 \\
\hline 02 & 72622 & 75.4998 & 10.3000 & 300.4 & 4 & 600.0 & 600.0 \\
\hline 48 & 72623 & 73.7502 & 10.1000 & 300.9 & $\Psi$ & 600.0 & 600.0 \\
\hline
\end{tabular}




\begin{tabular}{|c|c|c|c|c|c|c|c|}
\hline 02 & 72624 & 74.7499 & 9.1500 & 301.3 & 4 & 600.0 & 00.0 \\
\hline 02 & $\begin{array}{ll}727 & 1\end{array}$ & 84.9996 & 7.3500 & 302.0 & 4 & 600.0 & 600.0 \\
\hline 02 & 7272 & 108.7184 & 3.7750 & 301.0 & 4 & 600.0 & 00.0 \\
\hline 02 & 7273 & 90.1434 & 2.7000 & 300.2 & 4 & 600.0 & 600.0 \\
\hline 02 & $727 \quad 4$ & 96.8992 & 2.8750 & 299.9 & 5 & 600.0 & 00.0 \\
\hline 02 & 7275 & 143.6260 & 1.5750 & 295.6 & 6 & 600.0 & 00.0 \\
\hline 02 & 7276 & 79.7173 & 0.6250 & 297.1 & 6 & 600.0 & 00.0 \\
\hline 02 & $727 \quad 7$ & 145.1327 & 0.5250 & 299.8 & 5 & 600.0 & 00.0 \\
\hline 02 & 7278 & 214.6955 & 1.2250 & 300.3 & 4 & 600.0 & 00.0 \\
\hline 02 & 7279 & 258.8992 & 1.9750 & 300.6 & 3 & 600.0 & 600.0 \\
\hline 02 & 72710 & 192.7398 & 3.3500 & 300.0 & 2 & 600.0 & 00.0 \\
\hline 02 & 72711 & 197.9155 & 4.3750 & 300.7 & 3 & 600.0 & \\
\hline 02 & 72712 & 175.7502 & 5.0500 & 301.3 & 3 & 600.0 & 600.0 \\
\hline 02 & 72713 & 172.7499 & 5.2000 & 302.7 & 3 & 600.0 & 0 \\
\hline 02 & 72714 & 138.6457 & 750 & 304.3 & 3 & 600.0 & \\
\hline 02 & 72715 & 98.5000 & 250 & 305.2 & 3 & 600.0 & 60 \\
\hline 02 & 72716 & 87.5046 & 5.7000 & 304.6 & 3 & 600.0 & 600.0 \\
\hline 02 & 72717 & 85.4945 & 5.8500 & 303.8 & 3 & 600.0 & \\
\hline 02 & 72718 & 80.0001 & 7.8000 & 302.8 & 4 & 600.0 & .0 \\
\hline 02 & 72719 & 77.0000 & 8.3750 & 302.2 & 4 & 600.0 & 600 \\
\hline 02 & 72720 & 67.7489 & 8.72 & 300.2 & 4 & 600.0 & .0 \\
\hline 02 & 72721 & 71.2501 & 250 & 298.5 & 4 & 600.0 & \\
\hline 02 & 72722 & 77.0000 & 8.2000 & 299.2 & 4 & 600.0 & 600.0 \\
\hline 02 & 72723 & 80.7500 & 7.8250 & 300.0 & 4 & 600.0 & .0 \\
\hline 02 & 72724 & 80.0000 & 8 . & 299.2 & 4 & 600.0 & \\
\hline 02 & 7281 & 81.0007 & 500 & 299.4 & 4 & 600.0 & 60 \\
\hline 02 & 7282 & 85.0027 & 5.6000 & 299.0 & 4 & 600.0 & .0 \\
\hline 02 & 7283 & 81.4315 & 1.6 & 296.1 & 5 & 600.0 & \\
\hline 02 & 7284 & 70.2483 & 1.3750 & 294.2 & 6 & 600.0 & 60 \\
\hline 02 & 7285 & 142.7306 & 0.8750 & 293.6 & 6 & 600.0 & 600.0 \\
\hline 02 & 7286 & 87.9557 & 1 . & 294.1 & 6 & 600.0 & \\
\hline 02 & 7287 & 151.6820 & 1.7500 & 294.7 & 5 & 600.0 & 60 \\
\hline 02 & 7288 & 266.3818 & 1.2250 & 295.8 & 4 & 600.0 & 600.0 \\
\hline 02 & 7289 & 272.5093 & 500 & 295.9 & 3 & 600.0 & .0 \\
\hline 02 & 72810 & 275.1570 & 1.7250 & 297.6 & 2 & 600.0 & 60 \\
\hline 02 & 72811 & 253.7402 & 2.0750 & 299.0 & 1 & 600.0 & 600.0 \\
\hline 02 & 72812 & 86.2528 & 4.5000 & 299.7 & 2 & 600.0 & \\
\hline 02 & 72813 & 90.7488 & 5.4250 & 299.9 & 3 & 600.0 & 600.0 \\
\hline 02 & 72814 & 82.2446 & 6.6500 & 300.6 & 4 & 600.0 & 600.0 \\
\hline 02 & 72815 & 85.2535 & 7.1250 & 301.1 & 4 & 600.0 & \\
\hline 02 & 72816 & 75.5000 & 8.5750 & 301.0 & 4 & 600.0 & 600.0 \\
\hline 02 & 72817 & 79.0000 & 9.9750 & 299.5 & 4 & 600.0 & 600.0 \\
\hline 02 & 72818 & 75.0000 & 11.4250 & 298.0 & 4 & 600.0 & 600.0 \\
\hline 02 & 72819 & 74.7500 & 11.7500 & 296.3 & 4 & 600.0 & 600.0 \\
\hline 02 & 72820 & 80.5000 & 11.0250 & 294.2 & 4 & 600.0 & 600.0 \\
\hline 02 & 72821 & 78.7500 & 12.0000 & 292.2 & 4 & 600.0 & 600.0 \\
\hline 02 & 72822 & 80.9997 & 12.7250 & 29 & 4 & 600.0 & 600.0 \\
\hline 02 & 72823 & 74.4998 & 11.7500 & 292.8 & 4 & 600.0 & 600.0 \\
\hline
\end{tabular}




\begin{tabular}{|c|c|c|c|c|c|c|c|}
\hline 2 & 72824 & 75.7500 & 10.2500 & 293.9 & 4 & 600.0 & 600.0 \\
\hline 02 & 7291 & 74.4997 & 8.4750 & 294.0 & 4 & 600.0 & 600.0 \\
\hline 02 & 7292 & 70.0584 & 5.6500 & 291.6 & 4 & 600.0 & 00.0 \\
\hline 2 & 7293 & 81.2452 & 8.8000 & 291.1 & 4 & 600.0 & 00.0 \\
\hline 02 & 7294 & 78.9982 & 8.7000 & 290.6 & 4 & 600.0 & 00.0 \\
\hline 02 & 7295 & 75.5000 & 10.2500 & 292.4 & 4 & 600.0 & 00.0 \\
\hline 02 & 7296 & 94.5807 & 5.3250 & 293.2 & 4 & 600.0 & 0 \\
\hline 02 & 7297 & 193.7288 & 1.4250 & 292.7 & 3 & 600.0 & 00.0 \\
\hline 02 & 7298 & 259.5000 & 1.5250 & 293.7 & 2 & 600.0 & 00.0 \\
\hline 02 & 7299 & 247.9578 & 1.6500 & 293.9 & 1 & 60 & \\
\hline 02 & 72910 & 244.0149 & 2.1500 & 296.1 & 1 & 600.0 & \\
\hline 02 & 72911 & 241.8239 & 2.0750 & 297.8 & 1 & .0 & \\
\hline 02 & 72912 & 307.1949 & 2.6250 & 300.2 & 1 & 600.0 & .0 \\
\hline 02 & 72913 & 57.7545 & 3.2250 & 301.3 & 2 & 600.0 & \\
\hline 02 & 72914 & 89.9984 & 5 & 300.8 & 3 & .0 & \\
\hline 02 & 72915 & 79.5000 & 7.8500 & 300.5 & 4 & 600.0 & .0 \\
\hline 02 & 72916 & 75.0001 & 9.3500 & 300.3 & 4 & .0 & .0 \\
\hline 02 & 72917 & 77.5003 & 10 & 299.9 & 4 & .0 & \\
\hline 02 & 72918 & 88.7729 & 11.4500 & 298.8 & 4 & .0 & \\
\hline 02 & 72919 & 70.7493 & 11.2000 & 298.4 & 4 & .0 & .0 \\
\hline 02 & 72920 & 000 & 10 . & 297.8 & 4 & 6 & \\
\hline 02 & 72921 & 74.5000 & 9.2750 & 298.1 & 4 & .0 & \\
\hline 02 & 72922 & 81.7501 & 9.1250 & 298.3 & 4 & 600.0 & .0 \\
\hline 02 & 72923 & 81.4997 & 8 . & 298.1 & 4 & 0 & \\
\hline 02 & 72924 & 968 & 8 . & 297.9 & 4 & 0 & \\
\hline 02 & 7301 & 71.4991 & 7.7000 & 297.7 & 4 & .0 & \\
\hline 02 & 7302 & 78.7502 & 7.4000 & 297.5 & 4 & 6 & \\
\hline 02 & 7303 & 84.2500 & 6.7500 & 297.6 & 4 & .0 & \\
\hline 02 & 7304 & 84.5000 & 500 & 297.7 & 4 & .0 & \\
\hline 02 & 7305 & 82.5000 & 5.6250 & 297.7 & 4 & 6 & \\
\hline 02 & 7306 & 115.8631 & 3.4250 & 297.1 & 4 & 0 & \\
\hline 02 & 7307 & 13.9675 & 0 . & 295.7 & 3 & .0 & .0 \\
\hline 02 & 7308 & 264.0603 & 0.8500 & 296.2 & 2 & 600.0 & .0 \\
\hline 02 & $730 \quad 9$ & 245.2517 & 2.1500 & 296.2 & 2 & .0 & .0 \\
\hline 02 & 73010 & 235.9986 & & 297.3 & 1 & 600.0 & 0 \\
\hline 02 & 73011 & 185.2331 & 2.4000 & 299.3 & 1 & 600.0 & 600.0 \\
\hline 02 & 73012 & 173.7485 & 3 . & 300.7 & 2 & 600.0 & .0 \\
\hline 02 & 73013 & 172.0098 & 3.3000 & 301.7 & 2 & 600.0 & 600.0 \\
\hline 02 & 73014 & 183.6924 & 4.1750 & 303.0 & 3 & 600.0 & 600.0 \\
\hline 02 & 73015 & 170.2819 & 5 . & 304.0 & 3 & 600.0 & .0 \\
\hline 02 & 73016 & 115.1814 & 4.37 & 305.2 & 3 & 600.0 & 600.0 \\
\hline 02 & 73017 & 102.7563 & 5.4750 & 304.7 & 3 & 600.0 & 600.0 \\
\hline 02 & 73018 & 84.9992 & 8.5500 & 302.8 & 4 & 600.0 & 600.0 \\
\hline 02 & 73019 & 81.0000 & 10.0750 & 300.7 & 4 & 600.0 & 600.0 \\
\hline 02 & 73020 & 74.2501 & 9.5750 & 299.7 & 4 & 600.0 & 600.0 \\
\hline 02 & 73021 & 73.9998 & & 299.5 & . & 600.0 & 600.0 \\
\hline 02 & 73022 & 72.2500 & 9 . & 299.4 & 4 & 600.0 & 600.0 \\
\hline 02 & 73023 & 69.0000 & 8.975 & 299.8 & 4 & 600.0 & 600.0 \\
\hline
\end{tabular}




\begin{tabular}{|c|c|c|c|c|c|c|c|}
\hline 02 & 73024 & 68.5003 & 8.3000 & 299.8 & 4 & 600.0 & 600.0 \\
\hline 02 & 7311 & 60.7500 & 7.1500 & 300.4 & 4 & 600.0 & 600.0 \\
\hline 02 & 7312 & 65.2330 & 4.5000 & 299.9 & 4 & 600.0 & 00.0 \\
\hline 02 & 7313 & 56.7735 & 2.4500 & 298.6 & 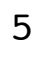 & 600.0 & 00.0 \\
\hline 02 & 7314 & 227.5024 & 1.1250 & 297.2 & 6 & 600.0 & 00.0 \\
\hline 02 & 7315 & 326.3543 & 0.8500 & 294.8 & 6 & 600.0 & 00.0 \\
\hline 02 & 7316 & 187.5891 & 0.2750 & 294.0 & 6 & 600.0 & 00.0 \\
\hline 02 & $\begin{array}{ll}731 & 7\end{array}$ & 356.4597 & 0.0750 & 297.4 & o & 600.0 & \\
\hline 02 & 7318 & 292.7898 & 0.6500 & 298.3 & 4 & 600.0 & 000 \\
\hline 02 & 7319 & 270.4320 & 1.3500 & 300.2 & 3 & 600.0 & \\
\hline 02 & 73110 & 237.5000 & 2.6250 & 299.3 & 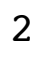 & 600.0 & \\
\hline 02 & 73111 & 233.4997 & 3.4250 & 299.8 & 3 & 600.0 & .0 \\
\hline 02 & 73112 & 232.2503 & 3.4250 & 300.9 & 2 & 600.0 & \\
\hline 02 & 73113 & 210.7508 & 5.4500 & 302.4 & 3 & 600.0 & 00.0 \\
\hline 02 & 73114 & 151.0000 & 50 & 304.2 & 2 & 600.0 & .0 \\
\hline 02 & 73115 & 90.4907 & 00 & 303.8 & 3 & 600.0 & .0 \\
\hline 02 & 73116 & 91.2427 & 500 & 303.6 & 4 & 600.0 & .0 \\
\hline 02 & 73117 & 88.0001 & 7 . & 303.0 & 4 & .0 & \\
\hline 02 & 73118 & 79.9931 & 9.3000 & 302.1 & 4 & 60 & \\
\hline 02 & 73119 & 72.7502 & 9 . & 301.1 & 4 & .0 & .0 \\
\hline 02 & 73120 & 71.2503 & 8 . & 299.7 & 4 & .0 & \\
\hline 02 & 73121 & 67.7502 & 8.7750 & 299.0 & 4 & .0 & \\
\hline 02 & 73122 & 70.7500 & 9.6000 & 298.4 & 4 & 60 & .0 \\
\hline 02 & 73123 & 71.7500 & 10.2250 & 298.1 & 4 & .0 & \\
\hline 02 & 73124 & 75.0000 & 9.4500 & 297.7 & 4 & .0 & 60 \\
\hline 02 & $\begin{array}{lll}8 & 1 & 1\end{array}$ & 71.4997 & 9.0500 & 297.8 & 4 & 600.0 & .0 \\
\hline 02 & 812 & 72.7500 & 10 . & 297.5 & 4 & 60 & .0 \\
\hline 02 & $\begin{array}{lll}8 & 1 & 3\end{array}$ & 73.7485 & 750 & 297.0 & 4 & .0 & .0 \\
\hline 02 & $\begin{array}{lll}8 & 1 & 4\end{array}$ & 71.7260 & 4.1000 & 295.0 & 4 & .0 & .0 \\
\hline 02 & 815 & 65.4991 & 8 . & 295.6 & 4 & .0 & .0 \\
\hline 02 & 816 & 72.7500 & 750 & 295.9 & 4 & .0 & \\
\hline 02 & $\begin{array}{lll}8 & 1 & 7\end{array}$ & 75.9998 & .000 & 296.8 & 4 & .0 & 600.0 \\
\hline 02 & $\begin{array}{lll}8 & 1 & 8\end{array}$ & 59.1267 & 3.0000 & 297.9 & 3 & .0 & 600.0 \\
\hline 02 & $\begin{array}{lll}8 & 1 & 9\end{array}$ & 247.0811 & 1.5750 & 298.4 & 2 & 600.0 & 60 \\
\hline 02 & 8110 & 235.4999 & 3.4750 & 297.9 & 3 & .0 & 600.0 \\
\hline 02 & 8111 & 210.0471 & 2.4750 & 299.7 & 2 & 600.0 & 600.0 \\
\hline 02 & 8112 & 6.8335 & 2 . & 301.7 & 1 & .0 & .0 \\
\hline 02 & 8113 & 76.1227 & 3.8750 & 302.1 & 2 & 600.0 & 600.0 \\
\hline 02 & 8114 & 90.7561 & 3.7500 & 303.2 & 2 & 600.0 & 600.0 \\
\hline 02 & 8115 & 99.2447 & 5.27 & 303.4 & 3 & 600.0 & 600.0 \\
\hline 02 & 8116 & 85.2688 & 6.3000 & 302.7 & 4 & 600.0 & 600.0 \\
\hline 02 & 8117 & 87.2505 & 7.9250 & 301.7 & 4 & 600.0 & 600.0 \\
\hline 02 & 8118 & 126.7173 & 8.8500 & 300.1 & 4 & 600.0 & 600.0 \\
\hline 02 & 8119 & 158.6739 & 9.9250 & 298.2 & 4 & 600.0 & 600.0 \\
\hline 02 & 8120 & 110.0485 & 9.9250 & 296.5 & 4 & 600.0 & 600.0 \\
\hline 02 & 8121 & 75.9998 & 9.3000 & 296.2 & 4 & 600.0 & \\
\hline 02 & 8122 & 72.2500 & 9.1000 & 296.2 & 4 & 600.0 & 600.0 \\
\hline 02 & $8 \quad 123$ & 73.7501 & 10.100 & 295.5 & 4 & 600.0 & 600.0 \\
\hline
\end{tabular}




\begin{tabular}{|c|c|c|c|c|c|c|c|c|}
\hline 2 & 8 & 124 & 71.9999 & 10.0000 & 294.9 & 4 & 600.0 & 00.0 \\
\hline 02 & 8 & 21 & 76.7485 & 9.2500 & 294.4 & 4 & 600.0 & 600.0 \\
\hline 02 & 8 & 22 & 80.4994 & 10.2750 & 293.3 & 4 & 600.0 & 00.0 \\
\hline 02 & 8 & 23 & 74.0000 & 11.2500 & 292.7 & 4 & 600.0 & 00.0 \\
\hline 02 & 8 & 24 & 77.2500 & 10.3250 & 293.0 & 4 & 600.0 & 00.0 \\
\hline 02 & 8 & 25 & 78.4999 & 9.8000 & 293.6 & 4 & 600.0 & 00.0 \\
\hline 02 & 8 & 26 & 80.0000 & 10.0750 & 293.0 & 4 & 600.0 & 600.0 \\
\hline 02 & 8 & 27 & 82.0001 & 9.4750 & 292.3 & 4 & 600.0 & 00.0 \\
\hline 02 & 8 & 28 & 95.4712 & 2.5500 & 294.2 & 3 & 600.0 & 00.0 \\
\hline 02 & 8 & 29 & 8.1046 & 2.2500 & 295.3 & 2 & 600.0 & 600.0 \\
\hline 02 & 8 & 210 & 121.9715 & 3.6000 & 296.5 & 2 & 600.0 & 0 \\
\hline 02 & 8 & 211 & 121.2554 & 1.9250 & 299.0 & 1 & 600.0 & \\
\hline 02 & 8 & 212 & 224.5401 & 2.8250 & 300.0 & 1 & 600.0 & 600.0 \\
\hline 02 & 8 & 213 & 85.2489 & 4.2750 & 300.4 & 2 & 600.0 & 600.0 \\
\hline 02 & 8 & 214 & 92.0000 & 5.4250 & 300.6 & 3 & 600.0 & \\
\hline 02 & 8 & 215 & 94.5014 & 6.0750 & 300.8 & 4 & 600.0 & 60 \\
\hline 02 & 8 & 216 & 88.0000 & 6.2750 & 300.9 & 4 & 600.0 & 600.0 \\
\hline 02 & 8 & 217 & 78.4975 & 6.9250 & 300.3 & 4 & 600.0 & \\
\hline 02 & 8 & 218 & 146.5505 & 7.7750 & 299.0 & 4 & 600.0 & 60 \\
\hline 02 & 8 & 219 & 174.2547 & 9.1250 & 297.2 & 4 & 600.0 & 600 \\
\hline 02 & 8 & 220 & 138.7206 & 8.2500 & 295.5 & 4 & 600.0 & .0 \\
\hline 02 & 8 & 221 & 87.8827 & 9.7250 & 293.3 & 4 & 600.0 & \\
\hline 02 & 8 & 222 & 79.5000 & 10.1750 & 291.9 & 4 & 600.0 & 60 \\
\hline 02 & 8 & 223 & 67.0032 & 7.1250 & 294.8 & 4 & 600.0 & .0 \\
\hline 02 & 8 & 224 & 52.1687 & 3.1000 & 294.2 & 5 & 600.0 & \\
\hline 02 & 8 & 31 & 57.0582 & 3.1250 & 294.1 & 5 & 600.0 & 60 \\
\hline 02 & 8 & 32 & 57.3697 & 2.7500 & 293.0 & 6 & 600.0 & 60 \\
\hline 02 & 8 & 33 & 85.4924 & 3.45 & 292.2 & 5 & 600.0 & \\
\hline 02 & 8 & 34 & 81.5006 & 4.1000 & 291.5 & 4 & 600.0 & 60 \\
\hline 02 & 8 & 35 & 90.2609 & 2.9500 & 291.0 & 5 & 600.0 & 600 \\
\hline 02 & 8 & 36 & 101.4754 & 2.67 & 291.0 & 6 & 600.0 & \\
\hline 02 & 8 & 37 & 246.1033 & 0.9000 & 290.6 & 5 & 600.0 & 60 \\
\hline 02 & 8 & 38 & 252.2002 & 1.4000 & 292.1 & 4 & 600.0 & 600.0 \\
\hline 02 & 8 & 39 & 243.2575 & 2.0000 & 292.8 & 3 & 600.0 & .0 \\
\hline 02 & 8 & 310 & 237.9159 & 2.25 & 29 & 2 & 600.0 & .0 \\
\hline 02 & 8 & 311 & 230.4982 & 2.9000 & 295.2 & 1 & 600.0 & 600.0 \\
\hline 02 & 8 & 312 & 215.3567 & 3.8000 & 296.7 & 2 & 600.0 & \\
\hline 02 & 8 & 313 & 210.7258 & 3.8500 & 298.0 & 2 & 600.0 & 600.0 \\
\hline 02 & 8 & 314 & 90.7491 & 4.9500 & 299.1 & 3 & 600.0 & 600.0 \\
\hline 02 & 8 & 315 & 116.5014 & 6.6000 & 298.0 & 4 & 600.0 & \\
\hline 02 & 8 & 316 & 118.2528 & 7.0750 & 297.6 & 4 & 600.0 & 600.0 \\
\hline 02 & 8 & 317 & 155.6328 & 6.1500 & 297.5 & 4 & 600.0 & 600.0 \\
\hline 02 & 8 & 318 & 141.7714 & 9.1500 & 295.1 & 4 & 600.0 & 600.0 \\
\hline 02 & 8 & 319 & 182.0000 & 10.9750 & 292.6 & 4 & 600.0 & 600.0 \\
\hline 02 & 8 & 320 & 103.5094 & 11.1250 & 290.5 & 4 & 600.0 & 600.0 \\
\hline 02 & 8 & 321 & 82.4986 & 11.1500 & 289.0 & 4 & 600.0 & 600.0 \\
\hline 02 & 8 & 322 & 76.9997 & 11.725 & 288.2 & 4 & .0 & 600.0 \\
\hline 02 & 8 & 323 & 80.0005 & 12.1500 & 287.7 & 4 & 600.0 & 600.0 \\
\hline
\end{tabular}




\begin{tabular}{|c|c|c|c|c|c|c|c|c|}
\hline D & & 24 & 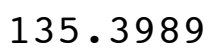 & $\$ 750$ & 38.1 & 4 & . & \\
\hline & 8 & 1 & 4708 & .8500 & 88.1 & & 00.0 & \\
\hline & 8 & 2 & 95.4802 & 500 & 287.1 & 4 & 00 & \\
\hline & 8 & 3 & 91.5541 & 3250 & 287.4 & & 00 & \\
\hline & 8 & 4 & 21 & 000 & 9.2 & & & \\
\hline & 8 & 5 & 176.0 & & 87.7 & & 0 & \\
\hline & 8 & 6 & 155.5436 & 00 & 286.6 & & 00 & \\
\hline & 8 & 7 & 6527 & & 87.9 & & & \\
\hline & 8 & 8 & 23 & 0 & 89.7 & & & \\
\hline & 8 & 9 & 102.4036 & 750 & 91.3 & & 0 & \\
\hline & 8 & 410 & 101.2501 & 000 & 292.0 & & & \\
\hline & 8 & 411 & 126 & & 93.2 & & & \\
\hline & 8 & 412 & 116.2859 & 4 . & 294.6 & & 0 & \\
\hline & 8 & 413 & 126.7477 & 9000 & 29 & & & \\
\hline & 8 & 414 & 123 & & 7 & & & \\
\hline & 8 & 415 & 84 . & & 297.8 & 4 & & \\
\hline & 8 & 416 & 86.0037 & 0 & 297 & 4 & & \\
\hline & 8 & 417 & 75 . & 0 & 3 & & & \\
\hline & 8 & 418 & 87 . & & 29 & 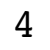 & & \\
\hline & 8 & 419 & 105.5065 & & 29 & 4 & & \\
\hline & 8 & 420 & 00 & 50 & 292.0 & 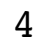 & 0 & \\
\hline & 8 & 421 & 0 & & 93.1 & 4 & & \\
\hline & 8 & 422 & 71.2595 & 0 & 292.6 & 4 & & \\
\hline & 8 & 423 & 47 & 50 & 292.1 & 4 & & \\
\hline & 8 & 424 & & & 292 & 4 & & \\
\hline 2 & 8 & 51 & 82 . & 50 & 292.6 & 4 & & \\
\hline 2 & 8 & 52 & 73 & 0 & 292.5 & 4 & & 0 \\
\hline & 8 & 53 & 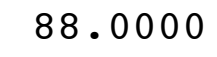 & & 292.1 & 4 & & \\
\hline 2 & 8 & 54 & 1 & 50 & 291.6 & 4 & & \\
\hline & 8 & 55 & 993 & & 291.0 & 4 & & . \\
\hline 5 & 8 & 56 & .2502 & 0 & 291.4 & 4 & & 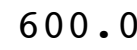 \\
\hline 2 & 8 & 57 & 0 & 0 & 291.8 & 4 & & \\
\hline & 8 & 58 & 87.6942 & 50 & 293.4 & 3 & & . \\
\hline 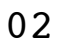 & 8 & 59 & 000 & 0 & 294.4 & 3 & 0 & \\
\hline & 8 & 510 & 91 & & & 3 & & \\
\hline 2 & 8 & 511 & 132 . & & 296.8 & 3 & & \\
\hline & 8 & 512 & 000 & & 297.1 & 3 & & \\
\hline 2 & 8 & 513 & 0 & 0 & 297.9 & 3 & & \\
\hline 02 & 8 & 514 & 127.5060 & 6.4750 & 298.7 & 4 & & 0 . \\
\hline ( & 8 & 515 & 109.2662 & & 298.6 & 4 & & \\
\hline 2 & 8 & 516 & 5000 & 50 & 297.9 & 4 & & \\
\hline ? & 8 & 517 & 82.9515 & & 297.0 & 4 & & \\
\hline 2 & 8 & 518 & 64.9988 & 250 & 295.7 & 4 & & 0 . \\
\hline 2 & 8 & 51 & & & 294.9 & 4 & & \\
\hline 02 & 8 & 520 & 7500 & 10 . & 293.7 & 4 & & \\
\hline ? & 8 & 521 & 72.5000 & 9.8000 & 292.9 & 4 & & 0 . \\
\hline & 8 & $5<2$ & & & 292 & 4 & & \\
\hline & & 523 & 64.2504 & 13.3750 & 291.1 & $I$ & 600.0 & $c 00$ \\
\hline
\end{tabular}




\begin{tabular}{|c|c|c|c|c|c|c|c|c|}
\hline 2 & 8 & 524 & 63.0000 & 12.8250 & 290.6 & 4 & 600.0 & 600.0 \\
\hline 02 & 8 & 61 & 64.2501 & 13.1000 & 290.3 & 4 & 600.0 & 00.0 \\
\hline 02 & 8 & 62 & 68.4992 & 11.9250 & 289.9 & 4 & 600.0 & 00.0 \\
\hline 02 & 8 & 63 & 103.4459 & 9.9750 & 289.7 & 4 & 600.0 & 00.0 \\
\hline 02 & 8 & 64 & 84.7587 & 10.4250 & 289.9 & 4 & 600.0 & 00.0 \\
\hline 02 & 8 & 65 & 180.2500 & 10.7000 & 288.7 & 4 & 600.0 & 00.0 \\
\hline 02 & 8 & 66 & 179.2527 & 10.1000 & 287.8 & 4 & 600.0 & 0 \\
\hline 02 & 8 & 67 & 180.7512 & 8.2000 & 287.7 & 4 & 600.0 & 00.0 \\
\hline 02 & 8 & 68 & 163.1826 & 4.7500 & 290.3 & 3 & 600.0 & 00.0 \\
\hline 02 & 8 & 69 & 146.7485 & 3.6750 & 293.0 & 2 & 600.0 & 0 \\
\hline 02 & 8 & 610 & 168.7525 & 4.5750 & 293.6 & 3 & 600.0 & \\
\hline 02 & 8 & 611 & 182.0034 & 6.1500 & 294.5 & 4 & 600.0 & \\
\hline 02 & 8 & 612 & 152.0016 & 7.0250 & 295.7 & 4 & 600.0 & 00.0 \\
\hline 02 & 8 & 613 & 164.2374 & 6.1500 & 296.5 & 4 & 600.0 & 00.0 \\
\hline 02 & 8 & 614 & 154.5202 & 750 & 297.7 & 3 & .0 & \\
\hline 02 & 8 & 615 & 152.5147 & 5.3000 & 298.2 & 3 & 600.0 & .0 \\
\hline 02 & 8 & 616 & 148.9871 & 5.1750 & 298.9 & 3 & 60 & .0 \\
\hline 02 & 8 & 617 & 135.7495 & 5.9 & 298.2 & 3 & .0 & \\
\hline 02 & 8 & 618 & 128.4232 & 6.7750 & 296.8 & 4 & 60 & .0 \\
\hline 02 & 8 & 619 & 156.4583 & 7.5750 & 295.1 & 4 & .0 & .0 \\
\hline 02 & 8 & 620 & 155.7295 & 8 . & 293.8 & 4 & 6 & \\
\hline 02 & 8 & 621 & 119.2038 & 7.7750 & 294.0 & 4 & .0 & \\
\hline 02 & 8 & 622 & 65.2498 & 7.4000 & 294.3 & 4 & 600.0 & .0 \\
\hline 02 & 8 & 623 & 69.4985 & 5.87 & 294.1 & 4 & 6 & \\
\hline 02 & 8 & 624 & 080 & 5.6 & 294.6 & 4 & .0 & \\
\hline 02 & 8 & 71 & 84.0000 & 4.9000 & 294.3 & 5 & .0 & 600.0 \\
\hline 02 & 8 & 72 & 120.1841 & 2.4750 & 293.9 & 4 & 60 & .0 \\
\hline 02 & 8 & 73 & 180.2513 & 0.90 & 292.9 & 5 & .0 & \\
\hline 02 & 8 & 74 & 148.4476 & 1.1000 & 292.2 & 5 & .0 & 0.0 \\
\hline 02 & 8 & 75 & 128.6777 & 1.7250 & 292.0 & 5 & 60 & .0 \\
\hline 02 & 8 & 76 & 129.8444 & 3.40 & 291.3 & 4 & .0 & \\
\hline 02 & 8 & 77 & 134.3897 & 4.00 & 292.5 & 3 & .0 & 600.0 \\
\hline 02 & 8 & 78 & 159.7524 & 6.1750 & 293.2 & 4 & 600.0 & 600.0 \\
\hline 02 & 8 & 79 & 159.7485 & 7.5750 & 294.2 & 4 & 600.0 & 0.0 \\
\hline 02 & 8 & 710 & 166.2878 & 6.5000 & 295.2 & 4 & 600.0 & 600.0 \\
\hline 02 & 8 & 711 & 170.2522 & 6.4250 & 296.2 & 4 & 600.0 & 600.0 \\
\hline 02 & 8 & 712 & 165.5000 & 5.3250 & 297.7 & 3 & 600.0 & 0.0 \\
\hline 02 & 8 & 713 & 175.4946 & 4.8750 & 299.1 & 3 & 600.0 & 600.0 \\
\hline 02 & 8 & 714 & 163.7483 & 5.9750 & 300.1 & 3 & 600.0 & 600.0 \\
\hline 02 & 8 & 715 & 179.7457 & 6.7000 & 300.6 & 4 & 600.0 & 600.0 \\
\hline 02 & 8 & 716 & 179.0037 & 5.2500 & 301.2 & 3 & 600.0 & 600.0 \\
\hline 02 & 8 & 717 & 178.2616 & 5.4000 & 301.6 & 3 & 600.0 & 600.0 \\
\hline 02 & 8 & 718 & 184.7499 & 4.7750 & 301.5 & 3 & 600.0 & 600.0 \\
\hline 02 & 8 & 719 & 135.5757 & 4.4500 & 300.6 & 4 & 600.0 & 600.0 \\
\hline 02 & 8 & 720 & 73.9998 & 5.6500 & 298.7 & 4 & 600.0 & 600.0 \\
\hline 02 & 8 & 721 & 76.5000 & 7.3250 & 297.7 & 4 & 600.0 & 600.0 \\
\hline 02 & 8 & 722 & 76.5000 & 7.5500 & 297.4 & 4 & 600.0 & 600.0 \\
\hline 02 & 0 & 723 & 73.0000 & 7.5000 & 297.6 & 4 & 600.0 & 600.0 \\
\hline
\end{tabular}




\begin{tabular}{|c|c|c|c|c|c|c|c|c|}
\hline 2 & & 24 & 000 & 5750 & 98.1 & 4 & 600.0 & \\
\hline 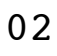 & 8 & 1 & 00.5475 & .0250 & 298.9 & \pm & 600.0 & \\
\hline & 8 & 2 & 103.2395 & 1500 & 299.2 & 4 & 00 & \\
\hline & 8 & 3 & 123.7826 & 5250 & 299.4 & & 00.0 & \\
\hline & 8 & 4 & 143.4990 & 5250 & 98.4 & & 00 & \\
\hline & 8 & 5 & 133.2563 & .8750 & 297.5 & & 00 & \\
\hline & 8 & 6 & 148.2496 & 3250 & 296.8 & & 00 & \\
\hline & 8 & 7 & 137.7425 & 6500 & 298.4 & & 0 & \\
\hline & 8 & 8 & 154.0000 & 9750 & 98.6 & & & \\
\hline & 8 & 9 & 5582 & 8750 & 299.4 & & 0 & 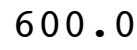 \\
\hline & 8 & 810 & 166.5009 & 7.4750 & 299.8 & & & \\
\hline & 8 & 11 & 165.2479 & 6.9000 & 300.5 & & & \\
\hline & 8 & 312 & 168.7497 & 9250 & 301.4 & 4 & 0 & \\
\hline & 8 & 813 & 168.2497 & 2500 & 302.4 & 4 & 0.0 & \\
\hline & 8 & 14 & 174.9947 & 0 & 30 & & & \\
\hline & 8 & 815 & 175.2544 & & 304 & 3 & & \\
\hline & 8 & 816 & 180.0005 & 6500 & 304.9 & 3 & .0 & \\
\hline & 8 & 17 & 182.7504 & 0 & 305.1 & & 0 & \\
\hline & 8 & 818 & 182.5043 & & 304.9 & 3 & & \\
\hline & 8 & 819 & 181.9011 & 2 . & 304.1 & 4 & & 8 \\
\hline 2 & 8 & 820 & 143.0184 & 2.6000 & 301.9 & 4 & 0 & \\
\hline & 8 & 821 & 012 & & 301.6 & 4 & & \\
\hline & 8 & 822 & 000 & 50 & 301.0 & 4 & & \\
\hline 2 & 8 & 823 & 72.7501 & 3250 & 301.0 & 4 & 0 & \\
\hline & 8 & 824 & 73.4994 & & 30 & 4 & & \\
\hline 2 & 8 & 91 & 76.7501 & 50 & 301.5 & 4 & & \\
\hline$?$ & 8 & 92 & 79.5005 & 7750 & 302.0 & 4 & 0 & . \\
\hline 2 & 8 & 93 & 81.5006 & 9250 & 302.2 & 4 & & \\
\hline 2 & 8 & 94 & 87.9989 & 50 & 302.2 & 4 & & \\
\hline 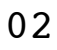 & 8 & 95 & 82.2838 & 250 & 301.4 & 5 & 0 & U. \\
\hline 2 & 8 & 96 & 41.7675 & 5500 & 300.9 & 4 & 0 & 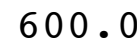 \\
\hline 2 & 8 & 97 & 168.1509 & 0 & 301.6 & 3 & & \\
\hline 2 & 8 & 98 & 182.9219 & & 302.7 & 2 & & . \\
\hline 2 & 8 & 99 & 194.9965 & 750 & 303.9 & 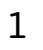 & 0 & \\
\hline 2 & 8 & 910 & 168.2500 & 0 & 302.8 & 2 & & \\
\hline 2 & 8 & 911 & 168.0000 & 500 & 303.0 & 3 & & . \\
\hline 2 & 8 & 912 & 170.5000 & 4000 & 304.1 & 3 & & 0 \\
\hline 2 & 8 & 913 & 177.5000 & 500 & 305.4 & 3 & & \\
\hline 02 & 8 & 914 & 172.7356 & 4.8250 & 306.2 & 3 & .0 & 0 . \\
\hline 2 & 8 & 915 & 191.0000 & 5250 & 307.1 & 3 & & 0 . \\
\hline 2 & 8 & 916 & 183.9969 & 4.7000 & 307.2 & 3 & & 0 \\
\hline 02 & 8 & 917 & 179.0355 & 4.4000 & 307.1 & 3 & & 0 . \\
\hline 2 & 8 & 918 & 168.2739 & 3.6750 & 306.8 & 3 & 0 & 0 . \\
\hline 02 & 8 & 919 & 91.2019 & 750 & 306.2 & 4 & & \\
\hline 02 & 8 & 920 & 71.2499 & 6.7500 & 304.5 & 4 & & 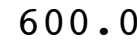 \\
\hline 2 & 8 & 921 & 71.4997 & 8.4000 & 303.2 & 4 & 600 & 600. \\
\hline & 8 & 922 & 500 & & 30 & 4 & & \\
\hline & & 923 & 77.2501 & 9.7000 & 302.3 & 7 & 600.0 & 600 \\
\hline
\end{tabular}




\begin{tabular}{|c|c|c|c|c|c|c|c|}
\hline 2 & 8924 & 74.0000 & 8.8750 & 302.3 & 4 & 600.0 & 600.0 \\
\hline 02 & 8101 & 72.0000 & 8.3500 & 302.6 & 4 & 600.0 & 600.0 \\
\hline 02 & 8102 & 73.2500 & 8.1500 & 302.5 & 4 & 600.0 & 00.0 \\
\hline 02 & 8103 & 77.5000 & 7.6500 & 302.6 & 4 & 600.0 & 00.0 \\
\hline 02 & 8104 & 78.5000 & 7.5250 & 302.5 & 4 & 600.0 & 00.0 \\
\hline 02 & 8105 & 76.2492 & 8.0750 & 302.2 & 4 & 600.0 & 00.0 \\
\hline 02 & 8106 & 76.2500 & 8.2500 & 301.9 & 4 & 600.0 & 600.0 \\
\hline 02 & 8107 & 77.5000 & 7.6000 & 302.2 & 4 & 600.0 & 00.0 \\
\hline 02 & 8108 & 79.7499 & 5.8250 & 303.4 & 4 & 600.0 & 00.0 \\
\hline 02 & 8109 & 243.2483 & 2.3500 & 304.7 & 3 & 600.0 & 600.0 \\
\hline 02 & 81010 & 235.7499 & 2.9250 & 304.2 & 3 & 600.0 & 0 \\
\hline 02 & 81011 & 207.6889 & 2.3500 & 305.9 & 2 & 600.0 & \\
\hline 02 & 81012 & 206.3236 & 3.9500 & 306.6 & 2 & 600.0 & 600.0 \\
\hline 02 & 81013 & 221.5000 & 4.2500 & 307.2 & 3 & 600.0 & 0 \\
\hline 02 & 81014 & 203.4816 & 4.2750 & 307.8 & 3 & 600.0 & \\
\hline 02 & 81015 & 186.0184 & 4.8000 & 308.0 & 3 & 600.0 & 60 \\
\hline 02 & 81016 & 197.5003 & 5.0500 & 308.0 & 3 & 600.0 & 600.0 \\
\hline 02 & 81017 & 180.5024 & 4.9500 & 308.0 & 3 & 600.0 & \\
\hline 02 & 81018 & 154.1260 & 4.4000 & 307.4 & 3 & 600.0 & .0 \\
\hline 02 & 81019 & 77.7416 & 5.7000 & 305.6 & 4 & 600.0 & 600 \\
\hline 02 & 81020 & 69.7504 & 8.75 & 302.7 & 4 & 600.0 & .0 \\
\hline 02 & 81021 & 68.5000 & 9.0000 & 302.4 & 4 & 0.0 & \\
\hline 02 & 81022 & 73.2500 & 8.7250 & 302.3 & 4 & 600.0 & 600.0 \\
\hline 02 & 81023 & 75.7500 & 9.0500 & 302.1 & 4 & 600.0 & .0 \\
\hline 02 & 81024 & 74.2501 & 9.4500 & 301.7 & 4 & 600.0 & \\
\hline 02 & 8111 & 75.2500 & 9.6250 & 301.3 & 4 & 600.0 & 60 \\
\hline 02 & 8112 & 77.7501 & 9.5750 & 301.1 & 4 & 600.0 & .0 \\
\hline 02 & 8113 & 78.0000 & 9.0750 & 300.8 & 4 & 600.0 & \\
\hline 02 & 8114 & 79.2500 & 8.7500 & 300.9 & 4 & 600.0 & 60 \\
\hline 02 & 8115 & 78.7500 & 8.8750 & 300.7 & 4 & 600.0 & 600.0 \\
\hline 02 & 8116 & 78.5000 & 8.25 & 301.0 & 4 & 600.0 & \\
\hline 02 & 8117 & 81.4983 & 5.0750 & 302.2 & 3 & 600.0 & 60 \\
\hline 02 & 8118 & 96.0215 & 1.4000 & 303.6 & 2 & 600.0 & 600.0 \\
\hline 02 & 8119 & 151.8957 & 2.17 & 304.9 & 1 & 600.0 & .0 \\
\hline 02 & 81110 & 233.3323 & 2.3000 & 305.1 & 1 & 600.0 & 60 \\
\hline 02 & 81111 & 197.2273 & 4.4500 & 304.8 & 2 & 600.0 & 600.0 \\
\hline 02 & 81112 & 178.5000 & 5.9500 & 305.4 & 3 & 600.0 & \\
\hline 02 & 81113 & 166.7499 & 5.0250 & 306.2 & 3 & 600.0 & 600.0 \\
\hline 02 & 81114 & 185.4914 & 5.1250 & 306.5 & 3 & 600.0 & 600.0 \\
\hline 02 & 81115 & 189.9998 & 5.6500 & 306.7 & 3 & 600.0 & \\
\hline 02 & 81116 & 174.7500 & 5.3000 & 307.2 & 3 & 600.0 & 600.0 \\
\hline 02 & 81117 & 163.1731 & 5.0750 & 307.4 & 3 & 600.0 & 600.0 \\
\hline 02 & 81118 & 92.9935 & 5.0750 & 306.9 & 3 & 600.0 & 600.0 \\
\hline 02 & 81119 & 85.7502 & 5.6500 & 305.0 & 4 & 600.0 & 600.0 \\
\hline 02 & 81120 & 80.0000 & 7.6500 & 302.8 & 4 & 600.0 & 600.0 \\
\hline 02 & 81121 & 76.5009 & 9.7000 & 301.8 & 4 & 600.0 & 600.0 \\
\hline 02 & 81122 & 75.5000 & 10.650 & 301.2 & 4 & 600.0 & 600.0 \\
\hline 02 & 81123 & 74.5000 & 11.3000 & 300.6 & 4 & 600.0 & 600.0 \\
\hline
\end{tabular}




\begin{tabular}{|c|c|c|c|c|c|c|c|}
\hline 02 & 81124 & 75.2504 & 11.1750 & 300.5 & 4 & 600.0 & 600.0 \\
\hline 02 & 8121 & 76.7500 & 10.5500 & 300.9 & 4 & 600.0 & 00,0 \\
\hline 02 & 8122 & 79.0001 & 10.0750 & 301.2 & 4 & 600.0 & 00.0 \\
\hline 02 & 8123 & 78.2500 & 10.4000 & 300.3 & 4 & 600.0 & 00.0 \\
\hline 02 & 8124 & 79.0000 & 10.4000 & 299.6 & 4 & 600.0 & 00.0 \\
\hline 02 & 8125 & 80.5000 & 9.1500 & 299.8 & 4 & 600.0 & 00.0 \\
\hline 02 & 8126 & 82.5002 & 8.6250 & 300.0 & 4 & 600.0 & 00.0 \\
\hline 02 & 8127 & 81.7500 & 7.8000 & 300.5 & 4 & 600.0 & 00.0 \\
\hline 02 & 8128 & 82.0000 & 5.1750 & 302.3 & 4 & 600.0 & 00.0 \\
\hline 02 & 8129 & 183.8826 & 250 & 303.8 & 3 & 600 & 0 \\
\hline 02 & 81210 & 178.0161 & 50 & 303.8 & 2 & 600.0 & \\
\hline 02 & 81211 & 167.2507 & 250 & 302.6 & 3 & 600.0 & \\
\hline 02 & 81212 & 168.0002 & 5.1250 & 303.2 & 3 & 600.0 & 00.0 \\
\hline 02 & 81213 & 171.2513 & 50 & 304.9 & 3 & 600.0 & 00.0 \\
\hline 02 & 81214 & 174.4992 & 50 & 306.3 & 3 & .0 & \\
\hline 02 & 81215 & 170.7552 & 50 & 307.4 & 3 & 600.0 & 00.0 \\
\hline 02 & 81216 & 168.4994 & 50 & 307.5 & 3 & 60 & .0 \\
\hline 02 & 81217 & 168.9973 & & 307.6 & 3 & .0 & \\
\hline 02 & 81218 & 116.6882 & 500 & 307.5 & 3 & 60 & .0 \\
\hline 02 & 81219 & 80.4982 & 00 & 305.8 & 4 & .0 & .0 \\
\hline 02 & 81220 & 78.5000 & 8 . & .9 & 4 & 6 & \\
\hline 02 & 81221 & 75.7500 & 8 . & 303.7 & 4 & .0 & \\
\hline 02 & 81222 & 76.0001 & 00 & 303.9 & 4 & 600.0 & .0 \\
\hline 02 & 81223 & 73.2500 & 8 . & 304.2 & 4 & 6 & \\
\hline 02 & 81224 & 74.9999 & 8 . & 304.2 & 4 & .0 & \\
\hline 02 & 8131 & 79.5000 & 7 . & 304.3 & 4 & .0 & .0 \\
\hline 02 & 8132 & 79.0000 & 6.6250 & 304.7 & 4 & 60 & .0 \\
\hline 02 & 8133 & 77.9999 & 5 & 304.6 & 4 & .0 & \\
\hline 02 & 8134 & 124.1928 & 2 . & 303.0 & 5 & .0 & .0 \\
\hline 02 & 8135 & 109.7086 & 750 & 302.0 & 5 & 60 & .0 \\
\hline 02 & 8136 & 119.4786 & 1. & 300.8 & 6 & .0 & \\
\hline 02 & 8137 & 159.9619 & 1 . & 300.3 & 5 & .0 & 600.0 \\
\hline 02 & 8138 & 231.7009 & 0.5250 & 302.6 & 4 & 600.0 & 600.0 \\
\hline 02 & 8139 & 246.0000 & 1.5250 & 303.1 & 3 & 0 & 0.0 \\
\hline 02 & 81310 & 241.9989 & 2.6750 & 303.0 & 3 & 600.0 & 600.0 \\
\hline 02 & 81311 & 195.7375 & 3.8750 & 303.2 & 2 & 600.0 & 600.0 \\
\hline 02 & 81312 & 189.0069 & 500 & 303.7 & 3 & 600.0 & 0.0 \\
\hline 02 & 81313 & 192.7485 & 5.4250 & 305.3 & 3 & 600.0 & 600.0 \\
\hline 02 & 81314 & 195.7509 & 5.7500 & 306.8 & 3 & 600.0 & 600.0 \\
\hline 02 & 81315 & 69.1149 & 3 . & 308.6 & 2 & 600.0 & 600.0 \\
\hline 02 & 81316 & 82.4982 & 000 & 308.4 & 3 & 600.0 & 600.0 \\
\hline 02 & 81317 & 75.7511 & 6.0500 & 307.7 & 4 & 600.0 & 600.0 \\
\hline 02 & 81318 & 78.7512 & 7.1250 & 306.6 & 4 & 600.0 & 600.0 \\
\hline 02 & 81319 & 79.5000 & 8.4000 & 305.1 & 4 & 600.0 & 600.0 \\
\hline 02 & 81320 & 73.2502 & 9.7750 & 303.7 & 4 & 600.0 & 600.0 \\
\hline 02 & 81321 & 71.9998 & 9.9500 & 303.5 & 4 & 600.0 & 600.0 \\
\hline 02 & 81322 & 76.4999 & 9.3750 & 303.2 & 4 & 600.0 & 600.0 \\
\hline $0<$ & 81323 & 77.2501 & 10.0250 & 303.1 & 4 & 600.0 & 600.0 \\
\hline
\end{tabular}




\begin{tabular}{|c|c|c|c|c|c|c|c|}
\hline 02 & 81324 & 77.2501 & 9.4750 & 302.8 & 4 & 600.0 & 600.0 \\
\hline 02 & 8141 & 74.9989 & 8.7250 & 302.6 & 4 & 600.0 & 600.0 \\
\hline 02 & 8142 & 76.0000 & 8.2000 & 302.6 & 4 & 600.0 & 00.0 \\
\hline 02 & 8143 & 75.2500 & 7.6750 & 302.4 & 4 & 600.0 & 00.0 \\
\hline 02 & 8144 & 73.0019 & 8.0250 & 302.4 & 4 & 600.0 & 00.0 \\
\hline 02 & 8145 & 70.2506 & 7.5250 & 302.3 & 4 & 600.0 & 00.0 \\
\hline 02 & 8146 & 71.2501 & 7.0000 & 302.2 & 4 & 600.0 & 600.0 \\
\hline 02 & 8147 & 71.2499 & 6.2500 & 302.4 & 4 & 600.0 & 00.0 \\
\hline 02 & 8148 & 77.5000 & 5.6000 & 303.3 & 4 & 600.0 & \\
\hline 02 & 8149 & 115.8823 & 3.2500 & 304.5 & 3 & 600.0 & 600.0 \\
\hline 02 & 81410 & 139.2898 & 2.6750 & 303.5 & 2 & 600.0 & 0 \\
\hline 02 & 81411 & 138.2490 & 4.3250 & 304.6 & 3 & 600.0 & \\
\hline 02 & 81412 & 108.2128 & 5.3500 & 305.5 & 3 & 600.0 & .0 \\
\hline 02 & 81413 & 102.4935 & 4.7250 & 306.7 & 3 & 600.0 & 0 \\
\hline 02 & 81414 & 68.4545 & 5.7500 & 307.7 & 3 & 600.0 & .0 \\
\hline 02 & 81415 & 73.2644 & 7.3250 & 307.2 & 4 & 600.0 & \\
\hline 02 & 81416 & 78.0005 & 8.4250 & 306.4 & 4 & 600.0 & 600.0 \\
\hline 02 & 81417 & 68.9985 & 8.8250 & 305.4 & 4 & 600.0 & .0 \\
\hline 02 & 81418 & 66.0000 & 9.6250 & 304.4 & 4 & 600.0 & \\
\hline 02 & 81419 & 68.0000 & 10.1500 & 303.3 & 4 & 600.0 & 600.0 \\
\hline 02 & 81420 & 70.2500 & 9.4250 & 303.0 & 4 & 600.0 & 600.0 \\
\hline 02 & 81421 & 66.7500 & 9.6750 & 303.0 & 4 & 600.0 & \\
\hline 02 & 81422 & 69.7500 & 9.9750 & 302.8 & 4 & 600.0 & 60 \\
\hline 02 & 81423 & 70.2502 & 9.4500 & 302.9 & 4 & 600.0 & .0 \\
\hline 02 & 81424 & 72.5000 & 9.8250 & 302.8 & 4 & 600.0 & \\
\hline 02 & 8151 & 70.2500 & 9.4750 & 302.7 & 4 & 600.0 & 60 \\
\hline 02 & 8152 & 70.5000 & 8.9500 & 302.7 & 4 & 600.0 & .0 \\
\hline 02 & 8153 & 76.0000 & 9.075 & 302.5 & 4 & 600.0 & .0 \\
\hline 02 & 8154 & 78.0000 & 7.3750 & 302.1 & 4 & 600.0 & .0 \\
\hline 02 & 8155 & 75.2495 & 5.9000 & 301.0 & 4 & 600.0 & .0 \\
\hline 02 & 8156 & 84.0000 & 6.4250 & 300.0 & 4 & 600.0 & 60 \\
\hline 02 & 8157 & 83.2498 & 6.4500 & 300.8 & 4 & 600.0 & \\
\hline 02 & 8158 & 68.4035 & 4.9750 & 301.8 & 3 & 600.0 & 60 \\
\hline 02 & 8159 & 294.4437 & 1.5750 & 302.7 & 2 & 600.0 & .0 \\
\hline 02 & 81510 & 241.2447 & 2.7500 & 299.8 & 3 & 600.0 & \\
\hline 02 & 81511 & 221.2777 & 2.4250 & 302.0 & 2 & 600.0 & 600.0 \\
\hline 02 & 81512 & 187.2143 & 3.2750 & 303.4 & 2 & 600.0 & 600.0 \\
\hline 02 & 81513 & 163.0086 & 2.9500 & 304.6 & 2 & 600.0 & 60 \\
\hline 02 & 81514 & 175.2620 & 4.0750 & 306.0 & 3 & 600.0 & 600.0 \\
\hline 02 & 81515 & 189.2495 & 4.8500 & 307.1 & 3 & 600.0 & 600.0 \\
\hline 02 & 81516 & 119.1329 & 4.4750 & 307.3 & 3 & 600.0 & 600.0 \\
\hline 02 & 81517 & 93.2517 & 5.0750 & 307.2 & 3 & 600.0 & 600.0 \\
\hline 02 & 81518 & 74.9936 & 5.9000 & 305.6 & 3 & 600.0 & 600.0 \\
\hline 02 & 81519 & 63.0000 & 9.3500 & 304.6 & 4 & 600.0 & 600.0 \\
\hline 02 & 81520 & 58.7505 & 6.7500 & 304.6 & 4 & 600.0 & 600.0 \\
\hline 02 & 81521 & 350.9544 & 0.9500 & 302.5 & 5 & 600.0 & 600.0 \\
\hline 02 & 81522 & 16.5041 & 1.375 & 300.8 & 4 & 600.0 & .0 \\
\hline 02 & 81523 & 15.7524 & 1.4000 & 301.7 & 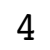 & 600.0 & 600.0 \\
\hline
\end{tabular}




\begin{tabular}{|c|c|c|c|c|c|c|c|}
\hline 02 & 81524 & 10.4925 & 0.8500 & 301.7 & 4 & 600.0 & 600.0 \\
\hline 02 & 8161 & 17.2185 & 1.3500 & 301.5 & 4 & 600.0 & 600.0 \\
\hline 02 & 8162 & 65.2706 & 0.2500 & 298.5 & 5 & 600.0 & 00.0 \\
\hline 02 & 8163 & 264.3204 & 0.2000 & 296.0 & 6 & 600.0 & 00.0 \\
\hline 02 & 8164 & 197.7062 & 0.3000 & 295.8 & 0 & 600.0 & 00.0 \\
\hline 02 & 8165 & 85.1428 & 0.5000 & 296.3 & 6 & 600.0 & 00.0 \\
\hline 02 & 8166 & 17.2745 & 0.5750 & 296.5 & 6 & 600.0 & 600.0 \\
\hline 02 & 8167 & 99.8914 & 0.9750 & 295.2 & 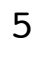 & 600.0 & 00.0 \\
\hline 02 & 8168 & 140.5968 & 0.7000 & 297.1 & 4 & 600.0 & \\
\hline 02 & 8169 & 350.9501 & 0.9750 & 298.6 & 3 & 600.0 & 600.0 \\
\hline 02 & 81610 & 289.8813 & 1.5000 & 300.0 & 2 & 600.0 & 0 \\
\hline 02 & 81611 & 242.7545 & 2.6500 & 299.9 & 2 & 600.0 & \\
\hline 02 & 81612 & 213.5402 & 2.8250 & 300.8 & 1 & 600.0 & .0 \\
\hline 02 & 81613 & 239.2498 & 2.9750 & 302.2 & 2 & 600.0 & 0 \\
\hline 02 & 81614 & 204.6316 & 3.3500 & 304.1 & 2 & 600.0 & .0 \\
\hline 02 & 81615 & 145.3717 & 2.6250 & 305.8 & 1 & 600.0 & .0 \\
\hline 02 & 81616 & 75.9941 & 4.1750 & 305.4 & 2 & 600.0 & 600.0 \\
\hline 02 & 81617 & 83.2501 & 7.2750 & 303.2 & 3 & 600.0 & .0 \\
\hline 02 & 81618 & 78.7482 & 8.1000 & 302.0 & 4 & 600.0 & \\
\hline 02 & 81619 & 77.7503 & 8.2500 & 301.6 & 4 & 600.0 & 600.0 \\
\hline 02 & 81620 & 79.7498 & 9.4750 & 300.9 & 4 & 600.0 & 600.0 \\
\hline 02 & 81621 & 83.2527 & 9.4250 & 301.1 & 4 & 600.0 & \\
\hline 02 & 81622 & 81.4994 & 9.1500 & 301.1 & 4 & 600.0 & 60 \\
\hline 02 & 81623 & 77.2499 & 8.6000 & 300.9 & 4 & 600.0 & .0 \\
\hline 02 & 81624 & 76.0004 & 8.5000 & 300.3 & 4 & 600.0 & \\
\hline 02 & 8171 & 74.5008 & 8.3500 & 301.0 & 4 & 600.0 & 60 \\
\hline 02 & 8172 & 71.2502 & 7.6750 & 301.8 & 4 & 600.0 & .0 \\
\hline 02 & 8173 & 69.0002 & 6.9000 & 302.5 & 4 & 600.0 & .0 \\
\hline 02 & 8174 & 74.9988 & 7.4000 & 302.5 & 4 & 600.0 & 60 \\
\hline 02 & 8175 & 69.0000 & 9.1000 & 301.5 & 4 & 600.0 & 600.0 \\
\hline 02 & 8176 & 70.0000 & 8.1000 & 300.8 & 4 & 600.0 & 60 \\
\hline 02 & 8177 & 74.9968 & 6 . & 299.9 & 4 & 600.0 & 60 \\
\hline 02 & 8178 & 79.7501 & 6.3500 & 300.8 & 4 & 600.0 & 600.0 \\
\hline 02 & 8179 & 76.5796 & 4.0000 & 301.2 & 3 & 600.0 & .0 \\
\hline 02 & 81710 & 86.5000 & 250 & 301.1 & 4 & 600.0 & \\
\hline 02 & 81711 & 106.5623 & 5.3250 & 300.8 & 3 & 600.0 & 600.0 \\
\hline 02 & 81712 & 76.7504 & 8.2750 & 301.1 & 4 & 600.0 & 600.0 \\
\hline 02 & 81713 & 73.0003 & 7.9000 & 302.4 & 4 & 600.0 & 60 \\
\hline 02 & 81714 & 77.4937 & 8.2500 & 302.5 & 4 & 600.0 & 600.0 \\
\hline 02 & 81715 & 69.5000 & 9.3250 & 302.4 & 4 & 600.0 & 600.0 \\
\hline 02 & 81716 & 64.7500 & 11.2750 & 302.0 & 4 & 600.0 & 600.0 \\
\hline 02 & 81717 & 65.2500 & 11.3500 & 301.3 & 4 & 600.0 & 600.0 \\
\hline 02 & 81718 & 74.0007 & 8.5500 & 300.9 & 4 & 600.0 & 600.0 \\
\hline 02 & 81719 & 72.2505 & 8.8250 & 299.1 & 4 & 600.0 & 600.0 \\
\hline 02 & 81720 & 67.7507 & 10.5500 & 298.5 & 4 & 600.0 & 600.0 \\
\hline 02 & 81721 & 64.7500 & 12.4750 & 298.0 & 4 & 600.0 & 600.0 \\
\hline 02 & 81722 & 62.5000 & 11.050 & 298.8 & 4 & 600.0 & .0 \\
\hline 02 & 81723 & 66.2506 & 8.4250 & 299.9 & $I$ & 600.0 & 600.0 \\
\hline
\end{tabular}




\begin{tabular}{|c|c|c|c|c|c|c|c|}
\hline 2 & 81724 & 72.4992 & 7.5750 & 299.7 & 4 & 600.0 & 00.0 \\
\hline 02 & 8181 & 93.5008 & 5.6750 & 299.3 & 4 & 600.0 & 600.0 \\
\hline 02 & 8182 & 105.3941 & 4.4250 & 297.2 & 4 & 600.0 & 00.0 \\
\hline 02 & 8183 & 129.3943 & 1.3500 & 293.6 & 5 & 600.0 & 00.0 \\
\hline 02 & 8184 & 316.5404 & 0.4250 & 292.5 & 0 & 600.0 & 00.0 \\
\hline 02 & 8185 & 106.1765 & 1.8000 & 292.7 & 6 & 600.0 & 00.0 \\
\hline 02 & 8186 & 171.0644 & 1.0750 & 292.7 & 6 & 600.0 & 600.0 \\
\hline 02 & 8187 & 155.7554 & 0.8250 & 293.1 & J & 600.0 & 00.0 \\
\hline 02 & 8188 & 300.0611 & 0.5500 & 295.3 & 4 & 600.0 & 00.0 \\
\hline 02 & 8189 & 304.9478 & 0.9750 & 296.8 & 3 & 600.0 & .0 \\
\hline 02 & 81810 & 182.8531 & 2.3000 & 296.7 & 2 & 600.0 & 00.0 \\
\hline 02 & 81811 & 209.8017 & 3.2250 & 297.8 & 2 & 600.0 & \\
\hline 02 & 81812 & 176.5009 & 5.0250 & 298.6 & 3 & 600.0 & 00.0 \\
\hline 02 & 81813 & 140.4205 & 4.9000 & 301.2 & 3 & 600.0 & 0 \\
\hline 02 & 81814 & 139.2590 & 5.9250 & 301.9 & 3 & 600.0 & \\
\hline 02 & 81815 & 90.5206 & 5.2250 & 302.2 & 3 & 600.0 & 60 \\
\hline 02 & 81816 & 88.2382 & 6.2000 & 302.0 & 4 & 600.0 & 600.0 \\
\hline 02 & 81817 & 81.0037 & 8.6750 & 300.5 & 4 & 600.0 & \\
\hline 02 & 81818 & 70.2502 & 9.2000 & 299.6 & 4 & 600.0 & .0 \\
\hline 02 & 81819 & 70.7494 & 10.7500 & 299.0 & 4 & 600.0 & .0 \\
\hline 02 & 81820 & 67.9993 & 1.55 & 298.6 & 4 & 600.0 & .0 \\
\hline 02 & 81821 & 71.2508 & 10.5750 & 298.2 & 4 & 0.0 & \\
\hline 02 & 81822 & 73.2501 & 10.7250 & 298.1 & 4 & 600.0 & 600.0 \\
\hline 02 & 81823 & 72.2500 & 10.4250 & 297.0 & 4 & 600.0 & .0 \\
\hline 02 & 81824 & 76.7497 & 9.5750 & 297.1 & 4 & 600.0 & \\
\hline 02 & 8191 & 77.5003 & 9.3500 & 297.6 & 4 & 600.0 & 60 \\
\hline 02 & 8192 & 77.4997 & 9.6750 & 296.1 & 4 & 600.0 & .0 \\
\hline 02 & 8193 & 76.9999 & 8.5500 & 295.6 & 4 & 0.0 & \\
\hline 02 & 8194 & 77.0001 & 8.5250 & 29 & 4 & 600.0 & 60 \\
\hline 02 & 8195 & 73.5005 & 9.7500 & 293.0 & 4 & 600.0 & .0 \\
\hline 02 & 8196 & 73.5015 & 9.67 & 292.2 & 4 & 600.0 & \\
\hline 02 & 8197 & 77.2500 & 11.1250 & 292.0 & 4 & 600.0 & .0 \\
\hline 02 & 8198 & 75.5009 & 13.0000 & 289.8 & 4 & 600.0 & 600.0 \\
\hline 02 & 8199 & 72.0000 & 12.400 & 290.7 & 4 & 600.0 & \\
\hline 02 & 81910 & 77.0000 & 9.7250 & 292.4 & 4 & 600.0 & .0 \\
\hline 02 & 81911 & 79.2498 & 9.1750 & 293.4 & 4 & 600.0 & 600.0 \\
\hline 02 & 81912 & 83.7487 & 9.2500 & 294.0 & 4 & 600.0 & \\
\hline 02 & 81913 & 82.7500 & 11.1000 & 294.4 & 4 & 600.0 & .0 \\
\hline 02 & 81914 & 72.2509 & 12.4000 & 294.2 & 4 & 600.0 & 600.0 \\
\hline 02 & 81915 & 88.7382 & 10.2250 & 294.5 & 4 & 600.0 & \\
\hline 02 & 81916 & 83.7540 & 10.1500 & 294.2 & 4 & 600.0 & 600.0 \\
\hline 02 & 81917 & 80.7390 & 11.1750 & 293.5 & 4 & 600.0 & 600.0 \\
\hline 02 & 81918 & 89.7431 & 11.8250 & 292.0 & 4 & 600.0 & 600.0 \\
\hline 02 & 81919 & 80.0000 & 13.0250 & 289.7 & 4 & 600.0 & 600.0 \\
\hline 02 & 81920 & 74.5000 & 13.1000 & 288.8 & 4 & 600.0 & 600.0 \\
\hline 02 & 81921 & 78.5000 & 12.6500 & 288.5 & 4 & 600.0 & 600.0 \\
\hline 02 & 81922 & 76.2492 & 11.425 & 288.4 & 4 & 600.0 & 600.0 \\
\hline 02 & 81923 & 77.9993 & 11.3750 & 289.3 & 4 & 600.0 & 600.0 \\
\hline
\end{tabular}




\begin{tabular}{|c|c|c|c|c|c|c|c|}
\hline 2 & 81924 & 77.0000 & 9.9000 & 290.5 & 4 & 600.0 & 600.0 \\
\hline 02 & 8201 & 78.5000 & 9.4500 & 289.8 & 4 & 600.0 & 600.0 \\
\hline 02 & 8202 & 81.7498 & 8.8250 & 290.3 & 4 & 600.0 & 00.0 \\
\hline 02 & 8203 & 90.7511 & 9.1250 & 291.3 & 4 & 600.0 & 00.0 \\
\hline 02 & 8204 & 90.2500 & 8.0500 & 292.1 & 4 & 600.0 & 00.0 \\
\hline 02 & 8205 & 91.5000 & 6.9500 & 292.3 & 4 & 600.0 & 00.0 \\
\hline 02 & 8206 & 90.0000 & 7.0750 & 292.4 & 4 & 600.0 & 0 \\
\hline 02 & 8207 & 84.0000 & 7.3000 & 292.8 & 4 & 600.0 & 00.0 \\
\hline 02 & 8208 & 94.0000 & 5.7250 & 294.6 & 3 & 600.0 & 00.0 \\
\hline 02 & 8209 & 110.9049 & 3.5500 & 296.6 & 2 & 600 & 0 \\
\hline 02 & 82010 & 119.8406 & 4.2000 & 296.8 & 3 & 600.0 & \\
\hline 02 & 82011 & 95.5058 & 5.3250 & 297.6 & 3 & 600.0 & \\
\hline 02 & 82012 & 82.7502 & 4.4000 & 299.4 & 3 & 600.0 & 00.0 \\
\hline 02 & 82013 & 84.2748 & 5.8250 & 299.9 & 3 & 600.0 & \\
\hline 02 & 82014 & 88.0000 & 00 & 299.7 & 4 & .0 & \\
\hline 02 & 82015 & 87.7328 & 8.0750 & 299.2 & 4 & 600.0 & .0 \\
\hline 02 & 82016 & 69.9693 & 7.5250 & 298.8 & 4 & 60 & .0 \\
\hline 02 & 82017 & 68.2483 & 7.3250 & 297.9 & 4 & .0 & \\
\hline 02 & 82018 & 65.9998 & 9.3500 & 296.1 & 4 & .0 & \\
\hline 02 & 82019 & 63.0000 & 10.2000 & 294.3 & 4 & .0 & .0 \\
\hline 02 & 82020 & 70.7478 & 9 . & 293.1 & 4 & 6 & \\
\hline 02 & 82021 & 72.9984 & 8.9250 & 293.5 & 4 & .0 & \\
\hline 02 & 82022 & 72.2500 & 9.4750 & 293.5 & 4 & 600.0 & .0 \\
\hline 02 & 82023 & 71.2500 & 9.35 & 293.3 & 4 & 6 & \\
\hline 02 & 82024 & 69.4995 & 9. & 292.8 & 4 & .0 & \\
\hline 02 & 8211 & 76.2502 & 8.8000 & 292.6 & 4 & .0 & \\
\hline 02 & 8212 & 79.0000 & 8.4750 & 292.7 & 4 & .0 & .0 \\
\hline 02 & 8213 & 80.0002 & 8.4250 & 292.3 & 4 & .0 & \\
\hline 02 & 8214 & 82.7499 & 7.2500 & 292.1 & 4 & .0 & .0 \\
\hline 02 & 8215 & 92.2515 & 6.1750 & 291.8 & 4 & 60 & .0 \\
\hline 02 & 8216 & 88.4945 & 6.9500 & 291.4 & 4 & .0 & \\
\hline 02 & 8217 & 105.4973 & 5.2250 & 292.7 & 4 & .0 & 600.0 \\
\hline 02 & 8218 & 152.2511 & 5.7250 & 293.3 & 3 & 600.0 & 600.0 \\
\hline 02 & 8219 & 151.9899 & 5.4750 & 293.7 & 3 & 0 & 0.0 \\
\hline 02 & 82110 & 168.5006 & 5.3250 & 294.7 & 3 & 600.0 & 600.0 \\
\hline 02 & 82111 & 170.4985 & 5.3500 & 295.7 & 3 & 600.0 & 600.0 \\
\hline 02 & 82112 & 178.0003 & 5.02 & 296.9 & 3 & 600.0 & 600.0 \\
\hline 02 & 82113 & 176.0032 & 4.4750 & 298.5 & 3 & 600.0 & 600.0 \\
\hline 02 & 82114 & 176.0024 & 5.0250 & 299.5 & 3 & 600.0 & 600.0 \\
\hline 02 & 82115 & 160.2125 & 6.0000 & 299.8 & 4 & 600.0 & 600.0 \\
\hline 02 & 82116 & 103.4062 & 4.4750 & 300.5 & 3 & 600.0 & 600.0 \\
\hline 02 & 82117 & 90.0004 & 6.0000 & 299.2 & 4 & 600.0 & 600.0 \\
\hline 02 & 82118 & 83.7508 & 7.7500 & 296.9 & 4 & 600.0 & 600.0 \\
\hline 02 & 82119 & 77.7502 & 8.4250 & 295.2 & 4 & 600.0 & 600.0 \\
\hline 02 & 82120 & 76.4995 & 10.2250 & 293.1 & 4 & 600.0 & 600.0 \\
\hline 02 & 82121 & 70.9999 & 11.3750 & 293.2 & 4 & 600.0 & 600.0 \\
\hline 02 & 82122 & 70.7500 & 11.5000 & 293.5 & 4 & 600.0 & 600.0 \\
\hline 02 & 82123 & 71.5000 & 10.5500 & 293.9 & 4 & 600.0 & 600.0 \\
\hline
\end{tabular}




\begin{tabular}{|c|c|c|c|c|c|c|c|}
\hline 2 & 82124 & 75.5000 & 9.8750 & 294.1 & 4 & 600.0 & 600.0 \\
\hline 02 & 8221 & 75.7500 & 10.4500 & 293.8 & 4 & 600.0 & 600.0 \\
\hline 02 & 8222 & 72.4994 & 500 & 293.5 & 4 & 600.0 & 00.0 \\
\hline 02 & 8223 & 68.7501 & 9.8000 & 292.7 & 4 & 600.0 & 0 \\
\hline 02 & 8224 & 67.9993 & 7.2250 & 291.7 & 4 & 600.0 & 00.0 \\
\hline 02 & 8225 & 73.7499 & 8.9250 & 290.2 & 4 & 600.0 & 00.0 \\
\hline 02 & 8226 & 77.0000 & 10.6750 & 289.0 & 4 & 600.0 & 0 \\
\hline 02 & 8227 & 76.0001 & 10.8000 & 289.0 & 4 & 600.0 & 00.0 \\
\hline 02 & 8228 & 86.0019 & 6.8750 & 290.5 & 4 & 600.0 & 00.0 \\
\hline 02 & 8229 & 106.6253 & 3.0750 & 291.9 & 3 & .0 & \\
\hline 02 & 82210 & 263.0455 & 1.8250 & 294.2 & - & 600.0 & \\
\hline 02 & 82211 & 237.4983 & 2.4000 & 295.2 & 1 & .0 & \\
\hline 02 & 82212 & 287.1443 & 2.5250 & 296.8 & 1 & 600.0 & \\
\hline 02 & 82213 & 65.3311 & 3.9500 & 297.2 & 2 & 600.0 & \\
\hline 02 & 82214 & 95.4626 & 5.1500 & 297.0 & 3 & .0 & \\
\hline 02 & 82215 & 136.3679 & 5.6000 & 297.2 & 3 & 600.0 & \\
\hline 02 & 82216 & 136.8681 & 7.5250 & 296.4 & 4 & 60 & .0 \\
\hline 02 & 82217 & 147.4698 & 9.0750 & 294.8 & 4 & .0 & \\
\hline 02 & 82218 & 118.8699 & 9.9500 & 292.7 & 4 & .0 & \\
\hline 02 & 82219 & 145.7082 & 10.4750 & 291.0 & 4 & .0 & .0 \\
\hline 02 & 82220 & 176.2658 & 11.5500 & 289.2 & 4 & 0 & \\
\hline 02 & 82221 & 117.3598 & 12.0250 & 288.6 & 4 & .0 & \\
\hline 02 & 82222 & 79.9989 & 12.3500 & 287.1 & 4 & 600.0 & .0 \\
\hline 02 & 82223 & 76.9999 & 12.70 & 286.9 & 4 & 0 & \\
\hline 02 & 82224 & 76.7518 & 12.8000 & 286.7 & 4 & 0 & \\
\hline 02 & 8231 & 73.5000 & 11.6750 & 286.6 & 4 & .0 & \\
\hline 02 & 8232 & 77.9999 & 11.2000 & 286.2 & 4 & 60 & \\
\hline 02 & 8233 & 80.4998 & 12.0500 & 285.8 & 4 & .0 & \\
\hline 02 & 8234 & 80.2501 & 12.7250 & 285.3 & 4 & .0 & .0 \\
\hline 02 & 8235 & 79.5000 & 12.3500 & 285.6 & 4 & .0 & \\
\hline 02 & 8236 & 80.5000 & 11.7500 & 285.1 & 4 & .0 & \\
\hline 02 & 8237 & 79.2500 & 11.1000 & 284.8 & 4 & .0 & .0 \\
\hline 02 & 8238 & 78.2498 & 10.4000 & 285.3 & 4 & 0 & .0 \\
\hline 02 & 8239 & 87.7468 & 8.4250 & 286.8 & 4 & .0 & .0 \\
\hline 02 & 82310 & 89.2499 & 6.9750 & 288.3 & 4 & 600.0 & 0.0 \\
\hline 02 & 82311 & 93.2676 & 4.8250 & 290.7 & 3 & 600.0 & 600.0 \\
\hline 02 & 82312 & 144.1363 & 4.65 & 292.6 & 3 & 600.0 & .0 \\
\hline 02 & 82313 & 148.7527 & 5.4750 & 293.4 & 3 & 600.0 & 600.0 \\
\hline 02 & 82314 & 156.2180 & 7.2500 & 293.9 & 4 & 600.0 & 600.0 \\
\hline 02 & 82315 & 141.2870 & 6.7750 & 294.5 & 4 & 600.0 & .0 \\
\hline 02 & 82316 & 99.2363 & 6.4250 & 295.3 & 4 & 600.0 & 0.0 \\
\hline 02 & 82317 & 97.2475 & 5.9250 & 295.5 & 3 & 600.0 & 600.0 \\
\hline 02 & 82318 & 76.9986 & 7.4250 & 294.5 & 4 & 600.0 & 600.0 \\
\hline 02 & 82319 & 73.0000 & 8.3250 & 293.3 & 4 & 600.0 & 600.0 \\
\hline 02 & 82320 & 74.7499 & 8.9000 & 292.5 & 4 & 600.0 & 600.0 \\
\hline 02 & 82321 & 76.0000 & 8.3000 & 292.9 & . & 600.0 & 600.0 \\
\hline 02 & 82322 & 81.5000 & 7.80 & 293.5 & 4 & 600.0 & 600.0 \\
\hline 02 & 82323 & 77.5000 & 9.075 & 292.7 & $\Psi$ & 600.0 & 600.0 \\
\hline
\end{tabular}




\begin{tabular}{|c|c|c|c|c|c|c|c|}
\hline 02 & 82324 & 80.9999 & 9.2250 & 292.8 & 4 & 600.0 & 600.0 \\
\hline 02 & 8241 & 80.7500 & 9.1750 & 292.4 & 4 & 600.0 & 600.0 \\
\hline 02 & 8242 & 79.7497 & 6.7750 & 292.9 & 4 & 600.0 & 00.0 \\
\hline 02 & 8243 & 84.5024 & 5.4250 & 293.7 & 4 & 600.0 & 600.0 \\
\hline 02 & 8244 & 79.2501 & 7.8000 & 292.8 & 4 & 600.0 & 00.0 \\
\hline 02 & 8245 & 81.0000 & 8.7000 & 292.8 & 4 & 600.0 & 600.0 \\
\hline 02 & 8246 & 82.4969 & 8.2750 & 292.8 & 4 & 600.0 & 600.0 \\
\hline 02 & 8247 & 103.5133 & 2.7500 & 292.0 & 3 & 600.0 & 600.0 \\
\hline 02 & 8248 & 94.4625 & 3.0000 & 290.8 & 2 & 600.0 & 00.0 \\
\hline 02 & 8249 & 186.9516 & 1.3250 & 293.2 & 1 & 600.0 & 600.0 \\
\hline 02 & 82410 & 309.8110 & 1.8000 & 294.5 & 1 & 600.0 & 600.0 \\
\hline 02 & 82411 & 329.6655 & 1.9500 & 296.2 & 1 & 600.0 & 00.0 \\
\hline 02 & 82412 & 253.0623 & 2.0750 & 297.5 & 1 & 600.0 & 600.0 \\
\hline 02 & 82413 & 89.4180 & 3.0000 & 299.3 & 2 & 600.0 & 600.0 \\
\hline 02 & 82414 & 82.1012 & 3.5750 & 300.3 & 2 & 600.0 & \\
\hline 02 & 82415 & 85.5284 & 000 & 300.0 & 3 & 600.0 & 60 \\
\hline 02 & 82416 & 99.2537 & 4.7500 & 300.2 & 3 & 600.0 & 600.0 \\
\hline 02 & 82417 & 85.7501 & 6.8000 & 299.0 & 4 & 600.0 & \\
\hline 02 & 82418 & 90.2498 & 6.4750 & 297.8 & 4 & 600.0 & 60 \\
\hline 02 & 82419 & 92.7446 & 5.4250 & 296.0 & 4 & 600.0 & 600.0 \\
\hline 02 & 82420 & 89.0049 & 5.4750 & 294.9 & 4 & 600.0 & .0 \\
\hline 02 & 82421 & 70.5000 & 000 & 294.9 & 4 & 600.0 & \\
\hline 02 & 82422 & 69.7505 & 8.6250 & 294.8 & 4 & 600.0 & 600.0 \\
\hline 02 & 82423 & 70.0000 & 8.8500 & 295.0 & 4 & 600.0 & .0 \\
\hline 02 & 82424 & 63.5000 & 9.4500 & 294.8 & 4 & 600.0 & \\
\hline 02 & 8251 & 67.9947 & 8.9000 & 294.7 & 4 & 600.0 & 600.0 \\
\hline 02 & 8252 & 77.5056 & 7.8000 & 294.3 & 4 & 600.0 & 600.0 \\
\hline 02 & 8253 & 75.4995 & 8.5000 & 293.9 & 4 & 600.0 & \\
\hline 02 & 8254 & 72.2501 & 8.0000 & 293.8 & 4 & 600.0 & 600.0 \\
\hline 02 & 8255 & 75.7497 & 8.0500 & 293.8 & 4 & 600.0 & 600.0 \\
\hline 02 & 8256 & 75.5000 & 7 . & 293.7 & 4 & 600.0 & .0 \\
\hline 02 & 8257 & 85.2505 & 7.6250 & 293.9 & 4 & 600.0 & 60 \\
\hline 02 & 8258 & 96.7405 & 4.8750 & 295.2 & 3 & 600.0 & 600.0 \\
\hline 02 & 8259 & 246.7613 & 1.8500 & 295.6 & 2 & 600.0 & .0 \\
\hline 02 & 82510 & 199.4408 & 2.9500 & 296.8 & 1 & 600.0 & 600.0 \\
\hline 02 & 82511 & 167.9989 & 6.0500 & 297.0 & 2 & 600.0 & 600.0 \\
\hline 02 & 82512 & 163.7513 & 6.4500 & 298.0 & 3 & 600.0 & .0 \\
\hline 02 & 82513 & 171.4997 & 7.7750 & 298.8 & 4 & 600.0 & 600.0 \\
\hline 02 & 82514 & 175.0000 & 8.0000 & 300.1 & 4 & 600.0 & 600.0 \\
\hline 02 & 82515 & 161.2508 & 7.5000 & 301.0 & 4 & 600.0 & \\
\hline 02 & 82516 & 138.5780 & 5.9750 & 301.2 & 3 & 600.0 & 600.0 \\
\hline 02 & 82517 & 92.5014 & 6.4000 & 300.8 & 4 & 600.0 & 600.0 \\
\hline 02 & 82518 & 80.5003 & 6.8000 & 299.5 & 4 & 600.0 & 600.0 \\
\hline 02 & 82519 & 75.7499 & 7.4500 & 297.8 & 4 & 600.0 & 600.0 \\
\hline 02 & 82520 & 76.7500 & 9.2250 & 296.9 & 4 & 600.0 & 600.0 \\
\hline 02 & 82521 & 73.9993 & 8.8250 & 297.1 & 4 & 600.0 & 600.0 \\
\hline 02 & 82522 & 72.2500 & 7500 & 297.3 & 4 & 600.0 & 600.0 \\
\hline 02 & 82523 & 74.5000 & 8.4000 & 298.1 & 4 & 600.0 & 600.0 \\
\hline
\end{tabular}




\begin{tabular}{|c|c|c|c|c|c|c|c|}
\hline 2 & 82524 & 79.0000 & 8.3000 & 298.3 & 4 & 600.0 & 600.0 \\
\hline 2 & 8261 & 80.5000 & 8.0250 & 298.2 & 4 & 600.0 & 600.0 \\
\hline 02 & 8262 & 5000 & 8.2500 & 297.6 & 4 & 600.0 & 00.0 \\
\hline 02 & 8263 & 82.7500 & 8.6000 & 297.2 & 4 & 600.0 & 00.0 \\
\hline 02 & 8264 & 83.2504 & 8.6000 & 296.3 & 4 & 600.0 & 00.0 \\
\hline 02 & 8265 & 90.7468 & 6.8000 & 296.1 & 4 & 600.0 & 00.0 \\
\hline 02 & 8266 & 112.5237 & 5.1500 & 295.6 & 4 & 600.0 & 0 \\
\hline 02 & 8267 & 139.2912 & 5.3000 & 295.6 & 4 & 600.0 & 00.0 \\
\hline 02 & 8268 & 149.5048 & 6.9250 & 295.7 & 4 & 600.0 & 00.0 \\
\hline 02 & 8269 & 152.7497 & 7.5000 & 295.8 & 4 & 60 & 0 \\
\hline 02 & 82610 & 159.2501 & 5.4750 & 297.5 & 3 & 600.0 & \\
\hline 02 & 82611 & 163.2455 & 5.2250 & 299.2 & 3 & 600.0 & \\
\hline 02 & 82612 & 160.2505 & 6.6000 & 300.1 & 4 & 600.0 & 00.0 \\
\hline 02 & 82613 & 166.4979 & 8.2250 & 301.1 & 4 & 600.0 & \\
\hline 02 & 82614 & 171.2501 & 00 & 302.5 & 4 & .0 & \\
\hline 02 & 82615 & 167.5000 & 6.6000 & 303.8 & 4 & 600.0 & 00.0 \\
\hline 02 & 82616 & 132.0042 & 6.4750 & 304.7 & 4 & .0 & .0 \\
\hline 02 & 82617 & 124.9968 & 6.5500 & 304.7 & 4 & .0 & \\
\hline 02 & 82618 & 93.2484 & 6.0500 & 303.8 & 4 & 60 & \\
\hline 02 & 82619 & 76.5000 & 6.6000 & 302.0 & 4 & .0 & .0 \\
\hline 02 & 82620 & 68.4998 & 7 . & 300.6 & 4 & 6 & \\
\hline 02 & 82621 & 67.9959 & 7.1750 & 300.6 & 4 & .0 & \\
\hline 02 & 82622 & 79.7498 & 8.0750 & 300.9 & 4 & 600.0 & .0 \\
\hline 02 & 82623 & 81.2491 & 7.2500 & 301.0 & 4 & 6 & \\
\hline 02 & 82624 & 78.7485 & 7.4500 & 300.8 & 4 & 0 & \\
\hline 02 & 8271 & 91.2496 & 7.3750 & 301.1 & 4 & .0 & \\
\hline 02 & 8272 & 111.2518 & 8.0500 & 300.9 & 4 & 60 & .0 \\
\hline 02 & 8273 & 135.0000 & 10.3000 & 300.6 & 4 & .0 & \\
\hline 02 & 8274 & 153.2503 & 500 & 299.8 & 4 & .0 & .0 \\
\hline 02 & 8275 & 161.2498 & 10.1500 & 298.8 & 4 & 6 & .0 \\
\hline 02 & 8276 & 160.0001 & 8.6500 & 298.2 & 4 & .0 & \\
\hline 02 & 8277 & 155.7488 & 8 . & 298.4 & 4 & .0 & 0.0 \\
\hline 02 & 8278 & 162.7500 & 10.2750 & 298.8 & 4 & 600.0 & 0.0 \\
\hline 02 & 8279 & 168.0004 & 12.7250 & 299.1 & 4 & .0 & 0.0 \\
\hline 02 & 82710 & 165.2503 & 10.6750 & 299.7 & 4 & 600.0 & 0.0 \\
\hline 02 & 82711 & 165.2480 & 8.7250 & 300.3 & 4 & 600.0 & 600.0 \\
\hline 02 & 82712 & 166.0008 & 8.8000 & 301.2 & 4 & 600.0 & 0.0 \\
\hline 02 & 82713 & 163.2526 & 7.2750 & 302.4 & 4 & 600.0 & 600.0 \\
\hline 02 & 82714 & 157.7478 & 6.3250 & 303.6 & 4 & 600.0 & 600.0 \\
\hline 02 & 82715 & 173.7506 & 000 & 304.6 & 3 & 600.0 & 600.0 \\
\hline 02 & 82716 & 177.9908 & 6.8250 & 305.1 & 4 & 600.0 & 600.0 \\
\hline 02 & 82717 & 184.7463 & 6.1250 & 305.2 & 4 & 600.0 & 600.0 \\
\hline 02 & 82718 & 195.5037 & 3.8500 & 305.0 & 3 & 600.0 & 600.0 \\
\hline 02 & 82719 & 149.9600 & 2.3250 & 304.2 & 4 & 600.0 & 600.0 \\
\hline 02 & 82720 & 87.4997 & 2.7750 & 303.1 & 4 & 600.0 & 600.0 \\
\hline 02 & 82721 & 81.4959 & 3.9500 & 302.6 & 4 & 600.0 & 600.0 \\
\hline 02 & 82722 & 77.4999 & 7.425 & 301.2 & 4 & 600.0 & 600.0 \\
\hline $0<$ & 82723 & 72.7500 & 7.7000 & 301.2 & 4 & 600.0 & 600.0 \\
\hline
\end{tabular}




\begin{tabular}{|c|c|c|c|c|c|c|c|}
\hline 2 & 82724 & 72.2500 & 8.1250 & 301.7 & 4 & 600.0 & 00.0 \\
\hline 02 & 8281 & 74.2499 & 8.0000 & 302.3 & 4 & 600.0 & 600.0 \\
\hline 02 & 8282 & 76.4990 & 7.2000 & 302.3 & 4 & 600.0 & 00.0 \\
\hline 02 & 8283 & 80.9973 & 6.3750 & 302.3 & 4 & 600.0 & 600.0 \\
\hline 02 & 8284 & 74.4957 & 6.4250 & 302.1 & 4 & 600.0 & 00.0 \\
\hline 02 & 8285 & 128.4386 & 3.1000 & 300.1 & 4 & 600.0 & 00.0 \\
\hline 02 & 8286 & 92.2503 & 5.8750 & 301.2 & 4 & 600.0 & 600.0 \\
\hline 02 & 8287 & 93.2483 & 5.6000 & 301.0 & 4 & 600.0 & 00.0 \\
\hline 02 & 8288 & 127.0139 & 5.8500 & 301.3 & 3 & 600.0 & 00.0 \\
\hline 02 & 8289 & 136.2515 & 6.2500 & 301.4 & 4 & 600.0 & .0 \\
\hline 02 & 82810 & 144.2512 & 7.1250 & 301.7 & 4 & 600.0 & 00.0 \\
\hline 02 & 82811 & 151.5024 & 6.5500 & 302.7 & 4 & 600.0 & \\
\hline 02 & 82812 & 120.1805 & 5.6750 & 303.7 & 3 & 600.0 & 00.0 \\
\hline 02 & 82813 & 108.5097 & 6.5750 & 304.1 & 4 & 600.0 & 0 \\
\hline 02 & 82814 & 92.2522 & 7.1000 & 304.6 & 4 & 600.0 & \\
\hline 02 & 82815 & 97.5000 & 7.5250 & 304.4 & 4 & 600.0 & 60 \\
\hline 02 & 82816 & 90.7494 & 7.9500 & 303.5 & 4 & 600.0 & 600.0 \\
\hline 02 & 82817 & 76.2498 & 8.5000 & 302.1 & 4 & 600.0 & \\
\hline 02 & 82818 & 71.5000 & 9.2750 & 300.4 & 4 & 600.0 & .0 \\
\hline 02 & 82819 & 72.0000 & 9.4500 & 298.8 & 4 & 600.0 & 600 \\
\hline 02 & 82820 & 76.5000 & 10.17 & 297.2 & 4 & 600.0 & .0 \\
\hline 02 & 82821 & 74.2500 & 10.3750 & 297.0 & 4 & 600.0 & \\
\hline 02 & 82822 & 72.9996 & 7.1250 & 299.1 & 4 & 600.0 & 600.0 \\
\hline 02 & 82823 & 73.2504 & 7.37 & 298.7 & 4 & 600.0 & .0 \\
\hline 02 & 82824 & 123.4625 & 2.2750 & 297.1 & 5 & 600.0 & \\
\hline 02 & 8291 & 103.7816 & 0.9500 & 292.8 & 6 & 600.0 & 60 \\
\hline 02 & 8292 & 62.6826 & 1.0000 & 290.4 & 6 & 600.0 & .0 \\
\hline 02 & 8293 & 20.7655 & 0.9750 & 290.3 & 6 & 600.0 & \\
\hline 02 & 8294 & 131.7371 & 1.2750 & 291.1 & 6 & 600.0 & 60 \\
\hline 02 & 8295 & 31.1879 & 0.8250 & 290.9 & 6 & 600.0 & .0 \\
\hline 02 & 8296 & 56.3347 & 1.00 & 291.9 & 6 & 600.0 & \\
\hline 02 & 8297 & 36.0311 & 1.1250 & 289.6 & 5 & 600.0 & 60 \\
\hline 02 & 8298 & 142.7492 & 2.0500 & 290.9 & 4 & 600.0 & 600.0 \\
\hline 02 & 8299 & 282.2019 & 1.50 & 293.7 & 3 & 600.0 & .0 \\
\hline 02 & 82910 & 241.2512 & 2.4000 & 294.0 & 2 & 600.0 & 60 \\
\hline 02 & 82911 & 237.5000 & 2.5750 & 295.3 & 1 & 600.0 & 600.0 \\
\hline 02 & 82912 & 238.7418 & 1.9250 & 297.8 & 1 & 600.0 & \\
\hline 02 & 82913 & 179.1973 & 3.5000 & 299.4 & 2 & 600.0 & .0 \\
\hline 02 & 82914 & 167.6126 & 5.8500 & 300.1 & 3 & 600.0 & 600.0 \\
\hline 02 & 82915 & 108.7514 & 4.775 & 300.7 & 3 & 600.0 & \\
\hline 02 & 82916 & 94.7492 & 4.6500 & 300.2 & 3 & 600.0 & 600.0 \\
\hline 02 & 82917 & 82.7509 & 4.9750 & 300.2 & 3 & 600.0 & 600.0 \\
\hline 02 & 82918 & 77.5000 & 6.1250 & 298.9 & 4 & 600.0 & 600.0 \\
\hline 02 & 82919 & 75.7505 & 7.7250 & 295.9 & 4 & 600.0 & 600.0 \\
\hline 02 & 82920 & 53.0011 & 5.0250 & 295.6 & 4 & 600.0 & 600.0 \\
\hline 02 & 82921 & 86.0295 & 3.1500 & 295.6 & 5 & 600.0 & 600.0 \\
\hline 02 & 82922 & 128.3008 & 1.225 & & 6 & 600.0 & 600.0 \\
\hline 02 & 82923 & 156.0942 & 0.8500 & 295.1 & 0 & 600.0 & 600.0 \\
\hline
\end{tabular}




\begin{tabular}{|c|c|c|c|c|c|c|c|}
\hline 2 & 2924 & & 6000 & 4.4 & & - & \\
\hline & 301 & 1 & .5000 & 93.9 & 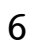 & 600.0 & \\
\hline & 8302 & 356.0208 & 2750 & 294.0 & 6 & 00 & \\
\hline & 8303 & 333.6905 & 3500 & 292.2 & & 00 & \\
\hline & 304 & 50.31 & .4000 & 91.8 & & 0 & \\
\hline & 8305 & 194.5751 & 3250 & 291.6 & & 00 & \\
\hline & 8306 & 17.2534 & 1.8250 & 293.0 & & 00 & \\
\hline & 307 & 27.9257 & 5500 & 94.8 & & & \\
\hline & 8308 & 329.8378 & 6750 & 95.0 & 3 & & \\
\hline & 309 & 292.5092 & 1.2750 & 296.1 & & 00 & 0 \\
\hline & 3010 & 242.0048 & 2.4250 & 295.4 & & & \\
\hline & 3011 & 217.2103 & 210 & 96.4 & 2 & & \\
\hline 2 & 3012 & 164.5000 & 3.07 & 298.2 & 2 & 0 & \\
\hline & 3013 & 165.7539 & 3.10 & 299.9 & & 0.0 & \\
\hline & 3014 & 174.50 & & 300.6 & 3 & & \\
\hline & 83015 & 172.00 & & 301.7 & 3 & & \\
\hline & 3016 & 171.5000 & 0 & 302.1 & 3 & .0 & . \\
\hline & 017 & 179 . & 5.20 & 2.1 & 3 & & \\
\hline & 83018 & 153. & 4 . & 01.1 & 4 & & \\
\hline & 83019 & 130.7541 & 2.9750 & 299.4 & 4 & 0 & 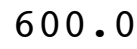 \\
\hline 2 & 83020 & 78.4977 & 4.2500 & 298.9 & 4 & 0 & \\
\hline & 83021 & 75. & & & 4 & & \\
\hline 2 & 83022 & 76.24 & 6.87 & 298.7 & 4 & & \\
\hline 2 & 83023 & 81.4991 & 5.45 & 299.7 & 4 & 0 & \\
\hline 2 & 83024 & 86.24 & & & 4 & & \\
\hline 2 & 8311 & 72.35 & 1.02 & & 5 & & \\
\hline 2 & 8312 & 92.7732 & .32 & 299.0 & 6 & 0 & J. \\
\hline 2 & 8313 & 105.15 & 0 & .6 & 6 & & \\
\hline 2 & 8314 & 188.0077 & 750 & 297.8 & 5 & & $\bullet$ \\
\hline 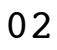 & 8315 & 205.7055 & 0.05 & 297.2 & 6 & 0 & 0. \\
\hline 2 & 8316 & 23.24 & 1.25 & 297.0 & 5 & & 0 . \\
\hline 2 & 8317 & 15 . & 0 & 298.8 & 4 & & \\
\hline 2 & 8318 & 300 . & 0 & .7 & 3 & & . \\
\hline 2 & 8319 & 224 . & 2.25 & 300.9 & 2 & 0 & 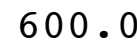 \\
\hline 2 & 83 & 164 . & 0 & & 3 & & \\
\hline 2 & & 164.7499 & 5.15 & 299.3 & 3 & & . \\
\hline 2 & 83112 & 175.4994 & 4.62 & 300.8 & 3 & & 0 . \\
\hline 2 & 83113 & 195.4750 & 3 . & 302.6 & 2 & & 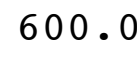 \\
\hline 02 & 83114 & 217.0091 & 2.9500 & 304.1 & 1 & .0 & 0 . \\
\hline 2 & 83115 & 196.4885 & 3500 & 304.3 & 2 & & 0 . \\
\hline 2 & & 186 . & 5 . & 304.2 & 3 & & 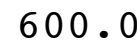 \\
\hline 02 & 83117 & 183.0011 & 3.8500 & 304.5 & 3 & & 0 . \\
\hline 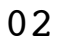 & 83118 & 180.5301 & 3.7000 & 304.3 & 3 & 0 & 0 . \\
\hline 02 & 83119 & 193.500 & 4 . & & 4 & & \\
\hline 02 & 83120 & 162.8773 & 2.4750 & 301.6 & 4 & & 0 . \\
\hline 2 & 83121 & 140.5008 & 2.4750 & 301.6 & 5 & 600 & 600. \\
\hline & & 129. & & & 5 & & \\
\hline & 83123 & 133.5000 & 2.9000 & 301.8 & 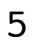 & 600.0 & 600 \\
\hline
\end{tabular}




\begin{tabular}{|c|c|c|c|c|c|c|c|c|}
\hline D & & 4 & 2 & $\$ 750$ & 01.7 & & 0 & \\
\hline & 9 & 1 & 04.7482 & 1250 & 301.6 & & 600.0 & \\
\hline & 9 & 2 & 129.7261 & 6000 & 301.5 & 4 & 00 & \\
\hline & 9 & 3 & 136.2501 & 7500 & 300.7 & & 00.0 & \\
\hline & & 4 & 112.2372 & 2750 & 99.9 & & & \\
\hline & 9 & 5 & 122.9651 & 5000 & 00.2 & & 0 & \\
\hline & a & 16 & 133.2481 & 1250 & 299.6 & & 00 & \\
\hline & $y$ & 7 & 132.1652 & 1.3500 & 300.5 & & & \\
\hline & 9 & 8 & 156.7818 & & 1.9 & & & \\
\hline & & 19 & 174.2518 & 500 & 303.3 & & 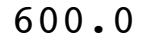 & \\
\hline & 9 & 110 & 166.0000 & 0 & 302.4 & & & \\
\hline & 9 & 111 & 185 & & 04.1 & & & \\
\hline & 9 & 112 & 5081 & 0 & 304.6 & & 0 & \\
\hline & 9 & 113 & 187.2498 & 0 & 30 & & & \\
\hline & 9 & 114 & 187 & & & & & \\
\hline & 9 & 115 & 189 & & & & & \\
\hline & 9 & 116 & 198.2470 & & 306.9 & & .0 & \\
\hline & 9 & 117 & 202.24 & & 7 & & & \\
\hline & 9 & 118 & 202.50 & & & & & \\
\hline & 9 & 119 & 160.3549 & & & 4 & & \\
\hline & 9 & 120 & 100.4424 & 0 & 304.0 & & .0 & \\
\hline & 9 & 121 & 66.2 & & & & & \\
\hline & 9 & 122 & 78.2501 & 50 & 30 & 5 & & \\
\hline & 9 & 123 & 79.74 & 0 & 30 & 4 & 0 & \\
\hline & 9 & 124 & 80.0 & & & 4 & & \\
\hline 2 & 9 & 21 & 82 . & 0 & 30 & 4 & & \\
\hline & 9 & 22 & 80.5000 & 0 & 30 & 4 & & 0 \\
\hline & 9 & 23 & 80.50 & 0 & & 4 & & \\
\hline 2 & 9 & 24 & 83. & 50 & & 5 & & \\
\hline & 9 & 25 & 81.5000 & 2000 & 5 & 4 & & J. \\
\hline 2 & 9 & 26 & 84.7500 & 3250 & .6 & 4 & & 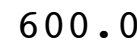 \\
\hline 2 & 9 & 27 & 93. & 0 & & 4 & & \\
\hline & 9 & 28 & 52.5323 & & 5.2 & 2 & & . \\
\hline 2 & 9 & 29 & 250.3423 & 750 & 307.2 & 2 & & \\
\hline & 9 & 210 & 188.60 & & & 2 & & \\
\hline 2 & 9 & 211 & 191.7509 & & 305.5 & 3 & & \\
\hline & 9 & 212 & 190.2506 & 2500 & 305.5 & 3 & & \\
\hline 2 & 9 & 213 & 185. & 4. & & 3 & & \\
\hline 2 & 9 & 214 & 193.7533 & & 307.1 & 3 & & 0 . \\
\hline & 9 & 215 & 191.0000 & 750 & 307.8 & 3 & & \\
\hline 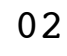 & 9 & 216 & 182.2577 & 50 & 308.2 & 3 & & \\
\hline 0 & 9 & 217 & 127.2358 & 500 & 308.1 & 3 & & 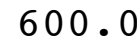 \\
\hline & 9 & 218 & 93.7931 & 6.3000 & 305.9 & 4 & & 0 . \\
\hline 2 & 9 & 219 & 71.2498 & 250 & & 4 & & \\
\hline 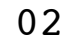 & 9 & 220 & 75.0000 & .3750 & 302.1 & 4 & & \\
\hline 20 & 9 & 221 & 70.7497 & 0.2500 & 301.2 & 4 & & 00 . \\
\hline & 9 & 222 & 74 & & & 4 & & \\
\hline & & 223 & 77.0001 & 8.3750 & 300.8 & $I$ & 600.0 & 60 \\
\hline
\end{tabular}




\begin{tabular}{|c|c|c|c|c|c|c|c|c|}
\hline 2 & 9 & 224 & 76.4998 & 7.5500 & 300.8 & 4 & 600.0 & 00.0 \\
\hline 2 & 9 & 31 & 56.4763 & 4.5000 & 302.1 & 4 & 600.0 & 600.0 \\
\hline 02 & 9 & 32 & 153.7850 & 1.1750 & 302.2 & 5 & 600.0 & 00.0 \\
\hline 02 & 9 & 33 & 139.4997 & 1.4500 & 300.8 & 5 & 600.0 & 00.0 \\
\hline 02 & 9 & 34 & 148.7508 & 1.4500 & 299.7 & & 600.0 & 00.0 \\
\hline 02 & 9 & 35 & 197.4244 & 1.4250 & 297.2 & 6 & 600.0 & 00.0 \\
\hline 02 & 9 & 36 & 184.9855 & 0.2750 & 295.9 & $\Gamma$ & 600.0 & 600.0 \\
\hline 02 & 9 & 37 & 131.5931 & 1.3250 & 298.0 & 6 & 600.0 & 00.0 \\
\hline 02 & 9 & 38 & 274.2856 & 0.2750 & 301.7 & 3 & 600.0 & \\
\hline 02 & 9 & 39 & 256.1935 & 1.0000 & 302.6 & 4 & 600.0 & 600.0 \\
\hline 02 & 9 & 310 & 238.5000 & 2.0000 & 302.8 & 3 & 600.0 & 0 \\
\hline 02 & 9 & 311 & 185.4773 & 3.6000 & 302.6 & 2 & 600.0 & \\
\hline 02 & 9 & 312 & 190.7537 & 4.1000 & 303.4 & 3 & 600.0 & .0 \\
\hline 02 & 9 & 313 & 177.7262 & 3.7000 & 304.2 & 2 & 600.0 & 0 \\
\hline 02 & 9 & 314 & 177.2555 & 5 . & 305.2 & 3 & 600.0 & .0 \\
\hline 02 & 9 & 315 & 117.6386 & 4. & 306.3 & 3 & 600.0 & \\
\hline 02 & 9 & 316 & 137.5070 & 5.6250 & 306.1 & 3 & 600.0 & 600.0 \\
\hline 02 & 9 & 317 & 85.2323 & 5.6750 & 305.3 & 3 & 600.0 & .0 \\
\hline 02 & 9 & 318 & 80.5064 & 6.9500 & 303.1 & 4 & 600.0 & \\
\hline 02 & 9 & 319 & 75.0012 & 8.5250 & 300.6 & 4 & 600.0 & 600.0 \\
\hline 02 & 9 & 320 & 77.5020 & 8.2500 & 298.8 & 4 & 600.0 & .0 \\
\hline 02 & 9 & 321 & 89.9999 & 8.1750 & 297.4 & 4 & 600.0 & \\
\hline 02 & 9 & 322 & 104.5031 & 4.3500 & 297.7 & 4 & 600.0 & 60 \\
\hline 02 & 9 & 323 & 130.0078 & 4.9250 & 297.0 & 4 & 600.0 & .0 \\
\hline 02 & 9 & 324 & 139.0000 & $4.2 .3 \cdot 2 \cdot 3$ & 296.6 & 4 & 600.0 & \\
\hline 02 & 9 & 41 & 140.2517 & 000 & 296.3 & 4 & 600.0 & 60 \\
\hline 02 & 9 & 42 & 107.2600 & 2.9250 & 296.5 & 4 & 600.0 & .0 \\
\hline 02 & 9 & 43 & 71.0001 & 5.27 & 296.3 & 4 & 600.0 & .0 \\
\hline 02 & 9 & 44 & 68.5000 & 7.2750 & 296.0 & 4 & 600.0 & 60 \\
\hline 02 & 9 & 45 & 65.7500 & 10.4500 & 296.0 & 4 & 600.0 & .0 \\
\hline 02 & 9 & 46 & 66.7500 & 11.0000 & 296.5 & 4 & 600.0 & 60 \\
\hline 02 & 9 & 47 & 53.4986 & 8 . & 296.9 & 4 & 600.0 & 60 \\
\hline 02 & 9 & 48 & 55.7701 & 000 & 298.0 & 3 & 600.0 & 60 \\
\hline 02 & 9 & 49 & 87.3775 & 2.7000 & 299.4 & 2 & 600.0 & .0 \\
\hline 02 & 9 & 410 & 19.7173 & 1 . & 299.6 & 1 & 600.0 & \\
\hline 02 & 9 & 411 & 257.9097 & 1.8000 & 299.8 & 1 & 600.0 & 600.0 \\
\hline 02 & 9 & 412 & 86.5014 & 4.7750 & 299.0 & 2 & 600.0 & 600.0 \\
\hline 02 & 9 & 413 & 85.0032 & 4.1250 & 301.4 & 3 & 600.0 & 60 \\
\hline 02 & 9 & 414 & 65.9982 & 5.4750 & 301.5 & 3 & 600.0 & 600.0 \\
\hline 02 & 9 & 415 & 83.4999 & 7.4750 & 300.1 & 4 & 600.0 & 600.0 \\
\hline 02 & 9 & 416 & 76.5161 & 7.7000 & 299.3 & 4 & 600.0 & 600.0 \\
\hline 02 & 9 & 417 & 76.5018 & 10.0250 & 297.7 & 4 & 600.0 & 600.0 \\
\hline 02 & 9 & 418 & 74.5000 & 12.0250 & 295.7 & 4 & 600.0 & 600.0 \\
\hline 02 & 9 & 419 & 77.2500 & 12.9750 & 293.7 & 4 & 600.0 & 600.0 \\
\hline 02 & 9 & 420 & 74.2500 & 10.7250 & 292.4 & 4 & 600.0 & 600.0 \\
\hline 02 & 9 & 421 & 65.0000 & 12.8000 & 290.9 & 4 & 600.0 & 600.0 \\
\hline 02 & 9 & 422 & 61.7502 & 12.35 & 29 & 4 & 600.0 & .0 \\
\hline 02 & ( & 423 & 60.0006 & 12.6000 & 290.2 & 4 & 600.0 & 600.0 \\
\hline
\end{tabular}




\begin{tabular}{|c|c|c|c|c|c|c|c|c|}
\hline 2 & 9 & 424 & 40.9968 & 13.4000 & 291.4 & 4 & 600.0 & 00.0 \\
\hline 2 & 9 & 51 & 67.9416 & 7.6250 & 291.0 & 4 & 600.0 & 600.0 \\
\hline 02 & 9 & 52 & 96.0011 & 4.9750 & 289.2 & 4 & 600.0 & 00.0 \\
\hline 02 & 9 & 53 & 104.7500 & 5.2250 & 288.6 & 4 & 600.0 & 00.0 \\
\hline 02 & 9 & 54 & 97.5000 & 6.0000 & 288.3 & 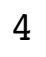 & 600.0 & 00.0 \\
\hline 02 & 9 & 55 & 102.0024 & 5.3750 & 288.3 & 4 & 600.0 & 00.0 \\
\hline 02 & 9 & 56 & 96.5006 & 5.4500 & 288.2 & 4 & 600.0 & 500.0 \\
\hline 02 & 9 & 57 & 111.2512 & 4.7750 & 288.7 & 4 & 600.0 & .0 \\
\hline 02 & 9 & 58 & 107.5009 & 5.4750 & 289.8 & 3 & 600.0 & \\
\hline 02 & 9 & 59 & 96.5125 & 5.2500 & 290.8 & 3 & 600.0 & 600.0 \\
\hline 02 & 9 & 510 & 84.3275 & 5.7500 & 292.0 & 3 & 600.0 & 0 \\
\hline 02 & 9 & 511 & 95.0000 & 7.5250 & 292.6 & 4 & 600.0 & \\
\hline 02 & 9 & 512 & 103.7442 & 7.6250 & 294.0 & 4 & 600.0 & .0 \\
\hline 02 & 9 & 513 & 89.2470 & 6.1750 & 295.8 & 4 & 600.0 & \\
\hline 02 & 9 & 514 & 72.4969 & 7.2750 & 296.3 & 4 & 600.0 & .0 \\
\hline 02 & 9 & 515 & 71.5001 & 8.10 & 295.7 & 4 & 600.0 & \\
\hline 02 & 9 & 516 & 69.7499 & 7.1250 & 296.0 & 4 & 600.0 & 600.0 \\
\hline 02 & 9 & 517 & 70.4991 & 7.4500 & 295.2 & 4 & 600.0 & .0 \\
\hline 02 & 9 & 518 & 73.0000 & 8.5000 & 293.4 & 4 & 600.0 & \\
\hline 02 & 9 & 519 & 72.5000 & 9.2250 & 291.3 & 4 & 600.0 & \\
\hline 02 & 9 & 520 & 76.0000 & 8.8500 & 290.4 & 4 & 600.0 & .0 \\
\hline 02 & 9 & 521 & 75.5023 & 8.1750 & 290.3 & 4 & 600.0 & \\
\hline 02 & 9 & 522 & 68.7499 & 7.1000 & 289.8 & 4 & 600.0 & 60 \\
\hline 02 & 9 & 523 & 75.7494 & 6.9250 & 289.0 & 4 & 600.0 & .0 \\
\hline 02 & 9 & 524 & 62.7500 & 9.0500 & 289.5 & 4 & 600.0 & \\
\hline 02 & 9 & 61 & 90.7381 & 6.6500 & 288.8 & 4 & 600.0 & 60 \\
\hline 02 & 9 & 62 & 69.2448 & 9.1000 & 287.9 & 4 & 600.0 & .0 \\
\hline 02 & 9 & 63 & 74.7485 & 5.80 & 287.7 & 4 & 600.0 & .0 \\
\hline 02 & 9 & 64 & 104.7590 & 5.3750 & 287.2 & 4 & 600.0 & 60 \\
\hline 02 & 9 & 65 & 71.2489 & 12.5750 & 286.8 & 4 & 600.0 & .0 \\
\hline 02 & 9 & 66 & 118.0389 & 11.4750 & 286.8 & 4 & 600.0 & .0 \\
\hline 02 & 9 & 67 & 118.5138 & 10.2750 & 286.9 & 4 & 600.0 & 60 \\
\hline 02 & 9 & 68 & 99.6891 & 9.4000 & 288.0 & 4 & 600.0 & 60 \\
\hline 02 & 9 & 69 & 96.2521 & 7.3750 & 289.2 & 4 & 600.0 & .0 \\
\hline 02 & 9 & 610 & 109.7620 & 6.0250 & 290.5 & 4 & 600.0 & \\
\hline 02 & 9 & 611 & 110.2707 & 6.3750 & 291.4 & 4 & 600.0 & 600.0 \\
\hline 02 & 9 & 612 & 90.7016 & 7.2000 & 292.1 & 4 & 600.0 & 600.0 \\
\hline 02 & 9 & 613 & 131.7947 & 8.4500 & 292.3 & 4 & 600.0 & 60 \\
\hline 02 & 9 & 614 & 93.4983 & 7.9500 & 293.1 & 4 & 600.0 & 600.0 \\
\hline 02 & 9 & 615 & 105.4986 & 8.1750 & 293.0 & 4 & 600.0 & 600.0 \\
\hline 02 & 9 & 616 & 126.5014 & 8.2250 & 292.3 & 4 & 600.0 & 600.0 \\
\hline 02 & 9 & 617 & 165.0103 & 8.3750 & 291.5 & 4 & 600.0 & 600.0 \\
\hline 02 & 9 & 618 & 176.9993 & 8.5000 & 290.3 & 4 & 600.0 & 600.0 \\
\hline 02 & 9 & 619 & 151.3719 & 10.6000 & 288.5 & 4 & 600.0 & 600.0 \\
\hline 02 & 9 & 620 & 71.8974 & 11.4000 & 287.2 & 4 & 600.0 & 600.0 \\
\hline 02 & 9 & 621 & 129.9881 & 11.2500 & 286.8 & 4 & 600.0 & 600.0 \\
\hline 02 & 9 & 622 & 88.5814 & 11.2500 & 28 & 4 & 600.0 & .0 \\
\hline 02 & ( & 623 & 150.1499 & 11.6000 & 286.5 & 4 & 600.0 & 600.0 \\
\hline
\end{tabular}




\begin{tabular}{|c|c|c|c|c|c|c|c|c|}
\hline & 9 & 24 & 1.2508 & .1500 & 86.8 & 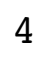 & 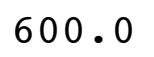 & \\
\hline 2 & 9 & 1 & 7.0002 & 8.2750 & 86.6 & & 00,0 & \\
\hline & 9 & 72 & 171.0100 & 6.9750 & 286.2 & & 00 & \\
\hline & 9 & 73 & 177.9995 & 7.9500 & 286.3 & & 00 & \\
\hline & 9 & 4 & 000 & 5750 & 36.9 & & & \\
\hline & 9 & 75 & 81.7500 & 9500 & 287.1 & & 0 & \\
\hline & 9 & 76 & 3608 & 9000 & 286.4 & & 00 & \\
\hline & 9 & 7 & 97.4104 & 8000 & 86.4 & & & \\
\hline & 9 & 78 & 81 & 2000 & 8 & & & \\
\hline & 9 & 79 & 58.4397 & 5.2250 & 288.3 & & 0 & \\
\hline & 9 & 710 & 5006 & 5.3250 & 289 & & & \\
\hline & 9 & 711 & 74.75 & 6.0250 & 90.2 & & & \\
\hline & 9 & 712 & 70.7498 & 500 & 291.4 & 4 & .0 & \\
\hline 2 & 9 & 713 & 02 & 6.2500 & 29 & & & \\
\hline & 9 & 714 & 7 & & 8 & & & \\
\hline & 9 & 715 & 4 & & 295 & & & \\
\hline 2 & 9 & 716 & 48 & 000 & 295 & & .0 & \\
\hline & 9 & 717 & 1 . & 0 & 29 & & & \\
\hline 2 & 9 & 718 & 57 & & 293.4 & $x$ & & \\
\hline 2 & 9 & 719 & 9 & 50 & 29 & 4 & & \\
\hline 02 & 9 & 720 & 57.5052 & 250 & 290 & 4 & .0 & \\
\hline 2 & 9 & 721 & 6 & & & $\mathbf{4}$ & & \\
\hline 2 & 9 & 722 & 46. & 00 & 29 & 4 & & \\
\hline 2 & 9 & 723 & 76 . & 250 & 29 & 4 & . 0 & \\
\hline & 9 & 724 & 70 & & 0 & 4 & & \\
\hline 02 & 9 & 81 & 76 . & & 29 & 4 & & \\
\hline 02 & 9 & 82 & 78 . & 500 & 291.2 & 4 & .0 & \\
\hline & 9 & 83 & 94 . & 4 . & 291.5 & 4 & & \\
\hline 02 & 9 & 84 & 22 & 0 & 291.3 & 4 & & \\
\hline . & 9 & 85 & 14. & 000 & 290 & 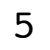 & & \\
\hline 02 & 9 & 86 & 96 & 3.3500 & 291.3 & 4 & & \\
\hline 02 & 9 & 87 & 39. & 0 & 291.5 & 5 & & \\
\hline 02 & 9 & 88 & 174 . & 500 & 291.8 & 4 & .0 & \\
\hline 02 & 9 & 89 & 174 . & 50 & 292.2 & 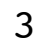 & 0 & \\
\hline 02 & 9 & 810 & 169 & & 29 & 3 & & \\
\hline 02 & 9 & 811 & 170 . & & 294.1 & 3 & & \\
\hline 0 & 9 & 812 & 170.999 & 750 & 294.9 & 3 & .0 & \\
\hline 2 & 9 & 813 & 177 . & 4 . & 296.1 & 3 & & \\
\hline 02 & 9 & 814 & 174.7851 & 250 & 297.0 & 3 & .0 & \\
\hline 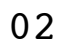 & 9 & 815 & 178.993 & 750 & 297.8 & 3 & & \\
\hline 2 & 9 & 816 & 176 . & 6 . & 298.2 & 4 & & \\
\hline 02 & 9 & 817 & 187 . & & 298.4 & 4 & .0 & 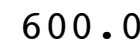 \\
\hline 02 & 9 & 818 & 192.2500 & 5.9750 & 297.8 & 4 & & 0 \\
\hline 02 & 9 & 819 & 123.8800 & 3 . & 296.6 & 4 & & \\
\hline 02 & 9 & 820 & 73.7507 & & 295.0 & 7 & & \\
\hline 02 & 9 & 821 & 74.0003 & 7.2250 & 294.3 & 4 & .0 & 500 \\
\hline 02 & 9 & 822 & & & 294.2 & 4 & & \\
\hline & r & 823 & 70.4998 & 8.4250 & 294.4 & & .0 & 600 \\
\hline
\end{tabular}




\begin{tabular}{|c|c|c|c|c|c|c|c|}
\hline 2 & 9824 & 70.9999 & 7.9000 & 294.6 & 4 & 600.0 & 600.0 \\
\hline 02 & $\begin{array}{lll}9 & 9 & 1\end{array}$ & 73.7498 & 8.1500 & 294.6 & 4 & 600.0 & 00.0 \\
\hline 02 & 992 & 70.7500 & 7.9000 & 295.0 & 4 & 600.0 & 00.0 \\
\hline 02 & $\begin{array}{lll}9 & 9 & 3\end{array}$ & 72.7502 & 7.0000 & 295.4 & 4 & 600.0 & 00.0 \\
\hline 02 & $\begin{array}{lll}9 & 9 & 4\end{array}$ & 83.2515 & .7250 & 295.8 & 5 & 600.0 & 00.0 \\
\hline 02 & 995 & 131.6593 & 2.1500 & 294.7 & 6 & 600.0 & 00.0 \\
\hline 02 & 996 & 45.5784 & 0.4500 & 293.0 & 6 & 600.0 & 00.0 \\
\hline 02 & $\begin{array}{lll}9 & 9 & 7\end{array}$ & 136.7493 & 1.8750 & 294.3 & 5 & 600.0 & 00.0 \\
\hline 02 & 998 & 152.4962 & 3.2500 & 295.9 & 4 & 600.0 & 00.0 \\
\hline 02 & $\begin{array}{lll}9 & 9 & 9\end{array}$ & 161.0097 & 4.3000 & 296.9 & 3 & 600.0 & 0 \\
\hline 02 & 9910 & 163.9988 & .6750 & 297.4 & 3 & 600.0 & \\
\hline 02 & 9911 & 168.2499 & 6.3500 & 298.0 & 4 & 600.0 & \\
\hline 02 & 9912 & 165.7500 & 7.0250 & 298.3 & 4 & 600.0 & 00.0 \\
\hline 02 & 9913 & 160.9989 & 5.3500 & 299.5 & 3 & 600.0 & 00.0 \\
\hline 02 & 9914 & 167.2467 & 00 & 300.8 & 3 & .0 & \\
\hline 02 & 9915 & 169.0023 & 5.8000 & 301.5 & 3 & 600.0 & 00.0 \\
\hline 02 & 9916 & 161.5015 & 6.3250 & 301.9 & 4 & 60 & .0 \\
\hline 02 & 9917 & 163.5083 & 5.22 & 302.2 & 4 & .0 & \\
\hline 02 & 9918 & 160.2393 & 3.9500 & 302.2 & 4 & 600.0 & .0 \\
\hline 02 & 9919 & 145.7627 & 2.6000 & 300.8 & 4 & .0 & .0 \\
\hline 02 & 9920 & 108.3077 & 3.12 & 299.4 & 4 & 60 & \\
\hline 02 & 9921 & 77.9998 & 6.4750 & 298.8 & 4 & .0 & \\
\hline 02 & 9922 & 76.2500 & 7.5500 & 298.5 & 4 & 600.0 & .0 \\
\hline 02 & 9923 & 77.2500 & 7.60 & 298.8 & 4 & 6 & \\
\hline 02 & 9924 & 77.7500 & 7.9000 & 298.8 & 4 & .0 & \\
\hline 02 & 9101 & 78.5000 & 7.6250 & 299.1 & 4 & 600.0 & 600.0 \\
\hline 02 & 9102 & 80.2501 & 7.1250 & 299.4 & 4 & 600.0 & .0 \\
\hline 02 & 9103 & 76.2500 & 6.3250 & 299.4 & 4 & .0 & \\
\hline 02 & 9104 & 76.7500 & 5.7250 & 299.6 & 4 & .0 & 0.0 \\
\hline 02 & 9105 & 85.7455 & 4.4250 & 299.7 & 4 & .0 & .0 \\
\hline 02 & 9106 & 85.5000 & 3.1250 & 299.1 & 4 & .0 & \\
\hline 02 & 9107 & 155.7644 & 0.3500 & 298.8 & 5 & .0 & 600.0 \\
\hline 02 & 9108 & 346.4061 & 0.8500 & 300.5 & 4 & 600.0 & 600.0 \\
\hline 02 & 9109 & 258.0437 & 1.3500 & 301.5 & 3 & 600.0 & 0.0 \\
\hline 02 & 91010 & 239.4924 & 2.5500 & 301.5 & 2 & 600.0 & 600.0 \\
\hline 02 & 91011 & 226.4914 & 2.2000 & 301.8 & 1 & 600.0 & 600.0 \\
\hline 02 & 91012 & 229.7510 & 2.8500 & 302.4 & 2 & 600.0 & 600.0 \\
\hline 02 & 91013 & 199.6911 & 3.7000 & 302.8 & 2 & 600.0 & 600.0 \\
\hline 02 & 91014 & 179.0105 & 4.0000 & 303.7 & 3 & 600.0 & 600.0 \\
\hline 02 & 91015 & 179.4973 & 4.3000 & 304.3 & 3 & 600.0 & 600.0 \\
\hline 02 & 91016 & 180.9986 & 5.8250 & 304.3 & 3 & 600.0 & 600.0 \\
\hline 02 & 91017 & 177.0005 & 5.5250 & 304.4 & 3 & 600.0 & 600.0 \\
\hline 02 & 91018 & 167.4589 & 5.2500 & 304.0 & 4 & 600.0 & 600.0 \\
\hline 02 & 91019 & 93.7472 & 6.2500 & 302.4 & 4 & 600.0 & 600.0 \\
\hline 02 & 91020 & 79.0080 & 6.3750 & 301.0 & 4 & 600.0 & 600.0 \\
\hline 02 & 91021 & 72.2501 & 6.2750 & 300.6 & 4 & 600.0 & 600.0 \\
\hline 02 & 91022 & 68.7500 & 6.7250 & 300.5 & 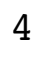 & 600.0 & 600.0 \\
\hline $0<$ & 91023 & 69.4999 & 6.9250 & 300.5 & 4 & 600.0 & 600.0 \\
\hline
\end{tabular}




\begin{tabular}{|c|c|c|c|c|c|c|c|}
\hline 02 & 91024 & 3.2500 & 6.7000 & 300.6 & 4 & 600.0 & 600.0 \\
\hline 2 & 9111 & 3.0000 & 7.2250 & 300.6 & 4 & 600.0 & 600.0 \\
\hline 02 & 9112 & 80.2498 & 7.0000 & 300.6 & 4 & 600.0 & 00.0 \\
\hline 02 & 9113 & 80.4999 & 6.9500 & 300.8 & 4 & 600.0 & 00.0 \\
\hline 02 & 9114 & 78.7500 & 6.5750 & 301.0 & 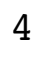 & 600.0 & 00.0 \\
\hline 02 & 9115 & 82.7460 & 5.5500 & 301.0 & 4 & 600.0 & 00.0 \\
\hline 02 & 9116 & 91.7492 & 3.9250 & 301.0 & 5 & 600.0 & 500.0 \\
\hline 02 & 9117 & 155.2183 & 1.4500 & 300.0 & 0 & 600.0 & 00.0 \\
\hline 02 & 9118 & 71.8339 & 0.6500 & 300.5 & 5 & 600.0 & \\
\hline 02 & 9119 & 176.5327 & 2.1750 & 301.5 & 4 & 600.0 & 600.0 \\
\hline 02 & 91110 & 165.7499 & 3.4750 & 300.7 & 4 & 600.0 & 0 \\
\hline 02 & 91111 & 165.2499 & 3.7500 & 301.7 & 4 & 600.0 & \\
\hline 02 & 91112 & 172.7527 & 000 & 302.5 & 3 & 600.0 & .0 \\
\hline 02 & 91113 & 179.0000 & 5.0250 & 303.6 & 3 & 600.0 & \\
\hline 02 & 91114 & 190.0000 & 5 . & 304.4 & 3 & 600.0 & .0 \\
\hline 02 & 91115 & 175.7503 & 5 . & 304.8 & 3 & 600.0 & \\
\hline 02 & 91116 & 177.7464 & 5.4000 & 305.0 & 3 & 600.0 & 600.0 \\
\hline 02 & 91117 & 134.4449 & 5.3250 & 304.9 & 3 & 600.0 & .0 \\
\hline 02 & 91118 & 82.7424 & 5. & 303.4 & 4 & 600.0 & \\
\hline 02 & 91119 & 72.2501 & 9.3500 & 301.0 & 4 & 600.0 & 600.0 \\
\hline 02 & 91120 & 71.7500 & 10.1500 & 300.5 & 4 & 600.0 & .0 \\
\hline 02 & 91121 & 71.7499 & 10. & 300.3 & 4 & 600.0 & \\
\hline 02 & 91122 & 72.2500 & 750 & 300.2 & 4 & 600.0 & 60 \\
\hline 02 & 91123 & 73.7499 & 10.0750 & 300.2 & 4 & 600.0 & .0 \\
\hline 02 & 91124 & 81.2500 & 8.57 & 300.0 & 4 & 600.0 & \\
\hline 02 & 9121 & 79.4995 & 500 & 299.9 & 4 & 600.0 & 60 \\
\hline 02 & 9122 & 83.0000 & 8.2750 & 299.9 & 4 & 600.0 & .0 \\
\hline 02 & 9123 & 79.9999 & 8.32 & 299.7 & 4 & 600.0 & .0 \\
\hline 02 & 9124 & 82.2500 & 8.1750 & 299.4 & 4 & 600.0 & .0 \\
\hline 02 & 9125 & 79.2501 & 8.6250 & 299.1 & 4 & 600.0 & .0 \\
\hline 02 & 9126 & 78.2501 & 8.5500 & 297.8 & 4 & 600.0 & .0 \\
\hline 02 & 9127 & 80.7505 & 7.9500 & 297.7 & 4 & 600.0 & \\
\hline 02 & 9128 & 81.2500 & 250 & 298.5 & 4 & 600.0 & 60 \\
\hline 02 & 9129 & 89.4976 & 5.1750 & 300.2 & 3 & 600.0 & .0 \\
\hline 02 & 91210 & 165.5659 & 2 . & 302.3 & 2 & 600.0 & \\
\hline 02 & 91211 & 221.8042 & 2.9000 & 302.0 & 2 & 600.0 & 600.0 \\
\hline 02 & 91212 & 181.2382 & 4.2500 & 302.8 & 3 & 600.0 & 600.0 \\
\hline 02 & 91213 & 176.5000 & 4.8500 & 303.6 & 3 & 600.0 & 60 \\
\hline 02 & 91214 & 114.1480 & 4.0500 & 304.9 & 3 & 600.0 & 600.0 \\
\hline 02 & 91215 & 86.5027 & 5.6000 & 303.9 & 3 & 600.0 & 600.0 \\
\hline 02 & 91216 & 79.7430 & 6.5750 & 303.2 & 4 & 600.0 & 600.0 \\
\hline 02 & 91217 & 74.2500 & 7.9000 & 302.6 & 4 & 600.0 & 600.0 \\
\hline 02 & 91218 & 72.7500 & 9.4000 & 300.8 & 4 & 600.0 & 600.0 \\
\hline 02 & 91219 & 74.9999 & 9.9500 & 298.7 & 4 & 600.0 & 600.0 \\
\hline 02 & 91220 & 73.0000 & 10.0500 & 298.2 & 4 & 600.0 & 600.0 \\
\hline 02 & 91221 & 74.5000 & 9.5000 & 298.6 & 4 & 600.0 & 600.0 \\
\hline 02 & 91222 & 73.4998 & & 29 & 4 & 600.0 & 600.0 \\
\hline 02 & 91223 & 67.5000 & 7.6750 & 299.7 & $I$ & 600.0 & 600.0 \\
\hline
\end{tabular}




\begin{tabular}{|c|c|c|c|c|c|c|c|}
\hline 02 & 91224 & 70.4998 & 6.5500 & 299.6 & 4 & 600.0 & 600.0 \\
\hline 02 & 9131 & 78.2500 & 7.1750 & 298.9 & 4 & 600.0 & 600.0 \\
\hline 02 & 9132 & 81.0000 & 6.9250 & 298.6 & 4 & 600.0 & 00.0 \\
\hline 02 & 9133 & 88.1731 & 3.9500 & 298.4 & 4 & 600.0 & 600.0 \\
\hline 02 & 9134 & 132.4583 & 1.2500 & 297.3 & 5 & 600.0 & 00.0 \\
\hline 02 & 9135 & 141.2692 & 0.2500 & 295.7 & 6 & 600.0 & 00.0 \\
\hline 02 & 9136 & 120.7491 & 1.0250 & 296.0 & 6 & 600.0 & 600.0 \\
\hline 02 & 9137 & 144.0561 & 1.4500 & 296.6 & 6 & 600.0 & 00.0 \\
\hline 02 & 9138 & 153.9612 & 1.4000 & 298.3 & 5 & 600.0 & 00.0 \\
\hline 02 & 9139 & 207.8085 & 0.9250 & 300.3 & 4 & 600.0 & 600.0 \\
\hline 02 & 91310 & 258.5630 & 1.6750 & 300.7 & 3 & 600.0 & 600.0 \\
\hline 02 & 91311 & 226.7940 & 3.2250 & 300.3 & 2 & 600.0 & \\
\hline 02 & 91312 & 173.2535 & 4.8250 & 300.0 & 3 & 600.0 & 600.0 \\
\hline 02 & 91313 & 171.2505 & 5.3000 & 301.0 & 3 & 600.0 & 600.0 \\
\hline 02 & 91314 & 182.2507 & 6.6000 & 302.5 & 4 & 600.0 & \\
\hline 02 & 91315 & 166.2498 & 5.3000 & 304.2 & 3 & 600.0 & 60 \\
\hline 02 & 91316 & 105.2383 & 5.2750 & 303.4 & 3 & 600.0 & 600.0 \\
\hline 02 & 91317 & 88.0149 & 5.5500 & 301.3 & 4 & 600.0 & \\
\hline 02 & 91318 & 74.7499 & 9.3750 & 299.8 & 4 & 600.0 & 60 \\
\hline 02 & 91319 & 73.5000 & 9.5750 & 299.9 & 4 & 600.0 & 600.0 \\
\hline 02 & 91320 & 68.5000 & 8.3250 & 300.4 & 4 & 600.0 & .0 \\
\hline 02 & 91321 & 68.9998 & 6.9500 & 300.3 & 4 & 600.0 & \\
\hline 02 & 91322 & 75.2501 & 8.7250 & 299.8 & 4 & 600.0 & 600.0 \\
\hline 02 & 91323 & 88.9745 & 6.9750 & 299.0 & 4 & 600.0 & .0 \\
\hline 02 & 91324 & 82.9982 & 6.7500 & 299.0 & 4 & 600.0 & \\
\hline 02 & 9141 & 81.2500 & 7.3000 & 299.3 & 4 & 600.0 & 600.0 \\
\hline 02 & 9142 & 90.5232 & 3.2500 & 299.6 & 5 & 600.0 & 600.0 \\
\hline 02 & 9143 & 110.2535 & 2.6500 & 298.0 & 5 & 600.0 & \\
\hline 02 & 9144 & 87.2299 & 1.9750 & 298.5 & 6 & 600.0 & 600.0 \\
\hline 02 & 9145 & 92.7600 & 2.8250 & 298.7 & 5 & 600.0 & 600.0 \\
\hline 02 & 9146 & 93.5607 & .9000 & 298.1 & 6 & 600.0 & .0 \\
\hline 02 & 9147 & 103.0679 & 0.4000 & 298.4 & 6 & 600.0 & 60 \\
\hline 02 & 9148 & 79.9678 & 0.6000 & 301.0 & 5 & 600.0 & 600.0 \\
\hline 02 & 9149 & 213.9613 & 0.4250 & 301.4 & 4 & 600.0 & .0 \\
\hline 02 & 91410 & 266.8719 & 1.6500 & 300.3 & 3 & 600.0 & 60 \\
\hline 02 & 91411 & 247.9634 & 2.7000 & 300.3 & 2 & 600.0 & 600.0 \\
\hline 02 & 91412 & 238.1937 & 1.6250 & 301.9 & 1 & 600.0 & .0 \\
\hline 02 & 91413 & 181.0035 & 3.5750 & 302.4 & 2 & 600.0 & 600.0 \\
\hline 02 & 91414 & 172.0048 & 4.4250 & 302.6 & 3 & 600.0 & 600.0 \\
\hline 02 & 91415 & 170.4973 & 4.5000 & 303.2 & 3 & 600.0 & \\
\hline 02 & 91416 & 167.7498 & 4.7250 & 303.5 & 3 & 600.0 & 600.0 \\
\hline 02 & 91417 & 147.3784 & 4.3500 & 303.3 & 3 & 600.0 & 600.0 \\
\hline 02 & 91418 & 81.7493 & 6.1000 & 300.9 & 4 & 600.0 & 600.0 \\
\hline 02 & 91419 & 74.2470 & 7.7250 & 299.0 & 4 & 600.0 & 600.0 \\
\hline 02 & 91420 & 75.4977 & 9.2750 & 298.1 & 4 & 600.0 & 600.0 \\
\hline 02 & 91421 & 80.5000 & 7.6000 & 298.5 & 4 & 600.0 & 600.0 \\
\hline 02 & 91422 & 84.7498 & 6.9500 & 298.4 & 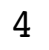 & 600.0 & 600.0 \\
\hline 02 & 91423 & 81.5002 & 7.7000 & 298.2 & 4 & 600.0 & 600.0 \\
\hline
\end{tabular}




\begin{tabular}{|c|c|c|c|c|c|c|c|}
\hline 02 & 91424 & 80.2500 & 8.0750 & 298.1 & 4 & 600.0 & 600.0 \\
\hline 02 & 9151 & 74.9998 & 8.2250 & 297.9 & 4 & 600.0 & 00,0 \\
\hline 02 & 9152 & 75.4998 & 8.9000 & 296.4 & 4 & 600.0 & 00.0 \\
\hline 02 & 9153 & 76.7500 & 10.8750 & 293.9 & 4 & 600.0 & 00.0 \\
\hline 02 & 9154 & 81.0002 & 250 & 294.0 & 4 & 600.0 & 00.0 \\
\hline 02 & 9155 & 78.2492 & 10.1250 & 294.0 & 4 & 600.0 & 00.0 \\
\hline 02 & 9156 & 74.7500 & 13.1000 & 291.6 & 4 & 600.0 & 0 \\
\hline 02 & 9157 & 73.7498 & 11.4750 & 290.7 & 4 & 600.0 & 00.0 \\
\hline 02 & 9158 & 67.0011 & 11.1000 & 292.4 & 4 & 600.0 & 00.0 \\
\hline 02 & 9159 & 68.7489 & 11.6000 & 292.6 & 4 & 60 & 0 \\
\hline 02 & 91510 & 68.2518 & 10.3250 & 293.8 & 4 & 600.0 & \\
\hline 02 & 91511 & 72.4931 & 6.6750 & 294.9 & 4 & 600.0 & \\
\hline 02 & 91512 & 98.7447 & 5.4000 & 295.4 & 3 & 600.0 & .0 \\
\hline 02 & 91513 & 104.7489 & 6.9000 & 294.8 & 4 & 600.0 & \\
\hline 02 & 91514 & 04.5046 & & 294.1 & 4 & .0 & \\
\hline 02 & 91515 & 101.7499 & 8.1250 & 294.2 & 4 & 600.0 & \\
\hline 02 & 91516 & 71.4524 & 9.4500 & 293.8 & 4 & 60 & .0 \\
\hline 02 & 91517 & 97.5277 & 11.4750 & 292.6 & 4 & .0 & \\
\hline 02 & 91518 & 131.7955 & 10.4250 & 291.7 & 4 & .0 & \\
\hline 02 & 91519 & 90.6271 & 5.4000 & 290.1 & 4 & .0 & .0 \\
\hline 02 & 91520 & 67.9890 & 9. & 289.1 & 4 & 6 & \\
\hline 02 & 91521 & 64.0000 & 13. & 288.4 & 4 & .0 & \\
\hline 02 & 91522 & 68.2499 & 10.2250 & 288.5 & 4 & 600.0 & .0 \\
\hline 02 & 91523 & 63.7494 & 11.35 & 288.3 & 4 & 0 & \\
\hline 02 & 91524 & 68.7497 & 10 & 288.5 & 4 & .0 & \\
\hline 02 & 9161 & 65.2500 & 10.8750 & 288.8 & 4 & .0 & \\
\hline 02 & 9162 & 67.2498 & 10.1250 & 288.5 & 4 & 60 & .0 \\
\hline 02 & 9163 & 75.2497 & 7.9000 & 288.7 & 4 & .0 & \\
\hline 02 & 9164 & 84.2540 & 9.7500 & 288.2 & 4 & .0 & .0 \\
\hline 02 & 9165 & 82.4982 & 9.9000 & 286.4 & 4 & 6 & \\
\hline 02 & 9166 & 75.9993 & 9.6250 & 285.7 & 4 & .0 & \\
\hline 02 & 9167 & 80.7499 & 9.2500 & 286.8 & 4 & .0 & 0 \\
\hline 02 & 9168 & 102.9694 & 6.0250 & 289.0 & 4 & 600.0 & 600.0 \\
\hline 02 & 9169 & 198.7630 & 3.0000 & 291.3 & 3 & .0 & 0.0 \\
\hline 02 & 91610 & 174.9087 & 3.2500 & 292.2 & 2 & 600.0 & 600.0 \\
\hline 02 & 91611 & 174.7492 & 4.2250 & 293.0 & 3 & 600.0 & 600.0 \\
\hline 02 & 91612 & 171.4997 & 5.9250 & 294.0 & 3 & 600.0 & .0 \\
\hline 02 & 91613 & 166.0018 & 6.7250 & 295.4 & 4 & 600.0 & 600.0 \\
\hline 02 & 91614 & 162.2535 & 5.5000 & 296.8 & 3 & 600.0 & 600.0 \\
\hline 02 & 91615 & 146.5037 & 5.3250 & 297.8 & - & 600.0 & 600.0 \\
\hline 02 & 91616 & 109.9342 & 5.2000 & 298.1 & 3 & 600.0 & 600.0 \\
\hline 02 & 91617 & 92.0000 & 6.2750 & 297.5 & 4 & 600.0 & 600.0 \\
\hline 02 & 91618 & 81.2707 & 7.3250 & 295.9 & 4 & 600.0 & 600.0 \\
\hline 02 & 91619 & 78.5075 & 8.9250 & 294.0 & 4 & 600.0 & 600.0 \\
\hline 02 & 91620 & 74.5003 & 8.7250 & 293.9 & 4 & 600.0 & 600.0 \\
\hline 02 & 91621 & 75.0000 & 9.2750 & 293.7 & 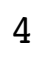 & 600.0 & 600.0 \\
\hline 02 & 91622 & 74.5000 & 8.6500 & 294.0 & 4 & 600.0 & 600.0 \\
\hline 48 & 91623 & 73.5000 & 9.125 & 293.7 & 4 & 600.0 & 600.0 \\
\hline
\end{tabular}




\begin{tabular}{|c|c|c|c|c|c|c|c|}
\hline 2 & 91624 & 71.2495 & 9.4750 & 293.4 & 4 & 600.0 & 00.0 \\
\hline 02 & 9171 & 73.2500 & 10.4250 & 292.8 & 4 & 600.0 & 00.0 \\
\hline 02 & 9172 & 71.7500 & 10.0250 & 292.4 & 4 & 600.0 & 00.0 \\
\hline 02 & 9173 & 78.4998 & 10.0750 & 292.4 & 4 & 600.0 & 00.0 \\
\hline 02 & 9174 & 82.2500 & 9.3500 & 292.1 & 4 & 600.0 & 00.0 \\
\hline 02 & 9175 & 81.0000 & 9.3500 & 291.1 & 4 & 600.0 & 00.0 \\
\hline 02 & 9176 & 77.2486 & 500 & 291.1 & 4 & 600.0 & 00.0 \\
\hline 02 & 9177 & 79.7489 & 8.0750 & 290.8 & 4 & 600.0 & 00.0 \\
\hline 02 & 9178 & 81.5000 & 6.2000 & 292.7 & 4 & 600.0 & 00.0 \\
\hline 02 & 9179 & 100.7496 & 4.5000 & 294.5 & 3 & 600.0 & .0 \\
\hline 02 & 91710 & 106.5000 & 4.4000 & 295.6 & 4 & 600.0 & 00.0 \\
\hline 02 & 91711 & 90.8114 & 3.2000 & 297.2 & 3 & 600.0 & \\
\hline 02 & 91712 & 94.9986 & 4.7250 & 298.0 & 3 & 600.0 & .0 \\
\hline 02 & 91713 & 103.0132 & 5.8000 & 298.2 & 3 & 600.0 & 600 \\
\hline 02 & 91714 & 103.2532 & 250 & 298.4 & 3 & 600.0 & \\
\hline 02 & 91715 & 92.4936 & 6.0250 & 298.8 & 4 & 600.0 & 60 \\
\hline 02 & 91716 & 81.0000 & 7.45 & 298.6 & 4 & 600.0 & 600.0 \\
\hline 02 & 91717 & 82.0103 & 8.1750 & 296.9 & 4 & 600.0 & \\
\hline 02 & 91718 & 64.4999 & 9.3000 & 295.8 & 4 & 600.0 & .0 \\
\hline 02 & 91719 & 68.7500 & 9.9 & 294.0 & 4 & 600.0 & .0 \\
\hline 02 & 91720 & 68.2501 & 10 . & 293.0 & 4 & 600.0 & .0 \\
\hline 02 & 91721 & 70.5002 & 10 . & 292.6 & 4 & 0.0 & \\
\hline 02 & 91722 & 75.5000 & 10.4250 & 292.7 & 4 & 600.0 & 60 \\
\hline 02 & 91723 & 80.2501 & 9.7 & 292.9 & 4 & 600.0 & .0 \\
\hline 02 & 91724 & 88.9992 & 8 . & 292.7 & 4 & 600.0 & \\
\hline 02 & 9181 & 94.7500 & 7.1500 & 293.3 & 4 & 600.0 & 60 \\
\hline 02 & 9182 & 135.7502 & 7.8000 & 294.3 & 4 & 600.0 & .0 \\
\hline 02 & 9183 & 139.2500 & 9. & 294.0 & 4 & 0.0 & \\
\hline 02 & 9184 & 147.7509 & 9.8750 & 293.3 & 4 & 600.0 & 60 \\
\hline 02 & 9185 & 148.5000 & 9.87 & 293.5 & 4 & 600.0 & .0 \\
\hline 02 & 9186 & 149.0000 & 11 . & 293.4 & 4 & 600.0 & \\
\hline 02 & 9187 & 152.7497 & 10 . & 293.5 & 4 & 600.0 & .0 \\
\hline 02 & 9188 & 161.2505 & 10.3000 & 293.9 & 4 & 600.0 & 600.0 \\
\hline 02 & 9189 & 167.2491 & 11 . & 294.3 & 4 & 600.0 & \\
\hline 02 & 91810 & 173.5046 & 10 . & 295.4 & 4 & 600.0 & .0 \\
\hline 02 & 91811 & 180.4999 & 10.4750 & 296.9 & 4 & 600.0 & 600.0 \\
\hline 02 & 91812 & 178.7529 & 10.3000 & 297.9 & 4 & 600.0 & \\
\hline 02 & 91813 & 180.7496 & 10.1250 & 298.8 & 4 & 600.0 & .0 \\
\hline 02 & 91814 & 181.2499 & 9.0250 & 299.7 & 4 & 600.0 & 600.0 \\
\hline 02 & 91815 & 184.5024 & 7 . & 300.3 & 4 & 600.0 & \\
\hline 02 & 91816 & 179.2552 & 5.9500 & 300.8 & 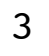 & 600.0 & 600.0 \\
\hline 02 & 91817 & 175.5011 & 5.6750 & 301.2 & 3 & 600.0 & 600.0 \\
\hline 02 & 91818 & 171.0349 & 3.9750 & 301.4 & 4 & 600.0 & 600.0 \\
\hline 02 & 91819 & 125.6878 & 2.1500 & 300.6 & 4 & 600.0 & 600.0 \\
\hline 02 & 91820 & 138.7668 & 4.1750 & 300.7 & 4 & 600.0 & 600.0 \\
\hline 02 & 91821 & 137.2484 & 5.2250 & 301.1 & 4 & 600.0 & 600.0 \\
\hline 02 & 91822 & 142.9992 & 7.175 & 301.2 & 4 & 600.0 & 600.0 \\
\hline 02 & 91823 & 113.4188 & 6.3250 & 300.5 & $\mathbf{4}$ & 600.0 & 600.0 \\
\hline
\end{tabular}




\begin{tabular}{|c|c|c|c|c|c|c|c|}
\hline 02 & 91824 & 96.2506 & 5.2000 & 299.8 & 4 & 600.0 & 600.0 \\
\hline 02 & 9191 & 102.2511 & 4.6250 & 299.0 & 4 & 600.0 & 600.0 \\
\hline 02 & 9192 & 88.1055 & 3.6000 & 298.6 & 4 & 600.0 & 00.0 \\
\hline 02 & 9193 & 94.7430 & 2.5000 & 297.8 & 5 & 600.0 & 600.0 \\
\hline 02 & 9194 & 74.5532 & 1.8000 & 297.1 & 0 & 600.0 & 00.0 \\
\hline 02 & 9195 & 311.1280 & 1.3250 & 295.2 & 6 & 600.0 & 00.0 \\
\hline 02 & 9196 & 335.2417 & 0.7750 & 294.4 & 6 & 600.0 & 600.0 \\
\hline 02 & 9197 & 211.3003 & 0.5500 & 294.6 & 6 & 600.0 & 00.0 \\
\hline 02 & 9198 & 342.2494 & 0.9250 & 296.5 & 5 & 600.0 & 00.0 \\
\hline 02 & 9199 & 265.6185 & 1.6250 & 298.1 & 4 & 600.0 & 600.0 \\
\hline 02 & 91910 & 277.7411 & 2.2250 & 299.0 & 3 & 600.0 & 600 \\
\hline 02 & 91911 & 260.2075 & 2.6000 & 299.9 & 2 & 600.0 & \\
\hline 02 & 91912 & 238.5239 & 2.1750 & 301.2 & 1 & 600.0 & 600.0 \\
\hline 02 & 91913 & 211.7498 & 2.8750 & 301.9 & 1 & 600.0 & 600.0 \\
\hline 02 & 91914 & 218.4677 & 2.5 & 302.7 & 1 & 600.0 & \\
\hline 02 & 91915 & 206.9050 & 3.1000 & 303.3 & 2 & 600.0 & 60 \\
\hline 02 & 91916 & 169.0339 & 3.3750 & 303.6 & 2 & 600.0 & 600.0 \\
\hline 02 & 91917 & 169.9801 & 2.9500 & 303.9 & 3 & 600.0 & \\
\hline 02 & 91918 & 171.2491 & 3.3250 & 303.3 & 4 & 600.0 & 60 \\
\hline 02 & 91919 & 146.6677 & 2.3750 & 301.7 & 5 & 600.0 & 600.0 \\
\hline 02 & 91920 & 76.7240 & 3 . & 302.1 & 5 & 600.0 & .0 \\
\hline 02 & 91921 & 70.7491 & 5 . & 301.9 & 4 & 600.0 & \\
\hline 02 & 91922 & 75.7500 & 000 & 300.9 & 4 & 600.0 & 600.0 \\
\hline 02 & 91923 & 75.7501 & 7.2500 & 300.6 & 4 & 600.0 & .0 \\
\hline 02 & 91924 & 75.2500 & 7 & 300.1 & 4 & 600.0 & \\
\hline 02 & 9201 & 74.7497 & 8.2000 & 299.8 & 4 & 600.0 & 600.0 \\
\hline 02 & 9202 & 73.7500 & 500 & 299.9 & 4 & 600.0 & 60 \\
\hline 02 & 9203 & 73.9999 & 6 . & 300.2 & 4 & 600.0 & \\
\hline 02 & 9204 & 96.6009 & 3.5250 & 299.8 & 5 & 600.0 & 60 \\
\hline 02 & 9205 & 66.2536 & 3.0000 & 300.6 & 4 & 600.0 & 600.0 \\
\hline 02 & 9206 & 35.2247 & 0 . & 299.5 & 5 & 600.0 & .0 \\
\hline 02 & 9207 & 11.1186 & 0 . & 299.8 & 4 & 600.0 & 60 \\
\hline 02 & 9208 & 129.5710 & 1.2000 & 300.4 & 3 & 600.0 & 600.0 \\
\hline 02 & 9209 & 242.4777 & 000 & 301.4 & 2 & 600.0 & .0 \\
\hline 02 & 92010 & 237.2447 & 1 . & 302.2 & 1 & 600.0 & 600.0 \\
\hline 02 & 92011 & 241.7851 & 1.9750 & 302.9 & 1 & 600.0 & 600.0 \\
\hline 02 & 92012 & 254.2890 & 2.5500 & 304.2 & 1 & 600.0 & .0 \\
\hline 02 & 92013 & 219.0568 & 3.8250 & 303.8 & 2 & 600.0 & 600.0 \\
\hline 02 & 92014 & 230.5469 & 2.4250 & 305.1 & 1 & 600.0 & 600.0 \\
\hline 02 & 92015 & 195.4791 & 3.6000 & 305.6 & 2 & 600.0 & \\
\hline 02 & 92016 & 180.2390 & 5.2000 & 305.5 & 3 & 600.0 & 600.0 \\
\hline 02 & 92017 & 161.5453 & 4.5250 & 305.4 & 3 & 600.0 & 600.0 \\
\hline 02 & 92018 & 125.7963 & 4.8000 & 304.6 & 4 & 600.0 & 600.0 \\
\hline 02 & 92019 & 84.7530 & 5.5000 & 302.3 & 4 & 600.0 & 600.0 \\
\hline 02 & 92020 & 69.4954 & 6.3000 & 301.5 & 4 & 600.0 & 600.0 \\
\hline 02 & 92021 & 61.7500 & 7.1000 & 301.7 & 4 & 600.0 & 600.0 \\
\hline 02 & 92022 & 66.2496 & 7.0000 & 301.6 & 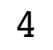 & 600.0 & 600.0 \\
\hline 02 & 92023 & 67.0000 & 7.5000 & 301.6 & 4 & 600.0 & 600.0 \\
\hline
\end{tabular}




\begin{tabular}{|c|c|c|c|c|c|c|c|}
\hline 02 & 92024 & 70.5010 & 7.9500 & 301.0 & 4 & 600.0 & 600.0 \\
\hline 02 & 9211 & 72.7500 & 8.4000 & 300.3 & 4 & 600.0 & 600.0 \\
\hline 02 & 9212 & 74.2500 & 8.4500 & 300.2 & 4 & 600.0 & 00.0 \\
\hline 02 & 9213 & 78.0000 & 8.2500 & 300.3 & 4 & 600.0 & 00.0 \\
\hline 02 & 9214 & 75.7500 & .5250 & 300.8 & 4 & 600.0 & 00.0 \\
\hline 02 & 9215 & 73.4982 & 6.7750 & 300.9 & 4 & 600.0 & 00.0 \\
\hline 02 & 9216 & 136.7685 & 1.9000 & 298.8 & 5 & 600.0 & 00.0 \\
\hline 02 & 9217 & 151.9312 & 1.4250 & 298.1 & 4 & 600.0 & 00.0 \\
\hline 02 & 9218 & 165.5270 & 0.1250 & 300.9 & 3 & 600.0 & 00.0 \\
\hline 02 & 9219 & 227.6198 & 0.6500 & 302.9 & 2 & 600.0 & 0 \\
\hline 02 & 92110 & 257.2619 & 2.0250 & 302.6 & 1 & 600.0 & \\
\hline 02 & 92111 & 230.6066 & 2.6500 & 302.8 & 1 & 600.0 & \\
\hline 02 & 92112 & 180.0102 & 3.4750 & 302.3 & 2 & 600.0 & 00.0 \\
\hline 02 & 92113 & 162.9940 & 3.7250 & 303.3 & 3 & 600.0 & 00.0 \\
\hline 02 & 92114 & 167.0014 & 5. & 303.6 & 3 & .0 & \\
\hline 02 & 92115 & 172.5006 & 5.3750 & 304.4 & 3 & 600.0 & 00.0 \\
\hline 02 & 92116 & 171.2499 & 6.2750 & 304.6 & 4 & 60 & .0 \\
\hline 02 & 92117 & 164.2506 & 5.1500 & 304.6 & 4 & .0 & \\
\hline 02 & 92118 & 118.4580 & 4.7750 & 304.4 & 4 & 600.0 & .0 \\
\hline 02 & 92119 & 77.2498 & 7.1500 & 302.7 & 4 & .0 & .0 \\
\hline 02 & 92120 & 71.0002 & 8.1 & 302.0 & 4 & 60 & \\
\hline 02 & 92121 & 73.7499 & 8.2500 & 301.5 & 4 & .0 & \\
\hline 02 & 92122 & 75.0000 & 7.8750 & 300.9 & 4 & 600.0 & .0 \\
\hline 02 & 92123 & 77.0000 & 7.8500 & 300.8 & 4 & 60 & .0 \\
\hline 02 & 92124 & 77.2500 & 7.9500 & 300.8 & 4 & .0 & \\
\hline 02 & 9221 & 77.0000 & 7.5750 & 301.0 & 4 & 600.0 & 600.0 \\
\hline 02 & 9222 & 77.0000 & 7.2500 & 301.4 & 4 & 600.0 & .0 \\
\hline 02 & 9223 & 77.7499 & 7.4750 & 301.4 & 4 & .0 & \\
\hline 02 & 9224 & 83.4989 & 7.3750 & 301.2 & 4 & .0 & 0.0 \\
\hline 02 & 9225 & 104.2489 & 4.7250 & 301.8 & 4 & .0 & .0 \\
\hline 02 & 9226 & 139.1233 & 0.9500 & 300.7 & 4 & .0 & \\
\hline 02 & 9227 & 132.7149 & 1.9000 & 301.4 & 5 & .0 & 600.0 \\
\hline 02 & 9228 & 159.7498 & 2.2500 & 301.6 & 4 & 600.0 & 600.0 \\
\hline 02 & 9229 & 177.2467 & 3.2000 & 302.1 & 3 & 600.0 & 0.0 \\
\hline 02 & 92210 & 166.9999 & 4.8500 & 301.5 & 3 & 600.0 & 600.0 \\
\hline 02 & 92211 & 166.7502 & 4.5500 & 301.6 & 4 & 600.0 & 600.0 \\
\hline 02 & 92212 & 165.5000 & 4.7000 & 302.3 & 4 & 600.0 & 600.0 \\
\hline 02 & 92213 & 165.7496 & 5.4000 & 303.6 & 4 & 600.0 & 600.0 \\
\hline 02 & 92214 & 165.2503 & 4.9500 & 304.4 & 4 & 600.0 & 600.0 \\
\hline 02 & 92215 & 166.7502 & 4.5000 & 305.3 & 3 & 600.0 & 600.0 \\
\hline 02 & 92216 & 164.7500 & 4.9750 & 305.8 & 4 & 600.0 & 600.0 \\
\hline 02 & 92217 & 164.0002 & 5.1750 & 305.8 & 4 & 600.0 & 600.0 \\
\hline 02 & 92218 & 158.4981 & 4.4250 & 304.9 & 5 & 600.0 & 600.0 \\
\hline 02 & 92219 & 113.9231 & 4.8250 & 304.3 & 4 & 600.0 & 600.0 \\
\hline 02 & 92220 & 83.0187 & 6.8750 & 303.6 & 4 & 600.0 & 600.0 \\
\hline 02 & 92221 & 74.0023 & 7.2000 & 302.5 & 4 & 600.0 & 600.0 \\
\hline 02 & 92222 & 70.5003 & 7.4000 & 302.5 & 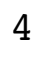 & 600.0 & 600.0 \\
\hline $0<$ & 92223 & 75.5000 & 7.1500 & 302.6 & 4 & 600.0 & 600.0 \\
\hline
\end{tabular}




\begin{tabular}{|c|c|c|c|c|c|c|c|}
\hline 02 & 92224 & 77.7499 & 6.7500 & 302.7 & 4 & 600.0 & 00.0 \\
\hline 02 & 9231 & 79.7505 & 6.8500 & 302.7 & 4 & 600.0 & 600.0 \\
\hline 02 & 9232 & 84.7500 & 6.0750 & 302.8 & 4 & 600.0 & 00.0 \\
\hline 02 & 9233 & 79.7499 & 6.0750 & 302.6 & 4 & 600.0 & 600.0 \\
\hline 02 & 9234 & 84.0000 & 5.7000 & 302.9 & 4 & 600.0 & 00.0 \\
\hline 02 & 9235 & 90.2882 & 5.0000 & 302.8 & 4 & 600.0 & 00.0 \\
\hline 02 & 9236 & 114.0000 & 3.6750 & 302.8 & 4 & 600.0 & 600.0 \\
\hline 02 & 9237 & 149.4968 & 1.7250 & 301.3 & 4 & 600.0 & 500.0 \\
\hline 02 & 9238 & 163.7506 & 1.3750 & 302.6 & 4 & 600.0 & 00.0 \\
\hline 02 & 9239 & 213.5413 & 1.1500 & 304.5 & 3 & 600.0 & 600.0 \\
\hline 02 & 92310 & 165.7500 & 3.8750 & 302.6 & 3 & 600.0 & 600.0 \\
\hline 02 & 92311 & 166.0001 & 3.9750 & 303.1 & 3 & 600.0 & \\
\hline 02 & 92312 & 164.0000 & 4.2250 & 304.2 & 3 & 600.0 & 600.0 \\
\hline 02 & 92313 & 173.0037 & 4.9000 & 305.1 & 3 & 600.0 & 600.0 \\
\hline 02 & 92314 & 173.4992 & 6.0250 & 305.5 & 4 & 600.0 & \\
\hline 02 & 92315 & 170.2508 & 5.2500 & 306.4 & 3 & 600.0 & 60 \\
\hline 02 & 92316 & 176.7446 & 5.1250 & 306.7 & 3 & 600.0 & 600.0 \\
\hline 02 & 92317 & 165.4872 & 4.5750 & 306.5 & 4 & 600.0 & \\
\hline 02 & 92318 & 168.2197 & 3.4000 & 305.2 & 4 & 600.0 & 60 \\
\hline 02 & 92319 & 95.2937 & 4.2250 & 303.8 & 4 & 600.0 & 600.0 \\
\hline 02 & 92320 & 70.2500 & 7.0750 & 303.0 & 4 & 600.0 & .0 \\
\hline 02 & 92321 & 69.2503 & 8.3250 & 302.7 & 4 & 600.0 & \\
\hline 02 & 92322 & 68.0000 & 9.0750 & 302.6 & 4 & 600.0 & 600.0 \\
\hline 02 & 92323 & 66.995 & 9.8000 & 302.6 & 4 & 600.0 & .0 \\
\hline 02 & 92324 & 64.5002 & 9.4000 & 302.3 & 4 & 600.0 & \\
\hline 02 & 9241 & 62.7500 & 9.1500 & 302.4 & 4 & 600.0 & 60 \\
\hline 02 & 9242 & 67.7439 & 8.2750 & 302.6 & 4 & 600.0 & .0 \\
\hline 02 & 9243 & 88.7252 & 2.1250 & 301.7 & 5 & 600.0 & \\
\hline 02 & $924 \quad 4$ & 67.4980 & 3.6000 & 302.2 & 4 & 600.0 & 60 \\
\hline 02 & 9245 & 86.4510 & 2.4000 & 301.6 & 5 & 600.0 & 600.0 \\
\hline 02 & 9246 & 105.2344 & 0.77 & 300.3 & 6 & 600.0 & .0 \\
\hline 02 & 9247 & 62.7624 & 0.2750 & 299.8 & 6 & 600.0 & 60 \\
\hline 02 & 9248 & 219.5423 & 0.8750 & 301.5 & 5 & 600.0 & 600.0 \\
\hline 02 & 9249 & 253.2475 & 0.8250 & 303.8 & 4 & 600.0 & .0 \\
\hline 02 & 92410 & 244.7445 & 1.8750 & 303.6 & 3 & 600.0 & 60 \\
\hline 02 & 92411 & 177.2718 & 3.2750 & 302.2 & 2 & 600.0 & 600.0 \\
\hline 02 & 92412 & 168.6812 & 2.8000 & 302.8 & 1 & 600.0 & .0 \\
\hline 02 & 92413 & 193.9889 & 3.3250 & 303.6 & 2 & 600.0 & 600.0 \\
\hline 02 & 92414 & 230.6908 & 2.1750 & 304.4 & 1 & 600.0 & 600.0 \\
\hline 02 & 92415 & 228.2492 & 3.0000 & 304.9 & 2 & 600.0 & \\
\hline 02 & 92416 & 169.6818 & 3.8250 & 304.8 & 3 & 600.0 & 600.0 \\
\hline 02 & 92417 & 201.7198 & 3.5000 & 304.5 & 2 & 600.0 & 600.0 \\
\hline 02 & 92418 & 119.1651 & 3.6500 & 304.4 & 3 & 600.0 & 600.0 \\
\hline 02 & 92419 & 102.4145 & 3.3250 & 302.5 & 4 & 600.0 & 600.0 \\
\hline 02 & 92420 & 60.2489 & 7.1000 & 301.8 & 4 & 600.0 & 600.0 \\
\hline 02 & 92421 & 56.7501 & 6.3750 & 301.4 & 4 & 600.0 & 600.0 \\
\hline 02 & 92422 & 69.2499 & 8.0500 & 300.8 & 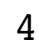 & 600.0 & 600.0 \\
\hline 02 & 92423 & 70.9989 & 7.6750 & 300.8 & 4 & 600.0 & 600.0 \\
\hline
\end{tabular}




\begin{tabular}{|c|c|c|c|c|c|c|c|}
\hline 2 & 92424 & 68.4954 & 8.0250 & 300.0 & 4 & 600.0 & 600.0 \\
\hline 02 & 9251 & 67.0000 & 8.5250 & 300.2 & 4 & 600.0 & 600.0 \\
\hline 02 & 9252 & 74.7326 & 1000 & 300.6 & 4 & 600.0 & 00.0 \\
\hline 02 & 9253 & 75.7500 & 7.1250 & 301.0 & 4 & 600.0 & 00.0 \\
\hline 02 & 9254 & 83.9871 & 6.1750 & 301.5 & 4 & 600.0 & 00.0 \\
\hline 02 & 9255 & 88.0184 & 5.5250 & 300.9 & 4 & 600.0 & 00.0 \\
\hline 02 & 9256 & 86.2505 & 5.9250 & 301.4 & 4 & 600.0 & 00.0 \\
\hline 02 & 9257 & 84.7494 & 5.5750 & 301.5 & 4 & 600.0 & 00.0 \\
\hline 02 & 9258 & 152.8222 & 2.5250 & 302.3 & 3 & 600.0 & 00.0 \\
\hline 02 & 9259 & 193.6158 & 3.2250 & 302.2 & 2 & 600.0 & 0 \\
\hline 02 & 92510 & 161.7507 & 6.0500 & 298.2 & 3 & 600.0 & \\
\hline 02 & 92511 & 166.5006 & 7.2500 & 298.3 & 4 & 600.0 & \\
\hline 02 & 92512 & 169.0001 & 6.7750 & 299.8 & 4 & 600.0 & 00.0 \\
\hline 02 & 92513 & 174.0005 & 7.2750 & 301.0 & 4 & 600.0 & 00.0 \\
\hline 02 & 92514 & 168.7500 & 6.2750 & 302.0 & 4 & .0 & \\
\hline 02 & 92515 & 165.0011 & 5.6750 & 303.3 & 3 & 600.0 & .0 \\
\hline 02 & 92516 & 168.4977 & 5.7250 & 304.0 & 3 & 60 & .0 \\
\hline 02 & 92517 & 162 . & 5.0500 & 303.9 & 4 & .0 & \\
\hline 02 & 92518 & 99.3943 & 4.8500 & 303.2 & 4 & 60 & .0 \\
\hline 02 & 92519 & 81.0000 & 6.3250 & 301.7 & 4 & .0 & .0 \\
\hline 02 & 92520 & 77.2487 & 6.95 & 1.3 & 4 & 6 & \\
\hline 02 & 92521 & 71.7501 & 8.1000 & .9 & 4 & .0 & \\
\hline 02 & 92522 & 74.5000 & 7.8500 & 300.1 & 4 & 600.0 & .0 \\
\hline 02 & 92523 & 76.0000 & 8.77 & .1 & 4 & 6 & \\
\hline 02 & 92524 & 76.7502 & 9.3250 & .3 & 4 & .0 & \\
\hline 02 & 9261 & 77.2501 & 9.2750 & 298.1 & 4 & .0 & .0 \\
\hline 02 & 9262 & 74.7500 & 9.0750 & 298.7 & 4 & 60 & .0 \\
\hline 02 & 9263 & 81.7513 & 8.6000 & 299.4 & 4 & .0 & \\
\hline 02 & 9264 & 82.2503 & 7.8000 & 299.0 & 4 & .0 & 0 \\
\hline 02 & 9265 & 76.9998 & 6.8250 & 297.9 & 4 & 6 & .0 \\
\hline 02 & 9266 & 80.7477 & 6.4750 & 297.7 & 4 & .0 & \\
\hline 02 & 9267 & 98.2549 & 4.7500 & .8 & 4 & .0 & 600.0 \\
\hline 02 & 9268 & 121.1349 & 2.9000 & 300.2 & 3 & 600.0 & 600.0 \\
\hline 02 & 9269 & 125.5643 & 1.1250 & 300.6 & 2 & 0 & 0.0 \\
\hline 02 & 92610 & 347.9163 & 1.3500 & 300.8 & 1 & 600.0 & 600.0 \\
\hline 02 & 92611 & 296.1074 & 1.1500 & 302.3 & 1 & 600.0 & 600.0 \\
\hline 02 & 92612 & 245.2887 & 1.8000 & 302.3 & 1 & 600.0 & 600.0 \\
\hline 02 & 92613 & 74.3512 & 2.6000 & 303.3 & 1 & 600.0 & 600.0 \\
\hline 02 & 92614 & 81.0001 & 6.2500 & 301.3 & 2 & 600.0 & 600.0 \\
\hline 02 & 92615 & 82.7528 & 8.7000 & 299.1 & 3 & 600.0 & 600.0 \\
\hline 02 & 92616 & 70.7499 & 9.8000 & 299.0 & 4 & 600.0 & 600.0 \\
\hline 02 & 92617 & 80.7505 & 9.1250 & 298.8 & 4 & 600.0 & 600.0 \\
\hline 02 & 92618 & 76.2499 & 9.9250 & 298.3 & 4 & 600.0 & 600.0 \\
\hline 02 & 92619 & 71.5000 & 10.1250 & 297.8 & 4 & 600.0 & 600.0 \\
\hline 02 & 92620 & 71.5000 & 9.6000 & 295.7 & 4 & 600.0 & 600.0 \\
\hline 02 & 92621 & 71.0002 & 9.7500 & 294.1 & 4 & 600.0 & 600.0 \\
\hline 02 & 92622 & 94.0288 & 7.1750 & 291.3 & 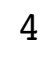 & 600.0 & 600.0 \\
\hline 02 & 92623 & 82.2418 & 11.4000 & 288.6 & 4 & 600.0 & 600.0 \\
\hline
\end{tabular}




\begin{tabular}{|c|c|c|c|c|c|c|c|}
\hline 02 & 92624 & 82.0076 & 10.1000 & 288.3 & 4 & 600.0 & 600.0 \\
\hline 2 & 9271 & 84.7492 & 10.4000 & 287.8 & 4 & 600.0 & 00,0 \\
\hline 02 & 9272 & 76.5018 & 10.2000 & 287.1 & 4 & 600.0 & 00.0 \\
\hline 2 & 9273 & 72.7533 & 9.3500 & 286.4 & 4 & 600.0 & 00.0 \\
\hline 02 & 9274 & 77.7500 & 12.5750 & 286.2 & 4 & 600.0 & 00.0 \\
\hline 02 & 9275 & 80.0000 & 10.9750 & 286.1 & 4 & 600.0 & 00.0 \\
\hline 02 & $927 \quad 6$ & 72.4997 & 3250 & 285.6 & 4 & 600.0 & 0 \\
\hline 02 & 9277 & 77.0012 & 7.9750 & 285.5 & 4 & 600.0 & 00.0 \\
\hline 02 & 9278 & 153.9061 & 3.1000 & 286.2 & 3 & 600.0 & 00.0 \\
\hline 02 & 9279 & 40.9866 & 1.8250 & 287.7 & 2 & 600 & 0 \\
\hline 02 & 92710 & 276.5069 & 1.6750 & 288.7 & 1 & 600.0 & \\
\hline 02 & 92711 & 295.6027 & 1.7750 & 290.1 & 1 & 600.0 & \\
\hline 02 & 92712 & 247.9447 & 2.6250 & 291.1 & 1 & 600.0 & \\
\hline 02 & 92713 & 211.2690 & 3.4750 & 291.7 & 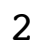 & 600.0 & 00.0 \\
\hline 02 & 92714 & 200.5957 & 00 & 291.8 & 3 & .0 & \\
\hline 02 & 92715 & 164.0269 & 6.5250 & 291.1 & 4 & 600.0 & .0 \\
\hline 02 & 92716 & 89.7204 & 3.5750 & 292.5 & 3 & .0 & .0 \\
\hline 02 & 92717 & 88.1230 & 3.4 & 292.0 & 2 & .0 & \\
\hline 02 & 92718 & 92.7403 & 4.1500 & 290.4 & 3 & 60 & \\
\hline 02 & 92719 & 84.2498 & 6.0 & 288.4 & 4 & .0 & .0 \\
\hline 02 & 92720 & 73.2505 & 6 . & 287.3 & 4 & 6 & \\
\hline 02 & 92721 & 77.0011 & 5.5 & 286.7 & 4 & .0 & \\
\hline 02 & 92722 & 71.0000 & 6.1000 & 286.7 & 4 & 600.0 & .0 \\
\hline 02 & 92723 & 67.2498 & $5 . \varepsilon$ & 286.8 & 4 & 6 & \\
\hline 02 & 92724 & 71.5000 & & 286.9 & 4 & .0 & \\
\hline 02 & 9281 & 75.9988 & 4.3 & 286.8 & 4 & 600.0 & .0 \\
\hline 02 & 9282 & 84.7500 & 4.0500 & 286.5 & 5 & 0.0 & .0 \\
\hline 02 & 9283 & 126.2448 & 2.5 & 286.4 & 5 & .0 & \\
\hline 02 & 9284 & 242.3841 & 1.0250 & 286.1 & 6 & .0 & .0 \\
\hline 02 & 9285 & 243.2545 & 0.9750 & 285.6 & 6 & 6 & \\
\hline 02 & 9286 & 290.0047 & 0.52 & 285.3 & 6 & .0 & \\
\hline 02 & 9287 & 356.4992 & 0.72 & 285.4 & 6 & .0 & 0.0 \\
\hline 02 & 9288 & 29.2541 & 1.0500 & 286.2 & 5 & 600.0 & 0.0 \\
\hline 02 & 9289 & 301.1105 & 0.8250 & 287.6 & 4 & 0 & 0.0 \\
\hline 02 & 92810 & 273.7800 & 2.1000 & 287.6 & 3 & 600.0 & 600.0 \\
\hline 02 & 92811 & 274.5126 & 2.4250 & 288.2 & 2 & 600.0 & 600.0 \\
\hline 02 & 92812 & 265.7507 & 3.15 & 289.1 & 2 & 600.0 & 600.0 \\
\hline 02 & 92813 & 257.2515 & 3.3750 & 289.4 & 2 & 600.0 & 600.0 \\
\hline 02 & 92814 & 258.7818 & 3.1500 & 290.9 & 2 & 600.0 & 600.0 \\
\hline 02 & 92815 & 251.8045 & 3.77 & 291.2 & 2 & 600.0 & 600.0 \\
\hline 02 & 92816 & 247.7550 & 4.05 & 291.3 & 3 & 600.0 & 600.0 \\
\hline 02 & 92817 & 260.9773 & 4.5750 & 290.7 & 3 & 600.0 & 600.0 \\
\hline 02 & 92818 & 297.9596 & 4.1000 & 290.5 & 4 & 600.0 & .0 \\
\hline 02 & 92819 & 333.2334 & 1.8500 & 289.8 & 4 & 600.0 & 600.0 \\
\hline 02 & 92820 & 291.8998 & 1.6250 & 289.5 & 5 & 600.0 & 600.0 \\
\hline 02 & 92821 & 110.8794 & 2.3000 & 288.9 & 6 & 600.0 & 600.0 \\
\hline 02 & 92822 & 97.2111 & 3.4500 & 288.3 & 5 & 600.0 & 600.0 \\
\hline 02 & 92823 & 79.7498 & 5.500 & 287.8 & 4 & 600.0 & 600.0 \\
\hline
\end{tabular}




\begin{tabular}{|c|c|c|c|c|c|c|c|}
\hline 2 & 92824 & 73.7497 & 6.4000 & 287.7 & 4 & 600.0 & 600.0 \\
\hline 02 & 9291 & 77.4994 & 6.8250 & 287.5 & 4 & 600.0 & 600.0 \\
\hline 02 & 9292 & 77.7497 & 6.8000 & 287.7 & 4 & 600.0 & 00.0 \\
\hline 02 & 9293 & 74.2501 & 6.9750 & 287.8 & 4 & 600.0 & 00.0 \\
\hline 02 & 9294 & 75.2496 & 4.4250 & 287.3 & 4 & 600.0 & 00.0 \\
\hline 02 & 9295 & 76.7535 & 6.9000 & 287.0 & 4 & 600.0 & 00.0 \\
\hline 02 & 9296 & 80.0000 & 7.1250 & 286.6 & 4 & 600.0 & 00.0 \\
\hline 02 & 9297 & 79.0012 & 7.4250 & 285.8 & 4 & 600.0 & 00.0 \\
\hline 02 & 9298 & 74.5001 & 7.3250 & 286.6 & 4 & 600.0 & 00.0 \\
\hline 02 & 9299 & 79.2502 & 5.4750 & 288.5 & 4 & 600 & 0 \\
\hline 02 & 92910 & 92.0030 & 3.9750 & 290.8 & 3 & 600.0 & \\
\hline 02 & 92911 & 83.5000 & 3.2750 & 291.8 & 2 & 600.0 & \\
\hline 02 & 92912 & 73.0827 & 2.8500 & 293.6 & 1 & 600.0 & .0 \\
\hline 02 & 92913 & 32.7677 & 3.6750 & 293.8 & - & 600.0 & 00.0 \\
\hline 02 & 92914 & 101.7286 & 3. & 294.8 & 2 & .0 & \\
\hline 02 & 92915 & 105.4798 & 5.4000 & 294.3 & 3 & 600.0 & .0 \\
\hline 02 & 92916 & 89.7209 & 5.8500 & 293.7 & 3 & 60 & .0 \\
\hline 02 & 92917 & 86.7504 & 7.2000 & 292.3 & 4 & .0 & \\
\hline 02 & 92918 & 75.7496 & 7.8750 & 290.1 & 4 & 60 & .0 \\
\hline 02 & 92919 & 73.2501 & 9.6500 & 288.0 & 4 & .0 & .0 \\
\hline 02 & 92920 & 75.5000 & 9 . & 287.7 & 4 & 6 & \\
\hline 02 & 92921 & 77.0002 & 9.4750 & 287.0 & 4 & .0 & \\
\hline 02 & 92922 & 71.2498 & 9.8500 & 285.7 & 4 & 600.0 & .0 \\
\hline 02 & 92923 & 69.9998 & 0.47 & 285.2 & 4 & 6 & \\
\hline 02 & 92924 & 65.4991 & 0 & 285.5 & 4 & .0 & \\
\hline 02 & 9301 & 73.2486 & 7.3750 & 286.4 & 4 & .0 & 600.0 \\
\hline 02 & 9302 & 80.1364 & 4.0250 & 287.6 & 4 & .0 & .0 \\
\hline 02 & 9303 & 79.7497 & 5.80 & 286.8 & 4 & .0 & \\
\hline 02 & 9304 & 129.3375 & 2.9250 & 285.7 & 5 & .0 & .0 \\
\hline 02 & 9305 & 147.3363 & 1.9750 & 284.1 & 6 & 6 & .0 \\
\hline 02 & 9306 & 87.2527 & $\triangle 15$ & 284.2 & 5 & .0 & \\
\hline 02 & 9307 & 81.5013 & 3.70 & 285.1 & 4 & .0 & 600.0 \\
\hline 02 & 9308 & 53.2875 & 1.9750 & 286.3 & 3 & 600.0 & 600.0 \\
\hline 02 & 9309 & 273.1051 & 160 & 288.5 & 2 & .0 & 0.0 \\
\hline 02 & 93010 & 193.5076 & 3.77 & 288.6 & 3 & 600.0 & 600.0 \\
\hline 02 & 93011 & 176.5000 & 5.2750 & 289.1 & 3 & 600.0 & 600.0 \\
\hline 02 & 93012 & 185.2360 & 4.8500 & 289.9 & 3 & 600.0 & 0.0 \\
\hline 02 & 93013 & 180.7496 & 5.3250 & 290.7 & 3 & 600.0 & 600.0 \\
\hline 02 & 93014 & 179.0000 & 5.7000 & 291.5 & 3 & 600.0 & 600.0 \\
\hline 02 & 93015 & 165.5000 & 4.1500 & 292.6 & 3 & 600.0 & 600.0 \\
\hline 02 & 93016 & 162.5416 & 5.4250 & 293.3 & 3 & 600.0 & 600.0 \\
\hline 02 & 93017 & 114.6571 & 5.0250 & 293.4 & 3 & 600.0 & 600.0 \\
\hline 02 & 93018 & 86.7476 & 5.7250 & 291.8 & 4 & 600.0 & 600.0 \\
\hline 02 & 93019 & 79.9989 & 5.7750 & 290.2 & 4 & 600.0 & 600.0 \\
\hline 02 & 93020 & 75.5000 & 6.4500 & 289.7 & 4 & 600.0 & 600.0 \\
\hline 02 & 93021 & 70.5014 & 7.4750 & 288.9 & 4 & 600.0 & 600.0 \\
\hline 02 & 93022 & 71.9989 & 8.7250 & 288.7 & 4 & 600.0 & 600.0 \\
\hline$U<$ & 93023 & 73.5038 & 7.325 & 288.8 & 4 & 600.0 & 600.0 \\
\hline
\end{tabular}




\begin{tabular}{|c|c|c|c|c|c|c|c|}
\hline & & 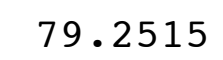 & 7.5500 & 289.2 & & & \\
\hline 210 & 1 & 7 חמחמ & 8.4500 & 288.8 & & & \\
\hline & 2 & 14 & 500 & 89.0 & & 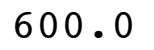 & \\
\hline 21 & 3 & 50.7519 & 1.6750 & 288.7 & & 00 & \\
\hline & 4 & & & & & & \\
\hline & 5 & 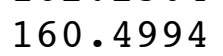 & 0 & 3 & & & \\
\hline & 6 & 157.7499 & .750 & 86.3 & & & \\
\hline & 7 & 160 & 00 & & & & \\
\hline & 8 & 164 & & 4 & & & \\
\hline 10 & 9 & 168.2503 & 2.7750 & 86 & & & \\
\hline 210 & 110 & 2494 & 0 & 7 & & & \\
\hline & 111 & $=72$ & & & & & \\
\hline 10 & 12 & 155 & 0 & 89 & & & \\
\hline 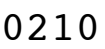 & 113 & 155 & 0 & 7 & & & \\
\hline & 171 & & & & & & \\
\hline 210 & 115 & 155 & & 2 & & & \\
\hline & 16 & 165 & 0 & 292 & & & \\
\hline & 117 & 168 & & 6 & & & \\
\hline 0 & 118 & 165 & & 291 & & & \\
\hline 210 & 119 & 154 & & 29 & & & \\
\hline & 120 & & & & & & \\
\hline & 121 & & & & & & \\
\hline 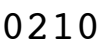 & 122 & 156 & & 28 & & & \\
\hline 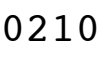 & 123 & & 0 & $2 \varepsilon$ & & & \\
\hline & 124 & & & & & & \\
\hline & 1 & 154 & & 28 & 4 & & \\
\hline 0 & 2 & 152 & & & & & \\
\hline & 3 & 155 . & & & & & \\
\hline 210 & 4 & 1 & 0 & 28 & 4 & & \\
\hline 210 & 25 & 158 . & & $2 \varepsilon$ & & & \\
\hline & 6 & 162 . & & & 4 & & \\
\hline 0 & 7 & 16 & 0 & 28 & 4 & & \\
\hline & 28 & 162.75 & & 285.8 & & & \\
\hline 210 & 9 & 166 & & & 4 & & \\
\hline & 210 & 1 & & & 4 & & \\
\hline & 211 & 171 . & & & 4 & & \\
\hline & 212 & 182.7498 & 0 & 29 & 4 & & \\
\hline 210 & 213 & 182 . & 0 & 290.8 & 1 & & \\
\hline 210 & 214 & 179 . & 10 . & 291.5 & 4 & & \\
\hline & 215 & 167. & & 292.3 & 4 & & \\
\hline & 216 & 165 . & & & 4 & & \\
\hline 0210 & 217 & 174.7803 & & 293.3 & 4 & & \\
\hline & 218 & 169. & & 292.4 & 4 & & \\
\hline & 219 & 135 . & & 291.7 & & & \\
\hline 0210 & 220 & 149.0012 & & 291.5 & $I$ & & \\
\hline & 221 & 149.5005 & & 291.6 & כ & 0 & \\
\hline & 222 & & & 29 & & & \\
\hline & 23 & 115.7502 & ou & 292.5 & & 600.0 & 600 \\
\hline
\end{tabular}




\begin{tabular}{|c|c|c|c|c|c|c|c|}
\hline $2+0$ & 24 & & 6.7000 & 2.7 & & & \\
\hline & 1 & 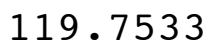 & 6.5000 & 92.3 & 4 & 6000 & \\
\hline & 32 & 116.7499 & .3250 & 291.9 & 4 & 00 & \\
\hline 21 & 33 & 133.1230 & 6.4500 & 291.4 & & 00.0 & \\
\hline & 4 & 49.9924 & .2000 & 90.5 & & 0 & \\
\hline & 5 & 155.0017 & 3.5250 & 289.3 & & $0 C$ & \\
\hline 2 & 36 & 145.2503 & 8000 & 290.2 & & 00 & U \\
\hline 2 & 7 & 46.5027 & 4.1750 & 289.2 & & .0 & \\
\hline 21 & 8 & 3.004 & 4.7500 & 90.4 & 3 & & \\
\hline 21 & 9 & 44.0000 & 8250 & 291.0 & & 00 & 0 \\
\hline 1 & 310 & 149.9969 & 8.1500 & 291.7 & & 00.0 & \\
\hline 2. & 11 & 34 & 9.1000 & 292.0 & $I$ & .0 & \\
\hline 21 & 12 & 161. & 7.12 & 292.7 & 4 & 00.0 & ( \\
\hline 21 & 13 & 160.7499 & 6.3500 & 294.0 & 4 & 0.0 & \\
\hline & 14 & 166 & & 95.2 & 3 & & \\
\hline 2 & 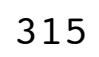 & 164 & & 296.1 & 3 & & \\
\hline 21 & 16 & 159.7981 & 4.1000 & 296.7 & 3 & 0.0 & 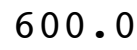 \\
\hline$z$ & 317 & 169 . & 3. & 296.8 & 4 & .0 & \\
\hline$?$ & 318 & 130 . & 4 . & 295.5 & 4 & & \\
\hline 21 & 319 & 86 . & 8250 & 294.2 & 4 & 0 & 0 \\
\hline 21 & 320 & 76 & .3750 & 293.4 & 4 & .0 & 00 \\
\hline 2 & 321 & 67 . & & 293.3 & 4 & & \\
\hline 2 & 322 & 62 & 000 & 293.4 & 4 & & \\
\hline 210 & 323 & 69.0000 & 7.5250 & 293.5 & 4 & 0 & 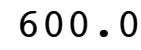 \\
\hline 2 & 324 & 54 . & 170 & 29 & 5 & & \\
\hline 0 & 41 & 19 & 2.4250 & 29 & 4 & & \\
\hline 2 & 42 & 44.8613 & 1.1500 & 293.5 & 5 & .0 & U. \\
\hline 2 & 43 & 159.24 & 3.42 & 291.8 & 4 & & \\
\hline 02 & 44 & 143. & 750 & 287.8 & 4 & & 0. \\
\hline 21 & 45 & 146 . & 3. & 287.9 & 5 & & 0 . \\
\hline 2 & 46 & 133.75 & 2.3750 & 288.2 & 4 & & 0 . \\
\hline 2 & 47 & 116.77 & 2 . & 289.0 & 5 & & \\
\hline & 48 & 149 . & 750 & 289.4 & 4 & & U • \\
\hline 2 & 49 & 165.2501 & 4.7500 & 290.4 & 4 & 0 & 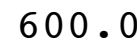 \\
\hline 2 & 410 & 167 & 750 & 291.0 & 4 & & \\
\hline & 411 & 164 & 4.4500 & 292.5 & 4 & & . \\
\hline . & 412 & 164.5000 & 4.8750 & 294.0 & 4 & .0 & 0 . \\
\hline 21 & 413 & 166.2479 & 750 & 295.4 & 3 & & 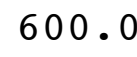 \\
\hline 02 & 414 & 173.2549 & 4.5000 & 296.3 & 3 & .0 & 600 . \\
\hline & 415 & 154.2086 & 4.6500 & 296.9 & 3 & .0 & 0 . \\
\hline & 416 & 154.5000 & 250 & 297.3 & 3 & & 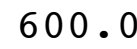 \\
\hline 02 & 417 & 161.2245 & 4.1250 & 297.5 & 4 & & 0 . \\
\hline 2 & 418 & 137.4796 & 1.5250 & 297.0 & 5 & 0 & 00 . \\
\hline & 419 & 74.2326 & 1.4750 & 296 & 4 & & \\
\hline 021 & 420 & 76.5001 & 5.6500 & & 4 & & 600. \\
\hline 2 & 421 & 79.9989 & 6.2250 & 294.9 & 4 & 600.0 & 600. \\
\hline & 422 & 2522 & & & 4 & & \\
\hline & 423 & 72.4999 & 7.6000 & 294.7 & 4 & 600.0 & 600 \\
\hline
\end{tabular}




\begin{tabular}{|c|c|c|c|c|c|c|c|}
\hline & 424 & 5.2507 & 7.0000 & 5.3 & 4 & 600.0 & \\
\hline 210 & 51 & 76.7500 & 7.4500 & 295.1 & 4 & 00.0 & \\
\hline 1 & 52 & 77.7500 & 7000 & 295.3 & 4 & 0 & \\
\hline & 3 & 79.2503 & .7000 & 295.3 & & 0 & \\
\hline 1 & 4 & 17.9727 & 500 & 294.7 & & 00 & \\
\hline 21 & 5 & 3.5740 & 3250 & 293.5 & & 00 & \\
\hline (1) & 6 & 74.9747 & 750 & 293.0 & & 0 & \\
\hline 1 & 7 & 48.0288 & 1.5000 & 93.2 & & & \\
\hline 21 & 8 & 141.9704 & 1.6750 & 294.7 & & 00.0 & 00 \\
\hline 21 & 9 & 5000 & 5.0000 & 295.1 & & & \\
\hline - & 10 & 159.4963 & 0 & 94.1 & & & \\
\hline 21 & 11 & 155 & 9 . & 294.2 & & 0 & 0 \\
\hline 210 & 512 & 7499 & 8.3500 & 294.9 & & & \\
\hline 210 & 513 & 164.2503 & 7.75 & 296.2 & & & \\
\hline 210 & 514 & 162 . & & 297.8 & 4 & & \\
\hline 210 & 515 & 168.2501 & .1000 & 298.7 & 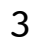 & 0 & \\
\hline 210 & 516 & 169.2609 & 0 & 299.1 & 3 & & \\
\hline 10 & 517 & 155.2494 & & 299.5 & 4 & & \\
\hline 210 & 518 & 164.7461 & 2 . & 298.6 & 4 & 0 & 500. \\
\hline 21 & 519 & 131.7738 & 1 . & 297.3 & 5 & & \\
\hline 210 & 520 & 89.7959 & & 297.6 & 4 & & \\
\hline 210 & 521 & 4320 & & $29^{\circ}$ & 4 & & \\
\hline 210 & 522 & 80.7500 & 0 & 296.4 & 4 & 0 & 1 \\
\hline 210 & 523 & 82.4994 & & 296.6 & 4 & & 0 . \\
\hline 210 & 524 & 94 & & 29 & 4 & & \\
\hline 210 & 61 & 81.4991 & 0 & 297.2 & 4 & 0 & c \\
\hline 210 & 62 & 930 & 0 & 297.5 & 4 & & \\
\hline 210 & 63 & 00 & 0 & $29^{\circ}$ & 4 & & \\
\hline 210 & 64 & 126.9773 & 2 . & 296.7 & 5 & 0 & 0 \\
\hline 210 & 65 & 150 . & & 294.3 & 4 & & \\
\hline 210 & 66 & 3 & & 29 & 4 & & \\
\hline 210 & 67 & 108 . & & 29 & 4 & & \\
\hline 210 & 68 & 116.5104 & 4.90 & 295.4 & 3 & 0 & \\
\hline 210 & 69 & 161. & 0 & 296.4 & 4 & & \\
\hline 210 & 610 & 167.2500 & & 297.2 & 4 & & \\
\hline 210 & 611 & 171.7501 & 4 . & 298.4 & 3 & & \\
\hline 210 & 612 & 173.4987 & 0 & 298 & 3 & & \\
\hline 0210 & 613 & 177.2498 & 4.4750 & 299.9 & 3 & .0 & 0 . \\
\hline 0210 & 614 & 177.0000 & 4.3500 & 300.5 & 3 & .0 & \\
\hline 210 & 615 & 169 . & 0 & 300.8 & 3 & & \\
\hline 0210 & 616 & 168.7406 & 3 . & 301.1 & 3 & & 00 . \\
\hline 0210 & 617 & 161.5064 & 3.5500 & 300.9 & 4 & 0 & 00 \\
\hline 0210 & 618 & 151.0069 & 2.72 & 300.0 & 5 & 0 & \\
\hline 0210 & 619 & 111.2829 & 1.9750 & 299.7 & 5 & 600.0 & 600 \\
\hline 0210 & 620 & 99.6669 & 1.7750 & 299.4 & 4 & 600.0 & 00 \\
\hline 010 & 621 & 107.7888 & & 299.7 & 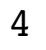 & & \\
\hline 210 & 622 & 112.7425 & 2 . & 299.1 & 4 & 600 & 600 \\
\hline 10 & 23 & 85.2496 & 5.0250 & 299. & 4 & 600.0 & 60 \\
\hline
\end{tabular}




\begin{tabular}{|c|c|c|c|c|c|c|c|}
\hline & 24 & 9.7500 & 7.2250 & 8.3 & 4 & 00.0 & \\
\hline 210 & 1 & 95.2522 & 4.4000 & 298.8 & 4 & 00.0 & \\
\hline 1 & 72 & 149.2188 & 0000 & 297.5 & & 0 & \\
\hline & 3 & 4.8119 & .9750 & 298.0 & & 00 & \\
\hline 1 & 4 & 95.9685 & 4500 & 297.3 & & 00 & \\
\hline 21 & 75 & 838 & 4500 & 297.6 & & 0 & \\
\hline 21 & 6 & 161 & .2500 & 295.7 & & 0 & \\
\hline & 7 & 28 & 50 & 9 & & & \\
\hline 21 & 8 & 151.2158 & 1.7750 & 296.5 & & 00.0 & 0 \\
\hline 21 & 79 & 165.2503 & 3.5000 & 296 & & & \\
\hline 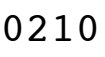 & 710 & 165.2489 & 3.1000 & 297.7 & & & \\
\hline 210 & 711 & 166. & 3.3250 & 299.1 & & 0 & \\
\hline 210 & 712 & 0002 & 5.0750 & 299.7 & & & \\
\hline 210 & 713 & 165.5000 & 7750 & 300.2 & & & \\
\hline 210 & 714 & 169. & .2500 & 300.9 & 3 & & \\
\hline 210 & 715 & 157.4973 & 4.6250 & 302.1 & 4 & 0 & \\
\hline 210 & 716 & 163. & 4.6750 & 302.4 & 3 & & \\
\hline 210 & 717 & 158 . & 4.5250 & 302.4 & 4 & & \\
\hline 210 & 718 & 154.2517 & 3.2000 & 301.2 & & 0 & 600 . \\
\hline 210 & 719 & 581 & 5000 & 9 & 4 & 0 & \\
\hline 210 & 720 & 136 . & 0 & & 4 & & \\
\hline 210 & 721 & 519 & 3500 & 29 & 4 & & \\
\hline 210 & 722 & 77.4997 & 5750 & 299.2 & 4 & 0 & 1 \\
\hline 210 & 723 & 76.2496 & 500 & 299.2 & 4 & & \\
\hline 210 & 724 & 999 & 750 & 29 & 4 & & \\
\hline 210 & 81 & 76.7500 & 8500 & 299.1 & 4 & 0 & c \\
\hline 210 & 82 & 76.4994 & 5250 & .7 & 4 & & \\
\hline 210 & 83 & 107 . & 500 & 299.7 & 4 & & \\
\hline 210 & 84 & 126.4045 & 1.6000 & 297.5 & 5 & 0 & 0 \\
\hline 210 & 85 & 44 & 3000 & 297.4 & 6 & & \\
\hline 210 & 86 & 117 & 5750 & 29 & 6 & & \\
\hline 210 & 87 & 069 & 500 & 296.1 & 5 & & \\
\hline 210 & 88 & 103.2773 & 0.6250 & 299.5 & 4 & 0 & \\
\hline 210 & 89 & 165 . & 3.1000 & 298.9 & 4 & & \\
\hline 210 & 810 & 166. & 5750 & 298.7 & 4 & & \\
\hline 210 & 811 & 163.7501 & 8500 & 298.4 & 4 & & \\
\hline 210 & 812 & 163. & 0 & 299.1 & 4 & & \\
\hline 0210 & 813 & 168.7552 & 2.6500 & 301.4 & 3 & & 0 . \\
\hline 210 & 814 & 182.9539 & 5250 & 302.0 & 2 & .0 & \\
\hline 210 & 815 & 167 . & 000 & 302.0 & 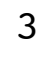 & & \\
\hline 0210 & 816 & 160.7498 & 3.6500 & 302.6 & 4 & & 00 . \\
\hline 210 & 817 & 158.7503 & 3.5000 & 302.5 & 4 & 0 & م \\
\hline 0210 & 818 & 151.2686 & 3.4000 & 300.8 & 4 & 0 & \\
\hline 0210 & 819 & 84.8719 & 3.5750 & 300.3 & 4 & 600.0 & 600. \\
\hline 0210 & 820 & 74.2429 & 5.6750 & 300.1 & 4 & 600.0 & 00 \\
\hline 010 & 821 & & 500 & 300.2 & 4 & & \\
\hline 0210 & 822 & 73.7502 & 6.7500 & 299.9 & 4 & 600 & 600 \\
\hline 210 & 823 & 76.9998 & 7.0500 & 299.6 & 4 & 60 & 60 \\
\hline
\end{tabular}




\begin{tabular}{|c|c|c|c|c|c|c|}
\hline 0 & - & 6750 & 98.5 & & 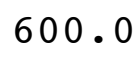 & \\
\hline 109 & 4.2501 & 8.5250 & 298.8 & & 00.0 & \\
\hline $\begin{array}{lll}0 & 9 & 2\end{array}$ & 78.7500 & 5000 & 299.5 & & 00 & \\
\hline 109 & 86.7504 & 7500 & 299.7 & & 00 & \\
\hline $\begin{array}{lll}0 & 9 & 4\end{array}$ & & 7250 & 9.0 & & & \\
\hline 09 & 0.7908 & 4000 & 98.6 & & 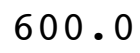 & \\
\hline 2109 & 2302 & 3500 & 298.3 & & 00 & \\
\hline $\begin{array}{lll}0 & 9 & 7\end{array}$ & 800 & 3500 & 97.5 & & & \\
\hline 09 & 745 & & 9 & & & \\
\hline 21099 & 5061 & 7500 & 97.8 & & 0 & \\
\hline 210910 & 162.75 & 4.00 & 298 & & & \\
\hline $0 \quad 911$ & 167.75 & & 99.1 & & & \\
\hline 210912 & 163. & 4. & 299.1 & & & \\
\hline 0913 & 168.7 & 0 & 4 & & & \\
\hline $0 \quad 914$ & & & & & & \\
\hline 915 & & & 01.1 & & & \\
\hline 0916 & 102.5 & & 7 & & & \\
\hline $0 \quad 917$ & 77 & & 3 & & & \\
\hline $0 \quad 918$ & 68. & 0 & 297 & & & \\
\hline $0 \quad 919$ & 71. & & 297 & 4 & & \\
\hline 10920 & 70. & 0 & 297.5 & 4 & & \\
\hline $0 \quad 921$ & & & 6 & 4 & & \\
\hline 922 & & & 297 & 4 & & \\
\hline $0 \quad 923$ & 81.2 & & 296 & 4 & & \\
\hline 924 & 80 . & & 29 & 4 & & \\
\hline 101 & 79. & & 29 & 4 & & \\
\hline 102 & 79.2503 & 0 & 296.2 & 4 & & 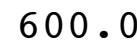 \\
\hline 103 & 71.7 & & 296 & 4 & & \\
\hline 104 & 79 . & & 29 & 4 & & \\
\hline 210105 & 76.7505 & & 292.8 & 4 & & . \\
\hline 210106 & 75.4997 & 50 & 290.9 & 4 & & . \\
\hline 210107 & 91.4 & & 293 & 5 & & \\
\hline 108 & & & & 4 & & \\
\hline 210109 & 77.5000 & 750 & 291.9 & 3 & & \\
\hline 010 & 59. & & 29 & 3 & & \\
\hline 011 & 72.8580 & & & 3 & & \\
\hline 012 & 620 & 1500 & 291.4 & 3 & & \\
\hline 013 & 72 . & 0 & 292 & 2 & & \\
\hline 1014 & 86.2511 & 5000 & 293.6 & 3 & & . \\
\hline 2101015 & 552 & .750 & 292.7 & 4 & & \\
\hline 016 & 75.2502 & 50 & 291.3 & 4 & & \\
\hline 017 & & 2750 & & 4 & & \\
\hline 018 & 68.5002 & 8.0750 & 290.2 & 4 & & 0 \\
\hline 02101019 & 74.9995 & 500 & & 4 & & \\
\hline 02101020 & 74.5000 & 0.1750 & 287.2 & 4 & & \\
\hline 02 & 74.5000 & 11.1500 & 286.6 & 4 & & 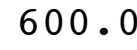 \\
\hline & & 0 & & 4 & & \\
\hline 1023 & 77.2506 & 11.7250 & 286.4 & & & s \\
\hline
\end{tabular}




\begin{tabular}{|c|c|c|c|c|c|c|}
\hline 024 & 84.7514 & 9.6750 & 286.6 & 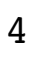 & 600.0 & \\
\hline 0111 & 84.7500 & 9.4250 & 86.2 & 4 & 600.0 & \\
\hline 10112 & 84.7382 & 7250 & 286.5 & 4 & 00 & \\
\hline 10113 & 164.0124 & 1.4750 & 285.3 & & 00 & \\
\hline 210114 & 140.8734 & .3500 & 83.5 & & 0 & \\
\hline 10115 & 100.2329 & 250 & 83.6 & & 00 & \\
\hline 210116 & 5008 & 7750 & 284.2 & & 00 & \\
\hline 210117 & 96.4987 & 8250 & 84.4 & & & \\
\hline 210118 & 94.7473 & 5500 & 1 & & & \\
\hline 210119 & 149.6808 & 5750 & 86.4 & & 0 & 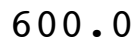 \\
\hline 2101110 & 157.7140 & 9000 & 287.8 & & & \\
\hline 11 & 168.7506 & 4.2000 & 89.0 & & & \\
\hline 21 & 157 . & 750 & 289.0 & 3 & 0 & \\
\hline 2101113 & 151.0000 & 5.0000 & 29 & & & \\
\hline 14 & 151. & 250 & & & & \\
\hline 5 & 154 & 0 & 293.5 & 4 & & \\
\hline 116 & 161.2504 & 9750 & 294.2 & 4 & .0 & \\
\hline 17 & 159.2502 & 000 & 0 & 4 & & \\
\hline 18 & 138.4382 & 500 & .4 & 4 & & \\
\hline 21 & 76.9876 & 1.6250 & 293.2 & 5 & 0 & $\bullet$ \\
\hline 21 & 793 & 1.9750 & 293.3 & 6 & 0 & \\
\hline 21 & 95 . & 750 & & 3 & & \\
\hline 22 & 350 & 1.9750 & 29 & 4 & & \\
\hline 123 & 60.5000 & 1.7250 & 293.7 & 4 & 0 & \\
\hline 021 & 62 . & & & 5 & & \\
\hline 21 & 103. & 000 & 29 & 6 & & \\
\hline 210122 & 155.9969 & 3500 & 292.0 & 5 & 0 & J. \\
\hline 210123 & 145 . & 500 & 291.5 & 4 & & \\
\hline 0210124 & 124 . & 500 & 29 & 4 & & \\
\hline 210125 & 152.7500 & 4.7000 & 291.1 & 4 & 0 & U. \\
\hline 210126 & 138.5023 & 1.9500 & 290.7 & 4 & & . \\
\hline 210127 & 126 . & 500 & 29 & 5 & & \\
\hline 210128 & 130 . & 000 & .9 & 4 & & . \\
\hline 210129 & 147 & 500 & 292.0 & 3 & 0 & 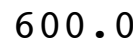 \\
\hline 021 & 149 . & 250 & 292.2 & 4 & & \\
\hline & 158.2502 & $\$ 750$ & 292.6 & 4 & & 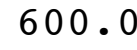 \\
\hline 2101212 & 167.7502 & 5250 & 293.0 & 4 & & 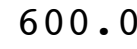 \\
\hline 0210 & 167.0000 & 50 & 293.9 & 4 & & 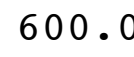 \\
\hline 021 & 166.2500 & 6.2500 & 295.1 & 4 & .0 & 0 . \\
\hline 2101215 & 166.2504 & 0750 & 295.8 & 3 & & \\
\hline 0210 & 160.5006 & 000 & 296.4 & 4 & & \\
\hline 021 & 155.2438 & 2.9750 & 296.3 & 4 & & 0 . \\
\hline 2101218 & 151.0032 & 1.9750 & 295.2 & 5 & 0 & 0 . \\
\hline 02101219 & 128.7344 & 250 & 294 & 5 & & \\
\hline 02101220 & 138.7171 & 1.0250 & 294.2 & 5 & & 0 . \\
\hline 2101221 & 106.3880 & 1.3250 & 294.4 & 6 & & 600 \\
\hline & 92 & & & 6 & & \\
\hline 2101223 & 114.5360 & 2.2000 & 294.9 & & .0 & 60 \\
\hline
\end{tabular}




\begin{tabular}{|c|c|c|c|c|c|c|}
\hline 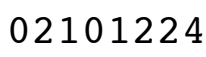 & (2) & 3.1750 & 295.0 & 4 & 600.0 & \\
\hline 0131 & 117.3083 & 1.3500 & 294.4 & & 600.0 & \\
\hline 10132 & 145.2415 & .8750 & 294.0 & 6 & 00 & \\
\hline 10133 & 103.5889 & 0250 & 292.4 & & 00 & \\
\hline 210134 & 44.6626 & .2250 & 92.6 & & 0 & \\
\hline 210135 & 150.0102 & 500 & 292.6 & & 00 & \\
\hline 210136 & 3360 & 250 & 293.0 & & 00 & \\
\hline 210137 & 105.2336 & 750 & 93.0 & & & \\
\hline 210138 & 153.8341 & 9250 & 94.9 & & & \\
\hline 210139 & 164 & 3.2500 & 293.0 & 4 & 0 & - \\
\hline 2101310 & 164.0006 & 4.4250 & 292.9 & & & \\
\hline 11 & 165.5000 & 5000 & 94.0 & 4 & & \\
\hline 21 & 165 & 750 & 294.8 & 4 & 0 & \\
\hline 21 & 167.5000 & 3.9250 & 296.1 & 3 & 0.0 & \\
\hline 14 & 167 . & 00 & 2 & & & \\
\hline 5 & 154 . & 2. & 298.3 & 3 & & \\
\hline 316 & 160.7501 & 3.5250 & 298.2 & 4 & .0 & . \\
\hline 17 & 151.4 & 50 & 5 & 4 & & \\
\hline 18 & 145 . & 000 & 296.7 & 5 & & \\
\hline 21 & 135 & 3750 & 296.3 & 4 & 0 & 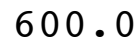 \\
\hline 21 & 135.2554 & 1.8250 & 296 & 5 & 0 & \\
\hline 1 & 114.7 & 500 & & 4 & & \\
\hline 21 & 97 . & 2.8250 & 297.1 & 4 & & \\
\hline 23 & 80.4 & 2.4000 & 297.1 & 4 & 0 & \\
\hline 21 & 85 . & & 297.1 & 4 & & \\
\hline 021 & 81 . & 4250 & 29 & 5 & & \\
\hline 210142 & 96.7316 & 3500 & 296.3 & 6 & 0 & J. \\
\hline 210143 & 154.24 & 3000 & 29 & 6 & & \\
\hline 021 & 164 & 000 & 295.3 & 5 & & \\
\hline 210145 & 167.5092 & 0.7000 & 294.3 & 5 & 0 & 0. \\
\hline 210146 & 133.7516 & 1.2000 & 294.0 & 4 & 0 & S \\
\hline 21 & 140.2498 & 1.2250 & 293.7 & 4 & & \\
\hline 210148 & 147.74 & 5500 & .4 & 4 & & . \\
\hline 0210149 & 150.9751 & 1.9750 & 293.4 & 4 & 0 & 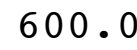 \\
\hline 21 & 162 . & 2 . & & 4 & & \\
\hline & 166.5000 & 3.6000 & 296.0 & 4 & & ( \\
\hline 210 & 167.2500 & 4.7000 & 296.3 & 4 & & 0 . \\
\hline 0210 & 165.0 & 0 & 29 & 3 & & 0 . \\
\hline 021 & 184.2503 & 4.0000 & 298.4 & 3 & .0 & 0 . \\
\hline 2101415 & 188.2539 & 2.8500 & 299.3 & 2 & & \\
\hline 0210 & 214.5037 & 750 & 299.5 & 1 & & \\
\hline 021 & 169.2727 & 2.3000 & 299.0 & 2 & & 0 . \\
\hline 02101418 & 137.5484 & 2.7500 & 297.5 & 3 & 0 & 0 . \\
\hline 0210 & 86.7499 & 250 & 296 & 4 & & \\
\hline 02101420 & 79.2500 & 5.8250 & 295.6 & 4 & & 0 . \\
\hline 210 & 78.0000 & 6.9500 & 294.9 & 4 & .0 & 00 . \\
\hline & 77 & & 294.4 & 4 & & \\
\hline 2101423 & 79.2500 & 8.7500 & 294.2 & $\Psi$ & 600.0 & 60 \\
\hline
\end{tabular}




\begin{tabular}{|c|c|c|c|c|c|c|}
\hline & 0.1500 & 9.3000 & 93.5 & 4 & - & \\
\hline 0151 & 0.0001 & 9.3000 & 293.3 & & 600.0 & \\
\hline 10152 & 5000 & 1000 & 293.2 & 4 & 00 & \\
\hline 10153 & 78.7500 & 6500 & 292.9 & & 00 & \\
\hline 0154 & 000 & .2000 & 92.9 & & & \\
\hline 10155 & 000 & 9750 & 92.7 & & 0 & \\
\hline 210156 & 82.5000 & $\$ 500$ & 292.3 & & 00 & \\
\hline 210157 & 84.5000 & & 92.7 & & & \\
\hline 10158 & 0.7497 & & 93.0 & & & \\
\hline 210159 & 51.5000 & 1.6500 & 293.7 & & 0 & \\
\hline 510 & 31.7421 & 1.60 & 295.3 & & & \\
\hline & 07.1343 & 12 & 94.2 & & & \\
\hline 21 & 86 & 6 . & 293.0 & & & \\
\hline 3 & 3 & 0.04 & 93.0 & & & \\
\hline & & & & & & \\
\hline & 86 & & 292.9 & 4 & & \\
\hline 6 & 82. & 9.0 & 292.3 & 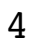 & & \\
\hline 17 & 79 & 0 & 1.2 & & & \\
\hline & 79 & & 289.2 & & & \\
\hline 9 & & 0 & 288.0 & 4 & & \\
\hline 20 & 80 . & 3.5500 & 286.8 & 4 & & \\
\hline & 0 & & & 4 & & \\
\hline 2 & 9 & & & 4 & & \\
\hline 23 & 80.7500 & 0 & & 4 & & \\
\hline 24 & 78 & & & 4 & & \\
\hline 61 & 76 . & 11 & & 4 & & \\
\hline 210162 & 502 & 0 & 7 & 4 & & \\
\hline 210163 & 83. & & & 4 & & \\
\hline 64 & 79 & 0 & & 4 & & \\
\hline 210165 & 82 . & & 8 & 4 & & . \\
\hline 210166 & 83.0000 & 3000 & 28 & 4 & & \\
\hline 67 & 81 & 1 & & 4 & & \\
\hline 168 & & & & 4 & & \\
\hline 210169 & 500 & & 28 & 4 & & \\
\hline & & & & 4 & & \\
\hline & 84 . & & 7.6 & 4 & & \\
\hline & 006 & 5500 & 289.1 & 3 & & \\
\hline 13 & 7 . & & & 4 & & \\
\hline 14 & 90.2498 & 6.7500 & 290.8 & 4 & & . \\
\hline 515 & 86.0021 & 7.1250 & 290.8 & 4 & & \\
\hline & 75.2517 & 7 . & & 4 & & \\
\hline & 71.7503 & 8.0750 & & 4 & & \\
\hline 618 & .5000 & 8.8250 & 288.3 & 4 & & 0 \\
\hline 210 & 503 & 0 & & 4 & & \\
\hline 02101620 & 69.7445 & 8.5250 & & 7 & & \\
\hline 210 & 79.2501 & 10.0500 & 285.7 & 4 & & 0 . \\
\hline & & & & 4 & & \\
\hline 2101623 & 85.2501 & 11.0000 & 284.5 & & & s \\
\hline
\end{tabular}




\begin{tabular}{|c|c|c|c|c|c|c|}
\hline$x$ & 1.2000 & 0.5000 & 83.9 & 4 & (6) & \\
\hline 0171 & 4.2501 & 10.7500 & 83.7 & 4 & 00.0 & \\
\hline 0172 & 82.4997 & 500 & 283.3 & 4 & 00 & \\
\hline 10173 & 79.7500 & 1250 & 283.3 & & 00 & \\
\hline 210174 & 32.2499 & 8.2750 & 33.0 & & & \\
\hline 10175 & 2.4999 & 500 & 82.9 & & 0 & \\
\hline 210176 & 7518 & 3500 & 82.6 & & 00 & \\
\hline 210177 & 80 . & 500 & 82.7 & & & \\
\hline 210178 & 0 & 9250 & 83.4 & & & \\
\hline 210179 & 3684 & 000 & 85.5 & & 0 & \\
\hline 2101710 & 296.0048 & 1.9750 & 287.6 & & & \\
\hline 711 & 260.6 & 1.5250 & 38.9 & & & \\
\hline .712 & 170 . & 3. & 89.5 & & & \\
\hline 2101713 & 108.7 & 4.0000 & 91.2 & & & \\
\hline 714 & 94 . & & & & & \\
\hline & 84 . & & .1 & 4 & & \\
\hline 716 & 83.2 & 7.5000 & 290.6 & 4 & & \\
\hline 717 & 77 . & 00 & 2 & & & \\
\hline & 42 . & & & - & & \\
\hline 19 & & 000 & 288.6 & 4 & & \\
\hline 720 & 56.73 & 3.5000 & 287.6 & 4 & 0 & \\
\hline 21 & 82. & & & 4 & & \\
\hline 22 & 83. & 00 & & 4 & & \\
\hline 723 & 82.0 & 00 & .7 & 4 & & \\
\hline 724 & 82.2 & & & 4 & & \\
\hline 81 & 77. & 50 & & 4 & & \\
\hline 210182 & 79.0001 & 250 & 2.7 & 4 & & . \\
\hline 210183 & 80.7500 & 0 & & 4 & & \\
\hline 184 & 77 . & 0 & & 4 & & \\
\hline 210185 & 83.2500 & 500 & 282.8 & 4 & & J. \\
\hline 210186 & 85.7499 & 000 & 282.7 & 4 & & . \\
\hline 187 & 84.2 & 0 & & 4 & & \\
\hline 210188 & 83.5000 & & 3 & 4 & & 0 . \\
\hline 210189 & 86.2467 & 500 & 28 & 4 & & \\
\hline 810 & 176.8 & & & 3 & & \\
\hline 311 & 210.4742 & & & 2 & & \\
\hline 812 & 248.5516 & 750 & 289.4 & 1 & & \\
\hline 813 & 328.1531 & 2 . & 29 & 1 & & \\
\hline 0210 & 122.6873 & 3.8500 & 292.2 & 2 & & ( \\
\hline 2101815 & 118.2435 & 4.4500 & 291.9 & 3 & & \\
\hline 816 & 136.0658 & & & 3 & & \\
\hline 021 & 103.7502 & 4.5750 & 291.8 & 4 & & \\
\hline 2101818 & 81.4997 & 5.6250 & 290.1 & 4 & & 0 . \\
\hline 0210 & 68.7472 & 750 & & 4 & & \\
\hline 02101820 & 63.7506 & & & 4 & & \\
\hline 2101821 & 77.2506 & 5.3500 & 288.3 & 4 & & 0 \\
\hline & & & & 4 & & \\
\hline 2101823 & 34.9289 & 0.8250 & 287.4 & & .0 & 0 \\
\hline
\end{tabular}




\begin{tabular}{|c|c|c|c|c|c|c|}
\hline 824 & 8.2636 & .0000 & 287.3 & 5 & 600.0 & \\
\hline 0191 & 0.5009 & 4.0000 & 286.6 & 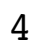 & 600.0 & \\
\hline 10192 & 79.7655 & 2.3750 & 286.6 & 5 & 00 & \\
\hline 10193 & 86.7499 & 2.8750 & 286.8 & & 00 & \\
\hline 210194 & 106.8226 & .2000 & 86.2 & & 0 & \\
\hline 10195 & 151.7583 & 1.3500 & 86.3 & & 00 & \\
\hline 210196 & 178.5021 & 7250 & 285.8 & & 00 & \\
\hline 210197 & 108.2485 & .5750 & 85.0 & & & \\
\hline 210198 & 17.5110 & 5000 & 87.4 & & & \\
\hline 210199 & 152.9733 & 1.2250 & 289.6 & & 00 & 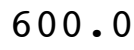 \\
\hline 2101910 & 166.5184 & 1.7500 & 289.7 & & 0 & \\
\hline 11 & 165.4989 & 3.5500 & 89.7 & & & \\
\hline 912 & 169.7502 & 4.2500 & 290.5 & & 0 & \\
\hline 913 & 165.7501 & 4.9500 & 291.6 & & 0.0 & \\
\hline 14 & 168 & & 7 & & & \\
\hline 15 & 164 . & & 293.3 & & & \\
\hline 916 & 166.0007 & 5.3000 & 293.5 & & .0 & 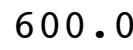 \\
\hline 17 & 143.7953 & 4 . & 293.4 & & & \\
\hline 18 & 83 . & & 291.9 & & & \\
\hline 21 & 78.0006 & 6.4750 & 290 & 4 & & 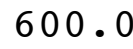 \\
\hline 20 & 79.5000 & 7.07 & 290.7 & & 0 & \\
\hline 21 & 77.9994 & & 290.5 & & & \\
\hline 22 & 79.0002 & 0 & 29 & 4 & & \\
\hline 23 & 76.2814 & 0 & 291.0 & & 0 & \\
\hline 24 & 70.7193 & & 29 & & & \\
\hline 021 & 167.8476 & 3750 & 290.3 & 5 & & \\
\hline 210202 & 261.9440 & 000 & 289.4 & & 0 & . \\
\hline 210203 & 202.1563 & 0 & 289.0 & 6 & & \\
\hline 0210204 & 109.2485 & 0 . & 288.3 & 6 & & \\
\hline 210205 & 165.5217 & 0 & 287.3 & 6 & 0 & 0 . \\
\hline 210206 & 179.8788 & 0 & 287.0 & 5 & & 0 . \\
\hline 210207 & 284.5052 & 0 & 287.3 & 6 & & \\
\hline 210208 & 165 . & 0 & 289.0 & 5 & 0 & 0 . \\
\hline 210209 & 294 . & 0 & 290.6 & 4 & 0 & 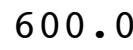 \\
\hline 021 & 244.7605 & 0 & 290.9 & 3 & & \\
\hline 021 & 229 . & 2.1500 & 291.4 & 2 & & 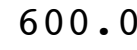 \\
\hline 012 & 149.7505 & 2.1250 & 292.3 & 1 & & 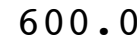 \\
\hline 0210 & 166.2506 & 4.17 & 292.9 & 2 & & 0 . \\
\hline 0210 & 172.0057 & 4.0750 & 293.8 & 3 & .0 & 0 . \\
\hline 02102015 & 176.2672 & 0250 & 294.3 & 3 & & \\
\hline 0210 & 167.0107 & 3. & 294.6 & 3 & & 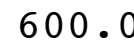 \\
\hline 0210 & 150.7506 & 2.1500 & 294.7 & 4 & & 0 . \\
\hline 02102018 & 148.3032 & 2.2000 & 293.1 & 4 & 0 & 0 \\
\hline 02102019 & 93.7662 & 000 & 292.8 & 4 & & \\
\hline 02102020 & 78.7495 & 5.8750 & 292.2 & 4 & & \\
\hline 2102021 & 76.7500 & 5.3750 & 292.7 & 4 & & 00 . \\
\hline & 02 & & 293.5 & 5 & & \\
\hline 2102023 & 114.8809 & 2.1000 & 293.1 & & 60 & 60 \\
\hline
\end{tabular}




\begin{tabular}{|c|c|c|c|c|c|c|}
\hline & & & & & & \\
\hline 021 & & 6500 & 0.7 & & 0 & \\
\hline 021 & 4.7836 & 3250 & 290.3 & & 00 & \\
\hline 1021 & 34.3703 & 3750 & 289.7 & & 0 & \\
\hline 021 & ? & 500 & 8.7 & & & \\
\hline 021 & & & 38.6 & & & \\
\hline 21021 & 68.2813 & 250 & 88.3 & & 0 & \\
\hline 21021 & 148 . & 500 & 87.4 & & & \\
\hline 1021 & 148 & 50 & 88.0 & & & \\
\hline 21021 & 163. & 500 & 89.8 & & & \\
\hline 210211 & 164 & 4.8750 & 90.6 & & & \\
\hline 1 & 158 & & 90.9 & & & \\
\hline 21 & 162 & 0 & 91.1 & & & \\
\hline 1021 & 163. & 7 . & 92.1 & & & \\
\hline 1 & & & 2 & & & \\
\hline & & & 294.3 & & & \\
\hline 021 & 163. & 0 & 294.7 & & 0 & \\
\hline 1 & 160 & 4. & 4.6 & & 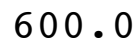 & \\
\hline & & & 293.4 & & & \\
\hline $2-1+2$ & 01 & 0 & 291.9 & & & \\
\hline 021 & 00 & 50 & 291.3 & & & \\
\hline 2. & & & & & & \\
\hline - & 05 & 0 & $2 \varepsilon$ & & & \\
\hline 210212 & 76 & 50 & 289.5 & 4 & & \\
\hline 12 & & & & & & \\
\hline 22 & 02 & 9. & & 4 & & \\
\hline 21022 & & 50 & 289.1 & 4 & & \\
\hline 21022 & 01 & 0 & $2 \varepsilon$ & 4 & & \\
\hline 21022 & 00 & 0 & $2 \xi$ & 4 & & \\
\hline 21022 & 492 & & 8.8 & 4 & & \\
\hline 21022 & 501 & & 286.7 & 4 & & \\
\hline 21022 & & 0 & 287.6 & 4 & & \\
\hline 21022 & 01 & & 287.4 & 4 & & \\
\hline 21022 & & 0 & 287.4 & 4 & & \\
\hline & & & & 4 & & \\
\hline & & & $2 \xi$ & 4 & & \\
\hline 100 & 01 & 7.8000 & 288.7 & 4 & & \\
\hline 0251 & & 7.1250 & 290.1 & 4 & & \\
\hline $\begin{array}{ll}2 \\
0\end{array}$ & & 7.5250 & 290.6 & 4 & & \\
\hline 02215 & 501 & 8250 & 290.9 & 4 & & \\
\hline & & 500 & 290.9 & 4 & & \\
\hline & & 9.5000 & 289.6 & 4 & & \\
\hline 10221 & 2498 & .5000 & 287.5 & 4 & 0 & \\
\hline 2102219 & & 2.7000 & 286.0 & 4 & & \\
\hline 2102220 & & & 285.0 & 4 & & \\
\hline 02221 & 5009 & 3.7000 & 284.1 & 4 & 600.0 & \\
\hline & & & & 4 & & \\
\hline 80 & 78.0001 & 12.6250 & 283.2 & $I$ & 600.0 & 6 \\
\hline
\end{tabular}




\begin{tabular}{|c|c|c|c|c|c|c|}
\hline- & .500 & 6500 & 83.3 & 4 & 000 & \\
\hline 210231 & 1.7500 & 11.6000 & 283.2 & 4 & 600.0 & \\
\hline 10232 & 79.5005 & 9.0000 & 283.2 & 4 & 00 & \\
\hline 10233 & 77.7500 & 8.7500 & 282.9 & & 00 & \\
\hline 210234 & 5.5011 & 9.3500 & 82.7 & & 0 & \\
\hline 210235 & 73.5002 & 500 & 82.7 & & 00 & \\
\hline 210236 & 71.7500 & 000 & 282.5 & & 00 & \\
\hline 210237 & 71.7500 & 500 & 82.4 & & & \\
\hline 210238 & 72.5000 & 0 & 82.6 & & & \\
\hline 210239 & 74.7499 & 250 & 83.7 & & 0 & 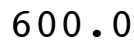 \\
\hline 2102310 & 84.2505 & 4.5750 & 285.1 & & & \\
\hline 11 & 91.0373 & 000 & 85.5 & & & \\
\hline 21 & 76. & 3.3500 & 286.7 & & 0 & \\
\hline 13 & 56.4 & 3.87 & 287.7 & & 0.0 & \\
\hline & 73. & & & & & \\
\hline & 84 . & & 287.3 & 3 & & \\
\hline 6 & 88 & 7.2250 & 286.2 & 4 & 0 & \\
\hline 17 & 77 . & 8 . & 6 & 4 & & \\
\hline 18 & 68. & 8 . & 283.6 & 4 & & \\
\hline 21 & 69 . & 8.4750 & 283.2 & 4 & & \\
\hline 20 & 70.5000 & 7.67 & 282.7 & 4 & 0 & \\
\hline 1 & 70.2 & & 32.5 & 4 & & \\
\hline 2 & 73. & 0 & 282.4 & 4 & & \\
\hline 23 & 76.4 & 8.60 & 282.3 & 4 & 0 & \\
\hline 24 & 74.7 & & 82.0 & 4 & & \\
\hline 021 & 72 . & 50 & 281.9 & 4 & & \\
\hline 210242 & 75.4995 & 8.0000 & 281.7 & 4 & 0 & U. \\
\hline 210243 & 74 . & 5000 & 31.5 & 4 & & \\
\hline $24 \quad 4$ & 72 . & 7 . & 281.4 & 4 & & \\
\hline 210245 & 73.7478 & 000 & 281.2 & 4 & & 0 • \\
\hline 210246 & 81.2500 & 6.8250 & 281.0 & 4 & & 0 . \\
\hline 247 & 76.4914 & 0 & 281.4 & 4 & & \\
\hline 210248 & 67.1765 & & 281.9 & 3 & & 0 . \\
\hline 210249 & 49.9780 & 4.8750 & 283.0 & 3 & 0 & \\
\hline & 64.23 & 0 & & 3 & & \\
\hline & 89.5061 & 3.6500 & 285.4 & 2 & & . \\
\hline 12 & 85.8189 & 2.7500 & 286.8 & 1 & & 0 . \\
\hline 21 & 356.1895 & 000 & 288.1 & 1 & & \\
\hline 0210 & 158.0014 & 3.9250 & 287.5 & 2 & .0 & 0 . \\
\hline 210 & 159.7420 & 4.6500 & 288.0 & 3 & & \\
\hline 021 & 160.2517 & 250 & 288.6 & 3 & & \\
\hline 021 & 153.2500 & 3.0750 & 288.5 & 4 & & 0 . \\
\hline 2102418 & 134.4769 & 1.1250 & 287.5 & 5 & 0 & 0 . \\
\hline 02102419 & 66.0055 & 2.6000 & & 5 & & \\
\hline 02102420 & 71.5000 & 5.2000 & & 4 & & \\
\hline 2102421 & 73.7500 & 6.4000 & 287.5 & 4 & & 600. \\
\hline & 12 & & & 4 & & \\
\hline 2102423 & 69.2500 & 7.8500 & 286.4 & & 60 & 60 \\
\hline
\end{tabular}




\begin{tabular}{|c|c|c|c|c|c|c|}
\hline 24 & 5.7505 & 7.6750 & 286.6 & . & 600.0 & \\
\hline 210251 & 9.7494 & 6.7750 & 286.8 & 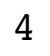 & 600.0 & \\
\hline 10252 & 3.4998 & 8750 & 287.1 & 4 & 00 & 0 \\
\hline 210253 & 5.9977 & 6.6500 & 287.4 & & 00 & \\
\hline 210254 & 2495 & .0000 & 87.6 & & 0 & \\
\hline 210255 & 85.2479 & 7500 & 87.3 & 4 & 00 & \\
\hline 210256 & 87.2515 & 7500 & 287.0 & 4 & 00 & \\
\hline 210257 & 5.7509 & 2500 & 85.8 & & & \\
\hline 210258 & 1.0000 & 2250 & 86.2 & & & \\
\hline 210259 & 0897 & 3250 & 287.4 & & 00 & 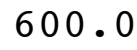 \\
\hline 2102510 & 284.4751 & 1.5250 & 288.6 & & 0 & \\
\hline 11 & 269.7253 & 1.4750 & 89.2 & & 00.0 & \\
\hline 21 & 148.3876 & 2.8000 & 285.9 & 1 & 0 & . \\
\hline 21 & 167.2382 & 3.2500 & 286.7 & & 0.0 & \\
\hline 14 & 014 & 50 & .5 & & & \\
\hline 5 & 420 & & 288.0 & 3 & & \\
\hline 16 & 160.2517 & 3.8250 & 288.6 & 3 & .0 & 8 \\
\hline 17 & .2500 & 0 & 38.5 & 4 & 0 & \\
\hline 18 & 134.4769 & 50 & 287.5 & 5 & & \\
\hline 21 & 055 & 2.6000 & 287.7 & 5 & 0 & 0 \\
\hline 21 & 71.5000 & 5.2000 & 287.7 & 4 & 0 & 8 \\
\hline 21 & 73.7500 & & 287.5 & 4 & & \\
\hline 22 & 2502 & 750 & 28 & 4 & & \\
\hline 23 & 69.2500 & 8500 & 286.4 & 4 & 0 & 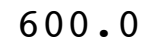 \\
\hline 24 & 7505 & & & 4 & & \\
\hline 61 & 7494 & 7750 & 286.8 & 4 & & \\
\hline 210262 & 63.4998 & 3750 & 287.1 & 4 & 0 & U. \\
\hline 210263 & 977 & 500 & 287.4 & 4 & & . \\
\hline 264 & 2495 & 00 & 287.6 & 4 & & \\
\hline 210265 & 85.2479 & 7500 & 287.3 & 4 & 0 & 0 • \\
\hline 210266 & 87.2515 & 5.7500 & 287.0 & 4 & 0 & 0 . \\
\hline 67 & 75.7509 & 0 & 285.8 & 4 & & \\
\hline 210268 & 71.0000 & 250 & 286.2 & 4 & & 0 . \\
\hline 210269 & 118.0897 & 3.3250 & 287.4 & 3 & 0 & . \\
\hline 021 & 284.4751 & 50 & 288.6 & 2 & & \\
\hline & 269.7253 & 1.4750 & 289.2 & 1 & & 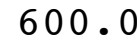 \\
\hline 512 & 243.0448 & 8000 & 289.9 & 1 & & . \\
\hline 21 & 251.3134 & 50 & 291.2 & 1 & & 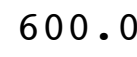 \\
\hline 0210 & 207.0757 & 1.9000 & 292.8 & 1 & .0 & 0 . \\
\hline 515 & 102.2148 & 4.3000 & 292.3 & 2 & 0 & o. \\
\hline 0210 & 71.7492 & 000 & 291.0 & 3 & & 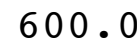 \\
\hline 021 & 75.4985 & 5.8750 & 290.4 & 4 & .0 & 600 . \\
\hline 2102618 & 72.4992 & 6.9250 & 288.8 & 4 & .0 & 00 . \\
\hline 02102619 & 68.2495 & 750 & 289.5 & 4 & & \\
\hline 02102620 & 61.4969 & 4.8500 & 288.8 & 4 & & 00 \\
\hline 2102621 & 67.7497 & 5.8750 & 287.2 & 4 & 600.0 & 600 \\
\hline & 023 & & 287.6 & 5 & & \\
\hline 2102623 & 23.5785 & 1.4500 & 287.7 & & 600.0 & 60 \\
\hline
\end{tabular}




\begin{tabular}{|c|c|c|c|c|c|c|}
\hline 24 & & 6250 & 7.0 & & & \\
\hline 271 & 1 & .1500 & 286.5 & 6 & 600.0 & \\
\hline 0272 & 3427 & 3000 & 284.4 & 6 & 00 & \\
\hline 10273 & 142.2515 & 3500 & 282.1 & & 00 & \\
\hline $21027 \quad 4$ & 61. & 1250 & 82.9 & & 0 & \\
\hline 10275 & 5365 & 1000 & 283.2 & & 00 & \\
\hline 210276 & 7501 & 2750 & 282.3 & & 00 & \\
\hline 210277 & 125 . & .3500 & 82.3 & & & \\
\hline 210278 & 76.02 & 0500 & 85.2 & 3 & & \\
\hline 210279 & 133.6400 & 0750 & 87.2 & & 0 & 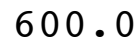 \\
\hline 2102710 & 161.7520 & 3.1250 & 288.0 & & & \\
\hline 2711 & 162.2435 & 117 & 88.9 & & & \\
\hline 2102712 & 159.74 & 4.3500 & 289.9 & & 0 & \\
\hline 2102713 & 156.4959 & 4.3750 & 291.4 & & 0.0 & \\
\hline 2714 & 154 & 0 & & & & \\
\hline 715 & 161 & 4. & 292.6 & 3 & & \\
\hline 2716 & 155 & 2 & 293.3 & 3 & .0 & . \\
\hline 2717 & 139. & 0 & 92.7 & 4 & & \\
\hline 2718 & 86 . & 0 & 291.7 & 4 & & \\
\hline 2719 & 75 & 87 & 290.6 & 4 & & $\bullet$ \\
\hline 2720 & 74.5000 & .7250 & 290.3 & 4 & 0 & 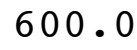 \\
\hline 721 & 76.2 & 0 & & 4 & & \\
\hline 722 & 77 . & 0 & 289 & 4 & & \\
\hline 723 & 78.2500 & 0 & 289.4 & 4 & 0 & \\
\hline 724 & 73.2 & & 289.3 & 4 & & \\
\hline 281 & 71.5 & 0 & 289.2 & 4 & & \\
\hline 210282 & 71.5000 & 1000 & 289.5 & 4 & 0 & . \\
\hline 210283 & 76 . & 0 & .7 & 5 & & \\
\hline 0210284 & 105 & 3 . & 28 & 4 & & \\
\hline 0210285 & 63.0 & 750 & 289.0 & 5 & 0 & 0 . \\
\hline 0210286 & 108.6122 & 2.0750 & 288.8 & 6 & 0 & s. \\
\hline 0210287 & 132.9 & 0 & 287.8 & 6 & & \\
\hline 0210288 & 55 . & 250 & 289.5 & 5 & & 0 . \\
\hline 0210289 & 94.6397 & 250 & 292.9 & 4 & 0 & \\
\hline 2810 & 168.0 & 3 . & 291.7 & 4 & & \\
\hline 811 & 161.9985 & 4.5250 & 291.7 & 4 & & . \\
\hline 2102812 & 156.5000 & 0250 & 291.6 & 3 & 0 & 0. \\
\hline 02102813 & 160.5014 & 4. & 29 & 3 & & 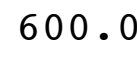 \\
\hline 02102814 & 155.5000 & 5.0500 & 293.9 & 4 & .0 & 0 . \\
\hline 2102815 & 157.5067 & 4.6750 & 294.7 & 4 & & \\
\hline 02102816 & 165.9995 & 250 & 295.0 & 3 & & 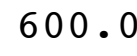 \\
\hline 021 & 134.7926 & 4.5500 & 294.4 & 4 & .0 & 0 . \\
\hline 2102818 & 75.2524 & 6.4000 & 293.1 & 4 & 0 & 00 . \\
\hline 02102819 & 64.2501 & 750 & 292.4 & 4 & & \\
\hline 02102820 & 68.2460 & 8.7250 & 292.4 & 4 & & 0 . \\
\hline 2102821 & 68.4945 & 8.6250 & 292.4 & 4 & 600 & 600 \\
\hline & & & 29 & 4 & & \\
\hline 2102823 & 66.5339 & 2.8500 & 291.2 & & .0 & $=0$ \\
\hline
\end{tabular}




\begin{tabular}{|c|c|c|c|c|c|c|}
\hline 324 & 0.5374 & 4.0750 & 90.4 & 4 & 600.0 & \\
\hline 0291 & 2.7474 & 5.8500 & 289.6 & 4 & 600.0 & \\
\hline 0292 & 84.7683 & 8750 & 289.3 & 4 & 00 & \\
\hline 10293 & 85.0000 & 1000 & 289.2 & & 00 & \\
\hline 210294 & 120.7981 & 5000 & 89.0 & & 0 & \\
\hline 10295 & 120.2446 & 250 & 88.7 & & 00 & \\
\hline 210296 & 130.2631 & 500 & 288.0 & & 00 & \\
\hline 210297 & 116.8762 & 500 & 87.4 & & & \\
\hline 210298 & 96.5086 & 000 & 88.8 & & & \\
\hline 210299 & 151.7032 & 5250 & 289.9 & 4 & 0 & 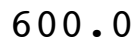 \\
\hline 2102910 & 166.7497 & 3.0750 & 290.7 & & & \\
\hline 911 & 166.0034 & 3.7250 & 90.7 & & & \\
\hline 912 & 165.2509 & 5 . & 291.4 & 4 & 0 & \\
\hline 2913 & 164.9994 & 5.4000 & 292.5 & 3 & 0.0 & \\
\hline 2914 & 166.7499 & 0 & & & & \\
\hline 15 & $135.6^{\circ}$ & 4 . & 29 & 3 & & \\
\hline 916 & 88.2415 & 4.6750 & 294.5 & 3 & .0 & . \\
\hline 917 & 65.2480 & 0 & .1 & 4 & & \\
\hline 18 & 72.4613 & 50 & 291.9 & 4 & & \\
\hline 919 & 64.7450 & 500 & 291 & 4 & & \\
\hline 920 & 66.5138 & 6.0500 & 291.6 & 4 & 0 & 00 \\
\hline 21 & 75.2524 & & & 4 & & \\
\hline 922 & 70.7502 & 50 & 290.2 & 4 & & \\
\hline 923 & 70.7497 & 3500 & 289.3 & 4 & 0 & \\
\hline 924 & 69.5009 & & 28 & 4 & & \\
\hline 301 & 68.5054 & 500 & 288.6 & 4 & & \\
\hline 210302 & 67.2501 & 1000 & 287.5 & 4 & 0 & U. \\
\hline 210303 & 73.0032 & 50 & 287.5 & 4 & & \\
\hline 0210304 & 73.7501 & 50 & 28 & 4 & & \\
\hline 210305 & 75.2503 & 3500 & 286.8 & 4 & 0 & U. \\
\hline 210306 & 75.0000 & 500 & 286.6 & 4 & 0 & 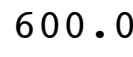 \\
\hline 210307 & 79.2470 & 50 & & 4 & & \\
\hline 210308 & 4.7646 & 50 & 286.7 & 4 & & . \\
\hline 0210309 & .7496 & 3000 & 286.1 & 4 & 0 & 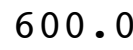 \\
\hline 010 & .2526 & 6 . & & 4 & & \\
\hline & 163.5000 & 6.7500 & 286.1 & 4 & & 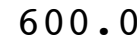 \\
\hline 2103012 & 160.7518 & 750 & 286.9 & 4 & & 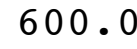 \\
\hline 021 & 165.2501 & 0 & 288.0 & 4 & & 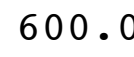 \\
\hline 02103014 & 163.5000 & 5.9500 & 289.3 & 4 & .0 & 0 . \\
\hline 2103015 & 163.7502 & 4000 & 290.1 & 4 & & \\
\hline 0210 & 166.2495 & 6 . & 289.9 & 4 & & 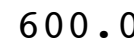 \\
\hline 0210 & 160.9998 & 5.1750 & 289.2 & 4 & & 0 . \\
\hline 02103018 & 151.2550 & 2.5500 & 287.8 & 5 & 0 & 0 . \\
\hline 02103019 & 124.1769 & 000 & 287.3 & 6 & & \\
\hline 02103020 & 127.5003 & 1.5250 & 287.4 & 5 & & 0 . \\
\hline 02103021 & 136.9997 & 1.2250 & 287.1 & 4 & & 00 . \\
\hline & 122. & & & 4 & & \\
\hline 2103023 & 115.9984 & 2.3750 & 287.2 & & .0 & 60 \\
\hline
\end{tabular}




\begin{tabular}{|c|c|c|c|c|c|c|}
\hline 03024 & & 0 & & & & \\
\hline 311 & 0 & 2.2000 & 286.5 & & & \\
\hline 10312 & 99 & 2750 & 286.0 & & 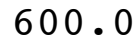 & \\
\hline 210313 & 41.7506 & 0750 & 285.5 & & 00 & \\
\hline 210314 & 38 & & & & & \\
\hline 10315 & 7521 & 9750 & & & 0 & \\
\hline 210316 & 1.5002 & 6.5250 & 83.7 & & & \\
\hline 210317 & 5000 & 1250 & & & & \\
\hline 210318 & 5.2479 & 5.2750 & 83.4 & & & \\
\hline 210319 & 6.2498 & 4.5750 & .9 & & 0 & \\
\hline 2103110 & 9972 & 9000 & 28 & & & \\
\hline 2111 & 011 & & & & & \\
\hline 2103112 & 009 & 0 & 286.1 & & 0 & \\
\hline 2103113 & 5003 & 0.0 & 287.4 & & 0 & \\
\hline 2111 & & & & & & \\
\hline 115 & 542 & & 289.5 & & & \\
\hline 03116 & 0000 & 0 & 289.8 & & & \\
\hline 3117 & 00 & 0 & 4 & & & \\
\hline 3118 & 00 & & 28 & & & \\
\hline 03119 & 000 & 0 & 287 & & & \\
\hline 3120 & 502 & 0 & 7 & & & \\
\hline 121 & 11 & & 7 & & & \\
\hline 3122 & 406 & & 28 & 4 & & \\
\hline 2103123 & 9.7446 & & 28 & 4 & & \\
\hline 2103124 & 4.2499 & & 28 & 4 & & \\
\hline 21111 & 7495 & 0 & 28 & 4 & & \\
\hline 21112 & 2.5021 & & 28 & 4 & & \\
\hline 021113 & 3.2369 & & 3 & 4 & & \\
\hline 021114 & 9 & & 28 & 4 & & \\
\hline 21115 & 000 & & 28 & 4 & & \\
\hline 21116 & 501 & & & 4 & & \\
\hline 021117 & 8 & 0 & $2 \varepsilon$ & 4 & & \\
\hline 021118 & 007 & & 282.7 & $x_{1}$ & 0 & \\
\hline 21119 & 982 & & 28 & 4 & & \\
\hline 211110 & 1 & & 28 & 4 & & \\
\hline 0211111 & 000 & & 284.4 & 4 & & \\
\hline 211112 & 495 & & 28 & 4 & & \\
\hline 0211113 & 003 & 0 & 5 & 3 & & \\
\hline $0211 \quad 114$ & 003 & 3000 & 287.3 & 4 & 0 & 0 . \\
\hline 21111 & 986 & 0 & $28 \varepsilon$ & 3 & & \\
\hline 0211116 & 982 & 4 . & $28 \varepsilon$ & 4 & & \\
\hline $0211 \quad 117$ & 148.7502 & 4.0250 & 287.7 & 5 & 60 & . \\
\hline 0211118 & 525 & & 287.0 & a & 0 & \\
\hline 211119 & 504 & & 286 & 4 & & \\
\hline $0211 \quad 120$ & 142.5000 & 2.9000 & 286.3 & 5 & 60 & 00 . \\
\hline $211 \quad 121$ & 345 & & 286.0 & 4 & 600 & \\
\hline 021112 & 1 & & 28 & 4 & & \\
\hline $211 \quad 123$ & 9995 & 1.9000 & 286.1 & & 600.0 & 500 \\
\hline
\end{tabular}




\begin{tabular}{|c|c|c|c|c|c|c|c|}
\hline د & 24 & & & & & & \\
\hline & 1 & 25 & .9250 & 6.2 & & 0 & \\
\hline 1 & 22 & 137.9989 & 1.5750 & 285.6 & & 00 . & \\
\hline 21 & 23 & 137.5000 & 1.5500 & 285.4 & & 00.0 & \\
\hline & 4 & 146.9993 & .4000 & 85.3 & & 0 & \\
\hline & 25 & 143.7505 & 3250 & 84.6 & & 00 & \\
\hline 21 & 26 & 146.7499 & 2250 & 283.9 & & 00 & U \\
\hline 21 & 7 & 49.7532 & 2.5000 & 0 & & .0 & \\
\hline 21 & 8 & 10.4673 & 1.4750 & 85.3 & & & \\
\hline 21 & 9 & 53.2781 & 0.8500 & 287.7 & & 600.0 & 00 \\
\hline 21 & 210 & 162.7500 & 3.2500 & 286.7 & & 00.0 & \\
\hline 2 & 211 & 61.4995 & 3.6000 & .9 & & .0 & \\
\hline 21 & 12 & 61.9999 & 3.7250 & 287.8 & & 00.0 & ( \\
\hline 21 & 213 & 63.5000 & 4.3500 & 288.0 & & 0.0 & \\
\hline 2. & 14 & 000 & & & & & \\
\hline 21 & 1 & 162 & & 28 & & & \\
\hline 21 & 216 & 156.2498 & 3.0250 & 289.4 & & .0 & $\bullet$ \\
\hline 21 & 217 & 150.0000 & 0 & 9 & & .0 & \\
\hline 21 & 18 & 136.5010 & 2 & 287.7 & & & \\
\hline 21 & 219 & 67.2007 & 4750 & 287.7 & & 0 & \\
\hline 21 & 220 & 139.0294 & 0.7250 & 287.5 & & 0 & 00 . \\
\hline 21 & 221 & 101.9614 & $\begin{array}{ll}0 & 72\end{array}$ & 288.2 & & & \\
\hline 21 & 222 & 665 & 0.3000 & 288.6 & 6 & & \\
\hline 21 & 223 & 40.6184 & 0.3750 & 288.9 & & . 0 & 0 • \\
\hline 21 & 224 & 126 . & & & & & \\
\hline 02 & 31 & 241 . & 0 . & $28^{\circ}$ & 6 & & \\
\hline 21 & 32 & 349.2742 & 0.4750 & 286.9 & 5 & .0 & U. \\
\hline 21 & 33 & 351.2158 & 1 . & $28^{\circ}$ & 4 & & ( \\
\hline 02 & 34 & 316.8235 & 00 & 28 & 5 & & $\bullet$ \\
\hline 21 & 35 & 268.7041 & 0.3000 & 286.4 & 6 & 0 & 0 . \\
\hline 021 & 36 & 154.5727 & 0.3750 & 286.4 & 5 & .0 & 0 . \\
\hline 21 & 37 & 183.5015 & 0 & 28 & 5 & & \\
\hline 021 & 38 & 164.7427 & 500 & 286.8 & 6 & & U. \\
\hline 021 & 39 & 95.3084 & 0.7250 & 289.1 & 5 & 0 & 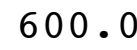 \\
\hline 21 & 310 & 182.6686 & 1.2000 & 289.1 & 4 & & \\
\hline 02 & 311 & 180 . & 1.5000 & 289.7 & 3 & & 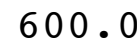 \\
\hline 21 & 312 & 167.4979 & 3.5750 & 290.5 & 3 & .0 & 0 . \\
\hline 21 & 313 & 157.4992 & 6.1500 & 290.3 & 4 & & ( \\
\hline 021 & 314 & 157.0000 & 5.3250 & 291.2 & 4 & .0 & 600 . \\
\hline 021 & 315 & 162.0002 & 5.7750 & 291.4 & 4 & .0 & o. \\
\hline 2 & 316 & 150.4987 & 5.2000 & 291.7 & 4 & & 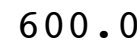 \\
\hline 021 & 317 & 130.4718 & 3.1000 & 291.9 & 4 & & 0 . \\
\hline 21 & 318 & 120.7516 & 2.5500 & 291.4 & 5 & 0 & 00 . \\
\hline 02 & 319 & 114.5821 & 1.4250 & 290 & 6 & & \\
\hline 021 & 320 & 124.3846 & 1.8750 & 290.0 & 6 & & 600. \\
\hline 2 & 321 & 73.4985 & 5.7000 & 290.6 & 5 & 600.0 & 600 \\
\hline & 32 & 0000 & & & 4 & & \\
\hline & 323 & 60.7499 & 8.5500 & 290.8 & $\mathbf{4}$ & 600.0 & 600 \\
\hline
\end{tabular}




\begin{tabular}{|c|c|c|c|c|c|c|c|}
\hline 1 & 24 & 0.0000 & 7.9500 & 291.0 & 4 & 000.0 & \\
\hline 211 & 41 & 7.7500 & 7.1750 & 290.9 & 4 & 600.0 & \\
\hline 1 & 42 & 74.2498 & 8000 & 290.5 & 4 & 00 & \\
\hline 21 & 43 & 76.2467 & 5.0750 & 290.3 & & 00.0 & \\
\hline & 44 & 3.2558 & 4.9000 & 90.1 & & 0 & \\
\hline 21 & 45 & 151.9633 & 1.8250 & 288.9 & & 00 & \\
\hline 21 & 46 & 315.9168 & 0.5750 & 287.0 & & 00 & \\
\hline 21 & 47 & 243.9491 & .2500 & 86.3 & & 0 & \\
\hline 21 & 48 & 29.5548 & 0.7500 & 287.0 & & 00 & \\
\hline 21 & 9 & 325.0000 & 0.6750 & 289.2 & & 0 & \\
\hline 21 & 410 & 281.9379 & 1 & 89.3 & & & \\
\hline 21 & 411 & 165.2475 & & 88.3 & 3 & & \\
\hline 21 & 12 & 163.5000 & 500 & 288.5 & & 00 & \\
\hline 2 & 413 & 801 & 0 & 90.9 & & & \\
\hline 21 & 414 & 222 & & 92 & 2 & & \\
\hline 21 & 15 & 104.6699 & 3 . & 292.8 & 2 & 0 & \\
\hline 21 & 416 & 114.4699 & 4.22 & 292.5 & 3 & 000 & \\
\hline 2 & 417 & 112.4977 & & 292.1 & 4 & & \\
\hline 21 & 18 & 269 & 0 & 290.5 & 5 & 0 & \\
\hline 21 & 419 & 43.7594 & 0 & 289.6 & 5 & 0 & \\
\hline 21 & 420 & 13.4991 & 0 & 288 & 4 & & \\
\hline 2 & 421 & 133 & 0 & 288.5 & 5 & & \\
\hline 21 & 422 & 352.7151 & 0 & 288.0 & 6 & 0 & \\
\hline 21 & 423 & 111.9771 & 0 & 287.8 & 5 & & \\
\hline 2. & 424 & 511 & & 287.7 & 4 & & \\
\hline 21 & 51 & 69.5003 & 50 & 288.6 & 4 & & \\
\hline 21 & 52 & 85.0002 & 4.8500 & 288.1 & 4 & & \\
\hline 21 & 53 & 94.5000 & 0 & 287.6 & 4 & & \\
\hline 21 & 54 & 141.0178 & 0 & 286.9 & 5 & & \\
\hline 21 & 55 & 6.2975 & 0 & 286.3 & 4 & & \\
\hline 021 & 56 & 329.4685 & & 286 & 5 & & \\
\hline 021 & 57 & 296 . & 0 . & 285.8 & 6 & & \\
\hline 021 & 58 & 351.2498 & 1.6500 & 286.5 & 5 & & \\
\hline 21 & 59 & 328.3606 & 0 & 287.3 & 4 & & \\
\hline 021 & 510 & 268 . & 1 . & 288.4 & 3 & & \\
\hline 021 & 11 & 277.9563 & 2.1500 & 288.3 & 2 & & \\
\hline 021 & 512 & 237.0000 & 2 . & 287.7 & 2 & & \\
\hline 021 & 513 & 256.4801 & 2 & 289.8 & 1 & .0 & 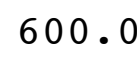 \\
\hline 021 & 514 & 265.7413 & 1.3000 & 291.4 & 1 & & 6 \\
\hline 02 & 515 & 291.1275 & & 292.3 & 1 & & \\
\hline 021 & 516 & 156.2151 & 2 . & 291.6 & 1 & & 6 \\
\hline 021 & 517 & 137.2509 & 1.7750 & 290.3 & 2 & 0 & 0 \\
\hline 021 & 518 & 126.5459 & & 288.9 & 3 & & \\
\hline 0211 & 519 & 18.2481 & 0.7500 & 289.5 & 4 & 600.0 & 600 \\
\hline 021 & 520 & 89.2444 & 0.2250 & 289.1 & 5 & 600.0 & 600 \\
\hline & 521 & 106.58 & & 289.0 & 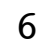 & & \\
\hline & 522 & 128.5055 & 0 . & 289.2 & 5 & & 600 \\
\hline & 523 & 46.7487 & 0.0000 & 288.3 & $\mathrm{~J}$ & 600.0 & 600 \\
\hline
\end{tabular}




\begin{tabular}{|c|c|c|c|c|c|c|c|}
\hline$\perp$ & 24 & 0.4799 & 0.8500 & 8.4 & & 00 & \\
\hline 11 & 1 & 502510 & 1.4250 & 288.3 & & & \\
\hline 21 & 62 & 270 & 250 & 287.4 & & 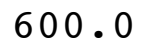 & \\
\hline & 3 & 330.8796 & 0.8250 & 87.1 & & 00 & \\
\hline & 4 & 16 . & 1.2250 & 7.0 & & & \\
\hline 1 & 5 & 367 & 250 & 86.5 & & $x^{2}+2$ & \\
\hline$-1+2+3$ & 6 & 111.8239 & 1.9000 & 5 & & 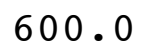 & \\
\hline 21 & 7 & 163. & .8000 & & & & \\
\hline t & 8 & 274.3218 & 0.6000 & 286.1 & & 0 & \\
\hline 21 & 9 & 329.2257 & 750 & & & & \\
\hline 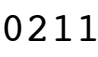 & 10 & 311.5093 & 500 & 4 & & & \\
\hline & 11 & 283. & & & & & \\
\hline 21 & 12 & 276.7481 & 500 & 288.3 & & 0 & \\
\hline 1 & 13 & 281.9851 & 000 & 5 & & & \\
\hline & 14 & 286 . & & & & & \\
\hline 21 & 15 & 266 . & 000 & 291.5 & & & \\
\hline 21 & 616 & 259.2847 & .4000 & 291 & & 0 & \\
\hline & 617 & 268.54 & & & & & \\
\hline 21 & 18 & 159. & 250 & $28 \varepsilon$ & & & \\
\hline 21 & 619 & 309.1842 & 7750 & 288.5 & & & \\
\hline & 620 & 17 . & & & & & \\
\hline 2 & 621 & 11 . & 750 & 5 & & & \\
\hline 21 & 622 & 355.9996 & 1.6000 & 290.8 & & & \\
\hline & 623 & 0 . & 500 & 0.4 & & & \\
\hline & 624 & 339. & & $2 \varepsilon$ & & & \\
\hline $19+2$ & 71 & 1.0186 & 500 & 290.0 & 4 & & \\
\hline 21 & 72 & 355 . & 7.0500 & 289.2 & 4 & & \\
\hline & 73 & 337 . & 0 & 287.1 & 4 & & \\
\hline $17+2$ & 74 & 302 . & 000 & 286.5 & 4 & & \\
\hline 1 & 75 & 323 . & .7500 & 284.5 & 4 & & \\
\hline 2. & 76 & 308 . & & 9 & 4 & & \\
\hline & 77 & 321. & 0 & $2 \varepsilon$ & 4 & & \\
\hline 21 & 78 & 311.9887 & 7.4500 & 283.9 & 4 & & \\
\hline $21+2$ & 79 & 305 . & 7 & 284.1 & 4 & & \\
\hline & 710 & 307 & & & 4 & & \\
\hline & 711 & 303.9979 & 7.4250 & 285.9 & 4 & & \\
\hline & 712 & 294 . & 500 & 287.4 & 4 & & \\
\hline & 713 & 313 . & 00 & 287.8 & 4 & & \\
\hline 1 & 714 & 309.0000 & .9250 & 287.9 & 4 & & \\
\hline & 715 & 326 . & 0 & 287.3 & 4 & & \\
\hline & 716 & 328 . & 0 & 287.2 & 1 & & \\
\hline 021 & 717 & 332.4990 & 9.0250 & 286.5 & 4 & & \\
\hline & 718 & 327 . & & 286.5 & 4 & & \\
\hline 021 & 719 & 331.4962 & 7.2000 & 286.3 & 4 & & \\
\hline 02 & 720 & 340.5000 & 7.8750 & 287.0 & 4 & 0 & 00 \\
\hline & 721 & 358 . & 00 & & 4 & & \\
\hline & 722 & 11.6929 & & 290.6 & & & \\
\hline & 123 & 42.7534 & & 290. & & .0 & 50 \\
\hline
\end{tabular}




\begin{tabular}{|c|c|c|c|c|c|c|c|}
\hline 1 & 24 & & 8.7750 & 0.1 & 4 & & \\
\hline 1 & 81 & .7535 & 9.4250 & 289.7 & 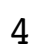 & 1000 & \\
\hline 1 & 82 & 40.2496 & 5750 & 289.4 & a & 0 & \\
\hline 21 & 3 & 30.7504 & 9.1250 & 289.4 & & 00.0 & \\
\hline & 4 & 7.9813 & 50 & 88.9 & & 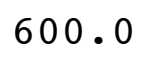 & \\
\hline 21 & 5 & 5596 & 1.4750 & 87.6 & & 0 & \\
\hline $2 \perp$ & 6 & 63.4303 & 1.0250 & 88.1 & & 00 & \\
\hline 21 & 7 & 034 & 3.0000 & 87.2 & & 0 & \\
\hline 21 & 8 & 00.7232 & 1.4750 & 286.3 & & .0 & \\
\hline 21 & 9 & 3399 & 2.0500 & 286.4 & & 0 & \\
\hline 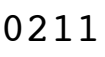 & 10 & 113. & 0 & 87 & & & \\
\hline 21 & 11 & 839 & & 89 & & & \\
\hline 21 & 12 & 501 & 9.4500 & 289.5 & & 0 & \\
\hline 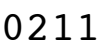 & 13 & 2503 & 9 . & 4 & & & \\
\hline 21 & 14 & 529 & & 5 & & & \\
\hline 21 & 15 & 573 & 0.2000 & 290.1 & 4 & 0 & \\
\hline 21 & 816 & 61.5000 & 9750 & 289.5 & & 0 & \\
\hline 2 & 817 & 63.9996 & 0 . & 88.7 & 4 & & \\
\hline 21 & 18 & 997 & 0 & 288.2 & 4 & & \\
\hline 2. & 819 & 48.7156 & 5000 & 288.7 & 4 & 0 & \\
\hline 2 & 20 & 36 & & & & & \\
\hline 2 & 21 & 72 & 9 . & $28^{\circ}$ & 4 & & \\
\hline 21 & 822 & 83.7506 & 3000 & 286.9 & 4 & & \\
\hline 2 & 823 & 75 & 0 & 9 & 4 & & \\
\hline 2 & 824 & 16 & & & 4 & & \\
\hline 21 & 91 & 032 & 750 & 286.3 & 4 & & \\
\hline 2. & 92 & 908 & 2.5250 & 285.7 & 5 & & \\
\hline 2 & 93 & 36 & 750 & 6 & 4 & & \\
\hline $21+1$ & 94 & 500 & 500 & 285.5 & 4 & & \\
\hline 21 & 95 & 320 & 500 & 285.0 & 4 & & \\
\hline 2. & 96 & & & & 4 & & \\
\hline-1 & 97 & 452 & 2 . & $2 \varepsilon$ & 4 & & \\
\hline 21 & 98 & 57.9338 & 1.5250 & 285.9 & 5 & & \\
\hline 21 & 99 & 308 & 1. & 4 & 4 & & \\
\hline & 910 & 5 & 1 . & & 3 & & \\
\hline 21 & 911 & 135.5103 & 2.1500 & 288.2 & & & \\
\hline & 912 & 318 . & & .3 & 1 & & \\
\hline 2 & 913 & 69.5979 & 2 . & 289.6 & 1 & & \\
\hline 021 & 914 & 60.7455 & 5250 & 289.2 & 2 & & \\
\hline & 915 & 67 . & 50 & 285 & 3 & & \\
\hline 2 & 916 & 62.5063 & 500 & 288.2 & 4 & & \\
\hline 021 & 917 & 62.0050 & 4.6000 & 287.4 & 4 & & 0 \\
\hline & 918 & 000 & 3000 & 286.9 & 4 & & \\
\hline 021 & 919 & 67.7524 & 5.2750 & 286.3 & 4 & & 600 \\
\hline 02 & 920 & 53.9998 & 5.0000 & 286.0 & 4 & 600.0 & 00 . \\
\hline & 921 & 539 & & & 5 & & \\
\hline & 922 & 40.9927 & 4.1250 & & 4 & & 600 \\
\hline & 923 & 54.8604 & 1.8500 & 285.7 & & 600.0 & 600 \\
\hline
\end{tabular}




\begin{tabular}{|c|c|c|c|c|c|c|}
\hline 11 & & 1.2500 & 5.5 & & & \\
\hline 211101 & 2.6115 & 1.5250 & 285.2 & & & \\
\hline 211102 & 33.0000 & 1.6500 & 285.1 & 6 & 00 & \\
\hline 211103 & 41.2660 & 3.9500 & 285.9 & & 00 & \\
\hline 211104 & 349.1790 & 50 & & & 0 & \\
\hline 11105 & 14.0860 & 1.7000 & 85.5 & & 00 & \\
\hline 211106 & 48.2735 & 4.4750 & 286.6 & & 00 & \\
\hline 211107 & 44.4895 & .8000 & & & & \\
\hline 211108 & 343.4514 & 1.6500 & 286.5 & & 0 & \\
\hline 211109 & 7.9425 & 2.0000 & 287.4 & & 00.0 & \\
\hline 010 & 46.9871 & 5.2500 & 288 & & 0 & \\
\hline 011 & 36.9992 & & 88.5 & & & \\
\hline 012 & 23.7403 & 5.5500 & 288.8 & & 0 & \\
\hline 1013 & 43.3913 & 3.1500 & 287.6 & & 0 & \\
\hline 014 & & & & & & \\
\hline 015 & 83.5086 & 4.3750 & 286.7 & & 0 & \\
\hline 016 & 67.4418 & 6.2750 & 287.2 & 4 & 0 & \\
\hline 017 & 73.7552 & 500 & 28 & - & & \\
\hline 018 & 65.0009 & 750 & 28 & 4 & 0 & \\
\hline 1019 & 72.2136 & 5500 & 28 & 4 & 0 & \\
\hline 020 & 68.4971 & 0 & 28 & 4 & & \\
\hline 021 & 69.00 & 0 & 2 & 4 & & \\
\hline 022 & 69.7370 & 5.2000 & 286.2 & 4 & 0 & \\
\hline 2111023 & 100.7413 & 4.8500 & 28 & 4 & 0 & \\
\hline 024 & 84.8792 & 0 & 28 & 4 & & \\
\hline 211111 & 72.2534 & 9250 & 285.2 & 5 & & \\
\hline 211112 & 69.7508 & 6000 & 285.2 & 4 & 0 & \\
\hline 0211113 & 80.0005 & 5 . & 28 & 4 & & \\
\hline 0211114 & 80.2492 & 750 & 28 & 5 & 0 & - \\
\hline 0211115 & 106.0154 & 4.5500 & 285.5 & 5 & 0 & \\
\hline 211116 & 129.5000 & 4. & 28 & 5 & & \\
\hline 0211117 & 144.7476 & 250 & 28 & 4 & & \\
\hline 0211118 & 146.2522 & 3.4000 & 284.6 & 4 & 0 & J. \\
\hline 0211119 & 124.0374 & 500 & 28 & 3 & 0 & \\
\hline 110 & 000 & 3. & 28 & 3 & & \\
\hline 111 & 163.2472 & 4.5500 & 286.2 & 4 & 0 & 0 . \\
\hline 1112 & 168.5008 & 1250 & 285.8 & 4 & 0 & \\
\hline 021 & 167.2501 & 50 & 286.3 & 4 & 0 & 0 . \\
\hline 1114 & 153.9793 & 2.5000 & 287.6 & 3 & .0 & 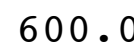 \\
\hline 1115 & 163.4890 & 250 & 287.8 & 3 & 6 & \\
\hline 116 & 157.2481 & 500 & 287 & 4 & & \\
\hline 1117 & 161.7501 & 2.1250 & 287.3 & 4 & 600.0 & 600 . \\
\hline 02111118 & 145.7501 & 1.3000 & 286.3 & 5 & 600 & 000 \\
\hline 1119 & 99.5230 & 0.6750 & 286.3 & 4 & 600 & 60 \\
\hline 1120 & 116.4531 & 0.3750 & 286.2 & 5 & 600.0 & 600 \\
\hline 1121 & 166.3651 & 0.0000 & 285.5 & 6 & 600.0 & 600 \\
\hline 122 & 317.5791 & 250 & 285.5 & 6 & 600 & 60 \\
\hline 1123 & 338.2570 & 1.6500 & 285.2 & & 600.0 & 600 \\
\hline
\end{tabular}




\begin{tabular}{|c|c|c|c|c|c|c|}
\hline $111<4$ & & & & & & \\
\hline 1121 & \pm & 3000 & 1 & 6 & 0 & \\
\hline 11122 & 45.9712 & 2000 & 85.3 & 6 & 00 & \\
\hline 211123 & 5221 & 7750 & 285.3 & 6 & 00 & \\
\hline 211124 & 8.4586 & 8000 & & & 0 & \\
\hline 211125 & 76 & 750 & & 6 & 0 & \\
\hline 211126 & 3211 & 3250 & 285.2 & 6 & 00 & \\
\hline 211127 & 993 & 2.8500 & & & & \\
\hline 211128 & 4.8158 & .6000 & 6 & 0 & & \\
\hline 211129 & 5092 & 1.8750 & 86.0 & & 00 & 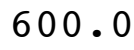 \\
\hline 2111210 & 19.8264 & 1.3250 & 287.9 & & & \\
\hline 21 & 174.7269 & 1.4250 & 9.0 & 3 & & \\
\hline 2111212 & 3153 & 2.65 & 289.5 & 3 & 0 & \\
\hline 2111213 & 9949 & 1.65 & 289.7 & 2 & & \\
\hline 214 & 296 & 1.92 & .2 & $\perp$ & & \\
\hline 215 & 237 & $1.9^{\circ}$ & 291.2 & 1 & & \\
\hline 1216 & 5037 & 2.4500 & 289.6 & 2 & .0 & \\
\hline 1217 & 145.5000 & 3.5000 & 0 & 3 & & \\
\hline 218 & 139. & 1.55 & 287.3 & 4 & & \\
\hline 219 & 128 & 107 & 288.6 & 4 & & \\
\hline 02111220 & 156.0009 & 6.0000 & 287.5 & 4 & 0 & \\
\hline 221 & 155 . & 752 & & 4 & & \\
\hline 222 & 152 & 4.60 & 28 & 5 & & \\
\hline 2111223 & 136.5107 & 3.07 & 9 & 4 & 0 & \\
\hline 224 & 155 & & & 5 & & \\
\hline 131 & 156 & 6.9000 & 28 & 4 & & \\
\hline 211132 & 151.9999 & 15 & 284.2 & 4 & 0 & J. \\
\hline 211133 & 149.0000 & 0 & 3 & 4 & & \\
\hline 0211134 & 149 & 6 . & 28 & 4 & & \\
\hline 211135 & 149.0006 & 80 & 2 & 4 & 0 & 0. \\
\hline 211136 & 142.6137 & 5.7500 & 28 & 4 & & s. \\
\hline 0211137 & 87.5023 & 33 & & 4 & & \\
\hline 0211138 & 120.5683 & & 28 & 4 & & . \\
\hline 0211139 & 144.5023 & 5.05 & 28 & 4 & 0 & 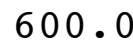 \\
\hline 1310 & 161.5014 & 0 & & 4 & & \\
\hline 021 & 169 & 5 & 5.3 & 3 & & 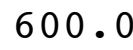 \\
\hline 2111312 & 157.5159 & 0 & 286.7 & 3 & 0 & 0 . \\
\hline 1313 & 168 & 4 . & 28 & 3 & & 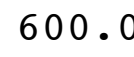 \\
\hline 02111314 & 150.5000 & 6.2250 & 287.6 & 4 & .0 & 0 \\
\hline 02111315 & 149.0012 & 7.2500 & 287.9 & 4 & & \\
\hline 1316 & 155.7565 & 6 . & 288 & 4 & & 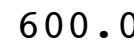 \\
\hline 1317 & 161.2535 & 4.5750 & 288.1 & 5 & .0 & 0 . \\
\hline 2111318 & 146.7649 & 2.8000 & 287.5 & 4 & 0 & 0 . \\
\hline 02111319 & 5718 & 3 . & & 4 & & \\
\hline 02111320 & 86.7312 & 4.4250 & & 4 & & 00 \\
\hline 02111321 & 102.5000 & 4.0750 & 287.9 & 5 & 600 & 600 \\
\hline & .029 & & & 4 & & \\
\hline 2111323 & 132 & 2.8250 & 287.4 & & .0 & 60 \\
\hline
\end{tabular}




\begin{tabular}{|c|c|c|c|c|c|c|}
\hline$<4$ & & & & & & \\
\hline $21114 \quad 1$ & 994 & 2250 & 287.9 & 4 & & \\
\hline 21114 & 72 & 1500 & 287.7 & 4 & r & \\
\hline 211143 & 129.2494 & 4.3750 & 287.5 & & 00 & \\
\hline $21114 \quad 4$ & & 50 & & & & \\
\hline 14 & 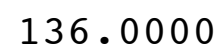 & 9000 & 2 & & 0 & \\
\hline 211146 & 5157 & 1750 & 285.8 & & 0 & \\
\hline 211147 & 2642 & 500 & 85.8 & & & \\
\hline 211148 & 162.7488 & 250 & 285.1 & & 0 & \\
\hline 211149 & 33.2638 & 500 & 285.1 & & 00 & \\
\hline 410 & 54.7685 & 000 & 86.6 & & 0 & \\
\hline & 27119 & & 1 & 4 & & \\
\hline 21 & 9993 & 000 & 287.9 & 4 & 0 & \\
\hline 3 & 147.2422 & 4 . & 288.9 & 3 & 0 & \\
\hline 14 & 52.0147 & & & - & & \\
\hline 21 & 57.2515 & 0 & 29 & 4 & & \\
\hline 6 & 52.0078 & 4.47 & 290.6 & 4 & 0 & \\
\hline & 160.9920 & 2 . & 28 & 4 & & \\
\hline 2 & 0.3808 & & 288.9 & 5 & 0 & \\
\hline 19 & 2.4988 & 2. & 289.6 & 4 & & \\
\hline 20 & 000 & 3. & 289.6 & 0 & & \\
\hline 21 & 820 & & .4 & 5 & & \\
\hline 22 & 59.1282 & 0 & 289.2 & 6 & 0 & \\
\hline 23 & 34.5194 & 0 & 288.8 & 5 & & \\
\hline 24 & 234.8739 & & 287.4 & 6 & & \\
\hline 51 & 7047 & 750 & 286 & 5 & & \\
\hline $21115 \quad 2$ & 147.4959 & 5000 & 286.4 & 4 & & \\
\hline 0211153 & 146.9938 & & 286.1 & 5 & & \\
\hline 0211154 & 716 & 1 . & 28 & 6 & & \\
\hline $21115 \quad 5$ & 355.5135 & 1.2250 & 285.8 & 5 & & \\
\hline 211156 & 300 . & 1. & 28 & 5 & & \\
\hline 157 & 774 & 0 & 28 & 6 & & \\
\hline 0211158 & 299.7739 & 1.8000 & 285.3 & 5 & 0 & 0 . \\
\hline 211159 & 255 . & 250 & 285.5 & 4 & & \\
\hline 10 & 280 . & 1. & 28 & 3 & & \\
\hline 21 & 302.9834 & 2.4500 & 283.7 & 3 & & \\
\hline 512 & 325.2498 & 500 & 284.5 & 3 & & \\
\hline 021 & 288.3150 & 0 & 284.6 & 3 & & 0 . \\
\hline 514 & 267.0763 & 1.4750 & 284.2 & 2 & 0 & 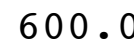 \\
\hline 515 & 239. & 500 & 283.9 & 2 & & \\
\hline 516 & 263 & 0 . & 284.5 & 1 & & \\
\hline 517 & 306.1004 & 1.0000 & 283.4 & 2 & 600.0 & 600 . \\
\hline 2111518 & 039 & 1.7250 & 282.6 & 3 & 600 & - \\
\hline 519 & 9.9830 & 1.5000 & 284.9 & 4 & & \\
\hline 021 & 351.2504 & 1.4250 & 287.3 & 4 & 600.0 & 00 \\
\hline 52 & 41.1391 & 500 & 288.0 & 5 & 600 & \\
\hline & 226 & 0.7250 & 287.7 & 6 & 60 & \\
\hline 523 & 298.6122 & 0.4750 & 288.0 & & 600.0 & 600 \\
\hline
\end{tabular}




\begin{tabular}{|c|c|c|c|c|c|c|}
\hline & & & & & & \\
\hline 211161 & 21 & 6000 & 6.4 & & 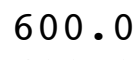 & \\
\hline 11162 & 325 & 1.9750 & 289.2 & 4 & 00 & \\
\hline 11163 & 0.4733 & 9000 & 289.8 & & 00 & \\
\hline 211164 & 12 . & 1.8250 & 90.3 & & & \\
\hline 11165 & 326. & & 89.0 & & 0 & \\
\hline $21116 \quad 6$ & 7924 & 8750 & 89.7 & & 00 & \\
\hline 211167 & 34.2495 & 2.7000 & 91.7 & & & \\
\hline 211168 & 0.5314 & 1.7250 & 89.7 & & & \\
\hline 211169 & 357.5073 & 2.6500 & 90.0 & & 0 & \\
\hline 2111610 & 1.8475 & 1.9000 & 294.2 & & & \\
\hline 11 & 260.7188 & 117 & 93.3 & & & \\
\hline 21 & 196.8511 & 0 & 293.0 & & & \\
\hline 613 & 160.4678 & 50 & 292.9 & & & \\
\hline 14 & 112 . & & & & & \\
\hline 21 & 155 . & 2 . & 29 & & & \\
\hline 616 & 338.5927 & 1.62 & 290.5 & & 0 & \\
\hline 17 & 136 . & 0 & 4 & & & \\
\hline 18 & 140 . & 50 & 5 & & & \\
\hline 19 & 129.3259 & 2.4000 & 287.2 & 4 & & \\
\hline 20 & 54.5018 & 3.9250 & 287.4 & 4 & 0 & \\
\hline 21 & 112.9443 & & & 4 & & \\
\hline 22 & 75 & 0 & 28 & 4 & & \\
\hline 523 & 84.4969 & 0 & 287 & 4 & 0 & \\
\hline 24 & 88 . & & & 4 & & \\
\hline 71 & 73. & 0 & 28 & 4 & & \\
\hline 211172 & 78.0015 & 0 & 28 & 4 & & . \\
\hline 211173 & 73.249 & 0 & 0 & 4 & & \\
\hline $21117 \quad 4$ & 72 . & 0 & $2 \varepsilon$ & 4 & & \\
\hline 211175 & 69.0000 & 50 & 2 & 4 & & J. \\
\hline $21117 \quad 6$ & 74.7505 & 7. & 284.3 & 4 & & 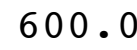 \\
\hline $21117 \quad 7$ & 93.9466 & 0 & & 4 & & \\
\hline 211178 & 123 . & 0 & 283.8 & 4 & & 0 . \\
\hline 211179 & 4.6642 & 0 & 28 & 4 & & \\
\hline 10 & 134.74 & 0 & & 3 & & \\
\hline .711 & 154.0024 & 8.8750 & $2 \varepsilon$ & 4 & & \\
\hline 2111712 & 160.4982 & .7750 & 285.8 & 4 & & \\
\hline .713 & 157 . & 0 & & 4 & & \\
\hline 02111714 & 158.0000 & 6.2250 & 286.3 & 4 & & 0 . \\
\hline 2111715 & 147.9853 & 4.9500 & 286.8 & 4 & & \\
\hline 716 & 152.2506 & 250 & 287.3 & 4 & & \\
\hline 021 & 131.0000 & 3.7250 & 287.4 & 4 & & 0 . \\
\hline 2111718 & 135.2609 & 3.8750 & 286.8 & 4 & & 0 . \\
\hline 719 & 130.2391 & 250 & & 4 & & \\
\hline 02111720 & 121.7504 & 4.1750 & & 4 & & 0 . \\
\hline 1721 & 126.0000 & 5.6500 & 286.9 & 4 & & 00 . \\
\hline & 132 . & & & 4 & & \\
\hline 2111723 & 130.2771 & 3.8000 & 287.2 & & .0 & 600 \\
\hline
\end{tabular}




\begin{tabular}{|c|c|c|c|c|c|c|}
\hline 24 & & & & & & \\
\hline 211181 & 1 & 8750 & 6.5 & & ) & \\
\hline 11182 & 151.0000 & 1250 & 286.3 & 4 & 00 & \\
\hline 211183 & 139.2541 & 5250 & 286.0 & & 00 & \\
\hline 211184 & 138.8774 & 1500 & 85.9 & & 0 & \\
\hline 211185 & 90.6309 & 0250 & 86.0 & & 0 & \\
\hline 211186 & 120.7668 & 500 & 285.7 & & 00 & \\
\hline 211187 & 101.5374 & 2.4500 & 85.5 & & & \\
\hline 211188 & 122.4893 & 3.5250 & 85.8 & & & \\
\hline 211189 & 128.7552 & 2500 & 86.1 & & 00.0 & 8 \\
\hline 2111810 & 154.2479 & 0750 & 285.6 & & & \\
\hline 21 & 158.0007 & 0 & 86.5 & & & \\
\hline 1812 & 162.2498 & 4. & 287.0 & 4 & 0 & \\
\hline 2111813 & 163.7504 & 4.1250 & 287.4 & & & \\
\hline 814 & 163.2506 & & & 4 & & \\
\hline 815 & 153.0046 & & 287.6 & 4 & & \\
\hline 1816 & 139.2693 & 500 & 287.0 & 4 & .0 & \\
\hline 817 & 138.9950 & 0 & 7 & 4 & & \\
\hline 818 & 130.7641 & & 285.3 & 5 & & \\
\hline 819 & 5144 & 0 & 285.2 & 4 & & \\
\hline 820 & 129.4992 & 2.9250 & 286.1 & 4 & 0 & \\
\hline 21 & 129.2509 & & & 4 & & \\
\hline 21 & 103.1944 & 1 . & 28 & 5 & & \\
\hline 2111823 & 113.7323 & 1.8000 & 286.3 & 5 & 0 & \\
\hline 824 & 138.7500 & & & 5 & & \\
\hline 021 & 145.0000 & & 28 & 5 & & \\
\hline 211192 & 128.9907 & 1.8000 & 283.6 & 4 & 0 & U. \\
\hline 211193 & 41.2455 & 1.35 & 284.2 & 5 & & \\
\hline 0211194 & 61.2654 & 0 . & 284 & 6 & & \\
\hline 0211195 & 42.9422 & & 28 & 6 & & 0 . \\
\hline 0211196 & 22.6053 & 750 & 284.9 & 6 & & 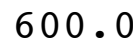 \\
\hline 0211197 & 97.7329 & 0 & 28 & 6 & & \\
\hline 0211198 & 133.9981 & & 285.9 & 5 & & 0 . \\
\hline 0211199 & 145.2505 & 1.7000 & 287.6 & 4 & 0 & \\
\hline 910 & 143.3387 & 0 & 287.9 & 4 & & \\
\hline 02 & 152.4998 & 6.4250 & 287.2 & 4 & & 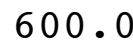 \\
\hline 02111912 & 156.2493 & 6.2500 & 287.7 & 4 & 0 & 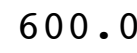 \\
\hline 1913 & 149 . & 500 & 288 & 4 & & 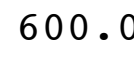 \\
\hline 021 & 149.0000 & 4.8750 & 289.9 & 4 & .0 & 0 . \\
\hline 02111915 & 137.9926 & 2.4250 & 291.3 & 3 & & \\
\hline 916 & 103.9993 & 250 & 293.0 & 2 & & 0 \\
\hline 021 & 97.7499 & 1.9750 & 292.9 & 3 & .0 & 0 . \\
\hline 2111918 & 112.7578 & 2.1000 & 292.2 & 4 & .0 & 00 . \\
\hline 0211 & 153.7448 & 1.4750 & 290.0 & 5 & & \\
\hline 02111920 & 174.4488 & 1.5250 & 288.4 & 6 & 0 & 600. \\
\hline 02111921 & 12.8289 & 0.5000 & 287.6 & 6 & 600.0 & 600. \\
\hline 922 & 322.9708 & & 287 & 6 & & \\
\hline 2111923 & 357.6165 & 1.7500 & 287.5 & & .0 & 60 \\
\hline
\end{tabular}




\begin{tabular}{|c|c|c|c|c|c|c|}
\hline 24 & $-c^{-}$ & .9250 & 88.2 & & - & \\
\hline 21120 & 6.7132 & .9250 & 88.6 & & 600.0 & \\
\hline 11202 & 147.2463 & 1.3000 & 286.6 & 5 & 00 & \\
\hline 211203 & 11.6684 & 0.2500 & 285.6 & & 00 & \\
\hline $21120 \quad 4$ & 279.1511 & .2000 & 35.0 & & 0 & \\
\hline 11205 & 287.7332 & 5500 & 84.7 & & 0 & \\
\hline 211206 & 304.6198 & 7000 & 284.2 & & 00 & \\
\hline 211207 & 274.2672 & 1000 & 83.4 & & & \\
\hline 211208 & 201.8098 & 0 & 4.3 & & & \\
\hline 211209 & 269.7102 & 3000 & 286.3 & & 0 & 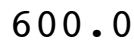 \\
\hline 2112010 & 288.0348 & 1.6000 & 287.6 & & & \\
\hline 011 & 252.5269 & 217 & 87.8 & & & \\
\hline 012 & 279.7685 & 2.5750 & 288.4 & & 0 & \\
\hline 2112013 & 273.2486 & $2 \cdot 13$ & 289 & & & \\
\hline 014 & 301.2494 & & 1 & & & \\
\hline 15 & 318.7500 & 1 . & 290.7 & & & \\
\hline 2016 & 308.7189 & 1.3500 & 290.4 & & 0 & \\
\hline 017 & 304.1570 & 0 & 7 & & & \\
\hline 018 & 339.4884 & 1.4 & 287.8 & 5 & & \\
\hline 019 & 10.2504 & 132 & 287.7 & & & \\
\hline 2020 & 352.2815 & 1.2750 & 289.1 & & 0 & \\
\hline 21 & 359.2740 & & 289.2 & 5 & & \\
\hline 022 & 313.0720 & 0 & 289.7 & 6 & & \\
\hline 2112023 & 203.6482 & .90 & 289.4 & 5 & 0 & \\
\hline 2024 & 204.5049 & & & 5 & & \\
\hline 211 & 292.6230 & 0 & $2 \varepsilon$ & 6 & & \\
\hline 211212 & 180.0909 & 0 & 287.3 & 6 & 0 & J. \\
\hline 211213 & 22.6429 & 0 & 287.3 & 6 & & \\
\hline 0211214 & 125.7993 & 50 & 28 & 6 & & \\
\hline 211215 & 134.0000 & 000 & 284.9 & 5 & & 0. \\
\hline 0211216 & 139.7501 & 3.1500 & 283.4 & 5 & & S \\
\hline 211217 & 147.7493 & 3 & & 5 & & \\
\hline 0211218 & 145.2501 & 0 & 284.5 & 5 & & 0 . \\
\hline 0211219 & 142.2576 & 8750 & 28 & 4 & 0 & \\
\hline 21 & 154.1618 & 0 & & 3 & & \\
\hline 021 & 202.5382 & 0.3500 & & 2 & & ( \\
\hline 2112112 & 144.9142 & 1.1500 & 288.5 & 1 & & 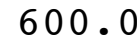 \\
\hline 2113 & 278.3176 & 0 . & 289.4 & 1 & & - \\
\hline 02112114 & 182.7389 & 1.5750 & 289.8 & 1 & .0 & 0 . \\
\hline 02112115 & 150.7505 & 2.4250 & 288.7 & 2 & & \\
\hline 02112116 & 142.9907 & 2.1750 & 289.0 & 3 & & 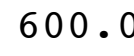 \\
\hline 021 & 116.2331 & 1.1250 & 288.5 & 4 & & 0 . \\
\hline 2112118 & 137.0263 & 0.8500 & 288.7 & 5 & 0 & 0 . \\
\hline 02112119 & 91.7869 & 0.7750 & 291.0 & 6 & & \\
\hline 02112120 & 138.9992 & 1.7750 & 291.7 & 5 & 0 & 0 . \\
\hline 02112121 & 136.7550 & 1.7750 & 290.7 & 5 & & 600. \\
\hline & 145 . & & & 4 & & \\
\hline 2112123 & 141.0037 & 3.7250 & 289.7 & & .0 & 60 \\
\hline
\end{tabular}




\begin{tabular}{|c|c|c|c|c|c|c|}
\hline 124 & & & & & & \\
\hline 1221 & & & 8.9 & & & \\
\hline 11222 & 2501 & 7250 & 289.2 & & 00 & \\
\hline 11223 & 5000 & 2.6750 & 288.3 & & 00 & \\
\hline 211224 & & 1.8250 & & & & \\
\hline 11225 & 687 & 8000 & 87.6 & & 0 & \\
\hline 211226 & 132.6187 & 1.3750 & 288.3 & & 00 & \\
\hline 211227 & 7 & 1.5250 & 88.3 & & & \\
\hline 211228 & 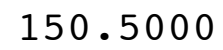 & 3750 & 88.2 & & & \\
\hline 211229 & 122.0518 & 0.6500 & 289.4 & & 00 & 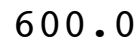 \\
\hline 2112210 & 268.83 & 0.4750 & 291 & & & \\
\hline 21 & 277 . & 1.4000 & 3 & & & \\
\hline 21 & 62 & 2.1750 & 287.8 & & 0 & \\
\hline 213 & 82 & 7.9750 & 28 & & & \\
\hline & 81 & 500 & & & & \\
\hline 21 & 77 & & 285.6 & & & \\
\hline 21 & 72 & 7.7750 & 285 & & & \\
\hline 17 & 76 & 500 & 2 & & & \\
\hline 21 & 80 & 00 & 28 & & & \\
\hline 21 & 72 . & 0.2750 & 28 & & & \\
\hline 20 & 67.0001 & 1.3250 & 28 & & 0 & \\
\hline 21 & 74.2 & & & & & \\
\hline 2 & 71 & 000 & & & & \\
\hline 23 & 70.5000 & .2750 & 28 & & 0 & \\
\hline 24 & 68 . & & & & & \\
\hline 31 & 69 & 750 & & 4 & & \\
\hline 211232 & 69.2501 & 3750 & 282 & 4 & & 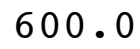 \\
\hline 211233 & 71.49 & 500 & 7 & 4 & & \\
\hline 211234 & 71 & 00 & 28 & 4 & & \\
\hline 211235 & 000 & 000 & & 4 & & \\
\hline 211236 & 74.2524 & 8.3750 & 282.8 & 4 & & \\
\hline 211237 & 79.4954 & 0 & 282 & 4 & & \\
\hline 211238 & & 000 & 282.8 & 4 & & \\
\hline 211239 & 501 & 000 & 283.5 & 4 & 0 & \\
\hline 21 & & 0 & & 4 & & \\
\hline & & 750 & 285.2 & 3 & & \\
\hline 12 & 98.9924 & 3.5000 & 286.1 & 2 & & \\
\hline 2 & 82.49 & 000 & 28 & 3 & & \\
\hline 021 & 87.7364 & 5.2750 & 286.6 & 4 & & 0 \\
\hline 211 & 84.7354 & 5.7250 & 286.5 & 4 & & \\
\hline & 90.6233 & 1.7000 & 287.7 & 3 & & \\
\hline 021 & 81.9796 & 2.1500 & 287.3 & 4 & & . \\
\hline 2112318 & 276.9685 & 1.3500 & 285.6 & 5 & 0 & 0 . \\
\hline 0211 & 335.6233 & 3750 & 284.7 & & & \\
\hline 02112320 & 45.5484 & 2.1000 & 284.7 & 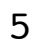 & & 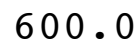 \\
\hline 21 & 33.0307 & 1.6000 & 284.4 & 5 & 600 & 00 . \\
\hline & & & & 4 & & \\
\hline 211 & 0903 & 2.4000 & 283.3 & & & $\sigma$ \\
\hline
\end{tabular}




\begin{tabular}{|c|c|c|c|c|c|c|}
\hline 2112324 & 6.1979 & 2.4750 & 83.3 & 6 & - & \\
\hline 1241 & 7.0576 & 1.7500 & 82.5 & 6 & 600.0 & \\
\hline 11242 & 6.7165 & 9000 & 282.7 & 5 & 00 & \\
\hline 11243 & 23.9271 & 4500 & 282.6 & & 00 & \\
\hline $21124 \quad 4$ & 8.4782 & & 83.0 & & & \\
\hline 11245 & 52 . & & 82.9 & & 0 & \\
\hline 211246 & 680 & 750 & 83.0 & & 00 & \\
\hline 211247 & 344 & 000 & 83.2 & & & \\
\hline 211248 & 156 & 50 & 83.0 & & & \\
\hline 21124 & 135 & 000 & 82.7 & & 0 & \\
\hline 2410 & 96.3996 & 1.7250 & 282.7 & & & \\
\hline 11 & 158.5847 & 0 & 83.4 & 3 & & \\
\hline 21 & 188 & 00 & 282.9 & 3 & & \\
\hline 21 & 150.9986 & 1.6750 & 282.7 & & & \\
\hline & 163. & & & & & \\
\hline 5 & 161 . & & .1 & 4 & & \\
\hline 21 & 154.9993 & 3.3250 & 284.1 & 4 & & \\
\hline 17 & 155 . & 4 . & & 4 & & \\
\hline 18 & 158 . & 50 & 282.4 & 5 & & \\
\hline 21 & 158 & 250 & 282.0 & 4 & & \\
\hline 21 & 156.5426 & 50 & 281.8 & 5 & 0 & \\
\hline 21 & 168.2415 & & & 4 & & \\
\hline 2 & 368 & 0 & 5 & 5 & & \\
\hline 23 & 91.9990 & 0 & 3 & 6 & 0 & \\
\hline 24 & 164 . & & & 6 & & \\
\hline 021 & 154 . & & .2 & 5 & & \\
\hline 211252 & 151.0975 & 2000 & 6 & 4 & & \\
\hline 211253 & 133.5000 & 0 & & 4 & & \\
\hline 25 & 89 . & 50 & & 4 & & \\
\hline $21125 \quad 5$ & 141.2708 & & 0.6 & 4 & & \\
\hline 211256 & 134.2716 & 4.7250 & 0.3 & 4 & & 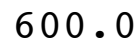 \\
\hline $21125 \quad 7$ & 140 . & 0 & & 4 & & \\
\hline 211258 & 134 . & & 1.0 & 4 & & \\
\hline 211259 & 144 . & 500 & 281.2 & 4 & & \\
\hline & 146 . & 0 & & 4 & & \\
\hline & 147.2473 & & 1.9 & 4 & & \\
\hline 12 & 141.5000 & 250 & 282.9 & 4 & & \\
\hline 13 & 150.2258 & 0 & & 4 & & \\
\hline 021 & 160.2443 & 5.8500 & 286.6 & 3 & & t \\
\hline 21 & 161.9984 & 8.2250 & 287.9 & 4 & & \\
\hline & 160.9995 & 7 . & & 4 & & \\
\hline 021 & 149.2492 & 000 & .3 & 4 & & \\
\hline 518 & 148.0007 & 0.5000 & 288.2 & 4 & & 0 \\
\hline 021 & 149.4998 & 500 & & 4 & & \\
\hline 0211 & 156.0001 & 12.4750 & & 4 & & \\
\hline 21 & 155.7499 & 10.0250 & 286.4 & 4 & & (2) \\
\hline & & & & 4 & & \\
\hline 211 & 128.7549 & 6.4000 & 286.0 & & & 600 \\
\hline
\end{tabular}




\begin{tabular}{|c|c|c|c|c|c|c|}
\hline 4 & & & & & & \\
\hline 211261 & 3997 & .4000 & 9 & & & \\
\hline 11262 & 128.4962 & 2500 & 84.6 & & 00 & \\
\hline 11263 & 142.7493 & 5750 & 284.5 & & 00 & \\
\hline 211264 & 39.2499 & & & & & \\
\hline 11265 & 0 & & 83 & & 0 & \\
\hline 211266 & 139.9975 & 2750 & 83.8 & & 00 & \\
\hline 21126 & 47.2503 & 250 & & & & \\
\hline 211268 & 9.0002 & 4750 & 3 & & & \\
\hline 211269 & 38.2488 & 1250 & 84.9 & & 0 & 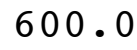 \\
\hline 2610 & 137.2491 & 8.0750 & 285 & & & \\
\hline 1 & 143.5000 & 000 & & & & \\
\hline 21 & 2480 & 5 . & 287.5 & & 0 & \\
\hline 21 & 146.7493 & 6.00 & 8 & & & \\
\hline 1 & 146.2501 & & & & & \\
\hline 21 & 40.2425 & & 4 & 4 & & \\
\hline 21 & 129.0000 & 4.9500 & 290.8 & 3 & 0 & \\
\hline 17 & 113.5213 & 0 & 6 & & & \\
\hline 2 & 134.5000 & & .2 & 4 & & \\
\hline 21 & 125.8734 & $\$ 500$ & 288.8 & 4 & & \\
\hline 21 & 104.4724 & 3.65 & 289.5 & 4 & 0 & \\
\hline 1 & 128.5021 & & & 4 & & \\
\hline 2 & 96.7332 & 0 & 288.6 & 4 & & \\
\hline 23 & 125.0419 & 0 & 287.7 & 4 & 0 & \\
\hline & 146 & & & 5 & & \\
\hline 1 & 146.2500 & 4 . & & 5 & & \\
\hline $27 \quad 2$ & 122.7384 & 1.7500 & 4 & 4 & 0 & . \\
\hline 211273 & 143.9890 & 1.55 & & 4 & & \\
\hline 274 & 150.9240 & 50 & & 5 & & \\
\hline $21127 \quad 5$ & 126.7549 & $\$ 500$ & 5.6 & 6 & 0 & J. \\
\hline $21127 \quad 6$ & 121.0836 & 1.5750 & 9 & 5 & & 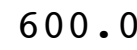 \\
\hline 277 & 157.7537 & 0 & & 4 & & \\
\hline 21 & 141.5322 & & 28 & 5 & & \\
\hline 211279 & 136.9931 & 1.5500 & 286.2 & 4 & 0 & \\
\hline 21 & 151.0381 & 0 & & 3 & & \\
\hline & 161.7623 & & 5.7 & 3 & & \\
\hline 712 & 185.4396 & 0000 & 286.4 & 2 & & \\
\hline 13 & 227.9234 & 1. & 28 & 1 & & \\
\hline 021 & 238.7514 & 1.6750 & 287.9 & 2 & & 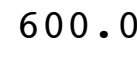 \\
\hline 211 & 169.4708 & 1.1250 & 288.4 & 1 & & \\
\hline & 317.5000 & 0 . & 289.1 & 2 & & \\
\hline 021 & 349.7703 & 1.4500 & 287.3 & 3 & & 0 . \\
\hline 2112718 & 82.4652 & 1.3000 & 286.1 & 4 & 0 & 0 . \\
\hline 211 & 64.2991 & 750 & & 5 & & \\
\hline 02112720 & 102.9974 & 3.1000 & & 4 & & 0 . \\
\hline 2112721 & 198.8352 & 1.4500 & 287.6 & 5 & & 00 . \\
\hline & 990 & & & b & & \\
\hline 21 & 7356 & 1.2000 & 286.4 & & 600.0 & 6 \\
\hline
\end{tabular}




\begin{tabular}{|c|c|c|c|c|c|c|}
\hline 2724 & & 1250 & & & & \\
\hline 211281 & 444 & 0.2750 & 286.7 & 6 & & \\
\hline 211282 & 59.2925 & 1750 & 286.3 & 6 & 00 & \\
\hline 211283 & 138.4850 & 1.2750 & 286.0 & & 00 & \\
\hline $21128 \quad 4$ & 102.5 & 1.4500 & & & 0 & \\
\hline 11285 & 7700 & 7500 & 84.0 & & 00 & \\
\hline 211286 & 344.4752 & 0.6500 & 284.3 & & 0 & \\
\hline 211287 & 13.2502 & L. 5500 & & & & \\
\hline 211288 & 354.7618 & 1.6000 & 285.3 & & 0 & \\
\hline 211289 & 328.1992 & 0.5250 & 287.0 & & 00 & \\
\hline 2810 & 277.0073 & 0.4500 & 287.9 & & & \\
\hline 811 & 244.0474 & & 87.8 & & & \\
\hline 812 & 264.5892 & 1.5250 & 286.2 & & 0 & \\
\hline 813 & 140.5000 & 2.4250 & 286.6 & & 0.0 & \\
\hline 14 & 134.5003 & & & 2 & & \\
\hline 21 & 134.2492 & 2.4500 & 287.4 & 3 & 0 & \\
\hline 316 & 131.3015 & 0 & 286 & 2 & 0 & \\
\hline 17 & 90.4899 & 0 & 28 & 3 & & \\
\hline 21 & 140 . & 0 & 285 & 4 & 0 & \\
\hline 319 & 145.7528 & 0 & 286.2 & 4 & & \\
\hline 20 & 124.0143 & 3. & 9 & 5 & & \\
\hline 21 & 121. & & 288.2 & 4 & & \\
\hline 322 & 107 & 50 & 288.5 & 5 & 0 & \\
\hline 21 & 99.2479 & 3 . & 289.3 & 4 & 0 & \\
\hline 24 & 129 . & & 28 & 5 & & \\
\hline 91 & 142.2515 & 000 & 287 & 4 & & \\
\hline 211292 & 76.0992 & 2.22 & 287.0 & 5 & 0 & \\
\hline 0211293 & 10.1079 & 12 & 7 & 6 & & $\mathrm{C}$ \\
\hline 0211294 & 117.9438 & 1 . & 28 & 6 & 0 & • \\
\hline 211295 & 249.8335 & 0.8000 & 286.7 & 6 & 0 & \\
\hline 211296 & 282.7961 & 0 & 28 & 6 & & \\
\hline 0211297 & 397 & 50 & 28 & 6 & & $\sigma_{0}$ \\
\hline 0211298 & 97.2468 & 2.1250 & 287.0 & 6 & 0 & J \\
\hline 0211299 & 144.9728 & 0 & $2 \varepsilon$ & 5 & 0 & \\
\hline 21 & 151. & 1 & 28 & 4 & & \\
\hline 021 & 127.6280 & 1 . & 289.8 & 3 & & 0 . \\
\hline 211 & 166.2438 & 2.5000 & 289.8 & 3 & 0 & \\
\hline 021 & 174.7561 & 2.92 & 288.9 & 3 & 0 & 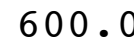 \\
\hline 021 & 176.9662 & 1.9750 & 288.6 & 3 & 60 & 0 . \\
\hline 2112915 & 157.0000 & 250 & 288.6 & 4 & 6 & \\
\hline 021 & 152.4991 & 000 & 288.1 & 4 & & \\
\hline 021 & 143.2776 & 1.3000 & 287.1 & 5 & 600.0 & 600 . \\
\hline 2112918 & 42.6158 & 1.7250 & 286.1 & 6 & 600 & 000 \\
\hline 021 & 4.1208 & 1.6250 & 286.4 & 5 & & 60 \\
\hline 021 & 346.9615 & 0.4500 & 286.6 & 4 & 600.0 & 00 . \\
\hline 211 & 321.5279 & 0.5750 & 287.0 & 5 & 600 & 600. \\
\hline 2 & 344.1997 & & 286.9 & 3 & 60 & \\
\hline 211 & 4.9887 & 1.1000 & 287.0 & $\mathbf{4}$ & 600.0 & 600 \\
\hline
\end{tabular}




\begin{tabular}{|c|c|c|c|c|c|c|c|}
\hline \multicolumn{2}{|c|}{02112924} & 9.7493 & 1.7500 & 286.7 & 4 & 600.0 & \\
\hline \multicolumn{2}{|c|}{0211301} & 276.4777 & 1.1250 & 285.8 & & & \\
\hline \multicolumn{2}{|c|}{0211302} & 3870 & 500 & 85.1 & & 0 & \\
\hline \multicolumn{2}{|c|}{021130} & 218.9449 & 250 & 4.8 & & 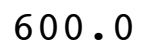 & \\
\hline \multicolumn{2}{|c|}{021130} & 41.7432 & 250 & & & & \\
\hline \multicolumn{2}{|c|}{021130} & 85.7398 & 250 & 87.4 & & 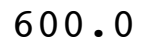 & \\
\hline \multicolumn{2}{|c|}{021130} & 201.5355 & 1250 & 85.1 & & 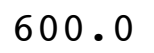 & \\
\hline \multicolumn{2}{|c|}{021130} & 133.7606 & 3750 & 32.5 & & 0 & \\
\hline \multicolumn{2}{|c|}{0211308} & 108.85 & 750 & 82.7 & & 0 & \\
\hline \multirow{2}{*}{\multicolumn{2}{|c|}{$\begin{array}{l}021130 \quad 9 \\
02113010\end{array}$}} & 94.1755 & 250 & 285.4 & & & \\
\hline & & 94.9552 & 000 & & & & \\
\hline \multicolumn{2}{|c|}{02113011} & 56.3512 & 000 & 0 & & & \\
\hline \multicolumn{2}{|c|}{02113012} & 189. & 3750 & & & 0 & \\
\hline \multicolumn{2}{|c|}{02113013} & 141 . & 000 & & & & \\
\hline \multicolumn{2}{|c|}{02113014} & & & & & & \\
\hline \multicolumn{2}{|c|}{02113015} & 355 . & 250 & & & & \\
\hline \multicolumn{2}{|c|}{02113016} & 114.73 & 0 & 4 & & & \\
\hline \multicolumn{2}{|c|}{02113017} & 122.6 & & & & & \\
\hline \multicolumn{2}{|c|}{02113018} & 291 & 0 & & & & \\
\hline \multicolumn{2}{|c|}{02113019} & 153.71 & 0 & & & & \\
\hline \multicolumn{2}{|c|}{02113020} & 82 . & & & & & \\
\hline \multicolumn{2}{|c|}{02113021} & 37 & & & 5 & & \\
\hline 02113 & 022 & 21.0193 & 0 & 0 & & & \\
\hline 21 & & 74 . & & & & & \\
\hline 21 & 24 & 195 . & & & 5 & & \\
\hline 21 & 11 & 168 . & 500 & & 5 & & \\
\hline 212 & 12 & 126.71 & 000 & & 6 & & \\
\hline 212 & 13 & 51. & 0 & & 6 & & \\
\hline 212 & 14 & 54 & & & 5 & & \\
\hline 212 & 15 & 125 . & 750 & & 5 & & \\
\hline 212 & 16 & 136 . & & & 4 & & \\
\hline 0212 & 17 & 136 & & & 5 & & \\
\hline 212 & 18 & 142 . & 9500 & 1.7 & 5 & & \\
\hline 212 & 19 & 124 . & 0 & & 4 & & \\
\hline 0212 & 110 & 158 & & & 4 & & \\
\hline 212 & 111 & 152 . & 250 & 283.8 & 4 & & \\
\hline 212 & 112 & 159. & & & 4 & & \\
\hline 0212 & 113 & 158 . & 750 & 28 & 3 & & \\
\hline 212 & 114 & 166.46 & 500 & 285.3 & 2 & & \\
\hline 212 & 115 & 162 & & & 3 & & \\
\hline 0212 & 116 & 151. & 2 . & & 4 & & \\
\hline 0212 & 117 & 135.74 & 1.9250 & 284.4 & 4 & & \\
\hline 0212 & 118 & 109. & 500 & 283 & 5 & & \\
\hline 0212 & 119 & 134.8995 & 0.6250 & & 5 & & 600. \\
\hline 0212 & 120 & 120.2652 & 0.5500 & 284.0 & 4 & 0 & 00 . \\
\hline 1212 & 121 & 126 . & & & 5 & & \\
\hline 212 & 122 & 145.6034 & 750 & & & & \\
\hline 212 & 120 & 198.1656 & 0.8750 & 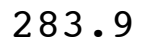 & & .0 & -0 \\
\hline
\end{tabular}




\begin{tabular}{|c|c|c|c|c|c|c|c|}
\hline & 24 & $\pm \cdot 2500$ & 1.0000 & 3.6 & & 600.0 & \\
\hline 1 & 21 & 8.3301 & 0.8750 & 283.8 & & $c 00$ & \\
\hline 12 & 22 & 46.0103 & 9250 & 83.2 & & 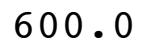 & \\
\hline 212 & 3 & 113.1955 & 0.5750 & 283.0 & & 00 & \\
\hline 12 & 4 & 1 & 0 & 82.4 & & & \\
\hline 212 & 5 & 134.1218 & 1.3500 & 282.8 & & 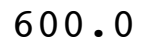 & \\
\hline 212 & 6 & 96.2906 & 0.4750 & 282.7 & & 0 & \\
\hline 212 & 7 & 145.7459 & 1.1750 & 82.9 & & 0 & \\
\hline 212 & 8 & 132.6277 & 1.1500 & 83.3 & & 0 & \\
\hline 212 & 9 & 154.0016 & 0.6250 & 285.0 & & 0 & \\
\hline 212 & 10 & 136 . & 500 & 84.5 & & & \\
\hline 212 & 11 & 146 . & & 85.6 & & & \\
\hline 212 & 12 & 153. & 2.8500 & 286.1 & & 0 & \\
\hline 212 & 213 & 163. & 3.2000 & 7 & & & \\
\hline 212 & 14 & 196. & & & & & \\
\hline 212 & 15 & 220 . & 1.3250 & 288.1 & & 0 & \\
\hline 212 & 216 & 217.2315 & 1.37 & 287.5 & & 0 & \\
\hline 212 & 217 & $200.1 \varepsilon$ & 120 & & & & \\
\hline 212 & 18 & 244 & $0 \quad 15$ & 285.2 & 4 & & \\
\hline 212 & 219 & 997 & 50 & 285.3 & & 0 & \\
\hline 212 & 220 & 26 & 0 & & & & \\
\hline 212 & 221 & 32 & 1. & 28 & 4 & & \\
\hline 212 & 222 & 40.7190 & 1.0000 & 286.2 & & 0 & \\
\hline 212 & 223 & 32 . & 0 & & & & \\
\hline 212 & 224 & 18 & & & 5 & & \\
\hline 212 & 31 & 259 & 000 & 284 & 6 & & \\
\hline 212 & 32 & 325.9323 & 0.77 & 284.8 & & & \\
\hline 212 & 33 & 338 . & 42 & 3 & 5 & & \\
\hline 212 & 34 & 321 . & 017 & 28 & 5 & & \\
\hline 212 & 35 & 673 & 0.42 & 28 & 6 & & \\
\hline 0212 & 36 & 151. & 0 & & 5 & & \\
\hline 0212 & 37 & 145 . & 0 & .4 & 5 & & \\
\hline 212 & 38 & 142.9981 & .77 & 282.0 & J & & \\
\hline 0212 & 39 & 143.2763 & 0 & 282 & 4 & & - \\
\hline 0212 & 310 & 175.6075 & 0.2750 & & 3 & & \\
\hline 0212 & 311 & 274.4369 & 0.8500 & 286.4 & & & \\
\hline 212 & 312 & 267 . & 1.60 & 28 & 2 & & \\
\hline 0212 & 313 & 276.2035 & 1.7000 & 5.4 & 1 & & 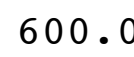 \\
\hline 0212 & 314 & 311.9951 & 1.9250 & 287.1 & 2 & & 6 \\
\hline 0212 & 315 & 296 . & 2 . & 287.3 & 2 & & \\
\hline 0212 & 316 & 292.5009 & 2 . & 287.1 & 3 & & \\
\hline 0212 & 317 & 297.0219 & 1.5000 & 286.3 & 4 & & 0 \\
\hline 0212 & 318 & 242.7464 & 0.21 & 285.0 & 5 & & \\
\hline 0212 & 319 & 270.3538 & 0.4250 & 284.5 & 6 & 600.0 & 600 \\
\hline 0212 & 320 & 353.2389 & 0.7250 & 284.3 & 6 & 600.0 & 00 \\
\hline & 321 & 52 & & & 6 & & \\
\hline 0212 & 322 & 140.2497 & 1.7750 & & & .0 & 600 \\
\hline 212 & 23 & 153.2508 & 1.2250 & 284.5 & & 600.0 & 600 \\
\hline
\end{tabular}




\begin{tabular}{|c|c|c|c|c|c|c|c|}
\hline 212 & 24 & & .5000 & $3 \cdot 3$ & & & \\
\hline 2 & 1 & 68.9396 & 0.9500 & 283.1 & & & \\
\hline$\perp$ & 2 & 356.1390 & 4250 & 283.1 & & 00 & \\
\hline 21 & 3 & 98.0037 & .1500 & 282.7 & & 00 & \\
\hline & 4 & 107.3035 & .8750 & 3.0 & & 0 & \\
\hline & 5 & 88.7639 & .0250 & 82.9 & & 00 & \\
\hline 21 & 6 & 144.9862 & 1.6500 & 283.0 & & 0 & \\
\hline 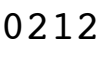 & 7 & 151.8069 & .6750 & 82.8 & & & \\
\hline 21 & 8 & 136.4982 & 1.1750 & 282.4 & & 0 & \\
\hline 21 & 9 & 141.2506 & 1.7750 & 282.7 & & 00.0 & \\
\hline 2 & 410 & 149.7478 & 3.5750 & 282.6 & & & \\
\hline 21 & 411 & 152.2506 & 3.5750 & 83.4 & & & \\
\hline 21 & 12 & 156.4998 & 2.7750 & 284.7 & 4 & 0 & \\
\hline 21 & 413 & 155.7504 & 2.6250 & 286.2 & 3 & 0.0 & \\
\hline 1 & 414 & 152.00 & 2.8250 & & & & \\
\hline 212 & 415 & 188.9539 & 2.4000 & 286.8 & 3 & 0 & \\
\hline 1 & 416 & 208.7492 & 1.4250 & 286.5 & & 0 & \\
\hline 212 & 417 & 143.76 & 1.4750 & 6 & & & \\
\hline 212 & 418 & 148.5000 & 1.2750 & 284.7 & 4 & 0 & \\
\hline 21 & 419 & 143.2067 & 8250 & 284.5 & 4 & 0 & \\
\hline 21 & 420 & 105.5593 & 250 & 284.2 & 5 & & \\
\hline 212 & 421 & 85 & 250 & 284.2 & 6 & & \\
\hline 21 & 422 & 69.4178 & 750 & 283 & 6 & 0 & \\
\hline 21 & 423 & 4. & 1 . & 284.3 & 5 & 0 & \\
\hline 212 & 424 & 320.25 & 250 & 284 & 5 & & \\
\hline 212 & 51 & 333.2587 & 1.2750 & 284.2 & 4 & & \\
\hline 212 & 52 & 306.7554 & 1.7250 & 284 & 5 & 0 & \\
\hline 212 & 53 & 342.5495 & 1.3250 & 283 & 5 & 0 & C \\
\hline 0212 & 54 & 305.5248 & 1.4750 & 28 & 4 & 0 & 0 . \\
\hline 212 & 55 & 321.5994 & 1.6250 & 283.8 & 5 & 0 & \\
\hline 21 & 56 & 304 . & 500 & 28 & 6 & & \\
\hline 021 & 57 & 266 . & 000 & 28 & 6 & & \\
\hline 0212 & 58 & 16.6225 & 0.4750 & 284.1 & 6 & 00 & U. \\
\hline 212 & 59 & 318 . & 3000 & 28 & 5 & 0 & \\
\hline 212 & 510 & 306 . & 00 & 28 & 4 & & \\
\hline 0212 & 511 & 325.5585 & 0.7750 & 285.6 & 3 & & 0 . \\
\hline 212 & 512 & 278.4861 & 1.0500 & 286.6 & 2 & 0 & \\
\hline 0212 & 513 & 276.4726 & 1.1750 & 286.6 & 2 & 0 & 0 \\
\hline 0212 & 514 & 243.2503 & 1.7500 & 286.1 & 3 & .0 & 0 . \\
\hline 212 & 515 & 281.8558 & 000 & 286.0 & 2 & $6 c$ & \\
\hline 0212 & 516 & 292.7936 & 1.7250 & & 3 & & \\
\hline 0212 & 517 & 293.1796 & 1.4750 & 284.6 & 4 & 600.0 & 600 . \\
\hline 212 & 518 & 289.0044 & 0.6250 & 283.7 & 4 & 600 & 600. \\
\hline 0212 & 519 & 264.2808 & 0.2500 & & 5 & & 60 \\
\hline 0212 & 520 & 162.5024 & 0.3500 & 283.0 & 4 & 600.0 & 00 \\
\hline 212 & 521 & 220.3995 & 0.2250 & 282.9 & 5 & 600.0 & 600 . \\
\hline 21 & 522 & 155.9498 & 250 & & 5 & .0 & 60 \\
\hline 21 & 523 & 197.7809 & 0.2000 & 282.5 & & 600.0 & 600 \\
\hline
\end{tabular}




\begin{tabular}{|c|c|c|c|c|c|c|c|}
\hline & 24 & & 0.3000 & & & & \\
\hline 1 & 1 & 80 . & 0.4500 & 282.1 & 0 & & \\
\hline I & 62 & 154.7276 & 4000 & 281.9 & 6 & 00 & \\
\hline 21 & 6 & 127.6005 & .2250 & 281.7 & & 00 & \\
\hline & 4 & 148.7 & .3250 & 81.1 & & 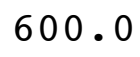 & \\
\hline & 5 & 134.7291 & 1.0500 & 281.3 & & 00 & \\
\hline 21 & 6 & 119.3665 & 0.3250 & 281.2 & & 0 & \\
\hline & 7 & 139.7247 & .6250 & 81.4 & & & \\
\hline 1 & 8 & 71.1423 & .0500 & 81.4 & 6 & 0 & \\
\hline 21 & 9 & 353.7045 & 0.2000 & 282.3 & & 00 & \\
\hline 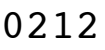 & 610 & 131.2145 & 0.6750 & 282.7 & 2 & 0 & \\
\hline & 611 & 229.1599 & 1.2250 & 83.4 & 3 & & \\
\hline 21 & 12 & 190.3755 & 1.1500 & 283.2 & 2 & 0 & \\
\hline 1 & 613 & 158.7429 & 2.4750 & 283.1 & 1 & 0.0 & \\
\hline - & 614 & 159.7390 & 2.3250 & 282.7 & 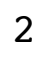 & & \\
\hline 21 & 61 & 138.5768 & 2.1750 & 283.0 & 1 & 0 & \\
\hline 212 & 616 & 158.2593 & 2.6250 & 282.8 & 2 & 0 & \\
\hline 12 & 617 & 146.9865 & 750 & 3.1 & 3 & & \\
\hline 21 & 18 & 85.4962 & 4.8250 & 284.4 & 4 & 0 & \\
\hline 21 & 619 & 26.6936 & .9250 & 285.1 & 5 & 0 & \\
\hline 212 & 620 & 116.4622 & 750 & 285.2 & 6 & & \\
\hline 1 & 621 & 130 . & 0 & 284.4 & 6 & & \\
\hline 21 & 622 & 287.1926 & 500 & 283.0 & 6 & 0 & \\
\hline 21 & 623 & 344.10 & 1.1000 & 282.9 & 5 & 0 & \\
\hline 212 & 624 & 43. & 1.2000 & 283.9 & 5 & & \\
\hline 21 & 71 & 2527 & 7750 & 285.0 & 6 & & \\
\hline 21 & 72 & 89.0861 & .5250 & 284.2 & 6 & 0 & \\
\hline 0212 & 73 & 81.4562 & 1.4250 & 285.0 & 6 & & C \\
\hline 0212 & 74 & 86 & 50 & 285.3 & 5 & 0 & 0 . \\
\hline 212 & 75 & 80.9997 & 000 & 285.4 & 4 & 0 & \\
\hline 212 & 76 & 138.4256 & 50 & .2 & 4 & & \\
\hline 0212 & 77 & 145 & 50 & 282.2 & 5 & & 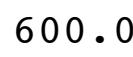 \\
\hline 0212 & 78 & 132.3218 & 2250 & 282.3 & 4 & 00 & U. \\
\hline 212 & 79 & 141.60 & 250 & 283.0 & 4 & 0 & \\
\hline 212 & 710 & 149 . & 50 & .5 & 3 & & \\
\hline 0212 & 711 & 148.9950 & 3000 & 283.7 & 4 & & 0 . \\
\hline 212 & 712 & 162.7511 & 3.8000 & 284.8 & 4 & 0 & \\
\hline 0212 & 713 & 159.5013 & 4.5250 & 285.2 & 4 & 0 & 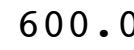 \\
\hline 0212 & 714 & 154.4994 & 4.6000 & 285.9 & 4 & .0 & 0 . \\
\hline 212 & 71 & 157 . & 3250 & 286.7 & 4 & 60 & \\
\hline 0212 & 716 & 146.2537 & 2.3000 & & 4 & & \\
\hline 0212 & 717 & 243.1047 & 0.4000 & 286.6 & 5 & 600.0 & 600 . \\
\hline 27 & 718 & 358.1890 & 1.0250 & 285.3 & 5 & 600 & 600. \\
\hline 0212 & 719 & 35.4718 & 1.2500 & & 6 & 600 & 60 \\
\hline 0212 & 720 & 109.4595 & 0.4250 & 285.6 & 6 & 600.0 & 00 \\
\hline 21 & 721 & 2767 & 0.8750 & 285.9 & 6 & 600.0 & 600. \\
\hline 21 & 722 & 97.4378 & 1.7250 & & 6 & 600 & 60 \\
\hline 21 & 723 & 136.6137 & 1.0000 & 285.8 & & 600.0 & 600 \\
\hline
\end{tabular}




\begin{tabular}{|c|c|c|c|c|c|c|c|}
\hline 212 & 24 & & .6250 & 84.9 & & & \\
\hline 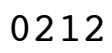 & 81 & 256.7196 & .3750 & 284.3 & 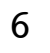 & & \\
\hline 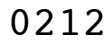 & 82 & 48.2643 & 3250 & 284.4 & 6 & 00 & \\
\hline 21 & 3 & 348.8466 & .6500 & 284.6 & & 00 & \\
\hline & 4 & 282.4803 & .6000 & & & 0 & \\
\hline & 5 & 263 & 7250 & 283.8 & 6 & 00 & \\
\hline 21 & 6 & 328.6051 & 0.4750 & 284.2 & & 0 & \\
\hline 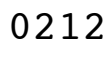 & 7 & 21.8032 & L. 0750 & 83.8 & & & \\
\hline 1 & 8 & 13.5017 & 0.7000 & 284.2 & 6 & 0 & \\
\hline 21 & 9 & 297.2109 & 0.5500 & 284.2 & & 00.0 & \\
\hline 1 & 810 & 312.5556 & 0.4250 & 286.7 & 4 & 0 & \\
\hline & 11 & 278.6079 & 1.2750 & 86.0 & 3 & & \\
\hline 21 & 12 & 265.9671 & 1.8500 & 284.7 & 2 & 00 & \\
\hline 21 & 813 & 292.9987 & 2.0500 & 283.9 & 2 & 0.0 & \\
\hline 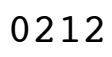 & 814 & 289.9973 & & & 2 & & \\
\hline 21 & 15 & 293.2654 & 1.8000 & 285.2 & 3 & 0 & \\
\hline 212 & 816 & 306.0000 & 1.4500 & 285.2 & 3 & 0 & \\
\hline 12 & 817 & 303. & 0 . & 284.3 & 4 & & \\
\hline 212 & 818 & 320 . & 0 . & 283.3 & 4 & 0 & \\
\hline 21 & 819 & 5108 & 3500 & 282.8 & 5 & & \\
\hline 212 & 820 & 141 . & 0 & 282.6 & 5 & & \\
\hline 212 & 821 & 139.4998 & & 282.5 & 5 & & \\
\hline 21 & 822 & 3100 & 500 & 282.2 & 6 & 0 & \\
\hline 21 & 823 & 23.9783 & 500 & 282.3 & 6 & 0 & \\
\hline 212 & 824 & 170.7367 & & 282.3 & 6 & & \\
\hline 212 & 91 & 118.7478 & 500 & 281.6 & 6 & & \\
\hline 21 & 92 & 6.0306 & 500 & 281.8 & 6 & 0 & \\
\hline 0212 & 93 & 07.3362 & 0 & 281.8 & 6 & & C \\
\hline 0212 & 94 & 180 & 000 & 282.5 & 6 & 0 & 0 . \\
\hline 212 & 95 & 066 & 5000 & 282.4 & 6 & 0 & \\
\hline 21 & 96 & 352 . & 0 & 280.8 & 5 & & \\
\hline 0212 & 97 & 336 . & 0 & 281.6 & 5 & & 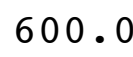 \\
\hline 0212 & 98 & 290.3055 & 3250 & 282.2 & 6 & 0 & J \\
\hline 212 & 99 & 229 . & 0 & 282.5 & 5 & 0 & \\
\hline 212 & 910 & 157. & & 282.6 & 4 & & \\
\hline 0212 & 911 & 155.7494 & 000 & 280.6 & 3 & & 0 . \\
\hline 212 & 912 & 154.4972 & 500 & 281.1 & 3 & 0 & \\
\hline 0212 & 913 & 155.4692 & 250 & 282.6 & 2 & 0 & \\
\hline 0212 & 914 & 153.5023 & 1.5500 & 283.7 & 2 & 60 & 0 . \\
\hline 212 & 915 & 683 & 250 & 284.4 & 3 & 60 & \\
\hline 0212 & 916 & 285.3326 & 250 & 283.4 & 2 & & \\
\hline 0212 & 917 & 349.4299 & 1.3500 & 283.5 & 3 & 600.0 & 600 . \\
\hline 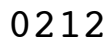 & 918 & 48.9989 & 000 & 285.3 & 4 & 600 & 600. \\
\hline 0212 & 919 & 29.4862 & 1.9000 & 284.8 & 5 & & 600 \\
\hline 0212 & 920 & 53.7122 & 3.0250 & 285.0 & 4 & 600.0 & 500 \\
\hline 212 & 921 & 36.9931 & 3.7500 & 284.9 & 4 & 600.0 & 600. \\
\hline 21 & 922 & 2508 & & 285.1 & 4 & .0 & 60 \\
\hline 21 & 923 & 32.5386 & 3.8250 & 284.9 & $\mathbf{4}$ & 600.0 & 600 \\
\hline
\end{tabular}




\begin{tabular}{|c|c|c|c|c|c|c|}
\hline 212 & & 2.2250 & 283.7 & 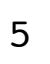 & 0.0 & \\
\hline 212101 & 112.1444 & 1.5000 & 283.8 & 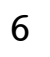 & 00.0 & \\
\hline 12102 & 3486 & 8250 & 284.6 & & 0 & \\
\hline 12103 & 336.6686 & .0500 & 84.3 & & 0 & \\
\hline 12104 & 36.6433 & .6750 & 284.8 & & 00 & \\
\hline 212105 & 998 & 4500 & 284.8 & & 0 & \\
\hline 212106 & 344.1997 & .3750 & & & & \\
\hline 212107 & 322.74 & .3500 & 83.8 & & & \\
\hline 212108 & 16.2301 & 0.7250 & 284.0 & & 00.0 & 0 \\
\hline 212109 & 18.1095 & 4250 & & & & \\
\hline 2121010 & 158.9351 & 1.2250 & & & & \\
\hline 2121011 & 246.5000 & 1.0750 & 284.9 & & 0 & 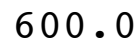 \\
\hline 2121012 & 87.5500 & 3.5500 & 286.8 & & & \\
\hline 2121013 & 93.9945 & .4000 & & & & \\
\hline 2121014 & 84.8033 & 4.3000 & 287.2 & & & \\
\hline 015 & 68.9696 & 3000 & 286.5 & & 0 & \\
\hline 016 & 72.4929 & 2500 & 0 & & & \\
\hline 017 & 68.9966 & 4250 & & & & \\
\hline 018 & 68.2481 & 6.2750 & 284.1 & & 0 & 0 \\
\hline 019 & 69.2559 & 5.9000 & $2 \varepsilon$ & & & \\
\hline 020 & 64.2500 & & & & & \\
\hline 021 & 012 & 000 & & 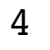 & & \\
\hline 2121022 & 78.2500 & 7.7250 & 283.5 & 4 & 0 & 1 \\
\hline 2121023 & 71.5002 & 7.2000 & & 4 & & \\
\hline 2121024 & 003 & 750 & & 4 & & \\
\hline 212111 & 78.7499 & 3500 & 2 & 4 & & 1 \\
\hline 212112 & 76.2491 & 5250 & & 4 & & \\
\hline 212113 & 78.9998 & 000 & $2 \varepsilon$ & 4 & & \\
\hline 212114 & 77.2540 & 2500 & 282.9 & 4 & & • \\
\hline 212115 & 77.5003 & 3000 & $2 \varepsilon$ & 4 & & \\
\hline 0212116 & 84.2492 & 0 & 28 & 4 & & \\
\hline 0212117 & 91.2512 & 500 & 282.9 & 5 & & \\
\hline 0212118 & 86.2875 & 4.9500 & 283.0 & 5 & 0 & \\
\hline 0212119 & 128.5735 & 2.4750 & 283.9 & 4 & & \\
\hline 02121110 & 109.2733 & 3250 & 283 & 3 & & \\
\hline 2121111 & 129.7173 & 3.8250 & 284.7 & 3 & & \\
\hline 02121112 & 168.2285 & 4 . & & 3 & & \\
\hline 02121113 & 176.7219 & 3.0750 & 283.8 & 3 & & 0 \\
\hline 02121114 & 190.9962 & 2.8250 & 284.0 & 2 & 0 & \\
\hline 2121115 & 162.2506 & 750 & & 3 & & \\
\hline 02121116 & 226.5654 & 1.2000 & 284.7 & 2 & & 00 . \\
\hline 02121117 & 48.9265 & 1.2000 & 283.8 & 3 & 0 & م0 \\
\hline 02121118 & 136.3003 & 1.6750 & 283.7 & 4 & & \\
\hline 02121119 & 100.2652 & 3.6000 & 284.6 & 4 & 600.0 & 500. \\
\hline 02121120 & 114.6896 & 3.2750 & 283.9 & 4 & 600.0 & 00 \\
\hline 2121121 & 979 & 00 & & 4 & & \\
\hline 02121122 & 145.0000 & 1.5500 & 283.4 & 5 & 600 & 600 \\
\hline 1211 & 127.6211 & 1.1750 & 280 & & 60 & 60 \\
\hline
\end{tabular}




\begin{tabular}{|c|c|c|c|c|c|c|}
\hline 21124 & 4.5498 & 3250 & 279.7 & 5 & 0.0 & \\
\hline 12121 & 13.5139 & 1.1500 & 280.8 & 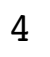 & 00.0 & \\
\hline 12122 & 337.2709 & 2500 & 281.5 & 1 & 0 & \\
\hline 12123 & 358.7571 & .7250 & 81.3 & & 0 & \\
\hline 12124 & 353.0624 & .4750 & 281.7 & & 00 & \\
\hline 212125 & 278 & 5750 & 281.1 & & 0 & \\
\hline 212126 & 289.0827 & & 279.2 & & & \\
\hline 212127 & 239.6553 & & 77.9 & & & \\
\hline 212128 & 224.0830 & 0.7250 & 277.2 & & 00.0 & . \\
\hline 212129 & 331.7752 & 02 & 277.4 & & & \\
\hline 2121210 & 200.5746 & 0 & 78.4 & & & \\
\hline 2121211 & 259.4591 & 0.8250 & 279.9 & & 0 & \\
\hline 2121212 & 231.2974 & 1.8000 & 279.5 & & & \\
\hline 2121213 & 289.0743 & 47 & 79.6 & & & \\
\hline 2121214 & 287 . & & 280.3 & & & \\
\hline 215 & 328 . & .0250 & 280.8 & & & \\
\hline 2121216 & 336.4908 & 1.32 & 280.8 & 1 & & \\
\hline 217 & 14.7772 & 2 . & 280.9 & 2 & & \\
\hline 218 & 14.7390 & 0 & 281.5 & & 0 & \\
\hline 2121219 & 142.0444 & .45 & 282.9 & 4 & & \\
\hline 220 & 220 . & & & 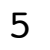 & & \\
\hline 221 & 306 & & $2 \varepsilon$ & 6 & & \\
\hline 2121222 & 343.5191 & 1.52 & 283.0 & 6 & 0 & \\
\hline 2121223 & 350.5000 & 77 & 283.5 & 6 & & \\
\hline 224 & 230 . & 1 . & $2 \varepsilon$ & 6 & & \\
\hline 212131 & 344.2587 & 0 & 286.2 & 5 & 0 & \\
\hline 212132 & 338.4985 & 0 & 2 & 4 & & \\
\hline 212133 & 321 . & 0 & $2 \varepsilon$ & 5 & & \\
\hline 212134 & 332.4197 & 0 & 5.9 & 5 & 0 & $\pi$ \\
\hline 212135 & 322 . & 0 & & 6 & & \\
\hline 212136 & 258 . & 0 & $2 \varepsilon$ & 6 & & \\
\hline 0212137 & 269.6979 & & 284.9 & 6 & & \\
\hline 212138 & 276.8080 & 6250 & 284.1 & 6 & 0 & \\
\hline 0212139 & 312.4976 & 0 & 283.8 & 5 & & \\
\hline 2121310 & 310 . & 9 . & $2 \varepsilon$ & 4 & & \\
\hline 2121311 & 310.7482 & 7.37 & 284.7 & 4 & & \\
\hline 02121312 & 311.4971 & 0 & & 4 & & \\
\hline 02121313 & 309.7634 & 5.5750 & 6.7 & 3 & & 0 . \\
\hline 2121314 & 292.6818 & 3000 & 286.3 & 3 & 0 & \\
\hline 2121315 & 298 . & 0 & & 3 & & \\
\hline 02121316 & 304.2480 & 5.4250 & .4 & 4 & & \\
\hline 02121317 & 312.5003 & 4.9000 & 284.4 & 4 & 0 & $0 c^{-1}$ \\
\hline 02121318 & 309.9988 & 7.3500 & & 4 & & \\
\hline 02121319 & 304.2500 & 7.3750 & 284.6 & 4 & 600.0 & 500 . \\
\hline 02121320 & 288.4959 & 5.4250 & 284.9 & 4 & 600 & 00 \\
\hline 02121321 & 282.5025 & & & 4 & & \\
\hline 02121322 & 304.0173 & 3.6000 & & 4 & & 600 \\
\hline 2121323 & 312.0592 & 5.1000 & 286.5 & & 60 & 60 \\
\hline
\end{tabular}




\begin{tabular}{|c|c|c|c|c|c|c|}
\hline & & & & & & \\
\hline 14 & 3 & & & & & \\
\hline 2142 & 90 & 3500 & 85.8 & & 00 & \\
\hline 12143 & 19.4994 & 8500 & 286.2 & & 0 & \\
\hline 214 & & & & & & \\
\hline 214 & & & & & & \\
\hline $21214 \quad 6$ & 000 & 7000 & 86.2 & & 00 & \\
\hline 21214 & 71 & & & & & \\
\hline 212148 & 94 & & & & & \\
\hline 21214 & 330.2498 & 500 & 86.4 & & 0 & \\
\hline 410 & 7514 & 6.0500 & 0 & & & \\
\hline 11 & 3127401 & 50 & & & & \\
\hline 21 & 73 & 3750 & 87.4 & & & \\
\hline 21 & 500 & 0.3250 & $28^{\circ}$ & & & \\
\hline & & & & & & \\
\hline & & 2 & & 4 & & \\
\hline 21 & 979 & 2.0000 & 285.5 & 4 & & \\
\hline 17 & 45 & 0 & & & & \\
\hline 21 & 36 & 11 . & & 4 & & \\
\hline 21 & 566 & & & 4 & & \\
\hline 21 & 26.7512 & 12.1250 & & 4 & & \\
\hline 21 & & & & $\mathrm{H}^{-}$ & & \\
\hline 21 & 44 & 10 . & & 4 & & \\
\hline 23 & 16.0217 & 0 & 2 & 4 & & \\
\hline 21 & & & & 4 & & \\
\hline 021 & 99 & 8. & & 4 & & \\
\hline $21215 \quad 2$ & 4985 & 500 & & 4 & & \\
\hline 212153 & 000 & & & 4 & & \\
\hline 54 & 64 & & & 4 & & \\
\hline 212155 & 273 & & & 4 & & 0 . \\
\hline 212156 & 329.2499 & 2.2000 & & 5 & & \\
\hline 212157 & 168 & 0 & & 4 & & \\
\hline 212158 & 2584 & & & 4 & & \\
\hline 212159 & 309.7632 & 0 & & 3 & & \\
\hline 212 & 326 & & & 4 & & \\
\hline & & & & 4 & & \\
\hline 512 & 300.3755 & 500 & 3 & 3 & & \\
\hline 513 & 332 . & 4. & & 3 & & \\
\hline 0212 & 343 . & 4 . & & 3 & & 0 . \\
\hline 2121515 & 340.9973 & & 286 & 4 & & \\
\hline 2121516 & 326 . & 5 . & & 4 & & \\
\hline 0212 & 325.7539 & 4 . & & 4 & & \\
\hline 2121518 & 335.0038 & 6.8000 & 284 & 4 & & 0 \\
\hline 02121519 & 334 . & & & 4 & & \\
\hline 02121520 & 328.2522 & & & 4 & & \\
\hline 2121521 & 324.2610 & 9.3750 & & 4 & & 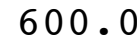 \\
\hline & & & & 4 & & \\
\hline 2121523 & 4968 & 1.0250 & & & & \\
\hline
\end{tabular}




\begin{tabular}{|c|c|c|c|c|c|c|}
\hline & & & & & & \\
\hline 2161 & 9945 & & 34.4 & & & \\
\hline 2162 & 082 & .5500 & 84.9 & 4 & 00 & \\
\hline 12163 & 354.2501 & 1.2750 & 283.5 & & 00 & \\
\hline 212164 & 2500 & 0.3750 & 3.1 & & & \\
\hline 12165 & 7502 & 13. & 83.6 & & 0 & \\
\hline 212166 & 5388 & 7750 & 84.7 & & 00 & \\
\hline 21216 & 205 & 6.9000 & 85.2 & & & \\
\hline 12168 & 3.7597 & 500 & 1 & & & \\
\hline 212169 & 9986 & 7.2000 & 85.1 & & 0 & 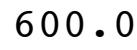 \\
\hline 1610 & 44.7437 & 6.2000 & 285.7 & & & \\
\hline 11 & 37.2374 & 8.3500 & & & & \\
\hline 21 & 4834 & 500 & 284 & & 0 & \\
\hline 613 & 57.2561 & 11.0750 & 28 & & & \\
\hline & & & & & & \\
\hline 21 & & & 9 & 4 & & \\
\hline 21 & 44.27 & 7.9000 & 284 & 4 & 0 & \\
\hline 17 & 8 & 500 & & 4 & & \\
\hline 21 & & & & 4 & & \\
\hline 21 & 5010 & 1750 & 282 & 4 & & \\
\hline 21 & 56.6537 & 4.2250 & $2 \varepsilon$ & 4 & 0 & \\
\hline 21 & & & & 4 & & \\
\hline 2 & 57. & $\$ 750$ & 7 & 5 & & \\
\hline 623 & 66.2750 & 2.7250 & 8 & 6 & & \\
\hline 24 & 64 & 5250 & & & & \\
\hline 7 & 0 & 50 & 28 & 4 & & \\
\hline 212172 & .5029 & 4000 & 6 & 4 & & . \\
\hline 212173 & 536 & 5000 & & 4 & & \\
\hline $21217 \quad 4$ & 53 & 00 & $2 \varepsilon$ & 4 & & \\
\hline 212175 & 16.4096 & 2.3500 & 9.9 & 5 & & J. \\
\hline 212176 & 49.4751 & 1.3250 & 9.7 & 6 & & 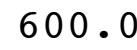 \\
\hline $21217 \quad 7$ & $34 \mathrm{c}$ & 50 & 8 & 5 & & \\
\hline 212178 & 62.2518 & 500 & 28 & 4 & & . \\
\hline 212179 & 83.4838 & 500 & 280.0 & 4 & & \\
\hline 212 & 56 & 0 & & 3 & & \\
\hline & & 6.1000 & & 4 & & \\
\hline 2121712 & 61.7386 & 8.1000 & 280.7 & 4 & & \\
\hline 212 & 73.3496 & 0 & 28 & 4 & & \\
\hline 02121714 & 98.0000 & 7.4750 & 281.8 & 4 & & 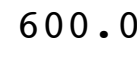 \\
\hline 2121715 & 89.8459 & 8.2000 & 282.0 & 4 & & \\
\hline 2121716 & 69.7390 & 5000 & 280 & 4 & & \\
\hline 02121717 & 64.2500 & 8.7500 & & 4 & & \\
\hline 2121718 & 67.7426 & 8.2000 & 280.7 & 4 & & 0 . \\
\hline 2121719 & 77.7426 & 250 & & 4 & & \\
\hline 02121720 & 103.2577 & & & 4 & & \\
\hline 2121721 & 91.9962 & 5.2250 & 280.1 & 4 & & 00 . \\
\hline & 00 & & & 4 & & \\
\hline 2121723 & 80.8293 & 4.5250 & 280.2 & & & 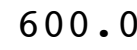 \\
\hline
\end{tabular}




\begin{tabular}{|c|c|c|c|c|c|c|}
\hline- & - & 3250 & 79.9 & 4 & 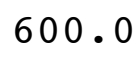 & \\
\hline 2181 & 2.2517 & 6.7250 & 279.5 & 4 & 600.0 & \\
\hline 12182 & 5092 & 7500 & 280.0 & 4 & 00 & \\
\hline 12183 & 5000 & 9250 & 279.6 & & 00 & \\
\hline 212184 & 855 & 2000 & 79.4 & & & \\
\hline 12185 & & 50 & 79.2 & & 0 & \\
\hline 212186 & 472 & 2250 & 279.1 & & 0 & \\
\hline 212187 & 503 & & 279.2 & & & \\
\hline 212188 & 3.7654 & 0 & 79.4 & & & \\
\hline 212189 & 2501 & 5750 & 280.1 & & 0 & \\
\hline 2121810 & 55.1892 & 0 & 281.1 & & & \\
\hline 11 & 88.5000 & 211 & 81.7 & & & \\
\hline 812 & 493 & 3. & 282.0 & & & \\
\hline 813 & 91.7122 & 0.00 & 282.6 & & & \\
\hline 14 & 84 . & & & & & \\
\hline 315 & 63. & & 1 & & & \\
\hline 816 & 297.4810 & 2.77 & 283.3 & & & \\
\hline 817 & 330.5018 & 0 & 0 & & & \\
\hline 818 & 333.2452 & & 280 & 1 & & \\
\hline 819 & 323.2192 & 2. & 280.1 & 4 & & \\
\hline 820 & 339.2616 & 2.62 & 279.7 & 4 & & \\
\hline 21 & 342.9976 & & 9.9 & 4 & & \\
\hline 21 & 349.2688 & 0 & 279 & 4 & & \\
\hline 823 & 337.7524 & 2.30 & 279.6 & 5 & & \\
\hline 324 & 308.7315 & & & 4 & & \\
\hline 91 & 312.1343 & 2 . & 279.8 & 5 & & \\
\hline 212192 & 244.5129 & 0 & 279.5 & 6 & & \\
\hline 212193 & 324.7791 & 0 & & 5 & & \\
\hline 212194 & 325 . & 6 . & 27 & 4 & & \\
\hline 212195 & 330.9963 & 0 & 279.5 & 4 & & . \\
\hline 212196 & 347.4612 & 2 . & 278.7 & 5 & & 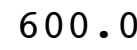 \\
\hline 212197 & 289.4925 & 0 & 278 & 5 & & \\
\hline 212198 & 315.5706 & & 27 & 4 & & 0 . \\
\hline 212199 & 330.2500 & 2.1000 & $2 \varepsilon$ & 4 & & \\
\hline 212 & 317.7467 & 0 & & 4 & & \\
\hline & 305.0004 & 0.7250 & 1.0 & 4 & & \\
\hline 2121912 & 281.2467 & 8.8000 & 280.2 & 4 & & \\
\hline 2121913 & 314.9331 & 7500 & 280.6 & 4 & & \\
\hline 02121914 & 340.2500 & 8.8000 & 281.4 & 4 & & 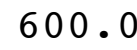 \\
\hline 2121915 & 357.7663 & 7500 & 281.0 & 3 & & \\
\hline 2121916 & 66.7169 & 250 & 277.3 & 4 & & \\
\hline 02121917 & 28.0252 & 4.2500 & 276.5 & 4 & & \\
\hline 2121918 & 19.8054 & 2.9750 & 277.6 & 4 & & 0 . \\
\hline 02121919 & 336.5057 & 750 & & 5 & & \\
\hline 02121920 & 36.1224 & 3.8250 & & 4 & & \\
\hline 2121921 & 358.8269 & 2.6750 & 279.7 & 5 & & 2 \\
\hline 12 & & & & 4 & & \\
\hline 2121923 & 345.4768 & 0.9500 & 279.6 & & & 00 \\
\hline
\end{tabular}




\begin{tabular}{|c|c|c|c|c|c|c|}
\hline- & & & & & & \\
\hline 201 & t & 500 & 279.6 & & 0 & \\
\hline 12202 & 6.9922 & 000 & 278.3 & & 00 & \\
\hline 12203 & 342.7497 & 500 & 277.8 & & 00 & \\
\hline $21220 \quad 4$ & 1 & 500 & 277.2 & & & \\
\hline 12205 & 0 & 750 & 278.2 & & 0 & \\
\hline 212206 & 4893 & 500 & 278.1 & & 00 & \\
\hline 212207 & 92 & & 278.4 & & & \\
\hline 212208 & 7652 & 4250 & 78.5 & & & \\
\hline 212209 & 2589 & 250 & 279.7 & & 0 & 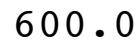 \\
\hline 2122010 & 347.7803 & 2.2500 & 281.4 & & & \\
\hline 2011 & 327.2782 & 2.5000 & 82.2 & & & \\
\hline 2122012 & 338.6657 & 4.2500 & 282.8 & & 0 & \\
\hline 2122013 & 356.2498 & 4.9750 & 283.4 & & & \\
\hline 2014 & 000 & & & & & \\
\hline 2015 & 6707 & & 281.9 & 2 & & \\
\hline 2122016 & 339.1075 & 3.8750 & 281.7 & 3 & .0 & \\
\hline 2017 & 343.3230 & 50 & 1.5 & 4 & & \\
\hline 2018 & 8.5064 & 00 & 1.5 & 5 & & \\
\hline 2019 & 349.1894 & 1.6250 & 1.6 & 6 & & \\
\hline 2122020 & 331.2091 & 1.9500 & 1.0 & 6 & 0 & 00. \\
\hline 021 & 346.8046 & & & 6 & & \\
\hline 022 & 321.5106 & 500 & .8 & 5 & & \\
\hline 2122023 & 332.0043 & 1.6750 & 280.9 & 6 & 0 & \\
\hline 2122024 & 339.3038 & & .9 & 6 & & \\
\hline 211 & 347.6666 & 500 & 281.3 & 5 & & \\
\hline 212212 & 341.4960 & 250 & 281.2 & 4 & 0 & J. \\
\hline 212213 & 348.5000 & 1 . & .7 & 5 & & \\
\hline 0212214 & 352.0368 & 00 & .5 & 4 & & \\
\hline 0212215 & 1.7504 & 750 & 0.8 & 5 & 0 & U. \\
\hline 212216 & 27.2379 & 3.1000 & 1.3 & 4 & & . \\
\hline 0212217 & 27.0276 & 0 & 1.2 & 4 & & \\
\hline 0212218 & 296.5000 & 1.3250 & 0.7 & 5 & & 0 . \\
\hline 0212219 & 322.5087 & 1.5000 & 280.9 & 4 & 0 & \\
\hline 02122110 & 334.3176 & 0 & 3.2 & 3 & & \\
\hline 0212 & 263.1985 & 1.1750 & 4.0 & 2 & & \\
\hline 2122112 & 243.3903 & .5000 & 283.6 & 1 & & 0 . \\
\hline 02122113 & 202.8978 & 0 & 282.4 & 2 & & 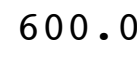 \\
\hline 02122114 & 230.1987 & 2.0500 & 283.1 & 2 & .0 & 0 . \\
\hline 02122115 & 187.0000 & 2.1250 & 283.4 & 2 & 0 & \\
\hline 02122116 & 181.8217 & 500 & 283.2 & 3 & & 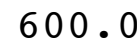 \\
\hline 02122117 & 155.2498 & 2.4250 & 282.2 & 4 & .0 & 0 . \\
\hline 2122118 & 148.2472 & 2.7750 & 281.6 & 5 & 0 & 00 . \\
\hline 02122119 & 134.2494 & 7500 & 281.8 & 4 & & \\
\hline 02122120 & 138.9699 & 4.7000 & 281.9 & 4 & & 0 . \\
\hline 2122121 & 162.7494 & 7.7750 & 280.4 & 4 & & 600. \\
\hline 122 & & & 279.8 & 4 & & \\
\hline 2122123 & 157.7511 & 8.6250 & 279.6 & & 60 & -1 \\
\hline
\end{tabular}




\begin{tabular}{|c|c|c|c|c|c|c|}
\hline & & & & & & \\
\hline 221 & & .7250 & 9.5 & & & \\
\hline 2222 & 074 & 4000 & 279.8 & & 00 & \\
\hline 12223 & 000 & 6.7000 & 279.8 & & 0 & \\
\hline 212224 & 00 & 500 & 80.0 & & & \\
\hline 12225 & 416 & & 279.7 & & & \\
\hline 212226 & 368 & 5750 & 279.6 & & 0 & \\
\hline 212227 & 95 & & 79.3 & & & \\
\hline 212228 & 75 & 0 & 79.1 & & & \\
\hline 212229 & 5000 & 0.0500 & 279.0 & & 0 & \\
\hline 2122210 & 5002 & 9500 & 279.7 & & & \\
\hline 211 & $5 \cap 01$ & 9.1250 & 80.3 & & & \\
\hline 21 & 2 & 0.7250 & 281.0 & & & \\
\hline 213 & 58 & 1.6000 & & & & \\
\hline 11 & & 0 & & & & \\
\hline & & 0 & 282.6 & 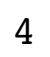 & & \\
\hline 21 & 166 & 1.1500 & 282.4 & 4 & & \\
\hline 217 & 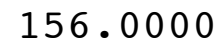 & 0 & 1.8 & & & \\
\hline & 0 & 0 & 281.4 & 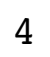 & & \\
\hline 9 & 152 & 5250 & & 4 & & \\
\hline 20 & 148.50 & 2.8000 & 281.7 & 4 & & \\
\hline & & & 1.3 & 4 & & \\
\hline 2 & 5 & 0 & $2 \varepsilon$ & 4 & & \\
\hline 23 & 163.4 & 7000 & 279.7 & 4 & & \\
\hline 24 & 162 & & & 4 & & \\
\hline 31 & 154 . & 50 & 277 & 4 & & \\
\hline 212232 & 147.7501 & .7250 & 277.3 & 4 & & \\
\hline 212233 & 143 & $\$ 250$ & & 4 & & \\
\hline 34 & 142 . & 00 & 27 & 4 & & \\
\hline 212235 & 139. & 750 & 2 & 4 & & 0 . \\
\hline 212236 & 133.2509 & 7500 & 6.5 & 4 & & \\
\hline 212237 & 135.2584 & 0 & 2 & 4 & & \\
\hline 212238 & 125 . & & 6.2 & 4 & & \\
\hline 212239 & 117.7552 & 750 & 27 & 4 & & \\
\hline 10 & 128 & 0 & & 4 & & \\
\hline & 131.4946 & & 8.0 & 4 & & \\
\hline 212 & 138.5276 & 750 & 278.8 & 4 & & \\
\hline 212 & 159 & 0 & 279.4 & 4 & & \\
\hline 212 & 154.0023 & 500 & 280.1 & 4 & & . \\
\hline 212231 & 155.5147 & 750 & 280.5 & 3 & & \\
\hline 212 & 149 . & 0 & 280.4 & 4 & & \\
\hline 0212 & 151.0003 & 3.6750 & 279.6 & 5 & & \\
\hline 2122318 & 132.0084 & 2.2000 & 278.7 & 5 & & 0 \\
\hline 212231 & 207 & 750 & & & & \\
\hline 02122320 & 54.4581 & 1.5500 & & J & & \\
\hline 21223 & 32.5480 & 2.0500 & 278.5 & 4 & & 00 . \\
\hline & & & & כ & & \\
\hline 2122323 & 53 & 0.5750 & 278.5 & & & \\
\hline
\end{tabular}




\begin{tabular}{|c|c|c|c|c|c|c|}
\hline $23<4$ & 9.7711 & 0.6000 & 278.6 & 6 & 000.0 & \\
\hline $224 \quad 1$ & 49.1554 & 0.3000 & 278.8 & & 600.0 & \\
\hline 12242 & 146.0016 & 1.8750 & 278.9 & 4 & 00 & \\
\hline 212243 & 139.8616 & 1.4500 & 278.5 & & 00 & \\
\hline $21224 \quad 4$ & 340. & .1000 & 277.8 & & 0 & \\
\hline 12245 & 12 & .4250 & 277.9 & 4 & 00 & \\
\hline 212246 & 8.5224 & 1.1000 & 278.1 & & 0 & \\
\hline 212247 & 62.5947 & .6250 & 78.5 & & & \\
\hline 212248 & 145.4991 & 1.5250 & 279.0 & 5 & 0 & \\
\hline 212249 & 147.5346 & 1.3750 & 279.3 & 4 & 0 & \\
\hline 2410 & 25.3369 & .8500 & 279.4 & 3 & 0 & \\
\hline & 47.67 & & & & & \\
\hline 212 & 227.2280 & 1.4000 & 280.2 & 2 & 0 & \\
\hline 2122413 & 169.5879 & 1.8250 & 279.0 & 1 & 0 & \\
\hline 14 & 207.97 & & & 1 & & \\
\hline 21 & 246 . & 1.5500 & 281.0 & 2 & & \\
\hline 416 & 284.3751 & 1.15 & 280.6 & 2 & 0 & \\
\hline 212 & 293. & 1.37 & 9.9 & 3 & & \\
\hline 212 & 264 . & 0.35 & 278.4 & 4 & 0 & \\
\hline 419 & 51.74 & 0 & 278.6 & 5 & & \\
\hline 20 & 149 . & 0 & 278.8 & 5 & & \\
\hline 21 & 151. & & 278.4 & 5 & & \\
\hline 2122422 & 184.7980 & 17 & 277.9 & 4 & 0 & U • \\
\hline 2122423 & 358 . & 0 & 7.5 & 5 & 0 & \\
\hline 2122424 & 72 . & & 7.6 & 6 & & \\
\hline 212251 & 145.2496 & 0 & 276.8 & 5 & & \\
\hline 212252 & 142.7476 & 0 & 5.9 & 4 & & \\
\hline 0212253 & 165.6100 & 0 & 27 & 5 & & \\
\hline 0212254 & 13. & 0.80 & .6 & 5 & & \\
\hline $21225 \quad 5$ & 9 . & 52 & 276.8 & 5 & & \\
\hline 212256 & 345 . & 0 & 5.8 & 6 & & \\
\hline 0212257 & 16 . & 0 & 277.0 & 5 & & \\
\hline 0212258 & 338.2470 & 92 & 276.9 & 4 & 0 & 0 . \\
\hline 212259 & 323 . & 137 & .8 & 3 & & \\
\hline 02122510 & 330 . & 0 & .7 & 3 & & \\
\hline 02122511 & 289. & 0500 & 278.5 & 2 & & 0 . \\
\hline 2122512 & 248.4635 & 0.9250 & 278.8 & 1 & & \\
\hline 02122513 & 186. & 2.07 & 278.4 & 2 & & \\
\hline 02122514 & 218 . & 1.6250 & 277.3 & 1 & 0 & 0 . \\
\hline 2122515 & 324 . & .6250 & 278.4 & 2 & & \\
\hline 02122516 & 302 . & 0 & 278.1 & 3 & & \\
\hline 02122517 & 312.2405 & 1.7500 & 278.1 & 4 & 600.0 & 00 . \\
\hline 2122518 & 130.5915 & 2.5000 & 278.1 & 5 & 600 & 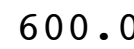 \\
\hline 02122519 & 111.4622 & 1.5250 & 278.3 & 6 & & \\
\hline 02122520 & 319.6311 & 0.6500 & 278.9 & 6 & 600 & 00 \\
\hline 2122521 & 127 & 1.5750 & 279.9 & 5 & 600 & \\
\hline 2122522 & 335 & 2.1000 & 280.2 & 6 & 60 & \\
\hline 2122523 & 356.2569 & 1.9500 & 281.2 & & 600.0 & 600 \\
\hline
\end{tabular}




\begin{tabular}{|c|c|c|c|c|c|c|}
\hline 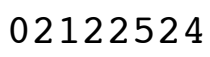 & & & & & & \\
\hline 261 & 1312 & 0 & 2.3 & 6 & ) & \\
\hline 12262 & 339.2541 & 000 & 282.9 & 5 & 00 & \\
\hline 12263 & 265.8123 & 0000 & 281.8 & & 00 & \\
\hline 212264 & 667 & 9500 & 80.9 & & 0 & \\
\hline 12265 & 49 & 2750 & 280.7 & & 0 & \\
\hline 212266 & 5010 & 9500 & 281.6 & 4 & 00 & \\
\hline 212267 & 2500 & 250 & 82.1 & & 0 & \\
\hline 212268 & 0.9993 & 4500 & 82.3 & 4 & & \\
\hline 212269 & 307.0000 & 7.8000 & 282.8 & 4 & 00.0 & 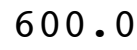 \\
\hline 2122610 & 309.7485 & 6.8000 & 283.4 & I & 0 & \\
\hline 21 & 300.2506 & 5.9500 & 83.7 & 4 & & \\
\hline 212 & 4962 & 250 & 284.2 & 3 & 0 & \\
\hline 212 & 294.4907 & 4.0500 & 28 & 3 & & \\
\hline 14 & 272 & & & & & \\
\hline 21 & 277 & 1 . & 285.0 & 1 & & \\
\hline 21 & 271.4590 & 2.6000 & 285.6 & 1 & 0 & \\
\hline 17 & 298 & 2.3250 & 4 & 2 & & \\
\hline 21 & 311 & 3 . & 285.1 & 3 & & \\
\hline 21 & 5000 & 000 & 285.1 & 4 & & \\
\hline 21 & 335.9977 & 3.3500 & 284.7 & 4 & 0 & 00. \\
\hline 21 & 337.2491 & & & 5 & & \\
\hline 21 & 329 & 750 & 28 & 4 & & \\
\hline 23 & 326.0000 & 3.6250 & 28 & 4 & 0 & \\
\hline 24 & 283 & & & 5 & & \\
\hline 021 & 335 . & 1.2250 & 28 & 6 & & \\
\hline $21227 \quad 2$ & 332.7206 & 2.4250 & 285 & 5 & 0 & . \\
\hline 212273 & 315.3364 & 9500 & 7 & 6 & & \\
\hline $021227 \quad 4$ & 280 & 250 & 28 & 6 & & \\
\hline 212275 & 238.3559 & 1.1000 & 286.1 & 6 & & 0 . \\
\hline 0212276 & 299.4564 & 2.1500 & 285.2 & 6 & & ( \\
\hline $21227 \quad 7$ & 341.8855 & 1.4250 & 28 & 6 & & \\
\hline 0212278 & 282.4236 & 500 & 28 & 6 & & 0 . \\
\hline 0212279 & 318.7759 & 2.3000 & 286.1 & 5 & 0 & \\
\hline 02122710 & 340.7441 & 0 & & 4 & & \\
\hline 0212 & 321.7857 & 2.5000 & 287.7 & 3 & & 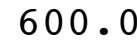 \\
\hline 212 & 275.6431 & 1.2250 & 287.8 & 2 & $\cdot 0$ & 0 . \\
\hline 0212 & 249.7461 & 1.2250 & 28 & 1 & & . \\
\hline 02122714 & 290.9283 & 1.0500 & 286.7 & 1 & .0 & 0 \\
\hline 02122715 & 353.2898 & 3.9000 & 288.4 & 2 & 0 & \\
\hline 02122716 & 259.2231 & 2.2500 & 288.0 & 1 & & 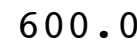 \\
\hline 02122 & 147.5779 & 1.4250 & 287.0 & 2 & .0 & 0 . \\
\hline 2122718 & 336.4885 & 4.5000 & 286.0 & 3 & 0 & 00 . \\
\hline 02122719 & 340.4880 & 2.4250 & & 4 & & \\
\hline 02122720 & 348.4983 & 3.7500 & 286.2 & 4 & 0 & 00 \\
\hline 02122721 & 343.7487 & 3.5750 & 286.3 & 4 & 600.0 & 600 \\
\hline 12 & 50 & & & 4 & & \\
\hline 2122723 & 351.5000 & 3.6500 & 287.3 & & .0 & 60 \\
\hline
\end{tabular}




\begin{tabular}{|c|c|c|c|c|c|c|}
\hline 2724 & 8.5028 & 2250 & 7.9 & 4 & 0.0 & \\
\hline 12281 & 337.4429 & 3000 & 287.3 & 5 & 00 & \\
\hline 12282 & 4212 & 1000 & 287.5 & & 70 & \\
\hline 2283 & 358.5009 & .2750 & 87.4 & & 0 & \\
\hline 12284 & 348.6945 & 2.7250 & 87.0 & & 0 & \\
\hline 212285 & 349 & 2.9750 & 286.9 & & 0 & \\
\hline 212286 & 341 . & & & & & \\
\hline 212287 & 343.5000 & 0 & & & & \\
\hline 212288 & 335.7506 & 4.4000 & 285.8 & & 0 & 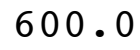 \\
\hline 212289 & 327.0048 & 0 & 28 & & & \\
\hline 2122810 & 308.9988 & & & & & \\
\hline 2122811 & 296 . & 5000 & 285.3 & & & \\
\hline 2122812 & 5023 & 3250 & 6 & & & \\
\hline 2122813 & 318.7232 & .5250 & 2 & & & \\
\hline 2122814 & 354 . & 0 & 288.1 & & & \\
\hline 2122815 & 49.2069 & .7500 & 281.5 & 4 & & \\
\hline 122816 & 32.9853 & L. 3500 & 5 & & & \\
\hline 122817 & 24.7497 & & 1 & 4 & & \\
\hline 2122818 & 11.0288 & 4.4250 & 280.2 & 4 & 0 & 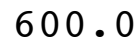 \\
\hline 122819 & 90 & 0 & .2 & & & \\
\hline 2122820 & 42 . & & & 4 & & \\
\hline 2122821 & 853 & 0 & & 4 & & \\
\hline 2122822 & 20.4965 & 4.55 & 281.4 & 4 & & \\
\hline 2122823 & 12.2363 & & & $I$ & & \\
\hline 2122824 & 147 & 50 & 28 & 4 & & \\
\hline 212291 & 19.7502 & 0 & 281.7 & 4 & & \\
\hline 212292 & 18.0184 & 0 & 28 & 4 & & \\
\hline 212293 & 15.2734 & 50 & 28 & 4 & & \\
\hline $21229 \quad 4$ & 51.7455 & 0 & 280.3 & 4 & & . \\
\hline 212295 & & & 27 & 4 & & \\
\hline 212296 & 922 & & 28 & 4 & & \\
\hline 212297 & 598 & 0 & & 4 & & \\
\hline 212298 & 323 & 0 & 280.5 & 4 & & \\
\hline 212299 & 61. & 0 & 28 & 4 & & \\
\hline 2122910 & 000 & & 281.4 & 4 & & \\
\hline 2122911 & 003 & 5500 & 282.1 & 4 & & \\
\hline 2122912 & 147 & 0 & 282 & 4 & & \\
\hline 02122913 & .2654 & 0 & 282.8 & 3 & & \\
\hline 2122914 & .2488 & 0 & 283.1 & 3 & & \\
\hline 2122915 & 387 & 0 & & 3 & & \\
\hline 02122916 & 61.4920 & 4.0000 & 283.4 & 3 & & \\
\hline 2122917 & 60.5013 & .1000 & 282.4 & 4 & & \\
\hline 02122918 & 54.2505 & 5.8500 & 281.5 & 4 & & \\
\hline 02122919 & 58.5000 & 5.4000 & 281.1 & 4 & & \\
\hline 2122920 & 57.5006 & 4.1500 & 280.8 & 5 & 0 & 00 \\
\hline 12292 & & & & & & \\
\hline 02122922 & 54.2501 & 5.87 & 280.8 & 4 & & 600 \\
\hline 22923 & 49.7500 & 5.0750 & 280.8 & & 60 & 60 \\
\hline
\end{tabular}




\begin{tabular}{|c|c|c|c|c|c|c|}
\hline-5 & 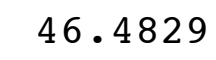 & .1250 & 80.6 & & & \\
\hline 2301 & 7.6924 & 2.6750 & 81.1 & 4 & 6000 & \\
\hline 12302 & 21.2508 & 250 & 281.1 & 5 & 00 & \\
\hline 12303 & 352.2907 & 1500 & 280.9 & & 00 & \\
\hline $21230 \quad 4$ & 5.9607 & .750 & & & & \\
\hline 12305 & 52.0090 & 0 & 81.3 & & 0 & \\
\hline 212306 & 90.7955 & 3250 & 281.5 & & 00 & \\
\hline 212307 & 347.0015 & 250 & 80.1 & & & \\
\hline 212308 & 33.5170 & 4250 & 80.7 & & & \\
\hline 212309 & 13.0188 & 5000 & 81.6 & & 0 & \\
\hline 010 & 3.5213 & 0.6250 & 282.5 & & & \\
\hline 11 & 335.5429 & 1.3250 & 82.6 & & & \\
\hline 21 & 250.4642 & 500 & 282.2 & & 0 & \\
\hline 21 & 157.5344 & 500 & 281.9 & & & \\
\hline & 158.6833 & & & & & \\
\hline & 187 . & & .7 & 3 & & \\
\hline 21 & 310.2471 & 2.1500 & 280.5 & 4 & 0 & \\
\hline 17 & 319.5000 & 2 & & 4 & & \\
\hline 18 & 330.4928 & & & 4 & & \\
\hline 19 & 316.4668 & & 280.8 & 4 & & \\
\hline 20 & 329.5915 & 6 . & 281.3 & 4 & 0 & \\
\hline 1 & 314.7761 & & & 4 & & \\
\hline 22 & 326.5014 & 00 & 81.0 & 4 & & \\
\hline 23 & 304.8840 & 4.2250 & .1 & 4 & 0 & \\
\hline 24 & 12.1722 & & & 4 & & \\
\hline 021 & 359.2500 & 0 & & 4 & & \\
\hline 212312 & 0.2500 & 750 & 3.2 & 4 & & . \\
\hline 212313 & 28.1291 & & 1.7 & 4 & & \\
\hline 0212314 & 21.3726 & 50 & & 4 & & \\
\hline 212315 & 78.0000 & 500 & 281.3 & 4 & & J. \\
\hline 212316 & 64.2503 & 50 & 281.9 & 4 & & 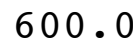 \\
\hline 212317 & 76.3560 & 0 & & 4 & & \\
\hline 212318 & 78.9481 & 0 & .3 & 4 & & 0 . \\
\hline 212319 & 67.9407 & 0 & 282.1 & 4 & & \\
\hline 212 & 70.4554 & & & 4 & & \\
\hline 021 & 65.7509 & & .2 & 3 & & \\
\hline 212 & 66.9952 & 750 & 283.0 & 4 & & \\
\hline 212 & 64.5092 & 0 & 284.0 & 3 & & 0 \\
\hline 0212 & 84.5001 & 3.1000 & 283.5 & 2 & & 0 . \\
\hline 2123115 & 224.8628 & 1.4750 & 282.6 & 1 & & \\
\hline 0212 & 23.2060 & 500 & 281.8 & 2 & & \\
\hline 0212 & 44.0000 & 250 & 281.5 & 3 & & 0 . \\
\hline 2123118 & 59.9919 & 2.7750 & 281.8 & 4 & & 0 . \\
\hline 02123119 & 53.8541 & 500 & & 4 & & \\
\hline 02123120 & 92.3017 & & & 4 & & \\
\hline 2123121 & 82.0359 & 5.0250 & 281.4 & 4 & & 00 . \\
\hline- & 97 & & & כ & & \\
\hline 2123123 & 74.5006 & 4.8000 & 281.1 & & .0 & 00 \\
\hline
\end{tabular}




\begin{tabular}{|c|c|c|c|c|c|c|c|c|}
\hline & & & 82.5015 & 5.1000 & 281.1 & 4 & 600.0 & \\
\hline 3 & 1 & 11 & 9.7502 & 5.1250 & 281.1 & & 600.0 & \\
\hline & 1 & 12 & 80.7548 & 6250 & 281.1 & 4 & 00 & 0 \\
\hline & 1 & 13 & 91.2506 & 5750 & 281.2 & & 00 & \\
\hline & 1 & 14 & 36.8963 & 6500 & 1.1 & & 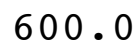 & \\
\hline & 1 & 15 & 150.2509 & 0 & 80.0 & & 0( & \\
\hline & 1 & 16 & 142.5006 & 6750 & 279.3 & & 00 & \\
\hline & 1 & 17 & 126.5874 & 7500 & 79.3 & & 0 & \\
\hline & 1 & 18 & 70.4245 & 4250 & 79.6 & & & \\
\hline & 1 & 19 & 129.4461 & 1.0750 & 80.7 & & 0 & U \\
\hline & 1 & 110 & 131.9799 & .4000 & 281.0 & & 0.0 & \\
\hline & 1 & 111 & 274.7685 & 0 & 82.8 & & & \\
\hline & 1 & 112 & 199.4579 & 2 . & 281.6 & & 0 & \\
\hline & 1 & 113 & 148.5024 & $0.1 v$ & 280.4 & & 0.0 & \\
\hline & 1 & 114 & 260 & 0 & 32.8 & & & \\
\hline & 1 & 11 & 238 . & & 282.5 & & & \\
\hline & 1 & 116 & 183.4390 & 0 & 283.6 & & .0 & \\
\hline & 1 & 117 & 151. & 0 & 1.6 & & & \\
\hline & 1 & 118 & 247 . & & 30.5 & 3 & & \\
\hline & 1 & 119 & 218.1891 & 0 & 280.1 & 4 & 0 & 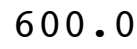 \\
\hline 3 & 1 & 120 & 252.7697 & 500 & 280.1 & & 0 & \\
\hline & 1 & 121 & 300.8555 & & 30.0 & & & \\
\hline & 1 & 122 & 348 . & 0 & 279.8 & 4 & & \\
\hline & 1 & 123 & 339.5387 & 0 & 280.4 & 4 & 0 & \\
\hline & 1 & 124 & 346.7501 & & .5 & 4 & & \\
\hline 3 & 1 & 21 & 338 . & 3750 & 280.7 & 5 & & \\
\hline & 1 & 22 & 172.4799 & 0 & 0.4 & 6 & 0 & J. \\
\hline & 1 & 23 & 227 . & 0 & 0.5 & 6 & & \\
\hline 3 & 1 & 24 & 169. & 00 & 279.6 & 5 & & \\
\hline & 1 & 25 & 176.6295 & 2500 & 279.6 & 6 & 0 & 0. \\
\hline & 1 & 26 & 24.2684 & 2.1000 & 279.3 & 6 & & 0 \\
\hline 3 & 1 & 27 & 160.0386 & 2 & 279.7 & 6 & & \\
\hline & 1 & 28 & 313.2182 & 0 & 279.4 & 6 & 0 & . \\
\hline & 1 & 29 & 13.5190 & 500 & 280.5 & 5 & 0 & 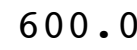 \\
\hline & 1 & 210 & 350.2512 & 0 & 281.6 & 4 & & \\
\hline 3 & 1 & 211 & 17.0063 & 2000 & 283.1 & 3 & & U \\
\hline & 1 & 212 & 243.3976 & 1250 & 283.6 & 2 & & 0 . \\
\hline 3 & 1 & 213 & 152.2495 & 4 . & .3 & 3 & & . \\
\hline 03 & 1 & 214 & 162.0023 & 3.8500 & 280.9 & 4 & .0 & 0 . \\
\hline & 1 & 215 & 172.0327 & 2.6500 & 281.8 & 3 & & \\
\hline & 1 & 216 & 236.9038 & 1.1500 & 281.7 & 2 & & 0 \\
\hline 3 & 1 & 217 & 155.0465 & 0.8250 & 281.5 & 3 & & 0 . \\
\hline & 1 & 218 & 190.7308 & 0.6000 & 281.4 & 4 & 0 & 0 \\
\hline 03 & 1 & 219 & 151.5000 & 2.7000 & & 5 & & \\
\hline 03 & 1 & 220 & 130.2267 & 2.3250 & & 6 & & 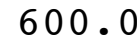 \\
\hline 0 & 1 & 221 & 112.6091 & 1.2250 & 280.4 & 6 & 600 & 00. \\
\hline & 1 & 222 & 30 & & & 6 & & \\
\hline & 1 & 223 & 117.9475 & 2.1250 & 280.7 & & 600.0 & 600 \\
\hline
\end{tabular}




\begin{tabular}{|c|c|c|c|c|c|c|c|c|}
\hline 3 & 1 & 224 & 68.7751 & 0.8500 & 283.4 & 6 & 600.0 & 600.0 \\
\hline 03 & 1 & 31 & 85.8698 & 0.6000 & 286.7 & 6 & 600.0 & 600.0 \\
\hline 03 & 1 & 32 & 29.7311 & 2.1250 & 283.8 & 6 & 600.0 & 00.0 \\
\hline 03 & 1 & 33 & 229.2993 & 1.9250 & 281.1 & 6 & 600.0 & 600.0 \\
\hline 03 & 1 & 34 & 3.9212 & 2.2500 & 281.4 & 5 & 600.0 & 00.0 \\
\hline 03 & 1 & 35 & 67.6907 & 1.5500 & 282.9 & 6 & 600.0 & 00.0 \\
\hline 03 & 1 & 36 & 152.2228 & 3.7250 & 279.5 & 5 & 600.0 & 600.0 \\
\hline 03 & 1 & 37 & 122.0752 & 3.3750 & 279.0 & 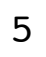 & 600.0 & 500.0 \\
\hline 03 & 1 & 38 & 150.0000 & 4.8500 & 278.8 & 5 & 600.0 & 00.0 \\
\hline 03 & 1 & 39 & 142.2322 & 3.5000 & 279.1 & 4 & 600.0 & 600.0 \\
\hline 03 & 1 & 310 & 322.6640 & 2.5750 & 280.9 & 3 & 600.0 & 600 \\
\hline 03 & 1 & 311 & 234.6628 & 1.7250 & 282.2 & 2 & 600.0 & \\
\hline 03 & 1 & 312 & 295.2586 & 1.6250 & 283.5 & 1 & 600.0 & 600.0 \\
\hline 03 & 1 & 313 & 190.2120 & 1.7000 & 284.3 & 1 & 600.0 & 600.0 \\
\hline 03 & 1 & 314 & 172.9848 & 2.2500 & 284.5 & 2 & 600.0 & \\
\hline 03 & 1 & 315 & 163.7500 & 3.3500 & 283.5 & 3 & 600.0 & 60 \\
\hline 03 & 1 & 316 & 158.5000 & 2.5750 & 283.2 & 4 & 600.0 & 600.0 \\
\hline 03 & 1 & 317 & 157.2673 & 0.9750 & 283.6 & 5 & 600.0 & .0 \\
\hline 03 & 1 & 318 & 283.2392 & 0.6250 & 282.1 & 6 & 600.0 & 60 \\
\hline 03 & 1 & 319 & 344.5286 & 1.7000 & 281.8 & 5 & 600.0 & 60 \\
\hline 03 & 1 & 320 & 357.9910 & 2.1000 & 281.6 & 5 & 600.0 & .0 \\
\hline 03 & 1 & 321 & 11.9951 & 1.9500 & 281.8 & 4 & 600.0 & \\
\hline 03 & 1 & 322 & 232.5337 & 0.7000 & 282.4 & 5 & 600.0 & 600.0 \\
\hline 03 & 1 & 323 & 181.2969 & 1.0500 & 282.2 & 6 & 600.0 & 60 \\
\hline 03 & 1 & 324 & 122.6744 & 1.2250 & 282.2 & 6 & 600.0 & \\
\hline 03 & 1 & 41 & 130.9952 & 1.0500 & 282.5 & 6 & 600.0 & 600.0 \\
\hline 03 & 1 & 42 & 346.7376 & 0.6750 & 282.8 & 6 & 600.0 & 600.0 \\
\hline 03 & 1 & 43 & 11.5851 & 1.4250 & 284.2 & 5 & 600.0 & .0 \\
\hline 03 & 1 & 44 & 74.6733 & 1.4000 & 284.2 & 6 & 600.0 & 600.0 \\
\hline 03 & 1 & 45 & 335.2000 & 1.1000 & 283.9 & 6 & 600.0 & 600.0 \\
\hline 03 & 1 & 46 & 247.6202 & 1.42 & 282.7 & 6 & 600.0 & .0 \\
\hline 03 & 1 & 47 & 141.5080 & 2.1750 & 283.3 & 6 & 600.0 & 600.0 \\
\hline 03 & 1 & 48 & 77.0529 & 2.5250 & 283.9 & 6 & 600.0 & 600.0 \\
\hline 03 & 1 & 49 & 109.5503 & 2.3000 & 284.4 & 5 & 600.0 & .0 \\
\hline 03 & 1 & 410 & 147.5640 & 2.2250 & 287.2 & 4 & 600.0 & 600.0 \\
\hline 03 & 1 & 411 & 154.2705 & 3.2000 & 287.2 & 3 & 600.0 & 600.0 \\
\hline 03 & 1 & 412 & 171.4960 & 2.8000 & 288.2 & 3 & 600.0 & 600.0 \\
\hline 03 & 1 & 413 & 138.5000 & 4.4500 & 288.1 & 3 & 600.0 & 600.0 \\
\hline 03 & 1 & 414 & 157.0435 & 1.5250 & 289.4 & 2 & 600.0 & 600.0 \\
\hline 03 & 1 & 415 & 110.7976 & 2.8500 & 289.4 & 2 & 600.0 & \\
\hline 03 & 1 & 416 & 97.7118 & 1.9500 & 288.9 & 1 & 600.0 & 600.0 \\
\hline 03 & 1 & 417 & 98.7769 & 2.8500 & 289.9 & 2 & 600.0 & 600.0 \\
\hline 03 & 1 & 418 & 108.2432 & 2.9500 & 288.4 & 3 & 600.0 & 600.0 \\
\hline 03 & 1 & 419 & 95.4982 & 5.2250 & 288.5 & 4 & 600.0 & 600.0 \\
\hline 03 & 1 & 420 & 94.7255 & 4.8000 & 288.2 & 4 & 600.0 & 600.0 \\
\hline 03 & 1 & 421 & 109.5335 & 4.2750 & 287.0 & 4 & 600.0 & 600.0 \\
\hline 03 & 1 & 422 & 105.3920 & 3.3000 & $28^{\circ}$ & 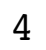 & 600.0 & 600.0 \\
\hline 03 & $\perp$ & 423 & 114.2565 & 2.2000 & 285.8 & 3 & 600.0 & 600.0 \\
\hline
\end{tabular}




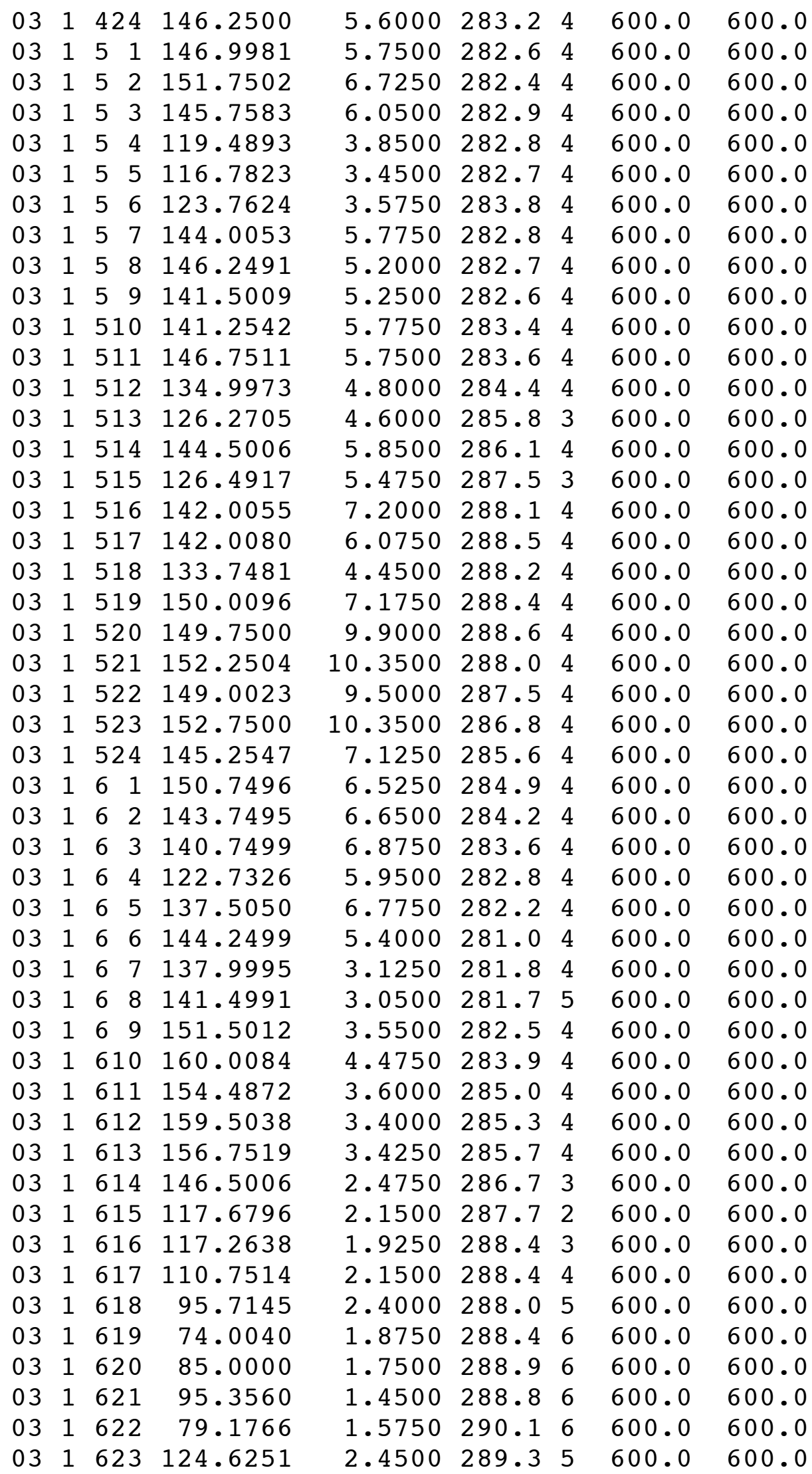




\begin{tabular}{|c|c|c|c|c|c|c|c|}
\hline & 24 & & 750 & 87.5 & & 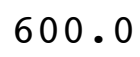 & \\
\hline & $\begin{array}{lll}1 & 7 & 1\end{array}$ & 103.5869 & .1500 & 284.7 & & 600.0 & \\
\hline & 172 & 160.0041 & 1.1750 & 285.0 & 5 & 00 & \\
\hline & $\begin{array}{lll}1 & 7 & 3\end{array}$ & 155.9969 & 1.5750 & 285.3 & & 00.0 & \\
\hline & $\begin{array}{lll}17 & 4\end{array}$ & 880 & 1250 & 85.3 & & 00 & \\
\hline & 175 & 501 & .8750 & 84.5 & & 00 & \\
\hline & 176 & 142.2502 & 000 & 281.9 & & 00 & 0 \\
\hline & $\begin{array}{lll}17 & 7\end{array}$ & 40.5000 & .8000 & 81.5 & & 0 & \\
\hline & 178 & 8.5003 & .6250 & 81.0 & & & \\
\hline & $\begin{array}{lll}1 & 7 & 9\end{array}$ & 5215 & 1.5250 & 282.3 & 4 & 500 & 0 \\
\hline & 1710 & 157.7550 & 2.5500 & 282.9 & & 0 & \\
\hline & 1711 & 158.0046 & 2.8750 & 83.1 & & .0 & \\
\hline & 1712 & 000 & 2.2500 & 283.9 & 3 & 00.0 & U \\
\hline & 1713 & 149 . & 3.5750 & 284.1 & & 0.0 & 00 \\
\hline & 1714 & 99 & 500 & & 4 & & \\
\hline & 1715 & 151. & 4.50 & .3 & 4 & & \\
\hline & 1716 & 136.7530 & 4.4250 & 287.1 & 4 & .0 & 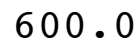 \\
\hline & 1717 & 1 & 4.1250 & .1 & 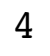 & 0 & \\
\hline & 1718 & 124 & 1.9500 & 287.7 & 5 & & \\
\hline & 1719 & 128.2110 & 2.6500 & 287.9 & 4 & .0 & $v$ \\
\hline & 1720 & 130.2492 & 2.4000 & 288.2 & 4 & 0 & \\
\hline & 1721 & 76.2559 & 1.4250 & & 5 & & \\
\hline & 1722 & 291 & 2.0000 & 289.6 & 5 & & \\
\hline & 1723 & 43 & 1.2000 & 290.3 & 5 & 0 & 00 \\
\hline & 1724 & & 2 . & 29 & 4 & & \\
\hline & $\begin{array}{lll}1 & 8 & 1\end{array}$ & 123 & 2.1000 & 287.1 & 5 & & \\
\hline & 182 & .2586 & 1.2500 & 281.3 & 6 & 0 & J. \\
\hline & 183 & 12.1695 & 0.9000 & 28 & 6 & & \\
\hline & 184 & 737 & 1.1000 & 28 & 5 & & $\bullet$ \\
\hline & 185 & 85.824 & 1.0750 & 286.8 & 6 & 0 & 0 • \\
\hline & 186 & 122.401 & 1.4000 & 279.0 & 6 & 0 & 0 . \\
\hline & $\begin{array}{lll}1 & 8 & 7\end{array}$ & $96^{\circ}$ & 0.8250 & 276.6 & 6 & & \\
\hline & 188 & 44.4387 & 1.1750 & 277.7 & 6 & & 0 • \\
\hline & 189 & 336.7194 & 1.0500 & 280.6 & 5 & 0 & . \\
\hline & 1810 & 307 & 0 & 279.4 & 4 & & 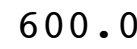 \\
\hline & 1811 & 251.8933 & 0.8250 & 278.7 & 3 & & 0 . \\
\hline & 1812 & 274.6234 & .7250 & 279.3 & 2 & .0 & 0 . \\
\hline & 1813 & 243.1767 & 0.7750 & 28 & 1 & & . \\
\hline 03 & 1814 & 138.1320 & 0.6500 & 280.3 & 1 & .0 & 0 . \\
\hline & 1815 & 338.9305 & 0.8000 & 280.9 & 1 & .0 & 0 . \\
\hline & 1816 & 22.2496 & 0.6500 & 280.9 & 1 & & 0 . \\
\hline & 1817 & 352.5954 & 1.8250 & 280.2 & 2 & .0 & 00 . \\
\hline & 1818 & 137.7360 & 0.8500 & 282.2 & 3 & 0 & 00 . \\
\hline & 1819 & 172.8661 & 1.1500 & 283.1 & 4 & & \\
\hline 03 & 1820 & 154.4960 & 0.8000 & 282.5 & 5 & & 600. \\
\hline & 1821 & 140.2788 & 1.0500 & 281.6 & 6 & 600.0 & 600 \\
\hline & 822 & & & & 6 & & \\
\hline & 823 & 18.5227 & 0.5000 & 283.9 & 0 & 600.0 & 600 \\
\hline
\end{tabular}




\begin{tabular}{|c|c|c|c|c|c|c|c|}
\hline 03 & 1824 & 349.3852 & 0.9500 & 284.2 & 6 & 600.0 & 600.0 \\
\hline 03 & $\begin{array}{lll}1 & 9 & 1\end{array}$ & 350.6980 & 0.7250 & 284.6 & 6 & 600.0 & 600.0 \\
\hline 03 & 192 & 285.4443 & 0.7750 & 284.5 & 6 & 600.0 & 600.0 \\
\hline 3 & 193 & 12.5000 & 1.9750 & 284.6 & 5 & 600.0 & 600.0 \\
\hline 03 & 194 & 19.0000 & 1.1250 & 285.2 & 4 & 600.0 & 600.0 \\
\hline 03 & 195 & 36.5616 & 0.2250 & 285.0 & 5 & 600.0 & 600.0 \\
\hline 03 & 196 & 136.5723 & 1.2750 & 283.1 & 6 & 600.0 & 600.0 \\
\hline 03 & $\begin{array}{lll}1 & 9 & 7\end{array}$ & 147.3415 & 1.2000 & 278.9 & 6 & 600.0 & 600.0 \\
\hline 03 & 198 & 140.8516 & 1.6500 & 280.2 & 6 & 600.0 & 600.0 \\
\hline 03 & 199 & 264.0000 & 0.8500 & 279.6 & 5 & 600.0 & 600.0 \\
\hline 3 & 1910 & 1.6795 & 0.6750 & 279.9 & 4 & 600.0 & 600.0 \\
\hline 03 & 1911 & 278.7324 & 0.5250 & 280.2 & 3 & 600.0 & 600.0 \\
\hline 03 & 1912 & 345.7666 & 1.7250 & 279.8 & 4 & 600.0 & 600.0 \\
\hline 03 & 1913 & 357.5105 & 0.8250 & 280.2 & 3 & 600.0 & 600.0 \\
\hline 03 & 1914 & 329.2116 & 0.9250 & 282.2 & 2 & 600.0 & 600.0 \\
\hline 03 & 1915 & 214.8436 & 2.1250 & 281.9 & 1 & 600.0 & 600.0 \\
\hline 03 & 1916 & 295.6985 & 1.7000 & 280.3 & 2 & 600.0 & 600.0 \\
\hline 03 & 1917 & 300.8065 & 1.0750 & 280.0 & 3 & 600.0 & 600.0 \\
\hline 03 & 1918 & 279.5527 & 0.7750 & 280.0 & 4 & 600.0 & 600.0 \\
\hline 03 & 1919 & 291.7574 & 0.6250 & 280.0 & 5 & 600.0 & 600.0 \\
\hline 03 & 1920 & 342.7546 & 1.4500 & 280.0 & 4 & 600.0 & 600.0 \\
\hline 03 & 1921 & 212.1943 & 0.9750 & 280.7 & 5 & 600.0 & 600.0 \\
\hline 03 & 1922 & 211.7517 & 0.7750 & 280.7 & 6 & 600.0 & 600.0 \\
\hline 03 & 1923 & 345.9237 & 1.5500 & 280.7 & 5 & 600.0 & 600.0 \\
\hline 03 & 1924 & 336.2532 & 2.2250 & 281.1 & 5 & 600.0 & 600.0 \\
\hline 03 & 1101 & 345.7295 & 3.1500 & 281.8 & 4 & 600.0 & 600.0 \\
\hline 03 & 1102 & 341.9998 & 2.9750 & 282.5 & 4 & 600.0 & 600.0 \\
\hline 03 & 1103 & 348.0118 & 2.6000 & 282.9 & 4 & 600.0 & 600.0 \\
\hline 03 & 1104 & 351.9977 & 2.7000 & 283.1 & 4 & 600.0 & 600.0 \\
\hline 03 & 1105 & 323.7325 & 1.7500 & 283.0 & 5 & 600.0 & 600.0 \\
\hline 03 & 1106 & 318.2374 & 1.0000 & 282.9 & 5 & 600.0 & 600.0 \\
\hline 03 & 1107 & 357.3547 & 0.8250 & 283.0 & 4 & 600.0 & 600.0 \\
\hline 03 & 1108 & 350.4590 & 0.9500 & 283.3 & 5 & 600.0 & 600.0 \\
\hline 03 & 1109 & 20.0115 & 0.3000 & 283.3 & 4 & 600.0 & 600.0 \\
\hline 03 & 11010 & 320.4646 & 1.5000 & 283.2 & 3 & 600.0 & 600.0 \\
\hline 03 & 11011 & 347.7518 & 1.1500 & 283.6 & 3 & 600.0 & 600.0 \\
\hline 03 & 11012 & 311.0030 & 2.3250 & 284.9 & 3 & 600.0 & 600.0 \\
\hline 03 & 11013 & 262.5160 & 1.3250 & 286.0 & 2 & 600.0 & 600.0 \\
\hline 03 & 11014 & 267.5138 & 1.5250 & 285.9 & 1 & 600.0 & 600.0 \\
\hline 03 & 11015 & 277.9184 & 1.5500 & 286.8 & 1 & 600.0 & 600.0 \\
\hline 03 & 11016 & 313.2829 & 2.1750 & 286.2 & 2 & 600.0 & 600.0 \\
\hline 03 & 11017 & 350.7339 & 1.4000 & 286.7 & 3 & 600.0 & 600.0 \\
\hline 03 & 11018 & 352.7506 & 2.0250 & 285.6 & 4 & 600.0 & 600.0 \\
\hline 03 & 11019 & 337.2492 & 3.1000 & 284.7 & 4 & 600.0 & 600.0 \\
\hline 03 & 11020 & 335.5023 & 2.8750 & 284.7 & 4 & 600.0 & 600.0 \\
\hline 03 & 11021 & 1.1959 & 1.5750 & 285.5 & 5 & 600.0 & 600.0 \\
\hline 03 & 11022 & 12.0034 & 2.0250 & 285.9 & 4 & 600.0 & 600.0 \\
\hline 03 & 11023 & 21.2206 & 1.7000 & 285.9 & 5 & 600.0 & 600.0 \\
\hline
\end{tabular}




\begin{tabular}{|c|c|c|c|c|c|c|c|}
\hline 3 & 1024 & & 4750 & 3 & & . & \\
\hline & 111 & 13 & .7250 & 4.9 & & 600.0 & \\
\hline & 1112 & 30.5363 & 1.2500 & 285.2 & 6 & 00 & \\
\hline & 113 & 12.0297 & 1.7750 & 285.4 & & 00.0 & \\
\hline & 114 & 37.0000 &. .4250 & 85.0 & & 00 & \\
\hline & 115 & 18.7488 & .9750 & 84.9 & & 00 & \\
\hline & 116 & 357.7509 & 1.7000 & 284.3 & 4 & 500 & \\
\hline & 117 & 346.4899 & L. 1750 & 84.2 & & 0 & \\
\hline & 118 & 26 & L. 6750 & 84.7 & 4 & & \\
\hline & 119 & 3088 & 1.6250 & 285.2 & 3 & 00 & 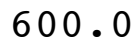 \\
\hline & 1110 & 36.0368 & 1.3750 & 285.9 & & 500.0 & \\
\hline & 1111 & 313.1254 & .4000 & 87.1 & & 00.0 & \\
\hline & 11 & 249 & 1.4000 & 285.5 & 3 & 00.0 & 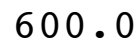 \\
\hline & 1113 & 237.0162 & 1.3250 & 284.4 & 3 & 00.0 & 0 \\
\hline & 114 & 273 & 0 & & & & \\
\hline & 1115 & 341 . & 0 . & 28 & 1 & & \\
\hline & 1116 & 343.24 & 2.0250 & 285.4 & 2 & 0.0 & 政 \\
\hline & 1117 & 339. & 1.5750 & 7 & 3 & 0 & \\
\hline & 1118 & 57 . & 1.05 & 28 & 4 & & \\
\hline & 1119 & 300.5707 & 0.5250 & 283 & 5 & 000.0 & 政 \\
\hline & 1120 & 8.2778 & 0.3250 & $2 \varepsilon$ & 6 & 600.0 & 0. \\
\hline & 1121 & 116.2081 & 0.6250 & & 6 & & \\
\hline & 1122 & 75.0895 & 1.6000 & 28 & 6 & & \\
\hline & 1123 & 9.5786 & 1.0250 & 282.8 & 6 & 0 & . \\
\hline & 11124 & 14.0084 & 0.5750 & 28 & 6 & & \\
\hline & 1121 & 122.1137 & 1.4750 & 28 & 6 & & \\
\hline & 1122 & 123.52 & 1.7750 & 1 & 5 & 0 & U. \\
\hline & 1123 & 204.96 & 0.5250 & 0 & 6 & & \\
\hline & 1124 & 348.7057 & 1.3750 & 28 & 6 & & $\bullet$ \\
\hline & 1125 & 7.0031 & 1.9750 & 282.9 & 5 & 0 & 0 • \\
\hline & 1126 & 343.7438 & 1.1000 & 284.5 & 6 & 0 & 0 . \\
\hline & 1127 & 203.52 & 1.2000 & 283.7 & 6 & & \\
\hline & 1128 & 140.5008 & 2.0250 & 283.0 & 5 & 0 & 0 • \\
\hline & 1129 & 135.3969 & 1.2750 & 28 & 4 & .0 & 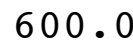 \\
\hline & 11210 & 133 & 0.7500 & & 3 & & \\
\hline & 11211 & 273.3190 & 0.8250 & 286.6 & 2 & & 0 . \\
\hline & 11212 & 191.7684 & 0.6750 & 286.8 & 1 & $\cdot 0$ & 0 . \\
\hline & 11213 & 200 . & 1.6500 & 28 & 1 & & 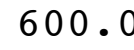 \\
\hline 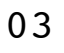 & 11214 & 208.8923 & 2.0500 & 287.2 & 1 & .0 & 0 . \\
\hline & 11215 & 194.2387 & 1.3750 & 287.3 & 1 & .0 & 0 \\
\hline & 11216 & 149.7499 & 2.4000 & 287.1 & 2 & & 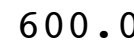 \\
\hline & 11217 & 147.4875 & 1.8250 & 287.0 & 3 & .0 & 600 . \\
\hline & 11218 & 150.0103 & 2.3750 & 286.2 & 4 & .0 & 00 . \\
\hline & 11219 & 176.9151 & 0.9500 & 285 & 5 & 600 & \\
\hline 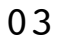 & 11220 & 345.5323 & 1.6250 & 285.4 & 4 & 600.0 & 00 . \\
\hline & 11221 & 343.7463 & 2.8500 & 285.5 & 4 & 600.0 & 500 . \\
\hline & 22 & 132 & & & 5 & & \\
\hline & 11223 & 95.5672 & 1.3500 & 287.4 & 0 & 600.0 & 600 \\
\hline
\end{tabular}




\begin{tabular}{|c|c|c|c|c|c|c|c|}
\hline 3 & 1224 & & 0 & 4 & & & \\
\hline & 1131 & 506 & .4000 & 286.9 & 4 & 00.0 & \\
\hline & 1132 & 111.0206 & 1.0500 & 285.8 & 5 & 00 & \\
\hline & 133 & 5431 & 1.0750 & 284.6 & & 00.0 & \\
\hline & 134 & 6.7295 & .6000 & 84.2 & & 00 & \\
\hline & 135 & 0.2503 & .2000 & 284.2 & & 00 & \\
\hline & 136 & 2.2494 & 2.0000 & 284.8 & & 500 & \\
\hline & 137 & 352.0522 & L. 2000 & 85.2 & & .0 & \\
\hline & 138 & 288.1692 & .7500 & 85.9 & & & \\
\hline & 139 & 151.5430 & 1.1250 & 86.1 & & 500.0 & 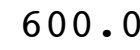 \\
\hline & 1310 & 303.8993 & 0.3750 & 287.6 & & 00.0 & \\
\hline & 1311 & 341.0000 & 0.4750 & 88.9 & & 0 & \\
\hline & 1312 & 191.1336 & 1.8250 & 288.9 & & 0 & \\
\hline & 1313 & 175.4472 & 2.42 & 288.7 & & 0.0 & 0 \\
\hline & 314 & 163. & 0 & & & & \\
\hline & 1315 & 192 . & 2.15 & 288.5 & & & \\
\hline & 1316 & 293.0021 & 0.8250 & 288.5 & & 0.0 & . \\
\hline & 317 & 160 . & 1.7000 & .4 & & 0 & \\
\hline & 318 & 77 . & 1.1 & .5 & & & \\
\hline & 1319 & 99.4 & 1.2000 & 286.2 & & .0 & . \\
\hline & 1320 & 142.0007 & 3.1250 & 285.2 & & 000 & 0 \\
\hline & 1 & 140.2 & 2.42 & & & & \\
\hline & 1322 & 107.9396 & 1.7500 & 284.6 & 6 & & \\
\hline & 1323 & 78.7506 & 4.1500 & 285.2 & & 0 & . \\
\hline & 11324 & 80.7496 & & & 5 & & \\
\hline & 1141 & 89.0 & 4.0750 & 28 & 5 & & \\
\hline & 1142 & 75.7518 & 4.5500 & 285.2 & 5 & 0 & U. \\
\hline & 1143 & 76.0004 & 4.72 & .3 & 5 & & \\
\hline & 1144 & 79.9 & 4.5250 & 28 & 5 & & $\bullet$ \\
\hline & 1145 & 88.0000 & 2.50 & .4 & 4 & 0 & 0 • \\
\hline & 1146 & 91.6055 & 1.60 & 285.0 & 5 & 0 & 0 . \\
\hline & 1147 & 124.4495 & $0 \quad 37$ & 28 & 6 & & \\
\hline & 1148 & 104.6837 & 3.60 & 283.8 & 5 & 0 & 0 . \\
\hline & 1149 & 78.7540 & 4 . & 28 & 4 & .0 & 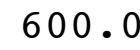 \\
\hline & 11410 & 80.6779 & 2 . & & 3 & & \\
\hline & 11411 & 8.2308 & 0.7250 & 286.2 & 2 & .0 & 0 . \\
\hline & 1412 & 309.6713 & 1.7250 & 286.9 & 1 & $\cdot 0$ & 0 . \\
\hline & 11413 & 264.0515 & 2.60 & 28 & 2 & & 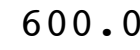 \\
\hline 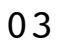 & 11414 & 225.4998 & 2.6000 & 284.9 & 3 & .0 & 0 . \\
\hline & 11415 & 307.7422 & 1.2500 & 285.2 & 2 & .0 & . \\
\hline & 11416 & 256.9791 & 1.1500 & 286.0 & 1 & & 600. \\
\hline & 11417 & 241.1878 & 0.8750 & 284.8 & 2 & .0 & 600 . \\
\hline & 11418 & 131.2437 & 0.6500 & 284.7 & 3 & 600 & 00 . \\
\hline & 11419 & 157.5590 & 0.5000 & 28 & 4 & & \\
\hline 03 & 11420 & 130.0412 & 0.7500 & & 5 & & 00 . \\
\hline & 11421 & 143.5032 & 1.9750 & 284.1 & 5 & 600.0 & 500. \\
\hline & & 158.7518 & & & 5 & & \\
\hline & 11423 & 152.4987 & 5.6250 & 283.8 & $\Psi$ & 600.0 & 600 \\
\hline
\end{tabular}




\begin{tabular}{|c|c|c|c|c|c|c|c|}
\hline 3 & 1424 & - & .7750 & 33.5 & & & \\
\hline 3 & 1151 & 157 . & 5.8500 & 282.8 & 4 & & \\
\hline & 1152 & 9998 & 9750 & 282.5 & 5 & 00 & \\
\hline & 153 & 159.5000 & 0000 & 282.2 & & 00 & \\
\hline & 154 & 148 . & .6250 & 82.0 & & 0 & \\
\hline & 155 & 135.5000 & .4250 & 81.9 & 5 & 00 & \\
\hline & 156 & 153.7519 & 500 & 281.5 & & 00 & \\
\hline & 157 & 138.5000 & 500 & 81.3 & & & \\
\hline & 158 & 141.4995 & 4.7000 & 281.3 & 4 & 00 & \\
\hline & 159 & 119.9895 & 3.9000 & 280.9 & & 00.0 & \\
\hline & 510 & 130.4997 & 0 & 281.7 & 1 & 0 & \\
\hline & 511 & 15100 & & 82.2 & 4 & & \\
\hline & 512 & 155.7539 & 0 & 283.0 & 3 & 0 & \\
\hline & 513 & 160.9464 & 2.1750 & 284.1 & & 0.0 & \\
\hline & $5=11$ & $160 \Omega 0$ & & 85.5 & 1 & & \\
\hline & 515 & 160.9227 & 2.6250 & 285.3 & 2 & 0 & \\
\hline & 516 & 140.4088 & 2.22 & 285.1 & 2 & 0 & \\
\hline & 517 & 351 & 1 . & 5.9 & 3 & & \\
\hline & 8 & 100 & 2.22 & 285.2 & 4 & 0 & \\
\hline & 519 & 110.2244 & 1.37 & 285.2 & 5 & 0 & \\
\hline & 520 & .2269 & 1.70 & 285.7 & 6 & & \\
\hline & 521 & 160.1707 & 1 . & 283.7 & 5 & & \\
\hline & 522 & 159.2558 & 1.1250 & 282.2 & 6 & 600 & 600 . \\
\hline & 523 & 308.79 & 0 . & 281.7 & 6 & 0 & \\
\hline & 524 & 325.10 & & 281.6 & 5 & & \\
\hline & 161 & 3.2498 & 0 & 281.6 & 5 & & \\
\hline & 162 & 319.0022 & 0.4250 & 281.4 & 4 & 0 & \\
\hline & 163 & 257.2415 & 0 & 281.2 & 5 & 0 & \\
\hline & 1164 & 343.46 & 0 & 280.7 & 6 & 0 & 0 \\
\hline & 165 & 3.0005 & $\$ 500$ & 281.1 & 5 & 0 & \\
\hline & 1166 & 56.9942 & 1. & 281.1 & 4 & & \\
\hline & 1167 & 6.99 & 2. & 281.4 & 4 & & • \\
\hline & 1168 & 296.7649 & 250 & 281.2 & 5 & 600 & 0 . \\
\hline & 1169 & 286.3631 & 50 & 282.4 & 4 & 0 & \\
\hline & 610 & 283. & 50 & 283.7 & 3 & & \\
\hline & 611 & 299.0839 & 1.6750 & 283.3 & 2 & 60 & 0 . \\
\hline & 1612 & 155.7539 & 250 & 283.0 & 3 & 600 & \\
\hline 3 & 11613 & 160.9464 & 2.1750 & 284.1 & 2 & 0 & . \\
\hline & 11614 & 160.0018 & 2.2250 & 285.5 & 1 & 600.0 & 0 . \\
\hline & 11615 & 160.9227 & 50 & 285.3 & 2 & 600 & \\
\hline & 11616 & 140.4088 & 250 & & 2 & & \\
\hline 03 & 11617 & 59.5000 & 0.7000 & 286.8 & 3 & 600.0 & 600 . \\
\hline & 11618 & 179.6951 & 1.6250 & 283.2 & 4 & 600.0 & 600. \\
\hline & 11619 & 51.1690 & 1.1250 & 284.8 & 5 & 600 & 600 \\
\hline 03 & 11620 & 250.1219 & 0.1250 & 285.4 & 6 & 600.0 & 00 . \\
\hline & 11621 & 280.9571 & 0.4250 & 285.4 & 6 & 600.0 & 600 . \\
\hline & 11622 & 335 & & & 5 & 600 & \\
\hline & 11623 & 262.5702 & 0.5750 & 285.0 & r & 600.0 & 600 \\
\hline
\end{tabular}




\begin{tabular}{|c|c|c|c|c|c|c|c|}
\hline 03 & 11624 & 23.7946 & 1.3000 & 285.5 & 5 & 600.0 & 600.0 \\
\hline 03 & 1171 & 31.7500 & 1.0750 & 287.2 & 4 & 600.0 & 600.0 \\
\hline 03 & 1172 & 150.1936 & 1.2500 & 286.4 & 5 & 600.0 & 00.0 \\
\hline 03 & 1173 & 141.9258 & 1.3500 & 283.6 & 5 & 600.0 & 00.0 \\
\hline 03 & 1174 & 45.0928 & 1.1750 & 284.7 & 6 & 600.0 & 00.0 \\
\hline 03 & 1175 & 135.8186 & 1.1250 & 285.5 & 5 & 600.0 & 00.0 \\
\hline 03 & 1176 & 149.4992 & 2.3250 & 284.2 & 5 & 600.0 & 00.0 \\
\hline 03 & 1177 & 150.2505 & 1.8000 & 283.7 & 5 & 600.0 & 00.0 \\
\hline 03 & 1178 & 138.6037 & 1.9000 & 282.9 & 6 & 600.0 & 00.0 \\
\hline 03 & 1179 & 53.1460 & 1.3750 & 283.4 & 5 & 600.0 & 00.0 \\
\hline 03 & 11710 & 272.0870 & 0.6000 & 285.2 & 4 & 600.0 & 00.0 \\
\hline 03 & 11711 & 337.0404 & 1.0750 & 284.6 & 3 & 600.0 & 00.0 \\
\hline 03 & 11712 & 300.6383 & 1.5000 & 286.1 & 2 & 600.0 & 00.0 \\
\hline 03 & 11713 & 282.4420 & 1.9250 & 284.4 & 2 & 600.0 & 00.0 \\
\hline 03 & 11714 & 293.0390 & 2.0750 & 283.9 & 3 & .0 & \\
\hline 03 & 11715 & 324.1142 & 1.2250 & 285.5 & 2 & 600.0 & 00.0 \\
\hline 03 & 11716 & 299.8591 & 0.7000 & 287.7 & 1 & 600.0 & 600.0 \\
\hline 03 & 11717 & 211.1746 & 0.4250 & 285.9 & 2 & .0 & \\
\hline 03 & 11718 & 25.8850 & 1.1000 & 282.9 & 3 & 600.0 & .0 \\
\hline 03 & 11719 & 153.0498 & 1.5000 & 280.1 & 4 & 60 & .0 \\
\hline 03 & 11720 & 1529 & 1.00 & 279.7 & 5 & 60 & \\
\hline 03 & 11721 & 20.0607 & 0.9500 & 282.5 & 6 & .0 & \\
\hline 03 & 11722 & 30.2618 & 1.6250 & 285.5 & 6 & 600.0 & 600.0 \\
\hline 03 & 11723 & 21.2337 & 1.57 & 286.4 & 5 & .0 & .0 \\
\hline 03 & 11724 & 150.5027 & 2.4250 & 282.5 & 6 & .0 & \\
\hline 03 & 1181 & 146.2639 & 3.0250 & 279.2 & 5 & 600.0 & 600.0 \\
\hline 03 & 1182 & 154.2199 & 2.0750 & 277.2 & 4 & 600.0 & .0 \\
\hline 03 & 1183 & 119.2708 & 1.0000 & 277.0 & 5 & 600.0 & \\
\hline 03 & 1184 & 156.9902 & 1.3500 & 276.2 & 5 & 600.0 & 600.0 \\
\hline 03 & 1185 & 170.0561 & 1.5750 & 276.3 & 6 & 600.0 & 600.0 \\
\hline 03 & 1186 & 139.2058 & 0.8500 & 276.6 & 6 & .0 & \\
\hline 03 & 1187 & 128.1519 & 1.0750 & 276.1 & 6 & 600.0 & 600.0 \\
\hline 03 & 1188 & 33.0002 & 0.9000 & 278.9 & 5 & 600.0 & 600.0 \\
\hline 03 & 1189 & 155.2518 & 2.4750 & 276.3 & 4 & 600.0 & 0.0 \\
\hline 03 & 11810 & 83.8785 & 1.1750 & 277.1 & 3 & 600.0 & 600.0 \\
\hline 03 & 11811 & 113.0847 & 0.8750 & 279.5 & 2 & 600.0 & 600.0 \\
\hline 03 & 11812 & 19.2272 & 0.5750 & 283.8 & 1 & 600.0 & 600.0 \\
\hline 03 & 11813 & 337.7222 & 1.1750 & 285.6 & 1 & 600.0 & 600.0 \\
\hline 03 & 11814 & 160.2685 & 0.9750 & 285.1 & 1 & 600.0 & 600.0 \\
\hline 03 & 11815 & 224.1214 & 1.3750 & 283.3 & 1 & 600.0 & 600.0 \\
\hline 03 & 11816 & 141.6295 & 2.0250 & 281.0 & 1 & 600.0 & 600.0 \\
\hline 03 & 11817 & 98.6891 & 1.3000 & 283.1 & 2 & 600.0 & 600.0 \\
\hline 03 & 11818 & 147.9984 & 1.8750 & 285.1 & 3 & 600.0 & 600.0 \\
\hline 03 & 11819 & 83.0545 & 0.7750 & 284.3 & 4 & 600.0 & 600.0 \\
\hline 03 & 11820 & 24.7603 & 0.1750 & 284.6 & 5 & 600.0 & 600.0 \\
\hline 03 & 11821 & 155.7834 & 0.8750 & 285.9 & 6 & 600.0 & 600.0 \\
\hline 03 & 11822 & 76.9904 & 0.7250 & 285.6 & 6 & 600.0 & 600.0 \\
\hline 4 & 11823 & 113.1979 & 1.0500 & 284.7 & 0 & 600.0 & 600.0 \\
\hline
\end{tabular}




\begin{tabular}{|c|c|c|c|c|c|c|c|}
\hline 3 & 824 & & 0 & 84.9 & & & \\
\hline & 1191 & 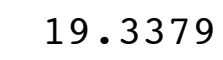 & .9250 & 80.2 & 6 & 600.0 & \\
\hline & 1192 & 306.9968 & 1.0000 & 276.7 & 6 & 00 & \\
\hline & 193 & 341.2467 & 1.3500 & 275.6 & & 00 & \\
\hline & 194 & 330 . & 5000 & 74.7 & & 0 & \\
\hline & 195 & 322.6782 & 5000 & 274.6 & & 0( & \\
\hline & 196 & 159.4962 & 1.9750 & 274.5 & & 00 & \\
\hline & 197 & .704 & .1500 & 275.1 & & & \\
\hline & 198 & 314.9237 & 50 & 74.9 & & & \\
\hline & 199 & 83.7078 & 1.0750 & 274.5 & & 0 & U \\
\hline & 1910 & 331.2828 & 1.1500 & 275.3 & & 00.0 & \\
\hline & 1911 & 277.3599 & 0 & 76.2 & & & \\
\hline & 1912 & 305.2953 & 750 & 276.9 & 2 & 0 & \\
\hline & 913 & 262.3965 & 0 & 277.7 & 1 & 0.0 & . \\
\hline & 914 & 274.9047 & & 9 & & & \\
\hline & 915 & 264.7032 & & 278.2 & 1 & & \\
\hline & 1916 & 317.9965 & 1.00 & 278.4 & 1 & .0 & 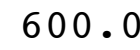 \\
\hline & 917 & 354.6955 & 0 & 278.3 & & 0 & \\
\hline & 918 & 357.7344 & & 277.9 & 3 & & \\
\hline & 919 & 4.9926 & 1.8750 & 277.4 & 4 & 00.0 & 0 \\
\hline & 920 & 13.5015 & 1.8250 & 277.1 & 4 & 0 & \\
\hline & 921 & 62.2029 & & 276.6 & 5 & & \\
\hline & 1922 & 345.2353 & 1.3750 & 276.5 & 6 & & \\
\hline & 923 & 350.0152 & 1.6000 & 276.4 & 5 & 0 & 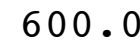 \\
\hline & 11924 & 10.9908 & & & 4 & & \\
\hline & 1201 & 304.2487 & 000 & 275.3 & 5 & & \\
\hline & 1202 & 313.1376 & 3000 & 275.3 & 6 & 0 & . \\
\hline & 1203 & 250.6294 & 5500 & 275.4 & 6 & & \\
\hline & 1204 & 359.9455 & 1.5 & 274.8 & 5 & & \\
\hline & 1205 & 85.5000 & 1.77 & 274.4 & 6 & 0 & 0 . \\
\hline & 1206 & 168.1461 & 2.1000 & 274.9 & 5 & & 0 . \\
\hline & 1207 & 339.9355 & 0 & 275.6 & 6 & & \\
\hline & 1208 & 343.1206 & 500 & 275.3 & 6 & 0 & . \\
\hline & 1209 & 337.2143 & 5750 & 275.1 & 5 & 0 & 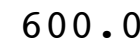 \\
\hline & 12010 & 239.7144 & 0 & 275.6 & 4 & & \\
\hline & 12011 & 174.7478 & 1.1750 & 276.5 & 3 & & 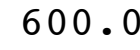 \\
\hline & 12012 & 270.4467 & 8250 & 277.8 & 2 & & 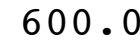 \\
\hline & 12013 & 219 . & 500 & 278.4 & 1 & & 0 . \\
\hline 03 & 12014 & 236.3059 & 1.3750 & 278.7 & 1 & .0 & 0 . \\
\hline & 12015 & 173.5000 & 1.8750 & 278.4 & 2 & & 0 . \\
\hline & 12016 & 239.6056 & 1.6000 & 278.4 & 1 & & 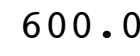 \\
\hline & 12017 & 344.7646 & 1.5500 & 278.3 & 2 & .0 & 0 . \\
\hline & 12018 & 221.4229 & 1.6250 & 278.2 & 3 & 0 & 0 . \\
\hline & 12019 & 296.2163 & 1.4750 & 278.0 & 4 & & \\
\hline 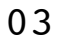 & 12020 & 324.1530 & 1.5500 & 277.8 & 5 & & 0 . \\
\hline & 12021 & 317.2495 & 1.8000 & 277.5 & 4 & 600.0 & 00 . \\
\hline & 12022 & 1200 & & & 5 & & \\
\hline & 12023 & 353.5981 & 1.4250 & 277.6 & r & 600.0 & 60 \\
\hline
\end{tabular}




\begin{tabular}{|c|c|c|c|c|c|c|c|}
\hline 3 & 2024 & & & 77.7 & & 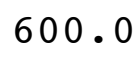 & \\
\hline & 1211 & & 2.8250 & 277.5 & 6 & 600.0 & \\
\hline & 1212 & 18.7955 & 7500 & 278.5 & 6 & 00 & \\
\hline & 213 & 341.0859 & 1.3500 & 278.0 & & 00 & \\
\hline & 214 & 15.4614 & .6750 & 279.0 & & 0 & \\
\hline & 1215 & 230 & .1500 & 280.4 & & 0 & \\
\hline & 216 & 1.6118 & 1500 & 279.1 & & 00 & \\
\hline & 217 & 16.7634 & .0250 & 281.7 & & 0 & \\
\hline & 1218 & 183.1313 & 8000 & 83.8 & 0 & & \\
\hline & 1219 & 6966 & 1.3250 & 284.5 & & 0 & U \\
\hline & 2110 & 154.3786 & 2.4500 & 282.2 & & 000 & \\
\hline & 2111 & 125.6684 & 1.6250 & 81.0 & 3 & & \\
\hline & 12112 & 272.6229 & 1.1500 & 281.7 & & 0 & \\
\hline & 2113 & 242.8996 & 1.7500 & 281.2 & 1 & 0 & 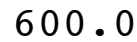 \\
\hline & 2114 & 163.7485 & 0 & 80.1 & & & \\
\hline & 121 & 152.7566 & 1 . & 280.6 & 1 & & \\
\hline & 12116 & 336.5000 & 1.1250 & 280.8 & 2 & 00.0 & 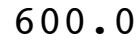 \\
\hline & 2117 & 341.8095 & .4500 & 281.2 & 3 & 0 & \\
\hline & 12118 & 216.5224 & 1.7500 & 280.8 & 4 & & \\
\hline & 12119 & 200.5074 & 0.6500 & 280.2 & 5 & .0 & . \\
\hline & 12120 & 356.7580 & 1.1750 & 280.6 & 6 & 0 & \\
\hline & 12121 & 8.5828 & & 281.0 & 6 & & \\
\hline & 12122 & 321.4593 & 1.1000 & 281.8 & 6 & 0 & 0 . \\
\hline & 12123 & 161.2252 & 7750 & 281.3 & 6 & .0 & 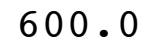 \\
\hline & 12124 & 315.4420 & .6250 & 0.9 & 6 & & \\
\hline & 1221 & 301.6347 & 500 & 280.2 & 6 & & \\
\hline & 1222 & 266.2252 & .1500 & 279.9 & 6 & 0 & . \\
\hline & 1223 & 329.1099 & 5750 & 279.9 & 6 & & \\
\hline & 1224 & 113.6756 & 250 & 280.1 & 6 & & \\
\hline & 1225 & 78.1377 & 500 & 281.0 & 6 & 0 & 0. \\
\hline & 1226 & 143.1999 & .8750 & 282.1 & 6 & & 0 . \\
\hline & 1227 & 191.6950 & 00 & 280.8 & 6 & & \\
\hline & 1228 & 335.7199 & 1.3750 & 279.3 & 6 & & . \\
\hline & 1229 & 355.8476 & 3000 & 282.0 & 5 & 0 & . \\
\hline & 12210 & 309 & 0 & 284.8 & 4 & & \\
\hline & 12211 & 304.5436 & 1.1750 & 285.6 & 3 & & 0 . \\
\hline & 12212 & 150.9709 & 6000 & 285.8 & 2 & & \\
\hline & 12213 & 122.1980 & 000 & 284.6 & 1 & & 0 . \\
\hline & 12214 & 152.1138 & 1.5500 & 285.4 & 1 & .0 & 0 . \\
\hline & 12215 & 163.4341 & 2.0500 & 286.2 & 1 & & \\
\hline & 12216 & 359.1132 & 500 & 286.3 & 1 & & 0 \\
\hline & 12217 & 255.5163 & 1.1500 & 286.3 & 2 & & 0 . \\
\hline & 12218 & 335.4418 & 0.7500 & 287.8 & 3 & 0 & 0 . \\
\hline & 12219 & 353.6727 & 1.2750 & 287.8 & 4 & & \\
\hline 03 & 12220 & 144.7605 & 2.5500 & 286.4 & 4 & & 0 . \\
\hline & 12221 & 137.5092 & 2.0500 & 287.0 & 4 & 600.0 & 00. \\
\hline & 22 & 102 . & & 287.7 & 5 & & \\
\hline & 12223 & 346.0000 & 0.7500 & 287.4 & & 600.0 & 60 \\
\hline
\end{tabular}




\begin{tabular}{|c|c|c|c|c|c|c|c|}
\hline 3 & 2224 & & 0.5000 & 87.2 & & & \\
\hline & 1231 & 302.8036 & 0.1750 & 287.0 & 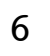 & & \\
\hline & 1232 & 6769 & 2750 & 287.1 & 6 & 00 & \\
\hline & 1233 & 321.2153 & .7500 & 285.9 & & 00 & \\
\hline & 1234 & 319.3882 & L. 3000 & & & 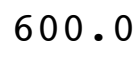 & \\
\hline & 1235 & 234.8379 & 7000 & 86.6 & 6 & 00 & \\
\hline & 1236 & 22.7465 & 0.7250 & 286.2 & & 0 & \\
\hline & 1237 & 20.1746 & .9000 & 86.4 & & & \\
\hline & 1238 & 116.5000 & 1.1750 & 286.4 & 6 & 0 & \\
\hline & 1239 & 100.2336 & 1.4000 & 286.7 & & 00.0 & \\
\hline & 2310 & 123.3742 & 1.5750 & 287.5 & & 00 & \\
\hline & 2311 & 217.2683 & & 87.2 & 3 & & \\
\hline & 2312 & 152.5040 & 500 & 286.0 & 3 & 00 & \\
\hline & 2313 & 253.2997 & 1.5500 & 288.3 & 2 & 0.0 & \\
\hline & 2314 & 163.75 & & & 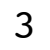 & & \\
\hline & 12315 & 159.76 & 1000 & 286.8 & 4 & 0 & \\
\hline & 2316 & 163.25 & 4.3250 & 285 & 4 & 0 & \\
\hline & 2317 & 165 . & 0 & 5 & 3 & & \\
\hline & 123 & 155 . & 4 . & 285.5 & 4 & 0 & \\
\hline & 2319 & 155.74 & 5500 & 285.3 & 4 & & \\
\hline & 2320 & 147.16 & 000 & 285.1 & 5 & & \\
\hline & 2321 & 137 . & 50 & 284.9 & 6 & & \\
\hline & 12322 & 117.9935 & 1.3250 & 28 & 6 & 0 & 0. \\
\hline & 12323 & 85 . & 1.2000 & 284.9 & 6 & 0 & \\
\hline & 12324 & 110 . & 250 & 284.7 & 6 & & \\
\hline & 1241 & 152.4999 & 500 & 283 & 5 & & \\
\hline & 1242 & 142.74 & 4.2500 & 283.4 & 4 & & \\
\hline & 1243 & 141.67 & 0 & 283.3 & 5 & & \\
\hline & 1244 & 11 & 0 & 28 & 4 & 0 & 0 . \\
\hline & $124 \quad 5$ & 111.46 & 0 & 283.1 & 5 & 0 & \\
\hline & 1246 & 129.22 & 0 & 283.2 & 4 & & \\
\hline & 1247 & 121. & 0 & 282.8 & 4 & & 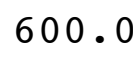 \\
\hline & 1248 & 125.9455 & 500 & 282.0 & 4 & 0 & U. \\
\hline & 1249 & 134.65 & 50 & 282.3 & 3 & 0 & \\
\hline & 12410 & 144 . & 0 & 282.0 & 4 & & \\
\hline & 12411 & 137.5000 & 3.3250 & 283.3 & 4 & & 0 . \\
\hline & 12412 & 155.7493 & 750 & 283.4 & 4 & 0 & \\
\hline 3 & 12413 & 146.28 & 250 & 284.8 & 3 & 0 & • \\
\hline & 12414 & 147.2589 & 2.2000 & 285.2 & 3 & 0 & 0 . \\
\hline & 12415 & 147.2562 & 500 & 285.6 & 2 & 0 & \\
\hline & 12416 & 220.11 & 750 & 286.2 & 1 & & \\
\hline 03 & 12417 & 261.9701 & 0.6250 & 285.2 & 2 & 600.0 & 600 . \\
\hline & 12418 & 11.4274 & 1.2500 & 284.6 & 3 & 600 & 600. \\
\hline & 12419 & 130.0741 & 1.1750 & 285.5 & 4 & & 10 \\
\hline 03 & 12420 & 83.4039 & 0.6750 & 285.6 & 5 & 600.0 & 00 . \\
\hline & 12421 & 277.4131 & 0.3250 & 286.5 & 6 & 600.0 & 500. \\
\hline & 12422 & & 0.7250 & 286.2 & 6 & 60 & \\
\hline & 12423 & 198.0564 & 0.9250 & 285.8 & & 600.0 & 600 \\
\hline
\end{tabular}




\begin{tabular}{|c|c|c|c|c|c|c|c|}
\hline 3 & 2424 & 140 & 9250 & & & 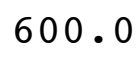 & \\
\hline 2 & 1251 & 785 & 1.1250 & 6.7 & 5 & 600.0 & \\
\hline & 1252 & 147.5182 & 1.9250 & 285.4 & 6 & 00 & 0 \\
\hline & 1253 & 163.2466 & 1.4250 & 285.4 & & 00 & \\
\hline & 254 & 148.9993 & 000 & 84.8 & & 0 & \\
\hline & 1255 & 136 & 000 & 83.8 & & 0 & \\
\hline & 256 & 149.7506 & 9500 & 283.5 & & 00 & \\
\hline & 1257 & 108.6752 & 9000 & & & 0 & \\
\hline & 1258 & 731 & 3.4250 & 84.7 & 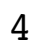 & 0 & \\
\hline & 259 & 111.8219 & 2.7750 & 86.1 & 3 & 0 & \\
\hline & 2510 & 157.2450 & 5.9000 & 283 & & 0 & \\
\hline & 2511 & 158 & 3.9750 & 82.9 & 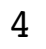 & & \\
\hline & 12512 & 154 & 000 & 283.1 & 2 & 0 & \\
\hline & 2513 & 018 & 3.3250 & 283.7 & 4 & 0 & \\
\hline & 12514 & 198 & 0 & & & & \\
\hline & 1251 & 224 & 0 . & 1 & & & \\
\hline & 2516 & 200 . & 1.4000 & 284.2 & & 0 & \\
\hline & 12517 & 162 & 1.3250 & 9 & & 0 & \\
\hline & 12518 & 141 & 0.7000 & 0 & 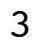 & & \\
\hline & 12519 & 329.1930 & 1.2000 & 28 & 4 & 0 & \\
\hline & 12520 & 341 & 2.7500 & 283.8 & 4 & 0 & \\
\hline & 12521 & 339 & 2.6250 & & 4 & & \\
\hline & 12522 & 343 & 1.5250 & $2 \varepsilon$ & 5 & 0 & \\
\hline & 12523 & 359 . & 2.3500 & 283.3 & 4 & 0 & \\
\hline & 12524 & 358 & 1.9500 & & 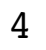 & & \\
\hline & 126 & 344 & 2.3000 & $2 \varepsilon$ & 5 & & \\
\hline & 1262 & 317 . & 1.9750 & 283.3 & 5 & 0 & \\
\hline & 1263 & 302 & 1.4250 & 2 & 5 & 0 & \\
\hline & 1264 & 25 & 1.1000 & 282.9 & 6 & 0 & \\
\hline & 1265 & 282 . & 1.4000 & 282.8 & 6 & 0 & \\
\hline & 1266 & 316 & 2.2500 & 282.6 & 5 & 0 & \\
\hline & 1267 & 32 & 3.4250 & 282.3 & 4 & & \\
\hline & 1268 & 296 & 1.5750 & 282.3 & 5 & 0 & \\
\hline & 1269 & 241 & 1.6750 & 282.5 & 4 & 0 & \\
\hline & 126 & 244 & 2.2000 & 282.8 & 3 & & \\
\hline & 12611 & 221 . & 2.8500 & 282.9 & 3 & 600.0 & \\
\hline & 12612 & 219.0221 & 3.8000 & 283.0 & 4 & م0 & \\
\hline & 12613 & 255 & 2.2000 & 283.2 & 3 & 0 & \\
\hline & 12614 & 273 . & 1.9000 & 283.4 & 2 & 600.0 & \\
\hline & 12615 & 252 & 1.6500 & 283.7 & 1 & 0 & \\
\hline & 12616 & 283 & 0.9250 & 284.0 & 1 & 600.0 & \\
\hline & 12617 & 222.4994 & 0.8250 & 284.1 & 2 & 600.0 & \\
\hline & 12618 & 139.3117 & 2.4500 & 283.5 & 3 & 600.0 & \\
\hline & 12619 & 747 & 1.8250 & 283 & 4 & 600 & \\
\hline & 12620 & 85.9659 & 1.5000 & 283.6 & 5 & 600.0 & \\
\hline & 1262 & 358.0000 & 1.7250 & 283.7 & 6 & 600.0 & \\
\hline & 12 & & 1 . & & 0 & & \\
\hline & 12623 & 357.4016 & 2.0250 & 284.1 & 0 & 600.0 & 10 \\
\hline
\end{tabular}




\begin{tabular}{|c|c|c|c|c|c|c|c|}
\hline 3 & 2624 & & 1.2500 & 84.0 & & & \\
\hline & 1271 & 41.1880 & 2.0500 & 283.9 & 0 & & \\
\hline & 1272 & 1500 & 1.6000 & 284.1 & 6 & 00 & \\
\hline & 1273 & 300.2102 & 1.3500 & 283.7 & & 00 & \\
\hline & 1274 & 345.7458 & 1.2750 & 83.2 & & 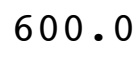 & \\
\hline & 1275 & 5603 & 1.1000 & 83.6 & 6 & 00 & \\
\hline & 1276 & 241.0874 & 0.8000 & 283.9 & & 0 & \\
\hline & 1277 & 62.3337 & L. 5250 & 85.0 & & & \\
\hline & 1278 & 155.5472 & 2.4250 & 285.9 & 6 & 0 & \\
\hline & 1279 & 114.1381 & 1.9000 & 285.2 & & 0 & \\
\hline & 2710 & 90.7519 & 3.5500 & 288.4 & & 0 & \\
\hline & 2711 & 94.0781 & 00 & & 3 & & \\
\hline & 12712 & 3035 & 2.3000 & 289.9 & 2 & 0 & \\
\hline & 12713 & 90.3845 & 3.0250 & 290.2 & 2 & 0.0 & \\
\hline & 12714 & 120.7409 & & & 2 & & \\
\hline & 12715 & 96.1949 & 250 & 288.8 & 3 & 0 & \\
\hline & 2716 & 68.0718 & 3.00 & 290.7 & 2 & 0 & \\
\hline & 2717 & 714 & 0 & 3 & 3 & & \\
\hline & 12718 & 082 & 4 . & 287.9 & 4 & 0 & \\
\hline & 2719 & 73.2510 & 0 & 287.2 & 4 & & \\
\hline & 2720 & 77 & 0 & 287.3 & 4 & & \\
\hline & 2721 & 101. & & 287.0 & 4 & & \\
\hline & 12722 & 153.2500 & 750 & 284.5 & 4 & 0 & 0 . \\
\hline & 12723 & 133.5242 & 6 . & 1 & 4 & 0 & \\
\hline & 12724 & 125.3316 & 0 & 285.2 & 4 & & \\
\hline & 1281 & 157.2522 & 750 & 284.2 & 5 & & \\
\hline & 1282 & 133.8102 & 3.3000 & 284.0 & 4 & & \\
\hline & 1283 & 120.4739 & & 284.2 & 4 & & \\
\hline & 1284 & 106 & 50 & 284.4 & 4 & 0 & 0 . \\
\hline & $128 \quad 5$ & 138.2197 & 0 & 284.1 & 4 & & \\
\hline & 1286 & 112.9921 & 0 & 28 & 5 & & \\
\hline & 1287 & 120 . & 0 & 28 & 5 & & 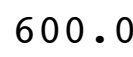 \\
\hline & 1288 & 135.4977 & 1500 & 283.5 & 5 & 0 & U. \\
\hline & 1289 & 101.2085 & 0 & & 4 & & \\
\hline & 12810 & 129.4749 & 0 & 28 & 3 & & \\
\hline & 12811 & 153.2508 & 500 & 284.2 & 4 & & • \\
\hline & 12812 & 152.2500 & 250 & 284.4 & 4 & & \\
\hline 3 & 12813 & 162.0034 & 0 & 28 & 4 & & • \\
\hline & 12814 & 175.9596 & 7500 & 286.1 & 3 & 0 & 0 . \\
\hline & 12815 & 191.2273 & 2000 & 286 & 3 & 0 & \\
\hline & 12816 & 164.5000 & 3 . & & 3 & & \\
\hline 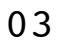 & 12817 & 159.4862 & 3.0000 & 285.9 & 4 & 600.0 & 600 . \\
\hline & 12818 & 205.4371 & 1.3750 & 284.3 & 5 & 600 & 800 \\
\hline & 12819 & 350.4527 & 1.2250 & 283.7 & 6 & & 10 \\
\hline 03 & 12820 & 353.5179 & 1.6500 & 283.4 & 5 & 600.0 & 00 \\
\hline & 12821 & 48.1531 & 1.9750 & 283.7 & 6 & 600.0 & 500. \\
\hline & 12822 & 101.3127 & 3000 & & 6 & 60 & \\
\hline & 12823 & 132.2239 & 1.5500 & 285.8 & $J$ & 600.0 & 600 \\
\hline
\end{tabular}




\begin{tabular}{|c|c|c|c|c|c|c|c|}
\hline 3 & 2824 & & .9500 & 84.9 & & & \\
\hline & 1291 & 181. & 0.7750 & 284.6 & & & \\
\hline & 1292 & 204.9675 & 7500 & 283.6 & & 00 & \\
\hline & 1293 & 130.3826 & .4000 & 283.6 & & 00.0 & \\
\hline & 1294 & 4 & L. 1000 & & & 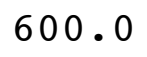 & \\
\hline & 1295 & 123.8352 & .7250 & 83.7 & & 00 & \\
\hline & 1296 & 6.4948 & 1.1750 & 283.4 & & 0 & \\
\hline & 1297 & 78.4779 & .2500 & & & & \\
\hline & 1298 & 81.3997 & 0.5000 & 283.0 & & 0 & \\
\hline & 1299 & 76.2811 & 0.9500 & 285.0 & & 0 & \\
\hline & 2910 & 288.1292 & .0250 & 286.1 & & 0 & \\
\hline & 2911 & 290.8875 & 1.3000 & 9 & & & \\
\hline & 12912 & 232.8509 & 1.7250 & 286.8 & & 0 & \\
\hline & 2913 & 247.8181 & 1.7500 & 286.4 & & 0.0 & 0 \\
\hline & 2914 & 286.2619 & 1.9250 & & & & \\
\hline & 12915 & 167.7579 & 4.3500 & 285.3 & & 0 & \\
\hline & 2916 & 118.9644 & 2.6250 & 285.1 & & 0 & \\
\hline & 2917 & 66.8694 & 000 & 0 & & & \\
\hline & 12918 & 144.5000 & 500 & 285.4 & & 0 & \\
\hline & 2919 & 138.7794 & 9750 & 285.1 & 4 & 0 & \\
\hline & 2920 & 86.0349 & 000 & 1 & & & \\
\hline & 2921 & 86. & 50 & 28 & 4 & & \\
\hline & 2922 & 83.7496 & 7250 & 287.2 & 4 & 0 & 00. \\
\hline & 12923 & 79.2501 & 2750 & 28 & 4 & 0 & \\
\hline & 12924 & 80 . & 00 & 28 & 4 & & \\
\hline & 1301 & 920 & 750 & 28 & 4 & & \\
\hline & 1302 & 85.7687 & 250 & 287.1 & 4 & 0 & \\
\hline & 1303 & 87.9693 & 0 & 28 & 5 & & \\
\hline & 1304 & 137.5704 & 000 & 285.3 & 4 & 0 & • \\
\hline & 1305 & 88.3028 & 4500 & 283.0 & 5 & 0 & \\
\hline & 1306 & 129.93 & 250 & 28 & 6 & & \\
\hline & 1307 & 118 . & 00 & 28 & 5 & & $\bullet$ \\
\hline & 1308 & 150.5000 & 2.2000 & 285.5 & 4 & 0 & J • \\
\hline & 1309 & 127.5031 & 750 & 28 & 3 & 0 & \\
\hline & 13010 & 145 . & 0 & 28 & 3 & & \\
\hline & 13011 & 145.3421 & 000 & 285.1 & 2 & & 0 . \\
\hline & 13012 & 178.8743 & 5000 & 285.9 & 2 & 0 & \\
\hline 2 & 13013 & 170.7095 & 250 & 287.2 & 1 & 0 & • \\
\hline & 13014 & 161.7180 & 2.8750 & 286.7 & 1 & 0 & 0 . \\
\hline & 13015 & 151.0021 & 3000 & 285.0 & 2 & 0 & \\
\hline & 13016 & 95.2042 & 1.4000 & & 1 & & \\
\hline 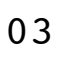 & 13017 & 138.7483 & 2.2750 & 286.5 & 2 & 600.0 & 600 . \\
\hline & 13018 & 130.0000 & 2.4500 & 285.7 & 3 & 600 & 600. \\
\hline & 13019 & 142.5000 & 3.3750 & 284.6 & 4 & & ( \\
\hline 3 & 13020 & 143.5003 & 3.3500 & 284.6 & 5 & 600.0 & 00 \\
\hline & 13021 & 148.7500 & 3.3750 & 284.6 & 5 & 600.0 & 60 \\
\hline & 13022 & 133.5789 & & & 5 & & \\
\hline & 13023 & 78.5180 & 0.7500 & 284.7 & & 600.0 & 600 \\
\hline
\end{tabular}




\begin{tabular}{|c|c|c|c|c|c|c|c|}
\hline 03 & 13024 & 32.7492 & 1.2000 & 285.7 & 5 & 600.0 & 00.0 \\
\hline 03 & 1311 & 28.0967 & 0.4000 & 285.9 & 4 & 600.0 & 600.0 \\
\hline 03 & 1312 & 200.7185 & 0.4750 & 286.0 & 5 & 600.0 & 00.0 \\
\hline 03 & 1313 & 277.1389 & 0.8250 & 285.7 & 6 & 600.0 & 600.0 \\
\hline 03 & 1314 & 13.5270 & 1.3000 & 286.7 & 5 & 600.0 & 00.0 \\
\hline 03 & 1315 & 37.4379 & 0.9250 & 286.0 & 6 & 600.0 & 00.0 \\
\hline 03 & 1316 & 156.2475 & 2.4000 & 282.8 & 5 & 600.0 & 600.0 \\
\hline 03 & 1317 & 275.7844 & 0.8250 & 281.9 & 0 & 600.0 & 00.0 \\
\hline 03 & 1318 & 316.4658 & 1.1250 & 283.2 & 6 & 600.0 & 00.0 \\
\hline 03 & 1319 & 191.9839 & 0.4250 & 284.5 & $\Gamma$ & 600.0 & .0 \\
\hline 03 & 3110 & 24.2448 & 1.2750 & 285.9 & 4 & 600.0 & 600.0 \\
\hline 03 & 3111 & 329.0789 & 0.7000 & 286.7 & 3 & 600.0 & \\
\hline 03 & 3112 & 328.1900 & 1.2750 & 286.3 & 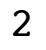 & 600.0 & 600.0 \\
\hline 03 & 3113 & 301.7397 & 1.4000 & 285.9 & 2 & 600.0 & 600.0 \\
\hline 03 & 3114 & 204.8180 & 2 & 284.0 & 2 & 600.0 & \\
\hline 03 & 13115 & 315.6050 & 1.0750 & 284.7 & 1 & 600.0 & 60 \\
\hline 03 & 13116 & 254.0755 & 1.3000 & 285.5 & 1 & 600.0 & 600.0 \\
\hline 03 & 13117 & 150 . & 2 & 2.5 & 2 & 600.0 & .0 \\
\hline 03 & 13118 & 164.5220 & 2.1250 & 281.5 & 3 & 600.0 & 60 \\
\hline 03 & 13119 & 160.0000 & 1.8250 & 281.2 & 4 & 600.0 & 60 \\
\hline 03 & 13120 & 75.6685 & 1. & 281.3 & 5 & 600.0 & .0 \\
\hline 03 & 13121 & 9.4972 & 1. & 281.4 & 5 & 600.0 & \\
\hline 03 & 13122 & 77.0666 & 0.4500 & 281.3 & 6 & 600.0 & 60 \\
\hline 03 & 13123 & 268.5088 & 0.9000 & 280.6 & 6 & 600.0 & .0 \\
\hline 03 & 13124 & 67.3049 & 750 & 281.8 & 6 & 600.0 & \\
\hline 03 & $\begin{array}{lll}2 & 1 & 1\end{array}$ & 117.4689 & 3.2750 & 285.9 & 5 & 600.0 & 600.0 \\
\hline 03 & 212 & 112.6210 & 2.5500 & 282.5 & 6 & 600.0 & 600.0 \\
\hline 03 & 213 & 78.7499 & 4.1250 & 286.3 & 5 & 600.0 & \\
\hline 03 & $\begin{array}{lll}2 & 1 & 4\end{array}$ & 79.7455 & 4.7000 & 287.4 & 4 & 600.0 & 600.0 \\
\hline 03 & 215 & 8.5286 & 1.8750 & 285.6 & 5 & 600.0 & 600 \\
\hline 03 & 216 & 178.0090 & 1 . & 282.8 & 6 & 600.0 & .0 \\
\hline 03 & $\begin{array}{lll}2 & 1 & 7\end{array}$ & 122.1175 & 4.5000 & 283.1 & 5 & 600.0 & 60 \\
\hline 03 & 218 & 75.7533 & 8.2000 & 284.4 & 4 & 600.0 & 600.0 \\
\hline 03 & 219 & 64.0005 & 8.775 & 284.2 & 4 & 600.0 & .0 \\
\hline 03 & 2110 & 61.5000 & 12 . & .2 & 4 & 600.0 & 60 \\
\hline 03 & 2111 & 62.2498 & 11.9000 & 285.1 & 4 & 600.0 & 600.0 \\
\hline 03 & 2112 & 106.7575 & 4.6250 & 286.1 & 3 & 600.0 & \\
\hline 03 & 2113 & 79.1142 & 7.4500 & 286.1 & 4 & 600.0 & 600.0 \\
\hline 03 & 2114 & 89.2310 & 7.6750 & 286.2 & 4 & 600.0 & 600.0 \\
\hline 03 & 2115 & 70.2524 & 7.7500 & 286.2 & 4 & 600.0 & \\
\hline 03 & 2116 & 92.0050 & 8.1000 & 28 & 4 & 600.0 & 600.0 \\
\hline 03 & 2117 & 91.0011 & 8.3000 & 285.3 & 4 & 600.0 & 600.0 \\
\hline 03 & 2118 & 97.5041 & 8.7750 & 284.6 & 4 & 600.0 & 600.0 \\
\hline 03 & 2119 & 111.4791 & 7.7500 & 284.2 & 4 & 600.0 & 600.0 \\
\hline 03 & 2120 & 97.4959 & 5.5750 & 283.6 & 4 & 600.0 & 600.0 \\
\hline 03 & 2121 & 123.2429 & 6.5750 & 283.8 & 4 & 600.0 & 600.0 \\
\hline 03 & 2122 & 135.8238 & 9 . & & 4 & 600.0 & 600.0 \\
\hline 03 & 2123 & 142.0000 & 10.7250 & 283.6 & 4 & 600.0 & 600.0 \\
\hline
\end{tabular}




\begin{tabular}{|c|c|c|c|c|c|c|c|c|}
\hline & 2 & 4 & & & & & & \\
\hline & 2 & 1 & 0 & .8250 & 2.7 & & 0 & \\
\hline & 2 & 22 & 153.7500 & 2.9000 & 282.1 & & 00 & \\
\hline & $?$ & 23 & 55.7501 & 3.1500 & 281.8 & & 00.0 & \\
\hline & 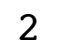 & 4 & 500 & .6750 & 1.2 & & & \\
\hline & 2 & 5 & 2500 & 3.2000 & 81.2 & & 0 & \\
\hline & 2 & 26 & 63.7499 & 2.3000 & 80.8 & & 00 & \\
\hline & 2 & 7 & 000 & 2.5250 & 80.3 & & & \\
\hline & 2 & 8 & 8.9998 & 1.95 & 80.2 & & & \\
\hline & 2 & 9 & 52.7500 & 11.3250 & 81.1 & & 00 & 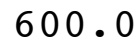 \\
\hline & 2 & 210 & 53.2496 & 9.8250 & 81.7 & & & \\
\hline & 2 & 211 & 97500 & 8.8 & 32.5 & & & \\
\hline & 2 & 212 & 99 & 8.8750 & 83.4 & & 00.0 & \\
\hline & 2 & 213 & 6.2506 & 10.20 & 0 & & & \\
\hline & 2 & 214 & & & & & & \\
\hline & 2 & 21 & & & 284.8 & & & \\
\hline & 2 & 216 & 807 & 8 . & 285.1 & 4 & .0 & \\
\hline & 2 & 217 & 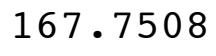 & 6 . & .1 & & & \\
\hline & 2 & 218 & & & .1 & 4 & & \\
\hline & 2 & 219 & 1228 & & 7 & 4 & & \\
\hline & 2 & 220 & 153.7513 & 3. & 283.3 & 4 & 0 & \\
\hline & 2 & 221 & 00 & & & 4 & & \\
\hline & 2 & 222 & 02 & 0 & .9 & 4 & & \\
\hline & 2 & 223 & 0.7506 & 5 . & 0 & 4 & 0 & \\
\hline & 2 & 224 & & & & 4 & & \\
\hline & 2 & 31 & 03 & & & 4 & & \\
\hline & 2 & 32 & 9.7530 & 0 & 282.2 & 4 & 0 & J. \\
\hline & 2 & 33 & 6240 & 0 & 1.6 & 4 & & \\
\hline & 2 & 34 & 48 & 0 & .1 & 4 & & \\
\hline & 2 & 35 & 626 & 0 & 0.9 & 4 & & J. \\
\hline & 2 & 36 & 117.9924 & 4.8000 & 280.4 & 4 & & 0 \\
\hline & 2 & 37 & 261 & & & 4 & & \\
\hline & 2 & 38 & 144 & & 280.4 & 4 & & 0 . \\
\hline & 2 & 39 & 000 & 3.2750 & 280.9 & 3 & & \\
\hline & 2 & 310 & 15 & & $2 \varepsilon$ & 4 & & \\
\hline & 2 & 311 & 162 . & & .1 & 3 & & \\
\hline & 2 & 312 & 153.504 & 4.4250 & 282.9 & 4 & & \\
\hline & 2 & 313 & 16 & 4. & & 4 & & \\
\hline & 2 & 314 & 158.5000 & 3750 & 3.2 & 4 & & 0 . \\
\hline & 2 & 31 & 157 . & 4.2500 & 283.6 & 4 & & \\
\hline & 2 & 316 & 038 & 750 & & 4 & & \\
\hline & 2 & 317 & 144.6588 & 0.6750 & .0 & 3 & & 0 . \\
\hline & 2 & 318 & 156.3554 & 0.5750 & 284.3 & 4 & & 0 . \\
\hline & 2 & 319 & 533 & 250 & & 3 & & \\
\hline 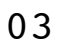 & 2 & 320 & 338.8466 & 0.6750 & 282.1 & 0 & & \\
\hline & 2 & 321 & 9231 & 1.2500 & 283.2 & 6 & 600 & 00 . \\
\hline & 2 & 322 & 17 & & & 6 & & \\
\hline & & 323 & 41.3691 & 1.2500 & 282.8 & r & 600.0 & 600 \\
\hline
\end{tabular}




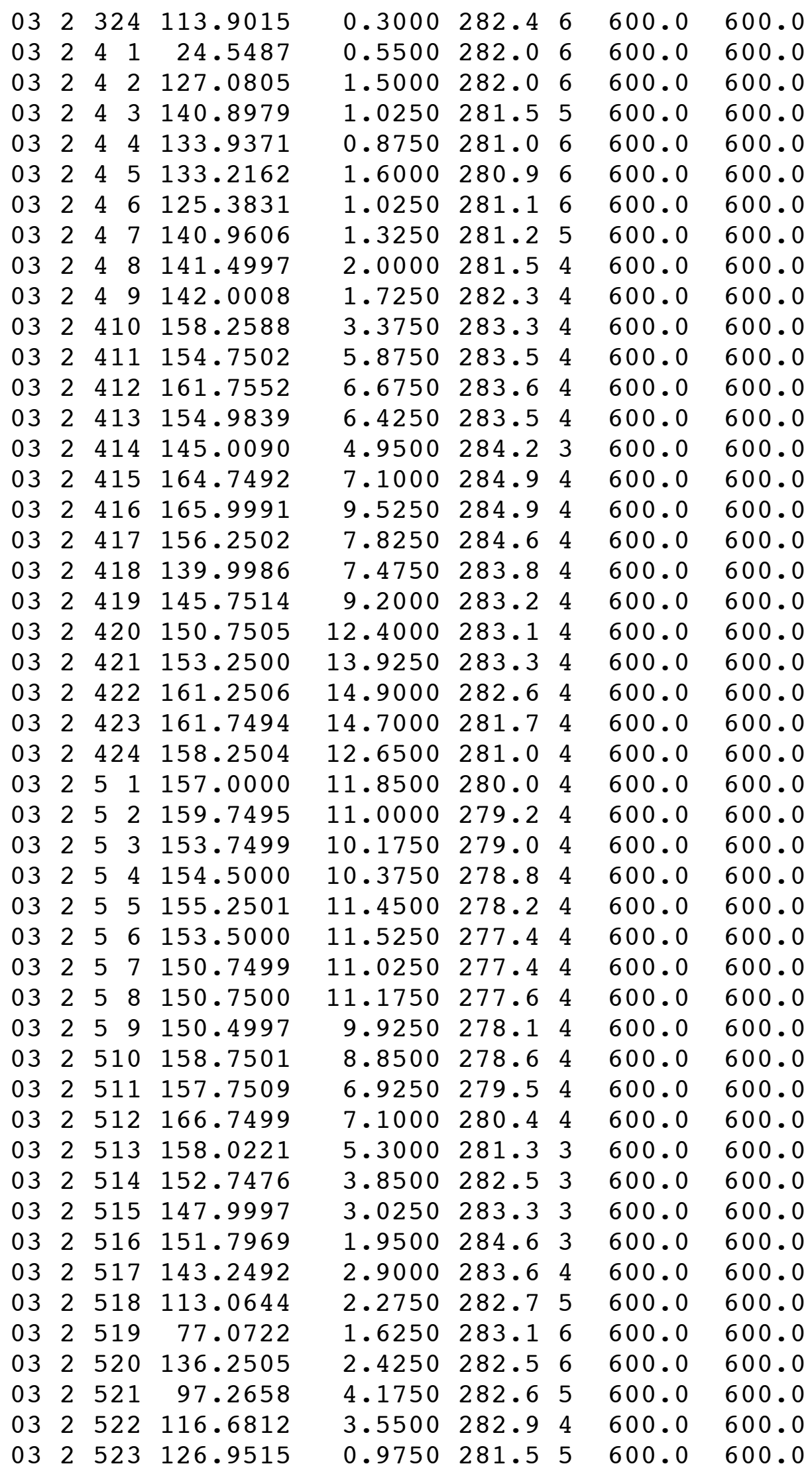




\begin{tabular}{|c|c|c|c|c|c|c|c|c|}
\hline 03 & 2 & 524 & 108.7571 & 1.7750 & 281.9 & 4 & 600.0 & 600.0 \\
\hline 3 & 2 & 61 & 140.7533 & 1.5500 & 281.7 & 5 & 600.0 & 600.0 \\
\hline 3 & 2 & 62 & 147.5683 & 0.8250 & 281.0 & 6 & 600.0 & 600.0 \\
\hline 3 & 2 & 63 & 91.4174 & 1.1500 & 281.7 & 5 & 600.0 & 600.0 \\
\hline 3 & 2 & 64 & 83.7464 & 0.7250 & 282.0 & 6 & 600.0 & 600.0 \\
\hline 3 & 2 & 65 & 87.9775 & 0.5250 & 281.9 & 6 & 600.0 & 600.0 \\
\hline 3 & 2 & 66 & 99.6717 & 0.3250 & 281.4 & 6 & 600.0 & 600.0 \\
\hline 03 & 2 & 67 & 150.7503 & 3.1750 & 279.1 & 5 & 600.0 & 600.0 \\
\hline 3 & 2 & 68 & 97.4321 & 1.9750 & 277.8 & 6 & 600.0 & 600.0 \\
\hline 3 & 2 & 69 & 147.7525 & 3.7000 & 278.3 & 5 & 600.0 & 600.0 \\
\hline 3 & 2 & 610 & 158.2518 & 3.9000 & 278.0 & 4 & 600.0 & 600.0 \\
\hline 3 & 2 & 611 & 230.1019 & 1.5500 & 281.0 & 3 & 600.0 & 600.0 \\
\hline 03 & 2 & 612 & 264.9835 & 2.9750 & 282.2 & 2 & 600.0 & 600.0 \\
\hline 03 & 2 & 613 & 171.2382 & 6.2500 & 280.9 & 3 & 600.0 & 600.0 \\
\hline 3 & 2 & 614 & 185.5000 & 5.0750 & 281.4 & 3 & 600.0 & 600.0 \\
\hline 03 & 2 & 615 & 200.2671 & 3.7250 & 282.3 & 2 & 600.0 & 600.0 \\
\hline 03 & 2 & 616 & 198.9997 & 3.3250 & 282.7 & 2 & 600.0 & 600.0 \\
\hline 03 & 2 & 617 & 189.9992 & 3.1000 & 282.7 & 3 & 600.0 & 600.0 \\
\hline 03 & 2 & 618 & 181.7998 & 2.3750 & 282.0 & 4 & 600.0 & 600.0 \\
\hline 03 & 2 & 619 & 155.7501 & 2.9500 & 281.2 & 5 & 600.0 & 600.0 \\
\hline 03 & 2 & 620 & 144.7020 & 1.3250 & 281.6 & 6 & 600.0 & 600.0 \\
\hline 03 & 2 & 621 & 38.7500 & 2.2750 & 281.7 & 5 & 600.0 & 600.0 \\
\hline 03 & 2 & 622 & 29.5008 & 1.6750 & 281.9 & 5 & 600.0 & 600.0 \\
\hline 03 & 2 & 623 & 23.9998 & 2.5500 & 282.1 & 5 & 600.0 & 600.0 \\
\hline 03 & 2 & 624 & 334.5488 & 1.3250 & 282.1 & 6 & 600.0 & 600.0 \\
\hline 03 & 2 & 71 & 151.5006 & 5.1750 & 279.4 & 5 & 600.0 & 600.0 \\
\hline 03 & 2 & 72 & 152.2495 & 6.8250 & 278.8 & 4 & 600.0 & 600.0 \\
\hline 03 & 2 & 73 & 147.5000 & 5.8500 & 278.2 & 4 & 600.0 & 600.0 \\
\hline 03 & 2 & 74 & 149.2498 & 6.3500 & 277.3 & 4 & 600.0 & 600.0 \\
\hline 03 & 2 & 75 & 149.5000 & 8.8500 & 277.0 & 4 & 600.0 & 600.0 \\
\hline 03 & 2 & 76 & 150.0000 & 10.0500 & 277.5 & 4 & 600.0 & 60 \\
\hline 03 & 2 & 77 & 150.2500 & 10.5000 & 277.5 & 4 & 600.0 & 600.0 \\
\hline 03 & 2 & 78 & 151.0000 & 10.5500 & 277.6 & 4 & 600.0 & 600.0 \\
\hline 03 & 2 & 79 & 154.2500 & 10.0250 & 278.1 & 4 & 600.0 & 600.0 \\
\hline 03 & 2 & 710 & 154.7500 & 9.8500 & 278.6 & 4 & 600.0 & 600.0 \\
\hline 03 & 2 & 711 & 152.2503 & 8.4000 & 279.7 & 4 & 600.0 & 600.0 \\
\hline 03 & 2 & 712 & 156.2455 & 7.5750 & 281.1 & 4 & 600.0 & 600.0 \\
\hline 03 & 2 & 713 & 163.2505 & 7.6750 & 282.4 & 4 & 600.0 & 600.0 \\
\hline 03 & 2 & 714 & 174.5021 & 8.5500 & 283.1 & 4 & 600.0 & 600.0 \\
\hline 03 & 2 & 715 & 173.2500 & 7.5250 & 283.4 & 4 & 600.0 & 600.0 \\
\hline 03 & 2 & 716 & 185.0006 & 6.5250 & 283.6 & 4 & 600.0 & 600.0 \\
\hline 03 & 2 & 717 & 173.2575 & 5.1750 & 283.4 & 3 & 600.0 & 600.0 \\
\hline 03 & 2 & 718 & 149.0021 & 3.5500 & 282.6 & 4 & 600.0 & 600.0 \\
\hline 03 & 2 & 719 & 121.9979 & 3.7500 & 282.6 & 4 & 600.0 & 600.0 \\
\hline 03 & 2 & 720 & 118.2511 & 3.8750 & 282.7 & 4 & 600.0 & 600.0 \\
\hline 03 & 2 & 721 & 120.5021 & 3.6500 & 282.8 & 4 & 600.0 & 600.0 \\
\hline 03 & 2 & 722 & 106.4917 & 3.1750 & 282.9 & 4 & 600.0 & 600.0 \\
\hline & 2 & 723 & 112.0118 & 3.5000 & 283.4 & 4 & 600.0 & 600.0 \\
\hline
\end{tabular}




\begin{tabular}{|c|c|c|c|c|c|c|c|}
\hline 32 & 724 & 2443 & 3.4000 & 283.0 & 4 & 600.0 & 600.0 \\
\hline 032 & 81 & 121.2580 & 2.7750 & 283.2 & 4 & 600.0 & 600.0 \\
\hline 032 & 82 & 105.8561 & 1.9250 & 282.9 & 5 & 600.0 & 600.0 \\
\hline 032 & 83 & 139.9612 & 1.5000 & 282.6 & 4 & 600.0 & 600.0 \\
\hline 032 & 84 & 126.1971 & 1.8500 & 282.3 & 5 & 600.0 & 600.0 \\
\hline 032 & 85 & 112.5398 & 3.5000 & 282.3 & 4 & 600.0 & 600.0 \\
\hline 032 & 86 & 151.0004 & 3.6500 & 280.1 & 4 & 600.0 & 600.0 \\
\hline 032 & 87 & 126.9893 & 4.6750 & 280.6 & 4 & 600.0 & 600.0 \\
\hline 032 & 88 & 134.2523 & 5.5750 & 280.9 & 4 & 600.0 & 600.0 \\
\hline 03 & 89 & 125.7728 & 4.6250 & 281.3 & 4 & 600.0 & 600.0 \\
\hline 32 & 810 & 156.2480 & 4.5000 & 281.4 & 4 & 600.0 & 600.0 \\
\hline 032 & 811 & 150.2498 & 4.8750 & 281.4 & 4 & 600.0 & 600.0 \\
\hline 032 & 812 & 152.4977 & 4.0500 & 282.2 & 3 & 600.0 & 600.0 \\
\hline 032 & 813 & 162.2499 & 5.7500 & 282.5 & 4 & 600.0 & 600.0 \\
\hline 032 & 814 & 161.5086 & 5.7500 & 283.3 & 4 & 600.0 & 600.0 \\
\hline 032 & 815 & 165.2524 & 6.0250 & 283.4 & 4 & 600.0 & 600.0 \\
\hline 032 & 816 & 160.2499 & 5.2500 & 283.5 & 4 & 600.0 & 600.0 \\
\hline 032 & 817 & 153.7499 & 4.2750 & 283.3 & 4 & 600.0 & 600.0 \\
\hline 032 & 818 & 146.0018 & 3.9000 & 282.6 & 4 & 600.0 & 600.0 \\
\hline 032 & 819 & 150.2509 & 3.3500 & 281.9 & 5 & 600.0 & 600.0 \\
\hline 032 & 820 & 147.0023 & 1.8250 & 282.0 & 5 & 600.0 & 600.0 \\
\hline 03 & 821 & 91.6199 & 1.3750 & 282.2 & 5 & 600.0 & 600.0 \\
\hline 032 & 822 & 82.5002 & 2.4750 & 283.5 & 4 & 600.0 & 600.0 \\
\hline 032 & 823 & 95.5649 & 2.1750 & 283.7 & 4 & 600.0 & 600.0 \\
\hline 032 & 824 & 122.2555 & 1.4500 & 283.3 & 5 & 600.0 & 600.0 \\
\hline 03 & 91 & 148.2534 & 2.4000 & 282.7 & 5 & 600.0 & 600.0 \\
\hline 032 & 92 & 49.4100 & 0.8250 & 280.8 & 6 & 600.0 & 600.0 \\
\hline 032 & 93 & 3.4963 & 0.7750 & 280.8 & 5 & 600.0 & 600.0 \\
\hline 03 & 94 & 21.5017 & 0.5750 & 280.8 & 5 & 600.0 & 600.0 \\
\hline 032 & 95 & 110.1727 & 0.0500 & 280.0 & 6 & 600.0 & 600.0 \\
\hline 032 & 96 & 70.7248 & 0.4250 & 280.0 & 6 & 600.0 & 600.0 \\
\hline 03 & 97 & 90.3456 & 0.5250 & 280.4 & 6 & 600.0 & 600.0 \\
\hline 032 & 98 & 237.2416 & 0.3000 & 280.7 & 6 & 600.0 & 600.0 \\
\hline 032 & 99 & 307.6232 & 0.6000 & 283.3 & 5 & 600.0 & 600.0 \\
\hline 03 & 910 & 328.3633 & 1.2250 & 284.2 & 4 & 600.0 & 600.0 \\
\hline 03 & 911 & 277.5165 & 1.8750 & 283.8 & 3 & 600.0 & 600.0 \\
\hline 032 & 912 & 271.6590 & 2.3500 & 284.3 & 2 & 600.0 & 600.0 \\
\hline 032 & 913 & 237.7503 & 2.5500 & 283.8 & 2 & 600.0 & 600.0 \\
\hline 03 & 914 & 221.0000 & 2.6500 & 284.2 & 2 & 600.0 & 600.0 \\
\hline 03 & 915 & 216.3158 & 2.2750 & 285.2 & 1 & 600.0 & 600.0 \\
\hline 03 & 916 & 170.2401 & 2.0500 & 285.1 & 2 & 600.0 & 600.0 \\
\hline 03 & 917 & 150.4969 & 2.5250 & 284.5 & 3 & 600.0 & 600.0 \\
\hline 032 & 918 & 151.9995 & 1.6750 & 283.3 & 4 & 600.0 & 600.0 \\
\hline 03 & 919 & 70.9726 & 1.3500 & 282.7 & 5 & 600.0 & 600.0 \\
\hline 03 & 920 & 60.0800 & 1.8750 & 283.6 & 5 & 600.0 & 600.0 \\
\hline 03 & 921 & 91.9955 & 2.5250 & 284.2 & 5 & 600.0 & 600.0 \\
\hline 03 & 922 & 82.3868 & 2.3000 & 284.1 & 6 & 600.0 & 600.0 \\
\hline 2 & 923 & 32.8541 & 1.6250 & 283.5 & 3 & 600.0 & 600.0 \\
\hline
\end{tabular}




\begin{tabular}{|c|c|c|c|c|c|c|c|}
\hline & 24 & 3.7556 & 1.1500 & 283.2 & 4 & 800 & \\
\hline 3 & 2101 & 2.8060 & 0.5500 & 282.8 & & ل & \\
\hline & 2102 & 34.6567 & 1.3250 & 282.9 & & 00 & \\
\hline & 2103 & 13.7498 & 2.0500 & 283.5 & & 00 & \\
\hline & 2104 & 1.2236 & 1.4000 & 83.4 & & 0 & \\
\hline & 2105 & 39.8690 & .4750 & 83.7 & & 00 & \\
\hline & 2106 & 63.2484 & 1.1500 & 283.3 & & 00 & \\
\hline & 2107 & 51.4762 & L. 6750 & 81.0 & & & \\
\hline & 2108 & 108.2426 & 0.9500 & 280.0 & & 0 & \\
\hline & 2109 & 126.0263 & 1.2250 & 281.9 & & 00 & \\
\hline & 1010 & 151.7813 & 4.2000 & .79 .9 & & 0 & \\
\hline & 011 & 168.1873 & 2.8000 & 81.7 & & & \\
\hline & 1012 & 238.9884 & 2.2500 & 283.6 & & 0 & \\
\hline & 21013 & 229.7499 & 2.5250 & 283.8 & & 0.0 & \\
\hline & 1014 & 229.80 & & & & & \\
\hline & 21015 & 251.2398 & 1.7000 & 285.0 & & 0 & \\
\hline & 21016 & 273.7010 & .8000 & 286.0 & & 0 & \\
\hline & 21017 & 262.2552 & 0.97 & 5.4 & & & \\
\hline & 21018 & 222.7325 & 1.3250 & 283.2 & & 0 & \\
\hline & 1019 & 331.7523 & 1.2000 & 282.5 & 3 & 0 & \\
\hline & 1020 & 4.4988 & 0 & 282.5 & & & \\
\hline & 21021 & 2.66 & 50 & 283.0 & 5 & & \\
\hline & 21022 & 0.7496 & 000 & 28 & 4 & 0 & 0 • \\
\hline & 21023 & 45.9853 & 2.2000 & 5 & 4 & 0 & \\
\hline & 21024 & 31 & 0 & & 4 & & \\
\hline & 2111 & 293.7416 & 7500 & 282.6 & 5 & & \\
\hline & 2112 & 224.6649 & .6500 & 282.2 & 6 & 0 & \\
\hline & 2113 & 131.8393 & 72 & 283.0 & 6 & & \\
\hline & 2114 & 149 . & 1.6750 & 28 & 5 & 0 & 0 . \\
\hline & 2115 & 159.5070 & 1.7250 & 282.6 & 2 & & \\
\hline & 2116 & 140 . & 0 & 281.3 & 5 & & \\
\hline & 2117 & 21. & 250 & 281.2 & 6 & & \\
\hline & 2118 & 1.0050 & 0.9250 & 281.6 & 5 & 0 & U. \\
\hline & 2119 & 0.8235 & 000 & $2 \varepsilon$ & 4 & & \\
\hline & 21110 & 252 . & 0 & 28 & 3 & & \\
\hline & 21111 & 110.8410 & 5250 & 281.0 & 2 & & • \\
\hline & 21112 & 208.1321 & 1.2500 & 282.9 & 1 & & \\
\hline 3 & 21113 & 255.5140 & 1.8250 & 283.7 & 1 & 0 & • \\
\hline & 21114 & 249.3923 & 1.3000 & 283.7 & 1 & 0 & 0 . \\
\hline & 21115 & 270.2909 & 250 & 28 & 1 & & \\
\hline & 21116 & 284.3071 & 500 & & 1 & & \\
\hline 03 & 21117 & 286.1949 & 2.2750 & 283.7 & 2 & 600.0 & 600 . \\
\hline & 21118 & 304.8404 & 0.9750 & 283.8 & 3 & 600 & 600. \\
\hline 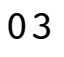 & 21119 & 68.0369 & 1.3750 & & 4 & & 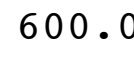 \\
\hline 03 & 21120 & 311.2434 & 0.8750 & 283.6 & 5 & 600.0 & 00 \\
\hline & 21121 & 306.1178 & 1.1500 & & 6 & 600.0 & 60 \\
\hline & 21122 & 343.4807 & 1.9250 & & 5 & 600 & \\
\hline & 21123 & 313.4607 & 0.9500 & 282.8 & & 600.0 & 600 \\
\hline
\end{tabular}




\begin{tabular}{|c|c|c|c|c|c|c|c|}
\hline 03 & 21124 & 5.5072 & 1.3250 & 282.9 & 5 & 600.0 & 600.0 \\
\hline 03 & 2121 & 283.5704 & 0.4750 & 282.7 & 6 & 600.0 & 600.0 \\
\hline 03 & 2122 & 156.2478 & 2.1750 & 282.2 & 5 & 600.0 & 00.0 \\
\hline 3 & 2123 & 141.7505 & 3.0250 & 281.9 & 4 & 600.0 & 00.0 \\
\hline 03 & 2124 & 146.2450 & 1.9500 & 281.8 & 4 & 600.0 & 00.0 \\
\hline 03 & 2125 & 143.9995 & 3.1750 & 281.5 & 4 & 600.0 & 00.0 \\
\hline 03 & 2126 & 148.4995 & 5.3500 & 281.3 & 4 & 600.0 & 00.0 \\
\hline 03 & 2127 & 141.5018 & 4.8750 & 281.3 & 4 & 600.0 & 00.0 \\
\hline 03 & 2128 & 140.0000 & 3.9500 & 281.5 & 4 & 600.0 & 00.0 \\
\hline 03 & 2129 & 144.7624 & 3.2000 & 280.9 & 4 & 600.0 & 00.0 \\
\hline 03 & 21210 & 133.0736 & 0.9000 & 280.5 & 3 & 600.0 & 00.0 \\
\hline 03 & 21211 & 314.0268 & 1.1750 & 281.5 & 2 & 600.0 & \\
\hline 03 & 21212 & 253.2193 & 1.0000 & 281.9 & 1 & 600.0 & 00.0 \\
\hline 03 & 21213 & 309.7475 & 2.5500 & 282.9 & 2 & 600.0 & 00.0 \\
\hline 03 & 21214 & 262.5092 & 50 & 282.0 & 2 & .0 & \\
\hline 03 & 21215 & 267.0002 & 4.7500 & 281.6 & 3 & 600.0 & 00.0 \\
\hline 03 & 21216 & 274.4669 & 6.6000 & 282.1 & 4 & 600.0 & 600.0 \\
\hline 03 & 21217 & 294.2503 & 6.37 & 282.2 & 4 & .0 & \\
\hline 03 & 21218 & 302.2461 & 5.5250 & 282.4 & 4 & 600.0 & .0 \\
\hline 03 & 21219 & 305.0024 & 3.7250 & 282.9 & 4 & 60 & .0 \\
\hline 03 & 21220 & 171.5240 & 1.7 & 283.0 & 5 & 60 & \\
\hline 03 & 21221 & 154.7499 & 3.5500 & 282.3 & 4 & .0 & \\
\hline 03 & 21222 & 150.2534 & 2.8000 & 282.0 & 4 & 600.0 & 500.0 \\
\hline 03 & 21223 & 148.5000 & 1.67 & 282.1 & 4 & 60 & .0 \\
\hline 03 & 21224 & 273.1937 & 0 & 282.2 & 5 & .0 & \\
\hline 03 & 2131 & 155.6241 & 0.6500 & 282.3 & 6 & 600.0 & 600.0 \\
\hline 03 & 2132 & 244.3766 & 0.5000 & 282.2 & 6 & 600.0 & .0 \\
\hline 03 & 2133 & 9.3196 & 0.7750 & 282.2 & 6 & 0 & \\
\hline 03 & 2134 & 316.6861 & 0.5500 & 282.1 & 6 & 600.0 & 600.0 \\
\hline 03 & 2135 & 326.8788 & 0.9250 & 282.2 & 6 & 600.0 & 600.0 \\
\hline 03 & 2136 & 0.4511 & 1.6 & 282.3 & 6 & .0 & \\
\hline 03 & 2137 & 12.7722 & 2.4750 & 282.9 & 5 & 600.0 & 600.0 \\
\hline 03 & 2138 & 22.4906 & 2.5750 & 285.4 & 4 & 600.0 & 600.0 \\
\hline 03 & 2139 & 20.0427 & 1.1250 & 286.2 & 3 & 600.0 & 0.0 \\
\hline 03 & 21310 & 236.8960 & 0.5500 & 288.8 & 2 & 600.0 & 600.0 \\
\hline 03 & 21311 & 177.4696 & 3.0250 & 286.1 & 2 & 600.0 & 600.0 \\
\hline 03 & 21312 & 136.9386 & 3.1750 & 284.6 & 2 & 600.0 & 600.0 \\
\hline 03 & 21313 & 180.0228 & 2.2250 & 285.6 & 1 & 600.0 & 600.0 \\
\hline 03 & 21314 & 182.0985 & 1.9250 & 286.0 & 2 & 600.0 & 600.0 \\
\hline 03 & 21315 & 112.1112 & 1.8250 & 286.8 & 1 & 600.0 & 600.0 \\
\hline 03 & 21316 & 119.0975 & 3.7000 & 285.9 & 2 & 600.0 & 600.0 \\
\hline 03 & 21317 & 82.0048 & 4.9500 & 286.6 & 3 & 600.0 & 600.0 \\
\hline 03 & 21318 & 90.5074 & 5.4000 & 286.1 & 4 & 600.0 & 600.0 \\
\hline 03 & 21319 & 94.0007 & 5.2250 & 285.5 & 4 & 600.0 & 600.0 \\
\hline 03 & 21320 & 69.4989 & 4.5500 & 285.1 & 4 & 600.0 & 600.0 \\
\hline 03 & 21321 & 78.9989 & 4.6500 & 284.7 & 4 & 600.0 & 600.0 \\
\hline 03 & 21322 & 69.7455 & 5.2500 & 283.9 & 4 & 600.0 & 600.0 \\
\hline & $213<3$ & 65.2501 & 5.275 & 283.5 & 4 & 600.0 & 600.0 \\
\hline
\end{tabular}




\begin{tabular}{|c|c|c|c|c|c|c|c|}
\hline 3 & 21324 & 59.0034 & 5.8250 & 283.0 & 4 & 600.0 & 600.0 \\
\hline 03 & $214 \quad 1$ & 60.2756 & 6.7250 & 282.7 & 4 & 600.0 & 600.0 \\
\hline 03 & 2142 & 57.0004 & 9.9250 & 282.6 & 4 & 600.0 & 00.0 \\
\hline 03 & 2143 & 55.5000 & 10.0750 & 282.6 & 4 & 600.0 & 600.0 \\
\hline 03 & $214 \quad 4$ & 57.9989 & 8.7000 & 282.4 & 4 & 600.0 & 00.0 \\
\hline 03 & 2145 & 65.5027 & 8.1250 & 282.3 & 4 & 600.0 & 00.0 \\
\hline 03 & 2146 & 64.2501 & 8.5750 & 282.2 & 4 & 600.0 & 600.0 \\
\hline 03 & 2147 & 68.4977 & 7.5500 & 282.4 & 4 & 600.0 & 500.0 \\
\hline 03 & 2148 & 71.9996 & 7.4000 & 282.6 & 4 & 600.0 & 00.0 \\
\hline 03 & 2149 & 75.7496 & 6.3000 & 282.8 & 4 & 600.0 & 600.0 \\
\hline 03 & 21410 & 78.0000 & 5.4750 & 283.1 & 4 & 600.0 & 600.0 \\
\hline 03 & 21411 & 75.0007 & 5.7000 & 283.4 & 4 & 600.0 & \\
\hline 03 & 21412 & 70.7474 & 4.3500 & 284.0 & 4 & 600.0 & 600.0 \\
\hline 03 & 21413 & 79.5009 & 3.5750 & 284.8 & 4 & 600.0 & 600 \\
\hline 03 & 21414 & 91.5000 & 4.0250 & 284.9 & 4 & 600.0 & \\
\hline 03 & 21415 & 155.7134 & 2.4500 & 286.3 & 3 & 600.0 & 60 \\
\hline 03 & 21416 & 149.1811 & 1.7750 & 286.9 & 2 & 600.0 & 600.0 \\
\hline 03 & 21417 & 85.9977 & 3.8500 & 285.0 & 3 & 600.0 & \\
\hline 03 & 21418 & 72.0211 & 3.9250 & 284.6 & 4 & 600.0 & 60 \\
\hline 03 & 21419 & 67.7117 & 3.7250 & 284.5 & 4 & 600.0 & 60 \\
\hline 03 & 21420 & 78.4990 & 5.8500 & 283.6 & 4 & 600.0 & .0 \\
\hline 03 & 21421 & 65.2499 & 6.2500 & 283.2 & 4 & 600.0 & \\
\hline 03 & 21422 & 26.3522 & 3.9000 & 283.2 & 4 & 600.0 & 600.0 \\
\hline 03 & 21423 & 350.2346 & 2.3250 & 283.1 & 5 & 600.0 & .0 \\
\hline 03 & 21424 & 355.1765 & 2.3500 & 283.2 & 5 & 600.0 & \\
\hline 03 & 2151 & 30.0369 & 2.4750 & 283.2 & 5 & 600.0 & 600.0 \\
\hline 03 & 2152 & 47.0506 & 2.5750 & 283.4 & 6 & 600.0 & 600.0 \\
\hline 03 & 2153 & 52.4740 & 2.5250 & 283.1 & 6 & 600.0 & \\
\hline 03 & 2154 & 90.5094 & 4.0250 & 282.3 & 5 & 600.0 & 600.0 \\
\hline 03 & 2155 & 79.2290 & 2.9500 & 282.3 & 5 & 600.0 & 600.0 \\
\hline 03 & 2156 & 323.6355 & 1.07 & 281.7 & 6 & 600.0 & .0 \\
\hline 03 & 2157 & 318.7427 & 2.7250 & 281.1 & 5 & 600.0 & 60 \\
\hline 03 & 2158 & 329.0111 & 2.6750 & 281.1 & 4 & 600.0 & 600.0 \\
\hline 03 & 2159 & 337.2893 & 2.3500 & 281.3 & 3 & 600.0 & .0 \\
\hline 03 & 21510 & 300.9389 & 1.0500 & 282.0 & 2 & 600.0 & 60 \\
\hline 03 & 21511 & 166.4510 & 1.9750 & 282.8 & 1 & 600.0 & 600.0 \\
\hline 03 & 21512 & 205.6686 & 1.3750 & 283.5 & 2 & 600.0 & .0 \\
\hline 03 & 21513 & 277.1018 & 1.6750 & 283.9 & 1 & 600.0 & 600.0 \\
\hline 03 & 21514 & 283.7393 & 1.8500 & 284.8 & 1 & 600.0 & 600.0 \\
\hline 03 & 21515 & 247.9696 & 1.2500 & 285.9 & 1 & 600.0 & \\
\hline 03 & 21516 & 206.7030 & 2.1500 & 285.6 & 2 & 600.0 & 600.0 \\
\hline 03 & 21517 & 164.3669 & 1.2000 & 285.2 & 1 & 600.0 & 600.0 \\
\hline 03 & 21518 & 103.7620 & 0.6500 & 285.0 & 2 & 600.0 & 600.0 \\
\hline 03 & 21519 & 336.1212 & 0.9500 & 285.0 & 3 & 600.0 & 600.0 \\
\hline 03 & 21520 & 17.7589 & 5.6250 & 285.4 & 4 & 600.0 & 600.0 \\
\hline 03 & 21521 & 356.5110 & 5.9750 & 285.4 & 4 & 600.0 & 600.0 \\
\hline 03 & 21522 & 337.0005 & 5.5500 & $28<$ & 1 & 600.0 & 600.0 \\
\hline 03 & 21523 & 332.7500 & 7.0000 & 285.0 & 4 & 600.0 & 600.0 \\
\hline
\end{tabular}




\begin{tabular}{|c|c|c|c|c|c|c|c|}
\hline 3 & 1524 & & 3000 & 84.6 & & & \\
\hline & 2161 & 523 & 4.6250 & 83.5 & 4 & 600.0 & \\
\hline & 2162 & 48.9416 & 7250 & 282.6 & 4 & 00 & \\
\hline & 163 & 74.9820 & 5.6000 & 282.2 & & 00 & \\
\hline & 164 & 000 & .8250 & 81.1 & & 0 & \\
\hline & 165 & 315.1561 & .4750 & 80.8 & & 00 & \\
\hline & 166 & 162. & 1.1500 & 281.1 & & 00 & \\
\hline & 167 & 776 & 2.1750 & 81.7 & & 0 & \\
\hline & 2168 & 41 & 4.0250 & 81.7 & & & \\
\hline & 169 & 351.6405 & 1.7250 & 282.5 & 4 & 00 & 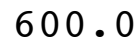 \\
\hline & 1610 & 341.1905 & 2.4000 & 283.7 & & 500.0 & \\
\hline & 1611 & 4.2231 & 1.2000 & 85.3 & & 0 & \\
\hline & 1612 & 311.37 & 1.6750 & 285.4 & 1 & 0 & . \\
\hline & 1613 & 296.5344 & 2.7500 & 285.1 & & 0.0 & 0 \\
\hline & 1614 & 275 . & 0 & & & & \\
\hline & 615 & 232 . & 1 . & 28 & 1 & & \\
\hline & 1616 & 241.7922 & 1.3500 & 286.7 & 1 & 0.0 & . \\
\hline & 617 & 185 . & 250 & .9 & 1 & 0 & \\
\hline & 1618 & 127 . & 3 . & 28 & 2 & & \\
\hline & 1619 & 107.5176 & 5.1250 & 283.8 & 3 & .0 & . \\
\hline & 21620 & 85.7683 & 750 & .0 & 4 & 0 & 0. \\
\hline & 21621 & 71.9 & & .3 & 4 & & \\
\hline & 622 & 71 . & 3250 & 282.1 & 4 & & 0 . \\
\hline & 623 & 81.5000 & 4.7250 & 282.1 & 5 & 0 & J. \\
\hline & 21624 & 85.7301 & & & כ & & \\
\hline & 2171 & 111. & 50 & 282.2 & 4 & & \\
\hline & 2172 & 116.7413 & 500 & 282.3 & 4 & 0 & U. \\
\hline & 2173 & 127 . & 000 & 282.1 & 4 & & \\
\hline & 2174 & 126 . & 500 & 282.1 & 4 & & $\bullet$ \\
\hline & 2175 & 123.9927 & 250 & 281.6 & 5 & 0 & 0 . \\
\hline & 2176 & 138.5000 & 2.3250 & 281.7 & 4 & 0 & 0 . \\
\hline & 2177 & 139.9993 & 2 . & 281.4 & 4 & & \\
\hline & 2178 & 149 . & 0 & 281.1 & 5 & 0 & 0 • \\
\hline & 2179 & 150.7491 & 50 & 281.5 & 4 & 0 & 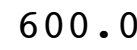 \\
\hline & 21710 & 160. & 0 & 281.2 & 4 & & \\
\hline & 21711 & 157.0007 & 6 . & 281.4 & 4 & & 0 . \\
\hline & 21712 & 164.5023 & 5250 & 281.5 & 4 & $\cdot 0$ & 0 \\
\hline & 21713 & 152.7499 & 5 . & 281.6 & 4 & & 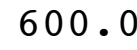 \\
\hline 03 & 21714 & 149.0371 & 3.7250 & 282.5 & 3 & .0 & 0 . \\
\hline & 21715 & 141.2830 & 0250 & 283.2 & 4 & & 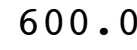 \\
\hline & 21716 & 154.0032 & 000 & 283.7 & 4 & & 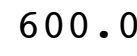 \\
\hline & 21717 & 151.5206 & 3.0500 & 283.8 & 4 & .0 & 00 . \\
\hline & 21718 & 154.2501 & 3.2250 & 283.1 & 5 & .0 & 00 . \\
\hline & 21719 & 105.1205 & 000 & 282.6 & 4 & & \\
\hline 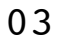 & 21720 & 67.7453 & 5.0750 & 282.5 & 4 & & 00 . \\
\hline & 21721 & 71.2512 & 6.2000 & 282.5 & 4 & 600.0 & 500 . \\
\hline & 21722 & 69.7499 & & & 4 & & \\
\hline & 21723 & 71.5000 & 7.0750 & 281.9 & 1 & 600.0 & 600 \\
\hline
\end{tabular}




\begin{tabular}{|c|c|c|c|c|c|c|c|}
\hline 03 & 21724 & 73.9998 & 6.3750 & 281.7 & 4 & 600.0 & 600.0 \\
\hline 03 & 2181 & 72.7499 & 6.4750 & 282.0 & 4 & 600.0 & 600.0 \\
\hline 03 & 2182 & 79.7501 & 6.4500 & 282.0 & 4 & 600.0 & 00.0 \\
\hline 03 & 2183 & 79.2500 & 6.6250 & 281.8 & 4 & 600.0 & 00.0 \\
\hline 03 & 2184 & 80.2501 & .4750 & 282.2 & 4 & 600.0 & 00.0 \\
\hline 03 & 2185 & 86.4895 & 4.9000 & 282.5 & 5 & 600.0 & 00.0 \\
\hline 03 & 2186 & 79.9999 & 7.7250 & 281.2 & 4 & 600.0 & 00.0 \\
\hline 03 & 2187 & 79.0000 & 8.1500 & 280.8 & 4 & 600.0 & 00.0 \\
\hline 03 & 2188 & 75.9979 & 8.4000 & 280.9 & 4 & 600.0 & 00.0 \\
\hline 03 & 2189 & 79.7502 & 7.8250 & 281.9 & 4 & 600.0 & 00.0 \\
\hline 03 & 21810 & 90.6040 & 3.6500 & 283.2 & 3 & 600.0 & 00.0 \\
\hline 03 & 21811 & 142.8563 & 1.5750 & 284.8 & 2 & 600.0 & 00.0 \\
\hline 03 & 21812 & 192.5370 & 2.7750 & 284.8 & 1 & 600.0 & 00.0 \\
\hline 03 & 21813 & 183.7562 & 3.2000 & 285.1 & 2 & 600.0 & 00.0 \\
\hline 03 & 21814 & 167.5145 & 4.5500 & 285.7 & 3 & .0 & \\
\hline 03 & 21815 & 159.0004 & 4.4000 & 286.2 & 3 & 600.0 & 00.0 \\
\hline 03 & 21816 & 149.9376 & 4.3750 & 286.3 & 4 & 600.0 & 600.0 \\
\hline 03 & 21817 & 162.2483 & 3.9250 & 286.0 & 4 & .0 & \\
\hline 03 & 21818 & 156.0000 & 3.8500 & 284.9 & 4 & 600.0 & .0 \\
\hline 03 & 21819 & 126.2387 & 2.7500 & 284.1 & 4 & 60 & .0 \\
\hline 03 & 21820 & 68.7519 & 5.3500 & 283.5 & 4 & 60 & \\
\hline 03 & 21821 & 68.2500 & 5.4750 & 283.2 & 4 & .0 & \\
\hline 03 & 21822 & 75.2487 & 6.1000 & 283.1 & 4 & 600.0 & 600.0 \\
\hline 03 & 21823 & 76.7501 & 7.30 & 283.1 & 4 & .0 & .0 \\
\hline 03 & 21824 & 78.2500 & 7.7000 & 282.8 & 4 & .0 & \\
\hline 03 & 2191 & 69.2500 & 7.5500 & 282.4 & 4 & 600.0 & 600.0 \\
\hline 03 & 2192 & 72.0000 & 8.0250 & 282.2 & 4 & 600.0 & .0 \\
\hline 03 & 2193 & 65.7500 & 8.8750 & 281.9 & 4 & 600.0 & \\
\hline 03 & 2194 & 67.5000 & 8.1750 & 281.6 & 4 & 600.0 & 600.0 \\
\hline 03 & 2195 & 65.7545 & 7.4500 & 281.6 & 4 & 600.0 & 600.0 \\
\hline 03 & 2196 & 41.0454 & 2.2000 & 281.5 & 5 & 600.0 & .0 \\
\hline 03 & 2197 & 58.9959 & 3.1250 & 282.0 & 4 & 600.0 & 600.0 \\
\hline 03 & 2198 & 63.1939 & 3.9250 & 281.9 & 4 & 600.0 & 600.0 \\
\hline 03 & 2199 & 78.7691 & 9.2250 & 281.1 & 4 & 600.0 & 0.0 \\
\hline 03 & 21910 & 86.2443 & 5.8500 & 280.5 & 3 & 600.0 & 600.0 \\
\hline 03 & 21911 & 72.3741 & 5.9500 & 280.8 & 3 & 600.0 & 600.0 \\
\hline 03 & 21912 & 77.2338 & 7.1000 & 280.7 & 4 & 600.0 & 600.0 \\
\hline 03 & 21913 & 93.7946 & 7.6250 & 281.8 & 4 & 600.0 & 600.0 \\
\hline 03 & 21914 & 100.9925 & 8.3500 & 282.8 & 4 & 600.0 & 600.0 \\
\hline 03 & 21915 & 99.2522 & 6.9500 & 283.3 & 4 & 600.0 & 600.0 \\
\hline 03 & 21916 & 99.5013 & 7.1750 & 283.5 & 4 & 600.0 & 600.0 \\
\hline 03 & 21917 & 98.7513 & 7.0750 & 283.2 & 4 & 600.0 & 600.0 \\
\hline 03 & 21918 & 96.0000 & 8.2500 & 282.5 & 4 & 600.0 & 600.0 \\
\hline 03 & 21919 & 91.5003 & 8.0250 & 282.1 & 4 & 600.0 & 600.0 \\
\hline 03 & 21920 & 91.2489 & 8.4750 & 281.7 & 4 & 600.0 & 600.0 \\
\hline 03 & 21921 & 91.9963 & 8.9750 & 281.1 & 4 & 600.0 & 600.0 \\
\hline 03 & 21922 & 88.7504 & 9.2500 & 280.6 & 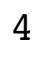 & 600.0 & 600.0 \\
\hline U. & 21923 & 92.2498 & 8.625 & 280.2 & 4 & 600.0 & 600.0 \\
\hline
\end{tabular}




\begin{tabular}{|c|c|c|c|c|c|c|c|}
\hline 3 & 1924 & 2.7508 & 8.0000 & 280.2 & 4 & 000.0 & \\
\hline & 2201 & 8.2438 & 8.6000 & 280.4 & & 1000 & \\
\hline & 2202 & 22.2522 & 000 & 31.5 & & מ & \\
\hline & 2203 & 20.5120 & 500 & 81.3 & & 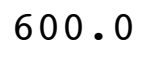 & \\
\hline & 204 & 7.4978 & & & & & \\
\hline & 2205 & 118 & 250 & 81.2 & & 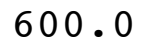 & \\
\hline & 2206 & 124.7501 & 9500 & 81.7 & & - & \\
\hline & 2207 & 134.2527 & & & & & \\
\hline & 2208 & 139.7492 & 0.2500 & 82.3 & & 0 & \\
\hline & 2209 & 140.5000 & 7750 & 82.9 & & 0 & \\
\hline & 2010 & 152 . & 0 & & & & \\
\hline & 2011 & 161 . & & 8 & & & \\
\hline & 2012 & 164 . & 7500 & 84.6 & & 0 & \\
\hline & 2013 & 159. & & & & & \\
\hline & 2014 & 160 & & & & & \\
\hline & 2015 & 159. & 0 & & & & \\
\hline & 22016 & 158.4886 & 0.12 & 281 & & U & \\
\hline & 22017 & 170 . & & & & & \\
\hline & 2018 & 132 . & & 28 & 4 & & \\
\hline & 22019 & 130 . & 0 & $2 \varepsilon$ & & & \\
\hline & 2020 & 125 & & & & & \\
\hline & 22021 & 114 . & & & 4 & & \\
\hline & 2022 & 144 . & 07 & 9 & & & \\
\hline & 2023 & 140 & & & & & \\
\hline & 22024 & 138 & & & 4 & & \\
\hline & 2211 & 135 & & & 4 & & \\
\hline & 2212 & 127 . & 0 & & 4 & & \\
\hline & 2213 & 152 . & & & 4 & & \\
\hline & 2214 & 149 . & & & 4 & & \\
\hline & 2215 & 141 . & 0 & 28 & a & & \\
\hline & 2216 & 143 . & & & & & \\
\hline & 2217 & 140 . & 0 & & 4 & & \\
\hline & 2218 & 146 . & .2000 & 284.2 & 4 & & \\
\hline & 2219 & 155 . & 0 & & 4 & & \\
\hline & 22110 & 158 & & & 3 & & \\
\hline & 22111 & 146.4991 & & 284.2 & 4 & & \\
\hline & 22112 & 151. & 0 & & 4 & & \\
\hline & 22113 & 145 . & 0 & .0 & 4 & & \\
\hline & 22114 & 142.9989 & & 286.3 & 4 & & \\
\hline & 22115 & & & & 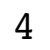 & & \\
\hline & 22116 & 150 . & & & 4 & & \\
\hline & 22117 & 142.5015 & 2.9750 & 287.5 & 4 & & \\
\hline & 22118 & 145 . & 250 & 287.7 & 4 & & \\
\hline & 22119 & 155.0002 & & & 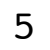 & & \\
\hline & 22120 & 97.0322 & 2.4250 & 286.6 & 4 & 0 & 00 \\
\hline & 22121 & 116 & & & J & & \\
\hline & 22122 & & 5.7750 & & & & \\
\hline & 2123 & 76.7500 & 6.1750 & 286.8 & & 600.0 & 50 \\
\hline
\end{tabular}




\begin{tabular}{|c|c|c|c|c|c|c|c|}
\hline & 2124 & 87.4988 & 5.7000 & 287.2 & 4 & 600.0 & \\
\hline & 2221 & 8.2298 & 3.2500 & 286.7 & 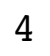 & 600.0 & \\
\hline & 2222 & 30.4501 & 0000 & 286.0 & 4 & 00.0 & \\
\hline & 2223 & 148.5000 & .9750 & 285.4 & & 00 & \\
\hline & 2224 & $\lfloor 95$ & .0750 & & & 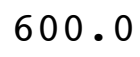 & \\
\hline & 2225 & 5516 & 1.9000 & 84.6 & & 00 & \\
\hline & 2226 & 120.7535 & 2.0750 & 284.8 & & 0 & \\
\hline & 2227 & 122.9952 & .3000 & 85.9 & & & \\
\hline & 2228 & 131.3531 & 2.7750 & 284.8 & & 0 & \\
\hline & 2229 & 131.6301 & 1.7000 & 286.9 & & 0 & \\
\hline & 22210 & 167.2595 & 4.1000 & 285 & & 0 & \\
\hline & 2211 & 167.4998 & & & 4 & & \\
\hline & 2212 & 162.2519 & 5.4250 & 285.8 & 4 & 0 & \\
\hline & 22213 & 157.7500 & 5.8250 & 286.2 & 2 & 0.0 & \\
\hline & 22214 & 162.7494 & & & 4 & & \\
\hline & 22215 & 167.4999 & 500 & 287.3 & 4 & & \\
\hline & 22216 & 158.2448 & 8000 & 287.2 & 4 & 0 & \\
\hline & 22217 & 151. & 0 & 0 & 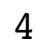 & & \\
\hline & 22218 & 144.4995 & 3 . & 28 & 4 & 0 & \\
\hline & 22219 & 132.0485 & 0500 & 286.1 & 4 & & \\
\hline & 22220 & 101.5485 & 0 & 28 & A & & \\
\hline & 22221 & 111.7517 & 50 & 28 & 5 & & \\
\hline & 22222 & 80.5052 & 250 & 286.4 & 5 & 0 & 0. \\
\hline & 22223 & 47.7266 & 500 & 28 & 5 & 0 & \\
\hline & 22224 & 29.7512 & 0 & 28 & 4 & & \\
\hline & 2231 & 58.3308 & 250 & 286.2 & 5 & & \\
\hline & 2232 & 82.7466 & 2000 & 285 & 6 & & \\
\hline & 2233 & 73.5701 & 0 & 28 & 5 & & \\
\hline & 2234 & 74.6081 & 0 & 285.8 & 6 & & • \\
\hline & 2235 & 107.7500 & 5000 & 285.6 & 5 & & \\
\hline & 2236 & 86.2048 & 0 & 285 & 6 & & \\
\hline & 2237 & 031 & 0 & 28 & 5 & & \\
\hline & 2238 & 101.5861 & 750 & 285.4 & 5 & 0 & 0 . \\
\hline & 2239 & 5.2268 & 0 & 28 & 4 & & \\
\hline & 22310 & 180 . & 0 & 28 & 3 & & \\
\hline & 22311 & 161.5109 & 750 & 287.6 & 2 & & 0 . \\
\hline & 22312 & 167.9993 & 500 & 288.2 & 2 & & \\
\hline 3 & 22313 & 198.1871 & 0 & 288.9 & 1 & & 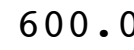 \\
\hline & 22314 & 191.0486 & 2.2000 & 288.9 & 1 & 0 & 0 . \\
\hline & 22315 & 216.4514 & 250 & 289.0 & 1 & & \\
\hline & 22316 & 239. & 750 & 289.0 & 2 & & \\
\hline 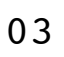 & 22317 & 319.8784 & 0.5750 & 288.8 & 2 & 600.0 & 600 . \\
\hline & 22318 & 337.0807 & 1.0250 & 287.5 & 3 & 600 & . \\
\hline & 22319 & 316.2566 & 0.8500 & 286.6 & 4 & & \\
\hline 03 & 22320 & 307.1188 & 1.0500 & 286.4 & 5 & 600.0 & 00 \\
\hline & 22321 & 357.4845 & 1.9750 & 286.3 & 5 & 600 & 50 \\
\hline & 22322 & 115.0800 & 50 & 286.5 & 6 & 600 & \\
\hline & 22323 & 86.4986 & 4.8250 & 285.5 & & 600.0 & 600 \\
\hline
\end{tabular}




\begin{tabular}{|c|c|c|c|c|c|c|c|}
\hline & 2324 & 9.3313 & 500 & 86.3 & 6 & 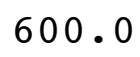 & \\
\hline & 2241 & 349.4813 & 8500 & 285.0 & 5 & 600.0 & \\
\hline & 2242 & 74.2627 & 250 & 284.9 & 6 & 00 & \\
\hline & 243 & 169.0795 & 7750 & 284.6 & & 00 & \\
\hline & 244 & $\$ 97$ & 250 & & & & \\
\hline & 2245 & 140 & & 83.6 & & 0 & \\
\hline & 2246 & 3012 & 3000 & 83.8 & & 00 & \\
\hline & 2247 & 148.4971 & 0750 & 82.7 & & & \\
\hline & 2248 & 7506 & 1000 & 82.0 & & & \\
\hline & 2249 & 038 & 5750 & 82.3 & 4 & 0 & \\
\hline & 22410 & 155 & 750 & 282.2 & & & \\
\hline & 2411 & 155 & & 7 & & & \\
\hline & 22412 & 153. & 4.6000 & 83.4 & 4 & & \\
\hline & 22413 & 4872 & 5250 & 1 & 4 & 0 & \\
\hline & 22414 & 168 & 0 & & & & \\
\hline & 224 & 174 . & & & 4 & & \\
\hline & 22416 & 162.7502 & 1750 & 4 & 4 & .0 & \\
\hline & 22417 & 188.8159 & 0 & & & & \\
\hline & 22418 & 177.9387 & 0 & & 4 & & \\
\hline & 22419 & 161.2397 & 9250 & & 5 & & 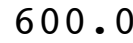 \\
\hline & 22420 & 168 & 000 & & 5 & 0 & \\
\hline & 22421 & 175 & & & 4 & & \\
\hline & 22422 & 181.2495 & 000 & 28 & 4 & & \\
\hline & 22423 & 034 & 000 & 7 & 4 & 0 & \\
\hline & 22424 & 190.4186 & & & 4 & & \\
\hline & 2251 & 5277 & 0 & $2 \varepsilon$ & 4 & & \\
\hline & 2252 & 127.7517 & 0 & 2.7 & 4 & & . \\
\hline & 2253 & 143.9947 & 0 & & 4 & & \\
\hline & 2254 & 53.1052 & 000 & & 5 & & \\
\hline & 2255 & 161.5117 & 5500 & 2.0 & 4 & & J. \\
\hline & 2256 & 137.9562 & 250 & 1.9 & 4 & & 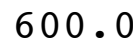 \\
\hline & 2257 & 143.2454 & 0 & & 4 & & \\
\hline & 2258 & 137.2493 & 0 & .3 & 4 & & 0 . \\
\hline & 2259 & 023 & 2500 & .2 & 4 & & \\
\hline & 225 & 7520 & 0 & & 4 & & \\
\hline & 22511 & 157.2400 & 1.3750 & & 4 & & 0 • \\
\hline & 22512 & 524 & 7500 & 282.7 & 4 & & \\
\hline & 22513 & 159. & 0 & 5 & 4 & & \\
\hline & 22514 & 153.7489 & 7.2250 & .6 & 4 & & 0 . \\
\hline & 22515 & 162 . & 7.2500 & 285.1 & 4 & & \\
\hline & 22516 & 156 . & 250 & 28 & 4 & & \\
\hline & 22517 & 158.0000 & 5.7750 & & 4 & & 0 . \\
\hline & 22518 & 137.4890 & 4.4500 & 285.0 & 4 & & 0 . \\
\hline & 22519 & 110 & 4.7000 & & 4 & & \\
\hline & 22520 & 85.2506 & & & 4 & & \\
\hline & 22521 & 0006 & 7.2500 & 282.4 & 4 & & 0 \\
\hline & & 1499 & & & 4 & & \\
\hline & 22523 & 76.2494 & 9.0250 & 281.5 & $\Psi$ & 600.0 & 60 \\
\hline
\end{tabular}




\begin{tabular}{|c|c|c|c|c|c|c|c|}
\hline & 2524 & 928 & 5000 & 280.8 & 4 & 000 & \\
\hline & 2261 & 7.2504 & 9.1750 & 280.8 & & 600.0 & \\
\hline & 2262 & 4.0000 & .6750 & 280.6 & 4 & 00 & \\
\hline & 2263 & 67.7499 & 0.3750 & 280.8 & & 00 & \\
\hline & 2264 & 1.5000 & 9.5750 & 81.2 & & 0 & \\
\hline & 2265 & 1.2500 & & 81.1 & & 0 & \\
\hline & 2266 & 5000 & 5000 & 281.2 & & 00 & \\
\hline & 2267 & 69.2500 & 500 & 81.1 & & 0 & \\
\hline & 2268 & 1.7509 & 0 & 81.3 & & & \\
\hline & 269 & 5012 & 1250 & 82.0 & & 0 & U \\
\hline & 2610 & 65.0000 & 8.2250 & 282.7 & & 00.0 & \\
\hline & 2611 & 67.2485 & 0 & 33.1 & & & \\
\hline & 2612 & 85.7468 & 4 . & 84.0 & 3 & 0 & \\
\hline & 2613 & 81.4446 & 4.8000 & 28 & & 0 & . \\
\hline & 2614 & 76 & & & & & \\
\hline & 2615 & 70 . & & 9 & 4 & & \\
\hline & 2616 & 70.0000 & 8.92 & 283.2 & 4 & 0.0 & 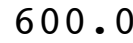 \\
\hline & 2617 & 71.5000 & 0 & .9 & $x^{2}+2$ & & \\
\hline & 2618 & 73.6514 & & .3 & 4 & & \\
\hline & 2619 & 68.94 & 6.3250 & 281.0 & 4 & .0 & . \\
\hline & 22620 & 58.0000 & 8 . & 280.8 & 4 & 0 & \\
\hline & 22621 & 46 . & & & 4 & & \\
\hline & 2622 & 33.2195 & 2 . & 28 & 5 & & \\
\hline & 2623 & 24.7839 & 1.75 & 280.8 & 5 & 0 & \\
\hline & 22624 & 40.53 & & & 6 & & \\
\hline & 2271 & 251. & 1 . & 27 & 6 & & \\
\hline & 2272 & 332.7501 & 2.52 & 278.5 & 5 & 0 & J. \\
\hline & 2273 & 337.5652 & 1.77 & 78.3 & 5 & & \\
\hline & 2274 & 875 & 50 & 27 & 5 & & \\
\hline & 2275 & 116.2293 & 1.65 & 279.3 & 5 & & 0. \\
\hline & 2276 & 127.0000 & 3.7750 & 279.8 & 5 & & 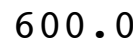 \\
\hline & 2277 & 149.4848 & 2 . & 279.5 & 4 & & \\
\hline & 2278 & 244.72 & 1 . & 279.3 & 5 & & . \\
\hline & 2279 & 146.5000 & 4.7750 & 279.6 & 4 & 0 & 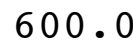 \\
\hline & 22710 & 104 . & & & 3 & & \\
\hline & 22711 & 120.6450 & 000 & 281.5 & 3 & & 0 . \\
\hline & 22712 & 129.2334 & 9500 & 281.8 & 2 & & \\
\hline & 22713 & 168.7497 & 5 . & 281.5 & 3 & & \\
\hline & 22714 & 153.7416 & 3000 & 282.4 & 3 & .0 & 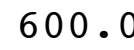 \\
\hline & 22715 & 165.7825 & 0 & 283.0 & 3 & & \\
\hline & 22716 & 134.7452 & 4 . & 283 & 4 & & 0 \\
\hline & 22717 & 149.5405 & 5.4250 & 283.8 & 4 & & 0 . \\
\hline & 22718 & 147.5000 & 4.6750 & 283.1 & 5 & 0 & 0 . \\
\hline & 22719 & 93.4624 & 250 & & 4 & & \\
\hline & 22720 & 73.7485 & 6.75 & & 4 & & \\
\hline & 22721 & 71.2503 & 7.4500 & 280.5 & 4 & & 00 . \\
\hline & 22722 & 75 & & & 4 & & \\
\hline & 22723 & 75.2500 & 7.4500 & 280.4 & 7 & 600.0 & 600 \\
\hline
\end{tabular}




\begin{tabular}{|c|c|c|c|c|c|c|c|}
\hline 3 & 22724 & 73.0000 & 7.6250 & 280.2 & 4 & 600.0 & 00.0 \\
\hline 03 & 2281 & 74.5003 & 8.2000 & 280.3 & 4 & 600.0 & 600.0 \\
\hline 03 & 2282 & 76.5000 & 8.4000 & 280.6 & 4 & 600.0 & 00.0 \\
\hline 03 & 2283 & 77.2499 & 7.4500 & 281.0 & 4 & 600.0 & 00.0 \\
\hline 03 & $228 \quad 4$ & 89.4430 & 5.9500 & 281.0 & 4 & 600.0 & 00.0 \\
\hline 03 & 2285 & 117.0041 & 4.9500 & 281.2 & 4 & 600.0 & 00.0 \\
\hline 03 & 2286 & 116.4960 & 4.5250 & 280.8 & 4 & 600.0 & 600.0 \\
\hline 03 & 2287 & 108.0034 & 4.8000 & 280.8 & 4 & 600.0 & 500.0 \\
\hline 03 & 2288 & 125.5107 & 5.8000 & 281.1 & 4 & 600.0 & 00.0 \\
\hline 03 & 2289 & 145.2674 & 7.6250 & 280.9 & 4 & 600.0 & 600.0 \\
\hline 03 & 22810 & 161.7502 & 6.8500 & 280.9 & 4 & 600.0 & 600.0 \\
\hline 03 & 22811 & 151.2472 & 4.8750 & 281.8 & 4 & 600.0 & \\
\hline 03 & 22812 & 154.0073 & 4.8250 & 283.1 & 3 & 600.0 & 600.0 \\
\hline 03 & 22813 & 157.0004 & 5.9250 & 283.9 & 4 & 600.0 & 600.0 \\
\hline 03 & 22814 & 158.5000 & 4.3750 & 284.6 & 3 & 600.0 & \\
\hline 03 & 22815 & 165.7509 & 3.4000 & 285.1 & 3 & 600.0 & 60 \\
\hline 03 & 22816 & 179.9943 & 2.7750 & 285.6 & 2 & 600.0 & 600.0 \\
\hline 03 & 22817 & 234.0373 & 1.0000 & 285.9 & 1 & 600.0 & \\
\hline 03 & 22818 & 93.5253 & 2.4500 & 285.0 & 2 & 600.0 & 60 \\
\hline 03 & 22819 & 70.4862 & 3.8000 & 284.1 & 3 & 600.0 & 600.0 \\
\hline 03 & 22820 & 75.7528 & 5.57 & 283.3 & 4 & 600.0 & .0 \\
\hline 03 & 22821 & 75.7500 & 7.2250 & 282.7 & 4 & 600.0 & \\
\hline 03 & 22822 & 73.4991 & 8.5500 & 281.9 & 4 & 600.0 & 600.0 \\
\hline 03 & 22823 & 71.0000 & 9.1750 & 281.4 & 4 & 600.0 & .0 \\
\hline 03 & 22824 & 73.5000 & 9.6750 & 281.4 & 4 & 600.0 & \\
\hline 03 & $\begin{array}{lll}3 & 1 & 1\end{array}$ & 66.5000 & 9.6000 & 281.2 & 4 & 600.0 & 600.0 \\
\hline 03 & $\begin{array}{lll}3 & 1 & 2\end{array}$ & 63.9998 & 8.5500 & 281.2 & 4 & 600.0 & 600.0 \\
\hline 03 & $\begin{array}{lll}3 & 1 & 3\end{array}$ & 63.0002 & 7.4500 & 281.0 & 4 & 600.0 & .0 \\
\hline 03 & $\begin{array}{lll}3 & 1 & 4\end{array}$ & 68.0002 & 7.1000 & 280.7 & 4 & 600.0 & 600.0 \\
\hline 03 & 315 & 70.7500 & 6.7250 & 280.4 & 4 & 600.0 & 600.0 \\
\hline 03 & 316 & 64.7500 & 6.8000 & 280.4 & 4 & 600.0 & .0 \\
\hline 03 & $\begin{array}{lll}3 & 1 & 7\end{array}$ & 69.2499 & 6.6500 & 280.4 & 4 & 600.0 & 600.0 \\
\hline 03 & $\begin{array}{lll}3 & 1 & 8\end{array}$ & 74.5000 & 5.6500 & 280.8 & 4 & 600.0 & 600.0 \\
\hline 03 & $\begin{array}{lll}3 & 1 & 9\end{array}$ & 77.9924 & 5.2250 & 281.8 & 4 & 600.0 & .0 \\
\hline 03 & 3110 & 98.8392 & 4.6000 & 282.8 & 3 & 600.0 & 60 \\
\hline 03 & 3111 & 160.2470 & 5.4500 & 282.7 & 3 & 600.0 & 600.0 \\
\hline 03 & 3112 & 160.2497 & 5.5500 & 283.1 & 3 & 600.0 & 600.0 \\
\hline 03 & 3113 & 157.7482 & 7.0500 & 283.3 & 4 & 600.0 & 600.0 \\
\hline 03 & 3114 & 155.0000 & 6.9500 & 283.7 & 4 & 600.0 & 600.0 \\
\hline 03 & 3115 & 164.2511 & 7.2750 & 284.3 & 4 & 600.0 & \\
\hline 03 & 3116 & 155.7539 & 6.3500 & 284.7 & 4 & 600.0 & 600.0 \\
\hline 03 & 3117 & 143.7770 & 4.8500 & 285.0 & 4 & 600.0 & 600.0 \\
\hline 03 & 3118 & 140.0000 & 4.0500 & 285.0 & 4 & 600.0 & 600.0 \\
\hline 03 & 3119 & 141.4994 & 3.3000 & 284.5 & 4 & 600.0 & 600.0 \\
\hline 03 & 3120 & 126.2499 & 4.5250 & 285.2 & 4 & 600.0 & 600.0 \\
\hline 03 & 3121 & 97.9977 & 5.0000 & 285.0 & 4 & 600.0 & 600.0 \\
\hline 03 & 3122 & 96.5018 & 5.6000 & 28 & 4 & 600.0 & 600.0 \\
\hline 03 & 3123 & 94.2504 & 5.8750 & 284.5 & 4 & 600.0 & 600.0 \\
\hline
\end{tabular}




\begin{tabular}{|c|c|c|c|c|c|c|c|}
\hline & 24 & - & 6.5500 & 284.7 & 4 & 年 & \\
\hline 3 & $\begin{array}{lll}3 & 2 & 1\end{array}$ & 133.4982 & 8.2000 & 285.6 & & 0 & \\
\hline & 322 & 141.9999 & 7500 & 284.9 & 4 & 00 & \\
\hline & 323 & 140.2498 & 7.5250 & 284.4 & & 00 & \\
\hline & 324 & 149.0018 & .7250 & 83.7 & & 0 & \\
\hline & 325 & 139.7725 & 7.4500 & 282.5 & 4 & 00 & \\
\hline & 326 & 144.5023 & 7.0500 & 281.6 & & 0 & \\
\hline & 3 & 050 & .1750 & 80.7 & & & \\
\hline & 32 & 103.3278 & 1.9500 & 282.5 & 3 & 0 & \\
\hline & 3 & 142.5008 & 3.4750 & 283.2 & & 00 & \\
\hline & 3210 & 138.7500 & 4.2500 & 283.8 & & 00 & \\
\hline & 211 & 135.7710 & 4.6000 & 4 & 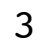 & & \\
\hline & 12 & 156.2501 & 5.6750 & 284.9 & 4 & 00 & \\
\hline & 3213 & 159.7502 & 3.9750 & 285.8 & 3 & 0.0 & \\
\hline & 214 & 160.2461 & & & $I$ & & \\
\hline & 15 & 166.2488 & 250 & 287.1 & 3 & 0 & \\
\hline & 216 & 148.3385 & 9000 & 288.0 & 3 & 0 & \\
\hline & 217 & 17.7129 & 00 & 288.6 & & & \\
\hline & 218 & 202.5682 & 250 & 287.2 & 3 & 0 & \\
\hline & 219 & 118.7270 & 3250 & 285.4 & 4 & 0 & \\
\hline & 3220 & 000 & 500 & 284.4 & 4 & & \\
\hline & 3221 & 504 & 50 & 283.5 & 4 & & \\
\hline & 22 & 76.7500 & 500 & 282.7 & 4 & 0 & 600 . \\
\hline & 3223 & 7494 & 000 & 282.7 & 4 & 0 & \\
\hline & 3224 & .7727 & & 282.4 & 4 & & \\
\hline & $\begin{array}{lll}3 & 3 & 1\end{array}$ & 62.1169 & 000 & 282.2 & 4 & & \\
\hline & $\begin{array}{lll}3 & 3 & 2\end{array}$ & . 9995 & 500 & 280.8 & 4 & 0 & \\
\hline & 333 & .4999 & 250 & 28 & 4 & & \\
\hline & 334 & 989 & 000 & 280.7 & 4 & 0 & e \\
\hline & 335 & 927 & 7.2000 & 280.8 & 4 & 0 & \\
\hline & 336 & .2508 & 500 & 280.7 & 4 & & \\
\hline & $\begin{array}{lll}3 & 3 & 7\end{array}$ & 934 & 50 & 280.1 & 4 & & 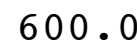 \\
\hline & $\begin{array}{lll}3 & 3\end{array}$ & 64.4495 & 500 & 280.1 & 4 & 0 & 0 . \\
\hline & 339 & 014 & 50 & 281.1 & 4 & 0 & \\
\hline & 3310 & 00 & 0 & 281.8 & 4 & & \\
\hline & 311 & 963 & 500 & 282.6 & 4 & 0 & 0 . \\
\hline & 3312 & 496 & 3250 & 283.3 & 3 & 0 & \\
\hline 3 & 3313 & .4916 & 250 & 283.1 & 4 & 0 & 60 \\
\hline & 3314 & .0152 & 1500 & 283.3 & 3 & .0 & 0 . \\
\hline & 3315 & 966 & 500 & 282.6 & 4 & 0 & \\
\hline & 3316 & 954 & 000 & & 3 & & \\
\hline J & 3317 & 70.7040 & 3.9000 & 283.4 & 2 & 600.0 & 600. \\
\hline & 3318 & 65.5012 & 3750 & 282.7 & 3 & 600 & 600. \\
\hline & 3319 & 72.5005 & 6.2750 & 281.4 & 4 & & 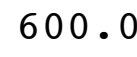 \\
\hline 03 & 3320 & 74.5011 & 7.5000 & 280.9 & 4 & 600.0 & 00 \\
\hline & 3321 & .7494 & 7.7250 & 280.6 & 4 & 600.0 & 600. \\
\hline & 3322 & 2498 & 000 & 280.3 & 4 & & \\
\hline & 3323 & 72.0004 & 6.5500 & 280.2 & 4 & 600.0 & 600 \\
\hline
\end{tabular}




\begin{tabular}{|c|c|c|c|c|c|c|c|c|}
\hline 03 & 3 & 324 & 75.5009 & 6.1250 & 280.1 & 4 & 600.0 & 600.0 \\
\hline 03 & 3 & 41 & 71.5011 & 5.2750 & 280.0 & 4 & 600.0 & 600.0 \\
\hline 03 & 3 & 42 & 75.2498 & 5.1500 & 280.1 & 4 & 600.0 & 00.0 \\
\hline 03 & 3 & 43 & 103.5142 & 3.6250 & 280.3 & 4 & 600.0 & 00.0 \\
\hline 03 & 3 & 44 & 113.2219 & 3.6500 & 280.4 & 4 & 600.0 & 00.0 \\
\hline 03 & 3 & 45 & 120.5207 & 4.1250 & 279.9 & 4 & 600.0 & 00.0 \\
\hline 03 & 3 & 46 & 113.9950 & 4.0000 & 279.8 & 5 & 600.0 & 00.0 \\
\hline 03 & 3 & 47 & 99.2503 & 3.2500 & 279.9 & 5 & 600.0 & 00.0 \\
\hline 03 & 3 & 48 & 95.7499 & 2.7750 & 280.1 & 4 & 600.0 & 500.0 \\
\hline 03 & 3 & 49 & 102.7223 & 2.8000 & 281.2 & 3 & 600.0 & 00.0 \\
\hline 03 & 3 & 410 & 157.5009 & 4.8500 & 281.8 & 3 & 600.0 & 00.0 \\
\hline 03 & 3 & 411 & 135.4223 & 3.8000 & 282.1 & 2 & 600.0 & 00.0 \\
\hline 03 & 3 & 412 & 157.4791 & 3.9000 & 282.6 & 2 & 600.0 & 00.0 \\
\hline 03 & 3 & 413 & 160.4816 & 3.3500 & 283.8 & 2 & 600.0 & 00.0 \\
\hline 03 & 3 & 414 & 160.9897 & 3.5000 & 284.3 & 2 & .0 & \\
\hline 03 & 3 & 415 & 161.6955 & 4.0750 & 284.2 & 3 & 600.0 & 00.0 \\
\hline 03 & 3 & 416 & 137.1928 & 3.5500 & 284.7 & 2 & 600.0 & 600.0 \\
\hline 03 & 3 & 417 & 90.7492 & 4.3500 & 284.8 & 3 & .0 & \\
\hline 03 & 3 & 418 & 88.2499 & 5.1750 & 284.0 & 4 & 600.0 & 00.0 \\
\hline 03 & 3 & 419 & 72.9893 & 6.5500 & 282.7 & 4 & .0 & .0 \\
\hline 03 & 3 & 420 & 72.2501 & 0 & 281.9 & 4 & 60 & \\
\hline 03 & 3 & 421 & 75.2498 & 7.5500 & 281.6 & 4 & .0 & .0 \\
\hline 03 & 3 & 422 & 74.5009 & 7.3250 & 281.7 & 4 & 600.0 & 600.0 \\
\hline 03 & 3 & 423 & 77.7500 & .1250 & 281.6 & 4 & .0 & .0 \\
\hline 03 & 3 & 424 & 74.7499 & 8.4750 & 281.0 & 4 & .0 & \\
\hline 03 & 3 & 51 & 75.0002 & 8.5500 & 280.8 & 4 & 600.0 & 600.0 \\
\hline 03 & 3 & 52 & 79.0000 & 8.0750 & 280.8 & 4 & 600.0 & .0 \\
\hline 03 & 3 & 53 & 77.0001 & 6.2000 & 282.5 & 4 & 600.0 & \\
\hline 03 & 3 & 54 & 82.9671 & 4.6500 & 283.0 & 4 & 600.0 & 600.0 \\
\hline 03 & 3 & 55 & 138.5285 & 2.8000 & 282.9 & 4 & 600.0 & 600.0 \\
\hline 03 & 3 & 56 & 151.4959 & 1.5500 & 282.5 & 5 & 600.0 & .0 \\
\hline 03 & 3 & 57 & 91.4748 & 1.3750 & 282.4 & 6 & 600.0 & 600.0 \\
\hline 03 & 3 & 58 & 47.3873 & 0.7750 & 283.8 & 5 & 600.0 & 600.0 \\
\hline 03 & 3 & 59 & 259.2356 & 0.7250 & 285.9 & 4 & 600.0 & 0.0 \\
\hline 03 & 3 & 510 & 287.2457 & 1.8250 & 284.5 & 3 & 600.0 & 600.0 \\
\hline 03 & 3 & 511 & 272.1758 & 1.5500 & 285.4 & 2 & 600.0 & 600.0 \\
\hline 03 & 3 & 512 & 270.7448 & 1.6250 & 286.2 & 1 & 600.0 & 600.0 \\
\hline 03 & 3 & 513 & 247.5875 & 2.4000 & 286.7 & 1 & 600.0 & 600.0 \\
\hline 03 & 3 & 514 & 279.7936 & 1.8500 & 288.2 & 1 & 600.0 & 600.0 \\
\hline 03 & 3 & 515 & 243.5924 & $1.5 / 50$ & 288.9 & 1 & 600.0 & 600.0 \\
\hline 03 & 3 & 516 & 260.9202 & 1.5750 & 289.4 & 1 & 600.0 & 600.0 \\
\hline 03 & 3 & 517 & 306.2495 & 1.8500 & 289.1 & 2 & 600.0 & 600.0 \\
\hline 03 & 3 & 518 & 302.9752 & 1.8000 & 288.0 & 3 & 600.0 & 600.0 \\
\hline 03 & 3 & 519 & 9.1101 & 1.5250 & 286.5 & 4 & 600.0 & 600.0 \\
\hline 03 & 3 & 520 & 76.7499 & 5.3000 & 285.8 & 4 & 600.0 & 600.0 \\
\hline 03 & 3 & 521 & 68.0031 & 4.4500 & 285.5 & 4 & 600.0 & 600.0 \\
\hline 03 & 3 & 522 & 51.6271 & 4.0500 & 284.8 & 4 & 600.0 & 600.0 \\
\hline U. & 0 & 523 & 15.4982 & 2.675 & 285.0 & 4 & 600.0 & 600.0 \\
\hline
\end{tabular}




\begin{tabular}{|c|c|c|c|c|c|c|c|c|}
\hline 03 & 3 & 524 & 12.0006 & 2.4500 & 284.9 & 4 & 600.0 & 600.0 \\
\hline 03 & 3 & 61 & 29.2110 & 1.6000 & 284.8 & 5 & 600.0 & 600.0 \\
\hline 03 & 3 & 62 & 45.9455 & 0.7250 & 284.2 & 6 & 600.0 & 00.0 \\
\hline 3 & 3 & 63 & 135.9138 & 1.3500 & 283.7 & 5 & 600.0 & 00.0 \\
\hline 03 & 3 & 64 & 137.2513 & 1.2750 & 283.0 & 4 & 600.0 & 00.0 \\
\hline 03 & 3 & 65 & 150.7502 & 1.4000 & 282.6 & 5 & 600.0 & 00.0 \\
\hline 03 & 3 & 66 & 128.6813 & 1.0250 & 282.4 & 6 & 600.0 & 00.0 \\
\hline 03 & 3 & 67 & 53.1118 & 2.2500 & 282.6 & 6 & 600.0 & 00.0 \\
\hline 03 & 3 & 68 & 126.4739 & 1.0500 & 284.0 & 5 & 600.0 & 00.0 \\
\hline 03 & 3 & 69 & 159.2220 & 0.7250 & 285.9 & 4 & 600.0 & .0 \\
\hline 03 & 3 & 610 & 243.7503 & 1.3750 & 286.9 & 3 & 600.0 & \\
\hline 03 & 3 & 611 & 253.6905 & 250 & 286.6 & 2 & 600.0 & \\
\hline 03 & 3 & 612 & 239.2374 & 1.6500 & 287.8 & 1 & 600.0 & 00.0 \\
\hline 03 & 3 & 613 & 126.3851 & 2.9000 & 288.8 & 1 & 600.0 & 00.0 \\
\hline 03 & 3 & 614 & 107.0107 & 50 & 289.1 & 2 & .0 & \\
\hline 03 & 3 & 615 & 110.2497 & 5. & 289.2 & 3 & 600.0 & 00.0 \\
\hline 03 & 3 & 616 & 100.5656 & 5.8000 & 289.4 & 3 & 600.0 & 600.0 \\
\hline 03 & 3 & 617 & 66.2498 & 6.2 & 289.3 & 4 & .0 & \\
\hline 03 & 3 & 618 & 62.7500 & 5.9250 & 288.5 & 4 & 600.0 & .0 \\
\hline 03 & 3 & 619 & 57.9968 & 6.9750 & 287.8 & 4 & 60 & .0 \\
\hline 03 & 3 & 620 & 68.2499 & 6. & 287.8 & 4 & 60 & \\
\hline 03 & 3 & 621 & 69.2500 & 7.4000 & 287.5 & 4 & .0 & \\
\hline 03 & 3 & 622 & 67.7496 & 8.0250 & 287.0 & 4 & 600.0 & .0 \\
\hline 03 & 3 & 623 & 65.7500 & 7.97 & 286.6 & 4 & 60 & \\
\hline 03 & 3 & 624 & 68.5001 & 7.2500 & 286.2 & 4 & .0 & \\
\hline 03 & 3 & 71 & 67.7500 & 8.5000 & 286.0 & 4 & 600.0 & 600.0 \\
\hline 03 & 3 & 72 & 68.2500 & 9.2000 & 285.7 & 4 & 600.0 & .0 \\
\hline 03 & 3 & 73 & 70.5000 & 10.0000 & 285.3 & 4 & .0 & \\
\hline 03 & 3 & 74 & 65.7500 & 8.7250 & 285.3 & 4 & 600.0 & 0.0 \\
\hline 03 & 3 & 75 & 68.7506 & 8.9500 & 285.1 & 4 & 60 & .0 \\
\hline 03 & 3 & 76 & 76.9966 & 8.1000 & 285.2 & 4 & .0 & \\
\hline 03 & 3 & 77 & 71.7502 & 7.7000 & 284.9 & 4 & 600.0 & 600.0 \\
\hline 03 & 3 & 78 & 79.0004 & 7.6000 & 283.6 & 4 & 600.0 & 600.0 \\
\hline 03 & 3 & 79 & 78.2503 & 7.7750 & 284.2 & 4 & 600.0 & 0.0 \\
\hline 03 & 3 & 710 & 77.7502 & 8.8750 & 283.7 & 4 & 600.0 & 600.0 \\
\hline 03 & 3 & 711 & 82.2496 & 6.9250 & 284.8 & 4 & 600.0 & 600.0 \\
\hline 03 & 3 & 712 & 91.7499 & 6.1750 & 286.0 & 4 & 600.0 & 0.0 \\
\hline 03 & 3 & 713 & 93.0000 & 6.0000 & 286.8 & 4 & 600.0 & 600.0 \\
\hline 03 & 3 & 714 & 94.5002 & 6.3250 & 287.1 & 4 & 600.0 & 600.0 \\
\hline 03 & 3 & 715 & 76.9981 & 9.2500 & 286.8 & 4 & 600.0 & 600.0 \\
\hline 03 & 3 & 716 & 71.2501 & 10.7250 & 286.5 & 4 & 600.0 & 600.0 \\
\hline 03 & 3 & 717 & 74.2500 & 10.8000 & 285.3 & 4 & 600.0 & 600.0 \\
\hline 03 & 3 & 718 & 66.7530 & 9.2500 & 283.9 & 4 & 600.0 & 600.0 \\
\hline 03 & 3 & 719 & 73.0000 & 10.4250 & 282.5 & 4 & 600.0 & 600.0 \\
\hline 03 & 3 & 720 & 71.2500 & 11.8750 & 281.8 & 4 & 600.0 & 600.0 \\
\hline 03 & 3 & 721 & 73.0000 & 10.6750 & 281.6 & . & 600.0 & 600.0 \\
\hline 03 & 3 & 722 & 75.5003 & 10.2250 & 281.3 & 4 & 600.0 & 600.0 \\
\hline 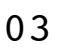 & 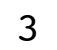 & 723 & 76.2500 & 10.750 & 281.3 & 4 & 600.0 & 600.0 \\
\hline
\end{tabular}




\begin{tabular}{|c|c|c|c|c|c|c|c|}
\hline & 24 & טלת ים & .0000 & 281.6 & 4 & ( & \\
\hline & $\begin{array}{lll}3 & 8 & 1\end{array}$ & 0.0000 & 8.8750 & 282.3 & & & \\
\hline & 382 & 5003 & 1000 & 282.9 & & ( & \\
\hline & 383 & 3.4710 & 1.3250 & 283.8 & & 00 & \\
\hline & 4 & 475 & & & & & \\
\hline & 38 & 81.3931 & 2500 & 82.6 & & s & \\
\hline & 38 & 73.2163 & 0.6250 & 282.8 & & & \\
\hline & 7 & 02.2928 & .2250 & 81.9 & & & \\
\hline & 3 & 8.8693 & 0.3750 & 84.0 & & & \\
\hline & 3 & 76.8406 & 0.4000 & 286.3 & & 0 & \\
\hline & 10 & 275.6228 & 1.2500 & 86.5 & & & \\
\hline & 3 & 233.4992 & 2.2000 & & & & \\
\hline & 12 & 227.1974 & 2.4000 & 86.1 & & 0 & \\
\hline & 3813 & 229.4232 & 1.5000 & 87.9 & & 0 & \\
\hline & 3814 & 268 & & & & & \\
\hline & 15 & 282.2784 & 1.8250 & 289.6 & & & \\
\hline & 16 & 207.2732 & 1.7000 & 290.4 & & & \\
\hline & 17 & 135 . & 0 & 0.2 & & & \\
\hline & 18 & 74 . & 2.95 & 289.2 & & & \\
\hline & 19 & 86.7503 & 9000 & 287.1 & & & \\
\hline & 3820 & 72 . & 0 & .4 & & & \\
\hline & 21 & 42 . & & & 5 & & \\
\hline & 22 & 15.9161 & 000 & 285.4 & 6 & & \\
\hline & 3823 & 19. & 1.70 & .3 & 5 & & \\
\hline & 3824 & 24 . & & & 4 & & \\
\hline & $\begin{array}{lll}3 & 9 & 1\end{array}$ & 989 & 0 & 285.4 & 4 & & \\
\hline & $\begin{array}{lll}3 & 9 & 2\end{array}$ & 70 . & 25 & 284.8 & 5 & & \\
\hline & 393 & 5 & 0 & & 6 & & \\
\hline & $\begin{array}{lll}3 & 9 & 4\end{array}$ & 41 & 1 . & .5 & 5 & & \\
\hline & 395 & 860 & 2250 & 282.4 & 0 & & \\
\hline & 396 & 821 & 0 & 282 & 6 & & \\
\hline & $\begin{array}{lll}3 & 9 & 7\end{array}$ & 99 & 0 & .9 & 5 & & \\
\hline & $\begin{array}{lll}3 & 9 & 8\end{array}$ & 62.5029 & 000 & 283.2 & 4 & 0 & \\
\hline & $\begin{array}{lll}3 & 9 & 9\end{array}$ & 49 & 0 & .8 & 3 & & \\
\hline & 10 & 2 & 0 & .4 & 2 & & \\
\hline & 11 & 212.8835 & 000 & 286.6 & 1 & & \\
\hline & 3912 & 308 & 0 & 286.6 & 1 & & \\
\hline & 3913 & 207 . & 2 . & 28 & 1 & & \\
\hline & 3914 & 225 . & 1.9000 & 289.5 & 1 & & \\
\hline & 3915 & & & 289.5 & 2 & & \\
\hline & 3916 & 111.2513 & & & 3 & & \\
\hline & 3917 & 90.2492 & 4.3000 & 288.9 & 4 & 0 & 0 . \\
\hline & 3918 & 78.4925 & & 287.7 & 4 & 0 & \\
\hline & 3919 & 72.4995 & 750 & & 4 & & \\
\hline 3 & 3920 & 68.2501 & 8.9000 & 285.2 & 4 & 60 & \\
\hline & 3921 & 74.0000 & 0.6250 & 285.2 & 4 & 600.0 & \\
\hline & 3922 & 72 & & & 4 & & \\
\hline & 923 & 79.0008 & 8.4250 & 284.5 & & 600.0 & 600 \\
\hline
\end{tabular}




\begin{tabular}{|c|c|c|c|c|c|c|c|}
\hline & 924 & 1 & 7.4250 & 284.4 & 4 & 600.0 & \\
\hline & 3101 & 5.9988 & 5.7000 & 284.4 & 4 & 600.0 & \\
\hline & 3102 & 82.2463 & 9000 & 284.3 & 4 & 00 & \\
\hline & 3103 & 87.2495 & 6.0500 & 284.5 & & 00.0 & \\
\hline & 3104 & 4.2500 & .5250 & 84.4 & & 00 & \\
\hline & 3105 & 0.7499 & 250 & 84.3 & & 00 & \\
\hline & 3106 & 102.4954 & 3500 & 284.8 & & 500 & \\
\hline & 3107 & 2512 & 50 & 85.2 & & .0 & \\
\hline & 3108 & 133.75 & 0000 & 85.6 & 4 & & \\
\hline & 3109 & 97.2475 & 4.6750 & 286.5 & & 00 & 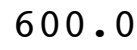 \\
\hline & 31010 & 159.2730 & 4.3500 & 287.5 & & 0 & \\
\hline & 31011 & 166.00 & 207 & 87.1 & 4 & .0 & \\
\hline & 31012 & 162.75 & 4.2500 & 288.5 & 3 & 00.0 & ( \\
\hline & 31013 & 164.75 & .9250 & 288.6 & & 0.0 & 0 \\
\hline & 31014 & 163.7 & & 0 & 3 & & \\
\hline & 31015 & 151.9 & & 290.3 & 4 & & \\
\hline & 31016 & 158.7 & 4.0750 & 290.4 & 4 & 0.0 & 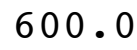 \\
\hline & 31017 & 175 . & 4 . & .1 & 4 & 0 & \\
\hline & 31018 & 150.7 & & 289.7 & 4 & & \\
\hline & 31019 & 118.2106 & 2.9250 & 288.8 & 4 & .0 & U. \\
\hline & 31020 & 89.9834 & 4.3500 & 288.6 & 4 & 0 & 0. \\
\hline & 31021 & 73.2 & & 288.4 & 4 & & \\
\hline & 31022 & 81.2 & 7.3250 & 288.1 & 4 & & \\
\hline & 31023 & 77.4 & .5000 & 287.4 & 4 & 0 & . \\
\hline & 31024 & 74.2 & 0 & 7.1 & 4 & & \\
\hline & 3111 & 78.9 & 250 & .9 & 4 & & \\
\hline & 3112 & 86.7502 & 6.2250 & 286.9 & 4 & 0 & U. \\
\hline & 3113 & 94.7 & 3.2250 & .2 & 4 & & \\
\hline & 3114 & 83.4 & 3.8750 & 287.2 & 5 & & $\bullet$ \\
\hline & 3115 & 79.7 & 750 & 286.0 & 4 & 0 & 0 • \\
\hline & 3116 & 78.4944 & 7.2000 & 284.9 & 4 & 0 & 0 . \\
\hline & 3117 & 85.2 & 750 & 285.9 & 4 & & \\
\hline & 3118 & 07.5067 & 1.2750 & 287.8 & 3 & 0 & 0 • \\
\hline & 3119 & 0.5896 & 4250 & 288.9 & 2 & 0 & . \\
\hline & 31110 & 1 & 0 & & 2 & & \\
\hline & 31111 & 167 . & 4.0000 & 288.3 & 3 & & 0 . \\
\hline & 31112 & 150.3093 & 5250 & 289.7 & 2 & $\cdot 0$ & . \\
\hline & 31113 & 142.5092 & 250 & 290.7 & 3 & & 0 . \\
\hline 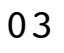 & 31114 & 94.2507 & 5.4750 & 291.2 & 3 & .0 & 0 . \\
\hline & 31115 & 97.2503 & 3500 & 291.3 & 4 & & 0 . \\
\hline & 31116 & 93.497 & 000 & 291.4 & 4 & & 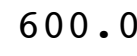 \\
\hline & 31117 & 86.0000 & 8.6000 & 290.9 & 4 & .0 & 0 . \\
\hline & 31118 & 80.2447 & 8.8000 & 289.4 & 4 & .0 & 00 . \\
\hline & 31119 & 70.5017 & 000 & 288.4 & 4 & & \\
\hline J & 31120 & 73.2527 & 9.1500 & 288.2 & 4 & & 00 . \\
\hline & 31121 & 68.5000 & 8.9500 & 288.0 & 4 & 600.0 & 500. \\
\hline & 31122 & 0. & & & 4 & & \\
\hline & 31123 & 66.2500 & 8.7250 & 287.3 & $\Psi$ & 600.0 & 600 \\
\hline
\end{tabular}




\begin{tabular}{|c|c|c|c|c|c|c|c|}
\hline 03 & 31124 & 70.5002 & 9.5000 & 286.5 & 4 & 600.0 & 600.0 \\
\hline 03 & 3121 & 74.5002 & 9.1000 & 287.0 & 4 & 600.0 & 600.0 \\
\hline 03 & 3122 & 73.5003 & 9.6000 & 286.1 & 4 & 600.0 & 00.0 \\
\hline 03 & 3123 & 73.4997 & 9.0250 & 286.1 & 4 & 600.0 & 600.0 \\
\hline 03 & 3124 & 71.7497 & 7.9000 & 286.1 & 4 & 600.0 & 00.0 \\
\hline 03 & 3125 & 65.7606 & 5.2000 & 287.5 & 4 & 600.0 & 000.0 \\
\hline 03 & 3126 & 77.7518 & 5.8750 & 287.2 & 4 & 600.0 & 600.0 \\
\hline 03 & 3127 & 85.7837 & 5.2250 & 287.7 & 4 & 600.0 & 00.0 \\
\hline 03 & 3128 & 80.4948 & 4.7500 & 287.8 & 4 & 600.0 & 00.0 \\
\hline 03 & 3129 & 349.5250 & 2.4500 & 288.4 & 3 & 600.0 & 600.0 \\
\hline 03 & 31210 & 293.5310 & 2.0000 & 289.1 & 2 & 600.0 & 600 \\
\hline 03 & 31211 & 253.1588 & 2.6750 & 289.2 & 1 & 600.0 & \\
\hline 03 & 31212 & 285.7571 & 2.0250 & 290.1 & 1 & 600.0 & 600.0 \\
\hline 03 & 31213 & 288.2537 & 2.7000 & 291.3 & 1 & 600.0 & 600.0 \\
\hline 03 & 31214 & 289.4680 & 3.1000 & 291.6 & 2 & 600.0 & \\
\hline 03 & 31215 & 291.2947 & 2.6500 & 293.1 & 1 & 600.0 & 60 \\
\hline 03 & 31216 & 313.2068 & 3.4500 & 293.2 & 2 & 600.0 & 600.0 \\
\hline 03 & 31217 & 308.5008 & 2.6000 & 292.9 & 3 & 600.0 & \\
\hline 03 & 31218 & 296.2894 & 1.4250 & 291.7 & 4 & 600.0 & 60 \\
\hline 03 & 31219 & 81.6867 & 4.5750 & 290.7 & 5 & 600.0 & 600.0 \\
\hline 03 & 31220 & 54.1188 & 2.9250 & 290.4 & 4 & 600.0 & .0 \\
\hline 03 & 31221 & 23.7491 & 1.1000 & 289.9 & 5 & 600.0 & \\
\hline 03 & 31222 & 355.2609 & 1.1250 & 289.5 & 4 & 600.0 & 600.0 \\
\hline 03 & 31223 & 359.3416 & 0.9000 & 289.2 & 5 & 600.0 & .0 \\
\hline 03 & 31224 & 357.7424 & 1.8500 & 288.5 & 4 & 600.0 & \\
\hline 03 & 3131 & 358.9904 & 4.0750 & 289.2 & 4 & 600.0 & 600.0 \\
\hline 03 & 3132 & 0.2501 & 3.6000 & 289.3 & 4 & 600.0 & 600.0 \\
\hline 03 & 3133 & 348.9977 & 2.9750 & 290.0 & 4 & 600.0 & .0 \\
\hline 03 & 3134 & 5.9913 & 1.1000 & 289.8 & 5 & 600.0 & 600.0 \\
\hline 03 & 3135 & 256.1664 & 0.7500 & 288.6 & 6 & 600.0 & 600.0 \\
\hline 03 & 3136 & 309.5671 & 1.25 & 287.9 & 6 & 600.0 & .0 \\
\hline 03 & 3137 & 251.2351 & 1.8000 & 285.8 & 6 & 600.0 & 600.0 \\
\hline 03 & 3138 & 141.4756 & 1.5500 & 284.5 & 5 & 600.0 & 600.0 \\
\hline 03 & 3139 & 294.3615 & 0.67 & 284.5 & 4 & 600.0 & .0 \\
\hline 03 & 31310 & 24.2904 & 1.1750 & 285.3 & 3 & 600.0 & 600.0 \\
\hline 03 & 31311 & 272.6554 & 2.0250 & 285.4 & 2 & 600.0 & 600.0 \\
\hline 03 & 31312 & 279.9342 & 1.8250 & 286.5 & 1 & 600.0 & 600.0 \\
\hline 03 & 31313 & 304.5177 & 1.6000 & 288.0 & 1 & 600.0 & 600.0 \\
\hline 03 & 31314 & 23.2528 & 7.5000 & 291.3 & 2 & 600.0 & 600.0 \\
\hline 03 & 31315 & 8.2509 & 7.7500 & 291.1 & 3 & 600.0 & \\
\hline 03 & 31316 & 2.9995 & 6.7250 & 290.2 & 4 & 600.0 & 600.0 \\
\hline 03 & 31317 & 0.0000 & 6.5250 & 288.4 & 4 & 600.0 & 600.0 \\
\hline 03 & 31318 & 356.2517 & 6.5000 & 287.5 & 4 & 600.0 & 600.0 \\
\hline 03 & 31319 & 338.2483 & 9.0250 & 287.8 & 4 & 600.0 & 600.0 \\
\hline 03 & 31320 & 349.8045 & 5.9750 & 288.8 & 4 & 600.0 & 600.0 \\
\hline 03 & 31321 & 331.5000 & 4.0500 & 287.7 & 4 & 600.0 & 600.0 \\
\hline 03 & 31322 & 319.5202 & 5.2000 & 286.9 & 4 & 600.0 & 600.0 \\
\hline 03 & 31323 & 255.0306 & 2.1750 & 286.4 & $\mathrm{~J}$ & 600.0 & 600.0 \\
\hline
\end{tabular}




\begin{tabular}{|c|c|c|c|c|c|c|c|}
\hline & 324 & & 0 & & & & \\
\hline & 141 & 5 & 10.2250 & 6.2 & 4 & 0 & \\
\hline & 3142 & 048 & 7.2500 & 286.1 & 4 & 00 & \\
\hline & 143 & 55.2507 & 7.5250 & 286.6 & & 00.0 & \\
\hline & 144 & 66 & 750 & & & & \\
\hline & 145 & 737 & 3000 & 86.2 & & 0( & \\
\hline & 146 & 049 & 1250 & 285.7 & & 00 & \\
\hline & 147 & 3165 & 500 & 85.7 & & 0 & \\
\hline & 148 & .75 & 8750 & 4 & 3 & & \\
\hline & 14 & 7800 & 5.2750 & 87.1 & & 00 & U \\
\hline & 1410 & 21.5105 & 6.7750 & 287.4 & & 00.0 & \\
\hline & 1411 & 1110 & 5.9000 & 88.3 & & & \\
\hline & 14 & 96 & 5.8250 & 289.3 & 3 & 0 & \\
\hline & 1413 & 98.77 & 6.2000 & 290.7 & & 0.0 & 00 \\
\hline & 14 & 29 & 0 & 6 & & & \\
\hline & & 251 & & 292.8 & 4 & & \\
\hline & 1416 & 7.5021 & 7.5500 & 291.9 & 4 & .0 & 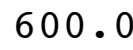 \\
\hline & 17 & 1.2500 & 250 & 5 & 4 & 0 & \\
\hline & 418 & 2.7499 & 000 & 7 & 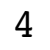 & & \\
\hline & 31419 & 350.2435 & 4.9000 & 288.3 & 4 & 00.0 & 0. \\
\hline & 31420 & 358.7495 & 5250 & 28 & 4 & 0.0 & \\
\hline & 421 & 283.16 & 0 & & 4 & & \\
\hline & 422 & 299 & 7.8500 & 28 & 4 & & ? \\
\hline & 31423 & 296.5032 & 750 & $2 \varepsilon$ & 4 & 0 & 0. \\
\hline & 31424 & 317.25 & & & 4 & & \\
\hline & 3151 & 328.5006 & 2750 & & 4 & & \\
\hline & 3152 & 322.7229 & 2250 & 0 & 4 & 0 & J. \\
\hline & 3153 & 325.0344 & 5.7500 & & 4 & & \\
\hline & 3154 & 302.5000 & 5.3500 & 28 & 4 & & 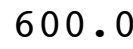 \\
\hline & 3155 & 350.0254 & 6500 & 287.1 & 4 & 0 & 0 . \\
\hline & 3156 & 57.5200 & 8.8750 & 285.4 & 4 & & 0 . \\
\hline & 3157 & 7504 & 7.1750 & 28 & 4 & & \\
\hline & 3158 & 75.9940 & 6.6750 & 282 & 4 & & . \\
\hline & 3159 & 67.2500 & 9.1500 & 28 & 4 & 0 & 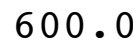 \\
\hline & 31510 & 000 & 0 & & 4 & & \\
\hline & 31511 & 64.5000 & 8.3250 & 5.5 & 4 & & 0 . \\
\hline & 31512 & 59.7511 & 7.8500 & 286.3 & 4 & & \\
\hline & 31513 & 66.1258 & 4.2750 & 28 & 3 & & \\
\hline & 31514 & 110.9975 & 5.3000 & 287.0 & 3 & & 0 . \\
\hline & 31515 & 116.5018 & 6.0250 & 287.1 & 4 & & 0 . \\
\hline & 31516 & 117.5080 & 6.3250 & 287.1 & 4 & & U. \\
\hline & 31517 & 102.7505 & 6.2250 & 286.8 & 4 & & 0 . \\
\hline & 31518 & .0199 & 7.0750 & 285.8 & 4 & 0 & 0 . \\
\hline & 31519 & 67.0002 & 7.7250 & 284 & 4 & & \\
\hline 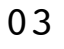 & 31520 & 70.2500 & 7.8500 & & 4 & & 0 . \\
\hline & 31521 & 67.2502 & 7.7500 & 282.6 & 4 & 0 & 00 . \\
\hline & & 31 & & & 4 & & \\
\hline & 31523 & 0037 & 7.1500 & 283.1 & 1 & 600.0 & 600 \\
\hline
\end{tabular}




\begin{tabular}{|c|c|c|c|c|c|c|c|}
\hline 03 & 31524 & 72.0000 & 9.0250 & 282.6 & 4 & 600.0 & 600.0 \\
\hline 03 & 3161 & 75.5002 & 9.1250 & 282.3 & 4 & 600.0 & 600.0 \\
\hline 03 & 3162 & 70.5193 & 8.1750 & 282.6 & 4 & 600.0 & 00.0 \\
\hline 3 & 3163 & 31.8718 & 1.1500 & 282.4 & 5 & 600.0 & 00.0 \\
\hline 03 & 3164 & 177.6830 & 0.9500 & 282.1 & 6 & 600.0 & 00.0 \\
\hline 03 & 3165 & 167.6476 & 0.9000 & 281.3 & 6 & 600.0 & 00.0 \\
\hline 03 & 3166 & 141.3384 & 1.6250 & 281.6 & 5 & 600.0 & 00.0 \\
\hline 03 & 3167 & 104.5280 & 1.9750 & 282.3 & 6 & 600.0 & 00.0 \\
\hline 03 & 3168 & 350.9827 & 0.6750 & 282.3 & 5 & 600.0 & 00.0 \\
\hline 03 & 3169 & 28.2410 & 1.9250 & 281.5 & 4 & 600.0 & .0 \\
\hline 03 & 31610 & 14.7026 & 0.7500 & 281.3 & 3 & 600.0 & \\
\hline 03 & 31611 & 5.9610 & 1.0750 & 281.4 & 2 & 600.0 & \\
\hline 03 & 31612 & 7.4034 & 1.5500 & 281.2 & 1 & 600.0 & 00.0 \\
\hline 03 & 31613 & 352.5000 & 1.4500 & 282.8 & 2 & 600.0 & 00.0 \\
\hline 03 & 31614 & 49.9083 & 3.2500 & 284.9 & 2 & .0 & \\
\hline 03 & 31615 & 83.7670 & 5.0500 & 285.4 & 3 & 600.0 & 00.0 \\
\hline 03 & 31616 & 66.2487 & 6.6750 & 285.5 & 4 & 600.0 & 600.0 \\
\hline 03 & 31617 & 133.0580 & 4.9250 & 284.2 & 3 & .0 & \\
\hline 03 & 31618 & 82.2896 & 5.1000 & 283.5 & 4 & 600.0 & .0 \\
\hline 03 & 31619 & 63.0002 & 8.5250 & 282.4 & 4 & 60 & .0 \\
\hline 03 & 31620 & 65.0005 & 7.3000 & 281.7 & 4 & 60 & \\
\hline 03 & 31621 & 64.5000 & 9.3500 & 281.5 & 4 & .0 & \\
\hline 03 & 31622 & 69.2452 & 8.7750 & 281.3 & 4 & 600.0 & 0 \\
\hline 03 & 31623 & 79.7494 & 6.5500 & 281.3 & 4 & 60 & \\
\hline 03 & 31624 & 92.0021 & 5.4250 & 281.3 & 4 & .0 & \\
\hline 03 & 3171 & 73.7346 & 7.4000 & 281.2 & 4 & 600.0 & 600.0 \\
\hline 03 & 3172 & 77.7529 & 7.3500 & 281.0 & 4 & 600.0 & .0 \\
\hline 03 & 3173 & 80.5000 & 7.4500 & 280.8 & 4 & .0 & \\
\hline 03 & 3174 & 87.0006 & 8.6250 & 280.7 & 4 & .0 & 0.0 \\
\hline 03 & 3175 & 89.0011 & 7.3250 & 280.4 & 4 & 60 & .0 \\
\hline 03 & 3176 & 97.2180 & 7.9250 & 280.2 & 4 & .0 & \\
\hline 03 & 3177 & 96.0000 & 8.3750 & 279.8 & 4 & .0 & 600.0 \\
\hline 03 & 3178 & 91.7539 & 7.1750 & 280.0 & 4 & 600.0 & 600.0 \\
\hline 03 & 3179 & 87.5000 & 6.1500 & 280.8 & 4 & 600.0 & 0.0 \\
\hline 03 & 31710 & 101.6945 & 5.4750 & 282.1 & 3 & 600.0 & 600.0 \\
\hline 03 & 31711 & 147.7507 & 7.8000 & 283.0 & 4 & 600.0 & 600.0 \\
\hline 03 & 31712 & 155.7499 & 8.9750 & 283.8 & 4 & 600.0 & 600.0 \\
\hline 03 & 31713 & 161.7466 & 9.2250 & 284.6 & 4 & 600.0 & 600.0 \\
\hline 03 & 31714 & 154.2502 & 8.8750 & 285.3 & 4 & 600.0 & 600.0 \\
\hline 03 & 31715 & 146.2476 & 10.3250 & 286.1 & 4 & 600.0 & 600.0 \\
\hline 03 & 31716 & 138.5230 & 9.9500 & 286.7 & 4 & 600.0 & 600.0 \\
\hline 03 & 31717 & 112.0027 & 9.0500 & 286.6 & 4 & 600.0 & 600.0 \\
\hline 03 & 31718 & 106.5003 & 7.5000 & 286.2 & 4 & 600.0 & 600.0 \\
\hline 03 & 31719 & 90.5000 & 7.2000 & 284.5 & 4 & 600.0 & 600.0 \\
\hline 03 & 31720 & 75.2535 & 9.0250 & 283.3 & 4 & 600.0 & 600.0 \\
\hline 03 & 31721 & 81.4997 & 9.5000 & & 4 & 600.0 & 600.0 \\
\hline 03 & 31722 & 75.2502 & 8.9750 & 283.0 & 4 & 600.0 & 600.0 \\
\hline 0 & 31723 & 95.9995 & 9.575 & 283.4 & 4 & 600.0 & 600.0 \\
\hline
\end{tabular}




\begin{tabular}{|c|c|c|c|c|c|c|c|}
\hline & 24 & 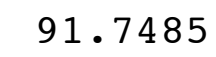 & 250 & 33.2 & 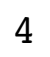 & 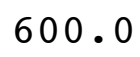 & \\
\hline & 3181 & 109.5000 & 0.0750 & 283.8 & & 00.0 & \\
\hline & 182 & 124.2500 & .1500 & 283.9 & & 00 & \\
\hline & 183 & 128.7502 & 1.2750 & 283.6 & & 00 & \\
\hline & 184 & 140.5002 & 1.5500 & & & & \\
\hline & 185 & 143.2500 & 0 & 3.0 & & 0 & \\
\hline & 186 & 149. & 1250 & 82.8 & & 00 & \\
\hline & 187 & 2500 & 0 & 82.5 & & & \\
\hline & 188 & 146 . & 0 & 82.7 & & & \\
\hline & 189 & 142 . & 0.5250 & 83.0 & & 0 & \\
\hline & 1810 & 143.0000 & 9.2250 & 283.8 & & & \\
\hline & 811 & 129 & & & & & \\
\hline & 1812 & 131.50 & & 85.5 & & & \\
\hline & 813 & 5128 & 7.87 & 7 & & 0 & \\
\hline & 814 & 150 & & & & & \\
\hline & 815 & 144 . & & .7 & 4 & & \\
\hline & 816 & 120.7602 & 8.31 & 288.1 & 4 & 0 & \\
\hline & 817 & 03 & 7 . & & & & \\
\hline & 818 & 88 . & & & & & \\
\hline & 819 & 81.2499 & & 5.7 & 4 & .0 & \\
\hline & 820 & 501 & 8 . & 3 & 4 & 0 & \\
\hline & 21 & 77 . & & & $\mathbf{4}$ & & \\
\hline & 2 & 77 & & 7 & 4 & & \\
\hline & 823 & 76.2 & 9 . & 9 & 4 & & \\
\hline & 31824 & 74 . & & & 4 & & \\
\hline & 3191 & 75 & 0 & & 4 & & \\
\hline & 3192 & 80.7456 & 0 & 8 & 4 & & 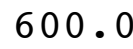 \\
\hline & 3193 & 105.7182 & & & 4 & & \\
\hline & 3194 & 101. & & & 4 & & \\
\hline & 3195 & 83.2499 & 0 & 2.9 & 4 & & . \\
\hline & 3196 & 81.2505 & 500 & 2.6 & 4 & & 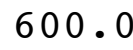 \\
\hline & 3197 & 76.7 & 0 & & 4 & & \\
\hline & 3198 & 73.5000 & & 282.8 & 4 & & 。 \\
\hline & 3199 & 002 & 750 & & 4 & & \\
\hline & 319 & 11 & & & 3 & & \\
\hline & & 105.9839 & & & 4 & & 0 • \\
\hline & 31912 & 104.0000 & 5750 & 36.8 & 3 & & \\
\hline & 31913 & 80 & 0 & & 3 & & \\
\hline & 31914 & 78.0000 & 4.4500 & .8 & 3 & & 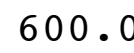 \\
\hline & 31915 & 75.7514 & 50 & 288.0 & 4 & & \\
\hline & 31916 & 82.7499 & 500 & 287 & 4 & & \\
\hline & & 94.2505 & 5.7250 & & 3 & & 0 . \\
\hline & 31918 & 87.7410 & 4.9500 & 286.4 & 4 & & 0 . \\
\hline & 31919 & 103.3044 & 750 & & 4 & & \\
\hline & & 87 & 4.2500 & & 4 & & \\
\hline & 31921 & 57.8654 & 4.3000 & 284.2 & 4 & & 0 \\
\hline & & 345 & & & 3 & & \\
\hline & 31923 & 702 & 1.4000 & 284.7 & & 600.0 & 600 \\
\hline
\end{tabular}




\begin{tabular}{|c|c|c|c|c|c|c|c|}
\hline 03 & 31924 & 62.1784 & 1.9250 & 284.0 & 6 & 600.0 & 600.0 \\
\hline 03 & 3201 & 55.7383 & 4.1500 & 283.8 & 5 & 600.0 & 600.0 \\
\hline 03 & 3202 & 72.5054 & 6.0500 & 283.6 & 4 & 600.0 & 00.0 \\
\hline 3 & 3203 & 89.4995 & 5.7250 & 282.8 & 4 & 600.0 & 00.0 \\
\hline 03 & 3204 & 104.2274 & 5.3750 & 282.2 & 4 & 600.0 & 00.0 \\
\hline 03 & 3205 & 130.7670 & 2.4750 & 282.0 & 5 & 600.0 & 00.0 \\
\hline 03 & 3206 & 109.9606 & 4.3250 & 282.2 & 4 & 600.0 & 00.0 \\
\hline 03 & 3207 & 96.0000 & 5.9750 & 282.0 & 4 & 600.0 & 00.0 \\
\hline 03 & 3208 & 103.2349 & 3.7000 & 282.2 & 3 & 600.0 & 00.0 \\
\hline 03 & 3209 & 86.7420 & 4.7750 & 283.1 & 3 & 600 & 0 \\
\hline 03 & 32010 & 90.2535 & 5.1500 & 284.4 & 3 & 600.0 & \\
\hline 03 & 32011 & 100.2575 & 5.3000 & 285.1 & 3 & 600.0 & \\
\hline 03 & 32012 & 110.6888 & 5.9250 & 285.5 & 3 & 600.0 & 00.0 \\
\hline 03 & 32013 & 117.1777 & 6.4000 & 285.9 & 4 & 600.0 & 00.0 \\
\hline 03 & 32014 & 84.5129 & 6.1250 & 286.7 & 4 & .0 & \\
\hline 03 & 32015 & 88.9986 & 6.1500 & 286.6 & 4 & 600.0 & 00.0 \\
\hline 03 & 32016 & 96.7540 & 5.8000 & 286.9 & 3 & 60 & 600.0 \\
\hline 03 & 32017 & 97.4675 & 5.3750 & 286.9 & 3 & .0 & \\
\hline 03 & 32018 & 88.7489 & 6.5750 & 285.9 & 4 & 60 & .0 \\
\hline 03 & 32019 & 78.5000 & 6.1000 & 284.3 & 4 & .0 & .0 \\
\hline 03 & 32020 & 72.7500 & 7.25 & 283.5 & 4 & 60 & \\
\hline 03 & 32021 & 73.7509 & 9.2500 & 283.5 & 4 & .0 & \\
\hline 03 & 32022 & 70.4999 & 8.8250 & 283.4 & 4 & 600.0 & .0 \\
\hline 03 & 32023 & 74.2500 & 9.10 & 283.8 & 4 & 6 & \\
\hline 03 & 32024 & 76.7500 & 9.1750 & 283.9 & 4 & .0 & \\
\hline 03 & 3211 & 72.7499 & 8.5000 & 283.7 & 4 & .0 & 600.0 \\
\hline 03 & 3212 & 76.5000 & 8.4750 & 283.9 & 4 & .0 & .0 \\
\hline 03 & 3213 & 78.5000 & 8.5750 & 283.9 & 4 & .0 & \\
\hline 03 & 3214 & 71.2500 & 10.0250 & 282.6 & 4 & .0 & .0 \\
\hline 03 & 3215 & 74.5000 & 8.6750 & 283.1 & 4 & 60 & .0 \\
\hline 03 & 3216 & 72.0007 & 8.0750 & 283.2 & 4 & .0 & \\
\hline 03 & 3217 & 80.5018 & 7.1250 & 283.8 & 4 & .0 & 0.0 \\
\hline 03 & 3218 & 84.0000 & 7.6500 & 284.2 & 4 & 600.0 & 600.0 \\
\hline 03 & 3219 & 87.0006 & 5.7000 & 285.0 & 4 & .0 & 0.0 \\
\hline 03 & 32110 & 93.4689 & 4.30 & 286.6 & 3 & 600.0 & 600.0 \\
\hline 03 & 32111 & 102.2600 & 2.8750 & 288.3 & 3 & 600.0 & 600.0 \\
\hline 03 & 32112 & 111.0584 & 3.1000 & 289.2 & 2 & 600.0 & .0 \\
\hline 03 & 32113 & 90.4686 & 2.8500 & 289.6 & 1 & 600.0 & 600.0 \\
\hline 03 & 32114 & 137.0190 & 3.9000 & 290.5 & 2 & 600.0 & 600.0 \\
\hline 03 & 32115 & 140.4950 & 4.6000 & 290.5 & 3 & 600.0 & 600.0 \\
\hline 03 & 32116 & 110.7506 & 3.9500 & 291.0 & 3 & 600.0 & 600.0 \\
\hline 03 & 32117 & 110.5000 & 4.6000 & 291.1 & 4 & 600.0 & 600.0 \\
\hline 03 & 32118 & 86.7494 & 5.5750 & 289.8 & 4 & 600.0 & 600.0 \\
\hline 03 & 32119 & 76.2511 & 7.3000 & 288.1 & 4 & 600.0 & 600.0 \\
\hline 03 & 32120 & 72.2532 & 8.9750 & 287.4 & 4 & 600.0 & 600.0 \\
\hline 03 & 32121 & 71.2511 & 7.9000 & 287.4 & 4 & 600.0 & 600.0 \\
\hline 03 & 32122 & 80.7512 & 7.0000 & 287.8 & 4 & 600.0 & 600.0 \\
\hline 0 & 32123 & 78.4999 & 6.875 & 287.5 & 4 & 600.0 & 600.0 \\
\hline
\end{tabular}




\begin{tabular}{|c|c|c|c|c|c|c|c|}
\hline 3 & 32124 & 75.0110 & 6.4500 & 286.9 & 4 & 600.0 & 00.0 \\
\hline 03 & 3221 & 78.7495 & 3.5000 & 287.3 & 4 & 600.0 & 600.0 \\
\hline 03 & 3222 & 78.7500 & 4.3000 & 286.8 & 4 & 600.0 & 00.0 \\
\hline 03 & 3223 & 73.2504 & 6.5500 & 286.5 & 4 & 600.0 & 600.0 \\
\hline 03 & 3224 & 56.3933 & 2.7750 & 286.4 & 5 & 600.0 & 00.0 \\
\hline 03 & 3225 & 62.3954 & 1.2500 & 285.2 & 6 & 600.0 & 00.0 \\
\hline 03 & 3226 & 44.5936 & 1.3000 & 285.1 & 6 & 600.0 & 600.0 \\
\hline 03 & 3227 & 143.7519 & 1.3250 & 284.6 & 3 & 600.0 & 00.0 \\
\hline 03 & 3228 & 122.3120 & 0.6000 & 286.3 & 4 & 600.0 & 00.0 \\
\hline 03 & 3229 & 258.3421 & 0.9250 & 288.4 & 3 & 600.0 & .0 \\
\hline 03 & 32210 & 290.2660 & 1.9250 & 288.1 & 2 & 600.0 & 00.0 \\
\hline 03 & 32211 & 239.7493 & 2.2000 & 288.0 & 2 & 600.0 & \\
\hline 03 & 32212 & 296.7816 & 2.0500 & 289.5 & 1 & 600.0 & 600.0 \\
\hline 03 & 32213 & 262.0000 & 2.1250 & 290.5 & 1 & 600.0 & 0 \\
\hline 03 & 32214 & 238.2779 & 2.7 & 290.7 & 1 & 600.0 & \\
\hline 03 & 32215 & 256.1272 & 2.4750 & 291.2 & 1 & 600.0 & 60 \\
\hline 03 & 32216 & 114.5395 & 2.9250 & 290.5 & 1 & 600.0 & 600.0 \\
\hline 03 & 32217 & 81.7505 & 4.9500 & 289.2 & 2 & 600.0 & \\
\hline 03 & 32218 & 76.9998 & 8.3500 & 286.6 & 3 & 600.0 & 60 \\
\hline 03 & 32219 & 77.2512 & 9.0250 & 285.6 & 4 & 600.0 & 600 \\
\hline 03 & 32220 & 76.0000 & 8 . & 284.4 & 4 & 600.0 & .0 \\
\hline 03 & 32221 & 70.2500 & 50 & 284.2 & 4 & 600.0 & \\
\hline 03 & 32222 & 67.7499 & 10.1750 & 284.5 & 4 & 600.0 & 600.0 \\
\hline 03 & 32223 & 74.5000 & 8.7 & 284.9 & 4 & 600.0 & .0 \\
\hline 03 & 32224 & 72.0000 & 0 & 285.6 & 4 & 600.0 & \\
\hline 03 & 3231 & 85.2575 & 3. & 284.5 & 4 & 600.0 & 60 \\
\hline 03 & 3232 & 94.4845 & 3.8000 & 284.3 & 4 & 600.0 & .0 \\
\hline 03 & 3233 & 78.0005 & 7.9 & 284.6 & 4 & 600.0 & \\
\hline 03 & 3234 & 90.9927 & 500 & 284.4 & 4 & 600.0 & 60 \\
\hline 03 & 3235 & 110.0112 & 3.7750 & 284.2 & 4 & 600.0 & 60 \\
\hline 03 & 3236 & 91.2446 & 3 . & 284.0 & 5 & 600.0 & \\
\hline 03 & 3237 & 91.0012 & 4 & 283.9 & 4 & 600.0 & 60 \\
\hline 03 & 3238 & 98.7496 & 3.8750 & 283.6 & 3 & 600.0 & 600.0 \\
\hline 03 & 3239 & 68.3667 & 5 . & 283.4 & 4 & 600.0 & .0 \\
\hline 03 & 32310 & 122.9970 & 2.2250 & 28 & 3 & 600.0 & 60 \\
\hline 03 & 32311 & 219.7685 & 1.3750 & 284.6 & 2 & 600.0 & 600.0 \\
\hline 03 & 32312 & 83.7093 & 1.5500 & 284.9 & 1 & 600.0 & \\
\hline 03 & 32313 & 96.0698 & 2 . & 285.5 & 1 & 600.0 & 600.0 \\
\hline 03 & 32314 & 75.2467 & 4.5750 & 285.7 & 2 & 600.0 & 600.0 \\
\hline 03 & 32315 & 63.7478 & 5.4250 & 287.0 & 3 & 600.0 & \\
\hline 03 & 32316 & 93.3321 & 4.1250 & 287.8 & 3 & 600.0 & 600.0 \\
\hline 03 & 32317 & 93.7545 & 3.4500 & 287.7 & 3 & 600.0 & 600.0 \\
\hline 03 & 32318 & 107.9989 & 3.0000 & 287.2 & 4 & 600.0 & 600.0 \\
\hline 03 & 32319 & 99.2334 & 4.9500 & 285.1 & 4 & 600.0 & 600.0 \\
\hline 03 & 32320 & 86.5000 & 6.6250 & 284.6 & 4 & 600.0 & 600.0 \\
\hline 03 & 32321 & 75.7500 & 7.0000 & 283.9 & 4 & 600.0 & 600.0 \\
\hline 03 & 32322 & 77.2500 & 6.900 & .5 & 4 & 600.0 & 600.0 \\
\hline 03 & 32323 & 79.0000 & 6.875 & 284.4 & 4 & 600.0 & 600.0 \\
\hline
\end{tabular}




\begin{tabular}{|c|c|c|c|c|c|c|c|}
\hline 3 & 32324 & 79.2498 & 7.8250 & 283.1 & 4 & 600.0 & 600.0 \\
\hline 03 & 3241 & 77.2503 & 8.6750 & 282.2 & 4 & 600.0 & 600.0 \\
\hline 03 & 3242 & 74.0000 & 8.4250 & 282.4 & 4 & 600.0 & 00.0 \\
\hline 03 & 3243 & 73.7497 & 7.5000 & 282.7 & 4 & 600.0 & 600.0 \\
\hline 03 & $324 \quad 4$ & 83.7430 & 5.2000 & 283.5 & 4 & 600.0 & 00.0 \\
\hline 03 & 3245 & 90.7500 & 3.3500 & 284.4 & 4 & 600.0 & 00.0 \\
\hline 03 & 3246 & 93.4940 & 3.4750 & 284.3 & 4 & 600.0 & 600.0 \\
\hline 03 & 3247 & 84.2549 & 3.3000 & 284.5 & 4 & 600.0 & 00.0 \\
\hline 03 & 3248 & 88.5457 & 1.0250 & 286.0 & 3 & 600.0 & 00.0 \\
\hline 03 & 3249 & 176.2561 & 1.9500 & 287.1 & 2 & 600.0 & 600.0 \\
\hline 03 & 32410 & 171.2501 & 2.9500 & 287.1 & 3 & 600.0 & 600.0 \\
\hline 03 & 32411 & 113.3595 & 5.0500 & 286.8 & 3 & 600.0 & \\
\hline 03 & 32412 & 68.6630 & 5.5000 & 286.9 & 3 & 600.0 & 600.0 \\
\hline 03 & 32413 & 140.8278 & 4.7500 & 288.1 & 3 & 600.0 & 600 \\
\hline 03 & 32414 & 132.0986 & 3.7750 & 288.9 & 2 & 600.0 & \\
\hline 03 & 32415 & 154.9966 & 4.9250 & 289.1 & 3 & 600.0 & 60 \\
\hline 03 & 32416 & 138.1373 & 5.3500 & 289.3 & 4 & 600.0 & 600.0 \\
\hline 03 & 32417 & 112.7512 & 5.3500 & 289.3 & 3 & 600.0 & \\
\hline 03 & 32418 & 94.4959 & 6.2750 & 288.3 & 4 & 600.0 & 60 \\
\hline 03 & 32419 & 78.2508 & 7.4000 & 286.4 & 4 & 600.0 & 600.0 \\
\hline 03 & 32420 & 78.0235 & 6.7250 & 285.3 & 4 & 600.0 & .0 \\
\hline 03 & 32421 & 61.7505 & 8.5250 & 285.2 & 4 & 600.0 & \\
\hline 03 & 32422 & 68.7502 & 9.2250 & 285.3 & 4 & 600.0 & 600.0 \\
\hline 03 & 32423 & 73.5000 & 7.82 & 286.0 & 4 & 600.0 & .0 \\
\hline 03 & 32424 & 78.5000 & 6.8250 & 286.6 & 4 & 600.0 & \\
\hline 03 & 3251 & 83.2496 & 7.1000 & 286.7 & 4 & 600.0 & 600.0 \\
\hline 03 & 3252 & 87.0002 & 7.2000 & 286.7 & 4 & 600.0 & 600.0 \\
\hline 03 & 3253 & 102.0919 & 6.8500 & 287.0 & 4 & 600.0 & \\
\hline 03 & 3254 & 124.2467 & 4.3500 & 287.7 & 4 & 600.0 & 600.0 \\
\hline 03 & 3255 & 130.7528 & 3.6000 & 287.6 & 4 & 600.0 & 600.0 \\
\hline 03 & 3256 & 120.4862 & 1.1250 & 286.6 & 5 & 600.0 & .0 \\
\hline 03 & 3257 & 109.7816 & 1.7000 & 287.3 & 6 & 600.0 & 60 \\
\hline 03 & 3258 & 88.7552 & 0.6500 & 288.1 & 5 & 600.0 & 600.0 \\
\hline 03 & 3259 & 39.7546 & 2.0250 & 288.4 & 4 & 600.0 & .0 \\
\hline 03 & 32510 & 187.7390 & 1.6250 & 290.0 & 3 & 600.0 & 60 \\
\hline 03 & 32511 & 261.3064 & 1.7000 & 289.8 & 2 & 600.0 & 600.0 \\
\hline 03 & 32512 & 195.9465 & 3.2250 & 290.8 & 2 & 600.0 & .0 \\
\hline 03 & 32513 & 147.1125 & 3.3500 & 291.7 & 2 & 600.0 & 600.0 \\
\hline 03 & 32514 & 93.7569 & 3.2000 & 292.6 & 2 & 600.0 & 600.0 \\
\hline 03 & 32515 & 94.5490 & 5.2250 & 292.9 & 3 & 600.0 & \\
\hline 03 & 32516 & 104.2431 & 4.3500 & 292.5 & 3 & 600.0 & 600.0 \\
\hline 03 & 32517 & 103.0103 & 4.6750 & 292.2 & 3 & 600.0 & 600.0 \\
\hline 03 & 32518 & 81.5000 & 7.4250 & 290.4 & 4 & 600.0 & 600.0 \\
\hline 03 & 32519 & 56.0000 & 6.1000 & 289.0 & 4 & 600.0 & 600.0 \\
\hline 03 & 32520 & 64.2563 & 8.1250 & 288.8 & 4 & 600.0 & 600.0 \\
\hline 03 & 32521 & 78.0110 & 7.1000 & 288.5 & 4 & 600.0 & 600.0 \\
\hline 03 & 32522 & 84.7100 & 5.4250 & 288.6 & 4 & 600.0 & 600.0 \\
\hline 03 & 32523 & 112.2294 & 3.2500 & 288.5 & 4 & 600.0 & 600.0 \\
\hline
\end{tabular}




\begin{tabular}{|c|c|c|c|c|c|c|c|}
\hline & 24 & & & & & & \\
\hline & 261 & 1 & & & & & \\
\hline & 3262 & 6.5691 & 5750 & 286.6 & & 00 & \\
\hline & 263 & 37.3222 & 9250 & 287.1 & & 0 & \\
\hline & 264 & 53 & & & & & \\
\hline & 265 & & & & & & \\
\hline & 266 & 5722 & .750 & 88.2 & & 00 & \\
\hline & 3267 & 4939 & 0 & 87.6 & & & \\
\hline & 268 & 116.1308 & 0 & 87.0 & & & \\
\hline & 269 & 9094 & 000 & 6 & & 0 & \\
\hline & 2610 & 50.7500 & 1500 & 8 & & & \\
\hline & 2611 & 58.2638 & & & & & \\
\hline & 26 & 66.9989 & 50 & 287 & & & \\
\hline & 2613 & 82.7473 & 6.6250 & $28 \varepsilon$ & & & \\
\hline & 14 & 73 & & & & & \\
\hline & 5 & 78 . & & & & & \\
\hline & 2616 & 58.2506 & 1500 & 288.3 & 4 & .0 & \\
\hline & 17 & 61.5002 & 0 & 7 & & & \\
\hline & 2618 & 62.0001 & 50 & & & & \\
\hline & 2619 & 61.2502 & 2250 & 28 & 4 & & \\
\hline & 32620 & 60.5000 & 3500 & 28 & & & \\
\hline & 3 & 63.7502 & 0 & & 4 & & \\
\hline & 326 & 67.7500 & 250 & 282 & 4 & & \\
\hline & 23 & 72.7505 & 0.20 & 282.4 & & 0 & \\
\hline & 32 & 69.5000 & & 82.2 & 4 & & \\
\hline & 3271 & 70.7497 & 0 & 28 & 4 & & \\
\hline & 3272 & 75.2498 & 0 & 281.5 & 4 & & . \\
\hline & 3273 & 79.74 & 0 & 281.3 & 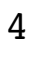 & & \\
\hline & 3274 & 100 . & 0 & 28 & 4 & & \\
\hline & 3275 & 132.2515 & 750 & 282.7 & $I$ & & 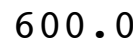 \\
\hline & 3276 & 138.7553 & 7.7000 & 282.5 & 4 & & \\
\hline & 3277 & 144.0 & 017 & 82.6 & 4 & & \\
\hline & 3278 & 146.0000 & 750 & 282.6 & 4 & & . \\
\hline & 3279 & 155.5052 & 500 & 283.0 & 4 & & \\
\hline & 32710 & 162.50 & 0 & & 4 & & \\
\hline & 32711 & 153.7498 & 0.4000 & 284.2 & 4 & & . \\
\hline & 32712 & 156.9998 & 9000 & 285.2 & 4 & & \\
\hline & 32713 & 154.2499 & 250 & 0 & 4 & & \\
\hline & 32714 & 153.7573 & 000 & 287.0 & 4 & & 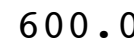 \\
\hline & 32715 & 152.4997 & 9000 & 287.9 & 4 & & \\
\hline & 32716 & 151.7501 & 750 & 288.6 & 4 & & \\
\hline & 32717 & 153.2496 & 11.1000 & 289.1 & 4 & & \\
\hline & 32718 & 153.7487 & 5250 & 288.9 & 4 & & 0 \\
\hline & 32719 & 151.2495 & 250 & 287.9 & 4 & & \\
\hline & 32720 & 152.7500 & 1.1500 & & 7 & & \\
\hline & 32721 & 148.5000 & 13.2500 & 287.4 & 4 & & 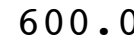 \\
\hline & & 150 . & & & & & \\
\hline & 32723 & 149.4998 & 14.1000 & 287.0 & & .0 & 0 \\
\hline
\end{tabular}




\begin{tabular}{|c|c|c|c|c|c|c|c|}
\hline & 2724 & & .6750 & 6.5 & & & \\
\hline & 3281 & 0.7500 & 0.9000 & & & & \\
\hline & 3282 & 52.2495 & 1.0500 & 285.5 & 4 & . & \\
\hline & 3283 & 53.5001 & 2.4000 & 284.9 & & 00 & \\
\hline & 284 & 0 & & & & & \\
\hline & 3285 & 0000 & 0 & 8 & & 0 & \\
\hline & 3286 & 52.7499 & 2.4500 & & & & \\
\hline & 3287 & 7500 & 1.30 & & & & \\
\hline & 3288 & 58.7499 & 1.47 & 84.5 & & & \\
\hline & 3289 & 170.2500 & 1.3750 & & & 0 & \\
\hline & 32810 & 69.5000 & 0 & 28 & & & \\
\hline & 2811 & 9.7493 & & & 4 & & \\
\hline & 2812 & 63.2763 & 0 & 287.6 & 4 & 0 & \\
\hline & 2813 & 63.0000 & 9.33 & 288.7 & 4 & 0 & \\
\hline & 32814 & 73.2526 & & & I & & \\
\hline & 32815 & 178.2511 & & 290 & 4 & & \\
\hline & 32816 & 70.7500 & 0 & 290.9 & 4 & & \\
\hline & 32817 & 982 & 0 & 1 & 4 & & \\
\hline & 32818 & 059 & & 29 & 4 & & \\
\hline & 32819 & 157.2498 & 0 & 289.5 & 4 & & \\
\hline & 32820 & 500 & 0 & 1 & 4 & & \\
\hline & 32821 & & & 28 & 4 & & \\
\hline & 32822 & 52.7446 & 4 . & 28 & 5 & 0 & U. \\
\hline & 32823 & 567 & 4 . & 28 & 4 & & \\
\hline & 32824 & $v$ & & 28 & 4 & & \\
\hline & 3291 & 869 & & 288.7 & 4 & & \\
\hline & 3292 & 0.2369 & 4.9 & 287 & 4 & & \\
\hline & 3293 & 5.7803 & & 28 & 4 & & \\
\hline & 3294 & 093 & 0 & 28 & 5 & & \\
\hline & 3295 & 5.8794 & & 286.2 & 4 & & \\
\hline & 3296 & .7626 & & 28 & 4 & & \\
\hline & 3297 & 492 & & 28 & 5 & & \\
\hline & 3298 & 52.0000 & & 286.9 & 4 & 0 & \\
\hline & 3299 & 827 & & 28 & 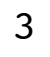 & & \\
\hline & 32910 & 266 & 0 & 29 & 2 & & \\
\hline & 32911 & 231.2105 & & 290.3 & 1 & & \\
\hline & 32912 & 840 & 2 . & 290.7 & 1 & & \\
\hline & 32913 & 23 & 0 & 292.3 & 1 & 0 & 。 \\
\hline & 32914 & 183. & & 292.3 & 2 & 0 & 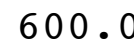 \\
\hline & 32915 & 69 & & 292.9 & 2 & & \\
\hline & 32916 & 162.8018 & 3 . & 293.3 & 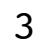 & & \\
\hline & 32917 & 156.2515 & 2.2250 & 294.1 & 3 & 600.0 & 8. \\
\hline & 32918 & 3.7499 & & 293.2 & 4 & 0 & \\
\hline & 32919 & 142.7485 & & 291.9 & 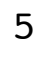 & & \\
\hline 3 & 32920 & 81.9823 & 2.5750 & 291.7 & 4 & 600 & 00 . \\
\hline & 32921 & 4998 & 5.2750 & 291.3 & 4 & 600 & \\
\hline & & 91 & & 290.7 & 4 & & \\
\hline & 32923 & 73.9992 & 6.5500 & 290.5 & 4 & 600.0 & 600 \\
\hline
\end{tabular}




\begin{tabular}{|c|c|c|c|c|c|c|c|}
\hline 03 & 32924 & 71.7500 & 7.2250 & 290.2 & 4 & 600.0 & 600.0 \\
\hline 3 & 3301 & 67.2500 & 7.6500 & 290.3 & 4 & 600.0 & 600.0 \\
\hline 03 & 3302 & 73.5023 & 6.6750 & 291.0 & 4 & 600.0 & 00.0 \\
\hline 03 & 3303 & 79.2395 & 4.1000 & 291.3 & 4 & 600.0 & 00.0 \\
\hline 03 & 3304 & 101.7736 & .2750 & 290.7 & 5 & 600.0 & 00.0 \\
\hline 03 & 3305 & 129.4052 & 0.8500 & 290.1 & 6 & 600.0 & 00.0 \\
\hline 03 & 3306 & 17.0922 & 0.9250 & 289.5 & 6 & 600.0 & 600.0 \\
\hline 03 & 3307 & 4.0430 & 0.8000 & 290.5 & 5 & 600.0 & 00.0 \\
\hline 03 & 3308 & 292.0546 & 0.7000 & 291.5 & 4 & 600.0 & .0 \\
\hline 03 & 3309 & 244.9924 & 1.3000 & 291.9 & 4 & 600.0 & 600.0 \\
\hline 03 & 33010 & 251.6952 & 1.9500 & 291.9 & 3 & 600.0 & 0 \\
\hline 03 & 33011 & 291.3036 & 2.4750 & 292.8 & 2 & 600.0 & 600.0 \\
\hline 03 & 33012 & 284.7027 & 2.2250 & 293.4 & 1 & 600.0 & 600.0 \\
\hline 03 & 33013 & 306.4586 & 1.9750 & 294.9 & 1 & 600.0 & 600.0 \\
\hline 03 & 33014 & 279.9390 & 1.9000 & 296.5 & 1 & 600.0 & .0 \\
\hline 03 & 33015 & 243.1839 & 2.4000 & 296.6 & 1 & 600.0 & .0 \\
\hline 03 & 33016 & 228.0038 & 2.5000 & 296.4 & 1 & 600.0 & 600.0 \\
\hline 03 & 33017 & 191.9984 & 2.3500 & 295.9 & 2 & 600.0 & .0 \\
\hline 03 & 33018 & 210.6508 & 1.3750 & 295.9 & 3 & 600.0 & .0 \\
\hline 03 & 33019 & 168.4322 & 0.2750 & 294.6 & 4 & 600.0 & 600.0 \\
\hline 03 & 33020 & 34.2779 & 0.6000 & 293.4 & 5 & 600.0 & 600.0 \\
\hline 03 & 33021 & 72.0000 & 4.2000 & 293.9 & 5 & 600.0 & \\
\hline 03 & 33022 & 76.0009 & 3.5750 & 293.9 & 5 & 600.0 & 60 \\
\hline 03 & 33023 & 79.2500 & 5.5250 & 293.5 & 4 & 600.0 & .0 \\
\hline 03 & 33024 & 77.0000 & 4 & 292.8 & 4 & 600.0 & \\
\hline 03 & 3311 & 78.4999 & 5.6250 & 292.1 & 4 & 600.0 & 60 \\
\hline 03 & 3312 & 77.0011 & 4.9500 & 291.9 & 5 & 600.0 & 600.0 \\
\hline 03 & 3313 & 64.0126 & 2.90 & 292.1 & 4 & 600.0 & .0 \\
\hline 03 & 3314 & 73.0002 & 2.0750 & 292.0 & 4 & 600.0 & 60 \\
\hline 03 & 3315 & 66.2532 & 2.2750 & 292.0 & 4 & 600.0 & 600.0 \\
\hline 03 & 3316 & 40.7457 & 1.3500 & 291.6 & 5 & 600.0 & 60 \\
\hline 03 & 3317 & 20.2460 & 2.0750 & 291.3 & 4 & 600.0 & 60 \\
\hline 03 & 3318 & 355.7407 & 1.5250 & 292.6 & 4 & 600.0 & 600.0 \\
\hline 03 & 3319 & 282.0139 & 0.9250 & 294.3 & 3 & 600.0 & 600.0 \\
\hline 03 & 33110 & 246.3514 & 1 . & 294.9 & 2 & 600.0 & 60 \\
\hline 03 & 33111 & 225.0000 & 2.6750 & 293.9 & 1 & 600.0 & 600.0 \\
\hline 03 & 33112 & 214.5283 & 2.5000 & 294.4 & 1 & 600.0 & 600.0 \\
\hline 03 & 33113 & 250.0770 & 2.8500 & 295.1 & 1 & 600.0 & 600.0 \\
\hline 03 & 33114 & 305.5690 & 2.4500 & 296.5 & 1 & 600.0 & 600.0 \\
\hline 03 & 33115 & 67.2551 & 4.5750 & 296.4 & 2 & 600.0 & 600.0 \\
\hline 03 & 33116 & 81.0062 & 5.4250 & 294.5 & 3 & 600.0 & 600.0 \\
\hline 03 & 33117 & 76.0000 & 5.7000 & 293.5 & 4 & 600.0 & 600.0 \\
\hline 03 & 33118 & 74.7545 & 4.0250 & 294.0 & 4 & 600.0 & 600.0 \\
\hline 03 & 33119 & 72.9941 & 5.5750 & 292.3 & 4 & 600.0 & 600.0 \\
\hline 03 & 33120 & 73.0018 & 9.2750 & 289.5 & 4 & 600.0 & 600.0 \\
\hline 03 & 33121 & 51.5055 & 8.0750 & 288.3 & 4 & 600.0 & 600.0 \\
\hline 03 & 33122 & 41.2398 & 7. & 28 & 4 & 600.0 & .0 \\
\hline 03 & 33123 & 67.4983 & 9.9250 & 285.8 & $\mathbf{4}$ & 600.0 & 600.0 \\
\hline
\end{tabular}




\begin{tabular}{|c|c|c|c|c|c|c|c|c|}
\hline 3 & & 124 & 60.0000 & 9.0250 & 285.0 & 4 & 600.0 & 600.0 \\
\hline 03 & 4 & 11 & 79.4936 & 8.3750 & 284.7 & 4 & 600.0 & 600.0 \\
\hline 03 & 4 & 12 & 71.0000 & 9.8750 & 284.3 & 4 & 600.0 & 00.0 \\
\hline 03 & 4 & 13 & 68.0011 & 11.3500 & 283.9 & 4 & 600.0 & 600.0 \\
\hline 03 & 4 & 14 & 72.7497 & 11.9750 & 283.0 & 4 & 600.0 & 00.0 \\
\hline 03 & 4 & 15 & 69.4973 & 12.6000 & 282.7 & 4 & 600.0 & 500.0 \\
\hline 03 & 4 & 16 & 62.2481 & 11.9250 & 283.2 & 4 & 600.0 & 600.0 \\
\hline 03 & 4 & 17 & 68.7637 & 9.6750 & 283.7 & 4 & 600.0 & 500.0 \\
\hline 03 & 4 & 18 & 61.7244 & 10.9750 & 282.6 & 4 & 600.0 & 00.0 \\
\hline 03 & 4 & 19 & 62.5000 & 10.6750 & 282.4 & 4 & 600.0 & 600.0 \\
\hline 03 & 4 & 110 & 65.5002 & 8.1500 & 282.6 & 4 & 600.0 & 600.0 \\
\hline 03 & 4 & 111 & 56.0147 & 7.9500 & 282.0 & 4 & 600.0 & \\
\hline 03 & 4 & 112 & 39.7425 & 7.9250 & 282.2 & 4 & 600.0 & 600.0 \\
\hline 03 & 4 & 113 & 44.2492 & 7.1500 & 282.8 & 4 & 600.0 & 600.0 \\
\hline 03 & 4 & 114 & 58.9945 & 9.2500 & 283.0 & 4 & 600.0 & \\
\hline 03 & 4 & 115 & 65.7556 & 7.5250 & 283.2 & 4 & 600.0 & 60 \\
\hline 03 & 4 & 116 & 70.0027 & 6.7000 & 283.9 & 4 & 600.0 & 600.0 \\
\hline 03 & 4 & 117 & 73.5028 & 6.9250 & 283.5 & 4 & 600.0 & \\
\hline 03 & 4 & 118 & 69.9814 & 6.1750 & 282.9 & 4 & 600.0 & 60 \\
\hline 03 & 4 & 119 & 59.2498 & 7.1000 & 281.6 & 4 & 600.0 & 60 \\
\hline 03 & 4 & 120 & 64.2621 & 5.5000 & 280.7 & 4 & 600.0 & .0 \\
\hline 03 & 4 & 121 & 68.2566 & 4.0750 & 280.1 & 4 & 600.0 & \\
\hline 03 & 4 & 122 & 72.4979 & 4.4750 & 280.0 & 4 & 600.0 & 600.0 \\
\hline 03 & 4 & 123 & 77.2811 & 5.6500 & 280.0 & 4 & 600.0 & .0 \\
\hline 03 & 4 & 124 & 58.2472 & 7.5750 & 279.9 & 4 & 600.0 & \\
\hline 03 & 4 & 21 & 57.7502 & 6.6000 & 279.8 & 4 & 600.0 & 600.0 \\
\hline 03 & 4 & 22 & 50.0278 & 6.1500 & 279.8 & 4 & 600.0 & 600.0 \\
\hline 03 & 4 & 23 & 34.5014 & 5.1750 & 279.9 & 4 & 600.0 & .0 \\
\hline 03 & 4 & 24 & 38.9369 & 4.0750 & 280.1 & 4 & 600.0 & 600.0 \\
\hline 03 & 4 & 25 & 30.9535 & 3.1250 & 279.6 & 4 & 600.0 & 600.0 \\
\hline 03 & 4 & 26 & 50.5684 & 2.60 & 279.3 & 4 & 600.0 & .0 \\
\hline 03 & 4 & 27 & 64.7445 & 4.3000 & 278.4 & 4 & 600.0 & 60 \\
\hline 03 & 4 & 28 & 69.9997 & 4.3500 & 279.0 & 4 & 600.0 & 600.0 \\
\hline 03 & 4 & 29 & 70.9973 & 3.2250 & 280.0 & 3 & 600.0 & .0 \\
\hline 03 & 4 & 210 & 64.7657 & 4.7000 & 281.4 & 3 & 600.0 & 60 \\
\hline 03 & 4 & 211 & 51.3869 & 5.6250 & 282.2 & 3 & 600.0 & 600.0 \\
\hline 03 & 4 & 212 & 53.4228 & 5.4000 & 281.0 & 3 & 600.0 & \\
\hline 03 & 4 & 213 & 31.9924 & 5.8250 & 281.8 & 3 & 600.0 & 600.0 \\
\hline 03 & 4 & 214 & 43.2910 & 5.5000 & 280.1 & 3 & 600.0 & 600.0 \\
\hline 03 & 4 & 215 & 47.5109 & 7.3000 & 282.3 & 4 & 600.0 & \\
\hline 03 & 4 & 216 & 58.2455 & 7.4250 & 282.9 & 4 & 600.0 & 600.0 \\
\hline 03 & 4 & 217 & 58.0000 & 7.2500 & 282.5 & 4 & 600.0 & 600.0 \\
\hline 03 & 4 & 218 & 101.3321 & 6.7500 & 279.1 & 4 & 600.0 & 600.0 \\
\hline 03 & 4 & 219 & 60.4977 & 6.6750 & 278.5 & 4 & 600.0 & 600.0 \\
\hline 03 & 4 & 220 & 69.2496 & 4.9000 & 279.0 & 4 & 600.0 & 600.0 \\
\hline 03 & 4 & 221 & 67.4998 & 7.0500 & 279.3 & 4 & 600.0 & 600.0 \\
\hline 03 & 4 & 222 & 73.7501 & 4.950 & 27 & 4 & 600.0 & 600.0 \\
\hline 03 & 4 & 223 & 63.4324 & 5.9750 & 278.9 & 4 & 600.0 & 600.0 \\
\hline
\end{tabular}




\begin{tabular}{|c|c|c|c|c|c|c|c|c|}
\hline 3 & 4 & 224 & 88.7025 & 4.0000 & 278.8 & 4 & 600.0 & 600.0 \\
\hline 03 & 4 & 31 & 68.2212 & 4.6500 & 278.7 & 4 & 600.0 & 600.0 \\
\hline 03 & 4 & 32 & 311.9224 & 1.6000 & 278.4 & 5 & 600.0 & 00.0 \\
\hline 03 & 4 & 33 & 309.9119 & 1.1000 & 278.2 & 6 & 600.0 & 00.0 \\
\hline 03 & 4 & 34 & 47.5942 & 1.7250 & 278.3 & 6 & 600.0 & 00.0 \\
\hline 03 & 4 & 35 & 56.7488 & 3.6000 & 278.4 & 5 & 600.0 & 00.0 \\
\hline 03 & 4 & 36 & 51.5107 & 2.6500 & 278.1 & 4 & 600.0 & 00.0 \\
\hline 03 & 4 & 37 & 17.6707 & 1.5500 & 277.7 & 5 & 600.0 & 00.0 \\
\hline 03 & 4 & 38 & 6.1837 & 1.7000 & 279.5 & 4 & 600.0 & 00.0 \\
\hline 03 & 4 & 39 & 52.0478 & 1.9500 & 281.4 & 3 & 600.0 & 00.0 \\
\hline 03 & 4 & 310 & 215.2781 & 1.4750 & 281.5 & 2 & 600.0 & 00.0 \\
\hline 03 & 4 & 311 & 213.0408 & 2.6500 & 282.5 & 1 & 600.0 & 00.0 \\
\hline 03 & 4 & 312 & 248.8331 & 2.2250 & 282.8 & 1 & 600.0 & 00.0 \\
\hline 03 & 4 & 313 & 32.2959 & 2.2750 & 283.8 & 1 & 600.0 & 00.0 \\
\hline 03 & 4 & 314 & 94.2757 & 4.6250 & 284.4 & 2 & .0 & \\
\hline 03 & 4 & 315 & 101.7468 & 5.8250 & 284.6 & 3 & 600.0 & 00.0 \\
\hline 03 & 4 & 316 & 75.4648 & 5.3750 & 285.0 & 3 & 600.0 & 600.0 \\
\hline 03 & 4 & 317 & 70.2485 & 6.5750 & 284.5 & 4 & .0 & \\
\hline 03 & 4 & 318 & 70.0000 & 6.6000 & 283.5 & 4 & 600.0 & .0 \\
\hline 03 & 4 & 319 & 72.5001 & 6.5250 & 282.2 & 4 & .0 & .0 \\
\hline 03 & 4 & 320 & 71.9998 & 7.3250 & 281.3 & 4 & 60 & \\
\hline 03 & 4 & 321 & 68.5000 & 8.0000 & 280.9 & 4 & 600.0 & \\
\hline 03 & 4 & 322 & 62.4983 & 6.8750 & 280.6 & 4 & 600.0 & 600.0 \\
\hline 03 & 4 & 323 & 42.0091 & 6.8000 & 280.9 & 4 & .0 & .0 \\
\hline 03 & 4 & 324 & 359.7497 & 3.3500 & 280.3 & 4 & .0 & \\
\hline 03 & 4 & 41 & 9.2562 & 4.0500 & 280.3 & 4 & 600.0 & 600.0 \\
\hline 03 & 4 & 42 & 336.5018 & 4.0000 & 279.6 & 4 & 600.0 & 600.0 \\
\hline 03 & 4 & 43 & 323.4085 & 2.2750 & 279.8 & 5 & 600.0 & \\
\hline 03 & 4 & 44 & 354.7433 & 2.2500 & 280.3 & 6 & 600.0 & 600.0 \\
\hline 03 & 4 & 45 & 42.7714 & 4.3500 & 280.0 & 5 & 600.0 & 600.0 \\
\hline 03 & 4 & 46 & 27.9779 & 3.9250 & 278.2 & 4 & .0 & .0 \\
\hline 03 & 4 & 47 & 55.6869 & 4.0500 & 278.7 & 4 & 600.0 & 600.0 \\
\hline 03 & 4 & 48 & 82.9651 & 4.6750 & 279.9 & 3 & 600.0 & 600.0 \\
\hline 03 & 4 & 49 & 97.1745 & 5.6750 & 279.1 & 3 & 600.0 & 0.0 \\
\hline 03 & 4 & 410 & 84.0271 & 4.7750 & 280.8 & 3 & 600.0 & 600.0 \\
\hline 03 & 4 & 411 & 93.2289 & 5.5750 & 282.0 & 3 & 600.0 & 600.0 \\
\hline 03 & 4 & 412 & 79.7754 & 5.7500 & 282.8 & 3 & 600.0 & 600.0 \\
\hline 03 & 4 & 413 & 67.0030 & 6.3750 & 283.6 & 4 & 600.0 & 600.0 \\
\hline 03 & 4 & 414 & 97.2364 & 6.9000 & 283.2 & 4 & 600.0 & 600.0 \\
\hline 03 & 4 & 415 & 87.9897 & 7.5250 & 283.5 & 4 & 600.0 & 600.0 \\
\hline 03 & 4 & 416 & 80.2658 & 7.3750 & 283.4 & 4 & 600.0 & 600.0 \\
\hline 03 & 4 & 417 & 84.0000 & 7.1500 & 282.9 & 4 & 600.0 & 600.0 \\
\hline 03 & 4 & 418 & 66.4977 & 7.4750 & 282.0 & 4 & 600.0 & 600.0 \\
\hline 03 & 4 & 419 & 65.9993 & 8.1250 & 280.8 & 4 & 600.0 & 600.0 \\
\hline 03 & 4 & 420 & 68.4998 & 7.9750 & 280.0 & 4 & 600.0 & 600.0 \\
\hline 03 & 4 & 421 & 65.2500 & 8.1250 & 279.7 & 4 & 600.0 & 600.0 \\
\hline 03 & 4 & 422 & 68.7442 & 6.3250 & 279.4 & 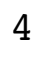 & 600.0 & 600.0 \\
\hline 03 & 4 & 423 & 76.4933 & 6.5750 & 279.2 & $\Psi$ & 600.0 & 600.0 \\
\hline
\end{tabular}




\begin{tabular}{|c|c|c|c|c|c|c|c|c|}
\hline 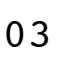 & 4 & 424 & 70.2515 & 6.2500 & 279.1 & 4 & 600.0 & 600.0 \\
\hline 03 & 4 & 51 & 71.2499 & 6.6000 & 278.9 & 4 & 600.0 & 600.0 \\
\hline 03 & 4 & 52 & 78.7502 & 7.5250 & 278.8 & 4 & 600.0 & 00.0 \\
\hline 03 & 4 & 53 & 79.7514 & 7.3250 & 278.5 & 4 & 600.0 & 00.0 \\
\hline 03 & 4 & 54 & 77.2486 & 7.3500 & 278.3 & 4 & 600.0 & 00.0 \\
\hline 03 & 4 & 55 & 84.2492 & 7.3250 & 278.5 & 4 & 600.0 & 00.0 \\
\hline 03 & 4 & 56 & 85.5002 & 7.3750 & 278.4 & 4 & 600.0 & 00.0 \\
\hline 03 & 4 & 57 & 82.2501 & 7.1250 & 278.4 & 4 & 600.0 & 00.0 \\
\hline 03 & 4 & 58 & 83.5000 & 5.8000 & 279.4 & 4 & 600.0 & 00.0 \\
\hline 03 & 4 & 59 & 87.4948 & 4.3000 & 280.6 & 3 & 600.0 & 00.0 \\
\hline 03 & 4 & 510 & 102.2473 & 3.4000 & 282.2 & 2 & 600.0 & 00.0 \\
\hline 03 & 4 & 511 & 149.4915 & 4.8000 & 282.8 & 3 & 600.0 & \\
\hline 03 & 4 & 512 & 116.5000 & 4.4500 & 283.6 & 3 & 600.0 & 00.0 \\
\hline 03 & 4 & 513 & 102.9977 & 5.1250 & 284.2 & 3 & 600.0 & 00.0 \\
\hline 03 & 4 & 514 & 112.5476 & 6.2250 & 284.5 & 4 & .0 & \\
\hline 03 & 4 & 515 & 90.5083 & 6.0000 & 285.0 & 4 & 600.0 & 00.0 \\
\hline 03 & 4 & 516 & 72.7461 & 7.1250 & 284.8 & 4 & 600.0 & 600.0 \\
\hline 03 & 4 & 517 & 62.5002 & 7.8250 & 284.2 & 4 & .0 & \\
\hline 03 & 4 & 518 & 63.9995 & 9.1500 & 283.1 & 4 & 600.0 & .0 \\
\hline 03 & 4 & 519 & 64.0000 & 9.3750 & 281.7 & 4 & 60 & .0 \\
\hline 03 & 4 & 520 & 61.2509 & 8. & 280.9 & 4 & 60 & \\
\hline 03 & 4 & 521 & 66.7500 & 10.0500 & 280.4 & 4 & .0 & \\
\hline 03 & 4 & 522 & 66.7500 & 8.2000 & 280.4 & 4 & 600.0 & 500.0 \\
\hline 03 & 4 & 523 & 65.5000 & 8.6500 & 280.5 & 4 & 60 & .0 \\
\hline 03 & 4 & 524 & 67.5000 & 250 & 280.5 & 4 & .0 & \\
\hline 03 & 4 & 61 & 64.7465 & 8.9250 & 280.4 & 4 & 600.0 & 600.0 \\
\hline 03 & 4 & 62 & 71.4945 & 9.3500 & 280.2 & 4 & 600.0 & .0 \\
\hline 03 & 4 & 63 & 75.7521 & 8.2750 & 280.0 & 4 & 0 & \\
\hline 03 & 4 & 64 & 78.0000 & 9.2000 & 280.2 & 4 & 600.0 & 600.0 \\
\hline 03 & 4 & 65 & 75.0000 & 8.9000 & 280.0 & 4 & 600.0 & 600.0 \\
\hline 03 & 4 & 66 & 70.5000 & 8.4000 & 280.1 & 4 & .0 & \\
\hline 03 & 4 & 67 & 75.2491 & 6.6750 & 280.6 & 4 & .0 & 600.0 \\
\hline 03 & 4 & 68 & 72.2512 & 6.8750 & 281.4 & 4 & 600.0 & 600.0 \\
\hline 03 & 4 & 69 & 74.7502 & 7.6000 & 281.8 & 4 & 600.0 & 0.0 \\
\hline 03 & 4 & 610 & 64.2551 & 7.95 & 283.1 & 4 & 600.0 & 600.0 \\
\hline 03 & 4 & 611 & 57.0000 & 8.1750 & 283.4 & 4 & 600.0 & 600.0 \\
\hline 03 & 4 & 612 & 62.9690 & 6.7750 & 283.5 & 4 & 600.0 & 600.0 \\
\hline 03 & 4 & 613 & 57.2494 & 6.7250 & 284.6 & 4 & 600.0 & 600.0 \\
\hline 03 & 4 & 614 & 83.5018 & 6.5000 & 285.9 & 4 & 600.0 & 600.0 \\
\hline 03 & 4 & 615 & 82.4750 & 6.2750 & 286.3 & 4 & 600.0 & 600.0 \\
\hline 03 & 4 & 616 & 92.5055 & 7.0000 & 286.2 & 4 & 600.0 & 600.0 \\
\hline 03 & 4 & 617 & 83.5152 & 7.0000 & 285.5 & 4 & 600.0 & 600.0 \\
\hline 03 & 4 & 618 & 66.7501 & 6.7250 & 284.9 & 4 & 600.0 & 600.0 \\
\hline 03 & 4 & 619 & 68.2496 & 7.2750 & 283.9 & 4 & 600.0 & 600.0 \\
\hline 03 & 4 & 620 & 71.7497 & 8.5250 & 282.8 & 4 & 600.0 & 600.0 \\
\hline 03 & 4 & 621 & 72.0002 & 8.3500 & 282.5 & 4 & 600.0 & 600.0 \\
\hline 03 & 4 & 622 & 74.0000 & 9.2000 & 282.2 & 4 & 600.0 & 600.0 \\
\hline 03 & 4 & 623 & 79.9998 & 10.175 & 282.1 & 4 & 600.0 & 600.0 \\
\hline
\end{tabular}




\begin{tabular}{|c|c|c|c|c|c|c|c|c|}
\hline 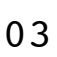 & 4 & 624 & 74.5000 & 9.0000 & 281.9 & 4 & 600.0 & 600.0 \\
\hline 03 & 4 & 71 & 80.0000 & 8.5000 & 281.9 & 4 & 600.0 & 600.0 \\
\hline 03 & 4 & 72 & 83.5000 & 7.7750 & 282.1 & 4 & 600.0 & 00.0 \\
\hline 03 & 4 & 73 & 80.7500 & 7.6000 & 282.1 & 4 & 600.0 & 00.0 \\
\hline 03 & 4 & 74 & 77.2500 & 7.7750 & 281.9 & 4 & 600.0 & 00.0 \\
\hline 03 & 4 & 75 & 81.9986 & 7.0500 & 281.8 & 4 & 600.0 & 00.0 \\
\hline 03 & 4 & 76 & 86.2495 & 6.7750 & 281.8 & 4 & 600.0 & 00.0 \\
\hline 03 & 4 & 77 & 86.2500 & 6.0500 & 282.1 & 4 & 600.0 & 00.0 \\
\hline 03 & 4 & 78 & 130.9410 & 4.3500 & 283.7 & 3 & 600.0 & 00.0 \\
\hline 03 & 4 & 79 & 162.9976 & 5.1000 & 284.1 & 3 & 600.0 & 00.0 \\
\hline 03 & 4 & 710 & 174.7483 & 3.3750 & 285.0 & 3 & 600.0 & 00.0 \\
\hline 03 & 4 & 711 & 170.7527 & 3.3750 & 286.2 & 2 & 600.0 & 00.0 \\
\hline 03 & 4 & 712 & 196.9192 & 3.1250 & 287.5 & 2 & 600.0 & 00.0 \\
\hline 03 & 4 & 713 & 206.5987 & 3.2250 & 288.4 & 2 & 600.0 & 00.0 \\
\hline 03 & 4 & 714 & 204.2413 & 2.9500 & 289.4 & 1 & .0 & \\
\hline 03 & 4 & 715 & 188.7497 & 3.9000 & 289.7 & 2 & 600.0 & 00.0 \\
\hline 03 & 4 & 716 & 198.2317 & 3.5000 & 290.2 & 2 & 600.0 & 600.0 \\
\hline 03 & 4 & 717 & 193.5093 & 3.5000 & 290.3 & 2 & .0 & \\
\hline 03 & 4 & 718 & 175.2576 & 3.1750 & 290.0 & 3 & 600.0 & .0 \\
\hline 03 & 4 & 719 & 158.7371 & 2.2000 & 289.1 & 4 & 60 & .0 \\
\hline 03 & 4 & 720 & 160.0034 & 1.40 & 288.6 & 5 & 60 & \\
\hline 03 & 4 & 721 & 119.1584 & 0.4250 & 288.0 & 6 & .0 & \\
\hline 03 & 4 & 722 & 329.3005 & 0.2250 & 287.1 & 5 & 600.0 & 00.0 \\
\hline 03 & 4 & 723 & 245.6691 & 0.5250 & 287.0 & 6 & .0 & .0 \\
\hline 03 & 4 & 724 & 21.5005 & 1.7250 & 286.9 & 5 & .0 & \\
\hline 03 & 4 & 81 & 1.2504 & 1.7750 & 286.8 & 4 & 600.0 & 600.0 \\
\hline 03 & 4 & 82 & 352.7501 & 1.2000 & 286.7 & 5 & 600.0 & 600.0 \\
\hline 03 & 4 & 83 & 297.2877 & 0.7750 & 286.2 & 6 & 600.0 & \\
\hline 03 & 4 & 84 & 321.5000 & 1.5000 & 286.1 & 5 & 600.0 & 600.0 \\
\hline 03 & 4 & 85 & 322.2472 & 1.5750 & 285.4 & 4 & 600.0 & 600.0 \\
\hline 03 & 4 & 86 & 296.8186 & 1.2500 & 285.0 & 5 & 600.0 & .0 \\
\hline 03 & 4 & 87 & 294.3658 & 1.4500 & 285.3 & 6 & 600.0 & 600.0 \\
\hline 03 & 4 & 88 & 290.8588 & 1.7500 & 286.8 & 5 & 600.0 & 600.0 \\
\hline 03 & 4 & 89 & 278.5020 & 1.9250 & 287.7 & 4 & 600.0 & 0.0 \\
\hline 03 & 4 & 810 & 266.2446 & 1.8250 & 289.0 & 3 & 600.0 & 600.0 \\
\hline 03 & 4 & 811 & 254.4920 & 2.0000 & 290.4 & 2 & 600.0 & 600.0 \\
\hline 03 & 4 & 812 & 274.5000 & 2.1250 & 291.6 & 1 & 600.0 & 600.0 \\
\hline 03 & 4 & 813 & 268.9728 & 2.1500 & 293.0 & 1 & 600.0 & 600.0 \\
\hline 03 & 4 & 814 & 208.0389 & 3.2250 & 293.7 & 2 & 600.0 & 600.0 \\
\hline 03 & 4 & 815 & 186.2246 & 3.4500 & 293.8 & 2 & 600.0 & 600.0 \\
\hline 03 & 4 & 816 & 174.7518 & 3.2500 & 294.5 & 2 & 600.0 & 600.0 \\
\hline 03 & 4 & 817 & 184.0081 & 1.9500 & 294.9 & 1 & 600.0 & 600.0 \\
\hline 03 & 4 & 818 & 140.2489 & 1.5500 & 294.9 & 2 & 600.0 & 600.0 \\
\hline 03 & 4 & 819 & 94.4359 & 4.5250 & 293.0 & 3 & 600.0 & 600.0 \\
\hline 03 & 4 & 820 & 72.0000 & 5.8500 & 291.6 & 4 & 600.0 & 600.0 \\
\hline 03 & 4 & 821 & 76.0000 & 7.0500 & 290.6 & 4 & 600.0 & 600.0 \\
\hline 03 & 4 & 822 & 74.5000 & 7.9750 & 289.6 & 4 & 600.0 & 600.0 \\
\hline 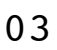 & 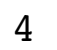 & 823 & 74.7499 & 8.650 & 288.9 & 4 & 600.0 & 600.0 \\
\hline
\end{tabular}




\begin{tabular}{|c|c|c|c|c|c|c|c|}
\hline & $4 \quad 824$ & 75.5000 & 7.5000 & 289.1 & 4 & 600.0 & 600.0 \\
\hline 3 & $\begin{array}{lll}4 & 9 & 1\end{array}$ & 76.0000 & 6.8250 & 289.2 & 4 & 600.0 & 600.0 \\
\hline 03 & $\begin{array}{lll}4 & 9 & 2\end{array}$ & 73.0000 & 4.1250 & 289.6 & 4 & 600.0 & 00.0 \\
\hline 03 & $\begin{array}{lll}4 & 9 & 3\end{array}$ & 70.2498 & 4.3250 & 289.2 & 4 & 600.0 & 00.0 \\
\hline 03 & $\begin{array}{lll}4 & 9 & 4\end{array}$ & 67.2523 & 2.5750 & 289.3 & 4 & 600.0 & 00.0 \\
\hline 03 & 495 & 72.2484 & 2.3000 & 289.3 & 5 & 600.0 & 00.0 \\
\hline 03 & 496 & 77.7501 & 2.6750 & 289.2 & 4 & 600.0 & 00.0 \\
\hline 03 & $\begin{array}{lll}4 & 9 & 7\end{array}$ & 75.2526 & 3.3500 & 289.2 & 4 & 600.0 & 00.0 \\
\hline 03 & $\begin{array}{lll}4 & 9 & 8\end{array}$ & 90.7275 & 2.4000 & 290.5 & 3 & 600.0 & 00.0 \\
\hline 03 & $\begin{array}{lll}4 & 9 & 9\end{array}$ & 225.9651 & 1.2250 & 293.2 & 2 & 600.0 & .0 \\
\hline 03 & 4910 & 254.5461 & 2.5000 & 292.5 & 1 & 600.0 & \\
\hline 03 & 4911 & 218.6988 & 3.3500 & 292.0 & 2 & 600.0 & \\
\hline 03 & 4912 & 195.2112 & 3.5500 & 292.7 & 2 & 600.0 & 00.0 \\
\hline 03 & 4913 & 170.9755 & 3.9000 & 293.4 & 2 & 600.0 & 00.0 \\
\hline 03 & $4 \quad 914$ & 165.7487 & 00 & 293.5 & 3 & .0 & \\
\hline 03 & 4915 & 169.7579 & 4.7750 & 293.5 & 3 & 600.0 & .0 \\
\hline 03 & 4916 & 170.4976 & 5.1750 & 293.7 & 3 & 60 & .0 \\
\hline 03 & 4917 & 138.0908 & 4. & 293.5 & 3 & .0 & \\
\hline 03 & 4918 & 95.0055 & 4.5750 & 292.2 & 4 & 60 & .0 \\
\hline 03 & 4919 & 76.0126 & 5.1500 & 290.9 & 4 & 60 & .0 \\
\hline 03 & 4920 & 71.5001 & 9 . & 290.2 & 4 & 60 & \\
\hline 03 & 4921 & 73.7499 & 8.2500 & 289.6 & 4 & .0 & \\
\hline 03 & 4922 & 75.2496 & 8.0750 & 289.2 & 4 & 600.0 & .0 \\
\hline 03 & 4923 & 71.4979 & 7.52 & 289.4 & 4 & 60 & \\
\hline 03 & $4 \quad 924$ & 74.7499 & 7 . & 289.4 & 4 & .0 & \\
\hline 03 & 4101 & 76.5000 & 8.9750 & 289.0 & 4 & .0 & 600.0 \\
\hline 03 & 4102 & 74.9998 & 9.6000 & 288.0 & 4 & 600.0 & .0 \\
\hline 03 & 4103 & 72.4999 & 9.8000 & 286.9 & 4 & .0 & \\
\hline 03 & 4104 & 70.7499 & 10.3500 & 287.0 & 4 & .0 & 0.0 \\
\hline 03 & 4105 & 68.7497 & 11.8750 & 286.4 & 4 & 60 & .0 \\
\hline 03 & 4106 & 66.7496 & 11.65 & 286.5 & 4 & .0 & \\
\hline 03 & 4107 & 44.2611 & 8.22 & 287.4 & 4 & .0 & 600.0 \\
\hline 03 & 4108 & 51.5284 & 4.2500 & 289.9 & 3 & 600.0 & 600.0 \\
\hline 03 & 4109 & 355.8334 & 3.3 & 290.3 & 2 & 600.0 & 0.0 \\
\hline 03 & 41010 & 270.0496 & 2.92 & 289.6 & 1 & 600.0 & 600.0 \\
\hline 03 & 41011 & 287.9989 & 2.5500 & 291.1 & 1 & 600.0 & 600.0 \\
\hline 03 & 41012 & 310.5131 & 2.2500 & 292.6 & 1 & 600.0 & 0.0 \\
\hline 03 & 41013 & 21.7030 & 3.6750 & 292.0 & 2 & 600.0 & 600.0 \\
\hline 03 & 41014 & 74.7508 & 8.8500 & 288.2 & 3 & 600.0 & 600.0 \\
\hline 03 & 41015 & 71.2500 & 10.1500 & 286.5 & 4 & 600.0 & 600.0 \\
\hline 03 & 41016 & 72.0000 & 9.8000 & 286.4 & 4 & 600.0 & 600.0 \\
\hline 03 & 41017 & 73.9994 & 9.8250 & 286.3 & 4 & 600.0 & 600.0 \\
\hline 03 & 41018 & 72.7571 & 7.1750 & 287.2 & 4 & 600.0 & 600.0 \\
\hline 03 & 41019 & 83.1996 & 6.9750 & 286.1 & 4 & 600.0 & 600.0 \\
\hline 03 & 41020 & 85.7356 & 5.2000 & 285.2 & 4 & 600.0 & 600.0 \\
\hline 03 & 41021 & 131.3234 & 3.9750 & 284.0 & . & 600.0 & 600.0 \\
\hline 03 & 41022 & 84.9973 & 7.0500 & 283.4 & 4 & 600.0 & 600.0 \\
\hline 0 & 41023 & 76.2515 & 8.675 & 283.1 & 4 & 600.0 & 600.0 \\
\hline
\end{tabular}




\begin{tabular}{|c|c|c|c|c|c|c|c|}
\hline 3 & 41024 & 74.2496 & 6.1750 & 283.0 & 4 & 600.0 & 00.0 \\
\hline 03 & 4111 & 74.5000 & 5.2750 & 282.8 & 4 & 600.0 & 600.0 \\
\hline 03 & 4112 & 68.7520 & 8.4250 & 283.0 & 4 & 600.0 & 00.0 \\
\hline 03 & 4113 & 54.1974 & 4.3500 & 283.1 & 4 & 600.0 & 600.0 \\
\hline 03 & 4114 & 26.6726 & 1.8750 & 281.8 & 5 & 600.0 & 00.0 \\
\hline 03 & 4115 & 77.9834 & 2.6500 & 281.5 & 6 & 600.0 & 00.0 \\
\hline 03 & 4116 & 87.0798 & 3.6500 & 281.3 & 5 & 600.0 & 600.0 \\
\hline 03 & 4117 & 91.4377 & 4.9000 & $281 \cdot 6$ & 4 & 600.0 & 00.0 \\
\hline 03 & 4118 & 70.7500 & 5.8250 & 282.3 & 4 & 600.0 & 00.0 \\
\hline 03 & 4119 & 76.2512 & 6.7250 & 283.1 & 4 & 600.0 & .0 \\
\hline 03 & 41110 & 66.3131 & 2.7250 & 286.3 & 3 & 600.0 & 00.0 \\
\hline 03 & 41111 & 336.5957 & 2.5750 & 287.7 & 2 & 600.0 & \\
\hline 03 & 41112 & 74.3699 & 3.6500 & 287.1 & 2 & 600.0 & 600.0 \\
\hline 03 & 41113 & 337.1349 & 2.7000 & 288.2 & 1 & 600.0 & 600 \\
\hline 03 & 41114 & 15.2363 & 2.6500 & 288.9 & 1 & 600.0 & \\
\hline 03 & 41115 & 3.2427 & 1.6750 & 289.5 & 1 & 600.0 & 60 \\
\hline 03 & 41116 & 51.6575 & 2.0750 & 289.2 & 1 & 600.0 & 600.0 \\
\hline 03 & 41117 & 358.7810 & 1.5750 & 289.4 & 1 & 600.0 & \\
\hline 03 & 41118 & 44.5586 & 1.2250 & 288.7 & 1 & 600.0 & 60 \\
\hline 03 & 41119 & 96.0000 & 4.0500 & 287.6 & 2 & 600.0 & 600 \\
\hline 03 & 41120 & 77.4999 & 5.45 & 286.2 & 3 & 600.0 & .0 \\
\hline 03 & 41121 & 76.2503 & 6. & 285.0 & 4 & 600.0 & \\
\hline 03 & 41122 & 77.4960 & 5.1500 & 284.3 & 4 & 600.0 & 600.0 \\
\hline 03 & 41123 & 30.7578 & 1.8000 & 284.4 & 5 & 600.0 & .0 \\
\hline 03 & 41124 & 328.9181 & 2.1500 & 285.3 & 6 & 600.0 & \\
\hline 03 & 4121 & 14.0986 & 5.1750 & 286.0 & 5 & 600.0 & 60 \\
\hline 03 & 4122 & 44.0362 & 3.6500 & 285.7 & 4 & 600.0 & .0 \\
\hline 03 & 4123 & 115.9501 & 4.2000 & 284.9 & 4 & 600.0 & \\
\hline 03 & 4124 & 130.2080 & 4.0750 & 284.8 & 4 & 600.0 & 60 \\
\hline 03 & 4125 & 51.0594 & 2.4000 & 284.5 & 5 & 600.0 & 600.0 \\
\hline 03 & 4126 & 334.8427 & 3.57 & 284.0 & 5 & 600.0 & \\
\hline 03 & 4127 & 337.7502 & 6.1000 & 284.3 & 4 & 600.0 & 60 \\
\hline 03 & 4128 & 349.2482 & 7.9000 & 284.5 & 4 & 600.0 & 600.0 \\
\hline 03 & 4129 & 329.2604 & 5.300 & 285.0 & 3 & 600.0 & .0 \\
\hline 03 & 41210 & 332.2550 & 7.5750 & 286.3 & 4 & 600.0 & 60 \\
\hline 03 & 41211 & 346.7538 & 7.4000 & 287.3 & 4 & 600.0 & 600.0 \\
\hline 03 & 41212 & 344.7520 & 9.4250 & 287.8 & 4 & 600.0 & \\
\hline 03 & 41213 & 357.5003 & 10.0750 & 286.3 & 4 & 600.0 & 600.0 \\
\hline 03 & 41214 & 358.2515 & 5.8000 & 284.9 & 3 & 600.0 & 600.0 \\
\hline 03 & 41215 & 18.9098 & 5.1750 & 284.7 & 3 & 600.0 & \\
\hline 03 & 41216 & 51.7738 & 4.1000 & 283.6 & 3 & 600.0 & 600.0 \\
\hline 03 & 41217 & 38.5025 & 4.7250 & 283.7 & 3 & 600.0 & 600.0 \\
\hline 03 & 41218 & 10.2501 & 8.7250 & 284.1 & 4 & 600.0 & 600.0 \\
\hline 03 & 41219 & 22.0023 & 12.9750 & 283.8 & 4 & 600.0 & 600.0 \\
\hline 03 & 41220 & 22.9993 & 9.4000 & 283.9 & 4 & 600.0 & 600.0 \\
\hline 03 & 41221 & 353.5456 & 3.8750 & 283.9 & 4 & 600.0 & 600.0 \\
\hline 03 & 41222 & 327.5505 & 2.7000 & 28 & 5 & 600.0 & 600.0 \\
\hline 03 & 41223 & 141.9419 & 1.7750 & 283.1 & 0 & 600.0 & 600.0 \\
\hline
\end{tabular}




\begin{tabular}{|c|c|c|c|c|c|c|c|}
\hline 3 & 1224 & 82 & 6000 & & & & \\
\hline & 4131 & 5 & 3.1500 & 2.1 & & 600.0 & \\
\hline & 4132 & 248.8817 & 1.8000 & 282.0 & 6 & 00 & \\
\hline & 133 & 294.7125 & 1.0500 & 281.9 & & 00.0 & \\
\hline & 134 & 287.1161 & .7000 & 81.9 & & 0 & \\
\hline & 135 & 209.800 & .4750 & 281.9 & & 00 & \\
\hline & 136 & 138.6742 & 9750 & 281.3 & & 00 & \\
\hline & 137 & 54.6551 & 3.9250 & 81.5 & & .0 & \\
\hline & 138 & 321.0166 & 2.4250 & 81.6 & & & \\
\hline & 139 & 326.4975 & 3.7000 & 83.8 & 3 & 500 & 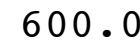 \\
\hline & 1310 & 4.2504 & 5.7750 & 284.9 & & 00.0 & \\
\hline & 1311 & 43.3721 & .8500 & 84.4 & & .0 & \\
\hline & 13 & 24.7490 & 6.0750 & 285.0 & 4 & 00.0 & ( \\
\hline & 1313 & 22.5110 & 8.8500 & 284.6 & 4 & 0.0 & ( \\
\hline & 314 & 21. & & & & & \\
\hline & & 33. & & 286.5 & 3 & & \\
\hline & 316 & 27.0029 & 5250 & 286.7 & 3 & 0.0 & 政 \\
\hline & 1317 & 11.4833 & 6 . & .4 & & 0 & \\
\hline & 318 & 40 & 4 . & 285.0 & 3 & & \\
\hline & 319 & 85.7605 & 3.9250 & 283.7 & 4 & 00.0 & 证。 \\
\hline & 41320 & 89.4041 & 4.3500 & 282.9 & 4 & 00.0 & 0. \\
\hline & 1 & 85.5199 & $1 \quad 17$ & 282.0 & 4 & & \\
\hline & 2 & 977 & 50 & 281.2 & 4 & & 0 . \\
\hline & 41323 & 25.7459 & 5.4750 & 281.4 & 4 & 0 & J. \\
\hline & 41324 & 341.4997 & & 80.9 & 4 & & \\
\hline & 414 & 51. & 1.4750 & .1 & 5 & & \\
\hline & 4142 & 124.0000 & 6750 & 280.9 & 6 & 0 & J. \\
\hline & 4143 & 311.7184 & 0 & 79.7 & 6 & & \\
\hline & 4144 & 12.2448 & 500 & 279.1 & 6 & & 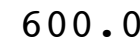 \\
\hline & 4145 & 344.6873 & 9500 & 279.1 & 6 & 0 & 0 • \\
\hline & 4146 & 14.9597 & 1.6250 & 279.3 & 5 & 0 & 0 . \\
\hline & 4147 & 14.7483 & 1.5250 & 280.2 & 4 & & \\
\hline & 4148 & 352.9977 & 1.7250 & 281.7 & 4 & 0 & 0 • \\
\hline & 4149 & 292.4330 & 1.4000 & 284.1 & 3 & 0 & 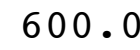 \\
\hline & 414 & 286.7736 & 0 & & 2 & & \\
\hline & 41411 & 201.0197 & 3 . & 284.9 & 2 & & 0 . \\
\hline & 41412 & 205.9963 & 3.2250 & 284.8 & 2 & & \\
\hline & 41413 & 176.9723 & 5000 & 28 & 3 & & 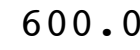 \\
\hline 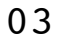 & 41414 & 154.4948 & 6.3750 & 284.1 & 4 & .0 & 0 . \\
\hline & 41415 & 105.1006 & 5750 & 283.4 & 3 & & 0 . \\
\hline & 41416 & 65.2487 & 500 & 282.3 & 4 & & 600. \\
\hline & 41417 & 76.7361 & 4.2250 & 283.5 & 3 & .0 & 600 . \\
\hline & 41418 & 92.2461 & 5.1500 & 283.3 & 3 & 608 & 00 . \\
\hline & 41419 & 71.7485 & 5.4500 & 282 & 4 & & 0 \\
\hline 03 & & 71.9952 & 7.2000 & 281.7 & 4 & & 00 . \\
\hline & 41421 & 67.0000 & 8.4750 & 281.1 & 4 & 600.0 & 600 . \\
\hline & & 68 & 50 & & 4 & & \\
\hline & 41423 & 66.5000 & 8.1250 & 280.8 & $\mathbf{4}$ & 600.0 & 600 \\
\hline
\end{tabular}




\begin{tabular}{|c|c|c|c|c|c|c|c|}
\hline 03 & 41424 & 63.7500 & 9.3250 & 280.8 & 4 & 600.0 & 600.0 \\
\hline 03 & 4151 & 70.2522 & 8.6250 & 280.7 & 4 & 600.0 & 00.0 \\
\hline 03 & 4152 & 70.7492 & 8.9750 & 280.9 & 4 & 600.0 & 00.0 \\
\hline 3 & 4153 & 70.2501 & 8.9000 & 280.7 & 4 & 600.0 & 00.0 \\
\hline 03 & 4154 & 69.0000 & 9.0250 & 280.4 & 4 & 600.0 & 00.0 \\
\hline 03 & 4155 & 68.2496 & 10.5000 & 280.2 & 4 & 600.0 & 00.0 \\
\hline 03 & 4156 & 63.5000 & 12.3000 & 279.9 & 4 & 600.0 & 00.0 \\
\hline 03 & 4157 & 66.7499 & 11.8500 & 280.4 & 4 & 600.0 & 00.0 \\
\hline 03 & 4158 & 66.0005 & 9.0250 & 281.2 & 4 & 600.0 & 00.0 \\
\hline 03 & 4159 & 69.0000 & 7.0500 & 282.4 & 4 & 600.0 & 0 \\
\hline 03 & 41510 & 92.5631 & 4.3500 & 283.8 & 3 & 600.0 & \\
\hline 03 & 41511 & 123.7485 & 4.1500 & 284.2 & 3 & 600.0 & \\
\hline 03 & 41512 & 256.8228 & 1.4000 & 285.9 & 2 & 600.0 & 00.0 \\
\hline 03 & 41513 & 248.0046 & 1.6500 & 286.6 & 1 & 600.0 & 00.0 \\
\hline 03 & 41514 & 242 . & 250 & 286.7 & 1 & .0 & \\
\hline 03 & 41515 & 257.9092 & 1.8000 & 286.7 & 1 & 600.0 & 00.0 \\
\hline 03 & 41516 & 290.2259 & 1.8250 & 286.7 & 1 & 60 & 600.0 \\
\hline 03 & 41517 & 352.2411 & 1.8750 & 286.9 & 1 & .0 & \\
\hline 03 & 41518 & 39.5553 & 1.7250 & 286.3 & 1 & 60 & .0 \\
\hline 03 & 41519 & 148.2380 & 2.0750 & 285.3 & 2 & 60 & .0 \\
\hline 03 & 41520 & 126.7898 & 1.75 & 283.9 & 3 & 60 & \\
\hline 03 & 41521 & 17.0777 & 1.9000 & 284.0 & 4 & .0 & \\
\hline 03 & 41522 & 15.5000 & 1.4250 & 283.8 & 5 & 600.0 & .0 \\
\hline 03 & 41523 & 321.9948 & 0.97 & 283.0 & 6 & 60 & \\
\hline 03 & 41524 & 343 . & 1.2000 & 282.7 & 6 & .0 & \\
\hline 03 & 4161 & 359.7559 & 1.0250 & 282.0 & 5 & .0 & 600.0 \\
\hline 03 & 4162 & 14.7498 & 1.1000 & 282.6 & 5 & 600.0 & .0 \\
\hline 03 & 4163 & 351.5386 & 1.3250 & 282.8 & 5 & .0 & \\
\hline 03 & 4164 & 293.2507 & 1.8000 & 281.8 & 6 & .0 & 600.0 \\
\hline 03 & 4165 & 312.0018 & 1.9500 & 281.1 & 5 & 60 & .0 \\
\hline 03 & 4166 & 333.4729 & 1.8500 & 280.6 & 5 & .0 & \\
\hline 03 & 4167 & 340.4584 & 1.3500 & 280.5 & 5 & .0 & 600.0 \\
\hline 03 & 4168 & 313.0019 & 1.4000 & 282.2 & 4 & 600.0 & 600.0 \\
\hline 03 & 4169 & 285.0096 & 2.2750 & 284.1 & 3 & 0 & 0.0 \\
\hline 03 & 41610 & 278.5480 & 2.2500 & 285.1 & 2 & 600.0 & 600.0 \\
\hline 03 & 41611 & 242.9574 & 1.5000 & 286.2 & 1 & 600.0 & 600.0 \\
\hline 03 & 41612 & 279.4044 & 1.1500 & 286.7 & 1 & 600.0 & 600.0 \\
\hline 03 & 41613 & 71.3147 & 2.2500 & 286.4 & 1 & 600.0 & 600.0 \\
\hline 03 & 41614 & 71.5008 & 2.9250 & 285.7 & 2 & 600.0 & 600.0 \\
\hline 03 & 41615 & 77.7509 & 3.1500 & 284.9 & 3 & 600.0 & 600.0 \\
\hline 03 & 41616 & 75.5000 & 2.9000 & 283.9 & 4 & 600.0 & 600.0 \\
\hline 03 & 41617 & 93.0000 & 2.0250 & 283.7 & 4 & 600.0 & 600.0 \\
\hline 03 & 41618 & 129.5043 & 2.0750 & 283.3 & 4 & 600.0 & 600.0 \\
\hline 03 & 41619 & 104.0185 & 2.4250 & 283.0 & 4 & 600.0 & 600.0 \\
\hline 03 & 41620 & 88.7512 & 3.5750 & 282.8 & 5 & 600.0 & 600.0 \\
\hline 03 & 41621 & 94.5017 & 2.8500 & 282.6 & 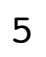 & 600.0 & 600.0 \\
\hline 03 & 41622 & 83.4794 & 4.3750 & 282.7 & 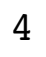 & 600.0 & 600.0 \\
\hline 0 & 41623 & 73.2540 & 5.925 & 282.7 & 4 & 600.0 & 600.0 \\
\hline
\end{tabular}




\begin{tabular}{|c|c|c|c|c|c|c|c|}
\hline 3 & 41624 & 72.7499 & 6.0250 & 282.6 & 4 & 600.0 & 600.0 \\
\hline 3 & $417 \quad 1$ & 5.5000 & 6.0750 & 282.5 & 4 & 600.0 & 600.0 \\
\hline 03 & 4172 & 71.5052 & 5.4500 & 282.4 & 4 & 600.0 & 00.0 \\
\hline 03 & 4173 & 99.0616 & 2.8750 & 282.2 & 4 & 600.0 & 00.0 \\
\hline 03 & $417 \quad 4$ & 132.0699 & 2.5000 & 282.1 & & 600.0 & 00.0 \\
\hline 03 & 4175 & 65.7497 & 4.3500 & 282.1 & $J$ & 600.0 & 00.0 \\
\hline 03 & 4176 & 62.2500 & 4.8000 & 282.0 & 4 & 600.0 & 500.0 \\
\hline 03 & 4177 & 69.9996 & 5.6500 & 282.0 & 4 & 600.0 & .0 \\
\hline 03 & 4178 & 79.5000 & 5.3250 & 282.3 & 4 & 600.0 & \\
\hline 03 & 4179 & 88.4990 & 4.9000 & 283.1 & 4 & 600.0 & 600.0 \\
\hline 03 & 41710 & 92.2044 & 4.6500 & 284.0 & 3 & 600.0 & 0 \\
\hline 03 & 41711 & 107.4741 & 4.6750 & 285.0 & 3 & 600.0 & \\
\hline 03 & 41712 & 105.0011 & 4.7500 & 285.3 & 3 & 600.0 & .0 \\
\hline 03 & 41713 & 75.4793 & 4.8750 & 286.0 & 3 & 600.0 & 0 \\
\hline 0 & 41714 & 70.7013 & 6 & 286.3 & 4 & 600.0 & .0 \\
\hline 03 & 41715 & 56.4997 & 8.4000 & 286.5 & 4 & 600.0 & \\
\hline 03 & 41716 & 63.0000 & 11.0500 & 285.5 & 4 & 600.0 & 600.0 \\
\hline 03 & 41717 & 64.2500 & 9.2500 & 284.7 & 4 & 600.0 & .0 \\
\hline 03 & 41718 & 63.0003 & 8.6750 & 283.7 & 4 & 600.0 & \\
\hline 03 & 41719 & 56.2497 & 9.3500 & 282.6 & 4 & 600.0 & 600.0 \\
\hline 03 & 41720 & 58.7500 & 10.2000 & 281.8 & 4 & 600.0 & .0 \\
\hline 03 & 41721 & 63.5001 & 11.90 & 281.5 & 4 & 600.0 & \\
\hline 03 & 41722 & 64.2500 & 10.8500 & 281.4 & 4 & 600.0 & 60 \\
\hline 03 & 41723 & 65.5000 & 10.3750 & 281.5 & 4 & 600.0 & .0 \\
\hline 03 & 41724 & 65.2501 & 10.5000 & 281.7 & 4 & 600.0 & \\
\hline 03 & 4181 & 61.0000 & 11.3250 & 281.6 & 4 & 600.0 & 60 \\
\hline 03 & 4182 & 61.0000 & 9.8750 & 281.5 & 4 & 600.0 & .0 \\
\hline 03 & 4183 & 62.0000 & 8.95 & 281.3 & 4 & 600.0 & .0 \\
\hline 03 & 4184 & 61.7500 & 9.4250 & 280.9 & 4 & 600.0 & .0 \\
\hline 03 & 4185 & 61.0002 & 9.6250 & 280.6 & 4 & 600.0 & .0 \\
\hline 03 & 4186 & 60.7500 & 8.8250 & 280.4 & 4 & 600.0 & 60 \\
\hline 03 & 4187 & 76.2589 & 7.4750 & 280.7 & 4 & 600.0 & \\
\hline 03 & 4188 & 82.5002 & 7.0000 & 281.5 & 4 & 600.0 & 600.0 \\
\hline 03 & 4189 & 83.4939 & 5.3500 & 282.7 & 3 & 600.0 & .0 \\
\hline 03 & 41810 & 117.7964 & 5.2250 & 283.5 & 3 & 600.0 & \\
\hline 03 & 41811 & 129.7726 & 6.1250 & 284.0 & 4 & 600.0 & 600.0 \\
\hline 03 & 41812 & 143.7452 & 6.2000 & 284.9 & 4 & 600.0 & 600.0 \\
\hline 03 & 41813 & 155.7499 & 7.00 & 286.0 & 4 & 600.0 & 60 \\
\hline 03 & 41814 & 161.0101 & 7.6250 & 286.7 & 4 & 600.0 & 600.0 \\
\hline 03 & 41815 & 159.7496 & 7.3000 & 287.1 & 4 & 600.0 & 600 \\
\hline 03 & 41816 & 150.7244 & 6.2750 & 287.7 & 4 & 600.0 & 600.0 \\
\hline 03 & 41817 & 160.5165 & 6.7250 & 288.0 & 4 & 600.0 & 600.0 \\
\hline 03 & 41818 & 109.1865 & 6.8500 & 287.3 & 4 & 600.0 & 600.0 \\
\hline 03 & 41819 & 73.5012 & 6.8750 & 285.3 & 4 & 600.0 & 600.0 \\
\hline 03 & 41820 & 75.2500 & 8.6750 & 284.5 & 4 & 600.0 & 600.0 \\
\hline 03 & 41821 & 78.0001 & 8.8750 & 284.6 & 4 & 600.0 & 600.0 \\
\hline 03 & 41822 & 74.4991 & 8 . & & 4 & 600.0 & .0 \\
\hline 03 & 41823 & 86.9981 & 7.3250 & 284.8 & $\mathbf{4}$ & 600.0 & 600.0 \\
\hline
\end{tabular}




\begin{tabular}{|c|c|c|c|c|c|c|c|}
\hline 03 & 41824 & 90.5000 & 6.7500 & 285.5 & 4 & 600.0 & 600.0 \\
\hline 03 & 4191 & 93.9966 & 7.4250 & 285.3 & 4 & 600.0 & 600.0 \\
\hline 03 & 4192 & 113.9910 & 7.8500 & 286.0 & 4 & 600.0 & 00.0 \\
\hline 3 & 4193 & 136.2511 & 9.5250 & 286.0 & 4 & 600.0 & 00.0 \\
\hline 03 & 4194 & 148.5000 & 9.8750 & 285.0 & 4 & 600.0 & 00.0 \\
\hline 03 & 4195 & 145.5000 & 10.0000 & 284.6 & 4 & 600.0 & 00.0 \\
\hline 03 & 4196 & 143.4999 & 8.2000 & 284.4 & 4 & 600.0 & 00.0 \\
\hline 03 & 4197 & 150.4979 & 7.1500 & 284.7 & 4 & 600.0 & 00.0 \\
\hline 03 & 4198 & 166.2417 & 7.6250 & 285.0 & 4 & 600.0 & 00.0 \\
\hline 03 & 4199 & 171.2500 & 7.8000 & 285.4 & 4 & 600 & 0 \\
\hline 03 & 41910 & 163.0016 & 6.2500 & 286.3 & 4 & 600.0 & \\
\hline 03 & 41911 & 153.2403 & 5.8750 & 287.2 & 3 & 600.0 & \\
\hline 03 & 41912 & 154.4960 & 4.6250 & 288.8 & 3 & 600.0 & .0 \\
\hline 03 & 41913 & 148.2524 & 3.8750 & 289.9 & 2 & 600.0 & \\
\hline 03 & 41914 & 163.7499 & 500 & 290.7 & 3 & .0 & \\
\hline 03 & 41915 & 151.8648 & 3.6000 & 291.3 & 2 & 600.0 & .0 \\
\hline 03 & 41916 & 151.5000 & 3.4250 & 291.5 & 2 & 60 & .0 \\
\hline 03 & 41917 & 156.0000 & 3.65 & 291.5 & 2 & .0 & \\
\hline 03 & 41918 & 148.7272 & 3.5000 & 291.0 & 3 & 60 & \\
\hline 03 & 41919 & 145.9682 & 3.4500 & 290.1 & 4 & .0 & .0 \\
\hline 03 & 41920 & 77.4893 & 5.55 & 288.2 & 4 & 6 & \\
\hline 03 & 41921 & 72.4991 & 6.7000 & 287.3 & 4 & .0 & \\
\hline 03 & 41922 & 74.2498 & 7.5250 & 286.5 & 4 & 600.0 & .0 \\
\hline 03 & 41923 & 77.5000 & 7.92 & 286.3 & 4 & 6 & \\
\hline 03 & 41924 & 75.7499 & 8.6500 & 286.3 & 4 & .0 & \\
\hline 03 & 4201 & 76.0000 & 8.2500 & 286.2 & 4 & .0 & .0 \\
\hline 03 & 4202 & 65.2806 & 5.9250 & 286.6 & 4 & 60 & .0 \\
\hline 03 & 4203 & 51.7501 & 2.1750 & 287.2 & 5 & .0 & \\
\hline 03 & $420 \quad 4$ & 33.5019 & 1.8750 & 287.2 & 5 & .0 & 0.0 \\
\hline 03 & $420 \quad 5$ & 31.2498 & 1.3000 & 286.7 & 6 & 60 & .0 \\
\hline 03 & 4206 & 67.7647 & 2.4000 & 285.8 & 6 & .0 & \\
\hline 03 & 4207 & 77.5003 & 3.4750 & 285.1 & 5 & .0 & 0.0 \\
\hline 03 & 4208 & 75.9823 & 3.0500 & 285.3 & 4 & 600.0 & 0.0 \\
\hline 03 & $420 \quad 9$ & 72.5040 & 2.8500 & 285.2 & 3 & 600.0 & 0.0 \\
\hline 03 & 42010 & 46.2279 & 2.27 & 286.6 & 2 & 600.0 & 600.0 \\
\hline 03 & 42011 & 68.2795 & 2.3250 & 289.2 & 1 & 600.0 & 600.0 \\
\hline 03 & 42012 & 75.0757 & 4.10 & 287.9 & 2 & 600.0 & 0.0 \\
\hline 03 & 42013 & 72.2482 & 5.7000 & 287.5 & 3 & 600.0 & 600.0 \\
\hline 03 & 42014 & 76.9954 & 5.6500 & 287.7 & 3 & 600.0 & 600.0 \\
\hline 03 & 42015 & 78.7495 & 5.400 & 288.3 & 3 & 600.0 & 600.0 \\
\hline 03 & 42016 & 71.7489 & 7.3500 & 288.0 & 4 & 600.0 & 600.0 \\
\hline 03 & 42017 & 73.7499 & 7.8250 & 287.2 & 4 & 600.0 & 600.0 \\
\hline 03 & 42018 & 75.0000 & 8.2500 & 285.6 & 4 & 600.0 & 600.0 \\
\hline 03 & 42019 & 72.0000 & 9.7750 & 284.7 & 4 & 600.0 & 600.0 \\
\hline 03 & 42020 & 70.0000 & 10.6250 & 284.3 & 4 & 600.0 & 600.0 \\
\hline 03 & 42021 & 69.2505 & 10.5250 & 283.7 & 4 & 600.0 & 600.0 \\
\hline 03 & 42022 & 62.5000 & 12.1250 & 283.5 & 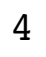 & 600.0 & 600.0 \\
\hline U. & 42023 & 62.5000 & 12.1000 & 283.3 & 4 & 600.0 & 600.0 \\
\hline
\end{tabular}




\begin{tabular}{|c|c|c|c|c|c|c|c|}
\hline 3 & 42024 & 61.7500 & 11.9500 & 283.2 & 4 & 600.0 & 00.0 \\
\hline 03 & 4211 & 62.2502 & 11.3500 & 282.5 & 4 & 600.0 & 00.0 \\
\hline 03 & 4212 & 63.2500 & 12.4000 & 282.3 & 4 & 600.0 & 00.0 \\
\hline 03 & 4213 & 59.7501 & 13.9750 & 281.8 & 4 & 600.0 & 00.0 \\
\hline 03 & 4214 & 58.2500 & 11.9500 & 282.0 & $I$ & 600.0 & 00.0 \\
\hline 03 & 4215 & 59.5003 & 8.6750 & 281.4 & 4 & 600.0 & 00.0 \\
\hline 03 & 4216 & 60.4908 & 4.9500 & 280.9 & 4 & 600.0 & 600.0 \\
\hline 03 & 4217 & 79.0000 & 4.6750 & 280.5 & 4 & 600.0 & 00.0 \\
\hline 03 & 4218 & 65.2499 & 7.7000 & 280.4 & 4 & 600.0 & 00.0 \\
\hline 03 & 4219 & 78.5124 & 6.6000 & 282.1 & 4 & 600.0 & .0 \\
\hline 03 & 42110 & 56.2495 & 6.6750 & 283.0 & 4 & 600.0 & 600.0 \\
\hline 03 & 42111 & 64.2755 & 00 & 282.8 & 3 & 600.0 & \\
\hline 03 & 42112 & 103.7229 & 6.9500 & 282.3 & 4 & 600.0 & .0 \\
\hline 03 & 42113 & 66.9954 & 7.3750 & 280.9 & 4 & 600.0 & 00.0 \\
\hline 03 & 42114 & 71.0000 & & 280.5 & 3 & 600.0 & \\
\hline 03 & 42115 & 72.0000 & 6.5750 & 281.7 & 4 & 600.0 & 60 \\
\hline 03 & 42116 & 64.9958 & 6.7000 & 282.6 & 4 & 600.0 & 600.0 \\
\hline 03 & 42117 & 72.7444 & 6 & 282.6 & 4 & 600.0 & \\
\hline 03 & 42118 & 81.7675 & 6.3750 & 281.6 & 4 & 600.0 & .0 \\
\hline 03 & 42119 & 75.9888 & 5.8000 & 280.9 & 4 & 600.0 & 600 \\
\hline 03 & 42120 & 76.9998 & 5. & 280.2 & 4 & 600.0 & .0 \\
\hline 03 & 42121 & 73.7528 & 4 . & 280.1 & 4 & 600.0 & \\
\hline 03 & 42122 & 61.5000 & 7.7500 & 280.1 & 4 & 600.0 & 600.0 \\
\hline 03 & 42123 & 60.0000 & 9.3250 & 280.2 & 4 & 600.0 & .0 \\
\hline 03 & 42124 & 62.2499 & 7 . & 280.2 & 4 & 600.0 & \\
\hline 03 & 4221 & 63.5000 & 6.9750 & 279.8 & 4 & 600.0 & 60 \\
\hline 03 & 4222 & 65.9968 & 6.5750 & 279.4 & 4 & 600.0 & .0 \\
\hline 03 & 4223 & 92.7470 & 4 . & 279.5 & 4 & 600.0 & \\
\hline 03 & 4224 & 66.6872 & 4.4000 & 279.0 & 4 & 600.0 & 60 \\
\hline 03 & 4225 & 151.0890 & 2.9750 & 279.0 & 4 & 600.0 & 60 \\
\hline 03 & 4226 & 184.6128 & 2 . & 278.9 & 5 & 600.0 & \\
\hline 03 & 4227 & 100.2514 & 500 & 278.7 & 6 & 600.0 & 60 \\
\hline 03 & 4228 & 161.7445 & 2.5000 & 279.7 & 5 & 600.0 & 600.0 \\
\hline 03 & 4229 & 161.5267 & 2 . & 281.7 & 4 & 600.0 & .0 \\
\hline 03 & 42210 & 168.5135 & $3.2^{\circ}$ & 282.6 & 3 & 600.0 & .0 \\
\hline 03 & 42211 & 181.0032 & 3.4500 & 284.5 & 2 & 600.0 & 600.0 \\
\hline 03 & 42212 & 135.5117 & 3.8750 & 285.1 & 2 & 600.0 & \\
\hline 03 & 42213 & 117.7606 & 4.3000 & 285.6 & 3 & 600.0 & 600.0 \\
\hline 03 & 42214 & 94.0118 & 5.7250 & 286.0 & 3 & 600.0 & 600.0 \\
\hline 03 & 42215 & 72.9893 & 5.8500 & 286.2 & 3 & 600.0 & \\
\hline 03 & 42216 & 61.7518 & 6.8250 & 286.3 & 4 & 600.0 & 60 \\
\hline 03 & 42217 & 64.0000 & 7.6000 & 285.8 & 4 & 600.0 & 600.0 \\
\hline 03 & 42218 & 65.7499 & 10.2500 & 284.4 & 4 & 600.0 & 600.0 \\
\hline 03 & 42219 & 64.7500 & 10.2250 & 283.0 & 4 & 600.0 & 600.0 \\
\hline 03 & 42220 & 68.9999 & 9.3750 & 282.1 & 4 & 600.0 & 600.0 \\
\hline 03 & 42221 & 70.2500 & 9.3500 & 281.8 & 4 & 600.0 & 600.0 \\
\hline 03 & 42222 & 68.7502 & 9.575 & 281 & 4 & 600.0 & 600.0 \\
\hline 03 & 42223 & 70.2500 & 9.7250 & 281.1 & 4 & 600.0 & 600.0 \\
\hline
\end{tabular}




\begin{tabular}{|c|c|c|c|c|c|c|c|}
\hline 3 & 42224 & 70.7501 & 10.2750 & 281.0 & 4 & 600.0 & 00.0 \\
\hline 03 & 4231 & 68.5002 & 9.3750 & 281.0 & 4 & 600.0 & 600.0 \\
\hline 03 & 4232 & 68.5000 & 11.0500 & 280.9 & 4 & 600.0 & 00.0 \\
\hline 03 & 4233 & 64.0000 & 11.3750 & 280.8 & 4 & 600.0 & 600.0 \\
\hline 03 & 4234 & 64.0000 & 10.4250 & 280.7 & 4 & 600.0 & 00.0 \\
\hline 03 & 4235 & 68.0000 & 9.3250 & 280.6 & 4 & 600.0 & 00.0 \\
\hline 03 & 4236 & 69.7500 & 8.2000 & 280.5 & 4 & 600.0 & 600.0 \\
\hline 03 & 4237 & 69.2506 & 7.8750 & 281.1 & 4 & 600.0 & 00.0 \\
\hline 03 & 4238 & 64.2500 & 6.6750 & 282.2 & 4 & 600.0 & 00.0 \\
\hline 03 & 4239 & 71.7565 & 5.0000 & 283.2 & 3 & 600.0 & 600.0 \\
\hline 03 & 42310 & 31.6343 & 2.9750 & 284.9 & 2 & 600.0 & 00.0 \\
\hline 03 & 42311 & 322.0107 & 2.1250 & 285.8 & 1 & 600.0 & \\
\hline 03 & 42312 & 306.9781 & 2.4750 & 286.4 & 1 & 600.0 & 600.0 \\
\hline 03 & 42313 & 292.2609 & 2.1750 & 287.3 & 1 & 600.0 & 600.0 \\
\hline 03 & 42314 & 265.8458 & 2.5000 & 288.2 & 1 & 600.0 & \\
\hline 03 & 42315 & 216.8542 & 2.3250 & 288.5 & 1 & 600.0 & 60 \\
\hline 03 & 42316 & 267.7393 & 1.9500 & 289.1 & 1 & 600.0 & 600.0 \\
\hline 03 & 42317 & 57.5128 & 3.6500 & 288.4 & 2 & 600.0 & \\
\hline 03 & 42318 & 71.4969 & 4.6250 & 287.4 & 3 & 600.0 & 60 \\
\hline 03 & 42319 & 73.5000 & 6.1250 & 286.0 & 4 & 600.0 & 600 \\
\hline 03 & 42320 & 70.7498 & 7 . & 284.7 & 4 & 600.0 & .0 \\
\hline 03 & 42321 & 75.2500 & 7 . & 284.6 & 4 & 600.0 & \\
\hline 03 & 42322 & 64.5092 & 6.1000 & 284.8 & 4 & 600.0 & 600.0 \\
\hline 03 & 42323 & 13.7636 & 2.7750 & 285.6 & 5 & 600.0 & .0 \\
\hline 03 & 42324 & 307.7689 & 250 & 285.2 & 6 & 600.0 & \\
\hline 03 & 4241 & 285.7274 & 1.2250 & 284.0 & 6 & 600.0 & 60 \\
\hline 03 & 4242 & 68.8503 & 1.8250 & 284.7 & 6 & 600.0 & 60 \\
\hline 03 & 4243 & 31.9635 & 2.8250 & 285.1 & 6 & 600.0 & \\
\hline 03 & $424 \quad 4$ & 15.9989 & 5.1250 & 285.7 & 5 & 600.0 & 60 \\
\hline 03 & 4245 & 327.8136 & 3.4250 & 285.5 & 5 & 600.0 & 600.0 \\
\hline 03 & 4246 & 358.7537 & 500 & 285.6 & 4 & 600.0 & \\
\hline 03 & 4247 & 3.4939 & 4.4500 & 285.1 & 4 & 600.0 & 60 \\
\hline 03 & 4248 & 54.9045 & 4.7250 & 284.0 & 3 & 600.0 & 600.0 \\
\hline 03 & 4249 & 88.5010 & 6.90 & 281.0 & 4 & 600.0 & .0 \\
\hline 03 & 42410 & 80.5009 & 6 . & 281.3 & 4 & 600.0 & 60 \\
\hline 03 & 42411 & 81.5005 & 7.5250 & 281.3 & 4 & 600.0 & 600.0 \\
\hline 03 & 42412 & 75.5000 & 7.3000 & 281.3 & 4 & 600.0 & .0 \\
\hline 03 & 42413 & 70.5018 & 4.3500 & 282.9 & 3 & 600.0 & 600.0 \\
\hline 03 & 42414 & 87.7129 & 4.6750 & 284.0 & 3 & 600.0 & 600.0 \\
\hline 03 & 42415 & 74.8297 & 4.6500 & 285.3 & 3 & 600.0 & \\
\hline 03 & 42416 & 93.4977 & 4.9500 & 284.7 & 3 & 600.0 & 600.0 \\
\hline 03 & 42417 & 63.9931 & 5.7250 & 284.9 & 3 & 600.0 & 600.0 \\
\hline 03 & 42418 & 71.2421 & 4.5250 & 284.6 & 3 & 600.0 & 600.0 \\
\hline 03 & 42419 & 82.0062 & 3.9250 & 283.5 & 4 & 600.0 & 600.0 \\
\hline 03 & 42420 & 89.9999 & 4.6000 & 282.7 & 4 & 600.0 & 600.0 \\
\hline 03 & 42421 & 78.2508 & 4.2750 & 281.8 & 4 & 600.0 & 600.0 \\
\hline 03 & 42422 & 80.2496 & 5.550 & 281.4 & 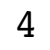 & 600.0 & 600.0 \\
\hline 03 & 42423 & 69.0595 & 5.7500 & 281.2 & 4 & 600.0 & 600.0 \\
\hline
\end{tabular}




\begin{tabular}{|c|c|c|c|c|c|c|c|}
\hline & 2424 & & & 0.7 & & & \\
\hline & 4251 & 337. & 0500 & 30.6 & & 00,0 & \\
\hline & 4252 & 20.0929 & 2.9500 & 281.2 & & 00 & \\
\hline & 4253 & 23.9972 & 0750 & 281.1 & & 00.0 & \\
\hline & 4254 & 328 . & .8000 & 80.6 & & 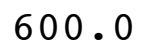 & \\
\hline & 4255 & 2.50 & .6750 & 81.1 & & 00 & \\
\hline & 4256 & 9920 & 1.7750 & 281.1 & & 00 & \\
\hline & 4257 & 305.8434 & L. 3750 & 82.2 & & 0 & \\
\hline & 4258 & 18.8038 & 3.1500 & 82.8 & & & \\
\hline & 4259 & 8.7415 & 4.4750 & 83.1 & & 00 & 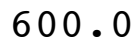 \\
\hline & 42510 & 353.4945 & 5.4250 & 284.0 & & 00.0 & \\
\hline & 42511 & 6.5013 & 0 & 84.5 & & .0 & \\
\hline & 42512 & 354.50 & 8.0750 & 284.6 & & 00.0 & 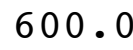 \\
\hline & 42513 & 358 . & 6.0750 & 284 & & 0.0 & , \\
\hline & 42514 & 354 . & & & & & \\
\hline & 42515 & 359 . & & 28 & & & \\
\hline & 42516 & 10.7336 & 3.52 & 284.5 & & 0.0 & 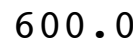 \\
\hline & 42517 & 70 . & 0 & $\cdot 1$ & & .0 & \\
\hline & 42518 & 66. & 2 . & .1 & & & \\
\hline & 42519 & 53.7088 & 3.9000 & 282.7 & & 00.0 & . \\
\hline & 42520 & 68.5015 & 4.7000 & 282.9 & & .0 & 0. \\
\hline & 42521 & 53.7121 & & 282.1 & & & \\
\hline & 42522 & 60.5000 & 0 & 281.7 & & & 0 . \\
\hline & 42523 & 60.4991 & 7.9500 & 281.6 & & .0 & 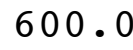 \\
\hline & 42524 & 26.8115 & & 81.3 & & & \\
\hline & 4261 & 2 . & 1.7500 & 281.1 & 5 & & \\
\hline & 4262 & 350.0023 & 1.1750 & 281.0 & & 0 & J \\
\hline & 4263 & 268.6390 & 1.10 & 8 & & & \\
\hline & 4264 & 255.1902 & 0 . & .2 & 6 & & $\bullet$ \\
\hline & 4265 & 16.7568 & 5250 & 280.2 & 6 & 0 & 0 • \\
\hline & 4266 & 97.5000 & 0.8250 & 280.4 & 6 & .0 & 0 . \\
\hline & 4267 & 78.4919 & 2 . & 281.3 & 6 & & \\
\hline & 4268 & 94.1965 & 3000 & 282.7 & 5 & 0 & 0 • \\
\hline & 4269 & 154.0246 & 3.3250 & 282.8 & 4 & .0 & 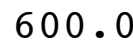 \\
\hline & 42610 & 148 . & 0 & & 3 & & \\
\hline & & 151.7582 & 3.9500 & 284.5 & 2 & & 0 . \\
\hline & 42612 & 148.9908 & .6000 & 285.4 & 3 & 0 & \\
\hline & 42613 & 177.7535 & 3 . & 286.6 & 2 & & . \\
\hline & 42614 & 171.4747 & 4.6500 & 286.7 & 3 & .0 & 0 . \\
\hline & 42615 & 185.7635 & 50 & 286.4 & 3 & & 0 . \\
\hline & 42616 & 183.1996 & 3. & 286.0 & 3 & & 60 \\
\hline & 42617 & 134.9882 & 3.9500 & 286.1 & 3 & .0 & 00 . \\
\hline & 42618 & 156.7617 & 4.0250 & 286.0 & 4 & 0 & 00 . \\
\hline & 42619 & 118.5576 & 000 & 285.3 & 4 & & \\
\hline 03 & 42620 & 79.9952 & 5.2000 & 283.9 & 4 & & 0 . \\
\hline & 42621 & 73.5000 & 6.0500 & 282.9 & 4 & 600.0 & 500 . \\
\hline & & 67.2502 & & & 4 & & \\
\hline & 42623 & 73.5000 & 7.0750 & 282.3 & $\mathbf{4}$ & 600.0 & 600 \\
\hline
\end{tabular}




\begin{tabular}{|c|c|c|c|c|c|c|c|}
\hline 3 & 42624 & 72.0002 & 7.3250 & 282.0 & 4 & 600.0 & 00.0 \\
\hline 03 & $427 \quad 1$ & 69.7501 & 8.6750 & 281.5 & 4 & 600.0 & 600.0 \\
\hline 03 & 4272 & 69.7504 & 7.7750 & 281.4 & 4 & 600.0 & 00.0 \\
\hline 03 & 4273 & 59.2500 & 8.2750 & 281.3 & 4 & 600.0 & 600.0 \\
\hline 03 & $427 \quad 4$ & 63.7499 & 6.4250 & 281.7 & 4 & 600.0 & 00.0 \\
\hline 03 & 4275 & 73.0000 & 6.1250 & 282.0 & 4 & 600.0 & 00.0 \\
\hline 03 & 4276 & 77.4999 & 7.6500 & 281.2 & 4 & 600.0 & 600.0 \\
\hline 03 & $427 \quad 7$ & 86.2505 & 4.1750 & 282.6 & 4 & 600.0 & 00.0 \\
\hline 03 & $427 \quad 8$ & 230.0197 & 1.8750 & 284.7 & 3 & 600.0 & 00.0 \\
\hline 03 & 4279 & 275.9966 & 1.8250 & 285.5 & 2 & 600.0 & .0 \\
\hline 03 & 42710 & 286.5010 & 2.5250 & 285.9 & 1 & 600.0 & 00.0 \\
\hline 03 & 42711 & 256.3974 & 2.5000 & 287.1 & 1 & 600.0 & \\
\hline 03 & 42712 & 233.3662 & 2.4250 & 288.3 & 1 & 600.0 & 600.0 \\
\hline 03 & 42713 & 267.9526 & 2.5750 & 289.2 & 1 & 600.0 & 600 \\
\hline 03 & 42714 & 196.8769 & 500 & 289.7 & 2 & 600.0 & \\
\hline 03 & 42715 & 317.1910 & 2.6750 & 290.6 & 1 & 600.0 & 60 \\
\hline 03 & 42716 & 253.3818 & 2.2250 & 291.2 & 1 & 600.0 & 600.0 \\
\hline 03 & 42717 & 21.3089 & 3.1000 & 291.1 & 2 & 600.0 & \\
\hline 03 & 42718 & 6.5000 & 4.1500 & 289.9 & 3 & 600.0 & 60 \\
\hline 03 & 42719 & 31.4954 & 6.2000 & 287.8 & 4 & 600.0 & 600 \\
\hline 03 & 42720 & 39.2516 & 8.7750 & 286.3 & 4 & 600.0 & .0 \\
\hline 03 & 42721 & 6.2248 & 250 & 286.8 & 4 & 600.0 & \\
\hline 03 & 42722 & 25.4572 & 8.5250 & 286.3 & 4 & 600.0 & 600.0 \\
\hline 03 & 42723 & 15.7505 & 10.2750 & 285.0 & 4 & 600.0 & .0 \\
\hline 03 & 42724 & 354.1385 & 5.6250 & 285.2 & 4 & 600.0 & \\
\hline 03 & 4281 & 308.9613 & 3.4250 & 284.1 & 5 & 600.0 & 60 \\
\hline 03 & 4282 & 77.5000 & 7.6500 & 281.1 & 4 & 600.0 & .0 \\
\hline 03 & 4283 & 54.0588 & 9.0500 & 280.8 & 4 & 600.0 & \\
\hline 03 & 4284 & 32.4875 & 4.0250 & 281.4 & 4 & 600.0 & 60 \\
\hline 03 & 4285 & 329.2538 & 2.3250 & 281.5 & 5 & 600.0 & 600.0 \\
\hline 03 & 4286 & 318.3435 & 2 . & 281.7 & 6 & 600.0 & \\
\hline 03 & 4287 & 274.4615 & 1.8000 & 280.8 & 6 & 600.0 & 60 \\
\hline 03 & 4288 & 283.1641 & 1.7750 & 281.2 & 5 & 600.0 & 600.0 \\
\hline 03 & 4289 & 285.5462 & 2.175 & 281.7 & 4 & 600.0 & .0 \\
\hline 03 & 42810 & 227.1000 & 250 & 282.7 & 3 & 600.0 & 60 \\
\hline 03 & 42811 & 306.5743 & 3.5750 & 283.4 & 2 & 600.0 & 600.0 \\
\hline 03 & 42812 & 50.6628 & 4.1000 & 284.2 & 3 & 600.0 & \\
\hline 03 & 42813 & 19.6981 & 2.2500 & 285.7 & 2 & 600.0 & 600.0 \\
\hline 03 & 42814 & 35.7497 & 7.2250 & 287.1 & 3 & 600.0 & 600.0 \\
\hline 03 & 42815 & 32.7965 & 6.0750 & 287.2 & 4 & 600.0 & \\
\hline 03 & 42816 & 75.7444 & 5.6750 & 28 & 3 & 600.0 & 600.0 \\
\hline 03 & 42817 & 84.5284 & 5.3750 & 285.9 & 3 & 600.0 & 600.0 \\
\hline 03 & 42818 & 76.4831 & 7.4000 & 284.0 & 4 & 600.0 & 600.0 \\
\hline 03 & 42819 & 62.2498 & 10.1000 & 283.0 & 4 & 600.0 & 600.0 \\
\hline 03 & 42820 & 55.2480 & 8.3500 & 282.8 & 4 & 600.0 & 600.0 \\
\hline 03 & 42821 & 45.4595 & 5.9500 & 282.5 & 4 & 600.0 & 600.0 \\
\hline 03 & 42822 & 36.0010 & 6.400 & 282.5 & 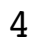 & 600.0 & 600.0 \\
\hline 03 & 42823 & 38.0112 & 6.6750 & 282.6 & 4 & 600.0 & 600.0 \\
\hline
\end{tabular}




\begin{tabular}{|c|c|c|c|c|c|c|c|}
\hline 03 & 42824 & 31.2505 & 7.4250 & 282.3 & 4 & 600.0 & 600.0 \\
\hline 03 & 4291 & 8.7352 & 4.2250 & 282.0 & 4 & 600.0 & 600.0 \\
\hline 03 & 4292 & 340.4117 & 1.0500 & 281.5 & 5 & 600.0 & 00.0 \\
\hline 3 & 4293 & 219.1638 & .5000 & 281.2 & 6 & 600.0 & 00.0 \\
\hline 03 & 4294 & 284.0836 & 0.8250 & 281.4 & 6 & 600.0 & 00.0 \\
\hline 03 & 4295 & 213.0871 & 0.4500 & 281.3 & 6 & 600.0 & 00.0 \\
\hline 03 & 4296 & 28.2912 & 0.5250 & 281.6 & 6 & 600.0 & 00.0 \\
\hline 03 & 4297 & 88.9156 & 1.0500 & 283.6 & 6 & 600.0 & 00.0 \\
\hline 03 & 4298 & 52.4058 & 2.8250 & 283.8 & 5 & 600.0 & 00.0 \\
\hline 03 & 4299 & 47.7324 & 3.8500 & 284.2 & 4 & 600.0 & 00.0 \\
\hline 03 & 42910 & 56.2375 & 3.9000 & 285.1 & 3 & 600.0 & 00.0 \\
\hline 03 & 42911 & 68.7350 & 250 & 285.0 & 3 & 600.0 & \\
\hline 03 & 42912 & 82.9534 & 4.6250 & 285.9 & 3 & 600.0 & 00.0 \\
\hline 03 & 42913 & 84.5467 & 4.0500 & 286.2 & 3 & 600.0 & 00.0 \\
\hline 03 & 42914 & 71.5000 & 250 & 284.8 & 4 & .0 & \\
\hline 03 & 42915 & 60.0002 & 7.0000 & 284.3 & 4 & 600.0 & 00.0 \\
\hline 03 & 42916 & 71.9724 & 6.7000 & 284.4 & 4 & 600.0 & 600.0 \\
\hline 03 & 42917 & 71.7270 & 7.0750 & 284.6 & 4 & .0 & \\
\hline 03 & 42918 & 95.0001 & 6.5000 & 284.4 & 4 & 600.0 & .0 \\
\hline 03 & 42919 & 103.5137 & 6.4000 & 283.4 & 4 & 60 & .0 \\
\hline 03 & 42920 & 84.7498 & 0 & 282.2 & 4 & 60 & \\
\hline 03 & 42921 & 78.2539 & 6.5250 & 281.8 & 4 & .0 & \\
\hline 03 & 42922 & 77.4969 & 5.1750 & 281.4 & 4 & 600.0 & 500.0 \\
\hline 03 & 42923 & 72.0000 & 6 . & 281.2 & 4 & 60 & .0 \\
\hline 03 & 42924 & 69.4991 & 7. & 281.1 & 4 & .0 & \\
\hline 03 & 4301 & 69.2501 & 7.2000 & 280.9 & 4 & 600.0 & 600.0 \\
\hline 03 & 4302 & 75.0007 & 6.7000 & 280.8 & 4 & 600.0 & .0 \\
\hline 03 & 4303 & 75.5000 & 6.6250 & 280.8 & 4 & 0 & \\
\hline 03 & 4304 & 70.7497 & 6.9000 & 280.5 & 4 & .0 & 600.0 \\
\hline 03 & $430 \quad 5$ & 69.5000 & 7.3000 & 280.4 & 4 & 600.0 & 0.0 \\
\hline 03 & 4306 & 61.2498 & 6.8000 & 280.4 & 4 & .0 & \\
\hline 03 & 4307 & 65.7514 & 000 & 281.1 & 4 & .0 & 600.0 \\
\hline 03 & 4308 & 71.2502 & 5.1750 & 282.4 & 3 & 600.0 & 600.0 \\
\hline 03 & $430 \quad 9$ & 88.5237 & 3.7750 & 283.9 & 2 & 600.0 & 0.0 \\
\hline 03 & 43010 & 114.2312 & 4.4250 & 284.7 & 3 & 600.0 & 600.0 \\
\hline 03 & 43011 & 141.4982 & 5.1000 & 285.1 & 3 & 600.0 & 600.0 \\
\hline 03 & 43012 & 139.4884 & 3.7250 & 286.7 & 2 & 600.0 & 600.0 \\
\hline 03 & 43013 & 163.2382 & 4.0500 & 287.6 & 3 & 600.0 & 600.0 \\
\hline 03 & 43014 & 166.2559 & 4.4000 & 288.3 & 3 & 600.0 & 600.0 \\
\hline 03 & 43015 & 166.0002 & 4.8750 & 289.0 & 3 & 600.0 & 600.0 \\
\hline 03 & 43016 & 166.7496 & 5.4750 & 289.2 & 3 & 600.0 & 600.0 \\
\hline 03 & 43017 & 170.5067 & 5.5750 & 289.3 & 3 & 600.0 & 600.0 \\
\hline 03 & 43018 & 160.7406 & 5.4500 & 288.9 & 3 & 600.0 & 600.0 \\
\hline 03 & 43019 & 156.2638 & 4.3250 & 287.9 & 4 & 600.0 & 600.0 \\
\hline 03 & 43020 & 83.4916 & 5.5250 & 286.1 & 4 & 600.0 & 600.0 \\
\hline 03 & 43021 & 73.0018 & 6.2750 & 284.9 & 4 & 600.0 & 600.0 \\
\hline 03 & 43022 & 73.2501 & 6.4250 & 284.3 & 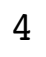 & 600.0 & 600.0 \\
\hline 03 & 43023 & 72.7500 & 6.9000 & 284.2 & 4 & 600.0 & 600.0 \\
\hline
\end{tabular}




\begin{tabular}{|c|c|c|c|c|c|c|c|}
\hline & \pm & 970 & 500 & 35.2 & 4 & 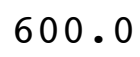 & \\
\hline & $\begin{array}{lll}5 & 1 & 1\end{array}$ & 30.2472 & 3000 & 285.3 & & 600.0 & \\
\hline & $\begin{array}{lll}5 & 1 & 2\end{array}$ & 98.7791 & 2.8000 & 285.9 & & 00 & \\
\hline & $\begin{array}{lll}5 & 1 & 3\end{array}$ & 115.9297 & 5500 & 286.1 & & 00 & \\
\hline & 14 & 109.2605 & 500 & & & & \\
\hline & $\begin{array}{lll}5 & 1 & 5\end{array}$ & 136.0000 & 3500 & 9 & & 0 & \\
\hline & $\begin{array}{lll}5 & 1 & 6\end{array}$ & 166.5435 & 500 & 85.1 & & 00 & \\
\hline & $\begin{array}{lll}5 & 1 & 7\end{array}$ & 172.4946 & 250 & & & & \\
\hline & $\begin{array}{lll}5 & 1 & 8\end{array}$ & 178.2191 & 0 & 6 & & & \\
\hline & $\begin{array}{lll}5 & 1 & 9\end{array}$ & 194.7511 & 1.2750 & 88.2 & & 0 & \\
\hline & 5110 & 232.0558 & 2.4750 & 288.9 & & & \\
\hline & $5 \quad 111$ & 213.2561 & 20 & & & & \\
\hline & 112 & 204.9993 & 3.7750 & 89.1 & & 0 & \\
\hline & 113 & 210.5309 & 2.9000 & & & .0 & \\
\hline & 114 & 178 & 0 & & & & \\
\hline & 5115 & 189. & & & & & \\
\hline & 5116 & 169.3545 & .70 & 39.8 & & 0 & \\
\hline & 5117 & 279 . & 0 & & & & \\
\hline & 5118 & 608 & & & & & \\
\hline & 119 & 92.6239 & 9750 & 288.3 & & 0 & \\
\hline & 5120 & 2756 & 1.2500 & 8.4 & 3 & 0 & \\
\hline & $5 \quad 121$ & 151. & & & I & & \\
\hline & 5122 & 136 & 0 & 287.7 & 5 & & \\
\hline & 5123 & 132.2466 & 0.7500 & 2 & 6 & 0 & \\
\hline & $5 \quad 124$ & 90.2065 & & & 6 & & \\
\hline & $\begin{array}{lll}5 & 2 & 1\end{array}$ & 78.5005 & 0 & $2 \varepsilon$ & 5 & & \\
\hline & 522 & 74.2500 & 0 & 287.0 & 4 & & 0 \\
\hline & 523 & 67.9712 & & & 4 & & \\
\hline & 524 & 61.1169 & 0 & & 4 & & \\
\hline & 525 & 357.0574 & 0 & 6.1 & 4 & 0 & J. \\
\hline & 526 & 329.0000 & & 28 & 4 & & 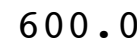 \\
\hline & $\begin{array}{lll}5 & 2 & 7\end{array}$ & 319 . & 0 & 9 & 4 & & \\
\hline & 528 & 320.7343 & 0 & 28 & 4 & & . \\
\hline & $\begin{array}{lll}5 & 2 & 9\end{array}$ & 300 . & 5250 & & 4 & 0 & \\
\hline & 5210 & 317 . & 0 & & 4 & & \\
\hline & 5211 & 332.2506 & 4000 & 5.8 & 4 & & . \\
\hline & 5212 & 311.9092 & 5250 & 7.1 & 4 & & \\
\hline & 5213 & 307.4998 & 0 & & 4 & & \\
\hline & $5 \quad 214$ & 305.5046 & 8.2500 & & 4 & & 0 . \\
\hline & 5215 & 307.8758 & 2500 & 285.9 & 4 & & \\
\hline & 5216 & 332.5000 & 0 & & 4 & & \\
\hline & $5 \quad 217$ & 326.5000 & 6.4500 & & 4 & & 0 . \\
\hline & 5218 & 319.7478 & 6.4000 & 285.8 & 4 & 0 & 0 . \\
\hline & 5219 & 328.5000 & 4. & & 4 & & \\
\hline $0 ?$ & 5220 & 333.2512 & 4.7000 & & 4 & & \\
\hline & $5 \quad 221$ & 314.5562 & 3.0750 & 284.0 & 4 & & 00 . \\
\hline & 222 & 318 & & & J & & \\
\hline & 223 & 323.5559 & 1.8750 & 283.7 & & 600.0 & 80 \\
\hline
\end{tabular}




\begin{tabular}{|c|c|c|c|c|c|c|c|}
\hline & 5 & & 750 & 84.0 & & & \\
\hline & 53 & & 1250 & 810 & & & \\
\hline & $\begin{array}{lll}5 & 3 & 2\end{array}$ & 125.3162 & 1.3000 & 83.7 & & 00 & \\
\hline & 53 & 204.1486 & 0.7250 & 283.7 & & 00 & \\
\hline & $\begin{array}{lll}5 & 3 & 4\end{array}$ & 0 & & & & & \\
\hline & $\begin{array}{lll}5 & 3 & 5\end{array}$ & 24 & 1.2250 & 83 & & v & \\
\hline & 53 & 08.8362 & 8500 & 83.0 & & 00 & \\
\hline & 5 & 87 & 1.20 & & & & \\
\hline & 5 & 24 & 1.0750 & 9 & & & \\
\hline & 5 & 42.9816 & 1.5500 & 84.0 & & 00 & \\
\hline & 5 & 7309 & 1.3250 & 5 & & & \\
\hline & 5 & 4 & 0 & 2 & & & \\
\hline & 5 & 1 & 3.10 & 86 & & .0 & \\
\hline & 5 & 02 & 2.22 & 4 & & & \\
\hline & 4 & & 2.2 & & & & \\
\hline & 53 & & 1.6 & 28 & & & \\
\hline & 5 & 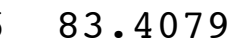 & 3.0 & 0 & & .0 & \\
\hline & 5 & & 4. & 7 & & & \\
\hline & 5 & & 5 . & 1 & & & \\
\hline & 5 & 65 & 7.42 & 286 & 4 & & \\
\hline & 5 & 62.7500 & 1.57 & 28 & 4 & . 0 & \\
\hline & $5 \quad 321$ & & & & 4 & & \\
\hline & 5 & 3 & 11 & 0 & 4 & & \\
\hline & 323 & 5013 & 8.8 & & 4 & .0 & \\
\hline & $5 \quad 324$ & & 4 . & & 4 & & \\
\hline & 54 & 0 & 4 & & 4 & & \\
\hline & $\begin{array}{ll}54 \\
4\end{array}$ & 5323 & 7.3 & 282.6 & 4 & .0 & \\
\hline & 54 & 40 & 8.7 & & 4 & & \\
\hline & 54 & 90 & 4. & 28 & 4 & & \\
\hline & 54 & 458 & 7.0 & 0 & 4 & .0 & \\
\hline & 546 & 826 & $7.5^{\circ}$ & 282.3 & 4 & & \\
\hline & $\begin{array}{lll}5 & 4 & 7\end{array}$ & 115 & 3.4 & 9 & 3 & & \\
\hline & 54 & & & & 3 & & \\
\hline & 54 & 005 & 500 & $2 \varepsilon$ & 4 & 0 & \\
\hline & 5410 & 2 & & & 4 & & \\
\hline & 5 & 495 & 8 . & & 4 & & \\
\hline & 5412 & 63.4992 & 8.0750 & 285.1 & 4 & .0 & \\
\hline & 5413 & 97 & 7 . & & 4 & & \\
\hline 03 & $5 \quad 414$ & 153 & 8.25 & $2 \varepsilon$ & 4 & .0 & \\
\hline & 54 & 520 & 7.3000 & 286.1 & 4 & & \\
\hline & 54 & 00 & 8. & & 4 & & \\
\hline 03 & 5417 & 128 & 6.72 & & 4 & .0 & \\
\hline & 5418 & 64.7493 & 8.2250 & 285.7 & 4 & & 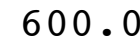 \\
\hline 03 & 5419 & 000 & 9.95 & & 4 & & \\
\hline 03 & 5420 & 64.2504 & 0.6000 & & 2 & .0 & \\
\hline 03 & $5 \quad 421$ & 65.7485 & 10.0000 & 282.9 & 4 & .0 & 500 \\
\hline & $5 \quad 422$ & & & & 4 & & \\
\hline & 423 & 68.7500 & 8.4500 & 282.8 & & .0 & 600 \\
\hline
\end{tabular}




\begin{tabular}{|c|c|c|c|c|c|c|c|c|}
\hline 3 & 5 & 424 & 65.0000 & 8.2250 & 282.8 & 4 & 600.0 & 600.0 \\
\hline 3 & 5 & 51 & 68.5000 & 9.0500 & 282.7 & 4 & 600.0 & 600.0 \\
\hline 03 & 5 & 52 & 66.0000 & 10.0750 & 282.7 & 4 & 600.0 & 00.0 \\
\hline 3 & 5 & 53 & 65.9999 & 9.4750 & 282.6 & 4 & 600.0 & 00.0 \\
\hline 03 & 5 & 54 & 72.5018 & 8.9750 & 282.7 & 4 & 600.0 & 00.0 \\
\hline 03 & 5 & 55 & 77.5000 & 9.3750 & 282.3 & 4 & 600.0 & 00.0 \\
\hline 03 & 5 & 56 & 78.2504 & 9.6250 & 281.7 & 4 & 600.0 & 00.0 \\
\hline 03 & 5 & 57 & 79.4998 & 8.9500 & 282.0 & 4 & 600.0 & 00.0 \\
\hline 03 & 5 & 58 & 70.4997 & 7.1000 & 282.9 & 4 & 600.0 & 00.0 \\
\hline 03 & 5 & 59 & 72.9596 & 5.3250 & 284.0 & 3 & 600.0 & .0 \\
\hline 03 & 5 & 510 & 91.2584 & 4.6250 & 285.2 & 3 & 600.0 & \\
\hline 03 & 5 & 511 & 96.7493 & 4.2500 & 286.4 & 3 & 600.0 & \\
\hline 03 & 5 & 512 & 100.2278 & 4.9000 & 287.0 & 3 & 600.0 & 00.0 \\
\hline 03 & 5 & 513 & 102.9671 & 4.1000 & 288.6 & 3 & .0 & 00.0 \\
\hline 03 & 5 & 514 & 98.0007 & 5.1250 & 289.2 & 3 & .0 & \\
\hline 03 & 5 & 515 & 95.5000 & 7.4750 & 288.8 & 4 & 600.0 & 00.0 \\
\hline 03 & 5 & 516 & 90.5000 & 7.8750 & 288.4 & 4 & 60 & 600.0 \\
\hline 03 & 5 & 517 & 96.0053 & 8.3000 & 287.1 & 4 & .0 & \\
\hline 03 & 5 & 518 & 76.7519 & 8.6500 & 285.9 & 4 & 60 & .0 \\
\hline 03 & 5 & 519 & 74.0000 & 10.0250 & 284.7 & 4 & .0 & .0 \\
\hline 03 & 5 & 520 & 72.7500 & 11.0000 & 283.8 & 4 & 6 & \\
\hline 03 & 5 & 521 & 77.9998 & 11.3000 & 283.5 & 4 & .0 & \\
\hline 03 & 5 & 522 & 71.7489 & 11.2750 & 283.0 & 4 & 600.0 & .0 \\
\hline 03 & 5 & 523 & 68.0002 & 10.67 & 283.4 & 4 & 6 & \\
\hline 03 & 5 & 524 & 71.5000 & 10.8500 & 283.1 & 4 & .0 & \\
\hline 03 & 5 & 61 & 74.2500 & 10.3250 & 282.7 & 4 & .0 & 600.0 \\
\hline 03 & 5 & 62 & 75.4991 & 11.7250 & 281.8 & 4 & .0 & .0 \\
\hline 03 & 5 & 63 & 70.4982 & 11.7500 & 281.4 & 4 & .0 & \\
\hline 03 & 5 & 64 & 65.7499 & 13.2500 & 281.1 & 4 & .0 & 600.0 \\
\hline 03 & 5 & 65 & 71.2500 & 12.4750 & 281.2 & 4 & 60 & 600.0 \\
\hline 03 & 5 & 66 & 76.7496 & 9.4250 & 282.0 & 4 & .0 & \\
\hline 03 & 5 & 67 & 81.0050 & 8.0500 & 281.8 & 4 & .0 & 600.0 \\
\hline 03 & 5 & 68 & 65.7504 & 10.4000 & 282.0 & 4 & 600.0 & 0.0 \\
\hline 03 & 5 & 69 & 67.7501 & 10.0750 & 283.0 & 4 & .0 & 0.0 \\
\hline 03 & 5 & 610 & 67.2501 & 10.0250 & 284.2 & 4 & 600.0 & 600.0 \\
\hline 03 & 5 & 611 & 69.9999 & 9.6500 & 285.3 & 4 & 600.0 & 600.0 \\
\hline 03 & 5 & 612 & 72.2498 & 9.2250 & 286.4 & 4 & 600.0 & .0 \\
\hline 03 & 5 & 613 & 75.4986 & 8.7000 & 287.1 & 4 & 600.0 & 600.0 \\
\hline 03 & 5 & 614 & 72.9793 & 9.3000 & 287.4 & 4 & 600.0 & 600.0 \\
\hline 03 & 5 & 615 & 71.5000 & 11.0250 & 286.7 & 4 & 600.0 & 0.0 \\
\hline 03 & 5 & 616 & 69.2500 & 10.8000 & 286.4 & 4 & 600.0 & 600.0 \\
\hline 03 & 5 & 617 & 67.9998 & 11.5750 & 285.4 & 4 & 600.0 & 600.0 \\
\hline 03 & 5 & 618 & 68.0000 & 11.4750 & 284.9 & 4 & 600.0 & 600.0 \\
\hline 03 & 5 & 619 & 71.0005 & 9.9500 & 283.5 & 4 & 600.0 & 600.0 \\
\hline 03 & 5 & 620 & 78.7502 & 8.5500 & 283.4 & 4 & 600.0 & 600.0 \\
\hline 03 & 5 & 621 & 71.4991 & 9.6750 & 282.9 & 4 & 600.0 & 600.0 \\
\hline 0 & 5 & 622 & 70.9999 & 9.8500 & 283.0 & 4 & 600.0 & 600.0 \\
\hline & & 623 & 66.2501 & 10.250 & 282.9 & & 600.0 & 600.0 \\
\hline
\end{tabular}




\begin{tabular}{|c|c|c|c|c|c|c|c|c|}
\hline 3 & 5 & 624 & 64.2500 & 10.2500 & 282.8 & 4 & 600.0 & 600.0 \\
\hline 03 & 5 & 71 & 5.2502 & 11.2500 & 282.6 & 4 & 600.0 & 600.0 \\
\hline 03 & 5 & 72 & 65.0001 & 9.8500 & 282.5 & 4 & 600.0 & 00.0 \\
\hline 03 & 5 & 73 & 67.7498 & 7.8000 & 282.5 & 4 & 600.0 & 600.0 \\
\hline 03 & 5 & 74 & 66.5009 & 7.4750 & 282.2 & 4 & 600.0 & 00.0 \\
\hline 03 & 5 & 75 & 63.7505 & 7.3000 & 282.1 & 4 & 600.0 & 500.0 \\
\hline 03 & 5 & 76 & 64.2496 & 6.8750 & 281.9 & 4 & 600.0 & 600.0 \\
\hline 03 & 5 & 77 & 66.7498 & 6.9250 & 282.8 & 4 & 600.0 & 500.0 \\
\hline 03 & 5 & 78 & 59.2492 & 6.7750 & 283.6 & 4 & 600.0 & 00.0 \\
\hline 03 & 5 & 79 & 74.2511 & 6.0000 & 284.5 & 4 & 600.0 & 600.0 \\
\hline 03 & 5 & 710 & 95.0096 & 5.9250 & 285.6 & 3 & 600.0 & 00.0 \\
\hline 03 & 5 & 711 & 93.4962 & 4.5000 & 286.9 & 3 & 600.0 & \\
\hline 03 & 5 & 712 & 93.0049 & 5.0750 & 287.6 & 3 & 600.0 & 600.0 \\
\hline 03 & 5 & 713 & 86.5165 & 5.4750 & 287.6 & 3 & 600.0 & 600.0 \\
\hline 03 & 5 & 714 & 65.9952 & 5.3250 & 287.4 & 3 & 600.0 & \\
\hline 03 & 5 & 715 & 70.2485 & 6.3000 & 288.8 & 4 & 600.0 & 60 \\
\hline 03 & 5 & 716 & 63.5000 & 9.0250 & 287.5 & 4 & 600.0 & 600.0 \\
\hline 03 & 5 & 717 & 64.2499 & 9.2250 & 286.7 & 4 & 600.0 & \\
\hline 03 & 5 & 718 & 66.7497 & 8.3500 & 285.9 & 4 & 600.0 & 60 \\
\hline 03 & 5 & 719 & 61.2500 & 12.2500 & 283.8 & 4 & 600.0 & 600.0 \\
\hline 03 & 5 & 720 & 56.5000 & 14.4750 & 282.3 & 4 & 600.0 & .0 \\
\hline 03 & 5 & 721 & 55.2504 & 14. & 282.0 & 4 & 600.0 & \\
\hline 03 & 5 & 722 & 59.7500 & 16.0750 & 281.6 & 4 & 600.0 & 600.0 \\
\hline 03 & 5 & 723 & 56.7500 & 15. & 281.5 & 4 & 600.0 & .0 \\
\hline 03 & 5 & 724 & 56.2503 & 14 . & 281.7 & 4 & 600.0 & \\
\hline 03 & 5 & 81 & 54.7557 & 13.3000 & 281.4 & 4 & 600.0 & 600.0 \\
\hline 03 & 5 & 82 & 58.7537 & 12.4750 & 281.4 & 4 & 600.0 & 60 \\
\hline 03 & 5 & 83 & 56.7514 & 8.9500 & 281.1 & 4 & 600.0 & .0 \\
\hline 03 & 5 & 84 & 68.2492 & 6.8750 & 280.9 & 4 & 600.0 & 60 \\
\hline 03 & 5 & 85 & 67.0005 & 8.2000 & 280.6 & 4 & 600.0 & 600.0 \\
\hline 03 & 5 & 86 & 67.0000 & 8.72 & 280.7 & 4 & 600.0 & .0 \\
\hline 03 & 5 & 87 & 67.5000 & 9.6 & 281.0 & 4 & 600.0 & 60 \\
\hline 03 & 5 & 88 & 76.5000 & 8.4500 & 281.4 & 4 & 600.0 & 600.0 \\
\hline 03 & 5 & 89 & 59.7499 & 9.1750 & 280.9 & 4 & 600.0 & .0 \\
\hline 03 & 5 & 810 & 69.0034 & 7. & 282.7 & 4 & 600.0 & 60 \\
\hline 03 & 5 & 811 & 64.4936 & 6.5750 & 284.1 & 4 & 600.0 & 600.0 \\
\hline 03 & 5 & 812 & 60.7497 & 7.6750 & 283.5 & 4 & 600.0 & 600.0 \\
\hline 03 & 5 & 813 & 58.7505 & 9.7250 & 284.5 & 4 & 600.0 & 600.0 \\
\hline 03 & 5 & 814 & 59.4998 & 11.2500 & 284.6 & 4 & 600.0 & 600.0 \\
\hline 03 & 5 & 815 & 59.4924 & 9.9000 & 284.7 & 4 & 600.0 & \\
\hline 03 & 5 & 816 & 60.2584 & 9.9000 & 284.3 & 4 & 600.0 & 600.0 \\
\hline 03 & 5 & 817 & 67.9973 & 8.8750 & 283.9 & 4 & 600.0 & 600.0 \\
\hline 03 & 5 & 818 & 79.4936 & 8.2000 & 283.1 & 4 & 600.0 & 600.0 \\
\hline 03 & 5 & 819 & 79.2465 & 8.1500 & 281.9 & 4 & 600.0 & 600.0 \\
\hline 03 & 5 & 820 & 66.5009 & 6.6000 & 281.1 & 4 & 600.0 & 600.0 \\
\hline 03 & 5 & 821 & 71.7517 & 7.9250 & 280.7 & 4 & 600.0 & 600.0 \\
\hline 03 & 5 & 822 & 68.5021 & 7.000 & 280.4 & 4 & 600.0 & 600.0 \\
\hline 03 & $J$ & 823 & 78.9995 & 7.4500 & 280.3 & 4 & 600.0 & 600.0 \\
\hline
\end{tabular}




\begin{tabular}{|c|c|c|c|c|c|c|c|}
\hline 03 & $5 \quad 824$ & 80.2496 & 7.9750 & 280.2 & 4 & 600.0 & 600.0 \\
\hline 03 & $\begin{array}{lll}5 & 9 & 1\end{array}$ & 85.5000 & 7.9250 & 280.1 & 4 & 600.0 & 600.0 \\
\hline 03 & 592 & 71.5008 & 7.6500 & 279.9 & 4 & 600.0 & 00.0 \\
\hline 03 & $\begin{array}{lll}5 & 9 & 3\end{array}$ & 69.7500 & 7.6500 & 279.6 & 4 & 600.0 & 00.0 \\
\hline 03 & $\begin{array}{lll}5 & 9 & 4\end{array}$ & 74.0002 & 6.8500 & 279.6 & 4 & 600.0 & 00.0 \\
\hline 03 & 595 & 69.7498 & 7.7250 & 279.5 & 4 & 600.0 & 00.0 \\
\hline 03 & 596 & 77.9998 & 6.7750 & 279.7 & 4 & 600.0 & 00.0 \\
\hline 03 & $\begin{array}{lll}5 & 9 & 7\end{array}$ & 80.0011 & 6.4500 & 280.5 & 4 & 600.0 & 00.0 \\
\hline 03 & $\begin{array}{lll}5 & 9 & 8\end{array}$ & 81.9950 & 3.9750 & 282.1 & 3 & 600.0 & 00.0 \\
\hline 03 & $\begin{array}{lll}5 & 9 & 9\end{array}$ & 77.0021 & 2.8750 & 284.1 & 2 & 600.0 & .0 \\
\hline 03 & 5910 & 106.5007 & 2.5750 & 285.1 & 1 & 600.0 & \\
\hline 03 & 5911 & 92.2450 & 3.1750 & 286.4 & 2 & 600.0 & \\
\hline 03 & 5912 & 127.2231 & 4.3250 & 286.6 & 3 & 600.0 & 00.0 \\
\hline 03 & 5913 & 113.2391 & 4.6250 & 287.3 & 3 & 600.0 & 00.0 \\
\hline 03 & 5914 & 110.7560 & 4.9750 & 287.7 & 3 & .0 & \\
\hline 03 & 5915 & 99.7514 & 6.5250 & 287.6 & 4 & 600.0 & 00.0 \\
\hline 03 & 5916 & 97.2524 & 8.6750 & 286.6 & 4 & 60 & 600.0 \\
\hline 03 & 5917 & 88.9975 & 9.3500 & 285.9 & 4 & .0 & \\
\hline 03 & 5918 & 74.4998 & 8.8250 & 285.0 & 4 & 600.0 & .0 \\
\hline 03 & 5919 & 67.7500 & 8.9250 & 283.7 & 4 & 60 & .0 \\
\hline 03 & 5920 & 70.5000 & 9. & 282.5 & 4 & 60 & \\
\hline 03 & $\begin{array}{ll}5 & 921\end{array}$ & 70.7500 & 10.7000 & 281.8 & 4 & .0 & \\
\hline 03 & 5922 & 71.7500 & 10.5500 & 281.8 & 4 & 600.0 & .0 \\
\hline 03 & 5923 & 70.5000 & 9.70 & 282.0 & 4 & 60 & .0 \\
\hline 03 & 5924 & 75.0004 & 9.0750 & 282.4 & 4 & .0 & \\
\hline 03 & 5101 & 75.7500 & 8.9500 & 282.3 & 4 & .0 & 600.0 \\
\hline 03 & 5102 & 77.2498 & 8.1000 & 282.2 & 4 & 600.0 & 600.0 \\
\hline 03 & 5103 & 76.2500 & 6.9750 & 282.2 & 4 & .0 & \\
\hline 03 & 5104 & 79.2429 & 5.9250 & 282.8 & 4 & .0 & 600.0 \\
\hline 03 & 5105 & 106.7562 & 3.2000 & 283.3 & 4 & 600.0 & 600.0 \\
\hline 03 & 5106 & 132.5438 & 2.1750 & 283.2 & 5 & .0 & \\
\hline 03 & 5107 & 160.7295 & 1.7750 & 284.5 & 4 & .0 & 600.0 \\
\hline 03 & 5108 & 178.7506 & 2.9000 & 285.7 & 4 & 600.0 & 600.0 \\
\hline 03 & 5109 & 181.9893 & 3.1250 & 287.4 & 3 & 0.0 & 0.0 \\
\hline 03 & 51010 & 167.2504 & 3.2000 & 288.1 & 2 & 600.0 & 600.0 \\
\hline 03 & 51011 & 191.5000 & 4.0500 & 288.8 & 3 & 600.0 & 600.0 \\
\hline 03 & 51012 & 182.6191 & 4.0750 & 289.7 & 3 & 600.0 & 0.0 \\
\hline 03 & 51013 & 195.5052 & 4.8000 & 290.1 & 3 & 600.0 & 600.0 \\
\hline 03 & 51014 & 158.5000 & 4.7000 & 290.9 & 3 & 600.0 & 600.0 \\
\hline 03 & 51015 & 150.4648 & 4.1750 & & 3 & 600.0 & 600.0 \\
\hline 03 & 51016 & 126.3958 & 5.1750 & 290.9 & 3 & 600.0 & 600.0 \\
\hline 03 & 51017 & 115.7666 & 5.1750 & 291.4 & 3 & 600.0 & 600.0 \\
\hline 03 & 51018 & 94.2429 & 5.4000 & 290.6 & 3 & 600.0 & 600.0 \\
\hline 03 & 51019 & 71.5000 & 6.3750 & 288.6 & 4 & 600.0 & 600.0 \\
\hline 03 & 51020 & 76.2499 & 7.0250 & 286.9 & 4 & 600.0 & 600.0 \\
\hline 03 & 51021 & 72.7508 & 8.2500 & 286.3 & 4 & 600.0 & 600.0 \\
\hline 03 & 51022 & 68.5000 & 9.1500 & 286.1 & 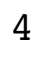 & 600.0 & 600.0 \\
\hline & 51023 & 67.7489 & 8.975 & 285.6 & 4 & 600.0 & 600.0 \\
\hline
\end{tabular}




\begin{tabular}{|c|c|c|c|c|c|c|c|}
\hline 03 & 51024 & 70.7500 & 9.3750 & 285.4 & 4 & 600.0 & 600.0 \\
\hline 03 & 5111 & 74.0000 & 9.5000 & 285.1 & 4 & 600.0 & 600.0 \\
\hline 03 & 5112 & 71.7500 & 7.9750 & 286.3 & 4 & 600.0 & 00.0 \\
\hline 03 & 5113 & 71.2511 & 6.3500 & 287.7 & 4 & 600.0 & 600.0 \\
\hline 03 & 5114 & 72.4994 & 5.6250 & 286.9 & 4 & 600.0 & 00.0 \\
\hline 03 & 5115 & 81.6113 & 4.0500 & 285.9 & 4 & 600.0 & 000.0 \\
\hline 03 & 5116 & 23.9962 & 1.2250 & 286.3 & 5 & 600.0 & 600.0 \\
\hline 03 & 5117 & 57.2127 & 0.3250 & 288.5 & 4 & 600.0 & 600.0 \\
\hline 03 & 5118 & 281.9884 & 1.0250 & 290.5 & 3 & 600.0 & 00.0 \\
\hline 03 & 5119 & 238.2427 & 2.2750 & 289.4 & 2 & 600.0 & 600.0 \\
\hline 03 & 51110 & 238.0000 & 2.8250 & 289.8 & 2 & 600.0 & 600.0 \\
\hline 03 & 51111 & 259.2529 & 3.3000 & 290.9 & 2 & 600.0 & .0 \\
\hline 03 & 51112 & 223.0205 & 3.5750 & 291.5 & 2 & 600.0 & 600.0 \\
\hline 03 & 51113 & 216.4987 & 3.3750 & 292.6 & 2 & 600.0 & 600.0 \\
\hline 03 & 51114 & 172.5883 & 3.3250 & 293.7 & 2 & 600.0 & \\
\hline 03 & 51115 & 162.7659 & 4.7750 & 293.5 & 3 & 600.0 & 60 \\
\hline 03 & 51116 & 166.5000 & 6.2750 & 293.5 & 4 & 600.0 & 600.0 \\
\hline 03 & 51117 & 113.0055 & 5.2000 & 293.4 & 3 & 600.0 & \\
\hline 03 & 51118 & 99.4673 & 5.9250 & 291.8 & 3 & 600.0 & 60 \\
\hline 03 & 51119 & 67.9989 & 7.4000 & 289.9 & 4 & 600.0 & 600.0 \\
\hline 03 & 51120 & 75.7497 & 8.57 & 288.1 & 4 & 600.0 & .0 \\
\hline 03 & 51121 & 71.7506 & 9 . & 287.8 & 4 & 600.0 & \\
\hline 03 & 51122 & 70.0000 & 9.8750 & 287.6 & 4 & 600.0 & 600.0 \\
\hline 03 & 51123 & 79.0000 & 8.9500 & 287.8 & 4 & 600.0 & .0 \\
\hline 03 & 51124 & 78.5001 & 6. & 288.6 & 4 & 600.0 & \\
\hline 03 & 5121 & 71.4997 & 4.7000 & 289.3 & 4 & 600.0 & 600.0 \\
\hline 03 & 5122 & 54.7390 & 3.5750 & 289.3 & 4 & 600.0 & 600.0 \\
\hline 03 & 5123 & 44.7519 & 2.5750 & 289.6 & 4 & 600.0 & .0 \\
\hline 03 & 5124 & 59.7335 & 2.1750 & 289.3 & 5 & 600.0 & 600.0 \\
\hline 03 & 5125 & 104.9533 & 1.2500 & 288.6 & 6 & 600.0 & 600.0 \\
\hline 03 & 5126 & 53.7086 & 1.0500 & 288.3 & 6 & 600.0 & .0 \\
\hline 03 & 5127 & 90.1027 & 0.5500 & 291.1 & 5 & 600.0 & 600.0 \\
\hline 03 & 5128 & 250.6614 & 1.1250 & 292.9 & 4 & 600.0 & 600.0 \\
\hline 03 & 5129 & 194.5842 & 3. & 292.0 & 3 & 600.0 & .0 \\
\hline 03 & 51210 & 184.7463 & 4 . & 291.5 & 3 & 600.0 & 60 \\
\hline 03 & 51211 & 181.5028 & 3.9750 & 292.3 & 2 & 600.0 & 600.0 \\
\hline 03 & 51212 & 170.5000 & 3.8500 & 293.8 & 2 & 600.0 & 600.0 \\
\hline 03 & 51213 & 175.2544 & 4.3250 & 294.7 & 3 & 600.0 & 600.0 \\
\hline 03 & 51214 & 175.7537 & 5.4000 & 295.4 & 3 & 600.0 & 600.0 \\
\hline 03 & 51215 & 175.9807 & 5.8250 & 296.0 & 3 & 600.0 & 600.0 \\
\hline 03 & 51216 & 178.7490 & 6.2750 & 296.4 & 4 & 600.0 & 600.0 \\
\hline 03 & 51217 & 183.5000 & 6.3000 & 296.4 & 4 & 600.0 & 600.0 \\
\hline 03 & 51218 & 172.9593 & 5.2750 & 296.3 & 3 & 600.0 & 600.0 \\
\hline 03 & 51219 & 148.5029 & 5.1250 & 295.2 & 4 & 600.0 & 600.0 \\
\hline 03 & 51220 & 97.2455 & 4.2750 & 293.5 & 4 & 600.0 & 600.0 \\
\hline 03 & 51221 & 98.7533 & 2.9500 & 293.6 & 4 & 600.0 & 600.0 \\
\hline 03 & 51222 & 83.2501 & 3.8000 & 293.3 & 5 & 600.0 & 600.0 \\
\hline 03 & 51223 & 78.7500 & 5.1000 & 292.9 & 4 & 600.0 & 600.0 \\
\hline
\end{tabular}




\begin{tabular}{|c|c|c|c|c|c|c|c|}
\hline 03 & 51224 & 78.2500 & 4.7000 & 293.2 & 5 & 600.0 & 600.0 \\
\hline 3 & 5131 & 77.5000 & 4.5250 & 293.5 & 5 & 600.0 & 00,0 \\
\hline 03 & 5132 & 101.3633 & 3.2250 & 293.8 & 4 & 600.0 & 00.0 \\
\hline 3 & 5133 & 130.7499 & 2.2500 & 292.9 & 4 & 600.0 & 00.0 \\
\hline 03 & 5134 & 141.7506 & 2.7500 & 292.0 & 5 & 600.0 & 00.0 \\
\hline 03 & 5135 & 148.0000 & 2.6500 & 291.1 & 5 & 600.0 & 00.0 \\
\hline 03 & 5136 & 130.2480 & 2.0750 & 291.2 & 4 & 600.0 & 0 \\
\hline 03 & 5137 & 120.9952 & 2.1250 & 292.0 & 4 & 600.0 & 00.0 \\
\hline 03 & 5138 & 174.3597 & 1.9250 & 293.9 & 3 & 600.0 & 00.0 \\
\hline 03 & 5139 & 170.0055 & 3.5000 & 294.6 & 3 & 60 & 0 \\
\hline 03 & 51310 & 171.4998 & 5.2500 & 294.7 & 4 & 600.0 & \\
\hline 03 & 51311 & 169.2492 & 5.3750 & 295.4 & 3 & .0 & \\
\hline 03 & 51312 & 165.4998 & 4.5250 & 296.2 & 3 & 600.0 & .0 \\
\hline 03 & 51313 & 147.4879 & 4.1000 & 297.2 & 3 & 600.0 & \\
\hline 03 & 51314 & 151.7294 & 5.0750 & 298.1 & 3 & .0 & \\
\hline 03 & 51315 & 149.0027 & 5.1500 & 298.1 & 3 & 600.0 & .0 \\
\hline 03 & 51316 & 112.7502 & 5.0000 & 298.0 & 3 & 60 & .0 \\
\hline 03 & 51317 & 103.7763 & 5.4250 & 297.6 & 3 & .0 & \\
\hline 03 & 51318 & 96.5161 & 5.6750 & 296.5 & 3 & .0 & \\
\hline 03 & 51319 & 73.0014 & 6.3000 & 295.1 & 4 & .0 & .0 \\
\hline 03 & 51320 & 70.7499 & 8 . & 293.7 & 4 & 6 & \\
\hline 03 & 51321 & 73.0001 & 9.1250 & 292.8 & 4 & .0 & \\
\hline 03 & 51322 & 71.5009 & 9.7000 & 292.7 & 4 & 600.0 & .0 \\
\hline 03 & 51323 & 73.7500 & 0.27 & 291.6 & 4 & 0 & \\
\hline 03 & 51324 & 69.2500 & 10 & 291.1 & 4 & 0 & \\
\hline 03 & 5141 & 72.0001 & 3750 & 291.2 & 4 & .0 & \\
\hline 03 & 5142 & 74.2500 & 10.5000 & 290.6 & 4 & 60 & .0 \\
\hline 03 & 5143 & 75.2500 & 10.2750 & 290.0 & 4 & .0 & \\
\hline 03 & 5144 & 76.0000 & 9.5500 & 289.8 & 4 & .0 & .0 \\
\hline 03 & $514 \quad 5$ & 77.7500 & 9.0500 & 290.0 & 4 & 6 & .0 \\
\hline 03 & 5146 & 77.2500 & 11.2750 & 286.5 & 4 & .0 & \\
\hline 03 & 5147 & 78.2500 & 12.7250 & 284.3 & 4 & .0 & .0 \\
\hline 03 & 5148 & 78.2500 & 13.3750 & 284.9 & 4 & 0 & 0.0 \\
\hline 03 & 5149 & 76.0000 & 12.9750 & 285.6 & 4 & .0 & .0 \\
\hline 03 & 51410 & 76.5000 & 12.7500 & 285.2 & 4 & .0 & 0.0 \\
\hline 03 & 51411 & 73.2495 & 9.9750 & 288.3 & 4 & 600.0 & 600.0 \\
\hline 03 & 51412 & 82.2500 & 10.3000 & 290.5 & 4 & 600.0 & .0 \\
\hline 03 & 51413 & 80.0000 & 10.7000 & 291.0 & 4 & 600.0 & 600.0 \\
\hline 03 & 51414 & 79.9998 & 11.1000 & 290.8 & 4 & 600.0 & 600.0 \\
\hline 03 & 51415 & 72.7498 & 11.5500 & 290.4 & 4 & 600.0 & .0 \\
\hline 03 & 51416 & 74.7494 & 11.3750 & 291.2 & 4 & 600.0 & 600.0 \\
\hline 03 & 51417 & 77.2501 & 12.7750 & 290.1 & 4 & 600.0 & 600.0 \\
\hline 03 & 51418 & 67.9998 & 11.9000 & 290.3 & 4 & 600.0 & 600.0 \\
\hline 03 & 51419 & 68.5002 & 12.6250 & 288.3 & 4 & 600.0 & 600.0 \\
\hline 03 & 51420 & 70.7499 & 14.4750 & 285.7 & 4 & 600.0 & 600.0 \\
\hline 03 & 51421 & 69.2501 & 14.6500 & 285.4 & 4 & 600.0 & 600.0 \\
\hline 0 & 51422 & 65.2500 & 14.8500 & 286.3 & 4 & 600.0 & 600.0 \\
\hline & $5 \perp 4<3$ & 68.5000 & 15.750 & 284.9 & 4 & 600.0 & 600.0 \\
\hline
\end{tabular}




\begin{tabular}{|c|c|c|c|c|c|c|c|}
\hline 3 & 51424 & 71.5005 & 16.0000 & 284.5 & 4 & 600.0 & 600.0 \\
\hline 03 & 5151 & 65.5000 & 14.9750 & 284.1 & 4 & 600.0 & 600.0 \\
\hline 03 & 5152 & 74.1433 & 7.7250 & 284.8 & 4 & 600.0 & 00.0 \\
\hline 03 & 5153 & 76.3869 & 11.3750 & 284.1 & 4 & 600.0 & 00.0 \\
\hline 3 & 5154 & 66.2500 & 12.8750 & 284.3 & 4 & 600.0 & 00.0 \\
\hline 03 & 155 & 68.7501 & 10.8250 & 284.8 & 4 & 600.0 & 00.0 \\
\hline 03 & 156 & 61.2492 & 10.9500 & 285.0 & 4 & 600.0 & 600.0 \\
\hline 03 & 157 & 86.1818 & 6.1500 & 286.2 & 4 & 600.0 & 000.0 \\
\hline 03 & 158 & 70.4516 & 10.1250 & 285.3 & 4 & 600.0 & .0 \\
\hline 03 & 159 & 61.7493 & 11.9750 & 284.4 & 4 & 600.0 & 600.0 \\
\hline 03 & 1510 & 72.9979 & 8.7500 & 285.3 & 4 & 600.0 & 0 \\
\hline 03 & 511 & 79.0000 & 7.6000 & 287.4 & 4 & 600.0 & \\
\hline 03 & 1512 & 70.7342 & 7.4250 & 288.8 & 4 & 600.0 & .0 \\
\hline 03 & 1513 & 66.2487 & 6.5750 & 290.4 & 4 & 600.0 & 0 \\
\hline 03 & 514 & 68.9998 & 8.1750 & 290.6 & 4 & 600.0 & .0 \\
\hline 03 & 515 & 71.2500 & 9.1250 & 290.2 & 4 & 600.0 & \\
\hline 03 & 516 & 71.0000 & 12.1500 & 288.5 & 4 & 600.0 & 600.0 \\
\hline 03 & 517 & 74.2500 & 14.3000 & 287.1 & 4 & 600.0 & .0 \\
\hline 03 & 518 & 78.5000 & 13.1250 & 286.5 & 4 & 600.0 & \\
\hline 03 & 519 & 78.7506 & 12.3500 & 285.7 & 4 & 600.0 & 600.0 \\
\hline 03 & 1520 & 75.2499 & 12.5000 & 284.6 & 4 & 600.0 & 600.0 \\
\hline 03 & 521 & 74.5000 & 11.8750 & 285.3 & 4 & 600.0 & \\
\hline 03 & 522 & 74.4998 & 9.6250 & 286.6 & 4 & 600.0 & 60 \\
\hline 03 & 1523 & 79.2498 & 8.3500 & 287.0 & 4 & 600.0 & .0 \\
\hline 03 & 1524 & 75.2501 & 7.2500 & 287.8 & 4 & 600.0 & \\
\hline 03 & 5161 & 73.7499 & 7.2750 & 288.0 & 4 & 600.0 & 60 \\
\hline 03 & 162 & 80.0021 & 6.7750 & 288.0 & 4 & 600.0 & .0 \\
\hline 03 & 5163 & 80.9995 & 5.7000 & 287.7 & 4 & 600.0 & .0 \\
\hline 03 & 5164 & 84.7504 & 7.3000 & 287.0 & 4 & 600.0 & 60 \\
\hline 03 & 5165 & 82.9979 & 5.6750 & 286.7 & 4 & 600.0 & 600.0 \\
\hline 03 & 5166 & 133.8166 & 2.5250 & 286.7 & 5 & 600.0 & 60 \\
\hline 03 & 5167 & 102.4406 & 3.8750 & 287.5 & 4 & 600.0 & 60 \\
\hline 03 & 5168 & 127.8415 & 4.3500 & 287.9 & 3 & 600.0 & 600.0 \\
\hline 03 & 5169 & 158.2511 & 6.9000 & 287.9 & 4 & 600.0 & 600.0 \\
\hline 03 & 51610 & 160.5008 & 5.0250 & 289.2 & 3 & 600.0 & 60 \\
\hline 03 & 51611 & 173.7495 & 4.3750 & 290.9 & 3 & 600.0 & 600.0 \\
\hline 03 & 51612 & 159.2656 & 4.4250 & 291.7 & 3 & 600.0 & 600.0 \\
\hline 03 & 51613 & 166.5320 & 5.2750 & 292.8 & 3 & 600.0 & 600.0 \\
\hline 03 & 51614 & 162.0088 & 5.2250 & 293.7 & 3 & 600.0 & 600.0 \\
\hline 03 & 51615 & 167.5000 & 5.3500 & 294.8 & 3 & 600.0 & 600.0 \\
\hline 03 & 51616 & 150.2464 & 5.3500 & 295.3 & 3 & 600.0 & 600.0 \\
\hline 03 & 51617 & 112.0511 & 5.6750 & 294.9 & 3 & 600.0 & 600.0 \\
\hline 03 & 51618 & 86.7500 & 6.4500 & 293.5 & 4 & 600.0 & 600.0 \\
\hline 03 & 51619 & 76.2496 & 6.8500 & 292.2 & 4 & 600.0 & 600.0 \\
\hline 03 & 51620 & 83.2544 & 9.0000 & 290.9 & 4 & 600.0 & 600.0 \\
\hline 03 & 51621 & 73.7459 & 8.1000 & 290.9 & 4 & 600.0 & 600.0 \\
\hline 03 & 51622 & 62.2498 & & & 4 & 600.0 & .0 \\
\hline 03 & 51623 & 69.7500 & 7.7750 & 291.4 & $\mathbf{4}$ & 600.0 & 600.0 \\
\hline
\end{tabular}




\begin{tabular}{|c|c|c|c|c|c|c|c|}
\hline 3 & 51624 & 68.2500 & 8.7250 & 290.8 & 4 & 600.0 & 00.0 \\
\hline 03 & 5171 & 72.9996 & 8.8000 & 290.2 & 4 & 600.0 & 00,0 \\
\hline 03 & 5172 & 77.9988 & 8.7500 & 290.0 & 4 & 600.0 & 00.0 \\
\hline 03 & 5173 & 79.0000 & 8.5750 & 289.4 & 4 & 600.0 & 00.0 \\
\hline 03 & 174 & 82.2495 & 8.8750 & 288.1 & $I$ & 600.0 & 00.0 \\
\hline 03 & 5175 & 102.2219 & 7.8000 & 287.8 & 4 & 600.0 & 00.0 \\
\hline 03 & 5176 & 139.2595 & 9.3250 & 288.2 & 4 & 600.0 & 00.0 \\
\hline 03 & 5177 & 154.4917 & 12.6500 & 287.2 & 4 & 600.0 & 00.0 \\
\hline 03 & 5178 & 159.9989 & 7.7250 & 287.7 & 4 & 600.0 & 00.0 \\
\hline 03 & 5179 & 161.4986 & 8.8750 & 287.7 & 4 & 600.0 & .0 \\
\hline 03 & 51710 & 154.5000 & 6.9500 & 288.3 & 4 & 600.0 & 00.0 \\
\hline 03 & 1711 & 154.0024 & 5.5750 & 289.7 & 3 & 600.0 & \\
\hline 03 & 51712 & 156.7485 & 4.9000 & 291.5 & 3 & 600.0 & .0 \\
\hline 03 & 51713 & 164.7502 & 4.8250 & 292.7 & 3 & 600.0 & 600 \\
\hline 03 & 51714 & 163.5000 & 5 & 293.8 & 3 & 600.0 & \\
\hline 03 & 51715 & 172.2893 & 5.3500 & 294.7 & 3 & 600.0 & 60 \\
\hline 03 & 51716 & 170.2497 & 5.8250 & 295.3 & 3 & 600.0 & .0 \\
\hline 03 & 51717 & 176.5035 & 6.7000 & 295.5 & 4 & 600.0 & \\
\hline 03 & 51718 & 146.3811 & 5.4250 & 295.0 & 3 & 600.0 & .0 \\
\hline 03 & 51719 & 86.4671 & 5.3000 & 293.2 & 4 & 600.0 & .0 \\
\hline 03 & 51720 & 73.5002 & 6.77 & 291.0 & 4 & 600.0 & .0 \\
\hline 03 & 51721 & 74.0000 & 6.77 & 290.2 & 4 & 0.0 & \\
\hline 03 & 51722 & 83.9384 & 5.9000 & 290.4 & 4 & 600.0 & \\
\hline 03 & 51723 & 111.7750 & 4.8000 & 291.5 & 4 & 600.0 & .0 \\
\hline 03 & 51724 & 137.2434 & 5.7750 & 291.8 & 4 & 00.0 & \\
\hline 03 & 5181 & 158.7496 & 9.2000 & 290.4 & 4 & 600.0 & 60 \\
\hline 03 & 5182 & 161.2506 & 11.9250 & 288.2 & 4 & 600.0 & .0 \\
\hline 03 & 5183 & 161.2500 & 12.6250 & 286.7 & 4 & 0.0 & \\
\hline 03 & 5184 & 168.5017 & 12.6000 & 285.9 & 4 & 600.0 & 60 \\
\hline 03 & 5185 & 166.5000 & 11.2000 & 285.2 & 4 & 600.0 & .0 \\
\hline 03 & 5186 & 168.0005 & 13.27 & 284.5 & 4 & 0.0 & \\
\hline 03 & 5187 & 169.5009 & 15.7250 & 284.3 & 4 & 600.0 & \\
\hline 03 & 5188 & 171.0001 & 16.0750 & 284.4 & 4 & 600.0 & 600.0 \\
\hline 03 & 5189 & 176.5000 & 14.75 & 285.2 & 4 & 600.0 & \\
\hline 03 & 51810 & 175.2465 & 14.1250 & 286.2 & 4 & 600.0 & .0 \\
\hline 03 & 51811 & 169.2501 & 12.7250 & 287.5 & 4 & 600.0 & 600.0 \\
\hline 03 & 51812 & 167.0000 & 10.8250 & 289.0 & 4 & 600.0 & \\
\hline 03 & 51813 & 167.2518 & 10.7250 & 290.3 & 4 & 600.0 & .0 \\
\hline 03 & 51814 & 170.2500 & 9.4000 & 291.6 & 4 & 600.0 & 600.0 \\
\hline 03 & 51815 & 168.2500 & 7.750 & 292.6 & 4 & 600.0 & \\
\hline 03 & 51816 & 173.0000 & 7.675 & 293.4 & 4 & 600.0 & 60 \\
\hline 03 & 51817 & 168.9979 & 8.1500 & 293.8 & 4 & 600.0 & 600.0 \\
\hline 03 & 51818 & 166.2500 & 7.8750 & 293.7 & 4 & 600.0 & 600.0 \\
\hline 03 & 51819 & 165.2532 & 6.2250 & 293.1 & 4 & 600.0 & 600.0 \\
\hline 03 & 51820 & 147.5000 & 5.0000 & 292.0 & 4 & 600.0 & 600.0 \\
\hline 03 & 51821 & 144.7502 & 5.3500 & 291.6 & 4 & 600.0 & 600.0 \\
\hline 03 & 51822 & 143.7498 & 6.725 & 1.5 & 4 & 600.0 & 600.0 \\
\hline 03 & 51823 & 147.0000 & 7.925 & 291.5 & 4 & 600.0 & 600.0 \\
\hline
\end{tabular}




\begin{tabular}{|c|c|c|c|c|c|c|c|}
\hline 3 & 1824 & & 500 & 5 & & & \\
\hline & 191 & 150 & 7.6500 & 1.1 & 4 & $\Leftrightarrow \cap 00$ & \\
\hline & 192 & 155.2499 & 7.9500 & 290.4 & 4 & 00.0 & \\
\hline & 193 & 159.2495 & 8.5750 & 290.0 & & 00.0 & \\
\hline & 194 & 55.2467 & .4250 & 289.5 & & & \\
\hline & 195 & 25 & 4750 & 289.2 & & $0 C$ & \\
\hline & 196 & 58.4997 & 9000 & 289.1 & & 00 & \\
\hline & 197 & 2496 & .6750 & 289.7 & & .0 & \\
\hline & 198 & 2.9989 & .7500 & 290.1 & & & \\
\hline & 199 & 67.9998 & 8.5750 & 290.6 & & 00 & 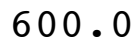 \\
\hline & 1910 & 160.0000 & 6.5250 & 291.7 & & & \\
\hline & 1911 & 165.2526 & 9.72 & 292.9 & & .0 & \\
\hline & 1912 & 993 & 9.0500 & 294.1 & 4 & 00.0 & \\
\hline & 913 & 54.7508 & 7.5250 & 295 & 4 & 0.0 & \\
\hline & 914 & 56.2 & 0 & 29 & 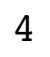 & & \\
\hline & 915 & 712 & & 297 & 4 & & \\
\hline & 1916 & 05 & 6.4000 & 298.2 & 4 & 00.0 & 000 \\
\hline & 917 & 87 & 500 & $29 \varepsilon$ & 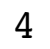 & .0 & \\
\hline & 918 & & 5 & 298 & 3 & & \\
\hline & 919 & 58 & 3500 & 298 & 4 & .0 & \\
\hline 3 & 1920 & 88.8702 & 5.7750 & 296 & 4 & $\cdot 0$ & 600 \\
\hline & 921 & 76 & 702 & & 4 & & \\
\hline & 1922 & 76 & 7.6000 & 295.4 & 4 & .0 & \\
\hline & 1923 & 79.7468 & 6.2500 & 29 & 4 & .0 & 000 \\
\hline & 51924 & 79.7 & 642 & 29 & 4 & & \\
\hline 0. & 5201 & 76 & 6.82 & 29 & 4 & & \\
\hline & 5202 & 81.5002 & 7.0250 & 295 & 4 & .0 & 000 \\
\hline & 5203 & 86 & 6 & 29 & 4 & .0 & \\
\hline 0 & 5204 & 4 & 0 & 29 & 4 & .0 & \\
\hline & 5205 & 132.9945 & 2.7500 & 294.4 & 4 & .0 & 60 \\
\hline & 5206 & 143.2505 & 3.1500 & 293.7 & 5 & .0 & \\
\hline & 5207 & 7889 & 3 . & 29 & 4 & & \\
\hline & 5208 & 168 . & 500 & 29 & 4 & .0 & \\
\hline & 5209 & 168.2535 & 7.8250 & 295.1 & 4 & .0 & \\
\hline ) & 52010 & 172 & 0 & 29 & 4 & & 0 \\
\hline 03 & 52011 & 17 & 3250 & 297.3 & 3 & .0 & \\
\hline & 52012 & 168.2415 & 5250 & 298.1 & 3 & .0 & \\
\hline & 52013 & 174 . & 0 & 29 & 3 & .0 & \\
\hline - & 52014 & 162 . & 5.0750 & 300.0 & 3 & .0 & 600 \\
\hline & 52015 & 161.0000 & 1500 & 300.8 & 3 & .0 & \\
\hline & 52016 & 164.7618 & 000 & 301.7 & 3 & & \\
\hline 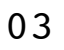 & 52017 & 134.0770 & 5.2500 & 301.8 & 3 & .0 & \\
\hline & 52018 & 81.1976 & 5.0500 & 300.7 & 3 & .0 & 00 \\
\hline 03 & 52019 & 2504 & 6.4250 & 299 & 4 & & \\
\hline 03 & 52020 & 73.4991 & 6.3500 & 297.2 & 4 & 600.0 & \\
\hline 03 & 52021 & 70.2502 & 6.7000 & 296.6 & 4 & 600.0 & 600 \\
\hline & 52022 & & & & 4 & & \\
\hline & 52023 & 73.5000 & 7.4500 & 296.7 & & .0 & 600 \\
\hline
\end{tabular}




\begin{tabular}{|c|c|c|c|c|c|c|c|}
\hline 3 & 52024 & 75.4998 & 6.6250 & 296.9 & 4 & 600.0 & 600.0 \\
\hline 03 & 5211 & 72.4999 & 7.2250 & 296.8 & 4 & 600.0 & 600.0 \\
\hline 03 & 5212 & 75.5000 & 7.8750 & 296.0 & 4 & 600.0 & 00.0 \\
\hline 03 & 5213 & 76.2494 & 7.3500 & 295.5 & 4 & 600.0 & 600.0 \\
\hline 03 & 5214 & 89.5000 & 3.9500 & 296.4 & 4 & 600.0 & 00.0 \\
\hline 03 & 5215 & 129.5067 & 2.1250 & 296.0 & 4 & 600.0 & 00.0 \\
\hline 03 & 5216 & 127.7919 & 2.4500 & 295.4 & 4 & 600.0 & 600.0 \\
\hline 03 & 5217 & 158.7581 & 4.1250 & 295.7 & 4 & 600.0 & 00.0 \\
\hline 03 & 5218 & 158.2499 & 6.1250 & 295.8 & 4 & 600.0 & 00.0 \\
\hline 03 & 5219 & 155.2501 & 6.9750 & 296.3 & 4 & 600.0 & 600.0 \\
\hline 03 & 52110 & 171.5003 & 7.8500 & 297.2 & 4 & 600.0 & 600.0 \\
\hline 03 & 52111 & 167.0002 & 6.6750 & 298.3 & 4 & 600.0 & \\
\hline 03 & 52112 & 169.7488 & 5.8000 & 299.6 & 3 & 600.0 & 600.0 \\
\hline 03 & 52113 & 171.2524 & 4.9750 & 300.9 & 3 & 600.0 & 600.0 \\
\hline 03 & 52114 & 166.7571 & 4.9250 & 302.0 & 3 & 600.0 & \\
\hline 03 & 52115 & 171.0554 & 4.7000 & 302.6 & 3 & 600.0 & 60 \\
\hline 03 & 52116 & 173.4876 & 6.0250 & 303.0 & 4 & 600.0 & 600.0 \\
\hline 03 & 52117 & 180.0011 & 5.8000 & 303.0 & 3 & 600.0 & \\
\hline 03 & 52118 & 145.8705 & 5.3750 & 302.6 & 3 & 600.0 & 60 \\
\hline 03 & 52119 & 80.9770 & 5.6250 & 300.8 & 4 & 600.0 & 600.0 \\
\hline 03 & 52120 & 71.9998 & 7.2250 & 298.5 & 4 & 600.0 & .0 \\
\hline 03 & 52121 & 71.2504 & 7.9250 & 297.6 & 4 & 600.0 & \\
\hline 03 & 52122 & 66.2496 & 7.9250 & 297.4 & 4 & 600.0 & 600.0 \\
\hline 03 & 52123 & 70.7500 & .4250 & 297.6 & 4 & 600.0 & .0 \\
\hline 03 & 52124 & 72.5000 & 750 & 297.8 & 4 & 600.0 & \\
\hline 03 & 5221 & 71.2499 & 9.2000 & 297.8 & 4 & 600.0 & 600.0 \\
\hline 03 & 5222 & 71.7500 & 8.2500 & 297.9 & 4 & 600.0 & .0 \\
\hline 03 & 5223 & 72.5000 & 7.2250 & 298.2 & 4 & 600.0 & \\
\hline 03 & 5224 & 76.2502 & 6.3750 & 297.8 & 4 & 600.0 & 60 \\
\hline 03 & 5225 & 81.2488 & 5.0500 & 297.9 & 4 & 600.0 & 600.0 \\
\hline 03 & 5226 & 102.0073 & 2.6500 & 298.3 & 4 & 600.0 & .0 \\
\hline 03 & 5227 & 129.4600 & 1.4250 & 299.4 & 3 & 600.0 & 60 \\
\hline 03 & 5228 & 210.7582 & 1.2500 & 301.2 & 2 & 600.0 & 600.0 \\
\hline 03 & 5229 & 176.5000 & 4.2000 & 299.9 & 3 & 600.0 & .0 \\
\hline 03 & 52210 & 174.9996 & 5.2500 & 299.8 & 4 & 600.0 & 60 \\
\hline 03 & 52211 & 172.7501 & 6.1000 & 300.3 & 4 & 600.0 & 600.0 \\
\hline 03 & 52212 & 167.7498 & 6.8250 & 300.5 & 4 & 600.0 & .0 \\
\hline 03 & 52213 & 165.2501 & 5.8250 & 301.7 & 3 & 600.0 & 600.0 \\
\hline 03 & 52214 & 170.5000 & 5.3250 & 302.6 & 3 & 600.0 & 600.0 \\
\hline 03 & 52215 & 162.0043 & 4.7750 & 303.2 & 3 & 600.0 & \\
\hline 03 & 52216 & 155.2740 & 4.9000 & 303.6 & 3 & 600.0 & 600.0 \\
\hline 03 & 52217 & 125.5901 & 4.1000 & 303.9 & 3 & 600.0 & 600.0 \\
\hline 03 & 52218 & 85.4997 & 5.0750 & 302.8 & 4 & 600.0 & 600.0 \\
\hline 03 & 52219 & 74.5000 & 6.2250 & 300.9 & 4 & 600.0 & 600.0 \\
\hline 03 & 52220 & 68.2503 & 6.9000 & 299.6 & 4 & 600.0 & 600.0 \\
\hline 03 & 52221 & 71.4997 & 8.1250 & 299.1 & 4 & 600.0 & 600.0 \\
\hline 03 & 52222 & 74.7500 & 8.225 & 298.9 & 4 & 600.0 & 600.0 \\
\hline 03 & 52223 & 78.2500 & 8.2750 & 299.4 & 4 & 600.0 & 600.0 \\
\hline
\end{tabular}




\begin{tabular}{|c|c|c|c|c|c|c|c|}
\hline 3 & 52224 & 78.7500 & 8.3250 & 299.8 & 4 & 600.0 & 600.0 \\
\hline 03 & 5231 & 80.5003 & 7.6000 & 300.1 & 4 & 600.0 & 600.0 \\
\hline 03 & 5232 & 7500 & 6.2000 & 300.1 & 4 & 600.0 & 00.0 \\
\hline 3 & 5233 & 80.5006 & 5.0750 & 299.6 & 4 & 600.0 & 0 \\
\hline 03 & 5234 & 78.7501 & 6.0000 & 299.5 & 4 & 00.0 & .0 \\
\hline 03 & 5235 & 80.0000 & 3.9500 & 300.3 & 5 & 600.0 & 00.0 \\
\hline 03 & 5236 & 117.6700 & 1.5250 & 300.1 & 4 & 600.0 & 0 \\
\hline 03 & 5237 & 137.1573 & 0.7750 & 301.4 & 3 & 600.0 & 00.0 \\
\hline 03 & 5238 & 166.7499 & 3.6250 & 299.8 & 3 & 600.0 & 00.0 \\
\hline 03 & 5239 & 168.2498 & 6.0000 & 299.6 & 4 & 60 & 0 \\
\hline 03 & 52310 & 165.7485 & 6.6250 & 300.5 & 4 & 600.0 & \\
\hline 03 & 52311 & 166.5032 & 6.3000 & 301.5 & 4 & .0 & \\
\hline 03 & 52312 & 169.5000 & 5.9500 & 302.8 & 3 & 600.0 & \\
\hline 03 & 52313 & 168.7420 & 6.6750 & 303.8 & 4 & 600.0 & \\
\hline 03 & 52314 & 153.0073 & 6.7 & 304.0 & 4 & .0 & \\
\hline 03 & 52315 & 107.2289 & 6.4750 & 303.6 & 4 & 600.0 & \\
\hline 03 & 52316 & 80.2504 & 7.2750 & 303.2 & 4 & 60 & .0 \\
\hline 03 & 52317 & 70.0000 & 10.22 & 301.1 & 4 & .0 & \\
\hline 03 & 52318 & 72.2503 & 11.2750 & 298.5 & 4 & 60 & \\
\hline 03 & 52319 & 77.9989 & 10.9000 & 297.4 & 4 & .0 & .0 \\
\hline 03 & 52320 & 72.7492 & 11. & 295.1 & 4 & 6 & \\
\hline 03 & 52321 & 72.5000 & 12.1000 & 294.9 & 4 & .0 & \\
\hline 03 & 52322 & 72.2500 & 11.9000 & 295.7 & 4 & 60 & .0 \\
\hline 03 & 52323 & 72.2499 & 11.30 & 294.7 & 4 & 0 & \\
\hline 03 & 52324 & 72.4992 & 12 . & 291.9 & 4 & 0 & \\
\hline 03 & 5241 & 76.5000 & 14.4250 & 291.3 & 4 & 60 & \\
\hline 03 & 5242 & 73.0000 & 15.6250 & 291.1 & 4 & 60 & \\
\hline 03 & 5243 & 77.0000 & 16.50 & 290.2 & 4 & .0 & \\
\hline 03 & $524 \quad 4$ & 79.2503 & 14.7750 & 289.8 & 4 & .0 & .0 \\
\hline 03 & $524 \quad 5$ & 73.7501 & 15.2500 & 289.3 & 4 & .0 & \\
\hline 03 & 5246 & 64.0000 & 15.3750 & 288.3 & 4 & .0 & \\
\hline 03 & 5247 & 66.9999 & 14.1750 & 288.1 & 4 & .0 & .0 \\
\hline 03 & 5248 & 69.9993 & 13.8250 & 288.5 & 4 & 6 & 0.0 \\
\hline 03 & 5249 & 69.2500 & 12.9750 & 290.2 & 4 & .0 & .0 \\
\hline 03 & 52410 & 65.2500 & 12.3250 & 291.7 & 4 & .0 & 0.0 \\
\hline 03 & 52411 & 68.5000 & 11.6750 & 292.5 & 4 & 600.0 & 0.0 \\
\hline 03 & 52412 & 65.0021 & 12.12 & 292.0 & 4 & 600.0 & .0 \\
\hline 03 & 52413 & 64.5000 & 13.4000 & 292.0 & 4 & 600.0 & 600.0 \\
\hline 03 & 52414 & 63.2504 & 15.2000 & 290.8 & 4 & 600.0 & 600.0 \\
\hline 03 & 52415 & 63.7500 & 12.37 & 292.0 & 4 & 600.0 & .0 \\
\hline 03 & 52416 & 65.2511 & 12.37 & 292.3 & 4 & 600.0 & 600.0 \\
\hline 03 & 52417 & 63.0000 & 13.3000 & 291.8 & 4 & 600.0 & 600.0 \\
\hline 03 & 52418 & 67.5000 & 13.3500 & 290.9 & 4 & 600.0 & 600.0 \\
\hline 03 & 52419 & 72.2501 & 14.2000 & 288.6 & 4 & 600.0 & 600.0 \\
\hline 03 & 52420 & 68.9993 & 15.0000 & 287.3 & 4 & 600.0 & 600.0 \\
\hline 03 & 52421 & 68.5000 & 15.4500 & 286.5 & 4 & 600.0 & .0 \\
\hline 03 & 52422 & 70.7499 & 14.20 & 286.7 & 4 & 600.0 & 600.0 \\
\hline & 52423 & 67.7502 & 15.1000 & 285.5 & $\Psi$ & 600.0 & 600.0 \\
\hline
\end{tabular}




\begin{tabular}{|c|c|c|c|c|c|c|c|}
\hline 3 & 52424 & 66.2500 & 13.5750 & 285.7 & 4 & 600.0 & 600.0 \\
\hline 3 & 5251 & 68.5000 & 13.7500 & 285.3 & 4 & 600.0 & 600.0 \\
\hline 03 & 5252 & 68.0014 & 13.8250 & 284.8 & 4 & 600.0 & 00.0 \\
\hline 3 & 5253 & 66.5003 & 13.0750 & 285.7 & 4 & 600.0 & 00.0 \\
\hline 03 & 5254 & 67.0000 & 11.7000 & 286.2 & 4 & 600.0 & 00.0 \\
\hline 03 & 5255 & 61.5009 & 11.8500 & 285.6 & 4 & 600.0 & 00.0 \\
\hline 03 & 5256 & 63.5000 & 12.9000 & 286.0 & 4 & 600.0 & 00.0 \\
\hline 03 & $\begin{array}{ll}525 & 7\end{array}$ & 63.2499 & 11.3250 & 286.6 & 4 & 600.0 & 00.0 \\
\hline 03 & 5258 & 63.7497 & 12.0000 & 287.0 & 4 & 600.0 & 00.0 \\
\hline 03 & 5259 & 63.7500 & 10.9000 & 288.5 & 4 & 600.0 & 0 \\
\hline 03 & 52510 & 67.7492 & 750 & 290.2 & 4 & 600.0 & \\
\hline 03 & 52511 & 79.5006 & 500 & 290.9 & 4 & 600.0 & \\
\hline 03 & 52512 & 73.9998 & 8.8750 & 291.1 & 4 & 600.0 & 00.0 \\
\hline 03 & 52513 & 74.9936 & 250 & 292.0 & 4 & 600.0 & 00.0 \\
\hline 03 & 52514 & 69.2482 & 00 & 292.2 & 4 & .0 & \\
\hline 03 & 52515 & 69.7487 & 750 & 292.8 & 4 & 600.0 & .0 \\
\hline 03 & 52516 & 67.0001 & 10.8500 & 293.0 & 4 & 60 & .0 \\
\hline 03 & 52517 & 66.9998 & 10. & 293.0 & 4 & .0 & \\
\hline 03 & 52518 & 78.7488 & 500 & 292.6 & 4 & 60 & .0 \\
\hline 03 & 52519 & 71.0017 & 10.8500 & 291.2 & 4 & .0 & .0 \\
\hline 03 & 52520 & 63.5000 & 12 . & 289.0 & 4 & 6 & \\
\hline 03 & 52521 & 55.7266 & 9 . & 288.8 & 4 & .0 & \\
\hline 03 & 52522 & 65.2517 & 8.1 & 289.0 & 4 & 600.0 & .0 \\
\hline 03 & 52523 & 66.5000 & 7 . & 288.4 & 4 & 6 & \\
\hline 03 & 52524 & 64.7525 & 6 . & 287.2 & 4 & .0 & \\
\hline 03 & 5261 & 67.7590 & 000 & 286.1 & 4 & .0 & 600.0 \\
\hline 03 & 5262 & 59.2213 & 7.2750 & 286.1 & 4 & .0 & .0 \\
\hline 03 & 5263 & 78.7504 & 7 . & 285.7 & 4 & .0 & \\
\hline 03 & 5264 & 67.7516 & 8 . & 285.7 & 4 & .0 & 0.0 \\
\hline 03 & 5265 & 68.2498 & 7.4500 & 285.4 & 4 & 60 & .0 \\
\hline 03 & 5266 & 78.0000 & 7. & 285.7 & 4 & .0 & \\
\hline 03 & 5267 & 77.2403 & 6 . & 286.1 & 4 & .0 & 0.0 \\
\hline 03 & 5268 & 94.0018 & 4.4500 & 288.4 & 3 & 600.0 & 0.0 \\
\hline 03 & 5269 & 130.1893 & 3. & 290.7 & 2 & .0 & .0 \\
\hline 03 & 52610 & 234.4901 & 1.8500 & 294.0 & 1 & 600.0 & 600.0 \\
\hline 03 & 52611 & 103.6366 & 3.0500 & 295.1 & 2 & 600.0 & 600.0 \\
\hline 03 & 52612 & 161.2438 & 7 . & 294.5 & 3 & 600.0 & 0.0 \\
\hline 03 & 52613 & 165.7500 & 7.8750 & 295.1 & 4 & 600.0 & 600.0 \\
\hline 03 & 52614 & 159.5153 & 7.5250 & 295.9 & 4 & 600.0 & 600.0 \\
\hline 03 & 52615 & 136.7742 & 7 . & 296.4 & 4 & 600.0 & 600.0 \\
\hline 03 & 52616 & 113.4977 & 5.4500 & 297.1 & 3 & 600.0 & 600.0 \\
\hline 03 & 52617 & 98.0001 & 5.4250 & 296.9 & 3 & 600.0 & 600.0 \\
\hline 03 & 52618 & 89.2482 & 5.8750 & 296.2 & 4 & 600.0 & 600.0 \\
\hline 03 & 52619 & 76.7461 & 6.6500 & 294.9 & 4 & 600.0 & 600.0 \\
\hline 03 & 52620 & 75.5000 & 8.1000 & 293.5 & 4 & 600.0 & 600.0 \\
\hline 03 & 52621 & 81.2500 & & 293.4 & 4 & 600.0 & 600.0 \\
\hline 03 & 52622 & 89.0000 & 7.6750 & 293.9 & 4 & 600.0 & 600.0 \\
\hline & 52623 & 90.2489 & 7.5250 & 294.3 & 4 & 600.0 & 600.0 \\
\hline
\end{tabular}




\begin{tabular}{|c|c|c|c|c|c|c|c|}
\hline 3 & 2624 & & 750 & 0 & & & \\
\hline & 5271 & 25 & 3.8250 & 5.1 & 4 & 0 & \\
\hline & 272 & 121.2489 & 4.6750 & 294.9 & 4 & 00 . & \\
\hline & 5273 & 128.7517 & 4.1500 & 294.9 & & 00.0 & \\
\hline & $527 \quad 4$ & 130.0000 & .9750 & 94.6 & & & \\
\hline & 5275 & 5.5018 & 9500 & 294.6 & & 00 & \\
\hline & 5276 & 16.5009 & 3.8250 & 295.0 & 4 & 00 & \\
\hline & 5277 & 34.8327 & 3.4750 & 95.9 & & .0 & \\
\hline & 5278 & 9.4847 & 5.5000 & 296.5 & 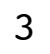 & & \\
\hline & 5279 & 67.7498 & 500 & 297.4 & 3 & 00 & \\
\hline & 2710 & 56.2491 & 4.7250 & 298.4 & & & \\
\hline & 2711 & 34.7415 & 3.9750 & 295 & & & \\
\hline & 2712 & 71.7473 & 500 & 300.7 & 3 & .0 & \\
\hline & 2713 & 77.8018 & 4.2500 & 301.6 & 3 & .0 & \\
\hline & 2714 & 77.2532 & 0 & 30 & 3 & & \\
\hline & 2715 & 74.5000 & & 303 & 3 & & \\
\hline & 2716 & 186.7640 & .1750 & 304.2 & 3 & 0.0 & 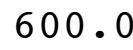 \\
\hline & 2717 & 175.0090 & 0 & 30 & 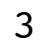 & .0 & \\
\hline & 52718 & 146.7902 & 50 & 304 & 3 & & \\
\hline & 52719 & 93.9992 & 250 & 302 & 4 & .0 & 000 \\
\hline & 52720 & 72.2500 & 750 & 301.2 & 5 & .0 & \\
\hline & 52721 & .7501 & & & 4 & & \\
\hline & 52722 & 7499 & 00 & 30 & 4 & & \\
\hline & 52723 & 72.2496 & 3500 & 30 & 4 & $\cdot 0$ & \\
\hline & 52724 & 72.2499 & & & 4 & & \\
\hline 0. & 5281 & 72.5000 & 7 . & 30 & 4 & & \\
\hline & 5282 & 75.9999 & 6.2250 & 30 & 4 & .0 & \\
\hline & 5283 & 62.5000 & 4.12 & 30 & 4 & .0 & \\
\hline 0 & 5284 & 973 & 0 & 30 & 5 & .0 & \\
\hline & 5285 & 61.0552 & 500 & 300.8 & 6 & 0.0 & \\
\hline & 5286 & 76.7499 & 750 & 300.4 & 5 & .0 & \\
\hline & 5287 & 73. & 0 & & 4 & & \\
\hline & 5288 & 211.6884 & 500 & 302 & 3 & .0 & 0 \\
\hline & 5289 & 232.7502 & 3.3250 & 301.2 & 2 & .0 & \\
\hline & 52810 & 215.5257 & 0 & 30 & 2 & & \\
\hline 03 & 52811 & 5063 & 000 & 30 & 3 & .0 & \\
\hline & 52812 & 194.5323 & 7250 & 302.7 & 3 & .0 & \\
\hline & 52813 & 190.7478 & 0 & & 3 & .0 & \\
\hline 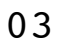 & 52814 & 187.9839 & 4.5500 & 304.8 & 3 & .0 & 60 \\
\hline & 52815 & 188.0220 & 2250 & 305.7 & 3 & .0 & \\
\hline & 52816 & 177.2516 & 750 & & 3 & & \\
\hline-1 & 52817 & 187.0021 & 4.5750 & 30 & 3 & .0 & \\
\hline & 52818 & 185.0002 & 4.4000 & 305.4 & 4 & .0 & 0 \\
\hline 03 & 52819 & 161.4810 & 250 & 30 & 5 & & \\
\hline 03 & 52820 & 141.1171 & & & 4 & 0 & \\
\hline 07 & 52821 & 86.7400 & 4.9250 & 302.6 & 5 & .0 & 600 \\
\hline & 52822 & $7^{\circ}$ & & & 4 & & \\
\hline & 52823 & 78.5000 & 5.2750 & 301.6 & & .0 & 600 \\
\hline
\end{tabular}




\begin{tabular}{|c|c|c|c|c|c|c|c|}
\hline 3 & 52824 & 78.2503 & 3.7750 & 302.2 & 5 & 600.0 & 00.0 \\
\hline 03 & 5291 & 71.5001 & 2.0000 & 302.6 & 4 & 600.0 & 600.0 \\
\hline 03 & 5292 & 69.2519 & 0.9250 & 302.4 & 5 & 600.0 & 00.0 \\
\hline 03 & 5293 & 79.0336 & 0.3250 & 301.5 & 6 & 600.0 & 00.0 \\
\hline 03 & 5294 & 170.1311 & 0.7000 & 301.3 & 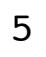 & 600.0 & 00.0 \\
\hline 03 & 5295 & 114.4470 & 0.6500 & 300.6 & 6 & 600.0 & 00.0 \\
\hline 03 & 5296 & 72.2731 & 0.6750 & 299.6 & 6 & 600.0 & 600.0 \\
\hline 03 & 5297 & 61.2337 & 1.0250 & 300.6 & 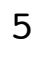 & 600.0 & 00.0 \\
\hline 03 & 5298 & 294.5869 & 1.3750 & 302.5 & 4 & 600.0 & 00.0 \\
\hline 03 & 5299 & 323.0377 & 2.8500 & 302.8 & 3 & 600.0 & 600.0 \\
\hline 03 & 52910 & 269.2501 & 3.8250 & 302.3 & 2 & 600.0 & 600.0 \\
\hline 03 & 52911 & 244.5194 & 4.1750 & 302.6 & 3 & 600.0 & \\
\hline 03 & 52912 & 181.2292 & 4.5750 & 302.5 & 3 & 600.0 & 600.0 \\
\hline 03 & 52913 & 102.9652 & 8.4500 & 299.4 & 4 & 600.0 & 600.0 \\
\hline 03 & 52914 & 74.7521 & 10.3750 & 297.8 & 4 & 600.0 & \\
\hline 03 & 52915 & 80.5000 & 12.0750 & 296.3 & 4 & 600.0 & 60 \\
\hline 03 & 52916 & 78.4994 & 12.4750 & 294.6 & 4 & 600.0 & 600.0 \\
\hline 03 & 52917 & 82.5000 & 12.0500 & 293.4 & 4 & 600.0 & \\
\hline 03 & 52918 & 83.7500 & 13.2250 & 291.5 & 4 & 600.0 & .0 \\
\hline 03 & 52919 & 79.0000 & 12.9250 & 288.3 & 4 & 600.0 & 600 \\
\hline 03 & 52920 & 80.7485 & 10.82 & 287.5 & 4 & 600.0 & .0 \\
\hline 03 & 52921 & 65.2500 & 14.1000 & 285.9 & 4 & 600.0 & \\
\hline 03 & 52922 & 61.2501 & 14.4000 & 285.5 & 4 & 600.0 & 600.0 \\
\hline 03 & 52923 & 64.5000 & 11.2750 & 285.3 & 4 & 600.0 & .0 \\
\hline 03 & 52924 & 69.6656 & 7.1500 & 285.1 & 4 & 600.0 & \\
\hline 03 & 5301 & 77.7578 & 8.0750 & 285.1 & 4 & 600.0 & 60 \\
\hline 03 & 5302 & 80.2494 & 9.9250 & 284.9 & 4 & 600.0 & .0 \\
\hline 03 & 5303 & 85.2473 & 7.87 & 284.6 & 4 & 600.0 & \\
\hline 03 & 5304 & 80.3360 & 8.5000 & 284.5 & 4 & 600.0 & 60 \\
\hline 03 & 5305 & 51.2532 & 9.77 & 284.5 & 4 & 600.0 & 60 \\
\hline 03 & 5306 & 61.2498 & 8.02 & 284.6 & 4 & 600.0 & \\
\hline 03 & 5307 & 65.7485 & 9.4750 & 284.8 & 4 & 600.0 & 60 \\
\hline 03 & 5308 & 76.4987 & 6.8250 & 285.9 & 4 & 600.0 & 600.0 \\
\hline 03 & 5309 & 67.5117 & 3.05 & 288.6 & 3 & 600.0 & .0 \\
\hline 03 & 53010 & 56.8902 & 2.7 & 290.3 & 2 & 600.0 & .0 \\
\hline 03 & 53011 & 94.2475 & 4.6500 & 291.1 & 3 & 600.0 & 600.0 \\
\hline 03 & 53012 & 151.0045 & 7.0250 & 290.3 & 4 & 600.0 & \\
\hline 03 & 53013 & 164.0000 & 8.2500 & 290.5 & 4 & 600.0 & 600.0 \\
\hline 03 & 53014 & 161.9981 & 6.8000 & 292.1 & 4 & 600.0 & 600.0 \\
\hline 03 & 53015 & 154.2505 & 6.2000 & 293.4 & 4 & 600.0 & \\
\hline 03 & 53016 & 153.4999 & 5.9750 & 294.5 & 3 & 600.0 & 600.0 \\
\hline 03 & 53017 & 141.3189 & 5.8000 & 295.3 & 3 & 600.0 & 600.0 \\
\hline 03 & 53018 & 107.0005 & 6.2000 & 294.1 & 4 & 600.0 & 600.0 \\
\hline 03 & 53019 & 88.9972 & 7.5500 & 292.0 & 4 & 600.0 & 600.0 \\
\hline 03 & 53020 & 72.4997 & 7.7250 & 291.7 & 4 & 600.0 & 600.0 \\
\hline 03 & 53021 & 76.2496 & 8.9000 & 290.5 & 4 & 600.0 & 600.0 \\
\hline 03 & 53022 & 79.4997 & 8.7500 & 291.1 & 4 & 600.0 & 600.0 \\
\hline 03 & 53023 & 79.2498 & 8.3500 & 292.3 & 4 & 600.0 & 600.0 \\
\hline
\end{tabular}




\begin{tabular}{|c|c|c|c|c|c|c|c|}
\hline 3 & 53024 & 2500 & 7.9000 & 292.6 & 4 & 600.0 & 00.0 \\
\hline 3 & 5311 & 80.2500 & 7.0500 & 293.0 & 4 & 600.0 & 600.0 \\
\hline 03 & 5312 & 81.0000 & 6.7000 & 293.5 & 4 & 600.0 & 00.0 \\
\hline 03 & 5313 & 81.4999 & 6.9500 & 293.1 & 4 & 600.0 & 00.0 \\
\hline 03 & 5314 & 79.7498 & 7.1250 & 292.4 & 4 & 600.0 & 00.0 \\
\hline 03 & 5315 & 81.2473 & 7.1500 & 292.2 & 4 & 600.0 & 00.0 \\
\hline 03 & 5316 & 93.2496 & 4.4000 & 292.5 & 4 & 600.0 & 600.0 \\
\hline 03 & 5317 & 101.2262 & 2.5500 & 293.1 & 3 & 600.0 & 00.0 \\
\hline 03 & 5318 & 112.9879 & 3.5000 & 294.6 & 2 & 600.0 & .0 \\
\hline 03 & 5319 & 181.2196 & 3.4000 & 295.8 & 2 & 600.0 & 600.0 \\
\hline 03 & 53110 & 251.4412 & 2.7500 & 297.2 & 1 & 600.0 & 0 \\
\hline 03 & 53111 & 190.9244 & 3.8500 & 298.4 & 2 & 600.0 & \\
\hline 03 & 53112 & 164.3487 & 5.5750 & 298.7 & 3 & 600.0 & .0 \\
\hline 03 & 53113 & 83.2935 & 2.8750 & 301.2 & 2 & 600.0 & 0 \\
\hline 03 & 53114 & 100.2506 & 4.9500 & 301.1 & 3 & 600.0 & .0 \\
\hline 03 & 53115 & 93.4914 & 6.4500 & 300.7 & 4 & 600.0 & \\
\hline 03 & 53116 & 97.2487 & 7.2000 & 299.5 & 4 & 600.0 & 600.0 \\
\hline 03 & 53117 & 84.4992 & 7.7500 & 299.3 & 4 & 600.0 & .0 \\
\hline 03 & 53118 & 83.5000 & 8.9250 & 298.2 & 4 & 600.0 & \\
\hline 03 & 53119 & 78.2500 & 9.6500 & 297.4 & 4 & 600.0 & \\
\hline 03 & 53120 & 70.4994 & 9.8000 & 297.0 & 4 & 600.0 & .0 \\
\hline 03 & 53121 & 73.2495 & 10.7000 & 297.1 & 4 & 600.0 & \\
\hline 03 & 53122 & 73.5000 & 9.9500 & 297.2 & 4 & 600.0 & 60 \\
\hline 03 & 53123 & 74.5002 & 8.6500 & 297.1 & 4 & 600.0 & .0 \\
\hline 03 & 53124 & 79.4991 & 8.3500 & 296.8 & 4 & 600.0 & \\
\hline 03 & $\begin{array}{lll}6 & 1 & 1\end{array}$ & 85.2501 & 8.4000 & 296.6 & 4 & 600.0 & 60 \\
\hline 03 & $\begin{array}{lll}6 & 1 & 2\end{array}$ & 82.0000 & 7.7500 & 296.2 & 4 & 600.0 & 600.0 \\
\hline 03 & 613 & 80.5000 & 5.9250 & 296.0 & 4 & 600.0 & .0 \\
\hline 03 & $\begin{array}{lll}6 & 1 & 4\end{array}$ & 93.8881 & 4.9250 & 296.2 & 4 & 600.0 & 60 \\
\hline 03 & $\begin{array}{lll}6 & 1 & 5\end{array}$ & 116.2494 & 4.7250 & 296.5 & 4 & 600.0 & 600.0 \\
\hline 03 & 616 & 117.4614 & 5.6500 & 295.6 & 4 & 600.0 & 60 \\
\hline 03 & $\begin{array}{lll}6 & 1 & 7\end{array}$ & 141.9992 & 7.4750 & 296.2 & 4 & 600.0 & 60 \\
\hline 03 & $\begin{array}{lll}6 & 1 & 8\end{array}$ & 164.5226 & 6.7750 & 297.0 & 4 & 600.0 & 60 \\
\hline 03 & $\begin{array}{lll}6 & 1 & 9\end{array}$ & 172.2461 & 7.2750 & 297.6 & 4 & 600.0 & 60 \\
\hline 03 & 6110 & 171.2502 & 7.7750 & 298.2 & 4 & 600.0 & 60 \\
\hline 03 & 6111 & 170.7474 & 8.1500 & 299.0 & 4 & 600.0 & 600.0 \\
\hline 03 & 6112 & 168.0000 & 7.1000 & 300.0 & 4 & 600.0 & 600.0 \\
\hline 03 & 6113 & 162.7910 & 6.4000 & 301.8 & 4 & 600.0 & 60 \\
\hline 03 & $6 \quad 114$ & 169.7666 & 5.6000 & 303.3 & 3 & 600.0 & 600.0 \\
\hline 03 & 6115 & 177.4998 & 6.3750 & 303.9 & 4 & 600.0 & 600.0 \\
\hline 03 & 6116 & 122.1046 & 5.6500 & 304.2 & 3 & 600.0 & 600.0 \\
\hline 03 & 6117 & 91.2376 & 6.1000 & 303.5 & 4 & 600.0 & 600.0 \\
\hline 03 & 6118 & 98.2570 & 6.3500 & 302.9 & 4 & 600.0 & 600.0 \\
\hline 03 & 6119 & 70.0005 & 5.7500 & 301.5 & 4 & 600.0 & 600.0 \\
\hline 03 & 6120 & 72.2501 & 7.6000 & 299.8 & 4 & 600.0 & 600.0 \\
\hline 03 & 6121 & 71.7501 & 7.8250 & 299.8 & 4 & 600.0 & 600.0 \\
\hline 03 & $6 \quad 122$ & 68.5000 & 7.90 & 29 & 4 & 600.0 & .0 \\
\hline 03 & 6123 & 65.0002 & 7.8250 & 299.6 & $\mathbf{4}$ & 600.0 & 600.0 \\
\hline
\end{tabular}




\begin{tabular}{|c|c|c|c|c|c|c|c|c|}
\hline 3 & 6 & 124 & 70.5000 & 8.1750 & 299.9 & 4 & 600.0 & 600 \\
\hline 03 & 6 & 21 & 70.0001 & 8.3000 & 300.1 & 4 & 600.0 & 600.0 \\
\hline 03 & 6 & 22 & 74.2479 & 7.1500 & 300.1 & 4 & 600.0 & 00.0 \\
\hline 03 & 6 & 23 & 104.9995 & 3.1000 & 300.3 & 4 & 600.0 & 600.0 \\
\hline 03 & 6 & 24 & 127.5015 & 2.6250 & 299.8 & 4 & 600.0 & 00.0 \\
\hline 03 & 6 & 25 & 137.4914 & 3.4000 & 299.0 & 4 & 600.0 & 00.0 \\
\hline 03 & 6 & 26 & 131.2839 & 5.6000 & 298.4 & 4 & 600.0 & 600.0 \\
\hline 03 & 6 & 27 & 151.3318 & 7.5500 & 298.9 & 4 & 600.0 & 00.0 \\
\hline 03 & 6 & 28 & 167.2502 & 7.8500 & 299.1 & 4 & 600.0 & 00.0 \\
\hline 03 & 6 & 29 & 164.2500 & 9.3000 & 299.5 & 4 & 600.0 & 600.0 \\
\hline 03 & 6 & 210 & 162.7496 & 7.9500 & 299.4 & 4 & 600.0 & 600 \\
\hline 03 & 6 & 211 & 162.7514 & 7.3250 & 300.2 & 4 & 600.0 & \\
\hline 03 & 6 & 212 & 163.2532 & 6.7250 & 301.8 & 4 & 600.0 & 600.0 \\
\hline 03 & 6 & 213 & 169.7500 & 6.6000 & 303.2 & 4 & 600.0 & 600.0 \\
\hline 03 & 6 & 214 & 167.2498 & 6.7000 & 304.4 & 4 & 600.0 & \\
\hline 03 & 6 & 215 & 173.2499 & 6.3000 & 305.1 & 4 & 600.0 & 60 \\
\hline 03 & 6 & 216 & 181.2486 & 6.5500 & 305.6 & 4 & 600.0 & 600.0 \\
\hline 03 & 6 & 217 & 182.0002 & 6.3250 & 305.8 & 4 & 600.0 & 0 \\
\hline 03 & 6 & 218 & 177.5001 & 6.6750 & 305.5 & 4 & 600.0 & 60 \\
\hline 03 & 6 & 219 & 131.4254 & 4.9000 & 304.9 & 4 & 600.0 & 60 \\
\hline 03 & 6 & 220 & 78.4963 & 5.2250 & 302.8 & 4 & 600.0 & 年 \\
\hline 03 & 6 & 221 & 70.7494 & 6.0750 & 301.6 & 4 & 600.0 & \\
\hline 03 & 6 & 222 & 77.2502 & 6.6750 & 301.5 & 4 & 600.0 & 60 \\
\hline 03 & 6 & 223 & 76.7496 & 6.9500 & 301.6 & 4 & 600.0 & 60 \\
\hline 03 & 6 & 224 & 76.0000 & 7.4500 & 301.4 & 4 & 600.0 & \\
\hline 03 & 6 & 31 & 73.7500 & 8.1500 & 300.6 & 4 & 600.0 & 600.0 \\
\hline 03 & 6 & 32 & 78.2501 & 8.1000 & 300.6 & 4 & 600.0 & 60 \\
\hline 03 & 6 & 33 & 77.2500 & 8.2000 & 300.9 & 4 & 600.0 & \\
\hline 03 & 6 & 34 & 77.5000 & 7.6250 & 301.2 & 4 & 600.0 & 60 \\
\hline 03 & 6 & 35 & 81.4954 & 6.1000 & 301.3 & 4 & 600.0 & 600 \\
\hline 03 & 6 & 36 & 79.2450 & 6.00 & 300.7 & 4 & 600.0 & 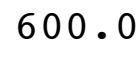 \\
\hline 03 & 6 & 37 & 73.2268 & 5.2500 & 301.7 & 4 & 600.0 & 60 \\
\hline 03 & 6 & 38 & 95.7748 & 2.0250 & 304.3 & 3 & 600.0 & 600.0 \\
\hline 03 & 6 & 39 & 193.0000 & 4.6000 & 303.1 & 3 & 600.0 & .0 \\
\hline 03 & 6 & 310 & 192.5009 & 5.07 & 302.3 & 4 & 600.0 & 60 \\
\hline 03 & 6 & 311 & 183.3109 & 4.8000 & 304.1 & 3 & 600.0 & 600.0 \\
\hline 03 & 6 & 312 & 158.2249 & 5.5250 & 304.6 & 3 & 600.0 & \\
\hline 03 & 6 & 313 & 97.7573 & 4.8750 & 304.8 & 3 & 600.0 & 600.0 \\
\hline 03 & 6 & 314 & 88.0012 & 6.0000 & 304.5 & 4 & 600.0 & 600.0 \\
\hline 03 & 6 & 315 & 85.7545 & 6.2000 & 304.5 & 4 & 600.0 & \\
\hline 03 & 6 & 316 & 72.2502 & 7.5750 & 303.9 & 4 & 600.0 & 600.0 \\
\hline 03 & 6 & 317 & 61.5001 & 9.3750 & 302.5 & 4 & 600.0 & 600.0 \\
\hline 03 & 6 & 318 & 64.0000 & 10.8500 & 300.8 & 4 & 600.0 & 600.0 \\
\hline 03 & 6 & 319 & 75.7496 & 10.8500 & 299.3 & 4 & 600.0 & 600.0 \\
\hline 03 & 6 & 320 & 74.5000 & 10.0500 & 298.2 & 4 & 600.0 & 600.0 \\
\hline 03 & 6 & 321 & 70.5000 & 11.1750 & 297.9 & 4 & 600.0 & 600.0 \\
\hline 03 & 6 & 322 & 68.9999 & 11.3250 & 29 & 4 & 600.0 & 600.0 \\
\hline 03 & 6 & 323 & 69.2500 & 10.6250 & 298.0 & 4 & 600.0 & 600.0 \\
\hline
\end{tabular}




\begin{tabular}{|c|c|c|c|c|c|c|c|c|}
\hline & 6 & 324 & 75.7502 & 9.8250 & 297.7 & 4 & 600.0 & 600.0 \\
\hline 3 & 6 & 41 & 77.7500 & 10.1500 & 296.7 & 4 & 600.0 & 600.0 \\
\hline 03 & 6 & 42 & 79.2500 & 10.5250 & 295.4 & 4 & 600.0 & 00.0 \\
\hline 3 & 6 & 43 & 78.5000 & 10.5250 & 294.1 & 4 & 600.0 & 00.0 \\
\hline 03 & 6 & 44 & 77.5000 & 11.0250 & 292.7 & 4 & 600.0 & 00.0 \\
\hline 03 & 6 & 45 & 78.0000 & 11.2000 & 290.5 & 4 & 600.0 & 00.0 \\
\hline 03 & 6 & 46 & 76.2501 & 12.2750 & 289.9 & 4 & 600.0 & 00.0 \\
\hline 03 & 6 & 47 & 76.9999 & 11.4000 & 290.2 & 4 & 600.0 & 00.0 \\
\hline 03 & 6 & 48 & 82.4990 & 10.0000 & 290.7 & 4 & 600.0 & 00.0 \\
\hline 03 & 6 & 49 & 103.5108 & 5.5000 & 293.2 & 3 & 600.0 & .0 \\
\hline 03 & 6 & 410 & 237.8727 & 2.1500 & 296.9 & - & 600.0 & \\
\hline 03 & 6 & 411 & 283.2171 & 2.3000 & 298.6 & 1 & 600.0 & \\
\hline 03 & 6 & 412 & 72.4840 & 3.8500 & 299.2 & 2 & 600.0 & 00.0 \\
\hline 03 & 6 & 413 & 86.9979 & 4.1750 & 300.2 & 3 & 600.0 & 00.0 \\
\hline 03 & 6 & 414 & 501 & 750 & 300.7 & 4 & .0 & \\
\hline 03 & 6 & 415 & 88.7500 & 7.0250 & 300.5 & 4 & 600.0 & .0 \\
\hline 03 & 6 & 416 & 80.2503 & 7.4000 & 300.4 & 4 & 60 & 600.0 \\
\hline 03 & 6 & 417 & 82.5006 & 8.4500 & 299.9 & 4 & .0 & \\
\hline 03 & 6 & 418 & 81.4999 & 9.2500 & 298.1 & 4 & 60 & .0 \\
\hline 03 & 6 & 419 & 81.7494 & 8.4500 & 297.5 & 4 & 60 & .0 \\
\hline 03 & 6 & 420 & 78 . & 9.4250 & 295.6 & 4 & 6 & \\
\hline 03 & 6 & 421 & 76.7500 & 11.5000 & 295.5 & 4 & .0 & \\
\hline 03 & 6 & 422 & 68.5000 & 10.6750 & 296.0 & 4 & 600.0 & .0 \\
\hline 03 & 6 & 423 & 79.5008 & 10.20 & 29 & 4 & 60 & \\
\hline 03 & 6 & 424 & 76.7499 & 10.5750 & 296.0 & 4 & .0 & \\
\hline 03 & 6 & 51 & 78.7518 & 9.7750 & 296.4 & 4 & .0 & 600.0 \\
\hline 03 & 6 & 52 & 68.0000 & 8.1750 & 294.6 & 4 & 600.0 & .0 \\
\hline 03 & 6 & 53 & 70.7500 & 8.6000 & 292.4 & 4 & .0 & \\
\hline 03 & 6 & 54 & 71.4998 & 10.2500 & 291.0 & 4 & .0 & 0.0 \\
\hline 03 & 6 & 55 & 77.2499 & 10.9500 & 289.6 & 4 & 60 & .0 \\
\hline 03 & 6 & 56 & 77.2498 & 10.3000 & 290.3 & 4 & .0 & \\
\hline 03 & 6 & 57 & 82.5000 & 9.1250 & 290.1 & 4 & .0 & 600.0 \\
\hline 03 & 6 & 58 & 86.4997 & 5.3000 & 290.4 & 3 & 600.0 & 600.0 \\
\hline 03 & 6 & 59 & 86.7513 & 5.1750 & 291.1 & 4 & 600.0 & 0.0 \\
\hline 03 & 6 & 510 & 89.5217 & 3.1000 & 294.0 & 3 & 600.0 & 600.0 \\
\hline 03 & 6 & 511 & 88.3942 & 2.7750 & 296.7 & 2 & 600.0 & 600.0 \\
\hline 03 & 6 & 512 & 31.0245 & 3.9500 & 297.6 & 2 & 600.0 & 0.0 \\
\hline 03 & 6 & 513 & 84.9687 & 4.7750 & 297.7 & 3 & 600.0 & 600.0 \\
\hline 03 & 6 & 514 & 89.7496 & 5.1250 & 298.5 & 3 & 600.0 & 600.0 \\
\hline 03 & 6 & 515 & 92.7499 & 6.1750 & & 4 & 600.0 & 600.0 \\
\hline 03 & 6 & 516 & 88.2504 & 7.3750 & 298.5 & 4 & 600.0 & 600.0 \\
\hline 03 & 6 & 517 & 83.7500 & 7.5750 & 298.1 & 4 & 600.0 & 600.0 \\
\hline 03 & 6 & 518 & 80.5000 & 9.7750 & 296.3 & 4 & 600.0 & 600.0 \\
\hline 03 & 6 & 519 & 80.0000 & 11.0250 & 294.0 & 4 & 600.0 & 600.0 \\
\hline 03 & 6 & 520 & 75.0001 & 11.8000 & 291.7 & 4 & 600.0 & 600.0 \\
\hline 03 & 6 & 521 & 75.7496 & 13.3000 & & 4 & 600.0 & 600.0 \\
\hline 03 & 6 & 522 & 79.0000 & 12.0250 & 290.2 & 4 & 600.0 & 600.0 \\
\hline 0 & 6 & 523 & 77.0000 & 13.275 & 288.8 & 4 & 600.0 & 600.0 \\
\hline
\end{tabular}




\begin{tabular}{|c|c|c|c|c|c|c|c|c|}
\hline & 6 & 524 & 75.7500 & 13.5000 & 288.2 & 4 & 600.0 & 600.0 \\
\hline 03 & 6 & 61 & 73.0000 & 12.2500 & 289.0 & 4 & 600.0 & 00.0 \\
\hline 03 & 6 & 62 & 78.0000 & 11.1750 & 288.5 & 4 & 600.0 & 00.0 \\
\hline 3 & 6 & 63 & 7.5000 & 12.8000 & 287.4 & 4 & 600.0 & 00.0 \\
\hline 03 & 6 & 64 & 77.2500 & 12.0250 & 286.7 & 4 & 600.0 & 00.0 \\
\hline 03 & 6 & 65 & 70.2498 & 12.4750 & 286.8 & 4 & 600.0 & 00.0 \\
\hline 03 & 6 & 66 & 73.7499 & 13.3250 & 286.9 & 4 & 600.0 & 00.0 \\
\hline 03 & 6 & 67 & 80.7499 & 10.0250 & 286.8 & 4 & 600.0 & 00.0 \\
\hline 03 & 6 & 68 & 83.7501 & 8.6750 & 287.4 & 4 & 600.0 & 00.0 \\
\hline 03 & 6 & 69 & 85.7499 & 6.8250 & 288.7 & 4 & 600.0 & 0 \\
\hline 03 & 6 & 610 & 6.7499 & 5.2750 & 290.7 & 3 & 600.0 & \\
\hline 03 & 6 & 611 & 92.2452 & 4.4500 & 292.7 & 3 & 600.0 & \\
\hline 03 & 6 & 612 & 91.5055 & 4.3250 & 294.7 & 3 & 600.0 & 00.0 \\
\hline 03 & 6 & 613 & 5.4972 & 4.2000 & 296.5 & 3 & 600.0 & 00.0 \\
\hline 03 & 6 & 614 & 73.2317 & 5.2750 & 297.6 & 3 & .0 & \\
\hline 03 & 6 & 615 & 77.5002 & 5.7750 & 297.7 & 3 & 600.0 & .0 \\
\hline 03 & 6 & 616 & 77.2504 & 7.3250 & 297.1 & 4 & 60 & 600.0 \\
\hline 03 & 6 & 617 & 75.2499 & 9.6750 & 295.6 & 4 & .0 & \\
\hline 03 & 6 & 618 & 73.2501 & 10.5250 & 294.5 & 4 & .0 & .0 \\
\hline 03 & 6 & 619 & 77.5002 & 11.9250 & 292.4 & 4 & .0 & .0 \\
\hline 03 & 6 & 620 & 70.0005 & 12.30 & 290.2 & 4 & 6 & \\
\hline 03 & 6 & 621 & 71.5000 & 13.7750 & 288.5 & 4 & .0 & \\
\hline 03 & 6 & 622 & 71.2502 & 13.7250 & 288.1 & 4 & 600.0 & .0 \\
\hline 03 & 6 & 623 & 8.4999 & 13.97 & 288.0 & 4 & 60 & \\
\hline 03 & 6 & 624 & 75 . & 13.1750 & 288.1 & 4 & .0 & \\
\hline 03 & 6 & 71 & 72.5000 & 12.9750 & 287.6 & 4 & .0 & 600.0 \\
\hline 03 & 6 & 72 & 72.5014 & 12.6250 & 287.6 & 4 & 600.0 & .0 \\
\hline 03 & 6 & 73 & 75.2499 & 13.4000 & 286.5 & 4 & .0 & \\
\hline 03 & 6 & 74 & 79.2504 & 12.7500 & 286.4 & 4 & .0 & 0.0 \\
\hline 03 & 6 & 75 & 79.0000 & 12.6500 & 286.2 & 4 & 60 & .0 \\
\hline 03 & 6 & 76 & 80.0000 & 12.8000 & 286.0 & 4 & .0 & \\
\hline 03 & 6 & 77 & 82.2500 & 11.7500 & 286.2 & 4 & .0 & 600.0 \\
\hline 03 & 6 & 78 & 86.2438 & 9.4000 & 287.2 & 4 & 600.0 & 600.0 \\
\hline 03 & 6 & 79 & 86.7501 & 7.5500 & 288.7 & 4 & 600.0 & 0.0 \\
\hline 03 & 6 & 710 & 87.0000 & 7.22 & 289.7 & 4 & 600.0 & 600.0 \\
\hline 03 & 6 & 711 & 88.2501 & 5.6500 & 291.5 & 3 & 600.0 & 600.0 \\
\hline 03 & 6 & 712 & 86.9989 & 5.8750 & 293.1 & 3 & 600.0 & 0.0 \\
\hline 03 & 6 & 713 & 88.5000 & 6.7250 & 294.3 & 4 & 600.0 & 600.0 \\
\hline 03 & 6 & 714 & 87.2500 & 7.4000 & 295.0 & 4 & 600.0 & 600.0 \\
\hline 03 & 6 & 715 & 83.4995 & 8.2750 & 295.9 & 4 & 600.0 & 600.0 \\
\hline 03 & 6 & 716 & 79.2506 & 9.5000 & 295.9 & 4 & 600.0 & 600.0 \\
\hline 03 & 6 & 717 & 76.2512 & 9.0500 & 295.9 & 4 & 600.0 & 600.0 \\
\hline 03 & 6 & 718 & 70.0009 & 10.3250 & 294.7 & 4 & 600.0 & 600.0 \\
\hline 03 & 6 & 719 & 74.2515 & 10.7500 & 293.9 & 4 & 600.0 & 600.0 \\
\hline 03 & 6 & 720 & 76.7500 & 11.7750 & 292.2 & 4 & 600.0 & 600.0 \\
\hline 03 & 6 & 721 & 78.7493 & 11.5500 & 292.8 & 4 & 600.0 & 600.0 \\
\hline 03 & 6 & 722 & 82.5000 & 10.3250 & 292.8 & 4 & 600.0 & 600.0 \\
\hline 0 & 6 & 723 & 66.7499 & 10.225 & 292.5 & & 600.0 & 600.0 \\
\hline
\end{tabular}




\begin{tabular}{|c|c|c|c|c|c|c|c|c|}
\hline & 6 & 724 & 72.0001 & 10.4750 & 292.3 & 4 & 600.0 & 600.0 \\
\hline 3 & 6 & 81 & 73.2501 & 10.2000 & 291.9 & 4 & 600.0 & 600.0 \\
\hline 03 & 6 & 82 & 79.5015 & 9.8500 & 291.7 & 4 & 600.0 & 00.0 \\
\hline 03 & 6 & 83 & 74.0000 & 9.8750 & 291.5 & 4 & 600.0 & 00.0 \\
\hline 03 & 6 & 84 & 75.4997 & 000 & 291.3 & 4 & 600.0 & 00.0 \\
\hline 03 & 6 & 85 & 77.0000 & 9.5750 & 291.3 & 4 & 600.0 & 00.0 \\
\hline 03 & 6 & 86 & 84.2555 & 7.4250 & 291.7 & 4 & 600.0 & 00.0 \\
\hline 03 & 6 & 87 & 85.7518 & 6.3500 & 291.6 & 4 & 600.0 & 00.0 \\
\hline 03 & 6 & 88 & 77.7439 & 4.8750 & 291.6 & 4 & 600.0 & 00.0 \\
\hline 03 & 6 & 89 & 96.6109 & 500 & 292.6 & 3 & 600.0 & 00.0 \\
\hline 03 & 6 & 810 & 82.7616 & 00 & 293.6 & 3 & 600.0 & 00.0 \\
\hline 03 & 6 & 811 & 80.0000 & 50 & 295.3 & 3 & 600.0 & \\
\hline 03 & 6 & 812 & 85.7507 & 500 & 295.9 & 3 & 600.0 & 00.0 \\
\hline 03 & 6 & 813 & 78.7498 & 5.3250 & 296.9 & 3 & 600.0 & 00.0 \\
\hline 03 & 6 & 814 & 78.0103 & & 297.6 & 4 & .0 & \\
\hline 03 & 6 & 815 & 96.5207 & 6 . & 298.4 & 4 & 600.0 & 00.0 \\
\hline 03 & 6 & 816 & 87.9931 & 7.7750 & 299.0 & 4 & 600.0 & 600.0 \\
\hline 03 & 6 & 817 & 80.2501 & 8.5 & 298.8 & 4 & .0 & \\
\hline 03 & 6 & 818 & 76.7500 & 9.6500 & 297.6 & 4 & 600.0 & .0 \\
\hline 03 & 6 & 819 & 73.5000 & 8.9500 & 296.8 & 4 & .0 & .0 \\
\hline 03 & 6 & 820 & 000 & 8 . & 296.9 & 4 & 60 & \\
\hline 03 & 6 & 821 & 73.2500 & 9. & 296.6 & 4 & .0 & \\
\hline 03 & 6 & 822 & 70.5000 & 9.4000 & 296.8 & 4 & 600.0 & .0 \\
\hline 03 & 6 & 823 & 68.7499 & 8.5 & 295.8 & 4 & 60 & \\
\hline 03 & 6 & 824 & 74.5000 & 7 . & 294.3 & 4 & .0 & \\
\hline 03 & 6 & 91 & 76.3163 & 6.2500 & 291.1 & 4 & 600.0 & 600.0 \\
\hline 03 & 6 & 92 & 72.0000 & 6.6000 & 292.1 & 4 & 600.0 & .0 \\
\hline 03 & 6 & 93 & 66.7497 & 9.4 & 293.5 & 4 & 0 & \\
\hline 03 & 6 & 94 & 67.7496 & 7 . & 293.9 & 4 & 600.0 & 600.0 \\
\hline 03 & 6 & 95 & 74.3560 & 5.6250 & 290.7 & 4 & 600.0 & 0.0 \\
\hline 03 & 6 & 96 & 77.9998 & 5 . & 290.7 & 4 & .0 & \\
\hline 03 & 6 & 97 & 352.0053 & 1.2 & 292.4 & 3 & .0 & 600.0 \\
\hline 03 & 6 & 98 & 170.8874 & 2.0250 & 293.0 & 2 & 600.0 & 600.0 \\
\hline 03 & 6 & 99 & 307.9650 & 1.5 & 293.7 & 1 & 600.0 & 0.0 \\
\hline 03 & 6 & 910 & 309.5585 & 1.7000 & 295.6 & 1 & 600.0 & 600.0 \\
\hline 03 & 6 & 911 & 295.1555 & 2.1000 & 297.0 & 1 & 600.0 & 600.0 \\
\hline 03 & 6 & 912 & 33.4853 & 2 . & 297.7 & 1 & 600.0 & 600.0 \\
\hline 03 & 6 & 913 & 47.3085 & 3.8750 & 298.4 & 2 & 600.0 & 600.0 \\
\hline 03 & 6 & 914 & 51.7518 & 5.6250 & 297.7 & 3 & 600.0 & 600.0 \\
\hline 03 & 6 & 915 & 58.2524 & 5 & 298.0 & 3 & 600.0 & 600.0 \\
\hline 03 & 6 & 916 & 69.5000 & 5.7500 & 298.1 & 3 & 600.0 & 600.0 \\
\hline 03 & 6 & 917 & 82.2500 & 7.6750 & 296.8 & 4 & 600.0 & 600.0 \\
\hline 03 & 6 & 918 & 82.2496 & 8.1500 & 295.3 & 4 & 600.0 & 600.0 \\
\hline 03 & 6 & 919 & 73.7496 & 9.0000 & 293.1 & 4 & 600.0 & 600.0 \\
\hline 03 & 6 & 920 & 73.9995 & 10.1000 & 290.5 & 4 & 600.0 & 600.0 \\
\hline 03 & 6 & 921 & 66.5000 & 12.1750 & 288.4 & 4 & 600.0 & 600.0 \\
\hline 03 & 6 & 922 & 72.7500 & 12.7250 & 287.3 & 4 & 600.0 & 600.0 \\
\hline 03 & 6 & 923 & 71.7499 & 12.7000 & 286.6 & 4 & 600.0 & 600.0 \\
\hline
\end{tabular}




\begin{tabular}{|c|c|c|c|c|c|c|c|}
\hline 3 & 6924 & 73.7500 & 12.0750 & 286.5 & 4 & 600.0 & 600.0 \\
\hline 3 & 6101 & 80.7499 & 9.5000 & 286.3 & 4 & 600.0 & 600.0 \\
\hline 03 & 6102 & 79.0000 & 7.7500 & 285.8 & 4 & 600.0 & 00.0 \\
\hline 03 & 6103 & 79.2500 & 6.4750 & 285.9 & 4 & 600.0 & 00.0 \\
\hline 3 & 6104 & 80.2499 & 6.5250 & 285.5 & 4 & 600.0 & 00.0 \\
\hline 03 & 6105 & 99.3956 & 3.9500 & 285.2 & 4 & 600.0 & .0 \\
\hline 03 & 6106 & 292.3328 & 0.4750 & 285.1 & 5 & 600.0 & 00.0 \\
\hline 03 & 6107 & 225.0008 & 1.2250 & 287.0 & 4 & 600.0 & .0 \\
\hline 03 & 6108 & 228.0000 & 2.0500 & 287.4 & 3 & 600.0 & \\
\hline 03 & 6109 & 228.6559 & 2.0000 & 288.4 & 2 & 600.0 & 600.0 \\
\hline 03 & 61010 & 242.1484 & 2.5750 & 289.7 & 1 & 600.0 & 0 \\
\hline 03 & 61011 & 259.4841 & 2.2250 & 291.7 & 1 & 600.0 & \\
\hline 03 & 61012 & 211.0588 & 2.8500 & 293.1 & 1 & 600.0 & .0 \\
\hline 03 & 61013 & 176.5092 & 4.4750 & 293.7 & 2 & 600.0 & 0 \\
\hline 0 & 61014 & 173.2472 & 4.45 & 294.1 & 3 & 600.0 & .0 \\
\hline 03 & 61015 & 163.1186 & 5. & 294.9 & 3 & 600.0 & \\
\hline 03 & 61016 & 158.7557 & 6.3000 & 294.4 & 4 & 600.0 & 600.0 \\
\hline 03 & 61017 & 109.5000 & 5.7250 & 293.9 & 3 & 600.0 & .0 \\
\hline 03 & 61018 & 88.6756 & 6.6000 & 292.0 & 4 & 600.0 & \\
\hline 03 & 61019 & 5000 & 7.8250 & 290.1 & 4 & 600.0 & 600.0 \\
\hline 03 & 61020 & 71.5000 & 9.9750 & 287.8 & 4 & 600.0 & .0 \\
\hline 03 & 61021 & 73.0000 & 10.25 & 286.3 & 4 & 600.0 & \\
\hline 03 & 61022 & 68.2500 & 10.9750 & 285.5 & 4 & 600.0 & 60 \\
\hline 03 & 61023 & 68.7500 & 11.5750 & 285.0 & 4 & 600.0 & .0 \\
\hline 03 & 61024 & 74.7505 & 10.45 & 284.9 & 4 & 600.0 & \\
\hline 03 & 6111 & 81.2501 & 9.0750 & 284.9 & 4 & 600.0 & 60 \\
\hline 03 & 6112 & 82.7497 & 8.7000 & 284.7 & 4 & 600.0 & .0 \\
\hline 03 & 6113 & 81.2492 & 8.40 & 284.4 & 4 & 600.0 & .0 \\
\hline 03 & 6114 & 77.7500 & 8.7500 & 284.3 & 4 & 600.0 & .0 \\
\hline 03 & 6115 & 74.5000 & 8.8000 & 284.1 & 4 & 600.0 & .0 \\
\hline 03 & 6116 & 74.5005 & 7.8750 & 284.1 & 4 & 600.0 & .0 \\
\hline 03 & 6117 & 79.0000 & 7 . & 285.0 & 4 & 600.0 & \\
\hline 03 & 6118 & 89.9866 & 4.42 & 286.6 & 3 & 600.0 & .0 \\
\hline 03 & 6119 & 247.6020 & 1.5750 & 289.9 & 2 & 600.0 & .0 \\
\hline 03 & 61110 & 239.8269 & 2 . & 291.2 & 1 & 600.0 & \\
\hline 03 & 61111 & 303.6568 & 2.9000 & 292.1 & 1 & 600.0 & 600.0 \\
\hline 03 & 61112 & 45.9970 & 4.4250 & 292.2 & 2 & 600.0 & 600.0 \\
\hline 03 & 61113 & 61.7631 & 4 . & 293.3 & 3 & 600.0 & 60 \\
\hline 03 & 61114 & 68.9993 & 6.3500 & 293.2 & 4 & 600.0 & 600.0 \\
\hline 03 & 61115 & 69.2500 & 8.0500 & 292.7 & 4 & 600.0 & 600 \\
\hline 03 & 61116 & 68.0000 & 9.2000 & 292.0 & 4 & 600.0 & 600.0 \\
\hline 03 & 61117 & 71.2501 & 10.7250 & .0 & 4 & 600.0 & 600.0 \\
\hline 03 & 61118 & 70.7499 & 12.9500 & 287.3 & 4 & 600.0 & 600.0 \\
\hline 03 & 61119 & 66.9998 & 14.6500 & 285.3 & 4 & 600.0 & 600.0 \\
\hline 03 & 61120 & 69.2499 & 15.7250 & 284.1 & 4 & 600.0 & 600.0 \\
\hline 03 & 61121 & 65.0000 & 14.3500 & 283.7 & 4 & 600.0 & 600.0 \\
\hline 03 & 61122 & 68.9992 & 14. & & 4 & 600.0 & .0 \\
\hline 03 & 61123 & 73.4997 & 14.9750 & 283.7 & $\mathbf{4}$ & 600.0 & 600.0 \\
\hline
\end{tabular}




\begin{tabular}{|c|c|c|c|c|c|c|c|}
\hline 3 & 61124 & 72.7500 & 14.5750 & 283.8 & 4 & 600.0 & 00.0 \\
\hline 3 & 6121 & 71.4998 & 13.3000 & 283.6 & 4 & 600.0 & 600.0 \\
\hline 03 & 6122 & 70.7498 & 13.1000 & 283.1 & 4 & 600.0 & 00.0 \\
\hline 03 & 6123 & 71.2499 & 12.5750 & 282.9 & 4 & 600.0 & 00.0 \\
\hline 13 & 6124 & 72.5002 & 12.2500 & 282.9 & . & 600.0 & 00.0 \\
\hline 03 & 6125 & 68.9999 & 11.5750 & 282.8 & 4 & 600.0 & .0 \\
\hline 03 & 6126 & 74.2500 & 11.0000 & 282.9 & 4 & 600.0 & 600.0 \\
\hline 03 & 6127 & 2.0000 & 11.0000 & 283.4 & 4 & 600.0 & .0 \\
\hline 03 & 6128 & 73.2499 & 11.1250 & 283.8 & 4 & 600.0 & \\
\hline 03 & 6129 & 81.7500 & 10.4500 & 284.5 & 4 & 600.0 & 600.0 \\
\hline 03 & 61210 & 75.7505 & 8.6000 & 286.3 & 4 & 600.0 & .0 \\
\hline 03 & 61211 & 81.2490 & 8.3250 & 287.2 & 4 & 600.0 & \\
\hline 03 & 61212 & 93.2526 & 8.0000 & 288.5 & 4 & 600.0 & .0 \\
\hline 03 & 61213 & 85.7502 & 7.8500 & 289.4 & 4 & 600.0 & 0 \\
\hline 03 & 61214 & 78.2501 & 8.1250 & 290.2 & 4 & 600.0 & .0 \\
\hline 03 & 61215 & 72.7518 & 8.4 & 290.5 & 4 & 600.0 & \\
\hline 03 & 61216 & 77.2499 & 11.3750 & 288.9 & 4 & 600.0 & 600.0 \\
\hline 03 & 61217 & 73.7504 & 12.2500 & 288.3 & 4 & 600.0 & .0 \\
\hline 03 & 61218 & 77.0001 & 13. & 287.3 & 4 & 600.0 & \\
\hline 03 & 61219 & 76.0000 & 12.8250 & 286.3 & 4 & 600.0 & 600.0 \\
\hline 03 & 61220 & 71.5003 & 12.0250 & 286.2 & 4 & 600.0 & .0 \\
\hline 03 & 61221 & 65.0001 & 12.9750 & 285.4 & 4 & 600.0 & \\
\hline 03 & 61222 & 67.7499 & 13.9000 & 283.9 & 4 & 600.0 & 60 \\
\hline 03 & 61223 & 68.5000 & 14.2250 & 284.5 & 4 & 600.0 & .0 \\
\hline 03 & 61224 & 70.7498 & 14.0500 & 284.7 & 4 & 600.0 & \\
\hline 03 & 6131 & 69.5009 & 15.0750 & 284.6 & 4 & 600.0 & 60 \\
\hline 03 & 6132 & 68.2506 & 14.9500 & 284.1 & 4 & 600.0 & .0 \\
\hline 03 & 6133 & 67.0000 & 15.42 & 283.7 & 4 & 600.0 & .0 \\
\hline 03 & 6134 & 65.2495 & 14.2750 & 283.8 & 4 & 600.0 & .0 \\
\hline 03 & 6135 & 68.7498 & 13.2000 & 284.9 & 4 & 600.0 & .0 \\
\hline 03 & 6136 & 73.7496 & 13.4500 & 285.1 & 4 & 600.0 & 60 \\
\hline 03 & 6137 & 70.2495 & 12.7000 & 285.4 & 4 & 600.0 & \\
\hline 03 & 6138 & 69.5001 & 10.6250 & 286.5 & 4 & 600.0 & .0 \\
\hline 03 & 6139 & 75.0002 & 87 & 287.7 & 4 & 600.0 & .0 \\
\hline 03 & 61310 & 73.0000 & 8 & 289.5 & 4 & 600.0 & \\
\hline 03 & 61311 & 71.7500 & 6.5500 & 292.2 & 4 & 600.0 & 600.0 \\
\hline 03 & 61312 & 87.7770 & 6.2500 & 293.9 & 4 & 600.0 & 600.0 \\
\hline 03 & 61313 & 89.2500 & 7.9500 & 294.0 & 4 & 600.0 & 60 \\
\hline 03 & 61314 & 82.2479 & 8.0000 & 294.5 & 4 & 600.0 & 600.0 \\
\hline 03 & 61315 & 83.7501 & 7.3750 & 295.8 & 4 & 600.0 & 600.0 \\
\hline 03 & 61316 & 88.0014 & 9.2750 & 295.3 & 4 & 600.0 & 600.0 \\
\hline 03 & 61317 & 79.2501 & 10.7750 & 294.8 & 4 & 600.0 & 600.0 \\
\hline 03 & 61318 & 72.0011 & 10.6750 & 293.4 & 4 & 600.0 & 600.0 \\
\hline 03 & 61319 & 70.2499 & 9.8750 & 291.9 & 4 & 600.0 & 600.0 \\
\hline 03 & 61320 & 67.4995 & 12.8750 & 290.7 & 4 & 600.0 & 600.0 \\
\hline 03 & 61321 & 65.5000 & 14.2500 & 290.5 & 4 & 600.0 & 600.0 \\
\hline 03 & 61322 & 998 & 13.52 & & 4 & 600.0 & .0 \\
\hline 03 & 61323 & 66.7500 & 14.0250 & 289.2 & 1 & 600.0 & 600.0 \\
\hline
\end{tabular}




\begin{tabular}{|c|c|c|c|c|c|c|c|}
\hline 3 & 61324 & 67.7501 & 12.8250 & 288.5 & 4 & 600.0 & 00.0 \\
\hline 3 & 6141 & 71.2499 & 12.0750 & 288.2 & 4 & 600.0 & 600.0 \\
\hline 03 & 6142 & 72.0001 & 11.1750 & 288.3 & 4 & 600.0 & 00.0 \\
\hline 03 & 6143 & 70.2500 & 10.9000 & 288.7 & 4 & 600.0 & 00.0 \\
\hline 13 & 6144 & 75.4991 & 10.1750 & 288.4 & 4 & 600.0 & 00.0 \\
\hline 03 & 6145 & 78.0000 & 11.2750 & 287.4 & 4 & 600.0 & .0 \\
\hline 03 & 6146 & 77.2500 & 12.0000 & 286.3 & 4 & 600.0 & 600.0 \\
\hline 03 & 6147 & 81.7501 & 10.9500 & 287.2 & 4 & 600.0 & .0 \\
\hline 03 & 6148 & 81.2532 & 6.1250 & 290.4 & 4 & 600.0 & \\
\hline 03 & 6149 & 268.1742 & 1.8500 & 292.8 & 3 & 600.0 & 600.0 \\
\hline 03 & 61410 & 267.0000 & 2.3500 & 293.9 & 2 & 600.0 & 0 \\
\hline 03 & 61411 & 210.7008 & 2.5250 & 295.3 & 1 & 600.0 & \\
\hline 03 & 61412 & 93.4936 & 4.5750 & 295.2 & 2 & 600.0 & .0 \\
\hline 03 & 61413 & 95.5014 & 5.6500 & 296.7 & 3 & 600.0 & 0 \\
\hline 0 & 61414 & 96.4994 & 500 & 297.6 & 4 & 600.0 & .0 \\
\hline 03 & 61415 & 80.2452 & 6 & 298.4 & 4 & 600.0 & \\
\hline 03 & 61416 & 86.5069 & 8.3000 & 297.6 & 4 & 600.0 & 600.0 \\
\hline 03 & 61417 & 88.4986 & 8.4500 & 296.5 & 4 & 600.0 & .0 \\
\hline 03 & 61418 & 73.5017 & 7.6750 & 295.9 & 4 & 600.0 & \\
\hline 03 & 61419 & 70.2511 & 9.6500 & 294.9 & 4 & 600.0 & 600.0 \\
\hline 03 & 61420 & 69.7503 & 9.8750 & 294.3 & 4 & 600.0 & .0 \\
\hline 03 & 61421 & 68.0000 & 9.9750 & 294.4 & 4 & 600.0 & \\
\hline 03 & 61422 & 69.2498 & 9.4750 & 294.5 & 4 & 600.0 & 60 \\
\hline 03 & 61423 & 68.5000 & 9.0250 & 294.5 & 4 & 600.0 & .0 \\
\hline 03 & 61424 & 71.5000 & 10.4000 & 294.0 & 4 & 600.0 & \\
\hline 03 & 6151 & 70.7505 & 11.5250 & 293.4 & 4 & 600.0 & 60 \\
\hline 03 & 6152 & 71.2500 & 11.4750 & 292.7 & 4 & 600.0 & .0 \\
\hline 03 & 6153 & 70.7496 & 9.8500 & 292.5 & 4 & 600.0 & .0 \\
\hline 03 & 6154 & 77.0000 & 9.6750 & 292.1 & 4 & 600.0 & .0 \\
\hline 03 & 6155 & 81.2499 & 9.3000 & 291.6 & 4 & 600.0 & .0 \\
\hline 03 & 6156 & 83.0000 & 10.0500 & 291.6 & 4 & 600.0 & 60 \\
\hline 03 & 6157 & 85.2499 & 8.5250 & 292.5 & 4 & 600.0 & \\
\hline 03 & 6158 & 99.2486 & 5.0250 & 294.8 & 4 & 600.0 & 600.0 \\
\hline 03 & 6159 & 173.1357 & 4.7000 & 296.5 & 3 & 600.0 & .0 \\
\hline 03 & 61510 & 171.5000 & 6.7000 & 295.9 & 4 & 600.0 & \\
\hline 03 & 61511 & 169.7466 & 6.3750 & 296.8 & 4 & 600.0 & 600.0 \\
\hline 03 & 61512 & 170.7499 & 6.2000 & 298.4 & 4 & 600.0 & 600.0 \\
\hline 03 & 61513 & 170.2604 & 4.87 & 300.0 & 3 & 600.0 & 60 \\
\hline 03 & 61514 & 165.0487 & 6.2500 & 301.0 & 4 & 600.0 & 600.0 \\
\hline 03 & 61515 & 151.7496 & 5.6750 & 301.5 & 3 & 600.0 & 600.0 \\
\hline 03 & 61516 & 126.0587 & 5.2750 & 302.2 & 3 & 600.0 & 600.0 \\
\hline 03 & 61517 & 90.9896 & 5.3500 & 301.9 & 3 & 600.0 & 600.0 \\
\hline 03 & 61518 & 82.0011 & 6.0000 & 300.6 & 4 & 600.0 & 600.0 \\
\hline 03 & 61519 & 75.7502 & 7.1250 & 298.2 & 4 & 600.0 & 600.0 \\
\hline 03 & 61520 & 72.2523 & 8.8000 & 296.6 & 4 & 600.0 & 600.0 \\
\hline 03 & 61521 & 69.2496 & 9.0250 & 296.4 & 4 & 600.0 & 600.0 \\
\hline 03 & 61522 & 72.2500 & 8.750 & 29 & 4 & 600.0 & .0 \\
\hline 03 & 61523 & 76.5002 & 9.0000 & 297.5 & $\mathbf{4}$ & 600.0 & 600.0 \\
\hline
\end{tabular}




\begin{tabular}{|c|c|c|c|c|c|c|c|}
\hline 3 & 61524 & 69.2500 & 8.5250 & 297.7 & 4 & 600.0 & 600.0 \\
\hline 03 & 6161 & 70.2499 & 8.6500 & 297.5 & 4 & 600.0 & 600.0 \\
\hline 03 & 6162 & 77.4992 & 7.8750 & 297.1 & 4 & 600.0 & 00.0 \\
\hline 03 & 6163 & 77.0000 & 7.5500 & 296.4 & 4 & 600.0 & 00.0 \\
\hline 03 & 6164 & 75.7500 & 8.8750 & 295.6 & 4 & 600.0 & 00.0 \\
\hline 03 & 6165 & 78.2481 & 8.4750 & 295.9 & 4 & 600.0 & 00.0 \\
\hline 03 & 6166 & 92.4991 & 7.4750 & 296.2 & 4 & 600.0 & 600.0 \\
\hline 03 & 6167 & 106.3818 & 7.7000 & 296.6 & 4 & 600.0 & 00.0 \\
\hline 03 & 6168 & 153.0000 & 6.2250 & 298.5 & 4 & 600.0 & 00.0 \\
\hline 03 & 6169 & 162.7498 & 6.0000 & 299.2 & 4 & 600.0 & 600.0 \\
\hline 03 & 61610 & 167.7497 & 6.6750 & 300.0 & 4 & 600.0 & 00.0 \\
\hline 03 & 61611 & 165.2392 & 6.2000 & 301.1 & 4 & 600.0 & \\
\hline 03 & 61612 & 172.4768 & 5.1250 & 302.6 & 3 & 600.0 & 600.0 \\
\hline 03 & 61613 & 172.5244 & 4.7250 & 303.9 & 3 & 600.0 & 600 \\
\hline 03 & 61614 & 149.7339 & 5.7500 & 304.6 & 3 & 600.0 & \\
\hline 03 & 61615 & 99.8240 & 5.9750 & 304.9 & 3 & 600.0 & 60 \\
\hline 03 & 61616 & 85.2550 & 6.9750 & 304.7 & 4 & 600.0 & 600.0 \\
\hline 03 & 61617 & 88.9973 & 7.5250 & 304.2 & 4 & 600.0 & \\
\hline 03 & 61618 & 67.4991 & 8.2500 & 303.4 & 4 & 600.0 & 60 \\
\hline 03 & 61619 & 63.2495 & 9.4500 & 302.4 & 4 & 600.0 & 600 \\
\hline 03 & 61620 & 58.9998 & 8.6250 & 302.3 & 4 & 600.0 & .0 \\
\hline 03 & 61621 & 62.7500 & 8.8500 & 301.6 & 4 & 600.0 & \\
\hline 03 & 61622 & 64.7499 & 8.5250 & 301.6 & 4 & 600.0 & 600.0 \\
\hline 03 & 61623 & 70.4989 & 8.2500 & 301.3 & 4 & 600.0 & .0 \\
\hline 03 & 61624 & 71.2500 & 8.2250 & 300.7 & 4 & 600.0 & \\
\hline 03 & 6171 & 72.5000 & 8.2500 & 300.8 & 4 & 600.0 & 60 \\
\hline 03 & 6172 & 75.4997 & 8.6250 & 300.2 & 4 & 600.0 & .0 \\
\hline 03 & 6173 & 73.7500 & 9.2250 & 299.7 & 4 & 600.0 & \\
\hline 03 & 6174 & 72.2500 & 7.8500 & 299.6 & 4 & 600.0 & 60 \\
\hline 03 & 6175 & 94.1241 & 1.9500 & 298.0 & 5 & 600.0 & 600 \\
\hline 03 & 6176 & 86.1148 & 1.150 & 297.1 & 6 & 600.0 & \\
\hline 03 & 6177 & 72.2496 & 3.6500 & 298.1 & 5 & 600.0 & 60 \\
\hline 03 & 6178 & 83.7175 & 5.8500 & 298.0 & 4 & 600.0 & 600.0 \\
\hline 03 & 6179 & 329.5823 & 2.27 & 300.3 & 3 & 600.0 & .0 \\
\hline 03 & 61710 & 139.0749 & 3.1750 & 300.8 & 2 & 600.0 & 60 \\
\hline 03 & 61711 & 158.2486 & 2.6000 & 302.8 & 1 & 600.0 & 600.0 \\
\hline 03 & 61712 & 89.4931 & 5.6250 & 301.1 & 2 & 600.0 & \\
\hline 03 & 61713 & 83.5038 & 8.2750 & 299.5 & 3 & 600.0 & 600.0 \\
\hline 03 & 61714 & 82.4786 & 8.3500 & 299.5 & 4 & 600.0 & 600.0 \\
\hline 03 & 61715 & 88.7649 & 8.7250 & 299.8 & 4 & 600.0 & \\
\hline 03 & 61716 & 80.2501 & 10.5750 & 299.4 & 4 & 600.0 & 600.0 \\
\hline 03 & 61717 & 77.7497 & 11.5000 & 298.7 & 4 & 600.0 & 600.0 \\
\hline 03 & 61718 & 85.2500 & 11.4250 & 296.8 & 4 & 600.0 & 600.0 \\
\hline 03 & 61719 & 74.7501 & 11.2500 & 297.8 & 4 & 600.0 & 600.0 \\
\hline 03 & 61720 & 73.7492 & 9.7750 & 296.9 & 4 & 600.0 & 600.0 \\
\hline 03 & 61721 & 68.2499 & 9.9750 & 296.4 & 4 & 600.0 & 600.0 \\
\hline 03 & 61722 & 74.5000 & 10.775 & 296.4 & 4 & 600.0 & 600.0 \\
\hline 03 & 61723 & 70.7499 & 11.7750 & 295.2 & 4 & 600.0 & 600.0 \\
\hline
\end{tabular}




\begin{tabular}{|c|c|c|c|c|c|c|c|}
\hline 3 & 61724 & 68.2500 & 11.8750 & 294.8 & 4 & 600.0 & 00.0 \\
\hline 3 & 6181 & 70.2501 & 12.1500 & 294.8 & 4 & 600.0 & 600.0 \\
\hline 03 & 6182 & 65.2500 & 12.0500 & 294.9 & 4 & 600.0 & 00.0 \\
\hline 03 & 6183 & 70.7499 & 11.4250 & 295.4 & 4 & 600.0 & 00.0 \\
\hline 3 & 6184 & 66.0000 & 12.2500 & 294.8 & 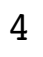 & 600.0 & 00.0 \\
\hline 03 & 6185 & 72.0000 & 12.0000 & 292.9 & 4 & 600.0 & .0 \\
\hline 03 & 6186 & 68.7506 & 11.5250 & 292.1 & 4 & 600.0 & 500.0 \\
\hline 03 & 6187 & 72.9998 & 11.3500 & 293.1 & 4 & 600.0 & .0 \\
\hline 03 & $618 \quad 8$ & 68.7513 & 11.0500 & 292.9 & 4 & 600.0 & \\
\hline 03 & 6189 & 68.7488 & 11.3750 & 292.2 & 4 & 600.0 & .0 \\
\hline 03 & 61810 & 71.0002 & 10.7250 & 293.1 & 4 & 600.0 & .0 \\
\hline 03 & 61811 & 71.7485 & 9.9500 & 294.2 & 4 & 600.0 & \\
\hline 03 & 61812 & 73.7497 & 8.3500 & 296.6 & 4 & 600.0 & .0 \\
\hline 03 & 61813 & 98.5335 & 8.1500 & 297.0 & 4 & 600.0 & \\
\hline 03 & 61814 & 118.2489 & 500 & 297.6 & 4 & 600.0 & \\
\hline 03 & 61815 & 109.2662 & 4 & 298.5 & 3 & 600.0 & \\
\hline 03 & 61816 & 70.0027 & 8.0500 & 297.8 & 4 & 600.0 & .0 \\
\hline 03 & 61817 & 62.0000 & 13.4000 & 296.4 & 4 & 600.0 & .0 \\
\hline 03 & 61818 & 61.7500 & 13.9500 & 295.8 & 4 & 00.0 & \\
\hline 03 & 61819 & 60.7499 & 13.3500 & 294.9 & 4 & 600.0 & 600.0 \\
\hline 03 & 61820 & 62.4997 & 13.1750 & 293.6 & 4 & 600.0 & .0 \\
\hline 03 & 61821 & 65.2500 & 11. & 293.6 & 4 & 600.0 & \\
\hline 03 & 61822 & 65.9999 & 11.9000 & 292.0 & 4 & 600.0 & .0 \\
\hline 03 & 61823 & 68.2495 & 11.4000 & 291.4 & 4 & 600.0 & .0 \\
\hline 03 & 61824 & 68.7501 & 11.17 & 290.9 & 4 & 600.0 & \\
\hline 03 & 6191 & 71.5000 & 11.3250 & 290.4 & 4 & 600.0 & 60 \\
\hline 03 & 6192 & 69.9998 & 12.7500 & 288.0 & 4 & 600.0 & .0 \\
\hline 03 & 6193 & 60.4998 & 11.52 & 286.6 & 4 & 600.0 & .0 \\
\hline 03 & 6194 & 77.9930 & 6.9250 & 286.7 & 4 & 600.0 & .0 \\
\hline 03 & 6195 & 82.5000 & 10.5500 & 286.2 & 4 & 600.0 & .0 \\
\hline 03 & 6196 & 75.9986 & 11.350 & 286.1 & 4 & 600.0 & .0 \\
\hline 03 & 6197 & 77.5000 & 9.7500 & 286.4 & 4 & 600.0 & \\
\hline 03 & 6198 & 79.0000 & 8.90 & 287.1 & 4 & 600.0 & .0 \\
\hline 03 & 6199 & 89.2492 & 5.6250 & 289.6 & 4 & 600.0 & .0 \\
\hline 03 & 61910 & 88.7483 & 3.55 & 291.6 & 3 & 600.0 & \\
\hline 03 & 61911 & 64.3748 & 1.6750 & 294.7 & 2 & 600.0 & 600.0 \\
\hline 03 & 61912 & 90.9966 & 4.6000 & 294.6 & 3 & 600.0 & 600.0 \\
\hline 03 & 61913 & 91.2680 & 5.925 & 295.3 & 3 & 600.0 & 60 \\
\hline 03 & 61914 & 82.7517 & 7.8250 & 295.7 & 4 & 600.0 & 600.0 \\
\hline 03 & 61915 & 77.7508 & 8.6000 & 295.4 & 4 & 600.0 & 600 \\
\hline 03 & 61916 & 79.5003 & 10.3500 & 293.7 & 4 & 600.0 & 600.0 \\
\hline 03 & 61917 & 73.9979 & 11.3500 & 292.6 & 4 & 600.0 & 600.0 \\
\hline 03 & 61918 & 76.4994 & 12.0750 & 291.7 & 4 & 600.0 & 600.0 \\
\hline 03 & 61919 & 77.2507 & 12.0750 & 290.4 & 4 & 600.0 & 600.0 \\
\hline 03 & 61920 & 76.0000 & 12.0250 & 289.2 & 4 & 600.0 & 600.0 \\
\hline 03 & 61921 & 79.7495 & 10.1500 & 288.7 & 4 & 600.0 & 600.0 \\
\hline 0 & 61922 & 72.0000 & 9 & .5 & 4 & 600.0 & .0 \\
\hline 03 & 61923 & 75.0002 & 11.6500 & 287.7 & 1 & 600.0 & 600.0 \\
\hline
\end{tabular}




\begin{tabular}{|c|c|c|c|c|c|c|c|}
\hline 3 & 61924 & 68.7501 & 11.3000 & 287.6 & 4 & 600.0 & 00.0 \\
\hline 03 & 6201 & 70.0001 & 12.3250 & 287.0 & 4 & 600.0 & 600.0 \\
\hline 03 & 6202 & 66.5003 & 11.7500 & 287.0 & 4 & 600.0 & 00.0 \\
\hline 03 & 6203 & 67.0000 & 12.5500 & 286.2 & 4 & 600.0 & 00.0 \\
\hline 03 & $620 \quad 4$ & 69.2497 & 11.3000 & 286.7 & 4 & 600.0 & 00.0 \\
\hline 03 & 6205 & 77.2502 & 9.8750 & 286.1 & 4 & 600.0 & 00.0 \\
\hline 03 & 6206 & 77.2500 & 11.8000 & 285.5 & 4 & 600.0 & 00.0 \\
\hline 03 & 6207 & 78.5000 & 10.6000 & 286.9 & 4 & 600.0 & 00.0 \\
\hline 03 & 6208 & 77.7494 & 8.3250 & 288.3 & 4 & 600.0 & 00.0 \\
\hline 03 & 6209 & 91.0008 & 7.1500 & 289.7 & 4 & 600.0 & .0 \\
\hline 03 & 62010 & 89.7500 & 4.8750 & 291.8 & 3 & 600.0 & 00.0 \\
\hline 03 & 62011 & 100.9460 & 50 & 294.1 & 2 & 600.0 & \\
\hline 03 & 62012 & 80.0592 & 4.7000 & 295.0 & 3 & 600.0 & 00.0 \\
\hline 03 & 62013 & 85.7519 & 5.7750 & 295.9 & 3 & 600.0 & .0 \\
\hline 03 & 62014 & 83.2513 & & 295.8 & 4 & 600.0 & \\
\hline 03 & 62015 & 77.7495 & 8.5250 & 295.2 & 4 & 600.0 & 60 \\
\hline 03 & 62016 & 73.5000 & 9.2500 & 295.2 & 4 & 600.0 & 600.0 \\
\hline 03 & 62017 & 65.7504 & 10.9750 & 294.1 & 4 & 600.0 & \\
\hline 03 & 62018 & 75.9999 & 12.4750 & 292.2 & 4 & 600.0 & .0 \\
\hline 03 & 62019 & 63.2468 & 12.2000 & 291.1 & 4 & 600.0 & .0 \\
\hline 03 & 62020 & 60.2500 & 12.20 & 289.1 & 4 & 600.0 & .0 \\
\hline 03 & 62021 & 66.2508 & 12 . & 288.2 & 4 & 0.0 & \\
\hline 03 & 62022 & 65.5000 & 13.5750 & 287.9 & 4 & 600.0 & 600.0 \\
\hline 03 & 62023 & 64.2500 & 13.3750 & 288.0 & 4 & 600.0 & .0 \\
\hline 03 & 62024 & 64.5006 & 13. & 287.2 & 4 & 00.0 & \\
\hline 03 & 6211 & 66.5000 & 10.8250 & 287.1 & 4 & 600.0 & 60 \\
\hline 03 & 6212 & 72.7552 & 11.6000 & 287.0 & 4 & 600.0 & .0 \\
\hline 03 & 6213 & 72.9998 & 12.7250 & 286.1 & 4 & 600.0 & \\
\hline 03 & 6214 & 75.2511 & 12.2500 & 285.2 & 4 & 600.0 & 60 \\
\hline 03 & 6215 & 78.2472 & 9000 & 285.7 & 4 & 600.0 & .0 \\
\hline 03 & 6216 & 77.5107 & 7 . & 285.4 & 4 & 600.0 & \\
\hline 03 & 6217 & 106.2492 & 250 & 286.2 & 4 & 600.0 & .0 \\
\hline 03 & 6218 & 83.9904 & 6.6250 & 287.1 & 4 & 600.0 & 600.0 \\
\hline 03 & 6219 & 93.0107 & 7 . & 288.1 & 4 & 600.0 & .0 \\
\hline 03 & 62110 & 95.7499 & 5 . & 290.0 & 3 & 600.0 & 60 \\
\hline 03 & 62111 & 88.5174 & 4.3750 & 292.4 & 3 & 600.0 & 600.0 \\
\hline 03 & 62112 & 96.0021 & 6.0750 & 293.4 & 4 & 600.0 & \\
\hline 03 & 62113 & 97.0004 & 6.5250 & 294.1 & 4 & 600.0 & 600.0 \\
\hline 03 & 62114 & 97.2504 & 7.1250 & 294.6 & 4 & 600.0 & 600.0 \\
\hline 03 & 62115 & 91.9993 & 7.925 & 294.9 & 4 & 600.0 & \\
\hline 03 & 62116 & 90.7496 & 7.8000 & 295.2 & 4 & 600.0 & 600.0 \\
\hline 03 & 62117 & 84.7489 & 7.6250 & 294.5 & 4 & 600.0 & 600.0 \\
\hline 03 & 62118 & 80.0000 & 7.6000 & 293.2 & 4 & 600.0 & 600.0 \\
\hline 03 & 62119 & 74.9998 & 8.2750 & 292.5 & 4 & 600.0 & 600.0 \\
\hline 03 & 62120 & 75.5003 & 8.4250 & 291.9 & 4 & 600.0 & 600.0 \\
\hline 03 & 62121 & 71.0000 & 9.4250 & 291.4 & 4 & 600.0 & 600.0 \\
\hline 03 & 62122 & 72.0000 & 9.325 & 292.0 & 4 & 600.0 & 600.0 \\
\hline 03 & 62123 & 68.4998 & 8.4500 & 292.3 & 4 & 600.0 & 600.0 \\
\hline
\end{tabular}




\begin{tabular}{|c|c|c|c|c|c|c|c|}
\hline 3 & 62124 & 72.7500 & 9.2250 & 292.0 & 4 & 600.0 & 600.0 \\
\hline 03 & 6221 & 69.2506 & 9.7250 & 291.8 & 4 & 600.0 & 600.0 \\
\hline 03 & 6222 & 74.2500 & 8.8000 & 291.6 & 4 & 600.0 & 00.0 \\
\hline 03 & 6223 & 75.5000 & 9.2500 & 290.8 & 4 & 600.0 & 600.0 \\
\hline 03 & 6224 & 78.0001 & 9.4500 & 289.7 & 4 & 600.0 & 00.0 \\
\hline 03 & 6225 & 78.9993 & 7.5250 & 290.4 & 4 & 600.0 & 00.0 \\
\hline 03 & 6226 & 76.0007 & 6.9000 & 289.6 & 4 & 600.0 & 600.0 \\
\hline 03 & 6227 & 76.9993 & 5.7750 & 290.0 & 4 & 600.0 & 00.0 \\
\hline 03 & 6228 & 87.0154 & 2.1750 & 292.0 & 3 & 600.0 & 00.0 \\
\hline 03 & 6229 & 102.7328 & 3.0750 & 292.8 & 2 & 600.0 & 600.0 \\
\hline 03 & 62210 & 57.8228 & 2.3750 & 295.2 & 1 & 600.0 & 600.0 \\
\hline 03 & 62211 & 127.5000 & 3.8000 & 295.4 & 2 & 600.0 & \\
\hline 03 & 62212 & 169.7501 & 7.4500 & 294.8 & 3 & 600.0 & 600.0 \\
\hline 03 & 62213 & 146.9635 & 5.7250 & 296.8 & 3 & 600.0 & 600.0 \\
\hline 03 & 62214 & 106.5276 & & 297.6 & 3 & 600.0 & \\
\hline 03 & 62215 & 101.2520 & 750 & 297.5 & 4 & 600.0 & 60 \\
\hline 03 & 62216 & 90.2479 & 7.0750 & 297.0 & 4 & 600.0 & 600.0 \\
\hline 03 & 62217 & 95.2180 & 5. & 296.8 & 3 & 600.0 & \\
\hline 03 & 62218 & 69.7502 & 8.0750 & 295.3 & 4 & 600.0 & 60 \\
\hline 03 & 62219 & 67.5018 & 250 & 294.1 & 4 & 600.0 & 600 \\
\hline 03 & 62220 & 58.9999 & 9 . & 294.1 & 4 & 600.0 & .0 \\
\hline 03 & 62221 & 73.7513 & 250 & 293.3 & 4 & 600.0 & \\
\hline 03 & 62222 & 71.7476 & 8.8750 & 292.2 & 4 & 600.0 & 600.0 \\
\hline 03 & 62223 & 66.0000 & 11. & 291.1 & 4 & 600.0 & .0 \\
\hline 03 & 62224 & 69.5000 & 10 . & 290.9 & 4 & 600.0 & \\
\hline 03 & 6231 & 66.7500 & 000 & 291.0 & 4 & 600.0 & 600.0 \\
\hline 03 & 6232 & 69.0000 & 9.7250 & 290.7 & 4 & 600.0 & 60 \\
\hline 03 & 6233 & 72.4995 & 9 . & 290.1 & 4 & 600.0 & \\
\hline 03 & 6234 & 69.7548 & 000 & 289.3 & 4 & 600.0 & 60 \\
\hline 03 & 6235 & 77.2502 & 9.6500 & 288.6 & 4 & 600.0 & 600.0 \\
\hline 03 & 6236 & 75.5003 & 9 . & 287.9 & 4 & 600.0 & .0 \\
\hline 03 & 6237 & 79.5003 & 500 & 288.4 & 4 & 600.0 & 60 \\
\hline 03 & 6238 & 81.0002 & 7.9500 & 289.6 & 4 & 600.0 & 600.0 \\
\hline 03 & 6239 & 92.0002 & 6 . & 290.9 & 4 & 600.0 & .0 \\
\hline 03 & 62310 & 102.7493 & 6.2250 & 292.5 & 4 & 600.0 & 60 \\
\hline 03 & 62311 & 108.2688 & 5.6750 & 293.8 & 3 & 600.0 & 600.0 \\
\hline 03 & 62312 & 109.5000 & 6.2250 & 294.8 & 4 & 600.0 & \\
\hline 03 & 62313 & 102.7690 & 6.4750 & 295.0 & 4 & 600.0 & 600.0 \\
\hline 03 & 62314 & 82.5008 & 7.2500 & 295.2 & 4 & 600.0 & 600.0 \\
\hline 03 & 62315 & 77.5000 & 8.3750 & 295.0 & 4 & 600.0 & \\
\hline 03 & 62316 & 72.5006 & 500 & 294.7 & 4 & 600.0 & 600.0 \\
\hline 03 & 62317 & 74.9993 & 8.0000 & 294.5 & 4 & 600.0 & 600.0 \\
\hline 03 & 62318 & 74.2500 & 9.2250 & 293.3 & 4 & 600.0 & 600.0 \\
\hline 03 & 62319 & 74.5000 & 8.8250 & 293.0 & 4 & 600.0 & 600.0 \\
\hline 03 & 62320 & 71.5000 & 9.3000 & 292.3 & 4 & 600.0 & 600.0 \\
\hline 03 & 62321 & 74.2500 & 8.8500 & 292.0 & 4 & 600.0 & 600.0 \\
\hline 03 & 62322 & 71.0001 & 9.2500 & 292.2 & 4 & 600.0 & 600.0 \\
\hline 03 & 62323 & 67.7500 & 9.7500 & 292.0 & 4 & 600.0 & 600.0 \\
\hline
\end{tabular}




\begin{tabular}{|c|c|c|c|c|c|c|c|}
\hline 3 & 62324 & 73.2499 & 9.8000 & 291.3 & 4 & 600.0 & 00.0 \\
\hline 03 & 6241 & 83.7496 & 8.9750 & 290.6 & 4 & 600.0 & 00.0 \\
\hline 03 & 6242 & 118.2562 & 7.7250 & 291.2 & 4 & 600.0 & 00.0 \\
\hline 03 & 6243 & 125.4792 & 8.8750 & 290.7 & 4 & 600.0 & 00.0 \\
\hline 03 & $624 \quad 4$ & 151.2501 & 11.1000 & 290.9 & 4 & 600.0 & 00.0 \\
\hline 03 & 6245 & 156.7500 & 10.3000 & 290.3 & 4 & 600.0 & 00.0 \\
\hline 03 & 6246 & 155.5000 & 9.6000 & 290.3 & 4 & 600.0 & 600.0 \\
\hline 03 & 6247 & 161.2504 & 10.0750 & 290.7 & 4 & 600.0 & 00.0 \\
\hline 03 & 6248 & 162.5000 & 9.9750 & 291.6 & 4 & 600.0 & 00.0 \\
\hline 03 & 6249 & 165.5009 & 9.9750 & 292.5 & 4 & 600.0 & .0 \\
\hline 03 & 62410 & 162.9992 & 9.2000 & 293.7 & 4 & 600.0 & 600.0 \\
\hline 03 & 62411 & 167.5013 & 9.1250 & 295.4 & 4 & 600.0 & \\
\hline 03 & 62412 & 168.5000 & 8.2500 & 297.0 & 4 & 600.0 & .0 \\
\hline 03 & 62413 & 174.4990 & 8.3000 & 298.2 & 4 & 600.0 & 600.0 \\
\hline 03 & 62414 & 173.7485 & 8.2750 & 299.1 & 4 & 600.0 & \\
\hline 03 & 62415 & 163.2455 & 7.7750 & 300.1 & 4 & 600.0 & 60 \\
\hline 03 & 62416 & 174.7461 & 8.1500 & 300.6 & 4 & 600.0 & 600.0 \\
\hline 03 & 62417 & 175.0000 & 7.1500 & 301.2 & 4 & 600.0 & \\
\hline 03 & 62418 & 124.2311 & 5.9250 & 301.0 & 3 & 600.0 & .0 \\
\hline 03 & 62419 & 86.0025 & 5.1750 & 299.6 & 4 & 600.0 & 600 \\
\hline 03 & 62420 & 951 & 5.20 & 298.1 & 4 & 600.0 & .0 \\
\hline 03 & 62421 & 78 . & 5.7750 & 297.5 & 4 & 600.0 & \\
\hline 03 & 62422 & 75.2500 & 6.1250 & 297.4 & 4 & 600.0 & 600.0 \\
\hline 03 & 62423 & 76.5003 & 5.95 & 297.0 & 4 & 600.0 & .0 \\
\hline 03 & 62424 & 76.2501 & 6.6750 & 297.1 & 4 & 600.0 & \\
\hline 03 & 6251 & 76.7499 & 6.8000 & 297.3 & 4 & 600.0 & 60 \\
\hline 03 & 6252 & 76.0000 & 7.1250 & 297.7 & 4 & 600.0 & .0 \\
\hline 03 & 6253 & 75.2501 & 6.2500 & 298.2 & 4 & 600.0 & \\
\hline 03 & 6254 & 78.0002 & 4.8250 & 298.4 & 5 & 600.0 & 60 \\
\hline 03 & 6255 & 123.5618 & 3.1750 & 297.9 & 4 & 600.0 & 60 \\
\hline 03 & 6256 & 149.5003 & 6.10 & 296.2 & 4 & 600.0 & \\
\hline 03 & 6257 & 155.7502 & 9.8000 & 296.8 & 4 & 600.0 & 60 \\
\hline 03 & 6258 & 164.0000 & 9.1500 & 296.8 & 4 & 600.0 & 600.0 \\
\hline 03 & 6259 & 166.7494 & 7.000 & 297.2 & 4 & 600.0 & .0 \\
\hline 03 & 62510 & 164.7514 & 5.4250 & 298.2 & 3 & 600.0 & 60 \\
\hline 03 & 62511 & 167.2898 & 5.0000 & 299.6 & 3 & 600.0 & 600.0 \\
\hline 03 & 62512 & 173.2323 & 4.7000 & 301.1 & 3 & 600.0 & \\
\hline 03 & 62513 & 173.0000 & 5.8000 & 302.3 & 3 & 600.0 & 600.0 \\
\hline 03 & 62514 & 180.0080 & 6.3500 & 303.2 & 4 & 600.0 & 600.0 \\
\hline 03 & 62515 & 177.7655 & 4.7000 & 304.2 & 3 & 600.0 & \\
\hline 03 & 62516 & 192.7530 & 5.0750 & 305.1 & 3 & 600.0 & 60 \\
\hline 03 & 62517 & 191.0000 & 5.7250 & 304.9 & 3 & 600.0 & 600.0 \\
\hline 03 & 62518 & 178.2475 & 5.5750 & 305.0 & 3 & 600.0 & 600.0 \\
\hline 03 & 62519 & 188.5069 & 5.2000 & 304.3 & 4 & 600.0 & 600.0 \\
\hline 03 & 62520 & 173.7339 & 3.4500 & 303.1 & 4 & 600.0 & 600.0 \\
\hline 03 & 62521 & 148.9994 & 2.9750 & 302.3 & 5 & 600.0 & 600.0 \\
\hline 03 & 62522 & 139.2506 & 2.550 & 302.1 & 5 & 600.0 & 600.0 \\
\hline 03 & 62523 & 126.4948 & 3.3250 & 301.9 & $\mathrm{~J}$ & 600.0 & 600.0 \\
\hline
\end{tabular}




\begin{tabular}{|c|c|c|c|c|c|c|c|}
\hline & 2524 & & & & & & \\
\hline & 6261 & 00 & .1500 & 1.5 & 4 & 0 & \\
\hline & 6262 & 90.2491 & 0000 & 301.6 & 4 & 00 & \\
\hline & 6263 & 2461 & 9000 & 301.2 & & 00.0 & \\
\hline & 6264 & 9 & 2000 & 1.4 & & & \\
\hline & 6265 & & 3250 & 1.6 & & 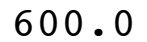 & \\
\hline & 6266 & 3146 & 7250 & 302.0 & & 00 & \\
\hline & 6267 & 163.5080 & 0 & 01.9 & & & \\
\hline & 6268 & 165.7502 & 0 & 1.7 & & & \\
\hline & 6269 & 171.2524 & 5250 & 302.4 & & 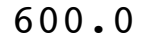 & \\
\hline & 2610 & 178.0007 & 0 & 302.8 & & .0 & \\
\hline & 2611 & 175.4983 & 0 & 03.7 & & & \\
\hline & 62612 & 180.7364 & 0 & 304.9 & 3 & 0 & \\
\hline & 2613 & 188.4979 & 0 & 306.4 & & 0.0 & 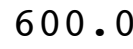 \\
\hline & 62614 & 209 . & & & & & \\
\hline & 26 & 202 . & & & 3 & & \\
\hline & 2616 & 189.5259 & 0 & 307.8 & 3 & .0 & 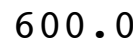 \\
\hline & 62617 & 188 . & 0 & & 1 & & \\
\hline & 62618 & 186.25 & & & 4 & & \\
\hline & 62619 & 181.7465 & 0 & 307.0 & 4 & .0 & 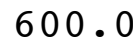 \\
\hline & 62620 & 105.2657 & 0 & 30 & 4 & 0 & \\
\hline & 626 & 69.7485 & & & 4 & & \\
\hline & 62622 & 71.5018 & & 30 & 4 & & \\
\hline & 62623 & 000 & & .0 & 4 & .0 & \\
\hline & 62624 & 66. & & & 4 & & \\
\hline & 6271 & 68 . & & .7 & 4 & & \\
\hline & 6272 & 67.2500 & 0 & 302.8 & 4 & 0 & J. \\
\hline & 6273 & 69 . & 0 & 2.8 & 4 & & \\
\hline & 6274 & 69.7459 & & .8 & 4 & & \\
\hline & $627 \quad 5$ & 74.7504 & & 2.8 & 4 & & J. \\
\hline & 6276 & 67.4997 & 0 & 302.9 & 4 & & . \\
\hline & 6277 & 70 . & 0 & & 4 & & \\
\hline & 6278 & 120 . & 0 & 5.4 & 3 & & 0 . \\
\hline & 6279 & 171.1559 & 0 & 5.3 & 2 & 0 & \\
\hline & 627 & 167 & & & 3 & & \\
\hline & 62711 & 174.2472 & 0 & 5.7 & 3 & & 0 • \\
\hline & 62712 & 175 & & 7.7 & 3 & & \\
\hline & 62713 & 177 . & 0 & 30 & 4 & & \\
\hline & 62714 & 180.2569 & & 308.6 & 4 & & 0 . \\
\hline & 62715 & 183.0000 & 2250 & 309.2 & 4 & & \\
\hline & 62716 & 119.2578 & 50 & & 4 & & \\
\hline & 62717 & 89.7472 & 8.4500 & 308.2 & 4 & & 0 . \\
\hline & 62718 & 90.2481 & 7.2250 & 307.3 & 4 & 0 & 0 . \\
\hline & 62719 & 73.7433 & 500 & & 4 & & \\
\hline & 62720 & 64.9996 & 9.3250 & & 4 & & \\
\hline & 62721 & 64.0000 & 0.7250 & 302.9 & 4 & & 0 . \\
\hline & & & & & 4 & & \\
\hline & 62723 & 63.0000 & 11.0000 & 302.7 & 1 & 600.0 & 600 \\
\hline
\end{tabular}




\begin{tabular}{|c|c|c|c|c|c|c|c|}
\hline 3 & 62724 & 63.5000 & 10.8250 & 302.7 & 4 & 600.0 & 600.0 \\
\hline 03 & 6281 & 61.7500 & 11.1000 & 302.6 & 4 & 600.0 & 00,0 \\
\hline 03 & 6282 & 64.2501 & 11.5250 & 302.6 & 4 & 600.0 & 00.0 \\
\hline 3 & 6283 & 64.2501 & 11.1000 & 302.6 & 4 & 600.0 & 00.0 \\
\hline 03 & 6284 & 63.5000 & 10.1750 & 302.5 & 4 & 600.0 & 00.0 \\
\hline 03 & 6285 & 63.7499 & 9.4250 & 302.3 & 4 & 600.0 & 00.0 \\
\hline 03 & 6286 & 65.2500 & 8.9750 & 302.4 & 4 & 600.0 & 00.0 \\
\hline 03 & 6287 & 74.7494 & 7.3500 & 302.9 & 4 & 600.0 & 00.0 \\
\hline 03 & 6288 & 109.2547 & 5.3750 & 303.7 & 3 & 600.0 & 00.0 \\
\hline 03 & 6289 & 115.4991 & 4.6500 & 304.4 & 3 & 60 & 0 \\
\hline 03 & 62810 & 112.7627 & 5.1750 & 305.0 & 3 & 600.0 & \\
\hline 03 & 62811 & 95.2219 & 5.3750 & 306.1 & 3 & 600.0 & \\
\hline 03 & 62812 & 82.2469 & 6.9000 & 306.3 & 4 & 600.0 & 00.0 \\
\hline 03 & 62813 & 102.7495 & 7.0250 & 306.8 & 4 & 600.0 & \\
\hline 03 & 62814 & 104.9943 & 7 . & 307.1 & 4 & .0 & \\
\hline 03 & 62815 & 84.5018 & 8.2500 & 307.0 & 4 & 600.0 & 00.0 \\
\hline 03 & 62816 & 85.0042 & 8.1000 & 306.5 & 4 & 60 & 0.0 \\
\hline 03 & 62817 & 94.7500 & 8.8 & 306.2 & 4 & .0 & \\
\hline 03 & 62818 & 81.2507 & 7.4250 & 305.2 & 4 & 60 & \\
\hline 03 & 62819 & 76.5000 & 8.4000 & 303.4 & 4 & .0 & .0 \\
\hline 03 & 62820 & 91.0300 & 5.82 & 301.4 & 4 & 6 & \\
\hline 03 & 62821 & 99.2487 & 5.2000 & 299.7 & 4 & .0 & \\
\hline 03 & 62822 & 105.4780 & 5.1500 & 299.0 & 4 & 600.0 & .0 \\
\hline 03 & 62823 & 123.0096 & 4.45 & 298.8 & 4 & 6 & \\
\hline 03 & 62824 & 116.2501 & 5. & 298.2 & 4 & .0 & \\
\hline 03 & 6291 & 98.5947 & 6.1750 & 297.7 & 4 & .0 & \\
\hline 03 & 6292 & 92.2513 & 5.4750 & 298.2 & 4 & 60 & .0 \\
\hline 03 & 6293 & 87.5010 & 5.92 & 298.7 & 4 & .0 & \\
\hline 03 & 6294 & 72.2503 & 8.1500 & 298.5 & 4 & .0 & .0 \\
\hline 03 & 6295 & 65.7500 & 7.9750 & 298.5 & 4 & .0 & .0 \\
\hline 03 & 6296 & 65.4997 & 8.07 & 298.6 & 4 & .0 & \\
\hline 03 & 6297 & 71.2494 & 6.92 & 299.6 & 4 & .0 & 0 \\
\hline 03 & 6298 & 85.4710 & 5.5500 & 300.7 & 4 & 600.0 & 0.0 \\
\hline 03 & 6299 & 97.7504 & 5.67 & 301.1 & 4 & 0.0 & .0 \\
\hline 03 & 62910 & 96.5097 & 6.32 & 301.3 & 4 & 600.0 & 0.0 \\
\hline 03 & 62911 & 77.5009 & 7.4000 & 301.0 & 4 & 600.0 & 600.0 \\
\hline 03 & 62912 & 82.9975 & 8.02 & 301.5 & 4 & 600.0 & .0 \\
\hline 03 & 62913 & 92.9988 & 8.0250 & 302.0 & 4 & 600.0 & 600.0 \\
\hline 03 & 62914 & 109.2490 & 7.6750 & 301.9 & 4 & 600.0 & 600.0 \\
\hline 03 & 62915 & 100.5193 & 7.3000 & 301.8 & 4 & 600.0 & 600.0 \\
\hline 03 & 62916 & 96.4991 & 7.6000 & 302.0 & 4 & 600.0 & 600.0 \\
\hline 03 & 62917 & 97.2370 & 7.1500 & 301.5 & 4 & 600.0 & 600.0 \\
\hline 03 & 62918 & 105.7794 & 6.9500 & 300.6 & 4 & 600.0 & 600.0 \\
\hline 03 & 62919 & 75.5000 & 6.3750 & 299.5 & 4 & 600.0 & 600.0 \\
\hline 03 & 62920 & 72.7552 & 7.7500 & 297.8 & 4 & 600.0 & 600.0 \\
\hline 03 & 62921 & 76.2602 & 6.3750 & 296.9 & 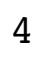 & 600.0 & 600.0 \\
\hline 03 & 62922 & 63.2498 & 10.3750 & 296.5 & 4 & 600.0 & 600.0 \\
\hline U. & 62923 & 65.4997 & 10.3250 & 296.3 & 4 & 600.0 & 600.0 \\
\hline
\end{tabular}




\begin{tabular}{|c|c|c|c|c|c|c|c|}
\hline 3 & 62924 & 67.0001 & 9.6500 & 295.7 & 4 & 600.0 & 600.0 \\
\hline 03 & 6301 & 67.0001 & 9.2000 & 295.9 & 4 & 600.0 & 600.0 \\
\hline 03 & 6302 & 67.2500 & 8.7750 & 296.0 & 4 & 600.0 & 00.0 \\
\hline 3 & 6303 & 70.2501 & 7.9250 & 296.0 & 4 & 600.0 & 00.0 \\
\hline 03 & 6304 & 75.4998 & 7.8000 & 295.5 & 4 & 600.0 & 00.0 \\
\hline 03 & 6305 & 90.2504 & 5.9750 & 295.0 & 4 & 600.0 & 00.0 \\
\hline 03 & 6306 & 85.9995 & 6.5250 & 294.9 & 4 & 600.0 & 00.0 \\
\hline 03 & 6307 & 81.9998 & 6.7250 & 294.9 & 4 & 600.0 & 00.0 \\
\hline 03 & 6308 & 82.7501 & 5.7750 & 296.1 & 4 & 600.0 & 00.0 \\
\hline 03 & 6309 & 197.9910 & 2.4500 & 297.7 & 3 & 600 & .0 \\
\hline 03 & 63010 & 253.2535 & 2.1000 & 297.3 & 2 & 600.0 & \\
\hline 03 & 63011 & 223.3595 & 2.2250 & 299.0 & 1 & 600.0 & \\
\hline 03 & 63012 & 99.4048 & 4.2000 & 299.5 & 2 & 600.0 & 00.0 \\
\hline 03 & 63013 & 86.2506 & 5.6750 & 299.8 & 3 & 600.0 & 00.0 \\
\hline 03 & 63014 & 82.5014 & 6.7000 & 299.8 & 4 & .0 & \\
\hline 03 & 63015 & 75.7497 & 6.4500 & 300.1 & 4 & 600.0 & .0 \\
\hline 03 & 63016 & 85.0000 & 7.5250 & 300.1 & 4 & 600.0 & 600.0 \\
\hline 03 & 63017 & 86.7500 & 7.8250 & 299.3 & 4 & .0 & \\
\hline 03 & 63018 & 84.2431 & 7.6250 & 297.8 & 4 & 60 & .0 \\
\hline 03 & 63019 & 79.0000 & 9.7250 & 296.0 & 4 & .0 & .0 \\
\hline 03 & 63020 & 75.0003 & 10.8000 & 293.7 & 4 & 60 & \\
\hline 03 & 63021 & 79.5003 & 8.7250 & 292.0 & 4 & .0 & \\
\hline 03 & 63022 & 73.5000 & 7.9000 & 291.0 & 4 & 600.0 & .0 \\
\hline 03 & 63023 & 69.5000 & 7.825 & 289.5 & 4 & 60 & \\
\hline 03 & 63024 & 64.3005 & 6.0250 & 291.5 & 4 & .0 & \\
\hline 03 & $\begin{array}{lll}7 & 1 & 1\end{array}$ & 71.7535 & 8.4750 & 292.9 & 4 & .0 & 600.0 \\
\hline 03 & $\begin{array}{lll}7 & 1 & 2\end{array}$ & 70.7498 & 9.0000 & 293.4 & 4 & 600.0 & .0 \\
\hline 03 & $\begin{array}{lll}7 & 1 & 3\end{array}$ & 68.7482 & 8.7000 & 292.9 & 4 & .0 & \\
\hline 03 & $\begin{array}{lll}7 & 1 & 4\end{array}$ & 72.2495 & 8.7750 & 292.5 & 4 & .0 & 0.0 \\
\hline 03 & $\begin{array}{lll}7 & 1 & 5\end{array}$ & 68.4977 & 9.0000 & 292.3 & 4 & 60 & .0 \\
\hline 03 & $\begin{array}{lll}7 & 1 & 6\end{array}$ & 70.7500 & 8.6500 & 292.8 & 4 & .0 & \\
\hline 03 & $\begin{array}{lll}7 & 1 & 7\end{array}$ & 66.0005 & 7.4500 & 294.3 & 4 & 600.0 & 600.0 \\
\hline 03 & $\begin{array}{lll}7 & 1 & 8\end{array}$ & 75.5005 & 7.5250 & 295.6 & 4 & 600.0 & 600.0 \\
\hline 03 & $\begin{array}{lll}7 & 1 & 9\end{array}$ & 80.9993 & 500 & 296.6 & 3 & 600.0 & 0.0 \\
\hline 03 & $7 \quad 110$ & 97.2498 & 3.9000 & 297.7 & 2 & 600.0 & 600.0 \\
\hline 03 & $7 \quad 111$ & 100.0772 & 4.7750 & 298.4 & 3 & 600.0 & 600.0 \\
\hline 03 & 7112 & 78.2328 & 4.2750 & 299.7 & 3 & 600.0 & 0.0 \\
\hline 03 & 7113 & 66.4699 & 4.9250 & 300.7 & 3 & 600.0 & 600.0 \\
\hline 03 & $7 \quad 114$ & 84.7287 & 5.2250 & 300.5 & 3 & 600.0 & 600.0 \\
\hline 03 & 7115 & 100.2499 & 5.7500 & 300.7 & 3 & 600.0 & 600.0 \\
\hline 03 & 7116 & 103.2415 & 5.9750 & 300.5 & 3 & 600.0 & 600.0 \\
\hline 03 & 7117 & 96.7640 & 5.8750 & 300.3 & 3 & 600.0 & 600.0 \\
\hline 03 & 7118 & 70.4997 & $6.6 / 50$ & 299.4 & 4 & 600.0 & 600.0 \\
\hline 03 & $7 \quad 119$ & 71.7528 & 7.3250 & 298.0 & 4 & 600.0 & 600.0 \\
\hline 03 & 7120 & 73.0000 & 8.2250 & 296.4 & 4 & 600.0 & 600.0 \\
\hline 03 & $7 \quad 121$ & 70.7502 & 9.2250 & 295.4 & 4 & 600.0 & 600.0 \\
\hline 03 & 7122 & 71.5002 & 9.5750 & 294.5 & 4 & 600.0 & 600.0 \\
\hline 03 & $7 \quad 123$ & 76.0006 & 9.8500 & 294.3 & 4 & 600.0 & 600.0 \\
\hline
\end{tabular}




\begin{tabular}{|c|c|c|c|c|c|c|c|c|}
\hline 3 & 7 & 124 & 77.2500 & 10.3500 & 294.1 & 4 & 600.0 & 600.0 \\
\hline 03 & 7 & 21 & 75.0000 & 9.2250 & 295.1 & 4 & 600.0 & 600.0 \\
\hline 03 & 7 & 22 & 75.9999 & 9.1500 & 294.5 & 4 & 600.0 & 00.0 \\
\hline 03 & 7 & 23 & 75.5001 & 9.4750 & 293.9 & 4 & 600.0 & 00.0 \\
\hline 03 & 7 & 24 & 74.2500 & 8.9750 & 294.3 & 4 & 600.0 & 00.0 \\
\hline 03 & 7 & 25 & 75.0000 & 8.7250 & 294.5 & 4 & 600.0 & 00.0 \\
\hline 03 & 7 & 26 & 74.7508 & 7.8250 & 294.5 & 4 & 600.0 & 00.0 \\
\hline 03 & 7 & 27 & 75.7506 & 5.0000 & 295.8 & 4 & 600.0 & 00.0 \\
\hline 03 & 7 & 28 & 89.2486 & 4.5250 & 297.2 & 3 & 600.0 & 00.0 \\
\hline 03 & 7 & 29 & 77.0662 & 2.8000 & 299.2 & 2 & 600.0 & .0 \\
\hline 03 & 7 & 210 & 232.5000 & 3.6750 & 299.5 & 3 & 600.0 & \\
\hline 03 & 7 & 211 & 256.6196 & 3.0750 & 300.6 & 2 & 600.0 & \\
\hline 03 & 7 & 212 & 211.4566 & 5.1500 & 301.1 & 3 & 600.0 & 00.0 \\
\hline 03 & 7 & 213 & 151.5984 & 4.7250 & 302.3 & 3 & .0 & .0 \\
\hline 03 & 7 & 214 & 100.5625 & 4.2250 & 302.4 & 3 & .0 & \\
\hline 03 & 7 & 215 & 111.2432 & 5.0500 & 302.1 & 3 & 600.0 & 00.0 \\
\hline 03 & 7 & 216 & 111.2570 & 5.7000 & 301.7 & 3 & 600.0 & 600.0 \\
\hline 03 & 7 & 217 & 87.4981 & 5.2500 & 301.3 & 3 & .0 & \\
\hline 03 & 7 & 218 & 75.7500 & 8.5000 & 299.7 & 4 & 600.0 & .0 \\
\hline 03 & 7 & 219 & 74.0000 & 8.4500 & 298.7 & 4 & .0 & .0 \\
\hline 03 & 7 & 220 & 72.9979 & 0.0500 & 297.2 & 4 & 60 & \\
\hline 03 & 7 & 221 & 71.5000 & 9.6750 & 296.5 & 4 & .0 & \\
\hline 03 & 7 & 222 & 70.0000 & 10.3500 & 296.2 & 4 & 600.0 & .0 \\
\hline 03 & 7 & 223 & 66.7520 & 9.22 & 296.1 & 4 & .0 & \\
\hline 03 & 7 & 224 & 61.2498 & 8.3250 & 295.7 & 4 & .0 & \\
\hline 03 & 7 & 31 & 68.7505 & 8.5000 & 294.8 & 4 & 600.0 & 600.0 \\
\hline 03 & 7 & 32 & 72.7504 & 9.1750 & 294.2 & 4 & 600.0 & .0 \\
\hline 03 & 7 & 33 & 73.7501 & 8.8000 & 294.7 & 4 & .0 & \\
\hline 03 & 7 & 34 & 60.2506 & 7.5750 & 294.4 & 4 & 600.0 & 0.0 \\
\hline 03 & 7 & 35 & 67.2705 & 8.4250 & 294.0 & 4 & 600.0 & .0 \\
\hline 03 & 7 & 36 & 76.0007 & 8.0750 & 295.2 & 4 & .0 & \\
\hline 03 & 7 & 37 & 78.2501 & 7.9750 & 295.5 & 4 & .0 & 600.0 \\
\hline 03 & 7 & 38 & 81.4999 & 7.7750 & 296.7 & 4 & 600.0 & 600.0 \\
\hline 03 & 7 & 39 & 85.5000 & 4.9500 & 299.1 & 3 & 600.0 & 0.0 \\
\hline 03 & 7 & 310 & 193.2373 & 3.0500 & 301.0 & 2 & 600.0 & 600.0 \\
\hline 03 & 7 & 311 & 285.7772 & 2.9000 & 301.8 & 1 & 600.0 & 600.0 \\
\hline 03 & 7 & 312 & 48.8924 & 3.47 & 302.9 & 2 & 600.0 & 0.0 \\
\hline 03 & 7 & 313 & 106.4957 & 4.7750 & 302.6 & 3 & 600.0 & 600.0 \\
\hline 03 & 7 & 314 & 138.3077 & 6.4750 & 302.3 & 4 & 600.0 & 600.0 \\
\hline 03 & 7 & 315 & 148.5015 & 500 & 302.3 & 4 & 600.0 & 0.0 \\
\hline 03 & 7 & 316 & 135.7255 & 6.3250 & 302.3 & 4 & 600.0 & 600.0 \\
\hline 03 & 7 & 317 & 118.6187 & 5.6750 & 302.1 & 3 & 600.0 & 600.0 \\
\hline 03 & 7 & 318 & 99.7780 & 5.7500 & 300.9 & 3 & 600.0 & 600.0 \\
\hline 03 & 7 & 319 & 78.7423 & 8.5750 & 299.2 & 4 & 600.0 & 600.0 \\
\hline 03 & 7 & 320 & 67.0002 & 9.5250 & 297.7 & 4 & 600.0 & 600.0 \\
\hline 03 & 7 & 321 & 61.7751 & 5.8250 & 297.3 & . & 600.0 & 600.0 \\
\hline 03 & 7 & 322 & 103.7920 & 3.8250 & 297.1 & 4 & 600.0 & 600.0 \\
\hline 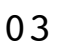 & 7 & 323 & 65.0005 & 9.975 & 296.9 & 4 & 600.0 & 600.0 \\
\hline
\end{tabular}




\begin{tabular}{|c|c|c|c|c|c|c|c|c|}
\hline 3 & 7 & 324 & 72.0019 & 9.6000 & 296.6 & 4 & 600.0 & 600.0 \\
\hline 3 & 7 & 41 & 71.7500 & 9.6250 & 296.8 & 4 & 600.0 & 00.0 \\
\hline 03 & 7 & 42 & 69.0000 & 8.9000 & 297.1 & 4 & 600.0 & 00.0 \\
\hline 03 & 7 & 43 & 69.7500 & 8.0250 & 297.1 & 4 & 600.0 & 00.0 \\
\hline 03 & 7 & 44 & 72.2488 & 7.2250 & 296.9 & 4 & 600.0 & 00.0 \\
\hline 03 & 7 & 45 & 83.5018 & 6.6000 & 296.9 & 4 & 600.0 & 00.0 \\
\hline 03 & 7 & 46 & 84.9998 & 5.3000 & 296.8 & 4 & 600.0 & 00.0 \\
\hline 03 & 7 & 47 & 94.7502 & 3.9000 & 297.9 & 4 & 600.0 & 00.0 \\
\hline 03 & 7 & 48 & 161.0865 & 4.4500 & 299.3 & 3 & 600.0 & 00.0 \\
\hline 03 & 7 & 49 & 171.0000 & 5.3000 & 299.8 & 4 & 600.0 & .0 \\
\hline 03 & 7 & 410 & 168.5017 & 5.7250 & 300.3 & 3 & 600.0 & \\
\hline 03 & 7 & 411 & 169.4973 & 5.7750 & 301.1 & 3 & 600.0 & \\
\hline 03 & 7 & 412 & 172.5008 & 6.2000 & 301.6 & 4 & 600.0 & 00.0 \\
\hline 03 & 7 & 413 & 176.2503 & 6.0250 & 302.5 & 4 & 600.0 & 00.0 \\
\hline 03 & 7 & 414 & 174.4991 & 250 & 303.6 & 4 & .0 & \\
\hline 03 & 7 & 415 & 175.2495 & 7.2250 & 304.6 & 4 & 600.0 & .0 \\
\hline 03 & 7 & 416 & 165.4072 & 6.8250 & 305.5 & 4 & 60 & 600.0 \\
\hline 03 & 7 & 417 & 120.1441 & 5.7500 & 305.7 & 3 & .0 & \\
\hline 03 & 7 & 418 & 87.2500 & 8.2500 & 304.1 & 4 & 600.0 & .0 \\
\hline 03 & 7 & 419 & 86.2498 & 7.8000 & 303.0 & 4 & 60 & .0 \\
\hline 03 & 7 & 420 & 73.2498 & 7.72 & 301.0 & 4 & 60 & \\
\hline 03 & 7 & 421 & 68.5003 & 8.0250 & 300.1 & 4 & .0 & \\
\hline 03 & 7 & 422 & 70.0000 & 8.5250 & 299.6 & 4 & 600.0 & .0 \\
\hline 03 & 7 & 423 & 73.0000 & 9.10 & 299.2 & 4 & 60 & \\
\hline 03 & 7 & 424 & 71.7499 & 9.7750 & 299.0 & 4 & .0 & \\
\hline 03 & 7 & 51 & 68.2501 & 10.0000 & 298.9 & 4 & .0 & 0 \\
\hline 03 & 7 & 52 & 63.0000 & 9.5000 & 299.0 & 4 & 600.0 & .0 \\
\hline 03 & 7 & 53 & 73.4982 & 8.6000 & 298.8 & 4 & .0 & \\
\hline 03 & 7 & 54 & 60.5000 & 9.4250 & 298.8 & 4 & .0 & 0.0 \\
\hline 03 & 7 & 55 & 68.2510 & 8.5250 & 298.9 & 4 & 60 & .0 \\
\hline 03 & 7 & 56 & 69.7500 & 8.8750 & 298.6 & 4 & .0 & \\
\hline 03 & 7 & 57 & 73.7502 & 9.5000 & 298.9 & 4 & .0 & 600.0 \\
\hline 03 & 7 & 58 & 79.9997 & 7.5000 & 299.5 & 4 & 600.0 & 600.0 \\
\hline 03 & 7 & & 82.2498 & 7.8750 & 300.0 & 4 & 600.0 & 0.0 \\
\hline 03 & 7 & 510 & 82.7577 & 6.5750 & 300.9 & 4 & 600.0 & 600.0 \\
\hline 03 & 7 & 511 & 82.7153 & 5.6750 & 301.9 & 3 & 600.0 & 600.0 \\
\hline 03 & 7 & 512 & 88.7501 & 6.2750 & 302.8 & 4 & 600.0 & 0.0 \\
\hline 03 & 7 & 513 & 87.0041 & 6.9000 & 303.3 & 4 & 600.0 & 600.0 \\
\hline 03 & 7 & 514 & 88.9993 & 8.6750 & 302.9 & 4 & 600.0 & 600.0 \\
\hline 03 & 7 & 515 & 84.7494 & 9.2500 & 302.8 & 4 & 600.0 & 600.0 \\
\hline 03 & 7 & 516 & 64.5008 & 9.3000 & 302.9 & 4 & 600.0 & 600.0 \\
\hline 03 & 7 & 517 & 70.9979 & 10.2000 & 302.5 & 4 & 600.0 & 600.0 \\
\hline 03 & 7 & 518 & 69.7504 & 9.7500 & 302.1 & 4 & 600.0 & 600.0 \\
\hline 03 & 7 & 519 & 65.7496 & 10.9250 & 300.0 & 4 & 600.0 & 600.0 \\
\hline 03 & 7 & 520 & 67.7501 & 10.6500 & 298.6 & 4 & 600.0 & 600.0 \\
\hline 03 & 7 & 521 & 67.5000 & 10.9000 & 297.8 & 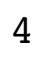 & 600.0 & 600.0 \\
\hline 03 & 7 & 522 & 66.7500 & 10.0250 & 298.3 & 4 & 600.0 & 600.0 \\
\hline 03 & 7 & 523 & 66.2500 & 9.5500 & 297.7 & 4 & 600.0 & 600.0 \\
\hline
\end{tabular}




\begin{tabular}{|c|c|c|c|c|c|c|c|c|}
\hline 3 & 7 & 524 & 84.5090 & 7.2250 & 296.5 & 4 & 600.0 & 00.0 \\
\hline 03 & 7 & 61 & 82.7519 & 8.3000 & 295.9 & 4 & 600.0 & 600.0 \\
\hline 03 & 7 & 62 & 62.0000 & 11.9000 & 295.1 & 4 & 600.0 & 00.0 \\
\hline 03 & 7 & 63 & 67.9996 & 10.5750 & 295.1 & 4 & 600.0 & 00.0 \\
\hline 03 & 7 & 64 & 67.9984 & 10.2250 & 295.1 & 4 & 600.0 & 00.0 \\
\hline 03 & 7 & 65 & 69.2500 & 10.4500 & 293.6 & 4 & 600.0 & 00.0 \\
\hline 03 & 7 & 66 & 71.7499 & 9.8250 & 292.5 & 4 & 600.0 & 600.0 \\
\hline 03 & 7 & 67 & 74.7506 & 11.1750 & 292.6 & 4 & 600.0 & 00.0 \\
\hline 03 & 7 & 68 & 81.4957 & 9.9000 & 293.2 & 4 & 600.0 & 00.0 \\
\hline 03 & 7 & 69 & 98.4977 & 7.1000 & 293.9 & 4 & 600.0 & .0 \\
\hline 03 & 7 & 610 & 103.7667 & 7.5750 & 295.0 & 4 & 600.0 & 00.0 \\
\hline 03 & 7 & 611 & 97.5005 & 7.7500 & 295.2 & 4 & 600.0 & \\
\hline 03 & 7 & 612 & 88.0092 & 7.6500 & 296.1 & 4 & 600.0 & 600.0 \\
\hline 03 & 7 & 613 & 79.7499 & 7.1250 & 297.6 & 4 & 600.0 & 600 \\
\hline 03 & 7 & 614 & 83.73 & & 299.3 & 4 & 600.0 & \\
\hline 03 & 7 & 615 & 78.5018 & 000 & 299.4 & 4 & 600.0 & 60 \\
\hline 03 & 7 & 616 & 67.0000 & 9.42 & 298.6 & 4 & 600.0 & 600.0 \\
\hline 03 & 7 & 617 & 63.2498 & 10. & 298.0 & 4 & 600.0 & .0 \\
\hline 03 & 7 & 618 & 62.5002 & 11. & 297.0 & 4 & 600.0 & 60 \\
\hline 03 & 7 & 619 & 70.2495 & 10.3000 & 296.0 & 4 & 600.0 & .0 \\
\hline 03 & 7 & 620 & 66.4997 & 10. & 295.2 & 4 & 600.0 & .0 \\
\hline 03 & 7 & 621 & 67.5000 & 10. & 295.2 & 4 & 0.0 & \\
\hline 03 & 7 & 622 & 67.9977 & 9.9750 & 295.5 & 4 & 600.0 & 600.0 \\
\hline 03 & 7 & 623 & 73.25 & 8.92 & 295.7 & 4 & 600.0 & .0 \\
\hline 03 & 7 & 624 & 66.25 & 10 & 295.5 & 4 & 00.0 & \\
\hline 03 & 7 & 71 & 64.0000 & 11.00 & 295.0 & 4 & 600.0 & 60 \\
\hline 03 & 7 & 72 & 64.9997 & 11.2250 & 294.4 & 4 & 600.0 & .0 \\
\hline 03 & 7 & 73 & 64.2479 & 11.95 & 293.8 & 4 & 00.0 & \\
\hline 03 & 7 & 74 & 66.2500 & 11.6750 & 293.1 & 4 & 600.0 & 60 \\
\hline 03 & 7 & 75 & 66.5000 & 11.2750 & 292.5 & 4 & 600.0 & 60 \\
\hline 03 & 7 & 76 & 65.5000 & 12.22 & 292.6 & 4 & 600.0 & \\
\hline 03 & 7 & 77 & 64.7499 & 12.8750 & 293.0 & 4 & 600.0 & .0 \\
\hline 03 & 7 & 78 & 70.0000 & 11.8000 & 293.1 & 4 & 600.0 & 600.0 \\
\hline 03 & 7 & 79 & 67.9999 & 10.52 & 294.1 & 4 & 600.0 & .0 \\
\hline 03 & 7 & 710 & 73.7494 & 9. & 295.2 & 4 & 600.0 & 60 \\
\hline 03 & 7 & 711 & 70.2499 & 10.0250 & 295.3 & 4 & 600.0 & 600.0 \\
\hline 03 & 7 & 712 & 70.0001 & 10.9500 & 295.7 & 4 & 600.0 & \\
\hline 03 & 7 & 713 & 68.5000 & 10. & 296.7 & 4 & 600.0 & 600.0 \\
\hline 03 & 7 & 714 & 72.0011 & 10.8500 & 297.0 & 4 & 600.0 & 600.0 \\
\hline 03 & 7 & 715 & 75.9706 & 10.7250 & 296.7 & 4 & 600.0 & \\
\hline 03 & 7 & 716 & 99.4967 & 8.6000 & 295.2 & 4 & 600.0 & 600.0 \\
\hline 03 & 7 & 717 & 95.7476 & 5.6500 & 295.5 & 3 & 600.0 & 600.0 \\
\hline 03 & 7 & 718 & 65.2405 & 11.1000 & 294.8 & 4 & 600.0 & 600.0 \\
\hline 03 & 7 & 719 & 74.2492 & 11.0000 & 293.6 & 4 & 600.0 & 600.0 \\
\hline 03 & 7 & 720 & 78.7500 & 11.8500 & 292.4 & 4 & 600.0 & 600.0 \\
\hline 03 & 7 & 721 & 76.0000 & 11.4000 & 293.1 & 4 & 600.0 & 600.0 \\
\hline 03 & 7 & 722 & 73.2499 & 11.175 & 294 & 4 & 600.0 & 600.0 \\
\hline 03 & 7 & 723 & 73.0000 & 12.3750 & 294.2 & 4 & 600.0 & 600.0 \\
\hline
\end{tabular}




\begin{tabular}{|c|c|c|c|c|c|c|c|c|}
\hline 3 & 7 & 724 & 74.0001 & 11.8250 & 294.1 & 4 & 600.0 & 600.0 \\
\hline 3 & 7 & 81 & 73.0001 & 10.6750 & 294.0 & 4 & 600.0 & 600.0 \\
\hline 03 & 7 & 82 & 66.9998 & 9.6750 & 294.4 & 4 & 600.0 & 00.0 \\
\hline 3 & 7 & 83 & 64.4987 & 9.1250 & 294.6 & 4 & 600.0 & 00.0 \\
\hline 03 & 7 & 84 & 70.4983 & 9.8750 & 294.6 & 4 & 600.0 & 00.0 \\
\hline 03 & 7 & 85 & 73.5002 & 10.9500 & 295.3 & 4 & 600.0 & 00.0 \\
\hline 03 & 7 & 86 & 74.2500 & 9.3750 & 295.8 & 4 & 600.0 & 00.0 \\
\hline 03 & 7 & 87 & 80.4997 & 8.5500 & 296.8 & 4 & 600.0 & 00.0 \\
\hline 03 & 7 & 88 & 95.2501 & 5.0500 & 297.6 & 3 & 600.0 & 00.0 \\
\hline 03 & 7 & 89 & 153.6437 & 5.3000 & 298.5 & 3 & 600.0 & .0 \\
\hline 03 & 7 & 810 & 176.0000 & 6.9750 & 297.9 & 4 & 600.0 & \\
\hline 03 & 7 & 811 & 169.7520 & 8.1500 & 298.0 & 4 & 600.0 & \\
\hline 03 & 7 & 812 & 169.2504 & 7.8250 & 298.9 & 4 & 600.0 & 00.0 \\
\hline 03 & 7 & 813 & 172.7508 & 7.0000 & 299.8 & 4 & 600.0 & 00.0 \\
\hline 03 & 7 & 814 & 168.2492 & 6.9500 & 301.3 & 4 & .0 & \\
\hline 03 & 7 & 815 & 149.6346 & 6.7500 & 303.1 & 4 & 600.0 & 00.0 \\
\hline 03 & 7 & 816 & 135.0011 & 6.8500 & 303.6 & 4 & 600.0 & 600.0 \\
\hline 03 & 7 & 817 & 95.2446 & 6.6750 & 302.9 & 4 & .0 & \\
\hline 03 & 7 & 818 & 78.7512 & 7.1500 & 301.5 & 4 & 600.0 & .0 \\
\hline 03 & 7 & 819 & 65.7499 & 8.3250 & 300.0 & 4 & .0 & .0 \\
\hline 03 & 7 & 820 & 68.9999 & 9.82 & 298.6 & 4 & 6 & \\
\hline 03 & 7 & 821 & 69.7500 & 10.3000 & 298.1 & 4 & .0 & \\
\hline 03 & 7 & 822 & 73.9999 & 9.9500 & 298.3 & 4 & 600.0 & .0 \\
\hline 03 & 7 & 823 & 71.5000 & 9.50 & 298.4 & 4 & 60 & \\
\hline 03 & 7 & 824 & 67.7500 & 8.82 & 298.2 & 4 & .0 & \\
\hline 03 & 7 & 91 & 65.0000 & 8.1750 & 298.3 & 4 & 600.0 & 600.0 \\
\hline 03 & 7 & 92 & 74.2504 & 7.9250 & 298.2 & 4 & 600.0 & .0 \\
\hline 03 & 7 & 93 & 75.2500 & 7.3750 & 297.7 & 4 & .0 & \\
\hline 03 & 7 & 94 & 76.2500 & 7.7000 & 297.1 & 4 & .0 & 0.0 \\
\hline 03 & 7 & 95 & 81.0000 & 5.7750 & 297.7 & 4 & 60 & .0 \\
\hline 03 & 7 & 96 & 78.0005 & 4.7750 & 298.1 & 4 & .0 & .0 \\
\hline 03 & 7 & 97 & 105.1762 & 1.0750 & 300.4 & 3 & 600.0 & 600.0 \\
\hline 03 & 7 & 98 & 309.0064 & 0.9500 & 302.8 & 2 & 600.0 & 600.0 \\
\hline 03 & 7 & 99 & 241.5493 & 1.9750 & 302.9 & 1 & 600.0 & 0.0 \\
\hline 03 & 7 & 910 & 169.0001 & 4.80 & 301.0 & 2 & 600.0 & 600.0 \\
\hline 03 & 7 & 911 & 176.4997 & 6.1000 & 301.9 & 3 & 600.0 & 600.0 \\
\hline 03 & 7 & 912 & 175.2498 & 7.70 & 303.1 & 4 & 600.0 & 0.0 \\
\hline 03 & 7 & 913 & 167.7502 & 6.3750 & 303.9 & 4 & 600.0 & 600.0 \\
\hline 03 & 7 & 914 & 178.5003 & 6.1750 & 304.5 & 4 & 600.0 & 600.0 \\
\hline 03 & 7 & 915 & 184.7483 & 6.40 & 305.3 & 4 & 600.0 & .0 \\
\hline 03 & 7 & 916 & 178.2501 & 6.0000 & 305.7 & 4 & 600.0 & 600.0 \\
\hline 03 & 7 & 917 & 122.7955 & 5.9000 & 305.8 & 3 & 600.0 & 600.0 \\
\hline 03 & 7 & 918 & 78.5360 & 5.6000 & 304.8 & 4 & 600.0 & 600.0 \\
\hline 03 & 7 & 919 & 76.0008 & 6.5750 & 303.4 & 4 & 600.0 & 600.0 \\
\hline 03 & 7 & 920 & 72.9995 & 7.7250 & 301.1 & 4 & 600.0 & 600.0 \\
\hline 03 & 7 & 921 & 71.7500 & 8.7500 & 300.0 & . & 600.0 & 600.0 \\
\hline 03 & 7 & 922 & 68.5001 & 9.8750 & 299.9 & 4 & 600.0 & 600.0 \\
\hline 03 & 7 & 923 & 72.5000 & 9.775 & 299.6 & 4 & 600.0 & 600.0 \\
\hline
\end{tabular}




\begin{tabular}{|c|c|c|c|c|c|c|c|}
\hline 3 & 7924 & 74.9999 & 9.4750 & 299.4 & 4 & 600.0 & 600.0 \\
\hline 3 & 7101 & 72.0080 & 10.5250 & 298.7 & 4 & 600.0 & 600.0 \\
\hline 03 & 7102 & 66.2496 & 11.8250 & 297.7 & 4 & 600.0 & 00.0 \\
\hline 3 & 7103 & 73.0000 & 11.4750 & 297.9 & 4 & 600.0 & 00.0 \\
\hline 03 & 7104 & 68.7499 & 10. & 298.3 & 4 & 600.0 & 00.0 \\
\hline 03 & 7105 & 72.7485 & 9.1000 & 298.2 & 4 & 600.0 & 00.0 \\
\hline 03 & 7106 & 72.0000 & 500 & 298.1 & 4 & 600.0 & 00.0 \\
\hline 03 & $\begin{array}{ll}710 & 7\end{array}$ & 70.7533 & 7.2000 & 298.9 & 4 & 600.0 & 00.0 \\
\hline 03 & 7108 & 64.7500 & 6.9250 & 300.1 & 4 & 600.0 & 00.0 \\
\hline 03 & 7109 & 66.2500 & 750 & 301.0 & 4 & 600 & 0 \\
\hline 03 & 71010 & 73.0000 & 50 & 301.7 & 4 & 600.0 & \\
\hline 03 & 71011 & 75.2545 & 50 & 303.3 & 3 & .0 & \\
\hline 03 & 71012 & 90.2429 & 500 & 303.9 & 4 & 600.0 & 00.0 \\
\hline 03 & 71013 & 86.2505 & 50 & 305.0 & 4 & 600.0 & 00.0 \\
\hline 03 & 71014 & 93.7497 & 7. & 305.1 & 4 & .0 & \\
\hline 03 & 71015 & 91.0000 & 250 & 305.3 & 4 & 600.0 & .0 \\
\hline 03 & 71016 & 76.2442 & 50 & 304.6 & 4 & 60 & .0 \\
\hline 03 & 71017 & 72.0006 & 10. & 303.7 & 4 & .0 & \\
\hline 03 & 71018 & 75.0002 & 10 . & 302.7 & 4 & 60 & \\
\hline 03 & 71019 & 66.7496 & 11.2500 & 300.8 & 4 & .0 & .0 \\
\hline 03 & 71020 & 64.7500 & 13. & 299.0 & 4 & 6 & \\
\hline 03 & 71021 & 68.5000 & 14. & 298.1 & 4 & .0 & \\
\hline 03 & 71022 & 67.0000 & 15.2750 & 297.7 & 4 & 600.0 & .0 \\
\hline 03 & 71023 & 67.9999 & 14 . & 297.9 & 4 & 6 & \\
\hline 03 & 71024 & 69.2500 & 13 & 298.0 & 4 & 6 & \\
\hline 03 & 7111 & 72.2500 & 11 . & 298.6 & 4 & .0 & \\
\hline 03 & 7112 & 75.0000 & 000 & 298.7 & 4 & .0 & .0 \\
\hline 03 & 7113 & 74.7501 & 9 . & 298.2 & 4 & .0 & \\
\hline 03 & 7114 & 73.7502 & & 298.1 & 4 & .0 & .0 \\
\hline 03 & 7115 & 78.0000 & 500 & 297.9 & 4 & .0 & .0 \\
\hline 03 & 7116 & 77.2500 & 00 & 298.4 & 4 & .0 & \\
\hline 03 & 7117 & 81.5000 & & 298.6 & 4 & .0 & 0.0 \\
\hline 03 & 7118 & 81.2500 & 7.9500 & 298.8 & 4 & 600.0 & 0.0 \\
\hline 03 & 7119 & 92.9703 & 6. & 300.3 & 4 & .0 & 0.0 \\
\hline 03 & 71110 & 135.0172 & & 301.1 & 3 & 600.0 & 600.0 \\
\hline 03 & 71111 & 57.7348 & 2.4000 & 303.4 & 2 & 600.0 & 600.0 \\
\hline 03 & 71112 & 98.7481 & 3 . & 304.0 & 2 & 600.0 & .0 \\
\hline 03 & 71113 & 105.7480 & 4.1500 & 305.0 & 3 & 600.0 & 600.0 \\
\hline 03 & 71114 & 166.5003 & 7.2500 & 304.9 & 4 & 600.0 & 600.0 \\
\hline 03 & 71115 & 157.5069 & 6 . & 304.8 & 4 & 600.0 & 600.0 \\
\hline 03 & 71116 & 96.5438 & 5.3250 & 305.0 & 3 & 600.0 & 600.0 \\
\hline 03 & 71117 & 84.2523 & 5.8250 & 304.5 & 3 & 600.0 & 600.0 \\
\hline 03 & 71118 & 76.7504 & 6.2500 & 303.6 & 4 & 600.0 & 600.0 \\
\hline 03 & 71119 & 64.5000 & 7.8750 & 302.1 & 4 & 600.0 & 600.0 \\
\hline 03 & 71120 & 64.4997 & 8.9000 & 301.0 & 4 & 600.0 & 600.0 \\
\hline 03 & 71121 & 76.7497 & & 300.8 & 4 & 600.0 & 600.0 \\
\hline 03 & 71122 & 76.7500 & 9.025 & 300.2 & 4 & 600.0 & 600.0 \\
\hline 0 & 71123 & 67.2498 & 9.125 & 300.2 & $\Psi$ & 600.0 & 600.0 \\
\hline
\end{tabular}




\begin{tabular}{|c|c|c|c|c|c|c|c|}
\hline 3 & 71124 & 67.2500 & 9.5250 & 299.6 & 4 & 600.0 & 00.0 \\
\hline 03 & 7121 & 71.2501 & 10.3000 & 298.8 & 4 & 600.0 & 600,0 \\
\hline 03 & 7122 & 71.2502 & 10.5000 & 298.6 & 4 & 600.0 & 00.0 \\
\hline 03 & 7123 & 71.0000 & 11.0500 & 298.0 & 4 & 600.0 & 00.0 \\
\hline 03 & 7124 & 70.0000 & 11.1500 & 298.1 & 4 & 600.0 & 00.0 \\
\hline 03 & 7125 & 74.2502 & 11.7750 & 297.7 & 4 & 600.0 & 00.0 \\
\hline 03 & 7126 & 74.5000 & 12.0250 & 298.1 & 4 & 600.0 & 00.0 \\
\hline 03 & 7127 & 70.5000 & 11.1500 & 298.8 & 4 & 600.0 & 00.0 \\
\hline 03 & 7128 & 69.0000 & 9.3250 & 299.7 & 4 & 600.0 & 00.0 \\
\hline 03 & 7129 & 77.5000 & 9.2500 & 300.0 & 4 & 600.0 & .0 \\
\hline 03 & 71210 & 84.2503 & 7.3750 & 301.9 & 4 & 600.0 & 600.0 \\
\hline 03 & 71211 & 73.2481 & 6.1250 & 303.2 & 4 & 600.0 & \\
\hline 03 & 71212 & 81.7598 & 7.1750 & 303.7 & 4 & 600.0 & 600.0 \\
\hline 03 & 71213 & 86.0001 & 7.7000 & 303.8 & 4 & 600.0 & .0 \\
\hline 03 & 71214 & 88.2457 & 7.6750 & 303.9 & 4 & 600.0 & \\
\hline 03 & 71215 & 86.2489 & 8.1000 & 303.8 & 4 & 600.0 & 60 \\
\hline 03 & 71216 & 78.0000 & 9.2500 & 302.9 & 4 & 600.0 & 600.0 \\
\hline 03 & 71217 & 67.7485 & 10.1000 & 302.2 & 4 & 600.0 & \\
\hline 03 & 71218 & 65.9996 & 10.8750 & 300.8 & 4 & 600.0 & .0 \\
\hline 03 & 71219 & 63.7502 & 12.9000 & 298.3 & 4 & 600.0 & 60 \\
\hline 03 & 71220 & 62.5001 & 15.00 & 297.4 & 4 & 600.0 & .0 \\
\hline 03 & 71221 & 64.4999 & 14. & 297.1 & 4 & 600.0 & \\
\hline 03 & 71222 & 69.7504 & 12.6750 & 296.8 & 4 & 600.0 & 600.0 \\
\hline 03 & 71223 & 67.7500 & 12.4250 & 296.3 & 4 & 600.0 & .0 \\
\hline 03 & 71224 & 66.2500 & 12.57 & 295.9 & 4 & 600.0 & \\
\hline 03 & 7131 & 67.7500 & 13.3000 & 295.1 & 4 & 600.0 & 60 \\
\hline 03 & 7132 & 67.0000 & 13.0750 & 294.7 & 4 & 600.0 & .0 \\
\hline 03 & 7133 & 65.4991 & 11.52 & 294.9 & 4 & 600.0 & \\
\hline 03 & 7134 & 72.7499 & 9.8500 & 295.0 & 4 & 600.0 & 60 \\
\hline 03 & 7135 & 71.7500 & 9.8500 & 295.0 & 4 & 600.0 & .0 \\
\hline 03 & 7136 & 74.5000 & 11. & 295.1 & 4 & 600.0 & \\
\hline 03 & 7137 & 73.2500 & 10.7000 & 295.6 & 4 & 600.0 & 60 \\
\hline 03 & 7138 & 72.2491 & 8.6250 & 296.9 & 4 & 600.0 & 600.0 \\
\hline 03 & 7139 & 76.5000 & 6 . & 298.1 & 4 & 600.0 & .0 \\
\hline 03 & 71310 & 80.7525 & 6. & 297.8 & 4 & 600.0 & 60 \\
\hline 03 & 71311 & 89.9991 & 5.4500 & 299.4 & 3 & 600.0 & 600.0 \\
\hline 03 & 71312 & 84.2549 & 4.7750 & 301.4 & 3 & 600.0 & \\
\hline 03 & 71313 & 95.9812 & 5.4250 & 301.8 & 3 & 600.0 & 600.0 \\
\hline 03 & 71314 & 84.7489 & 5.6750 & 302.7 & 3 & 600.0 & 600.0 \\
\hline 03 & 71315 & 90.7518 & 7.0000 & 302.6 & 4 & 600.0 & \\
\hline 03 & 71316 & 92.7688 & 7.4750 & 302.4 & 4 & 600.0 & 600.0 \\
\hline 03 & 71317 & 89.7498 & 8.4000 & 301.8 & 4 & 600.0 & 600.0 \\
\hline 03 & 71318 & 91.2513 & 8.2750 & 300.9 & 4 & 600.0 & 600.0 \\
\hline 03 & 71319 & 80.7493 & 7.8750 & 299.7 & 4 & 600.0 & 600.0 \\
\hline 03 & 71320 & 77.2501 & 8.7750 & 298.5 & 4 & 600.0 & 600.0 \\
\hline 03 & 71321 & 74.7500 & 8.5250 & 298.1 & 4 & 600.0 & 600.0 \\
\hline 03 & 71322 & 67.0000 & 8.100 & 298.2 & 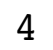 & 600.0 & 600.0 \\
\hline 03 & 71323 & 67.9999 & 8.1500 & 298.1 & 4 & 600.0 & 600.0 \\
\hline
\end{tabular}




\begin{tabular}{|c|c|c|c|c|c|c|c|}
\hline 03 & 71324 & 69.9999 & 8.3500 & 297.8 & 4 & 600.0 & 600.0 \\
\hline 03 & 7141 & 78.4992 & 8.9000 & 297.3 & 4 & 600.0 & 600.0 \\
\hline 03 & 7142 & 73.5000 & 9.1500 & 297.2 & 4 & 600.0 & 00.0 \\
\hline 3 & 7143 & 78.4998 & 6.6000 & 298.1 & 4 & 600.0 & 00.0 \\
\hline 03 & 7144 & 82.7497 & 4.5750 & 298.7 & 4 & 600.0 & 00.0 \\
\hline 03 & 7145 & 112.1600 & 2.0500 & 298.7 & 5 & 600.0 & 00.0 \\
\hline 03 & 7146 & 124.0199 & 0.5250 & 298.4 & 5 & 600.0 & 00.0 \\
\hline 03 & 7147 & 178.5474 & 1.0250 & 300.4 & 4 & 600.0 & 00.0 \\
\hline 03 & 7148 & 175.0008 & 3.4250 & 299.4 & 4 & 600.0 & 00.0 \\
\hline 03 & 7149 & 171.7492 & 4.3750 & 299.9 & 4 & 600.0 & 0 \\
\hline 03 & 71410 & 169.9993 & 5.0250 & 300.8 & 4 & 600.0 & \\
\hline 03 & 71411 & 171.4986 & 5.1750 & 301.9 & 3 & 600.0 & \\
\hline 03 & 71412 & 170.2646 & 4.8250 & 302.9 & 3 & 600.0 & 00.0 \\
\hline 03 & 71413 & 166.5024 & 5.4500 & 303.9 & 3 & 600.0 & 00.0 \\
\hline 03 & 71414 & 176.9940 & 00 & 304.9 & 3 & .0 & \\
\hline 03 & 71415 & 180.7626 & 5.6750 & 305.6 & 3 & 600.0 & 00.0 \\
\hline 03 & 71416 & 183.4987 & 6.1750 & 306.1 & 4 & 60 & 00.0 \\
\hline 03 & 71417 & 185.5000 & 5.32 & 306.0 & 3 & .0 & \\
\hline 03 & 71418 & 180.0414 & 4.4250 & 305.9 & 3 & 60 & .0 \\
\hline 03 & 71419 & 98.2441 & 4.0250 & 305.6 & 4 & .0 & .0 \\
\hline 03 & 71420 & 78.2499 & 5 . & 303.0 & 4 & 6 & \\
\hline 03 & 71421 & 73.5000 & 7.3500 & 301.4 & 4 & .0 & \\
\hline 03 & 71422 & 63.0014 & 8.0500 & 301.5 & 4 & 600.0 & .0 \\
\hline 03 & 71423 & 72.9996 & 6.2 & 301.5 & 4 & 6 & \\
\hline 03 & 71424 & 69.2488 & 7.7500 & 301.7 & 4 & .0 & \\
\hline 03 & 7151 & 74.0000 & 6.9250 & 301.7 & 4 & .0 & 600.0 \\
\hline 03 & 7152 & 63.2515 & 5.3750 & 302.0 & 4 & 600.0 & .0 \\
\hline 03 & 7153 & 74.5000 & 4.9000 & 302.0 & 5 & .0 & \\
\hline 03 & 7154 & 76.5003 & 7.8750 & 300.8 & 4 & .0 & 0.0 \\
\hline 03 & $715 \quad 5$ & 75.5003 & 8.5000 & 299.9 & 4 & .0 & .0 \\
\hline 03 & 7156 & 84.9903 & 4.6500 & 300.3 & 4 & .0 & \\
\hline 03 & 7157 & 263.3287 & 0.7250 & 300.7 & 3 & .0 & 0.0 \\
\hline 03 & 7158 & 349.3763 & 1.3000 & 302.1 & 2 & 600.0 & 0.0 \\
\hline 03 & 7159 & 357.9306 & 2.4750 & 302.4 & 1 & 600.0 & 0.0 \\
\hline 03 & 71510 & 71.8473 & 3.9500 & 301.2 & 2 & 600.0 & 600.0 \\
\hline 03 & 71511 & 81.5467 & 4.6250 & 303.6 & 3 & 600.0 & 600.0 \\
\hline 03 & 71512 & 90.9995 & 6.050 & 303.3 & 4 & 600.0 & 0.0 \\
\hline 03 & 71513 & 83.0096 & 5.9250 & 303.9 & 3 & 600.0 & 600.0 \\
\hline 03 & 71514 & 79.5055 & 7.8000 & 303.5 & 4 & 600.0 & 600.0 \\
\hline 03 & 71515 & 70.5000 & 8.7500 & 303.6 & 4 & 600.0 & 600.0 \\
\hline 03 & 71516 & 71.9973 & 7.9750 & 303.2 & 4 & 600.0 & 600.0 \\
\hline 03 & 71517 & 69.2498 & 9.4250 & 302.6 & 4 & 600.0 & 600.0 \\
\hline 03 & 71518 & 63.7497 & 11.0000 & 301.9 & 4 & 600.0 & 600.0 \\
\hline 03 & 71519 & 62.5000 & 12.7750 & 300.7 & 4 & 600.0 & 600.0 \\
\hline 03 & 71520 & 61.5000 & 12.7250 & 299.9 & 4 & 600.0 & 600.0 \\
\hline 03 & 71521 & 63.4991 & 12.5250 & 299.4 & 4 & 600.0 & 600.0 \\
\hline 03 & 71522 & 67.0000 & 11.8000 & 299.2 & 4 & 600.0 & 600.0 \\
\hline 0 & 71523 & 69.5000 & 11.1500 & 299.1 & $\Psi$ & 600.0 & 600.0 \\
\hline
\end{tabular}




\begin{tabular}{|c|c|c|c|c|c|c|c|}
\hline 3 & 71524 & 68.5000 & 10.1250 & 299.6 & 4 & 600.0 & 600.0 \\
\hline 03 & 7161 & 69.7502 & 10.0000 & 299.1 & 4 & 600.0 & 600.0 \\
\hline 03 & 7162 & 69.5001 & 0000 & 298.8 & 4 & 600.0 & 00.0 \\
\hline 03 & 7163 & 68.9993 & 8.8750 & 298.7 & 2 & 600.0 & 00.0 \\
\hline 03 & 7164 & 68.7498 & 8.8500 & 298.9 & 4 & 600.0 & 00.0 \\
\hline 03 & 7165 & 68.7500 & 8.6000 & 299.4 & 4 & 600.0 & 00.0 \\
\hline 03 & 7166 & 70.2499 & 7.5500 & 299.5 & 4 & 600.0 & 00.0 \\
\hline 03 & 7167 & 68.0000 & 6.7000 & 300.2 & 4 & 600.0 & .0 \\
\hline 03 & 7168 & 76.9984 & 5.9250 & 301.2 & 4 & 600.0 & 000 \\
\hline 03 & 7169 & 137.2915 & 3.7000 & 302.6 & 3 & 600.0 & 600.0 \\
\hline 03 & 71610 & 234.5782 & 3.1250 & 301.8 & 2 & 600.0 & \\
\hline 03 & 71611 & 200.7707 & 500 & 302.0 & 3 & 600.0 & 60 \\
\hline 03 & 71612 & 179.5023 & 6.3500 & 303.4 & 4 & 600.0 & 0 \\
\hline 03 & 71613 & 175.0003 & 7.8250 & 304.9 & 4 & 600.0 & 00.0 \\
\hline 03 & 71614 & 171.7494 & 000 & 305.7 & 4 & 600.0 & 60 \\
\hline 03 & 71615 & 177.5034 & 6.0 & 305.8 & 4 & 600.0 & 600.0 \\
\hline 03 & 71616 & 161.9873 & 5 . & 306.4 & 3 & 600.0 & .0 \\
\hline 03 & 71617 & 110.4414 & 5 . & 306.7 & 3 & .0 & \\
\hline 03 & 71618 & 82.0024 & 5.5500 & 305.8 & 3 & 600.0 & 600.0 \\
\hline 03 & 71619 & 79.0000 & 6 . & 304.3 & 4 & 600.0 & .0 \\
\hline 03 & 71620 & 72.9986 & 9 . & 302.4 & 4 & .0 & \\
\hline 03 & 71621 & 75.7499 & 000 & 302.0 & 4 & .0 & 60 \\
\hline 03 & 71622 & 69.4991 & 9.4250 & 302.3 & 4 & 60 & 600.0 \\
\hline 03 & 71623 & 71.0000 & 500 & 302.7 & 4 & .0 & \\
\hline 03 & 71624 & 75.2501 & 750 & 302.9 & 4 & .0 & 60 \\
\hline 03 & 7171 & 76.2500 & 7. & 303.0 & 4 & 600.0 & 600.0 \\
\hline 03 & 7172 & 74.2501 & 5 . & 302.9 & 4 & .0 & .0 \\
\hline 03 & 7173 & 82.5000 & 4.9250 & 303.0 & 5 & 600.0 & .0 \\
\hline 03 & 7174 & 83.5000 & 5.7750 & 302.6 & 4 & 600.0 & 600.0 \\
\hline 03 & 7175 & 81.7502 & 5 . & 302.3 & 4 & .0 & .0 \\
\hline 03 & 7176 & 82.0000 & 5 . & 302.5 & 4 & .0 & .0 \\
\hline 03 & 7177 & 99.4712 & 250 & 303.5 & 3 & .0 & 600.0 \\
\hline 03 & 7178 & 168.6743 & 2.0250 & 305.2 & 2 & 600.0 & 60 \\
\hline 03 & 7179 & 170.0000 & 5.9250 & 302.8 & 3 & 600.0 & 600.0 \\
\hline 03 & 71710 & 168.2495 & 500 & 302.5 & 4 & .0 & 600.0 \\
\hline 03 & 71711 & 171.0000 & 5.7250 & 303.9 & 3 & 600.0 & 600.0 \\
\hline 03 & 71712 & 172.7501 & 5.1750 & 305.2 & 3 & .0 & 00.0 \\
\hline 03 & 71713 & 180.4933 & 4.5500 & 306.7 & 3 & 600.0 & 600.0 \\
\hline 03 & 71714 & 192.2489 & 5.0000 & 307.5 & 3 & 600.0 & 600.0 \\
\hline 03 & 71715 & 176.7437 & 4 . & 308.0 & $\sigma$ & 600.0 & 600.0 \\
\hline 03 & 71716 & 179.7472 & 5.1500 & 308.7 & 3 & 600.0 & 600.0 \\
\hline 03 & 71717 & 172.2377 & 5.7750 & 308.5 & 3 & 600.0 & 600.0 \\
\hline 03 & 71718 & 171.2508 & 5.0250 & 308.1 & 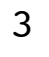 & 600.0 & 600.0 \\
\hline 03 & 71719 & 128.7697 & 4.7750 & 307.9 & 4 & 600.0 & 600.0 \\
\hline 03 & 71720 & 82.7489 & 6.0000 & 306.3 & 4 & 600.0 & 600.0 \\
\hline 03 & 71721 & 75.9977 & 5500 & 305.3 & 4 & 600.0 & \\
\hline 03 & 71722 & 81.5000 & 7.4000 & 304.6 & 4 & 600.0 & 600.0 \\
\hline 03 & 71723 & 76.0001 & 9.325 & 303.9 & 4 & 600.0 & 600. \\
\hline
\end{tabular}




\begin{tabular}{|c|c|c|c|c|c|c|c|}
\hline 03 & 71724 & 74.0000 & 10.0750 & 303.9 & 4 & 600.0 & 600.0 \\
\hline 03 & 7181 & 72.7500 & 9.7500 & 303.7 & 4 & 600.0 & 600.0 \\
\hline 03 & 7182 & 75.5000 & 9.0750 & 303.8 & 4 & 600.0 & 00.0 \\
\hline 3 & 7183 & 73.0001 & 8.8250 & 303.5 & 4 & 600.0 & 00.0 \\
\hline 03 & 7184 & 75.2499 & 8.8000 & 303.2 & 4 & 600.0 & 00.0 \\
\hline 03 & 7185 & 76.7500 & 8.4750 & 303.1 & 4 & 600.0 & 00.0 \\
\hline 03 & 7186 & 78.7500 & 8.0250 & 303.7 & 4 & 600.0 & 00.0 \\
\hline 03 & 7187 & 78.7502 & 7.2500 & 304.6 & 4 & 600.0 & 00.0 \\
\hline 03 & 7188 & 79.2489 & 5.2750 & 306.2 & 4 & 600.0 & 00.0 \\
\hline 03 & 7189 & 117.8217 & 3.8500 & 307.6 & 3 & 600.0 & 0 \\
\hline 03 & 71810 & 167.2501 & 6.1000 & 306.1 & 4 & 600.0 & \\
\hline 03 & 71811 & 176.7374 & 6.2250 & 305.9 & 4 & 600.0 & \\
\hline 03 & 71812 & 192.7506 & 5.7000 & 306.3 & 3 & 600.0 & 00.0 \\
\hline 03 & 71813 & 162.3000 & 5.6000 & 307.6 & 3 & 600.0 & 00.0 \\
\hline 03 & 71814 & 122.4849 & 750 & 309.0 & 3 & .0 & \\
\hline 03 & 71815 & 94.2329 & 6.4000 & 309.0 & 4 & 600.0 & 00.0 \\
\hline 03 & 71816 & 86.7713 & 7.2750 & 308.7 & 4 & 60 & 0.0 \\
\hline 03 & 71817 & 69.7501 & 8.9000 & 308.2 & 4 & .0 & \\
\hline 03 & 71818 & 66.2498 & 8.7000 & 307.4 & 4 & 60 & .0 \\
\hline 03 & 71819 & 63.7501 & 9.2750 & 306.7 & 4 & .0 & .0 \\
\hline 03 & 71820 & 65.2500 & 8 . & 5.1 & 4 & 60 & \\
\hline 03 & 71821 & 67.2500 & 9.0000 & 304.9 & 4 & .0 & \\
\hline 03 & 71822 & 69.5000 & 8.7250 & 304.8 & 4 & 600.0 & .0 \\
\hline 03 & 71823 & 76.7483 & 7.8000 & 305.3 & 4 & 60 & \\
\hline 03 & 71824 & 85.7485 & 5.1000 & 305.1 & 4 & .0 & \\
\hline 03 & 7191 & 78.0724 & 2.2750 & 304.8 & 5 & .0 & 600.0 \\
\hline 03 & 7192 & 71.2479 & 5.5500 & 304.4 & 4 & 600.0 & .0 \\
\hline 03 & 7193 & 72.2501 & 6.2500 & 303.9 & 4 & .0 & \\
\hline 03 & 7194 & 77.7500 & 7.7500 & 303.2 & 4 & .0 & 0.0 \\
\hline 03 & 7195 & 70.7499 & 9.4000 & 302.9 & 4 & 60 & .0 \\
\hline 03 & 7196 & 71.7493 & 8.4250 & 303.3 & 4 & .0 & \\
\hline 03 & 7197 & 76.1719 & 6.2000 & 304.4 & 4 & .0 & 600.0 \\
\hline 03 & 7198 & 242.4327 & 2.2000 & 302.4 & 3 & 600.0 & 600.0 \\
\hline 03 & 7199 & 284.3193 & 1.5250 & 302.8 & 2 & 600.0 & 0.0 \\
\hline 03 & 71910 & 250.4617 & 2.0250 & 304.8 & 1 & 600.0 & 600.0 \\
\hline 03 & 71911 & 214.7036 & 3.3750 & 306.2 & 2 & 600.0 & 600.0 \\
\hline 03 & 71912 & 222.4770 & 3.9000 & 306.4 & 2 & 600.0 & 600.0 \\
\hline 03 & 71913 & 228.9815 & 4.0750 & 307.6 & 3 & 600.0 & 600.0 \\
\hline 03 & 71914 & 248.3638 & 5.0250 & 308.5 & 3 & 600.0 & 600.0 \\
\hline 03 & 71915 & 78.9668 & 4.3250 & 308.8 & 3 & 600.0 & 600.0 \\
\hline 03 & 71916 & 94.9908 & 4.9750 & 308.5 & 3 & 600.0 & 600.0 \\
\hline 03 & 71917 & 84.7520 & 4.6000 & 308.6 & 3 & 600.0 & 600.0 \\
\hline 03 & 71918 & 127.2602 & 3.5000 & 307.1 & 2 & 600.0 & 600.0 \\
\hline 03 & 71919 & 115.0997 & 2.7000 & 305.7 & 3 & 600.0 & 600.0 \\
\hline 03 & 71920 & 71.4976 & 6.1250 & 304.1 & 4 & 600.0 & 600.0 \\
\hline 03 & 71921 & 73.2470 & 7.8250 & 302.8 & 4 & 600.0 & 600.0 \\
\hline 03 & 71922 & 68.3601 & 2.6500 & 302.5 & 5 & 600.0 & 600.0 \\
\hline 0 & 71923 & 28.5000 & 3.1250 & 303.7 & 4 & 600.0 & 600.0 \\
\hline
\end{tabular}




\begin{tabular}{|c|c|c|c|c|c|c|c|}
\hline 3 & 71924 & 71.3144 & 2.8250 & 304.3 & 5 & 600.0 & 00.0 \\
\hline 03 & 7201 & 19.7288 & 1.5000 & 303.2 & 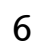 & 600.0 & 600.0 \\
\hline 03 & 7202 & 66.8837 & 2.2500 & 303.3 & 6 & 600.0 & 00.0 \\
\hline 03 & 7203 & 61.7902 & 3.6500 & 303.6 & - & 600.0 & 00.0 \\
\hline 03 & $720 \quad 4$ & 78.5057 & 6.2250 & 302.5 & 2 & 600.0 & 00.0 \\
\hline 03 & 7205 & 331.1613 & 2.4500 & 296.1 & J & 600.0 & 00.0 \\
\hline 03 & 7206 & 14.2275 & 1.7750 & 298.9 & $\sigma$ & 600.0 & .0 \\
\hline 03 & $\begin{array}{ll}720 & 7\end{array}$ & 347.0821 & 1.6250 & 301.7 & 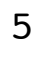 & 600.0 & 00.0 \\
\hline 03 & $720 \quad 8$ & 237.6608 & 1.5500 & 303.1 & 4 & 600.0 & 00.0 \\
\hline 03 & 7209 & 208.5000 & 2.8250 & 303.8 & 3 & 600.0 & .0 \\
\hline 03 & 72010 & 195.7546 & 3.6000 & 303.4 & 2 & 600.0 & 600.0 \\
\hline 03 & 72011 & 222.7151 & 3.0500 & 304.1 & 2 & 600.0 & \\
\hline 03 & 72012 & 225.8672 & 2.4250 & 306.0 & 1 & 600.0 & 00.0 \\
\hline 03 & 72013 & 196.5098 & 3.9000 & 307.0 & 2 & 600.0 & 600.0 \\
\hline 03 & 72014 & 185.0132 & 4.3250 & 307.8 & 3 & 600.0 & \\
\hline 03 & 72015 & 183.7481 & 4.5250 & 308.4 & 3 & 600.0 & 60 \\
\hline 03 & 72016 & 180.5052 & 5.0500 & 308.8 & 3 & 600.0 & .0 \\
\hline 03 & 72017 & 189.7468 & 4.9500 & 308.7 & 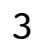 & 600.0 & .0 \\
\hline 03 & 72018 & 173.9654 & 4.9000 & 307.9 & 3 & 600.0 & .0 \\
\hline 03 & 72019 & 81.2500 & 6.0000 & 306.2 & 4 & 600.0 & .0 \\
\hline 03 & 72020 & 79.9998 & 8 . & 303.3 & 4 & 600.0 & .0 \\
\hline 03 & 72021 & 77.7500 & 10. & 302.2 & 4 & 0.0 & \\
\hline 03 & 72022 & 77.7500 & 10.9250 & 301.7 & 4 & 600.0 & 600.0 \\
\hline 03 & 72023 & 79.2500 & 8.97 & 302.8 & 4 & 600.0 & .0 \\
\hline 03 & 72024 & 75.2540 & 8 . & 304.0 & 4 & 00.0 & \\
\hline 03 & 7211 & 71.2554 & 6.6250 & 304.1 & 4 & 600.0 & 60 \\
\hline 03 & 7212 & 96.0482 & 4.6750 & 303.6 & 4 & 600.0 & .0 \\
\hline 03 & 7213 & 45.0438 & 0.80 & 300.3 & 5 & 600.0 & \\
\hline 03 & 7214 & 51.7837 & 2.7250 & 302.0 & 6 & 600.0 & 60 \\
\hline 03 & 7215 & 50.3727 & 3.5500 & 304.1 & 5 & 600.0 & .0 \\
\hline 03 & 7216 & 178.2416 & 2.35 & 297.3 & 6 & 600.0 & \\
\hline 03 & 7217 & 333.1206 & 1.0750 & 297.3 & 5 & 600.0 & .0 \\
\hline 03 & 7218 & 328.6193 & 0.9750 & 301.1 & 4 & 600.0 & 600.0 \\
\hline 03 & 7219 & 278.0111 & 1.27 & 304.8 & 3 & 600.0 & .0 \\
\hline 03 & 72110 & 196.6773 & 3.8000 & 304.8 & 2 & 600.0 & .0 \\
\hline 03 & 72111 & 183.7651 & 4.4000 & 304.6 & 3 & 600.0 & 600.0 \\
\hline 03 & 72112 & 171.2455 & 4.4000 & 305.8 & 3 & 600.0 & \\
\hline 03 & 72113 & 174.2382 & 4.9500 & 307.5 & 3 & 600.0 & .0 \\
\hline 03 & 72114 & 85.4599 & 3.7750 & 309.4 & 2 & 600.0 & 600.0 \\
\hline 03 & 72115 & 96.7769 & 5.0750 & 309.0 & 3 & 600.0 & \\
\hline 03 & 72116 & 109.5000 & 5.6250 & 309.0 & 3 & 600.0 & 60 \\
\hline 03 & 72117 & 107.4871 & 5.7250 & 308.8 & 3 & 600.0 & 600.0 \\
\hline 03 & 72118 & 106.7499 & 5.8500 & 307.5 & 3 & 600.0 & 600.0 \\
\hline 03 & 72119 & 81.4939 & 7.4750 & 305.4 & 4 & 600.0 & 600.0 \\
\hline 03 & 72120 & 74.0000 & 10.1500 & 304.1 & 4 & 600.0 & 600.0 \\
\hline 03 & 72121 & 68.7497 & 7.5250 & 304.3 & 4 & 600.0 & 600.0 \\
\hline 03 & 72122 & 71.2502 & 9.300 & 304.5 & 4 & 600.0 & 600.0 \\
\hline 03 & 72123 & 71.5009 & 8.8750 & 304.5 & 4 & 600.0 & 600.0 \\
\hline
\end{tabular}




\begin{tabular}{|c|c|c|c|c|c|c|c|}
\hline 3 & 72124 & 75.7500 & 9.4750 & 304.3 & 4 & 600.0 & 600.0 \\
\hline 3 & 7221 & 75.2500 & 9.7000 & 303.9 & 4 & 600.0 & 600.0 \\
\hline 03 & 7222 & 76.5000 & 8.7000 & 303.6 & 4 & 600.0 & 00.0 \\
\hline 3 & 7223 & 75.5018 & 8.8500 & 303.2 & 4 & 600.0 & 00.0 \\
\hline 03 & 7224 & 75.0000 & 9.4750 & 303.0 & 4 & 600.0 & 00.0 \\
\hline 03 & 7225 & 73.0000 & 9.1500 & 302.7 & 4 & 600.0 & 00.0 \\
\hline 03 & 7226 & 71.4998 & 8.7750 & 302.9 & 4 & 600.0 & 00.0 \\
\hline 03 & 7227 & 75.7506 & 7.5250 & 303.6 & 4 & 600.0 & 00.0 \\
\hline 03 & 7228 & 84.2504 & 6.8250 & 304.6 & 4 & 600.0 & 00.0 \\
\hline 03 & 7229 & 85.7496 & 000 & 305.4 & 4 & 600.0 & 0 \\
\hline 03 & 72210 & 106.7190 & 4.8750 & 306.7 & 3 & 600.0 & \\
\hline 03 & 72211 & 92.2501 & 500 & 307.5 & 3 & 600.0 & \\
\hline 03 & 72212 & 85.2877 & 5.1000 & 308.3 & 3 & 600.0 & 00.0 \\
\hline 03 & 72213 & 106.5268 & 5.1 & 308.8 & 3 & 600.0 & 00.0 \\
\hline 03 & 72214 & 100.2063 & 6 . & 308.9 & 4 & .0 & \\
\hline 03 & 72215 & 83.4992 & 750 & 308.1 & 4 & 600.0 & 00.0 \\
\hline 03 & 72216 & 72.2410 & 8.9 & 307.8 & 4 & 60 & .0 \\
\hline 03 & 72217 & 71.7499 & 9. & 307.8 & 4 & .0 & \\
\hline 03 & 72218 & 80.2519 & 8.4500 & 306.9 & 4 & 60 & .0 \\
\hline 03 & 72219 & 74.5086 & 7.8500 & 305.9 & 4 & 60 & .0 \\
\hline 03 & 72220 & 67.0005 & 9 . & 5.1 & 4 & 60 & \\
\hline 03 & 72221 & 62.5000 & 10. & 304.8 & 4 & .0 & \\
\hline 03 & 72222 & 64.2500 & 10.6 & 304.6 & 4 & 600.0 & .0 \\
\hline 03 & 72223 & 66.0000 & 10 . & 304.5 & 4 & 60 & \\
\hline 03 & 72224 & 72.9998 & 10 . & 304.4 & 4 & .0 & \\
\hline 03 & 7231 & 71.0000 & 9. & 304.3 & 4 & .0 & 0.0 \\
\hline 03 & 7232 & 71.5000 & 8.6500 & 304.3 & 4 & 600.0 & .0 \\
\hline 03 & 7233 & 68.7529 & 9. & 303.7 & 4 & .0 & \\
\hline 03 & 7234 & 64.2501 & 9.7 & 303.3 & 4 & .0 & 0.0 \\
\hline 03 & 7235 & 68.9996 & 9.1500 & 303.1 & 4 & 60 & .0 \\
\hline 03 & 7236 & 73.0000 & 8. & 303.8 & 4 & .0 & \\
\hline 03 & 7237 & 70.0021 & 8. & 304.2 & 4 & .0 & 600.0 \\
\hline 03 & 7238 & 71.2522 & 6.2000 & 305.2 & 4 & 600.0 & 600.0 \\
\hline 03 & 7239 & 174.1462 & 3.1 & 303.7 & 3 & 0 & 0.0 \\
\hline 03 & 72310 & 238.5542 & 1.1750 & 301.0 & 2 & 600.0 & 600.0 \\
\hline 03 & 72311 & 309.8995 & 1.4000 & 303.8 & 1 & 600.0 & 600.0 \\
\hline 03 & 72312 & 237.0617 & 1.8 & 302.9 & 1 & 600.0 & 0.0 \\
\hline 03 & 72313 & 192.4642 & 1.5500 & 304.4 & 1 & 600.0 & 600.0 \\
\hline 03 & 72314 & 118.9472 & 3.4500 & 306.3 & 2 & 600.0 & 600.0 \\
\hline 03 & 72315 & 233.4867 & 2 . & 304.7 & 1 & 600.0 & 600.0 \\
\hline 03 & 72316 & 237.6925 & 1.4250 & 304.7 & 1 & 600.0 & 600.0 \\
\hline 03 & 72317 & 247.5631 & 1.6250 & 304.9 & 1 & 600.0 & 600.0 \\
\hline 03 & 72318 & 146.7481 & 3.6500 & 303.3 & 2 & 600.0 & 600.0 \\
\hline 03 & 72319 & 88.1748 & 5.8500 & 304.5 & 3 & 600.0 & 600.0 \\
\hline 03 & 72320 & 72.2502 & 6.7250 & 303.6 & 4 & 600.0 & 600.0 \\
\hline 03 & 72321 & 70.5001 & & 303.9 & . & 600.0 & 600.0 \\
\hline 03 & 72322 & 61.2498 & 7.0000 & 304.5 & 4 & 600.0 & 600.0 \\
\hline 0 & 72323 & 69.5009 & 6.4000 & 304.8 & 4 & 600.0 & 600.0 \\
\hline
\end{tabular}




\begin{tabular}{|c|c|c|c|c|c|c|c|}
\hline 03 & 72324 & 70.2382 & 4.3500 & 304.9 & 4 & 600.0 & 600.0 \\
\hline 03 & 7241 & 74.0073 & 5.7500 & 304.5 & 4 & 600.0 & 600.0 \\
\hline 03 & 7242 & 81.0037 & 6.7250 & 304.9 & 4 & 600.0 & 00.0 \\
\hline 03 & 7243 & 1.4088 & 2.4000 & 302.7 & 5 & 600.0 & 600.0 \\
\hline 03 & $724 \quad 4$ & 278.2881 & 1.2750 & 298.0 & 0 & 600.0 & 00.0 \\
\hline 03 & 7245 & 348.0407 & 1.5250 & 296.9 & 6 & 600.0 & 00.0 \\
\hline 03 & 7246 & 130.3395 & 0.7250 & 298.1 & 6 & 600.0 & 600.0 \\
\hline 03 & 7247 & 47.9788 & 0.6000 & 300.6 & 5 & 600.0 & 00.0 \\
\hline 03 & $724 \quad 8$ & 265.2511 & 1.5000 & 302.8 & 4 & 600.0 & 00.0 \\
\hline 03 & 7249 & 308.8635 & 1.0250 & 304.1 & 3 & 600.0 & 600.0 \\
\hline 03 & 72410 & 231.9993 & 3.7500 & 303.7 & 2 & 600.0 & 600.0 \\
\hline 03 & 72411 & 239.0000 & 2.5000 & 304.2 & 1 & 600.0 & \\
\hline 03 & 72412 & 235.4956 & 2.8000 & 305.2 & 1 & 600.0 & 600.0 \\
\hline 03 & 72413 & 186.8178 & 4.6500 & 306.4 & 2 & 600.0 & 600.0 \\
\hline 03 & 72414 & 124.2586 & 5.2000 & 307.5 & 3 & 600.0 & \\
\hline 03 & 72415 & 88.7509 & 6.9500 & 307.2 & 4 & 600.0 & 60 \\
\hline 03 & 72416 & 99.7436 & 7.4500 & 306.5 & 4 & 600.0 & 600.0 \\
\hline 03 & 72417 & 80.0000 & 8.1250 & 305.8 & 4 & 600.0 & \\
\hline 03 & 72418 & 77.7500 & 8.7500 & 304.5 & 4 & 600.0 & 60 \\
\hline 03 & 72419 & 70.9995 & 8.5000 & 304.1 & 4 & 600.0 & 600.0 \\
\hline 03 & 72420 & 82.4871 & 5.1000 & 302.9 & 4 & 600.0 & .0 \\
\hline 03 & 72421 & 76.5108 & 6.8 & 303.1 & 4 & 600.0 & \\
\hline 03 & 72422 & 73.9989 & 7.1750 & 302.3 & 4 & 600.0 & 600.0 \\
\hline 03 & 72423 & 63.7503 & 6.9750 & 301.8 & 4 & 600.0 & .0 \\
\hline 03 & 72424 & 64.7499 & 750 & 302.0 & 4 & 600.0 & \\
\hline 03 & 7251 & 71.7502 & 7.3500 & 302.1 & 4 & 600.0 & 600.0 \\
\hline 03 & 7252 & 74.2500 & 7.3250 & 302.0 & 4 & 600.0 & 600.0 \\
\hline 03 & 7253 & 44.0027 & 4.2750 & 299.9 & 4 & 600.0 & .0 \\
\hline 03 & 7254 & 57.9569 & 1.3750 & 294.7 & 5 & 600.0 & 600.0 \\
\hline 03 & 7255 & 3.3172 & 1.5000 & 294.4 & 6 & 600.0 & 600.0 \\
\hline 03 & 7256 & 341.2934 & 1 . & 293.0 & 5 & 600.0 & .0 \\
\hline 03 & 7257 & 339.5348 & 0.9250 & 294.3 & 4 & 600.0 & 60 \\
\hline 03 & 7258 & 341.5210 & 1.6500 & 295.2 & 3 & 600.0 & 600.0 \\
\hline 03 & 7259 & 289.4902 & 1.9750 & 298.7 & 2 & 600.0 & .0 \\
\hline 03 & 72510 & 239.9897 & 2 . & 300.2 & 1 & 600.0 & 600.0 \\
\hline 03 & 72511 & 209.4185 & 4.6000 & 300.3 & 2 & 600.0 & 600.0 \\
\hline 03 & 72512 & 212.2334 & 4.5250 & 301.7 & 3 & 600.0 & 600.0 \\
\hline 03 & 72513 & 343.9926 & 2.8500 & 304.4 & 2 & 600.0 & 600.0 \\
\hline 03 & 72514 & 63.5419 & 4.2500 & 305.3 & 3 & 600.0 & 600.0 \\
\hline 03 & 72515 & 79.0533 & 4.8500 & 306.2 & 3 & 600.0 & \\
\hline 03 & 72516 & 74.7501 & 6.0250 & 305.9 & 4 & 600.0 & 600.0 \\
\hline 03 & 72517 & 72.0110 & 6.2750 & 305.2 & 4 & 600.0 & 600.0 \\
\hline 03 & 72518 & 89.2132 & 3.5750 & 304.6 & 3 & 600.0 & 600.0 \\
\hline 03 & 72519 & 352.8366 & 1.5750 & 302.9 & 4 & 600.0 & 600.0 \\
\hline 03 & 72520 & 45.4109 & 3.0500 & 300.1 & 5 & 600.0 & 600.0 \\
\hline 03 & 72521 & 25.8205 & 3.7500 & 298.2 & 4 & 600.0 & 600.0 \\
\hline 03 & 72522 & 57.4751 & 3.1500 & 299.6 & 5 & 600.0 & 600.0 \\
\hline 03 & 72523 & 137.5680 & 2.1750 & 299.3 & $\mathrm{~J}$ & 600.0 & 600.0 \\
\hline
\end{tabular}




\begin{tabular}{|c|c|c|c|c|c|c|c|}
\hline 3 & 72524 & 54.9337 & 1.7250 & 298.9 & 6 & 600.0 & 00.0 \\
\hline 03 & 7261 & 43.8191 & 1.1000 & 298.8 & 6 & 600.0 & 600.0 \\
\hline 03 & 7262 & 54.6426 & 0.7000 & 298.5 & 6 & 600.0 & 00.0 \\
\hline 03 & 7263 & 15.7838 & 0.6500 & 298.0 & 6 & 600.0 & 00.0 \\
\hline 03 & 7264 & 50.9491 & 0.9000 & 295.9 & 6 & 600.0 & 00.0 \\
\hline 03 & 7265 & 128.1397 & 0.9500 & 293.4 & 6 & 600.0 & 00.0 \\
\hline 03 & 7266 & 348.1074 & 0.8250 & 293.4 & 6 & 600.0 & 600.0 \\
\hline 03 & 7267 & 356.9848 & 1.6000 & 296.2 & 3 & 600.0 & 00.0 \\
\hline 03 & 7268 & 351.4890 & 1.1250 & 299.4 & 4 & 600.0 & 00.0 \\
\hline 03 & 7269 & 271.3025 & 1.4250 & 301.8 & 3 & 600.0 & .0 \\
\hline 03 & 72610 & 230.7328 & 2.8250 & 301.0 & 2 & 600.0 & 00.0 \\
\hline 03 & 72611 & 171.2250 & 3.8500 & 302.3 & 2 & 600.0 & \\
\hline 03 & 72612 & 160.5010 & 4.2000 & 303.0 & 3 & 600.0 & 600.0 \\
\hline 03 & 72613 & 171.9945 & 4.2750 & 304.3 & 3 & 600.0 & 0 \\
\hline 03 & 72614 & 174.0000 & 4.5000 & 305.2 & 3 & 600.0 & \\
\hline 03 & 72615 & 174.7500 & 5.7000 & 305.9 & 3 & 600.0 & 60 \\
\hline 03 & 72616 & 185.2551 & 6.9750 & 305.9 & 4 & 600.0 & 600.0 \\
\hline 03 & 72617 & 180.9920 & 6.3000 & 305.8 & 4 & 600.0 & .0 \\
\hline 03 & 72618 & 170.9975 & 5.8250 & 305.5 & 3 & 600.0 & .0 \\
\hline 03 & 72619 & 100.8293 & 5.3000 & 305.2 & 4 & 600.0 & 600 \\
\hline 03 & 72620 & 78.7626 & 6.22 & 302.5 & 4 & 600.0 & .0 \\
\hline 03 & 72621 & 65.9986 & 8.2250 & 301.7 & 4 & 600.0 & \\
\hline 03 & 72622 & 68.7499 & 8.5750 & 301.9 & 4 & 600.0 & 600.0 \\
\hline 03 & 72623 & 74.5003 & 7.7500 & 302.1 & 4 & 600.0 & .0 \\
\hline 03 & 72624 & 77.2500 & 8.0250 & 301.9 & 4 & 600.0 & \\
\hline 03 & 7271 & 78.7499 & 7.8000 & 302.4 & 4 & 600.0 & 60 \\
\hline 03 & 7272 & 80.2503 & 5.9000 & 303.0 & 4 & 600.0 & .0 \\
\hline 03 & 7273 & 80.5000 & 8.3750 & 301.5 & 4 & 600.0 & \\
\hline 03 & 7274 & 79.5000 & 7.4000 & 301.6 & 4 & 600.0 & 60 \\
\hline 03 & 7275 & 79.4998 & 7.8000 & 301.1 & 4 & 600.0 & .0 \\
\hline 03 & 7276 & 90.9931 & 6 . & 301.4 & 4 & 600.0 & \\
\hline 03 & $727 \quad 7$ & 97.0046 & 5.2000 & 302.2 & 4 & 600.0 & .0 \\
\hline 03 & 7278 & 136.4732 & 3.9000 & 303.7 & 3 & 600.0 & 600.0 \\
\hline 03 & 7279 & 170.2495 & 4 . & 304.2 & 4 & 600.0 & .0 \\
\hline 03 & 72710 & 170.7492 & 000 & 304.4 & 4 & 600.0 & .0 \\
\hline 03 & 72711 & 168.0000 & 6.0000 & 304.8 & 4 & 600.0 & 600.0 \\
\hline 03 & 72712 & 172.0000 & 4.9250 & 306.0 & 3 & 600.0 & \\
\hline 03 & 72713 & 177.2235 & 5.3000 & 307.0 & 3 & 600.0 & 600.0 \\
\hline 03 & 72714 & 174.8236 & 5.0750 & 307.9 & 3 & 600.0 & 600.0 \\
\hline 03 & 72715 & 164.2403 & 750 & 308.4 & 3 & 600.0 & \\
\hline 03 & 72716 & 92.9516 & 5.8750 & 308.1 & 3 & 600.0 & 600.0 \\
\hline 03 & 72717 & 85.9989 & 7.5500 & 306.4 & 4 & 600.0 & 600.0 \\
\hline 03 & 72718 & 76.0007 & 7.7750 & 305.3 & 4 & 600.0 & 600.0 \\
\hline 03 & 72719 & 73.7502 & 9.0750 & 304.1 & 4 & 600.0 & 600.0 \\
\hline 03 & 72720 & 70.0000 & 10.7000 & 302.6 & 4 & 600.0 & 600.0 \\
\hline 03 & 72721 & 66.2499 & 11.1500 & 302.1 & 4 & 600.0 & 600.0 \\
\hline 03 & 72722 & 68.7499 & 9500 & 302.0 & 4 & .0 & 600.0 \\
\hline 03 & 72723 & 72.2503 & 11.6500 & 302.5 & 4 & 600.0 & 600.0 \\
\hline
\end{tabular}




\begin{tabular}{|c|c|c|c|c|c|c|c|}
\hline 3 & 72724 & 72.5000 & 10.8750 & 302.6 & 4 & 600.0 & 600.0 \\
\hline 3 & 7281 & 69.7500 & 10.6500 & 303.0 & 4 & 600.0 & 00,0 \\
\hline 3 & 7282 & 70.0000 & 1750 & 303.4 & 4 & 600.0 & 00.0 \\
\hline 03 & 7283 & 70.0000 & 9.8750 & 302.3 & 4 & 600.0 & 00.0 \\
\hline 03 & 7284 & 77.2499 & 10.3000 & 302.0 & 4 & 600.0 & 00.0 \\
\hline 03 & 7285 & 78.7500 & 10.9750 & 301.4 & 4 & 600.0 & 00.0 \\
\hline 03 & 7286 & 73.5000 & 10.0750 & 300.5 & 4 & 600.0 & 0 \\
\hline 03 & 7287 & 76.7501 & 9.4500 & 301.5 & 4 & 600.0 & 00.0 \\
\hline 03 & 7288 & 85.7502 & 9.1250 & 302.9 & 4 & 600.0 & 00.0 \\
\hline 03 & 7289 & 91.2500 & 6.4250 & 303.6 & 4 & 60 & 0 \\
\hline 03 & 72810 & 169.2995 & 500 & 304.9 & 3 & 600.0 & \\
\hline 03 & 72811 & 163.5299 & 500 & 306.4 & 3 & .0 & \\
\hline 03 & 72812 & 160.4991 & 5.9250 & 307.4 & 3 & 600.0 & .0 \\
\hline 03 & 72813 & 164.2450 & 6.3000 & 308.6 & 4 & 600.0 & \\
\hline 03 & 72814 & 155.0248 & 000 & 309.5 & 4 & .0 & \\
\hline 03 & 72815 & 152.5003 & 7.0000 & 309.2 & 4 & 600.0 & \\
\hline 03 & 72816 & 138.1465 & 5.8250 & 309.7 & 3 & 60 & .0 \\
\hline 03 & 72817 & 93.2499 & 5.6500 & 309.4 & 3 & .0 & \\
\hline 03 & 72818 & 80.2500 & 7.7250 & 307.6 & 4 & 60 & \\
\hline 03 & 72819 & 78.5000 & 9.5000 & 305.9 & 4 & .0 & .0 \\
\hline 03 & 72820 & 76.4998 & 10 . & .1 & 4 & 6 & \\
\hline 03 & 72821 & 73.0000 & 9.9500 & .1 & 4 & .0 & \\
\hline 03 & 72822 & 70.0012 & 11.4000 & 304.6 & 4 & 60 & .0 \\
\hline 03 & 72823 & 70.7500 & 11.30 & 304.8 & 4 & 0 & \\
\hline 03 & 72824 & 70.2500 & 11.7250 & 304.6 & 4 & 0 & \\
\hline 03 & 7291 & 71.2500 & 10.1750 & 304.3 & 4 & .0 & \\
\hline 03 & 7292 & 74.7497 & 9.3500 & 304.0 & 4 & 60 & .0 \\
\hline 03 & 7293 & 70.4997 & 9.3750 & 304.1 & 4 & .0 & \\
\hline 03 & 7294 & 71.5001 & 9.8250 & 304.1 & 4 & .0 & \\
\hline 03 & 7295 & 75.0000 & 9.8000 & 304.0 & 4 & .0 & \\
\hline 03 & 7296 & 81.7500 & 9.4250 & 304.1 & 4 & .0 & \\
\hline 03 & 7297 & 87.2500 & 8.5000 & 304.5 & 4 & .0 & .0 \\
\hline 03 & 7298 & 87.0003 & 6.2000 & 304.9 & 4 & 0 & 0.0 \\
\hline 03 & 7299 & 143.4045 & 2.9500 & 306.4 & 3 & .0 & .0 \\
\hline 03 & 72910 & 179.6271 & 4.52 & 5.9 & 3 & 0 & 0 \\
\hline 03 & 72911 & 169.5000 & 5.4000 & 305.6 & 3 & 600.0 & 600.0 \\
\hline 03 & 72912 & 162.0002 & 7.20 & 307.4 & 4 & 600.0 & .0 \\
\hline 03 & 72913 & 165.2501 & 7.4000 & 307.8 & 4 & 600.0 & 600.0 \\
\hline 03 & 72914 & 134.7881 & 6.1000 & 308.8 & 4 & 600.0 & 600.0 \\
\hline 03 & 72915 & 88.7500 & 5.60 & 309.4 & 4 & 600.0 & .0 \\
\hline 03 & 72916 & 87.2499 & 6.8500 & 308.8 & 4 & 600.0 & 600.0 \\
\hline 03 & 72917 & 76.5000 & 7.6750 & 307.8 & 4 & 600.0 & 600.0 \\
\hline 03 & 72918 & 70.7504 & 7.6750 & 306.9 & 4 & 600.0 & 600.0 \\
\hline 03 & 72919 & 69.7468 & 7.6750 & 307.1 & 4 & 600.0 & 600.0 \\
\hline 03 & 72920 & 72.0000 & 8.4250 & 307.0 & 4 & 600.0 & 600.0 \\
\hline 03 & 72921 & 70.7500 & 10.0000 & 307.1 & 4 & 600.0 & .0 \\
\hline 03 & 72922 & 33.3486 & 6.800 & 306.1 & 4 & 600.0 & 600.0 \\
\hline 0 & 72923 & 8.0985 & 1.9750 & 302.4 & & 600.0 & 600.0 \\
\hline
\end{tabular}




\begin{tabular}{|c|c|c|c|c|c|c|c|}
\hline 03 & 72924 & 56.3457 & 3.5500 & 306.2 & 5 & 600.0 & 00.0 \\
\hline 03 & $\begin{array}{ll}730 & 1\end{array}$ & 57.5764 & 1.9250 & 304.9 & 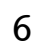 & 600.0 & 600.0 \\
\hline 03 & 7302 & 56.8994 & 4.0500 & 307.4 & 5 & 600.0 & 00.0 \\
\hline 03 & 7303 & 289.0863 & 3.6250 & 298.4 & 4 & 600.0 & 00.0 \\
\hline 03 & $730 \quad 4$ & 342.0172 & 3.2500 & 297.2 & 4 & 600.0 & 00.0 \\
\hline 03 & 7305 & 8.8999 & 2.7500 & 299.4 & כ & 600.0 & 00.0 \\
\hline 03 & 7306 & 100.5115 & 2.5250 & 299.9 & 6 & 600.0 & 600.0 \\
\hline 03 & $\begin{array}{ll}730 & 7\end{array}$ & 128.2957 & 3.9750 & 302.2 & 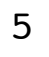 & 600.0 & 00.0 \\
\hline 03 & $730 \quad 8$ & 359.1538 & 1.6500 & 298.8 & 4 & 600.0 & 00.0 \\
\hline 03 & 7309 & 234.8954 & 2.2000 & 299.5 & 3 & 600.0 & 600.0 \\
\hline 03 & 73010 & 341.0393 & 1.2250 & 298.3 & 2 & 600.0 & 600 \\
\hline 03 & 73011 & 260.6840 & 2.0500 & 301.4 & 1 & 600.0 & \\
\hline 03 & 73012 & 213.2196 & 2.5750 & 303.8 & 1 & 600.0 & 600.0 \\
\hline 03 & 73013 & 202.4313 & 4.5500 & 302.3 & 2 & 600.0 & 600.0 \\
\hline 03 & 73014 & 198.9632 & 3.8000 & 302.8 & 2 & 600.0 & \\
\hline 03 & 73015 & 176.7507 & 5.4000 & 302.7 & 3 & 600.0 & 60 \\
\hline 03 & 73016 & 204.4295 & 2.6000 & 304.0 & 2 & 600.0 & 600.0 \\
\hline 03 & 73017 & 295.4954 & 3.37 & 304.1 & 2 & 600.0 & .0 \\
\hline 03 & 73018 & 294.7511 & 3.3000 & 302.3 & 3 & 600.0 & 60 \\
\hline 03 & 73019 & 337.2946 & 2.1250 & 300.5 & 4 & 600.0 & .0 \\
\hline 03 & 73020 & 53.0084 & 4.55 & 301.5 & 4 & 600.0 & .0 \\
\hline 03 & 73021 & 16.5954 & 3.4250 & 299.6 & 5 & 600.0 & \\
\hline 03 & 73022 & 256.3919 & 2.4000 & 296.3 & 6 & 600.0 & 600.0 \\
\hline 03 & 73023 & 325.9215 & 2.3500 & 294.9 & 6 & 600.0 & .0 \\
\hline 03 & 73024 & 36.2869 & 1.9750 & 295.0 & 6 & 600.0 & \\
\hline 03 & 7311 & 136.8045 & 1.8750 & 295.7 & 6 & 600.0 & 600.0 \\
\hline 03 & 7312 & 334.3694 & 3.4500 & 296.6 & 5 & 600.0 & .0 \\
\hline 03 & 7313 & 329.6478 & 1.85 & 289.5 & 6 & 600.0 & \\
\hline 03 & 7314 & 33.7705 & 2.4250 & 291.6 & 6 & 600.0 & 60 \\
\hline 03 & 7315 & 354.5633 & 2.3250 & 296.0 & 6 & 600.0 & 600.0 \\
\hline 03 & 7316 & 229.3715 & 2 . & 291.0 & 6 & 600.0 & .0 \\
\hline 03 & 7317 & 14.0041 & 1.8250 & 291.8 & 5 & 600.0 & 60 \\
\hline 03 & 7318 & 330.5408 & 1.3000 & 294.6 & 4 & 600.0 & 600.0 \\
\hline 03 & 7319 & 298.9148 & 1.2000 & 297.4 & 3 & 600.0 & .0 \\
\hline 03 & 73110 & 255.6386 & 1.6250 & 298.0 & 2 & 600.0 & 60 \\
\hline 03 & 73111 & 188.4878 & 3.7500 & 297.5 & 2 & 600.0 & 600.0 \\
\hline 03 & 73112 & 185.2448 & 4.0750 & 297.5 & 3 & 600.0 & .0 \\
\hline 03 & 73113 & 165.7498 & 3.8000 & 299.4 & 2 & 600.0 & 600.0 \\
\hline 03 & 73114 & 165.5032 & 5.3250 & 300.8 & 3 & 600.0 & 600.0 \\
\hline 03 & 73115 & 115.2479 & 4.7250 & 301.1 & 3 & 600.0 & \\
\hline 03 & 73116 & 145.2168 & 4.8000 & 300.0 & 3 & 600.0 & 600.0 \\
\hline 03 & 73117 & 190.7505 & 4.8250 & 298.0 & 3 & 600.0 & 600.0 \\
\hline 03 & 73118 & 154.4995 & 2.1500 & 297.7 & 3 & 600.0 & 600.0 \\
\hline 03 & 73119 & 244.2927 & 2.0250 & 297.3 & 4 & 600.0 & 600.0 \\
\hline 03 & 73120 & 176.1782 & 2.2000 & 295.2 & 5 & 600.0 & 600.0 \\
\hline 03 & 73121 & 48.9913 & 2.0000 & 296.1 & 6 & 600.0 & 600.0 \\
\hline 03 & 73122 & 68.7530 & 3.000 & 298.5 & 5 & 600.0 & 600.0 \\
\hline 03 & 73123 & 134.7877 & 1.3750 & 295.8 & 0 & 600.0 & 600.0 \\
\hline
\end{tabular}




\begin{tabular}{|c|c|c|c|c|c|c|c|c|}
\hline 03 & & 124 & 80.9902 & 4.6250 & 296.8 & 5 & 600.0 & 00.0 \\
\hline 03 & 8 & 11 & 91.5000 & 3.4250 & 297.0 & 2 & 600.0 & 600.0 \\
\hline 03 & 8 & 12 & 78.7502 & 6.1250 & 296.3 & 4 & 600.0 & 00.0 \\
\hline 03 & 8 & 13 & 74.7492 & 7.7250 & 296.4 & 4 & 600.0 & 600.0 \\
\hline 03 & 8 & 14 & 82.9982 & 6.0000 & 297.0 & $I$ & 600.0 & 00.0 \\
\hline 03 & 8 & 15 & 83.7500 & 5.3250 & 297.4 & 4 & 600.0 & 00.0 \\
\hline 03 & 8 & 16 & 86.0000 & 5.7250 & 297.4 & 4 & 600.0 & 600.0 \\
\hline 03 & 8 & 17 & 130.2190 & 2.9500 & 297.6 & 3 & 600.0 & 00.0 \\
\hline 03 & 8 & 18 & 33.1756 & 1.2250 & 296.8 & 2 & 600.0 & 00.0 \\
\hline 03 & 8 & 19 & 177.9339 & 2.7250 & 299.5 & 1 & 600.0 & .0 \\
\hline 03 & 8 & 110 & 152.3470 & 2.8500 & 300.3 & 1 & 600.0 & 00.0 \\
\hline 03 & 8 & 111 & 74.0980 & 1.6000 & 302.5 & 1 & 600.0 & \\
\hline 03 & 8 & 112 & 122.7214 & 4.3250 & 302.3 & 2 & 600.0 & 600.0 \\
\hline 03 & 8 & 113 & 173.7504 & 6.9750 & 302.1 & 3 & 600.0 & 600.0 \\
\hline 03 & 8 & 114 & 171.7476 & 6.2000 & 302.6 & 4 & 600.0 & \\
\hline 03 & 8 & 115 & 135.5097 & 4.7000 & 303.2 & 3 & 600.0 & 60 \\
\hline 03 & 8 & 116 & 90.5000 & 5.0750 & 303.0 & 3 & 600.0 & 600.0 \\
\hline 03 & 8 & 117 & 96.5017 & 5.8500 & 301.9 & 3 & 600.0 & \\
\hline 03 & 8 & 118 & 84.7507 & 5.8750 & 300.2 & 4 & 600.0 & 60 \\
\hline 03 & 8 & 119 & 78.7496 & 7.1250 & 298.3 & 4 & 600.0 & 60 \\
\hline 03 & 8 & 120 & 71.2500 & 9.57 & 297.1 & 4 & 600.0 & .0 \\
\hline 03 & 8 & 121 & 71.2501 & 11.1000 & 296.1 & 4 & 600.0 & \\
\hline 03 & 8 & 122 & 71.9999 & 11.8250 & 295.6 & 4 & 600.0 & 600.0 \\
\hline 03 & 8 & 123 & 73.5002 & 10.4500 & 295.5 & 4 & 600.0 & .0 \\
\hline 03 & 8 & 124 & 76.2500 & 9.5500 & 295.3 & 4 & 600.0 & \\
\hline 03 & 8 & 21 & 71.9995 & 10.4250 & 294.6 & 4 & 600.0 & 60 \\
\hline 03 & 8 & 22 & 80.7499 & 8.0250 & 295.3 & 4 & 600.0 & .0 \\
\hline 03 & 8 & 23 & 80.7499 & 7.9000 & 296.1 & 4 & 600.0 & \\
\hline 03 & 8 & 24 & 72.7502 & 6.7750 & 296.1 & 4 & 600.0 & 60 \\
\hline 03 & 8 & 25 & 73.5003 & 5.2500 & 295.0 & 4 & 600.0 & 60 \\
\hline 03 & 8 & 26 & 77.5031 & 7.42 & 291.9 & 4 & 600.0 & \\
\hline 03 & 8 & 27 & 109.1663 & 2.9250 & 291.1 & 3 & 600.0 & 60 \\
\hline 03 & 8 & 28 & 25.3400 & 1.6500 & 292.6 & 2 & 600.0 & 600.0 \\
\hline 03 & 8 & 29 & 10.8068 & 1.600 & 294.2 & 1 & 600.0 & .0 \\
\hline 03 & 8 & 210 & 196.5478 & 2.0500 & 295.0 & 1 & 600.0 & 60 \\
\hline 03 & 8 & 211 & 242.5262 & 2.0500 & 295.5 & 1 & 600.0 & 600.0 \\
\hline 03 & 8 & 212 & 241.5620 & 2.3250 & 296.5 & 1 & 600.0 & \\
\hline 03 & 8 & 213 & 220.3437 & 2.6250 & 296.5 & 1 & 600.0 & 600.0 \\
\hline 03 & 8 & 214 & 213.0152 & 3.6750 & 296.9 & 2 & 600.0 & 600.0 \\
\hline 03 & 8 & 215 & 224.4920 & 3.3750 & 298.0 & 2 & 600.0 & \\
\hline 03 & 8 & 216 & 215.9726 & 3.0500 & 299.0 & - & 600.0 & 600.0 \\
\hline 03 & 8 & 217 & 200.2810 & 3.6000 & 299.3 & 2 & 600.0 & 600.0 \\
\hline 03 & 8 & 218 & 156.0373 & 5.0250 & 298.7 & 3 & 600.0 & 600.0 \\
\hline 03 & 8 & 219 & 120.0239 & 4.6000 & 297.1 & 4 & 600.0 & 600.0 \\
\hline 03 & 8 & 220 & 79.2500 & 6.2750 & 294.0 & 4 & 600.0 & 600.0 \\
\hline 03 & 8 & 221 & 79.4997 & 7.1750 & 292.6 & 4 & 600.0 & 600.0 \\
\hline 03 & 8 & 222 & 77.7500 & 6.225 & 292.7 & 4 & 600.0 & 600.0 \\
\hline 03 & 8 & 223 & 77.7502 & 6.7250 & 292.7 & 4 & 600.0 & 600.0 \\
\hline
\end{tabular}




\begin{tabular}{|c|c|c|c|c|c|c|c|c|}
\hline 3 & 8 & 224 & 82.5002 & 6.5000 & 292.6 & 4 & 600.0 & 600.0 \\
\hline 3 & 8 & 31 & 87.0008 & 3.1750 & 293.6 & 4 & 600.0 & 00.0 \\
\hline 03 & 8 & 32 & 78.0000 & 6.6500 & 294.2 & 4 & 600.0 & 00.0 \\
\hline 3 & 8 & 33 & 75.0000 & 7.8500 & 293.2 & 4 & 600.0 & 00.0 \\
\hline 03 & 8 & 34 & 75.7500 & 8.8000 & 292.1 & 4 & 600.0 & 00.0 \\
\hline 03 & 8 & 35 & 75.7500 & 8.9250 & 292.5 & 4 & 600.0 & 00.0 \\
\hline 03 & 8 & 36 & 77.5000 & 7.5250 & 292.2 & 4 & 600.0 & 00.0 \\
\hline 03 & 8 & 37 & 79.2500 & 5.8000 & 292.5 & 4 & 600.0 & 00.0 \\
\hline 03 & 8 & 38 & 82.5000 & 4.9750 & 292.9 & 3 & 600.0 & 00.0 \\
\hline 03 & 8 & 39 & 126.2276 & 2.4500 & 295.3 & 2 & 600.0 & .0 \\
\hline 03 & 8 & 310 & 275.9249 & 1.9000 & 297.8 & 1 & 600.0 & \\
\hline 03 & 8 & 311 & 240.2051 & 2.0250 & 299.3 & 1 & 600.0 & \\
\hline 03 & 8 & 312 & 30.3932 & 2.3500 & 300.6 & 1 & 600.0 & 00.0 \\
\hline 03 & 8 & 313 & 67.3886 & 4.1500 & 299.9 & 2 & 600.0 & 00.0 \\
\hline 03 & 8 & 314 & 80.7489 & 4. & 300.1 & 3 & .0 & \\
\hline 03 & 8 & 315 & 95.7554 & 5.4750 & 300.2 & 3 & 600.0 & .0 \\
\hline 03 & 8 & 316 & 91.4997 & 5.4500 & 300.2 & 3 & 60 & 600.0 \\
\hline 03 & 8 & 317 & 89.7485 & 6.17 & 299.4 & 4 & .0 & \\
\hline 03 & 8 & 318 & 82.7500 & 6.8750 & 298.2 & 4 & 60 & .0 \\
\hline 03 & 8 & 319 & 74.4999 & 8.8250 & 295.9 & 4 & .0 & .0 \\
\hline 03 & 8 & 320 & 75 . & 0 & 293.7 & 4 & 6 & \\
\hline 03 & 8 & 321 & 71.7500 & 12.2750 & 292.3 & 4 & .0 & \\
\hline 03 & 8 & 322 & 73.0001 & 13.1250 & 291.2 & 4 & 600.0 & .0 \\
\hline 03 & 8 & 323 & 76.0000 & 13.42 & 290.9 & 4 & 60 & \\
\hline 03 & 8 & 324 & 76.0000 & 12.95 & 290.6 & 4 & .0 & \\
\hline 03 & 8 & 41 & 79.2500 & 11.6500 & 290.7 & 4 & .0 & 600.0 \\
\hline 03 & 8 & 42 & 76.0000 & 12.1250 & 289.8 & 4 & 60 & .0 \\
\hline 03 & 8 & 43 & 77.7500 & 12.3750 & 289.2 & 4 & .0 & \\
\hline 03 & 8 & 44 & 78.7500 & 12.1500 & 289.4 & 4 & .0 & 0.0 \\
\hline 03 & 8 & 45 & 78.7500 & 11.7250 & 289.0 & 4 & 60 & .0 \\
\hline 03 & 8 & 46 & 74.4997 & 12.3250 & 288.2 & 4 & .0 & \\
\hline 03 & 8 & 47 & 74.2501 & 12.0250 & 288.6 & 4 & .0 & 0.0 \\
\hline 03 & 8 & 48 & 71.7500 & 10.0500 & 290.0 & 4 & 600.0 & 0.0 \\
\hline 03 & 8 & 49 & 75.7497 & 8.60 & 291.2 & 4 & .0 & 0.0 \\
\hline 03 & 8 & 410 & 80.5000 & 6.9500 & 292.5 & 4 & 600.0 & 600.0 \\
\hline 03 & 8 & 411 & 88.7505 & 6.2000 & 293.8 & 4 & 600.0 & 600.0 \\
\hline 03 & 8 & 412 & 81.2461 & 7.62 & 295.0 & 4 & 600.0 & 600.0 \\
\hline 03 & 8 & 413 & 77.2504 & 7.7500 & 295.9 & 4 & 600.0 & 600.0 \\
\hline 03 & 8 & 414 & 77.5046 & 8.7750 & 296.5 & 4 & 600.0 & 600.0 \\
\hline 03 & 8 & 415 & 77.2068 & 10.8250 & 296.3 & 4 & 600.0 & 600.0 \\
\hline 03 & 8 & 416 & 73.7499 & 11.1250 & 295.6 & 4 & 600.0 & 600.0 \\
\hline 03 & 8 & 417 & 76.0002 & 10.7250 & 294.9 & 4 & 600.0 & 600.0 \\
\hline 03 & 8 & 418 & 76.4991 & 10.7000 & 293.9 & 4 & 600.0 & 600.0 \\
\hline 03 & 8 & 419 & 75.0000 & 10.7250 & 292.8 & 4 & 600.0 & 600.0 \\
\hline 03 & 8 & 420 & 69.2501 & 13.2250 & 291.3 & 4 & 600.0 & 600.0 \\
\hline 03 & 8 & 421 & 65.2528 & 12.7500 & 290.1 & 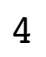 & 600.0 & 600.0 \\
\hline 03 & 8 & 422 & 58.2501 & 13.3500 & 289.2 & 4 & 600.0 & 600.0 \\
\hline - & 8 & 423 & 53.2504 & 11.425 & 289.2 & & 600.0 & 600.0 \\
\hline
\end{tabular}




\begin{tabular}{|c|c|c|c|c|c|c|c|c|}
\hline 3 & 8 & 424 & 59.0107 & 6.9250 & 289.1 & 4 & 600.0 & 600.0 \\
\hline 3 & 8 & 51 & 66.7478 & 8.3750 & 289.2 & 4 & 600.0 & 600.0 \\
\hline 03 & 8 & 52 & 60.2491 & 7.6500 & 289.3 & 4 & 600.0 & 00.0 \\
\hline 03 & 8 & 53 & 62.5000 & 8.3250 & 289.3 & 4 & 600.0 & 00.0 \\
\hline 03 & 8 & 54 & 64.0021 & 6.9000 & 289.2 & 4 & 600.0 & 00.0 \\
\hline 03 & 8 & 55 & 60.2501 & 6.2750 & 289.1 & 4 & 600.0 & 00.0 \\
\hline 03 & 8 & 56 & 69.2479 & 5.7500 & 289.3 & 4 & 600.0 & 600.0 \\
\hline 03 & 8 & 57 & 65.4998 & 5.3000 & 290.3 & 4 & 600.0 & 000.0 \\
\hline 03 & 8 & 58 & 58.5046 & 5.8250 & 291.6 & 3 & 600.0 & .0 \\
\hline 03 & 8 & 59 & 91.4890 & 4.9250 & 292.3 & 3 & 600.0 & 600.0 \\
\hline 03 & 8 & 510 & 136.4575 & 3.8250 & 293.6 & 2 & 600.0 & 0 \\
\hline 03 & 8 & 511 & 103.8124 & 2.7750 & 295.5 & 1 & 600.0 & 600.0 \\
\hline 03 & 8 & 512 & 90.3081 & 3.6500 & 296.3 & 2 & 600.0 & 60 \\
\hline 03 & 8 & 513 & 101.0000 & 4.9500 & 296.7 & 3 & 600.0 & .0 \\
\hline 03 & 8 & 514 & 125.2315 & 5.3000 & 297.0 & 3 & 600.0 & .0 \\
\hline 03 & 8 & 515 & 161.5000 & 7.2500 & 296.8 & 4 & 600.0 & .0 \\
\hline 03 & 8 & 516 & 139.4810 & 5.4750 & 296.9 & 3 & 600.0 & 600.0 \\
\hline 03 & 8 & 517 & 119.0000 & 5.5500 & 296.7 & 3 & 600.0 & .0 \\
\hline 03 & 8 & 518 & 110.7505 & 7.4000 & 295.2 & 4 & 600.0 & \\
\hline 03 & 8 & 519 & 84.7488 & 8.7250 & 293.4 & 4 & 600.0 & 60 \\
\hline 03 & 8 & 520 & 68.4991 & 9.0500 & 291.7 & 4 & 600.0 & .0 \\
\hline 03 & 8 & 521 & 67.2479 & 9.0750 & 290.8 & 4 & 600.0 & \\
\hline 03 & 8 & 522 & 67.0000 & 9.1500 & 290.3 & 4 & 600.0 & 60 \\
\hline 03 & 8 & 523 & 69.7506 & 8.9000 & 289.7 & 4 & 600.0 & .0 \\
\hline 03 & 8 & 524 & 69.4979 & 8.6750 & 289.9 & 4 & 600.0 & \\
\hline 03 & 8 & 61 & 73.7500 & 9.2250 & 290.4 & 4 & 600.0 & 60 \\
\hline 03 & 8 & 62 & 69.5000 & 9.3000 & 290.3 & 4 & 600.0 & .0 \\
\hline 03 & 8 & 63 & 73.7502 & 9.6500 & 289.7 & 4 & 600.0 & .0 \\
\hline 03 & 8 & 64 & 75.7500 & 10.2250 & 288.3 & 4 & 600.0 & 60 \\
\hline 03 & 8 & 65 & 77.5000 & 9.9750 & 288.3 & 4 & 600.0 & 600.0 \\
\hline 03 & 8 & 66 & 68.7497 & 10.1000 & 288.3 & 4 & 600.0 & 60 \\
\hline 03 & 8 & 67 & 71.5001 & 9.9750 & 288.4 & 4 & 600.0 & 60 \\
\hline 03 & 8 & 68 & 78.9997 & 7.8750 & 289.3 & 4 & 600.0 & 60 \\
\hline 03 & 8 & 69 & 76.7535 & 7.7750 & 290.3 & 4 & 600.0 & 600 \\
\hline 03 & 8 & 610 & 72.0000 & 7.9000 & 291.4 & 4 & 600.0 & 60 \\
\hline 03 & 8 & 611 & 80.7502 & 5.7500 & 293.2 & 3 & 600.0 & 600.0 \\
\hline 03 & 8 & 612 & 86.7499 & 6.5750 & 293.6 & 4 & 600.0 & 600.0 \\
\hline 03 & 8 & 613 & 93.2503 & 7.1500 & 294. & 4 & 600.0 & 60 \\
\hline 03 & 8 & 614 & 94.2475 & 6.7750 & 295.0 & 4 & 600.0 & 600.0 \\
\hline 03 & 8 & 615 & 85.0000 & 6.5500 & 295.7 & 4 & 600.0 & 600.0 \\
\hline 03 & 8 & 616 & 75.4990 & 7.3750 & 295.3 & 4 & 600.0 & 600.0 \\
\hline 03 & 8 & 617 & 73.5000 & 8.7750 & 294.3 & 4 & 600.0 & 600.0 \\
\hline 03 & 8 & 618 & 73.9995 & 10.2750 & 292.6 & 4 & 600.0 & 600.0 \\
\hline 03 & 8 & 619 & 72.2491 & 12.2250 & 290 & 4 & 600.0 & 600.0 \\
\hline 03 & 8 & 620 & 64.4991 & 14.9750 & 289.7 & 4 & 600.0 & 600.0 \\
\hline 03 & 8 & 621 & 64.5000 & 14.1500 & 289.1 & 4 & 600.0 & 600.0 \\
\hline 03 & 8 & 622 & 62.7318 & 8 . & & 4 & 600.0 & .0 \\
\hline 03 & 8 & 623 & 64.2423 & 8.9000 & 288.5 & 4 & 600.0 & 600.0 \\
\hline
\end{tabular}




\begin{tabular}{|c|c|c|c|c|c|c|c|c|}
\hline ح & 8 & 624 & 60.5000 & 7.8250 & 288.4 & 4 & 600.0 & 600.0 \\
\hline 3 & 8 & 71 & 57.7476 & 7.6250 & 288.5 & 4 & 600.0 & 00.0 \\
\hline 03 & 8 & 72 & 66.5003 & 8.9750 & 288.4 & 4 & 600.0 & 00.0 \\
\hline 3 & 8 & 73 & 68.4998 & 10.4250 & 288.3 & 4 & 600.0 & 00.0 \\
\hline 03 & 8 & 74 & 69.2463 & 9.8750 & 288.5 & 4 & 600.0 & 00.0 \\
\hline 03 & 8 & 75 & 70.7499 & 9.8000 & 288.7 & 4 & 600.0 & 00.0 \\
\hline 03 & 8 & 76 & 70.7500 & 9.9500 & 288.7 & 4 & 600.0 & 00.0 \\
\hline 03 & 8 & 77 & 72.0001 & 9.9500 & 288.9 & 4 & 600.0 & 00.0 \\
\hline 03 & 8 & 78 & 76.2486 & 9.1500 & 289.5 & 4 & 600.0 & 00.0 \\
\hline 03 & 8 & 79 & 75.7505 & 7.9000 & 290.7 & 4 & 600.0 & .0 \\
\hline 03 & 8 & 710 & 84.5005 & 7.0250 & 292.1 & 4 & 600.0 & \\
\hline 03 & 8 & 711 & 84.4994 & 6.1000 & 293.9 & 4 & 600.0 & \\
\hline 03 & 8 & 712 & 91.0000 & 5.1250 & 295.3 & 3 & 600.0 & 00.0 \\
\hline 03 & 8 & 713 & 100.2302 & 4.7500 & 296.4 & 3 & 600.0 & 00.0 \\
\hline 03 & 8 & 714 & 95.7511 & 250 & 296.8 & 4 & .0 & \\
\hline 03 & 8 & 715 & 109.2759 & 6.5750 & 297.0 & 4 & 600.0 & .0 \\
\hline 03 & 8 & 716 & 105.9871 & 6.7500 & 296.9 & 4 & 60 & 0.0 \\
\hline 03 & 8 & 717 & 104.4686 & 5.9000 & 296.4 & 3 & .0 & \\
\hline 03 & 8 & 718 & 76.1970 & 8.7000 & 295.0 & 4 & 60 & .0 \\
\hline 03 & 8 & 719 & 74.9973 & 9.7750 & 293.0 & 4 & .0 & .0 \\
\hline 03 & 8 & 720 & 63.7500 & 13.7750 & 291.0 & 4 & 6 & \\
\hline 03 & 8 & 721 & 65.2498 & 13.7250 & 289.8 & 4 & .0 & \\
\hline 03 & 8 & 722 & 66.7499 & 13.0000 & 288.6 & 4 & 600.0 & .0 \\
\hline 03 & 8 & 723 & 67.2499 & 12.75 & 288.5 & 4 & 60 & \\
\hline 03 & 8 & 724 & 70.2500 & 12.9500 & 288.4 & 4 & .0 & \\
\hline 03 & 8 & 81 & 73.0000 & 11.0250 & 290.5 & 4 & .0 & 0.0 \\
\hline 03 & 8 & 82 & 69.7500 & 9.1500 & 290.6 & 4 & 600.0 & .0 \\
\hline 03 & 8 & 83 & 67.0001 & 9.2500 & 290.0 & 4 & .0 & \\
\hline 03 & 8 & 84 & 77.5000 & 9.0000 & 289.9 & 4 & .0 & 600.0 \\
\hline 03 & 8 & 85 & 75.5000 & 9.3000 & 289.6 & 4 & 60 & 0.0 \\
\hline 03 & 8 & 86 & 74.7500 & 9.4000 & 289.8 & 4 & .0 & \\
\hline 03 & 8 & 87 & 76.9999 & 7.6500 & 291.0 & 4 & .0 & 0.0 \\
\hline 03 & 8 & 88 & 79.5000 & 7.1500 & 292.4 & 4 & 600.0 & 0.0 \\
\hline 03 & 8 & 89 & 91.4979 & 5.7000 & 293.6 & 4 & .0 & .0 \\
\hline 03 & 8 & 810 & 96.0000 & 5.2500 & 294.9 & 3 & 600.0 & 600.0 \\
\hline 03 & 8 & 811 & 97.0000 & 5.0500 & 296.5 & 3 & 600.0 & 600.0 \\
\hline 03 & 8 & 812 & 95.0008 & 5.2000 & 297.7 & 3 & 600.0 & 0.0 \\
\hline 03 & 8 & 813 & 92.0011 & 5.3500 & 298.7 & 3 & 600.0 & 600.0 \\
\hline 03 & 8 & 814 & 102.7528 & 5.9500 & 299.2 & 3 & 600.0 & 600.0 \\
\hline 03 & 8 & 815 & 104.7494 & 6.1500 & 299.1 & 4 & 600.0 & 600.0 \\
\hline 03 & 8 & 816 & 86.0000 & 6.0500 & 299.1 & 4 & 600.0 & 600.0 \\
\hline 03 & 8 & 817 & 73.2472 & 6.4500 & 298.8 & 4 & 600.0 & 600.0 \\
\hline 03 & 8 & 818 & 64.5000 & 7.8000 & 297.6 & 4 & 600.0 & 600.0 \\
\hline 03 & 8 & 819 & 67.7499 & 9.8750 & 295.9 & 4 & 600.0 & 600.0 \\
\hline 03 & 8 & 820 & 69.0000 & 10.0500 & 294.2 & 4 & 600.0 & 600.0 \\
\hline 03 & 8 & 821 & 72.5002 & 10.9500 & 293.6 & 4 & 600.0 & 600.0 \\
\hline 03 & 8 & 822 & 67.2500 & 11.0000 & 294.2 & 4 & 600.0 & 600.0 \\
\hline & 8 & 823 & 71.2503 & 11.025 & 293.9 & 4 & 600.0 & 600.0 \\
\hline
\end{tabular}




\begin{tabular}{|c|c|c|c|c|c|c|c|}
\hline 3 & $8 \quad 824$ & 74.7500 & 10.0500 & 293.6 & 4 & 600.0 & 600.0 \\
\hline 3 & $\begin{array}{lll}8 & 9 & 1\end{array}$ & 72.2504 & 10.3500 & 293.2 & 4 & 600.0 & 00.0 \\
\hline 03 & $\begin{array}{lll}8 & 9 & 2\end{array}$ & 72.2500 & 500 & 293.3 & 4 & 600.0 & 00.0 \\
\hline 3 & $\begin{array}{lll}8 & 9 & 3\end{array}$ & 75.7499 & 9.6750 & 293.1 & 4 & 600.0 & 00.0 \\
\hline 03 & $\begin{array}{lll}8 & 9 & 4\end{array}$ & 76.7499 & 250 & 293.1 & 4 & 600.0 & 00.0 \\
\hline 03 & 895 & 76.7498 & 9.1000 & 293.4 & 4 & 600.0 & 00.0 \\
\hline 03 & 896 & 75.5002 & 8.7750 & 293.5 & 4 & 600.0 & 00.0 \\
\hline 03 & $\begin{array}{lll}8 & 9 & 7\end{array}$ & 79.7496 & 8.3500 & 293.7 & 4 & 600.0 & 00.0 \\
\hline 03 & $\begin{array}{lll}8 & 9 & 8\end{array}$ & 80.0000 & 7.4750 & 294.8 & 4 & 600.0 & 00.0 \\
\hline 03 & $\begin{array}{lll}8 & 9 & 9\end{array}$ & 87.0024 & 6.2000 & 296.1 & 4 & 600.0 & 0 \\
\hline 03 & 8910 & 97.2526 & 4.7500 & 297.7 & 3 & 600.0 & \\
\hline 03 & 8911 & 103.2471 & 3.4250 & 298.9 & 2 & 600.0 & \\
\hline 03 & 8912 & 92.7511 & 4.3500 & 299.5 & 3 & 600.0 & 00.0 \\
\hline 03 & 8913 & 93.7453 & 4 & 299.7 & 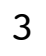 & 600.0 & 00.0 \\
\hline 03 & 8914 & 88.2497 & 50 & 300.4 & 3 & .0 & \\
\hline 03 & 8915 & 97.5000 & 6.3250 & 300.3 & 4 & 600.0 & .0 \\
\hline 03 & 8916 & 92.2489 & 6.3250 & 300.1 & 4 & 60 & .0 \\
\hline 03 & 8917 & 75.7519 & 7. & 299.3 & 4 & .0 & \\
\hline 03 & 8918 & 68.4997 & 8.8000 & 297.8 & 4 & 60 & .0 \\
\hline 03 & 8919 & 74.0002 & 10.9000 & 295.9 & 4 & .0 & .0 \\
\hline 03 & 8920 & 76 . & 10 . & 294.6 & 4 & 6 & \\
\hline 03 & 8921 & 76.7497 & 10.7750 & 293.4 & 4 & .0 & \\
\hline 03 & 8922 & 70.5005 & 10.0000 & 293.0 & 4 & 600.0 & .0 \\
\hline 03 & 8923 & 89.5024 & 9 . & 292.3 & 4 & 6 & \\
\hline 03 & 8924 & 000 & 10. & 292.4 & 4 & .0 & \\
\hline 03 & 8101 & 80.2500 & 11.6750 & 292.5 & 4 & .0 & \\
\hline 03 & 8102 & 73.9996 & 11.5750 & 291.8 & 4 & 60 & .0 \\
\hline 03 & 8103 & 73.7500 & 10.8000 & 292.5 & 4 & .0 & \\
\hline 03 & 8104 & 71.9998 & 10.5750 & 292.8 & 4 & .0 & .0 \\
\hline 03 & 8105 & 71.4986 & 9.5000 & 293.0 & 4 & 6 & .0 \\
\hline 03 & 8106 & 76.0000 & 9.5500 & 293.2 & 4 & .0 & \\
\hline 03 & 8107 & 78.2500 & 9.6250 & 293.6 & 4 & .0 & 0.0 \\
\hline 03 & 8108 & 78.0000 & 9.1750 & 294.4 & 4 & 600.0 & 0.0 \\
\hline 03 & 8109 & 79.2499 & 6. & 296.2 & 4 & .0 & .0 \\
\hline 03 & 81010 & 81.2504 & 5.5000 & 298.2 & 3 & 600.0 & 600.0 \\
\hline 03 & 81011 & 94.2589 & 4.6500 & 299.5 & 3 & 600.0 & 600.0 \\
\hline 03 & 81012 & 86.7416 & 4.77 & 300.3 & 3 & 600.0 & 600.0 \\
\hline 03 & 81013 & 91.2507 & 5.5750 & 300.5 & 3 & 600.0 & 600.0 \\
\hline 03 & 81014 & 85.4969 & 6.2500 & 301.0 & 4 & 600.0 & 600.0 \\
\hline 03 & 81015 & 73.0012 & 7.225 & 300.7 & 4 & 600.0 & 600.0 \\
\hline 03 & 81016 & 75.2517 & 8.2000 & 300.1 & 4 & 600.0 & 600.0 \\
\hline 03 & 81017 & 76.7514 & 9.4750 & 298.8 & 4 & 600.0 & 600.0 \\
\hline 03 & 81018 & 79.5000 & 11.4250 & 296.9 & 4 & 600.0 & 600.0 \\
\hline 03 & 81019 & 80.4994 & 10.5750 & 295.5 & 4 & 600.0 & 600.0 \\
\hline 03 & 81020 & 67.7511 & 11.0750 & 295.5 & 4 & 600.0 & 600.0 \\
\hline 03 & 81021 & 64.5000 & 12.8000 & & 4 & 600.0 & 600.0 \\
\hline 03 & 81022 & 68.2503 & 9.6750 & 296.1 & 4 & 600.0 & 600.0 \\
\hline & 81023 & 71.5000 & 10.425 & 295.8 & 4 & 600.0 & 600.0 \\
\hline
\end{tabular}




\begin{tabular}{|c|c|c|c|c|c|c|c|}
\hline 03 & 81024 & 69.0000 & 10.1250 & 295.5 & 4 & 600.0 & 600.0 \\
\hline 03 & 8111 & 69.5002 & 9.8000 & 295.1 & 4 & 600.0 & 600.0 \\
\hline 03 & 8112 & 71.5000 & 10.2250 & 294.7 & 4 & 600.0 & 00.0 \\
\hline 3 & 8113 & 72.0002 & 10.0000 & 294.5 & 4 & 600.0 & 00.0 \\
\hline 03 & 8114 & 60.9982 & 10. & 294.1 & 4 & 600.0 & 00.0 \\
\hline 03 & 8115 & 59.0000 & 10.9500 & 293.4 & 4 & 600.0 & 00.0 \\
\hline 03 & 8116 & 67.2500 & 11.0250 & 292.6 & 4 & 600.0 & 00.0 \\
\hline 03 & 8117 & 67.7502 & 10.7500 & 292.8 & 4 & 600.0 & 00.0 \\
\hline 03 & 8118 & 68.7500 & 9.5750 & 294.2 & 4 & 600.0 & 00.0 \\
\hline 03 & 8119 & 71.9995 & 000 & 295.4 & 4 & 600.0 & 0 \\
\hline 03 & 81110 & 76.7498 & 000 & 296.8 & 4 & 600.0 & \\
\hline 03 & 81111 & 83.2492 & 250 & 298.1 & 3 & 600.0 & \\
\hline 03 & 81112 & 90.0004 & 6.1500 & 299.0 & 4 & 600.0 & 00.0 \\
\hline 03 & 81113 & 91.5006 & 750 & 299.6 & 4 & 600.0 & \\
\hline 03 & 81114 & 89.2499 & 6 . & 300.3 & 4 & .0 & \\
\hline 03 & 81115 & 85.5011 & 6.2250 & 300.4 & 4 & 600.0 & .0 \\
\hline 03 & 81116 & 79.2797 & 6.9500 & 300.3 & 4 & 60 & .0 \\
\hline 03 & 81117 & 82.2541 & 7 . & 299.2 & 4 & .0 & \\
\hline 03 & 81118 & 78.2797 & 6.4000 & 297.8 & 4 & 60 & \\
\hline 03 & 81119 & 65.0000 & 12.6750 & 296.8 & 4 & .0 & .0 \\
\hline 03 & 81120 & 63.7497 & 12 . & 296.3 & 4 & 6 & \\
\hline 03 & 81121 & 70.2501 & 500 & 296.0 & 4 & .0 & \\
\hline 03 & 81122 & 70.0001 & 9.2250 & 296.0 & 4 & 600.0 & .0 \\
\hline 03 & 81123 & 68.0016 & 8 . & 295.5 & 4 & 6 & \\
\hline 03 & 81124 & 65.9999 & 9 . & 295.4 & 4 & .0 & \\
\hline 03 & 8121 & 72.9989 & 500 & 295.2 & 4 & .0 & \\
\hline 03 & 8122 & 77.2495 & 9.5000 & 294.6 & 4 & .0 & .0 \\
\hline 03 & 8123 & 73.2500 & 9.6500 & 294.2 & 4 & .0 & \\
\hline 03 & 8124 & 75.2500 & 9.4250 & 293.9 & 4 & .0 & 0.0 \\
\hline 03 & 8125 & 76.4998 & 9.3000 & 293.6 & 4 & .0 & .0 \\
\hline 03 & 8126 & 77.5000 & 8 . & 293.6 & 4 & .0 & \\
\hline 03 & 8127 & 82.0001 & 7.6750 & 293.9 & 4 & .0 & 600.0 \\
\hline 03 & 8128 & 84.0004 & 5.9000 & 295.2 & 4 & 600.0 & 0.0 \\
\hline 03 & 8129 & 138.9499 & 4.5000 & 297.3 & 3 & .0 & 0.0 \\
\hline 03 & 81210 & 174.7502 & 6.7750 & 297.4 & 4 & 600.0 & 600.0 \\
\hline 03 & 81211 & 176.5008 & 6.7500 & 298.2 & 4 & 600.0 & 600.0 \\
\hline 03 & 81212 & 176.9963 & 7.0000 & 299.5 & 4 & 600.0 & .0 \\
\hline 03 & 81213 & 99.6972 & 4.7000 & 302.0 & 3 & 600.0 & 600.0 \\
\hline 03 & 81214 & 103.2786 & 4.9500 & 302.5 & 3 & 600.0 & 600.0 \\
\hline 03 & 81215 & 111.4924 & 5.7000 & 302.5 & 3 & 600.0 & 600.0 \\
\hline 03 & 81216 & 91.7506 & 7.0750 & 301.9 & 4 & 600.0 & 600.0 \\
\hline 03 & 81217 & 81.9959 & 7.8000 & 300.6 & 4 & 600.0 & 600.0 \\
\hline 03 & 81218 & 77.0000 & 8.6750 & 298.9 & 4 & 600.0 & 600.0 \\
\hline 03 & 81219 & 75.0000 & 10.1250 & 297.5 & 4 & 600.0 & 600.0 \\
\hline 03 & 81220 & 69.5003 & 12.1750 & 295.7 & 4 & 600.0 & 600.0 \\
\hline 03 & 81221 & 67.5000 & 11.4750 & 295.4 & . & 600.0 & 600.0 \\
\hline 03 & 81222 & 65.2502 & 11.7750 & 295.4 & 4 & 600.0 & 600.0 \\
\hline & 81223 & 66.0000 & 11.1000 & 295.4 & 4 & 600.0 & 600.0 \\
\hline
\end{tabular}




\begin{tabular}{|c|c|c|c|c|c|c|c|}
\hline 03 & 81224 & 55.7508 & 7.8750 & 295.7 & 4 & 600.0 & 600.0 \\
\hline 03 & 8131 & 66.5000 & 7.9000 & 296.6 & 4 & 600.0 & 600.0 \\
\hline 03 & 8132 & 69.7500 & 8.1750 & 296.5 & 4 & 600.0 & 00.0 \\
\hline 3 & 8133 & 73.5000 & 8.4000 & 296.5 & 4 & 600.0 & 00.0 \\
\hline 03 & 8134 & 68.0000 & 7.6000 & 296.3 & 4 & 600.0 & 00.0 \\
\hline 03 & 8135 & 70.2500 & 7.5250 & 296.5 & 4 & 600.0 & 00.0 \\
\hline 03 & 8136 & 70.7499 & 7.7250 & 296.4 & 4 & 600.0 & 00.0 \\
\hline 03 & 8137 & 73.0000 & 8.8500 & 295.2 & 4 & 600.0 & 00.0 \\
\hline 03 & 8138 & 75.5000 & 8.5500 & 295.9 & 4 & 600.0 & 00.0 \\
\hline 03 & 8139 & 99.8332 & 5.8750 & 297.4 & 3 & 600.0 & 0 \\
\hline 03 & 81310 & 237.7499 & 3.1250 & 297.6 & 3 & 600.0 & \\
\hline 03 & 81311 & 242.1818 & 2.8250 & 298.8 & 2 & 600.0 & \\
\hline 03 & 81312 & 141.6029 & 3.3250 & 301.3 & 2 & 600.0 & 00.0 \\
\hline 03 & 81313 & 175.2496 & 7.4500 & 300.8 & 3 & 600.0 & 00.0 \\
\hline 03 & 81314 & 173.9977 & 7.2750 & 301.5 & 4 & .0 & \\
\hline 03 & 81315 & 167.7648 & 6.3750 & 302.3 & 4 & 600.0 & 00.0 \\
\hline 03 & 81316 & 144.0555 & 5.6750 & 303.0 & 3 & 600.0 & 600.0 \\
\hline 03 & 81317 & 116.4880 & 5.20 & 302.9 & 3 & .0 & \\
\hline 03 & 81318 & 97.7626 & 6.1000 & 301.4 & 4 & 600.0 & .0 \\
\hline 03 & 81319 & 69.9995 & 6.4750 & 299.4 & 4 & .0 & .0 \\
\hline 03 & 81320 & 73.5000 & 7.85 & 297.8 & 4 & 60 & \\
\hline 03 & 81321 & 71.9998 & 8.6750 & 297.0 & 4 & .0 & \\
\hline 03 & 81322 & 75.0000 & 8.2500 & 296.9 & 4 & 600.0 & .0 \\
\hline 03 & 81323 & 70.5010 & 7.92 & 297.0 & 4 & 60 & \\
\hline 03 & 81324 & 006 & 7.3250 & 296.9 & 4 & .0 & \\
\hline 03 & 8141 & 76.2500 & 7.4000 & 297.0 & 4 & 600.0 & 600.0 \\
\hline 03 & 8142 & 79.4998 & 7.8500 & 296.2 & 4 & 600.0 & .0 \\
\hline 03 & 8143 & 75.5000 & 6.1500 & 296.6 & 4 & .0 & \\
\hline 03 & 8144 & 72.7501 & 4.9250 & 297.1 & 4 & .0 & 600.0 \\
\hline 03 & 8145 & 71.8110 & 3.0750 & 296.0 & 4 & 60 & 600.0 \\
\hline 03 & 8146 & 30.3631 & 1.1750 & 295.3 & 5 & .0 & \\
\hline 03 & 8147 & 68.0891 & 0.9500 & 295.1 & 4 & .0 & 600.0 \\
\hline 03 & 8148 & 223.8452 & 1.3500 & 295.1 & 3 & 600.0 & 600.0 \\
\hline 03 & 8149 & 306.9709 & 1.5500 & 296.4 & 2 & 0 & 0.0 \\
\hline 03 & 81410 & 79.0246 & 3.0500 & 296.0 & 2 & 600.0 & 600.0 \\
\hline 03 & 81411 & 89.7301 & 3.3500 & 297.3 & 2 & 600.0 & 600.0 \\
\hline 03 & 81412 & 70.5764 & 4.2750 & 298.1 & 3 & 600.0 & 600.0 \\
\hline 03 & 81413 & 81.4985 & 5.9250 & 298.2 & 4 & 600.0 & 600.0 \\
\hline 03 & 81414 & 88.7485 & 5.2250 & 299.5 & 3 & 600.0 & 600.0 \\
\hline 03 & 81415 & 84.4995 & 6.7500 & 298.9 & 4 & 600.0 & 600.0 \\
\hline 03 & 81416 & 87.2506 & 7.7250 & 298.6 & 4 & 600.0 & 600.0 \\
\hline 03 & 81417 & 81.9973 & 7.7750 & 298.3 & 4 & 600.0 & 600.0 \\
\hline 03 & 81418 & 75.7496 & 9.2750 & 296.7 & 4 & 600.0 & 600.0 \\
\hline 03 & 81419 & 77.2500 & 10.1000 & 295.7 & 4 & 600.0 & 600.0 \\
\hline 03 & 81420 & 78.0001 & 11.3750 & 294.6 & 4 & 600.0 & 600.0 \\
\hline 03 & 81421 & 78.4992 & 11.6000 & 293.8 & 4 & 600.0 & 600.0 \\
\hline 03 & 81422 & 79.2506 & 9.6500 & 295.6 & 4 & 600.0 & 600.0 \\
\hline & $014<3$ & 66.5001 & 11.075 & 294.0 & 4 & 600.0 & 600.0 \\
\hline
\end{tabular}




\begin{tabular}{|c|c|c|c|c|c|c|c|}
\hline 3 & 81424 & 61.9999 & 12.0250 & 294.0 & 4 & 600.0 & 00.0 \\
\hline 03 & 8151 & 70.9973 & 12.0500 & 293.5 & 4 & 600.0 & 600.0 \\
\hline 03 & 8152 & 75.7501 & 10.6750 & 294.2 & 4 & 600.0 & 00.0 \\
\hline 03 & 8153 & 70.7501 & 10.8500 & 294.7 & 4 & 600.0 & 00.0 \\
\hline 03 & 8154 & 67.7501 & 10.1000 & 294.0 & 4 & 600.0 & 00.0 \\
\hline 03 & 8155 & 62.2501 & 9.6500 & 294.3 & 4 & 600.0 & 00.0 \\
\hline 03 & 8156 & 63.5000 & 9.4500 & 294.3 & 4 & 600.0 & 600.0 \\
\hline 03 & 8157 & 68.4994 & 9.1000 & 294.7 & 4 & 600.0 & 00.0 \\
\hline 03 & 8158 & 77.7497 & 7.2750 & 296.9 & 4 & 600.0 & 00.0 \\
\hline 03 & 8159 & 115.4414 & 4.0000 & 298.8 & 3 & 600.0 & 600.0 \\
\hline 03 & 81510 & 98.7500 & 4.1250 & 299.8 & 3 & 600.0 & 00.0 \\
\hline 03 & 81511 & 145.9492 & 3.6250 & 301.7 & 2 & 600.0 & \\
\hline 03 & 81512 & 108.5167 & 4.3000 & 302.1 & 3 & 600.0 & 600.0 \\
\hline 03 & 81513 & 89.2432 & 3.5000 & 302.9 & 2 & 600.0 & .0 \\
\hline 03 & 81514 & 91.4983 & 500 & 303.8 & 2 & 600.0 & \\
\hline 03 & 81515 & 112.4966 & 4.6750 & 303.7 & 3 & 600.0 & 60 \\
\hline 03 & 81516 & 108.5055 & 5.4750 & 303.3 & 3 & 600.0 & 600.0 \\
\hline 03 & 81517 & 81.0011 & 5.8250 & 303.0 & 3 & 600.0 & \\
\hline 03 & 81518 & 94.2571 & 6.9750 & 301.4 & 4 & 600.0 & .0 \\
\hline 03 & 81519 & 75.7504 & 8.2250 & 300.3 & 4 & 600.0 & 600 \\
\hline 03 & 81520 & 72.0000 & 10.1000 & 298.7 & 4 & 600.0 & .0 \\
\hline 03 & 81521 & 70.7474 & 9.7000 & 298.3 & 4 & 600.0 & \\
\hline 03 & 81522 & 71.0000 & 9.9250 & 298.4 & 4 & 600.0 & 600.0 \\
\hline 03 & 81523 & 72.2500 & 10.1750 & 298.3 & 4 & 600.0 & .0 \\
\hline 03 & 81524 & 68.5000 & 9.1250 & 298.8 & 4 & 600.0 & \\
\hline 03 & 8161 & 68.7496 & 7.7500 & 299.6 & 4 & 600.0 & 60 \\
\hline 03 & 8162 & 75.0021 & 6.6750 & 300.3 & 4 & 600.0 & .0 \\
\hline 03 & 8163 & 84.0000 & 5.3750 & 300.0 & 4 & 600.0 & \\
\hline 03 & 8164 & 75.2502 & 5.4250 & 299.8 & 4 & 600.0 & 60 \\
\hline 03 & 8165 & 75.4997 & 5.1000 & 299.0 & 4 & 600.0 & 60 \\
\hline 03 & 8166 & 77.0000 & 5.10 & 298.9 & 4 & 600.0 & \\
\hline 03 & 8167 & 116.4934 & 2.7000 & 298.9 & 3 & 600.0 & 60 \\
\hline 03 & 8168 & 150.0580 & 1.3750 & 300.2 & 2 & 600.0 & 600.0 \\
\hline 03 & 8169 & 200.5671 & 2.97 & 300.1 & 1 & 600.0 & .0 \\
\hline 03 & 81610 & 175.2498 & 4.3500 & 299.4 & 2 & 600.0 & 60 \\
\hline 03 & 81611 & 178.4991 & 4.9000 & 299.7 & 3 & 600.0 & 600.0 \\
\hline 03 & 81612 & 173.5018 & 4.6250 & 300.8 & 3 & 600.0 & \\
\hline 03 & 81613 & 174.5023 & 5.5250 & 301.6 & 3 & 600.0 & 600.0 \\
\hline 03 & 81614 & 184.0018 & 4.9500 & 302.9 & 3 & 600.0 & 600.0 \\
\hline 03 & 81615 & 177.0449 & 5.2250 & 303.7 & 3 & 600.0 & \\
\hline 03 & 81616 & 183.7502 & 5.3250 & 304.3 & 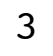 & 600.0 & 600.0 \\
\hline 03 & 81617 & 172.7511 & 5.3750 & 304.4 & 3 & 600.0 & 600.0 \\
\hline 03 & 81618 & 128.8004 & 5.3250 & 304.3 & 3 & 600.0 & 600.0 \\
\hline 03 & 81619 & 83.7478 & 6.4000 & 302.8 & 4 & 600.0 & 600.0 \\
\hline 03 & 81620 & 77.2502 & 8.1500 & 300.9 & 4 & 600.0 & 600.0 \\
\hline 03 & 81621 & 74.0000 & 7.9500 & 299.7 & 4 & 600.0 & 600.0 \\
\hline 03 & 81622 & 75.2503 & 9.775 & 299.1 & 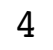 & 600.0 & 600.0 \\
\hline 03 & 81623 & 71.2501 & 9.8000 & 298.8 & 4 & 600.0 & 600.0 \\
\hline
\end{tabular}




\begin{tabular}{|c|c|c|c|c|c|c|c|}
\hline 3 & 81624 & 75.0000 & 9.5750 & 298.7 & 4 & 600.0 & 600.0 \\
\hline 03 & 8171 & 76.7500 & 9.4000 & 298.9 & 4 & 600.0 & 600.0 \\
\hline 03 & 8172 & 77.2500 & 8.8250 & 299.3 & 4 & 600.0 & 00.0 \\
\hline 03 & 8173 & 75.7500 & 7.6000 & 300.0 & 4 & 600.0 & 600.0 \\
\hline 03 & 8174 & 79.7509 & 5.4000 & 300.4 & 4 & 600.0 & 00.0 \\
\hline 03 & 8175 & 88.9989 & 2.7000 & 300.4 & 4 & 600.0 & 00.0 \\
\hline 03 & 8176 & 78.2483 & 2.1750 & 300.5 & 5 & 600.0 & 600.0 \\
\hline 03 & 8177 & 139.9828 & 1.3000 & 300.6 & 4 & 600.0 & 00.0 \\
\hline 03 & 8178 & 211.3728 & 0.8000 & 300.0 & 3 & 600.0 & 00.0 \\
\hline 03 & 8179 & 236.9973 & 1.9750 & 300.6 & 2 & 600.0 & .0 \\
\hline 03 & 81710 & 205.9166 & 3.3250 & 300.7 & 2 & 600.0 & 00.0 \\
\hline 03 & 81711 & 171.5000 & 4.6250 & 300.9 & 3 & 600.0 & \\
\hline 03 & 81712 & 161.7530 & 4.1000 & 302.0 & 3 & 600.0 & 00.0 \\
\hline 03 & 81713 & 165.2500 & 4.0750 & 303.4 & 3 & 600.0 & 0 \\
\hline 03 & 81714 & 176.7683 & & 304.4 & 3 & 600.0 & \\
\hline 03 & 81715 & 176.2528 & 4.7000 & 305.1 & 3 & 600.0 & 60 \\
\hline 03 & 81716 & 170.9993 & 5.4750 & 305.4 & 3 & 600.0 & 600.0 \\
\hline 03 & 81717 & 132.3552 & 5.00 & 305.8 & 3 & 600.0 & .0 \\
\hline 03 & 81718 & 94.2483 & 5.2500 & 305.0 & 3 & 600.0 & .0 \\
\hline 03 & 81719 & 79.4985 & 6.5750 & 302.8 & 4 & 600.0 & 600 \\
\hline 03 & 81720 & 73.9924 & 8.70 & 301.2 & 4 & 600.0 & .0 \\
\hline 03 & 81721 & 69.2499 & 9.5250 & 300.2 & 4 & 600.0 & \\
\hline 03 & 81722 & 70.2502 & 8.9750 & 299.4 & 4 & 600.0 & 600.0 \\
\hline 03 & 81723 & 69.2499 & 9.1500 & 298.6 & 4 & 600.0 & .0 \\
\hline 03 & 81724 & 68.5000 & 9.6 & 299.1 & 4 & 600.0 & \\
\hline 03 & 8181 & 68.9994 & 9.2500 & 299.7 & 4 & 600.0 & 60 \\
\hline 03 & 8182 & 75.9996 & 9.1500 & 300.0 & 4 & 600.0 & .0 \\
\hline 03 & 8183 & 80.5000 & 7.92 & 300.0 & 4 & 600.0 & \\
\hline 03 & 8184 & 76.4995 & 7.3500 & 299.7 & 4 & 600.0 & 60 \\
\hline 03 & 8185 & 73.4998 & 7.2250 & 299.9 & 4 & 600.0 & 60 \\
\hline 03 & 8186 & 81.7499 & 6.82 & 299.8 & 4 & 600.0 & \\
\hline 03 & 8187 & 84.7494 & 6.6000 & 300.1 & 4 & 600.0 & 60 \\
\hline 03 & 8188 & 133.0583 & 2.7500 & 301.7 & 3 & 600.0 & 600.0 \\
\hline 03 & 8189 & 279.5205 & 1.27 & 302.4 & 2 & 600.0 & .0 \\
\hline 03 & 81810 & 238.7499 & 2.70 & 300.1 & 3 & 600.0 & .0 \\
\hline 03 & 81811 & 254.1920 & 2.2750 & 301.9 & 2 & 600.0 & 600.0 \\
\hline 03 & 81812 & 311.5064 & 1.8000 & 305.4 & 1 & 600.0 & \\
\hline 03 & 81813 & 90.4982 & 4.1000 & 305.1 & 2 & 600.0 & 600.0 \\
\hline 03 & 81814 & 93.7342 & 5.2000 & 305.1 & 3 & 600.0 & 600.0 \\
\hline 03 & 81815 & 101.0016 & 5.5750 & 305.2 & 3 & 600.0 & \\
\hline 03 & 81816 & 92.3118 & 6.1000 & 304.9 & 4 & 600.0 & 600.0 \\
\hline 03 & 81817 & 73.5000 & 8.8500 & 303.6 & 4 & 600.0 & 600.0 \\
\hline 03 & 81818 & 73.7499 & 10.8500 & 302.2 & 4 & 600.0 & 600.0 \\
\hline 03 & 81819 & 76.5000 & 10.9750 & 299.6 & 4 & 600.0 & 600.0 \\
\hline 03 & 81820 & 73.2503 & 11.8000 & 298.1 & 4 & 600.0 & 600.0 \\
\hline 03 & 81821 & 68.0000 & 11.5250 & 297.8 & 4 & 600.0 & 600.0 \\
\hline 03 & 81822 & 69.2498 & 10.9500 & & 4 & .0 & 600.0 \\
\hline 03 & 81823 & 73.7500 & 9.9000 & 300.0 & $\mathbf{4}$ & 600.0 & 600.0 \\
\hline
\end{tabular}




\begin{tabular}{|c|c|c|c|c|c|c|c|}
\hline 03 & 81824 & 61.7505 & 8.1250 & 299.6 & 4 & 600.0 & 600.0 \\
\hline 03 & 8191 & 66.5086 & 6.6750 & 299.5 & 4 & 600.0 & 600.0 \\
\hline 03 & 8192 & 74.5000 & 8.3750 & 298.4 & 4 & 600.0 & 00.0 \\
\hline 03 & 8193 & 72.7344 & 2.7750 & 299.1 & 5 & 600.0 & 600.0 \\
\hline 03 & 8194 & 72.7502 & 5.4250 & 299.0 & 4 & 600.0 & 00.0 \\
\hline 03 & 8195 & 77.2500 & 6.9750 & 298.0 & 4 & 600.0 & 00.0 \\
\hline 03 & 8196 & 71.7496 & 4.7500 & 295.8 & 4 & 600.0 & 600.0 \\
\hline 03 & 8197 & 89.0000 & 1.3750 & 297.4 & 3 & 600.0 & 500.0 \\
\hline 03 & 8198 & 260.2781 & 1.3250 & 297.6 & 2 & 600.0 & 00.0 \\
\hline 03 & 8199 & 249.1985 & 1.6000 & 296.7 & 1 & 600.0 & 600.0 \\
\hline 03 & 81910 & 274.9617 & 1.7250 & 297.6 & 1 & 600.0 & 600.0 \\
\hline 03 & 81911 & 242.2935 & 2.5000 & 299.3 & 1 & 600.0 & \\
\hline 03 & 81912 & 253.7795 & 2.4250 & 300.8 & 1 & 600.0 & 600.0 \\
\hline 03 & 81913 & 242.0002 & 3.3000 & 301.8 & 2 & 600.0 & 600.0 \\
\hline 03 & 81914 & 268.3513 & 3.3750 & 303.1 & 2 & 600.0 & \\
\hline 03 & 81915 & 26.6851 & 3.4000 & 303.6 & 2 & 600.0 & 60 \\
\hline 03 & 81916 & 34.2700 & 4.2000 & 303.4 & 3 & 600.0 & 600.0 \\
\hline 03 & 81917 & 78.0069 & 6.3250 & 300.8 & 4 & 600.0 & \\
\hline 03 & 81918 & 78.7499 & 7.4250 & 297.8 & 4 & 600.0 & 60 \\
\hline 03 & 81919 & 76.0000 & 5.8500 & 296.6 & 4 & 600.0 & 600.0 \\
\hline 03 & 81920 & 75.3419 & 2.7250 & 296.3 & 5 & 600.0 & .0 \\
\hline 03 & 81921 & 69.1213 & 1.3750 & 297.0 & 6 & 600.0 & \\
\hline 03 & 81922 & 98.7532 & 0.4750 & 297.2 & 6 & 600.0 & 600.0 \\
\hline 03 & 81923 & 77.4406 & 0.7000 & 296.8 & 6 & 600.0 & .0 \\
\hline 03 & 81924 & 147.2975 & 0.9750 & 296.0 & 5 & 600.0 & \\
\hline 03 & 8201 & 42.9144 & 0.8750 & 296.3 & 6 & 600.0 & 600.0 \\
\hline 03 & 8202 & 89.6553 & 0.6250 & 296.9 & 6 & 600.0 & 60 \\
\hline 03 & 8203 & 234.6500 & 0.7250 & 295.3 & 6 & 600.0 & \\
\hline 03 & 8204 & 8.0571 & 0.7250 & 295.5 & 6 & 600.0 & 600.0 \\
\hline 03 & 8205 & 299.3762 & 0.7750 & 293.9 & 6 & 600.0 & 600.0 \\
\hline 03 & 8206 & 25.0000 & 1.9500 & 296.5 & 5 & 600.0 & .0 \\
\hline 03 & 8207 & 321.6399 & 0.8750 & 298.1 & 4 & 600.0 & 60 \\
\hline 03 & 8208 & 239.6176 & 0.7250 & 299.2 & 3 & 600.0 & 600.0 \\
\hline 03 & 8209 & 264.3109 & 1.3250 & 299.3 & 2 & 600.0 & .0 \\
\hline 03 & 82010 & 243.5644 & 2.2000 & 300.0 & 1 & 600.0 & 60 \\
\hline 03 & 82011 & 230.0042 & 3.2250 & 300.1 & 2 & 600.0 & 600.0 \\
\hline 03 & 82012 & 185.2400 & 2.7500 & 301.2 & 1 & 600.0 & 600.0 \\
\hline 03 & 82013 & 195.5000 & 5.0500 & 302.0 & 2 & 600.0 & 600.0 \\
\hline 03 & 82014 & 179.9661 & 4.7750 & 302.3 & 3 & 600.0 & 600.0 \\
\hline 03 & 82015 & 169.5031 & 5.8000 & 303.1 & 3 & 600.0 & \\
\hline 03 & 82016 & 156.9943 & 5.8750 & 303.7 & 3 & 600.0 & 600.0 \\
\hline 03 & 82017 & 128.8081 & 4.7000 & 304.0 & 3 & 600.0 & 600.0 \\
\hline 03 & 82018 & 87.5001 & 5.1500 & 302.7 & 4 & 600.0 & 600.0 \\
\hline 03 & 82019 & 76.5001 & 5.5500 & 300.8 & 4 & 600.0 & 600.0 \\
\hline 03 & 82020 & 75.9999 & 6.5750 & 299.3 & 4 & 600.0 & 600.0 \\
\hline 03 & 82021 & 75.7502 & 7.7250 & 298.8 & 4 & 600.0 & 600.0 \\
\hline 03 & 82022 & 77.5002 & 7.575 & 299.4 & 4 & 600.0 & 600.0 \\
\hline 03 & 82023 & 79.0000 & 6.6750 & 299.7 & 4 & 600.0 & 600.0 \\
\hline
\end{tabular}




\begin{tabular}{|c|c|c|c|c|c|c|c|}
\hline 3 & 82024 & 78.7500 & 6.4500 & 299.4 & 4 & 600.0 & 600.0 \\
\hline 03 & 8211 & 73.7500 & 7.3250 & 298.8 & 4 & 600.0 & 600.0 \\
\hline 03 & 8212 & 79.7367 & 3.7500 & 297.7 & 4 & 600.0 & 00.0 \\
\hline 03 & 8213 & 82.4113 & 1.6500 & 296.5 & 5 & 600.0 & 600.0 \\
\hline 03 & 8214 & 67.3205 & 0.9750 & 293.5 & 0 & 600.0 & 00.0 \\
\hline 03 & 8215 & 147.5244 & 1.0750 & 293.1 & 6 & 600.0 & 00.0 \\
\hline 03 & 8216 & 80.3532 & 2.4750 & 292.3 & 6 & 600.0 & 600.0 \\
\hline 03 & 8217 & 79.0000 & 6.3500 & 291.7 & 5 & 600.0 & 00.0 \\
\hline 03 & 8218 & 81.7244 & 4.3250 & 292.9 & 4 & 600.0 & 00.0 \\
\hline 03 & 8219 & 88.7494 & 2.6000 & 294.4 & 3 & 600.0 & 600.0 \\
\hline 03 & 82110 & 97.0381 & 2.2750 & 294.3 & 2 & 600.0 & 600.0 \\
\hline 03 & 82111 & 272.5228 & 2.1250 & 297.5 & 1 & 600.0 & \\
\hline 03 & 82112 & 26.5000 & 2.3000 & 297.1 & 1 & 600.0 & 600.0 \\
\hline 03 & 82113 & 81.5002 & 3.6000 & 294.4 & 2 & 600.0 & 600 \\
\hline 03 & 82114 & 74.9843 & 3.6250 & 293.9 & 2 & 600.0 & \\
\hline 03 & 82115 & 78.2363 & 7.1500 & 293.1 & 3 & 600.0 & 60 \\
\hline 03 & 82116 & 68.9995 & 10.2500 & 294.0 & 4 & 600.0 & 600.0 \\
\hline 03 & 82117 & 64.7496 & 8.9000 & 294.9 & 4 & 600.0 & \\
\hline 03 & 82118 & 76.7500 & 7.5500 & 294.5 & 4 & 600.0 & 60 \\
\hline 03 & 82119 & 72.0027 & 9.3750 & 292.2 & 4 & 600.0 & 600 \\
\hline 03 & 82120 & 74.5017 & 9.1250 & 291.6 & 4 & 600.0 & .0 \\
\hline 03 & 82121 & 80.7500 & 7.6750 & 292.3 & 4 & 600.0 & \\
\hline 03 & 82122 & 77.7501 & 8.7750 & 292.0 & 4 & 600.0 & 600.0 \\
\hline 03 & 82123 & 76.9998 & 9.8500 & 291.4 & 4 & 600.0 & .0 \\
\hline 03 & 82124 & 79.7499 & 750 & 291.3 & 4 & 600.0 & \\
\hline 03 & 8221 & 79.7499 & 9.3000 & 290.7 & 4 & 600.0 & 60 \\
\hline 03 & 8222 & 77.5003 & 9.6000 & 289.8 & 4 & 600.0 & .0 \\
\hline 03 & 8223 & 72.2500 & 10.0250 & 289.0 & 4 & 600.0 & \\
\hline 03 & 8224 & 73.5000 & 9.8250 & 288.5 & 4 & 600.0 & 60 \\
\hline 03 & 8225 & 75.7513 & 7.5750 & 288.7 & 4 & 600.0 & 600.0 \\
\hline 03 & 8226 & 78.5000 & 9 . & 288.5 & 4 & 600.0 & \\
\hline 03 & 8227 & 66.4971 & 7.3000 & 288.9 & 4 & 600.0 & 60 \\
\hline 03 & 8228 & 75.9989 & 6.2250 & 288.8 & 4 & 600.0 & 600.0 \\
\hline 03 & 8229 & 68.2501 & 5.800 & 289.0 & 4 & 600.0 & .0 \\
\hline 03 & 82210 & 75.7509 & 5 . & 289.0 & 4 & 600.0 & 60 \\
\hline 03 & 82211 & 74.2468 & 5.3250 & 289.2 & 4 & 600.0 & 600.0 \\
\hline 03 & 82212 & 74.2515 & 5.0000 & 290.1 & 3 & 600.0 & 600.0 \\
\hline 03 & 82213 & 75.3647 & 3.4500 & 293.5 & 2 & 600.0 & 600.0 \\
\hline 03 & 82214 & 54.7152 & 2.0250 & 295.7 & 1 & 600.0 & 600.0 \\
\hline 03 & 82215 & 13.0133 & 1.6500 & 297.2 & 1 & 600.0 & \\
\hline 03 & 82216 & 21.0876 & 3.1250 & 297.5 & 2 & 600.0 & 600.0 \\
\hline 03 & 82217 & 117.2077 & 5.2750 & 296.5 & 3 & 600.0 & 600.0 \\
\hline 03 & 82218 & 172.2791 & 7.1500 & 294.7 & 4 & 600.0 & 600.0 \\
\hline 03 & 82219 & 158.7948 & 5.1000 & 293.5 & 4 & 600.0 & 600.0 \\
\hline 03 & 82220 & 86.5021 & 6.3750 & 291.5 & 4 & 600.0 & 600.0 \\
\hline 03 & 82221 & 71.5005 & 6.5250 & 290.5 & 4 & 600.0 & 600.0 \\
\hline 03 & 82222 & 70.4997 & 7.300 & 289.9 & 4 & 600.0 & 600.0 \\
\hline 03 & 82223 & 71.2500 & 7.4000 & 289.4 & 4 & 600.0 & 600.0 \\
\hline
\end{tabular}




\begin{tabular}{|c|c|c|c|c|c|c|c|}
\hline 3 & 82224 & 69.2498 & 8.7250 & 289.2 & 4 & 600.0 & 600.0 \\
\hline 3 & 8231 & 69.7496 & 9.0500 & 289.5 & 4 & 600.0 & 600.0 \\
\hline 03 & 8232 & 74.5000 & 8.5250 & 289.6 & 4 & 600.0 & 00.0 \\
\hline 3 & 8233 & 75.4998 & 8.1250 & 289.9 & 4 & 600.0 & 00.0 \\
\hline 03 & 8234 & 76.7500 & 7.0000 & 290.1 & 4 & 600.0 & 00.0 \\
\hline 03 & 8235 & 78.0000 & 4.8750 & 290.6 & 4 & 600.0 & 00.0 \\
\hline 03 & 8236 & 81.7500 & 4.0000 & 290.7 & 4 & 600.0 & 00.0 \\
\hline 03 & 8237 & 90.5017 & 2.9500 & 291.4 & 4 & 600.0 & 00.0 \\
\hline 03 & 8238 & 119.7857 & 3.2500 & 293.0 & 3 & 600.0 & 00.0 \\
\hline 03 & 8239 & 168.4765 & 3.9000 & 294.5 & 3 & 600.0 & 0 \\
\hline 03 & 82310 & 171.4998 & 5.8000 & 294.7 & 4 & 600.0 & 00.0 \\
\hline 03 & 82311 & 175.4973 & 5.1750 & 295.4 & 3 & 600.0 & \\
\hline 03 & 82312 & 175.2515 & 4.6750 & 296.8 & 3 & 600.0 & 00.0 \\
\hline 03 & 82313 & 176.7507 & 5.5000 & 297.5 & 3 & 600.0 & 00.0 \\
\hline 03 & 82314 & 177.7487 & 750 & 298.5 & 3 & .0 & \\
\hline 03 & 82315 & 178.2527 & 5.1750 & 299.2 & 3 & 600.0 & 00.0 \\
\hline 03 & 82316 & 167.2501 & 5.0750 & 299.9 & 3 & 600.0 & 600.0 \\
\hline 03 & 82317 & 174.4944 & 5.0750 & 300.1 & 3 & .0 & \\
\hline 03 & 82318 & 186.2499 & 5.1000 & 299.9 & 3 & 600.0 & .0 \\
\hline 03 & 82319 & 110.8865 & 5.1250 & 298.7 & 4 & 60 & .0 \\
\hline 03 & 82320 & 77.0000 & 5.5500 & 297.0 & 4 & 60 & \\
\hline 03 & 82321 & 72.5000 & 6.7250 & 296.0 & 4 & .0 & \\
\hline 03 & 82322 & 75.5003 & 6.5500 & 295.9 & 4 & 600.0 & 00.0 \\
\hline 03 & 82323 & 74.7499 & 6.2250 & 296.3 & 4 & 60 & .0 \\
\hline 03 & 82324 & 78.2496 & 6.2750 & 296.4 & 4 & .0 & \\
\hline 03 & 8241 & 78.2508 & 3.8250 & 297.1 & 4 & 600.0 & 600.0 \\
\hline 03 & 8242 & 74.5014 & 2.4000 & 297.6 & 4 & 600.0 & .0 \\
\hline 03 & 8243 & 113.0817 & 1.0250 & 297.2 & 5 & 0 & \\
\hline 03 & 8244 & 150.2783 & 0.6250 & 296.6 & 6 & 600.0 & 600.0 \\
\hline 03 & 8245 & 118.1219 & 0.8250 & 296.1 & 6 & 600.0 & 600.0 \\
\hline 03 & 8246 & 123.5084 & 1.3000 & 296.2 & 5 & .0 & \\
\hline 03 & 8247 & 136.0000 & 1.6750 & 297.0 & 4 & .0 & 600.0 \\
\hline 03 & 8248 & 164.0053 & 3.9250 & 297.8 & 4 & 600.0 & 600.0 \\
\hline 03 & 8249 & 168.7500 & 4.3500 & 298.3 & 4 & .0 & 0.0 \\
\hline 03 & 82410 & 167.7511 & 4.4000 & 299.0 & 4 & 600.0 & 600.0 \\
\hline 03 & 82411 & 182.7518 & 5.2250 & 300.0 & 3 & 600.0 & 600.0 \\
\hline 03 & 82412 & 188.2503 & 4.9500 & 300.8 & 3 & 600.0 & 600.0 \\
\hline 03 & 82413 & 180.9991 & 4.6750 & 301.6 & 3 & 600.0 & 600.0 \\
\hline 03 & 82414 & 188.2452 & 4.4750 & 302.7 & 3 & 600.0 & 600.0 \\
\hline 03 & 82415 & 195.0000 & 4.5000 & 303.4 & 3 & 600.0 & 600.0 \\
\hline 03 & 82416 & 208.7721 & 4.8750 & 303.7 & 3 & 600.0 & 600.0 \\
\hline 03 & 82417 & 192.7883 & 4.2250 & 303.9 & 3 & 600.0 & 600.0 \\
\hline 03 & 82418 & 178.0021 & 4.8500 & 303.6 & 3 & 600.0 & 600.0 \\
\hline 03 & 82419 & 178.7573 & 5.2750 & 302.8 & 4 & 600.0 & 600.0 \\
\hline 03 & 82420 & 152.2648 & 2.9250 & 301.5 & 4 & 600.0 & 600.0 \\
\hline 03 & 82421 & 83.4948 & 4.4250 & 300.9 & 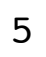 & 600.0 & 600.0 \\
\hline 03 & 82422 & 76.5000 & 4.8750 & 300.8 & 5 & 600.0 & 600.0 \\
\hline U. & 82423 & 69.7503 & 3.5000 & 301.3 & J & 600.0 & 600.0 \\
\hline
\end{tabular}




\begin{tabular}{|c|c|c|c|c|c|c|c|}
\hline 3 & 82424 & 75.2501 & 4.4500 & 301.1 & 5 & 600.0 & 00.0 \\
\hline 03 & 8251 & 78.7500 & 3.8750 & 301.3 & 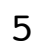 & 600.0 & 600.0 \\
\hline 03 & 8252 & 78.0000 & 2.4250 & 301.4 & 4 & 600.0 & 00.0 \\
\hline 03 & 8253 & 79.5000 & 1.4250 & 301.1 & 4 & 600.0 & 600.0 \\
\hline 03 & 8254 & 95.9677 & 1.3750 & 300.9 & 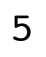 & 600.0 & 00.0 \\
\hline 03 & 8255 & 152.6313 & 0.9750 & 300.0 & 6 & 600.0 & 00.0 \\
\hline 03 & 8256 & 21.7281 & 0.8000 & 298.9 & 6 & 600.0 & 600.0 \\
\hline 03 & 8257 & 136.6414 & 0.7000 & 300.4 & & 600.0 & 00.0 \\
\hline 03 & 8258 & 168.3122 & 0.8750 & 303.4 & 4 & 600.0 & 00.0 \\
\hline 03 & 8259 & 174.0000 & 2.8500 & 303.2 & 4 & 600.0 & .0 \\
\hline 03 & 82510 & 165.9999 & 4.1500 & 303.1 & 4 & 600.0 & 00.0 \\
\hline 03 & 82511 & 167.9962 & 5.0750 & 303.2 & 4 & 600.0 & \\
\hline 03 & 82512 & 174.2500 & 5.0000 & 303.9 & 3 & 600.0 & 00.0 \\
\hline 03 & 82513 & 172.4983 & 4.3750 & 305.0 & 3 & 600.0 & 0 \\
\hline 03 & 82514 & 169.2493 & 4.1000 & 306.2 & 3 & 600.0 & \\
\hline 03 & 82515 & 185.2472 & 5.8000 & 306.1 & 3 & 600.0 & 60 \\
\hline 03 & 82516 & 192.4927 & 6.7250 & 306.0 & 4 & 600.0 & 600.0 \\
\hline 03 & 82517 & 191.2488 & 5.9000 & 305.5 & 4 & 600.0 & .0 \\
\hline 03 & 82518 & 176.7558 & 4.3000 & 305.4 & 3 & 600.0 & .0 \\
\hline 03 & 82519 & 113.2565 & 4.8000 & 304.6 & 4 & 600.0 & 600 \\
\hline 03 & 82520 & 80.5000 & 6.57 & 302.9 & 4 & 600.0 & .0 \\
\hline 03 & 82521 & 71.5000 & 7.0750 & 302.0 & 4 & 0.0 & \\
\hline 03 & 82522 & 69.5000 & 7.7500 & 301.6 & 4 & 600.0 & 600.0 \\
\hline 03 & 82523 & 70.2499 & 8.9250 & 301.4 & 4 & 600.0 & .0 \\
\hline 03 & 82524 & 71.0011 & 10.1750 & 300.8 & 4 & 00.0 & \\
\hline 03 & 8261 & 54.2149 & 8.5250 & 299.7 & 4 & 600.0 & 60 \\
\hline 03 & 8262 & 59.7073 & 6.2250 & 300.1 & 4 & 600.0 & .0 \\
\hline 03 & 8263 & 74.6630 & 4.8250 & 299.0 & 4 & 600.0 & \\
\hline 03 & 8264 & 61.9908 & 6.1500 & 300.3 & 4 & 600.0 & 60 \\
\hline 03 & 8265 & 183.8560 & 2.1750 & 299.5 & 5 & 600.0 & .0 \\
\hline 03 & 8266 & 332.7494 & 2.27 & 296.4 & 0 & 600.0 & \\
\hline 03 & 8267 & 17.8012 & 3.2750 & 296.2 & 5 & 600.0 & 60 \\
\hline 03 & 8268 & 346.1048 & 1.1500 & 300.5 & 4 & 600.0 & 600.0 \\
\hline 03 & 8269 & 333.4870 & 2.50 & 302.7 & 3 & 600.0 & .0 \\
\hline 03 & 82610 & 282.8781 & 4.3000 & 302.5 & 3 & 600.0 & .0 \\
\hline 03 & 82611 & 263.2494 & 3.2000 & 302.9 & 2 & 600.0 & 600.0 \\
\hline 03 & 82612 & 238.0814 & 2.0000 & 304.8 & 1 & 600.0 & \\
\hline 03 & 82613 & 53.9122 & 3.7250 & 305.6 & 2 & 600.0 & 600.0 \\
\hline 03 & 82614 & 83.0235 & 4.9750 & 305.7 & 3 & 600.0 & 600.0 \\
\hline 03 & 82615 & 99.2493 & 5.875 & 305.3 & 3 & 600.0 & \\
\hline 03 & 82616 & 91.2584 & 6.1000 & 305.1 & 4 & 600.0 & 600.0 \\
\hline 03 & 82617 & 86.9993 & 5.3250 & 303.9 & 3 & 600.0 & 600.0 \\
\hline 03 & 82618 & 73.7444 & 8.9000 & 301.6 & 4 & 600.0 & 600.0 \\
\hline 03 & 82619 & 67.4991 & 11.8000 & 300.2 & 4 & 600.0 & 600.0 \\
\hline 03 & 82620 & 67.7499 & 11.3250 & 298.5 & 4 & 600.0 & 600.0 \\
\hline 03 & 82621 & 64.7504 & 9.7000 & 298.5 & 4 & 600.0 & 600.0 \\
\hline 03 & 82622 & 61.5003 & 11.425 & 298.4 & 4 & 600.0 & 600.0 \\
\hline 03 & 82623 & 55.2652 & 8.9250 & 297.6 & $\mathbf{4}$ & 600.0 & 600.0 \\
\hline
\end{tabular}




\begin{tabular}{|c|c|c|c|c|c|c|c|}
\hline 3 & 82624 & 76.4779 & 4.6750 & 297.1 & 4 & 600.0 & 00.0 \\
\hline 03 & 8271 & 56.4998 & 9.8750 & 297.8 & 4 & 600.0 & 600.0 \\
\hline 03 & 8272 & 63.0000 & 9.9500 & 297.4 & 4 & 600.0 & 00.0 \\
\hline 03 & 8273 & 68.4997 & 9.0250 & 298.1 & 4 & 600.0 & 00.0 \\
\hline 03 & 8274 & 71.9998 & 8.4250 & 297.8 & 4 & 600.0 & 00.0 \\
\hline 03 & 8275 & 68.2501 & 8.2500 & 297.9 & 4 & 600.0 & 00.0 \\
\hline 03 & 8276 & 74.7500 & 8.4500 & 297.4 & 4 & 600.0 & 600.0 \\
\hline 03 & 8277 & 72.0000 & 8.3500 & 297.8 & 4 & 600.0 & 00.0 \\
\hline 03 & 8278 & 72.4990 & 7.1750 & 299.0 & 4 & 600.0 & 00.0 \\
\hline 03 & 8279 & 84.2503 & 5.0500 & 299.9 & 3 & 600.0 & .0 \\
\hline 03 & 82710 & 142.7613 & 2.9000 & 301.3 & 2 & 600.0 & 00.0 \\
\hline 03 & 82711 & 122.9696 & 3.6250 & 301.3 & 2 & 600.0 & \\
\hline 03 & 82712 & 100.7529 & 5.9250 & 300.8 & 3 & 600.0 & 600.0 \\
\hline 03 & 82713 & 85.7530 & 5.4000 & 301.9 & 3 & 600.0 & 0 \\
\hline 03 & 82714 & 87.5000 & 5.9500 & 302.2 & 3 & 600.0 & \\
\hline 03 & 82715 & 82.4986 & 7.3500 & 301.8 & 4 & 600.0 & 60 \\
\hline 03 & 82716 & 77.5005 & 8.9250 & 301.1 & 4 & 600.0 & 600.0 \\
\hline 03 & 82717 & 76.0019 & 7.5500 & 300.9 & 4 & 600.0 & \\
\hline 03 & 82718 & 73.7500 & 9.4250 & 299.5 & 4 & 600.0 & .0 \\
\hline 03 & 82719 & 74.9962 & 9.7000 & 297.7 & 4 & 600.0 & .0 \\
\hline 03 & 82720 & 76.5010 & 11.15 & 296.6 & 4 & 600.0 & .0 \\
\hline 03 & 82721 & 76.4992 & 10. & 296.6 & 4 & 0.0 & \\
\hline 03 & 82722 & 79.0000 & 9.6750 & 297.1 & 4 & 600.0 & 600.0 \\
\hline 03 & 82723 & 79.5000 & 10.07 & 297.3 & 4 & 600.0 & .0 \\
\hline 03 & 82724 & 76.0005 & 9 . & 296.9 & 4 & 600.0 & \\
\hline 03 & 8281 & 71.7500 & 11.0000 & 295.8 & 4 & 600.0 & 60 \\
\hline 03 & 8282 & 75.0005 & 10.5500 & 295.9 & 4 & 600.0 & .0 \\
\hline 03 & 8283 & 72.5000 & 11.9250 & 294.8 & 4 & 600.0 & \\
\hline 03 & 8284 & 72.7502 & 11.0750 & 295.6 & 4 & 600.0 & 60 \\
\hline 03 & 8285 & 73.0000 & 9.3000 & 296.4 & 4 & 600.0 & .0 \\
\hline 03 & 8286 & 74.2501 & 8 . & 296.9 & 4 & 600.0 & \\
\hline 03 & 8287 & 73.0002 & 9.1000 & 296.4 & 4 & 600.0 & 60 \\
\hline 03 & 8288 & 68.7504 & 7.8000 & 296.4 & 4 & 600.0 & 600.0 \\
\hline 03 & 8289 & 77.9979 & 6.25 & 297.1 & 4 & 600.0 & .0 \\
\hline 03 & 82810 & 101.8495 & 6.6250 & 296.4 & 4 & 600.0 & .0 \\
\hline 03 & 82811 & 97.7517 & 4.8500 & 297.5 & 3 & 600.0 & 600.0 \\
\hline 03 & 82812 & 93.0000 & 5.5500 & 297.6 & 3 & 600.0 & \\
\hline 03 & 82813 & 90.9927 & 7.8500 & 296.8 & 4 & 600.0 & 600.0 \\
\hline 03 & 82814 & 76.7498 & 9.2000 & 296.6 & 4 & 600.0 & 600.0 \\
\hline 03 & 82815 & 71.4995 & 10.5500 & 297.2 & 4 & 600.0 & \\
\hline 03 & 82816 & 70.5000 & 11.7750 & 296.9 & 4 & 600.0 & 600.0 \\
\hline 03 & 82817 & 71.2500 & 13.2250 & 296.5 & 4 & 600.0 & 600.0 \\
\hline 03 & 82818 & 71.5005 & 12.1250 & 296.0 & 4 & 600.0 & 600.0 \\
\hline 03 & 82819 & 70.5000 & 13.3250 & 294.9 & 4 & 600.0 & 600.0 \\
\hline 03 & 82820 & 70.5000 & 13.0500 & 294.3 & 4 & 600.0 & 600.0 \\
\hline 03 & 82821 & 70.7508 & 12.2000 & 293.9 & 4 & 600.0 & 600.0 \\
\hline 03 & 82822 & 71.2500 & 11.850 & 293.7 & 4 & 600.0 & 600.0 \\
\hline 03 & 82823 & 67.9998 & 11.5750 & 293.9 & $\mathbf{4}$ & 600.0 & 600.0 \\
\hline
\end{tabular}




\begin{tabular}{|c|c|c|c|c|c|c|c|}
\hline 3 & 82824 & 70.7502 & 10.4250 & 295.0 & 4 & 600.0 & 00.0 \\
\hline 03 & 8291 & 72.2500 & 9.8250 & 295.3 & 4 & 600.0 & 600.0 \\
\hline 03 & 8292 & 75.9931 & 8.5500 & 296.2 & 4 & 600.0 & 00.0 \\
\hline 03 & 8293 & 82.4985 & 6.9750 & 295.9 & 4 & 600.0 & 600.0 \\
\hline 03 & 8294 & 87.9988 & 7.3500 & 294.7 & 4 & 600.0 & 00.0 \\
\hline 03 & 8295 & 83.0000 & 9.1000 & 293.4 & 4 & 600.0 & 00.0 \\
\hline 03 & 8296 & 86.5008 & 7.1250 & 293.1 & 4 & 600.0 & 600.0 \\
\hline 03 & 8297 & 116.2319 & 4.2000 & 293.5 & 3 & 600.0 & 00.0 \\
\hline 03 & 8298 & 227.0775 & 1.2000 & 294.8 & 2 & 600.0 & 00.0 \\
\hline 03 & 8299 & 202.2990 & 2.9000 & 295.1 & 1 & 600.0 & 600.0 \\
\hline 03 & 82910 & 172.2499 & 4.6000 & 295.1 & 2 & 600.0 & 00.0 \\
\hline 03 & 82911 & 165.5008 & 6.9250 & 295.9 & 3 & 600.0 & \\
\hline 03 & 82912 & 159.4968 & 6.2750 & 297.0 & 4 & 600.0 & 600.0 \\
\hline 03 & 82913 & 164.9966 & 5.5000 & 298.9 & 3 & 600.0 & .0 \\
\hline 03 & 82914 & 164.2495 & 750 & 300.0 & 3 & 600.0 & \\
\hline 03 & 82915 & 155.7616 & 500 & 301.5 & 3 & 600.0 & 60 \\
\hline 03 & 82916 & 154.7520 & 4.7500 & 302.0 & 3 & 600.0 & 600.0 \\
\hline 03 & 82917 & 158.2503 & 000 & 301.6 & 3 & 600.0 & \\
\hline 03 & 82918 & 104.3786 & 000 & 301.2 & 3 & 600.0 & 60 \\
\hline 03 & 82919 & 79.7498 & 6.7250 & 298.4 & 4 & 600.0 & 600 \\
\hline 03 & 82920 & 75.0002 & 7 . & 297.5 & 4 & 600.0 & .0 \\
\hline 03 & 82921 & 75.2495 & 250 & 296.6 & 4 & 600.0 & \\
\hline 03 & 82922 & 77.2500 & 10.0500 & 296.0 & 4 & 600.0 & 600.0 \\
\hline 03 & 82923 & 74.2499 & 9.7250 & 296.2 & 4 & 600.0 & .0 \\
\hline 03 & 82924 & 70.7501 & 9 . & 296.6 & 4 & 600.0 & \\
\hline 03 & 8301 & 68.2500 & 000 & 297.2 & 4 & 600.0 & 60 \\
\hline 03 & 8302 & 74.5006 & 8.3750 & 297.5 & 4 & 600.0 & .0 \\
\hline 03 & 8303 & 81.0000 & 5. & 298.1 & 4 & 600.0 & \\
\hline 03 & 8304 & 88.0000 & 750 & 297.7 & 4 & 600.0 & 60 \\
\hline 03 & 8305 & 157.2050 & 1.4250 & 296.2 & 5 & 600.0 & .0 \\
\hline 03 & 8306 & 70.9979 & 4 . & 297.2 & 4 & 600.0 & \\
\hline 03 & 8307 & 72.1694 & 250 & 298.0 & 3 & 600.0 & 60 \\
\hline 03 & 8308 & 203.4321 & 1.4000 & 296.1 & 2 & 600.0 & 600.0 \\
\hline 03 & 8309 & 249.8016 & 1 . & 296.7 & 1 & 600.0 & .0 \\
\hline 03 & 83010 & 240.7439 & 2 . & 297.4 & 2 & 600.0 & .0 \\
\hline 03 & 83011 & 234.7919 & 2.5000 & 298.6 & 2 & 600.0 & 600.0 \\
\hline 03 & 83012 & 184.5063 & 750 & 300.1 & 2 & 600.0 & \\
\hline 03 & 83013 & 274.8958 & 500 & 302.4 & 1 & 600.0 & 600.0 \\
\hline 03 & 83014 & 36.3504 & 3.2250 & 304.0 & 2 & 600.0 & 600.0 \\
\hline 03 & 83015 & 55.1737 & 250 & 304.3 & 2 & 600.0 & \\
\hline 03 & 83016 & 91.2496 & 3.9500 & 303.8 & 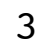 & 600.0 & 600.0 \\
\hline 03 & 83017 & 86.4288 & 5.3250 & 302.9 & 3 & 600.0 & 600.0 \\
\hline 03 & 83018 & 82.9998 & 5.8750 & 301.7 & 4 & 600.0 & 600.0 \\
\hline 03 & 83019 & 77.9995 & 6.2500 & 301.0 & 4 & 600.0 & 600.0 \\
\hline 03 & 83020 & 72.5000 & 7.3250 & 300.2 & 4 & 600.0 & 600.0 \\
\hline 03 & 83021 & 77.0001 & 7.5500 & 300.1 & 4 & 600.0 & 600.0 \\
\hline 03 & 83022 & 76.7500 & 7.550 & 300.0 & 4 & 600.0 & 600.0 \\
\hline 03 & 83023 & 77.7500 & 8.7000 & 299.0 & 4 & 600.0 & 600.0 \\
\hline
\end{tabular}




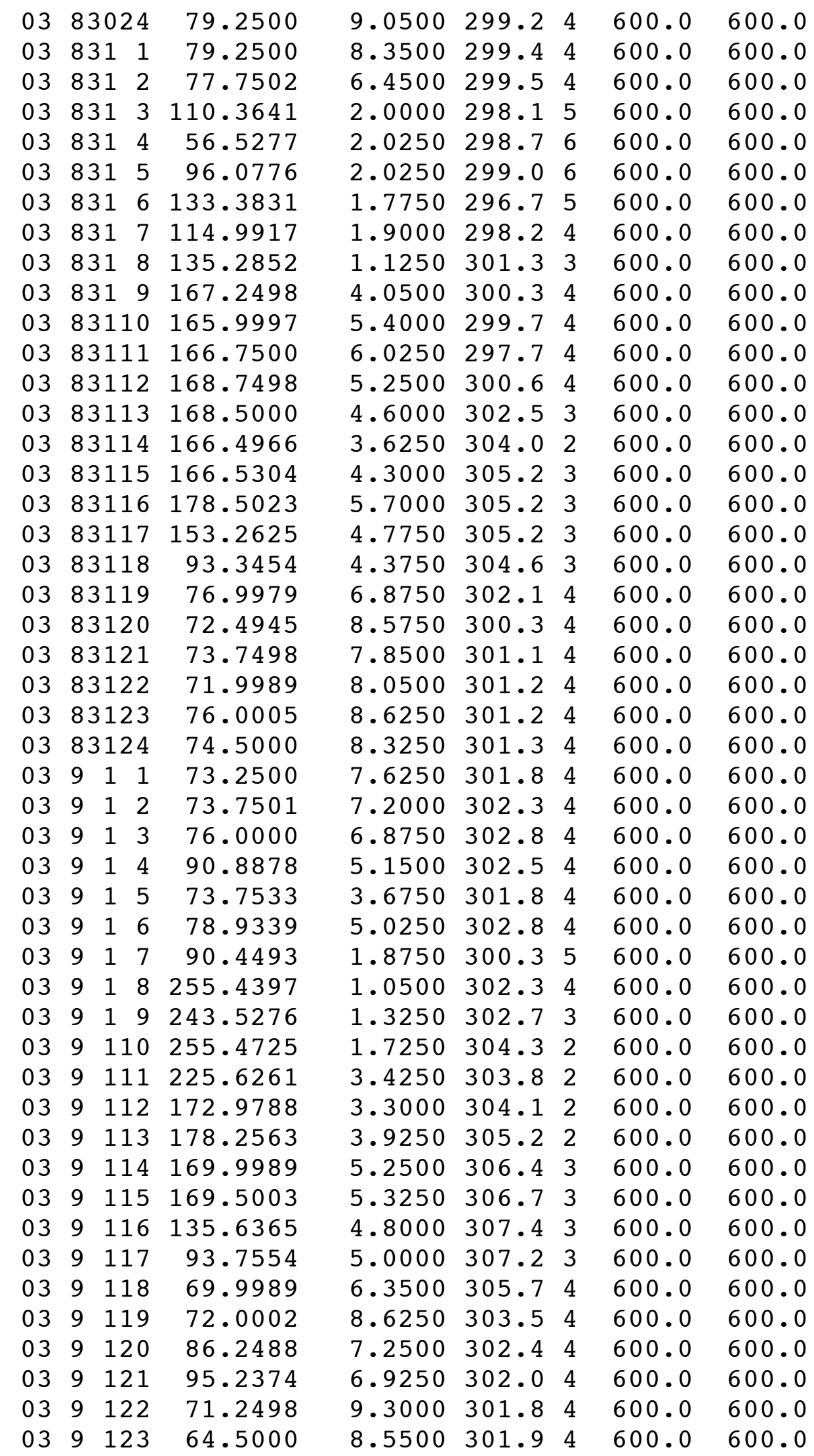




\begin{tabular}{|c|c|c|c|c|c|c|c|c|}
\hline 3 & 9 & 124 & 67.4997 & 8.0750 & 302.6 & 4 & 600.0 & 600 \\
\hline 03 & 9 & 21 & 71.7506 & 7.0750 & 303.1 & 4 & 600.0 & 600.0 \\
\hline 03 & 9 & 22 & 77.4991 & 6.3500 & 303.6 & 4 & 600.0 & 00.0 \\
\hline 03 & 9 & 23 & 81.7509 & 4.7500 & 303.6 & 4 & 600.0 & 600.0 \\
\hline 03 & 9 & 24 & 75.0073 & 3.0000 & 303.7 & 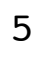 & 600.0 & 00.0 \\
\hline 03 & 9 & 25 & 92.2100 & 1.3500 & 302.4 & 6 & 600.0 & 00.0 \\
\hline 03 & 9 & 26 & 253.5678 & 0.6250 & 300.9 & 6 & 600.0 & 600.0 \\
\hline 03 & 9 & 27 & 266.4945 & 0.8000 & 301.7 & 0 & 600.0 & 00.0 \\
\hline 03 & 9 & 28 & 281.2226 & 0.6250 & 303.4 & 5 & 600.0 & 00.0 \\
\hline 03 & 9 & 29 & 228.4260 & 1.3500 & 305.0 & 4 & 600.0 & 600.0 \\
\hline 03 & 9 & 210 & 230.7576 & 2.3500 & 303.8 & 3 & 600.0 & 600.0 \\
\hline 03 & 9 & 211 & 167.0004 & 4.2500 & 303.6 & 4 & 600.0 & \\
\hline 03 & 9 & 212 & 164.7499 & 4.9250 & 304.4 & 4 & 600.0 & 600.0 \\
\hline 03 & 9 & 213 & 169.5014 & 5.1750 & 305.7 & 3 & 600.0 & 600.0 \\
\hline 03 & 9 & 214 & 167.7501 & 5.5000 & 306.4 & 3 & 600.0 & \\
\hline 03 & 9 & 215 & 166.2501 & 6.2500 & 306.7 & 4 & 600.0 & 60 \\
\hline 03 & 9 & 216 & 169.2532 & 5.7500 & 307.7 & 3 & 600.0 & 600.0 \\
\hline 03 & 9 & 217 & 157.7519 & 5.4000 & 307.7 & 3 & 600.0 & \\
\hline 03 & 9 & 218 & 105.5236 & 4.1250 & 306.8 & 4 & 600.0 & 60 \\
\hline 03 & 9 & 219 & 75.5000 & 6.3500 & 303.8 & 4 & 600.0 & 60 \\
\hline 03 & 9 & 220 & 73.9999 & 8.1500 & 302.1 & 4 & 600.0 & 年 \\
\hline 03 & 9 & 221 & 75.4998 & 9.8500 & 301.2 & 4 & 600.0 & \\
\hline 03 & 9 & 222 & 78.7500 & 10.1500 & 300.8 & 4 & 600.0 & 600.0 \\
\hline 03 & 9 & 223 & 77.5000 & 10.3250 & 300.7 & 4 & 600.0 & 60 \\
\hline 03 & 9 & 224 & 77.2500 & 10.2 & 301.2 & 4 & 600.0 & \\
\hline 03 & 9 & 31 & 75.5000 & 10.5000 & 301.1 & 4 & 600.0 & 60 \\
\hline 03 & 9 & 32 & 76.5000 & 9.8750 & 301.4 & 4 & 600.0 & 60 \\
\hline 03 & 9 & 33 & 79.2496 & 8.25 & 301.6 & 4 & 600.0 & \\
\hline 03 & 9 & 34 & 87.4977 & 5.9250 & 301.5 & 4 & 600.0 & 60 \\
\hline 03 & 9 & 35 & 54.1386 & 4.6750 & 299.4 & 4 & 600.0 & 600 \\
\hline 03 & 9 & 36 & 39.6476 & 2.07 & 297.5 & 5 & 600.0 & \\
\hline 03 & 9 & 37 & 76.8827 & 3.2000 & 299.9 & 5 & 600.0 & 60 \\
\hline 03 & 9 & 38 & 108.1607 & 2.4250 & 299.1 & 4 & 600.0 & 600.0 \\
\hline 03 & 9 & 39 & 91.9995 & 4.42 & 301.4 & 3 & 600.0 & 60 \\
\hline 03 & 9 & 310 & 148.7242 & 3.0250 & 303.1 & 2 & 600.0 & 60 \\
\hline 03 & 9 & 311 & 133.3815 & 5.4500 & 303.3 & 3 & 600.0 & 600.0 \\
\hline 03 & 9 & 312 & 160.7499 & 5.5750 & 303.5 & 3 & 600.0 & \\
\hline 03 & 9 & 313 & 157.0000 & 5.8250 & 303.9 & 3 & 600.0 & 600.0 \\
\hline 03 & 9 & 314 & 143.2506 & 6.2500 & 303.8 & 4 & 600.0 & 600.0 \\
\hline 03 & 9 & 315 & 134.0000 & 5.875 & 304.3 & 3 & 600.0 & \\
\hline 03 & 9 & 316 & 134.7530 & 5.6250 & 304.3 & 3 & 600.0 & 600.0 \\
\hline 03 & 9 & 317 & 104.7511 & 4.1500 & 303.7 & 3 & 600.0 & 600.0 \\
\hline 03 & 9 & 318 & 75.7403 & 3.8000 & 302.9 & 4 & 600.0 & 600.0 \\
\hline 03 & 9 & 319 & 74.2486 & 5.2000 & 301.2 & 4 & 600.0 & 600.0 \\
\hline 03 & 9 & 320 & 104.2509 & 1.7000 & 301.5 & 5 & 600.0 & 600.0 \\
\hline 03 & 9 & 321 & 72.0009 & 3.0750 & 302.2 & 4 & 600.0 & 600.0 \\
\hline 03 & 9 & 322 & 71.2539 & 6.7000 & 301.6 & 4 & 600.0 & 600.0 \\
\hline 03 & 9 & 323 & 101.0497 & 2.0500 & 301.3 & $\mathrm{~J}$ & 600.0 & 600.0 \\
\hline
\end{tabular}




\begin{tabular}{|c|c|c|c|c|c|c|c|c|}
\hline 03 & 9 & 324 & 81.7342 & 6.4000 & 298.6 & 4 & 600.0 & 600.0 \\
\hline 03 & 9 & 41 & 79.7500 & 10.4000 & 296.0 & 4 & 600.0 & 600.0 \\
\hline 03 & 9 & 42 & 79.2501 & 9.9250 & 296.1 & 4 & 600.0 & 00.0 \\
\hline 03 & 9 & 43 & 79.5001 & 9.1500 & 296.1 & 4 & 600.0 & 00.0 \\
\hline 03 & 9 & 44 & 80.5000 & 8.2750 & 295.9 & 4 & 600.0 & 00.0 \\
\hline 03 & 9 & 45 & 79.5002 & 6.0250 & 296.3 & 4 & 600.0 & 00.0 \\
\hline 03 & 9 & 46 & 79.5005 & 6.5250 & 295.8 & 4 & 600.0 & 00.0 \\
\hline 03 & 9 & 47 & 80.4998 & 7.0000 & 295.1 & 4 & 600.0 & 00.0 \\
\hline 03 & 9 & 48 & 79.7492 & 6.0750 & 295.9 & 4 & 600.0 & 00.0 \\
\hline 03 & 9 & 49 & 266.4176 & 2.2000 & 297.9 & 3 & 600.0 & .0 \\
\hline 03 & 9 & 410 & 277.7853 & 1.9500 & 299.1 & - & 600.0 & 00.0 \\
\hline 03 & 9 & 411 & 45.5810 & 000 & 299.8 & 1 & 600.0 & \\
\hline 03 & 9 & 412 & 55.8885 & 3.1000 & 300.8 & 2 & 600.0 & 00.0 \\
\hline 03 & 9 & 413 & 65.7433 & 4.3750 & 301.0 & 3 & 600.0 & 00.0 \\
\hline 03 & 9 & 414 & 63.7416 & & 301.6 & 3 & .0 & \\
\hline 03 & 9 & 415 & 82.0000 & 000 & 300.1 & 4 & 600.0 & 00.0 \\
\hline 03 & 9 & 416 & 86.0000 & 8.6750 & 299.7 & 4 & 600.0 & 600.0 \\
\hline 03 & 9 & 417 & 85.2500 & 8.8 & 298.9 & 4 & .0 & \\
\hline 03 & 9 & 418 & 82.7498 & 9.7000 & 297.2 & 4 & 600.0 & .0 \\
\hline 03 & 9 & 419 & 76.0000 & 9.4500 & 295.4 & 4 & 60 & .0 \\
\hline 03 & 9 & 420 & 76 . & 10 . & 293.9 & 4 & 60 & \\
\hline 03 & 9 & 421 & 75.7500 & 9 . & 293.8 & 4 & .0 & \\
\hline 03 & 9 & 422 & 76.7500 & 8.4000 & 294.9 & 4 & 600.0 & .0 \\
\hline 03 & 9 & 423 & 77.5000 & 7.5 & 295.4 & 4 & 60 & \\
\hline 03 & 9 & 424 & 77.2500 & 8 . & 294.1 & 4 & .0 & \\
\hline 03 & 9 & 51 & 77.0000 & 7.6250 & 294.0 & 4 & 600.0 & 600.0 \\
\hline 03 & 9 & 52 & 78.7500 & 6.8750 & 294.0 & 4 & 600.0 & .0 \\
\hline 03 & 9 & 53 & 79.7500 & 8. & 291.9 & 4 & .0 & \\
\hline 03 & 9 & 54 & 78.2500 & 7.0750 & 291.6 & 4 & .0 & 0.0 \\
\hline 03 & 9 & 55 & 76.2500 & 4.4500 & 292.0 & 4 & 60 & 0.0 \\
\hline 03 & 9 & 56 & 75.0095 & 1. & 291.9 & 5 & .0 & \\
\hline 03 & 9 & 57 & 30.1963 & 0 . & 293.2 & 6 & .0 & 600.0 \\
\hline 03 & 9 & 58 & 18.4866 & 1.1000 & 295.4 & 5 & 600.0 & 600.0 \\
\hline 03 & 9 & 59 & 113.6223 & 2. & 294.3 & 4 & 0.0 & 0.0 \\
\hline 03 & 9 & 510 & 92.3441 & 2.2500 & 295.5 & 3 & 600.0 & 600.0 \\
\hline 03 & 9 & 511 & 296.7457 & 2.4750 & 297.1 & 2 & 600.0 & 600.0 \\
\hline 03 & 9 & 512 & 264.0133 & 2.0000 & 298.9 & 1 & 600.0 & 0.0 \\
\hline 03 & 9 & 513 & 224.4279 & 3.0000 & 299.8 & 2 & 600.0 & 600.0 \\
\hline 03 & 9 & 514 & 179.0468 & 3.3500 & 300.7 & 2 & 600.0 & 600.0 \\
\hline 03 & 9 & 515 & 199.4440 & 4 . & 301.4 & 3 & 600.0 & 600.0 \\
\hline 03 & 9 & 516 & 64.2879 & 4.0750 & 301.5 & 3 & 600.0 & 600.0 \\
\hline 03 & 9 & 517 & 79.5000 & 4.5500 & 300.5 & 4 & 600.0 & 600.0 \\
\hline 03 & 9 & 518 & 79.5000 & 7.2500 & 297.8 & 4 & 600.0 & 600.0 \\
\hline 03 & 9 & 519 & 78.0000 & 9.9000 & 294.0 & 4 & 600.0 & 600.0 \\
\hline 03 & 9 & 520 & 79.7499 & 10.7250 & 291.7 & 4 & 600.0 & 600.0 \\
\hline 03 & 9 & 521 & 80.2500 & 11.3250 & 290.9 & 4 & 600.0 & 600.0 \\
\hline 03 & 9 & 522 & 78.5002 & 11.7250 & 289.8 & 4 & 600.0 & 600.0 \\
\hline & 9 & 523 & 77.2500 & 11.8000 & 289.3 & 4 & 600.0 & 600.0 \\
\hline
\end{tabular}




\begin{tabular}{|c|c|c|c|c|c|c|c|c|}
\hline & 9 & 524 & 77.0000 & 12.1250 & 288.7 & 4 & 600.0 & 600.0 \\
\hline 3 & 9 & 61 & 72.4997 & 12.1750 & 287.8 & 4 & 600.0 & 00.0 \\
\hline 03 & 9 & 62 & 71.5000 & 12.3750 & 287.2 & 4 & 600.0 & 00.0 \\
\hline 3 & 9 & 63 & 70.2500 & 11.6500 & 286.9 & 4 & 600.0 & 00.0 \\
\hline 03 & 9 & 64 & 67.7500 & 11.3750 & 286.8 & 4 & 600.0 & 00.0 \\
\hline 03 & 9 & 65 & 72.0011 & 11.1000 & 286.7 & 4 & 600.0 & 00.0 \\
\hline 03 & 9 & 66 & 73.5000 & 10.5500 & 286.6 & 4 & 600.0 & 00.0 \\
\hline 03 & 9 & 67 & 76.2500 & 9.9000 & 286.8 & 4 & 600.0 & 00.0 \\
\hline 03 & 9 & 68 & 80.2500 & 8.2500 & 287.9 & 4 & 600.0 & 00.0 \\
\hline 03 & 9 & 69 & 90.2472 & 5.2750 & 289.7 & 4 & 600.0 & .0 \\
\hline 03 & 9 & 610 & 86.9972 & 2.9750 & 291.9 & 3 & 600.0 & \\
\hline 03 & 9 & 611 & 70.8761 & 250 & 293.8 & 2 & 600.0 & \\
\hline 03 & 9 & 612 & 68.3759 & 3.9000 & 294.4 & 2 & 600.0 & 00.0 \\
\hline 03 & 9 & 613 & 89.3468 & 5.3000 & 295.2 & 3 & 600.0 & \\
\hline 03 & 9 & 614 & 94.5011 & 6 . & 295.4 & 4 & .0 & \\
\hline 03 & 9 & 615 & 86.2508 & 500 & 296.2 & 3 & 600.0 & .0 \\
\hline 03 & 9 & 616 & 74.7511 & 6.6500 & 295.7 & 4 & 60 & .0 \\
\hline 03 & 9 & 617 & 74.2501 & 7. & 294.5 & 4 & .0 & \\
\hline 03 & 9 & 618 & 70.0000 & 9.5750 & 292.9 & 4 & .0 & \\
\hline 03 & 9 & 619 & 69.5000 & 11.4250 & 290.7 & 4 & .0 & .0 \\
\hline 03 & 9 & 620 & 64.4997 & 11. & 289.3 & 4 & 6 & \\
\hline 03 & 9 & 621 & 74.0000 & 10 . & 288.7 & 4 & .0 & \\
\hline 03 & 9 & 622 & 71.7502 & 11.0000 & 288.1 & 4 & 600.0 & .0 \\
\hline 03 & 9 & 623 & 72.2500 & 11.75 & 287.8 & 4 & 6 & \\
\hline 03 & 9 & 624 & 75.5000 & 10.7250 & 288.2 & 4 & 60 & \\
\hline 03 & 9 & 71 & 72.0000 & 8.4250 & 289.5 & 4 & .0 & \\
\hline 03 & 9 & 72 & 81.0004 & 8.7000 & 288.9 & 4 & 60 & .0 \\
\hline 03 & 9 & 73 & 82.2501 & 10.9500 & 287.5 & 4 & .0 & \\
\hline 03 & 9 & 74 & 74.5001 & 11.0750 & 287.6 & 4 & .0 & 0.0 \\
\hline 03 & 9 & 75 & 73.2500 & 10.6500 & 288.0 & 4 & 60 & .0 \\
\hline 03 & 9 & 76 & 74.2500 & 10.3000 & 287.9 & 4 & .0 & \\
\hline 03 & 9 & 77 & 77.2501 & 9.5500 & 287.9 & 4 & .0 & 600.0 \\
\hline 03 & 9 & 78 & 79.0001 & 8.5250 & 289.3 & 4 & 600.0 & 0.0 \\
\hline 03 & 9 & 79 & 85.0303 & 5.5750 & 290.5 & 3 & 600.0 & 0 \\
\hline 03 & 9 & 710 & 241.9871 & 2.1250 & 294.9 & 2 & 600.0 & 600.0 \\
\hline 03 & 9 & 711 & 114.3634 & 3.6500 & 294.6 & 2 & 600.0 & 600.0 \\
\hline 03 & 9 & 712 & 98.7165 & 3.5250 & 295.4 & 2 & 600.0 & .0 \\
\hline 03 & 9 & 713 & 93.7512 & 5.0500 & 296.8 & 3 & 600.0 & 600.0 \\
\hline 03 & 9 & 714 & 86.9902 & 7.2750 & 297.1 & 4 & 600.0 & 600.0 \\
\hline 03 & 9 & 715 & 84.2499 & 8.0750 & 296.2 & 4 & 600.0 & .0 \\
\hline 03 & 9 & 716 & 86.5017 & 7.3500 & 296.8 & 4 & 600.0 & 600.0 \\
\hline 03 & 9 & 717 & 76.0000 & 8.4750 & 296.2 & 4 & 600.0 & 600.0 \\
\hline 03 & 9 & 718 & 77.4997 & 10.5500 & 294.3 & 4 & 600.0 & 600.0 \\
\hline 03 & 9 & 719 & 79.7544 & 9.2250 & 292.4 & 4 & 600.0 & 600.0 \\
\hline 03 & 9 & 720 & 63.5003 & 8.6500 & 292.0 & 4 & 600.0 & 600.0 \\
\hline 03 & 9 & 721 & 74.9004 & 5.7250 & 292.0 & 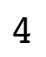 & 600.0 & 600.0 \\
\hline 03 & 9 & 722 & 62.2499 & 13.2750 & 291.2 & 4 & 600.0 & 600.0 \\
\hline U. & 9 & 723 & 63.5001 & 11.975 & 290.7 & 4 & 600.0 & 600.0 \\
\hline
\end{tabular}




\begin{tabular}{|c|c|c|c|c|c|c|c|c|}
\hline 3 & 9 & 724 & 62.5000 & 13.3750 & 289.2 & 4 & 600.0 & 00.0 \\
\hline 03 & 9 & 81 & 81.0607 & 9.6750 & 289.9 & 4 & 600.0 & 600.0 \\
\hline 03 & 9 & 82 & 124.7340 & 3.7000 & 288.9 & 4 & 600.0 & 00.0 \\
\hline 03 & 9 & 83 & 77.2461 & 9.1500 & 290.4 & 4 & 600.0 & 600.0 \\
\hline 03 & 9 & 84 & 67.2491 & 8.8250 & 289.5 & 4 & 600.0 & 00.0 \\
\hline 03 & 9 & 85 & 68.0000 & 9.9250 & 288.4 & 4 & 600.0 & 00.0 \\
\hline 03 & 9 & 86 & 71.5000 & 9.3000 & 287.1 & 4 & 600.0 & 600.0 \\
\hline 03 & 9 & 87 & 76.7449 & 8.6500 & 287.3 & 4 & 600.0 & 00.0 \\
\hline 03 & 9 & 88 & 86.4997 & 6.3500 & 289.0 & 4 & 600.0 & 00.0 \\
\hline 03 & 9 & 89 & 112.1090 & 6.6000 & 290.5 & 4 & 600.0 & 600.0 \\
\hline 03 & 9 & 810 & 148.5006 & 7.8000 & 291.1 & 4 & 600.0 & 00.0 \\
\hline 03 & 9 & 811 & 167.4596 & 4.1750 & 292.6 & 3 & 600.0 & \\
\hline 03 & 9 & 812 & 166.7505 & 4.6250 & 294.2 & 3 & 600.0 & 600.0 \\
\hline 03 & 9 & 813 & 168.7473 & 4.6000 & 295.4 & 3 & 600.0 & 600.0 \\
\hline 03 & 9 & 814 & 174.2481 & & 296.2 & 3 & 600.0 & \\
\hline 03 & 9 & 815 & 176.9986 & 6.1250 & 296.8 & 4 & 600.0 & 60 \\
\hline 03 & 9 & 816 & 170.7498 & 6.0500 & 297.1 & 4 & 600.0 & 600.0 \\
\hline 03 & 9 & 817 & 127.5897 & 6.5500 & 296.9 & 4 & 600.0 & \\
\hline 03 & 9 & 818 & 79.7466 & 7.0500 & 294.9 & 4 & 600.0 & 60 \\
\hline 03 & 9 & 819 & 69.2498 & 8.9000 & 292.2 & 4 & 600.0 & 60 \\
\hline 03 & 9 & 820 & 54.5169 & 9.62 & 291.5 & 4 & 600.0 & .0 \\
\hline 03 & 9 & 821 & 64.2501 & 750 & 292.3 & 4 & 600.0 & \\
\hline 03 & 9 & 822 & 67.7501 & 8.9500 & 292.6 & 4 & 600.0 & 60 \\
\hline 03 & 9 & 823 & 69.0002 & 8.8750 & 291.9 & 4 & 600.0 & .0 \\
\hline 03 & 9 & 824 & 75.7500 & 8 . & 292.1 & 4 & 600.0 & \\
\hline 03 & 9 & 91 & 74.4998 & 000 & 290.8 & 4 & 600.0 & 60 \\
\hline 03 & 9 & 92 & 67.9986 & 10.4500 & 290.0 & 4 & 600.0 & .0 \\
\hline 03 & 9 & 93 & 66.5000 & 10.6750 & 290.0 & 4 & 600.0 & \\
\hline 03 & 9 & 94 & 65.7499 & 3000 & 289.7 & 4 & 600.0 & 60 \\
\hline 03 & 9 & 95 & 67.5000 & 10.1500 & 290.0 & 4 & 600.0 & 600 \\
\hline 03 & 9 & 96 & 68.5000 & 9 . & 289.5 & 4 & 600.0 & \\
\hline 03 & 9 & 97 & 67.2498 & 10.5750 & 289.6 & 4 & 600.0 & .0 \\
\hline 03 & 9 & 98 & 77.2515 & 10.5000 & 288.8 & 4 & 600.0 & 600.0 \\
\hline 03 & 9 & 99 & 68.5153 & 9 . & 289.3 & 4 & 600.0 & .0 \\
\hline 03 & 9 & 910 & 71.7548 & 8 . & 290.5 & 4 & 600.0 & \\
\hline 03 & 9 & 911 & 65.9995 & 10.0500 & 291.8 & 4 & 600.0 & 600.0 \\
\hline 03 & 9 & 912 & 63.9977 & 10.3000 & 292.8 & 4 & 600.0 & \\
\hline 03 & 9 & 913 & 75.4768 & 9.0000 & 292.4 & 4 & 600.0 & 600.0 \\
\hline 03 & 9 & 914 & 97.2658 & 6.7750 & 292.4 & 4 & 600.0 & 600.0 \\
\hline 03 & 9 & 915 & 110.2738 & 6.2000 & 292.5 & 4 & 600.0 & \\
\hline 03 & 9 & 916 & 71.7472 & 6.1250 & 293.2 & 4 & 600.0 & 600.0 \\
\hline 03 & 9 & 917 & 88.7549 & 4.5750 & 293.8 & 3 & 600.0 & 600.0 \\
\hline 03 & 9 & 918 & 122.3683 & 4.6750 & 293.3 & 4 & 600.0 & 600.0 \\
\hline 03 & 9 & 919 & 89.3422 & 6.8250 & 291.5 & 4 & 600.0 & 600.0 \\
\hline 03 & 9 & 920 & 61.0004 & 8.7750 & 290.9 & 4 & 600.0 & 600.0 \\
\hline 03 & 9 & 921 & 67.2498 & 8.3750 & 290.6 & 4 & 600.0 & 600.0 \\
\hline 03 & 9 & 922 & 0004 & 8 . & 290.2 & 4 & 600.0 & 600.0 \\
\hline 03 & 9 & 923 & 65.2501 & 8.6000 & 290.1 & 4 & 600.0 & 600.0 \\
\hline
\end{tabular}




\begin{tabular}{|c|c|c|c|c|c|c|c|}
\hline 3 & 9924 & 75.2496 & 6.8000 & 290.5 & 4 & 600.0 & 600.0 \\
\hline 3 & 9101 & 76.0002 & 7.6750 & 289.8 & 4 & 600.0 & 600.0 \\
\hline 03 & 9102 & 70.2498 & .3000 & 289.1 & 4 & 600.0 & 00.0 \\
\hline 3 & 9103 & 82.2503 & 7.8250 & 289.4 & 4 & 600.0 & 00.0 \\
\hline 03 & 9104 & 101.2454 & 6.3250 & 289.6 & 4 & 600.0 & 00.0 \\
\hline 03 & 9105 & 123.5162 & 6.0250 & 289.5 & 4 & 600.0 & 00.0 \\
\hline 03 & 9106 & 142.2495 & 7.5250 & 289.2 & 4 & 600.0 & 00.0 \\
\hline 03 & 9107 & 141.6214 & 4.6250 & 288.3 & 4 & 600.0 & 00.0 \\
\hline 03 & 9108 & 116.2532 & 3.6500 & 289.4 & 3 & 600.0 & 00.0 \\
\hline 03 & 9109 & 146.0053 & 6.2000 & 290.5 & 4 & 600.0 & 0 \\
\hline 03 & 91010 & 160.9890 & 7.6000 & 292.4 & 4 & 600.0 & \\
\hline 03 & 91011 & 158.0083 & 7.0500 & 293.8 & 4 & 600.0 & \\
\hline 03 & 91012 & 164.2448 & 6.5250 & 295.3 & 4 & 600.0 & 00.0 \\
\hline 03 & 91013 & 163.7505 & .2750 & 297.0 & 4 & 600.0 & 00.0 \\
\hline 03 & 91014 & 165.2529 & .5750 & 298.3 & 4 & .0 & \\
\hline 03 & 91015 & 178.9920 & 6.9000 & 299.2 & 4 & 600.0 & 00.0 \\
\hline 03 & 91016 & 180.2496 & 6.2250 & 299.5 & 4 & 60 & 600.0 \\
\hline 03 & 91017 & 166.7512 & 5.9500 & 299.9 & 3 & .0 & \\
\hline 03 & 91018 & 141.4182 & 5.7750 & 299.3 & 4 & 600.0 & .0 \\
\hline 03 & 91019 & 66.0000 & 5.3000 & 297.0 & 4 & 60 & .0 \\
\hline 03 & 91020 & 70.2501 & 5. & 296.3 & 4 & 60 & \\
\hline 03 & 91021 & 77.2486 & 6.0500 & 296.1 & 4 & .0 & \\
\hline 03 & 91022 & 82.4997 & 5.7250 & 296.2 & 4 & 600.0 & .0 \\
\hline 03 & 91023 & 91.2141 & 4.45 & 296.9 & 4 & 60 & \\
\hline 03 & 91024 & 90.2300 & 5.2250 & 297.1 & 4 & .0 & \\
\hline 03 & 9111 & 136.7539 & 6.4000 & 299.0 & 4 & .0 & 600.0 \\
\hline 03 & 9112 & 153.5017 & 7.3500 & 298.2 & 4 & 600.0 & .0 \\
\hline 03 & 9113 & 151.7557 & 7.4250 & 297.2 & 4 & .0 & \\
\hline 03 & 9114 & 132.7295 & 6.9750 & 296.4 & 4 & .0 & 600.0 \\
\hline 03 & 9115 & 124.2496 & 6.1500 & 295.6 & 4 & 600.0 & 600.0 \\
\hline 03 & 9116 & 107.2548 & 6.1500 & 295.2 & 4 & .0 & \\
\hline 03 & 9117 & 109.9941 & 6.4250 & 295.3 & 4 & .0 & 600.0 \\
\hline 03 & 9118 & 143.4940 & 7.5250 & 295.6 & 4 & 600.0 & 600.0 \\
\hline 03 & 9119 & 152.7502 & 6.1750 & 296.1 & 4 & .0 & 0.0 \\
\hline 03 & 91110 & 156.7529 & 5.97 & 297.2 & 4 & 600.0 & 600.0 \\
\hline 03 & 91111 & 170.0002 & 4.8750 & 298.3 & 3 & 600.0 & 600.0 \\
\hline 03 & 91112 & 168.7488 & 5.1000 & 299.6 & 3 & 600.0 & 600.0 \\
\hline 03 & 91113 & 170.2500 & 5.8750 & 300.6 & 3 & 600.0 & 600.0 \\
\hline 03 & 91114 & 176.7503 & 5.5500 & 301.7 & 3 & 600.0 & 600.0 \\
\hline 03 & 91115 & 177.0000 & 4 . & 302.5 & 3 & 600.0 & 600.0 \\
\hline 03 & 91116 & 171.2471 & 5.2250 & 302.7 & 3 & 600.0 & 600.0 \\
\hline 03 & 91117 & 165.0007 & 4.7000 & 302.8 & 3 & 600.0 & 600.0 \\
\hline 03 & 91118 & 155.2501 & 3.3750 & 302.7 & 4 & 600.0 & 600.0 \\
\hline 03 & 91119 & 113.4185 & 2.8500 & 302.1 & 4 & 600.0 & 600.0 \\
\hline 03 & 91120 & 97.2325 & 5.1500 & 302.1 & 4 & 600.0 & 600.0 \\
\hline 03 & 91121 & 118.7509 & & 302.4 & 4 & 600.0 & 600.0 \\
\hline 03 & 91122 & 138.5017 & 6.1750 & 302.3 & 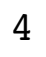 & 600.0 & 600.0 \\
\hline U. & 91123 & 140.7778 & 3.2250 & 300.9 & 4 & 600.0 & 600.0 \\
\hline
\end{tabular}




\begin{tabular}{|c|c|c|c|c|c|c|c|}
\hline & 24 & & & & & & \\
\hline & 121 & 88.3755 & 3.7750 & 1.0 & & & \\
\hline & 9122 & 95 & 4.8250 & 300.9 & & 00 & \\
\hline & 123 & 136.8018 & 250 & 301.3 & & $n$ & \\
\hline & 124 & 51.2532 & 0.9750 & & & & \\
\hline & 125 & 133.90 & 0 & 98.5 & & & \\
\hline & 126 & 104.0004 & 3500 & 297.8 & & ( & \\
\hline & 127 & 125.7391 & 4750 & 98.0 & & & \\
\hline & 128 & 152.7485 & 1750 & 1 & & & \\
\hline & 129 & 161.7474 & 8.4250 & 298.6 & & & \\
\hline & 1210 & 162.7507 & 2.7000 & 298.7 & & & \\
\hline & 1211 & 166.0145 & 0.4000 & 99.8 & & & \\
\hline & 1212 & 161.50 & 250 & 301.3 & & & \\
\hline & 1213 & 157.003 & 750 & 302.7 & & & \\
\hline & 1214 & 164 . & & & & & \\
\hline & 1215 & 167.49 & & 30 & & & \\
\hline & 1216 & 168.001 & 750 & 305.3 & & .0 & \\
\hline & 91217 & 162 . & 0 & 5 & & & \\
\hline & 1218 & 176. & & 30 & & & \\
\hline & 1219 & 156.50 & 750 & & & & \\
\hline & 91220 & 151.2489 & 750 & 302.9 & & .0 & \\
\hline & 91221 & 149.24 & & & & & \\
\hline & 912 & 133.74 & 0 & 30 & & & \\
\hline & 91223 & 126.7586 & 250 & 7 & & 0 & \\
\hline & 91224 & 123. & & & & & \\
\hline & 9131 & 155.2500 & & & 4 & & \\
\hline & 9132 & 153.7502 & 000 & 30 & 4 & 0 & \\
\hline & 9133 & 159.99 & 0 & & 4 & & \\
\hline & 9134 & 157 . & 12. & 29 & 4 & & \\
\hline & 9135 & 145.7499 & & 297.1 & $I$ & & \\
\hline & 9136 & 151.7499 & & 296.3 & 4 & & \\
\hline & 9137 & 12 & 0 & & 4 & & \\
\hline & 9138 & 157 . & & & 4 & & \\
\hline & 9139 & 155.5000 & 0 & 29 & 4 & 0 & \\
\hline & 91310 & 1 & & & 4 & & \\
\hline & 91 & 15 & & & 4 & & \\
\hline & 91312 & 501 & & 300.1 & 4 & & \\
\hline & 91313 & 15 & 7. & 30 & 4 & & \\
\hline & 91314 & 164.7505 & & 302.3 & 4 & .0 & \\
\hline & 91315 & 162.7496 & & 303.3 & 4 & & \\
\hline & 91316 & 164.4890 & & & 3 & & \\
\hline & 91317 & 175 . & & & 3 & & \\
\hline & 91318 & 182.2500 & & 303.3 & a & & 0 \\
\hline & 91319 & 344 & & & 5 & & \\
\hline 03 & & & & 302.0 & 1 & .0 & \\
\hline & 91321 & 133.0006 & 3.2000 & 301.9 & 5 & & 0 \\
\hline & & & & & 4 & & \\
\hline & 91323 & 125.2491 & 3.0750 & 301.9 & & & 600 \\
\hline
\end{tabular}




\begin{tabular}{|c|c|c|c|c|c|c|c|}
\hline & 24 & & & & & & \\
\hline & 141 & 117.9936 & 3.6000 & 2.2 & 4 & 0 & \\
\hline & 142 & 15.9958 & 4500 & 302.4 & 4 & 00 & \\
\hline & 143 & 142.7631 & 2.3250 & 301.3 & & 00.0 & \\
\hline & 144 & r & 500 & 00.3 & & & \\
\hline & 145 & 7 & & 99.5 & & 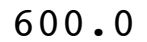 & \\
\hline & 146 & 27.2508 & 8500 & 299.2 & & 00 & \\
\hline & 147 & 5.2925 & 8000 & 99.1 & & & \\
\hline & 148 & 1.7857 & 0 & 99.7 & & & \\
\hline & 14 & 2346 & 500 & 300.3 & & 00 & \\
\hline & 1410 & 4.5023 & 9500 & 301.0 & & 00.0 & \\
\hline & 1411 & 4.7505 & 000 & 301.7 & & & \\
\hline & 14 & 73.7535 & 7.2500 & 302.7 & 4 & 0 & \\
\hline & 413 & 31.75 & 0 & 303.4 & 4 & 00 & 0 \\
\hline & 1414 & 8.75 & & & & & \\
\hline & 5 & 5 & & 30 & 3 & & \\
\hline & 416 & 75.7504 & 0 & 305.3 & 3 & .0 & 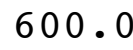 \\
\hline & 417 & 6.9023 & 0 & 2 & & & \\
\hline & 18 & & & 303.4 & 4 & & \\
\hline & 419 & 5003 & 7 . & 301.1 & 4 & .0 & 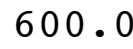 \\
\hline & 420 & 64.999 & 50 & 30 & 4 & .0 & \\
\hline & 21 & 68. & & & 4 & & \\
\hline & 22 & 2502 & 8.8250 & 30 & 4 & & \\
\hline & 91423 & 73.5000 & 2250 & 299.9 & 4 & 0 & \\
\hline & 91424 & 76.4997 & & & 4 & & \\
\hline & 9151 & 68 . & 9 . & 29 & 4 & & \\
\hline & 9152 & 68.2492 & 4000 & 298.4 & 4 & 0 & . \\
\hline & 9153 & 72.00 & 8.27 & 298 & 4 & & \\
\hline & 9154 & 68. & 50 & 29 & 4 & & \\
\hline & 9155 & 73.2495 & 000 & 298.6 & 4 & & J. \\
\hline & 9156 & 57.2360 & 5.7000 & 299.0 & 4 & & . \\
\hline & 9157 & 41.64 & 3.12 & 299 & 4 & & \\
\hline & 9158 & 76.6604 & 1.7500 & 297.8 & 3 & & 0 . \\
\hline & 9159 & 286.147 & 1.2750 & 297.4 & 2 & & \\
\hline & 91510 & 269.44 & 0 & & 1 & & \\
\hline & 91511 & 288.7684 & 1.7500 & 300.0 & 1 & & 0 . \\
\hline & 91512 & 115.3574 & 3.9000 & 301.3 & 2 & & \\
\hline & 91513 & 8076 & 500 & 30 & 3 & & \\
\hline & 91514 & 91.5000 & 6.1750 & 300.6 & 4 & & 0 . \\
\hline & 91515 & 89.709 & $\$ 750$ & 301.1 & 4 & & \\
\hline & 91516 & 73.9801 & 6 . & 301.6 & 4 & & \\
\hline & & 75.7416 & 7.5250 & 300.0 & 4 & & 0 . \\
\hline & 91518 & 73.7396 & 8.1750 & 297.8 & 4 & & 0 . \\
\hline & 91519 & 64.5000 & 750 & & 4 & & \\
\hline & & 68.5000 & 3.3250 & & 4 & & \\
\hline & 91521 & 63.2500 & 14.0750 & 295.1 & 4 & & 00 \\
\hline & & & & & 4 & & \\
\hline & 91523 & 59.0001 & 11.3750 & 295.9 & 1 & .0 & 600 \\
\hline
\end{tabular}




\begin{tabular}{|c|c|c|c|c|c|c|c|}
\hline 3 & 91524 & 61.4807 & 6.6500 & 295.4 & 4 & 600.0 & 00.0 \\
\hline 3 & 9161 & 86.2410 & 4.9750 & 294.6 & 4 & 600.0 & 600.0 \\
\hline 03 & 9162 & 59.0001 & 10.0750 & 294.6 & 4 & 600.0 & 00.0 \\
\hline 03 & 9163 & 63.0002 & 10.5000 & 294.4 & 4 & 600.0 & 00.0 \\
\hline 13 & 9164 & 54.7495 & 9.6750 & 294.4 & 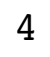 & 600.0 & 00.0 \\
\hline 03 & 9165 & 67.9619 & 7.6000 & 294.6 & 4 & 600.0 & .0 \\
\hline 03 & 9166 & 61.2470 & 10.8000 & 294.1 & 4 & 600.0 & 500.0 \\
\hline 03 & 9167 & 74.7502 & 7.5750 & 293.7 & 4 & 600.0 & .0 \\
\hline 03 & 9168 & 75.2501 & 8.3500 & 294.3 & 4 & 600.0 & \\
\hline 03 & 9169 & 74.7496 & 8.8500 & 293.2 & 4 & 600.0 & 600.0 \\
\hline 03 & 91610 & 73.2461 & 6.6000 & 295.2 & 4 & 600.0 & .0 \\
\hline 03 & 91611 & 70.7529 & 7.7250 & 295.5 & 4 & 600.0 & \\
\hline 03 & 91612 & 77.4769 & 6.8000 & 296.0 & 4 & 600.0 & .0 \\
\hline 03 & 91613 & 64.9758 & 8.4750 & 297.7 & 4 & 600.0 & \\
\hline 03 & 91614 & 80.2500 & 9.3000 & 297.6 & 4 & 600.0 & .0 \\
\hline 03 & 91615 & 79.7507 & 10.1500 & 297.1 & 4 & 600.0 & \\
\hline 03 & 91616 & 69.0057 & 10.0750 & 296.2 & 4 & 600.0 & 600.0 \\
\hline 03 & 91617 & 73.2501 & 9.6500 & 295.3 & 4 & 600.0 & .0 \\
\hline 03 & 91618 & 70.9992 & 9.3750 & 294.5 & 4 & 600.0 & \\
\hline 03 & 91619 & 70.2500 & 9.7750 & 293.3 & 4 & 600.0 & 600.0 \\
\hline 03 & 91620 & 72.2495 & 10.2500 & 292.5 & 4 & 600.0 & .0 \\
\hline 03 & 91621 & 72.0000 & 10.0500 & 291.8 & 4 & 600.0 & \\
\hline 03 & 91622 & 72.7500 & 10.5500 & 291.8 & 4 & 600.0 & 60 \\
\hline 03 & 91623 & 69.7499 & 10.5750 & 290.9 & 4 & 600.0 & .0 \\
\hline 03 & 91624 & 69.0000 & 11.0500 & 290.7 & 4 & 600.0 & \\
\hline 03 & 9171 & 76.0002 & 10.9000 & 290.4 & 4 & 600.0 & 60 \\
\hline 03 & 9172 & 72.7502 & 9.6000 & 290.8 & 4 & 600.0 & .0 \\
\hline 03 & 9173 & 75.4917 & 8.95 & 290.6 & 4 & 600.0 & .0 \\
\hline 03 & 9174 & 105.8979 & 8.5000 & 290.5 & 4 & 600.0 & 60 \\
\hline 03 & 9175 & 140.5021 & 10.4500 & 289.9 & 4 & 600.0 & .0 \\
\hline 03 & 9176 & 149.0076 & 12.0750 & 288.7 & 4 & 600.0 & .0 \\
\hline 03 & 9177 & 156 . & 11.3000 & 287.9 & 4 & 600.0 & 60 \\
\hline 03 & 9178 & 162.5000 & 10.8750 & 287.9 & 4 & 600.0 & 60 \\
\hline 03 & 9179 & 169.5009 & 10.9000 & 288.8 & 4 & 600.0 & .0 \\
\hline 03 & 91710 & 170.2504 & 10 . & 289.8 & 4 & 600.0 & \\
\hline 03 & 91711 & 163.2504 & 7.9500 & 291.3 & 4 & 600.0 & 600.0 \\
\hline 03 & 91712 & 157.0014 & 7.6000 & 292.8 & 4 & 600.0 & 600.0 \\
\hline 03 & 91713 & 160.6650 & 8.43 & 293.8 & 4 & 600.0 & 60 \\
\hline 03 & 91714 & 157.0014 & 7.6000 & 292.8 & 4 & 600.0 & 600.0 \\
\hline 03 & 91715 & 160.6650 & 8.433 & 293.8 & 4 & 600.0 & 600 \\
\hline 03 & 91716 & 169.0000 & 8.1000 & 297.1 & 4 & 600.0 & 60 \\
\hline 03 & 91717 & 165.5013 & 7.5750 & 297.2 & 4 & 600.0 & 600.0 \\
\hline 03 & 91718 & 178.7597 & 6.4750 & 296.8 & 4 & 600.0 & 600.0 \\
\hline 03 & 91719 & 162.0000 & 2.8000 & 295.6 & 4 & 600.0 & 600.0 \\
\hline 03 & 91720 & 153.2507 & 2.2750 & 295.1 & 4 & 600.0 & 600.0 \\
\hline 03 & 91721 & 112.9791 & 3.4500 & 295.5 & 4 & 600.0 & 600.0 \\
\hline 03 & 91722 & 142.7508 & & .1 & 4 & .0 & .0 \\
\hline 03 & 91723 & 133.7498 & 5.175 & 296.5 & 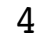 & 600.0 & 600.0 \\
\hline
\end{tabular}




\begin{tabular}{|c|c|c|c|c|c|c|c|}
\hline 3 & 1724 & 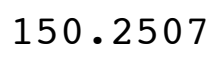 & 7.1000 & 96.5 & & . & \\
\hline 3 & 9181 & 153.5000 & 7.7750 & 296.1 & 4 & & \\
\hline & 9182 & 0002 & 1250 & 295.3 & 4 & 00 & \\
\hline & 9183 & 157.0001 & 1500 & 294.5 & & 00 & \\
\hline & 184 & 7477 & .3500 & 93.7 & & 0 & \\
\hline & 185 & 131.2438 & .1500 & 293.7 & 4 & 00 & \\
\hline & 186 & 138.4997 & 7.7500 & 293.7 & & 0 & \\
\hline & 187 & 062 & .6500 & 93.5 & & & \\
\hline & 9188 & 2486 & .9750 & 293.9 & & 0 & \\
\hline & 9189 & 156.2496 & 5250 & 294.4 & & 00 & \\
\hline & 1810 & 164.2330 & 7.4500 & 295.1 & & 00 & \\
\hline & 811 & 0.4969 & & 96.1 & 4 & & \\
\hline & 1812 & 167.5008 & 9500 & 297.5 & 3 & 0 & \\
\hline & 1813 & 61.4931 & 6.5750 & 298.7 & 2 & 0.0 & \\
\hline & 814 & 69.4885 & & 299 & 4 & & \\
\hline & 91815 & 995 & 750 & 300.7 & 4 & 0 & \\
\hline & L 816 & 165.7502 & 0 & 301.1 & 4 & 0 & \\
\hline & 1817 & 515 & 0 & 301.2 & 4 & & \\
\hline & 1818 & 164 . & 500 & 300.8 & 4 & 0 & \\
\hline & 91819 & 151.5138 & 3750 & 299.2 & 4 & 0 & \\
\hline & 820 & 930 & 0 & 299.4 & 4 & & \\
\hline & 91821 & 096 & 50 & 299.0 & 5 & & \\
\hline & 91822 & 97.8967 & 750 & 298.6 & 4 & 00 & 600 . \\
\hline & 91823 & 008 & 0 & 25 & 4 & 0 & \\
\hline & 91824 & 494 & 0 & 29 & 5 & & \\
\hline & 9191 & 2863 & 750 & 298 & 4 & & \\
\hline & 9192 & 98.2312 & 3.5500 & 298.8 & 4 & 0 & \\
\hline & 9193 & 89.2500 & 0 & 29 & 5 & & \\
\hline & 9194 & 704 & 750 & 298.2 & 4 & 0 & 0 . \\
\hline & 9195 & 42.5415 & 2750 & 297.8 & 5 & & \\
\hline & 9196 & 141.4995 & 000 & 296 & 5 & & \\
\hline & 9197 & 034 & 0 & 297.4 & 6 & & 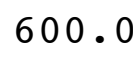 \\
\hline & 9198 & 147.7488 & 5750 & 299.6 & 5 & 0 & U. \\
\hline & 9199 & 17.9182 & 00 & 301.7 & 4 & 0 & \\
\hline & 91910 & 241 & 50 & 30 & 3 & & \\
\hline & 91911 & 943 & 750 & 300.4 & 2 & & • \\
\hline & 91912 & 246.2527 & 2.2250 & 302.0 & 1 & 0 & \\
\hline 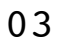 & 91913 & 224.2398 & 3.3500 & 302.6 & 2 & 0 & • \\
\hline & 91914 & 206.2479 & 3.4750 & 303.3 & 2 & .0 & 0 . \\
\hline & 91915 & 189.7521 & 500 & 303.6 & 3 & 0 & \\
\hline & 91916 & 187.7828 & 250 & 303 & 3 & & \\
\hline 03 & 91917 & 187.4962 & 4.0750 & 303.7 & 3 & 600.0 & 600 . \\
\hline & 91918 & 190.7497 & 750 & 302.9 & 4 & 600 & 600. \\
\hline 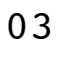 & 91919 & 162.2680 & 2.3750 & 301.1 & 4 & & 10 \\
\hline 03 & 91920 & 136.5110 & 1.9500 & 300.8 & 4 & 600.0 & 00 \\
\hline & 91921 & .4954 & 3.3250 & 300.6 & 5 & 600.0 & 600. \\
\hline & 91 & 000 & 250 & 300.6 & 5 & 60 & \\
\hline & 91923 & 98.0799 & 3.9750 & 300.5 & 4 & 600.0 & 600 \\
\hline
\end{tabular}




\begin{tabular}{|c|c|c|c|c|c|c|c|}
\hline & 1924 & & 2.1000 & 0.2 & & & \\
\hline 3 & 9201 & 131.2495 & 2.3500 & 0.1 & & & \\
\hline & 9202 & 7532 & 1250 & 298.8 & 4 & 00 & \\
\hline & 9203 & 29.0000 & . 4750 & 298.8 & & 00 & \\
\hline & 9204 & 0.2259 & .1750 & 98.9 & & 0 & \\
\hline & 9205 & 5000 & .4250 & 97.6 & 4 & 00 & \\
\hline & 9206 & 134.2494 & 2.5750 & 297.0 & & 0 & \\
\hline & 9207 & 5.2492 & .2000 & 97.0 & & & \\
\hline & 9208 & 50.0000 & 6.7000 & 297.6 & 4 & 0 & \\
\hline & 9209 & 53.5000 & 6.8500 & 298.0 & & 0 & \\
\hline & 92010 & 57.7468 & .4500 & 298.7 & $\mathbf{4}$ & 0 & \\
\hline & 92011 & 7.5031 & .2250 & 299.9 & 4 & & \\
\hline & 92012 & 57.7508 & 6.7000 & 300.9 & 4 & 0 & \\
\hline & 92013 & 65.7506 & 6.7750 & 302.0 & $I$ & 0.0 & \\
\hline & 92014 & 0.2461 & 5.5250 & & 3 & & \\
\hline & 92015 & 56.5000 & 5.3500 & 304.5 & 3 & 0 & \\
\hline & 92016 & 0.2497 & 27 & 305 & 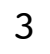 & 0 & \\
\hline & 92017 & 008 & 000 & 7 & 3 & & \\
\hline & 92018 & 870 & 2.3500 & .3 & 4 & 0 & \\
\hline & 92019 & 730 & 1.2500 & 30 & 5 & 0 & \\
\hline & 92020 & 000 & 500 & 0 & 4 & & \\
\hline & 92021 & 99 & & & 4 & & \\
\hline & 92022 & 81.2500 & 750 & 302.2 & 4 & 0 & 0. \\
\hline & 92023 & 496 & 0 & 2.1 & 4 & 0 & \\
\hline & 92024 & 000 & & & 4 & & \\
\hline & 9211 & 81.2500 & 7000 & & 4 & & \\
\hline & 9212 & 83.2498 & 0 & 30 & 4 & 0 & \\
\hline & 9213 & 86.0000 & 0 & 7 & 4 & & \\
\hline 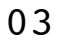 & 9214 & 387 & 0 & .6 & 4 & 0 & 0 . \\
\hline & 9215 & .1668 & 1000 & 299.7 & 5 & & \\
\hline & 9216 & 50.4723 & 0 & 2 & 6 & & \\
\hline 3 & 9217 & 693 & 50 & .7 & 5 & & \\
\hline & 9218 & 162.2504 & 500 & 300.1 & 4 & 0 & U. \\
\hline & 9219 & 519 & 250 & .2 & 4 & 0 & \\
\hline & 92110 & 516 & 3 . & .4 & 4 & & \\
\hline & 92111 & 158.4991 & 3.4500 & 302.2 & 3 & & 0 . \\
\hline & 92112 & 184.7854 & 250 & 3.8 & 2 & 0 & \\
\hline . & 92113 & 218.7826 & 3 . & .8 & 2 & & 0 \\
\hline & 92114 & 231.0064 & 2.7750 & 305.9 & 1 & 600.0 & 0 . \\
\hline & 92115 & 212.2518 & 750 & 30 & 2 & 600 & \\
\hline & 92116 & 214.2907 & 2 . & & 1 & & \\
\hline 03 & 92117 & 195.4121 & 2.1000 & 306.7 & 1 & 600.0 & 600 . \\
\hline & 92118 & 186.1426 & 1.7750 & 305.8 & 2 & 600 & 600. \\
\hline 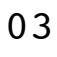 & 92119 & 114.2998 & 1.1750 & 303.9 & 3 & & 6 \\
\hline 03 & 92120 & 108.9452 & 0.9250 & 303.3 & 4 & 600.0 & 00 . \\
\hline & 92121 & 113.3382 & 0.8000 & 303.1 & 5 & 600.0 & 500. \\
\hline & 92122 & 123.3287 & & 30 & 4 & & \\
\hline & 92123 & 75.5000 & 4.0000 & 304.3 & & 600.0 & 600 \\
\hline
\end{tabular}




\begin{tabular}{|c|c|c|c|c|c|c|c|}
\hline 3 & 2124 & & $\$ 500$ & & & & \\
\hline & 9221 & 12 & 2.2250 & 3.2 & 4 & 00.0 & \\
\hline & 9222 & 148.9943 & 2.0000 & 302.7 & 5 & 00 & \\
\hline & 9223 & 141.5000 & 1.5000 & 302.6 & & 00 & \\
\hline & 9224 & 069 & 000 & 2.2 & & 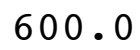 & \\
\hline & 9225 & 157 & 8500 & 01.6 & & 0( & \\
\hline & 9226 & 790 & 0.4750 & 299.9 & & 00 & \\
\hline & 9227 & 7041 & 9250 & 300.5 & & 0 & \\
\hline & 9228 & 146. & 0 & 00.4 & & & \\
\hline & 9229 & 226.1944 & 0.8000 & 303.3 & 4 & 0 & U \\
\hline & 92210 & 250.5018 & 1.6500 & 304.4 & & 500.0 & \\
\hline & 92211 & 189.74 & 0 & 303.9 & & & \\
\hline & 92212 & 78.7504 & 3.8000 & 304.4 & & 0 & \\
\hline & 92213 & 190.9979 & 5500 & 30 & & 0 & 8 \\
\hline & 92214 & 209.5198 & 0 & & & & \\
\hline & 92215 & 195.7477 & & 30 & 2 & & \\
\hline & 92216 & 182.4941 & 3.3750 & 307.5 & 2 & 00.0 & 0 \\
\hline & 92217 & 169.5000 & 0 & 3 & 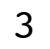 & 0 & \\
\hline & 92218 & 154.7617 & 2.7750 & 30 & 4 & & \\
\hline & 92219 & 145.5153 & 2.1250 & 305.2 & 5 & 00.0 & 00 \\
\hline & 92220 & 4366 & 0.8000 & 30 & 6 & 0 & \\
\hline & 92221 & 31 & & & 5 & & \\
\hline & 92222 & 2500 & 3750 & 30 & 4 & 0 & • \\
\hline & 92223 & .7500 & 750 & 3 & 4 & .0 & . \\
\hline & 92224 & .2504 & & & 4 & & \\
\hline & 9231 & 83.5000 & 500 & .2 & 4 & & \\
\hline & 9232 & 82.2501 & 5000 & 3 & 4 & 0 & . \\
\hline & 9233 & 81.7500 & 0 & 7 & 4 & & \\
\hline & 9234 & 000 & 50 & .7 & 4 & & \\
\hline & 9235 & 85.7501 & 0 & 3.1 & 4 & 0 & 0 . \\
\hline & 9236 & 121.5685 & 3.2000 & 301.6 & 5 & & 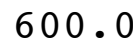 \\
\hline & 9237 & 144.7185 & 2 . & & 5 & & \\
\hline & 9238 & 125.2671 & 000 & .4 & 4 & 0 & . \\
\hline & 9239 & 309.6734 & 0500 & .5 & 3 & 0 & 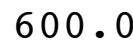 \\
\hline & 92310 & 266 & 2 . & & 2 & & \\
\hline & 92311 & 243.2492 & 2.5750 & .4 & 3 & .0 & 0 . \\
\hline & 92312 & 5005 & 7500 & 3.3 & 2 & & \\
\hline & 92313 & 258.2412 & 3250 & 30 & 1 & & 0 . \\
\hline & 92314 & 62.1380 & 4.0500 & 305.2 & 2 & .0 & 600 . \\
\hline & 92315 & 995 & 9500 & 304.6 & 3 & & \\
\hline & 92316 & 001 & 750 & & 4 & & 60 \\
\hline & 92317 & 80.7500 & 6.9750 & 303.0 & 4 & .0 & 0 . \\
\hline & 92318 & 82.4998 & 8.7500 & 300.8 & 4 & .0 & 0 . \\
\hline & 92319 & 83.0000 & 8.9500 & & 4 & & \\
\hline 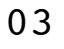 & 92320 & 83.9999 & 8.4000 & & 4 & & 0 . \\
\hline & 92321 & 84.7496 & 4.2250 & 300.9 & 4 & 600.0 & 500 \\
\hline & 92322 & 4987 & & & 4 & & \\
\hline & 92323 & 79.2496 & 7.4750 & 300.9 & $\mathbf{I}$ & 600.0 & 600 \\
\hline
\end{tabular}




\begin{tabular}{|c|c|c|c|c|c|c|c|}
\hline 3 & 92324 & 81.5000 & 7.5500 & 300.4 & 4 & 600.0 & 600.0 \\
\hline 03 & $924 \quad 1$ & 82.9999 & 7.6750 & 299.9 & 4 & 600.0 & 600.0 \\
\hline 03 & 9242 & 83.2500 & 8.7500 & 299.6 & 4 & 600.0 & 00.0 \\
\hline 3 & 9243 & 85.7500 & 9.3250 & 299.7 & 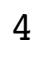 & 600.0 & 00.0 \\
\hline 03 & $924 \quad 4$ & 84.7496 & 9.3500 & 299.2 & 4 & 600.0 & 00.0 \\
\hline 03 & 9245 & 84.2515 & 9.9250 & 298.5 & 4 & 600.0 & 00.0 \\
\hline 03 & 9246 & 78.5000 & 11.5250 & 295.8 & 4 & 600.0 & 00.0 \\
\hline 03 & 9247 & 76.5000 & 12.0000 & 294.3 & 4 & 600.0 & \\
\hline 03 & 9248 & 77.9999 & 11.2000 & 294.6 & 4 & 600.0 & 00.0 \\
\hline 03 & 9249 & 77.0001 & 10.0000 & 294.7 & 4 & 600.0 & 00.0 \\
\hline 03 & 92410 & 78.9998 & 9.7500 & 294.8 & 4 & 600.0 & \\
\hline 03 & 92411 & 88.4917 & 7.7500 & 295.8 & 4 & 600.0 & 60 \\
\hline 03 & 92412 & 90.0014 & 7.4250 & 297.0 & 4 & 600.0 & \\
\hline 03 & 92413 & 82.5001 & 10.0250 & 295.9 & 4 & 0.0 & \\
\hline 03 & 92414 & 80.7500 & 10. & 295.9 & 4 & .0 & 60 \\
\hline 03 & 92415 & 75.0000 & 11.5000 & 295.5 & 4 & 600.0 & 0 \\
\hline 03 & 92416 & 75.5005 & 13.8000 & 294.6 & 4 & 600.0 & .0 \\
\hline 03 & 92417 & 75.0001 & 15 . & 293.9 & 4 & .0 & \\
\hline 03 & 92418 & 74.2500 & 15.6000 & 293.2 & 4 & 600.0 & 600.0 \\
\hline 03 & 92419 & 75.7500 & 15 . & 292.4 & 4 & .0 & .0 \\
\hline 03 & 92420 & 73.7501 & 14 . & 293.0 & 4 & .0 & \\
\hline 03 & 92421 & 74.0000 & 12.9750 & 293.7 & 4 & .0 & \\
\hline 03 & 92422 & 74.5001 & 13.0750 & 292.6 & 4 & .0 & 600.0 \\
\hline 03 & 92423 & 79.2500 & 12.6250 & 291.7 & 4 & .0 & \\
\hline 03 & 92424 & 82.9999 & 12.0750 & 291.5 & 4 & 60 & \\
\hline 03 & 9251 & 83.7502 & 10.6750 & 292.2 & 4 & 600.0 & .0 \\
\hline 03 & 9252 & 78.7500 & 10.47 & 291.0 & 4 & .0 & .0 \\
\hline 03 & 9253 & 78.2491 & 10.2750 & 290.5 & 4 & .0 & \\
\hline 03 & 9254 & 83.9993 & 11.2750 & 289.5 & 4 & .0 & 600.0 \\
\hline 03 & 9255 & 82.5000 & 10.5000 & 288.8 & 4 & .0 & .0 \\
\hline 03 & 9256 & 82.2500 & 750 & 289.1 & 4 & .0 & \\
\hline 03 & 9257 & 84.7509 & 7.1750 & 288.1 & 4 & .0 & 60 \\
\hline 03 & 9258 & 106.1902 & 2.85 & 290.1 & 3 & .0 & .0 \\
\hline 03 & 9259 & 143.6264 & 1.8000 & 292.2 & 2 & 600.0 & 60 \\
\hline 03 & 92510 & 178.4024 & 1.7250 & 294.1 & 1 & .0 & .0 \\
\hline 03 & 92511 & 254.9899 & 2.2750 & 294.6 & 1 & 600.0 & 600.0 \\
\hline 03 & 92512 & 268.2262 & 2 . & 295.9 & 1 & .0 & 00.0 \\
\hline 03 & 92513 & 35.0762 & 2.4250 & 298.3 & 1 & 600.0 & 600.0 \\
\hline 03 & 92514 & 92.2485 & 4.4500 & 297.3 & 2 & 600.0 & 600.0 \\
\hline 03 & 92515 & 87.9972 & 4 . & 29 & 3 & .0 & 600 \\
\hline 03 & 92516 & 83.5000 & 5.7500 & 297.9 & 4 & 600.0 & 600.0 \\
\hline 03 & 92517 & 81.7500 & 6.1750 & 296.8 & 4 & 600.0 & 600.0 \\
\hline 03 & 92518 & 80.7502 & 7.5000 & 294.9 & 4 & 600.0 & 600.0 \\
\hline 03 & 92519 & 82.4998 & 8.0000 & 293.9 & 4 & 600.0 & 600.0 \\
\hline 03 & 92520 & 77.9998 & 8.6250 & 294.6 & 4 & 600.0 & 600.0 \\
\hline 03 & 92521 & 4997 & 7 . & 29 & 4 & 600.0 & \\
\hline 03 & 92522 & 81.5000 & 8.0000 & 295.2 & $\Psi$ & 600.0 & 600.0 \\
\hline 0 & 92523 & 84.9999 & 8.225 & 295.2 & 4 & 600.0 & 600.0 \\
\hline
\end{tabular}




\begin{tabular}{|c|c|c|c|c|c|c|c|}
\hline 03 & 92524 & 81.0000 & 7.0000 & 293.3 & 4 & 600.0 & 600.0 \\
\hline 3 & 9261 & 58.7518 & 2.8500 & 292.7 & 4 & 600.0 & 600.0 \\
\hline 03 & 9262 & 78.7442 & 3000 & 293.0 & 5 & 600.0 & 00.0 \\
\hline 3 & 9263 & 83.7122 & .6250 & 291.9 & 6 & 600.0 & 00.0 \\
\hline 03 & 9264 & 107.1135 & .5500 & 292.5 & 5 & 600.0 & 00.0 \\
\hline 03 & 9265 & 148.2515 & 1.8000 & 289.6 & 4 & 600.0 & 00.0 \\
\hline 03 & 9266 & 130.5467 & 0.4000 & 289.0 & 5 & 600.0 & 00.0 \\
\hline 03 & 9267 & 311.4544 & 0.3250 & 288.9 & 6 & 600.0 & 00.0 \\
\hline 03 & 9268 & 329.0545 & 0.5000 & 292.1 & 5 & 600.0 & 00.0 \\
\hline 03 & 9269 & 295.6546 & 0.9750 & 293.8 & 4 & 600.0 & 0 \\
\hline 03 & 92610 & 243.9992 & 1.7250 & 294.7 & 3 & 600.0 & 00.0 \\
\hline 03 & 92611 & 207.9853 & 3.1500 & 294.5 & 2 & 600.0 & \\
\hline 03 & 92612 & 198.6216 & 3.1750 & 295.4 & 2 & 600.0 & 00.0 \\
\hline 03 & 92613 & 165.5073 & 3.9750 & 296.5 & 3 & 600.0 & 00.0 \\
\hline 03 & 92614 & 168.5023 & 4.2750 & 297.8 & 3 & .0 & \\
\hline 03 & 92615 & 169.5018 & 5.3250 & 298.6 & 4 & 600.0 & 00.0 \\
\hline 03 & 92616 & 172.9992 & 5.6750 & 299.1 & 4 & 600.0 & 600.0 \\
\hline 03 & 92617 & 171.7520 & 3.2250 & 299.4 & 3 & .0 & \\
\hline 03 & 92618 & 92.1116 & 2.7000 & 298.8 & 4 & 600.0 & .0 \\
\hline 03 & 92619 & 69.0000 & 4.9500 & 296.5 & 5 & 60 & .0 \\
\hline 03 & 92620 & 92.7506 & 4.2250 & 296.2 & 4 & 60 & \\
\hline 03 & 92621 & 87.2535 & 5.6250 & 296.5 & 4 & .0 & \\
\hline 03 & 92622 & 75.2499 & 7.8500 & 297.2 & 4 & 600.0 & 0 \\
\hline 03 & 92623 & 81.7496 & 6.8750 & 298.6 & 4 & 60 & .0 \\
\hline 03 & 92624 & 66.7120 & 5.8250 & 298.3 & 4 & .0 & \\
\hline 03 & 9271 & 114.2220 & 1.6500 & 295.8 & 5 & 600.0 & 600.0 \\
\hline 03 & 9272 & 60.0000 & 1.3500 & 295.7 & 6 & 600.0 & .0 \\
\hline 03 & 9273 & 13.4329 & 0.5500 & 293.2 & 6 & .0 & \\
\hline 03 & 9274 & 183.4845 & 0.3250 & 293.7 & 5 & 600.0 & 600.0 \\
\hline 03 & $927 \quad 5$ & 338.3342 & 0.3250 & 292.8 & 6 & 600.0 & 600.0 \\
\hline 03 & 9276 & 8.8828 & 1.5250 & 289.6 & 5 & .0 & \\
\hline 03 & 9277 & 54.9780 & 0.6250 & 291.1 & 6 & .0 & 600.0 \\
\hline 03 & 9278 & 146.5000 & 0.9750 & 293.5 & 5 & 600.0 & 600.0 \\
\hline 03 & 9279 & 297.8123 & 0.8000 & 295.8 & 4 & 600.0 & 0.0 \\
\hline 03 & 92710 & 256.2086 & 1.7500 & 295.3 & 3 & 600.0 & 600.0 \\
\hline 03 & 92711 & 245.5000 & 2.7000 & 295.4 & 3 & 600.0 & 600.0 \\
\hline 03 & 92712 & 232.7077 & 2.4250 & 297.1 & 2 & 600.0 & 600.0 \\
\hline 03 & 92713 & 211.5226 & 3.4500 & 298.0 & 2 & 600.0 & 600.0 \\
\hline 03 & 92714 & 165.7276 & 4.0000 & 298.7 & 3 & 600.0 & 600.0 \\
\hline 03 & 92715 & 168.9998 & 4.9250 & 299.1 & 4 & 600.0 & 600.0 \\
\hline 03 & 92716 & 165.2532 & 4.6000 & 299.6 & 4 & 600.0 & 600.0 \\
\hline 03 & 92717 & 151.5437 & 3.4500 & 300.0 & 3 & 600.0 & 600.0 \\
\hline 03 & 92718 & 83.2508 & 4.6500 & 299.1 & 4 & 600.0 & 600.0 \\
\hline 03 & 92719 & 82.5003 & 6.4500 & 297.9 & 4 & 600.0 & 600.0 \\
\hline 03 & 92720 & 93.8632 & 3.6750 & 299.4 & 4 & 600.0 & 600.0 \\
\hline 03 & 92721 & 129.7913 & 1.3250 & 298.3 & 5 & 600.0 & 600.0 \\
\hline 03 & 92722 & 82.6759 & 2.0000 & 299.4 & 5 & 600.0 & 600.0 \\
\hline 0 & 92723 & 91.2535 & 1.325 & 299.5 & 0 & 600.0 & 600.0 \\
\hline
\end{tabular}




\begin{tabular}{|c|c|c|c|c|c|c|c|}
\hline & 2724 & & & & & & \\
\hline & 9281 & 0 & .8000 & 7.6 & & & \\
\hline & 9282 & 498 & 3500 & 296.7 & & 00 & \\
\hline & 9283 & 121.3584 & 9000 & 295.7 & & 00 & \\
\hline & 284 & & 500 & 4.8 & & & \\
\hline & 9285 & & & 4 & & & \\
\hline & 9286 & 274 & 3250 & 293.1 & & 0 & \\
\hline & 9287 & 103 & 3500 & 92.8 & & & \\
\hline & 9288 & 68.8207 & 5000 & 95.0 & & & \\
\hline & 9289 & 118.1291 & 1.5750 & 295.7 & & 0 & \\
\hline & 92810 & 63.1281 & 2.17 & 295.7 & & & \\
\hline & 92811 & 337.6702 & 2.3 & 96.8 & & & \\
\hline & 92812 & 7581 & 3.8000 & 296.5 & & & \\
\hline & 2813 & 81.5001 & 6.07 & 296 & & & \\
\hline & 92814 & & & 3 & & & \\
\hline & 92815 & & & 29 & & & \\
\hline & 92816 & 84.2500 & 8.70 & 294.9 & 4 & & \\
\hline & 92817 & 502 & 5000 & 9 & & & \\
\hline & 92818 & 000 & 170 & 291.9 & 4 & & \\
\hline & 92819 & 7505 & .2250 & 290 & 4 & & \\
\hline & 92820 & 060 & 2.2000 & 290.3 & 4 & 0 & \\
\hline & 92821 & 98 & 0 & 290.1 & 4 & & \\
\hline & 92822 & 00 & 9500 & 29 & 4 & & \\
\hline & 92823 & 84.0000 & 12.62 & 289.5 & 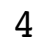 & & \\
\hline & 92824 & 505 & 0 & 289.0 & 4 & & \\
\hline & 9291 & 98 & 12.17 & 28 & 4 & & \\
\hline & 9292 & 74.5000 & 11.5000 & 290 & 4 & & . \\
\hline & 9293 & 2498 & 0 & 290 & 4 & & \\
\hline & 9294 & 499 & 1500 & 290.7 & 4 & & \\
\hline & 9295 & 2499 & 0 & 289.8 & 4 & & 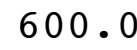 \\
\hline & 9296 & .5024 & 1500 & 289.2 & 4 & & \\
\hline & 9297 & 2499 & 0 & 288.6 & 4 & & \\
\hline & 9298 & 985 & 11.62 & 289.4 & 4 & & . \\
\hline & 9299 & 002 & 0 & 290.6 & 4 & & \\
\hline & 92910 & 494 & 0 & 293.1 & 4 & & \\
\hline & 92911 & & 4.4750 & 294.8 & 3 & & J \\
\hline & 92912 & 407 & 0 & 296.6 & 2 & & \\
\hline & 92913 & 926 & 4. & 297.4 & 3 & & \\
\hline & 92914 & 499 & 5.2750 & 297.8 & 3 & & 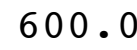 \\
\hline & 92915 & 048 & 750 & 297.4 & 3 & & \\
\hline & 92916 & 994 & 6.0500 & 296.9 & 4 & & \\
\hline & 92917 & 73.2501 & 7.0250 & 295.8 & 4 & & \\
\hline & 92918 & 72.2498 & .5000 & 294.3 & 4 & & 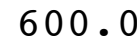 \\
\hline & 92919 & 500 & 750 & 293 & 4 & & \\
\hline & 92920 & 71.9999 & 8.4500 & 293.1 & 4 & & \\
\hline & 92921 & 71.7501 & 8.5750 & 293.5 & 4 & & 0 \\
\hline & 929 & & & 29 & 4 & & \\
\hline & 92923 & 78.7500 & 8.0000 & 293.6 & 1 & 600.0 & 6 \\
\hline
\end{tabular}




\begin{tabular}{|c|c|c|c|c|c|c|c|}
\hline 03 & 92924 & 81.0000 & 7.4250 & 294.1 & 4 & 600.0 & 600.0 \\
\hline 03 & 9301 & 83.7499 & 7.3000 & 294.0 & 4 & 600.0 & 600.0 \\
\hline 03 & 9302 & 83.9986 & 6.7750 & 293.9 & 4 & 600.0 & 00.0 \\
\hline 39 & 9303 & 83.7497 & 5.0250 & 293.7 & 4 & 600.0 & 0 \\
\hline 03 & 9304 & 90.9985 & 750 & 293.8 & 4 & 600.0 & .0 \\
\hline 03 & 9305 & 110.7408 & 3.0500 & 293.7 & 4 & 600.0 & 00.0 \\
\hline 03 & 9306 & 148.7645 & 500 & 293.3 & 4 & 600.0 & 0 \\
\hline 03 & 9307 & 153.7338 & 750 & 292.8 & 5 & 600.0 & 00.0 \\
\hline 03 & 9308 & 144.3229 & 1.6750 & 294.2 & 4 & 600.0 & 00.0 \\
\hline 03 & 9309 & 189.2551 & 2.2500 & 295.8 & 3 & 60 & 0 \\
\hline 03 & 93010 & 170.7500 & 4 & 294.8 & 4 & .0 & \\
\hline 03 & 93011 & 176.0048 & 4 . & 296.1 & 3 & .0 & \\
\hline 03 & 93012 & 176.7507 & 4.7 & 296.9 & 3 & 600.0 & \\
\hline 03 & 93013 & 172.9924 & 4.2 & 297.6 & 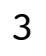 & 600.0 & \\
\hline 03 & 93014 & 171.2497 & & 298.8 & 3 & .0 & \\
\hline 03 & 93015 & 178.5668 & & 299.5 & 3 & 60 & \\
\hline 03 & 93016 & 172.4985 & 4.5 & 299.8 & 3 & .0 & .0 \\
\hline 03 & 93017 & 106.6383 & 4 . & 299.3 & 3 & .0 & \\
\hline 03 & 93018 & 78.9969 & 6.8 & 297.1 & 4 & .0 & \\
\hline 03 & 93019 & 76.7500 & 8.2250 & 295.7 & 4 & .0 & .0 \\
\hline 03 & 93020 & 75.2500 & 9 . & 294.9 & 4 & 6 & \\
\hline 03 & 93021 & 74.2494 & 10 . & 294.1 & 4 & .0 & \\
\hline 03 & 93022 & 72.5000 & 10.1 & 293.9 & 4 & 60 & .0 \\
\hline 039 & 93023 & 71.9998 & 10 . & 294.1 & 4 & 6 & \\
\hline 03 & 93024 & 76.2500 & & 294.5 & 4 & 60 & \\
\hline 0310 & $\begin{array}{lll}0 & 1 & 1\end{array}$ & 75.0002 & 10 & 293.7 & 4 & 600.0 & .0 \\
\hline 0310 & $\begin{array}{lll}0 & 1 & 2\end{array}$ & 75.0000 & 10.4250 & 292.6 & 4 & .0 & .0 \\
\hline 0310 & $\begin{array}{lll}0 & 1 & 3\end{array}$ & 74.7499 & 11 . & 292.2 & 4 & .0 & \\
\hline 0310 & $\begin{array}{lll}0 & 1 & 4\end{array}$ & 70.0000 & & 292.3 & 4 & .0 & .0 \\
\hline 0310 & $\begin{array}{lll}0 & 1 & 5\end{array}$ & 69.5009 & 10. & 292.0 & 4 & 6 & \\
\hline 0310 & $\begin{array}{lll}0 & 1 & 6\end{array}$ & 78.5006 & 11. & 289.4 & 4 & 0 & \\
\hline 0310 & $\begin{array}{lll}0 & 1 & 7\end{array}$ & 73.0004 & 10 . & 289.8 & 4 & .0 & .0 \\
\hline 0310 & $\begin{array}{lll}0 & 1 & 8\end{array}$ & 77.5021 & 11.0250 & 289.9 & 4 & .0 & 0 \\
\hline 0310 & $\begin{array}{lll}0 & 1 & 9\end{array}$ & 75.9968 & 11. & 290.2 & 4 & .0 & .0 \\
\hline 0310 & $0 \quad 110$ & 81.7507 & 7. & 291.3 & 4 & .0 & 0.0 \\
\hline 0310 & $0 \quad 111$ & 83.7488 & 6.1 & 293.5 & 4 & 600.0 & 600.0 \\
\hline 0310 & $0 \quad 112$ & 75.4991 & 6. & 294.2 & 4 & 600.0 & .0 \\
\hline 0310 & $0 \quad 113$ & 78.2502 & 7.4000 & 294.8 & 4 & 600.0 & 600.0 \\
\hline 0310 & $0 \quad 114$ & 70.2472 & 9.0750 & 295.5 & 4 & 600.0 & 600.0 \\
\hline 0310 & $0 \quad 115$ & 66.7502 & 9 . & 295.5 & 4 & 600.0 & .0 \\
\hline 0310 & 0116 & 64.0000 & 10. & 294.8 & 4 & 600.0 & 0.0 \\
\hline 0310 & $0 \quad 117$ & 65.5000 & 10.5500 & 294.0 & 4 & 600.0 & 600.0 \\
\hline 0310 & $0 \quad 118$ & 71.4786 & 10.2000 & 292.0 & 4 & 600.0 & 600.0 \\
\hline 0310 & $0 \quad 119$ & 67.2481 & 9.3250 & 291.0 & 4 & 600.0 & 600.0 \\
\hline 0310 & $0 \quad 120$ & 62.5000 & 10.8500 & 290.9 & 4 & 600.0 & 600.0 \\
\hline 0310 & $0 \quad 121$ & 63.7502 & 10.2750 & 291.1 & 4 & 600.0 & 600.0 \\
\hline 0310 & $0 \quad 122$ & 70.2504 & 9.80 & 291.7 & 4 & 600.0 & 600.0 \\
\hline 0310 & $0 \quad 123$ & 70.7500 & 9.975 & 291.8 & 4 & 600.0 & 600.0 \\
\hline
\end{tabular}




\begin{tabular}{|c|c|c|c|c|c|c|c|}
\hline 310 & 24 & 3.2501 & 9.0750 & 291.6 & 4 & 000.0 & \\
\hline 1 & 21 & $5 \quad 5001$ & 8.9750 & 291.4 & & 600.0 & \\
\hline & 22 & 501 & 500 & 91.1 & & 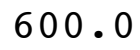 & \\
\hline & 3 & 6.9999 & 8.7500 & 90.6 & & 00 & \\
\hline & 4 & & & 90.7 & & & \\
\hline & 25 & 993 & 00 & 90.7 & & 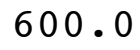 & \\
\hline 3 & 6 & 82.5005 & 00 & 290.1 & & 00 & \\
\hline & 7 & 000 & 8. & 90.3 & & & \\
\hline & 8 & 86.0005 & & 290.5 & & 0 & \\
\hline & 9 & 1.2481 & & 291.7 & & 0 & \\
\hline & 10 & 07 & & 92.6 & & & \\
\hline & 11 & 29 & & 95.4 & & & \\
\hline & 12 & 677 & 2 . & 296.2 & & 0 & \\
\hline 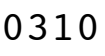 & 213 & 6 & & 8 & & & \\
\hline & 214 & & & 0 & & & \\
\hline & 215 & 82 . & 6 . & 295.9 & & & \\
\hline & 216 & 9943 & 6.82 & 295.8 & & 0 & \\
\hline & 217 & 85.2498 & & 294.3 & $H^{2}$ & & \\
\hline & 218 & 80 . & 0 & 292 & 4 & & \\
\hline & 219 & 78.7485 & 0 & 292.2 & & & \\
\hline & 220 & 00 & & 291.2 & & & \\
\hline & 221 & 02 & & 29 & 4 & & \\
\hline 3 & 222 & 73.7500 & 0 & 290.7 & 4 & & \\
\hline & 223 & 77.2499 & & 289 & 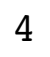 & & \\
\hline & 224 & & & 28 & 4 & & \\
\hline & 31 & 79.2501 & 0 & 289.0 & 4 & & \\
\hline 3 & 32 & 79.2501 & & 288.7 & 4 & & \\
\hline & 33 & 78. & & 3 & 4 & & \\
\hline & 34 & 84.7521 & & 290.0 & A & & \\
\hline & 35 & 121.9492 & & 289.8 & 5 & & \\
\hline & 36 & 68.5621 & & 287 & 6 & & \\
\hline & 37 & 7 & & .6 & 6 & & \\
\hline & 38 & 18.4189 & 1 . & 290.4 & 5 & & \\
\hline & 39 & 236 . & & 293 & 4 & & \\
\hline & 310 & 230.7537 & & & 3 & & \\
\hline & 311 & 208.6804 & & 293.9 & & & \\
\hline & 312 & 177 . & & 294.8 & 3 & & \\
\hline & 313 & 181. & & 296.7 & 3 & & \\
\hline$a_{2}>$ & 314 & 180.5096 & & 298.4 & 2 & & 0 . \\
\hline & 315 & 27 . & & & 1 & & \\
\hline & 316 & 84.0075 & & 297.9 & 2 & & \\
\hline & 317 & 83.2496 & & 295.8 & 3 & & \\
\hline & 318 & 80.7488 & & 296.0 & 4 & & \\
\hline & 319 & & & & 4 & & 600 \\
\hline 3 & 320 & 77.2501 & 0.5500 & 290.8 & 4 & .0 & م. \\
\hline & 321 & & & & 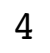 & & \\
\hline & 322 & 84.2408 & & & 4 & & \\
\hline & 203 & 82.6989 & 2.2750 & 294.3 & & 600.0 & 50 \\
\hline
\end{tabular}




\begin{tabular}{|c|c|c|c|c|c|c|c|}
\hline & 24 & & 1.4500 & 3.7 & & & \\
\hline & 1 & & 5.4250 & 92.9 & & 600.0 & \\
\hline & 42 & 79.7501 & 9.8750 & 289.6 & 4 & 00 & \\
\hline 31 & 43 & 79.7500 & 9.4750 & 289.3 & & 00.0 & \\
\hline & 4 & 75.7500 & & 88.2 & & 0 & \\
\hline & 45 & 79.5000 & & 88.4 & & 0 & \\
\hline & 46 & 76.7503 & 9.3250 & 289.3 & & 00 & \\
\hline & 47 & 79.5000 & 5.3750 & 90.6 & & & \\
\hline & 48 & 167.7408 & 1.0000 & 91.9 & & & \\
\hline & 49 & 47.2425 & 2.3000 & 92.4 & & 00.0 & 0 \\
\hline & 410 & 327.5000 & 1.8750 & 294.0 & & & \\
\hline & 411 & 196.2358 & 1.3000 & 6 & & 0 & \\
\hline & 412 & 244.84 & 1.9250 & 297.1 & & .0 & \\
\hline & 413 & 89.7502 & 4.1250 & 297.1 & & 0.0 & \\
\hline & 414 & 88 . & & 8 & & & \\
\hline & 415 & 86. & 6 . & 29 & 3 & & \\
\hline & 416 & 84.75 & 7.3000 & 296.5 & 4 & 0.0 & 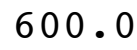 \\
\hline & 417 & 78 . & 8.5000 & .6 & 4 & & \\
\hline & 418 & 79. & 7.1750 & 294.6 & 4 & & \\
\hline & 419 & 69.2500 & 1.4250 & 293.8 & 4 & & \\
\hline & 420 & 68.7500 & 1.2750 & 293.3 & 4 & 0 & \\
\hline & 421 & 74.7499 & 0 & 292.3 & 4 & & \\
\hline & 422 & 78 & 5750 & 292.9 & 4 & & \\
\hline & 423 & 64. & 9000 & 292.7 & 4 & 0 & \\
\hline & 424 & 66. & 9 . & 292.1 & 4 & & \\
\hline & 51 & 73. & 9.2500 & 292.3 & 4 & & \\
\hline & 52 & 70.99 & .7250 & 292.1 & 4 & 0 & U. \\
\hline & 53 & 68.49 & 8.7500 & 292.2 & 4 & & \\
\hline & 54 & 69 . & 8.1500 & 292.1 & 4 & & \\
\hline & 55 & 73. & 000 & 291.6 & 4 & & 0 . \\
\hline & 56 & 72.2503 & 8.1500 & 291.4 & 4 & & 0 . \\
\hline & 57 & 75.2495 & 767 & 291.2 & 4 & & \\
\hline & 58 & 78 . & 6.3000 & 291.4 & 4 & & 0 . \\
\hline & 59 & 005 & 4.2000 & 293.3 & 3 & 0 & \\
\hline & 510 & 162 . & 0 & & 3 & & \\
\hline & 511 & 17 & 7500 & 294.4 & 4 & & 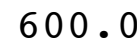 \\
\hline & 512 & 163.2499 & 6.3250 & 295.3 & 4 & & . \\
\hline & 513 & 170.5000 & 5 . & 296.8 & 3 & & \\
\hline & 514 & 179.5083 & 6.0000 & 297.5 & 4 & .0 & 0 . \\
\hline & 515 & 173.7521 & 5000 & 298.1 & 3 & & \\
\hline & 516 & 169.2778 & 7750 & 298.8 & 3 & & 0 \\
\hline & 517 & 169.2068 & 5.7000 & 298.4 & 4 & & 0 . \\
\hline & 518 & 101.0002 & 4.2000 & 297.2 & 4 & 0 & 0 \\
\hline & 519 & 88.5000 & 750 & 295 & 5 & & \\
\hline & 520 & 77.5000 & & & 4 & & 0 . \\
\hline & 521 & 75.7500 & 6.5000 & 295.0 & 4 & 600 & 600. \\
\hline & 522 & 76 & & & 4 & & \\
\hline & 523 & 76.5000 & 8.1750 & 294.9 & $\Psi$ & 600.0 & 600 \\
\hline
\end{tabular}




\begin{tabular}{|c|c|c|c|c|c|c|c|}
\hline D & 24 & 6.5000 & 7.5750 & 295.1 & 4 & 000 & \\
\hline 2. & 1 & 75005 & 6.3250 & 295.7 & 4 & & \\
\hline & 62 & 104.5237 & 6750 & 296.7 & 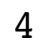 & 00 & \\
\hline 31 & 3 & 95.2395 & 5.2000 & 296.3 & & 00 & \\
\hline & 4 & 4.7550 & 00 & & & 0 & \\
\hline & 5 & 116.7521 & 500 & 1 & & 00 & \\
\hline & 6 & 144.2509 & 2000 & 295.6 & & 00 & \\
\hline & 7 & 108.7978 & 5000 & 5 & & & \\
\hline & 8 & 121.7162 & 2.8250 & 296.8 & & 0 & \\
\hline & 9 & 173.0002 & 3.2250 & 298.0 & & 00.0 & \\
\hline & 610 & 173.5000 & 4.97 & 298.2 & & 0 & \\
\hline & 611 & 177.6133 & & 99.1 & & & \\
\hline 3 & 12 & 174.3533 & 3.5250 & 300.8 & & 0 & \\
\hline 3. & 613 & 178.0001 & 4.9750 & 300.9 & & 0.0 & \\
\hline & 614 & 160.30 & & & & & \\
\hline & 61 & 138.1002 & 0 & 302.1 & 4 & 0 & \\
\hline & 616 & 97.7509 & 0 & 301.7 & 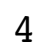 & 0 & \\
\hline & 617 & 89.74 & 0 & 30 & 4 & & \\
\hline & 618 & 88. & 6 . & 295 & 4 & 0 & \\
\hline & 619 & 91.4994 & 10 & 297.9 & 4 & & \\
\hline & 620 & 71.9982 & 0 & 29 & 4 & & \\
\hline & 621 & 79.44 & & 29 & 4 & & \\
\hline & 622 & 75.7390 & 0 & 296.7 & 4 & 0 & U. \\
\hline 3 & 623 & 64.49 & 8 . & 296.1 & 4 & & \\
\hline & 624 & 69. & & 29 & 4 & & \\
\hline & 71 & 81.7500 & 0 & 29 & 4 & & \\
\hline & 72 & 84.0000 & .50 & 294.0 & 4 & & \\
\hline & 73 & 77.2498 & 073 & 293 & 4 & & \\
\hline & 74 & 77 . & 0 & 29 & 4 & 0 & \\
\hline & 75 & 85.0104 & 0 & 295.4 & 4 & & \\
\hline & 76 & 89.24 & & 294 & 4 & & \\
\hline & 77 & 80 . & 0 & 292.3 & 4 & & \\
\hline & 78 & 75.9998 & 000 & 294.2 & 4 & 0 & 0 • \\
\hline & 79 & 92.50 & & 296 & 4 & & \\
\hline & 710 & 96. & 0 & 29 & 3 & & \\
\hline & 711 & 99.4915 & & 298.5 & 2 & & \\
\hline & 712 & 97 . & 0 & 299.6 & 3 & & \\
\hline & 713 & 90 . & 0 & 300.7 & 3 & 0 & 0 . \\
\hline & 714 & 99.7493 & 750 & 300.9 & 3 & 0 & 0 \\
\hline & 715 & 84.50 & 0 & 299.8 & 4 & & \\
\hline & 716 & 80.2452 & 7.55 & 298.5 & 4 & & \\
\hline 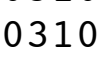 & 717 & 79.5003 & 8.4250 & 297.4 & 4 & 600.0 & 600 . \\
\hline & 718 & 79.2504 & 9.7250 & 295.4 & 4 & 600 & 000 \\
\hline & 719 & 78.0000 & 8.8250 & 294.7 & 4 & & 50 \\
\hline 3 & 720 & 80.0000 & 9.5000 & 295.0 & 4 & 600 & 0 \\
\hline & 721 & 74.7516 & 6.9250 & 295.9 & 4 & 600.0 & 600. \\
\hline & 722 & 73 & & 295.8 & 4 & 600 & \\
\hline & 723 & 75.7504 & 6.5750 & 296.2 & 4 & 600.0 & 600 \\
\hline
\end{tabular}




\begin{tabular}{|c|c|c|c|c|c|c|c|}
\hline 10 & 24 & 7.5003 & 5.9250 & 296.2 & 4 & 600.0 & \\
\hline . & 81 & 3 & 6.5250 & 296.1 & 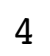 & $c 00$ & \\
\hline & 82 & 80 & 000 & 295.5 & 1 & 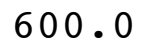 & \\
\hline & 3 & 2.7502 & 8.3500 & 94.9 & & 00.0 & \\
\hline & 4 & 1.7500 & & 4.6 & & & \\
\hline & 5 & 75.2488 & 9000 & 94.8 & & r & \\
\hline 3 & 6 & 84.7500 & 8.7000 & 94.7 & & 00 & \\
\hline & 7 & 5.7506 & .2500 & 95.1 & & 0 & \\
\hline & 8 & 86.4999 & 7.6250 & 295.6 & & 00.0 & \\
\hline & 9 & 5011 & 5750 & 295.7 & & 0 & \\
\hline & 10 & 504 & 0 & 95 & & & \\
\hline & 11 & & & 97 & & & \\
\hline & 12 & 93.7606 & 5.4750 & 298.1 & & 0 & \\
\hline & 813 & 2 & 0 & 97.9 & & & \\
\hline & 14 & & & 5 & & & \\
\hline & 815 & 77. & 9 . & 298.6 & 4 & & \\
\hline & 816 & 74.7530 & 9.87 & 298.4 & & U & \\
\hline & 817 & 517 & 0 & 297.3 & - & & \\
\hline & 818 & 65 . & 0 & 296 & 4 & & \\
\hline & 819 & 65.7501 & 2.02 & 294 & & 0 & \\
\hline & 820 & 63.49 & & 294 & & & \\
\hline & 821 & 63. & 12 . & 294 & 4 & & \\
\hline & 822 & 64.9999 & 12.30 & 293.2 & 4 & 0 & \\
\hline & 823 & 69 . & 12.72 & 291 & & & \\
\hline & 824 & 69 & & 29 & 4 & & \\
\hline & 91 & 70.2500 & 0 & 291.6 & 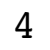 & & \\
\hline & 92 & 70.25 & 0 & 291 & 4 & & \\
\hline & 93 & 73.24 & & 291 & 4 & & \\
\hline & 94 & 79.5003 & & 290 & 4 & & \\
\hline & 95 & 71.24 & 9 . & 290.2 & 4 & & \\
\hline & 96 & 77.00 & 0 & 289 & 4 & & \\
\hline & 97 & 68 . & 0 & 289 & 4 & & \\
\hline & 98 & 77.7520 & .750 & 291.8 & 4 & & \\
\hline & 99 & 89.75 & 777 & 291.4 & 4 & & \\
\hline & 910 & & & & 4 & & \\
\hline & 911 & 96.0015 & 500 & 294.0 & 3 & & \\
\hline & 912 & 86.50 & 0 & 292 & 4 & & \\
\hline & 913 & 83. & 0 & 293.8 & 4 & & \\
\hline & 914 & 91.0000 & 8.2500 & 294.1 & 4 & & 0 . \\
\hline & 915 & 105.519 & & 294 & 4 & & \\
\hline & 916 & ר? 00 & & 293.3 & 4 & & \\
\hline & 917 & 75.2516 & 7.7250 & 292.4 & 4 & & \\
\hline & 918 & 71.2517 & 00 & 290 & 4 & & \\
\hline & 919 & & & & 4 & & 600. \\
\hline 3 & 920 & 73.0001 & 8.2750 & 290.1 & 4 & 600.0 & 00 . \\
\hline & 921 & & & & 4 & & \\
\hline & 922 & 77.5000 & 9.5750 & & & & 600 \\
\hline & 923 & 78.9710 & 8.2250 & 288.7 & & 600.0 & 60 \\
\hline
\end{tabular}




\begin{tabular}{|c|c|c|c|c|c|c|}
\hline 10 & & & & & & \\
\hline 1010 & 29.2 & 4000 & 290.0 & & 600 & \\
\hline 10 & 897 & 2750 & 9.5 & & 00 & \\
\hline 010 & 50.2508 & 0.2500 & 89.2 & 1 & 0 & 0 \\
\hline 0104 & 59.5000 & 2.5000 & 88.5 & & 0 & \\
\hline 105 & & 3750 & 88.0 & & & \\
\hline 106 & 500 & 12.1250 & 87.6 & & 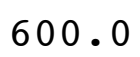 & \\
\hline 0107 & 000 & 12.5000 & 87.3 & & 0 & \\
\hline 0108 & & 1.9250 & 87.2 & & & \\
\hline 0109 & & 13.1000 & 87.5 & & & \\
\hline 1010 & 431 & 14.0750 & 88.3 & & & \\
\hline 1011 & 0 & 10.8750 & 39.5 & & & \\
\hline 012 & & & 90.9 & & & \\
\hline 013 & 71 . & 7.9750 & 92.0 & & & \\
\hline 1014 & 9 & 7.3250 & 92.7 & & & \\
\hline 015 & & 7.25 & & 4 & & \\
\hline 016 & 4 & 6.8250 & 294.0 & 4 & & \\
\hline 1017 & 72.2 & 6.8750 & 293.9 & 4 & 0 & \\
\hline 1018 & 15 & 4.8500 & 93.2 & 4 & & \\
\hline 019 & & 5.1000 & 291.8 & 4 & & \\
\hline 01020 & 5 & 4.2000 & 291.6 & 4 & & \\
\hline 021 & & 4.30 & . 1 & 4 & & \\
\hline 022 & & 5 . & 292.8 & 4 & & \\
\hline 01023 & 34 & 5.6250 & 293.1 & 4 & & \\
\hline .024 & & 5.8500 & 293.2 & 4 & & \\
\hline 111 & & 4 . & 292.8 & 4 & & \\
\hline 310112 & 08 & 3.1500 & 292.3 & 5 & & \\
\hline 310113 & 142 . & 2.7250 & 291.9 & 5 & & \\
\hline 10114 & 1 & 1.8750 & 291.9 & 5 & & \\
\hline 310115 & & 1.2750 & 291.2 & 4 & & \\
\hline 10116 & 18 & 0.5500 & 290.0 & 5 & & \\
\hline 310117 & & 1 . & 29 & 6 & & \\
\hline 310118 & & 0 & 29 & 5 & & \\
\hline 310119 & 90 & 1.6750 & 294.9 & 4 & & \\
\hline 1110 & & 2 . & 29 & 3 & & \\
\hline 111 & & 3 . & 294.6 & 2 & & \\
\hline 3101112 & 86 & 1.9500 & 296.3 & 1 & & \\
\hline 113 & 86 & 3.7000 & 296.6 & 2 & & \\
\hline .114 & & 4.2250 & 296.9 & 3 & & \\
\hline 3101115 & 529 & 3.6500 & 297.5 & 2 & 0 & \\
\hline 116 & 002 & 4.1000 & 297.9 & 3 & & \\
\hline 3101117 & 94 & 4.4750 & 297.6 & 4 & & \\
\hline 3101118 & 133 & 5.7000 & 296.2 & 4 & 600.0 & $=0$ \\
\hline 119 & & 6.6500 & 29 & 4 & & \\
\hline 3101120 & & 6.3250 & 295.9 & 4 & 600.0 & \\
\hline 3101121 & 005 & 7.6750 & 295.4 & 4 & 600.0 & 60 \\
\hline 122 & & & & 4 & & \\
\hline & 2491 & 8.8000 & 294.1 & 2 & 600.0 & \\
\hline
\end{tabular}




\begin{tabular}{|c|c|c|c|c|c|c|}
\hline $2-1$ & - & 8.5000 & 93.7 & 4 & 80.0 & \\
\hline 121 & 5.7545 & 8.7500 & 293.9 & 4 & 600.0 & \\
\hline 0122 & 0000 & 8500 & 292.8 & 4 & 00 & \\
\hline 10123 & 82.0027 & 6.4250 & 293.2 & & 00 & \\
\hline 0124 & 2562 & 3500 & 93.0 & & 0 & \\
\hline 0125 & 2 & & 92.5 & & 0 & \\
\hline 10126 & 9302 & 7500 & 292.3 & & 00 & \\
\hline 10127 & 510 & 50 & 91.6 & & & \\
\hline 0128 & 2500 & 250 & 91.4 & & & \\
\hline 310129 & 156.7528 & 7.0750 & 91.1 & & 0 & 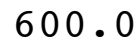 \\
\hline 1210 & 153.7504 & 6.3250 & 291.3 & & & \\
\hline 211 & 157.7 & 6.4500 & 92.8 & & & \\
\hline 212 & 170 & 3500 & 293.9 & & 0 & \\
\hline 1213 & 165.7 & 50 & 95.2 & & & \\
\hline 214 & 167 & & & & & \\
\hline & 161. & & 297.3 & 4 & & \\
\hline 216 & 162 . & 7 . & 297.8 & 4 & 0 & \\
\hline 217 & 156 . & 00 & .1 & 4 & & \\
\hline & 152 . & & 297.2 & 5 & & \\
\hline 19 & 155 . & 000 & 296.4 & 5 & & \\
\hline 220 & 162.25 & 4.5750 & 295.4 & 5 & 0 & \\
\hline & 147.9 & & & 4 & & \\
\hline 22 & 15 & 50 & 29 & 4 & & \\
\hline 223 & 158 . & 50 & 4 & 4 & 0 & \\
\hline 224 & 158 . & & & 4 & & \\
\hline 31 & 160 . & 00 & & 4 & & \\
\hline 310132 & 164.2500 & 000 & 29 & 4 & & . \\
\hline 310133 & 165 . & 0 & & 4 & & \\
\hline 134 & 162 . & 50 & 29 & 4 & & \\
\hline 310135 & 170.7499 & 000 & 29 & 4 & & J. \\
\hline 310136 & 165.4997 & 3500 & 25 & 4 & & 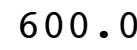 \\
\hline 310137 & 164 & 0 & & 4 & & \\
\hline 310138 & 166 . & & 290.5 & 3 & & 0 . \\
\hline 310139 & 164.7533 & 5000 & 291.0 & 4 & & \\
\hline & 151. & & & 4 & & \\
\hline & 151.2489 & 6.7500 & 292.4 & 4 & & \\
\hline 01312 & 159.7493 & 750 & 293.8 & 4 & & \\
\hline & 164 . & 5. & 29 & 3 & & \\
\hline 314 & 165.2498 & 3.8000 & 297.0 & 3 & & 0 . \\
\hline 01315 & 170.7496 & 3000 & 297.7 & 3 & & \\
\hline & 164.7515 & 500 & 298 & 3 & & \\
\hline & 162.2491 & 4.1250 & 297.8 & 4 & & 0 . \\
\hline 01318 & 153.7468 & 2.7000 & 296.5 & 5 & & 0 . \\
\hline 01319 & 90.9452 & 000 & 29 & 4 & & \\
\hline & 63.4949 & & & 5 & & \\
\hline 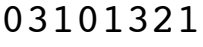 & 84.2499 & 4.8000 & 296.0 & 5 & & 00 \\
\hline & 82 & & & 3 & & \\
\hline 01323 & 79.7506 & 3.4750 & 296.1 & & 60 & 600 \\
\hline
\end{tabular}




\begin{tabular}{|c|c|c|c|c|c|c|}
\hline $8<4$ & 89.0073 & 2.3750 & 295.6 & 6 & 600.0 & \\
\hline 14 & 6.2506 & 4.0500 & 96.2 & 5 & 600.0 & \\
\hline 0142 & 82.5000 & 3750 & 295.5 & 4 & 00.0 & \\
\hline 10143 & 81.0002 & 6.9750 & 295.3 & 4 & 00 & \\
\hline 0144 & 86.9693 & 2000 & 95.3 & & 0 & \\
\hline 0145 & 127.7427 & 250 & 93.4 & 5 & 00 & \\
\hline 10146 & 115.5000 & 7750 & 293.6 & 4 & 00 & \\
\hline 0147 & 104.3044 & L. 6000 & 93.6 & & 0 & \\
\hline 0148 & 150.7380 & 0000 & 93.6 & & & \\
\hline 014 & 222.7334 & 000 & 297.2 & 3 & 00 & 0 \\
\hline 01410 & 281.2906 & 2.4000 & 295.8 & & 0 & \\
\hline & 228.2848 & 2.4250 & 96.5 & & & \\
\hline 2 & 239.0538 & 2.3500 & 297.5 & 1 & 0 & \\
\hline 13 & 176.3450 & 2.7500 & 297.7 & & 0.0 & \\
\hline & 211.1504 & & .8 & & & \\
\hline & 186.2550 & & 298.3 & 2 & & \\
\hline 416 & 168.7504 & 5.1500 & 298.0 & 3 & .0 & 8 \\
\hline 17 & 161.5002 & 4 & 6 & 4 & 0 & \\
\hline & 155.75 & & 296 & 4 & & \\
\hline 19 & 150.4995 & 5500 & 295.2 & 4 & 0 & 0 \\
\hline 20 & 119.1404 & 0.7750 & 294.7 & 5 & 0 & \\
\hline & 99.5836 & & & 5 & & \\
\hline 22 & 105.7550 & 0 & 29 & 4 & & \\
\hline 423 & 93.3334 & 4.2750 & 294.1 & 4 & 0 & \\
\hline 424 & 83.2334 & & 294 & 4 & & \\
\hline 51 & 74.0048 & 4250 & 29 & 4 & & \\
\hline 310152 & 52.2013 & 1250 & 29 & 5 & 0 & U. \\
\hline 310153 & 25.98 & 0 & 29 & 6 & & \\
\hline 0154 & 47.9847 & 250 & 29 & 6 & & \\
\hline 310155 & 238.0882 & 0.3000 & 292.0 & 6 & 0 & 0 . \\
\hline 310156 & 139.4001 & 1.3250 & 291.9 & 6 & & 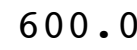 \\
\hline 310157 & 90.1626 & 0 & 290.6 & 5 & & \\
\hline 310158 & 79.7659 & 0 & 291.6 & 4 & & . \\
\hline 310159 & 66.6787 & 000 & 29 & 3 & 0 & 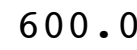 \\
\hline & 266.2838 & 2 . & & 2 & & \\
\hline 511 & 295.2965 & 1.9500 & 295.5 & 1 & & 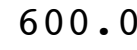 \\
\hline 101512 & 151.2840 & 3500 & 296.5 & 2 & & 0 . \\
\hline 3101513 & 88.0180 & 0 & 29 & 3 & & . \\
\hline 514 & 83.5000 & 8.5250 & 296.5 & 4 & .0 & 0 . \\
\hline 01515 & 87.7506 & 5250 & 295.5 & 4 & & 0 . \\
\hline & 73.2315 & 8 . & 29 & 4 & & 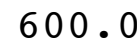 \\
\hline 310 & 69.7499 & 8.5750 & 295.2 & 4 & .0 & 0 . \\
\hline 3101518 & 78.7511 & 9.1250 & 293.1 & 4 & 0 & 0 . \\
\hline 3101519 & 74.5129 & 500 & 29 & 4 & & \\
\hline 03101520 & 82.0000 & & 292.5 & 4 & & 0 . \\
\hline 01521 & 73.7499 & 9.2250 & 293.1 & 4 & 600.0 & 00 . \\
\hline & & & & 4 & & \\
\hline 3101523 & 73.2498 & 8.4500 & 293.5 & $\Psi$ & 600.0 & $r 0$ \\
\hline
\end{tabular}




\begin{tabular}{|c|c|c|c|c|c|c|}
\hline 24 & 5.7499 & 8.5750 & 93.4 & 4 & 600.0 & \\
\hline 0161 & .5003 & 8.3500 & 293.0 & 4 & 600.0 & \\
\hline 0162 & 72.0005 & 7750 & 292.4 & 4 & 00 & \\
\hline 10163 & 75.7502 & 7.8250 & 292.3 & & 00 & \\
\hline 0164 & 81.7608 & .0000 & 92.2 & & 00 & \\
\hline 0165 & 76.5000 & 1250 & 292.2 & & 00 & \\
\hline 10166 & 99.5015 & 4750 & 292.3 & 4 & 00 & \\
\hline 0167 & 86.7476 & 4.7500 & 92.1 & & .0 & \\
\hline 0168 & 5075 & 2.9750 & 92.4 & 3 & & \\
\hline 10169 & 58.9961 & 4.0250 & 292.8 & & 00 & 8 \\
\hline 1610 & 170.7502 & 4.7500 & 294.2 & & 0 & \\
\hline & 176.0002 & 6.5000 & 93.7 & 4 & 00.0 & \\
\hline 2 & 177 . & 500 & 294.0 & 4 & 0 & \\
\hline 513 & 172.2475 & 5.9250 & 294.3 & 3 & 0.0 & \\
\hline & 169. & & 7 & & & \\
\hline & 166. & & 297.2 & 4 & & \\
\hline 616 & 174.7499 & 0000 & 297.7 & 4 & .0 & 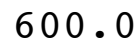 \\
\hline 17 & 173 & 3 . & 297.2 & 4 & 0 & \\
\hline & 170 . & 3750 & 295.8 & 5 & & \\
\hline 619 & 112.0448 & 1.1750 & 295.0 & 4 & 0 & $\checkmark$ \\
\hline 20 & 65.2513 & 4.3000 & 295.1 & 5 & .0 & 8 \\
\hline & 77 . & & 294.3 & 4 & & \\
\hline 2 & 78 & 7750 & 293.9 & 4 & & \\
\hline 23 & 79.7499 & 5.2500 & 294.5 & 4 & 0 & 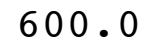 \\
\hline 24 & 70.4936 & & 29 & 4 & & \\
\hline $7 \quad 1$ & 63. & 500 & 29 & 5 & & \\
\hline 310172 & 92.7868 & 9000 & 294.6 & 6 & 0 & U. \\
\hline 310173 & 21.7581 & 000 & 294.4 & 5 & & \\
\hline 174 & 19. & 1.4250 & 294.2 & 4 & & \\
\hline 310175 & 1.2878 & 3250 & 293.4 & 4 & 0 & 0 • \\
\hline 310176 & 299.3152 & 1500 & 291.4 & 5 & 0 & 0 \\
\hline 177 & 38.2251 & 0 & 291.5 & 6 & & \\
\hline 310178 & 155.9839 & 0.4000 & 294.5 & 5 & 0 & 0 • \\
\hline 310179 & 288.6656 & 750 & 29 & 4 & 0 & 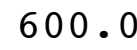 \\
\hline & 278.4016 & 0 & & 3 & & \\
\hline 711 & 264.2532 & 2.4250 & 295.2 & 2 & & 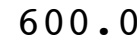 \\
\hline 101712 & 278.7519 & L. 9750 & 295.3 & 1 & & 0 . \\
\hline 713 & 289.0046 & 000 & 297.4 & 2 & & . \\
\hline 03101714 & 287.8585 & 2.7000 & 298.0 & 2 & .0 & 0 . \\
\hline 01715 & 279.0034 & 2.7500 & 298.6 & 1 & & 0 . \\
\hline & 283.8285 & 2.1500 & 29 & 1 & & 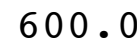 \\
\hline 310 & 269.5102 & 1.5250 & 298.8 & 2 & .0 & 0 . \\
\hline 3101718 & 272.0689 & 1.0250 & 297.2 & 3 & .0 & 00 . \\
\hline 03101719 & 355.0543 & 1.3000 & 296 & 4 & & \\
\hline 03101720 & 12.0005 & 0.6750 & 295.7 & 4 & & 600. \\
\hline 01721 & 14.2444 & 0.5500 & 295.2 & 5 & 600.0 & 00. \\
\hline & & 000 & 29 & 6 & & \\
\hline 101723 & 72.2138 & 0.4000 & 294.4 & & 600.0 & 5 \\
\hline
\end{tabular}




\begin{tabular}{|c|c|c|c|c|c|c|}
\hline 124 & & .0000 & 94.7 & & & \\
\hline 0181 & 6 & .3250 & 93.9 & & 600.0 & \\
\hline 0182 & 354.6882 & 1.1750 & 293.5 & 0 & 00 & \\
\hline 10183 & 21.2527 & 1.3500 & 294.0 & & 00 & \\
\hline 0184 & 137.08 & L. 1250 & 93.4 & & 0 & \\
\hline 0185 & 145.2 & .4000 & 292.3 & & 00 & \\
\hline 310186 & 9396 & 0.8250 & 292.5 & & 500 & \\
\hline 10187 & 324 & .7500 & 292.0 & & 0 & \\
\hline 310188 & 7 . & .0500 & 93.3 & & & \\
\hline 310189 & 268.5815 & 1.0750 & 295.7 & & 500.0 & 0 \\
\hline 3101810 & 299.6029 & 0.8750 & 294.7 & & 0 & \\
\hline 11 & 191.9753 & 2.1250 & .3 & & .0 & \\
\hline 812 & 161 & 3.2250 & 296.4 & & .0 & ( \\
\hline 813 & 163.0 & 3.7500 & 297.3 & & 0.0 & \\
\hline 14 & 172 . & .3250 & 298.3 & & & \\
\hline & 175 . & 0 & 298.7 & & & \\
\hline 816 & 171.7540 & .8250 & 297.9 & & 0.0 & 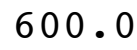 \\
\hline 17 & 109 . & .4250 & 9 & & 0 & \\
\hline & 62 . & 3.6250 & 29 & & & \\
\hline 19 & 63. & 2.5250 & 295.5 & & 0 & \\
\hline 320 & 62.7870 & 2.4750 & 293.7 & 6 & 0 & . \\
\hline 21 & 63.6 & 100 & 292.9 & & & \\
\hline 322 & 105 . & 1.6750 & 293 & 6 & & \\
\hline 323 & 2.8380 & 0.9500 & 293.5 & 6 & 0 & \\
\hline 324 & 45.1 & 3 . & 29 & 3 & & \\
\hline 91 & 67. & 5.2000 & 29 & 4 & & \\
\hline 310192 & 96.4897 & 7.3250 & 292.2 & 4 & 0 & U. \\
\hline 310193 & 69. & 5.1500 & 294.2 & 4 & & \\
\hline 194 & 119 & 3 . & 29 & 5 & & \\
\hline 310195 & 82.1047 & 3.1750 & 294.0 & 5 & .0 & 0 • \\
\hline 310196 & 82.5000 & 4.6000 & 294.3 & 4 & 0 & 0 . \\
\hline 310197 & 88.0 & 2.05 & 293 & 5 & & \\
\hline 310198 & 87.6482 & 9250 & 29 & 4 & & 0 • \\
\hline 310199 & 277.6804 & 1.2750 & 296.9 & 3 & 0 & 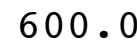 \\
\hline 10 & 272.4761 & 1.4250 & & 2 & & \\
\hline 911 & 218.3223 & 2.6500 & 297.9 & 1 & & . \\
\hline 912 & 186.4991 & 4.4500 & 297.2 & 2 & $\cdot 0$ & 0 . \\
\hline 13 & 187.7550 & 3.6250 & 298.3 & 2 & & 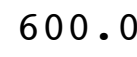 \\
\hline 14 & 172.7411 & 3.9750 & 299.0 & 3 & .0 & 0 . \\
\hline 01915 & 184.5005 & 4.2500 & 299.2 & 3 & & 0 . \\
\hline 916 & 185.7502 & 4.4750 & 299 & 3 & & 60 \\
\hline 0310 & 185.9957 & 5.3250 & 298.0 & 4 & .0 & 0 . \\
\hline 3101918 & 153.3367 & 2.2000 & 296.5 & 4 & .0 & 00 . \\
\hline 03101919 & 109.8980 & 0.8000 & 295 & 5 & & \\
\hline 03101920 & 55.3770 & 0.3250 & 295.4 & 6 & 0 & 600. \\
\hline 01921 & 124.4195 & 1.6250 & 296.6 & 5 & 600.0 & 600 \\
\hline & 141 . & & & 5 & & \\
\hline 3101923 & 45.5140 & 0.6500 & 296.2 & & 600.0 & 600 \\
\hline
\end{tabular}




\begin{tabular}{|c|c|c|c|c|c|c|}
\hline 924 & 7.7320 & 8250 & 96.1 & 5 & 600.0 & \\
\hline 20 & 8.5000 & 0.9750 & 296.5 & 4 & 600.0 & \\
\hline 0202 & 38.1155 & 3500 & 296.2 & 5 & 00 & \\
\hline 10203 & 152.5003 & 3.2500 & 294.4 & & 00.0 & \\
\hline $020 \quad 4$ & 47.5031 & .6500 & 93.1 & & 0 & \\
\hline 0205 & 108.0897 & 4500 & 292.7 & & 00 & \\
\hline 310206 & 120.5032 & 1.1250 & 293.0 & & 00 & \\
\hline 10207 & 79.8579 & .9250 & 94.0 & & 0 & \\
\hline 0208 & 15.4801 & 3000 & 97.7 & & & \\
\hline 310209 & 199. & 2.0500 & 297.0 & & 00 & 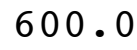 \\
\hline 3102010 & 163.0000 & 5.0000 & 293.5 & & 0 & \\
\hline 011 & 166.0000 & 5.5500 & 94.0 & & 00.0 & \\
\hline 012 & 170 . & 5 . & 295.8 & & 0 & \\
\hline 013 & 167.00 & 4.20 & 297.4 & & 0.0 & \\
\hline 014 & 170 . & & 98 & & & \\
\hline 15 & 163. & 4 . & 299.1 & & & \\
\hline 016 & 157.2505 & 3.7750 & 299.5 & & .0 & . \\
\hline 17 & 146.24 & 0 & 99.5 & & 0 & \\
\hline 18 & 150.0 & 3 . & 298.2 & & & \\
\hline 019 & 148.2501 & 3750 & 298.1 & & 0 & \\
\hline 020 & 130.4883 & 1.5500 & 298.0 & & 0 & 00 . \\
\hline 21 & 86.4317 & 0 & 298.1 & & & \\
\hline 22 & 57.25 & 1.10 & 298.5 & & & \\
\hline 023 & 47.5541 & .4500 & 298.1 & & 0 & 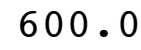 \\
\hline 024 & 113.6091 & 0 & 297.4 & & & \\
\hline 21 & 154.9873 & 500 & 297.3 & 4 & & \\
\hline 310212 & 159.7483 & 1.97 & 297.2 & 4 & 0 & U. \\
\hline 310213 & 162.500 & 0 & 296 & & & \\
\hline 214 & 131.6447 & 0.3750 & 29 & 6 & & $\bullet$ \\
\hline 310215 & 358.2512 & 1.0000 & 295.7 & & 0 & 0 . \\
\hline 310216 & 355.5000 & 1.2250 & 295.8 & & 0 & 0 \\
\hline 310217 & 348.2415 & 0 & 295.7 & 4 & & \\
\hline 310218 & 331.6523 & 9000 & 297.7 & 3 & 0 & 0 . \\
\hline 310219 & 265.5112 & 1250 & 298.6 & 2 & 0 & 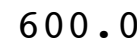 \\
\hline & 299.24 & 0 & 297.8 & 2 & & \\
\hline 11 & 264.5417 & 1.6500 & 298.6 & 1 & & 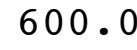 \\
\hline 112 & 245.0007 & 2.1000 & 299.7 & 1 & & 0 . \\
\hline 13 & 286.2555 & 1.6500 & 301.4 & 1 & & 0 . \\
\hline 3102 & 247.2072 & 1.6250 & 302.8 & 1 & .0 & 0 . \\
\hline 3102115 & 237.7364 & 1.6250 & 303.5 & 1 & & \\
\hline & 205.9666 & 250 & 302.9 & 2 & & 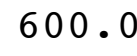 \\
\hline 310 & 155.2500 & 2.6000 & 301.9 & 3 & .0 & 0 . \\
\hline 3102118 & 138.4963 & 2.2250 & 299.7 & 4 & .0 & 00 . \\
\hline 03102119 & 100.1398 & 4.1250 & 299.6 & 4 & & \\
\hline 03102120 & 106.2310 & 2.5250 & 299.6 & 4 & & 0 . \\
\hline 02121 & 93.0007 & 5.0500 & 299.4 & 4 & 600.0 & 500 . \\
\hline & 75 & & & 4 & & \\
\hline 102123 & 45.6997 & 2.3500 & 298.3 & & 600.0 & 600 \\
\hline
\end{tabular}




\begin{tabular}{|c|c|c|c|c|c|c|}
\hline 2 & 0.2390 & 2.0500 & 297.8 & 4 & - & \\
\hline 221 & 75125 & 2.4250 & 297.7 & & 600.0 & \\
\hline 0222 & 7525 & 1.7750 & 298.0 & & 00 & \\
\hline 10223 & 15.7582 & 5250 & 297.4 & & 00 & \\
\hline 0224 & 677 & & & & & \\
\hline 0225 & 3 . & & 2 & & 0 & \\
\hline 10226 & 11.5038 & 3750 & 296.9 & & 00 & \\
\hline 0227 & 29.5000 & 500 & 96.7 & & & \\
\hline 0228 & 021 & & & & & \\
\hline 310229 & 485 & 1.6750 & 0 & & 0 & \\
\hline 2210 & 54.4844 & 2.3250 & 298 & & & \\
\hline 211 & 209.1 & 107 & 4 & & & \\
\hline 2 & 90 & 5 . & .8 & & & \\
\hline 213 & 83 . & 6.65 & 29 & & & \\
\hline & 83 & & & & & \\
\hline & 75 & & & & & \\
\hline 6 & 95 & 0.6500 & 294.0 & & & \\
\hline & 75 . & 0 & 5 & & & \\
\hline & 71 . & & & & & \\
\hline & 78 & 0 & 289.8 & 4 & & \\
\hline 20 & 72 . & 0 & 290.5 & 4 & & \\
\hline & 70 . & & & 4 & & \\
\hline 2 & 74 & 0 & 29 & 4 & & \\
\hline 23 & 73 & 0 & 291.7 & & & \\
\hline 24 & 77 . & & & 4 & & \\
\hline 31 & 79 & 9. & 29 & 4 & & \\
\hline 0232 & 507 & 3000 & 290.7 & $I$ & & \\
\hline 0233 & 93. & & .8 & 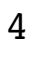 & & \\
\hline 34 & 130 . & 4 . & 29 & 4 & & \\
\hline 10235 & 126 . & 500 & 291.8 & 4 & & \\
\hline 310236 & 135.5005 & 1000 & 291.1 & 4 & & \\
\hline 37 & 1. & 8 . & .8 & 4 & & \\
\hline 310238 & 11 & 750 & 290.2 & 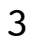 & & ? \\
\hline 310239 & 151.4902 & 250 & 291.6 & 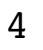 & & \\
\hline & 166. & 0 & 29 & 4 & & \\
\hline & 158.5001 & 500 & 292.5 & 4 & & \\
\hline & 158.0000 & 5250 & 294.3 & 4 & & \\
\hline & 172 . & 0 & .1 & 4 & & \\
\hline & 180.5011 & 10.9750 & 297.7 & 4 & & . \\
\hline 15 & 179.5072 & 9500 & 298.6 & 4 & & \\
\hline & 163. & 6 . & 299.1 & 4 & & \\
\hline & 159.7489 & 4. & 299.0 & 4 & & \\
\hline 02318 & 140.7667 & 2.8500 & 297.8 & 4 & & 0 \\
\hline & 148 . & 250 & 29 & 4 & & \\
\hline & 133.2464 & & & 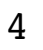 & & \\
\hline 02321 & 158.0000 & 8.9500 & 298.4 & 4 & & 0 \\
\hline & & & & 4 & & \\
\hline 02323 & 161.7500 & 9.8750 & 297.1 & & & \\
\hline
\end{tabular}




\begin{tabular}{|c|c|c|c|c|c|c|}
\hline $3<4$ & & & $5 \cdot 9$ & & & \\
\hline 241 & 00 & .5000 & 4.7 & 4 & 0 & \\
\hline 242 & 150.2495 & 3000 & 293.9 & 4 & 00 & \\
\hline 10243 & 128.9904 & 6.1750 & 293.6 & & 00 & \\
\hline $024 \quad 4$ & 132 & 8750 & 93.3 & & 0 & \\
\hline 0245 & & 750 & 92.7 & & 0 & \\
\hline 10246 & 125 & 6750 & 292.7 & & 00 & \\
\hline 310247 & 13 & 3250 & 92.3 & & 0 & \\
\hline 10248 & 39 & 1250 & 92.9 & & & \\
\hline 310249 & 140 . & 8.3500 & 293.3 & 4 & 00 & 0 \\
\hline 02410 & 145.992 & 7.8250 & 293.8 & & 0 & \\
\hline & 149.2 & 6.7500 & 94.4 & 4 & & \\
\hline 2 & 151 & 6.40 & 295.4 & 4 & 0 & \\
\hline & 155.25 & 7.15 & 296 & 4 & 0.0 & \\
\hline & & 0 & 5 & 4 & & \\
\hline & 155 & 5 . & 298.4 & 4 & & \\
\hline & 163 & 6.7750 & 298.6 & 4 & .0 & 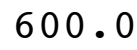 \\
\hline & 15 & 0 & 9 & 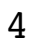 & & \\
\hline & 121 & 0 & 297.2 & 4 & & \\
\hline & & 0 & 297.7 & 4 & 0 & \\
\hline 20 & 127.53 & 3.07 & 297.7 & 4 & 0 & 0. \\
\hline & 129. & & 297.7 & 4 & & \\
\hline & & 0 & $29 \varepsilon$ & 4 & & \\
\hline 23 & 119 & 2.02 & 297.4 & 5 & 0 & \\
\hline 24 & 105 . & & 29 & 5 & & \\
\hline 51 & 157 . & 1 . & 29 & 5 & & \\
\hline 310252 & 138.74 & 1.8750 & 295.5 & 5 & 0 & . \\
\hline 310253 & 144.4 & 4.27 & 293.3 & 4 & & \\
\hline 54 & 105 & 1.3750 & 29 & 5 & & \\
\hline 310255 & 123.5293 & 1.9000 & 295.0 & 5 & 0 & 0 . \\
\hline 310256 & 123.2693 & 250 & 294.5 & 4 & & . \\
\hline 310257 & 144.5 & 0 & 293.6 & 4 & & \\
\hline 310258 & 148 . & & 293.8 & 4 & & 0 . \\
\hline 310259 & 146.7503 & 7.2250 & 294.5 & 4 & 0 & \\
\hline & 151.2522 & 0 & 3 & 4 & & \\
\hline & 156.7500 & 9.9500 & 295.2 & 4 & & . \\
\hline 12 & 156.7498 & 7.5000 & 296.3 & 4 & & 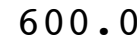 \\
\hline 13 & 157.2502 & 000 & 297.8 & 4 & & \\
\hline 14 & 161.008 & 5.2750 & 299.1 & 3 & .0 & 0 . \\
\hline 15 & 162.2515 & 6.8750 & 299.2 & 4 & & \\
\hline & 159.5000 & 000 & 299.4 & 4 & & 0 \\
\hline 17 & 153.2503 & 4.0750 & 299.5 & 4 & .0 & 0 . \\
\hline 3102518 & 105.8159 & 1.6500 & 298.8 & 5 & 0 & 0 . \\
\hline 3102519 & 112.8616 & 1.5750 & 299.1 & 6 & & \\
\hline & 103.5221 & 1.6000 & 299.5 & 6 & & 0 . \\
\hline 0252 & .1796 & 1.1250 & 299.8 & 5 & & 600. \\
\hline & & & 30 & 4 & & \\
\hline & 117 & 2.4500 & 300.5 & & 60 & -1 \\
\hline
\end{tabular}




\begin{tabular}{|c|c|c|c|c|c|c|}
\hline 4 & & & 9.4 & & & \\
\hline 0261 & 7 & .2250 & 98.9 & & & \\
\hline 0262 & 136.0874 & 7000 & 96.6 & 2 & 00 & \\
\hline 10263 & 106.6557 & 2000 & 296.2 & $\Lambda$ & 00 & 00 \\
\hline 0264 & 37.1286 & .8500 & 95.1 & & & \\
\hline 0265 & 95.8 & 3750 & 96.0 & & & \\
\hline 310266 & 26.8643 & .8750 & 297.1 & & 0 & 0 \\
\hline 310267 & 46.5002 & 3.8000 & 295.3 & & 0 & \\
\hline 310268 & 53.9977 & .7500 & 94.8 & & 0 & \\
\hline 310269 & 51.5018 & 4.3500 & 295.5 & & 0 & 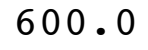 \\
\hline 3102610 & 164.5017 & 5.3250 & 296.2 & & & \\
\hline 02611 & 011 & 4.9750 & 297.0 & 3 & & \\
\hline 2612 & 165 & 3.9750 & 298.4 & 4 & & \\
\hline 3102613 & 014 & 5000 & 299.7 & 4 & & \\
\hline 02614 & 46 & 3750 & 299 & & & \\
\hline 02615 & 0 & & 30 & 3 & & \\
\hline 3102616 & 000 & 1000 & 300 & 3 & & \\
\hline 3102617 & 145.0381 & 4.4000 & 300.5 & 4 & 0 & \\
\hline 3102618 & & 3.1750 & 300.1 & 4 & & \\
\hline 3102619 & 60 & 000 & 300.3 & 4 & & \\
\hline 102620 & 86 & 2.6500 & 300.1 & 4 & 0 & \\
\hline 02621 & 6 & 250 & 30 & 5 & & \\
\hline 102622 & 77 & 500 & 30 & 5 & & \\
\hline 3102623 & 76.2522 & 3.0750 & 301.0 & 4 & 0 & 6 \\
\hline 102624 & - & 500 & 30 & 5 & & \\
\hline 10271 & 23 & 0 & 29 & 6 & & \\
\hline 310272 & 99 & 750 & 298.6 & 5 & & \\
\hline 310273 & 53.3276 & 0.6750 & 298.0 & 6 & 0 & 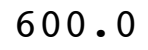 \\
\hline $31027 \quad 4$ & 154 . & 0 & 297.8 & 5 & & \\
\hline 31027 & & 500 & 297.4 & 6 & & \\
\hline 310276 & 120.7290 & 1.8250 & 296.8 & 6 & 0 & \\
\hline 310277 & 665 & 500 & 296.0 & 6 & & \\
\hline 310278 & 54 & 500 & 298.2 & 5 & & \\
\hline 310279 & 244.1446 & 0.6250 & 300.0 & 4 & 0 & \\
\hline 3102710 & & 250 & 299.0 & 3 & & \\
\hline 1 & & 1.4250 & 298.8 & 2 & & \\
\hline 3102712 & 201.1560 & 2.2250 & 300.6 & 1 & 0.0 & 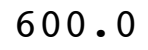 \\
\hline 3102713 & .5008 & 3750 & 300.4 & 2 & 0 & \\
\hline 3102 & 16 & 250 & 300.3 & 3 & .0 & \\
\hline 3102715 & 506 & 5.9500 & 300.6 & 4 & .0 & ח) \\
\hline 102716 & 515 & 250 & 301.1 & 3 & & \\
\hline 3102717 & 499 & 500 & 300.6 & 4 & & \\
\hline 3102718 & 134.7556 & 3.0750 & 300.4 & 4 & 600.0 & 600. \\
\hline 102719 & 13 & 750 & 300.1 & 4 & & \\
\hline 3102720 & 954 & 4.1250 & 300.2 & 4 & 600.0 & \\
\hline 03102721 & 2509 & 5.0500 & 300.8 & 4 & 600.0 & 600 \\
\hline 20 & & 4000 & 300.6 & 4 & 600.0 & \\
\hline & & 5.9500 & 300.1 & 4 & 600.0 & \\
\hline
\end{tabular}




\begin{tabular}{|c|c|c|c|c|c|c|}
\hline $1<4$ & 7.2465 & 4.3250 & 299.5 & 4 & 800 & \\
\hline 281 & 9.1041 & 1.3000 & 297.4 & & 600.0 & \\
\hline 110282 & 5280 & 0500 & 96.6 & 6 & 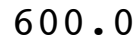 & \\
\hline 310283 & 14.9975 & 5000 & 296.6 & & 00 & \\
\hline 284 & 8.3805 & & & & & \\
\hline 0285 & 7001 & 0 & 6 & & 0 & \\
\hline 310286 & 5060 & 500 & 295.6 & & & \\
\hline 0287 & 7.5002 & 0 & & & & \\
\hline 310288 & 5183 & 0.7500 & 97.5 & & & \\
\hline 310289 & 270 . & 0.9000 & 298.6 & & 0 & \\
\hline 2810 & 267.1905 & 1.27 & .7 & & & \\
\hline 811 & 271.8733 & & & & & \\
\hline 812 & 295 & 0 & 299.2 & & 0 & \\
\hline 813 & 311.2505 & 2.20 & 300.6 & & 0 & \\
\hline & 301.2555 & & & & & \\
\hline 815 & 245.2202 & 0 & 302.1 & & & \\
\hline 2816 & 231 & 0 & 6 & & & \\
\hline 317 & 260 . & & 0 & & & \\
\hline 818 & 234 . & & 0 & & & \\
\hline 2819 & 11.1790 & 0 & 298.5 & & & \\
\hline 20 & 92 . & 0 & 2 & & & \\
\hline 21 & 80 . & & & & & \\
\hline 822 & 86.5003 & 0 & 30 & & & \\
\hline 23 & 86 . & 0 & 29 & & & \\
\hline 24 & 86 & & & 4 & & \\
\hline 310291 & 75 & & 29 & 4 & & \\
\hline 310292 & 97.78 & 0 & 298.6 & 4 & & \\
\hline 310293 & 77. & & 29 & 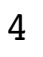 & & \\
\hline 310294 & 68 . & 0 & 25 & 4 & & \\
\hline 310295 & .9621 & & 296.6 & 5 & & \\
\hline 310296 & 7813 & & 29 & 0 & & \\
\hline 310297 & 10 & & 29 & 5 & & \\
\hline 310298 & 5076 & 2.5250 & 295.9 & 4 & 0 & \\
\hline 310299 & 823 & & 7 & 3 & & \\
\hline 10 & 88 & 0 & 29 & 4 & & \\
\hline & 72.4941 & & 296.2 & 4 & & \\
\hline 2 & 74.5000 & 0 & 297.2 & 4 & & \\
\hline & 692377 & 0 & 296.3 & 4 & & \\
\hline 914 & 502 & .8500 & 294.9 & 4 & & \\
\hline J & 69 . & 50 & 293.9 & 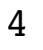 & & \\
\hline & 68. & 0 & 293.2 & 4 & & \\
\hline 3102 & 69.0000 & 12.6000 & 291.6 & 4 & 0 & 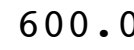 \\
\hline 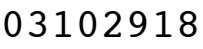 & 68 . & .2750 & 291.4 & 4 & 0 & \\
\hline & 67.2500 & 14.9500 & 288.5 & $I$ & & \\
\hline 03102920 & 63.0000 & 14.8250 & 288.3 & 4 & 600 & \\
\hline & 003 & .5500 & 286.9 & 4 & 0 & \\
\hline & 1533 & & .0 & & & \\
\hline 02923 & 60.7500 & 15.3750 & 284.8 & & 600.0 & 600 \\
\hline
\end{tabular}




\begin{tabular}{|c|c|c|c|c|c|c|}
\hline 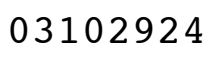 & 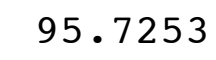 & 5250 & 83.6 & 4 & ( & \\
\hline 301 & 44696 & 7.9000 & 83.3 & 4 & 600.0 & \\
\hline 0302 & 7.1952 & 3250 & 282.9 & 4 & 00 & \\
\hline 10303 & 83.0107 & 1750 & 282.5 & & 00.0 & \\
\hline 0304 & 1.9984 & 7250 & 82.2 & & & \\
\hline 305 & 72.5006 & & 81.9 & & 0 & \\
\hline 0306 & 69.7500 & 8500 & 81.6 & & 00 & \\
\hline 0307 & 80.0000 & 5750 & 81.4 & & 0 & \\
\hline 0308 & 7496 & 3500 & 81.6 & & & \\
\hline 309 & 74.7502 & 5500 & 82.7 & & 0 & 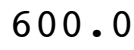 \\
\hline 010 & 80.4985 & 5.8000 & 284.0 & & & \\
\hline & 61.7749 & 4.4500 & 85.3 & & & \\
\hline 2 & 104 . & 3.2500 & 286.5 & & 0 & \\
\hline 13 & 113.2387 & 3.2500 & 87.0 & & 10.0 & \\
\hline & 94 . & & & & & \\
\hline & & & & 4 & & \\
\hline 6 & 4977 & .2000 & 285.2 & 4 & .0 & 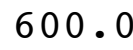 \\
\hline 17 & 70 . & 0 & & 4 & & \\
\hline & 68 . & 0 & 282.8 & 4 & & \\
\hline & 502 & 250 & 282.4 & 4 & & \\
\hline 20 & 63.7501 & .7250 & 282.0 & 4 & 0 & \\
\hline & 62.2500 & & 81.6 & 4 & & \\
\hline 2 & 7500 & 0 & 281.5 & 4 & & \\
\hline 23 & 61.7500 & .2000 & 281.4 & 4 & 0 & \\
\hline 24 & 54.7511 & & & 4 & & \\
\hline 11 & 60 & 8. & 281.5 & 4 & & \\
\hline 310312 & 65.7509 & 6.1250 & 281.4 & 4 & 0 & J. \\
\hline 310313 & 51.17 & 3500 & 1.0 & 5 & & \\
\hline 314 & 131.42 & 0 & $2 \varepsilon$ & 6 & & \\
\hline 310315 & 4.5693 & 2.4000 & 0.7 & 6 & 0 & J. \\
\hline 310316 & 262.9451 & 0.7250 & 280.5 & 6 & & 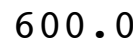 \\
\hline 317 & 279.0869 & .7750 & & 6 & & \\
\hline 310318 & 296 . & 250 & 280.8 & J & & 0 . \\
\hline 310319 & 322 & 250 & 280.9 & 4 & & \\
\hline & 303 . & 0 & & 3 & & \\
\hline & 299.4933 & & 282.7 & 2 & & \\
\hline 112 & 261.7662 & 6500 & 282.3 & 2 & & \\
\hline & 246.2523 & 4. & 282.0 & 3 & & \\
\hline 114 & 239.701 & 3.5500 & 281.6 & 2 & & 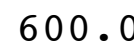 \\
\hline 103115 & $310.584 \varepsilon$ & 2.0750 & 281.4 & 1 & & \\
\hline & 351.2963 & 250 & 282.7 & 2 & & \\
\hline & 313.4612 & 1.2500 & 281.6 & 3 & & 0 . \\
\hline 3103118 & 207.0484 & 1.3750 & 280.7 & 4 & 0 & 0 . \\
\hline 3103119 & 184.6373 & 250 & & 5 & & \\
\hline 03103120 & 179.7762 & 1.7250 & & J & & \\
\hline 121 & 161.2498 & 4.2000 & 279.7 & 5 & & 00 \\
\hline & 137 & & & 4 & & \\
\hline 103123 & 130.7267 & 2.4250 & 279.2 & & .0 & s \\
\hline
\end{tabular}




\begin{tabular}{|c|c|c|c|c|c|c|c|}
\hline 27 & 21 & - & 1.1500 & 279.6 & 6 & 600.0 & \\
\hline & 11 & 151.2610 & 1.7000 & 279.6 & - & 600.0 & \\
\hline & 12 & 147.4983 & 2.7500 & 278.8 & 5 & 00 & 1 \\
\hline 31 & 13 & 139.0049 & 1.7500 & 278.3 & & 00.0 & \\
\hline & 14 & 146.2494 & .6000 & 278.1 & & 00 & \\
\hline & 15 & 154.7500 & 4.8500 & 277.8 & & 00 & \\
\hline & 16 & 150.0037 & 9000 & 278.0 & & 00 & \\
\hline & 17 & 104.8835 & L. 4500 & 277.7 & & 0 & \\
\hline & 18 & 127.1743 & .5750 & 78.8 & & & \\
\hline & 19 & 102.0582 & 1.9750 & 280.7 & & 00 & 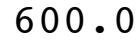 \\
\hline & 110 & 169.2462 & .1500 & 280.5 & & 00.0 & \\
\hline & 111 & 171.7500 & 4.4000 & 81.2 & & 0 & \\
\hline & 112 & 175.9975 & 4.4250 & 282.1 & & 0 & \\
\hline & 113 & 179.6873 & .7250 & 283.2 & & 0.0 & r \\
\hline & 114 & 171.7570 & & & & & \\
\hline & 11 & 190.2814 & & & & & \\
\hline & 116 & 164.2495 & 3.2750 & 284.9 & & 0.0 & . \\
\hline & 117 & 157.5000 & 3.3000 & .5 & & 0 & \\
\hline & 118 & 148.2506 & 2.7750 & 3.4 & & & \\
\hline & 119 & 127.0028 & 1.2500 & 282.9 & 4 & 0 & v. \\
\hline $3 .+3$ & 120 & 72.9693 & .5250 & 3.0 & 4 & 0 & \\
\hline & 121 & 74.7477 & 2.82 & & t & & \\
\hline & 122 & 93.5000 & 5000 & 3.2 & 5 & & \\
\hline 3 & 123 & 77.7500 & 5750 & 2.7 & 4 & 0 & \\
\hline & 124 & 80.4997 & & & 4 & & \\
\hline & 21 & 71.4998 & 000 & .5 & 4 & & \\
\hline & 22 & 70.7500 & 3750 & 2.5 & 4 & 0 & U. \\
\hline & 23 & 68.0009 & 750 & 2.6 & 4 & & \\
\hline & 24 & 70.2500 & 500 & .7 & 4 & & \\
\hline & 25 & 70.7499 & 2500 & 2.8 & 4 & 0 & 0 . \\
\hline & 26 & 69.7500 & 4.9000 & 2.7 & 4 & 0 & 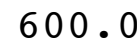 \\
\hline & 27 & 68.5000 & 50 & 2.4 & 4 & & \\
\hline & 28 & 75.5000 & 750 & 282.2 & 4 & 0 & 0 • \\
\hline & 29 & 70.7543 & 250 & 3.0 & 3 & 0 & 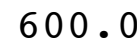 \\
\hline & 210 & 109.9816 & 0 & & 3 & & \\
\hline & 211 & 114.1484 & 3.2500 & 4.2 & 2 & & ( \\
\hline & 212 & 241.2494 & 250 & 4.4 & 2 & & t \\
\hline & 213 & 254.0440 & 000 & .6 & 1 & & 0 . \\
\hline J & 214 & 287.0762 & 2.1000 & 6.1 & 1 & .0 & 0 . \\
\hline & 215 & 77.7389 & $\$ 750$ & 6.1 & 1 & & 0 . \\
\hline & 216 & 92.8670 & 750 & 284.8 & 2 & & 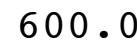 \\
\hline & 217 & 74.5041 & 4.8250 & 284.3 & 3 & .0 & 0 . \\
\hline & 218 & 68.2516 & 6.2500 & 283.0 & 4 & 0 & 00 . \\
\hline & 219 & 68.2499 & 6.7000 & 282.6 & 4 & & \\
\hline & 220 & 76.4998 & 6.2750 & 282.3 & 4 & & 00 . \\
\hline & 221 & 62.0085 & 4.2250 & 282.2 & 4 & 600.0 & 00. \\
\hline & 222 & 36 & 500 & 283.2 & 4 & & \\
\hline & 223 & 54.2588 & 3.3000 & 281.9 & 7 & 600.0 & $c 0$ \\
\hline
\end{tabular}




\begin{tabular}{|c|c|c|c|c|c|c|c|}
\hline- & 24 & & & & & & \\
\hline & 1 & 914 & 1.5250 & 1.4 & & 0 & \\
\hline & 32 & 52.8321 & 4.3750 & 281.6 & & 00 & \\
\hline 31 & 33 & 77.0281 & 4.8750 & 281.8 & & 00.0 & \\
\hline & 4 & 80.2567 & .6750 & 281.4 & & 0 & \\
\hline & 5 & 82.2743 & 4250 & 281.2 & & $0 C$ & \\
\hline & 6 & 73.7394 & 4750 & 281.0 & & 500.0 & 0 \\
\hline & 7 & 78.2212 & 6.6500 & 280.9 & & 00.0 & \\
\hline & 8 & 101.2486 & 4.9750 & 80.7 & & & \\
\hline & 9 & 89.5014 & 5.4250 & 280.8 & & 600.0 & 00 \\
\hline & 10 & 90.5017 & 4.1500 & 281.1 & & 0 & \\
\hline & 11 & 67.2487 & 1750 & 82.1 & & .0 & \\
\hline & 12 & 67.7402 & 3.2500 & 283.3 & & 00.0 & U \\
\hline & 13 & 131.0211 & 2.5750 & 285.1 & & 00.0 & 0 \\
\hline & 14 & 138.914 & 0 & & & & \\
\hline & & 197.9 & & 283.3 & 2 & & \\
\hline & 16 & 201.7496 & 3.2750 & 283.3 & 3 & 00.0 & 00 \\
\hline & 17 & 212.4933 & 1.90 & 3.3 & & 0 & \\
\hline & 18 & 175.734 & 1.2750 & 282.3 & 5 & & \\
\hline & 19 & 319.9425 & 0.5250 & 281.7 & 6 & 0 & \\
\hline 13. & 320 & 79.5250 & 1.2750 & 282.0 & 5 & 0 & 600. \\
\hline & 321 & 80.2491 & & 281.7 & 4 & & \\
\hline & 22 & 74.0001 & 4.3500 & 281.7 & 5 & & \\
\hline 3. & 323 & 101.8860 & 1.7250 & 281.4 & 6 & .0 & 0 。 \\
\hline & 324 & 102.1834 & 750 & 281.3 & 6 & & \\
\hline & 41 & 91.5000 & 1 . & 280.9 & 5 & & \\
\hline & 42 & 114.5571 & 1.5250 & 280.8 & 4 & 0 & U. \\
\hline & 43 & 148.7497 & 1.7000 & 280.3 & 5 & & . \\
\hline & 44 & 145 . & 1.2750 & 280.0 & 5 & & 0 . \\
\hline & 45 & 144.0023 & 1.1250 & 280.0 & 5 & .0 & 0 . \\
\hline 3 & 46 & 132.6117 & 0.7750 & 279.8 & 6 & 0 & 0 . \\
\hline & 47 & 51.5208 & 0 & 279.7 & 5 & & \\
\hline & 48 & 50.2080 & 50 & 281.1 & 6 & & 0 • \\
\hline 3. & 49 & 141.9415 & 0.8000 & 282.5 & 5 & 0 & 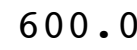 \\
\hline & 410 & 182.4230 & 0 & 282.2 & 4 & & 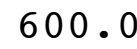 \\
\hline & 411 & 230.7629 & 0250 & 284.2 & 3 & & 0 . \\
\hline & 412 & 175.2467 & 3.6500 & 282.9 & 3 & 0 & 0 . \\
\hline & 413 & 174.4914 & 4 . & 283.7 & 3 & & 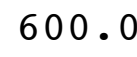 \\
\hline & 414 & 174.2494 & 3.7000 & 284.4 & 2 & .0 & 600 . \\
\hline & 415 & 184.0173 & 3.0750 & 285.1 & 2 & 0 & 0 . \\
\hline & 416 & 184.7465 & 500 & 284.8 & 3 & & 6 \\
\hline & 417 & 172.7482 & 3.3500 & 284.2 & 4 & .0 & 00 . \\
\hline & 418 & 173.2508 & 2.2750 & 283.1 & 4 & 600 & 00 . \\
\hline & 419 & 175.5021 & 1.5500 & 282.9 & 4 & 600 & 0 \\
\hline & 420 & 94.9310 & 0.9250 & 282.8 & 5 & 600.0 & 600 \\
\hline & 421 & 71.0069 & 2.4750 & 282.8 & 4 & 600.0 & 600 \\
\hline & 422 & 76 & & & 4 & & \\
\hline & 423 & 74.7519 & 4.1750 & 282.4 & $\mathbf{4}$ & 600.0 & 600 \\
\hline
\end{tabular}




\begin{tabular}{|c|c|c|c|c|c|c|c|}
\hline 11 & 24 & 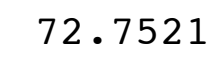 & 3.6750 & 82.6 & & & \\
\hline 21 & 51 & 1.7494 & 4.7750 & 282.2 & 4 & & \\
\hline 1 & 52 & 76.5000 & .6500 & 282.2 & 5 & 00 & \\
\hline 31 & 53 & 54.5764 & 3.4000 & 282.4 & & 00 & \\
\hline & 4 & 9.1415 & .8000 & 82.1 & & 0 & \\
\hline & 5 & 337.2728 & .6750 & 281.8 & & 00 & \\
\hline & 6 & 12.4899 & 1.0000 & 281.9 & & 00 & \\
\hline & 7 & 0.7963 & .2750 & 82.1 & & & \\
\hline & 8 & 158.4851 & 1.1750 & 281.7 & & 0 & \\
\hline & 9 & 171.7978 & 0.7750 & 283.6 & & 600.0 & \\
\hline S. & 10 & 188.7019 & 2.7000 & 282.8 & & 0 & \\
\hline & 11 & 169.2484 & 22 & 82.8 & 3 & & \\
\hline 3 & 12 & 216.2870 & 2.5500 & 284.3 & 2 & 00 & 0 \\
\hline 3 & 13 & 237.5000 & 1.77 & 284.9 & 1 & 0.0 & . \\
\hline & 14 & 253.6216 & & & 1 & & \\
\hline 3 & 15 & 323.4056 & 1 . & 285.6 & 1 & 0 & \\
\hline ? & 16 & 163.9934 & 1.12 & 284.5 & 1 & 00.0 & \\
\hline & 17 & 369 & 1.25 & 284.0 & 2 & & \\
\hline & 18 & 123. & 1.42 & 283.5 & 3 & 0 & \\
\hline 3 & 19 & 5087 & 1.10 & 282.9 & 4 & & \\
\hline 3 & 20 & 149 . & 2 . & 83.1 & 5 & 0 & \\
\hline & 21 & 98 & & 282.8 & 6 & & \\
\hline 3 & 22 & 125.9357 & 0 & 282.8 & 6 & 00 & 0. \\
\hline 3. & 523 & 68.2692 & 0 . & 282.9 & 6 & 0 & \\
\hline & 524 & 04 & & 282.9 & 5 & & \\
\hline & 61 & 2.9979 & 0 & 283.0 & 4 & & \\
\hline 3 & 62 & 327.7822 & 0.25 & 283.0 & 5 & 0 & \\
\hline & 63 & 258.3557 & 0 . & 282.9 & 6 & & \\
\hline 03 & 64 & 252.2531 & 0 . & 282.5 & 6 & 0 & 0 . \\
\hline 3 & 65 & 339.2168 & 1. & 282.5 & 5 & 0 & \\
\hline & 66 & 335 . & 1. & 282.6 & 4 & & \\
\hline & 67 & 307 . & 2 . & 282.0 & 4 & & 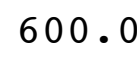 \\
\hline & 68 & 337.5000 & 1500 & 281.4 & 5 & 600 & 0 • \\
\hline & 69 & 308 . & 0 . & 282.6 & 4 & 0 & \\
\hline & 610 & 275 . & 1. & 283.7 & 3 & & \\
\hline & 611 & 288.4731 & & 285.0 & 2 & 60 & 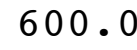 \\
\hline & 612 & 287 . & 2 . & 283.6 & 2 & 0 & \\
\hline & 613 & 293. & 2 . & 283.6 & 3 & 0 & 0 . \\
\hline 03 & 614 & 281.1129 & 1.7895 & 283.5 & 2 & 600.0 & 0 . \\
\hline & 615 & 260 . & & 283.2 & 3 & 600 & \\
\hline & 616 & 288.8000 & & 288.1 & 3 & & \\
\hline 03 & 617 & 244.6426 & 2.4953 & 287.4 & 4 & 600.0 & 600 . \\
\hline & 618 & 154.4252 & 3. & 285.4 & 4 & 600.0 & 600. \\
\hline & 619 & 145.6742 & 2.9878 & 284.8 & 5 & 600 & 600 \\
\hline 03 & 620 & 142.9751 & 3.4998 & 284.5 & 5 & 600.0 & 600 \\
\hline & 621 & 144.6500 & 3.4368 & 284.0 & 5 & 600.0 & 600 \\
\hline & 622 & 144.7748 & 8 & 283.7 & 5 & 600 & 60 \\
\hline & 623 & 148.6500 & 3.7768 & 283.1 & J & 600.0 & 600 \\
\hline
\end{tabular}




\begin{tabular}{|c|c|c|c|c|c|c|c|}
\hline 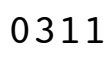 & 24 & & & & & & \\
\hline & 71 & 290 & 6018 & 81.9 & 4 & 00.0 & \\
\hline & 72 & 355.3275 & 1.8998 & 281.9 & כ & 00 & \\
\hline 31 & 73 & 353.6223 & 2.6220 & 282.1 & & 00.0 & \\
\hline & 74 & 301.5111 & .8418 & 82.6 & & 0 & \\
\hline & 75 & 228.305 & 1.6140 & 282.1 & & 00 & \\
\hline & 76 & 177.8995 & 2.9025 & 281.9 & & 500.0 & 0 \\
\hline & 77 & 58.6842 & 1.7200 & 282.0 & & 00.0 & \\
\hline & 78 & 284.119 & 1.1355 & 282.1 & & & \\
\hline & 79 & 295.2209 & 1.0178 & 282.4 & & 600.0 & 00 \\
\hline & 710 & 348.6092 & 1.3240 & 282.6 & & 00.0 & \\
\hline & 711 & 348.5843 & 1.4890 & 83.6 & & 00.0 & \\
\hline & 712 & 348 & 2.0718 & 284.4 & & 00.0 & 0 \\
\hline & 713 & 45.40 & 1.5953 & 285.3 & & 00.0 & . \\
\hline & 714 & + & 1 . & 1 & & & \\
\hline & 715 & 166. & 1.26 & 286.5 & & & \\
\hline & 716 & 320.4636 & 2.5683 & 286.5 & & 00.0 & 00 \\
\hline & 717 & 24 . & 1.30 & 1 & & .0 & 0 \\
\hline & 718 & 74 . & 2 . & 28 & & & \\
\hline & 719 & 40.21 & 2.0995 & 286.6 & 4 & 0 & U. \\
\hline 131 & 720 & 17.52 & 2.6093 & 286.2 & & .0 & 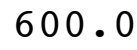 \\
\hline & 721 & 857 & 2.3553 & & & & \\
\hline & 722 & 9.64 & 1.4660 & 286.3 & & $\cdot 0$ & \\
\hline 3. & 723 & 16.2120 & 1.9078 & 285.7 & & .0 & 00 . \\
\hline & 724 & 154.97 & & & & & \\
\hline & 81 & 281.42 & 1.1 & 285.8 & 6 & & \\
\hline & 82 & 357.23 & 1.4755 & 285.8 & & .0 & U. \\
\hline & 83 & 28.89 & 1.90 & 28 & ; & 0 & 0 . \\
\hline & 84 & 32.30 & 2.26 & 28 & 5 & & • \\
\hline & 85 & 350.2830 & 1.7003 & 285.6 & 6 & .0 & 0 . \\
\hline & 86 & 344.0986 & 2.74 & 284.8 & 5 & .0 & 0 . \\
\hline & 87 & 346.7999 & 3.2 & 285.0 & 4 & & \\
\hline & 88 & 0.1241 & 2.96 & 285.0 & 4 & & 0 • \\
\hline 3 & 89 & 343.1235 & 3.2490 & 285.4 & 4 & 0 & o. \\
\hline & 810 & 333 & 0 & & 4 & & 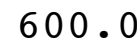 \\
\hline & 811 & 333.0974 & & 6.9 & 4 & & 0 . \\
\hline & 812 & 338.5001 & 6.7365 & 287.5 & 4 & .0 & 0 . \\
\hline & 813 & 337 . & 5.70 & 288.2 & 3 & & 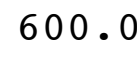 \\
\hline נט & 814 & 353.5017 & 6.3220 & 289.3 & 4 & .0 & 600 . \\
\hline & 815 & 342.9680 & 7.3713 & 290.2 & 4 & 0.0 & 0 . \\
\hline & 816 & 336.8715 & 7.5550 & 289.5 & 4 & & 6 \\
\hline & 817 & 334.2711 & 7.3308 & 288.3 & 4 & .0 & 600. \\
\hline & 818 & 332.8700 & 8.0435 & 287.1 & 4 & 600.0 & 600 . \\
\hline & 819 & 48.2464 & 3.7693 & 286.8 & 4 & 600 & 0 \\
\hline & 820 & 97.5339 & 4.7280 & 285.1 & 4 & 600.0 & 600 \\
\hline & 821 & 33.1481 & 3.3363 & 284.7 & 4 & 600.0 & 600 \\
\hline & 822 & 310 & & & 4 & & \\
\hline & 823 & 327.7287 & 4.0738 & 284.0 & & 600.0 & 600 \\
\hline
\end{tabular}




\begin{tabular}{|c|c|c|c|c|c|c|}
\hline 24 & & & & & & \\
\hline $\begin{array}{lll}11 & 9 & 1\end{array}$ & 825 & .9375 & 3.8 & & 0 & \\
\hline 192 & 5272 & 1.3858 & 283.9 & & 00 & \\
\hline 1193 & 301.1769 & 1.4778 & 284.3 & & 00.0 & \\
\hline 194 & 304.9073 & 9060 & & & 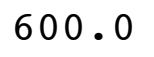 & \\
\hline 95 & 2.8754 & 9485 & 85 & & 0 & \\
\hline 196 & 9636 & 2.8435 & 284.9 & & 00 & \\
\hline $\begin{array}{lll}1 & 9 & 7\end{array}$ & 24.9156 & 4.0538 & 84.9 & & & \\
\hline 198 & 57.8222 & 5.36 & 84.4 & & & \\
\hline 1199 & 22.3884 & 3.3295 & 284.1 & & 00 & 0 \\
\hline 1910 & 3.2845 & 2.5665 & 283 & & & \\
\hline 1911 & 32.5468 & 2.3233 & 5 & & & \\
\hline 1912 & 308.4500 & 2.91 & 286.2 & & 00.0 & \\
\hline 1913 & 283.7579 & 3.03 & 286.9 & & 00.0 & \\
\hline 1914 & 286 . & 8 & & & & \\
\hline 1915 & 64 . & & 288.6 & & & \\
\hline 1916 & 133.4872 & 3.8080 & 287.3 & & .0 & 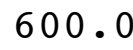 \\
\hline 1917 & 156 . & 3.4423 & .2 & & 0 & \\
\hline 1918 & 106.5992 & 2.07 & 2 & & & \\
\hline 1919 & 43.7433 & 2.6950 & 28 & & & $\bullet$ \\
\hline 11920 & 137.7051 & 1.8855 & $2 \varepsilon$ & 6 & 0 & 0 \\
\hline 1921 & 117.6417 & 160 & & 6 & & \\
\hline 1922 & 596 & 1.72 & 285.2 & 6 & & \\
\hline 1923 & 22.2127 & 1.5338 & .3 & 6 & . 0 & \\
\hline 1924 & 29 . & 1 . & & & & \\
\hline 101 & 89.4305 & 1.5198 & & 6 & & \\
\hline 1102 & 121.6175 & 2.5805 & .1 & 5 & 0 & J. \\
\hline 1103 & 130.6987 & 3.22 & & 4 & & \\
\hline 104 & 161.3599 & 3.2385 & .9 & 5 & & \\
\hline 11105 & 129.5592 & 3.0243 & 282.1 & 5 & 0 & 0 . \\
\hline 311106 & 97.1680 & 1.72 & 282.8 & 6 & 0 & s. \\
\hline 311107 & 97.3261 & 175 & 5 & 6 & & \\
\hline 311108 & 144.9918 & 3.55 & 282.2 & 5 & & 0 \\
\hline 311109 & 152.8503 & 5.9063 & 282.5 & 4 & 0 & \\
\hline 010 & 158.3246 & 871 & .3 & 4 & & \\
\hline 011 & 157 . & 6.42 & .9 & 4 & & . \\
\hline 3111012 & 150.9884 & 4.3828 & 283.8 & 4 & .0 & o. \\
\hline 1013 & 146 . & 4.3523 & & 3 & & 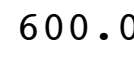 \\
\hline 1014 & 143 . & 4.5638 & .1 & 3 & .0 & 0 . \\
\hline 3111015 & 156.5991 & 4.6588 & 286.7 & 3 & & \\
\hline 1016 & 158.2824 & 318 & 28 & 2 & & 60 \\
\hline 017 & 145.1990 & 3.4530 & 286.6 & 3 & .0 & 0 . \\
\hline 3111018 & 146.6731 & 2.6495 & 285.8 & 4 & 0 & 00 . \\
\hline 1019 & 139.2500 & 2.7145 & & 5 & & \\
\hline 03111020 & 135.1750 & 2.9128 & & 4 & .0 & 600 \\
\hline 3111021 & 144.4502 & 3.7308 & 285.5 & 4 & 600.0 & 600 \\
\hline & 147 & 4.23 & & 4 & & \\
\hline 1023 & 120.7230 & 3.8075 & 285.8 & & .0 & 60 \\
\hline
\end{tabular}




\begin{tabular}{|c|c|c|c|c|c|c|}
\hline .024 & & & & & & \\
\hline 1111 & 126.6751 & 8640 & 5.8 & 4 & 0 & \\
\hline 1112 & 128.7003 & 2645 & 285.8 & 4 & 00 & \\
\hline 11113 & 141.2001 & 4135 & 285.3 & & 00 & \\
\hline 1114 & 31.3506 & 280 & & & 0 & \\
\hline 1115 & 132.8477 & & 84.4 & & 0 & \\
\hline 311116 & 143.6808 & 8.6523 & 84.0 & & 00 & \\
\hline 311117 & 55.4003 & 1. & 83.7 & & 0 & \\
\hline 11118 & 8496 & 0 & 83.4 & 4 & & \\
\hline 31111 & 47.3957 & 5485 & 83.9 & 4 & 00 & 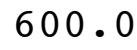 \\
\hline 10 & 148.8223 & 6.52 & 285 & & 0 & \\
\hline & 2.6250 & 9.48 & 35.6 & 4 & 0 & \\
\hline 2 & 3238 & 9.36 & 286.1 & 4 & 0 & \\
\hline 3 & 48.1478 & 7.33 & 7 & 4 & 0.0 & \\
\hline & 149.2000 & & & 4 & & \\
\hline & 150.5385 & & 2 & 3 & & \\
\hline 6 & 143.9645 & 4.6600 & 288.5 & 3 & .0 & 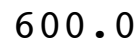 \\
\hline & 9.5945 & 4 . & 8 & 4 & & \\
\hline & 130 . & & 8 & 4 & & \\
\hline 9 & 4542 & 3 & 287.8 & 4 & 0 & $\bullet$ \\
\hline 20 & 139.9249 & 5.83 & 287.7 & 4 & 0 & \\
\hline & 126.6940 & & & 4 & & \\
\hline 2 & 682 & 0 & $28^{\circ}$ & 5 & & \\
\hline 23 & 131.0341 & 3.9955 & 287.6 & 4 & 0 & \\
\hline 24 & 140 & & & 4 & & \\
\hline 1 & 143. & 5 & 28 & 4 & & \\
\hline 311122 & 146.3249 & .9075 & 284 & 5 & 0 & J. \\
\hline 311123 & 132.0012 & 2.62 & 3 & 4 & & \\
\hline 311124 & 922 & 2 . & 28 & 4 & & \\
\hline 311125 & 136.4750 & 2.39 & 4 & 5 & 0 & 0 . \\
\hline 311126 & 133.5250 & 3.3068 & 284.3 & 5 & 0 & 0 \\
\hline 311127 & 139. & 301 & & 5 & & \\
\hline 311128 & 128 . & 2 . & 283.6 & 4 & & . \\
\hline 311129 & 143. & 2.79 & 5 & 3 & 0 & 0 . \\
\hline & 140 & 6 & & 4 & & \\
\hline & 147.4999 & & 5.8 & 4 & & . \\
\hline 11212 & 157.6250 & 8.10 & 287.4 & 4 & & 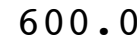 \\
\hline 13 & 150.7003 & 8 . & 3 & 4 & & . \\
\hline 14 & 157.8466 & 7.38 & 289.2 & 4 & .0 & 0 . \\
\hline 11215 & 180.6964 & 4.5690 & 288.8 & 3 & & \\
\hline & 155.8998 & $\$ 180$ & 288.8 & 4 & & \\
\hline 17 & 143.6590 & 3.3105 & 288.4 & 4 & & 0 . \\
\hline 11218 & 121.4923 & 2.9973 & 288.1 & 4 & 0 & 0 . \\
\hline 219 & 467 & 3. & & 4 & & \\
\hline & 68.4499 & 5.4475 & & 4 & & 0 . \\
\hline 1122 & 75.7521 & 5.3683 & 289.3 & 4 & & 00 \\
\hline & & & & 4 & & \\
\hline & 56.0534 & 2.3893 & 287.6 & & 600.0 & 600 \\
\hline
\end{tabular}




\begin{tabular}{|c|c|c|c|c|c|c|}
\hline $1<\angle 4$ & & .1545 & & & & \\
\hline 1131 & 151 & .9820 & 6.0 & & 0 & \\
\hline 11132 & 11.4346 & 2.0240 & 285.8 & 4 & 00 & \\
\hline 11133 & 359.0699 & 1.7063 & 286.0 & & 00 & \\
\hline 11134 & 14.4562 & .1178 & 86.6 & & 0 & \\
\hline 11135 & & 1.6788 & 86.4 & & 00 & \\
\hline 311136 & 101.6650 & 1.3440 & 286.2 & & 500.0 & 0 \\
\hline 311137 & 342.7842 & 1.72 & 85.8 & & 0 & \\
\hline 311138 & 12.8507 & 3.84 & 86.1 & & & \\
\hline 311139 & 3.5310 & 2.7620 & 286.6 & 4 & 00.0 & 0 \\
\hline 1310 & 315.9042 & 2.3700 & 287.6 & 3 & 0 & \\
\hline & 231.9602 & 2.52 & 87.6 & 2 & 0 & \\
\hline 2 & 174.8325 & 3.3928 & 287.5 & 3 & 0 & ( \\
\hline 13 & 186.3570 & 3.3593 & 288.3 & 3 & 0.0 & \\
\hline & 234 . & 2 . & & 3 & & \\
\hline & 238 & 1 . & 288.8 & 2 & & \\
\hline 316 & 191.6351 & 0.76 & 288.8 & 1 & 0.0 & . \\
\hline 17 & 154.0612 & 2.67 & .4 & 2 & 0 & \\
\hline & 89.6786 & 4.41 & 287.3 & 3 & & \\
\hline 9 & 76.2242 & 52 & 286.6 & 4 & 0 & U. \\
\hline 20 & 57.4248 & 5 . & 5.3 & 4 & 0 & 0 \\
\hline & 47.4961 & & & 5 & & \\
\hline 2 & 53.0492 & 5 & 28 & 4 & & \\
\hline 23 & 33.6100 & 4.35 & 28 & 4 & 0 & . \\
\hline 24 & 38.6167 & & & 4 & & \\
\hline 1 & 3.5309 & 1 & 28 & 5 & & \\
\hline 311142 & 25.1168 & 3.10 & 283 & 5 & 0 & U. \\
\hline 311143 & 10.7222 & 2 . & $2 \varepsilon$ & 4 & & \\
\hline $31114 \quad 4$ & 6.2006 & 1 . & 28 & 5 & & $\bullet$ \\
\hline 311145 & 6.3602 & 2.00 & 283.7 & 4 & .0 & 0 • \\
\hline 311146 & 11.9307 & 2.18 & 283.6 & 4 & 0 & 0 . \\
\hline 311147 & 18.1793 & 2 . & & 4 & & \\
\hline 311148 & 3.3037 & 8 & .7 & 4 & & 0 • \\
\hline 311149 & 336.2436 & 2.68 & 28 & 3 & 0 & 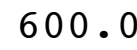 \\
\hline 10 & 276.6205 & 150 & & 2 & & \\
\hline 311 & 134.6867 & 2.47 & 7.0 & 1 & & 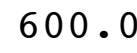 \\
\hline 3111412 & 338.1420 & 3.9868 & 288.1 & 2 & $\cdot 0$ & 0 . \\
\hline 13 & 10.6513 & 3 . & 287 & 2 & & 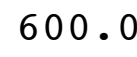 \\
\hline 0311 & 13.5256 & 5.1990 & 287.0 & 3 & .0 & 0 . \\
\hline 3111415 & 352.2754 & 4.1463 & 286.3 & 3 & .0 & 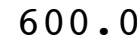 \\
\hline & 11.7867 & 623 & 286 & 3 & & 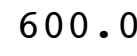 \\
\hline 311 & 31.4905 & 5.9483 & 285.5 & 4 & .0 & 600 . \\
\hline 3111418 & 64.9324 & 5.6738 & 283.2 & 4 & .0 & 00 . \\
\hline 0311 & 56.4246 & 6 . & & 4 & & \\
\hline 03111420 & 77.4218 & 4.5983 & 283.7 & 4 & 0 & 600 \\
\hline 114 & 64.0214 & 4.2720 & 284.0 & 4 & 600.0 & 500. \\
\hline & 71.9154 & & & 4 & & \\
\hline 311 & 64.8389 & 3.9543 & 283.8 & 4 & 600.0 & 600 \\
\hline
\end{tabular}




\begin{tabular}{|c|c|c|c|c|c|c|}
\hline 111424 & 1.7330 & 2.8800 & 282.9 & 5 & 600.0 & \\
\hline 1151 & 8.5158 & 1.8353 & 283.1 & 6 & 600 & \\
\hline 311152 & 307.9412 & 8885 & 283.3 & 6 & 00.0 & \\
\hline 11153 & 345.7104 & 1.3720 & 283.1 & & 00 & \\
\hline 1154 & 352.6808 & .8628 & 33.2 & & 0 & \\
\hline 1155 & 323.3865 & 1.2155 & 283.0 & & 00 & \\
\hline 311156 & 31.5728 & 0.9963 & 283.3 & & 00 & \\
\hline 1157 & 88.2505 & .2515 & 33.3 & & & \\
\hline 1158 & 91.7022 & 2.4173 & 282.9 & & 0 & \\
\hline 1159 & 65.4671 & 2.8878 & 282.3 & & 00 & \\
\hline 10 & 88.0169 & 2.3538 & 282.3 & & 0 & \\
\hline & 76.3496 & 3.4063 & 82.5 & & & \\
\hline 2 & 85.8866 & 2.1608 & 283.1 & & 0 & \\
\hline 13 & 26.2757 & 1.8120 & 283.6 & 1 & 0 & \\
\hline & 287.0715 & 2.1058 & & & & \\
\hline 5 & 293.2745 & 2.2920 & 283.6 & 3 & 0 & \\
\hline 516 & 281.1129 & 1.7895 & 283.5 & & 0 & \\
\hline 17 & 260.4251 & 1.0965 & .2 & 3 & & \\
\hline 8 & 59.1003 & 2.9563 & 283.3 & 4 & 0 & \\
\hline 19 & 64.8572 & 4.1260 & 283.1 & 4 & 0 & \\
\hline 20 & 74.3003 & 4.1088 & 282.7 & 4 & & \\
\hline 21 & 75.1253 & 4.2775 & 282.5 & 5 & & \\
\hline 22 & 73.6498 & 9093 & 282.3 & 5 & 500 & 600 . \\
\hline 23 & 81.9510 & 3.8500 & 282.0 & 5 & 0 & \\
\hline 24 & 76.2526 & 863 & 281.8 & 5 & & \\
\hline 61 & 75.3744 & 3.5085 & 282.0 & 5 & & \\
\hline 311162 & 83.8987 & .8153 & 281.9 & 4 & 0 & \\
\hline 311163 & 145.4496 & .3983 & 281.1 & 4 & & \\
\hline 164 & 135 . & .1248 & 281.0 & 5 & 0 & 0 . \\
\hline 311165 & 142.7000 & .0310 & 281.0 & 4 & 0 & \\
\hline 311166 & 119.9258 & 2.2038 & 28 & 4 & & \\
\hline 167 & 107.5073 & 1.7995 & 281.2 & 4 & & \\
\hline 311168 & 87.8231 & 2.6163 & 281.7 & 4 & 0 & 0 • \\
\hline 311169 & 151.2306 & .7960 & 281.6 & 4 & 0 & \\
\hline 510 & 54. & .6395 & 28 & 3 & & \\
\hline 511 & 147.5105 & 2.2788 & 284.8 & 2 & 0 & . \\
\hline 612 & 148.4043 & 3.2540 & 285.4 & 2 & 0 & \\
\hline 03 & 100.3376 & 3.6715 & & 2 & & $0^{-1}$ \\
\hline 14 & 85.9965 & 4.8303 & 286.4 & 3 & 600.0 & 0 . \\
\hline 615 & 898 & 4.4633 & 286.4 & 3 & 600 & \\
\hline 516 & 99. & 3.9700 & & 4 & & \\
\hline 031 & 73.5554 & 4.3528 & 286.1 & 4 & 600.0 & 600 . \\
\hline 11618 & 76.4603 & 4.9635 & 285.3 & 4 & 600.0 & 600 \\
\hline & 66.6618 & 4.6650 & 285.0 & 4 & & 60 \\
\hline 031 & 64.2500 & 4.9118 & 284.8 & 4 & 600.0 & 00 . \\
\hline 62 & 59.5006 & 6.6853 & 284.4 & 4 & 600.0 & 600. \\
\hline & 61.7750 & 7.6300 & & 4 & 600 & \\
\hline 523 & 56.3509 & 7.0295 & 284.9 & 4 & 600.0 & 600 \\
\hline
\end{tabular}




\begin{tabular}{|c|c|c|c|c|c|c|}
\hline $110<4$ & 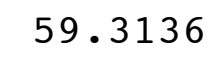 & .6580 & 285.0 & 4 & 600.0 & \\
\hline 1171 & 269.3635 & .1040 & 285.0 & & 600.0 & \\
\hline-1172 & 247.2331 & 1.6740 & 284.4 & 6 & 00 & \\
\hline 11173 & 328.1538 & 1.4413 & 284.5 & & 00 & \\
\hline 11174 & 46.9840 & .2275 & & & 0 & \\
\hline 11175 & 583 & 3.4028 & 85.0 & & 00 & \\
\hline 311176 & 91.2534 & 4.2743 & 284.5 & & 00 & \\
\hline 11177 & 79.7755 & .7438 & 84.4 & & 0 & \\
\hline 11178 & 82.6428 & 0493 & 84.4 & & & \\
\hline 311179 & 82.4427 & 6.2148 & 284.9 & 4 & 00 & 0 \\
\hline 3111710 & 82.8000 & 6.3198 & 285.4 & & 0 & \\
\hline .711 & 94.3257 & 5.6835 & & & & \\
\hline 1712 & 91.4987 & 5 . & 286.5 & 3 & 0 & \\
\hline 3111713 & 92.1472 & I. & 287.1 & & 0.0 & \\
\hline 714 & 118 & & & & & \\
\hline 715 & 140 & & 0 & 3 & & \\
\hline 1716 & 168.6070 & 9668 & 287.2 & 4 & .0 & . \\
\hline 1717 & 156.5248 & 4 . & 3 & 5 & & \\
\hline .718 & 153.65 & & 5 & 5 & & \\
\hline 1719 & 150.2254 & 3.4245 & 284.8 & 4 & 0 & 0. \\
\hline 3111720 & 140.8759 & 4.40 & 284.6 & 5 & 0 & \\
\hline .721 & 144.1998 & & & 3 & & \\
\hline 722 & 146 & 0 & 28 & 5 & & \\
\hline 3111723 & 144.7999 & 3.95 & 2 & 5 & 0 & \\
\hline 724 & 132 & & & 4 & & \\
\hline 81 & 113.5685 & 8 & & 4 & & \\
\hline 311182 & 129.0957 & 2.5378 & 6 & 4 & 0 & . \\
\hline 311183 & 126.6351 & 0 & & 4 & & \\
\hline 311184 & 156.7000 & 0 . & 28 & 4 & & \\
\hline 311185 & 190.9200 & 0.8085 & 2 & 5 & 0 & 0 . \\
\hline 311186 & 350.5916 & 0.9473 & 28 & 5 & & s. \\
\hline 311187 & 319.3160 & 3 & & 5 & & \\
\hline 311188 & 295.8437 & 42 & 28 & 5 & & 0 . \\
\hline 311189 & 101.4201 & 9323 & $2 \varepsilon$ & 4 & 0 & 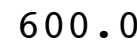 \\
\hline 810 & 250 & 8 & & 3 & & \\
\hline 811 & 265.1087 & 1.7143 & 5.6 & 2 & & 0 . \\
\hline 3111812 & 246.9698 & 4283 & 286.3 & 2 & & \\
\hline 1813 & 283.4715 & 2 . & 28 & 2 & & . \\
\hline 03111814 & 276.3964 & 2.6548 & 287.5 & 3 & .0 & 0 . \\
\hline 3111815 & 289.5147 & 2.5188 & 288.0 & 3 & & \\
\hline 1816 & 293.6219 & 680 & 288 & 3 & & 0 \\
\hline 317 & 301.8187 & 3.1733 & 287.2 & 4 & & 0 . \\
\hline 3111818 & 238.3015 & 1.7993 & 286.2 & 5 & 0 & 0 . \\
\hline 03111819 & 211.7441 & 1.1983 & & 6 & & \\
\hline 03111820 & 173.0009 & 1.1980 & 285.5 & 6 & & 0 . \\
\hline 11821 & 150.1740 & 2.0475 & 285.0 & 5 & .0 & 00 . \\
\hline & 142.77 & & & 4 & & \\
\hline 111823 & 93.9472 & 1.5025 & 284.2 & J & 600.0 & $\mathrm{r}^{\circ}$ \\
\hline
\end{tabular}




\begin{tabular}{|c|c|c|c|c|c|c|}
\hline 4 & & .4643 & 84.7 & & & \\
\hline 1191 & 301 . & 0438 & 284.6 & & 600.0 & \\
\hline 1192 & 302.2077 & 1703 & 284.2 & & 00 & \\
\hline 11193 & 11.3904 & 1.1275 & 284.6 & & 00 & \\
\hline 1194 & 79.4401 & 498 & & & & \\
\hline 1195 & 21.0888 & 3770 & 5 & & 0 & \\
\hline 311196 & 17.3157 & 2533 & 287.5 & & 00 & \\
\hline 11197 & 108.7516 & .71 & 84.1 & & & \\
\hline 11198 & 123.0648 & 47 & 0 & & & \\
\hline 311199 & 147.3006 & 2.8828 & 83.2 & & 0 & 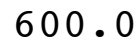 \\
\hline 1910 & 132.2474 & 2.1035 & 283.7 & & & \\
\hline & 314.3863 & 1.40 & & & & \\
\hline 2 & 161.9151 & 2.59 & 286.7 & & 0 & \\
\hline 913 & 158.8522 & 0 & 0 & & & \\
\hline & 158 . & & & & & \\
\hline & 146 . & & & & & \\
\hline 916 & 157.9047 & 2.92 & 287.8 & 4 & 0 & \\
\hline 917 & 129.8490 & 7 & 4 & & & \\
\hline & 128.5579 & & 8 & & & \\
\hline 919 & 144.4061 & 1 . & 287.8 & & 0 & \\
\hline 920 & 122.3302 & 2.0 & 287.7 & & 0 & \\
\hline 21 & 104.7103 & & & 6 & & \\
\hline 22 & 58.8527 & 5 & 28 & 5 & & \\
\hline 923 & 71.6769 & 3 & .1 & 4 & 0 & \\
\hline 24 & 027 & & & 4 & & \\
\hline 201 & 75.1499 & & 28 & 4 & & \\
\hline 311202 & 71.6161 & 3 & 7.9 & 4 & & o. \\
\hline 311203 & 67.6487 & 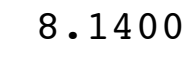 & 4 & 4 & & \\
\hline $31120 \quad 4$ & 69.7510 & 8 . & $2 \varepsilon$ & 4 & & \\
\hline 311205 & 66.8242 & 0 & 287.0 & 4 & & J. \\
\hline 311206 & 69.4217 & & 287.0 & 4 & & 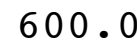 \\
\hline 311207 & 72.7753 & & 2 & 4 & & \\
\hline 311208 & 76.8000 & & 287.0 & 4 & & 0 . \\
\hline 311209 & 75.7252 & 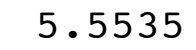 & .5 & 4 & 0 & 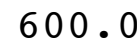 \\
\hline 12010 & 62.8150 & & & 3 & & \\
\hline 3112011 & 56.2366 & & 289.8 & 2 & & \\
\hline 12012 & 108.5632 & 5 & 290.5 & 2 & & \\
\hline 3112013 & 146.2304 & 3 . & 289.1 & 2 & & \\
\hline 03112014 & 100.9084 & 4.0663 & 288.7 & 3 & & 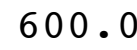 \\
\hline 12015 & 67.1260 & 3173 & 288.3 & 4 & & \\
\hline 2016 & 68.1714 & 6.6563 & 288.8 & 4 & & \\
\hline 3112017 & 73.4002 & 6.8308 & 287.2 & 4 & & 0 . \\
\hline 12018 & 73.6998 & 7.2238 & 286.4 & 4 & 0 & 0 . \\
\hline 3112019 & 272 & 9148 & 285.9 & 4 & & \\
\hline 112020 & 67.5500 & 0.2025 & 284.7 & 4 & & \\
\hline 1202 & 67.7246 & 7.7425 & 285.1 & 4 & & 0 . \\
\hline & 69 & & & 4 & & \\
\hline 112023 & 66.9342 & 7.9433 & 284.0 & & .0 & 600 \\
\hline
\end{tabular}




\begin{tabular}{|c|c|c|c|c|c|c|}
\hline 624 & 3.4001 & 0.1275 & 83.7 & & 0 & \\
\hline $\begin{array}{ll}21 & 1\end{array}$ & 8.1753 & 10.5800 & 82.6 & & & \\
\hline 11212 & 5003 & 925 & 282.2 & 4 & 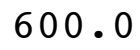 & \\
\hline 311213 & 76.4525 & 1550 & 281.6 & & 00 & \\
\hline $\begin{array}{ll}214 \\
21\end{array}$ & & & 1.0 & & & \\
\hline 215 & 554 & 450 & 81.2 & & b & \\
\hline 311216 & 763 & 613 & 280.6 & & & \\
\hline 1217 & 3808 & & 30.8 & & & \\
\hline 11218 & 9.3015 & & 80.3 & & & \\
\hline 311219 & 87.3977 & & 281.3 & & 0 & \\
\hline 10 & 87.8000 & & 82.1 & & & \\
\hline & 88 . & & 32.9 & & & \\
\hline 31 & 96.2753 & 0 & 282.9 & & 0 & \\
\hline 13 & 102.5325 & 9 & 33.6 & & 0 & \\
\hline & & & & & & \\
\hline & 121. & & 284.2 & 4 & & \\
\hline & 120 & & 3.4 & & & \\
\hline & 110 . & & & & & \\
\hline & 95 . & & 282.1 & 4 & & \\
\hline 19 & 922 & & 281.2 & 4 & & \\
\hline 20 & 88 . & & 7 & & & \\
\hline & 82 . & & & 4 & & \\
\hline 22 & 86.7999 & 3 & 1 & 1 & & \\
\hline 23 & 75.5903 & 325 & & 4 & & \\
\hline & 212 & & & 4 & & \\
\hline 21 & 677 & & 280 & 4 & & \\
\hline 311222 & 135.4750 & 0 & 0.1 & 4 & & \\
\hline 311223 & 144.8251 & & 7 & 4 & & \\
\hline 24 & 15 & 5 & 27 & 4 & & \\
\hline 311225 & 153. & & 5 & 4 & & \\
\hline 311226 & 000 & & & 4 & & \\
\hline 27 & 15 & 0 & 2 & 4 & & \\
\hline 311228 & 136.4497 & 5 & 278.1 & 4 & 0 & \\
\hline 311229 & 143 . & & 2 & 4 & & \\
\hline & 15 & 5 & 3 & 4 & & \\
\hline & 154.4501 & 850 & 280.2 & 4 & & \\
\hline & 150.3501 & 5 & 1.1 & 4 & & \\
\hline & 157.6742 & 5 & & 4 & & \\
\hline 14 & 161. & 600 & 282.0 & 4 & & \\
\hline & 176 . & 10. & 282.1 & 4 & & \\
\hline & 183. & & & 4 & & \\
\hline 17 & 165.3364 & 4.6208 & 281.0 & 4 & 0 & 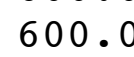 \\
\hline & 142 . & 078 & 280.3 & 4 & 0 & \\
\hline & 148.0256 & & & 4 & & \\
\hline 03112 & 148.0748 & 4.6830 & 279.8 & 3 & 600 & \\
\hline 221 & 13 & 5.4108 & & 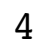 & & \\
\hline & 14 & & & & & \\
\hline 223 & 134.9004 & 6.0575 & 280.5 & & 600.0 & 600 \\
\hline
\end{tabular}




\begin{tabular}{|c|c|c|c|c|c|c|}
\hline 224 & & 0 & 9.9 & & & \\
\hline 1231 & 52 & .8550 & 279.3 & 4 & 0 & \\
\hline 1232 & 135.7004 & 5103 & 279.0 & 4 & 00 & \\
\hline 11233 & 112.8713 & 9123 & 278.4 & & 00 & \\
\hline 1234 & 08.9727 & .1903 & 278.3 & & 0 & \\
\hline 1235 & 103.5449 & 1198 & 278.4 & 4 & 00 & \\
\hline 311236 & 108.4203 & 470 & 277.8 & 1 & 00 & \\
\hline 311237 & 92.9514 & 1205 & 277.6 & & 0 & \\
\hline 11238 & 80.5502 & 8348 & 78.1 & 4 & & \\
\hline 311239 & 100.9931 & 5.6958 & 279.0 & 4 & 00.0 & 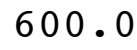 \\
\hline 3112310 & 135.3424 & 4.7695 & 279.9 & 4 & 0 & \\
\hline & 164.5303 & 3.5880 & 280.5 & 3 & 00.0 & \\
\hline 2 & 199.8182 & 2 . & 281.8 & 2 & 0 & ( \\
\hline 13 & 277.5008 & 3.09 & 282.5 & 2 & 0.0 & \\
\hline 14 & 3472 & & & 3 & & \\
\hline & 287 . & & 284.2 & 3 & & \\
\hline 6 & 250.9102 & 2 . & 283.7 & 3 & .0 & 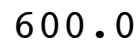 \\
\hline 17 & 179.4719 & 3 & 282.0 & 4 & 0 & \\
\hline & 153.5518 & & 281.1 & 5 & & \\
\hline 9 & 5077 & 2. & 280.9 & 4 & 0 & \\
\hline 20 & 24.6509 & 1.09 & 281.3 & 5 & 0 & 00 . \\
\hline & 19.8035 & & 1.4 & 4 & & \\
\hline 2 & 250 & 1 . & 281.4 & 4 & & \\
\hline 23 & 4.0000 & 2.04 & 281.2 & 5 & 0 & 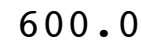 \\
\hline & 6.6719 & 1. & .5 & 4 & & \\
\hline 1 & 1.1446 & 1.4 & 1.4 & 5 & & \\
\hline 311242 & 262.3341 & 1.18 & 1.0 & 6 & 0 & U. \\
\hline 311243 & 316.0792 & 1.2 & .6 & 6 & & \\
\hline $31124 \quad 4$ & 160.7543 & 1.4 & .0 & 5 & & \\
\hline 311245 & 138.6004 & 1.86 & 279.7 & 5 & 0 & 0 • \\
\hline 311246 & 124.3747 & 1.24 & 280.0 & 6 & 0 & 0 . \\
\hline $31124 \quad 7$ & 82.8310 & 1 & 9.6 & 6 & & \\
\hline 311248 & 998 & & 0.0 & 5 & & 0 . \\
\hline 311249 & 125.0933 & 0.9458 & 280.2 & 4 & 0 & 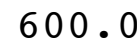 \\
\hline 10 & 22.5951 & 8 & 0.8 & 3 & & \\
\hline 311 & 199.5219 & & 2.0 & 2 & & 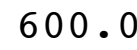 \\
\hline 311 & 217.9949 & 8498 & 283.1 & 1 & $\cdot 0$ & 0 . \\
\hline 3 & 166.7933 & 2 . & 3.2 & 1 & & . \\
\hline 0311 & 193.9307 & 3.91 & 282.7 & 2 & .0 & 0 . \\
\hline 112415 & 164.8623 & 4.0560 & 282.2 & 3 & & 0 . \\
\hline 0311 & 139.4787 & 2.1015 & 282.4 & 4 & & 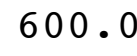 \\
\hline 0311 & 64.3282 & 2.0615 & 282.1 & 5 & .0 & 600 . \\
\hline 3112418 & 123.6563 & 2.3818 & 282.1 & 6 & 600 & 00 . \\
\hline 03112419 & 118.2783 & 3 . & 281.8 & 5 & & \\
\hline 03112420 & 82.7707 & 5.6813 & 281.4 & 4 & .0 & 600 \\
\hline 1242 & 71.4619 & 7.1210 & 281.3 & 4 & 600.0 & 00 \\
\hline & 809 & & 281.1 & 4 & & \\
\hline & 65.1271 & 5.7895 & 281.3 & 4 & 600.0 & 600 \\
\hline
\end{tabular}




\begin{tabular}{|c|c|c|c|c|c|c|}
\hline$-1-1$ & 6.5755 & 4.8735 & 281.2 & 5 & 600.0 & \\
\hline 1251 & 2.9250 & 5.0338 & 281.3 & 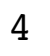 & 600.0 & \\
\hline 11252 & 76.2756 & 7175 & 279.9 & 4 & 00.0 & t \\
\hline 11253 & 78.2786 & 7.6750 & 279.7 & & 00.0 & \\
\hline 1254 & 80.4507 & .8680 & 279.9 & & 00 & \\
\hline 1255 & 105.5439 & .5308 & 279.5 & & 00 & \\
\hline 311256 & 125.7585 & 2.3718 & 279.0 & & 00.0 & 0 \\
\hline 11257 & 91.9250 & .4590 & 279.2 & & .0 & \\
\hline 11258 & 102.5750 & 930 & 279.0 & & & \\
\hline 311259 & 88.1734 & 4.6148 & 279.7 & & 00.0 & 8 \\
\hline 510 & 120.8859 & 3.9953 & 281.0 & & 00.0 & \\
\hline & 139.6995 & 4.8605 & 281.9 & & 00.0 & \\
\hline 2 & 150.3577 & 4.9055 & 282.9 & 3 & $\cdot 0$ & 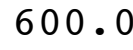 \\
\hline 13 & 102.5643 & 3.7755 & 284.3 & & 0.0 & . \\
\hline & 87.5590 & 5 & 84 & & & \\
\hline & 91.2047 & & 284.5 & & & \\
\hline 6 & 73.5864 & 5770 & 283.9 & 4 & 0.0 & 政 \\
\hline & 75.4903 & 6 . & 283.0 & 4 & 0 & \\
\hline & 63.6273 & & 282.3 & 4 & & \\
\hline 19 & 70.8759 & 6.7050 & 281.9 & 4 & .0 & . \\
\hline 20 & 74.3996 & 7.6925 & 281.7 & 4 & 0 & 0 \\
\hline & 68.4250 & & 281.4 & 4 & & \\
\hline 22 & 80.9253 & 958 & 281.6 & 4 & & \\
\hline 23 & 74.7941 & 975 & 281.5 & 4 & 0 & . \\
\hline 24 & 68.0203 & & 281.2 & 4 & & \\
\hline 1 & 72.3001 & 000 & 281.2 & 4 & & \\
\hline 311262 & 68.9766 & 415 & 281.1 & 4 & 0 & U. \\
\hline 311263 & 95.9205 & 743 & 281.1 & 4 & & \\
\hline 311264 & 71.9744 & 048 & 281.2 & 4 & & $\bullet$ \\
\hline 311265 & 72.5012 & 325 & 281.2 & 4 & 0 & 0 • \\
\hline 311266 & 75.8750 & 2470 & 281.0 & 4 & 0 & 0 . \\
\hline 67 & 73.9260 & 170 & 280.8 & 4 & & \\
\hline 311268 & 76.7513 & 8 & 280.7 & 4 & 0 & 0 • \\
\hline 311269 & 80.6059 & 5 & 281.1 & 3 & 0 & 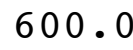 \\
\hline & 90.5259 & 0 & 281.7 & 3 & & \\
\hline & 134.8775 & 4.7468 & 282.4 & 3 & & 0 . \\
\hline 12 & 136.6556 & 5.1820 & 283.0 & 3 & $\cdot 0$ & 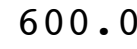 \\
\hline 13 & 136 & 180 & 283.9 & 3 & & . \\
\hline 0311 & 160.9866 & 6.2475 & 284.5 & 4 & .0 & 0 . \\
\hline 12615 & 166.8774 & 5350 & 284.4 & 3 & & 0 . \\
\hline & 150.9669 & 603 & 284.3 & 4 & & 60 \\
\hline 17 & 141.7238 & 4.9245 & 283.9 & 5 & .0 & 0 . \\
\hline 3112618 & 138.8255 & 4.6865 & 283.7 & 4 & 0 & 00 . \\
\hline 03112619 & 149.4250 & 960 & 282.9 & 4 & & \\
\hline & 148.2000 & 3.9580 & 282.5 & 5 & & 00 . \\
\hline 12621 & 137.2262 & 4.0838 & 282.6 & 4 & 600.0 & 00 \\
\hline & 127.1989 & & 6 & 4 & & \\
\hline 3112623 & 136.7460 & 2.9675 & 282.6 & & 600.0 & 600 \\
\hline
\end{tabular}




\begin{tabular}{|c|c|c|c|c|c|c|}
\hline 2624 & & 2618 & & & & \\
\hline 1271 & 1 & 4633 & 32.6 & 4 & 0 & \\
\hline 1272 & 149.0773 & 3545 & 282.3 & 4 & 00 & \\
\hline 11273 & 147.4756 & 1318 & 281.2 & & 00 & \\
\hline 11274 & 35.5405 & 8 & 80.3 & & 0 & \\
\hline 11275 & 31 & 5513 & 279.8 & 4 & 00 & \\
\hline 311276 & 22.7678 & 4363 & 279.9 & 4 & 00 & \\
\hline $31127 \quad 7$ & 32.2277 & 8 & 280.4 & & 0 & \\
\hline 311278 & 35.1810 & 0 & 80.0 & 4 & & \\
\hline 311279 & 60.4193 & 9405 & 279.9 & 4 & 500.0 & 0 \\
\hline 3112710 & 190.0453 & 2.6780 & 280.6 & 3 & 0 & \\
\hline 711 & 85.7666 & 3.2033 & 81.5 & & 00.0 & \\
\hline 2712 & 270 & 3. & 282.4 & 3 & 0 & 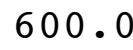 \\
\hline 3112713 & 233.2714 & 1.9803 & 283.5 & 2 & 0.0 & \\
\hline 714 & 223 & & 84.8 & 2 & & \\
\hline 715 & 216 & & 285.0 & 3 & & \\
\hline 2716 & 232.1017 & 2.8 & 284.9 & 3 & 0.0 & 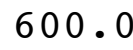 \\
\hline 717 & 33. & 3 & 284.2 & 4 & 0 & \\
\hline 718 & 185. & & 283.6 & 5 & & \\
\hline 719 & 185 & 1 . & 283.6 & 6 & 60 & \\
\hline 720 & 332.6989 & 1.2625 & 284.0 & 6 & 0 & 600. \\
\hline 721 & 138.5487 & & & 6 & & \\
\hline 722 & 5768 & 0 & 283.5 & 6 & & \\
\hline 723 & 144.7405 & 2.0743 & 282.4 & 5 & 0 & . \\
\hline 724 & 135.6754 & & 28 & 4 & & \\
\hline 81 & 95 . & 0.8 & 282.2 & 5 & & \\
\hline 311282 & 129.6989 & 2.0098 & 282.0 & 6 & 0 & J. \\
\hline 311283 & 43.0874 & 5 & 82.1 & 6 & & \\
\hline 284 & 173.2829 & 0 . & 282.3 & 6 & & $\bullet$ \\
\hline 311285 & 142.6783 & 2.5460 & 280.6 & 5 & .0 & 0 • \\
\hline 311286 & 113.3919 & 2.06 & 280.5 & 6 & 0 & 0 . \\
\hline 311287 & 143 . & 2 . & 281.0 & 5 & & \\
\hline 311288 & 123.2320 & 0 & 281.2 & 6 & & . \\
\hline 311289 & 46.3171 & 1.2755 & 282.0 & 5 & 0 & 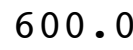 \\
\hline 810 & 195. & & & 4 & & \\
\hline & 278.8937 & 1.70 & 284.3 & 3 & & . \\
\hline 812 & 181.9813 & 2.2608 & 284.1 & 2 & $\cdot 0$ & 0 . \\
\hline 813 & 181.0220 & 1.66 & 284.4 & 1 & & . \\
\hline 314 & 164.8443 & 2.7288 & 283.8 & 2 & .0 & 0 . \\
\hline 3112815 & 139.4619 & 2.0275 & 282.9 & 1 & 0 & 0 . \\
\hline 12816 & 99.2923 & 1.9583 & 282.7 & 1 & & 600. \\
\hline 317 & 3.1092 & 1.1368 & 283.1 & 2 & .0 & 00 . \\
\hline 3112818 & 174.4729 & 1.3305 & 282.2 & 3 & 600 & 500 . \\
\hline 3112819 & 138.2213 & 1.9640 & & 4 & & 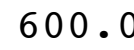 \\
\hline 03112820 & 140.3235 & 3.0195 & & 4 & .0 & 600. \\
\hline 3112821 & 143.7244 & 4.0138 & 280.4 & 5 & 60 & 600 \\
\hline & 140 & & & 5 & & \\
\hline 3112823 & 138.9001 & 2.8943 & 280.2 & & 60 & 600 \\
\hline
\end{tabular}




\begin{tabular}{|c|c|c|c|c|c|c|}
\hline 824 & & 4298 & & & & \\
\hline 1291 & 2 & .3358 & 0.2 & & 0.0 & \\
\hline 1292 & 140.0523 & $\$ 118$ & 280.2 & & 00 & \\
\hline 11293 & 141.7750 & 1608 & 280.2 & & 00.0 & \\
\hline 1294 & 003 & .5803 & 80.2 & & 0 & \\
\hline 1295 & 0498 & 3088 & 80.3 & & 00 & \\
\hline 311296 & 50.9000 & 7018 & 280.3 & & 500 & 0 \\
\hline 311297 & 48.3999 & 6.3025 & 80.1 & & 00.0 & \\
\hline 311298 & 5248 & 5468 & 79.9 & & & \\
\hline 311299 & 49.0500 & 4.2223 & 80.3 & & 600.0 & 0 \\
\hline 2910 & 46.2594 & 1.8670 & 282.1 & & 00.0 & \\
\hline 11 & 1.3275 & 4.2850 & 82.3 & & 00.0 & \\
\hline 912 & 742 & 5028 & 281.9 & & 00.0 & . \\
\hline 913 & 65.8253 & .5443 & 282.0 & & 00.0 & \\
\hline 914 & 480 & & 2.4 & & & \\
\hline 15 & & & 282.7 & & & \\
\hline 916 & 160.3401 & 9000 & 282.6 & & .0 & 0 \\
\hline 917 & 733 & 665 & 32.4 & & .0 & \\
\hline & 47 & & 282.0 & & & \\
\hline 19 & 3754 & 5173 & 281.5 & 4 & 0 & \\
\hline 920 & 116.3892 & 3.49 & 281.8 & & .0 & 600. \\
\hline 21 & 112.0435 & & 282.3 & & & \\
\hline 22 & 141 & 3 & 282.0 & & & \\
\hline 923 & 144.8501 & 3.7308 & 281.2 & & . 0 & 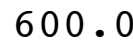 \\
\hline 24 & 147.4259 & 8 & 9 & & & \\
\hline 0 & 148.1754 & 4 . & 28 & 5 & & \\
\hline 311302 & 146.8498 & 8953 & 280.9 & 5 & .0 & U. \\
\hline 311303 & 120.5321 & 0 & 31.3 & 4 & & \\
\hline $31130 \quad 4$ & 163.6242 & 2 . & 282.7 & 5 & & 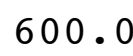 \\
\hline 311305 & 300.7281 & 2.3153 & 281.2 & 6 & & 0 • \\
\hline 311306 & 192.5119 & 1.7665 & 281.0 & 6 & & s. \\
\hline 311307 & 152.6034 & & 281.0 & 6 & & \\
\hline 311308 & 87.1616 & & 281.3 & 6 & & U. \\
\hline 311309 & 331.4368 & 2.79 & 281.1 & 5 & 0 & 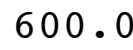 \\
\hline 10 & 216.2063 & 5 & 281.3 & 4 & & \\
\hline & 263.4751 & 2 . & 282.0 & 3 & & 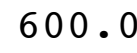 \\
\hline 311 & 273.3045 & 9320 & 282.5 & 2 & .0 & 0 . \\
\hline 13 & 296.3579 & 1 . & 281.7 & 1 & & ( \\
\hline 0311 & 274.3269 & 2.5755 & 282.0 & 1 & .0 & 0 . \\
\hline 3113015 & 278.2254 & 2.1765 & 281.7 & 1 & .0 & \\
\hline 13016 & 275.3201 & 145 & 281.9 & 1 & & 60 \\
\hline 0311 & 172.6611 & 2.9108 & 281.9 & 2 & .0 & 0 . \\
\hline 3113018 & 243.7999 & 2.2258 & 282.2 & 3 & 0 & 00 . \\
\hline 03113019 & 322.8638 & 918 & & 4 & & \\
\hline 03113020 & 316.2700 & 3.5555 & & 4 & 600.0 & 600. \\
\hline 1302 & 326.9404 & 2.4800 & 283.3 & 5 & 600.0 & 600 \\
\hline & 326 & & & 4 & & \\
\hline & 333.4552 & 3.1103 & 285.5 & & .0 & 60 \\
\hline
\end{tabular}




\begin{tabular}{|c|c|c|c|c|c|c|c|}
\hline & & & 3 & & & & \\
\hline & 1 & 288 & 2.2173 & 3.4 & 6 & 00.0 & \\
\hline & 12 & 147.8252 & 3.3790 & 281.5 & 5 & 00.0 & \\
\hline 12 & 13 & 139.0749 & 2.1783 & 281.4 & & 00.0 & \\
\hline & 14 & 8.1722 & .2150 & 81.4 & & 0 & \\
\hline & 15 & 353.3755 & .1383 & 81.3 & & $0 C$ & \\
\hline 1 & 16 & 352.5429 & 2.4978 & 281.0 & & 00 & 0 \\
\hline & 17 & 74.4173 & 1.9445 & 80.8 & & .0 & \\
\hline & 18 & 343.1269 & 2.4875 & 81.4 & & & \\
\hline & 19 & 319.0126 & 2.4495 & 282.5 & & 500.0 & 00 \\
\hline & 110 & 272.7841 & 1.5870 & 282.9 & & 00.0 & \\
\hline & 111 & 265.3613 & 2.1830 & 83.9 & & .0 & \\
\hline & 112 & 326.1476 & 5.60 & 286.5 & & 00.0 & ( \\
\hline & 113 & 332.1041 & 6.43 & 4 & & 0.0 & . \\
\hline & 114 & 341.4270 & 8 & .3 & & & \\
\hline & 115 & 348.9621 & & 289.1 & & & \\
\hline & 116 & 339.2266 & 5.2025 & 288.2 & & .0 & 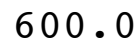 \\
\hline & 117 & 329.9334 & 3.68 & .3 & & .0 & \\
\hline & 118 & 333.6960 & 3.33 & 286.4 & 4 & & \\
\hline & 119 & 339.6252 & 2.55 & 286.3 & 4 & 0 & \\
\hline 312 & 120 & 356.2880 & 2.03 & 286.5 & & .0 & 00 . \\
\hline & 121 & 349.6513 & & & & & \\
\hline & 122 & 350.9868 & 0 & 28 & 6 & & \\
\hline 2 & 123 & 345.7685 & 1.44 & .8 & 6 & . 0 & 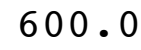 \\
\hline & 124 & 347.8088 & & & 6 & & \\
\hline & 21 & 5001 & 2 . & 28 & 6 & & \\
\hline 2 & 22 & 347.1313 & 1.48 & 9 & 6 & 0 & U. \\
\hline & 23 & 351.1951 & 2.62 & 6 & 5 & & \\
\hline & 24 & 6.3005 & 2.36 & 28 & 4 & & $\bullet$ \\
\hline 2 & 25 & 21.9061 & 2.79 & 6.3 & 4 & 0 & 0 • \\
\hline 312 & 26 & 48.8795 & 3.62 & 9 & 4 & .0 & 0 . \\
\hline & 27 & 76 . & 2.9 & & 4 & & \\
\hline & 28 & 92.2569 & 0 & 285.8 & 4 & & o. \\
\hline 312 & 29 & 42.7704 & 1.59 & 284.7 & 3 & 0 & 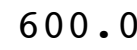 \\
\hline & 210 & 162.8155 & 0 & & 2 & & \\
\hline & 211 & 140.4927 & 1.63 & .4 & 1 & & 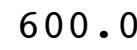 \\
\hline 312 & 212 & 133.1749 & 2.5020 & 284.4 & 2 & .0 & 0. \\
\hline 12 & 213 & 265.8575 & 2.17 & & 1 & & ( \\
\hline ט & 214 & 187.5123 & 3.31 & 284.9 & 2 & .0 & 0 . \\
\hline 312 & 215 & 143.0810 & 2.79 & 283.9 & 2 & & 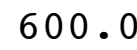 \\
\hline & 216 & 3.7346 & 1.12 & & 1 & & 0 \\
\hline & 217 & 296.8950 & 0.8543 & 285.4 & 2 & .0 & 0 . \\
\hline & 218 & 132.5540 & 2.1463 & 284.3 & 3 & 0 & 00 . \\
\hline & 219 & 131.1759 & 2.52 & & 4 & & \\
\hline & 220 & 134.3463 & 1.7568 & & 4 & & 00 \\
\hline & 221 & 124.8074 & 1.8673 & 284.7 & 5 & 600.0 & 600. \\
\hline & 222 & 115.3180 & 1 . & & 5 & & \\
\hline & 223 & 100.2937 & 2.4543 & 284.9 & & 600.0 & 600 \\
\hline
\end{tabular}




\begin{tabular}{|c|c|c|c|c|c|c|c|}
\hline- & 24 & 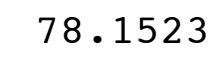 & 6.0243 & 84.2 & & - & \\
\hline & 31 & 1.5748 & 6.6350 & 284.0 & & 600.0 & \\
\hline & 32 & 68.2951 & 1903 & 283.8 & 4 & 00 & \\
\hline 1 & 33 & 66.0306 & 1410 & 284.0 & & 00.0 & \\
\hline & 4 & 96.0546 & 3.0990 & 84.2 & & 00 & \\
\hline & 5 & 122 . & 2.6550 & 83.9 & & 00 & \\
\hline 1 & 6 & 3694 & 1.5948 & 283.9 & & 500.0 & 0 \\
\hline & 7 & 138.7980 & 4.2433 & 82.4 & & 00.0 & \\
\hline & 8 & 137.6929 & 683 & 82.5 & & & \\
\hline & 9 & 116.0217 & 2.7983 & 282.7 & & 600.0 & 00 \\
\hline & 310 & 149.8945 & 4.0815 & 282.7 & & 0 & \\
\hline & 11 & 153.6998 & 511 & 82.8 & & .0 & \\
\hline & 12 & 142.90 & 4.7358 & 282.9 & & 00.0 & U \\
\hline & 13 & 142.2205 & 5.09 & 283.0 & & 00.0 & . \\
\hline & 14 & 153. & & 9 & & & \\
\hline & 15 & 154 . & & 284 & 4 & & \\
\hline & 16 & 140.5243 & 1.7988 & 285.8 & 3 & 00.0 & 00 \\
\hline & 317 & 140.63 & 1 . & .3 & & 0 & \\
\hline & 18 & & & 285.4 & 5 & & \\
\hline & 319 & 10.3185 & 1 . & 284.9 & 4 & 0 & 0 \\
\hline 31 & 320 & 3.2741 & 1.9243 & 284.4 & 5 & .0 & 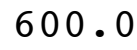 \\
\hline & 321 & 351.2743 & & & 4 & & \\
\hline & 322 & 344 . & & 28 & 4 & & \\
\hline 5 & 323 & 359.5004 & 2 . & 283.2 & 4 & 0 & . \\
\hline & 324 & 352.45 & & & 5 & & \\
\hline & 41 & 137 . & 1. & 282.6 & 6 & & \\
\hline & 42 & 139.9454 & 5073 & 281.9 & 6 & 0 & U. \\
\hline & 43 & 60.2358 & 3 & 282.2 & 6 & & \\
\hline & 44 & 349. & 0 . & 282 & 6 & & 0 \\
\hline & 45 & 169.1833 & 1.2340 & 281.0 & 6 & .0 & 0 . \\
\hline & 46 & 358.2537 & 0.9245 & 281.3 & 6 & 0 & 0 . \\
\hline & 47 & 18.52 & 0 & 282.0 & 6 & & \\
\hline & 48 & 26.0260 & 1.20 & 282.6 & 6 & & U • \\
\hline 21 & 49 & 99.5439 & 1.2715 & 283.9 & 5 & 0 & 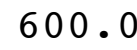 \\
\hline & 410 & 142.4970 & 2 . & 282.2 & 4 & & 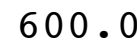 \\
\hline & 411 & 148.1004 & 2 . & 282.2 & 4 & & 0 . \\
\hline & 412 & 161.4732 & 1.3460 & 283.5 & 3 & $\cdot 0$ & 0 . \\
\hline 21 & 413 & 163.0528 & 2 . & 28 & 2 & & . \\
\hline 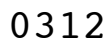 & 414 & 147.5411 & 2.8540 & 282.8 & 3 & .0 & 600 . \\
\hline & 415 & 263.9239 & 1.7148 & 282.7 & 2 & 0 & o. \\
\hline & 416 & 25.1771 & 2.2440 & 282.6 & 1 & & 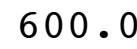 \\
\hline & 417 & 235.9602 & 1.9323 & 282.4 & 2 & .0 & 00 . \\
\hline & 418 & 79.5971 & 1.8783 & 282.8 & 3 & .0 & 00 . \\
\hline & 419 & 119.5999 & 120 & 283.8 & 4 & 600 & 0 \\
\hline & 420 & 95.6768 & 1.1858 & 284.4 & 5 & 600.0 & 600 \\
\hline & 421 & 274.9458 & 1.4923 & 283.2 & 6 & 600.0 & 600 \\
\hline & 422 & 274 . & & & 6 & & \\
\hline & 423 & 320.1409 & 1.0480 & 282.2 & & 600.0 & 600 \\
\hline
\end{tabular}




\begin{tabular}{|c|c|c|c|c|c|c|c|}
\hline & 4 & & & & & & \\
\hline & 1 & 341.9635 & .8403 & 2.2 & 6 & 600 & \\
\hline & 52 & 81.7163 & 0.9130 & 282.3 & 6 & 00 & \\
\hline 12 & 53 & 352.9070 & 1.5453 & 282.6 & & 00.0 & \\
\hline & 4 & 348.3697 & 1.3708 & 82.4 & & 0 & \\
\hline & 5 & 327.9554 & 1.5245 & 282.2 & & $0 C$ & \\
\hline & 56 & 351.8248 & 1.8145 & 282.1 & & 500.0 & 0 \\
\hline & 7 & 1.9253 & 2.1543 & 82.1 & & 00.0 & \\
\hline & 8 & 97.3710 & 2.5008 & 82.8 & & & \\
\hline & 9 & 94.0114 & 1.7545 & 283.5 & & 600.0 & 00 \\
\hline & 10 & 14.9975 & 1.2868 & 284.7 & & 0 & \\
\hline & 11 & 358.4999 & 1.34 & 86.7 & & .0 & \\
\hline & 12 & 131.7825 & 2.7985 & 287.7 & & 00.0 & U \\
\hline & 13 & 105.8590 & 2.7450 & 289.0 & 1 & 00.0 & 00 \\
\hline & 14 & 323 . & 2.12 & 5 & & & \\
\hline & 1 & 255 . & 1 . & 287.7 & 1 & & \\
\hline & 16 & 296.1770 & 1.6320 & 287.4 & 2 & 0.0 & 政 \\
\hline & 17 & 19.9307 & 1.61 & 287.2 & 3 & 0 & \\
\hline & 18 & 8.8247 & & 287.0 & 4 & & \\
\hline & 19 & 17.1216 & 2 . & 287.3 & 4 & 0 & \\
\hline 2 & 20 & 29.1055 & 2.3368 & 287.8 & 4 & 0 & 600. \\
\hline & 21 & 25.6782 & 1.92 & 287.6 & 5 & & \\
\hline & 22 & 25.6489 & 1 . & 287.6 & 6 & & \\
\hline & 523 & 43.0765 & 2.9598 & 288.1 & 5 & 0 & 00 • \\
\hline & 524 & 66.6393 & & & 6 & & \\
\hline & 61 & 329. & 1.04 & 28 & 6 & & \\
\hline & 62 & 185.1794 & 1.0303 & 286.6 & 6 & 0 & U. \\
\hline & 63 & 155.0189 & 1.14 & 28 & 6 & & \\
\hline & 64 & 123.3872 & 1.49 & 28 & 6 & & • \\
\hline & 65 & 336.4865 & 1.6323 & 286.4 & 6 & .0 & 0 . \\
\hline & 66 & 312.3505 & 1.5813 & 286.6 & 6 & 0 & 0 . \\
\hline & 67 & 165.1418 & 1.21 & 287.2 & 6 & & \\
\hline & 68 & 91.1743 & 1.7198 & 287.8 & 6 & & 0 • \\
\hline 2 & 69 & 38.5802 & 1.3908 & 287.8 & 5 & 0 & \\
\hline & 610 & 341.0988 & 2.1498 & 286.6 & 4 & & 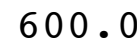 \\
\hline & 611 & 322.9563 & 3.1030 & 287.1 & 3 & & 0 . \\
\hline & 612 & 311.9041 & 2.0728 & 288.0 & 2 & $\cdot 0$ & 0 . \\
\hline & 613 & 153.7483 & 4.0243 & 288 & 3 & & . \\
\hline 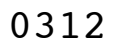 & 614 & 160.0998 & 5.1518 & 288.4 & 3 & .0 & 600 . \\
\hline & 615 & 251.5305 & 2.6108 & 287.4 & 2 & 0 & 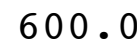 \\
\hline & 616 & 341.9778 & 078 & 288.2 & 2 & & 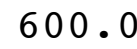 \\
\hline & 617 & 2.9252 & 5.2395 & 288.5 & 3 & .0 & 600. \\
\hline & 618 & 357.6500 & 5.1978 & 288.1 & 4 & .0 & 00 . \\
\hline & 619 & 346.3328 & 3.5590 & 288.1 & 4 & & \\
\hline & 620 & 15.1420 & 3.3843 & 287.6 & 5 & 600.0 & 600 \\
\hline & 621 & 36.1936 & 2.8988 & 287.0 & 6 & 600.0 & 600 \\
\hline & 622 & 352.1129 & & & 6 & & \\
\hline & 623 & 267.5257 & 2.1565 & 286.4 & r & 600.0 & 600 \\
\hline
\end{tabular}




\begin{tabular}{|c|c|c|c|c|c|c|c|}
\hline & 24 & & .6003 & $3 \cdot 3$ & & & \\
\hline 312 & 71 & 715 & 2.1943 & 285.1 & & & \\
\hline 1 & 72 & 77.0065 & .3035 & 285.5 & 6 & 00 & \\
\hline 31 & 73 & 72.1748 & 3.0485 & 285.7 & & 00.0 & \\
\hline & 74 & 106.1889 & 4.5623 & & & 0 & \\
\hline & 75 & 5249 & .2368 & 83.9 & 4 & 00 & \\
\hline 3 & 76 & 80.6847 & 7563 & 283.8 & & 0 & \\
\hline & 77 & 82.3648 & .8248 & 3.6 & & & \\
\hline & 7 & 76.3517 & 4.3253 & 283.4 & & .0 & \\
\hline 31 & 79 & 62.6001 & 470 & 283.5 & & 00.0 & \\
\hline 7 & 710 & 84.5949 & 5.1203 & 283.7 & 4 & 00.0 & \\
\hline & 711 & 60.4430 & 4.8508 & & 3 & & \\
\hline 31 & 712 & 83.0294 & 868 & 284.5 & & 00 & \\
\hline 31 & 713 & 104.7082 & 5.2338 & 284 & 3 & 0.0 & s \\
\hline & 714 & 77.6388 & & & & & \\
\hline 31 & 715 & 89.9251 & 6 & 285.3 & 4 & 0 & \\
\hline 31 & 716 & 90.5559 & 6 . & 284.8 & 4 & 0 & \\
\hline 31 & 717 & 84.2901 & 45 & 6 & 4 & & \\
\hline 3. & 718 & 64.8717 & 7. & 282.8 & 4 & 0 & \\
\hline (n) & 719 & 72.2005 & 75 & 282.2 & 4 & 0 & \\
\hline 3. & 720 & 74.4258 & 5 & 282.0 & 4 & & \\
\hline 3 & 721 & 86. & 25 & 281.7 & 4 & & \\
\hline 3 & 722 & 85.2001 & & 281.4 & 4 & 0 & \\
\hline 312 & 723 & 94.2520 & 0 & 281.1 & 4 & 0 & \\
\hline 3 & 724 & 81.84 & & & 4 & & \\
\hline & 81 & 504 & & 280.7 & 4 & & \\
\hline 3 & 82 & 124.2657 & 7. & 281.6 & 4 & 0 & \\
\hline 031 & 83 & 142.2765 & & 5 & 4 & & \\
\hline 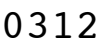 & 84 & 140 . & 6 . & 281.2 & 4 & 0 & 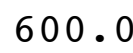 \\
\hline 31 & 85 & 143.2498 & 588 & 280.8 & 4 & 0 & \\
\hline 3 & 86 & 139.8251 & 4 & 280.4 & 4 & & \\
\hline 0312 & 87 & 134.5710 & 4 . & 28 & 4 & & . \\
\hline . & 88 & 123.5983 & 775 & 280.3 & 4 & 0.0 & U • \\
\hline 312 & 89 & 123.4261 & 5 & 280.8 & 4 & 0 & \\
\hline 312 & 810 & 142 . & & 281 & 4 & & \\
\hline 31 & 811 & 146.9971 & & 281.5 & 4 & 0 & 600 . \\
\hline 0 & 812 & 146.2498 & 850 & 282.2 & 3 & 0 & \\
\hline & 813 & 149.3746 & 495 & 282.5 & 4 & 0 & 60 \\
\hline 0312 & 814 & 144.2304 & 328 & 283.1 & 3 & 600.0 & 0 . \\
\hline 0312 & 815 & 147 . & 300 & 283.3 & 4 & 0 & \\
\hline 031 & 816 & 129.6502 & 425 & 283.4 & 3 & & \\
\hline 0312 & 817 & 125.8616 & 1.5363 & 282.9 & 4 & 600.0 & 600 . \\
\hline & 818 & 87.6121 & 0.9078 & 282.9 & 5 & 600.0 & 600. \\
\hline 31 & 819 & 64.7243 & 1.2663 & 282.7 & 4 & 600 & 600 \\
\hline 0312 & 820 & 29.5761 & 1.4728 & 282.3 & 4 & 600.0 & 600 \\
\hline & 821 & 34.5776 & 1.4603 & 282.3 & 5 & 600.0 & 600. \\
\hline & 822 & 359.5042 & 1.39 & 281.1 & 4 & .0 & 60 \\
\hline & 823 & 0.5163 & 1.8295 & 281.1 & & 600.0 & 600 \\
\hline
\end{tabular}




\begin{tabular}{|c|c|c|c|c|c|c|}
\hline 2 & & & & & & \\
\hline 129 & & & 1.3 & & 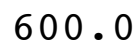 & \\
\hline 29 & 6.2748 & 2340 & 281.4 & & 00 & \\
\hline 1293 & 317.3401 & 1.5085 & 280.7 & & 00 & \\
\hline $\begin{array}{lll}2 & 9 & 4\end{array}$ & 289 . & 3693 & 80.9 & & & \\
\hline 295 & 322.3671 & 57 & 80.9 & & 0 & \\
\hline 1296 & 314.8458 & 2758 & 81.7 & & 00 & \\
\hline 1297 & 310.6960 & 1.6070 & 81.1 & & 0 & \\
\hline 298 & 74.2313 & 1.98 & 80.2 & & & \\
\hline 1299 & 149.1072 & 2.1353 & 80.3 & & 00 & 0 \\
\hline 2910 & 235.9190 & 1.19 & 80.7 & & & \\
\hline 2911 & 162.1744 & 1.73 & 30.7 & & & \\
\hline 2912 & 140.2372 & 2 . & 80.7 & & 0 & \\
\hline 2913 & 149.7020 & 2.47 & 282.5 & & 0.0 & \\
\hline 914 & 6.2665 & & & & & \\
\hline 915 & 14 & & 83.0 & & & \\
\hline 2916 & 357.4555 & 6.6 & 284.7 & & .0 & 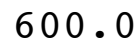 \\
\hline 2917 & 311.3564 & 7 . & .6 & & & \\
\hline 918 & 343 . & & .5 & & & \\
\hline 2919 & 4256 & & 283.7 & & & \\
\hline 2920 & 1.5388 & 3 . & 283.2 & & 0 & \\
\hline 2921 & 3.5184 & & 3.2 & & & \\
\hline 922 & 339.5345 & 4 & .3 & & & \\
\hline 2923 & 344.9541 & 8 & .5 & & 0 & \\
\hline 924 & 346 . & & & & & \\
\hline 101 & 352.8957 & 7 & 9 & 4 & & \\
\hline 102 & 22.4217 & 3 & .6 & 4 & 0 & 0 \\
\hline 103 & 37.2878 & 3 & .1 & 4 & & \\
\hline 104 & 354.2924 & 3 . & & 4 & & \\
\hline 12105 & 49.1766 & 7. & .6 & 4 & 0 & 0 • \\
\hline 312106 & 61.1753 & 2.31 & .4 & 4 & & 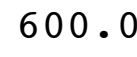 \\
\hline 312107 & 60.9007 & 9.3 & & 4 & & \\
\hline 312108 & 38.9799 & & 3.2 & 5 & & 0 • \\
\hline 312109 & 68.4556 & 5.4243 & 283.2 & 4 & 0 & \\
\hline 010 & 5001 & & & 3 & & \\
\hline 011 & 47.0485 & 4.77 & 1 & 3 & & 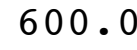 \\
\hline 3121012 & 11.7540 & 4.7423 & 284.1 & 3 & & 0 . \\
\hline 3121013 & .8481 & 4 . & & 3 & & \\
\hline 3121014 & 298.0339 & & 284.3 & 2 & .0 & 0 . \\
\hline 3121015 & .5890 & 4.56 & 285.2 & 3 & & \\
\hline 1016 & 74.0700 & 6.3458 & & 4 & & U. \\
\hline 017 & 70.4340 & 6.6758 & .8 & 4 & & 0 \\
\hline 3121018 & 61.6084 & 5.1245 & 283.4 & 4 & 0 & 0 \\
\hline 1019 & 49.4318 & 3 . & & 4 & & \\
\hline 03121020 & 1.7068 & 2.7010 & & 5 & & 0 . \\
\hline 2102 & 329.6550 & 1.5255 & 282.7 & 6 & & 600. \\
\hline & & & & 6 & & \\
\hline 3121023 & 9259 & 1.7533 & 282.0 & & .0 & 60 \\
\hline
\end{tabular}




\begin{tabular}{|c|c|c|c|c|c|c|}
\hline 024 & 2.0636 & 4.5595 & 282.2 & & 600.0 & \\
\hline 2111 & 109.8723 & 3.0275 & 281.3 & & 600.0 & \\
\hline 2112 & 86.9989 & 8.8850 & 280.9 & 4 & 00 & \\
\hline 12113 & 86.5025 & 7.7205 & 280.8 & & 00 & \\
\hline 2114 & 88.3772 & .7975 & 80.7 & & 0 & \\
\hline 2115 & 96.7493 & .1755 & 280.8 & & 00 & \\
\hline 12116 & 93.9896 & 7.6295 & 280.7 & & 00.0 & 0 \\
\hline 12117 & 74.9223 & 8.6025 & 80.7 & & 0 & \\
\hline 12118 & 84.7256 & .4375 & 80.3 & & & \\
\hline 312119 & 88.3412 & 8.3275 & 280.2 & & 00 & 8 \\
\hline 3121110 & 86.7506 & 8.3550 & 280.6 & & 0 & \\
\hline & 90.3974 & 7.8475 & 81.1 & & 00.0 & \\
\hline 2 & 102.0740 & 7.0575 & 281.4 & & 0 & \\
\hline 312 & 104.6782 & 6.4613 & 282.4 & & 0.0 & \\
\hline & 132.6823 & 8 & .8 & & & \\
\hline & 161.7011 & & 282.1 & & & \\
\hline 16 & 159.0251 & 9878 & 281.9 & 4 & .0 & 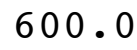 \\
\hline 17 & 154.7760 & 3 & 31.2 & & 0 & \\
\hline & 130.4864 & 3 . & 280.6 & & & \\
\hline 19 & 79.9759 & 4 & 280.8 & 4 & 0 & . \\
\hline 20 & 77.6502 & 96 & 281.0 & 4 & .0 & \\
\hline & 71.8752 & & & 4 & & \\
\hline 22 & 70.5250 & 8 & 280 & 4 & & \\
\hline 23 & 72.7752 & 6.6940 & 280.7 & 4 & 0 & 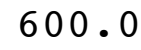 \\
\hline 24 & 74.0001 & & 8 & 4 & & \\
\hline 1 & 76.9527 & & 28 & 4 & & \\
\hline 312122 & 71.2743 & 4780 & 9 & 4 & 0 & U. \\
\hline 312123 & 74.6254 & 0 & 9 & 4 & & \\
\hline 24 & 73.8560 & 2 . & $2 \varepsilon$ & 5 & & \\
\hline 312125 & 90.4207 & 2.89 & 0.4 & 4 & 0 & 0 • \\
\hline 312126 & 27.9474 & 1.87 & 280.2 & 4 & & 0 . \\
\hline 312127 & 25.4660 & 0 & & 5 & & \\
\hline 312128 & 28.5530 & 0.97 & 0.7 & 6 & 0 & 0 • \\
\hline 312129 & 4.7232 & 1.2920 & 280.6 & 5 & 0 & 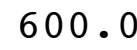 \\
\hline 10 & 359.0848 & 5 & & 4 & & \\
\hline & 19.4330 & 1.0413 & 282.6 & 3 & & 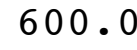 \\
\hline 212 & 237.4922 & 1.3513 & 282.7 & 2 & $\cdot 0$ & 0 . \\
\hline 13 & 289.6307 & 1.5258 & 282.7 & 1 & & 0 . \\
\hline 0312 & 292.1142 & 1.7078 & 282.9 & 2 & .0 & 0 . \\
\hline 3121215 & 247.9445 & 2.1830 & 282.8 & 3 & & \\
\hline 21216 & 278.6290 & 1.9873 & 282.0 & 3 & & 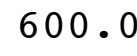 \\
\hline 312 & 345.3573 & 1.8395 & 282.1 & 4 & .0 & 0 . \\
\hline 3121218 & 47.2080 & 0.8910 & 282.8 & 5 & .0 & 00 . \\
\hline 3121219 & 8.3305 & 1.0770 & & 6 & & \\
\hline 03121220 & 116.8104 & 0.8048 & & 6 & & 00 . \\
\hline 3121221 & 140.4999 & 1.4925 & 283.1 & 6 & 600.0 & 00 \\
\hline & 64 & & & 6 & & \\
\hline 21223 & 16.9648 & 1.0030 & 283.7 & 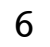 & 600.0 & 60 \\
\hline
\end{tabular}




\begin{tabular}{|c|c|c|c|c|c|c|}
\hline- & & & & & & \\
\hline 2131 & 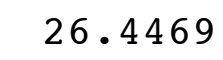 & 2.0398 & 1.9 & 6 & 0 & \\
\hline 2132 & 354.2254 & 1.6590 & 282.0 & 6 & 00 & \\
\hline 12133 & 4621 & 1.6405 & 283.3 & & 00 & \\
\hline 2134 & 533 & 1.2045 & 83.8 & & 0 & \\
\hline 2135 & 76 & 3 & 84.3 & & 0 & \\
\hline 12136 & 105. & 9025 & 284.5 & 5 & 00 & \\
\hline 12137 & 111 & 2.3153 & 84.6 & & 0 & \\
\hline 12138 & 1144 & 56 & 83.5 & 0 & & \\
\hline 31213 & 5252 & 1.5938 & 283.4 & & 00.0 & 8 \\
\hline 310 & 1380 & 1.8505 & 284.0 & & 0 & \\
\hline & 346.8158 & 1.5060 & 84.0 & 3 & & \\
\hline 2 & 278 & 190 & 285.2 & 2 & 0 & \\
\hline 3 & 308 & 1.5710 & 284.8 & & 0.0 & \\
\hline & 319 . & & & 3 & & \\
\hline & 301 . & & 284 & 4 & & \\
\hline 6 & 294 & 1.9095 & 285.2 & 3 & .0 & 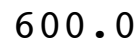 \\
\hline & 306 . & 1 . & & 4 & & \\
\hline & & & .0 & 5 & & \\
\hline & 52 & 83 & 285.2 & 6 & & \\
\hline 0 & 56.8060 & 2.9433 & 285.3 & 5 & 0 & \\
\hline & 19.2 & & & 6 & & \\
\hline 2 & 33 & 5 & 28 & 5 & & \\
\hline 23 & 351.4583 & 685 & $2 \varepsilon$ & 4 & 0 & \\
\hline & 356 . & & & 4 & & \\
\hline 1 & 356 . & 6 . & 28 & 4 & & \\
\hline 42 & 349.3809 & 488 & 284.8 & 4 & 0 & J. \\
\hline 312143 & 359 . & 3 & 2 & 4 & & \\
\hline 4 & 61 & 5 & $2 \varepsilon$ & 4 & & \\
\hline 312 & 356 & 928 & 1.4 & 4 & 0 & 0 . \\
\hline 312146 & 65.9989 & 3900 & 281.2 & 4 & & 0 . \\
\hline 312 & 67 . & 5 & & 4 & & \\
\hline 312 & 72.5007 & 625 & 0.6 & 4 & & . \\
\hline 312149 & .4008 & 975 & $2 \varepsilon$ & 4 & 0 & . \\
\hline & $\$ 97$ & 0 & & 4 & & \\
\hline & 83.0975 & 218 & 1.9 & 4 & & 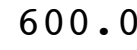 \\
\hline 12 & 89.5240 & 3943 & 282.6 & 4 & & 0 . \\
\hline 13 & 102.4778 & 718 & & 3 & & . \\
\hline 14 & 3249 & 3078 & 283.8 & 3 & .0 & 0 . \\
\hline 12 & 101.0405 & 795 & 284.2 & 3 & & \\
\hline & 102.8322 & 605 & 284.0 & 4 & & \\
\hline 312 & 62.0500 & 6.3555 & 282.8 & 4 & & 0 . \\
\hline 312141 & 63.3251 & 6.7678 & 281.7 & 4 & 0 & 0 . \\
\hline 312 & 64.8250 & 628 & & 4 & & \\
\hline 03121420 & 70.4495 & 6.6585 & & 4 & & 0 . \\
\hline 214 & 3001 & 6.4488 & 280.9 & 4 & 0 & 00 . \\
\hline & & & & 4 & & \\
\hline 121423 & 75.4257 & 5.6298 & 280.9 & & 600.0 & 600 \\
\hline
\end{tabular}




\begin{tabular}{|c|c|c|c|c|c|c|}
\hline 1424 & 95.6785 & 9030 & 281.0 & 4 & 600.0 & \\
\hline 2151 & 9.6245 & 4.1613 & 280.6 & 4 & תח & \\
\hline 12152 & 3088 & 1730 & 280.7 & 5 & 00 & \\
\hline 12153 & 81.8237 & 4.2360 & 280.5 & & 00 & \\
\hline 2154 & 93.9832 & 4.1238 & 0.5 & & 0 & \\
\hline 2155 & 6964 & 5105 & 79.8 & 4 & 00 & \\
\hline 312156 & 143.7360 & 2725 & 278.4 & & 0 & \\
\hline 2157 & 145.4262 & .7365 & 78.2 & & & \\
\hline 312158 & 145.1768 & 2635 & 277.3 & 4 & 0 & \\
\hline 312159 & 138.2251 & 3868 & 277.4 & 4 & 0 & \\
\hline 510 & 154.0508 & 855 & 278.9 & 4 & 0 & \\
\hline & 148.1023 & 33 & 78.8 & 4 & & \\
\hline 512 & 155.7526 & 7555 & 280.2 & 4 & 0 & \\
\hline 3121513 & 169.4591 & 3.6723 & 280.8 & 3 & 0 & \\
\hline & 214.3861 & & 81.3 & & & \\
\hline 5 & 282.6590 & 683 & 282.1 & 1 & 0 & \\
\hline 516 & 304.6636 & 1.69 & 282.4 & & 0 & \\
\hline 517 & 298 . & 1. & 281.8 & & & \\
\hline 31 & 214.5940 & 1 . & 281.2 & 4 & 0 & \\
\hline 519 & 149.1749 & 5 & 280 & 5 & 0 & \\
\hline 520 & 163. & 8 & 279.8 & 5 & & \\
\hline 521 & 139. & & 27 & 6 & & \\
\hline 522 & 9.3683 & 563 & 279.5 & 5 & 0 & 000. \\
\hline 523 & 346 . & 1 . & 279.2 & 6 & 0 & \\
\hline 524 & 346 . & & 279.1 & 5 & & \\
\hline 312161 & 345 & 140 & 279.2 & 4 & & \\
\hline 312162 & 323.1501 & 1.76 & 279.3 & 5 & 0 & \\
\hline 0312163 & 306.2967 & 5 & 279.2 & 6 & & \\
\hline 312164 & 291 & 3 & 27 & 5 & & 0 . \\
\hline 312165 & 284.7969 & 1 & 278.9 & 6 & & \\
\hline 312166 & 255.4247 & 3 & 27 & 6 & & \\
\hline 0312167 & 268 . & 8 & 0 & 6 & & \\
\hline 312168 & 349.8161 & 0 & 278.0 & 6 & 0 & J. \\
\hline 312169 & 211.8722 & 5 & 278.3 & 5 & & \\
\hline 610 & 216 . & & .8 & 4 & & \\
\hline 3121611 & 152.8494 & 4.45 & 278.2 & 4 & & • \\
\hline 21612 & 182.3490 & 2 & 279.8 & 3 & 0 & \\
\hline 03121613 & 168 . & 2 & 279.4 & 2 & & 0 . \\
\hline 03121614 & 154.0886 & 2.0780 & 280.8 & 1 & 0 & 0 . \\
\hline 21615 & 135.9986 & 125 & 281.0 & 2 & 0 & \\
\hline 3121616 & 151.5970 & & 281.0 & 1 & & \\
\hline 03121617 & 138.9244 & 2.8170 & 279.7 & 2 & 600.0 & 600. \\
\hline 3121618 & 142.7490 & 3.83 & 279.1 & 3 & 600 & 000 \\
\hline 03121619 & 122.5792 & 1.1020 & 279.7 & 4 & & \\
\hline 03121620 & 148.2447 & 1.2760 & 279.5 & 4 & 600.0 & 00 \\
\hline 21621 & 511 & 2.6338 & 279.8 & 5 & 600 & 00 \\
\hline 21622 & 138.7805 & 20 & 279.2 & 6 & 60 & \\
\hline 3121623 & 141.3215 & 3.2230 & 278.8 & & 600.0 & 600 \\
\hline
\end{tabular}




\begin{tabular}{|c|c|c|c|c|c|c|}
\hline 624 & & & & & & \\
\hline 2171 & 3 & 7703 & 278.9 & & 0 & \\
\hline 2172 & 123.8930 & 3448 & 278.9 & 4 & 00 & \\
\hline 12173 & 132.6493 & 3418 & 278.7 & & 00 & \\
\hline 2174 & 29.4074 & .2493 & 278.7 & & 0 & \\
\hline 2175 & 53.8450 & 56 & 279.7 & & 00 & \\
\hline 312176 & 5348 & 1.8900 & 280.8 & & 00 & \\
\hline $31217 \quad 7$ & 48.4535 & .5608 & 280.0 & & 0 & \\
\hline 312178 & 4.2499 & 5353 & 78.2 & & & \\
\hline 312179 & 0011 & 2185 & 279.6 & & 00 & 8 \\
\hline 3121710 & 136.2327 & 1.9943 & 280.8 & & 0 & \\
\hline 1711 & 141.4502 & 2.1303 & 81.7 & & 00.0 & \\
\hline 21712 & 148 & 1.54 & 282.9 & & 0 & . \\
\hline 3121713 & 262.2073 & 5 & 283.4 & & 0.0 & \\
\hline 1714 & 251.2233 & & & & & \\
\hline .715 & 271.76 & & 3.3 & & & \\
\hline 3121716 & 320.1452 & 1.4595 & 283.0 & & .0 & 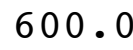 \\
\hline 1717 & 341.8756 & 8 & 2.7 & & 0 & \\
\hline 1718 & 322.7255 & & & 5 & & \\
\hline 1719 & 353.6512 & 1.6065 & 1.4 & 4 & .0 & . \\
\hline 3121720 & 4.6875 & .84 & 0.8 & 4 & 0 & 00. \\
\hline 1721 & 10.5481 & & & 4 & & \\
\hline .722 & 325.0471 & 128 & $2 \varepsilon$ & 5 & & \\
\hline 3121723 & 285.2368 & .5040 & 3.6 & 6 & 0 & 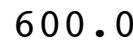 \\
\hline 3121724 & 186.8292 & 5288 & & 6 & & \\
\hline 81 & 137.6999 & 5 & .3 & 5 & & \\
\hline 312182 & 139.5737 & .7043 & .5 & 5 & 0 & . \\
\hline 312183 & 137.0001 & 54 & .6 & 5 & & \\
\hline 312184 & 142.8246 & 2 . & .8 & 5 & & \\
\hline 312185 & 137.2780 & 1.5593 & 0 & 5 & 0 & 0 • \\
\hline 312186 & 154.4803 & 2.1268 & 9.9 & 4 & 0 & 0 . \\
\hline 312187 & 182.7898 & 1.44 & .2 & 5 & & \\
\hline 312188 & 156.0432 & 5075 & 9.5 & 6 & 0 & 0 • \\
\hline 312189 & 178.3994 & 1.5343 & 279.8 & 5 & 0 & 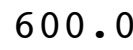 \\
\hline 3121810 & 184.5785 & 1.1425 & .4 & 4 & & \\
\hline 811 & 214.5118 & 1.32 & 1.2 & 3 & & 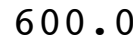 \\
\hline 3121812 & 247.5120 &. .3233 & 282.4 & 2 & $\cdot 0$ & 0 . \\
\hline 3121813 & 212.2781 & 1.71 & 282.9 & 1 & & . \\
\hline 03121814 & 212.2781 & 1.7197 & 282.9 & 1 & .0 & 0 . \\
\hline 3121815 & 155.6378 & 2.6560 & 283.1 & 2 & .0 & \\
\hline 03121816 & 157.5793 & 6698 & 282.9 & 3 & & 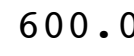 \\
\hline 03121817 & 304.7082 & 0.5983 & 283.1 & 4 & .0 & 0 . \\
\hline 3121818 & 23.4320 & 0.9338 & 282.7 & 5 & .0 & 00 . \\
\hline 03121819 & 16.2766 & 265 & 282.6 & 4 & & \\
\hline 03121820 & 10.1551 & 1.1485 & 283.6 & 5 & 600.0 & 00 . \\
\hline 2182 & 265.2896 & 1.1110 & 283.4 & 6 & 600.0 & 00 \\
\hline & 327.2121 & & 282.6 & 6 & & \\
\hline 121823 & 269.5206 & 1.3055 & 282.8 & & 600.0 & 60 \\
\hline
\end{tabular}




\begin{tabular}{|c|c|c|c|c|c|c|}
\hline $8<4$ & & & & & & \\
\hline 2191 & 7 & & 2.6 & 6 & 0 & \\
\hline 2192 & 156.1730 & 2318 & 282.8 & 6 & 00 & \\
\hline 12193 & 36.2292 & 5360 & 283.5 & & 00 & \\
\hline 2194 & 44.8257 & & 3.0 & & & \\
\hline 2195 & 3.4549 & 4078 & 82.2 & & , & \\
\hline 12196 & 179.9501 & 0158 & 82.7 & & 00 & \\
\hline 12197 & 09 . & 0 & 82.0 & & 0 & \\
\hline 12198 & 40.6742 & 5 & 81.7 & & & \\
\hline 312199 & 08.3992 & 1.8065 & 282.8 & & 0 & 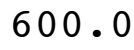 \\
\hline 1910 & 223.8237 & 1.4743 & 285.9 & & & \\
\hline 11 & 158.7235 & 2.6893 & 83.2 & & & \\
\hline 912 & 161 & 2.3858 & 282.4 & & 0 & \\
\hline 913 & 137.9879 & 2.9080 & 282.8 & & .0 & \\
\hline 914 & 136 & & & & & \\
\hline & 145 & & 281.1 & 2 & & \\
\hline 916 & 141.0249 & 3.9958 & 281.8 & 3 & .0 & . \\
\hline 917 & 142 . & 4 . & & 4 & & \\
\hline & 272 & & 282.3 & 5 & & \\
\hline 919 & 794 & 3 & 281 & 5 & 0 & 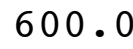 \\
\hline 920 & 298.5268 & 4.2388 & 280 & 4 & .0 & \\
\hline & 314.6686 & & & 4 & & \\
\hline 22 & 331 & 0 & 282 & 4 & & \\
\hline 923 & 338.1146 & 8233 & 282.7 & 4 & 0 & \\
\hline 924 & 309.5398 & & 2.7 & 5 & & \\
\hline 0 & 342.0878 & 3 & 282.6 & 5 & & \\
\hline 312202 & 71.4531 & 0638 & 0 & 6 & 0 & 0 • \\
\hline 312203 & 151.9336 & 0 & & 5 & & \\
\hline 204 & 135 . & 2.43 & $2 \varepsilon$ & 6 & & \\
\hline 312205 & 128.3992 & 1.72 & 3.6 & 6 & 0 & J. \\
\hline 312206 & 109.2034 & 1.60 & 283.8 & 6 & & 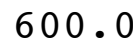 \\
\hline 312207 & 16.1456 & 0 & & 6 & & \\
\hline 312208 & 331.4147 & 3 & .7 & 5 & & 0 . \\
\hline 312209 & 344.8233 & 0 & & 4 & 0 & \\
\hline 3122010 & 20.6761 & 5 & & 4 & & \\
\hline 011 & 46.3394 & & 6.2 & 3 & & \\
\hline 122012 & 150.1630 & 0 & 284.3 & 2 & & \\
\hline 3122013 & 162.9131 & 3 . & 282.2 & 2 & & \\
\hline 3122014 & 160.1324 & 2.1703 & 281.4 & 3 & & 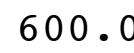 \\
\hline 3122015 & 139.1706 & 1.9740 & 281.4 & 2 & & \\
\hline 3122016 & 346.9338 & 1.0733 & 281.8 & 1 & & \\
\hline 3122017 & 348.9371 & 2.0458 & 282.3 & 2 & & 0 . \\
\hline $12201 \varepsilon$ & 270.8371 & 1.2603 & 282.3 & 3 & 0 & 0 . \\
\hline 3122019 & 422 & 0.9463 & & 4 & & \\
\hline 3122020 & 145.9444 & 1.8455 & 282.2 & 5 & & \\
\hline 2202 & 139.0031 & 2.7865 & 281.4 & 4 & & 00 \\
\hline & & & & 5 & & \\
\hline 12202 & 151.7240 & 3.6073 & 281.6 & & .0 & s \\
\hline
\end{tabular}




\begin{tabular}{|c|c|c|c|c|c|c|}
\hline 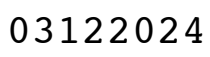 & & & & & & \\
\hline 221 & 1 & 3725 & 1.1 & 4 & 0 & \\
\hline 2212 & 153.1018 & 7305 & 281.1 & 4 & 00 & \\
\hline 12213 & 127.0264 & 2.8748 & 281.1 & & 00.0 & \\
\hline 2214 & 68.1887 & .2018 & 81.5 & & 0 & \\
\hline 2215 & 154.8644 & 5893 & 81.5 & & 0 & \\
\hline 312216 & 145.9492 & 1.8425 & 281.6 & & 00 & \\
\hline 32217 & 536 & .8968 & 81.5 & & & \\
\hline 12218 & 5775 & 8573 & 82.0 & & & \\
\hline 312219 & 172.1385 & 2.1683 & 282.1 & & 00 & 0 \\
\hline 3122110 & 156.5472 & 4.9223 & 281.7 & & 00.0 & \\
\hline & 155.1293 & 3.7530 & 81.8 & 4 & & \\
\hline 2 & 158.8799 & 4.5300 & 281.7 & 4 & 0 & \\
\hline 122113 & 157.0865 & 3.6423 & 281.9 & 4 & 0.0 & \\
\hline & 156 . & 3195 & 32.2 & 3 & & \\
\hline & 185 . & 1 . & 282.6 & 2 & & \\
\hline 31 & 269.3968 & 0.9550 & 282.7 & 1 & .0 & 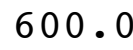 \\
\hline 17 & 9.3345 & 1.26 & 32.5 & & 0 & \\
\hline & 16.9921 & 1.42 & 282.1 & 3 & & \\
\hline 9 & 28.1491 & 1.0488 & 282.1 & 4 & 0 & 0 \\
\hline 20 & 128.7840 & 1.6773 & 281.8 & 5 & .0 & 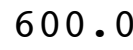 \\
\hline & 145.2060 & 2 . & 282.0 & 4 & & \\
\hline 22 & 77.0228 & 140 & 282.4 & 5 & & \\
\hline 23 & 349.4428 & 1.08 & 282.0 & 6 & . 0 & 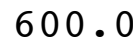 \\
\hline 24 & 319.4363 & & 281.9 & 6 & & \\
\hline 21 & 334.1995 & 4 & 281.9 & 5 & & \\
\hline 312222 & 345.9036 & 0 & 282.2 & 4 & 0 & . \\
\hline 312223 & 337.4555 & 2. & 82.5 & 4 & & \\
\hline 312224 & 252.3307 & 1 . & 282.3 & 5 & & $\bullet$ \\
\hline 312225 & 221.0001 & 1.58 & 281.9 & 6 & 0 & 0 . \\
\hline 312226 & 311.4845 & 1.7688 & 280.8 & 6 & 0 & 0 \\
\hline 312227 & 287.5057 & 2.31 & & 6 & & \\
\hline 312228 & 261.5769 & 8 & 280.1 & 6 & 0 & . \\
\hline 312229 & 312.1416 & 8 & 280.4 & 5 & 0 & 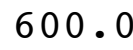 \\
\hline 3122210 & 299.7999 & 2 . & & 4 & & \\
\hline & 281.7063 & & 281.9 & 3 & & 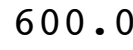 \\
\hline 212 & 277.8505 & 0 & 282.5 & 3 & 0 & 0 . \\
\hline 213 & 271.6400 & 2 . & 283.6 & 2 & & . \\
\hline 14 & 221.8887 & 1.8530 & 283.9 & 1 & .0 & 0 . \\
\hline 122215 & 255.4193 & 3.1418 & 283.3 & 2 & 0 & \\
\hline 22216 & 246.2410 & 2 . & 282.8 & 3 & & 0 \\
\hline 3122217 & 231.4131 & 2.2138 & 282.0 & 4 & .0 & 0 . \\
\hline 3122218 & 216.9132 & 1.7335 & 281.4 & 5 & 0 & 00 . \\
\hline 3122219 & 182.2567 & 2.9775 & & 4 & & \\
\hline 03122220 & 163.4845 & 4.3503 & 280.7 & 4 & 600.0 & 0 . \\
\hline 122221 & 159.0485 & 4.6803 & 280.6 & 4 & 0 & 00 \\
\hline & 15 & & & 4 & & \\
\hline 122223 & 153.6068 & 2.5405 & 280.5 & 1 & .0 & 60 \\
\hline
\end{tabular}




\begin{tabular}{|c|c|c|c|c|c|c|}
\hline $2<4$ & 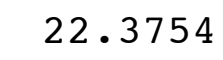 & & & & & \\
\hline 231 & 12 & .7938 & 0.2 & & 0 & \\
\hline 2232 & 147.7589 & 1.8348 & 280.1 & & 00 & \\
\hline 12233 & 162.4424 & 1.6590 & 280.3 & & 00 & \\
\hline 2234 & 37.1697 & 1.6840 & 81.0 & & & \\
\hline 2235 & 291.3681 & 100 & 82.2 & & 0 & \\
\hline 312236 & 276 & 2.7098 & 82.3 & & 00 & \\
\hline 12237 & 304.1521 & 4.0238 & 83.3 & & & \\
\hline 12238 & 324.0431 & 3125 & 84.7 & & & \\
\hline 312239 & 320.9900 & 7.6668 & 84.5 & & 0 & $2+$ \\
\hline 2310 & 318.9732 & 8.5450 & 284.2 & & & \\
\hline & 328.5493 & $7 \quad 10$ & 83.8 & & & \\
\hline 2 & 318 & 6 . & 284.4 & & 0 & \\
\hline 313 & 300.2142 & 5.60 & 3 & & 0.0 & \\
\hline & 305 & & & & & \\
\hline & 317 & 7 . & 7 & & & \\
\hline & 322.3021 & 5.74 & 4 & & 0 & 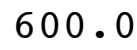 \\
\hline 17 & 327 . & 8 & & & & \\
\hline & 333. & & 3 & & & \\
\hline & 342.5352 & 2.41 & 283.8 & 6 & & \\
\hline 20 & 9.8161 & 2.72 & 1 & & 0 & \\
\hline & 245.1745 & 111 & & & & \\
\hline 2 & 344 & 248 & 3 & 6 & & \\
\hline 23 & 308.7308 & 2.55 & 9 & & 0 & \\
\hline & 296 & & & & & \\
\hline 1 & 336 & 8 . & 28 & 4 & & \\
\hline 312242 & 323.9284 & 5 & 1 & 4 & 0 & J. \\
\hline 312243 & 310.8755 & $6 c$ & & 4 & & \\
\hline 44 & 294 . & 7.21 & & 4 & & \\
\hline $31224 \quad 5$ & 278.8019 & 5 & 0 & 4 & & 0. \\
\hline 312246 & 299.8737 & 6.58 & & 4 & & s. \\
\hline 312247 & 299 . & 3 & & 4 & & \\
\hline 312248 & 295.4351 & 3 & 28 & 4 & & 0 . \\
\hline 312249 & 311.4727 & 4.49 & & 3 & 0 & 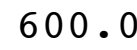 \\
\hline & 313 . & & & 3 & & \\
\hline & 1.4167 & 4.5770 & & 3 & & \\
\hline 412 & 141.4533 & 6.7163 & 284.3 & 4 & & \\
\hline 13 & 999 & 6.3200 & 28 & 4 & & \\
\hline 14 & 66.4688 & 3.2863 & .6 & 3 & & 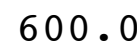 \\
\hline 122415 & 38.9416 & 2.9303 & 284.5 & 2 & & \\
\hline 3122416 & 354.5255 & 1.9998 & & 3 & & \\
\hline 3122417 & 47.8226 & 3.3528 & & 4 & & 0 . \\
\hline 3122418 & 359.4201 & 1.8103 & 283.5 & 5 & 0 & 0 . \\
\hline 3122419 & 21.7061 & 1.1675 & & 6 & & \\
\hline 03122420 & 0.5641 & 1.3160 & & 0 & & \\
\hline 224 & 87.6856 & 3.0980 & 284.1 & 5 & & 00 . \\
\hline & .751 & & & 4 & & \\
\hline 122423 & 73.3493 & 3.8763 & 283.8 & & 60 & -1 \\
\hline
\end{tabular}




\begin{tabular}{|c|c|c|c|c|c|c|}
\hline 2424 & 90.8446 & 2.6130 & 283.5 & 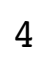 & 600.0 & \\
\hline 2251 & 5.1606 & 2.4988 & 283.4 & 4 & 600.0 & \\
\hline 12252 & 3322 & 2.8380 & 283.1 & 4 & 00.0 & \\
\hline 312253 & 92.6656 & 3.3405 & 282.7 & & 00 & \\
\hline 2254 & 1.9737 & 2.6740 & 32.5 & & 0 & \\
\hline 12255 & 2731 & 2.9093 & 82.2 & 4 & 00 & \\
\hline 312256 & 4932 & 4.6765 & 282.3 & & 0 & \\
\hline 312257 & 6.3968 & 4.7165 & 82.3 & & & \\
\hline 312258 & 93.7305 & 4.7913 & 281.6 & 4 & 0 & \\
\hline 312259 & 79.8248 & 6.2650 & 280.7 & & 00 & \\
\hline 510 & 81.7878 & 6.4603 & 280.3 & 4 & 0 & \\
\hline & 7.7947 & & 79.9 & 4 & & \\
\hline 31 & 81.9475 & 635 & 279.5 & 3 & 0 & \\
\hline 513 & 64.2682 & 5.1788 & 279.3 & $I$ & 0 & \\
\hline 14 & 67.8243 & & 79.8 & 4 & & \\
\hline 31 & 86.3749 & & 280.1 & 4 & 0 & \\
\hline 516 & 88.4497 & 5 & 280.7 & 4 & 0 & \\
\hline 17 & 92.1227 & 5 & 80.7 & 4 & & \\
\hline 31 & 81.9001 & 5 & 280.3 & 4 & 0 & \\
\hline 19 & 94.6228 & 0 & 280.1 & 4 & 0 & \\
\hline 20 & 90.7750 & 0 & 279.6 & 4 & & \\
\hline 21 & 745 & & 279.2 & 4 & & \\
\hline 312 & 66.4251 & 700 & 278.9 & 4 & 0 & 600 . \\
\hline 312 & 66.8501 & 0 & 278.5 & 4 & 0 & \\
\hline 24 & 248 & & 278.2 & 4 & & \\
\hline 312261 & 67.6488 & 550 & 277.9 & 4 & & \\
\hline 312262 & 73.2500 & 3.8100 & 277.7 & 4 & 0 & \\
\hline 0312263 & 78.0253 & 5 & 277.6 & 4 & & $0^{\circ}$ \\
\hline 312264 & 498 & 0 & 277.2 & 4 & 0 & 0 . \\
\hline 312265 & 64.3999 & 025 & 277.1 & 4 & 0 & \\
\hline 312266 & 64.3997 & 0 & 277.2 & 4 & & \\
\hline 0312267 & 500 & 8 . & 277.0 & 4 & & \\
\hline 312268 & 65.4249 & 8.7725 & 277.1 & 4 & 0 & 0 • \\
\hline 312269 & 60.5250 & 7.65 & 277.6 & 4 & 0 & \\
\hline 2610 & 476 & 6 & 278.4 & 4 & & \\
\hline 03122611 & 80.6498 & 663 & 279.1 & 4 & 0 & . \\
\hline 3122612 & 5937 & 5.2265 & 280.0 & 3 & 0 & \\
\hline 03122613 & 98.1931 & 5.4323 & 280.7 & 3 & & 0 . \\
\hline 03122614 & 101.1221 & 5.2408 & 281.1 & 3 & 0 & 0 . \\
\hline 3122615 & 97.2640 & 15 & 281.6 & 3 & 60 & \\
\hline 03122616 & 174.4639 & 3 . & 280 & 2 & & \\
\hline 03122617 & 159.8298 & 2.3680 & 279.9 & 3 & 600.0 & 600 . \\
\hline 3122618 & 148.4546 & 0.9043 & 280.0 & 4 & 600 & 600 \\
\hline 03122619 & 103.0396 & 1.4343 & 279.5 & 4 & 600 & $c_{0}$ \\
\hline 03122620 & 85.7880 & 3.6965 & 279.0 & 5 & 600.0 & 00 \\
\hline 122621 & 2757 & 3.7188 & 278.8 & 5 & 600.0 & 600. \\
\hline 122622 & 3495 & 228 & $27 \varepsilon$ & 5 & 600 & \\
\hline 3122623 & 75.0499 & 4.4495 & 278.5 & כ & 600.0 & 600 \\
\hline
\end{tabular}




\begin{tabular}{|c|c|c|c|c|c|c|}
\hline $2 x$ & 80.3001 & 4.6753 & 78.4 & & 80.0 & \\
\hline 2271 & 5.2999 & 5.3103 & 278.3 & & 600.0 & \\
\hline $227 \quad 2$ & 77.7483 & 9728 & 278.5 & 5 & 00 & \\
\hline 12273 & 98.7829 & 1.6545 & 278.2 & & 00 & \\
\hline $227 \quad 4$ & 112.5310 & 7708 & 278.2 & & 0 & \\
\hline 2275 & 6544 & .3755 & 278.0 & & 00 & \\
\hline 312276 & 84.5967 & 4.0748 & 277.9 & & 00 & \\
\hline 12277 & 217.9363 & .2405 & 277.0 & & 0 & \\
\hline 12278 & 326.1654 & 0938 & 75.9 & & & \\
\hline 312279 & 226.6096 & 7610 & 276.8 & & 00 & 0 \\
\hline 3122710 & 299.2859 & 1.0940 & 277.8 & & 0 & \\
\hline 711 & 293.5738 & 1.2825 & 278.6 & & & \\
\hline 2712 & 288.3593 & 665 & 279.6 & & 0 & \\
\hline 122713 & 252.8848 & 2.4258 & 279.7 & & .0 & \\
\hline 714 & 174.4 & 8 & & & & \\
\hline 715 & 172.1 & 2. & 279.7 & & & \\
\hline 2716 & 149.0231 & 2.2325 & 279.5 & & 0 & 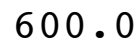 \\
\hline 717 & 137.1312 & 723 & 9.6 & & & \\
\hline 718 & 348.1331 & 555 & 279.2 & & & \\
\hline 719 & 12.56 & 0.5615 & 278.6 & & 0 & v. \\
\hline 720 & 29.18 & L. 2823 & 278.6 & & 0 & \\
\hline 721 & 105.0 & 5 & 278.9 & & & \\
\hline 722 & 98.4514 & 623 & 278.9 & & & \\
\hline 723 & 132.6508 & 1.3255 & 278.5 & & 0 & \\
\hline 724 & 158.1 & 210 & 8.4 & & & \\
\hline 81 & 11.3 & 1.1190 & 278.6 & 5 & & \\
\hline 312282 & 30.0758 & 1.6143 & 278.8 & & 0 & J. \\
\hline 312283 & 50.4704 & 13 & 3.7 & & & \\
\hline 284 & 153. & 353 & 278.2 & 6 & & \\
\hline 312285 & 192.5460 & 335 & 277.7 & 6 & 0 & 0 • \\
\hline 312286 & 17.2059 & 5 & 277.7 & 5 & & 0 . \\
\hline 312287 & 9.2875 & 0 & 277.7 & 5 & & \\
\hline 312288 & 100.5067 & 3 & 277.4 & 6 & 0 & 0 . \\
\hline 312289 & 329.4162 & 920 & 277.0 & 5 & 0 & 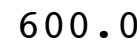 \\
\hline 810 & 270.24 & 0 & 7.5 & 4 & & \\
\hline & 286.1472 & 1.4385 & 277.7 & 3 & & ( \\
\hline 812 & 232.5211 & 8630 & 278.0 & 3 & & 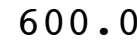 \\
\hline 3122813 & 252.5382 & 5 & 277.8 & 2 & & 0 . \\
\hline 814 & 290.2999 & 2.5210 & 278.7 & 2 & .0 & 0 . \\
\hline 3122815 & 281.3036 & 1.9705 & 278.9 & 3 & & \\
\hline 3122816 & 299.3747 & 2.7593 & 278.9 & 4 & & 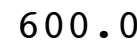 \\
\hline 3122817 & 309.4253 & 1.8333 & 278.6 & 4 & .0 & 0 . \\
\hline 3122818 & 336.7840 & 1.5800 & 278.5 & 5 & 0 & 0 . \\
\hline 3122819 & 17.9039 & 363 & 278.9 & 6 & & \\
\hline 03122820 & 64.7138 & 2.4648 & 279.7 & 6 & & 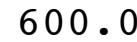 \\
\hline 122821 & 312.0973 & 1.0650 & 279.7 & 6 & & 00 . \\
\hline & 338 . & & 279.8 & 5 & & \\
\hline 122823 & 332.0254 & 3.0003 & 280.6 & & .0 & 600 \\
\hline
\end{tabular}




\begin{tabular}{|c|c|c|c|c|c|c|}
\hline $0<4$ & & & & & & \\
\hline 291 & 56 & 9405 & 0.8 & & 0 & \\
\hline 2292 & 23.4598 & 5688 & 280.5 & & 00 & \\
\hline 12293 & 337.5503 & 6.6148 & 281.2 & & 00 & \\
\hline 2294 & 311 . & 5.0928 & 81.4 & & & \\
\hline 2295 & 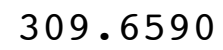 & 5803 & 81.6 & & 0 & \\
\hline 12296 & 394 & 6.2668 & 281.3 & & 00 & \\
\hline 12297 & 28.3219 & 6.0210 & 81.7 & & 0 & \\
\hline 12298 & 36 & 4725 & 80.9 & & & \\
\hline 312299 & 281.1135 & 8.5400 & 279.7 & & 00 & 8 \\
\hline 2910 & 279.77 & 7.7900 & 280.5 & & & \\
\hline 911 & $260-6211$ & 9.0700 & 9.3 & & & \\
\hline 912 & 87 & 5.2090 & 279.1 & & 0 & \\
\hline 913 & 290.9 & 6.3258 & 279.8 & & 0.0 & \\
\hline 914 & 09 & 23 & 2 & & & \\
\hline 5 & 80 & 8 . & 0 & & & \\
\hline 6 & 272 . & 0.3800 & 279.8 & & .0 & \\
\hline 917 & 306 . & 5 & 9 & & & \\
\hline 918 & 307 . & 550 & 279.2 & & & \\
\hline 19 & 315 & 7175 & 279.4 & & & \\
\hline 920 & 304.0755 & 7.6775 & 279.9 & & 0 & \\
\hline & 310 . & 7.4228 & & & & \\
\hline 22 & 294 & 7.5188 & 28 & 4 & & \\
\hline 923 & 302.4238 & 8.2375 & 280.2 & & 0 & \\
\hline 924 & 310 . & 550 & 279.9 & & & \\
\hline 0 & 30 & 50 & 279 & 4 & & \\
\hline 312302 & 310.1255 & 725 & 280.1 & 4 & 0 & J. \\
\hline 312303 & 322 . & 3230 & 5 & 4 & & \\
\hline 304 & 336 & 40 & 27 & 4 & & \\
\hline 312305 & 333. & 7615 & 279.7 & 4 & 0 & U. \\
\hline 312306 & 331.9471 & 3520 & 280.8 & 4 & 0 & 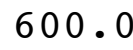 \\
\hline 312307 & 30 & 5 & 8 & 4 & & \\
\hline 312308 & & & 279.4 & 4 & & . \\
\hline 312309 & 156 & 6.9645 & 278.2 & 4 & 0 & \\
\hline 10 & 14 & 0 & .6 & 4 & & \\
\hline & 16 & & 278.8 & 4 & & \\
\hline 123012 & 159.8480 & 4423 & 278.8 & 4 & & 0 . \\
\hline 23013 & 163.1267 & 100 & 279.1 & 4 & & \\
\hline 014 & 153.7753 & 6.1285 & 279.3 & 4 & & 0 . \\
\hline 3123015 & 149.5535 & 0563 & 279.7 & 4 & & \\
\hline 3123016 & 983 & 635 & & 3 & & \\
\hline 3123017 & & 480 & 280.0 & 4 & & 0 . \\
\hline 3123018 & 123.2947 & 1.9915 & 279.6 & 5 & 0 & 0 . \\
\hline 3123019 & 531 & 3. & & 4 & & \\
\hline 03123020 & 142.8764 & 2.8103 & & 4 & & 0 . \\
\hline 2302 & 2526 & 1.5543 & 280.1 & 5 & & 00 . \\
\hline & & & & & & \\
\hline 12302 & 2993 & 3.8853 & 279.3 & & .0 & 60 \\
\hline
\end{tabular}




\begin{tabular}{|c|c|c|c|c|c|c|c|c|}
\hline \multicolumn{3}{|c|}{03123024} & & & & & & \\
\hline \multicolumn{3}{|c|}{0312311} & 3544 & .0863 & 9.5 & 6 & & \\
\hline \multicolumn{3}{|c|}{31231} & 194 & 8685 & 280.2 & $\sigma$ & 0 & \\
\hline \multicolumn{2}{|c|}{31231} & 13 & 39.2593 & 3168 & 280.7 & & 0 & \\
\hline \multicolumn{3}{|c|}{31231} & 324 . & & & & & \\
\hline \multicolumn{2}{|c|}{31231} & 15 & 143. & 8 & 80.2 & & & \\
\hline \multicolumn{3}{|c|}{031231} & 285 & 0 & 30.6 & & 0 & \\
\hline \multicolumn{2}{|c|}{31231} & 17 & 263 & & & & & \\
\hline \multicolumn{3}{|c|}{031231} & 103 & 5 & & & & \\
\hline \multicolumn{3}{|c|}{0312319} & 360 & 8 & 79.8 & & & \\
\hline \multicolumn{3}{|c|}{3123110} & 127.1127 & 1.18 & 1.0 & & & \\
\hline \multicolumn{3}{|c|}{03123111} & 323.2 & & & & & \\
\hline \multicolumn{3}{|c|}{03123112} & 188 & 1.4 & 2.7 & & & \\
\hline \multicolumn{3}{|c|}{03123113} & 212.1 & 3 & 2.8 & & & \\
\hline \multicolumn{3}{|c|}{03123114} & 254 & & & & & \\
\hline \multicolumn{3}{|c|}{03123115} & 247 . & & & & & \\
\hline \multicolumn{3}{|c|}{03123116} & 65.1 & 0 & 3.5 & 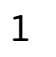 & & \\
\hline \multicolumn{3}{|c|}{03123117} & 310 . & 0 & & & & \\
\hline \multicolumn{3}{|c|}{03123118} & 328 . & & & 3 & & \\
\hline \multicolumn{3}{|c|}{03123119} & 316 & & 1 & 4 & & \\
\hline \multicolumn{3}{|c|}{03123120} & 340 . & 5 & & 5 & & \\
\hline & & & 52 . & & & 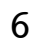 & & \\
\hline & & & & 3 & 0 & 5 & & \\
\hline & 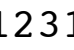 & 123 & 27.7582 & 3 & 5 & 5 & & \\
\hline & & 124 & 156. & & & 6 & & \\
\hline 4 & 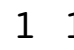 & 1 & 10 . & & & 4 & & \\
\hline & 11 & 12 & 328 . & 5 & 9 & 5 & & \\
\hline & 1 & 13 & 283 & 34 & & 5 & & \\
\hline 4 & 1 & 14 & 310 . & 5 & & 4 & & \\
\hline 4 & 1 & 15 & 273 . & 5 & 0 & 5 & & \\
\hline 4 & 11 & 16 & 300 . & 58 & 7 & 4 & & \\
\hline 4 & 1 & 17 & 1 & & & 4 & & \\
\hline 4 & 1 & 18 & 336 & 0 & 4 & 4 & & \\
\hline 4 & 1 & 19 & 304 . & 5 & & 5 & & \\
\hline & 1 & 110 & 0 & & & 4 & & \\
\hline 4 & 1 & 111 & 0 . & 0 & & 4 & & . \\
\hline 4 & 11 & 112 & 500 & 31 & 5 & 4 & & \\
\hline 4 & 1 & 113 & 44 . & 3 & & 4 & & \\
\hline 04 & 1 & 114 & 87 . & 8 & .1 & 4 & & \\
\hline 4 & 11 & 115 & 68 . & -5 & 1.9 & 4 & & \\
\hline 4 & 11 & 116 & 335.6554 & 1. & & 4 & & \\
\hline 4 & 11 & 117 & 52.3564 & 1.78 & .9 & 5 & & \\
\hline 4 & 11 & 118 & 99.2078 & $\lfloor .73$ & 281.6 & 5 & & \\
\hline 4 & 11 & 119 & 116 . & 8 & & 4 & & \\
\hline 04 & 11 & 120 & 153.6365 & 0.88 & & 5 & & \\
\hline 4 & 11 & 121 & 115.6068 & 1.7690 & 280.8 & 5 & & 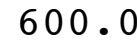 \\
\hline & 11 & 12 & & & & - & & \\
\hline 4 & 11 & 123 & 61.0833 & 4.2728 & 280.6 & 4 & .0 & 600 \\
\hline
\end{tabular}




\begin{tabular}{|c|c|c|c|c|c|c|c|c|}
\hline 04 & 1 & 124 & 77.2500 & 3.3688 & 280.7 & 4 & 600.0 & 600.0 \\
\hline 04 & 1 & 21 & 65.1163 & 2.8388 & 280.1 & 4 & 600.0 & 600.0 \\
\hline 04 & 1 & 22 & 31.9166 & 1.8803 & 279.9 & 5 & 600.0 & 00.0 \\
\hline 04 & 1 & 23 & 54.9855 & 1.0868 & 279.8 & 6 & 600.0 & 500.0 \\
\hline 04 & 1 & 24 & 89.4480 & 2.0895 & 279.8 & 5 & 600.0 & 00.0 \\
\hline 04 & 1 & 25 & 25.7632 & 1.8255 & 278.9 & 5 & 600.0 & 000 \\
\hline 04 & 1 & 26 & 22.8082 & 2.5870 & 279.2 & 4 & 600.0 & 00.0 \\
\hline 04 & 1 & 27 & 22.0778 & 2.0415 & 279.1 & 5 & 600.0 & 00.0 \\
\hline 04 & 1 & 28 & 50.4669 & 1.5020 & 279.4 & 5 & 600.0 & 600.0 \\
\hline 04 & 1 & 29 & 19.5086 & 1.8380 & 280.1 & 4 & 600.0 & 00.0 \\
\hline 04 & 1 & 210 & 337.6853 & 1.4685 & 280.9 & 3 & 600.0 & 00.0 \\
\hline 04 & 1 & 211 & 25.7240 & 3.6245 & 282.2 & 3 & 600.0 & 00.0 \\
\hline 04 & 1 & 212 & 47.6431 & 2.7033 & 281.9 & 2 & 600.0 & 00.0 \\
\hline 04 & 1 & 213 & 254.6831 & 2.8235 & 280.8 & 3 & 600.0 & 00.0 \\
\hline 04 & 1 & 214 & 287.5823 & 1.8290 & 280.9 & 3 & .0 & \\
\hline 04 & 1 & 215 & 236.4704 & 1.9225 & 281.4 & 4 & 600.0 & 00.0 \\
\hline 04 & 1 & 216 & 126.7772 & 6.8900 & 278.9 & 4 & 600.0 & 600.0 \\
\hline 04 & 1 & 217 & 74.3083 & 4.9968 & 278.6 & 4 & 600.0 & \\
\hline 04 & 1 & 218 & 96.1507 & 4.3598 & 279.0 & 4 & 600.0 & 00.0 \\
\hline 04 & 1 & 219 & 105.9117 & 5.6418 & 279.0 & 4 & 600.0 & 600.0 \\
\hline 04 & 1 & 220 & 105.6747 & 5.9753 & 278.8 & 4 & .0 & .0 \\
\hline 04 & 1 & 221 & 91.3750 & 6.9333 & 278.3 & 4 & 600.0 & .0 \\
\hline 04 & 1 & 222 & 79.9333 & 8.8000 & 278.1 & 4 & 600.0 & 600.0 \\
\hline 04 & 1 & 223 & 71.9500 & 8.1150 & 277.9 & 4 & 600.0 & .0 \\
\hline 04 & 1 & 224 & 78.8113 & 8.1850 & 277.6 & 4 & .0 & \\
\hline 04 & 1 & 31 & 91.2972 & 8.1675 & 277.2 & 4 & 600.0 & 600.0 \\
\hline 04 & 1 & 32 & 77.5135 & 7.3195 & 276.9 & 4 & 600.0 & 600.0 \\
\hline 04 & 1 & 33 & 81.5499 & 7.4200 & 276.8 & 4 & 600.0 & \\
\hline 04 & 1 & 34 & 76.7496 & 7.1973 & 276.5 & 4 & 600.0 & 600.0 \\
\hline 04 & 1 & 35 & 78.5000 & 7.0593 & 276.3 & 4 & 600.0 & 600.0 \\
\hline 04 & 1 & 36 & 71.8251 & 7.3175 & 276.2 & 4 & 600.0 & .0 \\
\hline 04 & 1 & 37 & 78.3252 & 7.2275 & 276.2 & 4 & 600.0 & 600.0 \\
\hline 04 & 1 & 38 & 77.8509 & 6.8630 & 276.0 & 4 & 600.0 & 600.0 \\
\hline 04 & 1 & 39 & 77.3464 & 6.0465 & 276.6 & 4 & 600.0 & 0.0 \\
\hline 04 & 1 & 310 & 83.9245 & 4.7523 & 277.4 & 3 & 600.0 & 600.0 \\
\hline 04 & 1 & 311 & 84.4336 & 3.8258 & 278.2 & 3 & 600.0 & 600.0 \\
\hline 04 & 1 & 312 & 148.9747 & 4.3983 & 278.5 & 3 & 600.0 & 600.0 \\
\hline 04 & 1 & 313 & 157.5278 & 3.9160 & 278.8 & 3 & 600.0 & 600.0 \\
\hline 04 & 1 & 314 & 162.2009 & 4.1033 & 278.9 & 3 & 600.0 & 600.0 \\
\hline 04 & 1 & 315 & 163.0393 & 4.9305 & 279.0 & 3 & 600.0 & 600.0 \\
\hline 04 & 1 & 316 & 169.6079 & 4.9043 & 278.5 & 3 & 600.0 & 600.0 \\
\hline 04 & 1 & 317 & 153.4508 & 4.1325 & 277.6 & 4 & 600.0 & 600.0 \\
\hline 04 & 1 & 318 & 148.4750 & 3.6205 & 276.9 & 4 & 600.0 & 600.0 \\
\hline 04 & 1 & 319 & 140.3249 & 3.2180 & 277.1 & 4 & 600.0 & 600.0 \\
\hline 04 & 1 & 320 & 129.1264 & 3.5025 & 277.0 & 4 & 600.0 & 600.0 \\
\hline 04 & 1 & 321 & 105.2849 & 4.1683 & 277.6 & 4 & 600.0 & 600.0 \\
\hline 04 & 1 & 322 & 90.8750 & 4.5613 & 277.8 & 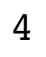 & 600.0 & 600.0 \\
\hline 14 & 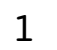 & 323 & 110.4180 & 3.8313 & 278.0 & 4 & 600.0 & 600.0 \\
\hline
\end{tabular}




\begin{tabular}{|c|c|c|c|c|c|c|c|}
\hline 4 & 24 & & 5 & 1.0 & & & \\
\hline $411-x-1$ & 41 & 91 & 2.6765 & 277.8 & & 00 & \\
\hline 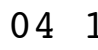 & 42 & 131.4749 & 8633 & 277.2 & 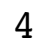 & 00 & \\
\hline 1 & 3 & 127.2975 & 3.0915 & 277.2 & & 00 & \\
\hline 1 & 44 & 142.6343 & 103 & 76.8 & & 0 & \\
\hline 1 & 45 & 81.5401 & 1920 & 277.4 & & v & \\
\hline & 46 & 127.1738 & 2.7123 & 276.8 & & 0 & \\
\hline 1 & 7 & 167.9349 & 0.8535 & 276.5 & & 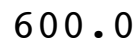 & \\
\hline 1 & 48 & 2.8969 & 1.4168 & 276.1 & & 0 & \\
\hline & 49 & 125.1303 & 1.4643 & 276.8 & & U & \\
\hline 1 & 410 & 155.4731 & 1.3083 & 277.4 & & 0 & \\
\hline 1 & 411 & 303 & 1.8095 & 278.7 & & & \\
\hline & 412 & 281 . & 2.26 & 279.5 & & 0 & \\
\hline 1 & 413 & 252.26 & 1.79 & 279.6 & & 0 & \\
\hline 1 & 414 & 21 & 3 . & 279.4 & & & \\
\hline & 415 & 16 & & 279.3 & & & \\
\hline 1 & 416 & 157.19 & 3.5930 & 278.9 & & 0 & \\
\hline 1 & 417 & 151 & $3 \cdot$ & 278.0 & & 0 & \\
\hline & 418 & 14 & & 277.3 & 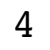 & & \\
\hline 1 & 419 & 145.94 & 3.0903 & 277.6 & 4 & 0 & \\
\hline 1 & 420 & 15 & 3 & 276.9 & 4 & 0 & \\
\hline & 421 & 15 & & 6.4 & 4 & & \\
\hline 1 & 422 & 14 & 3 & 276.1 & 4 & 0 & \\
\hline 1 & 423 & 142 & 5993 & 276.2 & 4 & 0 & \\
\hline 1 & 424 & 13 & & 27 & 4 & & \\
\hline 1 & 5 & 13 & 3. & 276.7 & 4 & & \\
\hline 1 & 52 & 117.2218 & 3.96 & 276.6 & 4 & 0 & \\
\hline 1 & 53 & 118 & 4 & 276.4 & 4 & 0 & \\
\hline 1 & 54 & 14 & 7 . & 276.5 & 4 & & \\
\hline 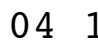 & 55 & 149.12 & 50 & 276.4 & 4 & 0 & \\
\hline 1 & 56 & 150.8249 & 5050 & 276.1 & 4 & 0 & \\
\hline 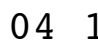 & 57 & 13 & 7. & 275.9 & 4 & & \\
\hline 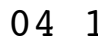 & 58 & 121.7608 & 6.7290 & 275.6 & 4 & 0 & \\
\hline 1 & 59 & 117.371 & 4380 & 276.8 & 3 & 0 & \\
\hline 1 & 510 & 14 & 5 & 277.2 & 3 & & \\
\hline & 511 & 14 & 4.6873 & 277.5 & 3 & 600.0 & \\
\hline 1 & 512 & 149.5000 & 4.2200 & 278.2 & 3 & 0 & \\
\hline 1 & 513 & 15 & 5.1720 & 278.9 & 4 & 0 & \\
\hline 1 & 514 & 159.5500 & 4.7090 & 279.0 & 3 & 600.0 & \\
\hline$\perp$ & 515 & 152.0742 & 3.3203 & 279.3 & 3 & 0 & \\
\hline 1 & 516 & 145 . & 2.8450 & 279.2 & 3 & 600 & \\
\hline 1 & 517 & 136.5743 & 2.0443 & 278.5 & 4 & 600.0 & \\
\hline 1 & 518 & 141.0002 & 3.0790 & 277.9 & 4 & 600.0 & \\
\hline 1 & 519 & 89.2981 & 1.1975 & 278.4 & 5 & 600 & \\
\hline 1 & 520 & 120.6516 & 1.0015 & 278.3 & 6 & 600.0 & \\
\hline 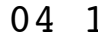 & 521 & 162.7524 & 1.3628 & 277.7 & 6 & 600.0 & \\
\hline$\perp$ & 322 & 27 & & 277 & 6 & & \\
\hline & 523 & 354.4953 & 1.4778 & 277.7 & J & 600.0 & 60 \\
\hline
\end{tabular}




\begin{tabular}{|c|c|c|c|c|c|c|c|}
\hline 4 & 1 & & 8 & 7.7 & & & \\
\hline 4 & 16 & 533 & 1.0285 & 277.5 & & 00 & \\
\hline 4 & 162 & 06.6828 & 1.3738 & 277.5 & & 00 & \\
\hline & 16 & 03.3385 & 1.4288 & 276.9 & & 00 & \\
\hline & 164 & 282 . & 3375 & 76.8 & & 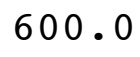 & \\
\hline & 16 & 50 & 515 & 276.8 & & $v$ & \\
\hline & 16 & 196.2223 & 1.4520 & 276.4 & & 00 & \\
\hline & 16 & 88.7765 & 1.4120 & 76.3 & & & \\
\hline & 16 & 663 & 1.0945 & 276.3 & & 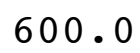 & \\
\hline & 16 & 316.0880 & 0.7323 & 276.9 & & 0 & \\
\hline & 1610 & 93.5243 & 1.4850 & 277.9 & & 0 & \\
\hline & 1611 & 290 & 1.7373 & 78.8 & & & \\
\hline & 1612 & 39 & 1.7743 & 279.1 & & 0 & \\
\hline & 1613 & 540 & 2.1948 & 279.1 & & 0 & \\
\hline & 1614 & 293 & 1 . & 78.5 & & & \\
\hline & 1615 & 291 & 3 . & 278.8 & & & \\
\hline & 16 & 166 & 1.3938 & 278.9 & & 0 & \\
\hline & 1617 & 262 & 2.3260 & 278.4 & & 0 & \\
\hline & 161 & & 2.7100 & 279.0 & & & \\
\hline & 19 & 353. & 2.6535 & 281.1 & & 0 & \\
\hline & 1620 & 322 & 2.8258 & 281.8 & & 0 & \\
\hline & 1621 & 332 & 2.8575 & 282.8 & & & \\
\hline & 22 & 35 & 3.9205 & 283.2 & & & \\
\hline & 1623 & 35 & 5.2445 & 283.4 & & 0 & \\
\hline & 1624 & & 2.6185 & 282.7 & & & \\
\hline & 17 & 295 & 3.6075 & 281.5 & & & \\
\hline & 17 & 316 & 5.0305 & 280.5 & & 0 & \\
\hline & 17 & 31 & 4.6310 & 281.1 & & 0 & \\
\hline & 174 & 31 & 3.6743 & 281.5 & 2 & & \\
\hline & 17 & 301 . & 4.6325 & 282.1 & & 0 & \\
\hline & 176 & 316 & 6.1168 & 282.1 & 4 & 0 & \\
\hline & $\begin{array}{lll}17 & 7\end{array}$ & 327 & 5.2553 & 283.4 & 2 & & \\
\hline & 17 & 331 & 4.1955 & 283.9 & - & 0 & \\
\hline & 179 & 321 & 6.2398 & 284.3 & 4 & 0 & \\
\hline & 10 & 30 & 2.8950 & 284.4 & 4 & & \\
\hline & 1711 & 167 & 2.3770 & 285.3 & 4 & 0 & \\
\hline & 1712 & 177.5521 & 2.5658 & 285.8 & 4 & 0 & \\
\hline & 1713 & 751 & 4.9273 & 286.3 & 4 & 0 & \\
\hline & 1714 & 232 . & 1.8970 & 284.9 & 4 & 600.0 & \\
\hline & 1715 & 232.4812 & 1.5120 & 284.2 & 4 & 0 & \\
\hline & 1716 & 162 & 2.3123 & 283.7 & 4 & 600.0 & \\
\hline & 1717 & 136 . & 2.2248 & 283.3 & 4 & 600.0 & \\
\hline & 1718 & 142.9072 & 2.3810 & 283.1 & 4 & 600.0 & \\
\hline & 1719 & 140 & 2.1968 & 282.4 & 5 & 600 & \\
\hline & 1720 & 46.6854 & 1.4573 & 283.0 & 0 & 600.0 & \\
\hline & 172 & 312.8247 & 1.7368 & 283.3 & 5 & 600.0 & \\
\hline & 72 & 29 & 1.7085 & 282 & 5 & & \\
\hline & 1723 & 320.2594 & 2.8695 & 283.6 & $\mathbf{4}$ & 600.0 & 0 \\
\hline
\end{tabular}




\begin{tabular}{|c|c|c|c|c|c|c|c|}
\hline 4 & 1 & & & & & & \\
\hline 4 & 18 & 23 & 1.3315 & 5.9 & & 00 & \\
\hline 4 & 182 & 138.9445 & 1.0478 & 285.8 & & 00 & \\
\hline & 18 & 118.7236 & 2.6203 & 282.2 & & 00 & \\
\hline & 184 & 10 & 0208 & 81.4 & & 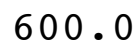 & \\
\hline & 185 & 325.6 & 1.8833 & 82.4 & & 0 & \\
\hline & 18 & 321.3376 & 2.5495 & 283.1 & & 00 & \\
\hline & 18 & 346.8674 & 2.88 & 84.4 & & 0 & \\
\hline & 18 & 333. & 1.72 & 85.6 & & 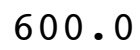 & \\
\hline & 18 & 350.3288 & 1.6805 & 86.9 & & 0( & \\
\hline & 1810 & 308.8669 & 2.3140 & 286 & & 0 & \\
\hline & 1811 & 240 & 208 & 3 & & & \\
\hline & 1812 & 966 & 1.68 & 284.5 & & 0 & \\
\hline & 1813 & 290.5055 & 1.93 & 286.1 & & 0 & \\
\hline & 1814 & 28 & 2 . & 7 & & & \\
\hline & 1815 & 282 & 1 . & 287.6 & & & \\
\hline & 1816 & 235.6681 & 2.0383 & 287.5 & & 0 & \\
\hline & 1817 & 140 & 3. & $\cdot 1$ & & 0 & \\
\hline & 1818 & 145 & 4 . & 283.6 & & & \\
\hline & 1819 & 142.7650 & 4.0 & 282.3 & & 0 & \\
\hline & 1820 & 141.3468 & 2.73 & 281.7 & & 0 & \\
\hline & 1821 & 123 & 2 . & 81.5 & & & \\
\hline & 1822 & 055 & 1 . & 281.2 & & 0 & \\
\hline & 1823 & 116 & 0.90 & 281.2 & & 0 & \\
\hline & 1824 & 19 & & 281.3 & & & \\
\hline & 19 & 357 & 1 . & 281.5 & & & \\
\hline & 19 & 4.2360 & 1.3 & 281.8 & & 0 & \\
\hline & 193 & 461 & 3. & 2 & & 0 & \\
\hline & 194 & 53. & 1.8 & 280.3 & 5 & 0 & \\
\hline & 19 & 117.4063 & 1.7 & 279.8 & & 0 & \\
\hline & 196 & 141.0992 & 2.19 & 280.2 & & 0 & \\
\hline & $\begin{array}{lll}1 & 9 & 7\end{array}$ & 226 & 1.1 & 280.7 & 6 & & \\
\hline & 19 & 171.4490 & 1.8 & 280.9 & 2 & 0 & \\
\hline & 199 & 675 & 1.0 & 282.2 & 4 & 0 & \\
\hline & 1910 & 31 & 1 . & 28 & 4 & & \\
\hline & 1911 & 32 & 2.7558 & 5.7 & 3 & 600.0 & \\
\hline & 1912 & 331.2270 & 3.6518 & 287.4 & 3 & مी & \\
\hline & 1913 & 323 & 5 . & 289 & 3 & 0 & \\
\hline & 1914 & 306.0577 & 4.04 & 290.3 & 3 & 600.0 & \\
\hline & 1915 & 151.0000 & 5.1210 & 285.7 & 4 & 0 & \\
\hline & 1916 & 142 . & 3.2 & 286.6 & 4 & 600 & \\
\hline & 1917 & 342.9748 & 1.4980 & 286.6 & 5 & 600.0 & \\
\hline & 1918 & 160.9976 & 1.2245 & 286.7 & 6 & 600.0 & \\
\hline & 1919 & 275.7934 & 1.7468 & 284 & 5 & 600 & \\
\hline & 1920 & 218.9455 & 1.4983 & 283.1 & 5 & 600.0 & \\
\hline & 1921 & 26.1028 & 0.8343 & 283.2 & 6 & 600.0 & \\
\hline & 1922 & 20 & 1.8 & & 3 & & \\
\hline & 1923 & 139.7003 & 2.3135 & 283.8 & $\mathbf{4}$ & 600.0 & 60 \\
\hline
\end{tabular}




\begin{tabular}{|c|c|c|c|c|c|c|c|}
\hline 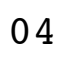 & 924 & & 1.4008 & 0 & & & \\
\hline 4 & 1101 & 7168 & 1.6225 & 284.0 & & & \\
\hline & 1102 & 72.4581 & 1.2318 & 283.7 & & 00 & \\
\hline & 1103 & 137.7010 & 1.7600 & 282.7 & & 00 & \\
\hline & 104 & 145.2027 & 3.3685 & 31.7 & & & \\
\hline & 105 & 150.9249 & 4.7320 & 81.8 & 4 & 00 & \\
\hline & 106 & 157.0244 & 2.9340 & 281.8 & & 0 & \\
\hline & 107 & 147.4750 & 6415 & 81.9 & & & \\
\hline & 1108 & 161.0766 & 3.8143 & 81.7 & 4 & 0 & \\
\hline & 1109 & 153.7736 & 3748 & 281.3 & & 0 & \\
\hline & 1010 & 163.3000 & 3.6730 & 281.3 & 4 & 0 & \\
\hline & 011 & 156.6025 & 2.4655 & 81.6 & 4 & & \\
\hline & 1012 & 150.7963 & 2.2058 & 282.4 & 3 & 0 & \\
\hline & 11013 & 165.4498 & 2.6550 & 282.6 & 3 & 0.0 & \\
\hline & 1014 & 151.4711 & & 82.4 & r & & \\
\hline & 11015 & 149.7750 & 4.4853 & 282.9 & 3 & 0 & \\
\hline & 1016 & 148.0504 & 53 & 283.0 & 3 & 0 & \\
\hline & 1017 & 117 . & 8 & .0 & 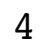 & & \\
\hline & 11018 & 144.1498 & 3.8070 & 283.1 & 4 & 0 & \\
\hline & 1019 & 145.6251 & 9493 & 283.2 & 4 & & \\
\hline & 1020 & 151.3747 & 30 & 282.8 & A & & \\
\hline & 1021 & 144.7730 & & 282.8 & 5 & & \\
\hline & 1022 & 140 & 2.0508 & 282.7 & 5 & 0 & \\
\hline 4 & 11023 & 131.9221 & 1.1318 & 283.1 & 6 & 0 & \\
\hline 4 & 11024 & 134.5969 & 3 & 282.9 & 6 & & \\
\hline & 1111 & 3745 & 1.7188 & 283.2 & 5 & & \\
\hline & 1112 & 154.5461 & 0763 & 282.8 & 6 & 0 & \\
\hline 4 & 1113 & 138.5217 & 2.1543 & 282.1 & 5 & 0 & \\
\hline 4 & 1114 & 500 & 00 & 282.2 & 4 & 0 & 0 . \\
\hline & 1115 & 148.9981 & 3.8623 & 281.9 & 4 & 0 & \\
\hline & 1116 & 132.5746 & 9090 & 282.3 & 5 & & \\
\hline 4 & 1117 & 183 & 50 & 283.0 & 6 & & \\
\hline & 1118 & 82.2758 & 1.3880 & 282.7 & 5 & 0 & J. \\
\hline 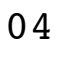 & 1119 & 107 & 5628 & 284.0 & 4 & 0 & \\
\hline & 11110 & 237 . & 35 & 28 & 3 & & \\
\hline & 11111 & 259.9747 & 895 & 285.2 & 2 & & 0 . \\
\hline & 11112 & 239.0030 & 2.6598 & 285.0 & 3 & 0 & \\
\hline 4 & 11113 & 242 . & 2.2490 & 284.7 & 3 & 0 & • \\
\hline 4 & 11114 & 238.7046 & 2.9435 & 284.2 & 3 & 0 & 0 . \\
\hline & 11115 & 229.3249 & 2.2870 & 283.3 & 4 & & \\
\hline 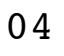 & 11116 & 262.0338 & 1.2883 & & 4 & & \\
\hline 4 & 11117 & 320.5678 & 1.8035 & 282.8 & 4 & 600.0 & 600 . \\
\hline & 11118 & 335.2552 & 1.8418 & 282.7 & 5 & 600 & 800 \\
\hline 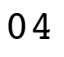 & 11119 & 309.3008 & 2.3525 & 282.6 & 4 & 600 & 10 \\
\hline 04 & 11120 & 29.4519 & 1.1445 & 282.4 & 5 & 600.0 & 00 . \\
\hline & 11121 & 20.2879 & 1.3918 & 282.0 & 6 & 600.0 & 600. \\
\hline & 11122 & 160.6001 & 1.8398 & 28 & 5 & 600 & 60 \\
\hline & 11123 & 143.0783 & 0.7655 & 280.3 & & 600.0 & 600 \\
\hline
\end{tabular}




\begin{tabular}{|c|c|c|c|c|c|c|c|}
\hline$-5+2+3$ & 1124 & 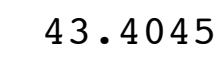 & 0.8818 & 80.4 & 5 & 800 & \\
\hline 4 & 1121 & 144.0602 & 1.4015 & 279.6 & 0 & ل & \\
\hline & 1122 & 217.5052 & 0105 & 279.6 & 6 & 00 & \\
\hline & 123 & 328.9243 & L. 2393 & 279.6 & & 00 & \\
\hline & 124 & 149.1104 & .4095 & 79.2 & & 0 & \\
\hline & 125 & 155.3003 & 2.4255 & 279.3 & & 00 & \\
\hline & 126 & 160.4000 & 1.8145 & 278.9 & & 0 & \\
\hline & 127 & 159.4504 & .1050 & 78.6 & & & \\
\hline & 128 & 151.4493 & 1.2315 & 278.6 & & 0 & \\
\hline & 129 & 174.1864 & 1.3435 & 278.7 & & 0 & \\
\hline & 1210 & 175.4869 & 2.6365 & 279.4 & & 00 & \\
\hline & 211 & 181.4545 & 152 & 80.3 & & & \\
\hline & 1212 & 176.6571 & 2.3245 & 280.6 & & 0 & \\
\hline & 1213 & 141.6264 & 1.2685 & 281.1 & & 0.0 & \\
\hline & 214 & 287.5704 & $1 \quad 10$ & & & & \\
\hline & 1215 & 153.1755 & 2.3025 & 280.9 & & 0 & \\
\hline & 216 & 359.2893 & L. 61 & 281.1 & 4 & 0 & \\
\hline & 217 & 54.7967 & 8 & 1.9 & & & \\
\hline & 218 & 51.9926 & 2.15 & 281.3 & 5 & 0 & \\
\hline & 219 & 17.4493 & .74 & 282.9 & 6 & & \\
\hline & 220 & 003 & 1.78 & 1.8 & & & \\
\hline & 221 & 136. & 3.22 & 279.2 & 4 & & \\
\hline & 222 & 126.8644 & 1.94 & 279.1 & 5 & 0 & • \\
\hline 4 & 223 & 3.1055 & 1.34 & 279.0 & 6 & 0 & \\
\hline & 224 & 357.0245 & 97 & 278.8 & 3 & & \\
\hline & 131 & 563 & 3 & 278.5 & 5 & & \\
\hline & 132 & 42.5722 & 53 & 278.2 & 5 & 0 & \\
\hline 4 & 133 & 334.8697 & 98 & 27 & 6 & & \\
\hline 4 & 1134 & 6 . & 1.87 & 277.4 & 5 & 0 & 0 . \\
\hline & 135 & 357.8680 & 91 & 277.3 & 5 & 0 & \\
\hline & 136 & 340 . & 0 & 277.4 & 4 & & \\
\hline 4 & 1137 & 359 . & 3 & 277.5 & 4 & & \\
\hline & 1138 & 322.1068 & 2.19 & 277.4 & 4 & 0 & J • \\
\hline 4 & 139 & 333. & .74 & 277.4 & 4 & & \\
\hline & 310 & 317 . & 3 & .7 & 4 & & \\
\hline & 11 & 271.4805 & 1.37 & 278.2 & 4 & & 0 . \\
\hline & 312 & 274.8175 & 1.3495 & 278.6 & 4 & & \\
\hline 4 & 11313 & 292.0228 & 1.43 & 278.6 & 3 & 0 & 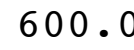 \\
\hline 4 & 11314 & 293.2324 & 1.6768 & 278.8 & 4 & .0 & 0 . \\
\hline & 315 & 281.7958 & 5 & 279.0 & 4 & & \\
\hline 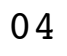 & 11316 & 311.7134 & 3 & 279.1 & 4 & & \\
\hline 4 & 11317 & 343.8575 & 2.4020 & 279.0 & 4 & 600.0 & 600 . \\
\hline & 318 & 339.1009 & 1.4023 & 278.6 & 5 & 600 & 400 \\
\hline & 11319 & 350.3504 & 1.3383 & 278.3 & 5 & 600 & 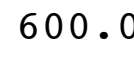 \\
\hline 04 & 11320 & 265.1213 & 1.1678 & 278.2 & 6 & 600.0 & 00 . \\
\hline & 321 & 109.8558 & 2.3828 & 277.7 & - & 600 & 60 \\
\hline & 11322 & 121.0209 & 1.9 & .5 & כ & 600 & \\
\hline & 1323 & 50.4498 & 1.2655 & 277.1 & & 600.0 & 600 \\
\hline
\end{tabular}




\begin{tabular}{|c|c|c|c|c|c|c|c|}
\hline$x$ & 1324 & & .7433 & 76.2 & & & \\
\hline 4 & 1141 & 171.1331 & 1.1628 & 276.3 & & & \\
\hline & 1142 & 4174 & 0783 & 276.5 & & 00 & \\
\hline & 143 & 7.1489 & L. 6080 & 276.6 & & 00 & \\
\hline & 144 & 356.6205 & L.3675 & 276.7 & & 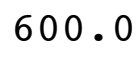 & \\
\hline & 145 & 231.7465 & .1613 & 276.5 & & 00 & \\
\hline & 146 & 40.4669 & 1.0663 & 276.4 & & 0 & \\
\hline & 147 & 14.2702 & 1.1060 & 276.2 & & & \\
\hline & 148 & 26.2770 & 1.3195 & 276.0 & & 0 & \\
\hline & 149 & 333.2150 & 0.8288 & 276.4 & & 00 & \\
\hline & 410 & 324.5643 & L. 1533 & 277.0 & & 00 & \\
\hline & 111 & 219.8112 & .9528 & 77.9 & & & \\
\hline & 2 & 179.3428 & 1.4835 & 278.8 & & 0 & \\
\hline & 413 & 252.5854 & 1.4783 & 278.8 & & 0.0 & \\
\hline & 414 & 223.5513 & 1.5355 & & & & \\
\hline & 5 & 147.1255 & 005 & 278.6 & 4 & 0 & \\
\hline & 416 & 143.9248 & 5265 & 279.5 & & 0 & \\
\hline & 417 & 151.9282 & 4.2470 & .2 & & & \\
\hline & 418 & 154.3511 & 3.2498 & .3 & 4 & 0 & \\
\hline & 419 & 144.4735 & 1183 & 280.0 & 4 & 0 & \\
\hline & 420 & 144.3607 & 458 & 9.8 & & & \\
\hline & 421 & 140.1492 & .3193 & 9.6 & 5 & & \\
\hline & 422 & 146.8015 & 2133 & 279.8 & 4 & 0 & . \\
\hline 4 & 1423 & 136.1622 & .3165 & 281.1 & 4 & 0 & \\
\hline 4 & 1424 & 144.3208 & 30 & 1.0 & 4 & & \\
\hline & 151 & 156.3013 & 9020 & $2 \varepsilon$ & 4 & & \\
\hline 4 & 152 & 96.3111 & 1195 & 280.7 & 5 & 0 & \\
\hline 1 & 1153 & 117.7479 & 253 & 281.3 & 5 & & \\
\hline 4 & 1154 & 86.3505 & 58 & 281.6 & 5 & 0 & 0 . \\
\hline & 1155 & 78.3021 & 7158 & 281.8 & 4 & 0 & \\
\hline & 1156 & 96.7981 & 725 & 282.0 & 4 & & \\
\hline 4 & 1157 & 113.3292 & 895 & 281.7 & 4 & & \\
\hline & 1158 & 105.1594 & 018 & 281.9 & 4 & 0 & J \\
\hline 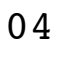 & 1159 & 207 & 095 & 282.7 & 3 & 0 & \\
\hline & 1510 & 84.2258 & 585 & 282.8 & 3 & & \\
\hline & 511 & 80.0063 & 245 & 283.3 & 3 & & 0 . \\
\hline & 512 & 151.1844 & 890 & 284.7 & 2 & 0 & \\
\hline 4 & 11513 & 138.7221 & 285 & 285.1 & 3 & 0 & • \\
\hline 4 & 11514 & 158.2711 & 3.8685 & 284.7 & 3 & 0 & 0 . \\
\hline & 11515 & 222.8042 & 2.3258 & 284.4 & 2 & 6 & \\
\hline 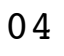 & 11516 & 241.8200 & 3.1415 & 282.6 & 3 & & \\
\hline 04 & 11517 & 252.3635 & 1.7758 & 281.5 & 4 & 600.0 & 600 . \\
\hline & 11518 & 282.3634 & 2.5653 & 280.9 & 4 & 600 & 600. \\
\hline- & 11519 & 290.5856 & 2.4695 & 280.6 & 4 & & 60 \\
\hline 04 & 11520 & 319.1769 & 2.9680 & 280.3 & 4 & 600.0 & 00 . \\
\hline & & 341.0629 & 1.6605 & 280.4 & 5 & 600.0 & 600 \\
\hline & 11522 & 296.7681 & 1.1105 & 280.4 & 0 & 600 & \\
\hline & 11523 & 285.2164 & 1.6948 & 280.3 & & 600.0 & 600 \\
\hline
\end{tabular}




\begin{tabular}{|c|c|c|c|c|c|c|c|}
\hline 4 & 524 & & 5 & & & & \\
\hline 1 & 161 & & .1263 & 0.8 & & 00.0 & \\
\hline & 1162 & 150.4038 & 1.1998 & 281.1 & 0 & 00 & \\
\hline & 163 & 171.8827 & 9205 & 280.8 & & 00 & \\
\hline & 164 & 287 & .0325 & 279.4 & & 0 & \\
\hline & 165 & 191.8136 & .1075 & 279.6 & & 0 & \\
\hline & 166 & 2350 & 0913 & 279.5 & & 00 & \\
\hline & 167 & 182.5307 & 155 & .79 .5 & & 0 & \\
\hline & 168 & 268.5222 & 2180 & 79.0 & & & \\
\hline & 169 & 224.2016 & 1.5870 & 278.8 & & 00 & 0 \\
\hline & 1610 & 258.8147 & 1.5098 & 279 & & 0.0 & \\
\hline & 1611 & 220.5130 & 2.0608 & 79.3 & & 0 & \\
\hline & 1612 & 187.8097 & 4.0555 & 279.2 & 4 & .0 & \\
\hline & 613 & 184.6591 & 4.0543 & 279.1 & 4 & 0.0 & \\
\hline & 614 & 174.8644 & 5 & 279.0 & 4 & & \\
\hline & 615 & 175.1898 & 2 . & 279.1 & 4 & & \\
\hline & 1616 & 182.7127 & .3223 & 279.0 & 4 & 0.0 & 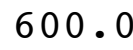 \\
\hline & 617 & 173.6833 & 5 & 279.3 & 5 & 0 & \\
\hline & 618 & 163.1435 & 960 & 279.4 & 5 & & \\
\hline & 1619 & 143.8400 & 2.3680 & 279.4 & 4 & .0 & U. \\
\hline & 620 & 149.9533 & 2.0835 & 279.7 & 5 & 0 & \\
\hline & 621 & 125.6549 & 120 & 280.0 & 6 & & \\
\hline & 622 & 125 & 9430 & 280.2 & 6 & & \\
\hline & 623 & 355.1655 & 1.6300 & 280.0 & 5 & 0 & 00 \\
\hline & 11624 & 290.1380 & 3 & 28 & 6 & & \\
\hline & 1171 & 132.9 & 1.4105 & 28 & 6 & & \\
\hline & 1172 & 324.9226 & 8433 & 280.1 & 6 & 0 & U. \\
\hline & 1173 & 133.1069 & 938 & 279.9 & 6 & & \\
\hline & 1174 & 268.2294 & 323 & 279 & 6 & & \\
\hline & 1175 & 221.4647 & 1.6913 & 279.7 & 6 & 0 & 0 • \\
\hline & 1176 & 290.0275 & 1.6338 & 279.2 & 6 & 0 & 0 . \\
\hline & 1177 & 268.9 & 8 & 278.9 & 6 & & \\
\hline & 1178 & 279.4130 & 5 & 278.6 & 6 & & 0 . \\
\hline & 1179 & 292.5520 & 2.5470 & 278.7 & 5 & 0 & 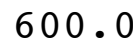 \\
\hline & 11710 & 296.3659 & 608 & 279.1 & 4 & & \\
\hline & 11711 & 256.1262 & & 279.2 & 4 & & 0 . \\
\hline & 11712 & 235.1450 & 1.8985 & 279.3 & 4 & $\cdot 0$ & . \\
\hline 4 & 11713 & 231.4499 & 1 . & 279.3 & 4 & & . \\
\hline 2 & 11714 & 228.3657 & 2.2035 & 279.2 & 4 & .0 & 0 . \\
\hline & 11715 & 212.5861 & 1.6913 & 279.2 & 4 & & 0 . \\
\hline & 11716 & 197.7437 & 1.5330 & 279.2 & 4 & & 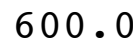 \\
\hline 4 & 11717 & 290.5357 & 1.7135 & 279.2 & 4 & .0 & 0 . \\
\hline & 11718 & 329.8483 & 2.7245 & 278.8 & 4 & 0 & 00 . \\
\hline th & 11719 & 321.5875 & 3.2043 & $27 \xi$ & 4 & & \\
\hline 4 & 11720 & 304.2642 & 2.4165 & 278.4 & 4 & & 00 . \\
\hline & 11721 & 220.3007 & 1.4773 & 278.2 & 5 & 600.0 & 600 \\
\hline & 11722 & 183.7762 & & & 4 & & \\
\hline & 11723 & 180.0352 & 2.4183 & 278.2 & & 600.0 & 600 \\
\hline
\end{tabular}




\begin{tabular}{|c|c|c|c|c|c|c|c|}
\hline 4 & 724 & & 2490 & 8.2 & & & \\
\hline & 181 & 293.7997 & 2.7015 & 278.2 & 4 & 0.0 & \\
\hline & 1182 & 290.9087 & 2.8923 & 278.1 & 4 & 00 & \\
\hline & 183 & 60.4126 & . 1098 & 278.1 & & 00 & \\
\hline & 184 & 4.2481 & 1260 & 278.0 & & 0 & \\
\hline & 185 & 276.9967 & .3765 & 277.9 & & 00 & \\
\hline & 186 & 219.3267 & 1.6640 & 277.9 & & 00 & \\
\hline & 187 & 93.5132 & .0668 & 277.8 & & 0 & \\
\hline & 188 & 52.3600 & 3 & 277.8 & & & \\
\hline & 189 & 37.3308 & 1.6683 & 278.3 & & 00 & 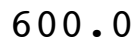 \\
\hline & 1810 & 196.3724 & 2.5108 & 278.9 & & 0 & \\
\hline & 1811 & 215.1247 & 2.3520 & 79.0 & & 0 & \\
\hline & 1812 & 228.3783 & 1.76 & 279.3 & 3 & 0 & \\
\hline & 1813 & 225.2144 & 1.74 & 279.5 & & 0.0 & 00 \\
\hline & 814 & 222.9875 & 2 . & 280.2 & & & \\
\hline & 1815 & 230.3611 & 2 . & 280.5 & 3 & & \\
\hline & 1816 & 210.1788 & $1.7^{\circ}$ & 280.4 & 4 & .0 & 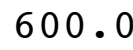 \\
\hline & 1817 & 222.7521 & 1 . & 0.1 & 1 & 0 & \\
\hline & 1818 & 142.0115 & & 279.3 & 5 & & \\
\hline & 819 & 57.8557 & 0.79 & 279.3 & 6 & .0 & 0 \\
\hline & 820 & 274.0699 & 0.45 & 279.0 & 6 & 0 & \\
\hline & 821 & 348.7580 & & 278.7 & 6 & & \\
\hline & 822 & 94.5763 & 3 & 278.7 & 5 & & \\
\hline & 823 & 150.7405 & 1.39 & 279.1 & 6 & 0 & 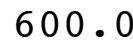 \\
\hline & 11824 & 146.6645 & & 279.3 & 6 & & \\
\hline & 1191 & 327.1022 & 1 . & 278.9 & 6 & & \\
\hline & 1192 & 27.3323 & 3043 & 279.1 & 6 & 0 & . \\
\hline & 1193 & 160.6908 & 1.74 & 278.6 & 5 & & \\
\hline & 1194 & 231.8134 & 0 . & 3.5 & 6 & & \\
\hline & 1195 & 108.4674 & 1.24 & 278.2 & 6 & 0 & 0 . \\
\hline & 1196 & 314.0069 & .91 & 278.1 & 6 & & 0 . \\
\hline & 1197 & 7.6378 & 0 & 278.0 & 6 & & \\
\hline & 1198 & 55.7795 & 3473 & 278.3 & 5 & 0 & . \\
\hline & 1199 & 109.2107 & 1 & 279.3 & 5 & 0 & 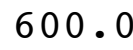 \\
\hline & 11910 & 224.8793 & 3 & 279.7 & 4 & & \\
\hline & 11911 & 188.8681 & 1.5735 & 279.8 & 4 & & U \\
\hline & 11912 & 176.6514 & 3.2605 & 279.7 & 4 & $\cdot 0$ & 0 . \\
\hline 4 & 11913 & 181.8197 & 3 . & 279.5 & 4 & & 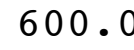 \\
\hline & 11914 & 185.2034 & 3.6498 & 279.2 & 4 & .0 & 0 \\
\hline & 11915 & 209.3027 & 1.8688 & 279.2 & 4 & & 0 . \\
\hline & 11916 & 209.6762 & 1.4628 & 279.3 & 4 & & 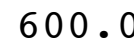 \\
\hline 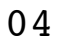 & 11917 & 166.3752 & 1.8888 & 279.2 & 5 & .0 & 0 . \\
\hline & 11918 & 133.8329 & 1.8448 & 279.4 & 5 & .0 & 00 . \\
\hline th & 11919 & 58.4771 & 2.6513 & 279.6 & 4 & & \\
\hline 04 & 11920 & 325.9192 & 1.7033 & 279.0 & 5 & & 600. \\
\hline & 11921 & 309.6337 & 1.7933 & 278.8 & 6 & 600.0 & 600 \\
\hline & & & & & 6 & & \\
\hline & 1923 & 175.8254 & 1.4070 & 279.1 & & 600.0 & 600 \\
\hline
\end{tabular}




\begin{tabular}{|c|c|c|c|c|c|c|c|}
\hline 4 & 924 & & 080 & 8.8 & & & \\
\hline & 1201 & 521 & 7873 & 278.8 & & 00.0 & \\
\hline & 1202 & 122.6539 & 3120 & 279.3 & 0 & 00 & \\
\hline & 1203 & 98.8619 & .0850 & 279.0 & & 00 & \\
\hline & 1204 & 117 & 3603 & 278.7 & & 0 & \\
\hline & 1205 & 228.4668 & .7640 & 278.5 & & 00 & \\
\hline & 206 & 6456 & -355 & 278.5 & & 00 & \\
\hline & 207 & 18.6380 & L. 2630 & 278.7 & & 0 & \\
\hline & 1208 & 4.1416 & 3 & 278.6 & & & \\
\hline & 1209 & 59.8552 & 2.0980 & 278.7 & & 00 & 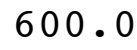 \\
\hline & 2010 & 161.1721 & 1.2370 & 279.1 & & & \\
\hline & 12011 & 205.1771 & 2.2990 & 79.5 & 4 & & \\
\hline & 12012 & 68.4223 & 2.1100 & 279.4 & 4 & .0 & \\
\hline & 2013 & 153.0656 & 2.4660 & 279.8 & 4 & 0.0 & 00 \\
\hline & 12014 & 7242 & 2.2 & 0.3 & & & \\
\hline & 12015 & 155.8472 & 2.5 & 280.7 & 3 & & \\
\hline & 12016 & 128.8766 & 2.0590 & 280.7 & 3 & .0 & 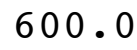 \\
\hline & 12017 & 745 & 1 . & 1.0 & 4 & & \\
\hline & 12018 & 76.7601 & 2 . & .8 & 4 & & \\
\hline & 12019 & 89.5711 & 2.8535 & .9 & 4 & 0 & 0 \\
\hline & 12020 & 226.0527 & 1.3058 & 280.3 & 5 & 0 & \\
\hline & 12021 & 338.7262 & 1.8 & 279.6 & 5 & & \\
\hline & 12022 & 359 & 1.5713 & 279.8 & 6 & & \\
\hline & 12023 & 346.5120 & 0.9283 & 280.2 & 6 & 0 & 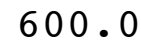 \\
\hline & 12024 & 354.1098 & 1.2 & .0 & 6 & & \\
\hline & 1211 & 2699 & 1.3 & .0 & 6 & & \\
\hline & 1212 & 164.6889 & 1.5455 & 8.8 & 5 & 0 & . \\
\hline & 1213 & 313.3543 & 1.20 & 3.8 & 6 & & \\
\hline & 1214 & 269.6041 & 1.0 & 3.7 & 6 & & \\
\hline & 1215 & 232.8853 & 1.0155 & 8.6 & 6 & 0 & 0 . \\
\hline & 1216 & 353.2454 & 1.6905 & 278.4 & 5 & & 0 . \\
\hline & 1217 & 347.4830 & 100 & 278.6 & 5 & & \\
\hline & 1218 & 177.2390 & 1.6013 & 278.7 & 5 & & . \\
\hline & 1219 & 5193 & 1.5793 & 279.6 & 4 & 0 & 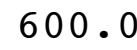 \\
\hline & 12110 & 208.9946 & 1.7133 & 0.6 & 3 & & \\
\hline & 12111 & 192.7402 & 1.92 & 1.5 & 2 & & . \\
\hline & 12112 & 157.7773 & 4.2818 & 1.5 & 3 & & 0 \\
\hline 4 & 12113 & 151. & 3.8940 & $2 \varepsilon$ & 3 & & 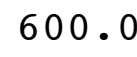 \\
\hline & 12114 & 142.6998 & 3.6570 & 284.0 & 3 & .0 & 0 . \\
\hline & 12115 & 130.0271 & 2.3975 & 285.1 & 2 & & 0 . \\
\hline & 12116 & 141.1255 & 2.7093 & 284.6 & 3 & & 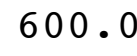 \\
\hline & 12117 & 143.3993 & 3.4110 & 283.5 & 4 & .0 & 0 . \\
\hline & 12118 & 150.0119 & 2.7555 & 283.4 & 4 & 0 & 00 . \\
\hline 04 & 12119 & 39.5848 & 1.5925 & 283.7 & 5 & & \\
\hline 04 & 12120 & 22.7594 & 1.3078 & 284.0 & 6 & & 600. \\
\hline & 12121 & 121.2323 & 1.6208 & 283.6 & 5 & 600.0 & 600 \\
\hline & 12122 & 41 & $2 . \varepsilon$ & & 4 & & \\
\hline & 12123 & 104.4786 & 3.2555 & 283.5 & $\Psi$ & 600.0 & 600 \\
\hline
\end{tabular}




\begin{tabular}{|c|c|c|c|c|c|c|c|}
\hline 4 & 2124 & & 8 & & & & \\
\hline & 1221 & 04 & .0170 & 3.3 & 4 & 0 & \\
\hline & 1222 & 80.8480 & 1960 & 283.3 & 4 & 00 & \\
\hline & 1223 & 83.0413 & 4.2560 & 283.0 & & 00 & \\
\hline & 1224 & 142 & 3493 & 81.7 & & 0 & \\
\hline & 1225 & 123.1071 & .2373 & 82.1 & & 0 & \\
\hline & 1226 & 132.7806 & 4355 & 281.1 & & 00 & \\
\hline & 1227 & 137.0429 & 4183 & 81.2 & & & \\
\hline & 1228 & 114.6002 & .22 & 81.8 & 4 & & \\
\hline & 1229 & 135.8732 & 2243 & 81.1 & & 0 & 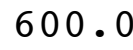 \\
\hline & 12210 & 148.7976 & 3.9748 & 281.9 & & & \\
\hline & 12211 & 163.1751 & 4.8630 & 82.2 & & 0 & \\
\hline & 12212 & 161.5751 & 5.01 & 282.0 & 3 & 00.0 & \\
\hline & 12213 & 159.4499 & 1010 & 282.6 & 3 & 0.0 & \\
\hline & 12214 & 159. & & 82.7 & & & \\
\hline & 12215 & 161.90 & & 282.0 & 3 & & \\
\hline & 12216 & 149.1711 & .33 & 281.5 & 3 & .0 & 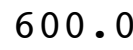 \\
\hline & 12217 & 132.2755 & 5 & 31.6 & 4 & & \\
\hline & 12218 & 67.7891 & & 282.1 & 5 & & \\
\hline & 12219 & 119.1347 & 2 . & 282.6 & 4 & & 0 \\
\hline & 12220 & 122.1320 & 2.05 & 282.6 & 5 & 0 & \\
\hline & 12221 & 121.5282 & & 282.2 & 5 & & \\
\hline & 12222 & 127.1011 & 3 & 281.5 & 5 & & \\
\hline & 12223 & 104.0814 & 8 & 281.7 & 6 & 0 & J • \\
\hline & 12224 & 108.4602 & & 81.8 & 6 & & \\
\hline & 1231 & 160 . & 1 . & 281.1 & 5 & & \\
\hline & 1232 & 15.0350 & 3 & 280.8 & 6 & 0 & . \\
\hline & 1233 & 345.6952 & 3 & 9 & 6 & & \\
\hline & 1234 & 12.1964 & 0 & 28 & 6 & & \\
\hline & 1235 & 287.0194 & 0 & 280.6 & 6 & 0 & 0 . \\
\hline & 1236 & 5.9646 & 0.70 & 280.1 & 6 & 0 & 0 . \\
\hline & 1237 & 325.4874 & 0 & 280.1 & 6 & & \\
\hline & 1238 & 226 . & 8 & 279.9 & 5 & & . \\
\hline & 1239 & 180.0300 & 0.8250 & 280.0 & 4 & 0 & 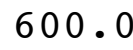 \\
\hline & 12310 & 270 . & 8 & & 3 & & \\
\hline & 12311 & 275.9316 & 2.23 & 281.2 & 2 & & 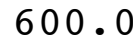 \\
\hline & 12312 & 223.9042 & .6998 & 281.3 & 2 & & 0 \\
\hline 4 & 12313 & 223. & 3 . & 280 & 3 & & . \\
\hline & 12314 & 198.3214 & 2.9673 & 280.8 & 3 & .0 & 0 . \\
\hline & 12315 & 177.2953 & 2.4338 & 280.9 & 2 & & 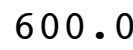 \\
\hline & 12316 & 185.6488 & 2.4640 & 279 & 3 & & 0 \\
\hline & 12317 & 313.1800 & 0.7378 & 279.6 & 4 & .0 & 0 . \\
\hline & 12318 & 106.5478 & 0.6888 & 280.1 & 5 & 0 & 0 . \\
\hline . & 12319 & 128.1443 & 1.7573 & 280.8 & 5 & & \\
\hline 04 & 12320 & 79.3442 & 3.3163 & 281.8 & 4 & & 600. \\
\hline & 12321 & 92.7457 & 3.0228 & 281.7 & 4 & 600.0 & 600 \\
\hline & 12322 & & & & 3 & & \\
\hline & 12323 & 88.3554 & 3.1990 & 281.8 & $\mathbf{4}$ & 600.0 & 600 \\
\hline
\end{tabular}




\begin{tabular}{|c|c|c|c|c|c|c|c|}
\hline 04 & 12324 & 14.6998 & 2.3895 & 280.1 & 4 & 600.0 & 600.0 \\
\hline 04 & 1241 & 43.1958 & 2.1738 & 280.7 & 5 & 600.0 & 600.0 \\
\hline 04 & 1242 & 85.9007 & 1.1925 & 281.1 & 6 & 600.0 & 00.0 \\
\hline 04 & 1243 & 114.9074 & 2.3530 & 280.6 & $\Gamma$ & 600.0 & 00.0 \\
\hline 04 & 1244 & 151.0803 & 2.4048 & 280.8 & o & 600.0 & 00.0 \\
\hline 04 & 1245 & 288.9608 & 2.0133 & 279.3 & 5 & 600.0 & 00.0 \\
\hline 04 & 1246 & 283.6983 & 1.6328 & 279.2 & 5 & 600.0 & 00.0 \\
\hline 04 & 1247 & 285.2417 & 2.1600 & 279.1 & 4 & 600.0 & 00.0 \\
\hline 04 & 1248 & 306.0780 & 1.3120 & 279.3 & 5 & 600.0 & 00.0 \\
\hline 04 & 1249 & 314.7312 & 1.1248 & 279.5 & 4 & 600.0 & 00.0 \\
\hline 04 & 12410 & 125.0623 & 1.2285 & 280.0 & 3 & 600.0 & 00.0 \\
\hline 04 & 12411 & 179.4789 & 1.7995 & 281.2 & 4 & 600.0 & \\
\hline 04 & 12412 & 127.2031 & 2.7528 & 281.7 & 3 & 600.0 & 00.0 \\
\hline 04 & 12413 & 152.8308 & 4.5243 & 280.6 & 3 & 600.0 & 00.0 \\
\hline 04 & 12414 & 156.6001 & 5. & 279.9 & 4 & .0 & \\
\hline 04 & 12415 & 162.5002 & 4.9075 & 279.7 & 4 & 600.0 & 00.0 \\
\hline 04 & 12416 & 160.2984 & 3.0878 & 280.0 & 4 & 600.0 & 600.0 \\
\hline 04 & 12417 & 119.3195 & 3.4803 & 280.3 & 4 & .0 & \\
\hline 04 & 12418 & 72.3603 & 4.4835 & 280.8 & 4 & 600.0 & .0 \\
\hline 04 & 12419 & 70.0246 & 4.8885 & 281.2 & 4 & 60 & .0 \\
\hline 04 & 12420 & 64.8750 & 8 & 282.2 & 4 & 60 & \\
\hline 04 & 12421 & 68.7752 & 6.5298 & 281.8 & 4 & .0 & \\
\hline 04 & 12422 & 77.0258 & 5.4600 & 281.5 & 4 & 600.0 & 0.0 \\
\hline 04 & 12423 & 40.1996 & 2.4565 & 281.1 & $J$ & 60 & .0 \\
\hline 04 & 12424 & 60.2549 & 5. & 281.1 & 4 & .0 & \\
\hline 04 & 1251 & 65.5261 & 6.1590 & 281.0 & 4 & 600.0 & 600.0 \\
\hline 04 & 1252 & 70.9741 & 6.2633 & 280.7 & 4 & 600.0 & .0 \\
\hline 04 & 1253 & 83.9907 & 6.1468 & 280.1 & 4 & .0 & \\
\hline 04 & 1254 & 135.7992 & 5.6200 & 279.9 & 4 & 600.0 & 600.0 \\
\hline 04 & 1255 & 149.7477 & 5.3073 & 279.6 & 4 & 600.0 & 600.0 \\
\hline 04 & 1256 & 166.8939 & 8.1265 & 279.4 & 4 & .0 & \\
\hline 04 & 1257 & 148.4830 & 198 & 278.5 & 4 & .0 & 600.0 \\
\hline 04 & 1258 & 142.9505 & 6.7983 & 278.6 & 4 & 600.0 & 600.0 \\
\hline 04 & 1259 & 126.7108 & 4.8028 & 279.0 & 3 & 600.0 & 0.0 \\
\hline 04 & 12510 & 140.4248 & 5.6075 & 279.9 & 3 & 600.0 & 600.0 \\
\hline 04 & 12511 & 153.5996 & 8.5550 & 280.6 & 4 & 600.0 & 600.0 \\
\hline 04 & 12512 & 151.1254 & 8.8225 & 281.1 & 4 & 600.0 & 600.0 \\
\hline 04 & 12513 & 156.8750 & 7.5525 & 281.7 & 4 & 600.0 & 600.0 \\
\hline 04 & 12514 & 157.5499 & 7.2715 & 282.1 & 4 & 600.0 & 600.0 \\
\hline 04 & 12515 & 157.4251 & 6.4695 & 282.4 & 4 & 600.0 & 600.0 \\
\hline 04 & 12516 & 150.6990 & 5.0370 & 282.5 & 3 & 600.0 & 600.0 \\
\hline 04 & 12517 & 140.7220 & 3.9995 & 282.2 & 4 & 600.0 & 600.0 \\
\hline 04 & 12518 & 148.5241 & 3.8410 & 281.3 & 4 & 600.0 & 600.0 \\
\hline 04 & 12519 & 155.7529 & 3.5855 & 280.3 & 4 & 600.0 & 600.0 \\
\hline 04 & 12520 & 141.8749 & 2.3995 & 280.5 & 4 & 600.0 & 600.0 \\
\hline 04 & 12521 & 135.1986 & 1.7753 & 280.2 & . & 600.0 & 600.0 \\
\hline 04 & 12522 & 22.7107 & 0.6615 & 280.6 & 6 & 600.0 & 600.0 \\
\hline 04 & 12523 & 133.8085 & 1.0198 & 280.5 & 0 & 600.0 & 600.0 \\
\hline
\end{tabular}




\begin{tabular}{|c|c|c|c|c|c|c|c|}
\hline- & 524 & & 4823 & & & & \\
\hline 4 & 1261 & 72 & 1.5229 & 30.6 & & & \\
\hline 4 & 1262 & 53.4042 & 1.0968 & 280.6 & & 00 . & \\
\hline & 263 & 147.3118 & .7320 & 280.7 & & 00.0 & \\
\hline & 264 & 200 & .2505 & 80.2 & & & \\
\hline & 1265 & 340 . & .3270 & 279.0 & & 0 & \\
\hline & 266 & 312.4127 & 2.2690 & 279.5 & & 00 & \\
\hline & 267 & 472 & .6688 & 280.5 & & & \\
\hline & 1268 & 347.7448 & .7545 & 79.7 & & & \\
\hline & 1269 & 128.8565 & 1.6790 & 281.5 & & 600.0 & . \\
\hline & 2610 & 98.1136 & 1.4968 & 282.4 & & & \\
\hline & 12611 & 172.0319 & 2.29 & 82.6 & & & \\
\hline & 12612 & 152.7130 & 3.74 & 282.5 & & .0 & \\
\hline & 2613 & 220.2885 & 2.8023 & 281.7 & & 0.0 & \\
\hline & 12614 & 171 . & 3 & 82.3 & & & \\
\hline & 12615 & 297 . & 2 & 281.4 & & & \\
\hline & 12616 & 296.5000 & 2.3020 & 281.4 & & 0.0 & 00 \\
\hline & 12617 & 304.4612 & 7 & 1.1 & & .0 & \\
\hline & 12618 & 111.6827 & 0 . & 281.2 & & & \\
\hline & 12619 & 328.8722 & 158 & 280.7 & & .0 & \\
\hline 4 & 12620 & 106.1987 & 1.3743 & 281.2 & & .0 & 000 \\
\hline & 12621 & 319.1469 & 12 & 281.2 & & & \\
\hline & 12622 & 13.0334 & 0 & 281.4 & 5 & & \\
\hline & 12623 & 318.2754 & 1.6053 & 281.7 & 6 & .0 & 000 \\
\hline & 12624 & 177.5111 & & & & & \\
\hline 4 & 1271 & 280.5845 & 1.3 & 28 & 5 & & \\
\hline & 1272 & 10.2168 & 2.6160 & 282.9 & 5 & .0 & 0 \\
\hline & 1273 & 17.7707 & 3 & 4 & 4 & .0 & \\
\hline & 1274 & 18.8986 & 3. & 28 & 4 & .0 & \\
\hline & 1275 & 349.0389 & 2.28 & & 4 & .0 & 00 \\
\hline 4 & 1276 & 322.2709 & 2.5130 & 283.2 & 4 & .0 & \\
\hline & 1277 & 334.0849 & 2.84 & 282.8 & 4 & & \\
\hline & 1278 & 340.8222 & 3.39 & 28 & 4 & .0 & \\
\hline 4 & 1279 & 333.7502 & 3.8028 & 282.6 & 4 & .0 & \\
\hline 1 & 12710 & 334.7751 & 4.1448 & 28 & 4 & & \\
\hline 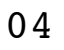 & 12711 & 328.2433 & 2.11 & & 4 & .0 & \\
\hline 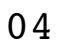 & 12712 & 223.3611 & 1.9183 & 284.6 & 3 & .0 & \\
\hline & 12713 & 295.1853 & 1 . & 28 & 3 & .0 & 6 \\
\hline 7 & 12714 & 241.5072 & 2.2660 & 284.9 & 2 & .0 & 60 \\
\hline 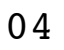 & 12715 & 85.5430 & 1645 & 286.2 & 3 & .0 & \\
\hline 4 & 12716 & 99.9198 & 745 & 285 & 3 & & \\
\hline 04 & 12717 & 76.2325 & 4.5788 & 284.1 & 4 & .0 & \\
\hline 04 & 12718 & 78.2500 & 5.1438 & 283.3 & 4 & .0 & 0 \\
\hline-2 & 12719 & 90.8296 & 6.1258 & 282.6 & 4 & .0 & \\
\hline 04 & 12720 & 91.0254 & 6.3265 & 282.2 & 4 & .0 & \\
\hline 04 & 12721 & 73.5505 & 6.7248 & 282.1 & 4 & .0 & 600 \\
\hline & 12722 & 84 & & & 4 & & \\
\hline & 12723 & 125.3325 & 4.8870 & 281.2 & & .0 & 600 \\
\hline
\end{tabular}




\begin{tabular}{|c|c|c|c|c|c|c|c|}
\hline$x_{1}$ & 2724 & & 7.8538 & 0.1 & & & \\
\hline 4 & 1281 & 149. & 7.1020 & 9.7 & 4 & & \\
\hline & 1282 & 9570 & 4190 & 279.6 & 4 & 00 & \\
\hline & 283 & 117.7883 & 5318 & 279.9 & & 00 & \\
\hline & 1284 & 129.37 & .0163 & 80.0 & & 0 & \\
\hline & 1285 & 151.3239 & .3953 & 279.3 & 4 & 00 & \\
\hline & 1286 & 151.2000 & 1940 & 279.0 & & 0 & \\
\hline & 1287 & 42.3749 & .6580 & 79.2 & & & \\
\hline & 1288 & 126.1613 & .0160 & 279.6 & 4 & 0 & \\
\hline & 1289 & 135.1999 & 2308 & 280.2 & & 00 & \\
\hline & 2810 & 50.9873 & 3.7133 & 280.4 & 4 & 0 & \\
\hline & 2811 & 3.5249 & 2.9038 & 81.0 & 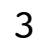 & & \\
\hline & 12812 & 33.1968 & 3.8288 & 282.2 & 3 & 0 & \\
\hline & 12813 & 57.6757 & 5.0645 & 283.1 & 3 & 00.0 & se \\
\hline & 12814 & 712 & & & & & \\
\hline & 12815 & 150.2680 & 978 & 284.4 & 3 & 0 & \\
\hline & 2816 & 122.0459 & 1330 & 284.6 & 3 & 00.0 & \\
\hline & 12817 & 4.5678 & 5 & 3.4 & 4 & & \\
\hline & 12818 & 104.5127 & 605 & 282.9 & 4 & 0 & \\
\hline & 12819 & 110.0113 & 1540 & 283.3 & 4 & 0 & \\
\hline & 12820 & 0 . & 5 & 3.2 & 4 & & \\
\hline & 12821 & 93. & 50 & 282.8 & 4 & & \\
\hline 4 & 12822 & 81 . & 45 & 282.4 & 4 & 0 & \\
\hline 4 & 12823 & 86.80 & 6.2955 & 282.3 & 4 & 0 & \\
\hline 4 & 12824 & 113.26 & 98 & 281.3 & 4 & & \\
\hline & 1291 & 10.0434 & 575 & 28 & 5 & & \\
\hline & 1292 & 346.6668 & 1.1625 & 279.7 & 6 & 0 & \\
\hline 4 & 1293 & 54.27 & 1.1578 & 279.6 & 6 & 0 & C \\
\hline 4 & 1294 & 34.65 & 1.3150 & 9.2 & 6 & 0 & 0 . \\
\hline & 1295 & 25.2609 & 1.8288 & 278.8 & 5 & 0 & \\
\hline & 1296 & 40.2560 & 995 & .7 & 5 & & \\
\hline 4 & 1297 & 357.6620 & 473 & .4 & 5 & & 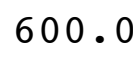 \\
\hline & 1298 & 335.4827 & 1.5545 & 277.9 & 4 & 0 & U • \\
\hline 4 & 1299 & 349 . & 713 & & 4 & 0 & \\
\hline & 12910 & 358 . & 418 & & 3 & & \\
\hline & 12911 & 254.8043 & 1.4913 & 280.5 & 2 & & 0 . \\
\hline & 12912 & 237.1894 & 583 & 281.4 & 2 & 0 & \\
\hline 4 & 12913 & 253.7188 & 530 & 282.4 & 2 & 0 & • \\
\hline 4 & 12914 & 236.6714 & 2.0145 & 282.5 & 2 & 600.0 & 0 . \\
\hline & 12915 & 244.93 & 620 & $2 \xi$ & 3 & 6 & \\
\hline- & 12916 & 187.1025 & 735 & & 3 & & \\
\hline 04 & 12917 & 147.3253 & 1.9570 & 282.7 & 4 & 600.0 & 600 . \\
\hline & 12918 & 75.4285 & 0.8773 & 282.5 & 5 & 600 & 000 \\
\hline . & 12919 & 321.8100 & 1.5305 & & 6 & 600 & 600 \\
\hline 04 & 12920 & 4.5882 & 1.0205 & 281.4 & 6 & 600.0 & 600 . \\
\hline & 12921 & 91.9025 & 3.3390 & 281.4 & 5 & 600.0 & 600. \\
\hline & 12922 & 32 & & & 4 & 60 & 60 \\
\hline & 12923 & 58.2993 & 3.1998 & 281.0 & $\mathbf{4}$ & 600.0 & 600 \\
\hline
\end{tabular}




\begin{tabular}{|c|c|c|c|c|c|c|c|}
\hline 04 & 12924 & 35.4331 & 2.9358 & 281.0 & 4 & 600.0 & 00.0 \\
\hline 04 & 1301 & 58.2285 & 3.0545 & 280.7 & 4 & 600.0 & 600.0 \\
\hline 04 & 1302 & 11.9720 & 2.9085 & 280.2 & 4 & 600.0 & 00.0 \\
\hline 04 & 1303 & 130.5396 & 1.7833 & 280.6 & 5 & 600.0 & 600.0 \\
\hline 04 & 1304 & 122.2147 & 1.0780 & 280.9 & 0 & 600.0 & 00.0 \\
\hline 04 & 1305 & 78.5863 & 1.9960 & 280.7 & 5 & 600.0 & 00.0 \\
\hline 04 & 1306 & 94.9746 & 7.6438 & 280.9 & 4 & 600.0 & 600.0 \\
\hline 04 & 1307 & 26.8430 & 5.0538 & 280.9 & 4 & 600.0 & 00.0 \\
\hline 04 & 1308 & 338.3386 & 2.0513 & 280.0 & 5 & 600.0 & 00.0 \\
\hline 04 & 1309 & 206.5775 & 1.2023 & 280.8 & 4 & 600.0 & 600.0 \\
\hline 04 & 13010 & 99.7683 & 3.9450 & 281.7 & 4 & 600.0 & .0 \\
\hline 04 & 13011 & 49.9108 & 385 & 282.3 & 4 & 600.0 & \\
\hline 04 & 13012 & 58.8895 & 4.6320 & 283.3 & 4 & 600.0 & 600.0 \\
\hline 04 & 13013 & 71.1008 & 6.5935 & 284.0 & 4 & 600.0 & 600.0 \\
\hline 04 & 13014 & 87.9047 & 63 & 283.8 & 4 & 600.0 & \\
\hline 04 & 13015 & 86.6272 & 6 . & 283.4 & 4 & 600.0 & 60 \\
\hline 04 & 13016 & 72.1216 & 6.2485 & 282.8 & 4 & 600.0 & 600.0 \\
\hline 04 & 13017 & 70.7202 & 6.6205 & 281.9 & 4 & 600.0 & .0 \\
\hline 04 & 13018 & 65.5512 & 6.2298 & 281.4 & 4 & 600.0 & 60 \\
\hline 04 & 13019 & 75.7998 & 8.8100 & 281.3 & 4 & 600.0 & 600 \\
\hline 04 & 13020 & 80.6257 & 7.9600 & 281.1 & 4 & 600.0 & .0 \\
\hline 04 & 13021 & 80.5001 & 8.1 & 280.9 & 4 & 600.0 & \\
\hline 04 & 13022 & 82.7250 & 9.1 & 280.5 & 4 & 600.0 & 600 \\
\hline 04 & 13023 & 81.1749 & 8.7850 & 280.1 & 4 & 600.0 & .0 \\
\hline 04 & 13024 & 80.8501 & 8 . & 279.5 & 4 & 600.0 & \\
\hline 04 & 1311 & 81.8500 & 8 . & 279.2 & 4 & 600.0 & 60 \\
\hline 04 & 1312 & 88.9203 & 7.9550 & 279.2 & 4 & 600.0 & 60 \\
\hline 04 & 1313 & 128.1046 & 7.8 & 280.0 & 4 & 600.0 & \\
\hline 04 & 1314 & 143.8783 & 8.8825 & 279.5 & 4 & 600.0 & 60 \\
\hline 04 & 1315 & 124.5552 & 8.0675 & 279.4 & 4 & 600.0 & 600.0 \\
\hline 04 & 1316 & 132.9275 & 9 . & 279.7 & 4 & 600.0 & \\
\hline 04 & 1317 & 142.2999 & 11.2900 & 279.4 & 4 & 600.0 & 60 \\
\hline 04 & 1318 & 140.5009 & 10.7425 & 279.2 & 4 & 600.0 & 600.0 \\
\hline 04 & 1319 & 137.6250 & 10.2600 & 279.5 & 4 & 600.0 & .0 \\
\hline 04 & 13110 & 130.3737 & 75 & 280.2 & 4 & 600.0 & .0 \\
\hline 04 & 13111 & 135.2752 & 8.7450 & 281.1 & 4 & 600.0 & 600.0 \\
\hline 04 & 13112 & 146.0252 & 8.4200 & 281.9 & 4 & 600.0 & \\
\hline 04 & 13113 & 161.6511 & 8.9675 & 282.1 & 4 & 600.0 & 600.0 \\
\hline 04 & 13114 & 158.8000 & 7.7750 & 282.6 & 4 & 600.0 & 600.0 \\
\hline 04 & 13115 & 161.6687 & 6.2043 & 282.9 & 4 & 600.0 & \\
\hline 04 & 13116 & 156.4892 & 4.1955 & 283.1 & 3 & 600.0 & 600.0 \\
\hline 04 & 13117 & 98.7660 & 5.7913 & 282.9 & 3 & 600.0 & 600.0 \\
\hline 04 & 13118 & 69.6479 & 6.6223 & 281.7 & 4 & 600.0 & 600.0 \\
\hline 04 & 13119 & 68.6247 & 7.6000 & 281.1 & 4 & 600.0 & 600.0 \\
\hline 04 & 13120 & 75.7000 & 7.1863 & 281.0 & 4 & 600.0 & 600.0 \\
\hline 04 & 13121 & 71.7248 & 7.6375 & 281.1 & 4 & 600.0 & 600.0 \\
\hline 04 & 13122 & 72.4750 & 7 . & 28 & 4 & 600.0 & 600.0 \\
\hline 04 & 13123 & 73.0502 & 7.0718 & 280.8 & 4 & 600.0 & 600.0 \\
\hline
\end{tabular}




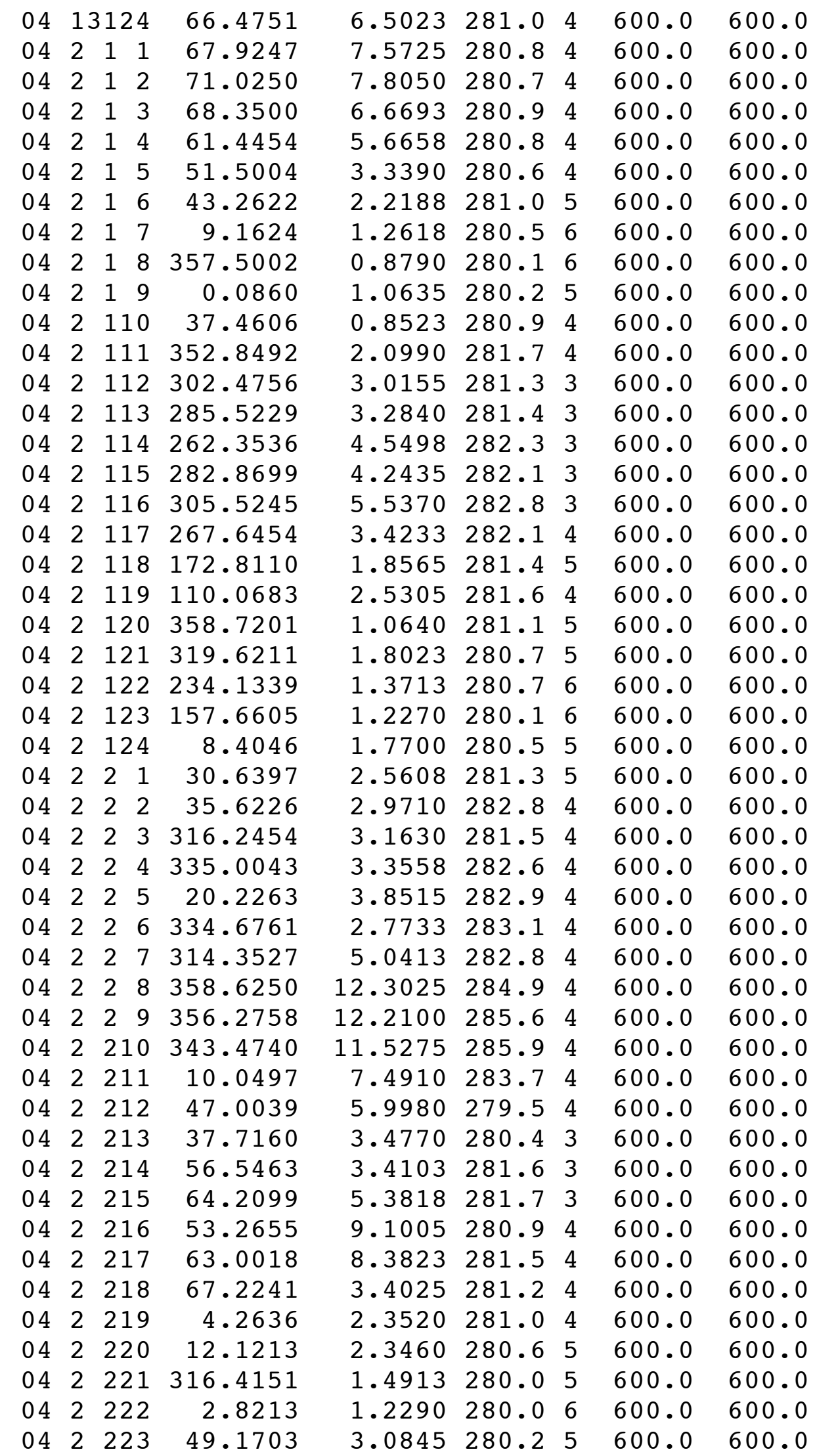




\begin{tabular}{|c|c|c|c|c|c|c|c|c|}
\hline 04 & 2 & 224 & 64.6750 & 3.5268 & 280.4 & 4 & 600.0 & 600.0 \\
\hline 04 & 2 & 31 & 39.0415 & 1.9013 & 280.0 & 5 & 600.0 & 600.0 \\
\hline 04 & 2 & 32 & 3.3551 & 1.4370 & 279.5 & 6 & 600.0 & 00.0 \\
\hline 04 & 2 & 33 & 3.8348 & 1.8015 & 279.5 & 5 & 600.0 & 500.0 \\
\hline 04 & 2 & 34 & 67.4690 & 1.2650 & 279.8 & 6 & 600.0 & 00.0 \\
\hline 04 & 2 & 35 & 20.0638 & 1.7400 & 279.3 & 5 & 600.0 & 00.0 \\
\hline 04 & 2 & 36 & 48.6266 & 3.2805 & 279.8 & 4 & 600.0 & 00.0 \\
\hline 04 & 2 & 37 & 60.2244 & 5.3768 & 280.4 & 4 & 600.0 & 00.0 \\
\hline 04 & 2 & 38 & 52.7435 & 2.9935 & 280.5 & 4 & 600.0 & 500.0 \\
\hline 04 & 2 & 39 & 52.7470 & 4.8523 & 280.7 & 4 & 600.0 & 00.0 \\
\hline 04 & 2 & 310 & 64.1830 & 5.2370 & 281.1 & 4 & 600.0 & 00.0 \\
\hline 04 & 2 & 311 & 93.9010 & 5 & 281.4 & 4 & 600.0 & 00.0 \\
\hline 04 & 2 & 312 & 90.1072 & 4.8233 & 281.7 & 3 & 600.0 & 00.0 \\
\hline 04 & 2 & 313 & 93.6278 & 3.8848 & 282.0 & 3 & 600.0 & 00.0 \\
\hline 04 & 2 & 314 & 82.7612 & 188 & 282.2 & 4 & .0 & \\
\hline 04 & 2 & 315 & 77.7425 & 4.7223 & 282.1 & 3 & 600.0 & 00.0 \\
\hline 04 & 2 & 316 & 73.4991 & 4.2818 & 282.1 & 3 & 600.0 & 600.0 \\
\hline 04 & 2 & 317 & 64.0505 & 4.7455 & 282.3 & 4 & 600.0 & \\
\hline 04 & 2 & 318 & 66.6985 & 5.1943 & 281.8 & 4 & 600.0 & 600.0 \\
\hline 04 & 2 & 319 & 76.2504 & 5.7363 & 281.8 & 4 & 600.0 & 600.0 \\
\hline 04 & 2 & 320 & 89.1308 & 4.6755 & 281.6 & 4 & .0 & \\
\hline 04 & 2 & 321 & 144.4372 & 2.5770 & 281.0 & 4 & 600.0 & .0 \\
\hline 04 & 2 & 322 & 134.7026 & 5.1923 & 281.5 & 4 & 600.0 & 600.0 \\
\hline 04 & 2 & 323 & 160.7000 & 4.1433 & 280.8 & 4 & 600.0 & .0 \\
\hline 04 & 2 & 324 & 157.0500 & 4 & 280.7 & 4 & .0 & \\
\hline 04 & 2 & 41 & 154.7006 & 4.8173 & 280.7 & 4 & 600.0 & 600.0 \\
\hline 04 & 2 & 42 & 156.9504 & 5.1085 & 280.5 & 4 & 600.0 & 600.0 \\
\hline 04 & 2 & 43 & 161.6225 & 5.4375 & 280.2 & 4 & 600.0 & .0 \\
\hline 04 & 2 & 44 & 161.1969 & 6.1808 & 279.7 & 4 & 600.0 & 600.0 \\
\hline 04 & 2 & 45 & 157.4749 & 6.6500 & 279.4 & 4 & 600.0 & 600.0 \\
\hline 04 & 2 & 46 & 159.2255 & 6 & 279.0 & 4 & 600.0 & .0 \\
\hline 04 & 2 & 47 & 158.4493 & 6.1053 & 278.6 & 4 & 600.0 & 600.0 \\
\hline 04 & 2 & 48 & 151.6011 & 5.9060 & 278.5 & 4 & 600.0 & 600.0 \\
\hline 04 & 2 & 49 & 148.9501 & 5.8280 & 278.9 & 4 & 600.0 & 0.0 \\
\hline 04 & 2 & 410 & 154.6000 & 5.0550 & 279.7 & 3 & 600.0 & 600.0 \\
\hline 04 & 2 & 411 & 146.9248 & 4.5560 & 280.6 & 3 & 600.0 & 600.0 \\
\hline 04 & 2 & 412 & 149.8250 & 108 & 281.1 & 4 & 600.0 & 600.0 \\
\hline 04 & 2 & 413 & 150.7900 & 5.2213 & 281.4 & 3 & 600.0 & 600.0 \\
\hline 04 & 2 & 414 & 149.7329 & 5.9435 & 282.1 & 4 & 600.0 & 600.0 \\
\hline 04 & 2 & 415 & 153.2233 & 6.5748 & .5 & 4 & 600.0 & 600.0 \\
\hline 04 & 2 & 416 & 146.5290 & 6.4198 & 283.0 & 4 & 600.0 & 600.0 \\
\hline 04 & 2 & 417 & 122.8426 & 5.4398 & 283.1 & 4 & 600.0 & 600.0 \\
\hline 04 & 2 & 418 & 124.8444 & 5.5278 & 282.9 & 4 & 600.0 & 600.0 \\
\hline 04 & 2 & 419 & 127.3576 & 6.2975 & 283.0 & 4 & 600.0 & 600.0 \\
\hline 04 & 2 & 420 & 109.6384 & 6.7013 & 282.7 & 4 & 600.0 & 600.0 \\
\hline 04 & 2 & 421 & 130.9401 & 7.3898 & 283.2 & 4 & 600.0 & 600.0 \\
\hline 04 & 2 & 422 & 125.4749 & 7.1628 & 283.4 & 4 & 600.0 & 600.0 \\
\hline 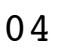 & 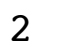 & 423 & 127.3646 & 6.9818 & 283.5 & 4 & 600.0 & 600.0 \\
\hline
\end{tabular}




\begin{tabular}{|c|c|c|c|c|c|c|c|}
\hline 4 & 24 & & 3 & 3.6 & & & \\
\hline 4 & 1 & 130. & 5.8108 & 3.5 & & 600.0 & \\
\hline 2 & 52 & 132.0484 & 4.8205 & 283.1 & 4 & 00 & \\
\hline & 53 & 118.0760 & 6648 & 282.9 & & 00 & \\
\hline 2 & 4 & 130.5090 & 365 & 82.4 & & 0 & \\
\hline 2 & 55 & 132.3471 & 2023 & 81.8 & & 0( & \\
\hline & 56 & 9274 & 7503 & 282.3 & & 00 & 0 \\
\hline 2 & 7 & 144.5452 & 6353 & 81.3 & & 0 & \\
\hline 2 & 8 & 30.3747 & 5918 & 81.6 & 4 & & \\
\hline & 59 & 40.4447 & 950 & 81.7 & & 500 & 00 \\
\hline$z$ & 510 & 154.0836 & 4.3485 & 282.1 & & 0 & \\
\hline 2 & 11 & 159.3739 & 4.6288 & 82.6 & & .0 & \\
\hline & 12 & 159.9495 & 5.4063 & 283.6 & 3 & 00.0 & 0 \\
\hline 4 & 13 & 54.5001 & 7.7925 & 283.7 & 4 & 00.0 & . \\
\hline 2 & 14 & 846 & & 3.9 & 4 & & \\
\hline 2 & 15 & 143 & & 284.5 & 3 & & \\
\hline 4 & 16 & 122.3422 & 610 & 284.8 & 3 & .0 & . \\
\hline 2 & 17 & 102.7638 & 0 & 4.7 & 4 & 0 & \\
\hline 2 & 18 & 106 . & & 284.2 & 4 & & \\
\hline 4 & 19 & 121.4502 & 33 & 283.9 & 4 & 0 & 0 \\
\hline 2 & 520 & 75.3011 & 1.6998 & 284.5 & 5 & 0 & 00 \\
\hline 2 & 521 & 37.4374 & & 284.2 & 5 & & \\
\hline & 22 & 31.4534 & 45 & 284.7 & 6 & & \\
\hline 4 & 523 & 8.8237 & 5378 & 283.7 & 6 & 0 & 00 • \\
\hline 2 & 524 & 6.2503 & & & 5 & & \\
\hline 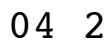 & 61 & 57.4391 & 0. & .5 & 6 & & \\
\hline 4 & 62 & 141.8249 & 890 & 283.2 & 5 & 0 & U. \\
\hline 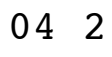 & 63 & 51.3386 & 1 . & 282.6 & 5 & & \\
\hline 2 & 64 & 204 & 0 . & 283.5 & 6 & & $\bullet$ \\
\hline 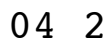 & 65 & 103.3603 & 1.1803 & 282.6 & 6 & 0 & 0 . \\
\hline 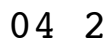 & 66 & 299.0474 & 1.8458 & 281.8 & 5 & 0 & 0 . \\
\hline 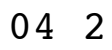 & 67 & 349.5162 & 2 . & 281.2 & 4 & & \\
\hline 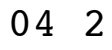 & 68 & 132.1426 & & 281.4 & 4 & & 0 • \\
\hline 42 & 69 & 151.2751 & 145 & 282.1 & 3 & 0 & \\
\hline 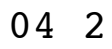 & 610 & 322 . & 5 & 283.9 & 2 & & 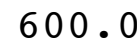 \\
\hline 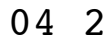 & 611 & 243.4842 & 2 . & 284.6 & 2 & & 0 . \\
\hline 42 & 612 & 262.7052 & 2.4263 & 284.8 & 2 & 0 & 0 . \\
\hline- & 613 & 273.5176 & 3 . & 28 & 3 & & ( \\
\hline 2 & 614 & 238.9728 & 2.7883 & 285.5 & 3 & .0 & 0 . \\
\hline $4 \quad 2$ & 615 & 228.3024 & 2.8448 & 285.7 & 3 & 0 & 0 . \\
\hline 2 & 616 & 267.2607 & 305 & 285 & 2 & & 600. \\
\hline 4 & 617 & 13.0322 & 2.1180 & 285.5 & 3 & .0 & 0 . \\
\hline 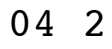 & 618 & 80.8736 & 2.0878 & 284.8 & 4 & 600 & 00 . \\
\hline 4 & 619 & 103.5778 & 1.3300 & & 5 & & 0 \\
\hline 04 & 620 & 88.0006 & 6.5225 & & 4 & & 600. \\
\hline 2 & 621 & 89.6914 & 5.5450 & 282.9 & 4 & 600.0 & 600 \\
\hline 2 & 622 & 94 & & & 4 & & \\
\hline & 623 & 80.8251 & 8.7000 & 281.3 & $\Psi$ & 600.0 & 600 \\
\hline
\end{tabular}




\begin{tabular}{|c|c|c|c|c|c|c|c|c|}
\hline \pm & 2 & 624 & 79.3008 & 10.0150 & 280.6 & 4 & 600.0 & 600.0 \\
\hline 04 & 2 & 71 & 83.4752 & 9.3750 & 280.5 & 4 & 600.0 & 00.0 \\
\hline 04 & 2 & 72 & 91.8998 & 7.4100 & 280.1 & 4 & 600.0 & 00.0 \\
\hline 04 & 2 & 73 & 89.5538 & 5.9100 & 279.5 & 4 & 600.0 & 00.0 \\
\hline 04 & 2 & 74 & 106.0934 & 5.5145 & 279.7 & 4 & 600.0 & 00.0 \\
\hline 04 & 2 & 75 & 34.2744 & 5.2003 & 280.0 & 4 & 600.0 & 00.0 \\
\hline 04 & 2 & 76 & 144.3793 & 4.6580 & 279.5 & 4 & 600.0 & 00.0 \\
\hline 04 & 2 & 77 & 143.5061 & 4.4760 & 279.6 & 4 & 600.0 & 00.0 \\
\hline 04 & 2 & 78 & 142.4073 & 3.8820 & 279.5 & 4 & 600.0 & 00.0 \\
\hline 04 & 2 & 79 & 128.7410 & 53 & 280.6 & 3 & 600.0 & .0 \\
\hline 04 & 2 & 710 & 158.3570 & 5.3828 & 281.2 & 3 & 600.0 & \\
\hline 04 & 2 & 711 & 151.7245 & 5.7518 & 281.7 & 4 & 600.0 & \\
\hline 04 & 2 & 712 & 144.9500 & 7.7233 & 282.6 & 4 & 600.0 & 00.0 \\
\hline 04 & 2 & 713 & 151.9750 & 7.5475 & 283.4 & 4 & .0 & 00.0 \\
\hline 04 & 2 & 714 & 154.5997 & 7. & 283.9 & 4 & .0 & \\
\hline 04 & 2 & 715 & 154.6503 & 7.7225 & 284.2 & 4 & 600.0 & 00.0 \\
\hline 04 & 2 & 716 & 156.1244 & 7.2428 & 284.1 & 4 & 60 & 600.0 \\
\hline 04 & 2 & 717 & 140.5498 & 5.6 & 283.5 & 4 & .0 & \\
\hline 04 & 2 & 718 & 132.6296 & 5.6980 & 283.4 & 4 & .0 & .0 \\
\hline 04 & 2 & 719 & 113.3232 & 5.7280 & 282.8 & 4 & 60 & .0 \\
\hline 04 & 2 & 720 & 129.8075 & 6.0 & 282.4 & 4 & 6 & \\
\hline 04 & 2 & 721 & 144.3423 & 4.9 & 281.3 & 4 & .0 & \\
\hline 04 & 2 & 722 & 118.8556 & 3.6853 & 281.3 & 4 & 600.0 & 00.0 \\
\hline 04 & 2 & 723 & 131.4248 & 5. & 281.5 & 4 & .0 & \\
\hline 04 & 2 & 724 & 140.5241 & 7.3 & 282.0 & 4 & .0 & \\
\hline 04 & 2 & 81 & 152.1238 & 8.4425 & 281.6 & 4 & 600.0 & 600.0 \\
\hline 04 & 2 & 82 & 154.4261 & 6.2853 & 280.5 & 4 & 600.0 & 600.0 \\
\hline 04 & 2 & 83 & 149.9250 & 7.1603 & 280.1 & 4 & .0 & \\
\hline 04 & 2 & 84 & 148.8996 & 7.5200 & 279.8 & 4 & .0 & 600.0 \\
\hline 04 & 2 & 85 & 157.4501 & 10.1475 & 279.6 & 4 & 60 & .0 \\
\hline 04 & 2 & 86 & 164.5016 & 10.56 & 278.5 & 4 & .0 & .0 \\
\hline 04 & 2 & 87 & 152.6250 & 9.2675 & 278.3 & 4 & 600.0 & 600.0 \\
\hline 04 & 2 & 88 & 143.7758 & 8.5425 & 278.0 & 4 & 600.0 & 600.0 \\
\hline 04 & 2 & 89 & 150.3795 & 11.4 & 278.5 & 4 & 0 & 0.0 \\
\hline 04 & 2 & 810 & 149.6500 & 10.01 & 279.3 & 4 & 600.0 & 600.0 \\
\hline 04 & 2 & 811 & 153.9016 & 7.3608 & 280.2 & 4 & 600.0 & 600.0 \\
\hline 04 & 2 & 812 & 147.5999 & 6 . & 281.4 & 4 & .0 & 0.0 \\
\hline 04 & 2 & 813 & 152.0004 & 7.3083 & 282.1 & 4 & 600.0 & 600.0 \\
\hline 04 & 2 & 814 & 154.7412 & 8.8925 & 282.4 & 4 & 600.0 & 600.0 \\
\hline 04 & 2 & 815 & 162.6737 & 7.55 & 282.6 & 4 & 600.0 & .0 \\
\hline 04 & 2 & 816 & 165.3510 & 7.3065 & 282.6 & 4 & 600.0 & 600.0 \\
\hline 04 & 2 & 817 & 150.3253 & 3.3678 & 282.4 & 3 & 600.0 & 600.0 \\
\hline 04 & 2 & 818 & 106.5679 & 2.0558 & 282.0 & 4 & 600.0 & 600.0 \\
\hline 04 & 2 & 819 & 88.3251 & 2.5608 & 281.6 & 4 & 600.0 & 600.0 \\
\hline 04 & 2 & 820 & 74.2421 & 3.5820 & 282.5 & 4 & 600.0 & 600.0 \\
\hline 04 & 2 & 821 & 105.5638 & 3.1058 & 282.3 & . & 600.0 & 600.0 \\
\hline $0<$ & 2 & 822 & 119.8746 & 3.3188 & 282.4 & 4 & 600.0 & 600.0 \\
\hline & 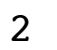 & 823 & 138.2511 & 4.250 & 282.0 & & 600.0 & 600.0 \\
\hline
\end{tabular}




\begin{tabular}{|c|c|c|c|c|c|c|c|}
\hline & 24 & & & & & & \\
\hline & $\begin{array}{lll}2 & 9 & 1\end{array}$ & 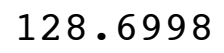 & .8635 & 31.9 & & & \\
\hline & 92 & 142.0255 & 9.7625 & 280.6 & & 00 & \\
\hline & $\begin{array}{lll}2 & 9 & 3\end{array}$ & 145.3253 & 0.5600 & 279.4 & & 00 & \\
\hline & 94 & & 8.1525 & 79.3 & & & \\
\hline & 295 & 90 & 5150 & 79.8 & & 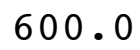 & \\
\hline & 296 & 5000 & 540 & 278.7 & & 00 & \\
\hline & $\begin{array}{lll}2 & 9 & 7\end{array}$ & 42.2605 & & 78.4 & & & \\
\hline & 298 & 0.6036 & & 78.0 & & & \\
\hline & $\begin{array}{lll}2 & 9 & 9\end{array}$ & 148.0015 & 700 & 78.2 & & 0 & 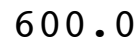 \\
\hline & 2910 & 6247 & & 279.1 & & & \\
\hline & 2911 & 54.7504 & 8 . & 80.3 & & & \\
\hline & 2912 & 2499 & 50 & 281.8 & & 0 & \\
\hline & 2913 & 58.7250 & 9.75 & 282.8 & & & \\
\hline & 2914 & 500 & & 8 & & & \\
\hline & 2915 & & & 284.3 & 4 & & \\
\hline & 2916 & 170.4967 & & 284.6 & 4 & & \\
\hline & 2917 & 206 & & 84.3 & & & \\
\hline & 2918 & 730 & & 283.8 & & & \\
\hline & 2919 & 561 & & 284.2 & 4 & & \\
\hline & 2920 & 139.8926 & 0 & 284.3 & 4 & 0 & \\
\hline & 2921 & 44.2132 & 5 & 84.8 & 4 & & \\
\hline & 2922 & 7000 & 0 & 28 & 4 & & \\
\hline & 2923 & 1.4001 & 0 & 282.6 & 4 & & \\
\hline & 2924 & 0 & & & 4 & & \\
\hline & 2101 & 000 & 0 & 28 & 4 & & \\
\hline & 2102 & 150.8000 & 0 & 280.3 & 4 & & . \\
\hline & 2103 & 139.9074 & & 9 & 4 & & \\
\hline & 2104 & 134.6035 & 0 & 279 & 4 & & \\
\hline & 2105 & 31.7777 & 8 & 279.5 & 4 & & J. \\
\hline & 2106 & 138.1583 & 475 & 278.9 & 4 & & \\
\hline & 2107 & 135.5246 & 5 & 278.6 & 4 & & \\
\hline & 2108 & 637 & & 278 & 4 & & \\
\hline & 2109 & 135.2265 & 65 & 279.2 & 4 & & \\
\hline & 21010 & 712 & & & 3 & & \\
\hline & 21011 & 143.7739 & & 1.6 & 3 & & \\
\hline & 21012 & 154.6912 & 568 & 282.5 & 3 & & \\
\hline & 21013 & 158 . & 4. & & 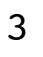 & & \\
\hline & 21014 & 159.8959 & & 283.6 & 3 & & 0 . \\
\hline & 21015 & 152.8741 & 3. & 284.6 & 3 & & \\
\hline & 21016 & 148.2749 & & & 3 & & \\
\hline & 21017 & 144.8499 & 3. & & 3 & & \\
\hline & 21018 & 121.7722 & 21013 & 283.4 & 4 & & 0 \\
\hline & 21019 & 113.7283 & & & & & \\
\hline & 21020 & 117.6131 & & & 3 & & \\
\hline & 21021 & 116.0075 & & 284.3 & 4 & & 00 . \\
\hline & 21 & & & & 4 & & \\
\hline & 1023 & 135.8526 & 2.2123 & 28 & & .0 & 60 \\
\hline
\end{tabular}




\begin{tabular}{|c|c|c|c|c|c|c|c|}
\hline- & 1024 & 0.1420 & 2680 & 6 & & & \\
\hline 4 & 2111 & 1 & 0.8743 & 3.9 & 5 & 600.0 & \\
\hline & 2112 & 30.3716 & 1.3685 & 284.1 & 6 & 00 & \\
\hline & 113 & 20.1728 & 8323 & 284.4 & & 00 & \\
\hline & 114 & 50.6432 & .8263 & & & 0 & \\
\hline & 115 & 122.5981 & .1080 & 83.7 & & 00 & \\
\hline & 116 & 7000 & 2.1710 & 282.8 & & 00 & \\
\hline & 117 & 138.4891 & .3428 & 82.5 & & 0 & \\
\hline & 118 & 128.7059 & .0960 & 82.5 & 4 & & \\
\hline & 119 & 152.2576 & 2.8430 & 280.3 & 3 & 00.0 & o \\
\hline & 1110 & 162.4127 & 2.3690 & 282.7 & & 0 & \\
\hline & 1111 & 153.7656 & 2.6068 & 83.6 & 3 & 0 & \\
\hline & 1112 & 210.3951 & 2.0373 & 284.9 & 2 & .0 & 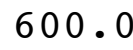 \\
\hline & 1113 & 232.8296 & 3.3090 & 285.0 & 3 & 0.0 & 00 \\
\hline & 1114 & 216 & 2.7 & & & & \\
\hline & 1115 & 211.1 & 1.42 & 285.6 & 2 & & \\
\hline & 1116 & 241.4284 & 2.0448 & 285.4 & 2 & 0.0 & . \\
\hline & 117 & 262.2186 & 1.7 & .1 & 3 & 0 & \\
\hline & 1118 & 345.1288 & 1.7 & 284.6 & 4 & & \\
\hline & 1119 & 353 & 2.0840 & 284.1 & 5 & 0 & 00 \\
\hline & 21120 & 8.6280 & 1.6585 & 284.1 & 6 & 0 & 00. \\
\hline & 21121 & 1.7135 & 1.3 & 284.2 & 6 & & \\
\hline & 1122 & 309.2137 & 1.1 & 284.0 & 6 & & \\
\hline & 21123 & 355.9010 & 1.7098 & .6 & 6 & 0 & 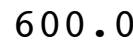 \\
\hline & 21124 & 342.2600 & & & 6 & & \\
\hline & 2121 & 277 . & 0 . & .8 & 6 & & \\
\hline & 2122 & 162.8886 & 1.7008 & 282.5 & 5 & 0 & U. \\
\hline & 2123 & 142.3232 & 1.7930 & 31.2 & 5 & & \\
\hline & 2124 & 16.6171 & 0.8675 & 281.7 & 6 & & • \\
\hline & 2125 & 3.59 & 0.6185 & 281.8 & 6 & 0 & 0 . \\
\hline & 2126 & 333.1445 & 0.8685 & 282.0 & 6 & 0 & 0 . \\
\hline & 2127 & 231.38 & 0.8740 & 281.5 & 6 & & \\
\hline & 2128 & 338 . & 0.7463 & 281.9 & 5 & & 0 • \\
\hline & 2129 & 253.957 & 1.1025 & 281.9 & 4 & 0 & . \\
\hline & 21210 & 263.46 & 1.6523 & 282.1 & 3 & & \\
\hline & 21211 & 265.1962 & 1.6375 & 283.1 & 2 & & 0 . \\
\hline & 21212 & 264.2563 & 2.8840 & 283.8 & 3 & $\cdot 0$ & t \\
\hline 4 & 21213 & 289.1788 & 3.3618 & 28 & 3 & & - \\
\hline 4 & 21214 & 272.7803 & 3.5563 & 286.1 & 3 & .0 & 0 . \\
\hline & 21215 & 247.9875 & 3.7090 & 287.6 & 3 & & 0 \\
\hline & 21216 & 221.9275 & 4.1165 & 287.3 & 3 & & 600. \\
\hline a & 21217 & 225.5625 & 3.1155 & 286.5 & 3 & .0 & 00 . \\
\hline & 21218 & 184.7106 & 1.8868 & 285.6 & 4 & .0 & 00 . \\
\hline 04 & 21219 & 204.9299 & 1.0145 & & 5 & & 0 \\
\hline 04 & 21220 & 16.0950 & 2.2703 & & 5 & 60 & 600. \\
\hline & 21221 & 14.7297 & 1.8455 & 286.4 & 5 & 600.0 & 600 \\
\hline & & 34 & & & 4 & & \\
\hline & & 305.6656 & 1.8920 & 286.0 & 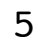 & .0 & 50 \\
\hline
\end{tabular}




\begin{tabular}{|c|c|c|c|c|c|c|c|}
\hline- & 224 & & 2.4175 & 6 & & & \\
\hline 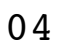 & 131 & 1 & 3.4043 & 5.1 & & 0 & \\
\hline 4 & 2132 & 1.5302 & 2.7960 & 284.8 & & 00 . & \\
\hline & 133 & 62.5987 & .4323 & 285.7 & & 00.0 & \\
\hline & 134 & 15.4294 & .3890 & & & & \\
\hline & 135 & 358.6479 & .2520 & 85.4 & & 00 & \\
\hline & 136 & 5.4453 & 2.6418 & 284.4 & & 00 & \\
\hline & 2137 & 14.8295 & 3.0330 & .1 & & .0 & \\
\hline & 2138 & 17.9957 & 2.3425 & 5 & 4 & & \\
\hline & 139 & 317.1312 & 3.6108 & 283.7 & 4 & 600.0 & 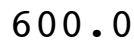 \\
\hline & 1310 & 307.5998 & 4.7363 & 285.0 & 1 & 00.0 & \\
\hline & 1311 & 300.1547 & 4.3425 & 3 & 4 & & \\
\hline & 1312 & 335.7492 & 5.39 & 287.4 & 3 & 00.0 & \\
\hline & 1313 & 322.8414 & 5.0318 & 288.8 & & 00.0 & \\
\hline & 21314 & 266.4136 & & & 3 & & \\
\hline & 21315 & 172.14 & & .6 & 4 & & \\
\hline & 1316 & 168.2584 & 4.1318 & 285.4 & 4 & 00.0 & 00 \\
\hline & 1317 & 343.9820 & 5 & .1 & 4 & .0 & \\
\hline & 21318 & 325.4647 & & 284.1 & 4 & & \\
\hline & 21319 & 234.2625 & 1 & 284 & 5 & .0 & \\
\hline 4 & 21320 & 257.8093 & 1.2228 & 284.1 & 6 & $\cdot 0$ & 000 \\
\hline & 213 & 297.5883 & 1.5583 & & 6 & & \\
\hline & 21322 & 346.9338 & 1 & 28 & 6 & .0 & \\
\hline & 21323 & 6.3775 & 1.9148 & 28 & 5 & .0 & 000 \\
\hline & 21324 & 327.1602 & & & 3 & & \\
\hline & 2141 & 136.2613 & 0 . & 28 & 6 & & \\
\hline & 2142 & 97.8296 & 1.5808 & .3 & 6 & 0.0 & 600 \\
\hline & 2143 & 150.2295 & 4 & 7 & 5 & .0 & \\
\hline & 2144 & 142.3489 & 8 & 28 & 4 & .0 & \\
\hline & 2145 & 143.0995 & 510 & 0.2 & 4 & .0 & 60 \\
\hline & 2146 & 143.1499 & 4.1858 & 280.1 & 4 & .0 & 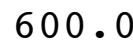 \\
\hline & 2147 & 90.1447 & 5 & 27 & 5 & & \\
\hline & 2148 & 136.3198 & 0 & 280.8 & 4 & .0 & \\
\hline 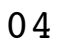 & 2149 & 125.3798 & 3 & 282.8 & 4 & .0 & \\
\hline & 21410 & 161.2000 & & & 3 & & 0 \\
\hline 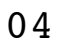 & 214 & 206.7369 & 2 & .4 & 2 & .0 & \\
\hline & 21412 & 218.4185 & 3.4088 & 285.7 & 2 & .0 & \\
\hline & 21413 & 217.5281 & 3 . & & 3 & .0 & 6 \\
\hline 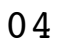 & 21414 & 211.0809 & 2.04 & .0 & 2 & .0 & 60 \\
\hline 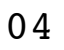 & 21415 & 238.6622 & 2.20 & 286.3 & 2 & .0 & \\
\hline 1 & 21416 & 229.7598 & 2 . & 28 & 3 & & 6 \\
\hline 04 & 21417 & 253.7068 & 1.5175 & .6 & 4 & .0 & 100 \\
\hline 04 & 21418 & 354.3573 & 1.6853 & 285.3 & 5 & .0 & 00 \\
\hline 04 & 21419 & 25.3044 & 2 . & & 5 & .0 & \\
\hline 04 & 21420 & 60.0871 & 2.4455 & & 5 & 600.0 & $r_{0}$ \\
\hline 04 & 21421 & 20.4237 & 1.9300 & 284.8 & 5 & 600.0 & 600 \\
\hline & & 61 & & & 5 & & \\
\hline & 21423 & 106.2020 & 3.1265 & 284.8 & & .0 & 600 \\
\hline
\end{tabular}




\begin{tabular}{|c|c|c|c|c|c|c|c|}
\hline 04 & 21424 & 98.6541 & 3.0270 & 284.1 & 4 & 600.0 & 00.0 \\
\hline 4 & 2151 & 60.3546 & 2.4488 & 284.3 & 5 & 600.0 & 600.0 \\
\hline 04 & 2152 & 4.7031 & 1.7835 & 283.4 & 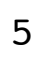 & 600.0 & 00.0 \\
\hline 04 & 2153 & 348.8758 & 1.5108 & 283.5 & 6 & 600.0 & 00.0 \\
\hline 04 & 2154 & 348.3845 & 2.3523 & 282.9 & & 600.0 & 00.0 \\
\hline 04 & 2155 & 342.7123 & 2.1333 & 283.3 & 3 & 600.0 & 00.0 \\
\hline 04 & 2156 & 338.7392 & 2.0628 & 283.1 & 5 & 600.0 & 600.0 \\
\hline 04 & 2157 & 5.7747 & 2.3105 & 283.5 & 4 & 600.0 & 000.0 \\
\hline 04 & 2158 & 12.1387 & 1.9328 & 284.4 & 4 & 600.0 & \\
\hline 04 & 2159 & 53.1652 & 1.3185 & 286.6 & 3 & 600.0 & 600.0 \\
\hline 04 & 21510 & 147.7244 & 0.9928 & 286.9 & 3 & 600.0 & 0 \\
\hline 04 & 21511 & 255.9199 & 1.6288 & 286.7 & 2 & 600.0 & .0 \\
\hline 04 & 21512 & 235.8412 & 2.9458 & 287.5 & 3 & 600.0 & 60 \\
\hline 04 & 21513 & 280.1541 & 2.6363 & 287.5 & 3 & 600.0 & 0 \\
\hline 04 & 21514 & 225.8711 & 3.3130 & 288.1 & 3 & 600.0 & .0 \\
\hline 04 & 21515 & 197.8590 & 3.4545 & 287.9 & 3 & 600.0 & \\
\hline 04 & 21516 & 176.1866 & 2.4358 & 287.6 & 4 & 600.0 & 600.0 \\
\hline 04 & 21517 & 136.4602 & 2.2075 & 287.3 & 4 & 600.0 & .0 \\
\hline 04 & 21518 & 113.3701 & 3.1208 & 286.5 & 4 & 600.0 & \\
\hline 04 & 21519 & 148.9413 & 1.9963 & 285.9 & 5 & 600.0 & 600.0 \\
\hline 04 & 21520 & 285.3960 & 1.4175 & 285.6 & 6 & 600.0 & 600.0 \\
\hline 04 & 21521 & 311.8000 & 1.7865 & 285.2 & 5 & 600.0 & \\
\hline 04 & 21522 & 318.1275 & 2.5228 & 285.3 & 4 & 600.0 & 60 \\
\hline 04 & 21523 & 302.5751 & 4.9110 & 286.5 & 4 & 600.0 & 60 \\
\hline 04 & 21524 & 210.1757 & 1.9280 & 286.9 & 5 & 600.0 & \\
\hline 04 & 2161 & 339.1602 & 2.8678 & 286.9 & 4 & 600.0 & 60 \\
\hline 04 & 2162 & 346.7510 & 4.0423 & 286.3 & 4 & 600.0 & .0 \\
\hline 04 & 2163 & 330.9416 & 6.3768 & 284.9 & 4 & 600.0 & .0 \\
\hline 04 & 2164 & 309.5230 & 6.7145 & 283.5 & 4 & 600.0 & 60 \\
\hline 04 & 2165 & 307.6750 & 8.5300 & 282.8 & 4 & 600.0 & 600.0 \\
\hline 04 & 2166 & 312.6999 & 9.8600 & 282.7 & 4 & 600.0 & 60 \\
\hline 04 & 2167 & 308.6999 & 725 & 282.3 & 4 & 600.0 & 60 \\
\hline 04 & 2168 & 308.0000 & 10.6700 & 282.3 & 4 & 600.0 & 60 \\
\hline 04 & 2169 & 316.9724 & 10.0700 & 282.5 & 4 & 600.0 & 60 \\
\hline 04 & 21610 & 320.6239 & 10 . & 283.0 & 4 & 600.0 & \\
\hline 04 & 21611 & 320.5287 & 9.1575 & 284.3 & 4 & 600.0 & 600.0 \\
\hline 04 & 21612 & 311.4780 & 6.2485 & 284.9 & 4 & 600.0 & 600.0 \\
\hline 04 & 21613 & 306.3844 & 3.7855 & 285.1 & 4 & 600.0 & 60 \\
\hline 04 & 21614 & 278.4905 & 4.2158 & 284.7 & 4 & 600.0 & 600.0 \\
\hline 04 & 21615 & 272.7896 & 3.1943 & 285.9 & 3 & 600.0 & 600.0 \\
\hline 04 & 21616 & 293.9833 & 3.8363 & 287.8 & 3 & 600.0 & 600.0 \\
\hline 04 & 21617 & 340.6331 & 8.3725 & 288.7 & 4 & 600.0 & 600.0 \\
\hline 04 & 21618 & 328.8500 & 8.3325 & 286.5 & 4 & 600.0 & 600.0 \\
\hline 04 & 21619 & 314.2502 & 9.0200 & 286.5 & 4 & 600.0 & 600.0 \\
\hline 04 & 21620 & 311.8250 & 8.5875 & 286.6 & 4 & 600.0 & 600.0 \\
\hline 04 & 21621 & 303.2502 & 7.3665 & 286.7 & 4 & 600.0 & 600.0 \\
\hline 04 & 21622 & 297.6000 & & 28 & 4 & 600.0 & .0 \\
\hline 04 & 21623 & 327.4303 & 3.8968 & 286.7 & $I$ & 600.0 & 600.0 \\
\hline
\end{tabular}




\begin{tabular}{|c|c|c|c|c|c|c|c|}
\hline- & 1624 & & 6013 & 86.7 & & & \\
\hline 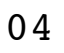 & 2171 & 0 & 6.6648 & 86.4 & \pm & $6 \cap 0$ & \\
\hline 4 & 2172 & 09.5250 & 0440 & 86.6 & & 00 & \\
\hline & 173 & 75.3967 & 2.3115 & 286.6 & & 00.0 & \\
\hline & 174 & 31.8432 & 1203 & 86.8 & & & \\
\hline & 175 & 5 & .66 & 286.4 & & 00 & \\
\hline & 176 & 3994 & 2.4575 & 286.2 & & 500 & \\
\hline & 177 & 52.7865 & 5 & 86.0 & & .0 & \\
\hline & 2178 & 1615 & .50 & 85.8 & & & \\
\hline & 179 & 30.4886 & 2.2328 & 286.1 & 4 & 600.0 & r \\
\hline & 21710 & 324.5682 & 2.9845 & 287.7 & & 00.0 & \\
\hline & 21711 & 5.3570 & 2.6163 & 88.5 & & & \\
\hline & 21712 & 7650 & 3.86 & 289.8 & & 00.0 & \\
\hline & 1713 & 47.8725 & 0.30 & 290.7 & & 0.0 & S \\
\hline & 21714 & 000 & & 9 & & & \\
\hline & 21715 & 000 & & 290.7 & 4 & & \\
\hline & 21716 & 180.0000 & 6.96 & 290.9 & 4 & 00.0 & 000 \\
\hline & 1717 & 180 . & 4 . & 6 & 4 & .0 & \\
\hline & 21718 & 229.2603 & & 28 & 4 & & \\
\hline & 21719 & 289.4196 & 8 & 289.1 & 5 & .0 & \\
\hline 4 & 21720 & 322.0414 & 2.74 & 289.1 & 5 & .0 & 000 \\
\hline & 21721 & 9.4246 & & 287.9 & 4 & & \\
\hline & 21722 & 6.1115 & & 287 & 4 & .0 & \\
\hline & 21723 & 282.0161 & 1. & 286 & 5 & .0 & 000 \\
\hline & 21724 & 169. & & & $J$ & & \\
\hline & 2181 & 186. & 2 . & 28 & 5 & & \\
\hline & 2182 & 179.9926 & 1.8800 & 284.8 & 5 & 0.0 & 600 \\
\hline & 2183 & 281.6220 & 1 . & 284 & 5 & .0 & \\
\hline & 2184 & 341.2749 & 5 . & 284 & 4 & .0 & \\
\hline & 2185 & 359.2135 & 4 . & 283.8 & 4 & .0 & 00 \\
\hline & 2186 & 44.4884 & 2.8088 & 283.6 & 4 & .0 & 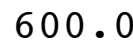 \\
\hline & 2187 & 110.5244 & 4 & 283.7 & 4 & .0 & \\
\hline & 2188 & 111.8315 & & 283 & 4 & .0 & \\
\hline a & 2189 & 95.5161 & 0 & 283.4 & 4 & .0 & \\
\hline & 21810 & 0 & & & 3 & & 0 \\
\hline 04 & 21811 & 683 & 8 & 4.2 & 4 & .0 & \\
\hline 04 & 21812 & 104.2997 & 6.7658 & 285.0 & 4 & .0 & \\
\hline & 21813 & 90.4254 & 7 . & 28 & 4 & .0 & 6 \\
\hline 2 & 21814 & 94.9756 & 8 . & 285.5 & 4 & .0 & 60 \\
\hline 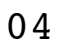 & 21815 & 109.4342 & 8.3450 & 285.7 & 4 & .0 & \\
\hline 1 & 21816 & 94.8459 & 7 . & 285.4 & 4 & & 6 \\
\hline 04 & 21817 & 111.1820 & 8. & 283.4 & 4 & .0 & 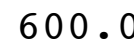 \\
\hline 04 & 21818 & 76.8949 & 7.9025 & 282.7 & 4 & .0 & 00 \\
\hline 04 & 21819 & 78.9971 & 000 & 283.2 & 4 & .0 & \\
\hline 04 & 21820 & 78.3700 & 8.6700 & 282.7 & 4 & 600.0 & 600 \\
\hline 04 & 21821 & 75.9500 & 9.2775 & 282.4 & 4 & 600.0 & 600 \\
\hline & 21822 & 75 & & .2 & 4 & & \\
\hline & 21823 & 69.8750 & 9.0200 & 282.1 & 1 & 600.0 & 600 \\
\hline
\end{tabular}




\begin{tabular}{|c|c|c|c|c|c|c|c|}
\hline 04 & 21824 & 72.0225 & 9.3100 & 281.8 & 4 & 600.0 & 00.0 \\
\hline 04 & 2191 & 72.6000 & 9.5875 & 281.4 & 4 & 600.0 & 600.0 \\
\hline 04 & 2192 & 74.4000 & 10.5800 & 280.9 & 4 & 600.0 & 00.0 \\
\hline 04 & 2193 & 70.7997 & 9.4850 & 280.6 & 4 & 600.0 & 600.0 \\
\hline 04 & 2194 & 74.6759 & 9.0325 & 280.7 & 4 & 600.0 & 00.0 \\
\hline 04 & 2195 & 71.8002 & 9.5825 & 280.1 & 4 & 600.0 & 00.0 \\
\hline 04 & 2196 & 72.2000 & 8.3375 & 280.2 & 4 & 600.0 & 600.0 \\
\hline 04 & 2197 & 71.8494 & 8.6400 & 280.4 & 4 & 600.0 & 500.0 \\
\hline 04 & 2198 & 71.8504 & 7.9750 & 280.6 & 4 & 600.0 & 00.0 \\
\hline 04 & 2199 & 72.0262 & 6.7485 & 281.2 & 4 & 600.0 & 600.0 \\
\hline 04 & 21910 & 87.3534 & 5.3550 & 281.9 & 3 & 600.0 & 600.0 \\
\hline 04 & 21911 & 94.2007 & 6.0990 & 282.6 & 4 & 600.0 & \\
\hline 04 & 21912 & 92.0331 & 5.2063 & 283.5 & 3 & 600.0 & 600.0 \\
\hline 04 & 21913 & 74.7285 & 4.6153 & 283.6 & 3 & 600.0 & 600.0 \\
\hline 04 & 21914 & 73.7899 & 4. & 284.0 & 3 & 600.0 & \\
\hline 04 & 21915 & 76.1249 & 5.4008 & 283.7 & 4 & 600.0 & 60 \\
\hline 04 & 21916 & 76.7242 & 5.2883 & 283.3 & 4 & 600.0 & 600.0 \\
\hline 04 & 21917 & 78.9019 & 4.9338 & 282.8 & 4 & 600.0 & \\
\hline 04 & 21918 & 83.0250 & 5.6583 & 282.2 & 4 & 600.0 & 60 \\
\hline 04 & 21919 & 77.5002 & 6.1968 & 281.6 & 4 & 600.0 & 600.0 \\
\hline 04 & 21920 & 74.7000 & $5.7 \varepsilon$ & 281.3 & 4 & 600.0 & .0 \\
\hline 04 & 21921 & 69.9750 & 4 . & 281.3 & 4 & 600.0 & \\
\hline 04 & 21922 & 63.2507 & 4.5565 & 281.4 & 4 & 600.0 & 600.0 \\
\hline 04 & 21923 & 62.7962 & 4.7260 & 281.3 & 4 & 600.0 & .0 \\
\hline 04 & 21924 & 61.0503 & 4. & 281.2 & 4 & 600.0 & \\
\hline 04 & 2201 & 56.1999 & 303 & 280.8 & 4 & 600.0 & 600.0 \\
\hline 04 & 2202 & 54.6115 & 3.2168 & 280.7 & 4 & 600.0 & 600.0 \\
\hline 04 & 2203 & 358.0559 & 1.66 & 279.9 & 5 & 600.0 & .0 \\
\hline 04 & 2204 & 0.3979 & 1.7500 & 280.3 & 6 & 600.0 & 600.0 \\
\hline 04 & 2205 & 293.9385 & 1.1640 & 280.4 & 6 & 600.0 & 600.0 \\
\hline 04 & 2206 & 349.2253 & 1.1 & 280.2 & 6 & 600.0 & .0 \\
\hline 04 & 2207 & 341.5009 & 1.3420 & 280.1 & 6 & 600.0 & 60 \\
\hline 04 & 2208 & 358.1215 & 1.2845 & 280.4 & 5 & 600.0 & 600.0 \\
\hline 04 & 2209 & 335.6767 & 1.18 & 280.5 & 4 & 600.0 & .0 \\
\hline 04 & 22010 & 351.2925 & 1 . & 28 & 3 & 600.0 & .0 \\
\hline 04 & 22011 & 261.6220 & 1.9593 & 281.5 & 2 & 600.0 & 600.0 \\
\hline 04 & 22012 & 253.5618 & 2.9340 & 282.2 & 3 & 600.0 & 600.0 \\
\hline 04 & 22013 & 247.8700 & 2.3310 & 282.5 & 3 & 600.0 & 600.0 \\
\hline 04 & 22014 & 69.7588 & 6.9438 & 286.6 & 4 & 600.0 & 600.0 \\
\hline 04 & 22015 & 58.3932 & 5.7938 & 286.7 & 3 & 600.0 & \\
\hline 04 & 22016 & 36.9398 & 5.1813 & 286.8 & 3 & 600.0 & 600.0 \\
\hline 04 & 22017 & 22.6119 & 4.6563 & 287.1 & 3 & 600.0 & 600.0 \\
\hline 04 & 22018 & 60.8868 & 4.9188 & 286.3 & 3 & 600.0 & 600.0 \\
\hline 04 & 22019 & 56.3793 & 4.3563 & 285.2 & 4 & 600.0 & 600.0 \\
\hline 04 & 22020 & 22.3111 & 5.2000 & 284.6 & 4 & 600.0 & 600.0 \\
\hline 04 & 22021 & 35.2856 & 5.0063 & 284.6 & 4 & 600.0 & 600.0 \\
\hline 04 & 22022 & 43.8347 & 4 . & 28 & 5 & 600.0 & 600.0 \\
\hline 04 & 22023 & 82.2917 & 2.7313 & 284.7 & $\mathrm{~J}$ & 600.0 & 600.0 \\
\hline
\end{tabular}




\begin{tabular}{|c|c|c|c|c|c|c|c|}
\hline$-5+2+3$ & 2024 & 1.3813 & 4.4563 & 284.4 & 5 & 000 & \\
\hline 4 & 2211 & 9.4914 & 5.2375 & 283.6 & & ل & \\
\hline & 2212 & 31.9785 & 4563 & 283.4 & 5 & 00 & \\
\hline & 2213 & 27.5703 & 6125 & 283.1 & & 00 & \\
\hline & 2214 & 4.6504 & 4.9813 & 33.1 & & 0 & \\
\hline & 2215 & 41.9950 & .5938 & 283.0 & & 00 & \\
\hline & 2216 & 18.9514 & 2125 & 282.9 & & 0 & \\
\hline & 2217 & 2.3417 & .1813 & 82.8 & & & \\
\hline & 2218 & 11.4333 & .8625 & 283.3 & & 0 & \\
\hline & 2219 & 9.1958 & 563 & 284.1 & & 0 & \\
\hline & 22110 & 0.4868 & .4250 & 284.9 & & 0 & \\
\hline & 22111 & 25.8736 & .2688 & 85.2 & & & \\
\hline & 22112 & 311.4939 & 7938 & 285.6 & & 0 & \\
\hline & 22113 & 328.8052 & 5.2938 & 286.3 & & 0.0 & 皮 \\
\hline & 22114 & 350.9299 & & 86.8 & & & \\
\hline & 22115 & 345.5087 & 4 . & 287.4 & & 0 & \\
\hline & 22116 & 6.5230 & 3.8688 & 287.3 & & 0 & \\
\hline & 22117 & 7.6501 & 0 & 6 & & & \\
\hline 4 & 22118 & 337.1560 & 438 & 286 & 4 & 0 & \\
\hline & 22119 & 61.2144 & 1.85 & 285.4 & 4 & 0 & \\
\hline & 22120 & 61.8138 & 1. & 285.1 & & & \\
\hline 4 & 22121 & 39.1744 & 1 . & 28 & 5 & & \\
\hline 4 & 22122 & 37.0820 & 438 & 284.8 & 5 & 00 & 600 . \\
\hline 4 & 22123 & 341.8011 & 500 & 0 & 5 & 0 & \\
\hline 4 & 22124 & 5.2409 & 3 & 28 & 3 & & \\
\hline & 2221 & 19.4280 & & 284.6 & 4 & & \\
\hline & 2222 & 31.6902 & 188 & 284.4 & 4 & 0 & \\
\hline 4 & 2223 & 44.1398 & 2 . & 283.9 & 5 & 0 & \\
\hline 4 & 2224 & 327.1262 & 8 & .5 & 5 & 0 & 0 . \\
\hline 4 & 2225 & 322.4781 & 0 & 283.4 & 5 & 0 & \\
\hline 4 & 2226 & 333.4426 & & $2 \varepsilon$ & 5 & & \\
\hline 4 & 2227 & 311 . & 5 & 28 & 5 & & \\
\hline & 2228 & 17.6796 & 5250 & 283.3 & 5 & 0 & U. \\
\hline 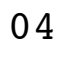 & 2229 & 51.4039 & 3 & .4 & 4 & 0 & \\
\hline & 22210 & 42 . & & 28 & 4 & & \\
\hline & 22211 & 44.4191 & & 284.1 & 3 & & 0 . \\
\hline & 22212 & 14.0804 & 4. & 284.2 & 3 & 0 & \\
\hline 4 & 22213 & 17.4700 & 0 & 284.4 & 3 & 0 & • \\
\hline 4 & 22214 & 82.3039 & 500 & 284.9 & 3 & 600.0 & 0 . \\
\hline 1 & 22215 & 17.3131 & 750 & 285.2 & 3 & 6 & \\
\hline 2 & 22216 & 27.8331 & 000 & & 3 & & \\
\hline 4 & 22217 & 41.7909 & 4.4938 & 285.1 & 3 & 600.0 & 600 . \\
\hline & 22218 & 49.8303 & 688 & 285.7 & 4 & 600 & 000 \\
\hline 2. & 22219 & 34.7895 & 4.7125 & 285.0 & 4 & 600 & $60+8$ \\
\hline 04 & 22220 & 40.1997 & 5.9063 & 283.3 & 4 & 600.0 & 00 . \\
\hline & 22221 & 42.4956 & 5.7875 & 282.6 & 4 & 600.0 & 500. \\
\hline & 22222 & 45.9963 & 4. & .5 & 4 & 600 & \\
\hline & 22223 & 54.9661 & 4.1813 & 281.6 & 4 & 600.0 & 600 \\
\hline
\end{tabular}




\begin{tabular}{|c|c|c|c|c|c|c|c|}
\hline 04 & 22224 & 36.7858 & 3.4500 & 281.2 & 4 & 600.0 & 600.0 \\
\hline 04 & 2231 & 59.1213 & 2.6688 & 281.2 & 5 & 600.0 & 600.0 \\
\hline 04 & 2232 & 36.6892 & 2.7563 & 281.3 & 5 & 600.0 & 00.0 \\
\hline 04 & 2233 & 62.4683 & 3.6250 & 281.2 & 5 & 600.0 & 600.0 \\
\hline 04 & 2234 & 45.3752 & 2.6625 & 281.0 & 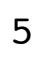 & 600.0 & 00.0 \\
\hline 04 & 2235 & 61.1736 & 2.9250 & 281.0 & 5 & 600.0 & 500.0 \\
\hline 04 & 2236 & 84.8377 & 3.3813 & 280.7 & 5 & 600.0 & 600.0 \\
\hline 04 & 2237 & 103.0851 & 3.5875 & 280.6 & J & 600.0 & 600.0 \\
\hline 04 & 2238 & 29.3876 & 3.4688 & 280.6 & 4 & 600.0 & 00.0 \\
\hline 04 & 2239 & 32.4285 & 3.7625 & 281.7 & 4 & 600.0 & 600.0 \\
\hline 04 & 22310 & 55.7658 & 4.1875 & 282.1 & 3 & 600.0 & 600.0 \\
\hline 04 & 22311 & 130.9711 & 4.2375 & 282.3 & 3 & 600.0 & 500.0 \\
\hline 04 & 22312 & 124.5604 & 4.0938 & 282.7 & 3 & 600.0 & 600.0 \\
\hline 04 & 22313 & 41.9655 & 4.5500 & 283.4 & 3 & 600.0 & 600.0 \\
\hline 04 & 22314 & 37.4902 & 3.9625 & 282.9 & 2 & 600.0 & \\
\hline 04 & 22315 & 48.9850 & 3.9563 & 283.8 & 3 & 600.0 & 60 \\
\hline 04 & 22316 & 59.2478 & 4.0188 & 283.7 & 3 & 600.0 & 600.0 \\
\hline 04 & 22317 & 55.4261 & 3.6000 & 283.7 & 3 & 600.0 & 00.0 \\
\hline 04 & 22318 & 58.5169 & 3.3063 & 283.0 & 3 & 600.0 & 60 \\
\hline 04 & 22319 & 46.1820 & 4.1625 & 282.2 & 4 & 600.0 & 600.0 \\
\hline 04 & 22320 & 43.9661 & 5.0813 & 281.9 & 4 & 600.0 & 600.0 \\
\hline 04 & 22321 & 52.8085 & $5 . \varepsilon$ & 281.6 & 4 & 600.0 & \\
\hline 04 & 22322 & 81.1485 & 5.9563 & 281.6 & 5 & 600.0 & 600.0 \\
\hline 04 & 22323 & 68.9963 & 5.8313 & 281.3 & 4 & 600.0 & 60 \\
\hline 04 & 22324 & 64.5772 & 813 & 281.4 & 5 & 600.0 & \\
\hline 04 & 2241 & 29.3044 & 3.2625 & 281.1 & 5 & 600.0 & 600.0 \\
\hline 04 & 2242 & 46.0954 & 3.2313 & 280.9 & 5 & 600.0 & 600.0 \\
\hline 04 & 2243 & 80.5194 & 4.4438 & 280.9 & 5 & 600.0 & .0 \\
\hline 04 & $224 \quad 4$ & 76.4043 & 4.5625 & 280.6 & 4 & 600.0 & 600.0 \\
\hline 04 & 2245 & 70.8715 & 5.0625 & 280.4 & 4 & 600.0 & 600.0 \\
\hline 04 & 2246 & 65.7670 & 5. & 280.4 & 4 & 600.0 & .0 \\
\hline 04 & 2247 & 68.3737 & 4.7688 & 279.8 & 5 & 600.0 & 600.0 \\
\hline 04 & 2248 & 64.8559 & 5.5125 & 279.8 & 4 & 600.0 & 600.0 \\
\hline 04 & 2249 & 71.9381 & 6.1 & 280.4 & 4 & 600.0 & .0 \\
\hline 04 & 22410 & 70.1283 & 6 . & 28 & 4 & 600.0 & 60 \\
\hline 04 & 22411 & 47.8334 & 5.1563 & 281.4 & 3 & 600.0 & 600.0 \\
\hline 04 & 22412 & 39.4364 & 5.4938 & 282.0 & 3 & 600.0 & 600.0 \\
\hline 04 & 22413 & 36.3677 & 4.2063 & 282.8 & 3 & 600.0 & 600.0 \\
\hline 04 & 22414 & 41.0211 & 3.4438 & 283.3 & 3 & 600.0 & 600.0 \\
\hline 04 & 22415 & 30.7802 & 3.1875 & 283.3 & 3 & 600.0 & 600.0 \\
\hline 04 & 22416 & 15.1231 & 2.5063 & 283.5 & 2 & 600.0 & 600.0 \\
\hline 04 & 22417 & 328.8609 & 2.0750 & 283.5 & 2 & 600.0 & 600.0 \\
\hline 04 & 22418 & 104.3473 & 2.0688 & 283.0 & 3 & 600.0 & 600.0 \\
\hline 04 & 22419 & 47.4317 & 2.6000 & 282.4 & 4 & 600.0 & 600.0 \\
\hline 04 & 22420 & 51.4623 & 3.0313 & 282.2 & 5 & 600.0 & 600.0 \\
\hline 04 & 22421 & 29.9950 & 2.9938 & 282.0 & 5 & 600.0 & 600.0 \\
\hline 04 & 22422 & 303.2476 & 3.362 & 281.7 & 5 & 600.0 & 600.0 \\
\hline 04 & 22423 & 39.4031 & 3.2813 & 281.9 & 4 & 600.0 & 600.0 \\
\hline
\end{tabular}




\begin{tabular}{|c|c|c|c|c|c|c|c|}
\hline 4 & 2424 & & 2188 & 0 & & & \\
\hline 4 & 2251 & 1.4337 & .0500 & 32.1 & & 0 & \\
\hline & 2252 & 38.6781 & 2.8125 & 282.1 & כ & 00 . & \\
\hline & 253 & 40.5913 & 2.5500 & 281.9 & & 00.0 & \\
\hline & 254 & 5252 & 7188 & 81.8 & & 0 & \\
\hline & 2255 & 440 & .2063 & 81.6 & & 00 & \\
\hline & 2256 & 5247 & 1.9563 & 281.7 & & 00 & \\
\hline & 2257 & 82.0549 & .7188 & 81.0 & & .0 & \\
\hline & 2258 & 17.9887 & 3.1625 & 81.2 & & & \\
\hline & 259 & 72.4271 & 3.7438 & 281.8 & & 00 & v \\
\hline & 2510 & 19.6195 & 4.7500 & 283.1 & & 00.0 & \\
\hline & 22511 & 18.8603 & .1875 & 84.0 & & .0 & \\
\hline & 2512 & 22.6693 & 4.9813 & 284.4 & & 00.0 & \\
\hline & 2513 & 15.1134 & 4.9000 & 284 & & 00.0 & . \\
\hline & 22514 & 13.3004 & 4 . & & & & \\
\hline & 22515 & 12.4462 & & 286.1 & & & \\
\hline & 22516 & 76.8729 & 4.1125 & 286.0 & & 0.0 & 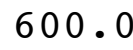 \\
\hline & 2517 & 85.1421 & 3 & 9 & & .0 & \\
\hline & 22518 & 86.0926 & 3 . & 285.6 & & & \\
\hline & 22519 & 98.8840 & 2.9375 & 284.9 & & .0 & 政 \\
\hline & 22520 & 92.8130 & 4.1375 & 284.8 & & 0 & 0. \\
\hline & 22521 & 106.6587 & & 4.4 & 4 & & \\
\hline & 22522 & 5342 & 3 & 284.2 & 4 & & \\
\hline & 22523 & 75.5751 & 5.4688 & 284.1 & 4 & . 0 & . \\
\hline & 22524 & 19.4161 & & & 4 & & \\
\hline & 2261 & 60.4653 & 38 & 28 & 5 & & \\
\hline & 2262 & 67.4694 & 2313 & 283.5 & 5 & 0 & U. \\
\hline & 2263 & 88.4811 & 5.5813 & 28 & 5 & & 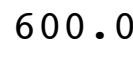 \\
\hline & 2264 & 20 . & 563 & 28 & 5 & & 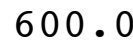 \\
\hline & 2265 & 38.8111 & 4.2500 & 283.4 & 5 & 0 & 0 . \\
\hline & 2266 & 93.1268 & 4.0313 & 283.4 & 4 & .0 & 0 . \\
\hline & 2267 & 72.2278 & 3.52 & .3 & 5 & & \\
\hline & 2268 & 125.9325 & 3.6938 & 283.1 & 5 & & 0 • \\
\hline & 2269 & 144.2860 & 3.5500 & 284.2 & 4 & 0 & 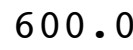 \\
\hline & 22610 & 4.3185 & 0 & & 4 & & \\
\hline & 22611 & 10.7116 & 4.1250 & 285.6 & 3 & & . \\
\hline & 22612 & 5.8351 & 3.8125 & 286.0 & 2 & .0 & 0 . \\
\hline 4 & 22613 & 350.4347 & 4.2500 & 28 & 2 & & . \\
\hline & 22614 & 145.8059 & 4.7188 & 286.9 & 3 & .0 & 0 . \\
\hline & 22615 & 143.5506 & 0938 & 287.1 & 3 & & 0 \\
\hline & 22616 & 142.0872 & 5.2750 & 286 & 3 & & 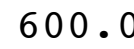 \\
\hline & 22617 & 91.4799 & 5.4125 & 286.8 & 4 & & 0 . \\
\hline & 22618 & 97.7353 & 3.7438 & 286.1 & 4 & 0 & 00 . \\
\hline . & 22619 & 44.4651 & 2.8938 & 28 & 5 & & 0 \\
\hline 4 & 22620 & 74.0282 & 4.1063 & 285.2 & 5 & & 00 . \\
\hline & 22621 & 66.2171 & 3.7938 & 285.3 & 4 & 600.0 & 600 \\
\hline & & 215 & & & 5 & & \\
\hline & 22623 & 50.4424 & 2.3875 & 285.5 & J & 600.0 & 600 \\
\hline
\end{tabular}




\begin{tabular}{|c|c|c|c|c|c|c|c|}
\hline 04 & 22624 & 62.8852 & 3.4750 & 284.7 & 5 & 600.0 & 600.0 \\
\hline 04 & 2271 & 20.7282 & 4.2063 & 284.2 & 5 & 600.0 & 600.0 \\
\hline 04 & 2272 & 55.3867 & 3.5625 & 283.8 & 5 & 600.0 & 00.0 \\
\hline 04 & 2273 & 50.5315 & 3.7938 & 283.4 & P & 600.0 & 00.0 \\
\hline 04 & 2274 & 77.8826 & 4.4438 & 283.5 & 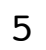 & 600.0 & 00.0 \\
\hline 04 & 2275 & 88.8755 & 3.6188 & 283.7 & 4 & 600.0 & 00.0 \\
\hline 04 & 2276 & 91.6330 & 4.5750 & 283.4 & - & 600.0 & 00.0 \\
\hline 04 & 2277 & 123.0533 & 4.4313 & 282.6 & & 600.0 & 00.0 \\
\hline 04 & 2278 & 57.7949 & 5.0625 & 282.1 & 5 & 600.0 & 00.0 \\
\hline 04 & 2279 & 104.5494 & 7.0688 & 282.2 & 4 & 600.0 & 0 \\
\hline 04 & 22710 & 105.0828 & 6.8875 & 283.2 & 4 & 600.0 & 00.0 \\
\hline 04 & 22711 & 121.0711 & 7.2313 & 283.6 & 4 & 600.0 & \\
\hline 04 & 22712 & 115.3081 & 6.6938 & 284.6 & 4 & 600.0 & 00.0 \\
\hline 04 & 22713 & 128.2361 & 7.0313 & 285.1 & 4 & 600.0 & 00.0 \\
\hline 04 & 22714 & 133.4822 & 38 & 285.6 & 4 & .0 & \\
\hline 04 & 22715 & 137.8638 & 7.1250 & 285.8 & 4 & 600.0 & 00.0 \\
\hline 04 & 22716 & 124.5009 & 6.7688 & 286.3 & 4 & 600.0 & 600.0 \\
\hline 04 & 22717 & 126.7601 & 6.1688 & 286.6 & 4 & .0 & \\
\hline 04 & 22718 & 120.3130 & 6.7438 & 286.1 & 4 & 600.0 & .0 \\
\hline 04 & 22719 & 113.0625 & 5.7500 & 285.2 & 4 & .0 & .0 \\
\hline 04 & 22720 & 106.8116 & 6.29 & 284.7 & 4 & 60 & \\
\hline 04 & 22721 & 103.4907 & 6.1563 & 284.3 & 4 & .0 & \\
\hline 04 & 22722 & 101.9884 & 5.3625 & 284.2 & 5 & 600.0 & 0 \\
\hline 04 & 22723 & 94.9981 & 5.27 & 284.0 & 4 & 60 & .0 \\
\hline 04 & 22724 & 98.3131 & 5.9313 & 283.9 & 4 & .0 & \\
\hline 04 & 2281 & 106.3740 & 7.1875 & 283.8 & 4 & 600.0 & 600.0 \\
\hline 04 & 2282 & 117.3741 & 7.4375 & 283.5 & 4 & 600.0 & .0 \\
\hline 04 & 2283 & 112.5001 & 6.6750 & 283.4 & 4 & 0 & \\
\hline 04 & 2284 & 107.7832 & 6.4688 & 283.3 & 4 & .0 & 600.0 \\
\hline 04 & 2285 & 115.3785 & 5.7813 & 283.2 & 4 & 600.0 & 600.0 \\
\hline 04 & 2286 & 119.6198 & 5.8000 & 282.6 & 4 & .0 & \\
\hline 04 & 2287 & 117.0863 & 6.3813 & 282.4 & 4 & .0 & 600.0 \\
\hline 04 & 2288 & 125.1928 & 7.3125 & 282.4 & 4 & 600.0 & 600.0 \\
\hline 04 & 2289 & 129.6925 & 7.2188 & 282.8 & 4 & 600.0 & 0.0 \\
\hline 04 & 22810 & 129.1299 & 6.7313 & 283.2 & 4 & 600.0 & 600.0 \\
\hline 04 & 22811 & 130.2491 & 7.4625 & 283.5 & 4 & 600.0 & 600.0 \\
\hline 04 & 22812 & 133.6892 & 7.3438 & 284.3 & 4 & 600.0 & 0.0 \\
\hline 04 & 22813 & 134.1258 & 7.3125 & 284.9 & 4 & 600.0 & 600.0 \\
\hline 04 & 22814 & 128.7339 & 6.6188 & 285.6 & 3 & 600.0 & 600.0 \\
\hline 04 & 22815 & 104.8125 & 6.0125 & 286.2 & 3 & 600.0 & 600.0 \\
\hline 04 & 22816 & 28.0260 & 4.9500 & 286.3 & 3 & 600.0 & 600.0 \\
\hline 04 & 22817 & 355.2601 & 4.3500 & 286.1 & 3 & 600.0 & 600.0 \\
\hline 04 & 22818 & 44.1468 & 3.3063 & 285.3 & 4 & 600.0 & 600.0 \\
\hline 04 & 22819 & 100.3791 & 3.9375 & 284.6 & 4 & 600.0 & 600.0 \\
\hline 04 & 22820 & 33.0528 & 4.6000 & 284.0 & 5 & 600.0 & 600.0 \\
\hline 04 & 22821 & 116.9464 & 5.2813 & 283.7 & 4 & 600.0 & 600.0 \\
\hline 04 & 22822 & 55.5878 & 5.5875 & 283.3 & 5 & 600.0 & 600.0 \\
\hline 04 & 22823 & 79.4997 & 6.9000 & 282.6 & 4 & 600.0 & 600.0 \\
\hline
\end{tabular}




\begin{tabular}{|c|c|c|c|c|c|c|c|}
\hline 4 & 22824 & 77.5556 & 8.5563 & 282.1 & 4 & 600.0 & 600.0 \\
\hline 04 & 2291 & 67.1640 & 8.3875 & 281.6 & 4 & 600.0 & 600.0 \\
\hline 04 & 2292 & 54.6210 & 7.5438 & 281.1 & 5 & 600.0 & 00.0 \\
\hline 04 & 2293 & 39.5624 & 6.7188 & 280.8 & 5 & 600.0 & 00.0 \\
\hline 04 & 2294 & 34.8981 & 5.0625 & 280.6 & o & 600.0 & 00.0 \\
\hline 04 & 2295 & 91.7591 & 6.2563 & 279.9 & 5 & 600.0 & 00.0 \\
\hline 04 & 2296 & 74.2152 & 6.3938 & 279.2 & 4 & 600.0 & 00.0 \\
\hline 04 & 2297 & 77.1517 & 5.7875 & 279.4 & 5 & 600.0 & 00.0 \\
\hline 04 & 2298 & 84.1233 & 7.0438 & 279.7 & 4 & 600.0 & 00.0 \\
\hline 04 & 2299 & 86.3279 & 6.5688 & 281.0 & 4 & 600.0 & 00.0 \\
\hline 04 & 22910 & 98.6317 & 5.4375 & 281.9 & 3 & 600.0 & 00.0 \\
\hline 04 & 22911 & 161.3262 & 5.5000 & 282.2 & 3 & 600.0 & .0 \\
\hline 04 & 22912 & 145.8092 & 5.8375 & 283.0 & 3 & 600.0 & 00.0 \\
\hline 04 & 22913 & 151.3105 & 6.5125 & 283.5 & 4 & 600.0 & 00.0 \\
\hline 04 & 22914 & 60.7431 & 7.0188 & 284.5 & 3 & .0 & \\
\hline 04 & 22915 & 78.4799 & 6.5688 & 285.0 & 3 & 600.0 & 00.0 \\
\hline 04 & 22916 & 82.9586 & 5.2813 & 285.3 & 3 & 600.0 & 600.0 \\
\hline 04 & 22917 & 138.7606 & 5.3000 & 285.2 & 3 & .0 & \\
\hline 04 & 22918 & 115.0477 & 4.6063 & 284.7 & 4 & 600.0 & .0 \\
\hline 04 & 22919 & 109.6851 & 4.4313 & 284.0 & 4 & 60 & .0 \\
\hline 04 & 22920 & 105.6875 & 6 . & 283.6 & 4 & 60 & \\
\hline 04 & 22921 & 98.3125 & 6.6813 & 283.4 & 4 & .0 & \\
\hline 04 & 22922 & 97.6250 & 6.5063 & 283.4 & 4 & 600.0 & 600.0 \\
\hline 04 & 22923 & 98.0002 & 7.06 & 283.4 & 4 & 60 & .0 \\
\hline 04 & 22924 & 96.1802 & 7.2000 & 283.4 & 4 & .0 & \\
\hline 04 & $\begin{array}{lll}3 & 1 & 1\end{array}$ & 68.7260 & 8.4375 & 281.8 & 4 & 600.0 & 600.0 \\
\hline 04 & $\begin{array}{lll}3 & 1 & 2\end{array}$ & 84.3136 & 7.7500 & 281.6 & 5 & 600.0 & .0 \\
\hline 04 & $\begin{array}{lll}3 & 1 & 3\end{array}$ & 80.5624 & 6.5750 & 281.3 & 5 & 600.0 & \\
\hline 04 & $\begin{array}{lll}3 & 1 & 4\end{array}$ & 80.2137 & 5.9250 & 281.1 & 5 & 600.0 & 600.0 \\
\hline 04 & 315 & 130.1343 & 6.4500 & 280.5 & 5 & 600.0 & 600.0 \\
\hline 04 & 316 & 117.0901 & 6.3875 & 279.5 & 4 & 600.0 & .0 \\
\hline 04 & $\begin{array}{lll}3 & 1 & 7\end{array}$ & 119.6501 & 5.5500 & 279.4 & 5 & 600.0 & 600.0 \\
\hline 04 & $\begin{array}{lll}3 & 1 & 8\end{array}$ & 124.3762 & 6.7750 & 279.9 & 4 & 600.0 & 600.0 \\
\hline 04 & 319 & 137.8928 & 5.8938 & 281.1 & 4 & 600.0 & 0.0 \\
\hline 04 & 3110 & 150.0232 & 5.7188 & 282.1 & 4 & 600.0 & 600.0 \\
\hline 04 & 3111 & 165.7488 & 5.4438 & 282.7 & 3 & 600.0 & 600.0 \\
\hline 04 & 3112 & 166.0574 & 5.7125 & 283.5 & 3 & 600.0 & 0.0 \\
\hline 04 & 3113 & 161.5456 & 6.0125 & 284.0 & 3 & 600.0 & 600.0 \\
\hline 04 & 3114 & 78.5524 & 6.8813 & 284.9 & 3 & 600.0 & 600.0 \\
\hline 04 & 3115 & 99.5041 & 6.1438 & 285.5 & 3 & 600.0 & 0.0 \\
\hline 04 & 3116 & 102.0810 & 4.7813 & 285.8 & 3 & 600.0 & 600.0 \\
\hline 04 & 3117 & 158.3266 & 4.6250 & 285.7 & 3 & 600.0 & 600.0 \\
\hline 04 & 3118 & 51.3076 & 3.9625 & 285.1 & 4 & 600.0 & 600.0 \\
\hline 04 & 3119 & 76.0583 & 3.5438 & 284.5 & 4 & 600.0 & 600.0 \\
\hline 04 & 3120 & 96.0946 & 5.1188 & 284.2 & 4 & 600.0 & 600.0 \\
\hline 04 & 3121 & 95.8767 & 6.0688 & 283.9 & 4 & 600.0 & 600.0 \\
\hline $0<$ & 3122 & 97.8135 & 6.1938 & 283.9 & 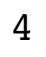 & 600.0 & 600.0 \\
\hline 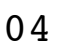 & 3123 & 92.5614 & 6.6938 & 283.9 & 4 & 600.0 & 600.0 \\
\hline
\end{tabular}




\begin{tabular}{|c|c|c|c|c|c|c|c|c|}
\hline 4 & 3 & 124 & 93.3072 & 7.3250 & 283.7 & 4 & 600.0 & 600.0 \\
\hline 04 & 3 & 21 & 88.1930 & 7.6000 & 283.2 & 4 & 600.0 & 600.0 \\
\hline 04 & 3 & 22 & 83.3151 & 7.2188 & 282.7 & 5 & 600.0 & 00.0 \\
\hline 04 & 3 & 23 & 107.4780 & 6.0938 & 282.1 & 5 & 600.0 & 00.0 \\
\hline 04 & 3 & 24 & 54.1730 & 5.3125 & 281.3 & $J$ & 600.0 & 00.0 \\
\hline 04 & 3 & 25 & 44.6818 & 4.8313 & 280.9 & 4 & 600.0 & 00.0 \\
\hline 04 & 3 & 26 & 52.4536 & 4.4188 & 280.5 & 4 & 600.0 & 00.0 \\
\hline 04 & 3 & 27 & 81.9222 & 3.9563 & 279.9 & 4 & 600.0 & 00.0 \\
\hline 04 & 3 & 28 & 35.8212 & 3.5500 & 280.3 & 4 & 600.0 & 00.0 \\
\hline 04 & 3 & 29 & 127.9622 & 4.6438 & 280.7 & 4 & 600.0 & 00.0 \\
\hline 04 & 3 & 210 & 54.0773 & 3.6375 & 281.5 & 3 & 600.0 & 00.0 \\
\hline 04 & 3 & 211 & 80.4049 & 3.4500 & 282.3 & 3 & 600.0 & \\
\hline 04 & 3 & 212 & 19.7552 & 3.4938 & 282.5 & 3 & 600.0 & 00.0 \\
\hline 04 & 3 & 213 & 30.2331 & 3.1000 & 283.3 & 2 & .0 & .0 \\
\hline 04 & 3 & 214 & 80.4670 & 3.6563 & 284.1 & 3 & .0 & \\
\hline 04 & 3 & 215 & 135.3163 & 3.9688 & 284.7 & 3 & 600.0 & 00.0 \\
\hline 04 & 3 & 216 & 130.6347 & 3.6313 & 285.2 & 3 & 600.0 & 600.0 \\
\hline 04 & 3 & 217 & 37.4428 & 10.6400 & 286.7 & 4 & .0 & \\
\hline 04 & 3 & 218 & 126.0150 & 10.6675 & 286.0 & 4 & 600.0 & .0 \\
\hline 04 & 3 & 219 & 226.0150 & 13.2150 & 285.9 & 4 & 60 & .0 \\
\hline 04 & 3 & 220 & 326.0150 & 13 & 285.6 & 4 & .0 & \\
\hline 04 & 3 & 221 & 66.0150 & 13.6250 & 285.2 & 4 & .0 & \\
\hline 04 & 3 & 222 & 166.0150 & 12.8100 & 284.8 & 4 & 600.0 & 00.0 \\
\hline 04 & 3 & 223 & 266.0150 & 11.5650 & 284.2 & 4 & .0 & \\
\hline 04 & 3 & 224 & 150 & - & .2 & 4 & 6 & \\
\hline 04 & 3 & 31 & 226.0150 & 8.9300 & 282.1 & 4 & 600.0 & 600.0 \\
\hline 04 & 3 & 32 & 326.0150 & 9.8350 & 281.6 & 4 & 600.0 & 600.0 \\
\hline 04 & 3 & 33 & 66.0150 & 9.7450 & 281.3 & 4 & .0 & \\
\hline 04 & 3 & 34 & 166.0150 & 9.0575 & 281.0 & 4 & 600.0 & 600.0 \\
\hline 04 & 3 & 35 & 266.0150 & 7.6718 & 280.5 & 4 & 600.0 & 600.0 \\
\hline 04 & 3 & 36 & 6.0150 & 9. & 280.8 & 4 & .0 & \\
\hline 04 & 3 & 37 & 106.0150 & 9.9675 & 280.8 & 4 & 600.0 & 600.0 \\
\hline 04 & 3 & 38 & 206.0150 & 8.2675 & 281.3 & 4 & 600.0 & 600.0 \\
\hline 04 & 3 & 39 & 306.0150 & 6. & 282.6 & 4 & 600.0 & .0 \\
\hline 04 & 3 & 310 & 46.0150 & 8.55 & 284.2 & 4 & 600.0 & 600.0 \\
\hline 04 & 3 & 311 & 146.0150 & 8.4800 & 284.5 & 4 & 600.0 & 600.0 \\
\hline 04 & 3 & 312 & 246.0150 & 7 . & 285.2 & 4 & 60 & 0.0 \\
\hline 04 & 3 & 313 & 346.0150 & 5.2535 & 285.4 & 3 & 600.0 & 600.0 \\
\hline 04 & 3 & 314 & 86.0150 & 3.9973 & 285.5 & 3 & 600.0 & 600.0 \\
\hline 04 & 3 & 315 & 186.0150 & 4.0968 & 285 & 3 & 600.0 & 0 \\
\hline 04 & 3 & 316 & 286.0150 & 2.4563 & 285.1 & 4 & 600.0 & 600.0 \\
\hline 04 & 3 & 317 & 26.0150 & 4.9275 & 284.7 & 4 & 600.0 & 600.0 \\
\hline 04 & 3 & 318 & 126.0150 & 4.7030 & 285.0 & 4 & 600.0 & 600.0 \\
\hline 04 & 3 & 319 & 226.0150 & 5.9235 & 284.2 & 4 & 600.0 & 600.0 \\
\hline 04 & 3 & 320 & 326.0150 & 6.2003 & 283.9 & 4 & 600.0 & 600.0 \\
\hline 04 & 3 & 321 & 66.0150 & & & 4 & 600.0 & 600.0 \\
\hline $0<$ & 3 & 322 & 166.0150 & 6.8723 & 284.0 & 4 & 600.0 & 600.0 \\
\hline 04 & 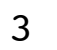 & 323 & 266.0150 & 6.889 & 283 & 4 & 600.0 & 600.0 \\
\hline
\end{tabular}




\begin{tabular}{|c|c|c|c|c|c|c|c|c|}
\hline 4 & 3 & 324 & 6.0150 & 7.9900 & 283.8 & 4 & 600.0 & 00.0 \\
\hline 04 & 3 & 41 & 226.0150 & 7.9625 & 283.7 & 4 & 600.0 & 600.0 \\
\hline 04 & 3 & 42 & 326.0150 & 8.6375 & 283.4 & 4 & 600.0 & 00.0 \\
\hline 04 & 3 & 43 & 66.0150 & 10.7750 & 283.5 & 4 & 600.0 & 600.0 \\
\hline 04 & 3 & 44 & 166.0150 & 11.6375 & 283.7 & 4 & 600.0 & 00.0 \\
\hline 04 & 3 & 45 & 266.0150 & 12.8525 & 283.7 & 4 & 600.0 & 00.0 \\
\hline 04 & 3 & 46 & 6.0150 & 14.0875 & 283.8 & 4 & 600.0 & 600.0 \\
\hline 04 & 3 & 47 & 106.0150 & 13.4825 & 284.1 & 4 & 600.0 & 500.0 \\
\hline 04 & 3 & 48 & 206.0150 & 12.6525 & 284.5 & 4 & 600.0 & 00.0 \\
\hline 04 & 3 & 49 & 306.0150 & 10.7325 & 285.1 & 4 & 600.0 & .0 \\
\hline 04 & 3 & 410 & 46.0150 & 8.5525 & 286.0 & 4 & 600.0 & 600.0 \\
\hline 04 & 3 & 411 & 146.0150 & 7.8738 & 286.6 & 4 & 600.0 & \\
\hline 04 & 3 & 412 & 246.0150 & 6.0113 & 287.5 & 3 & 600.0 & 600.0 \\
\hline 04 & 3 & 413 & 346.0150 & 5.0575 & 288.6 & 2 & 600.0 & 600.0 \\
\hline 04 & 3 & 414 & 86.0150 & 5.7198 & 288.8 & 3 & 600.0 & \\
\hline 04 & 3 & 415 & 186.0150 & 4.6783 & 289.2 & 3 & 600.0 & 60 \\
\hline 04 & 3 & 416 & 286.0150 & 5.7210 & 289.0 & 4 & 600.0 & 600.0 \\
\hline 04 & 3 & 417 & 26.0150 & 7.9305 & 288.0 & 4 & 600.0 & .0 \\
\hline 04 & 3 & 418 & 126.0150 & 10.5675 & 286.5 & 4 & 600.0 & 60 \\
\hline 04 & 3 & 419 & 226.0150 & 11.5325 & 285.7 & 4 & 600.0 & 600.0 \\
\hline 04 & 3 & 420 & 326.0150 & 10.3875 & .9 & 4 & 600.0 & .0 \\
\hline 04 & 3 & 421 & 66.0150 & 11.8200 & 285.3 & 4 & 600.0 & \\
\hline 04 & 3 & 422 & 166.0150 & 11.6225 & 284.5 & 4 & 600.0 & 600.0 \\
\hline 04 & 3 & 423 & 266.0150 & 10.4800 & 283.9 & 4 & 600.0 & .0 \\
\hline 04 & 3 & 424 & 6.0150 & 9.8825 & 283.7 & 4 & 600.0 & \\
\hline 04 & 3 & 51 & 226.0150 & 9.6125 & 283.3 & 4 & 600.0 & 600.0 \\
\hline 04 & 3 & 52 & 326.0150 & 6.9208 & 283.5 & 4 & 600.0 & 600.0 \\
\hline 04 & 3 & 53 & 66.0150 & 7.8323 & 283.1 & 4 & 600.0 & .0 \\
\hline 04 & 3 & 54 & 166.0150 & 7.7258 & 282.8 & 4 & 600.0 & 60 \\
\hline 04 & 3 & 55 & 266.0150 & 7.2625 & 283.9 & 4 & 600.0 & 600.0 \\
\hline 04 & 3 & 56 & 6.0150 & 7.7825 & 282.7 & 4 & 600.0 & .0 \\
\hline 04 & 3 & 57 & 106.0150 & 7.9100 & 283.0 & 4 & 600.0 & .0 \\
\hline 04 & 3 & 58 & 206.0150 & 6.2233 & 284.7 & 4 & 600.0 & 600.0 \\
\hline 04 & 3 & 59 & 306.0150 & 6.3150 & 285.6 & 4 & 600.0 & .0 \\
\hline 04 & 3 & 510 & 150 & 5.1750 & .7 & 3 & 600.0 & \\
\hline 04 & 3 & 511 & 146.0150 & 2.9680 & 287.6 & 2 & 600.0 & 600.0 \\
\hline 04 & 3 & 512 & 246.0150 & 3.2350 & 288.9 & 3 & 600.0 & \\
\hline 04 & 3 & 513 & 346.0150 & 2.4758 & 289.6 & 2 & 600.0 & 600.0 \\
\hline 04 & 3 & 514 & 86.0150 & 2.6123 & 289.8 & 3 & 600.0 & 600.0 \\
\hline 04 & 3 & 515 & 186.0150 & 3.9663 & 290.1 & 3 & 600.0 & \\
\hline 04 & 3 & 516 & 286.0150 & 3.9983 & 290.1 & 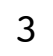 & 600.0 & 600.0 \\
\hline 04 & 3 & 517 & 26.0150 & 5.3473 & 289.5 & 4 & 600.0 & 600.0 \\
\hline 04 & 3 & 518 & 126.0150 & 6.2318 & 288.2 & 4 & 600.0 & 600.0 \\
\hline 04 & 3 & 519 & 226.0150 & 6.8843 & 287.2 & 4 & 600.0 & 600.0 \\
\hline 04 & 3 & 520 & 326.0150 & 6.4270 & 286.8 & 4 & 600.0 & 600.0 \\
\hline 04 & 3 & 521 & 66.0150 & 6.1328 & 286.9 & 4 & 600.0 & 600.0 \\
\hline 04 & 3 & 522 & 166.0150 & 9.1950 & 6 & 4 & 600.0 & 600.0 \\
\hline 04 & 3 & 523 & 266.0150 & 8.2625 & 285.2 & 4 & 600.0 & 600.0 \\
\hline
\end{tabular}




\begin{tabular}{|c|c|c|c|c|c|c|c|c|}
\hline 04 & 3 & 524 & 6.0150 & 8.4850 & 285.5 & 4 & 600.0 & 600.0 \\
\hline 04 & 3 & 61 & 226.0150 & 9.7600 & 284.3 & 4 & 600.0 & 600.0 \\
\hline 04 & 3 & 62 & 326.0150 & 8.2700 & 285.3 & 4 & 600.0 & 00.0 \\
\hline 04 & 3 & 63 & 66.0150 & 7.3175 & 285.9 & 4 & 600.0 & 600.0 \\
\hline 04 & 3 & 64 & 166.0150 & 7.6725 & 285.0 & 4 & 600.0 & 00.0 \\
\hline 04 & 3 & 65 & 266.0150 & 7.6450 & 284.7 & 4 & 600.0 & 00.0 \\
\hline 04 & 3 & 66 & 6.0150 & 4.8730 & 285.0 & 4 & 600.0 & 600.0 \\
\hline 04 & 3 & 67 & 106.0150 & 5.0695 & 285.0 & 4 & 600.0 & 600.0 \\
\hline 04 & 3 & 68 & 206.0150 & 4.1723 & 285.1 & 3 & 600.0 & 00.0 \\
\hline 04 & 3 & 69 & 306.0150 & 3.4185 & 286.2 & 3 & 600.0 & 600.0 \\
\hline 04 & 3 & 610 & 46.0150 & 4.5430 & 287.1 & 3 & 600.0 & 600.0 \\
\hline 04 & 3 & 611 & 146.0150 & 5.7965 & 286.7 & 4 & 600.0 & 00.0 \\
\hline 04 & 3 & 612 & 246.0150 & 4.7720 & 287.1 & 3 & 600.0 & 600.0 \\
\hline 04 & 3 & 613 & 346.0150 & 5.2763 & 287.8 & 2 & 600.0 & 600.0 \\
\hline 04 & 3 & 614 & 86.0150 & 5.9035 & 288.0 & 3 & 600.0 & \\
\hline 04 & 3 & 615 & 186.0150 & 5.0210 & 288.4 & 3 & 600.0 & 60 \\
\hline 04 & 3 & 616 & 286.0150 & 5.0615 & 288.4 & 3 & 600.0 & 600.0 \\
\hline 04 & 3 & 617 & 26.0150 & 4.8420 & 288.0 & 3 & 600.0 & 00.0 \\
\hline 04 & 3 & 618 & 126.0150 & 4.2505 & 287.6 & 4 & 600.0 & 600.0 \\
\hline 04 & 3 & 619 & 226.0150 & 5.2133 & 287.9 & 4 & 600.0 & 600.0 \\
\hline 04 & 3 & 620 & 326.0150 & 5.1248 & 287.7 & 4 & 600.0 & 600.0 \\
\hline 04 & 3 & 621 & 66.0150 & 4.5768 & 287.4 & 4 & 600.0 & .0 \\
\hline 04 & 3 & 622 & 166.0150 & 3.4458 & 286.9 & 4 & 600.0 & 600.0 \\
\hline 04 & 3 & 623 & 266.0150 & 3.7845 & 287.5 & 4 & 600.0 & 60 \\
\hline 04 & 3 & 624 & 6.0150 & 3.8123 & 287.4 & 4 & 600.0 & \\
\hline 04 & 3 & 71 & 226.0150 & 3.7965 & 287.8 & 4 & 600.0 & 600.0 \\
\hline 04 & 3 & 72 & 326.0150 & 3.4108 & 288.0 & 4 & 600.0 & 600.0 \\
\hline 04 & 3 & 73 & 66.0150 & 3.0320 & 287.4 & 4 & 600.0 & .0 \\
\hline 04 & 3 & 74 & 166.0150 & 4.1200 & 287.5 & 4 & 600.0 & 600.0 \\
\hline 04 & 3 & 75 & 266.0150 & 4.3985 & 287.2 & 4 & 600.0 & 600.0 \\
\hline 04 & 3 & 76 & 6.0150 & 4.6343 & 287.2 & 4 & 600.0 & 60 \\
\hline 04 & 3 & 77 & 106.0150 & 3.6423 & 287.3 & 4 & 600.0 & 600.0 \\
\hline 04 & 3 & 78 & 206.0150 & 5.1110 & 287.4 & 3 & 600.0 & 600.0 \\
\hline 04 & 3 & 79 & 306.0150 & 6.7605 & 287.7 & 4 & 600.0 & .0 \\
\hline 04 & 3 & 710 & 46.0150 & 8.5700 & 287.9 & 4 & 600.0 & 600.0 \\
\hline 04 & 3 & 711 & 146.0150 & 7.1963 & 288.6 & 4 & 600.0 & 600.0 \\
\hline 04 & 3 & 712 & 246.0150 & 6.1945 & 290.0 & 4 & 600.0 & 600.0 \\
\hline 04 & 3 & 713 & 346.0150 & 5.2028 & 290.8 & 3 & 600.0 & 600.0 \\
\hline 04 & 3 & 714 & 86.0150 & 4.3328 & 291.4 & 2 & 600.0 & 600.0 \\
\hline 04 & 3 & 715 & 186.0150 & 3.8998 & 292.2 & 3 & 600.0 & \\
\hline 04 & 3 & 716 & 286.0150 & 3.8920 & 292.3 & 3 & 600.0 & 600.0 \\
\hline 04 & 3 & 717 & 26.0150 & 4.4360 & 291.9 & 3 & 600.0 & 600.0 \\
\hline 04 & 3 & 718 & 126.0150 & 2.9088 & 291.3 & 4 & 600.0 & 600.0 \\
\hline 04 & 3 & 719 & 226.0150 & 2.9475 & 290.6 & 4 & 600.0 & 600.0 \\
\hline 04 & 3 & 720 & 326.0150 & 3.0010 & 290.0 & 4 & 600.0 & 600.0 \\
\hline 04 & 3 & 721 & 66.0150 & 3.7928 & 289.5 & 4 & 600.0 & 600.0 \\
\hline 04 & 3 & 722 & 166.0150 & 2.875 & 288.4 & 4 & 600.0 & 600.0 \\
\hline 04 & 3 & 723 & 266.0150 & 2.5948 & 288.5 & 4 & 600.0 & 600.0 \\
\hline
\end{tabular}




\begin{tabular}{|c|c|c|c|c|c|c|c|c|}
\hline 4 & 3 & 724 & 6.0150 & 1.1168 & 289.1 & 5 & 600.0 & 00.0 \\
\hline 04 & 3 & 81 & 226.0150 & 1.4585 & 289.1 & 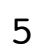 & 600.0 & 600.0 \\
\hline 04 & 3 & 82 & 326.0150 & 1.7000 & 289.2 & 5 & 600.0 & 00.0 \\
\hline 04 & 3 & 83 & 66.0150 & 1.1253 & 289.8 & 6 & 600.0 & 600.0 \\
\hline 04 & 3 & 84 & 166.0150 & 0.9265 & 289.2 & 0 & 600.0 & 00.0 \\
\hline 04 & 3 & 85 & 266.0150 & 0.9705 & 288.3 & 6 & 600.0 & 00.0 \\
\hline 04 & 3 & 86 & 6.0150 & 0.7613 & 287.7 & 6 & 600.0 & 600.0 \\
\hline 04 & 3 & 87 & 106.0150 & 0.8903 & 287.5 & 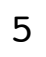 & 600.0 & 500.0 \\
\hline 04 & 3 & 88 & 206.0150 & 0.6135 & 288.1 & 4 & 600.0 & 00.0 \\
\hline 04 & 3 & 89 & 306.0150 & 1.1763 & 289.1 & 3 & 600.0 & 600.0 \\
\hline 04 & 3 & 810 & 20.2583 & 1.5038 & 291.2 & 2 & 600.0 & 600 \\
\hline 04 & 3 & 811 & 287.5572 & 2.3317 & 292.6 & 2 & 600.0 & \\
\hline 04 & 3 & 812 & 287.5572 & 2.3317 & 292.6 & 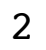 & 600.0 & 600.0 \\
\hline 04 & 3 & 813 & 357.4428 & 2.3750 & 293.0 & 2 & 600.0 & 600.0 \\
\hline 04 & 3 & 814 & 86.0150 & 2.6625 & 293.3 & 2 & 600.0 & \\
\hline 04 & 3 & 815 & 186.0150 & 2.1313 & 293.4 & 2 & 600.0 & 60 \\
\hline 04 & 3 & 816 & 286.0150 & 2.1233 & 293.5 & 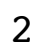 & 600.0 & 600.0 \\
\hline 04 & 3 & 817 & 26.0150 & 1.6820 & 293.1 & 2 & 600.0 & .0 \\
\hline 04 & 3 & 818 & 126.0150 & 1.0493 & 293.1 & 3 & 600.0 & 600.0 \\
\hline 04 & 3 & 819 & 226.0150 & 0.8740 & 292.8 & 4 & 600.0 & 600.0 \\
\hline 04 & 3 & 820 & 326.0150 & 0.9425 & 293.0 & 5 & 600.0 & .0 \\
\hline 04 & 3 & 821 & 66.0150 & 1.8093 & 293.9 & 5 & 600.0 & \\
\hline 04 & 3 & 822 & 166.0150 & 1.7115 & 294.4 & 6 & 600.0 & 600.0 \\
\hline 04 & 3 & 823 & 266.0150 & 1.2280 & 294.5 & 6 & 600.0 & .0 \\
\hline 04 & 3 & 824 & 6.0150 & 1.0180 & 293.9 & 6 & 600.0 & \\
\hline 04 & 3 & 91 & 226.0150 & 1.0603 & 293.2 & 6 & 600.0 & 600.0 \\
\hline 04 & 3 & 92 & 326.0150 & 1.3685 & 293.0 & 5 & 600.0 & 600.0 \\
\hline 04 & 3 & 93 & 66.0150 & 1.5485 & 291.5 & 6 & 600.0 & .0 \\
\hline 04 & 3 & 94 & 166.0150 & 0.9378 & 290.9 & 6 & 600.0 & 600.0 \\
\hline 04 & 3 & 95 & 266.0150 & 0.6888 & 290.8 & 6 & 600.0 & 600.0 \\
\hline 04 & 3 & 96 & 6.0150 & 483 & 291.3 & 6 & 600.0 & .0 \\
\hline 04 & 3 & 97 & 106.0150 & 1.3420 & 290.9 & 5 & 600.0 & 60 \\
\hline 04 & 3 & 98 & 206.0150 & 1.4600 & 290.9 & 4 & 600.0 & 600.0 \\
\hline 04 & 3 & 99 & 306.0150 & 1.9095 & 291.7 & 3 & 600.0 & .0 \\
\hline 04 & 3 & 910 & 150 & 1.6168 & .6 & 2 & 600.0 & 60 \\
\hline 04 & 3 & 911 & 146.0150 & 3.5368 & 293.4 & 2 & 600.0 & 600.0 \\
\hline 04 & 3 & 912 & 246.0150 & 5.2510 & 293.0 & 2 & 600.0 & 600.0 \\
\hline 04 & 3 & 913 & 346.0150 & 6.7718 & 293.6 & 3 & 600.0 & 600.0 \\
\hline 04 & 3 & 914 & 86.0150 & 6.3688 & 294.5 & 3 & 600.0 & 600.0 \\
\hline 04 & 3 & 915 & 186.0150 & 7.9055 & 294.5 & 4 & 600.0 & \\
\hline 04 & 3 & 916 & 286.0150 & 7.6893 & 294.6 & 4 & 600.0 & 600.0 \\
\hline 04 & 3 & 917 & 26.0150 & 4.8158 & 295.1 & 3 & 600.0 & 600.0 \\
\hline 04 & 3 & 918 & 126.0150 & 6.8008 & 295.0 & 4 & 600.0 & 600.0 \\
\hline 04 & 3 & 919 & 226.0150 & 6.6553 & 295.1 & 4 & 600.0 & 600.0 \\
\hline 04 & 3 & 920 & 326.0150 & 7.2760 & 295.7 & 4 & 600.0 & 600.0 \\
\hline 04 & 3 & 921 & 66.0150 & 7.4083 & 295.3 & 4 & 600.0 & 600.0 \\
\hline 04 & 3 & 922 & 166.0150 & 8.2575 & & 4 & 600.0 & 600.0 \\
\hline 04 & 3 & 923 & 266.0150 & 13.2050 & 295.8 & 4 & 600.0 & 600.0 \\
\hline
\end{tabular}




\begin{tabular}{|c|c|c|c|c|c|c|c|}
\hline 4 & 3924 & 6.0150 & 12.8000 & 294.0 & $x$ & 600.0 & 0.0 \\
\hline 04 & 3101 & 226.0150 & 12.2350 & 292.4 & 4 & 600.0 & 600,0 \\
\hline 04 & 3102 & 326.0150 & 13.2200 & 292.4 & 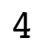 & 600.0 & 00.0 \\
\hline 04 & 3103 & 66.0150 & 12.4625 & 291.7 & 4 & 600.0 & 00.0 \\
\hline 04 & 3104 & 166.0150 & 13.8475 & 291.0 & 4 & 600.0 & 0.0 \\
\hline 04 & 3105 & 266.0150 & 14.2725 & 290.1 & 4 & 600.0 & 00.0 \\
\hline 04 & 3106 & 6.0150 & 13.5750 & 289.2 & 4 & 600.0 & 00.0 \\
\hline 04 & 3107 & 106.0150 & 13.7425 & 288.2 & 4 & 600.0 & 00.0 \\
\hline 04 & 3108 & 206.0150 & 14.7425 & 288.0 & 4 & 600.0 & .0 \\
\hline 04 & 3109 & 306.0150 & 12.5900 & 288.8 & 4 & 600.0 & \\
\hline 04 & 31010 & 46.0150 & 13.8100 & 289.9 & 4 & 600.0 & 00.0 \\
\hline 04 & 31011 & 146.0150 & 0 & 291.4 & 4 & 600.0 & \\
\hline 04 & 31012 & 246.0150 & 1.6100 & 292.5 & 4 & 600.0 & 00.0 \\
\hline 04 & 31013 & 346.0150 & 10.3200 & 293.6 & 4 & 600.0 & 0 \\
\hline 04 & 31014 & 86.0150 & $0 \quad 1$ & 294.3 & 4 & 600.0 & \\
\hline 04 & 31015 & 186.0150 & 9.2300 & 295.0 & 4 & 600.0 & 60 \\
\hline 04 & 31016 & 286.0150 & 8.0600 & 294.9 & 4 & 600.0 & 600.0 \\
\hline 04 & 31017 & 150 & 823 & 294.4 & 4 & 600.0 & .0 \\
\hline 04 & 31018 & 126.0150 & 5.9683 & 294.2 & 4 & 600.0 & .0 \\
\hline 04 & 31019 & 226.0150 & 335 & 293.9 & 4 & 600.0 & 60 \\
\hline 04 & 31020 & 326.0150 & 4 & 293.8 & 4 & 600.0 & .0 \\
\hline 04 & 31021 & 66.0150 & 3 & 294.2 & 4 & 600.0 & \\
\hline 04 & 31022 & 166.0150 & 9.9675 & 294.5 & 4 & 600.0 & 600 \\
\hline 04 & 31023 & 266.0150 & 11.43 & 294.3 & 4 & 600.0 & .0 \\
\hline 04 & 31024 & 6.0150 & 12 . & 293.5 & 4 & 600.0 & \\
\hline 04 & 3111 & 226.0150 & 850 & 292.1 & 4 & 600.0 & 60 \\
\hline 04 & 3112 & 326.0150 & 6775 & 291.1 & 4 & 600.0 & .0 \\
\hline 04 & 3113 & 66.0150 & .2158 & 290.4 & 4 & 600.0 & \\
\hline 04 & 3114 & 166.0150 & 7.2785 & 289.4 & 4 & 600.0 & 60 \\
\hline 04 & 3115 & 266.0150 & 9.6400 & 289.4 & 4 & 600.0 & .0 \\
\hline 04 & 3116 & 6.0150 & 11 . & 289.1 & 4 & 600.0 & \\
\hline 04 & 3117 & 106.0150 & 10 . & 288.5 & 4 & 600.0 & .0 \\
\hline 04 & 3118 & 206.0150 & 7.4895 & 288.7 & 4 & 600.0 & 600.0 \\
\hline 04 & 3119 & 306.0150 & 6 . & 289.7 & 4 & 600.0 & .0 \\
\hline 04 & 31110 & 150 & 5 . & 290.8 & 4 & 600.0 & .0 \\
\hline 04 & 31111 & 146.0150 & 5.6865 & 291.5 & 3 & 600.0 & 600.0 \\
\hline 04 & 31112 & 246.0150 & 5.9608 & 292.0 & 3 & 600.0 & \\
\hline 04 & 31113 & 346.0150 & 355 & 292.1 & 4 & 600.0 & 600.0 \\
\hline 04 & 31114 & 86.0150 & 5.7918 & 293.0 & 3 & 600.0 & 600.0 \\
\hline 04 & 31115 & 186.0150 & 5 . & 293.5 & 4 & 600.0 & \\
\hline 04 & 31116 & 286.0150 & $5.13^{\circ}$ & 293.7 & 3 & 600.0 & 600.0 \\
\hline 04 & 31117 & 26.0150 & 5.2028 & 293.1 & 4 & 600.0 & 600.0 \\
\hline 04 & 31118 & 126.0150 & 3.5678 & 292.1 & 4 & 600.0 & 600.0 \\
\hline 04 & 31119 & 226.0150 & 3.8330 & 291.6 & 4 & 600.0 & 600.0 \\
\hline 04 & 31120 & 326.0150 & 4.2778 & 291.9 & 4 & 600.0 & 600.0 \\
\hline 04 & 31121 & 66.0150 & 3.9403 & 291.7 & 4 & 600.0 & 600.0 \\
\hline 04 & 31122 & 166.0150 & & 29 & 4 & .0 & 600.0 \\
\hline 04 & 31123 & 266.0150 & 3.479 & 290.7 & 4 & 600.0 & 600.0 \\
\hline
\end{tabular}




\begin{tabular}{|c|c|c|c|c|c|c|c|}
\hline 04 & 31124 & 6.0150 & 4.5590 & 291.7 & 4 & 600.0 & 00.0 \\
\hline 04 & 3121 & 226.0150 & 4.2518 & 291.4 & 4 & 600.0 & 600.0 \\
\hline 04 & 3122 & 326.0150 & 5.7783 & 291.3 & 4 & 600.0 & 00.0 \\
\hline 04 & 3123 & 66.0150 & 4.1033 & 290.5 & 4 & 600.0 & 600.0 \\
\hline 04 & 3124 & 166.0150 & 3.6440 & 289.6 & 4 & 600.0 & 00.0 \\
\hline 04 & 3125 & 266.0150 & 3.5540 & 289.4 & 4 & 600.0 & 00.0 \\
\hline 04 & 3126 & 6.0150 & 3.2683 & 289.0 & 4 & 600.0 & 600.0 \\
\hline 04 & 3127 & 106.0150 & 2.9095 & 288.0 & 4 & 600.0 & 500.0 \\
\hline 04 & 3128 & 206.0150 & 3.0960 & 289.1 & 4 & 600.0 & 00.0 \\
\hline 04 & 3129 & 306.0150 & 5.1060 & 289.8 & 4 & 600.0 & 600.0 \\
\hline 04 & 31210 & 46.0150 & 4.2015 & 290.9 & 3 & 600.0 & 600.0 \\
\hline 04 & 31211 & 146.0150 & 4.4410 & 292.4 & 2 & 600.0 & \\
\hline 04 & 31212 & 246.0150 & 6.3338 & 293.0 & 3 & 600.0 & 600.0 \\
\hline 04 & 31213 & 346.0150 & 9.3150 & 293.1 & 4 & 600.0 & 600.0 \\
\hline 04 & 31214 & 86.0150 & 8 . & 293.5 & 4 & 600.0 & \\
\hline 04 & 31215 & 186.0150 & 6.9855 & 294.1 & 4 & 600.0 & 60 \\
\hline 04 & 31216 & 286.0150 & 6.9083 & 294.7 & 4 & 600.0 & 600.0 \\
\hline 04 & 31217 & 26.0150 & 6.2103 & 294.6 & 3 & 600.0 & \\
\hline 04 & 31218 & 126.0150 & 4.7810 & 294.0 & 4 & 600.0 & 60 \\
\hline 04 & 31219 & 226.0150 & 6.3785 & 293.9 & 4 & 600.0 & 600.0 \\
\hline 04 & 31220 & 326.0150 & 7.5710 & 293.3 & 4 & 600.0 & .0 \\
\hline 04 & 31221 & 66.0150 & 7.8175 & 294.0 & 4 & 600.0 & \\
\hline 04 & 31222 & 166.0150 & 6.4598 & 295.2 & 4 & 600.0 & 600.0 \\
\hline 04 & 31223 & 266.0150 & 5.4710 & 295.1 & 4 & 600.0 & .0 \\
\hline 04 & 31224 & 6.0150 & 7.0473 & 295.6 & 4 & 600.0 & \\
\hline 04 & 3131 & 226.0150 & 8.4450 & 295.2 & 4 & 600.0 & 600.0 \\
\hline 04 & 3132 & 326.0150 & 9.7500 & 294.2 & 4 & 600.0 & 600.0 \\
\hline 04 & 3133 & 66.0150 & 0.3000 & 293.2 & 4 & 600.0 & \\
\hline 04 & 3134 & 166.0150 & 12.9700 & 292.0 & 4 & 600.0 & 60 \\
\hline 04 & 3135 & 266.0150 & 9.4100 & 290.5 & 4 & 600.0 & 600.0 \\
\hline 04 & 3136 & 6.0150 & 4 . & 290.1 & 4 & 600.0 & .0 \\
\hline 04 & 3137 & 106.0150 & 5.2813 & 289.5 & 4 & 600.0 & 60 \\
\hline 04 & 3138 & 206.0150 & 6.5260 & 290.5 & 4 & 600.0 & 600.0 \\
\hline 04 & 3139 & 306.0150 & 8.797 & 291.0 & 4 & 600.0 & .0 \\
\hline 04 & 31310 & 150 & 10.9150 & 291.5 & 4 & 600.0 & 60 \\
\hline 04 & 31311 & 146.0150 & 11.0025 & 292.0 & 4 & 600.0 & 600.0 \\
\hline 04 & 31312 & 246.0150 & 7.0988 & 293.2 & 4 & 600.0 & 600.0 \\
\hline 04 & 31313 & 346.0150 & 7.1178 & 294.0 & 4 & 600.0 & 600.0 \\
\hline 04 & 31314 & 86.0150 & 5.3873 & 294.7 & 3 & 600.0 & 600.0 \\
\hline 04 & 31315 & 186.0150 & 348 & 295.5 & 3 & 600.0 & \\
\hline 04 & 31316 & 286.0150 & 6.2853 & 294.9 & 4 & 600.0 & 600.0 \\
\hline 04 & 31317 & 26.0150 & 4.1613 & 295.1 & 3 & 600.0 & 600.0 \\
\hline 04 & 31318 & 126.0150 & 3.7098 & 294.8 & 4 & 600.0 & 600.0 \\
\hline 04 & 31319 & 226.0150 & $2.850 \varepsilon$ & 294.6 & 4 & 600.0 & 600.0 \\
\hline 04 & 31320 & 326.0150 & 2.9868 & 293.9 & 4 & 600.0 & 600.0 \\
\hline 04 & 31321 & 66.0150 & 2.2223 & 293.8 & 4 & 600.0 & 600.0 \\
\hline 04 & 31322 & 166.0150 & 2.220 & 29 & 5 & 600.0 & 600.0 \\
\hline 04 & 31323 & 266.0150 & 2.5200 & 293.4 & 4 & 600.0 & 600.0 \\
\hline
\end{tabular}




\begin{tabular}{|c|c|c|c|c|c|c|c|}
\hline 04 & 31324 & 6.0150 & 3.4343 & 293.4 & 4 & 600.0 & 600.0 \\
\hline 04 & 3141 & 226.0150 & 5.2633 & 293.1 & 4 & 600.0 & 600.0 \\
\hline 04 & 3142 & 326.0150 & 3.0955 & 293.4 & 4 & 600.0 & 00.0 \\
\hline 04 & 3143 & 66.0150 & 0.9478 & 292.7 & 5 & 600.0 & 00.0 \\
\hline 04 & 3144 & 166.0150 & 1.8240 & 292.4 & o & 600.0 & 00.0 \\
\hline 04 & 3145 & 266.0150 & 0.7320 & 292.6 & 6 & 600.0 & 00.0 \\
\hline 04 & 3146 & 6.0150 & 0.9825 & 291.9 & 6 & 600.0 & 00.0 \\
\hline 04 & 3147 & 106.0150 & 2.7490 & 289.6 & 5 & 600.0 & 00.0 \\
\hline 04 & 3148 & 206.0150 & 2.5775 & 290.5 & 4 & 600.0 & 00.0 \\
\hline 04 & 3149 & 306.0150 & 2.6165 & 291.6 & 3 & 600.0 & 0 \\
\hline 04 & 31410 & 46.0150 & 100 & 292.2 & 3 & 600.0 & 00.0 \\
\hline 04 & 31411 & 146 . & 573 & 292.8 & 3 & 600.0 & \\
\hline 04 & 31412 & 246.0150 & 5.2258 & 293.5 & 3 & 600.0 & 00.0 \\
\hline 04 & 31413 & 346.0150 & 3.7745 & 294.8 & 2 & 600.0 & 00.0 \\
\hline 04 & 31414 & 86. & 45 & 295.5 & 2 & .0 & \\
\hline 04 & 31415 & 186.0150 & 140 & 296.9 & 3 & 600.0 & 00.0 \\
\hline 04 & 31416 & 286.0150 & 3.9148 & 297.6 & 3 & 60 & 600.0 \\
\hline 04 & 31417 & 26.01 & 4 . & 297.2 & 3 & .0 & \\
\hline 04 & 31418 & 126.0150 & 3.4205 & 296.8 & 4 & 60 & .0 \\
\hline 04 & 31419 & 226.0150 & 6 & 295.5 & 4 & 60 & .0 \\
\hline 04 & 31420 & 326 . & 7 . & 294.7 & 4 & 6 & \\
\hline 04 & 31421 & 66.0150 & 6. & 295.2 & 4 & .0 & \\
\hline 04 & 31422 & 166.0150 & 5.7293 & 295.5 & 4 & 600.0 & .0 \\
\hline 04 & 31423 & 266 . & 4 & 295.2 & 4 & 6 & \\
\hline 04 & 31424 & 6 . & 7. & 294.4 & 4 & .0 & \\
\hline 04 & 3151 & 226.0150 & 5.0683 & 293.9 & 4 & 600.0 & 600.0 \\
\hline 04 & 3152 & 326.0150 & 6.6080 & 293.7 & 4 & .0 & .0 \\
\hline 04 & 3153 & 66.0150 & 11.0000 & 292.9 & 4 & .0 & \\
\hline 04 & 3154 & 166.0150 & 11.9625 & 291.9 & 4 & .0 & 0.0 \\
\hline 04 & 3155 & 266.0150 & 11.5325 & 290.9 & 4 & 60 & .0 \\
\hline 04 & 3156 & 6.0150 & 11.0050 & 290.1 & 4 & .0 & \\
\hline 04 & 3157 & 106.0150 & 9. & 290.5 & 4 & 600.0 & 600.0 \\
\hline 04 & 3158 & 206.0150 & 11.4150 & 291.6 & 4 & 600.0 & 600.0 \\
\hline 04 & 3159 & 306.0150 & 10.2200 & 292.4 & 4 & 600.0 & 0.0 \\
\hline 04 & 31510 & 46.0150 & 8.2825 & 292.9 & 4 & 600.0 & 600.0 \\
\hline 04 & 31511 & 146.0150 & 8.5175 & 293.8 & 4 & 600.0 & 600.0 \\
\hline 04 & 31512 & 246.0150 & 9.967 & 294.7 & 4 & 600.0 & 600.0 \\
\hline 04 & 31513 & 355.8470 & 9.6150 & 295.4 & 4 & 600.0 & 600.0 \\
\hline 04 & 31514 & 86.0150 & 6.1513 & 295.5 & 3 & 600.0 & 600.0 \\
\hline 04 & 31515 & 186.0150 & 7.199 & 295.9 & 4 & 600.0 & 600.0 \\
\hline 04 & 31516 & 286.0150 & 7.1680 & 295.9 & 4 & 600.0 & 600.0 \\
\hline 04 & 31517 & 26.0150 & 5.8263 & 295.8 & 3 & 600.0 & 600.0 \\
\hline 04 & 31518 & 126.0150 & 5.7290 & 295.2 & 4 & 600.0 & 600.0 \\
\hline 04 & 31519 & 226.0150 & 6.0060 & 294.6 & 4 & 600.0 & 600.0 \\
\hline 04 & 31520 & 326.0150 & 7.1123 & 295.7 & 4 & 600.0 & 600.0 \\
\hline 04 & 31521 & 66.0150 & 9.9450 & 295.9 & 4 & 600.0 & 600.0 \\
\hline $0<$ & 31522 & 166.0150 & 9.9675 & 295.5 & 4 & 600.0 & 600.0 \\
\hline 04 & 31523 & 266.0150 & 9.955 & 294.8 & 4 & 600.0 & 600.0 \\
\hline
\end{tabular}




\begin{tabular}{|c|c|c|c|c|c|c|c|}
\hline 04 & 31524 & 6.0150 & 11.0350 & 294.0 & 4 & 600.0 & 00.0 \\
\hline 04 & 3161 & 226.0150 & 10.5225 & 293.3 & 4 & 600.0 & 600,0 \\
\hline 04 & 3162 & 326.0150 & 10.5050 & 292.3 & 4 & 600.0 & 00.0 \\
\hline 04 & 3163 & 66.0150 & 11.7200 & 291.9 & 4 & 600.0 & 600.0 \\
\hline 04 & 3164 & 166.0150 & 12.0325 & 291.1 & 4 & 600.0 & 00.0 \\
\hline 04 & 3165 & 266.0150 & 13.6050 & 289.9 & 4 & 600.0 & 00.0 \\
\hline 04 & 3166 & 6.0150 & 12.5550 & 289.2 & 4 & 600.0 & 600.0 \\
\hline 04 & 3167 & 106.0150 & 8.9100 & 288.7 & 4 & 600.0 & 500.0 \\
\hline 04 & 3168 & 206.0150 & 8.3350 & 289.4 & 4 & 600.0 & 00.0 \\
\hline 04 & 3169 & 306.0150 & 8.4300 & 290.2 & 4 & 600.0 & 600.0 \\
\hline 04 & 31610 & 46.0150 & 9.7275 & 291.3 & 4 & 600.0 & 00.0 \\
\hline 04 & 31611 & 146.0150 & 10.4250 & 292.5 & 4 & 600.0 & \\
\hline 04 & 31612 & 246.0150 & 5.6648 & 293.9 & 3 & 600.0 & 600.0 \\
\hline 04 & 31613 & 346.0150 & 5.6318 & 294.6 & 3 & 600.0 & 600.0 \\
\hline 04 & 31614 & 86.0150 & 6.3705 & 295.2 & 4 & 600.0 & \\
\hline 04 & 31615 & 186.0150 & 5.6443 & 295.6 & 3 & 600.0 & 60 \\
\hline 04 & 31616 & 286.0150 & 4.9970 & 296.0 & 3 & 600.0 & 600.0 \\
\hline 04 & 31617 & 26.0150 & 3.9058 & 295.9 & 3 & 600.0 & \\
\hline 04 & 31618 & 126.0150 & 3.8618 & 295.5 & 4 & 600.0 & 60 \\
\hline 04 & 31619 & 226.0150 & 2.7470 & 294.8 & 4 & 600.0 & 600.0 \\
\hline 04 & 31620 & 326.0150 & 3.3288 & 294.5 & 4 & 600.0 & .0 \\
\hline 04 & 31621 & 66.0150 & 4.7095 & 295.0 & 4 & 600.0 & \\
\hline 04 & 31622 & 166.0150 & 4.8750 & 295.2 & 4 & 600.0 & 600.0 \\
\hline 04 & 31623 & 266.0150 & 5.2728 & 295.5 & 4 & 600.0 & .0 \\
\hline 04 & 31624 & 6.0150 & 6.7938 & 295.9 & 4 & 600.0 & \\
\hline 04 & 3171 & 226.0150 & 6.9675 & 295.2 & 4 & 600.0 & 600.0 \\
\hline 04 & 3172 & 326.0150 & 6.9690 & 293.8 & 4 & 600.0 & 600.0 \\
\hline 04 & 3173 & 66.0150 & 8.6400 & 293.1 & 4 & 600.0 & \\
\hline 04 & 3174 & 166.0150 & 7.0805 & 292.2 & 4 & 600.0 & 60 \\
\hline 04 & 3175 & 266.0150 & 7.3323 & 291.5 & 4 & 600.0 & 600.0 \\
\hline 04 & 3176 & 6.0150 & 7.40 & 290.9 & 4 & 600.0 & .0 \\
\hline 04 & 3177 & 106.0150 & 7.0575 & 290.7 & 4 & 600.0 & 60 \\
\hline 04 & 3178 & 206.0150 & 6.2918 & 291.3 & 3 & 600.0 & 600.0 \\
\hline 04 & 3179 & 306.0150 & $4.39^{\circ}$ & 292.2 & 3 & 600.0 & .0 \\
\hline 04 & 31710 & 150 & 4.8838 & 293.1 & 3 & 600.0 & 60 \\
\hline 04 & 31711 & 146.0150 & 5.1625 & 294.4 & 2 & 600.0 & 600.0 \\
\hline 04 & 31712 & 246.0150 & 6.3185 & 294.9 & 3 & 600.0 & 600.0 \\
\hline 04 & 31713 & 346.0150 & 6.4310 & 295.4 & 3 & 600.0 & 600.0 \\
\hline 04 & 31714 & 86.0150 & 5.1450 & 296.2 & 2 & 600.0 & 600.0 \\
\hline 04 & 31715 & 186.0150 & 5.6995 & 296.7 & 3 & 600.0 & \\
\hline 04 & 31716 & 286.0150 & 5.2675 & 296.8 & 3 & 600.0 & 600.0 \\
\hline 04 & 31717 & 26.0150 & 4.8370 & 296.5 & 3 & 600.0 & 600.0 \\
\hline 04 & 31718 & 126.0150 & 5.0465 & 295.9 & 4 & 600.0 & 600.0 \\
\hline 04 & 31719 & 226.0150 & 4.5935 & 295.1 & 4 & 600.0 & 600.0 \\
\hline 04 & 31720 & 326.0150 & 3.6430 & 295.2 & 4 & 600.0 & 600.0 \\
\hline 04 & 31721 & 66.0150 & 3.1270 & 294.8 & 4 & 600.0 & 600.0 \\
\hline 04 & 31722 & 166.0150 & 3.317 & 29 & 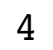 & 600.0 & 600.0 \\
\hline 04 & 31723 & 266.0150 & 3.1133 & 295.3 & 4 & 600.0 & 600.0 \\
\hline
\end{tabular}




\begin{tabular}{|c|c|c|c|c|c|c|c|}
\hline 4 & 31724 & 6.0150 & 3.1593 & 295.3 & 4 & 600.0 & 600.0 \\
\hline 04 & 3181 & 226.0150 & 2.2315 & 294.7 & 4 & 600.0 & 600.0 \\
\hline 04 & 3182 & 326.0150 & 1.2958 & 294.9 & 5 & 600.0 & 00.0 \\
\hline 4 & 3183 & 66.0150 & 0.8125 & 294.6 & 6 & 600.0 & 00.0 \\
\hline 04 & 3184 & 166.0150 & 1.3053 & 294.2 & 6 & 600.0 & 00.0 \\
\hline 04 & 3185 & 266.0150 & 1.5108 & 294.3 & 6 & 600.0 & 00.0 \\
\hline 04 & 3186 & 6.0150 & 0.9243 & 294.6 & 6 & 600.0 & 600.0 \\
\hline 04 & 3187 & 106.0150 & 1.1398 & 294.3 & 5 & 600.0 & 00.0 \\
\hline 04 & 3188 & 206.0150 & 1.0633 & 294.6 & 4 & 600.0 & 600.0 \\
\hline 04 & 3189 & 306.0150 & 1.0325 & 294.3 & 3 & 600.0 & 600 \\
\hline 04 & 31810 & 46.0150 & 1.8350 & 294.5 & 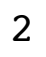 & 600.0 & \\
\hline 04 & 31811 & 146.0150 & 3.6365 & 294.1 & 2 & 600.0 & .0 \\
\hline 04 & 31812 & 246.0150 & 2.9870 & 295.4 & 2 & 600.0 & 600 \\
\hline 04 & 31813 & 346.0150 & 3.7125 & 296.3 & 2 & 600.0 & 600.0 \\
\hline 04 & 31814 & 150 & 4.4410 & 296.7 & 2 & 600.0 & 60 \\
\hline 04 & 31815 & 186.0150 & 3.5173 & 298.5 & 3 & 600.0 & 600.0 \\
\hline 04 & 31816 & 286.0150 & 2.8175 & 298.2 & 3 & 600.0 & .0 \\
\hline 04 & 31817 & 26.0150 & 3.0605 & 298.8 & 3 & .0 & \\
\hline 04 & 31818 & 126.0150 & 5.2038 & 296.4 & 4 & 600.0 & 600.0 \\
\hline 04 & 31819 & 226.0150 & 6.0 & 295.3 & 4 & 60 & .0 \\
\hline 04 & 31820 & 326.0150 & 7.1 & 295.7 & 4 & .0 & \\
\hline 04 & 31821 & 66.0150 & 7.9775 & 295.4 & 4 & .0 & 60 \\
\hline 04 & 31822 & 166.0150 & 9.1700 & 295.1 & 4 & .0 & 600.0 \\
\hline 04 & 31823 & 266.0150 & 10.24 & 294.6 & 4 & .0 & \\
\hline 04 & 31824 & 6.0150 & 11.0175 & 294.1 & 4 & .0 & 60 \\
\hline 04 & 3191 & 226.0150 & 9.1303 & 294.1 & 4 & 600.0 & 600.0 \\
\hline 04 & 3192 & 326.0150 & 4.9 & 293.4 & 4 & 600.0 & .0 \\
\hline 04 & 3193 & 66.0150 & 7.5018 & 292.9 & 4 & 600.0 & 60 \\
\hline 04 & 3194 & 166.0150 & 6.8183 & 292.5 & 4 & 600.0 & 600.0 \\
\hline 04 & 3195 & 266.0150 & 7.04 & 292.6 & 4 & 600.0 & .0 \\
\hline 04 & 3196 & 6.0150 & 3.8 & 292.3 & 4 & .0 & .0 \\
\hline 04 & 3197 & 106.0150 & 5.5883 & 292.8 & 4 & .0 & 600.0 \\
\hline 04 & 3198 & 206.0150 & 5.6540 & 293.7 & 4 & 600.0 & 600.0 \\
\hline 04 & 3199 & 306.0150 & 4.7968 & 295.2 & 3 & 600.0 & 600.0 \\
\hline 04 & 31910 & 46.0150 & 3.5368 & 297.0 & 3 & 600.0 & 600.0 \\
\hline 04 & 31911 & 146.0150 & 3.6808 & 297.2 & 2 & 600.0 & 600.0 \\
\hline 04 & 31912 & 246.0150 & 3.6230 & 295.4 & - & 600.0 & 600.0 \\
\hline 04 & 31913 & 346.0150 & 3.1245 & 296.7 & 2 & 600.0 & 600.0 \\
\hline 04 & 31914 & 86.0150 & 3.1973 & 298.2 & 2 & 600.0 & 600.0 \\
\hline 04 & 31915 & 186.0150 & 6.3273 & 297.2 & 3 & 600.0 & 600.0 \\
\hline 04 & 31916 & 286.0150 & 5.1120 & 297.1 & 3 & 600.0 & 600.0 \\
\hline 04 & 31917 & 26.0150 & 4.0123 & 296.4 & 3 & 600.0 & 600.0 \\
\hline 04 & 31918 & 126.0150 & 3.6533 & 295.0 & 4 & 600.0 & 600.0 \\
\hline 04 & 31919 & 226.0150 & 3.2595 & 293.9 & 4 & 600.0 & 600.0 \\
\hline 04 & 31920 & 326.0150 & 4.1380 & 294.1 & 4 & 600.0 & 600.0 \\
\hline 04 & 31921 & 66.0150 & 5.6778 & 294.0 & 4 & 600.0 & 600.0 \\
\hline 04 & 31922 & 166.0150 & 5.0025 & 294.1 & 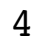 & 600.0 & 600.0 \\
\hline 4 & 31923 & 266.0150 & 5.8793 & 293.6 & 4 & 600.0 & 600.0 \\
\hline
\end{tabular}




\begin{tabular}{|c|c|c|c|c|c|c|c|}
\hline 4 & 31924 & 6.0150 & 5.8808 & 293.7 & 4 & 600.0 & 00.0 \\
\hline 04 & 3201 & 226.0150 & 1.9705 & 294.0 & 5 & 600.0 & 600,0 \\
\hline 04 & 3202 & 326.0150 & 1.2475 & 293.0 & 6 & 600.0 & 00.0 \\
\hline 04 & 3203 & 66.0150 & 1.4505 & 292.7 & 6 & 600.0 & 600.0 \\
\hline 04 & $320 \quad 4$ & 166.0150 & 0.9148 & 292.1 & 0 & 600.0 & 00.0 \\
\hline 04 & 3205 & 266.0150 & 2.5065 & 291.3 & 5 & 600.0 & 00.0 \\
\hline 04 & 3206 & 6.0150 & 3.9458 & 288.8 & 4 & 600.0 & 600.0 \\
\hline 04 & 3207 & 106.0150 & 4.2143 & 287.4 & 4 & 600.0 & 500.0 \\
\hline 04 & 3208 & 206.0150 & 4.0000 & 287.7 & 3 & 600.0 & 00.0 \\
\hline 04 & 3209 & 306.0150 & 3.3478 & 290.2 & 3 & 600.0 & .0 \\
\hline 04 & 32010 & 46.0150 & 3.3598 & 291.5 & 3 & 600.0 & 500.0 \\
\hline 04 & 32011 & 146.0150 & 3.5480 & 293.0 & 2 & 600.0 & \\
\hline 04 & 32012 & 246.0150 & 4.2875 & 294.1 & 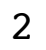 & 600.0 & 600.0 \\
\hline 04 & 32013 & 346.0150 & 5.0140 & 294.1 & 2 & 600.0 & 600.0 \\
\hline 04 & 32014 & 86.0150 & 4.3515 & 294.8 & 2 & 600.0 & \\
\hline 04 & 32015 & 186.0150 & 4.5073 & 295.3 & 2 & 600.0 & 60 \\
\hline 04 & 32016 & 286.0150 & 3.17 & 295.5 & 3 & 600.0 & 600.0 \\
\hline 04 & 32017 & 150 & 3.46 & 295.3 & 3 & 600.0 & .0 \\
\hline 04 & 32018 & 126.0150 & 2.5830 & 294.5 & 4 & 600.0 & 60 \\
\hline 04 & 32019 & 226.0150 & 1.1820 & 295.2 & 5 & 600.0 & 60 \\
\hline 04 & 32020 & 326.0150 & 1.3978 & 295.2 & 5 & 600.0 & .0 \\
\hline 04 & 32021 & 66.0150 & 3.7458 & 294.8 & 4 & 600.0 & \\
\hline 04 & 32022 & 166.0150 & 3.6283 & 294.9 & 4 & 600.0 & 600 \\
\hline 04 & 32023 & 266.0150 & 0.8195 & 295.4 & 5 & 600.0 & .0 \\
\hline 04 & 32024 & 6.0150 & 467 & 294.2 & 4 & 600.0 & \\
\hline 04 & 3211 & 226.0150 & 1.7218 & 294.8 & 5 & 600.0 & 60 \\
\hline 04 & 3212 & 326.0150 & 2.8543 & 294.8 & 4 & 600.0 & 60 \\
\hline 04 & 3213 & 66.0150 & 1.8088 & 294.4 & 5 & 600.0 & \\
\hline 04 & 3214 & 166.0150 & 1.0355 & 294.6 & 6 & 600.0 & 60 \\
\hline 04 & 3215 & 266.0150 & 1.2985 & 294.0 & 6 & 600.0 & 600.0 \\
\hline 04 & 3216 & 6.0150 & 0.9898 & 293.2 & 6 & 600.0 & \\
\hline 04 & 3217 & 106.0150 & 2.0863 & 291.5 & 5 & 600.0 & 60 \\
\hline 04 & 3218 & 206.0150 & 2.1555 & 292.3 & 4 & 600.0 & 600.0 \\
\hline 04 & 3219 & 306.0150 & 3.4160 & 293.3 & 3 & 600.0 & .0 \\
\hline 04 & 32110 & 150 & 5.8 & 292.1 & 3 & 600.0 & .0 \\
\hline 04 & 32111 & 146.0150 & 2.7478 & 295.0 & 2 & 600.0 & 600.0 \\
\hline 04 & 32112 & 246.0150 & 3.5783 & 295.3 & 2 & 600.0 & \\
\hline 04 & 32113 & 346.0150 & 4.9358 & 295.8 & 2 & 600.0 & 600.0 \\
\hline 04 & 32114 & 86.0150 & 5.9593 & 296.3 & 3 & 600.0 & 600.0 \\
\hline 04 & 32115 & 186.0150 & 5.0528 & 296.7 & 2 & 600.0 & \\
\hline 04 & 32116 & 286.0150 & 4.8698 & 295.9 & 3 & 600.0 & 600.0 \\
\hline 04 & 32117 & 26.0150 & 6.7130 & 294.7 & 4 & 600.0 & 600.0 \\
\hline 04 & 32118 & 126.0150 & 8.8950 & 294.0 & 4 & 600.0 & 600.0 \\
\hline 04 & 32119 & 226.0150 & 10.3375 & 293.3 & 4 & 600.0 & 600.0 \\
\hline 04 & 32120 & 326.0150 & 9.0275 & 293.7 & 4 & 600.0 & 600.0 \\
\hline 04 & 32121 & 66.0150 & 8.9425 & 293.2 & 4 & 600.0 & 600.0 \\
\hline 04 & 32122 & 166.0150 & 9.2575 & 292.4 & 4 & 600.0 & 600.0 \\
\hline 04 & 32123 & 266.0150 & 10.9575 & 291.3 & 4 & 600.0 & 600.0 \\
\hline
\end{tabular}




\begin{tabular}{|c|c|c|c|c|c|c|c|}
\hline & 32124 & 6.0150 & 10.7225 & 291.7 & 4 & 600.0 & 600.0 \\
\hline 04 & 3221 & 226.0150 & 10.6225 & 292.4 & 4 & 600.0 & 00,0 \\
\hline 4 & 3222 & 326.0150 & 9.8625 & 292.3 & 4 & 600.0 & 00.0 \\
\hline 04 & 3223 & 66.0150 & 10.6650 & 291.4 & 4 & 600.0 & 0 \\
\hline 04 & 3224 & 166.0150 & 125 & 291.5 & 4 & 00.0 & 0 \\
\hline 04 & 3225 & 266.0150 & 8.3000 & 291.8 & 4 & 600.0 & 00.0 \\
\hline 04 & 3226 & 6.0150 & 10.8550 & 289.0 & 4 & 600.0 & 0 \\
\hline 04 & 3227 & 106.0150 & 10.5700 & 289.2 & 4 & 600.0 & 00.0 \\
\hline 04 & 3228 & 206.0150 & 8.6800 & 290.2 & 4 & 600.0 & 00.0 \\
\hline 04 & 3229 & 306.0150 & 028 & 290.7 & 3 & .0 & \\
\hline 04 & 32210 & 46 . & 2.2580 & 291.3 & 3 & 600.0 & \\
\hline 04 & 32211 & 146 . & 805 & 292.6 & 2 & .0 & \\
\hline 04 & 32212 & 246.0150 & 2.1895 & 294.2 & 1 & 600.0 & \\
\hline 04 & 32213 & 346 . & 3.6133 & 294.4 & 1 & 600.0 & \\
\hline 04 & 32214 & 86. & 23 & 293.2 & 2 & .0 & \\
\hline 04 & 32215 & 186.0150 & 350 & 292.5 & 3 & 600.0 & \\
\hline 04 & 32216 & 286.0150 & 11.3900 & 291.7 & 4 & 60 & .0 \\
\hline 04 & 32217 & 26.01 & 11.6750 & 290.5 & 4 & .0 & \\
\hline 04 & 32218 & 126.0150 & 11.8825 & 288.6 & 4 & 60 & \\
\hline 04 & 32219 & 226.0150 & 12.3225 & 286.7 & 4 & .0 & .0 \\
\hline 04 & 32220 & 326 . & 13 & 286 & 4 & 0 & \\
\hline 04 & 32221 & 66 . & 12.7750 & 286.2 & 4 & .0 & \\
\hline 04 & 32222 & 166.0150 & 12.5425 & 286.2 & 4 & 60 & .0 \\
\hline 04 & 32223 & 266 . & 12.57 & 28 & 4 & 0 & \\
\hline 04 & 32224 & 6. & 12 . & 286 & 4 & 0 & \\
\hline 04 & 3231 & 226.0150 & 13.7350 & 284.7 & 4 & .0 & \\
\hline 04 & 3232 & 326.0150 & 14.3225 & 283.8 & 4 & 6 & \\
\hline 04 & 3233 & 66.0150 & 13.9000 & 284.6 & 4 & .0 & \\
\hline 04 & 3234 & 166.0150 & 14.0750 & 283.9 & 4 & .0 & \\
\hline 04 & 3235 & 266.0150 & 13.2450 & 283.8 & 4 & .0 & \\
\hline 04 & 3236 & 6.0150 & 13.4450 & 283.3 & 4 & 0 & \\
\hline 04 & 3237 & 106.0150 & 12.2250 & 284.2 & 4 & .0 & .0 \\
\hline 04 & 3238 & 206.0150 & 10.0975 & 286.8 & 4 & 6 & .0 \\
\hline 04 & 3239 & 306.0150 & 10.5750 & 287.0 & 4 & .0 & .0 \\
\hline 04 & 32310 & 46.0150 & 9.5725 & 287.9 & 4 & .0 & 0 \\
\hline 04 & 32311 & 146.0150 & 9.3950 & 288.2 & 4 & 600.0 & 0.0 \\
\hline 04 & 32312 & 246 . & 10.8125 & 288.1 & 4 & .0 & \\
\hline 04 & 32313 & 346.0150 & 10.1650 & 289.4 & 4 & 600.0 & 600.0 \\
\hline 04 & 32314 & 86.0150 & 9.8300 & 290.5 & 4 & 600.0 & 600.0 \\
\hline 04 & 32315 & 186. & 8.7600 & 291.0 & 4 & .0 & .0 \\
\hline 04 & 32316 & 286.0150 & 8.5350 & 289.9 & 4 & 600.0 & 600.0 \\
\hline 04 & 32317 & 26.0150 & 10.0950 & 288.6 & 4 & 600.0 & 600.0 \\
\hline 04 & 32318 & 126.0150 & 8.3500 & 286.9 & 4 & 600.0 & 600.0 \\
\hline 04 & 32319 & 226.0150 & 10.1625 & 285.3 & 4 & 600.0 & 600.0 \\
\hline 04 & 32320 & 326.0150 & 12.9900 & 284.1 & 4 & 600.0 & 600.0 \\
\hline 04 & 32321 & 66.0150 & 11.3025 & 284.4 & 4 & 600.0 & \\
\hline $0<$ & 32322 & 166.0150 & 11.917 & 285.5 & 4 & 600.0 & 600.0 \\
\hline & 32323 & 266.0150 & 12.5575 & 285.6 & & 600.0 & 600.0 \\
\hline
\end{tabular}




\begin{tabular}{|c|c|c|c|c|c|c|c|}
\hline 4 & 32324 & 6.0150 & 10.7200 & 285.5 & 4 & 600.0 & 600.0 \\
\hline 04 & 3241 & 226.0150 & 6.3105 & 285.0 & 4 & 600.0 & 600.0 \\
\hline 4 & 3242 & 326.0150 & 8.7500 & 285.4 & 4 & 600.0 & 00.0 \\
\hline 04 & 3243 & 66.0150 & 10.0225 & 284.9 & 4 & 600.0 & 00.0 \\
\hline 04 & 3244 & 166.0150 & 9.7200 & 283.6 & 4 & 600.0 & 00.0 \\
\hline 04 & 3245 & 266.0150 & 9.0325 & 283.4 & 4 & 600.0 & 00.0 \\
\hline 04 & 3246 & 6.0150 & 9.2225 & 283.4 & 4 & 600.0 & 00.0 \\
\hline 04 & 3247 & 106.0150 & 9.5525 & 283.2 & 4 & 600.0 & 00.0 \\
\hline 04 & 3248 & 206.0150 & 8.5325 & 283.9 & 4 & 600.0 & 00.0 \\
\hline 04 & 3249 & 306.0150 & 7.1120 & 285.5 & 4 & 600 & 0 \\
\hline 04 & 32410 & 46.0150 & 5.8848 & 287.5 & 3 & 600.0 & \\
\hline 04 & 32411 & 146 . & 6.1863 & 288.3 & 3 & 600.0 & \\
\hline 04 & 32412 & 246.0150 & 5.6913 & 289.0 & 2 & 600.0 & 00.0 \\
\hline 04 & 32413 & 346.0150 & 7.0070 & 289.9 & 3 & 600.0 & 00.0 \\
\hline 04 & 32414 & 86. & 768 & 290.6 & 3 & .0 & \\
\hline 04 & 32415 & 186.0150 & 6.9408 & 290.9 & 4 & 600.0 & .0 \\
\hline 04 & 32416 & 286.0150 & 6.7078 & 290.5 & 4 & 60 & 00.0 \\
\hline 04 & 32417 & 26.0150 & 8.2088 & 289.2 & 4 & .0 & \\
\hline 04 & 32418 & 126.0150 & 10.1550 & 287.7 & 4 & .0 & .0 \\
\hline 04 & 32419 & 226.0150 & 11.0075 & 286.2 & 4 & .0 & .0 \\
\hline 04 & 32420 & 326 . & 13.9600 & 285.0 & 4 & 6 & \\
\hline 04 & 32421 & 66.0150 & 14.7700 & 284.2 & 4 & .0 & \\
\hline 04 & 32422 & 166.0150 & 11.9600 & 284.4 & 4 & 600.0 & .0 \\
\hline 04 & 32423 & 266 . & 7.1058 & 284.6 & 4 & 6 & \\
\hline 04 & 32424 & 6 . & 4.0335 & 284.1 & 4 & .0 & \\
\hline 04 & 3251 & 226.0150 & 4.2475 & 284.1 & 4 & 600.0 & 600.0 \\
\hline 04 & 3252 & 326.0150 & 3.1908 & 283.6 & 4 & .0 & .0 \\
\hline 04 & 3253 & 66.0150 & 1.7910 & 283.0 & 5 & .0 & \\
\hline 04 & 3254 & 166.0150 & 1.8948 & 283.4 & 5 & .0 & .0 \\
\hline 04 & 3255 & 266.0150 & 2.9498 & 283.7 & 4 & .0 & .0 \\
\hline 04 & 3256 & 6.0150 & 3.6063 & 283.4 & 4 & 0 & \\
\hline 04 & 3257 & 106.0150 & 2.4085 & 283.7 & 4 & .0 & 0.0 \\
\hline 04 & 3258 & 206.0150 & 1.3463 & 284.1 & 3 & 600.0 & 0.0 \\
\hline 04 & 3259 & 306.0150 & 1.7415 & 285.4 & 2 & .0 & 0.0 \\
\hline 04 & 32510 & 46.0150 & 2.86 & 285.9 & 3 & 600.0 & 600.0 \\
\hline 04 & 32511 & 146.0150 & 3.7800 & 286.7 & 3 & 600.0 & 600.0 \\
\hline 04 & 32512 & 246.0150 & 3.7438 & 287.4 & 3 & 600.0 & .0 \\
\hline 04 & 32513 & 346.0150 & 1.8433 & 287.9 & 2 & 600.0 & 600.0 \\
\hline 04 & 32514 & 86.0150 & 4.4215 & 288.1 & 3 & 600.0 & 600.0 \\
\hline 04 & 32515 & 186. & 3.5603 & 287 & 4 & 600.0 & 600.0 \\
\hline 04 & 32516 & 286.0150 & 5.8625 & 286.0 & 4 & 600.0 & 600.0 \\
\hline 04 & 32517 & 26.0150 & 4.2278 & 286.0 & 4 & 600.0 & 600.0 \\
\hline 04 & 32518 & 126.0150 & 2.1463 & 286.2 & 5 & 600.0 & 600.0 \\
\hline 04 & 32519 & 226.0150 & 5.7958 & 284.9 & 4 & 600.0 & 600.0 \\
\hline 04 & 32520 & 326.0150 & 5.8433 & 282.9 & 4 & 600.0 & 600.0 \\
\hline 04 & 32521 & 66.0150 & 6.1975 & 282.7 & 4 & 600.0 & 600.0 \\
\hline $0<$ & 32522 & 166.0150 & 5.358 & 282.4 & 4 & 600.0 & 600.0 \\
\hline 04 & 32523 & 266.0150 & 6.3693 & 281.9 & 4 & 600.0 & 600.0 \\
\hline
\end{tabular}




\begin{tabular}{|c|c|c|c|c|c|c|c|}
\hline 4 & 32524 & 6.0150 & 7.1500 & 281.3 & 4 & 600.0 & 600.0 \\
\hline 04 & 3261 & 226.0150 & 4.8958 & 280.8 & 4 & 600.0 & 600.0 \\
\hline 04 & 3262 & 326.0150 & 393 & 280.4 & 4 & 600.0 & 00.0 \\
\hline 04 & 3263 & 66.0150 & .9370 & 280.5 & 4 & 600.0 & 00.0 \\
\hline 04 & 3264 & 166.0150 & .7303 & 280.6 & 4 & 600.0 & 00.0 \\
\hline 04 & 3265 & 266.0150 & 7.4775 & 280.5 & 4 & 600.0 & 00.0 \\
\hline 04 & 3266 & 6.0150 & 6.7325 & 280.3 & 4 & 600.0 & 00.0 \\
\hline 04 & 3267 & 106.0150 & 6.2235 & 280.5 & 4 & 600.0 & 00.0 \\
\hline 04 & 3268 & 206.0150 & 4.9033 & 281.3 & 3 & 600.0 & 00.0 \\
\hline 04 & 3269 & 306.0150 & 6.1808 & 282.3 & 4 & 600.0 & 00.0 \\
\hline 04 & 32610 & 46.0150 & 5.7738 & 283.4 & 4 & 600.0 & 00.0 \\
\hline 04 & 32611 & 146.0150 & 5.5410 & 284.0 & 4 & 600.0 & 00.0 \\
\hline 04 & 32612 & 246.0150 & 5.6435 & 284.8 & 3 & 600.0 & 00.0 \\
\hline 04 & 32613 & 346.0150 & 5.8760 & 285.9 & 3 & 600.0 & 00.0 \\
\hline 04 & 32614 & 86.0150 & 5.7105 & 285.6 & 3 & .0 & \\
\hline 04 & 32615 & 186.0150 & 5.4890 & 285.5 & 3 & 600.0 & 00.0 \\
\hline 04 & 32616 & 286.0150 & 6.5555 & 285.4 & 4 & 600.0 & 600.0 \\
\hline 04 & 32617 & 26.0150 & 5.3595 & 285.1 & 3 & .0 & \\
\hline 04 & 32618 & 126.0150 & 6.5148 & 284.1 & 4 & 600.0 & .0 \\
\hline 04 & 32619 & 226.0150 & 4.7173 & 283.4 & 4 & .0 & .0 \\
\hline 04 & 32620 & 326 . & 7.3630 & 283.0 & 4 & .0 & \\
\hline 04 & 32621 & 66.0150 & 4.7878 & 282.9 & 4 & 600.0 & \\
\hline 04 & 32622 & 166.0150 & 6.1520 & 282.7 & 4 & 600.0 & 500.0 \\
\hline 04 & 32623 & 266.0150 & 7.7273 & 282.7 & 4 & 60 & .0 \\
\hline 04 & 32624 & 6.0150 & 7.9100 & 282.7 & 4 & .0 & \\
\hline 04 & 3271 & 226.0150 & 7.8875 & 282.7 & 4 & 600.0 & 600.0 \\
\hline 04 & 3272 & 326.0150 & 7.5125 & 282.6 & 4 & 600.0 & .0 \\
\hline 04 & 3273 & 66.0150 & 6.5913 & 282.8 & 4 & 600.0 & \\
\hline 04 & 3274 & 166.0150 & 7.5300 & 283.1 & 4 & 600.0 & 600.0 \\
\hline 04 & 3275 & 266.0150 & 7.2675 & 282.9 & 4 & 600.0 & 600.0 \\
\hline 04 & 3276 & 6.0150 & 7.1390 & 283.0 & 4 & 600.0 & \\
\hline 04 & 3277 & 106.0150 & 5.4750 & 283.5 & 4 & 600.0 & 600.0 \\
\hline 04 & 3278 & 206.0150 & 6.1350 & 284.0 & 4 & 600.0 & 600.0 \\
\hline 04 & 3279 & 306.0150 & 4.4975 & 284.7 & 3 & 600.0 & 0.0 \\
\hline 04 & 32710 & 46.0150 & 4.7828 & 285.7 & 2 & 600.0 & 600.0 \\
\hline 04 & 32711 & 146.0150 & 5.7188 & 286.6 & 3 & 600.0 & 600.0 \\
\hline 04 & 32712 & 246.0150 & 6.6395 & 287.6 & 3 & 600.0 & 600.0 \\
\hline 04 & 32713 & 346.0150 & 7.3318 & 288.2 & 3 & 600.0 & 600.0 \\
\hline 04 & 32714 & 86.0150 & 6.7015 & 288.8 & 4 & 600.0 & 600.0 \\
\hline 04 & 32715 & 186.0150 & 7.6625 & 288.7 & 4 & 600.0 & 600.0 \\
\hline 04 & 32716 & 286.0150 & 7.4483 & 288.5 & 4 & 600.0 & 600.0 \\
\hline 04 & 32717 & 26.0150 & 7.1870 & 288.2 & 4 & 600.0 & 600.0 \\
\hline 04 & 32718 & 126.0150 & 6.5533 & 287.5 & 4 & 600.0 & 600.0 \\
\hline 04 & 32719 & 226.0150 & 4.9180 & 286.3 & 4 & 600.0 & 600.0 \\
\hline 04 & 32720 & 326.0150 & 3.9223 & 286.1 & 4 & 600.0 & 600.0 \\
\hline 04 & 32721 & 66.0150 & 4.2980 & 286.1 & 4 & 600.0 & 600.0 \\
\hline $0<$ & 32722 & 166.0150 & 4.9255 & 286.1 & 4 & 600.0 & 600.0 \\
\hline 04 & 32723 & 266.0150 & 6.1915 & 286.3 & 4 & 600.0 & 600.0 \\
\hline
\end{tabular}




\begin{tabular}{|c|c|c|c|c|c|c|c|}
\hline 04 & 32724 & 6.0150 & 5.7518 & 286.2 & 4 & 600.0 & 600.0 \\
\hline 04 & 3281 & 226.0150 & 6.1155 & 286.2 & 4 & 600.0 & 600.0 \\
\hline 04 & $328 \quad 2$ & 326.0150 & 4.7458 & 286.1 & 4 & 600.0 & 00.0 \\
\hline 04 & 3283 & 66.0150 & 5.2760 & 285.8 & 4 & 600.0 & 600.0 \\
\hline 04 & 3284 & 166.0150 & 7.8218 & 285.7 & 4 & 600.0 & 00.0 \\
\hline 04 & 3285 & 266.0150 & 8.5175 & 285.7 & 4 & 600.0 & 00.0 \\
\hline 04 & 3286 & 6.0150 & 8.4250 & 285.0 & 4 & 600.0 & 600.0 \\
\hline 04 & 3287 & 106.0150 & 7.7700 & 284.9 & 4 & 600.0 & 500.0 \\
\hline 04 & 3288 & 206.0150 & 7.4900 & 286.0 & 4 & 600.0 & 00.0 \\
\hline 04 & 3289 & 306.0150 & 6.7698 & 286.7 & 4 & 600.0 & 600.0 \\
\hline 04 & 32810 & 46.0150 & 6.9825 & 287.6 & 4 & 600.0 & 600.0 \\
\hline 04 & 32811 & 146.0150 & 5.6385 & 288.9 & 3 & 600.0 & \\
\hline 04 & 32812 & 246.0150 & 3.4913 & 290.2 & 2 & 600.0 & 600.0 \\
\hline 04 & 32813 & 346.0150 & 3.2935 & 292.0 & 2 & 600.0 & 600.0 \\
\hline 04 & 32814 & 86.0150 & 4.2598 & 292.4 & 2 & 600.0 & \\
\hline 04 & 32815 & 186.0150 & 2.6675 & 292.8 & 2 & 600.0 & 60 \\
\hline 04 & 32816 & 286.0150 & 2.2548 & 294.0 & 2 & 600.0 & 600.0 \\
\hline 04 & 32817 & 26.0150 & 2.4850 & 293.8 & 2 & 600.0 & .0 \\
\hline 04 & 32818 & 126.0150 & 3.1243 & 292.1 & 3 & 600.0 & 60 \\
\hline 04 & 32819 & 226.0150 & 1.5668 & 291.4 & 4 & 600.0 & 600.0 \\
\hline 04 & 32820 & 326.0150 & 1.6693 & 291.1 & 5 & 600.0 & .0 \\
\hline 04 & 32821 & 66.0150 & 1.6400 & 291.3 & 6 & 600.0 & \\
\hline 04 & 32822 & 166.0150 & 0.8340 & 291.7 & 6 & 600.0 & 600.0 \\
\hline 04 & 32823 & 266.0150 & 1.4773 & 291.3 & 6 & 600.0 & .0 \\
\hline 04 & 32824 & 6.0150 & 1.2850 & 291.0 & 6 & 600.0 & \\
\hline 04 & 3291 & 226.0150 & 1.4220 & 291.2 & 6 & 600.0 & 600.0 \\
\hline 04 & 3292 & 326.0150 & 1.5940 & 290.7 & 6 & 600.0 & 600.0 \\
\hline 04 & 3293 & 66.0150 & 1.6348 & 290.5 & 6 & 600.0 & .0 \\
\hline 04 & 3294 & 166.0150 & 1.8270 & 290.7 & 5 & 600.0 & 600.0 \\
\hline 04 & 3295 & 266.0150 & 1.7723 & 291.0 & 5 & 600.0 & 600.0 \\
\hline 04 & 3296 & 6.0150 & 0.9135 & 291.5 & 6 & 600.0 & .0 \\
\hline 04 & 3297 & 106.0150 & 1.8108 & 290.9 & 5 & 600.0 & 60 \\
\hline 04 & 3298 & 206.0150 & 0.6610 & 290.9 & 4 & 600.0 & 600.0 \\
\hline 04 & 3299 & 306.0150 & 1.6293 & 292.0 & 3 & 600.0 & .0 \\
\hline 04 & 32910 & 46.0150 & 2.3235 & 292.0 & 3 & 600.0 & 60 \\
\hline 04 & 32911 & 146.0150 & 2.6753 & 292.3 & 2 & 600.0 & 600.0 \\
\hline 04 & 32912 & 246.0150 & 2.7583 & 293.8 & 2 & 600.0 & 600.0 \\
\hline 04 & 32913 & 346.0150 & 2.8053 & 295.4 & 3 & 600.0 & 600.0 \\
\hline 04 & 32914 & 86.0150 & 3.2785 & 296.1 & 3 & 600.0 & 600.0 \\
\hline 04 & 32915 & 186.0150 & 2.8028 & 297.4 & 2 & 600.0 & \\
\hline 04 & 32916 & 286.0150 & 2.7500 & 297.6 & 3 & 600.0 & 600.0 \\
\hline 04 & 32917 & 26.0150 & 4.1953 & 297.5 & 3 & 600.0 & 600.0 \\
\hline 04 & 32918 & 126.0150 & 5.0675 & 295.6 & 4 & 600.0 & 600.0 \\
\hline 04 & 32919 & 226.0150 & 6.1233 & 294.0 & 4 & 600.0 & 600.0 \\
\hline 04 & 32920 & 326.0150 & 6.6185 & 292.7 & 4 & 600.0 & 600.0 \\
\hline 04 & 32921 & 66.0150 & 6.0603 & 293.9 & 4 & 600.0 & 600.0 \\
\hline 04 & 32922 & 166.0150 & 5.6633 & & 4 & 600.0 & 600.0 \\
\hline 04 & 32923 & 266.0150 & 4.2273 & 290.7 & 4 & 600.0 & 600.0 \\
\hline
\end{tabular}




\begin{tabular}{|c|c|c|c|c|c|c|c|}
\hline 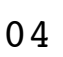 & 32924 & 6.0150 & 5.9323 & 287.0 & 4 & 600.0 & 600.0 \\
\hline 04 & 3301 & 226.0150 & 4.3053 & 288.5 & 4 & 600.0 & 600.0 \\
\hline 04 & 3302 & 326.0150 & 9.1580 & 285.6 & 4 & 600.0 & 00.0 \\
\hline 04 & 3303 & 66.0150 & 14.3675 & 282.6 & 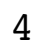 & 600.0 & 00.0 \\
\hline 04 & 3304 & 166.0150 & 14.4250 & 282.6 & 4 & 600.0 & 00.0 \\
\hline 04 & 3305 & 266.0150 & 025 & 282.8 & 4 & 600.0 & 00.0 \\
\hline 04 & 3306 & 6.0150 & 12.3575 & 283.0 & 4 & 600.0 & 600.0 \\
\hline 04 & 3307 & 106.0150 & 11.3100 & 283.5 & 4 & 600.0 & .0 \\
\hline 04 & 3308 & 206.0150 & 11.1175 & 283.8 & 4 & 600.0 & 600.0 \\
\hline 04 & 3309 & 306.0150 & 10.2925 & 284.3 & 4 & 600.0 & 00.0 \\
\hline 04 & 33010 & 46.0150 & 10.2775 & 284.4 & 4 & 600.0 & \\
\hline 04 & 33011 & 146.0150 & 9.9350 & 285.4 & 4 & 600.0 & 60 \\
\hline 04 & 33012 & 246.0150 & 8.4400 & 286.0 & 4 & 600.0 & 600 \\
\hline 04 & 33013 & 346.0150 & 6.5835 & 286.2 & 3 & 600.0 & 00.0 \\
\hline 04 & 33014 & 150 & 7.7018 & 286.8 & 4 & 600.0 & 60 \\
\hline 04 & 33015 & 186.0150 & 7.4638 & 286.3 & 4 & 600.0 & 600.0 \\
\hline 04 & 33016 & 286.0150 & 8.8425 & 285.3 & 4 & 600.0 & .0 \\
\hline 04 & 33017 & 26 . & 8 . & 285.3 & 4 & .0 & \\
\hline 04 & 33018 & 126.0150 & 8.6900 & 285.0 & 4 & 600.0 & 600.0 \\
\hline 04 & 33019 & 226 . & 8.44 & 284.2 & 4 & .0 & .0 \\
\hline 04 & 33020 & 326 . & 8 . & 284.0 & 4 & .0 & \\
\hline 04 & 33021 & 66.0150 & 8.6625 & 283.9 & 4 & .0 & 60 \\
\hline 04 & 33022 & 166.0150 & 8.5350 & 283.9 & 4 & .0 & 600.0 \\
\hline 04 & 33023 & 266.0150 & 9.6250 & 283.2 & 4 & .0 & \\
\hline 04 & 33024 & 6.0150 & 9.7900 & 282.8 & 4 & .0 & 60 \\
\hline 04 & 3311 & 226.0150 & 7.5175 & 282.7 & 4 & 600.0 & 600.0 \\
\hline 04 & 3312 & 326.0150 & 7.1188 & 282.8 & 4 & .0 & .0 \\
\hline 04 & 3313 & 66.0150 & 6.9870 & 282.4 & 4 & 600.0 & .0 \\
\hline 04 & 3314 & 166.0150 & 7.4400 & 282.2 & 4 & 600.0 & 600.0 \\
\hline 04 & 3315 & 266.0150 & 7.6400 & 281.7 & 4 & .0 & .0 \\
\hline 04 & 3316 & 6.0150 & 935 & 283.0 & 4 & .0 & \\
\hline 04 & 3317 & 106.0150 & 6.7035 & 283.3 & 4 & .0 & 600.0 \\
\hline 04 & 3318 & 206.0150 & 5.6170 & 283.7 & 3 & .0 & 600.0 \\
\hline 04 & 3319 & 306.0150 & 6.2135 & 284.8 & 4 & 600.0 & 600.0 \\
\hline 04 & 33110 & 46.0150 & 6 . & 285.9 & 4 & .0 & 600.0 \\
\hline 04 & 33111 & 146.0150 & 5.3995 & 287.2 & 3 & 600.0 & 600.0 \\
\hline 04 & 33112 & 246.0150 & 940 & 288.3 & 2 & .0 & 60 \\
\hline 04 & 33113 & 346.0150 & 5.7020 & 289.0 & 3 & 600.0 & 600.0 \\
\hline 04 & 33114 & 86.0150 & 6.4760 & 289.5 & 4 & 600.0 & 600.0 \\
\hline 04 & 33115 & 186 & 933 & 289.9 & 4 & 600.0 & 600.0 \\
\hline 04 & 33116 & 286.0150 & 6.2930 & 289.6 & 4 & 600.0 & 600.0 \\
\hline 04 & 33117 & 26.0150 & 6.6360 & 288.3 & 4 & 600.0 & 600.0 \\
\hline 04 & 33118 & 126.0150 & 7.1873 & 286.1 & 4 & 600.0 & 600.0 \\
\hline 04 & 33119 & 226.0150 & 7.2280 & 284.6 & 4 & 600.0 & 600.0 \\
\hline 04 & 33120 & 326.0150 & 9.5925 & 285.0 & 4 & 600.0 & 600.0 \\
\hline 04 & 33121 & 150 & 525 & 28 & 4 & 600.0 & \\
\hline 04 & 33122 & 166.0150 & 9.4650 & 284.8 & 4 & 600.0 & 600.0 \\
\hline 84 & 33123 & 0150 & 9.7725 & 284.2 & 4 & 600.0 & 600. \\
\hline
\end{tabular}




\begin{tabular}{|c|c|c|c|c|c|c|c|c|}
\hline 4 & & 3124 & 6.0150 & 9.3875 & 284.0 & 4 & 600.0 & 00.0 \\
\hline 04 & 4 & 11 & 78.1750 & 9.3350 & 283.7 & $x$ & 600.0 & 600.0 \\
\hline 04 & 4 & 12 & 80.5499 & 9.7175 & 283.4 & 4 & 600.0 & 00.0 \\
\hline 04 & 4 & 13 & 97.0312 & 8.2850 & 283.4 & 4 & 600.0 & 00.0 \\
\hline 04 & 4 & 14 & 138.0096 & 10.4675 & 284.4 & $x$ & 600.0 & 0.0 \\
\hline 04 & 4 & 15 & 149.0249 & 11.6750 & 283.9 & 4 & 600.0 & 00.0 \\
\hline 04 & 4 & 16 & 147.7250 & 10.5000 & 283.7 & 4 & 600.0 & .0 \\
\hline 04 & 4 & 17 & 149.6745 & 12.0575 & 284.1 & 4 & 600.0 & 00.0 \\
\hline 04 & 4 & 18 & 153.8250 & 13.5775 & 284.8 & 4 & 600.0 & .0 \\
\hline 04 & 4 & 19 & 156.2991 & 14.4925 & 285.3 & 4 & 600.0 & \\
\hline 04 & 4 & 110 & 151.2756 & 13.9925 & 286.2 & 4 & 600.0 & .0 \\
\hline 04 & 4 & 111 & 153.4243 & 16.3500 & 287.7 & 4 & 600.0 & \\
\hline 04 & 4 & 112 & 154.6249 & 15.3300 & 288.7 & 4 & 600.0 & .0 \\
\hline 04 & 4 & 113 & 161.9502 & 16.8075 & 289.4 & 4 & 600.0 & .0 \\
\hline 04 & 4 & 114 & 162.3500 & 16.4350 & 289.8 & 4 & 600.0 & \\
\hline 04 & 4 & 115 & 167.1747 & 275 & 290.1 & 4 & 600.0 & 60 \\
\hline 04 & 4 & 116 & 169.4507 & 16.4425 & 290.3 & 4 & 600.0 & .0 \\
\hline 04 & 4 & 117 & 165.5741 & 15 . & 290.1 & 4 & 600.0 & \\
\hline 04 & 4 & 118 & 167.5241 & 12.7925 & 289.6 & 4 & 600.0 & \\
\hline 04 & 4 & 119 & 153.1238 & 10.1875 & 288.2 & 4 & 600.0 & .0 \\
\hline 04 & 4 & 120 & 146.8497 & 11 . & 287.7 & 4 & 600.0 & .0 \\
\hline 04 & 4 & 121 & 154.4000 & 13. & 287.5 & 4 & 0.0 & \\
\hline 04 & 4 & 122 & 161.2513 & 11.9325 & 286.9 & 4 & 600.0 & \\
\hline 04 & 4 & 123 & 159.1746 & 11.3425 & 286.0 & 4 & 600.0 & \\
\hline 04 & 4 & 124 & 156.6000 & 13.5375 & 285.5 & 4 & 00.0 & \\
\hline 04 & 4 & 21 & 154.9000 & 300 & 285.0 & 4 & 600.0 & \\
\hline 04 & 4 & 22 & 151.0250 & 12.3750 & 284.0 & 4 & 600.0 & .0 \\
\hline 04 & 4 & 23 & 149.2499 & 12.8450 & 283.9 & 4 & 0.0 & \\
\hline 04 & 4 & 24 & 154.1750 & 13.7725 & 283.3 & 4 & 600.0 & 60 \\
\hline 04 & 4 & 25 & 157.2250 & 13.8275 & 282.9 & 4 & 600.0 & .0 \\
\hline 04 & 4 & 26 & 156.4250 & 12 . & 282.3 & 4 & 600.0 & \\
\hline 04 & 4 & 27 & 156.7500 & 13.2525 & 282.4 & 4 & 600.0 & .0 \\
\hline 04 & 4 & 28 & 162.6243 & 14.1125 & 283.4 & 4 & 600.0 & 600.0 \\
\hline 04 & 4 & 29 & 166.3748 & 13. & 284.6 & 4 & 600.0 & .0 \\
\hline 04 & 4 & 210 & 168.1249 & 325 & 286.5 & 4 & 600.0 & .0 \\
\hline 04 & 4 & 211 & 169.8497 & 10.9250 & 287.5 & 4 & 600.0 & 600.0 \\
\hline 04 & 4 & 212 & 171.8519 & 8.5075 & 288.9 & 4 & 600.0 & \\
\hline 04 & 4 & 213 & 173.0312 & 7.5800 & 289.7 & 4 & 600.0 & .0 \\
\hline 04 & 4 & 214 & 167.8400 & 6.1750 & 290.4 & 3 & 600.0 & 600.0 \\
\hline 04 & 4 & 215 & 165.6495 & 5.7580 & 291.0 & 3 & 600.0 & \\
\hline 04 & 4 & 216 & 160.3994 & 6.0020 & 291.2 & 2 & 600.0 & 60 \\
\hline 04 & 4 & 217 & 149.6489 & 5.3723 & 291.4 & 4 & 600.0 & 600.0 \\
\hline 04 & 4 & 218 & 142.9986 & 4.7875 & 290.9 & 4 & 600.0 & .0 \\
\hline 04 & 4 & 219 & 128.4839 & 2.9883 & 289.8 & 4 & 600.0 & 600.0 \\
\hline 04 & 4 & 220 & 108.4216 & 3.2720 & 289.5 & 4 & 600.0 & 600.0 \\
\hline 04 & 4 & 221 & 72.7000 & 5.9223 & 289.8 & 4 & 600.0 & 600.0 \\
\hline $0<$ & 4 & 222 & 70.9736 & 5.7008 & 290.1 & 4 & 600.0 & 600.0 \\
\hline 04 & 4 & 223 & 74.9000 & 6.2508 & 289.9 & 4 & 600.0 & 600.0 \\
\hline
\end{tabular}




\begin{tabular}{|c|c|c|c|c|c|c|c|c|}
\hline 4 & 4 & 224 & 70.8251 & 6.9280 & 289.9 & 4 & 600.0 & 600.0 \\
\hline 04 & 4 & 31 & 69.1258 & 7.8475 & 289.5 & 4 & 600.0 & 00.0 \\
\hline 04 & 4 & 32 & 73.3002 & 8.8275 & 288.8 & 4 & 600.0 & 00.0 \\
\hline 04 & 4 & 33 & 67.3490 & 8.3125 & 288.5 & 4 & 600.0 & 00.0 \\
\hline 04 & 4 & 34 & 84.8081 & 3.9433 & 288.3 & 4 & 600.0 & 00.0 \\
\hline 04 & 4 & 35 & 75.9740 & 6.8403 & 288.2 & 4 & 600.0 & 00.0 \\
\hline 04 & 4 & 36 & 75.2026 & 4.0815 & 288.0 & 4 & 600.0 & 00.0 \\
\hline 04 & 4 & 37 & 26.5303 & 2.1638 & 287.8 & 4 & 600.0 & 00.0 \\
\hline 04 & 4 & 38 & 332.3909 & 2.4075 & 288.7 & 3 & 600.0 & 00.0 \\
\hline 04 & 4 & 39 & 333.5033 & 2.3880 & 291.1 & 3 & 600.0 & 0 \\
\hline 04 & 4 & 310 & 85.2402 & 2.8890 & 293.6 & - & 600.0 & \\
\hline 04 & 4 & 311 & 83.1512 & 3.6668 & 294.6 & 2 & 600.0 & \\
\hline 04 & 4 & 312 & 74.6834 & 5.9003 & 295.0 & 3 & 600.0 & 00.0 \\
\hline 04 & 4 & 313 & 86.3816 & 6.6745 & 295.1 & 4 & 600.0 & 00.0 \\
\hline 04 & 4 & 314 & 79.2661 & 6.7760 & 295.5 & 4 & .0 & \\
\hline 04 & 4 & 315 & 72.5634 & 8.5000 & 295.1 & 4 & 600.0 & .0 \\
\hline 04 & 4 & 316 & 63.4000 & 10.1325 & 294.4 & 4 & 60 & .0 \\
\hline 04 & 4 & 317 & 59.4250 & 11.6150 & 292.9 & 4 & .0 & \\
\hline 04 & 4 & 318 & 58.3750 & 12.8350 & 291.5 & 4 & 60 & .0 \\
\hline 04 & 4 & 319 & 59.6250 & 12.0000 & 290.3 & 4 & .0 & .0 \\
\hline 04 & 4 & 320 & 60.2750 & 12.4900 & 289.6 & 4 & 6 & \\
\hline 04 & 4 & 321 & 60.8249 & 13.1100 & 289.1 & 4 & .0 & \\
\hline 04 & 4 & 322 & 63.4250 & 12.2600 & 289.1 & 4 & 600.0 & .0 \\
\hline 04 & 4 & 323 & 62.4250 & 12.0175 & 289.0 & 4 & 60 & \\
\hline 04 & 4 & 324 & 64.6499 & 12.8050 & 289.2 & 4 & .0 & \\
\hline 04 & 4 & 41 & 67.5500 & 11.3350 & 289.5 & 4 & .0 & 600.0 \\
\hline 04 & 4 & 42 & 68.2750 & 9.9100 & 289.5 & 4 & 600.0 & .0 \\
\hline 04 & 4 & 43 & 55.8132 & 5.6775 & 289.0 & 4 & .0 & \\
\hline 04 & 4 & 44 & 53.0715 & 4.8528 & 288.7 & 4 & .0 & 0.0 \\
\hline 04 & 4 & 45 & 56.6515 & 6.0453 & 287.5 & 4 & 60 & .0 \\
\hline 04 & 4 & 46 & 63.4234 & 6.6023 & 285.7 & 4 & .0 & \\
\hline 04 & 4 & 47 & 65.2008 & 5.4445 & 285.3 & 4 & .0 & 600.0 \\
\hline 04 & 4 & 48 & 119.4450 & 2.5090 & 287.2 & 3 & 600.0 & 600.0 \\
\hline 04 & 4 & 49 & 351.5159 & 1.7605 & 288.2 & 2 & 600.0 & 0.0 \\
\hline 04 & 4 & 410 & 174.7860 & 2.4275 & 289.7 & 2 & 600.0 & 600.0 \\
\hline 04 & 4 & 411 & 99.9240 & 3.5430 & 289.8 & 2 & 600.0 & 600.0 \\
\hline 04 & 4 & 412 & 87.2019 & 4.4108 & 290.1 & 2 & 600.0 & .0 \\
\hline 04 & 4 & 413 & 72.1240 & 4.5153 & 291.7 & 2 & 600.0 & 600.0 \\
\hline 04 & 4 & 414 & 63.6235 & 5.4433 & 291.9 & 2 & 600.0 & 600.0 \\
\hline 04 & 4 & 415 & 70.3001 & 8.2075 & 290.3 & 3 & 600.0 & 600.0 \\
\hline 04 & 4 & 416 & 76.8000 & 8.5800 & 289.2 & 4 & 600.0 & 600.0 \\
\hline 04 & 4 & 417 & 74.8251 & 10.1400 & 287.5 & 4 & 600.0 & 600.0 \\
\hline 04 & 4 & 418 & 74.6746 & 10.0050 & 285.7 & 4 & 600.0 & 600.0 \\
\hline 04 & 4 & 419 & 73.1536 & 12.0100 & 283.8 & 4 & 600.0 & 600.0 \\
\hline 04 & 4 & 420 & 73.8751 & 12.1500 & 283.3 & 4 & 600.0 & 600.0 \\
\hline 04 & 4 & 421 & 79.0000 & 13.8250 & 282.2 & 4 & 600.0 & 600.0 \\
\hline $0<$ & 4 & 422 & 66.9506 & 13.2675 & 281.7 & 4 & 600.0 & 600.0 \\
\hline 04 & 4 & 423 & 59.1239 & 14.9125 & 281.6 & 4 & 600.0 & 600.0 \\
\hline
\end{tabular}




\begin{tabular}{|c|c|c|c|c|c|c|c|c|}
\hline 4 & 4 & 424 & 60.4226 & 11.7050 & 281.7 & 4 & 600.0 & 600.0 \\
\hline 04 & 4 & 51 & 60.6999 & 10.8100 & 281.5 & 4 & 600.0 & 00.0 \\
\hline 04 & 4 & 52 & 77.6987 & 10.0950 & 281.2 & 4 & 600.0 & 00.0 \\
\hline 4 & 4 & 53 & 66.0500 & 13.1250 & 280.8 & 4 & 600.0 & 00.0 \\
\hline 04 & 4 & 54 & 68.3750 & 14.2400 & 280.6 & 4 & 600.0 & 00.0 \\
\hline 04 & 4 & 55 & 69.1000 & 14.2875 & 280.7 & 4 & 600.0 & 00.0 \\
\hline 04 & 4 & 56 & 69.0501 & 14.6250 & 280.7 & 4 & 600.0 & 00.0 \\
\hline 04 & 4 & 57 & 68.9750 & 14.0950 & 281.0 & 4 & 600.0 & 00.0 \\
\hline 04 & 4 & 58 & 69.9750 & 13.2350 & 281.5 & 4 & 600.0 & 00.0 \\
\hline 04 & 4 & 59 & 71.0246 & 10.9100 & 282.5 & 4 & 600.0 & .0 \\
\hline 04 & 4 & 510 & 67.4999 & 10.7325 & 283.5 & 4 & 600.0 & \\
\hline 04 & 4 & 511 & 74.8499 & 9.3800 & 284.7 & 4 & 600.0 & \\
\hline 04 & 4 & 512 & 72.9252 & 9.5450 & 285.8 & 4 & 600.0 & 00.0 \\
\hline 04 & 4 & 513 & 74.4501 & 9.1275 & 286.3 & 4 & 600.0 & 00.0 \\
\hline 04 & 4 & 514 & 71.1750 & 10.1325 & 286.8 & 4 & .0 & \\
\hline 04 & 4 & 515 & 72.0499 & 10.5850 & 287.1 & 4 & 600.0 & .0 \\
\hline 04 & 4 & 516 & 71.9769 & 10.5775 & 286.5 & 4 & 60 & .0 \\
\hline 04 & 4 & 517 & 67.8755 & 12.3600 & 285.2 & 4 & .0 & \\
\hline 04 & 4 & 518 & 69.5500 & 12.1825 & 283.6 & 4 & 60 & .0 \\
\hline 04 & 4 & 519 & 68.3510 & 12.4950 & 282.1 & 4 & .0 & .0 \\
\hline 04 & 4 & 520 & 69.7255 & 12.71 & 28 & 4 & 6 & \\
\hline 04 & 4 & 521 & 67.6251 & 12.0950 & 281.4 & 4 & .0 & \\
\hline 04 & 4 & 522 & 68.2507 & 11.7025 & 280.9 & 4 & 600.0 & .0 \\
\hline 04 & 4 & 523 & 63.2490 & 11.79 & $2 \varepsilon$ & 4 & 60 & \\
\hline 04 & 4 & 524 & 67.3500 & 12.3675 & 28 & 4 & .0 & \\
\hline 04 & 4 & 61 & 61.1744 & 12.3250 & 280.6 & 4 & .0 & .0 \\
\hline 04 & 4 & 62 & 63.0507 & 12.4050 & 280.6 & 4 & .0 & .0 \\
\hline 04 & 4 & 63 & 63.8750 & 12.4475 & 280.5 & 4 & .0 & \\
\hline 04 & 4 & 64 & 66.3750 & 12.1450 & 280.3 & 4 & .0 & 0.0 \\
\hline 04 & 4 & 65 & 66.2501 & 10.4300 & 280.0 & 4 & 60 & .0 \\
\hline 04 & 4 & 66 & 64.9754 & 10.67 & 279.9 & 4 & .0 & \\
\hline 04 & 4 & 67 & 63.6482 & 9.78 & 280.2 & 4 & .0 & 0.0 \\
\hline 04 & 4 & 68 & 80.0493 & 8.9350 & 280.9 & 4 & 600.0 & 600.0 \\
\hline 04 & 4 & & 81.5498 & 6. & 282.4 & 4 & 600.0 & 0.0 \\
\hline 04 & 4 & 610 & 95.0509 & 6.40 & 284.1 & 3 & 600.0 & 600.0 \\
\hline 04 & 4 & 611 & 91.9495 & 7.6925 & 285.0 & 4 & 600.0 & 600.0 \\
\hline 04 & 4 & 612 & 95.8556 & 6.42 & 286.5 & 4 & 600.0 & 600.0 \\
\hline 04 & 4 & 613 & 104.9998 & 6.7855 & 287.5 & 4 & 600.0 & 600.0 \\
\hline 04 & 4 & 614 & 111.3305 & 6.9140 & 288.2 & 4 & 600.0 & 600.0 \\
\hline 04 & 4 & 615 & 110.8874 & 7.61 & 28 & 4 & 600.0 & 600.0 \\
\hline 04 & 4 & 616 & 92.4743 & 7.9200 & 288.0 & 4 & 600.0 & 600.0 \\
\hline 04 & 4 & 617 & 73.8510 & 7.2438 & 287.1 & 4 & 600.0 & 600.0 \\
\hline 04 & 4 & 618 & 73.4756 & 7.3823 & 286.0 & 4 & 600.0 & 600.0 \\
\hline 04 & 4 & 619 & 79.7747 & 8.7050 & 284.7 & 4 & 600.0 & 600.0 \\
\hline 04 & 4 & 620 & 73.2993 & 10.8125 & 283.2 & 4 & 600.0 & 600.0 \\
\hline 04 & 4 & 621 & 72.1749 & & 284 & . & 600.0 & 600.0 \\
\hline $0<$ & 4 & 622 & 73.9001 & 9.6400 & 284.8 & 4 & 600.0 & 600.0 \\
\hline 84 & 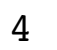 & 623 & 73.9748 & 8.790 & 285.0 & & 600.0 & 600.0 \\
\hline
\end{tabular}




\begin{tabular}{|c|c|c|c|c|c|c|c|c|}
\hline 4 & 4 & 624 & 76.2750 & 8.9550 & 285.2 & 4 & 600.0 & 600.0 \\
\hline 04 & 4 & 71 & 73.7500 & 10.4625 & 284.6 & 4 & 600.0 & 600.0 \\
\hline 04 & 4 & 72 & 70.7500 & 9.8250 & 284.6 & 4 & 600.0 & 00.0 \\
\hline 04 & 4 & 73 & 73.3738 & 8.5000 & 285.1 & 4 & 600.0 & 00.0 \\
\hline 04 & 4 & 74 & 81.1000 & 7.9575 & 285.5 & 4 & 600.0 & 00.0 \\
\hline 04 & 4 & 75 & 109.3120 & 5.0283 & 285.9 & 4 & 600.0 & 00.0 \\
\hline 04 & 4 & 76 & 128.9495 & 3.9128 & 285.9 & 4 & 600.0 & 00.0 \\
\hline 04 & 4 & 77 & 100.7152 & 3.9395 & 286.9 & 4 & 600.0 & 00.0 \\
\hline 04 & 4 & 78 & 153.9991 & 3.9423 & 287.9 & 3 & 600.0 & 00.0 \\
\hline 04 & 4 & 79 & 162.5762 & 5.3215 & 288.8 & 3 & 600.0 & 0 \\
\hline 04 & 4 & 710 & 159.0500 & 5.8613 & 289.8 & 3 & 600.0 & \\
\hline 04 & 4 & 711 & 158.7049 & 410 & 290.7 & 2 & 600.0 & \\
\hline 04 & 4 & 712 & 154.9336 & 4.2638 & 292.0 & 2 & 600.0 & 00.0 \\
\hline 04 & 4 & 713 & 154.2977 & 4.8870 & 293.0 & 2 & 600.0 & 00.0 \\
\hline 04 & 4 & 714 & 142.9152 & 33 & 294.1 & 3 & .0 & \\
\hline 04 & 4 & 715 & 126.1899 & 5.9620 & 294.5 & 3 & 600.0 & .0 \\
\hline 04 & 4 & 716 & 89.5955 & 6.3490 & 294.1 & 4 & 60 & 00.0 \\
\hline 04 & 4 & 717 & 72.1459 & 7. & 293.2 & 4 & .0 & \\
\hline 04 & 4 & 718 & 66.5750 & 8.4225 & 292.4 & 4 & 60 & .0 \\
\hline 04 & 4 & 719 & 67.9743 & 8.1075 & 291.4 & 4 & .0 & .0 \\
\hline 04 & 4 & 720 & 72.4248 & 8 . & 291.1 & 4 & 6 & \\
\hline 04 & 4 & 721 & 71.8738 & 8.2800 & 290.9 & 4 & .0 & \\
\hline 04 & 4 & 722 & 68.6000 & 9.1850 & 290.8 & 4 & 600.0 & .0 \\
\hline 04 & 4 & 723 & 67.5001 & 9. & 290.5 & 4 & 60 & \\
\hline 04 & 4 & 724 & 67.7246 & 9 . & 290.1 & 4 & .0 & \\
\hline 04 & 4 & 81 & 71.8749 & 9.6900 & 289.9 & 4 & .0 & 600.0 \\
\hline 04 & 4 & 82 & 76.1000 & 10.2700 & 289.3 & 4 & 600.0 & .0 \\
\hline 04 & 4 & 83 & 77.2252 & 9.0100 & 288.9 & 4 & .0 & \\
\hline 04 & 4 & 84 & 79.4498 & 7.3448 & 288.8 & 4 & 600.0 & 600.0 \\
\hline 04 & 4 & 85 & 96.9095 & 4.4473 & 289.0 & 4 & 60 & .0 \\
\hline 04 & 4 & 86 & 89.8745 & 1.1600 & 288.0 & 5 & .0 & \\
\hline 04 & 4 & 87 & 114.5246 & 1.8745 & 288.6 & 4 & 600.0 & 600.0 \\
\hline 04 & 4 & 88 & 104.0061 & 2.5940 & 291.1 & 3 & 600.0 & 600.0 \\
\hline 04 & 4 & 89 & 166.5750 & 5.6143 & 292.1 & 3 & 0 & 0.0 \\
\hline 04 & 4 & 810 & 154.4629 & 6.0653 & 292.4 & 3 & 600.0 & 600.0 \\
\hline 04 & 4 & 811 & 155.1999 & 6.4948 & 292.7 & 3 & 600.0 & 600.0 \\
\hline 04 & 4 & 812 & 144.8107 & 6.4990 & 293.7 & 4 & 600.0 & 0.0 \\
\hline 04 & 4 & 813 & 151.6268 & 6.8058 & 295.0 & 3 & 600.0 & 600.0 \\
\hline 04 & 4 & 814 & 157.9001 & 7.0935 & 295.5 & 3 & 600.0 & 600.0 \\
\hline 04 & 4 & 815 & 143.2846 & 5.7835 & 296.2 & 3 & 600.0 & 600.0 \\
\hline 04 & 4 & 816 & 124.2545 & 5.1790 & 296.3 & 3 & 600.0 & 600.0 \\
\hline 04 & 4 & 817 & 86.7794 & 5.2758 & 295.5 & 3 & 600.0 & 600.0 \\
\hline 04 & 4 & 818 & 78.9246 & 6.1393 & 294.1 & 4 & 600.0 & 600.0 \\
\hline 04 & 4 & 819 & 67.4000 & 7.6175 & 292.7 & 4 & 600.0 & 600.0 \\
\hline 04 & 4 & 820 & 68.9753 & 8.6975 & 292.3 & 4 & 600.0 & 600.0 \\
\hline 04 & 4 & 821 & 72.9999 & 8.9800 & 292.0 & 4 & 600.0 & 600.0 \\
\hline $0<$ & 4 & 822 & 71.7999 & 9.0700 & 291.6 & 4 & 600.0 & 600.0 \\
\hline 04 & 4 & 823 & 73.6500 & 10.2950 & 291.2 & & 600.0 & 600.0 \\
\hline
\end{tabular}




\begin{tabular}{|c|c|c|c|c|c|c|c|}
\hline 1 & $4 \quad 824$ & 5.5500 & 0.0225 & 291.7 & 4 & 00.0 & \\
\hline 4 & $\begin{array}{lll}4 & 9 & 1\end{array}$ & 0.8242 & 8.2800 & 292.0 & 4 & 00.0 & \\
\hline & $\begin{array}{lll}4 \quad 9 & 2\end{array}$ & 4514 & 130 & 292.7 & 1 & 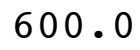 & \\
\hline & $\begin{array}{lll}4 & 9 & 3\end{array}$ & 02.8800 & 625 & 292.7 & & 00 & \\
\hline & $4 \quad 94$ & 69 & 08 & 92.4 & & & \\
\hline & $\begin{array}{lll}4 & 9 & 5\end{array}$ & 383 & 4.3543 & 91.8 & & U & \\
\hline & 496 & 183 & 998 & 291.1 & & 0 & \\
\hline & $\begin{array}{lll}4 & 9 & 7\end{array}$ & 38 & .4645 & 91.4 & & 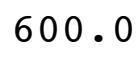 & \\
\hline & $\begin{array}{lll}4 & 9 & 8\end{array}$ & 752 & 3550 & 290.6 & & 0 & \\
\hline & $\begin{array}{lll}4 & 9 & 9\end{array}$ & 514 & 320 & 291.1 & & 0 & \\
\hline & 4910 & 152 . & 270 & 92.3 & & & \\
\hline & 4911 & 155. & 3673 & 93.8 & & & \\
\hline & 4912 & 154 . & 7273 & 295.2 & & 0 & \\
\hline & 4913 & 153. & .2578 & 3 & & & \\
\hline & 4914 & & & 8 & & & \\
\hline & 4915 & 503 & 010 & 297 & 3 & & \\
\hline & 4916 & 147.6258 & 5.7775 & 298.1 & 4 & 0 & \\
\hline & $4 \quad 917$ & 152.22 & 7.0663 & 9 & 4 & & \\
\hline 4 & 4918 & 147 . & 5 & 297 & 4 & & \\
\hline 4 & 4919 & 76.3225 & 2045 & 295.8 & 2 & 0 & \\
\hline & 4920 & $71 \cdot$ & 0 & & & & \\
\hline I & 4921 & 63. & 7 . & 29 & 4 & & \\
\hline 4 & 4922 & 66.7500 & 55 & 294.2 & 4 & & \\
\hline & 4923 & 72 & 5 & 294 & 4 & & \\
\hline 4 & $4 \quad 924$ & & & 29 & 4 & & \\
\hline & 4101 & 83.2456 & 5943 & 294 & 4 & & \\
\hline 4 & 4102 & 103.4762 & 4.90 & 295 & 4 & & \\
\hline 1 & 4103 & 82.1479 & 8 & 294 & 4 & & \\
\hline & $410 \quad 4$ & 116.4535 & 950 & 294 & 4 & & \\
\hline 4 & 4105 & 116.7644 & 35 & 293 & 4 & & \\
\hline & 4106 & 108 . & & 292 & 4 & & \\
\hline & 4107 & 118 . & 0 & 291 & 4 & & \\
\hline & 4108 & 140.0750 & 023 & 292.1 & 4 & & \\
\hline & 4109 & 145 . & 7 . & 292 & 4 & & \\
\hline & 41010 & 134 . & & & 3 & & \\
\hline & 41011 & 148.6734 & 7.2060 & 295.5 & 4 & & \\
\hline & 41012 & 156 . & 7.26 & 29 & 4 & & \\
\hline & 41013 & 152 . & 6.9808 & 297.7 & 4 & & \\
\hline & 41014 & 159.3919 & 7.9725 & 298.5 & 4 & & \\
\hline & 41015 & 158 . & 500 & & 4 & & \\
\hline & 41016 & 150.2248 & 7.7525 & 299 & 4 & & \\
\hline & 41017 & 151.8987 & 7.8125 & 299.3 & 4 & & \\
\hline & 41018 & 140.0186 & 6.6818 & 298 & 4 & & \\
\hline 4 & 41019 & 116.2204 & 6.1245 & 297.6 & 4 & & 600 \\
\hline 4 & 41020 & 101.5000 & 5.8800 & 296.7 & 4 & 0.0 & 00 . \\
\hline & 41021 & & & & 4 & & \\
\hline & 41022 & 67.8750 & 7.9650 & & & & 600 \\
\hline & 1023 & 69.8994 & 9.9375 & 292.7 & & 600.0 & 60 \\
\hline
\end{tabular}




\begin{tabular}{|c|c|c|c|c|c|c|c|}
\hline 04 & 41024 & 76.5750 & 9.4925 & 293.2 & 4 & 600.0 & 00.0 \\
\hline 04 & 4111 & 81.0250 & 9.1250 & 293.4 & 4 & 600.0 & 600.0 \\
\hline 04 & 4112 & 75.7750 & 8.5550 & 293.3 & 4 & 600.0 & 00.0 \\
\hline 04 & 4113 & 74.4500 & 7.4063 & 292.6 & 4 & 600.0 & 600.0 \\
\hline 04 & 4114 & 78.8989 & 6.5045 & 292.6 & 4 & 600.0 & 00.0 \\
\hline 04 & 4115 & 86.5242 & 6.3728 & 292.3 & 4 & 600.0 & 00.0 \\
\hline 04 & 4116 & 88.4998 & 6.1535 & 292.1 & 4 & 600.0 & 600.0 \\
\hline 04 & 4117 & 95.5930 & 4.8723 & 292.3 & 3 & 600.0 & 00.0 \\
\hline 04 & 4118 & 150.3416 & 2.6160 & 293.7 & 3 & 600.0 & 00.0 \\
\hline 04 & 4119 & 260.9823 & 2.1545 & 292.1 & 2 & 600.0 & 600.0 \\
\hline 04 & 41110 & 280.4818 & 2.6433 & 293.2 & 2 & 600.0 & 600.0 \\
\hline 04 & 41111 & 285.1141 & 2 . & 294.0 & 2 & 600.0 & \\
\hline 04 & 41112 & 254.1952 & 2.6138 & 295.6 & 2 & 600.0 & 600.0 \\
\hline 04 & 41113 & 260.3137 & 2.3858 & 297.0 & 2 & 600.0 & 600.0 \\
\hline 04 & 41114 & 88.6062 & & 297.9 & 2 & 600.0 & \\
\hline 04 & 41115 & 89.8498 & 6 . & 297.4 & 3 & 600.0 & 60 \\
\hline 04 & 41116 & 81.3008 & 7.58 & 296.4 & 4 & 600.0 & 600.0 \\
\hline 04 & 41117 & 79.1499 & 7.80 & 295.3 & 4 & 600.0 & \\
\hline 04 & 41118 & 70.8997 & 7.7200 & 294.3 & 4 & 600.0 & 60 \\
\hline 04 & 41119 & 58.6501 & 7.4 & 292.6 & 4 & 600.0 & 600.0 \\
\hline 04 & 41120 & 78.4668 & 4.58 & 291.3 & 4 & 600.0 & .0 \\
\hline 04 & 41121 & 41.6686 & 2 . & 292.2 & 4 & 600.0 & \\
\hline 04 & 41122 & 36.7749 & 3.1020 & 292.8 & 4 & 600.0 & 600.0 \\
\hline 04 & 41123 & 136.9718 & 1.4788 & 290.8 & 5 & 600.0 & .0 \\
\hline 04 & 41124 & 135.0790 & 1. & 289.5 & 5 & 600.0 & \\
\hline 04 & 4121 & 62.1213 & 1.0150 & 289.2 & 6 & 600.0 & 600.0 \\
\hline 04 & 4122 & 69.9518 & 1.2478 & 289.4 & 6 & 600.0 & 600.0 \\
\hline 04 & 4123 & 146.9090 & 1.9980 & 288.8 & 5 & 600.0 & \\
\hline 04 & 4124 & 107.7899 & 0.6618 & 287.9 & 6 & 600.0 & 60 \\
\hline 04 & 4125 & 26.0032 & 0.9690 & 287.5 & 6 & 600.0 & 600.0 \\
\hline 04 & 4126 & 62.7446 & 1 . & 287.5 & 5 & 600.0 & .0 \\
\hline 04 & 4127 & 56.1016 & 2.6250 & 287.3 & 4 & 600.0 & 60 \\
\hline 04 & 4128 & 78.1514 & 5.5555 & 286.8 & 3 & 600.0 & 600.0 \\
\hline 04 & 4129 & 82.1250 & 500 & 287.1 & 4 & 600.0 & .0 \\
\hline 04 & 41210 & 81.5713 & 5 . & 289.5 & 3 & 600.0 & 60 \\
\hline 04 & 41211 & 93.9269 & 4.6178 & 291.1 & 2 & 600.0 & 600.0 \\
\hline 04 & 41212 & 87.1207 & 5.6758 & 292.0 & 3 & 600.0 & 600.0 \\
\hline 04 & 41213 & 90.2756 & 5.5740 & 292.8 & 3 & 600.0 & 600.0 \\
\hline 04 & 41214 & 82.0553 & 5.7288 & 293.4 & 3 & 600.0 & 600.0 \\
\hline 04 & 41215 & 84.5996 & 7.0845 & 293.4 & 4 & 600.0 & \\
\hline 04 & 41216 & 84.3493 & 8.2425 & 292.7 & 4 & 600.0 & 600.0 \\
\hline 04 & 41217 & 75.8495 & 10.1050 & 290.5 & 4 & 600.0 & 600.0 \\
\hline 04 & 41218 & 71.9002 & 12.7625 & 287.0 & 4 & 600.0 & 600.0 \\
\hline 04 & 41219 & 67.8004 & 11.7225 & 286.0 & 4 & 600.0 & 600.0 \\
\hline 04 & 41220 & 70.3999 & 12.9150 & 284.2 & 4 & 600.0 & 600.0 \\
\hline 04 & 41221 & 60.8247 & 15.4000 & 282.9 & 4 & 600.0 & 600.0 \\
\hline 04 & 41222 & 56.4754 & 525 & 282.5 & 4 & 600.0 & 600.0 \\
\hline 04 & 41223 & 57.9749 & 10.7700 & 282.2 & 4 & 600.0 & 600.0 \\
\hline
\end{tabular}




\begin{tabular}{|c|c|c|c|c|c|c|c|}
\hline 4 & 41224 & 70.8293 & 10.0125 & 282.1 & 4 & 600.0 & 600.0 \\
\hline 04 & 4131 & 65.6705 & 10.5125 & 281.9 & 4 & 600.0 & 600.0 \\
\hline 04 & 4132 & 56.9246 & 14.2075 & 281.7 & 4 & 600.0 & 00.0 \\
\hline 04 & 4133 & 45.7986 & 11.4250 & 281.6 & 4 & 600.0 & 00.0 \\
\hline 04 & 4134 & 69.2069 & 8.3320 & 281.3 & 4 & 600.0 & 00.0 \\
\hline 04 & 4135 & 75.9646 & 7.0728 & 281.0 & 4 & 600.0 & 00.0 \\
\hline 04 & 4136 & 58.3754 & 11.0775 & 281.2 & 4 & 600.0 & 00.0 \\
\hline 04 & 4137 & 59.7002 & 14.5600 & 281.3 & 4 & 600.0 & 00.0 \\
\hline 04 & 4138 & 61.3502 & 13.5725 & 282.3 & 4 & 600.0 & 00.0 \\
\hline 04 & 4139 & 58.8250 & 12.9025 & 283.5 & 4 & 60 & 0 \\
\hline 04 & 41310 & 60.8750 & 11.0000 & 284.8 & 4 & 600.0 & \\
\hline 04 & 41311 & 58.4748 & 10. & 285.9 & 4 & .0 & \\
\hline 04 & 41312 & 57.3248 & 10.6150 & 286.9 & 3 & 600.0 & .0 \\
\hline 04 & 41313 & 63.8500 & 10.9450 & 287.3 & 3 & 600.0 & \\
\hline 04 & 41314 & 69.3725 & 11 & 287.6 & 4 & .0 & \\
\hline 04 & 41315 & 74.9174 & 225 & 287.7 & 4 & 600.0 & .0 \\
\hline 04 & 41316 & 71.7269 & 10.1575 & 287.7 & 4 & 60 & .0 \\
\hline 04 & 41317 & 67.2226 & 9.7 & 286.9 & 4 & .0 & \\
\hline 04 & 41318 & 70.9014 & 8.9 & 285.8 & 4 & 60 & \\
\hline 04 & 41319 & 75.1633 & 9.6500 & 284.9 & 4 & .0 & .0 \\
\hline 04 & 41320 & 63.7248 & 10 & 283.9 & 4 & 6 & \\
\hline 04 & 41321 & 65.4751 & 10.4 & 283.2 & 4 & .0 & \\
\hline 04 & 41322 & 66.3998 & 11.1800 & 282.9 & 4 & 600.0 & .0 \\
\hline 04 & 41323 & 81.1566 & 7.2 & 282.4 & 4 & 6 & \\
\hline 04 & 41324 & 70.8730 & 7 . & 282.1 & 4 & 6 & \\
\hline 04 & 4141 & 63.5501 & 7.6335 & 282.0 & 4 & .0 & 600.0 \\
\hline 04 & 4142 & 67.7214 & 4.8095 & 281.9 & 4 & 60 & .0 \\
\hline 04 & 4143 & 50.8156 & 3.5 & 281.9 & 4 & .0 & \\
\hline 04 & 4144 & 54.5998 & 4.1 & 282.0 & 4 & .0 & .0 \\
\hline 04 & 4145 & 54.8248 & 5.6025 & 282.1 & 4 & 60 & .0 \\
\hline 04 & 4146 & 57.8776 & 6 . & 282.1 & 4 & .0 & \\
\hline 04 & 4147 & 64.5250 & 3 . & 282.4 & 4 & .0 & 0.0 \\
\hline 04 & 4148 & 50.7397 & 5.0655 & 283.4 & 3 & .0 & 0.0 \\
\hline 04 & 4149 & 56.3752 & 5 & 285.0 & 3 & .0 & 0.0 \\
\hline 04 & 41410 & 43.8438 & 4.1833 & 286.5 & 2 & 600.0 & 600.0 \\
\hline 04 & 41411 & 101.3438 & 4.6320 & 287.4 & 2 & 600.0 & 600.0 \\
\hline 04 & 41412 & 121.5767 & 5.1540 & 288.4 & 2 & 600.0 & .0 \\
\hline 04 & 41413 & 115.5962 & 6.9910 & 288.8 & 3 & 600.0 & 600.0 \\
\hline 04 & 41414 & 104.7249 & 8.2600 & 289.2 & 4 & 600.0 & 600.0 \\
\hline 04 & 41415 & 91.2846 & 8.0663 & 289.9 & 4 & 600.0 & 600.0 \\
\hline 04 & 41416 & 72.8986 & 8.8430 & 289.8 & 4 & 600.0 & 600.0 \\
\hline 04 & 41417 & 63.5750 & 10.8325 & 288.9 & 4 & 600.0 & 600.0 \\
\hline 04 & 41418 & 63.0501 & 10.7875 & 287.9 & 4 & 600.0 & 600.0 \\
\hline 04 & 41419 & 66.8307 & 8.9000 & 286.2 & 4 & 600.0 & 600.0 \\
\hline 04 & 41420 & 62.5000 & 8.5025 & 284.9 & 4 & 600.0 & 600.0 \\
\hline 04 & 41421 & 65.2752 & & 284.3 & 4 & 600.0 & 600.0 \\
\hline $0<$ & 41422 & 70.0249 & 10.2950 & 284.0 & 4 & 600.0 & 600.0 \\
\hline 04 & 41423 & 66.8247 & 10.5725 & 283.6 & 4 & 600.0 & 600.0 \\
\hline
\end{tabular}




\begin{tabular}{|c|c|c|c|c|c|c|c|}
\hline 04 & 41424 & 68.6260 & 9.8220 & 283.5 & 4 & 600.0 & 600.0 \\
\hline 04 & 4151 & 71.4258 & 4.9873 & 283.3 & 4 & 600.0 & 600.0 \\
\hline 04 & 4152 & 80.6587 & 4.4430 & 283.0 & 4 & 600.0 & 00.0 \\
\hline 04 & 4153 & 82.8315 & 5.8020 & 282.7 & 4 & 600.0 & 00.0 \\
\hline 04 & 4154 & 70.1250 & 8.0775 & 282.1 & 4 & 600.0 & 00.0 \\
\hline 04 & 4155 & 93.2264 & 3.8100 & 281.5 & 4 & 600.0 & 00.0 \\
\hline 04 & 4156 & 122.4835 & 3.1460 & 280.7 & 4 & 600.0 & 00.0 \\
\hline 04 & 4157 & 116.2731 & 3.4475 & 281.2 & 4 & 600.0 & 00.0 \\
\hline 04 & 4158 & 106.1952 & 3.6605 & 282.0 & 3 & 600.0 & 00.0 \\
\hline 04 & 4159 & 103.0827 & 3.8073 & 282.8 & 3 & 600.0 & 00.0 \\
\hline 04 & 41510 & 83.5662 & 4.2070 & 284.1 & 2 & 600.0 & 00.0 \\
\hline 04 & 41511 & 110.3313 & 7.1008 & 285.2 & 3 & 600.0 & 00.0 \\
\hline 04 & 41512 & 107.1536 & 8.2300 & 286.5 & 3 & 600.0 & 00.0 \\
\hline 04 & 41513 & 106.3677 & 8.6000 & 286.5 & 4 & .0 & 00.0 \\
\hline 04 & 41514 & 100.5416 & 7.1000 & 287.0 & 4 & .0 & \\
\hline 04 & 41515 & 101.1302 & 6.1728 & 286.9 & 4 & 600.0 & 00.0 \\
\hline 04 & 41516 & 108.4550 & 6.2008 & 286.2 & 4 & 600.0 & 600.0 \\
\hline 04 & 41517 & 107.6265 & 5.4353 & 285.9 & 4 & .0 & \\
\hline 04 & 41518 & 99.7001 & 4.5115 & 285.4 & 3 & 600.0 & .0 \\
\hline 04 & 41519 & 78.4731 & 6.2675 & 283.9 & 4 & .0 & .0 \\
\hline 04 & 41520 & 64.6000 & 8.35 & 282.4 & 4 & .0 & \\
\hline 04 & 41521 & 70.6751 & 7.6925 & 281.7 & 4 & .0 & \\
\hline 04 & 41522 & 67.6250 & 8.2000 & 281.3 & 4 & 600.0 & 600.0 \\
\hline 04 & 41523 & 67.2500 & 8.99 & 281.0 & 4 & 60 & .0 \\
\hline 04 & 41524 & 66.7250 & 8.7850 & 280.9 & 4 & .0 & \\
\hline 04 & 4161 & 67.6500 & 8.6975 & 280.8 & 4 & 600.0 & 600.0 \\
\hline 04 & 4162 & 68.2498 & 7.9950 & 280.8 & 4 & 600.0 & 600.0 \\
\hline 04 & 4163 & 64.0500 & 8.7700 & 280.6 & 4 & 600.0 & \\
\hline 04 & 4164 & 65.7997 & 8.6650 & 280.5 & 4 & 600.0 & 600.0 \\
\hline 04 & 4165 & 61.4750 & 9.3825 & 280.5 & 4 & 600.0 & 600.0 \\
\hline 04 & 4166 & 60.0023 & 8.1350 & 280.4 & 4 & .0 & .0 \\
\hline 04 & 4167 & 63.2492 & 7.9050 & 280.9 & 4 & .0 & 600.0 \\
\hline 04 & 4168 & 66.8997 & 5.8580 & 282.3 & 4 & 600.0 & 600.0 \\
\hline 04 & 4169 & 76.8866 & 4.4255 & 283.6 & 3 & 600.0 & 0.0 \\
\hline 04 & 41610 & 77.5451 & 4.2688 & 285.0 & 2 & 600.0 & 600.0 \\
\hline 04 & 41611 & 115.5885 & 3.6593 & 286.4 & 2 & 600.0 & 600.0 \\
\hline 04 & 41612 & 118.7312 & 3.0403 & 286.4 & 3 & 600.0 & 600.0 \\
\hline 04 & 41613 & 75.3756 & 3.0980 & 286.6 & 3 & 600.0 & 600.0 \\
\hline 04 & 41614 & 121.4596 & 5.1015 & 286.6 & 3 & 600.0 & 600.0 \\
\hline 04 & 41615 & 129.7200 & 4.0703 & 286.6 & 3 & 600.0 & 600.0 \\
\hline 04 & 41616 & 138.9763 & 5.1895 & 286.0 & 4 & 600.0 & 600.0 \\
\hline 04 & 41617 & 109.8003 & 5.8230 & 285.6 & 4 & 600.0 & 600.0 \\
\hline 04 & 41618 & 76.2175 & 6.3953 & 284.5 & 4 & 600.0 & 600.0 \\
\hline 04 & 41619 & 77.1006 & 6.9323 & 283.7 & 4 & 600.0 & 600.0 \\
\hline 04 & 41620 & 67.4254 & 6.9735 & 283.2 & 4 & 600.0 & 600.0 \\
\hline 04 & 41621 & 62.0749 & 8.8500 & 282.5 & 4 & 600.0 & 600.0 \\
\hline $0<$ & 41622 & 71.3243 & 9.4500 & 281.8 & 4 & 600.0 & 600.0 \\
\hline 14 & 41623 & 68.0790 & 8.6375 & 281. & 4 & 600.0 & 600.0 \\
\hline
\end{tabular}




\begin{tabular}{|c|c|c|c|c|c|c|c|}
\hline 04 & 41624 & 66.8243 & 8.6175 & 281.3 & 4 & 600.0 & 600.0 \\
\hline 04 & 4171 & 4.9000 & 8.4900 & 281.3 & 4 & 600.0 & 600.0 \\
\hline 04 & 4172 & 60.3751 & 8.3000 & 281.2 & 4 & 600.0 & 00.0 \\
\hline 04 & 4173 & 79.7799 & 5.5655 & 281.2 & 4 & 600.0 & 600.0 \\
\hline 04 & $417 \quad 4$ & 71.1998 & 6.3193 & 281.0 & 4 & 600.0 & 00.0 \\
\hline 04 & 4175 & 67.4501 & 7.7413 & 280.7 & 4 & 600.0 & 00.0 \\
\hline 04 & 4176 & 68.7000 & 6.2643 & 280.5 & 4 & 600.0 & 600.0 \\
\hline 04 & $417 \quad 7$ & 63.3996 & 6.9095 & 280.8 & 4 & 600.0 & 00.0 \\
\hline 04 & 4178 & 64.5750 & 7.1993 & 281.7 & 4 & 600.0 & 00.0 \\
\hline 04 & 4179 & 57.9984 & 7.1485 & 283.0 & 4 & 600.0 & 600.0 \\
\hline 04 & 41710 & 78.7466 & 7.1288 & 284.4 & 4 & 600.0 & 600.0 \\
\hline 04 & 41711 & 88.1555 & 6.84 & 285.4 & 4 & 600.0 & \\
\hline 04 & 41712 & 100.8298 & 6.4615 & 285.3 & 3 & 600.0 & 600.0 \\
\hline 04 & 41713 & 82.8476 & 5.4315 & 285.2 & 3 & 600.0 & 600.0 \\
\hline 04 & 41714 & 78.6749 & 7.4725 & 287.0 & 3 & 600.0 & \\
\hline 04 & 41715 & 61.9469 & 7.9475 & 286.4 & 4 & 600.0 & 60 \\
\hline 04 & 41716 & 55.0991 & 9.3550 & 286.1 & 4 & 600.0 & 600.0 \\
\hline 04 & 41717 & 60.7752 & 9.7550 & 285.1 & 4 & 600.0 & \\
\hline 04 & 41718 & 68.5253 & 7.7258 & 284.1 & 4 & 600.0 & 60 \\
\hline 04 & 41719 & 63.5502 & 8.6000 & 283.0 & 4 & 600.0 & 600.0 \\
\hline 04 & 41720 & 59.0250 & 10.72 & 282.2 & 4 & 600.0 & .0 \\
\hline 04 & 41721 & 59.4500 & 12.0350 & 281.8 & 4 & 600.0 & \\
\hline 04 & 41722 & 57.2503 & 11.7475 & 281.7 & 4 & 600.0 & 600.0 \\
\hline 04 & 41723 & 54.7247 & 10.0550 & 281.4 & 4 & 600.0 & .0 \\
\hline 04 & 41724 & 46.2491 & 7.6 & 281.3 & 4 & 600.0 & \\
\hline 04 & 4181 & 50.0251 & 6.5 & 281.4 & 4 & 600.0 & 600.0 \\
\hline 04 & 4182 & 54.7386 & 5.5500 & 281.3 & 4 & 600.0 & 600.0 \\
\hline 04 & 4183 & 46.4500 & 5.86 & 281.0 & 4 & 600.0 & \\
\hline 04 & 4184 & 40.4748 & 4.5218 & 281.0 & 4 & 600.0 & 60 \\
\hline 04 & 4185 & 50.9748 & 4.6265 & 281.0 & 4 & 600.0 & 600.0 \\
\hline 04 & 4186 & 36.7013 & 3.4 & 280.6 & 4 & 600.0 & \\
\hline 04 & 4187 & 252.6882 & 1.1628 & 281.0 & 3 & 600.0 & 60 \\
\hline 04 & 4188 & 223.2121 & 1.6735 & 282.9 & 2 & 600.0 & 600.0 \\
\hline 04 & 4189 & 272.2904 & 2.88 & 284.2 & 3 & 600.0 & .0 \\
\hline 04 & 41810 & 263.8546 & 3.63 & 284.7 & 3 & 600.0 & 60 \\
\hline 04 & 41811 & 325.7364 & 2.3438 & 285.0 & 2 & 600.0 & 600.0 \\
\hline 04 & 41812 & 299.7169 & 2.2350 & 285.3 & 3 & 600.0 & 600.0 \\
\hline 04 & 41813 & 172.8326 & 2.59 & 285.2 & 3 & 600.0 & 600.0 \\
\hline 04 & 41814 & 298.8573 & 3.2898 & 285.6 & 3 & 600.0 & 600.0 \\
\hline 04 & 41815 & 87.4297 & 3.5420 & 283.9 & 3 & 600.0 & \\
\hline 04 & 41816 & 59.9288 & 2.8165 & 283.6 & 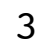 & 600.0 & 600.0 \\
\hline 04 & 41817 & 66.5128 & 2.8123 & 282.8 & 4 & 600.0 & 600.0 \\
\hline 04 & 41818 & 104.3145 & 3.2095 & 282.2 & 4 & 600.0 & 600.0 \\
\hline 04 & 41819 & 97.4798 & 3.8333 & $281 \cdot 6$ & 4 & 600.0 & 600.0 \\
\hline 04 & 41820 & 66.6763 & 3.0143 & 281.6 & 4 & 600.0 & 600.0 \\
\hline 04 & 41821 & 89.3544 & 2.0088 & 281.4 & 5 & 600.0 & 600.0 \\
\hline 04 & 41822 & 70.0875 & 2.4063 & 281.4 & 4 & 600.0 & 600.0 \\
\hline 04 & 41823 & 11.3425 & 0.9083 & 280.9 & $\mathrm{~J}$ & 600.0 & 600.0 \\
\hline
\end{tabular}




\begin{tabular}{|c|c|c|c|c|c|c|c|}
\hline 4 & 41824 & 15.7507 & 1.2313 & 280.9 & 6 & 600.0 & 600.0 \\
\hline 04 & 4191 & 7.2105 & 1.9428 & 280.9 & 5 & 600.0 & 600.0 \\
\hline 04 & 4192 & 54.4242 & 1.8643 & 280.9 & 5 & 600.0 & 00.0 \\
\hline 04 & 4193 & 48.6011 & 1.8618 & 280.9 & 5 & 600.0 & 00.0 \\
\hline 04 & 4194 & 2.4503 & 1.3785 & 281.1 & 6 & 600.0 & 00.0 \\
\hline 04 & 4195 & 27.6481 & 0.6498 & 281.5 & 6 & 600.0 & 00.0 \\
\hline 04 & 4196 & 338.8620 & 1.0840 & 281.5 & 6 & 600.0 & 600.0 \\
\hline 04 & 4197 & 328.7205 & 0.8990 & 281.8 & 5 & 600.0 & .0 \\
\hline 04 & 4198 & 26.5503 & 1.0623 & 282.6 & 4 & 600.0 & 600.0 \\
\hline 04 & 4199 & 281.1841 & 1.1813 & 283.4 & 3 & 600.0 & 600 \\
\hline 4 & 41910 & 260.5444 & 1.8773 & 284.9 & 2 & 600.0 & \\
\hline 04 & 41911 & 275.0607 & 2.4630 & 286.5 & 2 & 600.0 & .0 \\
\hline 04 & 41912 & 6.4982 & 3.3460 & 287.3 & 2 & 600.0 & .0 \\
\hline 04 & 41913 & 27.5269 & 3.9660 & 288.1 & 2 & 600.0 & 600.0 \\
\hline 04 & 41914 & 354.2043 & 3.8098 & 289.1 & 2 & 600.0 & 60 \\
\hline 04 & 41915 & 339.4730 & 2.7293 & 289.2 & 2 & 600.0 & 600 \\
\hline 04 & 41916 & 7.0684 & 3.4618 & 289.3 & 3 & 600.0 & .0 \\
\hline 04 & 41917 & 19.7260 & 5. & 289.1 & 3 & .0 & \\
\hline 04 & 41918 & 8.0744 & 5.6650 & 288.0 & 4 & 600.0 & 60 \\
\hline 04 & 41919 & 20.1203 & 5.9930 & 287.2 & 4 & .0 & .0 \\
\hline 04 & 41920 & 20.9025 & 4.6390 & 286.7 & 4 & .0 & \\
\hline 04 & 41921 & 9.8501 & 4.9490 & 286.2 & 4 & .0 & 60 \\
\hline 04 & 41922 & 8.3228 & 5.0388 & 286.5 & 4 & 60 & 600.0 \\
\hline 04 & 41923 & 5.7623 & 5.4118 & 286.6 & 4 & .0 & \\
\hline 04 & 41924 & 30.4494 & 445 & 286.7 & 4 & .0 & 60 \\
\hline 04 & 4201 & 25.0511 & 4.4940 & 286.7 & 4 & 600.0 & 600.0 \\
\hline 04 & $420 \quad 2$ & 73.7360 & 2.3695 & 286.0 & 4 & .0 & .0 \\
\hline 04 & 4203 & 4.1782 & 2.5760 & 286.8 & 4 & .0 & 60 \\
\hline 04 & $420 \quad 4$ & 358.2550 & 2.2223 & 286.6 & 5 & 600.0 & 600.0 \\
\hline 04 & $420 \quad 5$ & 4.6599 & 2.4898 & 286.5 & 4 & .0 & .0 \\
\hline 04 & 4206 & 291. & 1 . & 286.1 & 5 & .0 & \\
\hline 04 & 4207 & 183.7076 & 0.8358 & 286.5 & 4 & 600.0 & 600.0 \\
\hline 04 & $420 \quad 8$ & 122.9237 & 2.8048 & 287.0 & 3 & 600.0 & 600.0 \\
\hline 04 & 4209 & 240.6752 & 865 & 287.7 & 3 & 600.0 & 600.0 \\
\hline 04 & 42010 & 62.0832 & 4.0210 & 286.9 & 3 & .0 & 600.0 \\
\hline 04 & 42011 & 65.0932 & 4.2915 & 287.4 & 3 & 600.0 & 600.0 \\
\hline 04 & 42012 & 19.2266 & 2.6523 & 288.6 & 3 & .0 & .0 \\
\hline 04 & 42013 & 264.9878 & 2.7525 & 289.3 & 2 & 600.0 & 600.0 \\
\hline 04 & 42014 & 276.1265 & 3.2825 & 289.8 & 3 & 600.0 & 600.0 \\
\hline 04 & 42015 & 271.2160 & 900 & 290.9 & 2 & 600.0 & 600.0 \\
\hline 04 & 42016 & 73.8432 & 6.0155 & 290.5 & 3 & 600.0 & 600.0 \\
\hline 04 & 42017 & 78.2044 & 7.3235 & 288.5 & 4 & 600.0 & 600.0 \\
\hline 04 & 42018 & 67.3000 & 9.2050 & 286.8 & 4 & 600.0 & 600.0 \\
\hline 04 & 42019 & 77.0040 & 9.8750 & 285.7 & 4 & 600.0 & 600.0 \\
\hline 04 & 42020 & 79.9748 & 11.3300 & 284.5 & 4 & 600.0 & 600.0 \\
\hline 04 & 42021 & 76.9000 & 11.5275 & 284.1 & 4 & 600.0 & \\
\hline 04 & 42022 & 83.1690 & 8.458 & 283.8 & 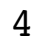 & 600.0 & 600.0 \\
\hline 04 & 42023 & 92.1775 & 7.5053 & 283.7 & 4 & 600.0 & 600.0 \\
\hline
\end{tabular}




\begin{tabular}{|c|c|c|c|c|c|c|c|}
\hline & 024 & $2+0$ & 145 & 33.8 & 4 & , & \\
\hline & 4211 & 5.7159 & 4.0698 & 84.0 & & ח & \\
\hline & 212 & 4898 & 800 & 83.7 & 4 & 00 & \\
\hline & 213 & 0.2970 & 505 & 283.6 & & 0 & \\
\hline & 214 & 723 & 590 & & & & \\
\hline & 215 & 7468 & & 2.6 & & 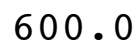 & \\
\hline & 4216 & 7333 & 00 & 82.9 & & 0 & \\
\hline & 217 & 6.1729 & 098 & & & & \\
\hline & 218 & 7.8760 & & & & & \\
\hline & 4219 & 127.9216 & 333 & 86.6 & & 0 & \\
\hline & 2110 & 93.5880 & 4.93 & 88.3 & & & \\
\hline & 42111 & 65.4250 & & & & & \\
\hline & 21 & 742 & 775 & 89.9 & & & \\
\hline & 21 & 65.2745 & 7.56 & .2 & & & \\
\hline & 421 & 695 & & & & & \\
\hline & $2]$ & 61 . & & 7 & & & \\
\hline & 21 & 61.3498 & 5 & 286.8 & 4 & & \\
\hline & 17 & 60.6756 & & & & & \\
\hline & 8 & 5748 & & & - & & \\
\hline & 21 & 4270 & 0 & .2 & 1 & & \\
\hline & 42120 & 251 & 0 & .0 & 4 & 0 & \\
\hline & $42]$ & 68.3250 & & & 4 & & \\
\hline & 2 & 749 & 0 & & 4 & & \\
\hline & 42123 & 66.7497 & 5 & & & & \\
\hline & 42124 & 76.7464 & & & 4 & & \\
\hline & 4221 & 110.6228 & & & 4 & & \\
\hline & 4222 & 134.9004 & 5 & 2 & 4 & & \\
\hline & 4223 & 141.9749 & & & 4 & & \\
\hline & 4224 & 250 & 5 & & 4 & & \\
\hline & 4225 & 146.4000 & 125 & .3 & 4 & & \\
\hline & 4226 & 150.6001 & 1 & .2 & 4 & & \\
\hline & 4227 & 151.7251 & 5 & & 4 & & \\
\hline & 4228 & 159.1750 & & 0 & 4 & & . \\
\hline & 4229 & 165.7502 & & & 4 & & \\
\hline & 42210 & 164.2236 & 5 & & 4 & & \\
\hline & & 181.2594 & & & 3 & & \\
\hline & 42212 & 3.9689 & 0 & 90.2 & 3 & & \\
\hline & 42213 & 173.3309 & 11 . & & 3 & & \\
\hline & & 177.4501 & & & 3 & & 0 . \\
\hline & 42215 & 175.1523 & 75 & 293.0 & 4 & & \\
\hline & 42216 & 171.1834 & U & & 4 & & \\
\hline & & 159.7508 & 10.9700 & & 4 & & \\
\hline & 42218 & 160.0000 & 6625 & 293.3 & 4 & & 0 \\
\hline & 42219 & 140.5257 & & & 4 & & \\
\hline & 42220 & 13 & & & 7 & & \\
\hline & 42221 & 145.5749 & 9.9225 & 292.2 & 4 & & 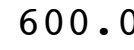 \\
\hline & & & & & & & \\
\hline & 2223 & 1000 & 9.092 & 292.1 & & & s \\
\hline
\end{tabular}




\begin{tabular}{|c|c|c|c|c|c|c|c|}
\hline- & 2224 & & .7050 & 1.1 & & & \\
\hline 4 & 4231 & 506 & .3200 & 290.4 & 4 & & \\
\hline & 4232 & ( & 7075 & 290.2 & 4 & 00 & \\
\hline & 4233 & 136.1985 & 6663 & 290.0 & & 00 & \\
\hline & 4234 & L 8 & .8610 & 89.6 & & 0 & \\
\hline & 4235 & 7.2750 & .6650 & 289.3 & 4 & 00 & \\
\hline & 4236 & 44.7572 & 7.6398 & 288.5 & & 0 & \\
\hline & 4237 & 31.9601 & .6253 & 88.3 & & & \\
\hline & 4238 & 131.0224 & .7513 & 289.1 & & 0 & \\
\hline & 4239 & 135.4965 & 7.1653 & 290.8 & & 0 & \\
\hline & 42310 & 53.8294 & 7.2525 & 292.5 & 4 & 0 & \\
\hline & 42311 & 7.2251 & 8.8325 & 93.9 & 4 & & \\
\hline & 42312 & 61.6500 & 9.0900 & 295.2 & 3 & 0 & \\
\hline & 42313 & 55.6097 & 8.1325 & 295.9 & 3 & 00.0 & se \\
\hline & 42314 & 76.1601 & & 296.6 & 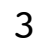 & & \\
\hline & 42315 & 173.5703 & 7.26 & 296.7 & 4 & 0 & \\
\hline & 42316 & 68.0612 & .9620 & 296.9 & 4 & .0 & \\
\hline & 42317 & 172.1 & $5 \cdot$ & 296.9 & 3 & & \\
\hline 4 & 42318 & 166.3099 & 4.56 & 296.1 & 3 & 0 & \\
\hline & 42319 & 74.8444 & 0 & 294.4 & 4 & 0 & \\
\hline & 42320 & 69.6 & 8 & 29 & 4 & & \\
\hline 4 & 42321 & 67. & & 293.0 & 4 & & \\
\hline 4 & 42322 & 64.7502 & 075 & 293.3 & 4 & 0 & \\
\hline 4 & 42323 & 64.8500 & 7.7525 & 293.6 & 4 & 0 & \\
\hline 4 & 42324 & 68.1 & 8 & 293.9 & 4 & & \\
\hline 4 & $424 \quad 1$ & 72.9504 & 793 & 293.8 & 4 & & \\
\hline & $424 \quad 2$ & 99.8624 & 4.3725 & 293.8 & 4 & 0 & \\
\hline 4 & 4243 & 18.8275 & 3 . & 293 & 4 & & \\
\hline 4 & $424 \quad 4$ & 52 & 05 & 292.6 & 4 & 0 & 0 \\
\hline & $424 \quad 5$ & 147.4762 & 070 & 289.8 & 4 & 0 & \\
\hline & 4246 & 144 . & 140 & 290 & 4 & & \\
\hline 4 & 4247 & 145 . & 0 & 29 & 4 & & . \\
\hline & 4248 & 142.5273 & 1700 & 291.4 & 4 & 0 & J \\
\hline 4 & 4249 & 144 . & 0 & 292.0 & 4 & 0 & \\
\hline & 42410 & 146 . & 8 & 293 & 4 & & \\
\hline & 42411 & 151.5500 & 938 & 294.4 & 4 & 0 & 0 . \\
\hline & 42412 & 163.6055 & 210 & 295.9 & 3 & 0 & \\
\hline 4 & 42413 & 169.3501 & 3 & 297.2 & 3 & 0 & • \\
\hline 4 & 42414 & 166.5562 & 6.4978 & 298.2 & 4 & 0 & 0 . \\
\hline & 42415 & 169.3055 & 735 & 298.8 & 3 & 0 & \\
\hline- & 42416 & 177.7264 & 238 & 298 & 4 & & \\
\hline 04 & 42417 & 178.5167 & 6.8853 & 298.7 & 4 & 600.0 & 600 . \\
\hline & 42418 & 176.9626 & 6.2948 & 298.0 & 4 & 600 & 800 \\
\hline- & 42419 & 169.6621 & 5.4998 & 296.4 & 4 & 600 & 600 \\
\hline 4 & 42420 & 105.1200 & 1.6183 & 296.8 & 5 & 600.0 & 600. \\
\hline & 42421 & 102.9341 & 1.2915 & 296.9 & 6 & 600.0 & 600 \\
\hline & 42422 & & 3.601 & 295.9 & 3 & 600 & \\
\hline & 42423 & 74.5999 & 6.8105 & 295.1 & $\mathbf{4}$ & 600.0 & 600 \\
\hline
\end{tabular}




\begin{tabular}{|c|c|c|c|c|c|c|c|}
\hline 4 & 42424 & 74.9250 & 7.0378 & 295.0 & 4 & 600.0 & 00.0 \\
\hline 04 & 4251 & 78.0250 & 6.2168 & 295.0 & 4 & 600.0 & 600.0 \\
\hline 04 & $425 \quad 2$ & 76.6250 & 6.3058 & 294.9 & 4 & 600.0 & 00.0 \\
\hline 04 & 4253 & 75.0500 & 7.1018 & 294.8 & 4 & 600.0 & 600.0 \\
\hline 04 & $425 \quad 4$ & 78.4750 & 5.3680 & 295.0 & 4 & 600.0 & 00.0 \\
\hline 04 & 4255 & 102.5960 & 2.3465 & 295.4 & 5 & 600.0 & 00.0 \\
\hline 04 & 4256 & 203.4083 & 0.7413 & 295.4 & 5 & 600.0 & 600.0 \\
\hline 04 & 4257 & 355.3049 & 0.8243 & 295.3 & 4 & 600.0 & 00.0 \\
\hline 04 & 4258 & 233.0227 & 1.4200 & 296.4 & 3 & 600.0 & 00.0 \\
\hline 04 & 4259 & 240.8081 & 2.5603 & 297.3 & 2 & 600.0 & 600.0 \\
\hline 04 & 42510 & 177.5280 & 5.2263 & 297.4 & 3 & 600.0 & 600.0 \\
\hline 04 & 42511 & 176.1621 & 6.1148 & 297.7 & 3 & 600.0 & \\
\hline 04 & 42512 & 175.7958 & 5.0118 & 298.8 & 2 & 600.0 & 600.0 \\
\hline 04 & 42513 & 187.1419 & 4.8233 & 300.2 & 2 & 600.0 & 600.0 \\
\hline 04 & 42514 & 186.8716 & 4.5545 & 300.9 & 2 & 600.0 & \\
\hline 04 & 42515 & 179.4424 & 4.6250 & 301.3 & 2 & 600.0 & 60 \\
\hline 04 & 42516 & 178.6620 & 4.3608 & 301.7 & 3 & 600.0 & 600.0 \\
\hline 04 & 42517 & 182.9189 & 4.5575 & 301.5 & 3 & 600.0 & \\
\hline 04 & 42518 & 166.6421 & 4.7653 & 300.8 & 3 & 600.0 & 60 \\
\hline 04 & 42519 & 158.6751 & 4.1950 & 299.0 & 4 & 600.0 & 600.0 \\
\hline 04 & 42520 & 149.8812 & 3.9168 & 297.7 & 4 & 600.0 & .0 \\
\hline 04 & 42521 & 86.7891 & 4.5713 & 297.9 & 4 & 600.0 & \\
\hline 04 & 42522 & 79.8994 & 3.5278 & 298.0 & 4 & 600.0 & 600.0 \\
\hline 04 & 42523 & 80.3496 & 3.7740 & 297.8 & 4 & 600.0 & .0 \\
\hline 04 & 42524 & 77.8732 & 4.8823 & 297.5 & 4 & 600.0 & \\
\hline 04 & 4261 & 91.7966 & 4.3865 & 297.6 & 4 & 600.0 & 600.0 \\
\hline 04 & 4262 & 92.4762 & 3.9378 & 297.7 & 4 & 600.0 & 60 \\
\hline 04 & 4263 & 98.7770 & 3.8600 & 297.5 & 4 & 600.0 & \\
\hline 04 & 4264 & 116.8241 & 3.4293 & 297.5 & 4 & 600.0 & 60 \\
\hline 04 & 4265 & 88.2499 & 4.3923 & 297.4 & 4 & 600.0 & 600.0 \\
\hline 04 & 4266 & 119.6348 & 2.9998 & 297.1 & 4 & 600.0 & .0 \\
\hline 04 & 4267 & 157.8482 & 3.0963 & 296.5 & 3 & 600.0 & 60 \\
\hline 04 & 4268 & 169.5500 & 3.7095 & 297.9 & 3 & 600.0 & 600.0 \\
\hline 04 & 4269 & 164.6750 & 4.8945 & 298.5 & 3 & 600.0 & .0 \\
\hline 04 & 42610 & 166.8499 & 4.1635 & 299.3 & 2 & 600.0 & 60 \\
\hline 04 & 42611 & 166.5770 & 4.2585 & 300.1 & 2 & 600.0 & 600.0 \\
\hline 04 & 42612 & 180.2884 & 4.9810 & 301.6 & 2 & 600.0 & .0 \\
\hline 04 & 42613 & 184.1500 & 4.5215 & 302.3 & 2 & 600.0 & 600.0 \\
\hline 04 & 42614 & 170.9518 & 4.7688 & 303.3 & 2 & 600.0 & 600.0 \\
\hline 04 & 42615 & 182.0135 & 6.6738 & 303.5 & 3 & 600.0 & \\
\hline 04 & 42616 & 177.0176 & 7.8350 & 303.5 & 4 & 600.0 & 600.0 \\
\hline 04 & 42617 & 170.8227 & 6.4573 & 303.5 & 4 & 600.0 & 600.0 \\
\hline 04 & 42618 & 172.6675 & 5.3115 & 302.9 & 4 & 600.0 & 600.0 \\
\hline 04 & 42619 & 141.1230 & 2.4078 & 301.7 & 5 & 600.0 & 600.0 \\
\hline 04 & 42620 & 112.9750 & 1.2993 & 301.5 & 6 & 600.0 & 600.0 \\
\hline 04 & 42621 & 123.4098 & 2.8860 & 300.6 & 5 & 600.0 & 600.0 \\
\hline 04 & 42622 & 124.4390 & 3.3968 & 300.7 & 4 & 600.0 & 600.0 \\
\hline 04 & 42623 & 131.4547 & 4.0690 & 300.7 & 4 & 600.0 & 600.0 \\
\hline
\end{tabular}




\begin{tabular}{|c|c|c|c|c|c|c|c|}
\hline & 24 & & & & & & \\
\hline & 4271 & & & 2 & & & \\
\hline & 4272 & 6.500 & 548 & 0.4 & 4 & 00 & \\
\hline & 273 & 20.2661 & 6885 & 300.0 & & 0 & \\
\hline & $427 \quad 4$ & 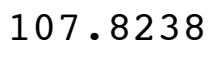 & & 0.5 & & & \\
\hline & 4275 & 102. & & 99.7 & & & \\
\hline & $427 \quad 6$ & 680 & 5 & 299.5 & & 00 & \\
\hline & 4277 & 5499 & & 99.6 & & & \\
\hline & 4278 & 143 & & 1.5 & & & \\
\hline & 4279 & 000 & 6623 & 302.4 & & 0 & \\
\hline & 42710 & 165.8009 & 1 & 302.3 & & & \\
\hline & 42711 & 71 & & & & & \\
\hline & 2712 & 58 & & 304.2 & & & \\
\hline & 42713 & 4756 & U & 30 & & & \\
\hline & 42714 & & & & & & \\
\hline & 42715 & 113 & & & 4 & & \\
\hline & 42716 & 95.24 & & 303 & 4 & & \\
\hline & 42717 & 94 & & & 4 & & \\
\hline & 42718 & & & & 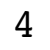 & & \\
\hline & 42719 & 62 & & 297.9 & 4 & .0 & \\
\hline & 42720 & 59 & & 6 & 4 & 0 & \\
\hline & 42721 & & & & 4 & & \\
\hline & 42722 & 51 & & 29 & 4 & & \\
\hline & 42723 & 490 & 5 & 296 & 4 & 0 & \\
\hline & 42724 & & & & 4 & & \\
\hline & 4281 & 66. & 0 & 29 & 4 & & \\
\hline & 4282 & 72.8899 & & 295 & 4 & & . \\
\hline & 4283 & 74.19 & & 29 & 4 & & \\
\hline & 4284 & 83.22 & & 29 & 4 & & \\
\hline & 4285 & 77.22 & & 294.2 & 4 & & 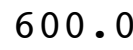 \\
\hline & 4286 & 83.625 & & 293.9 & 4 & & \\
\hline & 4287 & 75 & & 293 & 4 & & \\
\hline & 4288 & 72 . & & 5.2 & 4 & & . \\
\hline & 4289 & 67.907 & & 297.2 & 4 & & \\
\hline & 42810 & 88 & & 297.3 & 4 & & \\
\hline & 42811 & 102.8813 & & 297.0 & 4 & & 0 . \\
\hline & 42812 & 4.4371 & 5 & 297.1 & 3 & & \\
\hline & 42813 & 168 . & 5 & 297.2 & 3 & & \\
\hline & 42814 & 170.3217 & 275 & 297.7 & 4 & & 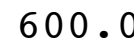 \\
\hline & 42815 & 168.2970 & 650 & 298.0 & 4 & & \\
\hline & 42816 & 178.3226 & 5 & 297.8 & 4 & & \\
\hline & 42817 & 175.0061 & 050 & 297.2 & 4 & & \\
\hline & 42818 & 178.6206 & 4.8725 & 296.2 & 4 & & 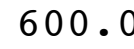 \\
\hline & 42819 & 157.8477 & & 294.8 & 4 & & \\
\hline & 42820 & 144.0709 & 12.6075 & 294.2 & 4 & & \\
\hline & 42821 & 140.6750 & 14.5350 & 293.8 & 4 & & 0 \\
\hline & 42822 & & & & 4 & & \\
\hline & 42823 & 145. & 12.2650 & 292.2 & & .0 & 0 \\
\hline
\end{tabular}




\begin{tabular}{|c|c|c|c|c|c|c|c|}
\hline 4 & 824 & & & & & & \\
\hline & 4291 & 3 & & 9.6 & & & \\
\hline & 4292 & 51.4750 & 1.2600 & 289.4 & & 00 & \\
\hline & 293 & 54.6754 & 1.1500 & 289.1 & & 0 & \\
\hline & 4294 & & 1.6925 & & & & \\
\hline & 4295 & & 12. & 87.6 & & 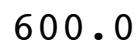 & \\
\hline & 4296 & .793 & 1.8450 & 287.6 & & 0 & \\
\hline & 297 & 5475 & 7650 & 88.0 & & & \\
\hline & 4298 & & 0 & 3 & & & \\
\hline & 299 & 61.4766 & 8.7875 & 90.6 & & 0 & \\
\hline & 2910 & 52.0007 & 9.4050 & 291.8 & & & \\
\hline & 42911 & 9.6236 & 982 & 92.8 & & & \\
\hline & 2912 & 55.3451 & 9.3200 & 293.7 & & 0 & \\
\hline & 2913 & 59.2750 & 9.41 & 294.8 & & 0.0 & \\
\hline & 2914 & 7087 & 9 . & & & & \\
\hline & 2915 & . 7288 & & 29 & 4 & & \\
\hline & 2916 & 49.6035 & 8.18 & 296.5 & 4 & 0 & \\
\hline & 2917 & 6.2439 & 8.29 & .6 & 4 & & \\
\hline & 2918 & 88.9696 & 7.73 & 294.8 & 4 & & \\
\hline & 2919 & 267 & 8.42 & 292.9 & 4 & 0 & \\
\hline & 42920 & 65.1498 & 8.9450 & 291.5 & 4 & 0 & \\
\hline & 42921 & 74.1761 & 9.84 & 291.3 & 4 & & \\
\hline & 2922 & 68.7997 & 933 & 291.1 & 4 & & \\
\hline & 42923 & 67.6498 & .2675 & 290.5 & 4 & 0 & \\
\hline & 42924 & 66.9750 & 0.3425 & 29 & 4 & & \\
\hline & 430 & 5000 & 10.57 & 29 & 4 & & \\
\hline & 4302 & 68.9254 & 10.0250 & 290.6 & 4 & & . \\
\hline & 4303 & 71.7000 & 9.06 & 29 & 4 & & \\
\hline & 4304 & 251 & 8 . & 29 & 4 & & \\
\hline & 4305 & 506 & 7.4175 & 290.7 & 4 & & . \\
\hline & 4306 & 75.6750 & 7.80 & 290.5 & 4 & & 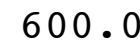 \\
\hline & 4307 & 74 . & 3 & 291.2 & 4 & & \\
\hline & 4308 & 732 & 3178 & 292.3 & 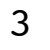 & & 0 . \\
\hline & 4309 & 11.5751 & 3.3518 & 294.3 & 3 & & \\
\hline & 43010 & 178 . & & & 2 & & \\
\hline & & 186.2695 & & 296.6 & 2 & & \\
\hline & 012 & 171.0750 & 7985 & 297.5 & 3 & & \\
\hline & 43013 & 153. & 848 & 298 & 3 & & \\
\hline & 14 & 155.9876 & 6.7370 & 298.6 & 3 & & . \\
\hline & 5 & 143.0617 & 110 & 298.8 & 2 & & \\
\hline & 43016 & 129.6011 & 55 & 299.0 & 3 & & \\
\hline & & 91.0738 & 6.0220 & 298.4 & 4 & & \\
\hline & 43018 & 78.1488 & 6.4045 & 297.0 & 4 & & 0 . \\
\hline & 43019 & 011 & 6.7668 & 295.3 & 4 & & \\
\hline & & 68.6000 & 8.9325 & 294.0 & 4 & & \\
\hline & & 68.5250 & 8.3050 & 293.6 & 4 & & 0 . \\
\hline & & & & & 4 & & \\
\hline & 3023 & 70.2999 & 8.8350 & 293.6 & & 600.0 & $c^{\circ}$ \\
\hline
\end{tabular}




\begin{tabular}{|c|c|c|c|c|c|c|c|}
\hline 4 & 43024 & 62.0996 & 8.9100 & 293.5 & 4 & 600.0 & 00.0 \\
\hline 04 & $\begin{array}{lll}5 & 1 & 1\end{array}$ & 62.7750 & 8.4000 & 294.0 & 4 & 600.0 & 600.0 \\
\hline 04 & $\begin{array}{lll}5 & 1 & 2\end{array}$ & 69.1500 & 8.1150 & 294.3 & 4 & 600.0 & 00.0 \\
\hline 04 & $\begin{array}{lll}5 & 1 & 3\end{array}$ & 69.9500 & 7.2073 & 294.4 & 4 & 600.0 & 600.0 \\
\hline 04 & $\begin{array}{lll}5 & 1 & 4\end{array}$ & 70.5750 & 6.8488 & 294.2 & 4 & 600.0 & 00.0 \\
\hline 04 & $\begin{array}{lll}5 & 1 & 5\end{array}$ & 72.6250 & 6.8138 & 294.3 & 4 & 600.0 & 500.0 \\
\hline 04 & 516 & 76.6000 & 6.1920 & 294.2 & 4 & 600.0 & 600.0 \\
\hline 04 & $\begin{array}{lll}5 & 1 & 7\end{array}$ & 59.0398 & 1.8718 & 294.1 & 3 & 600.0 & 500.0 \\
\hline 04 & $\begin{array}{lll}5 & 1 & 8\end{array}$ & 257.1634 & 2.1863 & 294.0 & 2 & 600.0 & 00.0 \\
\hline 04 & $\begin{array}{lll}5 & 1 & 9\end{array}$ & 201.3955 & 2.6145 & 295.0 & 2 & 600.0 & 600.0 \\
\hline 04 & 5110 & 221.1191 & 3.1163 & 296.9 & 2 & 600.0 & 600 \\
\hline 04 & $5 \quad 111$ & 175.2546 & 3.3033 & 298.6 & 2 & 600.0 & .0 \\
\hline 04 & 5112 & 182.8880 & 4.8578 & 300.4 & 2 & 600.0 & 600.0 \\
\hline 04 & $5 \quad 113$ & 179.9445 & 5.2618 & 300.8 & 2 & 600.0 & 600.0 \\
\hline 04 & $5 \quad 114$ & 175.1818 & 5.8 & 300.8 & 3 & 600.0 & \\
\hline 04 & 5115 & 188.0171 & 7.2000 & 301.0 & 4 & 600.0 & 60 \\
\hline 04 & 5116 & 182.4607 & 6.9865 & 300.9 & 4 & 600.0 & 600.0 \\
\hline 04 & $\begin{array}{ll}5 & 117\end{array}$ & 184.8251 & 6.2918 & 300.7 & 4 & 600.0 & .0 \\
\hline 04 & 5118 & 186.1124 & 6.1388 & 300.0 & 4 & 600.0 & 60 \\
\hline 04 & 5119 & 104.7789 & 5.1310 & 298.5 & 4 & 600.0 & 600.0 \\
\hline 04 & 5120 & 76.6253 & 5.9885 & 297.1 & 4 & 600.0 & .0 \\
\hline 04 & $5 \quad 121$ & 67.2000 & 6. & 296.5 & 4 & 600.0 & \\
\hline 04 & 5122 & 70.9253 & 7.5500 & 296.2 & 4 & 600.0 & 600.0 \\
\hline 04 & 5123 & 71.2002 & 8.2550 & 296.0 & 4 & 600.0 & 60 \\
\hline 04 & $5 \quad 124$ & 71.5001 & 7.8800 & 296.2 & 4 & 600.0 & \\
\hline 04 & $\begin{array}{lll}5 & 2 & 1\end{array}$ & 68.3250 & 7.7125 & 296.1 & 4 & 600.0 & 600.0 \\
\hline 04 & 522 & 71.3521 & 6.7623 & 296.0 & 4 & 600.0 & 600.0 \\
\hline 04 & 523 & 74.1250 & 5.4783 & 296.1 & 4 & 600.0 & .0 \\
\hline 04 & 524 & 94.4783 & 3.8395 & 296.1 & 4 & 600.0 & 600.0 \\
\hline 04 & 525 & 128.2643 & 3.2008 & 296.0 & 4 & 600.0 & 600.0 \\
\hline 04 & 526 & 146.2753 & 3.6518 & 293.9 & 4 & 600.0 & .0 \\
\hline 04 & $\begin{array}{lll}5 & 2 & 7\end{array}$ & 149.7501 & 4.5768 & 295.5 & 4 & 600.0 & 60 \\
\hline 04 & 528 & 161.4964 & 5.0890 & 297.0 & 3 & 600.0 & 600.0 \\
\hline 04 & 529 & 166.1996 & 5.1240 & 298.3 & 3 & 600.0 & .0 \\
\hline 04 & 5210 & 168.4252 & 4.7463 & 299.6 & 2 & 600.0 & 60 \\
\hline 04 & 5211 & 172.2923 & 4.9185 & 301.0 & 2 & 600.0 & 600.0 \\
\hline 04 & $5 \quad 212$ & 170.8343 & 4.2845 & 301.6 & 2 & 600.0 & 600.0 \\
\hline 04 & 5213 & 178.5515 & 5.3875 & 302.5 & 2 & 600.0 & 600.0 \\
\hline 04 & 5214 & 186.4717 & 4.8580 & 303.0 & 2 & 600.0 & 600.0 \\
\hline 04 & 5215 & 182.1951 & 5.2433 & 303.7 & 2 & 600.0 & \\
\hline 04 & 5216 & 188.9508 & 5.2015 & 303.7 & 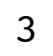 & 600.0 & 600.0 \\
\hline 04 & $5 \quad 217$ & 179.4603 & 5.1045 & 303.3 & 3 & 600.0 & 600.0 \\
\hline 04 & 5218 & 183.1910 & 6.4023 & 302.3 & 4 & 600.0 & 600.0 \\
\hline 04 & 5219 & 144.5965 & 6.0660 & 300.7 & 4 & 600.0 & 600.0 \\
\hline 04 & 5220 & 77.6513 & 6.0933 & 299.1 & 4 & 600.0 & 600.0 \\
\hline 04 & $5 \quad 221$ & 69.0497 & 7.8100 & 298.3 & 4 & 600.0 & 600.0 \\
\hline 04 & $5 \quad 222$ & 74.3765 & $81 \varepsilon$ & 297.9 & 4 & 600.0 & 600.0 \\
\hline 04 & $5 \quad 223$ & 67.4249 & 7.7225 & 297.9 & 4 & 600.0 & 600.0 \\
\hline
\end{tabular}




\begin{tabular}{|c|c|c|c|c|c|c|c|c|}
\hline 4 & 5 & 224 & 61.8991 & 5.7213 & 298.1 & 4 & 600.0 & 00.0 \\
\hline 04 & 5 & 31 & 61.9750 & 7.6800 & 298.2 & 4 & 600.0 & 600.0 \\
\hline 04 & 5 & 32 & 64.2003 & 7.4175 & 297.9 & 4 & 600.0 & 00.0 \\
\hline 04 & 5 & 33 & 66.6250 & 6.3585 & 297.5 & 4 & 600.0 & 600.0 \\
\hline 04 & 5 & 34 & 75.1993 & 5.4043 & 297.7 & 4 & 600.0 & 00.0 \\
\hline 04 & 5 & 35 & 86.2988 & 5.0075 & 297.6 & 4 & 600.0 & 00.0 \\
\hline 04 & 5 & 36 & 84.1478 & 5.9343 & 297.4 & 4 & 600.0 & 600.0 \\
\hline 04 & 5 & 37 & 81.7500 & 5.6168 & 297.7 & 4 & 600.0 & 500.0 \\
\hline 04 & 5 & 38 & 276.2524 & 2.5703 & 299.5 & 3 & 600.0 & 00.0 \\
\hline 04 & 5 & 39 & 237.1765 & 3.3313 & 300.0 & 3 & 600.0 & 600.0 \\
\hline 04 & 5 & 310 & 230.9719 & 3.9175 & 299.5 & 2 & 600.0 & 600.0 \\
\hline 04 & 5 & 311 & 231.3848 & 2.9503 & 300.7 & 2 & 600.0 & \\
\hline 04 & 5 & 312 & 216.7442 & 3.1760 & 302.5 & 1 & 600.0 & 600.0 \\
\hline 04 & 5 & 313 & 229.4254 & 3.0393 & 303.7 & 1 & 600.0 & 600.0 \\
\hline 04 & 5 & 314 & 206.5775 & 3.2653 & 304.7 & 2 & 600.0 & \\
\hline 04 & 5 & 315 & 127.0166 & 4.9400 & 305.9 & 2 & 600.0 & 60 \\
\hline 04 & 5 & 316 & 115.5338 & 5.6258 & 305.6 & 3 & 600.0 & 600.0 \\
\hline 04 & 5 & 317 & 98.6531 & 5.1978 & 304.3 & 3 & 600.0 & .0 \\
\hline 04 & 5 & 318 & 70.9722 & 5.6223 & 302.3 & 4 & 600.0 & 60 \\
\hline 04 & 5 & 319 & 70.5492 & 8.4750 & 301.1 & 4 & 600.0 & 600.0 \\
\hline 04 & 5 & 320 & 73.5050 & 7.0990 & 300.0 & 4 & 600.0 & .0 \\
\hline 04 & 5 & 321 & 87.3248 & 7.6930 & 299.1 & 4 & 600.0 & \\
\hline 04 & 5 & 322 & 78.5425 & 4.6318 & 298.8 & 4 & 600.0 & 600.0 \\
\hline 04 & 5 & 323 & 77.8982 & 3.8558 & 299.4 & 4 & 600.0 & .0 \\
\hline 04 & 5 & 324 & 105.2915 & 2.0950 & 299.2 & 5 & 600.0 & \\
\hline 04 & 5 & 41 & 57.7784 & 3.1325 & 299.7 & 4 & 600.0 & 600.0 \\
\hline 04 & 5 & 42 & 69.3989 & 5.4235 & 299.2 & 4 & 600.0 & 600.0 \\
\hline 04 & 5 & 43 & 74.5613 & 7.2770 & 297.3 & 4 & 600.0 & .0 \\
\hline 04 & 5 & 44 & 67.6266 & 6.9940 & 297.3 & 4 & 600.0 & 60 \\
\hline 04 & 5 & 45 & 68.3003 & 8.6825 & 296.5 & 4 & 600.0 & 600.0 \\
\hline 04 & 5 & 46 & 65.7248 & 9.2600 & 296.1 & 4 & 600.0 & .0 \\
\hline 04 & 5 & 47 & 69.0260 & 8.6175 & 296.7 & 4 & 600.0 & 60 \\
\hline 04 & 5 & 48 & 67.5999 & 7.9250 & 297.7 & 4 & 600.0 & 600.0 \\
\hline 04 & 5 & 49 & 76.6002 & 7.4925 & 298.5 & 4 & 600.0 & .0 \\
\hline 04 & 5 & 410 & 83.7004 & 7.1353 & 299.3 & 3 & 600.0 & 6 \\
\hline 04 & 5 & 411 & 76.4740 & 6.0685 & 299.7 & 3 & 600.0 & 600.0 \\
\hline 04 & 5 & 412 & 76.7522 & 5.7600 & 300.8 & 3 & 600.0 & 600.0 \\
\hline 04 & 5 & 413 & 77.3910 & 8.2425 & 299.9 & 3 & 600.0 & 600.0 \\
\hline 04 & 5 & 414 & 86.4268 & 8.6775 & 299.3 & 3 & 600.0 & 600.0 \\
\hline 04 & 5 & 415 & 72.3250 & 10.5375 & 298.3 & 4 & 600.0 & \\
\hline 04 & 5 & 416 & 68.5251 & 12.2575 & 296.6 & 4 & 600.0 & 600.0 \\
\hline 04 & 5 & 417 & 63.5743 & 12.4975 & 295.3 & 4 & 600.0 & 600.0 \\
\hline 04 & 5 & 418 & 66.4509 & 12.0500 & 294.1 & 4 & 600.0 & 600.0 \\
\hline 04 & 5 & 419 & 66.7752 & 11.1300 & 292.2 & 4 & 600.0 & 600.0 \\
\hline 04 & 5 & 420 & 73.1947 & 9.8475 & 289.4 & 4 & 600.0 & 600.0 \\
\hline 04 & 5 & 421 & 79.6720 & 12.3750 & 287.5 & 4 & 600.0 & 600.0 \\
\hline 04 & 5 & 422 & 75.9750 & 15.6450 & 287.3 & 4 & 600.0 & 600.0 \\
\hline 04 & 5 & 423 & 78.7000 & 14.8750 & 287.8 & 4 & 600.0 & 600.0 \\
\hline
\end{tabular}




\begin{tabular}{|c|c|c|c|c|c|c|c|c|}
\hline 4 & 5 & 424 & 71.3001 & 13.1025 & 287.3 & 4 & 600.0 & 600.0 \\
\hline 04 & 5 & 51 & 71.9507 & 13.6850 & 286.1 & 4 & 600.0 & 600.0 \\
\hline 04 & 5 & 52 & 63.1924 & 10.8725 & 285.9 & 4 & 600.0 & 00.0 \\
\hline 04 & 5 & 53 & 83.2485 & 7.9563 & 285.9 & 4 & 600.0 & 00.0 \\
\hline 04 & 5 & 54 & 73.3715 & 12.2700 & 285.4 & 4 & 600.0 & 00.0 \\
\hline 04 & 5 & 55 & 73.1752 & 11.1225 & 286.6 & 4 & 600.0 & 00.0 \\
\hline 04 & 5 & 56 & 72.7753 & 11.1100 & 286.9 & 4 & 600.0 & 00.0 \\
\hline 04 & 5 & 57 & 67.0250 & 10.7250 & 287.2 & 4 & 600.0 & 00.0 \\
\hline 04 & 5 & 58 & 72.6245 & 8.9475 & 288.0 & 4 & 600.0 & 00.0 \\
\hline 04 & 5 & 59 & 81.2738 & 5.8590 & 289.5 & 3 & 600.0 & 00.0 \\
\hline 04 & 5 & 510 & 88.2789 & 5.0638 & 290.7 & 2 & 600.0 & \\
\hline 04 & 5 & 511 & 93.0363 & 3.7930 & 292.7 & 2 & 600.0 & \\
\hline 04 & 5 & 512 & 58.0937 & 4.3378 & 294.1 & 2 & 600.0 & 00.0 \\
\hline 04 & 5 & 513 & 56.6541 & 5.5590 & 294.5 & 3 & 600.0 & 00.0 \\
\hline 04 & 5 & 514 & 61.2103 & 40 & 294.7 & 4 & .0 & \\
\hline 04 & 5 & 515 & 78.3286 & 7.3900 & 294.1 & 4 & 600.0 & 00.0 \\
\hline 04 & 5 & 516 & 83.1742 & 7.5113 & 294.0 & 4 & 60 & 600.0 \\
\hline 04 & 5 & 517 & 78.9006 & 8.1875 & 292.8 & 4 & .0 & \\
\hline 04 & 5 & 518 & 73.9999 & 9.1500 & 291.0 & 4 & 60 & .0 \\
\hline 04 & 5 & 519 & 76.7750 & 9.4900 & 289.2 & 4 & 60 & .0 \\
\hline 04 & 5 & 520 & 78.0749 & 10 & 287.7 & 4 & 60 & \\
\hline 04 & 5 & 521 & 77.3755 & 11.9125 & 286.7 & 4 & .0 & \\
\hline 04 & 5 & 522 & 73.0500 & 9.6875 & 286.6 & 4 & 600.0 & 0 \\
\hline 04 & 5 & 523 & 76.0750 & 9.29 & 286.1 & 4 & 60 & .0 \\
\hline 04 & 5 & 524 & 77.7250 & 9.7875 & 286.1 & 4 & .0 & \\
\hline 04 & 5 & 61 & 77.2000 & 10.2850 & 285.9 & 4 & .0 & 600.0 \\
\hline 04 & 5 & 62 & 77.5500 & 9.9300 & 285.7 & 4 & 600.0 & 600.0 \\
\hline 04 & 5 & 63 & 77.3000 & $9 . \varepsilon$ & 285.6 & 4 & .0 & \\
\hline 04 & 5 & 64 & 77.3500 & 8.9000 & 285.5 & 4 & 600.0 & 600.0 \\
\hline 04 & 5 & 65 & 74.8000 & 7.8425 & 285.5 & 4 & .0 & 0.0 \\
\hline 04 & 5 & 66 & 74.7751 & 7.7015 & 284.9 & 4 & 0 & \\
\hline 04 & 5 & 67 & 32.0047 & 2.8528 & 286.8 & 3 & 600.0 & 600.0 \\
\hline 04 & 5 & 68 & 279.0954 & 1.8120 & 288.4 & 2 & 600.0 & 600.0 \\
\hline 04 & 5 & 69 & 234.6597 & 2.2858 & 289.6 & 2 & 600.0 & 0.0 \\
\hline 04 & 5 & 610 & 204.2738 & 2.9233 & 291.1 & 2 & 600.0 & 600.0 \\
\hline 04 & 5 & 611 & 271.4923 & 3.0795 & 292.6 & 1 & 600.0 & 600.0 \\
\hline 04 & 5 & 612 & 259.1434 & 2.7405 & 293.5 & 1 & 60 & 0.0 \\
\hline 04 & 5 & 613 & 234.5366 & 3.0000 & 295.8 & 1 & 600.0 & 600.0 \\
\hline 04 & 5 & 614 & 187.1780 & 4.2165 & 297.6 & 2 & 600.0 & 600.0 \\
\hline 04 & 5 & 615 & 161.4791 & 5.9128 & 297.7 & 3 & 600.0 & 0 \\
\hline 04 & 5 & 616 & 143.6468 & 6.2913 & 296.8 & 4 & 600.0 & 600.0 \\
\hline 04 & 5 & 617 & 86.1780 & 5.9288 & 295.5 & 3 & 600.0 & 600.0 \\
\hline 04 & 5 & 618 & 85.3248 & 8.1000 & 293.6 & 4 & 600.0 & 600.0 \\
\hline 04 & 5 & 619 & 80.8015 & 8.7500 & 291.2 & 4 & 600.0 & 600.0 \\
\hline 04 & 5 & 620 & 77.7002 & 9.9600 & 289.5 & 4 & 600.0 & 600.0 \\
\hline 04 & 5 & 621 & 69.5750 & 9.2700 & 288.3 & 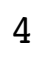 & 600.0 & 600.0 \\
\hline $0<$ & 5 & 622 & 66.7251 & 7.5325 & 288.1 & 4 & 600.0 & 600.0 \\
\hline 04 & & 623 & 59.7000 & 7.2060 & 288.1 & 4 & 600.0 & 600.0 \\
\hline
\end{tabular}




\begin{tabular}{|c|c|c|c|c|c|c|c|}
\hline 4 & 24 & 459 & 630 & 87.2 & 4 & 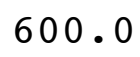 & \\
\hline 4 & 7 & 333.8857 & 498 & 285.3 & & 00.0 & \\
\hline & 72 & 43.7763 & 1.2678 & 285.0 & & 00 & \\
\hline & 73 & 77.7882 & 4.2103 & 285.6 & & 00 & \\
\hline 5 & 74 & 090 & 1330 & & & & \\
\hline & 75 & 119.3224 & 1390 & 8 & & 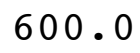 & \\
\hline & 76 & 157.4766 & 8415 & 83.3 & & 00 & \\
\hline & 7 & 334.7462 & 425 & & & & \\
\hline 5 & 78 & 188.1706 & 0158 & 9 & & & \\
\hline & 79 & 172.0781 & 418 & 88.5 & & 0 & \\
\hline & 710 & 188.1142 & 4.4685 & & & & \\
\hline 5 & 711 & 179.5649 & 0 & & & & \\
\hline & 712 & 175 . & 603 & 292.3 & & 0 & \\
\hline : & 713 & 167.2973 & 3 & 3 & & & \\
\hline 5 & 714 & 166 & & & & & \\
\hline 5 & 715 & 158 . & & 29 & & & \\
\hline 5 & 716 & 137.8001 & 3 & 294.2 & 4 & & \\
\hline 5 & 717 & 104 . & 3 & 5 & & & \\
\hline 5 & 718 & 98 . & & 5 & & & \\
\hline 4 & 719 & 7432 & & 290 & 4 & & \\
\hline 4 & 720 & 338 & 0 & 2 & 4 & 0 & \\
\hline 5 & 721 & 65 . & 0 & & 4 & & \\
\hline & 722 & 59 & 75 & 28 & 4 & & \\
\hline 4 & 723 & 62.7001 & 0 & 2 & & 0 & \\
\hline 5 & 724 & 60 & 0 & & & & \\
\hline 4 & 81 & 76 . & & & 4 & & \\
\hline 4 & 82 & 84 . & 3 & 0 & 4 & & 0 \\
\hline 5 & 83 & 93. & 0 & & 4 & & \\
\hline 4 & 84 & 96 & & & 4 & & \\
\hline 1 & 85 & 77 & & 0 & 4 & & 0 \\
\hline 5 & 86 & 110 . & 4. & & 4 & & \\
\hline 5 & 87 & 79 & & & 4 & & \\
\hline 5 & 88 & 86 & & 28 & 4 & & 0 . \\
\hline 5 & 89 & 80 & 5 & & 3 & & \\
\hline 5 & 810 & 7 & & & 4 & & \\
\hline 2 & 811 & 105 . & & .6 & 3 & & \\
\hline 5 & 812 & 108 . & 7923 & 291.7 & 3 & & \\
\hline 5 & 813 & 109. & 6. & & 3 & & \\
\hline- & 814 & 95 . & 6 . & .1 & 3 & & . \\
\hline 5 & 815 & 96. & 3775 & 293.1 & 4 & & \\
\hline 5 & 816 & 111 . & 600 & 292 & 4 & & \\
\hline 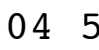 & 817 & & 8.9175 & & 4 & & \\
\hline 5 & 818 & 63.5000 & 9.9050 & 289.6 & 4 & & 0 \\
\hline 5 & 819 & 750 & .4925 & 28 & 4 & & \\
\hline 04 & 820 & 65.7998 & 1.6400 & & 4 & & \\
\hline 5 & 821 & 68.2254 & 10.1800 & 286.0 & 4 & & 00 . \\
\hline 5 & 822 & & & & 4 & & \\
\hline & 823 & 64.1002 & 9.7875 & 286.5 & & 600.0 & 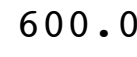 \\
\hline
\end{tabular}




\begin{tabular}{|c|c|c|c|c|c|c|c|}
\hline 4 & $5 \quad 824$ & 65.9495 & 9.7575 & 286.5 & 4 & 600.0 & 600.0 \\
\hline 04 & $\begin{array}{lll}5 & 9 & 1\end{array}$ & 66.2749 & 9.8700 & 286.5 & 4 & 600.0 & 00.0 \\
\hline 04 & 592 & 68.7250 & 9.7350 & 286.1 & 4 & 600.0 & 00.0 \\
\hline 4 & $\begin{array}{lll}5 & 9 & 3\end{array}$ & 75.8260 & 9.7925 & 285.8 & 4 & 600.0 & 00.0 \\
\hline 04 & $\begin{array}{lll}5 & 9 & 4\end{array}$ & 83.3001 & 9.2450 & 285.7 & 4 & 600.0 & 00.0 \\
\hline 04 & 595 & 83.3499 & 8.4750 & 285.9 & 4 & 600.0 & 00.0 \\
\hline 04 & 596 & 87.7997 & 7.2990 & 285.8 & 4 & 600.0 & 00.0 \\
\hline 04 & $\begin{array}{lll}5 & 9 & 7\end{array}$ & 94.4286 & 7.1020 & 286.7 & 4 & 600.0 & 00.0 \\
\hline 04 & $\begin{array}{lll}5 & 9 & 8\end{array}$ & 140.7766 & 7.1550 & 289.2 & 4 & 600.0 & 00.0 \\
\hline 04 & $\begin{array}{lll}5 & 9 & 9\end{array}$ & 149.6745 & 8.0925 & 290.0 & 4 & 600.0 & .0 \\
\hline 04 & 5910 & 148.2272 & 6.7978 & 291.2 & 4 & 600.0 & \\
\hline 04 & 5911 & 158.8476 & 5.5413 & 292.6 & 3 & 600.0 & \\
\hline 04 & 5912 & 161.9201 & 5.9018 & 294.1 & 3 & 600.0 & 00.0 \\
\hline 04 & 5913 & 150.8110 & 5.7505 & 294.9 & 2 & 600.0 & 00.0 \\
\hline 04 & 5914 & 132.9936 & 5.9925 & 295.6 & 3 & .0 & \\
\hline 04 & 5915 & 108.0327 & 6.9880 & 295.6 & 4 & 600.0 & .0 \\
\hline 04 & 5916 & 88.3668 & 7.1330 & 294.5 & 4 & 60 & .0 \\
\hline 04 & 5917 & 72.2001 & 9.7225 & 291.7 & 4 & .0 & \\
\hline 04 & 5918 & 70.9000 & 11.8800 & 289.5 & 4 & .0 & \\
\hline 04 & 5919 & 65.7883 & 14.6500 & 288.4 & 4 & .0 & .0 \\
\hline 04 & 5920 & 61.5500 & 15.5225 & 287.6 & 4 & 6 & \\
\hline 04 & $\begin{array}{ll}5 & 921\end{array}$ & 60.5251 & 12.5400 & 287.3 & 4 & .0 & \\
\hline 04 & 5922 & 59.3999 & 12.4925 & 287.6 & 4 & 600.0 & .0 \\
\hline 04 & 5923 & 65.8484 & 12.4950 & 286.3 & 4 & 6 & \\
\hline 04 & 5924 & 53.5905 & 11.4923 & 284.9 & 4 & 6 & \\
\hline 04 & 5101 & 54.3946 & 6.9105 & 284.4 & 4 & .0 & \\
\hline 04 & 5102 & 80.0444 & 5.9985 & 283.9 & 4 & 60 & .0 \\
\hline 04 & 5103 & 61.2000 & 13.7400 & 284.0 & 4 & .0 & \\
\hline 04 & 5104 & 61.7750 & 14.1675 & 283.4 & 4 & .0 & 0.0 \\
\hline 04 & 5105 & 62.4242 & 12.1425 & 282.9 & 4 & 60 & 0.0 \\
\hline 04 & 5106 & 69.2000 & 9.5825 & 282.8 & 4 & 0 & \\
\hline 04 & 5107 & 70.9643 & 10.1975 & 283.4 & 4 & .0 & 0 \\
\hline 04 & 5108 & 75.0055 & 7.2190 & 284.1 & 4 & 600.0 & 0.0 \\
\hline 04 & 5109 & 65.5648 & 7.7725 & 285.3 & 4 & .0 & 0 \\
\hline 04 & 51010 & 87.5418 & 6.5965 & 286.6 & 4 & 600.0 & 0.0 \\
\hline 04 & 51011 & 95.2249 & 6.2628 & 287.1 & 3 & 600.0 & 600.0 \\
\hline 04 & 51012 & 95.8670 & 4.8618 & 288.8 & 2 & 600.0 & .0 \\
\hline 04 & 51013 & 135.8522 & 5.1268 & 289.9 & 2 & 600.0 & 600.0 \\
\hline 04 & 51014 & 136.4191 & 5.3008 & 290.8 & 3 & 600.0 & 600.0 \\
\hline 04 & 51015 & 103.4200 & 6.5720 & 4 & 3 & 60 & 0.0 \\
\hline 04 & 51016 & 76.8457 & 6.8565 & 290.7 & 4 & 600.0 & 600.0 \\
\hline 04 & 51017 & 63.4744 & 10.1100 & 288.9 & 4 & 600.0 & 600.0 \\
\hline 04 & 51018 & 65.6252 & 10.9275 & 287.4 & 4 & 600.0 & 600.0 \\
\hline 04 & 51019 & 63.9000 & 10.3075 & 286.4 & 4 & 600.0 & 600.0 \\
\hline 04 & 51020 & 69.7499 & 9.4550 & 285.4 & 4 & 600.0 & 600.0 \\
\hline 04 & 51021 & 63.4790 & 9.8750 & & 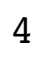 & 600.0 & 600.0 \\
\hline $0<$ & 51022 & 53.9269 & 10.5175 & & 4 & 600.0 & 600.0 \\
\hline & 51023 & 52.8000 & 10.1525 & 285.1 & 4 & 600.0 & 600.0 \\
\hline
\end{tabular}




\begin{tabular}{|c|c|c|c|c|c|c|c|}
\hline 04 & 51024 & 59.6980 & 10.2900 & 285.1 & 4 & 600.0 & 00.0 \\
\hline 04 & 5111 & 6.2986 & 10.6150 & 284.4 & 4 & 600.0 & 600,0 \\
\hline 04 & 5112 & 72.4248 & 10.3175 & 283.9 & 4 & 600.0 & 00.0 \\
\hline 04 & 5113 & 74.1001 & 10.2775 & 283.3 & 4 & 600.0 & 00.0 \\
\hline 04 & 114 & 70.6250 & 9.3925 & 283.0 & 4 & 600.0 & 00.0 \\
\hline 04 & 115 & 74.5500 & 10.0525 & 282.8 & 4 & 600.0 & 00.0 \\
\hline 04 & 5116 & 70.6750 & 9.6050 & 282.7 & 4 & 600.0 & 600.0 \\
\hline 04 & 5117 & 73.9498 & 9.8050 & 283.5 & 4 & 600.0 & 500.0 \\
\hline 04 & 5118 & 73.6251 & 10.3125 & 284.3 & 4 & 600.0 & 00.0 \\
\hline 04 & 5119 & 70.7439 & 9.0400 & 285.7 & 4 & 600.0 & 600.0 \\
\hline 04 & 51110 & 77.0996 & 7.3580 & 287.2 & 4 & 600.0 & 600.0 \\
\hline 04 & 51111 & 90.0002 & 8.3075 & 288.3 & 3 & 600.0 & \\
\hline 04 & 51112 & 82.6013 & 9.2500 & 289.2 & 3 & 600.0 & 600.0 \\
\hline 04 & 51113 & 80.5750 & 9.4625 & 289.0 & 3 & 600.0 & 600.0 \\
\hline 04 & 51114 & 71.5061 & 10. & 288.7 & 3 & 600.0 & \\
\hline 04 & 51115 & 79.0988 & 10.2875 & 288.3 & 4 & 600.0 & 60 \\
\hline 04 & 51116 & 71.2078 & 9.3800 & 288.2 & 4 & 600.0 & 600.0 \\
\hline 04 & 51117 & 63.8518 & 7.7800 & 288.6 & 4 & 600.0 & \\
\hline 04 & 51118 & 60.3000 & 6.7595 & 288.6 & 4 & 600.0 & 60 \\
\hline 04 & 51119 & 73.6758 & 7.5800 & 287.6 & 4 & 600.0 & 600.0 \\
\hline 04 & 51120 & 75.8249 & 950 & 286.3 & 4 & 600.0 & .0 \\
\hline 04 & 51121 & 72.8252 & 8 . & 286.0 & 4 & 600.0 & \\
\hline 04 & 51122 & 78.2250 & 8.6350 & 286.4 & 4 & 600.0 & 600.0 \\
\hline 04 & 51123 & 74.1250 & 8.2900 & 286.4 & 4 & 600.0 & .0 \\
\hline 04 & 51124 & 74.3249 & 8.6225 & 286.4 & 4 & 600.0 & \\
\hline 04 & 5121 & 81.4757 & 8.7875 & 286.5 & 4 & 600.0 & 60 \\
\hline 04 & 5122 & 100.6495 & 8.4800 & 287.3 & 4 & 600.0 & 600.0 \\
\hline 04 & 5123 & 132.3004 & 9.3325 & 287.6 & 4 & 600.0 & \\
\hline 04 & 5124 & 136.0530 & 9.9475 & 286.8 & 4 & 600.0 & 60 \\
\hline 04 & 5125 & 141.1500 & 11.2350 & 286.6 & 4 & 600.0 & 600.0 \\
\hline 04 & 5126 & 141.2749 & 10.27 & 286.8 & 4 & 600.0 & .0 \\
\hline 04 & 5127 & 147.0502 & 9 . & 287.6 & 4 & 600.0 & 60 \\
\hline 04 & 5128 & 160.1244 & 9.0450 & 289.1 & 4 & 600.0 & 600.0 \\
\hline 04 & 5129 & 167.6750 & 8 . & 290.3 & 4 & 600.0 & .0 \\
\hline 04 & 51210 & 166.9730 & 7 . & 291.6 & 4 & 600.0 & .0 \\
\hline 04 & 51211 & 163.6029 & 7.1028 & 292.9 & 3 & 600.0 & 600.0 \\
\hline 04 & 51212 & 137.1896 & 5.9665 & 294.0 & 2 & 600.0 & \\
\hline 04 & 51213 & 151.9084 & 6.5040 & 295.3 & 3 & 600.0 & 600.0 \\
\hline 04 & 51214 & 134.6676 & 6.1258 & 296.3 & 3 & 600.0 & 600.0 \\
\hline 04 & 51215 & 109.7718 & 6.0008 & 295.8 & 3 & 600.0 & \\
\hline 04 & 51216 & 88.6877 & 6 . & 295.3 & 7 & 600.0 & 600.0 \\
\hline 04 & 51217 & 62.8483 & 8.0625 & 293.9 & 4 & 600.0 & 600.0 \\
\hline 04 & 51218 & 66.7734 & 9.1175 & 292.5 & 4 & 600.0 & 600.0 \\
\hline 04 & 51219 & 70.0500 & 9.0025 & 291.1 & 4 & 600.0 & 600.0 \\
\hline 04 & 51220 & 74.5501 & 9.3375 & 290.0 & 4 & 600.0 & 600.0 \\
\hline 04 & 51221 & 79.0499 & 9.7625 & 290.0 & 4 & 600.0 & 600.0 \\
\hline 04 & 51222 & 78.0750 & 9 . & 290.4 & 4 & 600.0 & 600.0 \\
\hline 04 & 51223 & 76.2000 & 8.6525 & 290.7 & 4 & 600.0 & 600.0 \\
\hline
\end{tabular}




\begin{tabular}{|c|c|c|c|c|c|c|c|}
\hline 4 & 51224 & 75.0750 & 8.2275 & 290.7 & 4 & 600.0 & 00.0 \\
\hline 04 & 5131 & 72.4250 & 8.5775 & 290.5 & 4 & 600.0 & 600,0 \\
\hline 04 & 5132 & 76.4751 & 8.2975 & 290.4 & 4 & 600.0 & 00.0 \\
\hline 04 & 5133 & 76.9984 & 7.4048 & 290.2 & 4 & 600.0 & 00.0 \\
\hline 04 & 134 & 78.3250 & 5.8095 & 290.0 & 4 & 600.0 & 00.0 \\
\hline 04 & 135 & 77.5750 & 6.0755 & 289.1 & 4 & 600.0 & 00.0 \\
\hline 04 & 136 & 78.3470 & 4.1553 & 289.8 & 4 & 600.0 & 600.0 \\
\hline 04 & 137 & 275.7393 & 0.8405 & 291.2 & 3 & 600.0 & 00.0 \\
\hline 04 & 138 & 254.1887 & 1.9165 & 292.2 & 2 & 600.0 & 00.0 \\
\hline 04 & 5139 & 234.6326 & 3.6428 & 293.1 & 2 & 600.0 & 600.0 \\
\hline 04 & 51310 & 232.2099 & 3.2055 & 294.1 & 2 & 600.0 & 600 \\
\hline 04 & 1311 & 215.9354 & 2.9338 & 295.9 & 2 & 600.0 & \\
\hline 04 & 1312 & 185.5268 & 4.6440 & 297.7 & 2 & 600.0 & 600.0 \\
\hline 04 & 51313 & 169.3527 & 5.8225 & 298.3 & 3 & 600.0 & 600.0 \\
\hline 04 & 1314 & 143.0474 & 53 & 299.2 & 3 & 600.0 & \\
\hline 04 & 51315 & 103.5260 & 5.8510 & 299.3 & 3 & 600.0 & 60 \\
\hline 04 & 51316 & 98.9002 & 7.0323 & 298.5 & 4 & 600.0 & 600.0 \\
\hline 04 & 51317 & 87.6500 & 508 & 297.7 & 4 & 600.0 & \\
\hline 04 & 51318 & 72.8750 & 7.0180 & 296.1 & 4 & 600.0 & .0 \\
\hline 04 & 51319 & 66.3749 & 500 & 294.4 & 4 & 600.0 & 60 \\
\hline 04 & 51320 & 64.9001 & 12.2450 & 292.2 & 4 & 600.0 & .0 \\
\hline 04 & 51321 & 65.1509 & 11.3825 & 292.0 & 4 & 600.0 & \\
\hline 04 & 51322 & 67.7253 & 10.4400 & 292.8 & 4 & 600.0 & 600 \\
\hline 04 & 51323 & 67.8250 & 10.1650 & 293.3 & 4 & 600.0 & .0 \\
\hline 04 & 51324 & 61.7500 & 9.4550 & 293.1 & 4 & 600.0 & \\
\hline 04 & 5141 & 66.2246 & 9.3725 & 293.1 & 4 & 600.0 & 60 \\
\hline 04 & 5142 & 69.4498 & 8.8500 & 292.8 & 4 & 600.0 & 600.0 \\
\hline 04 & 5143 & 67.8712 & 9.2525 & 291.7 & 4 & 600.0 & \\
\hline 04 & 5144 & 63.4001 & 8.2600 & 291.6 & 4 & 600.0 & 60 \\
\hline 04 & 5145 & 66.2006 & 7.8150 & 291.7 & 4 & 600.0 & 600.0 \\
\hline 04 & 5146 & 69.5248 & 7.6530 & 291.7 & 4 & 600.0 & \\
\hline 04 & 5147 & 76.5231 & 7.2618 & 292.5 & 4 & 600.0 & 60 \\
\hline 04 & 5148 & 98.5423 & 6.1240 & 293.7 & 4 & 600.0 & 600.0 \\
\hline 04 & 5149 & 98.2242 & 6.7790 & 294.5 & 4 & 600.0 & .0 \\
\hline 04 & 51410 & 94.7501 & 638 & 295.4 & 4 & 600.0 & \\
\hline 04 & 51411 & 94.5140 & 6.8690 & 296.1 & 4 & 600.0 & 600.0 \\
\hline 04 & 51412 & 78.2486 & 7.3765 & 297.2 & 3 & 600.0 & \\
\hline 04 & 51413 & 80.7082 & 6.9995 & 298.6 & 3 & 600.0 & 600.0 \\
\hline 04 & 51414 & 76.9427 & 7.5425 & 298.4 & 4 & 600.0 & 600.0 \\
\hline 04 & 51415 & 70.8659 & 9.5000 & 297.6 & 4 & 600.0 & \\
\hline 04 & 51416 & 64.7247 & 11.4300 & 296.3 & 4 & 600.0 & 600.0 \\
\hline 04 & 51417 & 63.8500 & 12.1775 & 294.6 & 4 & 600.0 & 600.0 \\
\hline 04 & 51418 & 61.6250 & 13.6950 & 293.5 & 4 & 600.0 & 600.0 \\
\hline 04 & 51419 & 61.9500 & 13.1375 & 292.7 & 4 & 600.0 & 600.0 \\
\hline 04 & 51420 & 59.7496 & 12.9075 & 292.5 & 4 & 600.0 & 600.0 \\
\hline 04 & 51421 & 61.5002 & 12.8900 & 291.8 & 4 & 600.0 & 600.0 \\
\hline 04 & 51 & 66.0365 & 11.0025 & 29 & 4 & 600.0 & 600.0 \\
\hline 04 & 51423 & 66.7135 & 9.8175 & 291.1 & 4 & 600.0 & 600.0 \\
\hline
\end{tabular}




\begin{tabular}{|c|c|c|c|c|c|c|c|}
\hline 04 & 51424 & 63.7502 & 10.3225 & 290.8 & 4 & 600.0 & 600.0 \\
\hline 04 & 5151 & 68.2999 & 10.5100 & 291.0 & 4 & 600.0 & 600.0 \\
\hline 04 & 5152 & 69.1000 & 10.4650 & 290.6 & 4 & 600.0 & 00.0 \\
\hline 04 & 5153 & 67.1501 & 9.9950 & 291.3 & 4 & 600.0 & 00.0 \\
\hline 04 & 154 & 72.8029 & 11.8350 & 288.2 & 4 & 600.0 & 00.0 \\
\hline 04 & 155 & 68.5990 & 10.7550 & 287.4 & 4 & 600.0 & 00.0 \\
\hline 04 & 5156 & 63.5503 & 8.3350 & 290.3 & 4 & 600.0 & 600.0 \\
\hline 4 & 157 & 67.9271 & 8.5633 & 290.9 & 4 & 600.0 & .0 \\
\hline 04 & 158 & 72.0485 & 9.9525 & 288.1 & 4 & 600.0 & 600.0 \\
\hline 04 & 5159 & 71.6995 & 11.2350 & 289.0 & 4 & 600.0 & 600.0 \\
\hline 04 & 1510 & 80.4290 & 9.01 & 290.6 & 4 & 600.0 & \\
\hline 04 & 1511 & 78.3796 & 7.2668 & 292.7 & 3 & 600.0 & 60 \\
\hline 04 & 1512 & 72.1324 & 8.0350 & 294.3 & 3 & 600.0 & \\
\hline 04 & 1513 & 69.9499 & 10.3300 & 294.6 & 3 & 600.0 & 600.0 \\
\hline 04 & 1514 & 70.7250 & 9. & 295.4 & 3 & 600.0 & 60 \\
\hline 04 & 1515 & 73.7250 & 8.3900 & 295.2 & 4 & 600.0 & 600 \\
\hline 04 & 1516 & 66.2777 & 10.6350 & 294.6 & 4 & 600.0 & .0 \\
\hline 04 & 1517 & 65.6229 & 9.2125 & 294.6 & 4 & .0 & \\
\hline 04 & 1518 & 68.2968 & 8.4575 & 293.7 & 4 & 600.0 & 60 \\
\hline 04 & 1519 & 76.4248 & 7.51 & 293.1 & 4 & .0 & .0 \\
\hline 04 & 1520 & 68.1490 & 8. & 292.8 & 4 & .0 & \\
\hline 04 & 521 & 68.7751 & 9.3675 & 292.7 & 4 & .0 & 60 \\
\hline 04 & 1522 & 67.1250 & 10.7825 & 292.0 & 4 & .0 & 600.0 \\
\hline 04 & 1523 & 67.5250 & 10.8650 & 291.7 & 4 & .0 & \\
\hline 04 & 51524 & 67.0744 & 11.5125 & 291.2 & 4 & .0 & 60 \\
\hline 04 & 5161 & 67.8242 & 12.3925 & 290.5 & 4 & 600.0 & 600.0 \\
\hline 04 & 5162 & 69.9500 & $13.69^{\circ}$ & 289.4 & 4 & .0 & .0 \\
\hline 04 & 5163 & 72.5750 & 11.2150 & 289.0 & 4 & .0 & 60 \\
\hline 04 & 5164 & 72.9000 & 10.9925 & 288.1 & 4 & 600.0 & 600.0 \\
\hline 04 & 5165 & 74.5501 & 11.2050 & 287.5 & 4 & .0 & .0 \\
\hline 04 & 5166 & 500 & 10 . & 287.7 & 4 & .0 & \\
\hline 04 & 5167 & 80.0500 & 10.2900 & 288.2 & 4 & .0 & 600.0 \\
\hline 04 & 5168 & 77.5500 & 9.1775 & 288.6 & 4 & 600.0 & 600.0 \\
\hline 04 & 5169 & 78.7652 & 7.0918 & 290.2 & 3 & .0 & 600.0 \\
\hline 04 & 51610 & 85.8093 & 5.2903 & 292.0 & 2 & .0 & 600.0 \\
\hline 04 & 51611 & 96.5712 & 5.7090 & 293.8 & 3 & 600.0 & 600.0 \\
\hline 04 & 51612 & 95.7000 & 7.685 & 293.6 & 3 & 600.0 & 600.0 \\
\hline 04 & 51613 & 99.2735 & 7.2918 & 294.8 & 3 & 600.0 & 600.0 \\
\hline 04 & 51614 & 88.6747 & 7.2600 & 295.5 & 3 & 600.0 & 600.0 \\
\hline 04 & 51615 & 77.7503 & 8.812 & 295.3 & 4 & 600.0 & 600.0 \\
\hline 04 & 51616 & 65.4487 & 10.3325 & 294.1 & 4 & 600.0 & 600.0 \\
\hline 04 & 51617 & 60.0749 & 13.7025 & 292.4 & 4 & 600.0 & 600.0 \\
\hline 04 & 51618 & 62.7002 & 12.3375 & 291.1 & 4 & 600.0 & 600.0 \\
\hline 04 & 51619 & 61.6750 & 13.9225 & 289.1 & 4 & 600.0 & 600.0 \\
\hline 04 & 51620 & 64.3999 & 14.2900 & 287.7 & 4 & 600.0 & 600.0 \\
\hline 04 & 51621 & 63.0000 & 14.1400 & 287.4 & 4 & 600.0 & \\
\hline 04 & 51622 & 59.4000 & 14.997 & 287.0 & 4 & 600.0 & 600.0 \\
\hline 04 & 51623 & 60.1251 & 14.257 & 287.4 & 4 & 600.0 & 600.0 \\
\hline
\end{tabular}




\begin{tabular}{|c|c|c|c|c|c|c|c|}
\hline 4 & 51624 & 63.0998 & 14.1925 & 287.8 & 4 & 600.0 & 600.0 \\
\hline 04 & 5171 & 64.0502 & 13.1975 & 287.2 & 4 & 600.0 & 00.0 \\
\hline 4 & 5172 & 62.5250 & 13.5500 & 285.2 & 4 & 600.0 & 00.0 \\
\hline 04 & 5173 & 62.5986 & 13.5075 & 285.0 & 4 & 600.0 & 00.0 \\
\hline 04 & 5174 & 65.0250 & 575 & 284.1 & 4 & 600.0 & 00.0 \\
\hline 04 & 5175 & 61.3500 & 16.5775 & 283.6 & 4 & 600.0 & 00.0 \\
\hline 04 & 5176 & 65.5500 & 13.6675 & 283.7 & 4 & 600.0 & 0 \\
\hline 04 & 5177 & 62.2749 & 13.1100 & 284.5 & 4 & 600.0 & 00.0 \\
\hline 04 & 5178 & 64.3001 & 12.9200 & 285.3 & 4 & 600.0 & 00.0 \\
\hline 04 & 5179 & 58.6750 & 12.7325 & 286.0 & 4 & 60 & .0 \\
\hline 04 & 51710 & 57.5000 & 12.0700 & 287.0 & 4 & 600.0 & \\
\hline 04 & 51711 & 61.4502 & 575 & 287.6 & 3 & .0 & \\
\hline 04 & 51712 & 64.2500 & 11.7800 & 288.3 & 3 & 600.0 & 00.0 \\
\hline 04 & 51713 & 67.8497 & 10.7050 & 289.2 & J & 600.0 & \\
\hline 04 & 1714 & 60.9001 & 200 & 289.7 & 3 & .0 & \\
\hline 04 & 51715 & 65.0001 & 300 & 289.7 & 4 & 60 & .0 \\
\hline 04 & 51716 & 64.1748 & 13.3925 & 288.9 & 4 & 60 & .0 \\
\hline 04 & 51717 & 59.0000 & 14 . & 288.1 & 4 & .0 & \\
\hline 04 & 51718 & 61.4006 & 825 & 287.2 & 4 & .0 & \\
\hline 04 & 51719 & 60.3262 & 12.3600 & 286.2 & 4 & .0 & .0 \\
\hline 04 & 51720 & 50.4639 & 10 . & 285.1 & 4 & 6 & \\
\hline 04 & 51721 & 70.0507 & 14 . & 284.6 & 4 & .0 & \\
\hline 04 & 51722 & 64.2942 & 12.0150 & 284.4 & 4 & 600.0 & .0 \\
\hline 04 & 51723 & 60.7195 & 11 . & 284.5 & 4 & 6 & \\
\hline 04 & 51724 & 65.7978 & 13 & 284.5 & 4 & 6 & \\
\hline 04 & 5181 & 59.5249 & 975 & 284.4 & 4 & .0 & \\
\hline 04 & 5182 & 63.7851 & 6.4900 & 284.0 & 4 & 60 & .0 \\
\hline 04 & 5183 & 65.4001 & 7.3923 & 283.8 & 4 & .0 & \\
\hline 04 & 5184 & 92.8241 & 3.5055 & 283.6 & 4 & .0 & 0.0 \\
\hline 04 & 5185 & 72.6442 & 3.9305 & 283.5 & 4 & 6 & .0 \\
\hline 04 & 5186 & 73.3502 & 123 & 283.7 & 4 & .0 & \\
\hline 04 & 5187 & 75.5502 & 168 & 284.3 & 4 & .0 & 0 \\
\hline 04 & 5188 & 76.4250 & 7.0235 & 285.4 & 4 & 600.0 & 0.0 \\
\hline 04 & 5189 & 78.1251 & 755 & 286.8 & 4 & 600.0 & 0 \\
\hline 04 & 51810 & 78.2266 & 6.8193 & 28 & 4 & 0 & 0.0 \\
\hline 04 & 51811 & 87.6467 & 5.8738 & 289.9 & 3 & 600.0 & 600.0 \\
\hline 04 & 51812 & 96.2455 & 5.6983 & 291.0 & 2 & 600.0 & .0 \\
\hline 04 & 51813 & 98.6912 & 6.3443 & 291.5 & 3 & 600.0 & 600.0 \\
\hline 04 & 51814 & 95.2572 & 7.5413 & 291.8 & 3 & 600.0 & 600.0 \\
\hline 04 & 51815 & 80.0033 & 000 & 291.6 & 4 & 60 & .0 \\
\hline 04 & 51816 & 75.9079 & 8.1450 & 290.9 & 4 & 600.0 & 600.0 \\
\hline 04 & 51817 & 68.9347 & 8.7775 & 289.6 & 4 & 600.0 & 600.0 \\
\hline 04 & 51818 & 60.8999 & 11.6425 & 288.2 & 4 & 600.0 & 600.0 \\
\hline 04 & 51819 & 59.9250 & 12.8600 & 286.9 & 4 & 600.0 & 600.0 \\
\hline 04 & 51820 & 66.1500 & 12.1350 & 286.0 & 4 & 600.0 & 600.0 \\
\hline 04 & 51821 & 67.6665 & 9.8575 & 285.9 & 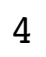 & 600.0 & \\
\hline $0<$ & 51822 & 71.2499 & 9.7100 & 286.2 & 4 & 600.0 & 600.0 \\
\hline & 1823 & 67.8999 & 9.9875 & 286.0 & 4 & 600.0 & 600.0 \\
\hline
\end{tabular}




\begin{tabular}{|c|c|c|c|c|c|c|c|}
\hline 4 & 51824 & 72.9501 & 8.6650 & 286.4 & 4 & 600.0 & 00.0 \\
\hline 04 & 5191 & 75.5000 & 8.0450 & 286.6 & 4 & 600.0 & 600.0 \\
\hline 04 & 5192 & 78.9983 & 7.6875 & 286.4 & 4 & 600.0 & 00.0 \\
\hline 04 & 5193 & 87.6500 & 6.6458 & 286.9 & 4 & 600.0 & 600.0 \\
\hline 04 & 194 & 81.2500 & 6.2983 & 286.8 & 4 & 600.0 & 00.0 \\
\hline 04 & 5195 & 80.1751 & 6.1793 & 286.7 & 4 & 600.0 & 00.0 \\
\hline 04 & 5196 & 94.6245 & 4.8395 & 287.0 & 4 & 600.0 & 600.0 \\
\hline 04 & 5197 & 121.1998 & 4.1435 & 288.8 & 3 & 600.0 & 00.0 \\
\hline 04 & 5198 & 150.2093 & 5.8783 & 289.8 & 4 & 600.0 & 00.0 \\
\hline 04 & 5199 & 154.5464 & 5.7498 & 290.9 & 3 & 600.0 & 600.0 \\
\hline 04 & 1910 & 157.0998 & 6.4000 & 292.1 & 3 & 600.0 & 600.0 \\
\hline 04 & 1911 & 159.8500 & 6.4443 & 293.1 & 3 & 600.0 & \\
\hline 04 & 1912 & 154.2251 & 5.8810 & 293.8 & 3 & 600.0 & 600.0 \\
\hline 04 & 51913 & 123.8964 & 4.9450 & 294.7 & 2 & 600.0 & 600.0 \\
\hline 04 & 1914 & 95.8148 & 6.14 & 294.8 & 3 & 600.0 & \\
\hline 04 & 51915 & 85.6134 & 6.85 & 294.9 & 4 & 600.0 & 60 \\
\hline 04 & 51916 & 92.6750 & 8.5800 & 293.9 & 4 & 600.0 & 600.0 \\
\hline 04 & 51917 & 65.8408 & 9.5975 & 292.4 & 4 & 600.0 & \\
\hline 04 & 51918 & 62.5751 & 12.1150 & 290.8 & 4 & 600.0 & .0 \\
\hline 04 & 51919 & 70.5000 & 12.1100 & 288.8 & 4 & 600.0 & 600 \\
\hline 04 & 51920 & 65.3751 & 14.3000 & 286.0 & 4 & 600.0 & .0 \\
\hline 04 & 51921 & 71.0500 & 14.4875 & 285.1 & 4 & 600.0 & \\
\hline 04 & 51922 & 70.0500 & 13.8450 & 285.3 & 4 & 600.0 & 600 \\
\hline 04 & 51923 & 74.3251 & 13.3900 & 285.1 & 4 & 600.0 & .0 \\
\hline 04 & 51924 & 70.5249 & 13.72 & 284.3 & 4 & 600.0 & \\
\hline 04 & 5201 & 69.7000 & 13.4250 & 284.2 & 4 & 600.0 & 60 \\
\hline 04 & 5202 & 74.7250 & 13.1125 & 284.0 & 4 & 600.0 & .0 \\
\hline 04 & 5203 & 75.9250 & 12.19 & 283.8 & 4 & 600.0 & \\
\hline 04 & $520 \quad 4$ & 74.9750 & 12.0275 & 28 & 4 & 600.0 & 60 \\
\hline 04 & 5205 & 77.1000 & 11.8425 & 283.2 & 4 & 600.0 & 600 \\
\hline 04 & 5206 & 78.8750 & 11.33 & 283.4 & 4 & 600.0 & \\
\hline 04 & $520 \quad 7$ & 77.8500 & 10.7575 & 284.3 & 4 & 600.0 & 60 \\
\hline 04 & 5208 & 78.4252 & 11.2750 & 285.2 & 4 & 600.0 & 600.0 \\
\hline 04 & 5209 & 76.8504 & 9 . & 286.9 & 4 & 600.0 & .0 \\
\hline 04 & 52010 & 77.7254 & 8.8475 & 288.2 & 4 & 600.0 & .0 \\
\hline 04 & 52011 & 70.8751 & 8.5825 & 289.5 & 3 & 600.0 & 600.0 \\
\hline 04 & 52012 & 74.4501 & 9.5075 & 290.4 & 3 & 600.0 & \\
\hline 04 & 52013 & 67.7988 & 10.07 & 290.5 & 3 & 600.0 & 600.0 \\
\hline 04 & 52014 & 74.1997 & 10.9075 & 290.3 & 3 & 600.0 & 600.0 \\
\hline 04 & 52015 & 73.0229 & 10.9625 & 290.4 & 4 & 600.0 & \\
\hline 04 & 52016 & 80.3793 & 9.7425 & 290.3 & 4 & 600.0 & 600.0 \\
\hline 04 & 52017 & 73.5000 & 11.7150 & 289.9 & 4 & 600.0 & 600.0 \\
\hline 04 & 52018 & 70.5500 & 11.6775 & 288.6 & 4 & 600.0 & 600.0 \\
\hline 04 & 52019 & 68.8000 & 11.5775 & 286.4 & 4 & 600.0 & 600.0 \\
\hline 04 & 52020 & 65.3251 & 13.7225 & 284.9 & 4 & 600.0 & 600.0 \\
\hline 04 & 52021 & 65.1999 & 16.3650 & 283.7 & 4 & 600.0 & 600.0 \\
\hline 04 & 52022 & 69.4000 & 16.6450 & 28 & 4 & 600.0 & 600.0 \\
\hline 04 & 52023 & 68.6500 & 15.8300 & 283.6 & 4 & 600.0 & 600.0 \\
\hline
\end{tabular}




\begin{tabular}{|c|c|c|c|c|c|c|c|}
\hline 4 & 52024 & 73.4750 & 16.2100 & 283.4 & 4 & 600.0 & 600.0 \\
\hline 04 & 5211 & 74.9750 & 15.3075 & 283.3 & 4 & 600.0 & 00,0 \\
\hline 04 & 5212 & 72.0250 & 14.0750 & 283.2 & 4 & 600.0 & 00.0 \\
\hline 04 & 5213 & 69.7000 & 14.5900 & 283.0 & 4 & 600.0 & 0 \\
\hline 04 & 5214 & 65.2250 & 13.0675 & 283.2 & 4 & 600.0 & .0 \\
\hline 04 & 5215 & 65.7750 & 12.7625 & 283.5 & 4 & 600.0 & 00.0 \\
\hline 04 & 5216 & 65.9495 & 12.1500 & 283.9 & 4 & 600.0 & 00.0 \\
\hline 04 & 5217 & 69.2750 & 11.6225 & 284.5 & 4 & 600.0 & 00.0 \\
\hline 04 & 5218 & 65.1750 & 10.9200 & 285.6 & 4 & 600.0 & 00.0 \\
\hline 04 & 5219 & 65.6248 & 9.8225 & 286.6 & 4 & 60 & 0 \\
\hline 04 & 52110 & 71.3016 & 9.9275 & 287.6 & 4 & 600.0 & \\
\hline 04 & 52111 & 74.8008 & 10.5375 & 288.5 & 3 & .0 & \\
\hline 04 & 52112 & 73.8000 & 10.2625 & 289.2 & 3 & 600.0 & .0 \\
\hline 04 & 52113 & 74.2000 & 11.8100 & 289.2 & 3 & 600.0 & \\
\hline 04 & 52114 & 73.52 & 12 . & 289.4 & 3 & .0 & \\
\hline 04 & 52115 & 70.4992 & 13. & 288.9 & 4 & 600.0 & .0 \\
\hline 04 & 52116 & 69.3500 & 14.2700 & 288.0 & 4 & 60 & .0 \\
\hline 04 & 52117 & 66.94 & 13.9825 & 287.2 & 4 & .0 & \\
\hline 04 & 52118 & 64.9750 & 13.6275 & 286.3 & 4 & .0 & \\
\hline 04 & 52119 & 69.4750 & 13.1300 & 285.4 & 4 & .0 & .0 \\
\hline 04 & 52120 & 68.54 & 14 . & 284.6 & 4 & 6 & \\
\hline 04 & 52121 & 67.5749 & 13. & 284.3 & 4 & .0 & \\
\hline 04 & 52122 & 70.3249 & 14.1750 & 284.2 & 4 & 60 & .0 \\
\hline 04 & 52123 & 74.1500 & 14. & 284.2 & 4 & 6 & \\
\hline 04 & 52124 & 75.2000 & 12. & 284.4 & 4 & 0 & \\
\hline 04 & 5221 & 73.9248 & 12.3275 & 284.3 & 4 & .0 & \\
\hline 04 & 5222 & 70.6501 & 12.7250 & 284.3 & 4 & 6 & .0 \\
\hline 04 & 5223 & 72.1254 & 12.0925 & 284.1 & 4 & .0 & \\
\hline 04 & 5224 & 73.7466 & 12.1600 & 283.7 & 4 & .0 & .0 \\
\hline 04 & 5225 & 72.8501 & 13.3025 & 283.5 & 4 & .0 & .0 \\
\hline 04 & 5226 & 71.0246 & 12. & 283.7 & 4 & .0 & \\
\hline 04 & 5227 & 69.1500 & 13. & 284.0 & 4 & .0 & .0 \\
\hline 04 & 5228 & 64.5487 & 13.1100 & 284.2 & 4 & 60 & 0.0 \\
\hline 04 & 5229 & 64.4499 & 13.3600 & 284.7 & 4 & 0 & .0 \\
\hline 04 & 52210 & 74.3231 & 11.2450 & 285.8 & 4 & .0 & 0.0 \\
\hline 04 & 52211 & 75.9250 & 11.8750 & 286.8 & 3 & 600.0 & 0.0 \\
\hline 04 & 52212 & 74 . & 10.8525 & 287.9 & 3 & 600.0 & .0 \\
\hline 04 & 52213 & 70.5745 & 11.9325 & 288.5 & 3 & 600.0 & 600.0 \\
\hline 04 & 52214 & 70.7999 & 10.1275 & 289.6 & 3 & 600.0 & 600.0 \\
\hline 04 & 52215 & 73.6229 & 750 & & 4 & 600.0 & 600.0 \\
\hline 04 & 52216 & 67.8751 & 10.2900 & 289.8 & 4 & 600.0 & 600.0 \\
\hline 04 & 52217 & 67.9250 & 12.6100 & 288.6 & 4 & 600.0 & 600.0 \\
\hline 04 & 52218 & 69.2000 & 13.5675 & 287.1 & 4 & 600.0 & 600.0 \\
\hline 04 & 52219 & 65.1501 & 13.6675 & 285.5 & 4 & 600.0 & 600.0 \\
\hline 04 & 52220 & 67.5253 & 14.0400 & 284.6 & 4 & 600.0 & 600.0 \\
\hline 04 & 52221 & 68.9000 & 15.0275 & 284.3 & 4 & 600.0 & \\
\hline $0<$ & 52222 & 69.8251 & 14.2875 & 284.2 & 4 & 600.0 & 600.0 \\
\hline 04 & 52223 & 67.3993 & 13.5500 & 283.8 & $\Psi$ & 600.0 & 600.0 \\
\hline
\end{tabular}




\begin{tabular}{|c|c|c|c|c|c|c|c|}
\hline 04 & 52224 & 65.1750 & 14.4825 & 283.6 & 4 & 600.0 & 600.0 \\
\hline 04 & 5231 & 67.2750 & 14.8125 & 283.5 & 4 & 600.0 & 600.0 \\
\hline 04 & 5232 & 66.5992 & 13.4800 & 283.6 & 4 & 600.0 & 00.0 \\
\hline 4 & 5233 & 65.0229 & 12.7550 & 283.4 & 4 & 600.0 & 00.0 \\
\hline 04 & 5234 & 68.4998 & 13.2275 & 283.2 & 4 & 600.0 & .0 \\
\hline 04 & 5235 & 71.8250 & 12.8125 & 283.2 & 4 & 600.0 & 00.0 \\
\hline 4 & 5236 & 69.4750 & 12.5475 & 283.4 & 4 & 600.0 & 00.0 \\
\hline 04 & 5237 & 73.9999 & 11.6950 & 284.0 & 4 & 600.0 & \\
\hline 04 & 5238 & 75.3751 & 11.0300 & 284.6 & 4 & 600.0 & 00.0 \\
\hline 04 & 5239 & 75.7488 & 10.0150 & 285.8 & 4 & 600.0 & 00.0 \\
\hline 4 & 52310 & 79.6994 & 8.5525 & 286.9 & 4 & 0.0 & \\
\hline 04 & 52311 & 95.3508 & 7.0150 & 288.5 & 3 & 600.0 & 60 \\
\hline 04 & 52312 & 102.2253 & 5.7298 & 290.7 & 3 & .0 & \\
\hline 04 & 52313 & 106.3749 & 6.0475 & 291.8 & 2 & 0 & \\
\hline 04 & 52314 & 104.7749 & 6.7450 & 292.3 & 3 & 60 & 0 \\
\hline 04 & 52315 & 93.6281 & 7.9725 & 292.4 & 4 & 600.0 & 0 \\
\hline 4 & 52316 & 98.6980 & 8.4150 & 291.9 & 4 & .0 & .0 \\
\hline 04 & 52317 & 107.2531 & 7.8120 & 291.0 & 4 & .0 & \\
\hline 04 & 52318 & 85.7560 & 7.0235 & 289.9 & 4 & 600.0 & 00.0 \\
\hline 04 & 52319 & 70.2250 & 10.8425 & 287.7 & 4 & .0 & .0 \\
\hline 04 & 52320 & 70.3500 & 12.78 & 285.8 & 4 & 0 & \\
\hline 04 & 52321 & 67.7240 & 10.3775 & 285.7 & 4 & .0 & \\
\hline 04 & 52322 & 70.8006 & 10.1725 & 285.1 & 4 & .0 & .0 \\
\hline 04 & 52323 & 71.9250 & 12.7150 & 284.4 & 4 & .0 & \\
\hline 04 & 52324 & 3500 & 12.3200 & 284.6 & 4 & 60 & \\
\hline 04 & 5241 & 74.2250 & 11.7175 & 284.4 & 4 & .0 & .0 \\
\hline 04 & 5242 & 73.8250 & 11.4825 & 284.6 & 4 & 0 & .0 \\
\hline 04 & 5243 & 74.8500 & 11.0750 & 284.5 & 4 & .0 & \\
\hline 04 & 5244 & 76.6000 & 10.8950 & 283.8 & 4 & .0 & .0 \\
\hline 04 & 5245 & 74.7502 & 9.7550 & 283.7 & 4 & .0 & \\
\hline 04 & 5246 & 71.5750 & 10.2800 & 283.9 & 4 & .0 & \\
\hline 04 & 5247 & 74.0976 & 9.0750 & 285.4 & 4 & .0 & .0 \\
\hline 04 & 5248 & 81.4966 & 6.7623 & 286.5 & 4 & .0 & .0 \\
\hline 04 & 5249 & 89.9257 & 4.6940 & 288.1 & 3 & .0 & .0 \\
\hline 04 & 52410 & 68.0591 & 2.9440 & 289.9 & 2 & .0 & .0 \\
\hline 04 & 52411 & 49.2696 & 3.0465 & 291.2 & 1 & 600.0 & 600.0 \\
\hline 04 & 52412 & 15.3763 & 3.5168 & 292.3 & 2 & .0 & 00.0 \\
\hline 04 & 52413 & 63.9654 & 5.1735 & 293.3 & 2 & 600.0 & 600.0 \\
\hline 04 & 52414 & 94.9746 & 7.2488 & 292.5 & 3 & 600.0 & 600.0 \\
\hline 04 & 52415 & 92.4240 & 7.8125 & 29 & 4 & .0 & 60 \\
\hline 04 & 52416 & 89.3140 & 8.5350 & 291.3 & 4 & 600.0 & 600.0 \\
\hline 04 & 52417 & 78.4010 & 8.2225 & 290.8 & 4 & 600.0 & 600.0 \\
\hline 04 & 52418 & 68.5248 & 9.0000 & 289.5 & 4 & 600.0 & 600.0 \\
\hline 04 & 52419 & 68.0000 & 10.7625 & 288.1 & 4 & 600.0 & 600.0 \\
\hline 04 & 52420 & 67.5500 & 11.3800 & 286.8 & 4 & 600.0 & 600.0 \\
\hline 04 & 52421 & 69.9250 & 10.8300 & & 4 & 600 & \\
\hline 04 & 52422 & 71.1753 & 11.5125 & 285.3 & $\Psi$ & 600.0 & 600.0 \\
\hline 04 & 52423 & 69.4500 & 11.0925 & 285.2 & 4 & 600.0 & 60 \\
\hline
\end{tabular}




\begin{tabular}{|c|c|c|c|c|c|c|c|}
\hline 04 & 52424 & 70.8250 & 11.7925 & 284.8 & 4 & 600.0 & 00.0 \\
\hline 04 & 5251 & 71.2007 & 11.6150 & 284.4 & 4 & 600.0 & 600,0 \\
\hline 04 & 5252 & 74.4000 & 10.3075 & 284.6 & 4 & 600.0 & 00.0 \\
\hline 04 & 5253 & 77.1998 & 9.1650 & 284.8 & 4 & 600.0 & 00.0 \\
\hline 04 & 5254 & 78.6751 & 7.8748 & 285.0 & 4 & 600.0 & 00.0 \\
\hline 04 & 5255 & 81.4001 & 9.9525 & 283.7 & 4 & 600.0 & 00.0 \\
\hline 04 & 5256 & 79.8251 & 9.9800 & 283.5 & 4 & 600.0 & 600.0 \\
\hline 04 & 5257 & 78.1751 & 9.5825 & 284.6 & 4 & 600.0 & 00.0 \\
\hline 04 & 5258 & 79.7250 & 8.4575 & 286.1 & 4 & 600.0 & 00.0 \\
\hline 04 & 5259 & 88.0760 & 6.1258 & 288.4 & 3 & 600.0 & .0 \\
\hline 04 & 2510 & 106.5960 & 4.2720 & 290.7 & 2 & 600.0 & 600.0 \\
\hline 04 & 52511 & 74.6684 & 4.3018 & 292.0 & 2 & 600.0 & \\
\hline 04 & 52512 & 72.2501 & 6.5268 & 292.9 & 3 & 600.0 & 500.0 \\
\hline 04 & 52513 & 83.2246 & 7.4210 & 294.2 & 3 & 600.0 & 0 \\
\hline 04 & 52514 & 81.7748 & 8.0175 & 294.6 & 3 & 600.0 & \\
\hline 04 & 52515 & 76.5973 & 8.9475 & 294.5 & 4 & 600.0 & 60 \\
\hline 04 & 52516 & 78.5253 & 8.4100 & 294.3 & 4 & 600.0 & 600.0 \\
\hline 04 & 52517 & 73.2747 & 8.7200 & 293.9 & 4 & 600.0 & \\
\hline 04 & 52518 & 78.6500 & 8.8000 & 293.1 & 4 & 600.0 & .0 \\
\hline 04 & 52519 & 64.9249 & 9.3725 & 291.9 & 4 & 600.0 & 60 \\
\hline 04 & 52520 & 68.8499 & 9.43 & 291.2 & 4 & 600.0 & .0 \\
\hline 04 & 52521 & 68.9000 & 10.9475 & 290.7 & 4 & 0.0 & \\
\hline 04 & 52522 & 67.7751 & 11.1800 & 290.6 & 4 & 600.0 & 600.0 \\
\hline 04 & 52523 & 70.3000 & 11.6000 & 290.6 & 4 & 600.0 & .0 \\
\hline 04 & 52524 & 69.3752 & 10.2750 & 291.1 & 4 & 600.0 & \\
\hline 04 & 5261 & 76.2500 & 10.1525 & 289.8 & 4 & 600.0 & 60 \\
\hline 04 & 5262 & 63.6497 & 9.8475 & 289.4 & 4 & 600.0 & .0 \\
\hline 04 & 5263 & 71.9251 & 10.1775 & 288.5 & 4 & 600.0 & \\
\hline 04 & 5264 & 68.7250 & 10.2250 & 287.1 & 4 & 600.0 & 60 \\
\hline 04 & 5265 & 78.4214 & 7.9745 & 287.3 & 4 & 600.0 & .0 \\
\hline 04 & 5266 & 141.9129 & 2 . & 285.4 & 4 & 600.0 & \\
\hline 04 & 5267 & 98.2140 & 645 & 287.1 & 3 & 600.0 & 60 \\
\hline 04 & 5268 & 85.4944 & 2.8153 & 289.7 & 3 & 600.0 & 600.0 \\
\hline 04 & 5269 & 0.3314 & 2.2580 & 291.5 & 2 & 600.0 & .0 \\
\hline 04 & 52610 & 274.4143 & 558 & 293.5 & 1 & 600.0 & .0 \\
\hline 04 & 52611 & 228.3272 & 3.4883 & 295.6 & 2 & 600.0 & 600.0 \\
\hline 04 & 52612 & 188.1341 & 3.6065 & 298.0 & 1 & 600.0 & \\
\hline 04 & 52613 & 344.6348 & 3.5655 & 299.8 & 1 & 600.0 & .0 \\
\hline 04 & 52614 & 89.9010 & 5.3628 & 299.6 & 2 & 600.0 & 600.0 \\
\hline 04 & 52615 & 83.2979 & 7.1695 & 299.2 & 3 & 600.0 & \\
\hline 04 & 52616 & 67.0000 & 8.5675 & 298.7 & 7 & 600.0 & 60 \\
\hline 04 & 52617 & 67.6750 & 7.5700 & 298.3 & 4 & 600.0 & 600.0 \\
\hline 04 & 52618 & 70.5998 & 9.0000 & 296.7 & 4 & 600.0 & 600.0 \\
\hline 04 & 52619 & 68.6500 & 9.2525 & 295.1 & 4 & 600.0 & 600.0 \\
\hline 04 & 52620 & 70.1750 & 10.0950 & 293.5 & 4 & 600.0 & 600.0 \\
\hline 04 & 52621 & 67.7499 & 11.7150 & 293.5 & 4 & 600.0 & 600.0 \\
\hline 04 & 52622 & 69.2506 & 10.1400 & & 4 & 600.0 & 600.0 \\
\hline 04 & 52623 & 72.2500 & 10.9275 & 291.1 & 4 & 600.0 & 600.0 \\
\hline
\end{tabular}




\begin{tabular}{|c|c|c|c|c|c|c|c|}
\hline 04 & 52624 & 73.0501 & 9.4550 & 291.6 & 4 & 600.0 & 600.0 \\
\hline 04 & 5271 & 73.1247 & 9.1300 & 290.4 & 4 & 600.0 & 00.0 \\
\hline 04 & 5272 & 71.9499 & 7.7790 & 290.3 & 4 & 600.0 & 00.0 \\
\hline 04 & 5273 & 73.9501 & 6.7678 & 290.5 & 4 & 600.0 & 00.0 \\
\hline 04 & 5274 & 72.4008 & 500 & 289.2 & 4 & 600.0 & 00.0 \\
\hline 04 & 5275 & 71.2750 & 9.5675 & 289.0 & 4 & 600.0 & 00.0 \\
\hline 04 & 5276 & 72.6500 & 875 & 288.5 & 4 & 600.0 & 00.0 \\
\hline 04 & 5277 & 75.6000 & 75 & 289.9 & 4 & 600.0 & 00.0 \\
\hline 04 & 5278 & 81.5741 & 8.0850 & 291.0 & 4 & 600.0 & 00.0 \\
\hline 04 & 5279 & 90.5999 & 68 & 293.5 & 4 & 600.0 & 0 \\
\hline 04 & 52710 & 85.3325 & 93 & 295.4 & 3 & 600.0 & \\
\hline 04 & 52711 & 98.3970 & 03 & 296.5 & 2 & 600.0 & \\
\hline 04 & 52712 & 89.4884 & 25 & 297.0 & 3 & 600.0 & 00.0 \\
\hline 04 & 52713 & 108.1750 & 7 . & 296.9 & 4 & 600.0 & 00.0 \\
\hline 04 & 52714 & 84.0926 & & 296.7 & 4 & .0 & \\
\hline 04 & 52715 & 71.2267 & 8 . & 295.4 & 4 & 600.0 & .0 \\
\hline 04 & 52716 & 71.6467 & 58 & 294.4 & 4 & 60 & 600.0 \\
\hline 04 & 52717 & 85.8276 & 7 . & 293.5 & 4 & .0 & \\
\hline 04 & 52718 & 77.7501 & 11. & 292.0 & 4 & .0 & \\
\hline 04 & 52719 & 68.3009 & 13. & 290.6 & 4 & .0 & .0 \\
\hline 04 & 52720 & 64.3250 & 13 & 290.3 & 4 & 6 & \\
\hline 04 & 52721 & 65.5751 & 13. & 289.9 & 4 & .0 & \\
\hline 04 & 52722 & 68.5251 & 12.8 & 290.5 & 4 & 600.0 & .0 \\
\hline 04 & 52723 & 70.9253 & 11 . & 290 & 4 & 60 & \\
\hline 04 & 52724 & 68.8221 & & 288.4 & 4 & .0 & \\
\hline 04 & 5281 & 94.3484 & & 287.0 & 4 & .0 & 0 \\
\hline 04 & 5282 & 99.2264 & 8. & 286.2 & 4 & .0 & .0 \\
\hline 04 & 5283 & 102.6691 & 7 . & 286.2 & 4 & .0 & \\
\hline 04 & 5284 & 78.8887 & 5 . & 286.5 & 4 & .0 & 0.0 \\
\hline 04 & $528 \quad 5$ & 80.4369 & 3. & 286.4 & 4 & 60 & .0 \\
\hline 04 & 5286 & 63.4879 & 9 . & 286.6 & 4 & .0 & \\
\hline 04 & 5287 & 59.8051 & 7 . & 286.8 & 3 & .0 & 600.0 \\
\hline 04 & 5288 & 57.9225 & 5.2 & 286.7 & 3 & 600.0 & 600.0 \\
\hline 04 & 5289 & 59.4287 & 6 . & 286.8 & 3 & 0.0 & 0.0 \\
\hline 04 & 52810 & 73.4725 & 5 . & 288.3 & 4 & 600.0 & 600.0 \\
\hline 04 & 52811 & 86.8720 & 6.6645 & 289.3 & 3 & 600.0 & 600.0 \\
\hline 04 & 52812 & 78.2132 & 8 . & 290.1 & 3 & 600.0 & 0.0 \\
\hline 04 & 52813 & 72.3152 & 8.5990 & 291.3 & 3 & 600.0 & 600.0 \\
\hline 04 & 52814 & 65.9732 & 8.9458 & 291.9 & 3 & 600.0 & 600.0 \\
\hline 04 & 52815 & 64.6250 & 12 . & 290.6 & 4 & 600.0 & 600.0 \\
\hline 04 & 52816 & 63.8500 & 14 & 289.6 & 4 & 600.0 & 600.0 \\
\hline 04 & 52817 & 59.6736 & 14.7600 & 288.4 & 4 & 600.0 & 600.0 \\
\hline 04 & 52818 & 54.3763 & 13.1775 & 287.4 & 4 & 600.0 & 600.0 \\
\hline 04 & 52819 & 46.6226 & 10.4775 & 286.3 & 4 & 600.0 & 600.0 \\
\hline 04 & 52820 & 61.0250 & 15.4425 & 285.1 & 4 & 600.0 & 600.0 \\
\hline 04 & 52821 & 57.8757 & 16.3000 & 284.6 & 4 & 600.0 & 600.0 \\
\hline 04 & 52822 & 54.5269 & 12.4400 & 284.7 & 4 & 600.0 & 600.0 \\
\hline 0 & 52823 & 56.1302 & 10.7525 & 284.8 & 4 & 600.0 & 600.0 \\
\hline
\end{tabular}




\begin{tabular}{|c|c|c|c|c|c|c|c|}
\hline 4 & 52824 & 67.0997 & 9.5200 & 284.8 & 4 & 600.0 & 600.0 \\
\hline 04 & 5291 & 67.1484 & 10.5525 & 284.5 & 4 & 600.0 & 600.0 \\
\hline 04 & 5292 & 76.5500 & 600 & 284.4 & 4 & 600.0 & 00.0 \\
\hline 4 & 5293 & 77.7000 & 9.6775 & 284.4 & 4 & 600.0 & 00.0 \\
\hline 04 & 5294 & 82.5750 & 700 & 284.1 & 4 & 600.0 & 00.0 \\
\hline 04 & 5295 & 84.5498 & 8.9375 & 284.0 & 4 & 600.0 & 00.0 \\
\hline 04 & 5296 & 98.9972 & 375 & 284.7 & 4 & 600.0 & 00.0 \\
\hline 04 & 5297 & 121.0597 & 7.8400 & 286.4 & 4 & 600.0 & 00.0 \\
\hline 04 & 5298 & 149.9943 & 11.9825 & 287.7 & 4 & 600.0 & 00.0 \\
\hline 04 & 5299 & 166.0254 & 12 . & 289.2 & 4 & 600 & 0 \\
\hline 04 & 52910 & 166.7750 & 700 & 290.6 & 4 & 600.0 & \\
\hline 04 & 52911 & 161.4220 & 00 & 292.3 & 3 & 600.0 & \\
\hline 04 & 52912 & 157.7035 & 390 & 294.0 & 3 & 600.0 & 00.0 \\
\hline 04 & 52913 & 155.2634 & 300 & 295.6 & J & 600.0 & 00.0 \\
\hline 04 & 52914 & 139.1443 & 13 & 296.4 & 3 & .0 & \\
\hline 04 & 52915 & 134.8931 & 393 & 296.8 & 3 & 600.0 & .0 \\
\hline 04 & 52916 & 128.7556 & 5.8428 & 296.8 & 3 & 60 & .0 \\
\hline 04 & 52917 & 96.5720 & 523 & 296.1 & 4 & .0 & \\
\hline 04 & 52918 & 73.4750 & 100 & 294.5 & 4 & .0 & \\
\hline 04 & 52919 & 72.1746 & 8.1725 & 293.0 & 4 & .0 & .0 \\
\hline 04 & 52920 & 71.0251 & 9 . & 291.9 & 4 & 6 & \\
\hline 04 & 52921 & 74.7250 & 10 . & 291.7 & 4 & .0 & \\
\hline 04 & 52922 & 70.3498 & 00 & 292.0 & 4 & 600.0 & .0 \\
\hline 04 & 52923 & 73.2000 & 8 . & 292.4 & 4 & 6 & \\
\hline 04 & 52924 & 69.9001 & 8 . & 292.8 & 4 & 6 & \\
\hline 04 & 5301 & 72.9751 & 625 & 292.5 & 4 & .0 & .0 \\
\hline 04 & 5302 & 75.1767 & 8.2175 & 292.5 & 4 & 60 & .0 \\
\hline 04 & 5303 & 71.2000 & 50 & 292.0 & 4 & .0 & \\
\hline 04 & 5304 & 70.8018 & 450 & 291.2 & 4 & .0 & .0 \\
\hline 04 & 5305 & 71.6512 & 9.3000 & 291.3 & 4 & .0 & .0 \\
\hline 04 & 5306 & 76.4250 & 978 & 292.2 & 4 & .0 & \\
\hline 04 & 5307 & 138.0367 & 255 & 293.4 & 3 & .0 & 0.0 \\
\hline 04 & 5308 & 214.3891 & 2.6270 & 293.9 & 2 & 600.0 & 0.0 \\
\hline 04 & 5309 & 203.7773 & 620 & 294.6 & 2 & 0.0 & .0 \\
\hline 04 & 53010 & 175.8129 & & 296.6 & 2 & 600.0 & 0.0 \\
\hline 04 & 53011 & 171.5250 & 5.3683 & 298.1 & 2 & 600.0 & 600.0 \\
\hline 04 & 53012 & 169.7500 & 5.2 & 299.2 & 3 & 600.0 & .0 \\
\hline 04 & 53013 & 167.1752 & 4.9073 & 300.1 & 2 & 600.0 & 600.0 \\
\hline 04 & 53014 & 175.8011 & 4.9680 & 301.1 & 2 & 600.0 & 600.0 \\
\hline 04 & 53015 & 171.5757 & 575 & 301.8 & 3 & 600.0 & 600.0 \\
\hline 04 & 53016 & 171.8011 & 6.1033 & 302.1 & 4 & 600.0 & 600.0 \\
\hline 04 & 53017 & 166.4555 & 6.5230 & 301.8 & 3 & 600.0 & 600.0 \\
\hline 04 & 53018 & 91.1007 & 5.0888 & 301.1 & 3 & 600.0 & 600.0 \\
\hline 04 & 53019 & 83.0532 & 5.4253 & 299.3 & 4 & 600.0 & 600.0 \\
\hline 04 & 53020 & 78.1250 & 6.9230 & 297.6 & 4 & 600.0 & 600.0 \\
\hline 04 & 53021 & 75.1000 & 8.0925 & 297.1 & 4 & 600.0 & \\
\hline 04 & 53022 & 71.4999 & 9.4325 & 296.5 & - & 600.0 & 600.0 \\
\hline 0 & 53023 & 74.4505 & 9.1250 & 296.1 & 4 & 600.0 & 600.0 \\
\hline
\end{tabular}




\begin{tabular}{|c|c|c|c|c|c|c|c|}
\hline 4 & 53024 & 74.8751 & 7.8325 & 296.7 & 4 & 600.0 & 00.0 \\
\hline 04 & 5311 & 72.8000 & 6.6333 & 296.7 & 4 & 600.0 & 600.0 \\
\hline 04 & 5312 & 75.3750 & 5.8948 & 296.7 & 4 & 600.0 & 00.0 \\
\hline 04 & 5313 & 80.2753 & 5.3733 & 296.5 & 4 & 600.0 & 00.0 \\
\hline 04 & 5314 & 76.8500 & 6.5280 & 296.0 & 4 & 600.0 & 00.0 \\
\hline 04 & 5315 & 76.1000 & 8.6250 & 294.9 & 4 & 600.0 & 00.0 \\
\hline 04 & 5316 & 78.0250 & 7.5720 & 295.3 & 4 & 600.0 & 600.0 \\
\hline 04 & 5317 & 86.3964 & 4.6228 & 296.9 & 3 & 600.0 & 00.0 \\
\hline 04 & 5318 & 113.6098 & 3.5443 & 298.6 & 3 & 600.0 & 00.0 \\
\hline 04 & 5319 & 189.5329 & 5.5078 & 299.3 & 3 & 600.0 & .0 \\
\hline 04 & 3110 & 183.6829 & 8.2200 & 300.2 & 4 & 600.0 & 500.0 \\
\hline 04 & 3111 & 172.3250 & 7.3483 & 301.1 & 3 & 600.0 & \\
\hline 04 & 3112 & 180.5929 & 7.0653 & 301.5 & 3 & 600.0 & 600.0 \\
\hline 04 & 53113 & 172.5251 & 6.5775 & 301.1 & 4 & 600.0 & 600.0 \\
\hline 04 & 53114 & 169.8748 & 5.5348 & 300.5 & 3 & 600.0 & \\
\hline 04 & 53115 & 155.1160 & 5.9338 & 301.9 & 3 & 600.0 & 60 \\
\hline 04 & 53116 & 111.3847 & 5.5968 & 303.0 & 3 & 600.0 & 600.0 \\
\hline 04 & 53117 & 97.7509 & 6.5590 & 302.7 & 4 & 600.0 & .0 \\
\hline 04 & 53118 & 78.9853 & 7.3228 & 301.0 & 4 & 600.0 & .0 \\
\hline 04 & 53119 & 69.6751 & 7.6400 & 299.0 & 4 & 600.0 & 600.0 \\
\hline 04 & 53120 & 61.6650 & 7.0950 & 298.5 & 4 & 600.0 & .0 \\
\hline 04 & 53121 & 73.3750 & 75 & 298.4 & 4 & 600.0 & \\
\hline 04 & 53122 & 66.2500 & 8.3600 & 298.7 & 4 & 600.0 & 600.0 \\
\hline 04 & 53123 & 69.7750 & 8.5300 & 298.5 & 4 & 600.0 & .0 \\
\hline 04 & 53124 & 71.6250 & 8.2650 & 298.3 & 4 & 600.0 & \\
\hline 04 & $\begin{array}{lll}6 & 1 & 1\end{array}$ & 79.2751 & 9.0125 & 297.6 & 4 & 600.0 & 60 \\
\hline 04 & $\begin{array}{lll}6 & 1 & 2\end{array}$ & 77.9256 & 9.2225 & 296.6 & 4 & 600.0 & 600.0 \\
\hline 04 & $\begin{array}{lll}6 & 1 & 3\end{array}$ & 70.9503 & 9.6675 & 295.7 & 4 & 600.0 & .0 \\
\hline 04 & $\begin{array}{lll}6 & 1 & 4\end{array}$ & 75.9245 & 9.4875 & 295.3 & 4 & 600.0 & 60 \\
\hline 04 & 615 & 74.2750 & 9.6825 & 295.0 & 4 & 600.0 & 600.0 \\
\hline 04 & 616 & 78.5494 & 275 & 295.2 & 4 & 600.0 & \\
\hline 04 & $\begin{array}{lll}6 & 1 & 7\end{array}$ & 90.3789 & 50 & 296.1 & 4 & 600.0 & 60 \\
\hline 04 & $\begin{array}{lll}6 & 1 & 8\end{array}$ & 84.3221 & 5.1463 & 297.1 & 3 & 600.0 & 600.0 \\
\hline 04 & $\begin{array}{lll}6 & 1 & 9\end{array}$ & 136.9709 & 4.9748 & 299.5 & 3 & 600.0 & .0 \\
\hline 04 & 6110 & 164.7740 & 815 & 30 & 4 & 600.0 & \\
\hline 04 & 6111 & 167.9454 & 8.3925 & 301.7 & 3 & 600.0 & 600.0 \\
\hline 04 & 6112 & 154.2502 & 7.6455 & 302.4 & 3 & 600.0 & \\
\hline 04 & 6113 & 131.1320 & 5.9498 & 303.5 & 3 & 600.0 & 600.0 \\
\hline 04 & $6 \quad 114$ & 99.7018 & 6.5538 & 303.8 & 3 & 600.0 & 600.0 \\
\hline 04 & 6115 & 93.1935 & 7.8925 & 303.2 & 4 & 600.0 & \\
\hline 04 & 6116 & 94.5592 & 7.4750 & 302.9 & 4 & 600.0 & 600.0 \\
\hline 04 & 6117 & 69.7187 & 8.4075 & 301.7 & 4 & 600.0 & 600.0 \\
\hline 04 & 6118 & 60.5475 & 10.6625 & 300.3 & 4 & 600.0 & 600.0 \\
\hline 04 & 6119 & 63.2236 & 10.8850 & 299.3 & 4 & 600.0 & 600.0 \\
\hline 04 & 6120 & 63.3004 & 10.3100 & 298.5 & 4 & 600.0 & 600.0 \\
\hline 04 & $6 \quad 121$ & 71.1492 & 9.4800 & 297.8 & 4 & 600.0 & 600.0 \\
\hline 04 & $6 \quad 122$ & 67.7499 & 10.0775 & 29 & 4 & 600.0 & 600.0 \\
\hline 04 & $6 \quad 123$ & 70.3003 & 9.9325 & 296.9 & 4 & 600.0 & 600.0 \\
\hline
\end{tabular}




\begin{tabular}{|c|c|c|c|c|c|c|c|c|}
\hline 4 & 6 & 124 & 69.1002 & 10.5400 & 296.3 & 4 & 600.0 & 600.0 \\
\hline 04 & 6 & 21 & 71.2486 & 11.0375 & 295.9 & 4 & 600.0 & 00.0 \\
\hline 04 & 6 & 22 & 72.4750 & 4475 & 295.2 & 4 & 600.0 & 00.0 \\
\hline 4 & 6 & 23 & 76.9500 & 10.3900 & 294.6 & 4 & 600.0 & 00.0 \\
\hline 04 & 6 & 24 & 73.1001 & 100 & 294.3 & 4 & 00.0 & 00.0 \\
\hline 04 & 6 & 25 & 77.0000 & 10.4750 & 293.6 & 4 & 600.0 & 00.0 \\
\hline 04 & 6 & 26 & 74.2250 & 9.4475 & 293.6 & 4 & 600.0 & 00.0 \\
\hline 04 & 6 & 27 & 85.7000 & 7.5725 & 294.6 & 4 & 600.0 & 00.0 \\
\hline 04 & 6 & 28 & 104.9401 & 5.3688 & 296.6 & 3 & 600.0 & 00.0 \\
\hline 04 & 6 & 29 & 158.1250 & 5.9453 & 298.9 & 4 & 60 & 0 \\
\hline 04 & 6 & 210 & 160.9251 & 6.8275 & 299.8 & 4 & 600.0 & \\
\hline 04 & 6 & 211 & 153.5633 & 7.5275 & 301.3 & 3 & .0 & \\
\hline 04 & 6 & 212 & 140.2552 & 7.5235 & 302.3 & 3 & 600.0 & 00.0 \\
\hline 04 & 6 & 213 & 124.9701 & 7.4013 & 303.2 & 3 & 600.0 & \\
\hline 04 & 6 & 214 & 101.7297 & 7.8050 & 303.3 & 3 & .0 & \\
\hline 04 & 6 & 215 & 75.1662 & 8.0275 & 303.1 & 4 & 600.0 & .0 \\
\hline 04 & 6 & 216 & 69.1730 & 9.3625 & 302.3 & 4 & 60 & .0 \\
\hline 04 & 6 & 217 & 66.3503 & 11.5150 & 301.2 & 4 & .0 & \\
\hline 04 & 6 & 218 & 66.7000 & 13.6475 & 299.0 & 4 & .0 & \\
\hline 04 & 6 & 219 & 64.4250 & 14.4200 & 298.3 & 4 & .0 & .0 \\
\hline 04 & 6 & 220 & 65.8749 & 13.0700 & 297.3 & 4 & 6 & \\
\hline 04 & 6 & 221 & 68.3501 & 12.7175 & 296.8 & 4 & .0 & \\
\hline 04 & 6 & 222 & 64.6750 & 12.3475 & 296.9 & 4 & 600.0 & .0 \\
\hline 04 & 6 & 223 & 63.5248 & 11.4575 & 296.5 & 4 & 6 & \\
\hline 04 & 6 & 224 & 66.7249 & 11.0000 & 296.5 & 4 & .0 & \\
\hline 04 & 6 & 31 & 79.8250 & 9.9475 & 294.4 & 4 & .0 & \\
\hline 04 & 6 & 32 & 73.3750 & 10.4325 & 294.7 & 4 & 60 & .0 \\
\hline 04 & 6 & 33 & 74.1753 & 10.5675 & 294.8 & 4 & .0 & \\
\hline 04 & 6 & 34 & 73.6000 & 10.7300 & 294.9 & 4 & .0 & .0 \\
\hline 04 & 6 & 35 & 80.9732 & 7.4528 & 294.9 & 4 & 6 & .0 \\
\hline 04 & 6 & 36 & 78.5252 & 10.2950 & 294.0 & 4 & 0 & \\
\hline 04 & 6 & 37 & 79.1750 & 11.0850 & 294.0 & 4 & .0 & 0 \\
\hline 04 & 6 & 38 & 83.1999 & 9.4025 & 295.4 & 4 & 600.0 & 0.0 \\
\hline 04 & 6 & & 83.5497 & 8.2025 & 296.2 & 4 & .0 & 0.0 \\
\hline 04 & 6 & 310 & 79.5233 & 8.1875 & 296.1 & 4 & 600.0 & 600.0 \\
\hline 04 & 6 & 311 & 79.3248 & 7.7800 & 297.8 & 3 & 600.0 & 600.0 \\
\hline 04 & 6 & 312 & 75.0499 & 8.0150 & 299.7 & 3 & 600.0 & .0 \\
\hline 04 & 6 & 313 & 79.2994 & 9.5150 & 300.3 & 3 & 600.0 & 600.0 \\
\hline 04 & 6 & 314 & 68.6000 & 10.8875 & 299.8 & 3 & 600.0 & 600.0 \\
\hline 04 & 6 & 315 & 72.7757 & 10.6550 & 299.8 & 4 & 600.0 & 600.0 \\
\hline 04 & 6 & 316 & 63.8749 & 13.0525 & 298.9 & 4 & 600.0 & 600.0 \\
\hline 04 & 6 & 317 & 62.6499 & 13.8950 & 297.9 & 4 & 600.0 & 600.0 \\
\hline 04 & 6 & 318 & 63.0499 & 12.4475 & 297.2 & 4 & 600.0 & 600.0 \\
\hline 04 & 6 & 319 & 62.2500 & 12.4475 & 296.1 & 4 & 600.0 & 600.0 \\
\hline 04 & 6 & 320 & 64.0750 & 12.4225 & 295.5 & 4 & 600.0 & 600.0 \\
\hline 04 & 6 & 321 & 65.7498 & 11.5175 & 295.6 & . & 600.0 & 600.0 \\
\hline $0<$ & 6 & 322 & 72.0500 & 12.3225 & 295.0 & 4 & 600.0 & 600.0 \\
\hline 0 & 0 & 323 & 67.5250 & 11.7125 & 295.3 & $\Psi$ & 600.0 & 600.0 \\
\hline
\end{tabular}




\begin{tabular}{|c|c|c|c|c|c|c|c|c|}
\hline 4 & 6 & 324 & 72.2499 & 10.1500 & 295.5 & 4 & 600.0 & 600.0 \\
\hline 04 & 6 & 41 & 73.1500 & 8.7200 & 295.7 & 4 & 600.0 & 00.0 \\
\hline 04 & 6 & 42 & 68.7001 & 9.9925 & 295.3 & 4 & 600.0 & 00.0 \\
\hline 4 & 6 & 43 & 67.1754 & 9.7475 & 294.9 & 4 & 600.0 & 00.0 \\
\hline 04 & 6 & 44 & 70.4252 & 10.5000 & 293.9 & 4 & 600.0 & 00.0 \\
\hline 04 & 6 & 45 & 64.2750 & 11.6575 & 293.8 & 4 & 600.0 & 00.0 \\
\hline 04 & 6 & 46 & 70.6648 & 9.6025 & 294.5 & 4 & 600.0 & 00.0 \\
\hline 04 & 6 & 47 & 80.5749 & 6.2863 & 295.8 & 4 & 600.0 & 00.0 \\
\hline 04 & 6 & 48 & 84.3043 & 5.0060 & 296.6 & 3 & 600.0 & 00.0 \\
\hline 04 & 6 & 49 & 162.5169 & 598 & 298.6 & 2 & 600.0 & .0 \\
\hline 04 & 6 & 410 & 168.6287 & 805 & 300.4 & 2 & 600.0 & \\
\hline 04 & 6 & 411 & 79.5512 & 640 & 300.8 & 2 & 600.0 & \\
\hline 04 & 6 & 412 & 80.6414 & 7.6243 & 300.3 & 3 & 600.0 & 00.0 \\
\hline 04 & 6 & 413 & 71.6724 & 8.6325 & 300.6 & 3 & 600.0 & \\
\hline 04 & 6 & 414 & 62.7750 & 00 & 299.9 & 3 & .0 & \\
\hline 04 & 6 & 415 & 67.3756 & 10 . & 300.1 & 4 & 600.0 & .0 \\
\hline 04 & 6 & 416 & 66.1750 & 10 & 299.6 & 4 & 60 & .0 \\
\hline 04 & 6 & 417 & 66.7993 & 10. & 299.3 & 4 & .0 & \\
\hline 04 & 6 & 418 & 68.4595 & 10 . & 298.8 & 4 & .0 & \\
\hline 04 & 6 & 419 & 67.9000 & 11.5225 & 297.8 & 4 & .0 & .0 \\
\hline 04 & 6 & 420 & 65.0252 & 10 . & 296.4 & 4 & 6 & \\
\hline 04 & 6 & 421 & 65.6247 & 12 . & 295.6 & 4 & .0 & \\
\hline 04 & 6 & 422 & 67.4502 & 13. & 295.2 & 4 & 600.0 & .0 \\
\hline 04 & 6 & 423 & 70.9500 & 12 . & 295.0 & 4 & 0 & \\
\hline 04 & 6 & 424 & 70.5250 & 12 . & 294.1 & 4 & 0 & \\
\hline 04 & 6 & 51 & 69.2751 & 12.4025 & 293.9 & 4 & .0 & \\
\hline 04 & 6 & 52 & 69.9750 & 11.8350 & 294.1 & 4 & .0 & .0 \\
\hline 04 & 6 & 53 & 67.9747 & 12 . & 293.8 & 4 & .0 & \\
\hline 04 & 6 & 54 & 61.7484 & 12.1550 & 293.6 & 4 & .0 & 0.0 \\
\hline 04 & 6 & 55 & 73.3725 & 8.2325 & 293.3 & 4 & 60 & .0 \\
\hline 04 & 6 & 56 & 66.7754 & 12 & 292.6 & 4 & 0 & \\
\hline 04 & 6 & 57 & 65.0507 & 12.4475 & 292.7 & 4 & .0 & 0.0 \\
\hline 04 & 6 & 58 & 65.6999 & 11.3400 & 293.2 & 4 & 600.0 & 0.0 \\
\hline 04 & 6 & 59 & 70.6257 & 8.8725 & 294.4 & 4 & 600.0 & .0 \\
\hline 04 & 6 & 510 & 83.1081 & 8.4 & 296.0 & 4 & 600.0 & 600.0 \\
\hline 04 & 6 & 511 & 81.5018 & 7.4893 & 297.4 & 4 & 600.0 & 00.0 \\
\hline 04 & 6 & 512 & 86.8750 & 7. & 299.0 & 3 & 600.0 & .0 \\
\hline 04 & 6 & 513 & 92.1247 & 8.8700 & 299.5 & 3 & 600.0 & 600.0 \\
\hline 04 & 6 & 514 & 86.5012 & 9.4025 & 299.4 & 3 & 600.0 & 600.0 \\
\hline 04 & 6 & 515 & 82.2254 & 10.6175 & 298.7 & 4 & 600.0 & 0.0 \\
\hline 04 & 6 & 516 & 80.7260 & 12.2775 & 298.3 & 4 & 600.0 & 600.0 \\
\hline 04 & 6 & 517 & 72.5999 & 10.5325 & 297.9 & 4 & 600.0 & 600.0 \\
\hline 04 & 6 & 518 & 68.5504 & 11.7350 & 297.0 & 4 & 600.0 & 600.0 \\
\hline 04 & 6 & 519 & 66.8245 & 11.1300 & 296.3 & 4 & 600.0 & 600.0 \\
\hline 04 & 6 & 520 & 74.8751 & 11.2050 & 296.1 & 4 & 600.0 & 600.0 \\
\hline 04 & 6 & 521 & 73.0492 & 11.7100 & 295.8 & 4 & 600.0 & \\
\hline $0<$ & 6 & 522 & 72.5696 & 10.5450 & 296.5 & 4 & 600.0 & 600.0 \\
\hline 0 & 0 & 523 & 68.6417 & 10.6625 & 296.0 & & 600.0 & 600.0 \\
\hline
\end{tabular}




\begin{tabular}{|c|c|c|c|c|c|c|c|c|}
\hline 4 & 6 & 524 & 62.9267 & 11.7850 & 294.6 & 4 & 600.0 & 600.0 \\
\hline 04 & 6 & 61 & 64.2255 & 11.7600 & 294.0 & 4 & 600.0 & 00.0 \\
\hline 04 & 6 & 62 & 71.6499 & 11.5625 & 293.2 & 4 & 600.0 & 00.0 \\
\hline 4 & 6 & 63 & 68.4002 & 10.9600 & 292.0 & 4 & 600.0 & 00.0 \\
\hline 04 & 6 & 64 & 72.0506 & 11.1175 & 291.0 & 4 & 600.0 & 00.0 \\
\hline 04 & 6 & 65 & 75.2993 & 11.6675 & 290.6 & 4 & 600.0 & 00.0 \\
\hline 04 & 6 & 66 & 78.7500 & 11.8225 & 291.3 & 4 & 600.0 & 00.0 \\
\hline 04 & 6 & 67 & 80.8499 & 10.3950 & 292.1 & 4 & 600.0 & 00.0 \\
\hline 04 & 6 & 68 & 81.9004 & 7.1353 & 294.6 & 4 & 600.0 & 00.0 \\
\hline 04 & 6 & 69 & 83.2492 & 7.3560 & 295.9 & 4 & 600.0 & .0 \\
\hline 04 & 6 & 610 & 85.5750 & 6.3570 & 297.1 & 3 & 600.0 & \\
\hline 04 & 6 & 611 & 82.9263 & 7.3455 & 298.5 & 3 & 600.0 & \\
\hline 04 & 6 & 612 & 85.8001 & 7.8723 & 300.1 & 3 & 600.0 & 00.0 \\
\hline 04 & 6 & 613 & 73.0270 & 9.9900 & 299.5 & - & 600.0 & \\
\hline 04 & 6 & 614 & 69.1481 & 11.4250 & 298.8 & 3 & .0 & \\
\hline 04 & 6 & 615 & 73.6247 & 11.8450 & 298.5 & 4 & 600.0 & .0 \\
\hline 04 & 6 & 616 & 79.7249 & 9.5275 & 298.9 & 4 & 60 & .0 \\
\hline 04 & 6 & 617 & 81.7251 & 9.9800 & 297.8 & 4 & .0 & \\
\hline 04 & 6 & 618 & 83.2781 & 8.9215 & 297.4 & 4 & .0 & .0 \\
\hline 04 & 6 & 619 & 61.3000 & 12.7875 & 295.1 & 4 & .0 & .0 \\
\hline 04 & 6 & 620 & 60.5755 & 14.9675 & 293.2 & 4 & 6 & \\
\hline 04 & 6 & 621 & 62.0501 & 14.8600 & 292.4 & 4 & .0 & \\
\hline 04 & 6 & 622 & 61.1252 & 16.0500 & 291.4 & 4 & 600.0 & .0 \\
\hline 04 & 6 & 623 & 64.8250 & 15.48 & 290.6 & 4 & 6 & \\
\hline 04 & 6 & 624 & 72.9957 & 300 & 289.9 & 4 & .0 & \\
\hline 04 & 6 & 71 & 64.0000 & 13.9450 & 289.1 & 4 & .0 & \\
\hline 04 & 6 & 72 & 61.6001 & 15.4150 & 287.7 & 4 & .0 & .0 \\
\hline 04 & 6 & 73 & 62.7998 & 13.9875 & 287.3 & 4 & .0 & \\
\hline 04 & 6 & 74 & 74.5804 & 6.5213 & 287.6 & 4 & .0 & 0.0 \\
\hline 04 & 6 & 75 & 56.4231 & 11.4550 & 287.0 & 4 & 6 & .0 \\
\hline 04 & 6 & 76 & 66.9263 & 12.0550 & 286.8 & 4 & 0 & \\
\hline 04 & 6 & 77 & 74.8874 & 10.7625 & 287.0 & 4 & .0 & 0.0 \\
\hline 04 & 6 & 78 & 75.9000 & 8.8875 & 287.8 & 4 & 600.0 & 0.0 \\
\hline 04 & 6 & 79 & 78.3747 & 8.532 & 288.7 & 4 & .0 & 0.0 \\
\hline 04 & 6 & 710 & 84.5507 & 8.0225 & 289.9 & 4 & .0 & 600.0 \\
\hline 04 & 6 & 711 & 86.9992 & 8.0525 & 290.8 & 3 & 600.0 & 600.0 \\
\hline 04 & 6 & 712 & 75.4438 & 8.9375 & 292.1 & 3 & 600.0 & .0 \\
\hline 04 & 6 & 713 & 73.0288 & 10.0750 & 293.0 & 3 & 600.0 & 600.0 \\
\hline 04 & 6 & 714 & 83.0238 & 9.8300 & 292.9 & 3 & 600.0 & 600.0 \\
\hline 04 & 6 & 715 & 78.4269 & 9.895 & 292.6 & 4 & 600.0 & 600.0 \\
\hline 04 & 6 & 716 & 51.8499 & 12.0475 & 293.1 & 4 & 600.0 & 600.0 \\
\hline 04 & 6 & 717 & 58.3751 & 15.4975 & 292.6 & 4 & 600.0 & 600.0 \\
\hline 04 & 6 & 718 & 63.4227 & 12.0275 & 290.3 & 4 & 600.0 & 600.0 \\
\hline 04 & 6 & 719 & 69.4949 & 9.3500 & 288.3 & 4 & 600.0 & 600.0 \\
\hline 04 & 6 & 720 & 63.9747 & 12.3550 & 287.3 & 4 & 600.0 & 600.0 \\
\hline 04 & 6 & 721 & 69.4525 & 9.3900 & 286.7 & . & 600.0 & 600.0 \\
\hline $0<$ & 6 & 722 & 65.0498 & 11.5225 & 286.1 & 4 & 600.0 & 600.0 \\
\hline 0 & 6 & 723 & 61.5750 & 11.375 & 286.0 & 4 & 600.0 & 600.0 \\
\hline
\end{tabular}




\begin{tabular}{|c|c|c|c|c|c|c|c|c|}
\hline 4 & 6 & 724 & 66.3499 & 10.6400 & 285.9 & 4 & 600.0 & 600.0 \\
\hline 04 & 6 & 81 & 77.2749 & 11.2550 & 285.5 & 4 & 600.0 & 00.0 \\
\hline 04 & 6 & 82 & 79.5031 & 10.0875 & 285.2 & 4 & 600.0 & 00.0 \\
\hline 4 & 6 & 83 & 80.8001 & 9.9250 & 284.8 & 4 & 600.0 & 00.0 \\
\hline 04 & 6 & 84 & 76.1500 & 9.7600 & 284.3 & 4 & 600.0 & 00.0 \\
\hline 04 & 6 & 85 & 74.5500 & 10.4800 & 283.9 & 4 & 600.0 & 00.0 \\
\hline 04 & 6 & 86 & 72.5757 & 9.7150 & 283.8 & 4 & 600.0 & 00.0 \\
\hline 04 & 6 & 87 & 74.3496 & 9.9275 & 284.6 & 4 & 600.0 & 00.0 \\
\hline 04 & 6 & 88 & 75.5499 & 8.3475 & 285.8 & 4 & 600.0 & 00.0 \\
\hline 04 & 6 & 89 & 76.1873 & 7.2865 & 287.8 & 4 & 600.0 & .0 \\
\hline 04 & 6 & 810 & 83.9000 & 6.8355 & 289.2 & 4 & 600.0 & \\
\hline 04 & 6 & 811 & 78.0989 & 7.3423 & 290.7 & 3 & 600.0 & \\
\hline 04 & 6 & 812 & 78.3993 & 8.8000 & 291.6 & 3 & 600.0 & 00.0 \\
\hline 04 & 6 & 813 & 72.0302 & 7.7245 & 292.0 & 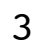 & 600.0 & \\
\hline 04 & 6 & 814 & 68.5500 & 10.2800 & 291.1 & 3 & .0 & \\
\hline 04 & 6 & 815 & 70.7497 & 11.9450 & 289.7 & 4 & 600.0 & .0 \\
\hline 04 & 6 & 816 & 67.2000 & 13.2425 & 288.5 & 4 & 60 & 600.0 \\
\hline 04 & 6 & 817 & 61.8774 & 13.0650 & 288.1 & 4 & .0 & \\
\hline 04 & 6 & 818 & 55.6000 & 13.7025 & 287.5 & 4 & 60 & .0 \\
\hline 04 & 6 & 819 & 60.1501 & 12.8650 & 286.4 & 4 & .0 & .0 \\
\hline 04 & 6 & 820 & 61.6250 & 9.79 & 286.1 & 4 & 6 & \\
\hline 04 & 6 & 821 & 69.4495 & 9.3700 & 286.2 & 4 & .0 & \\
\hline 04 & 6 & 822 & 73.7749 & 7.8775 & 286.4 & 4 & 600.0 & .0 \\
\hline 04 & 6 & 823 & 72.7800 & 7.74 & 286.5 & 4 & 60 & \\
\hline 04 & 6 & 824 & 72.7004 & 8.0425 & 285.9 & 4 & .0 & \\
\hline 04 & 6 & 91 & 72.1751 & 8.2275 & 286.2 & 4 & .0 & .0 \\
\hline 04 & 6 & 92 & 72.5000 & 8.0975 & 285.4 & 4 & .0 & .0 \\
\hline 04 & 6 & 93 & 76.3999 & 6.7778 & 286.0 & 4 & .0 & \\
\hline 04 & 6 & 94 & 73.9751 & 6.9553 & 285.7 & 4 & .0 & 0.0 \\
\hline 04 & 6 & 95 & 75.5747 & 7.2575 & 285.7 & 4 & 6 & .0 \\
\hline 04 & 6 & 96 & 84.1227 & 5.7008 & 285.8 & 4 & 0 & \\
\hline 04 & 6 & 97 & 110.4486 & 2.7785 & 286.8 & 3 & .0 & 0.0 \\
\hline 04 & 6 & 98 & 160.8721 & 2.0893 & 288.8 & 2 & 600.0 & 0.0 \\
\hline 04 & 6 & 99 & 193.6798 & 2.8268 & 290.6 & 2 & .0 & 0.0 \\
\hline 04 & 6 & 910 & 92.8502 & 2.2050 & 291.7 & 1 & 600.0 & 600.0 \\
\hline 04 & 6 & 911 & 215.6118 & 2.6970 & 293.9 & 2 & 600.0 & 600.0 \\
\hline 04 & 6 & 912 & 34.9579 & 3.1765 & 294.8 & 1 & 600.0 & .0 \\
\hline 04 & 6 & 913 & 104.5508 & 6.6250 & 295.0 & 2 & 600.0 & 600.0 \\
\hline 04 & 6 & 914 & 76.3943 & 6.7908 & 295.2 & 3 & 600.0 & 600.0 \\
\hline 04 & 6 & 915 & 89.5497 & 8.5275 & 294.6 & 4 & 600.0 & .0 \\
\hline 04 & 6 & 916 & 81.7680 & 9.5150 & 292.8 & 4 & 600.0 & 600.0 \\
\hline 04 & 6 & 917 & 71.3759 & 10.8575 & 291.1 & 4 & 600.0 & 600.0 \\
\hline 04 & 6 & 918 & 63.9503 & 12.0975 & 288.6 & 4 & 600.0 & 600.0 \\
\hline 04 & 6 & 919 & 59.0004 & 12.7600 & 287.2 & 4 & 600.0 & 600.0 \\
\hline 04 & 6 & 920 & 62.1250 & 13.1225 & 286.4 & 4 & 600.0 & 600.0 \\
\hline 04 & 6 & 921 & 65.7751 & 12.8400 & & 4 & 600.0 & 600.0 \\
\hline $0<$ & 6 & 922 & 66.5000 & 12.732 & & 4 & 600.0 & 600.0 \\
\hline 04 & 6 & 923 & 62.9751 & 13.430 & 285.5 & 4 & 600.0 & 600.0 \\
\hline
\end{tabular}




\begin{tabular}{|c|c|c|c|c|c|c|c|}
\hline 4 & 6924 & 64.0250 & 14.2025 & 285.4 & 4 & 600.0 & 600.0 \\
\hline 04 & 6101 & 62.8752 & 14.7300 & 285.1 & 4 & 600.0 & 600.0 \\
\hline 04 & 6102 & 70.7741 & 13.1200 & 284.9 & 4 & 600.0 & 00.0 \\
\hline 04 & 6103 & 71.2252 & 12.7125 & 284.8 & 4 & 600.0 & 00.0 \\
\hline 04 & 6104 & 72.3251 & 13.1550 & 284.6 & 4 & 600.0 & 00.0 \\
\hline 04 & 6105 & 76.0250 & 13.4975 & 284.4 & 4 & 600.0 & 00.0 \\
\hline 04 & 6106 & 72.7252 & 10.9075 & 284.8 & 4 & 600.0 & 0 \\
\hline 04 & 6107 & 67.8000 & 9.1375 & 285.5 & 4 & 600.0 & 00.0 \\
\hline 04 & 6108 & 65.8007 & 7.9450 & 286.7 & 4 & 600.0 & 00.0 \\
\hline 04 & 6109 & 82.9532 & 6.6370 & 288.4 & 4 & 60 & 0 \\
\hline 04 & 61010 & 75.6547 & 4.7185 & 290.4 & 3 & 600.0 & \\
\hline 04 & 61011 & 40.5995 & 3.3638 & 292.3 & 2 & .0 & \\
\hline 04 & 61012 & 50.7725 & 4.1058 & 293.5 & 2 & 600.0 & .0 \\
\hline 04 & 61013 & 86.9558 & 4.9808 & 294.7 & 2 & 600.0 & \\
\hline 04 & 61014 & 92.1490 & 460 & 295.3 & 3 & .0 & \\
\hline 04 & 61015 & 86.2252 & 6.9798 & 295.6 & 4 & 600.0 & .0 \\
\hline 04 & 61016 & 87.1748 & 7.5158 & 295.1 & 4 & 60 & .0 \\
\hline 04 & 61017 & 75.2715 & 7.3543 & 294.2 & 4 & .0 & \\
\hline 04 & 61018 & 61.4952 & 9.4525 & 292.5 & 4 & .0 & \\
\hline 04 & 61019 & 73.4751 & 10.3075 & 290.9 & 4 & .0 & .0 \\
\hline 04 & 61020 & 66.2001 & 11. & 290.0 & 4 & 6 & \\
\hline 04 & 61021 & 66.1750 & 10. & 290.3 & 4 & .0 & \\
\hline 04 & 61022 & 66.6750 & 10.3525 & 290.6 & 4 & 600.0 & .0 \\
\hline 04 & 61023 & 63.3750 & 10 . & 291.0 & 4 & 0 & \\
\hline 04 & 61024 & 63.7245 & 9 . & 291.1 & 4 & 0 & \\
\hline 04 & 6111 & 68.3741 & 425 & 291.2 & 4 & .0 & \\
\hline 04 & 6112 & 66.0000 & 8.4625 & 290.8 & 4 & 60 & .0 \\
\hline 04 & 6113 & 71.2751 & 450 & 290.4 & 4 & .0 & \\
\hline 04 & 6114 & 72.5996 & 050 & 289.8 & 4 & .0 & .0 \\
\hline 04 & 6115 & 74.2500 & 9.2400 & 288.9 & 4 & .0 & \\
\hline 04 & 6116 & 75.5502 & 7.2993 & 289.8 & 4 & .0 & \\
\hline 04 & 6117 & 86.2365 & 153 & 291.6 & 4 & .0 & .0 \\
\hline 04 & 6118 & 94.4736 & 4.8813 & 292.7 & 3 & 6 & 0 \\
\hline 04 & 6119 & 104.7289 & 3.2705 & 294.4 & 2 & 600.0 & .0 \\
\hline 04 & 61110 & 216.1710 & 3.1943 & 294.9 & 2 & 600.0 & 600.0 \\
\hline 04 & 61111 & 90.2235 & 5.3553 & 295.6 & 2 & 600.0 & 600.0 \\
\hline 04 & 61112 & 89.9517 & 6.8443 & 296.8 & 3 & 600.0 & .0 \\
\hline 04 & 61113 & 98.4498 & 7.9175 & 297.8 & 3 & 600.0 & 600.0 \\
\hline 04 & 61114 & 88.5754 & 9.9625 & 297.8 & 3 & 600.0 & 600.0 \\
\hline 04 & 61115 & 80.8157 & 000 & 297.4 & 4 & 600.0 & .0 \\
\hline 04 & 61116 & 70.6998 & 9.8550 & 296.5 & 4 & 600.0 & 600.0 \\
\hline 04 & 61117 & 64.1996 & 11.6775 & 295.7 & 4 & 600.0 & 600.0 \\
\hline 04 & 61118 & 65.3751 & 11.9250 & 294.4 & 4 & 600.0 & 600.0 \\
\hline 04 & 61119 & 61.4752 & 11.2325 & 293.4 & 4 & 600.0 & 600.0 \\
\hline 04 & 61120 & 61.1252 & 11.0225 & 292.7 & 4 & 600.0 & 600.0 \\
\hline 04 & 61121 & 65.1486 & 8.8300 & 292.7 & 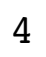 & 600.0 & 600.0 \\
\hline $0<$ & 61122 & 67.0518 & 9.0525 & 292.5 & 4 & 600.0 & 600.0 \\
\hline 04 & 61123 & 64.8250 & 9.1750 & 292.2 & 4 & 600.0 & 600.0 \\
\hline
\end{tabular}




\begin{tabular}{|c|c|c|c|c|c|c|c|}
\hline 4 & 61124 & 72.3001 & 10.0575 & 291.7 & 4 & 600.0 & 600.0 \\
\hline 04 & 6121 & 74.0752 & 9.7550 & 291.8 & 4 & 600.0 & 00.0 \\
\hline 04 & 6122 & 70.0000 & 9.4650 & 291.3 & 4 & 600.0 & 00.0 \\
\hline 04 & 6123 & 70.3733 & 9.5425 & 291.1 & 4 & 600.0 & 00.0 \\
\hline 04 & 6124 & 77.2250 & 10.0750 & 290.8 & 4 & 600.0 & 00.0 \\
\hline 04 & 6125 & 75.2995 & 10.3600 & 290.3 & 4 & 600.0 & 00.0 \\
\hline 04 & 6126 & 76.3500 & 9.3000 & 290.1 & 4 & 600.0 & 00.0 \\
\hline 04 & 6127 & 84.7752 & 8.6550 & 290.7 & 4 & 600.0 & 00.0 \\
\hline 04 & 6128 & 85.5471 & 6.8763 & 291.6 & 4 & 600.0 & 00.0 \\
\hline 04 & 6129 & 104.7509 & 5.5378 & 293.2 & 3 & 600 & 0 \\
\hline 04 & 61210 & 153.7946 & 108 & 295.5 & 3 & 600.0 & \\
\hline 04 & 61211 & 165.3000 & 550 & 296.2 & 3 & 600.0 & \\
\hline 04 & 61212 & 159.9016 & 6.6373 & 297.5 & 3 & 600.0 & 00.0 \\
\hline 04 & 61213 & 159.6723 & 5.3340 & 298.9 & 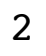 & 600.0 & \\
\hline 04 & 61214 & 174.0900 & 5 . & 300 & 3 & .0 & \\
\hline 04 & 61215 & 157.8096 & 6.2975 & 300.9 & 3 & 600.0 & .0 \\
\hline 04 & 61216 & 125.7729 & 6.3140 & 300.7 & 4 & 60 & .0 \\
\hline 04 & 61217 & 79.6433 & 6.9933 & 299.1 & 4 & .0 & \\
\hline 04 & 61218 & 72.2494 & 8.5275 & 297.4 & 4 & .0 & \\
\hline 04 & 61219 & 64.7748 & 9.2375 & 295.6 & 4 & .0 & .0 \\
\hline 04 & 61220 & 68.4747 & 8.82 & 29 & 4 & 6 & \\
\hline 04 & 61221 & 70.0002 & 8.7350 & 29 & 4 & .0 & \\
\hline 04 & 61222 & 69.4000 & 8.9200 & 295.8 & 4 & 600.0 & .0 \\
\hline 04 & 61223 & 69.1500 & 9.7300 & 29 & 4 & 0 & \\
\hline 04 & 61224 & 69.9499 & 11.52 & 294.2 & 4 & 6 & \\
\hline 04 & 6131 & 70.1247 & 11.9300 & 293.1 & 4 & .0 & \\
\hline 04 & 6132 & 70.4491 & 11.8825 & 292.7 & 4 & 60 & .0 \\
\hline 04 & 6133 & 65.8001 & 12.5000 & 292.2 & 4 & .0 & \\
\hline 04 & 6134 & 65.3997 & 11.2050 & 291.6 & 4 & .0 & .0 \\
\hline 04 & 6135 & 65.9974 & 10.7400 & 291.4 & 4 & 6 & .0 \\
\hline 04 & 6136 & 69.9749 & 9.6050 & 291.9 & 4 & .0 & \\
\hline 04 & 6137 & 76.6751 & 9.2025 & 292.4 & 4 & .0 & 0.0 \\
\hline 04 & 6138 & 74.3765 & 8.5575 & 292.8 & 4 & .0 & 0.0 \\
\hline 04 & 6139 & 77.2001 & 7.3508 & 294.2 & 4 & 0.0 & .0 \\
\hline 04 & 61310 & 104.1540 & 4.8133 & 297.4 & 3 & 0 & 600.0 \\
\hline 04 & 61311 & 120.6797 & 5.3873 & 299.3 & 3 & 600.0 & 600.0 \\
\hline 04 & 61312 & 79.7996 & 5.1553 & 300.0 & 2 & 600.0 & .0 \\
\hline 04 & 61313 & 85.4060 & 6.3070 & 301.2 & 3 & 600.0 & 600.0 \\
\hline 04 & 61314 & 88.1727 & 6.8078 & 301.6 & 3 & 600.0 & 600.0 \\
\hline 04 & 61315 & 86.5039 & 8.0733 & 301.2 & 4 & 600.0 & 600.0 \\
\hline 04 & 61316 & 75.4250 & 9.6625 & 300.1 & 4 & 600.0 & 600.0 \\
\hline 04 & 61317 & 66.6236 & 11.0150 & 298.6 & 4 & 600.0 & 600.0 \\
\hline 04 & 61318 & 65.6249 & 12.4350 & 296.6 & 4 & 600.0 & 600.0 \\
\hline 04 & 61319 & 62.8750 & 12.4525 & 295.9 & 4 & 600.0 & 600.0 \\
\hline 04 & 61320 & 62.4231 & 12.8425 & 295.5 & 4 & 600.0 & 600.0 \\
\hline 04 & 61321 & 67.1000 & 11.3625 & 295.3 & 4 & 600.0 & 600.0 \\
\hline $0<$ & 61322 & 69.7501 & 10.6475 & 294.6 & 4 & 600.0 & 600.0 \\
\hline 04 & 61323 & 69.4991 & 11.3225 & 294.2 & 4 & 600.0 & 600.0 \\
\hline
\end{tabular}




\begin{tabular}{|c|c|c|c|c|c|c|c|}
\hline 04 & 61324 & 75.2251 & 3850 & 293.7 & 4 & 600.0 & 600.0 \\
\hline 04 & $614 \quad 1$ & 74.5000 & 11.1750 & 293.4 & 4 & 600.0 & 600.0 \\
\hline 04 & 6142 & 72.0500 & 10.5000 & 293.2 & 4 & 600.0 & 00.0 \\
\hline 04 & 6143 & 73.4000 & 8.7525 & 293.3 & 2 & 600.0 & 00.0 \\
\hline 04 & 6144 & 81.8669 & 7.3775 & 293.6 & 4 & 600.0 & 00.0 \\
\hline 04 & 6145 & 92.0997 & 6.6208 & 293.8 & 4 & 600.0 & 00.0 \\
\hline 04 & 6146 & 120.7610 & 7.0265 & 295.4 & 4 & 600.0 & 600.0 \\
\hline 4 & 6147 & 140.2246 & 10.5550 & 295.7 & 4 & 600.0 & \\
\hline 04 & 6148 & 159.7743 & 12.2525 & 296.2 & 4 & 600.0 & 600.0 \\
\hline 04 & 6149 & 160.4752 & 9.8875 & 297.1 & 4 & 600.0 & 600.0 \\
\hline 4 & 61410 & 152.8997 & 8.5 & 297.9 & 4 & 600.0 & \\
\hline 04 & 61411 & 159.7837 & 10.2900 & 299.8 & 3 & 600.0 & 60 \\
\hline 04 & 61412 & 159.9529 & 8.4675 & 301.2 & 3 & 600.0 & \\
\hline 04 & 61413 & 151.6064 & 7.3450 & 302.7 & 3 & 600.0 & 00.0 \\
\hline 04 & 61414 & 158.4169 & 7. & 303.7 & 4 & 600.0 & 60 \\
\hline 04 & 61415 & 126.1043 & 5.7253 & 304.1 & 3 & 600.0 & 600.0 \\
\hline 04 & 61416 & 80.7447 & 5.8975 & 303.2 & 3 & 600.0 & .0 \\
\hline 04 & 61417 & 67.7249 & 6 . & 302.8 & 4 & .0 & \\
\hline 04 & 61418 & 68.7759 & 6.9785 & 302.5 & 4 & 600.0 & .0 \\
\hline 04 & 61419 & 64.2000 & 7.66 & 300.9 & 4 & .0 & .0 \\
\hline 04 & 61420 & 67.8749 & 8 . & 299.9 & 4 & .0 & \\
\hline 04 & 61421 & 73.0212 & 8 . & 299.6 & 4 & .0 & \\
\hline 04 & 61422 & 72.6537 & 9.11 & 299.7 & 4 & .0 & 60 \\
\hline 04 & 61423 & 76.1755 & 9.12 & 300.0 & 4 & .0 & \\
\hline 04 & 61424 & 75.9749 & 8.23 & 30 & 4 & 60 & \\
\hline 04 & 6151 & 94.9234 & 7.94 & 301.2 & 4 & 600.0 & .0 \\
\hline 04 & 6152 & 129.8737 & 8.18 & 302.0 & 4 & .0 & .0 \\
\hline 04 & 6153 & 142.2489 & 8.5450 & 301.3 & 4 & .0 & .0 \\
\hline 04 & 6154 & 144.5878 & 7.4338 & 299.8 & 4 & .0 & 600.0 \\
\hline 04 & 6155 & 134.2300 & 10.31 & 299.0 & 4 & .0 & .0 \\
\hline 04 & 6156 & 150.5251 & 12 . & 297.4 & 4 & .0 & \\
\hline 04 & 6157 & 154.4251 & 675 & 297.2 & 4 & .0 & 600.0 \\
\hline 04 & 6158 & 158.3255 & 8.0675 & 297.5 & 4 & .0 & 600.0 \\
\hline 04 & 6159 & 168.8250 & 9 . & 299.3 & 4 & .0 & 600.0 \\
\hline 04 & 61510 & 161.7157 & 7 . & 300.3 & 4 & .0 & 60 \\
\hline 04 & 61511 & 163.3533 & 7.2875 & 301.9 & 3 & 600.0 & 600.0 \\
\hline 04 & 61512 & 159.5746 & 7 . & 303.0 & 3 & .0 & 60 \\
\hline 04 & 61513 & 164.9500 & 6.8940 & 304.3 & 3 & 600.0 & 600.0 \\
\hline 04 & 61514 & 166.6780 & 6.8630 & 305.7 & 3 & 600.0 & 600.0 \\
\hline 04 & 61515 & 174.0638 & 7 . & 306.7 & 4 & .0 & 600.0 \\
\hline 04 & 61516 & 176.5358 & 7.6625 & 306.8 & 4 & 600.0 & 600.0 \\
\hline 04 & 61517 & 179.2099 & 7.7500 & 306.9 & 4 & 600.0 & 600.0 \\
\hline 04 & 61518 & 179.0696 & 6.2210 & 306.3 & 4 & 600.0 & 600.0 \\
\hline 04 & 61519 & 111.6325 & 4.7163 & 305.1 & 4 & 600.0 & 600.0 \\
\hline 04 & 61520 & 72.7281 & 7.3373 & 302.7 & 4 & 600.0 & 600.0 \\
\hline 04 & 61521 & 77.1000 & 9. & 301.4 & 4 & 600.0 & \\
\hline 04 & 61522 & 71.0499 & 10.3200 & 300.5 & 4 & 600.0 & 600.0 \\
\hline 04 & 61523 & 70.3250 & 10.355 & 300.2 & 4 & 600.0 & 600.0 \\
\hline
\end{tabular}




\begin{tabular}{|c|c|c|c|c|c|c|c|}
\hline 4 & 61524 & 71.8001 & 10.5550 & 300.5 & 4 & 600.0 & 00.0 \\
\hline 04 & 6161 & 69.9752 & 9.6525 & 300.8 & 4 & 600.0 & 600.0 \\
\hline 04 & 6162 & 76.0751 & 8.9825 & 301.3 & 4 & 600.0 & 00.0 \\
\hline 04 & 6163 & 85.6749 & 7.6448 & 301.3 & 4 & 600.0 & 00.0 \\
\hline 04 & 6164 & 107.4791 & 5.7023 & 301.6 & 4 & 600.0 & 00.0 \\
\hline 04 & 6165 & 125.8487 & 5.8558 & 301.2 & 4 & 600.0 & 00.0 \\
\hline 04 & 6166 & 144.5052 & 6.3555 & 300.4 & 4 & 600.0 & 600.0 \\
\hline 04 & 6167 & 153.4250 & 9.8550 & 300.6 & 4 & 600.0 & 00.0 \\
\hline 04 & 6168 & 159.1747 & 10.8525 & 301.0 & 4 & 600.0 & 00.0 \\
\hline 04 & 6169 & 160.0492 & 9.0700 & 301.7 & 4 & 600.0 & 600.0 \\
\hline 04 & 61610 & 155.0750 & 7.0818 & 302.3 & 4 & 600.0 & 0 \\
\hline 04 & 61611 & 155.1481 & 508 & 303.8 & 3 & 600.0 & \\
\hline 04 & 61612 & 161.2000 & 7.2865 & 305.0 & 3 & 600.0 & 00.0 \\
\hline 04 & 61613 & 150.7471 & 7.5348 & 305.5 & 3 & 600.0 & 600 \\
\hline 04 & 61614 & 137.9069 & 6.9215 & 306.2 & 3 & 600.0 & \\
\hline 04 & 61615 & 111.2882 & 6.6700 & 305.9 & 4 & 600.0 & 60 \\
\hline 04 & 61616 & 89.3988 & 6.3128 & 305.3 & 4 & 600.0 & 600.0 \\
\hline 04 & 61617 & 74.4750 & 7.09 & 304.3 & 4 & 600.0 & \\
\hline 04 & 61618 & 76.4481 & 8.5375 & 301.4 & 4 & 600.0 & .0 \\
\hline 04 & 61619 & 78.7499 & 10.5150 & 298.0 & 4 & 600.0 & .0 \\
\hline 04 & 61620 & 73.8000 & 11.1950 & 295.9 & 4 & 600.0 & .0 \\
\hline 04 & 61621 & 74.6250 & 10 . & 295.4 & 4 & 0.0 & \\
\hline 04 & 61622 & 70.7250 & 10.9450 & 294.8 & 4 & 600.0 & \\
\hline 04 & 61623 & 74.5280 & 7.9315 & 293.0 & 4 & 600.0 & .0 \\
\hline 04 & 61624 & 93.5507 & 528 & 293.2 & 4 & 00.0 & \\
\hline 04 & 6171 & 111.1329 & 2.6248 & 291.7 & 4 & 600.0 & 60 \\
\hline 04 & 6172 & 138.8500 & 1.6535 & 289.9 & 5 & 600.0 & .0 \\
\hline 04 & 6173 & 156.8298 & 1.2325 & 289.3 & 6 & 00.0 & \\
\hline 04 & 6174 & 151.9485 & 1.1093 & 288.9 & 6 & 600.0 & 60 \\
\hline 04 & 6175 & 82.2237 & 0.9080 & 288.3 & 6 & 600.0 & 600 \\
\hline 04 & 6176 & 96.5858 & 0 . & 289.6 & 5 & 600.0 & \\
\hline 04 & 6177 & 244.4515 & $1 . \varepsilon$ & 289.6 & 4 & 600.0 & .0 \\
\hline 04 & 6178 & 41.9706 & 2.9065 & 290.4 & 3 & 600.0 & 600.0 \\
\hline 04 & 6179 & 71.8333 & 3.2375 & 292.0 & 2 & 600.0 & \\
\hline 04 & 61710 & 236 . & 3.16 & 29 & 2 & 600.0 & \\
\hline 04 & 61711 & 220.7523 & 3.8993 & 296.3 & 2 & 600.0 & 600.0 \\
\hline 04 & 61712 & 215.6910 & 4.0915 & 298.1 & 2 & 600.0 & \\
\hline 04 & 61713 & 185.6878 & 6.6115 & 298.7 & 3 & 600.0 & .0 \\
\hline 04 & 61714 & 202.1100 & 6.2308 & 298.4 & 3 & 600.0 & 600.0 \\
\hline 04 & 61715 & 192.3368 & 6.2545 & 298.3 & 3 & 600.0 & \\
\hline 04 & 61716 & 182.7191 & 5.0993 & 298.1 & 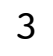 & 600.0 & 60 \\
\hline 04 & 61717 & 68.5493 & 4.7913 & 297.9 & 3 & 600.0 & 600.0 \\
\hline 04 & 61718 & 85.4237 & 6.2550 & 295.7 & 4 & 600.0 & 600.0 \\
\hline 04 & 61719 & 67.7242 & 8.4025 & 293.4 & 4 & 600.0 & 600.0 \\
\hline 04 & 61720 & 68.6999 & 9.9175 & 291.0 & 4 & 600.0 & 600.0 \\
\hline 04 & 61721 & 67.9754 & 10.9050 & 289.9 & 4 & 600.0 & 600.0 \\
\hline 04 & 61722 & 72.9751 & 9.6750 & 28 & 4 & 600.0 & 600.0 \\
\hline 04 & 61723 & 72.2251 & 7.5350 & 290.1 & 4 & 600.0 & 600.0 \\
\hline
\end{tabular}




\begin{tabular}{|c|c|c|c|c|c|c|c|}
\hline 04 & 61724 & 78.9466 & 5.6793 & 289.8 & 4 & 600.0 & 600.0 \\
\hline 04 & 6181 & 81.7501 & 7.0198 & 288.2 & 4 & 600.0 & 00,0 \\
\hline 04 & 6182 & 81.3250 & 7.0765 & 287.3 & 4 & 600.0 & 00.0 \\
\hline 04 & 6183 & 80.9000 & 8.4250 & 286.7 & 4 & 600.0 & 00.0 \\
\hline 04 & 6184 & 78.5500 & 9.3750 & 286.4 & 4 & 600.0 & 00.0 \\
\hline 04 & 6185 & 78.1001 & 9.2025 & 286.3 & 4 & 600.0 & 00.0 \\
\hline 04 & 6186 & 79.0000 & 7.1655 & 286.2 & 4 & 600.0 & 00.0 \\
\hline 04 & 6187 & 75.0750 & 6.0630 & 286.9 & 3 & 600.0 & 00.0 \\
\hline 04 & 6188 & 94.6333 & 2.8458 & 289.6 & 3 & 600.0 & 00.0 \\
\hline 04 & 6189 & 284.1724 & 2.4225 & 292.1 & 2 & 60 & .0 \\
\hline 04 & 61810 & 247.6127 & 2.9410 & 293.6 & 2 & 600.0 & \\
\hline 04 & 61811 & 235.5874 & 3.2713 & 295.0 & 2 & 600.0 & \\
\hline 04 & 61812 & 238.1967 & 3.6028 & 296.6 & 2 & 600.0 & 00.0 \\
\hline 04 & 61813 & 237.1131 & 3.2553 & 297.8 & 1 & .0 & 00.0 \\
\hline 04 & 61814 & 308.2613 & 3.2760 & 299.3 & 2 & .0 & \\
\hline 04 & 61815 & 68.7007 & 5.4565 & 299.2 & 2 & 600.0 & .0 \\
\hline 04 & 61816 & 89.4236 & 8.0475 & 297.2 & 3 & 60 & .0 \\
\hline 04 & 61817 & 86.4240 & 7.2955 & 296.4 & 4 & .0 & \\
\hline 04 & 61818 & 79.9252 & 7.8300 & 294.8 & 4 & .0 & \\
\hline 04 & 61819 & 73.4753 & 9.0475 & 293.1 & 4 & .0 & .0 \\
\hline 04 & 61820 & 66.1249 & 10.56 & 290.6 & 4 & 6 & \\
\hline 04 & 61821 & 71.2285 & 11. & 288.9 & 4 & .0 & \\
\hline 04 & 61822 & 76.3750 & 11.4650 & 288.4 & 4 & 600.0 & .0 \\
\hline 04 & 61823 & 75.0250 & 13.4 & 287.9 & 4 & 6 & \\
\hline 04 & 61824 & 76.4747 & 11. & 287.8 & 4 & 6 & \\
\hline 04 & 6191 & 80.2333 & 10.8300 & 287.5 & 4 & .0 & \\
\hline 04 & 6192 & 80.9500 & 12.0200 & 286.8 & 4 & .0 & .0 \\
\hline 04 & 6193 & 69.7273 & 12.5825 & 286.9 & 4 & .0 & \\
\hline 04 & 6194 & 74.8262 & 12.9325 & 286.6 & 4 & .0 & .0 \\
\hline 04 & 6195 & 74.8999 & 11.6225 & 286.9 & 4 & 60 & .0 \\
\hline 04 & 6196 & 78.4988 & 10.8175 & 287.1 & 4 & .0 & \\
\hline 04 & 6197 & 86.0743 & 5.14 & 289.2 & 3 & .0 & 0.0 \\
\hline 04 & 6198 & 90.0249 & 6.5585 & 289.4 & 4 & 600.0 & 600.0 \\
\hline 04 & 6199 & 80.7745 & 5. & 291.8 & 3 & 600.0 & .0 \\
\hline 04 & 61910 & 85.6250 & 6.06 & 293.3 & 3 & 600.0 & 0.0 \\
\hline 04 & 61911 & 83.3750 & 4.7515 & 295.1 & 2 & 600.0 & 600.0 \\
\hline 04 & 61912 & 78.1762 & 6.16 & 296.1 & 2 & 600.0 & .0 \\
\hline 04 & 61913 & 86.9258 & 7.0708 & 296.9 & 3 & 600.0 & 600.0 \\
\hline 04 & 61914 & 76.8774 & 8.8000 & 297.2 & 3 & 600.0 & 600.0 \\
\hline 04 & 61915 & 69.2749 & 9.23 & 297.9 & 4 & 60 & .0 \\
\hline 04 & 61916 & 69.7981 & 9.5150 & 297.7 & 4 & 600.0 & 600.0 \\
\hline 04 & 61917 & 69.0753 & 10.6975 & 296.7 & 4 & 600.0 & 600.0 \\
\hline 04 & 61918 & 65.4250 & 11.6800 & 296.4 & 4 & 600.0 & 600.0 \\
\hline 04 & 61919 & 65.9993 & 12.5650 & 294.7 & 4 & 600.0 & 600.0 \\
\hline 04 & 61920 & 64.0250 & 13.2325 & 293.9 & 4 & 600.0 & 600.0 \\
\hline 04 & 61921 & 64.3984 & 12.6125 & 292.8 & 4 & 600.0 & 600.0 \\
\hline $0<$ & 61922 & 68.0001 & 12.712 & 291.9 & 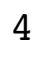 & 600.0 & 600.0 \\
\hline 04 & 61923 & 66.1751 & 12.387 & 291.6 & 4 & 600.0 & 600.0 \\
\hline
\end{tabular}




\begin{tabular}{|c|c|c|c|c|c|c|c|}
\hline 4 & 61924 & 70.9250 & 12.2250 & 290.2 & 4 & 600.0 & 600.0 \\
\hline 04 & 6201 & 74.5500 & 12.2575 & 289.6 & 4 & 600.0 & 600.0 \\
\hline 04 & 6202 & 76.7250 & 12.5550 & 288.3 & 4 & 600.0 & 00.0 \\
\hline 04 & 6203 & 76.9500 & 11.7175 & 288.6 & 4 & 600.0 & 00.0 \\
\hline 04 & 6204 & 75.4251 & 11.5150 & 288.5 & 4 & 600.0 & 00.0 \\
\hline 04 & 6205 & 75.4252 & 11.5575 & 288.8 & 4 & 600.0 & 00.0 \\
\hline 04 & 6206 & 76.9750 & 10.4350 & 289.5 & 4 & 600.0 & 0 \\
\hline 04 & 6207 & 80.4925 & 9.8000 & 290.0 & 4 & 600.0 & 00.0 \\
\hline 04 & 6208 & 82.6505 & 7.2583 & 292.4 & 4 & 600.0 & 00.0 \\
\hline 04 & 6209 & 90.7250 & 6.9450 & 293.6 & 4 & 60 & 0 \\
\hline 04 & 62010 & 102.1412 & 5.3365 & 296.1 & 3 & 600.0 & \\
\hline 04 & 62011 & 97.2530 & 343 & 297.3 & 2 & 600.0 & \\
\hline 04 & 62012 & 86.6756 & 5.1278 & 298.6 & 2 & 600.0 & .0 \\
\hline 04 & 62013 & 90.6242 & 7.1673 & 298.4 & 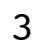 & 600.0 & \\
\hline 04 & 62014 & 84.8437 & & 298.8 & 3 & .0 & \\
\hline 04 & 62015 & 76.9251 & 00 & 298.2 & 4 & 600.0 & .0 \\
\hline 04 & 62016 & 70.9197 & 9.7975 & 297.4 & 4 & 60 & .0 \\
\hline 04 & 62017 & 76.2250 & 9.7625 & 297.2 & 4 & .0 & \\
\hline 04 & 62018 & 67.9750 & 10 & 296.1 & 4 & 60 & \\
\hline 04 & 62019 & 71.1250 & 9.7825 & 294.9 & 4 & .0 & .0 \\
\hline 04 & 62020 & 71.4255 & 11. & 292.8 & 4 & 6 & \\
\hline 04 & 62021 & 63.5250 & 13. & 291.4 & 4 & .0 & \\
\hline 04 & 62022 & 64.8000 & 13.5900 & 291.1 & 4 & 600.0 & .0 \\
\hline 04 & 62023 & 63.3751 & 13. & 291.4 & 4 & 6 & \\
\hline 04 & 62024 & 68.2250 & 13 & 29 & 4 & 6 & \\
\hline 04 & 6211 & 72.2000 & 13.2525 & 290.4 & 4 & .0 & \\
\hline 04 & 6212 & 72.8750 & 12.5550 & 289.4 & 4 & 60 & .0 \\
\hline 04 & 6213 & 73.5500 & 12.4250 & 289.1 & 4 & .0 & \\
\hline 04 & 6214 & 74.3249 & 12 . & 289.2 & 4 & .0 & .0 \\
\hline 04 & 6215 & 74.6751 & 11.9425 & 289.4 & 4 & 6 & .0 \\
\hline 04 & 6216 & 73.0250 & 11.7600 & 288.5 & 4 & .0 & \\
\hline 04 & 6217 & 74.4749 & 11 . & 28 & 4 & .0 & 0 \\
\hline 04 & 6218 & 77.8000 & 10.5225 & 290.6 & 4 & .0 & 0.0 \\
\hline 04 & 6219 & 86.7556 & 7.2850 & 293.5 & 4 & 0.0 & .0 \\
\hline 04 & 62110 & 90.0740 & 5.3798 & 295.7 & 3 & 600.0 & 600.0 \\
\hline 04 & 62111 & 88.7249 & 5.2865 & 297.3 & 2 & 600.0 & 600.0 \\
\hline 04 & 62112 & 84.8501 & 5.8718 & 298.1 & 3 & 600.0 & .0 \\
\hline 04 & 62113 & 85.6501 & 6.5033 & 299.7 & 3 & 600.0 & 600.0 \\
\hline 04 & 62114 & 89.0750 & 7.6550 & 300.2 & 3 & 600.0 & 600.0 \\
\hline 04 & 62115 & 85.3257 & 7.1390 & 300.2 & 4 & 600.0 & 600.0 \\
\hline 04 & 62116 & 73.2498 & 8.9150 & 299.7 & 4 & 600.0 & 600.0 \\
\hline 04 & 62117 & 67.9999 & 10.0175 & 298.2 & 4 & 600.0 & 600.0 \\
\hline 04 & 62118 & 74.5503 & 10.5600 & 297.4 & 4 & 600.0 & 600.0 \\
\hline 04 & 62119 & 70.9501 & 12.2375 & 296.2 & 4 & 600.0 & 600.0 \\
\hline 04 & 62120 & 65.5748 & 11.6575 & 294.5 & 4 & 600.0 & 600.0 \\
\hline 04 & 62121 & 72.8499 & 11.4050 & 294.2 & 4 & 600.0 & 600.0 \\
\hline $0<$ & 62122 & 70.6000 & 11.9900 & 293.9 & 4 & 600.0 & 600.0 \\
\hline 0 & 62123 & 72.1250 & 12.6850 & 292.2 & 4 & 600.0 & 600.0 \\
\hline
\end{tabular}




\begin{tabular}{|c|c|c|c|c|c|c|c|}
\hline 4 & 62124 & 72.5252 & 12.4525 & 292.6 & 4 & 600.0 & 600.0 \\
\hline 04 & 6221 & 72.5250 & 12.2100 & 291.6 & 4 & 600.0 & 00,0 \\
\hline 4 & 6222 & 76.9248 & 12.6500 & 291.0 & 4 & 600.0 & 00.0 \\
\hline 4 & 6223 & 73.7000 & 13.3800 & 290.1 & 4 & 600.0 & 0 \\
\hline 04 & 6224 & 76.9001 & 12 & 289.7 & 4 & 00.0 & .0 \\
\hline 04 & 6225 & 74.2000 & 11.6000 & 288.5 & 4 & 600.0 & 00.0 \\
\hline 04 & 6226 & 75.7747 & 10.9000 & 288.4 & 4 & 600.0 & 0 \\
\hline 04 & 6227 & 74.9999 & 9.8175 & 289.7 & 4 & 600.0 & 00.0 \\
\hline 04 & 6228 & 72.4997 & 7.1810 & 291.9 & 4 & 600.0 & 00.0 \\
\hline 04 & 6229 & 75.8745 & 03 & 292.7 & 4 & 60 & 0 \\
\hline 04 & 62210 & 69.0501 & 15 & 294.6 & 3 & 600.0 & \\
\hline 04 & 62211 & 67.6161 & 48 & 296.7 & 2 & .0 & \\
\hline 04 & 62212 & 71.5500 & 00 & 297.4 & 3 & 600.0 & .0 \\
\hline 04 & 62213 & 62.5999 & 9. & 298.0 & - & 600.0 & \\
\hline 04 & 62214 & 66.8250 & 11. & 297.4 & 3 & .0 & \\
\hline 04 & 62215 & 71.0744 & 11. & 297.4 & 4 & 60 & \\
\hline 04 & 62216 & 70.9000 & 11.7125 & 297.1 & 4 & .0 & .0 \\
\hline 04 & 62217 & 67.6003 & $13 . \varepsilon$ & 295.8 & 4 & .0 & \\
\hline 04 & 62218 & 63.8240 & 14. & 294.7 & 4 & 60 & \\
\hline 04 & 62219 & 64.6749 & 14.4300 & 294.4 & 4 & .0 & .0 \\
\hline 04 & 62220 & 500 & 15 . & 293.6 & 4 & 6 & \\
\hline 04 & 62221 & 68.4755 & 14 . & .0 & 4 & .0 & \\
\hline 04 & 62222 & 66.0001 & 12.3500 & 293.8 & 4 & 600.0 & .0 \\
\hline 04 & 62223 & 64.0749 & 11.5 & 294.2 & 4 & 0 & \\
\hline 04 & 62224 & 998 & 10 & 294.2 & 4 & 0 & \\
\hline 04 & 6231 & 75.4252 & 10 & 292.9 & 4 & .0 & .0 \\
\hline 04 & 6232 & 68.7745 & 10.5075 & 292.5 & 4 & 60 & .0 \\
\hline 04 & 6233 & 73.1998 & 10 . & 292.4 & 4 & .0 & \\
\hline 04 & 6234 & 75.7999 & 10 & 292.4 & 4 & .0 & \\
\hline 04 & 6235 & 70.9251 & 11.3025 & 292.1 & 4 & 6 & \\
\hline 04 & 6236 & 70.7250 & 11 & 292.2 & 4 & .0 & \\
\hline 04 & 6237 & 72.4498 & 25 & 293.5 & 4 & .0 & .0 \\
\hline 04 & 6238 & 79.7467 & 7.8475 & 294.9 & 4 & 6 & .0 \\
\hline 04 & 6239 & 114.5706 & 4 . & 296.6 & 3 & .0 & .0 \\
\hline 04 & 62310 & 90.6489 & & 29 & 3 & .0 & .0 \\
\hline 04 & 62311 & 91.0748 & 6.4515 & 296.9 & 3 & 600.0 & 0.0 \\
\hline 04 & 62312 & 87.4756 & 6.71 & 297.9 & 3 & 600.0 & .0 \\
\hline 04 & 62313 & 72.9246 & 5.9233 & 299.2 & 3 & 600.0 & 600.0 \\
\hline 04 & 62314 & 72.4390 & 7.8000 & 298.9 & 3 & 600.0 & 600.0 \\
\hline 04 & 62315 & 69.8500 & 9.2925 & 298.1 & 4 & .0 & .0 \\
\hline 04 & 62316 & 73.2751 & 10.9250 & 297.3 & 4 & 600.0 & 600.0 \\
\hline 04 & 62317 & 71.8249 & 11.6400 & 296.2 & 4 & 600.0 & 600.0 \\
\hline 04 & 62318 & 73.2998 & 11.7950 & 294.8 & 4 & 600.0 & 600.0 \\
\hline 04 & 62319 & 70.4750 & 13.0650 & 292.7 & 4 & 600.0 & 600.0 \\
\hline 04 & 62320 & 69.7750 & 14.7125 & 290.1 & 4 & 600.0 & 600.0 \\
\hline 04 & 62321 & 68.2750 & 14.7825 & 290.6 & 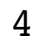 & 600.0 & \\
\hline 04 & 62322 & 69.8499 & 14.8475 & 291.3 & 4 & 600.0 & 600.0 \\
\hline 0 & 62323 & 70.4500 & 15.0050 & 291.4 & & 600.0 & 600.0 \\
\hline
\end{tabular}




\begin{tabular}{|c|c|c|c|c|c|c|c|}
\hline 4 & 62324 & 75.2500 & 14.1825 & 289.5 & 4 & 600.0 & 600.0 \\
\hline 04 & 6241 & 71.4245 & 13.6325 & 289.1 & 4 & 600.0 & 600.0 \\
\hline 04 & 6242 & 70.7500 & 12.6150 & 289.6 & 4 & 600.0 & 00.0 \\
\hline 04 & 6243 & 69.7000 & 11.7350 & 290.4 & 4 & 600.0 & 00.0 \\
\hline 04 & 6244 & 72.9000 & 11.3325 & 289.9 & 4 & 600.0 & 00.0 \\
\hline 04 & 6245 & 71.5500 & 11.5675 & 289.7 & 4 & 600.0 & 00.0 \\
\hline 04 & 6246 & 69.6240 & 11.9225 & 289.3 & 4 & 600.0 & 00.0 \\
\hline 04 & 6247 & 67.2752 & 10.7700 & 291.6 & 4 & 600.0 & 00.0 \\
\hline 04 & 6248 & 64.4486 & 9.5550 & 293.3 & 4 & 600.0 & 00.0 \\
\hline 04 & 6249 & 71.2757 & 8.7325 & 294.5 & 4 & 60 & 0 \\
\hline 04 & 62410 & 77.5251 & 7.6075 & 296.2 & 4 & 600.0 & \\
\hline 04 & 62411 & 79.2253 & 7.6780 & 296.8 & 3 & .0 & \\
\hline 04 & 62412 & 81.0779 & 7.7100 & 298.0 & 3 & 600.0 & 00.0 \\
\hline 04 & 62413 & 88.6159 & 7.8638 & 298.4 & - & 600.0 & \\
\hline 04 & 62414 & 90.7708 & 13 & 298.9 & 3 & .0 & \\
\hline 04 & 62415 & 90.2761 & 8.8675 & 298.4 & 4 & 600.0 & .0 \\
\hline 04 & 62416 & 94.3533 & 9.0675 & 298.4 & 4 & 60 & .0 \\
\hline 04 & 62417 & 67.3504 & 11.6700 & 297.8 & 4 & .0 & \\
\hline 04 & 62418 & 67.6500 & 12.0100 & 296.8 & 4 & .0 & \\
\hline 04 & 62419 & 62.9000 & 12.2975 & 295.1 & 4 & .0 & .0 \\
\hline 04 & 62420 & 65.2252 & 12.4950 & 294.2 & 4 & 6 & \\
\hline 04 & 62421 & 61.0000 & 12.9050 & 294.6 & 4 & .0 & \\
\hline 04 & 62422 & 60.4500 & 13.8625 & 295.1 & 4 & 600.0 & .0 \\
\hline 04 & 62423 & 57.7750 & 13.3300 & 294.8 & 4 & 6 & \\
\hline 04 & 62424 & 60.5500 & 12.1825 & 295.0 & 4 & 6 & \\
\hline 04 & 6251 & 63.7000 & 11.1075 & 294.8 & 4 & .0 & \\
\hline 04 & 6252 & 63.6500 & 11.6850 & 294.1 & 4 & 60 & .0 \\
\hline 04 & 6253 & 63.5748 & 10.7125 & 294.0 & 4 & .0 & \\
\hline 04 & 6254 & 64.1999 & 10.6150 & 293.6 & 4 & .0 & .0 \\
\hline 04 & 6255 & 65.5250 & 11.7700 & 292.6 & 4 & .0 & .0 \\
\hline 04 & 6256 & 63.4251 & 11.9325 & 292.5 & 4 & .0 & \\
\hline 04 & 6257 & 72.4998 & 13.3800 & 292.4 & 4 & .0 & 0 \\
\hline 04 & 6258 & 74.0001 & 10.9500 & 293.6 & 4 & .0 & 0.0 \\
\hline 04 & 6259 & 79.3000 & 8.5275 & 295.3 & 4 & 0 & .0 \\
\hline 04 & 62510 & 75.4989 & 8.4875 & 296.1 & 4 & .0 & 0.0 \\
\hline 04 & 62511 & 85.4589 & 6.8433 & 297.9 & 3 & 600.0 & 600.0 \\
\hline 04 & 62512 & 97.0500 & 7.0815 & 299.0 & 3 & 600.0 & .0 \\
\hline 04 & 62513 & 89.2007 & 7.6625 & 300.4 & 3 & 600.0 & 600.0 \\
\hline 04 & 62514 & 78.9489 & 8.6975 & 300.6 & 3 & 600.0 & 600.0 \\
\hline 04 & 62515 & 70.3980 & 9.7500 & 300.1 & 4 & 600.0 & 600.0 \\
\hline 04 & 62516 & 71.7252 & 9.4550 & 299.5 & 4 & 600.0 & 600.0 \\
\hline 04 & 62517 & 71.6751 & 10.1275 & 299.1 & 4 & 600.0 & 600.0 \\
\hline 04 & 62518 & 73.1000 & 10.6850 & 298.4 & 4 & 600.0 & 600.0 \\
\hline 04 & 62519 & 69.0750 & 11.1775 & 297.1 & 4 & 600.0 & 600.0 \\
\hline 04 & 62520 & 71.1002 & 10.9975 & 296.1 & 4 & 600.0 & 600.0 \\
\hline 04 & 62521 & 71.1247 & 11.4275 & 295.2 & 4 & 600.0 & 600.0 \\
\hline 04 & 62522 & 71.2000 & 11.8275 & 294.9 & 4 & 600.0 & 600.0 \\
\hline 0 & 62523 & 68.9250 & 11.0875 & 294.2 & 4 & 600.0 & 600.0 \\
\hline
\end{tabular}




\begin{tabular}{|c|c|c|c|c|c|c|c|}
\hline 4 & 62524 & 63.4731 & 11.9075 & 293.6 & 4 & 600.0 & 600.0 \\
\hline 04 & 6261 & 62.2000 & 12.2700 & 293.3 & 4 & 600.0 & 00,0 \\
\hline 4 & 6262 & 65.2500 & 12.4700 & 292.8 & 4 & 600.0 & 00.0 \\
\hline 4 & 6263 & 66.0250 & 12.6925 & 292.6 & 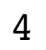 & 600.0 & 00.0 \\
\hline 04 & 6264 & 71.1250 & 11.7500 & 292.5 & 4 & 600.0 & 00.0 \\
\hline 04 & 6265 & 72.7001 & 11.6625 & 292.4 & 4 & 600.0 & 00.0 \\
\hline 04 & 6266 & 75.6500 & 12.1325 & 292.4 & 4 & 600.0 & 0 \\
\hline 04 & 6267 & 71.0228 & 10.9975 & 292.7 & 4 & 600.0 & 00.0 \\
\hline 04 & 6268 & 70.8508 & 10.3900 & 293.2 & 4 & 600.0 & 00.0 \\
\hline 04 & 6269 & 67.8500 & 9.5925 & 294.1 & 4 & 60 & 0 \\
\hline 04 & 62610 & 78.0739 & 6.5990 & 296.3 & 3 & 600.0 & \\
\hline 04 & 62611 & 96.0467 & 6.0875 & 298.0 & 3 & .0 & \\
\hline 04 & 62612 & 82.1005 & 7.3650 & 299.2 & 3 & 600.0 & 00.0 \\
\hline 04 & 62613 & 70.9750 & 9.3350 & 299.1 & - & 600.0 & \\
\hline 04 & 62614 & 70.2751 & 9. & 299.2 & 3 & .0 & \\
\hline 04 & 62615 & 76.7990 & 9.6950 & 299.3 & 4 & 600.0 & .0 \\
\hline 04 & 62616 & 67.5240 & 9.3800 & 299.1 & 4 & .0 & .0 \\
\hline 04 & 62617 & 58.0750 & 12.8475 & 298.4 & 4 & .0 & \\
\hline 04 & 62618 & 58.0749 & 12.4875 & 297.8 & 4 & .0 & \\
\hline 04 & 62619 & 62.2498 & 11.5725 & 296.3 & 4 & .0 & .0 \\
\hline 04 & 62620 & 750 & 11. & 294.5 & 4 & 6 & \\
\hline 04 & 62621 & 63.7250 & 12.0550 & 293.4 & 4 & .0 & \\
\hline 04 & 62622 & 66.9497 & 11.3375 & 293.2 & 4 & 600.0 & .0 \\
\hline 04 & 62623 & 67.6750 & 11.08 & 293.0 & 4 & 0 & \\
\hline 04 & 62624 & 70.7749 & 11.2625 & 292.8 & 4 & 0 & \\
\hline 04 & 6271 & 71.5500 & 11.7000 & 292.7 & 4 & 60 & .0 \\
\hline 04 & 6272 & 72.7750 & 10.5675 & 292.0 & 4 & 60 & .0 \\
\hline 04 & 6273 & 72.4259 & 9.6525 & 291.5 & 4 & .0 & \\
\hline 04 & 6274 & 75.8754 & 9.2475 & 291.7 & 4 & .0 & .0 \\
\hline 04 & 6275 & 78.2500 & 9.0425 & 291.8 & 4 & 6 & .0 \\
\hline 04 & 6276 & 82.6000 & 5.9920 & 292.8 & 4 & .0 & \\
\hline 04 & 6277 & 128.7844 & 3.1295 & 295.1 & 3 & .0 & .0 \\
\hline 04 & 6278 & 162.8789 & 4.1053 & 296.8 & 3 & 6 & 0.0 \\
\hline 04 & 6279 & 162.9497 & 5.4608 & 297.4 & 3 & .0 & .0 \\
\hline 04 & 62710 & 156.9236 & 5.9723 & 298.5 & 3 & 0 & 0 \\
\hline 04 & 62711 & 158.7261 & 7.1153 & 299.5 & 3 & 600.0 & 0.0 \\
\hline 04 & 62712 & 160.8014 & 6.3605 & 301.2 & 3 & 600.0 & .0 \\
\hline 04 & 62713 & 170.9995 & 5.9238 & 302.5 & 3 & 600.0 & 600.0 \\
\hline 04 & 62714 & 188.9344 & 6.8863 & 303.5 & 3 & 600.0 & 600.0 \\
\hline 04 & 62715 & 183.1550 & 6.5418 & 303.6 & 3 & 600.0 & .0 \\
\hline 04 & 62716 & 128.1098 & 5.4463 & 304.2 & 3 & 600.0 & 600.0 \\
\hline 04 & 62717 & 98.5404 & 5.7603 & 303.7 & 4 & 600.0 & 600.0 \\
\hline 04 & 62718 & 81.7002 & 6.4200 & 302.2 & 4 & 600.0 & .0 \\
\hline 04 & 62719 & 69.4749 & 6.8473 & 300.5 & 4 & 600.0 & 600.0 \\
\hline 04 & 62720 & 68.5250 & 8.6700 & 298.9 & 4 & 600.0 & 600.0 \\
\hline 04 & 62721 & 67.5248 & 9.1825 & 298.7 & 4 & 600.0 & \\
\hline 04 & 62722 & 70.8009 & 8.3850 & 298.7 & 4 & 600.0 & 600.0 \\
\hline 0 & 62723 & 70.7999 & 9.0900 & 298.7 & 4 & 600.0 & 600.0 \\
\hline
\end{tabular}




\begin{tabular}{|c|c|c|c|c|c|c|c|}
\hline 4 & 62724 & 70.2002 & 8.2175 & 298.2 & 4 & 600.0 & 600.0 \\
\hline 04 & 6281 & 72.3249 & 7.2283 & 298.3 & 4 & 600.0 & 600.0 \\
\hline 04 & 6282 & 59.1952 & 5.0558 & 298.2 & 4 & 600.0 & 00.0 \\
\hline 04 & 6283 & 46.8998 & 3.9360 & 298.6 & 4 & 600.0 & 0 \\
\hline 04 & 6284 & 42.6051 & 2.8285 & 298.4 & 4 & 600.0 & 00.0 \\
\hline 04 & 6285 & 55.7542 & 1.4445 & 297.6 & 5 & 600.0 & 00.0 \\
\hline 04 & 6286 & 73.8670 & 1.2390 & 297.4 & 4 & 600.0 & 0 \\
\hline 04 & 6287 & 171.0148 & 2.3095 & 296.6 & 3 & 600.0 & 00.0 \\
\hline 04 & 6288 & 210.7567 & 2.5570 & 297.7 & 3 & 600.0 & 00.0 \\
\hline 04 & 6289 & 234.7838 & 2.8910 & 298.4 & 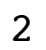 & 60 & 0 \\
\hline 04 & 62810 & 226.6565 & 4.4438 & 299.2 & 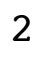 & 600.0 & \\
\hline 04 & 62811 & 226.4963 & 4.3210 & 300.2 & 2 & 600.0 & \\
\hline 04 & 62812 & 215.2379 & 4.1868 & 301.8 & 2 & 600.0 & 00.0 \\
\hline 04 & 62813 & 167.4034 & 4.3485 & 302.9 & 2 & 600.0 & \\
\hline 04 & 62814 & 152.4546 & & 302.8 & 3 & .0 & \\
\hline 04 & 62815 & 158.6704 & 6.6968 & 302.6 & 3 & 600.0 & .0 \\
\hline 04 & 62816 & 123.6666 & 5.0340 & 302.6 & 3 & 60 & .0 \\
\hline 04 & 62817 & 84.3463 & 6.0923 & 300.8 & 4 & .0 & \\
\hline 04 & 62818 & 81.9256 & 7.2918 & 299.1 & 4 & 60 & \\
\hline 04 & 62819 & 73.6251 & 9.5250 & 296.8 & 4 & .0 & .0 \\
\hline 04 & 62820 & 74.3000 & 1. & 294.8 & 4 & 6 & \\
\hline 04 & 62821 & 76.7743 & 575 & 294.9 & 4 & .0 & \\
\hline 04 & 62822 & 76.9000 & 7.3 & 293.9 & 4 & 600.0 & .0 \\
\hline 04 & 62823 & 74.6000 & 10 . & 291.7 & 4 & 0 & \\
\hline 04 & 62824 & 74.5500 & 10. & 292.1 & 4 & 0 & \\
\hline 04 & 6291 & 90.3690 & 7.8940 & 291.7 & 4 & .0 & \\
\hline 04 & 6292 & 79.2499 & 11.2150 & 289.0 & 4 & 60 & .0 \\
\hline 04 & 6293 & 79.6749 & 11.0050 & 288.7 & 4 & .0 & \\
\hline 04 & 6294 & 79.6249 & 11.9675 & 288.6 & 4 & .0 & .0 \\
\hline 04 & 6295 & 79.6250 & 13.91 & 287.6 & 4 & .0 & .0 \\
\hline 04 & 6296 & 82.8750 & 13. & 287.5 & 4 & .0 & \\
\hline 04 & 6297 & 76.2745 & 1 & 287.6 & 4 & .0 & .0 \\
\hline 04 & 6298 & 78.0505 & 10.9425 & 288.7 & 4 & .0 & 0.0 \\
\hline 04 & 6299 & 85.0771 & 8.1525 & 290.5 & 4 & 0 & .0 \\
\hline 04 & 62910 & 89.0250 & 6. & 292.4 & 4 & .0 & 0.0 \\
\hline 04 & 62911 & 87.9251 & 6.8235 & 294.3 & 4 & 600.0 & 600.0 \\
\hline 04 & 62912 & 86.1263 & 6 . & 296.1 & 3 & 600.0 & .0 \\
\hline 04 & 62913 & 70.3499 & 7.9530 & 296.6 & 3 & 600.0 & 600.0 \\
\hline 04 & 62914 & 69.8007 & 8.9750 & 297.0 & 3 & 600.0 & 600.0 \\
\hline 04 & 62915 & 67.8504 & 500 & 297.1 & 4 & 600.0 & .0 \\
\hline 04 & 62916 & 71.5499 & 9.7025 & 296.8 & 4 & 600.0 & 600.0 \\
\hline 04 & 62917 & 71.0238 & 10.8500 & 295.7 & 4 & 600.0 & 600.0 \\
\hline 04 & 62918 & 68.6751 & 10.9775 & 294.0 & 4 & 600.0 & 600.0 \\
\hline 04 & 62919 & 70.0999 & 13.1225 & 292.1 & 4 & 600.0 & 600.0 \\
\hline 04 & 62920 & 72.2254 & 13.3600 & 290.8 & 4 & 600.0 & 600.0 \\
\hline 04 & 62921 & 76.3750 & 12.3650 & 290.3 & 4 & 600.0 & \\
\hline $0<$ & 62922 & 78.1996 & 12.5775 & 289.7 & 4 & 600.0 & 600.0 \\
\hline 04 & 62923 & 77.8264 & 12.3750 & 288.9 & 4 & 600.0 & 600.0 \\
\hline
\end{tabular}




\begin{tabular}{|c|c|c|c|c|c|c|c|}
\hline 4 & 62924 & 69.5999 & 11.0450 & 288.5 & 4 & 600.0 & 00.0 \\
\hline 04 & 6301 & 76.6759 & 11.8325 & 288.3 & 4 & 600.0 & 600,0 \\
\hline 04 & 6302 & 78.0753 & 11.6200 & 288.3 & 4 & 600.0 & 00.0 \\
\hline 04 & 6303 & 68.2753 & 9.7575 & 287.8 & 4 & 600.0 & 00.0 \\
\hline 04 & $630 \quad 4$ & 68.2499 & 7.3368 & 287.3 & 4 & 600.0 & 00.0 \\
\hline 04 & 6305 & 74.0499 & 9.5925 & 287.0 & 4 & 600.0 & 00.0 \\
\hline 04 & 6306 & 77.7250 & 11.0300 & 287.2 & 4 & 600.0 & 00.0 \\
\hline 04 & 6307 & 77.4275 & 8.8975 & 287.9 & 4 & 600.0 & 00.0 \\
\hline 04 & 6308 & 77.5506 & 6.8535 & 289.1 & 4 & 600.0 & 00.0 \\
\hline 04 & 6309 & 85.3975 & 4.9778 & 291.1 & 3 & 600.0 & .0 \\
\hline 04 & 63010 & 96.2808 & 4.1433 & 293.2 & 2 & 600.0 & 500.0 \\
\hline 04 & 63011 & 92.8210 & 3.7695 & 295.1 & 2 & 600.0 & \\
\hline 04 & 63012 & 97.9748 & 3.8543 & 296.7 & 2 & 600.0 & 600.0 \\
\hline 04 & 63013 & 67.4839 & 4.4005 & 297.9 & 2 & 600.0 & .0 \\
\hline 04 & 63014 & 62.3713 & 4.9245 & 298.5 & 2 & 600.0 & \\
\hline 04 & 63015 & 69.9045 & 6.8223 & 297.7 & 3 & 600.0 & 60 \\
\hline 04 & 63016 & 71.7000 & 8.4175 & 296.7 & 4 & 600.0 & 600.0 \\
\hline 04 & 63017 & 70.4250 & 9.58 & 295.3 & 4 & 600.0 & \\
\hline 04 & 63018 & 61.5505 & 9.9300 & 293.6 & 4 & 600.0 & .0 \\
\hline 04 & 63019 & 62.9750 & 10.2600 & 292.0 & 4 & 600.0 & .0 \\
\hline 04 & 63020 & 68.4998 & 11.6700 & 289.6 & 4 & 600.0 & .0 \\
\hline 04 & 63021 & 73. & 13. & 288.2 & 4 & 600.0 & \\
\hline 04 & 63022 & 71.0082 & 13.2150 & 286.8 & 4 & 600.0 & 600.0 \\
\hline 04 & 63023 & 70.9253 & 13. & 286.4 & 4 & 600.0 & .0 \\
\hline 04 & 63024 & 69.0236 & 13. & 285.8 & 4 & 600.0 & \\
\hline 04 & $\begin{array}{lll}7 & 1 & 1\end{array}$ & 69.4250 & 13. & 285.5 & 4 & 600.0 & 60 \\
\hline 04 & $\begin{array}{lll}7 & 1 & 2\end{array}$ & 70.6249 & 13.2150 & 285.6 & 4 & 600.0 & .0 \\
\hline 04 & $\begin{array}{lll}7 & 1 & 3\end{array}$ & 73.2745 & 14 . & 285.8 & 4 & 600.0 & \\
\hline 04 & $\begin{array}{lll}7 & 1 & 4\end{array}$ & 78.0501 & 13. & 286.0 & 4 & 600.0 & 60 \\
\hline 04 & $\begin{array}{lll}7 & 1 & 5\end{array}$ & 77.2500 & 11.8925 & 285.9 & 4 & 600.0 & 60 \\
\hline 04 & $\begin{array}{lll}7 & 1 & 6\end{array}$ & 75.9250 & 10 . & 286.1 & 4 & 600.0 & \\
\hline 04 & $\begin{array}{lll}7 & 1 & 7\end{array}$ & 78.7998 & 9.4375 & 286.8 & 4 & 600.0 & 60 \\
\hline 04 & $\begin{array}{lll}7 & 1 & 8\end{array}$ & 82.3500 & 7.2178 & 288.6 & 4 & 600.0 & 600.0 \\
\hline 04 & $\begin{array}{lll}7 & 1 & 9\end{array}$ & 83.5458 & 4 . & 291.3 & 3 & 600.0 & .0 \\
\hline 04 & 7110 & 78.0254 & 4 . & 292.8 & 2 & 600.0 & .0 \\
\hline 04 & $7 \quad 111$ & 47.5417 & 4.2355 & 294.5 & 2 & 600.0 & 600.0 \\
\hline 04 & $7 \quad 112$ & 64.5271 & 533 & 295.7 & 2 & 600.0 & \\
\hline 04 & $7 \quad 113$ & 90.1763 & 528 & 297.0 & 2 & 600.0 & 600.0 \\
\hline 04 & $7 \quad 114$ & 91.7000 & 7.3075 & 297.6 & 3 & 600.0 & 600.0 \\
\hline 04 & 7115 & 89.3022 & 575 & 297.7 & 4 & 600.0 & \\
\hline 04 & 7116 & 80.8921 & 7.7428 & 297.7 & 4 & 600.0 & 60 \\
\hline 04 & 7117 & 79.2500 & 7.9625 & 297.0 & 4 & 600.0 & 600.0 \\
\hline 04 & 7118 & 75.4749 & 8.9150 & 295.7 & 4 & 600.0 & 600.0 \\
\hline 04 & 7119 & 75.5253 & 11.6000 & 293.5 & 4 & 600.0 & 600.0 \\
\hline 04 & $7 \quad 120$ & 77.3502 & 12.2050 & 292.5 & 4 & 600.0 & 600.0 \\
\hline 04 & $\begin{array}{ll}7 & 121\end{array}$ & 72.0750 & 11.7425 & 293.3 & 4 & 600.0 & 600.0 \\
\hline $0<$ & $7 \quad 122$ & 73.3750 & 11.4525 & 293.6 & 4 & 600.0 & 600.0 \\
\hline 04 & $7 \quad 123$ & 69.6500 & 10.9875 & 293.5 & 4 & 600.0 & 600.0 \\
\hline
\end{tabular}




\begin{tabular}{|c|c|c|c|c|c|c|c|}
\hline 4 & 24 & (1.02 & 9.7500 & 294.2 & 4 & ( & \\
\hline 4 & $\begin{array}{lll}7 & 2 & 1\end{array}$ & 80250 & .9600 & 94.3 & & & \\
\hline & $\begin{array}{lll}722 \\
7\end{array}$ & 249 & 6775 & 94.1 & & 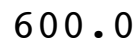 & \\
\hline & $\begin{array}{lll}72 & 3\end{array}$ & 4.9000 & 8.9850 & 293.8 & & 00 & \\
\hline & $7 \quad 24$ & & & 92.6 & & & \\
\hline & 72 & 97 & 75 & 92.7 & & 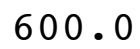 & \\
\hline & 72 & 257 & & 293.6 & & & \\
\hline & 7 & 433 & 763 & 94.0 & & & \\
\hline & 7 & 128 & 1.8338 & 94.3 & & & \\
\hline & 7 & 3270 & & 296.3 & & 0 & \\
\hline & 210 & 234.2117 & 2.2490 & 298.0 & & & \\
\hline & 211 & 3.6618 & & 3 & & & \\
\hline & 12 & 169.2739 & 320 & 301.5 & & 0 & \\
\hline 7 & $7 \quad 213$ & 85.1150 & 4.7423 & & & 0 & \\
\hline & $7 \quad 214$ & & & & & & \\
\hline & 15 & 89.1215 & 188 & 303 & & & \\
\hline 7 & 16 & 87.4999 & 5 & & & & \\
\hline & $7 \quad 217$ & 751 & & & & & \\
\hline 4 & $7 \quad 218$ & 71.2999 & 0 & 6 & 4 & & \\
\hline 4 & 219 & 5501 & 5 & & & & \\
\hline & 7220 & 6.2252 & & 2 & & & \\
\hline & $7 \quad 221$ & 68.6497 & & & 4 & & \\
\hline 7 & 22 & 7749 & 250 & 298 & 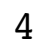 & & \\
\hline 7 & $7 \quad 223$ & 250 & & 298 & 4 & & \\
\hline & $7 \quad 224$ & 0 & & 29 & 4 & & \\
\hline 4 & $\begin{array}{lll}7 & 3 & 1\end{array}$ & 250 & & 29 & A & & \\
\hline 7 & $\begin{array}{lll}7 & 3 & 2\end{array}$ & 750 & & 297 & $x^{2}$ & & \\
\hline 7 & 733 & 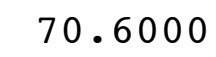 & 5 & 2 & 4 & & \\
\hline 7 & $\begin{array}{lll}7 & 3 & 4\end{array}$ & 70 & 725 & 29 & 4 & & \\
\hline & 735 & & 2900 & 29 & 4 & & \\
\hline 47 & 736 & 2 & & & 4 & & \\
\hline 7 & $\begin{array}{lll}7 & 3 & 7\end{array}$ & 525 & 8 & 29 & 3 & & \\
\hline & 738 & 17.0055 & 533 & 297.3 & & 0 & \\
\hline 47 & 739 & 953 & & 25 & 2 & & \\
\hline & $7 \quad 310$ & 2 & 8 & & 2 & & \\
\hline & $7 \quad 311$ & 227 . & & 300.3 & & & \\
\hline & $7 \quad 312$ & 221.8663 & 5 & 302.2 & 1 & & \\
\hline 7 & $7 \quad 313$ & 100125 & 5 & & & & \\
\hline & $7 \quad 314$ & 69.9473 & 335 & 303.6 & 2 & & \\
\hline & $\begin{array}{ll}7 & 31\end{array}$ & 7 & 993 & 30 & J & & \\
\hline 7 & 7316 & 508 & 7.8100 & & 4 & & \\
\hline 47 & $7 \quad 317$ & 77.0001 & 8.3525 & & 4 & 0 & . \\
\hline & $7 \quad 318$ & & 25 & & 4 & 0 & \\
\hline 47 & $7 \quad 319$ & 68.2500 & 11.8950 & 297.2 & 4 & & \\
\hline 47 & 7320 & 68.4250 & 9.9975 & 297.3 & 4 & 60 & \\
\hline & $7 \quad 321$ & 69.0251 & 1.0300 & 297.5 & 4 & 600 & \\
\hline & $7 \quad 322$ & .1750 & 10.34 & & & & \\
\hline & $7 \quad 323$ & 70.2250 & 9.8300 & 297.2 & & 600.0 & 600 \\
\hline
\end{tabular}




\begin{tabular}{|c|c|c|c|c|c|c|c|c|}
\hline 4 & 7 & 324 & 72.1750 & 9.4050 & 297.0 & 4 & 600.0 & 600.0 \\
\hline 04 & 7 & 41 & 68.3000 & 8.5375 & 297.4 & 4 & 600.0 & 600.0 \\
\hline 04 & 7 & 42 & 84.9931 & 4.9388 & 296.7 & 4 & 600.0 & 00.0 \\
\hline 04 & 7 & 43 & 69.3522 & 3.8098 & 296.6 & 4 & 600.0 & 00.0 \\
\hline 04 & 7 & 44 & 53.7618 & 0.8630 & 294.8 & 5 & 600.0 & 00.0 \\
\hline 04 & 7 & 45 & 92.9537 & 0.9540 & 294.6 & 6 & 600.0 & 00.0 \\
\hline 04 & 7 & 46 & 131.9239 & 1.3483 & 294.7 & 5 & 600.0 & 00.0 \\
\hline 04 & 7 & 47 & 168.7810 & 1.5855 & 294.5 & 4 & 600.0 & 00.0 \\
\hline 04 & 7 & 48 & 246.9208 & 2.2818 & 295.2 & 3 & 600.0 & 00.0 \\
\hline 04 & 7 & 49 & 241.6688 & 735 & 296.2 & 2 & 600.0 & 00.0 \\
\hline 04 & 7 & 410 & 233.2371 & $\mathbf{L} 13$ & 298.7 & 1 & 600.0 & 00.0 \\
\hline 04 & 7 & 411 & 230.3648 & 375 & 300.6 & 2 & 600.0 & \\
\hline 04 & 7 & 412 & 226.1386 & 100 & 302.6 & 2 & 600.0 & 00.0 \\
\hline 04 & 7 & 413 & 188.8010 & 5. & 304.0 & 2 & .0 & 00.0 \\
\hline 04 & 7 & 414 & 165.5908 & 3 & 304.6 & 3 & .0 & \\
\hline 04 & 7 & 415 & 90.3877 & 18 & 305.2 & 2 & 600.0 & 00.0 \\
\hline 04 & 7 & 416 & 106.4849 & 68 & 304.5 & 3 & 600.0 & 600.0 \\
\hline 04 & 7 & 417 & 92.2541 & 6. & 303.6 & 4 & .0 & \\
\hline 04 & 7 & 418 & 83.5752 & 6. & 302.5 & 4 & 600.0 & .0 \\
\hline 04 & 7 & 419 & 72.7501 & 7.4 & 300.8 & 4 & 60 & .0 \\
\hline 04 & 7 & 420 & 72.2500 & 9 . & 299.5 & 4 & 6 & \\
\hline 04 & 7 & 421 & 68.5000 & 10 . & 299.4 & 4 & .0 & \\
\hline 04 & 7 & 422 & 70.9000 & 10.3425 & 299.6 & 4 & 600.0 & 00.0 \\
\hline 04 & 7 & 423 & 70.8748 & 10 & 298.9 & 4 & .0 & \\
\hline 04 & 7 & 424 & 749 & 1 & 298.8 & 4 & 0 & \\
\hline 04 & 7 & 51 & 74.0996 & 10.1 & 298.5 & 4 & 600.0 & 600.0 \\
\hline 04 & 7 & 52 & 74.6500 & 8.9700 & 298.5 & 4 & 600.0 & 600.0 \\
\hline 04 & 7 & 53 & 76.1500 & 7 . & 299.2 & 4 & .0 & \\
\hline 04 & 7 & 54 & 75.9482 & 6.9763 & 298.9 & 4 & 600.0 & 00.0 \\
\hline 04 & 7 & 55 & 9.0850 & 1.4165 & 296.6 & 5 & .0 & .0 \\
\hline 04 & 7 & 56 & 34.7286 & 1 . & 296.6 & 4 & .0 & \\
\hline 04 & 7 & 57 & 269.9423 & 0.7 & 297.1 & 3 & 600.0 & 600.0 \\
\hline 04 & 7 & 58 & 272.2023 & 1.6003 & 299.1 & 2 & 600.0 & 600.0 \\
\hline 04 & 7 & 59 & 245.5196 & 2 . & 299.6 & 2 & 600.0 & .0 \\
\hline 04 & 7 & 510 & 197.1162 & 4 & 301.6 & 2 & 600.0 & 600.0 \\
\hline 04 & 7 & 511 & 177.7893 & 4.6808 & 304.2 & 2 & 600.0 & 600.0 \\
\hline 04 & 7 & 512 & 68.7000 & & 305.7 & 1 & 60 & 0.0 \\
\hline 04 & 7 & 513 & 88.0797 & 4.1025 & 306.2 & 2 & 600.0 & 600.0 \\
\hline 04 & 7 & 514 & 86.5508 & 5.1880 & 306.8 & 2 & 600.0 & 600.0 \\
\hline 04 & 7 & 515 & 84.8444 & & 306.8 & 3 & 60 & \\
\hline 04 & 7 & 516 & 77.9997 & 7.0980 & 306.1 & 3 & 600.0 & 600.0 \\
\hline 04 & 7 & 517 & 83.5504 & 7.4470 & 305.1 & 4 & 600.0 & 600.0 \\
\hline 04 & 7 & 518 & 74.4468 & 8.8025 & 303.5 & 4 & 600.0 & 600.0 \\
\hline 04 & 7 & 519 & 65.7501 & 10.5925 & 302.2 & 4 & 600.0 & 600.0 \\
\hline 04 & 7 & 520 & 68.6249 & 11.4950 & 301.0 & 4 & 600.0 & 600.0 \\
\hline 04 & 7 & 521 & 71.7746 & 11.5750 & 301.5 & 4 & 600.0 & 600.0 \\
\hline 04 & 7 & 522 & 71.2750 & 11.7275 & 301.4 & 4 & 600.0 & 600.0 \\
\hline 4 & 7 & 523 & 74.6000 & 11.0050 & 300.8 & $\Psi$ & 600.0 & 600.0 \\
\hline
\end{tabular}




\begin{tabular}{|c|c|c|c|c|c|c|c|c|}
\hline 4 & 7 & 524 & 72.7248 & 10.5025 & 300.7 & 4 & 600.0 & 600.0 \\
\hline 04 & 7 & 61 & 69.7249 & 11.0875 & 300.1 & 4 & 600.0 & 600.0 \\
\hline 04 & 7 & 62 & 66.7243 & .3125 & 299.6 & 4 & 600.0 & 00.0 \\
\hline 04 & 7 & 63 & 67.7268 & 9.8300 & 299.2 & 4 & 600.0 & 00.0 \\
\hline 04 & 7 & 64 & 75.9250 & 9.5350 & 299.4 & 4 & 600.0 & 00.0 \\
\hline 04 & 7 & 65 & 77.4249 & 8.7925 & 299.6 & 4 & 600.0 & 00.0 \\
\hline 04 & 7 & 66 & 73.9500 & 9.0675 & 299.3 & 4 & 600.0 & 00.0 \\
\hline 04 & 7 & 67 & 74.6000 & 8.5400 & 300.3 & 4 & 600.0 & 00.0 \\
\hline 04 & 7 & 68 & 83.3000 & 7.7225 & 301.5 & 4 & 600.0 & 00.0 \\
\hline 04 & 7 & 69 & 84.1745 & 7.9625 & 302.0 & 4 & 600.0 & .0 \\
\hline 04 & 7 & 610 & 88.7503 & 7.8500 & 302.5 & 4 & 600.0 & \\
\hline 04 & 7 & 611 & 85.3265 & 6.3188 & 303.5 & 3 & 600.0 & \\
\hline 04 & 7 & 612 & 69.7704 & 5.8925 & 305.0 & 3 & 600.0 & 00.0 \\
\hline 04 & 7 & 613 & 72.9532 & 8.5075 & 305.3 & 3 & 600.0 & 00.0 \\
\hline 04 & 7 & 614 & 69.9250 & 8. & 305.4 & 3 & .0 & \\
\hline 04 & 7 & 615 & 67.1001 & 8.9650 & 305.4 & 4 & 600.0 & 00.0 \\
\hline 04 & 7 & 616 & 63.8470 & 10.2925 & 304.3 & 4 & 60 & 600.0 \\
\hline 04 & 7 & 617 & 59.0250 & 10.7575 & 303.4 & 4 & .0 & \\
\hline 04 & 7 & 618 & 56.1500 & 10.2625 & 302.5 & 4 & 600.0 & .0 \\
\hline 04 & 7 & 619 & 65.1000 & 11.3025 & 301.3 & 4 & .0 & .0 \\
\hline 04 & 7 & 620 & 63.5498 & 11.59 & 301.0 & 4 & 60 & \\
\hline 04 & 7 & 621 & 62.8501 & 11.0725 & 30 & 4 & .0 & \\
\hline 04 & 7 & 622 & 65.6750 & 10.2375 & 300.8 & 4 & 600.0 & .0 \\
\hline 04 & 7 & 623 & 70.4500 & 10.2125 & 30 & 4 & 60 & .0 \\
\hline 04 & 7 & 624 & 66.6761 & 9.9 & 30 & 4 & .0 & \\
\hline 04 & 7 & 71 & 43.4356 & 6.8883 & 299.0 & 4 & 600.0 & 600.0 \\
\hline 04 & 7 & 72 & 52.7325 & 2.7343 & 296.6 & 4 & 600.0 & .0 \\
\hline 04 & 7 & 73 & 57.3495 & 5.2823 & 296.9 & 4 & .0 & \\
\hline 04 & 7 & 74 & 34.4711 & 2.5458 & 297.1 & 5 & 0 & 600.0 \\
\hline 04 & 7 & 75 & 91.4981 & 1.0473 & 296.0 & 6 & 60 & .0 \\
\hline 04 & 7 & 76 & 126.5340 & 1.1875 & 293.3 & 5 & .0 & \\
\hline 04 & 7 & 77 & 283.7999 & 1.1070 & 292.2 & 4 & 600.0 & 600.0 \\
\hline 04 & 7 & 78 & 268.9517 & 1.4825 & 293.9 & 3 & 600.0 & 600.0 \\
\hline 04 & 7 & 79 & 263.4260 & 2.2475 & 295.8 & 2 & 600.0 & 0.0 \\
\hline 04 & 7 & 710 & 242.8180 & 2.8148 & 296.7 & 2 & 600.0 & 600.0 \\
\hline 04 & 7 & 711 & 229.1835 & 3.0245 & 297.9 & 2 & 600.0 & 600.0 \\
\hline 04 & 7 & 712 & 286.8801 & 2.8198 & & 1 & 600.0 & 0.0 \\
\hline 04 & 7 & 713 & 36.1902 & 3.5855 & 301.3 & 1 & 600.0 & 600.0 \\
\hline 04 & 7 & 714 & 64.6464 & 4.7748 & 301.9 & 2 & 600.0 & 600.0 \\
\hline 04 & 7 & 715 & 57.3494 & 3.8033 & 302.4 & 2 & 600.0 & 0.0 \\
\hline 04 & 7 & 716 & 59.4955 & 6.2438 & 301.0 & 3 & 600.0 & 600.0 \\
\hline 04 & 7 & 717 & 62.4743 & 6.2573 & 300.3 & 4 & 600.0 & 600.0 \\
\hline 04 & 7 & 718 & 80.9433 & 6.4958 & 298.7 & 4 & 600.0 & 600.0 \\
\hline 04 & 7 & 719 & 74.7750 & 6.9408 & 296.7 & 4 & 600.0 & 600.0 \\
\hline 04 & 7 & 720 & 72.9251 & 8.6750 & 294.1 & 4 & 600.0 & 600.0 \\
\hline 04 & 7 & 721 & 72.8237 & 5.4265 & 294.0 & 4 & 600.0 & 600.0 \\
\hline 04 & 7 & 722 & 96.6083 & 3.4770 & 293.6 & 4 & 600.0 & 600.0 \\
\hline 84 & 7 & 723 & 123.1647 & 1.7720 & 292.9 & 3 & 600.0 & 600.0 \\
\hline
\end{tabular}




\begin{tabular}{|c|c|c|c|c|c|c|c|}
\hline 4 & 24 & & & & & & \\
\hline 4 & 81 & & 1.2683 & 90.2 & & 00.0 & \\
\hline 7 & 82 & 130.7726 & 1.4853 & 288.9 & 6 & 00 & \\
\hline 7 & 83 & 108.1262 & 1.0795 & 288.9 & & 00 & \\
\hline 7 & 84 & 48.0283 & 678 & 88.7 & & 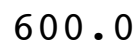 & \\
\hline 7 & 85 & 278.7360 & 1.2750 & 87.4 & & 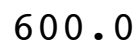 & \\
\hline 7 & 86 & 166.5374 & 8885 & 287.7 & & 00 & \\
\hline 7 & 87 & 97.2290 & 1.8375 & 88.0 & & & \\
\hline 7 & 88 & 265.6194 & 180 & 89.3 & & 0 & \\
\hline 7 & 89 & 222.0804 & 2.8803 & 91.3 & & 0 & \\
\hline 7 & 810 & 219.5590 & 3.2598 & 93.5 & & 0 & \\
\hline 7 & 811 & 180 & 3.3773 & 95.4 & & & \\
\hline 7 & 812 & 181 & 5.5680 & 297.1 & & 0 & \\
\hline 7 & 813 & 178.2630 & 7.3235 & 298.2 & & 0 & \\
\hline 7 & 814 & 1 & 8 & 97.8 & & & \\
\hline 7 & 815 & 19 & 6 & 298.1 & & & \\
\hline 7 & 816 & 193.9433 & 5.54 & 298.5 & & 0 & \\
\hline 7 & 817 & 287 & 5.64 & 8.5 & & 0 & \\
\hline 7 & 818 & 187 & 3. & 297.4 & & & \\
\hline 7 & 819 & 97.8991 & 5.8455 & 295.0 & & 0 & \\
\hline 7 & 820 & 4981 & 7.50 & 292.2 & 4 & 0 & \\
\hline 7 & 821 & 246 & 9.22 & 90.6 & $<$ & & \\
\hline 7 & 822 & 5745 & 9.3775 & 289.6 & 4 & 0 & \\
\hline 7 & 823 & 248 & 7.0428 & 289.7 & $I$ & 0 & \\
\hline 7 & 824 & 249 & 0 & 289.8 & 4 & & \\
\hline 7 & 9 & $7^{\circ}$ & 6.4 & 288.5 & 4 & & \\
\hline 7 & 92 & 4002 & 7.3480 & 288.1 & 4 & 0 & \\
\hline 7 & 93 & 999 & 6.43 & 287.6 & 4 & 0 & \\
\hline 7 & 94 & 751 & 6.5 & 286.9 & 4 & & \\
\hline 7 & 95 & 7252 & 6.5350 & 286.1 & 4 & 0 & \\
\hline 7 & 96 & 3750 & 4.63 & 286.5 & 4 & 0 & \\
\hline 7 & 97 & 249 & 6.5 & 286.5 & 4 & & \\
\hline 7 & 98 & 9510 & 4.1833 & 288.2 & 3 & 60 & \\
\hline 7 & 99 & 2648 & 2.4580 & 290.8 & 2 & 0 & \\
\hline 7 & 910 & 26 & 8 & 292.4 & 2 & & \\
\hline 7 & 911 & 27 & 3 . & 293.8 & 2 & .0 & \\
\hline 7 & 912 & 334.3902 & 3.9445 & 295.2 & 2 & مी & \\
\hline 7 & 913 & 952 & 2 . & 297.3 & 1 & 0 & \\
\hline 7 & 914 & 116 & 3.7460 & 298.3 & 1 & 600.0 & \\
\hline 1 & 915 & 2298 & 5.1923 & 298.2 & 2 & 0 & \\
\hline 7 & 916 & 694 & 4.9098 & 297.5 & 3 & 600.0 & \\
\hline 7 & 917 & 208.2863 & 4.9288 & 296.7 & 3 & 600.0 & \\
\hline 7 & 918 & 81.2088 & 5.4488 & 294.9 & 3 & 600.0 & \\
\hline I & 919 & 481 & 8.7125 & 292.0 & 4 & 600 & \\
\hline 7 & 920 & 67.5500 & 8.6600 & 290.0 & 4 & 600.0 & \\
\hline 7 & 921 & 77.5752 & 8.0475 & 289.1 & 4 & 600.0 & \\
\hline 7 & 922 & 30 & 0 & $2 \varepsilon$ & 4 & & \\
\hline & 923 & 74.3250 & 7.1480 & 289.2 & 4 & 600.0 & 60 \\
\hline
\end{tabular}




\begin{tabular}{|c|c|c|c|c|c|c|c|}
\hline 4 & 7924 & 74.6264 & 4.7315 & 290.3 & 4 & 600.0 & 600.0 \\
\hline 04 & 7101 & 79.7243 & 4.6655 & 289.9 & 4 & 600.0 & 600.0 \\
\hline 04 & 7102 & 75.0500 & 8.4725 & 289.6 & 4 & 600.0 & 00.0 \\
\hline 04 & 7103 & 72.6000 & 10.0375 & 289.6 & 4 & 600.0 & 00.0 \\
\hline 04 & 7104 & 67.1757 & 9.4150 & 289.9 & 4 & 600.0 & 00.0 \\
\hline 04 & 7105 & 72.1000 & 8.1000 & 289.9 & 4 & 600.0 & 00.0 \\
\hline 04 & 7106 & 79.2303 & 4.1783 & 290.5 & 4 & 600.0 & 00.0 \\
\hline 04 & 7107 & 141.8847 & 1.5580 & 292.3 & 3 & 600.0 & 00.0 \\
\hline 04 & 7108 & 299.2141 & 2.1485 & 292.1 & 2 & 600.0 & 00.0 \\
\hline 04 & 7109 & 266.6143 & 1.9745 & 293.6 & 2 & 600.0 & .0 \\
\hline 04 & 71010 & 233.1616 & 2.8510 & 295.7 & 2 & 600.0 & \\
\hline 04 & 71011 & 235.1191 & 2.9295 & 297.2 & 2 & 600.0 & \\
\hline 04 & 71012 & 37.4851 & 3.8135 & 299.1 & 1 & 600.0 & 00.0 \\
\hline 04 & 71013 & 88.6535 & 5.31 & 299.9 & 2 & .0 & 00.0 \\
\hline 04 & 71014 & 87.2310 & & 300.4 & 3 & .0 & \\
\hline 04 & 71015 & 75.3997 & 8.2150 & 300.1 & 4 & 600.0 & .0 \\
\hline 04 & 71016 & 70.6245 & 8.1075 & 300.0 & 4 & 60 & 600.0 \\
\hline 04 & 71017 & 65.9504 & 8.2 & 299.3 & 4 & .0 & \\
\hline 04 & 71018 & 68.0247 & 8.2543 & 298.2 & 4 & 60 & .0 \\
\hline 04 & 71019 & 69.2239 & 7.79 & 297.5 & 4 & 60 & .0 \\
\hline 04 & 71020 & 73.5501 & 9.06 & 296.2 & 4 & 6 & \\
\hline 04 & 71021 & 69.9749 & 10.68 & 295.8 & 4 & .0 & \\
\hline 04 & 71022 & 69.4500 & 11.5350 & 295.4 & 4 & 600.0 & .0 \\
\hline 04 & 71023 & 72.1500 & 11.00 & 295 & 4 & 60 & \\
\hline 04 & 71024 & 70.2002 & 10 & 29 & 4 & .0 & \\
\hline 04 & 7111 & 67.9248 & 8.6000 & 295.9 & 4 & .0 & .0 \\
\hline 04 & 7112 & 68.3750 & 8.0550 & 296.1 & 4 & .0 & .0 \\
\hline 04 & 7113 & 68.2984 & 7.6725 & 296.1 & 4 & .0 & \\
\hline 04 & 7114 & 74.6250 & 6.5320 & 296.3 & 4 & .0 & 0.0 \\
\hline 04 & 7115 & 72.8750 & 6.8863 & 296.1 & 4 & 60 & .0 \\
\hline 04 & 7116 & 72.0753 & 6.49 & 296.4 & 4 & .0 & \\
\hline 04 & $\begin{array}{ll}711 & 7\end{array}$ & 65.8554 & 4.11 & 297.2 & 3 & .0 & 0.0 \\
\hline 04 & 7118 & 274.5662 & 2.3518 & 296.1 & 2 & 600.0 & 0.0 \\
\hline 04 & 7119 & 235.6830 & 2.7035 & 297.4 & 2 & 600.0 & 0.0 \\
\hline 04 & 71110 & 230.7704 & 2.89 & & 2 & 600.0 & 600.0 \\
\hline 04 & 71111 & 164.5485 & 3.7183 & 300.6 & 2 & 600.0 & 600.0 \\
\hline 04 & 71112 & 173.4007 & 4.3813 & 302.0 & 2 & 600.0 & 0.0 \\
\hline 04 & 71113 & 176.3273 & 5.4783 & 303.1 & 2 & 600.0 & 600.0 \\
\hline 04 & 71114 & 191.7755 & 6.4885 & 303.9 & 3 & 600.0 & 600.0 \\
\hline 04 & 71115 & 199.3676 & 6.8043 & 304.0 & 4 & 600.0 & .0 \\
\hline 04 & 71116 & 183.7726 & 4.9855 & 304.3 & 3 & 600.0 & 600.0 \\
\hline 04 & 71117 & 113.0431 & 5.0038 & 304.6 & 3 & 600.0 & 600.0 \\
\hline 04 & 71118 & 91.5228 & 5.6975 & 303.3 & 4 & 600.0 & 600.0 \\
\hline 04 & 71119 & 72.1996 & 7.0765 & 301.1 & 4 & 600.0 & 600.0 \\
\hline 04 & 71120 & 64.0249 & 9.4050 & 299.4 & 4 & 600.0 & 600.0 \\
\hline 04 & 71121 & 65.1748 & 9.7650 & 298.7 & 4 & 600.0 & 600.0 \\
\hline $0<$ & 71122 & 70.3247 & 9.3325 & 298.5 & 4 & 600.0 & 600.0 \\
\hline 04 & 71123 & 75.8250 & 9.1800 & 298.3 & 4 & 600.0 & 600.0 \\
\hline
\end{tabular}




\begin{tabular}{|c|c|c|c|c|c|c|c|}
\hline 04 & 71124 & 72.2751 & 9.9950 & 297.7 & 4 & 600.0 & 00.0 \\
\hline 04 & 7121 & 72.9999 & 10.1475 & 297.4 & 4 & 600.0 & 600,0 \\
\hline 04 & 7122 & 70.9750 & 9.4250 & 297.8 & 4 & 600.0 & 00.0 \\
\hline 04 & 7123 & 72.6748 & 8.1250 & 298.3 & 4 & 600.0 & 00.0 \\
\hline 04 & 7124 & 75.9000 & 7.7525 & 298.2 & 4 & 600.0 & 00.0 \\
\hline 04 & 7125 & 73.9786 & 6.9463 & 298.6 & 4 & 600.0 & 00.0 \\
\hline 04 & 7126 & 71.2751 & 5.2223 & 298.5 & 4 & 600.0 & 600.0 \\
\hline 04 & 7127 & 76.0695 & 4.1368 & 299.9 & 3 & 600.0 & 00.0 \\
\hline 04 & 7128 & 238.6113 & 2.0860 & 300.2 & 2 & 600.0 & 00.0 \\
\hline 04 & 7129 & 234.9456 & 2.7273 & 301.0 & ? & 600.0 & .0 \\
\hline 04 & 71210 & 237.2907 & 3.4640 & 301.8 & 2 & 600.0 & 00.0 \\
\hline 04 & 71211 & 259.6961 & 3.2205 & 302.5 & 2 & 600.0 & \\
\hline 04 & 71212 & 42.8132 & 4.6173 & 303.4 & 2 & 600.0 & 600.0 \\
\hline 04 & 71213 & 84.0806 & 5.4810 & 303.3 & 2 & 600.0 & 600.0 \\
\hline 04 & 71214 & 81.2503 & 6.9835 & 302.9 & 3 & 600.0 & \\
\hline 04 & 71215 & 81.2000 & 6.7825 & 303.0 & 4 & 600.0 & 60 \\
\hline 04 & 71216 & 79.7176 & 8.05 & 302.5 & 4 & 600.0 & 600.0 \\
\hline 04 & 71217 & 76.2273 & 9.8800 & 300.4 & 4 & 600.0 & \\
\hline 04 & 71218 & 77.7967 & 7.5968 & 299.3 & 4 & 600.0 & .0 \\
\hline 04 & 71219 & 73.8251 & 11.4325 & 296.9 & 4 & 600.0 & 60 \\
\hline 04 & 71220 & 68.3502 & 11.51 & 294.0 & 4 & 600.0 & .0 \\
\hline 04 & 71221 & 68.0743 & 12.88 & 293.9 & 4 & 600.0 & \\
\hline 04 & 71222 & 62.5000 & 13.5950 & 294.6 & 4 & 600.0 & 600 \\
\hline 04 & 71223 & 64.4250 & 12.91 & 294.9 & 4 & 600.0 & .0 \\
\hline 04 & 71224 & 68.8501 & 12.15 & 294.8 & 4 & 600.0 & \\
\hline 04 & 7131 & 71.0000 & 13.2125 & 293.8 & 4 & 600.0 & 60 \\
\hline 04 & 7132 & 70.3000 & 11.0225 & 294.3 & 4 & 600.0 & 60 \\
\hline 04 & 7133 & 69.3000 & 11.13 & 294.5 & 4 & 600.0 & \\
\hline 04 & 7134 & 68.4500 & 10.7550 & 294.3 & 4 & 600.0 & 60 \\
\hline 04 & 7135 & 69.7501 & 11.2600 & 293.8 & 4 & 600.0 & 60 \\
\hline 04 & 7136 & 66.5750 & 11.88 & 292.9 & 4 & 600.0 & \\
\hline 04 & 7137 & 67.7000 & 11.1925 & 293.3 & 4 & 600.0 & 60 \\
\hline 04 & 7138 & 71.1250 & 10.0975 & 294.7 & 4 & 600.0 & 600.0 \\
\hline 04 & 7139 & 78.3249 & 7.53 & 295.7 & 3 & 600.0 & .0 \\
\hline 04 & 71310 & 86.9500 & 4. & 298.0 & 2 & 600.0 & .0 \\
\hline 04 & 71311 & 95.4068 & 3.29 & 299.6 & 1 & 600.0 & 600.0 \\
\hline 04 & 71312 & 84.1378 & 4.90 & 299.9 & 2 & 600.0 & \\
\hline 04 & 71313 & 80.2807 & 5.4683 & 300.8 & 2 & 600.0 & 600.0 \\
\hline 04 & 71314 & 85.6497 & 6.8378 & 300.9 & 3 & 600.0 & 600.0 \\
\hline 04 & 71315 & 88.0760 & 7.2295 & 300.9 & 4 & 600.0 & \\
\hline 04 & 71316 & 70.3909 & 7.4073 & 300.7 & 4 & 600.0 & 600.0 \\
\hline 04 & 71317 & 75.0250 & 8.8350 & 299.7 & 4 & 600.0 & 600.0 \\
\hline 04 & 71318 & 75.1000 & 11.0850 & 298.2 & 4 & 600.0 & 600.0 \\
\hline 04 & 71319 & 69.8500 & 11.5250 & 296.7 & 4 & 600.0 & 600.0 \\
\hline 04 & 71320 & 68.6756 & 9.2100 & 295.2 & 4 & 600.0 & 600.0 \\
\hline 04 & 71321 & 56.6998 & 11.0700 & 294.6 & 4 & 600.0 & 600.0 \\
\hline 04 & 71322 & 56.1499 & 11.9525 & 296.2 & 4 & 600.0 & 600.0 \\
\hline 04 & 71323 & 64.4751 & 10.9950 & 296.8 & 4 & 600.0 & 600.0 \\
\hline
\end{tabular}




\begin{tabular}{|c|c|c|c|c|c|c|c|}
\hline 4 & 71324 & 66.7500 & 10.5450 & 297.0 & 4 & 600.0 & 600.0 \\
\hline 04 & $714 \quad 1$ & 67.6998 & 9.6350 & 296.5 & 4 & 600.0 & 600.0 \\
\hline 04 & 7142 & 70.6237 & 8.0400 & 296.3 & 4 & 600.0 & 00.0 \\
\hline 04 & 7143 & 76.8507 & 8.9550 & 296.1 & 4 & 600.0 & 00.0 \\
\hline 04 & $714 \quad 4$ & 75.6250 & 8.4625 & 295.6 & 4 & 600.0 & 00.0 \\
\hline 04 & 7145 & 75.8747 & 8.4000 & 295.7 & 4 & 600.0 & 00.0 \\
\hline 04 & 7146 & 71.6998 & 8.5875 & 295.8 & 4 & 600.0 & 00.0 \\
\hline 04 & $\begin{array}{ll}714 & 7\end{array}$ & 80.5502 & 8.4475 & 296.5 & 4 & 600.0 & 00.0 \\
\hline 04 & 7148 & 78.0998 & 9.5250 & 296.3 & 4 & 600.0 & 00.0 \\
\hline 04 & 7149 & 81.4248 & 9.6150 & 295.8 & 4 & 600.0 & .0 \\
\hline 04 & 71410 & 95.3288 & 398 & 297.6 & 3 & 600.0 & \\
\hline 04 & 71411 & 89.0507 & 4. & 299.2 & 2 & 600.0 & \\
\hline 04 & 71412 & 82.4749 & 5.3195 & 300.6 & 2 & 600.0 & 00.0 \\
\hline 04 & 71413 & 86.3002 & 5.7703 & 301.2 & 3 & 600.0 & 00.0 \\
\hline 04 & 71414 & 88.0499 & & 301.4 & 3 & .0 & \\
\hline 04 & 71415 & 82.4023 & 7.7525 & 301.8 & 4 & 600.0 & .0 \\
\hline 04 & 71416 & 72.4746 & 7.9475 & 301.6 & 4 & 60 & 600.0 \\
\hline 04 & 71417 & 66.0234 & 8.9 & 300.4 & 4 & .0 & \\
\hline 04 & 71418 & 70.7746 & 11.6375 & 299.0 & 4 & 60 & \\
\hline 04 & 71419 & 66.7001 & 13.35 & 298.0 & 4 & .0 & .0 \\
\hline 04 & 71420 & 67.6034 & 10 & 297.1 & 4 & 6 & \\
\hline 04 & 71421 & 48.9295 & 7.7 & 295.6 & 4 & .0 & \\
\hline 04 & 71422 & 62.2979 & 9.2400 & 296.2 & 4 & 600.0 & .0 \\
\hline 04 & 71423 & 77.3745 & 10.1 & 296.7 & 4 & 60 & \\
\hline 04 & 71424 & 74.1198 & 8 . & 295.6 & 4 & .0 & \\
\hline 04 & 7151 & 82.2997 & 7.4028 & 294.7 & 4 & .0 & 600.0 \\
\hline 04 & 7152 & 63.9502 & 9.20 & 295.0 & 4 & 600.0 & .0 \\
\hline 04 & 7153 & 65.0501 & 9.3 & 295.1 & 4 & .0 & \\
\hline 04 & 7154 & 63.5498 & 8.69 & 295.7 & 4 & .0 & 0.0 \\
\hline 04 & $715 \quad 5$ & 64.7978 & 8.40 & 296.2 & 4 & 60 & .0 \\
\hline 04 & 7156 & 72.3751 & 8.28 & 296.6 & 4 & .0 & \\
\hline 04 & $\begin{array}{ll}715 & 7\end{array}$ & 77.1992 & 6.33 & 298.1 & 4 & .0 & 600.0 \\
\hline 04 & 7158 & 87.1392 & 4.5240 & 299.8 & 3 & 600.0 & 0.0 \\
\hline 04 & 7159 & 136.1835 & 4.20 & 301.1 & 3 & 600.0 & 0.0 \\
\hline 04 & 71510 & 235.6614 & 3.93 & 300.4 & 2 & 600.0 & 600.0 \\
\hline 04 & 71511 & 263.1463 & 3.0795 & 302.2 & 1 & 600.0 & 600.0 \\
\hline 04 & 71512 & 64.1939 & 4.15 & 303.3 & 2 & 600.0 & 0.0 \\
\hline 04 & 71513 & 74.9226 & 5.3903 & 303.6 & 3 & 600.0 & 600.0 \\
\hline 04 & 71514 & 99.5534 & 5.8815 & 303.8 & 3 & 600.0 & 600.0 \\
\hline 04 & 71515 & 81.5158 & 5. & 304.2 & 3 & 600.0 & 600.0 \\
\hline 04 & 71516 & 78.5293 & 7.39 & 303.5 & 4 & 600.0 & 600.0 \\
\hline 04 & 71517 & 70.1977 & 8.8150 & 302.1 & 4 & 600.0 & 600.0 \\
\hline 04 & 71518 & 67.3000 & 9.7925 & 300.7 & 4 & 600.0 & 600.0 \\
\hline 04 & 71519 & 68.0506 & 10.7800 & 299.3 & 4 & 600.0 & 600.0 \\
\hline 04 & 71520 & 59.1500 & 11.1900 & 298.2 & 4 & 600.0 & 600.0 \\
\hline 04 & 71521 & 62.8998 & 10.9100 & 296.9 & 4 & 600.0 & 600.0 \\
\hline $0<$ & 71522 & 64.6000 & 11.6100 & 296.8 & 4 & 600.0 & 600.0 \\
\hline 0 & 71523 & 63.6000 & 11.5450 & 296.9 & 4 & 600.0 & 600.0 \\
\hline
\end{tabular}




\begin{tabular}{|c|c|c|c|c|c|c|c|}
\hline 04 & 71524 & 7.8497 & 10.1300 & 297.0 & 4 & 600.0 & 600.0 \\
\hline 04 & 7161 & 66.0000 & 11.3600 & 296.9 & 4 & 600.0 & 600.0 \\
\hline 04 & 7162 & 71.8001 & 10.5550 & 296.8 & 4 & 600.0 & 00.0 \\
\hline 04 & 7163 & 70.9999 & 9.1050 & 297.0 & 4 & 600.0 & 00.0 \\
\hline 04 & 7164 & 71.2500 & 9.0200 & 297.4 & 4 & 600.0 & 00.0 \\
\hline 04 & 7165 & 66.1499 & 9.1475 & 297.7 & 4 & 600.0 & 00.0 \\
\hline 04 & 7166 & 67.1000 & 8.7575 & 297.7 & 4 & 600.0 & 00.0 \\
\hline 04 & 7167 & 62.4500 & 7.9 & 298.1 & 4 & 600.0 & 0 \\
\hline 04 & 7168 & 68.7882 & 6.9393 & 299.4 & 4 & 600.0 & 00.0 \\
\hline 04 & 7169 & 90.2980 & 5.6108 & 300.4 & 3 & 600.0 & 00.0 \\
\hline 04 & 71610 & 77.4668 & 88 & 301.4 & 2 & 600.0 & \\
\hline 04 & 71611 & 77.9132 & 5.3735 & 302.4 & 3 & 600.0 & 60 \\
\hline 04 & 71612 & 66.2021 & 4.8115 & 303.7 & 2 & 600.0 & \\
\hline 04 & 71613 & 83.0038 & 963 & 302.8 & 3 & 0 & \\
\hline 04 & 716 & 82.3250 & 33 & 302.5 & 3 & 60 & 0 \\
\hline 04 & 71615 & 86.2500 & 6.1555 & 303.3 & 3 & 600.0 & 600.0 \\
\hline 04 & 71616 & 8.4748 & 6.2 & 303.9 & 4 & .0 & .0 \\
\hline 04 & 71617 & 95.5250 & 7 . & 303.3 & 4 & .0 & \\
\hline 04 & 71618 & 79.4172 & 9.6525 & 301.7 & 4 & 600.0 & 600.0 \\
\hline 04 & 71619 & 72.5001 & 10 . & 299.9 & 4 & .0 & .0 \\
\hline 04 & 71620 & 69.9251 & 9. & 298.2 & 4 & 0 & \\
\hline 04 & 71621 & 70.0750 & 11. & 296.8 & 4 & .0 & .0 \\
\hline 04 & 71622 & 59.6749 & 12 . & 296.0 & 4 & 60 & .0 \\
\hline 04 & 71623 & 72.1248 & 11 . & 296.4 & 4 & .0 & \\
\hline 04 & 71624 & 73.9752 & 12 . & 296.1 & 4 & 60 & \\
\hline 04 & $\begin{array}{ll}717 & 1\end{array}$ & 72.4006 & 12.6700 & 296.2 & 4 & .0 & .0 \\
\hline 04 & $\begin{array}{ll}717 & 2\end{array}$ & 70.5500 & 11.22 & 296.5 & 4 & .0 & \\
\hline 04 & 7173 & 68.1752 & 10 . & 296.8 & 4 & .0 & .0 \\
\hline 04 & 7174 & 75.6755 & 9.7 & 296.4 & 4 & .0 & 600.0 \\
\hline 04 & 7175 & 68.2998 & 9.1 & 296.4 & 4 & .0 & .0 \\
\hline 04 & 7176 & 71.7754 & 7 . & 296.8 & 4 & .0 & \\
\hline 04 & $\begin{array}{ll}717 & 7\end{array}$ & 77.7003 & 7.7130 & 297.0 & 4 & .0 & .0 \\
\hline 04 & 7178 & 71.4250 & 8.0 & 298.0 & 4 & .0 & .0 \\
\hline 04 & 7179 & 71.7000 & 8. & 298.4 & 4 & 600.0 & 600.0 \\
\hline 04 & 71710 & 73.1493 & 9 . & 298.9 & 4 & .0 & .0 \\
\hline 04 & 71711 & 76.3246 & 9 . & 299.7 & 4 & .0 & .0 \\
\hline 04 & 71712 & 71.3999 & 9. & 301.2 & 3 & .0 & .0 \\
\hline 04 & 71713 & 73.5227 & 9.0750 & 301.5 & 3 & 600.0 & 600.0 \\
\hline 04 & 71714 & 72.1499 & 10.4150 & 301.6 & 3 & 600.0 & 600.0 \\
\hline 04 & 71715 & 72.7253 & 11 & 302.0 & 4 & .0 & 600.0 \\
\hline 04 & 71716 & 67.6996 & 10.8525 & 302.2 & 4 & 600.0 & 600.0 \\
\hline 04 & 71717 & 62.0257 & 10.8575 & 301.6 & 4 & 600.0 & 600.0 \\
\hline 04 & 71718 & 59.5003 & 10.8500 & 300.6 & 4 & 600.0 & 600.0 \\
\hline 04 & 71719 & 67.2990 & 11.0875 & 299.2 & 4 & 600.0 & 600.0 \\
\hline 04 & 71720 & 63.0750 & 12.3825 & 298.2 & 4 & 600.0 & 600.0 \\
\hline 04 & 71721 & 65.4999 & 11.4475 & 298.0 & 4 & 600.0 & \\
\hline 04 & 71722 & 71.5752 & 9.8700 & 297.6 & 4 & 600.0 & 600.0 \\
\hline 04 & 71723 & 69.3748 & 10.2500 & 297.7 & 4 & 600.0 & 600. \\
\hline
\end{tabular}




\begin{tabular}{|c|c|c|c|c|c|c|c|}
\hline 4 & 71724 & 56.5749 & 10.9000 & 296.7 & 4 & 600.0 & 600.0 \\
\hline 04 & 7181 & 68.9000 & 10.5875 & 296.7 & 4 & 600.0 & 600.0 \\
\hline 04 & 7182 & 65.7001 & 11.5825 & 295.8 & 4 & 600.0 & 00.0 \\
\hline 04 & 7183 & 4.4249 & 10.1600 & 295.6 & 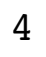 & 600.0 & 00.0 \\
\hline 04 & 7184 & 67.2252 & 10.2575 & 296.0 & 4 & 600.0 & 00.0 \\
\hline 04 & 7185 & 62.3749 & 9.2575 & 296.4 & 4 & 600.0 & 00.0 \\
\hline 04 & 7186 & 64.9750 & 9.1225 & 296.3 & 4 & 600.0 & 600.0 \\
\hline 04 & 7187 & 71.2000 & 9.6325 & 296.8 & 4 & 600.0 & \\
\hline 04 & 7188 & 65.8500 & 9.0500 & 297.4 & 4 & 600.0 & 000.0 \\
\hline 04 & 7189 & 70.6751 & 8.7275 & 298.3 & 4 & 600.0 & 0 \\
\hline 04 & 71810 & 70.3998 & 7.1925 & 299.8 & 4 & 600.0 & \\
\hline 04 & 71811 & 80.0927 & 7.2203 & 301.0 & 4 & 600.0 & 60 \\
\hline 04 & 71812 & 73.1953 & 6.2583 & 302.7 & 3 & 600.0 & \\
\hline 04 & 71813 & 84.1997 & 7.3045 & 303.1 & 3 & 0 & \\
\hline 04 & 71814 & 87.7751 & 7.8095 & 303.3 & 3 & .0 & .0 \\
\hline 04 & 71815 & 96.5995 & 8.4100 & 303.4 & 4 & 600.0 & .0 \\
\hline 04 & 71816 & 3.5495 & 7.4045 & 303.1 & 4 & 600.0 & .0 \\
\hline 04 & 71817 & 83.4999 & 9.3950 & 302.0 & 4 & .0 & \\
\hline 04 & 71818 & 85.2130 & 7.7023 & 300.9 & 4 & 600.0 & 600.0 \\
\hline 04 & 71819 & 79.4844 & 8.4825 & 299.8 & 4 & .0 & .0 \\
\hline 04 & 71820 & 66.7491 & 10.4 & 298.7 & 4 & .0 & \\
\hline 04 & 71821 & 65.4743 & 12.6475 & 297.5 & 4 & .0 & \\
\hline 04 & 71822 & 66.1996 & 12.1700 & 297.2 & 4 & .0 & .0 \\
\hline 04 & 71823 & 70.3754 & 11.9250 & 297.1 & 4 & .0 & \\
\hline 04 & 71824 & 73.4751 & 12.2800 & 296.7 & 4 & 60 & \\
\hline 04 & 7191 & 71.1500 & 13.3375 & 296.5 & 4 & 600.0 & .0 \\
\hline 04 & 7192 & 67.9500 & 12.8900 & 296.0 & 4 & 6 & .0 \\
\hline 04 & 7193 & 69.3751 & 12.6150 & 296.0 & 4 & .0 & \\
\hline 04 & 7194 & 69.5000 & 12.7225 & 295.7 & 4 & .0 & .0 \\
\hline 04 & 7195 & 71.8005 & 11.3750 & 295.3 & 4 & .0 & \\
\hline 04 & 7196 & 74.3752 & 10.8525 & 295.8 & 4 & .0 & \\
\hline 04 & 7197 & 72.3000 & 10.1375 & 296.9 & 4 & .0 & .0 \\
\hline 04 & 7198 & 73.0250 & 9.6050 & 298.0 & 4 & 60 & 600.0 \\
\hline 04 & 7199 & 74.2761 & 10.2925 & 297.5 & 4 & .0 & 600.0 \\
\hline 04 & 71910 & 72.8250 & 9.6700 & 298.2 & 4 & .0 & .0 \\
\hline 04 & 71911 & 71.7513 & 8.9150 & 299.5 & 4 & 600.0 & 600.0 \\
\hline 04 & 71912 & 78.0218 & 8.2393 & 300.3 & 3 & .0 & .0 \\
\hline 04 & 71913 & 65.1250 & 11.4600 & 300.3 & 3 & 600.0 & 600.0 \\
\hline 04 & 71914 & 62.1748 & 11.2275 & 300.9 & 3 & 600.0 & 600.0 \\
\hline 04 & 71915 & 63.4996 & 11.7475 & 300.8 & 4 & .0 & 600.0 \\
\hline 04 & 71916 & 61.0999 & 10.9400 & 300.5 & 4 & 600.0 & 600.0 \\
\hline 04 & 71917 & 59.6749 & 11.9150 & 299.1 & 4 & 600.0 & 600.0 \\
\hline 04 & 71918 & 57.7496 & 12.5400 & 297.8 & 4 & 600.0 & 600.0 \\
\hline 04 & 71919 & 56.6750 & 15.0175 & 296.2 & 4 & 600.0 & 600.0 \\
\hline 04 & 71920 & 62.6001 & 13.5750 & 295.5 & 4 & 600.0 & 600.0 \\
\hline $0<$ & 71921 & 60.4500 & 13.4150 & 29 & 4 & 600.0 & \\
\hline 04 & 71922 & 62.1251 & 12.2650 & 295.6 & 4 & 600.0 & 600.0 \\
\hline 4 & 71923 & 62.7501 & 11.3975 & 295.8 & 4 & 600.0 & 600.0 \\
\hline
\end{tabular}




\begin{tabular}{|c|c|c|c|c|c|c|c|}
\hline 04 & 71924 & 63.5000 & 11.1900 & 295.7 & 4 & 600.0 & 600.0 \\
\hline 04 & 7201 & 63.9500 & 10.7800 & 295.7 & 4 & 600.0 & 00,0 \\
\hline 04 & 7202 & 64.9750 & 10.0175 & 295.6 & 4 & 600.0 & 00.0 \\
\hline 04 & 7203 & 67.7250 & 9.6125 & 295.7 & 4 & 600.0 & 00.0 \\
\hline 04 & $720 \quad 4$ & 70.7000 & 10. & 295.3 & 4 & 600.0 & 00.0 \\
\hline 04 & 7205 & 73.2001 & 11.1325 & 295.0 & 4 & 600.0 & 00.0 \\
\hline 04 & 7206 & 71.7756 & 10.4150 & 295.4 & 4 & 600.0 & 0 \\
\hline 04 & $\begin{array}{ll}720 & 7\end{array}$ & 71.7500 & 9.3025 & 296.4 & 4 & 600.0 & 00.0 \\
\hline 04 & $720 \quad 8$ & 76.4000 & 7.7215 & 297.6 & 4 & 600.0 & 00.0 \\
\hline 04 & 7209 & 82.8000 & 5.7865 & 298.7 & 4 & 600 & 0 \\
\hline 04 & 72010 & 88.1745 & 5.6015 & 300.0 & 3 & 600.0 & \\
\hline 04 & 72011 & 87.6755 & 393 & 300.7 & 3 & 600.0 & \\
\hline 04 & 72012 & 88.9500 & 958 & 301.6 & 3 & 600.0 & 00.0 \\
\hline 04 & 72013 & 84.8557 & 083 & 302.7 & 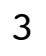 & 600.0 & 00.0 \\
\hline 04 & 72014 & 98.3634 & 98 & 303.5 & 3 & .0 & \\
\hline 04 & 72015 & 95.1269 & 495 & 303.1 & 4 & 600.0 & .0 \\
\hline 04 & 72016 & 90.3491 & 6.6203 & 303.1 & 3 & 60 & 600.0 \\
\hline 04 & 72017 & 83.8449 & 838 & 302.3 & 4 & .0 & \\
\hline 04 & 72018 & 71.9250 & 9.4650 & 300.9 & 4 & .0 & \\
\hline 04 & 72019 & 73.0750 & 11.3850 & 298.7 & 4 & .0 & .0 \\
\hline 04 & 72020 & 69.0256 & 13. & 298.0 & 4 & 6 & \\
\hline 04 & 72021 & 69.3665 & 10. & 298.1 & 4 & .0 & \\
\hline 04 & 72022 & 79.7754 & 8.3920 & 298.2 & 4 & 600.0 & .0 \\
\hline 04 & 72023 & 69.5253 & 9 . & 298.2 & 4 & 6 & \\
\hline 04 & 72024 & 67.6750 & 9 . & 299.2 & 4 & 6 & \\
\hline 04 & 7211 & 69.7252 & 150 & 299.8 & 4 & .0 & \\
\hline 04 & 7212 & 78.5517 & 6.2763 & 300.3 & 4 & .0 & .0 \\
\hline 04 & 7213 & 77.6500 & 6 & 300.4 & 4 & .0 & \\
\hline 04 & 7214 & 78.4250 & 725 & 300.1 & 4 & .0 & .0 \\
\hline 04 & 7215 & 77.7750 & 7 . & 299.9 & 4 & .0 & .0 \\
\hline 04 & 7216 & 62.0643 & 5. & 299.9 & 4 & .0 & \\
\hline 04 & $\begin{array}{ll}721 & 7\end{array}$ & 346.2024 & 1.9480 & 298.5 & 3 & .0 & 600.0 \\
\hline 04 & 7218 & 242.3119 & 1.8410 & 298.8 & 2 & 600.0 & 0.0 \\
\hline 04 & 7219 & 246.0703 & 1.9845 & 301.3 & 2 & 0.0 & 0.0 \\
\hline 04 & 72110 & 174.9083 & 5 . & 303.1 & 3 & 600.0 & 0.0 \\
\hline 04 & 72111 & 179.5076 & 6.8628 & 303.5 & 4 & 600.0 & 600.0 \\
\hline 04 & 72112 & 176.3251 & 6 . & 304.8 & 3 & 600.0 & 0.0 \\
\hline 04 & 72113 & 167.4962 & 6.4930 & 305.9 & 3 & 600.0 & 600.0 \\
\hline 04 & 72114 & 164.2009 & 6.1028 & 306.8 & 3 & 600.0 & 600.0 \\
\hline 04 & 72115 & 140.2494 & 683 & 307.2 & 4 & 600.0 & 600.0 \\
\hline 04 & 72116 & 98.2065 & 6.5295 & 307.0 & 3 & 600.0 & 600.0 \\
\hline 04 & 72117 & 100.9157 & 7.2815 & 306.2 & 4 & 600.0 & 600.0 \\
\hline 04 & 72118 & 72.4421 & 7.0995 & 305.1 & 4 & 600.0 & 600.0 \\
\hline 04 & 72119 & 67.7754 & 8.2800 & 303.5 & 4 & 600.0 & 600.0 \\
\hline 04 & 72120 & 65.6228 & 9.4350 & 301.8 & 4 & 600.0 & 600.0 \\
\hline 04 & 72121 & 67.3277 & 7.7700 & 300.3 & 4 & 600.0 & .0 \\
\hline $0<$ & 72122 & 68.3269 & 8.0100 & 300.2 & 4 & 600.0 & 600.0 \\
\hline 4 & 72123 & 52.0763 & 6.3038 & 300.1 & 4 & 600.0 & 600.0 \\
\hline
\end{tabular}




\begin{tabular}{|c|c|c|c|c|c|c|c|}
\hline 4 & 72124 & 47.9750 & 4.4405 & 299.9 & 4 & 600.0 & 600.0 \\
\hline 04 & 7221 & 40.6263 & 3.6753 & 299.5 & 4 & 600.0 & 600.0 \\
\hline 04 & 7222 & 59.1995 & 7.0118 & 300.4 & 4 & 600.0 & 00.0 \\
\hline 04 & 7223 & 67.4003 & 6.4100 & 300.6 & 4 & 600.0 & 00.0 \\
\hline 04 & 7224 & 77.5494 & 7.2218 & 300.3 & 4 & 600.0 & 00.0 \\
\hline 04 & 7225 & 74.1500 & 6.8185 & 299.7 & 4 & 600.0 & 00.0 \\
\hline 04 & 7226 & 80.5485 & 755 & 299.9 & 4 & 600.0 & 00.0 \\
\hline 04 & 7227 & 56.6044 & 3.7458 & 298.8 & 3 & 600.0 & 00.0 \\
\hline 04 & 7228 & 320.4394 & 1.7113 & 298.5 & 2 & 600.0 & 00.0 \\
\hline 04 & 7229 & 275.5682 & 1.9790 & 301.9 & 2 & 600.0 & 0 \\
\hline 04 & 72210 & 233.9096 & 3.8550 & 303.9 & 2 & 600.0 & \\
\hline 04 & 72211 & 299.1204 & 3.9125 & 304.0 & 2 & 600.0 & \\
\hline 04 & 72212 & 45.8761 & 4.3765 & 304.9 & 2 & 600.0 & 00.0 \\
\hline 04 & 72213 & 91.3765 & 6.2130 & 305.0 & 3 & 600.0 & \\
\hline 04 & 72214 & 82.7541 & 8. & 305.2 & 3 & .0 & \\
\hline 04 & 72215 & 81.5836 & 9.4875 & 305.0 & 4 & 600.0 & .0 \\
\hline 04 & 72216 & 74.8000 & 9.6925 & 304.8 & 4 & 60 & .0 \\
\hline 04 & 72217 & 68.9510 & 10.5975 & 303.8 & 4 & .0 & \\
\hline 04 & 72218 & 67.8251 & 10.8150 & 302.8 & 4 & .0 & \\
\hline 04 & 72219 & 68.2750 & 12.0350 & 301.6 & 4 & .0 & .0 \\
\hline 04 & 72220 & 67.1250 & 11. & 300.8 & 4 & 6 & \\
\hline 04 & 72221 & 68.0500 & 12. & 300.9 & 4 & .0 & \\
\hline 04 & 72222 & 67.2250 & 11.6075 & 301.0 & 4 & 600.0 & .0 \\
\hline 04 & 72223 & 67.9250 & 11.58 & 301.0 & 4 & 6 & \\
\hline 04 & 72224 & 66.0998 & 9. & 300.5 & 4 & 6 & \\
\hline 04 & 7231 & 67.1751 & 8.1600 & 300.3 & 4 & .0 & \\
\hline 04 & 7232 & 70.0500 & 7.8300 & 300.1 & 4 & 60 & .0 \\
\hline 04 & 7233 & 72.8250 & 7.9775 & 299.9 & 4 & .0 & \\
\hline 04 & 7234 & 72.7750 & 8.6175 & 298.5 & 4 & .0 & .0 \\
\hline 04 & 7235 & 78.5755 & 8.68 & 298.2 & 4 & 6 & .0 \\
\hline 04 & 7236 & 78.5250 & 9.30 & 297.7 & 4 & .0 & \\
\hline 04 & 7237 & 79.8250 & 9. & 298.7 & 4 & .0 & 0 \\
\hline 04 & 7238 & 77.2500 & 8.1025 & 300.2 & 4 & 600.0 & 0.0 \\
\hline 04 & 7239 & 89.4595 & 5.1525 & 300.9 & 3 & 0.0 & 0.0 \\
\hline 04 & 72310 & 240.0230 & 2.5373 & 299.8 & 2 & 600.0 & 600.0 \\
\hline 04 & 72311 & 112.9416 & 4.599 & 300.8 & 2 & 600.0 & 600.0 \\
\hline 04 & 72312 & 77.5202 & $6.71 \varepsilon$ & 301.3 & 3 & 600.0 & .0 \\
\hline 04 & 72313 & 70.7498 & 9.2450 & 301.5 & 3 & 600.0 & 600.0 \\
\hline 04 & 72314 & 72.8745 & 9.0250 & 303.0 & 3 & 600.0 & 600.0 \\
\hline 04 & 72315 & 72.9697 & 9.6900 & 303.1 & 4 & 600.0 & 600.0 \\
\hline 04 & 72316 & 73.3502 & 9.6350 & 302.9 & 4 & 600.0 & 600.0 \\
\hline 04 & 72317 & 70.7997 & 9.8350 & 302.2 & 4 & 600.0 & 600.0 \\
\hline 04 & 72318 & 74.0249 & 10.2325 & 300.8 & 4 & 600.0 & 600.0 \\
\hline 04 & 72319 & 74.8499 & 11.4525 & 298.9 & 4 & 600.0 & 600.0 \\
\hline 04 & 72320 & 76.2001 & 11.2350 & 297.9 & 4 & 600.0 & 600.0 \\
\hline 04 & 72321 & 70.4000 & 11.1175 & 298.0 & 4 & 600.0 & 600.0 \\
\hline 04 & 72322 & 69.9258 & 11.312 & 297.7 & 4 & 600.0 & 600.0 \\
\hline 04 & 72323 & 61.5244 & 10.477 & 297.8 & 4 & 600.0 & 600.0 \\
\hline
\end{tabular}




\begin{tabular}{|c|c|c|c|c|c|c|c|}
\hline 04 & 72324 & 66.7252 & 8.3525 & 296.6 & 4 & 600.0 & 600.0 \\
\hline 04 & $724 \quad 1$ & 69.4495 & 7.4875 & 298.1 & 4 & 600.0 & 600.0 \\
\hline 04 & 7242 & 66.7006 & 8.7925 & 297.1 & 4 & 600.0 & 00.0 \\
\hline 04 & 7243 & 63.8502 & 9.8450 & 296.5 & 4 & 600.0 & 600.0 \\
\hline 04 & $724 \quad 4$ & 64.9654 & 5.6220 & 295.8 & 4 & 600.0 & 00.0 \\
\hline 04 & 7245 & 83.1999 & 1.7608 & 296.1 & 5 & 600.0 & 00.0 \\
\hline 04 & 7246 & 134.3950 & 0.8208 & 296.3 & 5 & 600.0 & 600.0 \\
\hline 04 & 7247 & 124.4502 & 1.3013 & 295.3 & 4 & 600.0 & 600.0 \\
\hline 04 & 7248 & 351.6421 & 1.8195 & 296.5 & 3 & 600.0 & 00.0 \\
\hline 04 & 7249 & 104.1717 & 3.5353 & 297.2 & 2 & 600.0 & 600.0 \\
\hline 04 & 72410 & 91.9504 & 2.8190 & 298.6 & 2 & 600.0 & 600.0 \\
\hline 04 & 72411 & 179.2540 & 2.8453 & 300.7 & 2 & 600.0 & .0 \\
\hline 04 & 72412 & 30.6156 & 3.7910 & 301.3 & 1 & 600.0 & 600.0 \\
\hline 04 & 72413 & 39.0250 & 4.4668 & 302.4 & 2 & 600.0 & 600.0 \\
\hline 04 & 72414 & 50.3487 & 4.9855 & 302.9 & 2 & 600.0 & \\
\hline 04 & 72415 & 70.7844 & 5.6288 & 303.0 & 3 & 600.0 & 60 \\
\hline 04 & 72416 & 79.7494 & 5.7648 & 302.8 & 3 & 600.0 & 600.0 \\
\hline 04 & 72417 & 73.7751 & 5.9013 & 302.5 & 4 & 600.0 & \\
\hline 04 & 72418 & 69.3746 & 5.9408 & 301.4 & 4 & 600.0 & 60 \\
\hline 04 & 72419 & 77.3501 & 7.7125 & 298.6 & 4 & 600.0 & 600.0 \\
\hline 04 & 72420 & 76.3250 & 6.49 & 298.1 & 4 & 600.0 & .0 \\
\hline 04 & 72421 & 70.1145 & 8.1875 & 298.7 & 4 & 600.0 & \\
\hline 04 & 72422 & 73.6982 & 8.0325 & 298.4 & 4 & 600.0 & 600.0 \\
\hline 04 & 72423 & 69.5037 & 8.9950 & 297.3 & 4 & 600.0 & .0 \\
\hline 04 & 72424 & 71.5507 & 5.3985 & 297.9 & 4 & 600.0 & \\
\hline 04 & 7251 & 66.4286 & 1.6773 & 297.4 & 5 & 600.0 & 600.0 \\
\hline 04 & 7252 & 89.6576 & 1.9055 & 296.9 & 5 & 600.0 & 600.0 \\
\hline 04 & 7253 & 115.2242 & 1.2378 & 296.4 & 6 & 600.0 & .0 \\
\hline 04 & 7254 & 321.3759 & 0.9623 & 295.3 & 6 & 600.0 & 600.0 \\
\hline 04 & 7255 & 96.8193 & 0.7865 & 295.7 & 6 & 600.0 & 600.0 \\
\hline 04 & 7256 & 130.8836 & 1.0650 & 295.5 & 5 & 600.0 & .0 \\
\hline 04 & 7257 & 145.2656 & 0.9315 & 297.1 & 4 & 600.0 & 60 \\
\hline 04 & 7258 & 252.8465 & 1.4613 & 296.9 & 3 & 600.0 & 600.0 \\
\hline 04 & 7259 & 206.8724 & 3.0773 & 297.7 & 3 & 600.0 & .0 \\
\hline 04 & 72510 & 162.8255 & 3 . & 298.8 & 2 & 600.0 & 60 \\
\hline 04 & 72511 & 164.0001 & 4.3183 & 301.0 & 2 & 600.0 & 600.0 \\
\hline 04 & 72512 & 166.5002 & 4.3633 & 302.9 & 2 & 600.0 & 600.0 \\
\hline 04 & 72513 & 168.5253 & 5.1023 & 305.3 & 2 & 600.0 & 600.0 \\
\hline 04 & 72514 & 182.5445 & 5.2143 & 306.1 & 2 & 600.0 & 600.0 \\
\hline 04 & 72515 & 171.7495 & 5.6505 & 306.7 & 3 & 600.0 & \\
\hline 04 & 72516 & 174.7421 & 6.4408 & 307.0 & 4 & 600.0 & 600.0 \\
\hline 04 & 72517 & 182.8877 & 6.7098 & 306.3 & 4 & 600.0 & 600.0 \\
\hline 04 & 72518 & 166.2757 & 5.9163 & 305.7 & 3 & 600.0 & 600.0 \\
\hline 04 & 72519 & 108.8248 & 4.3880 & 304.6 & 4 & 600.0 & 600.0 \\
\hline 04 & 72520 & 78.2985 & 6.3845 & 303.0 & 4 & 600.0 & 600.0 \\
\hline 04 & 72521 & 75.2727 & 6.8503 & 302.3 & 4 & 600.0 & 600.0 \\
\hline 04 & 72522 & 70.2282 & 5.8100 & 303.1 & 4 & 600.0 & 600.0 \\
\hline 04 & 72523 & 70.0528 & 4.6770 & 303.3 & 4 & 600.0 & 600.0 \\
\hline
\end{tabular}




\begin{tabular}{|c|c|c|c|c|c|c|c|}
\hline 04 & 72524 & 75.7749 & 3.2868 & 303.8 & 4 & 600.0 & 600.0 \\
\hline 04 & 7261 & 59.0999 & 3.9308 & 303.1 & 4 & 600.0 & 00.0 \\
\hline 04 & 7262 & 53.4751 & 4.8570 & 302.8 & 4 & 600.0 & 00.0 \\
\hline 04 & 7263 & 71.6722 & 4.7878 & 302.9 & 4 & 600.0 & 00.0 \\
\hline 04 & 7264 & 122.9754 & 1.4815 & 300.8 & 5 & 600.0 & 00.0 \\
\hline 04 & 7265 & 148.8230 & 1.1805 & 300.8 & 6 & 600.0 & 00.0 \\
\hline 04 & 7266 & 204.8373 & 0.8955 & 300.6 & 5 & 600.0 & 00.0 \\
\hline 04 & 7267 & 260.6221 & 0.7855 & 301.1 & 4 & 600.0 & 00.0 \\
\hline 04 & 7268 & 203.4211 & 1.5510 & 301.6 & 3 & 600.0 & 00.0 \\
\hline 04 & 7269 & 212.0636 & 3.0005 & 302.9 & 3 & 600 & 0 \\
\hline 04 & 72610 & 200.2614 & 4.1435 & 302.4 & 2 & 600.0 & \\
\hline 04 & 72611 & 181.8425 & 3.4058 & 304.3 & 2 & 600.0 & \\
\hline 04 & 72612 & 228.2276 & 3.3825 & 305.3 & 1 & 600.0 & 00.0 \\
\hline 04 & 72613 & 170.5238 & 4.0373 & 307.0 & 2 & 600.0 & 00.0 \\
\hline 04 & 72614 & 149.9684 & 20 & 308.1 & 2 & .0 & \\
\hline 04 & 72615 & 98.8176 & 6.7785 & 307.9 & 3 & 600.0 & .0 \\
\hline 04 & 72616 & 112.0156 & 7.5618 & 307.6 & 4 & 60 & 600.0 \\
\hline 04 & 72617 & 81.9696 & 8.7650 & 306.5 & 4 & .0 & \\
\hline 04 & 72618 & 69.9998 & 8.7550 & 304.6 & 4 & 60 & \\
\hline 04 & 72619 & 63.5500 & 8.7475 & 302.9 & 4 & .0 & .0 \\
\hline 04 & 72620 & 64.7253 & 9.8125 & 2.1 & 4 & 6 & \\
\hline 04 & 72621 & 63.5498 & 9.7950 & 302.3 & 4 & .0 & \\
\hline 04 & 72622 & 66.7750 & 10.1750 & 302.0 & 4 & 600.0 & .0 \\
\hline 04 & 72623 & 67.3750 & 10 . & 301.7 & 4 & 6 & \\
\hline 04 & 72624 & 70.2500 & 600 & 301.1 & 4 & 60 & \\
\hline 04 & 7271 & 74.0000 & 10.5550 & 301.4 & 4 & .0 & \\
\hline 04 & 7272 & 64.6516 & 8.8650 & 301.7 & 4 & .0 & .0 \\
\hline 04 & 7273 & 53.2764 & 2.2513 & 301.3 & 5 & .0 & \\
\hline 04 & 7274 & 99.2929 & 1.7625 & 301.1 & 5 & .0 & .0 \\
\hline 04 & $727 \quad 5$ & 110.6440 & 2.0575 & 300.9 & 5 & 60 & .0 \\
\hline 04 & 7276 & 84.9440 & 1.4728 & 300.7 & 4 & .0 & \\
\hline 04 & 7277 & 330.3387 & 1.1130 & 297.2 & 3 & .0 & 0.0 \\
\hline 04 & 7278 & 300.6546 & 1.2588 & 297.1 & 2 & 600.0 & 0.0 \\
\hline 04 & 7279 & 249.3064 & 2.1448 & 299.0 & 2 & 600.0 & 0.0 \\
\hline 04 & 72710 & 232.7769 & 3.5815 & 300.9 & 2 & 600.0 & 600.0 \\
\hline 04 & 72711 & 226.0044 & 4.1700 & 301.8 & 2 & 600.0 & 600.0 \\
\hline 04 & 72712 & 223.4414 & 3.9818 & 303.6 & 2 & 600.0 & 0.0 \\
\hline 04 & 72713 & 265.9770 & 3.1225 & 305.0 & 1 & 600.0 & 600.0 \\
\hline 04 & 72714 & 347.5408 & 3.8595 & 305.8 & 2 & 600.0 & 600.0 \\
\hline 04 & 72715 & 71.6112 & 5.7568 & 305.1 & 3 & 600.0 & 600.0 \\
\hline 04 & 72716 & 85.2499 & 6.8718 & 304.4 & 3 & 600.0 & 600.0 \\
\hline 04 & 72717 & 73.8980 & 6.8225 & 303.4 & 4 & 600.0 & 600.0 \\
\hline 04 & 72718 & 73.9500 & 8.0425 & 302.0 & 4 & 600.0 & 600.0 \\
\hline 04 & 72719 & 71.1000 & 8.2875 & 299.7 & 4 & 600.0 & 600.0 \\
\hline 04 & 72720 & 58.4950 & 5.9485 & 299.3 & 4 & 600.0 & 600.0 \\
\hline 04 & 72721 & 86.3200 & 3.3665 & 299.7 & 4 & 600.0 & 600.0 \\
\hline $0<$ & 72722 & 70.2737 & 4.7485 & 300.4 & 4 & 600.0 & 600.0 \\
\hline 04 & 72723 & 68.0986 & 8.9925 & 300.1 & 4 & 600.0 & 600.0 \\
\hline
\end{tabular}




\begin{tabular}{|c|c|c|c|c|c|c|c|}
\hline 04 & 72724 & 74.8500 & 8.7825 & 300.8 & 4 & 600.0 & 600.0 \\
\hline 04 & 7281 & 75.6000 & 8.4400 & 301.0 & 4 & 600.0 & 600.0 \\
\hline 04 & 7282 & 75.7501 & 7.3183 & 300.5 & 4 & 600.0 & 00.0 \\
\hline 4 & 7283 & 106.3979 & 3.6753 & 299.4 & 4 & 600.0 & 00.0 \\
\hline 04 & 7284 & 48.1576 & 1.2683 & 297.7 & 5 & 600.0 & 00.0 \\
\hline 04 & 7285 & 73.1828 & 0.8620 & 297.0 & 6 & 600.0 & 00.0 \\
\hline 04 & 7286 & 136.6068 & 0.6395 & 296.7 & 5 & 600.0 & 00.0 \\
\hline 04 & 7287 & 92.7587 & 1.2988 & 295.2 & 4 & 600.0 & 00.0 \\
\hline 04 & $728 \quad 8$ & 309.9791 & 1.2928 & 295.7 & 3 & 600.0 & 00.0 \\
\hline 04 & 7289 & 277.2664 & 1.8180 & 297.0 & 2 & 600.0 & 0 \\
\hline 04 & 72810 & 244.8361 & 2.6183 & 299.0 & 2 & 600.0 & 00.0 \\
\hline 04 & 72811 & 237.4011 & 2.9065 & 301.0 & 2 & 600.0 & \\
\hline 04 & 72812 & 247.4190 & 2.8340 & 302.2 & 1 & 600.0 & 00.0 \\
\hline 04 & 72813 & 214.6325 & 2.8443 & 303.7 & 1 & 600.0 & 00.0 \\
\hline 04 & 72814 & 65.9811 & 5.0455 & 303.7 & 2 & .0 & \\
\hline 04 & 72815 & 77.9999 & 6.7793 & 303.1 & 3 & 600.0 & 00.0 \\
\hline 04 & 72816 & 71.9539 & 7.4635 & 302.6 & 4 & 60 & 600.0 \\
\hline 04 & 72817 & 72.0769 & 7.7850 & 302.1 & 4 & .0 & \\
\hline 04 & 72818 & 68.0746 & 7.2243 & 300.8 & 4 & 60 & .0 \\
\hline 04 & 72819 & 65.9497 & 7.2318 & 299.5 & 4 & .0 & .0 \\
\hline 04 & 72820 & 72.2565 & 5.6565 & 298.0 & 4 & 60 & \\
\hline 04 & 72821 & 78.8137 & 2.1768 & 298.6 & 5 & .0 & \\
\hline 04 & 72822 & 80.1495 & 1.9705 & 298.2 & 5 & 600.0 & .0 \\
\hline 04 & 72823 & 100.4474 & 1.3445 & 298.3 & 6 & 60 & .0 \\
\hline 04 & 72824 & 134.3336 & 1.4655 & 297.8 & 6 & .0 & \\
\hline 04 & 7291 & 105.8519 & 0.8945 & 294.5 & 6 & 600.0 & 600.0 \\
\hline 04 & 7292 & 191.3864 & 0.9730 & 293.6 & 6 & 600.0 & .0 \\
\hline 04 & 7293 & 160.8619 & 1.1153 & 292.1 & 6 & .0 & \\
\hline 04 & 7294 & 324.7985 & 1.3338 & 290.8 & 6 & .0 & 0.0 \\
\hline 04 & 7295 & 132.4208 & 1.0093 & 291.1 & 6 & .0 & .0 \\
\hline 04 & 7296 & 113.1688 & 1.1423 & 290.7 & 5 & 0 & \\
\hline 04 & 7297 & 185.7145 & 1.0540 & 291.4 & 4 & 600.0 & 600.0 \\
\hline 04 & 7298 & 285.4464 & 1.6390 & 293.2 & 3 & 600.0 & 600.0 \\
\hline 04 & 7299 & 257.2515 & 2.3615 & 294.6 & 2 & 600.0 & 0.0 \\
\hline 04 & 72910 & 240.3158 & 2.7903 & 296.1 & 2 & 600.0 & 600.0 \\
\hline 04 & 72911 & 232.0348 & 3.0040 & 297.7 & 2 & 600.0 & 600.0 \\
\hline 04 & 72912 & 254.2742 & 3.3190 & 299.7 & 2 & 600.0 & 600.0 \\
\hline 04 & 72913 & 279.5581 & 2.7908 & 301.2 & 1 & 600.0 & 600.0 \\
\hline 04 & 72914 & 320.2755 & 3.6543 & 303.1 & 2 & 600.0 & 600.0 \\
\hline 04 & 72915 & 14.0359 & 4.0173 & 303.5 & 2 & 600.0 & 600.0 \\
\hline 04 & 72916 & 58.7711 & 4.2358 & 303.5 & 2 & 600.0 & 600.0 \\
\hline 04 & 72917 & 71.8526 & 7.6345 & 302.0 & 3 & 600.0 & 600.0 \\
\hline 04 & 72918 & 74.2995 & 8.1800 & 300.6 & 4 & 600.0 & 600.0 \\
\hline 04 & 72919 & 69.2489 & 7.8800 & 298.6 & 4 & 600.0 & 600.0 \\
\hline 04 & 72920 & 67.4249 & 8.2800 & 297.2 & 4 & 600.0 & 600.0 \\
\hline 04 & 72921 & 66.0750 & 8.0815 & 297.8 & 4 & 600.0 & 600.0 \\
\hline 04 & 72922 & 71.3506 & 8.2438 & 298.8 & 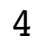 & 600.0 & 600.0 \\
\hline 4 & 72923 & 57.7667 & 4.4763 & 298.1 & $\Psi$ & 600.0 & 600.0 \\
\hline
\end{tabular}




\begin{tabular}{|c|c|c|c|c|c|c|c|}
\hline & 924 & 3.7128 & 5298 & 97.5 & & & \\
\hline & 301 & 190.8818 & .9090 & 295.3 & & $c 00 \Omega$ & \\
\hline & 302 & 34.5246 & 7785 & 294.3 & & 00 & \\
\hline & 303 & 99.3304 & 0.5993 & 294.0 & & 00.0 & \\
\hline & 304 & 104.4776 & 0.8118 & 92.7 & & & \\
\hline & 305 & 3088 & & 92.9 & & 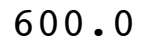 & \\
\hline & 306 & 119.7559 & 3543 & 292.6 & & 00 & \\
\hline & 307 & 61.3831 & 920 & 92.5 & & & \\
\hline & 308 & 336.3491 & 157 & 93.8 & & & \\
\hline & 309 & 257.8465 & 1.9780 & 295.8 & & 0 & \\
\hline & 3010 & 258.2783 & 2.7240 & 297.3 & & & \\
\hline & 3011 & 237.1909 & 3.1155 & 98.3 & & & \\
\hline & 3012 & 226.3345 & 3.0730 & 299.8 & & 0 & \\
\hline & 3013 & 215 & 3.2250 & 301.6 & & 00 & \\
\hline & 014 & 310 . & & & & & \\
\hline & 3015 & 48 . & & & & & \\
\hline & 3016 & 85.22 & 0 & 302.8 & & .0 & \\
\hline & 17 & 86 & 5 & .0 & & & \\
\hline & 3018 & & 0 & .8 & & & \\
\hline & 3019 & 72 & 8.4975 & 298.6 & & 0 & \\
\hline & 73020 & 61.1996 & 225 & 29 & & .0 & \\
\hline & 73021 & 62.17 & 5 & & & & \\
\hline & 73022 & 53 & 3.3155 & 295.4 & & & \\
\hline & 73023 & 86.80 & 1.6473 & 29 & & 0 & \\
\hline & 73024 & 92 & & 29 & & & \\
\hline & $\begin{array}{ll}731 & 1\end{array}$ & 76 & 2 . & 29 & 4 & & \\
\hline & 7312 & 80.1983 & $\$ 200$ & 294.3 & 4 & & . \\
\hline & 7313 & 71.4251 & 0 & 7 & 4 & & \\
\hline & 7314 & 7 & 0 & 28 & 4 & & \\
\hline & 7315 & 500 & 875 & 288.4 & 4 & & . \\
\hline & 7316 & & 9400 & 287.9 & 4 & & 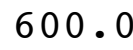 \\
\hline & 7317 & 5 & 5 & 288.4 & 4 & & \\
\hline & 7318 & 999 & 100 & 289.6 & 4 & & 。 \\
\hline & 7319 & 001 & 6 . & 291.7 & 4 & 0 & \\
\hline & & 86.7498 & 8 & 293.6 & 3 & & \\
\hline & & 87 . & 4.7170 & 295.4 & 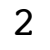 & & \\
\hline & 3112 & 62.3223 & 4.8115 & 297.4 & & & \\
\hline & 73113 & 65 . & 5.42 & 29 & 3 & & \\
\hline & 73114 & 71.7023 & 7.4345 & 298.2 & 3 & & . \\
\hline & 115 & 64.4253 & 100 & 298.2 & 4 & & \\
\hline & & 67.5248 & 675 & 297.3 & 4 & & \\
\hline & & 66.3245 & 12.2975 & 295.1 & 4 & & 0 . \\
\hline & 73118 & 69.5500 & 2.5550 & 293.5 & 4 & & 0 . \\
\hline & 73119 & 996 & 12.3400 & 291.7 & 4 & & \\
\hline & & 75.3250 & 13.1625 & 290.2 & $x^{2}$ & & \\
\hline & 73121 & 76.5496 & 13.8575 & 289.2 & 1 & & 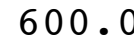 \\
\hline & & & & & & & \\
\hline & 73123 & 77.7248 & 15.8050 & 288.1 & & 600.0 & 600 \\
\hline
\end{tabular}




\begin{tabular}{|c|c|c|c|c|c|c|c|c|}
\hline 04 & & 124 & 73.8750 & 13.3800 & 287.9 & 4 & 600.0 & 00.0 \\
\hline 04 & 8 & 11 & 79.6502 & 13.8450 & 287.8 & 4 & 600.0 & 600.0 \\
\hline 04 & 8 & 12 & 79.1250 & 13.9950 & 287.5 & 4 & 600.0 & 00.0 \\
\hline 04 & 8 & 13 & 79.3750 & 12.3250 & 287.4 & 4 & 600.0 & 00.0 \\
\hline 04 & 8 & 14 & 30.6250 & 11.9675 & 287.1 & 4 & 600.0 & 00.0 \\
\hline 04 & 8 & 15 & 78.8500 & 11.4475 & 287.1 & 4 & 600.0 & 00.0 \\
\hline 04 & 8 & 16 & 73.9250 & 10.5075 & 287.2 & 4 & 600.0 & 600.0 \\
\hline 04 & 8 & 17 & 75.5001 & 9.5425 & 288.0 & 4 & 600.0 & 500.0 \\
\hline 04 & 8 & 18 & 82.0250 & 7.9025 & 289.5 & 4 & 600.0 & 00.0 \\
\hline 04 & 8 & 19 & 87.0748 & 5.9775 & 290.9 & 3 & 600.0 & .0 \\
\hline 04 & 8 & 110 & 79.3034 & 4.4553 & 292.9 & 2 & 600.0 & 500.0 \\
\hline 04 & 8 & 111 & 4.0617 & 4.4335 & 295.0 & 2 & 600.0 & \\
\hline 04 & 8 & 112 & 85.2105 & 4.9998 & 296.1 & 2 & 600.0 & 600.0 \\
\hline 04 & 8 & 113 & 98.1500 & 6.0370 & 296.9 & 3 & 600.0 & 600.0 \\
\hline 04 & 8 & 114 & 88.4747 & 7.0113 & 297.5 & 3 & 600.0 & \\
\hline 04 & 8 & 115 & 93.9002 & 8.0500 & 297.4 & 4 & 600.0 & 60 \\
\hline 04 & 8 & 116 & 83.9498 & 8.1075 & 297.1 & 4 & 600.0 & 600.0 \\
\hline 04 & 8 & 117 & 78.9739 & 9.2300 & 295.7 & 4 & 600.0 & \\
\hline 04 & 8 & 118 & 74.7250 & 10.4900 & 293.7 & 4 & 600.0 & 60 \\
\hline 04 & 8 & 119 & 71.4000 & 11.8700 & 291.0 & 4 & 600.0 & 60 \\
\hline 04 & 8 & 120 & 70.0251 & 13.74 & 288.6 & 4 & 600.0 & .0 \\
\hline 04 & 8 & 121 & 67.2000 & 12.8875 & 288.0 & 4 & 600.0 & \\
\hline 04 & 8 & 122 & 74.3250 & 11.7850 & 287.6 & 4 & 600.0 & 60 \\
\hline 04 & 8 & 123 & 74.1500 & 9.3625 & 288.6 & 4 & 600.0 & .0 \\
\hline 04 & 8 & 124 & 74.8250 & 11 & 287.6 & 4 & 600.0 & \\
\hline 04 & 8 & 21 & 75.4750 & 625 & 286.7 & 4 & 600.0 & 60 \\
\hline 04 & 8 & 22 & 74.5750 & 14.1000 & 286.5 & 4 & 600.0 & 60 \\
\hline 04 & 8 & 23 & 70.7532 & 12.5975 & 286.5 & 4 & 600.0 & .0 \\
\hline 04 & 8 & 24 & 60.9249 & 12.0300 & 286.2 & 4 & 600.0 & 60 \\
\hline 04 & 8 & 25 & 61.9751 & 12.1600 & 286.0 & 4 & 600.0 & 60 \\
\hline 04 & 8 & 26 & 67.5000 & 10. & 286.1 & 4 & 600.0 & \\
\hline 04 & 8 & 27 & 69.3235 & 10.2975 & 286.8 & 4 & 600.0 & 60 \\
\hline 04 & 8 & 28 & 78.5500 & 9.4800 & 287.8 & 4 & 600.0 & 600.0 \\
\hline 04 & 8 & 29 & 81.7738 & 8.472 & 288.8 & 4 & 600.0 & .0 \\
\hline 04 & 8 & 210 & 84.5012 & 6. & 290.4 & 4 & 600.0 & \\
\hline 04 & 8 & 211 & 85.9003 & 6.9270 & 291.4 & 4 & 600.0 & 600.0 \\
\hline 04 & 8 & 212 & 84.2010 & 6.5395 & 292.9 & 3 & 600.0 & \\
\hline 04 & 8 & 213 & 85.3000 & 7.9100 & 293.5 & 3 & 600.0 & 600.0 \\
\hline 04 & 8 & 214 & 78.8252 & 9.2250 & 293.7 & 3 & 600.0 & 600.0 \\
\hline 04 & 8 & 215 & 72.8249 & 9.9425 & 293.5 & 4 & 600.0 & \\
\hline 04 & 8 & 216 & 71.3997 & 12.3500 & 292.6 & 4 & 600.0 & 600.0 \\
\hline 04 & 8 & 217 & 70.9243 & 13.7175 & 291.3 & 4 & 600.0 & 600.0 \\
\hline 04 & 8 & 218 & 74.2250 & 13.5625 & 290.0 & 4 & 600.0 & 600.0 \\
\hline 04 & 8 & 219 & 73.4997 & 13.4900 & 288.7 & 4 & 600.0 & 600.0 \\
\hline 04 & 8 & 220 & 74.0250 & 13.8900 & 287.8 & 4 & 600.0 & 600.0 \\
\hline 04 & 8 & 221 & 77.5500 & 14.0375 & 287.8 & 4 & 600.0 & 600.0 \\
\hline 04 & 8 & 222 & 79.3000 & 13.867 & 287.7 & 4 & 600.0 & 600.0 \\
\hline 04 & 8 & 223 & 75.2500 & 12.5675 & 288.6 & 4 & 600.0 & 600.0 \\
\hline
\end{tabular}




\begin{tabular}{|c|c|c|c|c|c|c|c|c|}
\hline 4 & 8 & 224 & 77.9000 & 10.8400 & 289.5 & 4 & 600.0 & 600.0 \\
\hline 04 & 8 & 31 & 75.8000 & 9.8300 & 289.8 & 4 & 600.0 & 00.0 \\
\hline 04 & 8 & 32 & 76.6270 & 7.4753 & 289.8 & 4 & 600.0 & 00.0 \\
\hline 04 & 8 & 33 & 75.8750 & 8.5525 & 289.1 & 4 & 600.0 & 00.0 \\
\hline 04 & 8 & 34 & 79.0500 & 000 & 289.6 & 4 & 600.0 & 00.0 \\
\hline 04 & 8 & 35 & 79.1000 & 8.4325 & 289.4 & 4 & 600.0 & 00.0 \\
\hline 04 & 8 & 36 & 96.8870 & 3.5715 & 289.6 & 4 & 600.0 & 00.0 \\
\hline 04 & 8 & 37 & 82.5206 & 1.4385 & 290.2 & 3 & 600.0 & 00.0 \\
\hline 04 & 8 & 38 & 177.9578 & 2.1903 & 292.1 & 2 & 600.0 & 00.0 \\
\hline 04 & 8 & 39 & 246.7923 & 2.2398 & 293.9 & 2 & 600.0 & .0 \\
\hline 04 & 8 & 310 & 252.1232 & 2.6513 & 295.1 & 2 & 600.0 & \\
\hline 04 & 8 & 311 & 208.6866 & 4.1400 & 296.8 & 2 & 600.0 & \\
\hline 04 & 8 & 312 & 172.2293 & 5.2470 & 297.9 & 3 & 600.0 & 00.0 \\
\hline 04 & 8 & 313 & 170.4500 & 5.3568 & 299.4 & 2 & 600.0 & 00.0 \\
\hline 04 & 8 & 314 & 181.8693 & 6 . & 300.6 & 3 & .0 & \\
\hline 04 & 8 & 315 & 170.3736 & 5.9975 & 301.8 & 3 & 600.0 & .0 \\
\hline 04 & 8 & 316 & 156.0746 & 5.2260 & 302.3 & 3 & 60 & 00.0 \\
\hline 04 & 8 & 317 & 134.8575 & 5.2310 & 302.2 & 3 & .0 & \\
\hline 04 & 8 & 318 & 97.6036 & 5.5410 & 301.1 & 4 & 60 & .0 \\
\hline 04 & 8 & 319 & 80.5002 & 7.2740 & 298.8 & 4 & .0 & .0 \\
\hline 04 & 8 & 320 & 80.1253 & 10.3200 & 297.0 & 4 & 6 & \\
\hline 04 & 8 & 321 & 77.4780 & 11. & 296.6 & 4 & .0 & \\
\hline 04 & 8 & 322 & 65.8489 & 11.6800 & 295.4 & 4 & 600.0 & .0 \\
\hline 04 & 8 & 323 & 65.1998 & 11.00 & 295.3 & 4 & 6 & \\
\hline 04 & 8 & 324 & 65.9238 & 9 . & 296.2 & 4 & .0 & \\
\hline 04 & 8 & 41 & 68.9000 & 9.8250 & 296.6 & 4 & .0 & 600.0 \\
\hline 04 & 8 & 42 & 69.2748 & 7.6420 & 296.8 & 4 & 60 & .0 \\
\hline 04 & 8 & 43 & 99.7208 & 3.2383 & 295.5 & 4 & .0 & \\
\hline 04 & 8 & 44 & 83.1660 & 5.2248 & 296.2 & 4 & .0 & 0.0 \\
\hline 04 & 8 & 45 & 86.5214 & 4.3425 & 295.5 & 4 & 60 & 0.0 \\
\hline 04 & 8 & 46 & 60.4312 & 5.6103 & 295.1 & 4 & .0 & \\
\hline 04 & 8 & 47 & 73.6120 & 5.5330 & 294.8 & 3 & .0 & 0.0 \\
\hline 04 & 8 & 48 & 253.6039 & 1.7945 & 296.5 & 2 & 600.0 & 0.0 \\
\hline 04 & 8 & 49 & 331.8730 & 1.9750 & 296.8 & 2 & 600.0 & 0.0 \\
\hline 04 & 8 & 410 & 251.4536 & 2.61 & 298.5 & 2 & 600.0 & 600.0 \\
\hline 04 & 8 & 411 & 233.3541 & 3.2518 & 299.5 & 2 & 600.0 & 600.0 \\
\hline 04 & 8 & 412 & 129.9454 & 3.891 & 300.4 & 2 & 600.0 & .0 \\
\hline 04 & 8 & 413 & 72.5000 & 6.7373 & 301.0 & 3 & 600.0 & 600.0 \\
\hline 04 & 8 & 414 & 73.0498 & 8.5600 & 301.2 & 3 & 600.0 & 600.0 \\
\hline 04 & 8 & 415 & 72.2250 & 9.2500 & 300.7 & 4 & 600.0 & 600.0 \\
\hline 04 & 8 & 416 & 67.3754 & 8.7650 & 300.4 & 4 & 600.0 & 600.0 \\
\hline 04 & 8 & 417 & 62.6956 & 11.3175 & 299.3 & 4 & 600.0 & 600.0 \\
\hline 04 & 8 & 418 & 59.7249 & 11.9050 & 298.4 & 4 & 600.0 & 600.0 \\
\hline 04 & 8 & 419 & 57.7500 & 11.7675 & 297.1 & 4 & 600.0 & 600.0 \\
\hline 04 & 8 & 420 & 56.8250 & 13.2025 & 295.1 & 4 & 600.0 & 600.0 \\
\hline 04 & 8 & 421 & 58.2499 & 14.6225 & 294.0 & 4 & 600.0 & 600.0 \\
\hline $0<$ & 8 & 422 & 57.6500 & 13.8775 & 293.8 & 4 & 600.0 & 600.0 \\
\hline 04 & 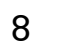 & 423 & 60.5500 & 12.7350 & 294.0 & 4 & 600.0 & 600.0 \\
\hline
\end{tabular}




\begin{tabular}{|c|c|c|c|c|c|c|c|c|}
\hline 4 & 8 & 424 & 61.8750 & 12.0100 & 294.3 & 4 & 600.0 & 600.0 \\
\hline 04 & 8 & 51 & 62.8000 & 10.5950 & 294.3 & 4 & 600.0 & 00.0 \\
\hline 04 & 8 & 52 & 64.6003 & 10.6875 & 294.2 & 4 & 600.0 & 00.0 \\
\hline 04 & 8 & 53 & 65.8988 & 10.1075 & 294.3 & 4 & 600.0 & 00.0 \\
\hline 04 & 8 & 54 & 67.7254 & 9.7225 & 293.8 & 4 & 600.0 & 00.0 \\
\hline 04 & 8 & 55 & 74.7517 & 8.7800 & 291.5 & 4 & 600.0 & 00.0 \\
\hline 04 & 8 & 56 & 69.8500 & 9.7225 & 291.6 & 4 & 600.0 & 00.0 \\
\hline 04 & 8 & 57 & 69.7251 & 9.2925 & 292.4 & 4 & 600.0 & 00.0 \\
\hline 04 & 8 & 58 & 75.4500 & 9.7875 & 292.9 & 4 & 600.0 & 00.0 \\
\hline 04 & 8 & 59 & 81.5601 & 8.0803 & 293.8 & 4 & 600.0 & 0 \\
\hline 04 & 8 & 510 & 90.8285 & 5.8788 & 295.2 & 3 & 600.0 & \\
\hline 04 & 8 & 511 & 75.3256 & 6.1015 & 297.1 & 3 & 600.0 & \\
\hline 04 & 8 & 512 & 83.2023 & 6.5333 & 298.0 & 3 & 600.0 & 00.0 \\
\hline 04 & 8 & 513 & 85.7497 & 8.6175 & 298.2 & 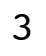 & 600.0 & 00.0 \\
\hline 04 & 8 & 514 & 101.6017 & 75 & 298.8 & 3 & .0 & \\
\hline 04 & 8 & 515 & 96.6501 & 8.1900 & 299.0 & 4 & 600.0 & .0 \\
\hline 04 & 8 & 516 & 73.4326 & 8.7005 & 298.6 & 4 & 60 & 0.0 \\
\hline 04 & 8 & 517 & 69.4501 & 10.8325 & 297.5 & 4 & .0 & \\
\hline 04 & 8 & 518 & 65.7999 & 11.2950 & 295.9 & 4 & 60 & .0 \\
\hline 04 & 8 & 519 & 58.5250 & 15.4850 & 294.4 & 4 & .0 & .0 \\
\hline 04 & 8 & 520 & 58.2749 & 14.2525 & 293.3 & 4 & 6 & \\
\hline 04 & 8 & 521 & 58.4000 & 12.9075 & 293.5 & 4 & .0 & \\
\hline 04 & 8 & 522 & 59.2000 & 11.8650 & 292.6 & 4 & 600.0 & .0 \\
\hline 04 & 8 & 523 & 56.6719 & 12.0 & 292.0 & 4 & 6 & \\
\hline 04 & 8 & 524 & 69.3004 & 10.5550 & 291.0 & 4 & .0 & \\
\hline 04 & 8 & 61 & 66.3748 & 11.1525 & 290.7 & 4 & .0 & 600.0 \\
\hline 04 & 8 & 62 & 61.8503 & 11.2525 & 291.7 & 4 & .0 & .0 \\
\hline 04 & 8 & 63 & 70.8929 & 10.6825 & 291.3 & 4 & .0 & \\
\hline 04 & 8 & 64 & 71.4969 & 10.2950 & 291.4 & 4 & .0 & 0.0 \\
\hline 04 & 8 & 65 & 71.2251 & 10.7975 & 290.7 & 4 & .0 & .0 \\
\hline 04 & 8 & 66 & 67.4784 & 11.1725 & 291.1 & 4 & .0 & \\
\hline 04 & 8 & 67 & 69.5756 & 10.0475 & 291.5 & 4 & .0 & 0.0 \\
\hline 04 & 8 & 68 & 68.1734 & 9.4800 & 291.8 & 4 & 600.0 & 600.0 \\
\hline 04 & 8 & & 66.2997 & 8.4400 & 293.4 & 4 & 600.0 & 0.0 \\
\hline 04 & 8 & 610 & 66.4742 & 7.9975 & 294.9 & 4 & 600.0 & 600.0 \\
\hline 04 & 8 & 611 & 77.9333 & 7.5620 & 295.9 & 3 & 600.0 & 600.0 \\
\hline 04 & 8 & 612 & 86.2498 & 7.3223 & 297.1 & 3 & 600.0 & 600.0 \\
\hline 04 & 8 & 613 & 91.4737 & 7.3900 & 298.2 & 3 & 600.0 & 600.0 \\
\hline 04 & 8 & 614 & 84.9939 & 6.9178 & 299.5 & 3 & 600.0 & 600.0 \\
\hline 04 & 8 & 615 & 96.1487 & 5.8998 & 299.9 & 3 & 600.0 & 600.0 \\
\hline 04 & 8 & 616 & 106.4348 & 6.8538 & 299.7 & 3 & 600.0 & 600.0 \\
\hline 04 & 8 & 617 & 79.7299 & 8.7400 & 298.2 & 4 & 600.0 & 600.0 \\
\hline 04 & 8 & 618 & 71.9504 & 8.1500 & 296.9 & 4 & 600.0 & 600.0 \\
\hline 04 & 8 & 619 & 65.0004 & 9.8925 & 295.3 & 4 & 600.0 & 600.0 \\
\hline 04 & 8 & 620 & 61.9524 & 9.9900 & 294.9 & 4 & 600.0 & 600.0 \\
\hline 04 & 8 & 621 & 65.0750 & 9.5175 & 294.8 & 4 & 600.0 & 600.0 \\
\hline $0<$ & 8 & 622 & 67.0249 & 9.6800 & 294.6 & 4 & 600.0 & 600.0 \\
\hline 04 & 8 & 623 & 69.7488 & 9.0850 & 294.5 & $\Psi$ & 600.0 & 600.0 \\
\hline
\end{tabular}




\begin{tabular}{|c|c|c|c|c|c|c|c|c|}
\hline 04 & 8 & 624 & 72.9511 & 8.9850 & 294.7 & 4 & 600.0 & 600.0 \\
\hline 04 & 8 & 71 & 75.2753 & 8.8625 & 294.8 & 4 & 600.0 & 600.0 \\
\hline 04 & 8 & 72 & 74.2997 & 8.2950 & 294.4 & 4 & 600.0 & 00.0 \\
\hline 04 & 8 & 73 & 72.1500 & 8.4175 & 294.1 & 4 & 600.0 & 600.0 \\
\hline 04 & 8 & 74 & 72.7750 & 7.4145 & 294.2 & 4 & 600.0 & 00.0 \\
\hline 04 & 8 & 75 & 80.9762 & 6.8110 & 294.4 & 4 & 600.0 & 00.0 \\
\hline 04 & 8 & 76 & 90.1254 & 5.9478 & 294.4 & 4 & 600.0 & 00.0 \\
\hline 04 & 8 & 77 & 99.2745 & 4.4168 & 295.8 & 3 & 600.0 & 00.0 \\
\hline 04 & 8 & 78 & 165.9798 & 4.1785 & 298.0 & 3 & 600.0 & 00.0 \\
\hline 04 & 8 & 79 & 169.7249 & 5.3903 & 298.5 & 4 & 600.0 & 00.0 \\
\hline 04 & 8 & 710 & 172.0751 & 5.7668 & 299.3 & 3 & 600.0 & 00.0 \\
\hline 04 & 8 & 711 & 167.8248 & 6.2518 & 300.1 & 3 & 600.0 & 00.0 \\
\hline 04 & 8 & 712 & 174.9069 & 6.6483 & 301.0 & 3 & 600.0 & 00.0 \\
\hline 04 & 8 & 713 & 181.7713 & 5.9600 & 302.2 & 3 & 600.0 & 00.0 \\
\hline 04 & 8 & 714 & 178.5418 & 5.7543 & 303.1 & 3 & .0 & \\
\hline 04 & 8 & 715 & 170.0258 & 5.0285 & 303.9 & 2 & 600.0 & 00.0 \\
\hline 04 & 8 & 716 & 172.0865 & 5.3665 & 303.9 & 3 & 600.0 & 600.0 \\
\hline 04 & 8 & 717 & 191.9272 & 5.5003 & 303.9 & 4 & .0 & \\
\hline 04 & 8 & 718 & 187.5539 & 4.9413 & 303.1 & 3 & 600.0 & 00.0 \\
\hline 04 & 8 & 719 & 143.2092 & 4.4443 & 302.1 & 4 & .0 & .0 \\
\hline 04 & 8 & 720 & 72.9249 & 5.78 & 300.6 & 4 & .0 & \\
\hline 04 & 8 & 721 & 71.9749 & 6.8833 & 300.0 & 4 & 600.0 & \\
\hline 04 & 8 & 722 & 68.1001 & 7.3525 & 299.7 & 4 & 600.0 & 600.0 \\
\hline 04 & 8 & 723 & 69.2001 & 7.77 & 299.5 & 4 & .0 & .0 \\
\hline 04 & 8 & 724 & 73.3000 & 8.1825 & 299.3 & 4 & .0 & \\
\hline 04 & 8 & 81 & 70.9750 & 7.5750 & 299.7 & 4 & 600.0 & 600.0 \\
\hline 04 & 8 & 82 & 76.6501 & 6.7513 & 300.1 & 4 & 600.0 & .0 \\
\hline 04 & 8 & 83 & 81.0253 & 5.0695 & 300.4 & 4 & 600.0 & \\
\hline 04 & 8 & 84 & 96.3399 & 0.9233 & 300.2 & 5 & 600.0 & 600.0 \\
\hline 04 & 8 & 85 & 36.5453 & 1.4995 & 299.8 & 6 & 600.0 & 600.0 \\
\hline 04 & 8 & 86 & 15.2869 & 1.0398 & 299.5 & 5 & 600.0 & .0 \\
\hline 04 & 8 & 87 & 310.4958 & 0.6518 & 299.6 & 4 & 600.0 & 600.0 \\
\hline 04 & 8 & 88 & 250.6740 & 1.4093 & 300.8 & 3 & 600.0 & 600.0 \\
\hline 04 & 8 & 89 & 257.2677 & 2.3823 & 302.2 & 3 & 600.0 & 0.0 \\
\hline 04 & 8 & 810 & 228.4585 & 3.2275 & 303.2 & 2 & 600.0 & 600.0 \\
\hline 04 & 8 & 811 & 209.2777 & 4.2285 & 304.6 & 2 & 600.0 & 600.0 \\
\hline 04 & 8 & 812 & 184.0079 & 4.5950 & 305.1 & 2 & 600.0 & 0.0 \\
\hline 04 & 8 & 813 & 177.6133 & 4.6868 & 305.8 & 2 & 600.0 & 600.0 \\
\hline 04 & 8 & 814 & 173.9733 & 5.0540 & 306.5 & 2 & 600.0 & 600.0 \\
\hline 04 & 8 & 815 & 178.5658 & 3.1393 & 306.9 & 2 & 600.0 & 600.0 \\
\hline 04 & 8 & 816 & 177.8000 & 5.9688 & 306.8 & 3 & 600.0 & 600.0 \\
\hline 04 & 8 & 817 & 107.1305 & 6.1465 & 306.2 & 4 & 600.0 & 600.0 \\
\hline 04 & 8 & 818 & 82.1296 & 6.4735 & 304.2 & 4 & 600.0 & 600.0 \\
\hline 04 & 8 & 819 & 78.6503 & 8.1475 & 301.8 & 4 & 600.0 & 600.0 \\
\hline 04 & 8 & 820 & 72.6749 & 9.3025 & 300.5 & 4 & 600.0 & 600.0 \\
\hline 04 & 8 & 821 & 72.9750 & 9.9100 & 300.0 & 4 & 600.0 & 600.0 \\
\hline 04 & 8 & 822 & 76.1750 & 9.242 & 300.1 & 4 & 600.0 & 600.0 \\
\hline 04 & 8 & 823 & 75.5750 & 9.137 & 300 . & 4 & 600.0 & 600.0 \\
\hline
\end{tabular}




\begin{tabular}{|c|c|c|c|c|c|c|c|}
\hline 4 & $8 \quad 824$ & 76.4000 & 8.5450 & 300.2 & 4 & 600.0 & 600.0 \\
\hline 04 & $\begin{array}{lll}8 & 9 & 1\end{array}$ & 77.5249 & 5.6018 & 300.9 & 4 & 600.0 & 00.0 \\
\hline 04 & 892 & 72.9765 & 4.4273 & 301.0 & 4 & 600.0 & 00.0 \\
\hline 04 & 893 & 72.3055 & 2.5333 & 301.3 & 4 & 600.0 & 00.0 \\
\hline 04 & 894 & 76.8750 & 4.9360 & 299.8 & 4 & 600.0 & 00.0 \\
\hline 04 & 895 & 76.9500 & 5.6163 & 299.0 & 4 & 600.0 & 00.0 \\
\hline 04 & 896 & 76.0501 & 4.8808 & 299.4 & 4 & 600.0 & 00.0 \\
\hline 04 & $\begin{array}{lll}8 & 9 & 7\end{array}$ & 70.3593 & 1.9818 & 301.1 & 3 & 600.0 & 00.0 \\
\hline 04 & 898 & 344.4055 & 1.5153 & 300.4 & 2 & 600.0 & 00.0 \\
\hline 04 & 899 & 298.5590 & 1.8393 & 300.5 & 2 & 600.0 & .0 \\
\hline 04 & 8910 & 260.8045 & 2.3728 & 301.8 & 2 & 600.0 & \\
\hline 04 & 8911 & 234.3969 & 73 & 302.9 & 2 & 600.0 & \\
\hline 04 & 8912 & 118.4343 & 3.0825 & 304.4 & 2 & 600.0 & 00.0 \\
\hline 04 & 8913 & 80.8000 & 6.2343 & 304.2 & 2 & 600.0 & 00.0 \\
\hline 04 & 8914 & 82.1507 & 7 . & 304.1 & 3 & .0 & \\
\hline 04 & 8915 & 75.2461 & 8 . & 303.8 & 4 & 600.0 & 00.0 \\
\hline 04 & 8916 & 68.1000 & 10.5625 & 302.5 & 4 & 60 & 600.0 \\
\hline 04 & 8917 & 70.7000 & 9. & 302.8 & 4 & .0 & \\
\hline 04 & 8918 & 69.2251 & 11. & 301.8 & 4 & 60 & .0 \\
\hline 04 & 8919 & 66.8745 & 12.6600 & 300.2 & 4 & .0 & .0 \\
\hline 04 & 8920 & 64.4499 & 12 . & 9.6 & 4 & 6 & \\
\hline 04 & 8921 & 70.3752 & 11. & 299.2 & 4 & .0 & \\
\hline 04 & 8922 & 64.0250 & 11.0275 & 299.3 & 4 & 600.0 & .0 \\
\hline 04 & 8923 & 66.9998 & 10 . & .7 & 4 & 6 & \\
\hline 04 & 8924 & 70.8249 & 10 . & .8 & 4 & .0 & \\
\hline 04 & 8101 & 74.5000 & 9.8475 & 299.8 & 4 & .0 & \\
\hline 04 & 8102 & 74.6250 & 9.82 & 299.2 & 4 & 60 & .0 \\
\hline 04 & 8103 & 76.6250 & 9. & 298.7 & 4 & .0 & \\
\hline 04 & 8104 & 76.9500 & 9. & 298.3 & 4 & .0 & 0.0 \\
\hline 04 & 8105 & 80.3251 & 8.0278 & 298.3 & 4 & 60 & .0 \\
\hline 04 & 8106 & 83.1250 & 7 . & 298.4 & 4 & .0 & \\
\hline 04 & 8107 & 146.2157 & 2 & .3 & 3 & .0 & 0.0 \\
\hline 04 & 8108 & 354.9840 & 1.0383 & 298.2 & 2 & 600.0 & 600.0 \\
\hline 04 & 8109 & 217.0564 & 2 . & 299.4 & 2 & 600.0 & 0.0 \\
\hline 04 & 81010 & 172.4271 & 5. & 300.2 & 2 & 600.0 & 600.0 \\
\hline 04 & 81011 & 164.3749 & 5.9405 & 301.6 & 3 & 600.0 & 600.0 \\
\hline 04 & 81012 & 166.3246 & $6 . \varepsilon$ & 302.9 & 3 & 600.0 & 0.0 \\
\hline 04 & 81013 & 164.2250 & 7.2398 & 304.3 & 3 & 600.0 & 600.0 \\
\hline 04 & 81014 & 167.8967 & 6.8850 & 305.8 & 3 & 600.0 & 600.0 \\
\hline 04 & 81015 & 168.1502 & 6.84 & 306.8 & 4 & 600.0 & .0 \\
\hline 04 & 81016 & 152.0989 & 5.7383 & 307.6 & 3 & 600.0 & 600.0 \\
\hline 04 & 81017 & 143.6423 & 5.8363 & 307.2 & 3 & 600.0 & 600.0 \\
\hline 04 & 81018 & 123.0529 & 5.2188 & 306.4 & 4 & 600.0 & 600.0 \\
\hline 04 & 81019 & 153.8852 & 3.1000 & 303.7 & 4 & 600.0 & 600.0 \\
\hline 04 & 81020 & 96.4433 & 3.6195 & 302.9 & 4 & 600.0 & 600.0 \\
\hline 04 & 81021 & 74.8000 & 6.9740 & 302.6 & 4 & 600.0 & 600.0 \\
\hline 04 & 81022 & 75.6251 & 8.1900 & 302.0 & 4 & 600.0 & 600.0 \\
\hline 04 & 81023 & 77.0000 & 9.4325 & 302.1 & $\Psi$ & 600.0 & 600.0 \\
\hline
\end{tabular}




\begin{tabular}{|c|c|c|c|c|c|c|c|}
\hline 04 & 81024 & 77.1750 & 8.5500 & 302.2 & 4 & 600.0 & 600.0 \\
\hline 04 & 8111 & 75.3000 & 7.8075 & 302.3 & 4 & 600.0 & 600.0 \\
\hline 04 & 8112 & 71.6761 & 3.9180 & 303.8 & 4 & 600.0 & 00.0 \\
\hline 04 & 8113 & 81.6505 & 1.0028 & 303.8 & 5 & 600.0 & 00.0 \\
\hline 04 & 8114 & 42.3719 & 1.9025 & 303.5 & 5 & 600.0 & 00.0 \\
\hline 04 & 8115 & 105.9265 & 0.7823 & 303.2 & 6 & 600.0 & 00.0 \\
\hline 04 & 8116 & 139.5386 & 1.7638 & 302.3 & 5 & 600.0 & 00.0 \\
\hline 04 & 8117 & 161.3186 & 2.8245 & 302.4 & 4 & 600.0 & 00.0 \\
\hline 04 & 8118 & 170.0503 & 4.1833 & 302.6 & 3 & 600.0 & 00.0 \\
\hline 04 & 8119 & 156.5913 & 4.7410 & 302.1 & 3 & 600.0 & 0 \\
\hline 04 & 81110 & 159.6570 & 5.5563 & 303.2 & 3 & 600.0 & 00.0 \\
\hline 04 & 81111 & 162.6500 & 6.7598 & 303.0 & 4 & 600.0 & \\
\hline 04 & 81112 & 164.7000 & 6.8998 & 304.6 & 3 & 600.0 & 00.0 \\
\hline 04 & 81113 & 162.5000 & 5.6573 & 306.5 & 3 & 600.0 & 00.0 \\
\hline 04 & 81114 & 166.3750 & 758 & 307.9 & 3 & .0 & \\
\hline 04 & 81115 & 169.5472 & 5.4975 & 308.8 & 3 & 600.0 & 00.0 \\
\hline 04 & 81116 & 172.7229 & 6.0110 & 309.2 & 3 & 600.0 & 600.0 \\
\hline 04 & 81117 & 178.3212 & 5.8078 & 308.9 & 4 & .0 & \\
\hline 04 & 81118 & 178.0544 & 4.7775 & 308.1 & 3 & 600.0 & .0 \\
\hline 04 & 81119 & 124.7690 & 3.9603 & 306.7 & 4 & .0 & .0 \\
\hline 04 & 81120 & 71.5751 & 5.8278 & 305.2 & 4 & .0 & \\
\hline 04 & 81121 & 71.6499 & 7.2078 & 304.5 & 4 & .0 & \\
\hline 04 & 81122 & 75.9000 & 8.2375 & 304.1 & 4 & 600.0 & 0 \\
\hline 04 & 81123 & 76.8996 & 8.3350 & 303.8 & 4 & 60 & .0 \\
\hline 04 & 81124 & 76.1250 & 8.3200 & 303.4 & 4 & .0 & \\
\hline 04 & 8121 & 75.0999 & 7.5903 & 302.9 & 4 & 600.0 & 600.0 \\
\hline 04 & 8122 & 74.7500 & 7.0655 & 302.8 & 4 & 600.0 & .0 \\
\hline 04 & 8123 & 73.6999 & 4.7333 & 303.5 & 4 & 600.0 & \\
\hline 04 & 8124 & 75.9000 & 7.1265 & 301.9 & 4 & .0 & 600.0 \\
\hline 04 & 8125 & 77.8000 & 8.7475 & 300.6 & 4 & 600.0 & 0.0 \\
\hline 04 & 8126 & 73.9500 & 8.0775 & 301.1 & 4 & .0 & \\
\hline 04 & 8127 & 93.0382 & 3.1288 & 303.2 & 3 & .0 & 600.0 \\
\hline 04 & 8128 & 322.2703 & 1.0718 & 301.6 & 2 & 600.0 & 600.0 \\
\hline 04 & 8129 & 299.8149 & 2.0580 & 301.6 & 2 & 600.0 & 0.0 \\
\hline 04 & 81210 & 237.9998 & 2.9413 & 303.2 & 2 & 600.0 & 600.0 \\
\hline 04 & 81211 & 240.6431 & 2.9230 & 304.0 & 2 & 600.0 & 600.0 \\
\hline 04 & 81212 & 275.7779 & 3.1378 & 305.0 & 1 & 600.0 & 600.0 \\
\hline 04 & 81213 & 50.1472 & 4.6395 & 306.8 & 2 & 600.0 & 600.0 \\
\hline 04 & 81214 & 83.1750 & 4.9933 & 306.6 & 2 & 600.0 & 600.0 \\
\hline 04 & 81215 & 85.9497 & 7.0330 & 305.9 & 3 & 600.0 & 600.0 \\
\hline 04 & 81216 & 82.0748 & 7.1358 & 305.2 & 4 & 600.0 & 600.0 \\
\hline 04 & 81217 & 71.8240 & 7.7000 & 304.0 & 4 & 600.0 & 600.0 \\
\hline 04 & 81218 & 71.3000 & 8.2425 & 302.2 & 4 & 600.0 & 600.0 \\
\hline 04 & 81219 & 68.4001 & 8.7775 & 300.0 & 4 & 600.0 & 600.0 \\
\hline 04 & 81220 & 64.7760 & 7.7435 & 300.1 & 4 & 600.0 & 600.0 \\
\hline 04 & 81221 & 60.2781 & 6.6713 & 300.8 & 4 & 600.0 & 600.0 \\
\hline 04 & 81222 & 57.8256 & 6.8835 & 300.8 & 4 & 600.0 & 600.0 \\
\hline 4 & 81223 & 58.0202 & 3.0675 & 299.9 & 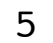 & 600.0 & 600.0 \\
\hline
\end{tabular}




\begin{tabular}{|c|c|c|c|c|c|c|c|}
\hline 4 & 224 & & & 9.3 & & & \\
\hline 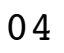 & 131 & 1 & 0260 & 96.3 & & & \\
\hline 4 & 132 & 31.7369 & 1.3643 & 296.3 & & 00 & \\
\hline & 133 & 58.4845 & 1.2510 & 297.3 & & 00.0 & \\
\hline & 134 & 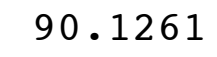 & 3868 & 8.3 & & & \\
\hline & 135 & 239 & 5 & 95.7 & & 0 & \\
\hline & 136 & 102.8543 & 0.6020 & 295.4 & & 00 & \\
\hline & 137 & 146 & 0.78 & 95.4 & & & \\
\hline & 138 & 260 & 5 & 96 & & & \\
\hline & 139 & 246.6274 & 2.0628 & 298.3 & & 0 & \\
\hline & 1310 & 0262 & 3.74 & 298.8 & & & \\
\hline & 1311 & 160.9541 & 202 & 4 & & & \\
\hline 4 & 13 & 192. & 4.93 & 302.5 & & & \\
\hline 4 & 1313 & 63.89 & 4.05 & 303 & & & \\
\hline & 1314 & 63 & & 30 & & & \\
\hline 4 & 1315 & 88 & & 30 & & & \\
\hline 4 & 1316 & 88.7250 & 6.35 & 303.2 & & .0 & \\
\hline 4 & 1317 & 501 & 5 & 302 & & & \\
\hline 4 & 1318 & 78 . & & 295 & 4 & & \\
\hline & 9 & 71 & & 297 & 4 & & \\
\hline 4 & 13 & 69.9741 & 7.64 & 296 & 4 & .0 & \\
\hline & 1 & & & & 4 & & \\
\hline 4 & 2 & 59 & 3 & 29 & 4 & & \\
\hline 4 & 81323 & 59.7000 & 2 & 29 & 4 & .0 & \\
\hline & 81324 & 12 & & 29 & 5 & & \\
\hline 4 & 8141 & 13 & 1 . & 29 & 6 & & \\
\hline & 8142 & 186.7322 & 1.08 & 292.8 & 6 & .0 & \\
\hline 4 & 8143 & 82.175 & & 29 & 5 & & \\
\hline 1 & 8144 & 775 & 1 . & 29 & 5 & & \\
\hline & 8145 & 139.1978 & & 291 & - & & \\
\hline 4 & 8146 & 2846 & 4.44 & 291 & 4 & & \\
\hline 4 & 8147 & .1225 & & 29 & 4 & & \\
\hline & 8148 & 5500 & & & 3 & & \\
\hline 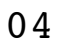 & 8149 & 464 & 5 . & 29 & 3 & 0 & \\
\hline 4 & 81410 & & & 29 & 2 & & \\
\hline 4 & 81 & 641 & & & 2 & & \\
\hline & 81412 & 78.9734 & 6 . & 296.6 & 3 & .0 & \\
\hline 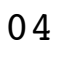 & 81413 & 78 . & 8 . & 29 & 3 & & \\
\hline 4 & 81 & 69.7501 & 9.98 & 29 & 3 & .0 & \\
\hline & 81415 & .6500 & 1.09 & 295.8 & 4 & & \\
\hline 4 & & 250 & 76 & 293 & 4 & & \\
\hline 4 & & 67.2506 & 14.77 & 291.8 & 4 & .0 & \\
\hline & 81418 & 79.1404 & 1.1375 & 291.3 & 4 & & 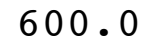 \\
\hline 04 & 81419 & 495 & 12.25 & 290 & 4 & & \\
\hline 04 & 81420 & 77.7000 & 13.6475 & 288.2 & 1 & & \\
\hline 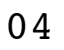 & 81421 & 71.2992 & 12.6400 & 289.0 & 4 & .0 & 500 \\
\hline & & & 12 . & & 4 & & \\
\hline & & 71.5249 & 13.507 & 289.1 & & .0 & 600 \\
\hline
\end{tabular}




\begin{tabular}{|c|c|c|c|c|c|c|c|}
\hline 04 & 81424 & 67.0506 & 12.9500 & 289.1 & 4 & 600.0 & 600.0 \\
\hline 04 & 8151 & 71.7500 & 14.0650 & 288.8 & 4 & 600.0 & 00.0 \\
\hline 04 & 8152 & 77.5246 & 100 & 288.6 & 4 & 600.0 & 00.0 \\
\hline 04 & 8153 & 83.4256 & 11.2475 & 289.6 & 4 & 600.0 & 00.0 \\
\hline 04 & 8154 & 77.5007 & 11.0650 & 289.0 & 4 & 600.0 & 00.0 \\
\hline 04 & 8155 & 85.6000 & 10.7050 & 288.5 & 4 & 600.0 & 00.0 \\
\hline 04 & 8156 & 86.4511 & 8.9500 & 289.6 & 4 & 600.0 & 00.0 \\
\hline 04 & 8157 & 90.4761 & 6.8223 & 291.6 & 4 & 600.0 & 00.0 \\
\hline 04 & 8158 & 82.8386 & 8.5650 & 290.8 & 4 & 600.0 & 00.0 \\
\hline 04 & 8159 & 144.4206 & 4.8075 & 292.9 & 3 & 600.0 & .0 \\
\hline 04 & 81510 & 237.1178 & 2.2630 & 293.3 & 2 & 600.0 & \\
\hline 04 & 81511 & 93.3304 & 365 & 296.6 & 2 & 600.0 & \\
\hline 04 & 81512 & 98.5521 & 4.6823 & 298.1 & 2 & 600.0 & 00.0 \\
\hline 04 & 81513 & 95.5204 & 6.5348 & 299.1 & 3 & 600.0 & 00.0 \\
\hline 04 & 1514 & 99.2769 & 60 & 299.4 & 4 & .0 & \\
\hline 04 & 81515 & 93.5748 & 300 & 299.0 & 4 & 600.0 & 00.0 \\
\hline 04 & 81516 & 87.9493 & 8.9125 & 298.6 & 4 & 60 & 600.0 \\
\hline 04 & 81517 & 81.2016 & 9. & 297.9 & 4 & .0 & \\
\hline 04 & 81518 & 75.0490 & 8.8200 & 296.6 & 4 & 60 & .0 \\
\hline 04 & 81519 & 64.8971 & 9.4175 & 295.6 & 4 & 60 & .0 \\
\hline 04 & 81520 & 69.3500 & 9. & 295.2 & 4 & 60 & \\
\hline 04 & 81521 & 68.9501 & 10.1825 & 295.0 & 4 & .0 & \\
\hline 04 & 81522 & 66.0791 & 10.4350 & 295.1 & 4 & 600.0 & .0 \\
\hline 04 & 81523 & 61.8750 & 10.06 & 295.4 & 4 & 60 & \\
\hline 04 & 81524 & 67.3752 & 10 . & 295.2 & 4 & .0 & \\
\hline 04 & 8161 & 67.1992 & 11.0925 & 294.8 & 4 & .0 & 0.0 \\
\hline 04 & 8162 & 77.1249 & 9.6350 & 294.4 & 4 & 600.0 & 600.0 \\
\hline 04 & 8163 & 76.0750 & 9.6150 & 294.1 & 4 & .0 & \\
\hline 04 & 8164 & 71.3495 & 9.3400 & 294.0 & 4 & .0 & 0.0 \\
\hline 04 & 8165 & 78.5754 & 8.0800 & 293.9 & 4 & 60 & 600.0 \\
\hline 04 & 8166 & 78.1003 & 6.4848 & 293.5 & 4 & .0 & \\
\hline 04 & 8167 & 77.8752 & 5.6948 & 293.9 & 4 & .0 & 600.0 \\
\hline 04 & 8168 & 118.5314 & 2.5595 & 295.9 & 3 & 600.0 & 600.0 \\
\hline 04 & 8169 & 202.9517 & 2.1668 & 297.2 & 2 & 600.0 & 0.0 \\
\hline 04 & 81610 & 233.1446 & 3.0723 & 297.6 & 2 & 600.0 & 600.0 \\
\hline 04 & 81611 & 243.1288 & 2.2075 & 299.1 & 1 & 600.0 & 600.0 \\
\hline 04 & 81612 & 43.9141 & 3.4698 & 301.1 & 2 & 600.0 & .0 \\
\hline 04 & 81613 & 78.0630 & 4.8508 & 301.7 & 2 & 600.0 & 600.0 \\
\hline 04 & 81614 & 98.4716 & 6.1915 & 302.0 & 3 & 600.0 & 600.0 \\
\hline 04 & 81615 & 85.4751 & 6.7790 & 301.9 & 4 & 600.0 & 0.0 \\
\hline 04 & 81616 & 83.5233 & 7.1840 & 301.6 & 4 & 600.0 & 600.0 \\
\hline 04 & 81617 & 75.8002 & 8.2925 & 300.6 & 4 & 600.0 & 600.0 \\
\hline 04 & 81618 & 76.4257 & 8.1475 & 299.7 & 4 & 600.0 & 600.0 \\
\hline 04 & 81619 & 69.4021 & 7.2510 & 298.8 & 4 & 600.0 & 600.0 \\
\hline 04 & 81620 & 65.1743 & 8.0100 & 298.3 & 4 & 600.0 & 600.0 \\
\hline 04 & 81621 & 66.5507 & 9.0175 & 298.4 & . & 600.0 & 600.0 \\
\hline $0<$ & 81622 & 70.4250 & 7.7175 & 298.4 & 4 & 600.0 & 600.0 \\
\hline 04 & 81623 & 70.3750 & 7.7850 & 298.6 & 4 & 600.0 & 600.0 \\
\hline
\end{tabular}




\begin{tabular}{|c|c|c|c|c|c|c|c|}
\hline 04 & 81624 & 70.6500 & 8.0525 & 298.5 & 4 & 600.0 & 600.0 \\
\hline 04 & 8171 & 71.9753 & 8.1600 & 298.0 & 4 & 600.0 & 00,0 \\
\hline 04 & 8172 & 69.3746 & 9.8025 & 297.0 & 4 & 600.0 & 00.0 \\
\hline 04 & 8173 & 68.1750 & 10.0550 & 296.2 & 4 & 600.0 & 00.0 \\
\hline 04 & 8174 & 68.4498 & 025 & 296.2 & 4 & 600.0 & 00.0 \\
\hline 04 & 8175 & 74.9000 & 10.0175 & 296.1 & 4 & 600.0 & 00.0 \\
\hline 04 & 8176 & 78.5250 & 075 & 296.0 & 4 & 600.0 & 00.0 \\
\hline 04 & 8177 & 81.0001 & 7.7725 & 296.2 & 4 & 600.0 & 00.0 \\
\hline 04 & 8178 & 96.5021 & 5.0010 & 297.5 & 3 & 600.0 & 00.0 \\
\hline 04 & 8179 & 196.8829 & 3.2580 & 299.8 & 3 & 600 & 0 \\
\hline 04 & 81710 & 174.9009 & 305 & 301.4 & 3 & 600.0 & \\
\hline 04 & 81711 & 166.1252 & 13 & 301.9 & 4 & 600.0 & \\
\hline 04 & 81712 & 162.8363 & 545 & 303.1 & 3 & 600.0 & 00.0 \\
\hline 04 & 81713 & 167.6250 & 28 & 304.4 & 3 & 600.0 & \\
\hline 04 & 81714 & 171.7038 & 73 & 304.8 & 3 & .0 & \\
\hline 04 & 81715 & 176.7280 & 6.2 & 305.4 & 4 & 600.0 & .0 \\
\hline 04 & 81716 & 155.2277 & 5.8 & 305.7 & 4 & 60 & .0 \\
\hline 04 & 81717 & 101.0066 & 6 . & 305.2 & 4 & .0 & \\
\hline 04 & 81718 & 79.5520 & 6 . & 303.3 & 4 & .0 & \\
\hline 04 & 81719 & 69.8708 & 7.4850 & 301.4 & 4 & .0 & .0 \\
\hline 04 & 81720 & 67.0758 & 8 . & 300.1 & 4 & 6 & \\
\hline 04 & 81721 & 71.6751 & 9. & 300.1 & 4 & .0 & \\
\hline 04 & 81722 & 66.9249 & 9.3 & 300.3 & 4 & 600.0 & .0 \\
\hline 04 & 81723 & 69.9503 & 9 . & 300.4 & 4 & 6 & \\
\hline 04 & 81724 & 250 & 9 . & 300.2 & 4 & 6 & \\
\hline 04 & 8181 & 76.7013 & 8.7225 & 300.1 & 4 & .0 & \\
\hline 04 & 8182 & 78.4012 & 7.6625 & 300.0 & 4 & .0 & .0 \\
\hline 04 & 8183 & 75.1746 & 7. & 299.6 & 4 & .0 & \\
\hline 04 & 8184 & 84.2263 & 6 . & 299.2 & 4 & .0 & 0.0 \\
\hline 04 & 8185 & 100.7022 & 2.9908 & 299.6 & 4 & 60 & .0 \\
\hline 04 & 8186 & 104.8002 & 0 . & 299.4 & 5 & .0 & \\
\hline 04 & 8187 & 153.5265 & 0 . & 299.0 & 4 & .0 & 0.0 \\
\hline 04 & 8188 & 268.1553 & 0.8390 & 300.4 & 3 & 600.0 & 0.0 \\
\hline 04 & 8189 & 235.3535 & 2. & 302.1 & 2 & 600.0 & 0.0 \\
\hline 04 & 81810 & 176.5769 & 3.6568 & 302.6 & 2 & 600.0 & 600.0 \\
\hline 04 & 81811 & 166.5483 & 4.7368 & 303.3 & 2 & 600.0 & 600.0 \\
\hline 04 & 81812 & 168.6713 & 5 & 304.3 & 2 & 600.0 & .0 \\
\hline 04 & 81813 & 176.9897 & 5.4703 & 305.7 & 2 & 600.0 & 600.0 \\
\hline 04 & 81814 & 165.0738 & 6.1645 & 306.7 & 3 & 600.0 & 600.0 \\
\hline 04 & 81815 & 129.4343 & 5 . & 307.6 & 2 & 600.0 & .0 \\
\hline 04 & 81816 & 115.8753 & 4.7670 & 307.5 & 3 & 600.0 & 600.0 \\
\hline 04 & 81817 & 91.6325 & 5.5163 & 306.8 & 3 & 600.0 & 600.0 \\
\hline 04 & 81818 & 73.5514 & 6.5683 & 305.1 & 4 & 600.0 & 600.0 \\
\hline 04 & 81819 & 68.9497 & 6.9375 & 303.2 & 4 & 600.0 & 600.0 \\
\hline 04 & 81820 & 72.2250 & 9.2425 & 301.5 & 4 & 600.0 & 600.0 \\
\hline 04 & 81821 & 66.5732 & 10.1725 & 301.0 & 4 & 600.0 & 600.0 \\
\hline $0<$ & 81822 & 67.4250 & 9.0800 & 301.7 & 4 & 600.0 & 600.0 \\
\hline 04 & 81823 & 66.7001 & 8.7625 & 301.8 & $\Psi$ & 600.0 & 600.0 \\
\hline
\end{tabular}




\begin{tabular}{|c|c|c|c|c|c|c|c|}
\hline 04 & 81824 & 62.7503 & 8.8425 & 302.1 & 4 & 600.0 & 600.0 \\
\hline 04 & 8191 & 62.6000 & 8.7000 & 301.9 & 4 & 600.0 & 600.0 \\
\hline 04 & 8192 & 46.6482 & 6.3673 & 301.3 & 4 & 600.0 & 00.0 \\
\hline 04 & 8193 & 41.9000 & 4.9613 & 301.4 & 4 & 600.0 & 600.0 \\
\hline 04 & 8194 & 45.9002 & 4.2605 & 301.3 & 4 & 600.0 & 00.0 \\
\hline 04 & 8195 & 94.7057 & 1.3493 & 300.3 & 5 & 600.0 & 00.0 \\
\hline 04 & 8196 & 14.6582 & 0.7038 & 297.8 & 5 & 600.0 & 00.0 \\
\hline 04 & 8197 & 47.6566 & 0.9910 & 298.6 & 4 & 600.0 & 00.0 \\
\hline 04 & 8198 & 192.5436 & 1.2020 & 299.1 & 3 & 600.0 & 00.0 \\
\hline 04 & 8199 & 230.4225 & 2.1180 & 300.1 & 2 & 600.0 & 00.0 \\
\hline 04 & 81910 & 232.3123 & 4.0065 & 300.8 & 2 & 600.0 & 00.0 \\
\hline 04 & 81911 & 224.9752 & 4.1725 & 300.6 & 2 & 600.0 & 00.0 \\
\hline 04 & 81912 & 224.4248 & 4.2220 & 302.3 & 2 & 600.0 & 00.0 \\
\hline 04 & 81913 & 217.4774 & 5.0848 & 304.0 & 2 & 600.0 & 00.0 \\
\hline 04 & 81914 & 77.6945 & 48 & 305.3 & 2 & .0 & \\
\hline 04 & 81915 & 88.5792 & 5.6498 & 304.9 & 3 & 600.0 & 00.0 \\
\hline 04 & 81916 & 82.3249 & 6.8013 & 303.9 & 4 & 600.0 & 600.0 \\
\hline 04 & 81917 & 69.4994 & 7.4675 & 302.5 & 4 & .0 & \\
\hline 04 & 81918 & 66.4503 & 7.6525 & 300.5 & 4 & 600.0 & .0 \\
\hline 04 & 81919 & 43.5697 & 5.6398 & 299.8 & 4 & 60 & .0 \\
\hline 04 & 81920 & 33.1772 & 4.0285 & 298.8 & 4 & .0 & \\
\hline 04 & 81921 & 31.3941 & 2.5123 & 299.5 & 5 & .0 & \\
\hline 04 & 81922 & 56.0326 & 1.9723 & 299.1 & 5 & 600.0 & 500.0 \\
\hline 04 & 81923 & 152.9914 & 1.0658 & 296.9 & 6 & 60 & .0 \\
\hline 04 & 81924 & 115.6576 & 1.3018 & 296.3 & 6 & .0 & \\
\hline 04 & 8201 & 222.5520 & 1.1135 & 294.9 & 6 & 600.0 & 600.0 \\
\hline 04 & 8202 & 8.0471 & 0.7455 & 294.3 & 6 & 600.0 & .0 \\
\hline 04 & 8203 & 55.6519 & 1.1948 & 294.2 & 6 & 600.0 & \\
\hline 04 & 8204 & 88.8934 & 0.7278 & 294.8 & 6 & 600.0 & 600.0 \\
\hline 04 & $820 \quad 5$ & 120.8579 & 1.0395 & 294.0 & 6 & 600.0 & 600.0 \\
\hline 04 & 8206 & 357.7734 & 1.1440 & 294.9 & 5 & .0 & .0 \\
\hline 04 & 8207 & 15.5009 & 2.0835 & 296.0 & 4 & .0 & 600.0 \\
\hline 04 & 8208 & 325.9108 & 1.1405 & 295.8 & 3 & 600.0 & 600.0 \\
\hline 04 & 8209 & 257.2378 & 2.1995 & 297.5 & 2 & 600.0 & 0.0 \\
\hline 04 & 82010 & 208.7431 & 2.7383 & 298.3 & 2 & 600.0 & 600.0 \\
\hline 04 & 82011 & 214.1969 & 3.7255 & 300.7 & 2 & 600.0 & 600.0 \\
\hline 04 & 82012 & 193.4076 & 5.9270 & 301.4 & 3 & 600.0 & 600.0 \\
\hline 04 & 82013 & 170.8979 & 5.2043 & 302.2 & 2 & 600.0 & 600.0 \\
\hline 04 & 82014 & 161.0975 & 6.3593 & 303.6 & 3 & 600.0 & 600.0 \\
\hline 04 & 82015 & 154.9500 & 6.1013 & 304.1 & 3 & 600.0 & 600.0 \\
\hline 04 & 82016 & 158.1402 & 5.9690 & 304.2 & 4 & 600.0 & 600.0 \\
\hline 04 & 82017 & 149.8371 & 4.5328 & 304.0 & 3 & 600.0 & 600.0 \\
\hline 04 & 82018 & 148.5174 & 3.8653 & 303.1 & 3 & 600.0 & 600.0 \\
\hline 04 & 82019 & 71.6000 & 5.1425 & 300.8 & 4 & 600.0 & 600.0 \\
\hline 04 & 82020 & 68.5995 & 6.7005 & 299.4 & 4 & 600.0 & 600.0 \\
\hline 04 & 82021 & 74.5888 & 4.8923 & 299.7 & 4 & 600.0 & 600.0 \\
\hline 04 & 82022 & 74.0500 & 4.3010 & 299.5 & 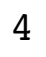 & 600.0 & 600.0 \\
\hline 4 & 82023 & 92.2820 & 1.6460 & 299.1 & 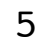 & 600.0 & 600.0 \\
\hline
\end{tabular}




\begin{tabular}{|c|c|c|c|c|c|c|c|}
\hline 04 & 82024 & 83.5130 & 1.6235 & 298.0 & 6 & 600.0 & 600.0 \\
\hline 04 & 8211 & 83.9638 & 1.6163 & 297.3 & 5 & 600.0 & 600.0 \\
\hline 04 & 8212 & 89.2442 & 1.5880 & 295.7 & 6 & 600.0 & 00.0 \\
\hline 4 & 8213 & 82.9203 & 5.8310 & 295.6 & 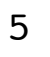 & 600.0 & 00.0 \\
\hline 04 & 8214 & 77.3003 & 8.3250 & 295.5 & 4 & 600.0 & 0 \\
\hline 04 & 8215 & 76.1000 & 9.2275 & 295.8 & 4 & 600.0 & 00.0 \\
\hline 4 & 8216 & 77.0500 & 8.6050 & 296.1 & 4 & 600.0 & 00.0 \\
\hline 4 & 8217 & 75.2501 & 6.8193 & 297.2 & 4 & 600.0 & \\
\hline 04 & 8218 & 82.7508 & 5.2695 & 299.3 & 3 & 600.0 & 000 \\
\hline 04 & 8219 & 255.1628 & 2.4890 & 299.4 & 3 & 600.0 & \\
\hline 04 & 82110 & 301.2344 & 2.4470 & 299.3 & 2 & 600.0 & \\
\hline 04 & 82111 & 186.4364 & 3.4105 & 300.8 & 2 & 600.0 & .0 \\
\hline 04 & 82112 & 94.8928 & 5.2380 & 300.0 & 3 & 600.0 & \\
\hline 04 & 82113 & 80.2502 & 6.7460 & 300.2 & 3 & 600.0 & \\
\hline 04 & 82114 & 78.5248 & 8.3450 & 299.9 & 4 & 0 & .0 \\
\hline 04 & 82115 & 70.8251 & 9.5975 & 299.6 & 4 & 600.0 & .0 \\
\hline 04 & 82116 & 68.5250 & 11.1250 & 298.4 & 4 & 600.0 & .0 \\
\hline 04 & 82117 & 68.1000 & 12.7800 & 296.3 & 4 & .0 & \\
\hline 04 & 82118 & 69.4500 & 13.3550 & 294.4 & 4 & 600.0 & 600.0 \\
\hline 04 & 82119 & 72.3756 & 13.05 & 292.7 & 4 & .0 & .0 \\
\hline 04 & 82120 & 77.9250 & 13.5275 & 291.5 & 4 & .0 & \\
\hline 04 & 82121 & 72.9753 & 13.3925 & 291.9 & 4 & .0 & \\
\hline 04 & 82122 & 74.7487 & 12.0650 & 293.1 & 4 & .0 & .0 \\
\hline 04 & 82123 & 73.3000 & 12.4125 & 292.4 & 4 & .0 & \\
\hline 04 & 82124 & 79.5533 & 12.2100 & 291.8 & 4 & 60 & \\
\hline 04 & 8221 & 75.4237 & 13.2775 & 290.7 & 4 & 600.0 & .0 \\
\hline 04 & 8222 & 77.6505 & 14.71 & 289.9 & 4 & 6 & .0 \\
\hline 04 & 8223 & 74.1502 & 14.0425 & 289.1 & 4 & .0 & \\
\hline 04 & 8224 & 73.7324 & 13.8775 & 288.3 & 4 & .0 & .0 \\
\hline 04 & 8225 & 70.5798 & 14.530 & 287.3 & 4 & .0 & \\
\hline 04 & 8226 & 71.2520 & 11 . & 288.7 & 4 & .0 & \\
\hline 04 & 8227 & 110.2920 & 4.5140 & 289.0 & 4 & .0 & .0 \\
\hline 04 & 8228 & 89.2890 & 6.6345 & 289.8 & 3 & .0 & 600.0 \\
\hline 04 & 8229 & 75.5725 & 350 & 290.4 & 4 & .0 & 600.0 \\
\hline 04 & 82210 & 70.9149 & 4.1868 & 294.1 & 3 & .0 & .0 \\
\hline 04 & 82211 & 132.0997 & 4.2790 & 294.4 & 3 & 600.0 & 600.0 \\
\hline 04 & 82212 & 905 & 4.42 & 296.0 & 2 & .0 & .0 \\
\hline 04 & 82213 & 79.9681 & 6.7420 & 295.4 & 3 & 600.0 & 600.0 \\
\hline 04 & 82214 & 82.9129 & 7.9148 & 296.3 & 4 & 600.0 & 600.0 \\
\hline 04 & 82215 & 92.0497 & 7.9865 & 296.5 & 4 & .0 & 600.0 \\
\hline 04 & 82216 & 89.8502 & 9.6725 & 296.1 & 4 & 600.0 & 600.0 \\
\hline 04 & 82217 & 89.9534 & 9.0550 & 296.0 & 4 & 600.0 & 600.0 \\
\hline 04 & 82218 & 69.7445 & 8.6525 & 295.3 & 4 & 600.0 & 600.0 \\
\hline 04 & 82219 & 84.4974 & 8.2275 & 293.7 & 4 & 600.0 & 600.0 \\
\hline 04 & 82220 & 63.2178 & 8.0110 & 292.7 & 4 & 600.0 & 600.0 \\
\hline 04 & 82221 & 62.8252 & 12.6925 & 292.0 & 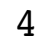 & 600.0 & \\
\hline 04 & 82222 & 63.8248 & 10.9800 & 291.9 & 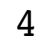 & 600.0 & 600.0 \\
\hline 04 & 82223 & 66.3734 & 11.302 & 291.4 & 4 & 600.0 & 600.0 \\
\hline
\end{tabular}




\begin{tabular}{|c|c|c|c|c|c|c|c|}
\hline 4 & 82224 & 62.3000 & 12.0725 & 290.9 & 4 & 600.0 & 600.0 \\
\hline 04 & 8231 & 63.7498 & 12.3950 & 290.6 & 4 & 600.0 & 600.0 \\
\hline 04 & 8232 & 67.4250 & 11.9025 & 290.3 & 4 & 600.0 & 00.0 \\
\hline 04 & 8233 & 67.1500 & 10.5375 & 290.0 & 4 & 600.0 & 00.0 \\
\hline 04 & 8234 & 68.7001 & 725 & 290.4 & 4 & 600.0 & 00.0 \\
\hline 04 & 8235 & 79.2752 & 7.4275 & 290.0 & 4 & 600.0 & 00.0 \\
\hline 04 & 8236 & 72.4224 & 8.9600 & 289.0 & 4 & 600.0 & 0 \\
\hline 04 & 8237 & 59.5247 & 8.9150 & 289.3 & 4 & 600.0 & 00.0 \\
\hline 04 & 8238 & 74.5001 & 7.7625 & 290.2 & 4 & 600.0 & 00.0 \\
\hline 04 & 8239 & 74.1771 & 7.4250 & 291.0 & 4 & 60 & 0 \\
\hline 04 & 82310 & 78.2539 & 5.8833 & 293.4 & 3 & 600.0 & \\
\hline 04 & 82311 & 93.7989 & 340 & 295.5 & 3 & 600.0 & \\
\hline 04 & 82312 & 97.7493 & 5.6473 & 296.9 & 3 & 600.0 & 00.0 \\
\hline 04 & 82313 & 91.7068 & 5. & 297.7 & 2 & 600.0 & \\
\hline 04 & 82314 & 84.7005 & 6. & 298.5 & 3 & .0 & \\
\hline 04 & 82315 & 86.6195 & 8.6725 & 298.6 & 4 & 600.0 & .0 \\
\hline 04 & 82316 & 79.2497 & 9.7200 & 297.8 & 4 & 60 & .0 \\
\hline 04 & 82317 & 69.9013 & 9.4 & 296.7 & 4 & .0 & \\
\hline 04 & 82318 & 63.2507 & 9.6825 & 295.7 & 4 & .0 & \\
\hline 04 & 82319 & 63.9748 & 11.0850 & 294.2 & 4 & .0 & .0 \\
\hline 04 & 82320 & 57.7750 & 12 . & 292.5 & 4 & 6 & \\
\hline 04 & 82321 & 66.2446 & 11.1925 & 292.0 & 4 & .0 & \\
\hline 04 & 82322 & 76.2763 & 10.8775 & 290.8 & 4 & 600.0 & .0 \\
\hline 04 & 82323 & 64.8750 & 11.62 & 290.9 & 4 & 6 & \\
\hline 04 & 82324 & 748 & 10 . & 291.3 & 4 & 6 & \\
\hline 04 & 8241 & 61.8000 & 10.8000 & 291.2 & 4 & 60 & \\
\hline 04 & 8242 & 65.6749 & 10.5875 & 290.6 & 4 & 60 & .0 \\
\hline 04 & 8243 & 71.3234 & 10. & 290.6 & 4 & .0 & \\
\hline 04 & 8244 & 72.9000 & 10.2725 & 290.3 & 4 & .0 & .0 \\
\hline 04 & 8245 & 69.8750 & 11.0650 & 289.9 & 4 & 6 & .0 \\
\hline 04 & 8246 & 72.8001 & 11.0375 & 290.4 & 4 & .0 & \\
\hline 04 & 8247 & 67.2221 & 9. & 291.2 & 4 & .0 & 0.0 \\
\hline 04 & 8248 & 66.5249 & 8.7175 & 293.0 & 4 & 600.0 & 0.0 \\
\hline 04 & 8249 & 62.7763 & 7.8375 & 294.4 & 4 & .0 & .0 \\
\hline 04 & 82410 & 58.0750 & 8.1413 & 295.9 & 4 & 600.0 & 0.0 \\
\hline 04 & 82411 & 69.3001 & 9.2325 & 296.2 & 4 & 600.0 & 600.0 \\
\hline 04 & 82412 & 72.9997 & 9.4925 & 296.7 & 4 & 600.0 & .0 \\
\hline 04 & 82413 & 76.9744 & 10.3550 & 297.1 & 4 & 600.0 & 600.0 \\
\hline 04 & 82414 & 74.9250 & 10.7125 & 297.1 & 4 & 600.0 & 600.0 \\
\hline 04 & 82415 & 69.0749 & 11.6750 & 296.7 & 4 & 600.0 & .0 \\
\hline 04 & 82416 & 70.0503 & 12.0900 & 296.2 & 4 & 600.0 & 600.0 \\
\hline 04 & 82417 & 61.4751 & 14.5350 & 295.2 & 4 & 600.0 & 600.0 \\
\hline 04 & 82418 & 63.6000 & 15.8675 & 293.6 & 4 & 600.0 & 600.0 \\
\hline 04 & 82419 & 68.0253 & 16.1375 & 292.1 & 4 & 600.0 & 600.0 \\
\hline 04 & 82420 & 66.7746 & 15.6975 & 291.3 & 4 & 600.0 & 600.0 \\
\hline 04 & 82421 & 68.7000 & 14.9350 & 290.8 & 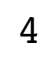 & 600.0 & \\
\hline $0<$ & 82422 & 66.5753 & 12.9450 & 290.7 & 4 & 600.0 & 600.0 \\
\hline 0 & 82423 & 65.4500 & 12.7800 & 290.7 & $\Psi$ & 600.0 & 600.0 \\
\hline
\end{tabular}




\begin{tabular}{|c|c|c|c|c|c|c|c|}
\hline 4 & 82424 & 67.1500 & 12.9875 & 290.4 & 4 & 600.0 & 600.0 \\
\hline 04 & 8251 & 67.6750 & 13.5700 & 290.6 & 4 & 600.0 & 00,0 \\
\hline 4 & 8252 & 72.1001 & 13.8350 & 290.5 & 4 & 600.0 & 00.0 \\
\hline 4 & 8253 & 72.7500 & 12.6025 & 290.4 & 4 & 600.0 & 00.0 \\
\hline 04 & 8254 & 74.6750 & 12.6475 & 290.1 & 4 & 600.0 & 00.0 \\
\hline 04 & 8255 & 72.9498 & 11.6400 & 289.6 & 4 & 600.0 & 00.0 \\
\hline 04 & 8256 & 68.3973 & 10.9200 & 289.9 & 4 & 600.0 & 00.0 \\
\hline 04 & 8257 & 64.8001 & 9.9875 & 290.6 & 4 & 600.0 & 00.0 \\
\hline 04 & 8258 & 61.3500 & 9.6675 & 291.5 & 4 & 600.0 & 00.0 \\
\hline 04 & 8259 & 59.9231 & 100 & 292.6 & 4 & 60 & 0 \\
\hline 04 & 82510 & 69.8001 & 8.5325 & 294.1 & 4 & 600.0 & \\
\hline 04 & 82511 & 75.0749 & 93 & 295.8 & 4 & 600.0 & \\
\hline 04 & 82512 & 81.3750 & 8.7795 & 296.4 & 4 & 600.0 & 00.0 \\
\hline 04 & 82513 & 72.7750 & 8.4800 & 297.5 & 4 & 600.0 & \\
\hline 04 & 82514 & 71.7638 & 25 & 298.4 & 4 & .0 & \\
\hline 04 & 82515 & 85.8253 & 8. & 298.5 & 4 & 600.0 & .0 \\
\hline 04 & 82516 & 78.6747 & 7.5413 & 298.5 & 4 & 60 & 600.0 \\
\hline 04 & 82517 & 84.9225 & 6.2240 & 298.3 & 4 & .0 & \\
\hline 04 & 82518 & 74.4138 & 5.8258 & 297.0 & 3 & .0 & \\
\hline 04 & 82519 & 55.1506 & 9.7875 & 295.1 & 4 & .0 & .0 \\
\hline 04 & 82520 & 58.8747 & 12.3 & 29 & 4 & 0 & \\
\hline 04 & 82521 & 57.7500 & 10.7700 & 29 & 4 & .0 & \\
\hline 04 & 82522 & 65.9249 & 12.2275 & 291.5 & 4 & 600.0 & .0 \\
\hline 04 & 82523 & 57.6000 & 12 & 29 & 4 & 6 & \\
\hline 04 & 82524 & 56.2257 & 11. & 291.4 & 4 & 6 & \\
\hline 04 & 8261 & 61.0005 & 11.8829 & 291.2 & 4 & .0 & \\
\hline 04 & 8262 & 69.2505 & 11.8275 & 290.6 & 4 & 60 & .0 \\
\hline 04 & 8263 & 68.3001 & 11.0 & 288.7 & 4 & .0 & \\
\hline 04 & 8264 & 64.9252 & 10.4700 & 288.6 & 4 & .0 & .0 \\
\hline 04 & 8265 & 68.9259 & 9.5350 & 288.9 & 4 & .0 & .0 \\
\hline 04 & 8266 & 77.6756 & 8.9550 & 288.4 & 4 & .0 & \\
\hline 04 & 8267 & 102.7584 & 6.7 & 290.5 & 4 & .0 & 0.0 \\
\hline 04 & 8268 & 137.8754 & 7.8708 & 292.2 & 4 & 0.0 & 0.0 \\
\hline 04 & 8269 & 150.2607 & 9.3150 & 293.8 & 4 & 0.0 & 0.0 \\
\hline 04 & 82610 & 160.9250 & 10.4475 & 294.6 & 4 & 0 & 0.0 \\
\hline 04 & 82611 & 158.5778 & 9.2775 & 295.9 & 4 & 600.0 & 600.0 \\
\hline 04 & 82612 & 165.4500 & 9.8400 & 297.3 & 4 & 600.0 & .0 \\
\hline 04 & 82613 & 165.1511 & 9.1175 & 298.1 & 4 & 600.0 & 600.0 \\
\hline 04 & 82614 & 163.1951 & 9.4225 & 298.8 & 4 & 600.0 & 600.0 \\
\hline 04 & 82615 & 177.4253 & 9.3775 & 29 & 4 & 600.0 & 600.0 \\
\hline 04 & 82616 & 178.5500 & 9.5650 & 299.5 & 4 & 600.0 & 600.0 \\
\hline 04 & 82617 & 180.7279 & 10.2000 & 299.2 & 4 & 600.0 & 600.0 \\
\hline 04 & 82618 & 182.9768 & 8.3650 & 298.6 & 4 & 600.0 & 600.0 \\
\hline 04 & 82619 & 170.0323 & 5.8673 & 297.5 & 4 & 600.0 & 600.0 \\
\hline 04 & 82620 & 127.1315 & 4.8203 & 296.9 & 4 & 600.0 & 600.0 \\
\hline 04 & 82621 & 113.9651 & & 297.0 & 4 & 600.0 & \\
\hline $0<$ & 82622 & 130.5749 & 8.2600 & 297.9 & 4 & 600.0 & 600.0 \\
\hline 04 & 82623 & 129.6003 & 9.3825 & 298.0 & & 600.0 & 600.0 \\
\hline
\end{tabular}




\begin{tabular}{|c|c|c|c|c|c|c|c|}
\hline & 624 & & & & & & \\
\hline & 271 & & 3.0550 & 7.2 & & & \\
\hline & 8272 & 42.2500 & 2650 & 296.5 & & 00 & \\
\hline & 273 & 3250 & 0.0375 & 295.7 & & 0 & \\
\hline & 274 & 999 & .24 & & & & \\
\hline & 8275 & .750 & L. 2200 & & & 0 & \\
\hline & $827 \quad 6$ & 3500 & 1.7725 & 294.8 & & 00 & \\
\hline & 277 & 750 & 9.8475 & 95.0 & & & \\
\hline & 8278 & 3747 & 5 & 9 & & & \\
\hline & 279 & 1935 & 7.3815 & 297.0 & & 0 & \\
\hline & 2710 & 57.5714 & 8.91 & 298.3 & & & \\
\hline & 2711 & 50.2502 & 8.1128 & 99.6 & & & \\
\hline & 82712 & 62.5276 & 7.34 & 300.8 & 4 & 0 & \\
\hline & 2713 & 58.9770 & 5.95 & 301.8 & & 0.0 & \\
\hline & 2714 & & & & & & \\
\hline & 2715 & & & & 3 & & \\
\hline & 2716 & 63.1224 & 6.32 & .7 & 4 & 0 & \\
\hline & 2717 & 1.8005 & 6 . & 7 & 4 & & \\
\hline & 2718 & 75.1862 & & 30 & 3 & & \\
\hline & 2719 & 9.6282 & 3.30 & 301.2 & 4 & & \\
\hline & 82720 & 121.0185 & 3 . & .4 & 4 & 0 & \\
\hline & 82721 & 73.8002 & & & 4 & & \\
\hline & 2722 & 234 & 8 & 29 & 4 & & \\
\hline & 82723 & 83.3250 & 4.66 & 29 & 4 & 0 & \\
\hline & 82724 & 78.1393 & & & 4 & & \\
\hline & 8281 & 7494 & 4 . & 29 & 4 & & \\
\hline & 8282 & 65.2501 & 5 & 300.2 & 4 & & 0 \\
\hline & 8283 & 60.4742 & 4.67 & .6 & 4 & & \\
\hline & 8284 & 08 & 2 . & .6 & 5 & & \\
\hline & 8285 & 129.6899 & 2 & .1 & 6 & & 0 \\
\hline & 8286 & 127.7516 & 2. & .1 & 5 & & 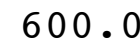 \\
\hline & 8287 & 4647 & 0 & 29 & 4 & & \\
\hline & 8288 & 168 . & 8 & .7 & 3 & & 。 \\
\hline & 8289 & 714 & 680 & .4 & 3 & & \\
\hline & 82810 & 16 & 0 & & 2 & & \\
\hline & 82811 & 170.6521 & & .4 & 2 & & \\
\hline & 82812 & 168.4997 & 70 & 304.3 & 3 & & \\
\hline & 82813 & 174 . & 5 . & 30 & 3 & & \\
\hline & 82814 & 193.4564 & 5 . & .7 & 3 & & 0 . \\
\hline & 82815 & 192 . & 678 & 305.9 & 3 & & \\
\hline & 82816 & 192.4587 & 0 & 30 & 4 & & \\
\hline & 82817 & 198.9140 & 5.1258 & & 3 & & \\
\hline & 82818 & 187.3458 & 4.5170 & 304.7 & 3 & & 0 . \\
\hline & 82819 & 132.1471 & 725 & & I & & \\
\hline 4 & 82820 & 3749 & 6.3278 & & 4 & & \\
\hline & 82821 & 69.5241 & 7.4320 & 302.3 & 4 & & 00 \\
\hline & 82 & & & & 4 & & \\
\hline & 82823 & 72.8500 & 8.5225 & 301.5 & 1 & 600.0 & 5 \\
\hline
\end{tabular}




\begin{tabular}{|c|c|c|c|c|c|c|c|}
\hline 4 & 82824 & 71.8000 & 8.3800 & 300.9 & 4 & 600.0 & 00.0 \\
\hline 04 & 8291 & 71.2751 & 8.8050 & 300.6 & 4 & 600.0 & 600,0 \\
\hline 04 & 8292 & 73.5499 & 9.1575 & 300.5 & 4 & 600.0 & 00.0 \\
\hline 04 & 8293 & 73.2001 & 8.7250 & 300.6 & 4 & 600.0 & 600.0 \\
\hline 04 & 8294 & 71.6251 & 8.0025 & 300.7 & 4 & 600.0 & 00.0 \\
\hline 04 & 8295 & 73.3500 & 7.7625 & 301.0 & 4 & 600.0 & 00.0 \\
\hline 04 & 8296 & 75.0750 & 6.8343 & 301.1 & 4 & 600.0 & 600.0 \\
\hline 04 & 8297 & 78.3250 & 5.0548 & 301.8 & 3 & 600.0 & 00.0 \\
\hline 04 & 8298 & 125.7638 & 2.1018 & 303.1 & 2 & 600.0 & 00.0 \\
\hline 04 & 8299 & 228.0994 & 2.1493 & 303.7 & 2 & 600.0 & 600.0 \\
\hline 04 & 82910 & 170.5610 & 5.2493 & 303.9 & 3 & 600.0 & .0 \\
\hline 04 & 82911 & 174.7497 & 6.0235 & 304.4 & 3 & 600.0 & \\
\hline 04 & 82912 & 169.4998 & 6.7468 & 305.5 & 4 & 600.0 & 600.0 \\
\hline 04 & 82913 & 167.0000 & 6.8683 & 306.1 & 4 & 600.0 & 600.0 \\
\hline 04 & 82914 & 163.5268 & 7. & 306.6 & 4 & 600.0 & \\
\hline 04 & 82915 & 142.9022 & 55 & 306.7 & 4 & 600.0 & 60 \\
\hline 04 & 82916 & 90.5001 & 6.0773 & 306.4 & 4 & 600.0 & 600.0 \\
\hline 04 & 82917 & 85.8909 & 6.7763 & 305.0 & 4 & 600.0 & \\
\hline 04 & 82918 & 75.5821 & 220 & 303.4 & 4 & 600.0 & 60 \\
\hline 04 & 82919 & 70.5250 & 9.4 & 301.4 & 4 & 600.0 & 600.0 \\
\hline 04 & 82920 & 65.9501 & 9.1650 & 300.6 & 4 & 600.0 & .0 \\
\hline 04 & 82921 & 66.8503 & 9. & 300.5 & 4 & 600.0 & \\
\hline 04 & 82922 & 67.4750 & 300 & 300.3 & 4 & 600.0 & 600 \\
\hline 04 & 82923 & 69.6750 & 10. & 300.4 & 4 & 600.0 & .0 \\
\hline 04 & 82924 & 71.7500 & 10. & 300.6 & 4 & 600.0 & \\
\hline 04 & 8301 & 71.6751 & 10. & 300.4 & 4 & 600.0 & 60 \\
\hline 04 & 8302 & 74.4500 & 075 & 300.5 & 4 & 600.0 & 60 \\
\hline 04 & 8303 & 74.3750 & 9 . & 300.2 & 4 & 600.0 & \\
\hline 04 & 8304 & 73.2000 & 625 & 300.0 & 4 & 600.0 & 60 \\
\hline 04 & 8305 & 73.5000 & 9.7325 & 299.8 & 4 & 600.0 & 60 \\
\hline 04 & 8306 & 72.6500 & 9 . & 299.4 & 4 & 600.0 & \\
\hline 04 & 8307 & 85.6144 & 6. & 300.0 & 3 & 600.0 & 60 \\
\hline 04 & 8308 & 329.1915 & 1.0790 & 298.9 & 2 & 600.0 & 600.0 \\
\hline 04 & 8309 & 254.1393 & 158 & 300.4 & 2 & 600.0 & .0 \\
\hline 04 & 83010 & 166.1019 & 4 . & 30 & 2 & 600.0 & \\
\hline 04 & 83011 & 179.9531 & 5.3690 & 303.4 & 3 & 600.0 & 600.0 \\
\hline 04 & 83012 & 62.4398 & 4.2653 & 305.4 & 2 & 600.0 & \\
\hline 04 & 83013 & 82.8315 & 508 & 305.9 & 2 & 600.0 & 600.0 \\
\hline 04 & 83014 & 91.9995 & 4.6113 & 306.0 & 2 & 600.0 & 600.0 \\
\hline 04 & 83015 & 106.3146 & 4.961 & 305.9 & 2 & 600.0 & \\
\hline 04 & 83016 & 102.8120 & 4.9415 & 305.6 & 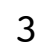 & 600.0 & 600.0 \\
\hline 04 & 83017 & 96.9648 & 5.2785 & 304.8 & 3 & 600.0 & 600.0 \\
\hline 04 & 83018 & 87.4610 & 6.2265 & 303.3 & 4 & 600.0 & 600.0 \\
\hline 04 & 83019 & 82.6183 & 7.9815 & 301.1 & 4 & 600.0 & 600.0 \\
\hline 04 & 83020 & 66.1985 & 10.7625 & 300.0 & 4 & 600.0 & 600.0 \\
\hline 04 & 83021 & 65.3251 & 11.1525 & 299.8 & 4 & 600.0 & 600.0 \\
\hline 04 & 83022 & 92.7259 & 7783 & & 4 & 600.0 & 600.0 \\
\hline 04 & 83023 & 77.8501 & 4.5238 & 299.9 & 4 & 600.0 & 600.0 \\
\hline
\end{tabular}




\begin{tabular}{|c|c|c|c|c|c|c|c|}
\hline 4 & 83024 & 81.8183 & 5.1543 & 299.4 & 4 & 600.0 & 00.0 \\
\hline 04 & 8311 & 63.3733 & 7.2488 & 299.4 & 4 & 600.0 & 600.0 \\
\hline 04 & 8312 & 75.1994 & 5.7908 & 299.0 & 4 & 600.0 & 00.0 \\
\hline 04 & 8313 & 55.6630 & 2.2660 & 299.4 & 5 & 600.0 & 00.0 \\
\hline 4 & 8314 & 45.1939 & 1.2920 & 299.9 & 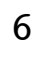 & 600.0 & 00.0 \\
\hline 04 & 8315 & 80.4220 & 1.1615 & 298.5 & 6 & 600.0 & 00.0 \\
\hline 04 & 8316 & 100.0074 & 0.8705 & 298.0 & 5 & 600.0 & 600.0 \\
\hline 04 & 8317 & 122.9602 & 1.0933 & 298.5 & 4 & 600.0 & .0 \\
\hline 04 & 8318 & 273.8094 & 0.9098 & 299.1 & 3 & 600.0 & \\
\hline 04 & 8319 & 295.4397 & 1.6943 & 299.4 & 2 & 600.0 & 60 \\
\hline 04 & 83110 & 252.7553 & 2.6770 & 300.7 & 2 & 600.0 & .0 \\
\hline 04 & 83111 & 237.3327 & 2.9510 & 301.7 & 2 & 600.0 & \\
\hline 04 & 83112 & 234.5009 & 2.7553 & 303.9 & 2 & 600.0 & .0 \\
\hline 04 & 83113 & 195.0986 & 5.6165 & 304.5 & 3 & 600.0 & \\
\hline 04 & 83114 & 187.0607 & 5 . & 305.3 & 2 & 600.0 & .0 \\
\hline 04 & 83115 & 145.8738 & 4 & 305.5 & 3 & 600.0 & \\
\hline 04 & 83116 & 60.1110 & 4.0398 & 305.4 & 3 & 600.0 & 600.0 \\
\hline 04 & 83117 & 81.7250 & 6.542 & 303.6 & 4 & 600.0 & .0 \\
\hline 04 & 83118 & 73.9751 & 8 & 301.0 & 4 & 600.0 & \\
\hline 04 & 83119 & 69.5253 & 9.8375 & 298.5 & 4 & 600.0 & \\
\hline 04 & 83120 & 68.7761 & 9.8500 & 297.3 & 4 & 600.0 & .0 \\
\hline 04 & 83121 & 60.0952 & 7.6095 & 298.1 & 4 & 600.0 & \\
\hline 04 & 83122 & 63.8489 & 4.4805 & 297.8 & 4 & 600.0 & 60 \\
\hline 04 & 83123 & 72.9224 & 2.3908 & 298.5 & 5 & 600.0 & .0 \\
\hline 04 & 83124 & 70.1230 & 1 . & 298.6 & 6 & 600.0 & \\
\hline 04 & $\begin{array}{lll}9 & 1 & 1\end{array}$ & 126.4201 & 1. & 298.8 & 6 & 600.0 & 60 \\
\hline 04 & 912 & 88.6873 & 0.8188 & 297.8 & 6 & 600.0 & 60 \\
\hline 04 & 933 & 118.6247 & 0.92 & 296.6 & 6 & 600.0 & .0 \\
\hline 04 & $\begin{array}{lll}9 & 1 & 4\end{array}$ & 101.2070 & 1.4828 & 295.9 & 6 & 600.0 & 60 \\
\hline 04 & 915 & 81.1163 & 5.2248 & 297.1 & 5 & 600.0 & .0 \\
\hline 04 & 916 & 100.1894 & 4.0018 & 297.9 & 4 & 600.0 & 60 \\
\hline 04 & $\begin{array}{lll}9 & 1 & 7\end{array}$ & 131.7452 & 1.2423 & 296.8 & 3 & 600.0 & 60 \\
\hline 04 & $\begin{array}{lll}9 & 1 & 8\end{array}$ & 339.4546 & 1.0260 & 297.3 & 2 & 600.0 & 60 \\
\hline 04 & $\begin{array}{lll}9 & 1 & 9\end{array}$ & 283.3555 & 1.8660 & 298.6 & 2 & 600.0 & 60 \\
\hline 04 & 9110 & 248.5391 & 728 & 299.3 & 2 & 600.0 & 60 \\
\hline 04 & $9 \quad 111$ & 246.2950 & 2.4640 & 301.1 & 2 & 600.0 & 600.0 \\
\hline 04 & 9112 & 245.9417 & 2.5945 & 302.9 & 2 & 600.0 & 600.0 \\
\hline 04 & 9113 & 103.3757 & 3.7160 & 304.7 & 2 & 600.0 & 60 \\
\hline 04 & $9 \quad 114$ & 70.0041 & 5.8225 & 304.2 & 3 & 600.0 & 600.0 \\
\hline 04 & 9115 & 82.9827 & 6.3533 & 304.2 & 3 & 600.0 & 600.0 \\
\hline 04 & 9116 & 72.1504 & 8.0925 & 303.9 & 4 & 600.0 & 600.0 \\
\hline 04 & $9 \quad 117$ & 70.0996 & 8.5203 & 303.4 & 4 & 600.0 & 600.0 \\
\hline 04 & 9118 & 64.7750 & 10.0575 & 302.0 & 4 & 600.0 & 600.0 \\
\hline 04 & 9119 & 66.7000 & 9.9825 & 301.2 & 4 & 600.0 & 600.0 \\
\hline 04 & 9120 & 65.2494 & 10.6500 & 301.0 & 4 & 600.0 & 600.0 \\
\hline 04 & $9 \quad 121$ & 63.3498 & 10.8800 & 300.7 & 4 & 600.0 & 600.0 \\
\hline 04 & $9 \quad 122$ & 60.0000 & 11.94 & .3 & 4 & 600.0 & .0 \\
\hline 04 & 9123 & 62.8000 & 11.5650 & 299.4 & $\mathbf{4}$ & 600.0 & 600.0 \\
\hline
\end{tabular}




\begin{tabular}{|c|c|c|c|c|c|c|c|c|}
\hline & 9 & 124 & 63.0750 & 10.7050 & 299.6 & 4 & 600.0 & 600.0 \\
\hline 04 & 9 & 21 & 64.9500 & 9.3525 & 299.5 & 4 & 600.0 & 00.0 \\
\hline 04 & 9 & 22 & 64.9780 & 10.1875 & 298.7 & 4 & 600.0 & 00.0 \\
\hline 04 & 9 & 23 & 67.1736 & 8.6975 & 297.4 & 4 & 600.0 & 00.0 \\
\hline 04 & 9 & 24 & 68.4266 & 875 & 296.8 & 4 & 00.0 & 00.0 \\
\hline 04 & 9 & 25 & 67.6764 & 8.9800 & 296.5 & 4 & 600.0 & 00.0 \\
\hline 04 & 9 & 26 & 77.9253 & 9.1650 & 296.2 & 4 & 600.0 & 0 \\
\hline 04 & 9 & 27 & 74.4775 & 9.6625 & 295.8 & 4 & 600.0 & 00.0 \\
\hline 04 & 9 & 28 & 82.3750 & 7.4965 & 296.0 & 4 & 600.0 & 00.0 \\
\hline 04 & 9 & 29 & 132.5859 & 003 & 297.4 & 4 & 60 & 0 \\
\hline 04 & 9 & 210 & 151.6762 & 685 & 297.5 & 4 & 600.0 & \\
\hline 04 & 9 & 211 & 156.3750 & 6 . & 298.4 & 4 & .0 & \\
\hline 04 & 9 & 212 & 172.4497 & 6.9290 & 299.5 & 3 & 600.0 & 00.0 \\
\hline 04 & 9 & 213 & 170.8930 & 7.0165 & 300.3 & 4 & 600.0 & \\
\hline 04 & 9 & 214 & 162.1839 & & 301.1 & 4 & .0 & \\
\hline 04 & 9 & 215 & 125.4281 & 5.4 & 301.7 & 3 & 600.0 & \\
\hline 04 & 9 & 216 & 124.9009 & 4.4800 & 301.5 & 3 & 60 & .0 \\
\hline 04 & 9 & 217 & 91.2751 & 5.66 & 300.8 & 3 & .0 & \\
\hline 04 & 9 & 218 & 77.8250 & 6.9750 & 299.1 & 4 & .0 & \\
\hline 04 & 9 & 219 & 72.9750 & 7.7625 & 297.2 & 4 & .0 & .0 \\
\hline 04 & 9 & 220 & 67.7662 & 8.26 & 296.4 & 4 & 6 & \\
\hline 04 & 9 & 221 & 65.9270 & 8.16 & 296.3 & 4 & .0 & \\
\hline 04 & 9 & 222 & 99.0531 & 7.8625 & 296.9 & 4 & 600.0 & .0 \\
\hline 04 & 9 & 223 & 150.8752 & $13.1 \varepsilon$ & 296.7 & 4 & 0 & \\
\hline 04 & 9 & 224 & 155.9250 & 11.1 & 295.1 & 4 & .0 & \\
\hline 04 & 9 & 31 & 156.2753 & 10.49 & 294.4 & 4 & .0 & \\
\hline 04 & 9 & 32 & 154.3500 & 11.7075 & 294.0 & 4 & 60 & .0 \\
\hline 04 & 9 & 33 & 143.3752 & 11.37 & 293.5 & 4 & .0 & \\
\hline 04 & 9 & 34 & 148.2536 & 11.2925 & 292.9 & 4 & .0 & .0 \\
\hline 04 & 9 & 35 & 154.5000 & 12.4100 & 292.4 & 4 & .0 & .0 \\
\hline 04 & 9 & 36 & 159.2253 & 14.2150 & 291.8 & 4 & .0 & \\
\hline 04 & 9 & 37 & 156.7997 & 15.01 & 291.9 & 4 & .0 & .0 \\
\hline 04 & 9 & 38 & 163.5249 & 16.33 & 292.8 & 4 & 60 & .0 \\
\hline 04 & 9 & 39 & 166.0255 & 16.44 & 293.7 & 4 & 600.0 & .0 \\
\hline 04 & 9 & 310 & 166.1500 & 17.08 & 294.8 & 4 & .0 & 0 \\
\hline 04 & 9 & 311 & 174.4754 & 16.5975 & 295.8 & 4 & 600.0 & 0.0 \\
\hline 04 & 9 & 312 & 169.3729 & 15.91 & 296.9 & 4 & 600.0 & .0 \\
\hline 04 & 9 & 313 & 163.2516 & 14.3100 & 297.8 & 4 & 600.0 & 600.0 \\
\hline 04 & 9 & 314 & 167.8252 & 13.1975 & 298.8 & 4 & 600.0 & 600.0 \\
\hline 04 & 9 & 315 & 171.5754 & 13.91 & 299.3 & 4 & 600.0 & .0 \\
\hline 04 & 9 & 316 & 167.3232 & 13.4075 & 299.4 & 4 & 600.0 & 600.0 \\
\hline 04 & 9 & 317 & 173.0760 & 14.1625 & 299.4 & 4 & 600.0 & 600.0 \\
\hline 04 & 9 & 318 & 175.5873 & 13.5875 & 299.0 & 4 & 600.0 & 600.0 \\
\hline 04 & 9 & 319 & 168.5183 & 10.4200 & 297.9 & 4 & 600.0 & 600.0 \\
\hline 04 & 9 & 320 & 154.0998 & 10.2050 & 297.8 & 4 & 600.0 & 600.0 \\
\hline 04 & 9 & 321 & 151.6997 & 10.987 & 297.7 & 4 & 600.0 & \\
\hline $0<$ & 9 & 322 & 157.2239 & 10.847 & 297.3 & 4 & 600.0 & 600.0 \\
\hline 04 & 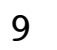 & 323 & 160.0259 & 8.872 & 295.9 & & 600.0 & 600.0 \\
\hline
\end{tabular}




\begin{tabular}{|c|c|c|c|c|c|c|c|}
\hline 4 & 24 & & 5715 & 4.8 & & 0 & \\
\hline 4 & 41 & 8.4439 & 4.4598 & 295.2 & 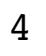 & 00 & \\
\hline 4 & 42 & 152.7925 & .1835 & 294.7 & 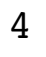 & 00 & \\
\hline 9 & 3 & 57.4750 & 0808 & 294.5 & 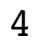 & 00 & \\
\hline 9 & 44 & 94 & 15 & 94.4 & & 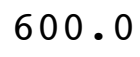 & \\
\hline 0 & 45 & 478 & 8 & 293.9 & & 0 & \\
\hline 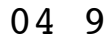 & 46 & 148.3748 & 550 & 294.0 & & 00 & \\
\hline 9 & 47 & 500 & 2090 & 294.0 & & & \\
\hline 9 & 48 & 250 & 043 & 294.4 & & 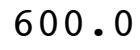 & \\
\hline 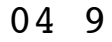 & 49 & 244 & 2700 & 295.7 & & U & \\
\hline 9 & 410 & 162 & 1475 & 297.2 & & 0 & \\
\hline 9 & 411 & 167 & 370 & 98.4 & & & \\
\hline 9 & 412 & 173 & 5.2028 & 299.4 & & 0 & \\
\hline 9 & 413 & 167 & 5.6585 & 300.5 & & 0 & \\
\hline 9 & 414 & 22 & 225 & 01.1 & & & \\
\hline 9 & 415 & & 5 . & 301.8 & & & \\
\hline y & 416 & 174 . & 4.8668 & 302.1 & & 0 & \\
\hline 9 & 417 & 37 & 3 . & 301.9 & & 0 & \\
\hline 9 & 418 & 89 & 2 . & 301.4 & . & & \\
\hline 9 & 419 & 1.7237 & 1.1755 & 300.9 & & 0 & \\
\hline 9 & 420 & 248 & 2.2000 & 300.2 & 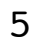 & 0 & \\
\hline 9 & 421 & & & 0.4 & 4 & & \\
\hline 9 & 422 & 996 & 3.3425 & 301.1 & 4 & 0 & \\
\hline 9 & 423 & 50 & 2.9935 & 300.2 & 4 & 0 & \\
\hline . & 424 & & & 300.6 & 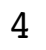 & & \\
\hline 9 & 51 & 71 & 1.9755 & 300.9 & 5 & & \\
\hline 9 & 52 & 933 & 1.2390 & 300.6 & 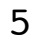 & 0 & \\
\hline 9 & 53 & 288 & 1.75 & 299.4 & 6 & & \\
\hline 0 & 54 & 16 & 1.1063 & 299.5 & 6 & & \\
\hline 9 & 55 & 197. & 0.6523 & 299.4 & 6 & 0 & \\
\hline 9 & 56 & 047 & 1.6460 & 299.3 & 6 & 0 & \\
\hline 0 & 57 & 6 & 2.2 & 298.5 & 5 & & \\
\hline 9 & 58 & 287 & 1.8298 & 300.1 & 4 & 0 & \\
\hline 9 & 59 & 347 & 2.6850 & 301.9 & 3 & 0 & \\
\hline 9 & 510 & 23 & 570 & 302.3 & 2 & & \\
\hline 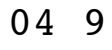 & 511 & & 5.3833 & 303.3 & 3 & 60 & \\
\hline 9 & 512 & 253 & 5.4000 & 304.6 & 3 & 0 & \\
\hline 9 & 513 & 25 & 615 & 305.6 & 3 & & \\
\hline 9 & 514 & 24 & 4.8770 & 305.7 & 2 & 600.0 & \\
\hline 9 & 515 & 212 & 4.4850 & 305.6 & 2 & 0 & \\
\hline 9 & 516 & 220 & 3.4670 & 305.4 & 3 & 600.0 & \\
\hline 9 & 517 & 226 . & 2.9563 & 305.1 & 3 & 600.0 & \\
\hline 9 & 518 & 203.1886 & 3.0733 & 304.0 & 3 & 600.0 & \\
\hline 9 & 519 & 607 & 2.0693 & 303.1 & 4 & 600 & \\
\hline 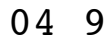 & 520 & 119.1773 & 1.4790 & 303.2 & 5 & 600.0 & \\
\hline 9 & 521 & 6023 & 3.5165 & 302.7 & 4 & 600.0 & \\
\hline$y$ & 522 & & 113 & .4 & 4 & & \\
\hline , & 523 & 69.7499 & 6.6393 & 301.8 & 4 & 600.0 & 60 \\
\hline
\end{tabular}




\begin{tabular}{|c|c|c|c|c|c|c|c|c|}
\hline 04 & 9 & 524 & 68.8750 & 6.8695 & 301.6 & 4 & 600.0 & 600.0 \\
\hline 04 & 9 & 61 & 71.0751 & 8.3900 & 300.4 & 4 & 600.0 & 600.0 \\
\hline 04 & 9 & 62 & 68.7000 & 8.6875 & 300.1 & 4 & 600.0 & 00.0 \\
\hline 04 & 9 & 63 & 73.0998 & 7.5775 & 300.4 & 4 & 600.0 & 600.0 \\
\hline 04 & 9 & 64 & 72.5497 & 6.6900 & 300.4 & 4 & 600.0 & 00.0 \\
\hline 04 & 9 & 65 & 78.4248 & 4.6273 & 300.7 & 4 & 600.0 & 500.0 \\
\hline 04 & 9 & 66 & 89.8399 & 2.5088 & 301.1 & 5 & 600.0 & 600.0 \\
\hline 04 & 9 & 67 & 109.1720 & 2.2570 & 300.9 & 4 & 600.0 & 500.0 \\
\hline 04 & 9 & 68 & 213.0950 & 0.9855 & 302.0 & 3 & 600.0 & 00.0 \\
\hline 04 & 9 & 69 & 225.8225 & 2.3028 & 302.5 & 2 & 600.0 & 600.0 \\
\hline 04 & 9 & 610 & 175.5023 & 3.8593 & 302.4 & 2 & 600.0 & 600.0 \\
\hline 04 & 9 & 611 & 221.7651 & 3.5148 & 303.6 & 2 & 600.0 & \\
\hline 04 & 9 & 612 & 179.1908 & 3.3090 & 304.8 & 2 & 600.0 & 600.0 \\
\hline 04 & 9 & 613 & 193.5945 & 3.8488 & 305.8 & 2 & 600.0 & 600.0 \\
\hline 04 & 9 & 614 & 205.0590 & 4.6913 & 306.8 & 2 & 600.0 & \\
\hline 04 & 9 & 615 & 188.9855 & 5.5613 & 306.9 & 3 & 600.0 & 60 \\
\hline 04 & 9 & 616 & 196.7745 & 5.4390 & 306.5 & 4 & 600.0 & 600.0 \\
\hline 04 & 9 & 617 & 184.9020 & 5.8328 & 306.0 & 4 & 600.0 & .0 \\
\hline 04 & 9 & 618 & 179.3517 & 4.8463 & 305.3 & 4 & 600.0 & 60 \\
\hline 04 & 9 & 619 & 101.4189 & 4.4975 & 304.1 & 4 & 600.0 & 600.0 \\
\hline 04 & 9 & 620 & 70.3497 & 5.8868 & 303.4 & 4 & 600.0 & .0 \\
\hline 04 & 9 & 621 & 67.8017 & 6.7388 & 302.6 & 4 & 600.0 & \\
\hline 04 & 9 & 622 & 62.6742 & 6.9748 & 302.1 & 4 & 600.0 & 600.0 \\
\hline 04 & 9 & 623 & 63.0496 & 8.2650 & 302.0 & 4 & 600.0 & 60 \\
\hline 04 & 9 & 624 & 70.0506 & 7.5650 & 302.0 & 4 & 600.0 & \\
\hline 04 & 9 & 71 & 65.2499 & 8.0325 & 301.5 & 4 & 600.0 & 600.0 \\
\hline 04 & 9 & 72 & 67.2250 & 8.1850 & 301.3 & 4 & 600.0 & 600.0 \\
\hline 04 & 9 & 73 & 69.8500 & 7.5000 & 301.3 & 4 & 600.0 & .0 \\
\hline 04 & 9 & 74 & 64.2256 & 7.7175 & 301.7 & 4 & 600.0 & 600.0 \\
\hline 04 & 9 & 75 & 67.1761 & 6.9180 & 301.6 & 4 & 600.0 & 600.0 \\
\hline 04 & 9 & 76 & 90.5644 & 4.6543 & 301.4 & 4 & 600.0 & .0 \\
\hline 04 & 9 & 77 & 80.8208 & 1.3345 & 299.5 & 3 & 600.0 & 60 \\
\hline 04 & 9 & 78 & 156.1053 & 1.6903 & 302.3 & 2 & 600.0 & 600.0 \\
\hline 04 & 9 & 79 & 165.4406 & 5.0460 & 302.0 & 3 & 600.0 & .0 \\
\hline 04 & 9 & 710 & 166.1000 & 833 & 302.8 & 2 & 600.0 & 60 \\
\hline 04 & 9 & 711 & 165.0437 & 4.0595 & 303.8 & 2 & 600.0 & 600.0 \\
\hline 04 & 9 & 712 & 163.3748 & 4.7435 & 304.7 & 2 & 600.0 & 600.0 \\
\hline 04 & 9 & 713 & 173.3999 & 5.5995 & 306.0 & 3 & 600.0 & 600.0 \\
\hline 04 & 9 & 714 & 175.8589 & 5.4130 & 306.4 & 3 & 600.0 & 600.0 \\
\hline 04 & 9 & 715 & 174.9065 & 5.0440 & 307.1 & 3 & 600.0 & \\
\hline 04 & 9 & 716 & 170.9338 & 5.2548 & 307.3 & 3 & 600.0 & 600.0 \\
\hline 04 & 9 & 717 & 160.9504 & 5.2725 & 307.0 & 4 & 600.0 & 600.0 \\
\hline 04 & 9 & 718 & 169.2589 & 4.4280 & 305.7 & 4 & 600.0 & 600.0 \\
\hline 04 & 9 & 719 & 105.9170 & 4.5010 & 304.7 & 4 & 600.0 & 600.0 \\
\hline 04 & 9 & 720 & 70.2980 & 6.2620 & 304.0 & 4 & 600.0 & 600.0 \\
\hline 04 & 9 & 721 & 68.2257 & 6.0275 & 303.6 & 4 & 600.0 & 600.0 \\
\hline 04 & 9 & 722 & 62.9497 & 7.4075 & 303.1 & 4 & 600.0 & 600.0 \\
\hline 04 & 9 & 723 & 68.3750 & 7.4625 & 303.3 & 4 & 600.0 & 600.0 \\
\hline
\end{tabular}




\begin{tabular}{|c|c|c|c|c|c|c|c|c|}
\hline 4 & 9 & 724 & 70.7499 & 7.9700 & 303.2 & 4 & 600.0 & 600.0 \\
\hline 04 & 9 & 81 & 72.0254 & 7.8175 & 303.3 & 4 & 600.0 & 00.0 \\
\hline 04 & 9 & 82 & 71.9001 & 7.3250 & 303.4 & 4 & 600.0 & 00.0 \\
\hline 04 & 9 & 83 & 68.7748 & 5.8763 & 303.4 & 4 & 600.0 & 00.0 \\
\hline 04 & 9 & 84 & 70.6943 & 2.5620 & 303.6 & $J$ & 600.0 & 00.0 \\
\hline 04 & 9 & 85 & 126.9131 & 1.4888 & 301.8 & 6 & 600.0 & 00.0 \\
\hline 04 & 9 & 86 & 63.2467 & 0.6723 & 301.7 & 6 & 600.0 & 00.0 \\
\hline 04 & 9 & 87 & 167.1020 & 1.0120 & 300.7 & 5 & 600.0 & 00.0 \\
\hline 04 & 9 & 88 & 205.7987 & 1.5520 & 300.3 & 4 & 600.0 & 00.0 \\
\hline 04 & 9 & 89 & 223.3465 & 2.2855 & 301.8 & 3 & 600.0 & 0 \\
\hline 04 & 9 & 810 & 218.2761 & 3.9363 & 302.6 & 2 & 600.0 & \\
\hline 04 & 9 & 811 & 245.0074 & 2.4043 & 303.3 & 2 & 600.0 & \\
\hline 04 & 9 & 812 & 232.7705 & 2.8455 & 304.9 & 2 & 600.0 & 00.0 \\
\hline 04 & 9 & 813 & 180.7478 & 3.4703 & 306.5 & 2 & 600.0 & 00.0 \\
\hline 04 & 9 & 814 & 166.3253 & 3.4233 & 307.2 & 2 & .0 & \\
\hline 04 & 9 & 815 & 181.0076 & 3.9915 & 307.8 & 2 & 600.0 & .0 \\
\hline 04 & 9 & 816 & 188.3893 & 5.5313 & 307.4 & 3 & 60 & 600.0 \\
\hline 04 & 9 & 817 & 175.9970 & 4.6115 & 306.5 & 3 & .0 & \\
\hline 04 & 9 & 818 & 125.6859 & 4.4130 & 305.9 & 3 & 600.0 & .0 \\
\hline 04 & 9 & 819 & 98.2678 & 4.8768 & 303.7 & 4 & .0 & .0 \\
\hline 04 & 9 & 820 & 74.7099 & 4.7568 & 302.6 & 4 & 60 & \\
\hline 04 & 9 & 821 & 47.3756 & 4.2423 & 303.0 & 4 & .0 & \\
\hline 04 & 9 & 822 & 55.4250 & 7.4188 & 303.1 & 4 & 600.0 & .0 \\
\hline 04 & 9 & 823 & 57.0756 & 7.0320 & 303.3 & 4 & 6 & \\
\hline 04 & 9 & 824 & 55.5505 & 6.3150 & 303.4 & 4 & .0 & \\
\hline 04 & 9 & 91 & 69.3767 & 4.5235 & 303.1 & 4 & 600.0 & 600.0 \\
\hline 04 & 9 & 92 & 88.3434 & 3.0088 & 303.5 & 4 & 600.0 & 0 \\
\hline 04 & 9 & 93 & 79.6527 & 2.9665 & 303.6 & 4 & .0 & \\
\hline 04 & 9 & 94 & 82.3998 & 3.7170 & 302.8 & 4 & .0 & 600.0 \\
\hline 04 & 9 & 95 & 134.5088 & 2.4323 & 300.6 & 5 & 60 & 0.0 \\
\hline 04 & 9 & 96 & 32.4898 & 1.5478 & 297.9 & 6 & .0 & \\
\hline 04 & 9 & 97 & 285.8837 & 0.8645 & 298.7 & 5 & .0 & 600.0 \\
\hline 04 & 9 & 98 & 162.7906 & 1.8925 & 300.2 & 4 & 600.0 & 600.0 \\
\hline 04 & 9 & 99 & 172.8244 & 4.3375 & 302.2 & 3 & 600.0 & 0.0 \\
\hline 04 & 9 & 910 & 203.1901 & 3.4175 & 303.3 & 2 & 600.0 & 600.0 \\
\hline 04 & 9 & 911 & 226.6958 & 3.3015 & 303.7 & 2 & 600.0 & 600.0 \\
\hline 04 & 9 & 912 & 180.7846 & 3.8360 & 304.8 & 2 & 600.0 & 600.0 \\
\hline 04 & 9 & 913 & 215.0477 & 4.1770 & 305.7 & 2 & 600.0 & 600.0 \\
\hline 04 & 9 & 914 & 210.2261 & 3.3795 & 306.3 & 2 & 600.0 & 600.0 \\
\hline 04 & 9 & 915 & 137.3919 & 4.8705 & 307.7 & 3 & 600.0 & 600.0 \\
\hline 04 & 9 & 916 & 99.6775 & 5.7258 & 307.2 & 4 & 600.0 & 600.0 \\
\hline 04 & 9 & 917 & 99.4229 & 6.0730 & 305.4 & 4 & 600.0 & 600.0 \\
\hline 04 & 9 & 918 & 88.2807 & 7.0998 & 302.7 & 4 & 600.0 & 600.0 \\
\hline 04 & 9 & 919 & 73.1998 & 9.3350 & 301.5 & 4 & 600.0 & 600.0 \\
\hline 04 & 9 & 920 & 65.6992 & 10.6750 & 300.5 & 4 & 600.0 & 600.0 \\
\hline 04 & 9 & 921 & 60.5501 & 10.3350 & 300.9 & 4 & 600.0 & 600.0 \\
\hline $0<$ & 9 & 922 & 56.9502 & 9.8725 & 301.1 & 4 & 600.0 & 600.0 \\
\hline 0 & $y$ & 923 & 58.1500 & 9.5175 & 301.6 & 4 & 600.0 & 600.0 \\
\hline
\end{tabular}




\begin{tabular}{|c|c|c|c|c|c|c|c|}
\hline 04 & 9924 & 57.7757 & 8.5675 & 301.8 & 4 & 600.0 & 600.0 \\
\hline 04 & 9101 & 60.9742 & 7.6165 & 301.4 & 4 & 600.0 & 600.0 \\
\hline 04 & 9102 & 75.0255 & 4.8008 & 300.0 & 4 & 600.0 & 00.0 \\
\hline 04 & 9103 & 56.5244 & 7.7525 & 300.3 & 4 & 600.0 & 00.0 \\
\hline 04 & 9104 & 64.2265 & 5.3818 & 299.7 & 4 & 600.0 & 00.0 \\
\hline 04 & 9105 & 79.9979 & 4.0163 & 299.4 & 4 & 600.0 & 00.0 \\
\hline 04 & 9106 & 87.9286 & 3.2888 & 299.2 & 4 & 600.0 & 00.0 \\
\hline 04 & 9107 & 87.2018 & 2.4388 & 299.8 & 3 & 600.0 & 00.0 \\
\hline 04 & 9108 & 57.6764 & 1.9200 & 301.2 & 2 & 600.0 & 00.0 \\
\hline 04 & 9109 & 321.8755 & 1.5058 & 300.7 & 2 & 600.0 & 00.0 \\
\hline 04 & 91010 & 245.0080 & 2.5105 & 301.6 & 2 & 600.0 & 00.0 \\
\hline 04 & 91011 & 234.2967 & 3.3750 & 302.2 & 2 & 600.0 & \\
\hline 04 & 91012 & 223.3571 & 3.0440 & 303.6 & 2 & 600.0 & 00.0 \\
\hline 04 & 91013 & 99.3767 & 3.3540 & 306.0 & 2 & 600.0 & 00.0 \\
\hline 04 & 91014 & 98.0647 & 248 & 305.6 & 2 & .0 & \\
\hline 04 & 91015 & 98.8871 & 6.3313 & 305.7 & 3 & 600.0 & 00.0 \\
\hline 04 & 91016 & 85.9513 & 7.4388 & 304.9 & 4 & 600.0 & 600.0 \\
\hline 04 & 91017 & 78.4726 & 7.6095 & 303.8 & 4 & .0 & \\
\hline 04 & 91018 & 91.5599 & 7.4000 & 302.6 & 4 & 600.0 & .0 \\
\hline 04 & 91019 & 72.9382 & 7.3618 & 301.6 & 4 & .0 & .0 \\
\hline 04 & 91020 & 73.3642 & 5.2560 & 30 & 4 & 60 & \\
\hline 04 & 91021 & 62.6052 & 5.6133 & 301.0 & 4 & .0 & \\
\hline 04 & 91022 & 63.8992 & 8.5825 & 300.7 & 4 & 600.0 & 00.0 \\
\hline 04 & 91023 & 57.9500 & 9.2125 & .2 & 4 & 60 & .0 \\
\hline 04 & 91024 & 61.8500 & 8.1725 & 300.0 & 4 & .0 & \\
\hline 04 & 9111 & 68.8253 & 8.0675 & 300.2 & 4 & 600.0 & 600.0 \\
\hline 04 & 9112 & 71.9502 & 7.1555 & 300.2 & 4 & 600.0 & .0 \\
\hline 04 & 9113 & 68.6502 & 6.5558 & 300.0 & 4 & 0 & \\
\hline 04 & 9114 & 68.9032 & 6.0248 & 299.2 & 4 & .0 & 600.0 \\
\hline 04 & 9115 & 86.6627 & 4.9763 & 299.2 & 4 & 60 & 600.0 \\
\hline 04 & 9116 & 113.6994 & 2.1808 & 297.7 & 5 & .0 & \\
\hline 04 & 9117 & 122.8634 & 1.3903 & 296.7 & 4 & .0 & 600.0 \\
\hline 04 & 9118 & 244.7314 & 1.6210 & 297.0 & 3 & 600.0 & 600.0 \\
\hline 04 & 9119 & 222.2297 & 1.6533 & 298.8 & 2 & 600.0 & 0.0 \\
\hline 04 & 91110 & 165.5243 & 4.9953 & 300.2 & 3 & 600.0 & 600.0 \\
\hline 04 & 91111 & 164.3997 & 4.4333 & 300.9 & 2 & 600.0 & 600.0 \\
\hline 04 & 91112 & 164.3533 & 5.0450 & 301.7 & 2 & 600.0 & 600.0 \\
\hline 04 & 91113 & 172.4648 & 6.3095 & 303.0 & 3 & 600.0 & 600.0 \\
\hline 04 & 91114 & 158.3786 & 7.0778 & 304.5 & 4 & 600.0 & 600.0 \\
\hline 04 & 91115 & 110.8929 & 5.4838 & 305.2 & 3 & 600.0 & 600.0 \\
\hline 04 & 91116 & 115.5743 & 5.9550 & 305.3 & 4 & 600.0 & 600.0 \\
\hline 04 & 91117 & 99.9500 & 9.7125 & 303.1 & 4 & 600.0 & 600.0 \\
\hline 04 & 91118 & 72.0751 & 9.4425 & 301.1 & 4 & 600.0 & 600.0 \\
\hline 04 & 91119 & 61.4481 & 9.5975 & 300.4 & 4 & 600.0 & 600.0 \\
\hline 04 & 91120 & 67.2236 & 8.2483 & 300.3 & 4 & 600.0 & 600.0 \\
\hline 04 & 91121 & 74.5750 & 7.3203 & 300.5 & 4 & 600.0 & 600.0 \\
\hline 04 & 91122 & 64.9453 & 8.5465 & 299.5 & 4 & 600.0 & 600.0 \\
\hline 4 & 91123 & 61.6750 & 9.9550 & 298.6 & 4 & 600.0 & 600.0 \\
\hline
\end{tabular}




\begin{tabular}{|c|c|c|c|c|c|c|c|}
\hline 4 & 91124 & 5248 & 11.2025 & 297.9 & 4 & 600.0 & 00.0 \\
\hline 04 & 9121 & 61.1998 & 13.3025 & 296.8 & 4 & 600.0 & 00,0 \\
\hline 04 & 9122 & 63.8644 & 11.7100 & 297.0 & 4 & 600.0 & 00.0 \\
\hline 04 & 9123 & 62.0753 & 11.4700 & 296.0 & 4 & 600.0 & 00.0 \\
\hline 04 & 9124 & 65.8000 & 11.6075 & 296.1 & 4 & 600.0 & 00.0 \\
\hline 04 & 9125 & 63.6005 & 13.1375 & 295.3 & 4 & 600.0 & 00.0 \\
\hline 04 & 9126 & 60.2497 & 12.2775 & 295.7 & 4 & 600.0 & 600.0 \\
\hline 04 & 9127 & 61.7000 & 11.0125 & 296.5 & 4 & 600.0 & 00.0 \\
\hline 04 & 9128 & 60.5000 & 11.7100 & 297.4 & 4 & 600.0 & 00.0 \\
\hline 04 & 9129 & 69.4486 & 10.1525 & 297.2 & 4 & 600.0 & .0 \\
\hline 04 & 91210 & 67.2249 & 11.8175 & 296.1 & 4 & 600.0 & 500.0 \\
\hline 04 & 91211 & 63.5515 & 10. & 296.9 & 4 & 600.0 & \\
\hline 04 & 91212 & 62.4218 & 10.6825 & 299.5 & 4 & 600.0 & .0 \\
\hline 04 & 91213 & 58.8493 & 10.7600 & 300.6 & 4 & 600.0 & 0 \\
\hline 04 & 91214 & 62.2253 & 11.3300 & 299.4 & 4 & 600.0 & \\
\hline 04 & 91215 & 60.8497 & 12 . & 298.5 & 4 & 600.0 & 60 \\
\hline 04 & 91216 & 67.7750 & 13.3175 & 298.0 & 4 & 600.0 & .0 \\
\hline 04 & 91217 & 63.7741 & 13.2200 & 295.6 & 4 & 600.0 & \\
\hline 04 & 91218 & 66.8500 & 13.3900 & 293.3 & 4 & 600.0 & .0 \\
\hline 04 & 91219 & 65.3249 & 000 & 293.1 & 4 & 600.0 & 60 \\
\hline 04 & 91220 & 60.5501 & 14 . & 293.9 & 4 & 600.0 & .0 \\
\hline 04 & 91221 & 71.2587 & 11. & 293.5 & 4 & 600.0 & \\
\hline 04 & 91222 & 66.4248 & 250 & 293.3 & 4 & 600.0 & 600.0 \\
\hline 04 & 91223 & 63.0252 & 13.6175 & 293.6 & 4 & 600.0 & .0 \\
\hline 04 & 91224 & 66.6500 & 12.2875 & 293.8 & 4 & 600.0 & \\
\hline 04 & 9131 & 67.4501 & 12.9300 & 292.8 & 4 & 600.0 & 60 \\
\hline 04 & 9132 & 66.9994 & 11.5475 & 291.7 & 4 & 600.0 & .0 \\
\hline 04 & 9133 & 65.0000 & 11.9750 & 291.2 & 4 & 0.0 & \\
\hline 04 & 9134 & 74.0749 & 5350 & 290.1 & 4 & 600.0 & 60 \\
\hline 04 & 9135 & 67.7503 & 12.1025 & 289.6 & 4 & 600.0 & .0 \\
\hline 04 & 9136 & 64.1736 & 11. & 289.9 & 4 & 600.0 & \\
\hline 04 & 9137 & 71.9250 & 11. & 290.2 & 4 & 600.0 & 60 \\
\hline 04 & 9138 & 71.9737 & 11.0500 & 291.2 & 4 & 600.0 & 600.0 \\
\hline 04 & 9139 & 75.3748 & 10.2700 & 292.5 & 4 & 600.0 & .0 \\
\hline 04 & 91310 & 77.0261 & 950 & 293.8 & 4 & 600.0 & .0 \\
\hline 04 & 91311 & 73.2252 & 8.6500 & 295.1 & 4 & 600.0 & 600.0 \\
\hline 04 & 91312 & 73.7305 & 6.3908 & 297.0 & 3 & 600.0 & \\
\hline 04 & 91313 & 89.5038 & 565 & 298.1 & 3 & 600.0 & 600.0 \\
\hline 04 & 91314 & 80.6436 & 6.7203 & 298.1 & 4 & 600.0 & 600.0 \\
\hline 04 & 91315 & 83.4018 & 7.1388 & 298.0 & 4 & 600.0 & \\
\hline 04 & 91316 & 75.7442 & 8.2275 & 296.8 & 4 & 600.0 & 600.0 \\
\hline 04 & 91317 & 80.3750 & 9.6400 & 294.9 & 4 & 600.0 & 600.0 \\
\hline 04 & 91318 & 80.7750 & 11.1500 & 293.2 & 4 & 600.0 & 600.0 \\
\hline 04 & 91319 & 69.4225 & 10.8525 & 292.5 & 4 & 600.0 & 600.0 \\
\hline 04 & 91320 & 70.7241 & 10.2350 & 292.7 & 4 & 600.0 & 600.0 \\
\hline 04 & 91321 & 77.7503 & 8.3625 & 293.6 & 4 & 600.0 & 600.0 \\
\hline 04 & 91322 & 76.6000 & 8.1975 & 294.0 & 4 & 600.0 & 600.0 \\
\hline 04 & 91323 & 75.7000 & 7.7300 & 294.1 & 4 & 600.0 & 600.0 \\
\hline
\end{tabular}




\begin{tabular}{|c|c|c|c|c|c|c|c|}
\hline & 24 & - & 910 & 94.1 & & & \\
\hline & 141 & 6.5526 & 4.4498 & 94.8 & & 0 & \\
\hline & 142 & 238 & 0518 & 294.8 & & 00 & \\
\hline & 143 & 150.2470 & 3143 & 293.7 & & 0 & \\
\hline & 44 & 55.4250 & & 92.7 & & & \\
\hline & 145 & 500 & & 92.2 & & & \\
\hline & 146 & 156.0249 & 5950 & 291.4 & & 0 & \\
\hline & 147 & 250 & & 91.2 & & & \\
\hline & 148 & 3752 & & 92.0 & & & \\
\hline & 149 & 168.7252 & 75 & 293.1 & & & \\
\hline & 410 & 171.9227 & 3.15 & 294 & & & \\
\hline & 11 & 166.0244 & & 7 & & & \\
\hline & 2 & 166 & 50 & 97.1 & & & \\
\hline & 413 & 165.7956 & 5 & 4 & & & \\
\hline & 4 & 160 & & & & & \\
\hline & & 167 . & & & & & \\
\hline & 6 & 148.0430 & 3 & 7 & & & \\
\hline & 17 & 152 . & & & & & \\
\hline & & 153. & & & & & \\
\hline & & 2500 & & & 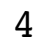 & & \\
\hline & 20 & 133.8546 & 4 . & 298.6 & & 0 & \\
\hline & & 98.0825 & & & $I$ & & \\
\hline & 2 & 127 . & 38 & 8 & 1 & & \\
\hline & 1423 & 131.6452 & 0 & & & & \\
\hline & 91424 & 141 & & & 4 & & \\
\hline & 9151 & 148.47 & & & 4 & & \\
\hline & 9152 & 151.7251 & 800 & 7.9 & 4 & & \\
\hline & 9153 & 154.5500 & 5 & & 4 & & \\
\hline & 9154 & 156. & 00 & & 4 & & \\
\hline & 9155 & 130.0179 & 913 & 29 & 4 & & 0 . \\
\hline & 9156 & 145.3259 & & & a & & \\
\hline & 9157 & 149 . & 25 & & 4 & & \\
\hline & 9158 & 157.2503 & & & 4 & & . \\
\hline & 9159 & 161.7499 & 850 & & a & & \\
\hline & & 165 . & 125 & & 4 & & \\
\hline & & 154.7456 & & .6 & 4 & & \\
\hline & 91512 & 154.1010 & 00 & 301.1 & 4 & & \\
\hline & 91513 & 153. & 10 & & 4 & & \\
\hline & 91514 & 153.4353 & 750 & .9 & 4 & & 0 . \\
\hline & 91515 & 151.5492 & 808 & 303.4 & 4 & & \\
\hline & & 158 . & 215 & & 4 & & \\
\hline & & 152.9752 & 908 & & 4 & & \\
\hline & 91518 & .6849 & 3485 & 302.1 & 4 & & 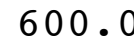 \\
\hline & & 249 & 348 & & 4 & & \\
\hline & & 66 & & & 4 & & \\
\hline & & 71.4478 & 6.9178 & 300.7 & 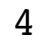 & & \\
\hline & & & & & & & \\
\hline & 1523 & 70 & 8.9500 & 299.8 & & 60 & 0 \\
\hline
\end{tabular}




\begin{tabular}{|c|c|c|c|c|c|c|c|}
\hline 4 & 91524 & 71.0754 & 10.1050 & 298.5 & 4 & 600.0 & 00.0 \\
\hline 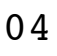 & 9161 & 70.1997 & 9.6425 & 298.2 & 4 & 600.0 & 600.0 \\
\hline 04 & 9162 & 77.7429 & 8.6525 & 298.3 & 4 & 600.0 & 00.0 \\
\hline 04 & 9163 & 115.7112 & 6.3170 & 296.4 & 4 & 600.0 & 00.0 \\
\hline 4 & 9164 & 75.4500 & 8.4600 & 295.1 & 4 & 600.0 & 00.0 \\
\hline 04 & 9165 & 87.6447 & 6.6608 & 295.7 & 4 & 600.0 & .0 \\
\hline 04 & 9166 & 146.5462 & 3.9920 & 295.7 & 4 & 600.0 & 00.0 \\
\hline 04 & 9167 & 143.8494 & 3.2310 & 296.2 & 4 & 600.0 & .0 \\
\hline 04 & 9168 & 154.7683 & 4.6455 & 297.3 & 3 & 600.0 & \\
\hline 04 & 9169 & 163.0002 & 6.9305 & 297.9 & 4 & 600.0 & .0 \\
\hline 04 & 91610 & 167.8749 & 7.0760 & 298.7 & 4 & 600.0 & .0 \\
\hline 04 & 91611 & 172.8334 & 6.4313 & 299.8 & 4 & 600.0 & \\
\hline 04 & 91612 & 171.4752 & 5.7998 & 300.8 & 3 & 600.0 & .0 \\
\hline 04 & 91613 & 188.2375 & 4.9068 & 301.7 & 2 & 600.0 & \\
\hline 04 & 91614 & 180.9437 & 4.4463 & 302.7 & 2 & 600.0 & \\
\hline 04 & 91615 & 172.0743 & 5.5838 & 303.2 & 3 & 600.0 & \\
\hline 04 & 91616 & 144.8593 & 5.8598 & 303.3 & 4 & 600.0 & 600.0 \\
\hline 04 & 91617 & 92.3906 & 5.8270 & 302.2 & 4 & 600.0 & .0 \\
\hline 04 & 91618 & 80.7516 & 7.0100 & 300.3 & 4 & 600.0 & \\
\hline 04 & 91619 & 70.5500 & 9.4400 & 298.8 & 4 & 600.0 & 600.0 \\
\hline 04 & 91620 & 67.7747 & 10.0975 & 298.3 & 4 & 600.0 & .0 \\
\hline 04 & 91621 & 67.6750 & 10.1125 & 298.7 & 4 & 600.0 & \\
\hline 04 & 91622 & 65.8408 & 6.9508 & 299.2 & 4 & 600.0 & 60 \\
\hline 04 & 91623 & 69.3254 & 6.6053 & 299.5 & 4 & 600.0 & .0 \\
\hline 04 & 91624 & 62.5246 & 6.7565 & 298.8 & 4 & 600.0 & \\
\hline 04 & 9171 & 67.9998 & 6.9700 & 298.5 & 4 & 600.0 & 60 \\
\hline 04 & 9172 & 71.8750 & 9.5250 & 297.0 & 4 & 600.0 & .0 \\
\hline 04 & 9173 & 68.7750 & 10.1500 & 296.0 & 4 & 600.0 & .0 \\
\hline 04 & $917 \quad 4$ & 73.8750 & 9.6750 & 295.7 & 4 & 600.0 & .0 \\
\hline 04 & 9175 & 73.4502 & 10.3575 & 295.0 & 4 & 600.0 & .0 \\
\hline 04 & 9176 & 72.2001 & 9.8150 & 294.5 & 4 & 600.0 & .0 \\
\hline 04 & 9177 & 67.5066 & 9.1075 & 294.1 & 4 & 600.0 & \\
\hline 04 & 9178 & 64.1269 & 9.0950 & 294.9 & 4 & 600.0 & .0 \\
\hline 04 & 9179 & 76.9997 & 7.3080 & 296.2 & 4 & 600.0 & .0 \\
\hline 04 & 91710 & 78.9004 & 7.2925 & 297.2 & 4 & 600.0 & \\
\hline 04 & 91711 & 68.9941 & 6.8143 & 299.0 & 4 & 600.0 & 600.0 \\
\hline 04 & 91712 & 64.2506 & 8.5525 & 299.7 & 4 & 600.0 & 600.0 \\
\hline 04 & 91713 & 59.4750 & 10.5650 & 300.1 & 4 & 600.0 & 60 \\
\hline 04 & 91714 & 53.9998 & 13.1050 & 299.5 & 4 & 600.0 & 600.0 \\
\hline 04 & 91715 & 57.8500 & 14.1625 & 297.0 & 4 & 600.0 & 600 \\
\hline 04 & 91716 & 60.8250 & 15.1250 & 295.7 & 4 & 600.0 & 600.0 \\
\hline 04 & 91717 & 57.1500 & 14.3975 & 295.8 & 4 & 600.0 & 600.0 \\
\hline 04 & 91718 & 56.3645 & 10.7050 & 294.1 & 4 & 600.0 & 600.0 \\
\hline 04 & 91719 & 104.5683 & 7.4910 & 292.0 & 4 & 600.0 & 600.0 \\
\hline 04 & 91720 & 79.6251 & 11.8475 & 290.3 & 4 & 600.0 & 600.0 \\
\hline 04 & 91721 & 73.6254 & 11.7600 & 289.1 & 4 & 600.0 & 600.0 \\
\hline 04 & 91722 & 88.5909 & 8.95 & 28 & 4 & .0 & .0 \\
\hline 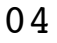 & 91723 & 78.3343 & 7.2533 & 288.2 & $I$ & 600.0 & 600.0 \\
\hline
\end{tabular}




\begin{tabular}{|c|c|c|c|c|c|c|c|}
\hline 04 & 91724 & 87.1278 & 7.4295 & 287.9 & 4 & 600.0 & 00.0 \\
\hline 04 & 9181 & 92.9497 & 10.0200 & 288.0 & 4 & 600.0 & 600.0 \\
\hline 04 & 9182 & 90.8500 & 8.8425 & 287.6 & 4 & 600.0 & 00.0 \\
\hline 04 & 9183 & 85.6000 & 8.8350 & 287.1 & 4 & 600.0 & 00.0 \\
\hline 04 & 9184 & 75.0751 & 9.0350 & 286.6 & 4 & 600.0 & 00.0 \\
\hline 04 & 9185 & 71.3759 & 9.8925 & 286.5 & 4 & 600.0 & 00.0 \\
\hline 04 & 9186 & 73.3179 & 9.1350 & 286.5 & 4 & 600.0 & 600.0 \\
\hline 04 & 9187 & 70.8202 & 7.9225 & 286.3 & 4 & 600.0 & 500.0 \\
\hline 04 & 9188 & 62.8998 & 8.0600 & 286.5 & 4 & 600.0 & 00.0 \\
\hline 04 & 9189 & 62.7000 & 8.3075 & 287.1 & 4 & 600.0 & 600.0 \\
\hline 04 & 91810 & 71.0692 & 9.2400 & 287.5 & 4 & 600.0 & 600 \\
\hline 04 & 91811 & 70.1489 & 8.1375 & 288.0 & 4 & 600.0 & \\
\hline 04 & 91812 & 69.7497 & 7.2443 & 288.7 & 4 & 600.0 & 600.0 \\
\hline 04 & 91813 & 71.0000 & 7.9865 & 289.0 & 4 & 600.0 & 600.0 \\
\hline 04 & 91814 & 72.0748 & 8.9675 & 289.0 & 4 & 600.0 & \\
\hline 04 & 91815 & 71.3740 & 8.3450 & 289.4 & 4 & 600.0 & 60 \\
\hline 04 & 91816 & 71.3245 & 9.6500 & 288.9 & 4 & 600.0 & 600.0 \\
\hline 04 & 91817 & 59.1255 & 8.9975 & 288.3 & 4 & 600.0 & .0 \\
\hline 04 & 91818 & 58.0002 & 10.9300 & 287.4 & 4 & 600.0 & 60 \\
\hline 04 & 91819 & 56.5000 & 11.8725 & 286.2 & 4 & 600.0 & .0 \\
\hline 04 & 91820 & 54.4749 & 12.5975 & 285.6 & 4 & 600.0 & .0 \\
\hline 04 & 91821 & 54.2250 & 12.1725 & 285.1 & 4 & 600.0 & \\
\hline 04 & 91822 & 59.1500 & 12.3500 & 284.7 & 4 & 600.0 & 600 \\
\hline 04 & 91823 & 55.9251 & 11.9650 & 284.7 & 4 & 600.0 & .0 \\
\hline 04 & 91824 & 49.7499 & 10.4125 & 284.7 & 4 & 600.0 & \\
\hline 04 & $919 \quad 1$ & 53.3001 & 10.5200 & 285.0 & 4 & 600.0 & 60 \\
\hline 04 & 9192 & 52.8001 & 8.3523 & 284.8 & 4 & 600.0 & 600.0 \\
\hline 04 & 9193 & 91.1244 & 4.9123 & 284.7 & 4 & 600.0 & \\
\hline 04 & 9194 & 100.6400 & 4.5180 & 285.0 & 4 & 600.0 & 60 \\
\hline 04 & 9195 & 63.8518 & 6.3015 & 285.2 & 4 & 600.0 & 600.0 \\
\hline 04 & 9196 & 70.2509 & 5.06 & 285.4 & 4 & 600.0 & \\
\hline 04 & 9197 & 54.5506 & 4.3785 & 285.7 & 4 & 600.0 & 60 \\
\hline 04 & 9198 & 60.0198 & 3.2148 & 286.1 & 4 & 600.0 & 600.0 \\
\hline 04 & 9199 & 166.9453 & 5.7690 & 283.7 & 4 & 600.0 & .0 \\
\hline 04 & 91910 & 5.4954 & 3.6185 & 282.6 & 3 & 600.0 & .0 \\
\hline 04 & 91911 & 12.6853 & 3.0255 & 285.0 & 2 & 600.0 & 600.0 \\
\hline 04 & 91912 & 52.2267 & 4.9045 & 286.7 & 3 & 600.0 & \\
\hline 04 & 91913 & 84.9748 & 4.8923 & 286.1 & 4 & 600.0 & 600.0 \\
\hline 04 & 91914 & 55.9747 & 7.6633 & 287.9 & 4 & 600.0 & 600.0 \\
\hline 04 & 91915 & 54.4777 & 7.7738 & 288.4 & 4 & 600.0 & \\
\hline 04 & 91916 & 51.5025 & 8.39 & 288.3 & 4 & 600.0 & 600.0 \\
\hline 04 & 91917 & 58.2250 & 9.0300 & 287.5 & 4 & 600.0 & 600.0 \\
\hline 04 & 91918 & 64.0909 & 7.6028 & 286.7 & 4 & 600.0 & 600.0 \\
\hline 04 & 91919 & 69.8673 & 7.3770 & 285.8 & 4 & 600.0 & 600.0 \\
\hline 04 & 91920 & 63.0483 & 7.4330 & 285.2 & 4 & 600.0 & 600.0 \\
\hline 04 & 91921 & 64.1736 & 7.2088 & 284.9 & 4 & 600.0 & 600.0 \\
\hline 04 & 91922 & 65.2753 & 7.845 & 285.1 & 4 & 600.0 & 600.0 \\
\hline 04 & 91923 & 71.4483 & 8.1050 & 285.5 & 4 & 600.0 & 600.0 \\
\hline
\end{tabular}




\begin{tabular}{|c|c|c|c|c|c|c|c|}
\hline 4 & 91924 & 69.8465 & 6.7020 & 285.3 & 4 & 600.0 & 600.0 \\
\hline 04 & 9201 & 69.3501 & 5.9910 & 285.3 & 4 & 600.0 & 600.0 \\
\hline 04 & 9202 & 75.9254 & 6.9190 & 284.7 & 4 & 600.0 & 00.0 \\
\hline 04 & 9203 & 72.5254 & 6.8335 & 284.7 & 4 & 600.0 & 00.0 \\
\hline 04 & 9204 & 60.2750 & 7.4120 & 285.1 & 4 & 600.0 & 00.0 \\
\hline 04 & 9205 & 71.8511 & 7.4550 & 285.2 & 4 & 600.0 & 00.0 \\
\hline 04 & 9206 & 75.8519 & 6.2720 & 284.8 & 4 & 600.0 & 00.0 \\
\hline 04 & 9207 & 75.9255 & 5.9545 & 284.9 & 4 & 600.0 & 00.0 \\
\hline 04 & 9208 & 121.3661 & 3.9870 & 285.9 & 3 & 600.0 & 00.0 \\
\hline 04 & 9209 & 157.3520 & 5.5458 & 286.5 & 3 & 600.0 & 0 \\
\hline 04 & 92010 & 160.8500 & 8.1325 & 286.6 & 4 & 600.0 & \\
\hline 04 & 92011 & 163.5509 & 8.6350 & 286.7 & 4 & 600.0 & \\
\hline 04 & 92012 & 163.3251 & 5.9023 & 287.5 & 3 & 600.0 & 00.0 \\
\hline 04 & 92013 & 172.4340 & 6.3633 & 288.8 & 3 & 600.0 & 00.0 \\
\hline 04 & 92014 & 165.8480 & 248 & 289.7 & 4 & .0 & \\
\hline 04 & 92015 & 168.8004 & 7.1705 & 290.6 & 3 & 600.0 & .0 \\
\hline 04 & 92016 & 174.8100 & 8.2325 & 290.9 & 4 & 60 & 0.0 \\
\hline 04 & 92017 & 173.4660 & 8.7675 & 291.1 & 4 & .0 & \\
\hline 04 & 92018 & 167.3075 & 7.4463 & 290.8 & 4 & 60 & .0 \\
\hline 04 & 92019 & 138.9495 & 6.0305 & 290.5 & 4 & .0 & .0 \\
\hline 04 & 92020 & 143.2562 & 5. & 290 & 4 & 6 & \\
\hline 04 & 92021 & 138.9692 & 7.2893 & 290.5 & 4 & .0 & \\
\hline 04 & 92022 & 130.7247 & 6.5665 & 290.7 & 4 & 600.0 & .0 \\
\hline 04 & 92023 & 140.9000 & 7.51 & 290.7 & 4 & 6 & \\
\hline 04 & 92024 & 147.1252 & 8.70 & 290.7 & 4 & .0 & \\
\hline 04 & 9211 & 151.3500 & 9.2975 & 290.2 & 4 & .0 & .0 \\
\hline 04 & 9212 & 145.7499 & 9.4325 & 289.7 & 4 & 60 & .0 \\
\hline 04 & 9213 & 144.3000 & 9.9575 & 289.2 & 4 & .0 & \\
\hline 04 & 9214 & 138.4563 & 9.2350 & 288.7 & 4 & .0 & 0.0 \\
\hline 04 & 9215 & 137.0757 & 8.9750 & 288.3 & 4 & .0 & .0 \\
\hline 04 & 9216 & 142.7505 & 10.3300 & 288.2 & 4 & .0 & \\
\hline 04 & 9217 & 146.8238 & 9.5350 & 288.5 & 4 & .0 & 0.0 \\
\hline 04 & 9218 & 156.7260 & 9.2050 & 289.4 & 4 & 600.0 & 600.0 \\
\hline 04 & 9219 & 166.5772 & 7.7195 & 289.9 & 4 & 600.0 & 0.0 \\
\hline 04 & 92110 & 150.6749 & 6.86 & & 4 & 600.0 & 600.0 \\
\hline 04 & 92111 & 147.9261 & 6.9510 & 292.4 & 4 & 600.0 & 600.0 \\
\hline 04 & 92112 & 145.9993 & 6.92 & 29 & 4 & 600.0 & 600.0 \\
\hline 04 & 92113 & 167.1110 & 7.3805 & 294.9 & 4 & 600.0 & 600.0 \\
\hline 04 & 92114 & 174.9045 & 6.2830 & 295.3 & 3 & 600.0 & 600.0 \\
\hline 04 & 92115 & 167.6175 & 5.1400 & & 3 & 600.0 & 600.0 \\
\hline 04 & 92116 & 157.0034 & 4.6220 & 296.1 & 3 & 600.0 & 600.0 \\
\hline 04 & 92117 & 162.7888 & 3.3870 & 296.0 & 3 & 600.0 & 600.0 \\
\hline 04 & 92118 & 159.0247 & 2.1680 & 295.3 & 4 & 600.0 & 600.0 \\
\hline 04 & 92119 & 102.8927 & 1.7240 & & 5 & 600.0 & 600.0 \\
\hline 04 & 92120 & 69.2024 & 4.8603 & 294.2 & 4 & 600.0 & 600.0 \\
\hline 04 & 92121 & 77.7000 & 5.2305 & & 4 & 600.0 & 600.0 \\
\hline 04 & 92122 & 76.6509 & 6.0600 & & 4 & 600.0 & 600.0 \\
\hline 4 & 92123 & 75.1250 & 6.5108 & 293.1 & 4 & 600.0 & 600.0 \\
\hline
\end{tabular}




\begin{tabular}{|c|c|c|c|c|c|c|c|}
\hline 4 & 92124 & 75.6000 & 5.9778 & 293.1 & 4 & 600.0 & 600.0 \\
\hline 04 & 9221 & 75.2750 & 5.4175 & 293.3 & 4 & 600.0 & 600.0 \\
\hline 04 & 9222 & 70.1745 & 4.4943 & 293.7 & 4 & 600.0 & 00.0 \\
\hline 04 & 9223 & 82.7188 & 3.7560 & 293.6 & 4 & 600.0 & 00.0 \\
\hline 04 & 9224 & 115.7621 & 1.2170 & 293.4 & o & 600.0 & 00.0 \\
\hline 04 & 9225 & 29.4052 & 1.9375 & 293.3 & 5 & 600.0 & 00.0 \\
\hline 04 & 9226 & 9.8270 & 1.7683 & 293.1 & 6 & 600.0 & 00.0 \\
\hline 04 & 9227 & 4.1292 & 1.3650 & 293.4 & 5 & 600.0 & 00.0 \\
\hline 04 & 9228 & 339.5272 & 1.2395 & 294.1 & 4 & 600.0 & 00.0 \\
\hline 04 & 9229 & 288.4144 & 2.7580 & 295.3 & 3 & 600.0 & 0 \\
\hline 04 & 92210 & 256.0043 & 3.8310 & 295.8 & 2 & 600.0 & \\
\hline 04 & 92211 & 256.6161 & 4.8615 & 296.1 & 2 & 600.0 & \\
\hline 04 & 92212 & 259.5235 & 4.9748 & 297.3 & 2 & 600.0 & 00.0 \\
\hline 04 & 92213 & 253.0053 & 4.3098 & 298.1 & 2 & 600.0 & 00.0 \\
\hline 04 & 92214 & 241.5187 & 3.7865 & 298.8 & 2 & .0 & \\
\hline 04 & 92215 & 224.5797 & 2.8190 & 298.7 & 3 & 600.0 & 00.0 \\
\hline 04 & 92216 & 227.3308 & 2.8708 & 298.6 & 3 & 60 & 600.0 \\
\hline 04 & 92217 & 223.6570 & 2.3343 & 298.3 & 3 & .0 & \\
\hline 04 & 92218 & 207.5339 & 2.2008 & 297.2 & 4 & 600.0 & .0 \\
\hline 04 & 92219 & 206.7905 & 1.5143 & 296.7 & 5 & 60 & .0 \\
\hline 04 & 92220 & 20.8968 & 2.8835 & 296.4 & 4 & 60 & \\
\hline 04 & 92221 & 66.4062 & 3.0343 & 296.9 & 4 & .0 & \\
\hline 04 & 92222 & 105.5771 & 3.3403 & 296.0 & 4 & 600.0 & .0 \\
\hline 04 & 92223 & 79.3242 & 5.9690 & 295.4 & 4 & 60 & \\
\hline 04 & 92224 & 74.3000 & 7.1350 & 294.9 & 4 & .0 & \\
\hline 04 & 9231 & 67.9732 & 6.5148 & 295.0 & 4 & .0 & 600.0 \\
\hline 04 & 9232 & 67.5009 & 6.2173 & 295.1 & 4 & .0 & .0 \\
\hline 04 & 9233 & 71.6501 & 5.4778 & 295.4 & 4 & .0 & \\
\hline 04 & 9234 & 97.8306 & 3.1388 & 295.5 & 4 & .0 & 0.0 \\
\hline 04 & 9235 & 126.0808 & 1.3735 & 295.3 & 5 & .0 & .0 \\
\hline 04 & 9236 & 45.2681 & 0.7780 & 294.6 & 6 & .0 & \\
\hline 04 & 9237 & 190.5352 & 0.7225 & 294.2 & 5 & .0 & 0.0 \\
\hline 04 & 9238 & 48.1522 & 1.1378 & 293.5 & 4 & 600.0 & 600.0 \\
\hline 04 & 9239 & 255.8373 & 2.1210 & 295.3 & 3 & 600.0 & 0.0 \\
\hline 04 & 92310 & 263.8480 & 2.1993 & 296.5 & 2 & 600.0 & 600.0 \\
\hline 04 & 92311 & 259.0638 & 2.5650 & 297.5 & 2 & 600.0 & 600.0 \\
\hline 04 & 92312 & 231.8423 & 2.7970 & 298.7 & 2 & 600.0 & 600.0 \\
\hline 04 & 92313 & 249.1029 & 2.4615 & 299.7 & 2 & 600.0 & 600.0 \\
\hline 04 & 92314 & 238.9313 & 2.7943 & 300.8 & 2 & 600.0 & 600.0 \\
\hline 04 & 92315 & 229.1424 & 3.0268 & 300.8 & 3 & 600.0 & 600.0 \\
\hline 04 & 92316 & 192.5928 & 3.8865 & 300.6 & 3 & 600.0 & 600.0 \\
\hline 04 & 92317 & 159.2804 & 3.5428 & 300.1 & 3 & 600.0 & 600.0 \\
\hline 04 & 92318 & 160.8503 & 3.7710 & 298.6 & 4 & 600.0 & 600.0 \\
\hline 04 & 92319 & 148.3001 & 2.1710 & 298.2 & 5 & 600.0 & 600.0 \\
\hline 04 & 92320 & 94.2676 & 1.2735 & 298.7 & 6 & 600.0 & 600.0 \\
\hline 04 & 92321 & 85.1024 & 1.2795 & 298.8 & 6 & 600.0 & 600.0 \\
\hline 04 & 92322 & 77.8714 & 1.4063 & 299.0 & 6 & 600.0 & 600.0 \\
\hline 4 & 92323 & 101.8875 & 1.8340 & 298.2 & 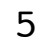 & 600.0 & 600.0 \\
\hline
\end{tabular}




\begin{tabular}{|c|c|c|c|c|c|c|c|}
\hline 04 & 92324 & 90.0077 & 2.7768 & 297.7 & 4 & 600.0 & 00.0 \\
\hline 04 & 9241 & 152.8583 & 1.2495 & 297.1 & 5 & 600.0 & 600.0 \\
\hline 04 & 9242 & 115.4893 & 0.5320 & 297.3 & 6 & 600.0 & 00.0 \\
\hline 04 & 9243 & 67.3044 & 1.3480 & 297.2 & 5 & 600.0 & 600.0 \\
\hline 04 & $924 \quad 4$ & 109.2504 & 0.8715 & 297.4 & 0 & 600.0 & 00.0 \\
\hline 04 & 9245 & 143.7893 & 0.7715 & 297.1 & 6 & 600.0 & 00.0 \\
\hline 04 & 9246 & 162.0556 & 0.9000 & 296.3 & 6 & 600.0 & 600.0 \\
\hline 04 & 9247 & 138.8277 & 1.3310 & 295.5 & 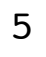 & 600.0 & 00.0 \\
\hline 04 & 9248 & 156.2373 & 2.6278 & 295.3 & 4 & 600.0 & 00.0 \\
\hline 04 & 9249 & 187.9848 & 2.6958 & 296.8 & 3 & 600.0 & 600.0 \\
\hline 04 & 92410 & 221.9417 & 1.7058 & 297.9 & 2 & 600.0 & 600.0 \\
\hline 04 & 92411 & 167.2230 & 4.0943 & 299.1 & 2 & 600.0 & \\
\hline 04 & 92412 & 161.0629 & 4.8678 & 299.5 & 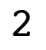 & 600.0 & 600.0 \\
\hline 04 & 92413 & 182.3392 & 3.8480 & 300.7 & 2 & 600.0 & 600.0 \\
\hline 04 & 92414 & 179.7910 & 3.8013 & 301.1 & 2 & 600.0 & \\
\hline 04 & 92415 & 170.8439 & 3.6510 & 301.7 & 3 & 600.0 & 60 \\
\hline 04 & 92416 & 185.9194 & 3.6155 & 301.7 & 3 & 600.0 & 600.0 \\
\hline 04 & 92417 & 185.3535 & 3.2285 & 301.1 & 3 & 600.0 & \\
\hline 04 & 92418 & 161.8224 & 2.0893 & 300.3 & 4 & 600.0 & 60 \\
\hline 04 & 92419 & 145.5061 & 445 & 299.5 & 5 & 600.0 & 600.0 \\
\hline 04 & 92420 & 123.2502 & 1.6 & 299.8 & 6 & 600.0 & .0 \\
\hline 04 & 92421 & 105.0737 & 1.8838 & 299.5 & 5 & 600.0 & \\
\hline 04 & 92422 & 92.7772 & 3.9280 & 298.6 & 4 & 600.0 & 600.0 \\
\hline 04 & 92423 & 74.2500 & 7.0360 & 297.7 & 4 & 600.0 & .0 \\
\hline 04 & 92424 & 74.2750 & 200 & 297.7 & 4 & 600.0 & \\
\hline 04 & 9251 & 75.2500 & 8.5600 & 297.6 & 4 & 600.0 & 60 \\
\hline 04 & 9252 & 75.3500 & 8.8100 & 297.0 & 4 & 600.0 & 600.0 \\
\hline 04 & 9253 & 75.7750 & 6.8910 & 297.1 & 4 & 600.0 & \\
\hline 04 & 9254 & 69.3000 & 3.3205 & 297.9 & 4 & 600.0 & 60 \\
\hline 04 & 9255 & 67.4595 & 3.1353 & 297.5 & 4 & 600.0 & 600.0 \\
\hline 04 & 9256 & 66.6734 & 713 & 297.4 & 4 & 600.0 & .0 \\
\hline 04 & 9257 & 72.0752 & 4.1970 & 297.2 & 4 & 600.0 & 60 \\
\hline 04 & 9258 & 135.4236 & 1.6203 & 298.5 & 3 & 600.0 & 600.0 \\
\hline 04 & 9259 & 255.5728 & 1.6803 & 298.2 & 2 & 600.0 & .0 \\
\hline 04 & 92510 & 252.2254 & 2.2835 & 298.7 & 3 & 600.0 & 60 \\
\hline 04 & 92511 & 191.6102 & 3.3080 & 299.9 & 2 & 600.0 & 600.0 \\
\hline 04 & 92512 & 174.6494 & 5.4750 & 300.6 & 3 & 600.0 & .0 \\
\hline 04 & 92513 & 138.3098 & 4.6758 & 302.3 & 2 & 600.0 & 600.0 \\
\hline 04 & 92514 & 161.9002 & 6.2763 & 302.2 & 3 & 600.0 & 600.0 \\
\hline 04 & 92515 & 143.3800 & 5.9565 & 302.6 & 4 & 600.0 & \\
\hline 04 & 92516 & 92.4362 & 5.4298 & 302.1 & 3 & 600.0 & 600.0 \\
\hline 04 & 92517 & 93.7499 & 4.9830 & 301.0 & 3 & 600.0 & 600.0 \\
\hline 04 & 92518 & 83.5297 & 5.3780 & 299.6 & 4 & 600.0 & 600.0 \\
\hline 04 & 92519 & 74.4765 & 7.8518 & 298.8 & 4 & 600.0 & 600.0 \\
\hline 04 & 92520 & 74.4498 & 7.3260 & 298.4 & 4 & 600.0 & 600.0 \\
\hline 04 & 92521 & 67.2498 & 8.5225 & 298.3 & 4 & 600.0 & 600.0 \\
\hline 04 & 92522 & 68.1751 & 8.7100 & 298.8 & 4 & 600.0 & 600.0 \\
\hline 04 & 92523 & 69.1250 & 7.9925 & 298.8 & 4 & 600.0 & 600.0 \\
\hline
\end{tabular}




\begin{tabular}{|c|c|c|c|c|c|c|c|}
\hline 04 & 92524 & 71.5497 & 6.9200 & 298.8 & 4 & 600.0 & 00.0 \\
\hline 04 & 9261 & 78.1575 & 5.4347 & 299.0 & 4 & 600.0 & 600.0 \\
\hline 04 & 9262 & 79.1748 & 6.2610 & 298.5 & 4 & 600.0 & 00.0 \\
\hline 04 & 9263 & 76.3998 & 6.1210 & 298.1 & 4 & 600.0 & 600.0 \\
\hline 04 & 9264 & 78.7751 & 4.9765 & 298.2 & 4 & 600.0 & 00.0 \\
\hline 04 & 9265 & 131.4873 & 2.2855 & 296.9 & 5 & 600.0 & 00.0 \\
\hline 04 & 9266 & 160.8424 & 1.0268 & 295.4 & 5 & 600.0 & 600.0 \\
\hline 04 & 9267 & 154.4149 & 3.9883 & 295.7 & 4 & 600.0 & 00.0 \\
\hline 04 & 9268 & 154.1501 & 5.9088 & 295.6 & 3 & 600.0 & 00.0 \\
\hline 04 & 9269 & 164.8001 & 5.1503 & 296.4 & 3 & 600.0 & 600.0 \\
\hline 04 & 92610 & 162.2000 & 5.7238 & 297.1 & 3 & 600.0 & 000 \\
\hline 04 & 92611 & 163.3998 & 5.4898 & 298.2 & 3 & 600.0 & \\
\hline 04 & 92612 & 166.9751 & 5.1565 & 299.3 & 2 & 600.0 & 600.0 \\
\hline 04 & 92613 & 159.4507 & 4.8098 & 300.3 & 2 & 600.0 & 600.0 \\
\hline 04 & 92614 & 169.2006 & 5.3473 & 301.0 & 3 & 600.0 & \\
\hline 04 & 92615 & 150.3232 & 4.6088 & 301.8 & 3 & 600.0 & 60 \\
\hline 04 & 92616 & 146.6000 & 4.7525 & 301.7 & 3 & 600.0 & 600.0 \\
\hline 04 & 92617 & 144.7330 & 4.0963 & 301.3 & 3 & 600.0 & \\
\hline 04 & 92618 & 146.8000 & 3.6395 & 299.6 & 4 & 600.0 & 60 \\
\hline 04 & 92619 & 102.5952 & 2.7815 & 299.1 & 4 & 600.0 & 600.0 \\
\hline 04 & 92620 & 56.9127 & 4.74 & 298.9 & 4 & 600.0 & .0 \\
\hline 04 & 92621 & 68.1583 & 6.1985 & 298.4 & 4 & 600.0 & \\
\hline 04 & 92622 & 67.3010 & 6.8128 & 297.8 & 4 & 600.0 & 600.0 \\
\hline 04 & 92623 & 53.0000 & 6.7725 & 297.4 & 4 & 600.0 & .0 \\
\hline 04 & 92624 & 35.7774 & 2.7210 & 297.2 & 5 & 600.0 & \\
\hline 04 & 9271 & 30.1668 & 2.4605 & 297.7 & 4 & 600.0 & 60 \\
\hline 04 & 9272 & 19.9749 & 3.0160 & 297.7 & 4 & 600.0 & .0 \\
\hline 04 & 9273 & 37.6758 & 2.7078 & 297.7 & 4 & 600.0 & \\
\hline 04 & 9274 & 69.7116 & 3.6525 & 297.2 & 4 & 600.0 & 60 \\
\hline 04 & 9275 & 68.6555 & 3.6160 & 296.8 & 4 & 600.0 & 600 \\
\hline 04 & 9276 & 75.1246 & 1.4710 & 296.4 & 5 & 600.0 & \\
\hline 04 & $927 \quad 7$ & 40.1927 & 635 & 295.0 & 4 & 600.0 & 60 \\
\hline 04 & 9278 & 131.0013 & 1.1368 & 294.9 & 3 & 600.0 & 600.0 \\
\hline 04 & 9279 & 195.5671 & 1.3280 & 295.8 & 2 & 600.0 & .0 \\
\hline 04 & 92710 & 349.1942 & 78 & 296.8 & 3 & 600.0 & .0 \\
\hline 04 & 92711 & 126.0638 & 2.7998 & 297.7 & 2 & 600.0 & 600.0 \\
\hline 04 & 92712 & 87.0958 & 5.4295 & 297.1 & 3 & 600.0 & \\
\hline 04 & 92713 & 79.8251 & 7.3873 & 296.2 & 4 & 600.0 & 600.0 \\
\hline 04 & 92714 & 72.1501 & 8.3250 & 296.0 & 4 & 600.0 & 600.0 \\
\hline 04 & 92715 & 71.2992 & 10.3225 & 294.9 & 4 & 600.0 & \\
\hline 04 & 92716 & 73.4500 & 14.3275 & 292.6 & 4 & 600.0 & 600.0 \\
\hline 04 & 92717 & 71.6999 & 14.3725 & 290.6 & 4 & 600.0 & 600.0 \\
\hline 04 & 92718 & 71.1000 & 14.0675 & 289.4 & 4 & 600.0 & 600.0 \\
\hline 04 & 92719 & 66.6499 & 15.5400 & 287.8 & 4 & 600.0 & 600.0 \\
\hline 04 & 92720 & 66.9058 & 14.1250 & 287.3 & 4 & 600.0 & 600.0 \\
\hline 04 & 92721 & 61.3750 & 16.0275 & 286.9 & 4 & 600.0 & 600.0 \\
\hline 04 & 92722 & 65.8001 & 15.9050 & 287.0 & 4 & 600.0 & 600.0 \\
\hline 04 & 92723 & 65.6750 & 14.7100 & 287.0 & 4 & 600.0 & 600.0 \\
\hline
\end{tabular}




\begin{tabular}{|c|c|c|c|c|c|c|c|}
\hline 4 & 92724 & 65.2500 & 13.8600 & 287.1 & 4 & 600.0 & 600.0 \\
\hline 04 & 9281 & 64.4750 & 14.9825 & 286.9 & 4 & 600.0 & 00,0 \\
\hline 4 & 9282 & 66.1750 & 15.1000 & 286.9 & 4 & 600.0 & 00.0 \\
\hline 04 & 9283 & 68.7500 & 13.5050 & 286.9 & 4 & 600.0 & 00.0 \\
\hline 04 & 9284 & 68.0751 & 100 & 286.8 & 4 & 600.0 & 00.0 \\
\hline 04 & 9285 & 69.6750 & 11.3850 & 286.8 & 4 & 600.0 & 00.0 \\
\hline 04 & 9286 & 65.6500 & 11.2300 & 286.7 & 4 & 600.0 & 00.0 \\
\hline 04 & 9287 & 66.8251 & 10.8500 & 286.7 & 4 & 600.0 & 00.0 \\
\hline 04 & 9288 & 61.8000 & 9.6525 & 287.5 & 4 & 600.0 & 00.0 \\
\hline 04 & 9289 & 72.2212 & 7.0548 & 288.8 & 4 & 60 & 0 \\
\hline 04 & 92810 & 94.8326 & 4.2413 & 290.5 & 3 & 600.0 & \\
\hline 04 & 92811 & 75.6019 & 4.2803 & 291.4 & 2 & .0 & \\
\hline 04 & 92812 & 85.5117 & 4.0255 & 292.8 & 2 & 600.0 & .0 \\
\hline 04 & 92813 & 69.4515 & 4.0268 & 293.9 & 2 & 600.0 & \\
\hline 04 & 92814 & 59.1513 & 0 & 294.7 & 2 & .0 & \\
\hline 04 & 92815 & 71.8816 & 6. & 294.3 & 3 & 600.0 & .0 \\
\hline 04 & 92816 & 74.4784 & 7.6500 & 293.2 & 4 & 60 & .0 \\
\hline 04 & 92817 & 68.1497 & 8.2 & 292.0 & 4 & .0 & \\
\hline 04 & 92818 & 65.8499 & 9.1600 & 290.4 & 4 & .0 & \\
\hline 04 & 92819 & 63.1500 & 9.77 & 288.9 & 4 & .0 & .0 \\
\hline 04 & 92820 & 70.0251 & 9 . & 288.1 & 4 & 6 & \\
\hline 04 & 92821 & 73.1000 & 10.54 & 287.5 & 4 & .0 & \\
\hline 04 & 92822 & 77.6756 & 10.2775 & 287.0 & 4 & 600.0 & .0 \\
\hline 04 & 92823 & 72.8750 & 10 . & 286.7 & 4 & 6 & \\
\hline 04 & 92824 & 76.1755 & 10 & 286.4 & 4 & 6 & \\
\hline 04 & 9291 & 76.5002 & 9.8400 & 286.1 & 4 & .0 & \\
\hline 04 & 9292 & 74.5250 & 8.8500 & 286.4 & 4 & 60 & .0 \\
\hline 04 & 9293 & 77.8250 & 10.4600 & 286.0 & 4 & .0 & \\
\hline 04 & 9294 & 78.6500 & 9.3325 & 285.9 & 4 & .0 & .0 \\
\hline 04 & 9295 & 80.4247 & 8.71 & 285.7 & 4 & 6 & .0 \\
\hline 04 & 9296 & 73.8250 & 9. & 285.3 & 4 & .0 & \\
\hline 04 & 9297 & 77.7249 & 8 . & 285.5 & 4 & .0 & 0 \\
\hline 04 & 9298 & 80.4476 & 6.2845 & 286.2 & 4 & .0 & 0.0 \\
\hline 04 & 9299 & 77.4493 & 4 . & 287.6 & 3 & 0 & .0 \\
\hline 04 & 92910 & 86.1227 & & 289.0 & 3 & 0 & 0.0 \\
\hline 04 & 92911 & 127.7200 & 2.8955 & 290.9 & 2 & 600.0 & 600.0 \\
\hline 04 & 92912 & 305.6641 & 2 . & 292.7 & 2 & 600.0 & .0 \\
\hline 04 & 92913 & 320.8831 & 3.3725 & 293.5 & 2 & 600.0 & 600.0 \\
\hline 04 & 92914 & 347.9514 & 3.0768 & 293.9 & 2 & 600.0 & 600.0 \\
\hline 04 & 92915 & 47.1519 & & 294.2 & 3 & 600.0 & .0 \\
\hline 04 & 92916 & 47.5961 & 4.0428 & 294.4 & 3 & 600.0 & 600.0 \\
\hline 04 & 92917 & 64.2356 & 4.8093 & 293.5 & 3 & 600.0 & 600.0 \\
\hline 04 & 92918 & 71.1997 & 6.4405 & 291.1 & 4 & 600.0 & 600.0 \\
\hline 04 & 92919 & 45.1652 & 4.7025 & 290.7 & 4 & 600.0 & 600.0 \\
\hline 04 & 92920 & 58.3958 & 6.1773 & 289.2 & 4 & 600.0 & 600.0 \\
\hline 04 & 92921 & 69.3500 & 10.8675 & 287.0 & 4 & 600.0 & 600.0 \\
\hline $0<$ & 92922 & 72.4252 & 9.742 & 286.8 & 4 & 600.0 & 600.0 \\
\hline 04 & 92923 & 76.5999 & 8.545 & 286.6 & 4 & 600.0 & 600.0 \\
\hline
\end{tabular}




\begin{tabular}{|c|c|c|c|c|c|c|c|}
\hline 04 & 92924 & 74.6254 & 8.0825 & 286.4 & 4 & 600.0 & 600.0 \\
\hline 04 & 9301 & 73.4751 & 7.6225 & 286.4 & 4 & 600.0 & 600.0 \\
\hline 04 & 9302 & 72.8012 & 7.7575 & 286.0 & 4 & 600.0 & 00.0 \\
\hline 04 & 9303 & 70.4999 & 7.4358 & 285.6 & 4 & 600.0 & 00.0 \\
\hline 04 & $930 \quad 4$ & 77.4498 & 6.0890 & 285.6 & 4 & 600.0 & 00.0 \\
\hline 04 & 9305 & 75.9998 & 5.8903 & 285.8 & 4 & 600.0 & 00.0 \\
\hline 04 & 9306 & 109.8678 & 3.8593 & 285.0 & 4 & 600.0 & 600.0 \\
\hline 049 & 9307 & 124.1610 & 2.7980 & 284.7 & 4 & 600.0 & 500.0 \\
\hline 04 & 9308 & 204.4164 & 3.0185 & 285.3 & 3 & 600.0 & 00.0 \\
\hline 04 & 9309 & 248.4063 & 2.0623 & 286.2 & 2 & 600.0 & 600.0 \\
\hline 04 & 93010 & 208.7252 & 2.1900 & 287.8 & 2 & 600.0 & 600.0 \\
\hline 04 & 93011 & 179.1397 & 2.4033 & 289.4 & 1 & 600.0 & \\
\hline 04 & 93012 & 145.9737 & 3.3090 & 290.8 & 2 & 600.0 & 600.0 \\
\hline 04 & 93013 & 164.4708 & 3.7340 & 291.6 & 2 & 600.0 & 600.0 \\
\hline 04 & 93014 & 155.3799 & 4.0155 & 292.6 & 2 & 600.0 & \\
\hline 04 & 93015 & 163.7980 & 3.7783 & 292.9 & 3 & 600.0 & 60 \\
\hline 04 & 93016 & 154.2495 & 4.3173 & 293.1 & 3 & 600.0 & 600.0 \\
\hline 04 & 93017 & 108.1049 & 4.7790 & 292.6 & 3 & 600.0 & \\
\hline 04 & 93018 & 87.2809 & 5.7883 & 290.9 & 4 & 600.0 & .0 \\
\hline 04 & 93019 & 76.6257 & 6.8838 & 289.7 & 4 & 600.0 & 600.0 \\
\hline 04 & 93020 & 71.8249 & 6.9680 & 288.4 & 4 & 600.0 & .0 \\
\hline 04 & 93021 & 69.1252 & 6.0375 & 287.7 & 4 & 600.0 & \\
\hline 04 & 93022 & 63.7953 & 6.0103 & 287.8 & 4 & 600.0 & 600.0 \\
\hline 04 & 93023 & 57.9117 & 3.9283 & 288.3 & 4 & 600.0 & .0 \\
\hline 04 & 93024 & 6.1412 & 0.7373 & 288.4 & 5 & 600.0 & \\
\hline 0410 & $\begin{array}{lll}0 & 1 & 1\end{array}$ & 14.0006 & 1.2638 & 288.0 & 6 & 600.0 & 600.0 \\
\hline 0410 & $\begin{array}{lll}0 & 1 & 2\end{array}$ & 108.0208 & 0.9095 & 286.3 & 6 & 600.0 & 600.0 \\
\hline 0410 & $\begin{array}{lll}0 & 1 & 3\end{array}$ & 116.0290 & 1.1675 & 284.9 & 6 & 600.0 & .0 \\
\hline 0410 & $\begin{array}{lll}0 & 1 & 4\end{array}$ & 93.1947 & 1.4915 & 284.8 & 6 & 600.0 & 600.0 \\
\hline 0410 & $\begin{array}{lll}0 & 1 & 5\end{array}$ & 128.3625 & 1.2033 & 285.2 & 6 & 600.0 & 600.0 \\
\hline 0410 & $\begin{array}{lll}0 & 1 & 6\end{array}$ & 95.1529 & 1.9040 & 284.8 & 5 & 600.0 & .0 \\
\hline 0410 & $\begin{array}{lll}0 & 1 & 7\end{array}$ & 94.8568 & 1.2960 & 285.7 & 4 & 600.0 & 60 \\
\hline 0410 & $\begin{array}{lll}0 & 1 & 8\end{array}$ & 7.1086 & 1.4523 & 286.3 & 3 & 600.0 & 600.0 \\
\hline 0410 & $\begin{array}{lll}0 & 1 & 9\end{array}$ & 297.4474 & 1.9173 & 287.4 & 2 & 600.0 & .0 \\
\hline 0410 & $0 \quad 110$ & 321.8806 & 1.8745 & 289.6 & 2 & 600.0 & 60 \\
\hline 0410 & $0 \quad 111$ & 218.9951 & 2.7288 & 290.9 & 2 & 600.0 & 600.0 \\
\hline 0410 & $0 \quad 112$ & 241.7536 & 2.4838 & 291.8 & 2 & 600.0 & 600.0 \\
\hline 0410 & $0 \quad 113$ & 218.2355 & 3.1265 & 293.2 & 2 & 600.0 & 600.0 \\
\hline 0410 & $0 \quad 114$ & 52.4910 & 3.9710 & 294.2 & 2 & 600.0 & 600.0 \\
\hline 0410 & $0 \quad 115$ & 94.0502 & 4.3393 & 294.2 & 3 & 600.0 & \\
\hline 0410 & 0116 & 92.4254 & 5.4653 & 293.9 & 4 & 600.0 & 600.0 \\
\hline 0410 & $0 \quad 117$ & 88.6499 & 5.4733 & 293.3 & 4 & 600.0 & 600.0 \\
\hline 0410 & $0 \quad 118$ & 82.4805 & 5.4585 & 291.8 & 4 & 600.0 & 600.0 \\
\hline 0410 & $0 \quad 119$ & 74.4510 & 6.5710 & 290.3 & 4 & 600.0 & 600.0 \\
\hline 0410 & $0 \quad 120$ & 74.4500 & 7.5233 & 289.7 & 4 & 600.0 & 600.0 \\
\hline 0410 & $0 \quad 121$ & 78.2229 & 7.7775 & 290.3 & 4 & 600.0 & 600.0 \\
\hline 0410 & 122 & 83.3249 & 7.1875 & 290.5 & 4 & 600.0 & 600.0 \\
\hline 0410 & $0 \quad 123$ & 77.2996 & 7.0420 & 290.7 & 4 & 600.0 & 600.0 \\
\hline
\end{tabular}




\begin{tabular}{|c|c|c|c|c|c|c|c|}
\hline & 24 & a & 5.7505 & 290.7 & 4 & & \\
\hline 11 & 21 & 25500 & 2.6875 & 291.7 & & & \\
\hline & 22 & 87.6856 & 4940 & 292.2 & & 5 & \\
\hline 4 & 23 & 22.0516 & 0.5083 & 291.7 & & 00 & \\
\hline & 24 & 42. & .8963 & 90.3 & & & \\
\hline & 25 & 3193 & 328 & 90.0 & & $0 c$ & \\
\hline & 6 & 949 & 1.1858 & 290.2 & & & \\
\hline & 7 & 512 & .9255 & 91.6 & & & \\
\hline & 8 & 177.5749 & 0.7030 & 292.4 & & & \\
\hline & 9 & 179.1769 & 1.4853 & 292.9 & & 00 & \\
\hline & 210 & 184.3025 & 2.9768 & 293 & & & \\
\hline & 211 & 179.9 & & 5 & & & \\
\hline & 12 & 154.2535 & 513 & 295.4 & & 0 & \\
\hline & 213 & 177.7567 & 3.7318 & 297.0 & & 0 & \\
\hline & 214 & 190.2848 & & 97.3 & & & \\
\hline & 215 & 188.0626 & 965 & 297.2 & & & \\
\hline & 216 & 193.4426 & 020 & 297.1 & & & \\
\hline & 217 & 167 . & & 296 & & & \\
\hline & 218 & 127.7734 & 2 . & 296 & & & \\
\hline & 219 & 100.1507 & 0 & 295 & & & \\
\hline & 220 & 76.4758 & & 295.2 & & & \\
\hline & 221 & 73.4247 & & 294 & 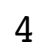 & & \\
\hline & 222 & 73.7249 & 775 & 29 & 4 & & \\
\hline 4 & 223 & 76 . & 0 & 294 & 4 & & \\
\hline & 224 & 74 . & & 293 & $I$ & & \\
\hline & 31 & 000 & 943 & 294 & 4 & & \\
\hline & 32 & 74.9250 & 943 & 293.6 & 4 & & \\
\hline & 33 & 75.1250 & & 293 & 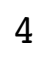 & & \\
\hline & 34 & 78 . & 3 & 294 & 4 & & \\
\hline & 35 & 94.9791 & 108 & 294.7 & 4 & & \\
\hline & 36 & 102 . & 3. & 294 & 4 & & \\
\hline & 37 & 84 & 3. & 29 & 4 & & \\
\hline & 38 & 154.2940 & 1.7160 & 294.9 & 3 & 0 & 0 . \\
\hline & 39 & 176 . & 965 & 29 & 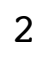 & & \\
\hline & 310 & 172 . & 2. & 29 & 3 & & \\
\hline & 311 & 160.7141 & 3573 & 296.3 & 2 & & \\
\hline & 312 & 170.2319 & 265 & 297.2 & 2 & & \\
\hline & 313 & 162.4487 & 010 & 297.9 & 2 & & 0 . \\
\hline & 314 & 160.3058 & 943 & 298.4 & 2 & & 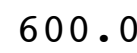 \\
\hline & 315 & 153. & 800 & 298.8 & 3 & & \\
\hline & 316 & 97 . & 503 & & 3 & & \\
\hline 4 & 317 & 82.5489 & 6.8795 & 297.2 & 4 & 600 & 0. \\
\hline & 318 & 74.7750 & 8.4600 & 295.1 & 4 & 0 & \\
\hline & 319 & 72.5250 & 9.3900 & 294.3 & 4 & & \\
\hline 04 & 320 & 70.6499 & 10.2050 & 293.8 & 4 & 60 & 00 . \\
\hline & 321 & 72.2000 & 9.8525 & 293.9 & 4 & 600.0 & \\
\hline & 322 & 71.9250 & 200 & & 4 & & \\
\hline & 323 & 74.2250 & 10.3925 & 293.7 & 4 & 600.0 & 600 \\
\hline
\end{tabular}




\begin{tabular}{|c|c|c|c|c|c|c|c|}
\hline 410 & 24 & 2.9999 & 12.7825 & 290.8 & 4 & 00.0 & \\
\hline 1 & 41 & 2.0250 & 12.2125 & 290.4 & & no & \\
\hline & 42 & 68.9987 & 2.3425 & 289.5 & & 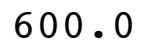 & \\
\hline & 43 & 5.2004 & 4.1100 & 286.9 & & 00 & \\
\hline & 44 & 8.2500 & .7175 & 5 & & & \\
\hline & 45 & 77.4750 & 2.0125 & 85.9 & & r & \\
\hline & 46 & 77.7502 & 8.7700 & 287.0 & & 00 & \\
\hline & 47 & 9.1507 & 6.7590 & 87.9 & & 0 & \\
\hline & 48 & 78.8998 & 6.6368 & 88.6 & & 00.0 & \\
\hline & 49 & 83.1960 & 7303 & 290.7 & & 0 & \\
\hline & 410 & 32.7499 & & 90 & & & \\
\hline & 411 & 77.9277 & & 93 & & & \\
\hline & 412 & 91.2754 & 600 & 294.4 & & 0 & \\
\hline & 413 & 82.54 & 7 . & 94 & & & \\
\hline & 414 & & & 94 & & & \\
\hline & 415 & 84.3002 & & 296.3 & 4 & & \\
\hline & 416 & 74.2500 & 6.2440 & 296.4 & 4 & 0.0 & \\
\hline & 417 & 78 & & 5 & 4 & & \\
\hline & 418 & 77 . & 5 & 292 & 4 & & \\
\hline & 419 & 73.27 & 3250 & 291.3 & 4 & 0 & \\
\hline & 420 & 72.42 & 0 & 1 & & & \\
\hline & 421 & 79 . & & 294 & 4 & & \\
\hline & 422 & 76.0003 & 6.8043 & 295.8 & 4 & 0 & \\
\hline & 423 & 75 . & 7. & 9 & 4 & & \\
\hline & 424 & 74 . & & 29 & 4 & & \\
\hline & 51 & 84.1512 & 175 & 295.8 & 4 & & \\
\hline & 52 & 80.65 & 8 . & 295.7 & 4 & & \\
\hline & 53 & 79. & & 295 & 4 & & \\
\hline & 54 & 85.32 & 005 & 295.1 & 4 & & \\
\hline & 55 & 77.72 & & 295.1 & 4 & & \\
\hline & 56 & 89. & & 295 & 4 & & \\
\hline & 57 & 84 . & & 294.8 & 4 & & \\
\hline & 58 & 60.8506 & 85 & 294.4 & 3 & & \\
\hline & 59 & 53.34 & 2. & 292.2 & 2 & & \\
\hline & 510 & 276 . & & 292.3 & 2 & & \\
\hline & 511 & 231.9287 & & 295.0 & & & \\
\hline & 512 & 171.38 & & 296 & 2 & & \\
\hline & 513 & 168.4532 & 470 & 297.6 & 2 & & \\
\hline 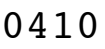 & 514 & 169.0644 & 6.1230 & 298.0 & 3 & & \\
\hline & 515 & 155 . & & 298 & 4 & & \\
\hline & 516 & 87.57 & 75 & 298.5 & 3 & & \\
\hline & 517 & 74.7701 & 5.7520 & 298.1 & 4 & & \\
\hline & 518 & 63.32 & 7.3248 & 296 & 4 & & \\
\hline 04 & 519 & 63.3252 & 8.2550 & 296.5 & 4 & & 600 \\
\hline 4 & 520 & 66.3755 & 8.1600 & 296.2 & 4 & 600.0 & 00 . \\
\hline & 521 & 80 & & & 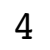 & & \\
\hline & 522 & 76.2853 & & 296.3 & 4 & & 600 \\
\hline & 523 & 72.5756 & 6.755 & 296.0 & & 600.0 & 600 \\
\hline
\end{tabular}




\begin{tabular}{|c|c|c|c|c|c|c|c|}
\hline & 24 & טלנים & 8.0800 & 295.7 & 4 & 0 & \\
\hline 11 & 1 & 8.5250 & 7.1463 & 295.4 & 4 & 0 & \\
\hline & 62 & 81.8250 & 6.6593 & 295.3 & 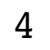 & 00 & \\
\hline 4 & 63 & 3.0991 & 5.7668 & 294.8 & & 00 & \\
\hline & 4 & & 8.6525 & 93.5 & & 0 & \\
\hline & 65 & 502 & 420 & 93.7 & & 00 & \\
\hline & 6 & 75.0499 & 3615 & 294.6 & & 0 & \\
\hline & 7 & 60 . & 1.0603 & 93.6 & & & \\
\hline & 8 & 1.2484 & 1.2493 & 294.1 & & 0 & \\
\hline & 69 & 283.8431 & 1.3380 & 294.9 & & 00 & \\
\hline & 610 & 212.2939 & 2.2318 & 295 & & 0 & \\
\hline & 611 & 198.34 & & 9 & & & \\
\hline & 12 & 161.5283 & 4.0293 & 297.6 & & 0 & \\
\hline 4 & 613 & 160.8500 & 5.9508 & 299.2 & 2 & 0.0 & \\
\hline & 614 & 165.3274 & & & & & \\
\hline & 615 & 175.1513 & 235 & 30 & 3 & & \\
\hline & 616 & 163.9971 & 4.8348 & 300.5 & 2 & 0 & \\
\hline & 617 & 113 & 415 & 9 & $\boldsymbol{J}$ & & \\
\hline & 618 & 75.1999 & 6.9848 & 297.8 & 4 & 0 & \\
\hline & 619 & 67.3248 & 525 & 296 & 4 & & \\
\hline & 620 & 59 . & 5 & 29 & 4 & & \\
\hline & 621 & 57. & 0 & $29^{\circ}$ & 4 & & \\
\hline & 622 & 62.8000 & 7050 & 29 & 4 & & U. \\
\hline 4 & 623 & 66 . & 0.2725 & 295 & 4 & & \\
\hline & 624 & 67 . & & 295 & 4 & & \\
\hline & 71 & 69. & 625 & 29 & 4 & & \\
\hline & 72 & 62.6207 & 5175 & 294.0 & 4 & & \\
\hline & 73 & 59.4221 & 9.9925 & 293.7 & 4 & & \\
\hline & 74 & 63. & 775 & 293.2 & 4 & 0 & \\
\hline & 75 & 57. & 7150 & 293.1 & 4 & & \\
\hline & 76 & 69. & 0 & 293.3 & 4 & & \\
\hline & 77 & 83. & 25 & 292.7 & 4 & & \\
\hline & 78 & 86.3753 & 5430 & 293.1 & 4 & 0 & J. \\
\hline & 79 & 83 & 448 & 294.0 & 4 & & \\
\hline & 710 & 9 & 3 & 294 & 4 & & \\
\hline & 711 & 94.4991 & 4.7933 & 296.3 & 3 & & \\
\hline & 712 & 595 & 4.7548 & 297.8 & 2 & & \\
\hline & 713 & 135. & 490 & 298.8 & 2 & 0 & 0 . \\
\hline 4 & 714 & 152.5496 & 5.4090 & 298.9 & 3 & 0 & 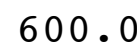 \\
\hline & 715 & 146 . & 818 & 299.0 & 4 & & \\
\hline & 716 & 137 . & 948 & 299.0 & 3 & & \\
\hline 04 & 717 & 90.5052 & 5.4675 & 297.8 & 4 & 600.0 & 600. \\
\hline & 718 & 69.8250 & 075 & 296.0 & 4 & 600 & - \\
\hline & 719 & 73.0500 & 8.8775 & 295.1 & 4 & & 50 \\
\hline 04 & 720 & 69.8250 & 7.8100 & 295.0 & 4 & 600 & 0 \\
\hline & 721 & 66.6502 & 9.5800 & 294.9 & 4 & 600.0 & 60 \\
\hline & 722 & 63 & 5225 & 294.9 & 4 & 600 & \\
\hline & 723 & 71.7246 & 7.7575 & 295.2 & 4 & 600.0 & 600 \\
\hline
\end{tabular}




\begin{tabular}{|c|c|c|c|c|c|c|c|}
\hline 0 & 24 & 5.6750 & 6.7065 & 295.0 & 4 & 00.0 & \\
\hline 1 & 81 & $2 \quad 3477$ & 5.7533 & 294.7 & & 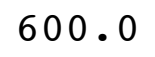 & \\
\hline & 82 & 001 & 4873 & 3.4 & & 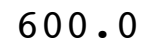 & \\
\hline & 3 & 1.6749 & 7.6460 & 93.2 & & 00 & \\
\hline & 4 & 5.7500 & .2585 & 94.1 & & & \\
\hline & 85 & 74.3467 & 3.4538 & 93.8 & & 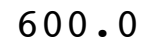 & \\
\hline & 6 & 77.5523 & 0335 & 292.7 & & 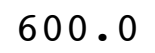 & \\
\hline & 7 & .4853 & 3 & 93.2 & & 0 & \\
\hline & 8 & 74.7756 & 230 & 294.6 & & 0 & \\
\hline & 9 & 13.6932 & & & & 0 & \\
\hline & 810 & 173 & & & & & \\
\hline & 811 & 826 & & 2 & & & \\
\hline & 12 & 230 . & 2 . & 297.7 & & 0 & \\
\hline & 813 & 4 & 4. & 9 & & & \\
\hline & 14 & & & & & & \\
\hline & 15 & 951 & & 298.3 & 4 & & \\
\hline & 816 & 78.6687 & 0 & 297.1 & 4 & 0 & \\
\hline & 817 & 71.7239 & 0 & 294.6 & 2 & & \\
\hline & 18 & 756 & & 293.0 & 4 & & \\
\hline & 819 & 61.5252 & & 291 & 4 & & \\
\hline & 820 & 252 & & & & & \\
\hline & 821 & 751 & & 28 & 4 & & \\
\hline & 822 & 88.0991 & 8 & 289.3 & 4 & & \\
\hline & 823 & 787 & & & 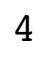 & & \\
\hline & 824 & & & & 4 & & \\
\hline & 91 & 72.7624 & & 289 & 4 & & \\
\hline & 92 & 3611 & & 289.2 & 4 & & \\
\hline & 93 & 68.4781 & & 289.5 & 4 & & \\
\hline & 94 & 616 & & 289 & 4 & & \\
\hline & 95 & 280 & 0 & 288.1 & 4 & & \\
\hline & 96 & 804 & & & 4 & & \\
\hline & 97 & 75 . & & 1 & 4 & & \\
\hline & 98 & 73. & 0 & 286.5 & 4 & & \\
\hline & 99 & 360 & & & 4 & & \\
\hline & 910 & 8 & & & 4 & & \\
\hline & 911 & 160 . & & 289.4 & 4 & & \\
\hline & 912 & 161. & & & 4 & & \\
\hline & 913 & 160 . & 5 & .7 & 4 & & \\
\hline & 914 & 156.0769 & 7700 & 292.4 & 4 & & 0 \\
\hline & 915 & 161. & & & 4 & & \\
\hline & 916 & 153.7744 & 6 . & 292.9 & 4 & & \\
\hline & 917 & 151.9762 & 6.8840 & 292.6 & 4 & & \\
\hline & 918 & 87.8905 & 280 & 291.0 & 4 & & \\
\hline & 919 & 74.1502 & 8.2700 & 289.8 & 4 & & \\
\hline 4 & 920 & 83.2344 & 8.3225 & 289.6 & 4 & 0 & 500 . \\
\hline & 921 & & & & 4 & & \\
\hline & 922 & 127.0952 & & 291.0 & & & \\
\hline & 923 & 144.0749 & 10.6000 & 290.7 & & 600.0 & 600 \\
\hline
\end{tabular}




\begin{tabular}{|c|c|c|c|c|c|c|}
\hline & & & & & & \\
\hline 101 & & & U & & & \\
\hline 0102 & 24 & 200 & 89.5 & & 00 & \\
\hline 0103 & 37.0752 & 2225 & 289.3 & & 00 & \\
\hline 104 & & 3.8925 & & & & \\
\hline 105 & & 4250 & 9.0 & & & \\
\hline 0106 & 3754 & 900 & 88.7 & & 00 & \\
\hline 0107 & 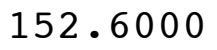 & 1.4250 & & & & \\
\hline 108 & & .6600 & 2 & & & \\
\hline 0109 & 3250 & 3.2400 & 90.1 & & 0 & \\
\hline 010 & 0252 & 2.4350 & 91.5 & & & \\
\hline 011 & 18959 & 2.8450 & 8 & & & \\
\hline 012 & 71 & 11.9525 & 94.2 & & & \\
\hline 013 & 9 & 12.6825 & 9 & & & \\
\hline 014 & & 5 & & & & \\
\hline & & 12 . & 9 & 4 & & \\
\hline 016 & 95 & 10.9125 & 296.2 & 4 & & \\
\hline 17 & 90 & 25 & 0 & & & \\
\hline 18 & & & & 4 & & \\
\hline 19 & 3517 & 00 & & 4 & & \\
\hline 020 & 144.40 & 11.9575 & 294.9 & 4 & 0 & \\
\hline 21 & & 2 . & & 4 & & \\
\hline 22 & 993 & 12 . & 29 & 4 & & \\
\hline 023 & 3500 & 13. & 293.0 & 4 & 0 & \\
\hline 024 & & & & 4 & & \\
\hline 11 & 1 & & 29 & 4 & & \\
\hline 0112 & 5950 & & 29 & 4 & & \\
\hline 113 & 7.15 & 7. & 5 & 4 & & \\
\hline 114 & 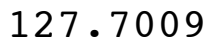 & 8 . & & 4 & & \\
\hline 0115 & 26.8483 & 25 & 8.7 & I & & \\
\hline 410116 & 141.2249 & 50 & 288.6 & 4 & & \\
\hline 117 & 11 & 75 & & 4 & & \\
\hline 410118 & & 0 & 288.5 & 4 & & \\
\hline 410119 & 139.8504 & & 289.3 & 4 & & \\
\hline & 1 & & & 4 & & \\
\hline & & & 1.6 & 3 & & \\
\hline 112 & 151. & 53 & 294.2 & 4 & & \\
\hline 113 & 157 . & 5. & 29 & 3 & & \\
\hline 114 & 161.84 & & 297.3 & 4 & & 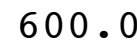 \\
\hline 115 & 176.315 & 448 & 298.1 & 3 & & \\
\hline & 182 . & 368 & 298.6 & 3 & & \\
\hline & 219.3602 & 1.7190 & 298.6 & 3 & & \\
\hline 118 & 111.0983 & 1.4465 & 298.0 & 4 & & 0 \\
\hline 119 & .4303 & 985 & & 4 & & \\
\hline 120 & 141.2321 & & & 4 & & \\
\hline 121 & 143.0000 & 6.5593 & 295.9 & 4 & & PO \\
\hline & & & & 4 & & \\
\hline 123 & 51.6524 & 1.6770 & 295.8 & & .0 & 600 \\
\hline
\end{tabular}




\begin{tabular}{|c|c|c|c|c|c|c|}
\hline 124 & 年 & .6865 & 96.1 & 5 & 600.0 & \\
\hline 0121 & 138.9861 & 1.7628 & 294.5 & & 600.0 & \\
\hline 0122 & 139.1005 & 3.8315 & 293.3 & & 00 & \\
\hline 10123 & 136.2372 & 3.3903 & 293.0 & & 00 & \\
\hline 0124 & 130.8882 & 7083 & 92.9 & & 0 & \\
\hline 0125 & 140.0250 & .8000 & 291.1 & & 0 & \\
\hline 10126 & 501 & 1433 & 291.9 & & 00 & \\
\hline 0127 & 134.3082 & .44 & 292.4 & & & \\
\hline 10128 & 141.6000 & 9625 & 93.1 & & & \\
\hline 410129 & 140.6176 & 9.4675 & 294.1 & & 00 & - \\
\hline 01210 & 148.2250 & 4750 & 295.3 & & & \\
\hline 211 & 149.4488 & 8.7850 & 96.8 & & & \\
\hline 1212 & 145.5501 & 7.2258 & 298.7 & & 0 & \\
\hline 1213 & 156.0046 & 7.1440 & 299.9 & & 0.0 & \\
\hline 214 & 154.2253 & & 300 & & & \\
\hline & 147.55 & & 301.4 & & & \\
\hline 1216 & 143.5000 & 3983 & 301.8 & & .0 & . \\
\hline 217 & 143.9759 & 5 & 301.2 & & & \\
\hline 218 & 106 . & 3 & 300.5 & & & \\
\hline 219 & 69.5225 & 50 & 299.2 & 4 & & • \\
\hline 1220 & 72.6750 & 5 & 298.7 & & 0 & \\
\hline 21 & 64.4000 & & & & & \\
\hline 222 & 67.4997 & 750 & 298.6 & 4 & & \\
\hline 1223 & 80.4500 & 700 & 299.2 & & 0 & \\
\hline 224 & 76.9748 & & 29 & & & \\
\hline 131 & 98.2735 & 70 & 299.0 & 4 & & \\
\hline 410132 & 113.0061 & 508 & 298.1 & 4 & 0 & J. \\
\hline 410133 & 108.6494 & 5943 & 297.2 & 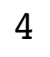 & & \\
\hline 410134 & 123.3204 & 4.7428 & 29 & 4 & & \\
\hline 410135 & 131.9250 & 4.4703 & 295.4 & 4 & 0 & 0. \\
\hline 410136 & 123.4499 & 3313 & 294.5 & 4 & & 0 \\
\hline 410137 & 119.3504 & 8 & 295.0 & 4 & & \\
\hline 0410138 & 123.2020 & & 294.9 & 3 & & 0 . \\
\hline 410139 & 133.3145 & 5.0073 & 295.7 & 3 & 0 & \\
\hline 310 & 133.9251 & 425 & 297.5 & 3 & & \\
\hline 04 & 149.5229 & 5.5368 & 298.7 & 3 & & ( \\
\hline 4101312 & 157.8999 & 3368 & 299.5 & 2 & & 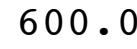 \\
\hline 313 & 158.8994 & 7170 & 300.3 & 2 & & 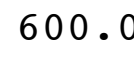 \\
\hline 04101314 & 162.5250 & 5.4355 & 301.1 & 3 & .0 & 0 . \\
\hline 4101315 & 160.8999 & 1838 & 301.1 & 4 & & \\
\hline & 156.4254 & 6.7318 & 301.3 & 4 & & 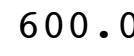 \\
\hline 17 & 146.8492 & 5.7515 & 300.7 & 4 & & 0 . \\
\hline 4101318 & 140.4431 & 3.1883 & 300.3 & 4 & 0 & 0 . \\
\hline 1319 & 98.8220 & 1.4943 & & 5 & & \\
\hline 04101320 & 67.1590 & 2.2370 & 301.3 & 5 & & 0 . \\
\hline 4101321 & 82.7793 & 2.3265 & 301.7 & 4 & 600 & 00 . \\
\hline & 97 & & & 4 & & \\
\hline 4101323 & 104.4841 & 2.7318 & 301.0 & & 60 & 60 \\
\hline
\end{tabular}




\begin{tabular}{|c|c|c|c|c|c|c|}
\hline $3<4$ & & .1300 & & & & \\
\hline 0141 & & .7040 & 0.0 & & 00.0 & \\
\hline 0142 & 128.1791 & 3.2478 & 299.9 & 4 & 00 & \\
\hline 10143 & 133.3468 & 2.6875 & 296.3 & & 00 & \\
\hline $014 \quad 4$ & 57. & .5263 & 96.3 & & 0 & \\
\hline 0145 & 75 & 410 & 297.0 & & 00 & \\
\hline 0146 & 123. & 2.8395 & 297.3 & & 00 & \\
\hline 0147 & 100 . & 7545 & 297.5 & & 0 & \\
\hline 0148 & 120.3745 & 2853 & 297.8 & 3 & & \\
\hline 014 & 172.9990 & 2.2365 & 297.9 & & 00 & 0 \\
\hline 1410 & 164.3750 & 4.0193 & 297.8 & & 0 & \\
\hline & 159.4571 & 2.8745 & 298.1 & & 00.0 & \\
\hline 2 & 173.7095 & 5308 & 299.6 & 2 & $\cdot 0$ & \\
\hline 413 & 161.9500 & 4.9085 & 299.5 & 3 & 0.0 & \\
\hline & 162 & & 300.1 & & & \\
\hline & 166 & & 300.1 & 3 & & \\
\hline 416 & 161.8250 & 3333 & 300.0 & 4 & .0 & 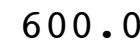 \\
\hline 17 & 151 . & 5.2300 & 298.8 & 4 & & \\
\hline & 142 . & 3.47 & 298.1 & 4 & & \\
\hline 9 & 136 & 428 & 298.5 & 4 & & \\
\hline 420 & 139.3988 & 2.7383 & 297.9 & 4 & 0 & 00 \\
\hline & 89.7010 & 4.2775 & 298.8 & 4 & & \\
\hline 4 & 86 . & 6.6785 & 298.8 & 4 & & \\
\hline 423 & 82.4500 & 5.4165 & 298.4 & 4 & 0 & \\
\hline 424 & 73.2967 & & 298.4 & 4 & & \\
\hline 51 & 102.3100 & 4 . & 298.0 & 4 & & \\
\hline 410152 & 108.9129 & 2.2228 & 297.6 & 5 & 0 & J. \\
\hline 410153 & 85.7555 & 4.6195 & 297.8 & 4 & & \\
\hline 0410154 & 135 & 2.5645 & 296.1 & 4 & & \\
\hline 410155 & 132.4332 & 9828 & 293.7 & 5 & & 0 • \\
\hline 410156 & 128.3670 & 3.2390 & 291.5 & 4 & & 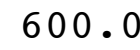 \\
\hline 0410157 & 97.20 & 2.4425 & 293.1 & 4 & & \\
\hline 0410158 & 148 . & 173 & 292.5 & 4 & & 0 . \\
\hline 0410159 & 89.7948 & 1.9410 & 293.7 & 3 & 0 & \\
\hline 41 & 251.3657 & 713 & 296.7 & 2 & & \\
\hline 041 & 162.6750 & 4.0350 & 296.1 & 3 & & 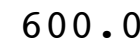 \\
\hline 4101512 & 160.3905 & 3.3183 & 297.3 & 3 & & o. \\
\hline 513 & 217.1184 & 575 & 298.3 & 2 & & . \\
\hline 041 & 235.3381 & 2.4390 & 298.2 & 2 & .0 & 600 \\
\hline 4101515 & 263.3684 & 1.5168 & 298.3 & 2 & & \\
\hline 516 & 282.3936 & 1.3453 & 298.9 & 2 & & 60 \\
\hline 041 & 123.8923 & 3.5483 & 298.0 & 3 & .0 & 00 . \\
\hline 04101518 & 93.0797 & 3.8543 & 297.0 & 4 & 0 & 00 . \\
\hline 0410 & 53.2248 & 668 & 295.8 & 4 & & \\
\hline 04101520 & 58.1007 & 7.5830 & 295.4 & 4 & 0 & 600. \\
\hline 410152 & 67.4249 & 8.8025 & 295.4 & 4 & 600.0 & 600 \\
\hline & & & 294.9 & 4 & & \\
\hline 41 & 70.6486 & 6.7973 & 294.7 & & 600.0 & 600 \\
\hline
\end{tabular}




\begin{tabular}{|c|c|c|c|c|c|c|}
\hline 24 & 2.2889 & 3.5925 & 93.9 & 4 & 0 & \\
\hline 410161 & .7298 & 5823 & 93.3 & 4 & 00.0 & \\
\hline 410162 & 314 & 3300 & 292.4 & 5 & 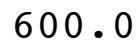 & \\
\hline 10163 & 0995 & 1.3578 & 292.1 & & 00 & \\
\hline 164 & & & 91.2 & & & \\
\hline 0165 & 130 & 4903 & 88.8 & & 00 & \\
\hline 0166 & 301 & 1.2718 & 287.1 & & 0 & \\
\hline 0167 & 160 & .7750 & 86.6 & & & \\
\hline 0168 & 118 & .5468 & 86.9 & & 0 & \\
\hline 0169 & 000 & 3985 & 287.4 & & 00 & \\
\hline 510 & 93.9323 & 038 & 88.7 & & 0 & \\
\hline & 169. & & 90.0 & & & \\
\hline 512 & 416 & 2.2463 & 291.9 & & 0 & \\
\hline 613 & 290 & 2.96 & 292.7 & & 0 & \\
\hline & 14 . & & 93.6 & & & \\
\hline 5 & 69. & 75 & 293.1 & 3 & & \\
\hline 516 & 73.6 & 3.68 & 292.8 & & 0 & \\
\hline 17 & 333. & 4.12 & 292.8 & & & \\
\hline 8 & 53. & & 290.7 & 4 & & \\
\hline 519 & 69.2 & 31 & 289.3 & 4 & & \\
\hline 20 & 49 . & 3 & 289.0 & 4 & & \\
\hline & 336 . & & 288.0 & 4 & & \\
\hline 522 & 280 & 2.4060 & 286.9 & 4 & 0 & U. \\
\hline 23 & 281.8516 & 3 & 286.4 & 5 & & \\
\hline 24 & 329. & & 28 & 4 & & \\
\hline 71 & 217.1114 & & 285.7 & 5 & & \\
\hline 0172 & 317.5484 & & 285.8 & 5 & & \\
\hline 410173 & 328.5268 & & 9 & 5 & & \\
\hline 174 & 350 & 0 & 28 & 5 & & \\
\hline 410175 & 335.20 & & 286.1 & 4 & & \\
\hline 0176 & 340.52 & & $2 \varepsilon$ & 4 & & \\
\hline 177 & 337 . & 2 . & $2 \varepsilon$ & 5 & & \\
\hline 410178 & 13.2566 & 1.20 & 287.5 & 4 & 0 & 0 . \\
\hline 410179 & .5406 & 1. & $2 \varepsilon$ & 3 & & \\
\hline & 18 & & .5 & 4 & & \\
\hline 711 & 348.2078 & & 289.2 & 3 & & \\
\hline & 589 & 0 & 291.0 & 3 & & \\
\hline 04 & 19 & 5 & 292.0 & 4 & & • \\
\hline 714 & 21 . & 2.3750 & 292.1 & 4 & & 0 . \\
\hline & 997 & 2.30 & 292.1 & 4 & & \\
\hline & 250 & 8 . & 290.5 & 4 & & \\
\hline 041 & 12.3741 & 5.3853 & 288.3 & 4 & 600.0 & 00 . \\
\hline & 15.4279 & 2.4068 & 288.2 & 4 & 600 & \\
\hline 1719 & 45.2650 & 4.6648 & 286.8 & 5 & & \\
\hline 04101720 & 61.1986 & 6.7993 & 284.8 & 4 & 600.0 & 00 \\
\hline 1721 & 994 & 7.9175 & 285.6 & 4 & 600 & \\
\hline & 1008 & & 28 & 4 & & \\
\hline 4101723 & 104 & 2.6280 & 285.2 & & 600.0 & 600 \\
\hline
\end{tabular}




\begin{tabular}{|c|c|c|c|c|c|c|}
\hline & & .5193 & & & & \\
\hline 10181 & 331. & .9755 & 4.6 & & 0 & \\
\hline 10182 & 45.2294 & 1.9605 & 285.1 & & 00 . & \\
\hline 10183 & 67.2800 & 7280 & 284.8 & & 00.0 & \\
\hline 0184 & 53.4502 & .5265 & & & & \\
\hline 0185 & 99 & & 84.1 & & 0 & \\
\hline 10186 & 87.8251 & 5033 & 84.0 & & 00 & \\
\hline 10187 & 78.9619 & 0 & & & .0 & \\
\hline 10188 & 54.8000 & 8 & 8 & & & \\
\hline 410189 & 57.8321 & 5200 & 85.5 & & 00 & 0 \\
\hline 1810 & 56.3130 & 4.6613 & 286.8 & & 00.0 & \\
\hline 11 & 69.5921 & 4.3723 & & & & \\
\hline 812 & 95.6 & 3 . & 288.1 & & 0 & \\
\hline 813 & 105.6764 & 101 & $28 \varepsilon$ & & 0.0 & \\
\hline 14 & 290 . & & & & & \\
\hline & 287 . & & & & & \\
\hline 816 & 273.5788 & 2.71 & 288.9 & & .0 & \\
\hline 17 & 306.1145 & 0 & 2 & & & \\
\hline & 303 . & & 4 & & & \\
\hline 19 & 324 & 3 & 286.8 & & & \\
\hline 320 & 25.8225 & 3.37 & 286.6 & & 0 & \\
\hline & 82.2453 & & & & & \\
\hline 22 & 18 & 3 & 28 & & & \\
\hline 323 & 350.6470 & 2.39 & 2 & & 0 & \\
\hline 24 & 351.0252 & & & & & \\
\hline 91 & 4 & 5 & 28 & & & \\
\hline 410192 & 353.1456 & 0 & $2 \varepsilon$ & 4 & 0 & J. \\
\hline 410193 & 323 & & 6 & & & \\
\hline 194 & 327 . & 6 . & 28 & 4 & & \\
\hline 410195 & 308.2135 & 6. & 286.2 & 4 & 0 & U. \\
\hline 410196 & 308.4488 & 5.7653 & 285.4 & 4 & & 0 . \\
\hline 97 & 297 . & 6 . & & 4 & & \\
\hline 410198 & 314 . & & 28 & 4 & & 0 . \\
\hline 410199 & 325.3781 & 3.53 & 284.0 & 4 & 0 & \\
\hline 10 & 325 . & 5 & & 4 & & \\
\hline 041 & 322 . & & 5.1 & 4 & & 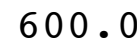 \\
\hline 912 & 306.0357 & 6900 & 284.2 & 4 & & 0 \\
\hline 13 & 324 . & & 28 & 4 & & \\
\hline 041 & 294.2483 & 3.8558 & 5.3 & 3 & & 0 . \\
\hline 915 & 128.1760 & 1.8163 & 286.0 & 3 & & \\
\hline 916 & 17.9927 & 9928 & 287 & 3 & & 0 \\
\hline 041 & 99.1884 & 5.8280 & 28 & 4 & & 0 . \\
\hline 4101918 & 73.7728 & 4.9538 & 285.4 & 4 & 0 & 0 . \\
\hline 919 & 868 & 2.7525 & & 5 & & \\
\hline 04101920 & 317.5152 & 1.5890 & & 0 & & 0 . \\
\hline 4101921 & 261.0695 & 2.1573 & 285.5 & 5 & & 600 \\
\hline & 34 & & & 5 & & \\
\hline 4101923 & 295.3740 & 2.2785 & 285.3 & & .0 & 6 \\
\hline
\end{tabular}




\begin{tabular}{|c|c|c|c|c|c|c|}
\hline $9<4$ & & 5478 & & & & \\
\hline 201 & 0 & 1.9643 & 4.3 & & 600.0 & \\
\hline 0202 & 317.5759 & 0383 & 283.5 & 4 & 00 & \\
\hline 10203 & 5.8643 & 1305 & 284.5 & 1 & 00 & \\
\hline 0204 & 337.9058 & 7208 & & & 0 & \\
\hline 0205 & 71.9874 & 4955 & 84.6 & 4 & 0 & \\
\hline 10206 & 112.3544 & 3630 & 283.2 & 4 & 00 & \\
\hline 410207 & 24.7352 & 8808 & 83.3 & & 0 & \\
\hline 10208 & 334.0824 & 1.0180 & 83.4 & 4 & & \\
\hline 410209 & 344.2675 & 1.3660 & 284.2 & 3 & 00 & 0 \\
\hline 2010 & 280.0157 & 1.5788 & 285.3 & & 0 & \\
\hline 011 & 253.8056 & 1.8723 & 85.8 & 2 & & \\
\hline 012 & 290.7904 & 3.3648 & 286.3 & 2 & 0 & ( \\
\hline 2013 & 260.1012 & 4.5125 & 285.5 & 3 & 00.0 & \\
\hline 014 & 248 . & & & 2 & & \\
\hline 15 & 273 & & 285.3 & 3 & & \\
\hline 016 & 294.8426 & 2.9370 & 284.6 & 4 & .0 & . \\
\hline 017 & 303.8297 & 8 & 6 & 4 & & \\
\hline 18 & 19.7073 & 0 & 28 & 5 & & \\
\hline 019 & 76.7673 & 5830 & 28 & 5 & & \\
\hline 020 & 153.1999 & 5.2883 & $2 \varepsilon$ & 4 & 0 & 00 . \\
\hline 21 & 146.6192 & & & 4 & & \\
\hline 022 & 166.7178 & 10 & 282.8 & 4 & & \\
\hline 023 & 147.4717 & 0 & 282.9 & 4 & 0 & \\
\hline 024 & 141.6998 & & & 4 & & \\
\hline 11 & 124.9942 & 4 . & 28 & 4 & & \\
\hline 410212 & 130.9486 & 3.2193 & 282.7 & 4 & 0 & J. \\
\hline 410213 & 146.0982 & 3. & .1 & 4 & & \\
\hline 214 & 139. & 4 . & 282.1 & 4 & & \\
\hline 410215 & 112.0194 & 4. & 282.2 & 4 & & 0 . \\
\hline 410216 & 108.7661 & 4.0425 & 281.9 & 4 & & s. \\
\hline 0410217 & 92.8499 & 305 & 282.0 & 4 & & \\
\hline 0410218 & 89.1416 & 0 & 282.9 & 3 & & 0 . \\
\hline 0410219 & 154.3123 & 3.3605 & 284.2 & 3 & 0 & \\
\hline 41 & 156.4151 & 3 & 28 & 3 & & \\
\hline 041 & 163.6995 & 5.5025 & 285.7 & 4 & & . \\
\hline 112 & 158.4750 & 5.2228 & 286.9 & 3 & 0 & . \\
\hline 41 & 88.5784 & 3.5548 & 28 & 2 & & 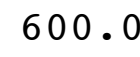 \\
\hline 041 & 119.4766 & 3.9240 & 289.2 & 3 & .0 & 0 . \\
\hline 04102115 & 118.5477 & 4.5868 & 289.5 & 3 & & \\
\hline 16 & 92.6264 & 995 & 289.2 & 3 & & 0 \\
\hline 041 & 68.4701 & 4.2260 & 288.6 & 4 & .0 & 0 . \\
\hline 04102118 & 63.2500 & 5.7590 & 287.0 & 4 & 0 & 00 . \\
\hline 119 & 72.3259 & 6.6043 & & 4 & & 0 \\
\hline 04102120 & 68.7243 & 7.2403 & 285.1 & 4 & & 600. \\
\hline 410212 & 72.0500 & 7.9325 & 284.7 & 4 & 600.0 & 600. \\
\hline & 999 & & & 4 & & \\
\hline 123 & 73.3280 & 7.9150 & 284.6 & $\Psi$ & 600.0 & 600 \\
\hline
\end{tabular}




\begin{tabular}{|c|c|c|c|c|c|c|}
\hline 4102124 & 80.2001 & 7.6600 & 284.2 & 4 & 600.0 & 600.0 \\
\hline 0410221 & 76.1000 & 7.3655 & 284.3 & 4 & 600.0 & 600.0 \\
\hline 0410222 & 79.0748 & 7.5000 & 284.2 & 4 & 600.0 & 00.0 \\
\hline 0410223 & 74.5495 & 7.6375 & 284.0 & 4 & 600.0 & 600.0 \\
\hline 0410224 & 73.6255 & 6.5155 & 284.0 & 4 & 600.0 & 00.0 \\
\hline 0410225 & 60.8758 & 5.4778 & 283.7 & 4 & 600.0 & 00.0 \\
\hline 0410226 & 15.4530 & 1.9578 & 283.4 & 5 & 600.0 & 600.0 \\
\hline 0410227 & 2.9444 & 1.0348 & 283.6 & 5 & 600.0 & 00.0 \\
\hline 0410228 & 40.7769 & 1.3278 & 284.1 & 4 & 600.0 & 00.0 \\
\hline 0410229 & 139.1272 & 2.1990 & 285.4 & 3 & 600.0 & 600.0 \\
\hline 04102210 & 184.2708 & 3.2133 & 286.0 & 3 & 600.0 & 600.0 \\
\hline 04102211 & 199.2026 & 3.2350 & 286.7 & 3 & 600.0 & \\
\hline 04102212 & 163.7171 & 3.5118 & 287.6 & 3 & 600.0 & 600.0 \\
\hline 04102213 & 181.5285 & 3.1228 & 288.3 & 3 & 600.0 & 600 \\
\hline 04102214 & 211.3137 & 2.8440 & 288.5 & 3 & 600.0 & \\
\hline 04102215 & 234.5638 & 2.2523 & 288.8 & 2 & 600.0 & 60 \\
\hline 04102216 & 240.1703 & 2.3128 & 288.8 & 2 & 600.0 & 600.0 \\
\hline 04102217 & 259.7998 & 1.6698 & 288.5 & 3 & 600.0 & \\
\hline 04102218 & 296.4366 & 1.1983 & 288.1 & 4 & 600.0 & 60 \\
\hline 04102219 & 60.2479 & 1.8753 & 287.6 & 5 & 600.0 & 600.0 \\
\hline 04102220 & 80.5055 & 2.4003 & 287.4 & 4 & 600.0 & .0 \\
\hline 04102221 & 84.8579 & 2.0050 & 287.5 & 5 & 600.0 & \\
\hline 04102222 & 38.4418 & 1.9448 & 287.1 & 5 & 600.0 & 600.0 \\
\hline 04102223 & 56.0956 & 1.9618 & 287.0 & 5 & 600.0 & .0 \\
\hline 04102224 & 72.6241 & 3.9503 & 286.0 & 4 & 600.0 & \\
\hline 0410231 & 73.6536 & 2.3303 & 286.7 & 5 & 600.0 & 600.0 \\
\hline 0410232 & 153.1150 & 0.8888 & 286.4 & 6 & 600.0 & 600.0 \\
\hline 0410233 & 72.9685 & 3.8943 & 285.8 & 5 & 600.0 & \\
\hline 0410234 & 24.0749 & 1.8750 & 285.9 & 5 & 600.0 & 600.0 \\
\hline 0410235 & 41.2528 & 1.2375 & 285.8 & 6 & 600.0 & 600.0 \\
\hline 0410236 & 98.4840 & 1.5840 & 285.7 & 5 & 600.0 & .0 \\
\hline 0410237 & 149.1450 & 1.1753 & 285.3 & 6 & 600.0 & 60 \\
\hline 0410238 & 223.1601 & 0.7338 & 285.3 & 5 & 600.0 & 600.0 \\
\hline 0410239 & 358.9321 & 1.2175 & 285.9 & 4 & 600.0 & .0 \\
\hline 04102310 & 4.2194 & 1.4640 & 286.2 & 4 & 600.0 & 60 \\
\hline 04102311 & 332.9627 & 1.3410 & 287.1 & 4 & 600.0 & 600.0 \\
\hline 04102312 & 341.6573 & 1.8120 & 286.8 & 4 & 600.0 & 600.0 \\
\hline 04102313 & 9.0835 & 1.2418 & 287.4 & 4 & 600.0 & 600.0 \\
\hline 04102314 & 250.9699 & 1.2180 & 287.9 & 4 & 600.0 & 600.0 \\
\hline 04102315 & 236.5984 & 1.5273 & 287.3 & 4 & 600.0 & \\
\hline 04102316 & 340.0414 & 1.7553 & 286.1 & 4 & 600.0 & 600.0 \\
\hline 04102317 & 339.9498 & 2.6343 & 286.0 & 4 & 600.0 & 600.0 \\
\hline 04102318 & 335.5899 & 1.3033 & 285.9 & 5 & 600.0 & 600.0 \\
\hline 04102319 & 286.2303 & 1.5088 & 285.3 & 5 & 600.0 & 600.0 \\
\hline 04102320 & 318.7352 & 1.8213 & 285.2 & 5 & 600.0 & 600.0 \\
\hline 04102321 & 262.9031 & 0.9873 & 285.3 & 6 & 600.0 & 600.0 \\
\hline 04102322 & 312.0316 & 1.1343 & 285.2 & 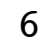 & 600.0 & 600.0 \\
\hline 04102323 & 279.8718 & 1.2645 & 285.1 & $J$ & 600.0 & 600.0 \\
\hline
\end{tabular}




\begin{tabular}{|c|c|c|c|c|c|c|}
\hline 04102324 & 4.8541 & 0.8473 & 285.1 & 6 & 600.0 & 600.0 \\
\hline 0410241 & 164.3448 & 2.3733 & 285.2 & 5 & 600.0 & 600.0 \\
\hline 0410242 & 180.0617 & 1.8703 & 284.8 & 5 & 600.0 & 00.0 \\
\hline 0410243 & 167.5500 & 4.2350 & 284.5 & 4 & 600.0 & 600.0 \\
\hline $041024 \quad 4$ & 165.9751 & 6.2435 & 284.4 & 4 & 600.0 & 00.0 \\
\hline 0410245 & 162.7251 & 6.6568 & 284.2 & 4 & 600.0 & 00.0 \\
\hline 0410246 & 174.7218 & 6.8548 & 284.0 & 4 & 600.0 & 600.0 \\
\hline 0410247 & 167.4439 & 7.5275 & 283.9 & 4 & 600.0 & 00.0 \\
\hline 0410248 & 153.9250 & 6.3940 & 283.8 & 4 & 600.0 & 00.0 \\
\hline 0410249 & 153.7243 & 5.5490 & 283.7 & 4 & 600.0 & 600.0 \\
\hline 04102410 & 150.9001 & 4.8740 & 283.9 & 4 & 600.0 & 600.0 \\
\hline 04102411 & 153.3749 & 5.9568 & 284.2 & 4 & 600.0 & \\
\hline 04102412 & 154.6500 & 4.8333 & 283.9 & 3 & 600.0 & 00.0 \\
\hline 04102413 & 153.1504 & 4.8435 & 286.3 & 3 & 600.0 & 600.0 \\
\hline 2414 & 151.8541 & 4.7170 & 287.2 & 3 & 600.0 & \\
\hline 04102415 & 153.2758 & 5.0728 & 287.7 & 3 & 600.0 & 60 \\
\hline 04102416 & 141.3211 & 4.0678 & 287.9 & 3 & 600.0 & 600.0 \\
\hline 04102417 & 139.5994 & 2.1940 & 287.5 & 4 & 600.0 & \\
\hline 04102418 & 130.3609 & 1.7498 & 286.8 & 5 & 600.0 & 60 \\
\hline 04102419 & 78.8500 & 3.8573 & 286.6 & 4 & 600.0 & 600.0 \\
\hline 04102420 & 71.0000 & 6.7613 & 285.5 & 4 & 600.0 & .0 \\
\hline 04102421 & 76.2501 & 6.6198 & 285.6 & 4 & 600.0 & \\
\hline 04102422 & 78.0250 & 6.8908 & 285.4 & 4 & 600.0 & 600.0 \\
\hline 04102423 & 75.7502 & 7.2425 & 285.2 & 4 & 600.0 & .0 \\
\hline 04102424 & 76.1751 & 7.0720 & 285.3 & 4 & 600.0 & \\
\hline 0410251 & 74.2999 & 6.7540 & 285.4 & 4 & 600.0 & 600.0 \\
\hline 0410252 & 70.7234 & 6.9708 & 285.2 & 4 & 600.0 & 60 \\
\hline 0410253 & 70.6741 & 6.6535 & 285.2 & 4 & 600.0 & \\
\hline 0410254 & 71.3756 & 6.8178 & 284.7 & 4 & 600.0 & 60 \\
\hline 0410255 & 68.4999 & 7.7975 & 283.5 & 4 & 600.0 & 600.0 \\
\hline 0410256 & 68.1997 & 7.7210 & 283.4 & 4 & 600.0 & .0 \\
\hline 0410257 & 35.9859 & 4.3693 & 283.9 & 4 & 600.0 & 60 \\
\hline 0410258 & 89.3998 & 1.9178 & 284.5 & 3 & 600.0 & 600.0 \\
\hline 0410259 & 281.3254 & 1.3133 & 285.1 & 2 & 600.0 & .0 \\
\hline 04102510 & 220.2842 & 1.7753 & 285.9 & 2 & 600.0 & 60 \\
\hline 04102511 & 95.8239 & 3.2698 & 286.1 & 3 & 600.0 & 600.0 \\
\hline 04102512 & 16.4687 & 2.1480 & 287.5 & 2 & 600.0 & .0 \\
\hline 04102513 & 260.1080 & 1.7470 & 287.4 & 2 & 600.0 & 600.0 \\
\hline 04102514 & 217.0331 & 2.2508 & 287.4 & 3 & 600.0 & 600.0 \\
\hline 04102515 & 182.6412 & 2.1503 & 287.7 & 3 & 600.0 & \\
\hline 04102516 & 82.4646 & 5.7780 & 287.4 & 4 & 600.0 & 600.0 \\
\hline 04102517 & 74.4750 & 7.9723 & 285.8 & 4 & 600.0 & 600.0 \\
\hline 04102518 & 54.0892 & 5.0680 & 285.5 & 4 & 600.0 & 600.0 \\
\hline 04102519 & 60.1372 & 6.5280 & 285.1 & 4 & 600.0 & 600.0 \\
\hline 04102520 & 40.8935 & 4.2050 & 285.3 & 4 & 600.0 & 600.0 \\
\hline 04102521 & 15.0375 & 2.7645 & 286.0 & 4 & 600.0 & 600.0 \\
\hline 04102522 & 151.1265 & 2.1218 & 285 & 5 & 600.0 & 600.0 \\
\hline 04102523 & 334.1090 & 3.5758 & 285.4 & 4 & 600.0 & 600.0 \\
\hline
\end{tabular}




\begin{tabular}{|c|c|c|c|c|c|c|}
\hline $8<4$ & & . 4585 & & & & \\
\hline 0261 & 972 & .9180 & 5.0 & 4 & 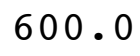 & \\
\hline 0262 & 3499 & 7610 & 285.7 & 4 & 00 & \\
\hline 10263 & 46.1022 & 6.1508 & 283.4 & & 00 & \\
\hline 0264 & 738 & .2650 & 82.5 & & 0 & \\
\hline 0265 & 501 & 4640 & 83.2 & 4 & 00 & \\
\hline 10266 & 37.8354 & 3240 & 283.3 & 4 & 00 & \\
\hline 10267 & 101.7604 & .3620 & 83.1 & 4 & 0 & \\
\hline 10268 & 74.5118 & 7388 & 83.3 & 4 & & \\
\hline 0269 & 90.4191 & 2.9698 & 283.1 & 3 & 600.0 & 0 \\
\hline 2610 & 88.5740 & 3.3548 & 283.4 & 3 & 0 & \\
\hline 611 & 41.4456 & 2.9385 & & 2 & 00.0 & \\
\hline 612 & 229.5093 & 4.3403 & 283.8 & 3 & 0 & . \\
\hline 2613 & 244.9545 & 4.8153 & 283.6 & 3 & 00.0 & \\
\hline 614 & 284 . & & & 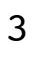 & & \\
\hline & 300 . & & 2 & 3 & & \\
\hline 616 & 320.4000 & 4.4540 & 284.0 & 3 & .0 & 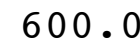 \\
\hline 617 & 312.4656 & 3 & .1 & 4 & 0 & \\
\hline & 295.2391 & 3 . & 282.4 & 4 & & \\
\hline 619 & 302 & 658 & 282.1 & 5 & 0 & \\
\hline 2620 & 5.5206 & 2.6228 & 282.2 & 4 & 0 & • \\
\hline & 68.8742 & 0 & 282.8 & 4 & & \\
\hline 622 & 70.8740 & 465 & 282.6 & 4 & & \\
\hline 2623 & 304.8814 & 1.0055 & 281.9 & 5 & 0 & 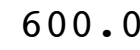 \\
\hline 24 & 82.2083 & & 4 & 5 & & \\
\hline 27 & 23.8887 & 1.22 & 281.5 & 6 & & \\
\hline 410272 & 102.6558 & 2.4225 & 281 & 5 & 0 & U. \\
\hline 410273 & 138.4007 & 623 & 3 & 4 & & \\
\hline $27 \quad 4$ & 137.5765 & 5 . & 279 & 4 & & \\
\hline 410275 & 160.6660 & 4.9055 & 280.2 & 4 & 0 & 0 • \\
\hline 410276 & 145.4053 & 1.8795 & 280.2 & 5 & 0 & 0 . \\
\hline 277 & 150.0433 & 3 . & 28 & 4 & & \\
\hline 410278 & 174.5101 & 5808 & 280.3 & 4 & & • \\
\hline 410279 & 183.1243 & 5.33 & 280.0 & 4 & 0 & \\
\hline 710 & 176.7386 & 0 & & 3 & & \\
\hline 711 & 162.0933 & 2.9195 & 280.3 & 3 & & 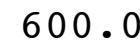 \\
\hline 4102712 & 141.9735 & 2.4538 & 281.5 & 2 & $\cdot 0$ & 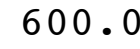 \\
\hline 713 & 152.3475 & 613 & 282.6 & 3 & & . \\
\hline 041 & 152.3990 & 3.0165 & 283.1 & 3 & .0 & 0 . \\
\hline 4102715 & 167.8260 & 3.1538 & 283.7 & 3 & & \\
\hline 716 & 160.1370 & 868 & 283.7 & 3 & & 60 \\
\hline 041 & 136.8434 & 1.7795 & 283.3 & 4 & .0 & 0 . \\
\hline 4102718 & 72.8261 & 2.9190 & 283.3 & 4 & .0 & 00 . \\
\hline 04102719 & 96.3437 & 433 & & 4 & & 0 \\
\hline 04102720 & 115.4509 & 2.3185 & 283.1 & 4 & 0 & 600. \\
\hline 4102721 & 131.2876 & 2.2860 & 282.7 & 5 & 600.0 & 600. \\
\hline & 80.1743 & 8 & & 4 & & \\
\hline & 71.3250 & 5.7783 & 282.3 & 7 & 600.0 & 600 \\
\hline
\end{tabular}




\begin{tabular}{|c|c|c|c|c|c|c|}
\hline 724 & 67.2000 & 5.7930 & 282.4 & 4 & 600.0 & \\
\hline 410281 & 9.3500 & 6.2220 & 282.6 & 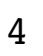 & 600.0 & \\
\hline 410282 & 67.8750 & .4385 & 282.4 & 4 & 00 & \\
\hline 410283 & 63.6754 & .2808 & 282.4 & & 00 & \\
\hline 0284 & 9.1250 & .2995 & 82.6 & & 0 & \\
\hline 0285 & 607 & 4070 & 82.7 & & 00 & \\
\hline 410286 & 72.9003 & 3000 & 281.9 & & 0 & \\
\hline 0287 & 71.3260 & .0728 & 81.6 & & & \\
\hline 410288 & 0099 & .7748 & 81.7 & & 0 & \\
\hline 410289 & 67.4461 & 8298 & 282.4 & 4 & 0 & \\
\hline 2810 & 337.0564 & 2.6730 & 283.3 & & & \\
\hline 811 & 277.3476 & 1.7855 & 83.4 & 2 & & \\
\hline 2812 & 234.5397 & 1.4503 & 284.5 & & 0 & \\
\hline 2813 & 242.2495 & 2.5958 & 284.8 & 3 & 0 & \\
\hline 814 & 228.5978 & 2.8063 & 85.1 & 3 & & \\
\hline 815 & 277.9698 & 1.9853 & 285.7 & 2 & & \\
\hline 2816 & 237.9772 & 35 & 285.5 & 3 & 0 & \\
\hline 817 & 307.7287 & 48 & 34.8 & 4 & & \\
\hline 818 & 330.2472 & 2.2543 & 284.2 & 5 & 0 & \\
\hline 2819 & 351.1862 & 1.6228 & 284.0 & 5 & & \\
\hline 820 & 76.1468 & 3688 & 284.3 & 5 & & \\
\hline 321 & 73.5713 & 2 . & 284.2 & 4 & & \\
\hline 822 & 71.8246 & 1.3365 & 284.5 & 5 & 0 & \\
\hline 823 & 83.2253 & 1.5050 & 284.7 & 5 & & \\
\hline 824 & 119.4711 & 0 & & 6 & & \\
\hline 410291 & 268 . & 553 & 283.8 & 6 & & \\
\hline 410292 & 181.4483 & 1.0048 & 283.1 & 6 & & \\
\hline 0410293 & 227.8321 & 3 & 282.9 & 6 & & 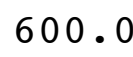 \\
\hline 0410294 & 260 . & 8 & 282.7 & 6 & & \\
\hline 410295 & 267.3247 & 1.3295 & 282.6 & 6 & & \\
\hline 410296 & 324.3150 & 5 & 282.6 & 6 & & \\
\hline 410297 & 206 . & 3 & 282.4 & 5 & & \\
\hline 0410298 & 315.9853 & 1.0493 & 282.2 & 4 & 0 & J. \\
\hline 410299 & 295 & 03 & 283.0 & 3 & & \\
\hline 910 & 239 & 28 & .5 & 2 & & \\
\hline 04102911 & 229.9330 & 2.6925 & 285.3 & 3 & & 0 . \\
\hline 2912 & 194.6041 & 4.2678 & 285.4 & 3 & & \\
\hline 041 & 203.4425 & 3.1908 & & 3 & & 0 . \\
\hline 04102914 & 183.2572 & 2.9963 & 286.3 & 3 & 0 & 0 . \\
\hline 2915 & 177.3449 & 710 & 286.5 & 3 & & \\
\hline 2916 & 170.4512 & 588 & & 3 & & \\
\hline 04102917 & 155.7181 & 2.7920 & 286.0 & 4 & 600.0 & 600 . \\
\hline 04102918 & 125.8871 & 2835 & 285.7 & 5 & 600 & 000 \\
\hline 04102919 & 78.1305 & 2.6490 & 285.8 & 4 & & s \\
\hline 04102920 & 105.7849 & 2.1023 & 285.4 & 5 & 600.0 & 00 \\
\hline 02921 & 126.1500 & 1.8468 & 285.2 & 5 & 600.0 & 600. \\
\hline & 136 & & & 4 & 60 & \\
\hline 4102923 & 128.5751 & 2.4983 & 285.3 & 4 & 600.0 & 600 \\
\hline
\end{tabular}




\begin{tabular}{|c|c|c|c|c|c|c|}
\hline $8<4$ & & 2.7788 & & & & \\
\hline 301 & 01 & 3.3053 & 5.6 & 4 & b & \\
\hline 0302 & 88.3793 & 2610 & 285.5 & 4 & 00 & \\
\hline 10303 & 76.9995 & 8093 & 285.7 & & 00 & \\
\hline 304 & 107 . & 2143 & & & & \\
\hline 0305 & 105.9612 & & & & 0 & \\
\hline 0306 & 9250 & 348 & 85.5 & & 00 & \\
\hline 0307 & 6998 & 7275 & & & 0 & \\
\hline 0308 & 9280 & 8398 & & & & \\
\hline 309 & 84.3022 & 1.7755 & 86.2 & & 00 & 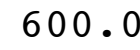 \\
\hline 010 & 313.2076 & 2.0795 & 287.1 & & & \\
\hline & 225.4768 & 2.9085 & 7.4 & & & \\
\hline 2 & 267.8218 & 1.7510 & 288.0 & & 0 & \\
\hline 3 & 234.7152 & 2.3990 & 5 & & & \\
\hline & 250.7719 & & & & & \\
\hline & 92.5013 & & & 3 & & \\
\hline 0 & 88.2507 & 8 & 288.6 & 4 & .0 & \\
\hline 17 & 89.2238 & 3 & & 4 & & \\
\hline & 76.236 & & & 4 & & \\
\hline & 1004 & 0 & 28 & 4 & & \\
\hline 20 & 74.0252 & 05 & 287 & 4 & 0 & \\
\hline & 76.3748 & & & 4 & & \\
\hline 22 & 239 & 0 & 28 & 4 & & \\
\hline 023 & 69.3259 & 0 & 2 & 4 & 0 & \\
\hline 24 & 72.8500 & & & 4 & & \\
\hline 311 & 77.8760 & 6 . & 28 & 4 & & \\
\hline 410312 & 106.4713 & 3 & 1 & 4 & & 0 \\
\hline 410313 & 137.149 & 0 & & 4 & & \\
\hline 314 & 139 & & & 4 & & \\
\hline 410315 & 146.4710 & 3 & 9 & 4 & & . \\
\hline 410316 & 152.6252 & 8 . & & 4 & & 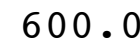 \\
\hline 317 & 145.9748 & & & 4 & & \\
\hline 410318 & 147 . & & & 4 & & \\
\hline 410319 & 146.9752 & 8.83 & 28 & 4 & & \\
\hline & 155 & 5 & & 4 & & \\
\hline & 154 & & & 4 & & \\
\hline 12 & 148.1737 & 9703 & 7.3 & 4 & & \\
\hline 13 & 161. & 0 & & 4 & & \\
\hline 14 & 163.5000 & 1.7625 & & 4 & & 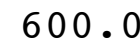 \\
\hline 115 & 168.5261 & 725 & 288.9 & 4 & & \\
\hline & 162.0750 & 125 & & 4 & & \\
\hline 17 & 155 . & 9.5125 & & 4 & & 0 . \\
\hline 03118 & 157.0501 & 6.1090 & 286.4 & 4 & & 0 . \\
\hline & 150 . & 418 & & 4 & & \\
\hline 041 & 139.7780 & 0.3550 & & 4 & & 0 . \\
\hline 121 & 138.2503 & 11.1675 & 286.6 & 4 & & 00 . \\
\hline & & 52 & & 4 & & \\
\hline 123 & 148.2253 & 11.3200 & 286.3 & & .0 & n \\
\hline
\end{tabular}




\begin{tabular}{|c|c|c|c|c|c|c|c|}
\hline & $21+>$ & & & & & & \\
\hline & 1 & 138.5448 & 6.8000 & 4.8 & 4 & 00 & \\
\hline & 12 & 3600 & 7000 & 284.6 & 4 & 00 & \\
\hline 41 & 1 & 147.5509 & 8.7750 & 284.9 & & 00 & \\
\hline & 14 & & 8.8250 & & & 0 & \\
\hline & 1 & & 250 & 84 & & 0 & \\
\hline & 1 & 152 . & 10.4750 & 83.6 & & 00 & \\
\hline & 17 & 151. & 0.6750 & 82.9 & & 0 & \\
\hline & 1 & $156 \quad 8391$ & 9.9500 & 83.2 & & & \\
\hline & 19 & 45.5137 & 5500 & 83.5 & & 00 & 0 \\
\hline & 110 & 142.5011 & 8.1750 & 284 & & 0 & \\
\hline & 111 & 111670 & 5.6000 & 85.4 & & & \\
\hline & 112 & 276 & 5.4000 & 286.7 & 4 & 0 & \\
\hline & 113 & 145.3504 & 4.9500 & 287.5 & 3 & 0.0 & \\
\hline & 114 & & 0 & & & & \\
\hline & 11 & & 4 . & & 3 & & \\
\hline 4 & 116 & 139.4608 & 4.0500 & 288.1 & 3 & .0 & \\
\hline & 117 & 114.5054 & 250 & 8 & 4 & & \\
\hline & 11 & 138.2318 & 000 & 0 & 5 & & \\
\hline 4 & 119 & 162 & 750 & 287 & 5 & & \\
\hline 4 & 120 & 317.7895 & 1.9250 & $2 \varepsilon$ & 5 & 0 & \\
\hline & 121 & 358.13 & 2 . & & 5 & & \\
\hline & 122 & 350 . & 0 & 28 & 5 & & \\
\hline 4 & 123 & 306.7668 & 1.4500 & $2 \xi$ & 5 & 0 & \\
\hline & 124 & 295 & & & 6 & & \\
\hline & 21 & 264 . & 1 . & 28 & 6 & & \\
\hline 4 & 22 & 232 & 3750 & 285.8 & 6 & 0 & J. \\
\hline 4 & 23 & 222 & 7500 & 6 & 6 & & \\
\hline 4 & 24 & 142 & 500 & 28 & 5 & & \\
\hline 4 & 25 & 139.4303 & 1.1250 & 28 & 5 & 0 & 0. \\
\hline 4 & 26 & 180.8425 & 1.1250 & 28 & 6 & & 0 . \\
\hline 4 & 27 & 132 . & 0 & 6 & 5 & & \\
\hline 4 & 28 & 159. & 000 & 1 & 4 & & 0 . \\
\hline 4 & 29 & 176.2605 & 500 & 28 & 3 & 0 & \\
\hline 1 & 210 & 262 & 0 & & 2 & & \\
\hline & 211 & 279 . & & 5.6 & 2 & & \\
\hline 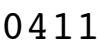 & 212 & 272.8326 & 2.4250 & 287.2 & 3 & & . \\
\hline 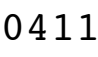 & 213 & 299.5778 & 2 . & 28 & 2 & & 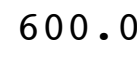 \\
\hline 04 & 214 & 293.6665 & 1.7750 & 3.8 & 2 & .0 & 0 . \\
\hline 4 & 215 & 243.0272 & 1.4000 & 288.9 & 2 & & \\
\hline 4 & 216 & 251.8427 & 1.5250 & 289 & 2 & & 0 \\
\hline 04 & 217 & 261.4355 & 0.8000 & 288.5 & 3 & & 0 . \\
\hline 4 & 218 & 340.3848 & 1.5750 & 287.9 & 4 & 0 & 0 . \\
\hline 4 & 219 & 353.8298 & 000 & & 5 & & \\
\hline 041 & 220 & 85.6607 & 2.5500 & & 5 & & 500. \\
\hline & 22 & 82.4734 & 6.3500 & 287.8 & 4 & 600.0 & 600. \\
\hline & 22 & 211 & & & 4 & & \\
\hline & $2 \pi$ & 80.2079 & 8.0750 & 287.5 & $\mathbf{4}$ & 600.0 & 60 \\
\hline
\end{tabular}




\begin{tabular}{|c|c|c|c|c|c|c|c|}
\hline & 24 & $1 \cdot 1452$ & 7.9250 & 287.6 & & 000.0 & \\
\hline 1 & 31 & $00<21$ & 7.3500 & 287.9 & & 0 & \\
\hline & 32 & 378 & 500 & 287.7 & & v & \\
\hline & 3 & 60.0949 & 50 & 287.4 & & 0 & \\
\hline & 4 & 2 & & 86.4 & & & \\
\hline & 35 & 59.0117 & 8.3250 & 85.4 & & 0 & \\
\hline & 6 & 57.5838 & 0.6500 & 284.5 & & 0 & \\
\hline & 7 & 63.3063 & 7.0750 & 84.4 & & 0 & \\
\hline & 8 & 63.6916 & 9.3500 & 284.1 & & 00.0 & \\
\hline & 9 & 65.4366 & 8.8000 & 283.6 & & 0 & \\
\hline & 10 & 67.1594 & 7 . & 82.7 & & & \\
\hline & 11 & 96.0631 & & 83.1 & & & \\
\hline & 12 & 02.7153 & 1000 & 283.1 & & 0 & \\
\hline & 313 & 24. & 5.42 & 84.1 & 4 & & \\
\hline & 14 & 0 & & 3 & & & \\
\hline & 15 & 163.7571 & 2000 & 283.0 & 4 & 0 & \\
\hline & 316 & 155.2950 & 6.4000 & 282.7 & 4 & 0.0 & \\
\hline & 317 & 127.57 & & 282.4 & 4 & & \\
\hline & 18 & 89.1385 & 5 . & 281.5 & 4 & & \\
\hline & 319 & 88.4169 & 4 . & 281.4 & 4 & 0 & \\
\hline & 20 & 127.16 & & .3 & 4 & & \\
\hline & 21 & 93.90 & & 281.0 & 4 & & \\
\hline & 322 & 146.1616 & 2.57 & 280.8 & 4 & 0 & \\
\hline & 323 & 263.00 & 0 & 280.2 & & & \\
\hline & 324 & 296 . & & 279.9 & 5 & & \\
\hline & 41 & 317.6394 & 000 & 279.7 & 4 & & \\
\hline & 42 & 312.64 & 500 & 279.6 & 4 & & \\
\hline & 43 & 330.48 & & 279.6 & 4 & & \\
\hline & 44 & 326.2700 & 0 & 279.5 & 4 & & \\
\hline & 45 & 310.53 & 0 & 279.9 & 4 & & \\
\hline & 46 & 269 . & & 279.5 & 5 & & \\
\hline & 47 & 268.88 & 3 . & 278.3 & 4 & & \\
\hline & 48 & 286.90 & 0 & 278.4 & 4 & & \\
\hline & 49 & 269.42 & & 279.2 & 4 & & \\
\hline & 410 & 267 . & & 280.6 & 3 & & \\
\hline & 411 & 266.9797 & & 281.2 & 3 & & \\
\hline & 412 & 262 . & & 282.1 & 3 & & \\
\hline & 413 & 274.3106 & 5. & 282.4 & 3 & & \\
\hline 4 & 414 & 269.5681 & & 282.9 & 3 & & \\
\hline & 415 & 289.43 & & 282.5 & 4 & & \\
\hline & 416 & 287.2937 & 0 & 281.2 & 4 & & \\
\hline 04 & 417 & 271.1997 & 3.6500 & 280.8 & 4 & & 0 \\
\hline & 418 & 306.5157 & & 281.0 & 4 & & \\
\hline 04 & 419 & 309.9159 & & 281.3 & 4 & & 600. \\
\hline 04 & 420 & 324.3393 & 4.5000 & 281.7 & 4 & 600.0 & 600. \\
\hline & 421 & 309 & & 282.2 & 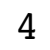 & & \\
\hline & 422 & 162.1915 & & 280.5 & 4 & & 600 \\
\hline & 423 & 170.5969 & 2.2750 & 280.5 & & 600.0 & 600 \\
\hline
\end{tabular}




\begin{tabular}{|c|c|c|c|c|c|c|c|}
\hline & 24 & & 2.0000 & 281.2 & & & \\
\hline 411 & 51 & 111 . & 2.1000 & 281.9 & & & \\
\hline & 52 & 93.1718 & 6000 & 282.8 & & 00 & \\
\hline & 53 & 117.4642 & 2.0000 & 283.5 & & 00.0 & \\
\hline & 4 & 135.40 & .7000 & 81.9 & & 0 & \\
\hline & 5 & 103 & 2.2500 & 282.9 & & 00 & \\
\hline & 6 & 109.2830 & 1.4250 & 285.1 & & 00 & \\
\hline & 7 & 84.8331 & L. 6000 & 85.3 & & & \\
\hline & 8 & 118.9264 & 2.2250 & 284.3 & & .0 & \\
\hline & 9 & 143.6460 & 3.0750 & 283.6 & & 00.0 & \\
\hline & 10 & 142.0119 & 2.7750 & 285.4 & & 00.0 & \\
\hline & 11 & 155.3 & 170 & 86.3 & & & \\
\hline 4 & 12 & 157.7449 & 5.7000 & 287.1 & & 00 & 0 \\
\hline 14 & 13 & 165.2346 & 4.97 & 287.5 & & 0.0 & \\
\hline & 14 & 159.6619 & & 287.9 & & & \\
\hline & 15 & 158.4380 & 4 . & 288.1 & 3 & 0 & \\
\hline & 16 & 128.9101 & 2.1500 & 288.4 & 3 & 0 & \\
\hline & 17 & 145 . & 3 . & 287.0 & 4 & & \\
\hline & 18 & 137.7457 & 3. & 286.9 & 4 & 0 & \\
\hline & 19 & 116.4339 & 0 & 287.7 & 4 & 0 & \\
\hline 14 & 20 & 93. & 3 . & 288.1 & 4 & 0 & \\
\hline & 21 & 76 . & & 88.3 & 4 & & \\
\hline 4 & 22 & 68.7868 & 0 & 289.3 & 4 & 0 & \\
\hline 4 & 523 & 168.8540 & 2 . & 289.2 & 5 & 0 & \\
\hline & 524 & 339. & & 287.2 & 3 & & \\
\hline & 61 & 11.9761 & 0 & 286.7 & 5 & & \\
\hline 4 & 62 & 152.4584 & 2 . & 286.5 & 5 & 0 & \\
\hline & 63 & 145 & 0 & 285.7 & 5 & & \\
\hline & 64 & 93. & 2 . & 285.8 & 4 & 0 & 0 . \\
\hline & 65 & 92.7332 & 20 & 286.3 & 4 & 0 & \\
\hline & 66 & 101.7624 & 0 & 286.5 & 4 & & \\
\hline & 67 & 108 . & 7. & 286.8 & 4 & & . \\
\hline 04 & 68 & 95.7391 & $\$ 750$ & 287.3 & 4 & 600.0 & 0 • \\
\hline 4 & 69 & 104.9220 & 0 & 288.3 & 4 & 0 & \\
\hline & 610 & 147 . & 6 . & 288.6 & 4 & & \\
\hline & 611 & 158.6002 & 5.4250 & 288.3 & 4 & 0 & 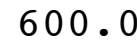 \\
\hline & 612 & 157.7692 & 500 & 288.5 & 3 & 0 & \\
\hline & 613 & 151.2521 & 0 & 289.6 & 3 & 0 & 0 . \\
\hline 04 & 614 & 145.3955 & 6 . & 290.2 & 4 & 600.0 & 0 . \\
\hline & 615 & 150.7914 & 0 & 290.6 & 4 & 0 & \\
\hline & 616 & 147.5955 & 3 . & 289.8 & 3 & & \\
\hline 04 & 617 & 162.6176 & 1.8750 & 289.3 & 4 & 600.0 & 600 . \\
\hline & 618 & 92.1495 & 3.7500 & 288.9 & 4 & 600.0 & 600. \\
\hline & 619 & 321.3402 & 1.9750 & 287.7 & 5 & 600 & 600 \\
\hline 04 & 620 & 347.2943 & 1.4250 & 287.0 & 6 & 600.0 & 600 \\
\hline & 621 & 337.8284 & 1.2250 & 287.2 & 6 & 600.0 & 600 \\
\hline & 622 & 354.6080 & 1.32 & 287.2 & 6 & .0 & 60 \\
\hline & 623 & 23.5044 & 1.1000 & 287.3 & & 600.0 & 600 \\
\hline
\end{tabular}




\begin{tabular}{|c|c|c|c|c|c|c|c|}
\hline & 24 & & 0250 & $36 \cdot 8$ & & & \\
\hline 1 & 1 & 133 & .7750 & 285.6 & 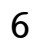 & & \\
\hline & 72 & 4063 & 8000 & 285.4 & 6 & 00 & \\
\hline 4. & 73 & 252.8793 & .9500 & 283.5 & & 00 & \\
\hline & 74 & 138.6652 & 2.8500 & 30.9 & & 0 & \\
\hline & 75 & 0137 & 500 & 81.1 & & 00 & \\
\hline & 76 & 128.3527 & 1.2500 & 281.3 & & 00 & \\
\hline & 77 & 62.8613 & .6500 & 81.8 & & & \\
\hline & 78 & 353.8000 & 1.2750 & 281.7 & J & 0 & \\
\hline & 79 & 336.7389 & 1.2500 & 282.6 & 4 & 00.0 & \\
\hline & 710 & 275.6650 & 2.1500 & 283.4 & 3 & 0 & \\
\hline & 711 & 260.9158 & 1.4250 & 84.6 & 2 & & \\
\hline 4 & 712 & 232.3524 & 2.2250 & 285.4 & 2 & 00 & 0 \\
\hline 14 & 713 & 257.9460 & 2.1000 & 285.9 & 2 & 0.0 & 0 \\
\hline & 714 & 203.1117 & 1.4500 & & 2 & & \\
\hline 4 & 715 & 143.9291 & 2.9250 & 288.4 & 3 & 0 & \\
\hline & 716 & 297.7137 & 2.9500 & 287.2 & 3 & 0 & \\
\hline & 717 & 348.7753 & 2 . & 286 & 4 & & \\
\hline & 718 & 82.3210 & 2.35 & 286.7 & 5 & 0 & \\
\hline & 719 & 84.3894 & 0 & 285.7 & 4 & 0 & \\
\hline 14 & 720 & 69.9311 & 7 . & .0 & 4 & & \\
\hline & 721 & 71.8831 & 50 & 28 & 4 & & \\
\hline 4 & 722 & 42.0782 & $\$ 750$ & 285.1 & 4 & 0 & \\
\hline 4 & 723 & 335.2727 & 1.82 & 284.9 & 5 & 0 & \\
\hline & 724 & 80.2724 & & 283.3 & 5 & & \\
\hline 4 & 81 & 6660 & 500 & 284.7 & 4 & & 0 . \\
\hline 4 & 82 & 64.8066 & 2.3250 & 285.0 & 5 & 0 & \\
\hline 4 & 83 & 73.6186 & 1.65 & 285.3 & 5 & & \\
\hline 04 & 84 & 94.4708 & 1.4000 & 284.7 & 6 & 0 & 0 \\
\hline A & 85 & 89.8488 & 4000 & 283.5 & 5 & 0 & \\
\hline & 86 & 74.7826 & 7. & 28 & 4 & & \\
\hline 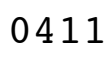 & 87 & 66.4037 & 7 . & .4 & 4 & & 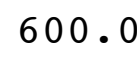 \\
\hline 04 & 88 & 54.1357 & 2.6250 & 283.9 & 4 & 600 & 0 . \\
\hline 04 & 89 & 326.1792 & 50 & & 4 & 0 & \\
\hline & 810 & 921 & 0 & 28 & 3 & & \\
\hline 04 & 811 & 108.3873 & 3.0250 & 284.7 & 3 & 60 & 600 . \\
\hline & 812 & 246.9852 & 2.2500 & 284.3 & 4 & 600 & \\
\hline 04 & 813 & 293.2557 & 1.8250 & 283.9 & 4 & 0 & 0 . \\
\hline 04 & 814 & 280.6197 & 1.8250 & 284.2 & 4 & 600.0 & 0 . \\
\hline & 815 & 292.0679 & 3250 & 284.3 & 4 & 600 & \\
\hline & 816 & 319.2838 & 750 & 28 & 4 & & \\
\hline 04 & 817 & 349.0872 & 1.7000 & 284.2 & 5 & 600.0 & 600 . \\
\hline & 818 & 347.9254 & 1.9000 & 284.1 & 6 & 600 & 600. \\
\hline & 819 & 348.2195 & 1.7750 & 284.3 & 5 & 600.0 & 600 \\
\hline 04 & 820 & 7.6806 & 1.3250 & 284.4 & 6 & 600.0 & 600 . \\
\hline & 821 & 9.5343 & 1.1250 & 284.4 & 6 & 600.0 & 600. \\
\hline & 822 & .5923 & & & 5 & & 600 \\
\hline & 823 & 169.3571 & 0.7750 & 284.4 & 0 & 600.0 & 600 \\
\hline
\end{tabular}




\begin{tabular}{|c|c|c|c|c|c|c|}
\hline 11824 & 90010 & 2.0500 & 284.1 & D & 00.0 & \\
\hline $\begin{array}{lll}119 & 1\end{array}$ & 0.3491 & 2.9750 & 283.8 & & 0 & \\
\hline $\begin{array}{lll}11 & 9 & 2\end{array}$ & 357 & 500 & 84.0 & & 0 & \\
\hline 193 & 216.3844 & 1.6500 & 83.3 & & 00 & \\
\hline 94 & 138.4868 & 1.6750 & 33.1 & & 0 & \\
\hline 195 & 136.7545 & 1.3000 & 83.1 & & 0 & \\
\hline 196 & 150.0571 & 1.7250 & 283.0 & & 00 & \\
\hline 197 & 323 . & .7250 & 83.1 & & 0 & \\
\hline 198 & 352.5004 & 0.7750 & 283.1 & & 00 & \\
\hline 199 & 310.7762 & 1.2250 & 283.6 & & 0 & \\
\hline 1910 & 266.2898 & .1250 & 3 & & & \\
\hline 1911 & 240.7784 & 50 & 85.0 & & & \\
\hline 1912 & 214.3740 & 2.3750 & 285.2 & & 0 & \\
\hline 1913 & 198.2288 & 50 & 35.3 & & & \\
\hline 1914 & 160.7853 & 50 & 9 & & & \\
\hline 1915 & 227.0750 & 250 & 285.3 & 4 & 0 & \\
\hline 1916 & 227.7065 & 0.7250 & 285.4 & & 0 & \\
\hline 1917 & 355.6252 & 0.5750 & & & & \\
\hline 1918 & 074 & 250 & 285 & 6 & & \\
\hline 1919 & 343.3612 & 5750 & 285.0 & & 0 & \\
\hline 920 & 340.4329 & & & & & \\
\hline 921 & 43. & 2 . & 28 & 4 & & \\
\hline 1922 & 114.6448 & .8000 & 285 & & 0 & \\
\hline 923 & 344.8760 & 4000 & 2 & & & \\
\hline 924 & 29. & & & 2 & & \\
\hline 101 & 38.2783 & 000 & 285.7 & 4 & & \\
\hline 1102 & 101.5877 & 3.2000 & 285.4 & 4 & & \\
\hline 1103 & 336.0707 & 250 & & 5 & & \\
\hline $41110 \quad 4$ & 122.0054 & 1.9750 & 283 & 5 & & \\
\hline 411105 & 143.1592 & 2.4000 & 282.8 & 4 & & \\
\hline 411106 & 139.1949 & & 282.6 & 4 & & \\
\hline 1107 & 165.8466 & 00 & .5 & 5 & & \\
\hline 411108 & 292.1047 & 0.9000 & 282.6 & 5 & & \\
\hline 411109 & 357.1376 & 1 . & 282 & 4 & & - \\
\hline 1010 & 328.5989 & 1.6000 & & 4 & & \\
\hline 1011 & 306.0851 & 1.8500 & 283.8 & 3 & & \\
\hline 012 & 284 . & 2 & 28 & 3 & & \\
\hline 1013 & 242.1985 & 1.8250 & 285.0 & 2 & & s \\
\hline 1014 & 177.4552 & 2.4250 & 285.7 & 3 & & 6 \\
\hline 015 & 217.4189 & & & 3 & & \\
\hline 1016 & 214.0877 & 50 & 28 & 3 & 0 & 600 . \\
\hline 1017 & 321.0725 & 1.3750 & 286.0 & 4 & 0 & 0 \\
\hline 1018 & 3.2453 & 50 & 285.9 & 5 & & \\
\hline 1019 & 32.5604 & 2.8750 & 286.0 & 4 & .0 & 600 \\
\hline 1020 & 53.4495 & 3.4500 & 285.4 & 4 & 600.0 & 500. \\
\hline 021 & 39 . & & & 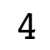 & & \\
\hline 022 & 42.3417 & 2.7500 & & 4 & & 600 \\
\hline 1023 & 36.8699 & 1.7250 & 285.6 & & 600.0 & 600 \\
\hline
\end{tabular}




\begin{tabular}{|c|c|c|c|c|c|c|}
\hline 04111024 & 8.4001 & 2.1000 & 285.0 & 5 & 600.0 & 600.0 \\
\hline 0411111 & 10.4754 & 2.1000 & 285.4 & 5 & 600.0 & 600.0 \\
\hline 0411112 & 255.0464 & 1.1250 & 285.2 & 6 & 600.0 & 00.0 \\
\hline 0411113 & 315.0000 & 0.9500 & 284.6 & 6 & 600.0 & 600.0 \\
\hline 0411114 & 342.1258 & 1.2750 & 284.3 & 6 & 600.0 & 00.0 \\
\hline 0411115 & 331.2021 & 1.6000 & 283.7 & 6 & 600.0 & 00.0 \\
\hline 0411116 & 351.5047 & 1.7250 & 283.3 & 5 & 600.0 & 600.0 \\
\hline 0411117 & 355.6677 & 2.0750 & 283.5 & 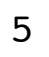 & 600.0 & 00.0 \\
\hline 0411118 & 1.8850 & 2.0500 & 283.8 & 4 & 600.0 & 00.0 \\
\hline 0411119 & 328.6270 & 1.4000 & 284.5 & 4 & 600.0 & 600.0 \\
\hline 04111110 & 308.4570 & 1.9000 & 285.1 & 3 & 600.0 & 600.0 \\
\hline 041 & 296.1753 & 2.6000 & 286.0 & 2 & 600.0 & \\
\hline 04111112 & 308.2304 & 2.4500 & 286.4 & 3 & 600.0 & 600.0 \\
\hline 04111113 & 265.9555 & 3.6000 & 286.2 & 3 & 600.0 & 600.0 \\
\hline 041 & 271.1486 & 500 & 286.2 & 3 & 600.0 & \\
\hline 041 & 275.7256 & 3.1750 & 286.3 & 3 & 600.0 & 60 \\
\hline 04111116 & 295.8505 & 1.8250 & 286.3 & 4 & 600.0 & 600.0 \\
\hline 041 & 303.2788 & 1.8000 & 285.8 & 5 & 600.0 & \\
\hline 041 & 333.8562 & 2.4750 & 285.4 & 5 & 600.0 & 60 \\
\hline 04111119 & 81.8903 & 7.6000 & 284.6 & 4 & 600.0 & 600.0 \\
\hline 041 & 105.8468 & 6.1000 & 284.3 & 4 & 600.0 & .0 \\
\hline 041 & 51.3402 & 3.7250 & 284.2 & 4 & 600.0 & \\
\hline 04111122 & 134.0049 & 1.8250 & 283.7 & 4 & 600.0 & 600.0 \\
\hline 0411 & 147.5177 & 1.8250 & 283.6 & 4 & 600.0 & .0 \\
\hline 041 & 104.6347 & 2.2000 & 283.6 & 4 & 600.0 & \\
\hline 0411121 & 65.3160 & 4.0250 & 283.4 & 4 & 600.0 & 600.0 \\
\hline 0411122 & 151.8584 & 5.1000 & 283.3 & 4 & 600.0 & 600.0 \\
\hline 0411123 & 154.3331 & 5.1000 & 283.2 & 4 & 600.0 & \\
\hline 0411124 & 166.1028 & 4.5750 & 283.2 & 4 & 600.0 & 600.0 \\
\hline 0411125 & 164.0347 & 5.2250 & 283.3 & 4 & 600.0 & 600.0 \\
\hline 0411126 & 168.9145 & 4.4000 & 283.2 & 4 & 600.0 & .0 \\
\hline 0411127 & 145.6698 & 3.6750 & 282.9 & 4 & 600.0 & 60 \\
\hline 0411128 & 142.4314 & 3.4000 & 283.6 & 4 & 600.0 & 600.0 \\
\hline 0411129 & 153.1780 & 4.6750 & 284.1 & 3 & 600.0 & .0 \\
\hline 041 & 139.4767 & 6.3250 & 284.5 & 4 & 600.0 & 60 \\
\hline 0411 & 145.0672 & 4.9500 & 285.8 & 3 & 600.0 & 600.0 \\
\hline 041 & 118.1741 & 5.7000 & 287.0 & 4 & 600.0 & 600.0 \\
\hline 04111213 & 104.5771 & 5.8250 & 287.9 & 4 & 600.0 & 600.0 \\
\hline 04111214 & 107.5620 & 5.8250 & 288.5 & 4 & 600.0 & 600.0 \\
\hline 041 & 116.0388 & 5.5500 & 289.0 & 3 & 600.0 & \\
\hline 041. & 81.5836 & 5.3000 & 288.6 & 3 & 600.0 & 600.0 \\
\hline 0411 & 65.5442 & 7.1500 & 286.8 & 4 & 600.0 & 600.0 \\
\hline 04111218 & 65.6870 & 8.0250 & 286.8 & 4 & 600.0 & 600.0 \\
\hline 0411 & 67.6666 & 7.9250 & 286.9 & 4 & 600.0 & 600.0 \\
\hline 0411 & 68.0652 & 8.0000 & 286.8 & 4 & 600.0 & 600.0 \\
\hline 0411 & 65.0350 & 7.2000 & 286.7 & 4 & 600.0 & 600.0 \\
\hline 0411 & 67.2917 & 7.650 & 28 & 4 & 600.0 & 600.0 \\
\hline 0411 & 64.8446 & 7.7250 & 286.2 & 4 & 600.0 & 600.0 \\
\hline
\end{tabular}




\begin{tabular}{|c|c|c|c|c|c|c|}
\hline 4111224 & 74.5683 & 7.0000 & 286.2 & 4 & 00.0 & \\
\hline 411131 & 0.4436 & 4.1250 & 285.7 & 4 & ח & \\
\hline 411132 & 521 & 3500 & 285.5 & 4 & 0 & \\
\hline 11133 & 69.1583 & .4000 & 284.3 & & 00.0 & \\
\hline 11134 & 4.3077 & .6000 & 84.8 & & 0.0 & \\
\hline 411135 & 5356 & 9000 & 84.9 & & 0 & \\
\hline 411136 & 43.8775 & 6.8000 & 284.6 & & 00 & \\
\hline 411137 & 375 & .5000 & 84.3 & & 0 & \\
\hline 411138 & 136.6192 & 5.6250 & 284.3 & & 00 & \\
\hline 411139 & 157.7692 & 500 & 284 & & 0 & \\
\hline 1310 & 147.4628 & 250 & 7 & & & \\
\hline 1311 & 127.4800 & & 284 & 4 & & \\
\hline 1312 & 142.2794 & 6.2500 & 285.5 & 1 & 0 & \\
\hline 313 & 161. & 500 & 36.9 & 4 & & \\
\hline & 139. & & 87.8 & 4 & & \\
\hline 315 & 139. & 000 & 288.8 & 3 & 0 & \\
\hline 1316 & 133.9733 & 4.5750 & 289.1 & 3 & 0 & \\
\hline & 99.1717 & & 288.7 & 4 & & \\
\hline 18 & 847 & 50 & 288.2 & 4 & & \\
\hline 1319 & 105.7476 & 0 & 288 & 4 & 0 & \\
\hline 20 & 110.7628 & & & 4 & & \\
\hline & 95.1814 & 0 & 28 & 4 & & \\
\hline 4111322 & 97.9072 & 2500 & 288.1 & 4 & 0 & \\
\hline 323 & 137.4604 & 0 & 6 & 4 & & \\
\hline 24 & 123. & & 28 & 4 & & \\
\hline 141 & 5806 & 0 & 287.4 & 5 & & \\
\hline $41114 \quad 2$ & 124.7879 & 2.4000 & 287.1 & 4 & & \\
\hline 411143 & 97.0715 & 0 & 87.1 & 4 & & \\
\hline $41114 \quad 4$ & 128.3773 & 3000 & 286.3 & 5 & & \\
\hline $41114 \quad 5$ & 132.7422 & 500 & 285.4 & 4 & & \\
\hline $41114 \quad 6$ & 144.3116 & & 9 & 4 & & \\
\hline 147 & 150 . & 0 & & 4 & & \\
\hline 0411148 & 38.3675 & 1.32 & 284.2 & 4 & & \\
\hline 149 & 355 . & 0 & & 3 & & \\
\hline 410 & 304 & & & 2 & & \\
\hline 1411 & 270.5891 & & 288.1 & & & \\
\hline 412 & 249.9779 & 1 . & $28^{\circ}$ & 2 & & \\
\hline 413 & 177.9228 & 2 . & 287.2 & 2 & & 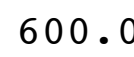 \\
\hline 04111414 & 128.9801 & 2.3000 & 286.0 & 2 & & 6 \\
\hline & 317.1591 & 2 . & 28 & 2 & & \\
\hline 416 & 210.4486 & 1.4000 & 286.5 & 3 & 0 & 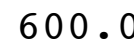 \\
\hline 04111417 & 71.8299 & 1.4500 & 284.4 & 4 & 0 & 0 \\
\hline 04 & 104.6288 & 500 & 284.5 & 5 & & \\
\hline 04111419 & 164.6167 & 1.7000 & 284.5 & 5 & 600.0 & 600 \\
\hline 04111420 & 242.1218 & 1.2750 & 284.4 & 5 & 600.0 & 600 \\
\hline & 312.8030 & & & - & & \\
\hline & 335.7520 & 1.5750 & 284.4 & 5 & & 600 \\
\hline 423 & 335.8908 & 1.6500 & 284.3 & & 600.0 & 600 \\
\hline
\end{tabular}




\begin{tabular}{|c|c|c|c|c|c|c|}
\hline$\pm<4$ & & .4000 & & & & \\
\hline 11151 & 3 & .4250 & 4.0 & & b & \\
\hline 11152 & 7009 & 1.6000 & 284.0 & & 00 & \\
\hline 11153 & 340.1410 & 1.3750 & 284.0 & & 00 & \\
\hline 1154 & 3349164 & .2500 & 83.9 & & 0 & \\
\hline 1155 & 71 &. .5250 & 283.9 & & 00 & \\
\hline 11156 & 5082 & 1.5000 & 283.8 & & 500 & U \\
\hline 11157 & 6.4064 & .9250 & 83.8 & & 00.0 & \\
\hline 11158 & 75.5055 & .6750 & 83.7 & & & \\
\hline 159 & 170.7308 & 1.3750 & 284.2 & & 600.0 & 0 \\
\hline 1510 & 159.2926 & 2.0000 & 285.3 & & 0 & \\
\hline & 255.7355 & 1.7750 & 86.1 & & 00.0 & \\
\hline 512 & 247.5022 & 1.7000 & 286.7 & & .0 & ( \\
\hline 513 & 267.6467 & 1.8500 & 286.7 & & 0.0 & \\
\hline & 286.1892 & 1.37 & & & & \\
\hline & 271.4441 & 1.50 & 286.7 & & & \\
\hline 516 & 350.2673 & 1.3000 & 286.3 & & .0 & 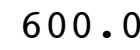 \\
\hline 517 & 42.3246 & 1.3000 & 9 & & 0 & \\
\hline & 74.0191 & 1.4000 & 28 & & & \\
\hline 519 & 3666 & 1.0750 & 28 & & & \\
\hline 1520 & 41.0275 & 1.5750 & 284.5 & & 0 & 00 . \\
\hline & 156.2864 & 11 & & & & \\
\hline 22 & 168.7463 & 0.8250 & 28 & 5 & & \\
\hline 523 & 183.2798 & 1.2250 & 282.9 & & 0 & \\
\hline 524 & 118.8013 & 1.10 & & & & \\
\hline 61 & 18.8384 & 0.6750 & 282.5 & 5 & & \\
\hline 411162 & 71.5651 & 1.3500 & 282.7 & 5 & 0 & J. \\
\hline 411163 & 92.0273 & 1.7000 & 281.8 & 5 & & \\
\hline 0411164 & 80.7667 & 2.4500 & 28 & 4 & & \\
\hline 411165 & 120.1578 & 1.0250 & 281.5 & 5 & 0 & 0. \\
\hline 411166 & 138.8545 & 1.8750 & 280.6 & 5 & 0 & 0 . \\
\hline 411167 & 138.3427 & 30 & 28 & 4 & & \\
\hline 411168 & 144.1947 & 3 . & 281.5 & 4 & & 0 \\
\hline 411169 & 160.2673 & 1.0500 & 282.5 & 3 & 0 & \\
\hline 41 & 202.6677 & 0.8 & 283.8 & 2 & & \\
\hline 04 & 176.6506 & 3.0750 & 283.7 & 2 & & 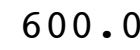 \\
\hline 4111612 & 159.6033 & 2.5500 & 283.7 & 2 & .0 & 0 \\
\hline 613 & 179.5637 & 1.9500 & 285 & 2 & & . \\
\hline 041 & 166.6938 & 3.3250 & 284.2 & 3 & .0 & 0 . \\
\hline 4111615 & 156.4444 & 3.7250 & 284.3 & 4 & & \\
\hline 616 & 121.3528 & 2.4500 & 284.5 & 4 & & 0 . \\
\hline 041 & 145.2320 & 2.1500 & 284.4 & 4 & .0 & 00 . \\
\hline 4111618 & 145.5137 & 3.1750 & 284.0 & 4 & .0 & 00 . \\
\hline 619 & 141.8990 & 3.9500 & 284.7 & 4 & & \\
\hline 04111620 & 142.5276 & 3.4500 & 284.5 & 4 & 600.0 & 600. \\
\hline 0411 & 135.1323 & 1.6250 & 285.2 & 5 & 600.0 & 600. \\
\hline & 124 . & & & 5 & & \\
\hline 41 & 83.5412 & 0.6000 & 284.4 & & 600.0 & 60 \\
\hline
\end{tabular}




\begin{tabular}{|c|c|c|c|c|c|c|}
\hline 1624 & (2) & 0.3000 & 284.1 & 5 & 600 & \\
\hline $41117 \quad 1$ & 134.5863 & 0.9000 & 84.1 & & 600 & \\
\hline 11172 & 5672 & 6250 & 284.4 & 5 & 00 & \\
\hline 11173 & 228.6127 & 4000 & 283.6 & & 00 & \\
\hline 1174 & 199.6538 & .6750 & 3.4 & & 0 & \\
\hline 175 & 136.1154 & 6750 & 83.8 & & 00 & \\
\hline 411176 & 155.1943 & 1.9250 & 284.0 & & 0 & \\
\hline 1177 & 99.0476 & .0250 & 83.8 & & & \\
\hline 1178 & 32.3394 & .6000 & 284.8 & & 0 & \\
\hline 411179 & 141.2954 & 2.0750 & 286.3 & & 00 & \\
\hline 1710 & 160.8803 & 2.3000 & 286.6 & & 0 & \\
\hline & 183.3274 & 1.6000 & & & & \\
\hline 1712 & 201.2886 & 2.2750 & 289.5 & & 0 & \\
\hline 1713 & 271.7075 & 2.17 & 289.5 & & 0 & \\
\hline 714 & 288.6186 & & & & & \\
\hline .715 & 266.2717 & 1.7750 & 289.5 & & 0 & \\
\hline 1716 & 293.3314 & 1.6750 & 288.5 & & 0 & \\
\hline 717 & 188.6156 & 0.72 & 86.8 & & & \\
\hline 718 & 49.8739 & 1. & 285.2 & & 0 & \\
\hline 1719 & 34.2711 & 8000 & 284.5 & & & \\
\hline 720 & 18.8432 & 0 & 284.3 & & & \\
\hline 721 & 106.6738 & & 285.1 & J & & \\
\hline .722 & 146.4292 & 2.4000 & 285.2 & 4 & 0 & • \\
\hline 723 & 134 & .2500 & 285.2 & & 0 & \\
\hline 724 & 105 . & & 284.6 & 5 & & \\
\hline 81 & 214.6320 & 0 & 28 & 5 & & \\
\hline 411182 & 139.4376 & 0 & 284.0 & 5 & 0 & \\
\hline 411183 & 114.1284 & 0 & 283.2 & & & \\
\hline 184 & 958 & 1 & .3 & 5 & & 0 . \\
\hline 1185 & 140.8922 & 1. & 281.8 & 5 & 0 & \\
\hline 186 & 126.0514 & 0 & 28 & 5 & & \\
\hline 187 & 112 . & 0 & 279.8 & 6 & & \\
\hline 0411188 & 103.4957 & 9500 & 283.2 & 2 & 0 & J. \\
\hline 189 & 161. & 0 & 280.6 & 4 & & \\
\hline 310 & 167 . & 1. & 281.3 & 3 & & \\
\hline 311 & 251.5026 & 1 . & 282.1 & 4 & & 0 . \\
\hline 812 & 352.7311 & 000 & 282.7 & 3 & 0 & \\
\hline 313 & 175.8935 & 0 & 282.6 & 3 & & 0 . \\
\hline 814 & 145.4077 & 250 & 282.4 & 3 & 0 & 0 . \\
\hline 1815 & 82.1467 & & 283.6 & 3 & 0 & \\
\hline 816 & 12.9528 & & 282.9 & 4 & & \\
\hline 817 & 7.4882 & 1.8500 & 282.7 & 4 & 600.0 & 600 . \\
\hline 1818 & 352.5686 & 1.7750 & 282.5 & 4 & 600 & 00 \\
\hline 319 & 189.1694 & 1.3250 & 282.1 & 5 & & 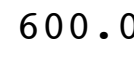 \\
\hline 820 & 10.0584 & 2.6500 & 281.7 & 4 & 600.0 & 00 \\
\hline 82 & 323.5158 & 1.9750 & 281.3 & 4 & 600.0 & 600 . \\
\hline & 237.2361 & 50 & 281.4 & 4 & 60 & \\
\hline 823 & 315.1661 & 1.7250 & 281.6 & 4 & 600.0 & 600 \\
\hline
\end{tabular}




\begin{tabular}{|c|c|c|c|c|c|c|}
\hline & & & & & & \\
\hline 1191 & 1260 & 6000 & 1.5 & & & \\
\hline 1192 & 669 & 1250 & 81.4 & & 00 & \\
\hline 11193 & 131.6726 & 2500 & 281.6 & & 0 & \\
\hline 1194 & 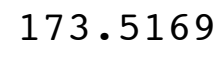 & & 1.5 & & & \\
\hline 1195 & 57 & & 81.3 & & & \\
\hline 11196 & 3908 & 6250 & 281.2 & & 00 & \\
\hline 11197 & 148 & 5500 & 81.1 & & & \\
\hline 1198 & & & 81.4 & & & \\
\hline 199 & 130 & 000 & 81.6 & & 0 & \\
\hline 1910 & 134 & 4.4500 & 282.1 & & & \\
\hline & 140 & 7.5750 & 82.7 & & & \\
\hline 912 & 149 & 9.3500 & 283.2 & & & \\
\hline 913 & 148 & 7.7500 & 283.7 & & & \\
\hline & 149 & & & & & \\
\hline & 151 & & 284.8 & 3 & & \\
\hline 916 & 130 & 7500 & 285.3 & & & \\
\hline 917 & 105 & 0 & .1 & & & \\
\hline & 14 & & .7 & & & \\
\hline 19 & 149 & 0 & 284 & 4 & & \\
\hline 1920 & 156.57 & 9000 & 284.3 & 4 & 0 & \\
\hline 21 & & & & 4 & & \\
\hline 922 & 159 & 50 & 283.9 & 4 & & \\
\hline 923 & 145 . & 50 & .3 & 4 & & \\
\hline 924 & 145 & & & 4 & & \\
\hline 0 & 12 & & 282.1 & 4 & & \\
\hline 411202 & 134.6936 & 500 & 281.5 & 4 & & \\
\hline 411203 & 123 & 0 & 31.4 & 4 & & \\
\hline $41120 \quad 4$ & 127 & & & 4 & & \\
\hline $41120 \quad 5$ & 145 . & & 0.0 & 4 & & \\
\hline 411206 & 145.9123 & 5000 & 279.2 & 4 & & \\
\hline $41120 \quad 7$ & 149 . & 0 & 79.1 & 4 & & \\
\hline 411208 & 142 & & 280.1 & 4 & & \\
\hline 411209 & 131. & 50 & 281.7 & 3 & & \\
\hline 2010 & 110 & 0 & 28 & 3 & & \\
\hline 011 & & & 3.7 & 4 & & \\
\hline 4112012 & 148.6660 & 9.4250 & 284.2 & 4 & & \\
\hline 2013 & 154 . & 9. & & 4 & & \\
\hline 2014 & 153. & 8.5250 & 286.4 & 4 & & \\
\hline 4112015 & 140.0662 & 1500 & 287.7 & 4 & & \\
\hline 2016 & 154.5923 & 000 & & 4 & & \\
\hline 2017 & 146.8677 & 12.0750 & .7 & 4 & & 0 . \\
\hline 411201 & 155.2950 & 13.3000 & 285.7 & 4 & & 0 \\
\hline 2019 & 160 . & 10 . & & 4 & & \\
\hline 04112020 & 162.9711 & 8.8750 & & 1 & & \\
\hline 411202 & 176.5492 & 10.2750 & 282.3 & 4 & 0 & 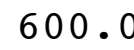 \\
\hline & 150 & & & 4 & & \\
\hline 411202 & 7128 & 9.1000 & 281.1 & & .0 & s \\
\hline
\end{tabular}




\begin{tabular}{|c|c|c|c|c|c|c|}
\hline & & & & & & \\
\hline 1211 & 181 & & 0 & & & \\
\hline 1212 & 171.504 & 7.5750 & 79.2 & & 0 & \\
\hline 11213 & 6150 & 9500 & 278.8 & & 0 & \\
\hline 1214 & & & & & & \\
\hline 1215 & 53 & 0 & 77.4 & & & \\
\hline 11216 & 3870 & 0750 & 277.6 & & 00 & \\
\hline 11217 & 548 & 0 & 277.8 & & & \\
\hline 11218 & 56 & 0 & 78.6 & & & \\
\hline 411219 & 158.1188 & 13.2500 & 279.2 & & 0 & \\
\hline 2110 & 162.1566 & 12.2000 & 279 & & & \\
\hline & 1648835 & 2.0500 & 80.9 & & & \\
\hline 2 & 152 & 11 . & 282.2 & & & \\
\hline 3 & 163.2870 & 2.2000 & 283 & & & \\
\hline & 164 & 0 & & & & \\
\hline & 171 & & 28 & 4 & & \\
\hline 16 & 157.6904 & 10.4750 & 284.9 & 4 & & \\
\hline & 145.6321 & & 4 & & & \\
\hline & 147.6606 & 0 & 28 & 4 & & \\
\hline$y$ & 148.6180 & 0 & 283 & 4 & & \\
\hline 20 & 150.1663 & 11.2750 & & 4 & & \\
\hline & 153.6916 & 11 . & & 4 & & \\
\hline 2 & 15 & 0 & 28 & 4 & & \\
\hline 23 & 151.4468 & 0 & 282.6 & 4 & & \\
\hline & 153. & & & 4 & & \\
\hline 1 & 149 . & & 28 & 4 & & \\
\hline 411222 & 147.6606 & & 282.1 & 4 & & \\
\hline 411223 & 146 . & & 281 & 4 & & \\
\hline 24 & 125 & & 28 & 4 & & \\
\hline 411225 & 97.7846 & & 1.1 & 4 & & \\
\hline 411226 & 129.3607 & 0 & 1.5 & 4 & & \\
\hline 411227 & 133.17 & 0 & & 4 & & \\
\hline 411228 & 137.74 & & 28 & 4 & & \\
\hline 411229 & 112.6199 & & 28 & 3 & & \\
\hline 10 & 145 & & & 2 & & \\
\hline & 193. & & .5 & 2 & & \\
\hline 12 & 271.10 & 00 & 287.7 & 2 & & \\
\hline 12 & 227 . & & 287 & 2 & & \\
\hline 41 & 149 . & & .1 & 3 & & \\
\hline & 139. & & 286.8 & 3 & & \\
\hline & 143.4495 & 00 & 287.6 & 3 & & \\
\hline 41 & 183.2397 & 1.4500 & 286.8 & 4 & & \\
\hline 1 & 224.3806 & 1.2250 & 284.0 & 5 & & 0 \\
\hline 219 & 353.6271 & 50 & & 5 & & \\
\hline 04112220 & & 1.5500 & & 5 & & \\
\hline 122 & 350.0845 & 1.6500 & 283.7 & 5 & & (20 \\
\hline & & & & כ & & \\
\hline & 14.3489 & 0.6500 & 283.5 & & & \\
\hline
\end{tabular}




\begin{tabular}{|c|c|c|c|c|c|c|}
\hline 2224 & & 5750 & & & & \\
\hline 11231 & 7811 & 2.4000 & 33.6 & 4 & & \\
\hline 11232 & 140.5796 & 1500 & 282.7 & 4 & 0 & \\
\hline 11233 & 139.7721 & 4.5250 & 281.8 & & 00 & \\
\hline 11234 & .5609 & 4.9500 & 0.6 & & 0 & \\
\hline 1235 & 8788 & .1500 & 80.1 & 4 & 00 & \\
\hline 411236 & 136.5371 & 4.0750 & 280.1 & & 00 & \\
\hline 411237 & L.9133 & 7500 & 80.6 & & & \\
\hline 411238 & 133.8846 & 3.2500 & 279.8 & 4 & 0 & \\
\hline 411239 & 136.1233 & 2.6750 & 280.3 & & 600.0 & \\
\hline 310 & 161.4287 & 3.85 & 281.7 & 3 & 0 & \\
\hline & 158.1986 & & 83.1 & 3 & & \\
\hline 41 & 159.2093 & 4 . & 283.7 & 3 & 00 & \\
\hline 313 & 156.3295 & 4.6250 & 284.0 & 3 & 0.0 & 0 \\
\hline & 156.2558 & & 285.1 & 3 & & \\
\hline 15 & 146.8261 & 4.32 & 285.6 & 3 & 0 & \\
\hline 316 & 143.4754 & 3.07 & 285.3 & 3 & 0 & \\
\hline 17 & 797 & 3 . & 284.8 & 4 & & \\
\hline 8 & 133.6725 & 4 . & 284.6 & 4 & 0 & \\
\hline 19 & 109.7933 & 2000 & 28 & 4 & & \\
\hline 20 & 100.8855 & 3 . & 284.9 & 4 & 0 & \\
\hline & 076 & & 284.7 & 4 & & \\
\hline 4 & 2578 & 000 & 28 & 4 & 0 & \\
\hline 23 & 320.8143 & 1 . & 283.4 & 5 & 0 & \\
\hline 24 & 304 . & & & 5 & & \\
\hline 41 & 7029 & 0 & 282.5 & 5 & & \\
\hline 411242 & 66.4768 & 1.32 & 282.5 & 5 & 0 & \\
\hline 0411243 & 188 & & 282.7 & 5 & 0 & $\mathrm{C}$ \\
\hline $041124 \quad 4$ & 374 & 0 & 282.0 & 5 & 0 & 0 . \\
\hline 0411245 & 104.6709 & 0 & 282.4 & 5 & 0 & \\
\hline 411246 & 10.2099 & & 282.1 & 5 & & \\
\hline 247 & 338.4824 & 0 & 282 & 5 & & 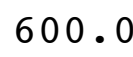 \\
\hline 0411248 & 253.4742 & 0 & 282.5 & 4 & 0 & 6 \\
\hline 411249 & 229.2758 & 0 & 28 & 3 & 0 & \\
\hline 10 & 251 . & 1. & 28 & 2 & & \\
\hline 041 & 274.0964 & 1.8250 & 287.2 & 2 & 0 & 0 . \\
\hline 41 & 231.0090 & 000 & 287.5 & 2 & 600 & \\
\hline 041 & 299.7630 & 2 & 288.0 & 2 & 0 & 0 . \\
\hline 041 & 297.1081 & 2.6500 & 288.4 & 3 & 600.0 & 0 . \\
\hline & 317.3416 & 2 . & 288 & 3 & 600 & \\
\hline 16 & 289.9204 & 1 . & & 3 & & \\
\hline 041 & 281.0235 & 1.3250 & 286.9 & 4 & 600.0 & 600 . \\
\hline 041 & 0.1504 & 1.8750 & 285.0 & 4 & 600 & 600. \\
\hline 041 & 76.6890 & 2.2750 & 284.8 & 4 & 600 & 600 \\
\hline 041 & 92.7609 & 3.6250 & 286.0 & 4 & 600.0 & 600 . \\
\hline 04 & 150.4014 & 1.6250 & 285.7 & 5 & 600.0 & 600 \\
\hline & 3046 & 0 . & & 5 & 600 & 60 \\
\hline 423 & 243.5538 & 0.9750 & 284.4 & $J$ & 600.0 & 600 \\
\hline
\end{tabular}




\begin{tabular}{|c|c|c|c|c|c|c|}
\hline 2424 & 39.35 & 0.9000 & 3.9 & D & 000.0 & \\
\hline $41125 \quad 1$ & 53.5836 & 2.6000 & 284.6 & 4 & 600.0 & \\
\hline 11252 & 9759 & 7750 & 85.1 & 5 & v & \\
\hline 11253 & 136.7419 & 2.4500 & 284.3 & & 00.0 & \\
\hline 11254 & 31.1416 & 2.1250 & 84.1 & & 0 & \\
\hline 411255 & 86.5318 & 1.1000 & 83.5 & & 0 & \\
\hline 411256 & 91.2825 & 1.4750 & 283.3 & & 00 & \\
\hline 411257 & 308.9483 & 1.4500 & 82.2 & & 0 & \\
\hline 411258 & 48.8925 & 0.9250 & 281.7 & & 00 & \\
\hline 411259 & 1.9043 & 1.8750 & 283.5 & & 0 & \\
\hline 2510 & 275 . & 1.5000 & 9 & & 0 & \\
\hline & 133. & 1.4250 & 286 & & & \\
\hline 12 & 99. & 3.3000 & 286.5 & & 00.0 & \\
\hline 13 & 87 . & .2500 & 4 & & & \\
\hline & 133. & & 285 & 3 & & \\
\hline 15 & 76 . & 2.6000 & 286.8 & & 0 & \\
\hline 16 & 89.7983 & 2.7500 & 286.0 & & 0 & \\
\hline & 79.5 & .8250 & & 4 & & \\
\hline 18 & 62 . & 0 & 286 & 4 & & \\
\hline 519 & 240 . & .0000 & 285.2 & 4 & 0 & \\
\hline 20 & 144 . & 0 & & 4 & & \\
\hline & 165. & 750 & 28 & 4 & & \\
\hline 522 & 156.8391 & .2000 & 282.8 & 4 & 0 & \\
\hline 23 & 156 . & .1000 & 5 & 4 & & \\
\hline & 155 . & 50 & 281.7 & 4 & & \\
\hline 61 & 138.6791 & 2429 & 281.2 & 4 & & \\
\hline 411262 & 153.1766 & 7.1500 & 280 & 4 & & \\
\hline 411263 & 145 . & 0 & & 4 & & \\
\hline $41126 \quad 4$ & 147.7500 & 0250 & 280.6 & 4 & & \\
\hline 411265 & 130.6324 & 4.2250 & 280.8 & 4 & & \\
\hline 411266 & 122 . & 50 & & 4 & & \\
\hline 267 & 126 . & 0 & L. 0 & 4 & & \\
\hline 411268 & 145.5948 & 5250 & 281.1 & 3 & & \\
\hline 411269 & 138 . & 0 & 283 & 2 & & \\
\hline & 158 . & & & 2 & & \\
\hline 511 & 157.8781 & 4.8000 & 284.3 & 3 & & \\
\hline 12 & 161.4287 & 5750 & & 4 & & \\
\hline 13 & 177 . & 500 & 285.0 & 3 & & 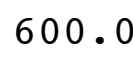 \\
\hline 04112614 & 205.3674 & 250 & 285.2 & 3 & & 6 \\
\hline & 227 . & & 28 & 4 & & \\
\hline 04112 & 204.5151 & 2 . & 284.8 & 4 & 0 & 6 \\
\hline 04112617 & 192.4424 & 1.6000 & 284.3 & 5 & 0 & 0 \\
\hline 04112618 & 297.9198 & 1.6000 & 283.7 & 4 & & \\
\hline 04112619 & 292.5609 & 1.0750 & 283.5 & 5 & 600.0 & 600. \\
\hline 04112620 & 341.6570 & 1.6500 & 283.2 & 4 & 600.0 & 600. \\
\hline & 354 & & & 4 & & \\
\hline & 333.3034 & 1.9000 & & 4 & & 600 \\
\hline 623 & 359.5593 & 1.2500 & 282.9 & & 600.0 & 600 \\
\hline
\end{tabular}




\begin{tabular}{|c|c|c|c|c|c|c|}
\hline 12624 & 8.0692 & 1.9750 & 283.0 & 4 & 600.0 & 00.0 \\
\hline $41127 \quad 1$ & 141.7233 & 2.7500 & 283.1 & 4 & 600.0 & 00,0 \\
\hline 0411272 & 9444 & 2.4000 & 282.9 & 4 & 600.0 & 00.0 \\
\hline 0411273 & 6.3228 & 1.4000 & 282.9 & 4 & 600.0 & 00.0 \\
\hline $041127 \quad 4$ & 308.7278 & 2.0500 & 282.9 & 4 & 600.0 & 0.0 \\
\hline 0411275 & 282.2953 & 1.7250 & 282.7 & 4 & 600.0 & 00.0 \\
\hline 0411276 & 354.9384 & 4.9500 & 284.4 & 4 & 600.0 & \\
\hline $041127 \quad 7$ & 20.0689 & 6.4000 & 285.9 & 4 & 600.0 & 00.0 \\
\hline 0411278 & 26.3721 & 6.3250 & 285.9 & 4 & 600.0 & .0 \\
\hline 0411279 & 107.1389 & 12.3000 & 283.5 & 4 & 600.0 & \\
\hline 04112710 & 160.7505 & 14.7500 & 279.3 & 4 & 600.0 & .0 \\
\hline 2711 & 154.3331 & 12.5 & 279.1 & 4 & 600.0 & \\
\hline 04112712 & 146.6278 & 11.3750 & 280.6 & 4 & 600.0 & .0 \\
\hline 04112713 & 145.1140 & 10.6750 & 282.8 & 4 & 600.0 & 0 \\
\hline 12714 & 153.4992 & & 283.8 & 4 & 00.0 & \\
\hline 2715 & 162.6545 & 15.4250 & 283.9 & 4 & 600.0 & \\
\hline 04112716 & 157.7992 & 14.9000 & 283.7 & 4 & 600.0 & \\
\hline 2717 & 155.4849 & 12.5 & 283.1 & 4 & 00.0 & \\
\hline 2718 & 149.9706 & 14.9000 & 282.6 & 4 & 600.0 & \\
\hline 04112719 & 148.5704 & 16.62 & 282.2 & 4 & 600.0 & \\
\hline 2720 & 152.4085 & 16.6 & 281.9 & 4 & 600.0 & \\
\hline 721 & 153.3071 & 16 . & 281.3 & 4 & .0 & \\
\hline 2722 & 150.7361 & 15.5000 & 280.9 & 4 & 600.0 & .0 \\
\hline 12723 & 149.2578 & 14 . & 280.6 & 4 & 600.0 & \\
\hline 724 & 146.9461 & 15 . & 280.1 & 4 & 0.0 & \\
\hline 281 & 148.3738 & 15 . & 279.5 & 4 & 600.0 & \\
\hline 0411282 & 149.0117 & 14.52 & 279.2 & 4 & 600.0 & .0 \\
\hline 0411283 & 146.3498 & 13.7 & 278.9 & 4 & 0.0 & \\
\hline $041128 \quad 4$ & 147.0664 & 13 & 278.6 & 4 & .0 & \\
\hline 0411285 & 150.6110 & 14.00 & 278.4 & 4 & 600.0 & \\
\hline 0411286 & 150.2907 & 13. & 278.1 & 4 & 0.0 & \\
\hline 0411287 & 150.5566 & 14 . & 277.7 & 4 & 600.0 & \\
\hline 0411288 & 150.5566 & 14.20 & 277.9 & 4 & 600.0 & .0 \\
\hline 0411289 & 148.2986 & 13 & 278.4 & 4 & 600.0 & \\
\hline 810 & 149.5272 & 12. & 27 & 4 & .0 & \\
\hline 04112811 & 154.2709 & 13.12 & 279.9 & 4 & 600.0 & 60 \\
\hline 04112812 & 160.8857 & 14.1000 & 280.8 & 4 & 600.0 & \\
\hline 04112813 & 160.8387 & 14.30 & 281.3 & 4 & 600.0 & .0 \\
\hline 04112814 & 158.6527 & 14.0250 & 281.8 & 4 & 600.0 & .0 \\
\hline 2815 & 159.7964 & 12.12 & 281.9 & 4 & 600.0 & \\
\hline 2816 & 156.7073 & 12.40 & 28 & 4 & 600.0 & 60 \\
\hline 04112817 & 149.1843 & 10.9250 & 280.8 & 4 & 600.0 & 600.0 \\
\hline 04112818 & 145.3955 & 10.8750 & 280.6 & 4 & 600.0 & \\
\hline 04112819 & 147.9794 & 12.1250 & 280.4 & 4 & 600.0 & 600.0 \\
\hline 04112820 & 151.4468 & 12.9250 & 280.4 & 4 & 600.0 & 600.0 \\
\hline 04112821 & 155.4849 & 10.7750 & 279.6 & 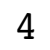 & 600.0 & 600.0 \\
\hline 04112822 & 147.2301 & 9.22 & & 4 & 600.0 & 600.0 \\
\hline 04112823 & 144.1623 & 10.625 & 278.4 & 4 & 600.0 & 600.0 \\
\hline
\end{tabular}




\begin{tabular}{|c|c|c|c|c|c|c|}
\hline 34 & & & 7.6 & & & \\
\hline 11291 & 142.9295 & .2750 & 277.2 & & & \\
\hline 1292 & 320 & 5250 & 277.0 & 4 & 00 & \\
\hline 11293 & 46.8677 & 10.2750 & 276.9 & & 00 & \\
\hline 1294 & & 0.0250 & 76.6 & & & \\
\hline 1295 & 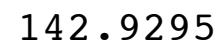 & 8.9250 & 276.3 & & 0 & \\
\hline 11296 & 42.4402 & 7.2500 & 276.1 & & 00 & \\
\hline 11297 & 584 & 6.5250 & 275.6 & & & \\
\hline 11298 & & 3750 & 75.6 & & & \\
\hline 11299 & 06.6291 & 5.5250 & 276.4 & & 00 & - \\
\hline 2910 & 131.8484 & 5.6250 & 277.1 & & & \\
\hline 11 & $150 \quad 2260$ & 5.9500 & 78.2 & 4 & & \\
\hline 2 & 161.33 & 5.2500 & 279.1 & 3 & 0 & \\
\hline 913 & 164.6065 & 5.0250 & 279.7 & & & \\
\hline 14 & 27 & 0 & 280.3 & & & \\
\hline & 0 & & 280.6 & 2 & & \\
\hline 6 & 311.6726 & 1.3500 & 281.5 & $a_{1}+2$ & 0 & \\
\hline 17 & 343.8958 & 1 . & 0.3 & 4 & & \\
\hline & & & 279.1 & 4 & & \\
\hline 19 & 2422 & 2 . & 279.2 & 4 & & \\
\hline 20 & 76.8153 & 0.82 & 278.8 & 5 & 0 & \\
\hline 21 & 129.0776 & & 278.4 & 3 & & \\
\hline 22 & 0 . & 0 & 277.8 & 5 & & \\
\hline 23 & 227.2906 & 0.87 & 277.8 & 5 & 0 & \\
\hline 24 & 145.7131 & & .7 & $\mathbf{u}^{2}$ & & \\
\hline 4 & 146 . & & 277.2 & 4 & & \\
\hline 411302 & 136.0276 & 1. & 276.9 & 4 & & \\
\hline 411303 & 101.6244 & & 276.5 & 5 & & \\
\hline $30 \quad 4$ & 266.1859 & 0 . & 276.3 & 5 & & \\
\hline 41130 & 134.2748 & 1 . & 276.0 & 5 & & \\
\hline 411306 & 137.4366 & 1.5000 & 276.6 & 5 & & \\
\hline $41130 \quad 7$ & 141.7456 & 162 & 276.7 & 5 & & \\
\hline 41130 & 126.1989 & 000 & 277.2 & 4 & & \\
\hline 411309 & 135.5125 & 1.4250 & 277.9 & 3 & 0 & \\
\hline 10 & 143.5609 & 000 & 279.9 & 2 & & \\
\hline 11 & 142.4402 & 1 . & 280.0 & 2 & & \\
\hline 12 & 175.5888 & 4.1250 & 280.6 & 3 & & \\
\hline 13 & 156.3295 & 3. & 280.1 & 3 & & \\
\hline 041 & 160.4721 & 4.6250 & 280.4 & 3 & & - \\
\hline 4113015 & 156.8391 & 4.2000 & 280.4 & 3 & & \\
\hline 4 & 151.2521 & 750 & 280.3 & 4 & & \\
\hline 041 & 146.7476 & 3.2000 & 279.7 & 4 & & \\
\hline 4113018 & 139.3676 & 2.8000 & 279.0 & 4 & & $a_{0}+2$ \\
\hline 019 & 135.6095 & 000 & 278.9 & 4 & & \\
\hline 04113020 & 146.3498 & 4.1750 & 278.5 & 4 & & . \\
\hline 4113 & 127.0161 & 2.2250 & 278.1 & 4 & & 00. \\
\hline & & & 278.6 & 4 & & \\
\hline 411 & 106.3004 & 3.8500 & 278.5 & & .0 & 60 \\
\hline
\end{tabular}




\begin{tabular}{|c|c|c|c|c|c|c|c|}
\hline 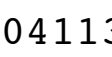 & & (2) & 000 & 278.9 & & & \\
\hline 412 & 11 & 3998 & 4.9515 & 278.9 & 4 & $6 \cap 00$ & \\
\hline 412 & 12 & 122 & 3205 & 278.7 & 4 & 00 & \\
\hline 12 & 13 & 84.0776 & 4.0850 & 278.8 & & 00.0 & \\
\hline & 14 & 103.2403 & 1.2363 & 279.3 & & 0 & \\
\hline & 15 & 5270 & 6243 & 279.6 & 4 & 00 & \\
\hline$x+2$ & 16 & 133.6578 & 3460 & 279.5 & & 0 & \\
\hline 1.0 & 17 & 150.5750 & .8688 & 279.4 & & & \\
\hline 12 & 18 & 111.8744 & .1645 & 279.2 & 4 & 0 & \\
\hline 41 & 19 & 106.4535 & 8593 & 280.8 & & 00.0 & \\
\hline 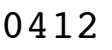 & 110 & 134.3824 & 5.8035 & 281.3 & & 0.0 & \\
\hline & 111 & 143.0753 & & 31.7 & $I$ & & \\
\hline 412 & 112 & 145.8646 & 578 & 282.0 & 4 & 0 & \\
\hline 412 & 113 & 147.7752 & 8.2130 & 282.3 & 4 & 00.0 & \\
\hline & 114 & 144.4752 & & 282.4 & 4 & & \\
\hline 412 & 115 & 146 & 8 & 282.3 & 3 & 0 & \\
\hline 2 & 116 & 143.5544 & 8 & 282.3 & 3 & 0 & \\
\hline 2 & 117 & 136.2528 & 3 & 1.3 & 4 & & \\
\hline 412 & 118 & 141.7127 & 4 . & .3 & 4 & 0 & \\
\hline 2 & 119 & 133 & 8 & & 4 & & \\
\hline 2 & 120 & 113.9555 & 3. & .6 & 4 & & \\
\hline 2 & 121 & 77.4724 & & & 4 & & \\
\hline 412 & 122 & 126.9121 & & .9 & 4 & 0 & \\
\hline 412 & 123 & 146.2994 & 3 . & .3 & 4 & 0 & \\
\hline 412 & 124 & 149.5246 & & 9.8 & 4 & & \\
\hline 412 & 21 & 3795 & 85 & 279.8 & 4 & & \\
\hline 412 & 22 & 113.0237 & 0 & 9.4 & 4 & 0 & \\
\hline 412 & 23 & 132.9463 & 55 & .7 & 4 & 0 & \\
\hline 0412 & 24 & 258 & 73 & 7.8 & 4 & 0 & 0 . \\
\hline 0412 & 25 & 132.1559 & 95 & 6.5 & 4 & 0 & \\
\hline 412 & 26 & 134.0771 & 3 & 2 & 4 & & \\
\hline 412 & 27 & 135 . & 35 & 5.7 & 4 & & . \\
\hline 0412 & 28 & 131.8298 & 325 & 276.6 & 4 & 0 & J \\
\hline 0412 & 29 & 123 & 928 & & 3 & & \\
\hline 412 & 210 & 735 & 40 & .3 & 2 & & \\
\hline 0412 & 211 & 151.2885 & 748 & 279.8 & 3 & & 0 . \\
\hline 412 & 212 & 146.8265 & 278 & 280.9 & 3 & 0 & \\
\hline 0412 & 213 & 152.1485 & 475 & 281.4 & 4 & 0 & 0 . \\
\hline 0412 & 214 & 163.2251 & 7.6585 & 281.7 & 4 & .0 & 0 . \\
\hline 412 & 215 & 162 . & 438 & 282.4 & 3 & 0 & \\
\hline 0412 & 216 & 136.3453 & 468 & 283.0 & 3 & & \\
\hline 0412 & 217 & 105.7899 & 2.1985 & 282.6 & 4 & 600.0 & 600 . \\
\hline 0412 & 218 & 126.3314 & 9483 & 281.6 & 4 & 600 & 600. \\
\hline 0412 & 219 & 141.6249 & 3.8850 & 281.7 & 4 & 600 & 600 \\
\hline 0412 & 220 & 141.1251 & 6.0405 & 281.9 & 4 & 600.0 & 600. \\
\hline & 221 & 136.7259 & 9.1200 & 281.8 & 4 & 600.0 & 600 . \\
\hline & 222 & 131.9263 & & 281.1 & 4 & & \\
\hline 412 & 223 & 120.0132 & 6.9550 & 280.2 & $I$ & 600.0 & 600 \\
\hline
\end{tabular}




\begin{tabular}{|c|c|c|c|c|c|c|c|}
\hline 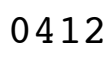 & 24 & & . 6458 & 9.5 & & & \\
\hline & 1 & 8 & .6865 & 279.0 & 4 & ח & \\
\hline & 32 & 122.7676 & 7753 & 278.5 & 4 & 00 & \\
\hline 41 & 3 & 134.7000 & 8.4475 & 278.4 & & 00 & \\
\hline & 4 & 124.4548 & 7025 & 278.3 & & 0 & \\
\hline & 5 & 103.2826 & 5715 & 277.7 & 4 & 00 & \\
\hline & 6 & 102.3729 & 4.6743 & 277.7 & 4 & 00 & \\
\hline & 7 & 124.3707 & 6.49 & 278.3 & & 00.0 & \\
\hline & 8 & 105.3527 & 0 & 278.3 & 4 & & \\
\hline & 9 & 115.6004 & 5.8068 & 279.8 & 4 & 00.0 & 0 \\
\hline & 10 & 126.6763 & 5.4100 & 281.0 & 4 & 0 & \\
\hline & 11 & 140.4500 & 5.5193 & 81.9 & 4 & 00.0 & \\
\hline & 12 & 146.7750 & 5 . & 282.5 & 4 & 00.0 & 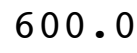 \\
\hline & 13 & 147.0503 & 5 . & 283.3 & 3 & 0.0 & \\
\hline & 14 & 151.6250 & & & & & \\
\hline & & 157.9788 & & 284.2 & 3 & & \\
\hline 4 & 16 & 153.5500 & 9638 & 283.3 & 4 & 0.0 & 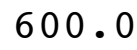 \\
\hline & 317 & 119.1173 & 3. & 282.4 & 4 & 0 & \\
\hline & 18 & 33.2754 & & 2.3 & 4 & & \\
\hline & 19 & 30.9746 & 2 . & 282.7 & 4 & 0 & \\
\hline 4 & 320 & 73.6333 & 1.5773 & 282.8 & 5 & 0 & • \\
\hline & 321 & 81.7810 & & 1.6 & 5 & & \\
\hline & 22 & 29.8236 & 0 & 281.7 & 5 & & \\
\hline 4 & 323 & 29.4711 & 925 & 1.9 & 5 & 0 & ) \\
\hline 4 & 324 & 355.1691 & & & 5 & & \\
\hline 041 & 41 & 150.7285 & & .2 & 4 & & \\
\hline 4 & 42 & 109.3424 & 58 & 9.9 & 5 & 0 & U. \\
\hline 4 & 43 & 15.9016 & 1 . & 9.8 & 5 & & \\
\hline 4 & 44 & 60.3311 & 0 . & 9.7 & 5 & & 0 \\
\hline 4 & 45 & 12.3506 & 1 . & 280.2 & 5 & .0 & 0 . \\
\hline 4$]$ & 46 & 9.3749 & 1.75 & 280.6 & 5 & 0 & 0 . \\
\hline 41 & 47 & 9.5507 & 1 & 0.8 & 5 & & \\
\hline 04 & 48 & 13.5500 & & 281.4 & 4 & & 0 • \\
\hline 41 & 49 & 110.5083 & 088 & 282.2 & 3 & 0 & \\
\hline - & 410 & 193.1867 & & 2.9 & 2 & & \\
\hline 04 & 411 & 318.7099 & & 4.8 & 2 & & 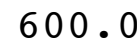 \\
\hline 41 & 412 & 218.6427 & 1.9968 & 284.7 & 2 & 0 & o. \\
\hline 4 & 413 & 243.9964 & 2 . & 3.7 & 2 & & . \\
\hline 041 & 414 & 158.0998 & 4.1713 & 282.6 & 3 & .0 & 600 . \\
\hline 41 & 415 & 158.3789 & 2.9855 & 282.2 & 3 & 0 & . \\
\hline 4 & 416 & 252.2143 & 1 . & 282.2 & 4 & & 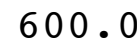 \\
\hline 041 & 417 & 334.1868 & 1.4398 & 281.4 & 4 & .0 & 00 . \\
\hline 4 & 418 & 351.0121 & 2.3558 & 280.6 & 4 & 0 & 00 . \\
\hline 04 & 419 & 356.7289 & 563 & 280.7 & 4 & & . \\
\hline 041 & 420 & 344.2001 & 1.8835 & 280.6 & 5 & & 600. \\
\hline 4 & 421 & 1.3294 & 2.2840 & 280.0 & 4 & 600.0 & 600 \\
\hline & 422 & 9226 & & 281 & 4 & & \\
\hline & 423 & 260.0562 & 1.4563 & 280.5 & & 600.0 & 60 \\
\hline
\end{tabular}




\begin{tabular}{|c|c|c|c|c|c|c|c|}
\hline & 24 & & & & & & \\
\hline & 1 & 4 & .6158 & 9.4 & & 0 & \\
\hline & 52 & 145.7459 & 3.0068 & 276.9 & & 00 . & \\
\hline 41 & 53 & 144.1730 & 5638 & 275.6 & & 00.0 & \\
\hline & 4 & 4.1250 & 0 & 275.4 & & & \\
\hline & 5 & & & 274.7 & & 0 & \\
\hline & 6 & 142.9500 & 4.4658 & 274.8 & & 00 & \\
\hline & 7 & 50 & & 274.9 & & .0 & \\
\hline & 8 & 7.7750 & 0 & 75.0 & & & \\
\hline & 9 & 49.7016 & 350 & 276.2 & & 00 & 0 \\
\hline & 10 & 151.1007 & 6.64 & 277.4 & & & \\
\hline & 11 & 145.7250 & 4.96 & 78.9 & & & \\
\hline & 12 & 148.0715 & 3.9395 & 281.1 & & 00.0 & \\
\hline & 13 & 53.9499 & 4 . & 282.2 & & 0.0 & \\
\hline & 14 & & & & & & \\
\hline & 1 & & & 28 & & & \\
\hline & 16 & 7.7502 & 3 . & 283.0 & & .0 & \\
\hline & 17 & 1093 & & 82.6 & & & \\
\hline & 18 & & & 282.6 & & & \\
\hline & 19 & 00 & & 282.0 & & & \\
\hline 4 & 20 & 62.1398 & 8 . & 281.7 & & .0 & \\
\hline & 21 & & & 281.7 & & & \\
\hline & 22 & 56 & 0 & 281.3 & 4 & & \\
\hline 4 & 523 & 1 & 0 & 281.4 & & 0 & \\
\hline & 524 & & & & & & \\
\hline & 61 & 88 & & 28 & 4 & & \\
\hline 4 & 62 & 3816 & 5 & 280.8 & 4 & 0 & . \\
\hline & 63 & 949 & 6. & 280.7 & 4 & & \\
\hline 4. & 64 & $6 c$ & 8 . & 28 & 4 & & \\
\hline 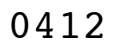 & 65 & 5236 & & 280.0 & 4 & & 0 . \\
\hline a. & 66 & 3344 & 6 . & 279.9 & 4 & & . \\
\hline 4 & 67 & 1 & 78 & 279.8 & 4 & & \\
\hline & 68 & 5222 & & 279.9 & 4 & & 0 . \\
\hline $1-$ & 69 & 503 & & 280.8 & 4 & 0 & \\
\hline $1-$ & 610 & 6 & & 282 & 3 & & \\
\hline & 611 & & & 283.6 & 2 & & \\
\hline 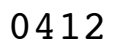 & 612 & 274.7970 & 8 & 283.7 & 3 & & \\
\hline & 613 & 282.4982 & 4. & 284.1 & 3 & & \\
\hline 041 & 614 & 287 . & 4.7535 & 284.5 & 3 & & 0 . \\
\hline & 615 & 288.3258 & 5968 & 284.3 & 3 & & \\
\hline & 616 & 342.5395 & 433 & 284.4 & 4 & & 0 \\
\hline 04 & 617 & 348.2257 & 763 & 284.3 & 4 & & 0 . \\
\hline & 618 & 341.5499 & 6.5370 & 283.6 & 4 & 0 & 0 . \\
\hline & 619 & 341.4250 & 355 & & 4 & & \\
\hline 041 & 620 & 323.1038 & & & 4 & & \\
\hline 41 & 621 & 329.5002 & 8.6800 & 283.3 & 4 & 600.0 & 00 \\
\hline & 622 & 27 & & & 4 & & \\
\hline & 623 & 283.9986 & 8.6750 & 282.7 & & 600.0 & 600 \\
\hline
\end{tabular}




\begin{tabular}{|c|c|c|c|c|c|c|c|}
\hline 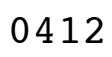 & 24 & & 7.4620 & & & & \\
\hline & 1 & 22 & 3.6250 & 2.3 & 4 & 600 & \\
\hline & 72 & 34.0058 & 7.8823 & 283.7 & 4 & 00 & \\
\hline 41 & 73 & 341.4356 & 7.5175 & 284.7 & & 00 & \\
\hline & 74 & 000 & .8313 & 85.3 & & 0 & \\
\hline & 75 & 5428 & 5 & 283.8 & 4 & 00 & \\
\hline & 76 & 182.3499 & 9538 & 281.3 & 4 & 500 & 0 \\
\hline & 77 & 99.1971 & 1.4513 & 281.3 & & 00.0 & \\
\hline & 78 & 3011 & 79 & 80.8 & 4 & & \\
\hline & 79 & 09.8932 & 0.8448 & 281.0 & 4 & 00 & 0 \\
\hline & 710 & 324.8848 & 1.1828 & 281.7 & 3 & 0 & \\
\hline & 711 & 289.8409 & 1.39 & 82.7 & & 00.0 & \\
\hline & 712 & 233.6309 & 2 . & 283.0 & 3 & 00.0 & 0 \\
\hline & 713 & 0843 & 2.34 & 283.2 & 3 & 00.0 & \\
\hline & 714 & & 2 . & & 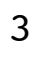 & & \\
\hline & 715 & & & 284.1 & 3 & & \\
\hline 4 & 716 & 3500 & 8 & 284.0 & 4 & 0.0 & 政 \\
\hline 4$\rfloor$ & 717 & 326.9250 & 5 & 2.1 & 4 & 0 & \\
\hline & 718 & 331. & & 281.7 & 4 & & \\
\hline 41 & 719 & 353 & 8 & 282.3 & 5 & 0 & \\
\hline 41 & 720 & 347.7963 & 2.15 & 282.7 & 5 & 0 & . \\
\hline & 721 & 352.7311 & & 283.2 & 4 & & \\
\hline & 722 & 3.80 & 3 . & 283.8 & 4 & & \\
\hline 4 & 723 & 334.4709 & 5.2723 & 283.7 & 4 & 0 & J • \\
\hline$\Delta$ & 724 & 308 . & & 8 & 4 & & \\
\hline 041 & 81 & 308.8247 & 5 . & .5 & 4 & & \\
\hline 4 & 82 & 317.3259 & 4 . & 283.3 & 4 & 0 & U. \\
\hline 4 & 83 & 339.2518 & 4 . & 284.2 & 4 & & \\
\hline 4 & 84 & 341.7246 & 3 . & 284.5 & 4 & & . \\
\hline 4 & 85 & 297.9609 & & 284.1 & 4 & & \\
\hline 4 & 86 & 289.2293 & 5.2553 & 283.3 & 4 & & \\
\hline 1 & 87 & 294.6752 & 25 & 283.2 & 4 & & \\
\hline 7 & 88 & 300.8684 & 6 . & 283.2 & 4 & & U • \\
\hline 41 & 89 & 309.2701 & 355 & 283.7 & 4 & 0 & \\
\hline 1 & 810 & 322 & & 284.6 & 4 & & 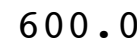 \\
\hline 04 & 811 & 317.8242 & & 285.2 & 4 & & 0 . \\
\hline 41 & 812 & 319.3237 & 5.1813 & 285.5 & 4 & 0 & o. \\
\hline 4 & 813 & 318.0317 & 4 . & .2 & 4 & & . \\
\hline 041 & 814 & 286.6934 & 2.59 & 287.0 & 4 & .0 & 600. \\
\hline 41 & 815 & 335.0059 & 2.1320 & 287.1 & 4 & & . \\
\hline 4 & 816 & 331.0754 & 715 & 286.3 & 4 & & 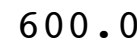 \\
\hline 041 & 817 & 319.1733 & 2.6958 & 285.9 & 4 & .0 & 600. \\
\hline 41 & 818 & 334.7996 & 3.7470 & 285.7 & 4 & .0 & 00 . \\
\hline 041 & 819 & 317.2414 & 2.2208 & 28 & 5 & & 0 \\
\hline 0412 & 820 & 11.3257 & 1.4010 & & 6 & 600.0 & 600. \\
\hline 041 & 821 & 9.0988 & 2.6398 & 284.7 & 5 & 600.0 & 600 \\
\hline & 822 & 62.3139 & & & 4 & & \\
\hline & 823 & 149.2551 & 2.7180 & 286.4 & & 600.0 & 600 \\
\hline
\end{tabular}




\begin{tabular}{|c|c|c|c|c|c|c|}
\hline 24 & & & & & & \\
\hline 1291 & 2.2741 & .7448 & 6.1 & 4 & 0 & \\
\hline 292 & 151.4576 & 1.1858 & 286.6 & 5 & 00 & \\
\hline 1293 & 353.9372 & 1.6383 & 285.7 & & 00 & \\
\hline 94 & 324 & 8025 & & & & \\
\hline 295 & 180 & 7963 & 86.3 & & 0 & \\
\hline 296 & 160 & 0.8495 & 286.4 & & 00 & \\
\hline $\begin{array}{lll}2 & 9 & 7\end{array}$ & 9887 & .8768 & 86.6 & & & \\
\hline 298 & 100 & .2390 & 1 & & & \\
\hline 299 & 144.5476 & 1.7700 & 86.1 & & 0 & 0 \\
\hline 2910 & 143.2372 & 2.4195 & 287.6 & & & \\
\hline 2911 & 113.8474 & 2.9385 & 88.5 & & & \\
\hline 2912 & 377 & 2.4693 & 288.7 & & 0 & \\
\hline 2913 & 0712 & 5.2260 & 288.3 & 3 & & \\
\hline 914 & 169 & & & & & \\
\hline 915 & 195 & & 0 & 3 & & \\
\hline 2916 & 214.1351 & 2.14 & 287.4 & 4 & .0 & \\
\hline 2917 & 149 . & 1 . & 3 & 5 & & \\
\hline 2918 & 66 & 1 . & 3 & 6 & & \\
\hline 2919 & 573 & 0 & 286.2 & 6 & & \\
\hline 12920 & 60.0892 & 0.8760 & 28 & 6 & 0 & \\
\hline 12921 & 38.9 & & & 6 & & \\
\hline 922 & 21 & 1.44 & 28 & 6 & & \\
\hline 923 & 355.5987 & 1.7793 & .2 & 5 & 0 & \\
\hline 924 & 485 & 1.86 & & 3 & & \\
\hline 101 & 346.2618 & 1 . & 28 & 6 & & \\
\hline 102 & 285.6339 & 1.4745 & 285.6 & 6 & 0 & U. \\
\hline 103 & 271.9541 & 1.8605 & $2 \varepsilon$ & 5 & & \\
\hline 104 & 273.8431 & 1.7265 & 28 & 6 & & 0 \\
\hline 412105 & 309.7661 & 1.6845 & 4.6 & 6 & & 0 . \\
\hline 412106 & 312.2423 & 1.9605 & 284.6 & 5 & & 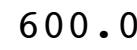 \\
\hline 412107 & 316.3339 & 2.72 & & 4 & & \\
\hline 412108 & 319.4905 & 2.6618 & 285.0 & 4 & & 0 \\
\hline 412109 & 316.0924 & 2.2858 & 28 & 3 & 0 & \\
\hline 010 & 283 & 2.7458 & & 3 & & \\
\hline 011 & 269 . & 2.48 & .2 & 3 & & 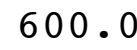 \\
\hline 4121012 & 281.9999 & 2.1305 & 285.3 & 2 & & 0 . \\
\hline 013 & 279.8890 & 1 . & .3 & 3 & & . \\
\hline 1014 & 278.1542 & 2.3268 & .6 & 4 & .0 & 0 . \\
\hline 4121015 & 294.6736 & 2.5645 & 285.7 & 4 & & \\
\hline 016 & 260.4501 & 2.4383 & 28 & 4 & & 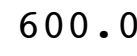 \\
\hline 017 & 275.5147 & 2.6825 & .1 & 4 & .0 & 0 . \\
\hline 4121018 & 305.3486 & 2.2943 & 285.9 & 4 & 0 & 00 . \\
\hline 1019 & 265.1246 & 1.5568 & & 5 & & \\
\hline 04121020 & 263.4151 & 1.6833 & 285.0 & 6 & & 600. \\
\hline 1021 & 262.4148 & 1.5323 & 284.7 & 6 & 600.0 & 600. \\
\hline 22 & 22 & & .3 & 6 & & \\
\hline 1023 & 2254 & 1.0743 & 284.2 & & 60 & 60 \\
\hline
\end{tabular}




\begin{tabular}{|c|c|c|c|c|c|c|}
\hline .024 & & & & & & \\
\hline 2111 & 281.3400 & .8288 & 3.9 & 5 & n & \\
\hline 12112 & 347.1057 & L. 1130 & 283.4 & 6 & 00 & \\
\hline 12113 & 288.0458 & 1.4840 & 283.0 & & 00 & \\
\hline 2114 & 298.4394 & 225 & 82.6 & & 0 & \\
\hline 2115 & 241.8 & 1450 & 82.7 & & 00 & \\
\hline 12116 & 6508 & 1605 & 282.4 & 6 & 500 & U \\
\hline 12117 & 44 & 1.2138 & 82.0 & & 00 & \\
\hline 12118 & 55.7477 & 3940 & 81.4 & & & \\
\hline 412119 & 265.4370 & 1.0963 & 281.6 & 4 & 600.0 & 0 \\
\hline 4121110 & 285.6526 & 1.6098 & 281.8 & 1 & 0 & \\
\hline & 239.3443 & 1.9015 & 82.0 & 4 & 0 & \\
\hline 2 & 293.3540 & 1.4508 & 282.1 & 4 & .0 & \\
\hline 4121113 & 286.3017 & 1.3870 & 282.2 & 4 & 0.0 & \\
\hline & 165 . & & 82.6 & 4 & & \\
\hline & 177 & 1.2 & 282.8 & 4 & & \\
\hline 41 & 196.0751 & 1.3333 & 282.8 & 4 & .0 & \\
\hline 17 & 216.7727 & 8 & 82.8 & 5 & & \\
\hline & 9.3782 & 30 & 282.5 & 6 & & \\
\hline 41 & 74.7060 & 1 . & 282.2 & 5 & & \\
\hline 41 & 3.3259 & 2.61 & 281.9 & 4 & 0 & . \\
\hline 21 & 347.8846 & & 281.6 & 5 & & \\
\hline 22 & 344 & 5 & 281.2 & 5 & & \\
\hline 23 & 346.1023 & 1.9058 & 281.3 & 5 & 0 & \\
\hline 24 & 356.2525 & & 281.2 & 5 & & \\
\hline 041 & 350.4996 & 2.1 & 281.1 & 5 & & \\
\hline 412122 & 334.7734 & 1.72 & 281.1 & 5 & 0 & J. \\
\hline 412123 & 335.1030 & .15 & 9 & 5 & & \\
\hline 24 & 287.6221 & 1 . & 28 & 5 & & \\
\hline 412125 & 340.4051 & 1.55 & 280.8 & 6 & 0 & U. \\
\hline 412126 & 200.2504 & 1.7628 & 280.6 & 5 & 0 & 0 . \\
\hline 412127 & 158.1239 & 105 & 280.4 & 5 & & \\
\hline 0412128 & 345.3500 & 1.48 & 280.2 & 5 & & o. \\
\hline 0412129 & 280.6960 & 1.57 & 280.6 & 4 & 0 & 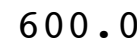 \\
\hline 412 & 160.4999 & 3 & & 3 & & \\
\hline 041 & 160.9165 & 3 . & 282.3 & 3 & & . \\
\hline 412 & 248.6455 & 2.8228 & 282.8 & 3 & $\cdot 0$ & 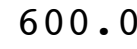 \\
\hline 41 & 303.6085 & 2.83 & 282.4 & 3 & & ( \\
\hline 041 & 73.1326 & 4.4485 & 283.1 & 3 & .0 & 0 . \\
\hline 215 & 167.5732 & 4.2738 & 283.3 & 3 & 0 & \\
\hline 216 & 200.9695 & 2.3088 & 283.7 & 3 & & 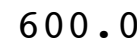 \\
\hline 0412 & 229.8901 & 1.9360 & 283.2 & 4 & .0 & 00 . \\
\hline 4121218 & 256.6738 & 0.7845 & 283.1 & 5 & .0 & 00 . \\
\hline 04121219 & 247.2686 & 1.4690 & 282.9 & 6 & & \\
\hline 04121220 & 255.1428 & 1.2265 & 282.7 & 5 & & 600. \\
\hline 4121221 & 323.5716 & 1.1448 & 282.7 & 5 & 600.0 & 600 \\
\hline & & & & 6 & & \\
\hline & 351.7597 & 0.9538 & 282.5 & & 600.0 & 600 \\
\hline
\end{tabular}




\begin{tabular}{|c|c|c|c|c|c|c|}
\hline 224 & & & & & & \\
\hline 2131 & 7179 & 2.2645 & 82.1 & & & \\
\hline 12132 & 4.3958 & 6398 & 281.8 & 4 & 0 & \\
\hline 12133 & 48.4208 & .7750 & 282.0 & & 00 & \\
\hline 12134 & 353.8147 & 1.4920 & 81.9 & & 0 & \\
\hline 12135 & 3688 & 1.0023 & 82.9 & 5 & 00 & \\
\hline 412136 & 14.2742 & 1.4985 & 282.9 & & 0 & \\
\hline 412137 & 29.3309 & L. 1600 & 83.7 & & & \\
\hline 412138 & 21.2824 & 1.0863 & 283.7 & 5 & 0 & \\
\hline 412139 & 9331 & 1.1755 & 283.9 & 4 & 00 & \\
\hline 310 & 333.9017 & 1.7063 & 284.7 & 3 & 0 & \\
\hline & 290.1126 & 2.3723 & 85.3 & 3 & & \\
\hline 312 & 296.0271 & 1.8073 & 285.8 & 2 & 0 & \\
\hline 313 & 256.6320 & 2.2995 & 286.2 & 3 & 0.0 & \\
\hline & 236.6292 & 100 & & 2 & & \\
\hline 15 & 196.0738 & 3.0435 & 285.8 & 3 & 0 & \\
\hline 316 & 179.7322 & 3.28 & 285.0 & 4 & 0 & \\
\hline 17 & 159.4796 & 3.10 & 4.3 & 4 & & \\
\hline 8 & 160.4000 & 1.70 & 283.6 & 5 & 0 & \\
\hline 319 & 164.8411 & .85 & 28 & 6 & 0 & \\
\hline 20 & 329.8330 & 1 . & 283.4 & 5 & & \\
\hline 21 & 346 . & 0 & 283.2 & 5 & & \\
\hline 322 & 5238 & 1.34 & 283 & 6 & 0 & \\
\hline 323 & 4.3806 & 2.00 & 282.9 & 5 & 0 & \\
\hline 324 & 284.8398 & 1.23 & 283.2 & 6 & & \\
\hline 412 & 182.7231 & 182 & 282.9 & 5 & & \\
\hline $41214 \quad 2$ & 259.8223 & 1.17 & 282.9 & 6 & 0 & \\
\hline 0412143 & 295.2531 & 166 & 282.9 & 6 & & 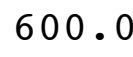 \\
\hline 44 & 2483 & 1.78 & 282.7 & 5 & 0 & 0 . \\
\hline $41214 \quad 5$ & 213.8165 & 1.12 & 282.7 & 6 & 0 & \\
\hline $41214 \quad 6$ & 195.4963 & 1.23 & 282.6 & 6 & & \\
\hline 47 & 290 & 0 & 282.5 & 5 & & \\
\hline $041214 \quad 8$ & 242.5836 & 1.7498 & 282.4 & 5 & 0 & U. \\
\hline 412149 & 248.7864 & 1.13 & 282.6 & 4 & 0 & \\
\hline 10 & 764 & 0 & 282.7 & 4 & & \\
\hline 0412 & 242.8720 & 2.2358 & 282.9 & 4 & & 0 . \\
\hline 412 & 227.4377 & 1953 & 282.7 & 4 & 0 & \\
\hline 041 & 212.2671 & 5 & 282.8 & 4 & 0 & 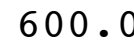 \\
\hline 0412 & 204.3387 & 1.9373 & 282.9 & 4 & .0 & 0 . \\
\hline 15 & 208.7997 & 738 & 282.7 & 4 & 60 & \\
\hline 16 & 251.2347 & 1.3620 & 282.6 & 4 & & \\
\hline 0412 & 288.7115 & 1.4315 & 282.5 & 5 & 600.0 & 600 . \\
\hline 04121418 & 306.0092 & 2330 & 282.1 & 6 & 600 & 000 \\
\hline 04121 & 202.4534 & 1.8820 & 281.8 & 5 & 600 & 600 \\
\hline 04121420 & 88.1364 & 1.5210 & 281.7 & 6 & 600.0 & 600 \\
\hline 4121421 & 118.7924 & 1.2665 & 281.3 & 6 & 600.0 & 600 . \\
\hline & 110.6796 & 1.1263 & 280.5 & 6 & 600 & 60 \\
\hline 4121423 & 166.6854 & 1.9075 & 280.7 & $J$ & 600.0 & 600 \\
\hline
\end{tabular}




\begin{tabular}{|c|c|c|c|c|c|c|}
\hline 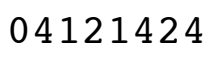 & 2.8291 & .2935 & 81.1 & & 600.0 & \\
\hline 2151 & 3.7004 & 2.4120 & 81.3 & & 600.0 & \\
\hline 12152 & 30.0494 & 2.9900 & 282.2 & 4 & 00.0 & \\
\hline 12153 & 69.9348 & 2.5968 & 282.6 & & 00 & \\
\hline 2154 & 08.7248 & .3225 & 83.6 & & 0 & \\
\hline 2155 & 107.5946 & 0648 & 84.1 & & 00 & \\
\hline 12156 & 126.7877 & 0123 & 283.7 & & 00 & U \\
\hline 12157 & 97.9247 & 2020 & 83.6 & & 00.0 & \\
\hline 12158 & 105.6283 & 4.3693 & 83.5 & & & \\
\hline 12159 & 107.3272 & 5635 & 283.5 & & 600.0 & 0 \\
\hline 21510 & 125.7052 & 5.6373 & 284.0 & & 0 & \\
\hline & 144.6774 & 5.1588 & 84.4 & & 00.0 & \\
\hline 512 & 146.5753 & 3.3940 & 285.1 & & 0 & . \\
\hline 21513 & 150.1981 & 2.8590 & 286.1 & & 0.0 & \\
\hline 14 & 163.5543 & & & & & \\
\hline & 153. & & 285.0 & & & \\
\hline 516 & 143.4000 & 685 & 284.7 & & 0.0 & 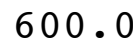 \\
\hline 517 & 143.40 & 4 . & 34.7 & & 0 & \\
\hline 518 & 108 . & & 285.3 & & & \\
\hline 519 & 103 & 685 & 285.5 & 4 & 0 & \\
\hline 520 & 84.0830 & 4.2150 & 286.4 & 4 & 0 & . \\
\hline 21 & 93.1521 & & & $I$ & & \\
\hline 522 & 102 & 3 & 286.2 & 4 & & \\
\hline 523 & 149 . & 2.23 & 284.0 & 5 & 0 & • \\
\hline 524 & 185 & & & 0 & & \\
\hline 61 & 92 . & & 28 & 6 & & \\
\hline 412162 & 136.0908 & 1.2533 & 282.8 & 6 & 0 & J. \\
\hline 412163 & 242.8333 & 1 . & 81.9 & 6 & & \\
\hline 412164 & 147 . & 1 . & 281.6 & 5 & & \\
\hline 412165 & 145.3983 & 1.4555 & 281.9 & 5 & 0 & 0 • \\
\hline 412166 & 284.7154 & 0.7150 & 282.2 & 6 & 0 & 0 . \\
\hline 412167 & 338 & 120 & 281.9 & 6 & & \\
\hline 412168 & 182 . & 127 & 281.0 & 5 & & 0 . \\
\hline 412169 & 191.5588 & 0.82 & 281.7 & 4 & 0 & 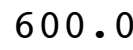 \\
\hline 41 & 309.8471 & 0 & 282.2 & 3 & & \\
\hline 041 & 252.8126 & 2.3705 & 283.0 & 2 & & . \\
\hline 4121612 & 256.3960 & .5790 & 283.1 & 3 & $\cdot 0$ & . \\
\hline 613 & 255.2095 & 933 & 283 & 3 & & ( \\
\hline 041 & 290.1180 & 2.4928 & 283.7 & 3 & .0 & 0 . \\
\hline 4121615 & 230.3665 & 2.3465 & 283.2 & 3 & & 0 . \\
\hline 1616 & 231.4976 & 1.9913 & 282.5 & 3 & & 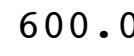 \\
\hline 0412 & 249.5850 & 1.4163 & 282.0 & 4 & .0 & 00 . \\
\hline 4121618 & 347.1929 & 1.9455 & 281.7 & 5 & 0 & 00 . \\
\hline 04121619 & 2.8748 & 1.9755 & 281.6 & 5 & & 0 \\
\hline 04121620 & 345.6853 & 1.6480 & 281.3 & 6 & .0 & 600. \\
\hline 4121621 & 350.0009 & 1.0398 & 281.5 & 6 & 600.0 & 600 \\
\hline & 28 & & & 6 & & \\
\hline 4121623 & 313.1064 & 1.0108 & 281.0 & & 600.0 & 60 \\
\hline
\end{tabular}




\begin{tabular}{|c|c|c|c|c|c|c|}
\hline $0<4$ & & & & & & \\
\hline 2171 & 962 & 1.5068 & 1.1 & & חס & \\
\hline 12172 & 4251 & 1.9810 & 281.6 & 5 & 00 & \\
\hline 12173 & 6709 & 2.0665 & 281.8 & & 00 & \\
\hline 2174 & 253 & .4248 & 82.4 & & 0 & \\
\hline 2175 & 250 & 49 & 82.7 & & 0 & \\
\hline 412176 & 31.5898 & 1.6755 & 282.3 & & 00 & \\
\hline $41217 \quad 7$ & 146.5800 & .1000 & 81.0 & & & \\
\hline 12178 & 150.7458 & 5778 & 81.5 & & & \\
\hline 412179 & 72.1446 & 0.8930 & 282.2 & & 00 & 0 \\
\hline 4121710 & 27.2436 & 1.4673 & 283.7 & & & \\
\hline 1711 & 232.2628 & 0.7505 & 85.3 & & & \\
\hline 1712 & 161.4579 & 1.4758 & 286.3 & & .0 & \\
\hline 4121713 & 149.2497 & 3.2363 & 285.8 & & & \\
\hline 1714 & 145.8758 & & & & & \\
\hline$\lfloor 715$ & 150 & & 285.2 & & & \\
\hline 4121716 & 144.9250 & 3 & 284.7 & & .0 & \\
\hline 4121717 & 137.2014 & 0 & .1 & & & \\
\hline 4121718 & 130.2349 & 5 & 286.0 & 5 & & \\
\hline 1719 & 150.0749 & 483 & 285.4 & 4 & & \\
\hline 4121720 & 115.7121 & 3.18 & 284.2 & 4 & 0 & 10 \\
\hline 1721 & 105.7652 & & & 5 & & \\
\hline .722 & 130.3647 & 3 & 285.7 & 5 & & \\
\hline 4121723 & 116.0637 & 2.04 & 286.3 & 5 & 0 & \\
\hline 724 & 149.935 & & & 6 & & \\
\hline 81 & 148.2159 & 2 . & 285.5 & 5 & & \\
\hline 412182 & 120.1836 & 3635 & 285.1 & 5 & 0 & . \\
\hline 412183 & 277.4462 & 3 & 5.6 & 6 & & \\
\hline 0412184 & 168.2778 & 1 . & 284.3 & 6 & & \\
\hline 0412185 & 10.4385 & 5 & 284.1 & 6 & 0 & U. \\
\hline 412186 & 316.4877 & 3 & 284.6 & 6 & & 0 . \\
\hline 412187 & 44.3743 & 0 & & 6 & & \\
\hline 0412188 & 16.5716 & 0 & 284.6 & 5 & & 0 . \\
\hline 0412189 & 330.3432 & 0.75 & 285.2 & 4 & 0 & \\
\hline 4121810 & 316.1192 & 3 & & 3 & & \\
\hline 811 & 317.9402 & 1.44 & 6.3 & 2 & & 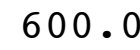 \\
\hline 4121812 & 271.8506 & 1 & 284.7 & 2 & & . \\
\hline 813 & 236.4730 & 1 . & .4 & 2 & & 0 . \\
\hline 041 & 254.4685 & 1.6205 & 282.5 & 2 & .0 & 0 . \\
\hline 4121815 & 296.2244 & 1.7118 & 282.3 & 2 & & \\
\hline 1816 & 320.6595 & 8 & 282.9 & 3 & & 0 \\
\hline 817 & 39.3645 & 0.8508 & 282.1 & 4 & .0 & 0 . \\
\hline 4121818 & 126.5005 & 1.2545 & 282.3 & 5 & 0 & 0 . \\
\hline 04121819 & 144.8730 & 1 . & 283.4 & 6 & & \\
\hline 04121820 & 109.0689 & & & 6 & & 500. \\
\hline 4121821 & 350.2241 & 0.4145 & 283.9 & 6 & 600.0 & 600. \\
\hline & 34 & & & 6 & & \\
\hline 4121823 & 154.2996 & 1.1750 & 282.8 & 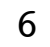 & 600.0 & 0 \\
\hline
\end{tabular}




\begin{tabular}{|c|c|c|c|c|c|c|}
\hline $0<4$ & & & & & & \\
\hline 2191 & 6 & 7053 & 1.0 & 6 & 00.0 & \\
\hline 2192 & 152.5239 & 7055 & 281.4 & 5 & 00 & \\
\hline 12193 & 52.7079 & 0.8790 & 281.2 & 6 & 00 & \\
\hline 2194 & 37.5944 & .7573 & 81.5 & & 0 & \\
\hline 2195 & 154.2534 & .8953 & 81.4 & 5 & 0 & \\
\hline 12196 & 148.3253 & 5140 & 281.2 & 4 & 00 & \\
\hline 12197 & 56.2284 & 3 & 281.5 & & 0 & \\
\hline 12198 & 149.6074 & 3 & 81.8 & & & \\
\hline 412199 & 144.4781 & 3.2898 & 280.9 & 4 & 00 & 0 \\
\hline 21910 & 156.9407 & 2.8575 & 282.5 & 3 & & \\
\hline 911 & 160.9032 & 20 & 83.5 & & & \\
\hline 912 & 145.8503 & 3 . & 284.5 & 3 & 0 & \\
\hline 21913 & 151.1750 & 4.7078 & 283.1 & 3 & 0.0 & \\
\hline 914 & 153 & 5 & & & & \\
\hline 915 & 143 . & & 28 & 3 & & \\
\hline 916 & 134.8888 & 5 & 283.9 & 3 & .0 & \\
\hline 917 & 126.7270 & 8 & 4 & 4 & & \\
\hline 918 & 113.8462 & 2 . & .1 & 4 & & \\
\hline 919 & 3576 & 3 & & 4 & & \\
\hline 920 & 88.1019 & 1.7945 & 284.5 & 5 & 0 & \\
\hline 921 & 136.9503 & & & 4 & & \\
\hline 922 & 104 & 1 . & 285.1 & 5 & & \\
\hline 4121923 & 100.4245 & 1.41 & 287.0 & 6 & 0 & \\
\hline 924 & 146 . & & & 5 & & \\
\hline 041 & 119. & 1 . & 28 & 5 & & \\
\hline 412202 & 135.6882 & 1.38 & 284.6 & 6 & 0 & J. \\
\hline 412203 & 113.7377 & 2.26 & 81.4 & 5 & & \\
\hline 0412204 & 106 . & 1.3 & 28 & 6 & & \\
\hline 412205 & 125.1099 & 1.8915 & 280.7 & 5 & 0 & 0. \\
\hline 412206 & 98.8161 & 2.5188 & 280.9 & 4 & & 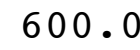 \\
\hline 412207 & 121.7098 & 2.31 & 279.1 & 5 & & \\
\hline 0412208 & 135 . & 1.17 & 278.1 & 5 & & . \\
\hline 412209 & 132.9652 & 2.3698 & 273.8 & 4 & 0 & \\
\hline 4122010 & 55.2720 & 112 & 277.3 & 3 & & \\
\hline 0412 & 151.5106 & 5.7260 & 279.8 & 3 & & . \\
\hline 4122012 & 85.9227 & 2.5658 & 277.9 & 2 & & 0 . \\
\hline 4122013 & 129.3144 & 2.31 & 284.0 & 2 & & . \\
\hline 0412 & 225.8211 & 2.6980 & 278.7 & 2 & .0 & 0 . \\
\hline 4122015 & 129.9429 & 4.2178 & 278.6 & 3 & & \\
\hline 04122016 & 77.8185 & 3.1915 & 279.9 & 3 & & 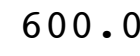 \\
\hline 04122017 & 112.0844 & 2.2945 & 280.3 & 4 & .0 & 0 . \\
\hline 4122018 & 108.2315 & 2.3550 & 282.8 & 4 & 0 & 0 . \\
\hline 04122019 & 64.1304 & 6533 & 276.9 & 5 & & \\
\hline 04122020 & 78.8501 & 3.2330 & 281.7 & 4 & & 0 . \\
\hline 412202 & 126.7222 & 3.4698 & 283.4 & 4 & 600.0 & 600. \\
\hline & 108 & & & 5 & & \\
\hline 412202 & 117.3163 & 4.0960 & 276.1 & & .0 & n \\
\hline
\end{tabular}




\begin{tabular}{|c|c|c|c|c|c|c|}
\hline $0<4$ & & & & & & \\
\hline 221 & 124.0725 & .7600 & 9.5 & & & \\
\hline 12212 & 141.0536 & 0.3325 & 79.0 & & 00 & \\
\hline 12213 & 26.1261 & 2900 & 279.0 & & 00 & \\
\hline 2214 & 9.5017 & 1.3225 & 9.6 & & & \\
\hline 2215 & 1747 & 2.2675 & 279.5 & & 0 & \\
\hline 12216 & 9999 & 9075 & 279.9 & & 00 & \\
\hline 41221 & 44.6005 & 750 & 280.3 & & & \\
\hline 412218 & 3000 & 5500 & 0.4 & & & \\
\hline 412219 & 1000 & 12.5200 & 280.8 & & 0 & \\
\hline 2110 & 1980 & 8.8575 & 281.4 & & & \\
\hline & 0.6592 & 71 & 2.0 & & & \\
\hline 4 & .750 & 6.36 & 83.1 & & .0 & \\
\hline 211 & 37 & 6.24 & & & & \\
\hline & 6 & & & & & \\
\hline & \pm 1 & & & 4 & & \\
\hline 16 & 995 & 6 . & 5.6 & 2 & .0 & \\
\hline 17 & 68 & 5. & & & & \\
\hline & 33. & & & 4 & & \\
\hline 9 & 06 & & & 4 & & \\
\hline 041 & 111.8771 & 5 . & 2 & 4 & • 0 & \\
\hline & 108 & & & 4 & & \\
\hline 2 & 103 & & & 4 & & \\
\hline 123 & 96.3700 & 6.86 & & 4 & . 0 & \\
\hline 124 & 121. & & & 4 & & \\
\hline 21 & 115 & & & 4 & & \\
\hline 412222 & 138.5027 & & 2 & 4 & .0 & \\
\hline 412223 & 123.0879 & & & 4 & & \\
\hline 224 & 135 & 6 . & & 4 & & \\
\hline 0412225 & 117.5340 & & & 4 & & \\
\hline 0412226 & 117.2521 & 5.5253 & & 4 & & \\
\hline 0412227 & 98.8170 & 5 & & 4 & & \\
\hline 0412228 & 008 & & & 4 & & \\
\hline 0412229 & 100.8213 & 6 . & & 4 & 0 & \\
\hline 2210 & 0 & & & 3 & & \\
\hline & 420 & & .6 & 3 & & \\
\hline 212 & 143.6830 & 6.38 & 2.6 & 4 & .0 & \\
\hline 213 & 145.4500 & & 8 & 3 & & \\
\hline 041 & 142 . & 4.98 & 2.9 & 3 & .0 & \\
\hline 22215 & 150.2518 & 5. & 2.7 & 4 & & \\
\hline 2216 & 494 & 4. & 282.2 & 3 & & \\
\hline 0412 & 144.9703 & & 282.2 & 4 & .0 & \\
\hline 4122218 & 141.7517 & 3.6580 & 281.9 & 4 & & 0 \\
\hline 04122219 & 050 & 3 . & 28 & 4 & & \\
\hline 04122220 & 111.7965 & 3.511 & 282.6 & 4 & & \\
\hline 04122221 & 107.7735 & 4.5215 & 283.0 & 4 & .0 & 00 \\
\hline & & & & 4 & & \\
\hline 412 & 15.7245 & 4.8783 & 282.3 & & & 600 \\
\hline
\end{tabular}




\begin{tabular}{|c|c|c|c|c|c|c|}
\hline 224 & & & & & & \\
\hline 2231 & 51 & .6610 & 1.4 & & & \\
\hline 2232 & 502 & 2625 & 280.8 & 4 & 00 & \\
\hline 12233 & 46.6255 & 5725 & 280.5 & & 00 & \\
\hline 2234 & 500 & 0.3225 & 30.1 & & & \\
\hline 2235 & & 1.7500 & 79.7 & & 0 & \\
\hline 122236 & 1751 & 12.6125 & 279.4 & & 00 & \\
\hline 12237 & 2.7747 & 11.9850 & 79.2 & & & \\
\hline 12238 & 9.9041 & 7075 & 79.4 & & & \\
\hline 412239 & 15.8001 & 7.0908 & 79.6 & & 0 & 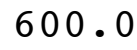 \\
\hline 22310 & 24.1748 & 7.1050 & 280.2 & & & \\
\hline 11 & 40.8509 & 7.4825 & 80.7 & & & \\
\hline 12 & 7748 & 6643 & 281.3 & 4 & 0 & \\
\hline 2313 & 3519 & 7.7548 & 282.3 & 4 & & \\
\hline & & & & & & \\
\hline & 250 & & 2 & 3 & & \\
\hline 16 & 132.2517 & 35 & 284.8 & 4 & & \\
\hline 17 & 5489 & 5 & .8 & 4 & & \\
\hline & 161 & 5 & .8 & 4 & & \\
\hline 9 & 250 & 700 & & 4 & & \\
\hline 20 & 133.4999 & 7.5250 & 284.1 & 4 & 0 & \\
\hline & & & & 4 & & \\
\hline 2 & 41 & 35 & 1 & 4 & & \\
\hline 323 & 90.9028 & 178 & .9 & 4 & 0 & \\
\hline 24 & & & .4 & 4 & & \\
\hline 41 & 58 & 5 & 282.3 & 5 & & \\
\hline $41224 \quad 2$ & 119.3770 & 1.7623 & 281.9 & 5 & & 0 \\
\hline 412243 & 198.7140 & 1.2613 & 31.0 & 6 & & \\
\hline 244 & 3 & 73 & 28 & 6 & & \\
\hline $41224 \quad 5$ & 83 & 888 & & 6 & & J. \\
\hline 412246 & 128.5904 & 565 & 279.3 & 6 & & 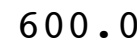 \\
\hline 412247 & 312.7967 & 0 & 277.9 & 6 & & \\
\hline 412248 & 661 & & .0 & 5 & & \\
\hline 412249 & 115.9892 & 055 & 278.8 & 4 & & \\
\hline 41 & 126 & 763 & & 3 & & \\
\hline 041 & & & 277.9 & 2 & & \\
\hline 12 & 308.0222 & 1.5613 & 278.3 & 2 & & \\
\hline 13 & 307 . & 1. & 280.4 & 2 & & \\
\hline 041 & 286.6074 & & 279.5 & 2 & & 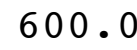 \\
\hline 415 & 181.3055 & 1.2250 & 281.1 & 2 & & \\
\hline 416 & 133.3942 & 623 & $27 \varepsilon$ & 3 & & \\
\hline 417 & 40.4149 & 403 & 279.6 & 4 & & 0 . \\
\hline 4122418 & 99.6209 & 1.2470 & 282.0 & 5 & & 0 . \\
\hline 04122419 & 30.6506 & 000 & 282 & 6 & & \\
\hline 04122420 & 129.4578 & 0.8643 & & 6 & & 0 . \\
\hline 4122421 & .4294 & 1.1895 & 282.5 & 6 & 600 & 500. \\
\hline & 18 & & & 6 & & \\
\hline 4122423 & 8.7754 & 1.4758 & 282.2 & & 600.0 & 600 \\
\hline
\end{tabular}




\begin{tabular}{|c|c|c|c|c|c|c|}
\hline & & .3995 & 32.1 & & & \\
\hline 251 & $5 \quad 1777$ & 3178 & 80.1 & & م & \\
\hline 2252 & 232.4604 & 1040 & 280.6 & & 00 & \\
\hline 12253 & 139.5091 & 5905 & 280.6 & & 00 & \\
\hline 2254 & 49 . & 8240 & 9.6 & & & \\
\hline 255 & 187. & & 79.4 & & 0 & \\
\hline 12256 & 42.9376 & 3985 & 279.2 & & 00 & \\
\hline 12257 & 110 . & & 80.5 & & & \\
\hline 12258 & 138.9846 & & 78.8 & & & \\
\hline 12259 & 335.6724 & 0.8148 & 80.3 & & 0 & \\
\hline 510 & 255.7129 & 1.41 & 279 & & & \\
\hline & 358.9188 & 150 & 80.7 & & & \\
\hline 2 & 332.3085 & 1 . & 282.5 & & & \\
\hline 3 & 130.9823 & 2.00 & & & & \\
\hline & 10 . & & & & & \\
\hline & 346 . & & 81.3 & & & \\
\hline 6 & 10.9688 & .72 & 281.3 & & & \\
\hline 17 & 357.3539 & & 9.3 & & & \\
\hline & 359.4294 & & 278.4 & & & \\
\hline 9 & 126.4135 & 0 & 281 & & & \\
\hline 20 & 270.9050 & 0.77 & 282.6 & & & \\
\hline & 280.7829 & & & 6 & & \\
\hline 2 & 110.2468 & 8 & 28 & 5 & & \\
\hline 23 & 98.3969 & 5.31 & 280.7 & 4 & & \\
\hline 24 & 671 & & & & & \\
\hline 4 & 11. & & $27 \xi$ & 4 & & \\
\hline 412262 & 4.1957 & 3 & 278.2 & 4 & & . \\
\hline 412263 & 16.5752 & & 7 & 5 & & \\
\hline 64 & 357.4716 & 1 . & 27 & 5 & & \\
\hline 412265 & 342.5546 & 2 . & 278.5 & 5 & & \\
\hline 412266 & 339.2733 & 2.54 & 279.0 & 4 & & \\
\hline 412267 & 248 . & & 279 & 5 & & \\
\hline 412268 & 908 & 1 & 280.4 & 4 & & 0 . \\
\hline 412269 & 331.9391 & 1 . & 280.0 & 4 & & \\
\hline 0 & 319 . & & & 4 & & \\
\hline 41 & 268.4340 & & 281.7 & 4 & & \\
\hline 12 & 277.5856 & 2 . & 283.2 & 3 & & \\
\hline 13 & 266 . & 5 . & & 3 & & \\
\hline 412 & 268.0758 & 6.2970 & 281.8 & 4 & & . \\
\hline 3 & 292.2889 & 4 . & 283.6 & 3 & & \\
\hline 616 & 329.4000 & 8.7600 & & 4 & & \\
\hline 0412 & 328.4669 & 8.2233 & 283.2 & 4 & & . \\
\hline 4122618 & 329.2750 & 1.0450 & 282.5 & 4 & & 0 . \\
\hline 4122619 & 332.6501 & 5 & & $\boldsymbol{I}$ & & \\
\hline 04122620 & 331.4494 & 0.7550 & 282.4 & 4 & & \\
\hline 4122621 & 330.8750 & 0.4100 & 282.5 & 4 & & 00 \\
\hline & 330 & 10 & & 4 & & \\
\hline 4122623 & 324.5113 & 8.9765 & 282.7 & & .0 & 600 \\
\hline
\end{tabular}




\begin{tabular}{|c|c|c|c|c|c|c|}
\hline 4 & & $\cdot 2080$ & & & & \\
\hline 271 & & & 2.7 & & & \\
\hline 2272 & 04 & 0275 & 283.2 & 4 & 00 & \\
\hline 12273 & 307.2498 & 1025 & 283.0 & & 00 & \\
\hline 2274 & & .8870 & 3.0 & & & \\
\hline 275 & 7.34 & 8.2200 & 83.5 & & 0 & \\
\hline 12276 & 4992 & 8.1425 & 282.8 & & 00 & \\
\hline 12277 & 53 & 7.4650 & 83.1 & & & \\
\hline 12278 & 1249 & 5 & 83.6 & & & \\
\hline 412279 & 39.1217 & 5700 & 84.0 & & 0 & 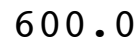 \\
\hline 22710 & 330.9990 & 4.7050 & 284 & & & \\
\hline 711 & 3221009 & 50 & 85.8 & & & \\
\hline 2712 & 250 & 300 & 285.2 & 4 & 0 & \\
\hline 2713 & 332.8500 & 13.9875 & 28 & & & \\
\hline 714 & 51 & 5 & & & & \\
\hline 715 & & 11. & 282.4 & 4 & & \\
\hline 716 & 3.2865 & 9.6100 & 282.6 & 4 & 0 & \\
\hline 717 & 980 & 6 . & & 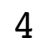 & & \\
\hline 71 & 663 & & & 4 & & \\
\hline 719 & 201 & 0 & 280.4 & 5 & & \\
\hline 720 & 143 & 1.76 & 280.4 & 5 & 0 & \\
\hline 721 & 249.5924 & & & 4 & & \\
\hline 722 & 142 & 0 & 3 & 4 & & \\
\hline 723 & 177.9342 & 1.5920 & 280.2 & 4 & 0 & \\
\hline 724 & 164 . & & 9.7 & 4 & & \\
\hline 81 & 26 & 5 & 279.9 & 4 & & \\
\hline 412282 & 271.2336 & 3 & 0.2 & 4 & & 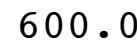 \\
\hline 412283 & 271.7022 & 3 & 1 & 4 & & \\
\hline 84 & 291 & 0 & & 4 & & \\
\hline 412285 & 292.0721 & 2.7020 & 0.5 & 4 & & 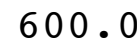 \\
\hline 412286 & 280.4991 & 3.85 & 0.7 & 4 & & 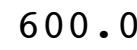 \\
\hline 412287 & 30 & 0 & & 4 & & \\
\hline 412288 & & & .1 & 4 & & \\
\hline 412289 & 319.2253 & 6.2185 & .4 & 4 & & \\
\hline 810 & 320 & & & 4 & & \\
\hline 41 & & & 2.8 & 4 & & \\
\hline 812 & 311.1023 & 9685 & 3.3 & 4 & & \\
\hline 41 & 270.4845 & 755 & 2 & 4 & & \\
\hline 814 & 288.6892 & 3.8190 & 1.8 & 4 & & . \\
\hline 815 & 314.8488 & 5.4668 & 280.9 & 4 & & \\
\hline 816 & 306 . & 3060 & & 4 & & \\
\hline 0412 & 320.1652 & 5.5323 & L. 0 & 4 & & 0 . \\
\hline 4122818 & 324.5760 & 4.2205 & 280.9 & 4 & & 0 . \\
\hline 4122819 & 2619 & 4.7173 & & 4 & & \\
\hline 04122820 & 195.2391 & 1.8670 & & 5 & & 0 . \\
\hline 4122821 & 316.9884 & 2.0815 & 281.2 & 5 & & 00 . \\
\hline & 21 & & & & & \\
\hline 4122823 & 337.4582 & 3.1998 & 281.3 & & .0 & 600 \\
\hline
\end{tabular}




\begin{tabular}{|c|c|c|c|c|c|c|}
\hline 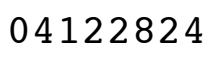 & & & & & & \\
\hline 2291 & . & .5485 & 1.8 & & & \\
\hline 2292 & 36.2206 & 7538 & 281.8 & 4 & 00 & \\
\hline 12293 & 291.5745 & 2.1858 & 281.1 & & 00 & \\
\hline 2294 & 234 . & 38 & 30.2 & & & \\
\hline 2295 & 165 & 3 & 80.2 & & 0 & \\
\hline 12296 & 285 & 0233 & 280.2 & & 00 & \\
\hline 12297 & 87.2556 & 8 & 80.0 & & & \\
\hline 12298 & 137.0747 & 0 & 80.1 & & & \\
\hline 12299 & 300.6215 & 458 & 80.2 & & 0 & 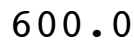 \\
\hline 2910 & 349.1673 & 1.3928 & 280.8 & & & \\
\hline 911 & 322.2503 & & 32.7 & & & \\
\hline 912 & 203.5704 & 2.3703 & 283.1 & & 0 & \\
\hline 913 & 28.1399 & 1.9985 & 28 & & & \\
\hline 914 & 260.3393 & & & & & \\
\hline 5 & 321.9604 & & & 3 & & \\
\hline 16 & 11.0751 & 715 & 284.8 & 4 & .0 & \\
\hline 917 & 4.1480 & 4. & & 4 & & \\
\hline 918 & 340.1425 & & 3 & 4 & & \\
\hline 19 & 312.8696 & & 281.9 & 4 & & \\
\hline 920 & 312.6217 & 6 . & 281.9 & 4 & .0 & \\
\hline 21 & 343.8270 & & & 4 & & \\
\hline 22 & 358.5263 & & 6 & 4 & & \\
\hline 923 & 356.4999 & 0 & 5 & 4 & 0 & \\
\hline 924 & 350.2255 & & & 4 & & \\
\hline 41 & 344.0010 & & & 4 & & \\
\hline 412302 & 347.8303 & 193 & 7 & 4 & & 0 \\
\hline 412303 & 158.6229 & & & 4 & & \\
\hline 304 & 322.0525 & 8 & & 4 & & \\
\hline 41230 & 5.2501 & & 2.0 & 4 & & J. \\
\hline 412306 & 10.4718 & 5 & 282.5 & 4 & & \\
\hline $41230 \quad 7$ & 39.8544 & & 32.2 & 4 & & \\
\hline 412308 & 58.4181 & & 281.6 & 4 & & \\
\hline 412309 & 37.2847 & 0 & 281.2 & 4 & & \\
\hline 41 & 29.4187 & & & 3 & & \\
\hline 041 & 34.5205 & & 5.2 & 3 & & \\
\hline 012 & 72.6401 & 748 & 283.9 & 3 & & \\
\hline 4123013 & 352.4960 & 5 & & 3 & & \\
\hline 04123014 & 318.8141 & 4.6768 & 282.4 & 4 & & 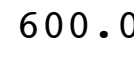 \\
\hline 4123015 & 344.4913 & 163 & 283.8 & 4 & & \\
\hline 3016 & 298.3095 & 903 & & 4 & & \\
\hline 04123017 & 318.8661 & 3.9335 & 282.9 & 4 & & . \\
\hline 4123018 & 325.5765 & 3.1845 & 282.3 & 4 & & 0 . \\
\hline 04123019 & 313.0263 & 303 & & 4 & & \\
\hline 04123020 & 328.0320 & 2.9283 & 282.4 & 4 & & 0 . \\
\hline 412302 & 2.5860 & 3.8380 & 283.0 & 4 & 600 & 00 . \\
\hline & 328.9642 & & & 4 & & \\
\hline 412302 & 79.7849 & 7.8925 & 281.2 & & .0 & 60 \\
\hline
\end{tabular}




$\begin{array}{lrrrrrrr}04123024 & 79.2756 & 5.6138 & 281.0 & 4 & 600.0 & 600.0 \\ 041231 & 1 & 8.7098 & 2.2700 & 280.8 & 4 & 600.0 & 600.0 \\ 041231 & 2 & 43.6280 & 3.2988 & 280.9 & 4 & 600.0 & 600.0 \\ 0412313 & 40.3502 & 3.6095 & 280.9 & 4 & 600.0 & 600.0 \\ 041231 & 4 & 45.7293 & 3.1103 & 280.7 & 4 & 600.0 & 600.0 \\ 041231 & 5 & 104.1222 & 4.7095 & 280.6 & 4 & 600.0 & 600.0 \\ 041231 & 6 & 71.8884 & 4.5900 & 279.7 & 4 & 600.0 & 600.0 \\ 041231 & 7 & 90.7803 & 5.5858 & 280.0 & 4 & 600.0 & 600.0 \\ 0412318 & 91.3954 & 3.8858 & 280.2 & 4 & 600.0 & 600.0 \\ 0412319 & 128.8545 & 2.7405 & 280.3 & 4 & 600.0 & 600.0 \\ 04123110 & 99.6846 & 3.1043 & 281.2 & 3 & 600.0 & 600.0 \\ 04123111 & 64.3623 & 4.5538 & 282.4 & 3 & 600.0 & 600.0 \\ 04123112 & 37.6297 & 4.4338 & 283.2 & 3 & 600.0 & 600.0 \\ 04123113 & 22.0287 & 4.2868 & 283.9 & 3 & 600.0 & 600.0 \\ 04123114 & 342.2963 & 2.7585 & 283.7 & 3 & 600.0 & 600.0 \\ 04123115 & 304.3494 & 2.5503 & 284.6 & 2 & 600.0 & 600.0 \\ 04123116 & 349.0503 & 2.7770 & 284.3 & 3 & 600.0 & 600.0 \\ 04123117 & 52.7633 & 3.2923 & 282.8 & 4 & 600.0 & 600.0 \\ 04123118 & 5.0815 & 4.5475 & 281.8 & 4 & 600.0 & 600.0 \\ 04123119 & 3.5011 & 3.6538 & 281.5 & 4 & 600.0 & 600.0 \\ 04123120 & 0.7615 & 3.9793 & 281.2 & 4 & 600.0 & 600.0 \\ 04123121 & 13.4005 & 3.3725 & 280.8 & 4 & 600.0 & 600.0 \\ 04123122 & 46.9085 & 2.0145 & 280.3 & 4 & 600.0 & 600.0 \\ 04123123 & 311.5179 & 2.2663 & 279.8 & 4 & 600.0 & 600.0 \\ 04123124 & 233.9425 & 1.8060 & 280.0 & 4 & 600.0 & 600.0\end{array}$




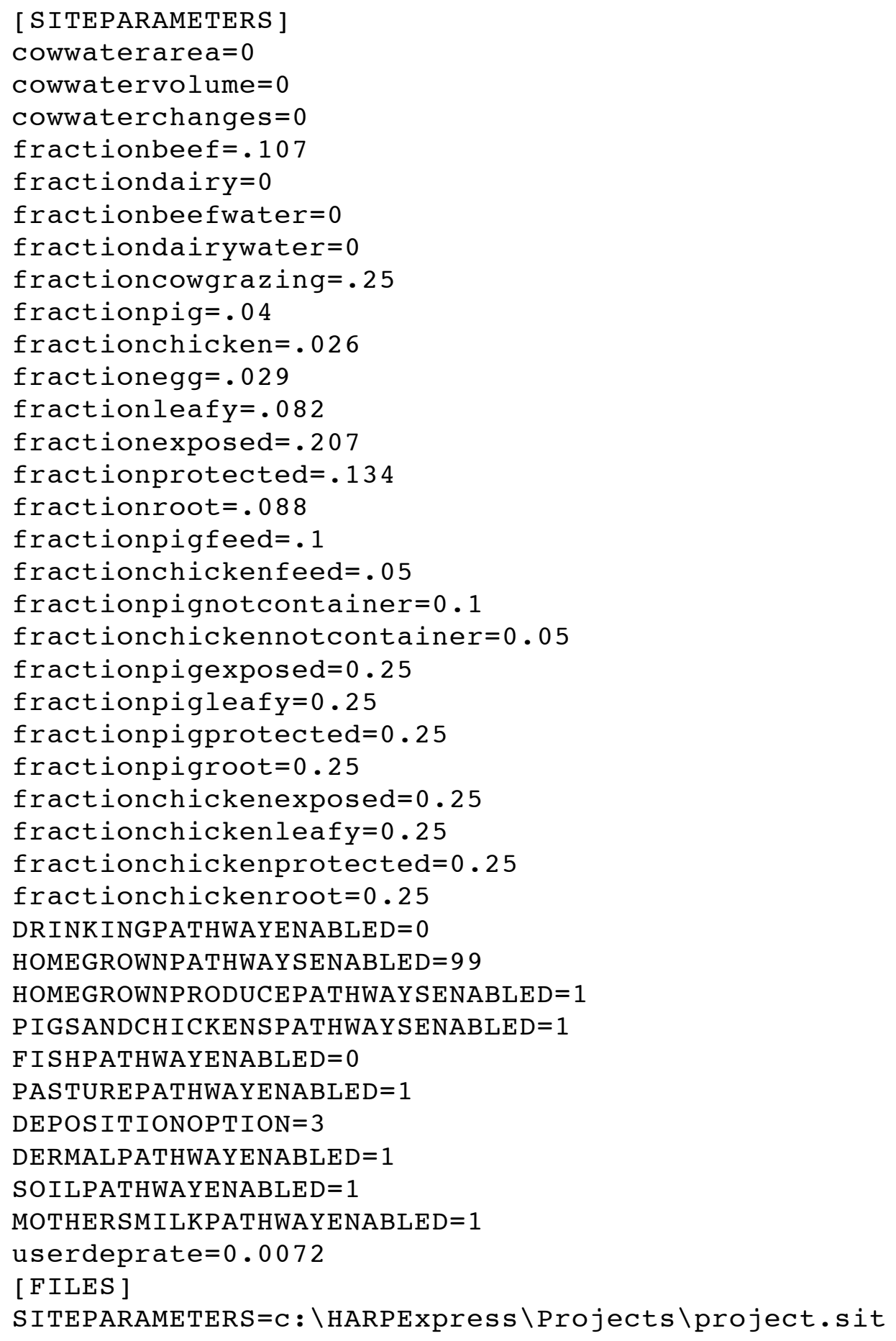




\section{HARP Output Folder/Bystander Worker Folder}

Calculation_note.txt

Rep_Acu_rect_AllSrc_AllCh_BySrc_Site.txt

Rep_Can_WRK_Avg_Rec5_AllSrc_AllCh_BySrc_Bychem_Site.txt

Rep_Can_WRK_Avg_Rec5_AllSrc_AllCh_bySrc_Site.txt

Rep_Chr_Wrk_PtEst_Rec5_AllSrc_AllCh_BySrc_Site.txt 
Calculation_note.txt 
Note to reviewers of bystander output files.

The output in the bystander worker folder is directly from HARP. Because the worker calculations in HARP are for an outdoor worker, the incidental soil ingestion rate is twice the rate used in the risk assessment for an indoor bystander worker. Consequently, the output for incidental soil ingestion rate should be divided by 2 to obtain the risk value for that pathway. 
Rep_Acu_rect_AllSrc_AllCh_BySrc_Site.tx 


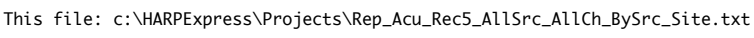

Created by HARP Version 1.0 Build 23.02.10

Uses ISC Version 99155

Uses BPIP Version 95086

Creation date: 10/24/2005 2:11:19 PM

EXCEPTION REPORT

(there have been no changes or exceptions)

INPUT FILES:

Source-Receptor file: c: \HARPExpress \Projects \EWTF.mta

Averaging period adjustment factors file: not applicable

Emission rates file: none

Site parameters file: C:\HARPExpress \Projects $\backslash$ Site300. sit

Screening mode is OFF

USER-DEFINED WORKER ADJUSTMENT AND EXPOSURE PARAMETERS

GLC Adjustment Factor:

Exposure Duration (years): 25

Exposure Frequency (days/year): 245

Explanatory Note: U.S. EPA value

Analysis method: Point Estimate

Health effect: Acute HI Simple (Concurrent Max.)

$\operatorname{Receptor}(\mathrm{s}): \quad 5$

$\begin{array}{ll}\text { Sources(s): } & \text { All } \\ \text { Chemicals(s): } & \text { All }\end{array}$

CHEMICAL CROSS-REFERENCE TABLE AND BACKGROUND CONCENTRATIONS

CHEM CAS

001 106990 $1,3-$ Butadiene $1,3-$ Butadiene

$000267562394 \quad 1-4,6-8 \mathrm{HpCDF} \quad 1,2,3,4,6,7,8$-Heptachlorodibenzofuran

$000355673897 \quad 1-4,7-9 \mathrm{HpCDF} \quad 1,2,3,4,7,8,9$-Heptachlorodibenzofuran

$000470648269 \quad 1-4,7,8 \mathrm{HXCDF}$

$000557117449 \quad 1-3,6-8 \mathrm{HxCDF} \quad 1,2,3,6,7,8$-Hexachlorodibenzofuran

$000635822469 \quad 1-4,6-8 \mathrm{HpCDD} \quad 1,2,3,4,6,7,8$-Heptachlorodibenzo-p-dioxin

$0007 \quad 121142$ 2,4-DiNitToluen 2,4-Dinitrotoluene

0008606202 2,6-DiNitToluen 2,6-Dinitrotoluene

000995578 2-CHLOROPHENOL 2-CHLOROPHENOL

$0010107051 \quad$ AllylChlor Allyl chlorid

$\begin{array}{llll}0011 & 7429905 & \text { Aluminum } & \text { Aluminum } \\ 0012 & 7440360 & \text { Antimony } & \text { Antimony }\end{array}$

00137440393 Barium

$001471432 \quad$ Benzene $\quad$ Benzene

00157440439 Cadmium Cadmium

001656235 CCl4 $\quad$ Carbon tetrachloride

$\begin{array}{llll}0016 & 56235 & \text { CCL4 } & \text { Carbon tetract } \\ 0017 & 67663 & \text { Chloroform } & \text { Chloroform }\end{array}$

$\begin{array}{llll}0017 & 67663 & \text { Chloroform } & \text { Chloroform } \\ 0018 & 7440473 & \text { Chromium } & \text { Chromium }\end{array}$

00197782505 Chlorine Chlorine

0020630080 Carbon Monoxide Carbon monoxide

00217440508 Copper Copper

0022110827 Cyclohexane Cyclohexane

002384662 DiethylPhthalat Diethyl phthalate

$0024122394 \quad$ Diphenylam Diphenylamine

$\begin{array}{llll}0025 & 75003 & \text { Ethyl Chloride Ethyl chloride \{Chlorethane } \\ 0026 & 100414 & \text { Ethyl Benzene Ethyl benzene }\end{array}$

$0027206440 \quad$ Fluoranthene Fluoranthene

$00287647010 \mathrm{HCl} \quad$ Hydrochloric acid

$\begin{array}{llll}0029 & 98828 & \text { Cumene } & \text { Cumene } \\ 0030 & 7439921 & \text { Lead } & \text { Lead }\end{array}$

03174873

Methyl Chloride Methyl chloride \{Chloromethane\}

1,1,1-TCA Methyl chloroform $\{1,1,1-\mathrm{TCA}\}$

0033108872 MethCyHex Methylcyclohexane

003475092 Methylene Chlor Methylene chloride \{Dichloromethane\}

003591203 Naphthalene Naphthalene

0036110543 Hexane Hexane

$\begin{array}{llll}0036 & 110543 & \text { Hexane } & \text { Hexane } \\ 0037 & 10102440 & \text { NITROGEN DIOXID NITROGEN DIOXIDE }\end{array}$

$\begin{array}{llll}0037 & 10102440 & \text { NITROGEN DIOXID NITROGEN DIOXIDE } \\ 0038 & 39001020 & 1-80 \mathrm{ctaCDF} & 1,2,3,4,6,7,8,9 \text {-0ctachlorodibenzofuran }\end{array}$

$003978115 \quad$ PETN

0040108952 Phenol Phenol

$0041115071 \quad$ Propylene Propylen

$\begin{array}{llll}0042 & 121824 & \text { RDX } & \text { RDX }\end{array}$

$\begin{array}{llll}0043 & 100425 & \text { Styrene } & \text { Styrene } \\ 0044 & 7446095 & \text { SULFUR DIOXIDE } & \text { SULFUR DIOXIDE }\end{array}$

0045127184 Perc $\quad$ Perchloroethylene \{Tetrachloroethene\}

0046108883 Toluene Toluene

$004775014 \quad$ Vinyl Chloride Vinyl chloride

00487440666 Zinc Zinc

004910101 NapthSur Naphthalene surrogate

005010102 EthBzSur Ethylbenzene surrogate

005110103 nHexSur n-Hexane surrogate

BACKGROUND (ug $/ \mathrm{m} \wedge 3)$

$0.000 \mathrm{E}+00$

$0.000 \mathrm{E}+00$

$0.000 \mathrm{E}+00$

$0.000 \mathrm{E}+00$

$0.000 \mathrm{E}+00$

$0.000 \mathrm{E}+00$

$0.000 \mathrm{E}+00$

$0.000 \mathrm{E}+00$

$0.000 \mathrm{E}+00$

$0.000 \mathrm{E}+00$

$0.000 \mathrm{E}+00$

$0.000 \mathrm{E}+00$

$0.000 \mathrm{E}+00$

$0.000 \mathrm{E}+00$

$0.000 \mathrm{E}+00$

$0.000 \mathrm{E}+00$

$0.000 \mathrm{E}+00$

$0.000 \mathrm{E}+00$

$0.000 \mathrm{E}+00$

$0.000 \mathrm{E}+00$

$0.000 \mathrm{E}+00$

$0.000 \mathrm{E}+00$

$0.000 \mathrm{E}+00$

$0.000 \mathrm{E}+00$

$0.000 \mathrm{E}+00$

$0.000 \mathrm{E}+00$

$0.000 \mathrm{E}+00$

$0.000 \mathrm{E}+00$

$0.000 \mathrm{E}+00$

$0.000 \mathrm{E}+00$

$0.000 \mathrm{E}+00$

$0.000 \mathrm{E}+00$

$0.000 \mathrm{E}+00$

$0.000 \mathrm{E}+00$

$0.000 \mathrm{E}+00$

$0.000 \mathrm{E}+00$

$0.000 \mathrm{E}+00$

$0.000 \mathrm{E}+00$

$0.000 \mathrm{E}+00$

$0.000 \mathrm{E}+00$

$0.000 \mathrm{E}+00$

$0.000 \mathrm{E}+00$

$0.000 \mathrm{E}+00$

$0.000 \mathrm{E}+00$

$0.000 \mathrm{E}+00$

$0.000 \mathrm{E}+00$

$0.000 \mathrm{E}+00$

$0.000 \mathrm{E}+00$

$0.000 \mathrm{E}+00$

$0.000 \mathrm{E}+00$

$0.000 \mathrm{E}+00$

$0.000 \mathrm{E}+00$

$0.000 \mathrm{E}+00$

DATA SOURCE:

CHEMICALS ADDED OR DELETED: none

EMISSIONS FOR FACILITY FAC=Site300 CO=* DEV=PR1 PRO=0BPan STK=1 NAME=LLNL Site 300 EMS (lbs/yr) SOURCE MULTIPLIER=1

CAS

1069901 1,3-Butadiene

$67562394 \quad 1-4,6-8 \mathrm{HpCDF}$

$55673897 \quad 1-4,7-9 \mathrm{HpCDF}$

$\begin{array}{ll}70648269 & 1-4,7,8 \mathrm{HxCDF} \\ 57117449 & 1-3,6-8 \mathrm{HxCDF}\end{array}$

$\begin{array}{rrrr}\text { MULTIPLIER } & \text { BG (ug/m^3) } & \text { AVRG (lbs/yr) } & \text { MAX (lbs/hr) } \\ 1 & 0 & 0.017 & 0.00017 \\ 1 & 0 & 0 & 0 \\ 1 & 0 & 0 & 0 \\ 1 & 0 & 0 & 0 \\ 1 & 0 & 0 & 0\end{array}$




\begin{tabular}{ll}
35822469 & 1-4,6-8HpCDD \\
121142 & 2,4-DiNitToluen \\
606202 & 2,6-DiNitToluen \\
95578 & 2-CHLOROPHENOL \\
107051 & AllylChlor \\
7429905 & Aluminum \\
7440360 & Antimony \\
7440393 & Barium \\
71432 & Benzene \\
7440439 & Cadmium \\
56235 & CCl4 \\
67663 & Chloroform \\
7440473 & Chromium \\
7782505 & Chlorine \\
630080 & Carbon Monoxide \\
7440508 & Copper \\
110827 & Cyclohexane \\
84662 & DiethylPhthalat \\
122394 & Diphenylam \\
75003 & Ethyl Chloride \\
100414 & Ethyl Benzene \\
206440 & Fluoranthene \\
7647010 & HCl \\
98828 & Cumene \\
7439921 & Lead \\
74873 & Methyl Chloride \\
71556 & 1,1,1-TCA \\
108872 & MethCyHex \\
75092 & Methylene Chlor \\
91203 & Naphthalene \\
110543 & Hexane \\
10102440 & NITROGEN DIOXID \\
39001020 & 1-80ctaCDF \\
78115 & PETN \\
108952 & Phenol \\
115071 & Propylene \\
121824 & RDX \\
100425 & Styrene \\
7446095 & SULFUR DIOXIDE \\
127184 & Perc \\
108883 & Toluene \\
75014 & Vinyl Chloride \\
7440666 & Zinc \\
10101 & NapthSur \\
10102 & EthBzSur \\
10103 & nHexSur \\
& \\
\hline
\end{tabular}

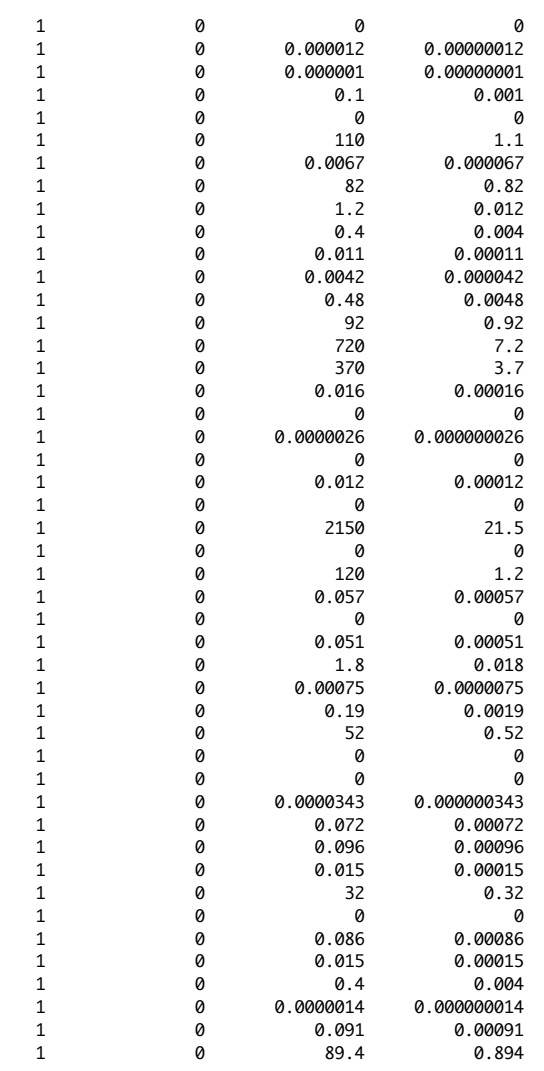

EMISSIONS FOR FACILITY FAC=Site300 CO=* DEV=PR1 PRO=0BCage3 STK=1 NAME=LLNL Site $300 \quad$ EMS (lbs/yr) SOURCE MULTIPLIER=1

\begin{tabular}{ll} 
CAS & ABBREV \\
106990 & $1,3-$ Butadiene \\
67562394 & $1-4,6-8$ HpCDF \\
55673897 & $1-4,7-9 H p C D F$ \\
70648269 & $1-4,7,8$ HXCDF \\
57117449 & $1-3,6-8$ HxCDF \\
35822469 & $1-4,6-8$ HpCDD \\
121142 & $2,4-$ DiNitToluen \\
606202 & $2,6-$ DiNitToluen \\
95578 & 2-CHLOROPHENOL \\
107051 & AllylChlor \\
7429905 & Aluminum \\
7440360 & Antimony \\
7440393 & Barium \\
71432 & Benzene \\
7440439 & Cadmium \\
56235 & CCl4 \\
67663 & Chloroform \\
7440473 & Chromium \\
7782505 & Chlorine \\
630080 & Carbon Monoxide \\
7440508 & Copper \\
110827 & Cyclohexane \\
84662 & DiethylPhthalat \\
122394 & Diphenylam \\
75003 & Ethyl Chloride \\
100414 & Ethyl Benzene \\
206440 & Fluoranthene \\
7647010 & HCl \\
98828 & Cumene \\
7439921 & Lead \\
74873 & Methyl Chloride \\
71556 & $1,1,1-$ TCA \\
108872 & MethCyHex \\
75092 & Methylene Chlor \\
91203 & Naphthalene \\
110543 & Hexane \\
10102440 & NITROGEN DIOXID \\
39001020 & $1-80$ ctaCDF \\
78115 & PETN \\
108952 & Phenol \\
115071 & Propylene \\
121824 & RDX \\
100425 & Styrene \\
7446095 & SULFUR DIOXIDE \\
127184 & Perc \\
108883 & Toluene \\
75014 & Vinyl Chloride \\
7440666 & Zinc \\
& \\
\hline &
\end{tabular}

\begin{tabular}{|c|c|c|c|}
\hline MULTIPLIER & BG $\left(u g / m^{\wedge} 3\right)$ & AVRG (lbs/yr) & $\operatorname{MAX}(\mathrm{lbs} / \mathrm{hr})$ \\
\hline 1 & 0 & 0 & 0 \\
\hline 1 & 0 & 0.00017 & 0.0000017 \\
\hline 1 & 0 & 0.0000395 & 0.000000395 \\
\hline 1 & 0 & 0.000105 & 0.00000105 \\
\hline 1 & 0 & 0.0000475 & 0.000000475 \\
\hline 1 & 0 & 0 & 0 \\
\hline 1 & 0 & 0 & 0 \\
\hline 1 & 0 & 0 & 0 \\
\hline 1 & 0 & 0 & 0 \\
\hline 1 & 0 & 0 & 0 \\
\hline 1 & 0 & 180 & 1.8 \\
\hline 1 & 0 & 0 & 0 \\
\hline 1 & 0 & 0.43 & 0.0043 \\
\hline 1 & 0 & 2.25 & 0.0225 \\
\hline 1 & 0 & 0 & 0 \\
\hline 1 & 0 & 0.028 & 0.00028 \\
\hline 1 & 0 & 0.0115 & 0.000115 \\
\hline 1 & 0 & 0 & 0 \\
\hline 1 & 0 & 1 & 0.01 \\
\hline 1 & 0 & 100 & 1 \\
\hline 1 & 0 & 0.075 & 0.00075 \\
\hline 1 & 0 & 0.01 & 0.0001 \\
\hline 1 & 0 & 0 & 0 \\
\hline 1 & 0 & 0 & 0 \\
\hline 1 & 0 & 0 & 0 \\
\hline 1 & 0 & 0.012 & 0.00012 \\
\hline 1 & 0 & 1 & 0.01 \\
\hline 1 & 0 & 415 & 4.15 \\
\hline 1 & 0 & 0 & 0 \\
\hline 1 & 0 & 1.4 & 0.014 \\
\hline 1 & 0 & 0.1 & 0.001 \\
\hline 1 & 0 & 0 & 0 \\
\hline 1 & 0 & 0.04 & 0.0004 \\
\hline 1 & 0 & 0.06 & 0.0006 \\
\hline 1 & 0 & 0 & 0 \\
\hline 1 & 0 & 0.024 & 0.00024 \\
\hline 1 & 0 & 0.033 & 0.00033 \\
\hline 1 & 0 & 0.0002 & 0.000002 \\
\hline 1 & 0 & 0 & 0 \\
\hline 1 & 0 & 0 & 0 \\
\hline 1 & 0 & 0.13 & 0.0013 \\
\hline 1 & 0 & 0 & 0 \\
\hline 1 & 0 & 0 & 0 \\
\hline 1 & 0 & 4.3 & 0.043 \\
\hline 1 & 0 & 0.0085 & 0.000085 \\
\hline 1 & 0 & 0.14 & 0.0014 \\
\hline 1 & 0 & 0 & 0 \\
\hline 1 & 0 & 2.85 & 0.0285 \\
\hline
\end{tabular}




$\begin{array}{rlrrrr}10101 & \text { NapthSur } & 1 & 0 & 0.8 & 0.008 \\ 10102 & \text { EthBzSur } & 1 & 0 & 0.038 & 0.00038 \\ 10103 & \text { nHexSur } & 1 & 0 & 9.83 & 0.0983\end{array}$
EMISSIONS FOR FACILITY FAC $=S i t e 300 \quad C 0=* \quad$ DEV=PR1 $\quad$ PRO=0BCage4 $\quad$ STK=1 NAME=LLNL Site $300 \quad$ EMS (lbs/yr)
SOURCE MULTIPLIER $=1$

$\begin{array}{ll}\text { SOURCE MULTIPLIER=1 } \\ \text { CAS } & \text { ABBREV } \\ 106990 & 1,3-\text { Butadiene } \\ 67562394 & 1-4,6-8 \text { HpCDF } \\ 55673897 & 1-4,7-9 H p C D F \\ 70648269 & 1-4,7,8 \text { HXCDF } \\ 57117449 & 1-3,6-8 \text { HCDF } \\ 35822469 & 1-4,6-8 \text { HpCDD } \\ 121142 & 2,4-\text { DiNitToluen } \\ 606202 & 2,6-\text { DiNitoluen } \\ 95578 & \text { 2-CHLOROPHENOL } \\ 107051 & \text { AllylChlor } \\ 7429905 & \text { Aluminum } \\ 7440360 & \text { Antimony } \\ 7440393 & \text { Barium } \\ 71432 & \text { Benzene } \\ 7440439 & \text { Cadmium } \\ 56235 & \text { CCl4 } \\ 67663 & \text { Chloroform } \\ 7440473 & \text { Chromium } \\ 7782505 & \text { Chlorine } \\ 630080 & \text { Carbon Monoxide } \\ 7440508 & \text { Copper } \\ 110827 & \text { Cyclohexane } \\ 84662 & \text { DiethylPhthalat } \\ 122394 & \text { Diphenylam } \\ 75003 & \text { Ethyl Chloride } \\ 100414 & \text { Ethyl Benzene } \\ 206440 & \text { Fluoranthene } \\ 7647010 & \text { HCl } \\ 98828 & \text { Cumene } \\ 7439921 & \text { Lead } \\ 74873 & \text { Methyl Chloride } \\ 71556 & 1,1,1-T C A \\ 108872 & \text { MethCyHex } \\ 75092 & \text { Methylene Chlor } \\ 91203 & \text { Naphthalene } \\ 110543 & \text { Hexane } \\ 10102440 & \text { NITROGEN DI0XID } \\ 39001020 & 1-80 \text { ctaCDF } \\ 78115 & \text { PETN } \\ 108952 & \text { Phenol } \\ 115071 & \text { Propylene } \\ 121824 & \text { RDX } \\ 100425 & \text { Styrene } \\ 7446095 & \text { SULFUR DIOXIDE } \\ 127184 & \text { Perc } \\ 108883 & \text { Toluene } \\ 75014 & \text { Vinyl Chloride } \\ 7440666 & \text { Zinc } \\ 10101 & \text { NapthSur } \\ 10102 & \text { EthBzSur } \\ 10103 & \text { nHexSur } \\ & \end{array}$

MULTIPLIER

$B G\left(u g / m^{\wedge} 3\right)$

$\begin{array}{rrrr}\text { MULTIPLIER } & \text { BG (ug/m^3) } & \text { AVRG }(\mathrm{lbs} / \mathrm{yr}) & \text { MAX }(\mathrm{lbs} / \mathrm{hr}) \\ 1 & 0 & 0.42 & 0.0042 \\ 1 & 0 & 0 & 0 \\ 1 & 0 & 0 & 0 \\ 1 & 0 & 0 & 0 \\ 1 & 0 & 0 & 0 \\ 1 & 0 & 0.0000214 & 0.000000214 \\ 1 & 0 & 0 & 0 \\ 1 & 0 & 0 & 0 \\ 1 & 0 & 0 & 0 \\ 1 & 0 & 0.63 & 0.0063 \\ 1 & 0 & 875 & 8.75 \\ 1 & 0 & 0.0235 & 0.000235 \\ 1 & 0 & 287 & 2.87 \\ 1 & 0 & 3.85 & 0.0385 \\ 1 & 0 & 2.07 & 0.385 \\ 1 & 0 & 0.266 & 0.00266 \\ 1 & 0 & 0.0133 & 0.000133 \\ 1 & 0 & 5.25 & 0.0525 \\ 1 & 0 & 0 & 0 \\ 1 & 0 & 3850 & 38.5 \\ 1 & 0 & 910 & 9.1 \\ 1 & 0 & 0.263 & 0.00263 \\ 1 & 0 & 2.7 & 0.027 \\ 1 & 0 & 0 & 0 \\ 1 & 0 & 0.0242 & 0.000242 \\ 1 & 0 & 0.161 & 0.00161 \\ 1 & 0 & 0 & 0 \\ 1 & 0 & 0 & 0 \\ 1 & 0 & 0.0256 & 0.000256 \\ 1 & 0 & 38.5 & 0.385 \\ 1 & 0 & 0.0595 & 0.000595 \\ 1 & 0 & 0.116 & 0.00116 \\ 1 & 0 & 0.245 & 0.00245 \\ 1 & 0 & 30.5 & 0.305 \\ 1 & 0 & 0 & 0 \\ 1 & 0 & 0.665 & 0.006655 \\ & 0 & & \end{array}$




$\begin{array}{lllrrr}10102440 & \text { NITROGEN DIOXID } & 1 & 0 & 154 & 1.54 \\ 39001020 & \text { 1-80ctaCDF } & 1 & 0 & 0 & 0 \\ 78115 & \text { PETN } & 1 & 0 & 19.6 & 0.196 \\ 108952 & \text { Phenol } & 1 & 0 & 0 & 0 \\ 115071 & \text { Propylene } & 1 & 0 & 2.56 & 0.0256 \\ 121824 & \text { RDX } & 1 & 0 & 259 & 2.59 \\ 100425 & \text { Styrene } & 1 & 0 & 1.47 & 0.0147 \\ 7446095 & \text { SULFUR DIOXIDE } & 1 & 0 & 455 & 4.55 \\ 127184 & \text { Perc } & 1 & 0 & 0.63 & 0.0063 \\ 108883 & \text { Toluene } & 1 & 0 & 1.05 & 0.0105 \\ 75014 & \text { Vinyl Chloride } & 1 & 0 & 0.0805 & 0.000805 \\ 7440666 & \text { Zinc } & 1 & 0 & 560 & 5.6 \\ 10101 & \text { NapthSur } & 1 & 0 & 0 & 0 \\ 10102 & \text { EthBzSur } & 1 & 0 & 0.62 & 0.0062 \\ 10103 & \text { nHexSur } & 1 & 0 & 119 & 1.19\end{array}$

ACUTE HI REPORT

RECEPTOR 5

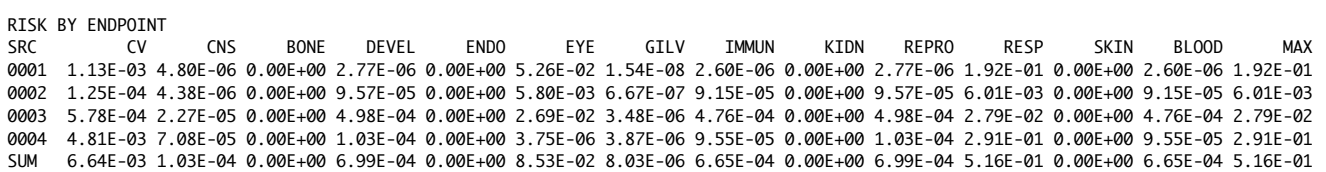


Rep_Can_WRK_Avg_Rec5_AllSrc_AllCh_BySrc_Bychem_Site.txt 
This file: c: \HARPExpress \Projects \Rep_Can_WRK_Avg_Rec5_AllSrc_AllCh_BySrC_ByChem_Site.txt

Created by HARP Version 1.0 Build 23.02.10

Uses ISC Version 99155

Uses BPIP Version 95086

Creation date: 10/24/2005 2:30:02 PM

EXCEPTION REPORT

(there have been no changes or exceptions)

INPUT FILES:

Source-Receptor file: c: \HARPExpress \Projects \EWTF.mta

Averaging period adjustment factors file: not applicable

Averaging period adjustment factors file: not applicable

Site parameters file: C:\HARPExpress $\backslash$ Projects $\backslash$ Site300. sit

Screening mode is OFF

USER-DEFINED WORKER ADJUSTMENT AND EXPOSURE PARAMETERS

GLC Adjustment Factor: 1

Exposure Duration (years): 25

Exposure Frequency (days/year): 245

Explanatory Note: U.S. EPA value

Analysis method: Point estimat

Heal th effect: Cancer Risk

Receptor(s): $\quad 5$

Sources(s): All

Chemicals(s): Al

SITE PARAMETERS

DEPOSITION

Deposition rate $(\mathrm{m} / \mathrm{s})$

0.0072

DRINKING WATER

*** Pathway disabled ***

FISH

*** Pathway disabled $* * *$

PASTURE

\begin{tabular}{|c|c|}
\hline \multicolumn{2}{|l|}{ ANIMALS' WATER } \\
\hline Water surface area $\left(m^{\wedge} 2\right)$ & 0 \\
\hline Water volume $(\mathrm{L})$ & 0 \\
\hline Volume changes per year & 0 \\
\hline \multicolumn{2}{|l|}{ Fraction of beef cows' water } \\
\hline \multicolumn{2}{|l|}{$\begin{array}{l}\text { Fraction of dairy cows' water } \\
\text { from pasture source }\end{array}$} \\
\hline \multicolumn{2}{|l|}{ ANIMALS' FEED } \\
\hline $\begin{array}{l}\text { Fraction of cows' feed } \\
\text { from grazing }\end{array}$ & 0.25 \\
\hline \multicolumn{2}{|l|}{ HUMAN INGESTION } \\
\hline $\begin{array}{l}\text { Fraction of ingested beef } \\
\text { from contaminated source }\end{array}$ & 0.107 \\
\hline \multicolumn{2}{|l|}{ Fraction of ingested dairy } \\
\hline from contaminated source & 0 \\
\hline \multicolumn{2}{|l|}{ ME GROWN PRODUCE } \\
\hline \multicolumn{2}{|l|}{ HUMAN INGESTION } \\
\hline \multicolumn{2}{|c|}{ Fraction of ingested leafy vegetable } \\
\hline \\
\hline $\begin{array}{l}\text { Fraction of ingested protected } \\
\text { from home grown source }\end{array}$ & $\begin{array}{l}\text { getable } \\
0.134\end{array}$ \\
\hline Fraction of ingested root vege & \\
\hline from home grown source & 0.088 \\
\hline
\end{tabular}

PIGS, CHICKENS AND EGGS

HUMAN INGESTION

Fraction of ingested pig

from home grown source

Fraction of ingested chicken

0.04

from home ingested chi

0.026

Fraction of ingested egg

0.026

from home grown source

0.029

ANIMALS' FEED

Fraction of pigs' feed

Fraction of chickens' feed

0.1

from home grown crop

0.05

SOIL INGESTION

Fraction of pigs' feed 


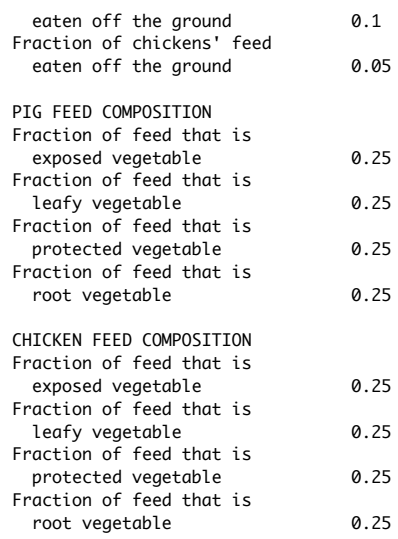

DERMAL ABSORPTION

*** Pathway disabled $* * *$

SOIL INGESTION

*** Pathway enabled ***

MOTHER'S MILK

*** Pathway enabled ***

CHEMICAL CROSS-REFERENCE TABLE AND BACKGROUND CONCENTRATIONS ABBREVIATION POLLUTANT NAME

0001106990 1,3-Butadiene 1,3-Butadiene

000267562394 1-4,6-8HpCDF 1,2,3,4,6,7,8-Heptachlorodibenzofuran

$000355673897 \quad 1-4,7-9 \mathrm{HpCDF} \quad 1,2,3,4,7,8,9$-Heptachlorodibenzofuran

$000470648269 \quad 1-4,7,8 \mathrm{HxCDF} \quad 1,2,3,4,7,8$-Hexachlorodibenzofuran

$000557117449 \quad 1-3,6-8 \mathrm{HxCDF} \quad 1,2,3,6,7,8$-Hexachlorodibenzofuran

000635822469 1-4,6-8HpCDD 1,2,3,4,6,7,8-Heptachlorodibenzo-p-dioxin

0007121142 2,4-DiNitToluen 2,4-Dinitrotoluene

0008606202 2,6-DiNitToluen 2,6-Dinitrotoluen

000995578 2-CHLOROPHENOL 2-CHLOROPHENOL

0010107051 AllylChlor Allyl chloride

00117429905 Aluminum Aluminum

00127440360 Antimony Antimony

$\begin{array}{llll}0013 & 7440393 & \text { Barium } & \text { Barium } \\ 0014 & 71432 & \text { Benzene } & \text { Benzene }\end{array}$

00157440439 Cadmium Cadmium

001656235 CC14 Carbon tetrachloride

$001767663 \quad$ Chloroform Chloroform

00187440473 Chromium Chromium

00197782505 Chlorine Chlorine

0020630080 Carbon Monoxide Carbon monoxide

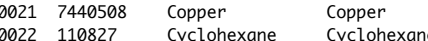

002384662 DiethylPhthalat Diethyl phthalate

$0024122394 \quad$ Diphenylam Diphenylamine

002575003 Ethyl Chloride Ethyl chloride \{Chlorethan

$0026100414 \quad$ Ethyl Benzene Ethyl benzene

$0027206440 \quad$ Fluoranthene Fluoranthene

$00287647010 \quad \mathrm{HCl} \quad$ Hydrochloric acid

002998828 Cumene Cumene

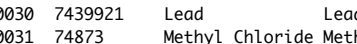

Methyl Chloride Methyl chloride \{Chloromethane

1,1,1-TCA Methyl chloroform $\{1,1,1-\mathrm{TCA}\}$

MethCyHex Methylcyclohexan

Methylene Chlor Methylene chloride \{Dichloromethane\}

$0035 \quad 91203$

0036110543

$0037 \begin{array}{ll}10102440 \\ 0038 & 39001020\end{array}$

Naphthalene

Hexane Hexane

NITROGEN DIOXID NITROGEN DIOXIDE

$\begin{array}{llll}0038 & 39001020 & 1-80 \text { ctaCDF } & 1,2,3,4,6,7,8,9 \text {-0ctachlorodibenzofuran } \\ 0039 & 78115 & \text { PETN } & \text { PETN (same as RDX) }\end{array}$

$\begin{array}{lll}108952 & \text { Phenol Phenol }\end{array}$

Propylene Propylen

$043 \quad 100425 \quad$ Styrene $\quad$ RDX

00447446095 SULFUR DIOXIDE SULFUR DIOXIDE

0045127184 Perc Perchloroethylene \{Tetrachloroethene\}

0046108883 Toluene Toluene

$0047 \quad 75014 \quad$ Vinyl Chloride Vinyl chloride

$0048 \quad 7440666 \quad$ Zinc

004910101 NapthSur Naphthalene surrogate

005010102 EthBzSur Ethylbenzene surrogate

$005110103 \quad$ nHexSur n-Hexane surrogate

BACKGROUND (ug/m^3)

$0.000 \mathrm{E}+00$

$0.000 \mathrm{E}+00$

$0.000 \mathrm{E}+00$

$0.000 \mathrm{E}+00$

$0.000 \mathrm{E}+00$

$0.000 \mathrm{E}+00$

$0.000 \mathrm{E}+00$

$0.000 \mathrm{E}+00$

$0.000 \mathrm{E}+00$

$0.000 \mathrm{E}+00$

$0.000 \mathrm{E}+00$

$0.000 E+00$

$0.000 E+00$

$0.000 E+00$

$0.000 \mathrm{E}+00$

$0.000 \mathrm{E}+00$

$0.000 \mathrm{E}+00$

$0.000 \mathrm{E}+00$

$0.000 \mathrm{E}+00$

$0.000 \mathrm{E}+00$

$0.000 \mathrm{E}+00$

$0.000 \mathrm{E}+00$

$0.000 \mathrm{E}+00$

$0.000 \mathrm{E}+00$

$0.000 \mathrm{E}+00$

$0.000 \mathrm{E}+00$

$0.000 \mathrm{E}+00$

$0.000 \mathrm{E}+00$

$0.000 \mathrm{E}+00$

$0.000 \mathrm{E}+00$

$0.000 \mathrm{E}+00$

$0.000 \mathrm{E}+00$

$0.000 \mathrm{E}+00$

$0.000 \mathrm{E}+00$

$0.000 \mathrm{E}+00$

$0.000 \mathrm{E}+00$

$0.000 \mathrm{E}+00$

$0.000 \mathrm{E}+00$

$0.000 \mathrm{E}+00$

$0.000 \mathrm{E}+00$

$0.000 \mathrm{E}+00$

$0.000 \mathrm{E}+00$

$0.000 \mathrm{E}+00$

$0.000 \mathrm{E}+00$

$0.000 \mathrm{E}+00$

$0.000 \mathrm{E}+00$

$0.000 \mathrm{E}+00$

$0.000 \mathrm{E}+00$

$0.000 \mathrm{E}+00$

$0.000 \mathrm{E}+00$

$0.000 \mathrm{E}+00$

$0.000 \mathrm{E}+00$

$0.000 \mathrm{E}+00$

EMISSIONS DATA SOURCE:

CHEMICALS ADDED OR DELETED: none EMISSIONS FOR FACILITY FAC=Site300 CO=* DEV=PR1 PRO=0BPan $\quad$ STK=1 NAME=LLNL Site $300 \quad$ EMS $\quad(l \mathrm{lbs} / \mathrm{yr}$ )
SOURCE MULTIPLIER=1

CAS

ABBREV

MULTIPLIER

BG (ug/m^3) AVRG (lbs/yr) MAX (lbs $/ \mathrm{hr}$ ) 


\begin{tabular}{|c|c|}
\hline 106990 & 1,3-Butadiene \\
\hline 67562394 & $1-4,6-8 \mathrm{HpCDF}$ \\
\hline 55673897 & $1-4,7-9 \mathrm{HpCDF}$ \\
\hline 70648269 & $1-4,7,8 \mathrm{HxCDF}$ \\
\hline 57117449 & $1-3,6-8 \mathrm{H} \times \mathrm{CDF}$ \\
\hline 35822469 & $1-4,6-8 \mathrm{HpCDD}$ \\
\hline 121142 & 2,4-DiNitToluen \\
\hline 606202 & 2,6-DiNitToluen \\
\hline 95578 & 2-CHLOROPHENOL \\
\hline 107051 & AllylChlor \\
\hline 7429905 & Aluminum \\
\hline 7440360 & Antimony \\
\hline 7440393 & Barium \\
\hline 71432 & Benzene \\
\hline 7440439 & Cadmium \\
\hline 56235 & CCl4 \\
\hline 67663 & Chloroform \\
\hline 7440473 & Chromium \\
\hline 7782505 & Chlorine \\
\hline 630080 & Carbon Monoxide \\
\hline 7440508 & Copper \\
\hline 110827 & Cyclohexane \\
\hline 84662 & DiethylPhthalat \\
\hline 122394 & Diphenylam \\
\hline 75003 & Ethyl Chloride \\
\hline 100414 & Ethyl Benzene \\
\hline 206440 & Fluoranthene \\
\hline 7647010 & $\mathrm{HCl}$ \\
\hline 98828 & Cumene \\
\hline 7439921 & Lead \\
\hline 74873 & Methyl Chloride \\
\hline 71556 & $1,1,1$-TCA \\
\hline 108872 & MethCyHex \\
\hline 75092 & Methylene Chlor \\
\hline 91203 & Naphthalene \\
\hline 110543 & Hexane \\
\hline 10102440 & NITROGEN DIOXID \\
\hline 39001020 & $1-80 \mathrm{ctaCDF}$ \\
\hline 78115 & PETN \\
\hline 108952 & Phenol \\
\hline 115071 & Propylene \\
\hline 121824 & RDX \\
\hline 100425 & Styrene \\
\hline 7446095 & SULFUR DIOXIDE \\
\hline 127184 & Perc \\
\hline 108883 & Toluene \\
\hline 75014 & Vinyl Chloride \\
\hline 7440666 & Zinc \\
\hline 10101 & NapthSur \\
\hline 10102 & EthBzSur \\
\hline 10103 & nHexSur \\
\hline
\end{tabular}

EMISSIONS FOR FACILITY FAC $=$ Site300 SOURCE MULTIPLIER $=1$

\begin{tabular}{|c|c|}
\hline CAS & ABBREV \\
\hline 106990 & 1,3-Butadiene \\
\hline 67562394 & $1-4,6-8 \mathrm{HpCDF}$ \\
\hline 55673897 & $1-4,7-9 \mathrm{HpCDF}$ \\
\hline 70648269 & $1-4,7,8 \mathrm{H} \times C D F$ \\
\hline 57117449 & $1-3,6-8 \mathrm{H} \times C D F$ \\
\hline 35822469 & $1-4,6-8 \mathrm{Hp} C D D$ \\
\hline 121142 & 2,4-DiNitToluen \\
\hline 606202 & 2,6-DiNitToluen \\
\hline 95578 & 2-CHLOROPHENOL \\
\hline 107051 & AllylChlor \\
\hline 7429905 & Aluminum \\
\hline 7440360 & Antimony \\
\hline 7440393 & Barium \\
\hline 71432 & Benzene \\
\hline 7440439 & Cadmium \\
\hline 56235 & CCl4 \\
\hline 67663 & Chloroform \\
\hline 7440473 & Chromium \\
\hline 7782505 & Chlorine \\
\hline 630080 & Carbon Monoxide \\
\hline 7440508 & Copper \\
\hline 110827 & Cyclohexane \\
\hline 84662 & DiethylPhthalat \\
\hline 122394 & Diphenylam \\
\hline 75003 & Ethyl Chloride \\
\hline 100414 & Ethyl Benzene \\
\hline 206440 & Fluoranthene \\
\hline 7647010 & $\mathrm{HCl}$ \\
\hline 98828 & Cumene \\
\hline 7439921 & Lead \\
\hline 74873 & Methyl Chloride \\
\hline 71556 & $1,1,1-\mathrm{TCA}$ \\
\hline 108872 & MethCyHex \\
\hline 75092 & Methylene Chlor \\
\hline 91203 & Naphthalene \\
\hline 110543 & Hexane \\
\hline 10102440 & NITROGEN DIOXID \\
\hline 39001020 & 1-80ctaCDF \\
\hline 78115 & PETN \\
\hline 108952 & Phenol \\
\hline 115071 & Propylene \\
\hline 121824 & $\mathrm{RDX}$ \\
\hline 100425 & Styrene \\
\hline
\end{tabular}

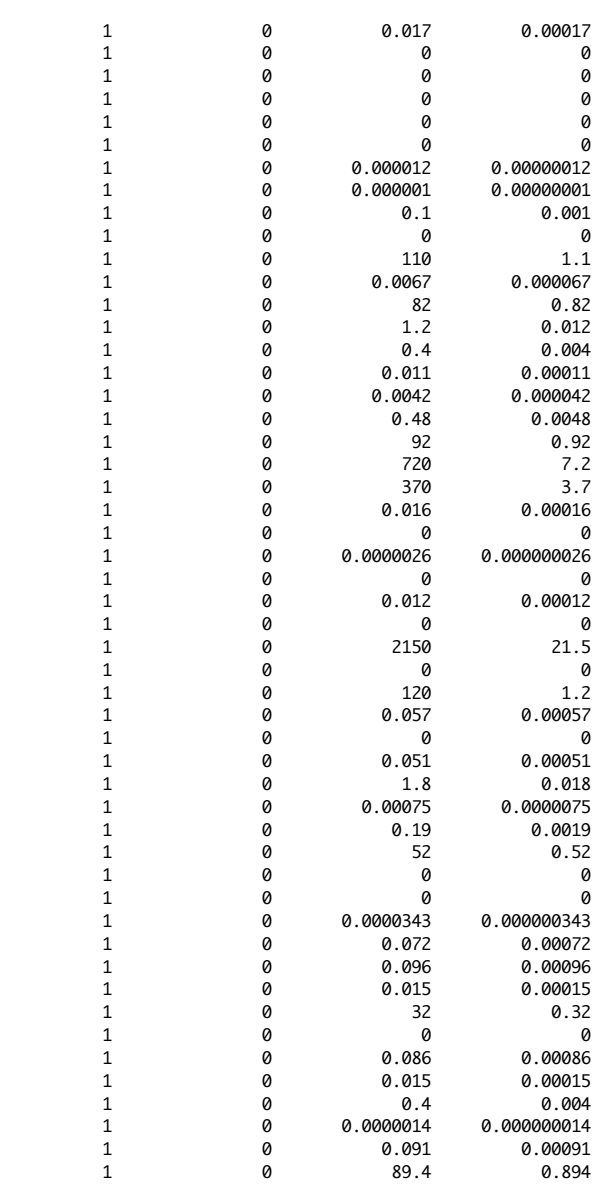

$\mathrm{C} 0=* \quad \mathrm{DEV}=\mathrm{PR} 1 \quad \mathrm{PRO}=0 \mathrm{BCage} 3 \quad \mathrm{STK}=1 \quad \mathrm{NAME}=\mathrm{LLNL}$ Site $300 \quad \mathrm{EMS}(\mathrm{lbs} / \mathrm{yr}$ )

BG $\left(u g / m^{\wedge} 3\right)$ AVRG (lbs/yr) MAX (lbs/hr)

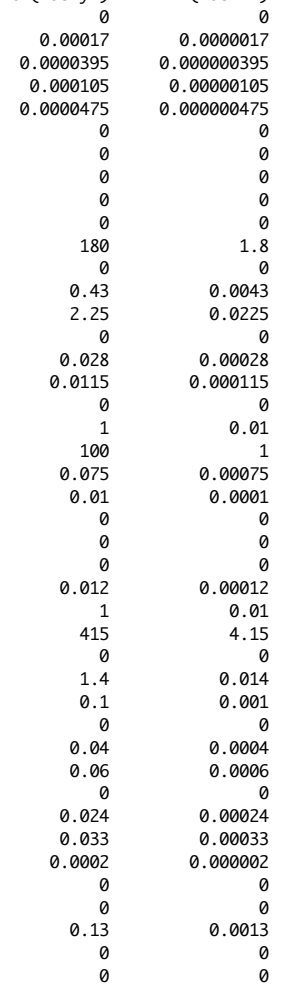




$\begin{array}{ll}7446095 & \text { SULFUR DIOXIDE } \\ 127184 & \text { Perc } \\ 108883 & \text { Toluene } \\ 75014 & \text { Vinyl Chloride } \\ 7440666 & \text { Zinc } \\ 10101 & \text { NapthSur } \\ 10102 & \text { EthBzSur } \\ 10103 & \text { nHexSur }\end{array}$

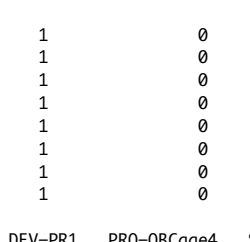

EMISSIONS FOR FACILITY FAC=Site300 SOURCE MULTIPLIER $=1$

\begin{tabular}{ll} 
CAS & ABBREV \\
106990 & $1,3-$ Butadiene \\
67562394 & $1-4,6-8$ HpCDF \\
55673897 & $1-4,7-9 H p C D F$ \\
70648269 & $1-4,7,8$ HXCDF \\
57117449 & $1-3,6-8 H x$ CDF \\
35822469 & $1-4,6-8$ HpCDD \\
121142 & $2,4-$ DiNitToluen \\
606202 & $2,6-$ DiNitToluen \\
95578 & 2-CHLOROPHENOL \\
107051 & AllylChlor \\
7429905 & Aluminum \\
7440360 & Antimony \\
7440393 & Barium \\
71432 & Benzene \\
7440439 & Cadmium \\
56235 & CCl4 \\
67663 & Chloroform \\
7440473 & Chromium \\
7782505 & Chlorine \\
630080 & Carbon Monoxide \\
7440508 & Copper \\
110827 & Cyclohexane \\
84662 & DiethylPhthalat \\
122394 & Diphenylam \\
75003 & Ethyl Chloride \\
100414 & Ethyl Benzene \\
206440 & Fluoranthene \\
7647010 & HCl \\
98828 & Cumene \\
7439921 & Lead \\
74873 & Methyl Chloride \\
71556 & $1,1,1-T C A$ \\
108872 & MethCyHex \\
75092 & Methylene Chlor \\
91203 & Naphthalene \\
110543 & Hexane \\
10102440 & NITROGEN DIOXID \\
39001020 & $1-80$ ctaCDF \\
78115 & PETN \\
108952 & Phenol \\
115071 & Propylene \\
121824 & RDX \\
100425 & Styrene \\
7446095 & SULFUR DIOXIDE \\
127184 & Perc \\
108883 & Toluene \\
75014 & Vinyl Chloride \\
7440666 & Zinc \\
10101 & NapthSur \\
10102 & EthBzSur \\
10103 & nHexSur \\
& \\
\hline
\end{tabular}

$\mathrm{CO}=* \quad$ DEV $=$ PR1 PRO=OBCage4 STK=1 NAME=LLNL Site $300 \quad$ EMS (lbs $/ \mathrm{yr})$

MULTIPLIER

BG $\left(\mathrm{ug} / \mathrm{m}^{\wedge} 3\right) \quad$ AVRG

$\begin{array}{rr}4.3 & 0.043 \\ 0.0085 & 0.000085 \\ 0.14 & 0.0014 \\ 0 & 0 \\ 2.85 & 0.0285 \\ 0.8 & 0.008 \\ 0.038 & 0.00038 \\ 9.83 & 0.0983\end{array}$

\begin{tabular}{|c|c|c|c|}
\hline -TIPLIER & BG $\left(u g / m^{\wedge} 3\right)$ & AVRG (lb & MAX (lbs/hr) \\
\hline 1 & 0 & 0 & 0 \\
\hline 1 & 0 & 0.000884 & 0.00000884 \\
\hline 1 & 0 & 0.000205 & 0.00000205 \\
\hline 1 & 0 & 0.000546 & 0.00000546 \\
\hline 1 & 0 & 0.000247 & 0.00000247 \\
\hline 1 & 0 & 0 & 0 \\
\hline 1 & 0 & 0 & 0 \\
\hline 1 & 0 & 0 & 0 \\
\hline 1 & 0 & 0 & 0 \\
\hline 1 & 0 & 0 & 0 \\
\hline 1 & 0 & 936 & 9.36 \\
\hline 1 & 0 & 0 & 0 \\
\hline 1 & 0 & 2.24 & 0.0224 \\
\hline 1 & 0 & 11.7 & 0.117 \\
\hline 1 & 0 & 0 & 0 \\
\hline 1 & 0 & 0.146 & 0.00146 \\
\hline 1 & 0 & 0.0598 & 0.000598 \\
\hline 1 & 0 & 0 & 0 \\
\hline 1 & 0 & 5.2 & 0.052 \\
\hline 1 & 0 & 520 & 5.2 \\
\hline 1 & 0 & 0.39 & 0.0039 \\
\hline 1 & 0 & 0.052 & 0.00052 \\
\hline 1 & 0 & 0 & 0 \\
\hline 1 & 0 & 0 & 0 \\
\hline 1 & 0 & 0 & 0 \\
\hline 1 & 0 & 0.0624 & 0.000624 \\
\hline 1 & 0 & 5.2 & 0.052 \\
\hline 1 & 0 & 2160 & 21.6 \\
\hline 1 & 0 & 0 & 0 \\
\hline 1 & 0 & 7.28 & 0.0728 \\
\hline 1 & 0 & 0.52 & 0.0052 \\
\hline 1 & 0 & 0 & 0 \\
\hline 1 & 0 & 0.208 & 0.00208 \\
\hline 1 & 0 & 0.312 & 0.00312 \\
\hline 1 & 0 & 0 & 0 \\
\hline 1 & 0 & 0.125 & 0.00125 \\
\hline 1 & 0 & 0.172 & 0.00172 \\
\hline 1 & 0 & 0.00104 & 0.0000104 \\
\hline 1 & 0 & 0 & 0 \\
\hline 1 & 0 & 0 & 0 \\
\hline 1 & 0 & 0.676 & 0.00676 \\
\hline 1 & 0 & 0 & 0 \\
\hline 1 & 0 & 0 & 0 \\
\hline 1 & 0 & 22.4 & 0.224 \\
\hline 1 & 0 & 0.0442 & 0.000442 \\
\hline 1 & 0 & 0.728 & 0.00728 \\
\hline 1 & 0 & 0 & 0 \\
\hline 1 & 0 & 14.8 & 0.148 \\
\hline 1 & 0 & 4.16 & 0.0416 \\
\hline 1 & 0 & 0.198 & 0.00198 \\
\hline 1 & 0 & 51.1 & 0.511 \\
\hline
\end{tabular}

EMISSIONS FOR FACILITY FAC=Site300 CO=* DEV=PR1 PRO=OD STK=1 NAME=LLNL Site 300 EMS (lbs/yr) SOURCE MULTIPLIER $=1$

$\begin{array}{ll}\text { CAS } & \text { ABBREV } \\ 106990 & 1,3-\text { Butadiene } \\ 67562394 & 1-4,6-8 \text { HpCDF } \\ 55673897 & 1-4,7-9 H \text { HCDF } \\ 70648269 & 1-4,7,8 \text { HCCDF } \\ 57117449 & 1-3,6-8 \text { HxCDF } \\ 35822469 & 1-4,6-8 \text { HpCDD } \\ 121142 & 2,4-\text { DiNitToluen } \\ 606202 & 2,6-\text { DiNitToluen } \\ 95578 & \text { 2-CHLOROPHENOL } \\ 107051 & \text { AllylChlor } \\ 7429905 & \text { Aluminum } \\ 7440360 & \text { Antimony } \\ 7440393 & \text { Barium } \\ 71432 & \text { Benzene } \\ 7440439 & \text { Cadmium } \\ 56235 & \text { CCl4 } \\ 67663 & \text { Chloroform } \\ 7440473 & \text { Chromium } \\ 7782505 & \text { Chlorine } \\ 630080 & \text { Carbon Monoxide } \\ 7440508 & \text { Copper } \\ 110827 & \text { Cyclohexane } \\ 84662 & \text { DiethylPhthalat } \\ 122394 & \text { Diphenylam } \\ 75003 & \text { Ethyl Chloride } \\ 100414 & \text { Ethyl Benzene } \\ 206440 & \text { Fluoranthene } \\ 7647010 & \text { HCl } \\ 98828 & \text { Cumene } \\ 7439921 & \text { Lead } \\ 74873 & \text { Methyl Chloride } \\ & \end{array}$

$\begin{array}{rrrr}\text { MULTIPLIER } & \text { BG (ug/m^3) } & \text { AVRG }(\mathrm{lbs} / \mathrm{yr}) & \text { MAX }(\mathrm{lbs} / \mathrm{hr}) \\ 1 & 0 & 0.42 & 0.0042 \\ 1 & 0 & 0 & 0 \\ 1 & 0 & 0 & 0 \\ 1 & 0 & 0 & 0 \\ 1 & 0 & 0 & 0 \\ 1 & 0 & 0.0000214 & 0.0000000214 \\ 1 & 0 & 0 & 0 \\ 1 & 0 & 0 & 0 \\ 1 & 0 & 0 & 0 \\ 1 & 0 & 0.63 & 0.0063 \\ 1 & 0 & 875 & 8.75 \\ 1 & 0 & 0.0235 & 0.000235 \\ 1 & 0 & 287 & 2.87 \\ 1 & 0 & 3.85 & 0.0385 \\ 1 & 0 & 2.07 & 0.385 \\ 1 & 0 & 0.266 & 0.00266 \\ 1 & 0 & 0.0133 & 0.000133 \\ 1 & 0 & 5.25 & 0.0525 \\ 1 & 0 & 0 & 0 \\ 1 & 0 & 3850 & 38.5 \\ 1 & 0 & 910 & 9.1 \\ 1 & 0 & 0.263 & 0.00263 \\ 1 & 0 & 2.7 & 0.027 \\ 1 & 0 & 0 & 0 \\ 1 & 0 & 0.0242 & 0.000242 \\ 1 & 0 & 0.161 & 0.00161 \\ 1 & 0 & 0 & 0 \\ 1 & 0 & 0 & 0 \\ 1 & 0 & 0.0256 & 0.000256 \\ 1 & 0 & 38.5 & 0.385 \\ 1 & 0 & 0.0595 & 0.000595\end{array}$




$\begin{array}{llllrr}71556 & \text { 1,1,1-TCA } & 1 & 0 & 0.116 & 0.00116 \\ 108872 & \text { MethCyHex } & 1 & 0 & 0.245 & 0.00245 \\ 75092 & \text { Methylene Chlor } & 1 & 0 & 30.5 & 0.305 \\ 91203 & \text { Naphthalene } & 1 & 0 & 0 & 0 \\ 110543 & \text { Hexane } & 1 & 0 & 0.665 & 0.00665 \\ 10102440 & \text { NITROGEN DIOXID } & 1 & 0 & 154 & 1.54 \\ 39001020 & 1-80 \text { ctaCDF } & 1 & 0 & 0 & 0 \\ 78115 & \text { PETN } & 1 & 0 & 19.6 & 0.196 \\ 108952 & \text { Phenol } & 1 & 0 & 0 & 0 \\ 115071 & \text { Propylene } & 1 & 0 & 2.56 & 0.0256 \\ 121824 & \text { RDX } & 1 & 0 & 259 & 2.59 \\ 100425 & \text { Styrene } & 1 & 0 & 1.47 & 0.0147 \\ 7446095 & \text { SULFUR DIOXIDE } & 1 & 0 & 455 & 4.55 \\ 127184 & \text { Perc } & 1 & 0 & 0.63 & 0.0063 \\ 108883 & \text { Toluene } & 1 & 0 & 1.05 & 0.0105 \\ 75014 & \text { Vinyl Chloride } & 1 & 0 & 0.0805 & 0.000805 \\ 7440666 & \text { Zinc } & 1 & 0 & 560 & 5.6 \\ 10101 & \text { NapthSur } & 1 & 0 & 0 & 0 \\ 10102 & \text { EthBzSur } & 1 & 0 & 0.62 & 0.0062 \\ 10103 & \text { nHexSur } & 1 & 0 & 119 & 1.19\end{array}$

CANCER RISK REPORT

RECEPTOR 5

SOURCE 1 RISK BY PATHWAY

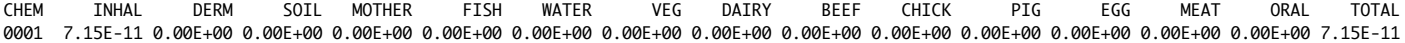

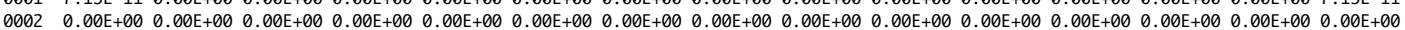
$00030.00 \mathrm{E}+000.00 \mathrm{E}+000.00 \mathrm{E}+000.00 \mathrm{E}+000.00 \mathrm{E}+000.00 \mathrm{E}+000.00 \mathrm{E}+000.00 \mathrm{E}+000.00 \mathrm{E}+00 \quad 0.00 \mathrm{E}+000.00 \mathrm{E}+000.00 \mathrm{E}+000.00 \mathrm{E}+000.00 \mathrm{E}+000.00 \mathrm{E}+00$

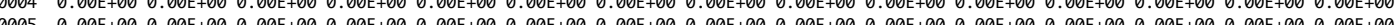

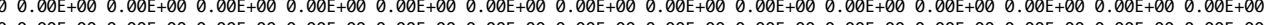

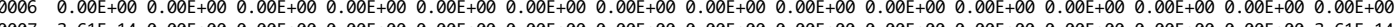
$0072.61 E-14$. 0008 ( $4.77 E-15$ a. 作 (1)

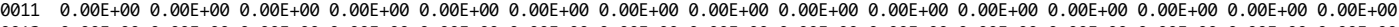

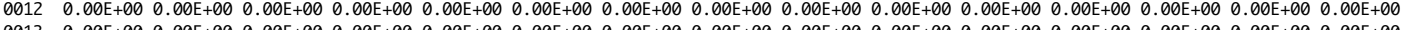

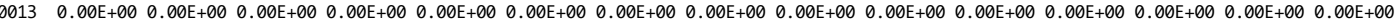

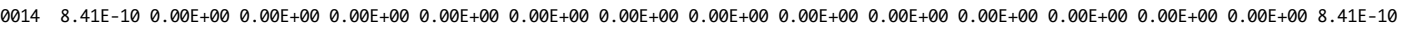
$0015 \quad 4.21 \mathrm{E}-08 \quad 0.00 \mathrm{E}+00 \quad 0.00 \mathrm{E}+00 \quad 0.00 \mathrm{E}+00 \quad 0.00 \mathrm{E}+00 \quad 0.00 \mathrm{E}+00 \quad 0.00 \mathrm{E}+00 \quad 0.00 \mathrm{E}+00 \quad 0.00 \mathrm{E}+00 \quad 0.00 \mathrm{E}+00 \quad 0.00 \mathrm{E}+00 \quad 0.00 \mathrm{E}+00 \quad 0.00 \mathrm{E}+00 \quad 0.00 \mathrm{E}+00 \quad 4.21 \mathrm{E}-08$

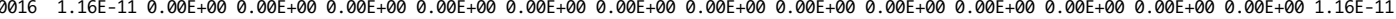

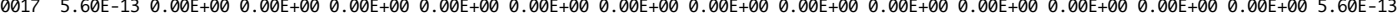
$0018 \quad 0.00 \mathrm{E}+00 \quad 0.00 \mathrm{E}+00 \quad 0.00 \mathrm{E}+00 \quad 0.00 \mathrm{E}+00 \quad 0.00 \mathrm{E}+00 \quad 0.00 \mathrm{E}+00 \quad 0.00 \mathrm{E}+00 \quad 0.00 \mathrm{E}+00 \quad 0.00 \mathrm{E}+00 \quad 0.00 \mathrm{E}+00 \quad 0.00 \mathrm{E}+00 \quad 0.00 \mathrm{E}+00 \quad 0.00 \mathrm{E}+00 \quad 0.00 \mathrm{E}+00 \quad 0.00 \mathrm{E}+00$ $0019 \quad 0.00 \mathrm{E}+00 \quad 0.00 \mathrm{E}+00 \quad 0.00 \mathrm{E}+00 \quad 0.00 \mathrm{E}+00 \quad 0.00 \mathrm{E}+00 \quad 0.00 \mathrm{E}+00 \quad 0.00 \mathrm{E}+00 \quad 0.00 \mathrm{E}+00 \quad 0.00 \mathrm{E}+00 \quad 0.00 \mathrm{E}+00 \quad 0.00 \mathrm{E}+00 \quad 0.00 \mathrm{E}+00 \quad 0.00 \mathrm{E}+00 \quad 0.00 \mathrm{E}+00 \quad 0.00 \mathrm{E}+00$ $0020 \quad 0.00 \mathrm{E}+00 \quad 0.00 \mathrm{E}+00 \quad 0.00 \mathrm{E}+00 \quad 0.00 \mathrm{E}+00 \quad 0.00 \mathrm{E}+00 \quad 0.00 \mathrm{E}+00 \quad 0.00 \mathrm{E}+00 \quad 0.00 \mathrm{E}+00 \quad 0.00 \mathrm{E}+00 \quad 0.00 \mathrm{E}+00 \quad 0.00 \mathrm{E}+00 \quad 0.00 \mathrm{E}+00 \quad 0.00 \mathrm{E}+00 \quad 0.00 \mathrm{E}+00 \quad 0.00 \mathrm{E}+00$

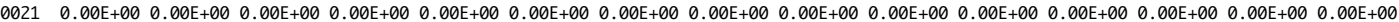
$00220.00 \mathrm{E}+000.00 \mathrm{E}+000.00 \mathrm{E}+000.00 \mathrm{E}+000.00 \mathrm{E}+000.00 \mathrm{E}+000.00 \mathrm{E}+000.00 \mathrm{E}+000.00 \mathrm{E}+000.00 \mathrm{E}+000.00 \mathrm{E}+000.00 \mathrm{E}+000.00 \mathrm{E}+000.00 \mathrm{E}+000.00 \mathrm{E}+00$ 0023 0.00E 0023

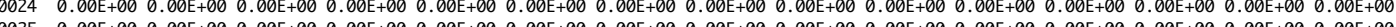
$00260.00 \mathrm{E}+00 \quad 0.00 \mathrm{E}+00 \quad 0.00 \mathrm{E}+00 \quad 0.00 \mathrm{E}$ 0027 D.00E $0028-0.00 E+00$. $\begin{array}{llllllllllllllllllllll}0029 & 0.00 \mathrm{E}+00 & 0.00 \mathrm{E}+00 & 0.00 \mathrm{E}+00 & 0.00 \mathrm{E}+00 & 0.00 \mathrm{E}+00 & 0.00 \mathrm{E}+00 & 0.00 \mathrm{E}+00 & 0.00 \mathrm{E}+00 & 0.00 \mathrm{E}+00 & 0.00 \mathrm{E}+00 & 0.00 \mathrm{E}+00 & 0.00 \mathrm{E}+00 & 0.00 \mathrm{E}+00 & 0.00 \mathrm{E}+00 & 0.00 \mathrm{E}+00\end{array}$

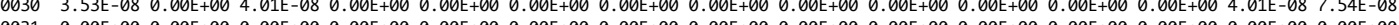

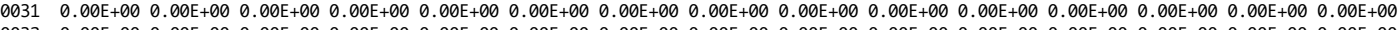

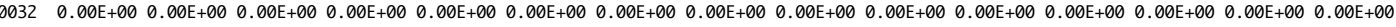

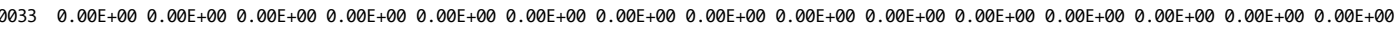
$0034 \quad 4.42 \mathrm{E}-11 \quad 0.00 \mathrm{E}+00 \quad 0.00 \mathrm{E}+00 \quad 0.00 \mathrm{E}+00 \quad 0.00 \mathrm{E}+00 \quad 0.00 \mathrm{E}+00 \quad 0.00 \mathrm{E}+00 \quad 0.00 \mathrm{E}+00 \quad 0.00 \mathrm{E}+00 \quad 0.00 \mathrm{E}+00 \quad 0.00 \mathrm{E}+00 \quad 0.00 \mathrm{E}+00 \quad 0.00 \mathrm{E}+00 \quad 0.00 \mathrm{E}+00 \quad 4.42 \mathrm{E}-11$

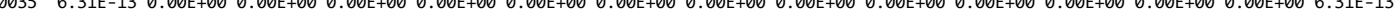

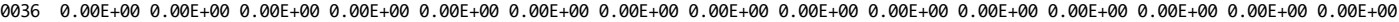
$0037 \quad 0.00 \mathrm{E}+00 \quad 0.00 \mathrm{E}+00 \quad 0.00 \mathrm{E}+00 \quad 0.00 \mathrm{E}+00 \quad 0.00 \mathrm{E}+00 \quad 0.00 \mathrm{E}+00 \quad 0.00 \mathrm{E}+00 \quad 0.00 \mathrm{E}+00 \quad 0.00 \mathrm{E}+00 \quad 0.00 \mathrm{E}+00 \quad 0.00 \mathrm{E}+00 \quad 0.00 \mathrm{E}+00 \quad 0.00 \mathrm{E}+00 \quad 0.00 \mathrm{E}+00 \quad 0.00 \mathrm{E}+00$

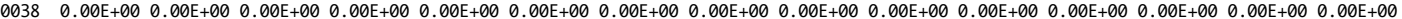
$00390.00 \mathrm{E}+00 \quad 0.00 \mathrm{E}+00 \quad 0.00 \mathrm{E}+00 \quad 0.00 \mathrm{E}+00 \quad 0.00 \mathrm{E}+00 \quad 0.00 \mathrm{E}+00 \quad 0.00 \mathrm{E}+00 \quad 0.00 \mathrm{E}+00 \quad 0.00 \mathrm{E}+00 \quad 0.00 \mathrm{E}+00 \quad 0.00 \mathrm{E}+00 \quad 0.00 \mathrm{E}+00 \quad 0.00 \mathrm{E}+00 \quad 0.00 \mathrm{E}+00 \quad 0.00 \mathrm{E}+00$ $0040 \quad 0.00 \mathrm{E}+00 \quad 0.00 \mathrm{E}+00 \quad 0.00 \mathrm{E}+00 \quad 0.00 \mathrm{E}+00 \quad 0.00 \mathrm{E}+00 \quad 0.00 \mathrm{E}+00 \quad 0.00 \mathrm{E}+00 \quad 0.00 \mathrm{E}+00 \quad 0.00 \mathrm{E}+00 \quad 0.00 \mathrm{E}+00 \quad 0.00 \mathrm{E}+00 \quad 0.00 \mathrm{E}+00 \quad 0.00 \mathrm{E}+00 \quad 0.00 \mathrm{E}+00 \quad 0.00 \mathrm{E}+00$

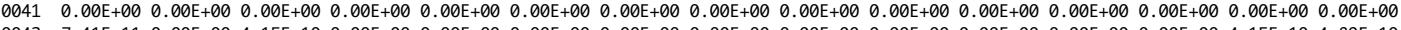

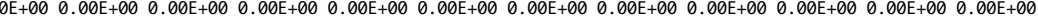
$00440.00 \mathrm{E}+00 \quad 0.00 \mathrm{E}+00 \quad 0.00 \mathrm{E}+00 \quad 0.00 \mathrm{E}+00 \quad 0.00 \mathrm{E}+00 \quad 0.00 \mathrm{E}+00 \quad 0.00 \mathrm{E}+00 \quad 0.00 \mathrm{E}+00 \quad 0.00 \mathrm{E}+00 \quad 0.00 \mathrm{E}+00 \quad 0.00 \mathrm{E}+00 \quad 0.00 \mathrm{E}+000.00 \mathrm{E}+000.00 \mathrm{E}+000.00 \mathrm{E}+00$ $0045 \quad 0.00 \mathrm{E}+00 \quad 0.00 \mathrm{E}+00 \quad 0.00 \mathrm{E}+00 \quad 0.00 \mathrm{E}+00 \quad 0.00 \mathrm{E}+00 \quad 0.00 \mathrm{E}+00 \quad 0.00 \mathrm{E}+00 \quad 0.00 \mathrm{E}+00 \quad 0.00 \mathrm{E}+00 \quad 0.00 \mathrm{E}+00 \quad 0.00 \mathrm{E}+00 \quad 0.00 \mathrm{E}+00 \quad 0.00 \mathrm{E}+00 \quad 0.00 \mathrm{E}+00 \quad 0.00 \mathrm{E}+00$ $00460.00 \mathrm{E}+000.00 \mathrm{E}+000.00 \mathrm{E}+000.00 \mathrm{E}+000.00 \mathrm{E}+000.00 \mathrm{E}+000.00 \mathrm{E}+000.00 \mathrm{E}+000.00 \mathrm{E}+00 \quad 0.00 \mathrm{E}+000.00 \mathrm{E}+000.00 \mathrm{E}+000.00 \mathrm{E}+000.00 \mathrm{E}+000.00 \mathrm{E}+00$

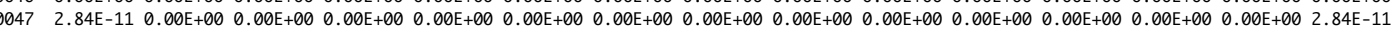
$00480.00 \mathrm{E}+00 \quad 0.00 \mathrm{E}+00 \quad 0.00 \mathrm{E}+00 \quad 0.00 \mathrm{E}+00 \quad 0.00 \mathrm{E}+00 \quad 0.00 \mathrm{E}+00 \quad 0.00 \mathrm{E}+00 \quad 0.00 \mathrm{E}+00 \quad 0.00 \mathrm{E}+00 \quad 0.00 \mathrm{E}+00 \quad 0.00 \mathrm{E}+00 \quad 0.00 \mathrm{E}+000.00 \mathrm{E}+000.00 \mathrm{E}+000.00 \mathrm{E}+00$

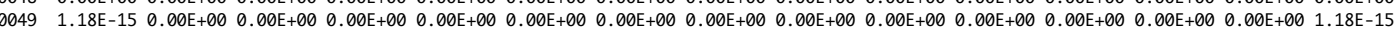

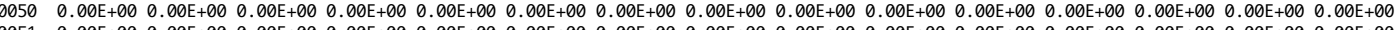

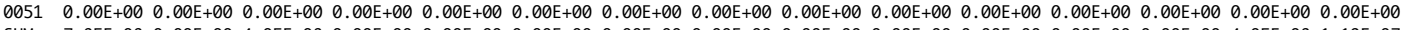

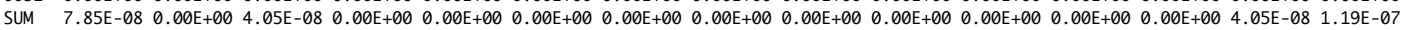

\section{SOURCE 2 RISK BY PATHWAY}

CHEM INHAL DERM SOIL MOTHER FISH WATER VEG DAIRY BEEF CHICK PIG EGG MEAT ORAL TOTAL

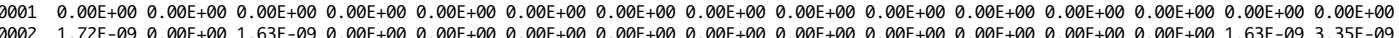

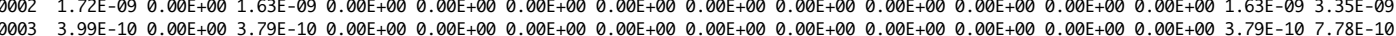
$0004 \quad 1.06 \mathrm{E}-080.00 \mathrm{E}+001.01 \mathrm{E}-080.00 \mathrm{E}+00 \quad 0.00 \mathrm{E}+000.00 \mathrm{E}+00 \quad 0.00 \mathrm{E}+000.00 \mathrm{E}+000.00 \mathrm{E}+00 \quad 0.00 \mathrm{E}+00 \quad 0.00 \mathrm{E}+00 \quad 0.00 \mathrm{E}+000.00 \mathrm{E}+001.01 \mathrm{E}-08 \quad 2.07 \mathrm{E}-08$ $0005 \quad 4.80 \mathrm{E}-090.00 \mathrm{E}+00 \quad 4.56 \mathrm{E}-090.00 \mathrm{E}+000.00 \mathrm{E}+000.00 \mathrm{E}+000.00 \mathrm{E}+000.00 \mathrm{E}+0000.00 \mathrm{E}+00 \quad 0.00 \mathrm{E}+00 \quad 0.00 \mathrm{E}+000.00 \mathrm{E}+000.00 \mathrm{E}+004.56 \mathrm{E}-09 \quad 9.35 \mathrm{E}-09$

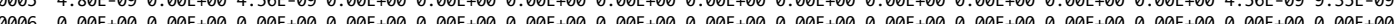
0006 (a. 0008 . 0008 (1) D. $0010-0.00 E-0.00 E$ E 0011 O.DOE+0O

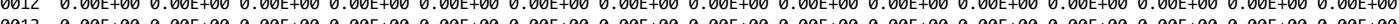

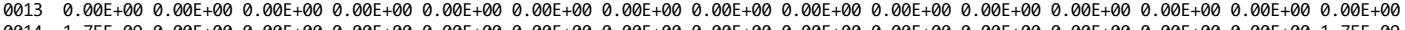
$0014 \quad 1.75 \mathrm{E}-09 \quad 0.00 \mathrm{E}+00 \quad 0.00 \mathrm{E}+00 \quad 0.00 \mathrm{E}+00 \quad 0.00 \mathrm{E}+00 \quad 0.00 \mathrm{E}+00 \quad 0.00 \mathrm{E}+00 \quad 0.00 \mathrm{E}+00 \quad 0.00 \mathrm{E}+00 \quad 0.00 \mathrm{E}+00 \quad 0.00 \mathrm{E}+00 \quad 0.00 \mathrm{E}+00 \quad 0.00 \mathrm{E}+00 \quad 0.00 \mathrm{E}+00 \quad 1.75 \mathrm{E}-09$

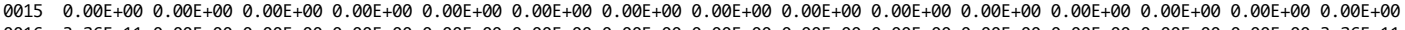

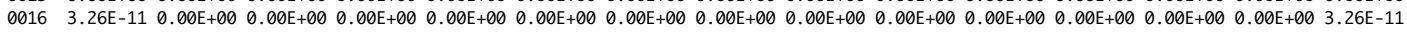


$0017 \quad 1.70 \mathrm{E}-12 \quad 0.00 \mathrm{E}+00 \quad 0.00 \mathrm{E}+00 \quad 0.00 \mathrm{E}+00 \quad 0.00 \mathrm{E}+00 \quad 0.00 \mathrm{E}+00 \quad 0.00 \mathrm{E}+00 \quad 0.00 \mathrm{E}+00 \quad 0.00 \mathrm{E}+00 \quad 0.00 \mathrm{E}+00 \quad 0.00 \mathrm{E}+00 \quad 0.00 \mathrm{E}+00 \quad 0.00 \mathrm{E}+00 \quad 0.00 \mathrm{E}+00 \quad 1.70 \mathrm{E}-12$

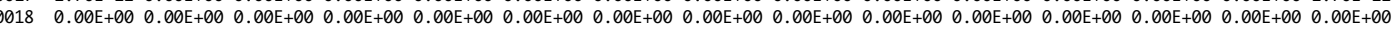
$0019 \quad 0.00 \mathrm{E}+00 \quad 0.00 \mathrm{E}+00 \quad 0.00 \mathrm{E}+00 \quad 0.00 \mathrm{E}+00 \quad 0.00 \mathrm{E}+00 \quad 0.00 \mathrm{E}+00 \quad 0.00 \mathrm{E}+00 \quad 0.00 \mathrm{E}+00 \quad 0.00 \mathrm{E}+00 \quad 0.00 \mathrm{E}+00 \quad 0.00 \mathrm{E}+00 \quad 0.00 \mathrm{E}+00 \quad 0.00 \mathrm{E}+00 \quad 0.00 \mathrm{E}+00 \quad 0.00 \mathrm{E}+00$ $0020 \quad 0.00 \mathrm{E}+00 \quad 0.00 \mathrm{E}+00 \quad 0.00 \mathrm{E}+00 \quad 0.00 \mathrm{E}+00 \quad 0.00 \mathrm{E}+00 \quad 0.00 \mathrm{E}+00 \quad 0.00 \mathrm{E}+00 \quad 0.00 \mathrm{E}+00 \quad 0.00 \mathrm{E}+00 \quad 0.00 \mathrm{E}+00 \quad 0.00 \mathrm{E}+00 \quad 0.00 \mathrm{E}+00 \quad 0.00 \mathrm{E}+00 \quad 0.00 \mathrm{E}+00 \quad 0.00 \mathrm{E}+00$

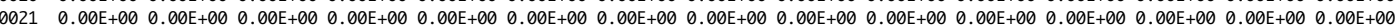
$0022 \quad 0.00 \mathrm{E}+00 \quad 0.00 \mathrm{E}+00 \quad 0.00 \mathrm{E}+00 \quad 0.00 \mathrm{E}+00 \quad 0.00 \mathrm{E}+00 \quad 0.00 \mathrm{E}+00 \quad 0.00 \mathrm{E}+00 \quad 0.00 \mathrm{E}+00 \quad 0.00 \mathrm{E}+00 \quad 0.00 \mathrm{E}+00 \quad 0.00 \mathrm{E}+00 \quad 0.00 \mathrm{E}+00 \quad 0.00 \mathrm{E}+00 \quad 0.00 \mathrm{E}+00 \quad 0.00 \mathrm{E}+00$

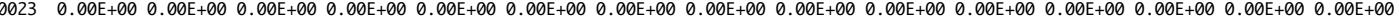
$0024 \quad 0.00 \mathrm{E}+00 \quad 0.00 \mathrm{E}+00 \quad 0.00 \mathrm{E}+00 \quad 0.00 \mathrm{E}+00 \quad 0.00 \mathrm{E}+00 \quad 0.00 \mathrm{E}+00 \quad 0.00 \mathrm{E}+00 \quad 0.00 \mathrm{E}+00 \quad 0.00 \mathrm{E}+00 \quad 0.00 \mathrm{E}+00 \quad 0.00 \mathrm{E}+00 \quad 0.00 \mathrm{E}+00 \quad 0.00 \mathrm{E}+00 \quad 0.00 \mathrm{E}+00 \quad 0.00 \mathrm{E}+00$ $0025 \quad 0.00 \mathrm{E}+00 \quad 0.00 \mathrm{E}+00 \quad 0.00 \mathrm{E}+00 \quad 0.00 \mathrm{E}+00 \quad 0.00 \mathrm{E}+00 \quad 0.00 \mathrm{E}+00 \quad 0.00 \mathrm{E}+00 \quad 0.00 \mathrm{E}+00 \quad 0.00 \mathrm{E}+00 \quad 0.00 \mathrm{E}+00 \quad 0.00 \mathrm{E}+00 \quad 0.00 \mathrm{E}+00 \quad 0.00 \mathrm{E}+00 \quad 0.00 \mathrm{E}+00 \quad 0.00 \mathrm{E}+00$

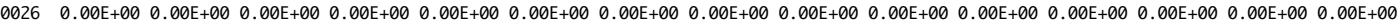
$0027 \quad 0.00 \mathrm{E}+00 \quad 0.00 \mathrm{E}+00 \quad 0.00 \mathrm{E}+00 \quad 0.00 \mathrm{E}+00 \quad 0.00 \mathrm{E}+00 \quad 0.00 \mathrm{E}+00 \quad 0.00 \mathrm{E}+00 \quad 0.00 \mathrm{E}+00 \quad 0.00 \mathrm{E}+00 \quad 0.00 \mathrm{E}+00 \quad 0.00 \mathrm{E}+00 \quad 0.00 \mathrm{E}+000.00 \mathrm{E}+00 \quad 0.00 \mathrm{E}+00 \quad 0.00 \mathrm{E}+00$ $00280.00 \mathrm{E}+000.00 \mathrm{E}+000.00 \mathrm{E}+000.00 \mathrm{E}+00.00 \mathrm{E}+00$. $00290.00 \mathrm{E}$ (1)

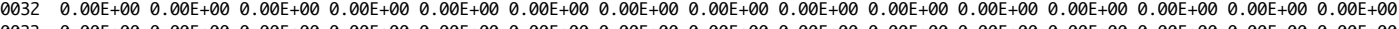

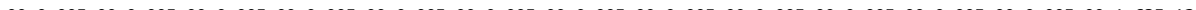

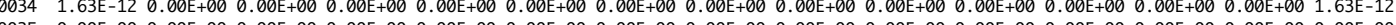

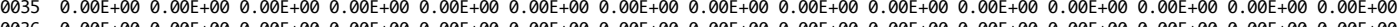

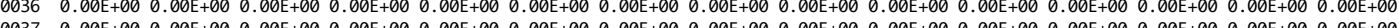

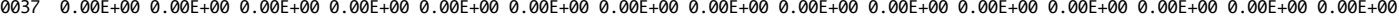

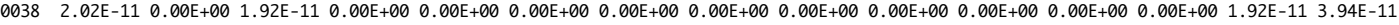
$0039 \quad 0.00 \mathrm{E}+00 \quad 0.00 \mathrm{E}+00 \quad 0.00 \mathrm{E}+00 \quad 0.00 \mathrm{E}+00 \quad 0.00 \mathrm{E}+00 \quad 0.00 \mathrm{E}+00 \quad 0.00 \mathrm{E}+00 \quad 0.00 \mathrm{E}+00 \quad 0.00 \mathrm{E}+00 \quad 0.00 \mathrm{E}+00 \quad 0.00 \mathrm{E}+00 \quad 0.00 \mathrm{E}+00 \quad 0.00 \mathrm{E}+00 \quad 0.00 \mathrm{E}+00 \quad 0.00 \mathrm{E}+00$

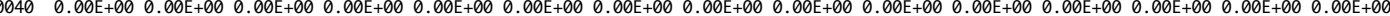
$0041 \quad 0.00 \mathrm{E}+00 \quad 0.00 \mathrm{E}+00 \quad 0.00 \mathrm{E}+00 \quad 0.00 \mathrm{E}+00 \quad 0.00 \mathrm{E}+00 \quad 0.00 \mathrm{E}+00 \quad 0.00 \mathrm{E}+00 \quad 0.00 \mathrm{E}+00 \quad 0.00 \mathrm{E}+00 \quad 0.00 \mathrm{E}+00 \quad 0.00 \mathrm{E}+00 \quad 0.00 \mathrm{E}+00 \quad 0.00 \mathrm{E}+00 \quad 0.00 \mathrm{E}+00 \quad 0.00 \mathrm{E}+00$ $0042 \quad 0.00 \mathrm{E}+00 \quad 0.00 \mathrm{E}+00 \quad 0.00 \mathrm{E}+00 \quad 0.00 \mathrm{E}+00 \quad 0.00 \mathrm{E}+00 \quad 0.00 \mathrm{E}+00 \quad 0.00 \mathrm{E}+00 \quad 0.00 \mathrm{E}+00 \quad 0.00 \mathrm{E}+00 \quad 0.00 \mathrm{E}+00 \quad 0.00 \mathrm{E}+00 \quad 0.00 \mathrm{E}+00 \quad 0.00 \mathrm{E}+00 \quad 0.00 \mathrm{E}+00 \quad 0.00 \mathrm{E}+00$

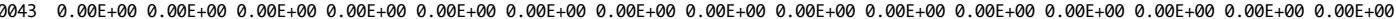

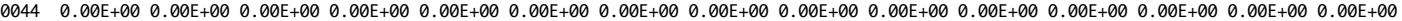
$0045 \quad 1.39 \mathrm{E}-12 \quad 0.00 \mathrm{E}+00 \quad 0.00 \mathrm{E}+00 \quad 0.00 \mathrm{E}+00 \quad 0.00 \mathrm{E}+00 \quad 0.00 \mathrm{E}+00 \quad 0.00 \mathrm{E}+00 \quad 0.00 \mathrm{E}+00 \quad 0.00 \mathrm{E}+00 \quad 0.00 \mathrm{E}+00 \quad 0.00 \mathrm{E}+00 \quad 0.00 \mathrm{E}+00 \quad 0.00 \mathrm{E}+00 \quad 0.00 \mathrm{E}+00 \quad 1.39 \mathrm{E}-12$ $00460.00 \mathrm{E}+00 \quad 0.00 \mathrm{E}+00 \quad 0.00 \mathrm{E}+00 \quad 0.00 \mathrm{E}+00 \quad 0.00 \mathrm{E}+000.00 \mathrm{E}+00 \quad 0.00 \mathrm{E}+00 \quad 0.00 \mathrm{E}+00 \quad 0.00 \mathrm{E}+00 \quad 0.00 \mathrm{E}+00 \quad 0.00 \mathrm{E}+00 \quad 0.00 \mathrm{E}+00 \quad 0.00 \mathrm{E}+000.00 \mathrm{E}+000.00 \mathrm{E}+00$

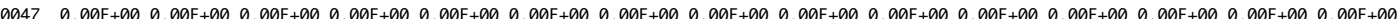
00478

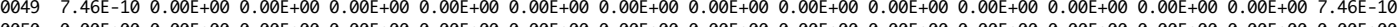

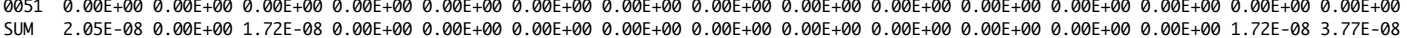

\section{SOURCE 3 RISK BY PATHWAY}

CHEM INHAL DERM SOIL MOTHER FISH WATER VEG DAIRY BEEF CHICK PIG EGG MEAT ORAL TOTAL $0001 \quad 0.00 \mathrm{E}+00 \quad 0.00 \mathrm{E}+00 \quad 0.00 \mathrm{E}+00 \quad 0.00 \mathrm{E}+00 \quad 0.00 \mathrm{E}+00 \quad 0.00 \mathrm{E}+00 \quad 0.00 \mathrm{E}+00 \quad 0.00 \mathrm{E}+00 \quad 0.00 \mathrm{E}+00 \quad 0.00 \mathrm{E}+00 \quad 0.00 \mathrm{E}+00 \quad 0.00 \mathrm{E}+00 \quad 0.00 \mathrm{E}+00 \quad 0.00 \mathrm{E}+00 \quad 0.00 \mathrm{E}+00$ $0002 \quad 8.23 \mathrm{E}-09 \quad 0.00 \mathrm{E}+00 \quad 7.82 \mathrm{E}-09 \quad 0.00 \mathrm{E}+00 \quad 0.00 \mathrm{E}+00 \quad 0.00 \mathrm{E}+00 \quad 0.00 \mathrm{E}+00 \quad 0.00 \mathrm{E}+00 \quad 0.00 \mathrm{E}+00 \quad 0.00 \mathrm{E}+00 \quad 0.00 \mathrm{E}+00 \quad 0.00 \mathrm{E}+00 \quad 0.00 \mathrm{E}+00 \quad 7.82 \mathrm{E}-09 \quad 1.61 \mathrm{E}-08$ $0003 \quad 1.91 \mathrm{E}-09 \quad 0.00 \mathrm{E}+00 \quad 1.81 \mathrm{E}-09 \quad 0.00 \mathrm{E}+00 \quad 0.00 \mathrm{E}+00 \quad 0.00 \mathrm{E}+00 \quad 0.00 \mathrm{E}+00 \quad 0.00 \mathrm{E}+00 \quad 0.00 \mathrm{E}+00 \quad 0.00 \mathrm{E}+00 \quad 0.00 \mathrm{E}+00 \quad 0.00 \mathrm{E}+00 \quad 0.00 \mathrm{E}+00 \quad 1.81 \mathrm{E}-09 \quad 3.72 \mathrm{E}-09$

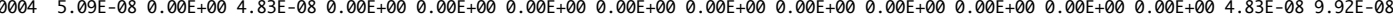

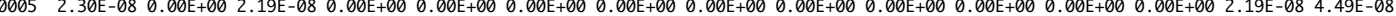

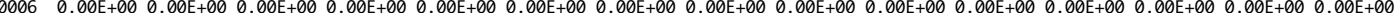
$0007 \quad 0.00 \mathrm{E}+00 \quad 0.00 \mathrm{E}+00 \quad 0.00 \mathrm{E}+00 \quad 0.00 \mathrm{E}+00 \quad 0.00 \mathrm{E}+00 \quad 0.00 \mathrm{E}+00 \quad 0.00 \mathrm{E}+00 \quad 0.00 \mathrm{E}+00 \quad 0.00 \mathrm{E}+00 \quad 0.00 \mathrm{E}+00 \quad 0.00 \mathrm{E}+00 \quad 0.00 \mathrm{E}+00 \quad 0.00 \mathrm{E}+00 \quad 0.00 \mathrm{E}+00 \quad 0.00 \mathrm{E}+00$ $0008 \quad 0.00 \mathrm{E}+00 \quad 0.00 \mathrm{E}+00 \quad 0.00 \mathrm{E}+00 \quad 0.00 \mathrm{E}+00 \quad 0.00 \mathrm{E}+00 \quad 0.00 \mathrm{E}+00 \quad 0.00 \mathrm{E}+00 \quad 0.00 \mathrm{E}+00 \quad 0.00 \mathrm{E}+00 \quad 0.00 \mathrm{E}+00 \quad 0.00 \mathrm{E}+00 \quad 0.00 \mathrm{E}+00 \quad 0.00 \mathrm{E}+00 \quad 0.00 \mathrm{E}+00 \quad 0.00 \mathrm{E}+00$ $0009 \quad 0.00 \mathrm{E}+00 \quad 0.00 \mathrm{E}+00 \quad 0.00 \mathrm{E}+00 \quad 0.00 \mathrm{E}+00 \quad 0.00 \mathrm{E}+00 \quad 0.00 \mathrm{E}+00 \quad 0.00 \mathrm{E}+00 \quad 0.00 \mathrm{E}+00 \quad 0.00 \mathrm{E}+00 \quad 0.00 \mathrm{E}+00 \quad 0.00 \mathrm{E}+00 \quad 0.00 \mathrm{E}+00 \quad 0.00 \mathrm{E}+00 \quad 0.00 \mathrm{E}+00 \quad 0.00 \mathrm{E}+00$ $0010 \quad 0.00 \mathrm{E}+00 \quad 0.00 \mathrm{E}+00 \quad 0.00 \mathrm{E}+00 \quad 0.00 \mathrm{E}+00 \quad 0.00 \mathrm{E}+00 \quad 0.00 \mathrm{E}+00 \quad 0.00 \mathrm{E}+00 \quad 0.00 \mathrm{E}+00 \quad 0.00 \mathrm{E}+00 \quad 0.00 \mathrm{E}+00 \quad 0.00 \mathrm{E}+00 \quad 0.00 \mathrm{E}+00 \quad 0.00 \mathrm{E}+00 \quad 0.00 \mathrm{E}+00 \quad 0.00 \mathrm{E}+00$

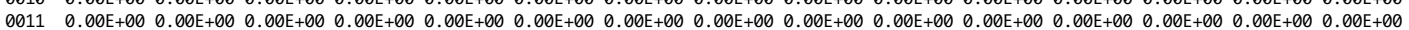

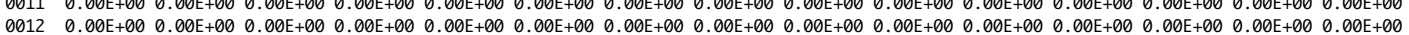

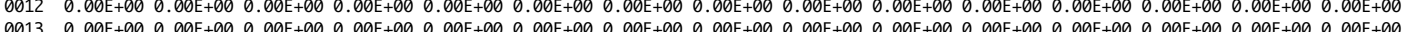

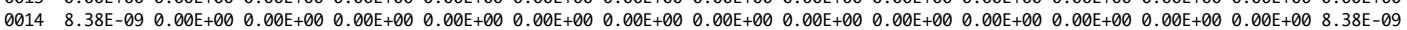
0015 .

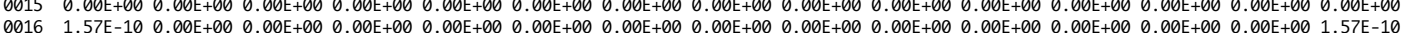

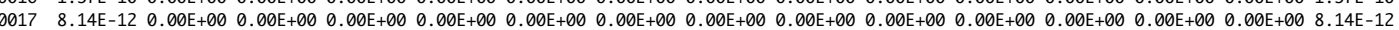

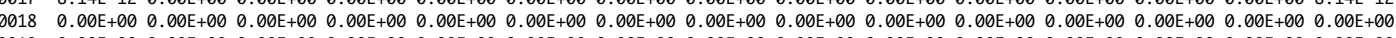
$0019 \quad 0.00 \mathrm{E}+00 \quad 0.00 \mathrm{E}+00 \quad 0.00 \mathrm{E}+00 \quad 0.00 \mathrm{E}+00 \quad 0.00 \mathrm{E}+00 \quad 0.00 \mathrm{E}+00 \quad 0.00 \mathrm{E}+00 \quad 0.00 \mathrm{E}+00 \quad 0.00 \mathrm{E}+00 \quad 0.00 \mathrm{E}+00 \quad 0.00 \mathrm{E}+00 \quad 0.00 \mathrm{E}+00 \quad 0.00 \mathrm{E}+00 \quad 0.00 \mathrm{E}+00 \quad 0.00 \mathrm{E}+00$ $0020 \quad 0.00 \mathrm{E}+00 \quad 0.00 \mathrm{E}+00 \quad 0.00 \mathrm{E}+00 \quad 0.00 \mathrm{E}+00 \quad 0.00 \mathrm{E}+00 \quad 0.00 \mathrm{E}+00 \quad 0.00 \mathrm{E}+00 \quad 0.00 \mathrm{E}+00 \quad 0.00 \mathrm{E}+00 \quad 0.00 \mathrm{E}+00 \quad 0.00 \mathrm{E}+00 \quad 0.00 \mathrm{E}+00 \quad 0.00 \mathrm{E}+00 \quad 0.00 \mathrm{E}+00 \quad 0.00 \mathrm{E}+00$

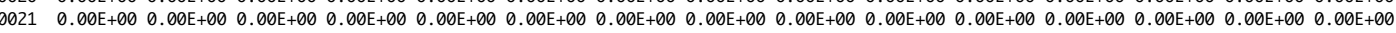

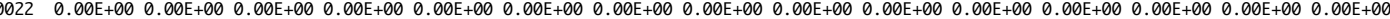

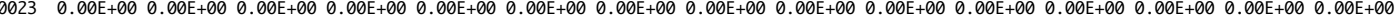
$\begin{array}{lll} & \end{array}$ $0025 \quad 0.00 \mathrm{E}+00 \quad 0.00 \mathrm{E}+00 \quad 0.00 \mathrm{E}+00 \quad 0.00 \mathrm{E}+00 \quad 0.00 \mathrm{E}+00 \quad 0.00 \mathrm{E}+00 \quad 0.00 \mathrm{E}+00 \quad 0.00 \mathrm{E}+00 \quad 0.00 \mathrm{E}+00 \quad 0.00 \mathrm{E}+00 \quad 0.00 \mathrm{E}+00 \quad 0.00 \mathrm{E}+00 \quad 0.00 \mathrm{E}+00 \quad 0.00 \mathrm{E}+00 \quad 0.00 \mathrm{E}+00$ $0026 \quad 0.00 \mathrm{E}+00 \quad 0.00 \mathrm{E}+00 \quad 0.00 \mathrm{E}+00 \quad 0.00 \mathrm{E}+00 \quad 0.00 \mathrm{E}+00 \quad 0.00 \mathrm{E}+00 \quad 0.00 \mathrm{E}+00 \quad 0.00 \mathrm{E}+00 \quad 0.00 \mathrm{E}+00 \quad 0.00 \mathrm{E}+00 \quad 0.00 \mathrm{E}+00 \quad 0.00 \mathrm{E}+00 \quad 0.00 \mathrm{E}+00 \quad 0.00 \mathrm{E}+00 \quad 0.00 \mathrm{E}+00$ $0027 \quad 0.00 \mathrm{E}+00 \quad 0.00 \mathrm{E}+00 \quad 0.00 \mathrm{E}+00 \quad 0.00 \mathrm{E}+00 \quad 0.00 \mathrm{E}+00 \quad 0.00 \mathrm{E}+00 \quad 0.00 \mathrm{E}+00 \quad 0.00 \mathrm{E}+00 \quad 0.00 \mathrm{E}+00 \quad 0.00 \mathrm{E}+00 \quad 0.00 \mathrm{E}+00 \quad 0.00 \mathrm{E}+00 \quad 0.00 \mathrm{E}+00 \quad 0.00 \mathrm{E}+00 \quad 0.00 \mathrm{E}+00$ $0028 \quad 0.00 \mathrm{E}+00 \quad 0.00 \mathrm{E}+00 \quad 0.00 \mathrm{E}+00 \quad 0.00 \mathrm{E}+00 \quad 0.00 \mathrm{E}+00 \quad 0.00 \mathrm{E}+00 \quad 0.00 \mathrm{E}+00 \quad 0.00 \mathrm{E}+00 \quad 0.00 \mathrm{E}+00 \quad 0.00 \mathrm{E}+00 \quad 0.00 \mathrm{E}+00 \quad 0.00 \mathrm{E}+00 \quad 0.00 \mathrm{E}+00 \quad 0.00 \mathrm{E}+00 \quad 0.00 \mathrm{E}+00$ $0029 \quad 0.00 \mathrm{E}+00 \quad 0.00 \mathrm{E}+00 \quad 0.00 \mathrm{E}+00 \quad 0.00 \mathrm{E}+00 \quad 0.00 \mathrm{E}+00 \quad 0.00 \mathrm{E}+00 \quad 0.00 \mathrm{E}+00 \quad 0.00 \mathrm{E}+00 \quad 0.00 \mathrm{E}+00 \quad 0.00 \mathrm{E}+00 \quad 0.00 \mathrm{E}+00 \quad 0.00 \mathrm{E}+00 \quad 0.00 \mathrm{E}+00 \quad 0.00 \mathrm{E}+00 \quad 0.00 \mathrm{E}+00$

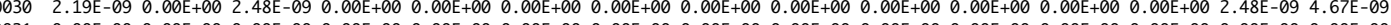
$0031 \quad 0.00 \mathrm{E}+00 \quad 0.00 \mathrm{E}+00 \quad 0.00 \mathrm{E}+00 \quad 0.00 \mathrm{E}+00 \quad 0.00 \mathrm{E}+00 \quad 0.00 \mathrm{E}+00 \quad 0.00 \mathrm{E}+00 \quad 0.00 \mathrm{E}+00 \quad 0.00 \mathrm{E}+00 \quad 0.00 \mathrm{E}+00 \quad 0.00 \mathrm{E}+00 \quad 0.00 \mathrm{E}+00 \quad 0.00 \mathrm{E}+00 \quad 0.00 \mathrm{E}+00 \quad 0.00 \mathrm{E}+00$ $00320.00 \mathrm{E}+000.00 \mathrm{E}+00 \quad 0.00 \mathrm{E}+00 \quad 0.00 \mathrm{E}+000.00 \mathrm{E}+000.00 \mathrm{E}+00 \quad 0.00 \mathrm{E}+00 \quad 0.00 \mathrm{E}+00 \quad 0.00 \mathrm{E}+00 \quad 0.00 \mathrm{E}+00 \quad 0.00 \mathrm{E}+000.00 \mathrm{E}+000.00 \mathrm{E}+000.00 \mathrm{E}+000.00 \mathrm{E}+00$ $00330.00 \mathrm{E}+00 \quad 0.00 \mathrm{E}+000.00 \mathrm{E}+000.00 \mathrm{E}+00 \quad 0.00 \mathrm{E}+00 \quad 0.00 \mathrm{E}+00 \quad 0.00 \mathrm{E}+00 \quad 0.00 \mathrm{E}+00 \quad 0.00 \mathrm{E}+00 \quad 0.00 \mathrm{E}+00 \quad 0.00 \mathrm{E}+00 \quad 0.00 \mathrm{E}+000.00 \mathrm{E}+00 \quad 0.00 \mathrm{E}+000.00 \mathrm{E}+00$ $0034 \quad 7.82 \mathrm{E}-12 \quad 0.00 \mathrm{E}+00 \quad 0.00 \mathrm{E}+00 \quad 0.00 \mathrm{E}+00 \quad 0.00 \mathrm{E}+00 \quad 0.00 \mathrm{E}+00 \quad 0.00 \mathrm{E}+00 \quad 0.00 \mathrm{E}+00 \quad 0.00 \mathrm{E}+00 \quad 0.00 \mathrm{E}+00 \quad 0.00 \mathrm{E}+00 \quad 0.00 \mathrm{E}+00 \quad 0.00 \mathrm{E}+00 \quad 0.00 \mathrm{E}+00 \quad 7.82 \mathrm{E}-12$

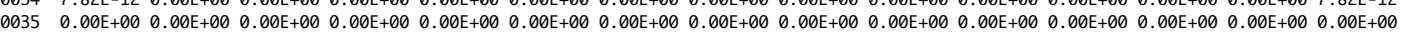

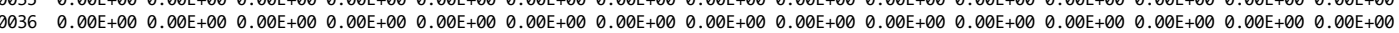

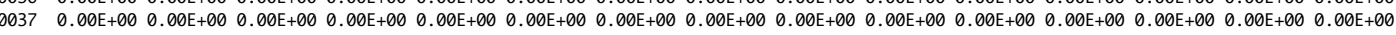

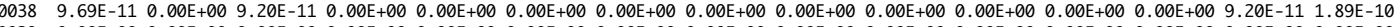

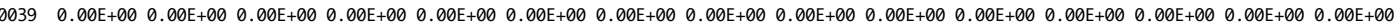
$0040 \quad 0.00 \mathrm{E}+00 \quad 0.00 \mathrm{E}+00 \quad 0.00 \mathrm{E}+00 \quad 0.00 \mathrm{E}+00 \quad 0.00 \mathrm{E}+00 \quad 0.00 \mathrm{E}+00 \quad 0.00 \mathrm{E}+00 \quad 0.00 \mathrm{E}+00 \quad 0.00 \mathrm{E}+00 \quad 0.00 \mathrm{E}+00 \quad 0.00 \mathrm{E}+00 \quad 0.00 \mathrm{E}+00 \quad 0.00 \mathrm{E}+00 \quad 0.00 \mathrm{E}+00 \quad 0.00 \mathrm{E}+00$

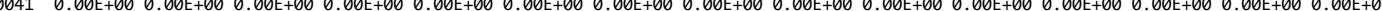
$0042 \quad 0.00 \mathrm{E}+00 \quad 0.00 \mathrm{E}+00 \quad 0.00 \mathrm{E}+00 \quad 0.00 \mathrm{E}+00 \quad 0.00 \mathrm{E}+00 \quad 0.00 \mathrm{E}+00 \quad 0.00 \mathrm{E}+00 \quad 0.00 \mathrm{E}+00 \quad 0.00 \mathrm{E}+00 \quad 0.00 \mathrm{E}+00 \quad 0.00 \mathrm{E}+00 \quad 0.00 \mathrm{E}+00 \quad 0.00 \mathrm{E}+00 \quad 0.00 \mathrm{E}+00 \quad 0.00 \mathrm{E}+00$

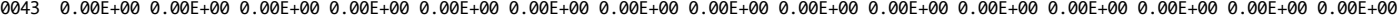
$0044 \quad 0.00 \mathrm{E}+00 \quad 0.00 \mathrm{E}+00 \quad 0.00 \mathrm{E}+00 \quad 0.00 \mathrm{E}+00 \quad 0.00 \mathrm{E}+00 \quad 0.00 \mathrm{E}+00 \quad 0.00 \mathrm{E}+00 \quad 0.00 \mathrm{E}+00 \quad 0.00 \mathrm{E}+00 \quad 0.00 \mathrm{E}+00 \quad 0.00 \mathrm{E}+00 \quad 0.00 \mathrm{E}+00 \quad 0.00 \mathrm{E}+00 \quad 0.00 \mathrm{E}+00 \quad 0.00 \mathrm{E}+00$

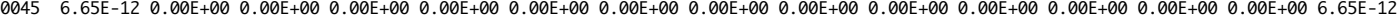
$0046 \quad 0.00 \mathrm{E}+00 \quad 0.00 \mathrm{E}+00 \quad 0.00 \mathrm{E}+00 \quad 0.00 \mathrm{E}+00 \quad 0.00 \mathrm{E}+00 \quad 0.00 \mathrm{E}+00 \quad 0.00 \mathrm{E}+00 \quad 0.00 \mathrm{E}+00 \quad 0.00 \mathrm{E}+00 \quad 0.00 \mathrm{E}+00 \quad 0.00 \mathrm{E}+00 \quad 0.00 \mathrm{E}+00 \quad 0.00 \mathrm{E}+00 \quad 0.00 \mathrm{E}+00 \quad 0.00 \mathrm{E}+00$ $0047 \quad 0.00 \mathrm{E}+00 \quad 0.00 \mathrm{E}+00 \quad 0.00 \mathrm{E}+00 \quad 0.00 \mathrm{E}+00 \quad 0.00 \mathrm{E}+00 \quad 0.00 \mathrm{E}+00 \quad 0.00 \mathrm{E}+00 \quad 0.00 \mathrm{E}+00 \quad 0.00 \mathrm{E}+00 \quad 0.00 \mathrm{E}+00 \quad 0.00 \mathrm{E}+00 \quad 0.00 \mathrm{E}+00 \quad 0.00 \mathrm{E}+00 \quad 0.00 \mathrm{E}+00 \quad 0.00 \mathrm{E}+00$

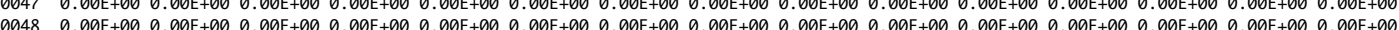

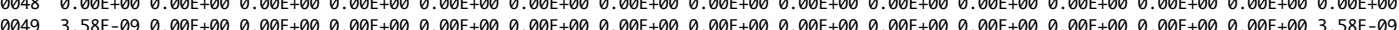

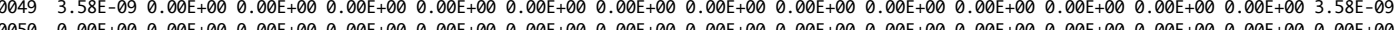

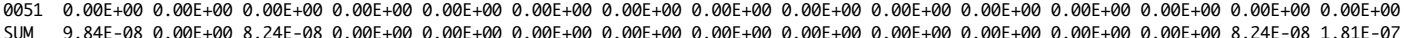
SOURCE 4 RISK BY PATHWAY

CHEM INHAL DERM SOIL MOTHER FISH WATER VEG DAIRY BEEF CHICK PIG EGG MEAT ORAL TOTAL $0001 \quad 7.64 \mathrm{E}-10 \quad 0.00 \mathrm{E}+00 \quad 0.00 \mathrm{E}+00 \quad 0.00 \mathrm{E}+00 \quad 0.00 \mathrm{E}+00 \quad 0.00 \mathrm{E}+00 \quad 0.00 \mathrm{E}+00 \quad 0.00 \mathrm{E}+00 \quad 0.00 \mathrm{E}+00 \quad 0.00 \mathrm{E}+00 \quad 0.00 \mathrm{E}+00 \quad 0.00 \mathrm{E}+00 \quad 0.00 \mathrm{E}+00 \quad 0.00 \mathrm{E}+00 \quad 7.64 \mathrm{E}-10$ $00020.00 \mathrm{E}+000.00 \mathrm{E}+000.00 \mathrm{E}+000.00 \mathrm{E}+\mathrm{L}$ $0003 \quad 0.00 \mathrm{E}+00 \quad 0.00 \mathrm{E}+00 \quad 0.00 \mathrm{E}+00 \quad 0.00 \mathrm{E}+00 \quad 0.00 \mathrm{E}+00 \quad 0.00 \mathrm{E}+00 \quad 0.00 \mathrm{E}+00 \quad 0.00 \mathrm{E}+00 \quad 0.00 \mathrm{E}+00 \quad 0.00 \mathrm{E}+00 \quad 0.00 \mathrm{E}+00 \quad 0.00 \mathrm{E}+00 \quad 0.00 \mathrm{E}+00 \quad 0.00 \mathrm{E}+00 \quad 0.00 \mathrm{E}+00$

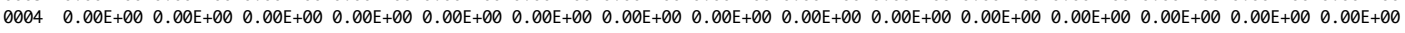


$0005 \quad 0.00 \mathrm{E}+00 \quad 0.00 \mathrm{E}+00 \quad 0.00 \mathrm{E}+00 \quad 0.00 \mathrm{E}+00 \quad 0.00 \mathrm{E}+00 \quad 0.00 \mathrm{E}+00 \quad 0.00 \mathrm{E}+00 \quad 0.00 \mathrm{E}+00 \quad 0.00 \mathrm{E}+00 \quad 0.00 \mathrm{E}+00 \quad 0.00 \mathrm{E}+00 \quad 0.00 \mathrm{E}+00 \quad 0.00 \mathrm{E}+00 \quad 0.00 \mathrm{E}+00 \quad 0.00 \mathrm{E}+00$ $0006 \quad 8.43 \mathrm{E}-11 \quad 0.00 \mathrm{E}+00 \quad 8.01 \mathrm{E}-11 \quad 0.00 \mathrm{E}+00 \quad 0.00 \mathrm{E}+00 \quad 0.00 \mathrm{E}+00 \quad 0.00 \mathrm{E}+00 \quad 0.00 \mathrm{E}+00 \quad 0.00 \mathrm{E}+00 \quad 0.00 \mathrm{E}+00 \quad 0.00 \mathrm{E}+00 \quad 0.00 \mathrm{E}+00 \quad 0.00 \mathrm{E}+00 \quad 8.01 \mathrm{E}-11 \quad 1.64 \mathrm{E}-10$ $0007 \quad 0.00 \mathrm{E}+00 \quad 0.00 \mathrm{E}+00 \quad 0.00 \mathrm{E}+00 \quad 0.00 \mathrm{E}+00 \quad 0.00 \mathrm{E}+00 \quad 0.00 \mathrm{E}+00 \quad 0.00 \mathrm{E}+00 \quad 0.00 \mathrm{E}+00 \quad 0.00 \mathrm{E}+00 \quad 0.00 \mathrm{E}+00 \quad 0.00 \mathrm{E}+00 \quad 0.00 \mathrm{E}+00 \quad 0.00 \mathrm{E}+00 \quad 0.00 \mathrm{E}+00 \quad 0.00 \mathrm{E}+00$ $0008 \quad 0.00 \mathrm{E}+00 \quad 0.00 \mathrm{E}+00 \quad 0.00 \mathrm{E}+00 \quad 0.00 \mathrm{E}+00 \quad 0.00 \mathrm{E}+00 \quad 0.00 \mathrm{E}+00 \quad 0.00 \mathrm{E}+00 \quad 0.00 \mathrm{E}+00 \quad 0.00 \mathrm{E}+00 \quad 0.00 \mathrm{E}+00 \quad 0.00 \mathrm{E}+00 \quad 0.00 \mathrm{E}+00 \quad 0.00 \mathrm{E}+00 \quad 0.00 \mathrm{E}+00 \quad 0.00 \mathrm{E}+00$

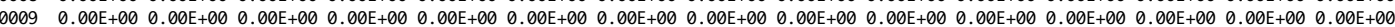
$0010 \quad 4.01 \mathrm{E}-11 \quad 0.00 \mathrm{E}+00 \quad 0.00 \mathrm{E}+00 \quad 0.00 \mathrm{E}+00 \quad 0.00 \mathrm{E}+00 \quad 0.00 \mathrm{E}+00 \quad 0.00 \mathrm{E}+00 \quad 0.00 \mathrm{E}+00 \quad 0.00 \mathrm{E}+00 \quad 0.00 \mathrm{E}+00 \quad 0.00 \mathrm{E}+00 \quad 0.00 \mathrm{E}+00 \quad 0.00 \mathrm{E}+00 \quad 0.00 \mathrm{E}+00 \quad 4.01 \mathrm{E}-11$ $0011 \quad 0.00 \mathrm{E}+00 \quad 0.00 \mathrm{E}+00 \quad 0.00 \mathrm{E}+00 \quad 0.00 \mathrm{E}+00 \quad 0.00 \mathrm{E}+00 \quad 0.00 \mathrm{E}+00 \quad 0.00 \mathrm{E}+00 \quad 0.00 \mathrm{E}+00 \quad 0.00 \mathrm{E}+00 \quad 0.00 \mathrm{E}+00 \quad 0.00 \mathrm{E}+00 \quad 0.00 \mathrm{E}+00 \quad 0.00 \mathrm{E}+00 \quad 0.00 \mathrm{E}+00 \quad 0.00 \mathrm{E}+00$ $0012 \quad 0.00 \mathrm{E}+00 \quad 0.00 \mathrm{E}+00 \quad 0.00 \mathrm{E}+00 \quad 0.00 \mathrm{E}+00 \quad 0.00 \mathrm{E}+00 \quad 0.00 \mathrm{E}+00 \quad 0.00 \mathrm{E}+00 \quad 0.00 \mathrm{E}+00 \quad 0.00 \mathrm{E}+00 \quad 0.00 \mathrm{E}+00 \quad 0.00 \mathrm{E}+00 \quad 0.00 \mathrm{E}+00 \quad 0.00 \mathrm{E}+00 \quad 0.00 \mathrm{E}+00 \quad 0.00 \mathrm{E}+00$ $0013 \quad 0.00 \mathrm{E}+00 \quad 0.00 \mathrm{E}+00 \quad 0.00 \mathrm{E}+00 \quad 0.00 \mathrm{E}+00 \quad 0.00 \mathrm{E}+00 \quad 0.00 \mathrm{E}+00 \quad 0.00 \mathrm{E}+00 \quad 0.00 \mathrm{E}+00 \quad 0.00 \mathrm{E}+00 \quad 0.00 \mathrm{E}+00 \quad 0.00 \mathrm{E}+00 \quad 0.00 \mathrm{E}+00 \quad 0.00 \mathrm{E}+00 \quad 0.00 \mathrm{E}+00 \quad 0.00 \mathrm{E}+00$ $0014 \quad 1.17 \mathrm{E}-09 \quad 0.00 \mathrm{E}+00 \quad 0.00 \mathrm{E}+00 \quad 0.00 \mathrm{E}+00 \quad 0.00 \mathrm{E}+00 \quad 0.00 \mathrm{E}+00 \quad 0.00 \mathrm{E}+00 \quad 0.00 \mathrm{E}+00 \quad 0.00 \mathrm{E}+00 \quad 0.00 \mathrm{E}+00 \quad 0.00 \mathrm{E}+00 \quad 0.00 \mathrm{E}+00 \quad 0.00 \mathrm{E}+00 \quad 0.00 \mathrm{E}+00 \quad 1.17 \mathrm{E}-09$ $0015 \quad 9.41 \mathrm{E}-08 \quad 0.00 \mathrm{E}+00 \quad 0.00 \mathrm{E}+00 \quad 0.00 \mathrm{E}+00 \quad 0.00 \mathrm{E}+00 \quad 0.00 \mathrm{E}+00 \quad 0.00 \mathrm{E}+00 \quad 0.00 \mathrm{E}+00 \quad 0.00 \mathrm{E}+00 \quad 0.00 \mathrm{E}+00 \quad 0.00 \mathrm{E}+00 \quad 0.00 \mathrm{E}+00 \quad 0.00 \mathrm{E}+00 \quad 0.00 \mathrm{E}+00 \quad 9.41 \mathrm{E}-08$

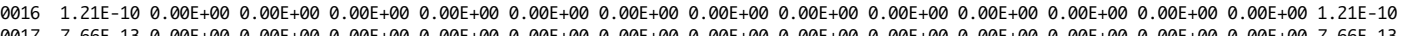
$0017 \quad 7.66 \mathrm{E}-13 \quad 0.00 \mathrm{E}+00 \quad 0.00 \mathrm{E}+00 \quad 0.00 \mathrm{E}+00 \quad 0.00 \mathrm{E}+00 \quad 0.00 \mathrm{E}+00 \quad 0.00 \mathrm{E}+00 \quad 0.00 \mathrm{E}+00 \quad 0.00 \mathrm{E}+00 \quad 0.00 \mathrm{E}+00 \quad 0.00 \mathrm{E}+00 \quad 0.00 \mathrm{E}+00 \quad 0.00 \mathrm{E}+00 \quad 0.00 \mathrm{E}+00 \quad 7.66 \mathrm{E}-13$ OOD (1)

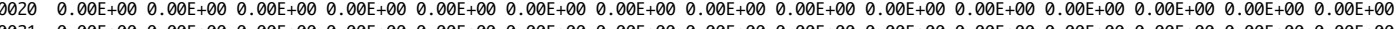
0021 .

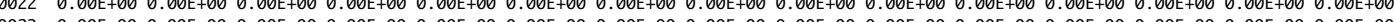

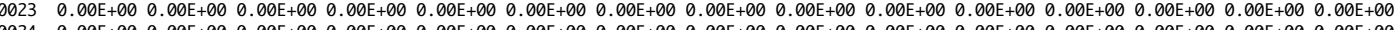

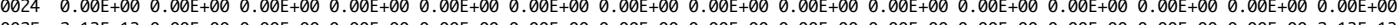

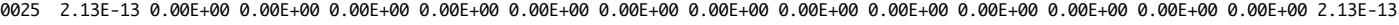
$0026 \quad 0.00 \mathrm{E}+00 \quad 0.00 \mathrm{E}+00 \quad 0.00 \mathrm{E}+00 \quad 0.00 \mathrm{E}+00 \quad 0.00 \mathrm{E}+00 \quad 0.00 \mathrm{E}+00 \quad 0.00 \mathrm{E}+00 \quad 0.00 \mathrm{E}+00 \quad 0.00 \mathrm{E}+00 \quad 0.00 \mathrm{E}+00 \quad 0.00 \mathrm{E}+00 \quad 0.00 \mathrm{E}+00 \quad 0.00 \mathrm{E}+00 \quad 0.00 \mathrm{E}+00 \quad 0.00 \mathrm{E}+00$ $0027 \quad 0.00 \mathrm{E}+00 \quad 0.00 \mathrm{E}+00 \quad 0.00 \mathrm{E}+00 \quad 0.00 \mathrm{E}+00 \quad 0.00 \mathrm{E}+00 \quad 0.00 \mathrm{E}+00 \quad 0.00 \mathrm{E}+00 \quad 0.00 \mathrm{E}+00 \quad 0.00 \mathrm{E}+00 \quad 0.00 \mathrm{E}+00 \quad 0.00 \mathrm{E}+00 \quad 0.00 \mathrm{E}+00 \quad 0.00 \mathrm{E}+00 \quad 0.00 \mathrm{E}+00 \quad 0.00 \mathrm{E}+00$

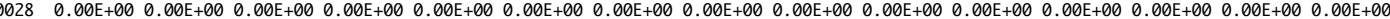
$00290.00 \mathrm{E}+00 \quad 0.00 \mathrm{E}+00 \quad 0.00 \mathrm{E}+00 \quad 0.00 \mathrm{E}+00 \quad 0.00 \mathrm{E}+00 \quad 0.00 \mathrm{E}+00 \quad 0.00 \mathrm{E}+00 \quad 0.00 \mathrm{E}+00 \quad 0.00 \mathrm{E}+00 \quad 0.00 \mathrm{E}+00 \quad 0.00 \mathrm{E}+00 \quad 0.00 \mathrm{E}+00 \quad 0.00 \mathrm{E}+00 \quad 0.00 \mathrm{E}+00 \quad 0.00 \mathrm{E}+00$

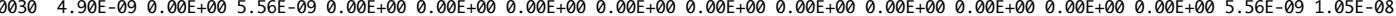
$0031 \quad 0.00 \mathrm{E}+00 \quad 0.00 \mathrm{E}+00 \quad 0.00 \mathrm{E}+00 \quad 0.00 \mathrm{E}+00 \quad 0.00 \mathrm{E}+00 \quad 0.00 \mathrm{E}+00 \quad 0.00 \mathrm{E}+00 \quad 0.00 \mathrm{E}+00 \quad 0.00 \mathrm{E}+00 \quad 0.00 \mathrm{E}+00 \quad 0.00 \mathrm{E}+00 \quad 0.00 \mathrm{E}+00 \quad 0.00 \mathrm{E}+00 \quad 0.00 \mathrm{E}+00 \quad 0.00 \mathrm{E}+00$ $0032 \quad 0.00 \mathrm{E}+00 \quad 0.00 \mathrm{E}+00 \quad 0.00 \mathrm{E}+00 \quad 0.00 \mathrm{E}+00 \quad 0.00 \mathrm{E}+00 \quad 0.00 \mathrm{E}+00 \quad 0.00 \mathrm{E}+00 \quad 0.00 \mathrm{E}+00 \quad 0.00 \mathrm{E}+00 \quad 0.00 \mathrm{E}+00 \quad 0.00 \mathrm{E}+00 \quad 0.00 \mathrm{E}+00 \quad 0.00 \mathrm{E}+000.00 \mathrm{E}+000.00 \mathrm{E}+00$

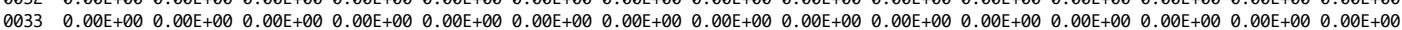
$00343.24 \mathrm{E}-100.00 \mathrm{E}+00 \quad 0.00 \mathrm{E}+00 \quad 0.00 \mathrm{E}+00 \quad 0.00 \mathrm{E}+00 \quad 0.00 \mathrm{E}+00 \quad 0.00 \mathrm{E}+00 \quad 0.00 \mathrm{E}+00 \quad 0.00 \mathrm{E}+00 \quad 0.00 \mathrm{E}+00 \quad 0.00 \mathrm{E}+00 \quad 0.00 \mathrm{E}+000.00 \mathrm{E}+00 \quad 0.00 \mathrm{E}+00 \quad 3.24 \mathrm{E}-10$ 0035 0.00E

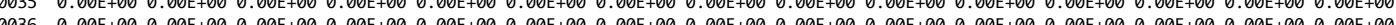

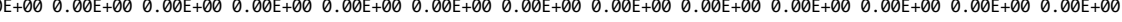

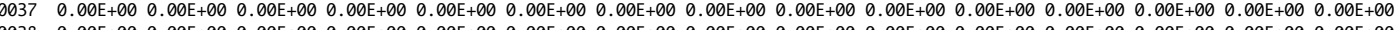

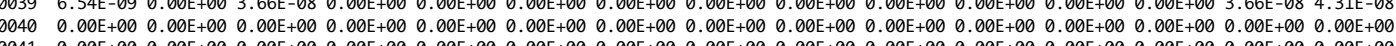

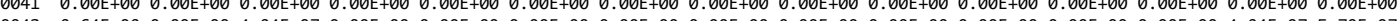

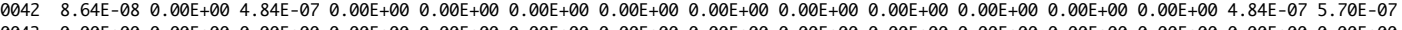

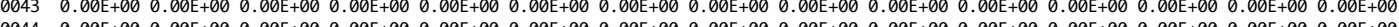
$0044 \quad 0.00 \mathrm{E}+00 \quad 0.00 \mathrm{E}+00 \quad 0.00 \mathrm{E}+00 \quad 0.00 \mathrm{E}+00 \quad 0.00 \mathrm{E}+00 \quad 0.00 \mathrm{E}+00 \quad 0.00 \mathrm{E}+00 \quad 0.00 \mathrm{E}+00 \quad 0.00 \mathrm{E}+00 \quad 0.00 \mathrm{E}+00 \quad 0.00 \mathrm{E}+00 \quad 0.00 \mathrm{E}+00 \quad 0.00 \mathrm{E}+00 \quad 0.00 \mathrm{E}+00 \quad 0.00 \mathrm{E}+00$

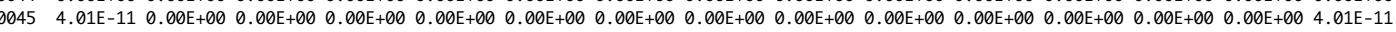

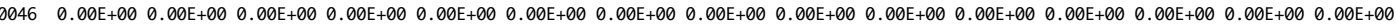

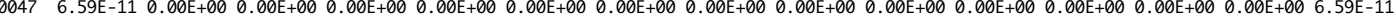

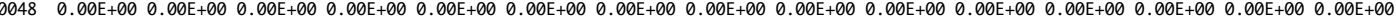
$0049 \quad 0.00 \mathrm{E}+00 \quad 0.00 \mathrm{E}+00 \quad 0.00 \mathrm{E}+00 \quad 0.00 \mathrm{E}+00 \quad 0.00 \mathrm{E}+00 \quad 0.00 \mathrm{E}+00 \quad 0.00 \mathrm{E}+00 \quad 0.00 \mathrm{E}+00 \quad 0.00 \mathrm{E}+00 \quad 0.00 \mathrm{E}+00 \quad 0.00 \mathrm{E}+00 \quad 0.00 \mathrm{E}+00 \quad 0.00 \mathrm{E}+00 \quad 0.00 \mathrm{E}+00 \quad 0.00 \mathrm{E}+00$ $0050 \quad 0.00 \mathrm{E}+00 \quad 0.00 \mathrm{E}+00 \quad 0.00 \mathrm{E}+00 \quad 0.00 \mathrm{E}+00 \quad 0.00 \mathrm{E}+00 \quad 0.00 \mathrm{E}+00 \quad 0.00 \mathrm{E}+00 \quad 0.00 \mathrm{E}+00 \quad 0.00 \mathrm{E}+00 \quad 0.00 \mathrm{E}+00 \quad 0.00 \mathrm{E}+00 \quad 0.00 \mathrm{E}+00 \quad 0.00 \mathrm{E}+00 \quad 0.00 \mathrm{E}+00 \quad 0.00 \mathrm{E}+00$

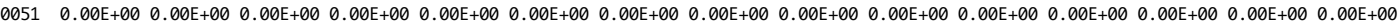
SUM $1.95 \mathrm{E}-07 \quad 0.00 \mathrm{E}+00 \quad 5.26 \mathrm{E}-07 \quad 0.00 \mathrm{E}+00 \quad 0.00 \mathrm{E}+00 \quad 0.00 \mathrm{E}+00 \quad 0.00 \mathrm{E}+00 \quad 0.00 \mathrm{E}+00 \quad 0.00 \mathrm{E}+00 \quad 0.00 \mathrm{E}+00 \quad 0.00 \mathrm{E}+00 \quad 0.00 \mathrm{E}+00 \quad 0.00 \mathrm{E}+00 \quad 5.26 \mathrm{E}-07 \quad 7.21 \mathrm{E}-07$

CHEM INHAL DERM SOIL MOTHER FISH WATER VEG DAIRY BEEF CHICK PIG EGG MEAT ORAL TOTAL $00018.35 \mathrm{E}-100.00 \mathrm{E}+000.00 \mathrm{E}+000.00 \mathrm{E}+000.00 \mathrm{E}+000.00 \mathrm{E}+000.00 \mathrm{E}+000.00 \mathrm{E}+000.00 \mathrm{E}+000.00 \mathrm{E}+000.00 \mathrm{E}+000.00 \mathrm{E}+000.00 \mathrm{E}+000.00 \mathrm{E}+008.35 \mathrm{E}-10$

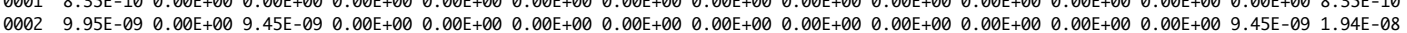

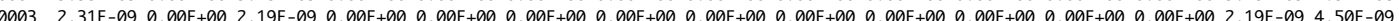

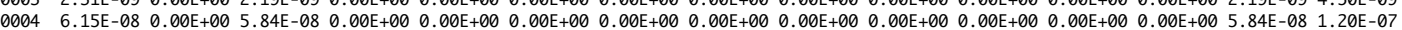

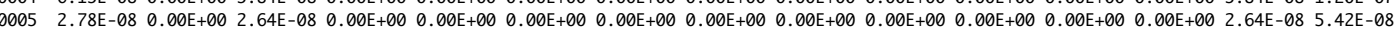

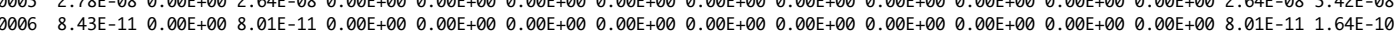

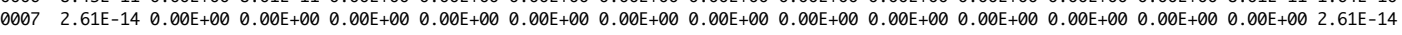
$0.00 \mathrm{E}+00 \quad 0.00 \mathrm{E}+00 \quad 0.00 \mathrm{E}+00 \quad 0.00 \mathrm{E}+00 \quad 0.00 \mathrm{E}+00 \quad 4.77 \mathrm{E}-15$ $0009 \quad 0.00 \mathrm{E}+00 \quad 0.00 \mathrm{E}+00 \quad 0.00 \mathrm{E}+00 \quad 0.00 \mathrm{E}+00 \quad 0.00 \mathrm{E}+00 \quad 0.00 \mathrm{E}+00 \quad 0.00 \mathrm{E}+00 \quad 0.00 \mathrm{E}+00 \quad 0.00 \mathrm{E}+00 \quad 0.00 \mathrm{E}+00 \quad 0.00 \mathrm{E}+00 \quad 0.00 \mathrm{E}+00 \quad 0.00 \mathrm{E}+00 \quad 0.00 \mathrm{E}+00 \quad 0.00 \mathrm{E}+00$

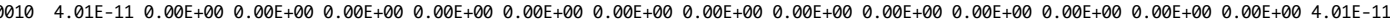
$0011 \quad 0.00 \mathrm{E}+00 \quad 0.00 \mathrm{E}+00 \quad 0.00 \mathrm{E}+00 \quad 0.00 \mathrm{E}+00 \quad 0.00 \mathrm{E}+00 \quad 0.00 \mathrm{E}+00 \quad 0.00 \mathrm{E}+00 \quad 0.00 \mathrm{E}+00 \quad 0.00 \mathrm{E}+00 \quad 0.00 \mathrm{E}+00 \quad 0.00 \mathrm{E}+00 \quad 0.00 \mathrm{E}+00 \quad 0.00 \mathrm{E}+00 \quad 0.00 \mathrm{E}+00 \quad 0.00 \mathrm{E}+00$

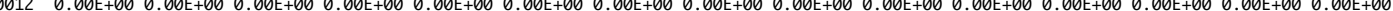

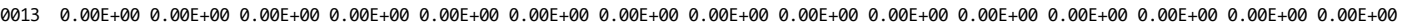

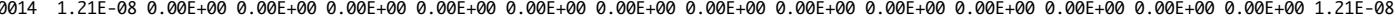
$0015 \quad 1.36 \mathrm{E}-07 \quad 0.00 \mathrm{E}+00 \quad 0.00 \mathrm{E}+00 \quad 0.00 \mathrm{E}+00 \quad 0.00 \mathrm{E}+00 \quad 0.00 \mathrm{E}+00 \quad 0.00 \mathrm{E}+00 \quad 0.00 \mathrm{E}+00 \quad 0.00 \mathrm{E}+00 \quad 0.00 \mathrm{E}+00 \quad 0.00 \mathrm{E}+00 \quad 0.00 \mathrm{E}+00 \quad 0.00 \mathrm{E}+00 \quad 0.00 \mathrm{E}+00 \quad 1.36 \mathrm{E}-07$ $0016 \quad 3.22 \mathrm{E}-10 \quad 0.00 \mathrm{E}+00 \quad 0.00 \mathrm{E}+00 \quad 0.00 \mathrm{E}+00 \quad 0.00 \mathrm{E}+00 \quad 0.00 \mathrm{E}+00 \quad 0.00 \mathrm{E}+00 \quad 0.00 \mathrm{E}+00 \quad 0.00 \mathrm{E}+00 \quad 0.00 \mathrm{E}+00 \quad 0.00 \mathrm{E}+00 \quad 0.00 \mathrm{E}+00 \quad 0.00 \mathrm{E}+00 \quad 0.00 \mathrm{E}+00 \quad 3.22 \mathrm{E}-10$ $0017 \quad 1.12 \mathrm{E}-11 \quad 0.00 \mathrm{E}+00 \quad 0.00 \mathrm{E}+00 \quad 0.00 \mathrm{E}+00 \quad 0.00 \mathrm{E}+00 \quad 0.00 \mathrm{E}+00 \quad 0.00 \mathrm{E}+00 \quad 0.00 \mathrm{E}+00 \quad 0.00 \mathrm{E}+00 \quad 0.00 \mathrm{E}+00 \quad 0.00 \mathrm{E}+00 \quad 0.00 \mathrm{E}+00 \quad 0.00 \mathrm{E}+00 \quad 0.00 \mathrm{E}+00 \quad 1.12 \mathrm{E}-11$ $0018 \quad 0.00 \mathrm{E}+00 \quad 0.00 \mathrm{E}+00 \quad 0.00 \mathrm{E}+00 \quad 0.00 \mathrm{E}+00 \quad 0.00 \mathrm{E}+00 \quad 0.00 \mathrm{E}+00 \quad 0.00 \mathrm{E}+00 \quad 0.00 \mathrm{E}+00 \quad 0.00 \mathrm{E}+00 \quad 0.00 \mathrm{E}+00 \quad 0.00 \mathrm{E}+00 \quad 0.00 \mathrm{E}+00 \quad 0.00 \mathrm{E}+00 \quad 0.00 \mathrm{E}+00 \quad 0.00 \mathrm{E}+00$ $0019 \quad 0.00 \mathrm{E}+00 \quad 0.00 \mathrm{E}+00 \quad 0.00 \mathrm{E}+00 \quad 0.00 \mathrm{E}+00 \quad 0.00 \mathrm{E}+00 \quad 0.00 \mathrm{E}+00 \quad 0.00 \mathrm{E}+00 \quad 0.00 \mathrm{E}+00 \quad 0.00 \mathrm{E}+00 \quad 0.00 \mathrm{E}+00 \quad 0.00 \mathrm{E}+00 \quad 0.00 \mathrm{E}+00 \quad 0.00 \mathrm{E}+00 \quad 0.00 \mathrm{E}+00 \quad 0.00 \mathrm{E}+00$ $0020 \quad 0.00 \mathrm{E}+00 \quad 0.00 \mathrm{E}+00 \quad 0.00 \mathrm{E}+00 \quad 0.00 \mathrm{E}+00 \quad 0.00 \mathrm{E}+00 \quad 0.00 \mathrm{E}+00 \quad 0.00 \mathrm{E}+00 \quad 0.00 \mathrm{E}+00 \quad 0.00 \mathrm{E}+00 \quad 0.00 \mathrm{E}+00 \quad 0.00 \mathrm{E}+00 \quad 0.00 \mathrm{E}+00 \quad 0.00 \mathrm{E}+00 \quad 0.00 \mathrm{E}+00 \quad 0.00 \mathrm{E}+00$ $00210.00 \mathrm{E}+00 \quad 0.00 \mathrm{E}+00 \quad 0.00 \mathrm{E}+00 \quad 0.00 \mathrm{E}+00 \quad 0.00 \mathrm{E}+00 \quad 0.00 \mathrm{E}+00 \quad 0.00 \mathrm{E}+00 \quad 0.00 \mathrm{E}+00 \quad 0.00 \mathrm{E}+00 \quad 0.00 \mathrm{E}+00 \quad 0.00 \mathrm{E}+000.00 \mathrm{E}+00 \quad 0.00 \mathrm{E}+00 \quad 0.00 \mathrm{E}+00 \quad 0.00 \mathrm{E}+00$

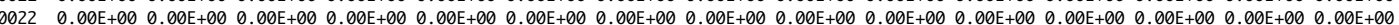

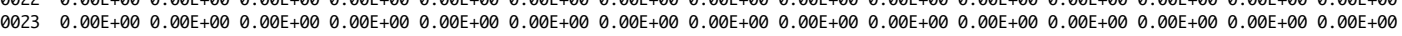
$00240.00 \mathrm{E}+00 \quad 0.00 \mathrm{E}+00 \quad 0.00 \mathrm{E}+00 \quad 0.00 \mathrm{E}+00 \quad 0.00 \mathrm{E}+00 \quad 0.00 \mathrm{E}+00 \quad 0.00 \mathrm{E}+00 \quad 0.00 \mathrm{E}+00 \quad 0.00 \mathrm{E}+00 \quad 0.00 \mathrm{E}+00 \quad 0.00 \mathrm{E}+00 \quad 0.00 \mathrm{E}+000.00 \mathrm{E}+000.00 \mathrm{E}+000.00 \mathrm{E}+00$

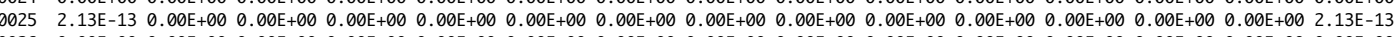

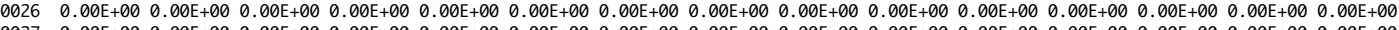
$0027 \quad 0.00 \mathrm{E}+00 \quad 0.00 \mathrm{E}+00 \quad 0.00 \mathrm{E}+00 \quad 0.00 \mathrm{E}+00 \quad 0.00 \mathrm{E}+00 \quad 0.00 \mathrm{E}+00 \quad 0.00 \mathrm{E}+00 \quad 0.00 \mathrm{E}+00 \quad 0.00 \mathrm{E}+00 \quad 0.00 \mathrm{E}+00 \quad 0.00 \mathrm{E}+00 \quad 0.00 \mathrm{E}+00 \quad 0.00 \mathrm{E}+00 \quad 0.00 \mathrm{E}+00 \quad 0.00 \mathrm{E}+00$

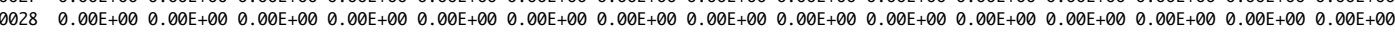

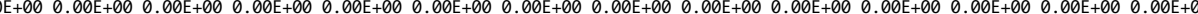
$0030 \quad 4.29 \mathrm{E}-08 \quad 0.00 \mathrm{E}+00 \quad 4.86 \mathrm{E}-08 \quad 0.00 \mathrm{E}+00 \quad 0.00 \mathrm{E}+00 \quad 0.00 \mathrm{E}+00 \quad 0.00 \mathrm{E}+00 \quad 0.00 \mathrm{E}+00 \quad 0.00 \mathrm{E}+00 \quad 0.00 \mathrm{E}+00 \quad 0.00 \mathrm{E}+00 \quad 0.00 \mathrm{E}+00 \quad 0.00 \mathrm{E}+00 \quad 4.86 \mathrm{E}-08 \quad 9.15 \mathrm{E}-08$

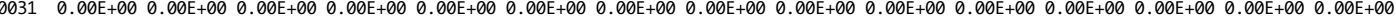
$0032 \quad 0.00 \mathrm{E}+00 \quad 0.00 \mathrm{E}+00 \quad 0.00 \mathrm{E}+00 \quad 0.00 \mathrm{E}+00 \quad 0.00 \mathrm{E}+00 \quad 0.00 \mathrm{E}+00 \quad 0.00 \mathrm{E}+00 \quad 0.00 \mathrm{E}+00 \quad 0.00 \mathrm{E}+00 \quad 0.00 \mathrm{E}+00 \quad 0.00 \mathrm{E}+00 \quad 0.00 \mathrm{E}+00 \quad 0.00 \mathrm{E}+00 \quad 0.00 \mathrm{E}+00 \quad 0.00 \mathrm{E}+00$ $0033 \quad 0.00 \mathrm{E}+00 \quad 0.00 \mathrm{E}+00 \quad 0.00 \mathrm{E}+00 \quad 0.00 \mathrm{E}+00 \quad 0.00 \mathrm{E}+00 \quad 0.00 \mathrm{E}+00 \quad 0.00 \mathrm{E}+00 \quad 0.00 \mathrm{E}+00 \quad 0.00 \mathrm{E}+00 \quad 0.00 \mathrm{E}+00 \quad 0.00 \mathrm{E}+00 \quad 0.00 \mathrm{E}+00 \quad 0.00 \mathrm{E}+00 \quad 0.00 \mathrm{E}+00 \quad 0.00 \mathrm{E}+00$ $0034 \quad 3.77 \mathrm{E}-10 \quad 0.00 \mathrm{E}+00 \quad 0.00 \mathrm{E}+00 \quad 0.00 \mathrm{E}+00 \quad 0.00 \mathrm{E}+00 \quad 0.00 \mathrm{E}+00 \quad 0.00 \mathrm{E}+00 \quad 0.00 \mathrm{E}+00 \quad 0.00 \mathrm{E}+00 \quad 0.00 \mathrm{E}+00 \quad 0.00 \mathrm{E}+00 \quad 0.00 \mathrm{E}+00 \quad 0.00 \mathrm{E}+00 \quad 0.00 \mathrm{E}+00 \quad 3.77 \mathrm{E}-10$ $0035 \quad 6.31 \mathrm{E}-130.00 \mathrm{E}+00 \quad 0.00 \mathrm{E}+00 \quad 0.00 \mathrm{E}+00 \quad 0.00 \mathrm{E}+00 \quad 0.00 \mathrm{E}+00 \quad 0.00 \mathrm{E}+00 \quad 0.00 \mathrm{E}+00 \quad 0.00 \mathrm{E}+00 \quad 0.00 \mathrm{E}+00 \quad 0.00 \mathrm{E}+00 \quad 0.00 \mathrm{E}+000.00 \mathrm{E}+00 \quad 0.00 \mathrm{E}+00 \quad 6.31 \mathrm{E}-13$

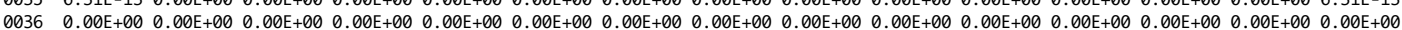
0036 (1)

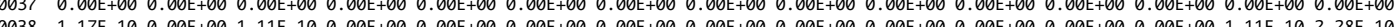

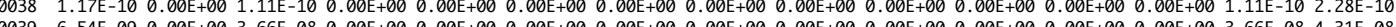

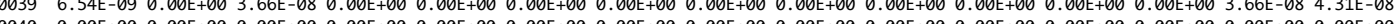

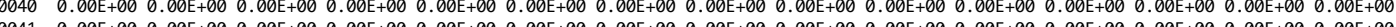
0041 $0.00 \mathrm{E}$ 0042 8.64E-08

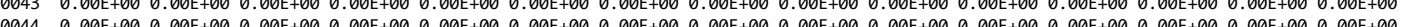

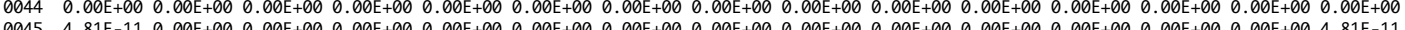
$0045 \quad 4.81 \mathrm{E}-11 \quad 0.00 \mathrm{E}+00 \quad 0.00 \mathrm{E}+00 \quad 0.00 \mathrm{E}+00 \quad 0.00 \mathrm{E}+00 \quad 0.00 \mathrm{E}+00 \quad 0.00 \mathrm{E}+00 \quad 0.00 \mathrm{E}+00 \quad 0.00 \mathrm{E}+00 \quad 0.00 \mathrm{E}+00 \quad 0.00 \mathrm{E}+00 \quad 0.00 \mathrm{E}+00 \quad 0.00 \mathrm{E}+00 \quad 0.00 \mathrm{E}+00 \quad 4.81 \mathrm{E}-11$

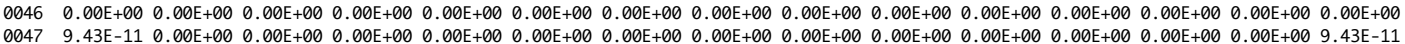




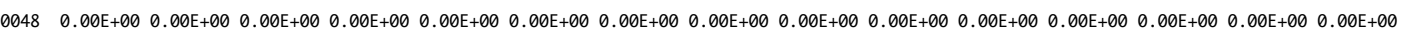

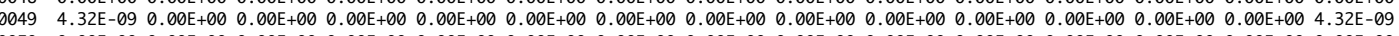
$0050 \quad 0.00 \mathrm{E}+00 \quad 0.00 \mathrm{E}+00 \quad 0.00 \mathrm{E}+00 \quad 0.00 \mathrm{E}+00 \quad 0.00 \mathrm{E}+00 \quad 0.00 \mathrm{E}+00 \quad 0.00 \mathrm{E}+00 \quad 0.00 \mathrm{E}+00 \quad 0.00 \mathrm{E}+00 \quad 0.00 \mathrm{E}+00 \quad 0.00 \mathrm{E}+00 \quad 0.00 \mathrm{E}+00 \quad 0.00 \mathrm{E}+00 \quad 0.00 \mathrm{E}+00 \quad 0.00 \mathrm{E}+00$

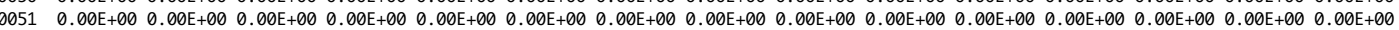

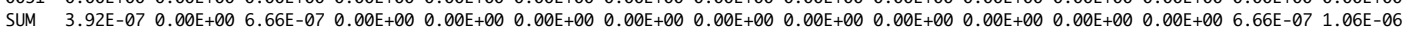


Rep_Can_WRK_Avg_Rec5_AllSrc_AllCh_bySrc_Site.txt 


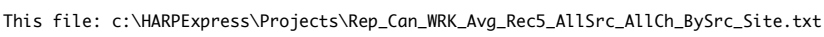

Created by HARP Version 1.0 Build 23.02.10

Uses ISC Version 9915

Uses BPIP Version 95086

Creation date: 10/24/2005 2:06:29 PM

EXCEPTION REPORT

(there have been no changes or exceptions)

INPUT FILES:

Source-Receptor file: c: \HARPExpress \Projects $\backslash$ EWTF.mta

Averaging period adjustment factors file: not applicable

Emission rates file: none

Site parameters file: C: $\backslash$ HARPExpress $\backslash$ Projects $\backslash$ Site300. sit

Screening mode is OFF

USER-DEFINED WORKER ADJUSTMENT AND EXPOSURE PARAMETERS

GLC Adjustment Factor:

Exposure Duration (years): 25

Exposure Frequency (days/year): 245

Explanatory Note: U.S. EPA value

Analysis method: Point estimat

Health effect: Cancer Risk

Receptor(s): $\quad 5$

Sources(s): $\quad$ All

SITE PARAMETERS

DEPOSITION

Deposition rate $(\mathrm{m} / \mathrm{s})$

0.0072

DRINKING WATER

*** Pathway disabled ***

FISH

*** Pathway disabled ***

PASTURE

ANIMALS' WATER

Water volume (L)

Fraction of beef cows' water

from pasture source 0

Fraction of dairy cows' water 0

ANIMALS' FEED

Fraction of cows' feed

from grazing

0.25

HUMAN INGESTION

Fraction of ingested beef

from contaminated source

Fraction of ingested dairy

from contaminated source

0.107

HOME GROWN PRODUCE

HUMAN INGESTION

Fraction of ingested leafy vegetable

Faction of ingested exposed vegetable

from home grown source 0.207

Fraction of ingested protected vegetable

from home grown source 0.134

Fraction of ingested root vegetable
from home grown source

PIGS, CHICKENS AND EGGS

HUMAN INGESTION

Fraction of ingested pig

from home grown source

Fraction of ingested chicken

0.04

from home ingested chick

Fraction of ingested egg

0.026

from home grown source

0.029

ANIMALS' FEED

Fraction of pigs' feed

from home grown crop

raction of chickens' feed

OIL INGESTION

Fraction of pigs' feed

eaten off the ground 


\begin{tabular}{|c|c|}
\hline $\begin{array}{l}\text { Fraction of chickens' feed } \\
\text { eaten off the ground }\end{array}$ & 0.05 \\
\hline PIG FEED COMPOSITION & \\
\hline $\begin{array}{l}\text { Fraction of feed that is } \\
\text { exposed vegetable }\end{array}$ & 0.25 \\
\hline $\begin{array}{l}\text { Fraction of feed that is } \\
\text { leafy vegetable }\end{array}$ & 0.25 \\
\hline $\begin{array}{l}\text { Fraction of feed that is } \\
\text { protected vegetable }\end{array}$ & 0.25 \\
\hline $\begin{array}{l}\text { Fraction of feed that is } \\
\text { root vegetable }\end{array}$ & 0.25 \\
\hline CHICKEN FEED COMPOSITION & \\
\hline $\begin{array}{l}\text { Fraction of feed that is } \\
\text { exposed vegetable }\end{array}$ & 0.25 \\
\hline $\begin{array}{l}\text { Fraction of feed that is } \\
\text { leafy vegetable }\end{array}$ & 0.25 \\
\hline $\begin{array}{l}\text { Fraction of feed that is } \\
\text { protected vegetable }\end{array}$ & 0.25 \\
\hline $\begin{array}{l}\text { Fraction of feed that is } \\
\text { root vegetable }\end{array}$ & 0.25 \\
\hline
\end{tabular}

DERMAL ABSORPTION

*** Pathway disabled $* * *$

SOIL INGESTION

*** Pathway enabled $* * *$

MOTHER'S MILK

*** Pathway enabled $* * *$

CHEMICAL CROSS-REFERENCE TABLE AND BACKGROUND CONCENTRATIONS

$\begin{array}{llll}\text { CHEM } & \text { CAS } & \text { ABBREVIATION } & \text { POLLUTANT NAME } \\ 0001 & 106990 & 1,3 \text {-Butadiene } & 1,3 \text {-Butadiene }\end{array}$

$000267562394 \quad 1-4,6-8 \mathrm{HpCDF} \quad 1,2,3,4,6,7,8$-Heptachlorodibenzofuran

$1,2,3,4,7,8,9-$ Heptachlorodibenzofuran

$1,2,3,4,7,8$ -

$000557117449 \quad 1-3,6-8 \mathrm{HxCDF} \quad 1,2,3,6,7,8$-Hexachlorodibenzofuran

$000635822469 \quad 1-4,6-8 \mathrm{HpCDD} \quad 1,2,3,4,6,7,8$-Heptachlorodibenzo-p-dioxin

$0007 \quad 121142$ 2,4-DiNitToluen 2,4-Dinitrotoluene

$0008606202 \quad 2,6$-DiNitioluen 2,6-Dinitrotolu

000995578 2-CHLOROPHENOL 2-CHLOROPHENOL

0010107051 AllylChlor Allyl chloride

00117429905 Aluminum Aluminum

00127440360 Antimony Antimony

00137440393 Barium Barium

$\begin{array}{llll}0014 & 71432 & \text { Benzene } & \text { Benzene } \\ 0015 & 7440439 & \text { Cadmium } & \text { Cadmium }\end{array}$

(0015 7440439 Cadmium

$\begin{array}{llll}0016 & 56235 & \text { CCl4 } & \text { Carbon tetract } \\ 0017 & 67663 & \text { Chloroform } & \text { Chloroform }\end{array}$

00187440473 Chromium Chromium

00197782505 Chlorine Chlorine

$0020630080 \quad$ Carbon Monoxide Carbon monoxide

00217440508 Copper Copper

0022110827 Cyclohexane Cyclohexane

002384662 DiethylPhthalat Diethyl phthalate

$0024122394 \quad$ Diphenylam $\quad$ Diphenylamine

$002575003 \quad$ Ethyl Chloride Ethyl chloride \{Chlorethane

$0026100414 \quad$ Ethyl Benzene Ethyl benzene

$\begin{array}{llll}0027 & 206440 & \text { Fluoranthene } & \text { Fluoranthene } \\ 0028 & 7647010 & \text { HCl } & \text { Hydrochloric acid }\end{array}$

$\begin{array}{llll}0028 & 7647010 & \mathrm{HCl} & \text { Hydroch } \\ 0029 & 98828 & \text { Cumene } & \text { Cumene }\end{array}$

$002998828 \quad$ Cumene Cumene

$\begin{array}{llll}0030 & 7439921 & \text { Lead } & \text { Lead } \\ 0031 & 74873 & \text { Methyl Chloride Methyl chloride \{Chloromethane } \\ 0032 & 71556 & 1,1,1-T C A & \text { Methyl chloroform }\{1,1,1-T C A\}\end{array}$

003271556 1,1,1-TCA Methyl chloroform $\{1,1,1-\mathrm{TCA}\}$

0033108872 MethCyHex Methylcyclohexane

003475092 Methylene Chlor Methylene chloride \{Dichloromethane\}

003591203 Naphthalene Naphthalene

0036110543 Hexane Hexane

003710102440 NITROGEN DIOXID NITROGEN DIOXIDE

003839001020 1-80ctaCDF $1,2,3,4,6,7,8,9$-0ctachlorodibenzofuran

003978115 PETN PETN (same as RDX)

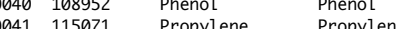

$\begin{array}{llll}0041 & 115071 & \text { Propylene } & \text { Propyle } \\ 0042 & 121824 & \text { RDX } & \text { RDX }\end{array}$

0043100425 Styrene Styrene

00447446095 SULFUR DIOXIDE SULFUR DIOXIDE

0045127184 Perc Perchloroethylene \{Tetrachloroethene\}

$0046 \quad 108883 \quad$ Toluene

$0047 \quad 75014$

$\begin{array}{llll}0047 & 75014 & \text { Vinyl Chloride } & \text { Vin } \\ 0048 & 7440666 & \text { Zinc } & \text { Zinc }\end{array}$

Toluene Torchlone

004910101 NapthSur Naphthalene surrogate

0050 10102 EthBzSur Ethylbenzene surrogate

005110103 nHexSur n-Hexane surrogate

BACKGROUND (ug/m^3)

$0.000 \mathrm{E}+00$

$0.000 \mathrm{E}+00$

$0.000 \mathrm{E}+00$

$0.000 \mathrm{E}+00$

$0.000 \mathrm{E}+00$

$0.000 \mathrm{E}+00$

$0.000 E+00$

$0.000 E+00$

$0.000 \mathrm{E}+00$

$0.000 \mathrm{E}+00$

$0.000 \mathrm{E}+00$

$0.000 \mathrm{E}+00$

$0.000 \mathrm{E}+00$

$0.000 E+00$

$0.000 \mathrm{E}+00$

$0.000 \mathrm{E}+00$

$0.000 \mathrm{E}+00$

$0.000 \mathrm{E}+00$

$0.000 \mathrm{E}+00$

$0.000 \mathrm{E}+00$

$0.000 \mathrm{E}+00$

$0.000 \mathrm{E}+00$

$0.000 \mathrm{E}+00$

$0.000 \mathrm{E}+00$

$0.000 \mathrm{E}+00$

$0.000 E+00$

$0.000 E+00$

$0.000 \mathrm{E}+00$

$0.000 \mathrm{E}+00$

$0.000 \mathrm{E}+00$

$0.000 \mathrm{E}+00$

$0.000 \mathrm{E}+00$

$0.000 \mathrm{E}+00$

$0.000 E+00$

$0.000 \mathrm{E}+00$

$0.000 \mathrm{E}+00$

$0.000 \mathrm{E}+00$

$0.000 \mathrm{E}+00$

$0.000 \mathrm{E}+00$

$0.000 \mathrm{E}+00$

$0.000 \mathrm{E}+00$

$0.000 \mathrm{E}+00$

$0.000 \mathrm{E}+00$

$0.000 \mathrm{E}+00$

$0.000 \mathrm{E}+00$

$0.000 \mathrm{E}+00$

$0.000 \mathrm{E}+00$

$0.000 \mathrm{E}+00$

$0.000 \mathrm{E}+00$

$0.000 E+00$

EMISSIONS DATA SOURCE:

CHEMICALS ADDED OR DELETED: none

EMISSIONS FOR FACILITY FAC $=S i t e 300 \quad$ CO=* DEV=PR1 PRO=OBPan STK=1 NAME=LLNL Site $300 \quad$ EMS (lbs/yr) SOURCE MULTIPLIER=1

$67562394 \quad 1,3-$ Butadien

MULTIPLIER

$\begin{array}{rr}\text { AVRG (lbs/yr) } & \text { MAX (lbs/hr) } \\ 0.017 & 0.00017\end{array}$ 


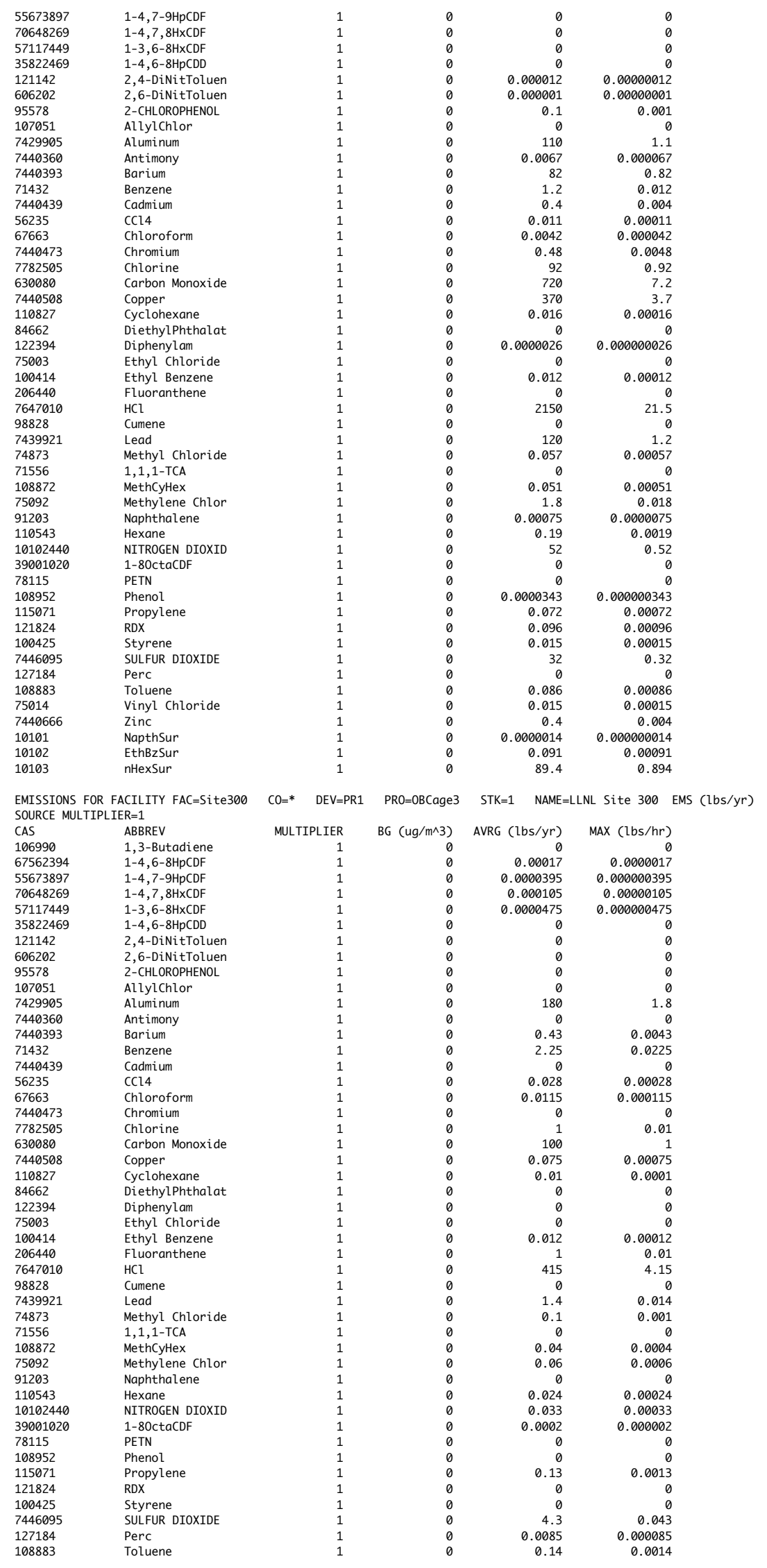




$\begin{array}{llllrr}75014 & \text { Vinyl Chloride } & 1 & 0 & 0 & 0 \\ 7440666 & \text { Zinc } & 1 & 0 & 2.85 & 0.0285 \\ 10101 & \text { NapthSur } & 1 & 0 & 0.8 & 0.008 \\ 10102 & \text { EthBzSur } & 1 & 0 & 0.038 & 0.00038 \\ 10103 & \text { nHexSur } & 1 & 0 & 9.83 & 0.0983\end{array}$

EMISSIONS FOR FACILITY FAC=Site300 CO=* DEV=PR1 PRO=0BCage4 STK=1 NAME=LLNL Site $300 \quad$ EMS (lbs/yr) SOURCE MULTIPLIER $=1$

CAS MULTIPLIER

$106990 \quad 1,3$-Butadiene

$\begin{array}{ll}1-4,6-8 \mathrm{HpCDF} \\ 556733897 & 1-4,7-9 \mathrm{HpCDF}\end{array}$

$70648269 \quad 1-4,7,8 \mathrm{H} \times \mathrm{CDF}$

$57117449 \quad 1-3,6-8 \mathrm{H} \times \mathrm{CDF}$

$35822469 \quad 1-4,6-8 \mathrm{HpCDD}$

$\begin{array}{ll}35822469 & 1-4,6-8 \mathrm{HpCDD} \\ 121142 & 2,4-\text {-DiNitToluen }\end{array}$

606202 2,6-DiNitToluen

95578 2-CHLOROPHENOL

107051 AllylChlor

$7429905 \quad$ Aluminum

7440360 Antimony

$7440393 \quad$ Barium

71432 Benzene

$\begin{array}{ll}7440439 & \text { Cadmiu } \\ 56235 & \text { CCl4 }\end{array}$

$67663 \quad$ Chloroform

$7440473 \quad$ Chromium

$7782505 \quad$ Chlorine

630080 Carbon Monoxide

$7440508 \quad$ Copper

$110827 \quad$ Cyclohexane

84662 DiethylPhthalat

122394 Diphenylam

75003 Ethyl Chloride

206440 Fluoranthene

$7647010 \quad \mathrm{HCl}$

$98828 \quad$ Cumene

$\begin{array}{ll}7439921 & \text { Lead } \\ 74873 & \text { Methyl Chloride }\end{array}$

715561 Methyl Ch

$\begin{array}{ll}108872 & \text { MethCyHex } \\ 75092 & \text { Methylene Chlor }\end{array}$

75092

$\begin{array}{ll}91203 & \text { Naphthal } \\ 110543 & \text { Hexane }\end{array}$

10102440 NITROGEN DIOXID

$39001020 \quad 1-80$ ctaCDF

$\begin{array}{ll}78115 & \text { PETN } \\ 108952 & \text { Phenol }\end{array}$

$\begin{array}{ll}108952 & \text { Phenol } \\ 115071 & \text { Propylene }\end{array}$

$121824 \quad$ RDX

$\begin{array}{ll}100425 & \text { Styrene } \\ 7446095 & \text { SULFUR DIOXIDE }\end{array}$

$127184 \quad$ Perc

$\begin{array}{ll}108883 & \text { Toluene } \\ 75014 & \text { Vinyl Chloride }\end{array}$

$7440666 \quad$ Ziny

10101 NapthSur

10102 EthBzSur

BG (ug/m^3)

AVRG (lbs/yr) MAX (lbs/hr)

nHexSur

EMISSIONS FOR FACIL

ITY $F A C=S i t e 300$

CAS ABBREV

ABBREV

$675623941-4,6-8 \mathrm{HpCDF}$

55673897 1-4,7-9HpCDF

$70648269 \quad 1-4,7,8 \mathrm{HxCDF}$

$57117449 \quad 1-3,6-8 \mathrm{HxCDF}$

$\begin{array}{ll}35822469 & 1-4,6-8 \mathrm{HpCDD} \\ 121142 & 2,4-D i N i t T o l u e n\end{array}$

606202 2,6-DiNitToluen

95578 2-CHLOROPHENOL

107051 AllylChlor

$7429905 \quad$ Aluminum

7440360 Antimony

$7440393 \quad$ Barium

71432 Benzene

$56235 \quad$ CCl4

$67663 \quad$ Chloroform

$\begin{array}{ll}7440473 & \text { Chromium } \\ 7782505 & \text { Chlorine }\end{array}$

630080 Carbon Monoxide

$7440508 \quad$ Copper

110827 Cyclohexane

84662 DiethylPhthalat

$122394 \quad$ Diphenylam

75003 Ethyl Chloride

100414 Ethyl Benzene

$206440 \quad$ Fluoranthene

$7647010 \quad \mathrm{HCl}$

$98828 \quad$ Cumene

$\begin{array}{ll}7439921 & \text { Lead } \\ 74873 & \text { Methyl Chloride }\end{array}$

$\begin{array}{ll}74873 & \text { Methyl Ch } \\ 71556 & 1,1,1-\mathrm{TCA}\end{array}$

108872

$\begin{array}{ll}75092 & \text { Methylene Chlo } \\ 91203 & \text { Naphthalene }\end{array}$

$C O=* \quad$ DEV $=$ PR 1

$P R 0=0 D \quad S T K=1 \quad$ NAME $=L L N L$ Site 300 EMS (lbs/yr)

MULTIPLIER

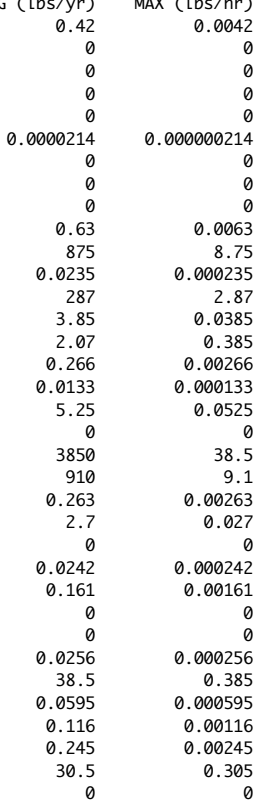




$\begin{array}{lllrrr}110543 & \text { Hexane } & 1 & 0 & 0.665 & 0.00665 \\ 10102440 & \text { NITROGEN DIOXID } & 1 & 0 & 154 & 1.54 \\ 39001020 & \text { 1-80ctaCDF } & 1 & 0 & 0 & 0 \\ 78115 & \text { PETN } & 1 & 0 & 19.6 & 0.196 \\ 108952 & \text { Phenol } & 1 & 0 & 0 & 0 \\ 115071 & \text { Propylene } & 1 & 0 & 2.56 & 0.0256 \\ 121824 & \text { RDX } & 1 & 0 & 259 & 2.59 \\ 100425 & \text { Styrene } & 1 & 0 & 1.47 & 0.0147 \\ 7446095 & \text { SULFUR DIOXIDE } & 1 & 0 & 455 & 4.55 \\ 127184 & \text { Perc } & 1 & 0 & 0.63 & 0.0063 \\ 108883 & \text { Toluene } & 1 & 0 & 1.05 & 0.0105 \\ 75014 & \text { Vinyl Chloride } & 1 & 0 & 0.0805 & 0.000805 \\ 7440666 & \text { Zinc } & 1 & 0 & 560 & 5.6 \\ 10101 & \text { NapthSur } & 1 & 0 & 0 & 0 \\ 10102 & \text { EthBzSur } & 1 & 0 & 0.62 & 0.0062 \\ 10103 & \text { nHexSur } & 1 & 0 & 119 & 1.19\end{array}$

CANCER RISK REPORT

RECEPTOR 5

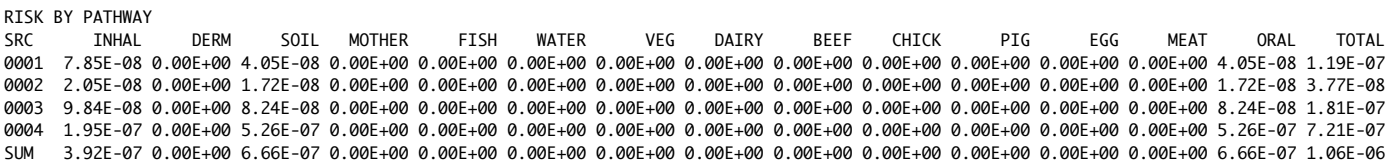

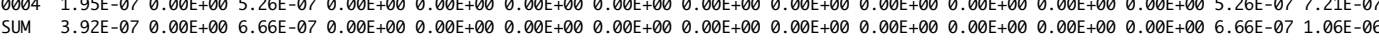


Rep_Chr_Wrk_PtEst_Rec5_AllSrc_AllCh_BySrc_Site.txt 
This file: c: \HARPExpress $\backslash$ Projects $\backslash$ Rep_Chr_Wrk_PtEst_Rec5_AllSrc_AllCh_BySrc_Site.txt

Created by HARP Version 1.0 Build 23.02.10

Uses ISC Version 99155

Uses BPIP Version 95086

Creation date: 10/24/2005 2:08:29 PM

EXCEPTION REPORT

(there have been no changes or exceptions)

INPUT FILES:

Source-Receptor file: c: \HARPExpress $\backslash$ Projects $\backslash$ EWTF.mta

Averaging period adjustment factors file: not applicable

Emission rates file: none

Site parameters file: C: \HARPExpress $\backslash$ Projects $\backslash$ Site300.sit

Screening mode is OFF

USER-DEFINED WORKER ADJUSTMENT AND EXPOSURE PARAMETERS

GLC Adjustment Factor: 1

Exposure Duration (years): 25

Exposure Frequency (days/year): 245

Explanatory Note: U.S. EPA value

Analysis method: Point estimate

Health effect: Chronic HI

Receptor(s): 5

Sources(s): Al

Chemicals(s):

All

SITE PARAMETERS

DEPOSITION

Deposition rate $(\mathrm{m} / \mathrm{s})$

0.0072

DRINKING WATER

*** Pathway disabled ***

FISH

*** Pathway disabled $* * *$

PASTURE

ANIMALS' WATER

Water surface area (nis)

Water volume (L)

Volume changes per year

from pasture source

Fraction of dairy cows' water

from pasture source

ANIMALS' FEED

Fraction of cows' feed

from grazing

HUMAN INGESTION

Fraction of ingested beef

from contaminated source

raction of ingested dairy

from contaminated source

0.107

OME GROWN PRODUCE

HUMAN INGESTION

Fraction of ingested leafy vegetable

from home grown source 0.082

Fraction of ingested exposed vegetable

from home grown source 0.207

Fraction of ingested protected vegetable

from home grown source 0.134

Fraction of ingested root vegetable

from home grown source 0.088

PIGS, CHICKENS AND EGGS

HUMAN INGESTION

Fraction of ingested pig

Fraction of ingested chicken

from home grown source

from home grown source

from home grown source

0.04

0.026

ANIMALS' FEED

Fraction of pigs' feed

from home grown crop

Fraction of chickens' feed

from home grown crop 


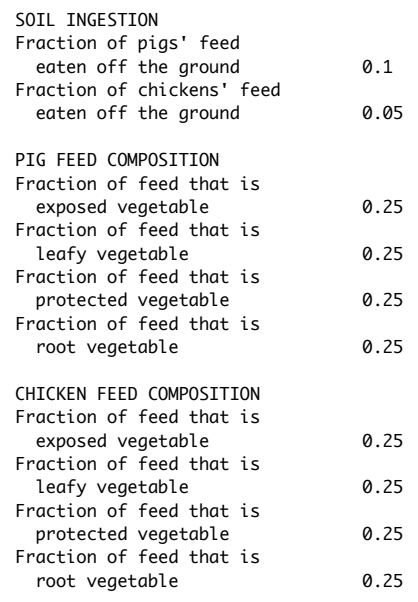

DERMAL ABSORPTION

*** Pathway disabled ***

SOIL INGESTION

*** Pathway enabled ***

MOTHER'S MILK

*** Pathway enabled $* * *$

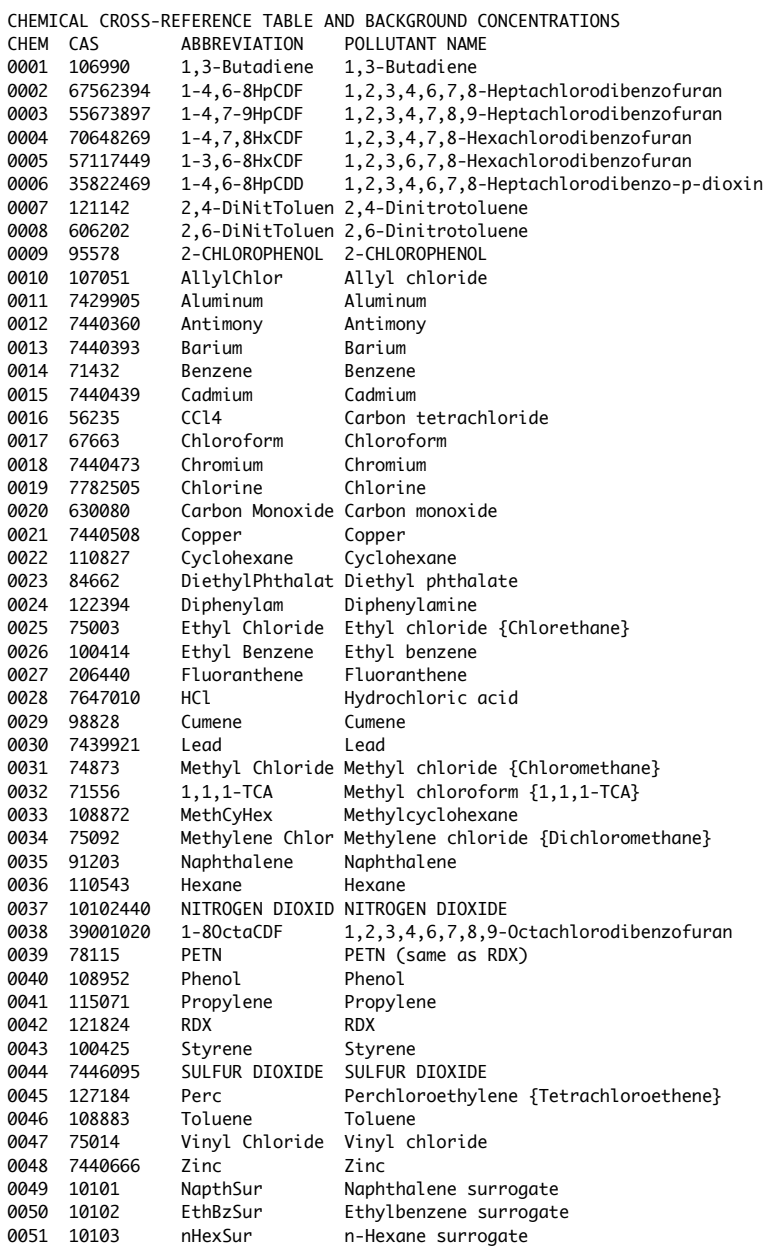

$0.000 \mathrm{E}+00$

$0.000 \mathrm{E}+00$

$0.000 E+00$

$0.000 \mathrm{E}+00$

$0.000 \mathrm{E}+00$

$0.000 \mathrm{E}+00$

$0.000 \mathrm{E}+00$

$0.000 \mathrm{E}+00$

$0.000 \mathrm{E}+00$

$0.000 \mathrm{E}+00$

$0.000 \mathrm{E}+00$

$0.000 \mathrm{E}+00$

$0.000 \mathrm{E}+00$

$0.000 \mathrm{E}+00$

$0.000 \mathrm{E}+00$

$0.000 \mathrm{E}+00$

$0.000 E+00$

$0.000 \mathrm{E}+00$

$0.000 \mathrm{E}+00$

$0.000 \mathrm{E}+00$

$0.000 \mathrm{E}+00$

$0.000 \mathrm{E}+00$

$0.000 \mathrm{E}+00$

$0.000 \mathrm{E}+00$

$0.000 \mathrm{E}+00$

$0.000 \mathrm{E}+00$

$0.000 \mathrm{E}+00$

$0.000 \mathrm{E}+00$

$0.000 \mathrm{E}+00$

$0.000 E+00$

$0.000 E+00$

$0.000 \mathrm{E}+00$

$0.000 \mathrm{E}+00$

$0.000 \mathrm{E}+00$

$0.000 \mathrm{E}+00$

$0.000 \mathrm{E}+00$

$0.000 \mathrm{E}+00$

$0.000 \mathrm{E}+00$

EMISSIONS DATA SOURCE :

CHEMICALS ADDED OR DELETED: none 


\begin{tabular}{|c|c|c|c|c|c|}
\hline $\begin{array}{l}\text { EMISSIONS FOR } \\
\text { SOURCE MULTIP }\end{array}$ & $\begin{array}{l}\text { EACILITY } \quad F A C=S i t e 300 \\
\text { IER }=1\end{array}$ & $C 0=* \quad D E V=P R 1$ & PRO=OBPan & $S T K=1 \quad$ NAME $=L L N L$ & Site 300 EMS \\
\hline CAS & ABBREV & MULTIPLIER & $B G\left(u g / m^{\wedge} 3\right)$ & AVRG (lbs/yr) & $\operatorname{MAX}(\mathrm{lbs} / \mathrm{hr})$ \\
\hline 106990 & 1,3-Butadiene & 1 & 0 & 0.017 & 0.00017 \\
\hline 67562394 & $1-4,6-8 \mathrm{HpCDF}$ & 1 & 0 & 0 & 0 \\
\hline 55673897 & $1-4,7-9 \mathrm{HPCDF}$ & 1 & 0 & 0 & 0 \\
\hline 70648269 & $1-4,7,8 \mathrm{H} \times C D F$ & 1 & 0 & 0 & 0 \\
\hline 57117449 & $1-3,6-8 \mathrm{H} \times C D F$ & 1 & 0 & 0 & 0 \\
\hline 35822469 & $1-4,6-8 \mathrm{HpCDD}$ & 1 & 0 & 0 & 0 \\
\hline 121142 & 2,4-DiNitToluen & 1 & 0 & 0.000012 & 0.00000012 \\
\hline 606202 & 2,6-DiNitToluen & 1 & 0 & 0.000001 & 0.00000001 \\
\hline 95578 & 2-CHLOROPHENOL & 1 & 0 & 0.1 & 0.001 \\
\hline 107051 & AllylChlor & 1 & 0 & 0 & 0 \\
\hline 7429905 & Aluminum & 1 & 0 & 110 & 1.1 \\
\hline 7440360 & Antimony & 1 & 0 & 0.0067 & 0.000067 \\
\hline 7440393 & Barium & 1 & 0 & 82 & 0.82 \\
\hline 71432 & Benzene & 1 & 0 & 1.2 & 0.012 \\
\hline 7440439 & Cadmium & 1 & 0 & 0.4 & 0.004 \\
\hline 56235 & CCl4 & 1 & 0 & 0.011 & 0.00011 \\
\hline 67663 & Chloroform & 1 & 0 & 0.0042 & 0.000042 \\
\hline 7440473 & Chromium & 1 & 0 & 0.48 & 0.0048 \\
\hline 7782505 & Chlorine & 1 & 0 & 92 & 0.92 \\
\hline 630080 & Carbon Monoxide & 1 & 0 & 720 & 7.2 \\
\hline 7440508 & Copper & 1 & 0 & 370 & 3.7 \\
\hline 110827 & Cyclohexane & 1 & 0 & 0.016 & 0.00016 \\
\hline 84662 & DiethylPhthalat & 1 & 0 & 0 & 0 \\
\hline 122394 & Diphenylam & 1 & 0 & 0.0000026 & 0.0000000026 \\
\hline 75003 & Ethyl Chloride & 1 & 0 & 0 & 0 \\
\hline 100414 & Ethyl Benzene & 1 & 0 & 0.012 & 0.00012 \\
\hline 206440 & Fluoranthene & 1 & 0 & 0 & 0 \\
\hline 7647010 & $\mathrm{HCl}$ & 1 & 0 & 2150 & 21.5 \\
\hline 98828 & Cumene & 1 & 0 & 0 & 0 \\
\hline 7439921 & Lead & 1 & 0 & 120 & 1.2 \\
\hline 74873 & Methyl Chloride & 1 & 0 & 0.057 & 0.00057 \\
\hline 71556 & 1,1,1-TCA & 1 & 0 & 0 & 0 \\
\hline 108872 & MethCyHex & 1 & 0 & 0.051 & 0.00051 \\
\hline 75092 & Methylene Chlor & 1 & 0 & 1.8 & 0.018 \\
\hline 91203 & Naphthalene & 1 & 0 & 0.00075 & 0.0000075 \\
\hline 110543 & Hexane & 1 & 0 & 0.19 & 0.0019 \\
\hline 10102440 & NITROGEN DIOXID & 1 & 0 & 52 & 0.52 \\
\hline 39001020 & $1-80 \mathrm{ctaCDF}$ & 1 & 0 & 0 & 0 \\
\hline 78115 & PETN & 1 & 0 & 0 & 0 \\
\hline 108952 & Phenol & 1 & 0 & 0.0000343 & 0.0000000343 \\
\hline 115071 & Propylene & 1 & 0 & 0.072 & 0.00072 \\
\hline 121824 & RDX & 1 & 0 & 0.096 & 0.00096 \\
\hline 100425 & Styrene & 1 & 0 & 0.015 & 0.00015 \\
\hline 7446095 & SULFUR DIOXIDE & 1 & 0 & 32 & 0.32 \\
\hline 127184 & Perc & 1 & 0 & 0 & 0 \\
\hline 108883 & Toluene & 1 & 0 & 0.086 & 0.00086 \\
\hline 75014 & Vinyl Chloride & 1 & 0 & 0.015 & 0.00015 \\
\hline 7440666 & Zinc & 1 & 0 & 0.4 & 0.004 \\
\hline 10101 & NapthSur & 1 & 0 & 0.0000014 & 0.000000014 \\
\hline 10102 & EthBzSur & 1 & 0 & 0.091 & 0.00091 \\
\hline 10103 & nHexSur & 1 & 0 & 89.4 & 0.894 \\
\hline
\end{tabular}

EMISSIONS FOR FACILITY FAC=Site300 CO=* DEV=PR1 PRO=0BCage3 STK=1 NAME=LLNL Site 300 EMS (lbs/yr) SOURCE MULTIPLIER=1

\begin{tabular}{ll} 
CAS & ABBREV \\
106990 & $1,3-$ Butadiene \\
67562394 & $1-4,6-8$ HPCDF \\
55673897 & $1-4,7-9 H$ HCDF \\
70648269 & $1-4,7,8$ HCDF \\
57117449 & $1-3,6-8$ HXCDF \\
35822469 & $1-4,6-8$ HpCDD \\
121142 & $2,4-$ DiNitToluen \\
606202 & $2,6-$ DiNitToluen \\
95578 & 2-CHLOROPHENOL \\
107051 & AllylChlor \\
7429905 & Aluminum \\
7440360 & Antimony \\
7440393 & Barium \\
71432 & Benzene \\
7440439 & Cadmium \\
56235 & CCl4 \\
67663 & Chloroform \\
7440473 & Chromium \\
7782505 & Chlorine \\
630080 & Carbon Monoxide \\
7440508 & Copper \\
110827 & Cyclohexane \\
84662 & DiethylPhthalat \\
122394 & Diphenylam \\
75003 & Ethyl Chloride \\
100414 & Ethyl Benzene \\
206440 & Fluoranthene \\
7647010 & HCl \\
98828 & Cumene \\
7439921 & Lead \\
74873 & Methyl Chloride \\
71556 & $1,1,1-$ TCA \\
108872 & MethCyHex \\
75092 & Methylene Chlor \\
91203 & Naphthalene \\
110543 & Hexane \\
10102440 & NITROGEN DI0XID \\
& \\
\hline
\end{tabular}

$\begin{array}{rrrr}\text { MULTIPLIER } & \text { BG (ug/m^3) } & \text { AVRG (lbs/yr) } & \text { MAX }(\mathrm{lbs} / \mathrm{hr}) \\ 1 & 0 & 0 & 0 \\ 1 & 0 & 0.00017 & 0.0000017 \\ 1 & 0 & 0.0000395 & 0.000000395 \\ 1 & 0 & 0.000105 & 0.00000105 \\ 1 & 0 & 0.0000475 & 0.000000475 \\ 1 & 0 & 0 & 0 \\ 1 & 0 & 0 & 0 \\ 1 & 0 & 0 & 0 \\ 1 & 0 & 0 & 0 \\ 1 & 0 & 0 & 0 \\ 1 & 0 & 180 & 1.8 \\ 1 & 0 & 0 & 0 \\ 1 & 0 & 0.43 & 0.0043 \\ 1 & 0 & 2.25 & 0.0225 \\ 1 & 0 & 0 & 0 \\ 1 & 0 & 0.028 & 0.00028 \\ 1 & 0 & 0.0115 & 0.000115 \\ 1 & 0 & 0 & 0 \\ 1 & 0 & 1 & 0.01 \\ 1 & 0 & 100 & 1 \\ 1 & 0 & 0.075 & 0.00075 \\ 1 & 0 & 0.01 & 0.0001 \\ 1 & 0 & 0 & 0 \\ 1 & 0 & 0 & 0 \\ 1 & 0 & 0 & 0 \\ 1 & 0 & 0.012 & 0.00012 \\ 1 & 0 & 1 & 0.01 \\ 1 & 0 & 415 & 4.15 \\ 1 & 0 & 0 & 0 \\ 1 & 0 & 1.4 & 0.014 \\ 1 & 0 & 0.1 & 0.001 \\ 1 & 0 & 0 & 0 \\ 1 & 0 & 0.04 & 0.0004 \\ 1 & 0 & 0.06 & 0.0006 \\ 1 & 0 & 0 & 0 \\ 1 & 0 & 0.024 & 0.00024 \\ 1 & 0 & 0.033 & 0.00033\end{array}$




$\begin{array}{ll}39001020 & \text { 1-80ctaCDF } \\ 78115 & \text { PETN } \\ 108952 & \text { Phenol } \\ 115071 & \text { Propylene } \\ 121824 & \text { RDX } \\ 100425 & \text { Styrene } \\ 7446095 & \text { SULFUR DIOXIDE } \\ 127184 & \text { Perc } \\ 108883 & \text { Toluene } \\ 75014 & \text { Vinyl Chloride } \\ 7440666 & \text { Zinc } \\ 10101 & \text { NapthSur } \\ 10102 & \text { EthBzSur } \\ 10103 & \text { nHexSur }\end{array}$

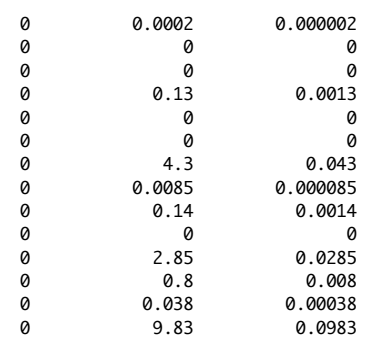

EMISSIONS FOR FACILITY FAC $=S i$ te $300 \quad C 0=* \quad$ DEV $=P R 1 \quad$ PRO $=0 B$ Cage 4 SOURCE MULTIPLIER $=1$

\begin{tabular}{ll} 
CAS & ABBREV \\
106990 & $1,3-$ Butadiene \\
67562394 & $1-4,6-8$ HpCDF \\
55673897 & $1-4,7-9 H p C D F$ \\
70648269 & $1-4,7,8$ HxCDF \\
57117449 & $1-3,6-8$ HXCDF \\
35822469 & $1-4,6$-8HpCDD \\
121142 & $2,4-$-DiNitToluen \\
606202 & 2,6 -DiNitToluen \\
95578 & 2-CHLOROPHENOL \\
107051 & AllylChlor \\
7429905 & Aluminum \\
7440360 & Antimony \\
7440393 & Barium \\
71432 & Benzene \\
7440439 & Cadmium \\
56235 & CCl4 \\
67663 & Chloroform \\
7440473 & Chromium \\
7782505 & Chlorine \\
630080 & Carbon Monoxide \\
7440508 & Copper \\
110827 & Cyclohexane \\
84662 & DiethylPhthalat \\
122394 & Diphenylam \\
75003 & Ethyl Chloride \\
100414 & Ethyl Benzene \\
206440 & Fluoranthene \\
7647010 & HCl \\
98828 & Cumene \\
7439921 & Lead \\
74873 & Methyl Chloride \\
71556 & $1,1,1-T C A$ \\
108872 & MethCyHex \\
75092 & Methylene Chlor \\
91203 & Naphthalene \\
110543 & Hexane \\
10102440 & NITROGEN DIOXID \\
39001020 & $1-80$ ctaCDF \\
78115 & PETN \\
108952 & Phenol \\
115071 & Propylene \\
121824 & RDX \\
100425 & Styrene \\
7446095 & SULFUR DIOXIDE \\
127184 & Perc \\
108883 & Toluene \\
75014 & Vinyl Chloride \\
7440666 & Zinc \\
10101 & NapthSur \\
10102 & EthBzSur \\
10103 & nHexSur \\
& \\
\hline
\end{tabular}

MULTIPLIER

LIER

BG $\left(u g / \mathrm{m}^{\wedge} 3\right)$

STK=1 NAME=LLNL Site 300 EMS (lbs/yr)

AVRG (lbs/yr) MAX (lbs/hr)

$\begin{array}{rr}0.000884 & 0.00000884 \\ 0.000205 & 0.00000205 \\ 0.000546 & 0.00000546 \\ 0.000247 & 0.00000247 \\ 0 & 0 \\ 0 & 0 \\ 0 & 0 \\ 0 & 0 \\ 0 & 0 \\ 936 & 9.36 \\ 0 & 0 \\ 2.24 & 0.0224 \\ 11.7 & 0.117 \\ 0 & 0 \\ 0.146 & 0.00146 \\ 0.0598 & 0.000598 \\ 0 & 0 \\ 5.2 & 0.052 \\ 520 & 5.2 \\ 0.39 & 0.0039 \\ 0.052 & 0.00052 \\ 0 & 0 \\ 0 & 0 \\ 0 & 0 \\ 0.0624 & 0.000624 \\ 5.2 & 0.052 \\ 2160 & 21.6 \\ 0 & 0 \\ 7.28 & 0.0728 \\ 0.52 & 0.0052 \\ 0 & 0 \\ 0.208 & 0.00208 \\ 0.312 & 0.00312 \\ 0 & 0 \\ 0.125 & 0.00125 \\ 0.172 & 0.00172 \\ 0.00104 & 0.0000104 \\ 0 & 0 \\ 0 & 0 \\ 0.676 & 0.00676 \\ 0 & 0 \\ 0 & 0 \\ 22.4 & 0.224 \\ 0.0442 & 0.000442 \\ 0.728 & 0.00728 \\ 0 & 0 \\ 14.8 & 0.148 \\ 4.16 & 0.0416 \\ 0.198 & 0.00198 \\ 51.1 & 0.511\end{array}$

EMISSIONS FOR FACILITY FAC $=$ Site300 SOURCE MULTIPLIER=1

$\begin{array}{ll}\text { CAS } & \text { ABBREV } \\ 106990 & 1,3-\text { Butadiene } \\ 67562394 & 1-4,6-8 \text { HpCDF } \\ 55673897 & 1-4,7-9 H p C D F \\ 70648269 & 1-4,7,8 \text { HxCDF } \\ 57117449 & 1-3,6-8 \text { HxCDF } \\ 35822469 & 1-4,6-8 \text { HpCDD } \\ 121142 & 2,4-\text { DiNitToluen } \\ 606202 & 2,6-\text { - DiNitToluen } \\ 95578 & \text { 2-CHLOROPHENOL } \\ 107051 & \text { AllylChlor } \\ 7429905 & \text { Aluminum } \\ 7440360 & \text { Antimony } \\ 7440393 & \text { Barium } \\ 71432 & \text { Benzene } \\ 7440439 & \text { Cadmium } \\ 56235 & \text { CCl4 } \\ 67663 & \text { Chloroform } \\ 7440473 & \text { Chromium } \\ 7782505 & \text { Chlorine } \\ 630080 & \text { Carbon Monoxide } \\ 7440508 & \text { Copper } \\ 110827 & \text { Cyclohexane } \\ 84662 & \text { DiethylPhthalat }\end{array}$

$\mathrm{CO}=* \quad \mathrm{DEV}=\mathrm{PR} 1$

$P R 0=0 D \quad S T K=1 \quad$ NAME $=L L N L$ Site 300 EMS (lbs/yr)

MULTIPLIER

$B G\left(u g / m^{\wedge} 3\right)$

AVRG (lbs/yr) MAX (lbs/hr)

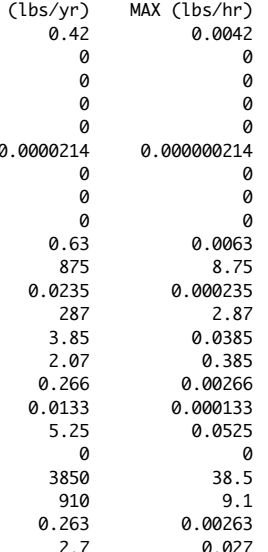




$\begin{array}{llrrrr}122394 & \text { Diphenylam } & 1 & 0 & 0 & 0 \\ 75003 & \text { Ethyl Chloride } & 1 & 0 & 0.0242 & 0.000242 \\ 100414 & \text { Ethyl Benzene } & 1 & 0 & 0.161 & 0.00161 \\ 206440 & \text { Fluoranthene } & 1 & 0 & 0 & 0 \\ 7647010 & \text { HCl } & 1 & 0 & 0 & 0 \\ 98828 & \text { Cumene } & 1 & 0 & 0.0256 & 0.000256 \\ 7439921 & \text { Lead } & 1 & 0 & 38.5 & 0.385 \\ 74873 & \text { Methyl Chloride } & 1 & 0 & 0.0595 & 0.000595 \\ 71556 & \text { 1,1,1-TCA } & 1 & 0 & 0.116 & 0.00116 \\ 108872 & \text { MethCyHex } & 1 & 0 & 0.245 & 0.00245 \\ 75092 & \text { Methylene Chlor } & 1 & 0 & 30.5 & 0.305 \\ 91203 & \text { Naphthalene } & 1 & 0 & 0 & 0 \\ 110543 & \text { Hexane } & 1 & 0 & 0.665 & 0.00665 \\ 10102440 & \text { NITROGEN DIOXID } & 1 & 0 & 154 & 1.54 \\ 39001020 & \text { 1-80ctaCDF } & 1 & 0 & 0 & 0 \\ 78115 & \text { PETN } & 1 & 0 & 19.6 & 0.196 \\ 108952 & \text { Phenol } & 1 & 0 & 0 & 0 \\ 115071 & \text { Propylene } & 1 & 0 & 2.56 & 0.0256 \\ 121824 & \text { RDX } & 1 & 0 & 259 & 2.59 \\ 100425 & \text { Styrene } & 1 & 0 & 1.47 & 0.0147 \\ 7446095 & \text { SULFUR DIOXIDE } & 1 & 0 & 455 & 4.55 \\ 127184 & \text { Perc } & 1 & 0 & 0.63 & 0.0063 \\ 108883 & \text { Toluene } & 1 & 0 & 1.05 & 0.0105 \\ 75014 & \text { Vinyl Chloride } & 1 & 0 & 0.0805 & 0.000805 \\ 7440666 & \text { Zinc } & 1 & 0 & 560 & 5.6 \\ 10101 & \text { NapthSur } & 1 & 0 & 0 & 0 \\ 10102 & \text { EthBzSur } & 1 & 0 & 0.62 & 0.0062 \\ 10103 & \text { nHexSur } & 1 & 0 & 119 & 1.19 \\ & & & & & \end{array}$

CHRONIC HI REPORT

RECEPTOR 5

RISK BY ENDPOINT CNS BONE DEVEL ENDO EYE GILV IMMUN KIDN REPRO RESP SKIN BLOOD

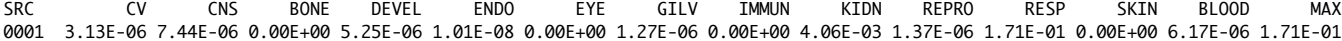

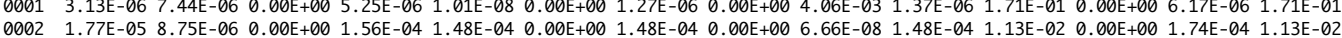

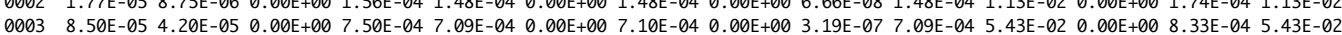

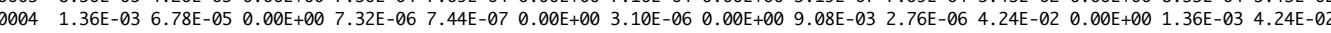

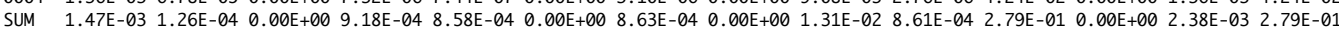


HARP Output Folder/Carnegie Resident Folder Rep_Acu_Rec2_AllSrc_AllCh_BySrc_Site.txt

Rep_Can_9yrC_Hi_Rec2_AllSrc_AllCh_BySrc_Site.txt Rep_Can_30yr_Hi_Rec2_AllSrc_AllCh_BySrc_Bychem_Site.txt

Rep_Can_30yr_Hi_Rec2_AllSrc_AllCh_bySrc_site.txt

Rep_Chr_Res_Hi_Rec2_AllSrc_AllCh_BySrc_Site.txt 
Rep_Acu_Rec2_AllSrc_AllCh_BySrc_Site.txt 


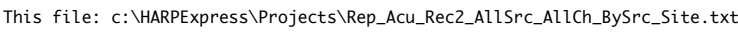

Created by HARP Version 1.0 Build 23.02.10

Uses ISC Version 99155

Uses BPIP Version 95086

Creation date: 10/24/2005 2:23:29 PM

EXCEPTION REPORT

(there have been no changes or exceptions)

INPUT FILES:

Source-Receptor file: c: \HARPExpress \Projects $\backslash E W T F . m t a$

Averaging period adjustment factors file: not applicable

Emission rates file: none

Site parameters file: C:\HARPExpress \Projects $\backslash$ Site300.sit

Screening mode is OFF

Analysis method: Point Estimate

Health effect: Acute HI Simple (Concurrent Max.)

Receptor(s): $\quad 2$

$\begin{array}{ll}\text { Sources(s): } & \text { All } \\ \text { Chemicals(s): } & \text { All }\end{array}$

CHEMICAL CROSS-REFERENCE TABLE AND BACKGROUND CONCENTRATIONS

CHEM CAS

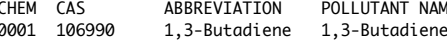

$000267562394 \quad 1-4,6-8 \mathrm{HpCDF} \quad 1,2,3,4,6,7,8$-Heptachlorodibenzofuran

$000355673897 \quad 1-4,7-9 \mathrm{HpCDF} \quad 1,2,3,4,7,8,9$-Heptachlorodibenzofuran

$000470648269 \quad 1-4,7,8 \mathrm{HxCDF} \quad 1,2,3,4,7,8$-Hexachlorodibenzofuran

$000557117449 \quad 1-3,6-8 \mathrm{HxCDF} \quad 1,2,3,6,7,8$-Hexachlorodibenzofuran

$000635822469 \quad 1-4,6-8 \mathrm{HpCDD} \quad 1,2,3,4,6,7,8$-Heptachlorodibenzo-p-dioxin

0007121142 2,4-DiNitToluen 2,4-Dinitrotoluene

$0008606202 \quad 2,6$-DiNitToluen 2,6-Dinitrotoluene

000995578 2-CHLOROPHENOL 2-CHLOROPHENOL

0010107051 AllylChlor Allyl chloride

00117429905 Aluminum Aluminum

00127440360 Antimony Antimony

00137440393 Barium Barium

$001471432 \quad$ Benzene Benzene

00157440439 Cadmium Cadmium

$001656235 \quad$ CCl4 Carbon tetrachloride

$001767663 \quad$ Chloroform Chloroform

00187440473 Chromium Chromium

$0020630080 \quad$ Carbon Monoxide Carbon monoxide

00217440508 Copper Copper

$0022110827 \quad$ Cyclohexane Cyclohexane

$\begin{array}{lll}0023 & 84662 & \text { DiethylPhthalat Diethyl phthala } \\ 0024 & 122394 & \text { Diphenylam }\end{array}$

$\begin{array}{lll}0025 & 75003 & \text { Diphenyl Chloride Ethyl chloride } \text { Chlorethan }\end{array}$

$0026100414 \quad$ Ethyl Benzene Ethyl benzene

$0027 \quad 206440$ Fluoranthene Fluoranthene

HCl Hydrochloric acid

$002998828 \quad$ Cumene Cumene

00307439921 Lead Lead

$\begin{array}{llll}0031 & 74873 & \text { Methyl Chloride Methyl chloride }\{\text { Chloromethane }\} \\ 0032 & 71556 & 1,1,1-\text { TCA } & \text { Methyl chloroform }\{1,1,1-\mathrm{TCA}\}\end{array}$

003271556

MethCyHex Methylcyclohexane

Methylene Chlor Methylene chloride \{Dichloromethane\}

$0035 \quad 91203$

$\begin{array}{llll}0036 & 110543 & \text { Hexane } & \text { Hexane } \\ 0037 & 10102440 & \text { NITROGEN DIOXID NITROGEN DIOXIDE }\end{array}$

Naphthalene Naphthalene

0038390010201 -80ctaCDF 1,2,3,4,6,7,8,9-0ctachlorodibenzofuran

$003978115 \quad$ PETN

0040108952 Phenol Phenol

$0041115071 \quad$ Propylene Propylene

$0042121824 \quad$ RDX

0043100425 Styrene Styrene

00447446095 SULFUR DTOXIDE SULFUR DIOXIDE

0045127184

Perc

Perchloroethylene \{Tetrachloroethene\}

$0047 \quad 75014$

8440666

$\begin{array}{llll}0048 & 7440666 & \text { Zinc } & \text { Zinc } \\ 0049 & 10101 & \text { NapthSur } & \text { Naphthalene surrogate }\end{array}$

Toluen

EthBzSur Ethylbenzene surrog

EMISSIONS DATA SOURCE:

CHEMICALS ADDED OR DELETED: none

\begin{tabular}{|c|c|c|c|c|c|c|c|c|}
\hline \multicolumn{2}{|c|}{$\begin{array}{l}\text { EMISSIONS FOR FACILITY FAC }=\text { Site } 300 \\
\text { SOURCE MULTIPLIER }=1\end{array}$} & $\mathrm{CO}=*$ & $D E V=P R 1$ & $\mathrm{PRO}=0 \mathrm{BPan}$ & STK $=1$ & $\mathrm{NAME}=\mathrm{LLNL}$ & Site 300 EMS & Is (lbs/yr) \\
\hline CAS & ABBREV & MULTIP & DIER & BG $(u g / m \wedge 3)$ & AVRG & (lbs/yr) & $\operatorname{MAX}(\mathrm{lbs} / \mathrm{hr})$ & \\
\hline 106990 & 1,3-Butadiene & & 1 & 0 & & 0.017 & 0.00017 & \\
\hline 67562394 & $1-4,6-8 \mathrm{HpCDF}$ & & 1 & 0 & & 0 & 0 & \\
\hline 55673897 & 1-4,7-9HрCDF & & 1 & 0 & & 0 & 0 & \\
\hline 70648269 & $1-4,7,8 \mathrm{HxCDF}$ & & 1 & 0 & & 0 & 0 & \\
\hline 57117449 & $1-3,6-8 \mathrm{H} \times C D F$ & & 1 & 0 & & 0 & 0 & \\
\hline 35822469 & $1-4,6-8 \mathrm{HpCDD}$ & & 1 & 0 & & 0 & 0 & \\
\hline 121142 & 2,4-DiNitToluen & & 1 & 0 & & 0.000012 & 0.00000012 & \\
\hline 606202 & 2,6-DiNitToluen & & 1 & 0 & & 0.000001 & 0.00000001 & \\
\hline 95578 & 2-CHLOROPHENOL & & 1 & 0 & & 0.1 & 0.001 & \\
\hline 107051 & AllylChlor & & 1 & 0 & & 0 & 0 & \\
\hline 7429905 & Aluminum & & 1 & 0 & & 110 & 1.1 & \\
\hline 7440360 & Antimony & & 1 & 0 & & 0.0067 & 0.000067 & \\
\hline 7440393 & Barium & & 1 & 0 & & 82 & 0.82 & \\
\hline 1432 & Benzene & & 1 & 0 & & 1.2 & 0.012 & \\
\hline
\end{tabular}

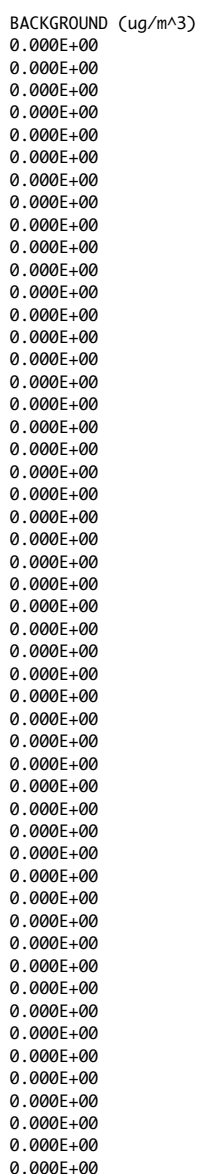




\begin{tabular}{|c|c|c|c|c|c|}
\hline 7440439 & Cadmium & 1 & 0 & 0.4 & 0.004 \\
\hline 56235 & CCl4 & 1 & 0 & 0.011 & 0.00011 \\
\hline 67663 & Chloroform & 1 & 0 & 0.0042 & 0.000042 \\
\hline 7440473 & Chromium & 1 & 0 & 0.48 & 0.0048 \\
\hline 7782505 & Chlorine & 1 & 0 & 92 & 0.92 \\
\hline 630080 & Carbon Monoxide & 1 & 0 & 720 & 7.2 \\
\hline 7440508 & Copper & 1 & 0 & 370 & 3.7 \\
\hline 110827 & Cyclohexane & 1 & 0 & 0.016 & 0.00016 \\
\hline 84662 & DiethylPhthalat & 1 & 0 & 0 & 0 \\
\hline 122394 & Diphenylam & 1 & 0 & 0.0000026 & 0.000000026 \\
\hline 75003 & Ethyl Chloride & 1 & 0 & 0 & 0 \\
\hline 100414 & Ethyl Benzene & 1 & 0 & 0.012 & 0.00012 \\
\hline 206440 & Fluoranthene & 1 & 0 & 0 & 0 \\
\hline 7647010 & $\mathrm{HCl}$ & 1 & 0 & 2150 & 21.5 \\
\hline 98828 & Cumene & 1 & 0 & 0 & 0 \\
\hline 7439921 & Lead & 1 & 0 & 120 & 1.2 \\
\hline 74873 & Methyl Chloride & 1 & 0 & 0.057 & 0.00057 \\
\hline 71556 & $1,1,1-\mathrm{TCA}$ & 1 & 0 & 0 & 0 \\
\hline 108872 & MethCyHex & 1 & 0 & 0.051 & 0.00051 \\
\hline 75092 & Methylene Chlor & 1 & 0 & 1.8 & 0.018 \\
\hline 91203 & Naphthalene & 1 & 0 & 0.00075 & 0.0000075 \\
\hline 110543 & Hexane & 1 & 0 & 0.19 & 0.0019 \\
\hline 10102440 & NITROGEN DIOXID & 1 & 0 & 52 & 0.52 \\
\hline 39001020 & 1-80ctaCDF & 1 & 0 & 0 & 0 \\
\hline 78115 & PETN & 1 & 0 & 0 & 0 \\
\hline 108952 & Phenol & 1 & 0 & 0.0000343 & 0.000000343 \\
\hline 115071 & Propylene & 1 & 0 & 0.072 & 0.00072 \\
\hline 121824 & $\mathrm{RDX}$ & 1 & 0 & 0.096 & 0.00096 \\
\hline 100425 & Styrene & 1 & 0 & 0.015 & 0.00015 \\
\hline 7446095 & SULFUR DIOXIDE & 1 & 0 & 32 & 0.32 \\
\hline 127184 & Perc & 1 & 0 & 0 & 0 \\
\hline 108883 & Toluene & 1 & 0 & 0.086 & 0.00086 \\
\hline 75014 & Vinyl Chloride & 1 & 0 & 0.015 & 0.00015 \\
\hline 7440666 & Zinc & 1 & 0 & 0.4 & 0.004 \\
\hline 10101 & NapthSur & 1 & 0 & 0.0000014 & 0.000000014 \\
\hline 10102 & EthBzSur & 1 & 0 & 0.091 & 0.00091 \\
\hline 10103 & nHexSur & 1 & 0 & 89.4 & 0.894 \\
\hline
\end{tabular}

EMISSIONS FOR FACILITY FAC $=$ Site300 CO=* DEV=PR1 PRO=0BCage3 STK=1 NAME=LLNL Site $300 \quad$ EMS (lbs/yr) SOURCE MULTIPLIER=1

\begin{tabular}{|c|c|}
\hline CAS & ABBREV \\
\hline 106990 & 1,3-Butadiene \\
\hline 67562394 & $1-4,6-8 \mathrm{HpCDF}$ \\
\hline 55673897 & $1-4,7-9 \mathrm{HpCDF}$ \\
\hline 70648269 & $1-4,7,8 \mathrm{HxCDF}$ \\
\hline 57117449 & $1-3,6-8 \mathrm{H} \times \mathrm{CDF}$ \\
\hline 35822469 & $1-4,6-8 \mathrm{HpCDD}$ \\
\hline 121142 & 2,4-DiNitToluen \\
\hline 606202 & 2,6-DiNitToluen \\
\hline 95578 & 2-CHLOROPHENOL \\
\hline 107051 & AllylChlor \\
\hline 7429905 & Aluminum \\
\hline 7440360 & Antimony \\
\hline 7440393 & Barium \\
\hline 71432 & Benzene \\
\hline 7440439 & Cadmium \\
\hline 56235 & CCl4 \\
\hline 67663 & Chloroform \\
\hline 7440473 & Chromium \\
\hline 7782505 & Chlorine \\
\hline 630080 & Carbon Monoxide \\
\hline 7440508 & Copper \\
\hline 110827 & Cyclohexane \\
\hline 84662 & DiethylPhthalat \\
\hline 122394 & Diphenylam \\
\hline 75003 & Ethyl Chloride \\
\hline 100414 & Ethyl Benzene \\
\hline 206440 & Fluoranthene \\
\hline 7647010 & $\mathrm{HCl}$ \\
\hline 98828 & Cumene \\
\hline 7439921 & Lead \\
\hline 74873 & Methyl Chloride \\
\hline 71556 & $1,1,1-\mathrm{TCA}$ \\
\hline 108872 & MethCyHex \\
\hline 75092 & Methylene Chlor \\
\hline 91203 & Naphthalene \\
\hline 110543 & Hexane \\
\hline 10102440 & NITROGEN DIOXID \\
\hline 39001020 & $1-80 \mathrm{ctaCDF}$ \\
\hline 78115 & PETN \\
\hline 108952 & Phenol \\
\hline 115071 & Propylene \\
\hline 121824 & RDX \\
\hline 100425 & Styrene \\
\hline 7446095 & SULFUR DIOXIDE \\
\hline 127184 & Perc \\
\hline 108883 & Toluene \\
\hline 75014 & Vinyl Chloride \\
\hline 7440666 & Zinc \\
\hline 10101 & NapthSur \\
\hline 10102 & EthBzSur \\
\hline 10103 & nHexSur \\
\hline
\end{tabular}

\begin{tabular}{|c|c|c|c|}
\hline MULTIPLIER & BG $(u g / m \wedge 3)$ & AVRG (lbs/yr) & $\operatorname{MAX}(\mathrm{lbs} / \mathrm{hr}$ ) \\
\hline 1 & 0 & 0 & 0 \\
\hline 1 & 0 & 0.00017 & 0.0000017 \\
\hline 1 & 0 & 0.0000395 & 0.000000395 \\
\hline 1 & 0 & 0.000105 & 0.000000105 \\
\hline 1 & 0 & 0.0000475 & 0.000000475 \\
\hline 1 & 0 & 0 & 0 \\
\hline 1 & 0 & 0 & 0 \\
\hline 1 & 0 & 0 & 0 \\
\hline 1 & 0 & 0 & 0 \\
\hline 1 & 0 & 0 & 0 \\
\hline 1 & 0 & 180 & 1.8 \\
\hline 1 & 0 & 0 & 0 \\
\hline 1 & 0 & 0.43 & 0.0043 \\
\hline 1 & 0 & 2.25 & 0.0225 \\
\hline 1 & 0 & 0 & 0 \\
\hline 1 & 0 & 0.028 & 0.00028 \\
\hline 1 & 0 & 0.0115 & 0.000115 \\
\hline 1 & 0 & 0 & 0 \\
\hline 1 & 0 & 1 & 0.01 \\
\hline 1 & 0 & 100 & 1 \\
\hline 1 & 0 & 0.075 & 0.00075 \\
\hline 1 & 0 & 0.01 & 0.0001 \\
\hline 1 & 0 & 0 & 0 \\
\hline 1 & 0 & 0 & 0 \\
\hline 1 & 0 & 0 & 0 \\
\hline 1 & 0 & 0.012 & 0.00012 \\
\hline 1 & 0 & 1 & 0.01 \\
\hline 1 & 0 & 415 & 4.15 \\
\hline 1 & 0 & 0 & 0 \\
\hline 1 & 0 & 1.4 & 0.014 \\
\hline 1 & 0 & 0.1 & 0.001 \\
\hline 1 & 0 & 0 & 0 \\
\hline 1 & 0 & 0.04 & 0.0004 \\
\hline 1 & 0 & 0.06 & 0.0006 \\
\hline 1 & 0 & 0 & 0 \\
\hline 1 & 0 & 0.024 & 0.00024 \\
\hline 1 & 0 & 0.033 & 0.00033 \\
\hline 1 & 0 & 0.0002 & 0.000002 \\
\hline 1 & 0 & 0 & 0 \\
\hline 1 & 0 & 0 & 0 \\
\hline 1 & 0 & 0.13 & 0.0013 \\
\hline 1 & 0 & 0 & 0 \\
\hline 1 & 0 & 0 & 0 \\
\hline 1 & 0 & 4.3 & 0.043 \\
\hline 1 & 0 & 0.0085 & 0.000085 \\
\hline 1 & 0 & 0.14 & 0.0014 \\
\hline 1 & 0 & 0 & 0 \\
\hline 1 & 0 & 2.85 & 0.0285 \\
\hline 1 & 0 & 0.8 & 0.008 \\
\hline 1 & 0 & 0.038 & 0.00038 \\
\hline 1 & 0 & 9.83 & 0.0983 \\
\hline
\end{tabular}

EMISSIONS FOR FACILITY FAC=Site300 CO=* DEV=PR1 PRO=0BCage4 STK=1 NAME=LLNL Site $300 \quad$ EMS (lbs/yr) SOURCE MULTIPLIER $=1$

$\begin{array}{ll}\text { CAS } & \text { ABBREV } \\ 106990 & 1,3-\text { Butadiene } \\ 67562394 & 1-4,6-8 \mathrm{HpCDF} \\ 55673897 & 1-4,7-9 \mathrm{HPCDF} \\ 70648269 & 1-4,7,8 \mathrm{HxCDF}\end{array}$

MULTIPLI
BG (ug/m^3) AVRG (lbs/yr) MAX (lbs/hr)
0.00088
0.0000000205




\begin{tabular}{|c|}
\hline 57117449 \\
\hline 35822469 \\
\hline 121142 \\
\hline 606202 \\
\hline 95578 \\
\hline 107051 \\
\hline 7429905 \\
\hline 7440360 \\
\hline 7440393 \\
\hline 71432 \\
\hline 7440439 \\
\hline 56235 \\
\hline 67663 \\
\hline 7440473 \\
\hline 7782505 \\
\hline 630080 \\
\hline 7440508 \\
\hline 110827 \\
\hline 84662 \\
\hline 122394 \\
\hline 75003 \\
\hline 100414 \\
\hline 206440 \\
\hline 7647010 \\
\hline 98828 \\
\hline 7439921 \\
\hline 74873 \\
\hline 71556 \\
\hline 108872 \\
\hline 75092 \\
\hline 91203 \\
\hline 110543 \\
\hline 10102440 \\
\hline 39001020 \\
\hline 78115 \\
\hline 108952 \\
\hline 115071 \\
\hline 121824 \\
\hline 100425 \\
\hline 7446095 \\
\hline 127184 \\
\hline 108883 \\
\hline 75014 \\
\hline 7440666 \\
\hline 10101 \\
\hline 10102 \\
\hline 10103 \\
\hline $\begin{array}{l}\text { EMISSIONS FOR } \\
\text { SOURCE MULTIPL }\end{array}$ \\
\hline CAS \\
\hline 106990 \\
\hline 67562394 \\
\hline 55673897 \\
\hline 70648269 \\
\hline 57117449 \\
\hline 35822469 \\
\hline 121142 \\
\hline 606202 \\
\hline 95578 \\
\hline 107051 \\
\hline 7429905 \\
\hline 7440360 \\
\hline 7440393 \\
\hline 71432 \\
\hline 7440439 \\
\hline 56235 \\
\hline 67663 \\
\hline 7440473 \\
\hline 7782505 \\
\hline 630080 \\
\hline 7440508 \\
\hline 110827 \\
\hline 84662 \\
\hline 122394 \\
\hline 75003 \\
\hline 100414 \\
\hline 206440 \\
\hline 7647010 \\
\hline 98828 \\
\hline 7439921 \\
\hline 74873 \\
\hline 71556 \\
\hline 108872 \\
\hline 75092 \\
\hline 91203 \\
\hline 110543 \\
\hline 10102440 \\
\hline 39001020 \\
\hline 78115 \\
\hline 108952 \\
\hline 115071 \\
\hline 121824 \\
\hline 100425 \\
\hline 7446095 \\
\hline 127184 \\
\hline 108883 \\
\hline 75014 \\
\hline 7440666 \\
\hline 10101 \\
\hline
\end{tabular}




$\begin{array}{rlrrrr}10102 & \text { EthBzSur } & 1 & 0 & 0.62 & 0.0062 \\ 10103 & \text { nHexSur } & 1 & 0 & 119 & 1.19\end{array}$

ACUTE HI REPORT

RECEPTOR 2

SRC CV CNS BONE DEVEL ENDO EYE GILV IMMUN KIDN REPRO RESP SKIN BLOOD MAX

$0001 \quad 8.54 \mathrm{E}-05 \quad 3.71 \mathrm{E}-07 \quad 0.00 \mathrm{E}+00 \quad 4.13 \mathrm{E}-07 \quad 0.00 \mathrm{E}+00 \quad 3.99 \mathrm{E}-03 \quad 2.30 \mathrm{E}-09 \quad 3.94 \mathrm{E}-07 \quad 0.00 \mathrm{E}+00 \quad 4.13 \mathrm{E}-07 \quad 1.45 \mathrm{E}-02 \quad 0.00 \mathrm{E}+00 \quad 3.94 \mathrm{E}-07 \quad 1.45 \mathrm{E}-02$

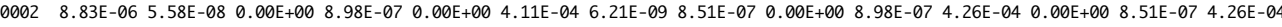

$0003 \quad 4.15 \mathrm{E}-05 \quad 2.82 \mathrm{E}-07 \quad 0.00 \mathrm{E}+00 \quad 4.66 \mathrm{E}-06 \quad 0.00 \mathrm{E}+00 \quad 1.93 \mathrm{E}-03 \quad 3.24 \mathrm{E}-08 \quad 4.43 \mathrm{E}-06 \quad 0.00 \mathrm{E}+00 \quad 4.66 \mathrm{E}-06 \quad 2.00 \mathrm{E}-03 \quad 0.00 \mathrm{E}+00 \quad 4.43 \mathrm{E}-06 \quad 2.00 \mathrm{E}-03$

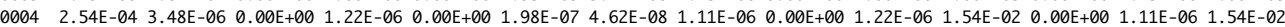

SUM $\quad 3.90 \mathrm{E}-04 \quad 4.19 \mathrm{E}-06 \quad 0.00 \mathrm{E}+00 \quad 7.20 \mathrm{E}-06 \quad 0.00 \mathrm{E}+00 \quad 6.33 \mathrm{E}-03 \quad 8.71 \mathrm{E}-08 \quad 6.78 \mathrm{E}-06 \quad 0.00 \mathrm{E}+00 \quad 7.20 \mathrm{E}-06 \quad 3.23 \mathrm{E}-02 \quad 0.00 \mathrm{E}+00 \quad 6.78 \mathrm{E}-06 \quad 3.23 \mathrm{E}-02$ 
Rep_Can_9yrC_Hi_Rec2_AllSrc_AllCh_BySrc_Site.txt 
This file: c: \HARPExpress $\backslash$ Projects $\backslash$ Rep_Can_9yrC_Hi_Rec2_AllSrc_AllCh_BySrc_Site.txt

Created by HARP Version 1.0 Build 23.02.10

Uses ISC Version 9915

Uses BPIP Version 95086

Creation date: 10/24/2005 2:19:02 PM

EXCEPTION REPORT

(there have been no changes or exceptions)

INPUT FILES:

Source-Receptor file: c: \HARPExpress \Projects $\backslash E W T F . m t a$

Averaging period adjustment factors file: not applicable

Emission rates file: none

Site parameters file: C:\HARPExpress \Projects $\backslash$ Site300. sit

Screening mode is OFF

Exposure duration: 9 year (child resident)

Analysis method: High-end Point Estimate

Health effect: Cancer Risk

Receptor(s): $\quad 2$

Sources(s):

All

SITE PARAMETERS

DEPOSITION

Deposition rate $(\mathrm{m} / \mathrm{s})$

0.0072

DRINKING WATER

*** Pathway disabled ***

FISH

*** Pathway disabled ***

PASTURE

ANIMALS' WATER

Water surface area $(\mathrm{m} \wedge 2)$

Water surface area

Volume changes per yea

Volume changes per year

from pasture source water

from pasture source

Fraction of dairy cows' water
from pasture source

ANIMALS' FEED

Fraction of cows' feed

from grazing

HUMAN INGESTION

Fraction of ingested beef

from contaminated sourc

Fraction of ingested dairy

from contaminated source

0.107

HOME GROWN PRODUCE

HUMAN INGESTION

Fraction of ingested leafy vegetable

from home grown source $\quad 0.082$

Fraction of ingested exposed vegetable

from home grown source 0.207

Fraction of ingested protected vegetabl

from home grown source 0.134

Fraction of ingested root vegetable

PIGS, CHICKENS AND EGGS

HUMAN INGESTION
Fraction of ingested pig
$\quad$ from home grown source
Fraction of ingested chicken
$\quad$ from home grown source
Fraction of ingested egg
$\quad$ from home grown source




\begin{tabular}{|c|c|}
\hline $\begin{array}{l}\text { leafy vegetable } \\
\text { Fraction of feed that is } \\
\text { protected vegetable }\end{array}$ & 0.25 \\
\hline $\begin{array}{l}\text { Fraction of feed that is } \\
\text { root vegetable }\end{array}$ & 0.25 \\
\hline $\begin{array}{l}\text { CHICKEN FEED COMPOSITION } \\
\text { Fraction of feed that is } \\
\text { exposed vegetable }\end{array}$ & 0.25 \\
\hline $\begin{array}{l}\text { Fraction of feed that is } \\
\text { leafy vegetable }\end{array}$ & 0.25 \\
\hline $\begin{array}{l}\text { Fraction of feed that is } \\
\text { protected vegetable } \\
\text { Fraction of feed that is }\end{array}$ & 0.25 \\
\hline root vegetable & 0.25 \\
\hline
\end{tabular}

DERMAL ABSORPTION

*** Pathway enabled $* * *$

SOIL INGESTION

*** Pathway enabled ***

MOTHER'S MILK

*** Pathway enabled ***

CHEMICAL CROSS-REFERENCE TABLE AND BACKGROUND CONCENTRATIONS

0001106990 1,3-Butadiene 1,3-Butadiene

1-4,6-8HpCDF $1,2,3,4,6,7,8$-Heptachlorodibenzofuran

$1,2,3,4,7,8,9$-Heptachlorodibenzofuran

$000470648269 \quad 1-4,7,8 \mathrm{HxCDF}$

$000557117449 \quad 1-3,6-8 \mathrm{HxCDF} \quad 1,2,3,6,7,8$-Hexachlorodibenzofuran

$000635822469 \quad 1-4,6-8 \mathrm{HpCDD} \quad 1,2,3,4,6,7,8$-Heptachlorodibenzo-p-dioxin

0007121142 2,4-DiNitToluen 2,4-Dinitrotoluene

0008606202 2,6-DiNitToluen 2,6-Dinitrotoluen

000995578 2-CHLOROPHENOL 2-CHLOROPHENOL

0010107051 AllylChlor Allyl chloride

00117429905 Aluminum Aluminum

00127440360 Antimony Antimony

$\begin{array}{llll}0013 & 7440393 & \text { Barium } & \text { Barium } \\ 0014 & 71432 & \text { Benzene } & \text { Benzene }\end{array}$

$001471432 \quad$ Benzene Benzene

$\begin{array}{llll}0015 & 7440439 & \text { Cadmium } & \text { Cadmium } \\ 0016 & 56235 & \text { CCl4 } & \text { Carbon tetrachloride }\end{array}$

$001656235 \quad$ CCl4 $\quad$ Carbon tetrac

$\begin{array}{llll}0017 & 67663 & \text { Chloroform } & \text { Chlorofor } \\ 0018 & 7440473 & \text { Chromium } & \text { Chromium }\end{array}$

00197782505 Chlorine Chlorine

0020630080 Carbon Monoxide Carbon monoxide

$00217440508 \quad$ Copper

0022110827 Cyclohexane Cyclohexane

002384662 DiethylPhthalat Diethyl phthalate

0024122394 Diphenylam Diphenylamine

$\begin{array}{llll}0025 & 75003 & \text { Ethyl Chloride Ethyl chloride \{Chlorethane\} } \\ 0026 & 100414 & \text { Ethyl Benzene Ethyl benzene }\end{array}$

$0027206440 \quad$ Fluoranthene Fluoranthene

$00287647010 \quad \mathrm{HCl} \quad$ Hydrochloric acid

$\begin{array}{llll}0029 & 98828 & \text { Cumene } & \text { Cumene } \\ 0030 & 7439921 & \text { Lead } & \text { Lead }\end{array}$

$\begin{array}{llll}0030 & 7439921 & \text { Lead } & \text { Lead } \\ 0031 & 74873 & \text { Methyl Chloride Methyl chloride \{Chloromethane\} }\end{array}$

003271556 1,1,1-TCA Methyl chloroform $\{1,1,1-\mathrm{TCA}\}$

$0033108872 \quad$ MethCyHex Methylcyclohexane

003475092 Methylene Chlor Methylene chloride \{Dichloromethane\}

003591203 Naphthalene Naphthalene

0036110543 Hexane

003710102440 NITROGEN DIOXID NITROGEN DIOXIDE

003839001020 1-80ctaCDF $1,2,3,4,6,7,8,9$-0ctachlorodibenzofuran

003978115 PETN

0040108952 Phenol Phenol

$0041115071 \quad$ Propylene Propylene

0042121824 RDX $\quad$ RDX

$\begin{array}{llll}0043 & 100425 & \text { Styrene } & \text { Styrene } \\ 0044 & 7446095 & \text { SULFUR DIOXIDE } & \text { SULFUR DIOXIDE }\end{array}$

$\begin{array}{llll}0044 & 7446095 & \text { SULFUR DIOXIDE } & \text { SULFUR DIOXIDE } \\ 0045 & 127184 & \text { Perc } & \text { Perchloroethylene } \text { \{Tetrachloroethene }\end{array}$

$\begin{array}{llll}0045 & 127184 & \text { Perc } & \text { Perchlor } \\ 0046 & 108883 & \text { Toluene } & \text { Toluene }\end{array}$

$\begin{array}{llll}0046 & 108883 & \text { Toluene } & \text { Toluene } \\ 0047 & 75014 & \text { Vinyl Chloride } & \text { Vinyl chloride }\end{array}$

00487440666 Zinc Zinc

004910101 NapthSur Naphthalene surrogate

005010102 EthBzSur Ethylbenzene surrogat

$n$-Hexane surrogate

BACKGROUND $(\mathrm{ug} / \mathrm{m} \wedge 3)$

$0.000 \mathrm{E}+00$

$0.000 \mathrm{E}+00$

$0.000 \mathrm{E}+00$

$0.000 \mathrm{E}+00$

$0.000 \mathrm{E}+00$

$0.000 \mathrm{E}+00$

$0.000 \mathrm{E}+00$

$0.000 \mathrm{E}+00$

$0.000 \mathrm{E}+00$

$0.000 \mathrm{E}+00$

$0.000 \mathrm{E}+00$

$0.000 \mathrm{E}+00$

$0.000 \mathrm{E}+00$

$0.000 \mathrm{E}+00$

$0.000 \mathrm{E}+00$

$0.000 \mathrm{E}+00$

$0.000 \mathrm{E}+00$

$0.000 E+00$

$0.000 E+00$

$0.000 E+00$

$0.000 \mathrm{E}+00$

$0.000 \mathrm{E}+00$

$0.000 \mathrm{E}+00$

$0.000 \mathrm{E}+00$

$0.000 \mathrm{E}+00$

$0.000 \mathrm{E}+00$

$0.000 \mathrm{E}+00$

$0.000 \mathrm{E}+00$

$0.000 \mathrm{E}+00$

$0.000 E+00$

$0.000 \mathrm{E}+00$

$0.000 \mathrm{E}+00$

$0.000 \mathrm{E}+00$

$0.000 \mathrm{E}+00$

$0.000 \mathrm{E}+00$

$0.000 \mathrm{E}+00$

$0.000 E+00$

$0.000 \mathrm{E}+00$

$0.000 \mathrm{E}+00$

$0.000 E+00$

$0.000 \mathrm{E}+00$

$0.000 \mathrm{E}+00$

$0.000 E+00$

$0.000 \mathrm{E}+00$

$0.000 E+00$

$0.000 E+00$

$0.000 \mathrm{E}+00$

$0.000 \mathrm{E}+00$

$0.000 E+00$

$0.000 \mathrm{E}+00$

$0.000 \mathrm{E}+00$

$0.000 \mathrm{E}+00$
$0.000 \mathrm{E}+00$

EMISSIONS DATA SOURCE:

CHEMICALS ADDED OR DELETED: none

\begin{tabular}{|c|c|}
\hline $\begin{array}{l}\text { EMISSION } \\
\text { SOURCE MM }\end{array}$ & $\begin{array}{l}\text { ACILITY FA } \\
E R=1\end{array}$ \\
\hline CAS & ABBREV \\
\hline 106990 & 1,3-Butadiene \\
\hline 67562394 & $1-4,6-8 \mathrm{HpCDF}$ \\
\hline 55673897 & $1-4,7-9 \mathrm{Hp} C \mathrm{CDF}$ \\
\hline 70648269 & $1-4,7,8 \mathrm{HxCDF}$ \\
\hline 57117449 & $1-3,6-8 \mathrm{H} \times \mathrm{CDF}$ \\
\hline 35822469 & $1-4,6-8 \mathrm{HpCDD}$ \\
\hline 121142 & 2,4-DiNitToluen \\
\hline 606202 & 2,6-DiNitToluen \\
\hline 95578 & 2-CHLOROPHENOL \\
\hline & Allyl \\
\hline
\end{tabular}

$C 0=* \quad$ DEV $=P R 1 \quad P R 0=0 B P a n \quad S T K=1 \quad$ NAME $=L L N L$ Site $300 \quad$ EMS $(l b s / y r)$

MULTIPLIER

BG (ug/m^3) AVRG (lbs/yr)

$\operatorname{MAX}(\mathrm{lbs} / \mathrm{hr})$

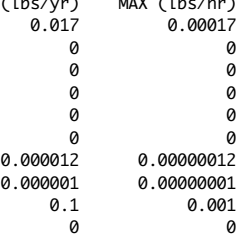




\begin{tabular}{ll}
7429905 & Aluminum \\
7440360 & Antimony \\
7440393 & Barium \\
71432 & Benzene \\
7440439 & Cadmium \\
56235 & CCl4 \\
67663 & Chloroform \\
7440473 & Chromium \\
7782505 & Chlorine \\
630080 & Carbon Monoxide \\
7440508 & Copper \\
110827 & Cyclohexane \\
84662 & DiethylPhthalat \\
122394 & Diphenylam \\
75003 & Ethyl Chloride \\
100414 & Ethyl Benzene \\
206440 & Fluoranthene \\
7647010 & HCl \\
98828 & Cumene \\
7439921 & Lead \\
74873 & Methyl Chloride \\
71556 & 1,1,1-TCA \\
108872 & MethCyHex \\
75092 & Methylene Chlor \\
91203 & Naphthalene \\
110543 & Hexane \\
10102440 & NITROGEN DIOXID \\
39001020 & 1-80ctaCDF \\
78115 & PETN \\
108952 & Phenol \\
115071 & Propylene \\
121824 & RDX \\
100425 & Styrene \\
7446095 & SULFUR DIOXIDE \\
127184 & Perc \\
108883 & Toluene \\
75014 & Vinyl Chloride \\
7440666 & Zinc \\
10101 & NapthSur \\
10102 & EthBzSur \\
10103 & nHexSur \\
& \\
\hline
\end{tabular}

$\begin{array}{rrrr}1 & 0 & 110 & 1.1 \\ 1 & 0 & 0.0067 & 0.000067 \\ 1 & 0 & 82 & 0.82 \\ 1 & 0 & 1.2 & 0.012 \\ 1 & 0 & 0.4 & 0.004 \\ 1 & 0 & 0.011 & 0.00011 \\ 1 & 0 & 0.0042 & 0.000042 \\ 1 & 0 & 0.48 & 0.0048 \\ 1 & 0 & 92 & 0.92 \\ 1 & 0 & 720 & 7.2 \\ 1 & 0 & 370 & 3.7 \\ 1 & 0 & 0.016 & 0.00016 \\ 1 & 0 & 0 & 0 \\ 1 & 0 & 0.0000026 & 0.000000026 \\ 1 & 0 & 0 & 0 \\ 1 & 0 & 0.012 & 0.00012 \\ 1 & 0 & 0 & 0 \\ 1 & 0 & 2150 & 21.5 \\ 1 & 0 & 0 & 0 \\ 1 & 0 & 120 & 1.2 \\ 1 & 0 & 0.057 & 0.00057 \\ 1 & 0 & 0 & 0 \\ 1 & 0 & 0.051 & 0.00051 \\ 1 & 0 & 1.8 & 0.018 \\ 1 & 0 & 0.00075 & 0.0000075 \\ 1 & 0 & 0.19 & 0.0019 \\ 1 & 0 & 52 & 0.52 \\ 1 & 0 & 0 & 0 \\ 1 & 0 & 0 & 0 \\ 1 & 0 & 0.0000343 & 0.000000343 \\ 1 & 0 & 0.072 & 0.00072 \\ 1 & 0 & 0.096 & 0.00096 \\ 1 & 0 & 0.015 & 0.00015 \\ 1 & 0 & 32 & 0.32 \\ 1 & 0 & 0 & 0 \\ 1 & 0 & 0.086 & 0.00086 \\ 1 & 0 & 0.015 & 0.00015 \\ 1 & 0 & 0.4 & 0.004 \\ 1 & 0 & 0.0000014 & 0.000000014 \\ 1 & 0 & 0.091 & 0.00091 \\ 1 & 0 & 89.4 & 0.894 \\ & 0 & & \end{array}$

EMISSIONS FOR FACILITY FAC $=$ Site300 SOURCE MULTIPLIER $=1$

ABBREV

67562394 1,3-Butadiene

1-4,6-8HpCDF

$57117449-1-4,7,8 \mathrm{HXCDF}$

$35822469 \quad 1-4,6-8 \mathrm{HpCDD}$

$\begin{array}{ll}121142 & \text { 2,4-DiNitToluen } \\ 606202 & \text { 2,6-DiNitToluen }\end{array}$

$\begin{array}{ll}606202 & \text { 2,6-DiNitToluen } \\ 95578 & \text { 2-CHLOROPHENOL }\end{array}$

107051 AllylChlor

7429905

7440360 Antimony

$\begin{array}{ll}7440393 & \text { Barium } \\ 71432 & \text { Benzene }\end{array}$

$7440439 \quad$ Cadmium

56235

$67663 \quad$ Chloroform

$7440473 \quad$ Chromium

630080 Carbon Monoxide

$7440508 \quad$ Copper

110827 Cyclohexane

$122394 \quad$ Diphenylam

$75003 \quad$ Ethyl Chloride

$100414 \quad$ Ethyl Benzene

206440 Fluoranthene

$7647010 \quad \mathrm{HCl}$

$98828 \quad$ Cumene

$7439921 \quad$ Methyl Chloride

715561 1,1,1-TCA

$\begin{array}{ll}108872 & \text { MethCyHex } \\ 75092 & \text { Methylene Chlor }\end{array}$

75092

$\begin{array}{ll}91203 & \text { Naphthal } \\ 110543 & \text { Hexane }\end{array}$

10102440 NITROGEN DIOXID

39001020 1-80ctaCDF

$\begin{array}{ll}78115 & \text { PETN } \\ 108952 & \text { Phenol }\end{array}$

115071 Propylene

$\begin{array}{ll}121824 & \text { RDX } \\ 100425 & \text { Styrene }\end{array}$

7446095 SULFUR DIOXIDE

$127184 \quad$ Perc

$108883 \quad$ Toluene

$75014 \quad$ Viny

$10101 \quad$ NapthSur

$\begin{array}{ll}10102 & \text { EthBzSur } \\ 10103 & \text { nHexSur }\end{array}$

MULTIPLIER BG $\left(u g / \mathrm{m}^{\wedge} 3\right)$

STK $=1 \quad$ NAME $=$ LLNL Site 300 EMS (lbs/yr)

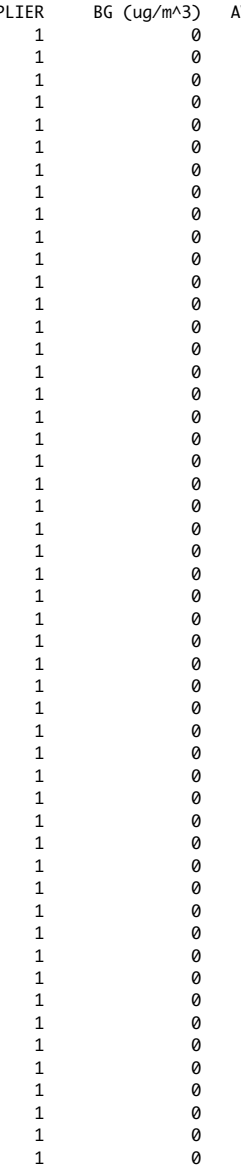

AVRG (lbs/yr) MAX (lbs/hr)

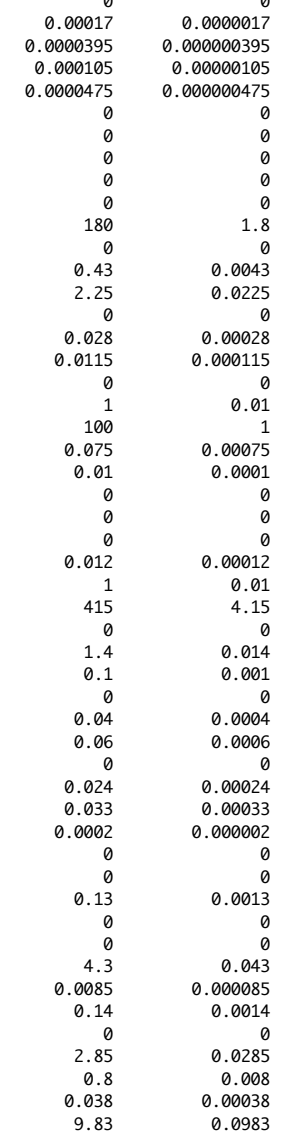

CAS ABBREV

MULTIPLIER

$\mathrm{PRO}=0 \mathrm{BCage} 4$

STK=1 NAME=LLNL Site 300 EMS (lbs/yr) 


\begin{tabular}{|c|c|}
\hline 106990 & 1,3-Butadiene \\
\hline 67562394 & $1-4,6-8 \mathrm{HpCDF}$ \\
\hline 55673897 & $1-4,7-9 \mathrm{HрCDF}$ \\
\hline 70648269 & $1-4,7,8 \mathrm{H} \times \mathrm{CDF}$ \\
\hline 57117449 & $1-3,6-8 \mathrm{H} \times \mathrm{CDF}$ \\
\hline 35822469 & $1-4,6-8 \mathrm{HpCDD}$ \\
\hline 121142 & 2,4-DiNitToluen \\
\hline 606202 & 2,6-DiNitToluen \\
\hline 95578 & 2-CHLOROPHENOL \\
\hline 107051 & AllylChlor \\
\hline 7429905 & Aluminum \\
\hline 7440360 & Antimony \\
\hline 7440393 & Barium \\
\hline 71432 & Benzene \\
\hline 7440439 & Cadmium \\
\hline 56235 & CCl4 \\
\hline 67663 & Chloroform \\
\hline 7440473 & Chromium \\
\hline 7782505 & Chlorine \\
\hline 630080 & Carbon Monoxide \\
\hline 7440508 & Copper \\
\hline 110827 & Cyclohexane \\
\hline 84662 & DiethylPhthalat \\
\hline 122394 & Diphenylam \\
\hline 75003 & Ethyl Chloride \\
\hline 100414 & Ethyl Benzene \\
\hline 206440 & Fluoranthene \\
\hline 7647010 & $\mathrm{HCl}$ \\
\hline 98828 & Cumene \\
\hline 7439921 & Lead \\
\hline 74873 & Methyl Chloride \\
\hline 71556 & $1,1,1$-TCA \\
\hline 108872 & MethCyHex \\
\hline 75092 & Methylene Chlor \\
\hline 91203 & Naphthalene \\
\hline 110543 & Hexane \\
\hline 10102440 & NITROGEN DIOXID \\
\hline 39001020 & 1-80ctaCDF \\
\hline 78115 & PETN \\
\hline 108952 & Phenol \\
\hline 115071 & Propylene \\
\hline 121824 & $\mathrm{RDX}$ \\
\hline 100425 & Styrene \\
\hline 7446095 & SULFUR DIOXIDE \\
\hline 127184 & Perc \\
\hline 108883 & Toluene \\
\hline 75014 & Vinyl Chloride \\
\hline 7440666 & Zinc \\
\hline 10101 & NapthSur \\
\hline 10102 & EthBzSur \\
\hline 10103 & nHexSur \\
\hline
\end{tabular}

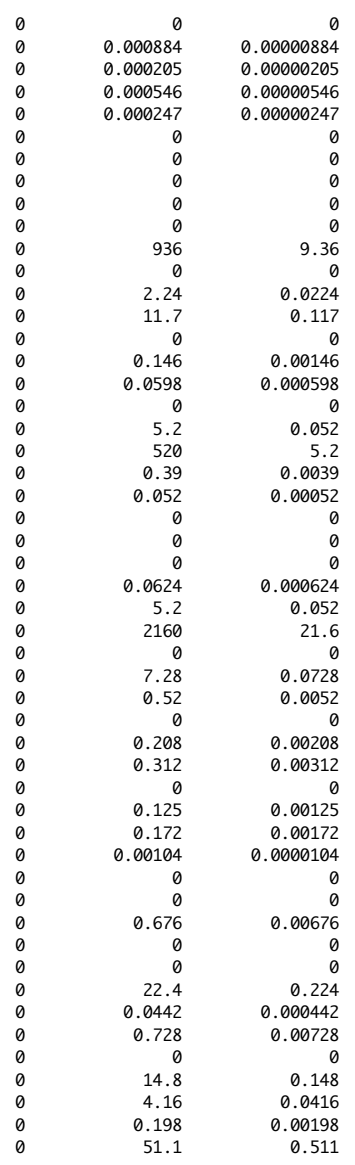

EMISSIONS FOR FACILITY FAC $=$ Site 300 SOURCE MULTIPLIER=1

$\begin{array}{ll}106990 & 1,3-\text { Butadiene } \\ 67562394 & 1-4,6-8 \mathrm{HpCDF}\end{array}$

$55673897 \quad 1-4,7-9 \mathrm{HpCDF}$

$70648269 \quad 1-4,7,8 \mathrm{HxCDF}$

$\begin{array}{ll}57117449 & 1-3,6-8 \mathrm{H} \times \mathrm{CDF} \\ 35822469 & 1-4,6-8 \mathrm{HpCDD}\end{array}$

121142 2,4-DiNitToluen

606202 2,6-DiNitToluen

95578 2-CHLOROPHENOL

107051 AllylChlor

$7429905 \quad$ Aluminum

7440360 Antimony

71432 Barium

$7440439 \quad$ Cadmium

$56235 \quad$ CCl4

$67663 \quad$ Chloroform

$7440473 \quad$ Chromium

630080 Carbon Monoxide

$7440508 \quad$ Copper

$110827 \quad$ Cyclohexane

84662 DiethylPhthalat

122394 Diphenylam

75003 Ethyl Chloride

100414 Ethyl Benzen

$206440 \quad$ Fluoranthene

$7647010 \quad \mathrm{HCl}$

$98828 \quad$ Cumene

$7439921 \quad$ Lead

74873 Methyl Chlor

$\begin{array}{ll}71556 & 1,1,1-\text { TCA } \\ 108872 & \text { MethCyHex }\end{array}$

75092 Methylene Chlo

91203 Naphthalen

110543

$10102440 \quad$ NITROGEN DIOXID

$39001020 \quad 1-80$ ctaCDF

78115 PETN

115071 Propylene

121824 RDX

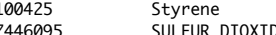

$127184 \quad$ Perc

$C O=* \quad$ DEV $=P R 1 \quad P R 0=0 D \quad S T K=1 \quad$ NAME $=L L N L$ Site 300 EMS (lbs/yr)

MULTIPLIER

$B G(u g / m \wedge 3)$
RRG (lbs/yr)

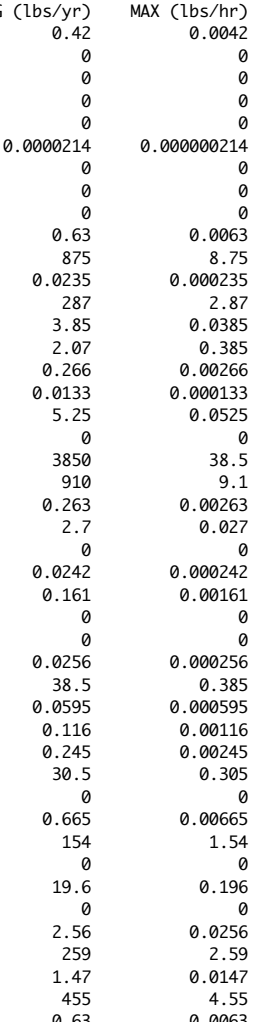




$\begin{array}{llllrr}108883 & \text { Toluene } & 1 & 0 & 1.05 & 0.0105 \\ 75014 & \text { Vinyl Chloride } & 1 & 0 & 0.0805 & 0.000805 \\ 7440666 & \text { Zinc } & 1 & 0 & 560 & 5.6 \\ 10101 & \text { NapthSur } & 1 & 0 & 0 & 0 \\ 10102 & \text { EthBzSur } & 1 & 0 & 0.62 & 0.0062 \\ 10103 & \text { nHexSur } & 1 & 0 & 119 & 1.19\end{array}$

CANCER RISK REPORT

RECEPTOR 2

RISK BY PATHWAY

SRC INHAL DERM SOIL MOTHER FISH WATER VEG DAIRY BEEF CHICK PIG EGG MEAT ORAL TOTAL

$\begin{array}{llllllllllllllllllll}0001 & 9.45 \mathrm{E}-09 & 1.52 \mathrm{E}-09 & 7.77 \mathrm{E}-09 & 0.00 \mathrm{E}+00 & 0.00 \mathrm{E}+00 & 0.00 \mathrm{E}+00 & 3.00 \mathrm{E}-09 & 0.00 \mathrm{E}+00 & 5.87 \mathrm{E}-12 & 4.40 \mathrm{E}-16 & 4.71 \mathrm{E}-14 & 1.06 \mathrm{E}-15 & 5.92 \mathrm{E}-12 & 1.23 \mathrm{E}-08 & 2.17 \mathrm{E}-08 \\ 0002 & 2.63 \mathrm{E}-09 & 3.09 \mathrm{E}-09 & 3.50 \mathrm{E}-09 & 3.76 \mathrm{E}-09 & 0.00 \mathrm{E}+00 & 0.00 \mathrm{E}+00 & 1.16 \mathrm{E}-09 & 0.00 \mathrm{E}+00 & 5.16 \mathrm{E}-09 & 4.72 \mathrm{E}-13 & 4.80 \mathrm{E}-11 & 1.14 \mathrm{E}-12 & 5.21 \mathrm{E}-09 & 1.67 \mathrm{E}-08 & 1.93 \mathrm{E}-08\end{array}$

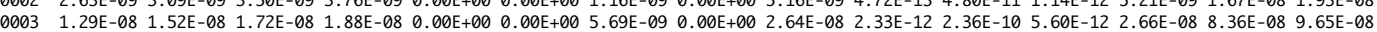

0004 3. $51 \mathrm{E}-08$ 4. $40 \mathrm{E}-08$ 1.51E-07 2.64E-11 0.00E 00 0.00E 00 4.57E-08 0.00E 00 5.

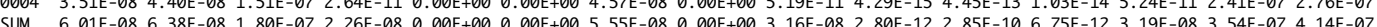


Rep_Can_30yr_Hi_Rec2_AllSrc_AllCh_BySrc_Bychem_Site.txt 


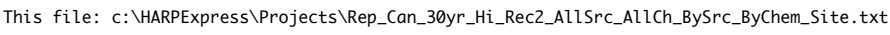

Created by HARP Version 1.0 Build 23.02.10

Uses ISC Version 9915

Uses BPIP Version 95086

Creation date: 10/24/2005 2:25:58 PM

EXCEPTION REPORT

(there have been no changes or exceptions)

INPUT FILES:

Source-Receptor file: c: \HARPExpress \Projects $\backslash E W T F$.mta

Averaging period adjustment factors file: not applicable

Emission rates file: none

Site parameters file: C: \HARPExpress $\backslash$ Projects $\backslash$ Site300. sit

Screening mode is OFF

Exposure duration: 30 year (adult resident)

Analysis method: High-end Point Estimate Health effect: Cancer Risk

Receptor(s):

Sources $(s):$
Chemicals $(s):$

SITE PARAMETERS

DEPOSITION

Deposition rate $(\mathrm{m} / \mathrm{s})$

0.0072

DRINKING WATER

*** Pathway disabled ***

FISH

*** Pathway disabled ***

PASTURE

ANIMALS' WATER

Water surface area $(\mathrm{m} n \mathrm{z})$

ter surface ared

Water volume $(L)$

Volume changes per year

from pasture cows' water

from pasture source

Fraction of dairy cows' water

from pasture source

ANIMALS' FEED

Fraction of cows' feed

from grazing

0.25

HUMAN INGESTION

Fraction of ingested beef

from contaminated source

Fraction of ingested dairy

from contaminated source

0.107

HOME GROWN PRODUCE

HUMAN INGESTION

Fraction of ingested leafy vegetable

from home grown source

Fraction of ingested exposed vegetable

from home grown source

Fraction of ingested protected veget 0.20

Fraction of ingested protected vegetable

from hom grown source

0.134

fraction of ingested root vegetable

PIGS, CHICKENS AND EGGS

HUMAN INGESTION
Fraction of ingested pig
$\quad$ from home grown source
Fraction of ingested chicken
$\quad$ rom home grown source
Fraction of ingested egg
$\quad$ from home grown source




Fraction of feed that is
leafy vegetable
Fraction of feed that is
protected vegetable
Fraction of feed that is
root vegetable

DERMAL ABSORPTION

*** Pathway enabled $* * *$

SOIL INGESTION

*** Pathway enabled ***

MOTHER'S MILK

*** Pathway enabled ***

\begin{tabular}{|c|c|c|c|}
\hline CHEM & CAS & ABBREVIATION & POLLUTANT NAME \\
\hline 0001 & 106990 & 1,3-Butadiene & 1,3-Butadiene \\
\hline 0002 & 67562394 & $1-4,6-8 \mathrm{HpCDF}$ & $1,2,3,4,6,7,8$-Heptachlorodibenzofuran \\
\hline 0003 & 55673897 & $1-4,7-9 \mathrm{HPCDF}$ & $1,2,3,4,7,8,9$-Heptachlorodibenzofuran \\
\hline 0004 & 70648269 & $1-4,7,8 \mathrm{H} \times \mathrm{CDF}$ & $1,2,3,4,7,8$-Hexachlorodibenzofuran \\
\hline 0005 & 57117449 & $1-3,6-8 \mathrm{H} \times \mathrm{CDF}$ & $1,2,3,6,7,8$-Hexachlorodibenzofuran \\
\hline 0006 & 35822469 & $1-4,6-8 \mathrm{HpCDD}$ & $1,2,3,4,6,7,8$-Heptachlorodibenzo-p-dioxin \\
\hline 0007 & 121142 & 2,4-DiNitToluen & 2,4-Dinitrotoluene \\
\hline 0008 & 606202 & 2,6-DiNitToluen & 2,6-Dinitrotoluene \\
\hline 0009 & 95578 & 2-CHLOROPHENOL & 2-CHLOROPHENOL \\
\hline 0010 & 107051 & AllylChlor & Allyl chloride \\
\hline 0011 & 7429905 & Aluminum & Aluminum \\
\hline 0012 & 7440360 & Antimony & Antimony \\
\hline 0013 & 7440393 & Barium & Barium \\
\hline 0014 & 71432 & Benzene & Benzene \\
\hline 0015 & 7440439 & Cadmium & Cadmium \\
\hline 0016 & 56235 & $\mathrm{CCl} 4$ & Carbon tetrachloride \\
\hline 0017 & 67663 & Chloroform & Chloroform \\
\hline 0018 & 7440473 & Chromium & Chromium \\
\hline 0019 & 7782505 & Chlorine & Chlorine \\
\hline 0020 & 630080 & Carbon Monoxide & Carbon monoxide \\
\hline 0021 & 7440508 & Copper & Copper \\
\hline 0022 & 110827 & Cyclohexane & Cyclohexane \\
\hline 0023 & 84662 & DiethylPhthalat & Diethyl phthalate \\
\hline 0024 & 122394 & Diphenylam & Diphenylamine \\
\hline 0025 & 75003 & Ethyl Chloride & Ethyl chloride \{Chlorethane\} \\
\hline 0026 & 100414 & Ethyl Benzene & Ethyl benzene \\
\hline 0027 & 206440 & Fluoranthene & Fluoranthene \\
\hline 0028 & 7647010 & $\mathrm{HCl}$ & Hydrochloric acid \\
\hline 0029 & 98828 & Cumene & Cumene \\
\hline 0030 & 7439921 & Lead & Lead \\
\hline 0031 & 74873 & Methyl Chloride & Methyl chloride \{Chloromethane\} \\
\hline 0032 & 71556 & $1,1,1-\mathrm{TCA}$ & Methyl chloroform $\{1,1,1-\mathrm{TCA}\}$ \\
\hline 0033 & 108872 & MethCyHex & Methylcyclohexane \\
\hline 0034 & 75092 & Methylene Chlor & Methylene chloride \{Dichloromethane\} \\
\hline 0035 & 91203 & Naphthalene & Naphthalene \\
\hline 0036 & 110543 & Hexane & Hexane \\
\hline 0037 & 10102440 & NITROGEN DIOXID & NITROGEN DIOXIDE \\
\hline 0038 & 39001020 & $1-80 \mathrm{ctaCDF}$ & $1,2,3,4,6,7,8,9$-0ctachlorodibenzofuran \\
\hline 0039 & 78115 & PETN & PETN (same as RDX) \\
\hline 0040 & 108952 & Phenol & Phenol \\
\hline 0041 & 115071 & Propylene & Propylene \\
\hline 0042 & 121824 & $\mathrm{RDX}$ & $\mathrm{RDX}$ \\
\hline 0043 & 100425 & Styrene & Styrene \\
\hline 0044 & 7446095 & SULFUR DIOXIDE & SULFUR DIOXIDE \\
\hline 0045 & 127184 & Perc & Perchloroethylene \{Tetrachloroethene\} \\
\hline 0046 & 108883 & Toluene & Toluene \\
\hline 0047 & 75014 & Vinyl Chloride & Vinyl chloride \\
\hline 0048 & 7440666 & Zinc & Zinc \\
\hline 004 & 10101 & NapthSur & Naphthalene surrogate \\
\hline 0050 & 10102 & EthBzSur & Ethylbenzene surrogate \\
\hline 51 & 10103 & nHexSur & $\mathrm{n}$-Hexane surrogate \\
\hline
\end{tabular}

BACKGROUND $(\mathrm{ug} / \mathrm{m} \wedge 3)$

$0.000 \mathrm{E}+00$

$0.000 \mathrm{E}+00$

$0.000 \mathrm{E}+00$

$0.000 \mathrm{E}+00$

$0.000 \mathrm{E}+00$

$0.000 \mathrm{E}+00$

$0.000 \mathrm{E}+00$

$0.000 \mathrm{E}+00$

$0.000 \mathrm{E}+00$

$0.000 \mathrm{E}+00$

$0.000 \mathrm{E}+00$

$0.000 \mathrm{E}+0$

$0.000 \mathrm{E}+00$

$0.000 E+00$

$0.000 \mathrm{E}+00$

$0.000 \mathrm{E}+00$

$0.000 \mathrm{E}+00$

$0.000 \mathrm{E}+00$

$0.000 \mathrm{E}+00$

$0.000 \mathrm{E}+00$

$0.000 \mathrm{E}+00$

$0.000 \mathrm{E}+00$

$0.000 \mathrm{E}+00$

$0.000 \mathrm{E}+00$

$0.000 \mathrm{E}+00$

$0.000 \mathrm{E}+00$

$0.000 \mathrm{E}+00$

$0.000 \mathrm{E}+00$

$0.000 \mathrm{E}+00$

$0.000 \mathrm{E}+00$

$0.000 \mathrm{E}+00$

$0.000 \mathrm{E}+00$

$0.000 \mathrm{E}+00$

$0.000 \mathrm{E}+00$

$0.000 \mathrm{E}+00$

$0.000 \mathrm{E}+00$

$0.000 \mathrm{E}+00$

$0.000 E+00$

$0.000 \mathrm{E}+00$

$0.000 E+00$

$0.000 \mathrm{E}+00$

$0.000 \mathrm{E}+00$

$0.000 \mathrm{E}+00$

$0.000 \mathrm{E}+00$

$0.000 \mathrm{E}+00$

$0.000 \mathrm{E}+00$

$0.000 \mathrm{E}+00$

$0.000 \mathrm{E}+00$

$0.000 \mathrm{E}+00$

$0.000 \mathrm{E}+00$

$0.000 \mathrm{E}+00$

$0.000 \mathrm{E}+00$

$0.000 \mathrm{E}+00$

EMISSIONS DATA SOURCE:

CHEMICALS ADDED OR DELETED: none

\begin{tabular}{|c|c|c|c|c|c|c|}
\hline $\begin{array}{l}\text { EMISSIONS } \\
\text { SOURCE MU }\end{array}$ & $\begin{array}{l}\text { ACILITY } F A C=\text { Site } 300 \\
E R=1\end{array}$ & $\mathrm{C} 0=* \quad \mathrm{DEV}=\mathrm{PR} 1$ & $P R 0=0 B P a n$ & STK $=1$ & NAME $=$ LLNL & Site 300 EMS \\
\hline CAS & ABBREV & MULTIPLIER & $B G(u g / m \wedge 3)$ & AVRG & (lbs/yr) & $\operatorname{MAX}(\mathrm{lbs} / \mathrm{hr})$ \\
\hline 106990 & 1,3-Butadiene & 1 & 0 & & 0.017 & 0.00017 \\
\hline 67562394 & $1-4,6-8 \mathrm{HpCDF}$ & 1 & 0 & & 0 & 0 \\
\hline 55673897 & $1-4,7-9 \mathrm{HpCDF}$ & 1 & 0 & & 0 & 0 \\
\hline 70648269 & $1-4,7,8 \mathrm{H} \times \mathrm{CDF}$ & 1 & 0 & & 0 & \\
\hline 57117449 & $1-3,6-8 \mathrm{H} \times C D F$ & 1 & 0 & & 0 & a \\
\hline 35822469 & $1-4,6-8 \mathrm{HpCDD}$ & 1 & 0 & & 0 & 0 \\
\hline 121142 & 2,4-DiNitToluen & 1 & 0 & & 0.000012 & 0.00000012 \\
\hline 606202 & 2,6-DiNitToluen & 1 & 0 & & 0.000001 & 0.00000001 \\
\hline
\end{tabular}




\begin{tabular}{ll}
95578 & 2-CHLOROPHENOL \\
107051 & AllylChlor \\
7429905 & Aluminum \\
7440360 & Antimony \\
7440393 & Barium \\
71432 & Benzene \\
7440439 & Cadmium \\
56235 & CCl4 \\
67663 & Chloroform \\
7440473 & Chromium \\
7782505 & Chlorine \\
630080 & Carbon Monoxide \\
7440508 & Copper \\
110827 & Cyclohexane \\
84662 & DiethylPhthalat \\
122394 & Diphenylam \\
75003 & Ethyl Chloride \\
100414 & Ethyl Benzene \\
206440 & Fluoranthene \\
7647010 & HCl \\
98828 & Cumene \\
7439921 & Lead \\
74873 & Methyl Chloride \\
71556 & 1,1,1-TCA \\
108872 & MethCyHex \\
75092 & Methylene Chlor \\
91203 & Naphthalene \\
110543 & Hexane \\
10102440 & NITROGEN DIOXID \\
39001020 & 1-80ctaCDF \\
78115 & PETN \\
108952 & Phenol \\
115071 & Propylene \\
121824 & RDX \\
100425 & Styrene \\
7446095 & SULFUR DIOXIDE \\
127184 & Perc \\
108883 & Toluene \\
75014 & Vinyl Chloride \\
7440666 & Zinc \\
10101 & NapthSur \\
10102 & EthBzSur \\
10103 & nHexSur \\
& \\
\hline
\end{tabular}

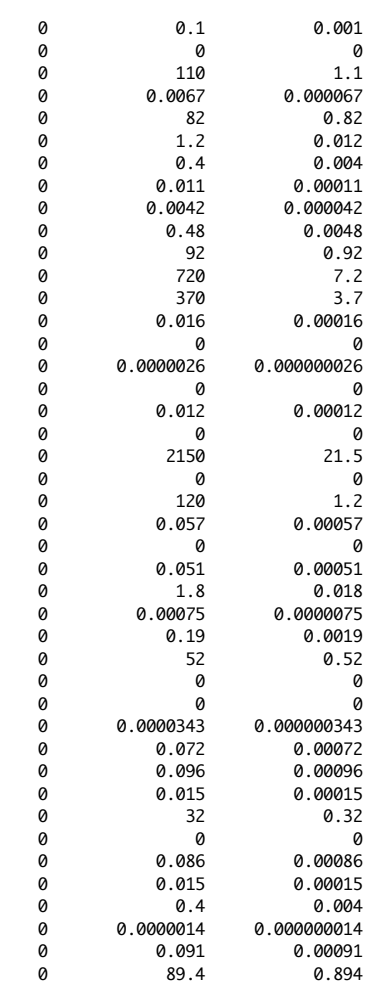

EMISSIONS FOR FACILITY FAC $=$ Site 300

$C 0=* \quad$ DEV $=P R 1 \quad P R O=0 B$ Cage 3

STK $=1 \quad$ NAME $=$ LLNL Site 300 EMS (lbs/yr) SOURCE MULTIPLIER $=1$

\begin{tabular}{ll} 
CAS & ABBREV \\
106990 & $1,3-$ Butadiene \\
67562394 & $1-4,6-8 H$ HCDF \\
55673897 & $1-4,7-9 H$ HCDF \\
70648269 & $1-4,7,8$ HCDF \\
57117449 & $1-3,6-8$ HCDF \\
35822469 & $1-4,6-8$ HpCDD \\
121142 & $2,4-$ DiNitToluen \\
606202 & $2,6-$ DiNitToluen \\
95578 & 2-CHLOROPHENOL \\
107051 & AllylChlor \\
7429905 & Aluminum \\
7440360 & Antimony \\
7440393 & Barium \\
71432 & Benzene \\
7440439 & Cadmium \\
56235 & CCl4 \\
67663 & Chloroform \\
7440473 & Chromium \\
7782505 & Chlorine \\
630080 & Carbon Monoxide \\
7440508 & Copper \\
110827 & Cyclohexane \\
84662 & DiethylPhthalat \\
122394 & Diphenylam \\
75003 & Ethyl Chloride \\
100414 & Ethyl Benzene \\
206440 & Fluoranthene \\
7647010 & HCl \\
98828 & Cumene \\
7439921 & Lead \\
74873 & Methyl Chloride \\
71556 & $1,1,1-T C A$ \\
108872 & MethCyHex \\
75092 & Methylene Chlor \\
91203 & Naphthalene \\
110543 & Hexane \\
10102440 & NITROGEN DI0XID \\
39001020 & 1 -80ctaCDF \\
78115 & PETN \\
108952 & Phenol \\
115071 & Propylene \\
121824 & RDX \\
100425 & Styrene \\
7446095 & SULFUR DIOXIDE \\
127184 & Perc \\
108883 & Toluene \\
75014 & Vinyl Chloride \\
7440666 & Zinc \\
10101 & NapthSur \\
10102 & EthBzSur \\
10103 & nHexSur \\
& \\
\hline
\end{tabular}

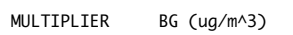

AVRG (lbs/yr) MAX (lbs/hr)

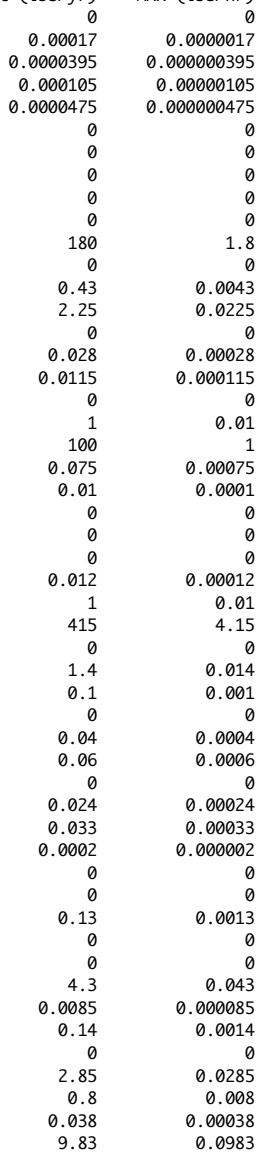




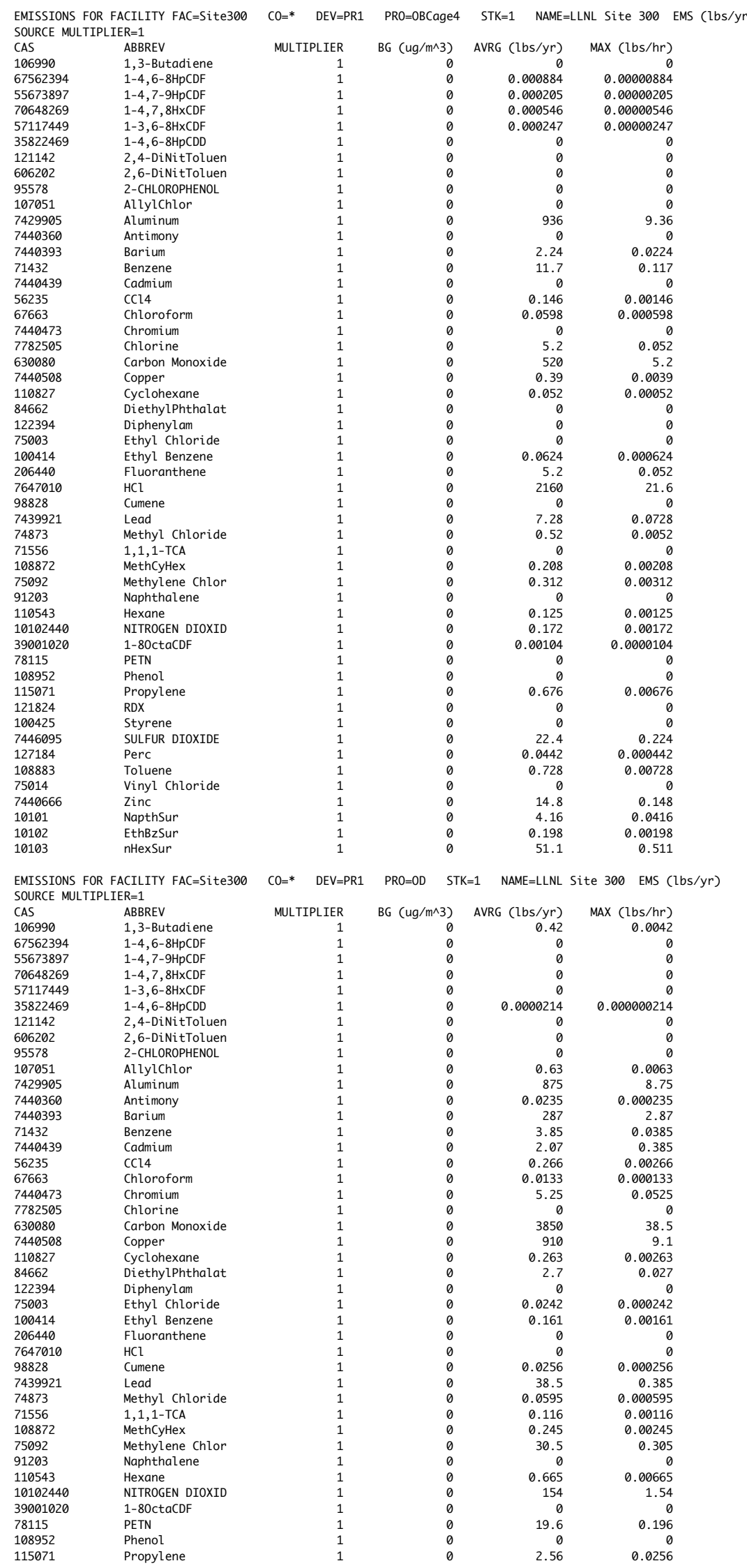




$\begin{array}{llllrr}121824 & \text { RDX } & 1 & 0 & 259 & 2.59 \\ 100425 & \text { Styrene } & 1 & 0 & 1.47 & 0.0147 \\ 7446095 & \text { SULFUR DIOXIDE } & 1 & 0 & 455 & 4.55 \\ 127184 & \text { Perc } & 1 & 0 & 0.63 & 0.0063 \\ 108883 & \text { Toluene } & 1 & 0 & 1.05 & 0.0105 \\ 75014 & \text { Vinyl Chloride } & 1 & 0 & 0.0805 & 0.000805 \\ 7440666 & \text { Zinc } & 1 & 0 & 560 & 5.6 \\ 10101 & \text { NapthSur } & 1 & 0 & 0 & 0 \\ 10102 & \text { EthBzSur } & 1 & 0 & 0.62 & 0.0062 \\ 10103 & \text { nHexSur } & 1 & 0 & 119 & 1.19\end{array}$

CANCER RISK REPORT

RECEPTOR 2

$\begin{array}{lllllllllll}\text { SOURCE } 1 & \text { RISK BY PATHWAY } \\ \text { CHEM INHAL DERM SOIL MOTHER FISH WATER VEG DAIRY BEEF CHICK PIG EGG MEAT ORAL TOTAL }\end{array}$

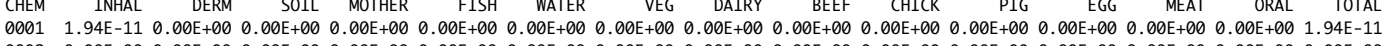

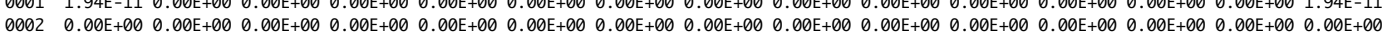

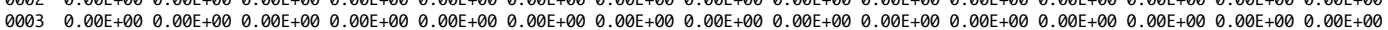

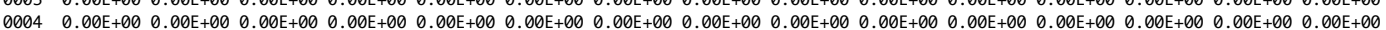
(.)

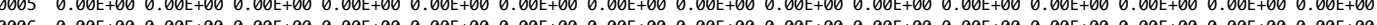
D007 $7.08 E-15$ 0.00E+00 0.00E 007 T.

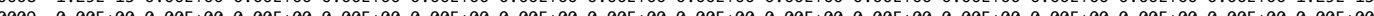

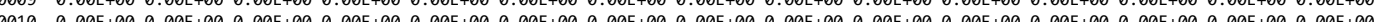

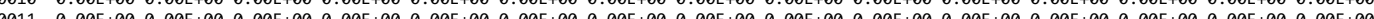

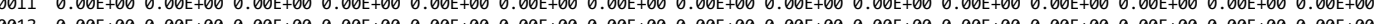

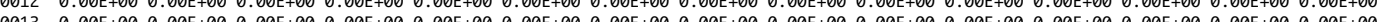

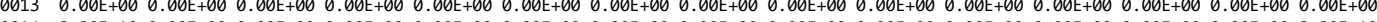
$0014 \quad 2.28 \mathrm{E}-10 \quad 0.00 \mathrm{E}+00 \quad 0.00 \mathrm{E}+00 \quad 0.00 \mathrm{E}+00 \quad 0.00 \mathrm{E}+00 \quad 0.00 \mathrm{E}+00 \quad 0.00 \mathrm{E}+00 \quad 0.00 \mathrm{E}+00 \quad 0.00 \mathrm{E}+00 \quad 0.00 \mathrm{E}+00 \quad 0.00 \mathrm{E}+00 \quad 0.00 \mathrm{E}+00 \quad 0.00 \mathrm{E}+00 \quad 0.00 \mathrm{E}+00 \quad 2.28 \mathrm{E}-10$

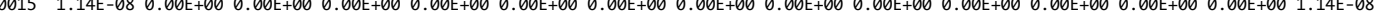

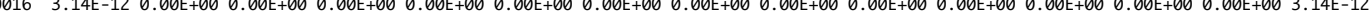

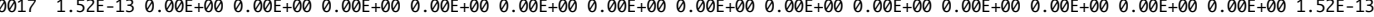

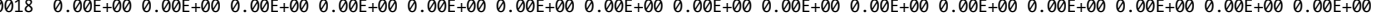

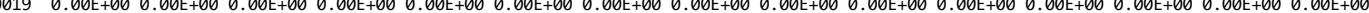

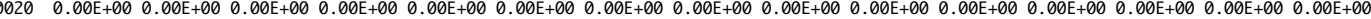

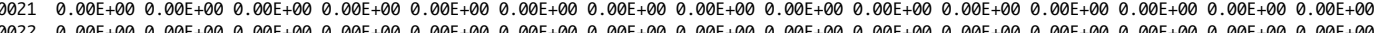

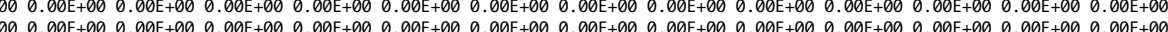
0024 . $0.00 \mathrm{E}+000.00 \mathrm{E}+000.00 \mathrm{E}+000.00 \mathrm{E}+000.00 \mathrm{E}+000.00 \mathrm{E}+000.00 \mathrm{E}+000.00 \mathrm{E}+000.00 \mathrm{E}+000.00 \mathrm{E}+000.00 \mathrm{E}+000.00 \mathrm{E}+000.00 \mathrm{E}+000.00 \mathrm{E}+000.00 \mathrm{E}+00$

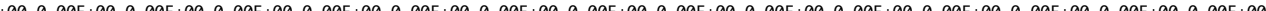

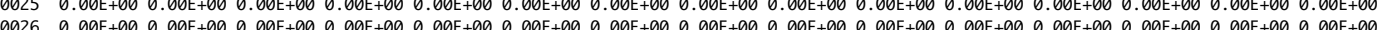
0026 D. $00278.0 .00 .00 E$ (1) $00290.00 E$ (1)

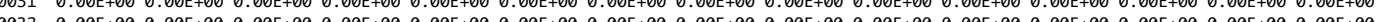
(1) 0033 D.00E .

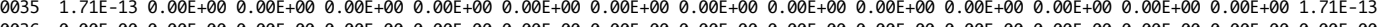

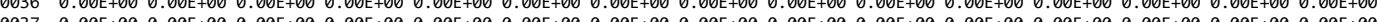

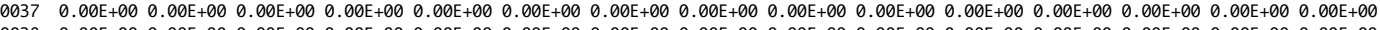

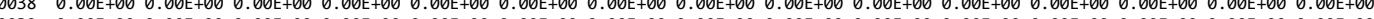
$0039 \quad 0.00 \mathrm{E}+00 \quad 0.00 \mathrm{E}+00 \quad 0.00 \mathrm{E}+00 \quad 0.00 \mathrm{E}+00 \quad 0.00 \mathrm{E}+00 \quad 0.00 \mathrm{E}+00 \quad 0.00 \mathrm{E}+00 \quad 0.00 \mathrm{E}+00 \quad 0.00 \mathrm{E}+00 \quad 0.00 \mathrm{E}+00 \quad 0.00 \mathrm{E}+00 \quad 0.00 \mathrm{E}+00 \quad 0.00 \mathrm{E}+00 \quad 0.00 \mathrm{E}+00 \quad 0.00 \mathrm{E}+00$ $0040 \quad 0.00 \mathrm{E}+00 \quad 0.00 \mathrm{E}+00 \quad 0.00 \mathrm{E}+00 \quad 0.00 \mathrm{E}+00 \quad 0.00 \mathrm{E}+00 \quad 0.00 \mathrm{E}+00 \quad 0.00 \mathrm{E}+00 \quad 0.00 \mathrm{E}+00 \quad 0.00 \mathrm{E}+00 \quad 0.00 \mathrm{E}+00 \quad 0.00 \mathrm{E}+00 \quad 0.00 \mathrm{E}+00 \quad 0.00 \mathrm{E}+00 \quad 0.00 \mathrm{E}+00 \quad 0.00 \mathrm{E}+00$

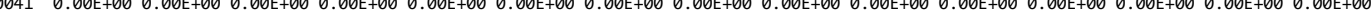

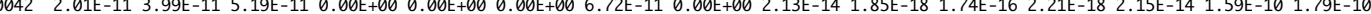

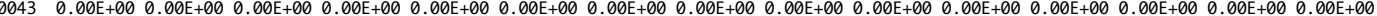

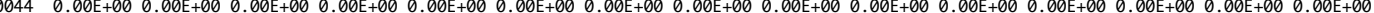
$0045 \quad 0.00 \mathrm{E}+00 \quad 0.00 \mathrm{E}+00 \quad 0.00 \mathrm{E}+00 \quad 0.00 \mathrm{E}+00 \quad 0.00 \mathrm{E}+00 \quad 0.00 \mathrm{E}+00 \quad 0.00 \mathrm{E}+00 \quad 0.00 \mathrm{E}+00 \quad 0.00 \mathrm{E}+00 \quad 0.00 \mathrm{E}+00 \quad 0.00 \mathrm{E}+00 \quad 0.00 \mathrm{E}+00 \quad 0.00 \mathrm{E}+00 \quad 0.00 \mathrm{E}+00 \quad 0.00 \mathrm{E}+00$

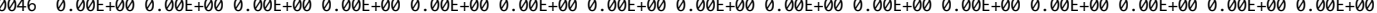
$0047 \quad 7.71 \mathrm{E}-12 \quad 0.00 \mathrm{E}+00 \quad 0.00 \mathrm{E}+00 \quad 0.00 \mathrm{E}+00 \quad 0.00 \mathrm{E}+00 \quad 0.00 \mathrm{E}+00 \quad 0.00 \mathrm{E}+00 \quad 0.00 \mathrm{E}+00 \quad 0.00 \mathrm{E}+00 \quad 0.00 \mathrm{E}+00 \quad 0.00 \mathrm{E}+00 \quad 0.00 \mathrm{E}+00 \quad 0.00 \mathrm{E}+00 \quad 0.00 \mathrm{E}+00 \quad 7.71 \mathrm{E}-12$ $0048 \quad 0.00 \mathrm{E}+000.00 \mathrm{E}+00 \quad 0.00 \mathrm{E}+00 \quad 0.00 \mathrm{E}+00 \quad 0.00 \mathrm{E}+00 \quad 0.00 \mathrm{E}+00 \quad 0.00 \mathrm{E}+000.00 \mathrm{E}+000.00 \mathrm{E}+00 \quad 0.00 \mathrm{E}+000.00 \mathrm{E}+000.00 \mathrm{E}+000.00 \mathrm{E}+000.00 \mathrm{E}+00 \quad 0.00 \mathrm{E}+00$ $0049 \quad 3.20 \mathrm{E}-16 \quad 0.00 \mathrm{E}+00 \quad 0.00 \mathrm{E}+00 \quad 0.00 \mathrm{E}+00 \quad 0.00 \mathrm{E}+00 \quad 0.00 \mathrm{E}+00 \quad 0.00 \mathrm{E}+00 \quad 0.00 \mathrm{E}+00 \quad 0.00 \mathrm{E}+00 \quad 0.00 \mathrm{E}+00 \quad 0.00 \mathrm{E}+00 \quad 0.00 \mathrm{E}+00 \quad 0.00 \mathrm{E}+00 \quad 0.00 \mathrm{E}+00 \quad 3.20 \mathrm{E}-16$ .0050 0051 1 $0.00 \mathrm{E}+00.00 \mathrm{E}$

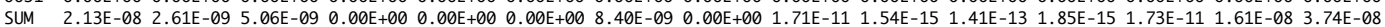

\section{SOURCE 2 RISK BY PATHWAY}

CHEM INHAL DERM SOIL MOTHER FISH WATER VEG DAIRY BEEF CHICK PIG EGG MEAT ORAL TOTAL $\begin{array}{lllllllllllllllll}0.001 & 0.00 \mathrm{E}+00 & 0.00 \mathrm{E}+00 & 0.00 \mathrm{E}+00 & 0.00 \mathrm{E}+00 & 0.00 \mathrm{E}+00 & 0.00 \mathrm{E}+00 & 0.00 \mathrm{E}+00 & 0.00 \mathrm{E}+00 & 0.00 \mathrm{E}+00 & 0.00 \mathrm{E}+00 & 0.00 \mathrm{E}+00 & 0.00 \mathrm{E}+00 & 0.00 \mathrm{E}+00 & 0.00 \mathrm{E}+00 & 0.00 \mathrm{E}+00\end{array}$

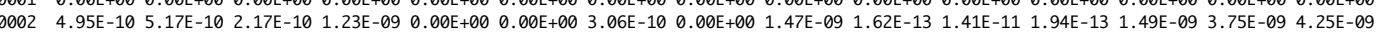

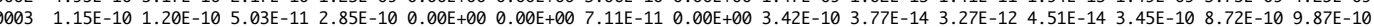

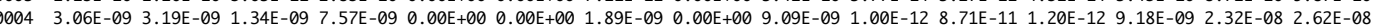

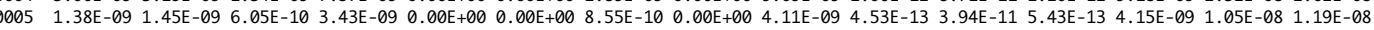

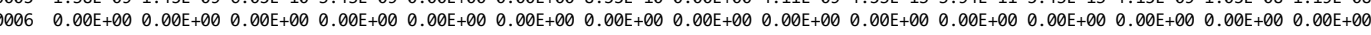

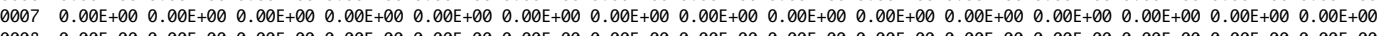

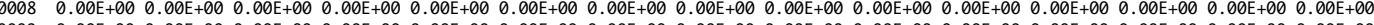

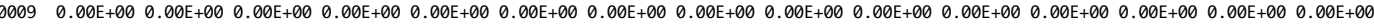
$0010 \quad 0.00 \mathrm{E}+00 \quad 0.00 \mathrm{E}+00 \quad 0.00 \mathrm{E}+00 \quad 0.00 \mathrm{E}+00 \quad 0.00 \mathrm{E}+00 \quad 0.00 \mathrm{E}+00 \quad 0.00 \mathrm{E}+00 \quad 0.00 \mathrm{E}+00 \quad 0.00 \mathrm{E}+00 \quad 0.00 \mathrm{E}+00 \quad 0.00 \mathrm{E}+00 \quad 0.00 \mathrm{E}+00 \quad 0.00 \mathrm{E}+00 \quad 0.00 \mathrm{E}+00 \quad 0.00 \mathrm{E}+00$ $0011 \quad 0.00 \mathrm{E}+00 \quad 0.00 \mathrm{E}+00 \quad 0.00 \mathrm{E}+00 \quad 0.00 \mathrm{E}+00 \quad 0.00 \mathrm{E}+00 \quad 0.00 \mathrm{E}+00 \quad 0.00 \mathrm{E}+00 \quad 0.00 \mathrm{E}+00 \quad 0.00 \mathrm{E}+00 \quad 0.00 \mathrm{E}+00 \quad 0.00 \mathrm{E}+00 \quad 0.00 \mathrm{E}+00 \quad 0.00 \mathrm{E}+00 \quad 0.00 \mathrm{E}+00 \quad 0.00 \mathrm{E}+00$ $0012 \quad 0.00 \mathrm{E}+00 \quad 0.00 \mathrm{E}+00 \quad 0.00 \mathrm{E}+00 \quad 0.00 \mathrm{E}+00 \quad 0.00 \mathrm{E}+00 \quad 0.00 \mathrm{E}+00 \quad 0.00 \mathrm{E}+00 \quad 0.00 \mathrm{E}+00 \quad 0.00 \mathrm{E}+00 \quad 0.00 \mathrm{E}+00 \quad 0.00 \mathrm{E}+00 \quad 0.00 \mathrm{E}+00 \quad 0.00 \mathrm{E}+00 \quad 0.00 \mathrm{E}+00 \quad 0.00 \mathrm{E}+00$

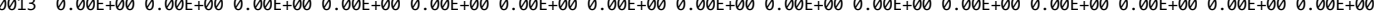
$0014 \quad 5.04 \mathrm{E}-10 \quad 0.00 \mathrm{E}+00 \quad 0.00 \mathrm{E}+00 \quad 0.00 \mathrm{E}+00 \quad 0.00 \mathrm{E}+00 \quad 0.00 \mathrm{E}+00 \quad 0.00 \mathrm{E}+00 \quad 0.00 \mathrm{E}+00 \quad 0.00 \mathrm{E}+00 \quad 0.00 \mathrm{E}+00 \quad 0.00 \mathrm{E}+00 \quad 0.00 \mathrm{E}+00 \quad 0.00 \mathrm{E}+00 \quad 0.00 \mathrm{E}+00 \quad 5.04 \mathrm{E}-10$

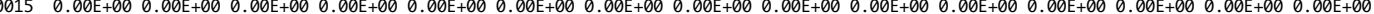

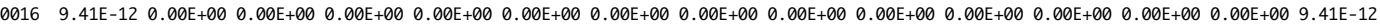

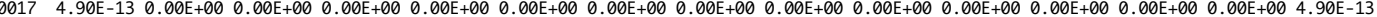

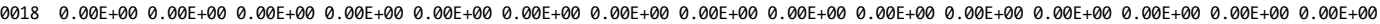

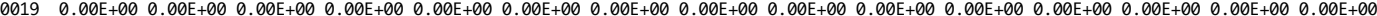

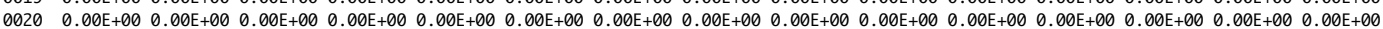
$00210.00 \mathrm{E}+00 \quad 0.00 \mathrm{E}+00 \quad 0.00 \mathrm{E}+00 \quad 0.00 \mathrm{E}+00 \quad 0.00 \mathrm{E}+00 \quad 0.00 \mathrm{E}+00 \quad 0.00 \mathrm{E}+00 \quad 0.00 \mathrm{E}+00 \quad 0.00 \mathrm{E}+00 \quad 0.00 \mathrm{E}+00 \quad 0.00 \mathrm{E}+00 \quad 0.00 \mathrm{E}+00 \quad 0.00 \mathrm{E}+00 \quad 0.00 \mathrm{E}+00 \quad 0.00 \mathrm{E}+00$

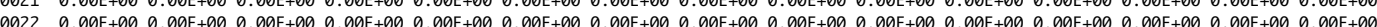
0023 . $0.00 \mathrm{E}+000.00 \mathrm{E}+000.00 \mathrm{E}+000.00 \mathrm{E}+00.00 \mathrm{E}+000.00 \mathrm{E}+000.00 \mathrm{E}+000.00 \mathrm{E}+000.00 \mathrm{E}+000.00 \mathrm{E}+000.00 \mathrm{E}+000.00 \mathrm{E}+000.00 \mathrm{E}+000.00 \mathrm{E}+000.00 \mathrm{E}+00$ 0023 . $\begin{array}{llll}0 & 0.024\end{array}$

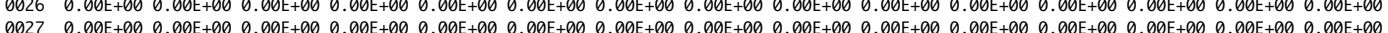


$0028 \quad 0.00 \mathrm{E}+00 \quad 0.00 \mathrm{E}+00 \quad 0.00 \mathrm{E}+00 \quad 0.00 \mathrm{E}+00 \quad 0.00 \mathrm{E}+00 \quad 0.00 \mathrm{E}+00 \quad 0.00 \mathrm{E}+00 \quad 0.00 \mathrm{E}+00 \quad 0.00 \mathrm{E}+00 \quad 0.00 \mathrm{E}+00 \quad 0.00 \mathrm{E}+00 \quad 0.00 \mathrm{E}+00 \quad 0.00 \mathrm{E}+00 \quad 0.00 \mathrm{E}+00 \quad 0.00 \mathrm{E}+00$

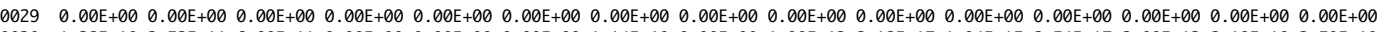

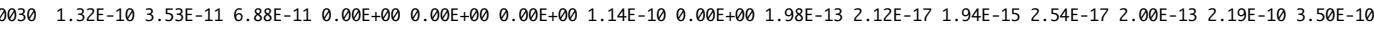

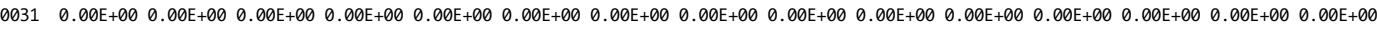

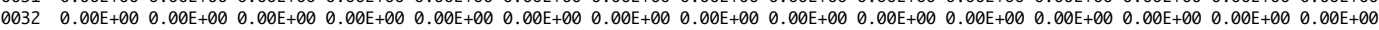
$0033 \quad 0.00 \mathrm{E}+00 \quad 0.00 \mathrm{E}+00 \quad 0.00 \mathrm{E}+00 \quad 0.00 \mathrm{E}+00 \quad 0.00 \mathrm{E}+00 \quad 0.00 \mathrm{E}+00 \quad 0.00 \mathrm{E}+00 \quad 0.00 \mathrm{E}+00 \quad 0.00 \mathrm{E}+00 \quad 0.00 \mathrm{E}+00 \quad 0.00 \mathrm{E}+00 \quad 0.00 \mathrm{E}+00 \quad 0.00 \mathrm{E}+00 \quad 0.00 \mathrm{E}+00 \quad 0.00 \mathrm{E}+00$ $0034 \quad 4.71 \mathrm{E}-13 \quad 0.00 \mathrm{E}+00 \quad 0.00 \mathrm{E}+00 \quad 0.00 \mathrm{E}+00 \quad 0.00 \mathrm{E}+00 \quad 0.00 \mathrm{E}+00 \quad 0.00 \mathrm{E}+00 \quad 0.00 \mathrm{E}+00 \quad 0.00 \mathrm{E}+00 \quad 0.00 \mathrm{E}+00 \quad 0.00 \mathrm{E}+00 \quad 0.00 \mathrm{E}+00 \quad 0.00 \mathrm{E}+00 \quad 0.00 \mathrm{E}+00 \quad 4.71 \mathrm{E}-13$

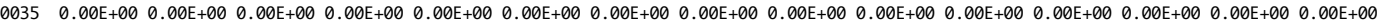

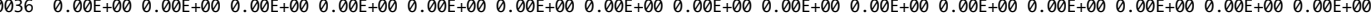

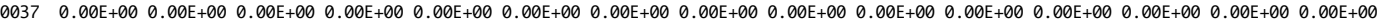

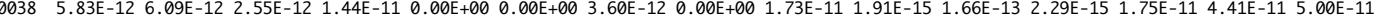
$00390.00 \mathrm{E}+00 \quad 0.00 \mathrm{E}+00 \quad 0.00 \mathrm{E}+00 \quad 0.00 \mathrm{E}+00 \quad 0.00 \mathrm{E}+00 \quad 0.00 \mathrm{E}+00 \quad 0.00 \mathrm{E}+00 \quad 0.00 \mathrm{E}+00 \quad 0.00 \mathrm{E}+00 \quad 0.00 \mathrm{E}+00 \quad 0.00 \mathrm{E}+00 \quad 0.00 \mathrm{E}+00 \quad 0.00 \mathrm{E}+00 \quad 0.00 \mathrm{E}+00 \quad 0.00 \mathrm{E}+00$

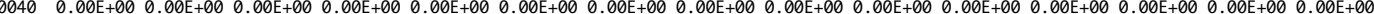

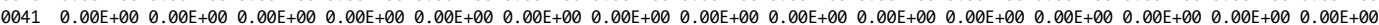
$00420.00 \mathrm{E}+00 \quad 0.00 \mathrm{E}+00 \quad 0.00 \mathrm{E}+00 \quad 0.00 \mathrm{E}+00 \quad 0.00 \mathrm{E}+00 \quad 0.00 \mathrm{E}+00 \quad 0.00 \mathrm{E}+00 \quad 0.00 \mathrm{E}+00 \quad 0.00 \mathrm{E}+00 \quad 0.00 \mathrm{E}+00 \quad 0.00 \mathrm{E}+00 \quad 0.00 \mathrm{E}+00 \quad 0.00 \mathrm{E}+00 \quad 0.00 \mathrm{E}+00 \quad 0.00 \mathrm{E}+00$ $00430.00 \mathrm{E}+000.00 \mathrm{E}+000.00 \mathrm{E}+000.00 \mathrm{E}+000.00 \mathrm{E}+000.00 \mathrm{E}+000.00 \mathrm{E}+000.00 \mathrm{E}+000.00 \mathrm{E}+000.00 \mathrm{E}+000.00 \mathrm{E}+000.00 \mathrm{E}+000.00 \mathrm{E}+000.00 \mathrm{E}+000.00 \mathrm{E}+00$ 0044 0.00E+00 0.00E 00 0.00E+00 0.00E 00 0.00E

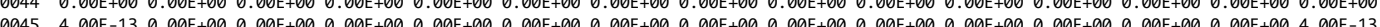

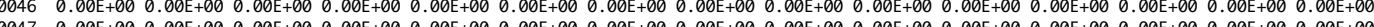
0047 OOA

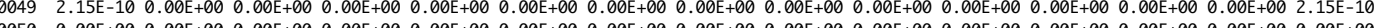
ODS1 $\begin{array}{lllllllllllllllllllll}0051 & 0.00 \mathrm{E}+00 & 0.00 \mathrm{E}+00 & 0.00 \mathrm{E}+00 & 0.00 \mathrm{E}+00 & 0.00 \mathrm{E}+00 & 0.00 \mathrm{E}+00 & 0.00 \mathrm{E}+00 & 0.00 \mathrm{E}+00 & 0.00 \mathrm{E}+00 & 0.00 \mathrm{E}+00 & 0.00 \mathrm{E}+00 & 0.00 \mathrm{E}+00 & 0.00 \mathrm{E}+00 & 0.00 \mathrm{E}+00 & 0.00 \mathrm{E}+00 \\ \text { SUM } & 5.92 \mathrm{E}-09 & 5.32 \mathrm{E}-09 & 2.28 \mathrm{E}-09 & 1.25 \mathrm{E}-08 & 0.00 \mathrm{E}+00 & 0.00 \mathrm{E}+00 & 3.24 \mathrm{E}-09 & 0.00 \mathrm{E}+00 & 1.50 \mathrm{E}-08 & 1.66 \mathrm{E}-12 & 1.44 \mathrm{E}-10 & 1.98 \mathrm{E}-12 & 1.52 \mathrm{E}-08 & 3.85 \mathrm{E}-08 & 4.45 \mathrm{E}-08\end{array}$

\section{SOURCE 3 RISK BY PATHWAY}

CHEM INHAL DERM SOIL MOTHER FISH WATER VEG DAIRY BEEF CHICK PIG EGG MEAT ORAL TOTAL $0001 \quad 0.00 \mathrm{E}+00 \quad 0.00 \mathrm{E}+00 \quad 0.00 \mathrm{E}+00 \quad 0.00 \mathrm{E}+00 \quad 0.00 \mathrm{E}+00 \quad 0.00 \mathrm{E}+00 \quad 0.00 \mathrm{E}+00 \quad 0.00 \mathrm{E}+00 \quad 0.00 \mathrm{E}+00 \quad 0.00 \mathrm{E}+00 \quad 0.00 \mathrm{E}+00 \quad 0.00 \mathrm{E}+00 \quad 0.00 \mathrm{E}+00 \quad 0.00 \mathrm{E}+00 \quad 0.00 \mathrm{E}+00$ $0002 \quad 2.44 \mathrm{E}-09 \quad 2.55 \mathrm{E}-09 \quad 1.07 \mathrm{E}-09 \quad 6.13 \mathrm{E}-09 \quad 0.00 \mathrm{E}+00 \quad 0.00 \mathrm{E}+00 \quad 1.51 \mathrm{E}-09 \quad 0.00 \mathrm{E}+00 \quad 7.52 \mathrm{E}-09 \quad 7.99 \mathrm{E}-13 \quad 6.94 \mathrm{E}-11 \quad 9.57 \mathrm{E}-13 \quad 7.60 \mathrm{E}-09 \quad 1.88 \mathrm{E}-08 \quad 2.13 \mathrm{E}-08$

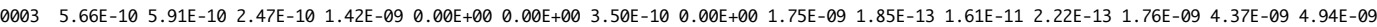

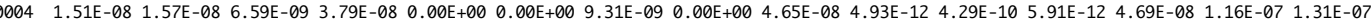
$0005 \quad 6.81 \mathrm{E}-09 \quad 7.12 \mathrm{E}-09 \quad 2.98 \mathrm{E}-09 \quad 1.71 \mathrm{E}-08 \quad 0.00 \mathrm{E}+00 \quad 0.00 \mathrm{E}+00 \quad 4.21 \mathrm{E}-09 \quad 0.00 \mathrm{E}+00 \quad 2.10 \mathrm{E}-08 \quad 2.23 \mathrm{E}-12 \quad 1.94 \mathrm{E}-10 \quad 2.67 \mathrm{E}-12 \quad 2.12 \mathrm{E}-08 \quad 5.27 \mathrm{E}-08 \quad 5.95 \mathrm{E}-08$

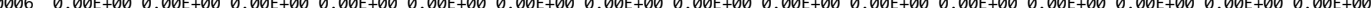
$0007 \quad 0.00 \mathrm{E}+00 \quad 0.00 \mathrm{E}+00 \quad 0.00 \mathrm{E}+00 \quad 0.00 \mathrm{E}+00 \quad 0.00 \mathrm{E}+00 \quad 0.00 \mathrm{E}+00 \quad 0.00 \mathrm{E}+00 \quad 0.00 \mathrm{E}+00 \quad 0.00 \mathrm{E}+00 \quad 0.00 \mathrm{E}+00 \quad 0.00 \mathrm{E}+00 \quad 0.00 \mathrm{E}+00 \quad 0.00 \mathrm{E}+00 \quad 0.00 \mathrm{E}+00 \quad 0.00 \mathrm{E}+00$

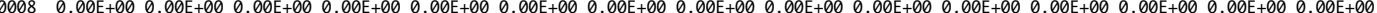
$0009 \quad 0.00 \mathrm{E}+00 \quad 0.00 \mathrm{E}+00 \quad 0.00 \mathrm{E}+00 \quad 0.00 \mathrm{E}+00 \quad 0.00 \mathrm{E}+00 \quad 0.00 \mathrm{E}+00 \quad 0.00 \mathrm{E}+00 \quad 0.00 \mathrm{E}+00 \quad 0.00 \mathrm{E}+00 \quad 0.00 \mathrm{E}+00 \quad 0.00 \mathrm{E}+00 \quad 0.00 \mathrm{E}+00 \quad 0.00 \mathrm{E}+00 \quad 0.00 \mathrm{E}+00 \quad 0.00 \mathrm{E}+00$

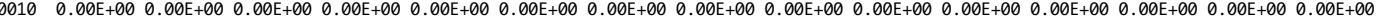

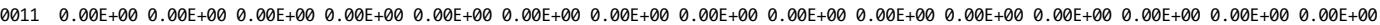
$0012 \quad 0.00 \mathrm{E}+00 \quad 0.00 \mathrm{E}+00 \quad 0.00 \mathrm{E}+00 \quad 0.00 \mathrm{E}+00 \quad 0.00 \mathrm{E}+00 \quad 0.00 \mathrm{E}+00 \quad 0.00 \mathrm{E}+00 \quad 0.00 \mathrm{E}+00 \quad 0.00 \mathrm{E}+00 \quad 0.00 \mathrm{E}+00 \quad 0.00 \mathrm{E}+00 \quad 0.00 \mathrm{E}+00 \quad 0.00 \mathrm{E}+00 \quad 0.00 \mathrm{E}+00 \quad 0.00 \mathrm{E}+00$ $00130.00 \mathrm{E}+000.00 \mathrm{E}+000.00 \mathrm{E}+000.00 \mathrm{E}+000.00 \mathrm{E}+000.00 \mathrm{E}+000.00 \mathrm{E}+000.00 \mathrm{E}+000.00 \mathrm{E}+00 \quad 0.00 \mathrm{E}+000.00 \mathrm{E}+000.00 \mathrm{E}+000.00 \mathrm{E}+000.00 \mathrm{E}+000.00 \mathrm{E}+00$ $00142.48 \mathrm{E}-090.00 \mathrm{E}+000.00 \mathrm{E}+000.00 \mathrm{E}+000.00 \mathrm{E}$

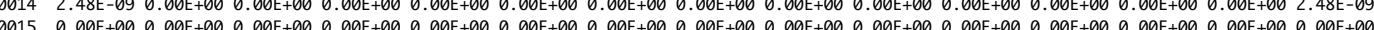

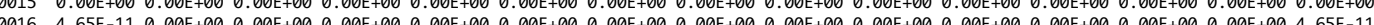

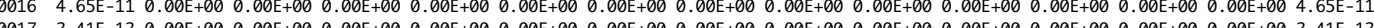

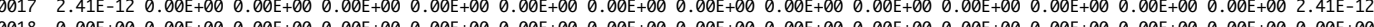
018 (D. (1)

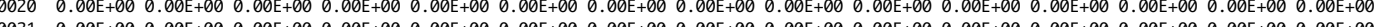
(DOEE 0022 D. 0023 0.00E

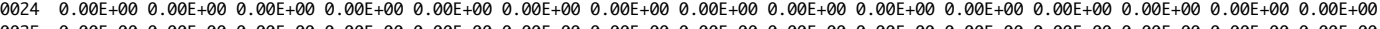

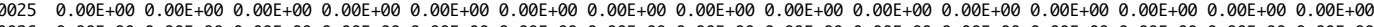

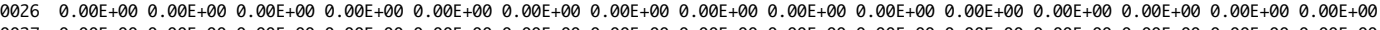

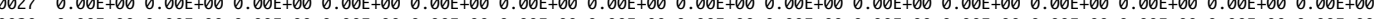
$0028 \quad 0.00 \mathrm{E}+00 \quad 0.00 \mathrm{E}+00 \quad 0.00 \mathrm{E}+00 \quad 0.00 \mathrm{E}+00 \quad 0.00 \mathrm{E}+00 \quad 0.00 \mathrm{E}+00 \quad 0.00 \mathrm{E}+00 \quad 0.00 \mathrm{E}+00 \quad 0.00 \mathrm{E}+00 \quad 0.00 \mathrm{E}+00 \quad 0.00 \mathrm{E}+00 \quad 0.00 \mathrm{E}+00 \quad 0.00 \mathrm{E}+00 \quad 0.00 \mathrm{E}+00 \quad 0.00 \mathrm{E}+00$

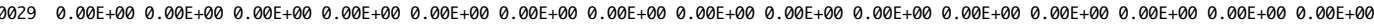

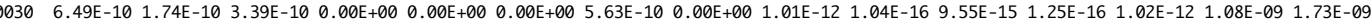

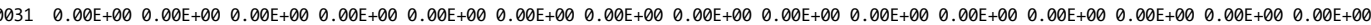
$0032 \quad 0.00 \mathrm{E}+00 \quad 0.00 \mathrm{E}+00 \quad 0.00 \mathrm{E}+00 \quad 0.00 \mathrm{E}+00 \quad 0.00 \mathrm{E}+00 \quad 0.00 \mathrm{E}+00 \quad 0.00 \mathrm{E}+00 \quad 0.00 \mathrm{E}+00 \quad 0.00 \mathrm{E}+00 \quad 0.00 \mathrm{E}+00 \quad 0.00 \mathrm{E}+00 \quad 0.00 \mathrm{E}+00 \quad 0.00 \mathrm{E}+00 \quad 0.00 \mathrm{E}+00 \quad 0.00 \mathrm{E}+00$

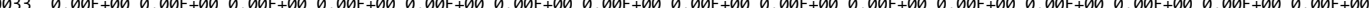

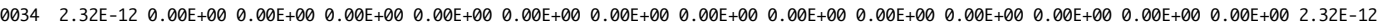

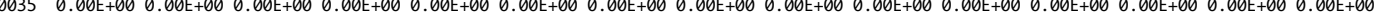
$0036 \quad 0.00 \mathrm{E}+00 \quad 0.00 \mathrm{E}+00 \quad 0.00 \mathrm{E}+00 \quad 0.00 \mathrm{E}+00 \quad 0.00 \mathrm{E}+00 \quad 0.00 \mathrm{E}+00 \quad 0.00 \mathrm{E}+00 \quad 0.00 \mathrm{E}+00 \quad 0.00 \mathrm{E}+00 \quad 0.00 \mathrm{E}+00 \quad 0.00 \mathrm{E}+00 \quad 0.00 \mathrm{E}+00 \quad 0.00 \mathrm{E}+00 \quad 0.00 \mathrm{E}+00 \quad 0.00 \mathrm{E}+00$ $00370.00 \mathrm{E}+00 \quad 0.00 \mathrm{E}+00 \quad 0.00 \mathrm{E}+00 \quad 0.00 \mathrm{E}+00 \quad 0.00 \mathrm{E}+000.00 \mathrm{E}+000.00 \mathrm{E}+000.00 \mathrm{E}+000.00 \mathrm{E}+00 \quad 0.00 \mathrm{E}+000.00 \mathrm{E}+000.00 \mathrm{E}+000.00 \mathrm{E}+000.00 \mathrm{E}+000.00 \mathrm{E}+00$

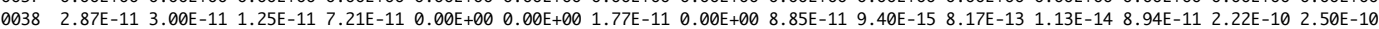

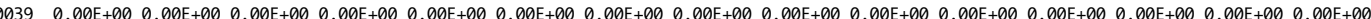

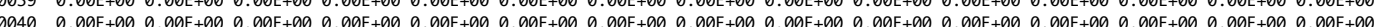

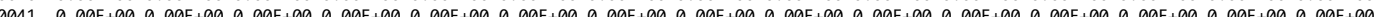
0041 .

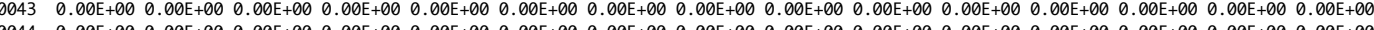
(0045 $1.97 \mathrm{E}-12$ O.00E

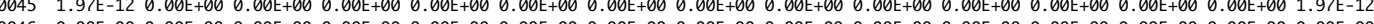
047 O.00E 0047 . OOH

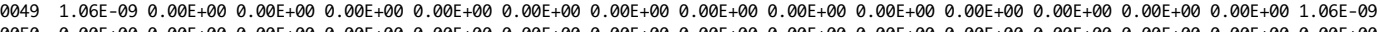

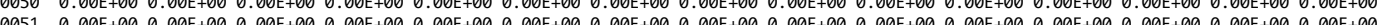

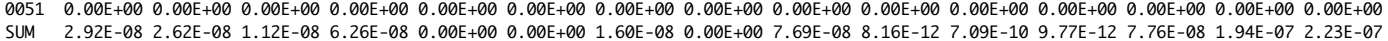

\section{SOURCE 4 RISK BY PATHWAY}

CHEM INHAL DERM SOIL MOTHER FISH WATER VEG DAIRY BEEF CHICK PIG EGG MEAT ORAL TOTAL

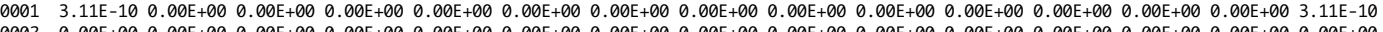

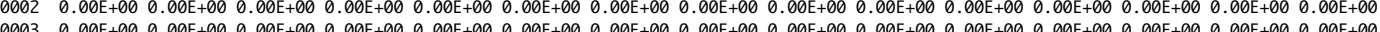

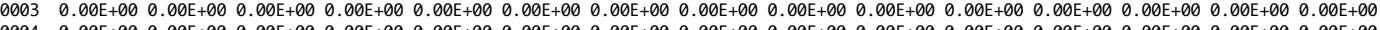

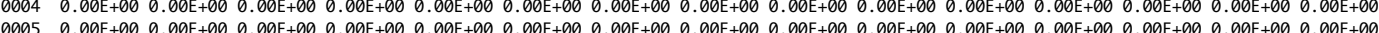

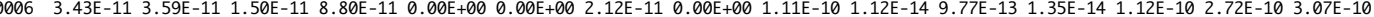
$0007 \quad 0.00 \mathrm{E}+00 \quad 0.00 \mathrm{E}+00 \quad 0.00 \mathrm{E}+00 \quad 0.00 \mathrm{E}+00 \quad 0.00 \mathrm{E}+00 \quad 0.00 \mathrm{E}+00 \quad 0.00 \mathrm{E}+00 \quad 0.00 \mathrm{E}+00 \quad 0.00 \mathrm{E}+00 \quad 0.00 \mathrm{E}+00 \quad 0.00 \mathrm{E}+00 \quad 0.00 \mathrm{E}+00 \quad 0.00 \mathrm{E}+00 \quad 0.00 \mathrm{E}+00 \quad 0.00 \mathrm{E}+00$ $0008 \quad 0.00 \mathrm{E}+00 \quad 0.00 \mathrm{E}+00 \quad 0.00 \mathrm{E}+00 \quad 0.00 \mathrm{E}+00 \quad 0.00 \mathrm{E}+00 \quad 0.00 \mathrm{E}+00 \quad 0.00 \mathrm{E}+00 \quad 0.00 \mathrm{E}+00 \quad 0.00 \mathrm{E}+00 \quad 0.00 \mathrm{E}+00 \quad 0.00 \mathrm{E}+00 \quad 0.00 \mathrm{E}+000.00 \mathrm{E}+00 \quad 0.00 \mathrm{E}+000.00 \mathrm{E}+00$

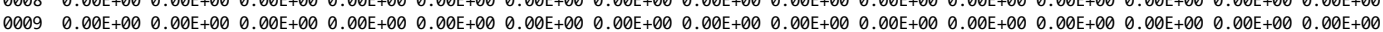
$0010 \quad 1.63 \mathrm{E}-11 \quad 0.00 \mathrm{E}+00 \quad 0.00 \mathrm{E}+00 \quad 0.00 \mathrm{E}+00 \quad 0.00 \mathrm{E}+00 \quad 0.00 \mathrm{E}+00 \quad 0.00 \mathrm{E}+00 \quad 0.00 \mathrm{E}+00 \quad 0.00 \mathrm{E}+00 \quad 0.00 \mathrm{E}+00 \quad 0.00 \mathrm{E}+00 \quad 0.00 \mathrm{E}+00 \quad 0.00 \mathrm{E}+00 \quad 0.00 \mathrm{E}+00 \quad 1.63 \mathrm{E}-11$

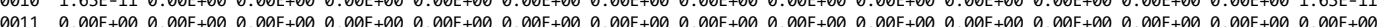

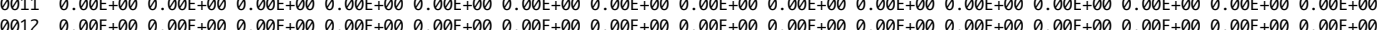

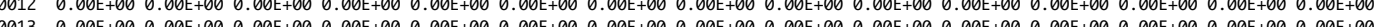
$\begin{array}{llllllllllllllllll}0013 & 0.00 E+00 & 0.00 \mathrm{E}+00 & 0.00 \mathrm{E}+00 & 0.00 \mathrm{E}+00 & 0.00 \mathrm{E}+00 & 0.00 \mathrm{E}+00 & 0.00 \mathrm{E}+00 & 0.00 \mathrm{E}+00 & 0.00 \mathrm{E}+00 & 0.00 \mathrm{E}+00 & 0.00 \mathrm{E}+00 & 0.00 \mathrm{E}+00 & 0.00 \mathrm{E}+00 & 0.00 \mathrm{E}+00 & 0.00 \mathrm{E}+00\end{array}$

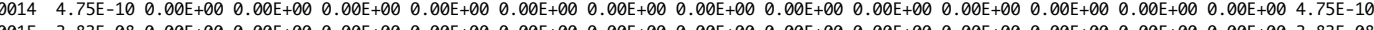
$\begin{array}{lllllllllllllllllllllllll}016 & 4.92 \mathrm{E}-11 & 0.00 \mathrm{E}+00 & 0.00 \mathrm{E}+00 & 0.00 \mathrm{E}+00 & 0.00 \mathrm{E}+00 & 0.00 \mathrm{E}+00 & 0.00 \mathrm{E}+00 & 0.00 \mathrm{E}+00 & 0.00 \mathrm{E}+00 & 0.00 \mathrm{E}+00 & 0.00 \mathrm{E}+00 & 0.00 \mathrm{E}+00 & 0.00 \mathrm{E}+00 & 0.00 \mathrm{E}+00 & 4.92 \mathrm{E}-11\end{array}$ 
$0017 \quad 3.12 \mathrm{E}-13 \quad 0.00 \mathrm{E}+00 \quad 0.00 \mathrm{E}+00 \quad 0.00 \mathrm{E}+00 \quad 0.00 \mathrm{E}+00 \quad 0.00 \mathrm{E}+00 \quad 0.00 \mathrm{E}+00 \quad 0.00 \mathrm{E}+00 \quad 0.00 \mathrm{E}+00 \quad 0.00 \mathrm{E}+00 \quad 0.00 \mathrm{E}+00 \quad 0.00 \mathrm{E}+00 \quad 0.00 \mathrm{E}+00 \quad 0.00 \mathrm{E}+00 \quad 3.12 \mathrm{E}-13$

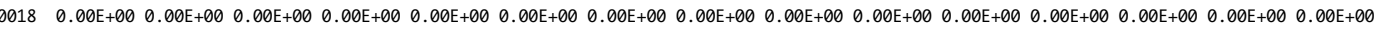
$0019 \quad 0.00 \mathrm{E}+00 \quad 0.00 \mathrm{E}+00 \quad 0.00 \mathrm{E}+00 \quad 0.00 \mathrm{E}+00 \quad 0.00 \mathrm{E}+00 \quad 0.00 \mathrm{E}+00 \quad 0.00 \mathrm{E}+00 \quad 0.00 \mathrm{E}+00 \quad 0.00 \mathrm{E}+00 \quad 0.00 \mathrm{E}+00 \quad 0.00 \mathrm{E}+00 \quad 0.00 \mathrm{E}+00 \quad 0.00 \mathrm{E}+00 \quad 0.00 \mathrm{E}+00 \quad 0.00 \mathrm{E}+00$

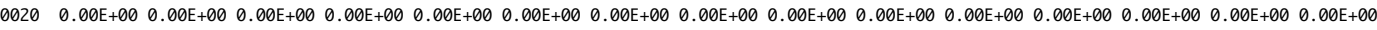

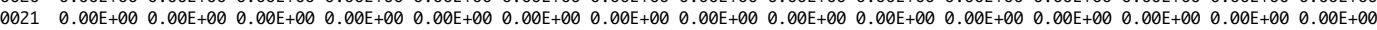
$0022 \quad 0.00 \mathrm{E}+00 \quad 0.00 \mathrm{E}+00 \quad 0.00 \mathrm{E}+00 \quad 0.00 \mathrm{E}+00 \quad 0.00 \mathrm{E}+00 \quad 0.00 \mathrm{E}+00 \quad 0.00 \mathrm{E}+00 \quad 0.00 \mathrm{E}+00 \quad 0.00 \mathrm{E}+00 \quad 0.00 \mathrm{E}+00 \quad 0.00 \mathrm{E}+00 \quad 0.00 \mathrm{E}+00 \quad 0.00 \mathrm{E}+00 \quad 0.00 \mathrm{E}+00 \quad 0.00 \mathrm{E}+00$

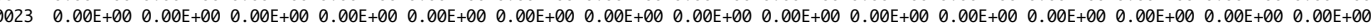

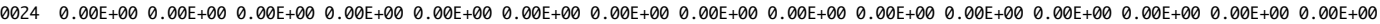

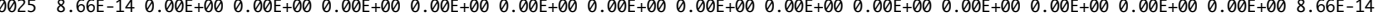
$0026 \quad 0.00 \mathrm{E}+00 \quad 0.00 \mathrm{E}+00 \quad 0.00 \mathrm{E}+00 \quad 0.00 \mathrm{E}+00 \quad 0.00 \mathrm{E}+00 \quad 0.00 \mathrm{E}+00 \quad 0.00 \mathrm{E}+00 \quad 0.00 \mathrm{E}+00 \quad 0.00 \mathrm{E}+00 \quad 0.00 \mathrm{E}+00 \quad 0.00 \mathrm{E}+00 \quad 0.00 \mathrm{E}+00 \quad 0.00 \mathrm{E}+00 \quad 0.00 \mathrm{E}+00 \quad 0.00 \mathrm{E}+00$ $0027 \quad 0.00 \mathrm{E}+00 \quad 0.00 \mathrm{E}+00 \quad 0.00 \mathrm{E}+00 \quad 0.00 \mathrm{E}+00 \quad 0.00 \mathrm{E}+00 \quad 0.00 \mathrm{E}+00 \quad 0.00 \mathrm{E}+00 \quad 0.00 \mathrm{E}+00 \quad 0.00 \mathrm{E}+00 \quad 0.00 \mathrm{E}+00 \quad 0.00 \mathrm{E}+00 \quad 0.00 \mathrm{E}+00 \quad 0.00 \mathrm{E}+00 \quad 0.00 \mathrm{E}+00 \quad 0.00 \mathrm{E}+00$ $0028 \quad 0.00 \mathrm{E}+00 \quad 0.00 \mathrm{E}+00 \quad 0.00 \mathrm{E}+00 \quad 0.00 \mathrm{E}+00 \quad 0.00 \mathrm{E}+00 \quad 0.00 \mathrm{E}+00 \quad 0.00 \mathrm{E}+00 \quad 0.00 \mathrm{E}+00 \quad 0.00 \mathrm{E}+00 \quad 0.00 \mathrm{E}+00 \quad 0.00 \mathrm{E}+00 \quad 0.00 \mathrm{E}+00 \quad 0.00 \mathrm{E}+00 \quad 0.00 \mathrm{E}+00 \quad 0.00 \mathrm{E}+00$ $00290.00 \mathrm{E}+00 \quad 0.00 \mathrm{E}+00 \quad 0.00 \mathrm{E}+00 \quad 0.00 \mathrm{E}+00 \quad 0.00 \mathrm{E}+00 \quad 0.00 \mathrm{E}+00 \quad 0.00 \mathrm{E}+00 \quad 0.00 \mathrm{E}+00 \quad 0.00 \mathrm{E}+00 \quad 0.00 \mathrm{E}+00 \quad 0.00 \mathrm{E}+00 \quad 0.00 \mathrm{E}+00 \quad 0.00 \mathrm{E}+00 \quad 0.00 \mathrm{E}+00 \quad 0.00 \mathrm{E}+00$ $0030 \quad 2.00 \mathrm{E}-09 \quad 5.35 \mathrm{E}-10 \quad 1.04 \mathrm{E}-09 \quad 0.00 \mathrm{E}+00 \quad 0.00 \mathrm{E}+00 \quad 0.00 \mathrm{E}+00 \quad 1.73 \mathrm{E}-09 \quad 0.00 \mathrm{E}+00 \quad 3.27 \mathrm{E}-12 \quad 3.21 \mathrm{E}-16 \quad 2.94 \mathrm{E}-14 \quad 3.84 \mathrm{E}-16 \quad 3.30 \mathrm{E}-12 \quad 3.31 \mathrm{E}-09 \quad 5.31 \mathrm{E}-09$ $00310.00 \mathrm{E}+000.00 \mathrm{E}+000.00 \mathrm{E}+000.00 \mathrm{E}+000.00 \mathrm{E}+000.00 \mathrm{E}+000.00 \mathrm{E}+000.00 \mathrm{E}+000.00 \mathrm{E}+000.00 \mathrm{E}+000.00 \mathrm{E}+000.00 \mathrm{E}+000.00 \mathrm{E}+000.00 \mathrm{E}+000.00 \mathrm{E}+00$ 0032 (0.00E

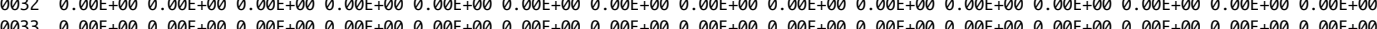
$\begin{array}{llllllll}0 & 0.00 \mathrm{E}+00 & 0.00 \mathrm{E}+00 & 0.00 \mathrm{E}+00 & 0.00 \mathrm{E}+00 & 0.00 \mathrm{E}+00 & 0.00 \mathrm{E}+00 & 0.00 \mathrm{E}+00\end{array}$

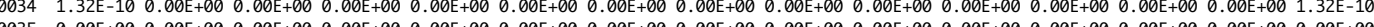
(1) 0036 . (1)

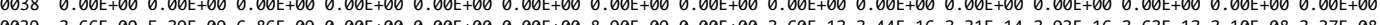
(1)

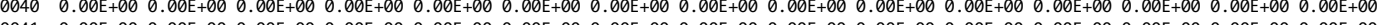

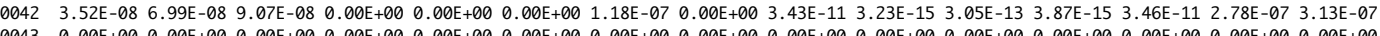

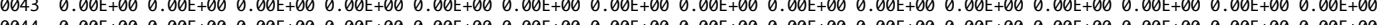

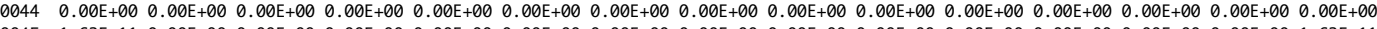
$0045 \quad 1.63 \mathrm{E}-11 \quad 0.00 \mathrm{E}+00 \quad 0.00 \mathrm{E}+00 \quad 0.00 \mathrm{E}+00 \quad 0.00 \mathrm{E}+00 \quad 0.00 \mathrm{E}+00 \quad 0.00 \mathrm{E}+00 \quad 0.00 \mathrm{E}+00 \quad 0.00 \mathrm{E}+00 \quad 0.00 \mathrm{E}+00 \quad 0.00 \mathrm{E}+00 \quad 0.00 \mathrm{E}+00 \quad 0.00 \mathrm{E}+00 \quad 0.00 \mathrm{E}+00 \quad 1.63 \mathrm{E}-11$

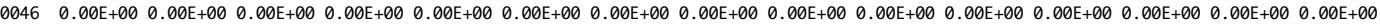

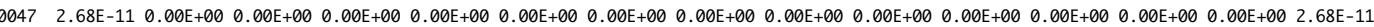

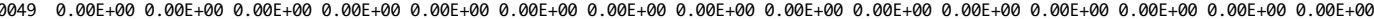
$\begin{array}{llllllllllllllll}0050 & 0.00 \mathrm{E}+00 & 0.00 \mathrm{E}+00 & 0.00 \mathrm{E}+00 & 0.00 \mathrm{E}+00 & 0.00 \mathrm{E}+00 & 0.00 \mathrm{E}+00 & 0.00 \mathrm{E}+00 & 0.00 \mathrm{E}+00 & 0.00 \mathrm{E}+00 & 0.00 \mathrm{E}+00 & 0.00 \mathrm{E}+00 & 0.00 \mathrm{E}+00 & 0.00 \mathrm{E}+00 & 0.00 \mathrm{E}+00 & 0.00 \mathrm{E}+00\end{array}$

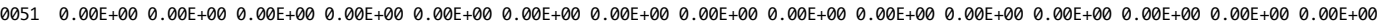
SUM 7.92E-08 7.57E-08 9.86E-08 $\quad 8.80 \mathrm{E}-11 \quad 0.00 \mathrm{E}+00 \quad 0.00 \mathrm{E}+00 \quad 1.28 \mathrm{E}-07 \quad 0.00 \mathrm{E}+00 \quad 1.51 \mathrm{E}-10 \quad 1.50 \mathrm{E}-14 \quad 1.33 \mathrm{E}-12 \quad 1.80 \mathrm{E}-14 \quad 1.53 \mathrm{E}-10 \quad 3.03 \mathrm{E}-07 \quad 3.82 \mathrm{E}-07$

CHEM INHAL DERM SOIL MOTHER FISH WATER VEG DAIRY BEEF CHICK PIG EGG MEAT ORAL TOTAL $\begin{array}{llllllllllllllll}0002 & 2.93 \mathrm{E}-09 & 3.06 \mathrm{E}-09 & 1.00 \mathrm{E}+00 & 0.00 \mathrm{E}+00 & 0.00 \mathrm{E}+00 & 0.00 \mathrm{E}+00 & 0.00 \mathrm{E}+00 & 0.00 \mathrm{E}+00 & 0.00 \mathrm{E}+00 & 0.00 \mathrm{E}+00 & 0.00 \mathrm{E}+00 & 0.00 \mathrm{E}+00 & 0.00 \mathrm{E}+00 & 0.00 \mathrm{E}+00 & 3.30 \mathrm{E}-10 \\ 0.36 \mathrm{E}-09 & 0.00 \mathrm{E}+00 & 0.00 \mathrm{E}+00 & 1.81 \mathrm{E}-09 & 0.00 \mathrm{E}+00 & 9.00 \mathrm{E}-09 & 9.61 \mathrm{E}-13 & 8.35 \mathrm{E}-11 & 1.15 \mathrm{E}-12 & 9.08 \mathrm{E}-09 & 2.26 \mathrm{E}-08 & 2.55 \mathrm{E}-08\end{array}$ $\begin{array}{llllll} & \end{array}$

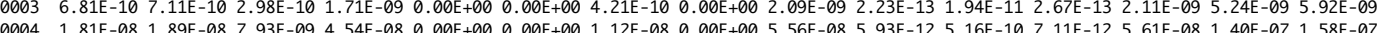

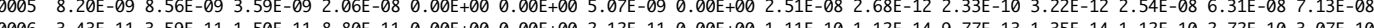
$\begin{array}{llllllll}0.000 & & \end{array}$ 0008 1.29E-15 0.00E

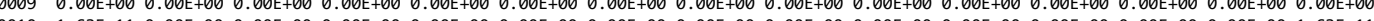
(1) 0011 O..0EE

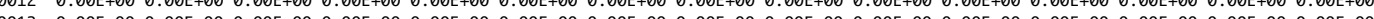

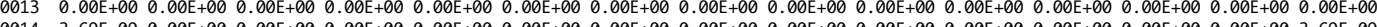

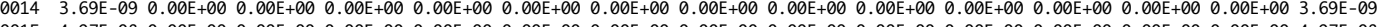

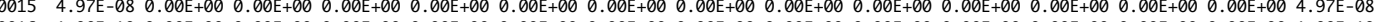

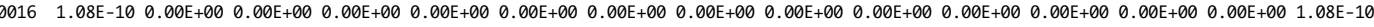
$0017 \quad 3.36 \mathrm{E}-12 \quad 0.00 \mathrm{E}+00 \quad 0.00 \mathrm{E}+00 \quad 0.00 \mathrm{E}+00 \quad 0.00 \mathrm{E}+00 \quad 0.00 \mathrm{E}+00 \quad 0.00 \mathrm{E}+00 \quad 0.00 \mathrm{E}+00 \quad 0.00 \mathrm{E}+00 \quad 0.00 \mathrm{E}+00 \quad 0.00 \mathrm{E}+00 \quad 0.00 \mathrm{E}+00 \quad 0.00 \mathrm{E}+00 \quad 0.00 \mathrm{E}+00 \quad 3.36 \mathrm{E}-12$

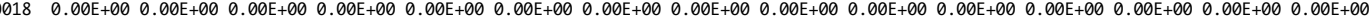
$0019 \quad 0.00 \mathrm{E}+00 \quad 0.00 \mathrm{E}+00 \quad 0.00 \mathrm{E}+00 \quad 0.00 \mathrm{E}+00 \quad 0.00 \mathrm{E}+00 \quad 0.00 \mathrm{E}+00 \quad 0.00 \mathrm{E}+00 \quad 0.00 \mathrm{E}+00 \quad 0.00 \mathrm{E}+00 \quad 0.00 \mathrm{E}+00 \quad 0.00 \mathrm{E}+00 \quad 0.00 \mathrm{E}+00 \quad 0.00 \mathrm{E}+00 \quad 0.00 \mathrm{E}+00 \quad 0.00 \mathrm{E}+00$

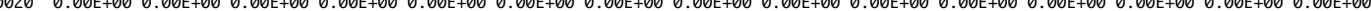

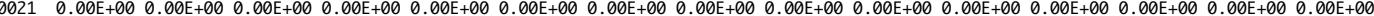

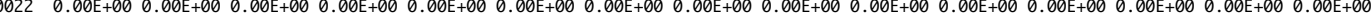

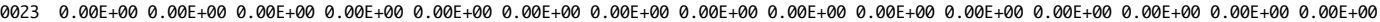

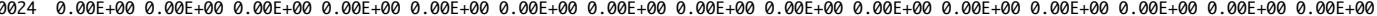

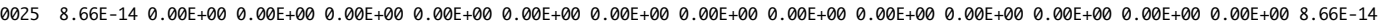
$00260.00 \mathrm{E}+000.00 \mathrm{E}+00 \quad 0.00 \mathrm{E}+00 \quad 0.00 \mathrm{E}+00 \quad 0.00 \mathrm{E}+000.00 \mathrm{E}+000.00 \mathrm{E}+000.00 \mathrm{E}+000.00 \mathrm{E}+00 \quad 0.00 \mathrm{E}+000.00 \mathrm{E}+00 \quad 0.00 \mathrm{E}+000.00 \mathrm{E}+000.00 \mathrm{E}+000.00 \mathrm{E}+00$ $0027 \quad 0.00 \mathrm{E}+00 \quad 0.00 \mathrm{E}+00 \quad 0.00 \mathrm{E}+00 \quad 0.00 \mathrm{E}+00 \quad 0.00 \mathrm{E}+00 \quad 0.00 \mathrm{E}+00 \quad 0.00 \mathrm{E}+00 \quad 0.00 \mathrm{E}+00 \quad 0.00 \mathrm{E}+00 \quad 0.00 \mathrm{E}+00 \quad 0.00 \mathrm{E}+00 \quad 0.00 \mathrm{E}+00 \quad 0.00 \mathrm{E}+00 \quad 0.00 \mathrm{E}+00 \quad 0.00 \mathrm{E}+00$ $00280.00 \mathrm{E}+000.00 \mathrm{E}+00 \quad 0.00 \mathrm{E}+00 \quad 0.00 \mathrm{E}+00 \quad 0.00 \mathrm{E}+00 \quad 0.00 \mathrm{E}+000.00 \mathrm{E}+000.00 \mathrm{E}+00 \quad 0.00 \mathrm{E}+00 \quad 0.00 \mathrm{E}+000.00 \mathrm{E}+000.00 \mathrm{E}+000.00 \mathrm{E}+000.00 \mathrm{E}+000.00 \mathrm{E}+00$ 0029 . $0.00 \mathrm{E}+000.00 \mathrm{E}+00.00 \mathrm{E}+000.00 \mathrm{E}+00.00 \mathrm{E}+000.00 \mathrm{E}+000.00 \mathrm{E}+000.00 \mathrm{E}+000.00 \mathrm{E}+000.00 \mathrm{E}+000.00 \mathrm{E}+000.00 \mathrm{E}+000.00 \mathrm{E}+000.00 \mathrm{E}+000.00 \mathrm{E}+00$

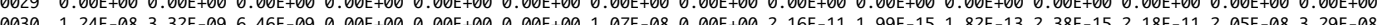
$00301.25 \mathrm{E}$

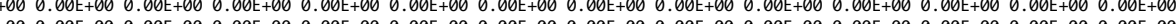

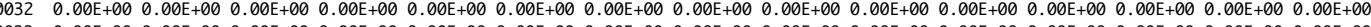
(1)

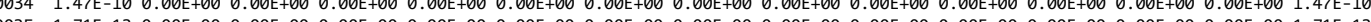
(1) 0036 D.VOE

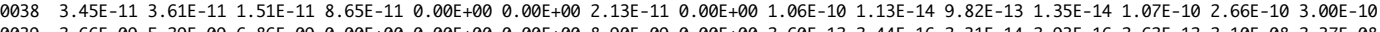

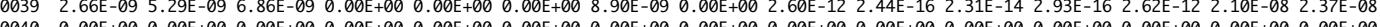

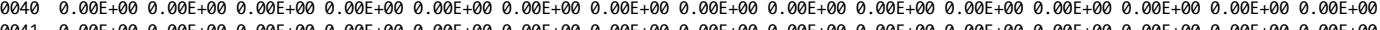

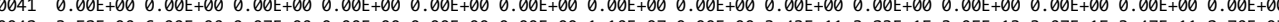

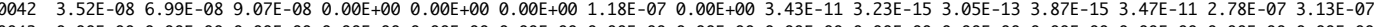
$0043 \quad 0.00 \mathrm{E}+00 \quad 0.00 \mathrm{E}+00 \quad 0.00 \mathrm{E}+00 \quad 0.00 \mathrm{E}+00 \quad 0.00 \mathrm{E}+00 \quad 0.00 \mathrm{E}+00 \quad 0.00 \mathrm{E}+00 \quad 0.00 \mathrm{E}+00 \quad 0.00 \mathrm{E}+00 \quad 0.00 \mathrm{E}+00 \quad 0.00 \mathrm{E}+00 \quad 0.00 \mathrm{E}+00 \quad 0.00 \mathrm{E}+00 \quad 0.00 \mathrm{E}+00 \quad 0.00 \mathrm{E}+00$ $0044 \quad 0.00 \mathrm{E}+00 \quad 0.00 \mathrm{E}+00 \quad 0.00 \mathrm{E}+00 \quad 0.00 \mathrm{E}+00 \quad 0.00 \mathrm{E}+00 \quad 0.00 \mathrm{E}+00 \quad 0.00 \mathrm{E}+00 \quad 0.00 \mathrm{E}+00 \quad 0.00 \mathrm{E}+00 \quad 0.00 \mathrm{E}+00 \quad 0.00 \mathrm{E}+00 \quad 0.00 \mathrm{E}+00 \quad 0.00 \mathrm{E}+00 \quad 0.00 \mathrm{E}+00 \quad 0.00 \mathrm{E}+00$ $0045 \quad 1.87 \mathrm{E}-11 \quad 0.00 \mathrm{E}+00 \quad 0.00 \mathrm{E}+00 \quad 0.00 \mathrm{E}+00 \quad 0.00 \mathrm{E}+00 \quad 0.00 \mathrm{E}+00 \quad 0.00 \mathrm{E}+00 \quad 0.00 \mathrm{E}+00 \quad 0.00 \mathrm{E}+00 \quad 0.00 \mathrm{E}+00 \quad 0.00 \mathrm{E}+00 \quad 0.00 \mathrm{E}+00 \quad 0.00 \mathrm{E}+00 \quad 0.00 \mathrm{E}+00 \quad 1.87 \mathrm{E}-11$

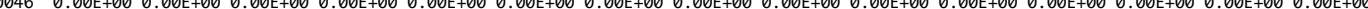

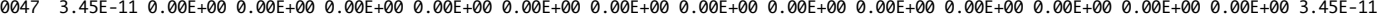

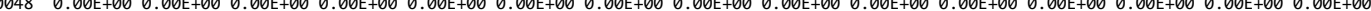
$0049 \quad 1.27 \mathrm{E}-09 \quad 0.00 \mathrm{E}+00 \quad 0.00 \mathrm{E}+00 \quad 0.00 \mathrm{E}+00 \quad 0.00 \mathrm{E}+00 \quad 0.00 \mathrm{E}+00 \quad 0.00 \mathrm{E}+00 \quad 0.00 \mathrm{E}+00 \quad 0.00 \mathrm{E}+00 \quad 0.00 \mathrm{E}+00 \quad 0.00 \mathrm{E}+00 \quad 0.00 \mathrm{E}+00 \quad 0.00 \mathrm{E}+00 \quad 0.00 \mathrm{E}+00 \quad 1.27 \mathrm{E}-09$

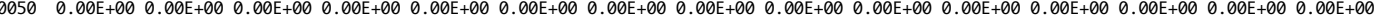

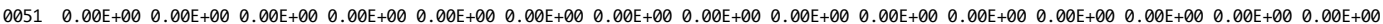
SUM $1.36 \mathrm{E}-07 \quad 1.10 \mathrm{E}-07 \quad 1.17 \mathrm{E}-07 \quad 7.52 \mathrm{E}-08 \quad 0.00 \mathrm{E}+00 \quad 0.00 \mathrm{E}+00 \quad 1.56 \mathrm{E}-07 \quad 0.00 \mathrm{E}+00 \quad 9.21 \mathrm{E}-08 \quad 9.83 \mathrm{E}-12 \quad 8.54 \mathrm{E}-10 \quad 1.18 \mathrm{E}-11 \quad 9.29 \mathrm{E}-08 \quad 5.51 \mathrm{E}-07 \quad 6.87 \mathrm{E}-07$ 
Rep_Can_30yr_Hi_Rec2_AllSrc_AllCh_bySrc_site.txt 
This file: c: \HARPExpress $\backslash$ Projects $\backslash$ Rep_Can_30yr_Hi_Rec2_AllSrc_AllCh_BySrc_Site.txt

Created by HARP Version 1.0 Build 23.02.10

Uses ISC Version 9915

Uses BPIP Version 95086

Creation date: 10/24/2005 2:14:20 PM

EXCEPTION REPORT

(there have been no changes or exceptions)

INPUT FILES:

Source-Receptor file: c: \HARPExpress \Projects $\backslash E W T F . m t a$

Averaging period adjustment factors file: not applicable

Emission rates file: none

Site parameters file: C:\HARPExpress \Projects $\backslash$ Site300. sit

Screening mode is OFF

Exposure duration: 30 year (adult resident)

Analysis method: High-end Point Estimate

Health effect: Cancer Risk

Receptor(s):

Sources(s):

Chemicals(s):

All

SITE PARAMETERS

DEPOSITION

Deposition rate $(\mathrm{m} / \mathrm{s})$

0.0072

DRINKING WATER

*** Pathway disabled ***

FISH

*** Pathway disabled ***

PASTURE

ANIMALS' WATER

Water surface area $(\mathrm{m} \wedge 2)$

Water surface area

Volume changes per yea

Volume changes per year

from pasture source water

from pasture source

raction of dairy cows' water

NIMALS' FEED

Fraction of cows' feed

from grazing

HUMAN INGESTION

Fraction of ingested beef

from contaminated source

Fraction of ingested dairy

from contaminated source

0.107

HOME GROWN PRODUCE

HUMAN INGESTION

Fraction of ingested leafy vegetable

from home grown source $\quad 0.082$

Fraction of ingested exposed vegetable

from home grown source 0.207

Fraction of ingested protected vegetable

from home grown source 0.134

Fraction of ingested root vegetable

PIGS, CHICKENS AND EGGS

HUMAN INGESTION
Fraction of ingested pig
$\quad$ from home grown source
Fraction of ingested chicken
$\quad$ from home grown source
Fraction of ingested egg
$\quad$ from home grown source




\begin{tabular}{|c|c|}
\hline $\begin{array}{l}\text { leafy vegetable } \\
\text { Fraction of feed that is } \\
\text { protected vegetable }\end{array}$ & 0.25 \\
\hline $\begin{array}{l}\text { Fraction of feed that is } \\
\text { root vegetable }\end{array}$ & 0.25 \\
\hline $\begin{array}{l}\text { CHICKEN FEED COMPOSITION } \\
\text { Fraction of feed that is } \\
\text { exposed vegetable }\end{array}$ & 0.25 \\
\hline $\begin{array}{l}\text { Fraction of feed that is } \\
\text { leafy vegetable }\end{array}$ & 0.25 \\
\hline $\begin{array}{l}\text { Fraction of feed that is } \\
\text { protected vegetable } \\
\text { Fraction of feed that is }\end{array}$ & 0.25 \\
\hline root vegetable & 0.25 \\
\hline
\end{tabular}

DERMAL ABSORPTION

*** Pathway enabled $* * *$

SOIL INGESTION

*** Pathway enabled ***

MOTHER'S MILK

*** Pathway enabled ***

CHEMICAL CROSS-REFERENCE TABLE AND BACKGROUND CONCENTRATIONS

0001106990 1,3-Butadiene 1,3-Butadiene

1-4,6-8HpCDF $1,2,3,4,6,7,8$-Heptachlorodibenzofuran

$1,2,3,4,7,8,9$-Heptachlorodibenzofuran

$000470648269 \quad 1-4,7,8 \mathrm{HxCDF}$

$000557117449 \quad 1-3,6-8 \mathrm{HxCDF} \quad 1,2,3,6,7,8$-Hexachlorodibenzofuran

$000635822469 \quad 1-4,6-8 \mathrm{HpCDD} \quad 1,2,3,4,6,7,8$-Heptachlorodibenzo-p-dioxin

0007121142 2,4-DiNitToluen 2,4-Dinitrotoluene

0008606202 2,6-DiNitToluen 2,6-Dinitrotoluen

000995578 2-CHLOROPHENOL 2-CHLOROPHENOL

0010107051 AllylChlor Allyl chloride

00117429905 Aluminum Aluminum

00127440360 Antimony Antimony

$\begin{array}{llll}0013 & 7440393 & \text { Barium } & \text { Barium } \\ 0014 & 71432 & \text { Benzene } & \text { Benzene }\end{array}$

$001471432 \quad$ Benzene Benzene

$\begin{array}{llll}0015 & 7440439 & \text { Cadmium } & \text { Cadmium } \\ 0016 & 56235 & \text { CCl4 } & \text { Carbon tetrachloride }\end{array}$

$001656235 \quad$ CCl4 $\quad$ Carbon tetrac

$\begin{array}{llll}0017 & 67663 & \text { Chloroform } & \text { Chlorofor } \\ 0018 & 7440473 & \text { Chromium } & \text { Chromium }\end{array}$

00197782505 Chlorine Chlorine

0020630080 Carbon Monoxide Carbon monoxide

$00217440508 \quad$ Copper

0022110827 Cyclohexane Cyclohexane

002384662 DiethylPhthalat Diethyl phthalate

0024122394 Diphenylam Diphenylamine

$\begin{array}{llll}0025 & 75003 & \text { Ethyl Chloride Ethyl chloride \{Chlorethane\} } \\ 0026 & 100414 & \text { Ethyl Benzene Ethyl benzene }\end{array}$

$0027206440 \quad$ Fluoranthene Fluoranthene

$00287647010 \quad \mathrm{HCl} \quad$ Hydrochloric acid

$\begin{array}{llll}0029 & 98828 & \text { Cumene } & \text { Cumene } \\ 0030 & 7439921 & \text { Lead } & \text { Lead }\end{array}$

$\begin{array}{llll}0030 & 7439921 & \text { Lead } & \text { Lead } \\ 0031 & 74873 & \text { Methyl Chloride Methyl chloride \{Chloromethane\} }\end{array}$

003271556 1,1,1-TCA Methyl chloroform $\{1,1,1-\mathrm{TCA}\}$

$0033108872 \quad$ MethCyHex Methylcyclohexane

003475092 Methylene Chlor Methylene chloride \{Dichloromethane\}

003591203 Naphthalene Naphthalene

0036110543 Hexane

003710102440 NITROGEN DIOXID NITROGEN DIOXIDE

003839001020 1-80ctaCDF $1,2,3,4,6,7,8,9$-0ctachlorodibenzofuran

003978115 PETN

0040108952 Phenol Phenol

$0041115071 \quad$ Propylene Propylene

0042121824 RDX $\quad$ RDX

$\begin{array}{llll}0043 & 100425 & \text { Styrene } & \text { Styrene } \\ 0044 & 7446095 & \text { SULFUR DIOXIDE } & \text { SULFUR DIOXIDE }\end{array}$

$\begin{array}{llll}0044 & 7446095 & \text { SULFUR DIOXIDE } & \text { SULFUR DIOXIDE } \\ 0045 & 127184 & \text { Perc } & \text { Perchloroethylene } \text { \{Tetrachloroethene }\end{array}$

$\begin{array}{llll}0045 & 127184 & \text { Perc } & \text { Perchlor } \\ 0046 & 108883 & \text { Toluene } & \text { Toluene }\end{array}$

$\begin{array}{llll}0046 & 108883 & \text { Toluene } & \text { Toluene } \\ 0047 & 75014 & \text { Vinyl Chloride } & \text { Vinyl chloride }\end{array}$

00487440666 Zinc Zinc

004910101 NapthSur Naphthalene surrogate

005010102 EthBzSur Ethylbenzene surrogat

$n$-Hexane surrogate

BACKGROUND $(\mathrm{ug} / \mathrm{m} \wedge 3)$

$0.000 \mathrm{E}+00$

$0.000 \mathrm{E}+00$

$0.000 \mathrm{E}+00$

$0.000 \mathrm{E}+00$

$0.000 \mathrm{E}+00$

$0.000 \mathrm{E}+00$

$0.000 \mathrm{E}+00$

$0.000 \mathrm{E}+00$

$0.000 \mathrm{E}+00$

$0.000 \mathrm{E}+00$

$0.000 \mathrm{E}+00$

$0.000 \mathrm{E}+00$

$0.000 \mathrm{E}+00$

$0.000 \mathrm{E}+00$

$0.000 \mathrm{E}+00$

$0.000 \mathrm{E}+00$

$0.000 \mathrm{E}+00$

$0.000 E+00$

$0.000 E+00$

$0.000 E+00$

$0.000 \mathrm{E}+00$

$0.000 \mathrm{E}+00$

$0.000 \mathrm{E}+00$

$0.000 \mathrm{E}+00$

$0.000 \mathrm{E}+00$

$0.000 \mathrm{E}+00$

$0.000 \mathrm{E}+00$

$0.000 \mathrm{E}+00$

$0.000 \mathrm{E}+00$

$0.000 E+00$

$0.000 \mathrm{E}+00$

$0.000 \mathrm{E}+00$

$0.000 \mathrm{E}+00$

$0.000 \mathrm{E}+00$

$0.000 \mathrm{E}+00$

$0.000 \mathrm{E}+00$

$0.000 E+00$

$0.000 \mathrm{E}+00$

$0.000 \mathrm{E}+00$

$0.000 E+00$

$0.000 \mathrm{E}+00$

$0.000 \mathrm{E}+00$

$0.000 E+00$

$0.000 \mathrm{E}+00$

$0.000 E+00$

$0.000 E+00$

$0.000 \mathrm{E}+00$

$0.000 \mathrm{E}+00$

$0.000 E+00$

$0.000 \mathrm{E}+00$

$0.000 \mathrm{E}+00$

$0.000 \mathrm{E}+00$
$0.000 \mathrm{E}+00$

EMISSIONS DATA SOURCE:

CHEMICALS ADDED OR DELETED: none

\begin{tabular}{|c|c|}
\hline $\begin{array}{l}\text { EMISSION } \\
\text { SOURCE MM }\end{array}$ & $\begin{array}{l}\text { ACILITY FA } \\
E R=1\end{array}$ \\
\hline CAS & ABBREV \\
\hline 106990 & 1,3-Butadiene \\
\hline 67562394 & $1-4,6-8 \mathrm{HpCDF}$ \\
\hline 55673897 & $1-4,7-9 \mathrm{Hp} C \mathrm{CDF}$ \\
\hline 70648269 & $1-4,7,8 \mathrm{HxCDF}$ \\
\hline 57117449 & $1-3,6-8 \mathrm{H} \times \mathrm{CDF}$ \\
\hline 35822469 & $1-4,6-8 \mathrm{HpCDD}$ \\
\hline 121142 & 2,4-DiNitToluen \\
\hline 606202 & 2,6-DiNitToluen \\
\hline 95578 & 2-CHLOROPHENOL \\
\hline & Allyl \\
\hline
\end{tabular}

$C 0=* \quad$ DEV $=P R 1 \quad P R 0=0 B P a n \quad S T K=1 \quad$ NAME $=L L N L$ Site $300 \quad$ EMS $(l b s / y r)$

MULTIPLIER

BG (ug/m^3) AVRG (lbs/yr)

$\operatorname{MAX}(\mathrm{lbs} / \mathrm{hr})$

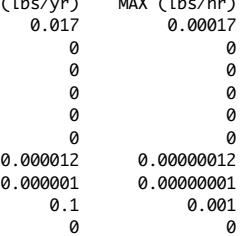




\begin{tabular}{ll}
7429905 & Aluminum \\
7440360 & Antimony \\
7440393 & Barium \\
71432 & Benzene \\
7440439 & Cadmium \\
56235 & CCl4 \\
67663 & Chloroform \\
7440473 & Chromium \\
7782505 & Chlorine \\
630080 & Carbon Monoxide \\
7440508 & Copper \\
110827 & Cyclohexane \\
84662 & DiethylPhthalat \\
122394 & Diphenylam \\
75003 & Ethyl Chloride \\
100414 & Ethyl Benzene \\
206440 & Fluoranthene \\
7647010 & HCl \\
98828 & Cumene \\
7439921 & Lead \\
74873 & Methyl Chloride \\
71556 & 1,1,1-TCA \\
108872 & MethCyHex \\
75092 & Methylene Chlor \\
91203 & Naphthalene \\
110543 & Hexane \\
10102440 & NITROGEN DIOXID \\
39001020 & 1-80ctaCDF \\
78115 & PETN \\
108952 & Phenol \\
115071 & Propylene \\
121824 & RDX \\
100425 & Styrene \\
7446095 & SULFUR DIOXIDE \\
127184 & Perc \\
108883 & Toluene \\
75014 & Vinyl Chloride \\
7440666 & Zinc \\
10101 & NapthSur \\
10102 & EthBzSur \\
10103 & nHexSur \\
& \\
\hline
\end{tabular}

$\begin{array}{rrrr}1 & 0 & 110 & 1.1 \\ 1 & 0 & 0.0067 & 0.000067 \\ 1 & 0 & 82 & 0.82 \\ 1 & 0 & 1.2 & 0.012 \\ 1 & 0 & 0.4 & 0.004 \\ 1 & 0 & 0.011 & 0.00011 \\ 1 & 0 & 0.0042 & 0.000042 \\ 1 & 0 & 0.48 & 0.0048 \\ 1 & 0 & 92 & 0.92 \\ 1 & 0 & 720 & 7.2 \\ 1 & 0 & 370 & 3.7 \\ 1 & 0 & 0.016 & 0.00016 \\ 1 & 0 & 0 & 0 \\ 1 & 0 & 0.0000026 & 0.000000026 \\ 1 & 0 & 0 & 0 \\ 1 & 0 & 0.012 & 0.00012 \\ 1 & 0 & 0 & 0 \\ 1 & 0 & 2150 & 21.5 \\ 1 & 0 & 0 & 0 \\ 1 & 0 & 120 & 1.2 \\ 1 & 0 & 0.057 & 0.00057 \\ 1 & 0 & 0 & 0 \\ 1 & 0 & 0.051 & 0.00051 \\ 1 & 0 & 1.8 & 0.018 \\ 1 & 0 & 0.00075 & 0.0000075 \\ 1 & 0 & 0.19 & 0.0019 \\ 1 & 0 & 52 & 0.52 \\ 1 & 0 & 0 & 0 \\ 1 & 0 & 0 & 0 \\ 1 & 0 & 0.0000343 & 0.000000343 \\ 1 & 0 & 0.072 & 0.00072 \\ 1 & 0 & 0.096 & 0.00096 \\ 1 & 0 & 0.015 & 0.00015 \\ 1 & 0 & 32 & 0.32 \\ 1 & 0 & 0 & 0 \\ 1 & 0 & 0.086 & 0.00086 \\ 1 & 0 & 0.015 & 0.00015 \\ 1 & 0 & 0.4 & 0.004 \\ 1 & 0 & 0.0000014 & 0.000000014 \\ 1 & 0 & 0.091 & 0.00091 \\ 1 & 0 & 89.4 & 0.894 \\ & 0 & & \end{array}$

EMISSIONS FOR FACILITY FAC $=$ Site300 SOURCE MULTIPLIER $=1$

ABBREV

67562394 1,3-Butadiene

1-4,6-8HpCDF

$57117449-1-4,7,8 \mathrm{HXCDF}$

$35822469 \quad 1-4,6-8 \mathrm{HpCDD}$

$\begin{array}{ll}121142 & \text { 2,4-DiNitToluen } \\ 606202 & \text { 2,6-DiNitToluen }\end{array}$

$\begin{array}{ll}606202 & \text { 2,6-DiNitToluen } \\ 95578 & \text { 2-CHLOROPHENOL }\end{array}$

107051 AllylChlor

7429905

7440360 Antimony

$\begin{array}{ll}7440393 & \text { Barium } \\ 71432 & \text { Benzene }\end{array}$

$7440439 \quad$ Cadmium

56235

$67663 \quad$ Chloroform

$7440473 \quad$ Chromium

630080 Carbon Monoxide

$7440508 \quad$ Copper

110827 Cyclohexane

$122394 \quad$ Diphenylam

$75003 \quad$ Ethyl Chloride

$100414 \quad$ Ethyl Benzene

206440 Fluoranthene

$7647010 \quad \mathrm{HCl}$

$98828 \quad$ Cumene

$7439921 \quad$ Methyl Chloride

715561 1,1,1-TCA

$\begin{array}{ll}108872 & \text { MethCyHex } \\ 75092 & \text { Methylene Chlor }\end{array}$

75092

$\begin{array}{ll}91203 & \text { Naphthal } \\ 110543 & \text { Hexane }\end{array}$

10102440 NITROGEN DIOXID

39001020 1-80ctaCDF

$\begin{array}{ll}78115 & \text { PETN } \\ 108952 & \text { Phenol }\end{array}$

115071 Propylene

$\begin{array}{ll}121824 & \text { RDX } \\ 100425 & \text { Styrene }\end{array}$

7446095 SULFUR DIOXIDE

$127184 \quad$ Perc

$108883 \quad$ Toluene

$75014 \quad$ Viny

$10101 \quad$ NapthSur

$\begin{array}{ll}10102 & \text { EthBzSur } \\ 10103 & \text { nHexSur }\end{array}$

MULTIPLIER BG $\left(u g / \mathrm{m}^{\wedge} 3\right)$

STK $=1 \quad$ NAME $=$ LLNL Site 300 EMS (lbs/yr)

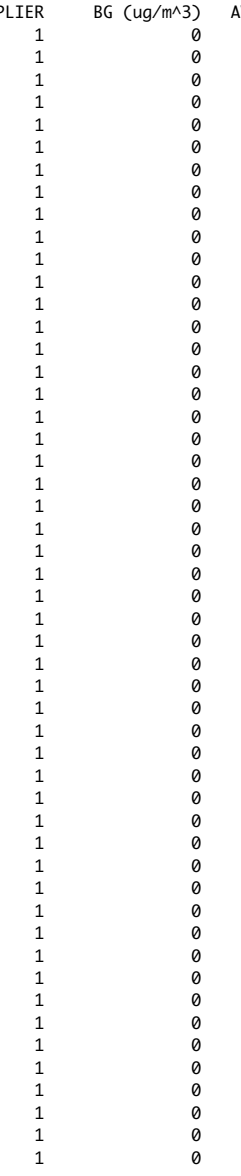

AVRG (lbs/yr) MAX (lbs/hr)

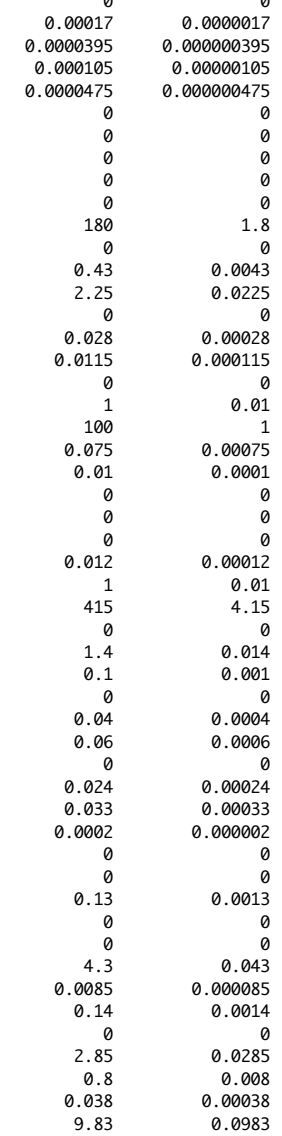

CAS ABBREV

MULTIPLIER

$\mathrm{PRO}=0 \mathrm{BCage} 4$

STK=1 NAME=LLNL Site 300 EMS (lbs/yr) 


\begin{tabular}{|c|c|}
\hline 106990 & 1,3-Butadiene \\
\hline 67562394 & $1-4,6-8 \mathrm{HpCDF}$ \\
\hline 55673897 & $1-4,7-9 \mathrm{HрCDF}$ \\
\hline 70648269 & $1-4,7,8 \mathrm{H} \times \mathrm{CDF}$ \\
\hline 57117449 & $1-3,6-8 \mathrm{H} \times \mathrm{CDF}$ \\
\hline 35822469 & $1-4,6-8 \mathrm{HpCDD}$ \\
\hline 121142 & 2,4-DiNitToluen \\
\hline 606202 & 2,6-DiNitToluen \\
\hline 95578 & 2-CHLOROPHENOL \\
\hline 107051 & AllylChlor \\
\hline 7429905 & Aluminum \\
\hline 7440360 & Antimony \\
\hline 7440393 & Barium \\
\hline 71432 & Benzene \\
\hline 7440439 & Cadmium \\
\hline 56235 & CCl4 \\
\hline 67663 & Chloroform \\
\hline 7440473 & Chromium \\
\hline 7782505 & Chlorine \\
\hline 630080 & Carbon Monoxide \\
\hline 7440508 & Copper \\
\hline 110827 & Cyclohexane \\
\hline 84662 & DiethylPhthalat \\
\hline 122394 & Diphenylam \\
\hline 75003 & Ethyl Chloride \\
\hline 100414 & Ethyl Benzene \\
\hline 206440 & Fluoranthene \\
\hline 7647010 & $\mathrm{HCl}$ \\
\hline 98828 & Cumene \\
\hline 7439921 & Lead \\
\hline 74873 & Methyl Chloride \\
\hline 71556 & $1,1,1$-TCA \\
\hline 108872 & MethCyHex \\
\hline 75092 & Methylene Chlor \\
\hline 91203 & Naphthalene \\
\hline 110543 & Hexane \\
\hline 10102440 & NITROGEN DIOXID \\
\hline 39001020 & 1-80ctaCDF \\
\hline 78115 & PETN \\
\hline 108952 & Phenol \\
\hline 115071 & Propylene \\
\hline 121824 & $\mathrm{RDX}$ \\
\hline 100425 & Styrene \\
\hline 7446095 & SULFUR DIOXIDE \\
\hline 127184 & Perc \\
\hline 108883 & Toluene \\
\hline 75014 & Vinyl Chloride \\
\hline 7440666 & Zinc \\
\hline 10101 & NapthSur \\
\hline 10102 & EthBzSur \\
\hline 10103 & nHexSur \\
\hline
\end{tabular}

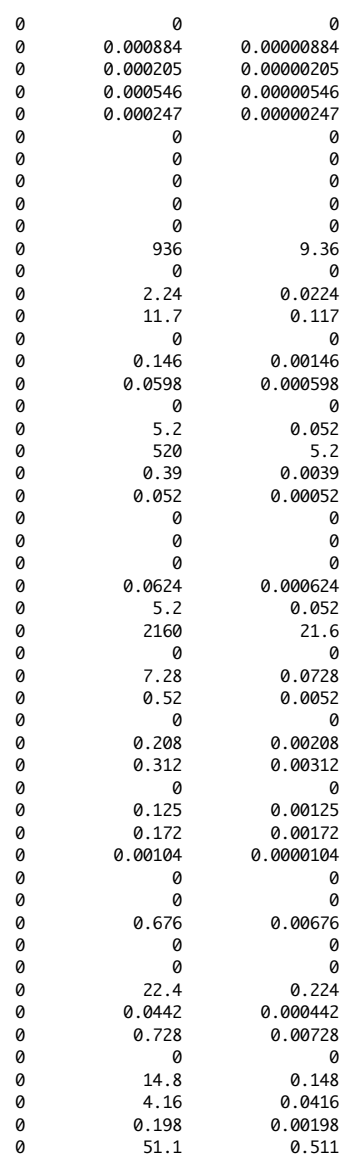

EMISSIONS FOR FACILITY FAC $=$ Site 300 SOURCE MULTIPLIER=1

$\begin{array}{ll}106990 & 1,3-\text { Butadiene } \\ 67562394 & 1-4,6-8 \mathrm{HpCDF}\end{array}$

$55673897 \quad 1-4,7-9 \mathrm{HpCDF}$

$70648269 \quad 1-4,7,8 \mathrm{HxCDF}$

$\begin{array}{ll}57117449 & 1-3,6-8 \mathrm{H} \times \mathrm{CDF} \\ 35822469 & 1-4,6-8 \mathrm{HpCDD}\end{array}$

121142 2,4-DiNitToluen

606202 2,6-DiNitToluen

95578 2-CHLOROPHENOL

107051 AllylChlor

$7429905 \quad$ Aluminum

7440360 Antimony

71432 Barium

$7440439 \quad$ Cadmium

$56235 \quad$ CCl4

$67663 \quad$ Chloroform

$7440473 \quad$ Chromium

630080 Carbon Monoxide

$7440508 \quad$ Copper

$110827 \quad$ Cyclohexane

84662 DiethylPhthalat

122394 Diphenylam

75003 Ethyl Chloride

100414 Ethyl Benzen

$206440 \quad$ Fluoranthene

$7647010 \quad \mathrm{HCl}$

$98828 \quad$ Cumene

$7439921 \quad$ Lead

74873 Methyl Chlor

$\begin{array}{ll}71556 & 1,1,1-\text { TCA } \\ 108872 & \text { MethCyHex }\end{array}$

75092 Methylene Chlo

91203 Naphthalen

110543

$10102440 \quad$ NITROGEN DIOXID

$39001020 \quad 1-80$ ctaCDF

78115 PETN

115071 Propylene

121824 RDX

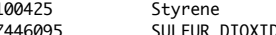

$127184 \quad$ Perc

$C O=* \quad$ DEV $=P R 1 \quad P R 0=0 D \quad S T K=1 \quad$ NAME $=L L N L$ Site 300 EMS (lbs/yr)

MULTIPLIER

$B G(u g / m \wedge 3)$
RRG (lbs/yr)

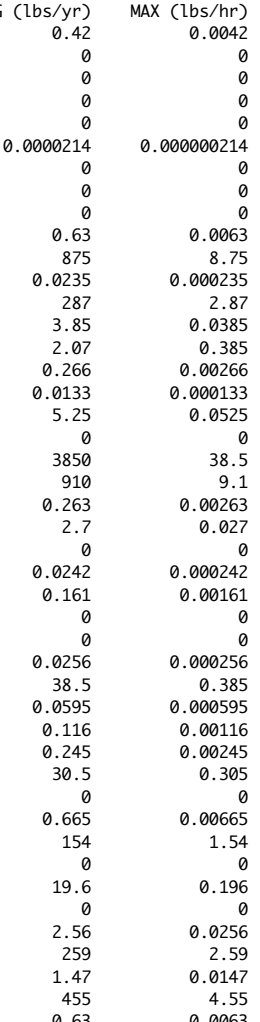




$\begin{array}{llllrr}108883 & \text { Toluene } & 1 & 0 & 1.05 & 0.0105 \\ 75014 & \text { Vinyl Chloride } & 1 & 0 & 0.0805 & 0.000805 \\ 7440666 & \text { Zinc } & 1 & 0 & 560 & 5.6 \\ 10101 & \text { NapthSur } & 1 & 0 & 0 & 0 \\ 10102 & \text { EthBzSur } & 1 & 0 & 0.62 & 0.0062 \\ 10103 & \text { nHexSur } & 1 & 0 & 119 & 1.19\end{array}$

CANCER RISK REPORT

RECEPTOR 2

RISK BY PATHWAY

SRC INHAL DERM SOIL MOTHER FISH WATER VEG DAIRY BEEF CHICK PIG EGG MEAT ORAL TOTAL

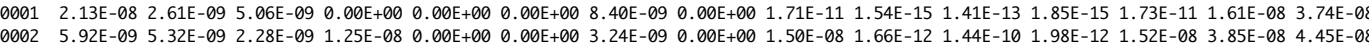

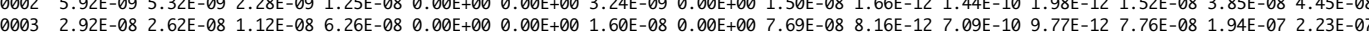

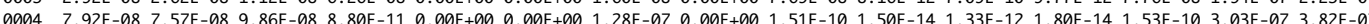

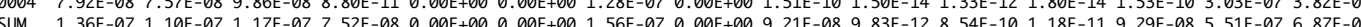


Rep_Chr_Res_Hi_Rec2_AllSrc_AllCh_BySrc_Site.txt 
This file: c: \HARPExpress $\backslash$ Projects $\backslash$ Rep_Chr_Res_Hi_Rec2_AllSrc_AllCh_BySrc_Site.txt

Created by HARP Version 1.0 Build 23.02.10

Uses ISC Version 99155

Uses BPIP Version 95086

Creation date: 10/24/2005 2:21:13 PM

EXCEPTION REPORT

(there have been no changes or exceptions)

INPUT FILES:

Source-Receptor file: c: \HARPExpress \Projects $\backslash$ EWTF.mta

Source-Receptor file: c: A
Averaging period adjustment factors file: not applicable

Emission rates file: none

Site parameters file: C:\HARPExpress \Projects \Site300.sit

Screening mode is OFF

Exposure duration: resident

Analysis method: High-end Point Estimat

Health effect: Chronic HI

Receptor(s): $\quad 2$

Sources(s): $\quad$ All

SITE PARAMETERS

DEPOSITION

Deposition rate $(\mathrm{m} / \mathrm{s})$

0.0072

DRINKING WATER

*** Pathway disabled ***

FISH

*** Pathway disabled $* * *$

PASTURE

ANIMALS' WATER

Water surface area $(m \wedge 2)$
Water volume $(L)$

Volume changes per year 0

Fraction of beef cows' water
from pasture source

Fraction of dairy cows' water
from pasture source

ANIMALS' FEED

Fraction of cows' feed
from grazing

HUMAN INGESTION

Fraction of ingested beef

from contaminated source $\quad 0.107$

Fraction of ingested dairy

from contaminated source

HOME GROWN PRODUCE

HUMAN INGESTION

Fraction of ingested leafy vegetable from home grown source $\quad 0.082$ Fraction of ingested exposed vegetable Fraction of ingested protected vegetable from home grown source 0.134 Fraction of ingested root vegetable

from home grown source 0.088

PIGS, CHICKENS AND EGGS

HUMAN INGESTION

Fraction of ingested pig

from home grown source

raction of ingested chicken

0.04

from home grown source
Fraction of ingested egg

0.026

from home grown source

0.029

ANIMALS' FEED

Fraction of pigs' feed

from home grown crop

Fraction of chickens' feed

from home grown crop

0.05

SOIL INGESTION

Fraction of pigs' feed

eaten off the ground

Fraction of chickens' feed

eaten off the ground

PIG FEED COMPOSITION

Fraction of feed that is 


\begin{tabular}{|c|c|}
\hline exposed vegetable & 0.25 \\
\hline $\begin{array}{l}\text { Fraction of feed that is } \\
\text { leafy vegetable }\end{array}$ & 0.25 \\
\hline $\begin{array}{l}\text { Fraction of feed that is } \\
\text { protected vegetable }\end{array}$ & 0.25 \\
\hline $\begin{array}{l}\text { Fraction of feed that is } \\
\text { root vegetable }\end{array}$ & 0.25 \\
\hline CHICKEN FEED COMPOSITION & \\
\hline Fraction of feed that is & 0.25 \\
\hline $\begin{array}{l}\text { Fraction of feed that is } \\
\text { leafy vegetable }\end{array}$ & 0.25 \\
\hline $\begin{array}{l}\text { Fraction of feed that is } \\
\text { protected vegetable }\end{array}$ & 0.25 \\
\hline $\begin{array}{l}\text { Fraction of feed that is } \\
\text { root vegetable }\end{array}$ & 0.25 \\
\hline
\end{tabular}

DERMAL ABSORPTION

*** Pathway enabled ***

SOIL INGESTION

*** Pathway enabled ***

MOTHER'S MILK

*** Pathway enabled ***

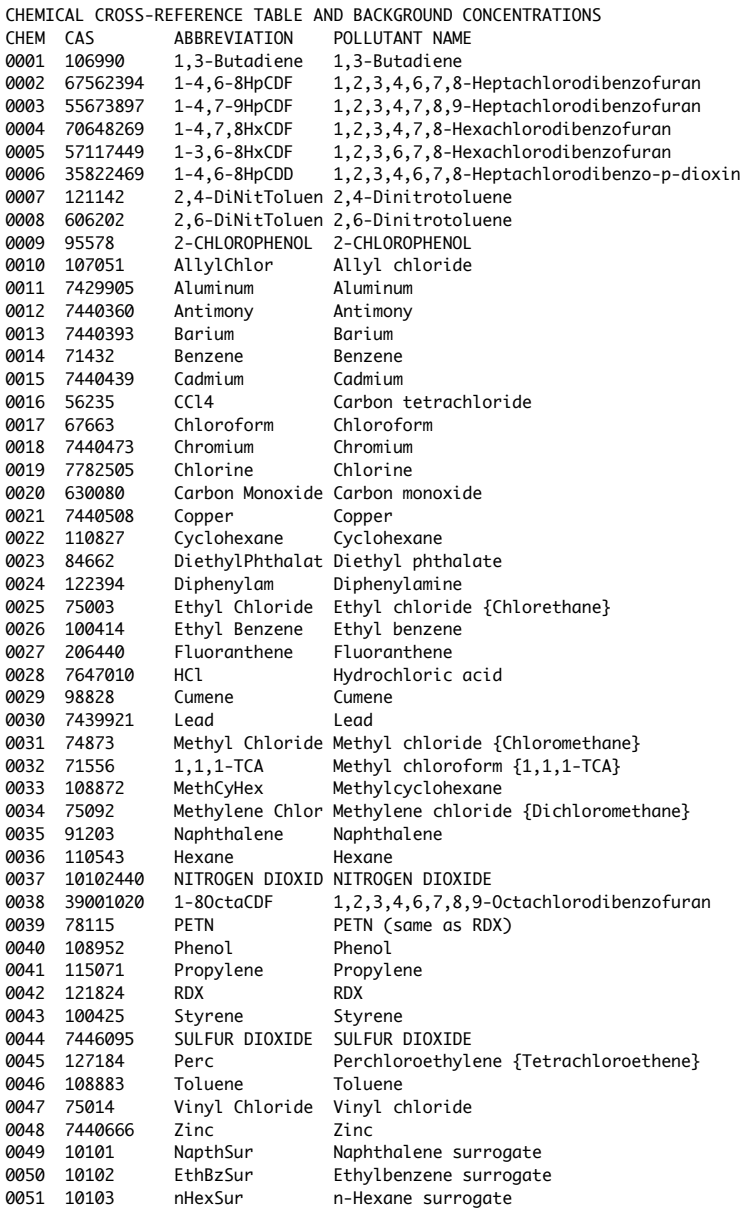

BACKGROUND ( $\left.\mathrm{ug} / \mathrm{m}^{\wedge} 3\right)$

$0.000 \mathrm{E}+00$

$.000 \mathrm{E}+00$

$0.000 \mathrm{E}+00$

$0.000 E+00$

$0.000 \mathrm{E}+00$

$0.000 \mathrm{E}+00$

$0.000 \mathrm{E}+00$

$0.000 \mathrm{E}+00$

$0.000 \mathrm{E}+00$

$0.000 \mathrm{E}+00$

$0.000 \mathrm{E}+00$

$0.000 \mathrm{E}+00$

$0.000 \mathrm{E}+00$

$0.000 \mathrm{E}+00$

$0.000 \mathrm{E}+00$

$0.000 \mathrm{E}+00$

$0.000 \mathrm{E}+00$

$0.000 \mathrm{E}+00$

$0.000 \mathrm{E}+00$

$0.000 \mathrm{E}+00$

$0.000 \mathrm{E}+00$

$0.000 E+00$

$0.000 E+00$

$0.000 E+00$

$0.000 \mathrm{E}+00$

$0.000 \mathrm{E}+00$

$0.000 \mathrm{E}+00$

$0.000 \mathrm{E}+00$

$0.000 \mathrm{E}+00$

$0.000 \mathrm{E}+00$

$0.000 \mathrm{E}+00$

$0.000 \mathrm{E}+00$

$0.000 \mathrm{E}+00$

$0.000 \mathrm{E}+00$

$0.000 \mathrm{E}+00$

$0.000 \mathrm{E}+00$

$0.000 \mathrm{E}+00$

$0.000 \mathrm{E}+00$

$0.000 \mathrm{E}+00$

$0.000 \mathrm{E}+00$

$0.000 \mathrm{E}+00$

$0.000 \mathrm{E}+00$

$0.000 E+00$

$0.000 \mathrm{E}+00$

$.000 \mathrm{E}+00$

$0.000 \mathrm{E}+00$

$0.000 \mathrm{E}+00$

$0.000 \mathrm{E}+00$

$0.000 \mathrm{E}+00$

$0.000 \mathrm{E}+00$

$0.000 \mathrm{E}+00$

$0.000 \mathrm{E}+00$

$0.000 \mathrm{E}+00$

EMISSIONS DATA SOURCE:

CHEMICALS ADDED OR DELETED: none

EMISSIONS FOR FACILITY FAC=Site300 CO=* DEV=PR1 PRO=0BPan STK=1 NAME=LLNL Site 300 EMS (lbs/yr) SOURCE MULTIPLIER $=1$

CAS ABBREV

CAS

1,3-Butadien

$67562394 \quad 1-4,6-8 \mathrm{HpCDF}$

$55673897 \quad 1-4,7-9 \mathrm{HpCDF}$

$70648269 \quad 1-4,7,8 \mathrm{HxCDF}$

$57117449 \quad 1-3,6-8 \mathrm{HxCDF}$

$35822469 \quad 1-4,6-8 \mathrm{HpCDD}$

MULTIPLIE

BG (ug/m^3) AVRG (lbs/yr)

$\begin{array}{rr}\text { bs } / \mathrm{yr}) & \text { MAX (lbs/hr) } \\ 0.017 & 0.00017 \\ 0 & 0 \\ 0 & 0 \\ 0 & 0 \\ 0 & 0 \\ 0 & 0\end{array}$




\begin{tabular}{|c|c|}
\hline 121142 & 2,4-DiNitToluen \\
\hline 606202 & 2,6-DiNitToluen \\
\hline 95578 & 2-CHLOROPHENOL \\
\hline 107051 & AllylChlor \\
\hline 7429905 & Aluminum \\
\hline 7440360 & Antimony \\
\hline 7440393 & Barium \\
\hline 71432 & Benzene \\
\hline 7440439 & Cadmium \\
\hline 56235 & $\mathrm{CCl} 4$ \\
\hline 67663 & Chloroform \\
\hline 7440473 & Chromium \\
\hline 7782505 & Chlorine \\
\hline 630080 & Carbon Monoxide \\
\hline 7440508 & Copper \\
\hline 110827 & Cyclohexane \\
\hline 84662 & DiethylPhthalat \\
\hline 122394 & Diphenylam \\
\hline 75003 & Ethyl Chloride \\
\hline 100414 & Ethyl Benzene \\
\hline 206440 & Fluoranthene \\
\hline 7647010 & $\mathrm{HCl}$ \\
\hline 98828 & Cumene \\
\hline 7439921 & Lead \\
\hline 74873 & Methyl Chloride \\
\hline 71556 & $1,1,1-\mathrm{TCA}$ \\
\hline 108872 & MethCyHex \\
\hline 75092 & Methylene Chlor \\
\hline 91203 & Naphthalene \\
\hline 110543 & Hexane \\
\hline 10102440 & NITROGEN DIOXID \\
\hline 39001020 & $1-80 \mathrm{ctaCDF}$ \\
\hline 78115 & PETN \\
\hline 108952 & Phenol \\
\hline 115071 & Propylene \\
\hline 121824 & $\mathrm{RDX}$ \\
\hline 100425 & Styrene \\
\hline 7446095 & SULFUR DIOXIDE \\
\hline 127184 & Perc \\
\hline 108883 & Toluene \\
\hline 75014 & Vinyl Chloride \\
\hline 7440666 & Zinc \\
\hline 10101 & NapthSur \\
\hline 10102 & EthBzSur \\
\hline 10103 & nHexSur \\
\hline
\end{tabular}

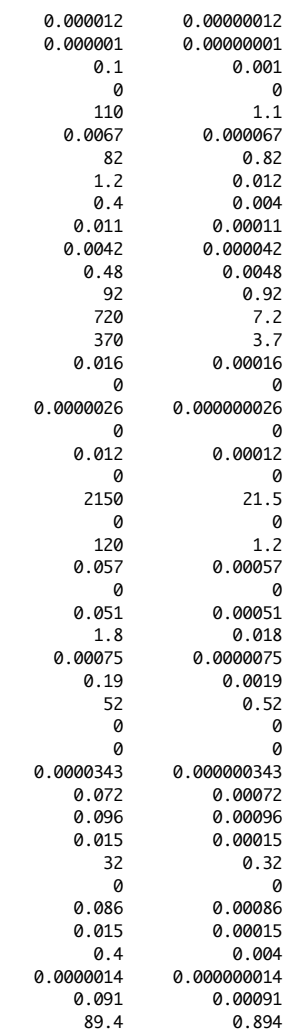

EMISSIONS FOR FACILITY FAC=Site300 CO=* DEV=PR1 PRO=0BCage3 STK=1 NAME=LLNL Site $300 \quad$ EMS (lbs/yr) SOURCE MULTIPLIER $=1$

\begin{tabular}{|c|c|}
\hline CAS & ABBREV \\
\hline 106990 & 1,3-Butadiene \\
\hline 67562394 & $1-4,6-8 \mathrm{HpCDF}$ \\
\hline 55673897 & $1-4,7-9 \mathrm{HpCDF}$ \\
\hline 70648269 & $1-4,7,8 \mathrm{H} \times \mathrm{CDF}$ \\
\hline 57117449 & $1-3,6-8 \mathrm{H} \times \mathrm{CDF}$ \\
\hline 35822469 & $1-4,6-8 \mathrm{HpCDD}$ \\
\hline 121142 & 2,4-DiNitToluen \\
\hline 606202 & 2,6-DiNitToluen \\
\hline 95578 & 2-CHLOROPHENOL \\
\hline 107051 & AllylChlor \\
\hline 7429905 & Aluminum \\
\hline 7440360 & Antimony \\
\hline 7440393 & Barium \\
\hline 71432 & Benzene \\
\hline 7440439 & Cadmium \\
\hline 56235 & CCl4 \\
\hline 67663 & Chloroform \\
\hline 7440473 & Chromium \\
\hline 7782505 & Chlorine \\
\hline 630080 & Carbon Monoxide \\
\hline 7440508 & Copper \\
\hline 110827 & Cyclohexane \\
\hline 84662 & DiethylPhthalat \\
\hline 122394 & Diphenylam \\
\hline 75003 & Ethyl Chloride \\
\hline 100414 & Ethyl Benzene \\
\hline 206440 & Fluoranthene \\
\hline 7647010 & $\mathrm{HCl}$ \\
\hline 98828 & Cumene \\
\hline 7439921 & Lead \\
\hline 74873 & Methyl Chloride \\
\hline 71556 & 1,1,1-TCA \\
\hline 108872 & MethCyHex \\
\hline 75092 & Methylene Chlor \\
\hline 91203 & Naphthalene \\
\hline 110543 & Hexane \\
\hline 10102440 & NITROGEN DIOXID \\
\hline 39001020 & $1-80 \mathrm{ctaCDF}$ \\
\hline 78115 & PETN \\
\hline 108952 & Phenol \\
\hline 115071 & Propylene \\
\hline 121824 & RDX \\
\hline 100425 & Styrene \\
\hline 7446095 & SULFUR DIOXIDE \\
\hline 127184 & Perc \\
\hline 108883 & Toluene \\
\hline 75014 & Vinyl Chloride \\
\hline 7440666 & Zinc \\
\hline 10101 & NapthSur \\
\hline
\end{tabular}

\begin{tabular}{|c|c|c|c|}
\hline $\begin{array}{r}\text { MULTIPLIER } \\
1\end{array}$ & $\begin{array}{r}\text { BG (ug/m^3) } \\
0\end{array}$ & AVRG (lbs/yr) & $\operatorname{MAX}(\mathrm{lbs} / \mathrm{hr})$ \\
\hline $\begin{array}{l}1 \\
1\end{array}$ & $\begin{array}{l}0 \\
0\end{array}$ & 0.00017 & $\begin{array}{r}0 \\
0.0000017\end{array}$ \\
\hline & 0 & & 0.0000017 \\
\hline 1 & 0 & 0.0000395 & 0.000000395 \\
\hline 1 & 0 & 0.000105 & 0.00000105 \\
\hline 1 & 0 & 0.0000475 & 0.000000475 \\
\hline 1 & 0 & 0 & 0 \\
\hline 1 & 0 & 0 & 0 \\
\hline 1 & 0 & 0 & 0 \\
\hline 1 & 0 & 0 & 0 \\
\hline 1 & 0 & 0 & 0 \\
\hline 1 & 0 & 180 & 1.8 \\
\hline 1 & 0 & 0 & 0 \\
\hline 1 & 0 & 0.43 & 0.0043 \\
\hline 1 & 0 & 2.25 & 0.0225 \\
\hline 1 & 0 & 0 & 0 \\
\hline 1 & 0 & 0.028 & 0.00028 \\
\hline 1 & 0 & 0.0115 & 0.000115 \\
\hline 1 & 0 & 0 & 0 \\
\hline 1 & 0 & 1 & 0.01 \\
\hline 1 & 0 & 100 & 1 \\
\hline 1 & 0 & 0.075 & 0.00075 \\
\hline 1 & 0 & 0.01 & 0.0001 \\
\hline 1 & 0 & 0 & 0 \\
\hline 1 & 0 & 0 & 0 \\
\hline 1 & 0 & 0 & 0 \\
\hline 1 & 0 & 0.012 & 0.00012 \\
\hline 1 & 0 & 1 & 0.01 \\
\hline 1 & 0 & 415 & 4.15 \\
\hline 1 & 0 & 0 & 0 \\
\hline 1 & 0 & 1.4 & 0.014 \\
\hline 1 & 0 & 0.1 & 0.001 \\
\hline 1 & 0 & 0 & 0 \\
\hline 1 & 0 & 0.04 & 0.0004 \\
\hline 1 & 0 & 0.06 & 0.0006 \\
\hline 1 & 0 & 0 & 0 \\
\hline 1 & 0 & 0.024 & 0.00024 \\
\hline 1 & 0 & 0.033 & 0.00033 \\
\hline 1 & 0 & 0.0002 & 0.000002 \\
\hline 1 & 0 & 0 & 0 \\
\hline 1 & 0 & 0 & 0 \\
\hline 1 & 0 & 0.13 & 0.0013 \\
\hline 1 & 0 & 0 & 0 \\
\hline 1 & 0 & 0 & 0 \\
\hline 1 & 0 & 4.3 & 0.043 \\
\hline 1 & 0 & 0.0085 & 0.000085 \\
\hline 1 & 0 & 0.14 & 0.0014 \\
\hline 1 & 0 & 0 & 0 \\
\hline 1 & 0 & 2.85 & 0.0285 \\
\hline 1 & 0 & 0.8 & 0.008 \\
\hline
\end{tabular}




\begin{tabular}{|c|c|c|c|}
\hline 10102 & EthBzSur & & 1 \\
\hline 10103 & nHexSur & & 1 \\
\hline $\begin{array}{l}\text { EMISSIONS FOR } \\
\text { SOURCE MULTIPL }\end{array}$ & $\begin{array}{l}\text { FACILITY FAC }=\text { Site } 300 \\
\text { LIER }=1\end{array}$ & $\mathrm{CO}=*$ & $\mathrm{DEV}=\mathrm{PR} 1$ \\
\hline CAS & ABBREV & MULTIF & PLIER \\
\hline 106990 & 1,3-Butadiene & & 1 \\
\hline 67562394 & $1-4,6-8 \mathrm{HpCDF}$ & & 1 \\
\hline 55673897 & $1-4,7-9 \mathrm{HpCDF}$ & & 1 \\
\hline 70648269 & $1-4,7,8 \mathrm{HxCDF}$ & & 1 \\
\hline 57117449 & $1-3,6-8 \mathrm{H} \times \mathrm{CDF}$ & & 1 \\
\hline 35822469 & $1-4,6-8 \mathrm{HpCDD}$ & & 1 \\
\hline 121142 & 2,4-DiNitToluen & & 1 \\
\hline 606202 & 2,6-DiNitToluen & & 1 \\
\hline 95578 & 2-CHLOROPHENOL & & 1 \\
\hline 107051 & AllylChlor & & 1 \\
\hline 7429905 & Aluminum & & 1 \\
\hline 7440360 & Antimony & & 1 \\
\hline 7440393 & Barium & & 1 \\
\hline 71432 & Benzene & & 1 \\
\hline 7440439 & Cadmium & & 1 \\
\hline 56235 & CCl4 & & 1 \\
\hline 67663 & Chloroform & & 1 \\
\hline 7440473 & Chromium & & 1 \\
\hline 7782505 & Chlorine & & 1 \\
\hline 630080 & Carbon Monoxide & & 1 \\
\hline 7440508 & Copper & & 1 \\
\hline 110827 & Cyclohexane & & 1 \\
\hline 84662 & DiethylPhthalat & & 1 \\
\hline 122394 & Diphenylam & & 1 \\
\hline 75003 & Ethyl Chloride & & 1 \\
\hline 100414 & Ethyl Benzene & & 1 \\
\hline 206440 & Fluoranthene & & 1 \\
\hline 7647010 & $\mathrm{HCl}$ & & 1 \\
\hline 98828 & Cumene & & 1 \\
\hline 7439921 & Lead & & 1 \\
\hline 74873 & Methyl Chloride & & 1 \\
\hline 71556 & 1,1,1-TCA & & 1 \\
\hline 108872 & MethCyHex & & 1 \\
\hline 75092 & Methylene Chlor & & 1 \\
\hline 91203 & Naphthalene & & 1 \\
\hline 110543 & Hexane & & 1 \\
\hline 10102440 & NITROGEN DIOXID & & 1 \\
\hline 39001020 & 1-80ctaCDF & & 1 \\
\hline 78115 & PETN & & 1 \\
\hline 108952 & Phenol & & 1 \\
\hline 115071 & Propylene & & 1 \\
\hline 121824 & $\mathrm{RDX}$ & & 1 \\
\hline 100425 & Styrene & & 1 \\
\hline 7446095 & SULFUR DIOXIDE & & 1 \\
\hline 127184 & Perc & & 1 \\
\hline 108883 & Toluene & & 1 \\
\hline 75014 & Vinyl Chloride & & 1 \\
\hline 7440666 & Zinc & & 1 \\
\hline 10101 & NapthSur & & 1 \\
\hline 10102 & EthBzSur & & 1 \\
\hline 10103 & nHexSur & & 1 \\
\hline EMISSIONS FOR & FACILITY FAC $=$ Site 300 & $\mathrm{CO}=*$ & $\mathrm{DEV}=\mathrm{PR} 1$ \\
\hline SOURCE MULTIPL & IER $=1$ & & \\
\hline CAS & ABBREV & MULTIF & PLIER \\
\hline 106990 & 1,3-Butadiene & & 1 \\
\hline 67562394 & $1-4,6-8 \mathrm{HpCDF}$ & & 1 \\
\hline 55673897 & $1-4,7-9 \mathrm{HpCDF}$ & & 1 \\
\hline 70648269 & $1-4,7,8 \mathrm{HxCDF}$ & & 1 \\
\hline 57117449 & $1-3,6-8 \mathrm{H} \times \mathrm{CDF}$ & & 1 \\
\hline 35822469 & $1-4,6-8 \mathrm{HpCDD}$ & & 1 \\
\hline 121142 & 2,4-DiNitToluen & & 1 \\
\hline 606202 & 2,6-DiNitToluen & & 1 \\
\hline 95578 & 2-CHLOROPHENOL & & 1 \\
\hline 107051 & AllylChlor & & 1 \\
\hline 7429905 & Aluminum & & 1 \\
\hline 7440360 & Antimony & & 1 \\
\hline 7440393 & Barium & & 1 \\
\hline 71432 & Benzene & & 1 \\
\hline 7440439 & Cadmium & & 1 \\
\hline 56235 & CCl4 & & 1 \\
\hline 67663 & Chloroform & & 1 \\
\hline 7440473 & Chromium & & 1 \\
\hline 7782505 & Chlorine & & 1 \\
\hline 630080 & Carbon Monoxide & & 1 \\
\hline 7440508 & Copper & & 1 \\
\hline 110827 & Cyclohexane & & 1 \\
\hline 84662 & DiethylPhthalat & & 1 \\
\hline 122394 & Diphenylam & & 1 \\
\hline 75003 & Ethyl Chloride & & 1 \\
\hline 100414 & Ethyl Benzene & & 1 \\
\hline 206440 & Fluoranthene & & 1 \\
\hline 7647010 & $\mathrm{HCl}$ & & 1 \\
\hline 98828 & Cumene & & 1 \\
\hline 7439921 & Lead & & 1 \\
\hline 74873 & Methyl Chloride & & 1 \\
\hline 71556 & 1,1,1-TCA & & 1 \\
\hline 108872 & MethCyHex & & 1 \\
\hline 75092 & Methylene Chlor & & 1 \\
\hline 91203 & Naphthalene & & 1 \\
\hline 110543 & Hexane & & 1 \\
\hline 10102440 & NITROGEN DIOXID & & 1 \\
\hline
\end{tabular}




$\begin{array}{llllrr}39001020 & \text { 1-80ctaCDF } & 1 & 0 & 0 & 0 \\ 78115 & \text { PETN } & 1 & 0 & 19.6 & 0.196 \\ 108952 & \text { Phenol } & 1 & 0 & 0 & 0 \\ 115071 & \text { Propylene } & 1 & 0 & 2.56 & 0.0256 \\ 121824 & \text { RDX } & 1 & 0 & 259 & 2.59 \\ 100425 & \text { Styrene } & 1 & 0 & 1.47 & 0.0147 \\ 7446095 & \text { SULFUR DIOXIDE } & 1 & 0 & 455 & 4.55 \\ 127184 & \text { Perc } & 1 & 0 & 0.63 & 0.0063 \\ 108883 & \text { Toluene } & 1 & 0 & 1.05 & 0.0105 \\ 75014 & \text { Vinyl Chloride } & 1 & 0 & 0.0805 & 0.000805 \\ 7440666 & \text { Zinc } & 1 & 0 & 560 & 5.6 \\ 10101 & \text { NapthSur } & 1 & 0 & 0 & 0 \\ 10102 & \text { EthBzSur } & 1 & 0 & 0.62 & 0.0062 \\ 10103 & \text { nHexSur } & 1 & 0 & 119 & 1.19\end{array}$

CHRONIC HI REPORT

RECEPTOR 2

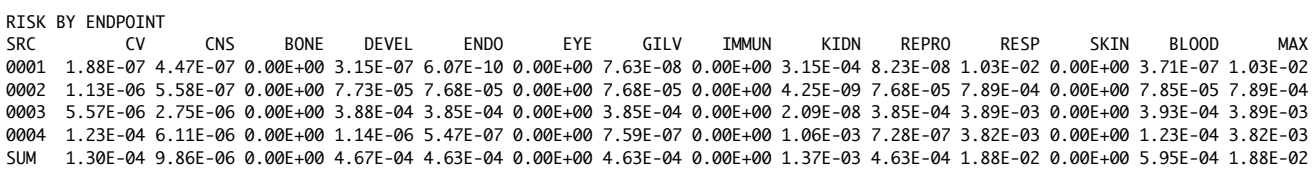


HARP Out Folder/Screening Output Folder

Rep_Acu_AllRec_AllSrc_AllCh_ByRec_Site.txt

Rep_Can_30yr_Hi_AllRec_AllSrc_Allch_ByRec_Site.txt

Rep_Can_WRK_Ave_AllSrc_Allch_ByRec_Site.txt

Rep_chr_Res_Hi_AllRec_AllSrc_AllCh_ByRec_Site.txt

Rep_Chr_Wrk_PtEst_AllRec_AllSrc_AllCh_byRec_Site.txt 
Rep_Acu_AllRec_AllSrc_AllCh_ByRec_Site.txt 


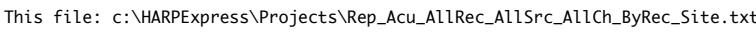

Created by HARP Version 1.0 Build 23.02.10

Uses ISC Version 99155

Uses BPIP Version 95086

Creation date: 10/24/2005 1:48:00 PM

EXCEPTION REPORT

(there have been no changes or exceptions)

INPUT FILES:

Source-Receptor file: c: \HARPExpress \Projects $\backslash$ EWTF.mta

Averaging period adjustment factors file: not applicable

Emission rates file: none

Site parameters file: c: \HARPExpress \Projects $\backslash$ Site300. sit

Screening mode is OFF

$\begin{array}{ll}\text { Analysis method: } & \text { Point Estimate } \\ \text { Health effect: } & \text { Acute HI Simple (Concurrent Max.) } \\ \text { Receptor(s): } & \text { All } \\ \text { Sources(s): } & \text { All } \\ \text { Chemicals(s): } & \text { All }\end{array}$

CHEMICAL CROSS-REFERENCE TABLE AND BACKGROUND CONCENTRATIONS

\begin{tabular}{|c|c|c|c|}
\hline CHEM & CAS & ABBREVIATION & POLLUTANT NAME \\
\hline 0001 & 106990 & 1,3-Butadiene & 1,3-Butadiene \\
\hline 0002 & 67562394 & $1-4,6-8 \mathrm{HpCDF}$ & $1,2,3,4,6,7,8$-Heptachlorodibenzofuran \\
\hline 0003 & 55673897 & $1-4,7-9 \mathrm{HPCDF}$ & $1,2,3,4,7,8,9$-Heptachlorodibenzofuran \\
\hline 0004 & 70648269 & $1-4,7,8 \mathrm{H} \times \mathrm{CDF}$ & $1,2,3,4,7,8$-Hexachlorodibenzofuran \\
\hline 0005 & 57117449 & $1-3,6-8 \mathrm{H} \times \mathrm{CDF}$ & $1,2,3,6,7,8$-Hexachlorodibenzofuran \\
\hline 0006 & 35822469 & $1-4,6-8 \mathrm{HpCDD}$ & $1,2,3,4,6,7,8$-Heptachlorodibenzo-p-dioxin \\
\hline 0007 & 121142 & 2,4-DiNitToluen & 2,4-Dinitrotoluene \\
\hline 0008 & 606202 & 2,6-DiNitToluen & 2,6-Dinitrotoluene \\
\hline 0009 & 95578 & 2-CHLOROPHENOL & 2-CHLOROPHENOL \\
\hline 0010 & 107051 & AllylChlor & Allyl chloride \\
\hline 0011 & 7429905 & Aluminum & Aluminum \\
\hline 0012 & 7440360 & Antimony & Antimony \\
\hline 0013 & 7440393 & Barium & Barium \\
\hline 0014 & 71432 & Benzene & Benzene \\
\hline 0015 & 7440439 & Cadmium & Cadmium \\
\hline 0016 & 56235 & $\mathrm{CCl} 4$ & Carbon tetrachloride \\
\hline 0017 & 67663 & Chloroform & Chloroform \\
\hline 0018 & 7440473 & Chromium & Chromium \\
\hline 0019 & 7782505 & Chlorine & Chlorine \\
\hline 0020 & 630080 & Carbon Monoxide & Carbon monoxide \\
\hline 0021 & 7440508 & Copper & Copper \\
\hline 0022 & 110827 & Cyclohexane & Cyclohexane \\
\hline 0023 & 84662 & DiethylPhthalat & Diethyl phthalate \\
\hline 0024 & 122394 & Diphenylam & Diphenylamine \\
\hline 0025 & 75003 & Ethyl Chloride & Ethyl chloride \{Chlorethane\} \\
\hline 0026 & 100414 & Ethyl Benzene & Ethyl benzene \\
\hline 0027 & 206440 & Fluoranthene & Fluoranthene \\
\hline 0028 & 7647010 & $\mathrm{HCl}$ & Hydrochloric acid \\
\hline 0029 & 98828 & Cumene & Cumene \\
\hline 0030 & 7439921 & Lead & Lead \\
\hline 0031 & 74873 & Methyl Chloride & Methyl chloride \{Chloromethane\} \\
\hline 0032 & 71556 & $1,1,1-\mathrm{TCA}$ & Methyl chloroform $\{1,1,1-\mathrm{TCA}\}$ \\
\hline 0033 & 108872 & MethCyHex & Methyl cyclohexane \\
\hline 0034 & 75092 & Methylene Chlor & Methylene chloride \{Dichloromethane\} \\
\hline 0035 & 91203 & Naphthalene & Naphthalene \\
\hline 0036 & 110543 & Hexane & Hexane \\
\hline 0037 & 10102440 & NITROGEN DIOXID & NITROGEN DIOXIDE \\
\hline 0038 & 39001020 & $1-80 \mathrm{ctaCDF}$ & $1,2,3,4,6,7,8,9$-0ctachlorodibenzofuran \\
\hline 0039 & 78115 & PETN & PETN (same as RDX) \\
\hline 0040 & 108952 & Phenol & Phenol \\
\hline 0041 & 115071 & Propylene & Propylene \\
\hline 0042 & 121824 & RDX & RDX \\
\hline 0043 & 100425 & Styrene & Styrene \\
\hline 0044 & 7446095 & SULFUR DIOXIDE & SULFUR DIOXIDE \\
\hline 0045 & 127184 & Perc & Perchloroethylene \{Tetrachloroethene\} \\
\hline 0046 & 108883 & Toluene & Toluene \\
\hline 0047 & 75014 & Vinyl Chloride & Vinyl chloride \\
\hline 0048 & 7440666 & Zinc & Zinc \\
\hline 0049 & 10101 & NapthSur & Naphthalene surrogate \\
\hline 0050 & 10102 & EthBzSur & Ethylbenzene surrogate \\
\hline 005 & 10103 & nHexSur & n-Hexane surrogate \\
\hline
\end{tabular}

BACKGROUND (ug/m^3)

$0.000 \mathrm{E}+00$

$0.000 \mathrm{E}+00$

$0.000 E+00$

$0.000 \mathrm{E}+00$

$0.000 \mathrm{E}+00$

$0.000 \mathrm{E}+00$

$0.000 \mathrm{E}+00$

$0.000 \mathrm{E}+00$

$0.000 \mathrm{E}+00$

$0.000 \mathrm{E}+00$

$0.000 \mathrm{E}+00$

$0.000 \mathrm{E}+00$

$0.000 \mathrm{E}+00$

$0.000 E+00$

$0.000 \mathrm{E}+00$

$0.000 \mathrm{E}+00$

$0.000 \mathrm{E}+00$

$0.000 \mathrm{E}+00$

$0.000 \mathrm{E}+00$

$0.000 \mathrm{E}+00$

$0.000 \mathrm{E}+00$

$0.000 \mathrm{E}+00$

$0.000 E+00$

$0.000 \mathrm{E}+00$

$0.000 \mathrm{E}+00$

$0.000 \mathrm{E}+00$

$0.000 \mathrm{E}+00$

$0.000 \mathrm{E}+00$

$0.000 E+00$

$0.000 \mathrm{E}+00$

$0.000 \mathrm{E}+00$

$0.000 \mathrm{E}+00$

$0.000 \mathrm{E}+00$

$0.000 \mathrm{E}+00$

$0.000 \mathrm{E}+00$

$0.000 \mathrm{E}+00$

$0.000 \mathrm{E}+00$

$0.000 \mathrm{E}+00$

$0.000 \mathrm{E}+00$

$0.000 \mathrm{E}+00$

$0.000 \mathrm{E}+00$

$0.000 \mathrm{E}+00$

$0.000 \mathrm{E}+00$

$0.000 \mathrm{E}+00$

$0.000 \mathrm{E}+00$

$0.000 \mathrm{E}+00$

$0.000 \mathrm{E}+00$

$0.000 \mathrm{E}+00$

$0.000 \mathrm{E}+00$

$0.000 \mathrm{E}+00$

$0.00 \mathrm{E}+00$

$0.000 \mathrm{E}+00$

EMISSIONS DATA SOURCE:

CHEMICALS ADDED OR DELETED: none

EMISSIONS FOR FACILITY FAC=Site300 CO=* DEV=PR1 PRO=0BPan STK=1 NAME=LLNL Site $300 \quad$ EMS (lbs/yr) SOURCE MULTIPLIER=1

1069901 1,3-Butadien

$67562394 \quad 1-4,6-8 \mathrm{HpCDF}$

$55673897 \quad 1-4,7-9 \mathrm{HpCDF}$

$70648269 \quad 1-4,7,8 \mathrm{HxCDF}$

$57117449 \quad 1-3,6-8 \mathrm{HxCDF}$

$35822469 \quad 1-4,6-8 \mathrm{HpCDD}$

121142 2,4-DiNitToluen

606202 2,6-DiNitToluen

107051

7429905

$7440360 \quad$ Antimony

$7440393 \quad$ Barium

MULTIPLIER
1
1
1
1
1
1
1
1
1
1
1
1
1
1

BG $(u g / m \wedge 3$

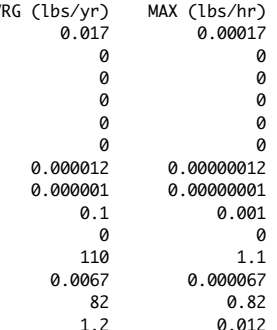




\begin{tabular}{|c|c|c|c|c|c|}
\hline 7440439 & Cadmium & 1 & 0 & 0.4 & 0.004 \\
\hline 56235 & $\mathrm{CCl}_{4}$ & 1 & 0 & 0.011 & 0.00011 \\
\hline 67663 & Chloroform & 1 & 0 & 0.0042 & 0.000042 \\
\hline 7440473 & Chromium & 1 & 0 & 0.48 & 0.0048 \\
\hline 7782505 & Chlorine & 1 & 0 & 92 & 0.92 \\
\hline 630080 & Carbon Monoxide & 1 & 0 & 720 & 7.2 \\
\hline 7440508 & Copper & 1 & 0 & 370 & 3.7 \\
\hline 110827 & Cyclohexane & 1 & 0 & 0.016 & 0.00016 \\
\hline 84662 & DiethylPhthalat & 1 & 0 & 0 & 0 \\
\hline 122394 & Diphenylam & 1 & 0 & 0.0000026 & 0.000000026 \\
\hline 75003 & Ethyl Chloride & 1 & 0 & 0 & 0 \\
\hline 100414 & Ethyl Benzene & 1 & 0 & 0.012 & 0.00012 \\
\hline 206440 & Fluoranthene & 1 & 0 & 0 & 0 \\
\hline 7647010 & $\mathrm{HCl}$ & 1 & 0 & 2150 & 21.5 \\
\hline 98828 & Cumene & 1 & 0 & 0 & 0 \\
\hline 7439921 & Lead & 1 & 0 & 120 & 1.2 \\
\hline 74873 & Methyl Chloride & 1 & 0 & 0.057 & 0.00057 \\
\hline 71556 & $1,1,1$-TCA & 1 & 0 & 0 & 0 \\
\hline 108872 & MethCyHex & 1 & 0 & 0.051 & 0.00051 \\
\hline 75092 & Methylene Chlor & 1 & 0 & 1.8 & 0.018 \\
\hline 91203 & Naphthalene & 1 & 0 & 0.00075 & 0.0000075 \\
\hline 110543 & Hexane & 1 & 0 & 0.19 & 0.0019 \\
\hline 10102440 & NITROGEN DIOXID & 1 & 0 & 52 & 0.52 \\
\hline 39001020 & 1-80ctaCDF & 1 & 0 & 0 & 0 \\
\hline 78115 & PETN & 1 & 0 & 0 & 0 \\
\hline 108952 & Phenol & 1 & 0 & 0.0000343 & 0.000000343 \\
\hline 115071 & Propylene & 1 & 0 & 0.072 & 0.00072 \\
\hline 121824 & $\mathrm{RDX}$ & 1 & 0 & 0.096 & 0.00096 \\
\hline 100425 & Styrene & 1 & 0 & 0.015 & 0.00015 \\
\hline 7446095 & SULFUR DIOXIDE & 1 & 0 & 32 & 0.32 \\
\hline 127184 & Perc & 1 & 0 & 0 & 0 \\
\hline 108883 & Toluene & 1 & 0 & 0.086 & 0.00086 \\
\hline 75014 & Vinyl Chloride & 1 & 0 & 0.015 & 0.00015 \\
\hline 7440666 & Zinc & 1 & 0 & 0.4 & 0.004 \\
\hline 10101 & NapthSur & 1 & 0 & 0.0000014 & 0.000000014 \\
\hline 10102 & EthBzSur & 1 & 0 & 0.091 & 0.00091 \\
\hline 10103 & nHexSur & 1 & 0 & 89.4 & 0.894 \\
\hline
\end{tabular}

EMISSIONS FOR FACILITY FAC=Site300 CO=* DEV=PR1 PRO=0BCage3 STK=1 NAME=LLNL Site $300 \quad$ EMS (lbs/yr) SOURCE MULTIPLIER

\begin{tabular}{|c|c|c|c|c|c|}
\hline CAS & ABBREV & MULTIPLIER & BG $\left(u g / m^{\wedge} 3\right)$ & AVRG (lbs/yr) & $\operatorname{MAX}(\mathrm{lbs} / \mathrm{hr})$ \\
\hline 106990 & 1,3-Butadiene & 1 & 0 & 0 & 0 \\
\hline 67562394 & $1-4,6-8 \mathrm{HpCDF}$ & 1 & 0 & 0.00017 & 0.0000017 \\
\hline 55673897 & $1-4,7-9 \mathrm{HPCDF}$ & 1 & 0 & 0.0000395 & 0.000000395 \\
\hline 70648269 & $1-4,7,8 \mathrm{H} \times \mathrm{CDF}$ & 1 & 0 & 0.000105 & 0.00000105 \\
\hline 57117449 & $1-3,6-8 \mathrm{H} \times \mathrm{CDF}$ & 1 & 0 & 0.0000475 & 0.000000475 \\
\hline 35822469 & $1-4,6-8 \mathrm{Hp} C D D$ & 1 & 0 & 0 & 0 \\
\hline 121142 & 2,4-DiNitToluen & 1 & 0 & 0 & 0 \\
\hline 606202 & 2,6-DiNitToluen & 1 & 0 & 0 & 0 \\
\hline 95578 & 2-CHLOROPHENOL & 1 & 0 & 0 & 0 \\
\hline 107051 & AllylChlor & 1 & 0 & 0 & 0 \\
\hline 7429905 & Aluminum & 1 & 0 & 180 & 1.8 \\
\hline 7440360 & Antimony & 1 & 0 & 0 & 0 \\
\hline 7440393 & Barium & 1 & 0 & 0.43 & 0.0043 \\
\hline 71432 & Benzene & 1 & 0 & 2.25 & 0.0225 \\
\hline 7440439 & Cadmium & 1 & 0 & 0 & 0 \\
\hline 56235 & CCl4 & 1 & 0 & 0.028 & 0.00028 \\
\hline 67663 & Chloroform & 1 & 0 & 0.0115 & 0.000115 \\
\hline 7440473 & Chromium & 1 & 0 & 0 & 0 \\
\hline 7782505 & Chlorine & 1 & 0 & 1 & 0.01 \\
\hline 630080 & Carbon Monoxide & 1 & 0 & 100 & 1 \\
\hline 7440508 & Copper & 1 & 0 & 0.075 & 0.00075 \\
\hline 110827 & Cyclohexane & 1 & 0 & 0.01 & 0.0001 \\
\hline 84662 & DiethylPhthalat & 1 & 0 & 0 & 0 \\
\hline 122394 & Diphenylam & 1 & 0 & 0 & 0 \\
\hline 75003 & Ethyl Chloride & 1 & 0 & 0 & 0 \\
\hline 100414 & Ethyl Benzene & 1 & 0 & 0.012 & 0.00012 \\
\hline 206440 & Fluoranthene & 1 & 0 & 1 & 0.01 \\
\hline 7647010 & $\mathrm{HCl}$ & 1 & 0 & 415 & 4.15 \\
\hline 98828 & Cumene & 1 & 0 & 0 & 0 \\
\hline 7439921 & Lead & 1 & 0 & 1.4 & 0.014 \\
\hline 74873 & Methyl Chloride & 1 & 0 & 0.1 & 0.001 \\
\hline 71556 & $1,1,1$-TCA & 1 & 0 & 0 & 0 \\
\hline 108872 & MethCyHex & 1 & 0 & 0.04 & 0.0004 \\
\hline 75092 & Methylene Chlor & 1 & 0 & 0.06 & 0.0006 \\
\hline 91203 & Naphthalene & 1 & 0 & 0 & 0 \\
\hline 110543 & Hexane & 1 & 0 & 0.024 & 0.00024 \\
\hline 10102440 & NITROGEN DIOXID & 1 & 0 & 0.033 & 0.00033 \\
\hline 39001020 & 1-80ctaCDF & 1 & 0 & 0.0002 & 0.000002 \\
\hline 78115 & PETN & 1 & 0 & 0 & 0 \\
\hline 108952 & Phenol & 1 & 0 & 0 & 0 \\
\hline 115071 & Propylene & 1 & 0 & 0.13 & 0.0013 \\
\hline 121824 & $\mathrm{RDX}$ & 1 & 0 & 0 & 0 \\
\hline 100425 & Styrene & 1 & 0 & 0 & 0 \\
\hline 7446095 & SULFUR DIOXIDE & 1 & 0 & 4.3 & 0.043 \\
\hline 127184 & Perc & 1 & 0 & 0.0085 & 0.000085 \\
\hline 108883 & Toluene & 1 & 0 & 0.14 & 0.0014 \\
\hline 75014 & Vinyl Chloride & 1 & 0 & 0 & 0 \\
\hline 7440666 & Zinc & 1 & 0 & 2.85 & 0.0285 \\
\hline 10101 & NapthSur & 1 & 0 & 0.8 & 0.008 \\
\hline 10102 & EthBzSur & 1 & 0 & 0.038 & 0.00038 \\
\hline 10103 & nHexSur & 1 & 0 & 9.83 & 0.0983 \\
\hline
\end{tabular}

EMISSIONS FOR FACILITY FAC=Site300 CO=* DEV=PR1 PRO=0BCage4 STK=1 NAME=LLNL Site 300 EMS (lbs/yr) SOURCE MULTIPLIER $=1$

\begin{tabular}{|c|c|c|c|c|c|}
\hline CAS & ABBREV & MULTIPLIER & BG $\left(u g / m^{\wedge} 3\right)$ & AVRG (lbs/yr) & $\operatorname{MAX}(\mathrm{lbs} / \mathrm{hr}$ ) \\
\hline 106990 & 1,3-Butadiene & 1 & 0 & 0 & 0 \\
\hline 67562394 & $1-4,6-8 \mathrm{HpCDF}$ & 1 & 0 & 0.000884 & 0.00000884 \\
\hline 55673897 & $1-4,7-9 \mathrm{HpCDF}$ & 1 & 0 & 0.000205 & 0.00000205 \\
\hline 70648269 & $1-4,7,8 \mathrm{HxCDF}$ & 1 & 0 & 0.000546 & 0.00000546 \\
\hline
\end{tabular}




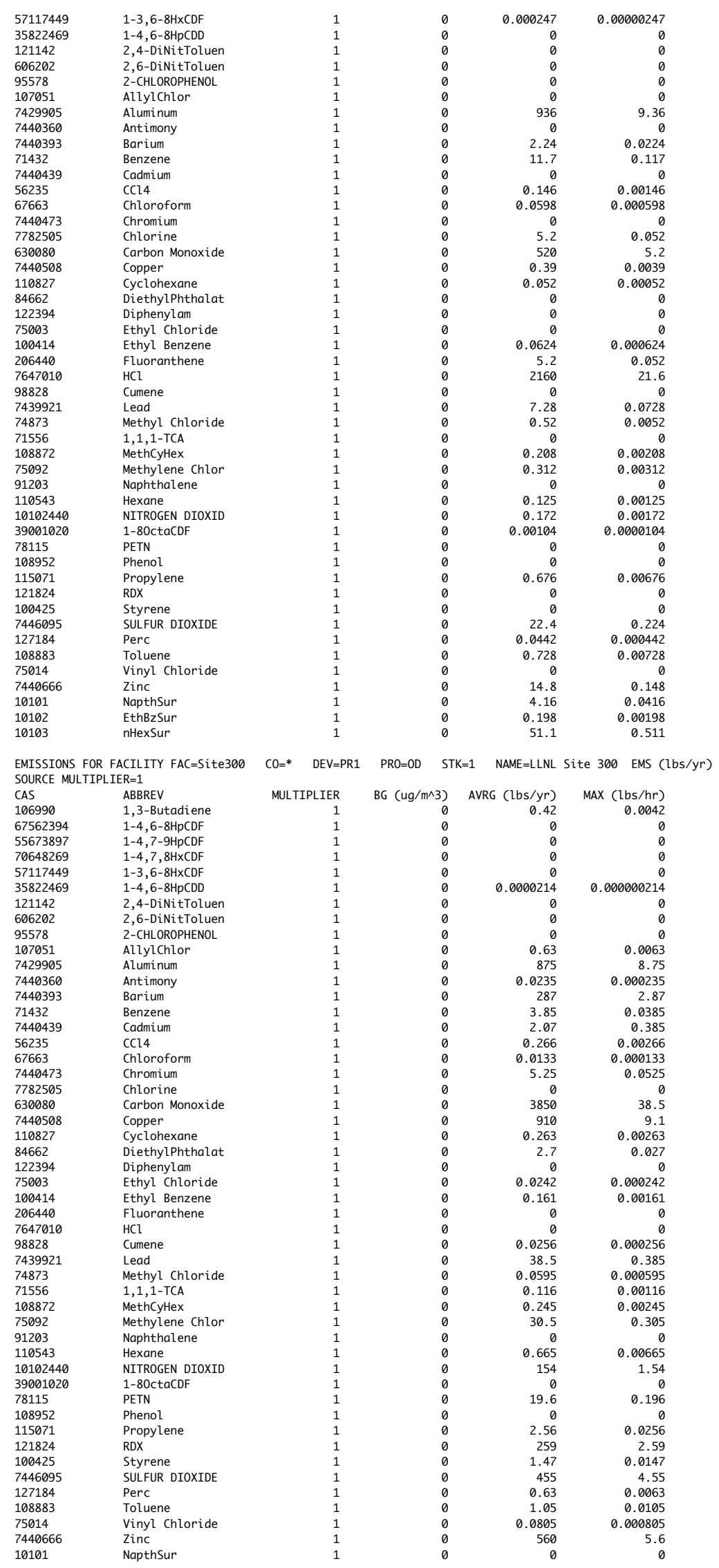




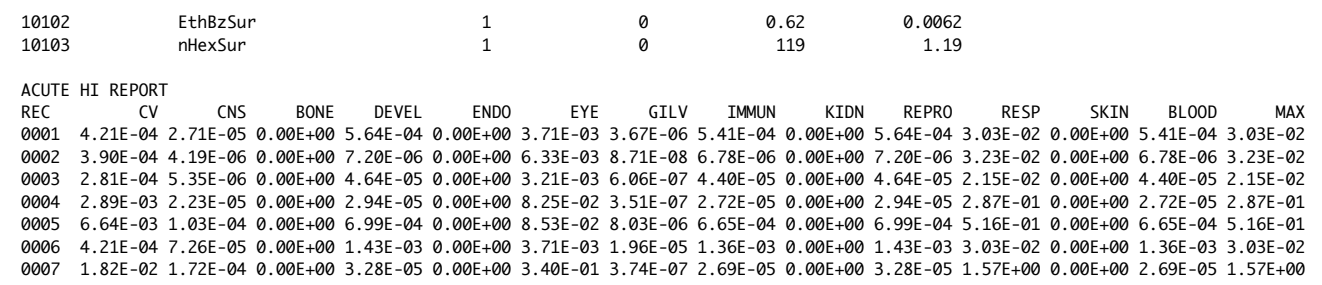


Rep_Can_30yr_Hi_AllRec_AllSrc_Allch_ByRec_Site.txt 
This file: c: \HARPExpress \Projects $\backslash$ Rep_Can_30yr_Hi_AllRec_AllSrc_AllCh_ByRec_Site.txt

Created by HARP Version 1.0 Build 23.02.10

Uses ISC Version 99155

Uses BPIP Version 95086

Creation date: 10/24/2005 1:43:03 PM

EXCEPTION REPORT

(there have been no changes or exceptions)

INPUT FILES:

Source-Receptor file: c: \HARPExpress $\backslash$ Projects $\backslash$ EWTF.mta

Averaging period adjustment factors file: not applicable

Emission rates file: none

Site parameters file: c: \HARPExpress \Projects\Site300.sit

Screening mode is OFF

Exposure duration: 30 year (adult resident)

Analysis method: High-end Point Estimate

Health effect: Cancer Risk

Receptor(s): All

Sources(s): All

SITE PARAMETERS

DEPOSITION

Deposition rate $(\mathrm{m} / \mathrm{s})$

0.0072

DRINKING WATER

*** Pathway disabled $* * *$

FISH

*** Pathway disabled ***

PASTURE

ANIMALS' WATER

Water surface area (min)

Water volume (L)

Volume changes per yea

Fraction of beef cows' water

from pasture source

Fraction of dairy cows' water

ANIMALS' FEED

Fraction of cows' feed

from grazing

HUMAN INGESTION

Fraction of ingested beef

from contaminated source

Fraction of ingested dairy

0.107

from contaminated source

HOME GROWN PRODUCE

HUMAN INGESTION

Fraction of ingested leafy vegetable

from home grown source $\quad 0.082$

Fraction of ingested exposed vegetable

from home grown source 0.207

Fraction of ingested protected vegetable

from home grown source 0.134

Fraction of ingested root vegetable

from home grown source $\quad 0.088$

PIGS, CHICKENS AND EGGS

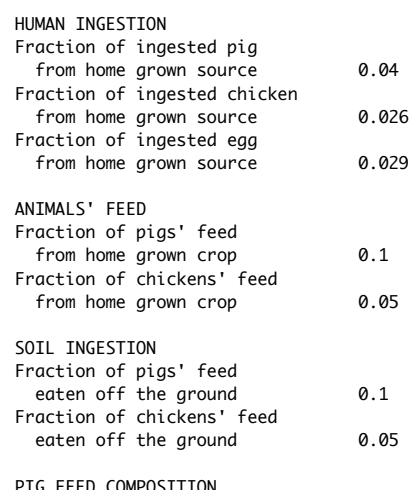

PIG FEED COMPOSITION 
DERMAL ABSORPTION

*** Pathway enabled ***

SOIL INGESTION

*** Pathway enabled ***

MOTHER'S MILK

*** Pathway enabled ***

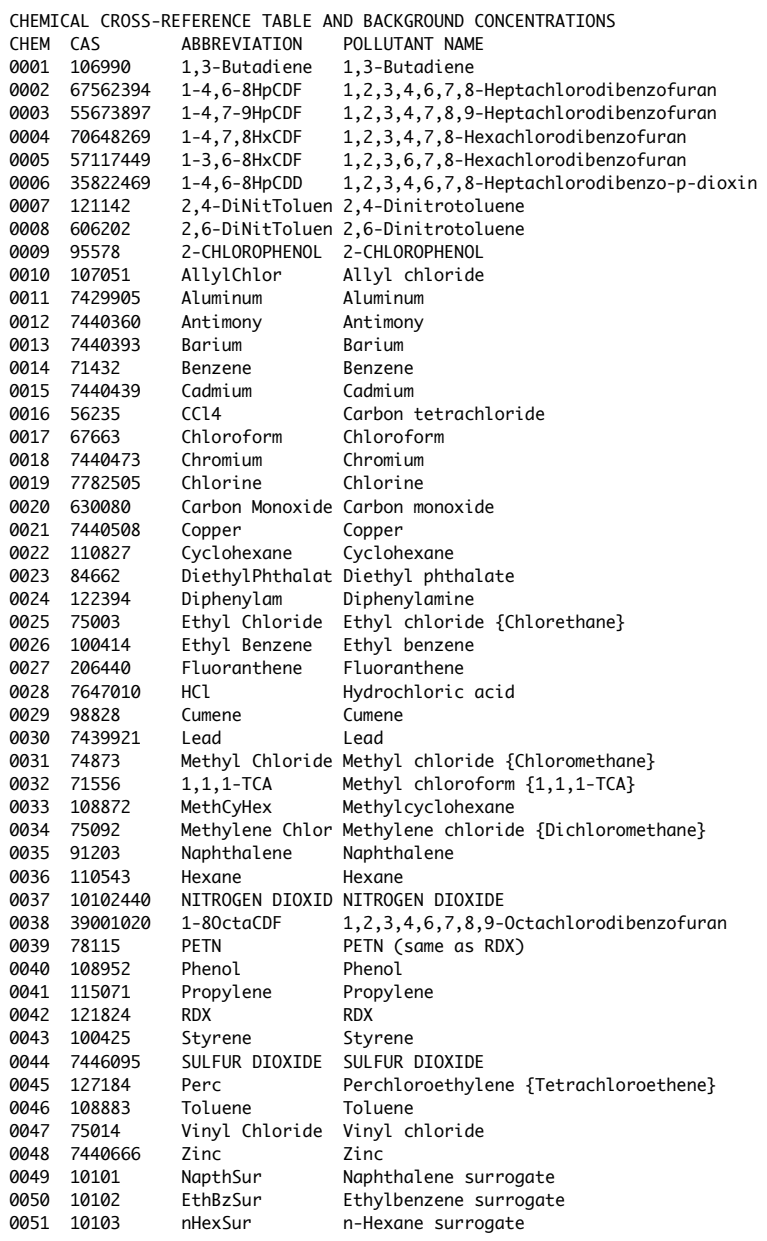

BACKGROUND (ug $/ \mathrm{m} \wedge 3$ )

$0.000 \mathrm{E}+00$

$0.000 \mathrm{E}+00$

$0.000 \mathrm{E}+00$

$0.000 \mathrm{E}+00$

$0.000 \mathrm{E}+00$

$0.000 \mathrm{E}+00$

$0.000 \mathrm{E}+00$

$0.000 \mathrm{E}+00$

$0.000 \mathrm{E}+00$

$0.000 \mathrm{E}+00$

$0.000 \mathrm{E}+00$

$0.000 \mathrm{E}+00$

$0.000 \mathrm{E}+00$

$0.000 \mathrm{E}+00$

$0.000 \mathrm{E}+00$

$0.000 \mathrm{E}+00$

$0.000 \mathrm{E}+00$

$0.000 \mathrm{E}+00$

$0.000 \mathrm{E}+00$

$0.000 \mathrm{E}+00$

$0.000 \mathrm{E}+00$

$0.000 \mathrm{E}+00$

$0.000 \mathrm{E}+00$

$0.000 \mathrm{E}+00$

$0.000 \mathrm{E}+00$

$0.000 \mathrm{E}+00$

$0.000 \mathrm{E}+00$

$0.000 \mathrm{E}+00$

$0.000 \mathrm{E}+00$

$0.000 \mathrm{E}+00$

$0.000 \mathrm{E}+00$

$0.000 \mathrm{E}+00$

$0.000 \mathrm{E}+00$

$0.000 \mathrm{E}+00$

$0.000 \mathrm{E}+00$

$0.000 \mathrm{E}+00$

$0.000 \mathrm{E}+00$

$0.000 \mathrm{E}+00$

$0.000 \mathrm{E}+00$

$0.000 \mathrm{E}+00$

$0.000 \mathrm{E}+00$

$0.000 \mathrm{E}+00$

$0.000 \mathrm{E}+00$

$0.000 \mathrm{E}+00$

$0.000 \mathrm{E}+00$

$0.000 \mathrm{E}+00$

$0.000 \mathrm{E}+00$

$0.000 \mathrm{E}+00$

$0.000 \mathrm{E}+00$

$0.000 \mathrm{E}+00$

$0.000 \mathrm{E}+00$

$0.000 \mathrm{E}+00$

$0.000 \mathrm{E}+00$

EMISSIONS DATA SOURCE:

CHEMICALS ADDED OR DELETED: none

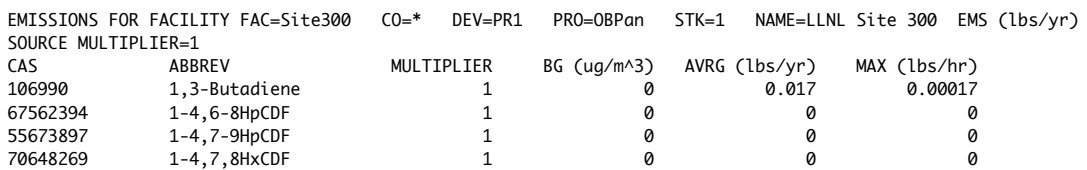




\begin{tabular}{|c|c|}
\hline 57117449 & $1-3,6-8 \mathrm{H} \times \mathrm{CDF}$ \\
\hline 35822469 & $1-4,6-8 \mathrm{HpCDD}$ \\
\hline 121142 & 2,4-DiNitToluen \\
\hline 606202 & 2,6-DiNitToluen \\
\hline 95578 & 2-CHLOROPHENOL \\
\hline 107051 & AllylChlor \\
\hline 7429905 & Aluminum \\
\hline 7440360 & Antimony \\
\hline 7440393 & Barium \\
\hline 71432 & Benzene \\
\hline 7440439 & Cadmium \\
\hline 56235 & CCl4 \\
\hline 67663 & Chloroform \\
\hline 7440473 & Chromium \\
\hline 7782505 & Chlorine \\
\hline 630080 & Carbon Monoxide \\
\hline 7440508 & Copper \\
\hline 110827 & Cyclohexane \\
\hline 84662 & DiethylPhthalat \\
\hline 122394 & Diphenylam \\
\hline 75003 & Ethyl Chloride \\
\hline 100414 & Ethyl Benzene \\
\hline 206440 & Fluoranthene \\
\hline 7647010 & $\mathrm{HCl}$ \\
\hline 98828 & Cumene \\
\hline 7439921 & Lead \\
\hline 74873 & Methyl Chloride \\
\hline 71556 & $1,1,1$-TCA \\
\hline 108872 & MethCyHex \\
\hline 75092 & Methylene Chlor \\
\hline 91203 & Naphthalene \\
\hline 110543 & Hexane \\
\hline 10102440 & NITROGEN DIOXID \\
\hline 39001020 & $1-80 \mathrm{ctaCDF}$ \\
\hline 78115 & PETN \\
\hline 108952 & Phenol \\
\hline 115071 & Propylene \\
\hline 121824 & $\mathrm{RDX}$ \\
\hline 100425 & Styrene \\
\hline 7446095 & SULFUR DIOXIDE \\
\hline 127184 & Perc \\
\hline 108883 & Toluene \\
\hline 75014 & Vinyl Chloride \\
\hline 7440666 & Zinc \\
\hline 10101 & NapthSur \\
\hline 10102 & EthBzSur \\
\hline 10103 & nHexSur \\
\hline
\end{tabular}

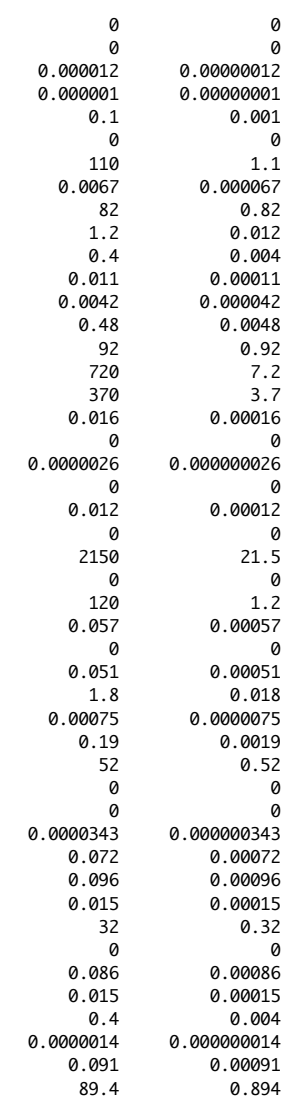

EMISSIONS FOR FACILITY FAC $=S i$ te $300 \quad$ CO $=* \quad$ DEV=PR1 PRO=0BCage 3 SOURCE MULTIPLIER $=1$

\begin{tabular}{|c|c|}
\hline CAS & ABBREV \\
\hline 106990 & 1,3-Butadiene \\
\hline 67562394 & $1-4,6-8 \mathrm{HpCDF}$ \\
\hline 55673897 & $1-4,7-9 \mathrm{HpCDF}$ \\
\hline 70648269 & $1-4,7,8 \mathrm{HxCDF}$ \\
\hline 57117449 & $1-3,6-8 \mathrm{H} \times \mathrm{CDF}$ \\
\hline 35822469 & $1-4,6-8 \mathrm{HpCDD}$ \\
\hline 121142 & 2,4-DiNitToluen \\
\hline 606202 & 2,6-DiNitToluen \\
\hline 95578 & 2-CHLOROPHENOL \\
\hline 107051 & AllylChlor \\
\hline 7429905 & Aluminum \\
\hline 7440360 & Antimony \\
\hline 7440393 & Barium \\
\hline 71432 & Benzene \\
\hline 7440439 & Cadmi um \\
\hline 56235 & CCl4 \\
\hline 67663 & Chloroform \\
\hline 7440473 & Chromium \\
\hline 7782505 & Chlorine \\
\hline 630080 & Carbon Monoxide \\
\hline 7440508 & Copper \\
\hline 110827 & Cyclohexane \\
\hline 84662 & DiethylPhthalat \\
\hline 122394 & Diphenylam \\
\hline 75003 & Ethyl Chloride \\
\hline 100414 & Ethyl Benzene \\
\hline 206440 & Fluoranthene \\
\hline 7647010 & $\mathrm{HCl}$ \\
\hline 98828 & Cumene \\
\hline 7439921 & Lead \\
\hline 74873 & Methyl Chloride \\
\hline 71556 & $1,1,1$-TCA \\
\hline 108872 & MethCyHex \\
\hline 75092 & Methylene Chlor \\
\hline 91203 & Naphthalene \\
\hline 110543 & Hexane \\
\hline 10102440 & NITROGEN DIOXID \\
\hline 39001020 & $1-80 \mathrm{ctaCDF}$ \\
\hline 78115 & PETN \\
\hline 108952 & Phenol \\
\hline 115071 & Propylene \\
\hline 121824 & RDX \\
\hline 100425 & Styrene \\
\hline 7446095 & SULFUR DIOXIDE \\
\hline 127184 & Perc \\
\hline 108883 & Toluen \\
\hline
\end{tabular}

MULTIPLIER BG $(u g / m \wedge 3) \quad A V R$

STK $=1 \quad$ NAME $=$ LLNL Site 300 EMS (lbs/yr)

RRG (lbs/yr)

$\operatorname{MAX}(\mathrm{lbs} / \mathrm{hr}$ )

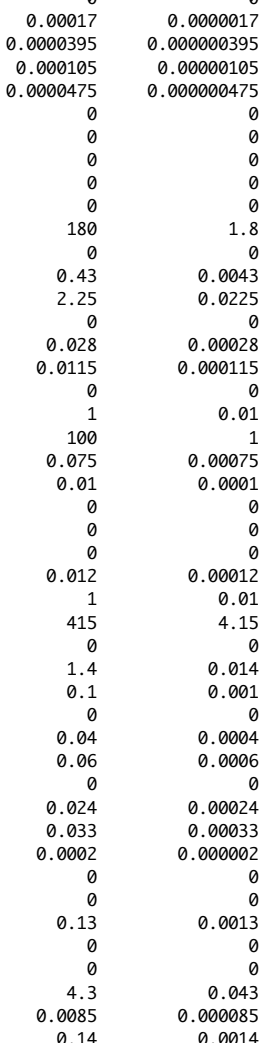




$\begin{array}{llllrr}75014 & \text { Vinyl Chloride } & 1 & 0 & 0 & 0 \\ 7440666 & \text { Zinc } & 1 & 0 & 2.85 & 0.0285 \\ 10101 & \text { NapthSur } & 1 & 0 & 0.8 & 0.008 \\ 10102 & \text { EthBzSur } & 1 & 0 & 0.038 & 0.00038 \\ 10103 & \text { nHexSur } & 1 & 0 & 9.83 & 0.0983\end{array}$

EMISSIONS FOR FACILITY FAC=Site300 CO=* DEV=PR1 PRO=0BCage4 STK=1 NAME=LLNL Site 300 EMS (lbs/yr)

SOURCE MULTIPLIER=1

\begin{tabular}{|c|c|}
\hline CAS & ABBREV \\
\hline 106990 & 1,3-Butadiene \\
\hline 67562394 & $1-4,6-8 \mathrm{HpCDF}$ \\
\hline 55673897 & 1-4,7-9HрCDF \\
\hline 70648269 & $1-4,7,8 \mathrm{H} \times \mathrm{CDF}$ \\
\hline 57117449 & $1-3,6-8 \mathrm{H} \times \mathrm{CDF}$ \\
\hline 35822469 & $1-4,6-8 \mathrm{HpCDD}$ \\
\hline 121142 & 2,4-DiNitToluen \\
\hline 606202 & 2,6-DiNitToluen \\
\hline 95578 & 2-CHLOROPHENOL \\
\hline 107051 & AllylChlor \\
\hline 7429905 & Aluminum \\
\hline 7440360 & Antimony \\
\hline 7440393 & Barium \\
\hline 71432 & Benzene \\
\hline 7440439 & Cadmium \\
\hline 56235 & CCl4 \\
\hline 67663 & Chloroform \\
\hline 7440473 & Chromium \\
\hline 7782505 & Chlorine \\
\hline 630080 & Carbon Monoxide \\
\hline 7440508 & Copper \\
\hline 110827 & Cyclohexane \\
\hline 84662 & DiethylPhthalat \\
\hline 122394 & Diphenylam \\
\hline 75003 & Ethyl Chloride \\
\hline 100414 & Ethyl Benzene \\
\hline 206440 & Fluoranthene \\
\hline 7647010 & $\mathrm{HCl}$ \\
\hline 98828 & Cumene \\
\hline 7439921 & Lead \\
\hline 74873 & Methyl Chloride \\
\hline 71556 & $1,1,1$-TCA \\
\hline 108872 & MethCyHex \\
\hline 75092 & Methylene Chlor \\
\hline 91203 & Naphthalene \\
\hline 110543 & Hexane \\
\hline 10102440 & NITROGEN DIOXID \\
\hline 39001020 & 1-80ctaCDF \\
\hline 78115 & PETN \\
\hline 108952 & Phenol \\
\hline 115071 & Propylene \\
\hline 121824 & RDX \\
\hline 100425 & Styrene \\
\hline 7446095 & SULFUR DIOXIDE \\
\hline 127184 & Perc \\
\hline 108883 & Toluene \\
\hline 75014 & Vinyl Chloride \\
\hline 7440666 & Zinc \\
\hline 10101 & NapthSur \\
\hline 10102 & EthBzSur \\
\hline 10103 & nHexSur \\
\hline
\end{tabular}

MULTIPLIER
1

$B G\left(u g / m^{\wedge} 3\right)$

AVRG (lbs/yr) MAX (lbs/hr)

1,3-Butadiene

4,6-8HpCDF

$1-4,7,8 \mathrm{HxCDF}$

$\begin{array}{ll}70648269 & 1-3,6-8 \mathrm{HxCDF} \\ 37117449 & 1-4,6-8 \mathrm{HpCDD}\end{array}$

$\begin{array}{ll}35822469 & 1-4,6-8 H p C D D \\ 121142 & 2,4-D i N i t T o l u e n\end{array}$

$\begin{array}{ll}606202 & \text { 2,6-DiNitToluen } \\ 95578 & \text { 2-CHLOROPHENOL }\end{array}$

107051 AllylChlor

7429905 Aluminum

$7440393 \quad$ Barium

7440439 Cadmium

56235

$7440473 \quad$ Chromium

630080 Carbon Monoxide

$7440508 \quad$ Copper

84662 DiethylPhthalat

$122394 \quad$ Diphenylam

$100414 \quad$ Ethyl Benzene

$206440 \quad$ Fluor

98828 Cumene

7439921 Lead

74873 Methyl Chloride

71556 1,1,1-TCA

75092 Methylene Chlor

$\begin{array}{ll}91203 & \text { Naphthal } \\ 110543 & \text { Hexane }\end{array}$

10102440 NITROGEN DIOXID

$39001020 \quad 1-80$ ctaCDF

108952 Pheno

$115071 \quad$ Propylene

$121824 \quad$ RDX

$7446095 \quad$ SULFUR DIOXIDE

$127184 \quad$ Perc

$\begin{array}{ll}108883 & \text { Toluene } \\ 75014 & \text { Vinyl Chloride }\end{array}$

$7440666 \quad$ Zinc

$\begin{array}{ll}10102 & \text { EthBzSur } \\ 10103 & \text { nHexSur }\end{array}$

EMISSIONS FOR FACILITY FAC $=$ Site 300

SOURCE MULTIPLIER $=1$

$\begin{array}{ll}\text { CAS } & \text { ABBREV } \\ 106990 & 1,3-\text { Butadiene } \\ 67562394 & 1-4,6-8 \text { HPCDF } \\ 55673897 & 1-4,7-9 \text { HPCDF } \\ 70648269 & 1-4,7,8 \text { HCCDF } \\ 57117449 & 1-3,6-8 \text { HCDF } \\ 35822469 & 1-4,6-8 \text { HCDD } \\ 121142 & 2,4-\text { DiNitToluen } \\ 606202 & 2,6 \text {-DiNitToluen } \\ 95578 & 2-\text { CHLOROPHENOL } \\ 107051 & \text { AllylChlor } \\ 7429905 & \text { Aluminum } \\ 7440360 & \text { Antimony } \\ 7440393 & \text { Barium } \\ 71432 & \text { Benzene } \\ 7440439 & \text { Cadmium } \\ 56235 & \text { CCl4 } \\ 67663 & \text { Chloroform } \\ 7440473 & \text { Chromium } \\ 7782505 & \text { Chlorine } \\ 630080 & \text { Carbon Monoxide } \\ 7440508 & \text { Copper } \\ 110827 & \text { Cyclohexane } \\ 84662 & \text { DiethylPhthalat } \\ 122394 & \text { Diphenylam } \\ 75003 & \text { Ethyl Chloride } \\ 100414 & \text { Ethyl Benzene } \\ 206440 & \text { Fluoranthene } \\ 7647010 & \text { HCl } \\ 98828 & \text { Cumene } \\ 7439921 & \text { Lead } \\ 74873 & \text { Methyl Chloride } \\ 71556 & 1,1,1-\text { CCA } \\ 108872 & \text { MethCyHex } \\ & \end{array}$

$\mathrm{CO}=* \quad \mathrm{DEV}=\mathrm{PR} 1$

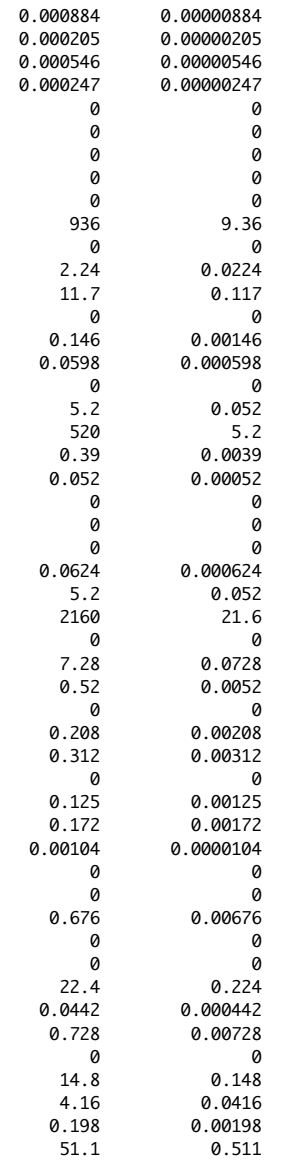

$\begin{array}{rrrr}\text { MULTIPLIER } & \text { BG (ug/m^3) } & \text { AVRG }(\mathrm{lbs} / \mathrm{yr}) & \text { MAX }(\mathrm{lbs} / \mathrm{hr}) \\ 1 & 0 & 0.42 & 0.0042 \\ 1 & 0 & 0 & 0 \\ 1 & 0 & 0 & 0 \\ 1 & 0 & 0 & 0 \\ 1 & 0 & 0 & 0 \\ 1 & 0 & 0.0000214 & 0.000000214 \\ 1 & 0 & 0 & 0 \\ 1 & 0 & 0 & 0 \\ 1 & 0 & 0 & 0 \\ 1 & 0 & 0.63 & 0.0063 \\ 1 & 0 & 875 & 8.75 \\ 1 & 0 & 0.0235 & 0.000235 \\ 1 & 0 & 287 & 2.87 \\ 1 & 0 & 3.85 & 0.0385 \\ 1 & 0 & 2.07 & 0.385 \\ 1 & 0 & 0.266 & 0.00266 \\ 1 & 0 & 0.0133 & 0.000133 \\ 1 & 0 & 5.25 & 0.0525 \\ 1 & 0 & 0 & 0 \\ 1 & 0 & 3850 & 38.5 \\ 1 & 0 & 910 & 9.1 \\ 1 & 0 & 0.263 & 0.00263 \\ 1 & 0 & 2.7 & 0.027 \\ 1 & 0 & 0 & 0 \\ 1 & 0 & 0.0242 & 0.000242 \\ 1 & 0 & 0.161 & 0.00161 \\ 1 & 0 & 0 & 0 \\ 1 & 0 & 0 & 0 \\ 1 & 0 & 0.0256 & 0.000256 \\ 1 & 0 & 38.5 & 0.385 \\ 1 & 0 & 0.0595 & 0.000595 \\ 1 & 0 & 0.116 & 0.00116 \\ 1 & 0 & 0.245 & 0.00245\end{array}$




$\begin{array}{lllrrr}75092 & \text { Methylene Chlor } & 1 & 0 & 30.5 & 0.305 \\ 91203 & \text { Naphthalene } & 1 & 0 & 0 & 0 \\ 110543 & \text { Hexane } & 1 & 0 & 0.665 & 0.00665 \\ 10102440 & \text { NITROGEN DIOXID } & 1 & 0 & 154 & 1.54 \\ 39001020 & \text { 1-80ctaCDF } & 1 & 0 & 0 & 0 \\ 78115 & \text { PETN } & 1 & 0 & 19.6 & 0.196 \\ 108952 & \text { Phenol } & 1 & 0 & 0 & 0 \\ 115071 & \text { Propylene } & 1 & 0 & 2.56 & 0.0256 \\ 121824 & \text { RDX } & 1 & 0 & 259 & 2.59 \\ 100425 & \text { Styrene } & 1 & 0 & 1.47 & 0.0147 \\ 7446095 & \text { SULFUR DIOXIDE } & 1 & 0 & 455 & 4.55 \\ 127184 & \text { Perc } & 1 & 0 & 0.63 & 0.0063 \\ 108883 & \text { Toluene } & 1 & 0 & 1.05 & 0.0105 \\ 75014 & \text { Vinyl Chloride } & 1 & 0 & 0.0805 & 0.000805 \\ 7440666 & \text { Zinc } & 1 & 0 & 560 & 5.6 \\ 10101 & \text { NapthSur } & 1 & 0 & 0 & 0 \\ 10102 & \text { EthBzSur } & 1 & 0 & 0.62 & 0.0062 \\ 10103 & \text { nHexSur } & 1 & 0 & 119 & 1.19\end{array}$

CANCER RISK REPORT

REC INHAL DERM SOIL MOTHER FISH WATER VEG DAIRY BEEF CHICK PIG EGG MEAT ORAL TOTAL $\begin{array}{llllllllllllllll}0001 & 1.30 \mathrm{E}-07 & 1.04 \mathrm{E}-07 & 1.12 \mathrm{E}-07 & 7.10 \mathrm{E}-08 & 0.00 \mathrm{E}+00 & 0.00 \mathrm{E}+00 & 1.49 \mathrm{E}-07 & 0.00 \mathrm{E}+00 & 9.21 \mathrm{E}-08 & 8.91 \mathrm{E}-12 & 7.74 \mathrm{E}-10 & 1.07 \mathrm{E}-11 & 9.29 \mathrm{E}-08 & 5.28 \mathrm{E}-07 & 6.57 \mathrm{E}-07\end{array}$

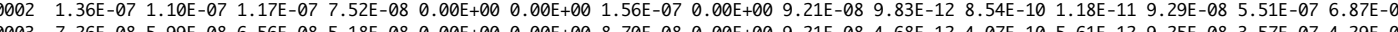

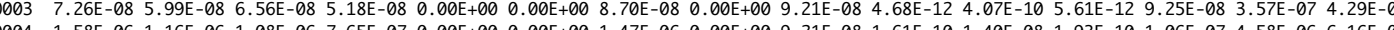

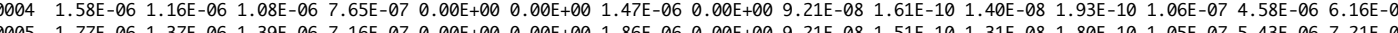
(1. $\begin{array}{lllllllllllllllllll}0006 & 1.30 \mathrm{E}-07 & 1.04 \mathrm{E}-07 & 1.12 \mathrm{E}-07 & 7.10 \mathrm{E}-08 & 0.00 \mathrm{E}+00 & 0.00 \mathrm{E}+00 & 1.49 \mathrm{E}-07 & 0.00 \mathrm{E}+00 & 9.21 \mathrm{E}-08 & 8.91 \mathrm{E}-12 & 7.74 \mathrm{E}-10 & 1.07 \mathrm{E}-11 & 9.29 \mathrm{E}-08 & 5.28 \mathrm{E}-07 & 6.57 \mathrm{E}-07 \\ 0007 & 1.13 \mathrm{E}-05 & 8.08 \mathrm{E}-06 & 7.74 \mathrm{E}-06 & 4.92 \mathrm{E}-06 & 0.00 \mathrm{E}+00 & 0.00 \mathrm{E}+00 & 1.05 \mathrm{E}-05 & 0.00 \mathrm{E}+00 & 9.21 \mathrm{E}-08 & 1.08 \mathrm{E}-09 & 9.35 \mathrm{E}-08 & 1.29 \mathrm{E}-09 & 1.88 \mathrm{E}-07 & 3.14 \mathrm{E}-05 & 4.27 \mathrm{E}-05\end{array}$ 
Rep_Can_WRK_Ave_AllSrc_Allch_ByRec_Site.txt 
This file: c: \HARPExpress \Projects \Rep_Can_WRK_Avg_AllRec_AllSrc_AllCh_ByRec_Site.txt

Created by HARP Version 1.0 Build 23.02.10

Uses ISC Version 9915

Uses BPIP Version 95086

Creation date: 10/24/2005 1:56:09 PM

EXCEPTION REPORT

(there have been no changes or exceptions)

INPUT FILES:

Source-Receptor file: c: \HARPExpress \Projects $\backslash E W T F . m t a$

Averaging period adjustment factors file: not applicable

Emission rates file: none

Site parameters file: C: $\backslash$ HARPExpress $\backslash$ Projects $\backslash$ Site300. sit

Screening mode is OFF

USER-DEFINED WORKER ADJUSTMENT AND EXPOSURE PARAMETERS

GLC Adjustment Factor: 1

Exposure Duration (years): 25

Exposure Frequency (days/year): 245

Explanatory Note: U.S. EPA value

Analysis method: Point estimate

Health effect: Cancer Risk

Receptor(s): All

$\begin{array}{ll}\text { Sources(s): } & \text { All } \\ \text { Chemicals(s): } & \text { All }\end{array}$

SITE PARAMETERS

DEPOSITION

Deposition rate $(\mathrm{m} / \mathrm{s})$

0.0072

DRINKING WATER

*** Pathway disabled $* * *$

FISH

*** Pathway disabled ***

PASTURE

ANIMALS' WATER

Water surface area $\left(\mathrm{m}^{\wedge} \mathrm{2}\right) \quad 0$

Water volume (L)

Volume changes per year

Fraction of beef cows' water

from pasture source

Fraction of dairy cows' water

Fraction of dairy cows' water
from pasture source

ANIMALS' FEED

Fraction of cows' feed

from grazing

HUMAN INGESTION

Fraction of ingested beef

from contaminated source

Fraction of ingested dairy

from contaminated source

0.107

HOME GROWN PRODUCE

HUMAN INGESTION

Fraction of ingested leafy vegetable

from home grown source 0.082

Fraction of ingested exposed vegetable

from home grown source $\quad 0.207$

Fraction of ingested protected vegetable

from home grown source 0.134

Fraction of ingested root vegetable

from home grown source 0.088

PIGS, CHICKENS AND EGGS

HUMAN INGESTION

Fraction of ingested pig

Fraction of ingested chicken

from home grown source

raction of ingested egg

0.04

from home grown source

0.026

ANIMALS' FEED

Fraction of pigs' feed

from home grown crop

Fraction of chickens' feed

0.029

from home grown crop

0.1

SOIL INGESTION

Fraction of pigs' feed

eaten off the ground

0.05 


\begin{tabular}{|c|c|}
\hline $\begin{array}{l}\text { Fraction of chickens' feed } \\
\text { eaten off the ground }\end{array}$ & 0.05 \\
\hline PIG FEED COMPOSITION & \\
\hline $\begin{array}{l}\text { Fraction of feed that is } \\
\text { exposed vegetable }\end{array}$ & 0.25 \\
\hline $\begin{array}{l}\text { Fraction of feed that is } \\
\text { leafy vegetable }\end{array}$ & 0.25 \\
\hline $\begin{array}{l}\text { Fraction of feed that is } \\
\text { protected vegetable }\end{array}$ & 0.25 \\
\hline $\begin{array}{l}\text { Fraction of feed that is } \\
\text { root vegetable }\end{array}$ & 0.25 \\
\hline CHICKEN FEED COMPOSITION & \\
\hline $\begin{array}{l}\text { Fraction of feed that is } \\
\text { exposed vegetable }\end{array}$ & 0.25 \\
\hline Fraction of feed that is & 0.25 \\
\hline $\begin{array}{l}\text { Fraction of feed that is } \\
\text { protected vegetable }\end{array}$ & 0.25 \\
\hline $\begin{array}{l}\text { Fraction of feed that is } \\
\text { root vegetable }\end{array}$ & 0.25 \\
\hline
\end{tabular}

DERMAL ABSORPTION

*** Pathway disabled ***

SOIL INGESTION

*** Pathway enabled ***

MOTHER'S MILK

*** Pathway enabled $* * *$

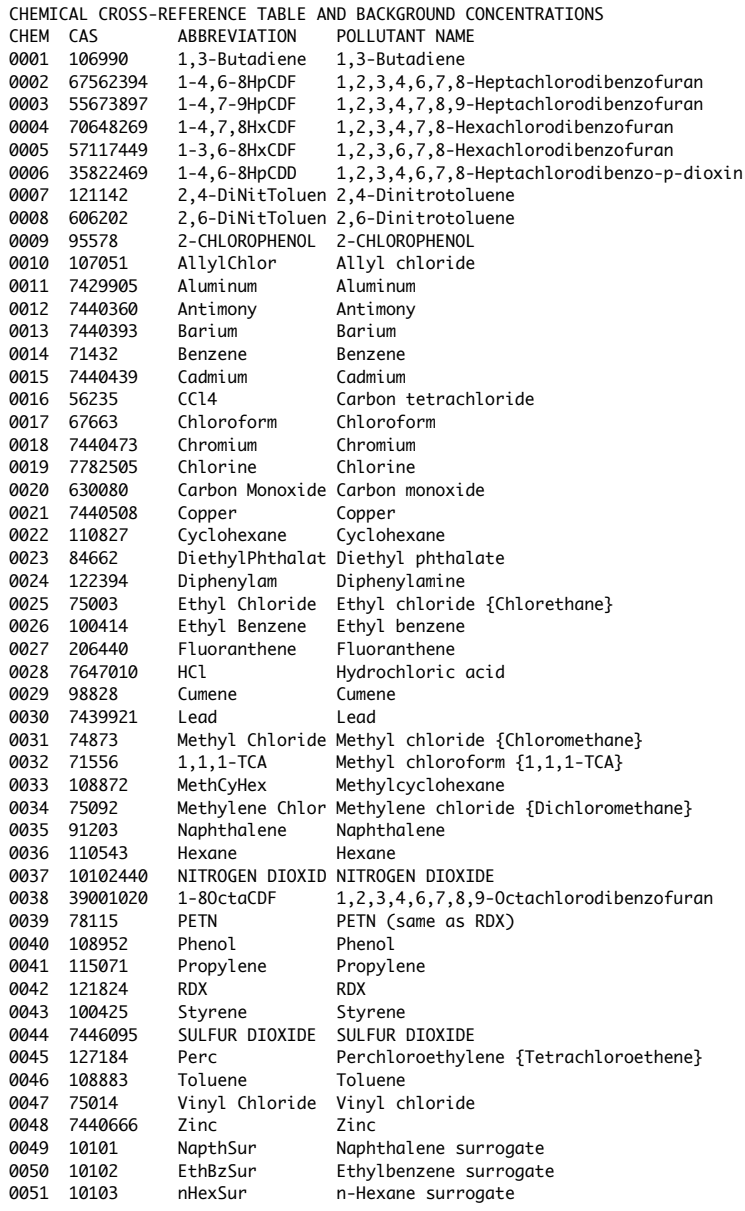

BACKGROUND (ug/ $\mathrm{m} \wedge 3$ )

$0.000 \mathrm{E}+00$

$0.000 \mathrm{E}+00$

$0.000 \mathrm{E}+00$

$0.000 \mathrm{E}+00$

$0.000 \mathrm{E}+00$

$0.000 \mathrm{E}+00$

$0.000 \mathrm{E}+00$

$0.000 \mathrm{E}+00$

$0.000 \mathrm{E}+00$

$0.000 \mathrm{E}+00$

$0.000 \mathrm{E}+00$

$0.000 \mathrm{E}+00$

$0.000 \mathrm{E}+00$

$0.000 \mathrm{E}+00$

$0.000 \mathrm{E}+00$

$0.000 \mathrm{E}+00$

$0.000 \mathrm{E}+00$

$0.000 \mathrm{E}+00$

$0.000 \mathrm{E}+00$
$0.000 \mathrm{E}+00$

$0.000 E+00$

$0.000 \mathrm{E}+00$

$0.000 \mathrm{E}+00$

$0.000 \mathrm{E}+00$

$0.000 E+00$

$0.000 \mathrm{E}+00$

$0.000 \mathrm{E}+00$

$0.000 \mathrm{E}+00$

$0.000 \mathrm{E}+00$

$0.000 \mathrm{E}+00$

$0.000 \mathrm{E}+00$

$0.000 \mathrm{E}+00$

$0.000 \mathrm{E}+00$

$0.000 \mathrm{E}+00$

$0.000 \mathrm{E}+00$

$0.000 \mathrm{E}+00$

$0.000 \mathrm{E}+00$

$0.000 \mathrm{E}+00$

$0.000 \mathrm{E}+00$

$0.000 \mathrm{E}+00$

$0.000 \mathrm{E}+00$

$0.000 \mathrm{E}+00$

$0.000 \mathrm{E}+00$

$0.000 \mathrm{E}+00$

$0.000 \mathrm{E}+00$

$0.000 \mathrm{E}+00$

$0.000 \mathrm{E}+00$

$0.000 \mathrm{E}+00$

$0.000 \mathrm{E}+00$

$0.000 \mathrm{E}+00$

$0.000 \mathrm{E}+00$

$0.000 \mathrm{E}+00$

$0.000 \mathrm{E}+00$

$0.000 \mathrm{E}+00$

EMISSIONS DATA SOURCE:

CHEMICALS ADDED OR DELETED: none

EMISSIONS FOR FACILITY FAC=Site300 CO=* DEV=PR1 PRO=0BPan STK=1 NAME=LLNL Site 300 EMS (lbs/yr) SOURCE MULTIPLIER $=1$
CAS
ABBREV
1069901 1,3-Butadiene
MULTIPLIER
$\begin{array}{rrr}\text { BG }(\mathrm{ug} / \mathrm{m} \wedge 3) & \text { AVRG (lbs/yr) } & \text { MAX (lbs/hr) } \\ 0 & 0.017 & 0.00017 \\ 0 & 0 & 0\end{array}$
$\begin{array}{rrr}\text { BG }\left(\mathrm{ug} / \mathrm{m}^{\wedge} 3\right) & \text { AVRG (lbs/yr) } & \text { MAX (lbs/hr) } \\ 0 & 0.017 & 0.00017 \\ 0 & 0 & 0\end{array}$
$67562394 \quad 1-4,6-8 \mathrm{HpCDF}$
1-4,6-8HpCDF 


\begin{tabular}{|c|c|}
\hline 55673897 & $1-4,7-9 \mathrm{HpCDF}$ \\
\hline 70648269 & $1-4,7,8 \mathrm{H} \times \mathrm{CDF}$ \\
\hline 57117449 & $1-3,6-8 \mathrm{H} \times \mathrm{CDF}$ \\
\hline 35822469 & $1-4,6-8 \mathrm{HpCDD}$ \\
\hline 121142 & 2,4-DiNitToluen \\
\hline 606202 & 2,6-DiNitToluen \\
\hline 95578 & 2-CHLOROPHENOL \\
\hline 107051 & AllylChlor \\
\hline 7429905 & Aluminum \\
\hline 7440360 & Antimony \\
\hline 7440393 & Barium \\
\hline 71432 & Benzene \\
\hline 7440439 & Cadmium \\
\hline 56235 & CCl4 \\
\hline 67663 & Chloroform \\
\hline 7440473 & Chromium \\
\hline 7782505 & Chlorine \\
\hline 630080 & Carbon Monoxide \\
\hline 7440508 & Copper \\
\hline 110827 & Cyclohexane \\
\hline 84662 & DiethylPhthalat \\
\hline 122394 & Diphenylam \\
\hline 75003 & Ethyl Chloride \\
\hline 100414 & Ethyl Benzene \\
\hline 206440 & Fluoranthene \\
\hline 7647010 & $\mathrm{HCl}$ \\
\hline 98828 & Cumene \\
\hline 7439921 & Lead \\
\hline 74873 & Methyl Chloride \\
\hline 71556 & 1,1,1-TCA \\
\hline 108872 & MethCyHex \\
\hline 75092 & Methylene Chlor \\
\hline 91203 & Naphthalene \\
\hline 110543 & Hexane \\
\hline 10102440 & NITROGEN DIOXID \\
\hline 39001020 & $1-80 \mathrm{ctaCDF}$ \\
\hline 78115 & PETN \\
\hline 108952 & Phenol \\
\hline 115071 & Propylene \\
\hline 121824 & $\mathrm{RDX}$ \\
\hline 100425 & Styrene \\
\hline 7446095 & SULFUR DIOXIDE \\
\hline 127184 & Perc \\
\hline 108883 & Toluene \\
\hline 75014 & Vinyl Chloride \\
\hline 7440666 & Zinc \\
\hline 10101 & NapthSur \\
\hline 10102 & EthBzSur \\
\hline 10103 & nHexSur \\
\hline
\end{tabular}

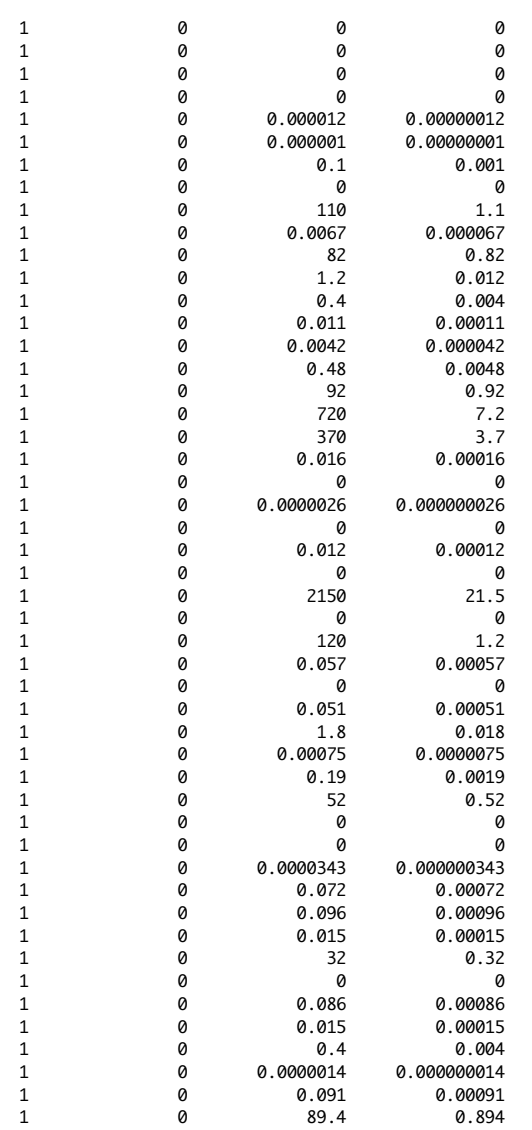

EMISSIONS FOR FACILITY FAC=Site300 CO=* DEV=PR1 PRO=0BCage3 STK=1 NAME=LLNL Site $300 \quad$ EMS (lbs/yr) SOURCE MULTIPLIER $=1$

$\begin{array}{ll}\text { CAS } & \text { ABBREV } \\ 106990 & 1,3-\text { Butadiene } \\ 67562394 & 1-4,6-8 \text { HpCDF } \\ 55673897 & 1-4,7-9 H \text { HCDF } \\ 70648269 & 1-4,7,8 \text { HXCDF } \\ 57117449 & 1-3,6-8 \text { HXCDF } \\ 35822469 & 1-4,6-8 \text { HPCDD } \\ 121142 & 2,4-\text { DiNitToluen } \\ 606202 & 2,6-\text { DiNitToluen } \\ 95578 & 2-\text { CHLOROPHENOL } \\ 107051 & \text { AllylChlor } \\ 7429905 & \text { Aluminum } \\ 7440360 & \text { Antimony } \\ 7440393 & \text { Barium } \\ 71432 & \text { Benzene } \\ 7440439 & \text { Cadmium } \\ 56235 & \text { CCl4 } \\ 67663 & \text { Chloroform } \\ 7440473 & \text { Chromium } \\ 7782505 & \text { Chlorine } \\ 630080 & \text { Carbon Monoxide } \\ 7440508 & \text { Copper } \\ 110827 & \text { Cyclohexane } \\ 84662 & \text { DiethylPhthalat } \\ 122394 & \text { Diphenylam } \\ 75003 & \text { Ethyl Chloride } \\ 100414 & \text { Ethyl Benzene } \\ 206440 & \text { Fluoranthene } \\ 7647010 & \text { HCl } \\ 98828 & \text { Cumene } \\ 7439921 & \text { Lead } \\ 74873 & \text { Methyl Chloride } \\ 71556 & 1,1,1-\text { CCA } \\ 108872 & \text { MethCyHex } \\ 75092 & \text { Methylene Chlor } \\ 91203 & \text { Naphthalene } \\ 110543 & \text { Hexane } \\ 10102440 & \text { NITROGEN DIOXID } \\ 39001020 & 1-80 \text { ctaCDF } \\ 78115 & \text { PETN } \\ 108952 & \text { Phenol } \\ 115071 & \text { Propylene } \\ 121824 & \text { RDX } \\ 100425 & \text { Styrene } \\ 7446095 & \text { SULFUR DIOXIDE } \\ 127184 & \text { Perc } \\ 108883 & \text { Toluene } \\ & \\ & \\ & \end{array}$

$\begin{array}{rrrr}\text { MULTIPLIER } & \text { BG (ug/m^3) } & \text { AVRG (lbs/yr) } & \text { MAX (lbs/hr) } \\ 1 & 0 & 0 & 0 \\ 1 & 0 & 0.00017 & 0.0000017 \\ 1 & 0 & 0.0000395 & 0.000000395 \\ 1 & 0 & 0.000105 & 0.00000105 \\ 1 & 0 & 0.0000475 & 0.000000475 \\ 1 & 0 & 0 & 0 \\ 1 & 0 & 0 & 0 \\ 1 & 0 & 0 & 0 \\ 1 & 0 & 0 & 0 \\ 1 & 0 & 0 & 0 \\ 1 & 0 & 180 & 1.8 \\ 1 & 0 & 0 & 0 \\ 1 & 0 & 0.43 & 0.0043 \\ 1 & 0 & 2.25 & 0.0225 \\ 1 & 0 & 0 & 0 \\ 1 & 0 & 0.028 & 0.00028 \\ 1 & 0 & 0.0115 & 0.000115 \\ 1 & 0 & 0 & 0 \\ 1 & 0 & 1 & 0.01 \\ 1 & 0 & 100 & 1 \\ 1 & 0 & 0.075 & 0.00075 \\ 1 & 0 & 0.01 & 0.0001 \\ 1 & 0 & 0 & 0 \\ 1 & 0 & 0 & 0 \\ 1 & 0 & 0 & 0 \\ 1 & 0 & 0.012 & 0.00012 \\ 1 & 0 & 1 & 0.01 \\ 1 & 0 & 415 & 4.15 \\ 1 & 0 & 0 & 0 \\ 1 & 0 & 1.4 & 0.014 \\ 1 & 0 & 0.1 & 0.001 \\ 1 & 0 & 0 & 0 \\ 1 & 0 & 0.04 & 0.0004 \\ 1 & 0 & 0.06 & 0.0006 \\ 1 & 0 & 0 & 0 \\ 1 & 0 & 0.024 & 0.00024 \\ 1 & 0 & 0.033 & 0.00033 \\ 1 & 0 & 0.0002 & 0.000002 \\ 1 & 0 & 0 & 0 \\ 1 & 0 & 0 & 0 \\ 1 & 0 & 0.13 & 0.0013 \\ 1 & 0 & 0 & 0 \\ 1 & 0 & 0 & 0 \\ 1 & 0 & 4.3 & 0.043 \\ 1 & 0 & 0.0085 & 0.000085 \\ 1 & 0 & 0.14 & 0.0014\end{array}$




$\begin{array}{llllrr}75014 & \text { Vinyl Chloride } & 1 & 0 & 0 & 0 \\ 7440666 & \text { Zinc } & 1 & 0 & 2.85 & 0.0285 \\ 10101 & \text { NapthSur } & 1 & 0 & 0.8 & 0.008 \\ 10102 & \text { EthBzSur } & 1 & 0 & 0.038 & 0.00038 \\ 10103 & \text { nHexSur } & 1 & 0 & 9.83 & 0.0983\end{array}$

EMISSIONS FOR FACILITY FAC $=S i t e 300 \quad$ CO $=* \quad$ DEV=PR1 PRO=0BCage4 STK=1 NAME=LLNL Site $300 \quad$ EMS (lbs/yr) SOURCE MULTIPLIER=1

\begin{tabular}{ll} 
SOURCE MULTIPLIER=1 \\
CAS & ABBREV \\
106990 & $1,3-$ Butadiene \\
67562394 & $1-4,6-8$ HpCDF \\
55673897 & $1-4,7-9 H p C D F$ \\
70648269 & $1-4,7,8$ HXCDF \\
57117449 & $1-3,6-8$ HXCDF \\
35822469 & $1-4,6-8$ HpCDD \\
121142 & $2,4-$ DiNitToluen \\
606202 & $2,6-$ DiNitToluen \\
95578 & 2-CHLOROPHENOL \\
107051 & AllylChlor \\
7429905 & Aluminum \\
7440360 & Antimony \\
7440393 & Barium \\
71432 & Benzene \\
7440439 & Cadmium \\
56235 & CCl4 \\
67663 & Chloroform \\
7440473 & Chromium \\
7782505 & Chlorine \\
630080 & Carbon Monoxide \\
7440508 & Copper \\
110827 & Cyclohexane \\
84662 & DiethylPhthalat \\
122394 & Diphenylam \\
75003 & Ethyl Chloride \\
100414 & Ethyl Benzene \\
206440 & Fluoranthene \\
7647010 & HCl \\
98828 & Cumene \\
7439921 & Lead \\
74873 & Methyl Chloride \\
71556 & $1,1,1-$ TCA \\
108872 & MethCyHex \\
75092 & Methylene Chlor \\
91203 & Naphthalene \\
110543 & Hexane \\
10102440 & NITROGEN DIOXID \\
39001020 & $1-80$ ctaCDF \\
78115 & PETN \\
108952 & Phenol \\
115071 & Propylene \\
121824 & RDX \\
100425 & Styrene \\
7446095 & SULFUR DIOXIDE \\
127184 & Perc \\
108883 & Toluene \\
75014 & Vinyl Chloride \\
7440666 & Zinc \\
10101 & NapthSur \\
10102 & EthBzSur \\
10103 & nHexSur \\
& \\
\hline
\end{tabular}

\begin{tabular}{|c|c|c|c|}
\hline MULTIPLIER & BG $(u g / m \wedge 3)$ & AVRG (lbs/yr) & MAX (lbs/hr) \\
\hline 1 & 0 & 0 & 0 \\
\hline 1 & 0 & 0.000884 & 0.00000884 \\
\hline 1 & 0 & 0.000205 & 0.00000205 \\
\hline 1 & 0 & 0.000546 & 0.00000546 \\
\hline 1 & 0 & 0.000247 & 0.00000247 \\
\hline 1 & 0 & 0 & 0 \\
\hline 1 & 0 & 0 & 0 \\
\hline 1 & 0 & 0 & 0 \\
\hline 1 & 0 & 0 & 0 \\
\hline 1 & 0 & 0 & 0 \\
\hline 1 & 0 & 936 & 9.36 \\
\hline 1 & 0 & 0 & 0 \\
\hline 1 & 0 & 2.24 & 0.0224 \\
\hline 1 & 0 & 11.7 & 0.117 \\
\hline 1 & 0 & 0 & 0 \\
\hline 1 & 0 & 0.146 & 0.00146 \\
\hline 1 & 0 & 0.0598 & 0.000598 \\
\hline 1 & 0 & 0 & 0 \\
\hline 1 & 0 & 5.2 & 0.052 \\
\hline 1 & 0 & 520 & 5.2 \\
\hline 1 & 0 & 0.39 & 0.0039 \\
\hline 1 & 0 & 0.052 & 0.00052 \\
\hline 1 & 0 & 0 & 0 \\
\hline 1 & 0 & 0 & 0 \\
\hline 1 & 0 & 0 & 0 \\
\hline 1 & 0 & 0.0624 & 0.000624 \\
\hline 1 & 0 & 5.2 & 0.052 \\
\hline 1 & 0 & 2160 & 21.6 \\
\hline 1 & 0 & 0 & 0 \\
\hline 1 & 0 & 7.28 & 0.0728 \\
\hline 1 & 0 & 0.52 & 0.0052 \\
\hline 1 & 0 & 0 & 0 \\
\hline 1 & 0 & 0.208 & 0.00208 \\
\hline 1 & 0 & 0.312 & 0.00312 \\
\hline 1 & 0 & 0 & 0 \\
\hline 1 & 0 & 0.125 & 0.00125 \\
\hline 1 & 0 & 0.172 & 0.00172 \\
\hline 1 & 0 & 0.00104 & 0.0000104 \\
\hline 1 & 0 & 0 & 0 \\
\hline 1 & 0 & 0 & 0 \\
\hline 1 & 0 & 0.676 & 0.00676 \\
\hline 1 & 0 & 0 & 0 \\
\hline 1 & 0 & 0 & 0 \\
\hline 1 & 0 & 22.4 & 0.224 \\
\hline 1 & 0 & 0.0442 & 0.000442 \\
\hline 1 & 0 & 0.728 & 0.00728 \\
\hline 1 & 0 & 0 & 0 \\
\hline 1 & 0 & 14.8 & 0.148 \\
\hline 1 & 0 & 4.16 & 0.0416 \\
\hline 1 & 0 & 0.198 & 0.00198 \\
\hline 1 & 0 & 51.1 & 0.511 \\
\hline
\end{tabular}

EMISSIONS FOR FACILITY FAC=Site300 CO=* DEV=PR1 PRO=0D STK=1 NAME=LLNL Site $300 \quad$ EMS (lbs/yr) SOURCE MULTIPLIER=1

$\begin{array}{ll}\text { CAS } & \text { ABBREV } \\ 106990 & 1,3-\text { Butadiene } \\ 67562394 & 1-4,6-8 \text { HpCDF } \\ 55673897 & 1-4,7-9 H \text { HCDF } \\ 70648269 & 1-4,7,8 \text { HxCDF } \\ 57117449 & 1-3,6-8 \text { HxCDF } \\ 35822469 & 1-4,6-8 \text { HpCDD } \\ 121142 & 2,4-\text { DiNitToluen } \\ 606202 & 2,6-\text { DiNitToluen } \\ 95578 & 2-\text { CHLOROPHENOL } \\ 107051 & \text { AllylChlor } \\ 7429905 & \text { Aluminum } \\ 7440360 & \text { Antimony } \\ 7440393 & \text { Barium } \\ 71432 & \text { Benzene } \\ 7440439 & \text { Cadmium } \\ 56235 & \text { CCl4 } \\ 67663 & \text { Chloroform } \\ 7440473 & \text { Chromium } \\ 7782505 & \text { Chlorine } \\ 630080 & \text { Carbon Monoxide } \\ 7440508 & \text { Copper } \\ 110827 & \text { Cyclohexane } \\ 84662 & \text { DiethylPhthalat } \\ 122394 & \text { Diphenylam } \\ 75003 & \text { Ethyl Chloride } \\ 100414 & \text { Ethyl Benzene } \\ 206440 & \text { Fluoranthene } \\ 7647010 & \text { HCl } \\ 98828 & \text { Cumene } \\ 7439921 & \text { Lead } \\ 74873 & \text { Methyl Chloride } \\ 71556 & 1,1,1-\text { CA } \\ 108872 & \text { MethCyHex } \\ 75092 & \text { Methylene Chlor } \\ 91203 & \text { Naphthalene } \\ & \\ & \end{array}$

MULTIPLIER

BG $(\mathrm{ug} / \mathrm{m} \wedge 3)$

RG (lbs/yr)

$\operatorname{MAX}(\mathrm{lbs} / \mathrm{hr})$

$\begin{array}{rr}\text { (lbs/yr) } & \text { MAX (lbs/hr) } \\ 0.42 & 0.0042 \\ 0 & 0 \\ 0 & 0 \\ 0 & 0 \\ 0 & 0 \\ 0.0000214 & 0.000000214 \\ 0 & 0 \\ 0 & 0 \\ 0 & 0 \\ 0.63 & 0.0063 \\ 875 & 8.75 \\ 0.0235 & 0.000235 \\ 287 & 2.87 \\ 3.85 & 0.0385 \\ 2.07 & 0.385 \\ 0.266 & 0.00266 \\ 0.0133 & 0.000133 \\ 5.25 & 0.0525 \\ 0 & 0 \\ 3850 & 38.5 \\ 910 & 9.1 \\ 0.263 & 0.00263 \\ 2.7 & 0.027 \\ 0 & 0 \\ 0.0242 & 0.000242 \\ 0.161 & 0.00161 \\ 0 & 0 \\ 0 & 0 \\ 0.0256 & 0.000256 \\ 38.5 & 0.385 \\ 0.0595 & 0.000595 \\ 0.116 & 0.00116 \\ 0.245 & 0.00245 \\ 30.5 & 0.305 \\ 0 & 0\end{array}$




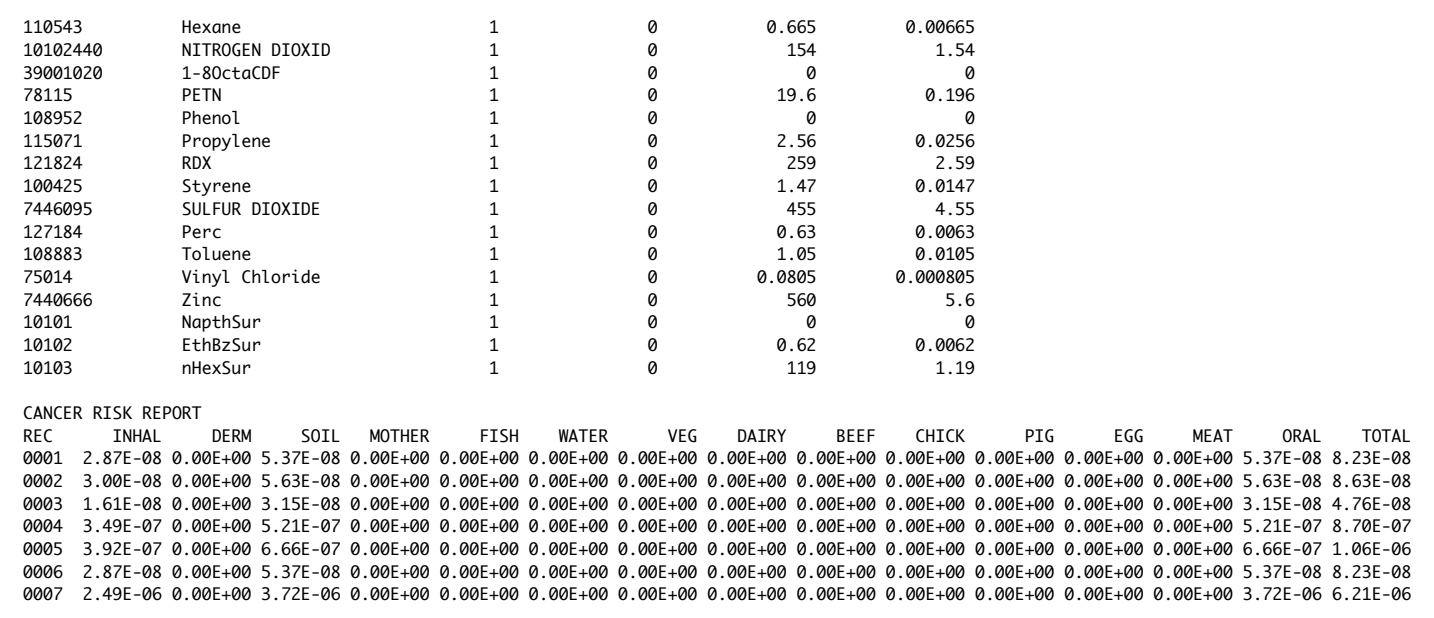


Rep_chr_Res_Hi_AllRec_AllSrc_AllCh_ByRec_Site.txt 
This file: c: \HARPExpress \Projects $\backslash$ Rep_Chr_Res_Hi_AllRec_AllSrc_AllCh_ByRec_Site.txt

Created by HARP Version 1.0 Build 23.02.10

Uses ISC Version 9915

Uses BPIP Version 95086

Creation date: 10/24/2005 1:45:36 PM

EXCEPTION REPORT

(there have been no changes or exceptions)

INPUT FILES:

Source-Receptor file: c: \HARPExpress \Projects \EWTF.mta

Source-Receptor file: c: $\backslash$ AARPExpress $\backslash$ Projects $\backslash$ WWTF.mta
Averaging period adjustment factors file: not applicable

Emission rates file: none

Site parameters file: c: \HARPExpress $\backslash$ Projects $\backslash$ Site300. sit

Screening mode is OFF

Exposure duration: resident

Analysis method: High-end Point Estimat

Health effect: Chronic HI

Receptor(s): All

Sources(s): $\quad$ All

SITE PARAMETERS

DEPOSITION

Deposition rate $(\mathrm{m} / \mathrm{s})$

0.0072

DRINKING WATER

*** Pathway disabled ***

FISH

*** Pathway disabled ***

PASTURE

ANIMALS' WATER

Water surface area $(\mathrm{m} \wedge 2) \quad 0$

Water volume (L)

Volume changes per year

from pasture source

Fraction of dairy cons' water

from pasture source

ANIMALS' FEED

Fraction of cows' feed

from grazing

HUMAN INGESTION

Fraction of ingested beef

from contaminated sourc

Fraction of ingested dairy

from contaminated source

0.107

HOME GROWN PRODUCE

HUMAN INGESTION

Fraction of ingested leafy vegetable

from home grown source 0.082

Fraction of ingested exposed vegetable

$\begin{array}{ll}\text { from home grown source } & 0.207\end{array}$

Fraction of ingested protected vegetable

from home grown source

0.134
0.088

from home grown source vegetable

PIGS, CHICKENS AND EGGS

$\begin{array}{ll}\text { HUMAN INGESTION } & \\ \text { Fraction of ingested pig } & \\ \quad \text { from home grown source } & 0.04 \\ \text { Fraction of ingested chicken } & \\ \quad \text { from home grown source } & 0.026 \\ \text { Fraction of ingested egg } & \\ \quad \text { from home grown source } & 0.029 \\ \text { ANIMALS' FEED } & \\ \text { Fraction of pigs' feed } \\ \quad \text { from home grown crop } \\ \text { Fraction of chickens' feed } \\ \quad \text { from home grown crop } \\ \begin{array}{l}\text { SOIL INGESTION } \\ \text { Fraction of pigs' feed } \\ \quad \text { eaten off the ground }\end{array} \\ \begin{array}{l}\text { Fraction of chickens' feed } \\ \text { eaten off the ground }\end{array} \\ \begin{array}{l}\text { PIG FEED COMPOSITION } \\ \text { Fraction of feed that is }\end{array} \\ \quad 0.1 \\ \end{array}$




\begin{tabular}{|c|c|}
\hline $\begin{array}{l}\text { exposed vegetable } \\
\text { Fraction of feed that is }\end{array}$ & 0.25 \\
\hline $\begin{array}{l}\text { Fraction of teed that is } \\
\text { leafy vegetable }\end{array}$ & 0.25 \\
\hline $\begin{array}{l}\text { Fraction of feed that is } \\
\text { protected vegetable }\end{array}$ & 0.25 \\
\hline $\begin{array}{l}\text { Fraction of feed that is } \\
\text { root vegetable }\end{array}$ & 0.25 \\
\hline CHICKEN FEED COMPOSITION & \\
\hline $\begin{array}{l}\text { Fraction of feed that is } \\
\text { exposed vegetable }\end{array}$ & 0.25 \\
\hline Fraction of feed that is & \\
\hline $\begin{array}{l}\text { Fraction of feed that is } \\
\text { protected vegetable }\end{array}$ & 0.25 \\
\hline $\begin{array}{l}\text { Fraction of feed that is } \\
\text { root vegetable }\end{array}$ & 0.25 \\
\hline
\end{tabular}

DERMAL ABSORPTION

*** Pathway enabled ***

SOIL INGESTION

*** Pathway enabled ***

MOTHER'S MILK

*** Pathway enabled ***
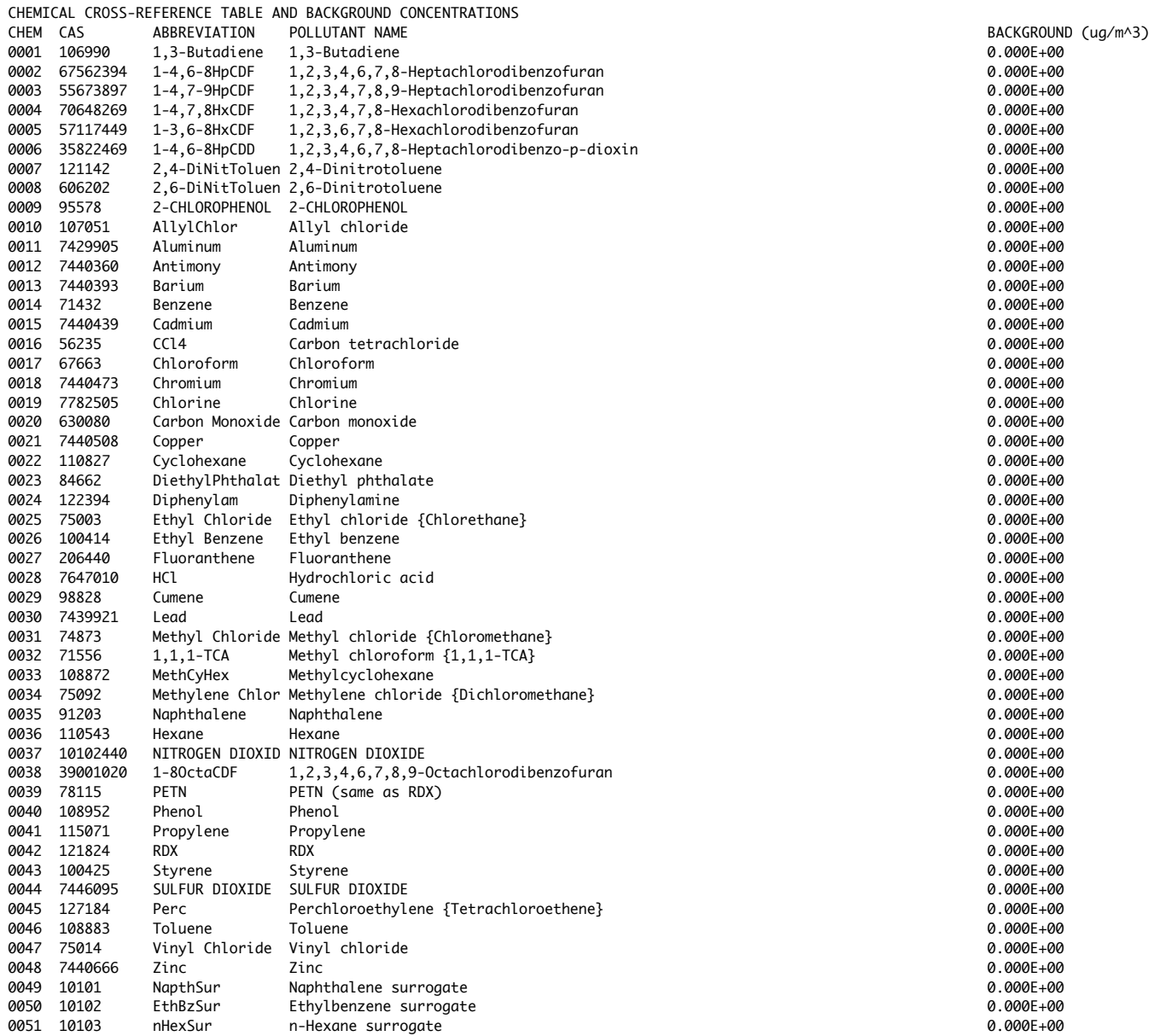

EMISSIONS DATA SOURCE:

CHEMICALS ADDED OR DELETED: none

\begin{tabular}{|c|c|c|c|c|c|c|c|c|}
\hline & \multicolumn{6}{|c|}{ SOURCE MULTIPLIER $=1$} & MS (lbs/yr) \\
\hline \multicolumn{9}{|c|}{$\begin{array}{l}\text { SOURCE } \\
\text { CAS }\end{array}$} \\
\hline 106990 & 1,3-Butadiene & & 1 & 0 & & 0.017 & 0.000 & 017 \\
\hline 67562394 & $1-4,6-8 \mathrm{HpCDF}$ & & 1 & 0 & & 0 & & 0 \\
\hline 55673897 & $1-4,7-9 \mathrm{HpCDF}$ & & 1 & 0 & & 0 & & 0 \\
\hline 70648269 & $1-4,7,8 \mathrm{H} \times C D F$ & & 1 & 0 & & 0 & & 0 \\
\hline 57117449 & $1-3,6-8 \mathrm{HxCDF}$ & & 1 & 0 & & 0 & & 0 \\
\hline 35822469 & $1-4,6-8 \mathrm{HpCDD}$ & & 1 & 0 & & 0 & & 0 \\
\hline
\end{tabular}




\begin{tabular}{|c|c|c|c|c|c|}
\hline 121142 & 2,4-DiNitToluen & 1 & 0 & 0.000012 & 0.00000012 \\
\hline 606202 & 2,6-DiNitToluen & 1 & 0 & 0.000001 & 0.00000001 \\
\hline 95578 & 2-CHLOROPHENOL & 1 & 0 & 0.1 & 0.001 \\
\hline 107051 & AllylChlor & 1 & 0 & 0 & 0 \\
\hline 7429905 & Aluminum & 1 & 0 & 110 & 1.1 \\
\hline 7440360 & Antimony & 1 & 0 & 0.0067 & 0.000067 \\
\hline 7440393 & Barium & 1 & 0 & 82 & 0.82 \\
\hline 71432 & Benzene & 1 & 0 & 1.2 & 0.012 \\
\hline 7440439 & Cadmium & 1 & 0 & 0.4 & 0.004 \\
\hline 56235 & CCl4 & 1 & 0 & 0.011 & 0.00011 \\
\hline 67663 & Chloroform & 1 & 0 & 0.0042 & 0.000042 \\
\hline 7440473 & Chromium & 1 & 0 & 0.48 & 0.0048 \\
\hline 7782505 & Chlorine & 1 & 0 & 92 & 0.92 \\
\hline 630080 & Carbon Monoxide & 1 & 0 & 720 & 7.2 \\
\hline 7440508 & Copper & 1 & 0 & 370 & 3.7 \\
\hline 110827 & Cyclohexane & 1 & 0 & 0.016 & 0.00016 \\
\hline 84662 & DiethylPhthalat & 1 & 0 & 0 & 0 \\
\hline 122394 & Diphenylam & 1 & 0 & 0.0000026 & 0.000000026 \\
\hline 75003 & Ethyl Chloride & 1 & 0 & 0 & 0 \\
\hline 100414 & Ethyl Benzene & 1 & 0 & 0.012 & 0.00012 \\
\hline 206440 & Fluoranthene & 1 & 0 & 0 & 0 \\
\hline 7647010 & $\mathrm{HCl}$ & 1 & 0 & 2150 & 21.5 \\
\hline 98828 & Cumene & 1 & 0 & 0 & 0 \\
\hline 7439921 & Lead & 1 & 0 & 120 & 1.2 \\
\hline 74873 & Methyl Chloride & 1 & 0 & 0.057 & 0.00057 \\
\hline 71556 & $1,1,1$-TCA & 1 & 0 & 0 & 0 \\
\hline 108872 & MethCyHex & 1 & 0 & 0.051 & 0.00051 \\
\hline 75092 & Methylene Chlor & 1 & 0 & 1.8 & 0.018 \\
\hline 91203 & Naphthalene & 1 & 0 & 0.00075 & 0.0000075 \\
\hline 110543 & Hexane & 1 & 0 & 0.19 & 0.0019 \\
\hline 10102440 & NITROGEN DIOXID & 1 & 0 & 52 & 0.52 \\
\hline 39001020 & $1-80 \mathrm{ctaCDF}$ & 1 & 0 & 0 & 0 \\
\hline 78115 & PETN & 1 & 0 & 0 & 0 \\
\hline 108952 & Phenol & 1 & 0 & 0.0000343 & 0.000000343 \\
\hline 115071 & Propylene & 1 & 0 & 0.072 & 0.00072 \\
\hline 121824 & RDX & 1 & 0 & 0.096 & 0.00096 \\
\hline 100425 & Styrene & 1 & 0 & 0.015 & 0.00015 \\
\hline 7446095 & SULFUR DIOXIDE & 1 & 0 & 32 & 0.32 \\
\hline 127184 & Perc & 1 & 0 & 0 & 0 \\
\hline 108883 & Toluene & 1 & 0 & 0.086 & 0.00086 \\
\hline 75014 & Vinyl Chloride & 1 & 0 & 0.015 & 0.00015 \\
\hline 7440666 & Zinc & 1 & 0 & 0.4 & 0.004 \\
\hline 10101 & NapthSur & 1 & 0 & 0.0000014 & 0.000000014 \\
\hline 10102 & EthBzSur & 1 & 0 & 0.091 & 0.00091 \\
\hline 10103 & nHexSur & 1 & 0 & 89.4 & 0.894 \\
\hline
\end{tabular}

EMISSIONS FOR FACILITY FAC=Site300 CO=* DEV=PR1 PRO=0BCage3 STK=1 NAME=LLNL Site $300 \quad$ EMS (lbs/yr) SOURCE MULTIPLIER $=1$

\begin{tabular}{ll} 
SOURCE MULTIPLIER=1 \\
CAS & ABBREV \\
106990 & $1,3-$ Butadiene \\
67562394 & $1-4,6-8 H$ HCDF \\
55673897 & $1-4,7-9 H$ HCDF \\
70648269 & $1-4,7,8 H \times$ CDF \\
57117449 & $1-3,6-8 H x C D F$ \\
35822469 & $1-4,6-8 H$ HCDD \\
121142 & $2,4-$ DiNitToluen \\
606202 & $2,6-$ DiNitToluen \\
95578 & 2-CHLOROPHENOL \\
107051 & AllylChlor \\
7429905 & Aluminum \\
7440360 & Antimony \\
7440393 & Barium \\
71432 & Benzene \\
7440439 & Cadmium \\
56235 & CCl4 \\
67663 & Chloroform \\
7440473 & Chromium \\
7782505 & Chlorine \\
630080 & Carbon Monoxide \\
7440508 & Copper \\
110827 & Cyclohexane \\
84662 & DiethylPhthalat \\
122394 & Diphenylam \\
75003 & Ethyl Chloride \\
100414 & Ethyl Benzene \\
206440 & Fluoranthene \\
7647010 & HCl \\
98828 & Cumene \\
7439921 & Lead \\
74873 & Methyl Chloride \\
71556 & $1,1,1-T C A$ \\
108872 & MethCyHex \\
75092 & Methylene Chlor \\
91203 & Naphthalene \\
110543 & Hexane \\
10102440 & NITROGEN DIOXID \\
39001020 & $1-80$ ctaCDF \\
78115 & PETN \\
108952 & Phenol \\
115071 & Propylene \\
121824 & RDX \\
100425 & Styrene \\
7446095 & SULFUR DIOXIDE \\
127184 & Perc \\
108883 & Toluene \\
75014 & Vinyl Chloride \\
7440666 & Zinc \\
10101 & NapthSur \\
& \\
\hline
\end{tabular}

\begin{tabular}{|c|c|c|c|}
\hline MULTIPLIER & BG $(u g / m \wedge 3)$ & AVRG (lbs/yr) & $\operatorname{MAX}(\mathrm{lbs} / \mathrm{hr})$ \\
\hline 1 & 0 & 0 & 0 \\
\hline 1 & 0 & 0.00017 & 0.0000017 \\
\hline 1 & 0 & 0.0000395 & 0.000000395 \\
\hline 1 & 0 & 0.000105 & 0.00000105 \\
\hline 1 & 0 & 0.0000475 & 0.000000475 \\
\hline 1 & 0 & 0 & 0 \\
\hline 1 & 0 & 0 & 0 \\
\hline 1 & 0 & 0 & 0 \\
\hline 1 & 0 & 0 & 0 \\
\hline 1 & 0 & 0 & 0 \\
\hline 1 & 0 & 180 & 1.8 \\
\hline 1 & 0 & 0 & 0 \\
\hline 1 & 0 & 0.43 & 0.0043 \\
\hline 1 & 0 & 2.25 & 0.0225 \\
\hline 1 & 0 & 0 & 0 \\
\hline 1 & 0 & 0.028 & 0.00028 \\
\hline 1 & 0 & 0.0115 & 0.000115 \\
\hline 1 & 0 & 0 & 0 \\
\hline 1 & 0 & 1 & 0.01 \\
\hline 1 & 0 & 100 & 1 \\
\hline 1 & 0 & 0.075 & 0.00075 \\
\hline 1 & 0 & 0.01 & 0.0001 \\
\hline 1 & 0 & 0 & 0 \\
\hline 1 & 0 & 0 & 0 \\
\hline 1 & 0 & 0 & 0 \\
\hline 1 & 0 & 0.012 & 0.00012 \\
\hline 1 & 0 & 1 & 0.01 \\
\hline 1 & 0 & 415 & 4.15 \\
\hline 1 & 0 & 0 & 0 \\
\hline 1 & 0 & 1.4 & 0.014 \\
\hline 1 & 0 & 0.1 & 0.001 \\
\hline 1 & 0 & 0 & 0 \\
\hline 1 & 0 & 0.04 & 0.0004 \\
\hline 1 & 0 & 0.06 & 0.0006 \\
\hline 1 & 0 & 0 & 0 \\
\hline 1 & 0 & 0.024 & 0.00024 \\
\hline 1 & 0 & 0.033 & 0.00033 \\
\hline 1 & 0 & 0.0002 & 0.000002 \\
\hline 1 & 0 & 0 & 0 \\
\hline 1 & 0 & 0 & 0 \\
\hline 1 & 0 & 0.13 & 0.0013 \\
\hline 1 & 0 & 0 & 0 \\
\hline 1 & 0 & 0 & 0 \\
\hline 1 & 0 & 4.3 & 0.043 \\
\hline 1 & 0 & 0.0085 & 0.000085 \\
\hline 1 & 0 & 0.14 & 0.0014 \\
\hline 1 & 0 & 0 & 0 \\
\hline 1 & 0 & 2.85 & 0.0285 \\
\hline 1 & 0 & 0.8 & 0.008 \\
\hline
\end{tabular}




\begin{tabular}{|c|c|c|c|}
\hline 10102 & EthBzSur & & 1 \\
\hline 10103 & nHexSur & & 1 \\
\hline $\begin{array}{l}\text { EMISSIONS FOR } \\
\text { SOURCE MULTIPL }\end{array}$ & $\begin{array}{l}\text { FACILITY } \quad F A C=S i t e 300 \\
\text { IER }=1\end{array}$ & $\mathrm{CO}=*$ & $\mathrm{DEV}=\mathrm{PR} 1$ \\
\hline CAS & ABBREV & MULTIP & PLIER \\
\hline 106990 & 1,3-Butadiene & & 1 \\
\hline 67562394 & $1-4,6-8 \mathrm{HpCDF}$ & & 1 \\
\hline 55673897 & $1-4,7-9 \mathrm{Hp} C \mathrm{CDF}$ & & 1 \\
\hline 70648269 & $1-4,7,8 \mathrm{HxCDF}$ & & 1 \\
\hline 57117449 & $1-3,6-8 \mathrm{H} \times C D F$ & & 1 \\
\hline 35822469 & $1-4,6-8 \mathrm{HpCDD}$ & & 1 \\
\hline 121142 & 2,4-DiNitToluen & & 1 \\
\hline 606202 & 2,6-DiNitToluen & & 1 \\
\hline 95578 & 2-CHLOROPHENOL & & 1 \\
\hline 107051 & AllylChlor & & 1 \\
\hline 7429905 & Aluminum & & 1 \\
\hline 7440360 & Antimony & & 1 \\
\hline 7440393 & Barium & & 1 \\
\hline 71432 & Benzene & & 1 \\
\hline 7440439 & Cadmium & & 1 \\
\hline 56235 & CCl4 & & 1 \\
\hline 67663 & Chloroform & & 1 \\
\hline 7440473 & Chromium & & 1 \\
\hline 7782505 & Chlorine & & 1 \\
\hline 630080 & Carbon Monoxide & & 1 \\
\hline 7440508 & Copper & & 1 \\
\hline 110827 & Cyclohexane & & 1 \\
\hline 84662 & DiethylPhthalat & & 1 \\
\hline 122394 & Diphenylam & & 1 \\
\hline 75003 & Ethyl Chloride & & 1 \\
\hline 100414 & Ethyl Benzene & & 1 \\
\hline 206440 & Fluoranthene & & 1 \\
\hline 7647010 & $\mathrm{HCl}$ & & 1 \\
\hline 98828 & Cumene & & 1 \\
\hline 7439921 & Lead & & 1 \\
\hline 74873 & Methyl Chloride & & 1 \\
\hline 71556 & 1,1,1-TCA & & 1 \\
\hline 108872 & MethCyHex & & 1 \\
\hline 75092 & Methylene Chlor & & 1 \\
\hline 91203 & Naphthalene & & 1 \\
\hline 110543 & Hexane & & 1 \\
\hline 10102440 & NITROGEN DIOXID & & 1 \\
\hline 39001020 & $1-80 \mathrm{ctaCDF}$ & & 1 \\
\hline 78115 & PETN & & 1 \\
\hline 108952 & Phenol & & 1 \\
\hline 115071 & Propylene & & 1 \\
\hline 121824 & RDX & & 1 \\
\hline 100425 & Styrene & & 1 \\
\hline 7446095 & SULFUR DIOXIDE & & 1 \\
\hline 127184 & Perc & & 1 \\
\hline 108883 & Toluene & & 1 \\
\hline 75014 & Vinyl Chloride & & 1 \\
\hline 7440666 & Zinc & & 1 \\
\hline 10101 & NapthSur & & 1 \\
\hline 10102 & EthBzSur & & 1 \\
\hline 10103 & nHexSur & & 1 \\
\hline EMISSIONS FOR & FACILITY $F A C=S i t e 300$ & $\mathrm{CO}=*$ & $D E V=P R 1$ \\
\hline SOURCE MULTIPL & IER $=1$ & & \\
\hline CAS & ABBREV & MULTIP & PLIER \\
\hline 106990 & 1,3-Butadiene & & 1 \\
\hline 67562394 & $1-4,6-8 \mathrm{HpCDF}$ & & 1 \\
\hline 55673897 & 1-4,7-9HрCDF & & 1 \\
\hline 70648269 & $1-4,7,8 \mathrm{HxCDF}$ & & 1 \\
\hline 57117449 & $1-3,6-8 \mathrm{H} \times \mathrm{CDF}$ & & 1 \\
\hline 35822469 & $1-4,6-8 \mathrm{HpCDD}$ & & 1 \\
\hline 121142 & 2,4-DiNitToluen & & 1 \\
\hline 606202 & 2,6-DiNitToluen & & 1 \\
\hline 95578 & 2-CHLOROPHENOL & & 1 \\
\hline 107051 & AllylChlor & & 1 \\
\hline 7429905 & Aluminum & & 1 \\
\hline 7440360 & Antimony & & 1 \\
\hline 7440393 & Barium & & 1 \\
\hline 71432 & Benzene & & 1 \\
\hline 7440439 & Cadmium & & 1 \\
\hline 56235 & CCl4 & & 1 \\
\hline 67663 & Chloroform & & 1 \\
\hline 7440473 & Chromium & & 1 \\
\hline 7782505 & Chlorine & & 1 \\
\hline 630080 & Carbon Monoxide & & 1 \\
\hline 7440508 & Copper & & 1 \\
\hline 110827 & Cyclohexane & & 1 \\
\hline 84662 & DiethylPhthalat & & 1 \\
\hline 122394 & Diphenylam & & 1 \\
\hline 75003 & Ethyl Chloride & & 1 \\
\hline 100414 & Ethyl Benzene & & 1 \\
\hline 206440 & Fluoranthene & & 1 \\
\hline 7647010 & $\mathrm{HCl}$ & & 1 \\
\hline 98828 & Cumene & & 1 \\
\hline 7439921 & Lead & & 1 \\
\hline 74873 & Methyl Chloride & & 1 \\
\hline 71556 & $1,1,1$-TCA & & 1 \\
\hline 108872 & MethCyHex & & 1 \\
\hline 75092 & Methylene Chlor & & 1 \\
\hline 91203 & Naphthalene & & 1 \\
\hline 110543 & Hexane & & 1 \\
\hline 10102440 & NITROGEN DIOXID & & 1 \\
\hline
\end{tabular}




$\begin{array}{llllrr}39001020 & \text { 1-80ctaCDF } & 1 & 0 & 0 & 0 \\ 78115 & \text { PETN } & 1 & 0 & 19.6 & 0.196 \\ 108952 & \text { Phenol } & 1 & 0 & 0 & 0 \\ 115071 & \text { Propylene } & 1 & 0 & 2.56 & 0.0256 \\ 121824 & \text { RDX } & 1 & 0 & 259 & 2.59 \\ 100425 & \text { Styrene } & 1 & 0 & 1.47 & 0.0147 \\ 7446095 & \text { SULFUR DIOXIDE } & 1 & 0 & 455 & 4.55 \\ 127184 & \text { Perc } & 1 & 0 & 0.63 & 0.0063 \\ 108883 & \text { Toluene } & 1 & 0 & 1.05 & 0.0105 \\ 75014 & \text { Vinyl Chloride } & 1 & 0 & 0.0805 & 0.000805 \\ 7440666 & \text { Zinc } & 1 & 0 & 560 & 5.6 \\ 10101 & \text { NapthSur } & 1 & 0 & 0 & 0 \\ 10102 & \text { EthBzSur } & 1 & 0 & 0.62 & 0.0062 \\ 10103 & \text { nHexSur } & 1 & 0 & 119 & 1.19 \\ & & & & & \end{array}$

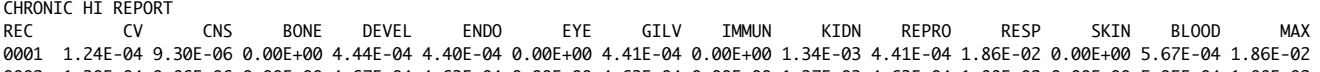

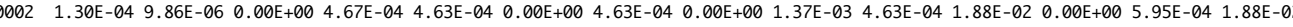
$0003 \quad 7.40 \mathrm{E}-05 \quad 5.31 \mathrm{E}-06 \quad 0.00 \mathrm{E}+00 \quad 3.41 \mathrm{E}-04 \quad 3.39 \mathrm{E}-04 \quad 0.00 \mathrm{E}+00 \quad 3.39 \mathrm{E}-04 \quad 0.00 \mathrm{E}+00 \quad 7.62 \mathrm{E}-04 \quad 3.39 \mathrm{E}-04 \quad 9.53 \mathrm{E}-03 \quad 0.00 \mathrm{E}+00 \quad 4.15 \mathrm{E}-04 \quad 9.53 \mathrm{E}-03$ $0004 \quad 1.08 \mathrm{E}-03 \quad 1.10 \mathrm{E}-04 \quad 0.00 \mathrm{E}+00 \quad 4.16 \mathrm{E}-03 \quad 4.10 \mathrm{E}-03 \quad 0.00 \mathrm{E}+00 \quad 4.10 \mathrm{E}-03 \quad 0.00 \mathrm{E}+00 \quad 1.39 \mathrm{E}-02 \quad 4.10 \mathrm{E}-03 \quad 2.86 \mathrm{E}-01 \quad 0.00 \mathrm{E}+00 \quad 5.23 \mathrm{E}-03 \quad 2.86 \mathrm{E}-01$ 0005 1.47E-03 $1.26 \mathrm{E}-04 \quad 0.00 \mathrm{E}+00 \quad 3.90 \mathrm{E}-03 \quad 3.84 \mathrm{E}-03 \quad 0.00 \mathrm{E}$

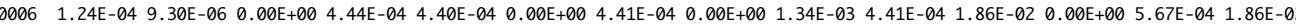

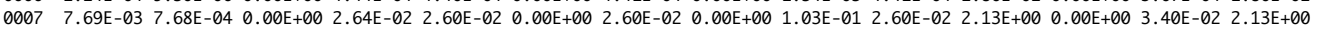


Rep_Chr_Wrk_PtEst_AllRec_AllSrc_AllCh_byRec_Site.txt 
This file: c: \HARPExpress $\backslash$ Projects $\backslash$ Rep_Chr_Wrk_PtEst_AllRec_AllSrc_AllCh_ByRec_Site.txt

Created by HARP Version 1.0 Build 23.02.10

Uses ISC Version 99155

Uses BPIP Version 95086

Creation date: 10/24/2005 1:57:22 PM

EXCEPTION REPORT

(there have been no changes or exceptions)

INPUT FILES:

Source-Receptor file: c: \HARPExpress \Projects $\backslash E W T F$.mta

Averaging period adjustment factors file: not applicable

Emission rates file: none

Site parameters file: C: \HARPExpress $\backslash$ Projects $\backslash$ Site300. sit

Screening mode is OFF

USER-DEFINED WORKER ADJUSTMENT AND EXPOSURE PARAMETERS

GLC Adjustment Factor:

Exposure Duration (years): 25

Exposure Frequency (days/year): 245

Explanatory Note: U.S. EPA value

Analysis method: Point estimat

Health effect: Chronic HI

Receptor(s): All

Sources(s):

Chemicals(s):

All

SITE PARAMETERS

DEPOSITION

Deposition rate $(\mathrm{m} / \mathrm{s})$

0.0072

DRINKING WATER

*** Pathway disabled ***

FISH

*** Pathway disabled ***

PASTURE

ANIMALS' WATER

Water surface area $\left(m^{\wedge} 2\right) \quad 0$

Water volume (L)

Volume changes per ye

Fraction of beef cows' water

from pasture source

Fraction of dairy cows' water

from pasture source

ANIMALS' FEED

Fraction of cows' feed

from grazing

HUMAN INGESTION

Fraction of ingested beef

Fraction of ingested dair

from contaminated source

0.107

0

HOME GROWN PRODUCE

HUMAN INGESTION

Fraction of ingested leafy vegetable

from home grown source 0.082 Fraction of ingested exposed vegetable from home grown source 0.207

Fraction of ingested protected vegetable

from home grown source 0.134

Fraction of ingested root vegetable

from home grown source $\quad 0.088$

PIGS, CHICKENS AND EGGS

HUMAN INGESTION

Fraction of ingested pig

from home grown source

Fraction of ingested chicken

0.04

from home grown source

0.02

raction of ingested egg

0.029

ANIMALS' FEED

Fraction of pigs' feed

raction of chickens' feed

from home grown crop

SOIL INGESTION

Fraction of pigs' feed

eaten off the ground 


\begin{tabular}{|c|c|}
\hline $\begin{array}{l}\text { Fraction of chickens' feed } \\
\text { eaten off the ground }\end{array}$ & 0.05 \\
\hline PIG FEED COMPOSITION & \\
\hline $\begin{array}{l}\text { Fraction of feed that is } \\
\text { exposed vegetable }\end{array}$ & 0.25 \\
\hline $\begin{array}{l}\text { Fraction of feed that is } \\
\text { leafy vegetable }\end{array}$ & 0.25 \\
\hline $\begin{array}{l}\text { Fraction of feed that is } \\
\text { protected vegetable }\end{array}$ & 0.25 \\
\hline $\begin{array}{l}\text { Fraction of feed that is } \\
\text { root vegetable }\end{array}$ & 0.25 \\
\hline CHICKEN FEED COMPOSITION & \\
\hline $\begin{array}{l}\text { Fraction of feed that is } \\
\text { exposed vegetable }\end{array}$ & 0.25 \\
\hline $\begin{array}{l}\text { Fraction of feed that is } \\
\text { leafy vegetable }\end{array}$ & 0.25 \\
\hline $\begin{array}{l}\text { Fraction of feed that is } \\
\text { protected vegetable }\end{array}$ & 0.25 \\
\hline $\begin{array}{l}\text { Fraction of feed that is } \\
\text { root vegetable }\end{array}$ & 0.25 \\
\hline
\end{tabular}

DERMAL ABSORPTION

*** Pathway disabled $* * *$

SOIL INGESTION

*** Pathway enabled $* * *$

MOTHER'S MILK

*** Pathway enabled $* * *$

CHEMICAL CROSS-REFERENCE TABLE AND BACKGROUND CONCENTRATIONS

CHEM CAS ABBREVIATION POLLUTA

$0001106990 \quad 1,3$-Butadiene 1,3-Butadiene

$000267562394 \quad 1-4,6-8 \mathrm{HpCDF} \quad 1,2,3,4,6,7,8$-Heptachlorodibenzofuran

$000355673897 \quad 1-4,7-9 H p C D F \quad 1,2,3,4,7,8,9$-Heptachlorodibenzofuran

$000470648269 \quad 1-4,7,8 \mathrm{HxCDF} \quad 1,2,3,4,7,8$-Hexachlorodibenzofuran

$000557117449 \quad 1-3,6-8 \mathrm{HxCDF} \quad 1,2,3,6,7,8$-Hexachlorodibenzofuran

$000635822469 \quad 1-4,6-8 \mathrm{HpCDD} \quad 1,2,3,4,6,7,8$-Heptachlorodibenzo-p-dioxin

0007121142 2,4-DiNitToluen 2,4-Dinitrotoluene

0008606202 2,6-DiNitToluen 2,6-Dinitrotoluen

000995578 2-CHLOROPHENOL 2-CHLOROPHENOL

0010107051 AllylChlor Allyl chloride

$\begin{array}{llll}0011 & 7429905 & \text { Aluminum } & \text { Aluminum } \\ 0012 & 7440360 & \text { Antimony } & \text { Antimony }\end{array}$

$\begin{array}{llll}0012 & 7440360 & \text { Antimony } & \text { Antimony } \\ 0013 & 7440393 & \text { Barium } & \text { Barium }\end{array}$

$\begin{array}{llll}0013 & 7440393 & \text { Barium } & \text { Barium } \\ 0014 & 71432 & \text { Benzene } & \text { Benzene }\end{array}$

00157440439 Cadmium Cadmium

001656235 CCl4 Carbon tetrachloride

$001767663 \quad$ Chloroform Chloroform

00187440473 Chromium Chromium

00197782505 Chlorine Chlorine

$0020630080 \quad$ Carbon Monoxide Carbon monoxide

00217440508 Copper $\quad$ Copper

$0023 \quad 84662$ DiethylPhthalat Diethyl phthalate

0024122394 Diphenylam Diphenylamine

$002575003 \quad$ Ethyl Chloride Ethyl chloride \{Chlorethane\}

$0026100414 \quad$ Ethyl Benzene Ethyl benzene

$0027206440 \quad$ Fluoranthene Fluoranthene

$00287647010 \quad \mathrm{HCl} \quad$ Hydrochloric acid

002998828 Cumene Cumene

$\begin{array}{llll}0030 & 7439921 & \text { Lead } & \text { Lead } \\ 0031 & 74873 & \text { Methyl Chloride Methyl chloride \{Chloromethane }\end{array}$

$003271556 \quad 1,1,1-$ TCA Methyl chloroform $\{1,1,1-\mathrm{TCA}\}$

108872 - MethCyHex

Methylene Chlor Methylene chloride \{Dichloromethane\}

003591203

$0036 \quad 110543$

$0037 \quad 10102440$

aphthalene Naphthalen

Hexane Hexane

003978115 PETN PETN (same as RDX)

0040108952

$0041 \quad 115071$

$0042 \quad 121824$

$\begin{array}{ll}0043 & 100425 \\ 0044 & 7446095\end{array}$

$0044 \quad 7446095$

Phenol

Phenol

Propylene Propylen

RDX RDX

$\begin{array}{ll}\text { Styrene } & \text { Styrene } \\ \text { SULFUR DIOXIDE } & \text { SULFUR DIOXIDE }\end{array}$

O045 $127184 \quad$ Per

004775014

$0048 \quad 7440666$

004910101

Perc

$\begin{array}{ll}\text { Vinyl Chloride } & \text { Vinyl chloride }\end{array}$

Zinc

$005110103 \quad$ nHexSur n-Hexane surrogate

BACKGROUND $(\mathrm{ug} / \mathrm{m} \wedge 3)$

$0.000 \mathrm{E}+00$

$0.000 \mathrm{E}+00$

$0.000 \mathrm{E}+00$

$0.000 \mathrm{E}+00$

$0.000 \mathrm{E}+00$

$0.000 \mathrm{E}+00$

$0.000 \mathrm{E}+00$

$0.000 \mathrm{E}+00$

$0.000 \mathrm{E}+00$

$0.000 \mathrm{E}+00$

$0.000 \mathrm{E}+00$

$0.000 \mathrm{E}+00$

$0.000 \mathrm{E}+00$

$0.000 \mathrm{E}+00$

$0.000 \mathrm{E}+00$

$0.000 \mathrm{E}+00$

$0.000 \mathrm{E}+00$

$0.000 \mathrm{E}+00$

$0.000 \mathrm{E}+00$

$0.000 \mathrm{E}+00$

$0.000 \mathrm{E}+00$

$0.000 \mathrm{E}+00$

$0.000 \mathrm{E}+00$

$0.000 \mathrm{E}+00$

$0.000 \mathrm{E}+00$

$0.000 \mathrm{E}+00$

$0.000 \mathrm{E}+00$

$0.00 \mathrm{E}$

$0.000 \mathrm{E}+00$

$0.000 E+00$

$0.000 \mathrm{E}+00$

$0.000 \mathrm{E}+00$

$0.000 \mathrm{E}+00$

$0.000 \mathrm{E}+00$

$0.000 \mathrm{E}+00$

$0.000 E+00$

$0.000 \mathrm{E}+00$

$0.000 \mathrm{E}+00$

$0.000 \mathrm{E}+00$

$0.000 \mathrm{E}+00$

$0.000 \mathrm{E}+00$

$0.000 \mathrm{E}+00$

$0.000 \mathrm{E}+00$

$0.000 \mathrm{E}+00$

$0.000 \mathrm{E}+00$

$0.000 \mathrm{E}+00$

$0.000 \mathrm{E}+00$

$0.000 \mathrm{E}+00$

$0.000 \mathrm{E}+00$

$0.000 \mathrm{E}+00$

$0.000 \mathrm{E}+00$

$0.000 \mathrm{E}+00$

EMISSIONS DATA SOURCE:

CHEMICALS ADDED OR DELETED: none

EMISSIONS FOR FACILITY FAC $=S i t e 300 \quad C 0=* \quad$ DEV=PR1 PRO=0BPan $\quad$ STK=1 NAME=LLNL Site $300 \quad$ EMS (lbs/yr) SOURCE MULTIPLIER $=1$

CAS ABBREV

$106990 \quad 1,3$-Butadien

MULTIPLIER

AVRG (lbs/yr) MAX (lbs/hr)

$\begin{array}{lrrrr}1-4,6-8 \mathrm{HpCDF} & 1 & 0 & 0.017 & 0.00017 \\ & 1 & 0 & 0 & 0\end{array}$ 


\begin{tabular}{|c|c|}
\hline 55673897 & $1-4,7-9 \mathrm{HpCDF}$ \\
\hline 70648269 & $1-4,7,8 \mathrm{H} \times C D F$ \\
\hline 57117449 & $1-3,6-8 \mathrm{H} \times C D F$ \\
\hline 35822469 & $1-4,6-8 \mathrm{HpCDD}$ \\
\hline 121142 & 2,4-DiNitToluen \\
\hline 606202 & 2,6-DiNitToluen \\
\hline 95578 & 2-CHLOROPHENOL \\
\hline 107051 & AllylChlor \\
\hline 7429905 & Aluminum \\
\hline 7440360 & Antimony \\
\hline 7440393 & Barium \\
\hline 71432 & Benzene \\
\hline 7440439 & Cadmium \\
\hline 56235 & CCl4 \\
\hline 67663 & Chloroform \\
\hline 7440473 & Chromium \\
\hline 7782505 & Chlorine \\
\hline 630080 & Carbon Monoxide \\
\hline 7440508 & Copper \\
\hline 110827 & Cyclohexane \\
\hline 84662 & DiethylPhthalat \\
\hline 122394 & Diphenylam \\
\hline 75003 & Ethyl Chloride \\
\hline 100414 & Ethyl Benzene \\
\hline 206440 & Fluoranthene \\
\hline 7647010 & $\mathrm{HCl}$ \\
\hline 98828 & Cumene \\
\hline 7439921 & Lead \\
\hline 74873 & Methyl Chloride \\
\hline 71556 & $1,1,1-\mathrm{TCA}$ \\
\hline 108872 & MethCyHex \\
\hline 75092 & Methylene Chlor \\
\hline 91203 & Naphthalene \\
\hline 110543 & Hexane \\
\hline 10102440 & NITROGEN DIOXID \\
\hline 39001020 & $1-80 \mathrm{ctaCDF}$ \\
\hline 78115 & PETN \\
\hline 108952 & Phenol \\
\hline 115071 & Propylene \\
\hline 121824 & $\mathrm{RDX}$ \\
\hline 100425 & Styrene \\
\hline 7446095 & SULFUR DIOXIDE \\
\hline 127184 & Perc \\
\hline 108883 & Toluene \\
\hline 75014 & Vinyl Chloride \\
\hline 7440666 & Zinc \\
\hline 10101 & NapthSur \\
\hline 10102 & EthBzSur \\
\hline 10103 & nHexSur \\
\hline $\begin{array}{l}\text { EMISSIONS FOR } \\
\text { SOURCE MULTIPL }\end{array}$ & $\begin{array}{l}\text { FACILITY } F A C=\text { Site } 300 \\
\text { IER }=1\end{array}$ \\
\hline CAS & ABBREV \\
\hline 106990 & 1,3-Butadiene \\
\hline 67562394 & $1-4,6-8 \mathrm{HpCDF}$ \\
\hline 55673897 & $1-4,7-9 \mathrm{HpCDF}$ \\
\hline 70648269 & $1-4,7,8 \mathrm{H} \times C D F$ \\
\hline 57117449 & $1-3,6-8 \mathrm{H} \times C D F$ \\
\hline 35822469 & $1-4,6-8 \mathrm{HpCDD}$ \\
\hline 121142 & 2,4-DiNitToluen \\
\hline 606202 & 2,6-DiNitToluen \\
\hline 95578 & 2-CHLOROPHENOL \\
\hline 107051 & AllylChlor \\
\hline 7429905 & Aluminum \\
\hline 7440360 & Antimony \\
\hline 7440393 & Barium \\
\hline 71432 & Benzene \\
\hline 7440439 & Cadmium \\
\hline 56235 & CCl4 \\
\hline 67663 & Chloroform \\
\hline 7440473 & Chromium \\
\hline 7782505 & Chlorine \\
\hline 630080 & Carbon Monoxide \\
\hline 7440508 & Copper \\
\hline 110827 & Cyclohexane \\
\hline 84662 & DiethylPhthalat \\
\hline 122394 & Diphenylam \\
\hline 75003 & Ethyl Chloride \\
\hline 100414 & Ethyl Benzene \\
\hline 206440 & Fluoranthene \\
\hline 7647010 & $\mathrm{HCl}$ \\
\hline 98828 & Cumene \\
\hline 7439921 & Lead \\
\hline 74873 & Methyl Chloride \\
\hline 71556 & $1,1,1$-TCA \\
\hline 108872 & MethCyHex \\
\hline 75092 & Methylene Chlor \\
\hline 91203 & Naphthalene \\
\hline 110543 & Hexane \\
\hline 10102440 & NITROGEN DIOXID \\
\hline 39001020 & $1-80 \mathrm{ctaCDF}$ \\
\hline 78115 & PETN \\
\hline 108952 & Phenol \\
\hline 115071 & Propylene \\
\hline 121824 & $\mathrm{RDX}$ \\
\hline 100425 & Styrene \\
\hline 7446095 & SULFUR DIOXIDE \\
\hline 127184 & Perc \\
\hline 108883 & Toluene \\
\hline
\end{tabular}




$\begin{array}{llllrr}75014 & \text { Vinyl Chloride } & 1 & 0 & 0 & 0 \\ 7440666 & \text { Zinc } & 1 & 0 & 2.85 & 0.0285 \\ 10101 & \text { NapthSur } & 1 & 0 & 0.8 & 0.008 \\ 10102 & \text { EthBzSur } & 1 & 0 & 0.038 & 0.00038 \\ 10103 & \text { nHexSur } & 1 & 0 & 9.83 & 0.0983\end{array}$

EMISSIONS FOR FACILITY FAC=Site300 CO=* DEV=PR1 PRO=0BCage4 STK=1 NAME=LLNL Site $300 \quad$ EMS (lbs/yr) SOURCE MULTIPLIER=1

\begin{tabular}{|c|c|c|c|c|c|}
\hline CAS & ABBREV & MULTIPLIER & BG $(u g / m \wedge 3)$ & AVRG (lbs/yr) & $\operatorname{MAX}(\mathrm{lbs} / \mathrm{hr})$ \\
\hline 106990 & 1,3-Butadiene & 1 & 0 & 0 & 0 \\
\hline 67562394 & $1-4,6-8 \mathrm{HpCDF}$ & 1 & 0 & 0.000884 & 0.00000884 \\
\hline 55673897 & $1-4,7-9 \mathrm{HpCDF}$ & 1 & 0 & 0.000205 & 0.00000205 \\
\hline 70648269 & $1-4,7,8 \mathrm{HxCDF}$ & 1 & 0 & 0.000546 & 0.00000546 \\
\hline 57117449 & $1-3,6-8 \mathrm{H} \times C D F$ & 1 & 0 & 0.000247 & 0.00000247 \\
\hline 35822469 & $1-4,6-8 \mathrm{HpCDD}$ & 1 & 0 & 0 & 0 \\
\hline 121142 & 2,4-DiNitToluen & 1 & 0 & 0 & 0 \\
\hline 606202 & 2,6-DiNitToluen & 1 & 0 & 0 & 0 \\
\hline 95578 & 2-CHLOROPHENOL & 1 & 0 & 0 & 0 \\
\hline 107051 & AllylChlor & 1 & 0 & 0 & 0 \\
\hline 7429905 & Aluminum & 1 & 0 & 936 & 9.36 \\
\hline 7440360 & Antimony & 1 & 0 & 0 & 0 \\
\hline 7440393 & Barium & 1 & 0 & 2.24 & 0.0224 \\
\hline 71432 & Benzene & 1 & 0 & 11.7 & 0.117 \\
\hline 7440439 & Cadmium & 1 & 0 & 0 & 0 \\
\hline 56235 & CCl4 & 1 & 0 & 0.146 & 0.00146 \\
\hline 67663 & Chloroform & 1 & 0 & 0.0598 & 0.000598 \\
\hline 7440473 & Chromium & 1 & 0 & 0 & 0 \\
\hline 7782505 & Chlorine & 1 & 0 & 5.2 & 0.052 \\
\hline 630080 & Carbon Monoxide & 1 & 0 & 520 & 5.2 \\
\hline 7440508 & Copper & 1 & 0 & 0.39 & 0.0039 \\
\hline 110827 & Cyclohexane & 1 & 0 & 0.052 & 0.00052 \\
\hline 84662 & DiethylPhthalat & 1 & 0 & 0 & 0 \\
\hline 122394 & Diphenylam & 1 & 0 & 0 & 0 \\
\hline 75003 & Ethyl Chloride & 1 & 0 & 0 & 0 \\
\hline 100414 & Ethyl Benzene & 1 & 0 & 0.0624 & 0.000624 \\
\hline 206440 & Fluoranthene & 1 & 0 & 5.2 & 0.052 \\
\hline 7647010 & $\mathrm{HCl}$ & 1 & 0 & 2160 & 21.6 \\
\hline 98828 & Cumene & 1 & 0 & 0 & 0 \\
\hline 7439921 & Lead & 1 & 0 & 7.28 & 0.0728 \\
\hline 74873 & Methyl Chloride & 1 & 0 & 0.52 & 0.0052 \\
\hline 71556 & $1,1,1$-TCA & 1 & 0 & 0 & 0 \\
\hline 108872 & MethCyHex & 1 & 0 & 0.208 & 0.00208 \\
\hline 75092 & Methylene Chlor & 1 & 0 & 0.312 & 0.00312 \\
\hline 91203 & Naphthalene & 1 & 0 & 0 & 0 \\
\hline 110543 & Hexane & 1 & 0 & 0.125 & 0.00125 \\
\hline 10102440 & NITROGEN DIOXID & 1 & 0 & 0.172 & 0.00172 \\
\hline 39001020 & 1-80ctaCDF & 1 & 0 & 0.00104 & 0.0000104 \\
\hline 78115 & PETN & 1 & 0 & 0 & 0 \\
\hline 108952 & Phenol & 1 & 0 & 0 & 0 \\
\hline 115071 & Propylene & 1 & 0 & 0.676 & 0.00676 \\
\hline 121824 & RDX & 1 & 0 & 0 & 0 \\
\hline 100425 & Styrene & 1 & 0 & 0 & 0 \\
\hline 7446095 & SULFUR DIOXIDE & 1 & 0 & 22.4 & 0.224 \\
\hline 127184 & Perc & 1 & 0 & 0.0442 & 0.000442 \\
\hline 108883 & Toluene & 1 & 0 & 0.728 & 0.00728 \\
\hline 75014 & Vinyl Chloride & 1 & 0 & 0 & 0 \\
\hline 7440666 & Zinc & 1 & 0 & 14.8 & 0.148 \\
\hline 10101 & NapthSur & 1 & 0 & 4.16 & 0.0416 \\
\hline 10102 & EthBzSur & 1 & 0 & 0.198 & 0.00198 \\
\hline 10103 & nHexSur & 1 & 0 & 51.1 & 0.511 \\
\hline
\end{tabular}

EMISSIONS FOR FACILITY FAC=Site300 CO=* DEV=PR1 PR0=0D STK=1 NAME=LLNL Site 300 EMS (lbs/yr) SOURCE MULTIPLIER $=1$

$\begin{array}{ll}\text { CAS } & \text { ABBREV } \\ 106990 & 1,3-\text { Butadiene } \\ 67562394 & 1-4,6-8 \text { HpCDF } \\ 55673897 & 1-4,7-9 H p C D F \\ 70648269 & 1-4,7,8 \text { HxCDF } \\ 57117449 & 1-3,6-8 \text { HxCDF } \\ 35822469 & 1-4,6-8 \text { HpCDD } \\ 121142 & 2,4-\text {-iNitToluen } \\ 606202 & 2,6-\text { - NiNitToluen } \\ 95578 & 2-\text { CHLOROPHENOL } \\ 107051 & \text { AllylChlor } \\ 7429905 & \text { Aluminum } \\ 7440360 & \text { Antimony } \\ 7440393 & \text { Barium } \\ 71432 & \text { Benzene } \\ 7440439 & \text { Cadmium } \\ 56235 & \text { CCl4 } \\ 67663 & \text { Chloroform } \\ 7440473 & \text { Chromium } \\ 7782505 & \text { Chlorine } \\ 630080 & \text { Carbon Monoxide } \\ 7440508 & \text { Copper } \\ 110827 & \text { Cyclohexane } \\ 84662 & \text { DiethylPhthalat } \\ 122394 & \text { Diphenylam } \\ 75003 & \text { Ethyl Chloride } \\ 100414 & \text { Ethyl Benzene } \\ 206440 & \text { Fluoranthene } \\ 7647010 & \text { HCl } \\ 98828 & \text { Cumene } \\ 7439921 & \text { Lead } \\ 74873 & \text { Methyl Chloride } \\ 71556 & 1,1,1-\text { TCA } \\ 108872 & \text { MethCyHex } \\ 75092 & \text { Methylene Chlor } \\ 91203 & \text { Naphthalene } \\ & \end{array}$

$\begin{array}{rrrr}\text { MULTIPLIER } & \text { BG (ug/m^3) } & \text { AVRG (lbs/yr) } & \text { MAX }(\mathrm{lbs} / \mathrm{hr}) \\ 1 & 0 & 0.42 & 0.0042 \\ 1 & 0 & 0 & 0 \\ 1 & 0 & 0 & 0 \\ 1 & 0 & 0 & 0 \\ 1 & 0 & 0 & 0 \\ 1 & 0 & 0.0000214 & 0.000000214 \\ 1 & 0 & 0 & 0 \\ 1 & 0 & 0 & 0 \\ 1 & 0 & 0 & 0 \\ 1 & 0 & 0.63 & 0.0063 \\ 1 & 0 & 875 & 8.75 \\ 1 & 0 & 0.0235 & 0.000235 \\ 1 & 0 & 287 & 2.87 \\ 1 & 0 & 3.85 & 0.0385 \\ 1 & 0 & 2.07 & 0.385 \\ 1 & 0 & 0.266 & 0.00266 \\ 1 & 0 & 0.0133 & 0.000133 \\ 1 & 0 & 5.25 & 0.0525 \\ 1 & 0 & 0 & 0 \\ 1 & 0 & 3850 & 38.5 \\ 1 & 0 & 910 & 9.1 \\ 1 & 0 & 0.263 & 0.00263 \\ 1 & 0 & 2.7 & 0.027 \\ 1 & 0 & 0 & 0 \\ 1 & 0 & 0.0242 & 0.000242 \\ 1 & 0 & 0.161 & 0.00161 \\ 1 & 0 & 0 & 0 \\ 1 & 0 & 0 & 0 \\ 1 & 0 & 0.0256 & 0.000256 \\ 1 & 0 & 38.5 & 0.385 \\ 1 & 0 & 0.0595 & 0.000595 \\ 1 & 0 & 0.116 & 0.00116 \\ 1 & 0 & 0.245 & 0.00245 \\ 1 & 0 & 30.5 & 0.305 \\ 1 & 0 & 0 & 0 \\ & 0 & & 0\end{array}$




\begin{tabular}{|c|c|c|c|c|c|c|c|c|c|c|c|}
\hline 110543 & Hexane & & 1 & 0 & 0.6 & 665 & 0.00665 & & & & \\
\hline 10102440 & NITROGEN DIOXID & & 1 & 0 & & 154 & 1.54 & & & & \\
\hline 39001020 & $1-80 \mathrm{ctaCDF}$ & & 1 & 0 & & 0 & 0 & 0 & & & \\
\hline 78115 & PETN & & 1 & 0 & & 9.6 & 0.196 & & & & \\
\hline 108952 & Phenol & & 1 & 0 & & 0 & & 0 & & & \\
\hline 115071 & Propylene & & 1 & 0 & & .56 & 0.0256 & & & & \\
\hline 121824 & $\operatorname{RDX}$ & & 1 & 0 & & 259 & 2.59 & & & & \\
\hline 100425 & Styrene & & 1 & 0 & & .47 & 0.0147 & & & & \\
\hline 7446095 & SULFUR DIOXIDE & & 1 & 0 & & 455 & 4.55 & & & & \\
\hline 127184 & Perc & & 1 & 0 & & .63 & 0.0063 & & & & \\
\hline 108883 & Toluene & & 1 & 0 & & .05 & 0.0105 & & & & \\
\hline 75014 & Vinyl Chloride & & 1 & 0 & 0.08 & 805 & 0.000805 & & & & \\
\hline 7440666 & Zinc & & 1 & 0 & & 560 & 5.6 & & & & \\
\hline 10101 & NapthSur & & 1 & 0 & & 0 & 0 & $\theta$ & & & \\
\hline 10102 & EthBzSur & & 1 & 0 & & .62 & 0.0062 & & & & \\
\hline 10103 & nHexSur & & 1 & 0 & & 119 & 1.19 & & & & \\
\hline \multicolumn{12}{|c|}{ CHRONIC HI REPORT } \\
\hline REC & BONE & DEVEL & ENDO & GILV & IMMUN & KIDN & REPRO & RESP & SKIN & BLOOD & MAX \\
\hline $0001 \quad 1.24 \mathrm{E}-04$ & $9.30 \mathrm{E}-06 \quad 0.00 \mathrm{E}+00$ & $5.45 \mathrm{E}-05$ & $5.07 \mathrm{E}-05 \quad 0.00 \mathrm{E}+00$ & $5.10 \mathrm{E}-05$ & $0.00 \mathrm{E}+00$ & $1.03 \mathrm{E}-03$ & $5.09 \mathrm{E}-05$ & $1.82 \mathrm{E}-02$ & $0.00 \mathrm{E}+00$ & $1.77 \mathrm{E}-04$ & $1.82 \mathrm{E}-02$ \\
\hline $0002 \quad 1.30 \mathrm{E}-04$ & $9.86 \mathrm{E}-06 \quad 0.00 \mathrm{E}+00$ & $6.00 \mathrm{E}-05$ & $5.60 \mathrm{E}-05 \quad 0.00 \mathrm{E}+00$ & $5.63 \mathrm{E}-05$ & $0.00 \mathrm{E}+00$ & $1.06 \mathrm{E}-03$ & $5.62 \mathrm{E}-05$ & $1.84 \mathrm{E}-02$ & $0.00 \mathrm{E}+00$ & $1.89 \mathrm{E}-04$ & $1.84 \mathrm{E}-02$ \\
\hline $0003 \quad 7.40 \mathrm{E}-05$ & $5.31 \mathrm{E}-06 \quad 0.00 \mathrm{E}+00$ & $2.87 \mathrm{E}-05$ & $2.67 \mathrm{E}-05 \quad 0.00 \mathrm{E}+00$ & $2.69 \mathrm{E}-05$ & $0.00 \mathrm{E}+00$ & $5.88 \mathrm{E}-04$ & $2.68 \mathrm{E}-05$ & $9.22 \mathrm{E}-03$ & $0.00 \mathrm{E}+00$ & $1.02 \mathrm{E}-04$ & $9.22 \mathrm{E}-03$ \\
\hline $0004 \quad 1.08 \mathrm{E}-03$ & $1.10 \mathrm{E}-04 \quad 0.00 \mathrm{E}+00$ & $9.82 \mathrm{E}-04$ & $9.19 \mathrm{E}-04 \quad 0.00 \mathrm{E}+00$ & $9.23 \mathrm{E}-04$ & $0.00 \mathrm{E}+00$ & $1.07 \mathrm{E}-02$ & $9.22 \mathrm{E}-04$ & 2. $83 \mathrm{E}-01$ & $0.00 \mathrm{E}+00$ & $2.05 \mathrm{E}-03$ & 2. $83 \mathrm{E}-01$ \\
\hline $0005 \quad 1.47 \mathrm{E}-03$ & $1.26 \mathrm{E}-04 \quad 0.00 \mathrm{E}+00$ & $9.18 \mathrm{E}-04$ & $8.58 \mathrm{E}-04 \quad 0.00 \mathrm{E}+00$ & 8. $63 \mathrm{E}-04$ & $0.00 \mathrm{E}+00$ & $1.31 \mathrm{E}-02$ & $8.61 \mathrm{E}-04$ & $2.79 \mathrm{E}-01$ & $0.00 \mathrm{E}+00$ & $2.38 \mathrm{E}-03$ & $2.79 \mathrm{E}-01$ \\
\hline $0006 \quad 1.24 \mathrm{E}-04$ & $9.30 \mathrm{E}-06 \quad 0.00 \mathrm{E}+00$ & $5.45 \mathrm{E}-05$ & $5.07 \mathrm{E}-05 \quad 0.00 \mathrm{E}+00$ & $5.10 \mathrm{E}-05$ & $0.00 \mathrm{E}+00$ & $1.03 \mathrm{E}-03$ & $5.09 \mathrm{E}-05$ & $1.82 \mathrm{E}-02$ & $0.00 \mathrm{E}+00$ & $1.77 \mathrm{E}-04$ & $1.82 \mathrm{E}-02$ \\
\hline $0007 \quad 7.69 \mathrm{E}-03$ & $7.68 \mathrm{E}-04 \quad 0.00 \mathrm{E}+00$ & $6.55 \mathrm{E}-03$ & $6.12 \mathrm{E}-03 \quad 0.00 \mathrm{E}+00$ & $6.15 \mathrm{E}-03$ & $0.00 \mathrm{E}+00$ & $7.98 \mathrm{E}-02$ & $6.14 \mathrm{E}-03$ & $2.11 \mathrm{E}+00$ & $0.00 \mathrm{E}+00$ & $1.42 \mathrm{E}-02$ & $2.11 \mathrm{E}+00$ \\
\hline
\end{tabular}


OBODM Output Folder

FORM12.OUT

FORM34.OUT 
FORM12.OUT 
OBODM V 1.3-

$10 / 24 / 2005 \mathrm{pg}$
6 receptors; $\mathrm{Ba}$ fctr $=0.0082$

Table 1

- Program Input Data -

- Program Models Selected -

- Time-average concentration

- Using final cloud rise ht. for all calc. distances.

- Complex terrain is being used.

- Grid system origin is not added to source rectangular coordinates

- Instantaneous sources can use both stable and adiabatic plume rise.

- Calculations restricted to daytime hours (sunrise+1hr to sunset-1hr)

- Hours with calm wind speeds use $1.0 \mathrm{~m} / \mathrm{s}$ and previous hours wind direction

- Missing hours use previous hours data or defaults.

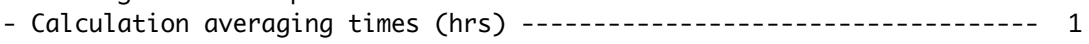

- Grid receptor system is ---_--- Rectangular

$$
\text { - Print Output Options - }
$$

- Print and save data using Summary processing mode

- Print highest and second highest at each receptor

- Print annual table

- Print source groups

- Print concentration output units ---_---_------- Micrograms/Cubic Meter

- Receptor Grid System Geometry -

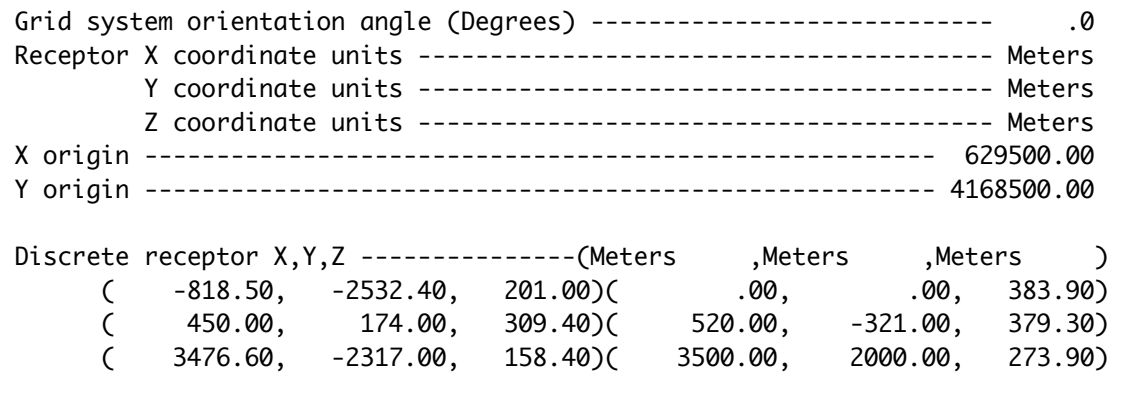

- Source Geometry/Emission Strength -

Material or fuel/explosive ---_--- TNT $(2,4,6$-Trinitrotoluene)

Pollutant/species -

Total number of sources -- 4

\begin{tabular}{|c|c|c|c|c|}
\hline $\begin{array}{l}\text { Source } \\
\text { Number }\end{array}$ & Ident & $\begin{array}{c}\text { Reference } \\
\text { System }\end{array}$ & $\begin{array}{c}\text { Source } \\
\text { Type }\end{array}$ & $\begin{array}{c}\text { Emission } \\
\text { Type }\end{array}$ \\
\hline
\end{tabular}

1 OB PAN

2 OB Cage (form 3)

3 OB Cage (form 4)

OBODM V 1.3-

$10 / 24 / 2005 \mathrm{pg}$
Rectangular Volume Quasi-continuous Rectangular Volume Quasi-continuous Rectangular Volume Quasi-continuous

6 receptors; $\mathrm{Ba}$ fctr $=0.0082$

Table 1 (cont.)

- Program Input Data - 
- Source Geometry/Emission Strength -

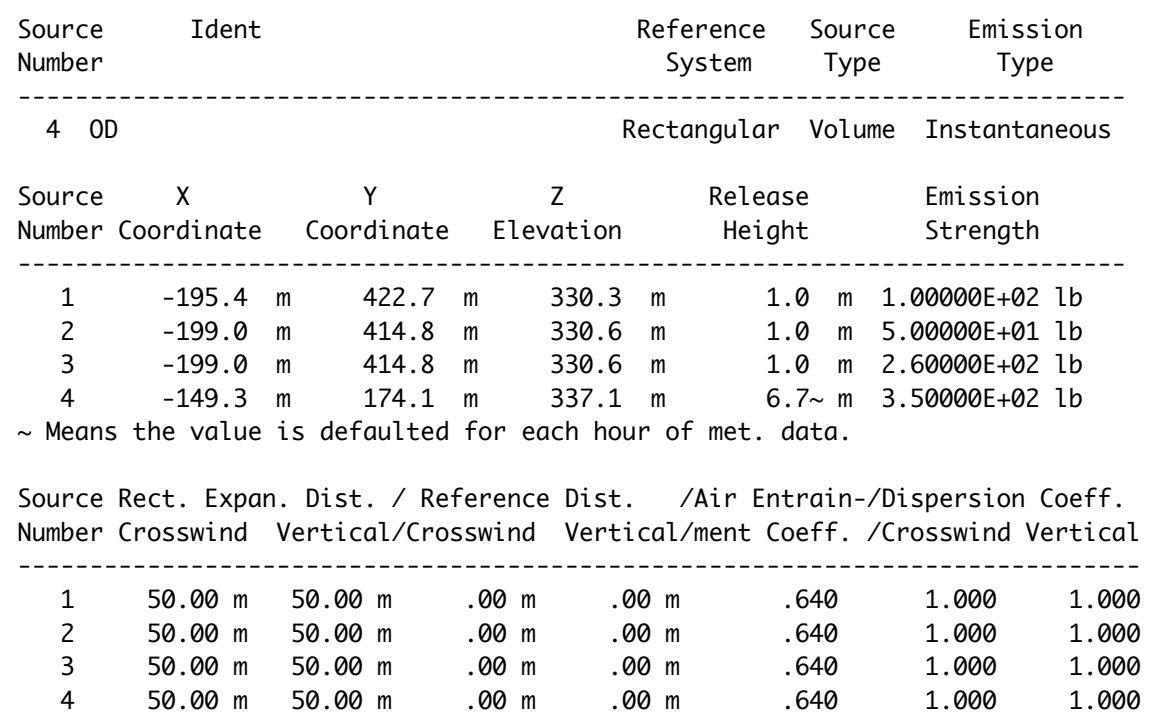

Source Initial Dimensions / Angle from North

Number Long side Short side Vertical / to Longside

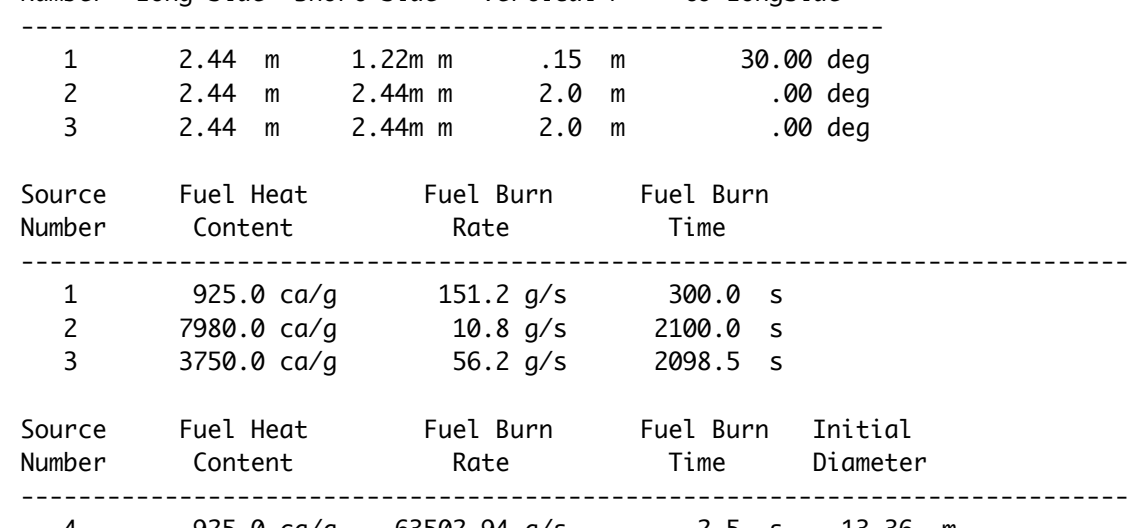

$4 \quad 925.0 \mathrm{ca} / \mathrm{g} \quad 63502.94 \mathrm{~g} / \mathrm{s} \quad 2.5 \mathrm{~s} \quad 13.36 \sim \mathrm{m}$

$\sim$ Means the value is defaulted for each hour of met. data.

Source Hours in which source is burned or detonated

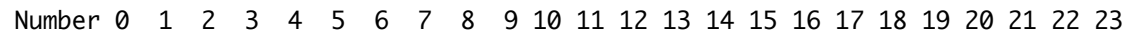

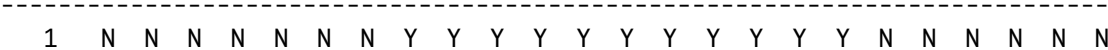

OBODM V 1.3-

6 receptors; $\mathrm{Ba}$ fctr $=0.0082$

$10 / 24 / 2005 \mathrm{pg} \quad 3$

Table 1 (cont.)

- Program Input Data -

- Source Geometry/Emission Strength -

Source Hours in which source is burned or detonated

Number $0 \begin{array}{llllllllllllllllllllllll} & 1 & 2 & 3 & 4 & 5 & 6 & 7 & 8 & 9 & 10 & 11 & 12 & 13 & 14 & 15 & 16 & 17 & 18 & 19 & 20 & 21 & 22 & 23\end{array}$

-

$\begin{array}{llllllllllllllllllllllllll}2 & N & N & N & N & N & N & N & N & Y & Y & Y & Y & Y & Y & Y & Y & Y & Y & Y & N & N & N & N & N & N\end{array}$

$\begin{array}{lllllllllllllllllllllllll}3 & N & N & N & N & N & N & N & Y & Y & Y & Y & Y & Y & Y & Y & Y & Y & Y & N & N & N & N & N & N\end{array}$ 


$\begin{array}{ll}\begin{array}{l}\text { Group } \\ \text { Number }\end{array} & \\ & \\ 1 & 1 \\ 2 & 2, \\ 3 & 3 \\ 4 & 4,\end{array}$

- Pollutant/Species Material Characteristics -

Pollutant/species name
Pollutant/species is
Particle material half-life
Molecular weight $(\mathrm{g} / \mathrm{g}-\mathrm{mol})$
Density of species ( $\mathrm{g} / \mathrm{cm} * * 3)$
Fraction of exhaust cloud constituting pollutant/species

$$
\begin{aligned}
& \text { - Meteorological Data - } \\
& \text { ( [\#-\#] min-max limits ) }
\end{aligned}
$$

Year [1900-2099]--- 2000

Month [ 1-12]

Day [ 1-31]

Hour (PST) [00-23]

Minute -.-.-.-.-. 0

Julian day $[1$ 366]

West longitude (degrees)

North latitude (degrees) - 37.00000

Time zone difference from GMT (hours)

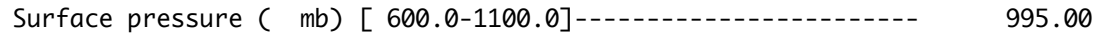

Cloud cover (8ths) [0-8]-.00

Cloud ceiling $(\mathrm{m})$ [ .0-20000.0]-1.0 9999.00

Net radiation index [-2- 4]-- 3 .

Pasquill stability category $[\mathrm{A}-\mathrm{F}]$

OBODM $\vee 1.3-$

$10 / 24 / 2005 \mathrm{pg} \quad 4$

6 receptors; $\mathrm{Ba}$ fctr $=0.0082$

Table 1 (cont.)

- Program Input Data -

- Meteorological Data -

( $[\#-\#]$ min-max limits)

Wind speed reference height $(\mathrm{m})$

Surface roughness length $(\mathrm{m})[\mathrm{m}$.00-1.00]-100

Vertical grad. of pot. temp. ( $\mathrm{C} / \mathrm{m})[-5.000-5.000]---1 .-0$

Reference wind speed $(\mathrm{m} / \mathrm{s})[1.0-50.0] \ldots \ldots$

Minimum (at $2 \mathrm{~m}$ ) wind speed $(\mathrm{m} / \mathrm{s})$

Air temperature (K) [213.1-333.1]

Standard dev. of wind direction angle (d) [1.0000-80.0000]----- 20.0000

Standard dev. wind elevation angle (d) [1.0000-50.0000]-.-.-. 7.0000

Longitudinal turbulence intensity (d) [1.0000-106.4000]---.--- 26.6000

Measurement time for std. dev. wind dir. angle ( s) [2.5-3600.0]- 600.00

Air humidity (\%) (.0-100.0)

Surface mixing layer height ( $\mathrm{m}$ ) [1.0-20000.0]-............. 1800

Wind direction (Vect) (deg) $[\quad .0-360.0]-270.0$

Wind-direction shear $(\mathrm{d} / \mathrm{m})\left[\begin{array}{ll}-45.0 & 45.0\end{array}\right] .00$

Wind-speed power law exponent [.000-5.000]--.-.-.-.-.-.-.-.

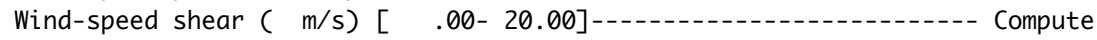

Elevation of meteorological data measurement site ( m) ------- $\quad 384.8$ 


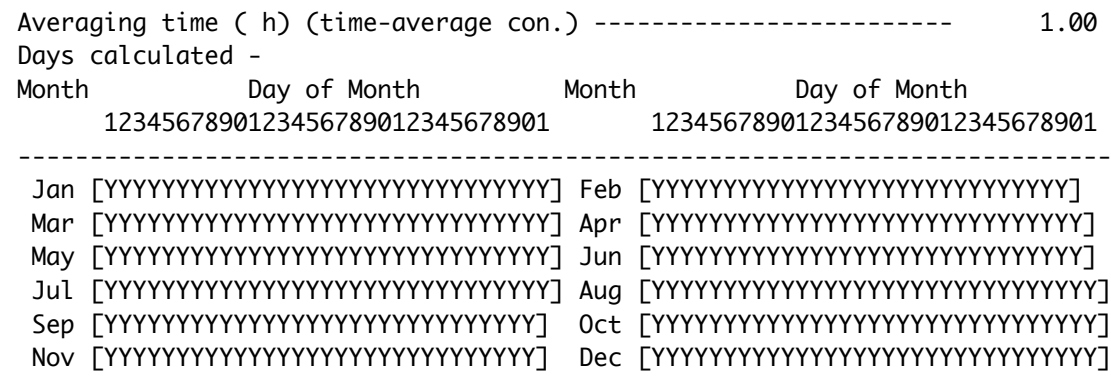

(Due to source group 1, sources: 1 )

(Maximum $=.13364$ at $X, Y, Z=629500.00,4168500.00,383.90)$

\begin{tabular}{|c|c|c|c|c|c|c|c|}
\hline $\begin{array}{c}x \\
\text { (Meters) }\end{array}$ & $\begin{array}{c}Y \\
\text { (Meters) }\end{array}$ & $\begin{array}{c}\mathrm{Z} \\
\text { (Meters) }\end{array}$ & Time-Avg. Con. & $\begin{array}{c}x \\
\text { (Meters) }\end{array}$ & $\begin{array}{c}\mathrm{Y} \\
\text { (Meters) }\end{array}$ & $\begin{array}{c}\text { Z } \\
\text { (Meters) }\end{array}$ & Time-Avg. Con. \\
\hline 628681.500 & 4165968.000 & 201.000 & $.966539 E-03$ & 629500.000 & 4168500.000 & 383.900 & .133635 \\
\hline 629950.000 & 4168674.000 & 309.400 & $.171665 E-01$ & 630020.000 & 4168179.000 & 379.300 & $.160983 E-01$ \\
\hline 632976.600 & 4166183.000 & 158.400 & $.467618 \mathrm{E}-03$ & 633000.000 & 4170500.000 & 273.900 & $.100429 E-02$ \\
\hline $\begin{array}{l}\text { 30DM V 1.3- } \\
/ 24 / 2005 \mathrm{pg}\end{array}$ & 7 & & 6 recep & ; Ba fctr & 0.0082 & & \\
\hline
\end{tabular}


Annual Average Barium Time-Average $\{1-\mathrm{hr}\}$ Concentration (Micrograms/Cubic Meter)

(Due to source group 2, sources: 2)

(Maximum $=.66794 \mathrm{E}-01$ at $\mathrm{X}, \mathrm{Y}, \mathrm{Z}=629500.00,4168500.00,383.90$ )

\begin{tabular}{|c|c|c|c|c|c|c|c|}
\hline $\begin{array}{c}\mathrm{X} \\
\text { (Meters) }\end{array}$ & $\begin{array}{c}Y \\
\text { (Meters) }\end{array}$ & $\begin{array}{c}\mathrm{z} \\
\text { (Meters) }\end{array}$ & Time-Avg. Con. & $\begin{array}{c}x \\
\text { (Meters) }\end{array}$ & $\begin{array}{c}Y \\
\text { (Meters) }\end{array}$ & $\begin{array}{c}\text { Z } \\
\text { (Meters) }\end{array}$ & Time-Avg. Con. \\
\hline 628681.500 & 4165968.000 & 201.000 & $.568851 \mathrm{E}-03$ & 629500.000 & 4168500.000 & 383.900 & $.667940 \mathrm{E}-01$ \\
\hline 629950.000 & 4168674.000 & 309.400 & $.105102 \mathrm{E}-01$ & 630020.000 & 4168179.000 & 379.300 & $.891566 \mathrm{E}-02$ \\
\hline 632976.600 & 4166183.000 & 158.400 & $.266714 E-03$ & 633000.000 & 4170500.000 & 273.900 & .499437E-03 \\
\hline
\end{tabular}

Table 4

Annual Average Barium Time-Average $\{1-\mathrm{hr}\}$ Concentration (Micrograms/Cubic Meter)

(Due to source group 3, sources: 3)

(Maximum $=.30209$ at $X, Y, Z=629500.00,4168500.00,383.90)$

\begin{tabular}{|c|c|c|c|c|c|c|c|}
\hline $\begin{array}{c}x \\
\text { (Meters) }\end{array}$ & $\begin{array}{c}Y \\
\text { (Meters) }\end{array}$ & $\begin{array}{c}\mathrm{Z} \\
\text { (Meters) }\end{array}$ & Time-Avg. Con. & $\begin{array}{c}\mathrm{x} \\
\text { (Meters) }\end{array}$ & $\begin{array}{c}\mathrm{Y} \\
\text { (Meters) }\end{array}$ & $\begin{array}{c}\mathrm{Z} \\
\text { (Meters) }\end{array}$ & Time-Avg. Con. \\
\hline 628681.500 & 4165968.000 & 201.000 & $.280150 \mathrm{E}-02$ & 629500.000 & 4168500.000 & 383.900 & .302095 \\
\hline 629950.000 & 4168674.000 & 309.400 & $.448804 \mathrm{E}-01$ & 630020.000 & 4168179.000 & 379.300 & $.427665 \mathrm{E}-01$ \\
\hline 632976.600 & 4166183.000 & 158.400 & $.133857 \mathrm{E}-02$ & 633000.000 & 4170500.000 & 273.900 & $.255351 \mathrm{E}-02$ \\
\hline
\end{tabular}

Table 5

Annual Average Barium Time-Average $\{1-\mathrm{hr}\}$ Concentration (Micrograms/Cubic Meter)

(Due to source group 4, sources: 4)

(Maximum $=.12371$ at $X, Y, Z=629500.00,4168500.00,383.90)$

\begin{tabular}{|c|c|c|c|c|c|c|c|}
\hline $\begin{array}{c}x \\
\text { (Meters) }\end{array}$ & $\begin{array}{c}\mathrm{Y} \\
\text { (Meters) }\end{array}$ & $\begin{array}{c}\mathrm{Z} \\
\text { (Meters) }\end{array}$ & Time-Avg. Con. & $\begin{array}{c}x \\
\text { (Meters) }\end{array}$ & $\begin{array}{c}\mathrm{Y} \\
\text { (Meters) }\end{array}$ & $\begin{array}{c}\mathrm{Z} \\
\text { (Meters) }\end{array}$ & Time-Avg. Con. \\
\hline 628681.500 & 4165968.000 & 201.000 & $.219330 \mathrm{E}-02$ & 629500.000 & 4168500.000 & 383.900 & .123706 \\
\hline 629950.000 & 4168674.000 & 309.400 & $.171942 \mathrm{E}-01$ & 630020.000 & 4168179.000 & 379.300 & $.243576 E-01$ \\
\hline 632976.600 & 4166183.000 & 158.400 & $.126254 \mathrm{E}-02$ & 633000.000 & 4170500.000 & 273.900 & $.209602 \mathrm{E}-02$ \\
\hline
\end{tabular}

Table 6

Highest Barium Time-Average \{1-hr\} Concentration (Micrograms/Cubic Meter)

(Due to source group 1, sources: 1)

(Maximum $=11.877$ at $X, Y, Z=629500.00,4168500.00,383.90)$

\begin{tabular}{|c|c|c|c|}
\hline $\begin{array}{c}\mathrm{X} \\
\text { (Meters) }\end{array}$ & $\begin{array}{c}Y \\
\text { (Meters) }\end{array}$ & $\begin{array}{c}\text { Z } \\
\text { (Meters) }\end{array}$ & Time-Avg. Con. Mo/Dy/Yr Jdy \\
\hline
\end{tabular}




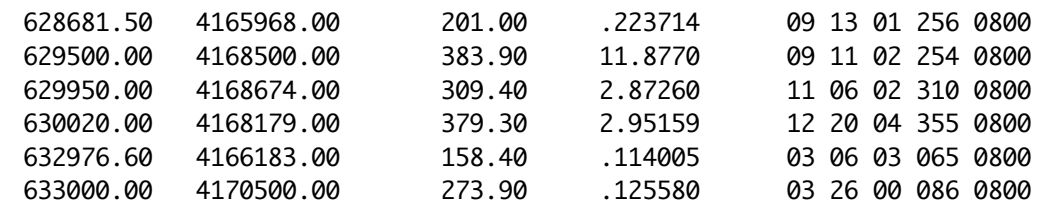

OBODM $\vee 1.3-$ $10 / 24 / 2005$ pg 11

6 receptors; $\mathrm{Ba}$ fctr $=0.0082$

Table 7

Second Highest Barium Time-Average \{1-hr\} Concentration (Micrograms/Cubic Meter)

(Due to source group 1, sources: 1)

(Maximum $=11.577$ at $X, Y, Z=629500.00,4168500.00,383.90)$

\begin{tabular}{|c|c|c|c|c|c|}
\hline $\begin{array}{c}x \\
\text { (Meters) }\end{array}$ & $\begin{array}{c}Y \\
\text { (Meters) }\end{array}$ & $\begin{array}{c}\text { Z } \\
\text { (Meters) }\end{array}$ & Time-Avg. Con. & Mo/Dy/Yr Jdy & $\mathrm{Hr}$ \\
\hline 628681.50 & 4165968.00 & 201.00 & .206617 & $\begin{array}{llll}11 & 05 & 01 & 309\end{array}$ & 0800 \\
\hline 629500.00 & 4168500.00 & 383.90 & 11.5772 & $\begin{array}{llll}12 & 23 & 00 & 358\end{array}$ & 0800 \\
\hline 629950.00 & 4168674.00 & 309.40 & 2.86891 & $\begin{array}{llll}12 & 25 & 04 & 360\end{array}$ & 0800 \\
\hline 630020.00 & 4168179.00 & 379.30 & 2.95108 & $\begin{array}{llll}02 & 01 & 02 & 032\end{array}$ & 0800 \\
\hline 632976.60 & 4166183.00 & 158.40 & $.962193 \mathrm{E}-01$ & $\begin{array}{llll}02 & 10 & 03 & 041\end{array}$ & 0900 \\
\hline 633000.00 & 4170500.00 & 273.90 & .124480 & $\begin{array}{llll}04 & 09 & 02 & 099\end{array}$ & 0800 \\
\hline
\end{tabular}

OBODM $\vee 1.3-$ $10 / 24 / 2005 \mathrm{pg} \quad 12$

6 receptors; $\mathrm{Ba}$ fctr $=0.0082$

Table 8

Highest Barium Time-Average $\{1-\mathrm{hr}\}$ Concentration (Micrograms/Cubic Meter)

(Due to source group 2, sources: 2)

(Maximum $=5.0540$ at $X, Y, Z=629500.00,4168500.00,383.90)$

$\begin{array}{cccc}\mathrm{X} & \mathrm{Y} & \mathrm{Z} & \text { Time-Avg. Con. Mo/Dy/Yr Jdy } \mathrm{Hr} \\ \text { (Meters) } & \text { (Meters) } & \text { (Meters) } & \\ \end{array}$

$\begin{array}{lllllllll}628681.50 & 4165968.00 & 201.00 & .832762 \mathrm{E}-01 & 09 & 13 & 01 & 256 & 0800 \\ 629500.00 & 4168500.00 & 383.90 & 5.05396 & 09 & 11 & 02 & 254 & 0800 \\ 629950.00 & 4168674.00 & 309.40 & 1.37170 & 01 & 19 & 04 & 019 & 0900 \\ 630020.00 & 4168179.00 & 379.30 & 1.17555 & 11 & 25 & 00 & 330 & 0900 \\ 632976.60 & 4166183.00 & 158.40 & .406607 \mathrm{E}-01 & 03 & 06 & 03 & 065 & 0800 \\ 633000.00 & 4170500.00 & 273.90 & .500138 \mathrm{E}-01 & 12 & 06 & 02 & 340 & 0800\end{array}$

OBODM V 1.3-

$10 / 24 / 2005$ pg 13

6 receptors; $\mathrm{Ba}$ fctr $=0.0082$

Table 9

Second Highest Barium Time-Average \{1-hr\} Concentration (Micrograms/Cubic Meter)

(Due to source group 2, sources: 2)

(Maximum $=5.0405$ at $X, Y, Z=629500.00,4168500.00,383.90)$

\begin{tabular}{cccc}
$\mathrm{X}$ & $\mathrm{Y}$ & $\mathrm{Z}$ & Time-Avg. Con. Mo/Dy/Yr Jdy Hr \\
(Meters) & (Meters) & (Meters) & \\
\hline
\end{tabular}




$\begin{array}{lllllllll}628681.50 & 4165968.00 & 201.00 & .802451 \mathrm{E}-01 & 11 & 05 & 01 & 309 & 0800 \\ 629500.00 & 4168500.00 & 383.90 & 5.04053 & 09 & 01 & 01 & 244 & 0800 \\ 629950.00 & 4168674.00 & 309.40 & 1.30104 & 12 & 25 & 04 & 360 & 0800 \\ 630020.00 & 4168179.00 & 379.30 & 1.15562 & 12 & 20 & 04 & 355 & 0800 \\ 632976.60 & 4166183.00 & 158.40 & .346644 \mathrm{E}-01 & 02 & 10 & 03 & 041 & 0900 \\ 633000.00 & 4170500.00 & 273.90 & .495244 \mathrm{E}-01 & 03 & 26 & 00 & 086 & 0800\end{array}$

OBODM $\vee 1.3$

$10 / 24 / 2005 \mathrm{pg} \quad 14$
Table 10

Highest Barium Time-Average \{1-hr\} Concentration (Micrograms/Cubic Meter)

(Due to source group 3, sources: 3)

(Maximum $=21.001$ at $X, Y, Z=629500.00,4168500.00,383.90)$

$\begin{array}{ccc}X & Y & Z\end{array}$ Time-Avg. Con. Mo/Dy/Yr Jdy Hr

$\begin{array}{lllllllll}628681.50 & 4165968.00 & 201.00 & .391287 & 09 & 13 & 01 & 256 & 0800 \\ 629500.00 & 4168500.00 & 383.90 & 21.0008 & 09 & 11 & 02 & 254 & 0800 \\ 629950.00 & 4168674.00 & 309.40 & 4.95688 & 01 & 19 & 04 & 019 & 0900 \\ 630020.00 & 4168179.00 & 379.30 & 5.44730 & 11 & 25 & 00 & 330 & 0900 \\ 632976.60 & 4166183.00 & 158.40 & .198177 & 03 & 06 & 03 & 065 & 0800 \\ 633000.00 & 4170500.00 & 273.90 & .246753 & 12 & 06 & 02 & 340 & 0800\end{array}$

OBODM $\vee 1.3-$ $10 / 24 / 2005$ pg 15

6 receptors; $\mathrm{Ba}$ fctr $=0.0082$

Table 11

Second Highest Barium Time-Average \{1-hr\} Concentration (Micrograms/Cubic Meter)

(Due to source group 3, sources: 3)

(Maximum $=20.977$ at $X, Y, Z=629500.00,4168500.00,383.90$ )

\begin{tabular}{|c|c|c|c|}
\hline $\begin{array}{c}\mathrm{x} \\
\text { (Meters) }\end{array}$ & $\begin{array}{c}Y \\
\text { (Meters) }\end{array}$ & $\begin{array}{c}\text { Z } \\
\text { (Meters) }\end{array}$ & Time-Avg. Con. Mo/Dy/Yr Jdy \\
\hline
\end{tabular}

$\begin{array}{lllllllll}628681.50 & 4165968.00 & 201.00 & .377467 & 11 & 05 & 01 & 309 & 0800 \\ 629500.00 & 4168500.00 & 383.90 & 20.9770 & 09 & 01 & 01 & 244 & 0800 \\ 629950.00 & 4168674.00 & 309.40 & 4.76711 & 12 & 25 & 04 & 360 & 0800 \\ 630020.00 & 4168179.00 & 379.30 & 5.31551 & 12 & 20 & 04 & 355 & 0800 \\ 632976.60 & 4166183.00 & 158.40 & .168996 & 02 & 10 & 03 & 041 & 0900 \\ 633000.00 & 4170500.00 & 273.90 & .243581 & 03 & 26 & 00 & 086 & 0800\end{array}$

OBODM V 1.3-

$10 / 24 / 2005$ pg 16

6 receptors; $\mathrm{Ba}$ fctr $=0.0082$

Table 12

Highest Barium Time-Average \{1-hr\} Concentration (Micrograms/Cubic Meter)

(Due to source group 4, sources: 4)

(Maximum $=18.767$ at $X, Y, Z=629500.00,4168500.00,383.90)$

$\begin{array}{cccc}X & Y & Z & \text { Time-Avg. Con. Mo/Dy/Yr Jdy Hr } \\ \text { (Meters) } & \text { (Meters) } & \text { (Meters) } & \\ \end{array}$

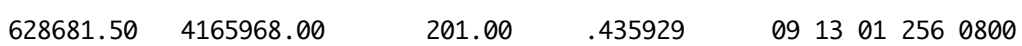




$\begin{array}{lllllllll}629500.00 & 4168500.00 & 383.90 & 18.7670 & 02 & 18 & 00 & 049 & 0800 \\ 629950.00 & 4168674.00 & 309.40 & 1.92837 & 01 & 01 & 00 & 001 & 0900 \\ 630020.00 & 4168179.00 & 379.30 & 8.25488 & 03 & 06 & 03 & 065 & 0800 \\ 632976.60 & 4166183.00 & 158.40 & .364497 & 10 & 16 & 01 & 289 & 0800 \\ 633000.00 & 4170500.00 & 273.90 & .585447 & 12 & 08 & 00 & 343 & 0900\end{array}$

OBODM V 1.3-

$10 / 24 / 2005 \mathrm{pg}$

17

6 receptors; $\mathrm{Ba}$ fctr $=0.0082$

Table 13

Second Highest Barium Time-Average \{1-hr\} Concentration (Micrograms/Cubic Meter)

(Due to source group 4, sources: 4)

(Maximum $=18.657$ at $X, Y, Z=629500.00,4168500.00,383.90)$

\begin{tabular}{|c|c|c|c|c|c|}
\hline $\begin{array}{c}\mathrm{x} \\
\text { (Meters) }\end{array}$ & $\begin{array}{c}\mathrm{Y} \\
\text { (Meters) }\end{array}$ & $\begin{array}{c}\mathrm{Z} \\
\text { (Meters) }\end{array}$ & Time-Avg. Con. & Mo/Dy/Yr Jdy & $\mathrm{Hr}$ \\
\hline 628681.50 & 4165968.00 & 201.00 & .425015 & 112604331 & 1700 \\
\hline 629500.00 & 4168500.00 & 383.90 & 18.6575 & $\begin{array}{llll}02 & 05 & 02 & 036\end{array}$ & 0800 \\
\hline 629950.00 & 4168674.00 & 309.40 & 1.92688 & $\begin{array}{llll}01 & 01 & 00 & 001\end{array}$ & 0800 \\
\hline 630020.00 & 4168179.00 & 379.30 & 7.99107 & $\begin{array}{llll}11 & 18 & 03 & 322\end{array}$ & 0800 \\
\hline 632976.60 & 4166183.00 & 158.40 & .347269 & $\begin{array}{llll}10 & 05 & 00 & 279\end{array}$ & 0800 \\
\hline 633000.00 & 4170500.00 & 273.90 & .532794 & $\begin{array}{llll}04 & 09 & 02 & 099\end{array}$ & 0800 \\
\hline
\end{tabular}

Elapsed run time $=\quad 1$ hours, .47 minutes.

$* * *$ end of $\mathrm{OBODM} * * *$ 
FORM34.OUT 
- Time-average concentration

- Using final cloud rise ht. for all calc. distances.

- Complex terrain is being used.

- Grid system origin is not added to source rectangular coordinates

- Instantaneous sources can use both stable and adiabatic plume rise.

- Calculations restricted to daytime hours (sunrise+1hr to sunset-1hr)

- Hours with calm wind speeds use $1.0 \mathrm{~m} / \mathrm{s}$ and previous hours wind direction

- Missing hours use previous hours data or defaults.

- Calculation averaging times (hrs) --

- Grid receptor system is ---_-nectangular

$$
\text { - Print Output Options - }
$$

- Print and save data using Summary processing mode

- Print highest and second highest at each receptor

- Print annual table

- Print source groups

- Print concentration output units ---------------- Micrograms/Cubic Meter

- Receptor Grid System Geometry -

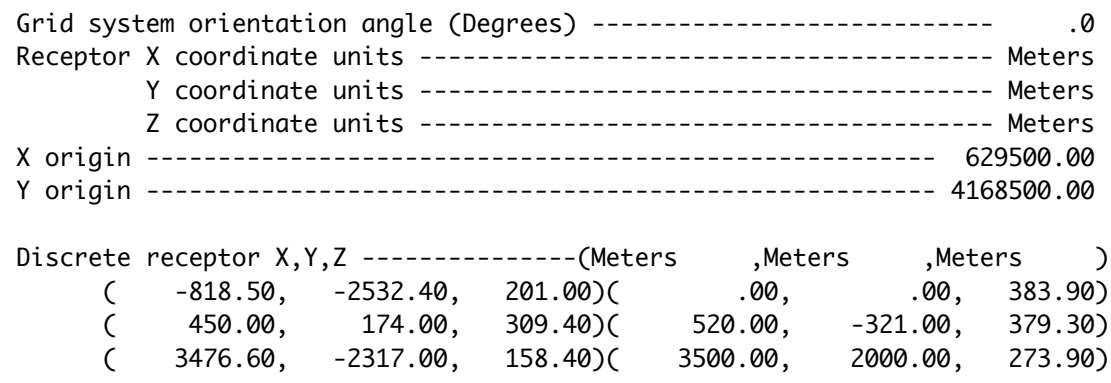

- Source Geometry/Emission Strength -

Material or fuel/explosive ----12,4,6-Trinitrotoluene)

Pollutant/species ---

Total number of sources - 4

\begin{tabular}{|c|c|c|c|c|}
\hline $\begin{array}{l}\text { Source } \\
\text { Number }\end{array}$ & Ident & $\begin{array}{c}\text { Reference } \\
\text { System }\end{array}$ & $\begin{array}{l}\text { Source } \\
\text { Type }\end{array}$ & $\begin{array}{c}\text { Emission } \\
\text { Type }\end{array}$ \\
\hline $10 \mathrm{~B}$ & B PAN & Rectangular & Volume & nuous \\
\hline $20 \mathrm{~B}$ & B Cage (form 3) & Rectangular & Volume & Quasi-continuous \\
\hline $30 B$ & B Cage (form 4) & Rectangular & Volume & Quasi-continuous \\
\hline
\end{tabular}

OBODM $\vee 1.3-$

$10 / 24 / 2005 \mathrm{pg}$ 
- Source Geometry/Emission Strength -

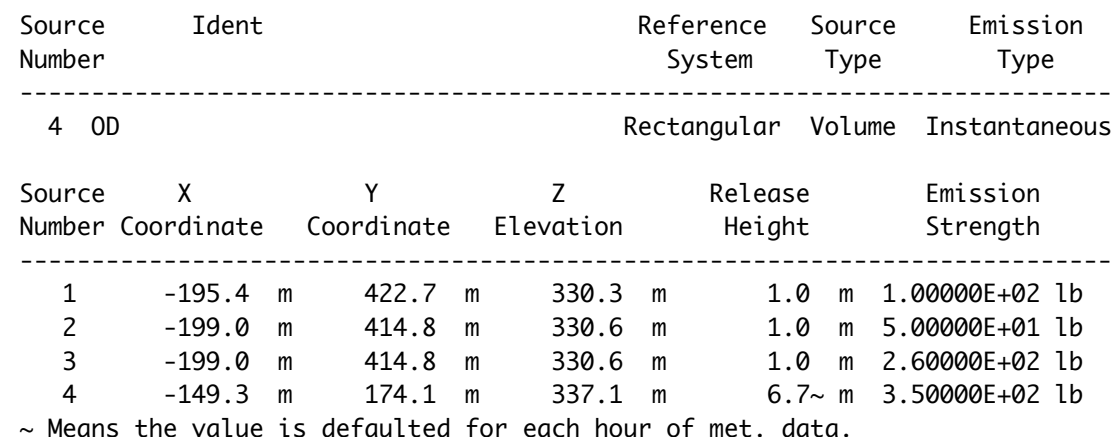

Source Rect. Expan. Dist. / Reference Dist. /Air Entrain-/Dispersion Coeff. Number Crosswind Vertical/Crosswind Vertical/ment Coeff. /Crosswind Vertical

$\begin{array}{llllllll}1 & 50.00 \mathrm{~m} & 50.00 \mathrm{~m} & .00 \mathrm{~m} & .00 \mathrm{~m} & .640 & 1.000 & 1.000 \\ 2 & 50.00 \mathrm{~m} & 50.00 \mathrm{~m} & .00 \mathrm{~m} & .00 \mathrm{~m} & .640 & 1.000 & 1.000 \\ 3 & 50.00 \mathrm{~m} & 50.00 \mathrm{~m} & .00 \mathrm{~m} & .00 \mathrm{~m} & .640 & 1.000 & 1.000 \\ 4 & 50.00 \mathrm{~m} & 50.00 \mathrm{~m} & .00 \mathrm{~m} & .00 \mathrm{~m} & .640 & 1.000 & 1.000\end{array}$

Source Initial Dimensions / Angle from North

Number Long side Short side Vertical / to Longside

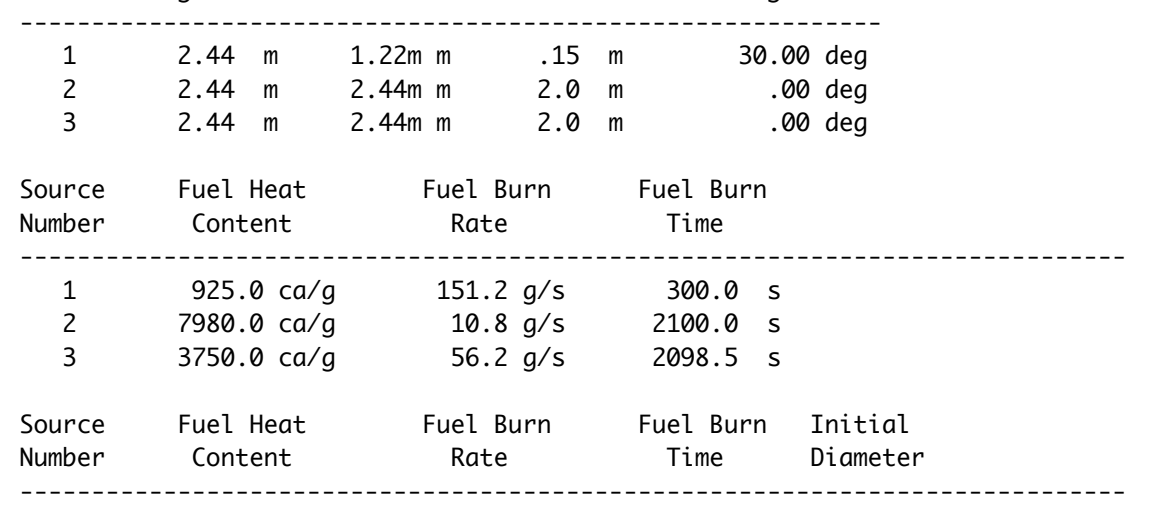

$4 \quad 925.0 \mathrm{ca} / \mathrm{g} \quad 63502.94 \mathrm{~g} / \mathrm{s} \quad 2.5 \mathrm{~s} \quad 13.36 \sim \mathrm{m}$

$\sim$ Means the value is defaulted for each hour of met. data.

Source Hours in which source is burned or detonated

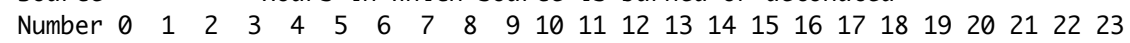

$\begin{array}{lllllllllllllllllllllllll}1 & N & N & N & N & N & N & N & Y & Y & Y & Y & Y & Y & Y & Y & Y & Y & Y & N & N & N & N & N & N\end{array}$

OBODM V 1.3-

$10 / 24 / 2005 \mathrm{pg} \quad 3$

6 receptors; $\mathrm{Ba}$ fctr $=0.000086$

Table 1 (cont.)

- Program Input Data -

- Source Geometry/Emission Strength -

Source Hours in which source is burned or detonated

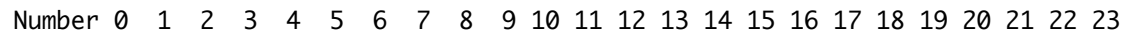

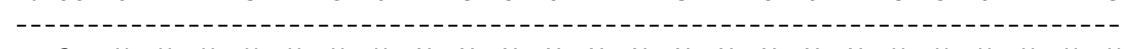

$\begin{array}{lllllllllllllllllllllllllll}2 & N & N & N & N & N & N & N & Y & Y & Y & Y & Y & Y & Y & Y & Y & Y & Y & N & N & N & N & N & N\end{array}$

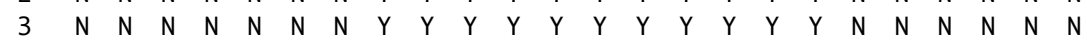


$\begin{array}{llllllllllllllllllllllllllll}4 & N & N & N & N & N & N & N & Y & Y & Y & Y & Y & Y & Y & Y & Y & Y & Y & N & N & N & N & N & N\end{array}$ Source grouping -

$\begin{array}{ll}\begin{array}{l}\text { Group } \\ \text { Number }\end{array} & \\ & \\ 1 & 1 \\ 2 & 2, \\ 3 & 3 \\ 4 & 4,\end{array}$

- Pollutant/Species Material Characteristics -

Pollutant/species name
Pollutant/species is
Particle material half-life
Molecular weight $(\mathrm{g} / \mathrm{g}-\mathrm{mol})$
Density of species ( $\mathrm{g} / \mathrm{cm} * * 3)$
Fraction of exhaust cloud constituting pollutant/species

- Meteorological Data -

( [\#-\#] min-max limits)

Year [1900-2099]-- 2000

Month [ 1-12]

Day [ 1-31]-...-...-. 0

Hour (PST) [00-23]

Minute --.-. 0

Julian day $[$ 1-366]-n-n

West longitude (degrees)

North latitude (degrees) - 37.00000

Time zone difference from GMT (hours)

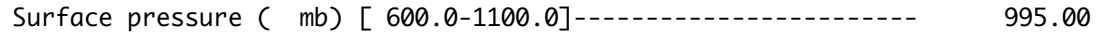

Cloud cover (8ths) [0-8]-.00

Cloud ceiling ( m) [ .0-20000.0]-..... 9999

Net radiation index [-2-4]-- 3.

Pasquill stability category $[\mathrm{A}-\mathrm{F}]-\mathrm{n}_{-1}$

OBODM V 1.3-

$10 / 24 / 2005 \mathrm{pg} \quad 4$

6 receptors; $\mathrm{Ba}$ fctr $=0.000086$

Table 1 (cont.)

- Program Input Data -

- Meteorological Data -

( [\#-\#] min-max limits)

Wind speed reference height $(\mathrm{m})$

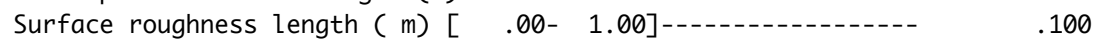

Vertical grad. of pot. temp. ( $\mathrm{C} / \mathrm{m})[-5.000-5.000]-\cdots$

Reference wind speed $(\mathrm{m} / \mathrm{s})[1.0-50.0] \ldots \ldots$

Minimum (at $2 \mathrm{~m}$ ) wind speed $(\mathrm{m} / \mathrm{s}$ )

Air temperature (K) [213.1-333.1]

Standard dev. of wind direction angle (d) [1.0000-80.0000]----- 20.0000

Standard dev. wind elevation angle (d) [1.0000-50.0000]--.-.-- 7.0000

Longitudinal turbulence intensity (d) [1.0000-106.4000]------- 26.6000

Measurement time for std. dev. wind dir. angle ( s) [2.5-3600.0]- 600.00

Air humidity (\%) (.0-100.0)-..... 70

Surface mixing layer height ( $\mathrm{m}$ ) [ 1.0-20000.0]-.......... 1800.00

Wind direction (Vect) (deg) $[\quad .0-360.0]$

Wind-direction shear $(\mathrm{d} / \mathrm{m})\left[\begin{array}{ll}-45.0 & 45.0]\end{array}\right.$

Wind-speed power law exponent [.000-5.000]--... 1

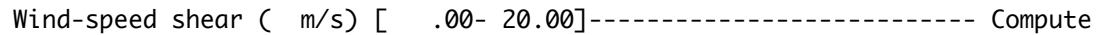

Elevation of meteorological data measurement site $(\mathrm{m})$ 


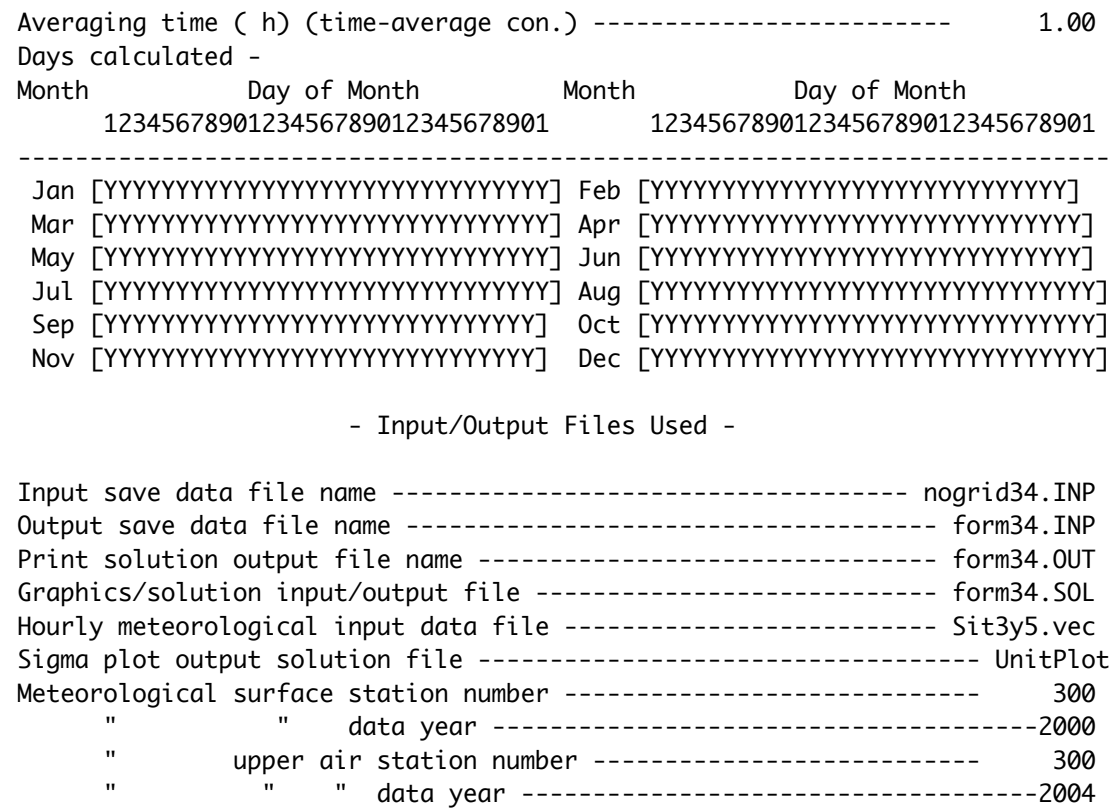

(Due to source group 1, sources: 1)

\begin{tabular}{|c|c|c|c|c|c|c|c|}
\hline $\begin{array}{c}\mathrm{x} \\
\text { (Meters) }\end{array}$ & $\begin{array}{c}\mathrm{Y} \\
\text { (Meters) }\end{array}$ & $\begin{array}{c}\mathrm{Z} \\
\text { (Meters) }\end{array}$ & Time-Avg. Con. & $\begin{array}{c}x \\
\text { (Meters) }\end{array}$ & $\begin{array}{c}\mathrm{Y} \\
\text { (Meters) }\end{array}$ & $\begin{array}{c}\mathrm{Z} \\
\text { (Meters) }\end{array}$ & Time-Avg. Con. \\
\hline 628681.500 & 4165968.000 & 201.000 & $.101369 E-04$ & 629500.000 & 4168500.000 & 383.900 & $.140154 \mathrm{E}-02$ \\
\hline 629950.000 & 4168674.000 & 309.400 & $.180039 E-03$ & 630020.000 & 4168179.000 & 379.300 & $.168836 \mathrm{E}-03$ \\
\hline 632976.600 & 4166183.000 & 158.400 & $.490429 \mathrm{E}-05$ & 633000.000 & 4170500.000 & 273.900 & $.105328 \mathrm{E}-04$ \\
\hline
\end{tabular}

OBODM V 1.310/24/2005 pg 7

6 receptors; $\mathrm{Ba}$ fctr $=0.000086$ 
Annual Average Barium Time-Average $\{1-\mathrm{hr}\}$ Concentration (Micrograms/Cubic Meter)

(Due to source group 2, sources: 2)

(Maximum $=.70052 \mathrm{E}-03$ at $\mathrm{X}, \mathrm{Y}, \mathrm{Z}=629500.00,4168500.00,383.90)$

\begin{tabular}{cccccccc}
$\begin{array}{c}X \\
\text { (Meters) }\end{array}$ & $\begin{array}{c}Y \\
\text { (Meters) }\end{array}$ & $\begin{array}{c}Z \\
\text { (Meters) }\end{array}$ & Time-Avg. Con. $\begin{array}{c}X \\
\text { (Meters) }\end{array}$ & $\begin{array}{c}Y \\
\text { (Meters) }\end{array}$ & $\begin{array}{c}\text { Z } \\
\text { (Meters) }\end{array}$ & Time-Avg. Con. \\
\hline 628681.500 & 4165968.000 & 201.000 & $.596601 \mathrm{E}-05$ & 629500.000 & 4168500.000 & 383.900 & $.700522 \mathrm{E}-03$ \\
629950.000 & 4168674.000 & 309.400 & $.110229 \mathrm{E}-03$ & 630020.000 & 4168179.000 & 379.300 & $.935060 \mathrm{E}-04$ \\
632976.600 & 4166183.000 & 158.400 & $.279725 \mathrm{E}-05$ & 633000.000 & 4170500.000 & 273.900 & $.523800 \mathrm{E}-05$
\end{tabular}

OBODM $\vee 1.3-$

6 receptors; Ba fctr $=0.000086$

$10 / 24 / 2005 \mathrm{pg} \quad 8$

Table 4

Annual Average Barium Time-Average $\{1-\mathrm{hr}\}$ Concentration (Micrograms/Cubic Meter)

(Due to source group 3, sources: 3)

(Maximum $=.31683 \mathrm{E}-02$ at $\mathrm{X}, \mathrm{Y}, \mathrm{Z}=629500.00,4168500.00,383.90)$

\begin{tabular}{|c|c|c|c|c|c|c|c|}
\hline $\begin{array}{c}x \\
\text { (Meters) }\end{array}$ & $\begin{array}{c}\mathrm{Y} \\
\text { (Meters) }\end{array}$ & $\begin{array}{c}\mathrm{Z} \\
\text { (Meters) }\end{array}$ & Time-Avg. Con. & $\begin{array}{c}x \\
\text { (Meters) }\end{array}$ & $\begin{array}{c}\mathrm{Y} \\
\text { (Meters) }\end{array}$ & $\begin{array}{c}\mathrm{Z} \\
\text { (Meters) }\end{array}$ & Time-Avg. Con. \\
\hline 628681.500 & 4165968.000 & 201.000 & $.293816 \mathrm{E}-04$ & 629500.000 & 4168500.000 & 383.900 & $.316831 \mathrm{E}-02$ \\
\hline 629950.000 & 4168674.000 & 309.400 & $.470697 \mathrm{E}-03$ & 630020.000 & 4168179.000 & 379.300 & $.448528 \mathrm{E}-03$ \\
\hline 632976.600 & 4166183.000 & 158.400 & $.140386 \mathrm{E}-04$ & 633000.000 & 4170500.000 & 273.900 & $.267807 E-04$ \\
\hline $\begin{array}{l}\text { JM V 1.3- } \\
24 / 2005 \mathrm{pg}\end{array}$ & 9 & & 6 recept & $\mathrm{Ba}$ fctr $=$ & 000086 & & \\
\hline
\end{tabular}

Table 5

Annual Average Barium Time-Average $\{1-\mathrm{hr}\}$ Concentration (Micrograms/Cubic Meter)

(Due to source group 4, sources: 4)

(Maximum $=.12974 \mathrm{E}-02$ at $\mathrm{X}, \mathrm{Y}, \mathrm{Z}=629500.00,4168500.00,383.90$ )

\begin{tabular}{|c|c|c|c|c|c|c|c|}
\hline $\begin{array}{c}\mathrm{x} \\
\text { (Meters) }\end{array}$ & $\begin{array}{c}\mathrm{Y} \\
\text { (Meters) }\end{array}$ & $\begin{array}{c}\mathrm{Z} \\
\text { (Meters) }\end{array}$ & Time-Avg. Con. & $\begin{array}{c}\mathrm{X} \\
\text { (Meters) }\end{array}$ & $\begin{array}{c}\mathrm{Y} \\
\text { (Meters) }\end{array}$ & $\begin{array}{c}\mathrm{Z} \\
\text { (Meters) }\end{array}$ & Time-Avg. Con. \\
\hline 628681.500 & 4165968.000 & 201.000 & $.230029 E-04$ & 629500.000 & 4168500.000 & 383.900 & $.129741 \mathrm{E}-02$ \\
\hline 629950.000 & 4168674.000 & 309.400 & $.180330 \mathrm{E}-03$ & 630020.000 & 4168179.000 & 379.300 & $.255458 \mathrm{E}-03$ \\
\hline 632976.600 & 4166183.000 & 158.400 & $.132412 E-04$ & 633000.000 & 4170500.000 & 273.900 & $.219826 \mathrm{E}-04$ \\
\hline
\end{tabular}

Highest Barium Time-Average $\{1-\mathrm{hr}\} \begin{gathered}\text { Table } \\ 6\end{gathered}$

(Due to source group 1, sources: 1)

(Maximum $=.12456$ at $X, Y, Z=629500.00,4168500.00,383.90)$

$\begin{array}{cccc}X & Y & Z & \text { Time-Avg. Con. Mo/Dy/Yr Jdy Hr } \\ \text { (Meters) } & \text { (Meters) } & \text { (Meters) } & \\ \end{array}$




$\begin{array}{lllllllll}628681.50 & 4165968.00 & 201.00 & .234627 \mathrm{E}-02 & 09 & 13 & 01 & 256 & 0800 \\ 629500.00 & 4168500.00 & 383.90 & .124563 & 09 & 11 & 02 & 254 & 0800 \\ 629950.00 & 4168674.00 & 309.40 & .301273 \mathrm{E}-01 & 11 & 06 & 02 & 310 & 0800 \\ 630020.00 & 4168179.00 & 379.30 & .309557 \mathrm{E}-01 & 12 & 20 & 04 & 355 & 0800 \\ 632976.60 & 4166183.00 & 158.40 & .119566 \mathrm{E}-02 & 03 & 06 & 03 & 065 & 0800 \\ 633000.00 & 4170500.00 & 273.90 & .131706 \mathrm{E}-02 & 03 & 26 & 00 & 086 & 0800\end{array}$

OBODM $\vee 1.3-$ $10 / 24 / 2005$ pg 11

6 receptors; $\mathrm{Ba}$ fctr $=0.000086$

Table 7

Second Highest Barium Time-Average \{1-hr\} Concentration (Micrograms/Cubic Meter)

(Due to source group 1, sources: 1)

(Maximum $=.12142$ at $\mathrm{X}, \mathrm{Y}, \mathrm{Z}=629500.00,4168500.00,383.90$ )

\begin{tabular}{ccccccccc}
$\begin{array}{c}\mathrm{X} \\
\text { (Meters) }\end{array}$ & $\begin{array}{c}\mathrm{Y} \\
\text { (Meters) }\end{array}$ & $\begin{array}{c}\mathrm{Z} \\
\text { (Meters) }\end{array}$ & Time-Avg. Con. Mo/Dy/Yr Jdy $\mathrm{Hr}$ \\
\hline 628681.50 & 4165968.00 & 201.00 & $.216696 \mathrm{E}-02$ & 11 & 05 & 01 & 309 & 0800 \\
629500.00 & 4168500.00 & 383.90 & .121420 & 12 & 23 & 00 & 358 & 0800 \\
629950.00 & 4168674.00 & 309.40 & $.300886 \mathrm{E}-01$ & 12 & 25 & 04 & 360 & 0800 \\
630020.00 & 4168179.00 & 379.30 & $.309503 \mathrm{E}-01$ & 02 & 01 & 02 & 032 & 0800 \\
632976.60 & 4166183.00 & 158.40 & $.100913 \mathrm{E}-02$ & 02 & 10 & 03 & 041 & 0900 \\
633000.00 & 4170500.00 & 273.90 & $.130552 \mathrm{E}-02$ & 04 & 09 & 02 & 099 & 0800
\end{tabular}

OBODM V 1.3$10 / 24 / 2005$ pg 12

6 receptors; $\mathrm{Ba}$ fctr $=0.000086$

Table 8

Highest Barium Time-Average \{1-hr\} Concentration (Micrograms/Cubic Meter)

(Due to source group 2, sources: 2)

(Maximum $=.53005 \mathrm{E}-01$ at $\mathrm{X}, \mathrm{Y}, \mathrm{Z}=629500.00,4168500.00,383.90)$

\begin{tabular}{|c|c|c|c|c|c|}
\hline $\begin{array}{c}\mathrm{X} \\
\text { (Meters) }\end{array}$ & $\begin{array}{c}\mathrm{Y} \\
\text { (Meters) }\end{array}$ & $\begin{array}{c}\mathrm{Z} \\
\text { (Meters) }\end{array}$ & Time-Avg. Con. & Mo/Dy/Yr Jdy & $\mathrm{Hr}$ \\
\hline 628681.50 & 4165968.00 & 201.00 & $.873384 \mathrm{E}-03$ & $\begin{array}{llll}09 & 13 & 01 & 256\end{array}$ & 0800 \\
\hline 629500.00 & 4168500.00 & 383.90 & $.530049 E-01$ & $091102 \quad 254$ & 0800 \\
\hline 629950.00 & 4168674.00 & 309.40 & $.143861 \mathrm{E}-01$ & $\begin{array}{llll}01 & 19 & 04 & 019\end{array}$ & 0900 \\
\hline 630020.00 & 41681 & 379.30 & $.123289 \mathrm{E}-01$ & 112500330 & 0900 \\
\hline 632976.60 & 4166183.00 & 158.40 & $.426442 \mathrm{E}-03$ & $\begin{array}{llll}03 & 06 & 03 & 065\end{array}$ & 0800 \\
\hline 633000.00 & 4170500.00 & 273.90 & $.524535 \mathrm{E}-03$ & $\begin{array}{llll}12 & 06 & 02 & 340\end{array}$ & 0800 \\
\hline
\end{tabular}

OBODM $\vee 1.3-$ $10 / 24 / 2005$ pg 13

6 receptors; $\mathrm{Ba}$ fctr $=0.000086$

Table 9

Second Highest Barium Time-Average \{1-hr\} Concentration (Micrograms/Cubic Meter)

(Due to source group 2, sources: 2)

(Maximum $=.52864 \mathrm{E}-01$ at $\mathrm{X}, \mathrm{Y}, \mathrm{Z}=629500.00,4168500.00,383.90)$

\begin{tabular}{cccc}
$\mathrm{X}$ & $\mathrm{Y}$ & $\mathrm{Z}$ & Time-Avg. Con. Mo/Dy/Yr Jdy Hr \\
(Meters) & (Meters) & (Meters) & \\
\hline
\end{tabular}




$\begin{array}{lllllllll}628681.50 & 4165968.00 & 201.00 & .841595 \mathrm{E}-03 & 11 & 05 & 01 & 309 & 0800 \\ 629500.00 & 4168500.00 & 383.90 & .528641 \mathrm{E}-01 & 09 & 01 & 01 & 244 & 0800 \\ 629950.00 & 4168674.00 & 309.40 & .136451 \mathrm{E}-01 & 12 & 25 & 04 & 360 & 0800 \\ 630020.00 & 4168179.00 & 379.30 & .121199 \mathrm{E}-01 & 12 & 20 & 04 & 355 & 0800 \\ 632976.60 & 4166183.00 & 158.40 & .363553 \mathrm{E}-03 & 02 & 10 & 03 & 041 & 0900 \\ 633000.00 & 4170500.00 & 273.90 & .519402 \mathrm{E}-03 & 03 & 26 & 00 & 086 & 0800\end{array}$

OBODM V 1.3-

$10 / 24 / 2005 \mathrm{pg} \quad 14$

6 receptors; $\mathrm{Ba}$ fctr $=0.000086$

Table 10

Highest Barium Time-Average \{1-hr\} Concentration (Micrograms/Cubic Meter)

(Due to source group 3, sources: 3)

(Maximum $=.22025$ at $X, Y, Z=629500.00,4168500.00,383.90)$

\begin{tabular}{|c|c|c|c|c|c|}
\hline $\begin{array}{c}\mathrm{X} \\
\text { (Meters) }\end{array}$ & $\begin{array}{c}\mathrm{Y} \\
\text { (Meters) }\end{array}$ & $\begin{array}{c}\mathrm{Z} \\
\text { (Meters) }\end{array}$ & Time-Avg. Con. & Mo/Dy/Yr Jdy & $\mathrm{Hr}$ \\
\hline 628681.50 & 4165968.00 & 201.00 & $.410374 \mathrm{E}-02$ & $\begin{array}{llll}09 & 13 & 01 & 256\end{array}$ & 0800 \\
\hline 629500.00 & 4168500.00 & 383.90 & .220252 & $\begin{array}{llll}09 & 11 & 02 & 254\end{array}$ & 0800 \\
\hline 629950.00 & 4168674.00 & 309.40 & $.519868 \mathrm{E}-01$ & $\begin{array}{llll}01 & 19 & 04 & 019\end{array}$ & 0900 \\
\hline 630020.00 & 4168179.00 & 379.30 & $.571302 \mathrm{E}-01$ & $\begin{array}{llll}11 & 2500 & 330\end{array}$ & 0900 \\
\hline 632976.60 & 4166183.00 & 158.40 & $.207845 \mathrm{E}-02$ & $\begin{array}{llll}03 & 06 & 03 & 065\end{array}$ & 0800 \\
\hline 633000.00 & 4170500.00 & 273.90 & $.258790 \mathrm{E}-02$ & $1206 \quad 02 \quad 340$ & 0800 \\
\hline
\end{tabular}

OBODM $\vee 1.3-$

$10 / 24 / 2005 \mathrm{pg} \quad 15$

6 receptors; $\mathrm{Ba}$ fctr $=0.000086$

Table 11

Second Highest Barium Time-Average $\{1-\mathrm{hr}\}$ Concentration (Micrograms/Cubic Meter)

(Due to source group 3, sources: 3)

(Maximum $=.22000$ at $X, Y, Z=629500.00,4168500.00,383.90)$

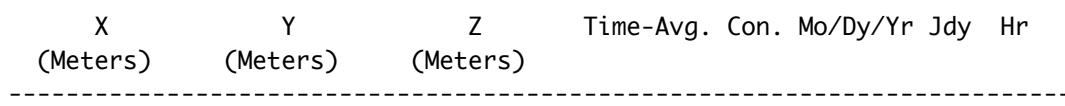

$\begin{array}{lllllllll}628681.50 & 4165968.00 & 201.00 & .395880 \mathrm{E}-02 & 11 & 05 & 01 & 309 & 0800 \\ 629500.00 & 4168500.00 & 383.90 & .220002 & 09 & 01 & 01 & 244 & 0800 \\ 629950.00 & 4168674.00 & 309.40 & .499965 \mathrm{E}-01 & 12 & 25 & 04 & 360 & 0800 \\ 630020.00 & 4168179.00 & 379.30 & .557481 \mathrm{E}-01 & 12 & 20 & 04 & 355 & 0800 \\ 632976.60 & 4166183.00 & 158.40 & .177240 \mathrm{E}-02 & 02 & 10 & 03 & 041 & 0900 \\ 633000.00 & 4170500.00 & 273.90 & .255463 \mathrm{E}-02 & 03 & 26 & 00 & 086 & 0800\end{array}$

OBODM V 1.3-

$10 / 24 / 2005 \mathrm{pg} \quad 16$

6 receptors; $\mathrm{Ba}$ fctr $=0.000086$

Table 12

Highest Barium Time-Average \{1-hr\} Concentration (Micrograms/Cubic Meter)

(Due to source group 4, sources: 4)

(Maximum $=.19682$ at $X, Y, Z=629500.00,4168500.00,383.90)$

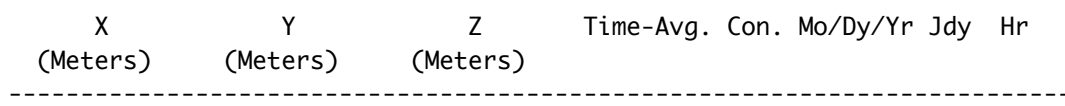

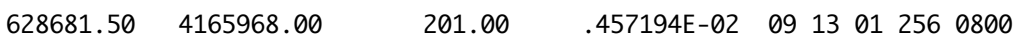




$\begin{array}{lllllllll}629500.00 & 4168500.00 & 383.90 & .196825 & 02 & 18 & 00 & 049 & 0800 \\ 629950.00 & 4168674.00 & 309.40 & .202244 \mathrm{E}-01 & 01 & 01 & 00 & 001 & 0900 \\ 630020.00 & 4168179.00 & 379.30 & .865755 \mathrm{E}-01 & 03 & 06 & 03 & 065 & 0800 \\ 632976.60 & 4166183.00 & 158.40 & .382277 \mathrm{E}-02 & 10 & 16 & 01 & 289 & 0800 \\ 633000.00 & 4170500.00 & 273.90 & .614005 \mathrm{E}-02 & 12 & 08 & 00 & 343 & 0900\end{array}$

OBODM V 1.3-

$10 / 24 / 2005 \mathrm{pg}$

17

6 receptors; $\mathrm{Ba}$ fctr $=0.000086$

Table 13

Second Highest Barium Time-Average \{1-hr\} Concentration (Micrograms/Cubic Meter)

(Due to source group 4, sources: 4)

(Maximum $=.19568$ at $X, Y, Z=629500.00,4168500.00,383.90)$

\begin{tabular}{ccccccccc}
$\begin{array}{c}X \\
\text { (Meters) }\end{array}$ & $\begin{array}{c}Y \\
\text { (Meters) }\end{array}$ & $\begin{array}{c}Z \\
\text { (Meters) }\end{array}$ & Time-Avg. Con. Mo/Dy/Yr Jdy & $\mathrm{Hr}$ \\
\hline & & & & & & & & \\
628681.50 & 4165968.00 & 201.00 & $.445747 \mathrm{E}-02$ & 11 & 26 & 04 & 331 & 1700 \\
629500.00 & 4168500.00 & 383.90 & .195676 & 02 & 05 & 02 & 036 & 0800 \\
629950.00 & 4168674.00 & 309.40 & $.202088 \mathrm{E}-01$ & 01 & 01 & 00 & 001 & 0800 \\
630020.00 & 4168179.00 & 379.30 & $.838088 \mathrm{E}-01$ & 11 & 18 & 03 & 322 & 0800 \\
632976.60 & 4166183.00 & 158.40 & $.364208 \mathrm{E}-02$ & 10 & 05 & 00 & 279 & 0800 \\
633000.00 & 4170500.00 & 273.90 & $.558784 \mathrm{E}-02$ & 04 & 09 & 02 & 099 & 0800
\end{tabular}

Elapsed run time $=0$ hours, 20.48 minutes.

*** end of OBODM *** 